\title{
The $44^{\text {th }}$ Annual Meeting of the European Society for Blood and Marrow Transplantation: Physicians Poster Sessions
}

\author{
18-21 March 2018 Lisbon, Portugal \\ Published online: 19 November 2018 \\ (c) Springer Nature Limited 2018
}

Copyright: Modified and published with permission from http://www.ebmt2018.org/

Sponsorship Statement: Publication of this supplement is sponsored by the European Society for Blood and Marrow Transplantation.

\section{PHYSICIANS - POSTER SESSION}

\section{Acute leukaemia}

\section{P001}

\section{A pilot study of daunorubicin-augmented hyper-CVAD induction chemotherapy followed by allogeneic hematopoietic cell transplantation for adults with acute lymphoblastic leukemia}

Sung-Yong Kim, Ji Hyun Park, So Young Yoon, Yo-Han Cho, Mark Hong Lee

Konkuk University Medical Center, Konkuk University School of Medicine, Seoul, Korea, Republic of

Background: Induction of complete remission (CR) is imperative for long-term survival in adult acute lymphoblastic leukemia (ALL) patients who are eligible for transplantation. Hyper-CVAD chemotherapy is one of the widely-used frontline remission induction regimens for these patients. This combination regimen showed a CR rate of $74 \%$ and mortality of $6 \%$ after one course of therapy (cycle 1). This indicates room for improvement. Prior studies suggest that daunorubicin has a lower frequency of cardiomyopathy and other severe complications in leukemia patients than doxorubicin, and that a high induction dose of daunorubicin could contribute to a favorable treatment outcome in adult ALL patients. Based on these observations, a pilot trial was conducted with adult ALL patients using a daunorubicin-augmented hyper-CVAD (hyperCVDD) regimen in which the doxorubicin in the standard
hyper-CVAD regimen was replaced with an increased dose of daunorubicin to improve the efficacy of the regimen.

Methods: We conducted a pilot trial of frontline remission induction using daunorubicin-augmented hyperCVAD regimen (hyper-CVDD) in adult ALL patients ( $\mathrm{n}=$ 14). From the standard hyper-CVAD regimen, doxorubicin was replaced with daunorubicin. Daunorubicin was administrated on days 4,11 , and 12 at a dose of $45 \mathrm{mg} / \mathrm{m}^{2} /$ day. If the study patients were less than 65 years of age and achieved CR, they proceeded to allo-HCT after one course of consolidation chemotherapy. A total of 14 patients were enrolled in the trial. The median age was 41.5 (range, 18-62). Six patients (42.9\%) were positive for BCR/ABL transcript and received tyrosine kinase inhibitor until transplantation. Four patients $(28.6 \%)$ had mixed phenotype acute leukemia; $B+$ myeloid $(n=2), T+$ myeloid $(n=1)$, and $B+T(n=1)$. Nine patients had B cell and two had T cell ALL.

Results: All patients completed the hyper-CVDD treatment as per protocol. All 14 patients (100\%) achieved CR. The median time to neutrophil recovery and platelet recovery was 20 days (range, 13-30) and 25 days (range, 20-40) from treatment, respectively. Two patients died of infection before and after the first consolidation, respectively. There were no deaths related to non-infectious toxicity including cardiotoxicity. Two patients relapsed after the first consolidation; one received salvage chemotherapy before allo-HCT and the other proceeded to transplantation without salvage chemotherapy because the percentage of blasts in bone marrow was 7\%. Thus, twelve patients among 14 transplant-eligible patients were able to proceed to allo-HCT. Overall survival (OS) and event-free 

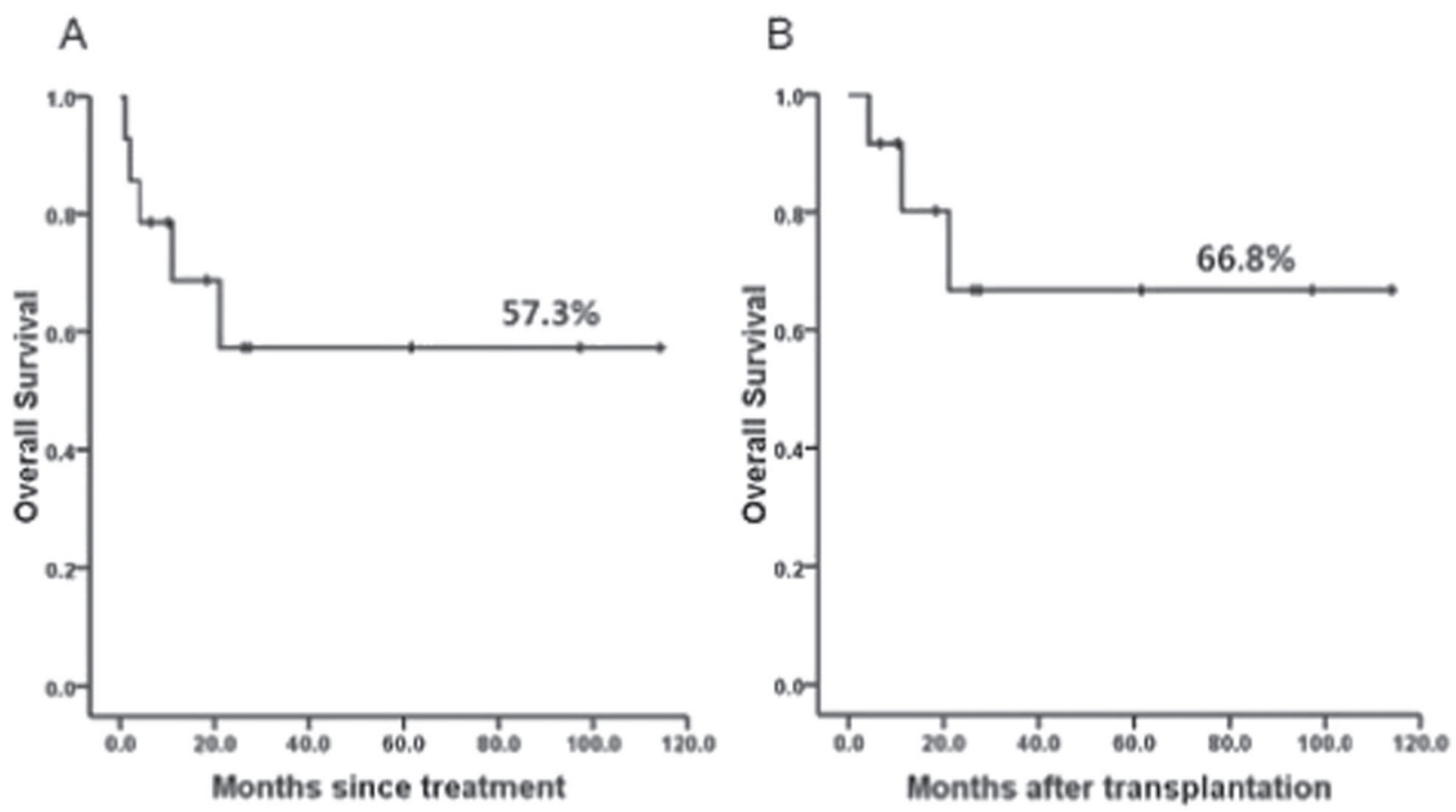

[P001 Figure] [Overall survival of all the study patients (A) and the transplanted patients $(B)]$

survival (EFS) of the study patients was $57.3 \%$ and $42.9 \%$ at three years. OS and relapse-free survival of transplanted patients was $66.8 \%$ and $55.0 \%$ at three years.

Conclusions: This pilot trial suggests the favorable efficacy of the hyper-CVDD chemotherapy as a frontline remission induction regimen before transplantation. Further clinical trials using this regimen are warranted.

Conflict of interest: There is no conflict of interest to declare.

\section{P002}

Allogeneic hematopoietic cell transplantation for primary refractory acute leukemia patients: a retrospective GITMO score based analysis

Ilaria Cutini, Chiara Nozzoli, Antonella Gozzini, Stefano Guidi, Irene Donnini, Riccardo Saccardi

AOU Careggi, Cellular Therapies and Transfusional Medicine Unit, Florence, Italy

Background: Patients with acute leukemia (AL) who fail to achieve complete remission (CR) have a dismal prognosis. Only few of them can be rescue after allogeneic hematopoietic cell transplantation (HCT). We retrospectively applied the GITMO score for PIF AL patients, that divides patients in 3 different categories; low, intermediate and high risk (Todisco E, BMT 2017).
Methods: The study population included 25 patients with $\mathrm{AL}$ presented as primary induction failure (PIF) who had received an allogeneic HCT between 1 March 2014 and 30 September 2017 at our institution. Median age was 51 yo. Disease characteristics and prior history of hematological diseased and gender are summarized in Table 1. Patients received myeloablative $(n=16)$ or reduced-intensity conditioning (RIC) regimens $(n=9)$. Median time from diagnosis to transplant was 7 months, (range 0-9 months).

Results: Among PIF AL population 16 belong to low risk group; 7 to intermediate group and 2 to high risk group respectfully. 18 out of 25 were evaluable on +30 days from transplant: 16 patients achieved complete hematological remission (CHR), 2 patients shown refractory disease. 7 patients died within 2 months (range 0-4 months) from transplant due to multiorgan failure (2 pts), sepsis ( $3 \mathrm{pts})$ and engraftment failure (2pts). 12 out of 25 (35\%) died due to TRM. Mean OS was 521 days, median OS was 331 days. Only 7 out of 25 patients (28\%) are still alive without active disease, mean follow up was 337 days (79-1348 days). Among the three GITMO categories, the mean and median OS was 527 and 364 days, 581 and 341 days, and 262 days for low, intermediate and high risk group respectively $(\mathrm{p}=0.126) .8$ patients experienced aGVHD and 7 patients cGVHD. All patients had received corticosteroids as frontline treatment. Those unresponsive were treated with second-line immunosuppressors. Mean time for developing GVHD was 2 and 4 months for aGVHD and cGVHD respectively. GVHD characteristic was shown in table 1. A 
trend to a better OS was shown in patients who developed $\operatorname{aGVHD}(p=0.380)$ and cGVHD $(p=0.219)$. No impact was shown for CMV sierological status. $(p=0,651)$. No impact was shown for myeloablative versus RIC regimens $(p=0.983)$.

Conclusions: The clinical outcome of PIF AL patients is poor and only a minor proportion of patients is rescued by HSCT. GITMO score can be used to create a risk score that helps to identify patients who most likely benefit from the procedure. The availability of reliable prognostic factor is particularly important in the era of alternative donor, such as haploidentical source. The small sample size may prevent to assess more significant differences across the population.

Conflict of interest: Nothing to disclose

\begin{tabular}{|c|c|c|}
\hline & $n^{\circ} 25$ & $\%$ \\
\hline Age & 51 уо (20-66) & \\
\hline Gender M F & 1015 & 4060 \\
\hline Diagnosis AML ALL & 241 & 964 \\
\hline $\begin{array}{l}\text { De novo Prior history } \\
\text { of: MDS ET IMF } \\
\text { Chemo Chemo+RT } \\
\text { HSCT }\end{array}$ & 16221112 & $\begin{array}{l}6488 \\
4448\end{array}$ \\
\hline $\begin{array}{l}\text { AML RISK (ELN 2017) } \\
\text { LOW RISK } \\
\text { INTERMEDIATE } \\
\text { RISK HIGH RISK }\end{array}$ & 21112 & $\begin{array}{l}844 \\
48\end{array}$ \\
\hline $\begin{array}{l}\text { CMV STATUS } \\
\text { NEGATIVE POSITIVE }\end{array}$ & 520 & 2080 \\
\hline $\begin{array}{l}\text { PIF GITMO SCORE } \\
\text { LOW } \\
\text { INTERMEDIATE } \\
\text { HIGH }\end{array}$ & 169 & 6436 \\
\hline $\mathrm{aGVHD}$ & $\begin{array}{l}\text { Skin (8) Global grade } 1 \\
\text { (5) Global grade } 2 \text { (3) } \\
\text { Intestine (2) Global grade } \\
1(0) \text { Global grade } 2 \text { (1) } \\
\text { Global grade } 3 \text { (1) liver } \\
\text { (0) }\end{array}$ & $\begin{array}{l}6337 \\
050 \\
500\end{array}$ \\
\hline cGVHD & Mild Moderate Severe & 241 \\
\hline
\end{tabular}

[[P002 Table] Table 1]

\section{P003}

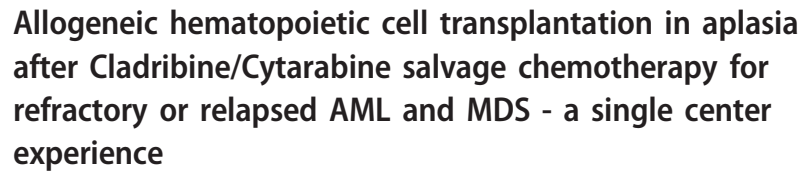

Allogeneic hematopoietic cell transplantation in aplasia after Cladribine/Cytarabine salvage chemotherapy for refractory or relapsed AML and MDS - a single center experience

Jan Vydra, Markéta Št’astná Marková, Veronika Válková, Ludmila Nováková, Barbora Čemusová, Petr Soukup, Michal Kolář, Antonín Vitek, Petr Cetkovský

Institute of Hematology and Blood Transfusion, Prague, Czech Republic

Background: Patients with chemorefractory acute myeloid leukemia (AML) and myelodysplastic syndromes (MDS) have poor prognosis. Results of standard HCT in such setting have been unsatisfactory and several sequential treatment regimens have been published consisting of cytoreductive chemotherapy followed by HCT in aplasia, without awaiting the recovery of normal hematopoiesis. The rationale for this approach is to reduce the tumor burden immediately prior to transplantation.

Methods: We performed a retrospective analysis of the outcome of treatment of patients with refractory or relapsing AML or MDS who received allogeneic HCT in aplasia after cladribine/cytarabine salvage chemotherapy at Institute of Hematology and Blood Transfusion in Prague. Patient characteristics and outcomes were retrieved from our database, which contains prospectively collected information on all patients who receive HCT at our institution. All patients signed informed consent with data collection and analysis and the study was reviewed by the institutional ethical committee.

Cladribine $(5 \mathrm{mg} / \mathrm{m} 2 /$ day IV) and Cytarabine $(2000 \mathrm{mg} /$ $\mathrm{m} 2 /$ day IV) were administered on days -15 to -11 before HCT, the conditioning regimen started on day -7 . Unmanipulated PBSC grafts were infused on day 0. GvHD prophylaxis consisted of post-transplant high dose cyclophosphamide $(50 \mathrm{mg} / \mathrm{kg}$ on days +3 and 4$)$, cyclosporine and mycophenolate mofetil.

Results: 21 patients (17 with AML and 4 with MDS) treated between 2015 and 2017 were included. 5 patients (24\%) relapsed after previous HCT, 10(48\%) had primary induction failure, $4(19 \%)$ had relapse refractory to salvage chemotherapy and 2 (9\%) had early molecular relapse of poor risk AML. Median followup time is $216(11-822)$ days, median age 53 (35-61) years. HCT-CI score was 0-1 in $12(56 \%), 2-3$ in $5(24 \%)$ and $>3$ in $4(20 \%)$. The conditioning regimen was Flu/Cy/Mel in 18 (85.7\%), Flu/ $\mathrm{Bu}[12.8 \mathrm{mg}$ iv] in $2(9.5 \%)$ and Flu/Cy/2GyTBI in $1(4.8 \%)$ patient. Donors were haploidentical in 19 (90\%), matched sibling in $1(5 \%)$ and mismatched unrelated in $1(5 \%)$ patient.

Neutrophil engraftment was prompt, $90.5 \%$ of patients engrafted and achieved CR, 2 (9.5\%) patients died before engraftment. Median time to engraftment was 19.5 days. $85.7 \%$ of patients engrafted platelets, median time to platelet engraftment was 31 days. 
Estimated OS at two years is 36,5\% (95\%CI 19.6 $68.9 \%$ ), RFS $29 \%(95 \%$ CI 14-60.4\%). Cumulative incidence of NRM and relapse were $25.9 \%$ and $45 \%$, respectively. In subgroup of patients with HCT-CI score $<=3$, incidence of NRM was $13.3 \%$.

Incidence of acute GVHD of any grade was $53 \%$. Incidence of grade 3 and 4 aGVHD were $19.6 \%$ and $9.5 \%$, respectively. Incidence of chronic GVHD was $36.7 \%$.

$14(66 \%)$ patients had fever in the first days following haploidentical graft infusion. Oral mucositis was usually mild, only one patient had grade 3 oral mucositis (after Flu/ $\mathrm{Bu}$ conditioning). Mild or moderate SOS syndrome was seen in $7(33 \%)$ of patients. Two patients died early before engraftment due to multiorgan failure.

Conclusions: Allogeneic HCT in aplasia after cladribine/ cytarabine salvage chemotherapy for AML or MDS is a feasible and active treatment regimen, which needs to be studied in a prospective trial.

Conflict of interest: None of the authors has anything to disclose

\section{P004}

\section{Allogeneic HSCT in Acute Lymphoblastic Leukemia: long- term outcome of 100 adult patients in 15 years}

Silvia Pierini, Fabio Giglio, Daniele Mannina, Massimo Bernardi, Jacopo Peccatori, Consuelo Corti, Andrea Assanelli, Gabriele Casirati, Raffaella Greco, Maria Teresa Lupo Stanghellini, Magda Marcatti, Sarah Marktel, Simona Piemontese, Fabio Ciceri

San Raffaele Scientific Institute IRCCS, Milano, Italy, Hematology and Bone Marrow Transplantation Unit, Milano, Italy

Background: Allogeneic hematopoietic stem cell transplantation (allo-HSCT) is an effective curative option to treat adult patients with high-risk acute lymphoblastic leukemia (ALL). source of HSCT and type of conditioning continue to be debated. The objective of this study is to perform a retrospective survey on allo-HSCT in adult ALL patients, in our Institution, over the past 15 years.

Methods: A total of 116 allo-HSCT on 100 patients affected by ALL have been performed between 2001 and 2017 in our Institution. Our analysis was then restricted to 72 patients at their first allo-HSCT and all data presented refer this cohort.

Results: Patients and HSCT characteristics are shown in table 1 .

3 conditioning regimes were performed using high dose of total dose irradiation(10-12 Gy) plus alkylating agent, treosulfan-based plus low dose TBI (2-4Gy) or treosulfanbased without TBI. GvHD prophylaxis was cyclosporine based in 25 patients, rapamycin based in 31 and cyclophosphamide plus rapamycin in 16 . With a median follow up of
43 months, the estimated probabilities of overall survival (OS) were $52+/-6 \%$ and $34+/-6 \%$ respectively at 1 and 5 years; the estimated probabilities of leukemia free survival (LFS) were $42+/-6 \%$ and $26+/-6 \%$, respectively at 1 and 5 years and the non-relapse mortality (NRM) probabilities were $8+/-3 \%$ at 100 days and $39+/-7 \%$ at 3 years. The cumulative incidence of aGvHD grade III-IV at 100 days was $20+/-5 \%$ and the cumulative incidence of cGvHD moderate-severe at 2 years was $25+1-7 \%$.

In univariate analysis, factors significantly associated with better overall survival were complete remission at HSCT $(\mathrm{p}=0.001)$, and a low dose of TBI ( 2 or $4 \mathrm{~Gy})(\mathrm{p}=0.005)$. The same results were confirmed in multivariate analysis (low dose TBI with $\mathrm{p}=0.022$ and CR at HSCT with $\mathrm{p}=$ $0.001)$.

No differences in OS and LFS were observed between MMRD and MUD ( $p=0.143$ for OS) $(p=0.286$ for OS). MRD had a better OS compared to MMRD/MUD $(\mathrm{p}=0.083)$.

Conclusions: in this small retrospective series of HSCT we could confirm that the state of disease at transplant is the most significant prognostic factor.

I our cohort, TBI is associated with better OS and LFS but only at low dose, in fact a high dose of TBI increases the NRM and aGvHD with statistical significance, finally impacting on OS. Low dose of TBI appear to have better outcome than high dose TBI and non TBI conditioning. In patients with ALL at their first transplant no significant differences in OS and LFS emerged between MUD and MMRD transplants, confirming that haplo-HSCT is an effective option in patients lacking a matched donor.

Conflict of interest: Nothing to disclose

\begin{tabular}{|c|c|c|c|c|c|}
\hline & $\begin{array}{l}116 \\
\text { all } \\
\text { HSCT }\end{array}$ & $\begin{array}{l}72 \\
\text { first } \\
\text { HSCT }\end{array}$ & & $\begin{array}{l}116 \\
\text { all } \\
\text { HSCT }\end{array}$ & $\begin{array}{l}72 \\
\text { first } \\
\text { HSCT }\end{array}$ \\
\hline $\begin{array}{l}\text { Median } \\
\text { age at } \\
\text { HSCT }\end{array}$ & $\begin{array}{l}37.2 \\
(18- \\
68)\end{array}$ & $\begin{array}{l}35.50 \\
(18- \\
68)\end{array}$ & $\begin{array}{l}\text { High dose TBI } \\
(10-12 \mathrm{~Gy})\end{array}$ & 17 & 12 \\
\hline B-ALL & 78 & 49 & $\begin{array}{l}\text { Treosulfan-based } \\
\text { and low dose TBI } \\
\text { (2-4Gy) }\end{array}$ & 32 & 18 \\
\hline T-ALL & 28 & 23 & Treosulfan-based & 67 & 42 \\
\hline CR1 & 37 & 32 & MUD & 23 & 21 \\
\hline $\mathrm{CR} \geq 2$ & 20 & 11 & MMRD & 58 & 30 \\
\hline \multirow[t]{2}{*}{ Rel } & 59 & 29 & MRD & 25 & 21 \\
\hline & & & UCB & 10 & 0 \\
\hline
\end{tabular}

[[P004 Table] Tab 1. Patients and HSCT characteristics] 


\section{P005}

\section{Allogeneic Transplantation for Acute Myeloid Leukemia with Monosomy 7 or Deletion 7q: a Study from the Acute Leukemia Working Party (ALWP) of the EBMT}

Xavier Poiré ${ }^{1}$, Myriam Labopin ${ }^{2}$, Emmanuelle Polge $^{2}$, Liisa Volin ${ }^{3}$, Jürgen Finke, Arnold Ganser, Didier Blaise ${ }^{6}$, Ibrahim YakoubAgha $^{7}$, Dietrich Beelen ${ }^{8}$, Noël Milpied ${ }^{9}$, Marie-Pierre Ledoux ${ }^{10}$, Gérard Socie $^{11}$, Dietger Niederwieser ${ }^{12}$, Mauricette Michallet ${ }^{13}$, Johan Maertens ${ }^{14}$, Jan Cornelissen ${ }^{15}$, Charles Craddock ${ }^{16}$, Mohamad Mohty ${ }^{2}$,Jordi Esteve ${ }^{17}$, Arnon Nagler ${ }^{18}$

${ }^{1}$ Cliniques Universitaires St-Luc, Hematology, Brussels, Belgium;

RI

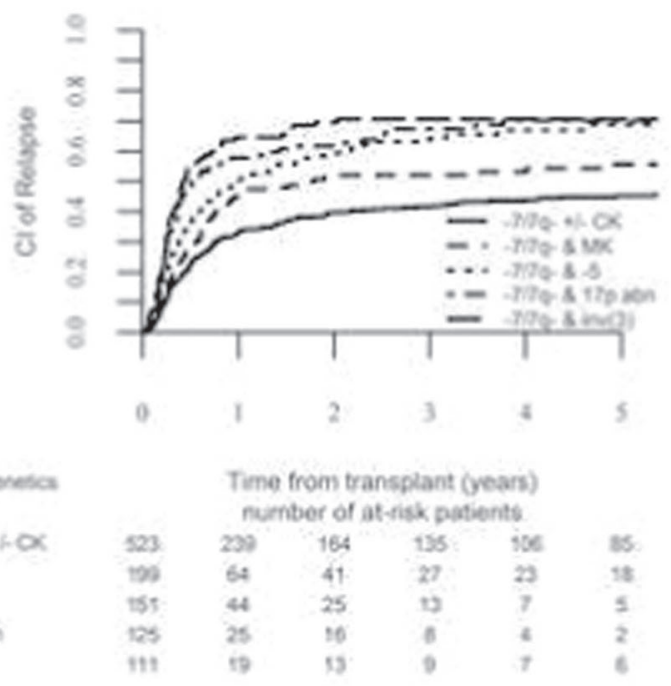

LFS

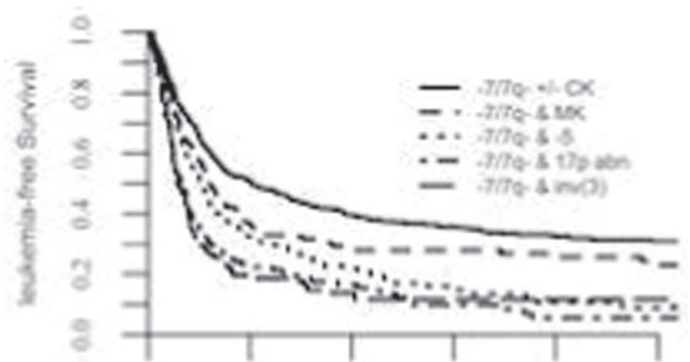

\begin{tabular}{|c|c|c|c|c|c|c|}
\hline \multirow{2}{*}{$\begin{array}{l}\text { Cysogenesca } \\
\text { mo: } \alpha\end{array}$} & \multicolumn{6}{|c|}{$\begin{array}{l}\text { Time from transplant (years) } \\
\text { number of at-risk pationts }\end{array}$} \\
\hline & 593 & 200 & 164 & t3s & t6e. & 85 \\
\hline$\omega x$ & 72 & 64 & $4 t$ & 27 & 23 & ts \\
\hline 5 & tst & 44 & 25 & 13 & 7 & $s$ \\
\hline Thp abe & 125 & 25 & 16 & 8 & 4 & 2 \\
\hline ing) & 111 & 10 & 13 & 0 & 7 & 4 \\
\hline
\end{tabular}

[P005 Figure] [RI, NRM, LFS and OS by cytogenetic subgroups.]
${ }^{2}$ Hôpital Saint Antoine, INSERM UMR 938, Paris, France; ${ }^{3} \mathrm{HUCH}$ Comprehensive Cancer Center, Stem Cell Transplantation Unit, Helsinki, Finland; ${ }^{4}$ University of Freiburg, Freiburg, Germany; ${ }^{5}$ Hannover Medical School, Department of Haematology, Hemostasis, Oncology and Stem Cell Transplantation, Hannover, Germany; ${ }^{6}$ Institut Paoli Calmettes, Department of Hematology, Centre de Recherche en Cancérologie de Marseille (CRCM), Marseille, France; ${ }^{7}$ Hôpital HURIEZ UAM allo-CSH, CHRU, Lille, France; ${ }^{8}$ Univeristy Hospital Essen, Essen, Germany; ${ }^{9} \mathrm{CHU}$ Bordeaux Hôpital Hautleveque, Pessac, France; ${ }^{10}$ Nouvel Hopital Civil, Strasbourg, France; ${ }^{11}$ Hôpital Saint Louis \& Université Paris 7, Denis Diderot, Paris, France; ${ }^{12}$ University Hospital Leipzig, Division of Haematology \& Oncology, Leipzig, Germany; ${ }^{13}$ Hôpital Lyon Sud, Lyon, France;

${ }^{14}$ University Hospital Gasthuisberg, Department of Hematology,

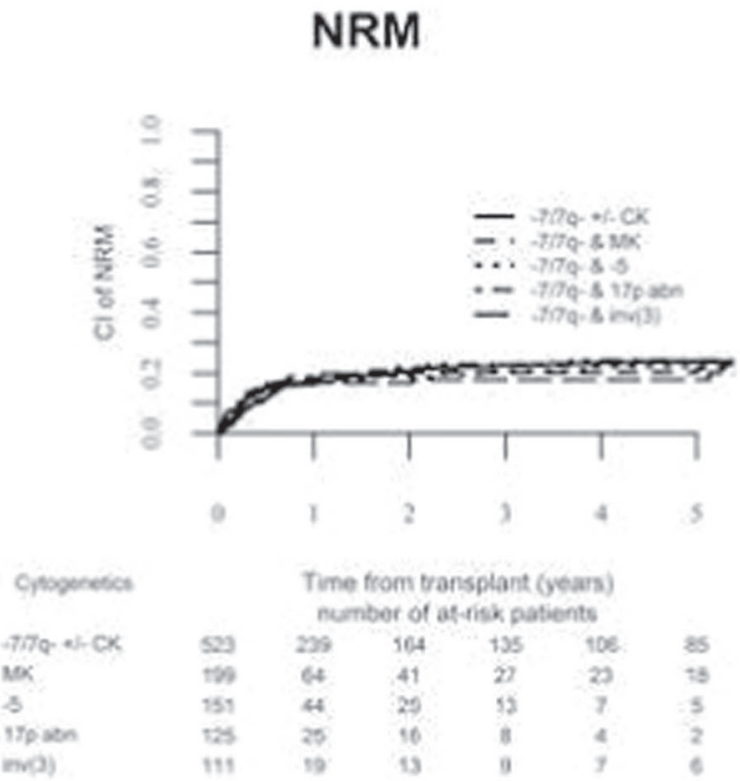


Leuven, Belgium; ${ }^{15}$ Erasmus MC Cancer Institute, University Medical Center, Rotterdam, Netherlands; ${ }^{16}$ Queen Elizabeth Hospital, Birmingham, United Kingdom; ${ }^{17}$ Hospital Clinic, Barcelona, Spain;

${ }^{18}$ Chaim Sheba Medical Center, Tel Hashomer, Israel

Background: Monosomy 7 or deletion $7 \mathrm{q}(-7 / 7 \mathrm{q}-)$ is the most frequent cytogenetic feature reported in acute myeloid leukemia (AML) and is associated with a dismal outcome. Allogeneic stem cell transplantation (SCT) allows to significantly improve their survival. Nevertheless, -7/7qoccurs frequently with other high-risk cytogenetic abnormalities, which may further influence the outcomes after SCT. Our aim was to evaluate the role of SCT in AML with -7/ $7 \mathrm{q}$ - in the context of additional cytogenetic abnormalities.

Methods: We selected adult with AML and $-7 / 7 \mathrm{q}-$ reported to the EBMT registry with a complete cytogenetic report. We included only first SCT from a sibling or unrelated donor (UD) performed between 2000 and 2016.

Results: A total of 1109 patients has been allocated. Median age was 52-year-old (range, 18-76) and median follow-up was 34 months (range, 0.7-193). At the time of SCT, 702 patients (63\%) were in first remission (CR1), 58 $(5 \%)$ in subsequent remission and $349(32 \%)$ had active disease. An UD was used in 613 patients (55\%). A myeloablative conditioning regimen was administered in $50 \%$.

The 2-year leukemia-free survival (LFS) and overall survival (OS) were $30 \%$ and $36 \%$, respectively. The 2-year non-relapse mortality (NRM) was $20 \%$ and the 2-year cumulative incidence of relapse (RI) was $50 \%$. The 2-year graft-versus-host disease (GvHD) and relapse-free survival (GRFS) was $21 \%$. In multivariate analysis, active disease was significantly associated with increased NRM and RI, which translated into worse LFS, OS and GRFS.

To evaluate the impact of additional cytogenetic abnormalities, we have been able to sort out 5 different groups. The first group included 523 patients with -7/7q- with or without complex karyotype (CK), but no monosomal karyotype (MK), monosomy 5 or deletion $5 \mathrm{q}(-5 / 5 \mathrm{q}-), 17 \mathrm{p}$ abnormalities (abn(17p)) or inversion of chromosome 3 (inv(3)).

Group 2 included 199 patients with -7/7q- within MK but without $-5 / 5 q-$, abn(17p) or inv(3).

Group 3 included 151 patients with $-7 / 7 \mathrm{q}$ - in association with $-5 / 5 q-$ but no abn(17p) or inv(3).

Group 4 included 125 patients with $-7 / 7 q-$ and abn(17p) but without inv(3). Finally, 111 patients with the combination of $-7 / 7 q-$ and $\operatorname{inv}(3)$ were assigned to the fifth group. NRM was similar across the groups. The 2-year LFS was $39.7 \%, 28.5 \%, 22.5 \%, 16.4 \%$ and $13.7 \%$ from group 1 to group 5 , respectively $(\mathrm{p}<0.001)$. The 2 -year OS in the group 5 were $47.7 \%, 34.9 \%, 28.2 \%, 15.9 \%$ and $19.7 \%$, respectively $(\mathrm{p}<0.001)$. The 2 -year GRFS was $29.7 \%$, $16.6 \%, 14.5 \%, 8.1 \%$ and $8.9 \%$ from group 1 to group 5 $(\mathrm{p}<0.001)$. Multivariate analysis confirmed those significant differences across groups, even when the analysis was focused on CR1 patients.

Conclusions: SCT in -7/7q- AML provides durable response in one third of the patients. Disease status at the time of transplantation remains the strongest prognostic factor for worse outcomes. The presence of $-7 / 7 \mathrm{q}$ - with or without CK in the absence of MK, $-5 / 5 q-$, abn(17p) or inv (3) was associated with a better survival after SCT. On the contrary, addition of $-5 / 5 q-$, abn(17p) or inv(3) identifies a sub-group with a limited benefit from SCT, who may be candidate for post-SCT interventions.

Conflict of interest: Nothing to disclose

\section{P006}

Abstract previously published

P007

Analysis of three salvage treatment regimens after relapsed/refractory acute myeloi leukemia (AML) (FLAG-Ida, FLAGO-IDA and PLERIFLAG)

Juan Bergua ${ }^{1}$, David Martinez-Cuadron ${ }^{2}$, Beatriz Boluda ${ }^{2}$, Pilar Martinez ${ }^{3}$, Rebecca Rodriguez-Veiga ${ }^{2}$,Jordi Esteve ${ }^{4}$, Susana Vives, Josefina Serrano ${ }^{6}$, Belen Vidriales, ${ }^{7}$ Olga Salamero ${ }^{8}$, Carlos Carretero $^{2}$, Ana Jimenez-Ubieto ${ }^{9}$, Julio Prieto ${ }^{10}$, Marina Díaz-Beyá ${ }^{4}$, Salut Brunet ${ }^{11}$, Celina Benavente ${ }^{12}$, Jose Antonio Perez-Simon ${ }^{13}$, Sara Suarez-Varela ${ }^{1}$, Federico Moscado ${ }^{14}$, Miguel Angel Sanz ${ }^{2}$, Pau Montesinos $^{2}$

${ }^{1}$ Hospital San Pedro de Alcántara, Hematologia, Caceres, Spain; ${ }^{2}$ Hospital La Fe, Hematologia, Valencia, Spain; ${ }^{3}$ Hospital 12 de Octubre, Hematologia, Madrid, Spain; ${ }^{4}$ Hospital Clinic de Barcelona, Hematologia, Barcelona, Spain; ${ }^{5}$ Hospital Trias y Pujol, Badalona, Badalona, Spain; ${ }^{6}$ Hospital Reina Sofia, Hematology, Cordoba, Spain; ${ }^{7}$ Hospital Clinico de Salamanca, Hematologia, Salamanca, Spain; ${ }^{8}$ Hospital Vall d'Hebron, Hematology, Barcelona, Spain; ${ }^{9}$ Hospital 12 de Octubre, Hematology, Madrid, Spain; ${ }^{10}$ Hospital de Don Benito, Hematologia, Don Benito, Spain; ${ }^{11}$ Hospital Sant Pau, Hematologia, Barcelona, Spain; ${ }^{12}$ Hospital Clinico San Carlos, Hematologia, Madrid, Spain; ${ }^{13}$ Hospital Virgen del Rocio, Hematologia, Sevilla, Spain; ${ }^{14}$ Hospital La Fe, Hematologia, Valenica, Spain

Background: We retropectively compare three salvage therapies based in FLAG-IDA scheme: FLAG-IDA (Fludarabine, Idarubicine, Cytarabine G-CSF as priming agent), FLAGO-IDA (Gemtuzumab plus FLAG-IDA, and PLERIFLAG (Plerixafor as priming factor). We use to compared two risk-criteria classifications HOVON (De Breems, 2005) and SALFLAGE (Bergua, 2016).

Methods: Statistical analysis: Differences between groups were tested using Fisher exact test. We compare differences onCR + Cri between treatments adjusting prognostic survival score groups using Mantel-Haenszel test 
using HOVON criteria (de Breems et al) and SALFLAGE criteria. Homogeneity of variables was tested using Woolftest. Survival analysis by Kaplan-Maier stratified analysis. To compare PLERIFLAG against the other two types of salvage treatment we performed match-pair analysis adjusting variables as age, previous allogenic transplant, time to relapse (refractory, $<12$ months and $>12$ months), karyotype using MRC, and FLT3 status. Kariotype risk was considered by HOVON criteria (inv16, $\mathrm{t}(8 ; 21)$ and all the others Kariotype), and SALFLAGE (inv(16), unfavourable risk by MRC risk plus $\mathrm{t}(8 ; 21)$, intermediate risk.

Patients: We analysed 300 patients relapsed or resistant to induction therapy, 251 treated with FLAG-IDA, 41 patientas with FLAGO-Ida, and 42 patients treated with PLERIFLAG. Analysis was conducted in different times; FLAG-Ida was the regiment of treatment of relapsed/ resitant patients in PETHEMA studies, FLAGO was used in a short period before withdraw Gemtuzumab use by EMEA agencies, and PLERIFLAG was a phase 2 trial on relapsed/ refractory patients. Both treatments were similar in terms of Age, Sex and previous myelodisplasia. The three types of treatment differed in characteristics. There were more unfavourable characteristics in PLERIFLAG vs. FLAGIda and FLAGO-IDA treatment: high risk karyotype (55\% in PLERIFLAG,vs 41 in FLAG-IDA vs. $33 \%$ in FLAGOIda), FLT3 (10\% in PLERIFLAG, 22\% in FLAG-Ida, 20\% in FLAGO-ida), time to relapse less than 12 months, or previous allogenic stem-cell transplantation. There were more patients classified as high risk using SALFLAGE criteria in PLERIFLAG 33(80\%),vs FLAG-Ida (93, 48\%) vs. FLAGO-Ida $(12,36 \%)$.

Results: Complete remission (CR) was achieved in 20patients (48.7\%) treated with Pleriflag, in 96 (48\%) treated with FLAG-IDA, and in $22(61 \%)$ of the patients treated with treated with FLAGO-IDA. Adjusted by Hovon score andSalflage SALFLAGE score does not show statatisitical differences in $\mathrm{CR}+\mathrm{Cri}$ rate $(\mathrm{p}=0.23$, Odds ratio between PLERIFLAG and FLAG-Ida and FLAGO-Ida (0.27, CI: -1.1-0.5) . The number of patients who received allogenic- stem cell transplantation were more in the case of PLERIFLAG $(25(60 \%), 9(22 \%)$ of them sequential vs FLAG-IDA $(85(34 \%))$ vs. FLGO-IDA $(7(18 \%))$. There no were differences in overall survival (OS) between PLERIFLAG (median 10.56 monts, CI(6.7-NR), FLAG-IDA (medina 8.45 months, $\mathrm{CI}(7.2-13)$ and FLAGO-IDA (median 9.53 months, $\mathrm{CI}(3.78-25)(\mathrm{p}=0.9)$ and EFS between the treatments, but there were differences when adjusted by SALFLAGE risk categories $(\mathrm{p}=0.02)$.

Conclusions: No differences between groups could be detected. These results may can be influenced by the high rate of Allegenic stem cell transplant in PLERIFLAG group; also supports the need of Allogenic stem trasplantation in sequential time schedule even if no CR has been achieved.

Clinical Trial Registry: eudrat 2011-000971-15

Conflict of interest: No conflict of interest

\section{P008}

\section{Antibodies to red blood cells antigens in patients of hematological clinic}

Elena Butina ${ }^{1}$, Galina Zaitseva ${ }^{1}$, Filipp Sherstnev ${ }^{2}$, Oleg Maximov ${ }^{3}$, Natalia Minaeva ${ }^{3}$, Igor Paramonov ${ }^{3}$

${ }^{1}$ Kirov Research Institute of Hematology and Blood Transfusion of the Federal Medical and Biological Agency of Russia,

Immunohematology, Kirov, Russian Federation; ${ }^{2}$ Kirov Research Institute of Hematology and Blood Transfusion of the Federal Medical and Biological Agency of Russia, Transfusiology, Kirov, Russian

Federation; ${ }^{3}$ Kirov Research Institute of Hematology and Blood Transfusion of the Federal Medical and Biological Agency of Russia, Hematology, Kirov, Russian Federation

Background: Screening of red blood cell (RBC) alloantibodies is mandatory for every hospitalization of hematological patient. In case of antibodies detection, their specificity is established and, if necessary, individual selection of RBC containing blood components is carried out. Aim. To study frequency of detection of RBC alloantibodies in hematological patients.

Methods: Analysis of results of the immunohematological examination of 3132 patients of hematological clinic was carried out. Screening and identification of antibodies were performed with equipment and reagents from BioRad (USA).

Results: Specific RBC alloantibodies were detected in $2.1 \%$ of hematological patients: $2.28 \%$ of men, $2.44 \%$ of women and $0.48 \%$ of children. The most frequently detected antibodies were anti-D $(0.32 \%),-\mathrm{E}(0.32 \%)$ and $-\mathrm{K}(0.32 \%)$. Antibodies to antigens C $(0.19 \%)$, c $(0.13 \%)$, $\mathrm{M}(0.13 \%), \mathrm{Le}^{\mathrm{a}}(0.13 \%), \mathrm{DC}(0.1 \%), \mathrm{Fy}^{\mathrm{a}}(0.1 \%)$ were also detected. The most uncommon were anti- $\mathrm{C}^{\mathrm{W}},-\mathrm{S},-\mathrm{Jk}^{\mathrm{a}},-\mathrm{Jk}^{\mathrm{b}}$, $-\mathrm{Le}^{\mathrm{b}},-\mathrm{Lu}^{\mathrm{a}},-\mathrm{Lu}^{\mathrm{b}}$ antibodies, their frequency varied from $0.06 \%$ to $0.03 \%$.

The maximum level of alloimmunization was observed in the group of patients with hemophilia A $-12.0 \%$ of patients. Relatively more frequently, antibodies are detected in patients with acute leukemia (3.5\%), myelodysplastic syndrome $(4.7 \%)$ and von Willebrand disease (4.9\%). In the remaining groups of patients, alloantibodies were detected at a frequency of $0.1 \%$ to $2.3 \%$.

Conclusions: Given that patients with blood diseases receive intensive transfusion therapy, it is extremely necessary to select donors that are compatible with recipient both for RBC antigens of ABO, Rhesus (C, c, E), Kell (K) systems and for results of indirect antiglobulin test. 
Conflict of interest: None of the authors has anything to disclose.

\section{P009}

\section{Assessing the prognostic impact of FLT3-ITD in acute myeloid leukemia patients undergoing haploidentical stem cell transplantation. An Acute Leukemia Working Party/ EBMT analysis}

Jonathan Canaani ${ }^{1}$, Myriam Labopin ${ }^{2}$, Xiao-Jun Huang ${ }^{3}$, William Arcese $^{4}$, Fabio Ciceri ${ }^{5}$, Didier Blaise ${ }^{6}$, Giuseppe Irrera ${ }^{7}$, Lucia Lopez Corral $^{8}$, Benedetto Bruno ${ }^{9}$, Stella Santarone ${ }^{10}$, Maria Teresa Van Lint $^{11}$, Antonin Vitek ${ }^{12}$, Jordi Esteve ${ }^{13}$, Mohamad Mohty ${ }^{2}$, Arnon Nagler $^{2,14}$

${ }^{1}$ Chaim Sheba Medical Center, Hematology Division, Ramat Gan, Israel; ${ }^{2}$ EBMT Paris Study Office, Paris, France; ${ }^{3}$ Peking University People's Hospital, Peking, China; ${ }^{4}$ Tor Vergata University, Rome, Italy; ${ }^{5}$ San Raffaele Scientific Institute, Hematology Division, Milano, Italy; ${ }^{6}$ Institut Paoli Calmettes, Marseille, France; ${ }^{7}$ Azienda Ospedaliera, Centro Unico Regionale Trapianti, Alberto Neri, Reggio Calabria, Italy; ${ }^{8}$ Hospital Clinic de Salamanca, Salamanca, Spain;

${ }^{9}$ Citta della Salute e della Scienza di Torino, Torino, Italy; ${ }^{10}$ Ospedale Civile, Pescara, Italy; ${ }^{11}$ Ospedale San Martino, Genova, Italy;

${ }^{12}$ Institute of Hematology and Blood Transfusion, Prague, Czech Republic; ${ }^{13}$ Hospital Clínic de Barcelona, Barcelona, Spain; ${ }^{14}$ Chaim Sheba Medical Center, Ramat Gan, Israel

Background: Fms-like tyrosine kinase 3 internal tandem duplication (FLT3-ITD) mutation designates patients with acute myeloid leukemia as having a particularly high risk for disease relapse. It is currently unclear whether FLT3ITD retains a significant prognostic role in haploidentical stem cell transplantation (haplo-SCT).

Methods: To assess whether FLT3-ITD is prognostically meaningful in T-cell replete haplo-SCT transplanted AML patients, we performed a comparative analysis between $F L T 3^{\mathrm{wt}}$ and $F L T 3-I T D^{\mathrm{mut}}$ using the multicenter registry of the acute leukemia working party of the European society for blood and marrow transplantation. Patients included in this study were AML patients over the age of 18 who underwent a first T-cell replete haplo-SCT in first remission.

Results: In all, a cohort consisting of 293 de-novo AML patients $\left(202 F\right.$ LT $^{\mathrm{wt}}$ and 91 FLT3-ITD $\left.^{\mathrm{mut}}\right)$ transplanted in first remission with T-cell replete haplo-SCT between 20052016 was analyzed. Both groups did not differ to a significant degree in terms of patient age, donor age, performance status, cytomegalovirus donor-recipient matching, and conditioning intensity. FLT3-ITD ${ }^{\mathrm{mut}}$ patients were more likely to be nucleophosminl (NPM1) mutated as well as be in the Medical Research Council (MRC) intermediate risk cytogenetic risk category. In multivariate analysis, patients with FLT3-ITD had comparable rates of relapse incidence [Hazard ratio $(\mathrm{HR})=1.34$, confidence interval (CI) 95\%, 0.67-2.7; $P=0.9$ ] and leukemia-free survival (HR=0.99, CI 95\%, 0.62-1.57; $P=0.9)$ to those of $F L T 3^{\mathrm{wt}}$ patients. Survival was not significantly impacted by FLT3-ITD status (HR=0.96, CI 95\%, 0.58-1.59; $P=0.8$ ), nor were the incidence of non-relapse mortality ( $\mathrm{HR}=0.78$, CI 95\%, 0.41-1.46; $P=0.44$ ), and graft versus host diseasefree/relapse-free survival $(\mathrm{HR}=0.84$, CI 95\%, 0.54-1.28; $P=0.42$ ). An analysis for patients treated either on antithymocyte globulin (ATG) protocols or post-transplant cyclophosphamide (PTCy) did not significantly correlate FLT3-ITD status with clinical outcome in either of these groups. Finally, a focused subset analysis of patients with MRC intermediate risk cytogenetics confirmed the absence of a prognostic impact of FLT3-ITD also for this group of patients.

Conclusions: The FLT3-ITD mutation possibly does not retain its prognostic significance in AML patients undergoing haplo-HCT, suggesting that haplo-SCT may conceivably overcome the negative prognostic impact of FLT3ITD in AML.

\section{Clinical Trial Registry: N/A}

Conflict of interest: All authors declare nothing to disclose.

\section{P010}

Azacitidine and escalating doses of DLI is an effective combinatin treatment for patients relapsing post allogeneic bone marrow transplantation

Shereef Elmoamly ${ }^{1}$, Dimitra Oikonomopoulou ${ }^{2}$, Maria Kaparou ${ }^{3}$, Evgenia Xenou, Amy Gudger ${ }^{3}$, Rebecca Pryor ${ }^{4}$, Hannah Giles ${ }^{3}$, Claire Horgan $^{3}$, Alexandros Kanellopoulos ${ }^{3}$, Shankara Paneesha ${ }^{3}$, Bhuvan Kishore, Richard Lovell', Kathy Holder ${ }^{3}$, Lynn Ryan ${ }^{3}$, Katie O'Collins ${ }^{3}$, Hara Giatra ${ }^{2}$, Ioannis Baltadakis ${ }^{2}$, Dimitrios Karakasis ${ }^{2}$, Emmanouil Nikolousis ${ }^{3}$

${ }^{1}$ Heart of England NHS Trust, Haematology, Birmingham, United Kingdom; ${ }^{2}$ Evaggelismos General Hospital, Athens, Greece; ${ }^{3}$ Heart of England NHS Trust, Birmingham, United Kingdom; ${ }^{4}$ Heart of England NHS Foundation Trust, Birmingham, United Kingdom

Background: Patients with AML have a significant risk of relapse even after undergoing haematopoetic stem cell transplantation.In such patients the outlook is very poor with the majority of the patients surviving less than a year. Donor lymphocyte infusions are unable to tackle the kinetics of haematological relapse while intensive chemotherapy could result in mortality post allogeneic transplant leaving a significant treatment gap for this group of patients

Methods: Between 20011 and 2017, a total 25 patients [15 males, 10 females, median age 59.05 (40-73)] received a myeloablative or RIC allograft (17 from fully matched unrelated donors, and 8 from an identical sibling) for standard or high risk AML in 2 centres and subsequently 
relapsed.14 patients relapsed within first year while 10 patients relapsed more than a year post allogeneic stem cell transplant. The conditioning regimes included fludarabine, together with melphalan and Campath or ATG (13 patients), Fludarabine Busulphan and Campath (5 patients) and TBI/ $\mathrm{Cy}$ or $\mathrm{Bu} / \mathrm{Cy}(7$ patients). Graft versus host disease prophylaxis was Ciclosporin for patients receiving Alemtuzumab or ATG but for patients who had a T-replete allograft Ciclosporin and low dose methotrexate was the preferred prophylaxis.All but four patients were transplanted in CR1. Patients who relapsed receive 2 courses of Azacitidine of $75 \mathrm{mg} / \mathrm{m} 2$ followed by DLI in repeated cycles with escalating doses of DLI.Progression free survival(PFS) and overall survival(OS) were assessed using SPSS software

Results: Median follo up was 19.3months (range 3-53).) 7 patients remain alive. Causes of death (18 cases) include relapse or progression of the original disease (13 cases), and infection (4 cases). 12 patients achieved CR while 9 achieved PR and 4 had ongoing disease post treatment with Aza and DLI Median PFS was 11.3 months for patients who relapsed within a year post transplant receiving Azacitidine and DLI but for those who relapsed more than one year median PFS hasn't been reached. The influence of timing of relapse on PFS and OS was analysed, showing a trend towards better PFS for patients relapsing more than 12 months post allogeneic stem cell transplant and a significantly better OS for those patients who developed GvHD after Azacitidine and DLI administration. Disease risk was not significant on PFS or OS post relapse which might be due to the low number of patients in each risk group All patients in ongoing complete remission managed to achieve full donor chimerism in both whole blood and $\mathrm{T}$ cells

Conclusions: Our results indicate that the combination of Azacitidine with escalating doses of DLI could potentially restore remission in patients relapsing post allogeneic stem cell transplantation for a reasonable amount of time in this difficult cohort of patients especially for patients who relapse more than a year post transplant. Our findings should be evaluated in prospective clinical trials

Our results indicate that the combination of Azacitidine with escalating doses of DLI could potentially restore remission in patients relapsing post allogeneic stem cell transplantation for a reasonable amount of time in this difficult cohort of patients especially for patients who relapse more than a year post transplant.Our findings should be evaluated in prospective clinical trials.

Clinical Trial Registry: NA

Conflict of interest: No conflicts of interest to disclose
P011

Better outcome in children compared to AYAs and young adults after allogeneic HSCT for AML: a multicenter retrospective study from the SFGMTC

Cecile Pochon ${ }^{1}$, Marie Detrait ${ }^{2}$, Eliane Albuisson ${ }^{3}$, Jean-Hugues Dalle $^{4}$, Didier Blaise, Gérard Michel ${ }^{6}$, Ibrahim Yakoub-Agha ${ }^{7}$, Jacques Olivier Bay ${ }^{8}$, Patrice Chevallier', Mohamad Mohty ${ }^{10}$, Noel Milpied $^{11}$, Mauricette Michallet ${ }^{12}$, Yves Bertrand ${ }^{13}$, Ali Bazarbachi ${ }^{14}$, Yves Chalandon ${ }^{15}$, Yves Beguin ${ }^{16}$, Stéphanie Nguyen Quoc ${ }^{17}$, Regis Peffault de Latour ${ }^{18}$, Marie-Thérèse Rubio ${ }^{2}$

${ }^{1}$ University Hospital of Nancy, Pediatric Onco-Hematology, Vandoeuvre-les-Nancy, France; ${ }^{2}$ University Hospital of Nancy, Hematology, Vandoeuvre-les-Nancy, France; ${ }^{3}$ University Hospital of Nancy, Plateforme d'Aide à la Recherche Clinique (PARC),

Vandoeuvre-les-Nancy, France; ${ }^{4}$ University Hospital Robert Debré, Hemato-Immunology, Paris, France; ${ }^{5}$ Paoli-Calmette University Institute, Hematology, Marseille, France; ${ }^{6}$ University Hospital La Timone, Pediatric Onco-Hematology, Marseille, France; ${ }^{7}$ University Hospital of Lille, Claude Huriez Hospital, Hematology, Lille, France; ${ }^{8}$ Clermont-Ferrand University Hospital (Estaing Hospital), Hematology, Clermont Ferrand, France; ${ }^{9}$ Nantes University Hospital, Hematology, Nantes, France; ${ }^{10}$ Saint Antoine University Hospital, Hematology, Paris, France; ${ }^{11}$ Bordeaux University Hospital (HautLévèque Hospital), Hematology, Pessac, France; ${ }^{12}$ Centre de Lutte contre le Cancer Régional Léon Bérard, Lyon, France; ${ }^{13}$ IHOP University Hospital, Pediatric Hematology, Lyon, France; ${ }^{14}$ American University of Beirut Medical Center, Beirut, Lebanon; ${ }^{15}$ Geneva's University Hospitals, Hematology, Stem Cell Transplant Unit, Geneva, Switzerland; ${ }^{16}$ University Hospital of Liège, Hematology, Liege, Belgium, ${ }^{17}$ La Pitié Salpétrière University Hospital (AP-HP), Hematology, Paris, France; ${ }^{18}$ Saint Louis Lariboisière University Hospital, Hematology, Paris, France

Background: *The 2 first authors contributed equally

Data on the outcome of AML in adolescents and young adults (AyAs) after allogeneic HSCT are scarce. In this retrospective study from the SFGM-TC, we analyzed the outcome of AML patients classified in 3 groups according to the age at transplantation: children $(<15$ years), AyAs (15-25 years) and adults (26-40 years).

Methods: Patients aged between 0 and 40 years who received a first allogeneic HSCT for AML between 2005 and 2015, reported in the SFGM-TC registry, were included.

Results: This study included 2240 patients from 43 centers, of whom 481 children (21.5\%), 545 AyAs (24.3\%) and 1214 adults $(54.2 \%)$.

According to the 2016 WHO classification, children had the highest rate $(31.8 \%)$ of adverse risk disease, followed by AyAs $(25.2 \%)$ and adults $(21.3 \%)(\mathrm{p}=0.0001)$. Extramedullary disease was observed in $42.8 \%, 24 \%$ and $21.4 \%$ of children, AyAs and adults $(\mathrm{p}=0.0001)$. Among patients in complete remission (CR), younger age was associated more frequently with advanced disease $(29.9 \% \geq \mathrm{CR} 2$ for children, $23 \%$ for AyAs and $18.2 \%$ for adults) $(\mathrm{p}=0.0001)$. 
Donors were matched siblings in $36.4 \%$ of children, $36.7 \%$ of AyAs and $40.3 \%$ of adults, and matched unrelated in $29.1 \%, 33.4 \%$ and $35.7 \%$ respectively. Children had more mismatched unrelated donors $(31.6 \%)$, compared to AyAs $(25.3 \%)$, and adults $(19.5 \%)(p=0.0001)$. Haploidentical HSCT counted for less than $4 \%$ of transplantations in each group.

Stem cell source diverged between the 3 groups $(\mathrm{p}=0.0001)$ : bone marrow $(\mathrm{BM})$ was the main source for children (60.9\% of grafts) followed by cord blood (CB) (28.9\%), whereas peripheral blood stem cells (PBSC) was the main source for adults (60\%), followed by BM $(29.7 \%)$ and $\mathrm{CB}(10.2 \%)$. AyAs were transplanted with $\mathrm{BM}$ in $44.4 \%$ of cases, PBSC in $40.4 \%$ or CB in $15.2 \%$.

The intensity of the conditioning was myeloablative (MAC) in $93 \%$ of children, $79 \%$ of AYAs and $77.7 \%$ of adults, while a reduced-intensity regimen was used in $4 \%$ of children, $12.3 \%$ of AyAs and $14.1 \%$ of adults $(\mathrm{p}=0.0001)$ at a median time of 150,166 and 170 days respectively after diagnosis.

With a median follow-up of 7 years, 5y-overall survival (OS) was higher in children (64\%), compared to AyAs $(54 \%)$ and adults $(52 \%)(\mathrm{p}=0.001)$. One-year cumulative incidence (CI) of relapse was comparable in the 3 groups: $27 \%$ for children, $33 \%$ for AYAs and 28\% for adults $(\mathrm{p}=0.19)$. There was no difference in the CI of grade II-IV acute GvHD at day +100 between the 3 groups: $37.7 \%$, $35.8 \%$ and $35 \%$ respectively $(\mathrm{p}=0.58)$. By contrast, oneyear CI of chronic GvHD was higher in adults $(37 \%)$ and AyAs $(31 \%)$ compared to children $(17 \%)(\mathrm{p}=0.0001)$.

In multivariate analysis, independent factors associated with better OS were younger age (HR $=1.37,95 \%$ CI_1.07-1.75, $\mathrm{p}=0.013), \mathrm{BM}(\mathrm{HR}=1.46,95 \%$ CI_1. 5$1.87, \mathrm{p}=0.002)$, MAC $(\mathrm{HR}=1.98,95 \%$ CI_1.5-2.61, $\mathrm{p}=$ $0.0001)$ and low-risk cytogenetics $(\mathrm{HR}=1.93,95 \%$ CI_1.53-2.45, $\mathrm{p}=0.0001)$.

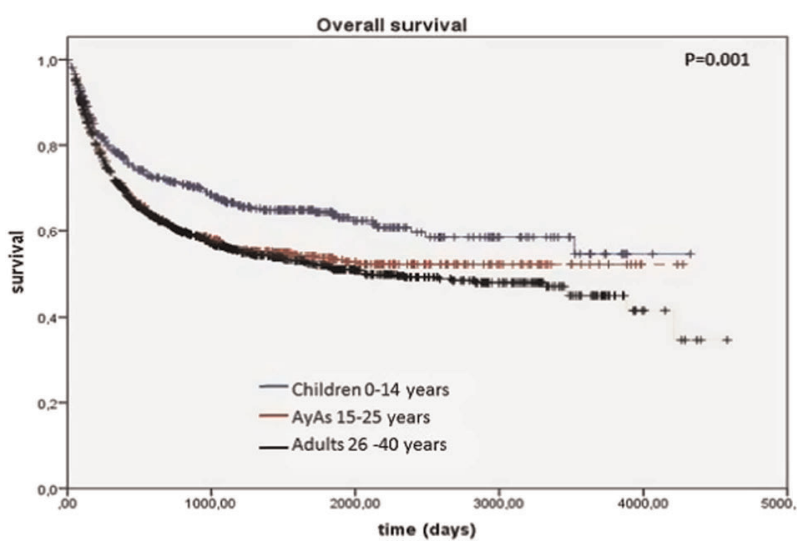

[P011 Figure] [Overall survival according to age group]
Conclusions: Children have a better OS than AyAs and adults. The other factors associated with a better outcome were MAC regimen, absence of poor cytogenetics, and BM as stem cells source.

Conflict of interest: We have to declare no conflict of interest

\section{P012}

Abstract previously published

\section{P013}

Characterization of Veno-Occlusive Disease (VOD) in Adult Patients With Acute Myeloid Leukemia (AML) Receiving Gemtuzumab Ozogamicin (GO) Before Allogeneic Stem Cell Transplant (SCT)

Vincent T. Ho ${ }^{1}$, Andrew St. Martin ${ }^{2}$, Waleska Perez $z^{2}$, Patricia Steinert ${ }^{2}$, Mei-Jie Zhang ${ }^{2}$, Deborah Chirnomas ${ }^{3}$, Caroline J. Hoang ${ }^{3}$, Fausto R. Loberiza, $\mathrm{Jr}^{3}$, Wael Saber ${ }^{2}$

${ }^{1}$ Dana-Farber Cancer Institute, Boston, MA, United States; ${ }^{2}$ Center for International Blood \& Marrow Transplant Research, Milwaukee, WI, United States; ${ }^{3}$ Pfizer Inc, New York, NY, United States

Background: Hepatic VOD has been reported in AML patients exposed to the CD33-directed antibody-drug conjugate GO before SCT.

Methods: To determine VOD risk and outcomes after GO exposure and subsequent SCT, we analyzed data from a subset of patients randomly selected for research level reporting to the Center for International Blood \& Marrow Transplant Research (CIBMTR). Adults with AML and GO exposure at any time before first myeloablative allogeneic SCT were matched 1:4 by age at SCT and disease status before SCT to patients without GO exposure. Marginal Cox regression and marginal logistic regression modeling were used to determine overall survival and incidence of VOD at 100-days, respectively. A stepwise model building approach was used to identify risk factors associated with VOD and death.

Results: 141 patients with and 564 patients without GO exposure underwent SCT between 2008 and 2011. Median (range) age for patients with and without GO exposure before SCT was 42 (18-73) years and 38 (18-74) years, respectively. Myeloablative conditioning (MAC) with chemotherapy (with GO, 61\%; without GO, 57\%) was utilized more frequently than MAC with total body irradiation (with, 39\%; without, 43\%). Incidence of VOD at 100-days between patients with/without GO exposure was similar $(4 \% / 3 \%)$, as was the incidence of severe VOD $(3 \% / 1 \%)$. Survival probabilities were similar in patients with/without GO exposure at 100 days $(81 \% / 81 \%)$, 
6 months $(67 \% / 69 \%)$, and 1 year $(52 \% / 58 \%)$ from SCT and 100-days $(50 \% / 43 \%)$, 6-months $(33 \% / 30 \%)$, and 1 -year $(33 \% / 26 \%)$ from onset of VOD. In multivariate analysis (MVA), GO exposure was not associated with an increased risk of VOD (OR, 1.05; 95\% CI, 0.41-2.67) or death (HR, 1.08; 95\% CI, 0.86-1.36).

Conclusions: The incidence of VOD was similar in AML patients with and without GO exposure before SCT. Moreover, GO exposure was not associated with an increased risk of VOD or death. Limitations of the study include selection bias associated with the retrospective design. Future analyses will evaluate the impact of dose on VOD risk and survival outcomes in this cohort.

Encore of BMT Tandem 2018. Funding: Pfizer. Data from the Coordinating Center of the CIBMTR are preliminary.

\section{Conflict of interest:}

V. Ho: advisory boards and consultant for Jazz Pharmaceuticals

D. Chirnomas, C. Hoang, F. Loberiza: employees of and own stocks in Pfizer Inc.

A. Martin, W. Perez, P. Steinert, M. Zhang, and W. Saber: nothing to disclose

\section{P014}

\section{Chemotherapy, donor lymphocyte infusions or second allogeneic stem cell transplantation (SCT) for post-allograft relapsed acute myeloid leukemia (AML) in adults}

Matthias Eder, Michael Stadler, Elke Dammann, Catherina Lueck, Victoria Panagiota, Lothar Hambach, Souheila Tayeb, Maleen Beck, Munir Rababah, David Dorn, Christian Koenecke, Steve Ehrlich, Arnold Ganser, Gernot Beutel

Hannover Medical School, Dept. of Hematology, Hemostasis, Oncology, and Stem Cell Transplantation, Hannover, Germany

Background: Relapse rates of acute myeloid leukemia (AML) after allogeneic stem cell transplantation (SCT) remain high with limited treatment options and low survival rates. We performed a retrospective single center analysis of adult AML patients with post- allograft relapse treated with (1) chemotherapy only without cellular therapy (CHX), (2) donor lymphocyte infusions (DLI) or (3) second SCT (2. TX).

Methods: One hundred seventy five AML patients transplanted between 01/1994 and 08/2017 with systemic $(\mathrm{n}=123)$, both systemic and extramedullary $(\mathrm{EM})(\mathrm{n}=31)$, and EM only $(n=21)$ post-allograft relapse were included in this analysis. Patients were treated with chemotherapy + - irradiation without further cellular therapy (CHX, $\mathrm{n}=61)$, with DLI $(\mathrm{n}=90)$ alone $(\mathrm{n}=12)$ or after chemo/ radiotherapy $(n=78)$, or received a second SCT with $(\mathrm{n}=15)$ or without previous DLI $(\mathrm{n}=9)$ from the same $(n=8)$ or an alternative $(n=16)$ donor (2.TX). Chemotherapies included conventional cytarabine-based regimens $+I-$ targeted therapies or hypomethylating agents. Treatment groups were compared for age, de novo or secondary AML, EM manifestation, CR rate at SCT, donor type, myeloablative (MAC) or reduced intensity conditioning (RIC) at initial SCT, and kind of chemotherapy for postallograft relapse. With a median follow-up of 0.5 years (range 0-21) we analyzed overall survival (OS) and non relapse mortality (NRM) after first post-allograft relapse, as well as acute and chronic GvHD incidence after start of cellular therapy for the DLI and 2.TX groups using KaplanMeier statistics. Variables with impact on OS were
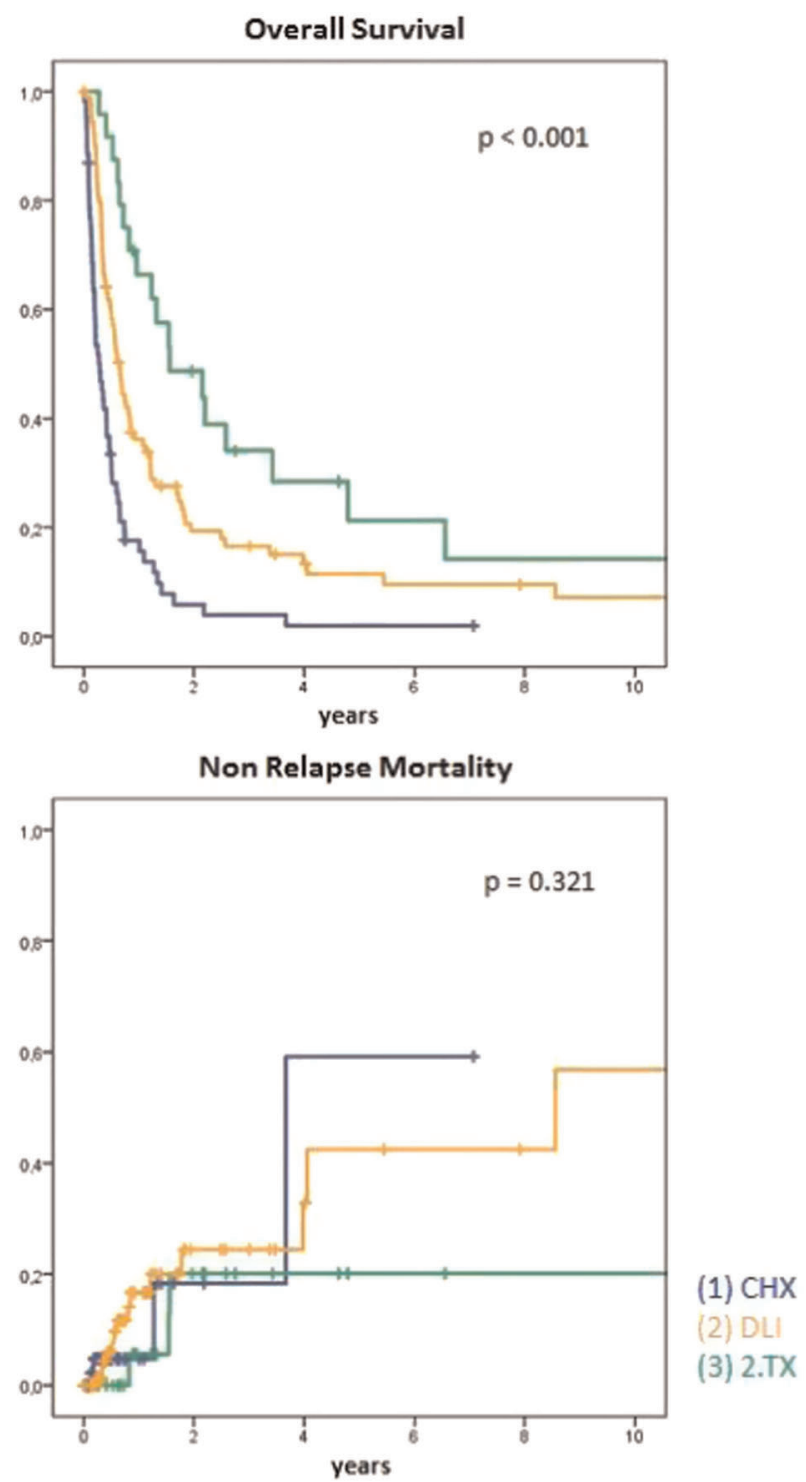

[P014 Figure] [Figure 1] 
identified by Cox regression analysis.

Results: Five year OS was significantly lower in the CHX group $(2.0 \%)$ as compared to the DLI $(11.5 \%)$ and 2.TX (21.3\%) groups, respectively. The CHX, DLI and 2.TX groups were different for age (mean 50, 49, and 39 years, $p$ $=0.001)$, rate of 1 . CR $(42.6 \%, 40 \%$ and $75 \%, p=0.008)$, rate of matched related donors $(39.3 \%, 20.0 \%$ and $58.3 \%$, p $=0.002)$ and rate of $\operatorname{MAC}(37.7 \%, 34.5 \%$ and $66.7 \%, \mathrm{p}=$ 0.005 ) all at initial SCT as well as use of hypomethylating agents for therapy at relapse $(18 \%, 24.4 \%$, and $4.2 \%, \mathrm{p}=$ $0.002)$. In contrast, EM manifestation $(41 \%, 27.8 \%$, and $33.3 \%, \mathrm{p}=0.29)$ and de novo AML $(77 \%, 67.8 \%$, and $70.8 \%, \mathrm{p}=0.46$ ) were equally distributed. OS was highest in the 2.TX group $(\mathrm{p}<0.001)$, whereas NRM was not different between the groups $(p=0.321)$ (Figure 1). Incidence of aGvHD (III-IV ${ }^{\circ}$ ) was $18.9 \%$ and $8.3 \%$ $(p=0.198)$ and of extensive cGvHD $22.2 \%$ and $8.3 \%$ $(\mathrm{p}=0.035)$ in the DLI and 2.TX groups, respectively. In multivariate analysis only MAC as compared to RIC for first SCT ( $p=0.028)$ but not age, CR rate at initial SCT, donortype, EM manifestation, de novo AML, and kind of chemotherapy after SCT was relevant for OS after postallograft relapse.

Conclusions: Our data show improved survival for adult patients with post-allograft relapsed AML treated by second SCT. Obviously, candidates for second SCT are a highly selected group with favorable characteristics. In contrast, patients treated with chemotherapy only have a very poor outcome. With the limitations of a single-center retrospective analysis these data suggest that second SCT may be considered for highly selected adult AML patients with post-allograft relapse.

Conflict of interest: The authors have no conflict of interest to disclose.

\section{P015}

Comparison of autologous stem cell transplantation versus haploidentical donor stem cell transplantation for favorable- and intermediate-risk acute myeloid leukemia patients in first complete remission

Jia Chen ${ }^{1,2,3}$, Lingyi Yang ${ }^{4}$, Yi Fan $^{1,2}$, Yang X ${ }^{1,2,3}$, Yue Han ${ }^{1,2,3}$,

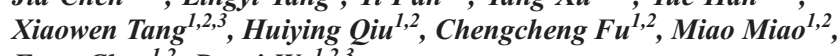
Feng Chen ${ }^{1,2}$, Depei $\mathrm{Wu}^{1,2,3}$

${ }^{I}$ the First Affiliated Hospital of Soochow University, Hematology, Suzhou, China; ${ }^{2}$ Jiangsu Institute of Hematology, Suzou, China;

${ }^{3}$ Soochow University, Institute of Blood and Marrow Transplantation, Suzhou, China: ${ }^{4}$ the First Affiliated Hospital of Soochow University, Respiratory, Suzhou, China

Background: Stem cell transplantation (SCT) is an attractive post-remission treatment option for patients with intermediate-risk acute myeloid leukemia (AML) and for some favorable-risk AML patients with additional nongenetic risk factors. Autologous SCT (auto-SCT) and haploidentical donor SCT (haplo-SCT) are the widely used alternatives in case of a lack of a human leukocyte antigen (HLA)-matched donor. However, limited data have been published on the direct comparison between these two transplant types.

Methods: Based on the transplantation database in our center, we conducted a retrospective study involving patients with favorable- and intermediate-risk AML in first complete remission (CR1), according to the NCCN guideline. Patients with APL, extramedullary disease or those achieving $\mathrm{CR}$ by more than two cycles were excluded.

Results: In total, 196 patients were included in the study, 88 of whom underwent auto-SCT and 107 underwent haplo-SCT. Patients in the auto-SCT group were older than those in the haplo-SCT group, with significant difference (P $=0.004)$. According to NCCN guidelines version 1.2017, 58 patients $(65.9 \%)$ in the auto-SCT group were classified as patients with favorable-risk AML, while only $30.8 \%$ of patients in the haplo-SCT group were classified as patients with favorable-risk AML $(\mathrm{P}<0.001)$. Core binding factor (CBF) AML presented more frequently in the auto-SCT group $(\mathrm{P}=0.005)$. Both the interval from diagnosis to transplantation and from remission to transplantation were longer for patients in the auto-SCT group $(\mathrm{P}<0.001)$.

In the whole cohort analyses, the impact of high relapse incidence in the auto-SCT group was compensated by low non-relapse mortality (NRM), which resulted in a comparable overall survival (OS) $(79.0 \pm 4.6 \%$ versus $80.1 \pm 5.0 \%$, $\mathrm{P}=0.769)$ and relapse-free survival (RFS) $(66.1 \pm 5.2 \%$ versus $77.4 \pm 4.8 \%, \mathrm{P}=0.079$ ) compared to those observed in the haplo-SCT group.

However, for patients with intermediate-risk AML, NRM was similar between the groups, and haplo-SCT exhibited superior survival. In case of post-SCT relapse, patients with intermediate-risk AML showed markedly inferior 3-year OS compared to that shown by patients with favorable-risk AML $(23.3 \pm 9.8 \%$ versus $60.8 \pm 14.3 \%, P=0.011)$. In the multivariate analyses, minimal residual disease (MRD) measured by flow cytometry, and gene mutation status before transplantation were independent predictors for both OS and RFS.

Conclusions: We concluded that both auto-SCT and haplo-SCT were acceptable options for post-remission treatment of patients with favorable- and intermediate-risk AML. Haplo-SCT yielded a better outcome in patients with intermediate-risk AML, but the relapse after SCT still led to a poor outcome. Clearance of MRD before SCT could improve the prognosis after transplantation. 


\begin{tabular}{|c|c|c|c|c|c|c|c|c|c|}
\hline & \multirow[b]{2}{*}{ Auto-SCT } & \multicolumn{2}{|l|}{ Whole cohort } & \multicolumn{3}{|c|}{ Favorable-risk patients } & \multirow[b]{2}{*}{ Auto-SCT } & \multicolumn{2}{|c|}{ Intermediate-risk patients } \\
\hline & & Haplo-SCT & $P$ value & Auto-SCT & Haplo-SCT & $P$ value & & Haplo-SCT & $\mathrm{P}$ value \\
\hline $3 y-N R M$ & $5.3 \pm 2.6 \%$ & $14.1 \pm 4.1 \%$ & 0.032 & $2.3 \pm 2.2 \%$ & $6.9 \pm 4.7 \%$ & 0.015 & $12.1 \pm 6.6 \%$ & $16.3 \pm 5.0 \%$ & 0.647 \\
\hline $3 y-C I R$ & $31.0 \pm 5.1 \%$ & $9.2 \pm 3.2 \%$ & $<0.001$ & $25.0 \pm 5.8 \%$ & $4.3 \pm 4.3 \%$ & 0.037 & $41.7 \pm 9.3 \%$ & $10.9 \pm 4.0 \%$ & $<0.001$ \\
\hline $3 y-O S$ & $79.0 \pm 4.6 \%$ & $80.1 \pm 5.0 \%$ & 0.769 & $88.3 \pm 5.2 \%$ & $93.1 \pm 4.7 \%$ & 0.318 & $56.1 \pm 9.2 \%$ & $76.7 \pm 5.9 \%$ & 0.018 \\
\hline $3 y-R F S$ & $66.1 \pm 5.2 \%$ & $77.4 \pm 4.8 \%$ & 0.079 & $75.0 \pm 5.8 \%$ & $89.0 \pm 6.0 \%$ & 0.448 & $50.0 \pm 9.1 \%$ & $73.8 \pm 5.8 \%$ & 0.006 \\
\hline
\end{tabular}

Conflict of interest: None of the authors has anything to disclose.

[[P015 Table] Comparisons of transplantation outcomes]
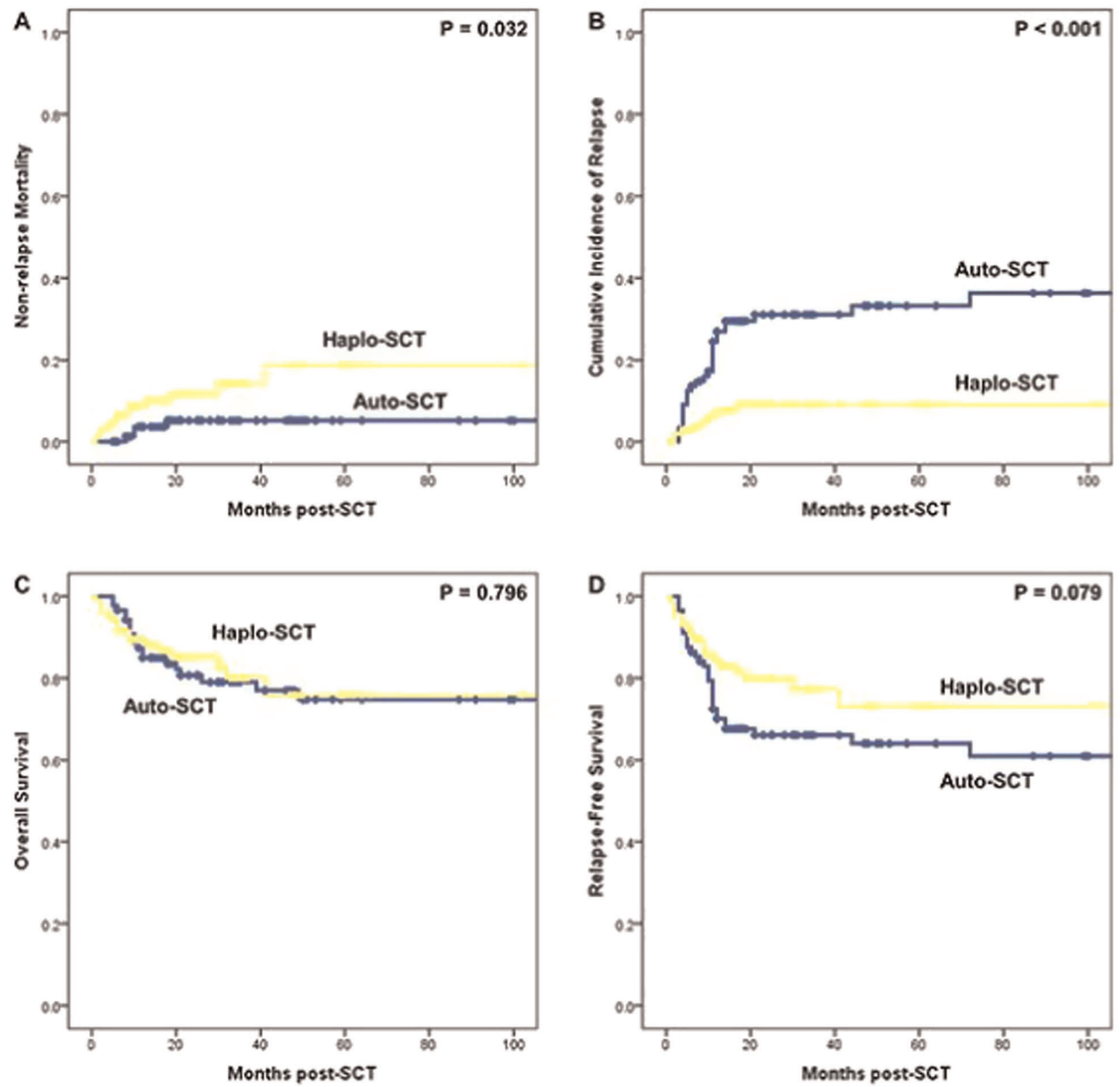

[P015 Figure] [Transplant outcome of the whole cohort] 


\section{P016}

Comparison of TBI- and busulfan in the occurrence of long term side effects after HSCT in pediatric acute leukaemia: a report from the AIEOP Registry

\section{Paola Quarello ${ }^{1}$, Franca Fagioli ${ }^{1}$, Franco Locatelli ${ }^{2}$, Claudio Favre $^{3}$, Maura Faraci ${ }^{4}$, Chiara Messina ${ }^{5}$, Arcangelo Prete ${ }^{6}$, Mimmo Ripaldi $^{7}$, Attilio Rovelli ${ }^{8}$, Marco Zecca ${ }^{9}$}

${ }^{1}$ Pediatric Onco-Hematology and Stem Cell Transplantation Division, Torino, Italy; ${ }^{2}$ Department of Pediatric Hematology-Oncology, Istituto di Ricovero e Cura a Carattere Scientifico (IRCCS), Bambino Gesù Children's Hospital, Roma, Italy; ${ }^{3}$ Department of Pediatric Oncology, Hematology, and Transplants, Anna Meyer Children's Hospital, Firenze, Italy; ${ }^{4}$ Department of Pediatric Hematology Oncology, G. Gaslini Institute, Genova, Italy; ${ }^{5}$ Department of Pediatric Hematology and Oncology, University of Padova, Padova, Italy; ${ }^{6}$ Paediatric Oncology and Haematology Unit "Lalla Seragnoli", Department of Paediatrics, University of Bologna Sant'Orsola-Malpighi Hospital, Bologna, Italy; ' BMT Unit, Department of Pediatric HematoOncology, Santobono-Pausilipon Hospital, Napoli, Italy; ${ }^{8}$ Department of Pediatric Hematology, San Gerardo Hospital, Monza, Italy;

${ }^{9}$ Department of Pediatric Onco-Hematology, IRCCS, Policlinico San Matteo Foundation, Pavia, Italy

Background: The outcome of hematopoietic stem cell transplant (HSCT) as treatment of pediatric patients with high-risk acute leukemia is increased in the last decades. However, this group of patients requires a long term followup due to late complications and second malignancies. An increased morbidity has often been attributed to the late toxicity of myeloablative conditioning regimens that include either Total Body Irradiation (TBI) or Busulfan (BU) in the majority of pediatric patients (Cohen, BMT 2008, Faraci, BMT 2008). Recently, Bernard et al. showed that late events seem less frequent after BU when compared with TBI (Bernard, BMT 2014).

The purpose of our retrospective multicenter study was to compare the long-term impact of BU or TBI on occurrence of late complications and second malignancies in childhood acute leukemia treated with HSCT.

Methods: We retrospectively evaluated the incidence of late effects in 646 pediatric patients (median age 10 years) undergoing HSCT in a AIEOP transplant Centre for acute lymphoid or myeloid leukemia (ALL or AML) between 2000 and 2013. All patients received TBI- or BU-based conditioning regimen and underwent HSCT in complete morphological remission.

Late effects incidences were calculated as a cumulative incidence to adjust the analysis for competing risks. Association between categorical variables were assessed with Fisher's exact test. P values $<0.05$ were considered to be statistically significant. Statistical analysis was performed using NCSS (Hintze, 2001; NCSS PASS, Number Crunched Statistical System, Kaysville, UT, USA).
Results: In total, 472 patients (73\%) had received TBI (95\% ALL and 5\% AML), when 174 (27\%) had a BUbased conditioning regimen (80\% AML and 20\% ALL). Median follow up from HSCT was 7 years. The 38\% (247/ 646) of patients developed at least one long-term late effects and $14 \%(88 / 646)$ of patients showed more than two complications. The incidence of at least one late complication was higher in TBI patients than in BU patients $(48 \%$ vs $28 \%, p=0.001)$. Similarly, the occurrence of more than two late effects was higher in the TBI group (16\% vs 7\%, p $=0.006$ ).

Specifically, univariate analyses revealed that adverse effects on height and cataract were significantly more frequent after a TBI-based conditioning regimen $(12 \%$ vs $7 \%, \mathrm{p}=0.04,19 \%$ vs $2 \%, \mathrm{p}<0.0001$ ) while no significant differences were observed in occurrence of gonadal dysfunction (33\% vs 20\%), hypothyroidism (17\% vs $12 \%)$ and pulmonary function impairment (6\% vs 6\%) between TBI- and BU- group. A significant higher incidence of second tumors was observed in TBI group $(19 \%$ vs $0 \%, \mathrm{p}=0.02)$.

Conclusions: Our retrospective analysis in a large cohort of patients suggests that late complications seem more frequent after TBI-based conditioning regimen in comparison with BU-based regimen and that the pattern of late side effects is different between these two groups. These data are important both in the choice of HSCT conditioning regimen and in the development of follow up measures. Thus, prospective and randomized studies aimed at better understanding the role of conditioning regimens on occurrence of different late effects are warranted.

Clinical Trial Registry: NA

Conflict of interest: The authors have no commercial, proprietary or financial interests in the products or companies described in this article.

\section{P017}

Dasatinib-induced Cytomegalovirus Reactivation Induces Earlier Molecular Response in Patients with Philadelphia Chromosome-Positive Acute Lymphoblastic Leukemia without Improvement of Transplant Outcome

Masahide Osaki, Motohito Okabe, Yuka Kawaguchi, Yoonha Lee, Marie Ohbiki, Miyo Yoshino, Hiroaki Araie, Seara Ikeno, Takahiko Sato, Takanobu Morishita, Yukiyasu Ozawa, Koichi Miyamura

Japanese Red Cross Nagoya Daiichi Hospital, Hematology, Nagoya, Japan

Background: Cytomegalovirus (CMV) infection during dasatinib therapy is reported to be correlated with a better prognosis in patients with chronic myeloid leukemia. However, this observation in patients with Philadelphia 
chromosome-positive acute lymphoblastic leukemia $(\mathrm{Ph}+$ ALL) who need hematopoietic stem cell transplantation is not yet clarified.

Methods: We retrospectively assessed 46 patients who were newly diagnosed with $\mathrm{Ph}+\mathrm{ALL}$ between 2008 and 2017 in our hospital. All patients received TKI combined chemotherapies (JALSG Ph+ALL208 (imatinib-based therapy) and JALSG Ph+ALL213 (dasatinib-based therapy)), respectively depends on the period). All protocol was composed of induction phase and 4 consolidation phases. We define early molecular complete remission (eCMR) as one that entered CMR after the first consolidation. According to the CMV infection status (seropositive or not) at diagnosis and reactivation/infection during therapies, we divided patients into 4 groups: CMV seropositive and reactivated group $(+/+) \quad(\mathrm{n}=5)$, not reactivated but seropositive group $(+/-)(\mathrm{n}=33)$ and CMV seronegative and no infection (group $(-/-)(\mathrm{n}=2)$ ), CMV status was not tested (unknown group, $\mathrm{n}=6$ ).

Results: Median age at diagnosis was 42 years. Eighteen patients were treated with dasatinib-based therapy and 28 patients were treated with imatinib-based therapy.

Neither of 2 seronegative patients developed CMV infection; group (-/-). Five of 16 seropositive patients who received dasatinib-based therapy developed CMV reactivation (CMV antigenemia in 3 and colitis in 2). On the other hand, none of 21 patients who were seropositive during imatinib treatment $(31 \%$ vs $0 \%, \mathrm{p}=0.01)$.

We analyzed the time of achieving CMR among patients treated with dasatinib-based therapy. All patients with CMV reactivation (group $(+/+)$ ) achieved eCMR. Seven of 11 patients with infected CMV without reactivation (group $(+/-))$ during dasatinib-based therapy achieved eCMR. Neither of 2 patients never infected with CMV (group $(-/-))$ achieved eCMR. Group $(+/+)$ tends to advance CMR in comparison with group $(-/-)(\mathrm{p}=0.0668$, Fisher's exact test) among patients who received dasatinib-based therapy.

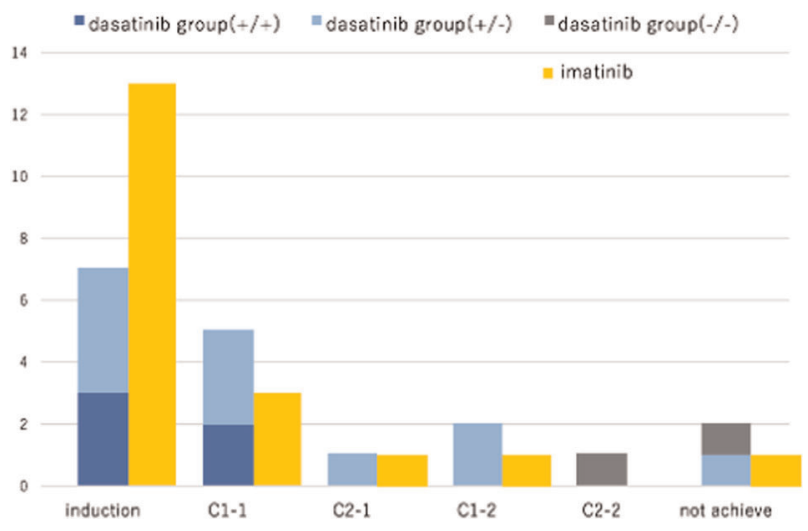

[P017 Figure] [The number of patients achieving CMR after induction and each consolidation]
34 patients received allogeneic hematopoietic stem cell transplantation. Overall survival at 2 years was not different between the patients with eCMR and patients without eCMR $(76.2 \%$ vs $81.8 \%$ p $=0.903)$. DFS and NRM at 2 years were 76.5 vs $72.7(\mathrm{p}=0.636)$ and 14.2 vs 18.2 $(\mathrm{p}=0.661)$, respectively. There is no difference between dasatinib patients and imatinib patients about CMV reactivation after transplantation $(50 \%$ vs $65 \%, \mathrm{p}=$ 0.487). A patient of group (+/-) died of CMV colitis after allogeneic hematopoietic stem cell transplantation.

Conclusions: We have demonstrated that CMV reactivation can achieve CMR rapidly exclusively among dasatinibbased therapy patients. Some study reported that earlier CMR induced better OS after allogeneic hematopoietic stem cell transplantation. But our study found that eCMR did not yield a favorable prognosis. The CMV reactivation group can develop CMV infection after HSCT and that may reduce overall survival.

Conflict of interest: K. Miyamura: Novartis, BristolMyers Squibb

\section{P018}

\section{Decitabine plus DLI is effective for TP 53 Mutated Patients with Relapse after Allogeneic Stem Cell Transplantation}

Christine Wolschke, Raissa Adjalle, Francis Ayuk, Ute-Marie von Pein, Gaby Zeck, Nikolaus Kröger

University Hospital Hamburg Eppendorf, Clinic for Stem cell Transplantation, Hamburg, Germany

Background: Patients with AML or MDS harbouring TP53 mutation have a risk of up to $60 \%$ for relapse after allogeneic stem cell transplantation (alloHSCT). Relapse after alloHSCT have a poor outcome, resulting in overall survival of 10-20\%. Remission appears to rely on therapies that maximise the graft versus leukemia effect. It is unclear whether chemotherapy is a prerequisite to donor cell therapy. Patient with myeloid neoplasm and TP 53 mutation have a poor remission rate after intensive chemotherapy. More recent data suggest efficacy of decitabine in TP53 mutated AML/MDS patients.

Here we describe 7 AML/MDS pts with TP53 mutation who relapse after alloHSCT and received decitabine (DAC) in combination with donor lymphocyte transfusion (DLI) as salvage therapy.

Methods: We retrospectively analyzed data of 7 TP53 mutated pts. with acute myeloid leukemia $(n=3)$ or MDS $(n=4)$ who relapsed after alloHSCT and were treated with a median of 4 cycles DAC (range 1 to 9). 6 pts. received a dose of $20 \mathrm{mg} / \mathrm{m}^{2}$ for 5 days every 28 days, 1 pt. $20 \mathrm{mg} / \mathrm{m}^{2}$ for 3 days every 21 days. DAC was the first salvage therapy in 5 pts. after allogeneic stem cell transplantation. 2 pts 
failed after a therapy with azacytidine. In 5 pts donor lymphocyte transfusion was administrated in addition to DAC. The median of DLI was 2 per patient (range 1 to 2 ) with a median $\mathrm{T}$ cell dosage of $5 \times 10^{5}$ to $5 \times 10^{6} \mathrm{CD} 3+$ cells/kg pt. 1 pat. also received lenalidomide in combination.

Results: As a result, overall response rate was $71 \%$, including 3 complete cytogenetic remission (cCR, 42\%) and 2 partial remission (PR, 29\%). 2 patients within the first line group achieved CR, 1 patient received DAC as second line treatment after previous Aza failure reached CR. Median duration of $\mathrm{CR}$ was 6 months (range 5 to 7 ) and 2 patients relapsed so far. Incidence of acute or chronic graft-versushost disease was low.

Conclusions: DAC in combination with exerts clinical efficacy in patients with AML or MDS with TP 53 Mutation and is able to induce complete remission in nearly half of the patients. These data suggest an alternative relapse regime in combination with DLI after an allogeneic stem cell transplantation for patients with TP 53 mutation. The role of decitabine as pre-transplant and maintenance strategy post transplantation for patients with high risk AML or MDS and TP53 Mutation should be evaluate in prospective clinical trials.

Conflict of interest: C. Wolschke: nothing to disclose

\section{P019}

Abstract previously published

\section{P020}

\section{Disease Progression is Main Barrier to Allogeneic Hematopoietic Stem Cell Transplantation (HCT) in Patients with Newly Diagnosed and Relapsed Acute Leukemia}

Elaina V. Preston ${ }^{1}$, Stephanie Chinapen ${ }^{1}$, Taylor Borrill ${ }^{1}$, Eric Davis $^{2}$, Brian C. Shaffer ${ }^{1,3}$, Roni Tamari, ${ }^{1,3}$, Ann A. Jakubowski, ${ }^{1,3}$, Miguel-Angel Perales ${ }^{1,3}$, Doris M. Ponce ${ }^{1,3}$, Gunjan Shah ${ }^{1,3}$, Parastoo B. Dahi ${ }^{1,3}$, Juliet N. Barker ${ }^{1,3}$, Ellin Berman ${ }^{3,4}$, Martin Tallman $^{3,4}$, Sergio A. Giralt ${ }^{1,3}$, Eytan Stein ${ }^{3,4}$, Boglarka Gyurkocza ${ }^{1,3}$

${ }^{1}$ Memorial Sloan Kettering Cancer Center, Dept. of Medicine, Adult Bone Marrow Transplant Service, New York, NY, United States;

${ }^{2}$ Memorial Sloan Kettering Cancer Center, Dept. of Pediatrics, Bone Marrow Transplant Service, New York, NY, United States; ${ }^{3}$ Weill Cornell Medical College, Dept. of Medicine, New York, NY, United States; ${ }^{4}$ Memorial Sloan Kettering Cancer Center, Dept. of Medicine, Leukemia Service, New York, NY, United States

Background: Multiple studies demonstrated the curative effect of HCT for patients with acute leukemia (AL), but it is estimated that only 1 of 3 eligible patients undergo this procedure. Historically, lack of available donor was among the most common reasons for not proceeding to allogeneic $\mathrm{HCT}$, but this is no longer a major limitation to transplant with widespread use of cord blood and haploidentical HCT.

Our aim is to prospectively determine the rates in which $\mathrm{AL}$ patients proceed to $\mathrm{HCT}$ based on disease risk using National Comprehensive Cancer Network guidelines and to identify reasons for not proceeding to HCT in the era of alternative donor sources.

Methods: All patients with newly diagnosed or relapsed AL admitted to our center's Adult Leukemia Service for induction or re-induction therapy were approached to enroll on this prospective observational study.

Results: Between April 2016 and December 2017, 90 patients were enrolled and have sufficient follow up (180 days from initiation of induction or re-induction).

While $52 \%$ of patients identified as White European, there was substantial representation from groups at high risk of not finding an 8/8 HLA-matched donor in the international registries.

Twenty-two patients had HLA-identical sibling donors. Fifty-three patients had at least one potential 8/8 HLAmatched unrelated donor identified through preliminary donor searches. In 4 cases suitable umbilical cord units or HLA-haploidentical donors were identified. The remaining $11 \mathrm{pts}$ did not have searches due to early death or known matched related donor.

Allogeneic HCT was recommended in 71 cases $(78 \%)$ based on disease-related risk via a consensus decision of Leukemia and Bone Marrow Transplant Services. Of the 71 patients, $46(65 \%)$ underwent HCT. The median time between starting induction or re-induction chemotherapy and transplant was 109 (range 41-545) days. Donors were HLA-identical siblings $(\mathrm{n}=10)$, HLA-matched unrelated donors $(\mathrm{n}=27)$, mismatched unrelated donor $(\mathrm{n}=1)$, and umbilical cords $(n=8)$.

Of the 25 patients for whom HCT was recommended but was not performed, 16 had progressive disease (11 have since died), 1 patient died of induction-related complications, 3 had severe comorbidities, 4 patients declined HCT and 1 patient was lost to follow up. There weren't any cases where donor availability was the barrier to transplant.

Conclusions: Based on interim results of our prospective observational trial $65 \%$ of patients underwent allogeneic HCT in whom it was recommended based on diseaserelated risk factors. A suitable donor was identified for all patients in whom allogeneic HCT was recommended. The main barrier to transplant was early disease progression and death.

Conflict of interest: None of the authors has anything to disclose. 

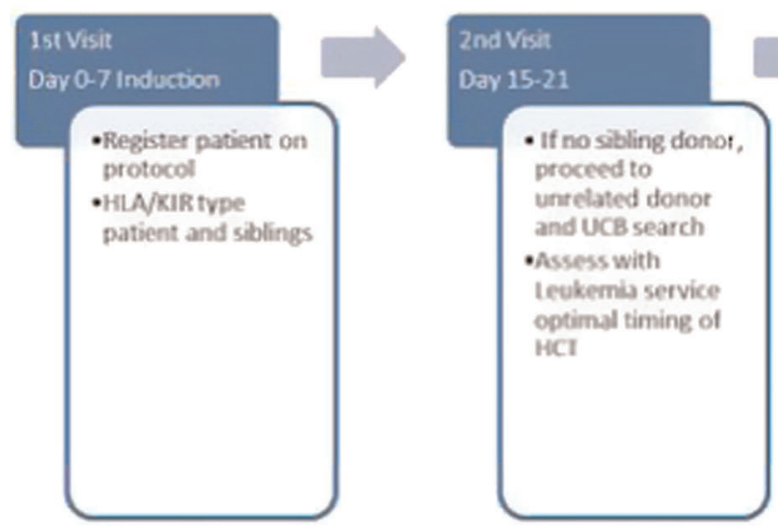

[P020 Figure] [Figure 1]

\begin{tabular}{lcc}
\hline Male Patients & 53 & $59 \%$ \\
Female Patients & 37 & $41 \%$ \\
Median Age & 58 & \\
Age Range & $24-74$ & \\
DISEASE STATUS AT DIAGNOSIS & \\
Newly Diagnosed & 66 & $73 \%$ \\
RES & 7 & $8 \%$ \\
First Relapse & 15 & $17 \%$ \\
Relapsed Refractory & 2 & $2 \%$ \\
\hline
\end{tabular}

\section{[[P020 Table] Patient Characteristics]}

\section{P021}

Donor lymphocyte infusion after salvage chemotherapy in relapsed acute myeloblastic leukemia patients after allogeneic stem cell transplantation

Atilla Uslu, Guldane Cengiz, Seval, Sinem Civriz Bozdag, Gunhan Gurman, Taner Demirer, Onder Arslan, Muhit Ozcan, Selami Kocak Toprak, Pervin Topcuoglu, Osman Ilhan

Ankara University School of Medicine, Department of Hematology and Stem Cell Transplant Unit, Ankara, Turkey

Background: Although allogeneic HSCT(AHSCT) can offer cure to acute myeloblastic leukemia (AML) patients, relapse still remains to be a limiting factor. The optimal treatment to improve the outcome of this poor prognostic group is controversial. We aimed to search the impact of donor lymphocyte infusion (DLI) after salvage treatment in relapsed AML patients after allogeneic HSCT.

Methods: We retrospectively included twenty AML patients who had hematological relapse after AHSCT, in Ankara University Faculty of Medicine Bone Marrow Transplantation Unit. Donor lymphocyte infusions have

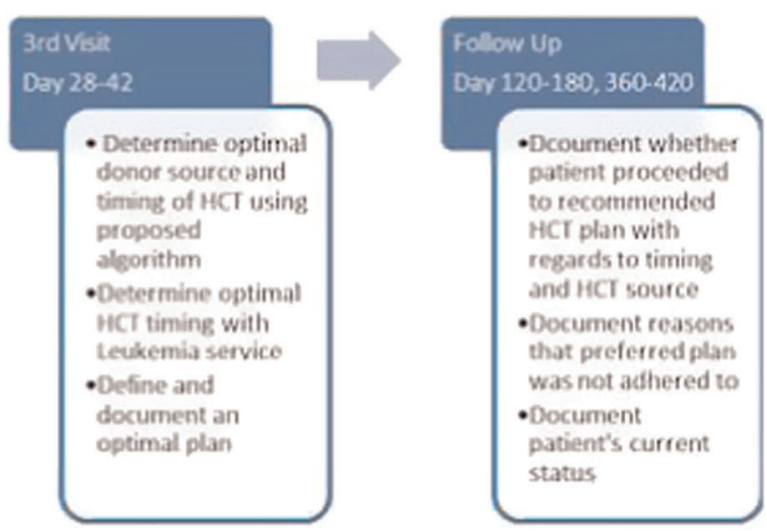

\begin{tabular}{|c|c|}
\hline Patients $(n=20)$ & \\
\hline Median Age (years) & $36(18-61)$ \\
\hline Sex, n (\%) & \\
\hline Male & $13(65 \%)$ \\
\hline Female & $7(35 \%)$ \\
\hline Cytogenetic risk & \\
\hline Standart risk & $10(50 \%)$ \\
\hline High risk & $6(30 \%)$ \\
\hline NA & $4(20 \%)$ \\
\hline Stem cell source, $\mathrm{n}(\%)$ & \\
\hline Peripheral blood & $18(90 \%)$ \\
\hline Bone marrow & $1(5 \%)$ \\
\hline Peripheral blood + bone marrow & $1(5 \%)$ \\
\hline Pretransplant disease status & \\
\hline Remission & $15(75 \%)$ \\
\hline Refractory & $5(25 \%)$ \\
\hline Patient CMV serological status, n & \\
\hline $\operatorname{Ig} \mathbf{G}(-)$ & $6(30 \%)$ \\
\hline $\operatorname{Lg} \mathbf{G}(+)$ & $14(70 \%)$ \\
\hline Conditioning regimens. & \\
\hline Myeloablative & $18(90 \%)$ \\
\hline Reduced intensity & $2(10 \%)$ \\
\hline Salyage therapy after relapse & \\
\hline EMA & 9 \\
\hline ElutARAC+/_Mitox/Ida & 4 \\
\hline IdatARAC & 4 \\
\hline Etoposid/ARAC & 1 \\
\hline Azacytidine & 2 \\
\hline Median Donor Age & $34(6-56)$ \\
\hline Donor gender, n (\%) & \\
\hline Male & $14(70 \%)$ \\
\hline Female & $6(30 \%)$ \\
\hline HLA match & \\
\hline Sibling 10/10 & $17(85 \%)$ \\
\hline Unrelated $10 / 10$ & $1(5 \%)$ \\
\hline Haploidentic. & $1(5 \%)$ \\
\hline Sibling $9 / 10$ & $1(5 \%)$ \\
\hline Sex mismatch & $10(50 \%)$ \\
\hline Blood group missmatch & $12(60 \%)$ \\
\hline
\end{tabular}

[P021 Figure] [Table 1: Demographic features of the patients]

been scheduled at the end of salvage treatments. We statistically analyzed the relationship between response to DLI and overall remission duration with patient/ DLI characteristics. 
Results: Demographic features of the patients have been summarized in Table 1. All but two patients have been treated with salvage chemotherapy. Donor lymphocyte infusion has been performed at the end of chemotherapy. Two patients have been treated with azacytidine and DLI has been given at the end of 2 cycles. DLI features of the patients have been shown in Table 2. Eight patients recieved DLI in complete remission whereas 5 out of 12 patients achieved remission after DLI. Response duration was not found to be related with gender, CMV status, cytogenetic risk factor, blood percent at relapse, remission duration before relapse, pretransplant acute or chronic GVHD before relapse. Also, donor age, gender, sex mismatch or blood group mismatch with recipient did not have a statistically significant effect on response duration after DLI. The only statistically significant factor was remission status before donor lymphocyte infusion. Acute GVHD was observed in $32 \%$ of the patients. Median survival in patients who achieved remission and refractory disease after DLI treatment were 2.2 years and 1.4 months respectively $(\mathrm{p}=0.93)$ Estimated overall survivals at 1 year who achieved remission were $60 \%$ respectively.

Conclusions: In AML patients who relapsed after AHSCT, donor lymphocyte infusion after salvage chemotherapy is a feasible treatment choice if remission can be achieved before infusion.

Conflict of interest: All authors: nothing to disclose

\begin{tabular}{|c|c|}
\hline Disease status at DLI (n (\%)) & $8(40 \%) 12(60 \%)$ \\
\hline Remission Active disease & \\
\hline $\begin{array}{l}\text { Median Infused CD3 + cell count } \\
(\mathrm{x} 108) \text { (median) }\end{array}$ & $1,38(0,27-11,60)$ \\
\hline
\end{tabular}

Total DLI number(n (\%)) 1234 9(52,0\%) 4(20,0\%) 6 $(24,0 \%) 1(4,0 \%)$

\section{[[P021 Table] Table 2: DLI features of the patients]}

\section{P022}

\section{Early molecular dagnosis and preventive therapy of expected cytological post-transplant relapses in patients with acute myeloid leukemia}

Yana Gudozhnikova, Nikolay Mamaev, Sergey Bondarenko, Ildar Barkhatov, Valeriya Katerina, Alena Shakirova, Tatiana Gindina, Valentina Kravtsova

Pavlov First St. Petersburg State Medical University, R.M. Gorbacheva Memorial Institute of Children Oncology, Hematology and Transpantation, St. Petersburg, Russian Federation

Background: Allogeneic hematopoietic stem cell transplantation (allo-HSCT) is now considered the best treatment of AML with poor prognosis, however, often followed by post-transplant relapses (PTR). Among PTR, molecular and cytological variants (mPTR and cPTR, respectively) were recognized, and WTl gene was an important independent prognostic indicator of acute leukemia following alloHSCT.

Methods: Ninty AML patients aged 2-68 years (median 38.8) who underwent allo-HSCT at our University were enrolled in the study. WT1 gene expression levels by realtime PCR as well as bone marrow blast counts were measured before transplantation as well as on days $\mathrm{D} 30+$, D60+, and D100+ after HSCT. The WT1 threshold value was determined as $250 \mathrm{WT} 1$ copies $/ 10^{4}$ copies of BCR gene. Overall survival (OS), relapse-free survival (RFS), and cumulative incidence of relapse (CIR) were determined for the WT1+ and WT1- patient groups. Besides, we have allocated time intervals between MPTR and CPTR in about one-third of patients.

Results: The levels of WT1 gene expression at allo-HSCT ranged from 0 to $56884 W T 1 / 10^{4} A B L$ copies (median 209). There was a significant difference in 2-year OS, RFS, and CIR between WT1-positive and WT1-negative groups $(34.1 \%, 24.4 \%, 43.9 \%$ vs $74.5 \%, 66 \%$, and $72.3 \%$, at $\mathrm{p}<$ $0.0001, \mathrm{p}<0.0001$ and $\mathrm{p}=0.003$, respectively). On the day $\mathrm{D} 30+$, the 250-copy threshold recognized difference only for CIR $(46.2 \%, 38.5 \%, 38.5 \%$ vs $60.9 \%, 50 \%$, and $59.4 \%$ at $\mathrm{p}=0.18, \mathrm{p}=0.17$ and $\mathrm{p}=0.04$, respectively). Meanwhile, the differences in these parameters between WT1and $\mathrm{WT} 1+$ groups were maximal at the time points of D60 $+(30.8 \%, 30.8 \%$ and $30.8 \%$ vs $66.1 \%, 53.2 \%$ and $61.3 \%$, at $\mathrm{p}=0.005, \mathrm{p}=0.009$ and 0.006 , respectively), and $\mathrm{D} 100+$ $(25 \%, 15 \%$ and $20 \%$ vs $74.5 \%, 63.6 \%$ and $69.1 \%$, at $\mathrm{p}<$ $0.0001, \mathrm{p}<0.0001$ and $\mathrm{p}<0.0001$, respectively). In general, these data showed a prognostic significance of WTI level normalization measured on D0, as well as on days D60+ and D100+ after allo-HSCT. In our cases, mPTR and cPTR were registered in 57/86 (66.2\%) and 33/86 (38.4\%) patients, respectively. In $33.3 \%$ of the patients (11/ 33), mPTR and cPTR were noted simultaneously, whereas in 22 cases, cPTR occurred with a delay after mPTR. This period ranged from 13 up to 321 days with a median of 35 days. It should be mentioned that our patients, upon detection of overexpressed WT1 obtained different preventive treatments that should be kept in mind explaining the increased interval between MPTR and cPTR. However, the prolonged interval also may be due to molecular and cytogenetic features of these leukemias. New investigations on the topic are needed to test this hypothesis.

Conclusions: Thus, in AML patients, WTI levels in bone marrow cells at time points of D0, D60+ and D100+ were predictive for OS, EFS and CIR, which agrees well with recently published data. The unusual phenomenon of cPTR delay after mPTR detection may be useful in clinical 
practice for the controlled molecular study of new medical drugs and therapeutic approaches.

Conflict of interest: The authors have stated that they have no conflicts of interest.

\section{P023}

\section{Efficacy of active surveillance in reducing mortality due to multidrug resistant bacteria in acute leukemia}

\author{
Christelle Castañon ${ }^{1}$, Alejandro Zuazua ${ }^{2}$, Adan Rodriguez ${ }^{2}$, Teresa \\ Bernal $^{1}$, Sara Alonso ${ }^{1}$, Enrique Colado ${ }^{\prime}$ \\ ${ }^{1}$ Hospital Universitario Central de Asturias, Oviedo, Spain; \\ ${ }^{2}$ Universidad de Oviedo, Oviedo, Spain
}

Background: Multidrug resistant infections (MRD), specially those caused by gram negative bacteria (GNB), are associated with poor outcomes in acute myeloblastic leukemia (AML) and could make hinder patients from completing their optimal treatment, such stem cell transplantation. Active surveillance (AS), where patients are screened for colonization with MDR-GNB, may serve as a tool to decrease bacterial transmission and guide the choice of empirical antibiotic treatment. The aim of the study was to analyze the impact of active surveillance in the incidence of MDR and GNB infections and infection related mortality in AML patients.

Methods: This was a prospective observational study including all consecutive adult patients with an AML diagnosis, treated with intensive chemotherapy at Hospital Universitario Central de Asturias (HUCA) from January 2015 to October 2017. The cohort of patients in whom active surveillance was performed (cohort 1) was compared to a cohort of AML patients in whom AS was not done (cohort 2). Empiric antibiotic therapy was considered appropriate if at least one of the empiric antibiotics was effective for the bacteria detected in surveillance cultures. All patients received fluoroquinolone prophylaxis. Data are presented as mean \pm standard deviation or as median (range). Univariate comparisons were done using the chisquare test (categorical variables) or Fisher's exact test (for contingency tables with frequencies below 5).

Results: From January 2015 to October 2017, seventythree AML patients received 172 intensive chemotherapy cycles. In cohort 1 , active surveillance was performed along 86 cycles. General characteristics were similar in both cohorts: median age 57 (43-64) years, $48 \%$ females, European-Leukemia-Net genetic risk favorable in $44 \%$, intermediate in $27 \%$ and high in $29 \%$ patients. Gram negative multidrug resistant bacteria colonization was detected in $55 \%$ of the cycles, without differences between the different phases of treatment $(46 \%$ during induction cycle-1, $70 \%$ during induction-2, 70\%, during consolidation-1, 44\% during consolidation-2 and 50\% during consolidation-3). Regarding the epidemiology of bacterial infections, the more frequent isolates in both cohorts were Gram negative $(45 \%)$ followed by gram positive (33\%) and polimicrobial infections (15\%). Infection due to multidrug resistant bacteria was detected in $50 \%$ of cycles. Empirical antibiotic treatment was considered appropriate according previous surveillance cultures in $86 \%$ of infectious episodes. Gram negative infections were statistically associated with higher mortality compared to other infections ( $21 \%$ vs $6.6 \%, \mathrm{p}=0.03$ ) and the main cause of death in complete remission prior to stem cell transplant. A non-significant reduction in thirty-day mortality was observed between cohort 1 and cohort 2 (7,5\% vs $12 \%)$.

Conclusions: In our population of acute leukemic patients, colonization due to GN resistant bacteria occurs in a high proportion of patients and is an early event during treatment. Gram negative bacterial infections remain the most frequent cause of morbidity and mortality. Although active surveillance may help in the choice of appropriate empiric antibiotic treatment in the era of multidrug resistance, larger studies are needed to demonstrate its efficacy in reducing mortality.

Conflict of interest: None of the authors has anything to disclose.

\section{P024}

Efficacy of allogeneic haematopoietic transplantation in older patients with myeloid malignancies assessed by Graft-versus-Host Disease Free, Relapse-Free Survival (GRFS). Multivariable Analysis from a Single Centre

Pavel Jindra, Michal Karas, Daniel Lysák, Alexandra Jungova, Katerina Steinerova, Hrabětová Marcela, Jiři Šrámek, Tereza Zábranská, Lekka Mohammadová, Veronika Bergerová

University Hospital Pilsen, Haematology \& Oncology, Pilsen, Czech Republic

Background: Older patients with high risk myeloid malignancies (AML/MDS) have dismal prognosis and the only curative option is alloHCT. The decision to offer HCT for such older pts is challenging given their high rate of transplant related morbidity/mortality. Recently, a composite endpoint of GRFS was proposed to incorporate the most significant complications of allogeneic transplantation and can be used as a meaningful parameter to define transplant success. However, the main variables affecting this endpoint in this particular population are poorly defined. To address this question, we have performed single centre retrospective analysis with long-term follow-up.

Methods: 91 consecutive pts $\geq 60$ years with AML ( $\mathrm{n}=$ 82) or MDS $(n=9)$ who received RIC alloHCT in our 


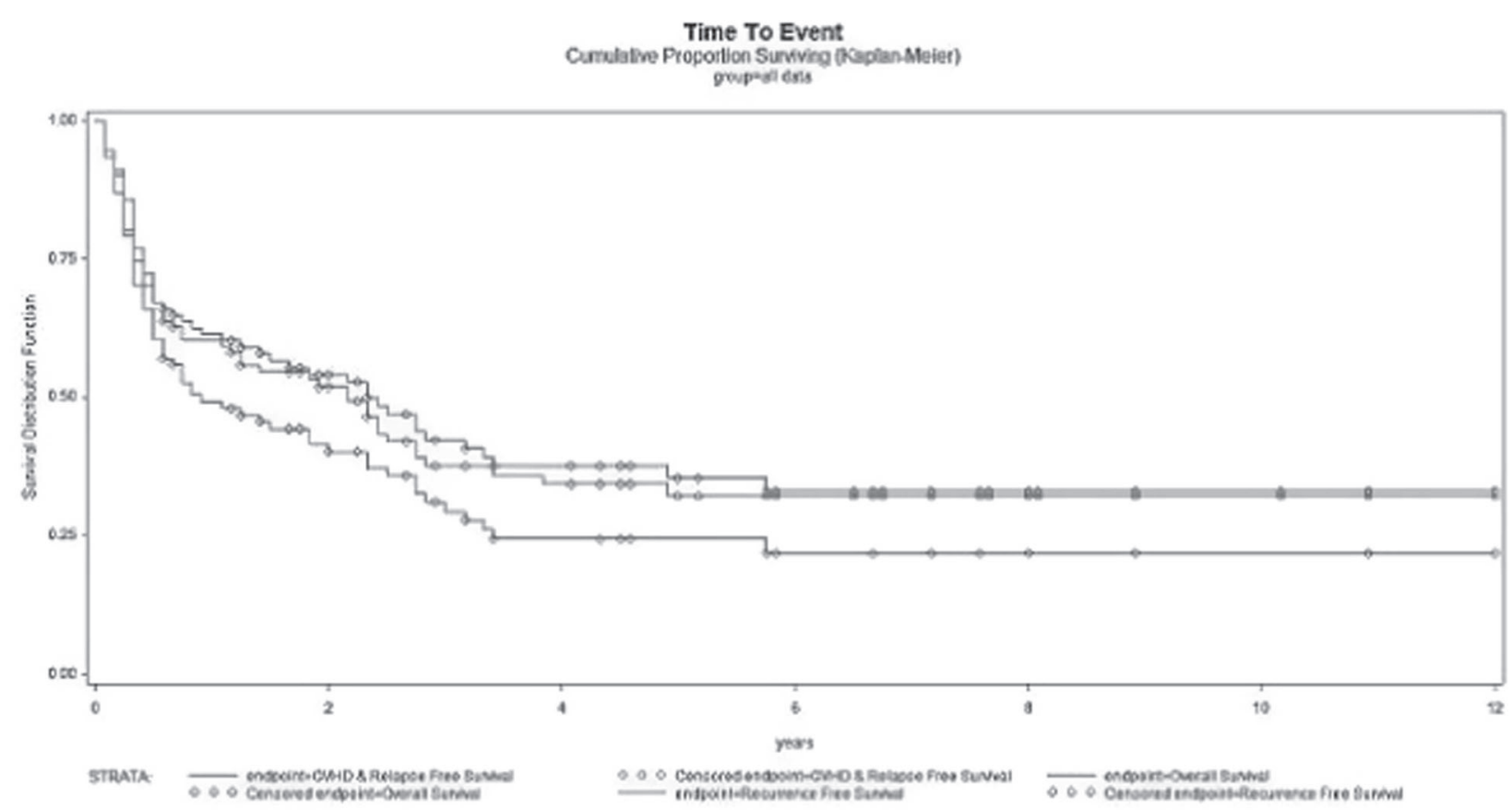

[P024 Figure] [OS, RFS and GRFS]

institution between 11/2001 and 11/2016 were analysed. GRFS events were defined as acute GVHD $\geq$ gr. III, chronic GVHD (requiring systemic therapy or extensive stage), relapse, or death whichever comes 1st during follow-up. Donors were matched sibling (MSD, $\mathrm{n}=25,27 \%$ ), matched unrelated donors (MUD, $\mathrm{n}=41,45 \%$ ), mismatched MUD $(\mathrm{n}=22,24 \%)$ or haploidentical donors $(\mathrm{n}=$ $3 \%$ ). The median age of cohort was 64 years (range 60-74), $62 \%$ were males. 32 pts $(35 \%)$ had advanced disease $(=$ $>\mathrm{CR} 1 / \mathrm{PR} 1$ ) at HCT. Disease risk index (DRI) at HCT was low/intermediate in $47 \mathrm{pts}(52 \%)$ and high in 44 pts $(48 \%)$. GVHD prophylaxis was uniformly based on calcineurin inhibitors and short MTX.

Results: With median follow-up for survivors of 52 months (range 7-144) a total of 54 (59\%) died, 20 (22\%) from relapse and 34 (37\%) from NRM. Estimated OS, RFS and GRFS at 1 year were $61 \%, 60 \%$, and $49 \%$, whereas corresponding probabilities at 5-years were $36 \%, 32 \%$ and $24 \%$, respectively (Figure 1). Cox regression hazard model identified high DRI as the strongest factor predicting extremely poor OS/RFS (HR 2.121, $\mathrm{p}=0.0049$ / HR 1.924, $\mathrm{p}=0.0123$ ), whereas recipient-donor sex mismatch was protective for both the outcomes (OS:HR 0.513, $\mathrm{p}=0.0131 / \mathrm{RFS}$ : HR 0.555, $\mathrm{p}=0.0249)$. A donor age $\geq 62$ year was associated with adverse OS (HR 2.110, p $=0,0345)$ as well as (yet borderline) CMV match (HR $1.791 \mathrm{p}=0.0392$ ). Recipient age $\geq 69$ years and $<2,5$ CD34 + cells/kg transplanted increase NRM (HR 4.493, p = 0.0068/HR 2.960, $\mathrm{p}=0.0078$ ).
In a multivariate analysis for GRFS endpoint, high DRI retains the most significant impact (HR 2.652, 95\% CI $1.562-4.563, \mathrm{p}=0.0003)$, followed by donor age $\geq 62$ years ((HR 2.304, 95\% CI 1.106-4.797, $\mathrm{p}=0.0268)$.

Conclusions: Our data are limited by retrospective nature and inherent selection bias. Despite this we found that with long-term follow up, significant portion of older patients with myeloid malignancies survive alloHCT without experiencing at least 1 GFRS event - $49 \%$ at 1year and $24 \%$ at 5years. In addition, we observe clear trend for plateau since 3-year time point. The negative impact of DRI and high donor age suggests that HCT should be performed early during the course of disease with the preference of young donor (regardless whether related or unrelated).

Conflict of interest: nothing to disclose

\section{P025}

Flag-Ida and donor lymphocyte infusion (DLI) for acute leukemia relapse after allogeneic stem cell transplantation (alloSCT) - single center experience

Jakub Radocha, Alzbeta Zavrelova, Miriam Lanska, Benjamin Visek, Jan M Horacek, Pavel Zak

University Hospital Hradec Kralove and Faculty of Medicine Hradec Kralove, Charles University, Department of Internal Medicine Haematology, Hradec Kralove, Czech Republic

Background: Prognosis of patients with relapsed acute myeloid (AML) or lymphoblastic (ALL) leukemia after 
alloSCT remains dismal. The treatment options are few with short overall survival. We analyzed the outcome of patients with AML and ALL who received Flag-Ida regimen for relapse after alloSCT.

Methods: All patients with relapse of AML and ALL who received at least one cycle of Flag-Ida (Idarubicin 12 $\mathrm{mg} / \mathrm{m} 2$ D1-3, Cytosinarabinoside $2000 \mathrm{mg} / \mathrm{m} 2 \mathrm{D} 1-5$ and Fludarabine $30 \mathrm{mg} / \mathrm{m} 2 \mathrm{D} 1-5)$ from 2006 to 2017 were eligible for analysis. The outcome in terms of progression free and overall survival was analyzed. The impact of DLI administered after Flag-Ida was analyzed.

Results: Overall 36 patients received 42 cycles of FlagIda. There were $20(55.5 \%)$ females and $16(44.5 \%)$ males with mean age 41 years (18-64 years). 25 (69\%) patients had AML and 11 (31\%) ALL. 20 (55.5\%) patients received Flag-Ida for $1^{\text {st }}$ relapse and $16(44.5 \%)$ for $2^{\text {nd }}$ and further relapse. CR was reached after 34 cycles $(81 \%)$, PR after 2 cycles (5\%), progressive disease after 4 cycles (9\%), 2 (5\%) were not evaluable due to early death. 10 (28\%) patients received $2^{\text {nd }}$ allogeneic transplantation from different donor. $22(61 \%)$ patients received DLI after Flag-Ida. Median follow-up from administration of treatment was 8.9 months (1.0-90.3 months). There was no difference in PFS and OS in patients with AML and ALL (14.3 months and 29.5 months, $\mathrm{p}=0.34$ for OS, 10.7 months and 12.1 months, $\mathrm{p}=0.51$ for PFS). Patients who received post treatment DLI after Flag-Ida showed significantly better OS (6.7 versus 23 months, $\mathrm{p}=0.0036$ ).

Conclusions: Flag-Ida plus DLI remain a valid clinical option for treatment of relapse after alloSCT with some patients reaching long term remission when post treatment DLI can be administred.

Conflict of interest: All authors: nothing to disclose

\section{P026}

\section{Haploidentical Stem Cell Transplantation with Post- Transplantation Cyclophosphamide for Pediatric Acute Lymphoblastic Leukemia: A Curative Option}

\section{Satya Prakash Yadav, Sagar Nivargi, Prashant Chabra, Anil Sharma, Neha Rastogi}

Medanta Medicity Hospital, Pediatric Hematology Oncology \& Bone Marrow Transplant, Gurgaon, India

Background: T-cell replete haploidentical stem cell transplantation(SCT) with post-transplantation cyclophosphamide(PTCy) has shown encouraging results for the treatment of hematologic malignancies in adults. However data regarding its application for curing pediatric acute lymphoblastic leukemia(ALL) is meagre. Here we present our experience using the same in 8 children suffering from ALL.
Methods: The conditioning was Non-myeloablative in 4 (Fludarabine $160 \mathrm{mg} / \mathrm{m}^{2}$, Cyclophosphamide $29 \mathrm{mg} / \mathrm{kg}$, total body irradiation TBI 2Gy) and Myeloablative in 4 (Thiotepa10 mg/kg, Fludarabine $160 \mathrm{mg} / \mathrm{m}^{2}$, Cyclophosphamide $29 \mathrm{mg} / \mathrm{kg}$, TBI $2 \mathrm{~Gy}$ in 3 and Fludarabine $160 \mathrm{mg} / \mathrm{m}^{2}$, Busulfan $12.8 \mathrm{mg} / \mathrm{kg}$ in 1). All received PTCy $50 \mathrm{mg} / \mathrm{kg}$ on day 3 and 4 as graft vs. host disease(GVHD) prophylaxis along with tacrolimus and mycophenolate mofetil. After informed consent, 8 children with ALL were allografted; median age was 10 years (range 4-14); Male: Female $=1: 1$. All were in complete remission $(\mathrm{CR})$ prior to SCT: CR1-1, CR2-5 and CR3-2. Donors were mobilized with Granulocyte colony stimulating factor 10 microgram/ $\mathrm{kg}$ daily for four days, the PBSC were collected with one large volume apheresis procedure. All the donors shared 5 out of 10 alleles with the recipient; all donors were parents except one who was a younger sibling. Only one was sexmismatched SCT. A median of 6 million of CD34cells $/ \mathrm{kg}$ was infused (range 5-23 million $/ \mathrm{kg}$ ).

Results: The engraftment rate was $100 \%$, median time of neutrophil engraftment was 18 days (range 13-23) and platelet engraftment was 15 days (range 9-20). Chimerism at day +30 was fully donor in all 8 children. One child relapsed in bone marrow on day +48 . He did not respond to Inotuzumab and died with progressive disease. Remaining 7 patients are alive at the median follow-up of 20 months (range 6-39). Three children relapsed at 6 months, 11 months and 15 months post SCT. One with isolated central nervous system (CNS) relapse achieved CR4 with chemotherapy followed by donor lymphocyte infusions (DLI) and is alive and disease free at follow up of 10 months . Escalating doses of DLI were given and dose of CD3 cells 1 million $/ \mathrm{kg}$ induced acute GVHD grade II in this patient. Second child with isolated CNS relapse failed to respond to DLI but achieved CR4 with cisplatin $75 \mathrm{mg} / \mathrm{m} 2$ and Bevacizumab $5 \mathrm{mg} / \mathrm{kg}$ once a month therapy and is alive and disease free at 4 months. Third child with isolated bone marrow relapse with blasts expressing CD20 achieved CR3 after treatment with a single dose of Rituximab $100 \mathrm{mg} / \mathrm{m} 2$ and is in CR at 15 months on maintenance Rituximab therapy. The cumulative incidence of graft versus host disease (GVHD) acute and chronic extensive was $12.5 \%$ and $36.5 \%$ respectively. Grade-I acute GVHD was seen in 1 patient. All three children with chronic graft vs. host disease are alive and disease free. Three out of 4 children receiving non-myeloablative conditioning relapsed. Overall survival is $88 \%$ and event free survival is $50 \%$ at a median follow up of 20 months.

Conclusions: Haploidentical SCT with PTCy is safe for children with ALL. Myeloablative conditioning, post transplant DLI and/or targeted therapies can help improve survival. 
Conflict of interest: None of the authors has anything to disclose.

\section{P027}

High efficacy of R-HyperCVAD plus Dasatinib followed by allogeneic stem cell transplantation then maintenance with Dasatinib in adults with philadelphia postive acute lymphoblastic leukemia

\begin{abstract}
Ahmad Ibrahim ${ }^{1}$, Rima Moghnieh', Charbel Khalit ${ }^{3}$, Ali Youssef ${ }^{4}$, Ghada Awad ${ }^{5}$, Kamal Zahran', Tamima Jisr ${ }^{7}$, Anas Mogharbel ${ }^{4}$

${ }^{l}$ Makassed and Middle East Institute of Health University HospitalsLebanese University, Hematology Oncology-BMT, Beirut, Lebanon; ${ }^{2}$ Makassed and Middle East Institute of Health University HospitalsLebanese University, Internal Medicine, Beirut, Lebanon; ${ }^{3}$ Middle East Institute of Health University Hospital-Saint Joseph UniversityLebanese University, Cellular Therapy, Beirut, Lebanon; ${ }^{4}$ Makassed University Hospitals, Hematology Oncology-BMT, Beirut, Lebanon;

${ }^{5}$ Makassed University Hospitals, BMT, Beirut, Lebanon; ${ }^{6}$ Middle East Institute of Health University Hospital, BMT, Beirut, Lebanon;

${ }^{7}$ Makassed University Hospitals, Laboratory Medicine, Beirut,

Lebanon
\end{abstract}

Background: Philadelphia $(\mathrm{Ph}+)$ chromosome acute lymphoblastic leukemia (ALL) represents approximately $25-40 \%$ of adults ALL patients (pts). $\mathrm{Ph}+\mathrm{ALL}$ is historically considered as having poor prognosis. Allogeneic hematopoietic stem cell transplantation (AHSCT) has been the gold standard therapy for maintenance of complete remission (CR) in $\mathrm{Ph}+\mathrm{ALL}$ pts. Pre and post AHSCT administration of first generation thyrosine kinase inhibitors (TKIs) Imatinib has dramatically improve the outcome of $\mathrm{Ph}+\mathrm{ALL}$ with reducing disease recurrence with a long term leukemia-free-survival reaching approximately $60 \%$, with no evidence that Imatinib had an adverse effect on transplant-related morbidity and mortality. The usage of second generation TKIs in the same setting improved the results in lower number of pts. The role of Ponatinib, a third generation of TKIs given as bridge to AHSCT or after AHSCT is under investigation. The achievement of molecular remission before AHSCT in adults' pts with $\mathrm{Ph}$ +ALL had a positive impact on relapse and long term outcome after AHSCT. We present the results of our experience in adults' pts with $\mathrm{Ph}+\mathrm{ALL}$ who underwent AHSCT in complete molecular response after induction therapy including Dasatinib, a second generation TKIs, and receiving a maintenance therapy after AHSCT with Dasatinib

Methods: All the pts received induction therapy according to R-HyperCVAD regimen associated to Dasatinib. AHSCT was performed from a related donor. Dasatinib was given in pre and post-transplant at the dose of $70 \mathrm{mg} /$ day.
Evaluation of molecular response was done by qPCR, In particular every 3 to 6 months after AHSCT.

Results: From 1/2010 to 3/2017, 6 pts were included in the study. Median age was 40yo (23-43). There were 3 males and 3 females. Four pts underwent allogeneic peripheral stem cell transplantation from an identical donor with a conditioning associating total body irradiation and Cyclophosmamide in 2 and TBF (Thiotepa, Bisulfex, Fludarabine) in 2 other pts. Two pts underwent haploidentical allogeneic bone marrow transplantation from their mothers after a conditioning with TBF. The pts started Dasatinib as maintenance therapy approximately 3 months after AHSCT except one who died at 1 month after AHSCT of CMV pneumonia. Median duration of Dasatinib administration as maintenance therapy was 19 months (6-48). No modification of dose of Dasatinib was done because of no major toxicity. Five pts are alive in complete molecular response with a median of $21 \mathrm{~m}$ (9-95).

Conclusions: AHSCT after R- HyperCVAD with Dasatinib followed by Dasatinib maintenance is highly efficient and well tolerated in pts with $\mathrm{Ph}+\mathrm{ALL}$.

Conflict of interest: Nothing to disclose

\section{P028}

High TAL1 expression predicts a poor clinical outcome in pediatric T-cell acute lymphoblastic leukemia

\section{Xinran Chu, Sh H}

Children's Hospital of Soochow University, Suzhou, China

Background: To explore the clinical characteristics and prognosis of pediatric T-cell acute lymphoblastie leukemia (T-ALL) enrolled in protocol CCLG-2008. The present study was also designed to investigate the clinical significance of TAL1 noncoding mutation and TAL1 expression in childhood T-ALL.

Methods: From July 2008 to November 2016, 150 consecutive patients aged $\leq 15$ years were treated with CCLG-2008 in Children's Hospital of Soochow University and Department of Pediatric, Institute of Hematology and Blood Diseases Hospital. Predictive values of clinical characteristics and early treatment responses,including prednisone response, bone marrow morphology on day 15 and 33 during induction chemotherapy and week 12 before consolidation therapy were analyzed. The incidence of TAL1 noncoding mutation was analyzed by polymerase chain reaction(PCR) and sanger sequencing in 89 children. TAL1 expression was anlayzed by real-time quantitative PCR in 67 patients.

Results: The 5-year overall survival (OS) and event free survival (EFS) rates for 150 patients were 64.5\% (SE,4.6) 
and $58.7 \%$ (SE, 4.5), respectively. Prednisone poor responder was strongly associated with decreased survival rates (5-year EFS rate, 47.1\% (SE,7.4)). And patients with $\geq 5 \%$ blast cells in bone marrow morphology at D33 tend to have lower 5-year EFS. Patients received bone marrow transplantation in high risk group showed no significant difference in overall survival rates than others who didn't ( $P$ $=0.146)$. We have eight patients $(9 \%)$ presented with heterozygous somatic mutation in noncoding elements of TAL1. All eight patients showed resistance to prednisone treatment. In TAL1 expression analysis, all TAL1 mutation patients were in high expression group(P75-P100). Cox analysis for 3-year OS identified high TAL1 expession and prednisone response as independent prognostic factors (Table 1). Survival analysis showed patients with high TAL1 expression had lower OS and EFS (Figures 1,2).

Conclusions: It is concluded that TAL1 noncoding mutation is not rare in pediatric T-ALL patients. And patients with high TAL1 expression (P75-P100) tend to have a poor clinical outcome.

Conflict of interest: The authors declared that they have no conflicts of interest to this work. We declare that we do not have any commercial or associative interest that represents a conflict of interest in connection with the work submitted.

\section{P029}

\section{HLA-haploidentical peripheral blood stem cell transplantation following reduced-intensity conditioning (haplo RIC-PBSCT) with very low-dose ATG for refractory/ relapsed pediatric acute leukemia}

\section{Kohei Higuchi, Akihisa Sawada, Mariko Shimizu, Aya Ioi, Tatsuro Nakanishi, Hiroshi Tsujimoto, Maho Sato, Masahiro Yasui, Masami Inoue \\ Osaka Women's and Children's Hospital, Department of Hematology/ Oncology, Izumi, Osaka, Japan}

Background: The prognosis of refractory/relapsed pediatric acute leukemia is dismal. To enhance graftversus-leukemia effect, HLA-haploidentical peripheral blood stem cell transplantation has been performed in our institute. In this setting, it is difficult to determine the optimal GVHD prophylaxis.

Methods: We retrospectively reviewed 17 pediatric patients with refractory/relapsed acute leukemia who underwent HLA-haploidentical peripheral blood stem cell transplantation following reduced-intensity conditioning (haplo RIC-PBSCT) with very low-dose ATG between 2012 and 2016.

Results: Six patients had acute lymphoblastic leukemia and 11 had acute myeloid leukemia. Four patients who relapsed after allogeneic stem cell transplantation (alloSCT) were in complete remission and 13 were on disease at haplo RIC-PBSCT. The median age at haplo RIC-PBSCT was 8 years (range, 1 to 17 years). The median follow-up duration was 7 months (range, 0 to 68 months). All patients received HLA-haploidentical graft from related donors, and received reduced-intensity conditioning consisting of fludarabine/clofarabine, melphalan, and etoposide. GVHD prophylaxis consisted of cyclosporine/tacrolimus, shortterm methotrexate, methylprednisolone, and ATG $1.25 \mathrm{mg} / \mathrm{kg}$ on day -2 . Median follow-up for survivors was 44 months (range, 15-68 months). The 2-year overall survival (OS) rate and event free survival (EFS) rate were $49.4 \%$ and $34.3 \%$, respectively. The 2-year cumulative transplant-related mortality (TRM) rate and relapse rate were $11.8 \%$ and $60.7 \%$, respectively. Among 17 patients, $12(70.5 \%)$ developed grade $3-4$ acute GVHD, 2 developed mild chronic GVHD, and 2 developed moderate chronic GVHD.

Conclusions: Although the sample size is small and follow-up duration is short, the 2-year OS/EFS of patients in this analysis including those who relapsed after allo-SCT was encouraging. The incidence of severe acute GVHD was high, but we could manage severe GVHD by adding therapeutic dose of immunosuppressant. For further improvement of outcome, it should be considered using combination therapy to enhance anti-leukemic effect such as molecular target therapeutic agents before and/or after transplantation.

Conflict of interest: nothing to disclose.

\section{P030}

Implementation of EBMT risk score in patients with Acute myeloid Leukemia undergoing allogeneic hematopoietic stem cell transplantation. What can we expect? - Single center experience

Lazar Chadievski, Irina Panovska, Aleksandra Pivkova Veljanovska, Zlate Stojanoski, Sonja Genadieva Stavric, Lidija Cevreska, Marija Popova Labacevska, Gazmend Amzai, Borce Georgievski

University Clinic for Hematology, Skopje, Macedonia, the Former Yugoslav Republic of

Background: Allogeneic hematopoietic stem cell transplantation (SCT) is the treatment that has the highest curative potential in treatment of patients with hematological malignancies, or to be more precise in patients with acute myeloid leukemia (AML). But as it is known to be probably the most complicated biological procedure in the medicine, it has own risks of complications, some of which can have worst outcome. Transplant related mortality (TRM) is higher in some patients depending on various 
factors. The European Group for Blood and Marrow Transplantation (EBMT) provides a simple and usable risk score that can be efficiently used to evaluate the risk in patients with AML undergoing allogeneic SCT.

Methods: We used the EBMT score to evaluate the pretransplantation risk in patients diagnosed with AML undergoing allogeneic SCT. We analyzed five factors, age of the patient $(<20$ years 0 points, $20-40$ years 1 point; over 40years 2 points), stage of the disease (early 0 points, intermediate 1 point, late 2 points), time from diagnosis ( $<12$ months 0 points, $>12$ month 1 point), donor type (sibling 0 point, unrelated 1 point), and donor recipient gender combination (female donor male recipient 1 point, any other combination 0 points). The result could augment the risk for an individual patient with increasing score from 0 as best to 7 as worst. We made a retrospective analysis of 30 patients with AML in the last 5 years all treated with allogeneic SCT. In our group, 15 were male (50\%), 15 were female patients (50\%). The average age of the patients was 38.7 (18-67).

Results: In $27(90 \%)$ patients we performed sibling allogeneic SCT, while in $3(10 \%)$ matched unrelated donor (MUD) allogeneic SCT. The mostly used conditioning regimen was $\mathrm{Bu}-\mathrm{Cy}(43.3 \%)$, then Bu-Cy-ATG (16.7\%), Bu-Cy-Mel (10\%). We used unmanipulated allografts. The median number of used stem cells was $3.41 \times 10^{8} / \mathrm{kg}$ TT mononuclear cells (1.5-8.0). The average day to engraftment was 13.8 (10-20). In the majority of patients the period to allogeneic SCT was $<12$ months (25 patients; $83 \%$ ). Using the EBMT score we divided the patients into two groups: those with score $<2$ (lower risk, favourable, 12 $(40 \%)$ and $\geq 3$ (higher risk, unfavourable, $18(60 \%)$ ). In the low risk group Transplant related mortality (TRM) was $16 \%$ ( 2 patients), while in the higher risk group 38\% (7 patients), but the Non relapse mortality was NRM 33\% (6 patients). The Overal Survival (OS) for 3 years in the group with score $<2$ was $85 \%$, while in the group with score $\geq 3$ was $57 \%$. But we must note that in the latter group two patients were transplanted in active disease, one of them was with haploidentical donor. Two of them died because of acute steroid refractory GvHD.

Conclusions: The EBMT score is a valuable tool to make adequate identification of patients with AML and favourable risk score that could benefit from allogeneic SCT as treatment option.

Conflict of interest: No disclosures

\section{P031}

Improvement of relapse-free survival by allogeneic stem cell transplantation in first complete remission in ELN favorable-risk adult AML patients with initial hyperleukocytosis
Feng-Ming Tien ${ }^{1,2}$, Cheng-Hong Tsai ${ }^{1,2}$, Hsin-An Hou ${ }^{2}$, Jih-Luh Tang ${ }^{1,2}$

${ }^{1}$ Tai-Cheng Stem Cell Therapy Center, National Taiwan University, Taipei, Taiwan, Republic of China; ${ }^{2}$ National Taiwan University Hopsital, Taipei, Taiwan, Republic of China

Background: Acute myeloid leukemia (AML) patients of the 2017 European Leukemia Net (ELN) favorable-risk group generally do not require allogeneic stem cell transplantation (allo-SCT) in first complete remission (CR1). However, a substantial portion of these patients still relapses and dies of disease progression. In this study, we aimed to refine the risk assessment in ELN favorablerisk patients and to identify a subgroup of patients who might benefit from allo-SCT in CR1.

Methods: The study population comprised 176 ELN favorable-risk AML patients. Hyperleukocytosis (HL), defined as initial white blood cell counts above 50,000/uL, was observed in $35.2 \%$ of patients $(n=62)$. We compared postremission treatment with chemotherapy and allo-SCT in

(A)
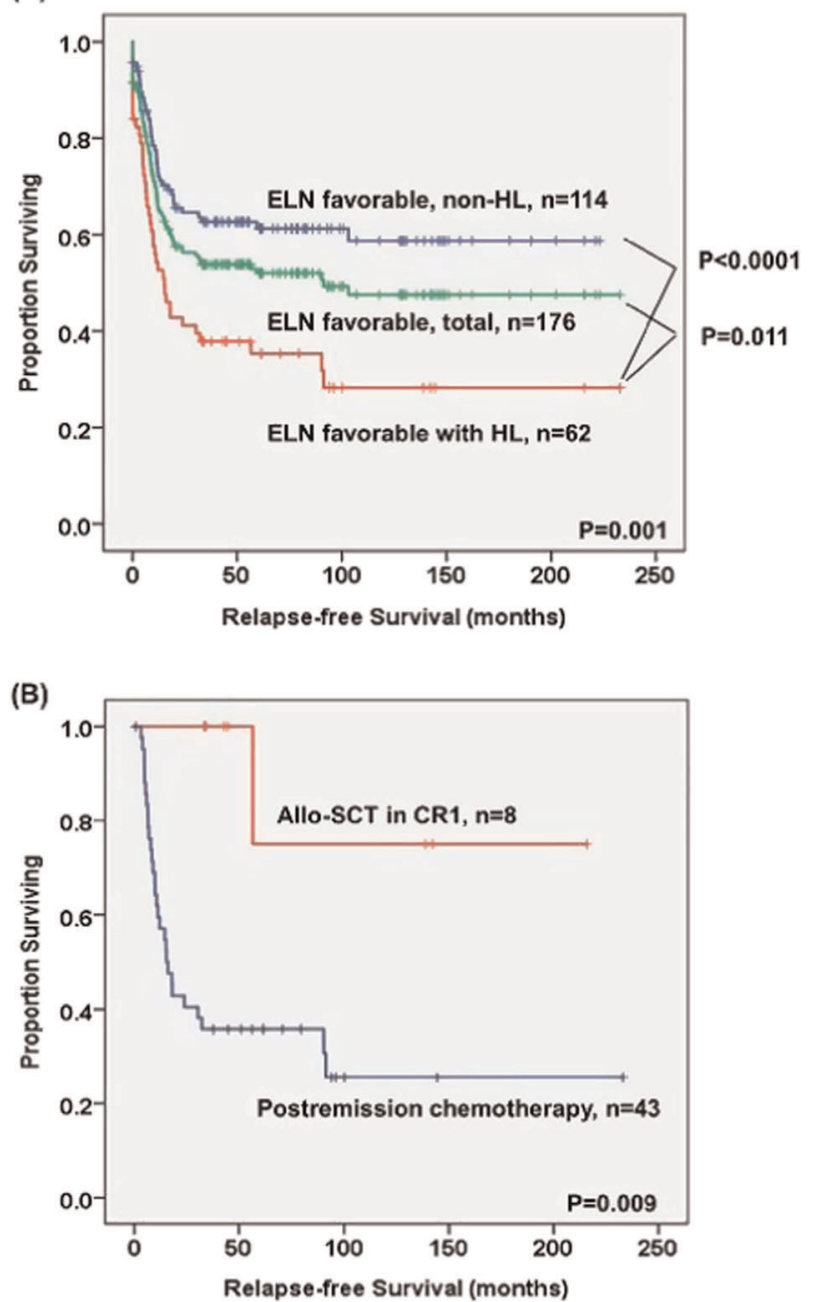

[P031 Figure] [Kaplan-Meier plots for Relapse-free Survival] 
patients with HL who achieved CR1 after induction chemotherapy. To minimize time to transplantation selection bias, survival analysis was limited to patients who remained in CR1 for at least 3 months (median time to allo-SCT) after achieving CR1.

Results: Among the HL and non-HL groups, there was no significant difference in gender, age, hemoglobin level and platelet counts at initial diagnosis. HL patients had higher incidences of inv(16), NPMI+/FLT3-ITD- and CEBPA double mutations, but lower incidence of $\mathrm{t}(8 ; 21)$ than non-HL patients. HL patients had a lower CR rate (85.5\% vs. $95.6 \%, \mathrm{P}=0.037)$ and a higher relapse rate (54.7\% vs. $29.4 \%, \mathrm{P}=0.002$ ). The median follow-up from the date of initial diagnosis was 76.8 months. HL patients had an inferior overall survival (OS) (median, 103.1 months vs. not reached (NR), $\mathrm{P}=0.024$ ) and relapse-free survival (RFS) (median, 15.2 months vs. NR, $\mathrm{P}<0.0001$ ) than nonHL patients (Figure 1A). Among the HL patients who attained CR1 for at least 3 months $(n=51), 8$ patients receive allo-SCT and 43 patients had postremission chemotherapy. The relapse rate was higher in chemotherapy group than in allo-SCT group (65.1\% vs. $0 \%, \mathrm{P}=0.001)$. Allo-SCT in CR1 was associated with a significantly better RFS (median, NR vs. 15.5 months, $\mathrm{P}=0.009$ ) (Figure $1 \mathrm{~B}$ ) and a trend toward better OS (median, NR vs. 103.3 months, $\mathrm{P}=0.092$ ). On the contrary, in non-HL group, patients receiving postremission chemotherapy alone had similar OS $(\mathrm{P}=0.816)$ and RFS $(\mathrm{P}=0.402)$ when compared to those with allo-SCT in CR1.

Conclusions: HL predicts a higher relapse rate and poorer survival in ELN favorable-risk AML patients. Hyperleukocytotic AML patients of the ELN favorablerisk group benefit from allo-SCT in CR1 with respect to RFS. Our results provide evidence for new transplantation strategies in hyperleukocytotic ELN favorable-risk patients.

Conflict of interest: The authors declare no relevant conflict of interest.

\section{P032}

\section{Influence of FLT3-ITD and NPM1 status on allogeneic hematopoietic cell transplant outcomes in patients with cytogenetically normal AML}

Ivan Pasic ${ }^{1}$, Waleed Da'na ${ }^{2}$, Wilson Lam ${ }^{1}$, Arjun Law ${ }^{1}$, Jeffrey Lipton $^{1}$, Auro Viswabandya ${ }^{1}$, Dennis Kim ${ }^{1}$, Santhosh Thyagu ${ }^{1}$, Hans Messner ${ }^{1}$, Fotios Michelis ${ }^{1}$

${ }^{1}$ Princess Margaret Cancer Centre, Medical Oncology \& Haematology, Toronto, Canada; ${ }^{2}$ King Hussein Cancer Center, Amman, Jordan

Background: In patients with cytogenetically normal (CN) AML, prognostic information based on the molecular status of NPMI and FLT3 genes is used to guide treatment. Individuals with an NPM1 mutation but without FLT3 internal tandem duplication $\left(N P M 1^{+} / F L T 3-I T D^{-}\right)$have favourable risk disease and are offered allogeneic HCT following relapse, while those with $N P M 1^{-} / F L T 3-I T D^{-}$or FLT3-ITD $^{+}$are considered at higher risk of relapse and are offered transplant in CR1. However, data regarding the impact of NPM1 and FLT3-ITD status post-allogeneic HCT are limited. We examined the effect of NPM1 and FLT3ITD status on outcomes post allogeneic HCT in patients with CN AML.

Methods: This retrospective single-center study included CN AML patients who underwent allogeneic HCT at the Princess Margaret Cancer Centre between 2006 and 2017. Overall survival (OS) was calculated using Kaplan-Meier analysis and multivariable Cox proportional hazards regression was performed. Cumulative incidence of relapse (CIR) and non-relapse mortality (NRM) were calculated using competing risk regression (Fine and Gray method). This study was approved by the University Health Network Research Ethics Board.

Results: A total of 131 patients were included: of these 63 (48\%) were $N P M 1^{-} /$FLT3-ITD ${ }^{-}$and 51 (39\%) FLT3-ITD ${ }^{+}$ (irrespective of NPM1 mutational status, all transplanted in CR1) while 17 (13\%) were $N P M 1^{+} / F L T 3-I T D^{-}$who had relapsed following conventional chemotherapy and were transplanted in CR2. Seventy-two patients (55\%) were female. Median age at HCT was 54 (range 19-72) and median follow-up of survivors was 36 months (range 2120). AML diagnosis was de novo in 117 (89\%) and secondary to another hematological disorder or other conditions in $14(11 \%)$ patients. Sixty-two (47\%) patients had related donors, $64(49 \%)$ had matched unrelated and 5 (4\%) had haploidentical donors. Peripheral blood stem cells were used for all patients. CMV serostatus was negative for both donor and recipient in 20 (15\%) of transplants. Myeloablative conditioning was used in 69 (53\%) patients, $62(47 \%)$ patients received reduced intensity regimens.

Univariate analysis demonstrated a 3 -year OS of $51 \%$ (95\% CI $=41-60$ ) for the entire cohort. When stratified by molecular status, NPM1-/FLT3-ITD ${ }^{-}$and FLT3-ITD ${ }^{+}$ patients demonstrated 3-year OS of 53\% (95\% CI $=39$ 66) and 46\% (95\% CI $=31-60)$, respectively, while NPM1 ${ }^{+} /$FLT3-ITD ${ }^{-}$patients transplanted in CR2 demonstrated 3year OS of $56 \%(95 \% \mathrm{CI}=29-76)(\mathrm{p}=0.52)$ (Figure 1). CIR at 3-years for NPM1-/FLT3-ITD ${ }^{-}$and FLT3-ITD ${ }^{+}$ patients was $14 \%(95 \% \mathrm{CI}=6-26)$ and $15 \%(95 \% \mathrm{CI}=6-$ 27) respectively, while no relapses occurred in the $\mathrm{NPMI}^{+}$/ FLT3-ITD $^{-}$group. NRM at 3-years for NPM1 $1^{-} / F L T 3-I T D^{-}$ and FLT3-ITD ${ }^{+}$patients was $38 \%(95 \% \mathrm{CI}=25-50)$ and $43 \% \quad(95 \% \mathrm{CI}=28-57)$ respectively, while for NPMI ${ }^{+} /$FLT3-ITD ${ }^{-}$patients 3-year NRM was $44 \%(95 \% \mathrm{CI}=$ 19-67) ( $\mathrm{p}=0.89)$. Multivariable analysis for OS and NRM 


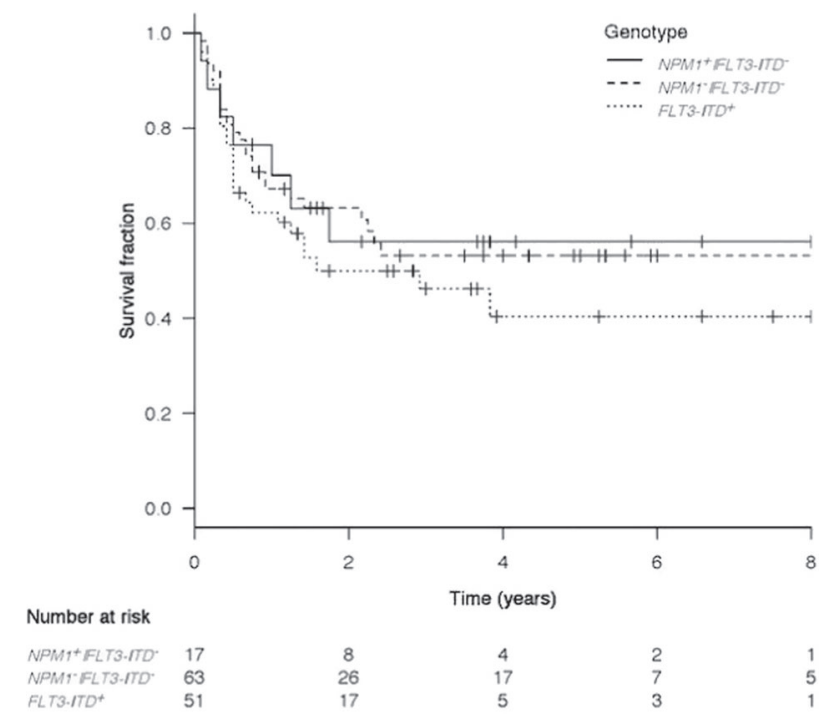

[P032 Figure] [Figure 1. Overall survival by molecular status.]

confirms that there is no significant difference between the molecular subgroups.

Conclusions: In patients with CN AML who undergo allogeneic HCT, the NPM1-/FLT3-ITD- and FLT3-ITD+ subgroups have similar post-transplant outcomes. Favourable risk patients $\left(\mathrm{NPMI}^{+} /\right.$FLT3-ITD $\left.{ }^{-}\right)$that undergo transplant in CR2 have similar outcomes to the NPM1-/ FLT3-ITD- and FLT3-ITD+ subgroups transplanted in CR1.

Clinical Trial Registry: Not applicable

Conflict of interest: None of the authors has anything to disclose.

\section{P033}

Inotuzumab-Ozogamicin induces remissions and enables allogeneic SCT in patients with Blinatumomab-refractory Bprecursor acute lymphoblastic leukemia

Veit Bücklein $^{1,2}$, Alessia Fraccaroli ${ }^{1}$, Dusan Prevalsek ${ }^{1}$, Sarah Häbe $^{1}$, Christoph Schulz ${ }^{1}$, Anna-Katharina Zoellner ${ }^{1}$, Karsten Spiekermann $^{1}$, Marion Subklewe ${ }^{1,2}$, Johanna Tischer ${ }^{1}$

${ }^{1}$ University of Munich, Department of Medicine III, University Hospital, Munich, Germany; ${ }^{2}$ University of Munich, Translational Cancer Immunology, Gene Center, Munich, Germany

Background: Allogeneic stem cell transplantation (alloSCT) so far is the only curative approach for relapsed/ refractory B-precursor ALL. Outcomes of allo-SCT improve with deep remission prior to conditioning. In the past years, treatment options for $\mathrm{r} / \mathrm{r}$ B-ALL have been widened significantly by the approval of Blinatumomab (Blina) and Inotuzumab-Ozogamicin (InO), immunotherapeutic agents with impressive activity in chemotherapy-refractory disease. However, a significant proportion of patients still shows refractory disease or early relapse, and the most effective sequence of treatments is yet unknown. Here, we assessed the activity of $\mathrm{InO}$ in patients with Blina-refractory $\mathrm{r} / \mathrm{r}$ ALL as bridge-to-transplant salvage concept.

Methods: Patients $\geq 18$ years eligible for allo-SCT with $\mathrm{r} /$ r B-precursor ALL after Blina treatment failure were treated with $\mathrm{InO}$ and included in this retrospective analysis. Response to treatment, toxicity (with special attention to veno-occlusive disease), frequency of subsequent allo-SCT, relapse-free and overall survival were evaluated.

Results: Eight patients (7 male, 1 female, median age 36 years, range 22-56 years) with BCR-ABL negative Bprecursor ALL were treated with $\mathrm{InO}$ after a median of four (range 3-5) prior anti-neoplastic treatment lines. Two of the patients had undergone previous allo-SCT. All patients had been treated with Blina prior to InO. Seven patients had Blina-refractory disease with no response to treatment, whereas one patient achieved a MRD-negative CR with two cycles of Blina, but had early relapse after 95 days. Median time from Blina initiation to start of InO treatment was 79 days (range 34-295 days). Short-term toxicity of $\mathrm{InO}$ was mainly hematologic and manageable, with thrombocytopenia (grade 1 in one, grade 2 in one, grade 4 in six patients) and neutropenia (grade 2 in one, grade 4 in seven patients) as most prominent adverse events. Apart from atypical pneumonia in one patient, no further infectious complications were recorded. VOD was suspected in two patients undergoing subsequent allo-SCT and successfully treated with defibrotide. No liver-associated consecutive morbidity or mortality was observed. Five of the eight patients $(62,5 \%)$ responded to $\mathrm{InO}$ treatment (median 1 cycle, range 1-2 cycles). CR was achieved in one (MRD+), $\mathrm{CRi}$ in three (2 MRD-, $1 \mathrm{MRD}+$ ) and PR in one patient. All responding patients proceeded to allo-SCT $(62,5 \%)$. Three of five patients responding to $\mathrm{InO}$ relapsed, with two relapses occurring before (56 and 60 days after $\mathrm{InO}$ initiation, respectively) and one relapse after subsequent allo-SCT (265 days after InO initiation). Median relapsefree survival was 58 days (range 16-367 days). Of the two patients with ongoing remission after $\mathrm{InO}$, one remains relapse-free after 97 days, and one patient died relapse-free due to sepsis on day 367 after InO initiation. At the time of analyses, three patients are alive. Median overall survival was 121 days (range 27-550 days).

Conclusions: By inducing remissions in a significant proportion of patients with Blina-refractory B-precursor ALL, InO represents a valuable therapy option for those patients with otherwise fatal prognosis. Subsequent alloSCT can be enabled in heavily pretreated patients with limited hematological toxicity, while observed VOD was 
manageable. If $\mathrm{InO}$ can induce long-lasting remissions without subsequent allo-SCT remains to be evaluated.

Conflict of interest: None of the authors has anything to disclose.

\section{P034} Isolated extramedullary relapse after hematopoietic cell
transplantation in acute leukemia patients

Annalisa Natale, Stella Santarone, Paola Olioso, Mauro Di Ianni, Gabriele Papalinetti, Corrado Colasante, Simone Ciavarella, Stefano Angelini, Paolo Di Bartolomeo

UTIE, Ospedale Civile, Dipartimento di Ematologia, Medicina Trasfusionale e Biotecnologie, Pescara, Italy

Background: Isolated extramedullary (IEM) relapse is a rare event after allogeneic HCT and its characteristics and prognosis are poorly defined. The aim of this study is to evaluate incidence, risk factors and outcome of IEM relapse in acute leukemia (AL) patients after HCT.

Methods: We retrospectively analyzed 458 consecutive AL patients (294 AML, 164 ALL) who underwent allogeneic HCT in our Center between March 1983 and June 2017.

Results: After a median follow-up of 140 months (range 7-420), 191 (41\%) patients are living. The 35-yr overall survival (OS) was $33.7+3 \% .178$ patients $(39 \%)$ showed AL relapse after a median follow-up of 188 days (range 18 -3980). Among them, 155 (87\%) died for disease progression. The 30-yr cumulative incidence (CI) of relapse was 40.4 $+0.06 \%(33.6+0.08 \%$ in AML and $52.4+0.16 \%$ in ALL, $\mathrm{P}<$ 0.0001). IEM relapse was histologically documented in 15 patients (8\%). IEM relapse involved testis (1), CNS (2), bone (2), breast (1), skin (1), lymphonode (2) in AML patients and CNS (2), skin (2), lymphnode (1), eye (1) in ALL patients. The 30-yr CI of IEM and bone marrow (BM) relapse was 3.4 $\pm 0.008 \%$ and $36.9 \pm 0.05 \%$ respectively. The median age of patients with IEM relapse and BM relapse was 22 years (266) and 32 (1-67) respectively. Table 1 shows some characteristics of patient population.

We analyzed the impact of age, gender, AL type (AML vs ALL), presence of EM disease at diagnosis, CIBMTR risk factor, disease status, conditioning (chemotherapy vs TBI and MAC vs RIC), graft source (BM vs PBSC), donor (sibling, MUD, haploidentical), acute and chronic GvHD on the occurence of IEM relapse. EM disease at diagnosis was the only factor associated with increased risk of IEM relapse both in univariate and multivariate analysis ( $\mathrm{HR} 4.14 ; \mathrm{P}=0.0257)$. The 13 ys OS rate was $22.2 \pm 12 \%$ for IEM relapse and $9+2.4 \%$ in $\mathrm{BM}$ relapse $(\mathrm{P}=0.141)$. Among 15 patients with $\mathrm{IEM}$ relapse, 11 died (10 for disease progression and 1 for acute GvHD after
DLI). 4 patients are living. Of them, 2 received a second allogeneic HCT, 1 chemotherapy and 1 sorafenib.

Conclusions: IEM relapse is a rare event compared to $\mathrm{BM}$ relapse in $\mathrm{AL}$ patients, seems to occur in younger patients and is associated with poor prognosis. Patients with EM disease at diagnosis are at higher risk of developing IEM relapse and need a close follow-up after HCT to detect early evidence of EM disease.

Conflict of interest: No conflict of interest.

\begin{tabular}{|c|c|c|}
\hline & $\begin{array}{l}\text { BM relapse } \\
(\mathrm{n}=163)\end{array}$ & $\begin{array}{l}\text { IEM relapse } \\
(\mathrm{n}=15)\end{array}$ \\
\hline $\begin{array}{l}\text { Median age,years; Gender,M/ } \\
\text { F; EM at AL diagnosis }\end{array}$ & $\begin{array}{l}32 ; 85 / 78 ; \\
11\end{array}$ & $22 ; 5 / 10 ; 3$ \\
\hline $\begin{array}{l}\text { Disease risk: CIBMTR2, } \\
\text { CIBMTR3 }\end{array}$ & 77,60 & 10,3 \\
\hline $\begin{array}{l}\text { Status at HCT:CR1, CR2, } \\
\mathrm{CR}>2 \text {, Active disease }\end{array}$ & $79,40,11,33$ & $6,8,0,1$ \\
\hline Time to relapse,days & $\begin{array}{l}185 \\
(18-3980)\end{array}$ & $\begin{array}{l}208 \\
(56-1900)\end{array}$ \\
\hline Chemotherapy/TBI; MAC/RIC & $\begin{array}{l}105 / 58 ; 153 / \\
10\end{array}$ & $12 / 3 ; 13 / 2$ \\
\hline BM/PBSC; Sib/MUD/Haplo & $\begin{array}{l}107 / 53 ; 101 / \\
37 / 25\end{array}$ & $9 / 6 ; 9 / 3 / 3$ \\
\hline aGvHD:no,grade1/2,grade $3 / 4$ & $\begin{array}{l}102,38 / 20,2 / \\
1\end{array}$ & $11,0 / 4,0 / 0$ \\
\hline cGvHD:yes,no,NV & $19,133,11$ & $1,13,1$ \\
\hline Living/Dead & $17 / 146$ & $4 / 11$ \\
\hline
\end{tabular}

[[P034 Table] Table 1]

\section{P035}

\section{Leukemia in Kosovo: a post-war concern}

Hatixhe Latifi-Pupovci, Miranda Selmanaj, Driton Vela, Violeta Grajcevci

University of Prishtina, Prishtina, Kosovo, Republic of

Background: During the Kosovo conflict, March through June 1999, NATO air-forces fired around 30000 rounds with depleted uranium (DU) in 85 locations. The 4-fold increased incidence of lymphoblastic leukaemias in Iraqi children during the war period 1989-1993, attributed to the use of DU rounds, rised concerns about the posible link between the use of DU rounds and post-war incidence of leukemia in Kosovo. 
The aim of this study was to compare the incidence rate of acute leukemia in the seven regions of Kosovo (Prishtina, Prizren, Peja, Gjakova, Mitrovica, Ferizaj and Gjilan) in pre-war period 1996-1998, with periods 2001-2003 and 2013-2015, and explore possible links between the number of sites hit by DU rounds and incidence of acute leukemia in Kosovo regions.

Methods: Acute leukemia cases diagnosed during period 1996-2015 were extracted from patients records in the University Clinical Centre of Kosovo. The number of sites hit by DU rounds by region was determined from accurate NATO records containing geographic coordinates. Incidence rates per 100,000 persons were calculated for each region and three periods (1996-1998, 2001-2003 and 20132015 ) based on population census data.

Results: Incidence rate of acute leukemia in Kosovo increased from 2.8 in 1996-1998 to 4.0 in 2001-2003 and 5.9 in 2013-2015. Also, there were significant differences between the regions. The biggest difference between incidence rates in the periods 1996-1998 and 2001-2003 were found in the Gjakova Region were incidence increased from 0 to 6.3, Prizren Region from 1.1 to 3.2, Peja Region from 1.4 to 2.7 and Mitrovica Region from 2.6 to 6.2. No significant changes were observed in Prishtina, Gjilan and Ferizaj. When differences between incidence rate for periods 1996-1998 and 2013-2015 were analysed, again, the biggest difference was found in Gjakova, from 0 to 6.3 followed by Peja from 1.4 to 6.8 and Mitrovica from 2.6 to 8.3, in Prizren from 1.1 to 3.2, Gjilan from 2.7 to 5.8, Ferizaj 3.7 to 5.8 and Prishtina from 5.8 to 6.5 .

The regions with the largest number of sites hit by DU rounds were the Gjakova Region - 25, Prizren Region - 25, Peja Region - 16 whereas with smaller number of sites hit were regions of Mitrovica - 5, Prishtina - 4, Gjilan - 3, Ferizaj - 10.

Conclusions: The biggest difference in incidence of acute leukemia in the periods 1996-1998, 2001-2003 and 20132015 was observed in regions with the biggest number of sites hit by DU rounds. One exception is the Mitrovica Region which is known for high concentration of lead in the air and topsoil.

Conflict of interest: Hatixhe Latifi-Pupovci have nothing to disclose

\section{P036}

Long-term outcomes of hematopoietic cell transplantation in adults with acute lymphoblastic leukemia during first remission according to the available donor types

Jae-Ho Yoon, Nack-Gyun Chung, Sung-Soo Park, Young-Woo Jeon, Sung-Eun Lee, Byung-Sik Cho, Ki-Seong Eom, Yoo-Jin Kim, HeeJe Kim, Chang-Ki Min, Seok-Goo Cho, Dong-Wook Kim, Jong-
Wook Lee, Woo-Sung Min, Seok Lee

Catholic BMT Center, The Catholic University of Korea, Hematology, Seoul, Korea, Republic of

Background: Adult patients with high-risk acute lymphoblastic leukemia (ALL) have a poor outcome with standard chemotherapy and usually undergo hematopoietic cell transplantation (HCT) from a matched sibling donor (MSD) or matched unrelated donor (MUD). When a matched donor is not available, HCT from a partiallymismatched unrelated donor (MMUD), cord blood (CB), or familial haploidentical donor is considered. We tried to analyze the long-term HCT outcomes according to the donor types including MSD, MUD, MMUD, CB, and autologous (AUTO) sources.

Methods: We enrolled 661 consecutive adult ALL patients who underwent transplantation from 1995 to 2015 in their first remission (CR1). Median age was 33 years (range, 15-65 years). The myeloablative conditioning regimen consisted of cyclophosphamide $(120 \mathrm{mg} / \mathrm{kg})$ and total body irradiation (TBI; $13.2 \mathrm{~Gy}$ ), and for double CB transplantation (DCBT), TBI (12 Gy), cytarabine $\left(9 \mathrm{~g} / \mathrm{m}^{2}\right)$, and fludarabine $\left(150 \mathrm{mg} / \mathrm{m}^{2}\right)$. Patients aged $\geq 50$ years or those with comorbid conditions were given an identical reduced-intensity conditioning regimen consisting of fludarabine $\left(150 \mathrm{mg} / \mathrm{m}^{2}\right)$ and melphalan $\left(140 \mathrm{mg} / \mathrm{m}^{2}\right)$. Conditioning regimen for AUTO was TBI (12 Gy), cytarabine $\left(9 \mathrm{~g} / \mathrm{m}^{2}\right)$, and melphalan $\left(100 \mathrm{mg} / \mathrm{m}^{2}\right)$. Graftversus-host disease (GVHD) prophylaxis was attempted by administering calcineurin inhibitors (cyclosporine for MSD transplants, tacrolimus for unrelated donor transplants) plus methotrexate or mycophenolate mofetil (for DCBT). Antithymocyte globulin (ATG) was administered to the patients who received MMUD grafts.

Results: After a median follow-up of 80.4 months (range, 6.5-259.1 months), the 7-year overall survival (OS) for the entire 661 patients was $57.0 \%$. In detail, the 7-year OS for MSD, MUD, MMUD, DCBT, and AUTO were $56.9 \%$, $56.8 \%, 62.6 \%, 65.1 \%$, and $48.6 \%$, respectively $(P=0.423)$. MSD, MUD and MMUD showed similar relapse rate $(27.5 \%)$ and non-relapse mortality (NRM) rate $(20.6 \%)$, but DCBT showed higher NRM rate $(29.6 \%)$ and lower relapse rate $(7.2 \%)$. AUTO showed higher relapse rate $(41.6 \%)$. Interestingly, calculated GVHD and relapse-free survival (GRFS) at 7 years for MSD, MUD, MMUD, and DCBT were $34.1 \%, 15.0 \%, 40.9 \%$, and $50.3 \%$, respectively, which was related with higher incidence of severe chronic GVHD in MUD (27.6\%) and MSD (14.1\%) compared to DCBT (4.2\%, $P<0.001)$. In high-risk subgroup of patients $(\mathrm{n}=$ 517) including 233 Philadelphia chromosome-positive ALL, the 7-year OS for MSD, MUD, MMUD, DCBT, and AUTO were $54.9 \%, 58.1 \%, 57.8 \%, 60.7 \%$, and $33.3 \%$, 
respectively. The 7-year GRFS for MSD, MUD, MMUD, and DCBT were $30.7 \%, 14.5 \%, 35.3 \%$, and $47.7 \%$, respectively, which revealed lower relapse $(8.7 \%)$ and severe chronic GVHD (2.6\%) rates of DCBT.

Conclusions: Our long-term data showed that outcomes are similar for transplantation using MSD, MUD, MMUD, or CB sources in adult patients with ALL in CR1. However, GRFS was rather superior in patients treated with DCBT than those received other donor sources. Further studies with a sizable population are needed to clarify the role of alternative donor transplantation for adult ALL in the context of GRFS.

Conflict of interest: The authors declare no conflict of interest.

\section{P037}

Long-term results of allogeneic hematopoietic stem cell transplantation in 533 patients with various hematological malignancies: single centre experience

\section{Marta Krejci, Michael Doubek, Miroslav Tomiska, Zdenek Racil, Andrea Janikova, Blanka Robesova, Jirina Prochazkova, Andrea Zmijakova, Katarina Ksenakova, Zdenek Kral, Jiri Mayer}

University Hospital Brno, Dpt. of Internal Medicine, Hematology and Oncology, Brno, Czech Republic

Background: Allogeneic stem cell transplantation (SCT) has been considered as the treatment of choice for many hematological disorders, mainly for hematological malignancies. Role of allogeneic SCT is widely discussed in era of novel drugs. Here we present our long-term experience with allogeneic SCT in cohort of 533 pts with various hematological malignancies.

Methods: We analyzed 533 pts undergoing allogeneic SCT in our centre from November 1996 to June 2017. The diagnoses of pts were as follows: AML (187 pts; 35\%), ALL (73 pts, 14\%), lymphomas (66 pts, 12\%), MDS+MPN (52 pts, 10\%), CML (74 pts, 14\%), CLL (47 pts, 9\%), other diagnoses (34 pts, 6\%). Median age of pts was 43 years (range 18-64). Types of donors and used grafts were as follows: HLA identical sibling, $\mathrm{n}=256(48 \%)$; unrelated donor, $\mathrm{n}=275$ (52\%); PBSCs, $\mathrm{n}=492$ (92\%); BM, $\mathrm{n}=41$ $(8 \%)$. Median follow-up from SCT was 26.6 months (range 0-254), median follow-up from SCT for surviving pts was 83.2 months (range 5-254). Myeloablative conditioning (MAC) was used in 253 pts (47\%), reduced intensity conditioning (RIC) was used in 280 pts (53\%). Disease status at SCT was remission in 351 pts (67\%) and active disease in 182 pts (33\%). Median time from diagnosis to SCT was 7.4 months.

Results: The overall response rate after allogeneic SCT was $85 \%$, including CR in $78 \%$ of pts and PR in $7 \%$ of pts.
The incidence of acute GvHD was $42 \%$ (grade $\mathrm{I}+\mathrm{II}$ in $32 \%$, grade III+IV in $10 \%$ ), the incidence of chronic GvHD was $40 \%$ (limited in 30\%, extensive in 10\%). Non-relapse mortality (NRM) was $18 \%, 19 \%$ and $20 \%$ at 1 year, 2 years and 4 years from SCT, respectively. Relapse incidence was $22 \%, 27 \%$ and $31 \%$ at 1 year, 2 years and 4 years from SCT, respectively. Median PFS was 31.5 months, median OS was 85.6 months for all 533 pts. Gender, age at SCT, type of donor, type of graft, time from diagnosis to SCT and type of conditioning did not significantly influence PFS and OS in our cohort of pts. Patients with AML have got significantly shorter PFS than pts with other diagnoses (median PFS 18.9 months versus 43.2 months, $\mathrm{p}=0.031$, HR 1.32), pts with active disease at SCT have got significantly shorter PFS than pts with remission at SCT (median PFS 9.6 months versus 73.7 months, p under 0.001, HR 1.79). In a multivariate analysis, diagnosis of AML versus other diagnoses (median OS 29.7 months versus 197.2 months, p under 0.001, HR 1.63) and active disease at SCT versus remission at SCT (median OS 25.0 months versus 186.5 months, $\mathrm{p}$ under 0.001 , HR 1.93) were significant predictors of poor OS.

Conclusions: Allogeneic transplantation still remains the standard treatment option predominantly for various hematological malignancies. According to our results, type of diagnosis and disease status at allogeneic SCT are the simple, but important prognostic factors for PFS and OS. Patients in remission of disease at allogeneic SCT have got significantly longer PFS and OS.

Conflict of interest: Nothing to disclose for all authors.

\section{P038}

Monitoring of chimerism on sorted peripheral CD34+ cells in patients with acute leukemia receiving allogeneic bone marrow transplant

Maria Ida Bonetti ${ }^{1}$, Benedetta Peruzzi ${ }^{2}$, Francesco Mannelli ${ }^{1}$, Anna Lari $^{3}$, Tommaso Rondelli ${ }^{2}$, Sara Bencini ${ }^{1}$, Ilaria Cutini ${ }^{1}$, Roberto Caporale ${ }^{2}$, Anna Maria Grazia Gelli ${ }^{2}$, Francesca Torricelli, ${ }^{3}$, Alberto Bosi $i^{I}$

${ }^{1}$ Università di Firenze, SOD Ematologia AOU Careggi, Firenze, Italy; ${ }^{2} S O D$ Laboratorio Generale, Settore Citometria Clinica, AOU Careggi, Firenze, Italy; ${ }^{3} S O D$ Diagnostica Genetica, AOU Careggi, Firenze, Italy

Background: An early detection of neoplastic cells after allogeneic hematopoietic stem cells transplantation (HSCT) in adult patients affected by acute leukemia provides the opportunity to manage and control the potential reemergence of malignant clone. Several groups have reported as rapidly increasing mixed chimerism correlates with higher risk of relapse. Some predictors of impending 
relapse are Minimal Residual Disease (MRD) monitoring by flow cytometry (FC) and PCR-based chimerism on both Bone Marrow (BM) or Peripheral Blood (PB) samples at specific time-points after HSCT. Restricting chimerism analysis on sorted CD34+ cells could be an early predictor of relapse after HSCT.

Methods: At diagnosis of acute leukemia, the most useful leukemia-associated aberrant immuno-phenotype (LAIP) was established for each patient to be investigated at specific time-points during treatment plan to detect LAIPpositive cells. DNA was extracted from PB of donor and recipient before HSCT and chimerism assessment was performed on whole $\mathrm{PB}$ and sorted $\mathrm{CD} 34+$ cells of recipient after HSCT at specific time points: day 30, 60, 100, 180, month 12 and 18 from HSCT.

Results: From January 2012 to August 2015, 124 patients affected by acute leukemia received allogeneic transplantation. 47 out of 124 patients have been enrolled in the project. 14 patients were not evaluated due to toxicity after transplantation. Hitherto, data about 33 patients (in whom at least 1 evaluation has been carried out) are available. In most cases, the results were concordant: MRD-negative status and full donor chimerism both in the whole PB and in the CD34+ sorted fraction occurred. We found discordance in 7 patients: 2 of them showed a transitory loss of chimerism which immediately converted to $100 \%$ donor at the next detection and always MRD negative; 3 patients showed always full chimerism in CD34+ fraction, whereas MRD was positive at some time-points without relapse; in the other 2 patients, the chimerism analysis on sorted CD34 + cells allowed an early diagnosis of disease recurrence by highlighting the presence of the patient $\hat{A}$ 's pathological cells with concomitant "negative signals" from FC-MRD and whole PB chimerism.

Conclusions: The last 2 patients clearly represent the basic rationale of this study: chimerism assessment may provide the opportunity of an early detection of neoplastic cells after transplantation. Regarding discordant results, the slightly loss of chimerism which immediately converted to $100 \%$ mandates for a confirmation with a further detection as soon as possible since one single assessment turns out to be inadequate to predict actual disease relapse. It would be interesting to address the significance of these fluctuations in chimerism both on whole PB and on sorted CD34+ cells on a larger patients' cohort. Chimerism assessment on CD34 + sorted fraction is an early predictor of relapse and might be useful to drive therapeutic strategy in the post transplantation setting.

Conflict of interest: The project was funded by Ministero della Salute and Regione Toscana (CUP D11J09000190003) and by Fondazione Biagioni-Borgogni and Legato Zottola Donation.

The authors declare no competing financial interests.
P039

\section{Outcome of haploidentical haematopoietic stem cell transplant in pediatric acute leukemia - a single center experience from India}

Sunil Bhat ${ }^{l}$, Sohini Chakraborty ${ }^{1}$, Shobha Badiger ${ }^{1}$, Pooja Mallya ${ }^{1}$, Ruchi Chaudhary, Ravi Joshi ${ }^{1}$, Archana $M V^{l}$, Nataraj $K S^{2}$, Sharat Damodar ${ }^{2}$

${ }^{1}$ Mazumdar Shaw Cancer Center, Narayana Health City, Pediatric Hematology Oncology and Bone Marrow Transplant, Bangalore, India; ${ }^{2}$ Mazumdar Shaw Cancer Center, Narayana Health City, Bangalore, India

Background: Haematopoietic stem cell transplant (HSCT) remains the potentially curative treatment modality for patients of acute leukemia in first complete remission or beyond. However, fully HLA matched donors are unavailable for majority of them and haploidentical transplant has emerged as a feasible option in such a scenario.

Methods: Retrospective analysis of all pediatric patients with acute leukemia undergoing haploidentical stem cell transplant at our Center from August 2014 to June 2017.

Results: Thirty two patients with a median age of 71.5 months (range 26 to 204) formed the study group. 21 $(65.6 \%)$ cases of acute lymphoblastic leukemia (ALL) (B cell $=16$, $\mathrm{T}$ cell $=4$ and biphenotypic $=1)$ and $11(34.4 \%)$ cases of acute myeloid leukemia (AML) were included in the study. Among the ALL patients 1 was in CR1 while remaining 21 were in CR2, whereas, among the AML patients 4, 6 and 1 patients were in CR1, CR2 and CR3 respectively. Myeloablative conditioning was used in all the cases. TBI based conditioning was used in ALL patients while Fludarabine-Busulphan based conditioning was used in AML patients .GVHD prophylaxis was provided in the form of TCR $\alpha \beta$ and CD19 depletion in 12 patients (37.5\%) while the remaining $20(62.5 \%)$ received post transplant cyclophosphamide (PTCY) along with calcineurin inhibitors and mycophenolate mofetil. Peripheral blood was the source of stem cells in majority of the patients with a median infused CD34+ cell dose of $19.95 \times 10^{6} / \mathrm{kg}$ in TCR depletion group and $6.4 \times 10^{6} / \mathrm{kg}$ in the PTCY group. Thirty one patients achieved haematological recovery with neutrophil engraftment at a median of 12 days vs 15 days ( $P$ $<0.01$ ) and platelet engraftment at a median of 10 days vs 21.5 days $(\mathrm{P}<0.05)$ in the TCR depletion and PTCY group respectively. The transplant outcomes are described in the table given below (table 1). The cumulative incidences of grade 2-4 GVHD, grade 3-4 GVHD, relapse and non relapse mortality were $20 \%$ vs $68.8 \%, 20 \%$ vs $16.7 \%, 30 \%$ vs $43.4 \%$ and $25 \%$ vs $34.4 \%$ in the TCR depletion group vs PTCY group respectively $(\mathrm{P}>0.05)$. The overall survival was $65.6 \%$ vs $42.4 \%$ in the TCR depletion and PTCY group 
Overall survival

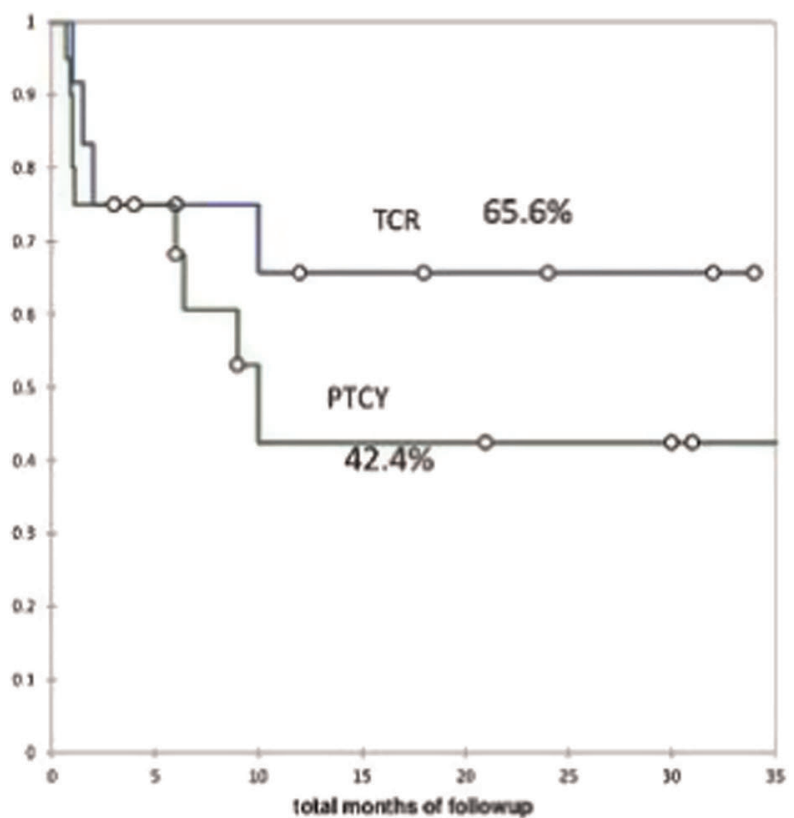

[P039 Figure] [Graph 1]

while the disease free survival was 5 Graph $12.5 \%$ vs $42.4 \%$ in the two groups (figure 1).

Conclusions: Haploidentical HSCT is a feasible approach in paediatric acute leukemia patients lacking a fully matched donor.

Conflict of interest: none

\begin{tabular}{lll}
\hline Transplant outcomes & Number & Percentage \\
\hline Acute GVHD & & \\
TCR depletion & $4 / 12$ & $33.3 \%$ \\
Grade I-II / III-IV & $2 / 2$ & \\
PTCY & $13 / 20$ & $65 \%$ \\
Grade I-II / III-IV & $10 / 3$ & \\
Relapse & $6 / 32$ & $18.8 \%$ \\
TCR depletion & 2 & \\
PTCY & 4 & $31.2 \%$ \\
CMV reactivation & $10 / 32$ & \\
\hline
\end{tabular}

[[P039 Table] Table 1]

\section{P040}

Outcomes of allogeneic hematopoietic stem cell transplantation in patients with B-cell acute lymphoblastic leukemia harboring $t(4 ; 11)(q 21 ; q 23)$

Jun Aoki', Shin Fujisawa ${ }^{1}$, Shuichi Mizuta ${ }^{2}$, Yukiyasu Ozawa ${ }^{3}$, Kazuteru Ohashi ${ }^{4}$, Shuichi Ota ${ }^{5}$, Hiroyasu Ogawa ${ }^{6}$, Takahiro Fukuda $^{7}$, Junji Tanaka ${ }^{8}$, Yoshiko Atsuta, ${ }^{9,10}$, Shinichi Kako ${ }^{11}$
Disease free survival

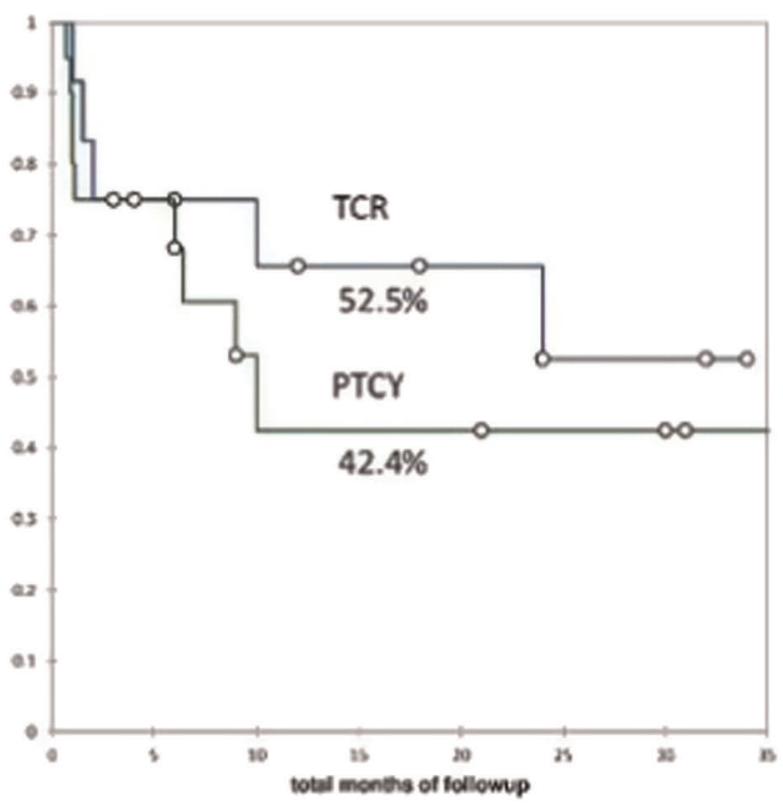

${ }^{l}$ Yokohama City University Medical Center, Yokohama, Japan;

${ }^{2}$ Toyohashi Medical Center, Toyohashi, Japan; ${ }^{3}$ Japanese Red Cross Nagoya First Hospital, Nagoya, Japan; ${ }^{4}$ Tokyo Metropolitan Cancer and Infectious Diseases Center, Komagome Hospital, Tokyo, Japan; ${ }^{5}$ Sapporo Hokuyu Hospital, Sapporo, Japan; ${ }^{6}$ Hyogo College of Medicine, Nishinomiya, Japan; ${ }^{7}$ National Cancer Center Hospital, Tokyo, Japan; ${ }^{8}$ Tokyo Women's Medical University, Tokyo, Japan;

${ }^{9}$ Japanese Data Center for Hematopoietic Cell Transplantation, Nagoya, Japan; ${ }^{10}$ Nagoya University Graduate School of Medicine, Nagoya, Japan; ${ }^{11}$ Saitama Medical Center, Jichi Medical University, Omiya, Japan

Background: Acute lymphoblastic leukemia (ALL) with $\mathrm{t}(4 ; 11)(\mathrm{q} 21 ; \mathrm{q} 23)$ accounts for approximately $8 \%-10 \%$ of adult B-cell ALL, and is characterized by poor outcomes. Therefore, the presence of this chromosomal abnormality in ALL is an indication for allogeneic hematopoietic stem cell transplantation (allo-HSCT). However, transplantation outcomes of ALL with $\mathrm{t}(4 ; 11)(\mathrm{q} 21 ; \mathrm{q} 23)$ remain unclear. We retrospectively examined the impact of $\mathrm{t}(4 ; 11)(\mathrm{q} 21 ; \mathrm{q} 23)$ on the outcomes of allo-HSCT in patients with B-cell ALL.

Methods: Clinical data were collected from the registry database of the Japan Society for Hematopoietic Cell Transplantation. We selected B-cell ALL patients who were aged $\geq 16$ years and underwent their first transplantation between January 2000 and December 2015. We compared clinical features and transplantation outcomes between the patients with $\mathrm{t}(4 ; 11)(\mathrm{q} 21 ; \mathrm{q} 23)$ and the patients with a normal karyotype. Overall survival (OS) was estimated using the Kaplan-Meier method and compared using the log lank test. In a multivariate analysis, the Cox proportional 
hazard model was used to analyze OS, using the following covariates: age, sex, disease status, donor source, conditioning regimen, transplantation year, time taken for alloHSCT from diagnosis and extramedullary disease.

Results: Number of cases with $\mathrm{t}(4 ; 11)(\mathrm{q} 21 ; \mathrm{q} 23)$ and a normal karyotype was 107 and 1196 respectively. The median age of the $\mathrm{t}(4 ; 11)(\mathrm{q} 21 ; \mathrm{q} 23)$ group was lower than the normal karyotype group ( 35 years vs. 40 years, $\mathrm{P}<$ $0.001)$. Higher proportion of the $t(4 ; 11)(\mathrm{q} 21 ; \mathrm{q} 23)$ group received allo-HSCT within 180 days of diagnosis $(52.3 \%$ vs. $25.9 \%, \mathrm{P}<0.001)$. The probabilities of OS the $\mathrm{t}(4 ; 11)$ (q21;q23) group was worse than the normal karyotype group (4ys, $46.2 \%$ vs. $52.0 \%, \mathrm{P}=0.024$ ). Subgroup analysis stratified by disease status revealed that the probabilities of OS was comparable between the $\mathrm{t}(4 ; 11)(\mathrm{q} 21 ; \mathrm{q} 23)$ group and the normal karyotype group in the CR patients $(61.7 \%$ vs. $61.1 \%, \mathrm{P}=0.519)$. In contrast, the $\mathrm{t}(4 ; 11)(\mathrm{q} 21 ; \mathrm{q} 23)$ group exhibited poorer OS in the non-CR patients $(10.9 \%$ vs. $21.8 \%, \mathrm{P}=0.011)$. After adjusting for covariates, $\mathrm{t}$ (4;11)(q21;q23) had no significant impact on OS (HR, 1.27; $\mathrm{P}=0.100)$. Subgroup analysis showed $\mathrm{t}(4 ; 11)(\mathrm{q} 21 ; \mathrm{q} 23) \mathrm{did}$ not affect on $\mathrm{OS}$ in the $\mathrm{CR}$ patients $(\mathrm{HR}, 1.06 ; \mathrm{P}=0.77)$. Multivariate analysis in the $\mathrm{t}(4 ; 11)(\mathrm{q} 21 ; \mathrm{q} 23)$ group revealed that non-CR at transplantation $(\mathrm{HR}, 4.32, \mathrm{P}<0.001)$ was significant risk factor for poor OS.

Conclusions: These findings suggested that allo-HSCT in CR may overcome poor prognosis of ALL patients with $t$ $(4 ; 11)(\mathrm{q} 21 ; \mathrm{q} 23)$.

Conflict of interest: All authors: nothing to disclose

\section{P041}

\section{Outcomes of high risk myeloid malignancies undergoing Hematopoietic Cell transplant in a tertiary Care facility: real world evidence from India}

Vivek S Radhakrishnan, Sanket Shah, Mita Roychowdhury, Jeevan Kumar, Saurabh Jayant Bhave, Reena Nair, Mammen Chandy

Tata Medical Center, Clinical Haematology and Bone Marrow Transplant, Kolkata, India

Background: Hematopoietic Cell transplantation (HCT) remains the cornerstone of treatment of high risk myeloid malignancies. Real world evidence from middle income countries are few.

Methods: We undertook a retrospective chart review of 103 HCT undertaken for 98 patients with high risk myeloid malignancies between Dec 2011 and Oct 2017 (M:69, F:29). Median age: 31 yrs (range: 7-60 yrs). These included Acute Myeloid Leukaemia (64), Acute Pro-myelocytic leukemia (APL: 5), MDS (11), CML (Chronic phase:3, Lymphoid Blast crisis:6, Myeloid blast crisis:5), Myelofibrosis (2), Chronic myelomonocytic Leukemia (1), and
Blastic Plasmacytoid dendritic cell neoplasm (1). High risk disease was on account of morphologic criteria (e.g myelodysplasia), high risk cytogenetics, molecular profile or clinical indications (induction failure, relapsed disease, etc.). 34 patients were in CR1, 19 in CR2 or CR3, and 45 patients with active disease (30 primary refractory, and 15 relapsed refractory) at the time of transplant referral. Amongst 34 patients who underwent transplant for relapsed disease: 7 relapsed in $<6$ months from CR1, 8 within 6-12 months and 19 relapsed after 12 months. All patients underwent allogeneic HCT, except 5 with relapsed APL (autologous HCT). Allogeneic HCT donor source included 53 Matched sibling donors, 35 haploidentical related donors, and 10 Matched unrelated donors. Conditioning therapy was myeloablative in 83 allogeneic and 5 autologous HCT. Reduced intensity conditioning was undertaken in 15 allogenic HCT. All patients received peripheral blood stem cell transplant (PBSCT). Throat and faecal surveillance culture for Multi-Drug Resistant Organisms (MDRO) was done for all 98 patients. GVHD prophylaxis varied, depending on type of allogeneic transplant, and included either $\mathrm{CsA}+\mathrm{mMtx} \pm \mathrm{ATG}$ or PTCy+Tac+MMF approach (34 patients).

Results: The median CD34 cell dose was $6.1 \times 10^{\wedge} 6$ cells/ $\mathrm{kg}$. Primary graft failure, 100-day mortality, Acute GVHD, Gr III/IV aGVHD, and cGVHD rates were 7\%, 17\%, 25\%, $10 \%$ and $33 \%$ patients respectively. At a median follow up of 344 days for the entire cohort, 5 year Overall Survival (OS) and Relapse Free Survival (RFS) were $48 \%$ and $40 \%$ respectively. Median overall survival: 35.1 months. MDRO colonization was observed in stool surveillance of 92 patients and throat surveillance culture of 30 patients [Among 116 species of MDRO identified in stool samples: 36 were ESBL producing gram negative bacilli(GNB), 38 carbapenem resistant GNB, and 7 vancomycin resistant enterococci]. In this single center retrospective series, the type of transplant, status of disease at the time of transplant, duration of remission in case of relapsed disease, and Blood group or sex mismatch between donor and recipient had no impact on OS or RFS. The only factor which was found to be significantly affecting outcome was gender: females had better outcome compared to males.

Conclusions: In a middle income country with a high prevalence of MDRO colonization, hematopoietic cell transplantation of high risk myeloid malignancies in a tertiary care center yields modest outcomes

Conflict of interest: No relevant conflicts of interest 


\section{P042}

\section{Patient-Reported Quality of Life (QoL) Following Tisagenlecleucel Infusion in Adult Patients with Relapsed/ Refractory ( $r / r)$ Diffuse Large B-Cell Lymphoma (DLBCL)}

\begin{abstract}
Richard T. Maziarz ${ }^{1}$, Michael R. Bishop ${ }^{2}$, Constantine S. Tam ${ }^{3}$, Peter Borchmann ${ }^{4}$, Ulrich Jaeger, Joseph McGuirk ${ }^{6}$, Edmund K. Waller $^{7}$, Samantha Jaglowski ${ }^{8}$, Charalambos Andreadis ${ }^{9}$, Stephen Ronan Foley ${ }^{10}$, Isabelle Fleury ${ }^{11}$, Stephan Mielke ${ }^{12}$, Jason R. Westin $^{13}$, Phoebe Joy Ho ${ }^{14}$, Veronika Bachanova ${ }^{15}$, Harald Holte ${ }^{16}$, John M. Magenau ${ }^{17}$, Oezlem Anak ${ }^{18}$, Lida Bubuteishvili Pacaud ${ }^{19}$, Siegbert Guenther ${ }^{18}$, Jie Zhang ${ }^{19}$, Lawrence Rasouliyan ${ }^{20}$, Feng Tai $^{19}$, Gilles A. Salles ${ }^{21}$, Stephen J. Schuster ${ }^{22}$
\end{abstract}

${ }^{1}$ Oregon Health \& Science Knight Cancer Institute, Center for Hematologic Malignancies, Portland, OR, United States; ${ }^{2}$ University of Chicago, Hematopoietic Stem Cell Transplantation Program, Chicago, IL, United States; ${ }^{3}$ St Vincent's Hospital and University of Melbourne, Peter MacCallum Cancer Centre, Melbourne, Australia;

${ }^{4}$ University Hospital of Cologne, Department of Haematology and Oncology, Cologne, Germany; ${ }^{5}$ Medical University of Vienna, Department of Medicine, Vienna, Austria; ${ }^{6}$ University of Kansas Medical Center, Department of Blood and Bone Marrow Transplant, Kansas City, KS, United States; ${ }^{7}$ Winship Cancer Institute of Emory University, Bone Marrow and Stem Cell Transplant Center, Atlanta, GA, United States; ${ }^{8}$ James Cancer Hospital and Solove Research Institute of Ohio State University Comprehensive Cancer Center, Columbus, OH, United States; ${ }^{9}$ University of California San Francisco Helen Diller Family Comprehensive Cancer Center, Department of Hematology and Blood and Marrow Transplant, San Francisco, CA, United States; ${ }^{10}$ McMaster University, Juravinski Hospital and Cancer Centre, Hamilton, Canada; ${ }^{11}$ University of Montreal, Maisonneuve-Rosemont Hospital, Montreal, Canada; ${ }^{12}$ Würzburg University Medical Center, Center for Allogeneic Stem Cell Transplantation, Würzburg, Germany; ${ }^{13}$ University of Texas MD Anderson Cancer Center, Department of Lymphoma and Myeloma, Division of Cancer Medicine, Houston, TX, United States; ${ }^{14}$ The University of Sydney, Department of Medicine, Sydney, Australia;

${ }^{15}$ University of Minnesota, Department of Medicine, Division of Hematology, Oncology and Transplantation, Minneapolis, MN, United States; ${ }^{16}$ Oslo University Hospital, Department of Oncology, Oslo,

Norway; ${ }^{17}$ University of Michigan Comprehensive Cancer Center, Ann Arbor, MI, United States; ${ }^{18}$ Novartis Pharma AG, Basel, Switzerland; ${ }^{19}$ Novartis Pharmaceuticals Corporation, East Hanover, NJ, United States; ${ }^{20}$ RTI Health Solutions, Barcelona, Spain; ${ }^{21}$ CHU Lyon-Sud Hospital, Hospices Civils de Lyon, Department of Hematology, Lyon, France; ${ }^{22}$ University of Pennsylvania, Lymphoma Program, Abramson Cancer Center, Philadelphia, PA, United States

Background: JULIET (NCT02445248) is evaluating a single infusion of tisagenlecleucel (CTL019) in adult patients with $\mathrm{r} / \mathrm{r}$ DLBCL. Primary analyses showed a best overall response rate of 53\% (complete response [CR], $40 \%)$.

Methods: Patients were aged $\geq 18$ years with chemorefractory DLBCL after $\geq 2$ lines of therapy and either failed or were otherwise ineligible for autologous hematopoietic stem cell transplant (SCT). At baseline, month-3 and month-6, patients completed the Short Form-36 Health Survey v2 (SF-36) and the Functional Assessment of
Cancer Therapy-Lymphoma (FACT-Lym). Higher scores indicate better QoL.

Results: 99/147 patients were infused before data cutoff; $95 \%$ had previously received $\geq 2$ chemotherapy treatments and $48 \%$ had relapsed after SCT. 43 pts with a best response of $\mathrm{CR}$ or partial response (PR) were followed $\geq 6$ months prior to data cutoff or discontinued earlier, and were eligible for analysis. QoL instruments were completed by 76 patients (94\%) at baseline and 40 patients who had CR or PR. 26 and 16 of patients with CR or PR completed the assessments at month-3 and -6, respectively. Scores of patients with CR or PR (mean baseline, mean change at month-3, mean change at month-6) indicated sustained improvements over baseline in all categories (Table 1). Similar trends were observed for SF-36.

Conclusions: Improvements in QoL were observed at month-3; scores at month-6 suggested sustained improvements. These results suggest that tisagenlecleucel therapy may improve QoL among responders despite patients experiencing early toxicity.

Clinical Trial Registry: ClinicalTrials.gov NCT02445248

\section{Conflict of interest:}

R. Maziarz: Novartis: research funding, honoraria, membership on entity's board of directors/advisory committees; Incyte: consultancy, honoraria; Juno, Kite: honoraria; Athersys: patents and royalties

M. Bishop: Novartis: consultancy: includes expert testimony; other: steering committee; Kite, Battersea: Consultancy: includes expert testimony; Celgene: honoraria, speakers bureau

C. Tam: Novartis: honoraria.

P. Borchmann: Novartis: Honoraria.

U. Jaeger: Roche, Janssen, Novartis: Honoraria; Membership on Entity's BOD/AC Novartis: RF

J. McGuirk: Novartis: No payment PI CAR-T Study.

E. Waller: Novartis: Consultancy: Includes ET, RF; Cambium Medical: Patents and Royalties, Membership on Entity's BOD/AC; Celldex, PRA, Amgen: Consultancy: Includes ET.

S. Jaglowski: Novartis, Kite: Consultancy, RF; Unum, Pharmacyclics: RF

C. Andreadis: Novartis: RF, Honoraria; Cellerant, Incyte, Pharmacyclics, Amgen: RF;Genentech:Honoraria; Equity Ownership-Spouse; Seattle Genetics, Gilead Astellas: Honoraria.

S. Foley: Novartis: Consultancy

I. Fleury: Merck, Janssen, Roche, Seattle Genetics, Gilead, Amgen, Lundbeck, Novartis:Consultancy.

S. Mielke: KIADIS: Travel Grants; Jazz Pharma:Speakers Bureau; Novartis: Consultancy; Celgene, Cellex GmbH: Speakers Bureau,Travel Grants;MSD: Consultancy,Travel 


\begin{tabular}{|c|c|c|c|c|c|}
\hline \multirow[b]{2}{*}{ FACT-Lym } & \multirow[b]{2}{*}{ MID } & \multicolumn{2}{|c|}{$\begin{array}{l}\text { Baseline (BL) } \\
\text { Mean (SD) }\end{array}$} & \multirow{2}{*}{$\begin{array}{c}\text { Change from BL } \\
\text { Month } 3 \\
\text { Mean (SD) } \\
\text { Patients with CR/PR } \\
(\mathrm{n}=27)^{\mathrm{a}}\end{array}$} & \multirow{2}{*}{$\begin{array}{c}\text { Change from BL } \\
\text { Month } 6 \\
\text { Mean (SD) } \\
\text { Patients with CR/PR } \\
\text { (n=16) }\end{array}$} \\
\hline & & $\begin{array}{l}\text { All patients } \\
(\mathrm{N}=76)\end{array}$ & $\begin{array}{l}\text { Patients with } C R / P R \\
\qquad(n=40)\end{array}$ & & \\
\hline FACT-G TS & $3-7$ & $77.4(16.0)$ & $78.5(15.7)$ & $7.1(11.9)$ & $3.8(14.8)$ \\
\hline FACT-Lym S & $2.9-5.4$ & $44.2(9.0)$ & $44.9(9.2)$ & $3.9(7.5)$ & $3.0(6.0)$ \\
\hline FACT-Lym TOI & $5.5-11$ & $81.4(19.0)$ & $83.1(19.1)$ & $8.1(14.6)$ & $5.9(14.2)$ \\
\hline FACT-Lym TS & $6.5-11.2$ & $121.1(23.8)$ & $123.4(23.9)$ & $11.8(17.2)$ & $6.8(19.8)$ \\
\hline
\end{tabular}

[P042 Figure] [Table 1]

Grants;Gilead:Travel Grants; DGHO: Travel Support; ISCT: Travel Support.

J. Westin: Novartis, Apotex, Kite, Celgene:Membership on Entity's BOD/AC.

P. Ho: Novartis, Amgen, BMS, Celgene, Janssen, Takeda: Honoraria, Membership on Entity's BOD/AC; Janssen,Takeda: Consultancy,.

V. Bachanova: Novartis: RF; Seattle Genetics, Juno, Zymogen: Membership on Entity's BOD/AC; Oxis: RF.

H. Holte: Novartis:Membership on Entity's BOD/AC.

J. Magenau: Nothing to Disclose.

O. Anak, S. Guenther: Novartis Pharma AG: Employment.

L. Pacaud, J. Zhang, F. Tai: Novartis Pharmaceuticals: Employment

L. Rasouliyan: RTI Health Solutions:Employment.

G. Salles: Amgen, BMS, Celgene, Gilead, Janssen, Kite, Merck, Morphosys, Novartis, Servier, Roche: Consultancy, Participation Symposia/AB; Roche: Consultancy, Research Grant;

S. Schuster: Novartis, Celgene, Genentech, Merck, Janssen R\&D, HoffmanLaRoche, BMS:RF; Novartis, Celgene, Genentech, Seattle Genetics, Nordic Nanovector: Consultancy: Includes ET

\section{P043}

Prognostic Effect of Pre-Treatment Cytogenetic Abnormalities In Iranian Adults Acute Myeloid Leukemia After Allogeneic Hematopoietic Stem Cell Transplantation

Marjan Yaghmaie ${ }^{1}$, Amirabbas Rashidi ${ }^{1}$, Kamran Alimoghadam ${ }^{1}$, Amir Kasaeian ${ }^{1}$, Mohammad Jahani ${ }^{1}$, Seyed Asadollah Mousavi, Mohammad Vaezi ${ }^{1}$, Hossein Kamranzadeh ${ }^{2}$, Ardeshir Ghavamzadeh $^{2}$

${ }^{1}$ Tehran University of Medical Sciences, Hematology, Oncology and Stem Cell Transplantation Research Center, Tehran, Iran, Islamic Republic of; ${ }^{2}$ tehran University of Medical Sciences, Hematology,
Oncology and Stem Cell Transplantation Research Center, Tehran, Iran, Islamic Republic of

Background: Several studies have revealed the significance of pre-treatment cytogenetic findings in tumor cells as a strong clinical predictor. We aimed to evaluate the effect of pre-treatment cytogenetic abnormality on treatment outcomes in Iranian adults with acute myeloid leukemia (AML) after allogeneic hematopoietic stem cell transplantation (HSCT).

Methods: A total of 323 adult patients with non-M3 AML underwent allogeneic HSCT from March 2011 to March 2015 in Shariati General Hospital, from which 206 patients with pre-treatment cytogenetic data were included in our study with median follow-up time of 47 months. Overall survival (OS) and Disease-free-survival (DFS) rate of different cytogenetic abnormalities were compared with normal karyotype.

Results: Five-year OS rate for all patients were $58.54 \%$. OS and DFS rates were significantly lower in abn $(3 q)$, inv (3)(q21;q26),t(3;3)(q21;q26), del(5q)/add(5q), t(6;9)(p23; q34), -7 , abn(7q), +8, 11q23 and abn(11q) (excluding $(11 ; 19))(\mathrm{p} 21 ; \mathrm{q} 23)$ and $(11 ; 19)(\mathrm{q} 23 ; \mathrm{p} 13))$, abn(17p), complex karyotype (more than 3 unrelated abnormalities) and monosomal karyotype. None of the cytogenetic abnormalities were associated with favorable outcomes.

Conclusions: Our study suggested that age and pretreatment cytogenetic abnormalities are independent predictors of treatment outcome after HSCT in AML patients. Unlike previous studies, in our study $\mathrm{t}(8 ; 21)$ and Inversion (16) were not associated with favorable outcomes and were classified as intermediate risk groups.

Conflict of interest: nothing to disclose

\section{P044}

Prognostic significance of pretransplant minimal residual disease in AML patients with mutated NPM1 
Veronika Valkova ${ }^{1}$, Jan Vydra ${ }^{1}$, Antonín Vitek ${ }^{1}$, Marketa Markova ${ }^{1}$, Jaroslav Polak ${ }^{2}$, Radka Petrbokova ${ }^{2}$, Ela Cerovska ${ }^{2}$, Ludmila Novakova ${ }^{1}$, Barbora Cemusova ${ }^{1}$, Michal Kolar ${ }^{1}$, Petr Cetkovsky ${ }^{1}$

${ }^{1}$ Institute of Haematology and Blood Transfusion, BMT, Prague, Czech Republic; ${ }^{2}$ Institute of Haematology and Blood Transfusion, Dpt of Genomics, Prague, Czech Republic

Background: There is increasing evidence that in AML, minimal residual disease (MRD) persisting after standard induction therapy is associated with increased risk of relapse and poor survival. Allogeneic hematopoietic stem cell transplantation (allo-HSCT) is considered to be the most effective strategy in the treatment of high risk AML. The aim was to evaluate the prognostic impact of pretransplant MRD measured by real-time quantitative PCR in patients with NPM1-mutated AML receiving allo-HSCT.

Methods: From 3/2004 to 6/2017, we evaluated 60 patients (pts) with a median age of 52 years (range; 24-66 years), 38 (63\%) women. Median follow-up for survival from time of allo-HSCT was 19 months. Twenty-six (43\%) pts were FLT3-ITD positive. At allo-HSCT, 44 (73\%) pts were in first, $12(20 \%)$ in second complete remission (CR) and 4 had refractory disease (RD). Forty-two (70\%) pts underwent myeloablative, 18 reduced-intensity alloHSCT. The donors were: identical sibling in $10 \mathrm{pts}$, haploidentical related in 4 pts, matched unrelated donor in 31 pts and mismatched UD in 15 pts. In 53 pts were used PBPC, in 7 pts bone marrow. All but one pts had intermediate risk prognostic score. Pre-transplant NPM1 MRD levels were measured in peripheral blood in all 60 pts. Pts with pretransplant MRD level of $\leq 10$ mutant copies of NPM1 per 10,000 ABL copies were considered as MRD negative. At the time of allo-HSCT 47 pts were MRD negative and 13 positive (4 refractory patients were excluded from further analysis).

Results: The estimated probabilities of 3-year relapse incidence (RI), non-relapse mortality (NRM), event-free survival (EFS), overall survival (OS) and chronic GVHD for the whole cohort were $21 \%, 32 \%, 43 \%, 53 \%$ and $84 \%$ respectively. Thirty-one (52\%) of pts developed acute GVHD. Significantly better results were observed in MRD neg pts with respect to RI and EFS (3-year RI and EFS 19\% and $54 \%$ and $64 \%$ and $13 \%$ for MRD neg and MRD positive pts resp, $p=0,001$ ), similar prognostic significance was observed in FLT3 -ITD positivity (3-year RI and EFS $10 \%$ and $53 \%$ and $55 \%$ and $29 \%$ for FLT3-ITD neg and positive pts resp., $\mathrm{p}=0,0035$ and 0,0005 ). The difference was not significant in terms of OS, however, there was no difference in OS even in pts with RD or CR. In multivariable analysis, increasing age was the only statistically significant predictor of poor OS (HR 1,8 for every 10 years of age, 95\% CI 1,03-3.17; $\mathrm{p}=0.04)$. In terms of EFS we observed inferior outcome in FLT3-ITD positive pts (HR 2,34; $95 \%$ CI 1,08- 5,07; $\mathrm{p}=0.03$ ). FLT3ITD positivity (HR $7.76(1.5-40), \mathrm{p}=0.014)$ and NPM1 negativity (HR $0.17(0.05-0.61) \mathrm{p}=0.006)$ were associated with higher relapse risk.

Conclusions: In AML patients with NPM1 mutation, MRD positivity before allo-HSCT is clearly associated with a higher incidence of relapse. The NRM in our cohort was relatively high, most likely due to GVHD. However, our data suggest that allo-HSCT and its associated development of GVL effect, mostly linked just to GVHD, may overcome the negative prognostic impact of MRD positivity.

Conflict of interest: None

\section{P045}

Prophylactic administration of Azacitidine in patients with AML after haploidentical stem cells transplantation increases overall survival

Renat Badaev, Darina Zammoeva, Larisa Girshova, Diana Babenetskaya, Natalia Ilyina, Yulia Alexeeva, Dmitriy Motorin

Federal Almazov North-West Medical Research Centre, St. Petersburg, Russian Federation

Background: Haplo-SCT is an appropriate alternative, when match donor unavailable.

Although, GvHD and relapses could potentially decrease the survival. Prophylactic administration of Azacitidine, performing antileukemic and immunomodulating activity, can help to overcome these obstacles.

Methods: 18 patients with AML after haplo-SCT were included in the study. All patients achieved MRD-negative remission on day 30 after SCT. MRD was assessed by measurement of WT1-gene expression. Administration of Azacitidine began not earlier than 2 months after SCT in case of full engraftment and absence of active GVHD. Dose of Azacitidine was $100 \mathrm{mg}$ D1-D5 every 28 days during 1 year after SCT. DLI were added in case of molecular relapse.

Results: 11 patients received prophylactic administration of Azacitidine and 7 were in control group. Median time of beginning of Azacitidine - 4 months (2-10). Median number of courses - 3,5 (1-9). The most frequent toxicity was hematological: anemia 1-2 - 27,8\% (N=5), thrombocytopenia $3-4-11,1 \%(\mathrm{~N}=2)$, neutropenia $3-4-27,8 \%(\mathrm{~N}=5)$. No case of secondary graft failure occurred.

9 patients $(81,8 \%)$ had acute GVHD before treatment with Azacitidine and only 5 (45,4\%) - after Aza-treatment. Four patients had relapse of aGVHD (3 - skin, 1 - gut) and only one case of gut-GVHD occurred de novo after treatment. Two patients required systemic immunosuppression with methylprednisolone. In control group aGVHD occurred in 5 patients $(71,4 \%)$ and 2 required systemic 
immunosuppression. Difference was not statistically significant due to small number of patients.

Rate of chronic GVHD in Aza-group and control group was comparable $9,1 \%(\mathrm{~N}=1)$ и $14,3 \%(\mathrm{~N}=1)$. All cases were skin-chGVHD accompanying with hepatic-GVHD in one patient.

Molecular relapse didn`t observe in Aza-group. In control group two patients $(28,6 \%)$ had molecular relapse and were treated by Azacitidine with DLI. The first patient died because of severe aGVHD and another one achieved MRDnegative remission and still alive. We didn't see any case of bone marrow relapse, probably because of intense GVL after haplo-SCT and short period of observation.

Currently, 10 patients in Aza-group (90,9\%) and 3 patients in control group $(42,9 \%)$ are alive with median observation period 9,5 and 7 months respectively. In Azagroup one patient died of aGVHD and in control group - 2 of infection and 2 of aGVHD. We saw significantly better overall survival in Aza-group ( $\mathrm{p}=0,021)$.

Conclusions: Prophylactic administration of Aza after haplo-SCT in patient with AML is safe and well tolerated. Aza prophylaxis significantly improves overall survival. This improvement could be due to the decrease of aGvHD and relapses, although, the differences appeared to be statistically nonsignificant in our cohort of patients.

Conflict of interest: None

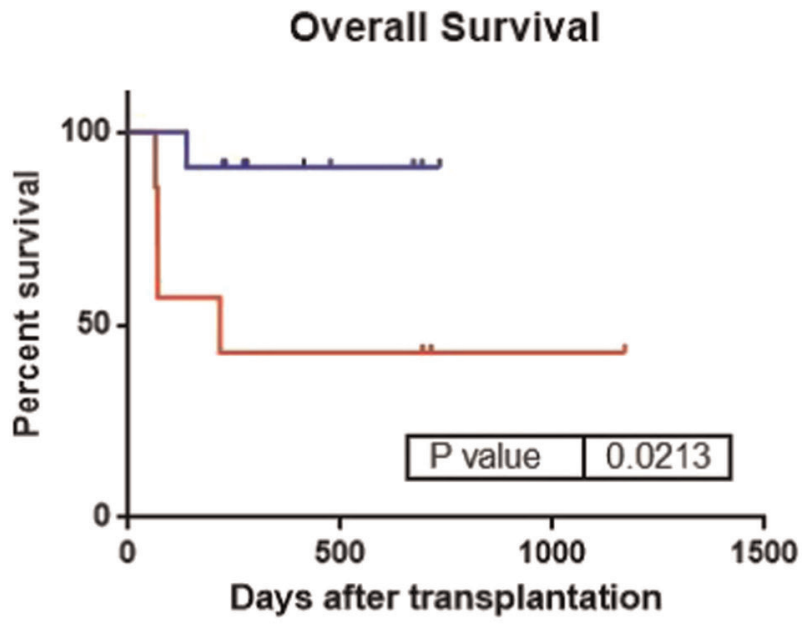

With Azacitidine prop
$(\mathrm{N}=11)$

[P045 Figure] [Overall survival]
Number of Haplo-SCT

18

Age

18-57 (median 42)

Sex : M/F

$7 / 11$

Status before SCT:

CR MRD-negative 11

CR MRD-positive

No CR

4

Graft source:

Bone marrow

2

Peripheral blood

16

$\mathrm{CD} 34+\mathrm{mln} / \mathrm{kg}$

$1,9-10,4$ (median $4,18)$

Conditioning regimens:

$\mathrm{Flu} / \mathrm{Cy} / \mathrm{Mel} 13$

$\mathrm{Bu} / \mathrm{Flu} / \mathrm{Cy} \quad 3$

Conditioning with previous

2

cytoreduction

GVHD prophylaxis

PT-Cy, MMF and CsA

[[P045 Table] Characteristics of Patients]

P046

Prospective single center experience of allogeneic stem cell transplantation in adult patients with NPM1 acute myeloid leukemia

Federico Lussana ${ }^{1}$, Chiara Caprioli ${ }^{1}$, Paola Stefanoni ${ }^{1}$, Chiara Pavoni $^{1}$, Orietta Spinelli ${ }^{1}$, Ksenija Buklijas ${ }^{1}$, Pamela Zanghi ${ }^{1}$, Alessandra Algarotti ${ }^{1}$, Caterina Micò ${ }^{1}$, Anna Grassi ${ }^{1}$, Tamara Intermesoli, Alessandro Rambaldi, ${ }^{1,2}$

${ }^{I}$ Ospedale Papa Giovanni XXIII, Hematology and Bone Marrow Transplant Unit, Bergamo, Italy; ${ }^{2}$ Università degli Studi di Milano, Department of Oncology and Hematology, Milan, Italy

Background: Nucleophosmin (NPM1) is one of the most commonly mutated genes in acute

myeloid leukemia (AML), present in $20-30 \%$ of cases. Mutations in NPM1 represent a distinct entity in the World Health Organization (WHO) classification and commonly the prognosis of patients with $N P M 1$ mutation is considered favorable. Nevertheless, recent studies showed a correlation between the persistence of NPM1 MRD level and patient adverse outcome. This association has generated substantial interest in using results of MRD-testing for the decision to transplant stratifying patients, but the benefit associated 
Disease Free Survival according to allo transplant

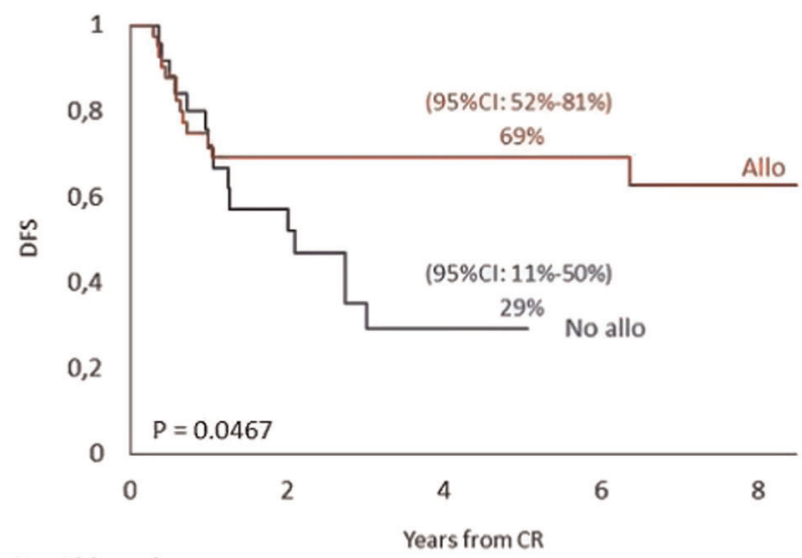

$\mathrm{N}$ at risk (events)

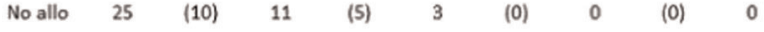

$\begin{array}{lllllllll}\text { Allo } & 41 & \text { (12) } & 21 & \text { (0) } & 19 & \text { (0) } & 11 & \text { (1) } 5\end{array}$

[P046 Figure] [Figure 1]

with allogeneic stem cell transplantation (alloHSCT) in first remission remains to be investigated.

Methods: From year 2006, 72 AML patients bearing the NPM1 mutation, with a median age of 53 (range 16-82) were consecutively treated according to the Northern Italy Leukaemia Group (NILG) protocol 02/06 [ClinicalTrials. gov Identifier: NCT00495287]. Patients were considered eligible to allogeneic transplant in first remission if, at diagnosis, they were FLT3 ITD positive, or had a high white blood cells (WBC) count $\left(>50 \times 10^{9} / \mathrm{L}\right)$, or they showed a persistent minimal residual disease (MRD) as molecularly detected after consolidation with high dose cytarabine.

Results: The main clinical findings of patients analyzed are summarized in Table 1.

Patients receiving alloHSCT were younger, had a higher WBC count with a FLT-ITD mutation at diagnosis, and showed more frequently a persistent MRD postivity after consolidation compared to patients not undergoing tranplantation (Table 1). After induction course, 66 patients (92\%) achieved a complete hematological remission (CR) and $41(62 \%)$ underwent an alloHSCT in first complete remission. MRD status after consolidation chemotherapy was available for 61 patients (85\%). Forty-one patients received an alloHSCT, the donors being 8 siblings, 27 unrelated, 1 haploidentical and 5 cord blood units. The 5years disease free survival (DFS) was significantly better for patients receiving an alloHSCT $69 \%$ (95\% CI: $52-81 \%$ ) vs $29 \%$ (95\% CI 11-50\%), $\mathrm{p}=0.046$ (Figure 1).

However, the 5 years OS was not significantly different being 67\% (95\% CI: $50 \%-80 \%)$ in patients receiving alloHSCT compared to 55\% (95\%CI:30-75\%) in patients not undergoing alloHSCT $(\mathrm{p}=0.705)$ in first remission.
For the whole patients' cohort $(\mathrm{n}=72)$, the 5 years overall survival (OS) was 59\% (95\%CI: 46\%-71\%). By multivariate analysis, the presense of FLT3-ITD mutation and the persistence of MRD after consolidation chemotherapy were associated with a shorter survival indipendently from the transplant consolidation.

Conclusions: Our results shows that alloHSCT improve DFS compared to standard chemotherapy also in patients with NPM1 acute myeloid leukemia at higher risk of leukemia relapse for the presence of FLT-ITD mutation and persistence of MRD postivity before transplant.

Conflict of interest: None of the authors has anything to disclose.

\begin{tabular}{|c|c|c|}
\hline & Allo HSCT (N=41) & $\begin{array}{l}\text { No allo HSCT } \\
(\mathrm{N}=25)\end{array}$ \\
\hline Age years, median (range) & $50(16-75)$ & $60(21-82)$ \\
\hline Sex, N (\%) Male Female & $17(41 \%) 24(59 \%)$ & $\begin{array}{l}13(52 \%) 12 \\
(48 \%)\end{array}$ \\
\hline LDH, U/L median (range) & $1157(725-2294)$ & $\begin{array}{l}1089(757- \\
1566)\end{array}$ \\
\hline WBC, X 109/L, median (range) & $36(16-86)$ & $15(3-56)$ \\
\hline $\begin{array}{l}\text { Molecular abnormalities None } \\
\text { FLT3ITD Others }\end{array}$ & $\begin{array}{l}16(39 \%) 17(41 \%) \\
8(20 \%)\end{array}$ & $\begin{array}{l}13(52 \%) 5 \\
(20 \%) 7(28 \%)\end{array}$ \\
\hline $\begin{array}{l}\text { MRD post-consolidation, N (\%) } \\
\text { Positive Negative }\end{array}$ & $22(55 \%) 18(45 \%)$ & $\begin{array}{l}14(67 \%) 7 \\
(33 \%)\end{array}$ \\
\hline $\begin{array}{l}\text { Donor type, N(\%) Sibling Unrelated } \\
\text { Cord blood HLA Haploidentical }\end{array}$ & $\begin{array}{l}8(20 \%) 27(66 \%) 5 \\
(12 \%) 1(2 \%)\end{array}$ & $--\cdot$ \\
\hline
\end{tabular}

[[P046 Table] Patients' characteristics]

\section{P047}

Reduced-intensity transplantation in patients with acute myeloid leukemia beyond complete remission - single centre experience

Michal Karas, Katerina Steinerova, Pavel Jindra, Daniel Lysak, Marcela Hrabetova, Jiri Sramek

Charles University Hospital Pilsen, Hemato-Oncology Dpt., Pilsen, Czech Republic

Background: reduced-intensity transplantation (RIT) for acute myeloid leukemia (AML) beyond complete remission (CR) is associated with higher relapse incidence, lower progression-free survival (PFS) and overall survival (OS). But elderly patients (pts) or pts with comorbidities can rarely benefit from myeloablative conditioning or intensive chemotherapy (CHT) for AML due to high risk of treatment-related mortality (TRM). To evaluate the role of RIT and potential effect of pre-transplantation characteristics in treatment of pts with AML beyond CR we retrospectively analysed the outcome of such pts undergoing RIT in our centre. 
Methods: since 200268 pts with median of age 60 years (22-74 years) with AML beyond CR underwent RIT. HCTCI was $\geq 3$ in $26(38 \%)$ pts. 29 pts were in the $1^{\text {st }}$ or $2^{\text {nd }}$ untreated relapse of AML, 20 pts had chemotherapyresistant disease and 19 pts underwent up-front RIT without previous AML therapy, all pts had more than $5 \%$ blasts in the bone marrow (BM). Donors were in 28\% HLA identical related, 10\% HLA haploidentical related, 40\% HLA matched unrelated and 22\% HLA mismatched unrelated. The source of stem cells was peripheral blood and the median of infused CD $34+$ cells was $6,55 \times 10^{\wedge} 6 / \mathrm{kg}(1,74-$ $14,34 \times 10^{\wedge} 6 / \mathrm{kg}$ ). The conditioning regimen consisted of fludarabine and melphalan in $65 \%$ pts and $35 \%$ pts underwent RIT after sequential regimen of CHT and RIC.

Results: 65 (96\%) pts engrafted and achieved complete remission (CR) with full donor chimerism (FDC) at day +30 after RIT. 45 pts (69\%) developed acute GVHD (20\% grade III-IV) and among 56 evaluable pts 22 (39\%) of them developed chronic GVHD (9 mild, 5 moderate, 8 severe). With median follow-up 86 months (3-179 months) 21 pts (31\%) are alive (20 pts in continuing CR with FDC). 24 pts (35\%) relapsed with median of time from RIT to relapse 6 months (1-27 months) and 23 of them died. 24 pts (35\%) died due to TRM and $9(13 \%)$ of them till day +100 after RIT. The estimated probabilities of 3-years PFS and OS are $31 \%$ and $34 \%$. Only presence of blasts in PB $(p=0.0001)$, more than $20 \%$ of BM blasts $(\mathrm{p}=0.0285)$ and HCT-CI $\geq 3$ $(\mathrm{p}=0.039)$ statistically significantly negatively affected the outcome of RIT.

Conclusions: in spite of relatively small number of evaluated pts and retrospective type of analysis our data suggest that RIT can achieved continuing long-term CR in about third of pts with AML beyond CR not eligible for myeloablative conditioning or intensive CHT of AML. The most important pre-transplantation factors significantly influencing RIT outcome were finding of circulating blasts in PB, percentage of BM blasts and HCT-CI. Status of disease, diagnostic cytogenetic findings, age, type of donor and type of conditioning regimen did not significantly affect the outcome of RIT.

Conflict of interest: The authors declare no conflict of interest.

\section{P048}

\section{Relapse after allogeneic hematopoietic stem cell transplantation for acute leukemia in children: a survey by the Turkish Pediatric Bone Marrow Study Group of 255 cases}

Volkan Hazar ${ }^{1}$, Gülsün Tezcan Karasu ${ }^{2}$, Gülyüz Öztürk ${ }^{3}$, Alphan Küpesiz, , Savaş Kansoy ${ }^{5}$, Namık Özbek ${ }^{6}$, Vedat Uygun ${ }^{7}$, Talia İleri ${ }^{8}$, Fatma Visal Okur ${ }^{9}$, Ülker Koçak ${ }^{10}$, Suar Çakı Kilic ${ }^{2}$, Arzu Akçay $^{3}$,
Elif Güler ${ }^{4}$, Salih Gözmen ${ }^{5}$, Musa Karakükcï̈ ${ }^{11}$, Ibrahim Bayram ${ }^{12}$, Tekin Aksu ${ }^{6}$, Akif Yeșilipek ${ }^{7}$, Barboros Şahin Karagün ${ }^{13}$, Sebnem Yllmaz Bengoa ${ }^{14}$, Mehmet Ertem ${ }^{8}$, Duygu Uçkan" ${ }^{9}$, Zühre Kaya ${ }^{10}$, Tunç Fışkınn ${ }^{15}$, Didem Atay ${ }^{3}$, Funda Tayfun Küpesiz ${ }^{4}$, Orhan Gürsel $^{16}$, Yöntem Yaman ${ }^{17}$, Ceyhun Bozkurt ${ }^{18}$, Müge Gökçe ${ }^{19}$, Serap Aksoylar $^{5,20}$

${ }^{1}$ Medical Park Göztepe Hospital, Pediatric Hematology \& Oncology and BMT Unit, Istanbul, Turkey; ${ }^{2}$ Medical Park Göztepe Hospital, Istanbul, Turkey; ${ }^{3}$ Acıbadem University Faculty of Medicine, Atakent Hospital, Istanbul, Turkey; ${ }^{4}$ Akdeniz University Faculty of Medicine, Antalya, Turkey; ${ }^{5}$ Ege University Faculty of Medicine, Izmir, Turkey; ${ }^{6}$ Ankara Child Health and Diseases Hematology Oncology Training and Research Hospital, Ankara, Turkey; ${ }^{7}$ Medical Park Antalya Hospital, Antalya, Turkey; ${ }^{8}$ Ankara University Faculty of Medicine, Ankara, Turkey; ${ }^{9}$ Hacettepe University Faculty of Medicine, Ankara, Turkey; ${ }^{10}$ Gazi University Faculty of Medicine, Ankara, Turkey;

${ }^{11}$ Erciyes University Faculty of Medicine, Kayseri, Turkey; ${ }^{12}$ Çukurova University Faculty of Medicine, Adana, Turkey; ${ }^{13}$ Acrbadem University Faculty of Medicine, Adana Hospital, Adana, Turkey;

${ }^{14}$ Dokuz Eylül University Faculty of Medicine, Izmir, Turkey;

${ }^{15}$ Medical Park Samsun Hospital, Samsun, Turkey; ${ }^{16}$ Health Science University Gülhane Medical Faculty, Ankara, Turkey; ${ }^{17}$ Istanbul Medipol University Faculty of Medicine, Istanbul, Turkey; ${ }^{18}$ Medical Park Bahçelievler Hospital, Istanbul, Turkey; ${ }^{19}$ Yeni Yüzyll University Faculty of Medicine, Gaziosmanpaşa Hospital, Istanbul, Turkey;

${ }^{20}$ Chief of the Turkish Pediatric BMT Group, Istanbul, Turkey

Background: Relapse is an important cause of death after hematopoietic stem cell transplantation (HSCT) for acute leukemia which represents a challenging management problem.

Methods: A questionnaire was sent to all Turkish pediatric transplant centers reporting leukemic relapse in a patient cohort of 938 HSCT for acute leukemia registered by the Turkish Pediatric Bone Marrow Registry between $1^{\text {st }}$ of January, 2004 and $31^{\text {st }}$ of December, 2015. Evaluable data was obtained in 255 patients (154 ALL, 94 AML, 5 bilineage leukemia and 2 indifferentiated leukemia) who underwent allogeneic HSCT and then relapsed.

Results: Median interval from transplantation and relapse was 180 days (range, 0-1740). Two hundred and twentyeight patients (139 ALL, 82 AML, 5 bilineage leukemia and 2 indifferentiated leukemia) received further treatment after relapse. Of these patients, 105 achieved a complete remission and had a higher cumulative overall survival of 12 months compare with patients not achieving complete remission $[49.3 \%(95 \%$ CI $39.5-59.1)$ vs $7.4 \%$ (95\% CI 2.6-11.2), $\mathrm{p}<0.001]$. Factors predictive for successful remission induction after relapse were age $(\leq 10$ years at first transplantation) [OR: 2.6, 95\% CI 1.5-4.6, $\mathrm{p}=0.001$ ], isolated extramedullary relapse (OR: 3.5, 95\% CI 1.6-7.7, $\mathrm{p}=0.002)$ and interval ( $>6$ months) between first HSCT and relapse (OR: 1.8, 95\% CI 1.0-3.1, p = 0.043). Fifty-five patients, 51 of whom were in complete remission, received a second HSCT. Of these patients, 14 were alive without disease with a median follow-up time of 990 days (range, 528-2982). 
HSCT for acute leukemia, n:942

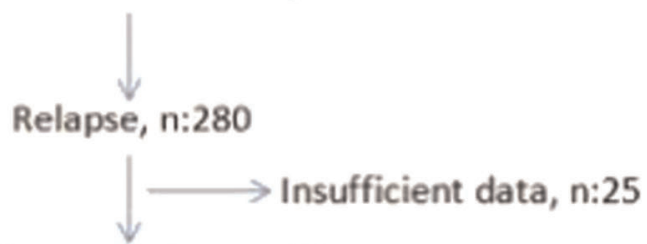

Study population, n:255

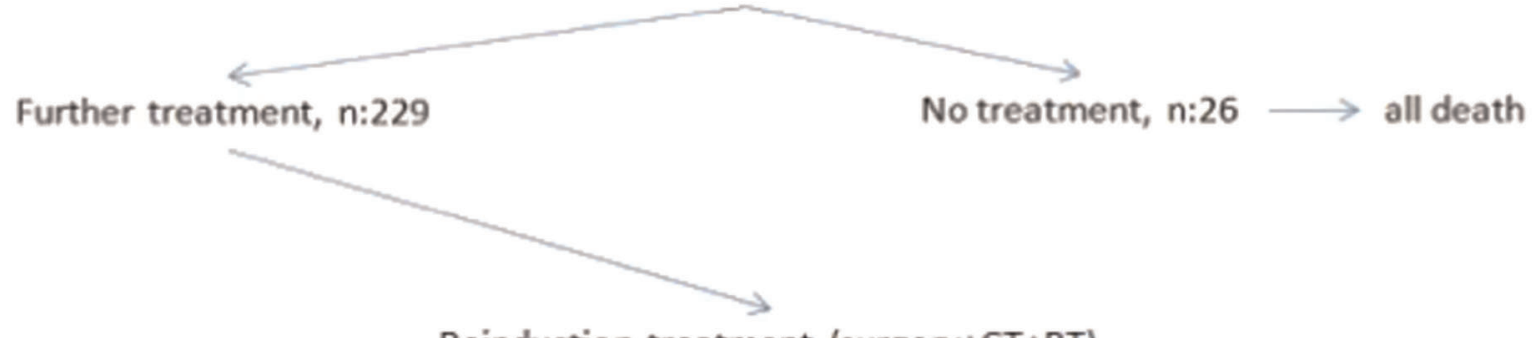

Reinduction treatment (surgery $\pm C T \pm R T$ )

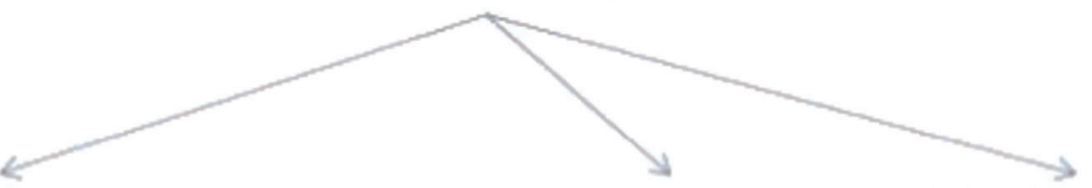

$\mathrm{CR}, \mathrm{n}: 105$

No CR, n:122

Reinduction death, $\mathrm{n}: 2$
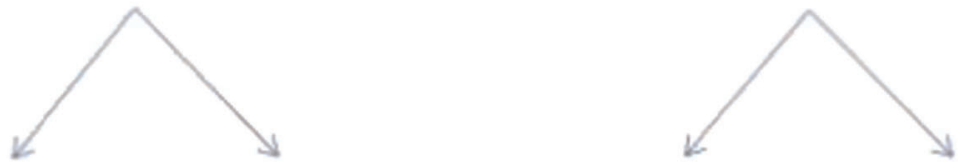

$2^{\text {nd }}$ HSCT, n:51 No HSCT, n:54

$2^{\text {nd }}$ HSCT, n:4 No HSCT, n:118
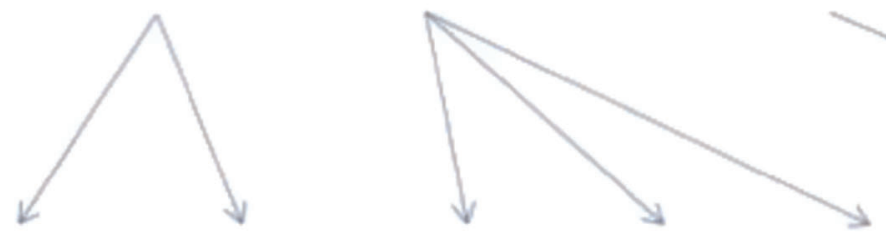

Alive, $\mathrm{n}: 16$ Death, $\mathrm{n}: 35$ Alive, $\mathrm{n}: 12$ Lost, $\mathrm{n}: 1$ Death, $\mathrm{n}: 41$

(14 NED)

(9 NED)

CR, complete remission; CT, chemotherapy; RT, radiotherapy; NED, no evidence of disease

[P048 Figure] [The flow diagram of the study population]

Conclusions: The results show that further treatment can prolong survival in pediatric patients who relapsed after HSCT for acute leukemia. In selected patients, long-term leukemia-free survival could be achieved by second HSCT.

Conflict of interest: The authors declare no conflict of interest.

\section{P049}

Safety and Efficacy of Blinatumomab in Nine Month Old ALL infantile with Persistent MRD Following Chemotherapy

Suleimman Al-Sweedan
King Faisal Specialist Hospital \& Research Center, Riyadh, Saudi Arabia

Background: Blinatumomab (Blincyto ${ }^{\circledR}$, Amgen Inc.), a bispecific CD19-directed CD-3 T-cell engager has produced remarkable responses in various B-cell malignancies, mainly for patients with refractory/relapsed acute lymphoblastic leukemia (ALL) but also in patients with refractory and heavily pretreated B-cell lymphomas. Though it is well established that Blinatumomab is an effective and tolerable treatment option for pediatric ALL, there is an insufficient evidence on its safety and efficacy in patients who are younger a year. 
Methods: We herein present a 14 months-old female patient, diagnosed with infantile acute lymphoblastic leukemia (ALL) at the age of 6 months. She presented with a white blood cell count of 1 million, platelets of 15,000, and a hemoglobin of $3.5 \mathrm{~g} / \mathrm{dL}$ with mild hepatosplenomegaly. She underwent leukapheresis, and peripheral flow cytometry confirmed the diagnosis of ALL. Cytogenetics is positive for MLL. CNS 1.

Results: BM immunophenotype results (03/7/2017) are consistent with positive B-ALL Minimal Residual Disease ( $0.02 \%$ of Mononuclear Cells) after the first cycle, 2nd BM immunophenotype after second cycle was negative.

Conclusions: In conclusion, in our patient, Blinatumomab found to be effective in terms of disease control before allo-SCT and showed an extremely safe profile.

Conflict of interest: NA

\section{P050}

\section{Salvage chemotherapy and donor lymphocyte infusion induces durable remissions in AML relapsing following allogeneic stem cell transplantation}

Marcin Lubowiecki ${ }^{1}$, Katalin Balassa ${ }^{1,2}$, Edmund Watson ${ }^{1}$, Rebecca Cash $^{1}$, Daja Barton ${ }^{1}$, Mandy Ellis ${ }^{1}$, Lara Rowley ${ }^{1}$, Denise Wareham $^{1}$, Claudia Costa ${ }^{1}$, Rachel Miller ${ }^{1}$, Rachel Pawson ${ }^{1,2}$, Angela Hamblin ${ }^{1}$, Lynn Quek ${ }^{1}$, Paresh Vyas ${ }^{1}$, Vanderson Rocha ${ }^{1,2}$, Andy Peniket ${ }^{1}$, Robert Danby ${ }^{1}$

${ }^{1}$ Oxford University Hospitals NHS Foundation Trust, Oxford, United Kingdom; ${ }^{2}$ NHS Blood and Transplant, Oxford, United Kingdom

Background: Allogeneic haematopoietic stem cell transplantation (HSCT) is an established curative treatment for myeloid malignancies. Nonetheless, relapse occurs in up to $40 \%$ of patients and outcomes post-allograft remain poor, with overall survival at 1 year reported around $20 \%$ (Bejanyan, 2015; Devillier, 2013). There is evidence showing that the use of a cytotoxic approach in selected patients together with donor lymphocyte infusions (DLI) or second allograft can achieve meaningful response rates of around 20-30\% (Lim, 2017; Vrhovac, 2016; Schroeder, 2013;).

Methods: We analysed the outcome of AML patients who developed morphological relapse after allogeneic HSCT performed between April 2011 and August 2017 at our centre. Patients underwent 3 monthly bone marrow assessments in the first year post transplant and thereafter when relapse was suspected. Patients with relapsed AML were offered treatment with either intensive AML chemotherapy (CT) or Azacitidine followed by DLI. In appropriate cases palliative chemotherapy or best supportive care was given. Overall survival (OS) data were

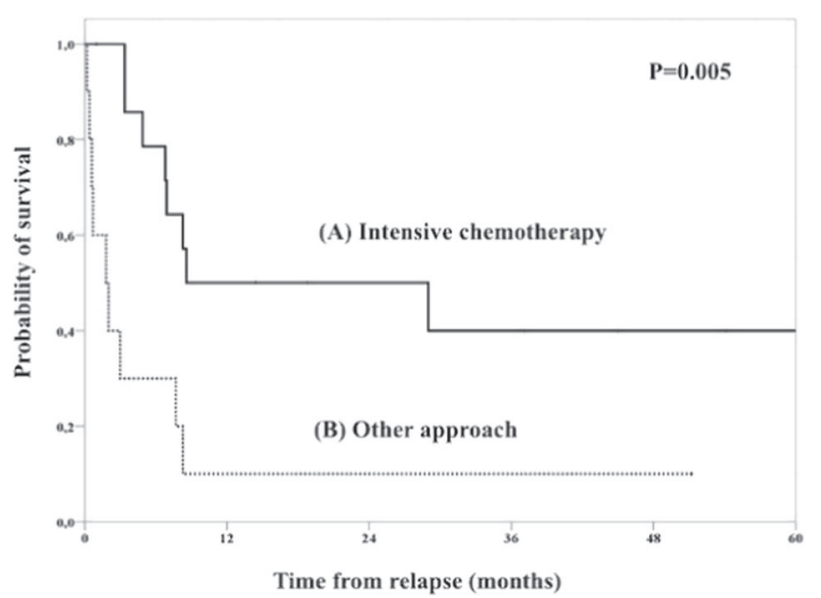

[P050 Figure] [Overall survival following high dose CT (A) or other treatment (B) using Kaplan-Meier estimates]

analysed by the log-rank test and Kaplan-Meier estimates were computed.

Results: Median age at transplantation was 54 years (range 18-69). The majority of patients received reduced intensity conditioning $(84 \%)$. The stem cells originated from MUD in 67\%, MRD in 28\%, UCB source in $4 \%$ and a haploidentical family donor in $1 \%$. Median follow-up time was 632 days (range from 8 to 2427). 109 patients were transplanted for AML of whom $25(23 \%)$ relapsed at time of follow-up. Post-transplant relapse occurred at a median of 238 days (range 48-825). Following relapse we were able to treat 19 patients (76\%) with salvage chemotherapy: 15 patients (60\% of relapsed patients) were treated with intensive AML CT (usually FLAG-Ida or FLA). Patients who were considered not to be fit for intensive therapy were treated with Azacitidine $(n=4 ; 16 \%)$, although this therapy was not available throughout the study period. Two patients $(8 \%)$ received palliative chemotherapy $(8 \%)$ and four $(16 \%)$ best supportive care. Only one patient underwent a second allograft following salvage chemotherapy. The majority of patients in the salvage CT/Azacitidine group (79\%) received DLI following count recovery.

Overall survival for all relapsed patients was $34 \%$ at 1 year and $28 \%$ at 2 years post-relapse. For those treated with intensive chemotherapy 1-year OS was 50\% compared to $10 \%$ if another treatment approach was used $(\mathrm{p}=0.005)$

(Figure 1). Of the 19 patients treated with high dose CT or Azacitidine, 53\% achieved complete remission and of those, $67 \%$ were alive in ongoing complete remission by the time of last follow-up.

Conclusions: We were able to offer salvage chemotherapy to the majority (76\%) of patients relapsing following HSCT for AML. Our data suggest that this approach is worthwhile and leads to durable remissions without the use of second stem cell transplant. 


\section{References:}

Bejanyan, N., et al. (2015) Biol Blood Marrow Transplant, March (21(3)), 454-459.

Devillier, R., et al. (2013) Leukemia \& Lymphoma, 54:6, 1228-1234

Lim, A.B.M., et al. (2017 [Epub ahead-of-print]) Internal Medicine Journal, June 19, doi: 10.1111/imj.13522.

Schroeder, T et al. (2013) Leukemia, 27, 1229-1235.

Vrhovac, R., et al. (2016) Bone Marrow Transplantation, 51, 186-193.

Conflict of interest: [All authors]: nothing to disclose

\section{P051}

\section{Sequential conditioning in unfavorable AML: a single center experience}

\section{Magalie Joris ${ }^{1}$, Amandine Charbonnier ${ }^{1}$, Bérengère Gruson ${ }^{1}$, Caroline Delette ${ }^{1}$, Marie-Noëlle Lacassagne ${ }^{2}$, Nicolas Guillaume ${ }^{3}$, Lö̈c Garçon ${ }^{4}$, Jean-Pierre Marolleau ${ }^{1}$, Delphine Lebon ${ }^{1}$ \\ ${ }^{1}$ CHU Amiens, Hématologie Clinique, Amiens, France; ${ }^{2}$ CHU Amiens, Laboratoire de Thérapie Cellulaire, Amiens, France; ${ }^{3} \mathrm{CHU}$ Amiens, Laboratoire HLA, Amiens, France; ${ }^{4} \mathrm{CHU}$ Amiens, Laboratoire Hématologie, Amiens, France}

Background: Response rates to common salvage regimens reach up to $10-15 \%$ in patients with refractory acute myeloid leukemia (AML) or early relapse. Without allogeneic HSCT, the long-term overall survival (OS) for patients with refractory AML hardly reaches 5\%. Several studies have demonstrated that salvage chemotherapy with sequential conditioning could reduce leukemia relapse risk with an acceptable toxicity profile for unfavorable acute myeloid leukemias (AML) and myelodysplastic syndromes (MDS). Therefore, we decided to assess this procedure in our center at Amiens University Hospital.

Methods: We conducted a monocentric retrospective study, including 34 patients aged over 18 years undergoing a hematopoietic stem cell transplant (HSCT) with sequential conditioning between January 2012 and December 2016, for refractory or high risk AML or MDS. 32/34 patients received sequential conditioning containing clofarabine (SET-RIC), the 2 others received Amsacrine based conditioning (FLAMSA).

Results: The median age was 48 years (range 27-70). Before conditioning, 19 patients $(56 \%)$ had active disease and $15(44 \%)$ were in complete remission (CR) including 6 with positive MRD. 18 patients had no or 1 prior treatment and 14 had at least 2 previous courses of chemotherapy. 11 (32\%) patients had HLA-identical sibling donors, 14 (41\%) match unrelated donors, $4(12 \%)$ mismatch unrelated donors and $5(15 \%)$ haploidentical donors. Majority of patients $(85 \%)$ received peripheral blood stem cell (PBSC)
PBSC with median CD34+ count of $7,71.10^{6} / \mathrm{kg}(1,84-$ $8,44)$. Acute GvHD prophylaxis with Ciclosporin A, in combination with Mycophenolate mofetil for unrelated donors, was withdrawal with a median time of 90 days.

With a median follow-up of 32 months, overall survival (OS) at 1 and 2 years was 70,6\% and 46\%. Median OS was 18 months (0-48 months) and median disease free survival (DFS) was 16 months (0-48 months). 11 patients (32\%) experienced relapse after HSCT with a median time from HSCT to relapse of 5 months (1-28 months). $56 \%$ of patients presented with grade I-II acute graft versus host disease (GVHD)and 12,5\% with grade III IV aGVHD . GVHD free relapse free survival at 1 and 2 years was $44 \%$ and $29 \%$. One-year cumulative incidence of disease related death and non-relapse mortality was $17,6 \%$ and $11,8 \%$ respectively. 14 patients $(44 \%)$ received immunomodulation with 5 Azacitidine and donor lymphocyte infusion (DLI) if no GVHD occurred within day 120 . OS was $86 \%$ in the 14 patients receiving DLI. In univariate analysis, relapse after HSCT and immunomodulation post HSCT (Figure) were significantly associated with overall survival ( $\mathrm{p}<0,0001$ and $\mathrm{p}=0,016$ respectively) but not the disease status before HSCT $(\mathrm{p}=0,47)$ neither aGVHD occurrence ( $\mathrm{p}$ $=0,14)$. In a multivariate analysis, only relapse after HSCT remained predictive of outcome $(\mathrm{p}=0,034)$.

Conclusions: According to previous publications, our study show interesting results of sequential conditioning regimen on refractory AML with an acceptable toxicity

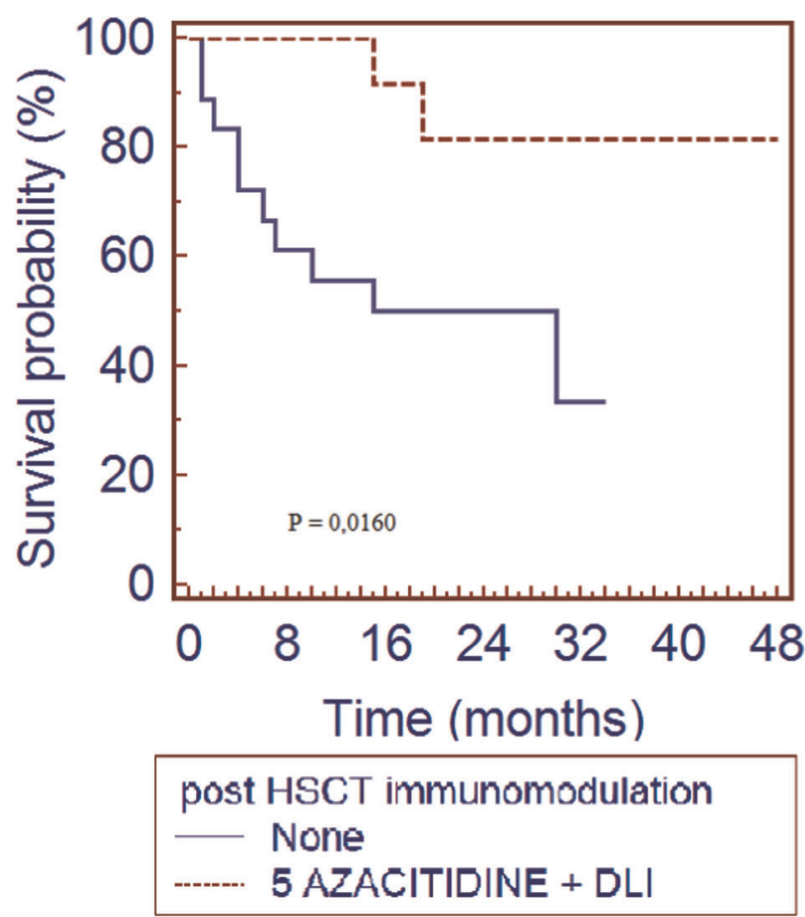

[P051 Figure] [Overall survival according to post HSCT immunomodulation with 5 Azacitidine + DLI administration] 
profile. In addition with post HSCT immunomodulation, we achieve very good OS and DFS whatever cytogenetic and disease status before HSCT. Unfortunately, we failed to identify some predictive factors for outcome like GVHD occurrence which was describe elsewhere probably because of the small size of our cohort.

Conflict of interest: JORIS: nothing to disclose

\section{P052}

\section{Significance of minimal residual disease before allogeneic hematopoietic cell transplantation for acute myeloid leukemia}

Sergey Bondarenko, Boris Afanasyev

Pavlov First Saint-Petersburg State Medical University, R.M. Gorbacheva Memorial Institute of Oncology, Haematology and Transplantation, Saint-Petersburg, Russian Federation

Background: Allogeneic hematopoietic stem cell transplantation (alloHSCT) is an effective therapy for many patients with acute myeloid leukemia (AML). The relapse after alloHSCT is the most common cause of treatment failure. However, multiparameter flow cytometry, cytogenetic and molecular studies in the onset of the disease makes it possible to detect the minimal residual disease (MRD) in AML remission in order to determine the prognosis and the therapy strategy.

Methods: We analysed 195 consecutive patients with AML since 2012 to 2016. Median age was 36(18-70) years. The median follow-up was 23(23-467) months. Disease status before alloHSCT: CR1 - 148 (76\%), CR2 - 47(24\%) patients. Molecular and genetic low-risk group was defined in $31(17 \%)$, standard risk in 119(59\%) and 45(24\%) highrisk patients. MRD was not determined (MRD (-)) in 155 (80\%) patients. A positive result of MRD (MRD (+)) before alloHSCT was in $40(20 \%)$ patients, including mutations, gene overexpression and leukemia associated immunophenotypes.

Results: Disease-free survival (DFS) after alloHSCT in CR1MRD(-) was 67\% (95\% CI 57-77), and in MRD(+) $54 \%(95 \%$ CI 36-72) $(\mathrm{p}=.03)$, whereas in patients with CR2MRD(-) 57\% (95\% CI 41-73) and CR2MRD(+) - 33\% (95\% CI 1-88) ( $\mathrm{p}=\mathrm{ns})$. The relapse incidence (RI) in the CR1MRD(-) was $22 \%$ (95\% CI 13-32), and in MRD(+) $36 \%$ (95\% CI 19-53), whereas in the CR2MRD(-) $17 \%$ (95\% CI 7-32), and in MRD(+) 66\% (95\% CI 15-91). With myeloablative conditioning (MAC), the DFS and RI after alloHSCT in CR1 did not depended on MRD: patients with MRD(-) 70\% (95\% CI 59-81) vs MRD(+) 64\% (95\% CI $36-92$ ) and $17 \%$ (95\% CI 7-39) vs $23 \%$ (95\% CI $12-35$ ) respectively. Whereas after reduced intensity conditioning (RIC), the DFC was higher, and the RI was lower in patients with MRD(-): $67 \%$ (95\% CI 54-80) vs 50\% (95\% CI $27-$ $77)(\mathrm{p}=.03)$ and $50 \%(95 \% \mathrm{CI} 25-70)$ vs $23 \%(95 \% \mathrm{CI}$ $12-35)(\mathrm{p}<.000)$. The cytogenetic risk group (intermediate and high) and the donor type (related and unrelated) had no effect on DFS and RI: $74 \%$ vs $63 \%$ and $47 \%$ vs $38 \%, 16 \%$ vs $19 \%$ and $59 \%$ vs $63 \% ; 68 \%$ vs $57 \%$ and $65 \%$ vs $42 \%$, $20 \%$ vs $29 \%$ and $29 \%$ vs $58 \%$. In the absence of chronic graft-versus-host disease(cGVHD), DFS and RI were better in patients with MRD(-) 64\% (95\% CI 45-83) vs 52\% (95\% CI $32-72)(\mathrm{p}=0.01)$ and $34 \%(95 \%$ CI $15-54)$ vs $44 \%$ $(95 \%$ CI $23-63)(\mathrm{p}=.03)$. In patients with cGVHD, the difference was insignificant: $70 \%$ vs $80 \%$ and $17 \%$ vs $20 \%$. In multivariate analysis, the impact on the RI has had the status of the disease $(\mathrm{MRD}(+) / \mathrm{MRD}(-))(\mathrm{HR} 2.3,95 \% \mathrm{CI}$, $1.1-4.9, \mathrm{p}=.03$ ) and the presence of $\operatorname{cGVHD}(\mathrm{yes} / \mathrm{no})$ (HR0.3, 95\% CI, 0.2-1.0, = .05). DFS was lower with $\operatorname{MRD}(+)(\mathrm{HR} 1.8,95 \% \mathrm{CI}, 1.0-3.5, \mathrm{p}=.05)$.

Conclusions: Immunophenotypic, cytogenetic and molecular data in the onset of the disease is necessary for monitoring the MRD in AML remission. The presence of MRD has a negative effect on DFS and RI after alloHSCT. $\mathrm{MRD}(+)$ status in front of the alloHSCT is an indication for the intensification of the conditioning regimen.

Conflict of interest: The authors declare no competing financial interests.

\section{P053}

Standard-NORMOXIC versus physiological HYPOXIC culture of AML-patientsÂ' (pts) whole blood (WB) samples with immunemodulatory Kits yields comparable proportions of Dendritic cells and functional results

\section{Fatemeh Doraneh Gard}

University Hospital of LMU, Munich, Germany

Background: For a clinical treatment strategy $\mathrm{DC}_{\text {leu }}$ could be generated ex vivo and transferred to the pts or alternatively blasts modulated to leukemia-derived DC $\left(\mathrm{DC}_{\text {leu }}\right)$ in vivo-resulting in T-cell activation. Combinations of GM-CSF+PICIBANIL (I), or +PGE2 (K) or + PGE1 (M) are kits that convert blasts into a ' $\mathrm{DC}_{\text {leu-vaccine' and }}$-vat induce antileukemic T-cell (memory) without induction of blast proliferation in a WB-culture model, in the presence of soluble and cellular components of the pts. To simulate physiological conditions most we compared normoxic $\left(\mathrm{N}^{*}\right.$; $\left.21 \% 0_{2}\right)$ vs physiological hypoxic $\left(\mathrm{H}^{*} ; 10 \% \mathrm{O}_{2}\right)$ culture conditions.

Methods: 15 AML- and 8 healthy WB-samples were cultured in parallel in $\mathrm{N}^{*}$ and $\mathrm{H}^{*}$ conditions treated with "I", "K" and "M" kits which followed by MLC with the probands' T-cells and blast-cytotoxicity-assays in AML- 
samples. Cellular compositions (DC-, blast-subtypes and immunestatus) and functional results were compared.

Results: Generation of $\mathrm{DC} / \mathrm{DC}_{\text {leu }}$ from WB in normoxia vs hypoxia with different kits: Under $\mathrm{N}^{*}$ vs $\mathrm{H}^{*}$ conditions comparable proportions of DC were generated with Kit I, K, $\mathrm{M}(9-13 \%)$ from 8 healthy- and 10-11\% from 15 AML WBsamples. $\% \mathrm{DC}_{\text {leu }}$ were also comparable (7-10\%) as well as\% $\mathrm{DC}_{\text {mature }}(9-11 \%)$. Success of DC-generation $(>10 \%$ DC, $>5 \% \mathrm{DC}_{\text {leu }}$ ) was comparable under $\mathrm{N}^{*} \mathrm{v} \mathrm{s} \mathrm{H} \mathrm{H}^{*}$ in healthy and AML-WB. Controls without added kits ("w/o") failed to produce $\mathrm{DC} / \mathrm{DC}_{\mathrm{leu}}$. Furthermore, treatments with Kits did not induce blasts' proliferation under $\mathrm{N}^{*}$ or $\mathrm{H}^{*}$ conditions.

After mixed lymphocyte culture (MLC) of T-cells with kit-treated AML-WB under $\mathrm{N}^{*}$ vs $\mathrm{H}^{*}$ with $\mathrm{I}, \mathrm{K}, \mathrm{M}$ comparable\% were generated for $\mathrm{T}_{\text {prolif }}(17-40 \%)$ and $\mathrm{T}_{\mathrm{em}}{ }^{-}$ eff $(30-48 \%)$, however I and $\mathrm{M}$, but not K-treated WB increased\% of $\mathbf{T}_{\text {non-naive }}$ (I,M:55-62, K:44\%), (I,M: 30-48, $\mathrm{K}: 37 \%$ ) and $\mathrm{T}_{\mathrm{cm}}$ (I,M:7-24, K:3\%), under $\mathrm{N}^{*}$ and $\mathrm{H}^{*}$ significantly.

Antileukemic activity of T-cells (with or w/o previous kittreatment of WB) in normoxia vs hypoxia: Cytotoxicityassays after T-cells' MLC with kit-treated (vs untreated) WB showed in 8 of 10 cases with I- and in 6 of 8 cases with M treated WB (in $\mathrm{N}^{*}$ vs $\mathrm{H}^{*}$ ) an improved blast-lysis (by 75$80 \%)$. Results obtained with parallel analysed cases under $\mathrm{N}^{*}$ vs $\mathrm{H}^{*}$ were comparable in 11 of $18(61 \%)$ cases. In some cases (especially after I-treatment) differences in antileukemic functionality in $\mathrm{N}^{*}$ vs $\mathrm{H}^{*}$ settings were found.

Conclusions: Cultivation of PB-cells with kits under $\mathrm{N}^{*}$ vs $\mathrm{H}^{*}$ conditions were comparable (DC-types, T-cell subtypes after MLC, mediation of antileukemic reactivity). This means, that standard $\mathrm{N}^{*}$ culture conditions (of WB) simulate reactions in the body well. Therefore production of cellular products (e.g for adoptive cell transfer) under $\mathrm{H}^{*}$ is not necessary. Our data show, that pts' treatment with $\mathrm{M}$ and I might improve antileukemic reactivity in vivo by a $\mathrm{DC}_{\text {leu }}{ }^{-}$ mediated mechanism.

Conflict of interest: [F. D. Gard]: nothing to disclose

\section{P054}

\section{Strategy for monitoring minimal residual disease using immunoglobulin clonality in patients with B-cell lymphoblastic leukemia}

Nack-Gyun Chung ${ }^{1}$, Seok Lee ${ }^{1}$, Irine Jo ${ }^{2}$, Seongkoo Kim ${ }^{1}$, Jae Wook $\mathrm{Lee}^{1}$, Jae-Ho Yoon ${ }^{1}$, Bin Cho ${ }^{1}$, Kyungja Han ${ }^{2}$, Yonggoo Kim ${ }^{2,3}$, Myungshin Kim ${ }^{2,3}$

${ }^{1}$ The Catholic Blood and Marrow Transplantation Center, St. Mary's Hospital, College of Medicine, The Catholic University of Korea, Seoul, Korea, Republic of; ${ }^{2}$ St. Mary's Hospital, College of Medicine, The Catholic University of Korea, Department of Laboratory Medicine, Seoul, Korea, Republic of; ${ }^{3}$ Catholic Genetic Laboratory
Center, College of Medicine, The Catholic University of Korea, Seoul, Korea, Republic of

Background: Minimal residual disease (MRD) monitoring is one of the most powerful tools to predict the risk of relapse. In this study we validate a feasible MRD monitoring strategy for B-lymphoblastic leukemia (BALL) using high-throughput sequencing (HTS) of immunoglobulin (Ig) clonality before implementation into routine clinical tests.

Methods: We selected $60 \mathrm{BM}$ specimens from 34 patients who were diagnosed with B-ALL and followed after treatment at the Catholic Blood and Marrow Transplantation Center, Seoul St. Mary's Hospital, The Catholic University of Korea. Twelve B-ALL with $\mathrm{t}(12 ; 21)$ (p13.2;q22.1); ETV6-RUNX1, 11 B-ALL with $\mathrm{t}(9 ; 22)$ (q34.1;q11.2); BCR-ABL1 and 11 B-ALL not otherwise specified were included. To quantification of leukemiaspecific fusion transcripts, the fusion transcript of ETV6$R U N X 1$ and $B C R-A B L 1$ was measured via reverse transcription quantitative-PCR (RT-qPCR) using the Real-Q ETV6-RUNX1 and Real-Q BCR-ABL1 quantification kit (Biosewoom Inc., Seoul, Korea), respectively. We used the LymphoTrack IGH FR1/2/3 assay panel (InVivoScribe Technologies) to assess the clonal $I G H$ rearrangement via HTS. When clonal $I G H$ rearrangement was negative in the initial specimen after using the primers targeting the conserved framework region (FR) 1 within the variable and joining region, a test using FR2, FR3 and the IGK assay panel (InVivoScribe Technologies) was performed.

Results: All diagnostic BM specimens in the study contained more than $70 \%$ leukemic blasts, except for 2 cases (median 92.5\%; range 27-99\%). IGH clonality was observed in all included B-ALL( $\mathrm{n}=34)$ after using primers targeting three framework regions, and the sum of the $I G H$ clonal burden varied(median $79.47 \%$; range $9.47-96.77 \%$ ). $I G K$ clonality was identified in $70 \%$ of patients and availed in cases with low IGH clonal burden. The total $I G H$ clonal burden was significantly correlated with the proportion of leukemic blasts in bone marrow and the quantity of leukemia-specific fusion transcripts including ETV6$R U N X 1$ and $B C R-A B L 1$. We could recognize the different response of each clone and the emerging clones originating from the trace of Ig rearrangement present in the initial specimen. The $I G H$ clonal burden after induction chemotherapy represented patient outcomes well. The IGH assay also provided information of repertoire diversity of $I G H$ rearrangement for which the low initial value increased after treatment.

Conclusions: The Ig clonality assay via HTS will be a promising tool for MRD monitoring of B-ALL through an adequate strategy to identify all significant clones, monitor 
individual clones, and determine repertoire diversity. Further studies to accumulate prospective data with clinical implication will help refine the strategy.

\section{Conflict of interest: None}

\section{P055}

\section{Systematic review and meta-analysis on the role of transplant in infant leukemia}

\section{Joy Mburu, Muthoni Mburu, Tony Truong, Arun Narendran}

University of Calgary, Hematology Oncology, Calgary, Canada

Background: Infants with leukemia have a poor prognosis despite advances in therapy in childhood leukemia. This is especially so for those with acute lymphoblastic leukemia associated with rearrangements of the KMT2A gene, and are less than 6 months of age at diagnosis. Role of transplant in high risk childhood leukemia's is well defined, while in infants with leukemia impact of transplant has not been clearly defined.

Methods: A review of the published literature was carried out in CINAHL, EMBASE, MEDLINE, PubMed, Cochrane databases and the grey literature, unto the period of September 2016., with inclusion of both retrospective and prospective studies. A Librarian did a search of various databases. Information retrieval from the articles was done by 2 reviewers. Inclusion criteria, also key words were based on PICOS. Population - infants (less than a year old at diagnosis of leukemia), leukemia (including both myeloid and lymphoid). Intervention - chemotherapy or hematopoietic stem cell transplant. Outcome - Event free survival and Overall survival. We excluded studies not in English. We utilized the Preferred Reporting Items for Systematic Reviews and Meta-Analyses (PRISMA) for the study methodology. High risk of bias in articles assessed, this was graded according to Newcastle-Ottawa Scale nonrandomized studies.

Results: There were 1848 articles identified from the database searched. Following duplicate removal, 1202 titles were evaluated for appropriateness. 492 abstracts were screened, and a total of 163 articles were assessed for eligibility. Studies included for analysis of infant leukemia were 31.20 studies on acute lymphoblastic leukemia and 11 on acute myeloid leukemia. For acute Lymphoblastic leukemia, 8 prospective trials, failed to outline the benefit in both event free survival and overall survival, that is similar to results from 12 retrospective studies. This was considered in the background of the differences in characteristics of the infants, time to transplant and choice of conditioning regimes. For acute myeloid leukemia, only prospective studies were used as they included outcome data beyond 3 years. Infants with AML have good survival data though similar to ALL, there was a small number of patients in the studies like in lymphoblastic leukemia. The event free survival of infants following transplant was no different from those who had continuous chemotherapy. Meta analysis was done using Revman software. In both myeloid and lymphoid leukemia's in infant and comparison of the chemotherapy and transplant group did not show any statistical signifance and there was a high rate of incontinency of the data ranging from 50-95\%.

Conclusions: High variation in studies leading to heterogeneity of data, that is including differences in time to transplant, either in complete remission one or two, small sample sizes and choice of chemotherapy and condition regimens make it difficult to carry out a meta- analysis and draw any conclusions. There is a need for randomized control trials to be done for a clear role to be drawn for both myeloid and lymphoblastic leukemia in infants.

No conflict of interest to declare

Conflict of interest: No conflict of interest to disclose

\section{P056}

The clinical implication of cytogenetic clonal evolution pattern in relapsed adult acute lymphoblastic leukemia patients

\section{Ji Hyun Lee ${ }^{1}$, Yoo Jin Lee ${ }^{2}$}

${ }^{1}$ Dong-A University, Department of Internal Medicine, Busan, Korea, Republic of; ${ }^{2}$ Kyungpook National University School of Medicine, Department of Internal Medicine, Daegu, Korea, Republic of

Background: Adult acute lymphoblastic leukemia (ALL) patients commonly relapse after allogeneic stem cell transplantation (Allo-SCT) and chemotherapy. ALL relapse has been related to clonal cytogenetic evolution, but there is no study which focuses on the cytogenetic pattern or its clinical implications after treatment.

Methods: Two hundred and twenty-four ALL was diagnosed as ALL in two centers in South Korea between January, 2000 and December, 2016. Among these patients, 181 patients were able to proceed to treatment $(80.8 \%)$ and among 104 patients $(57.5 \%)$ achieved complete remission (CR) after allo-SCT or chemotherapy only. 63 patients (60.6\%) relapsed after CR and finally 51 patients who had cytogenetic results both at initial diagnosis and at relapse were analyzed.

Results: Eighteen and 33 patients relapsed after alloHSCT and conventional chemotherapy using chromosome banding analysis combined with fluorescent in situ hybridization. The male to female ratio was 31:20 and the median age in allo-SCT group and conventional chemotherapy only group was 34.0 years (20-54 years) and 46 years (20-76 
years) respectively. At diagnosis, aberrant karyotypes were more frequent

in the HSCT than in the chemotherapy cohort (14 of 18; $77.8 \%$ versus 16 of $33 ; 48.5 \%$; $\mathrm{P}=0.042$ ).

Clonal changes from diagnosis and relapse were more frequent in the allo-SCT group

( 15 of $18 ; 83.3 \%$ versus 20 of $31 ; 64.5 \%$ ) compared with the conventional chemotherapy only group (not significant), mostly due to the clonal evolution. Appearance of new $\geq 3$ cytogenetic alterations was more frequent in the allo-SCT group (4 of $18 ; 22.2 \%$ versus 3 of $33 ; 9.1 \%$, not significant). The mean number of cytogenetic alterations was increased from 1.94 (standard deviation, $\mathrm{SD} \pm 1.47$ ) at diagnosis to $4.39(\mathrm{SD} \pm 1.47)$ at relapse in the allo-SCT group, but in the conventional chemotherapy only group, $2.06(\mathrm{SD} \pm 5.12)$ to 2.35 ( $\mathrm{SD} \pm 3.21$ ) (at diagnosis, $\mathrm{P}=0.047$, at relapse, not significant). Clonal change did not correlate to overall survival.

Conclusions: Clonal change and cytogenetic complexity was more frequently observed in the hematopoietic stem cell recipient group. The change of karyotype did not correlate to the overall survival of adult ALL patients, which might be affected the innate poor prognosis in this group of patients.

Conflict of interest: No conflict of interest

\section{P057}

Abstract previously published

\section{P058}

\section{The outcome of allogeneic stem cell transplantation in patients with high risk MDS and AML: single center experience}

\section{Khalid Halahleh ${ }^{1}$, Sarah Odaily, ${ }^{2}$ Abdallah Al Tell ${ }^{2}$, Tala Al- Awabdeh', Mohammad Ma'kosa ${ }^{3}$, Rula Najjar', Rawad Rihani, Mayada Abu Shanab ${ }^{6}$, Nilly Hussein ${ }^{7}$, Eman Khattab ${ }^{8}$, Shanta Sharma ${ }^{9}$, Ahmad Abu Khader, Dana Yousef ${ }^{10}$, Husam Abu-Jazar', Abdelghani Tbakhi ${ }^{11}$,Mahmoud Sarhan ${ }^{12}$}

\footnotetext{
${ }^{1}$ King Hussein Cancer Center, Adult Medical Oncology Hematology, Bone Marrow Transplantation Program, Amman, Jordan; ${ }^{2}$ King Hussein Cancer Center, Adult Medical Oncology Hematology, Amman, Jordan; ${ }^{3}$ King Hussein Cancer Center, Adult Medical Oncology Hematology, Bone Marrow Transplantation, Amman, Jordan; ${ }^{4}$ King Hussein Cancer Center, Clinical Pharmacy Department, Bone Marrow Transplantation Service, Amman, Jordan; ${ }^{5}$ King Hussein Cancer Center, Pediatric Medical Oncology Hematology, Bone Marrow Transplantation Program, Amman, Jordan; ${ }^{6}$ King Hussein Cancer Center, P. Bone Marrow Transplantation Program, Amman, Jordan; ${ }^{7}$ King Hussein Cancer Center, Amman, Jordan; ${ }^{8}$ King Hussein Cancer Center, Pediatric Onolcogy Hematology, Bone Marrow Transplantation Program, Amman, Jordan; ${ }^{9}$ King Hussein Cancer Center, Department of Cell Therapy and Applied Genomics, Amman, Jordan; ${ }^{10}$ King Hussein
}

Cancer Center, Nursing Department, Amman, Jordan; ${ }^{11}$ King Hussein Cancer Center, Department of Cell Theray and Applied Genomics, Amman, Jordan; ${ }^{12}$ King Hussein Cancer Center, Bone Marrow Transplantation, Amman, Jordan

Background: Allogeneic hematopoietic cell transplantation (HCT) is an effective, potentially curative treatment for advanced or high-risk AML and myelodysplastic syndrome (MDS) using different intensity conditioning regimens (1). Myeloablative conditioning is associated with a reduced risk of relapse,but does not translate to improved survival due to increased transplant-related mortality(TRM). Reduced-intensity conditioning (RIC) allowed extension of HCT to a much wider patient population by reducing the toxicity without compromising HCTefficacy. In this retrospective study, we report our experience on 64 patients with high risk MDS and AML using different conditioning regimens and report their long term outcome.

Methods: We performed a single center retrospective analysis on 64 adult patients with high risk myelodysplastic syndrome (MDS) or acute myeloid leukemia (AML), who underwent allogeneic HCT at King Hussein Cancer Center between January-2008 till December-2016.Using KaplanMeier method, we reported the outcome of patients in regard to overall and progression free survival, cumulative incidence of relapse, TRM, GvHD and identification of predictive factors for poor outcome. 16 patients with MDS, fourty-eight with de novo and secondary AML.41/19/4 were in CR1/CR2,CR3/active MDS respectively. Seventeen were males and 47 were females. Sixty-one received mached related donor, one MUD, one with one allele mismatched related donor and one umbilical cord blood transplantation.Fourty-one and twenty-three received combination of calcineurin inhibitor with either methotrexate and MMF respectively.Fourty-three cases received MAC and twenty-one reduced intensity conditioning before transplantation.

Results: Total of sixty-four patients were included. The median age at time of HCT was 42 years (range: 17-69). All patient received PBSC as graft sourse.90\% of patients engrafted on time and $88 \%$ had sustained full donor chimerism. $38.2 \%$ developed aGvHD grade II-IV, $11 \%$ grade III- IV.51.5\% developed cGvHD.After a median follow up time of 27.9 months, the probability of 3-year and 5 -year progression free survival and overall survival was $49.8 \%+8.2 \%$ and $41.5 \%+10.2 \%$ and $41 \% \pm 6.6 \%$ and $34 \% \pm 8.3 \%$ respectively. The probability of 3 -year and 5year cumulative incidence of relapse $39.1 \%$ and $42.8 \%$. The probability of 5 -year TRM $55.1 \% \pm 11.7 \%$.

After analyzing the data, there was significant improved survival outcome in those who had cGvHD (P-0.0001), but there was only trend toward improved progression free survival and overall survival in patients transplanted in CR1 
with de novo AML and who had an intermediate-good risk cytogenetics compared with poor risk group (P-0.2).

Conclusions: Allogeneic HCT is potentially curative approach in patients with high risk AML and MDS with improved survival in patients, who developed cGvHD and only trend toward improved survival outcome in patients transplanted in CR1 and had intermediate risk.

Conflict of interest: No conflict of interest

\section{P059}

\section{The Role and Mechanism of PTPN21 Mutation in Acute Lymphoblastic Leukemia Relapse after allo-HSCT}

\section{Huafang Wang, Ni Zhu, Binsheng Wang, Limengmeng Wang, He Huang}

Zhejiang University School of Medicine, Bone Marrow Transplantation Center, The First Affiliated Hospital, Hangzhou, China

Background: Disease relapse is one of the leading causes of death in patients with acute lymphoblastic leukemia (ALL) after allogeneic hematopoietic stem cell transplantation (allo-HSCT). The mechanism of leukemia relapse after allo-HSCT involve in the biological characteristics of leukemia cells .In our previous study, we performed exome sequencing analyses on matched diagnosis and relapse sample to explore genetic basis of relapse. We found that cells responsible for ALL relapse after allo-HSCT showed different gene mutations to the primary leukemia cells at diagnosis and acquired new gene mutations, including PTPN21 gene mutation happened in exon13(exon13: c. 1514 C>A: p. P505Q).

Methods: We designed several small guide RNA(also called sgRNA) located in the exon 13 of PTPN21 near the mutation site, synthesizing the sgRNA-CAS9 plasmids and donor template plasmid by molecule cloning technology and Screening sgRNA-CAS9 plasmid with the highest shear efficiency by T7E1 method. NALM6, a type of $\mathrm{PH}^{-} \mathrm{ALL}$ cell line, was used to construct PTPN21 mutation model in vitro. Using Crispr-Cas9 technology, we successfully gained the NALM6 cell line with PTPN21 mutation in exon13(exon13: c. 1514 C>A: p. P505Q). The proliferation of NALM6 cell lines was detected by CCK8 method. Cell cycle, apoptosis and drug resistance of NALM6 cell lines with mutation or not were detected through flow cytometry, and signal pathway associated proteins were detected by Western Blot and Immunofluorescence.

\section{Results:}

1. Compared with negative control group, the proliferation of NALM6 cell line with PTPN21 above mutation was increased significantly.
2. PTPN21 mutation in NALM6 cell line can promote cells' cell cycle transition from $\mathrm{G} 0$ phase to $\mathrm{S}$ phase.

3. There is no significant effect of PTPN21 mutation on the apoptosis and drug resistance of NALM6 cells.

4. The mutation of PTPN21 gene in NALM6 cell line enhances the activation level of PTPN21-SRC-ERK pathway, and acts directly or indirectly on downstream P21-CDK2 pathway, thereby regulating cell cycle. See graph below.

Conclusions: PTPN21 gene mutation (exon13: c. 1514 C $>$ A : p. P505Q) can significantly affect the proliferation
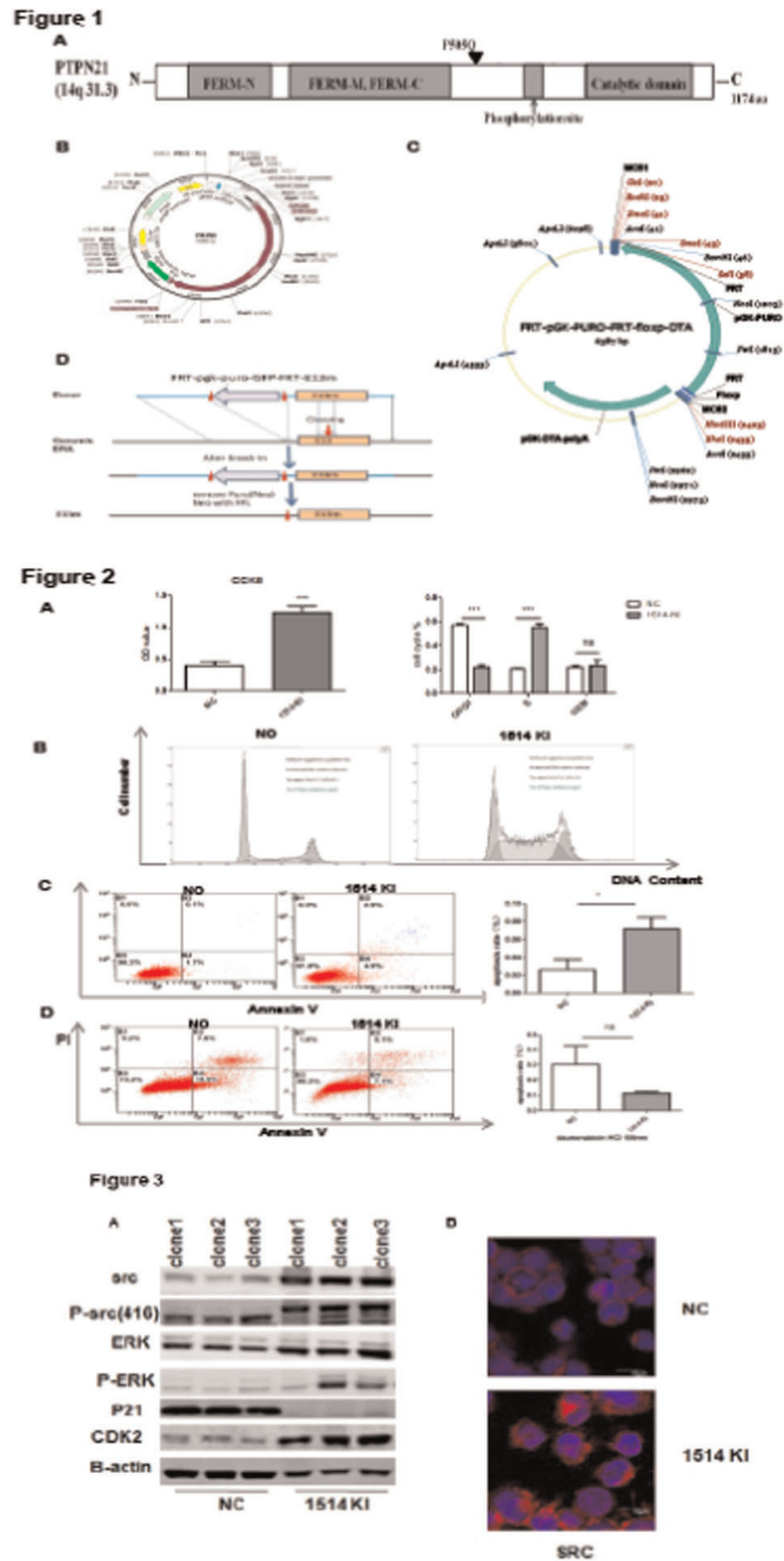

[P059 Figure] [Detection of biological activity in NALM6 cell line with PTPN21 mutation] 
and the progress of cell cycle in ALL. On the mechanism, PTPN21 mutation on above site may potentially activate downstream SRC-ERK signalling pathway and the P21CDK2 signalling pathway. which may be closely associated with the recurrence of ALL after allo-HSCT.

Conflict of interest: None of the authors has anything to disclose.

Figure 1: A. The specific site of PTPN21 gene mutation (exon13: c. 1514 C>A : p. P505Q).B-D. Flow chart of PTPN21 mutation cell line construction. Figure 2: The comparision in proliferation, cell cycle, apoptosis between negative control and PTPN21 mutation groups. Figure 3. The signal pathway associated proteins of PTPN21 downstream were detected by Western Blot and Immunofluorescence.

\section{P060}

The role of high-dose cytarabine as consolidation therapy before allogeneic hematopoietic cell transplantation for acute myeloid leukaemia without core-binding factor in the first complete remission

Yoo Jin Lee ${ }^{1}$, Sang Kyun Sohn ${ }^{1}$, Joon Ho Moon ${ }^{1}$, Hyeoung-Joon Kim $^{2}$, Jae Sook Ahn', Seo-Yeon Ahn', Young Rok Do ${ }^{3}$, Ji Hyun Lee ${ }^{4}$

${ }^{1}$ Kyungpook National University Hospital, Daegu, Korea, Republic of; ${ }^{2}$ Chonnam National University Hwasun Hospital, Hwasun, Jeollanamdo, Korea, Republic of; ${ }^{3}$ Keimyung University Dongsan Medical Center, Daegu, Korea, Republic of; ${ }^{4}$ Dong-A Medical Center, Pusan, Korea, Republic of

Background: The optimal number of high-dose cytarabine (HDAraC) consolidation cycles before allogeneic hematopoietic cell transplantation (allo-HCT) for acute myeloid leukaemia (AML) is not fully standardised. This study evaluated the therapeutic value of the number of HDAraC consolidation cycles before transplantation for patients with AML in the first complete remission (CR).

Methods: We retrospectively reviewed the medical records of 241 patients who received allo-HCT for AML without the core-binding factor (CBF) between 1998 and 2014 in Korea. All the patients received induction chemotherapy consisting of idarubicin $\left(12 \mathrm{mg} / \mathrm{m}^{2} /\right.$ day on days $1-3)$ and cytarabine $\left(100 \mathrm{mg} / \mathrm{m}^{2}\right.$ every 12 hours on days 1-7). After 186 patients (77.2\%) achieved first CR; they were reclassified into three subgroups: the $\mathrm{C} 0$ ( 0 cycle of HDAraC), C1 (1 cycle), and C2 (2 cycles) groups, according to the number of HDAraC consolidation cycles before transplantation.

Results: The median age at diagnosis was 43 years (range, 15-67 years). Thirty-two patients (17.2\%) had highrisk cytogenetics and 154 (82.8\%) had intermediate-risk cytogenetics by ELN. Among 186 patients in first CR, 21
(11.1\%), $116(61.4 \%)$, and $52(27.5 \%)$ patients were in the $\mathrm{C} 0, \mathrm{C} 1$, and $\mathrm{C} 2$ groups, respectively. The cumulative HDAraC dose was $3 \mathrm{~g} / \mathrm{m}^{2}$ (range, 3-9 $\mathrm{g} / \mathrm{m}^{2}$ ) in the $\mathrm{C} 1$ and $12 \mathrm{~g} / \mathrm{m}^{2}\left(6-18 \mathrm{~g} / \mathrm{m}^{2}\right)$ in the $\mathrm{C} 2$ groups. HDAraC-related toxicity included febrile neutropenia ( $19.6 \%$ vs. $41.7 \%)$ and documented infection $(19.6 \%$ vs. $29.2 \%, \mathrm{C} 1$ vs. $\mathrm{C} 2, \mathrm{p}=$ $0.041)$. Ten patients $(5.4 \%)$ had a relapse before transplantation: $3(14.3 \%)$ in the $\mathrm{C} 0,3(2.6 \%)$ in the $\mathrm{C} 1$, and 4 (7.8\%) in the $\mathrm{C} 2$ groups ( $\mathrm{p}=0.064)$.

The median time from CR to allo-HCT was 126 days (range, 60-270 days), 118 days (range, 60-174) in C0, 113 days (range, 84-214) in $\mathrm{C} 1$, and 158 days (range, 75270 ) in C2. The pre-transplant status was CR1 in 176 $(94.6 \%)$, er CR2 in $4(2.2 \%)$, and relapse in $6(3.2 \%)$. Ninety-nine patients $(53.2 \%)$ received myeloablative conditioning regimens. The median dose of $\mathrm{CD} 34+$ cells was $3.00 \times 10^{6} / \mathrm{kg}$ (range, $0.46-20.60 \times 10^{6} / \mathrm{kg}$ ). Ninety-nine percent of the patients achieved neutrophil engraftment at a median time of 12 days (range, 9-30 days). The incidence rates of acute and chronic graft-versus-host disease (GVHD) were $48.9 \%$ and $41.9 \%$, respectively. The median follow-up duration was 1135 days (range, 140-6774 days). The 2-year relapse-free survival (RFS) was $47.2 \% \pm 11.7 \%$, $71.6 \% \pm 4.4 \%$, and $79.9 \% \pm 5.7 \%$ in the $\mathrm{C} 0, \mathrm{C} 1$, and $\mathrm{C} 2$ groups, respectively $(\mathrm{p}=0.032)$. The 2 -year overall survival and non-relapse mortality did not significantly differ according to the number of HDAraC consolidation cycles. The adverse cytogenetic risk group had a benefit from HDAraC consolidation (hazard ratio $[\mathrm{HR}]=0.467 ; 95 \%$ confidence interval $[\mathrm{CI}], 0.221-0.988 ; \mathrm{p}=0.015)$. In the multivariate analysis, the following factors were associated with RFS: ENL adverse risk group (HR, 2.407; 95\% CI, 1.372-5.930; $\mathrm{p}=0.002$ ), no HDAraC consolidation (HR,

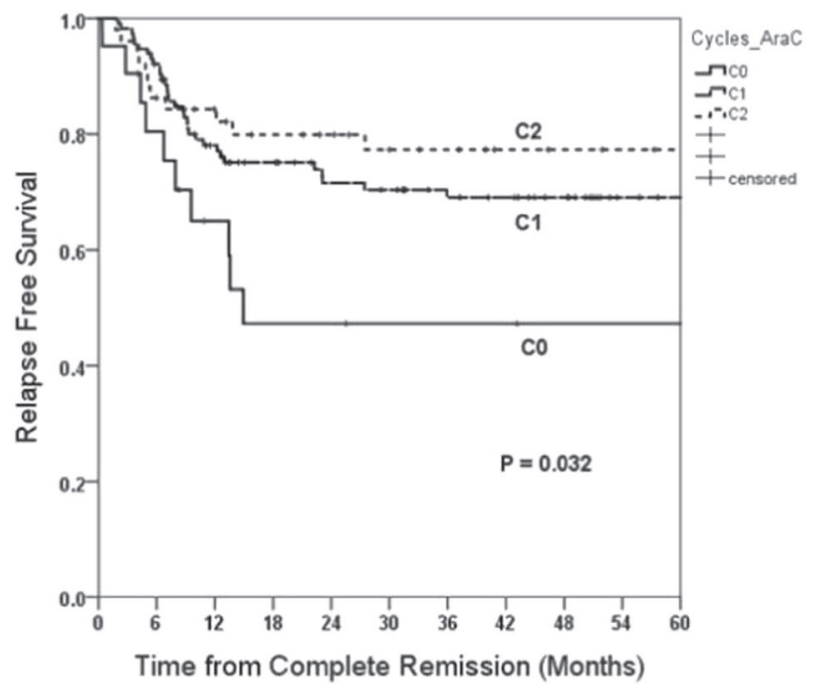

[P060 Figure] [Relapse-free survival according to the number of HDAraC consolidation cycles before transplantation] 
3.008; 95\% CI, 1.381-6.550; $\mathrm{p}=0.006$ ), and chronic GVHD (HR, 0.361; 95\% CI, 0.166-0.783; $\mathrm{p}=0.010$ ).

Conclusions: The use of HDAraC consolidation as a preHCT strategy had a positive role in AML without CBF. One or two cycle of HDAraC seemed to maximize the following HCT outcome in AML patients.

Conflict of interest: The authors have declared no conflicts of interest.

\section{P061}

Treosulfan, fludarabine and cytarabine (FLAT) myeloablative conditioning for autologous stem cell transplantation in elderly patients with acute myeloid leukemia in first complete remission

Massimo Bernardi, Francesca Pavesi, Carlo Messina, Francesca Lorentino, Jacopo Peccatori, Andrea Assanelli, Luca Vago, Bernhard Gentner, Raffaella Milani, Gabriele Casirati, Elisa Sala, Sara Mastaglio, Fabio Ciceri

San Raffaele Scientific Institute, Milano, Italy

Background: Consolidation of complete remission (CR) with autoHSCT is an option for patients (pts) with AML, in particular for elderly ones, as an alternative to alloHSCT. Up to now, no conditioning regimen for autoHSCT has proved preferable in terms of toxicities and improvement of leukemia free survival. Treosulfan is an alkylating agent which demonstrated to achieve maximum disease control with minimal toxicity in combination with fludarabine prior to alloHSCT in pts with high risk or relapsed/refractory AML. We designed a new conditioning regimen including treosulfan combined with fludarabine and cytarabine (FLAT) and tested prior to autoHSCT in AML pts. Data on feasibility and outcome are here reported.

Methods: Period 7/2006-10/2017, 27 pts with de novo AML, median age 67 (18-76). Cytogenetics: favourable 1, normal 22, intermediate 1 , complex 1 , not evaluable 2 . Molecular (18 pts evaluated): CBFB-MYH11 1, NPM1mut or CEBPAmut alone 8, FLT3ITD 5, negative 4. Prognostic risk (ELN 2017): favourable 8, intermediate 7, adverse 3, not evaluable 9. All pts were in CR1 after a median number of 2 chemo cycles (2-4) before autoHSCT. FLAT regimen: treosulfan $10 \mathrm{gr} / \mathrm{sqm}$ for 3 days, fludarabine $30 \mathrm{mg} / \mathrm{sqm}$ for 5 days, cytarabine $2 \mathrm{gr} / \mathrm{sqm}$ for 5 days, PEG filgrastim 1 s.c. vial after autoHSCT. Graft: PBSC, median CD34+ 6x106\% $\mathrm{kg}$ of BW (3.8-8.5). Time from CR1 to autoHSCT: median 92 days (44-135).

Results: 26 pts were alive in CR1 at day $+30,1$ patient died, in G4 pancytopenia, at day +31 because of invasive fungal infection (IFI). Median time to hematopoietic recovery: neutrophils $(>500)$ day +11 (10-38), platelets $(>20.000)$ day +21 (13-103). Extra-hematologic toxicities

\section{EFS from autoSCT}

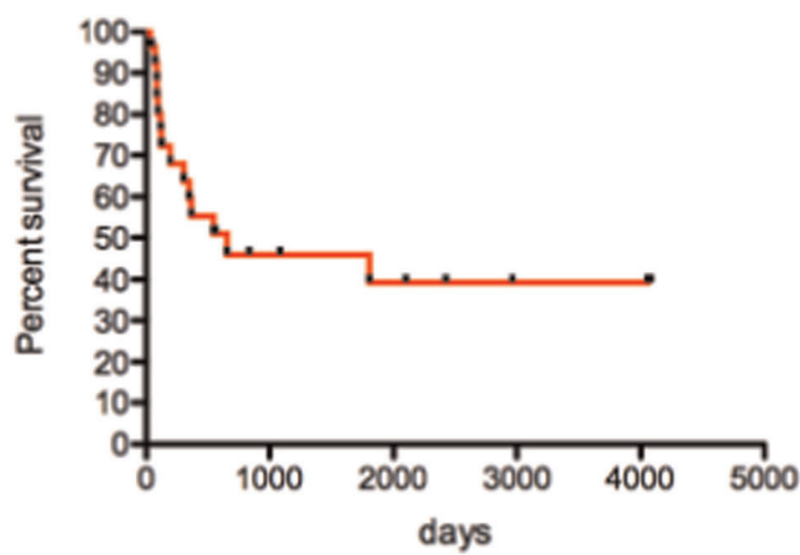

[P061 Figure] [EFS]

(CTCAE v4.0): median grade $2(0-4)$, see also table 1 . At last follow up (FU) 13 (48\%) pts are alive in CR1, with a median FU from autoHSCT of 641 days (33-4083). Thirteen pts have relapsed at a median of 547 days (311808) after transplant. Median EFS from autoHSCT is 368 days (33-4083), median OS from diagnosis is 1004 days (165-4258). Fourteen pts were $>65$ yrs old (10 older than $70 \mathrm{yrs}$ ): 5 (36\%) are alive in CR1, with a median FU from autoSCT of 454 days (33-4064). Overall non-relapse mortality was $4 \%$.

Conclusions: Consolidation of CR1 in AML pts with autoHSCT after conditioning with the new myeloablative FLAT regimen is feasibile and well tolerated. Prolonged OS from diagnosis and EFS from transplant have been obtained, also in elderly pts. A phase II study in pts older than 65 is ongoing in our Center to confirm these preliminary data.

Conflict of interest: None of the authors has anything to disclose.

\begin{tabular}{llll}
\hline & All pts & Pts $\leq 65$ yrs & Pts $>65$ yrs \\
\hline All pts & 27 & 12 & 15 \\
FUO $\left(\mathrm{N}^{\circ}\right)$ & $7(26 \%)$ & $4(33 \%)$ & $3(20 \%)$ \\
Sepsis $\left(\mathrm{N}^{\circ}\right)$ & 11 & $4(33 \%)$ & $7(47 \%)$ \\
& $(41 \%)$ & & \\
Gram negative/positive/molds & $3 / 7 / 1$ & $1 / 3 / 0$ & $2 / 4 / 1$ \\
Extra-Hematologic toxicities $\left(\mathrm{N}^{\circ}\right)$ & 15 & $7(58 \%)$ & $8(53 \%)$ \\
& $(55 \%)$ & & \\
hepatic/cardiac/neurologic/other & $5 / 5 / 1 / 4$ & $2 / 2 / 0 / 3$ & $3 / 3 / 1 / 1$ \\
$\begin{array}{l}\text { Neutrophils }<500\left(\mathrm{~N}^{\circ} \text { days, median/ }\right. \\
\text { range) }\end{array}$ & $11 / 9-38$ & $11 / 10-38$ & $10 / 9-34$ \\
$\begin{array}{l}\text { Platelets }<20.000\left(\mathrm{~N}^{\circ} \text { days, median/ }\right. \\
\text { range })\end{array}$ & $21 / 9-103$ & $24 / 9-100$ & $16 / 12-103$ \\
\hline
\end{tabular}

[[P061 Table] Table 1] 


\section{P062}

Umbilical Cord Blood Transplantation for Secondary AML: A Retrospective Study from the Acute Leukemia Working Party of the EBMT and Eurocord

\author{
Frederic Baron ${ }^{1}$, Myriam Labopin ${ }^{2}$, Annalisa Ruggeri ${ }^{3}$, Mohamad \\ Mohty $^{4}$, Didier Blaise ${ }^{5}$, Patrice Chevallier ${ }^{6}$, Jaime Sanz, Nathalie \\ Fegueux $^{8}$, Jan Cornelissen ${ }^{9}$, Alessandro Rambaldi ${ }^{10}$, Bipin \\ Savani $^{11}$, Eliane Gluckman ${ }^{3}$, Arnon Nagler ${ }^{12}$ \\ ${ }^{1}$ University of Liege, Liege, Belgium; ${ }^{2}$ EBMT Paris Office, CEREST- \\ TC, Paris, France; ${ }^{3}$ Eurocord, Hopital Saint Louis, Paris, France; \\ ${ }^{4}$ Hopital Saint Antoine, Universite Pierre \& Marie Curie, INSERM, \\ UMRs 938, Paris, France; ${ }^{5}$ Institut Paoli Calmettes, Marseille, \\ France; ${ }^{6} \mathrm{CHU}$ of Nantes, Nantes, France; ${ }^{7}$ Hospital Universitario la \\ $\mathrm{Fe}$, Valencia, Spain; ${ }^{8} \mathrm{CHU}$ Lapeyronie, Montpellier, France; \\ ${ }^{9}$ Erasmus Medical Center Rotterdam, Rotterdam, Netherlands; \\ ${ }^{10}$ Azienda Ospedaliera Papa Giovanni XXIII, Bergamo, Italy; ${ }^{11}$ bipin. \\ savani@Vanderbilt.Edu, Nashville, TN, United States; ${ }^{12}$ The Chaim \\ Sheba Medical Center, Tel-Hashomer, Israel
}

Background: Allogeneic hematopoietic stem cell transplantation is the only curative option for most patients with secondary acute myeloid leukemia (sAML). Umbilical cord blood transplantation (UCBT) is a treatment option for patients (pts) with primary AML who lack an HLA-matched donor. No systematic large analysis has evaluated risk factors and transplantation outcomes of UCBT for sAML. Therefore, the Acute Leukemia Working Party of the EBMT and Eurocord performed a retrospective registry study on sAML pts given UCBT.

Methods: Inclusion criteria included age $>$ or $=$ to 18 years, secondary AML, first UCBT between 2002-2016, first complete remission (CR1) or active disease at transplantation, and no ex vivo manipulation of the UCB.

Results: 146 pts (58\% female) met the study inclusion criteria. Previous diagnoses included myelodysplastic syndrome/myeloproliferative disorder $(\mathrm{n}=97)$, solid tumor $(\mathrm{n}=29$, including 23 breast cancer pts), lymphoma $(\mathrm{n}=$ 13), acute leukemia $(\mathrm{n}=5)$, chronic lymphocytic leukemia $(\mathrm{n}=1)$ and multiple myeloma $(\mathrm{n}=1)$. Status at transplantation was CR1 in 97 pts, primary refractory in 30 pts and relapsed sAML in the remaining 19 pts. The proportion of pts with good-, intermediate- and high-risk cytogenetics was $3 \%, 38 \%$, and $17 \%$, respectively (missing $42 \%$ ). Fiftyeight pts were given a single CBT (sCBT) and the remaining 88 pts a double $\mathrm{CBT}$ (dCBT). Conditioning was myeloablative (MAC) in $51(35 \%)$ pts and reducedintensity (RIC) in the remaining 95 (65\%) pts. Forty percent of the pts received ATG. One hundred and eighteen pts (83\%) achieved neutrophil engraftment while $25(17 \%)$ pts failed to engraft (including 13\% of patients transplanted in CR1 versus $30 \%$ of patients transplanted with active disease $(\mathrm{P}=0.008)$. The 100-day cumulative incidences of grade II-IV and grade III-IV acute GVHD were 35\% and 19\%, respectively. Two- year cumulative incidences of chronic and extensive chronic GVHD were $25 \%$ and $12 \%$, respectively. Two-year incidences of relapse and of nonrelapse mortality were $25 \%$ and $35 \%$, respectively, in pts in $\mathrm{CR} 1$ versus $36 \%(\mathrm{P}=0.06)$ and $49 \%(\mathrm{P}=0.03)$ in pts with active disease at transplantation. Two-year OS, LFS and GVHD-free relapse free survival (GRFS) were 42\%, 40\% and $26 \%$, respectively, in pts in $\mathrm{CR} 1$ versus $19 \%(\mathrm{P}<$ $0.001), 16 \%(\mathrm{P}<0.001)$ and $12 \%(\mathrm{P}=0.001)$, respectively, in pts with active disease at transplantation. Factors associated with OS in multivariate analyses included better survival in female recipients $(\mathrm{HR}=0.55, \mathrm{P}=0.004)$ and worse survival in pts with active disease at transplantation $(\mathrm{HR}=2.4, \mathrm{P}<0.001)$.

Conclusions: This is the first analysis of CBT in rather large group of pts with sAML .Our results suggest that CBT may rescue about $40 \%$ of patients with secondary AML in CR1 in the absence of an HLA-identical sibling donor. Future attempts should focus on reducing NRM and improving engraftment. Post-transplant strategies aiming in reducing relapse rates in these very high risk group of patients are also warranted.

Clinical Trial Registry: NA

Conflict of interest: No conflicts of interest

\section{P063}

Veno-Occlusive Disease (VOD) Characteristics in Pediatric Patients With Acute Myeloid Leukemia (AML) Receiving Gemtuzumab Ozogamicin (GO) Before Allogeneic Stem Cell Transplant (SCT)

Christine Duncan ${ }^{1}$, Andrew St. Martin ${ }^{2}$, Waleska Perez ${ }^{2}$, Patricia Steinert ${ }^{2}$, Mei-Jie Zhang ${ }^{2}$, Deborah Chirnomas ${ }^{3}$, Caroline J. Hoang ${ }^{3}$, Fausto R. Loberiza, $\mathrm{Jr}^{3}$, Wael Saber ${ }^{2}$

${ }^{1}$ Dana-Farber Cancer Institute, Boston, MA, United States; ${ }^{2}$ Center for International Blood \& Marrow Transplant Research, Milwaukee, WI, United States; ${ }^{3}$ Pfizer Inc, New York, NY, United States

Background: VOD has been reported in AML patients receiving $\mathrm{GO}$, an anti-CD33 antibody-drug conjugate. This analysis characterized VOD risk in pediatric patients receiving $\mathrm{GO}$ before $\mathrm{SCT}$.

Methods: Data were from a subset of patients randomly selected for research level reporting to the Center for International Blood \& Marrow Transplant Research (CIBMTR). Comparisons of the incidence of VOD at 100 days and overall survival were assessed using logistic regression and Cox regression models, respectively. A stepwise model building approach was used, with variables attaining $P \leq 0.05$ retained in the final model.

Results: This analysis included 152 pediatric patients who previously received GO and 348 control patients without GO 
exposure who underwent first allogeneic SCT between 20082011. Median (range) age was $8(<1-18)$ years in patients with and without GO exposure. Most patients with/without GO exposure had achieved first complete remission (with, 43\%; without, $57 \%$ ) or $\geq$ second complete remission (with, $38 \%$; without, $27 \%$ ). Donor source was balanced between patients with/without GO exposure. Overall incidence of VOD and severe VOD at 100 days was higher in patients with (16\% and $8 \%$, respectively) than without GO exposure (10\% and $3 \%$ ). Overall survival probabilities were generally comparable in patients with and without GO exposure at 100 days ( $84 \%$ vs $87 \%$ ), 6 months ( $78 \%$ vs $80 \%$ ), and 1 year (69\% vs 69\%) from SCT and at 100 days (64\% vs 68\%), 6 months (54\% vs 62\%), and 1 year (39\% vs 54\%) from VOD onset. Based on multivariate analyses, GO exposure was associated with a significant risk of VOD (OR, 2.15; 95\% CI, 1.25-3.70; $P=0.01)$ but not death $(\mathrm{HR}, 1.13 ; 95 \% \mathrm{CI}$, $0.86-1.49 ; P=0.39$ ).

Conclusions: In pediatric AML patients, GO exposure before SCT was associated with a higher incidence of VOD. However, GO exposure was not associated with an increased risk of death. Limitations of the study include selection bias associated with the retrospective design. Future analyses will evaluate the impact of dose on VOD risk and survival outcomes in this cohort.

Encore of BMT Tandem 2018. Funding: Pfizer. Data from the Coordinating Center of the CIBMTR are preliminary.

\section{Conflict of interest:}

C. Duncan, A. St. Martin, W. Perez, P. Steinert, M. Zhang, W. Saber: nothing to disclose

D. Chirnomas, C. Hoang, F. Loberiza: employees of and own stocks in Pfizer Inc.

\section{P064}

\section{With cGVHD but without intervention therapy had better survival in salvage allo-HSCT for AML in NR status}

Yue Lu, Yan LI Zhao, Xing Yu Cao, De Yan Liu, Ming Xiong, Jia Rui Zhou, Rui Juan Sun, Zhi Jie Wei, Jian Ping Zhang, Dao Pei Lu

\section{Ludaopei Hospital, Langfang, China}

Background: Salvage allo-HSCT appears to be the only possible cure way for refractory or relapsed AMLin NR status. However, leukemia relapse remains a major cause of transplant failure . the outcome still disappointed .In order to improved the outcome, we optimization our transplantation protocol, now we reported the results.

Methods: we retrospectively analyzed 136 patients with refractory or relapsed AML who underwent allo-HSCT from April 2012 to April 2015. The median percent of blasts was $25(5-90) \%, 55$ cases of them $\geq 5 \% \sim 20 \%, 47$ cases $\geq 20 \% \sim<50 \%, 22$ cases $\geq 50 \% \sim<80 \%, 12$ cases $\geq 80 \%$. Male to female $85 / 51$.the median age were 27 (2-60) years. 57 of them are primary refractory and 79 failured in remission after relapsed. 23 patients received MSD-HSCT, 14 received MUD-HSCT and 99 received Haplo-HSCT.the conditioning regimen based with busulfan $(3.2 \mathrm{mg} / \mathrm{kg}$ per day) for 4 days/ Cy $\left(1.8 \mathrm{~g} / \mathrm{m}^{2}\right.$ per day) for 2 days/ and ATG,19 of them combinated with HD-Ara-c $\left(2 \sim 3 \mathrm{~g} / \mathrm{m}^{2}\right.$ per day) for 3 days; 7 combinated with IDA $\left(10 \sim 12 \mathrm{mg} / \mathrm{m}^{2}\right.$ per days) and Ara-c $\left(3 \mathrm{~g} / \mathrm{m}^{2} \mathrm{q} 12 \mathrm{~h}\right.$ per days) for 3 days respectively; 22 cases combinated with Decitabine $(20 \mathrm{mg} /$ $\mathrm{m}^{2}$ per days and Ara-c $\left(2 \mathrm{~g} / \mathrm{m}^{2}\right.$ per days) for 5 days recpectively \pm IDA $\left(10 \mathrm{mg} / \mathrm{m}^{2}\right.$ per day)for 3 days; 77 cased combinated with Fludarabine $\left(30 \mathrm{mg} / \mathrm{m}^{2}\right.$ per days) and Ara-c $\left(2 \mathrm{~g} / \mathrm{m}^{2}\right.$ per days) for 5 days recpectively \pm IDA $\left(10 \mathrm{mg} / \mathrm{m}^{2}\right.$ per day) for 3 days; 11 cases combinated with Amsacrine $\left(100 \mathrm{mg} / \mathrm{m}^{2}\right.$ per day) and Ara-c $\left(2 \mathrm{~g} / \mathrm{m}^{2}\right.$ per day) for 4 days.136 of 29 patients received pre-emptive intervention,15 patients received MRD-directed intervention; 32 patients received chemotherapy and immunotherapy, while other 60 patients without intervention therapy . intervention therapy including donor lymphocyte infused and donor lymphocyte natural kill cell or cytotoxicity lymphocyte cell infused.

Results: The median follow up time were 24 (3-57) months; the 3-years-OS,DFS, Relapsed related mortality, TRM were $44.7 \pm 4.4 \%, 43.1 \pm 4.4 \%, 44.4 \pm 4.7 \%$ and 16.3 $\pm 3.8 \%$ respectively.Univariate analysis of factors with survival showed: lower percent of blasts pre-HSCT has better OS $[\geq 5 \% \sim 20 \%(55.9 \pm 6.8 \%)$ vs $\geq 20 \% \sim<50 \%(43.9$ $\pm 7.8 \%)$ vs $\geq 50 \% \sim<80 \%(31.8 \pm 6.8 \%)$ vs $\geq 80 \%(25.0 \pm 4.3 \%)$, $\mathrm{P}=0.038$ ), conditioning regimen including IDA has better OS compared with other [(HD-Ara-c $(26.3 \pm 10.1 \%)$ vs IDA/ Ara-c $(85.7 \pm 13.2 \%)$ vs Dec/Ara-c \pm IDA $(59.1 \pm 10.5 \%)$ vs FLAG \pm IDA $(50.0 \pm 5.8 \%)$ vs Amsa/Ara-c $(18.2 \pm 4.2 \%), \mathrm{P}=$ $0.026)$ ], post-HSCT without intervention therapy had best OS [(pre-emptive intervention vs MRD-directed intervention vs intervention after relapsed vs without intervention, $\mathrm{P}$ $=0.000)$ ], with II IV aGVHD has lower OS compared to $0 \sim \mathrm{IaGVHD}(34.2 \pm 7.2 \%$ vs $53.6 \pm 5.4 \%, \mathrm{P}=0.034)$, with cGVHD has better OS $(53.6 \pm 5.6 \%$ vs $18.2 \pm 4.2 \%, \mathrm{P}=$ 0.000 ), but there were no significant difference with age ( $\leq 14 \mathrm{y}$ vs $>14 \mathrm{y}, \mathrm{P}=0.752$ ), sex (male vs female, $\mathrm{P}=0.981$ ), HCT-CI ( $0-1$ vs $\geq 2, \mathrm{P}=0.549)$, type of disease (Primary vs Secondary, $\mathrm{P}=0.564$ ), with extramedullary lesions (Yes vs No, $\mathrm{P}=0.444$ ), Donor-patient sex match (male - male vs male - female vs female - female vs female- male, $\mathrm{P}=$ $0.823), \mathrm{MNC}(\times 108 / \mathrm{kg})(<8.76$ vs $\geq 8.76, \mathrm{P}=0.229), \mathrm{CD} 34$ cell counts $(\times 106 / \mathrm{kg})(<4$ vs $\geq 4, \mathrm{P}=0.512), \mathrm{CD} 3$ cell counts $(\times 108 / \mathrm{kg})(<1.67$ vs $\geq 1.67, \mathrm{P}=0.748)$, Donor type (MSD vs MUD vs Haplo-D,P $=0.313$ ). Multivariate analysis factor showed: post-HSCT without intervention 
has higher OS and DFS, with cGVHD not only has higher OS and DFS, but also had lower incidence of relapsed mortality.to analysed the impacted of with cGVHD on the patients who post-HSCT without intervention showed,the patients with cGVHD who has higher OS $(86.8 \pm 5.5 \%$ vs $59.1 \pm 10.5 \%, \mathrm{P}=0.01)$, but the patients with II IVaGVHD has higher TRM $(\mathrm{P}=0.007)$ and lower OS $(\mathrm{P}=0.002)$.

Conclusions: the patients With cGVHD but without intervention therapy had better survival in salvage alloHSCT for AML in NR status.

Conflict of interest: nothing to disclose

\section{P065}

WT1-specific T-cells in healthy donors and leukemia patients and the possibility of improved detection of the immune response using WT1 peptide-pulsed dendritic cells

Julia Panchenko ${ }^{1,2}$, Victoriia Kiseleva ${ }^{1}$, Vladimir Zhogov ${ }^{1}$, Natalya Khripkova ${ }^{1}$, Pavel Trakhtman ${ }^{1}$, Nikolay Starostin ${ }^{1}$, Michael Maschan $^{1}$, Elena Osipova ${ }^{1}$

${ }^{1}$ Dmitry Rogachev National Center of Pediatric Hematology, Oncology and Immunology, Moscow, Russian Federation; ${ }^{2}$ Moscow Institute of Physics and Technology, Moscow, Russian Federation

Background: WT1 antigen is a promising target for cancer immunotherapy because it has a high immunogenicity and is hyperexpressed in various types of leukemia and solid tumors. The complexity of determining WT1-specific T-lymphocytes is associated with a low frequency of these cells in the peripheral blood. In this regard, the study of the possibility of improved detection of the immune response against WT1 antigen may assist in development of immunotherapeutic approach to cancer.

Methods: The frequency of WT1-specific $\mathrm{T}$ cells in peripheral blood of healthy donors $(n=68)$ and leukemia patients (AML, $\mathrm{n}=4$, ALL, $\mathrm{n}=6$ ) after allogenic stem cells transplantation were monitored by dextramer staining and enzyme-linked immunospot (ELISPOT) assays with the WT1-overlapping peptide pool. Samples with more than 5 specific lymphocytes per 500000 mononuclear cells considered to be positive.

Monocyte-derived dendritic cells (DC) were generated from mononuclear (MNC) adherent cells from healthy donors $(n=10)$ in the presence of IL-4 and granulocytemacrophage colony-stimulating factor. On day 7 , immature DC were matured with a cytokine cocktail consisting of IL4, granulocyte-macrophage colony-stimulating factor, tumor necrosis factor (TNF) $\alpha$, harvested after $48 \mathrm{~h}$, pulsed with WT1 peptide pool and washed from peptide. Control DC were generated using the same method without incubation with WT1. The mature phenotype of generated DC were determined by Flow Cytometry with HLA-DR,
CD83, HLA-ABC, CD86 antibodies. Donor MNC were cultivated alone or with autologous DC (WT1 specific and control) to analyze the number of WT1 specific Tlymphocytes by ELISPOT-assay.

Results: WT1 specific T-lymphocytes above $0,001 \%$ from mononuclear cells were detected in 22 of $68(32,4 \%)$ donors. The frequency of anti-WT1 lymphocytes in healthy donors ranged from 6 to 328 per $500000 \mathrm{MNC}$ (median $16,5)$. In $6 \%$ of donors WT1 specific lymphocytes were detected in more than $0,01 \%$ of MNC. Specific anti-WT1 Tcells were also detected in periferical blood of 2 of 6 patients with ALL (12 and 20 WT1 specific lymphocytes per $500000 \mathrm{MNC}$ ), and 2 of 4 of AML (8 and 45 WT1 specific lymphocytes per $500000 \mathrm{MNC}$ ) patients after allo HSCT.

After 24-hour incubation of MNC with WT1-pulsed dendritic cells (10MNC: 1DC) the number of anti-WT1 Tlymphocytes (as detected by IFN gamma ELISPOT) increased by 2.8 to 20.3 times. Control cultures with DC only (pulsed or not pulsed) were negative in ELISPOTassay. Such a large range of values reflects the heterogeneity of the population of donors in relation to the immune response to the WT1 antigen, which emphasizes the importance of personalized approach when planning immunotherapy of tumors. Using cultural inserts (Carrier Plate System, Nunc) for 12-well plate we have demonstrated that WT1-specific DC influence the T-lymphocytes reactivity to antigen by direct contact.

Conclusions: The incubation of MNC with WT1-specific DC leads to a significant increase of T-cell immune response against the WT1 antigen. These results can be used to develop new approaches of dendritic cell vaccination against WT1 antigen.

Conflict of interest: Nothing to disclose

Aplastic anaemia

\section{P066}

Allogeneic bone marrow transplantation for children and adolescents with severe aplastic anemia in Brazil: A multicenter study on behalf of the Brazil-Seattle Consortium Study Group

Luiz Darrigo $\mathrm{Jr}^{1}$, Vergilio Coulturato ${ }^{2}$, Mair Souza ${ }^{2}$, Ederson Mattos $^{2}$, Gisele Loth ${ }^{3}$, Rodolfo Calixto ${ }^{4}$, Adriana Seber ${ }^{5}$, Victor Zecchin $^{6}$, Liane Daudt ${ }^{7}$, Alessandra Paz ${ }^{7}$, Rita Barbosa Tavares ${ }^{8}$, Leonardo Arcuri ${ }^{8}$, Antonio Macedo ${ }^{9}$, Ana Karine Vieira ${ }^{9}$, Cilmara Kuwahara $^{10}$, Roseane Gouveia ${ }^{5}$, Lisandro Ribeiro ${ }^{11}$, Juliana Fernandes $^{12}$, Mary Flowers ${ }^{13}$, Ricardo Pasquini ${ }^{3}$, Carmem Bonfim ${ }^{3}$

${ }^{1}$ Ribeirão Preto Medical School, University of São Paulo, Ribeirão Preto, Brazil; ${ }^{2}$ Hospital Amaral de Carvalho, Jahu, Brazil;

${ }^{3}$ Universidade Federal do Paraná, Curitiba, Brazil; ${ }^{4}$ Real Hospital Português de Beneficência, Recife, Brazil; ${ }^{5}$ Hospital Samaritano de São Paulo, São Paulo, Brazil; ${ }^{6}$ Graacc, São Paulo, Brazil; ${ }^{7}$ Hospital 
das Clínicas de Porto Alegre, Porto Alegre, Brazil; ${ }^{8}$ INCA, Rio de Janeiro, Brazil; ${ }^{9}$ Universidade Federal de Minas Gerais, Belo Horizonte, Brazil; ${ }^{10}$ Hospital Pequeno Príncipe, Curitiba, Brazil; ${ }^{11}$ Hospital Nossa Senhora das Graças, Curitiba, Brazil; ${ }^{12}$ ITACI, São Paulo, Brazil; ${ }^{13}$ Fred Hutchinson Cancer Research Center, Seattle, WA, United States

Background: Idiopathic severe aplastic anemia (SAA) is a rare life-threatening disease and bone marrow transplantation (BMT) from a matched sibling donor is the treatment of choice for pediatric patients. The aim of this study is to determine major transplants outcomes after BMT for children with SAA transplanted in Brazil.

Methods: This is a retrospective study that analyzed 115 consecutive patients under the age of 18 years who underwent a first allogeneic BMT for SAA between 2010 and 2014 in 12 Brazilian BMT centers. The median age was 10 years and $69 \%$ were male. Graft was an unmanipulated marrow from a matched related (MRD; sibling $\mathrm{n}=66$ and other relative $\mathrm{n}=3$ ) or unrelated (URD; HLA-matched $\mathrm{n}=$ 37 , and mismatched $n=9$ ) donor. Conditioning regimen widely varied among institutions. In MRD transplants, 44\% received Cyclophosphamide \pm r-ATG, 33\% Busulfanbased, and $20 \%$ Fludarabine-based regimen. Most URD BMT (63\%) were Fludarabine-based and 34\% Busulfanbased. In vivo T-cell depletion included r-ATG $(\mathrm{n}=31)$ or Campath $(\mathrm{n}=4)$ in $50 \%$ of the transplantswith MRD and all but one from URD. Calcineurin inhibitor and methotrexate were used in $89 \%$ of BMT. Acute and chronic graft-versushost disease (GVHD) were graded using Glucksberg and NIH scales, respectively.

Results: With a median follow-up of 4.5 years, 87 of 115 patients are alive. The 4-year overall survival (OS) was $77 \%$ (95\% CI: 70-86\%), not significantly different from MRD $(81 \%)$ or URD $(71 \% ; p=0.11)$. Seven patients died before Day +21 and were not evaluable for engraftment. No MRD had primary graft failure (GF), in contrast with 7/46 with URD (cumulative incidence 16\%; 95\% CI 4-26\%). Out of the 101 patients who engrafted, 11 had graft rejection, after a median of 228 days (cumulative incidence: $11 \%$ (95\% CI: 5-17\%), not significantly different between donor types. Thirteen patients had a 2nd BMT and 7 were alive at last follow-up. The cumulative incidence of grades III-IV acute GVHD was 7\% (95\% CI:2-12\%) and of chronic GVHD at 4 years was $17 \%$ (95\% CI:10-24\%), with no significant difference between donor types. Twenty-seven patients died after a median of 2.9 months after BMT. Major causes of death included infections $(\mathrm{n}=16)$, bleeding $(\mathrm{n}=3)$, secondary neoplasia $(n=2)$, and GVHD $(n=2)$. The 6month transplant related mortality was higher after URD than MRD (OR=2.4, 95\%CI: 0.9-6.4) although it did not reach significance $(\mathrm{p}=0.09)$.
Conclusions: The OS of pediatric patients transplanted for SAA in Brazil is 77\% (95\% CI: 70-86\%), similar among MRD or URD, but lower than expected in other international reports. Our group is working to standardize a national pediatric BMT protocol to improve results of allogeneic BMT for pediatric SAA in Brazil.

Conflict of interest: There is no conflict of interest to report.

\section{P067}

\section{ALLOGENEIC HEMATOPOEITIC STEM CELL TRANSPLANTATION (HSCT) AFTER FLUDARABINE BASED CONDITIONING IN 61 PATIENTS WITH FANCONI ANEMIA}

Sara Zerkout, Malek Benakli, Nadia Rahmoune, Hanane Bouarab, Dina Ait Ouali, Mounira Baazizi, Farih Mehdid, Rachida Belhadj, Chahrazed Benchouk, Kahina Tarhi, Rose-Marie Hamladji, Redhouane Ahmed Nacer

Hematology and Bone Marrow Transplantation Department, Algiers, Algeria

Background: Fanconi anemia (FA) is the most common constitutional aplasia with haematological and extrahaematological abnormalities, and high risk of transformation into myelodysplasia (MDS) or acute leukemia (AL). The only curative treatment is HSCT. We report the results of allogeneic HSCT in 61 patients (pts) with FA performed during a period of 15 years.

Methods: From January 2002 to December 2016, 61 pts with FA underwent allogeneic HSCT including 55 sibling HLA identical, 05 phenoidentical and one from an unrelated cord blood. The median age is 11 years ( 4.5 to 25$)$ of whom $34(55 \%)$ have less than 10 years. The sex ratio $(\mathrm{M} / \mathrm{F})$ is 1.1. The average diagnosis-transplant time is 26 months (4 to 102$)$. Fourty-eight pts $(78.7 \%)$ had a transfusion history of which $06(9.8 \%)$ had more than 20 red blood units transfusions. Thirteen $(22.4 \%)$ pts received previous treatment with androgens, corticosteroids or a combination of both. Two types of conditioning regimen were used successively in time: fludarabine-cyclophosphamideantithymocyte globulin (FLU-CY-ATG: 44 pts) and fludarabine-cyclophosphamide (FLU-CY: 17 pts). GVHD prophylaxis included association of ciclosporin and methotrexate.

The grafts used were peripheral blood stem cells in 56 pts (91,8\%) with an average CD34 + cell count: 9.42 106/kg (2.06-36.31), bone marrow transplant in 4 pts with an average level of nucleated cells $3.67 \times 108 / \mathrm{kg}(1.83-4.96)$ and cord blood with CD34 + cells: $1.33 \times 105 / \mathrm{kg}$ and NC: $7.73 \times 107 / \mathrm{kg}$. At July 2017 , the minimal follow up is 7 months and maximal is 187 months. 
Results: The median time to graft $\left(\mathrm{PNC}>0.5 \times 10^{9} / \mathrm{l}\right)$ was 14 days (7-36). One patient had severe veino-occlusive disease (VOD). Acute GVHD was observed in 9 pts (14.7\%), of which 03 (4.9\%) grade III-IV. Chronic GVHD in 13 pts $(25.5 \%)$, including 06 of extensive form. CMV reactivation is noted in 9 pts $(16 \%)$. Six cases $(10,9 \%)$ of rejection were observed of which 2 are alive, one after a boost and the other after a second allograft. After a median follow-up of 98 months (7-186), 45 pts (74\%) are alive with a strictly normal blood count and 16 pts (26\%) died, of which $11(18 \%)$ due to TRM (infection 5, GVHA 2, capillary leak syndrome 1 , hemorrhage 2 , VOD 1). The 1year TRM according to the type of conditioning is estimated at $21 \%$ and $11.3 \%$ respectively. One pt showed malignant transformation (acute leukemia at 5 months after rejection). After 15,5 years, Overall survival (OS) and event-free survival (EFS) are $72.5 \%$ and $62.8 \%$ respectively. The OS according to the type of conditioning at the same time (72 months) is 68,2\% (FLU-CY-ATG) and 87\% (FLU-CY) without significant difference $(0,2$ difference (p:10-5).

Conclusions: Allogeneic HSCT remains the only therapeutic alternative in FA. It permits to restore hematopoietic and to prevent progression to MDS or AL. The FLU-CY conditioning, adopted from 5 years in our center, is well tolerated and has given better results in terms of EFS and TRM.

Conflict of interest: nothing to disclose

\section{P068}

Effect of stem cells on Allogeneic Hematopoietic Stem Cell Transplantation in Adult Patients with Idiopathic Aplastic Anemia

Hawk Kim ${ }^{1}$, Kyoo-Hyung Lee ${ }^{2}$, Sang Kyun Sohn ${ }^{3}$, Seok Jin Kim ${ }^{4}$ Inho Kim ${ }^{5}$, Sung Hyun Kim ${ }^{6}$, Yong Park ${ }^{7}$, Jae Yong Kwak ${ }^{3}$, Min Kyoung Kim ${ }^{8}$, Sung Hwa Bae, Ho Jin Shin ${ }^{9}$, Jong Ho Won ${ }^{11}$, Won Sik Lee ${ }^{12}$, Yunsuk Choi ${ }^{13}$

${ }^{1}$ Gachon University Gil Medical Center, Gachon University College of Medicine, Division of Hematology, Incheon, Korea, Republic of; ${ }^{2}$ Asan Medical Center, University of Ulsan College of Medicine, Seoul, Korea, Republic of; ${ }^{3}$ Kyungpook National University Hospital, Daegu, Korea, Republic of; ${ }^{4}$ Samsung Medical Center, Sungkyunkwan University School of Medicine, Seoul, Korea, Republic of; ${ }^{5}$ Seoul National University Hospital, Seoul, Korea, Republic of; ${ }^{6}$ Dong-A University Medical Center, Busan, Korea, Republic of; ${ }^{7}$ Korea University Anam Hospital, Seoul, Korea, Republic of; ${ }^{8}$ Yeungnam University Medical Center, Daegu, Korea, Republic of; ${ }^{9}$ Daegu Catholic University Hospital, Daegu, Korea, Republic of; ${ }^{10}$ Pusan National University Hospital, Busan, Kosovo, Republic of; ${ }^{11}$ Soon Chun Hyang University Hospital, Seoul, Korea, Republic of; ${ }^{12}$ Inje University Busan Paik Hospital, Busan, Korea, Republic of; ${ }^{13}$ Ulsan University Hospital, University of Ulsan College of Medicine, Division of Hematology and Cellular Therapy, Ulsan, Korea, Republic of

Background: Many sAA patients receive hematopoietic stem cells from PBSC even though they receive stem cell from
MSD. We need to know which additional factors are affecting on transplantation outcomes when BM or PBSC is infused.

Methods: We retrospectively investigated the effect of stem cells on allogeneic hematopoietic cell transplantation (alloHCT) in idiopathic adult aplastic anemia (AA). Mismatched donors (MMD) included haplo-identical family donor, mismatched unrelated donor or partially matched family donor. Alternative donor (AD) referred either MUD or MMD.

Results: Total 267 patients were included in this analysis. $\mathrm{BM}$ was associated with low incidence of acute graft versus host disease (GvHD; $p<0.001$ ) but not in G3/4 acute GvHD $(\mathrm{p}=0.427)$. BM, however, had no impact on other transplantation outcomes including chronic GvHD $(\mathrm{p}=0.673)$ and primary/secondary graft failure $(\mathrm{p}=0.774)$. Higher stem cell dose had no impact on other transplantation outcomes except for low incidence of extensive chronic GvHD in MSD $(\mathrm{p}=0.025)$. Multivariate analysis on overall survival in MSD revealed that only Age at alloHSCT $<31$ years old $(\mathrm{p}=0.010)$ and prior platelet transfusion less than $86 \mathrm{U}(\mathrm{p}=0.046)$ were the favorable prognostic factors. On the other hand, multivariate analysis in $\mathrm{AD}$ revealed that higher stem cell dose $(\mathrm{HR}=2.596 ; 95 \%$ CI $1.020-6.609 ; \mathrm{p}=0.045)$ was the only significant favorable factors on overall.

Conclusions: PBSCs can be preferable in AD because higher stem cell dose can be easily achieved for longer overall survival in expense of acute GvHD. However, BM stem cells are preferred in MSD.

Conflict of interest: There is no conflict of interest to disclose

\section{P069}

Haploidentical hematopoietic stem cell transplantation with selection of CD34+ and CD45RA + cells depletion as novel approach for Severe Aplastic Anaemia in pediatric patients

Mercedes Gasior Kabat ${ }^{1}$, Laura Sissini ${ }^{2}$, David Bueno ${ }^{3}$, Raquel De Paz Arias $^{1}$, Isabel Badell, Montserrat Torrent ${ }^{5}$, Sergi Querol ${ }^{2}$, Sonsoles Sanroman ${ }^{1}$, Diego Plaza ${ }^{6}$, Antonio Marcos ${ }^{1}$, Ana Belen Romero $^{1}$, Ana Sastre ${ }^{6}$, Jaime Valentin ${ }^{7}$, Antonio Pérez-Martínez ${ }^{6}$

${ }^{1}$ Hospital Universitario La Paz, Hematology, Madrid, Spain; ${ }^{2}$ Hospital Universitario Sant Pau, Hematology, Barcelona, Spain; ${ }^{3}$ Hospital Universitario La Paz, Pediatric Hemato-oncology, Madrid, Spain; ${ }^{4}$ Hospital Universitario Sant Pau, Pediatric Onco-Hematology, Barcelona, Spain; ${ }^{5}$ Hospital Universitario Sant Pau, Pediatric Hemato-Oncology, Barcelona, Spain; ${ }^{6}$ Hospital Universitario La Paz, Pediatric Onco-Hematology, Madrid, Spain; ${ }^{7}$ Hospital Universitario La Paz, IDIPAZ, Madrid, Spain

Background: Haploidentical stem cell transplantation (HSCT) has been occasionally carried out in the setting of aplastic anaemia (AA) for patients lacking proper-matched 
donor. Haplo-HSCT in the paediatric setting requires T-cell depletion, which carries profound immunosuppression and high risk of graft failure. Naïve T-cells identified by CD45RA expression are believed to cause graft-versushost-disease (GvHD), while CD45RA- T-cells are memory cells that provide anti-infection and anti-tumoral effects. Depleting CD45RA + naïve cells and retaining memory Tcells in the graft is a novel approach to haploidentical HSCT for children with severe AA.

Methods: Three children with severe AA received CD45RA-depleted haplo-HSCT following nonmyeloablative conditioning. Cell-selection performed on G-CSF-mobilized peripheral blood. Two cellular products obtained using CliniMACS device. Patients (detailed in Table 1) have received 2 haploidentical cellular products. First product was result of a CD34-positive selection; second product was the result of CD45RA depletion.

Results: Three patients received haplo-HSCT from February to July 2017. Two of 3 patients were male. Median age at transplantation was 11 years (range 9-12). Graft composition detailed in Table 1. Neutrophil and platelet engraftment achieved at median of 10 days (range 9-11) and 12 days (range 10-13) post-transplant respectively. No graft failure was seen. Two patients developed aGvHD>grade II with gastrointestinal tract and skin involvement, all steroids responsive. One patient presented clinical features of probable cGvHD. All patients (55.5\%) remain alive with mean follow-up 129 (range 70-228) days post-transplant. Chimerism at day +30 was full donor. All patients presented cytomegalovirus reactivation, none progressed to CMV disease. No severe infectious complications seen.

Conclusions: CD45RA-depleted haplo-HCT showed a rapid and sustained neutrophil and platelet engraftment as well as a full donor chimerism. Acute GvHD was esteroidresponsive. No severe infections were seen. CD45RAdepleted haplo-HCT can be a novel approach for transplantation in patients with SAA lacking proper-matched family or unrelated donor.

\section{Conflict of interest: None}

\begin{tabular}{llll}
\hline Sex & $\begin{array}{l}\text { Patient } 1 \\
\text { Male }\end{array}$ & $\begin{array}{l}\text { Patient } 2 \\
\text { Female }\end{array}$ & $\begin{array}{l}\text { Patient 3 } \\
\text { Male }\end{array}$ \\
\hline $\begin{array}{l}\text { Age at transplantation (years) } \\
\text { Donor }\end{array}$ & 9 & 12 & 11 \\
Graf composition & Mother & Mother & $\begin{array}{l}\text { Brother } \\
\text { CD34+ (x106/Kg) }\end{array}$ \\
CD45RA $+/ \mathrm{Kg}$ & 6,26 & 7,73 & 6,41 \\
CD45RO $+/ \mathrm{Kg}$ & $1,09 \times 102$ & $0,5 \times 102$ & $1,8 \times 103$ \\
Log CD45RA+ depletion & $5 \times 107$ & $5 \times 107$ & $8,34 \times 106$ \\
CD20+/Kg & $>5$ & 4,69 & 3,83 \\
\hline & $9,3 \times 104$ & $3,3 \times 104$ & $1,40 \times 105$ \\
\hline
\end{tabular}

[[P069 Table] Table 1. Patient details and grafts composition]
P070

Hematopoietic Stem cell transplant in Aplastic anemia in patients older than $\mathbf{4 0}$ years: The experience of the Spanish Group of Hematopoietic Stem Cell Transplant (GETH)

Lucrecia Yañez ${ }^{1}$, Jaime Sanz ${ }^{2}$, Irene Garcia Cadenas ${ }^{3}$, Lourdes Vazquez $^{4}$, Ildefonso Espigado ${ }^{5}$, Isabel Sanchez Ortega ${ }^{6}, \mathrm{Mi} \mathrm{Kwon}^{7}$, Carlos Solano ${ }^{8}$

${ }^{1}$ Hospital Universitario Marqués de Valdecilla, Santander, Spain; ${ }^{2}$ Hospital Universitario la Fe, Valencia, Spain; ${ }^{3}$ Hospital Santa Creu I Sant Pau, Barcelona, Spain; ${ }^{4}$ Hospital Universitario de Salamanca, Salamanca, Spain; ${ }^{5}$ Hospital Universitario Virgen del Rocio, Sevilla, Spain; ${ }^{6}$ ICO - Hospital Duran i Reynals, Barcelona, Spain; ${ }^{7}$ Hospital Gregorio Marañón, Madrid, Spain; ${ }^{8}$ Hospital Clinico de Valencia, Hematology, Valencia, Spain

Background: Although survival after HLA identical bone marrow transplant or immunosuppressive therapy (IST) is similar in patients older than 40 years (55\% vs. $58 \%$ ), current guidelines recommend the latter for these group of patients (Bacigalupo, Blood 2017).

The aim of this retrospective study is to evaluate the role of hematopoietic stem cell transplant (SCT) in older patients with Aplastic anemia and to know if previous IST affects negatively SCT ouctomes.

Methods: Seven Spanish centers reported a total of 28 patients who underwent a SCT between 1998 and 2015. Patients with $2^{\text {nd }}$ transplant or further were excluded. Median age at diagnosis was 48 years (43-59) and 16 $(57.1 \%)$ were male. Seventeen patients had information at diagnosis about etiology (idiopathic 13, secondary to toxic or drug 1 , secondary to other disease 1 ), severity (moderate 3 , severe 6 , very severe 8 ), chromosome analysis (normal 8 , abnormal 1, not done or failed 8) and clinical features (hemorrhage 5, systemic infection 5). Two patients had a PNH clone $\geq 5 \%$.

Results: Previous IST: At least one course of cyclosporine and ATG was given to 23 patients (82.1\%). Time from IST to SCT was 6 months (4-11). Transplant characteristics: Median age at SCT was 50 years (45-60). Despite it was not statistically significant, time from diagnosis to SCT was delayed more than 3 months in those patients who received IST ( 6.8 vs. 3.1 ). The conditioning regimen mainly consisted of cyclophosphamide alone (16) or combined with fludarabine (10). Three patients (10.7\%) did not receive ATG. The GVHD prophylaxis was quite different between centers being the most frequent the combination of cyclosporine with methotrexate (5), mycophenolate (4) or corticosteroids (7). Stem cell source was bone marrow in 15 cases $(53.6 \%)$ being the donor an identical sibling in 21 (75.0\%). One patient underwent a haploidentical transplant. Transplant complications: Two patients $(7.1 \%)$ did not engraft and 2 patients presented an early graft loss (7.7\%). 


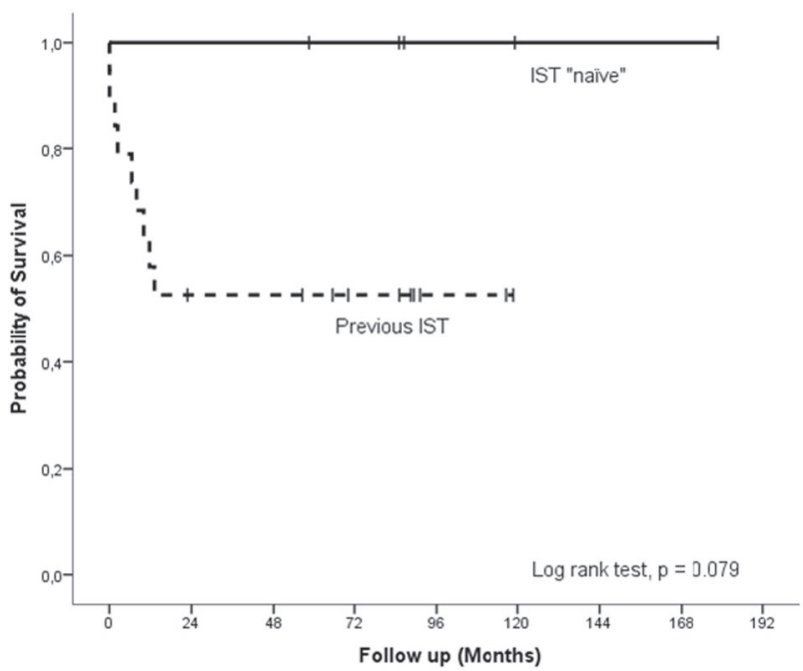

[P070 Figure] [Figure 1. Probability of OS after SCT in patients older than 40 years according to previous IST]

Nine patients (34.6\%) developed acute GVHD (3 grade I, 5 grade II, 1 grade III) and six (26.1\%) developed chronic GVHD (4 limited, 2 extensive). Outcome: Five patients $(17.8 \%)$ died before day +100 due to infections in 4 . With a median follow up of 59 moths (8-89), 16 patients (57.1\%) are alive, 12 in complete remission and 4 in partial remission. The probability of survival was higher in those who did not receive previous IST ( $\mathrm{p}=0.079$ ), Figure 1 .

Conclusions: In line with prior reports, our study recognizes SCT as a suitable option for older patients diagnosed with aplastic anemia. The better outcomes observed in patients who received an upfront SCT from an identical sibling need further confirmation in larger series.

Conflict of interest: The authors have nothing to disclose.

\section{P071}

Abstract previously published

\section{P072}

Rescue Haploidentical Transplantation for Aplastic Anaemia Following Primary Graft Failure

\author{
Santanu Sen ${ }^{1}$, Sameer Tulpule ${ }^{2}$
}

${ }^{1}$ Kokilaben Dhirubhai Ambani Hospital, Department of Paediatric Oncology \& Stem Cell Transplantation, Mumbai, India; ${ }^{2}$ Kokilaben Dhirubhai Ambani Hospital, Department of Stem Cell

Transplantation, Mumbai, India

Background: Primary graft failure remains one the important causes of mortality and morbidity in cases of stem cell transplantation. We report on a case of Aplastic Anaemia where an immediate rescue haploidentical stem cell transplantation from a different donor resulted in a successful engraftment. We suggest that an immediate transplantation with repeat conditioning with chemoradiation therapy can result in a successful engraftment in these cases of primary graft failure.

Methods: A 3yr old male child was diagnosed to have Severe Aplastic Anemia. Unfortunately there were no matched sibling donors and matched unrelated donor search did not yield any acceptable matches. We elected to undertake a haploidentical transplant using his fathers stem cells. Father was 5/10 HLA matched by high resolution typing and was DSA negative and had the same blood group. He was conditioned with ATG $(10 \mathrm{mg} / \mathrm{kg} / \mathrm{day} \mathrm{x}$ 3 days) Fludarabine (30 mg/kg x 5 days), Cyclophosphamide (14.5 mg/kg x 2 days), and low dose TBI at 200cGy and GVHD prophylaxis with Post Transplant Cyclophosphamide. CD34 dose used was $8.9 \times 10^{\wedge} 6 / \mathrm{kg}$.

Unfortunately he failed to engraft with counts remaining very low at Day +21 and a marrow examination was severely hypocellular. Hence at day +21 we undertook a second rescue haploidentical transplant for the primary graft failure. As he had rejected his fathers stem cells, we decided not to use the father the second time around as we surmised that the patient might have developed effective antibodies again him. Hence this time we decided to re-transplant him the second time using his mother stem cells. Though he was profoundly cytopenic \& neutropenic, we decided to give him full repeat conditioning with the same protocol as we surmised that he still had a active immune system that had rejected the primary graft. Repeat conditioning using the same protocol was started from Day +22 and mother's stem cells was infused on Day +28 .

Results: He engrafted successfully by Day +12 of the second transplant and though the transplant was complicated by a cutaneous fungal infection and CMV reactivation, child was discharged home with $100 \%$ donor engraftment. Currently the child is Day +182 and though he had a mild gut GVHD, this was easily managed with oral budesonide.

Conclusions: We suggest that a urgent second haploidentical transplant using repeat conditioning can be used successfully in cases of primary graft failure even in the face of severe cytopenia.

Conflict of interest: S. Sen: Nothing to disclose

\section{P073}

Results of allogeneic stem cell transplantation in patients affected by Shwachman-Diamond syndrome. A retrospective study of SAA-EBMT 
Simone Cesaro ${ }^{1}$, Gloria Tridello ${ }^{1}$, Christian Kratz ${ }^{2}$, Marta Pillon $^{3}, S$ Halkes $^{4}$, Edoardo Lanino ${ }^{5}$, Regis Peffault De Latour ${ }^{6}$, C Diaz De Heredia $^{7}$, Robert Wynn ${ }^{8}$, Johann Greil', Franco Locatelli ${ }^{10}$, Paul Veys $^{11}$, Anne Uyttebroeck ${ }^{12}$, Per Ljungman ${ }^{13}$, Patrice Chevalier ${ }^{14}$, Marc Ansari ${ }^{15}$, Isabel Badell ${ }^{16}$, Tayfun Gungor ${ }^{17}$, Salim Rahuman ${ }^{18}$, Johanna Tischer ${ }^{19}$, Cristina Tecchio ${ }^{20}$, Nigel Russel ${ }^{21}$, Alicja Chybicka $^{22}$, K Kallay ${ }^{23}$, Owen Smith ${ }^{24}$, B Afanasyev ${ }^{25}$, J Stein ${ }^{26}$, Jan Styczynski $^{27}$, Paul Bosman ${ }^{28}$, Carlo Dufour

${ }^{1}$ Azienda Ospedaliera Universitaria Integrata, Verona, Italy; ${ }^{2}$ Hannover Medical University, Hannover, Germany; ${ }^{3}$ Clinica di Oncoematologia Pediatrica, Padova, Italy; ${ }^{4}$ Leiden University Medical Hospital, Leiden, Netherlands; ${ }^{5}$ Istituto G. Gaslini, Genova, Italy; ${ }^{6}$ Hopital St. Louis, Paris, France; ${ }^{7}$ Hospital Vall d'Hebron, Barcelona, Spain; ${ }^{8}$ Central Manchester NHS Trust, Manchester, Manchester, United Kingdom; ${ }^{9}$ University of Heidelberg, Heidelberg, Germany; ${ }^{10}$ IRRCS Ospedale Pediatrico Bambino Gesu, Rome, Italy;

${ }^{11}$ Great Ormond Street Hospital, London, United Kingdom;

${ }^{12}$ University Hospital Leuven, Leuven, Belgium; ${ }^{13}$ Karolinska University Hospital, Stockholm, Sweden; ${ }^{14} \mathrm{CHU}$ Nantes, Nantes,

France; ${ }^{15}$ Hopitaux Universitaires de Geneve, Geneva, Switzerland;

${ }^{16}$ Hospital de la Santa Creu i Sant Pau, Barcelona, Spain; ${ }^{17}$ University Children's Hospital, Zurich, Switzerland; ${ }^{18}$ Royal Liverpool University Hospital, Liverpool, United Kingdom; ${ }^{19}$ Klinikum Grosshadem,

Munich, Germany; ${ }^{20}$ University Hematology, Verona, Italy;

${ }^{21}$ Nottingham University Hospitals NHS Trust, Nottingham, United Kingdom; ${ }^{22}$ Wroclaw Medical University, Wroclaw, Poland; ${ }^{23}$ United St. Istvan and St. Laszlo Hospital, Budapest, Hungary; ${ }^{24}$ Our Ladys Childrens Hospital, Dubllin, Ireland; ${ }^{25}$ Pavlov Medical University of St. Petersburg, St. Petersburg, Russian Federation; ${ }^{26}$ Schneider Children's Medical Center, Petach-Tikva, Israel; ${ }^{27}$ University Hospital, Collegium Medicum UMK, Bydgoszcz, Poland; ${ }^{28}$ EBMT Data Office, Leiden, Italy

Background: Shwachman-Diamond syndrome (SDS) is a rare multisystemic disease that affects $1 / 77.000$ children. SDS is the third cause of congenital bone marrow dysfunction, after Fanconi anemia and Blackfan-Diamond anemia, and the second cause of pancreatic insufficiency after cystic fibrosis. The transmission of SDS is autosomal recessive. The long-term survival of SDS patients is threatened by the occurrence of bone marrow failure that can evolve to severe aplastic anemia, myelodysplastic syndrome, and acute leukemia. In these cases, allogeneic haematopoietic stem cell transplant (HSCT) is the only valuable therapeutic option. In this study, we analyzed the outcome of SDS patients given HSCT and registered in the EBMT registry.

Methods: Data on HSCT in SDS patients were retrieved retrospectively from the EBMT-Promise database. Data collection was completed with a specific case report form sent to the centers.

Results: 74 SDS patients, F/M 39/35, were transplanted in 43 EBMT centers over the period 1988-2016 for SAA (60) or MDS/AML (14), after a median time from diagnosis of 3.4 yrs (0.2-30.6). Median age at HSCT was 8.7 yrs (0.842.9). Donor was a sibling or a family donor in $32 \%$ of cases (sibling 18, matched family donor 4 , haploidentical parent 2), or an unrelated donor in the remaining 68\% (21 matched, 14 mismatched, 15 matching not known). Donor source was BM (70\% of cases), PB (19\%) and CB (11\%). The D/R gender pair was $22 \% \mathrm{~F} / \mathrm{M}, 78 \%$ other combination; the CMV R/D serostatus was: 39\% neg/neg, 20\% neg/ pos, $20 \%$ pos/neg, and $20 \%$ pos/pos. The conditioning regimen was myeloablative and of reduced intensity in $73 \%$ and $27 \%$ of patients, respectively. TBI and TLI were used in 8 and 2 patients, respectively. GVHD prophylaxis was based on ex-vivo T-depletion in 9 patients and on in-vivo Tdepletion in 49 patients (36 ATG, 13 alemtuzumab). Moreover, 64 patients $(86 \%)$ received a pharmacological prophylaxis for GVHD based mainly on CSA and short methotrexate or on CSA plus other drugs. The 60-day C.I. of PMN engraftment was $85 \%$ after a median time of 17.5 days (10-102), the rate of graft failure being $15 \%$. Grade I-II and II-IV acute GVHD occurre in 28\% and 24\% of patients, respectively, while chronic GVHD was diagnosed in $21 \%$. After a median f-up of 7.3 years, the 5 -year OS was $63 \%$ (CI, 51 -73). The 5-year OS was $71 \%$ for SAA and 29\% for MSD/AML, p 0.0004. The mortality rate was 38\% (10\% relapse of MDS/AML or graft failure, $28 \%$ toxicity or other causes not specified).

Conclusions: This is the largest series reported so far of SDS patients given HSCT . HSCT showed to be a valuable therapeutic option in SDS patients, especially when transplanted for SAA. The safety and tolerability of HSCT is improved compared to previous historical series although the type of donor selection, intensity of conditioning regimen, and type of GVHD prophylaxis are still a matter of investigation.

Conflict of interest: Nothing to disclose for all authors

\section{P074}

\section{Results of haploidentical hematopoietic stem cell transplantation in patients with aplastic anemia: A single center experience}

Zafer Gulbas, Demet Çekdemir, İmran Dora, Eda Er, Çiğdem Eren

Anadolu Medical Center Hospital, Bone Marrow Transplantation, Kocaeli, Turkey

Background: New transplant approaches are necessary for patients with refractory severe aplastic anemia (SAA) who lack a matched sibling or unrelated donor (UD) or who have failed UD. We report our results with haploidentical hematopoietic stem cell transplantation (haploHSCT) using with posttransplant high-dose cyclophosphamide.

Methods: We retrospectively summarized 11patients who failed to respond to previous therapies with antithymocyte immunoglobulin and CsA and underwent haploHSCT from 2010 to 2017. The conditioning regimen before 
HSCT included cyclophosphamide, fludarabine, rabbit ATG. The recipients for graft versus host disease (GVHD) prophylaxis received posttransplant high-dose cyclophosphamide. Subsequent to successful allo-HSCT, the hematopoietic reconstitution was observed, a median interval from diagnosis to transplantation of 1.5 years (male, 6 patients; female, 5 patients; median age range, 18-61.

Results: We found that hematopoietic reconstruction was achieved in 9 patients . Graft failure occured in 2 patients and those patients underwent second transplantation from other family haplo donor and both engrafted. Two patients died in 100 days due to sepsis. We did not observed severe (grade 3 -4)acute or chronic graft versus host disease any of the patients.

Conclusions: Our findings show that haploHSCT can successfully rescue refractory SAA patients who lack donor. Delayed for the haploidentic transplantation increase the sepsis related death rate.

Conflict of interest: No conflicts of interest

\section{P075}

\section{Screening for telomere disease is crucial to improve the outcome of HSCT for pediatric patients suffering from severe aplastic anemia}

\section{Iris Nederlof ${ }^{1}$, Caroline Lindemans ${ }^{1}$, Dorine Bresters ${ }^{2}$, Marije Bartels $^{1}$}

${ }^{1}$ University Medical Center Utrecht, Pediatric Hematology and Stem Cell Transplantation, Utrecht, Netherlands; ${ }^{2}$ Leiden University

Medical Center, Pediatric Hematology/Oncology, Leiden, Netherlands

Background: Telomeropathies compromise a complex spectrum of diseases caused by unprotected and shortened telomeres, resulting in bone marrow failure, and other organ disease, including lung fibrosis and liver cirrhosis. Patients with telomeropathies, of which dyskeratosis congenita (DC) is the best-known example, are at increased risk of hematological stem cell transplantation (HSCT)- associated complications, predominantly significant organ damage following myeloablative conditioning regimen. In patients suffering from severe aplastic anemia (SAA), the preexistence of an underlying congenital bone marrow failure (BMF) disorders, including Fanconi anemia (FA) and DC, should be considered. However, telomeropathies encompass a wide spectrum and clinical presentation can therefore be subtle. Determining telomere length in patients with SAA can be used to predict the likelihood of an underlying telomeropathy (characterized by telomeres $<\mathrm{p} 1$ ) and to adjust treatment regimen if needed. The objective of this study is to investigate clinical characteristics of patients suffering from SAA or severe BMF with respect to a proven or suspected telomeropathy and outcome of allogeneic HSCT.

Methods: We investigated 46 patients (0-18 years) undergoing allogeneic HSCT at two Dutch pediatric HSCT departments between 2009 and September 2017. Patients suffering from SAA $(n=29)$, refractory cytopenia of childhood ( $\mathrm{RCC}, \mathrm{n}=11$ ), or BMF and a proven telomeropathy $(n=6)$ were included. Two patients suffering from telomeropathy-associated BMF that were not yet scheduled for SCT were included in the analysis on characteristics preSCT. A telomeropathy was diagnosed based on telomere length $<$ p1 in lymphocytes and granulocytes determined by Flow FISH (www.repeatdiagnostics.com). A retrospective analysis on general clinical characteristics, radiological and laboratory parameters associated with BMF, liver dysfunction or pulmonary disorder, complications and toxicity was performed for all patients. Mann-Whitney and Fisher's exact tests were used to analyze the differences between groups.

Results: In the telomeropathy group, congenital malformations were reported more frequently (4/8 versus 4/43, $\mathrm{p}=0.0147)$. We also observed elevated levels of ASAT and ALAT (both $>1 \times \mathrm{ULN})$, and $\gamma \mathrm{GT}(>2 \mathrm{x}$ ULN) in the telomeropathy group $(\mathrm{p}<0.0001, \mathrm{p}=0.0245)$. Abnormal radiological appearances of the liver were more frequently reported pre-HSCT in the telomeropathy group $(6 / 8)$ compared to the control group $(5 / 43, p=0.0006)$. Our data suggest a trend for macrocytic anemia in the telomeropathy group (4/8) compared to the non-tested group (4/43) $(p=0.076)$. There was no difference in reported radiological abnormalities of the lungs before HSCT. Four patients with a telomeropathy $(4 / 6,66 \%)$ died following HSCT complications, compared to $11(26 \%)$ in the non-tested group. Interestingly, based on our findings in the telomeropathy group, we have identified 12 patients in the non-tested group suggestive for an underlying telomeropathy, of which $5 / 12$ (42\%) died from severe HSCT-associated complications.

Conclusions: SAA/severe BMF can be the result of an underlying telomeropathy other than classical DC. In patients that will undergo allogeneic HSCT, determining telomere length can help to identify patients that have an increased risk for HSCT-associated complications. This will contribute to a better outcome of HSCT for all patients with $\mathrm{SAA} /$ severe BMF and to a better understanding of telomere disease in general.

Conflict of interest: None of the authors has anything to disclose.

\section{P076}

Very long-term follow up of aplastic anemia treated with allogeneic stem cell transplantation and immunosuppressive treatment 
Felicitas Zurbriggen, Jakob R. Passweg, Beatrice Drexler

University and University Hospital of Basel, Hematology, Basel, Switzerland

Background: Aplastic anemia (AA) historically was a fatal condition. Since the 1970s patients benefited from treatments consisting mainly of immunosuppression (IST, originally horse ATG) and hematopoietic stem cell transplantation (HSCT). Although the response rate and survival after treatment improved, problems remain, including incomplete response, relapse, disease- and treatment-related complications, late effects and clonal evolution. In order to assess very long-term outcome in aplastic anemia we analysed our patients in a single - center study over 44 years.

Methods: We analysed long term outcome of 302 patients treated between 1973 and 2017 at the University Hospital Basel.

Results: Median age at diagnosis was 29.9 years (range 0.71-80). Eight patients were diagnosed with moderate AA, 208 with severe AA and 23 with very severe AA. Horse ATG was first-line treatment in 224 patients, 74 patients received allogeneic HSCT and 4 had other first line therapy. With a median follow up of 17 years, overall survival at 30 years after initial treatment was $49+12 \%$ in transplanted patients and $41 \pm 9 \%$ in patients with initial ATG treatment. There were more early deaths in the HSCT group and more late deaths in the ATG group. Death rate was particular high in the first 10 years posttransplant, whereas transplant patients afterwards mostly survived with stable hematopoiesis (see figure). Patients with immunosuppressive treatment showed less early deaths but had a lower overall survival after 30 years. At last follow up most of long-term survivors are in complete or partial remission. More details will be presented at the conference.

Conclusions: Most of aplastic anemia survivors show long-term hematopoesis, whereas death occurs mostly early in the HSCT group and more late in the ATG group.

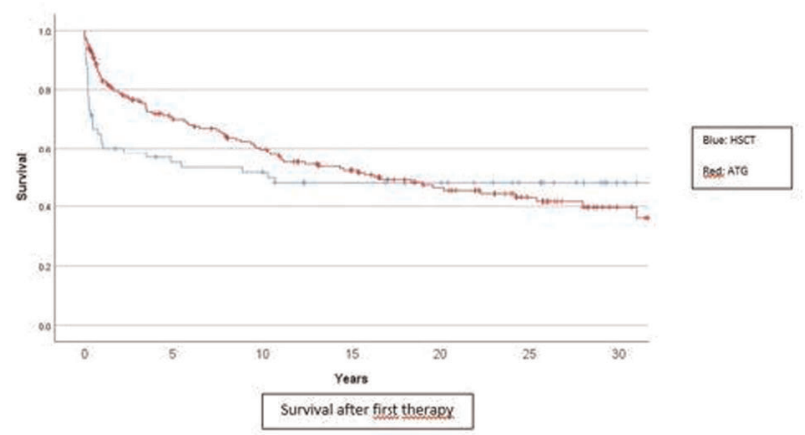

Conflict of interest: None of the authors has anything to disclose.

\section{Autoimmune diseases}

\section{P077}

\section{A retrospective analysis of false positive antibodies in patients receiving intravenous immunoglobulin for immune mediated neuropathies}

\author{
Allison Clendenan ${ }^{1}$, Jacquelyn Henry ${ }^{2}$, Carri Alldredge ${ }^{1}$, Richard \\ Burt $^{2}$ \\ ${ }^{l}$ Northwestern University, Division of Immunotherapy, Chicago, IL, \\ United States; ${ }^{2}$ Northwestern University, Division of Immunotherapy \\ for Autoimmune Diseases, Chicago, IL, United States
}

Background: Intravenous immunoglobulin (IVIg) is pooled preparation of $\mathrm{IgG}$ from thousands of donors and can possibly cause false positive antibodies in patients receiving IVIg potentially leading to improper diagnoses.

Methods: Records of 115 patients with immune mediated neuropathies who underwent evaluation at Northwestern Medicine in Chicago, IL were reviewed. Data collected included whether or not the patient was receiving IVIg at the time of evaluation, antibodies for hepatitis B core (anti$\mathrm{HBc}$ ), human T-lymphotropic virus (HTLV), human immunodeficiency virus (HIV), syphilis, and anti-glutamic acid decarboxylase (anti-GAD).

Results: The graph below depicts the percentage of patients with false positive antibodies divided into patients who were receiving IVIg versus those who were not receiving IVIg at the time of evaluation. Twenty-seven patients were not receiving IVIg. In the group not receiving IVIg there were two false positives $(7 \%, 2 / 27)$ identified (one anti-HBc, one syphilis). Eighty-eight patients were receiving IVIg. One hundred and forty-seven false positives were identified in this group. Of these false positives $73 \%$ (64/88) were anti-HBc, $1 \%$ (1/88) was HIV, 40\% (35/88) were HTLV, 82\% (23/28) were syphilis, and 92\% (23/26) were anti-GAD.

Conclusions: IVIg increases the incidence of false positive antibodies for infections, specifically, anti-HBc, HTLV, and syphilis. Confirmatory testing should be completed if the antibodies are positive and a patient is receiving IVIg. Anti-GAD can also be falsely elevated due to IVIg. Diagnoses supported by high anti-GAD in the blood, such as type 1 diabetes and stiff person syndrome, should be used with caution in patients receiving IVIg due to the likelihood of it being falsely positive.

Conflict of interest: None of the authors has anything to disclose.

[P076 Figure] [Survival after first therapy] 


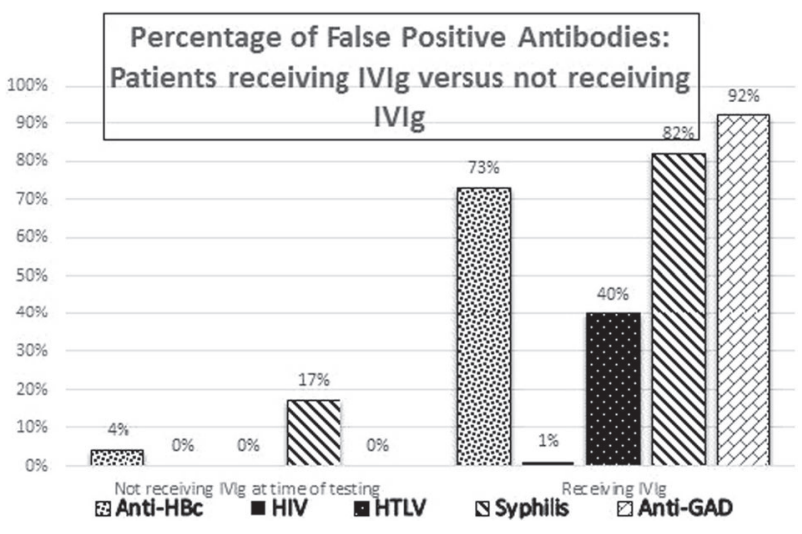

[P077 Figure] [Percentage of False Positive Antibodies: Patients receiving IVIg versus not receiving IVIg]

\section{P078}

Analysis of Immune Reconstitution by Multicolour Flow Cytometry in Multiple Sclerosis and Non-Autoimmune Patients Following Autologous Haematopoietic Stem Cell Transplantation

Carole D Ford ${ }^{1}$, Melissa Khoo ${ }^{1}$, John Zaunders ${ }^{2}$, Jennifer Massey ${ }^{3}$, Kevin Hendrawan ${ }^{1}$, Ian Sutton ${ }^{3}$, David D F Ma ${ }^{1,3}$, John J Moore

${ }^{1}$ St Vincent's Centre for Applied Medical Research, Sydney, Australia;

${ }^{2}$ The Kirby Institute, Sydney, Australia; ${ }^{3}$ St Vincent's Hospital,

Darlinghurst, Australia

Background: Autologous haematopoietic stem cell transplantation (ASCT) is a promising strategy for Multiple Sclerosis (MS) patients that do not respond to conventional treatments but the mechanisms able to achieve clinical improvement of MS in transplant recipients are not fully understood.

Methods: We hypothesised that ASCT eliminates the pro-inflammatory disease-causing $\mathrm{T}$ cells to halt disease progress, and promotes tolerance via multiple mechanisms, including reconstitution of immune-regulatory suppressive cell subsets, to achieve sustained disease control. We designed polychromatic flow cytometry panels with 9-14 colours to characterise over 30 subpopulations of peripheral blood cells (PBMC) of MS patients, undergoing ASCT in a Phase II study at our institute. Various differentiation markers, chemokine receptors, and trafficking markers were used to identify populations unique to autoimmune disease and compared these findings with those obtained from nonautoimmune Lymphoma patients treated with ASCT. Analysis included $t$-test, Wilcoxon signed rank test and tSNE.

Results: Immune reconstitution was assessed by multicolour flow cytometry in eighteen MS patients at pre-ASCT and 3, 6, and 12 months post-ASCT time points. Pro- inflammatory disease-causing $\mathrm{T}$ cells, such as CCR6 + CD161hi mucosal-associated invariant T (MAIT) cells (a Th17 subset with brain-homing abilities) and CD49d +CCR6+ CNS-homing cells, significantly decreased following treatment in both MS $(p<0.05)$ and NHL patients $(p$ $<0.05)$. Interestingly in MS patients, regulatory $\mathrm{T}$ cells increased significantly at 3 months $(p<0.05)$ post ASCT while in Lymphoma patients, regulatory $\mathrm{T}$ cells decreased at 6 months post ASCT $(\mathrm{p}<0.05)$. Expression of cytotoxic CD8+CD57+ T cells tripled at 3 months post ASCT $(p<$ 0.0001) in MS patients while no significant change occurred in Lymphoma patients. Natural killer (CD56hi) expression doubled $(p<0.05)$ by 3 months post ASCT in MS patients but in Lymphoma patients there was no significant change post transplantation.

Conclusions: ASCT depleted pro-inflammatory T cells in both MS and Lymphoma patients. However, we found the pattern of expression in other immune-regulatory cell subsets differed in these patient cohorts post transplantation. These distinct patterns of immune reconstitution between MS and Lymphoma post-ASCT provide tantalising indication that these changes may relate to the different underlying pathogenic process and aid to pinpoint changes specific to immune deregulation in MS. These findings have the potential to allow us to track disease progression and treatment outcomes by monitoring the presence of specific cell types, which may help to improve the management of patients living with MS.

Conflict of interest: Nothing to disclose

\section{P079}

Autologous Haematopoietic Stem Cell Transplantation (AHSCT) for Anti-Neutrophil Cytoplasmic Antibody (ANCA)associated Vasculitis (AAV): Results of a EBMT Retrospective Survey

Clare Samuelson ${ }^{1}$, Tobias Alexander ${ }^{2}$, Thomas Daikeler ${ }^{3}$, Manuela Badoglio $^{4}$, Jörg Henes ${ }^{5}$, Mohamed Akil ${ }^{1}$, Lars Skagerlind ${ }^{6}$, Gerhard Ehninger $^{7}$, Esa Jantunen ${ }^{8}$, Dominique Farge ${ }^{9}$, John A Snowden ${ }^{1}$

${ }^{1}$ Sheffield Teaching Hospitals NHS Foundation Trust, Sheffield, United Kingdom; ${ }^{2}$ Charite - University Medicine Berlin, Department of Rheumatology and Clinical Immunology, Berlin, Germany;

${ }^{3}$ University Hospital Basel, Department of Rheumatology, Basel, Switzerland; ${ }^{4}$ Saint Antoine Hospital, EBMT Study Office, Paris, France; ${ }^{5}$ Medizinische Klinik II, Universitätsklinikum Tübingen, Tübingen, Germany; ${ }^{6}$ University Hospital Umea, Department of Hematology, Umea, Sweden; ${ }^{7}$ Medizinische Klinik und Poliklinik I, Universitätsklinikum der Technischen Universität Dresden, Dresden, Germany; ${ }^{8}$ University Hospital Kuopio, Department of Internal Medicine, Kuopio, Finland; ${ }^{9}$ St. Louis Hospital, Internal Medicine, Paris, France

Background: ANCA-associated vasculitides (AAV) are chronic autoimmune diseases, which can present with life- 
threatening multi-system involvement. Current treatment options are still suboptimal with risks of disease progression and treatment-related toxicity. Autologous haematopoietic stem cell transplantation (AHSCT) has been used in a number of such cases but patient outcomes following such management are unclear.

Methods: Adults who received AHSCT for AAV and whose data were registered with the EBMT were identified retrospectively through the EBMT database. Treating physicians were surveyed to produce a retrospective evaluation of outcomes.

Results: 7 patients underwent AHSCT primarily for AAV between 1999-2014 in 6 centres across Europe. 5 females and 2 males were transplanted; 6 had a diagnosis of granulomatosis with polyangiitis and 1 eosoniphilic granulomatosis with polyangiitis. Median age was 39 years (range 32-55 years). Patients had received 4-6 prior lines of therapy, including cyclophosphamide (CYC, with median cumulative dose of $80 \mathrm{~g}$ ) and steroids in every case, and rituximab in 4 cases. Stem cell source was peripheral blood in every case; CD34-selection was performed in 4 cases, mean $\mathrm{CD}_{3} 4^{+}$cell dose was $4.2 \times 10^{6} / \mathrm{kg}$ (range $0.6-7.9 \times 10^{6} \%$ $\mathrm{kg}$ ). Conditioning regimen was CYC/ATG in 5 patients and CYC in 2 patients. Median follow-up was 86 months (range 1-204 months). Transplant-related mortality (TRM) occurred in 2 causes. All but one patient went into remission but 3 later relapsed at 6, 12 and 36 months, respectively, and required further treatment for disease control. At time of last clinical follow-up, 3 patients had drug-dependent partial response; 1 had drug-dependent complete remission and 1 had drug-free complete remission.

Conclusions: Outcomes of AHSCT for these heavily pretreated AAV patients were variable. Only 1 patient achieved drug-free complete remission and TRM was observed in a quarter. Nevertheless, AHSCT had the potential to stabilize AAV in patients who initially failed to respond to conventional therapies. These data do not support AHSCT for advanced stage ANCA-positive vasculitis, although it may have a place as salvage treatment in otherwise refractory patients. As for other autoimmune diseases, AHSCT may provide better outcomes when performed at early stage of disease. Overall, AHSCT should only performed in clinical trial settings in experienced centers.

\section{Conflict of interest:}

J. Snowden declares honoraria for speaking from Sanofi and Jazz.

T. Alexander declares honoria and/or traval support from Abbvie, Amgen, Neovii and Pfizer.

The other authors declare no conflicts of interest.
P080

Autologous Haematopoietic Stem Cell Transplantation (Auto-HSCT) in Stiff Person Syndrome (SPS): The Sheffield Experience

Alice Elizabeth Thorpe, Lewis Kass-Iliyya, Helen Jessop, Andrew Chantry, Marios Hadjivassiliou, Basil Sharrack, John A Snowden

Sheffield Teaching Hospitals NHS Foundation Trust, Sheffield, United Kingdom

Background: Stiff Person Syndrome (SPS) is a rare (1/ $1,000,000)$ immune-mediated neurological disorder characterised by muscle rigidity, spasms and high glutamic acid decarboxylase (GAD) antibodies. Auto-HSCT has successfully been used to treat limited numbers of SPS patients (Sanders S et al, JAMA Neurology, 2014). We describe our single centre experience of assessing referrals of SPS for potential auto-HSCT.

Methods: Between 2015-2017 we reviewed six patients with SPS from across the UK as summarised in table 1. One patient (Patient 6) was declined auto-HSCT as they were found to be stable on Mycophenolate and five were deemed suitable for autograft (Patients 1-5). Two patients have been treated. Patient 1 had the Progressive Encephalomyelitis with Rigidity and Myoclonus (PERM) variant of SPS with ataxia. Patient 2 had Classical SPS. Both patients were significantly disabled and had failed multiple immunotherapies including intravenous immunoglobulin (IVIG) and plasmapheresis. The patients were mobilised with Cyclophosphamide (Cy) $2 \mathrm{~g} / \mathrm{m}^{2}+$ G-CSF and conditioned with Cy $200 \mathrm{mg} / \mathrm{kg}+$ ATG followed by auto-HSCT, as per EBMT Guidelines (Snowden JA et al, Bone Marrow Transplantation, 2012). A third patient will be scheduled for auto-HSCT pending funding authorisation. Two patients have been declined funding.

Results: Despite significantly reduced performance status, both patients tolerated the procedure with routine toxicities. Patient 1 engrafted on day +10 and was hospitalised until day +16 . He developed EBV reactivation but did not require treatment. Subsequent neurological assessment showed disease stabilisation with normalisation of anti-GAD titres and fortnightly IVIG was discontinued. At 12 months post-transplant, MR spectroscopy of the cerebellum showed significant increase in the NAA/Cr ratio reflecting improved metabolic activity. Patient 2 engrafted on day +10 and was discharged on day +16 . She discontinued fortnightly IVIG and assessment at 6-months showed resolution of neurological symptoms and normalisation of clinical examination. Her anti-GAD titre remained high.

Conclusions: Auto-HSCT may be a viable treatment option in patients with severe SPS, supporting previous 


\begin{tabular}{|c|c|c|c|c|c|c|c|c|}
\hline Patient & Age & Gender & $\begin{array}{l}\text { SPS } \\
\text { Phenotype }\end{array}$ & $\begin{array}{l}\text { Disease duration } \\
\text { prior to auto- } \\
\text { HSCT assessment } \\
\text { (years) }\end{array}$ & $\begin{array}{l}\text { Baseline } \\
\text { anti-GAD } \\
\text { titre (U/ } \\
\mathrm{ml})\end{array}$ & Baseline Neurophysiology & $\begin{array}{l}\text { Prior } \\
\text { Immunomodulatory } \\
\text { Therapy }\end{array}$ & Outcome \\
\hline 1 & 52 & Male & $\begin{array}{l}\text { PERM \& } \\
\text { ataxia }\end{array}$ & 5 & 372 & Abnormal blink reflex. & $\begin{array}{l}\text { Plasmapheresis, } \\
\text { IVIG }\end{array}$ & $\begin{array}{l}\text { Auto-HSCT (NHS } \\
\text { funded) }\end{array}$ \\
\hline 2 & 36 & Female & $\begin{array}{l}\text { Classical } \\
\text { SPS }\end{array}$ & 8 & $>2000$ & $\begin{array}{l}\text { Brainstem hyper-excitability, } \\
\text { exaggerated startle reflex. }\end{array}$ & $\begin{array}{l}\text { Plasmapheresis, } \\
\text { IVIG }\end{array}$ & $\begin{array}{l}\text { Auto-HSCT (Self- } \\
\text { funded) }\end{array}$ \\
\hline 3 & 37 & Female & $\begin{array}{l}\text { Classical } \\
\text { SPS }\end{array}$ & 9 & $>2000$ & $\begin{array}{l}\text { Continuous motor unit activity, } \\
\text { brainstem hyper-excitability. }\end{array}$ & $\begin{array}{l}\text { IVIG, Rituximab, } \\
\text { Azathioprine }\end{array}$ & $\begin{array}{l}\text { Awaiting NHS } \\
\text { funding }\end{array}$ \\
\hline 4 & 53 & Female & $\begin{array}{l}\text { Classical } \\
\text { SPS }\end{array}$ & 16 & $>2000$ & Not available. & IVIG & $\begin{array}{l}\text { NHS funding } \\
\text { declined }\end{array}$ \\
\hline 5 & 52 & Male & PERM & 8 & $<5$ & $\begin{array}{l}\text { Exaggerated startle reflex with } \\
\text { variable delays. }\end{array}$ & $\begin{array}{l}\text { IVIG, } \\
\text { Plasmapheresis, } \\
\text { Mycophenolate, } \\
\text { Azathioprine, } \\
\text { Steroids }\end{array}$ & $\begin{array}{l}\text { NHS funding } \\
\text { declined }\end{array}$ \\
\hline 6 & 42 & Male & $\begin{array}{l}\text { Classical } \\
\text { SPS }\end{array}$ & 7 & $>2000$ & $\begin{array}{l}\text { Failure of suppression of } 2 \text { nd } \mathrm{R} 2 \\
\text { response in paired blink reflex. } \\
\text { Continuous motor unit activity. }\end{array}$ & $\begin{array}{l}\text { IVIG, } \\
\text { Plasmapheresis, } \\
\text { Mycophenolate }\end{array}$ & $\begin{array}{l}\text { Did not progress to } \\
\text { auto-HSCT as stable } \\
\text { on Mycophenolate }\end{array}$ \\
\hline
\end{tabular}

[[P080 Table] Table 1]

work (Sanders S et al, JAMA Neurology, 2014). It may also prove to be a more cost effective treatment in patients requiring regular treatment with expensive modalities, such as IVIG. Further work is warranted to establish long-term safety, efficacy and cost-effectiveness of auto-HSCT in SPS, along with optimising patient selection and transplant technique.

Conflict of interest: J. A. Snowden declares honoraria for speaking from Sanofi and Jazz.

\section{P081}

\section{High Dose Chemotherapy and Autologous Stem Cell Rescue For Multiple Sclerosis: Preliminary Data From India}

Gaurav Kharya ${ }^{1}$, Silky Jain ${ }^{1}$, Divya Bansal ${ }^{1}$, Anil Khetrapal, Vikrant Bhar ${ }^{3}$, Manish Mahajan ${ }^{4}$,Sumit Singh ${ }^{4}$

${ }^{1}$ Artemis Hospital, Pediatric Hematology Oncology Immunology and Bone Marrow Transplant, Gurugram, India; ${ }^{2}$ Artemis Hospital, Department of Transfusion Medicine, Gurugram, India, ${ }^{3}$ Artemis Hospital, Department of Lab Hematology, Gurugram, India; ${ }^{4}$ Artemis Hospital, Agrim Institute of Neurosciences, Gurugram, India

Background: Multiple sclerosis is a debilitating autoimmune disease leading to progressive neurological deterioration. It is categorized into relapsing remitting (RRMS), secondary progressive (SPMS) or primary progressive (PPMS). Extended disability status scale (EDSS) decides the extent of disability ranging from 0-10. Disease modifying agents (DMA) have been used to treat symptoms with variable results but they are limited by cost and adverse effects. There is new found interest in high dose chemotherapy and autologous stem cell rescue (HDC + ASCR) for treatment of MS. We report our initial experience from India.

Methods: Retrospective data from 37 patients suffering from MS who underwent HDC+ASCR between January 2017 till date was analyzed. Initial 10 patients received cyclophosphamide + GCSF based mobilization but subsequent patients underwent GCSF primed peripheral blood stem cells collected on day 5 with a target pre CD34 count of $>20 \%$. One patient required plerixifor as GCSF mobilization was suboptimal. Stem cell product was cryopreserved with DMSO while patient received conditioning chemotherapy. All 37 were conditioned using cyclophosphamide@50mg/kg/day x 3 or 4 days (day -5 to -2) and rATG (Thymoglobulin)@2.5mg/kg/day x 3 days (day -4 to -2 ). Median CD34 count infused was $7.3 \times 10^{6} / \mathrm{kg}$ (range 3.82 to 13.84 ).

Results: Median age at transplant was 47 years (range 33 to 67 ). 23 were $<50$ yrs whereas $14>50$ yrs of age. 22 patients had EDSS $<6$ whereas 15 had an EDSS of $>6.13$ were RRMS, 18 SPMS and 5 PPMS. 4 were drug naïve whereas 33 received at least 2 DMA before entering into transplant. At a median follow up of 200 days (range 16 to 357 ) overall survival is $92 \%$. 3 patients died due to transplant related complications (2 sepsis, 1 drug toxicity). Two patients developed DMSO induced kidney damage whereas one developed neurotoxicity. At the last follow up none of the patients were on any DMA with no progression of symptoms. A small subset of patients reported symptom reversal which needs to be substantiated by a neurologist.

Conclusions: High dose chemotherapy with autologous stem cell rescue (HDC + ASCR) is a useful modality to treat 
multiple sclerosis both in drug naïve and drug refractory cases. Larger studies and longer follow up is required to further consolidate this claim.

Clinical Trial Registry: None.

Conflict of interest: None.

\section{P082}

Impact of Autologous Haematopoietic Stem Cell Transplantation (AHSCT) in Multiple Sclerosis on Treatment Costs - Real World Analysis in a Nationwide Study

Katarzyna Orlewska ${ }^{1}$, Krzysztof Bogusz ${ }^{1}$, Monika Nojszewska ${ }^{2}$, Miroslaw Markiewicz ${ }^{3}$, Robert Liwoch ${ }^{3}$, Grzegorz Helbig ${ }^{3}$, Andrzej Śliwczyński ${ }^{4}$ Emilian Snarski ${ }^{1}$

${ }^{1}$ Medical University of Warsaw, Hematology, Oncology and Internal Diseases, Warszawa, Poland; ${ }^{2}$ Medical University of Warsaw, Department of Neurology, Warszawa, Poland; ${ }^{3}$ Medical University of Silesia, Department of Hematology and Bone Marrow

Transplantation, School of Medicine in Katowice, Katowice, Poland;

${ }^{4}$ Central Office of the National Health Fund, Warszawa, Poland

Background: Autologous haematopoietic stem cell transplantation (AHSCT) is an extremely effective therapeutic option for patients with severe multiple sclerosis (MS). The AHSCT is expensive and there is limited data on impact on real cost of treatment of MS patients outside of clinical trials. We collaborated with the National Health Fund (NHF) which collects all data on costs associated with treatment to assess the nationwide impact of AHSCT on costs of MS treatment among over 40000 MS patients.

Methods: This is a retrospective observational study based on healthcare administrative data from the National Health Fund (NHF), covering an entire Polish population of about 38 million inhabitants and all treatment costs within the system. Statistical analysis was performed using paired t-test in MedCalc. The costs were calculated from public payer's perspective in Polish złoty and are presented in Euro (1 EUR = 4.2 PLN, 2017).

Results: According to data from the NHF, the prevalence of MS in Poland was 103.06 and 113.06 per a population of 100,000 in 2008 and 2016, respectively. During the years 2008-2016, 102 patients suffering from MS underwent AHSCT in Poland and the treatment was covered by NHF. We have analysed all 102 patients comprehensively, taking into consideration all available data before and after AHSCT. According to the NHF data, the average treatment-related costs were about 4520 EUR per year before the transplantation, and were reduced to an average of 810 EUR per year after the transplantation $(\mathrm{p}<0.0001)$. The cost of AHSCT from NHF perspective is about 12380 EUR. During the studied period only 3 out of 102 patients required treatment with disease modifying drugs after AHSCT - two patients were treated with dimethyl fumarate approximately 2 years after the procedure (one dose and two doses within a month, respectively), one patient was treated with interferon beta-1b within a month after the procedure. Moreover, there were no AHSCT related deaths among patients in the studied group.

Conclusions: There is limited data on the impact of AHSCT on costs of treatment of MS. In a real world analysis of nationwide medical data, we present that AHSCT significantly reduces the use of disease modifying drugs among MS patients after AHSCT and leads to rapid reduction of treatment costs of these patients for the public payer. If the amount of treatment cost remains stable for the 3 years after AHSCT the expense of transplantation may be compensated within that time. The NHF data does not allow the assessment of clinical progression of the disease.

Clinical Trial Registry: Not applicable

Conflict of interest: Nothing to disclose

\section{P083}

Impact of homeostatic proliferation, telomere attrition, PD1 expression and CMV-reactivity on TCR diversity and relapse after AHSCT in systemic sclerosis patients

Lucas C M Arruda ${ }^{1,2}$, Emmanuel Clave ${ }^{3,4}$, João R. Lima-Júnior ${ }^{2}$, Kelen C. R. Malmegrim ${ }^{2,5}$, Corinne Douay ${ }^{4}$, Antônio José Alberdi ${ }^{3,6}$, Maria Carolina Oliveiral, ${ }^{1,2,7}$, Antoine Toubert ${ }^{3,4}$

${ }^{1}$ Ribeirão Preto Medical School, University of São Paulo, Department of Biochemistry and Immunology, Ribeirão Preto, Brazil; ${ }^{2}$ Center for Cell-based Therapy, Regional Hemotherapy Center of the Ribeirão Preto Medical School, University of São Paulo, Ribeirão Preto, Brazil; ${ }^{3}$ Université Paris Diderot, Sorbonne Paris Cité, Paris, France; ${ }^{4}$ INSERM UMR-1160, Institut Universitaire d'Hématologie, Hôpital Saint-Louis - APHP, Paris, France; ${ }^{5}$ School of Pharmaceutical Sciences of Ribeirão Preto, University of São Paulo, Department of Clinical, Toxicological and Bromatological Analysis, Ribeirão Preto, Brazil; " ${ }^{6}$ Plateforme Technologique, Institut Universitaire

d'Hématologie, Paris, France; ' Division of Clinical Immunology, Ribeirão Preto Medical School, University of São Paulo, Department of Internal Medicine, Ribeirão Preto, Brazil

Background: Autologous hematopoietic stem cell transplantation (AHSCT) has been used to treat severe and progressive systemic sclerosis (SSc) patients, however, exactly how AHSCT reshapes the immune system still need to be completely clarified. We assessed the role of replicative senescent and exhausted cells reconstitution and TCR characteristics on clinical outcome post-AHSCT in SSc patients.

Methods: Twenty-five SSc patients were clinically monitored for skin thickness (modified Rodnan's skin score, mRSS) and lung function (Forced Vital Capacity, FVC) before and semiannually until 3-years post-AHSCT. Patients were retrospectively classified as responders $(\mathrm{n}=$ 19) and non-responders $(n=6)$ according to clinical 
outcomes. Following EBMT guidelines, blood samples were collected for quantification of telomere length by RTqPCR, evaluation of TCR characteristics by New Generation Sequencing, reconstitution of senescent $\mathrm{CD} 8^{+} \mathrm{CD} 28^{-}$ $\mathrm{CD}_{57}{ }^{+}$and exhausted $\mathrm{PD}^{+}$cells by FACS, and serum cytokines level measurement by CBA-Flex. VDJTools was used to assess the TCR diversity (Shannon index), the relative TCR usage frequency (\%) of each $\mathrm{V} \beta$ and $\mathrm{J} \beta$ family, and the changes in V $\beta$-J $\beta$ segment pairing in CDR3 junctions. VDJviz and VDJdb databases were screened to annotate the frequency of CMV-specific TCR sequence motifs.

Results: From 6 months post-AHSCT until the end of follow-up, mRSS decreased $(P \leq 0.001)$ and the pulmonary function stabilized when compared to pre-AHSCT. In parallel, inflammatory cytokine levels (IL-6, IL-1 $\beta$ ) decreased transiently at 6 months $(P<0.05)$. Relative T/S telomere length ratio decreased by $41 \%$ at 6 months when compared to baseline $(P=0.015)$, correlating with the increase of $\mathrm{CD} 8^{+} \mathrm{CD} 28^{-}$cells expressing CD57 and FoxP3 $\left(\mathrm{r}_{\mathrm{s}}=-0.76, P=0.036\right)$. Consequently, there was an increase of $\mathrm{CD} 8{ }^{+} \mathrm{CD} 28^{-} \mathrm{CD} 57^{+}(P=0.004)$ and $\mathrm{CD} 8^{+} \mathrm{CD} 28^{-}{ }^{-}$oxP $3{ }^{+}$ $(P=0.001)$ percentages, with the CD57 expression by CD8 ${ }^{+} \mathrm{CD} 28^{-}$cells correlating negatively with the telomere length $\left(\mathrm{r}_{\mathrm{s}}=-0.34, P=0.015\right)$. PD-1 expression on both $\mathrm{CD}^{+}{ }^{+}$and $\mathrm{CD}^{+}{ }^{+}$-cells increased at 6 months $(P \leq 0.01)$. After AHSCT, responder patients presented lower TGF- $\beta$, IL-6, G-CSF $(P \leq 0.01)$ and IL-1, IL-17A, MIP-1 and IL-12 $(P<0.05)$ levels than non-responders, as well as higher PD1 expression on T- $(P<0.05)$ and $\mathrm{B}-(P \leq 0.01)$ cells. A strong shift in $\mathrm{V} \beta$-segments usage was observed for some families exclusively in responder patients. The TRBV13 segment usage increased post-AHSCT when compared to baseline $(P=0.031)$, while there was a significant decrease $(P<0.05)$ of the families TRBV18, TRBV24-1, TRBV29-1, TRBV4-1, TRBV4-3 and TRBV61 percentage usage. No such changes are present for $J \beta$ segments. For non-responding patients, no significant shift on $\mathrm{V} \beta$ - neither $\mathrm{J} \beta$-segments was observed following AHSCT. V-J pairing changes in responder patients resulted in increase of TCR diversity post-AHSCT as compared to baseline $(P=0.030)$, while decreased in non-responder patients. Among responders, only one patient presented CMV-specific sequences, that remained stable post-AHSCT ( $0.18 \%$ to $0.19 \%)$. The non-responder patients experienced an increase of CMV-specific sequences from $0.11 \pm 0.19 \%$ to $0.32 \pm 0.41 \% \quad(P=0.031)$, in parallel, all these patients presented a decrease of TCR diversity.

Conclusions: Homeostatic proliferation post-AHSCT results in transient telomere attrition and increased numbers of senescent and exhausted cells, leading to high PD-1 expression in patients with better clinical outcomes after AHSCT. Additionally, our results indicate a possible relationship between CMV infection and reduced TCR diversity and disease relapse after AHSCT in SSc patients.

Conflict of interest: All the authors have nothing to disclose

\section{P084}

Peripheral blood stem cell mobilisation with Cyclophosphamide and G-CSF in patients with Multiple Sclerosis: A safe and predictable procedure

Sara Lozano, Philippa Woolley, Ian Gabriel, Renuka Palanicawandar, Maria Teresa Cencioni, Paolo Muraro, Richard Nicholas, Malik Omar, Sinju Thomas, Tsembayena Dlamini, Rosalinda Elio, Eduardo Olavarria

Imperial College London, Haematology, London, United Kingdom

Background: Autologous hematopoietic stem cell transplantation (HSCT) has been utilised for the treatment of severe multiple sclerosis (MS). It results in significant improvement of neurological function, although patients could experience exacerbations of MS-related symptoms during the procedure. However, there is a lack of precise information regarding complications of the stem cell mobilisation and collection procedure in this patient population.

Methods: We analysed 45 patients diagnosed with MS that underwent a stem cell mobilization and collection from March 2016 to November 2017. The median age was 38 years (17-625). 21 patients (47\%) were male. The interval from diagnosis to HSCT was 114.3 months (range 11.6128.3). 24 patients (53\%) had relapsing-remitting (RRMS), 16 patients (35\%) secondary-progressive (SPMS) and 6 patients (12\%) primary-progressive (PPMS) Multiple Sclerosis. The median number of previous therapies was 2 (range 0-4). Only 2 patients (12\%) had not received any prior treatment. The median Expanded Disability Status Scale (EDSS) score was 6 (range 2-7). Peripheral blood stem cells were mobilized with cyclophosphamide (CY) $2 \mathrm{~g} /$ $\mathrm{m} 2$ in day +1 and daily GCSF ( $5 \mu \mathrm{g} / \mathrm{kg}$ subcutaneously) starting from day +3 from $\mathrm{CY}$ until the completion of the harvest.

Results: Of the total cohort, 35 patients (79\%) underwent mobilization with $\mathrm{CY}+\mathrm{GCSF}$ uneventfully. Only 2 patients (4\%) had an exacerbation of MS (one with fatigue and increase of spasticity, other with worsening weakness) during the mobilisation period. Five patients required hospital admission before or during the mobilization procedure: four patients developed neutropenic fever (including one septic episode) and one patient developed intractable G-CSF associated back pain. Two additional patients were hospitalised because of non-neutropenic 
Correlation between PB CD34 concentration and collection yield

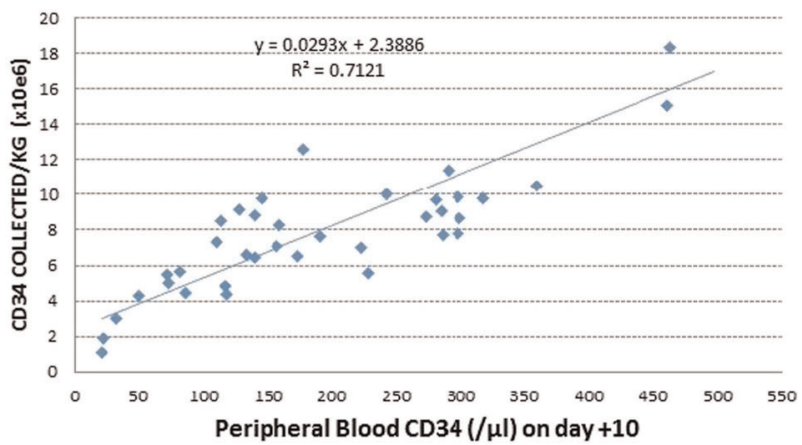

[P084 Figure] [Correlation between PB CD34 concentration and collection yield]

fevers and flare-up of MS symptoms after the stem cell collection.

The median CD34+ cell dose was $7.75 \times 10^{6} / \mathrm{kg}$ (range 2.2-24.3). The median number of apheresis was 1 (range 12 ), with only 2 patients (4\%) needing a second day of collection. All but one patient mobilised very predictably sufficient stem cells on day +10 after Cyclophosphamide allowing for a scheduling of Cyclophosphamide on a Friday and stem cell collection on a Monday. The peripheral blood CD34+ cells concentration on day +10 correlated with the final CD34+ cells yield of the collection (figure 1).

Since February 2017, all Cyclophosphamide infusions were given as an out-patients procedure. There were no differences in post mobilisation complications or stem cell collection efficiency between 20 (44\%) in-patient and 25 (56\%) out-patient mobilisations.

Conclusions: In our hands, after CY and GCSF mobilization there is a very low rate of exacerbations of MS symptoms and an acceptable rate of post mobilisation complications. Exacerbations of MS symptoms could occur after the stem cell collection procedure has been completed, raising the need for a prolonged follow up.

Conflict of interest: Nothing to disclose

\section{P085}

\section{Primary Immunodeficiency Disorders behind haematological cytopenia: early recognition for a prompt treatment strategy}

Sara Ciullini Mannurita ${ }^{1}$, Maddalena Bagni ${ }^{1}$, Marina Vignoli ${ }^{1}$, Fabio Tucci ${ }^{2}$, Veronica Tintori ${ }^{3}$, Stefano Frenos ${ }^{3}$, Maria Chiara Sanvito $^{3}$, Marinella Veltroni ${ }^{2}$, Claudio Favre $^{2}$, Eleonora Gambineri $^{1,3}$

${ }^{1}$ University of Florence, Department of 'NEUROFARBA': Section of Child's Health, Firenze, Italy; ${ }^{2}$ Anna Meyer' Children's Hospital, Department of Haematology-Oncology, Florence, Italy; 'Anna Meyer'
Children's Hospital, Department of Haematology-Oncology: BMT Unit, Florence, Italy

Background: Cytopenia, defined as the reduction of one or more mature blood cell types (eg. neutropenia, anemia, or thrombocytopenia) in the peripheral blood, may be a typical first symptom of primary immunodeficiency disorders (PIDDs). Possible causes of cytopenia in PIDDs comprise mainly immune dysregulation, bone marrow failure (BMF) and myelodysplasia. Our goal is to investigate possible immune mediated mechanisms underlying chronic cytopenia in children in order to to achieve an early diagnosis and consequently offer timely and appropriate therapy.

Methods: Among the patients referred to our Paediatric Haematology/Oncology Unit, we selected thirty-five patients affected by chronic cytopenia. These patients were characterized throughout clinical, immunological, haematological and genetic investigations. Nineteen patients were further evaluated with immunophenotyping by flow cytometry and Next Generation Sequencing (NGS) analysis of a gene panel including genes frequently implicated in immunodeficiency or immunodysregulation.

Results: Among thirty-five patients, seven patients showed a distinctive early onset clinical and haematological phenotypes and were promptly diagnosed with Fanconi Anemia, Shwachman-Diamond syndrome and severe congenital neutropenia; four patients were defined as affected by uncharacterized BMF and twenty-four affected by immune-mediated cytopenia (fourteen cases of isolated autoimmune neutropenia, one case of neutropenia associated with thrombocytopenia, two cases of autoimmune pancytopenia and seven patients with different combinations of cytopenia and multi-organ autoimmunity).

Immune phenotype analysis was performed on nineteen patients with immune mediated cytopenia. A specific pattern was found with abnormalities affecting T CD8 and B subtypes. We observed a decrease of CD8 effector memory (EM) and terminally differentiated (TEMRA) cell populations, while an increase of CD8 naive and central memory (CM) cells levels. B lymphocytic populations showed a decrease in total B cells, B switched cells and B memory cells and an increase in CD21low cells. Absence of plasmacytoid and monocytoid dendritic cells was observed in one case.

Preliminary results from NGS studies in 4 patients revealed mutations in the following genes: STAT3 (gainof-function mutation), IKAROS, GATA2 and a novel gene not previously associated with autoimmunity and immune dysregulation. Further validation studies are ongoing.

Based on our immunological and genetic studies four patients were successfully treated with haematopoietic stem cell transplantation (HSCT) in haploidentical, matched- 
related and unrelated setting using reduce intensity conditioning.

Conclusions: Our findings show how the presence of specific lymphocyte subpopulation patterns can be an important indicator of an immune-mediated pathogenesis of cytopenia and a helpful warning sign of PIDDs that should promptly be investigated with genetic analysis. Based on our experience an early diagnosis offers timely and appropriate therapy. HSCT reverses most of the inherited conditions with good long-term outcomes.

Conflict of interest: None of the authors has anything to disclose.

\section{P086}

Prognostic factors for clinical response in systemic lupus erythematosus patients treated by allogeneic mesenchymal stem cell

Lihui Wen ${ }^{1}$, Myriam Labopin ${ }^{2}$, Manuela Badoglio ${ }^{3}$, Dandan Wang ${ }^{1}$, Dominique Farge ${ }^{4}$, Lingyun Sun ${ }^{1}$

${ }^{1}$ The Affiliated Drum Tower Hospital of Nanjing University Medical School, Department of Rheumatology and Immunology, Nanjing, China; ${ }^{2}$ iEBMT Data Office, Sant Antoine Hospital, Department of Haematology, INSERM UMR 938, Université Pierre et Marie Curie, Paris, France; ${ }^{3}$ Hopital Saint-Antoine, EBMT Data Office, INSERM UMR 938, Paris, France; ${ }^{4}$ Hôpital St-Louis, AP-HP Assistance Publique des Hôpitaux de Paris, CRMR Centre de Référence des Maladies Auto-Immunes Systémiques Rares d'Iles-de-France, Filière 'FAI2R', Paris Denis Diderot University, Unité Clinique de Médecine Interne, Maladies Auto-Immunes et Pathologie Vasculaire, UF 04, Paris, France

Background: Using a single-center cohort of Chinese adult patients with systemic lupus erythematosus (SLE), defined by ACR criteria, we analyzed the predictors of the clinical response observed during the year after bone marrow-derived (BM) and/or umbilical cord-derived (UC) allogeneic mesenchymal stem cell (MSC) treatment.

Methods: Inclusion criteria were : adult active SLE patients (age $>18$ years) with a SLE Disease Activity Index (SLEDAI) score $\geq 8$, despite prior treatment (oral cyclophosphamide (CYC) $\left(500-700 \mathrm{mg} / \mathrm{m}^{2}\right)$ for $\geq 6$ months or mycophenolate mofetil (MMF) $(\geq 1000 \mathrm{mg} / \mathrm{d}$ ) or leflunomide (LEF) $(20 \mathrm{mg} / \mathrm{d})$ for $\geq 3$ months, or prednisone $\geq 20$ $\mathrm{mg} / \mathrm{d}$ with or without hydroxychloroquine (HCQ)) who received at least one intravenous injection of allogeneic BM and/or UC-MSC (1 million/kilo bodyweight). SLE clinical and biological symptoms, SLEDAI score were assessed at baseline, 1, 3, 6, and 12 months during follow-up. Clinical remission (CR) was defined as SLEDAI $<3$ without major organ activity, and $\leq 5 \mathrm{mg} /$ day prednisone with or without maintenance oral CYC, MMF, LEF. Low disease activity (LDA) was defined by SLEDAI score $\leq 4$ without major organ activity, and prednisone $\leq 7.5 \mathrm{mg} / \mathrm{day}$, with or without maintenance oral CYC, MMF, LEF. Prognostic factors for CR and LDA were identified by univariate and multivariate logistic regression. All variables associated with response in univariate analysis $(\mathrm{p} \leq 0.10)$ were included in the model, then a stepwise selection was used.

Results: Among 69 (61 female) patients, median aged 32y (range: 20-54), baseline SLEDAI was 13 (8-34), 39\% were anti-dsDNA positive, $43.5 \%$ had low serum complement levels and SLE duration before MSC injection was 62 months (range: 2-264). Prior treatment consisted of CYC (68.1\%), MMF (27.5\%), LEF (20.3\%), prednisone (100\%) or HCQ (44.9\%), 42 CYC induction (60.9\%). All patients received at least one (18 BM/51 UC) MSC injections or two within 1 month, using the same MSC dose and source in 24 patients (9 BM/15 UC). Median (range) of SLEDAI at 1, 3, 6, 12 months after MSC injection were respectively 9 (420), 8 (0-16), $6(0-18)$ and $5(0-18)$ (figure 1). CR was achieved for $16(23.2 \%)$ patients and LDA in $40(58 \%)$. Using multivariate analysis, older age $(\mathrm{OR}=0.90, p=$ $0.025)$, no arthralgia/arthritis ( $\mathrm{OR}=0.06, p=0.002)$, no prior oral CYC $(\mathrm{OR}=0.08, p=0.005)$ or $\mathrm{HCQ}(\mathrm{OR}=$ $0.08, p=0.008)$ were associated with higher CR rate. No association was found between $\mathrm{CR}$ and anti-dsDNA, cardiorespiratory disorder, prior prednisone, $\mathrm{CYC}$ induction or a second MSC injection. By univariate analysis, older age and no arthralgia/arthritis were associated with higher rate of LDA $(p=0.006$ and $p=0.03)$. All patients with neurological disorder $(\mathrm{n}=5)$ at baseline achieved LDA.

Conclusions: In active SLE patients, one intravenous injection of allogeneic MSC allowed 23\% CR and 58\% LDA within a year. Further studies, using unique immunosuppressive regimen and MSC sources in

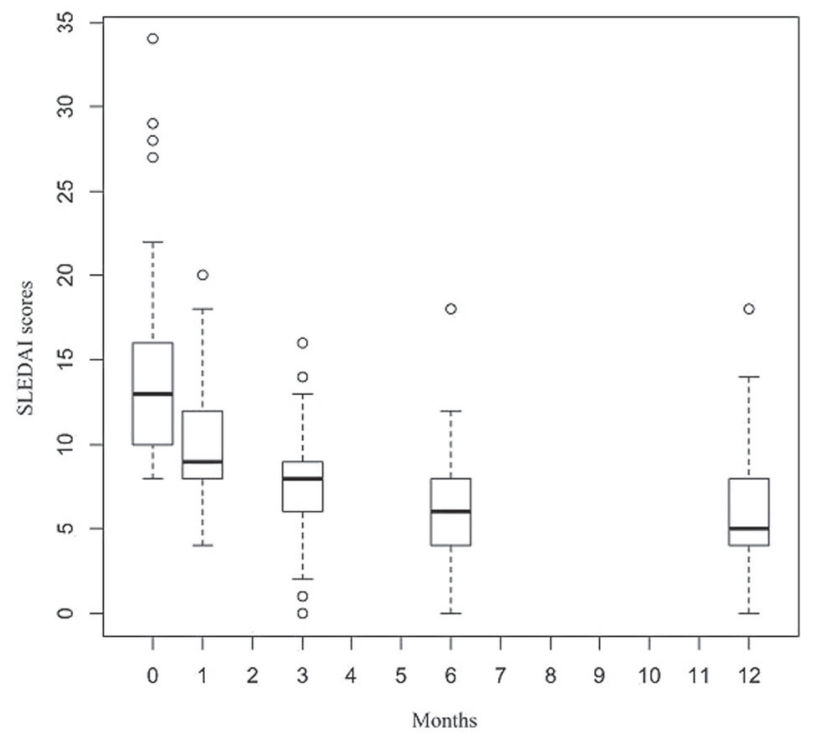

[P086 Figure] [Improvement of SLEDAI score during the 12 months after allogeneic MSC injection] 
collaboration with EBMT will allow refined analysis for the determinants of clinical response to allogenic MSC, a promising therapeutic option in multi-systemic SLE.

Conflict of interest: The authors declare no conflict of interest.

\section{P087}

\section{Reconstitution of natural or vaccination-driven immunity after aHSCT in multiple sclerosis}

Chiara Innocenti ${ }^{1}$, Maria Pia Amato ${ }^{2}$, Alessandro Barilaro ${ }^{2}$, Gaetano Bianchini, Teresa Capobianco ${ }^{3}$, Maria Antonia Calia ${ }^{3}$, Romina Ceschini ${ }^{3}$, Arianna Fani ${ }^{1}$, Marta Giannini ${ }^{2}$, Antonella Gozzini $^{1}$, Stefano Guidi ${ }^{1}$, Alice Mariottini ${ }^{2}$, Luca Massacesi ${ }^{2}$, Chiara Nozzoli $^{1}$, Anna Repice ${ }^{2}$, Gianmaria Rossolini ${ }^{3,4}$, Riccardo Saccardi ${ }^{1}$

${ }^{1}$ Careggi University Hospital of Florence, Cell Therapy and Blood Bank, Florence, Italy; ${ }^{2}$ Careggi University Hospital of Florence, Neurology, Florence, Italy; ${ }^{3}$ Careggi University Hospital of Florence, Microbiology and Virology Unit, Florence, Italy; ${ }^{4}$ Careggi University Hospital of Florence, Department of Experimental and Clinical Medicine, Florence, Italy

Background: In the last 20 years, high dose chemotherapy with autologous hematopoietic stem cell transplantation (aHSCT) has emerged as an effective and safe treatment for aggressive forms of multiple sclerosis (MS). Nevertheless, no data have been published about reconstitution of natural or vaccination-driven immunity after aHSCT and the indication to re-vaccinate patients. We report an analysis of antigen-specific immune recovery in a series of MS patients after aHSCT.

Methods: Blood samples from 22 MS patients who underwent aHSCT in our Center between 2006 and 2017 were analyzed. Each patient underwent mobilization with cyclophosphamide (CTX) 4gr/sqm + GCS- F and was conditioned with BEAM/ATG regimen. Antibody titres for varicella-zoster, measles, rubella, polio, hepatitis B viruses, and tetanus and diphtheria toxins were analysed before mobilization (baseline) and then 2 years after aHSCT. Chemiluminescent Microparticle Immunoassays (CMIA) were performed to determine Rubella and hepatitis B surface antigen antibodies (anti-HBsAg). Enzyme immunoassays were performed to assess Varicella Zoster, measles, diphtheria, tetanus IgG antibodies and $\operatorname{IgG}$ against polioviruses. All patients received prophylaxis with acyclovir and Thrimethoprim-Sulphametoxazole for six months after aHSCT. Patients did not receive re-vaccinations in the follow-up period.

Results: All patients showed a complete and sustained engraftment: median (range) time to $\mathrm{PMN} \geq 0.5 \times 10^{9} / \mathrm{L}$ and platelets $\geq 20 \times 10^{9} / \mathrm{L}$ were $12(9-15)$ and $11(7-14)$ days, respectively. Patients showed a complete return to a normal lymphocyte subsets counts within two years from transplant. No case of measles, rubella, or chickenpox occurred after transplant, with median follow-up of 40(17124) months. Table 1 reports antibodies titres at baseline and 2 years after aHSCT. A loss of serum protective immunity was observed in a variable rate of patients with all the tested agents, ranging between $11 \%$ in measles and $44 \%$ in diphtheria.

Conclusions: Re-vaccination in patients who underwent an autologous HSCT for a severe autoimmune disease is a common practice in many centers and is also recommended in the EBMT guidelines. However, some concerns about this practice was raised for the putative role of vaccinations as a autoimmunity trigger; indeed, no evidences about the loss of protective antibody titer in either natural or vaccinationdriven immunity were reported in patients transplanted for autoimmune diseaseas. In our series, a high variability in antibodies titers at baseline was shown; however, most patients showing a protective titre against the tested agents showed a trend to maintain it. A possible approach might be to revaccinate the patients with unprotective titre at 2 years after HSCT; a larger series of data is necessary to provide conclusive evidences on this topic.

Conflict of interest: None of the authors has anything to disclose.

\begin{tabular}{|c|c|c|c|c|c|c|c|c|}
\hline & $\begin{array}{l}\text { pre } \\
\text { aHSCT } \\
V Z V I g\end{array}$ & $\begin{array}{l}+2 \\
\text { years }\end{array}$ & $\begin{array}{l}\text { pre } \\
\text { aHSCT } \\
R U B E L L\end{array}$ & $\begin{array}{l}+2 \\
\text { years } \\
A \operatorname{Ig} G\end{array}$ & $\begin{array}{l}\text { pre } \\
\text { aHSCT } \\
M E A S L E\end{array}$ & $\begin{array}{l}+2 \\
\text { years } \\
S \operatorname{Ig} G\end{array}$ & $\begin{array}{l}\text { pre } \\
\text { aHSCT } \\
\text { antiHBS }\end{array}$ & $\begin{array}{l}+2 \\
\text { years }\end{array}$ \\
\hline negative & 1 & 3 & 2 & 3 & 18 & 16 & 11 & 13 \\
\hline positive & 20 & 16 & 20 & 17 & 3 & 6 & 11 & 9 \\
\hline \multirow[t]{2}{*}{ uncertain } & 1 & 3 & 0 & 2 & 1 & 0 & & \\
\hline & \multicolumn{2}{|c|}{ POLIO IgG } & \multicolumn{2}{|c|}{$\begin{array}{l}\text { TETANUS } \\
\text { IgG }\end{array}$} & \multicolumn{2}{|c|}{$\begin{array}{l}\text { DIPHTHERIA } \\
\text { IgG }\end{array}$} & & \\
\hline negative & 1 & 4 & 0 & 0 & 8 & 11 & & \\
\hline positive & 17 & 10 & 15 & 9 & 9 & 5 & & \\
\hline uncertain & 4 & 8 & 7 & 13 & 5 & 6 & & \\
\hline
\end{tabular}

[[P087 Table] Antibody titres at baseline and 2 years]

P088

Successful unrelated umbilical cord blood transplantation with reduce intensity conditioning for very early onset Inflammatory bowel disease: an innovative report from China

Xiaowen Zhai, Xiaowen Qian

Children's Hospital of Fudan University, Department of Hematology/ Oncology and Bone Marrow Transplant Unit, Shanghai, China

Background: Inflammatory bowel disease (IBD) is a chronic disorder of the gastrointestinal tract. Very early onset IBD (VEO-IBD) represents those severe children with 
disease onset occurring before 6 years old. Interleukin-10 receptors (IL-10RA, IL-10RB) mutation are considered to be one of the very important genes for VEO-IBD. Currently variant treatment, such as steroid medication, immunosuppressive agents, biological agents could not get complete remission. Allogeneic hematopoietic stem cell transplantation (allo-HSCT) was reported to induce remission in those with VEO-IBD.

Methods: We performed unrelated umbilical cord blood transplantation (UCBT) in 13 consecutive children with VEO-IBD due to IL-10 receptor mutation between 2015 and 2017. Median age of 13 children was 11 months (range, 4 to 46 months), and median body weight was $7 \mathrm{~kg}$ (range, 3.2 to $10 \mathrm{~kg}$ ). All patients received reduced intensity conditioning (RIC) regimen consisting of busulfan, fludarabine and cytarabine. Prophylaxis for graft-versus-host disease (GVHD) was tacrolimus. All patients received a $\leq 2$ HLA alleles-mismatched cord unit, 2 were HLA fully matched, 3 were $9 / 10$ matched, 8 were $8 / 10$ matched. Median nucleated cells of the cord blood were $14.29 \times 10^{7} / \mathrm{kg}$ (range, 7.06 to $51.5 \times 10^{7} / \mathrm{kg}$ ), and median $\mathrm{CD} 34+$ cells were $4.99 \times 10^{5} / \mathrm{kg}$ (range, 1.55 to $18.39 \times 10^{5} / \mathrm{kg}$ ).

Results: 10/13 (76.9\%) patients engrafted, median time of neutrophil engraftment was 25.5 days, and median time of platelet engraftment was 27 days. Median follow-up time was 6 months (range, 2 to 29 months), 9/13 (69.2\%) patients were alive with continuous donor engraftment, and achieved complete clinical remissions. Colonoscopy at 6-12 months after transplantation in 6 children revealed the mucosa healing. 2 patients had grade III/IV acute graftversus-host disease (GVHD). 4/13 (30.8\%) patients died, and the causes of death in 3 patients was infection leading to multi-organ failure after graft failure that occurred at 1-2 months after transplant, 1 patient died of HHV6 virus infection in the lungs and bronchiolitis obliterans.

Conclusions: It is the first clinical trial that unrelated UCBT was performed in VEO-IBD children in China. Our data should unrelated UCBT with RIC should be considered as a potentially curative therapeutic option in children with VEO-IBD.

Conflict of interest: The author declare that they have no conflict of interest.

\section{Cell therapy / cellular therapy}

\section{P089}

\section{Adoptive immunotherapy with virus specific $T$ cell: Experience of 2 Spanish cell-therapy laboratories}

Laura Alonso ${ }^{1}$, Alba Perez ${ }^{2}$, Maria Isabel Benitez ${ }^{1}$, Luisa Sisinni ${ }^{3}$, Eduardo Lopez Granados ${ }^{4,5}$, Guillermo Orti ${ }^{6}$, Pere Soler-Palacin ${ }^{7}$, Raquel De Paz Arias ${ }^{8}$, Margarita Codinach ${ }^{9}$, Francesc Rudilla, Cristina Diaz de Heredia ${ }^{1}$, Antonio Perez-Martinez ${ }^{2}$, Sergio Querol ${ }^{9}$
${ }^{1}$ Hospital Universitario Materno Infantil Vall d'Hebron, Pediatric Hematology and Oncology Department, Barcelona, Spain; ${ }^{2}$ Hospital Infantil Universitario La Paz, Servicio de Hemato-Oncología Pediátrica, Madrid, Spain; ${ }^{3}$ Santa Creu i Sant Pau HospitalUniversitat Autònoma, Pediatric Hematology, Oncology and HSCT Unit, Barcelona, Spain; ${ }^{4}$ Hospital Universitario La Paz, Clinical Immunology Department, Madrid, Spain; ${ }^{5}$ La Paz Biomedical Research Institute-IdiPAZ, Madrid, Spain; ${ }^{6}$ Hospital Universitario Vall d'Hebron, Hematology, Barcelona, Spain; ${ }^{7}$ Hospital Universitario Materno Infantil Vall d'Hebron, Unitat de Patologia Infecciosa $i$ Immunodeficiències de Pediatria, Barcelona, Spain; ${ }^{8}$ Hospital Universitario La Paz, Servicio de Hematología, Madrid, Spain; ${ }^{9}$ Banc de Sang $i$ Teixits, Barcelona, Spain

Background: Viral infections are a leading fatal complication for immunosuppressed patients, such as patients after hematopoietic stem cell transplantation (HSCT) and patients with primary immunodeficiencies (PIDs). Use of virus-specific T lymphocytes (VSTs) has been successful for the treatment and prevention of viral infections in these settings. Here we describe our experience with the clinical use of VSTs.

Methods: Retrospective review of the lymphoapheresis procedure, IFN $\gamma$ selection yield and clinical results of the VSTs produced in the Banc de Sang i Teixits and Hospital La Paz between 2014 and 2017.

Results: We assessed the outcome of 15 patients who experienced 17 different viral infections and were treated with virus specific T cells. A total of 27 VST infusions were analysed. Median age was 4.9 years (0.8-36).

Eleven patients received the infusion in the context of post-haematopoietic stem cell transplant viral infections. The indication in the rest of the patients was immunodeficiency- associated CMV infection in 3 patients and EBV chronic active disease in 1.

Donors: Donors were deemed fit to donate and were chosen depending on IFN- $\gamma$ secretion capacity and HLA match. For 10 of the 11 transplant patients, third-party donors were used. When multiple infusions were required for an episode of infection because of relapse or lack of response, the same donor was used in 5 patients and a different donor in 1 .

Toxicity: There were no complications associated to the apheresis procedure. Only one of the donors required the placement of a central venous catheter.

Two patients experienced infusion reaction and one developed aplasia. One of the patients experienced grade 2 acute GVHD secondary to the treatment and died of sepsis.

Response:

1. HSCT patients

EBV VST: One of the patients with EBV PTLD and CSF cleared infection in the CSF but his symptoms did not improve significantly and subsequently relapsed. Two patients did not show any response and received treatment with brentuximab. 
CMV VST: Four of the 5 patients with CMV infection cleared the virus, the other patient died of progression.

Adenovirus VST: one patient with viremia responded and cleared the viremia within 3 weeks, the patient with adenovirus disease progressed and died of disease.

EBV + CMV VST: both patients showed response

2. Non-HSCT patients

None of the non-transplant patients with CMV infection responded, 2 of them died of disease. The patient with EBV chronic active disease progressed and died of disease.

Donor cells were detected by chimerism in 4 patients.

Overall outcome: 6 patients died of progression and 1 patient died of sepsis.

Conclusions: The production of VSTs derived from either stem cell donors or third-party donors is feasible and this treatment is likely safe and effective especially for the treatment of viral infections after HSCT.

Clinical Trial Registry: No

Conflict of interest: None

\begin{tabular}{|c|c|c|c|c|}
\hline $\begin{array}{l}\text { Viral } \\
\text { specificity }\end{array}$ & $\begin{array}{l}\text { Number of } \\
\text { patients }\end{array}$ & $\begin{array}{l}\text { Number of } \\
\text { infusions }\end{array}$ & $\begin{array}{l}\text { Mean CD3 IFN } \gamma / \\
\mathrm{Kg}\end{array}$ & $\begin{array}{l}\text { Mean CD3 } \\
\text { nonIFN } \gamma / \mathrm{Kg}\end{array}$ \\
\hline CMV & 9 & 16 & $\begin{array}{l}2,23 \mathrm{E}+05(2,29 \mathrm{E} \\
+03-6,20 \mathrm{E}+05)\end{array}$ & $\begin{array}{l}9,89 \mathrm{E}+03(5,66 \mathrm{E} \\
+03-1,40 \mathrm{E}+04)\end{array}$ \\
\hline EBV & 4 & 6 & $\begin{array}{l}1,51 \mathrm{E}+04(2,57 \mathrm{E} \\
+03-3,25 \mathrm{E}+04)\end{array}$ & $\begin{array}{l}9,38 \mathrm{E}+03(2,01 \mathrm{E} \\
+03-2,50 \mathrm{E}+04)\end{array}$ \\
\hline Adenovirus & 2 & 3 & $\begin{array}{l}6,63 \mathrm{E}+02(4,02 \mathrm{E} \\
+02-9,86 \mathrm{E}+02)\end{array}$ & $\begin{array}{l}3,28 \mathrm{E}+03(2,10 \mathrm{E} \\
+03-4,46 \mathrm{E}+03)\end{array}$ \\
\hline $\begin{array}{l}\mathrm{CMV}+ \\
\mathrm{EBV}\end{array}$ & 2 & 3 & $\begin{array}{l}1,90 \mathrm{E}+04(7,79 \mathrm{E} \\
+03-3,02 \mathrm{E}+04)\end{array}$ & $\begin{array}{l}9,22 \mathrm{E}+03(8,51 \mathrm{E} \\
+03-9,92 \mathrm{E}+03)\end{array}$ \\
\hline
\end{tabular}

\section{[[P089 Table] Product characteristics]}

\section{P090}

Autologous umbilical cord blood in children with autism or in children with cerebral palsy - first applications in Poland

\author{
Anna Mucha ${ }^{1}$, Magda Chroscinska-Krawczyk ${ }^{2}$, Monika Kotarska ${ }^{2}$, \\ Krystyna Mitosek - Szewczyk ${ }^{2}$, Kamila Poteralska ${ }^{1}$, Joanna \\ Placzkowska ${ }^{1}$, Julia Piskula ${ }^{1}$, Agnieszka Orczykowska ${ }^{1}$, Julia \\ Walędzik $^{1}$, Karolina Zagórska ${ }^{1}$, Mariusz Grudniak ${ }^{1}$, Dominika \\ Gtadysz $^{1}$, Iwona Marszatek ${ }^{1}$, Maciej Boruczkowski ${ }^{3}$, Tomasz Oldak ${ }^{1}$, \\ Dariusz, Boruczkowski ${ }^{1}$ \\ ${ }^{1}$ PBKM, Warsaw, Poland; ${ }^{2}$ Lublin Medical University, Department of \\ Pediatric Neurology, Lublin, Poland; ${ }^{3}$ University of Medical Sciences, \\ Poznan, Poland
}

Background: Autologous umbilical cord blood (UCB) represents a potentially revolutionary therapy for neurological disorders such as autism spectrum disorders (ASD) or cerebral palsy $(\mathrm{CP})$. Patients with ASD reveal hyperactivity of the immune system, irregular neuronal growth and increased size and number of microglia. Cerebral palsy is the most common cause of physical disability in children. It may occur due to perinatal hypoxic insults, developmental brain abnormalities, genetic diseases, traumatic or infectious causes. The Polish Stem Cell Bank (PBKM) has provided autologous UCB for neuroprotective and neuroregenerative treatment in children with ASD and CP.

Methods: Eleven patients aged from 4 years and 9 months to 10 years and 3 months (medium age: 5 years and 8 months) were enrolled in this medical treatment. Among all patients, there were 4 Romanian children diagnosed with ASD, who received their own UCB collected in years 20102012. The UCB units were transferred from Biogenis to PBKM in 2017. The remaining 7 patients were Polish (3 patients with ASD, 4 with $\mathrm{CP}$ ) and were given own UCB collected in years 2007-2012.

All Romanian patients and 2 Polish patients had 1 UCB injection, the remaining 5 children received 2 UCB injections. Characteristics of autologous cord blood units (median and range) are in the table below.

All patients' parents provided signed consent before the start of UCB administration.

Results: All Polish patients have been examined by the same psychologist with PEDI-CAT test after 1 and 3 months of UCB administration. Some of the patients are still waiting for the final test (after 6 months), therefore the comprehensive results will be available in 2018. No adverse events were recorded.

Conclusions: The administration, collection and reinfusion of umbilical cord blood (USB) have proven to be safe and well-tolerated treatment possibility for ASD and CP patients.

\section{Conflict of interest:}

A. Mucha, K. Poteralska, J. Płaczkowska, J. Piskuła, A. Orczykowska, J. Walędzik, K. Zagórska, M. Grudniak, D. Gladysz, I. Marszalek, T. Oldak, D. Boruczkowski: PBKM employees

\begin{tabular}{lllll}
\hline $\mathrm{WBC}\left[10^{\wedge} 3 / \mu \mathrm{l}\right]$ & $\begin{array}{l}\mathrm{CD} 34+[\% \\
\mathrm{WBC}]\end{array}$ & $\begin{array}{l}\mathrm{RBC}\left[10^{\wedge} 6 /\right. \\
\mu \mathrm{l}]\end{array}$ & $\begin{array}{l}\mathrm{NRBC}\left[10^{\wedge} 3 /\right. \\
\mu l]\end{array}$ & $\mathrm{HCT}[\%]$ \\
\hline $26,7(15,45-$ & $0,23(0,07-$ & $\begin{array}{l}0,49(0,11- \\
0,94)\end{array}$ & $\begin{array}{l}0,36(0,02- \\
1,24)\end{array}$ & $\begin{array}{l}7,11(1,8-14 \\
42,39)\end{array}$ \\
\hline
\end{tabular}

\section{[[P090 Table] Table 1]}

\section{P091}

Automated manufacturing of gene-engineered T cells under serum free conditions 
Nadine Mockel-Tenbrinck, Carola Barth, Daniela Mauer, Janina Brauner, Waél Al Rawashdeh, Silke Schult, Olaf Hardt, Georg Rauser, Marion Jurk, Mario Assenmacher, Andrew Kaiser

Miltenyi Biotec GmbH, Bergisch Gladbach, Germany

Background: Manufacturing gene-modified $\mathrm{T}$ cells requires robust and reproducible processes that depend on materials and reagents fulfilling specific safety requirements. Generally these processes require the use of human $\mathrm{AB}$ serum, a reagent that is commercially limited. For most culture system, it is mandatory to ensure potent $\mathrm{T}$ cell expansion yielding high numbers of viable cells. Removing the need for human AB serum, especially in fully automated manufacturing systems during gene-engineered $\mathrm{T}$ cell preparation will reduce costs, dependence on reagents of limited availability and reduce potential risks of viral contaminations while increase commercial scalability to wide demand.

Methods: The CliniMACS Prodigy T Cell Transduction process enables the robust generation of gene-modified $\mathrm{T}$ cells without the need for serum supplementation when using MACS GMP T Cell TransAct in combination with TexMACS GMP Medium (from Miltenyi Biotec). Furthermore, implementation of this potent soluble polyclonal $\mathrm{T}$ cell stimulation reagent, allows for process simplification whereby a manipulation step such as a "bead removal" is rendered unnecessary.

Results: Further developing on a process requiring 3\% human $\mathrm{AB}$ serum, we now demonstrate the robustness of a serum free process capable of yielding comparably high expansion of viable and functional chimeric-antigenreceptor (CAR) modified $\mathrm{T}$ cells. Furthermore, $\mathrm{T}$ cell phenotype analysis indicated, that differentiation of selected and activated $\mathrm{T}$ cells does not differ for the serum free or the process using serum supplementation. Interestingly, significant higher transduction efficiencies and final CAR T cell numbers were observed using serum free condition. Additionally, no differences in the CAR T cell specificity and functionality, analyzed in vitro and in vivo, were observed.

Conclusions: The fully-automated CliniMACS Prodigy $\mathrm{T}$ Cell Transduction process minimize user interactions and enables the preparation of high numbers of functional CAR $\mathrm{T}$ cells in a serum free process. Thereby classical process risks, such as viral contamination due to human $A B$ serum, use of different devices or unnecessary manipulations in a non-automated system are reduced to a minimal. Accordingly, these improvements are the next step towards a commercial fully-automated manufacturing of CAR T cells for the treatment of a large number of patients.

Conflict of interest: This project has received funding from the European Union's Horizon 2020 research and innovation programme under grant agreement No 667980 (CARAT).

\section{P092}

\section{Bad Enemies, Best Weapons! Cell Therapy in Resistant Cytomegalovirus (CMV) Infection in a Child after Stem Cell Transplant (SCT)}

\section{Barbara Torres Guerola ${ }^{1}$, Juan Montoro Gómez ${ }^{2}$, Eduardo Lopez Briz $^{3}$, Sandra Carreño Sánchez ${ }^{1}$, José María Fernández Navarro ${ }^{4}$ \\ ${ }^{1}$ La Fe, Pediatric SCT Unit, Valencia, Spain; ${ }^{2}$ La Fe, Hematology, Valencia, Spain; ${ }^{3} \mathrm{La} F$, Pharmacy, Valencia, Spain; ${ }^{4} \mathrm{La} \mathrm{Fe}, \mathrm{SCT}$ Unit, Valencia, Spain}

Background: Infections remain a major source of morbidity and mortality in the allogeneic stem cell transplant arena. Cord blood transplant recipients account for higher rates of viral infections. Pharmacological treatment is not always successful.

Methods: We present the case of our first patient successfully treated with third party cellular therapy against refractory CMV infection.

4-year-old male with high risk B-ALL, candidate for allogeneic SCT in his second complete remission (CR). No MSD neither MUD available. A 5/8 cord blood unit was chosen. Both donor and recipient were CMV negative. Donor $/$ Receptor $=0+/ 0+$, female/male. Cell dosage $=$ TNC: $14 \times 10 \mathrm{e} 7 / \mathrm{Kg}, \mathrm{CD} 34: 6,5 \times 10 \mathrm{e} 5 / \mathrm{Kg}$. He received prophylactic acyclovir since admission. PCR for CMV detection was performed once a week since conditioning. A positive test in our center is defined by a threshold of 400 copies/mL.

Myeloablative conditioning regimen with thiotepa+ busulfan+fludarabine and ATG (total dose $6 \mathrm{mg} / \mathrm{Kg}$ ). GVHD prophylaxis: tacrolimus and short-course steroids. No important complications in his early post-transplant period. Engraftment day +16 . Discharged day +18 . As per local policy, all blood products were irradiated and filtered before transfusion.

Primary CMV infection on day +30 . Treated with ganciclovir but CMV viremia increased after 2 weeks of adequate therapy. He was then changed to foscarnet. At the same time he presented diarrhea and adenovirus was found in faeces plus mild haematuria with $\mathrm{JC} / \mathrm{BK}$ viruria, so weekly cidofovir was started in combination with foscarnet. Simultaneously tacrolimus was tapered (by day +60 ) and discontinued. No evidence of CMV end-organ disease. Blood counts and biochemistry were normal. His leukemia remained in complete remission and the patient had stable full donor chimerism.

The study of CMV resistances showed: CMV Mutations (UL54) A505V, K513R, A688V. CMV antiviral resistance 
(UL54) FOSCARNET Sensible. GANCICLOVIR Resistent. CIDOFOVIR Resistent.

Facing CMV infection resistant to first and second line treatment, we decided to test the benefit of third party cell therapy. As per local regulations, informed consent was obtained. We contacted with Memorial Sloane Kettering Cancer Center, NY, NY, USA. A suitable cell line of CMV specific Cytotoxic T Lymphocytes (CTL) was identified

Results: Five months post-transplant, our patient received cell therapy. The dose of anti CMV specific CTLs administered was $1 \times 10 \mathrm{e} 6 / \mathrm{kg}$ per day, three doses on days 1,8 and 15. No immediate side effects registered. A quick decrement on his CMV viral load was observed after second CTL infusion. Further controls have remained negative.

No GVHD has been detected so far nine months posttransplant, four months after specific anti CMV CTL infusion. Regarding immune reconstitution CD4, CD8 and immunoglobulin levels are normal. He remains in complete remission and full donor chimerism. He's back to school and his performance status is excellent (Lansky 100\%).

Conclusions: Specific third party CTLs have played a key role in our patient's treatment. In the future early inclusion in the management of difficult CMV infections should be considered.

Clinical Trial Registry: NA

Conflict of interest: NA

\section{P093}

CAR-T cells targeting $\alpha_{v} \beta_{3}$ integrin confer complete remission of epithelial cancers in pre-clinical models in vivo

\section{Lars Wallstabe ${ }^{1}$, Christoph Rader ${ }^{2}$, Hermann Einsele ${ }^{1}$, Michael Hudecek $^{1}$}

${ }^{1}$ Universitätsklinikum Würzburg, Medizinische Klinik und Poliklinik II, Würzburg, Germany; ${ }^{2}$ The Scripps Research Institute, Department of Immunology and Microbiology, Jupiter, FL, United States

Background: Integrins are heterodimeric transmembrane receptors that act in cell-cell and cell-matrix interactions. Expression of the $\alpha_{\mathrm{v}} \beta_{3}$ integrin (vitronectin receptor; $\alpha_{\mathrm{v}}$ : $\left.\mathrm{CD} 51=I T G A V \& \beta_{3}: \mathrm{CD} 61=I T G B 3\right)$ has been documented in several entities including melanoma, breast and colorectal cancer and glioblastoma where it enhances tumor cell survival and metastasis. Here we evaluated the antitumor function of $\alpha_{v} \beta_{3}$-specific CAR-T cells in vitro and a murine xenograft model in vivo.

Methods: Expression of $\alpha_{v} \beta_{3}$ integrin was analyzed by flow cytometry. CARs with a targeting domain derived from humanized mAB LM609 (higher vs. lower affinity clone v7 \& v11), equipped with either a long or short IgG$\mathrm{Fc}$ spacer (Hinge-CH2-CH3 vs. Hinge-only), each with a signaling module of $\mathrm{CD} 28 / \mathrm{CD} 3 z e t a$ (4 constructs) were expressed in lentiviral vectors for gene-transfer into $\mathrm{CD}^{+}$ and $\mathrm{CD}^{+} \mathrm{T}$ cells $(\mathrm{n}=3$ donors $) . \mathrm{CAR}^{+} \mathrm{T}$ cells were enriched to $>90 \%$ purity prior to functional testing.

Results: We confirmed high-level expression of $\alpha_{\mathrm{v}} \beta_{3}$ integrin on A375 melanoma and MDA-MB-231 breast cancer, and to a lower extent on Jurkat T-cell lymphoma cell lines. We observed specific and very potent lysis mediated by $\alpha_{v} \beta_{3}$ CAR-T cells, e.g. A375 there was $>60 \%$ specific lysis within a 4-hour co-culture with $\mathrm{CD} 8^{+} \mathrm{CAR}-\mathrm{T}$ cells $(\mathrm{E}: \mathrm{T}$ ratio $=20: 1)$. Further, we observed high-level production of IFN- $\gamma$ by $\mathrm{CD} 8^{+}$and $\mathrm{CD} 4^{+}$CAR-T cells, and production of IL- 2 by $\mathrm{CD} 4^{+}$CAR-T cells after stimulation with tumor cells, which translated into sustained viability and productive proliferation of CAR-T cells in vitro. Overall, we observed maximum antitumor reactivity with the $\alpha_{v} \beta_{3}$ CAR with short spacer and high-affinity targeting domain (v7).

We engrafted firefly luciferase positive A375 into NSG mice, which led to metastatic tumor growth in all animals. Mice were then treated with a single dose of $\alpha_{v} \beta_{3}$ CAR-T cells $\left(5 \times 10^{6}, \mathrm{CD} 8: \mathrm{CD} 4\right.$ ratio $\left.=1: 1\right)$, which led to complete resolution of all tumor lesions, and complete survival of the CAR-T mouse cohort until the end of the observation period. Mice treated with control $\mathrm{T}$ cells showed rapid tumor progression and inferior survival $(n=6$ mice per group; $\mathrm{p}<0.05)$. CAR-T cell engraftment and in vivo persistence for $>30$ days after adoptive transfer was documented by flow cytometry, and cytokine production was documented by multiplex cytokine analysis from mouse serum. The strongest antitumor effect in vivo was mediated by the $\alpha_{v} \beta_{3}$ CAR with short spacer and lowaffinity targeting domain (v11).

Conclusions: Our data demonstrate that CAR-T cells targeting $\alpha_{v} \beta_{3}$ integrin confer potent antitumor functions in vitro and in vivo. In line with our previous work, we found that extracellular spacer design and receptor affinity affected tumor recognition and were optimized in order to derive a CAR construct with maximum reactivity. Intriguingly, $\alpha_{v} \beta_{3}$ is also expressed on tumor vasculature and cancer-associated fibroblasts, suggesting $\alpha_{v} \beta_{3}$ CAR-T cells may be capable of efficiently migrating through endothelial and connective tissue barriers in the tumor microenvironment to exert their antitumor effect.

Conflict of interest: None

\section{P094}

CAR-T therapy shapes T cell repertoire in B-cell acute lymphocytic leukemia

Xiujian Wang ${ }^{1}$, Tao Sun ${ }^{2}$, Xiao Liu ${ }^{3}$, Pengfei Xu ${ }^{4}$, Chao Jin ${ }^{4}$, Lijuan Ding ${ }^{1}$, Fang $\mathrm{Ni}^{1}$, Yongxian $\mathrm{Hu}^{1}$, Jian $\mathrm{Yu}^{1}$, Yunyun Deng ${ }^{4}$, Hao Zhang $^{1}$, Zuyu Liang ${ }^{1}$, He Huang ${ }^{1}$ 

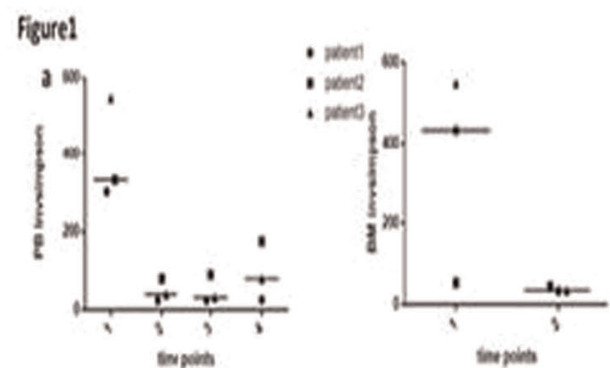

$b$
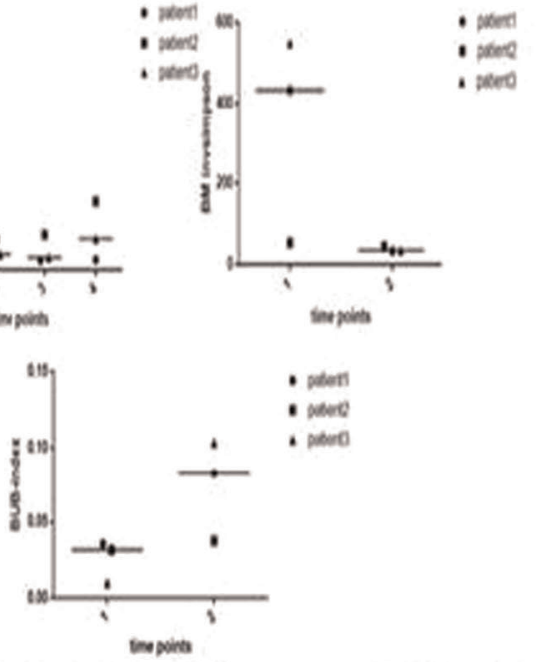
Figurel. a the change of peripheral blood (PB) and bone marrow(BM) T cell reperties
mexsared by insimsson. less invimpson showed narower $T$ cell mapertoires. b the simlanty of $T$ cell noperioires between BM and PB was increased by CAR.T therapy messured by BUS insex Bioger BUB inder indicated more similatity. The number 1 below the $X$ ars represerted timepoint before treztment and the number $2,3,4$ below the $X$ aws represented timepoirt after treatment.

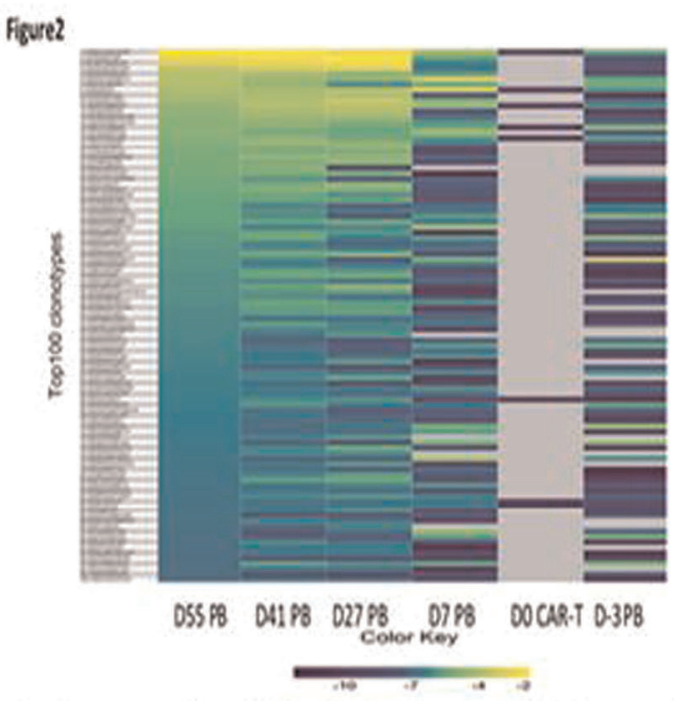

Figurez the represestative graph of done tracing. The graph taded the frequascy of top 100 doose of OSSPB in 0.3 07,027 and DS1PB and DOCART, showing the the TCR sequences of the eniched $\mathrm{T}$ cell dones in blood atter CAR-T thenoy were nx found in CAR.T cell pool and were at lowes frequency in pre-treatenent amples d.3 represented 3 dop betor Car.T thency,d represented the doy when Car.T wa infused and 07,027,041 and 055 recpesented 7,27,4 and 55 das atter CAR-T theno\%, respectiveth.

Figure?

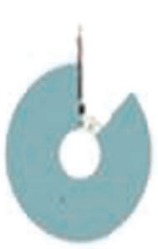

OOCART

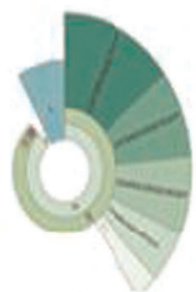

$027 \%$

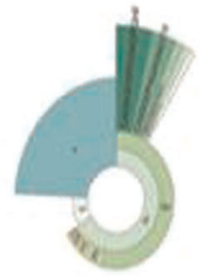

0.396

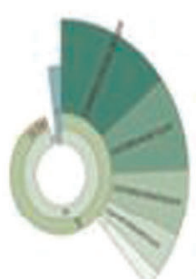

OA:PB

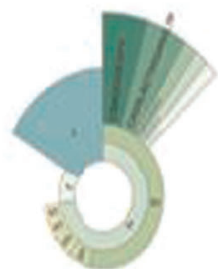

ONP

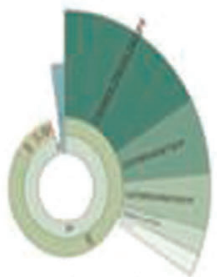

OSSPB

Figuresthe represetative sall gaph. The rasan of 1,2 and $3+$ sugzoted the frequency of TCQ secuences in PB having 1,2 and 3 or 3 more ereds. The amino rod sequence of $T$ cell receptor:TCR) complementary determination region 3 (CDR3) of top 5 dones was

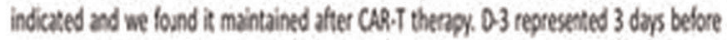

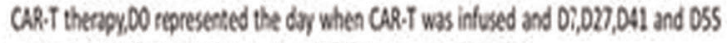
represented 7,27,4 and 55 deps atter CAR.T therop, respectively.

[P094 Figure] [The 3 figures of this abstract] 
${ }^{1}$ Zhejiang University School of Medicine, Bone Marrow Transplantation Center, The First Affiliated Hospital, Hangzhou, China; ${ }^{2}$ University of Chicago, Department of Surgery, Chicago, IL, United States; ${ }^{3}$ University of Chicago, Institute for Molecular

Engineering, Chicago, IL, United States; ${ }^{4}$ Immuquad Biotechnologies, Hangzhou, China

Background: Chimeric antigen receptor (CAR) T cells targeting the B-cell antigen CD19 have displayed potent anti-leukemia activities in refractory/relapsed acute lymphocytic leukemia (ALL). However, the influence on autologous immune system caused by CAR-T cells has not been well examined and elucidated. Here, we applied high-throughput TCR sequencing technology to assess the systemic and dynamic change of $\mathrm{T}$ cell repertoire induced by CAR-T cell therapy in Chinses B-ALL patients.

Methods: 3 female patients in our clinical trial were under observation before and after CAR-T administration, and achieved complete remission (CR) as clinical termination. During CAR-T treatment, they all experienced grade 2 to 3 cytokine release syndrome (CRS). The average age of the patient is 36 years old. The peripheral blood sample, bone marrow sample and the CAR-T sample before infusion were obtained to be tested. The TCR full length mRNAs were deeply sequenced using the ImmunHub ${ }^{\mathrm{TM}}$ TCR profiling system (ImmunQuad Biotech). Briefly, a 5' RACE unbiased amplification protocol was used. An algorithm was applied to raw sequencing data for PCR and sequencing errors correction and $\mathrm{V}, \mathrm{D}, \mathrm{J}, \mathrm{C}$ gene segments mapping with IMGT ${ }^{\circ}$.

Results: We observed the decreased diversity and increased clonality of $\mathrm{T}$ cell repertoire in peripheral blood and bone marrow compared to the pre-treatment controls measured by invsimpson. The TCR sequences of the enriched $\mathrm{T}$ cell clones in blood and bone marrow after CAR-T therapy were not found in CAR-T cell pool. Meanwhile, we found these treatment-associated top TCR sequences were at lower frequency in pre-treatment samples. What's more, The TCR sequences of the enriched $\mathrm{T}$ cell clones could maintain after CAR- $\mathrm{T}$ therapy. Additionally, CAR-T therapy increased the similarity, which was calculated using BUB index, of T cell repertoires between bone marrow and blood and substantially influenced the diversity and clonality of $\mathrm{T}$ cell repertoire.

Conclusions: Our investigation showed the big changes of T-cell diversity and clonality in patient's immune system and the post-treatment homogenization of $\mathrm{T}$ cell repertoires with clonal expansion in bone marrow and blood companying CAR-T cell administration. It implied that the lysis of tumor by CAR-T cell therapy might facilitate the priming and clonal expansion of patient's tumor antigen-specific $\mathrm{T}$ cells systematically. These enriched autologous $\mathrm{T}$ cells may enlarge the therapeutic effect with synergy.
Conflict of interest: None of the authors has anything to disclose.

\section{P095}

changes of T lymphocyte subsets after CAR-T treatment and the possible mechanisms

\author{
Lijuan Ding ${ }^{1}$, Zuyu Liang ${ }^{1}$, Yongxian $\mathrm{Hu}^{2}$, Jian $\mathrm{Yu}^{2}$, He Huang ${ }^{2}$ \\ ${ }^{1}$ Zhejiang University, Hangzhou, China; ${ }^{2}$ Bone Marrow \\ Transplantation Center, The First Affiliated Hospital, School of \\ Medicine, Zhejiang University, Hangzhou, China
}

Background: Chimeric antigen receptor-modified $\mathrm{T}$ cell (CAR-T) therapy, as one of the most promising targeted immunotherapies, has drawn extensive attention and resulted in multiple applications. According to published studies, CD19-directed CAR-T cells (CD19 CAR-T) can reach a complete remission (CR) rate of $93 \%$ and $63 \%$ in refractory/relapsed ALL and lymphoma, respectively. In tumor cell immunity, $\mathrm{T}$ cells play a central regulatory role. $\mathrm{T}$ cells are divided into several major subclasses according to different cell surface molecules, maintaining the body $\hat{A}$ 's immune balance.

Methods: We collected peripheral blood samples from patients with refractory relapsed acute lymphoblastic leukemia and lymphoma at different stages before and after CAR-T treatment. Flow cytometry was used to detect $\mathrm{T}$ lymphocyte subsets in peripheral blood, in order to investigate the impact of CAR-T treatment on tumor cell immunity and its possible mechanisms.

Results: The figures below show the changes of $\mathrm{T}$ lymphocyte subsets after CD19 CAR-T

therapy. Figure A: CD4+ and CD8+ T lymphocytes did not increase significantly, while activated CD4+ and CD8+ $\mathrm{T}$ lymphocytes increased, with more obvious elevation in CD8+ subset. Figure B: Th1 was the only helper T lymphocyte subset during CRS, which explained why IFN$\gamma$ became the dominant cytokine.

Conclusions: Activated CD4+ and CD8 + T lymphocyte were significantly increased during CRS, as well as Th1 subset. The three cell subsets bear the task of killing tumor cells and releasing cytokines.

Conflict of interest: None of the authors has anything to disclose.

\section{P096}

clinical-grade Aspergillus-specific T cells: Discontinued production of the lysate enforces the use of peptide pools and the Switch from magnetic selection to short term expansion 
A

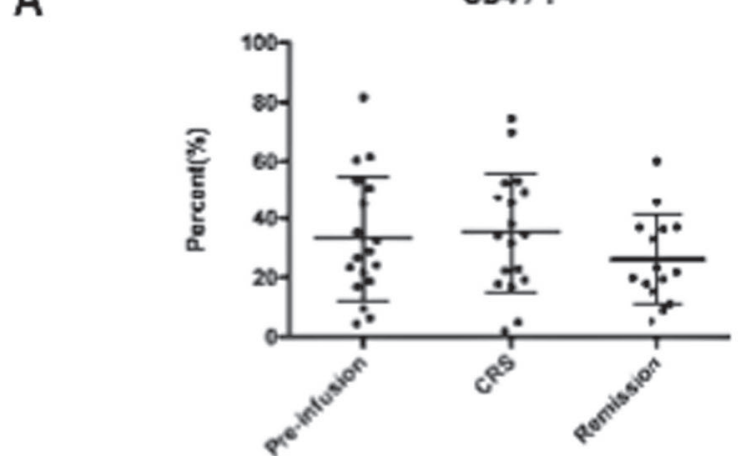

Activated CD4
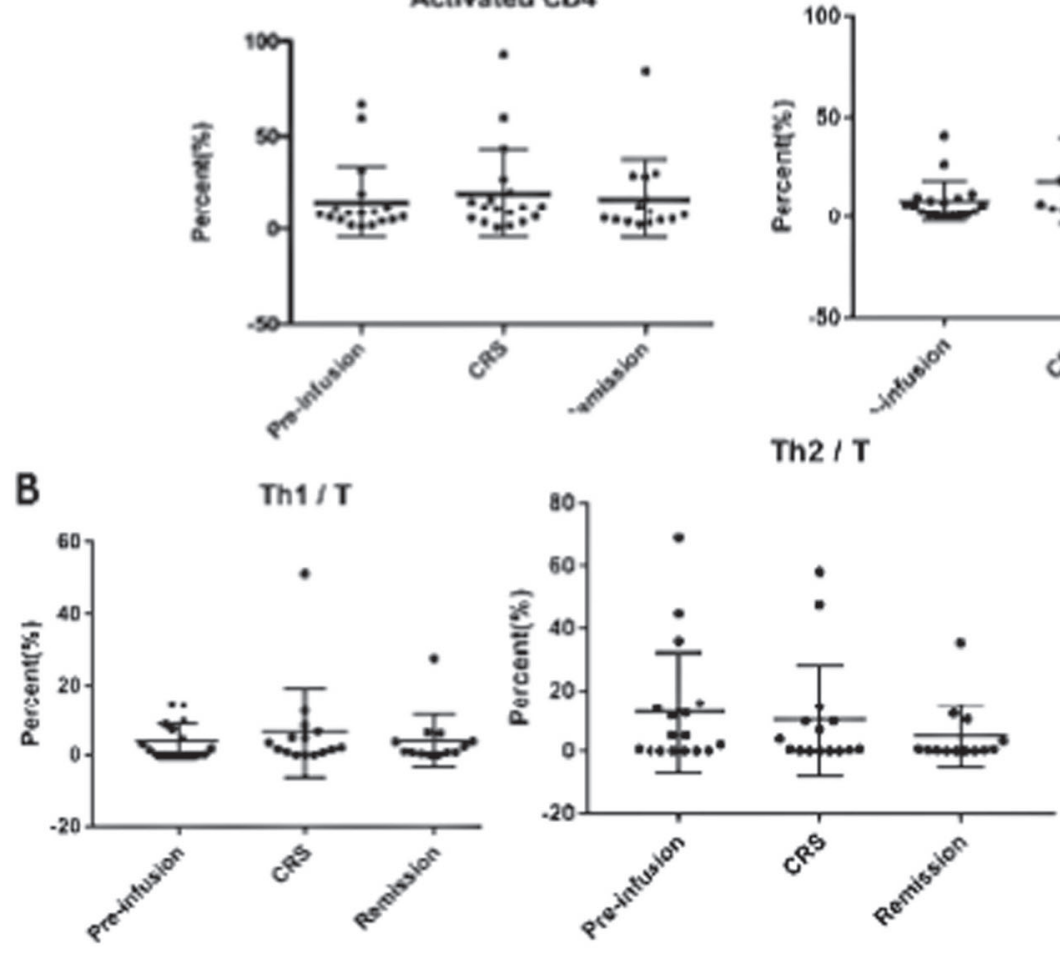

$\operatorname{CDB} / \mathrm{T}$

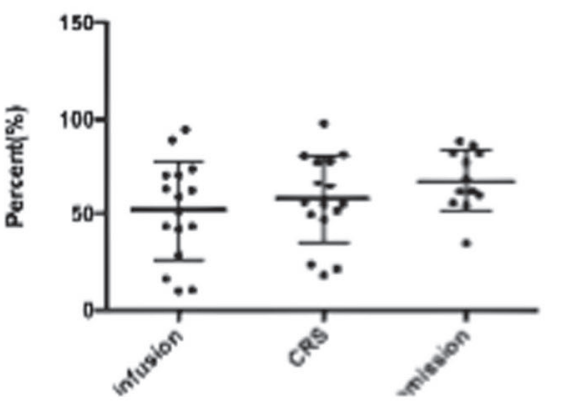

activated $\mathrm{CD} 8$

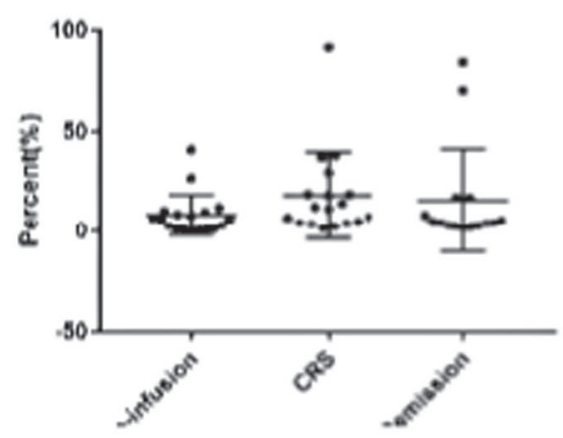

Th2 I T

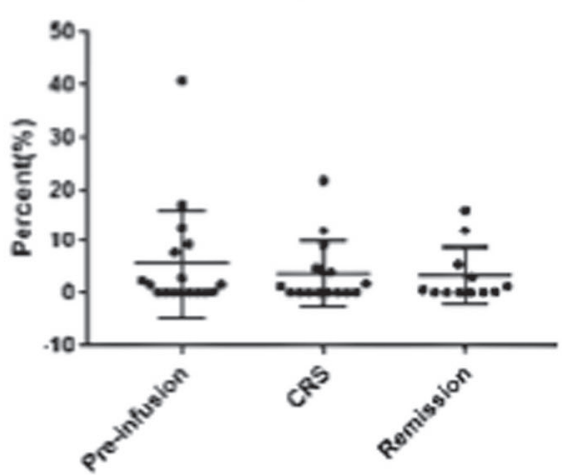

[P095 Figure] [Changes of T lymphocyte subsets]

René Geyeregger ${ }^{1,2}$, Sabine Tischer ${ }^{3,4}$, Nelli Frank ${ }^{1}$, Christine Hoffmann-Freimüller ${ }^{1}$, Julia Stemberger ${ }^{1}$, Britta MaeckerKolhoff $f^{4,5}$, Rainer Blasczyk ${ }^{3,4}$, Gerhard Fritsch ${ }^{1,2}$, Britta EizVesper $^{3,4}$

${ }^{1}$ Children's Cancer Research Institute, Clinical Cell Biology and FACS Core Unit, Vienna, Austria; ${ }^{2}$ Medical University of Vienna, Vienna, Austria; ${ }^{3}$ Hannover Medical School, Institute for Transfusion Medicine, Hannover, Germany; ${ }^{4}$ Hannover Medical School, Integrated Research and Treatment Center (IFB-TX), Hannover, Germany; ${ }^{5}$ Hannover Medical School, Department of Pediatric Hematology and Oncology, Hannover, Germany

Background: Aspergillus-fumigatus (Asp) infections constitute a major cause of morbidity and mortality in patients after haematopoietic stem cell transplantation. We obtained the manufactering liscence to generate clinicalgrade Asp-specific T cells via the Interferon-gamma (IFN-g) Cytokine Capture System(CCS) using a GMP-compliant
Asp-specific lysate. Due to an unexpected discontinued production of the lysate with the end of 2017, we started to find alternatives and tested the use of Asp-specific peptidepools for cytokine secretion assay (CSA) as well as with the already established GMP-coform short term expansion (STE) method.

Methods: Healthy volunteers of the alloCELL registry were screened for the presence of Asp-T-cells via IFN-g Elispot assay by stimulating PBMCs with either lysate $(\mathrm{n}=$ 320) or 7 different Asp-specific overlapping peptide-pools $(\mathrm{n}=30)$ (Cat, Crf1, f22, Gel1, pmp20, SHMT and SOD). PBMCs from leukapheresis $(n=8)$ were prepared in Hannover for the IFN-g-CSA, remaining cells were sent to Vienna to perform STE. For the IFN-g-CSA, $1 \times 10^{7}$ cells were stimulated for $16 \mathrm{~h}$ with GMP-conform lysate or for $4 \mathrm{~h}$ peptide-pools followed by magnetic selection of IFN-g- 
producing Tcells. Cells were characterized for phenotype and function by flow cytometry. For the STE, $2 \times 10^{7}$ cells were stimulated for 12 days with either the lysate or peptide-pools and IL-15. The final cell products were characterized via flow cytometry, IFN-g-EliSpot and IFN-g/ granzyme-B FluroSpot assays.

Results: Asp-T-cells were detectable (detection limit: $>2$ Spots $/ 2.5 \times 10 \mathrm{E} 5 \mathrm{PBMCs})$ in $63 \%$ of all volunteers after lysate stimulation but undetectable in $>85 \%$ of healthy donors if peptide-pools were used. The purity of lysatestimulated IFN-g+ Asp-T-cells after magnetic enrichment using the IFN-g CSA was 49\% (range 17.8\%-84.5\%), while stimulation with the overlapping peptide pools resulted in a non sastisfactory enrichment. In contrast, the STE revealed highly specific Asp-T-cell spot forming colonies(SFC)/10 cells) for both the lysate $\left(863 \pm 153 / 10^{5}\right.$ cells) and combined peptide-pools $\left(833 \pm 138 \mathrm{SFC} / 10^{5}\right.$ cells), respectively. Independent of the stimuli used, predominantly CD4+ T-cells were expanded $(80 \% \pm 3,3$ vs $78 \% \pm 6,3)$ compared to CD8+ T-cells $(17,1 \% \pm 3,1$ vs $18,4 \% \pm 5,6)$ among CD3. Whereas CD4+ T-cells were mainly of central-memory type, CD8+ T-cells comprise mainly effector-memory T-cells. Target cells were highly functional and cytotoxic as determined by secretion of granzyme-B and IFN-g.

Conclusions: Despite the discontinuation of lysate production and consequently enrichment of Asp-T-cells via the IFN-g CCS, clinical-scale production of peptide pool-expanded Asp-T-cells using the STE method might enable an efficient adoptive T-cell immunotherapy.

Conflict of interest: No conflict of interest

\section{P097}

\section{Clinical-grade depletion of T-cell receptor alpha/beta and CD19 positive cells from stem cell products with the CliniMACS Plus and CliniMACS Prodigy devices}

Marienn Reti ${ }^{1}$, Attila Szederjesi ${ }^{1}$, Szabolcs Tasnady ${ }^{1}$, Zita Farkas ${ }^{1}$, Laszlo Gopcsa ${ }^{1}$, Arpad Batai ${ }^{1}$, Aniko Barta ${ }^{1}$, Csaba Kassa ${ }^{2}$, Krisztian Kallay ${ }^{2}$, Gergely Krivan ${ }^{2}$, Tamas Masszi ${ }^{3}$, Peter Remenyi ${ }^{1}$

${ }^{1}$ St Istvan \& St.Laszlo Hospital, Hematology and Stem Cell Transplantation, Budapest, Hungary; ${ }^{2}$ St. Istvan \& St.Laszlo Hospital, Pediatric Hematology \& Stem Cell Transplantation, Budapest, Hungary; ${ }^{3}$ Semmelweis University, 3rd Clinic of Internal Medicine, Budapest, Hungary

Background: The hematopoietic stem cell transplatation is an increasingly used technique for treatment of several hematologic diseases. Cells harvested from peripherial blood (PBSC) or bone marrow (BM) contain cells, which can cause life-threatening graft versus host disease (GvHD). Donor immune cells responsible for GVHD can be selectively removed from the graft using immunomagnetic cell separation. For clinical grade cell depletion, the CliniMACS $^{\circledR}$ Plus (Miltenyi Biotec, Bergisch-Gladbach, Germany) has been the gold standard method so far. However, as a disadvantage, time consuming, extensive manual manipulation is necessary before starting the CliniMACS ${ }^{\circledR}$ Plus separation. Recently, a fully automated, functionally closed system technique - CliniMACS Prodigy - was developed, avoiding any manual manipulation requirement. The aim of our study is to analize and compare the results of $\mathrm{TCR} \alpha \beta^{+} / \mathrm{CD} 19^{+}$cell depletion obtained with the CliniMACS Plus (CM) and the CliniMACS Prodigy Systems (PD).

Methods: During the last 4 years (Jul 30, 2014 - Dec 13, 2017) $31 \mathrm{TCR} \alpha \beta^{+} / \mathrm{CD} 19^{+}$cell depletions (CM:6, PD:25; BM:1, PBSC:30) were performed for 28 patients (pts) (pediatric :12, adult:16).

Results: The results (median values and ranges are shown) were comparable for nucleated cell viability: CM:98\% (93-99), PD:97\% (84-99), $\mathrm{p}=0,56$; $\mathrm{TCR} \gamma \delta^{+}$cell recovery: CM:65\% (31-124), PD:69\% (21-97), $\mathrm{p}=0,81$; NK cell recovery: CM:69\% (46-94), PD: 70\% (33-92), $\mathrm{p}=$ 0,$95 ; \mathrm{TCR} \alpha \beta^{+}$cell depletion: $\mathrm{CM}: 4,1^{\log }(2,6-4,6)$, PD: $3,9^{\log }(3,4-4,6), \quad \mathrm{p}=0,99 ;$ and $\mathrm{CD} 20^{+}$cell depletion: $\mathrm{CM}: 3,0^{\log }(2,0-3,1)$, PD: $2,9^{\log }(1,9-3,6), \mathrm{p}=0,80$; respectively. However we obtained significantly higher CD34 ${ }^{+}$ cell recovery using PD system: CM:70\% (67-85), PD:81\% (57-95), $\mathrm{p}=0,05$.

The cell compositions of the final products were comparable without statistical difference. The median graft cell counts were the following ( $\times 10^{6} /$ recipient body weight $\mathrm{kg})$ : $\mathrm{CD} 34^{+}$cell content: CM:4,7 (2,03-95,82), PD:7,6 $(1,63-24,9) \quad \mathrm{p}=0,51 ; \mathrm{TCR} \alpha \beta^{+}$cell content: CM:0,016 (0,000-0,0879), PD:0,042 (0,016-0,042) p =0,47; TCR $\gamma \delta$ + cell content: CM:4,5 (1,0-10,8), PD:4,8 $(1,0-57,0) \mathrm{p}=$ $0,33 \mathrm{CD}^{\mathrm{C}} \mathrm{C}^{+}$cell content: CM:0,046 (0,031-0,308), PD:0,088 (0,011-0,442) P=0,57.

$30 / 31$ grafts were transfused, 1 pt died just before finishing graft manipulation. 5 pts (CM:3, PD:2) needed a $2^{\text {nd }}$ graft $\left(\mathrm{CD} 34^{+}\right.$selected, only) due to suboptimal stem cell content.

There were 5 non-takes and 6 deaths. 22/28 pts are alive with a median follow up of 89 days (range:3-1205).

Conclusions: The TCR $\alpha \beta / C D 19$ depletion is a increasingly used separation technique in our clinical practice. It could be shown that both, CM and the recently developed fully automated PD system are highly efficient techniques for graft manipulation. PD proved to be at least as good or slightly better than the old CM system. Due to its simplicity and the comparable cost, PD system seems to be the first choice technique in the future.

Conflict of interest: Congress support for M. Reti, A. Szederjesi and S. Tasnady by the Hungarian distributor of Miltenyi Biotec. 


\section{P098}

Comprehensive standards for extended quality controls of hematopoietic stem and progenitor cells in the context of ATMP production

Lisa Olfe, Marlene Luther, Mohamed El-Missiry, Katy Haussmann, Carola Tietze-Bürger, Lena Oevermann, Kamran Movassaghi, Lutz Uharek

Charité Campus Virchow Berlin, Berlin, Germany

Background: Hematopoietic stem and progenitor cells (HSPCs) are increasingly used as raw material for advanced therapy medical products (ATMPs), i.e. genetic engineered HSPCs for treatment of hemoglobinopathies. However, there are yet no comprehensive and clearly defined standards for extended quality controls. Moreover, successful ATMP development and in process controls depend on optimal subset definition.

Methods: A standardized multicolor flow cytometry panel and gating strategy was developed based on the work of Fritsch (Transfusion, 2017). We validated this method by characterizing the subset distribution of CD34+ HSPCs in different compartments: bone marrow (BM, $\mathrm{n}=$ 5 , median age $=8 \mathrm{yr}$ ), autologous stem cell grafts mobilized with G-CSF alone (autoG, $\mathrm{n}=18$, median age $=52 \mathrm{yr}$ ) or GCSF plus Plerixafor (autoGP, $\mathrm{n}=10$, median age $=56 \mathrm{yr}$ ) and G-CSF mobilized healthy allogeneic stem cell donors (alloG, $\mathrm{n}=5$, median age $=34 \mathrm{yr}$ ).

Results: Most importantly, our data confirmed the feasibility, robustness and reproducibility of the approach. In particular, we found significant differences in the distribution of CD34+ subsets: the percentage of CD10+ B-lymphoid precursors was high in BM while negligible in mobilized peripheral blood stem cells (61\% in BM vs. $0,3 \%$ in autoG, 3,4\% in autoGP and 0,6\% in alloG, $\mathrm{p}<0,05$ ). Frequency of primitive multi-potent progenitors (MPP) was significantly higher in autoG and alloG compared to BM $(52 \%$ and $64 \%$ vs. $7,5 \%, p<0,05)$. Interestingly, frequencies of MPPs also differed between autoG and alloG ( $p<0,05)$. Frequencies of CD38-CD45RA-CD90+ long-term repopulating cells were comparable in autoG $(9,9 \%)$, autoGP $(15,5 \%)$ and alloG $(12,2 \%)$, but appeared to be lower in BM $(5,1 \%)$. CD184 expression was significantly higher on BM CD34+ cells $(63,7 \%)$ compared to peripheral blood stem cells (autoG 4\%, autoGP 12,9\%, alloG 5,3\%, $\mathrm{p}<0,05)$ and significantly higher in autoGP compared to autoG $(\mathrm{p}<0,05)$.

Conclusions: Our approach allows to access differences in subset distribution of HSPCs (i.e CD34+CD38CD45RA-CD90+ HSCs) for quality control and product characterization in the context of ATMP production. So far, our data indicate that there are important differences in subset composition with regard to source and mobilization agent. Furthermore, our data revealed differences between healthy donors and pretreated patients which might be of critical impact for further processing/genetic engineering and in vivo functionality.

Conflict of interest: None of the authors has anything to disclose.

The research being reported in this abstract was supported by Beckman Coulter and may support development of a commercial product. Beckman Coulter neither has influenced nor takes responsibility for the content of this abstract. The terms of this collaboration have been reviewed and approved by the Charite Berlin in accordance with its policy on objectivity in research.

\section{P099}

Cord blood donor lymphocyte infusion to boost immune reconstitution and the outcome of an infant ALL with MLL rearrangements after transplantation

Laura Alonso ${ }^{1}$, Cristina Diaz de Heredia ${ }^{1}$, Laura Soria Guerrero ${ }^{2}$, Isabel Badell Serra ${ }^{3}$, Guillermo Orti, Rodrigo Martino Bufarull, Christelle Ferrà Coll ${ }^{6}$, Isabel Sanchez-Ortega Sanchez ${ }^{7}$, Laura Medina Marrero ${ }^{8}$, Nerea Castillo ${ }^{8}$, Ramón Gimeno Martínez ${ }^{2}$, Sergio Querol Giner ${ }^{8}$

${ }^{1}$ Hospital Materno-Infantil Vall d'Hebron, Barcelona, Spain, ${ }^{2}$ Hospital del Mar \& IMIM (Hospital del Mar Medical Research Institute), Immunologia, Barcelona, Spain; ${ }^{3}$ Hospital de la Santa Creu i Sant Pau, Pediatrics Service, Barcelona, Spain; ${ }^{4}$ Hospital Vall d'Hebron, Hematology Service, Barcelona, Spain; ${ }^{5}$ Hospital de la Santa Creu i Sant Pau, Hematology Service, Barcelona, Spain; ${ }^{6}$ Institut Català d'Oncologia Trias i Pujol, Badalona, Spain; ${ }^{7}$ Institut Català d'Oncologia Duran i Reynals, L'Hospitalet de Llobregat, Spain; ${ }^{8}$ Banc de Sang $i$ Teixits, Cell Therapy Services, Barcelona, Spain

Background: Donor lymphocyte infusion (DLI) is commonly used to treat incipient relapse after transplantation but not possible using cord blood as a source of cells. We have developed a phase I-II clinical trial (NCT02328885) to assess the ability of cord blood donor lymphocyte infusion (CB-DLI) to boost immune vigilance using the $20 \%$ fraction when a $80 / 20$ cryobag is available. This clinical trial is under evaluation. Here, we present a case report to show a potential effect of the $\mathrm{CB}$ lymphocytes in this setting.

Methods: Clinical case. Three month-old girl diagnosed with Infant ALL with MLL rearrangement was made to proceed to allogeneic hematopoietic stem cell transplant in first CR, when she was 8 months old, after poor response to chemotherapy. The source was a 5/6 matched unrelated cord blood unit. Patient fulfilled inclusion criteria to this clinical trial Conditioning regimen consisted of ATG, thiotepa, busulfan and fludarabine. Cells infused using the $80 \%$ 
fraction of a $\mathrm{CB}$ unit were 22.6E7 TNC and $6.8 \mathrm{E} 5 \mathrm{CD} 34 / \mathrm{kg}$ resulting in an early engraftment. Post-transplant course was complicated with grade 2 acute skin and gut GVHD which responded to methylprednisolone. Disease assessment at 3 months post-transplant demonstrated full donor chimerism; flow cytometry was negative and MLL PCR was $0.2 \%$. Immunosuppression was successfully weaned off and 4 months after HSCT, she was free of GVHD and off immunosuppression, she had persistent full donor chimerism and flow cytometry on bone marrow aspirates was negative for leukemic cells. However, MLL PCR was still detectable at a level of $0.067 \%$ and her CD4 counts were very low (0.08 $\mathrm{x} 10 \mathrm{E} 9 / \mathrm{l})$. Given the poor immune reconstitution and the risk of relapse, decision was made to infuse the $20 \%$ fraction of the cord blood unit $($ day +140$)$. CD3 + cells $/ \mathrm{kg}$ infused were adjusted to $1 \mathrm{E} 6 \mathrm{CD} 3 / \mathrm{kg}$.

Results: A rapid expansion on the $\mathrm{T}$ lymphocyte compartment was observed as early as 15 days after infusion. Three-weeks after infusion patient developed gut GVHD that required treatment with corticosteroids and cyclosporine until 6 months after the infusion. This $\mathrm{T}$ lymphocyte expansion was transient and numbers went back to previous levels. A second wave of expansion was noted two months later and was equally of short length. In both cases a KI67+ population was observed. The expansion was dominated by the CD4 subset of $\mathrm{T}$ lymphocytes, mostly by those bearing memory markers. Interestingly, her MLL PCR levels have fluctuated and became negative after 6-months of cord blood DLI. She is now leukaemia-free almost 3 years post-HSCT. An ELISPOT assay was routinely performed to assess reactivity in front of a panel of defined antigens. Interestingly, reactivity against a tumor peptide collection was detected along the follow-up.

Conclusions: Cord blood DLI seems to be feasible when a CBU is stored in a $80 / 20$ bag and there is sufficient cell dose in the $80 \%$ fraction. In spite there is a risk for GVHD that may need an adjustment of CD3 dose, this strategy could be an easy way to boost immunity to control minimal residual disease after engraftment.

Clinical Trial Registry: ClinicalTrials.gov Identifier: NCT02328885

https://www.clinicaltrials.gov/ct2/show/NCT02328885? term $=$ NCT02328885\&rank $=1$

Conflict of interest: Authors declare no conflict of interest

\section{P100}

Co-transplantation of third party donors-derived mesenchymal stem cells in hematopoietic stem cell transplantation in patients with severe aplastic anemia: A single institution experience
Arzu Akcay ${ }^{1}$, Didem Atay ${ }^{1}$, Fatih Erbey ${ }^{1}$, Ercument Ovali ${ }^{2}$, Gulyuz Ozturk $^{1}$

${ }^{1}$ Acibadem University, Istanbul, Turkey; ${ }^{2}$ Acibadem Altunizade HospitalLabcell, Istanbul, Turkey

Background: Hematopoietic stem cell transplantation (HSCT) for severe aplastic anemia (SAA) is mainly limited by the high incidence of graft failure and GvHD. Mesenchymal stem cells (MSCs) have been shown to support hematopoiesis in vivo and to display potent immunosuppressive effects to prevent or treat GvHD after HSCT. In this study, we aimed to determine the potential benefits of MSC co-transplantation in patients with SAA undergoing HSCT, regard to the outcomes engraftment and GVHD.

Methods: A total of 10 patients (median age 117 months, range 21-213) were carried out: 9 allogenic (match sibling/ family donor $=4$, match unrelated donor $=5$, haploidentical donor $=1)$. The conditioning regimen included fludarabin, cyclophosphamide in 7 patients. Thymoglobulin (ATG) were used in all patients. The recipients received cyclosporin A (CsA) and short-term methotrexate for GvHD prophylaxis in 7 patients, the other drugs in 3 patients. MSCs $\left(1 \times 10^{6}\right.$ cells $\left./ \mathrm{kg}\right)$ were administered by venous infusion $24 \mathrm{~h}$ before the hematopoietic graft. Bone marrow (in 6 pts) and peripheral blood (in 4 pts) $\mathrm{CD}^{+} 4^{+}$cells were infused intravenously. Median CD $34^{+}$counts were $4.18 \times$ $10^{6} / \mathrm{kg}$.

Results: No patients experienced infusional toxicity during the infusion of MSCs. In all patients achieved hematopoietic reconstitution and sustained full donor chimerism in 9 pts, mix chimerism in 1 pts. The median time for myeloid engraftment was 13 days (range 9-25 days) and for platelet engraftment was 23 days (range 10108 days). The incidence was $10 \%$ for grade I- II, $20 \%$ for III-IV acute GvHD and 10\% for chronic limited GvHD. Nine patients were alive, but a patient died from infection. Patients were followed-up for a median of 15,5-month (range 2-60).

Conclusions: Despite limited cases enrolled in the study, our findings support previous studies showing that HSCT combined with MSCs infusion could be an effective approach to reduce the risk of graft failure and GvHD in HSCT for SAA. MSC occupy a hematopoiesis-supportive capacity.

Conflict of interest: None of the authors has anything to disclose.

\section{P101}

Dissecting EBV-specific T-cell responses after successful allogeneic EBV-specific T-cell transfer for CNS PTLD 
Britta Maecker-Kolhoff, ${ }^{1,2}$, Rebecca E. Schultze-Florey ${ }^{1,2}$, Sabine Tischer $^{2,3}$, Leonie Kuhlmann ${ }^{4}$, Patrick Hundsdoerfer ${ }^{5,6}$, Arend Koch $^{6,7}$, Sarina Ravens ${ }^{4}$, Lilia Goudeva ${ }^{3}$, Christian Schultze-Florey ${ }^{8}$, Christian Koenecke ${ }^{8}$, Rainer Blasczyk ${ }^{3}$, Ulrike Koeht ${ }^{2,9}$, Hans-Gert Heuft ${ }^{3}$, Immo Prinz ${ }^{4}$, Britta Eiz-Vesper ${ }^{2,3}$

${ }^{1}$ Hannover Medical School, Pediatric Hematology and Oncology, Hannover, Germany; ${ }^{2}$ Integrated Research and Treatment Center Transplantation (IFB-Tx), Hannover, Germany; ${ }^{3}$ Hannover Medical School, Institute for Transfusion Medicine, Hannover, Germany; ${ }^{4}$ Hannover Medical School, Institute of Immunology, Hannover, Germany; ${ }^{5}$ Charité Universitaetsmedizin Berlin, Pediatric Hematology and Oncology, Berlin, Germany; ${ }^{6}$ Berlin Institute of Health, Berlin, Germany; ${ }^{7}$ Charité Universitaetsmedizin Berlin, Neuropathology, Berlin, Germany; ${ }^{8}$ Hannover Medical School, Hematology, Oncology, Hemostaseology and Stem Cell

Transplantation, Hannover, Germany; ${ }^{9}$ Hannover Medical School, Cell Therapy Center, Hannover, Germany

Background: EBV-associated post-transplant lymphoproliferative disease (PTLD) with CNS involvement is a severe complication after solid organ transplantation. Standard treatment with reduction of immunosuppression, anti-CD20 antibody application and/or chemotherapy often fails leading to poor outcome. Transfer of EBV-directed Tcells has shown promise, however, contributions of transferred and endogenous T-cells are largely unknown.

Methods: Manufacturing of clinical-grade EBV-specific CD4+ and CD8+ T-cells from a 5/10 HLA-matched third party donor was conducted using ppEBNA-1 and ppSelect and the IFN- $\gamma$ Cytokine Capture System (Miltenyi Biotech) on a CliniMACS device.

Monitoring of EBV load was performed by routine qPCR. EBV-specific T-cell frequencies pre/post transfer were quantified by IFN- $\gamma$ ELISpot using the peptide pools ppEBNA1, ppSelect, ppLMP2a, ppBZLF1 (Miltenyi Biotec). To enhance sensitivity analysis was repeated after 7day-expansion in the presence of ppEBNA1 or ppConsenus and 50U/ml IL-2 (Peprotec).

For TCR $\beta$ chain repertoire analysis expanded PBMCs were stained with antibodies against DAPI, hCD45, hCD3, hTCR $\alpha \beta$ and hCD8. Cells were sorted into TCR $\alpha \beta+/ C D 8$ + T-cells on a FACS Aria Fusion flow cytometer. RNA extraction (RNeasy Plus Micro Kit, QIAGEN) and reverse transcription (SMARTer RACE 5'-3' PCR Kit, Clontech) were carried out according to the manufacturer's recommendation. For combined amplification of the TCR $\beta$ CDR3-region and Illumina adaptor sequences the Advantage-2 PCR Kit (Clontech) was used. Indexing of the samples was performed with Nextera Primer Kit (Illumina) and the product was purified with the Agencourt AMPure XP Kit. Denaturation and dilution of the pool was done as described (Illumina MiSeq Dilution and Denaturation Guide). Next generation sequencing was performed on the Illumina MiSeq System. For analysis the FastQ files were annotated at IMGT/HighV-Quest database and processed with tcR-package and VDJtools.

Results: An 11-year-old boy developed multilocular EBV-positive CNS PTLD ten years after liver transplantation. Complete remission was achieved by repeated intravenous and intrathecal anti-CD20 antibody rituximab administration combined with intrathecal chemotherapy (methotrexate, cytarabine, prednisone) over a time period of three months. Due to the poor prognosis of CNS PTLD and lack of EBV-specific T-cells in patient's blood, we decided to perform EBV-directed T-cell immunotherapy as a consolidating treatment. The patient received five infusions of allogeneic EBV-specific T-cells $(25.000 / \mathrm{kg}$ bw each) manufactured by Cytokine Capture System from a 5/10 HLA-matched unrelated third party donor. No relevant acute toxicity was observed. EBV-specific T-cells became detectable after first injection and increased during the treatment course. NGS TCR-profiling verified the persistence and expansion of donor-derived EBV-specific clones. After two transfers epitope spreading to unrelated EBV antigens occurred suggesting onset of endogenous T-cell production, which was supported by detection of recipientderived clones in NGS TCR-profiling. Continuous complete remission was confirmed 27 months after initial diagnosis.

Conclusions: Repetitive transfer of partially HLAmatched EBV-specific T-cells from a third party donor evoked robust EBV-specific T-cell responses in a high risk PTLD patient. While T-cell specificity broadened over the treatment course, individual TCR $\beta$ sequences could be attributed to both donor and recipient progeny. Molecular tracking of individual T-cells will help understand the contributions of transferred vs. endogenous virus-specific $\mathrm{T}$-cells in patients receiving adoptive T-cell transfer.

Conflict of interest: The authors declare no conflict of interest. This work was supported by the German children's cancer fund (DKS 2011.05) and the German Federal Ministry of Education and Research (01EO1302). PH is participant in the BIH-Charité Clinical Fellows Program funded by the Charité - Universitätsmedizin Berlin and the Berlin Institute of Health.

\section{P102}

Abstract previously published

\section{P103}

Flow cytometric analysis of HLA disparities as a highly efficient tool to detect chimerism in rare cell populations after non-identical transplantation and adoptive cell transfers 
Rebecca Schultze-Florey ${ }^{1}$, Sabine Tischer ${ }^{2}$,Wolfgang Kühnau ${ }^{3}$, Britta Eiz-Vesper ${ }^{2}$, Britta Maecker-Kolhoff ${ }^{1}$

${ }^{1}$ Hannover Medical School, Ped. Hematology Oncology, Hannover, Germany; ${ }^{2}$ Hannover Medical School, Transfusion Medecine,

Hannover, Germany; ${ }^{3}$ Hannover Medical School, Institut for Human Genetics, Hannover, Germany

Background: Chimerism analysis after HLAmismatched stem cell transplantation is an important diagnostic tool for clinical decisions regarding modification of immunosuppression. In particular, flow-cytometry based detection of HLA disparities offers an attractive option to detect chimerism in lymphocyte subsets following HLAmismatched cell transfers. Here we present a broadening of the technique to detect rare virus-specific $\mathrm{T}$ cells in peripheral blood after adoptive cell transfer by flow cytometry.

Methods: PBMCs from residual blood samples from platelet (PLT) apheresis HLA-A01 ${ }^{+} / \mathrm{A} 02^{-} \mathrm{CMV}^{+}$(donor 1) and HLA-A01 $/ \mathrm{AO}^{+} \mathrm{CMV}^{+}$(donor 2 ) individuals from the alloCELL donor registry were isolated by Ficoll density centrifugation. $1 \times 10^{\wedge} 7 \mathrm{PBMC}$ from donor 1 and 2 were stimulated separately with pp65 and incubated overnight in a 24-well plate. After stimulation total cells of the donor 1 and 2 were mixed in ratios of 1:1 and 1:10 (donor 2: donor 1). The IFN- $\gamma$ positive $T$ cells were isolated via cytokine secretion assay (CSA, Miltenyi biotech) and analyzed via flow cytometry (mAb against CD45, CD3, CD4, CD8, IFN$\gamma$, HLA-A01, HLA-A02). Simultaneously a sample of the enriched IFN- $\gamma+$ CMV specific T cells was analyzed for molecular chimerism by STR PCR.

Results: Before magnetic enrichment the 1:1 mixed IFN$\gamma$ positive $\mathrm{T}$ cells $(\mathrm{CD} 45+, \mathrm{CD} 3+, \mathrm{CD} 56-)$ displayed a $17 \%$ HLA-A01+ (donor 1) phenotype and 59\% HLA-A02 + (donor 2) phenotype. After magnetic enrichment the distribution of HLA-A01+/HLA-A02+ cells was similar (17\% and $57 \%$, respectively; $1 \times 10^{\wedge} 6$ cells analyzed). The 1:10 (donor 2 : donor 1 ) mixed IFN- $\gamma$ positive cell suspension showed before enrichment a distribution of $12 \%$ and $75 \%$ (after enrichment $11 \%$ and $71 \%$ ). In comparison, STR analysis of enriched cells $\left(1 \times 10^{\wedge} 4\right.$ cells analyzed) revealed for the 1:1 mixture $30 \%$ of the donor 1 cells and $70 \%$ of the donor 2 cells. For the 1:10 mixture cells from donor 1 were $70 \%$ and from donor $230 \%$. Similar results were obtained in a second HLA-A2 mismatched donor pair. While results of FACS chimerism were available within 36 hours, molecular chimerism after magnetic enrichment took 4 days.

Conclusions: The increasing usage of HLA-mismatched transplantations requires fast and reliable determinations of cell chimerism. FACS analysis of mismatched HLA alleles provides a rapid technique to detect chimerism in subpopulations avoiding the need for cell purification.
Sensitivity is comparable to STR chimerism analysis. FACS chimerism can be combined with techniques of functional single cell identification such as cytokine secretion, which provides a valuable tool for monitoring of antigen-specific cells after partially HLA-matched transplantation and adoptive cell transfer. Prospective studies are needed to determine the fate of partially HLA-matched antigenspecific $\mathrm{T}$ cells in adoptive transfer situations.

Conflict of interest: All authors declare that there is no conflict of interest.

\section{P104}

Flow cytometry or Luminescence assay: Which the best quality control of extracorporeal photopheresis ?

Judith Desoutter, Christèle Ossart, Aline Regnier, Lavinia Merlusca, Delphine Lebon, Jean Pierre Marolleau, Marie Noëlle Lacassagne

CHU Amiens Picardie, Amiens Cedex, France

Background: In agreement with good practices for therapeutic use of human cells, quality control has to be performed to valid the process of extracorporeal photopheresis (ECP). Currently, the reference method to highlight inhibition of cell division after ECP, remains used of tritiated thymidine incorporation that requires a suitable environment for radioactivity assays. In last few years, alternative methods such as CFSE staining by flow cytometry or luminescent cell viability assay had been developed. Here, we report results, advantages and drawbacks about this both assays designed in our laboratory.

Methods: To perform CFSE staining by flow cytometry, we opted for CellTrace ${ }^{\mathrm{TM}}$ CFSE kit (Invitrogen) that, by binding covalently to intracellular amines, used to assess cell proliferation. For the luminescent cell viability test, we used CellTiter-Glo ${ }^{\circledast}$ Assay (Promega) which allows determining number of viable cells in culture, based on quantitation by a luminometer of the ATP present for reporting the presence of metabolically active cells. Both methods were performed using patients cells collected by cytapheresis at the beginning (PRE-ECP) and after 8-MOP (Metoxsalene, SALF ; Macopharma) irradiation cells (POST-ECP) reinjection to patient. Cells obtained were typed initially by flow cytometry to appreciate their viability (7-AAD) and their distribution of mononuclear cells (MNCs) subpopulations. Then, they were plated and incubated at $37^{\circ} \mathrm{C}$ with $5 \% \mathrm{CO}_{2}$ in divided two groups : non stimulated cells and stimulated cells with a solution of $10 \mu \mathrm{g} / \mathrm{mL}$ PHA (Phytohemagglutinin-P, Sigma-Aldrich) and $50 \mathrm{ng} / \mathrm{mL}$ of IL-2 (Human IL-2, Miltenyi Biotec). To demonstrate ECP efficacy, to our specifications, cell proliferation difference between stimulated and non- 
stimulated PRE-PCE cells (PROLIF) must be greater than $3 \%$ and in the same time, inhibition of cell division between PRE-PCE and POST-PCE cells (INH) will be greater than $97 \%$.

Results: CFSE assays were performed, specifically to $\mathrm{CD}^{+}$PRE-ECP and POST-ECP viable cells $\left(\mathrm{CD}^{+} / 7\right.$ $\mathrm{AAD}^{-}$) and were read at day 0 , to verify CFSE labelling, and at day 2-3 after ECP. In our 9 patients already analyzed, median of PROLIF was at $54.6 \pm 8.8 \%$ and median of INH was $98.8 \pm 1.0 \%$. In contrast, luminescent tests analyzed all type of viable MNCs (7-AAD) collected and were read at day 5-6. In our 9 patients studying, median of cell PROLIF was at $3.9 \pm 0.6 \%$ and median of INH was $100 \pm 0.17 \%$. To note, in CFSE test, one patient was out of INH specification ( $\mathrm{INH}=90.9 \%$ ), with a hematocrit at $2.1 \%$ (range to $0.5 \%$ to $2.1 \%$ across assays).

Conclusions: We show a low PROLIF in luminescent assay, staying in our specification, caused by entire MNCs analyzed without targeting, whereas our stimulation by PHA/IL-2 focuses on $\mathrm{CD}^{+}$lymphocytes, whose rate is highly heterogeneous $(9.7 \%$ to $71 \%)$ in our cohort. As for the patient out of INH specification, this observation is probably due to hematocrit greater than $2.0 \%$, known to reduce irradiation efficacy. However, it turns out that, both methods answer our laboratory specifications criteria. The choice of assay will depended on laboratory objectives, ECP pathology treated and further progress in understanding POST-ECP cells mechanisms of action in vivo.

Conflict of interest: No conflict of interest to disclose
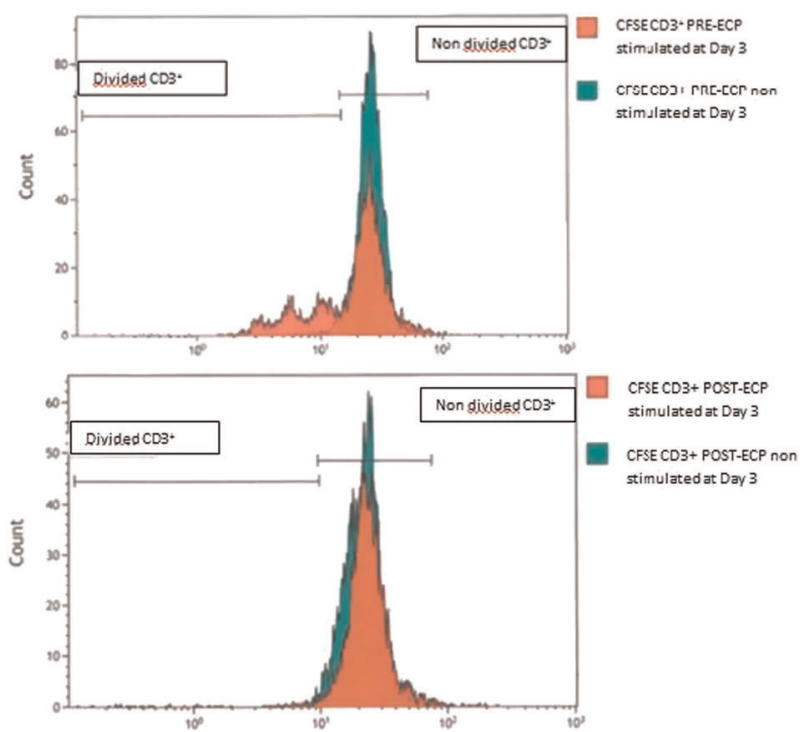

[P104 Figure] [Example of CFSE histograms obtained at Day 3 in CD3 + PRE-ECP and POST-ECP viable cells (CD3+/7-AAD-)]
P105

Fluorescence Based Reporter Cells Rapidly Identify and Distinguish Functional Chimeric Antigen Receptors (CARs) based on NF-KB and NFAT signaling

\section{Julian Rydzek ${ }^{1}$, Sabrina Jutz ${ }^{2}$, Judith Leitner ${ }^{2}$, Lars Wallstabe ${ }^{1}$, Thomas Nerreter ${ }^{1}$, Johannes Huppa ${ }^{3}$, Peter Steinberger ${ }^{2}$, Hermann Einsele $^{1}$, Michael Hudecek ${ }^{1}$ \\ ${ }^{1}$ Medizinische Klinik II, Universitätsklinikum, Würzburg, Germany; ${ }^{2}$ Institut für Immunologie, Medizinische Universität, Wien, Austria; ${ }^{3}$ Institut für Hygiene und Angewandte Immunologie, Medizinische Universität, Wien, Austria}

Background: Immunotherapy with chimeric antigen receptor (CAR)-modified T-cells is under intense preclinical and clinical investigation involving a rapidly increasing spectrum of new CAR designs and target antigens. Here, we present a novel dual parameter reporter (DPR) cell line that enables rapid high-throughput testing and functional characterization of CARs.

Methods: DPRs were derived from the Jurkat T-cell lymphoma line which was modified with NF-KB- and NFAT-inducible CFP and GFP reporter genes. CARs were expressed by lentiviral gene-transfer and CAR positive DPRs enriched to $>90 \%$ purity using an EGFRt selection marker. CAR DPRs were co-cultured with antigen positive stimulator cells and reporter gene-activation was analyzed by flow cytometry. Stimulation using T-cell stimulator (TCS) cells with membrane-bound anti-CD3 $\mathrm{scFv}$ that triggers CD3 on DPRs was used as reference.

Results: We transduced DPRs with a prototypic ROR1specific CAR with 4-1BB co-stimulation and detected highest NF-KB and NFAT reporter gene-induction after 24 to 48 hours of co-culture with ROR1 positive stimulator cells. Activation of NF- $\mathrm{kB}$ and NFAT by CAR engagement was also confirmed in primary T-cells using Western Blot. Next, we expressed a panel of ROR1-CARs with variable spacer length (derived from IgG4-Fc) in DPRs based on our previous work in primary T-cells showing the importance of a particular spacer design for optimal anti-tumor function. DPRs rapidly identified the optimal construct from this panel, shortening the time required for analysis from $\sim 3$ weeks to $\sim 6$ days. We were also interested in determining the NF- $\mathrm{KB}$ and NFAT activation signature in DPRs modified with a 1st (no co-stimulation), 2nd (CD28 or 4-1BB) and 3rd generation (CD28 plus 4-1BB) ROR1CAR. We detected similar levels of NFAT induction by each of these receptors (Figure 1). However, the ROR1CAR construct comprising a 4-1BB rather than CD28 costimulatory moiety showed significantly stronger induction of NF-kB $(\mathrm{n}=3, \mathrm{p}<0.05)$. Notably, the 2 nd generation ROR1-CAR with CD28 did not induce higher levels of NF- 
$N F-k B$

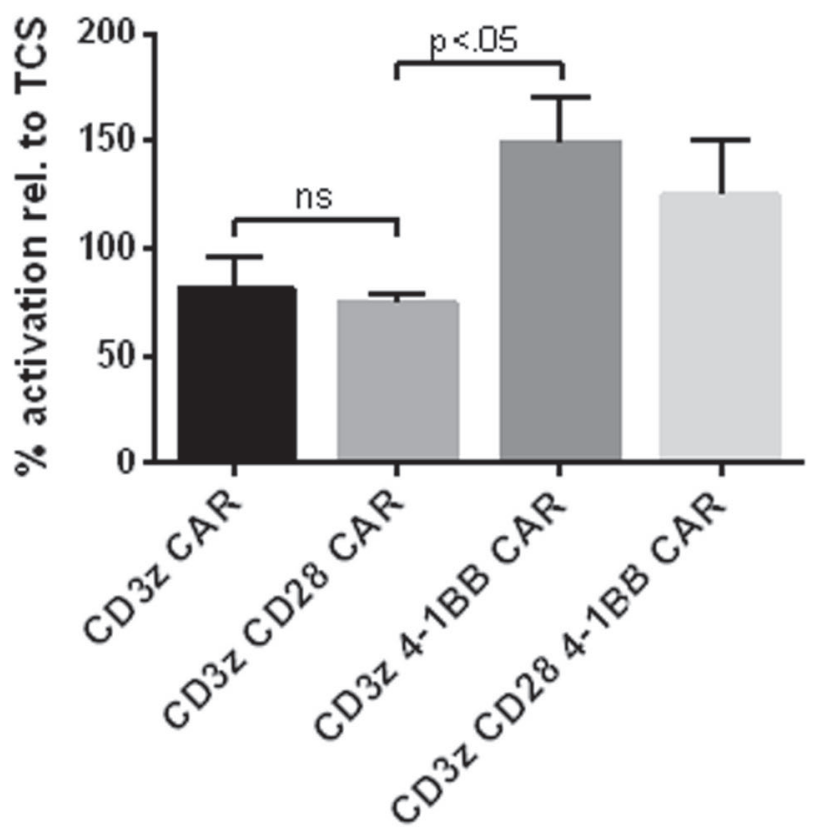

NFAT

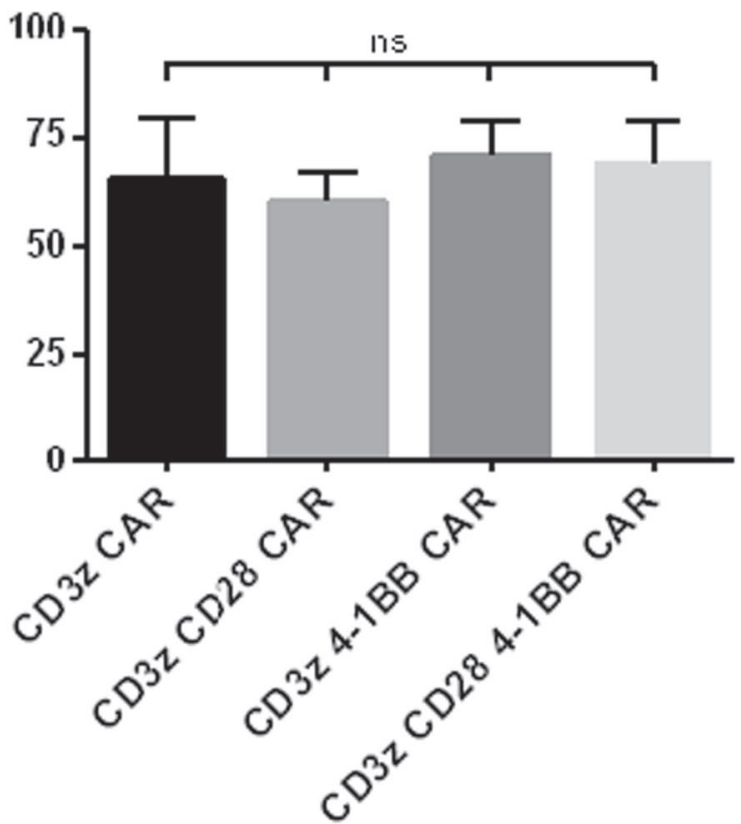

[P105 Figure] [NF- $K B$ and NFAT reporter gene-induction in DPRs expressing a panel of ROR1-CARs.]

$\kappa \mathrm{B}$ than the 1 st generation construct and the 3 rd generation construct did not induce higher levels of NF- $\mathrm{B}$ than the 2nd generation ROR1-CAR with 4-1BB. This indicated that the CD28 co-stimulatory domain in the CAR format did not contribute to NF- $\kappa \mathrm{B}$ signaling. To corroborate these data, we confirmed that stimulation of DPRs through TCR and endogenous CD28 (independent from the CAR) readily induced a NF- $\mathrm{KB}$ signal.

Conclusions: Our data demonstrate the potential to use DPRs to rapidly evaluate panels of CAR constructs based on NFAT and NF- $\kappa$ B signaling. Our data suggest that the conventional position of the CD28 co-stimulatory moiety in the CAR construct, located between transmembrane domain and CD3 zeta, is suboptimal for fully exploiting its costimulatory capacity. This finding has triggered the design of novel CAR formats that are currently being evaluated in our laboratory. Further, we are currently integrating DPRs into the analysis of CAR libraries with different specificity, affinity and receptor designs and are confident this novel DPR platform will accelerate the pre-clinical development and selection of CARs with optimal anti-tumor function for clinical translation.

Conflict of interest: None

\section{P106}

Immune reconstitution and graft correlations of innate effector cells after hematopoietic stem cell transplantation
Lia Minculescu ${ }^{1}$, Hanne Vibeke Marquart ${ }^{1}$, Lars Peter Ryder ${ }^{1}$, Anne Fischer-Nielsen ${ }^{1}$, Eva Kannik Haastrup ${ }^{1}$, Ida Schjodt ${ }^{2}$, Soren Lykke Petersen $^{2}$, Lone Smidstrup Friis ${ }^{2}$, Brian Thomas Kornblit ${ }^{2}$, Niels Smedegaard Andersen ${ }^{2}$, Henrik Sengelov ${ }^{2}$

${ }^{1}$ Copenhagen University Hospital, Rigshospitalet, Department of Clinical Immunology, Copenhagen, Denmark; ${ }^{2}$ Copenhagen University Hospital, Rigshospitalet, Department of Hematology, Copenhagen, Denmark

Background: Immune reconstitution of $\gamma \delta \mathrm{T}$ cells and NK cells are recognized as important for clinical outcomes after hematopoietic stem cell transplantation (HSCT) and could be dependent on contents and subset distribution of these cells in the stem cell graft.

Methods: From 2015 to 2017108 consecutive patients with hematological malignancies were transplanted at the Stem Cell Transplant Unit, Rigshospitalet, Denmark. The conditioning was myeloablative in 51 patients and nonmyeloablative in 57 patients. Donors were HLA-identical sibling (24), haploidentical (2) and 9/10 or 10/10 matched unrelated (82). T cell replete grafts were derived from bone marrow (17) and peripheral blood (91). Fresh graft samples and patient blood samples on day 28, 56, 91 and 180 after HSCT were analyzed for absolute concentrations of T- and NK cells (BD TruCount ${ }^{\mathrm{TM}}$ beads) and subset phenotypes by multicolor flow cytometry panels performed on BD FACSCanto $^{\text {TM }}$ II. Subset markers included TCR $\alpha \beta$, TCR 


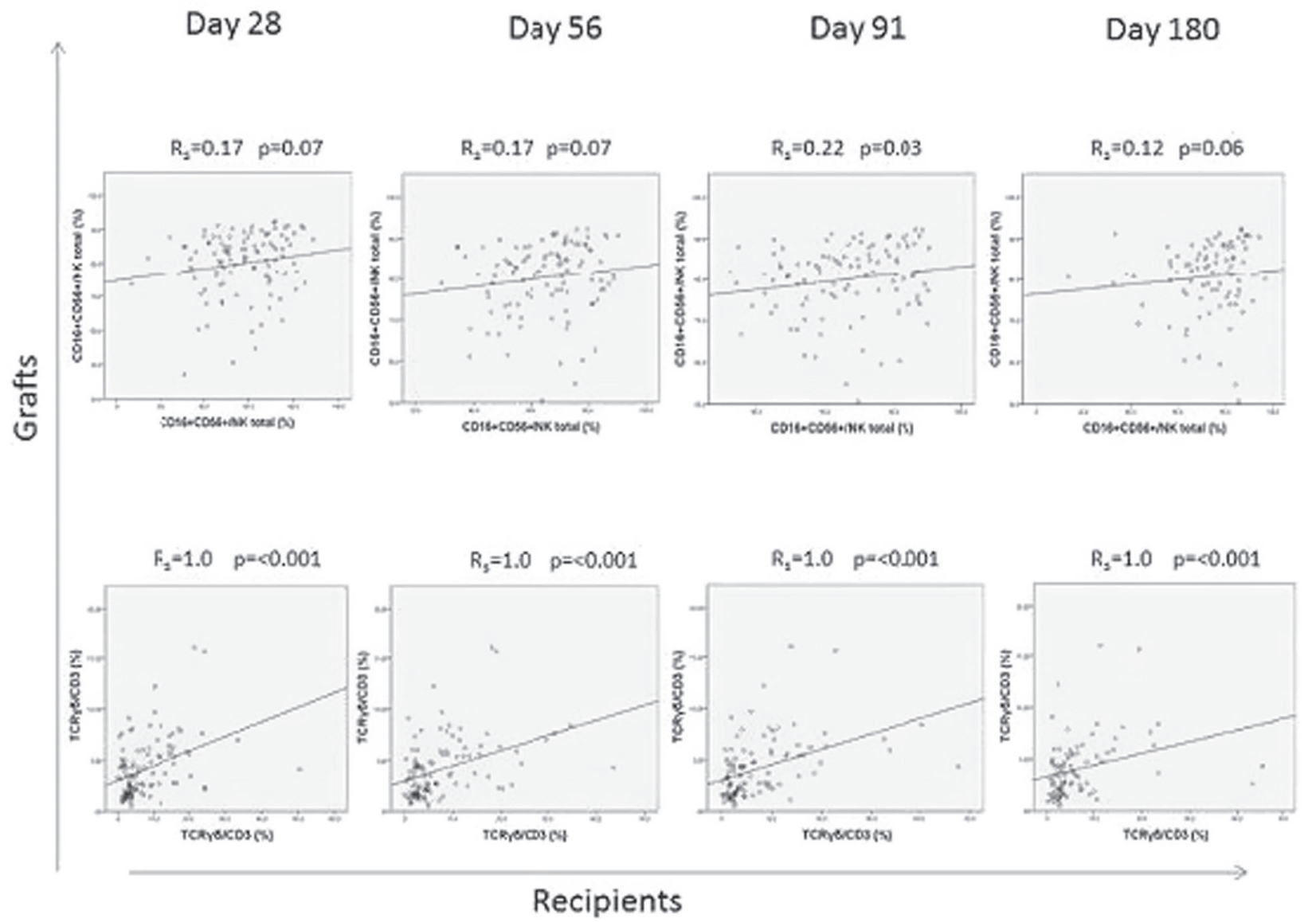

[P106 Figure] [Figure 1. Correlations between graft contents and immune reconstitution of NK and TCRgd cells.]

$\gamma \delta$, CD3, CD4, CD8, Vd1, Vd2, CD16, CD56, HLA-DR, CD45RO, CD45RA, CD197, CD314 and CD337.

Results: Median concentrations of CD3 T cells reached normal range by day 180 after transplantation while NK cells remained within normal range at all time points.

The fraction of $\gamma \delta \mathrm{T}$ cells out of CD3 T cells was stable, while the distribution of V $\delta 1$ and V82 showed increasing fractions of V 81 from day 28 towards day 180. Within the NK cell compartment, the CD16bright fraction remained stable, while the CD56bright fraction decreased along with a parallel increase in the $\mathrm{CD} 16+\mathrm{CD} 56+$ fraction towards day 180 . Immune reconstitution of $\gamma \delta \mathrm{T}$ cells, $\mathrm{V} \delta 1$ and $\mathrm{V} 2 \delta$ fractions correlated highly significantly with the graft contents of the corresponding cell subsets, $\mathrm{p}<0.001$ at all analyzed time points. The correlation to graft contents within the NK cell compartment showed greater variations; CD16bright cells only correlated on day $28(\mathrm{p}=0.05)$, CD56bright cells only on day $28(\mathrm{p}<0.01)$ and day 56 $(\mathrm{p}=0.01)$, and $\mathrm{CD} 16+\mathrm{CD} 56+$ cells only significantly on day $91(\mathrm{p}=0.03)$

Conclusions: Immune reconstitution showed compelling correlation to graft contents; the correlation was stronger for $\gamma \delta \mathrm{T}$ cells than for NK cells. The results are relevant for the increasing focus on innate effector cells and graft manipulation in HSCT.

Conflict of interest: All authors: nothing to disclose.

\begin{tabular}{|c|c|c|c|c|c|c|c|c|}
\hline $\begin{array}{l}\text { Cell concentrations, } \\
10 \times 7 / \mathrm{L} \text {, median } \\
\text { (range) }\end{array}$ & $\mathrm{n}$ & $\begin{array}{l}\text { Day } \\
28\end{array}$ & $\mathrm{n}$ & Day 56 & $\mathrm{n}$ & Day 91 & $\mathrm{n}$ & $\begin{array}{l}\text { Day } \\
180\end{array}$ \\
\hline $\begin{array}{l}\text { CD3 (normal range } \\
69-270 \text { ) }\end{array}$ & 108 & $\begin{array}{l}41.5 \\
(0- \\
200)\end{array}$ & 107 & $\begin{array}{l}43.0(0- \\
340)\end{array}$ & 103 & $\begin{array}{l}44.0(0- \\
980)\end{array}$ & 88 & $\begin{array}{l}80.5 \\
(5.6- \\
410)\end{array}$ \\
\hline $\begin{array}{l}\text { NK (normal range } \\
8.0-56 \text { ) }\end{array}$ & 108 & $\begin{array}{l}28.0 \\
(2.0- \\
92.0)\end{array}$ & 107 & $\begin{array}{l}19.0(0- \\
160)\end{array}$ & 103 & $\begin{array}{l}16.0(0- \\
90.0)\end{array}$ & 88 & $\begin{array}{l}23.0 \\
(2.0- \\
78.0)\end{array}$ \\
\hline \multicolumn{9}{|c|}{ Relative concentrations percent, median (range) } \\
\hline TCRgd/CD3 & 106 & $\begin{array}{l}4.2(0- \\
50.4)\end{array}$ & 106 & $\begin{array}{l}4.3(0- \\
43.6)\end{array}$ & 101 & $\begin{array}{l}4.0 \\
(0.1- \\
47.8)\end{array}$ & 88 & $\begin{array}{l}3.7 \\
(0.4- \\
45.3)\end{array}$ \\
\hline Vd1/TCRgd & 105 & $\begin{array}{l}10.0 \\
(0- \\
73.8)\end{array}$ & 105 & $\begin{array}{l}11.6(0- \\
82.4)\end{array}$ & 101 & $\begin{array}{l}12.8(0- \\
89.3)\end{array}$ & 87 & $\begin{array}{l}26.3(0- \\
95.8)\end{array}$ \\
\hline Vd2/TCRgd & 105 & $\begin{array}{l}87.6 \\
(0- \\
100)\end{array}$ & 105 & $\begin{array}{l}85.7 \\
(5.1- \\
100)\end{array}$ & 101 & $\begin{array}{l}82.8 \\
(3.7- \\
100)\end{array}$ & 87 & $\begin{array}{l}67.5 \\
(2.4- \\
98.9)\end{array}$ \\
\hline CD16 $++/$ NK cells & 108 & $\begin{array}{l}10.1 \\
(0.5- \\
72.2)\end{array}$ & 106 & $\begin{array}{l}14.2 \\
(2.1- \\
51.8)\end{array}$ & 102 & $\begin{array}{l}14.7 \\
(0.7- \\
59.1)\end{array}$ & 87 & $\begin{array}{l}12.3 \\
(1.3- \\
84.8)\end{array}$ \\
\hline
\end{tabular}




\begin{tabular}{|c|c|c|c|c|c|c|c|c|}
\hline $\begin{array}{l}\text { Cell concentrations, } \\
10 \times 7 / \mathrm{L}, \text { median } \\
\text { (range) }\end{array}$ & $\mathrm{n}$ & $\begin{array}{l}\text { Day } \\
28\end{array}$ & $\mathrm{n}$ & Day 56 & $\mathrm{n}$ & Day 91 & $\mathrm{n}$ & $\begin{array}{l}\text { Day } \\
180\end{array}$ \\
\hline $\begin{array}{l}\text { CD16+56+/NK } \\
\text { cells }\end{array}$ & 108 & $\begin{array}{l}58.3 \\
(6.7- \\
88.7)\end{array}$ & 106 & $\begin{array}{l}67.8 \\
(28.9- \\
90.4)\end{array}$ & 102 & $\begin{array}{l}67.8 \\
(32.4- \\
90.7)\end{array}$ & 87 & $\begin{array}{l}75.8 \\
(13.6- \\
96.7)\end{array}$ \\
\hline CD56++/NK cells & 108 & $\begin{array}{l}23.4 \\
(3.8- \\
71.7)\end{array}$ & 106 & $\begin{array}{l}14.8 \\
(3.4- \\
56.4)\end{array}$ & 102 & $\begin{array}{l}12.5 \\
(1.2- \\
59.8)\end{array}$ & 87 & $\begin{array}{l}8.7 \\
(1.5- \\
51.8)\end{array}$ \\
\hline
\end{tabular}

[[P106 Table] Table 1.]

\section{P107}

\section{Immuno-T, a motion comic educating patients and caregivers on how immunotherapy works}

Chiel Gharakhani Nia', Stijn Calis ${ }^{2}$, Yves Bertrand ${ }^{2}$, Lieve Achten ${ }^{3}$, Jasmien Soetens ${ }^{4}$, Barbara Casteels ${ }^{4}$, Daisy Flamez, ${ }^{5}$, Bruno Bollaert $^{6}$, Tessa Kerre ${ }^{1}$

${ }^{1}$ Ghent University Hospital, Hematology, Gent, Belgium; ${ }^{2}$ Holofarm, Gent, Belgium; ${ }^{3}$ Telma, Gent, Belgium; ${ }^{4}$ Ghent University Hospital, Cancer Centre, Gent, Belgium; ${ }^{5}$ Ghent University, Biomarked IOF

Consortium and CRIG, Gent, Belgium; ${ }^{6}$ WahWah vzw, Gent, Belgium

Background: The introduction of immunotherapy has been a major breakthrough in the treatment of cancer. In the general public and the average patient populations, the overall knowledge of the immune system and its role in cancer is very limited. Moreover, explaining the immune system and how immunotherapy works to patients and their caregivers, is challenging because of the extreme complexity.

Serious games and virtual aids have only been scarcely used for cancer patients or patients in general. However, the limited amount of literature evinces the benefit patients and caregivers experience when these services are employed.

We now have developed Immuno-T: a motion comic, explaining the genesis of cancer and the working mechanisms of three types of immunotherapy. A motion comic combines a digital comic with animation, sound effects, voice-over and/or a musical score.

Methods: The scenario, story board and basic character design were developed by a hematologist, executed by game developers, and further refined by small focus groups consisting of hematologists-oncologists, psychologists, study coordinators, medical students, nurses and communication managers. The result was a first usable version. One hundred testers were asked to give their opinion in a anonimous questionnaire. The first version was eventually evaluated by 40 people of different backgrounds (hematologists-oncologists (both pediatric and adult), psychologists, study coordinators, medical students, nurses, communication managers, game developers, laymen and a small number of patients). Taking all their remarks and observations into account, Immuno-T was further adapted and improved into the final version.

Results: Immuno-T explains three types of immunotherapy. In an engaging, interactive and easy-to-understand way, the complex theory of checkpoint inhibitors, CAR Tcells and BiTEs is illustrated. The majority of the evaluators $(94.9 \%)$ considered the motion comic a good tool to explain immunotherapy to patients, in addition to the physician consultation. In the first version, the level of understanding differed depending on the type of immunotherapy that was explained: BiTes were the least clear to the evaluators, prompting us to adapt this part of the motion comic. Almost $40 \%$ of the evaluators felt hopeful after watching the motion comic, 30\% experienced other positive emotions (happy, well-informed, strengthened).

Conclusions: Although motion comics carry important advantages (a clear and graphic presentation of complex information and a more engaging way of delivering information), they have only rarely been used in healthcare settings. Immuno-T is ready to be rolled out in the first real hospital setting. We are currently preparing a clinical trial to evaluate the effectiveness of information transmission using Immuno-T: does our motion book lead to patients' better understanding of the given treatment? Increasing patients' knowledge of disease and treatment has a proven impact on hope, strength and empowerment, and on adherence to the treatment.

Meanwhile, Immuno-T also keeps evolving: the motion book will be enriched with an augmented reality function to improve the understanding and deepen the user experience. Furthermore multiple translations are forthcoming, to facilitate the international career of our motion comic. Lastly, Immuno-T will also be evaluated in the context of education at different levels.

Clinical Trial Registry: Non applicable

Conflict of interest: The development of the motion comic was supported partly by a number of pharmaceutical companies having some forms of immunotherapy in their protfolio (Celgene, Celyad, Amgen, BMS, Roche, Novartis, MSD), but the major part was supported by the Innovation Fund of the Ghent University Hospital. The supporting companies had no influence on the development of the motion comic.

The individual authors have nothing to disclose.

\section{P108}

Improved Efficacy of Granulocyte Collections Obviating the Need for Addition of Hydroxyethyl Starch by Use of the Terumo BCT Spectra Optia Device 
Dimitra Oikonomopoulou ${ }^{1}$, Ioannis Tsonis ${ }^{1}$, Vassilis Anyfantis ${ }^{2}$, Chara Giatra $^{1}$, Maria Vardaka ${ }^{1}$, Zoi Poulopoulou ${ }^{1}$, Eirini Bika ${ }^{1}$ Zois Mellios $^{1}$, Ioannis Konstantellos ${ }^{1}$, Tatiana Tzenou ${ }^{1}$, Stavros Gigantes $^{1}$, Ioannis Baltadakis ${ }^{1}$, Nicholas Harhalakis ${ }^{1}$, Dimitrios Karakasis $^{1}$

${ }^{1}$ Evaggelismos General Hospital, Department of Haematology and Bone Marrow Transplantation Unit, Athens, Greece; ${ }^{2}$ Rontis Hellas $S$. A., Athens, Greece

Background: Granulocyte transfusions (GTs) represent a potentially life-saving intervention in patients with severe neutropenic infections and anticipated bone marrow recovery as it applies in the setting of hematopoietic stem cell transplantation (HSCT). However, their use has been limited by lack of urgent donor availability as well as by aspects pertaining to the collection and administration of adequate granulocyte doses in a safe manner. We explored the feasibility and safety of GTs in intensive chemotherapy and/or allogeneic HSCT recipients by the use of the Spectra Optia device. Our aim was to optimize collection efficacy, while avoiding the addition of Hydroxyethyl starch (HES) for sedimentation of red blood cells to prevent anaphylactoid reactions in recipients.

Methods: A total of 28 granulocyte collections (GCs) were obtained for 5 patients with grade IV neutropenia and life-threatening infections in the context of induction chemotherapy for acute leukemia $(n=1)$ or allogeneic HSCT $(n=4)$. Granulocyte donors (4 females/24 males) were either volunteer donors or friends or relatives of the patient, selected on the basis of ABO compatibility. For granulocyte mobilization, granulocyte-colony stimulating factor (G-CSF, 6-8 $\mu \mathrm{g} / \mathrm{kg}$, subcutaneously) plus dexamethasone (8 mg, intramuscularly) were administered 10-12 hours prior to collection. All collections were performed with the Spectra Optia device, v11.2 PMN collection protocol, without using HES. Peripheral veins were used as vascular access in all procedures. Calcium supplementation was given orally to all donors. The target dose was 3.5$5.0 \times 10^{\wedge} 10$ granulocytes per transfusion. Granulocyte collection products were irradiated prior to infusion to patients.

Results: The median granulocyte yield per collection procedure was 3.78 (range, $1.55-8.42$ ) $\times 10^{\wedge} 10$, with a median volume of 455 (range, 165-650) $\mathrm{ml}$ and a median hematocrit of $19.55 \%$ (range, $7.5-33.5 \%$ ). The median blood volume processed was 6000 (range, 2528-8418) $\mathrm{ml}$ or 1.17 (range 0.39-1.62) total blood volumes (TBV). There was a median platelet count reduction in donors of $29 \%$ (range 11-51\%) from baseline values, which was mainly dependent on blood volume processed. No remarkable side effects were recorded in donors in relation to granulocyte mobilization and collection. GTs were well tolerated by recipients without febrile or anaphylactoid reactions. A clinical benefit, defined as control of neutropenic infection until hematopoietic recovery or engraftment, was achieved by granulocyte transfusions in 3 out of 5 patients.

Conclusions: Granulocyte collection with the fully automated Spectra Optia device is a safe procedure for donors, and results in transfusion of adequately high doses of granulocytes. Comparable yields of granulocytes can be achieved with or without the addition of HES suggesting enhanced safety for recipients. Based on the above data, a broader application of GTs may be warranted especially in the context of HSCT.

Conflict of interest: None of the authors has anything to disclose.

\section{P109}

In vivo patterns of dendritic cell and monocyte populations in paediatric patients with acute GVHD treated with ECP

Aisling M Flinn, Tom J Altmann, Venetia Bigley, Xiao Nong Wang, Andrew R Gennery

Newcastle University, Institute of Cellular Medicine, Newcastle upon Tyne, United Kingdom

Background: Extracorporeal photopheresis (ECP) is a second line immune-modulating therapy used in the treatment of acute GVHD. Previous studies suggest a role of dendritic cells (DC) in the underlying tolerance-inducing mechanisms of action, and monocyte differentiation into DCs during the ECP process, although little is known about the true in vivo effects on DC and monocyte populations.

Methods: Six paediatric patients received ECP for the treatment of corticosteroid-refractory/dependent acute GVHD. Four patients have completed therapy, one was withdrawn after 16 cycles of ECP and one remains on ECP (week 72). Whole blood samples were analysed before ECP cycles at regular intervals and absolute numbers of myeloid $\mathrm{DC} \quad\left(\mathrm{mDC}, \quad \mathrm{CD}^{-}{ }^{-} \mathrm{CD} 19^{-} \mathrm{CD} 2{ }^{-} \mathrm{HLA}^{-} \mathrm{DR}^{+} \mathrm{CD} 14^{-} \mathrm{CD} 16^{-}\right.$ $\mathrm{CD}_{11 \mathrm{c}^{+}}$cells), plasmacytoid $\mathrm{DC}\left(\mathrm{pDC}, \mathrm{CD}^{-} \mathrm{CD} 19^{-}\right.$ CD20-HLA-DR ${ }^{+} \mathrm{CD} 14^{-} \mathrm{CD} 16^{-} \mathrm{CD} 11 \mathrm{c}^{-\mathrm{CD}} 123^{+}$cells), and monocytes populations (including classical monocytes, $\mathrm{CD}^{-} \mathrm{CD} 19^{-} \mathrm{CD} 20^{-} \mathrm{HLA}-\mathrm{DR}^{+} \mathrm{CD} 14^{+} \mathrm{CD} 16^{-}$cells), were enumerated in TruCOUNT $^{\circledR}$ (BD Biosciences) tubes using flow cytometry. Results were compared to two control groups at 4, 8 and 12 months post HSCT; paediatric patients post HSCT who did not develop acute GVHD (group 1, n $=6$ ) and paediatric patients with acute GVHD who did not receive ECP (group 2, $\mathrm{n}=6$ ).

Results: Among the patients who have completed ECP therapy, an overall increase in pDC numbers was observed, with a reduction in the $\mathrm{mDC} / \mathrm{pDC}$ ratio. In contrast, the patient who remains on ECP exhibits consistently low pDCs, with an increasing $\mathrm{mDC} / \mathrm{pDC}$ ratio. There was an overall downward trend of the classical monocyte 
Figure A
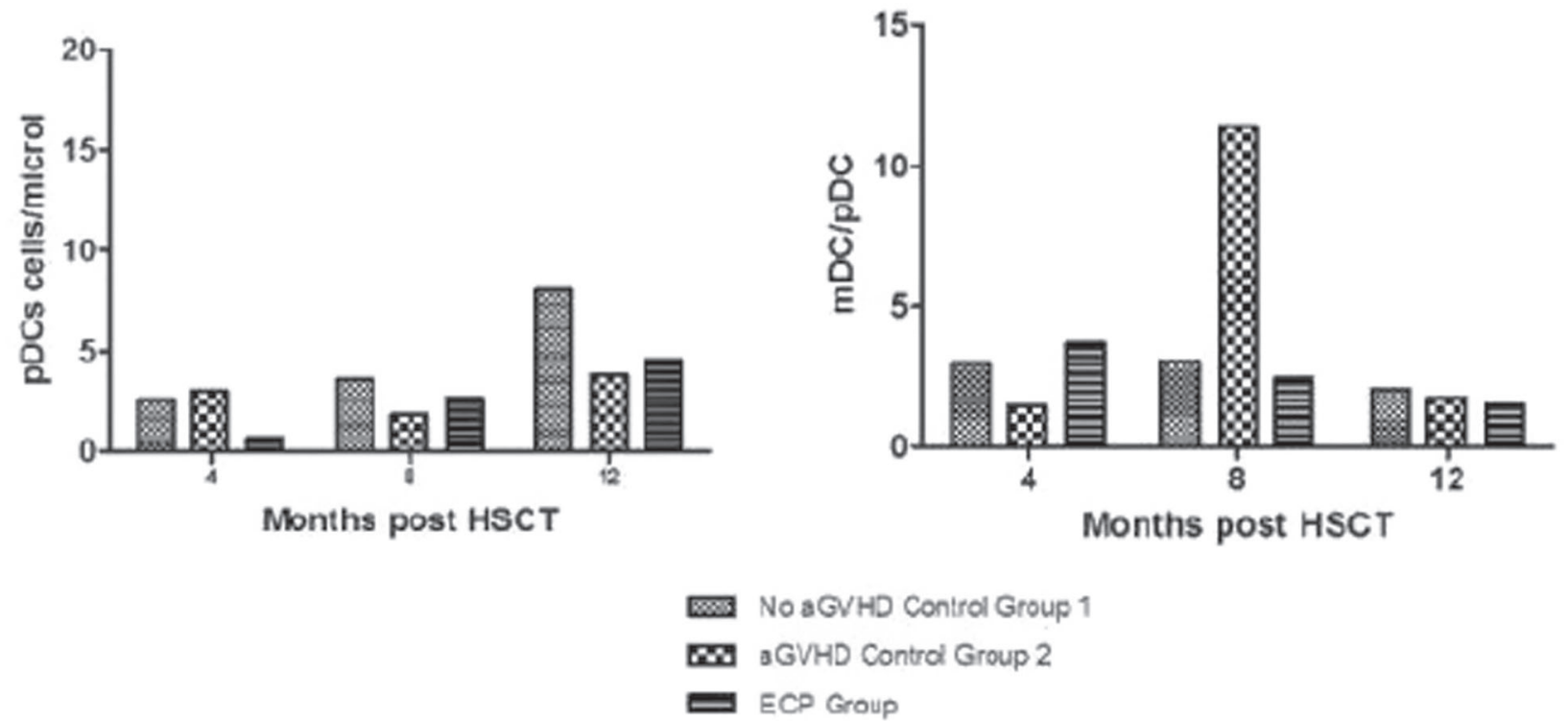

[P109 Figure] [Figure A. Dendritic cell populations at 4, 8 and 12 months post HSCT]

population seen in all ECP patients. The median $\mathrm{mDC} / \mathrm{pDC}$ ratio was higher in the ECP group at 4 months but higher in the acute GVHD control group 2 at 8 months (Figure A). A steady rise was seen in $\mathrm{mDC}$ and $\mathrm{pDC}$ numbers in both the no acute GVHD control group 1 and ECP groups, while this was more static in the acute GVHD control group 2 (Figure A).

Conclusions: Increasing the pDC population, and decreasing the $\mathrm{mDC} / \mathrm{pDC}$ ratio, overall promoting a favourable pDC environment may be an underlying mechanism of action behind ECP. Plasmacytoid DCs have been reported to have tolerance-inducing effects, including stimulation of regulatory T-lymphocytes and causing Tlymphocyte anergy. Decreasing classical monocyte populations may be due to DC differentiation and/or deviation from an inflammatory environment. Further research examining the phenotype of the DC populations and the concurrent impact on regulatory T-lymphocytes is needed.

Conflict of interest: None

\section{P110}

\section{Individual differences of multipotent mesenchymal stromal cells detected by interaction with lymphocytes}

Nikolay Kapranov, Yulia Davydova, Irina Galtzeva, Nataliya Petinati, Natalia Sats, Nina Drize, Larisa Kuzmina, Elena Parovichnikova, Valeriy Savchenko
National Research Center for Hematology, Moscow, Russian Federation

Background: Multipotent mesenchymal stromal cells (MSCs) are used for the treatment and prevention of acute graft versus host disease (GVHD). Not all MSCs samples are effective. The study of MSCs interaction with lymphocytes in vitro revealed 2 types of changes in the subpopulations of lymphocytes. The expression of HLADR on lymphocytes was significantly increased in the cocultivation with a part of the MSCs samples (group A), whereas co-cultivation of the same lymphocytes with other MSCs samples did not (group B). The aim of the study was to investigate the differences in the two identified groups of MSCs.

Methods: MSCs were derived from bone marrow (BM) of 26 donors ( 15 male and 11 female aged 11 to 62 years, median 27 years) and on $2^{\text {nd }}-3^{\text {rd }}$ passages were co-cultured with non-activated and activated with $5 \mathrm{mg} / \mathrm{ml}$ of PHA (PHA-lymphocytes) allogeneic lymphocytes in a ratio of about 1:10 for 4 days. Subpopulations of lymphocytes and MSCs surface markers were analyzed over time by flow cytometry. Determination of gene expression levels (REL) was performed by real time PCR.

Results: There were no differences between BM donors for groups A and B by sex, age and concentration of CFUs in their BM. Significant differences in the characteristics of MSCs were found in the total cell production for 4 passages, which was in group A 2.5 times lower than in group B $(8.1 \pm 1.5$ vs. $20.3 \pm 3.2)$. After a day of co- 
cultivation with lymphocytes, the REL of IDOI in group A was 6.5 times higher than group B $(10058 \pm 2695$ vs. 1554 $\pm 716)$, and REL of PPARg was 3.4 times lower $(0.35 \pm 0.06$ vs. 1.18 \pm 0.32 ). After 4 days of co-cultivation in group A, the REL of $I C A M 1$ was 3.2 times higher $(11.3 \pm 2.8$ vs. 3.5 \pm 0.9 ). Thus, the immunomodulatory ability of MSCs from group A should be significantly higher than from group B. This is also indicated by differences in the subpopulations of lymphocytes co-cultivated with MSCs from these groups.

The following significant differences in the subpopulations of lymphocytes were revealed. After 4 days of cocultivation with MSCs of group A, the fraction of CD4 + HLA-DR + was higher 2.8 times $(20.4 \pm 0.4$ vs. $7.4 \pm 1.0)$; CD8+HLA-DR+ 3.5 times $(35.5 \pm 1.3$ vs. $10.0 \pm 2.9)$; CD4 $+\mathrm{EM}$ (effector memory cells) 1.2 times ( $20.2 \pm 0.9$ vs. 16.6 \pm 1.6 ); CD4+TM ("transitional" memory cells) 3.5 times $(1.2 \pm 0.01$ vs. $0.3 \pm 0.2)$ for non-activated lymphocytes, for activated lymphocytes proportion of CD4+HLA-DR+ was higher 2.4 times (72.1 \pm 3.6 vs. $29.7 \pm 9.3)$; CD8+HLA-DR+ 2.2 times $(76.2 \pm 4.6$ vs. $35.0 \pm 13.1), \mathrm{CD} 4+\mathrm{CM}$ (central memory cells) 1.6 times $(53.4 \pm 2.7$ vs. $32.7 \pm 5.5)$; $\mathrm{CD} 8+\mathrm{CM}$ 2.4 times (35.8 \pm 2.7 vs. $15.1 \pm 6.0)$; CD4+TM 6.5 times $(1.8$ \pm 0.2 vs. $0.2 \pm 0.1)$; CD $8+$ EM 1.6 times $(16.3 \pm 2.3$ vs. 10.1 \pm 1.4 ); $\mathrm{CD} 4+\mathrm{PD} 1+2.5$ times (44.2 \pm 5.6 vs. $17.6 \pm 3.2)$; CD8 $+\mathrm{PD} 1+2.2$ times $(23.4 \pm 3.5$ vs. $10.6 \pm 2.6)$ and $\mathrm{CD} 8+\mathrm{TE}$ (terminal effector cells) was 2.5 times lower ( $6.2 \pm 0.7$ vs. 14.1 \pm 2.9 ) compared with lymphocytes co-cultured with MSCs from group B.

Conclusions: The revealed individual differences in MSCs can explain why not all MSC samples are effective for the treatment of autoimmune diseases, acute GVHD and others.

Conflict of interest: The study was financially supported through a grant from the Russian Science Foundation, Project № 16-15-00102. The individual authors have nothing to disclose.

\section{P111}

Induction of donor-specific tolerance in living donor kidney transplant recipients through clinical MIC cell infusion - a phase I study (TOL-1)

Christian Morath $^{1}$, Anita Schmitt ${ }^{2}$, Christian Kleist ${ }^{3}$, Volker Daniel ${ }^{4}$, Gerhard Opelz ${ }^{4}$, Caner Süsal ${ }^{4}$, Florian Kälble ${ }^{1}$, Claudia Sommerer ${ }^{1}$, Lei Wang ${ }^{2}$, Angela Hückelhoven ${ }^{2}$, Arianeb Mehrabi ${ }^{5}$, Uta Merle ${ }^{6}$, Luiza Pego da Silva ${ }^{1}$, Carsten Müller-Tidow ${ }^{2}$, Martin Zeier ${ }^{1}$, Matthias Schaier ${ }^{1}$, Michael Schmitt ${ }^{2}$, Peter Terness ${ }^{4}$

${ }^{1}$ University Clinic Heidelberg, Division of Nephrology, Heidelberg, Germany; ${ }^{2}$ University Clinic Heidelberg, Department of Internal Medicine V, Heidelberg, Germany; ${ }^{3}$ University Clinic Heidelberg, Department of Nuclear Medicine, Heidelberg, Germany; ${ }^{4}$ University of Heidelberg, Institute of Immunology, Heidelberg, Germany;

${ }^{5}$ University Clinic Heidelberg, Allgemein-, Viszeral- und
Transplantationschirurgie, Heidelberg, Germany; ${ }^{6}$ University Clinic Heidelberg, Department of Internal Medicine IV, Heidelberg, Germany

Background: Major limitations of long-term allograft survival are life-threatening side-effects by immunosuppressive medication and chronic allograft injury. Therefore there is a fervent need for the induction of a donor-specific tolerance without broad unspecific immunosuppression. To this aim a clinical phase I study with so called MIC cells was performed, i.e. donor-derived monocytes that gain immunosuppressive properties after incubation with the proliferation inhibitor mitomycin C.

Methods: For 10 kidney transplant recipients, MIC cells were manufactured under Good Manufacturing Practice (GMP) conditions out of leukapheresis products from the living donors. The amount of MIC cells administered to the patient was escalated from $1.5 \times 10^{\wedge} 6$ MIC cells per $\mathrm{kg}$ body weight at day $-2(\mathrm{~N}=3$, group $\mathrm{A})$, to $1.5 \times 10^{\wedge} 8 \mathrm{MIC}$ cells per $\mathrm{kg}$ body weight at day $-2(\mathrm{~N}=3$, group $\mathrm{B})$ or on day -7 $(\mathrm{N}=4$, group $\mathrm{C}$ ) before living donor kidney transplantation. After kidney transplantation, patients received a standard triple drug immunosuppressive therapy. The frequency of adverse events (AE) was measured as primary outcome at day 30 .

Results: Clinically, all kidney transplant recipients had a median serum creatinine of $1.4 \mathrm{mg} / \mathrm{dL}$ at day 30 and remained stable with a median creatinine of $1.48 \mathrm{mg} / \mathrm{dL}$ at day 180 without significant proteinuria (median $10 \mathrm{~g} / \mathrm{mol}$ creatinine at day 180) and without rejection episode. The patients experienced in total 70 AEs including four severe AEs not related to the MIC cell infusion. Besides two infectious complications no other AEs like positive cross match results, de novo donor-specific antibodies or rejection episodes were recorded.

In patients of group $\mathrm{C}$, a reduction of immunosuppressive therapy was effective in the observational phase with lowdose cyclosporine A and low-dose enteric-coated mycophenolate sodium.

Major immunological changes were observed in patients of group $\mathrm{C}$ with an increase of $\mathrm{CD} 19+\mathrm{B}$ cells up to a median of $300 / \mu \mathrm{L}$ until day 30 and a decrease thereafter to a median of $35 / \mu \mathrm{L}$ at day 180 . Furthermore CD19 $+\mathrm{CD} 24$ highCD38high transitional Bregs increased from a median of $2 \%$ at day 30 to a median of $20 \%$ of the total CD19+ B cell pool on day 180. And the plasma IL-10/ TNFalpha ratio increased from a median of 0.05 before cell therapy to a median of 0.11 at day 180 . In an in vitro mixed lymphocyte culture assay lymphocytes of these patients showed no or only minimal reactivity against irradiated donor lymphocytes while reactivity against 3rd party lymphocytes was preserved. 
Furthermore the analysis of MIC cell products in in vitro assays demonstrated the capability of MIC cells to induce tolerogenic dendritic cells by down-regulating costimulatory molecules CD80 and CD86 and the maturation molecule CD83 while up-regulating the immunosuppressive molecule CD103.

Conclusions: All kidney transplant recipients receiving the MIC cell product showed a stable renal function without any allograft injury nor rejection episodes even under reduction of conventional therapy with immunosuppressive drugs. Therefore MIC cells represent an innovative and extremely promising option for donor-specific immunosuppression in living donor kidney transplantation and beyond.

Clinical Trial Registry: EudraCT number 2014-00208630

Conflict of interest: C. Morath, A. Schmitt, C. Kleist, G. Opelz, M. Zeier, M. Schaier, M. Schmitt, and P. Terness are founders of Tolerogenix $\mathrm{X} \mathrm{GmbH}$. L. Wang is an employee of the company.

\section{P112}

\section{Infusion of Donor Memory T-Cells as a safe strategy to improve immune reconstitution after Haploidentical Stem Cell Transplant}

Andreina Figueira ${ }^{1}$, Alba Perez ${ }^{1}$, Blanca Rosich ${ }^{1}$, Yasmina Mozo ${ }^{1}$, David Bueno ${ }^{1}$, Sonsoles San Roman ${ }^{1}$, Berta Gonzalez ${ }^{1}$, Pedro Rubio ${ }^{1}$, Diego Plaza ${ }^{1}$, Ana Sastre ${ }^{1}$, Raquel De Paz ${ }^{2}$, Antonio Perez Martinez $^{1}$

${ }^{1}$ Hospital Universitario La Paz, Pediatric Hemato-Oncology and Stem Cell Transplantation Unit, Madrid, Spain; ${ }^{2}$ Hospital Universitario La Paz, Hematology Department, Madrid, Spain

Background: The use of T-cell depleted grafts in haploidentical stem cell transplantation (HSCT) has been proven efficient in preventing graft versus host disease (GvHD), although it is associated with a delay in early Tcell recovery which increases the risk of viral infections, leukemia relapse or graft rejection. Conventional donor lymphocyte infusion (DLI) after HSCT transplantation has proven to be an effective strategy; nevertheless, it is conditioned because of a high prevalence of GvHD even with low dose of T-cells. The infusion of selected lymphocyte subpopulations with low aloreactivity is emerging as an effective strategy to rectify this issue. The depletion of CD45RA+ naïve lymphocytes, while preserving $\mathrm{CD} 45 \mathrm{RO}+$ memory $\mathrm{T}$-cells, could provide a safe source of functional lymphocytes with anti-infection, antileukemic and anti-rejection properties, and a lower rate of adverse effects.

Methods: We present data of CD45RO+ memory T-cells DLI (mDLI) performed after HSCT in cases of mixed chimerism, persistent lymphopenia, presence of viral/ opportunistic infections or as a strategy to accelerate immune reconstitution.

Results: Fifteen patients with diagnosis of Acute Lymphoblastic Leukemia $(\mathrm{n}=6)$, Acute Myeloblastic Leukemia $(\mathrm{n}=3)$, Myelodisplastic Syndrome $(\mathrm{n}=1)$, Severe Aplastic Anemia $(n=3)$, Sideroblastic Anemia ( $=1)$ and Chronic Granulomatous Disease $(n=1)$, received one or more mDLI after HSCT. Of the fifteen patients, twelve received a HSCT with a graft consisting of CD34+ and CD45RA- cells, and three with a TCRab + cells depleted graft.

A total of forty-three mDLI were infused. The median dose of CD45RO+ memory T-cells infused was $5.00 \times 10^{7} \%$ $\mathrm{kg}$ (range: $4.8 \times 10^{4}-4.25 \times 10^{8} / \mathrm{Kg}$ ), with a median dose of CD45RA + naïve T-cells of $3.90 \times 10^{2} / \mathrm{kg}$ (range:0-1.3 $\times 10^{4} /$ $\mathrm{Kg}$ ).

The mDLI were infused at a median of seventy-seven days after HSCT (range:14-407 days), with a median interval between mDLI of thirty-four days (range:4159 days). $60 \%$ of patients received $2-3 \operatorname{mDLI}(n=9)$, while four patients $(26.67 \%)$ were infused in more than 3 occasions and two patients $(13.33 \%)$ were infused only once.

Twenty-one mDLI (49\%) were administered because of lymphopenia, fourteen of them (33\%) in patients with concomitant viral/opportunistic infections (CMV, EBV, $\mathrm{BKV}$, Adenovirus, VZV, toxoplasmosis). Mixed chimerism/graft failure was the motive of $37 \%$ of the $\mathrm{mDLI}(\mathrm{n}=$ 16) and six (14\%) were administered as an attempt to accelerate immune reconstitution.

All infusions were well tolerated without any side effects during the infusion or appearance/worsening of GvHD.

A progressive increase in T-cell counts was observed following six mDLI (28.57\%), although it was a transitory response (3-8 weeks) in five cases. Viral/opportunistic infections were controlled in five cases $(35.71 \%)$, requiring a median of $2 \mathrm{mDLI}$ to achieve this response. As for the mDLI administered in cases of mixed chimerism/graft failure, none were effective in reverting this situation.

Conclusions: Our preliminary data suggests that mDLI, is a safe adoptive immunotherapy strategy even with high dose of T-cells without infusion side effects or GvHD complications. Some efficacy has been observed in patients with lymphopenia and opportunistic infections, while there have been no positive results in patients with mixed chimerism/graft failure, up to date. However, to determine the real efficacy of this strategy, prospective studies are required.

Conflict of interest: None of the authors has anything to disclose. 


\section{P113}

\section{Long lasting responses to virus-targeted T-cell therapy in} oligometastatic EBV-related nasopharyngeal carcinoma

\author{
Simona Secondino ${ }^{1}$, Antonella Gurrado ${ }^{2}$, Sabrina Basso ${ }^{2}$, Gianpiero \\ Rizzo $^{1}$, Ilaria Imarisio ${ }^{1}$, Ilaria Pisani ${ }^{2}$, Jessica Bagnarino ${ }^{2}$, Marco \\ Zecca $^{3}$, Paolo Pedrazzoli ${ }^{1}$, Patrizia Comoli ${ }^{2}$ \\ ${ }^{1}$ Fondazione IRCCS Policlinico San Matteo, Oncology Unit, Pavia, \\ Italy; ${ }^{2}$ Fondazione IRCCS Policlinico San Matteo, Pediatric \\ Hematology/Oncology \& Cell Factory, Pavia, Italy; ${ }^{3}$ Fondazione \\ IRCCS Policlinico San Matteo, Pediatric Hematology/Oncology, \\ Pavia, Italy
}

Background: Nasopharyngeal carcinoma (NPC) is a Epstein-Barr virus (EBV)-related, highly chemoradiosensitive malignancy. However, one-third of patients are considered to be incurable because of metastatic or recurrence disease. Published experiences reported 7-14\% metastatic NPC patients surviving $\geq 2$ years. Expression of antigenic viral proteins by malignant cells constitutes a good target for immunotherapeutic strategies, and we have previously showed disease control by autologous EBVtargeted cell therapy in refractory/relapsed patients failing conventional treatment.

Methods: We describe the results of a T-cell therapy program for patients with oligometastatic relapsed NPC patients, treated at completion of second-line chemotherapy. The patients received 2 administrations of EBV-specific cytotoxic T lymphocytes (CTL) at a total cell dose/infusion of 1.5-3 $\times 10^{8}$, repeatable in case of objective response.

Results: Starting from 12/2011, we have treated 12 patients with refractory/metastatic disease. Six patients had more than 2 organ involved and $>3$ metastatic lesions, while 6 patients had oligometastatic disease, with less than 3 lesions. The best response after second-line treatment was progressive disease (PD) in 2 patients, stable disease (SD) in 3 patients, and partial (PR) or complete (CR) response in 4 and 3 patients, respectively. After CTL therapy, no severe adverse events were observed. The 5 patients treated with progressive or stable disease and more than 9 metastatic lesions showed NPC progression and died of their disease. The patient with $>3$ matastases who reached PR after second-line chemotherapy, stabilized disease for 2 years after T-cell therapy. Upon disease progression, she was treated with third-line capecitabine-based chemotherapy and reached long-lasting CR. She is alive without evidence of disease at 85 months from diagnosis. Of the 3 patients who reached CR after second-line chemotherapy and received adjuvant CTL treatment, 2 persist in CR at 5- and 3-year follow-up, while one patient had disease relapse, and reached long-lasting $\mathrm{CR}$ after treatment with $3^{\text {rd }}$-line gemcitabine-based chemotherapy. Of the 3 patients with oligometastatic disease who were treated with EBV-CTL while in PR after second-line chemotherapy, one reached long-lasting $\mathrm{CR}$, while the other 2 had disease progression after initial stabilization of PR. They received conventional treatments (one patient radiotherapy on site of disease and the other $3^{\text {rd }}$ line chemotherapy) followed by $\mathrm{T}$ cell therapy, achieving a CR. At a median follow-up of 58 months, 7/12 patients are alive with no evidence of disease.

Conclusions: EBV-specific CTL therapy is safe and associated with clinical benefit in patients with refractory or metastatic NPC. Combination of second-line chemotherapy and CTL therapy seems to yield the best results, which compare favourably to the outcomes described in the literature.

Clinical Trial Registry: N/A

Conflict of interest: none to declare

\section{P114}

Mixed chimerism reversal after cord blood donor lymphocyte infusion using a $20 \%$ fraction of the original CB unit

Albert Esquirol Sanfeliu ${ }^{1}$, Rodrigo Martino Bufarull ${ }^{1}$, Laura Soria Guerrero $^{2}$, Guillermo Orti ${ }^{3}$, Christelle Ferrà Coll ${ }^{4}$, Isabel SanchezOrtega Sanchez ${ }^{5}$, Cristina Diaz de Heredia ${ }^{6}$, Isabel Badell Serra ${ }^{7}$, Laura Medina Marrero ${ }^{8}$, Nerea Castillo ${ }^{8}$, Ramón Gimeno Martínez $^{2}$, Sergio Querol Giner ${ }^{8}$

${ }^{1}$ Hospital de la Santa Creu i Sant Pau, Hematology Service, Barcelona, Spain; ${ }^{2}$ Hospital del Mar \& IMIM (Hospital del Mar Medical Research Institute), Immunologia, Barcelona, Spain;

${ }^{3}$ Hospital Vall d'Hebron, Hematology Service, Barcelona, Spain; ${ }^{4}$ Institut Català d'Oncologia Trias i Pujol, Badalona, Spain; ${ }^{5}$ Institut Català d'Oncologia Duran i Reynals, L'Hospitalet de Llobregat, Spain; ${ }^{6}$ Hospital Materno-Infantil Vall d'Hebron, Barcelona, Spain; ${ }^{7}$ Hospital de la Santa Creu i Sant Pau, Pediatrics Service, Barcelona, Spain; ${ }^{8}$ Banc de Sang $i$ Teixits, Cell Therapy Services, Barcelona, Spain

Background: Donor lymphocyte infusion (DLI) has not been tested in CB transplantation. We have developed a phase I-II clinical trial (NCT02328885). to assess the ability of DLI from a $20 \%$ spared fraction after engraftment of the $80 \%$ original product. This clinical trial is under evaluation. Here, we present a case report to show a potential effect of the $\mathrm{CB}$ lymphocytes in reverting mixed chimera.

Methods: Clinical case: A 46 years old male was diagnosed with ALL and chemotherapy treatment was started using a high risk ALL scheme (PETHEMA group). The most critical complication during the treatment was a central vein thrombosis as a consequence of L-asparaginase coagulopathies. The evaluation at the end of chemotherapy was a first $\mathrm{CR}$ with a negative minimal residual disease (MRD). He received umbilical CB transplant due to lack of 
a related and unrelated suitable donor. Patient fulfilled criteria to enter this clinical trial.

Conditioning regiment consisted of thiotepa $(10 \mathrm{mg} / \mathrm{kg})$, fludarabine $(150 \mathrm{mg} / \mathrm{m} 2)$ and busulphan $(12 \mathrm{mg} / \mathrm{kg}$, oral administration). GvHD prophylaxis was late administration of ATG $(6 \mathrm{mg} / \mathrm{kg})$, cyclosporine and short pulses of prednisone thereafter. The first part $(80 \%)$ of umbilical CB containing 2.3E7 $\mathrm{TNC}$ and $1.1 \mathrm{E} 5 \mathrm{CD} 34 / \mathrm{kg}$ was infused. Neutrophils $\left(0.5 \times 10^{6} / \mathrm{L}\right)$ and platelets $\left(>20 \times 10^{6} / \mathrm{L}\right)$ recovery were +21 and +47 days respectively. The main complication was a cytomegalovirus reactivation at day +55 that required a valganciclovir/ganciclovir treatment associated with corticoids due to a differential diagnosis with GvHD. Corticotherapy was fastly decreased and stopped before a second part infusion of CB.

Results: Bone marrow aspirates showed CR with negative MRD. However, donor lymphocytes population in the chimerism decreased (see figure) and the DLI was scheduled for day +106 . The second part $(20 \%)$ of umbilical cord blood was infused containing 7E5 CD3/kg. Already one month after the infusion a 4-fold increase in the number of $T$ cells was apparent. These numbers amplified further in the next 3 months (from $70 \mathrm{~T}$ cells/microliter at the moment of infusion to $255 \mathrm{~T}$ cells/microliter 30 days later, or $933 \mathrm{~T}$ cells/microliter 3 months post-DLI). Concomitantly, an intense proliferative activity was noted. It was detected on CD4 $+\mathrm{T}$ cells and with a certain delay also on CD8+ cells. In this period a reversal in the CD4/ CD8 ratio was observed, and even although most circulating $\mathrm{T}$ cells were $\mathrm{CD} 8+$ before the infusion (ratio equal to 0.1 ), a normal ratio was achieved in only 2 months At all time points analyzed, $\mathrm{T}$ cell memory phenotypes predominated.

Conclusions: In summary, we observed a vigorous increase in the presence of circulating $\mathrm{T}$ lymphocytes after DLI. The consequence of second $\mathrm{CB}$ infusion was a chimerism improvement until achieving total donor lymphocytes chimerism that was maintained. Also, and a new cutaneous and digestive grade II GvHD that responded to medium doses of corticosteroids was shown. In the last

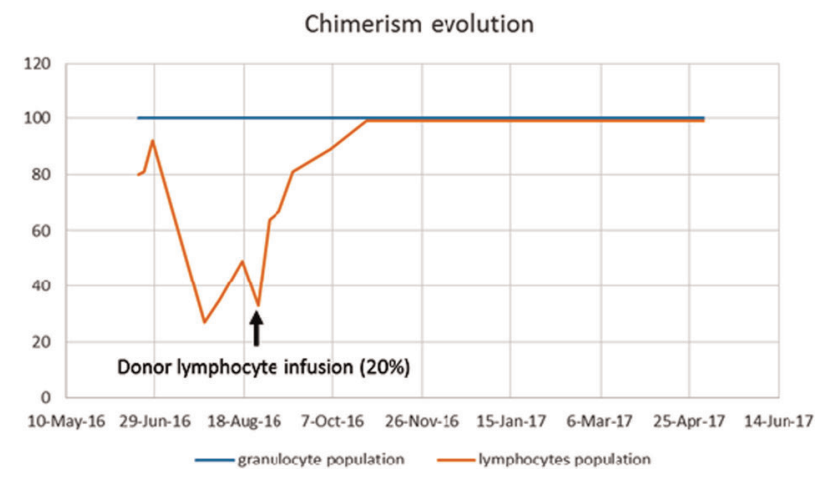

[P114 Figure] [Figure 1: T cell chimerism evolution] follow up, the patient was alive, in CR with full donor chimerism. Therefore, a potential role for this cell therapy intervention is proposed.

Clinical Trial Registry: NCT02328885

https://www.clinicaltrials.gov/ct2/results?term= NCT02328885\&Search $=$ Search

Conflict of interest: Authors declare no conflict of interest

\section{P115}

NK cell specific checkpoint inhibition improves anti-tumor efficacy of patient derived NK cells against autologous Multiple Myeloma

Sara Tognarelli, Ivana Von Metzler ${ }^{2}$, Sebastian Wirsching ${ }^{1}$, Benedikt Jakobs ${ }^{3}$, Andreas Mackensen ${ }^{3}$, Hubert Serve ${ }^{2}$, Peter Bader $^{1}$, Evelyn Ullrich ${ }^{1}$

${ }^{1}$ Goethe University Frankfurt am Main, Division for Stem Cell Transplantation and Immunology, Department for Children and Adolescents, Frankfurt, Germany; ${ }^{2}$ Goethe University Frankfurt am Main, Department of Hematology and Oncology, Frankfurt, Germany; ${ }^{3}$ University Hospital Erlangen, Department of Medicine 5, Erlangen, Germany

Background: Natural Killer (NK) cells are innate lymphocytes with a strong anti-tumor ability. In tumor patients, such as multiple myeloma (MM) patients, an elevated number of NK cells after stem cell transplantation (SCT) has been reported to have benefits for the patients. We recently showed that, at an early time point after autoSCT, CD56 ${ }^{\text {bright }} \mathrm{CD} 16^{-1+}$ NK cells represented the main NK cell subset. Remarkably, this NK cell population, considered to be immature, expressed maturation markers and exerted important cytotoxic functions, when challenged with K562 tumor cells, indicating a potential impact on antitumor immunity.

Methods: In this preclinical immune monitoring and scientific research study, NK cells have been isolated kits from peripheral blood and bone marrow of healthy donors and MM patients at defined time points using NK cell negative isolation. Also patient-derived tumor cells have been sorted from MM patient bone marrow samples at first diagnosis. Phenotypic and functional characterization has been performed by in vitro multi-color flow cytometry assays.

Results: With the aim of the NK cell use for adoptive cell therapy, we also addressed the cytotoxicity of patientderived, cytokine-stimulated NK cells against MM cells at diagnosis, before and after auto-SCT. We detected changes in NK cell phenotype as well as cytotoxic function before and after expansion. Remarkably, after cytokine stimulation patient NK cells did not significantly differ from healthy donor NK cells, showed a highly activated phenotype and 
were able to significantly enhance the lysis of MM cells. In a smaller cohort of MM patients we were able to isolate autologous tumor cells, as well as bone marrow NK cells and we could show that our cytokine-based ex vivo activation protocol was able to significantly improve even the lysis of autologous tumor cells, suggesting a potential use of NK cells as adoptive therapy for MM patients. Moreover, in an effort to further enhance the lysis of MM cells, we also used an "NK check point inhibitor" antibody, targeting the inhibitory NKG2A receptor. Blocking the NKG2A-HLA-E pathway induced a significant increase in the NK cell mediated cytotoxicity against Multiple Myeloma.

Conclusions: In addition to autologous stem cell transplantation, adoptive NK cell therapeutic protocols that imply a cytokine-based ex vivo NK cell expansion procedure might be of high interest for the treatment of Multiple Myeloma patients. Furthermore, our functional assays revealed NKG2A blocking antibodies as promising "NK check point inhibitors".

Conflict of interest: The authors have nothing to disclose.

\section{P116}

Abstract previously published

\section{P117}

Abstract previously published.

\section{P118}

Potent anti-leukemia activities of humanized chimeric antigen receptor modified T(CAR-T) cell therapy in Chinese patients with relapsed/refractory acute lymphoblast leukemia

Lei Xiao ${ }^{1}$, Qing Zhang ${ }^{2}$, Xiaofan $Z^{2} u^{3}$, Zhao $W^{1}$

${ }^{1}$ Innovative Cellular Therapeutics Co.,Ltd., Shanghai, China;

${ }^{2}$ Guangdong No.2 Province People 's Hospital,, Guangzhou, China;

${ }^{3}$ Institute of Hematology\& Blood Diseases Hospital, Chinese Academy of Medical Sciences and Peking Union Medical College, Tianjin, China

Background: Although highlighted breakthrough of CD19 targeted chimeric antigen receptor (CAR) T cell on blood malignancies, high relapse rate is a big challenge for CART cells with single chain variable fragment $(\mathrm{scFv})$ domains of murine origin. Our previously murine CART cells achieved high complete remissions (CRs) on patients with relapsed/refractory hematological malignances, as $83 \%$ complete remission rate in acute lymphoblastic leukemia and over $57 \%$ complete remission rate in non-hodgkin's lymphoma (NCT 02813837). However, unsatisfactory relapse rate was encountered as with most CAR with single chain variable fragment $(\mathrm{scFv})$ domains of murine origin. Human anti-mouse antibody (HAMA) may be the important culprit. Therefore, we constructed CD19-directed CAR T cells with a humanized $\mathrm{scFv}$ domain to overcome the potential for anti-murine immune-mediated rejection.

Methods: We have constructed humanized anti-CD19 CAR with lentiviral vector encoding a CAR composed of murine or humanized anti-CD19 scFv, CD3z, and 4-1BB domains. To estimate the anti-tumor activity and safety in vitro, they were co-cultured with tumor cell lines with or without CD19 expression. To evaluate the safety and efficiency in vivo, human tumor cell transplanted immune deficient mice were divided into four groups including humanized CAR-T cells, murine derived CART cells, normal $\mathrm{T}$ cells and buffer. What's more, a preliminary clinical trial on four patients with refractory or relapsed acute lymphoblast leukemia were conducted afterwards. Four subjects with $\mathrm{r} / \mathrm{r}$ B-ALL were treated with humanized CAR-T cells from December 16, 2016 to December 27, 2016. Both the murine and humanized CAR-T cells were infused with dose range between $0.45 \times 10 \mathrm{E} 6 \mathrm{CAR}-\mathrm{T}$ cells/ $\mathrm{kg}$ and $10.51 \times 10 \mathrm{E} 6 \mathrm{CAR}-\mathrm{T}$ cells $/ \mathrm{kg}$. All subjects were monitored closely during the trial.

Results: CD19 positive tumor cells co-cultured with CAR-T cells showed that most of the tumor cells were killed by CAR-T cells while CD19 negtive tumor cells remained alive. For tumor cell transplantation experiment, the group of humanized CD19 CAR achieved a longer survival. Pre-clinical experiments showed the antimalignancy ability and safety of CD19-directed CAR $\mathrm{T}$ cells with a humanized $\mathrm{scFv}$ domain. In the preliminary clinical trial, all four subjects with B-ALL treated by humanized CAR-T cells achieved CR as well as MRD negative, between 14 to 41 days after CART cell infusion. And none of them suffered from irreversible neurotoxicity.

Conclusions: This is the first multicentre report to our knowledge of successful treatment of $\mathrm{r} / \mathrm{r}$ B-ALL with humanized CD19 CAR T cells in China. Even r/r B-ALL with high-burden leukemia patients also was effective and associated with a high remission rate after autologous CD19 CAR-T infusion. Though the follow-up is short, CD19 humanized CAR-T cell demonstrated its anti-malignances activity in subjects with ALL.

Clinical Trial Registry: NCT 02813837

\section{Conflict of interest:}

Q. Zhang and X. Zhu: nothing to disclose;

Z. Wu and L. Xiao: stockholders of Innovative Cellular Therapeutics Co., Ltd. 


\section{P119}

\section{Processing of Peripheral Blood Hematopoietic Stem Cell Using a GMP- Automated Closed System in a Clean Room Facility}

Naziha Menasria ${ }^{1}$, Mohammed Bakr ${ }^{2}$, Rola Sami El Chahab ${ }^{1}$, Wedad S Hamdi', Shondalyn Tan Daquila ${ }^{1}$, Ajaeb Dakhilalla M H Al-Nabet ${ }^{3}$

${ }^{1}$ Hamad Medical Corporation, GMP Cellular Therapy Lab, Department Laboratory Medecine \& Pathology, Doha, Qatar; ${ }^{2}$ National Center for Cancer Care and Research (NCCCR), Doha, Qatar; ${ }^{3}$ Hamad Medical Corporation, Department Laboratory Medecine and Pathology, Doha, Qatar

Background: Autologous hematopoietic progenitor cells collected through apheresis (HPC-A) must be cryopreserved and stored before transplantation. Cell processing before HPC-A cryopreservation includes volume reduction that reduces the amount of dimethyl sulfoxide used as cryoprotectant, and decrease the needs for storage space in liquid nitrogen tanks.

Methods: We retrospectively compared the Pericell protocol performed with an automated GMP closed system: the Sepax 2 device (Biosafe SA / GE Healthcare) and the common manual technique that uses refrigerated centrifugation at $850 \times \mathrm{g}$ prior to plasma removal and volume adjustment. We comparatively evaluated Total Nucleated Cells (TNC) and CD34+ cell recovery \& viability, as well as technologist time needed to complete the procedure. A total of 19 procedures were carried out, to process apheresis products obtained from adult patients: eleven HPC-A units were processed with the historical technique and eight HPCA units were processed with the Pericell protocol in an attempt to validate this technique.

Results: Automated processing required 15 minutes per procedure whereas manual processing required 20 to 25 minutes. The percentage volume reduction using the Sepax 2 Technology was $58.3 \%$ (range, 36.7-76.0\%; SD, $\mathrm{SD}, \pm 13.8 \%$ ), starting from $301 \mathrm{~mL}$ (range, $190-430 \mathrm{~mL}$; $\mathrm{SD}, \pm 76.9 \mathrm{~mL}$ ) to reach a final value of $126.5 \mathrm{~mL}$ (range, 60- $200 \mathrm{ml}$; SD, $\pm 53.2 \mathrm{~mL}$ ). This is to be compared with the performances of the manual method :mean, $45.2 \%$ (range, 20.3-63.1\%; SD, $\pm 16.7 \%$ ), downsizing the volume of the cell suspension from $260.5 \mathrm{~mL}$ (range, $141-340 \mathrm{~mL}$; $\mathrm{SD}, \pm 72.3 \mathrm{~mL}$ ) to $140.3 \mathrm{~mL}$ (range, $60-240 \mathrm{ml} ; \mathrm{SD}, \pm 54.1$ $\mathrm{mL})),(\mathrm{P}$ value $=0.088)$. There was no significant differences in the recovery of nucleated cells (TNC) for cell products processed with the Sepax 2Technology (mean 97.4\%; range from $89.4 \%-110.9 \%$; $\mathrm{SD}, \pm 6.8 \%)$, CD34 ${ }^{+}$ cells (mean $105.2 \%$; range from $98.6 \%-119 \%$; SD, $\pm 7.0 \%$ ) or cell viability (mean, $99.8 \%$; range from $98.5-101$; $\mathrm{SD} \pm$ $0.83 \%$ ) compared with the centrifuge method:100.3\% (range from 92.3\%- $112.5 \%$; SD $\pm 5.7 \%$ ), mean; $107.2 \%$ (range, 91.3\%-132.1\%; SD, $\pm 13.4 \%$ ) and mean :100.1\% (range from 99-101.6; $\mathrm{SD} \pm 0.86 \%),(P$ value $>0.3$ ). Moreover, the automated processing demonstrated time effective results compared with a semi-automated centrifuge processing method. Although it is not significant, there was an increase in the volume reduction percentage using Sepax 2 Technology.

Conclusions: Our data demonstrates the effectiveness and suitability of an automated procedure for volume reduction of HPC-A collected by apheresis, prior to addition of the cryoprotectant and controlled-rate freezing. The Pericell protocol performed with Sepax 2 Technology shortens processing time, includes features that facilitate compliance with FACT-JACIE standards, can be operated in a GMP environment, and provides similar cell recovery and viability than the reference technique.

Conflict of interest: This study has been done without conflict of interest. The authors acknowledge the support of members of the Cell Processing Facility (Centre de Thérapie Cellulaire) at Institut Paoli-Calmettes and Inserm CBT-1409, Marseille, France for personnel training and support.

\section{P120}

Quantitative determination of virus-specific (CMV, EBV, Adeno) lymphocytes in donor peripheral blood and in CD45RA-depleted T-cell products before and after cryopreservation

Viktoriia Kiseleva, Svetlana Glushkova, Sergei Blagov, Elena Kurnikova, Yakov Muzalevskii, Aleksei Kazachenok, Yana Baizyanova, Rimma Khismatullina, Julia Panchenko, Natalia Khripkova, Elena Osipova, Michael Maschan

Dmitry Rogachev National Research Center of Pediatric Hematology, Oncology and Immunology, Moscow, Russian Federation

Background: Viral infections remain one of the leading cause of morbidity and mortality after hematopoietic stem cell transplantation (HSCT). Although pharmacotherapy may help to prevent or treat viral disease, these drugs are expensive, toxic, and often ineffective due to primary or secondary resistance. These deficiencies in conventional therapeutics have increased interest in adoptive cell therapy. Adoptive transfer of virus-specific cytotoxic T lymphocytes (VSTs) can rapidly reconstitute antiviral immunity without causing graft-versus-host disease. In this regard, evaluation of the numbers of VSTs in memory T-cell infusions is important to control viral infections before recovery of broad repertoire of T-cells after T-depleted haploidentical and unrelated transplantation. 
Methods: T-cells were derived from G-CSF stimulated $(\mathrm{n}=74)$ or unstimulated $(\mathrm{n}=5)$ apheresis of healthy donors. Apheresis product was processed with single-step CD45RA depletion procedure on CliniMACS Plus or Prodigy instrument. CD45RA-depleted fraction was aliquoted and cryopreserved for further use. The frequency of CMV, EBV, ADV -specific $\mathrm{T}$ cells in donor peripheral blood ( $\mathrm{n}=142$ ), CD45RA-depleted T-cells products before $(n=20)$ and after $(n=79)$ cryopreservation were monitored by gamma-interferon enzyme-linked immunospot (ELISPOT) assays with CMVpp65, AdV5 Hexon, EBV consensus respectively. Excess monocytes from the cell suspension were depleted by adhesion on plastic dishes for 2 hours. The CD3 proportion and monocyte /CD3 (Mon/ $\mathrm{CD} 3$ ) ratio in the cell suspension were determined by flow cytometry.

Results: In CD45RA-depleted T-cell products median Mon/CD3 was 0,5 and the efficiency of VSTs detection was dramatically lower in comparison with VSTs detection in peripheral blood (Median Mon/CD3=0,14). The median frequency per $300000 \mathrm{MNC}$ of CMV, EBV, ADV-specific $\mathrm{T}$ cells in the peripheral blood was 148; 54; 30 and in CD45RA-depleted T-cells products 4,0; 3,0; 0 respectively. Monocytes contained in CD45RA-depleted T-cells products can mediate suppression of interferon-gamma production by $\mathrm{T}$ cells after G-CSF stimulation of donors. After depletion of monocytes median Mon/CD3 ratio in cell suspension was $0,05(\mathrm{P}<0,0001)$ and the detection of VSTs significantly increased to $119 ; 88$ and 16 , respectively ( $\mathrm{p}<0,0001$ ). Among 79 DLI products after cryopreservation the median frequency of CMV, EBV, ADV-specific T cells was 43; 16; 6 per 300000 CD3 lymphocytes, respectively, median Mon/CD3 was 0,13. After adhesion on plastic the number of monocytes decreased by 4,8 times (Mon/CD3=0,027) and the frequency of VSTs detection increased approximately 5 times $(p<0,0001)$. The median frequency of CMV, EBV, ADV-specific T cells after monocyte depletion was 255; 153; 11 per 300000 CD3 lymphocytes, respectively $(\mathrm{p}<0,0001)$.

Conclusions: The excess of monocytes in cell suspension leads to inefficient detection of VSTs by ELISPOT assay and prior adhesion of monocytes can be recommended for correct VSTs detection in CD45RA-depleted T-cells products. The analysis of DLI aliquots after cryopreservation allows accurate quantification of specific antiviral Tlymphocytes administered to a patient and optimize antiviral therapy.

Conflict of interest: nothing to disclose

\section{P121}

Recovery of thymopoiesis in a paediatric cohort with acute GVHD - Can ECP help?
Aisling M Flinn, Catherine F Roberts, Xiao Nong Wang, Andrew R Gennery

Newcastle University, Institute of Cellular Medicine, Newcastle upon Tyne, United Kingdom

Background: Thymic damage inflicted by acute GVHD, corticosteroids and other non-selective immunosuppressive agents negatively impacts T-lymphocyte reconstitution following HSCT, essential for a successful clinical outcome. Extracorporeal photopheresis (ECP) is an alternative immune-modulating therapy that could, by promoting immune tolerance, reducing acute GVHD and allowing weaning of concurrent immunosuppression, facilitate thymic recovery and restoration of normal thymopoiesis.

Methods: Whole blood samples were taken from 8 paediatric patients with corticosteroid-refractory/dependent acute GVHD before ECP cycles at regular intervals. Thymopoiesis was prospectively assessed quantitatively by sequential monitoring of $\mathrm{CD}^{+} \mathrm{CD} 4^{+} \mathrm{CD} 45 \mathrm{RA}^{+} \mathrm{CD} 31^{+}$ naïve T-lymphocytes using flow cytometry and T-cell receptor excision circles (TRECs) using PCR, and qualitatively using T-cell receptor (TCR) DNA spectratyping analysis. Serum IL-7 was measured using ELISA as an indirect reflection of thymic output. Thymic recovery was compared to two control groups at 4, 8 and 12 months post HSCT; paediatric patients post HSCT who did not develop acute GVHD (group 1, $\mathrm{n}=6$ ) and paediatric patients with acute GVHD who did not receive ECP (group 2, $\mathrm{n}=6$ ). Statistical analysis was performed using the Kruskal-Wallis test, with $\mathrm{p}<0.05$ being significant.

Results: All ECP patients (median age at HSCT $=5.8$ years), with grade II-IV acute GVHD, demonstrated low thymic output at the beginning of ECP treatment, regardless of timing post HSCT (median absolute count of naïve Tlymphocytes $=5$ cells $/ \mu \mathrm{L}$, median number of TRECs $=$ 191/ml of blood) and a highly abnormal TCR repertoire. Four patients have completed ECP therapy (median number of ECP cycles $=21$ ), treatment is ongoing for 3, and one patient has been withdrawn from treatment. Qualitative and quantitative improvement in thymic output is seen in those who have completed therapy, with an inverse relationship with IL-7 evident (patient example in Figure A). Faster thymic recovery is evident in those who started ECP earlier. Among those who continue on ECP, one patient shows evidence of improving thymic output to date. One patient has demonstrated ongoing negligible thymic output 20 months post HSCT despite 72 weeks of ECP therapy, suggesting irreversible thymic damage. Median age at HSCT of control group 1 was 7.3 years and group 2 was 8.1 years. Comparing thymic recovery between the three groups showed no significant difference in naïve T-lymphocyte 

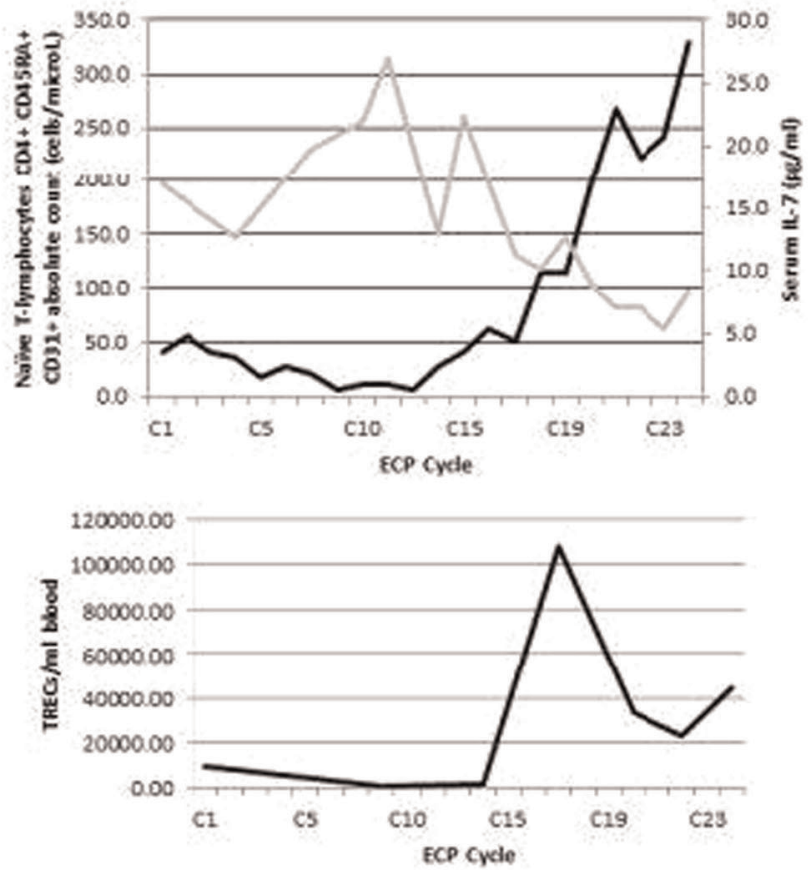

Pre ECP TCR DNA Spectratype

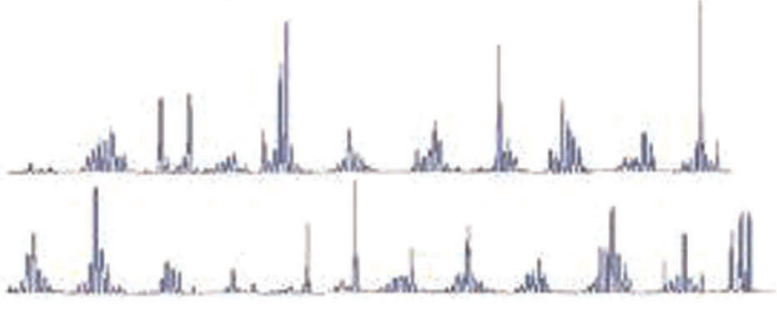

Post ECP TCR DNA Spectratype

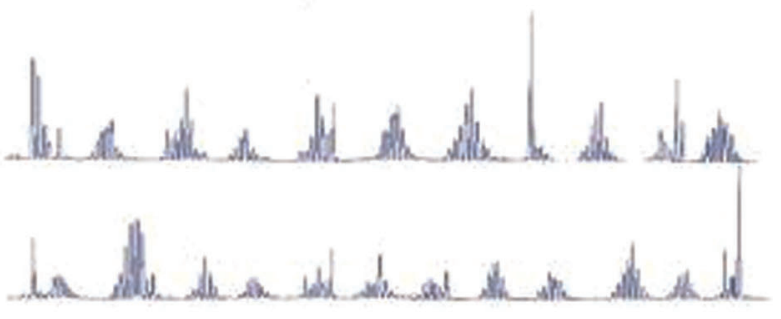

[P121 Figure] [Figure A. Example of qualitative and quantitative improvement seen in one ECP patient]

absolute counts $(\mathrm{p}=0.61,0.31,0.84$ at 4,8 and 12 months respectively).

Conclusions: This demonstrates, for the first time, that ECP can facilitate thymopoiesis in paediatric patients with aGVHD, potentially to the same rate as patients without acute GVHD, although further prospective data are needed. Promoting an immune tolerant environment post HSCT may be more conducive to faster T-lymphocyte reconstitution and further approaches to achieve this are needed, rather than our current armamentarium of immunosuppressive agents. Improvement is not seen in all patients, suggesting a point of thymic 'no return'; when excessive damage sustained by the thymus results in loss of intrinsic regenerative capacity and persistently inadequate $\mathrm{T}$ lymphocyte reconstitution. A biomarker to diagnose and monitor for thymic damage would be valuable.

\section{Conflict of interest: None}

\section{P122}

\section{Retrospective comparison of DLI and $\mathbf{g}$-DLI for lymphoid malignancies, myeloma and myeloid malignancies}

Sylvain Lamure ${ }^{1,2}$, Franciane Paul ${ }^{1,2}$, Anne-Laure Gagez ${ }^{1}$, Jérémy Delage $^{1,3}$, Nathalie Fegueux ${ }^{1}$, Guillaume Cartron ${ }^{1,2}$, Lu ZahoYang $^{4}$, Jean-Luc Veyrune ${ }^{4}$, Patrice Ceballos ${ }^{1}$, John De Vos ${ }^{2,4,5}$

${ }^{1}$ Montpellier University Hospital, Clinical Hematology, Montpellier, France; ${ }^{2}$ University of Montpellier, Montpellier, France; ${ }^{3}$ Clinique $d u$ Parc, Montpellier, France; ${ }^{4}$ Montpellier University Hospital, Unit for Cell Therapy, Montpellier, France; ${ }^{5}$ Montpellier University Hospital,
Institute for Regenerative Medicine and Biotherapy, Montpellier, France

Background: Donor lymphocyte infusion (DLI) can be use to prevent or cure hematological malignancies relapse after allogeneic stem cell transplantation, using antitumoral effect of donor T and NK cells. DLI's efficacy is correlated with low tumour burden, hence prophylactic and preemptive use for mixed chimerism and detectable minimal residual disease had been developped. This procedure is effective alone or associated with antitumoral chemotherapy or targeted therapy.

Recombinant human granulocyte colony stimulated factor (G-CSF) primed DLI (gDLI) derives from frozen aliquots of the initial peripheral hematological stem cell (PHSC) bag collection. PHSC often exceeds the required amount for transplantation ( 5 to $10 \times 10^{6} / \mathrm{kg}$ ), and cryoconservation is not pernicious for $\mathrm{T}$ lymphocyte. Based on anti-tumour outcome of T lymphocyte infused with PHSC, some team described efficacy and safety of gDLI in different situation. Biological effects of G-CSF on peripheral blood stem cells suggests a decrease in anti-tumoral activity of such primed DLI, such as: Th2 polarization, promotion of regulatory $\mathrm{T}$ cell and tolerogenic dendritic cell differentiation. Two retrospective studies compared the clinical equivalence for activity and toxicity of gDLI and classical DLI. They included 63 and 67 patients; mainly suffering of AML/ MDS, and no difference had been shown on clinical outcome. 
In this monocentric retrospective study, we aim to compare the response rate, the overall survival (OS), the GVHD rate and toxicity for patient who received gDLI and classical DLI. We intend to compare clinical outcome for different malignancies (ALL, myeloid malignancies, lymphoid malignancies and myeloma) and different situation (clinical relapse, molecular relapse and mixed chimerism).

Methods: We performed systematic review of medical charts of patient who underwent hematological stem cell transplantation and donor lymphocyte infusion for hematological malignancies in Montpellier University Hospital between 1998 and 2016.

Results: We retrospectively evaluated data of 140 patients. Initial diseases were myeloid malignancies $(\mathrm{N}=56)$, lymphoid malignancies $(\mathrm{N}=27)$, acute lymphoid leukemia $(\mathrm{N}=21)$ and myeloma $(\mathrm{N}=36)$. Indications for DLI were relapse $(\mathrm{N}=95)$, pre-emptive (detectable minimal residual disease, $\mathrm{N}=18$, mixed chimerism, $\mathrm{N}=23$ ) and prophylaxis $(\mathrm{N}=4)$. Sixty for patients of them had classical DLI, 66 had gDLI, and 10 had successively gDLI and classical DLI. Response rate was $37.4 \%$, median overall survival (OS) was 21.8 month, and 5-years OS was $29.7 \%$. Graft versus host disease occurred for $47,9 \%$ of them, treatment-requiring GVHD for $24.3 \%$, and $9,4 \%$ died from toxicity. There was no differences between classical and gDLI in term of response ( $\mathrm{p}=0.31)$, OS $(\mathrm{p}=0.8)$, GVHD $(\mathrm{p}=0,44)$, treated GVHD $(\mathrm{p}=0.78)$ and cause for mortality $(p=0.14)$. Factor associated with poor response were 3month mixed chimerism and relapse indication for DLI, myeloid malignancies and relapse indication were associated with shorter OS. Sub-group analysis shows no difference between classical and gDLI in outcome for lymphoid or myeloid malignancies, myeloma or ALL, neither in curative or pre-emptive indication.

Conclusions: Taken together our results and those previously published strongly suggests similar efficiency and toxicity of gDLI and classical DLI, for myeloid malignancies as well as multiple myeloma, ALL and lymphoma, to treat or prevent clinical or molecular relapse. G-DLI may substitute classical DLI.

Conflict of interest: No conflict of interest to declare.

\section{P123}

\section{Risk factors and novel biomarkers for cytokine release syndrome after CD19-targeted chimeric antigen receptor T cell therapy}

Yongxian Hu${ }^{1}$, Lijuan Ding ${ }^{1}$, Lei Xiao ${ }^{2}$, AlexH Zhang ${ }^{3}, H_{e} H_{u a n g}{ }^{1}$
${ }^{1}$ Zhejiang University, Hangzhou, China; ${ }^{2}$ Shanghai Innovative Cellular Therapy, CO LMD, Shanghai, China, ${ }^{3}$ Tongji University, School of Medicine, Shanghai, China

Background: CD19-targeted chimeric antigen receptor $\mathrm{T}$ cells (CART19s) are a highly effective novel immunotherapy for relapsed/refractory B-cell malignancies. Cytokine release syndrome (CRS) is the most significant and life-threatening complication. Our understanding of CRS continues to evolve, and identification of risk factors and novel laboratory biomarkers are needed to evaluate strategies to mitigate toxicity.

Methods: Patient clinical characteristics were analyzed in 42 patients who received CART19 therapy. A multiplex cytokine array was performed to detect 900 cytokine levels in sera from 3 stages (pre-CART19 infusion, peak CRS and CRS restoration) of 3 patients with grade 3 CRS. Based on these data, 16 selected cytokines in 42 patients undergoing CART19 therapy were evaluated by ELISA.

Results: CRS developed in $81 \%$ of patients (grade 1, $14.3 \%$; grade 2, $28.6 \%$; grade $3,38.1 \%$ ). Multivariable analysis of patient clinical characteristics identified high tumor burden, thrombocytopenia before lymphodepletion and number of previous relapse as independent risk factors of CRS. 16 cytokines (TECK, TGF $\alpha$, IP-10, MCP-2, FCAR, CXCL13, XCL1, IL-1 R4, AGRP, BAFF-R, BDNF, BRCK, CCL2, CCL3, GPC2, MMP-13) were identified as possible novel biomarkers by cytokine array (Figure 1A). IL-1 R4, MCP-1 and XCL1 were confirmed as novel biomarkers of CRS by ELISA. At stages of pre-infusion, peak CRS and CRS restoration, mean serum levels of IL-1 $\mathrm{R} 4$ were $56.6 \pm 49.2 \mathrm{pg} / \mathrm{ml}, 2473.8 \pm 449.2 \mathrm{pg} / \mathrm{ml}$ and 45 $\pm 39.5 \mathrm{pg} / \mathrm{ml}$ respectively; mean serum levels of MCP-1 were $16.6 \pm 15.2 \mathrm{pg} / \mathrm{ml}, 476.9 \pm 341.2 \mathrm{pg} / \mathrm{ml}$ and $21.5 \pm 27.6 \mathrm{pg} /$ $\mathrm{ml}$ respectively; mean serum levels of XCL1 were 9.6 $\pm 7.2 \mathrm{pg} / \mathrm{ml}, 77.9 \pm 45.8 \mathrm{pg} / \mathrm{ml}$ and $5.9 \pm 3.5 \mathrm{pg} / \mathrm{ml}$ respectively. All the above mean cytokine levels at stage of peak CRS were significantly higher than stages of pre-infusion and CRS restoration respectively ( $\mathrm{P}<0.01$, Figure 1B). IFN- $\gamma$ is one of the classical cytokine signature for CRS. Thus paired peak serum levels of IFN- $\gamma$, IL-1 R4, MCP-1 and XCL1 showed strong correlations (IFN- $\gamma$ VS IL-1 R4, Spearman $\mathrm{r}=0.342, \mathrm{p}=0.023$; IFN- $\gamma$ VS MCP-1, Spearman $\mathrm{r}=0.234, \mathrm{p}=0.035$; IFN- $\gamma$ VS XCL1, Spearman $\mathrm{r}=0.532, \mathrm{p}=0.014)$ implying that MCP-1, IL-1 R4 and XCL1 are novel biomarkers for CRS similar with IFN- $\gamma$.

Conclusions: Our data provide novel clues for prevention and treatment of CRS after CART19 therapy. Also our study lay the foundation for clarification of CRS pathophysiology.

Conflict of interest: None of the authors has anything to disclose. 

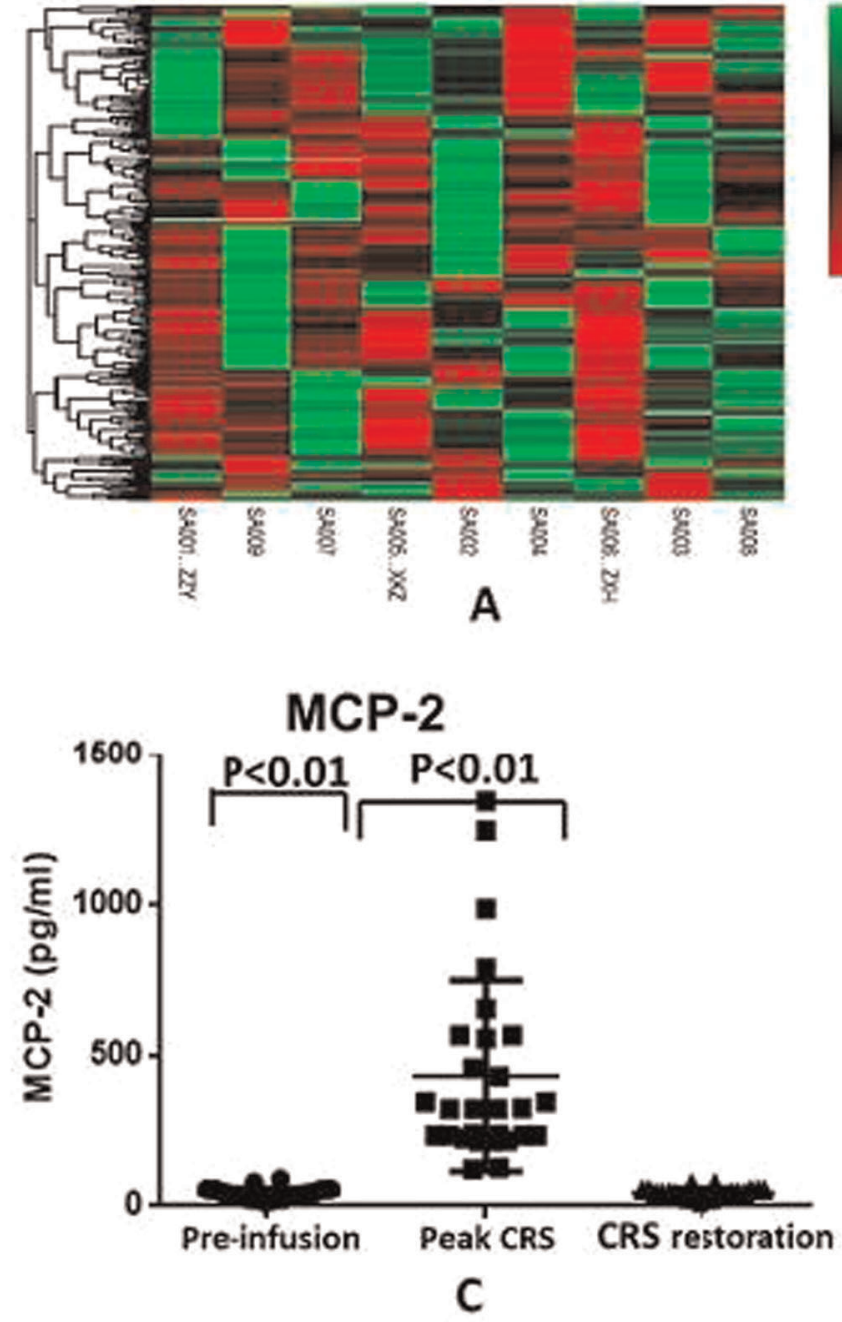

[P123 Figure] [Figure 1]

\section{P124}

Safety and feasibility of extracorporeal photopheresis (ECP) as second-line therapy for treatment of pediatric patients with acute GvHD

Marlene Luther ${ }^{1}$, Lisa Olfe ${ }^{2}$, Kamran Movassaghi ${ }^{2}$, Ina Lange ${ }^{2}$, Annette Künkele ${ }^{2}$,Johannes Schulte ${ }^{2}$, Angelika Eggert', Agnieszka Blum $^{2}$, Lutz Uharek', Carola Tietze-Bürger ${ }^{2}$

${ }^{1}$ Charité Campus Virchow-Klinikum, Stem Cell Facility, Berlin, Germany; ${ }^{2}$ Charité Campus Virchow-Klinikum, Berlin, Germany

Background: Only $30-60 \%$ of patients with severe acute GvHD respond to the first-line therapy with systemic steroid treatment. In adult patients, ECP has been established as a promising second-line therapy with an immunomodulatory but no generalized immunosuppressive effect. As there is so far only limited experience in pediatric

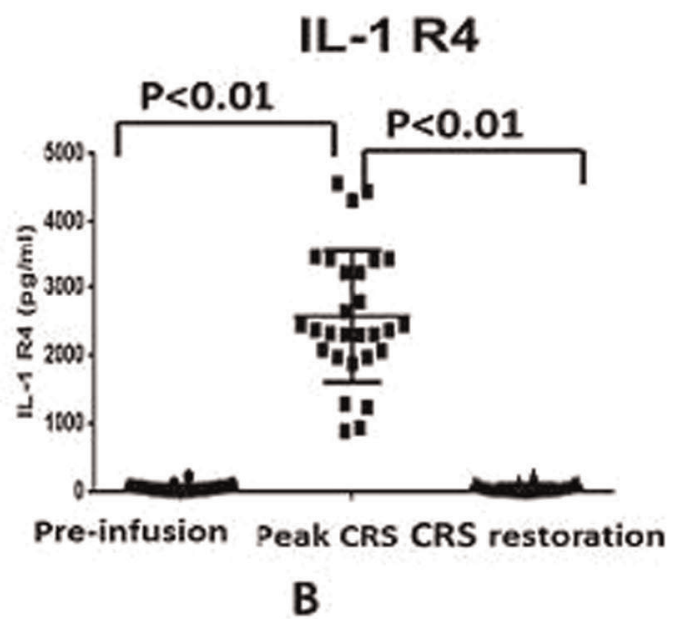

XCL1

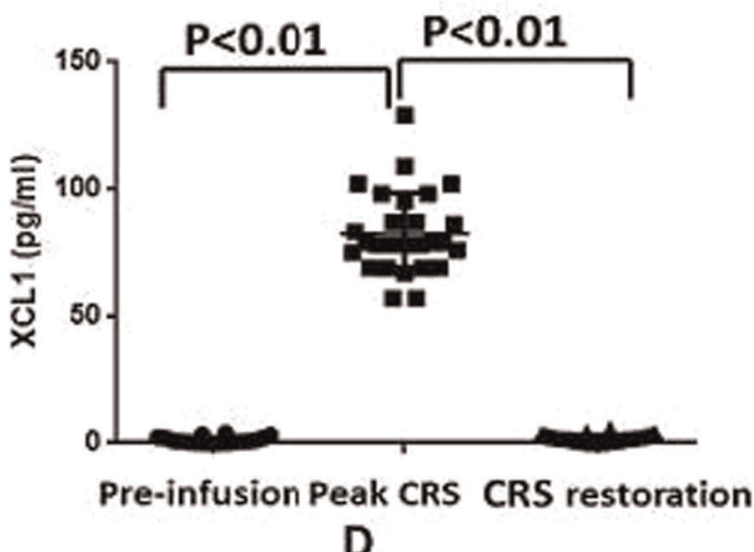

patients, we evaluated safety and outcome in a retrospective study.

Methods: Between 2014 and 2017 we treated 10 children with at least 3 cycles of ECP (median 9 cycles) for aGvHD. The median weight was $25 \mathrm{~kg}$ (range $11-91 \mathrm{~kg}$ ) with a median age of $11 \mathrm{yr}$ (range $1-15 \mathrm{yr}$ ). For children weighing less than $25 \mathrm{~kg}$ the leukapheresis circuit was primed with RBC. A total of 89 cycles were analyzed.

Results: We saw no adverse events CTC grade 3/4 during ECP treatment, in particular regarding hypotension and anaphylactic reactions. A severe sepsis which was unrelated to ECP, resulted in a fatal outcome in one patient. During 89 cycles of ECP only 3 RBC transfusions and 4 PLT transfusions had to be given because of anemia or thrombocytopenia. At the end of ECP treatment, 9 of 10 children improved with 4 complete remissions of GvHD and 5 partial responses with at least 50\% improvement. Also, $a \geq 50 \%$ reduction in steroid dose at the end of ECP 
treatment was possible in 9 out of 10 patients. The median time from onset of ECP treatment to end of immunosuppressive medication was 5 months for steroids and 9 months for all immunosuppressive medication, respectively.

Conclusions: ECP is a feasible and sufficiently safe treatment for pediatric patients with acute refractory GvHD. However, treatment of younger children requires special operating standards, a well trained staff, and is technically and logistically more demanding as compared to ECP in adults. Our preliminary clinical data confirm previous studies and suggest that the effort is justified because most children clearly improved after ECP.

Conflict of interest: None of the authors has anything to disclose.

\section{P125}

\section{Small scale production of clinical grade MSCs for a single center setting}

Adomas Bukauskas ${ }^{1}$, Mindaugas Stoskus ${ }^{1}$, Vilma Valceckiene ${ }^{1}$, Laimonas Griskevicius ${ }^{1,2}$

${ }^{1}$ Vilnius University Hospital Santaros Klinikos, Hematology, Oncology and Transfusion Medicine Center, Vilnius, Lithuania; ${ }^{2}$ Vilnius University, Department of Internal, Family Medicine and Oncology, Faculty of Medicine, Vilnius, Lithuania

Background: Classic cell culture flask method is an effective way to expand mesenchymal stromal cells (MSCs) but the demand for dedicated facilities, personnel and timeto-production is a significant hurdle for the small transplant center. Commercially available self-contained bioreactors increase cell yield and decrease manual work yet at the increased cost of disposables and culture medium. Herein we report a successful integration of both methods for clinical scale MSCs expansion following bone marrow aspirate processing through a filter-based device.

Methods: Human bone marrow was collected from healthy donors after informed consent was obtained. To retrieve MSCs fraction, filter-based Bone Marrow MSC Separation Device (Kaneka Corporation, Osaka, Japan) was used. All cells were grown in high glucose DMEM medium supplemented with $5 \%$ human platelet lysate and $5 \mathrm{U} / \mathrm{ml}$ heparin. Cell medium was exchanged every 3 days. After initial culture in flasks (Passage 0), cells were harvested and loaded on Quantum Cell Expansion System (Terumo BCT, Inc, Lakewood, CO, USA) (Passage 1). Following expansion in bioreactor MSCs were harvested and cryopreserved for therapeutic use. Samples were taken for quality control (sterility, mycoplasma DNA, endotoxin, genomic integrity and immunophenotype).

Results: 18 donors were 25.5 (range 22-35) years of age. The median of aspirated bone marrow fluid volume was only $20 \mathrm{ml}$ (range 15-35). Bone marrow mononuclear cells (BM MNCS) culture in flasks (Passage 0) took a median of 14 days (range 13-24). Of note, 3 of 18 (17\%) BM MNSCS cultures did not expand. The median yield of MSCs expanded in T-flasks was $38.4 \times 106$ (range $10-85 \times 10^{6}$ ). MSCs expansion in bioreactor took a median of 9 days (range 6-17). Doubling times ranged from 36.83 to $80.24 \mathrm{~h}$ with the median of $57.12 \mathrm{~h}$ (or 2.38 days). The final yield ranged from 275 to $1800 \times 10^{6}$ cells with the median of 550 $\times 10^{6}$ cells per culture. The median time from bone marrow aspiration to final yield was 23 days (range 20-41). At harvesting, cell viability was $\geq 95.9 \%$. All cells were compliant with flow cytometric ISCT criteria and were negative for both mycoplasma and endotoxin. One batch was contaminated with Staphylococcus Warneri and was not released for clinical use. 14 out of $18(78 \%)$ batches were released for clinical use.

Conclusions: Filter based device allows initial reduction of flask surface area (up to $600 \mathrm{~cm}^{2}$ ) required for culture of MSCs and simplifies dramatically Passage 0 process. Withdrawal of small amount of bone marrow fluid may decrease the pain associated with aspiration procedure and the bone marrow dilution with peripheral blood. This approach could be successfully adapted in small-scale hospital laboratories for clinical expansion of MSCs.

\section{Conflict of interest: None}

\section{P126}

\section{SUCCESSFUL MANAGEMENT OF SEVERE ADVERSE EFFECTS DURING AUTOLOGOUS PERIPHERAL BLOOD STEM CELL INFUSION AND POLICY IMPLEMENTATION REGARDING WASHING PRODUCTS}

Laura Medina Marrero ${ }^{1}$, José García-Arroba Peinado ${ }^{1}$, Carme Talarn Forcadell ${ }^{2}$, Laia Ramiro Infante ${ }^{1}$, Virginia Callao Molina ${ }^{1}$, Carmen Azqueta Molluna ${ }^{1}$, Nuria Martínez Llonch ${ }^{1}$, Elena Valdivia García $^{1}$, Margarita Codinach Creus ${ }^{1}$, Margarita Blanco García ${ }^{1}$, Susana García Gómez ${ }^{1}$, Luciano Rodríguez Gómez ${ }^{1}$, Lourdes Escoda Teigell', Sergi Querol Giner ${ }^{1}$

\section{${ }^{1}$ Banc de Sang i Teixits, Barcelona, Spain; ${ }^{2}$ Hospital Joan XXIII,} Tarragona, Spain

Background: Thawing and immediate infusion is the most common method used for autologous stem cell transplantation. Adverse reactions associated to these infusions have been well described and mainly associated to DMSO, despite other origins have also been reported, such as granulocyte contain or red blood cells osmotic lysis. Developing an infusion policy including post-thaw cell washing is important to allow administration of cell graft at special risk. 
Methods: We present two cases of severe adverse reactions occurring during infusion of autologous stem cell grafts in two patients with non-Hodgkin's lymphoma.

Case 1 was a 58 -year-old, $76 \mathrm{~kg} \mathrm{~b} / \mathrm{w}$ patient, that experienced itchy and sore throat after infusion of half of the first bag. The reaction was partially solved by reducing the rate of infusion. Thereafter, facial flashing and erythema suddenly occurred, which were accompanied by low blood pressure $(60 / 30 \mathrm{mmHg})$, tachycardia (110/minute), dizziness, nausea and vomits, and the infusion was interrupted. Immediately, a Trendelemburg position was adopted while saline solution, hydrocortisone and oxygen were administrated. One hour was required to recover normal vital signs, so one third of the bag could not be infused.

Case 2 was a 65 -year-old, $58 \mathrm{~kg}$ b/w patient that after ten minutes of infusion of the first bag developed low blood pressure $(70 / 40 \mathrm{mmHg}$ ), profuse sweating and dizziness. Saline solution and hydrocortisone were administered with successful resolution of the clinical picture.

Results: In both cases, ward physicians immediately contacted with the cell processing facility to report the severe adverse effects, so that an emergency strategy was considered. Teams agreed returning the remaining cryopreserved bags to the core cell therapy lab for graft washing. Procedure was performed using the Sepax ${ }^{\circledR}$ device using an automated protocol (Smartwash) that resulted in a satisfactory cell yield and viability. Afterwards, patient 1 did not develop any further complication; meanwhile, patient 2 exhibited a mild cutaneous reaction that disappeared after hydrocortisone, but allowing the infusion of the whole volume in the bag. Both patients had a successful engraftment.

In both cases, the quantity of DMSO was compliant with our infusion policy (less than 1 gr per kg patient b/w) and other quality variables including viability and colony growth were correct. However, the amount of granulocytes per $\mathrm{kg}$ in the collected products was high, 6.07E8 and $6.59 \mathrm{E} 8 / \mathrm{kg}$, respectively. After revision, this was considered as the most probable cause of the SARs observed.

Conclusions: Post-thaw washing allowed that almost all cryopreserved cells were finally infused. Consequently, after reviewing scientific literature and our own experience, we established a policy to prevent these severe reactions. Cell products with more than 4E8 granulocytes/kg are now recommended for post-thaw washing or multi-day infusion according CD34 cell numbers.

Conflict of interest: Nothing to disclose

\begin{tabular}{llllll}
\hline & $\begin{array}{l}\mathrm{CN} / \mathrm{kg} \\
(\mathrm{E} 8)\end{array}$ & $\begin{array}{l}\mathrm{CMN} / \mathrm{kg} \\
(\mathrm{E} 8)\end{array}$ & $\begin{array}{l}\mathrm{CD} 34 / \mathrm{kg} \\
(\mathrm{E} 6)\end{array}$ & $\begin{array}{l}\mathrm{GM} / \mathrm{kg} \\
\text { (E5) }\end{array}$ & $\begin{array}{l}\text { CFU/CD34 (\% } \\
\text { eclone) }\end{array}$ \\
\hline $\begin{array}{l}\text { CASE } \\
1\end{array}$ & 16.80 & 10.73 & 8.45 & 11.5 & 20 \\
$\begin{array}{l}\mathrm{CASE} \\
2\end{array}$ & 15.58 & 8.99 & 2.19 & 2.62 & 49 \\
\hline
\end{tabular}

\section{[[P126 Table] PRODUCTS CHARACTERISTICS]}

\section{P127}

Validation of a highly scalable CD34 selection procedure to support cell therapy strategies in haematopoietic progenitor cell transplantation

Carmen Azqueta Molluna ${ }^{1}$, Nuria Martinez Llonch ${ }^{1}$, Elena Valdivia Garcia $^{1}$, Margarita Codinach Creus ${ }^{1}$, Margarita Blanco Garcia ${ }^{1}$, Pere Barba ${ }^{2}$, Maria Isabel Benitez Carabante ${ }^{3}$, Luisa Sisinni ${ }^{4}$, Júlia Marsal $l^{5}$, Cristina Diaz de Heredia ${ }^{3}$, Isabel Badell Serra ${ }^{4}$, Rodrigo Martino Bufarull, Rocio Parody Porras ${ }^{7}$, Christelle Ferrà Coll ${ }^{8}$, Laura Medina Marrero ${ }^{1}$, Susana G Gómez ${ }^{1}$, Sergio Querol ${ }^{1}$

${ }^{1}$ Banc de Sang i Teixits, Cell Therapy Services, Barcelona, Spain, ${ }^{2}$ Hospital Vall d'Hebron, Hematology Service, Barcelona, Spain; ${ }^{3}$ Hospital Materno-Infantil Vall d'Hebron, Barcelona, Spain; ${ }^{4}$ Hospital de la Santa Creu i Sant Pau, Pediatrics Service, Barcelona, Spain; ${ }^{5}$ Hospital Sant Joan de Deu, Barcelona, Spain; ${ }^{6}$ Hospital de la Santa Creu i Sant Pau, Hematology Service, Barcelona, Spain; ${ }^{7}$ Institut Català d'Oncologia Duran i Reynals, L'Hospitalet de Llobregat, Spain; ${ }^{8}$ Institut Català d'Oncologia Trias i Pujol, Badalona, Spain

Background: CD34+ selection remains a very attractive method for different interventions in hematopitic stem cell transplantation. We currently use this methodology as a method of GVHD prevention or to boost haematopoiesis in the treatment of poor graft function (PGF) after transplantation. In order to standardize a high-throughput methodology for a core cell therapy lab, we developed and validated an automated cell processing procedure combining the Sepax ${ }^{\circledR}$ and the $\mathrm{CliniMACS}^{\circledR}$ systems.

Methods: We included 44 consecutive procedures. This new, automatic procedure is applied to peripheral blood apheresis. After product reception, first step consist on platelet depletion, and volume adjustment using the Smartredux program for Sepax device. Then, the cell product was incubated with the anti-CD34 monoclonal antibody and washed out using again the Sepax washing system, to further adjust the volume to this required by CliniMACS. Finally, CliniMACS tubing set was installed and the cells were introduced for $\mathrm{CD} 34$ selection procedure using the CD34 Selection program. Indications for CD34 selection were: CD34 enrichment, first step of CD45RA depletion both in the context of GVHD prophylaxis, and $\mathrm{CD} 34+$ cell boost related to PGF. 
Results: Median time from starting to end was 4 hours. Table 1 shows the post-Sepax cells recovered wit purities and yields. Before selection, platelet depletion and monoclonal antibody washing using Sepax device achieved extensive platelet depletion (up to a median of $68 \%$ ) with almost complete recovery of TNC and CD34 cells. Then, cells were adjusted to the required volume for immunomagnetic selection that achieved a consistent final product with high CD34 purity and a very low CD3 contain (5.2 CD3 log depletion).

Importantly, CD34 potency was maintained. CFUGM recovered after selection were 1.4E7 (0.1-5). Median clonogenic efficiency of seeded CD34 was $18 \%$ (5-38) that resulted in an optimal engraftment.

Conclusions: Here, we present a standardized procedure that resulted in a robust and fast, large-scale CD34 selection using the combination of two automated devices. This approach allowed an improvement of our previous results with a reproducible output of purity and yield, maintaining optimal levels of cell viability and potency that resulted in an efficient myeloid engraftment. This new automatic procedure allows a high throughput in a central core cell therapy lab.

Conflict of interest: The authors declare no conflict of interest

\begin{tabular}{lll}
\hline Variable & Post-Sepax & Post-CliniMACS \\
\hline Total CD34+ cells & $4.8 \mathrm{E} 8(2.0-7.6)$ & $3.3 \mathrm{E} 8(0.5-6.3)$ \\
CD34+ cell purity & $0.92 \%(0.74-0.94)$ & $94 \%(65-99)$ \\
CD34+ cell viability & $99 \%(94-100)$ & $95 \%(83-100)$ \\
CD34+ cell yield & $93 \%(76-107)$ & $81 \%(36-108)$ \\
Platelet yield & $32 \%(4-80)$ & N.A. \\
Total CD3+ cells & N.A. & $8 \mathrm{E} 4(0.5-16)$ \\
\hline
\end{tabular}

[[P127 Table] Cell purity and yields during positive selection]

\section{P128}

Abstract previously published

\section{P129}

Wharton's jelly-derived mesenchymal stem cells treatment in adult patients in Poland

Anna Mucha ${ }^{1}$, Mariusz Grudniak ${ }^{1}$, Magda Murzyn ${ }^{1}$, Jolanta Koryga ${ }^{1}$, Artur Olkowicz ${ }^{1}$, Karolina Poplawska ${ }^{1}$, Dominika

Gtadysz $^{1}$, Iwona Marszatek ${ }^{1}$, Maciej Boruczkowski ${ }^{2}$, Tomasz Otdak ${ }^{1}$, Dariusz, Boruczkowski ${ }^{1}$
${ }^{1}$ PBKM, Warszawa, Poland; ${ }^{2}$ University of Medical Sciences, Poznań, Poland

Background: Wharton's jelly-derived mesenchymal stem cells (WJ-MSCs) are a type of cell population with significant self-renewal and multi-lineage differentiation properties. Their high proliferation rate, immune privileged status, nontumorigenic properties make them ideal for both autologous and allogeneic use in regenerative medicine applications. WJ-MSCs therapy, based on cells prepared by Polish Bank of Stem Cells (PBKM), has shown promising effects in the treatment of many diseases and injuries.

Methods: From 2007 to 2017 PBKM has delivered WJMSCs products for 371 adult patients aged from 19 years and 2 months to 86 years and 5 months (median age: 47 years and 2 months). The patients after Bioethical Committee approval, received intravenous or intrathecal injections of WJ-MSCs, obtained from third party donor (TPD). The cells were collected from healthy newborns, then processed, screened for bacterial contamination and endotoxin content, and finally frozen in liquid nitrogen vapours. WJ-MSCs immunophenotype was confirmed using flow cytometry assay. The patients received from 1 to 5 injections in one medical course. The average cell dose per infusion was $1 \times 10^{\wedge} 6 / \mathrm{kg}$ of body weight. All patients were monitored for the procedure related adverse events.

Results: WJ-MSCs have been used in adult patients for more than 50 diseases and were most often used in neurology (66\% of all patients), ophthalmology (19\%) as well as orthopedics (13\%). The stem cells were also given to patients in hematology (4 patients) and gynecology (4 patients). The most common indications were amyotrophic lateral sclerosis (153 patients), retinitis pigmentosa (29 patients) and spinal cord injury (27 patients). All WJ-MSCs administrations took place in Poland. Immediately after stem cell infusion 16 adverse events (AE) occured and another $40 \mathrm{AE}$ at least 24 hours after WJ-MSC administration were observed. All AE were mild or moderate, eg. the most common was headache or nausea, which were easily managed with medications.

Conclusions: WJ-MSCs have a wide implementation in the medical experimental procedures and show promising preliminary results with high therapeutic value in adult patients. However further research is needed and long-term results have to be collected.

Conflict of interest: All the authors are employed at PBKM.

\section{P130}

Wharton's jelly-derived mesenchymal stem cells treatment in children with neurological diseases 
Anna Mucha ${ }^{1}$, Mariusz Grudniak ${ }^{1}$, Magda Chrościńska-Krawczyk ${ }^{2}$, Monika Kotarska ${ }^{2}$, Krystyna Mitosek - Szewczyk ${ }^{2}$, Magda Murzyn ${ }^{1}$, Jolanta Koryga ${ }^{1}$, Artur Olkowicz ${ }^{1}$, Karolina Poplawska ${ }^{1}$, Dominika Gładysz ${ }^{1}$, Iwona Marszałek ${ }^{1}$, Maciej Boruczkowski ${ }^{3}$, Tomasz Otdak ${ }^{1}$, Dariusz, Boruczkowski ${ }^{4}$

${ }^{1}$ PBKM, Warsaw, Poland; ${ }^{2}$ Lublin Medical University, Department of Pediatric Neurology, Lublin, Poland; ${ }^{3}$ University of Medical Sciences, Poznan, Poland; ${ }^{4}$ PBKM, Warszawa, Poland

Background: Many neurological diseases cannot be efficiently treated, because of their unknown pathogenesis and the lack of appropriate medications. The concept of repairing the nervous system by stem cells has recently evolved from hypothesis that tissue regeneration could have been achieved through stem cell differentiation into neural cells, acting mainly by the means of paracrine mechanisms. Transplantations of stem cells capable of producing neurotrophic factors may be an efficient method for improving brain function. Wharton's jelly mesenchymal stem cells (WJ-MSCs), thanks to Polish Bank of Stem Cells (PBKM), have been in use in children's neurology in Poland since 2014.

Methods: Two hundred and forty three neurological patients aged from 5 months to 18 years and 8 months (median age: 7 years and 2 months), after Bioethical Committee approval, received intravenous or intrathecal injections of WJ-MSCs, obtained from third-party donor (TPD). The cells were previously collected from healthy newborns, then processed, screened for bacterial contamination as well as endotoxin content, and frozen in liquid nitrogen vapour. WJ-MSCs immunophenotype was confirmed using flow cytometry assay. The patients received from 1 to 10 injections (5 in one medical course). The average cell dose per intravenous infusion was $1 \times 10^{\wedge} 6 / \mathrm{kg}$ of body weight. Each patient was examined by the same neurologist at the day of infusion.

Results: Neurological patients constisted of $87 \%$ of all pediatric patients listed in PBKM in years 2011- 2017. The most common indications were cerebral palsy (74 patients), autism (41 patients) and spina bifida (22 patients). The remaining 86 patients have been diagnosed with other neurological diseases (total number of diagnosis: 46). All WJ-MSCs administrations took place in Poland. Adverse events (AE) recorded during injections were easily managed with medications and occured 8 times (headache -4 , nausea - 2, lumbar puncture headache -1 , blood pressure increase $-1)$.

Conclusions: The administrations of third-party donor WJ-MSCs seem to be safe and efficient procedure with promising preliminary results in neurological patients. However more insight in the study will be beneficial, therefore long-term results still need to be collected.
Conflict of interest: All the authors are employed at PBKM.

Chronic leukaemia and other myeloproliferative disorders

P131

Allogeneic stem cell transplantation in Philadelphianegative chronic myeloproliferative neoplasms. A centre experience

Alejandro Avendaño Pita ${ }^{1}$, Javier Carrillo Checa ${ }^{1}$, Daniel Rivera Delgado $^{1}$, Veiga Vaz Alvaro ${ }^{2}$, Arratibel Zalacain Nerea ${ }^{2}$, Baile González Mónica ${ }^{2}$, Félix López Cadenas ${ }^{2}$, Perez López Estefania ${ }^{2}$, Lucia López Corral ${ }^{1}$, Cabrero Calvo Monica ${ }^{1}$, Jose Ramón González Porras $^{1}$, Maria Dolores Caballero Barrigon ${ }^{1}$, Ana Africa Martin Lopez $^{1}$

${ }^{1}$ Hospital Universitario de Salamanca/IBSAL, Salamanca, Spain; ${ }^{2}$ Hospital Universitario de Salamanca, Salamanca, Spain

Background: Nowadays, allogeneic stem cell transplantation (ASCT) is the only curative strategy in patients with high risk Philadelphia-negative chronic myeloproliferative neoplasms (CMN).

Methods: From 867 patients who underwent to an ASCT in our centre, $21(2.42 \%)$ were CMN different from chronic myeloid leukaemia. We retrospectively analysed this subgroup of patients: $8(38.1 \%)$ primary myelofibrosis (MF), $11(52.4 \%$ ) secondary MF (6 post PV, 4 post ET and 1 post other $\mathrm{CMN}$ ) and $2 \mathrm{CMN}$ different from MF (1 CMN JAK2+ and 1 CMN/MDS JAK2+ with fibrosis).

Results: The clinical and biological features and ASCT characteristics are summarized in table 1.

All patients, except two that were not evaluable, achieved neutrophil and platelet engraftment (only two patients did not reach 50.000 platelets). Concerning the complications related to ASCT: $71.4 \%$ of patients suffered from mucositis (86.7\% grade I and II), $23.8 \%$ developed CMV reactivation, 9.5\% haemorrhagic cystitis, $4.8 \%$ obstruction sinusoidal syndrome and none developed transplant associated microangiopathy. A total of 16 patients (76.2\%) developed acute graft versus host disease (GVHD) (18.8\% grades III-IV) with a median of presentation of 26 days (15-141) and 11 patients $(52.4 \%)$ developed chronic GVHD (63.6\% extensive) with a median of 203 days (120-566). Transplant related mortality (TRM) at day +100 was $4.8 \%$ and the global TRM.

At day +100 post ASCT, 17 patients $(80.9 \%)$ achieved complete remission (CR) (two of them with minimal residual disease) and $3(14.3 \%)$ patients were in relapse situation (one case not evaluable because of early dead). Six $(28.6 \%)$ of these 20 patients relapsed after a median of 150 days (53-2295) from ASCT. In four of them, the 
approach of relapse was modulating immunosuppression +/- donor lymphocyte infusion and hypometilating agents in the cases of MRD, reaching CR and complete chimerism.

In the last follow-up, 13 patients $(61.9 \%)$ were alive (11 in $\mathrm{CR}$ and 2 with active disease) and $8(38.1 \%)$ were dead from different causes: relapse in 1 case $(4.8 \%)$, infection in $5(23.8 \%)$, bleeding in $1(4.8 \%)$ and the last one from another causes (hepatic failure).

With a median follow-up of 35 months (16-121) the overall survival (OS) and event free survival (EFS) at 3 years was $61 \%$ and $57 \%$, respectively.

Conclusions: The results of our series confirms the potential curative of ASCT. Taking into account that TRM is not minimal, we observe that more than a half of patients are long survivors. Modulating immunosuppression plays an important role in the setting of post ASCT relapse.

Conflict of interest: Nothing to disclose

\begin{tabular}{|c|c|c|c|c|c|}
\hline $\begin{array}{l}\text { Clinical and } \\
\text { biological } \\
\text { characteristics } \\
(\mathrm{N}=21)\end{array}$ & $\mathrm{N}(\%)$ & $\begin{array}{l}\text { Median } \\
\text { (range) }\end{array}$ & $\begin{array}{l}\text { ASCT } \\
\text { characteristics }\end{array}$ & $\mathrm{N}(\%)$ & $\begin{array}{l}\text { Median } \\
\text { (range) }\end{array}$ \\
\hline Age (years) & & $\begin{array}{l}60(29- \\
68)\end{array}$ & Age & & $\begin{array}{l}61(30- \\
69)\end{array}$ \\
\hline $\begin{array}{l}\text { Sex: Male } \\
\text { Female }\end{array}$ & $\begin{array}{l}11(52.4) \\
10(47.6)\end{array}$ & & $\begin{array}{l}\text { Status at ASCT: } \\
\text { CR PR Clinical } \\
\text { improvement } \\
\text { Active disease } \\
\text { Progression }\end{array}$ & $\begin{array}{l}1(4.8) 5 \\
(23.8) 3 \\
(14.3) 8 \\
(38.1) 4 \\
(19.0)\end{array}$ & \\
\hline $\begin{array}{l}\text { AML } \\
\text { transformation } \\
\text { pre-ASCT }\end{array}$ & $5(23.8)$ & & $\begin{array}{l}\text { Peripheral blood } \\
\text { as source of stem } \\
\text { cells }\end{array}$ & $21(100)$ & \\
\hline $\begin{array}{l}\text { Time to AML } \\
\text { transformation } \\
\text { (months) }\end{array}$ & & $\begin{array}{l}8.8 \\
(2.7- \\
117.2)\end{array}$ & $\begin{array}{l}\text { Number of CD34 } \\
+(106 / \mathrm{kg})\end{array}$ & 6.59 & \\
\hline $\begin{array}{l}\text { Driver mutations } \\
(\mathrm{N}=18) \text { : JAK2 } \\
\text { CALR MPL } \\
\text { Triple negative }\end{array}$ & $\begin{array}{l}13(72.2) \\
2(11.1) 2 \\
(11.1) 1 \\
(5.6)\end{array}$ & & $\begin{array}{l}\text { Type of donor: } \\
\text { Sibling donor } \\
\text { Not sibling } \\
\text { donor } \\
\text { Haploidentical }\end{array}$ & $\begin{array}{l}15(71.4) \\
4(19) 2 \\
(9.5)\end{array}$ & \\
\hline $\begin{array}{l}\text { IPSS ( } N=18) \text { : } \\
\text { Low/int-1 Int-2/ } \\
\text { high }\end{array}$ & $\begin{array}{l}4(22.2) \\
14(77.8)\end{array}$ & & $\begin{array}{l}\text { Donor median } \\
\text { age }\end{array}$ & & $\begin{array}{l}51(30- \\
65)\end{array}$ \\
\hline $\begin{array}{l}\text { DIPSS-plus } \\
(\mathrm{N}=18) \text { Low/int- } \\
1 \text { Int-2/high }\end{array}$ & $\begin{array}{l}3(16.7) \\
15(83.3)\end{array}$ & & $\begin{array}{l}\text { Conditioning: } \\
\text { MAC RIC }\end{array}$ & $\begin{array}{l}5(23.8) \\
16(76.2)\end{array}$ & \\
\hline $\begin{array}{l}\text { Lines of } \\
\text { treatment pre- } \\
\text { ASCT }\end{array}$ & & $1(0-4)$ & $\begin{array}{l}\text { Status CMV: } \\
+/+-/+-/-\end{array}$ & $\begin{array}{l}16(76.2) \\
4(19) 1 \\
(4.8)\end{array}$ & \\
\hline $\begin{array}{l}\text { Type of } \\
\text { treatment: } \\
\text { Hidroxyurea } \\
\text { Splenectomy } \\
\text { JAK inhibitor } \\
\text { Chemotherapy } \\
\text { Previous } \\
\text { trasplant }\end{array}$ & $\begin{array}{l}9(42.9) 4 \\
(19) 11 \\
(52.4) 6 \\
(28.6) 2 \\
(9.5)\end{array}$ & & $\begin{array}{l}\text { ABO: ABO } \\
\text { identical Major } \\
\text { incomp. Minor } \\
\text { incomp. Major \& } \\
\text { Minor }\end{array}$ & $\begin{array}{l}15(71.4) \\
2(9.5) 1 \\
(4.8) 3 \\
(14.3)\end{array}$ & \\
\hline
\end{tabular}

[[P131 Table] Table 1]
P132

Chronic Neutrophilic Leukemia, An Extremely Rare Cause Of Neutrophilia In Childhood: Cure With Hematopoetic Stem Cell Transplantation

Akif Yeşilipek ${ }^{1}$, Vedat Uygun ${ }^{1}$, Seda Öztürkmen ${ }^{1}$, Hayriye Daloğlu ${ }^{1}$, Neşe Yaralı', Gülsün Karasu ${ }^{1}$

${ }^{1}$ MedicalPark Antalya, Pediatric Bone Marrow Transplantation Unit, Antalya, Turkey; ${ }^{2}$ Ankara Child Health and Diseases Haematology Oncology Training and Research Hospital, Department of Paediatric Haematology and Oncology, Ankara, Turkey

Background: Chronic neutrophilic leukemia (CNL) is a rare myeloproliferative disorder that occurs frequently after the mutations in gene for receptor of colony-stimulating factor 3 (CSF3R). Here we report a case of an Fanconi Anemia (FA) patient who developed CNL during follow-up and was cured with Hematopoetic Stem Cell Transplantation (HSCT).

Methods: The patient was referred to pediatric hematology department at the age of 8 years because of recurrent pulmonary infections and accompanying leukopenia. A workup revealed an elevated DEB-induced chromosome breakage leading to diagnosis of FA. At 12 years of age, WBC count increased to $15 \times 10^{9} / \mathrm{L}$; however, at the next visit 2 months later, the WBC count was increased to $63.8 \times 109 / \mathrm{L}$, which required further examination. Infection and reactive causes were investigated because of the presence of neutrophils representing $70 \%$ of the leukocytes in the peripheral smear, but no causative agent was detected. T618I mutation was detected in the CSF3R gene, which is frequently reported in patients with CNL. HSCT was planned because the patient had FA, which could accelerate the progression to AML.

Results: The patient was scheduled for transplantation from a $9 / 10$ compatible unrelated donor. In the preparation regimen, $180 \mathrm{mg} / \mathrm{m}^{2}$ fludarabine, $40 \mathrm{mg} / \mathrm{kg}$ cyclophosphamide, and $30 \mathrm{mg} / \mathrm{kg}$ anti-thymocyte globulin (ATG fresenius) were used. Cyclosporine and mycophenolate were used for graft versus host disease (GVHD) prophylaxis.GCSF was not used due to the CSF3R mutation history; the neutrophils and platelets engrafted at +18 and +9 , respectively. Grade II acute GVHD developed at the third week and resolved with methylprednisolone. However, chronic GVHD findings appeared on the eye, mouth, and skin at month 7 of the transplant, which was accepted as moderate chronic GVHD. Currently, at month 9 of the transplant, the patient is still experiencing mild chronic GVHD with full chimerism and is receiving tacrolimus and methylprednisolone. He now has no CSF3R mutation and has a leukocyte level of $7.5 \times 10^{9} / \mathrm{L}$. 
Conclusions: The association between FA and CSF3R mutation was not reported thus far. To our knowledge, the patient studied is the youngest CNL patient with a possible acquired mutation; moreover, it is the first case of $\mathrm{CNL}$ reported in a patient with FA. The CSF3R mutation should not be overlooked in patients with FA who have longstanding neutrophilia, because the mutation is a predisposing factor for developing AML.

Conflict of interest: Nothing to disclose

\section{P133}

CML: Stem Cell Transplantation in Children and Adolescents with Reduced Intensity Conditioning - a Multicentric Prospective Trial

Susanne Matthes ${ }^{1,2}$, Petr Sedlacek ${ }^{3}$, Roland Meisel ${ }^{4}$, Rita Beier $^{5}$, Maura Faraci ${ }^{6}$, Krzysztof Kalwak ${ }^{7}$, Marianne Ifversen ${ }^{8}$, Ingo Müller ${ }^{9}$, Jerry Stein ${ }^{10}$, Kim Vettenranta ${ }^{11}$, Gabriele Kropshofer ${ }^{12}$, Sabina Sufliarska ${ }^{13}$, Adriana Balduzzi ${ }^{14}$

${ }^{1}$ Medical Center - University of FreiburgUniversity, Vienna, Stem Cell Transplantation, Vienna, Austria; ${ }^{2}$ St Anna Childrens Hospital, Vienna, Austria; ${ }^{3}$ Charles University Motol Prague, Pediatric Hemato-Oncology, Prague, Czech Republic; ${ }^{4}$ Universitätsklinikum Düsseldorf, Pediatric Hemato-Oncology, Düsseldorf, Germany;

${ }^{5}$ University Children HospitalChildrens Hospital, Essen, Germany;

${ }^{6}$ Istituto di Ematologia, Università Cattolica del S. CuoreG. Gaslini, Genova, Italy; ${ }^{7}$ Wroclaw Medical University, Pediatric BMT, Wroclaw, Poland; ${ }^{8}$ Children and Adolescent Medicine Rigshosptilet,

Copenhagen, Denmark; ${ }^{9}$ University Medical Center HamburgEppendorf, Pediatric Stem Cell Transplantation, Hamburg, Germany; ${ }^{10}$ Schneider Children's Medical Center, BMT Unit, Petach Tikva, Israel; ${ }^{11}$ University of Adelaide, Hospital for Children and Adolescents, Helsinki, Finland; ${ }^{12}$ University Children Hospital, Hematology Oncology, Innsbruck, Austria; ${ }^{13}$ University Childrens Hospital, Pediatric Hematology/Oncology, Bratislava, Slovakia;

${ }^{14}$ Clinica di Oncoematologia Pediatrica Ospedale San Gerardo, Monza, Italy

Background: In the era of TKIs life expectancy of adult/ elderly patients with CML, responding to Imatinib approaches that of the general population. Only selected patients are being treated with SCT, although PFS and OS $>80 \%$ have been reported for patients with an EBMT-score of $0-2$.

In children and adolescents with CML treated with Imatinib, 10-year PFS is $60 \%$ and TKI-free survival is $6 \%$ (Giona et al 2015). Response rates in children treated with second line TKIs are comparable to those observed with Imatinib. Besides the fact that life-long TKI for children with CML implicates treatment for 50-70 years, non-target effects of Imatinib such as growth deficiency and chronic immunosuppression, treatment failures due to noncompliance in adolescents remain major concerns. In a prospective multicentric trial (ClinicalTrials.gov NCT02707393) the SCT commettee of the IBFM study group evaluated the safety and efficacy of SCT following a reduced intensity conditioning regimen (RIC) in children and adolescents with CML.

Methods: In order to reduce TRM, late effects and chronic GvHD the protocol was based on an ATGcontaining RIC regimen (Fludarabin 4 x $40 \quad \mathrm{mg} / \mathrm{m}^{2}$, Thiotepa 2 x $5 \mathrm{mg} / \mathrm{kg}$, Melphalan $140 \mathrm{mg} / \mathrm{m}^{2}$ ). The protocol included frequent BCR/ABL Monitoring post SCT and treatment with Imatinib in the first year in case of increasing BCR/ABL. Patients with persisting BCR/ABL > 0,02\% received DLI in the second year after SCT.

Results: A total of 30 patients have been included. Donors were matched related (10) and matched unrelated (20). Median age at SCT was 14 years. All patients were in $\mathrm{CP} 1$ and had received Imatinib or second line TKI. BCR/ $\mathrm{ABL}$ at the time of SCT ranged from $0,001-33 \%$. Indication for SCT was insufficient molecular response/secondary response loss in 19 patients and patient/physician choice for the remaining patients. All patients engrafted, 7/30 patients displayed mixed chimerism (8-80\% donor chimerism). TRM was $4 \%$ (16 year old boy with BCR/ABL > 30\% at time of SCT who died on day +180 of invasive adenovirus infection). Moderate/severe chronic GvHD was observed in $6 / 29$ patients which resolved in all cases, with no patients receiving immunosuppression beyond 2 years after SCT. There is no correlation between mixed chimerism and the need of secondary intervention. In six patients Imatinib treatment was restarted after a median of 4 months. Three patients received additional DLI in the second year after SCT, 2/3 being off TKI and in MR 5.5 at the time of analysis. No DLI-associated GvHD ocurred.

After a median follow-up of 52 months, current survival with $\mathrm{BCR} / \mathrm{ABL}<0,01 \%$ is $97 \%$ and current TKI-free survival is $90 \%$.

Conclusions: SCT following RIC is feasible in children and adolescents with CML and associated with a diseasefree and TKI-free survival of $>90 \%$. Patients with insufficient molecular Response or secondary response loss can be treated successfully with RIC-SCT. RIC-SCT may be a valid alternative to life-long treatment with TKIs in children and adolescents with CML.

Clinical Trial Registry: ClinicalTrials.gov NCT02707393

Conflict of interest: None of the authors has anything to disclose.

\section{P134}

GETH-CLL4: Long-term follow-up of a pilot trial evaluating the addition of ofatumumab to the conditioning regimen of patients with chronic lymphocytic leukemia undergoing allogeneic HCT 
Pau Montesinos ${ }^{1}$, Lucia Lopez-Corral ${ }^{2}$, David Valcarcel ${ }^{3}$, Montserrat Rovira ${ }^{4}$, Jose Antonio Garcia-Marco ${ }^{5}$, Javier Loscertales $^{6}$, Carol Moreno ${ }^{7}$, Rafael Duarte ${ }^{8}$, Maria Jose Terol', Pau Abrisqueta ${ }^{3}$, Monica Cabrero ${ }^{2}$, Jaime Sanz ${ }^{1}$, Julio Delgado $^{4}$

${ }^{1}$ Hospital La Fe, Valencia, Spain; ${ }^{2}$ Hospital Clinico, Salamanca, Spain; ${ }^{3}$ Hospital Vall d'Hebron, Barcelona, Spain; ${ }^{4}$ Hospital Clinic, Barcelona, Spain; ${ }^{5}$ Hospital Puerta de Hierro, Madrid, Spain;

${ }^{6}$ Hospital La Princesa, Madrid, Spain; ${ }^{7}$ Hospital Santa Creu i Sant Pau, Barcelona, Spain; ${ }^{8}$ ICO - Hospital Duran i Reynals, Barcelona, Spain; ${ }^{9}$ Hospital Clinic, Valencia, Spain

Background: Patients with CLL whose tumor cells harbor TP53 aberrations or relapsing early after chemoimmunotherapy are considered to have an adverse prognosis with conventional agents. These patients have been traditionally offered an alloHCT according to EBMT guidelines. The addition of rituximab to the typical fludarabine-based conditioning regimen has apparently improved the outcome of patients with CLL. In this investigator-initiated trial we hypothesized that the addition of ofatumumab, a novel anti-CD20 monoclonal antibody with greater anti-CLL activity, to the conditioning regimen could also improve the outcome of these patients.

Methods: Patients with CD20-positive CLL were eligible in case of TP53 aberrations or disease relapse within 12 months of the last chemoimmunotherapy. The disease had to be in complete or partial response at the time of transplantation. Donors were siblings or unrelated volunteers with 9-10/10 HLA compatibility. The conditioning regimen comprised ofatumumab (300 $\mathrm{mg}$ on day $-20,2000$ $\mathrm{mg}$ on days -13 and -6 and $1000 \mathrm{mg}$ on days +1 and +8 ); fludarabine $(150 \mathrm{mg} / \mathrm{m} 2)$; and melphalan $(70-140 \mathrm{mg} / \mathrm{m} 2)$. GvHD prophylaxis was with cyclosporine A and mycophenolate mofetil. Patients with one-allele mismatch also received rabbit thymoglobulin $(8 \mathrm{mg} / \mathrm{kg})$. The primary end point of the study was progression-free survival (PFS) at 3 years.

Results: Median age of the 18 recruited patients was 57 (range, 35-62) years, and 13 (72\%) were male. Donors were HLA-matched in all but two cases. All patients proceeded to alloHCT except one whose donor became pregnant after initiation of ofatumumab therapy and was withdrawn from the study. The remaining 17 patients completed all ofatumumab infusions. All patients who underwent alloHCT engrafted. The cumulative incidence of developing any kind of GvHD was $76 \%$ at 1 and 3 years, respectively. Moreover, the cumulative incidence of developing severe GvHD (defined as grade 3-4 acute or extensive chronic GvHD) was 53\% and $65 \%$ at 1 and 3 years, respectively. With a median follow-up of 53 (range, 36-60) months for survivors, there have been no relapses on study. As no relapses were observed in the study, both PFS and overall survival were identical: $47 \%$ (95\% CI $28-78 \%$ ) and $40 \%$ (95\% CI $22-73 \%)$ at 3 and 5 years from transplantation,

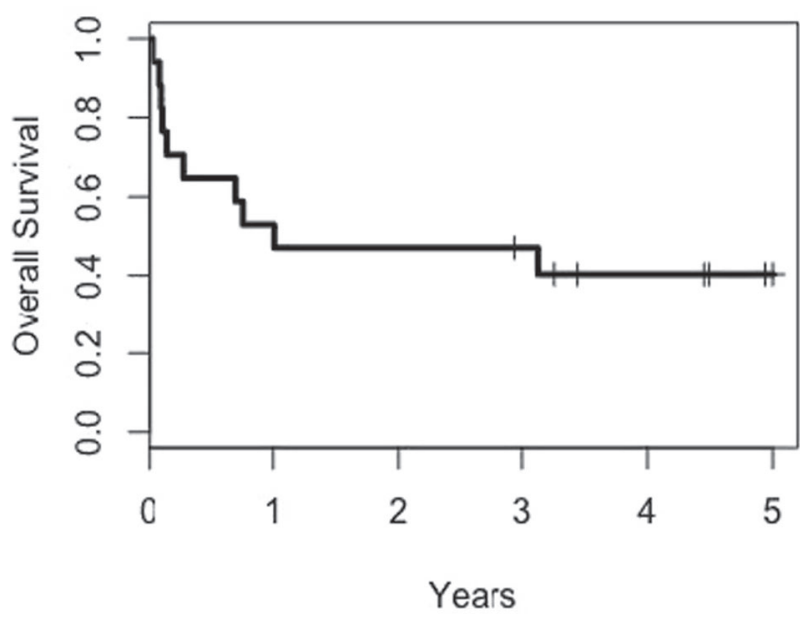

[P134 Figure] [Long-term overall survival of the entire population]

respectively (Figure 1). Causes of death were septicemia (3), pneumonia (2), GvHD (2), cerebral hemorrhage (1), PML (1) and hemoptysis (1).

Conclusions: The addition of ofatumumab to the conditioning regimen of CLL patients undergoing alloHCT reduced the relapse rate completely, but was not able to reduce the GvHD rate, thus leading to a very high nonrelapse mortality. However, the long-term follow-up of this trial shows a clear plateau in the survival curve and therefore alloHCT may be still recommended in a restricted group of patients. There is, however, a clear need to identify those patients with a low risk of non-relapse mortality, as has been the aim of recent EBMT initiatives.

Clinical Trial Registry: This study was registered at clinicaltrials.gov (NCT01455051) and performed in accordance with the Declaration of Helsinki and the International Conference on Harmonized Guidelines for Good Clinical Practice.

Conflict of interest: For the purpose of this trial, ofatumumab was provided free of charge together with an unrestricted grant from GSK/Novartis. None of the authors has anything to disclose.

\section{P135}

Impact of target therapy on outcome after allogeneic stem cell transplantation in patients with $B C R-A B L$-negative myeloproliferative neoplasms: a single center experience

Maria Barabanshikova, Elena Morozova, Ivan Moiseev, Julia Vlasova, Vadim Baikov, Ildar Barkhatov, Elena Darskaya, Sergey Bondarenko, Boris Afanasyev 
First Pavlov State Medical University of Saint Petersburg, Raisa Gorbacheva Memorial Institute of Children Oncology, Hematology and Transplantation, Saint Petersburg, Russian Federation

Background: Post-polycythemia vera (post-PVMF), post-essential thrombocythemia (post-ETMF), primary myelofibrosis (PMF) and myelodysplastic/myeloproliferative neoplasms (MDS/MPN) are $B C R-A B L$-negative hematopoietic stem cell disorders with generally poor outcome. The aim of the study was to compare the results of allogeneic hematopoietic stem cell transplantation (alloHSCT) in patients with myelofibrosis (MF) and MDS/MPN and evaluate if target therapy with ruxolitinib improves the outcome in MF.

Methods: We compared 3 groups of patients: MDS/ MPN, MF without history of ruxolitinib, and MF with preand post-transplant ruxolitininb. 40 patients aged 49 (30-61) years were included in the analysis. 26 patients were diagnosed with MF (3 - post-ETMF, 6 - post-PVMF, 20 PMF), 11 - MDS/MPN (7 - CMML, 1 - aCML, 3 - MDS/ MPN unclassifiable). All patients received conditioning regimen with fludarabine $180 \mathrm{mg} / \mathrm{m}^{2}$ plus busulfan $8-10$ $\mathrm{mg} / \mathrm{kg}$. In $24 \mathrm{MFand}$ MDS/MPN-patients without ruxolitinib graft versus host disease prophylaxis (GVHD) consisted of antithymocytic globulin (ATGAM $60 \mathrm{mg} / \mathrm{kg}$ or thymoglobuline $5 \mathrm{mg} / \mathrm{kg}$ ) and tacrolimus/mophethyl-mycophenolate. $16 \mathrm{MF}$-patients received ruxolitinib $30-45 \mathrm{mg}$ daily at least 3 months until D- 1 and $10-15 \mathrm{mg}$ from $\mathrm{D}+5$ to $\mathrm{D}+100$ and GVHD prophylaxis with cyclophosphamide $50 \mathrm{mg} / \mathrm{kg}$ $\mathrm{D}+3,+4$. Among them disease stabilization before alloHSCT occurred in 11 patients, disease progression - 1, clinical improvement - 4 according to ELN criteria. All MDS/MPN-patients had active disease at the moment of alloHSCT. AlloHSCT was performed from unrelated (29), related (7) and haploidentical (4) donor. Stem cell source was granulocyte colony-stimulating factor mobilized peripheral blood progenitor cell (PBSC) (29) and bone marrow (11). Median number of CD34+cells/kg was $6,5 \times 10^{6}(3,0$ - 11,9) in PBSC group and 2,7 x $10^{6}(1,0-8,0)$ in bone marrow group.

Results: Median follow up was 13,7 months $(3,6-126,1)$ Primary engraftment was documented in 30 patients. The rate of primary graft failure was significantly higher in MDS/MPN- compared to MF-patients, $54 \%$ versus $16 \%$ subsequently $(\mathrm{p}=0.014)$. All of them died due to infection (8) or disease progression (2).

Acute GVHD grade 2-4 and chronic GVHD was documented in $33 \%$ and $50 \%$ of MF and MDS/MPNpatients without history of ruxolitinib therapy, $26 \%$ and $33 \%$ of MF-patients who received ruxolitinib $(\mathrm{p}=\mathrm{ns})$. The highest incidence of severe sepsis was observed in MDS/ MPN-patients - $63 \%$ compared to MF-patients with or

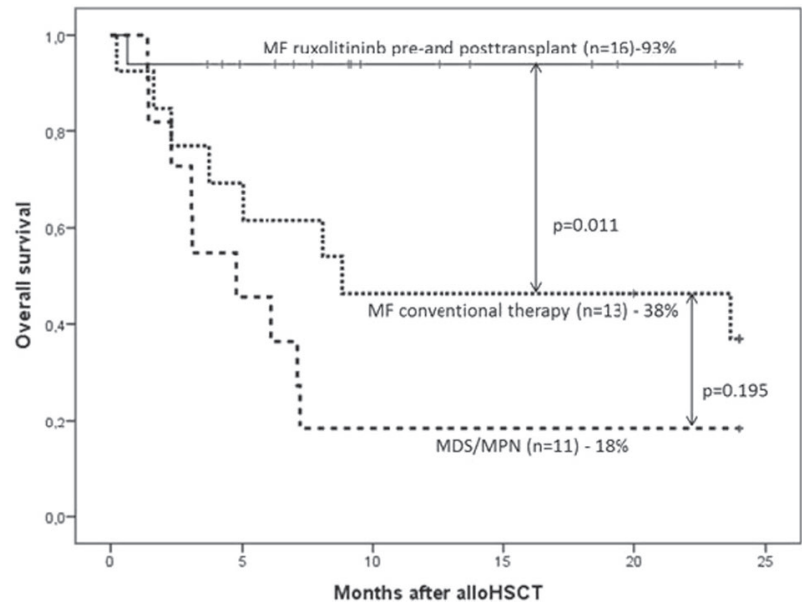

[P135 Figure] [Figure 1.]

without history of target therapy $-24 \%(\mathrm{p}=0.029)$. The rate of toxic hepatitis grade 3-4 and venoocclusive liver disease was similar between groups: $21 \%$ and $12 \%$ in patients with no history of ruxolitinib, $25 \%$ and $13 \%$ in patients treated with ruxolitinib.

We found no significant difference in 2-year OS between MDS/MPN- and MF-patients treated with conventional therapy ( $38 \%$ vs $18 \%, p=0.195$ ), but the 2 -year OS of MFpatients treated with ruxolitinib pre- and posttransplant was significantly superior $(93 \%$ vs $38 \%, p=0.011)$ (figure 1 ).

Conclusions: In our small patient cohort there was no difference between MPN and MF on conventional therapy. However introduction of JAK inhibitor in the pre- and posttransplant period might improve results of alloHSCT in such unfavorable disease as MF. Larger multicenter studies are required to confirm this assumption.

Conflict of interest: None of the authors has anything to disclose.

\section{P136}

Abstract previously published

\section{P137}

Abstract previously published

\section{P138}

Outcome of Allogeneic Haematopoietic Stem Cell Transplantation in Myeloproliferative NeoplasmsUnclassified: A Retrospective Study by the Chronic Malignancies Working Party of the EBMT 
Donal McLornan ${ }^{1,2}$, Richard Szydlo ${ }^{3}$, Marie Robin $^{4}$, Linda Koster ${ }^{5}$, Gernot Stuhler $^{6}$, Renate Arnold ${ }^{7}$, Dietger Niederwieser ${ }^{8}$, Ghulam Mufti, Dietrich Beelen ${ }^{10}$, Arnold Ganser ${ }^{11}$, Aleks Radujkovic ${ }^{12}$, Donald Bunjes ${ }^{13}$, Henrik Sengeloev ${ }^{14}$, Ibrahim Yakoub-Agha ${ }^{15}$, Ram Malladi $^{16}$, Mahmoud Aljurf ${ }^{7}$, Igor Wolfgang Blau ${ }^{7}$, Peter Kalhs ${ }^{18}$, Stig Lenhoff ${ }^{19}$, Antonio Campos ${ }^{20}$, Gerald $G$ Wulf $^{21}$, Mareike Verbeek $^{22}$, Matthias Stelljes ${ }^{23}$, Yves Beguin ${ }^{24}$, Vves Chalandon ${ }^{25}$, Nicolaus Kroger ${ }^{26}$

${ }^{1}$ Guy's and St.Thomas NHS Foundation Trust, Haematology, London, United Kingdom; ${ }^{2}$ King's College London, London, United Kingdom; ${ }^{3}$ Imperial College Healthcare NHS Trust, Department of Clinical Sciences, London, United Kingdom; ${ }^{4}$ Hôpital Saint-Louis, Service d'Hématologie-Greffe, Paris, France; ${ }^{5}$ EBMT Data Office, Leiden, Netherlands; ${ }^{6}$ Deutsche Klinik für Diagnostik, Wiesbade, Germany; ${ }^{7}$ Charité Universitaetsmedizin Berlin, Berlin, Germany; ${ }^{8}$ University Hospital Leipzig, Division of Haematology \& Oncology, Leipzig, Germany; ${ }^{9}$ King's College Hospital/ King's College London, London, United Kingdom; ${ }^{10}$ Univeristy Hospital Essen, Haematology, Essen, Germany; ${ }^{11}$ University of Hannover, Hannover, Germany; ${ }^{12}$ University of Heidelberg, Medizinische Klinik u. Poliklinik V, Heidelberg, Germany; ${ }^{13}$ Universitätsklinikum Ulm, Ulm, Germany;

${ }^{14}$ Rigshospitalet, Copenhagen, Denmark; ${ }^{15} \mathrm{CHU}$ de Lille, LIRIC, INSERM U 995, Universite de Lille, Lille, France; ${ }^{16}$ Queen Elizabeth Hospital, Haematology, Birmingham, United Kingdom; ${ }^{17}$ King Faisal Specialist Hospital \& Research Center, Riyadh, Saudi Arabia;

${ }^{18}$ Medizinische Universitaet Wien, Vienna, Austria; ${ }^{19}$ Skanes

University Hospital, Lund, Sweden; ${ }^{20}$ Inst. Português de Oncologia do Porto, Porto, Portugal; ${ }^{21}$ Universitaetsklinikum Goettingen,

Goettingen, Germany; ${ }^{22}$ Klinkum Rechts der Isar, Munich, Germany; ${ }^{23}$ University of Münster, Munster, Germany; ${ }^{24}$ University of Liege,

Liege, Belgium; ${ }^{25}$ Hôpitaux Universitaires de Genève, Geneva,

Switzerland; ${ }^{26}$ University Hospital Eppendorf, Hamburg, Germany

Background: Myeloproliferative Neoplasm (MPN), unclassified (MPN-U) is a rare and inherently heterogeneous disease entity that presents with an MPN-type clinical/ histological phenotype yet fails to meet the diagnostic criteria for the other MPN variants. The clinical course is highly varied, no well-defined treatment algorithm exists and the outcome of allogeneic haematopoietic stem cell transplantation (allo-HSCT) has never been documented in detail. We hereby report on a multicentre, EBMTregistry based study of adult MPN-U patients.

Methods: All patients who underwent allo-HSCT for MPN-U between 2000-2015 (Reduced Intensity Conditioning (RIC) or Myeloablative Conditioning (MAC)) utilising either bone marrow or peripheral blood stem cells were selected. Statistical analyses were performed with SPSS 22 (SPSS Inc./IBM, Armonk, NY). All patients provided informed consent according to the Declaration of Helsinki.

Results: A total of 229 patients were analysed. MAC regimens, as defined by EBMT criteria, were utilised in 111 patients while 118 received RIC. Median age at allo-HSCT was 51 years (range(r), 19-71) in MAC and $58(\mathrm{r}, 24-74)$ in RIC cohorts. Median time from diagnosis to allo-HSCT in the MAC and RIC cohorts= 12 months (r, 2-282) and 16 months $(r, 2-244)$ respectively. Donor types were as follows: MAC; matched sibling donors (MSD) $n=40$
(36\%), unrelated donors (URD) $\mathrm{n}=65$ (59\%) and mismatched related (MMR) $\mathrm{n}=6(5 \%)$; RIC cohort MSD; $\mathrm{n}=36$ (31\%), URD; $\mathrm{n}=75$ (63\%) and MMR $\mathrm{n}=$ $7(6 \%)$. Median times to neutrophil engraftment for MAC and RIC platforms were identical at 18 days $(\mathrm{p}=0.97)$. Acute (a) and Chronic (c) Graft Versus Host Disease (GVHD) status was documented in $96 \%$ and $64 \%$ of the entire cohort. Rates of grade II-IV aGVHD were higher in the MAC cohort (33\%) when compared to the RIC cohort $(21 \% ; \mathrm{p}=0.05)$ whereas cGVHD rates (limited/ extensive) were similar: $21 \% / 30 \%$ in the MAC cohort versus $18 \%$ / $20 \%$ in the RIC cohort $(\mathrm{p}=0.87)$. The median follow up period of surviving patients was 30 months (range 1-182). Non-relapse mortality (NRM) probabilities at 1,3 and 5years were considerable in both groups: $32 \%, 42 \%$, and $52 \%$ (MAC) and 35\%, 44\% and 44\% (RIC); $\mathrm{p}=0.81$. Cumulative incidence of relapse (CIR) at 1,3 and 5-years was $22 \%, 35 \%$ and $39 \%$ (MAC) and 24\%, $41 \%$ and $41 \%$ (RIC) $(\mathrm{p}=0.59)$. Moreover, the 1,3 and 5 year overall survivals (OS) rates for MAC and RIC were $64 \%, 46 \%$ and $34 \%$ and $59 \%, 40 \%$ and $39 \%$ respectively $(\mathrm{p}=0.68)$. Regarding the best time to transplant in the disease course, there was a near significant trend for improved outcome in the MAC setting when recipients underwent allo-HSCT within the first year of diagnosis compared to those transplanted later $(\mathrm{p}=0.08)$.

Conclusions: This is the largest study to date reporting on outcomes of allo-HSCT for MPN-U. Given the heterogeneity of the disease course, OS rates appear acceptable but it is of note that NRM and CIR rates in both settings are considerable. In the MAC setting, transplanting earlier in the disease course appears to be associated with improved survival. Of note, there was no significant difference in relapse rates between RIC and MAC. Multivariate and subgroup analyses will be presented.

Clinical Trial Registry: Not relevant

Conflict of interest: Nothing relevant to disclose and no conflict of interests for the authors

\section{P139}

\section{Outcome of Allogeneic Transplantation in CML in the Tyrosinkinase Inhibitors Era, Single Centre Evaluation}

Markéta Št'astná-Marková, Jan Vydra, Veronika Válková, Hana Klamová, Ludmila Nováková, Barbora Čemusová, Michal Kolár̆, Petr Cetkovský, Antonín Vitek

Institute of Haematology and Blood Transfusion, Praha, Czech Republic

Background: The introduction of tyrosinkinase inhibitors (TKIs) to the CML treatment has been a destiny changing event for the majority of patients. Allogeneic 
transplantation then remains a therapeutic option for patients, in which the use of TKIs is not suitable for any reason.

Methods: 46 patients with the diagnosis of CML transplanted between the years 2005-2017, which means in the period when at least imatinib was available in the front line therapy, were evaluated. 11 patients were transplanted before the full effect of TKI was waited for, in the era of transition from up front transplantation to TKIs treatment only, 8 patients were diagnosed in advanced phase of the disease, 5 patients showed severe, otherwise not resolvable TKIs toxicity and 18 patients progressed on TKIs treatment. Except of 2 patients transplanted in RIC all the others were prepared with full conditioning either with busulfan or TBI 12 Gy, using ATG in unrelated donors. The overall survival and incidence of relapse were evaluated considering the reason for transplantation indication, disease status and GVHD occurrence.

Results: The overall survival in 2 and 10 years was $75 \%$ resp. $70 \%$. There was naturally a significant difference in overall survival between up-front transplanted patients (who would not be transplanted at present) and the patients with advanced phase or not sufficient TKIs response, in 5 years $100 \%$ resp. $64 \%$ and in 10 years $92 \%$ resp. $62 \%$. The survival of patients diagnosed in advanced phase (AP), who all received induction chemotherapy together with TKIs before entering the transplantation, in the comparison to the patients diagnosed in chronic phase (CP) but failing the TKIs response was in 2 years $88 \%$ resp. $65 \%$, in 5 years $85 \%$ resp. $58 \%$ and in 10 years $63 \%$ resp. 58\%. Statistically in these small numbers not significant. When comparing patients diagnosed in $\mathrm{CP}$ and staying still in $\mathrm{CP}$ before transplant (excluding up-front transplants) (CP1), those diagnosed in $\mathrm{CP}$ and progressing to AP during TKIs treatment (CP2) and those diagnosed as late as $\mathrm{AP}(\mathrm{AP})$, the statistically worst surviving group was the prior transplant accelerating one. (92\%- $\mathrm{CP} 1,27 \%-\mathrm{CP} 2,87 \% \mathrm{AP})$ in 2 years.

In the occurrence of aGVHD gr. 2-3 during the treatment the survival in one year was $76 \%$ vs $96 \%$ without the complication, $\mathrm{p}=0,07$, on the contrary the occurrence of cGVHD improved the survival in 5 years to $83 \%$ vs $70 \%$ (not significant in this cohort).

Conclusions: The introduction of TKIs to the CML treatment has selected the patients with biologically poor character of the disease, for whom the allogeneic transplantation is the only curative approach. It seems that the patients diagnosed in the advanced phase but transplanted in chemotherapy and TKIs induced remission have better results than those primary resistant or who have lost the treatment response to TKIs. It looks like the graft versus leukaemia effect connected with the occurrence of cGVHD, known to be effective in CML in the pre-TKIs era, has a role also in these patients, although we do not statistically prove it in this small cohort.

Conflict of interest: Nothing to disclose

\section{P140}

\section{Outcomes of Allogenic Hematopoietic Cell Transplantation for Childhood Chronic Myeloid Leukemia: Single Center Experience}

Hanafy Hafez $^{1,2}$, Amr Abdalla, ${ }^{1,2}$,Mahmoud Hammad ${ }^{1,2}$, Nayera Hamdy $^{3,4}$, Dina Yassin ${ }^{4}$, Sherin Ibrahim ${ }^{4}$, Lama El Halaby, Alaa Elhaddad $^{1,2}$

${ }^{1}$ National Cancer Institute, Cairo University, Pediatric Oncology and BMT, Cairo, Egypt; ${ }^{2}$ Children Cancer Hospital Egypt CCHE 57357 , Pediatric Oncology and BMT, Cairo, Egypt; ${ }^{3}$ National Cancer Institute, Cairo University, Clinical Pathology, Cairo, Egypt; ${ }^{4}$ Children Cancer Hospital Egypt CCHE 57357, Clinical Pathology, Cairo, Egypt; ${ }^{5}$ Children Cancer Hospital Egypt CCHE 57357, Research Department, Cairo, Egypt

Background: Despite the apparent efficacy and favorable toxicity profile of TKIs, allogeneic SCT remains the only curative treatment for CML especially in younger patients, but transplant related morbidity and mortality should be considered. We evaluated the clinical characteristics and outcomes of pediatric CML patients who had SCT in our center.

Methods: This retrospective study included pediatric patients (below 18 years) with confirmed CML, who received an allogeneic SCT at the stem cell transplantation unit, Children Cancer Hospital Egypt (CCHE 57357) between August 2007 and June 2017 with follow up till December 2017. All patients received Myeloablative conditioning chemotherapy containing Busulfan/Cyclophosphamide followed by stem cell infusion from matched related donors.

Results: From the whole 110 patients diagnosed with CML, 41 had available matched related donors and subjected to HSCT during the study period while 69 patients continued TKI therapy. The median age at diagnosis was 12 years and M/F ratio was 1.4:1. Thirtyseven patients were diagnosed with chronic phase, one case with accelerated disease and 3 patients with blastic crises and average duration before transplant was 12 months. Bone marrow was the stem cell source in 30 patients and peripheral blood was used in 11 cases and the average stem cell dose was $4.5 \times 10^{6} / \mathrm{kg}$. The 3 -year overall survival and event free survival were $97 \%$ and $86 \%$ respectively, and the transplant related mortality at $\mathrm{d} 100$ and one year were $0 \%$ each. The incidence of chronic graft versus- host disease (cGVHD) was $82 \%$ in cases with peripheral blood source compared to $30 \%$ in cases who received bone marrow stem cells (P. value 0.005), also cGVHD was higher in F/M 
donor/recipient transplant (62\%) as compared to $\mathrm{M} / \mathrm{F}(46 \%)$ and $\mathrm{M} / \mathrm{M}$ or $\mathrm{F} / \mathrm{F}(35 \%)$ but not statistically significant.

Conclusions: Considering the good survival rates and very low TRM associated with HSCT in our pediatric CML patients, our data suggest offering transplant for all patients with available matched related donor, without need for prolonged exposure to TKIs. Bone marrow stem cell source should be preferred in those young patients to avoid a significant risk of cGVHD and its related complications.

Conflict of interest: All authors declare that nothing to disclose

\section{P141}

\section{Patients with T315I mutation CML: characteristics and outcomes of the treatment}

Julia Vlasova, Elena Morozova, Maria Barabanshchikova, Tatyana Gindina, Ildar Barhatov, Ivan Moiseev, Alexander Alyanskiy, Elena Darskaya, Boris Afanasyev

R.M. Gorbacheva Institute of Pediatric Oncology Hematology and Transplantation, I.P.Pavlov First St. Petersburg State Medical University, Hematology, St. Petersburg, Russian Federation

Background: Resistance to tyrosine kinase inhibitors (TKI) in patients with chronic myeloid leukemia (CML) is frequently caused by point mutations in the BCR-ABL kinase domain, including the gatekeeper mutant T315I, which confers a high degree of resistance to all currently approved tyrosine kinase inhibitors except ponatinib.

Methods: Retrospective analysis of 79 BCR-ABL ${ }^{\mathrm{T} 315 \mathrm{I}}$ -positive CML patients (pts) was done. Allogeneic bone marrow transplantation (allo-HSCT) was made in 20 pts, 59 pts received only pharmacological therapy (33 pts received TKI as monotherapy or in combination with other drugs other 26 pts received hydroxyurea, interferon- $\alpha$ or chemotherapy). At the time of T315I detection $51(65 \%)$ pts were in CP, 22 (28\%) pts had AP and 6 (7\%) pts were in BC. Median $(\mathrm{Me})$ age at the time of mutation detected was 42 years (13-75) (38 years in HSCT-group). In allo-HSCT group $12(60 \%)$ pts had unrelated donors, 17 (85\%) pts received more than 2 lines TKIs before HSCT, 2 pts were in $\mathrm{BC}$ at the time of HSCT, 5 pts were in AP, 8 pts were in $\mathrm{CP} \geq 2,5$ pts were in $\mathrm{CP} 1$. The number of points on EBMT scale: 3-4 points - 16(80\%) pts, 5-7 points - 4(20\%) pts. Conditioning regimen in $14(70 \%)$ pts had reduced intensity. Me time to HSCT after T315I detection was 7 months (0-72). Mutation analysis was performed by Sanger sequencing. Overall survival (OS) was estimated by Kaplan-Meier method with log-rank test for comparison between groups. Cox regression was used for multivariate survival analysis that included next covariates: age, phase on the time of mutation detection, performance of alloHSCT, time to T315I detection from TKI start.

Results: Me follow-up time after T315I detection was 21 months (1-100). 5-years OS in whole group was $42 \%$ (fig.1A). According to multivariate analysis only CML phase at the time of mutation detection significantly affect to survival in whole group. All pts in BC $(n=5,2$ in HSCT group and 3 in non-HSCT group) died within first year after T315I indication wherein Me survival time was 1,3 month (fig.1B). 5-years OS in non-HSCT group $(n=59)$ was $42 \%$ with Me survival time 2,8 years. 5-years OS after alloHSCT $(n=20)$ was $37 \%$ with Me survival time 5 months (fig.1C). All living patients after allo-HSCT are in deep molecular response. There was no significant difference in 5 -years OS between TKI $(n=17)$ and non-TKI $(n=42)$ pharmacological therapy (non-HSCT) groups (42\% and $47 \%$ respectively, $\mathrm{p}=0,53$ ) (fig.1D).

Conclusions: Detection of T315I mutation in TKIresistant patients is extremely unfavorable factor for survival, especially in the advanced phase CML, and it is a great reason for switching to ponatinib or other new potential investigated drugs if possible. Allo-HSCT can be a potential option for this group of patients in case of good selection taking into consideration transplant risk, especially for patients in $\mathrm{CP} \geq 2$.

Conflict of interest: Nothing to disclose

\section{P142}

Abstract previously published

\section{P143}

Secondary chronic neutrophilic leukemia (CNL) after severe aplastic anaemia (SAA) - a case report

Laurent Schmied ${ }^{1}$, Pontus Lundberg ${ }^{1}$, Alexander Tzankov ${ }^{2}$, Martina Kleber $^{1}$, Jakob Passweg ${ }^{1}$, Beatrice Drexler ${ }^{1}$

${ }^{1}$ University Hospital Basel, Hematology, Basel, Switzerland;

${ }^{2}$ University Hospital Basel, Pathology, Basel, Switzerland

Background: Chronic neutrophilic leukemia (CNL) and aplastic anemia (AA) are both rare diseases. Here we report the case of a 42 years old male patient with a medical history of 20 years of SAA, who developed a secondary $\mathrm{CNL}$ and subsequently received an allogeneic hematopoietic stem cell transplantation (HSCT). To the best of our knowledge a secondary CNL after SAA has not yet been described.

Methods: Case report

Results: After diagnosis of severe aplastic anemia at the age of 22 the subsequent treatment encompassed three cycles of horse ATG and cyclosporine resulting in disease 


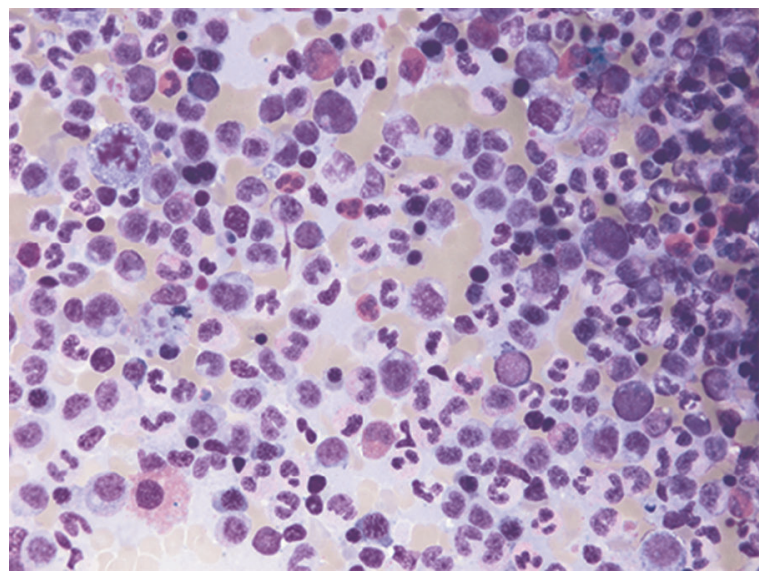

[P143 Figure] [Fig. 1. Bone marrow aspirate showing hypercellular bone marrow with neutrophilic proliferation.]

control for nine, three and seven years, respectively. After the third cycle of intensive immunosuppressive therapy, cyclosporine was maintained as long-term therapy. Nineteen years after the initial diagnosis neutrophilia was observed without signs of infection, inflammation or solid tumor. Bone marrow examination revealed hypercellularity with neutrophilic proliferation and discrete dysplasia of the myeloid cell lineage. Molecular genetics detected a SETBP1, EZH2 and CSF3R mutation (T618I). Integrative diagnosis was made according to WHO classification confirming the diagnosis of CNL. Subsequently, the patient was first treated with Ruxolitinib, showing normalization of the neutrophil counts within 3 months, but treatment had to be stopped due to thrombocytopenia after 6 months. Then allogeneic hematopoietic stem cell transplantation from a 12/12 HLA identical unrelated donor after conditioning with Cyclophosphamid $(60 \mathrm{mg} / \mathrm{Kg} /$ day for two days) and Busulphan (4 x $0.8 \mathrm{mg} / \mathrm{Kg} / \mathrm{day}$ for four days) could be performed without relevant toxicity, complications or graft versus host disease (GvHD). The posttransplant follow up (3 months) shows complete remission without any signs of GvHD or other complications.

Conclusions: We describe the first case of CNL that has evolved secondary to SAA nineteen years after the initial diagnosis. Possible pathophysiological mechanisms could include damage of the hematopoetic stem cells by previous cytotoxic therapy or selection of a myeloid clone occurring in aplastic anemia.

Conflict of interest: The authors have no conflicts of interests to declare

\section{P144}

Validation and comparison of prognostic systems in secondary (post-PV/ET) myelofibrosis after allogeneic stemcell transplantation. A report of the CMWP-EBMT
Nico Gagelmann ${ }^{1}$, Dirk-Jan Eikema ${ }^{2}$, Linda Koster ${ }^{2}$, Christine Wolschke ${ }^{1}$, Renate Arnold ${ }^{3}$, Lothar Kanz, Grant McQuaker ${ }^{5}$, Ibrahim Yakoub-Agha ${ }^{6}$, Thierry Lamy ${ }^{7}$, Gerard Socie ${ }^{8}$, Jean Henri Bourhis $^{9}$, Mohamad Mohty ${ }^{10}$, Jan J Cornelissen ${ }^{11}$, Patrice Chevallier $^{12}$, Paolo Bernasconi ${ }^{13}$, Matthias Stelljes ${ }^{14}$, Pierre-Simon Rohrlich $^{15}$, Renato Fanin ${ }^{16}$, Jürgen Finke ${ }^{17}$, Johan Maertens ${ }^{18}$, Didier Blaise ${ }^{19}$, Maija Itälä-Remes ${ }^{20}$, Hélène Labussière-Wallet ${ }^{21}$, Marie Robin $^{8}$, Yves Chalandon ${ }^{22}$, Nicolaus Kröger ${ }^{1}$

${ }^{1}$ University Medical Center Hamburg Eppendorf, Hamburg, Germany; ${ }^{2}$ EBMT Data Office, Leiden, Netherlands; ${ }^{3}$ Charité Universitaetsmedizin Berlin, Berlin, Germany; ${ }^{4}$ University Hospital, Tübingen, Germany; ${ }^{5}$ Gartnaval General Hospital, Glasgow, United Kingdom, ${ }^{6}$ Hospital CHU de Lille, LIRIC, INSERM U 995, Université de Lille, Lille, France; ${ }^{7}$ University Hospital, Rennes, France; ${ }^{8}$ Hopital St. Louis, Paris, France; ${ }^{9}$ Gustave Roussy, Institut de Cancérologie, Villejuif, France; ${ }^{10}$ Hospital Saint Antoine, Paris, France; ${ }^{11}$ Erasmus MC Cancer Institute, Rotterdam, Netherlands; ${ }^{12}$ CHU Nantes, Nantes, France; ${ }^{13}$ Fondazione IRCCS Policlinico San Matteo, Pavia, Italy;

${ }^{14}$ University of Münster, Münster, Germany; ${ }^{15} \mathrm{CHU}$ Nice - Hôpital de I ARCHET I, Nice, Germany; ${ }^{16}$ Azienda Ospedaliero Universitaria di Udine, Udine, Italy; ${ }^{17}$ University of Freiburg, Freiburg, Germany;

${ }^{18}$ UZ Leuven, Leuven, Belgium; ${ }^{19}$ Institut Paoli Calmettes, Marseille, France; ${ }^{20}$ HUCH Comprehensive Cancer Center, Helsinki, Finland;

${ }^{21}$ Centre Hospitalier Lyon Sud, Lyon, France; ${ }^{22}$ Hôpitaux

Universitaires de Genève, Geneva, Switzerland

Background: A recent model using clinical-molecular features of patients with secondary myelofibrosis (sMF) was developed by MYSEC to predict outcome from diagnosis. However, it is unclear whether scores, such as the MYSEC or DIPSS are prognostic in the transplant setting in sMF. We aimed to validate and to compare these models in sMF patients who underwent allogeneic hematopoietic stem-cell transplantation.

Methods: We identified 159 sMF patients who received stem-cell transplant from related $(\mathrm{n}=59)$ or unrelated donors $(\mathrm{n}=100)$ between 2007 and 2015 with available data on blood levels at transplant and presence of mutations at diagnosis. The MYSEC model was calculated as follows: one point was assigned to presence of constitutional symptoms and platelets $<150 \times 10^{9} / \mathrm{L}$. Two points were assigned to hemoglobin $<11 \mathrm{~g} / \mathrm{dl}$, circulating blasts $\geq 3 \%$ and a CALR-unmutated genotype, whereas 0.15 points were assigned for each year of age. Risk groups (number of patients) according to MYSEC and DIPSS were: low ( $\mathrm{n}=$ 27 and $\mathrm{n}=16)$, intermediate- $1(\mathrm{n}=70$ and $\mathrm{n}=59)$, intermediate- $2(\mathrm{n}=40$ and $\mathrm{n}=70)$, and high $(\mathrm{n}=22$ and $\mathrm{n}$ $=14$ ). Scores were validated using Kaplan-Meier estimates while C-statistics were applied to evaluate their discriminatory power.

Results: Median follow-up was 41 months (range, 29 to 52) while the median time between diagnosis and transplant was 128 months (range, 2 to 526). The median age of sMF patients was 52 years (range, 32 to 75 ). Overall survival at three years was $55.9 \%$ (95\% confidence interval [CI], 47.5 to 64.3). By stratifying sMF according to evolution from 


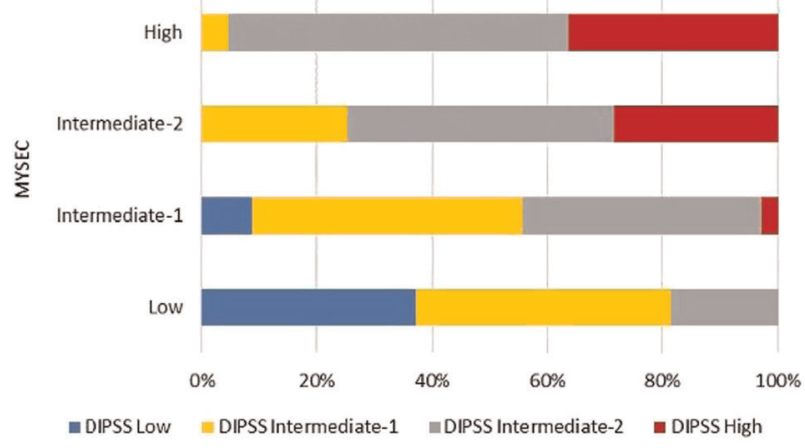

[P144 Figure] [Risk categorization of DIPSS vs. MYSEC]

either polycythemia vera (post-PV) or essential thrombocytopenia (post-ET), no difference was found in survival at three years being $57.7 \%$ (95\% CI, 45.5 to 70.0 ) for post-PV and $54.6 \%(95 \% \mathrm{CI}, 43.0$ to $66.2 ; \mathrm{p}=0.92)$ for post-ET.

Overall survival rates at three years according to each risk group of the DIPSS were as follows: $79.5 \%$ (95\% CI, 58.5 to 100 ) for the low-risk, $56.3 \%$ (95\% CI, 42.6 to 70.0 ) for the intermediate-1-risk, $53.9 \%$ (95\% CI, 41.2 to 66.6) for the intermediate-2-risk, and $40.8 \%$ (95\% CI, 14.1 to 67.5 ) for the high-risk group. Overall, DIPSS was not predictive of outcome $(\mathrm{p}=0.30)$.

Regarding MYSEC, probabilities of survival at three years were $69.3 \%$ (95\% CI, 51.5 to 87.1 ) for the low-risk, $64.5 \%$ (95\% CI, 52.5 to 76.5$)$ for the intermediate-1-risk, 46.8 (95\% CI, 29.7 to 63.9) for the intermediate-2-risk, and 21.7\% (95\% $\mathrm{CI}, 0$ to 45.4) for the high-risk group. The MYSEC model was predictive of survival overall $(\mathrm{p}=0.03)$.

When used to assign patients to the four discrete risk categories, the test retained moderate predictivitability $(\mathrm{C}$ index $=0.585$ ). However, prognostic ability was improved in comparison with DIPSS (C-index $=0.546$ ). MYSEC significantly re-classified patients according to risk ( $\mathrm{p}<$ $0.001)$.

Conclusions: In comparison to DIPSS, which is validated for primary myelofibrosis but currently also used for risk stratification in sMF patients receiving transplant, the clinical-molecular system by MYSEC provides significant re-classification of patients leading to improved prognostic capability.

Conflict of interest: No conflicts of interest related to the abstract

\section{Conditioning regimen}

\section{P145}

12 month CD4 + T-cell count and DLI treatment for mixed T-cell chimerism is associated with favorable outcomes in Reduced-Intensity Alemtuzumab-based transplant for low grade lymphoma
Claire Horgan, Shereef Elmoamly, Maria Kaparou, David Davies, Bhuvan Kishore, Manos Nikolousis, Richard Lovell, Shankara Paneesha, Katie Randall, Kathy Holder, Julie Suhr, Alex Kanellopoulos

Heart of England NHS Foundation Trust, Haematology, Birmingham, United Kingdom

Background: Upfront reduced intensity conditioned (RIC) allogeneic stem cell transplant (allo-SCT) may confer survival advantage for selected patients with heavily pretreated low grade lymphoma, although data on Alemtuzumab use for graft versus host disease prophylaxis in this setting is limited. We conducted a retrospective study of patients with heavily pre-treated low-grade lymphoma undergoing RIC-Alemtuzumab allo-SCT at the Heart of England NHS Trust.

Methods: We analyzed 22 consecutive patients, median age 57.5 years, 9 female and 13 male, with a diagnosis of follicular lymphoma, marginal zone lymphoma, or waldenstrom's macroglobulinaemia who underwent Alemtuzumabbased RIC allo-SCT, 8 (36\%) sibling-donor and the remaining $14(64 \%)$ volunteer-unrelated donor (VUD), at Heart of England NHS Trust between January 2010 and October 2017. 10 patients were conditioned with FMC whereas 12 patients had BEAM-Campath. Total Alemtuzumab dose was $25 \mathrm{mg}$ (d-1) and 50mg (d-2, d-1) for sibling and unrelated donor transplants respectively.10 patients $(45 \%)$ were in complete remission pre-transplant, with the remaining 12 patients $(55 \%)$ being in partial remission. Patients had received a median of 3 (1-6) lines of treatment prior to transplant, and 2 of the 22 patients had previously received an autologous transplant.

Results: With a median follow up of 27.4 months, choice of conditioning did not seem to affect progression-free (PFS) $(\mathrm{p}=0.48)$, or overall survival (OS) $(\mathrm{p}=0.91)$, albeit statistically significant improvements in OS for DLI (donor lymphocyte infusion)-treated patients were observed $(\mathrm{p}=0.001$, figure 1). Overall, 7 patients $(32 \%)$ underwent DLI, with the majority (71\%) being for mixed chimerism at a median day of +196 post transplant. In terms of immune reconstitution, CD4+ T-cell count $>200 / \mu \mathrm{l}$ at 12 months was associated with better OS (mean OS for CD4+ >200/ $\mu 1$ patients at 12 months $=61$ months vs 18 months for patients with $\mathrm{CD} 4+<200 / \mu \mathrm{l}$ at 12 months, $(\mathrm{p}=0.017)$ ). Besides, median OS for patients who had CD4+ T-cell count $>200 / \mu \mathrm{l}$ at 12 months was 61.7 months. Acute GVHD affected 50\% (11) patients, with 6 patients (27\% of total) having grade 3 or 4 , the remaining 5 (23\% of total patients) having grade 1 or 2 disease. 4 individuals (18\%) exhibited chronic GVHD, with only 1 of these having extensive disease. Interestingly, CD4 + T-cell count $<200 / \mu \mathrm{l}$ at 12 months did not have any association with either acute or 


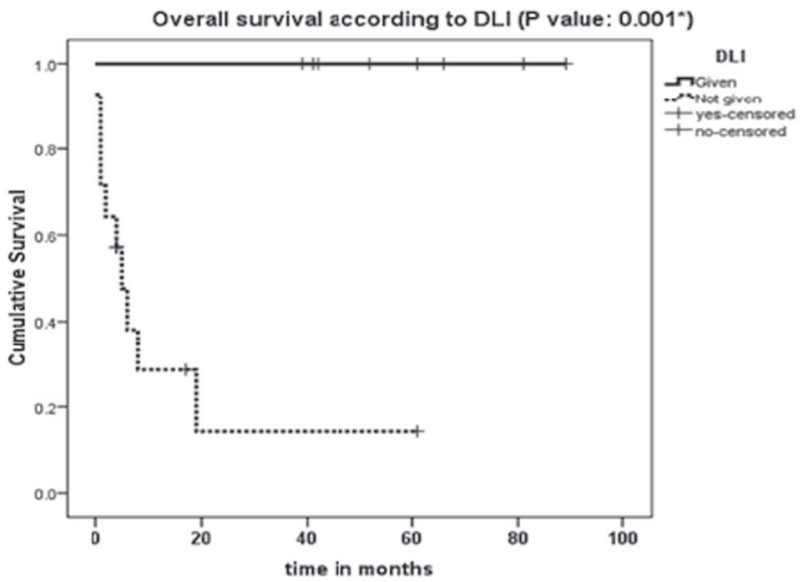

[P145 Figure] [Overall Survival according to DLI]

chronic GVHD $(\mathrm{p}=0.56$ and $\mathrm{p}=0.84$ respectively). At 3 months, whole blood chimerism was not predictive of either PFS $(p=0.31)$ or OS $(0.9)$, although there was a trend towards better outcomes for patients with mixed Tcell chimerism at 3 months $(\mathrm{p}=0.1)$. Finally, transplant related mortality was $23 \%$.

Conclusions: In our series of Alemtuzumab based RIC allo-SCT for low grade lymphomas, three-month mixed Tcell chimerism necessitating DLI correlates with a favorable outcome. CD4+ T-cell immune reconstitution at 12 months seems to affect overall survival but CD4+ T-cell count $<$

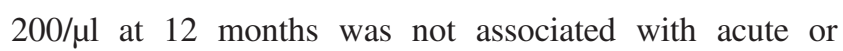
chronic GVHD. In summary, upfront RIC allo-SCT with Alemtuzumab is valid curative treatment for high risk low grade lymphoid malignancies, but prospective studies are needed to identify key determinants of its success.

Clinical Trial Registry: $\mathrm{n} / \mathrm{a}$

Conflict of interest: None of the listed authors has anything to disclose

\section{P146}

A case of a Myelodisplastic disease derived from a previously HLA-haploidentical transplanted marrow followed by a second HLA-haploidentical transplantation completely mismatched respect to the first

Matteo Parma ${ }^{1}$, Elisabetta Terruzzi ${ }^{1}$, Elisa Diral ${ }^{1}$, Andrea Aroldi ${ }^{1}$,
Marilena Fedele ${ }^{1}$, Pietro Enrico Pioltelli ${ }^{1}$, Carlo Gambacorti-
Passerini $^{2}$
${ }^{1}$ Ospedale San Gerardo, Monza, Italy; ${ }^{2}$ Ospedale San Gerardo,
Clinica Ematologica dell Universita Milano-Bicocca, Monza, Italy

Background: In recent years Haploidentical Bone Marrow Transplantation (Haplo-BMT) has been widely used in patients with high risk hematological malignancies who lack a matched related donor (MRD) and cannot obtain a matched unrelated donor (MUD) in a reasonable time. Here we describe a singular case of a patient who was submitted to Haplo-BMT for an Acute Myeloid Leukemia (AML) and who developed 5 years after a mielodysplastic syndrome. He was submitted to a second Haplo-BMT from a different donor, whose HLA was completely different respect to the first donor.

Methods: A 46-year-old male patient with undifferentiated AML was submitted to Haplo-BMT from his brother in first complete remission in 2011. The outcome was favorable, no major complications occurred, only a mild self-limiting chronic GvHD it was observed.

After 5 years of wellness, the patient presented worsening anaemia and a platelet decrease. A bone marrow aspirate was diagnostic for a three-lineage myelodisplastic syndrome, with $10 \%$ blast infiltration and a monosomal karyotype (7-). The chimerism status evaluated on bone marrow and peripheral mononuclear cells remained "full donor" on multiple controls. We concluded for a second haematologic neoplasm derived from the transplanted marrow. It is worth noting that the donor is actually fine.

Based on the good conditions the patient was submitted to a second BMT from a different donor (his son) who was HLA-Haploidentical respect to him but completely mismatched respect to the first donor. Considering the chimaerism status at the transplant, we were in the following situation: Haplo in GvHD direction, completely mismatched in reject direction but also in Graft versus Leukemia direction. In order to reduce the risk of rejection we performed an ATG $(7,5 \mathrm{mg} / \mathrm{Kg})$ priming 18 days before BMT. Anti HLA-antibody research revealed only a low title of anti-HLA of class-II. Conditioning regimen was a reduced-intensity Tiotepa-Busulfan-Fludarabine regimen, GvHD prophylaxis was performed with Cyclosporin-A, Micofenolate-Mofetil and post transplant Cyclophosphamide.

Results: No significant complication was observed during the procedure; platelet and neutrophil engraft occurred at day +28 and +32 respectively from BMT. Actually after 1 year the patient is in good conditions, no infections, viral reactivations, GvHD nor other complications appeared during the follow-up. He shows a complete and stable cytogenetic remission and chimaerism analysis reveals a $100 \%$ donor engraft either on marrow or on peripheral mono-nucleated cells since day +30 .

Conclusions: Here we report our singular experience of a double Haplo-BMT with two completely HLA different donors performed for a secondary neoplasm occurred on the transplanted cells. In our knowledge there is not similar case reported on literature.

Conflict of interest: C. Giambacorti-Passerini: consultancy, honoraria and research founding from Pfizer; 
consultancy from BMS. The other authors have nothing to declare.

\section{P147}

A comparison of FLAMSA vs Thio/Bu/Flu (TBF) vs Flu/Treo (FT) conditioning for allogeneic transplantation in active acute myeloid leukemia. A study from the ALWP-EBMT

RI

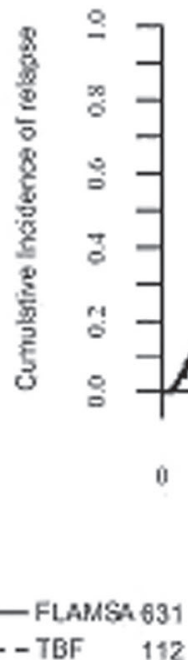

$$
\begin{aligned}
& \begin{array}{ll}
\text { - } & \text { TBF } \\
\text {-.. Trea-Fu } 112
\end{array}
\end{aligned}
$$

Francesco Saraceni ${ }^{1}$, Myriam Labopin ${ }^{2}$, Arne Brecht ${ }^{3}$, Nicolaus Kröger ${ }^{4}$, Matthias Eder ${ }^{5}$, Johanna Tischer ${ }^{6}$, Hélène LabussièreWallet $^{7}$, Herman Einsele ${ }^{8}$, Dietrich Beelen ${ }^{9}$, Donald Bunjes ${ }^{10}$, Dietger Niederwieser ${ }^{11}$, Tilmann Bochtler ${ }^{12}$, Bipin Savani ${ }^{13}$, Mohamad Mohty ${ }^{14}$, Arnon Nagler ${ }^{15}$

${ }^{1}$ Ravenna Hospital, Ravenna, Italy; ${ }^{2}$ EBMT Paris Study Office CEREST-TC, Saint Antoine Hospital, Department of Haematology, Paris, France; ${ }^{3}$ Deutsche Klinik fuer Diagnostik, KMT Zentrum, Wiesbaden, Germany; ${ }^{4}$ University Hospital Eppendorf, Bone Marrow Transplantation Centre, Hamburg, Germany; ${ }^{5}$ Hannover Medical School, Department of Haematology, Hemostasis, Oncology and Stem

\section{LFS}

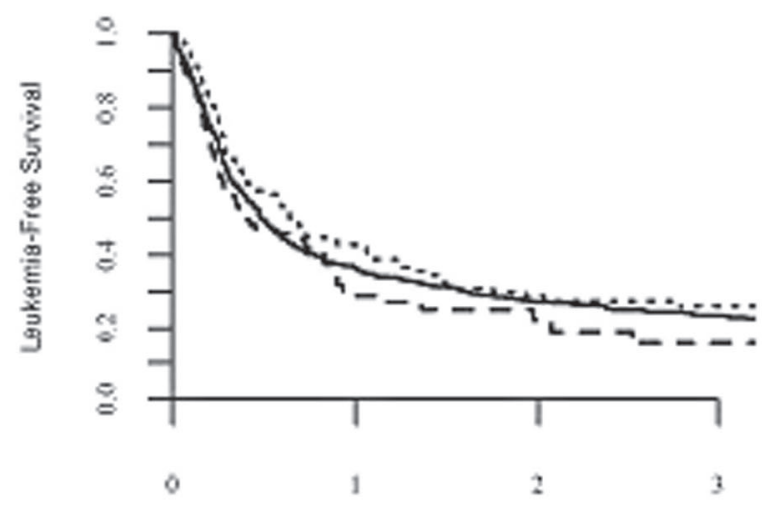

Time tach uansplans (years)

number of s:-rak paoonts

\begin{tabular}{lcccc}
- ELAJUSA & 631 & 190 & 123 & 89 \\
\hline-- TBF & 112 & 20 & 8 & 3 \\
$\ldots$ Treo-Flu & 113 & 44 & 24 & 19
\end{tabular}

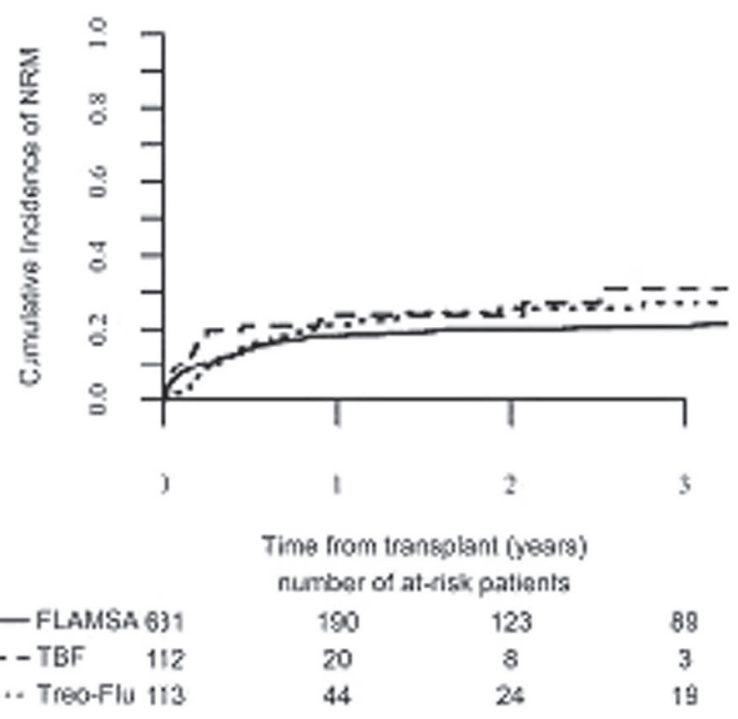

\section{NRM}

\section{OS}

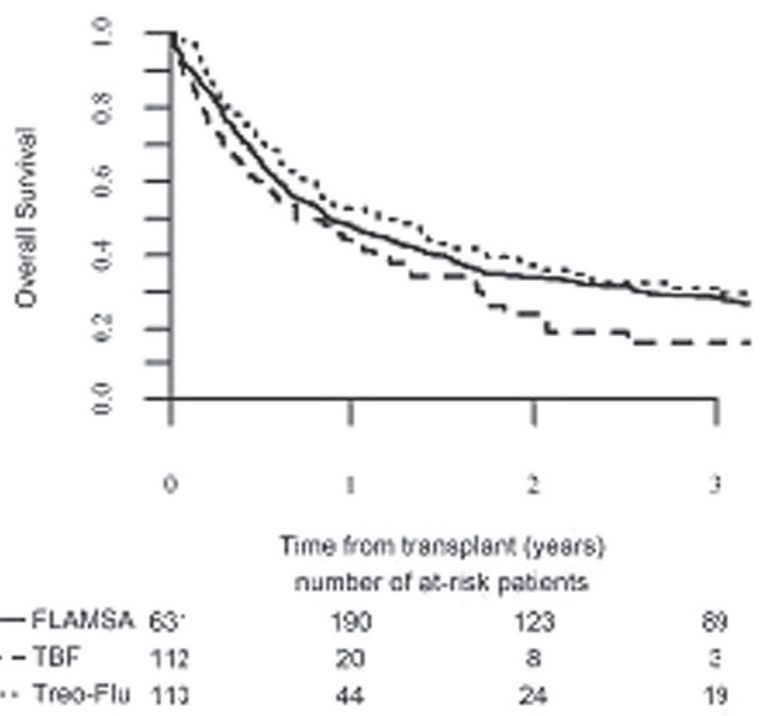

[P147 Figure] [Transplant outcome following FLAMSA vs TBF vs FT] 
Cell Transplantation, Hannover, Germany; ${ }^{6}$ Klinikum Grosshadern Med. Klinik III, Munich, Germany; ${ }^{7}$ Centre Hospitalier Lyon Sud, Pavillon Marcel Bérard -Bat 1G, Service Hematologie, Lyon, France; ${ }^{8}$ Universitaetsklinikum Wuerzburg, Med. Klinik und Poliklinik II, Wuerzburg, Germany; ${ }^{9}$ University Hospital, Department of Bone Marrow Transplantation, Essen, Germany; ${ }^{10}$ Klinik fuer Innere Medzin III, Universitätsklinikum Ulm, Ulm, Germany; ${ }^{11}$ University Hospital Leipzig, Division of Haematology \& Oncology, Leipzig Germany; ${ }^{12}$ University of Heidelberg, Medizinische Klinik u. Poliklinik V, Heidelberg, Germany; ${ }^{13}$ Vanderbilt University Medical Center, Nashville, TN, United States; ${ }^{14}$ Saint Antoine Hospital, Department of Haematology, Paris, France; ${ }^{15}$ Chaim Sheba Medical Center, Department of Bone Marrow Transplantation, Tel Hashomer, Israel

Background: The choice of conditioning regimen is particularly crucial in the setting of active acute myeloid leukemia (AML), as the need for a powerful anti-leukemic activity should not negate a good toxicity profile of the protocol.The sequential FLAMSA regimen (fludarabinecytosine arabinoside-amsacrine combined with TBI or busulfan and cyclophosphamide),TBF (thiotepa-busulfanfludarabine) and FT (fludarabine-treosulfan) are three possible options.

Methods: We analysed transplantation outcomes of 856 adult pts with AML in active disease phase (primary refractory or first or second relapse) that underwent a first SCT from matched sibling (MSD) or unrelated donor (UD) between 2005 and 2016 and reported to the ALWP of the EBMT.Six hundred and thirty-one pts received FLAMSA,112 received TBF, while 113 pts were transplanted following the FT protocol.According to institutional policy,FLAMSA was busulfan or TBI -based in $32 \%$ and $68 \%$ of the cases, respectively.Pts who received oral busulfan,manipulated grafts, or transplant from $<9 / 10$ HLA-matched donor were excluded.

Results: Median follow-up was 53 months in FLAMSA and FT groups and 16 months in the TBF group.Median age at transplant was 52 years in FLAMSA and TBF and 58 years in the FT groups, respectively $(\mathrm{p}=0.001)$. At 100 days, cumulative incidence of grade II-IV aGVHD was $28 \%$, 29\% and $24 \%(\mathrm{p}=0.7)$, while incidence of grade III-IV aGVHD was $11 \%, 12 \%$ and $10 \%$ for FLAMSA, TBF and FT, respectively $(\mathrm{p}=0.9)$. The 2 -year cumulative incidence of cGVHD and severe cGVHD was $26 \%, 26 \%, 33 \%(\mathrm{p}=0.4)$ and $11 \%, 19 \%, 13 \%(\mathrm{p}=0.5)$ for FLAMSA, TBF and FT, respectively. Complete remissions cumulative incidence for pts that reached day 100 was $88 \%, 80 \%, 92 \%$ for FLAMSA, TBF and FT, respectively $(\mathrm{p}=0.13)$. No significant difference was observed between the three conditioning regimens in 2 year non-relapse mortality (NRM), being 20\%, 24\% and 26\% in FLAMSA,TBF and FT, respectively $(\mathrm{p}=0.24)$ (Figure 1).Similarly, the 2-year relapse incidence (RI) rates were 53\%, 54\%, 46\%, respectively $(p=0.33)$.No significant difference was observed in 2 year leukemia-free survival (LFS), with rates of $27 \%, 22 \%, 29 \%$ for FLAMSA, TBF and FT, respectively $(\mathrm{p}=0.28)$. OS following TBF conditioning $(24 \%)$ was comparable to FLAMSA $(34 \%)$ and FT $(37 \%, \mathrm{p}=0.10)$. Finally, the composite endpoint graft-vs-host disease-free, relapse-free survival (GRFS) at 2 years was $20 \%, 13 \%$, $23 \%$ for FLAMSA, TBF and FT, respectively $(\mathrm{p}=0.15)$.In multivariate analysis no significant difference was observed between the three preparative regimens in terms of transplant outcome.Karnofsky performance score (KPS) above $80 \%$ was independently associated with better OS (HR 0.7, $\mathrm{p}=0.01$ ) and GRFS (HR 0.7, $\mathrm{p}=0.01$ ). Importantly, in-vivo T-cell depletion (TCD) was associated with lower incidence of grade III-IV aGVHD (HR 0.4, p = 0.018 ), chronic (HR 0.4, p =0.006) and severe cGVHD (HR 0.4, $\mathrm{p}=0.005$ ).

Conclusions: These results suggest that the three preparative regimens namely FLAMSA, TBF and FT provide similar anti-leukemic effect in AML pts with active disease.Transplant related complications,TRM and outcome did not differ, as well. Karnofsky performance score emerged as a strong predictor of GRFS and overall survival. In-vivo TCD was associated with lower incidence of acute and chronic GVHD.In conclusion all three conditioning protocols are valid options for transplantation in pts with relapsed/refractory AML to be compared in future prospective studies.

Conflict of interest: [All authors]: nothing to declare

\section{P148}

Abstract previously published

\section{P149}

\section{Alemtuzumab is feasible as substitute for ATG in the conditioning regimen FBM (Fludarabine, BCNU, Melphalan) - a single center study}

\section{Merle Weniger ${ }^{1}$, Natalie Schub ${ }^{1}$, Thomas Spanberger ${ }^{1}$, Martin Gramatzki $^{1}$, Andreas Günther ${ }^{1,2}$}

${ }^{1}$ Division of Stem Cell Transplantation and Immunotherapy, Medical Department 2, Kiel, Germany; ${ }^{2}$ Helios Kliniken Schwerin,

Hematology, Oncology and Stem Cell Transplantation, Schwerin, Germany

Background: In recent years conditioning regimens with reduced or intermediate intensity were developed to extend the curative approach of allogenic stem cell transplantation to elderly and unfit patients. The combination of fludarabine, BCNU and melphalan (FBM) ${ }^{1}$ has shown activity in acute myeloblastic leukemia (AML), myelodysplastic syndrome (MDS) and multiple myeloma (MM). However, 
this conditioning regimen can be combined with different methods of in vivo $\mathrm{T}$ cell depletion and immunosuppression. In our center two different approaches for $\mathrm{T}$ cell depletion are in use according to physician's discretion: the monoclonal CD52 antibody alemtuzumab or rabbit anti Tlymphocyte globulin (Neovii). This retrospective single center study analyzes the impact of the used in vivo $\mathrm{T}$ cell depletion on acute toxicity of the conditioning regimen as well as on the long term outcome.

1 Marks et al. Blood. 2008;112:415-25

Methods: All consecutive patients transplanted in our center between 2010 and 2016, where FBM was used as conditioning regimen, were included in the study. Most of the 163 patients were treated with ATG, while 36 received alemtuzumab. According to our institutional standard patients transplanted from a non-related HLA matched donor (NRMD, 9/10 or 10/10) were treated with ATG in a dose of $40 \mathrm{~g} / \mathrm{kg}$, while in cases with HLA matched related donors (MRD) the dose was reduced to $30 \mathrm{~g} / \mathrm{kg}$. In contrast, the equivalent dose for alemtuzumab calls for $30 \mathrm{mg}$ (absolute) for transplants from NRMD and $20 \mathrm{mg}$ in MRD transplantation. To address acute toxicity of the conditioning regimen clinical symptoms were recorded according to the common terminology criteria for adverse events. The endpoints for long term outcome were overall survival, rate of relapse and acute GvHD.

Results: The median age in both patient groups was comparable (alemtuzumab: 60 years, ATG: 58 years), but the patients treated with alemtuzumab had a significantly higher comorbidity score compared to the ATG cohort (median HCT-CI: 3 versus 1). All patients in the alemtuzumab group were diagnosed with AML or MDS, whereas ATG was also used in MM and ALL cases. Interestingly, the tolerability of alemtuzumab in regard of hypotension, tachycardia, rash, fever and chills was better than with ATG. Notably, with a median follow up of 584 months we observed no significant difference in overall survival in both groups (alemtuzumab: 56\%, ATG: 59\%). The rate of acute GvHD was significantly lower in the alemtuzumab group ( $44 \%$ vs $68 \%, \mathrm{p}=0.01$ ). In AML and MDS patients the rate of relapse tended to be higher in alemtuzumab treated patients than in the ATG cohort (39 versus 29\%), although no statistical significance was achieved.

Conclusions: The conditioning regimen FBM showed impressive activity in our single center study with both drugs, ATG and alemtuzumab. The more tolerable drug was alemtuzumab, although the achieved level of immunosuppression seemed to be deeper at the chosen dose levels of both drugs. Since the outcome of both cohorts was comparable, alemtuzumab is found to be a feasible substitute for ATG in patients, where acute toxicity of the conditioning regimen should be avoided.

\section{Conflict of interest: No}

P150

\section{Allogeneic stem cell transplantation for elderly patients (60-70 years of age): RIC, chronic GVHD and treatment response improve survival}

Kari Remes, Suvi Jokela, Maija Itälä-Remes, Mervi Putkonen, Marjut Kauppila, Tommi Salmi, Soile Salomäki, Urpu Salmenniemi

Turku University Hospital, Dept of Clinical Haematology \& Stem Cell Transplantation Unit, Turku, Finland

Background: Due to poor results with chemotherapy in elderly patients and improvements in the control of complications of allogeneic stem cell transplantation (ASCT) the use of ASCT has extended to patients over 60 years of age, and the upper age limit has increased to 7075 years of age. The assessment of transplant outcomes is critical to test the feasibility of this treatment modality in the older age groups.

Methods: Our institutional transplant registry data were retrospectively analysed for patients aged 61-70 years, transplanted between 1.1.2010 and 30.6.2015 $(\mathrm{N}=59)$. The analysis is as of 30.6.2016 when a minimum follow-up of one year was fulfilled. The primary end point is overall survival (OS). Kaplan-Meier curves were produced, and Cox regression model was used to identify factors affecting OS.

Results: The median age is 63 (range: 61-69) years; 17 (29\%) were in the age group $66-70$ years. $66 \%$ of patients had AML/MDS, $21 \%$ lymphatic/plasma cell malignancies, $8 \%$ other myeloid malignancies, and $5 \%$ aplastic anemia. The disease status at transplant was CR in half of the patients, and the Gratwohl scoring identified an early disease in $46 \%$, intermediate in $15 \%$ and advanced in $39 \%$ of the patients.

The donor was unrelated in $80 \%$; source of stem cells was blood in $85 \%$ and marrow in $15 \%$. RIC conditioning was used for $59 \%$ and MA for $41 \%$ of patients. HLA-matches were full in $29 \%$, DPB1-mm in $35 \%$, and HLA-A, C or DRB3 $\mathrm{mm}$ in $12 \%$. Severe acute GVHD (Gr3-4) occurred in $18(30 \%)$, and extensive chronic GVHD in $7(12.5 \%)$ patients. There is no significant difference in OS for patients aged 61-65 vs 66-70 years even if the survival curve seems better for those of 61-65 years (Fig). In univariate analysis significant factors for OS were conditioning (RIC > MA; P $=0.001$ ), occurrence of chronic GVHD (yes $>$ no; $\mathrm{P}=$ $0.014)$ and best response to ASCT (CR $>$ less than CR; $\mathrm{P}=$ 0.037).

Conclusions: ASCT is a feasible treatment modality for patients aged 61-70 years with relatively good OS. Patients over 65 years may have a worse outcome but their small 


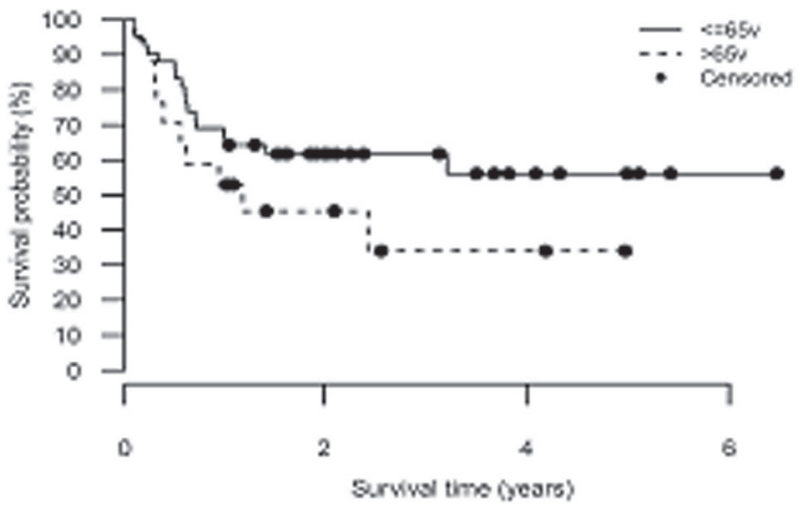

[P150 Figure] [Overall survival of patients aged 61-65 years $(N=42)$ vs 66-69 years $(N=17)]$

number excludes meaningful statistics. However, in univariate analysis the age seems not to be a significant factor for OS in opposite to intensity of conditioning, chronic GVHD, and best response to ASCT.

Conflict of interest: All authors: nothing to disclose.

\section{P151}

BCNU-EAM vs non-BCNU alternative alkylator -EAM conditioning regimens for autologous stem cell transplantation in lymphoma patients

Deniz Goren Sahin ${ }^{1,2}$, Guldane Seval Cengiz ${ }^{3}$, Ender Soydan ${ }^{4}$, Emine Tulay Ozcelik ${ }^{1,2}$, Ayla Gokmen ${ }^{4}$, Zafer Gokgoz ${ }^{4}$, Pervin Topçuoğlu ${ }^{3}$, Onder Arslan ${ }^{3}$, Gunhan Gurman ${ }^{3}$, Muhit Ozcan ${ }^{3}$, Mutlu Arat $^{2}$

${ }^{1}$ Istanbul Bilim University, Hematology Department, Istanbul, Turkey; ${ }^{2}$ Sisli Florence Nightingale Hospital, Hematopoietic Stem Cell Transplantation Unit, Istanbul, Turkey; ${ }^{3}$ Ankara University School of Medicine, Ankara, Turkey; ${ }^{4}$ Medicana Ankara International Hospital, Ankara, Turkey

Background: Autologous hematopoietic stem cell transplantation (HSCT) is a potentially curative therapeutic approach for relapsed/refractory lymphomas. Although carmustine, etoposide, cytarabine, and melphalan (BEAM) is the most commonly used conditioning regimen for high-dose therapy (HDT) before auto-HSCT, to date, there is no published prospective randomized study comparing different types of conditioning regimen. The cost and availability of BCNU forced many centres to search for alternatives. Therefore, gold standard regimens have not been established and clinical rehearsal differs substantially among institutions. Here we aim to compare between B-EAM and alternative alkylator -EAM backbone based regimen on the potential toxicities and survival in lymphoma patients.
Kaplan-Meier Survival Analysis

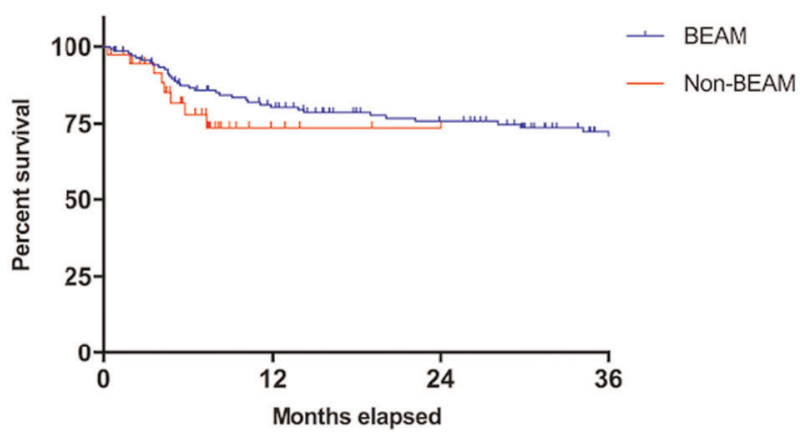

[P151 Figure] [Kaplan-Meier survival analysis of two groups]

Methods: Patients with relapsed refractory lymphoma, who underwent autologous HSCT between 2012-2017, were evaluated from three different highly active transplant institutions in Turkey. One hundred eighty adult lymphoma patients (age: 18-65 years) with a follow up time at least 6 months were included. Patients with B-EAM $(\mathrm{n}=139)$ high dose chemotherapy were compared with patients having received non-BEAM regimen; T- thiotepa (T) -EAM $(\mathrm{n}=16)$, Bendamustine (Be)-EAM $(\mathrm{n}=12)$, Lomustine (L) -EAM $(\mathrm{n}=13)$ using the following matching factors: age, gender, lymphoma subtype, disease status at transplant, toxicity, overall survival, and causes of death.

Results: Totally 180 patients (109 males, 71 females) were identified: 139 patients and 41 patients were treated with BEAM and non-BEAM regimen, respectively. Patients' baseline characteristics are detailed in Table 1.

There was no statistically significant difference between mean age and gender between groups. With regards to toxicities among groups; BEAM group had a significantly lower rate of mucositis and gastrointestinal toxicity than non-BEAM group $(\mathrm{p}=0.01$ and $\mathrm{p}=0.002$, respectively). Renal toxicity did not show difference among groups $(p=0.92)$. Although BEAM group showed a lower rate of transplant related mortality at $100^{\text {th }}$ day $(5.5 \%$ vs. $11.4 \%)$, the difference did not reach to statistical significance. Estimated mean survival time for BEAM and Non-BEAM was $52.1 \pm 2.5$ months and $18.8 \pm 1.5$ months, respectively $(\mathrm{p}=0.2, \log$-rank test) (Figure 1).

The significant difference in median follow/up times has to be taken into account.

Conclusions: Our study showed that EAM backbone (non-BEAM) conditioning regimens with alternative alkylating agents have similar results compared to B-EAM regimen in terms of efficacy, and toxicity. There is still need for an optimal conditioning regimen with lower toxicity, better efficacy and less cost than BEAM. Prospective randomized controlled studies with large number of patients 
are warranted to find out the optimal backbone combination, which may establish as a standard regimen for HSCT in lymphoma patients.

Conflict of interest: Nothing to disclose

\begin{tabular}{|c|c|c|c|}
\hline Patients & B-EAM & Non-BEAM & p-value \\
\hline $\begin{array}{l}\text { Median follow-up } \\
\text { (months) }\end{array}$ & 29.2 & 6.5 & $<0.0001$ \\
\hline $\begin{array}{l}\text { Age at HSCT, median } \\
\text { (SD) (years) }\end{array}$ & $46.6( \pm 15.1)$ & $47.7( \pm 14.3)$ & 0.67 \\
\hline Gender male (n)\% & $87(62.6 \%)$ & $22(53.7 \%)$ & 0.36 \\
\hline Entities (n) (\%) NHL/HL & $\begin{array}{l}94(67.6 \%) / 45 \\
(32.4 \%)\end{array}$ & $\begin{array}{l}30(73.2 \%) / 11 \\
(26.8 \%)\end{array}$ & 0.56 \\
\hline $\begin{array}{l}\text { Disease status at } \\
\text { transplant, } \mathrm{n}(\%) \text { CR1/ } \\
\text { CR2/PR/Refractory }\end{array}$ & $\begin{array}{l}20(15.9 \%) / 24 \\
(19.0 \%) / 53(42.1 \%) / \\
29(23.0 \%)\end{array}$ & $\begin{array}{l}6(14.6 \%) / 6 \\
(14.6 \%) / 20 \\
(48.8 \%) / 9(22.0 \%)\end{array}$ & 0.74 \\
\hline Mucositis, n (\%) & $69(49.6 \%)$ & $31(75.6 \%)$ & 0.01 \\
\hline Renal, n (\%) & $6(4.3 \%)$ & $2(4.8 \%)$ & 0.92 \\
\hline $\begin{array}{l}\text { Gastrointestinal system, } \mathrm{n} \\
(\%)\end{array}$ & $71(51.0 \%)$ & $32(78.0 \%)$ & 0.002 \\
\hline $\begin{array}{l}\text { Transplant related } \\
\text { mortality at 100th day Yes } \\
\text { (n) }(\%)\end{array}$ & $6(5.5 \%)$ & $4(11.4 \%)$ & 0.25 \\
\hline
\end{tabular}

\section{[[P151 Table] Table 1]}

\section{P152}

\section{BEAM AND FEAM CONDITIONING FOR HODGKIN DISEASE: A RETROSPECTIVE MULTICENTER STUDY FROM FONDAZIONE ITALIANA LINFOMI (FIL)}

\author{
Matteo Pelosini ${ }^{1}$, Jacopo Olivieri ${ }^{2}$, Federico Mosna ${ }^{3}$, Angelo Fama ${ }^{4}$, \\ Simone Ferrero ${ }^{5}$, Giuseppe Carli, Margherita Giannoccaro ${ }^{6}$, \\ Nicola Sgherza ${ }^{8}$, Sara Rattotti ${ }^{9}$, Dario Marino ${ }^{10}$, Giacomo Loseto ${ }^{11}$, \\ Anna Maria Mazzone ${ }^{12}$, Teresa Calimeri ${ }^{13}$, Maria Chiara Tisi, ${ }^{6,14}$, \\ Gabriella Tomei ${ }^{15}$, P Chiusolo ${ }^{14}$, G Perali $^{2}$, A. Billio $^{3}$, Enrico \\ Orciuolo $^{16}$, K Codeluppi $^{17}$, P.M. Stefani ${ }^{18}$, P. Ghione ${ }^{19}$, F. \\ Gherlinzoni $^{18}$, Attilio Olivieri ${ }^{20}$, I. Federici ${ }^{20}, R$ Centurioni $^{21}, R$. \\ Matera $^{7}$, S Finotto ${ }^{22}$, N Cascavilla ${ }^{8}$, Renato Fanin ${ }^{2}$, Francesco \\ $Z_{a j a}{ }^{2}$
}

${ }^{1}$ Azienda Ospedaliera Universitaria Pisana, U.O. Ematologia, Pisa, Italy; ${ }^{2}$ A.O.U. Santa Maria della Misericordia di Udine, Ematologia, Udine, Italy; ${ }^{3}$ Ospedale Regionale 'S. Maurizio', Ematologia e Centro Trapianto di Midollo Osseo, Bolzano, Italy; ${ }^{4}$ IRCCS, Ematologia $A S M N$, Reggio Emilia, Italy; ${ }^{5}$ AOU Città della Salute e della Scienza di Torino, - Ematologia 1, Dipartimento di Biotecnologie Molecolari e Scienze per la Salute, Università di Torino, Torino, Italy; ${ }^{6}$ Ospedale San Bortolo, Ematologia, Vicenza, Italy; ${ }^{7}$ ASL Le/1 P.O. Vito Fazzi,

Ematologia, Lecce, Italy; ${ }^{8}$ IRCCS Ospedale Casa Sollievo Sofferenza, Ematologia, San Giovanni Rotondo, Italy; ${ }^{9}$ Fondazione IRCCS Policlinico San Matteo, Ematologia, Pavia, Italy; ${ }^{10}$ IOV, IRCCS, UOC di Oncologia Medica 1, Padova, Italy; ${ }^{11}$ IRCCS - Ospedale Oncologico, Ematologia, Bari, Italy; ${ }^{12}$ Ospedale 'S. Giuseppe Moscati, Ematologia, Taranto, Italy; ${ }^{13}$ IRCCS Ospedale San Raffaele, Divisione di Ematologia, Milano, Italy; ${ }^{14}$ Università Cattolica del Sacro Cuore, Istituto di Ematologia, Roma, Italy; ${ }^{15}$ Ospedale di IvreaASL TO4, Ematologia, Ivrea, Italy; ${ }^{16}$ Azienda Ospedaliera

Universitaria Pisana, U.O. Ematologia Universitaria, Pisa, Italy;

${ }^{17}$ ASMN - IRCCS, Ematologia, Reggio Emilia, Italy; ${ }^{18}$ Azienda ULSS
9, Ematologia, Treviso, Italy; ${ }^{19}$ AOU Città della Salute e della Scienza di Torino, Ematologia 1, Dipartimento di Biotecnologie Molecolari e Scienze per la Salute, Università di Torino, Torino, Italy; ${ }^{20}$ Azienda Ospedali Riuniti di Ancona, Ematologia, Ancona, Italy; ${ }^{21}$ ASUR AV3, UOC Medicina Interna ed Ematologia, Civitanova Marche, Italy; ${ }^{22}$ IOV, IRCCS, UOC di Oncologia Medica 1, I, Padova, Italy

Background: High dose chemotherapy with stem cell rescue is the standard of care for relapse refractory Hodgkin disease (HD) patients even if newer treatment are nowadays available. The most used conditioning regimen is the BEAM scheme. In last years carmustine shortage has been an issue worldwide and in Italy as well. Fotoemustine emerged as a possible alternative with the FEAM scheme.

Methods: We retrospectively analyzed data of 1038 consecutive patients transplanted in 16 different Italian centers from 2008 to 2015 with BEAM and FEAM regimen. Here we report data about HD patients $(n=278)$.

Results: Median age was 39 for both groups. There were $42,7 \%$ female patients in BEAM and $45,6 \%$ in FEAM. Patient characteristics at diagnosis were comparable with stage II (BEAM 36\% vs 41,6\% FEAM), stage III (BEAM $28 \%$ vs $23,8 \%$ FEAM) and stage IV (33,5\% BEAM vs $32 \%$ FEAM). B symptoms were present respectively in $48,2 \%$ (BEAM) and 56,6\% (FEAM). First line treatment was ABVD in $92 \%$ in both groups. Radiotherapy before transplant (as consolidation or in 2nd line) was used in $36,6 \%$ case in BEAM cohort vs $14 \%$ in FEAM. Disease assessment before SCT showed in the BEAM CR in 63\%, PR 26,8\% and resistant disease (RD) in 9,1\%, metabolic CR (PET negative at transplant) in $59,8 \%$ patients. In the FEAM group CR 53,5\%, PR 34,1\%, RD 11,\%. metabolic CR in $47,3 \%$. BEAM patients had an higher Sorror score at transplant (Sorror $>314,6 \%$ vs 2,63). Neutrophil engraftment was similar in both groups (10 days). Grade III-IV mucositis occurred in $28 \%$ of BEAM and $34 \%$ of FEAM. Febrile neutropenia grade III-IV were observed respectively in 46\% (BEAM) and 57\% (FEAM). Response status did not differ at day 100 post ASCT (CR 85\% BEAM vs 75,\% FEAM) and neither at last follow-up (continuous CR 67,7\% BEAM vs $62,2 \%$ FEAM). Non-relapse mortality at 1 year (BEAM 0,6\% vs FEAM 2,6\%) was comparable. Overall survival (OS) at 2 years was not different (BEAM 92\% vs FEAM $88,6 \%, \mathrm{p}=0.16$ ). Death occurred respectively in $16,4 \%$ and $16,6 \%$ for BEAM and FEAM due to disease progression in the majority of patients. However, the FEAM group had a trend to and inferior progression-free survival at 1 year (BEAM 74,6\% vs FEAM 65\%,4 $\mathrm{p}=0.075$ ) and higher relapse incidence $(18,6 \%$ BEAM vs $26,4 \%$ FEAM, $\mathrm{p}=0,051$ )

Conclusions: Substitution with Fotemustine did not increase general toxicities or infectious episodes; however we observed a sligher higher rate of gastrointestinal and 
infectious toxicities in FEAM patients that did not lead to higher NRM neither significantly worsened OS. Although the retrospective design of the study should caution against definitive conclusions we observed a trend to a better PFS and relapse incidence in BEAM patients, that could be related a better disease control status at the time of transplant.

Conflict of interest: None

\section{P153}

BEAM versus LEAM conditioning chemotherapy for autologous transplantation in lymphoma. A retrospective study from the BSBMT

Nick Morley ${ }^{1}$, John Snowden ${ }^{1}$, Rachel Pearce ${ }^{2}$, Julia Perry ${ }^{2}$, Ruth Paul $^{2}$, Keiren Kirkland ${ }^{2}$, Jonathan Lambert ${ }^{3}$, Ram Malladi ${ }^{4}$, Andy Peniket $^{5}$

${ }^{1}$ Sheffield Teaching Hospitals NHS Foundation Trust, Sheffield, United Kingdom; ${ }^{2}$ British Society of Blood and Marrow Transplantation, London, United Kingdom; ${ }^{3}$ University College London Hospitals NHS Foundation Trust, London, United Kingdom; ${ }^{4}$ University Hospitals Birmingham NHS Foundation Trust, Birmingham, United Kingdom; ${ }^{5}$ Oxford University Hospitals NHS Foundation Trust, Oxford, United Kingdom

Background: Since Linch et. al. published the results of a BNLI trial comparing BEAM with autologous rescue with mini-BEAM in the Lancet in 1993, BEAM has been considered the 'gold standard' conditioning regimen for patients with relapsed Hodgkin lymphoma in the UK. Not long afterwards the Parma Study also reported significant benefit from the Carmustine containing high dose chemotherapy (the BEAC regimen) for patients with NonHodgkin Lymphoma. Over recent years there have been intermittent shortages of the drug Carmustine leading to increasing use of the drug Lomustine as a substitute.

Methods: In order to compare the safety and efficacy of BEAM with LEAM conditioning chemotherapy we conducted a retrospective registry survey of patients with Hodgkin and Non-Hodgkin Lymphoma for first autologous transplant in the UK with a Day 0 between January 2009 and December 2013 and at least 100 days follow up available. Data to be sourced through Med A/B forms. Overall outcome to be assessed through Overall Survival (OS). Efficacy through progression free survival (PFS) and relapse rate (RR) and Safety through non-relapse mortality (NRM).

Results: Overall 1060 patients were identified from 30 centres with a median follow up of nearly 4 years. 650 (61\%) patients had received BEAM and 410 (39\%) LEAM conditioning. Both groups were well matched for year of transplant, gender, age, diagnosis, presence of comorbidity, lines of prior therapy, disease status at transplant and

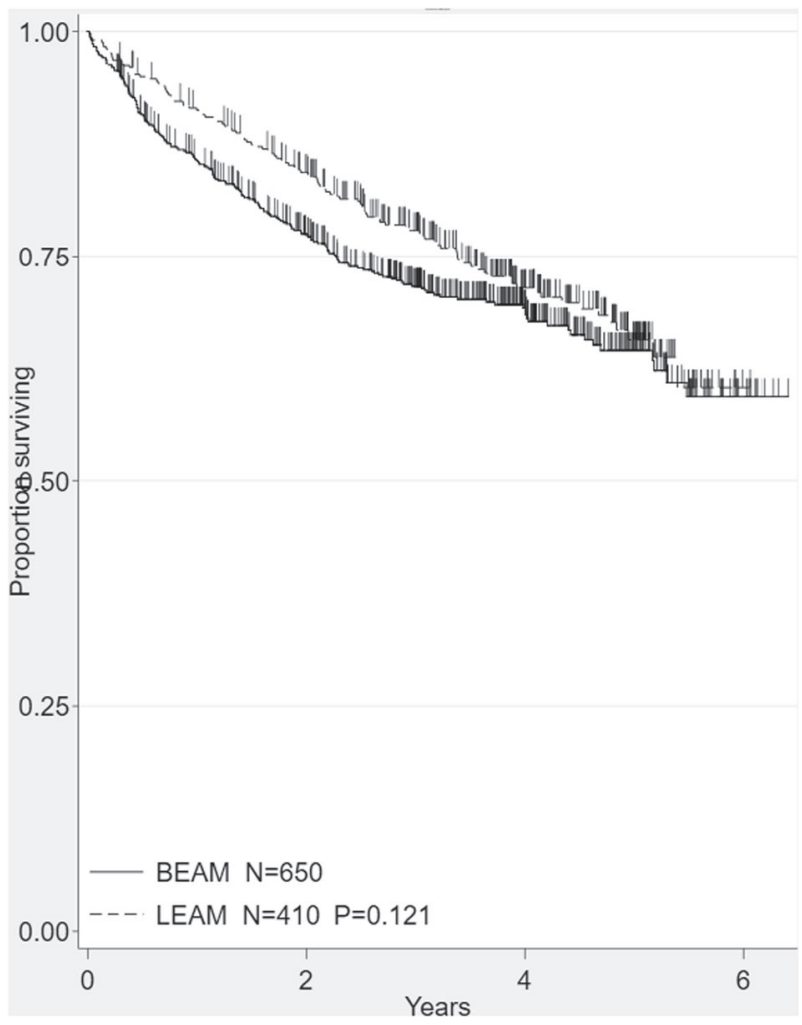

[P153 Figure] [Overall Survival by Conditioning Regimen]

performance status. Median time to neutrophil (Median 11 days) and platelet (Median 14-15 days) engraftment were equivalent, as was disease response to transplant (CR 74$78 \%$ ). Assessing efficacy, PFS was equivalent at 1 year $(86 \%$ v $91 \%)$ and 5 years $(51 \%$ v $55 \%), \mathrm{p}=0.12$, as was RR at both 1 year (20\% v 16\%) and 5 years ( $41 \%$ v $39 \%)$, p $=0.246$. In terms of safety, NRM was equivalent at

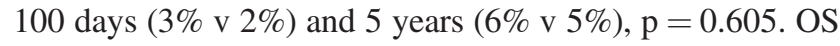
was also equivalent at 1 year $(86 \% \mathrm{v} 91 \%)$ and 5 years (64\% v 66\%), $\mathrm{p}=0.121$.

Conclusions: Comparison of BEAM with LEAM conditioning chemotherapy for first autologous transplant in patients with Hodgkin and Non-Hodgkin Lymphoma showed equivalent safety, efficacy and overall survival. Lomustine can be safely substituted in place of Carmustine chemotherapy.

\section{Clinical Trial Registry: BSBMT CTCR 15-01}

Conflict of interest:

J. Snowden: Honoraria for speaking/chairing Sanofi and Jazz

N. Morley: Conference support and advisory board fees, Roche 


\section{P154}

BEAM versus single agent High Dose Melphalan (HDM) conditioning regimen for autologous hematopoietic stem cell transplant (ASCT): a retrospective matched analysis in relapse/refractory Hodgkin lymphoma

MS Rauf ${ }^{1}$, Panayotis Kaloyannidis ${ }^{2}$, Irfan Maghfoor ${ }^{1}$, Solaf Kafnar ${ }^{2}$, John Apostolidis', Khalid Al Anezi ${ }^{2}$, Harbi Salman', Shaibani Eshrak ${ }^{2}$, Jenifer Bacal', Tusneem A.M. Elhassan ${ }^{1}$, Saad Akhtar ${ }^{1}$, Hani Al Hashmi ${ }^{2}$

${ }^{1}$ King Faisal Hospital and Research Center, Oncology Center, Riyadh, Saudi Arabia; ${ }^{2}$ King Fahad Specialist Hospital, Adult Hematology and Stem Cell Transplantation Department, Dammam, Saudi Arabia

Background: The ideal conditioning regimen still remains a challenge for patients undergoing autologous stem cell transplantation (ASCT) for relapsed/refractory Hodgkin Lymphoma (RR-HL). The commonly used regimen is BEAM but single agent high-dose Melphalan (HDM) has also been used, however so far, there are limited experience and data comparing BEAM vs. HDM.

Methods: Medical records of 112 RR-HL patients, from two different institutions, who evaluated with chemosensitive disease after salvage chemotherapy and underwent ASCT from 2008 onwards, were reviewed retrospectively after Institutional Review Board Approval. A relatively smaller cohort of patients conditioned with HDM $(n=28)$ was compared in a matched-paired analysis (1:3) with a larger cohort of patients who received BEAM $(n=84)$. The patient groups (BEAM vs. HDM) had similar median age (30ys) and sex (M:F 1.7:1 vs. 1.8:1) and were matched for disease status before salvage (late relapse: 36 vs. 12 and early relapse/primary refractory: 48 vs.16) and disease status pre-ASCT [complete remission (CR): 39 vs. 13 and partial remission (PR): 45 vs.15). BEAM-regimen was given in the standard infusion instructions and doses over 6 days, while HDM $\left(200 \mathrm{mg} / \mathrm{m}^{2}\right)$ was given in a single day infusion. All patients received prophylaxis against microbial, fungal and viral infections; GCSF was routinely administered at the dose of $5 \mathrm{mcg} / \mathrm{kg}$ at +1 day (BEAMgroup) and at +5 day (HDM-group). Kaplan-Meier method was used to calculate overall survival (OS) and progression free survival (PFS).

Results: The engraftment was successful; the median day for neutrophils $>500 / \mathrm{mm} 3$ was +11 for both groups whilst for platelets $>20000 / \mathrm{mm} 3$ a faster recovery was noticed for HDM-group: +13 vs. +22 days $(\mathrm{p}<0.001)$. We had previously reported (Leuk Lymphoma.2008;49:769-78) platelet recovery at 15 days with BEAM, now 22 days because of logistic reasons and extra platelet transfusions for inpatient perma-catheter removal. After a median follow-up of approximately 2 years, 64 patients are alive (59 disease-free) in BEAM-group while 24 (19 disease-free) in the HDM-group. For the whole cohort of patients the $5 y$ y-OS was $68 \%$ vs. $80 \%$ and 5ys-PFS was $52 \%$ vs. $65 \%$ for the BEAM and HDM-group respectively. The 100-day transplant related mortality was similar and acceptable for both groups: 2/84 (2.3\%) in BEAM-group vs.1/28 (3.5\%) in the HDM-group (MERS-CoV outbreak). From 1998-2015, 13/334 (3.8\%) patients who received BEAM at KFSHRC died of TRM.

Conclusions: This study, though retrospective, demonstrates that for RR-HL patients, the conditioning regimen consisting of single-dose HDM may have similar efficacy as compared to the BEAM-regimen. Importantly, difference in the duration of chemotherapy administration (6 days for BEAM vs. 1 day for HDM), results in a shorter hospitalization period, and the shorter period of GCSF administration post-ASCT, may contribute to a better-cost effectiveness for the HDM-regimen. However, additional
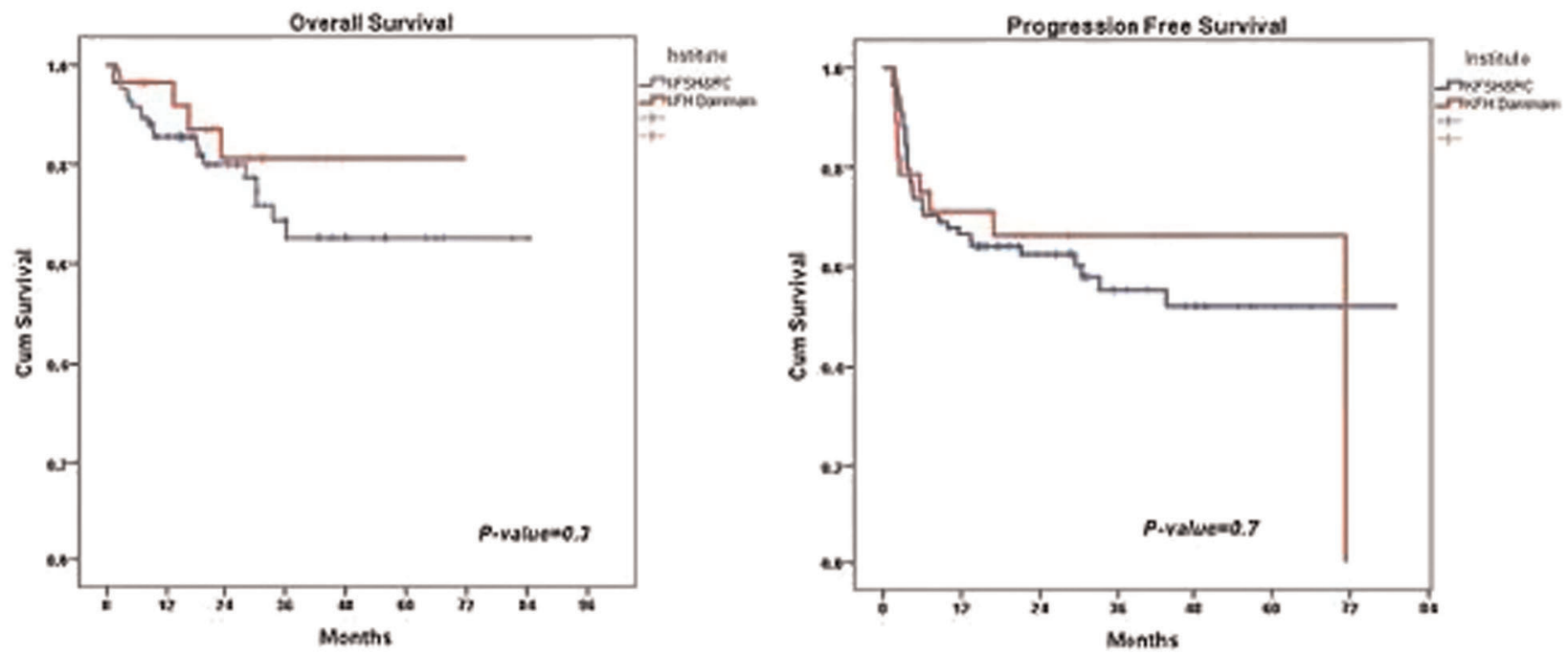

[P154 Figure] [Graph 1] 
studies with larger cohort and longer follow-up are needed. Long term disease outcome, toxicity and meticulous comparative cost analysis are needed.

Conflict of interest: Nothing to disclose

\section{P155}

BEAM vs FEAM: a retrospective comparative study of conditioning regimens in lymphoma patients undergoing autologous stem cell transplant

Francesco Marchesi ${ }^{1}$, Saveria Capria ${ }^{2}$, Diana Giannerelli ${ }^{3}$, Silvia Maria Trisolini ${ }^{2}$, Michela Ansuinelli, Maria Denise Caputo ${ }^{2}$, Alessandra Serrao ${ }^{2}$, Svitlana Gumenyuk ${ }^{1}$, Daniela Renzi ${ }^{1}$, Livio Pupo $^{4}$, Francesca Palombi ${ }^{1}$, Ida Provenzano ${ }^{4}$, Alice Di Rocco ${ }^{2}$, Francesco Pisani ${ }^{1}$, Atelda Romano ${ }^{1}$, Antonio Spadea ${ }^{1}$, Elena Papa ${ }^{1}$, Marco Canfora ${ }^{5}$, Maria Cantonetti ${ }^{4}$, Andrea Mengarelli ${ }^{1}$

${ }^{1}$ Regina Elena National Cancer Institute, Hematology and Stem Cell Transplant Unit, Rome, Italy; ${ }^{2}$ University La Sapienza, Department of Cellular Biotechnologies and Hematology, Rome, Italy; ${ }^{3}$ Regina Elena National Cancer Institute, Biostatistical Unit, Rome, Italy; ${ }^{4}$ Tor

Vergata University, Hematology, Rome, Italy; ${ }^{5}$ Regina Elena National Cancer Institute, Scientific Direction, Rome, Italy

Background: BEAM is the standard conditioning for lymphoma patients undergoing ASCT. However, in Italy, BCNU is often substituted by Fotemustine (FEAM) due to difficult supplying. Comparison studies are lacking.

Methods: From Jan-2007 to Jan-2017, 362 consecutive lymphoma patients undergoing ASCT in two JACIE accredited Institutions were reviewed. Selection for BEAM (200) or FEAM (162) was based on BCNU availability. Median age was 48 years (13-69). ASCT was performed for aggressive NHL in 225 cases (62\%), indolent NHL in 37 (10\%) and HL in 100 (28\%), and after first line in 72 cases (20\%), after first salvage treatment in $214(59 \%)$ and in more advanced phase in the remaining $76(21 \%)$. Pre-ASCT response status was CR in 64\% (first CR $11 \%$, $\geq$ second CR $53 \%$ ), PR in $32 \%$ and refractory disease in $4 \%$ of cases. The two groups of patients were well-balanced for all analyzed parameters, expect for median follow-up (BEAM: 72 months, FEAM: 25 months; $\mathrm{P}<0.0001$ ).

Results: After FEAM, a significant higher incidence of grade 3-4 oral mucositis (52\% vs $30 \% ; \mathrm{P}<0.0001)$ and documented infections ( $46 \%$ vs $36 \% ; \mathrm{P}=0.05$ ) were observed. Non-relapse mortality and pulmonary toxicities were similar in the two groups. Response status at day-100 post-ASCT was significantly better for BEAM group (CR: $87 \%$ vs $75 \% ; \mathrm{P}=0.005)$. This difference was evident both in DLBCL (CR: $83 \%$ vs $64 \% ; \mathrm{P}=0.008$ ) and HL patients (CR: $87 \%$ vs $76 \%$; $\mathrm{P}=0.13$ ). At multivariate analysis, conditioning was the only independent factor able to affect post-transplant CR (BEAM vs FEAM ORs: 2.26, 95\% CI: 1.28-3.99; $\mathrm{P}=0.005)$, together with pre-transplant disease status (CR vs no CR ORs: 4.72, 95\%CI: 2.66-8.35; P< 0.0001). PFS and OS were not significantly different (2yPFS BEAM: $76 \%$ vs FEAM: $74 \%$; $=0.211 ; 2 \mathrm{yOS}$ BEAM: $87 \%$ vs FEAM: $84 \%$; $\mathrm{P}=0.578$ ).

Conclusions: Despite limitations due to the nonrandomized nature of this study, BEAM conditioning regimen seems better tolerated. Although PFS and OS are similar at this time, considering the higher proportion of CR post-ASCT and the longer median follow-up, BEAM should remain the regimen of choice in these patients. A longer follow-up of FEAM patients will allow to evaluate whether the survival curves will differ in the next future.

Conflict of interest: None of the authors has any conflicts of interest to report.

\section{P156}

BeEAC conditioning regimen for autologous hematopoietic stem-cell transplantation in malignant lymphoma patients

Yulia Dubinina, Vladislav Sarzhevskiy, Anastasiya Samoylova, Nikita Mochkin, Vladimir Melnichenko, Dina Kolesnikova, Vladimir Bogatyrev, Elena Smirnova, Diana Ivanova, Anna Bannikova

State Budgetary Healthcare Institution, National Medical Surgical Center N.A. N.I. Pirogov, Ministry of Health of Russia, Moscow, Russian Federation

Background: Nowadays there are no randomized trials for comparison the effectiveness and tolerability of different conditioning regimens in malignant lymphoma (ML) patients.

Aim of the study is to assess the safety and toxicity profile, overall (OS) and progressive-free survival (PFS) in patients who received BeEAC, the novel conditioning regimen for autoHSCT in primary-refractory and relapsed ML patients (ClinicalTrials.gov NCT03315520).

Methods: Between January 2016 and August 201793 patients received autoHSCT: Hodgkin lymphoma (HL) - 60, non-Hodgkin lymphoma - 33 patients; 42 women and 51 men. Median follow-up was 12 months. The median age was 33 years (18-65). Before autoHSCT 43 patients $(45,3 \%)$ had CR, $47(50,5 \%)$ - PR, 3 patients $(3,2 \%)$ were in a stable disease (SD). Median number of chemotherapy lines was 3 (1-9). The conditioning regimen BeEAC: increasing doses of bendamustine $160 \mathrm{mg} / \mathrm{m} * 2,180 \mathrm{mg} /$ $\mathrm{m} * 2,200 \mathrm{mg} / \mathrm{m} * 2 \mathrm{D}-6, \mathrm{D}-5$, combined with fixed-dose cytarabine $400 \mathrm{mg} / \mathrm{M} 2 \mathrm{D}-4$ - D-1; etoposide $400 \mathrm{mg} / \mathrm{M} 2 \mathrm{D}-4$ - D-1; cyclophosphamide $140 \mathrm{mg} / \mathrm{M} 2$ totally, divided in 4 days (D-4 - D-1). In a phase I study patients were divided into 3 cohorts with 3 patients in each, where bendamustine dose increased from 160 to $200 \mathrm{mg} / \mathrm{m} * 2$ D-6D-5. 
Results: A Phase I study showed no dose limited toxicity was described. The following patients (84) received bendamustine $200 \mathrm{mg} / \mathrm{m} * 2$.

Hematological toxicity is presented in table 1 .

Cardiotoxic effects were detected in 7 patients $(7.5 \%)$ and were following: hydropericardium, dry pericarditis, postcytostatic cardiomyopathy with acute biventricular heart failure and paroxysmal atrial fibrillation. 41 (44\%) patients had no signs of oral mucositis (OM, WHO classification), $22(23.65 \%)$ had grade $1 \mathrm{OM}, 24(25.8 \%)$ - grade 2, 5 $(5.37 \%)$ - grade 3 and 1 (1.1\%) patient had grade 4. Intestinal mucositis (NCI-CTC) was determined in 35 patients: grade $1-16(17.2 \%)$ patients, grade $2-6$ (6.4\%), grade $3-13(14 \%)$.

$22(23.65 \%)$ patients developed liver toxicity (CTCAE $4.03)$, but in majority of cases it was grade 1 .

$5(5.37 \%)$ patients developed renal toxicity (CTCAE 4.03), 1 patient grade 2.

The CR rate increased from $45.3 \%$ to $59 \%$ during 100 days after HSCT. 18 (20\%) patients remained in PR, 1 $(1.05 \%)$ - SD.

37 patients relapsed or progressed after HSCT. The frequency of relapse depended on the number of chemotherapy lines (CL). 3 (8.1\%) patients had $1 \mathrm{CL}, 11$ (29.7\%) - 2 lines, $11(29.7 \%)-3$ lines and 12 (32.4\%)

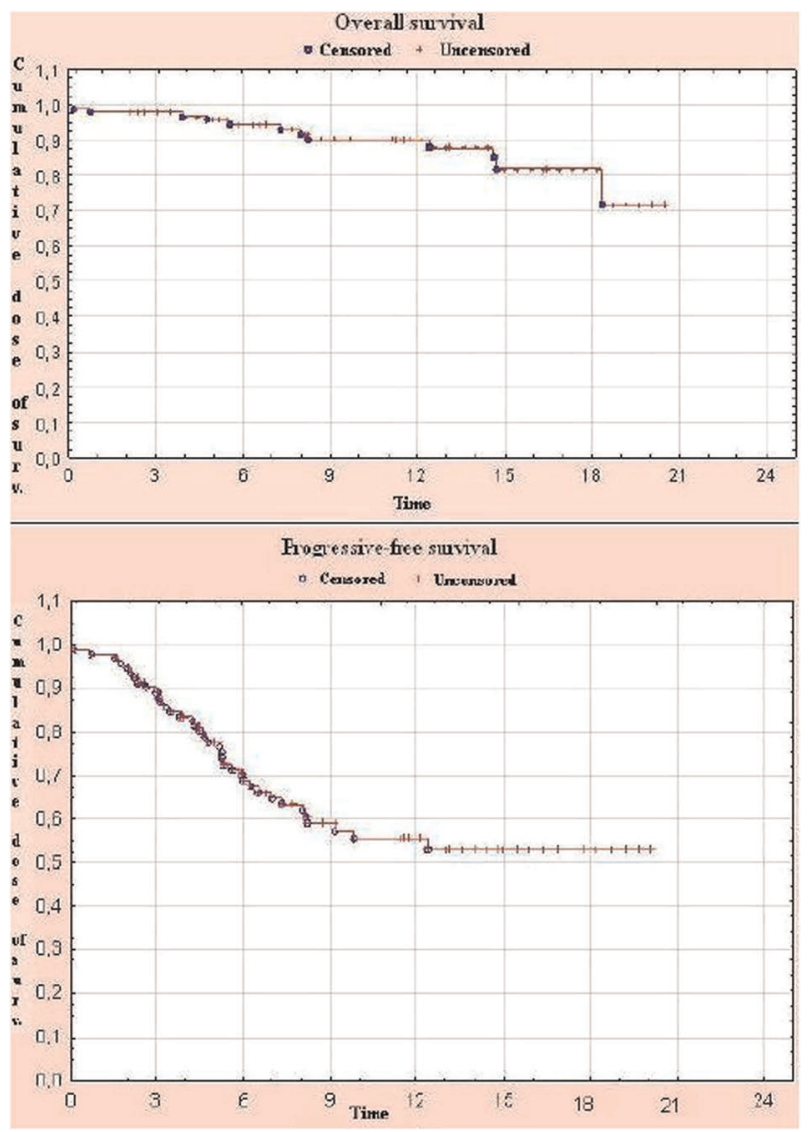

[P156 Figure] [Progressive-free and overall survival] patients had $4 \mathrm{CL}$ and even more. 2 patients $(2,1 \%)$ with NHL B-cell lymphoma relapsed in CNS.

Overall mortality was $12,9 \%$ (12 patients). 4 of them died from progressive disease, 3 - from cardiotoxicity, 1 - from sepsis, 2 - from bleeding, 1 - from pulmonary edema during radiation therapy and 1 from postoperative complications not-related with ML. Between them 2 patients $(2,1 \%)$ died in the early post-transplant period (before $\mathrm{D}+30$ ): 1 from sepsis and 1 from acute cardiotoxicity.

Median PFS and OS at 1 year were $54 \%$ and $86 \%$, respectively

Conclusions: Data analysis showed that BeEAC is a safety conditioning regimen. Further investigation needs for assessment of long-term survival.

Clinical Trial Registry: ClinicalTrials.gov NCT03315520

Conflict of interest: No disclosure

P157

Bendamustine as an alternative to $\mathrm{BCNU}$ as conditioning regimen for autologous stem cell transplantation in patients with aggressive lymphoma: Results phase 2 study from GELTAMO

Alba María Redondo ${ }^{1}$, D Valcárcel ${ }^{2}$, A.P González ${ }^{3}$, M Suárez-

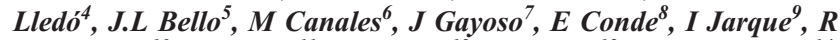
Del Campo ${ }^{10}$, R Arranz ${ }^{11}$, M.J Terol ${ }^{12}$, J.J Rifón ${ }^{13}$, M.J Rodríguez ${ }^{14}$, M.J Ramirez ${ }^{15}$, N Castro ${ }^{16}$, A Sánchez ${ }^{17}$, J López-Jiménez ${ }^{18}$, J Briones $^{19}$, A López $^{20}$, L Palomera ${ }^{21}$, M Baile ${ }^{1}$, Dolores Caballero ${ }^{1}$, Alejandro Martín ${ }^{1}$

${ }^{1}$ Hospital Universitario de Salamanca/IBSAL, Hematology, Salamanca, Spain; ${ }^{2}$ Hospital Vall d'Hebron, Hematology, Barcelona, Spain; ${ }^{3}$ Hospital Central de Asturias, Hematology, Oviedo, Spain; ${ }^{4}$ Hospital Clinic, Hematology, Barcelona, Spain; ${ }^{5}$ Complejo Hospitalario Universitario de Santiago de Compostela, Hematology, Santiago de Compostela, Spain; ${ }^{6}$ Hospital Universitario La Paz, Hematology, Madrid, Spain; ${ }^{7}$ Hospital General Universitario Gregorio Maranon, Hematology, Madrid, Spain; ${ }^{8}$ Hospital Universitario Marqués de Valdecilla, Hematology, Santander, Spain; ${ }^{9}$ Hospital Universitario la Fe, Hematology, Valencia, Spain;

${ }^{10}$ Hospital Son LLátzer, Hematology, Palma, Spain; ${ }^{11}$ Hospital la Princesa, Hematology, Madrid, Spain; ${ }^{12}$ Hospital Universitario de Valencia, Hematology, Valencia, Spain; ${ }^{13}$ Clínica Universidad de Navarra, Hematology, Pamplona, Spain; ${ }^{14}$ Hospital Universitario de Canarias, Hematology, San Cristóbal de La Laguna, Spain;

${ }^{15}$ Hospital de Jeréz, Hematology, Jerez de la Frontera, Spain;

${ }^{16}$ Hospital 12 de Octubre, Hematology, Madrid, Spain; ${ }^{17}$ Hospital Virgen de la Arreixaca, Hematology, Murcia, Spain; ${ }^{18}$ Hospital Ramón y Cajal, Hematology, Madrid, Spain; ${ }^{19}$ Hospital Santa Creu I Sant Pau, Hematology, Barcelona, Spain; ${ }^{20}$ Hospital Arnau de Villanoba, Hematology, Valencia, Spain; ${ }^{21}$ Hospital Clínico de Zaragoza, Hematology, Zaragoza, Spain

Background: BEAM is the most employed conditioning regimen for lymphoma patients in Europe. However, BCNU is hardly available and it has been related to 
pulmonary complications. We conducted a phase 2 trial to evaluate the safety and efficacy of bendamustine instead of $\mathrm{BCNU}$ in the BEAM regimen as conditioning for autologous stem-cell transplantation (ASCT) in patients with aggressive lymphomas.

Methods: Inclusion criteria were: histologic diagnosis of i) relapsed or refractory diffuse large B-cell lymphoma (DLBCL) or grade 3B follicular lymphoma (FL) in partial response (PR) or complete remission (CR) after salvage therapy, or ii) transformed DLBCL or peripheral T-cell lymphoma (PTCL) in first or subsequent PR or CR. Conditioning regimen consisted of bendamustine (200 $\mathrm{mg} / \mathrm{m}^{2}$, days -7 and -6$)$, etoposide $\left(200 \mathrm{mg} / \mathrm{m}^{2}\right.$, days -5 to $-2)$, cytarabine (400 $\mathrm{mg} / \mathrm{m}^{2}$, days -5 to -2$)$, and melphalan $\left(140 \mathrm{mg} / \mathrm{m}^{2}\right.$, day -1$)$ (BendaEAM regimen). Primary endpoint was progression-free survival (PFS) at 3 years. Secondary endpoints were toxicity, response to transplant, and overall survival (OS).

Results: Sixty patients (median age 55 [28-71] years, $50 \%$ male) from 22 Spanish hospitals were included from May 2011 to November 2012. Histologies were as follows: 40 DLBCL, 3 grade 3B FL, 13 transformed DLBCL and 7 PTCL. $82 \%$ of patients had received two or more lines of treatment prior to ASCT. Thirty-seven patients $(62 \%)$ were in metabolic CR (assessed by PET/CT) at the time of transplant and 23 (38\%) in PR.

All patients (except one who died early) engrafted after a median of 11 (9-72) and 14 (4-53) days, respectively, to achieve $>0.5 \times 10^{9} / 1$ neutrophils and $>20 \times 10^{9} / 1$ platelets. A total of 39 serious adverse events were reported before day +100 , including 14 infectious episodes, 2 of them resulting in respiratory failure and death of the patient $(3.3 \%$ of transplant-related mortality), and 5 episodes of renal failure after bendamustine administration, reversible in all cases. Non-relapse mortality after day +100 was $3.3 \%$ (1 patient from WernickeÂ's encephalopathy and 1 from infectious complications).

Regarding response to transplant, 45 patients $(75 \%)$ achieved CR and 6 (10\%) PR. With a median follow-up of 67 months (40-77), 24 patients had relapsed disease, 3 had secondary neoplasms (2 myelodysplastic syndrome and 1 cholangiocarcinoma) and 20 patients died. Estimated 5-y PFS and OS in the global series were $51 \%$ and $65 \%$, respectively. Patients with PET + disease at study entry had significantly worse PFS (26 vs $67 \%$ at 5 years, $p=0.001$ ) and OS ( 56 vs $75 \%$ at 5 years, $\mathrm{p}=0.036$ ) than patients who underwent the ASCT in metabolic CR, and this was the only prognostic factor affecting both PFS (RR 0.27 [0.12$0.56]$ ) and OS (RR 0.40 [0.17-0.97]) in the multivariate analysis.

Conclusions: BendaEAM conditioning is a safe, feasible and active regimen in patients with aggressive lymphomas. Infectious and renal toxicities should be carefully monitored. Our long-term results indicate that efficacy is similar to that previously reported with other regimens most commonly used like BEAM. PET status at the time of transplant is the key prognostic factor for survival.

Clinical Trial Registry: ClinicalTrials.gov Identifier: NCT01296256.

Conflict of interest: No conflicts of interest related to the present abstract in the last 12 months.

\section{P158}

Busulfan and melphalan versus high-dose melphalan as a conditioning regimen for autologous stem cell transplantation in patients with multiple myeloma

Sung-Hoon Jung ${ }^{1}$, Je-Jung Lee ${ }^{1}$, Jin Seok Kim², Chang-Ki Min ${ }^{3}$, Kihyun Kim ${ }^{4}$, Yunsuk Choi ${ }^{5}$, Hyeon-Seok Eom ${ }^{6}$, Young Don Joo ${ }^{7}$, Sung-Hyun Kim ${ }^{8}$, Jae-Yong Kwak ${ }^{9}$, Hye Jin Kang ${ }^{10}$, Jae Hoon $L_{e e^{11}}$, Ho Sup Lee ${ }^{12}$, Yeung-Chul Mun ${ }^{13}$, Joon Ho Moon ${ }^{14}$, Sang Kyun Sohn ${ }^{14}$, Seong Kyu Park ${ }^{15}$, Yong Park ${ }^{16}$, Ho-Jin Shin ${ }^{17}$, SungSoo Yoon $^{18}$

${ }^{1}$ Chonnam National University Hwasun Hospital, Hwasun, Korea, Republic of; ${ }^{2}$ Severance Hospital, Yonsei University College of Medicine, Seoul, Korea, Republic of; ${ }^{3}$ Seoul St. Mary's Hospital, Catholic University of Korea, Seoul, Korea, Republic of: ${ }^{4}$ Samsung Medical Center, Seoul, Korea, Republic of; ${ }^{5}$ Ulsan University Hospital, Ulsan, Korea, Republic of; ${ }^{6}$ National Cancer Center, Goyang-si, Korea, Republic of; ${ }^{7}$ Inje University Busan Paik Hospital, Busan, Korea, Republic of; ${ }^{8}$ Dong-A Medical Center, Busan, Korea, Republic of; ${ }^{9}$ Chonbuk National University Medical School, Jeonju, Korea, Republic of; ${ }^{10}$ Korea Cancer Center Hospital, Korea Institute of Radiological and Medical Sciences, Seoul, Korea, Republic of;

${ }^{11}$ Gachon University Gil Hospital, Incheon, Korea, Republic of;

${ }^{12}$ Kosin University Gospel Hospital, Busan, Korea, Republic of;

${ }^{13}$ Ewha Woman's University School of Medicine, Seoul, Korea, Republic of; ${ }^{14}$ Kyungpook National University Hospital, Daegu, Korea, Republic of; ${ }^{15}$ Soonchunhyang University Bucheon Hospital, Bucheon, Korea, Republic of; ${ }^{16}$ Korea University College of Medicine, Seoul, Korea, Republic of; ${ }^{17}$ Pusan National University Hospital, Busan, Korea, Republic of; ${ }^{18}$ Seoul National University Hospital, Seoul, Korea, Republic of

Background: Recent studies have suggested that intravenous busulfan and melphalan (BUMEL) might be an effective and tolerable conditioning regimen for autologous stem cell transplantation (ASCT) in patients with multiple myeloma (MM). This study compared the efficacy and toxicity of BUMEL and high-dose melphalan (HDMEL) as conditioning regimens in transplant- eligible patients with MM.

Methods: We retrospectively analyzed the records of 270 patients with newly diagnosed MM who received BUMEL or HDMEL as conditioning regimens followed by early ASCT (less than 12 months from the initial diagnosis). To reduce selection bias and its effect on the treatment results, we performed a 1:1 propensity score-matched analysis according to age, sex, International Staging System disease 
stage, lactate dehydrogenase level, and inclusion of cyclophosphamide in the induction regimen.

Results: Seventy-six patients received BUMEL, which consisted of intravenous busulfan administered at $3.2 \mathrm{mg} / \mathrm{kg}$ once a day on days -6 to -4 followed by melphalan administered at $70 \mathrm{mg} / \mathrm{m}^{2}$ on days -3 and -2 . The same number of patients received HDMEL consisting of melphalan at $100 \mathrm{mg} / \mathrm{m}^{2}$ on days -3 and -2 . All patients were treated with thalidomide-based regimens as the induction therapy. No statistically significant difference in the complete response (CR) and overall response rate (ORR) after ASCT was observed between BUMEL and HDMEL (CR $38.2 \%$ vs. $50.0 \%, P=0.191$; ORR $92.1 \%$ vs. 94.7\%, $P=0.745$ ). After a median follow-up of 25.2 months in the BU-MEL group and 60.8 months in the HDMEL group, the progression-free survival was 34.3 (20.9-47.8) months and 25.1 (12.3-38.0) months, respectively $(P=0.729)$. With respect to non-hematologic toxicities, infection was more frequently reported in the BUMEL group $(P<0.001)$, but no statistically significant differences in the incidence of grade 3 infections was observed (BUMEL $22.4 \%$ vs. HDMEL 15.8\%, $\mathrm{P}=0.459$ ). Venous-occlusive disease occurred in three patients who received BUMEL and recovered with supportive therapy.

Conclusions: In conclusion, BUMEL is an effective alternative conditioning regimen with acceptable toxicity for early ASCT in patients who were treated with thalidomide-based induction therapy.

Conflict of interest: The authors declare that they have no conflict of interest

\section{P159}

Abstract previously published

\section{P160}

\section{Busulfan PK-guided dose-adjustment in adult allogeneic stem cell transplantation - single-center experience}

Claudia Langebrake ${ }^{1,2}$, Christine Wolschke ${ }^{1}$, Maximilian Christopeit $^{1}$, Alexander Müller ${ }^{3}$, Nicolaus M. Kröger ${ }^{1}$

${ }^{1}$ University Medical Center Hamburg-Eppendorf, Department of Stem Cell Transplantation, Hamburg, Germany; ${ }^{2}$ University Medical Center Hamburg Eppendorf, Hospital Pharmacy, Hamburg, Germany;

${ }^{3}$ University Medical Center Hamburg Eppendorf, Department of Legal Medicine, Hamburg, Germany

Background: It is well known, that there is a high interindividual variety between dosing and exposition of busulfan $(\mathrm{Bu})$ on the one hand and the correlation between exposition and clinical outcome on the other hand. Therapeutic drug monitoring (TDM) has been considered to optimise patient outcome. However, due to technical and logistical reasons, pharmacokinetics (PK) -guided doseadjustment has not been established in the majority of haematopoietic stem cell (HSCT) centres in Europe yet.

Methods: Single-centre retrospective analysis of adult patients undergoing allogeneic HSCT with initial $\mathrm{Bu}$ dosing of $3.2 \mathrm{mg} / \mathrm{kg} \mathrm{Bu}$ intravenously once daily (based on adjusted ideal body weight [AIBW] for obese patients). Busulfan concentration was measured with gas chromatography-mass spectrometry (GC-MS) and AUC was calculated using Bayesian curve fitting. If the calculated AUC was outside the pre-defined target AUC, dose adjustment was undertaken for the subsequent doses.

Results: In a 14 months period a total of 95 patients (male/female: 44/51) received $\mathrm{Bu}$ TDM with varying conditioning regimens, e.g. Bu/fludarabine, Bu/cyclophosphamide, thiotepa/Bu/ \pm fludarabine with either antithymocyte globuline (ATG) $(\mathrm{n}=82)$ or post-transplantation cyclophosphamide $(\mathrm{n}=13)$.

Median age was 58.4 years (range 19.7-73.1), median body mass index was $25.1 \mathrm{~kg} / \mathrm{m}^{2}$ (range: 15.6-36). AIBW for initial dose calculation was used in 33 (34.5\%) patients. Mean AUC at the first day of quantification was $16.4 \mathrm{~h} * \mathrm{mg} /$ 1 ( \pm 4.0, range: $9.7-31.9 \mathrm{~h} * \mathrm{mg} / \mathrm{l})$. The calculated busulfan AUC before dose adjustment was in median $20.5 \%$ (mean $18.1 \pm 20.5 \%$ ) below the target AUC. This resulted in a dose adjustment in $71(74.7 \%)$ patients with an increase of busulfan dose for the subsequent days in $61(64.9 \%)$ and a dose decrease in $10(10.6 \%)$ patients, respectively. After dose adjustment, the median deviation from the received AUC to the target AUC was $-2.4 \%$ (mean $-5.1 \% \pm 10.4 \%$ ), with $73(76.8 \%)$ of patients being within in the predefined cumulative AUC target range $( \pm 10 \%)$.

Conclusions: Our data confirm the importance of patient individual dose adjustment of $\mathrm{Bu}$ during conditioning in adult allo SCT patients. The implementation of Bu-TDM within clinical routine care is feasible and results in a better target attainment of cumulative $\mathrm{Bu}$ exposition.

Conflict of interest: No conflicts of interest.

\section{P161}

Chemo-conditioning before allo-HSCT for children below two years of age with acute leukemia - an EBMT-PDWP study

Andre Willasch ${ }^{1}$, Christina Peters ${ }^{2}$, Petr Sedláček ${ }^{3}$, Jean-Hugues Dalle $^{4}$, Stelios Graphakos ${ }^{5}$, Akif Yesilipek ${ }^{6}$, Jacek Wachowiak ${ }^{7}$, Arjan Lankester ${ }^{8}$, Arcangelo Prete ${ }^{9}$, Amir Ali Hamidieh ${ }^{10}$, Marianne Ifversen $^{11}$, Jochen Buechner ${ }^{12}$, Gergely Kriván ${ }^{13}$, Rose-Marie Hamladji $^{14}$, Cristina Diaz de Heredia ${ }^{15}$, Elena Skorobogatova ${ }^{16}$, Gérard Michel $^{17}$, Franco Locatelli ${ }^{18}$, Alice Bertaina ${ }^{18}$, Paul Veys ${ }^{19}$, Sophie Dupont ${ }^{20}$, Reuven Or ${ }^{21}$, Tayfun Güngör ${ }^{22}$, Olga Aleinikova ${ }^{23}$, Sabina Sufliarska ${ }^{24}$, Mikael Sundin $^{25}$, Jelena Rascon ${ }^{26}$, Ain Kaare ${ }^{27}$, 
Damir Nemet ${ }^{28}$, Franca Fagiloi ${ }^{29}$, Thomas Klingebiel ${ }^{1}$, Jan Styczynski ${ }^{30}$, Marc Bierings ${ }^{31}$, Kálmán Nagy ${ }^{32}$, Manuel Abecasis ${ }^{33}$, Boris Afanasyev ${ }^{34}$, Marc Ansari ${ }^{35}$, Arnaud Dalissier ${ }^{36}$, Myriam Labopin $^{37}$, Eric Beohou ${ }^{36}$, Peter Bader ${ }^{1}$

${ }^{I}$ EBMT Paediatric Diseases Working Party and Division for Stem Cell Transplantation and Immunology, Department for Children and Adolescents, University Hospital, Goethe University, Frankfurt am Main, Germany; ${ }^{2}$ St Anna Children's Hospital, Vienna, Austria; ${ }^{3}$ University Hospital Motol, Department of Pediatric Hematology and Oncology, Prague, Czech Republic; ${ }^{4}$ Hôpital Robert Debré and ParisDiderot University, Department of Pediatric Hemato-Immunology,

Paris, France; ${ }^{5}$ Stem Cell Transplant Unit, Aghia Sophia Children's Hospital, Thivon and Papadiamantopoulou, Athens, Greece; ${ }^{6}$ School of Medicine, Bahcesehir University, Department of Pediatric Hematology-Oncology, Istanbul, Turkey; ${ }^{7}$ University of Medical Sciences, Department and Clinic of Pediatric Oncology, Hematology and Transplantology, Poznan, Poland; ${ }^{8}$ Leiden University Medical Center, Department of Pediatrics, Leiden, Netherlands; ${ }^{9}$ HematologyOncology Unit 'Lalla Seràgnoli', Department of Pediatrics, University of Bologna, Bologna, Italy; ${ }^{10}$ Hematology-Oncology and Stem Cell Transplantation Research Center, Tehran University of Medical Sciences, Tehran, Iran, Islamic Republic of; ${ }^{11}$ Copenhagen University Hospital, Rigshospitalet, Department of Pediatrics, Copenhagen, Denmark; ${ }^{12}$ Oslo University Hospital Rikshospitalet, Department of Pediatric Medicine, Oslo, Norway; ${ }^{13}$ United St. Istvan and St. László Hospital, Department of Paediatric Haematology and Stem Cell Transplantation, Budapest, Hungary; ${ }^{14}$ Centre Pierre et Marie Curie, Service Hématologie Greffe de Moelle, Alger, Algeria; ${ }^{15}$ Servicio de Hematologia y Oncologia Pediátricas, Hospital Universitario Vall d'Hebron, Barcelona, Spain; ${ }^{16}$ Russian Children's Hospital, BMT Department, Moscow, Russian Federation; ${ }^{17}$ Timone Enfants Hospital, AP-HM and Aix-Marseille University, Department of Pediatric Hematology and Oncology and Research Unit EA 3279, Marseille, France; ${ }^{18}$ IRCCS Ospedale Pediatrico Bambino Gesù, Università di Pavia, Dipartimento di Onco-Ematologia Pediatrica e Medicina Trasfusionale, Rome, Italy; ${ }^{19}$ Great Ormond Street Hospital for Children NHS Foundation Trust, London, United Kingdom;

${ }^{20}$ Cliniques Universitaires Saint-Luc, Department of Pediatric Hematology and Oncology, Brussels, Belgium; ${ }^{21}$ Hadassah-Hebrew University Medical Center, Department of Bone Marrow

Transplantation, Jerusalem, Israel; ${ }^{22}$ Division of Stem Cell Transplantation, Children's Research Center (CRC), University Children's Hospital, Zurich, Switzerland; ${ }^{23}$ Republic Clinical Research Centre for Pediatric Oncology and Hematology, Minsk, Belarus; ${ }^{24}$ BMT Unit, Department of Pediatric Hematology and Oncology, Comenius University Medical School, Limbová, Bratislava, Slovakia; ${ }^{25}$ Pediatric Blood Disorders, Immunodeficiency and Stem Cell Transplantation, Astrid Lindgren Children's Hospital, Karolinska University Hospital, Stockholm, Sweden; ${ }^{26}$ Center of Pediatric Oncology and Hematology, Bone Marrow Transplantation Unit, Children's Hospital, Affiliate of Vilnius University Hospital Santariskiu Klinikos, Vilnius, Lithuania; ${ }^{27}$ Tartu University Hospital, Tartu, Estonia; ${ }^{28}$ Internal Clinic, University Hospital Centre, Department of Haematology, Zagreb, Croatia; ${ }^{29}$ Paediatric Onco-Haematology, City of Science and Health of Turin, Regina Margherita Children's Hospital, Turino, Italy; ${ }^{30}$ Nicolaus Copernicus University Torun, Department of Pediatric Hematology and Oncology, Collegium Medicum, Bydgoszcz, Poland; ${ }^{31}$ BMT-Unit, University Medical Centre Utrecht Pediatrics, Utrecht, Netherlands; ${ }^{32}$ Child Welfare Center, Borsod County Teaching Hospital, Department of Hematology, Miskolc, Hungary; ${ }^{33}$ Bone Marrow Transplant Program, Instituto Portugues Oncologia, Lisbon, Portugal; ${ }^{34}$ Raisa Gorbacheva Memorial Research Institute for Pediatric Oncology, Haematology and Transplantation, Saint Petersburg State Medical I.P. Pavlov University, Saint Petersburg, Russian Federation; ${ }^{35}$ Geneva
University Hospital, Geneva University, Department of Pediatrics, Onco-Hematology Unit, Geneva, Switzerland; ${ }^{36}$ EBMT Paediatric Diseases Working Party, Paris, France; ${ }^{37}$ EBMT Paris Study Office, Paris, France

Background: Paediatric patients (pts) below two years (y) of age with acute leukemia commonly receive chemotherapy for myeloablative conditioning (MAC) before allogeneic haematopoietic stem cell transplantation (allo-HSCT). It is unknown, which combination of chemotherapeutics offers the best outcome.

Methods: To investigate the outcomes of different chemotherapy-based conditioning-regimens, we performed a retrospective Paediatric Diseases Working Party (PDWP)EBMT-registry based study. Children below two y of age after MAC for first allo-HSCT of bone marrow (BM), peripheral blood SC (PBSC) or cord blood (CB) from matched siblings (MSD) or unrelated donors (UD) in first remission (CR1) between 2000 and 2014 were included. Propensity score weighting was used to consider the bias due to confounding variables, e.g. different size and composition of subgroups. Univariate analysis and multivariable Cox model were weighted on diagnosis, age at HSCT, y of HSCT, interval between diagnosis and HSCT, donor type and SC source. Cox model was clustered on EBMT centers.

\section{Results:}

In total 400 pts (AML: 218 (54\%), ALL: 182 (46\%)) were included. 215 pts (54\%) received BM, 128 pts (32\%) CB and 57 (14\%) PBSC from UD (254 (64\%)) or MSD (146 $(36 \%))$. Thirty-six different conditioning-regimens were used. Busulfan/Cyclophosphamide+/-Melphalan+/-ATG $(\mathrm{Bu} / \mathrm{Cy}+/-\mathrm{Mel}+/$-ATG) was the most frequently applied regimen $(255(64 \%))$. The remaining conditionings were summarized as "others" (145 (36\%)). Patients treated with these two conditioning groups had a median age of $1.2 \mathrm{y}$ (interquartile range (IR) 0.8-1.6 y) and $1.1 \mathrm{y}$ ((IR) 0.8-1.4 y) and a median follow-up of 3.8 and $2.4 \mathrm{y}$.

No significant differences between the two conditioning cohorts $\mathrm{Bu} / \mathrm{Cy}+/-\mathrm{Mel}+/$-ATG and "others" were detected for LFS (5-y-LFS, 61.6 vs. 64.7\%), relapse incidence (5-yRI, 27.0 vs. $24.7 \%$ ), OS (5-y-OS, 70.5 vs. $66.8 \%$ ), nonrelapse mortality (5-y-NRM, 11.3 vs. $10.6 \%$ ), aGvHD grade III-IV (day 100) 12.6 vs. $13.0 \%$, cGvHD (5-y) 16.2 vs. $16.9 \%$ and for GvHD-free/relapse-free survival (5-y-GRFS, 54.4 vs. $58.0 \%)$.

In multivariable Cox model, no significant differences between the two conditioning cohorts were found as well.

Conclusions: Allo-HSCT for children below two years of age with high relapse-risk acute leukemia offers an impressive chance of cure. The most frequently applied regimen $\mathrm{Bu} / \mathrm{Cy}+/$-Mel+/-ATG did not demonstrate superiority compared to other conditionings. This retrospective 
data is currently re-evaluated in a prospective, randomized, international trial (ALL SCTped 2012 FORUM).

Conflict of interest: The authors declare no relationships to disclose.

\section{P162}

CMV status predicts survival in refractory/relapsed myeloid patients after a clofarabine-based sequential regimen: a retrospective study on behalf of the SFGM-TC

\begin{abstract}
Amandine Le Bourgeois ${ }^{1}$, Myriam Labopin ${ }^{2}$, Felipe Suarez ${ }^{3}$, Regis Peffault De Latour ${ }^{4}$, Didier Blaise ${ }^{5}$, Sylvain Chantepie ${ }^{6}$, Stephanie Nguyen $^{7}$, Natacha Maillard ${ }^{8}$, Reza Tabrizi ${ }^{9}$, Ibrahim YakoubAgha $^{10}$, Anne Huynh ${ }^{11}$, Tony Marchand ${ }^{12}$, Karin Bilger ${ }^{13}$, Patrice Ceballos $^{14}$, Amandine Charbonnier ${ }^{15}$, Pascal Turlure ${ }^{16}$, MarieTherèse Rubio ${ }^{17}$, Mohamad Mohty ${ }^{2}$, Patrice Chevallier ${ }^{1}$

${ }^{1}$ Nantes Hospital, Hematology, Nantes, France; ${ }^{2}$ Saint Antoine Hospital, Hematology, Paris, France; ${ }^{3}$ Necker Hospital, Hematology, Paris, France; ${ }^{4}$ Saint Louis Hospital, Hematology, Paris, France; ${ }^{5}$ Institut Paoli Calmettes, Hematology, Marseille, France; ${ }^{6}$ Caen Hospital, Hematology, Caen, France; ${ }^{7}$ Salpétrière Hospital, Hematology, Paris, France; ${ }^{8}$ Poitiers Hospital, Hematology, Poitiers, France; ${ }^{9}$ Bordeaux Hospital, Hematology, Bordeaux, France; ${ }^{10}$ Lille

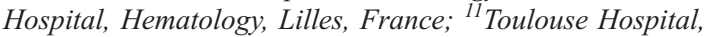
Hematology, Toulouse, France; ${ }^{12}$ Rennes Hospital, Hematology, Rennes, France; ${ }^{13}$ Strasbourg Hospital, Hematology, Strasbourg, France; ${ }^{14}$ Montpellier Hospital, Hematology, Montpellier, France; ${ }^{15}$ Amiens Hospital, Hematology, Amiens, France; ${ }^{16}$ Limoges Hospital, Hematology, Limoges, France; ${ }^{17}$ Nancy Hospital, Hematology, Nancy, France
\end{abstract}

Background: Recently, we have published the prospective results of a sequential regimen using a clofarabine (clo), cytosine arabinoside and reduced-intensity conditioning (RIC) regimen in 24 cases with primary refractory acute myeloid leukemia (AML) showing encouraging results with a very low non relapse mortality (Mohty, 2017). Here we report the outcomes after such sequential regimen in a larger cohort of patients.

Methods: This was a retrospective study including all patients reported within the SFGM-TC registry having received a clo-based sequential conditioning regimen before allotransplant for active myeloid disease. Data were obtained through PROMISE, an internet-based system shared by all European transplantation centers. All patients gave informed consent allowing to collect their personal data from this data base.

The sequential regimen consisted of clo $(30 \mathrm{mg} / \mathrm{m} 2 /$ day $)$ and cytosine arabinoside ( $1 \mathrm{~g} / \mathrm{m} 2 /$ day $)$ for 5 days, followed, after a 3-day rest, by RIC combining cyclophosphamide (60 $\mathrm{mg} / \mathrm{kg})$, iv busulfan $(3.2 \mathrm{mg} / \mathrm{kg} /$ day $)$ for 2 days and antithymocyte globulin $(2.5 \mathrm{mg} / \mathrm{kg} / \mathrm{day})$ for 2 days.

The primary objective of the study was to report the main outcomes at 1 year: overall survival (OS), disease free survival (DFS), relapse incidence (RI), NRM and GVHD
Relapse free survival (GRFS). Secondary objectives were to identify prognostic factors for patient's survival.

Results: Between January 2007 and December 2016, 131 patients (males $\mathrm{n}=75$, median age: 52.6 years, range: 18 71) met the inclusion criteria. There were 111 AML patients, including 9 secondary AML, and 20 cases with myelodysplastic or myeloproliferative syndrome. Status at transplant was as follows: primary refractory $n=81$, relapse 1 or $2 \mathrm{n}=46$, missing $\mathrm{n}=4$. The majority of patients received peripheral blood stem cell as source of graft $(n=$ 127 , bone marrow $n=4)$. All patients received a graft from a matched donor (sibling $n=64$, unrelated $n=67$ ). Donor (D)/recipient (R) CMV status was as follows: D-/R-: $\mathrm{n}=$ 55, $\mathrm{D}+/ \mathrm{R}-: \mathrm{n}=14, \mathrm{D}-/ \mathrm{R}+: 24, \mathrm{D}+/ \mathrm{R}+: 38$.

Engraftment was observed in 105/122 assessable cases $(86 \%)$ and patients achieving complete remission (CR) after transplant were $63 \%(n=72 / 114$ evaluable cases). With a median follow-up of 12 months (range: 2.7-74.3) for alive patients, 1-year OS, DFS, RI, NRM and GRFS were 39.2\% [30.2-48.2], 28.1\% [19.8-36.5], 41\% [32.1-49.8], 30.8\% [22.7-39.3], and 22.2\% [14.3-30], respectively.

In multivariate analysis, $\mathrm{D} / \mathrm{R}$ CMV negative status was associated with lower OS (HR: 1.74, 95\%CI: 1.10-2.76, p $=0.016)$, DFS (HR: 1.70; 95\%CI: 1.09-2.66, $\mathrm{p}=0.018$ ) and GRFS (HR: 1.76 ; 95\%CI: $1.15-2.71, \mathrm{p}=0.009$ ) and higher RI (HR: 2.48; 95\%CI: 1.37-4.50, $\mathrm{p}=0.002$ ). D/R CMV status was not associated with NRM.

Conclusions: Despite high CR achievement, this large cohort of patients confirmed the relatively poor outcome of patients with active myeloid disease at transplant and receiving a clofarabine-based sequential regimen. The favorable impact on survivals of positive D or R CMV status may be related to a potential graft-vs-leukemia effect of CMV reactivation (thanks to $\mathrm{NK}$ or $\mathrm{T}$ cells stimulation) after transplant. The validation of this hypothesis is on-going.

Conflict of interest: no conflict of interest

\section{P163}

Combined use of Treosulfan and epigenetic agent in highdose conditioning regimen in patients with very high-risk solid tumors

Igor Dolgopolov, George Mentkevich, Nataly Subbotina, Vasily Boyarshinov, Vidmante Daylidite

Institute for Pediatric Oncology \& Hematology, Pediatric BMT, Moscow, Russian Federation

Background: In attempt to improve the treatment results in patients with very high-risk Ewing's sarcoma (ES) and neuroblastoma (NB) we initiated a study of HDCT with autologous SCT including epigenetic therapy (in order to increase tumor cell chemosensitivity) and split conditioning 
regimen based on a near maximum of tolerated dose of Treosulfan (Treo).

Methods: Two patients with solid tumor with multiple bone involvement were included. One with stage 4 ES after 5 courses and one with stage $4 \mathrm{NB}$ after 7 courses of induction therapy. Both patients were over age 2 and in VGPR. Split conditioning regimen consisted of treosulfan $10000 \mathrm{mg} / \mathrm{m}^{2} / \mathrm{d}$ on $\mathrm{d}-11,-10$, and $-4,-3\left(40000 \mathrm{mg} / \mathrm{m}^{2} /\right.$ course $)$, melphalan 70 $\mathrm{mg} / \mathrm{m}^{2} / \mathrm{d}$, on d-3,-2 (140 mg/ $/ \mathrm{m}^{2} /$ course $)$ and 5-azacitidine 75 $\mathrm{mg} / \mathrm{m}^{2} / \mathrm{d}$, given on $\mathrm{d}-14$ through -12 and d -9 through $-3(8$ injections, $600 \mathrm{mg} / \mathrm{m}^{2} /$ course). On $\mathrm{d} 0$ patients were transplanted with autologous PBSC with 5.2 and 5.0x $10^{6} \mathrm{CD} 34+$ cells/kg. G-CSF was given from $\mathrm{d} 0$.

Results: Regimen related toxicity was moderate and manageable. Both pts developed grade 2-3 GI (stomatitis with painful erythema requiring parenteral nutrition and narcotic drugs, diarrhea with 4-6 stool/day, moderate dyspepsia and/or dysphagia) and febrile neutropenia requiring IV antibiotics. In both cases transient liver toxicity of grade 2-3 with AST/ALT elevation and hemorrhage of grade 2 were observed. Patients received 1 and 3 RBC, and 3 and 14 PLT transfusions, respectively. Both patients engrafted with $\mathrm{WBC}>1.0 \times 10^{9} / 1$ on $\mathrm{d}+10$ and +16 and PLT $>20 \times 10^{9} / 1$ on $\mathrm{d}+14$ and +30 , respectively. Patient with NB is alive without disease 10 mos. after HDCT. Patient with ES relapsed 19 mos. after HDCT and stated on a research protocol.

Conclusions: We can speculate that Treo based split regimen with addition of 5-azacitidine is feasible with acceptable toxicity profile. This regimen seems to be reasonable for solid tumors with low cell growth fraction. More pts and longer follow-up period are needed to make further conclusions.

Conflict of interest: No conflict of interest.

\section{P164}

\section{Comparable Outcomes after Allogeneic Stem-cell Transplantation with Myeloablative BuFlu or BuCy Conditioning Regimens in Acute Myeloid Leukemia - Analysis of the Polish Adult Leukemia Group}

\section{Anna Lojko-Dankowska ${ }^{1}$, Barbara Nasilowska-Adamska ${ }^{2}$, Patrycja Mensah-Glanowska ${ }^{3}$, Agnieszka Piekarska ${ }^{4}$, Magdalena Matuszak ${ }^{1}$, Beata Piatkowska-Jakubas ${ }^{3}$, Kazimierz Halaburda ${ }^{2}$, Tomasz Wrobel $^{5}$, Mieczyslaw Komarnicki ${ }^{1}$, Anna $C_{z y z}{ }^{5}$ \\ ${ }^{1}$ University of Medical Sciences, Poznan, Poland; ${ }^{2}$ Institute of Hematology and Transfusion Medicine, Warsaw, Poland; \\ ${ }^{3}$ Jagiellonian University Medical Collage, Krakow, Poland; ${ }^{4}$ Medical University of Gdansk, Gdansk, Poland; ${ }^{5}$ Wroclaw Medical University, Wroclaw, Poland}

Background: Busulfan (Bu)-based myeloablative conditioning regimen is widely used for patients with acute myeloid leukemia (AML) treated with allogeneic hematopoietic stem cell transplantation (alloHCT). Busulfan is most frequently used in combination with either cyclophosphamide (Cy) or fludarabine (Flu). The aim of this multicenter retrospective analysis was to compare the outcome of alloHCT in adults patients with AML in first complete remission (CR1) or beyond CR1 who were conditioned with myeloablative BuCy or reduced toxicity BuFlu regimen.

Methods: Adult patients with AML $(n=128)$ treated with alloHCT from either HLA-identical sibling $(\mathrm{n}=37)$ or unrelated donor $(\mathrm{n}=91)$, in CR1 $(\mathrm{n}=99)$ or beyond CR1 $(\mathrm{n}=29)$, who were conditioned with iv busulfan given in myeloablative dose $(9,6-12,8 \mathrm{mg} / \mathrm{kg})$ in combination with Flu $(\mathrm{n}=72)$ or Cy $(\mathrm{n}=56)$, between year 2000 and 2017, were included in the analysis. Patients treated with BuFlu were older (median 44 y. vs. 34 y., p < 0.001) and received more frequently stem cells collected from peripheral blood ( $94 \%$ vs. $43 \%, \mathrm{p}<0.001$ ).

Results: Engraftment was observed in all patients. The median time to neutrophil count $(0.5 \mathrm{G} / \mathrm{L})$ and platelet count $(20 \mathrm{G} / \mathrm{L})$ recovery was shorter after BuFlu in comparison with $\mathrm{BuCy}$ (18 days vs 21 days; $\mathrm{p}<0.001$ and 11 days vs. 18 days; $p<0.001$ ). Febrile neutropenia occurred in $67 \%$ of patients in the BuFlu group and $84 \%$ in the $\mathrm{BuCy}$ group $(\mathrm{p}=0.06)$. Non-infectious complications grade 2-4 were observed in $11 \%$ and $9 \%$ of patients, respectively $(\mathrm{p}=0.13)$. Acute GvHD grade II-IV was present in $14 \%$ of patients in the BuFlu group and $20 \%$ patients in the BuCy group ( $\mathrm{p}=$ $0.58)$. The respective frequency of extensive moderate/ severe chronic GvHD was $24 \%$ and $14 \%(\mathrm{p}=0.13)$. The median follow up time among survivors is 22 months (range, 2-80). The 2-year OS for patients conditioned with BuFlu was $65 \%$ and for those conditioned with $\mathrm{BuCy} 67 \%$ (log-rank $\mathrm{p}=0.61$ ). The respective 2-year LFS was $62 \%$ and $64 \%(\mathrm{p}=0.56)$. In addition, the OS and LFS for patients transplanted in CR1 and beyond CR1 did not differ significantly between the BuFlu and BuCy group. Similarly, the 1-year cumulative incidence (CI) of non-relapse mortality and the 2-year CI of relapse were comparable between two groups (11\% vs $9 \%$ and $21 \%$ vs $22 \%$, respectively) (p ns). In multivariate analysis faster lymphocyte recovery favorably influenced OS (HR 0.47, p= 0.026).

Conclusions: Myeloablative reduced toxicity conditioning regimen based on iv busulfan combined with fludarabine appears as effective as the BuCy combination in adult patients with AML, both in first and subsequent CR. The results of our study indicate that Cy may be replaced by fludarabine in busulfan-based myeloablative conditioning regimen without negative impact on disease control.

Conflict of interest: None of the authors has anything to declare. 


\section{P165}

Comparison between Equine and Rabbit anti-thymocyte globulin (ATG) used in conditioning for pediatric patients undergoing Hematopoietic stem cell transplantation (HSCT) for thalassemia and aplastic anaemia

Sunil Bhat ${ }^{1}$, Ruchi Chaudhary ${ }^{1}$, Shobha Badiger ${ }^{1}$, Pooja Mallya ${ }^{1}$, Sohini Chakraborty ${ }^{1}$, Archana $M V^{1}$, Ravi Joshi ${ }^{1}$, Nataraj $K S^{2}$, Sharat Damodar ${ }^{2}$

${ }^{1}$ Mazumdar Shaw Cancer Center, Narayana Health City, Pediatric Hematology Oncology and Bone Marrow Transplant, Bangalore, India; ${ }^{2}$ Mazumdar Shaw Cancer Center, Narayana Health City, Bangalore, India

Background: HSCT related complications and disease relapse are major obstacles in successful transplantation. Addition of anti-thymocyte globulin (ATG) has reduced rejection and GVHD incidence significantly. This study was conducted to look for relative efficacy of rabbit and equine ATG in pediatric HSCT conditioning.

Methods: Single centre retrospective analysis of thalassemia major and severe aplastic anemia (SAA) patients who underwent HSCT (January 2013-May 2017) and received ATG during conditioning regimen.

Results: Rabbit or Equine ATG were used as a part of conditioning regimen in 79 thalassemia (Pesaro class I/class II/class III: 2/61/16) and 21 patients of SAA (frontline transplant/after failure of immunosuppression: 18/3). Equine ATG group was labeled as group-1 and rabbit ATG as group-2.

Median CD34 dose $\left(10^{6} / \mathrm{kg}\right)$ used in group-1 and 2 were 6 and 5.55 in thalassemia while 2.95 and 5.54 in SAA respectively. CSA-MTx was used as GVHD prophylaxis. Median total dose of equine ATG was $90 \mathrm{mg} / \mathrm{kg}$ and rabbit ATG $7.5 \mathrm{mg} / \mathrm{kg}$. Flu/Bu/CY/ATG was the conditioning regimen in class I and II of thalassemia while treosulphan based conditioning was used in class III. Both groups were analyzed for transplant related complications and outcome. Outcomes are shown in table-1 and were comparable except Group-1 had more cases of proven bacterial infection $(p=0.07)$ and veno-occlusive disease $(p=0.03)$. Acute GVHD was seen in $40 \%$ patients in both groups $(p=0.985)$ and chronic GVHD in $8.5 \%$ in group- 1 and $6.6 \%$ cases of group-2 $(p=1)$. Median chimerism at day +30 and day +90 in group- 1 were $96 \%$ and $97 \%$ while $96 \%$ and $95.5 \%$ in group- $2(p$ value $0.92 \& 0.86)$. Overall survival (OS) were $85 \%$ and $84 \%$ in group-1 and group-2 over a median period of follow up 29months (as shown in figure1 ) and event free survival (EFS) $80.8 \%$ in group-1 and $78 \%$ in group-2 respectively ( $p$-value $0.9 \& 0.77$ ).

In SAA, Flu/Bu/ATG was used for conditioning and CSA-MTx as GVHD prophylaxis. Bone marrow was used

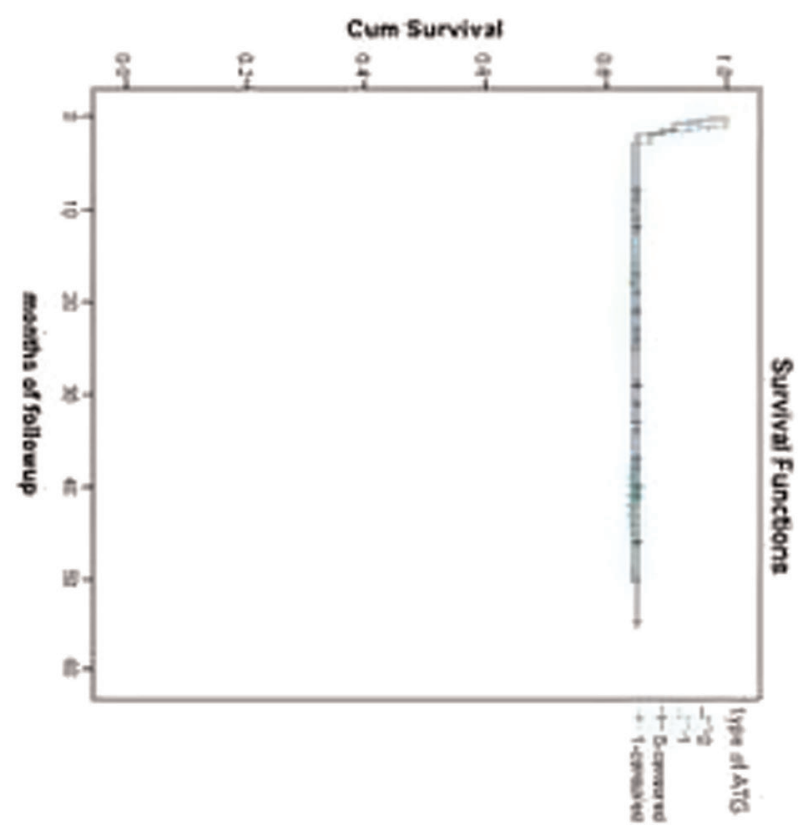

[P165 Figure] [Graph 1]

as stem cell source in 10 patients and PBSC in 11. Group-1 had early neutrophil and platelet engraftment with less number of proven bacterial infections. Median chimerism at day +30 and day +90 were $90 \%$ and $93 \%$ in group- 1 while it was $93 \%$ in group- $2(p=0.91)$ at both time intervals. Acute GVHD was seen in $22 \%$ in group- 1 and in $25 \%$ cases in group-2. OS was $67 \%$ in both groups.

Conclusions: Effectiveness of rabbit and horse ATG were similar in thalassemia patients while in SAA equine ATG use was associated with early neutrophil engraftment and less incidence of bacterial infection but OS in both groups were comparable.

Conflict of interest: None

\begin{tabular}{|c|c|c|c|c|c|c|}
\hline & \multicolumn{3}{|c|}{ Thalassemia } & \multicolumn{3}{|c|}{ Aplastic anemia } \\
\hline & $\begin{array}{l}\text { Rabbit } \\
(\mathrm{N}=32)\end{array}$ & $\begin{array}{l}\text { equine } \\
(\mathrm{N}=47)\end{array}$ & $\begin{array}{l}\mathrm{P} \\
\text { value }\end{array}$ & $\begin{array}{l}\text { Rabbit } \\
(\mathrm{N}=12)\end{array}$ & $\begin{array}{l}\text { equine } \\
(\mathrm{N}=9)\end{array}$ & $\begin{array}{l}\mathrm{P} \\
\text { Value }\end{array}$ \\
\hline $\begin{array}{l}\text { Type of transplant MSD/ } \\
\text { MRD/HAPLO/MUD }\end{array}$ & $\begin{array}{l}20 / 2 / 3 / \\
7\end{array}$ & $\begin{array}{l}38 / 2 / 1 / \\
6\end{array}$ & 0.23 & $7 / 2 / 3$ & $7 / 2 / 0$ & 0.63 \\
\hline $\begin{array}{l}\text { Neutrophil/ Platelet } \\
\text { engraftment(median) }\end{array}$ & $14 / 20$ & $\begin{array}{l}13.5 / \\
18.5\end{array}$ & $\begin{array}{l}0.24 / \\
0.24\end{array}$ & $15 / 22$ & $13 / 14$ & $\begin{array}{l}0.75 / \\
0.68\end{array}$ \\
\hline Proven bacterial infection & 16 & 5 & 0.07 & 6 & 2 & 0.21 \\
\hline CMV reactivation & 30 & 21 & 0.1 & 6 & 6 & 0.46 \\
\hline Invasive fungal infection & 3 & 3 & 0.62 & 1 & 2 & 0.38 \\
\hline $\begin{array}{l}\text { Acute GVHD Grade I - II } \\
\text { Grade III - IV }\end{array}$ & 109 & 49 & 0.958 & 03 & 20 & 0.19 \\
\hline Chronic GVHD & 1 & 2 & 1 & 2 & 0 & 0.21 \\
\hline
\end{tabular}

[[P165 Table] Table 1] 


\section{P166}

Comparison of non-myeloablative and reduced-intensity conditioning prior to allogeneic stem cell transplantation in older patients with myelodysplastic syndrome

Madlen Jentzsch, Christian Pfrepper, Richard Linke, Christine Döhring, Juliane Grimm, Stefanie Beinicke, Janine Häntschel, Andrea Hille, Wolfram Pönisch, Vladan Vucinic, Georg-Nikolaus Franke, Gerhard Behre, Dietger Niederwieser, Sebastian Schwind

Universitätsklinik Leipzig, Hematology and int. Oncology, Leipzig, Germany

Background: Myelodysplastic syndrome (MDS) is a clinically heterogeneous disease. Allogeneic stem cell transplantation (HSCT) remains the only curative treatment. The most appropriate conditioning intensity remains to be determined. In younger MDS patients a lower non relapse mortality (NRM) but higher cumulative incidence of relapse (CIR) was shown in reduced-intensity (RIC) compared to myeloablative conditioning HSCT. Dose reduced regimens, including RIC and non-myeloablative (NMA) regimens, allow HSCT in older individuals representing the majority of MDS patients. Data comparing RIC and NMA conditioning for HSCT in older MDS patients are lacking.

Methods: We retrospectively analyzed 117 MDS patients older than 50 (median 61.3, range 50.1-74.9) years who received NMA ( $3 \times 30 \mathrm{mg} / \mathrm{qm}$ fludarabine with $2 \mathrm{~Gy}[\mathrm{n}=70]$ or $3 \mathrm{~Gy}[\mathrm{n}=1]$ total body irradiation [TBI]) or RIC $(5 \times 30 \mathrm{mg} / \mathrm{qm}$ fludarabine with $8 \mathrm{mg} / \mathrm{kg}$ oral $[\mathrm{n}=41]$ or $6.4 \mathrm{mg} / \mathrm{kg}$ intravenous $[\mathrm{n}=5$ ] busulfan, including $3 \times 2 \mathrm{mg} / \mathrm{kg}$ thymoglobulin in unrelated HSCT) HSCT. While patients < 65 years received NMA (51\%) or RIC (49\%) conditioning, patients $>=65$ years predominantly received NMA $(85 \%)$. Thus, outcomes for patients younger and older than 65years at HSCT were analyzed separately. Karyotype analyses were performed centrally at our institution. IPSS-R was $0 \%$ very low, $8 \%$ low, $24 \%$ intermediate, $28 \%$ high, $40 \%$ very high.

Results: Overall, NMA patients were older $(\mathrm{P}=.007)$, but age did not differ between RIC and NMA conditioned patients when patients younger $(\mathrm{P}=.96)$ or older $(\mathrm{P}=.55)$ than 65 years were compared separately. The percentage of patients receiving therapy (demethylating agents and/or chemotherapy) prior to HSCT did not differ between RIC and NMA conditioning ( $68 \%$ vs. $56 \%, \mathrm{P}=.24$ ), however due to predominant application of NMA conditioning before 2012, NMA patients less often received demethylating agents alone ( $27 \%$ vs. $64 \%, \mathrm{P}=.005)$. The proportion of patients with blast excess $(\mathrm{P}=.27)$ and complex karyotype $(25 \%$ vs. $24 \%, \mathrm{P}=1)$ as well as scores for IPSS-R risk $(\mathrm{P}=.57)$, IPSS- $\mathrm{R}$ genetic risk $(\mathrm{P}=.64)$ and HCT-CI $(\mathrm{P}=.80)$ did not differ between NMA- or RIC-
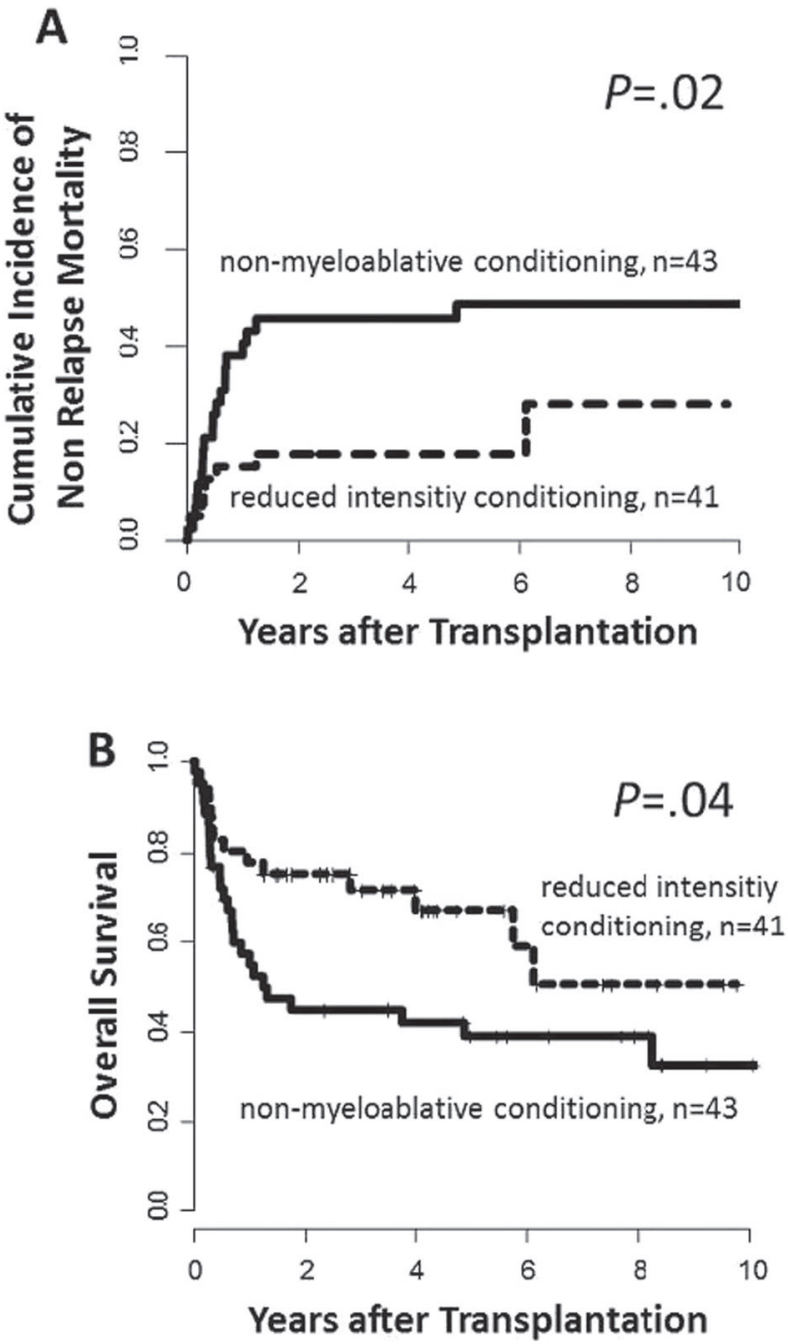

[P166 Figure] [(A) NRM and (B) OS in MDS patients aged 50- $<65$ years according to NMA vs. RIC conditioning]

conditioned patients. NMA-conditioned patients had more graft rejections $(11 \%$ vs. $0 \%, \mathrm{P}=.02)$ or chronic graftversus-host disease by trend (cGvHD, $87 \%$ vs. $49 \%, \mathrm{P}$ $=.08)$. In patients younger than 65years $(\mathrm{n}=84)$, NMA associated with higher NRM $(\mathrm{P}=.02$, Figure $1 \mathrm{~A})$ and shorter overall survival (OS, $\mathrm{P}=.04$, Figure $1 \mathrm{~B})$. In contrast, there was no difference in $\mathrm{CIR}(\mathrm{P}=.22)$ or secondary acute myeloid leukemia (CIsAML, $\mathrm{P}=.93$ ) between NMA or RIC conditioning. In patients older than 65years at HSCT $(\mathrm{n}=33)$, there were no differences in NRM $(\mathrm{P}=.96)$, OS $(\mathrm{P}=.49)$, CIR $(\mathrm{P}=.25)$ or CIsAML $(\mathrm{P}=.59)$ with regard to the conditioning regime.

Conclusions: Patients aged 50-65years receiving NMAHSCT had more graft rejection, more cGVHD by trend, higher NRM and shorter OS. Our study is limited by its retrospective nature and a possible selection bias towards NMA in older or less fit patients. While age and the comorbidity index HCT-CI did not differ between NMA 
and RIC, some comorbidities might not be appropriately reflected in the HSCT-CI scoring. Patients younger than 65years might benefit from the more intensive RIC or intensification of NMA (e.g. 3 Gy TBI) conditioning. However, NMA conditioning in older patients above 65years allows curative treatment with long-term survival.

Conflict of interest: G. Franke: Takeda: consultancy; Pfizer: honoraria; Novartis: honoraria, research funding.

S. Schwind: Novartis: Consultancy, research funding. All other authors have nothing to disclose.

\section{P167}

Comparison of Safety and Efficacy of Bendamustine versus Carmustine in the conditioning regimen for autologous stem cell transplantation in patients with relapsed/ refractoy lymphoma

Munira Moosajee, Samad Jehangir, Sobia Sawani, Wajeeha Tariq, Natasha Ali, Mohammed Usman Sheikh, Salman Adil

Aga Khan University, Karachi, Pakistan

Background: Bendamustine and carmustine have shown have both been used as part of conditioning regimen for autologous stem cell transplant (SCT) for relapsed/ refractory lymphoma. However, bendamustine has a higher toxicity profile as compared to carmustine in some studies. Carmustine availability is sporadic in many countries and therefore bendamustine is being used more frequently. We report our experience with bendamustine as part of conditioning regimen (BeEAM) because of non availability of carmustine in Pakistan.

Methods: This is a retrospective study to compare the safety and efficacy of bendamustine at $150 \mathrm{mg} / \mathrm{m} 2$ on day -5 and -4 coupled with etoposide $200 \mathrm{mg} / \mathrm{m} 2$ day -5 to -2 , cytarabine $200 \mathrm{mg} / 2$ on day -5 to -2 and melphalan $140 \mathrm{mg} /$ $\mathrm{m} 2$ on day -1 (BeEAM) versus carmustine $300 \mathrm{mg} / \mathrm{m} 2$ on day -6 coupled with the same agents (BEAM) as conditioning regimen to autologous SCT.

Results: Total 45 patients were included in the study. 14 patients in the BeEAM group and 31 patients in the BEAM group. The median age was 28 years in both groups. $49 \%$ $(\mathrm{n}=22)$ had Hodgkin's lymphoma and $28 \%(\mathrm{n}=17)$ were diagnosed with non hodgkins lymphoma $(\mathrm{n}=15)$, predominantly diffuse large $\mathrm{B}$ cell and mantle cell lymphoma. $57 \%$ vs $6 \%$ of the patients were in CR at time of transplant in the BeEAM and BEAM groups respectively. The median number of CD 34+ cells infused was 5.5 x 10(6) CD 34+ cells/ kg and was similar in both groups. Median time to absolute neutrophil count engrafted of 17 days (BeEAM) vs 13 days (BEAM). Median time to platelet engraftment of 25 days (BeEAM) versus 16 days
(BEAM). The 100 day transplantation mortality was $28.6 \%$ (4 patients) vs $19.3 \%$ (6 patients) . 8 patients (57.2\%) vs 5 patients $(16 \%)$ had GII - III acute kidney injury, 5 patients (35.7\%) vs 9 patients(29\%) had grade II-III hyperbilirubinemia and 7 patients $(50 \%)$ vs 7 patients $(22 \%)$ had GII diarrhea. At the end of 3 months, $35.7 \%$ (5 patients) vs 15 patients $(48 \%)$ were in complete response. The median disease free survival with 3 months vs 15.4 months $(\mathrm{p}=0.0420)$ and overall survival was 30 months vs. 44 months $(\mathrm{p}=0.02)$ in the BeEAM and BEAM cohorts respectively.

Conclusions: Our study shows that bendamustine is a potentially toxic agent in the conditioning regimen when compared to BCNU for autologous SCT with significant liver, kidney and GI toxicity. In addition, the overall survival and disease free survival is inferior to the BEAM conditioning regimen. Prospective trials need to be done to determine the efficacy and safety of bendamustine in conditioning regimen for ASCT.

Conflict of interest: None of the authors has anything to disclose.

\section{P168}

Cyclophosphamide, bendamustine and etoposide ( $\mathrm{CBeV})$, a new CBV conditioning regimen for lymphoma autotransplants

Gregorio Jaimovich ${ }^{1}$, Belen Rosales Ostriz ${ }^{2}$, Martin Castro ${ }^{2}$, Nicolas Fernendez Escobar ${ }^{3}$, Agustina Cia $^{3}$, Leandro Riera ${ }^{4}$, Patricio Duarte $^{4}$, Cecilia Foncuberta ${ }^{5}$, Adriana Vitriu ${ }^{5}$, Alejandra Banchieri $^{6}$, Hector Longoni ${ }^{6}$

${ }^{1}$ Favaloro University Hospital / Sanatorio Anchorena, BMT, Buenos Aires, Argentina; ${ }^{2}$ Sanatorio Anchorena, BMT, Buenos Aires, Argentina; ${ }^{3}$ Favaloro University Hospital, BMT, Buenos Aires, Argentina; ${ }^{4}$ Cemic, BMT, Buenos Aires, Argentina; ${ }^{5}$ Fleming Institute, BMT, Buenos Aires, Argentina; ${ }^{6}$ Clinicas Hospital Buenos Aires, BMT, Buenos Aires, Argentina

Background: CBV (cyclophosphamide, carmustine [BCNU] and etoposide) is commonly used for pretransplant conditioning for lymphoma autotransplants. However, carmustine is associated with lung toxicity, high cost and variable availability.

Methods: We developed a new $\mathrm{CBV}$ regimen $(\mathrm{CBeV})$ swapping bendamustine for carmustine and conducted a multi-center phase-2 study evaluating the safety and efficacy of $\mathrm{CBeV}$ (cyclophosphamide, 4.8-6.0 g/mE +2 , bendamustine, $400 \mathrm{mg} / \mathrm{mE}+2$, etoposide $1.0-2.4 \mathrm{~g} / \mathrm{mE}+2$ ) in 100 consecutive subjects receiving autotransplants for lymphomas. Median age was 49 years (range, 18-74 years). 61 were male. 36 had Hodgkin lymphoma, 24, diffuse large B-cell lymphoma (DLBCL), 18, mantle cell lymphoma (MCL) and 22, other lymphoma diagnoses. 78 subjects 


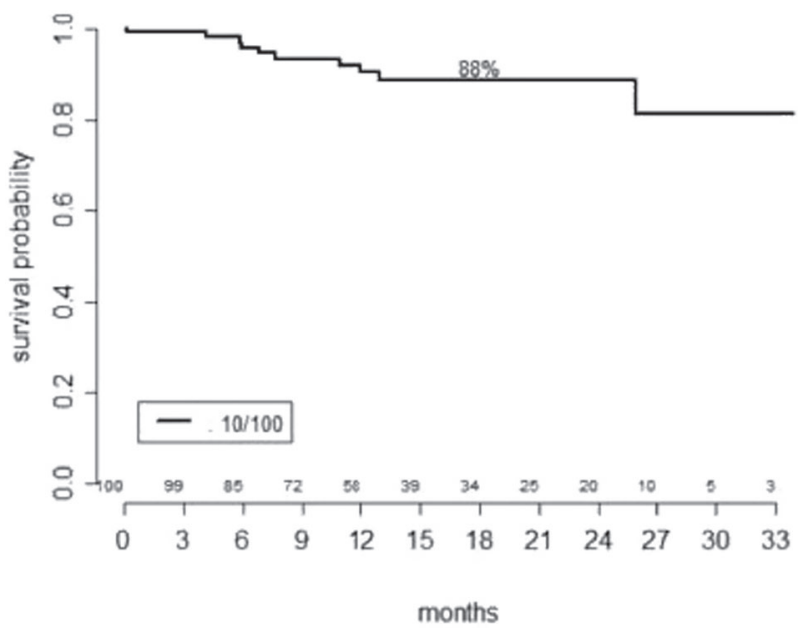

[P168 Figure] [Overall survival]

failed $\geq 2$ treatment lines pretransplant. At transplant 33 were in $1^{\text {st }}$ complete remission (CR), 42 in $2^{\text {nd }} C R$ and 25 in partial remission (PR). Subjects received a median of $3.9 \mathrm{X}$ $10 \mathrm{E}+6 \mathrm{CD} 34-$ postve cells $/ \mathrm{kg}$ (range, 1,2-27 x10E+6/kg). Median follow-up is 13 months (range, 3-34 months).

Results: All evaluable subjects had bone marrow recovery after a median of 11 days (range, 9-23 days) for neutrophils $>0.5 \times 10 \mathrm{E}+9 / \mathrm{L}$ and 14 days (range, 7-40 days) days for platelets $>20 \times 10 \mathrm{E}+9 / \mathrm{L}$. Grade- 3- 4 toxicities were fever $(\mathrm{N}=75)$, gastrointestinal $(\mathrm{N}=36)$, heart $(\mathrm{N}=10)$, renal $(\mathrm{N}: 4)$ and pulmonary $(\mathrm{N}=6)$, but in contrast to the lung toxicity of carmustine toxicity after $\mathrm{CBeV}$ was reversible. 1 subject died of renal and multi-organ failure. 90 subjects are alive; 10 died including 9 from lymphoma progression. Overall survival at 1.5 years was $88 \%$ (confidence interval (CI) 95\% (81.4 - 96.1) including 92\% (CI: $86,99 \%$ ) for subjects transplanted in $1^{\text {st }}$ or $2^{\text {nd }} \mathrm{CR}$ and $77 \%$ (CI: 59, 100\%); for those transplanted in PR, $\mathrm{p}=$ 0.168 . Cumulative incidence of relapse at 1.5 years was $25 \%$ (CI: $12,37 \%$ ) for subjects transplanted $1^{\text {st }}$ or $2^{\text {nd }} C R$ and $32 \%$ (CI: $3,52 \%$ ); for those transplanted in PR, p = 0.42 .

Conclusions: The safety and efficacy profiles of $\mathrm{CBeV}$ are similar to that of $\mathrm{CBV}$ but $\mathrm{CBeV}$ has the advantage of lower cost and more consistent availability. Preliminary efficacy results are encouraging but longer follow-up is needed to assess safety and efficacy of this regimen. A randomized trial comparing $\mathrm{CBeV}$ to $\mathrm{CBV}$ would be needed to definitively address this question.

Conflict of interest: None of the authors has anything to disclose.
P169

Excellent outcome for 91 Fanconi Anemia patients
undergoing matched related transplants using
Cyclophosphamide $60 \mathrm{mg} / \mathrm{kg}$ in Curitiba, Brazil

Carmem Bonfim ${ }^{1,2,3}$, Samantha Nichele ${ }^{1}$, Lisandro Ribeiro ${ }^{1,3}$, Gisele Loth $^{1}$, Daniela Pilonetto ${ }^{1}$, Ana Luiza Fabro ${ }^{2}$, Cilmara Kuwahara ${ }^{2}$, Fernanda Lara Benini', Daniela Marinho ${ }^{1}$, Marco Antonio Bitencourt ${ }^{1}$, Rebeca Toassa Gomes Mousquer ${ }^{1}$, Noemi Farah Pereira $^{1}$, Ricardo Pasquini, ${ }^{1,3}$

${ }^{1}$ BMT Unit Federal University of Parana, Curitiba, Brazil; ${ }^{2}$ Hospital Infantil Pequeno Principe, Curitiba, Brazil; ${ }^{3}$ Hospital Nossa Senhora das Graças, Curitiba, Brazil

Background: Fanconi anemia(FA) is a rare inherited disorder characterized by congenital abnormalities, progressive bone marrow failure and cancer predisposition. Survival after Hematopoietic Cell transplantation (HCT) improved dramatically over the past decade due to the use of better preparatory regimens and supportive care.

Methods: In this retrospective study we will analyze data on clinical presentation and outcome of 91 patients(pts) with FA transplanted between 06/1999 and 02/2017. Median age at transplantation was 9ys (range: 7 - 23). $57 \%$ were male. $87 \%$ were CMV positive. 77 pts $(85 \%)$ received a median of 7 blood transfusions (range: 1-151) before transplant. All pts were transplanted in marrow failure and received bone marrow from matched siblings (MSD) $(n=74)$ or other matched related donors (MRD) (n $=17$ ). All pts received a preparatory regimen with Cyclophosphamide $60 \mathrm{mg} / \mathrm{kg}(\mathrm{CY} 60)$ without $(\mathrm{n}=82)$ or with rabbit-ATG $5 \mathrm{mg} / \mathrm{kg}(\mathrm{n}=9)$ and GVHD prophylaxis with cyclosporine and methotrexate.

Results: One patient died before $\mathrm{D}+21$ and was not evaluable for engraftment. Primary graft failure occurred in one patient in the MSD group and this patient was successfully rescued after a $3^{\text {rd }}$ transplant using a haploidentical donor with post-transplantation CY. Ninety pts engrafted and secondary graft failure occurred in 5pts (MSD=3pts; MRD=2pts) between 49 and 742 days after transplant (M: 156). Four out of these 5 pts are alive and well with full donor chimerism after a $2^{\text {nd }}$ or $3^{\text {rd }}$ HCT. Mucositis grade II-III occurred in more than $70 \%$ of pts. Acute GVHD occurred in 10/89 evaluable pts; grade II (n $=5)$; grade III $(\mathrm{n}=2)$ and grade IV $(\mathrm{n}=3)$ while chronic GVHD occurred in 21/89 evaluable pts; NIH global severity score was mild $(\mathrm{n}=10)$; moderate $(\mathrm{n}=5)$ and severe $(\mathrm{n}=$ 6). Seven pts died at a median of 906 days after transplant (range: 12- 5088). Causes of death include oral squamous cell carcinoma $(\mathrm{n}=2)$; GVHD $(\mathrm{n}=3)$; central nervous system bleeding $(\mathrm{n}=1)$ and sepsis $(\mathrm{n}=1)$. Transplant related mortality at one year was very low $(n=3)$. Eighty- 
four pts are alive with a median follow-up of 7 ys (Range: 10 months - 18 years) and an overall survival of $95 \%$ at $5 \mathrm{y}$. There was no difference in survival according to the type of donor MSD(91\%) vs MRD(94\%). In the univariate analysis survival was much better for pts without acute-GVHD compared to pts with this complication $(98.7 \%$ vs $50 \%$ p < 0.001). 14 pts did not receive any transfusions before transplant and all of them are alive and well, none developed acute-GVHD and 2 had mild chronic GVHD.

Conclusions: In this study, the use of CY $60 \mathrm{mg} / \mathrm{kg}$ with or without ATG was associated with an excellent survival, good engraftment and very low mortality rate. This highly successful and low-cost preparatory regimen can be used all over the world to treat patients with FA in aplastic phase.

Conflict of interest: All authors declare no conflict of interest

\section{P170}

Favorable efficacy of TBI-based compared with high-dose chemotherapy conditioning for auto-SCT in patients with peripheral T-cell lymphomas: An analysis by the Polish Lymphoma Research Group

Tomasz Czerw ${ }^{1}$, Andrzej Smagur ${ }^{1}$, Anna Czyz ${ }^{2}$, Maria SadusWojciechowska ${ }^{1}$, Jacek Najda ${ }^{1}$, Wlodzimierz Mendrek ${ }^{1}$, Malgorzata Sobczyk-Kruszelnicka ${ }^{1}$, Joanna Romejko-Jarosinska ${ }^{3}$, Grzegorz, Helbig ${ }^{4}$, Agnieszka Piekarska ${ }^{5}$, Lidia Poplawska ${ }^{3}$, Beata Piatkowska-Jakubas ${ }^{6}$, Dorota Hawrylecka ${ }^{6,7}$, Barbara NasilowskaAdamska ${ }^{8}$, Anna Lojko-Dankowska ${ }^{9}$, Anna Kopinska ${ }^{4}$, Jan Walewski ${ }^{3}$, Mieczyslaw Komarnicki ${ }^{9}$, Leszek Miszczyk ${ }^{10}$, Jerzy Holowiecki $^{1}$, Sebastian Giebel ${ }^{1}$

${ }^{1}$ Maria Sklodowska-Curie Memorial Cancer Center and Institute of Oncology, Gliwice Branch, Department of Bone Marrow

Transplantation and Oncohematology, Gliwice, Poland; ${ }^{2}$ Wroclaw Medical University, Wroclaw, Poland; ${ }^{3}$ Maria Sklodowska-Curie Cancer Centre and Institute of Oncology, Warsaw, Poland; ${ }^{4}$ Silesian Medical University, Katowice, Poland; ${ }^{5}$ Medical University of Gdansk, Gdansk, Poland; ${ }^{6}$ Jagiellonian University Medical College, Cracow, Poland; ${ }^{7}$ Oncology Center, Brzozow, Poland; ${ }^{8}$ Institute of Hematology and Blood Transfusion, Warsaw, Poland; ${ }^{9}$ Poznan University of Medical Sciences, Poznan, Poland; ${ }^{10}$ Maria SklodowskaCurie Memorial Cancer Center and Institute of Oncology, Gliwice Branch, Radiotherapy Department, Gliwice, Poland

Background: High-dose consolidation therapy with autologous stem cell support (autoSCT) is considered beneficial for transplant-eligible patients with newly diagnosed and relapsed peripheral T-cell lymphomas (PTCL). The evidence is based on retrospective analyses which included heterogeneity of mainly chemotherapybased conditioning regimens. Achieving complete remission (CR) before autoSCT is one of the strongest predictors of the outcome. There are few data regarding the comparison of Total Body Irradiation (TBI) and non-TBI based approaches, which was the goal of this study.
Methods: The current analysis included patients with PTCL who were autografted in CR. We retrospectively analyzed the outcome of 18 patients conditioned with $12 \mathrm{~Gy}$ TBI (given in 3 fractions of 4 Gy on 3 consecutive days) combined with $120 \mathrm{mg} / \mathrm{kg}$ cyclophosphamide with 35 patients who received high-dose chemotherapy regimens (BEAM (BCNU, etoposide, cytarabine, melphalan), $\mathrm{n}=25$; $\mathrm{CBV}$ (BCNU, etoposide, cyclophosphamide), $\mathrm{n}=8$; other, $\mathrm{n}=2$ ). All patients who received TBI-based regimen were transplanted in a single institution (Department of BMT in Gliwice) whereas the remaining autoSCTs were performed in the other Polish Lymphoma Research Group registered centers. The groups characteristics were as follows (TBI and non-TBI, respectively): the median age at transplantation: 42 years (22-65) and 44 (15-63); PTCL subtype (PTCL NOS-6; AITL-2; ALCL ALK(-)-4, ALK(+)-6 and PTCL NOS-21; AITL-5; ALCL ALK(-)-4, ALK(+)-1, ALK status unknown-4); stage at diagnosis (II-5; III-10; IV-3 and II-5; III-10; IV-20); the median number of pre-transplant chemotherapy lines: 1 (1-2) and 2 (1-3); year of transplantation: 2010-2016 and 1998-2011; the median observation time 43 months (8-85) and 61 (9-199). Patients in the non-TBI group received more pre-transplant lines of therapy and were transplanted in the earlier years. All the remaining factors were comparable between the studied groups.

Results: All patients in the TBI group engrafted whereas one patient in the chemotherapy group did not engraft. There were no early and late non-relapse mortality cases noted in the TBI group. In the chemotherapy group four patients died due to non-relapse causes (2-sepsis; 1secondary neoplasm; 1-heart failure). The probability of overall survival at 3 years and 5 years after autoSCT was significantly higher for those conditioned with TBI-based regimen compared with chemotherapy-based approach $(100 \%$ vs $74 \%$ and $100 \%$ and $66 \% ; \mathrm{p}=0.025$, respectively). The probability of progression-free-survival at 3 years and 5 years was $94 \%$ vs $67 \%$ and $81 \%$ vs $63 \%$; $p=$ 0.15 , respectively.

Conclusions: TBI seems to be an effective part of conditioning before autoSCT for PTCL allowing potentially to obtain better outcomes compared with high-dose chemotherapy regimens for patients transplanted in CR.

Conflict of interest: The authors declare no potential conflicts of interest.

\section{P171}

Favorable outcome of post-transplantation cyclophosphamide haploidentical peripheral blood stem 
cell transplantation with a targeted busulfan-based myeloablative conditioning regimen using intensive pharmacokinetic monitoring for pediatric patients

Kyung Taek Hong ${ }^{1}$, Hyoung Jin Kang ${ }^{1}$, Jung Yoon Choi ${ }^{1}$, Che Ry Hong $^{1}$, Sang Hoon Song ${ }^{2}$, Kyung-Sang Yu ${ }^{3}$, In-Jin Jang ${ }^{3}$, Hee Young Shin ${ }^{1}$

${ }^{1}$ Seoul National University Hospital, Seoul National University College of Medicine, Pediatrics, Seoul, Korea, Republic of; ${ }^{2}$ Seoul National University Hospital, Seoul National University College of Medicine, Laboratory Medicin, Seoul, Korea, Republic of; ${ }^{3}$ Seoul National University Hospital, Seoul National University College of Medicine, Pharmacology and Clinical Pharmacology, Seoul, Korea, Republic of

Background: The use of a haploidentical donor for hematopoietic stem cell transplantation has shown promisssing results for hematologic malignancies and nonmalignant diseases, particularly in cases without HLA-matched donor. Unfortunately, data on haploidentical hematopoietic stem cell transplantation (haplo-HSCT) with post-transplantation cyclophosphamide (PTCy), in children, are rare and conflicting. Wer had been used an intensive daily pharmacokinetic (PK) monitoring method for busulfan dosing, and optimized intensity of conditioning regimen by calculating the total exposure of busulfan. Here we applied this targeted-busulfan method using daily PK monitoring to haplo-HSCT using PTCy in pediatric patients, and the safety and outcomes were evaluated.

Methods: We retrospectively studied 34 patients who underwent haplo-HSCT (23 malignant diseases and 11 nonmalignant diseases) at the Seoul National University Children's Hospital, from February 2014 to April 2017. The conditioning regimen of the haplo-HSCT was composed of busulfan, fludarabine $\left(40 \mathrm{mg} / \mathrm{m}^{\wedge} 2\right.$ once daily via IV from days -8 to -4$)$, and cyclophosphamide $(14.5 \mathrm{mg} / \mathrm{kg}$ once daily via IV from days -3 to -2 . Busulfan $\left(120 \mathrm{mg} / \mathrm{m}^{\wedge} 2\right.$ for patients aged $\geq 1$ year and $80 \mathrm{mg} / \mathrm{m} 2$ for patients aged $<1$ year) was administered as a starter dose on day -8 and administered once daily, thereafter, and a subsequent targeted dose of busulfan was analyzed according to the daily intensive therapeutic drug monitoring results from days -7 to -5 . PTCy $(50 \mathrm{mg} / \mathrm{kg})$ was given on day 3 and 4 .

Results: The median follow-up period was 23 months (range, 1-47 months), and the median value of the total infused busulfan AUC was 74,078 $\mu \mathrm{g} \times \mathrm{h} / \mathrm{L}$ (range, 67,30278,478 ). The median days of neutrophil and platelet engraftment were 15 days and 29 days, respectively, and the cumulative incidence $(\mathrm{CI})$ rates of engraftment were $97.1 \%$ and $96.6 \%$, respectively. The CI rates of grade II-IV, grade III-IV acute and extensive chronic graft versus host disease were $38.2 \%, 5.9 \%$, and $9.4 \%$, respectively. The overall survival rate, event-free survival rate, and treatmentrelated mortality rate at the time of median follow-up were $84.6 \%, 79.4 \%$, and $2.4 \%$, respectively. Based on the subgroup analysis of patients with malignancies $(n=23)$, the relapse incidence rate at the time of median follow-up was $21.7 \%$.

Conclusions: Haplo-HSCT using PTCy, along with a targeted busulfan-based myeloablative conditioning regimen and peripheral blood as a stem cell source, was a safe and promising therapeutic option for children with hematologic malignancies and nonmalignant diseases.

Conflict of interest: The authors declare no competing financial interests

\section{P172}

Fit Elderly Patients with aggressive lymphoma do not need dose reduction of conditioning regimen

Osman Ilhan, Güldane Cengiz Seval, Atilla Uslu, Sinem Civriz, Bozdag, Selami Koçak Toprak, Meltem Kurt Yuksel, Pervin Topcuoglu, Onder Arslan, Muhit Ozcan, Taner Demirer, Hamdi Akan, Meral Beksac, Gunhan Gurman

Ankara University School of Medicine, Department of Hematology and Stem Cell Transplant Unit, Ankara, Turkey

Background: High-dose chemotherapy followed by autologous hematopoietic cell transplantation (auto HSCT) may provide survival benefit in patients with non-hodgkin lymphoma (NHL). Retrospective analyses suggest that the benefit of HDT extends to elderly patients with NHL, which is an important finding considering that the median age at diagnosis is 67 years (range: 65-74) for NHL in United States, using 2010-2014 US SEER data. We aimed to define the efficacy and toxicity of auto HSCT in patients $>60$ years with NHL.

Methods: From January 2005 through August 2017, data from 26 patients with aggressive NHL above 60 years of age who were eligible for auto HSCT according to geriatric assessment (GA) were evaluated. All these data were obtained from the Ankara University Faculty of Medicine, Department of Hematology and Bone Marrow Transplant Unit. Their diagnosis were as following; 16 diffuse large B cell lymphoma (primary refractory or relapsed disease), 7 mantle cell lymphoma (first complete remission), 2 follicular lymphoma and 1 anaplastic large cell lymphoma. We compared the toxicity profile and outcome between the research group: patient aged 60 years and above and the control group: patient $<60$ years. 
Results: All of the patients were stage III or IV at diagnosis; ten out of 26 elderly patients had active disease at the time of auto HSCT. The median follow-up was 20.5 months (range, 1-60 mos). Prior to transplantation majority $(85 \%)$ of the elderly patients received BEAM protocol as conditioning treatment. Bone marrow stem cell was used in only 1 patient None of the patient had mobilization failure, the median peripheral CD34 level was $5.24 \times 10^{6} / \mathrm{kg}$. Forty-eight percent of the patients experienced grade 3-4 mucositis and $77 \%$ of the patients had microbiology-documented infection. Sixty-two percent of the patients had diarrhea with median duration of 8 days (range, 5-20 days). Renal toxicity was occurred in 7 (27\%) patients while hepatic toxicity in $1(10 \%)$ patients. Median time to neutrophil recovery was 10 days (range, 8-18 days) and platelet recovery 11 days (range, 10-32 days). Overall response was obtained from all patients $(23 \% \mathrm{CR})$. At the time of data collection, 4 patients $(15 \%)$ of patients' $\geq 60$ years have deceased. Relapse $(n=3)$ was the main course of death. The probability of 4-year progression free survival (PFS) and estimated overall survival (OS) in elderly patients were $44.4 \%$ and $39.4 \%$, respectively.

Conclusions: Based on this single center study, autoHSCT is safe and efficacious in the treatment of elderly lymphoma patients. We emphasize the need for further research in order to determine the risk-benefit threshold for HSCT based on age coupled with comorbidity and fragility.

Conflict of interest: None of the authors has anything to disclose

\section{P173}

\section{Fludarabin/Busulfan/Thiotepa/ATG is as an effective myeloablative conditioning protocol in adults undergoing unrelated donor stem cell transplantation}

Edmund Watson ${ }^{1}$, Katalin Balassa ${ }^{1,2}$, Giancarlo Fatobene ${ }^{3}$, Marcin Lubowiecki ${ }^{1}$, Rebecca Cash ${ }^{1}$, Daja Barton ${ }^{1}$, Mandy Ellis ${ }^{1}$, Lara Rowley $^{1}$, Denise Wareham ${ }^{1}$, Claudia Costa ${ }^{1}$, Rachel Miller ${ }^{1}$, Robert Danby ${ }^{1}$, Vanderson Rocha ${ }^{1,2,3}$, Andy Peniket ${ }^{1}$

${ }^{1}$ Oxford University Hospitals NHS Foundation Trust, Clinical Haematology, Oxford, United Kingdom; ${ }^{2}$ NHS Blood and Transplant, Oxford, United Kingdom; ${ }^{3}$ Universidade de São Paulo, Department of Haematology, Sao Paulo, Brazil

Background: TBI-based myeloablative conditioning (MAC) is well-established in the treatment of haematologic malignancies. However, radiotherapy can cause logistical problems for transplant scheduling and is associated with significant long- and short-term toxicity. Non-TBI based MAC protocols are now available but there are limited outcome data. We report the use of a Fludarabin/Busulfan/ Thiotepa/ATG regimen - originally developed for umbilical cord transplantation - in adult unrelated donor haematopoietic stem cell transplantation (HSCT).

Methods: We performed an analysis of fourteen consecutive patients undergoing Thiotepa-based allogeneic HSCT at two institutions. Primary diagnoses were AML ( $n$ =9), MDS (2), ALL (1), extranodal NK/T cell lymphoma (1), and ALCL (1). At conditioning, eleven patients were in complete remission, one had primary refractory AML, one had AML in partial remission, and one had MDS RAEB1. The recipients' age range was from 17 to 52 years (median 27.5); eight were male.

All patients received the myeloablative combination of Thiotepa $(10 \mathrm{mg} / \mathrm{kg})$, Busulfan $(9.6 \mathrm{mg} / \mathrm{kg})$, Fludarabine $\left(150 \mathrm{mg} / \mathrm{m}^{2}\right)$ and Rabbit ATG $(8 \mathrm{mg} / \mathrm{kg})$, adapted from Sanz et al (2012). Thirteen allografts were from a matched unrelated donor (MUD) and one was from a mismatched related donor. Ten allografts were 10/10 HLA allele matched and four were 9/10. Twelve of the recipients were seropositive for EBV prior to transplant.

Results: Median follow-up for live patients was 40 months (range 6 to 59). Neutrophil engraftment occurred in thirteen patients, with a median of nineteen days (from 15 to 30); two patients experienced delayed engraftment (at 29 and 30 days). The patient with primary refractory AML died prior to engraftment.

Overall survival and progression-free survival at three years were 61 and $62 \%$, respectively. Transplant-related mortality was $21 \%$ at 100 days and $29 \%$ at one year. Five patients died in follow-up: three from sepsis (at 21, 64 and 212 days from transplant), one from disease relapse (at 497 days) and one from EBV-related PTLD (at 76 days). Acute GvHD occurred in six patients (57.1\%), with five episodes of Grade I and one of Grade III. Chronic GvHD was reported in two patients (14.3\%).

Of the twelve EBV-positive recipients, six (50\%) experienced asymptomatic EBV reactivation of at least $10^{3}$ and those whose titre reached $10^{6}$ were all successfully treated with Rituximab $(n=3)$. An additional three patients (25\%) had EBV reactivation and post-transplant lymphoproliferative disorder (PTLD). Two of these achieved CR with radio- or immunochemotherapy, whereas one was diagnosed four days prior to death and was not fit for treatment; this patient had underlying EBV positive NK/T cell lymphoma.

Seven recipients were seropositive for CMV prior to transplant and five $(71.4 \%)$ experienced CMV reactivation. All CMV seronegative recipients received cells from seronegative donors.

Conclusions: This Thiotepa-based MAC regimen is an effective alternative to TBI-based conditioning in allogeneic HSCT and is associated with successful engraftment and a low rate of GvHD. We describe a high incidence of EBV reactivation and advise careful monitoring of EBV titres in 
patients treated with this regimen. Overall, our outcomes suggest this is a promising protocol for use in patients requiring myeloablative conditioning.

Conflict of interest: None of the authors has anything to disclose.

\section{P174}

Fludarabine with either $8 \mathrm{~Gy}$ or $12 \mathrm{~Gy}$ total body irradiation as conditioning regimen before allogenic hematopoietic cell transplantation in patients with AML. A single center experience

Malgorzata Sobczyk-Kruszelnicka ${ }^{1}$, Malgorzata Ociepa ${ }^{1}$, Tomasz Czerw $^{1}$, Wlodzimierz Mendrek ${ }^{1}$, Jacek Najda ${ }^{1}$, Maria SadusWojciechowska $^{1}$, Katarzyna Michalak ${ }^{1}$, Andrzej Frankiewicz ${ }^{1}$, Magdalena Glowala-Kosinska ${ }^{1}$, Agata Chwieduk ${ }^{1}$, Wojciech Fidyk ${ }^{1}$, Slawomir Blamek ${ }^{2}$ Leszek Miszczyk ${ }^{2}$, Jerzy Holowiecki ${ }^{1}$, Sebastian Giebel $^{1}$

${ }^{1}$ Maria Sklodowska-Curie Institute - Cancer Center, Gliwice Branch, Department of Bone Marrow Transplantation and Oncohematology, Gliwice, Poland; ${ }^{2}$ Maria Sklodowska-Curie Institute - Cancer Center, Gliwice Branch, Radiotherapy Department, Gliwice, Poland

Background: Although acute myeloid leukemia (AML) is the most frequent indication for allogenic hematopoietic cell transplantation (alloHCT) the optimal conditioning has not been established so far. Among regimens based on total body irradiation (TBI), the combination of TBI $8 \mathrm{~Gy}+$ fludarabine(Flu) was prospectively compared with TBI 12 Gy + cyclophosphamide, showing similar efficacy while reduced toxicity. In our center patients with comorbidities or older age were treated with TBI $8 \mathrm{~Gy} / \mathrm{Flu}$ while for younger, „fit" patients the dose of TBI in combination with Flu was escalated to $12 \mathrm{~Gy}$. The letter regimen has not been reported. The aim of the stydy was to evaluate its toxicity and efficacy.

Methods: The analysis included 54 patients (women - 31, men - 23), 27 treated with TBI 8Gy/Flu and 27 treated with TBI 12Gy/Flu, between years 2011-2016. The median age was $54(20$ - 67) and $36(20-61)$, respectively. The disease stage before the procedure was as follows: CR1-44, CR2-6, CR3-1, PR-1 and NR-2. Patients were treated with HSCT from either HLA-matched siblings $(n=22)$ or unrelated donors $(n=32)$. TBI was administered in 4 Gy once daily fractions on days (-3), -2, -1. The immunosuppressive therapy consisted of cyclosporine + methotrexate $+/$ - ATG. Peripheral blood was used as a source of stem cells.

Results: Median neutrophil recovery time (ANC $>0.5 \mathrm{G} /$ 1) was 17 days in the $8 \mathrm{~Gy}$ group and 16 days in the $12 \mathrm{~Gy}$ group. The incidence of grade 2-4 acute GvHD was $11 \%$ and $15 \%$, while extensive chronic GvHD $0 \%$ and $4 \%$, respectively. The rate of grade 3 non-hematological toxicities was $11 \%$ and $18 \%$, respectively. Grade 4 adverse events were not reported. The probability of OS after 2 years was $62 \%(+/-10 \%)$ in the $8 \mathrm{~Gy}$ group and $80 \%(+/-$ $11 \%)$ in the $12 \mathrm{~Gy}$ group $(\mathrm{p}=0.14)$. The PFS probability was $58 \%(+/-10 \%)$ and $77 \%(+/-11 \%)$ respectively $(\mathrm{p}=0.16)$. The rates of relapse were $32 \%$ and $7 \%(p=0.11)$, while nonrelapse mortality was $10 \%$ and $13 \%(\mathrm{p}=\mathrm{NS})$, respectively.

Conclusions: The combination of fludarabine with TBI is characterized by good tolerance and enhancing efficacy. Unless there are any contraindications, the TBI 12Gy seems to be the preferred dose. However, the efficacy and safety of the TBI 12Gy conditioning regimen with fludarabine requires verification in prospective clinical trial.

Conflict of interest: nothing to disclose

\section{P175}

Haploidentical hematopoietic stem cell transplantation with post-transplant high-dose cyclophosphamide in highrisk children: A single-center study

Vedat Uygun ${ }^{1}$, Gülsün Karasu ${ }^{1}$, Seda Ö̈türkmen ${ }^{1}$, Hayriye Daloğlu ${ }^{1}$, Suar Kılıç ${ }^{2}$,Volkan Hazar ${ }^{2}$, Akif Yeşilipek ${ }^{1}$

${ }^{1}$ MedicalPark Antalya, Pediatric Bone Marrow Transplantation Unit, Antalya, Turkey; ${ }^{2}$ MedicalPark Göztepe, Pediatric Bone Marrow Transplantation Unit, İstanbul, Turkey

Background: Graft-versus-host disease (GVHD) remains a major impediment to the widespread applicability of allogeneic blood and marrow transplantation. Haploidentical hematopoetic stem cell transplantations are increasing steadily due to immediate availability of a donor and better results. In this study, we present the results of 74 transplantations of 72 children who received haploidentical HSCT in our center with unmanipulated bone marrow or peripheral blood stem cells and GVHD prophylaxis with posttransplant cyclophosphamide.

Methods: Most patients received a busulfan-based myeloablative conditioning regimen with weight adjusted doses. G-CSF-primed, unmanipulated BM with additional next-day collected PBSC was the most common stem cell source. Posttransplant CY (50 mg/kg IV) was given on days three and five post-HSCT in case the transplantation performed on the same day. However CY was given on the fourth and fifth day if the stem cell infusion was performed on day 0 and 1. CsA or tacrolimus with MMF or MP was also used for GVHD prophylaxis.

Results: The majority of patients were transplanted for malignancy (84\%) and the median age was eight yr (range: 0.4-20.1 yr). Seventy (95\%) of the 74 patients engrafted, and 67 (95\%) of 70 evaluable subjects achieved full donor chimerism by day +30 . Only one patient experienced with Grade IV GVHD. Grades II-III acute GVHD developed in thirty-four patients (46\%), and chronic GVHD was found in 
fourteen patients (19\%). Fourteen patients died within the first 100 days mostly due to infections (TRM 19\%). Forty patients are currently alive, with a median follow-up of 27 months (range 6-56 months). The 12-month OS and DFS were 58\% (95\% CI: $45.2-68.0)$ and $52 \%$ (95\% CI: 39.8$62.8)$, respectively.

Conclusions: Our results with these high-risk patients are encouraging for haploidentical HSCT in pediatric patients. Given the growing data on the similarity of outcomes after HLA-matched and haploidentical HSCT, further studies are required to determine whether others factors may be more important for donor selection than HLA-matching in pediatric population.

Conflict of interest: Nothing to disclose

\section{P176}

Abstract previously published

\section{P177}

Impact of $\mathrm{CD}_{3} 4^{+}$and $\mathrm{CD}^{+}$cell doses on outcomes in alemtuzumab-based reduced intensity transplantation for high-risk MDS and AML: outcomes of a single UK centre

Nuno M. Borges, Louise Duncan, Erin Hurst

Freeman Hospital, Haematology, Newcastle upon Tyne, United Kingdom

Background: The impact of cellular composition on transplant outcomes remains controversial. Others have assessed the role of cell doses on outcome in mixed populations of myeloablative, reduced intensity and T-cell depleted or non-depleted transplants. In this study we have assessed the impact of $\mathrm{CD} 34^{+}$and $\mathrm{CD} 3^{+}$doses in a welldefined cohort of MDS/AML patients, the commonest transplant indications. Patients were transplanted using alemtuzumab-based reduced intensity conditioning (RIC) protocols. To our knowledge, this is the first time exclusively alemtuzumab T-cell depleted transplant population outcomes in relation to cellular composition have been evaluated.

Methods: Outcomes pertaining to consecutive MDS/ AML patients transplanted with alemtuzumab-based RIC protocols over a 2 -year period in a single centre were retrospectively analysed, including demographics, cell doses, engraftment, chimerism, donor lymphocyte infusions (DLI), and graft-versus-host disease.

Results: Between May 2015 and May 2017, 44 patients underwent RIC HSCT for MDS/AML. Twenty-two (50\%) were male, and the average age at transplant was 56 years (31-71). Conditioning consisted of fludarabine and melphalan in $22(50 \%)$ of patients, with the remainder receiving busulfan instead of the latter. All patients received T-cell depletion with alemtuzumab, 30mg in sibling (SIB) transplants, $60 \mathrm{mg}$ in matched unrelated donor (MUD) transplants, and $90 \mathrm{mg}$ in mismatched $(<10 / 10)$ unrelated donor transplants, and received ciclosporin for GVHD prophylaxis. Thirty-two (73\%) patients received MUD, and $12(27 \%)$ received sibling donor cells. The average infused cell doses were $6 \times 10^{6}(2.2-11.6)$ and $264 \times 10^{6}(86-660)$ for $\mathrm{CD} 4^{+}$and $\mathrm{CD}^{+}$cells, respectively. The average time to engraftment was 15.3 days (9-31). Twenty-two (50\%) patients received DLI (average cell dose $1.3 \times 10^{6}$, range 0.1 17 ), and each patient received on average 1.6 fractions (14), either prophylactically (73\%) or therapeutically (27\%). Fourteen $(32 \%)$ patients developed acute GVHD grades IIIV and 27 (61\%) chronic GVHD (including progressive and de novo). In the acute group, the incidence of grades I, II, and III were $11(44 \%), 11(44 \%)$, and $3(12 \%)$, respectively (no patients developed grade IV). In the chronic group, the incidence of $\mathrm{NIH}$ severity grades mild, moderate, and severe were 12 (44\%), 9 (33\%), and 6 (22\%), respectively. Overall mortality was $17 / 44$ (39\%). Relapse mortality was $6 / 44(14 \%)$ and non-relapse mortality was $11 / 44(25 \%)$. There was no significant association between $\mathrm{CD} 34^{+}$or $\mathrm{CD}^{+}$cell dose on outcomes including engraftment, number of DLI infusions, survival or the development of acute or chronic GVHD. There was a trend towards higher GVHD rates in MUDs compared to Sib's, $(\mathrm{p}=0.08$ for acute GVHD, and $\mathrm{p}=0.74$ for chronic GVHD).

Conclusions: In a uniformly treated MDS/AML cohort, we have demonstrated no impact of cell doses on transplant outcomes for the first time in an exclusively RIC alemtuzumab-based transplant group.

Conflict of interest: None of the authors has anything to disclose.

\section{P178}

Impact of reduced-intensity conditioning on engraftment and transfusion needs in ABO-incompatible bone marrow allogeneic transplantation

Gala Aglaia Méndez ${ }^{1}$, Cristina Amunárriz ${ }^{2}$, José Luis Arroyo ${ }^{2}$, Iñigo Romón ${ }^{1}$

${ }^{1}$ Hospital Universitario Marqués de Valdecilla, Santander, España, Hematology, Santander, Spain; ${ }^{2}$ Banco de Sangre y Tejidos de Cantrabria, Liencres, Spain

Background: Current evidence on reduced intensity conditioning (RIC) regimens in ABO-mismatched allogeneic transplantation is limited. It is hypothesized that this kind of conditioning provoke a longer survival of receptor's plasma cells, thus, producing isohemagglutinins against 
donor erythrocytes, which may cause delayed erythrocyte recovery, higher transfusion needs and pure red cell aplasia (PRCA). Although some studies have supported these associations, there is still controversy surrounding it [1].

We have carried out this study, therefore, to describe our experience in major $\mathrm{ABO}-$ mismatched bone marrow $(\mathrm{BM})$ allogeneic transplantation in the setting of myeloablative (MA) and RIC conditionings.

Methods: We retrospectively studied major and bidirectional ABO-incompatible allogeneic BM transplants performed in or center from 2006 to 2017. We analyzed neutrophil and platelet engraftments, red blood cell (RBC) recovery, $\mathrm{RBC}$ units transfused until day +100 , platelet units transfused until day +60 , and PRCA.

Data was analyzed with IBM SPSS software ${ }^{\circledR}$, version 24. Descriptive statics are reported as median (and range). Shapiro Wilks test was used for testing normality and mean comparisons were performed with Mann Whitney's U test.

Results: A total of 63 transplants were included: $65 \%$ MA and $35 \%(n=22)$ RIC. The latter were mostly Fludarabine-Melphalan (63.6\%) conditionings and, in the remaining, Busulphan-Fludarabine (18\%) or schemes based on cyclophosphamide (18\%). Diagnoses were mainly AML/ MDS (43,4\%), ALL (14,3\%) and NHL (11.1\%). They were HLA identical $(n=13)$, unrelated $(n=46)$ and haploidentical (n 4). An average of $0,13 \mathrm{ml} / \mathrm{kg}$ recipient body weight (0.03-0.37) ABO-mismatched erythrocytes were infused, without differences between groups.

Median RBC recovery was 30 days (40 in RIC and 29 in MA, $p=0.6$ NS). We identified 6 cases of APSR, all of them on group $\mathrm{O}$ recipients from a group $\mathrm{A}$ donor but, contrary to expectations, mainly in myeloablative group.

Medians to reach neutrophil and platelet engraftments were 19 and 24 days respectively, with 5 platelet units transfused until day +60 and 6 RBC units transfused until day +100 . There weren't any differences between both groups.

Conclusions: In our series, we have observed a tendency to a delayed RBC recovery in the RIC group, although not statistically significant. We haven't found differences in terms of transfusion requirements nor in PRCA, unlike some of previous reports. This could be due to the fact that in our reduced intensity conditioning group, it predominated regimens based on alkylating agents, which may present higher activity against host plasma cells than other agents as cyclophosphamide, as previously hypothesized [2].

In conclusion, studies with more homogeneous groups of patients are required to identify which RIC conditionings are more likely to cause immunohematological complications in ABO- incompatible transplants.

\section{References:}

1. Hefazy M et al. Transfusion 2016 Feb;56(2):518-27.

2. Kanda et al. Br J Heamatol 2002; 118, 128-131.

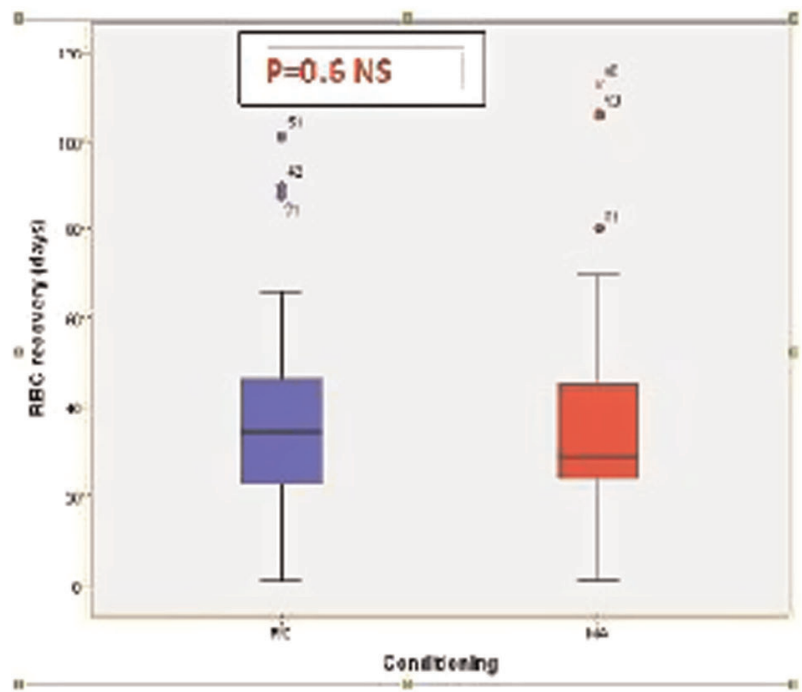

[P178 Figure] [Image 1: $R B C$ recovery (days) in $R I C$ and $M A$ conditioning]

Conflict of interest: None of the authors has disclosed any conflicts of interest.

\section{P179}

Incorporation of GSTA1 genetic variants into a population pharmacokinetic model for IV Busulfan in paediatric hematopoietic stem cell transplantation

Tiago Nava ${ }^{1,2}$, Nastya Kassir ${ }^{3}$, Mohamed Azis Rezgui ${ }^{4}$, Chakradhara Rao S. Uppugunduri ${ }^{1,5}$, Patricia Huezo-Diaz Curtis ${ }^{1,5}$, Yves Théoret ${ }^{4}$, Liane Esteves Daudt ${ }^{6}$, Catherine Litalien ${ }^{4}$, Marc Ansari, ${ }^{1,5}$, Maja Krajinovic $^{4}$, Henrique Bittencourt ${ }^{4}$

${ }^{1}$ CANSEARCH Research Laboratory, University of Geneva, Faculty of Medicine, Geneva, Switzerland; ${ }^{2}$ University Hospital of Geneval University of Geneva, Department of Pediatrics, Geneve, Switzerland; ${ }^{3}$ Certara Strategic Consulting, Montreal, Canada; ${ }^{4}$ Centre Hospitalier Universitaire Sainte-Justine, Université de Montréal, Montreal, Canada; ${ }^{5}$ University Hospital of Geneva/University of Geneva, Pediatrics, Geneve, Switzerland; ${ }^{6}$ Hospital de Clínicas de Porto Alegre, Porto Alegre, Brazil

Background: Busulfan $(\mathrm{Bu})$ is an alkylating agent used before hematopoietic stem cell transplantation (HSCT). In children, it is associated with high pharmacokinetic (PK) variability, in spite of using the intravenous (IV) formulation. As such, population PK (PopPK) models using anthropometric data such as weight and/or age are needed for $\mathrm{Bu}$ dosing in children and adolescents. Genetic functional variants in GSTA1, gene coding for (the main metabolizing enzyme of $\mathrm{Bu}$ ) have been consistently associated with $\mathrm{Bu} \mathrm{PK}$ and were recently successfully introduced into an adult PopPK model. Hence, the 
investigators aim was to develop a paediatric PopPK model for IV Bu to investigate the effect of the GSTAl genotype on PK parameters.

Methods: Bu concentration-time data was collected from 112 patients from 0.1 to 20 years of age (median 5.4 years) who received IV $\mathrm{Bu}$ during the conditioning regimen prior to HSCT in a single center. Both $\mathrm{q} 6 \mathrm{~h}$ and $\mathrm{q} 24 \mathrm{~h}$ administration schedules were included. Weight, sex, baseline disease (malignant vs. non-malignant), age, conditioning regimen ( $\geq 2$ alkylating agents $v s$. others) and GSTA1 haplotypes, grouped as diplotypes, were evaluated as covariates of PK parameters by using non-linear mixed effects analysis. The ability to achieve the target $\mathrm{AUC}_{24 \mathrm{~h}}$ (3,600 - 6,000 $\mu \mathrm{M} \cdot \mathrm{min}$ or its equivalent for q6h infusions) after the first dose was assessed by estimating the first dose based on the present PopPK model. Results were compared to other available models in children, including current dosing recommendations from regulatory agencies.

Results: A total of 115 transplantations were included. Raw Bu concentrations from six- (q6h) and eight-timepoint (q24h) PK profiles were available for the analysis, from the first dose (100\%) up to the fourth day of drug administration $(22 \%)$. A one-compartment model with first-order elimination best described the data. Allometric scaling of weight and an age-based factor of $\mathrm{Bu}$ metabolism maturation (Fmat) were included in the base model. GSTA1 diplotypes were found to be the only significant covariate of $\mathrm{Bu}$ clearance (CL) and explained $27 \%$ of the between-subject variability. Bu CL was $7 \%$ faster and $12 \%$ slower for rapid and poor metabolizers, respectively, in comparison with normal metabolizers. $\mathrm{Bu}$ doses calculated using the parameters of the proposed PopPK model were estimated to achieve the target AUC in $85.2 \%$ of the cases, against $75 \%$ with the currently available most performing models and $37.4 \%$ and $42.6 \%$ when doses were calculated according to the current recommendations from European (EMA) and American (FDA), respectively.

Conclusions: The present study provides the first PopPK model for IV Bu in children and adolescents that incorporates a genetic background (GSTAl genetic variants) explaining the variability in PK parameters. The present model, by adapting the treatment to an individualized metabolic potential, intends to minimize fluctuations of the drug exposure, which may decrease the occurrence of treatment-related toxicities in more susceptible individuals. A prospective study is required to validate the current findings.

Conflict of interest: The authors declare no conflict of interest

\section{P180}

Intensified conditioning with fludarabine/melphalan plus total body irradiation for acute myeloid leukemia with minimal residual or active disease

Sarah Lindner, Fabian Lang, Tobias Berg, Julia Riemann, Zuzana Jedlickova, Saskia Gueller, Rosa Toenges, Juliane Steinmann, Hans Martin, Hubert Serve, Gesine Bug

University Hospital Frankfurt, Goethe University, Frankfurt, Germany

Background: In acute myeloid leukemia (AML), outcome after allogeneic hematopoietic stem cell transplantation (HSCT) in patients with active or minimal residual disease (MRD) at transplantation remains poor, but may be improved by providing highly efficient antileukemic drug(s) shortly before a reduced intensity conditioning regimen.

Methods: We retrospectively analyzed the impact of melphalan $\left(140 \mathrm{mg} / \mathrm{m}^{2}\right)$ followed by fludarabine $(120 \mathrm{mg} /$ $\mathrm{m}^{2}$ ) and total body irradiation (TBI, 4-8 Gy; FLU/MEL/ TBI, $\mathrm{n}=27)$ compared to fludarabine $\left(100-120 \mathrm{mg} / \mathrm{m}^{2}\right)$, cytarabine $\left(4000-8000 \mathrm{mg} / \mathrm{m}^{2}\right)$, amsacrine $\left(400 \mathrm{mg} / \mathrm{m}^{2}\right)$ in addition to fludarabin $\left(50-60 \mathrm{mg} / \mathrm{m}^{2}\right)$ and intravenous busulfan $(6.4-9.6 \mathrm{mg} / \mathrm{kg}$; FLAMSA-RIC, $\mathrm{n}=12)$ on the outcome of 39 consecutively transplanted AML patients with MRD (FLU/MEL/TBI: $\mathrm{n}=5$ ) or active disease (FLU/ MEL/TBI: $\mathrm{n}=22$; FLAMSA-RIC: $\mathrm{n}=12$ ) between $01 /$ 2011-06/2017. Disease status prior to transplant was primary refractory $(n=27)$ or relapsed AML $(n=12)$. MRD was determined by quantitative polymerase chain reaction (qPCR) using mutated NPM1 $(\mathrm{n}=4)$ and CBFBMYH11 $(n=1)$. Active disease was defined as $\geq 5 \%$ bone marrow (BM) blasts or extramedullary disease. All patients received a T cell-replete, HLA-matched donor graft, mostly peripheral blood stem cells (95\%). Graft-versus-hostdisease (GvHD) prophylaxis consisted of cyclosporine A and mycophenolate mofetil; additional antithymocyte globulin was applied in the unrelated donor setting.

Results: In the FLU/MEL/TBI group, patients were significantly younger ( 48 vs. 67 years, $p=0.008$ ) and had a significantly lower median Hematopoietic Cell Transplantation-Comorbidity Index (0 vs. $3, \mathrm{p}=0.001)$. Patients in the FLU/MEL/TBI group were more likely to have an adverse-risk karyotype ( $45 \%$ vs. $17 \%, \mathrm{p}=0.049$ ), whereas more patients in the FLAMSA-RIC group presented with secondary AML (58\% vs. $10 \%, \mathrm{p}=$ 0.004). Median BM blast count (23 vs. $28 \%, \mathrm{p}=0.907$ ) and time from diagnosis/relapse to transplant (2.5 vs. 2.4 months, $\mathrm{p}=0.871$ ) did not differ between groups. Six patients $(15 \%)$ were grafted from a matched related donor, 29 patients $(74 \%)$ from a 10/10 matched unrelated donor (MUD), 4 patients (10\%) from a 9/10 MUD. All patients engrafted. With a median follow-up of 19 months (range, 267), 2-year overall survival (OS) was $81 \pm 7 \%$ and significantly superior in the FLU/MEL/TBI vs. FLAMSARIC group $(86 \pm 6 \%$ vs. $66 \pm 14 \%, p=0.043$, Figure 1$)$, relapse-free survival was $82 \pm 9 \%$ vs. $58 \pm 14 \%(\mathrm{p}=0.015)$. 


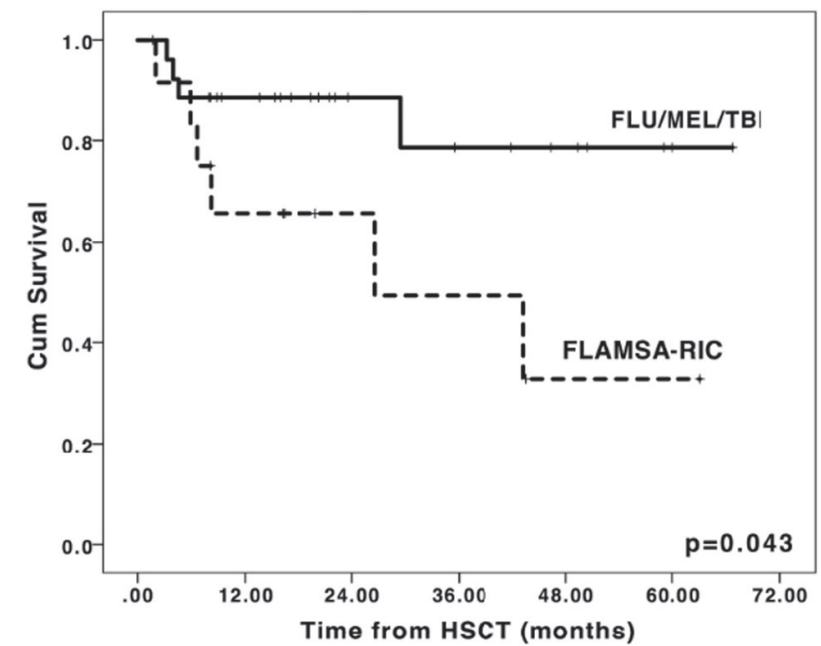

[P180 Figure] [Figure 1 Probability of overall survival by conditioning regimen]

Relapse after FLU/MEL/TBI occurred in 4 patients with high-risk genetics (complex aberrant karyotype with loss of TP53 ( $\mathrm{n}=2)$, RUNX1 mutation $(\mathrm{n}=1)$, FLT3-ITD $(\mathrm{n}=$ 1)) and 1 patient with extramedullary disease. No relapse was seen in patients with MRD positive disease at transplantation. There was no non-relapse mortality after 2 years in both groups. Cumulative incidences of acute GvHD grade 3-4 at day +100 and chronic severe GvHD at 1 year did not differ between groups $(3 \pm 0 \%$ vs. $8 \pm 1 \%, \mathrm{p}=0.895$ and $11 \pm 0 \%$ vs. $8 \pm 0 \%, \mathrm{p}=0.589$ ).

Conclusions: Our data suggest that addition of TBI to FLU/MEL is well tolerated and associated with favorable OS in patients with active or MRD positive AML, who face a similarly high risk of relapse. Thus, an intensified conditioning regimen such as FLU/MEL/TBI may also be considered for MRD positive AML.

\section{Conflict of interest:}

F. Lang received support from the Frankfurter Förderung "Nachwuchswissenschaftler" and the EUTOS funding program, had advisory roles at Novartis, Ariad, Sanofi Aventis, and Bristol-Myers Squibb and received funding from Novartis.

T. Berg received funding by Alexion, Abbvie, Astellas, Celgene and had advisory roles at Riemser.

G. Bug received honoraria from Eurocept and funding by the Alfred und Angelika Gutermuth-Stiftung and the Verein Knochenmarktransplantation/Gentherapie Frankfurt (KGF).

The other authors have nothing to disclose.

\section{P181}

\section{BUSULFAN THERAPEUTIC AUC IN CHILDREN UNDERGOING ALLOGENIC HAEMATOPOIETIC STEM CELL TRANSPLANTATION}

Hemalatha Doss ${ }^{1}$, Adam Gassas ${ }^{2}$, Patricia Breslin' ${ }^{2}$, Michelle Cummins $^{2}$, Colin Steward ${ }^{2}$, Christina Moris ${ }^{2}$, Jessica Fuidge ${ }^{2}$, Stephen Palmer ${ }^{1}$, Ponni Sivaprakasam ${ }^{2}$

${ }^{1}$ University of Bristol, Haem/Onc/BMT, Bristol, United Kingdom;

${ }^{2}$ University of Bristol, Bristol, United Kingdom

Background: In children, age or weight based Busulfan $(\mathrm{Bu})$ dosing is used with drug monitoring to achieve therapeutic area under the curve (AUC). Achieving a specific target is important for the success of allogeneic HSCT and also to reduce hepatic toxicity.

Objectives: 1.This study looks at the accuracy of achieving target AUC with the standard weight based intravenous (IV) $\mathrm{Bu}$ dose. 2. The accuracy of achieving target AUC after dose adjustment. 3. Toxicity, in particular veno-occlusive disease (VOD), in relation to Bu AUC.

Methods: Weight based dosing for Busulfan were used as follows: $2 \mathrm{mg} / \mathrm{kg}$ for $<9 \mathrm{~kg} ; 2.4 \mathrm{mg} / \mathrm{kg}$ for $9-<16 \mathrm{~kg} ; 2.2 \mathrm{mg} /$ $\mathrm{kg}$ for $16-23 \mathrm{~kg} ; 1.9 \mathrm{mg} / \mathrm{kg}$ for $>23-34 \mathrm{~kg}$ and $1.6 \mathrm{mg} / \mathrm{kg}$ for $>34 \mathrm{~kg}$. Bu was administered every 12 hours as a 3 hour infusion with a maximum of 8 doses. AUC levels were measured after the first dose and $\mathrm{Bu}$ dose was adjusted, if necessary. If dose adjustment was made, a repeat level after dose 5 was measured in some patients. The maximum allowed increment was $50 \%$ of the first dose. The different AUC target ranges were $70-100 \mathrm{mg} / \mathrm{L}$.hr for myeloablative conditioning (MAC), 60-65mg/L.hr for reduced intensity conditioning (RIC) and 45-65mg/L.hr for children with haemophagocytic lymphohistiocytosis (HLH). Study design was a retrospective analysis.

Results: Between October 2014 to November 2017, we identified 34 children with a median age of 8.4 yrs (range: $0.7 \mathrm{yrs}-16.2 \mathrm{yrs}$ ) who received Bu-based conditioning for allogeneic HSCT. Fourteen out of the 34 patients $(41 \%)$ achieved target AUC without requiring dose modification. All of these children had no VOD.

Dose modification was done in the remaining 59\% children. Out of the 20 children who needed dose modification, 17 required dose increment and had received MAC. 13/17 had repeat AUC to confirm the achievement of final AUC. Nine of the 13 achieved the target AUC based on the repeat level at dose 5. Four out of these 9 patients developed VOD requiring treatment with Defibrotide and 1 patient died from VOD. The remaining 4 out of the 13 patients (30\%) didn't achieve the target in spite of maximum dose increment and none of them developed VOD. All patients engrafted except for one child with early death due to severe, progressive VOD at $\mathrm{D}+18$.

Conclusions: Only $41 \%$ of the patients achieved target AUC based on the standard weight dosing. Those who required dose increase and achieved AUC target at dose 5 had a high incidence of VOD (45\%). A different model of 
$\mathrm{Bu}$ dose adjustment that also includes genetic factors such as the glutathione S-transferase (GST) gene variation may be required for accurate $\mathrm{Bu}$ dosing without increasing toxicity.

Conflict of interest: nothing to declare

\section{P182}

\section{Long Term Outcome of Patients Receiving Nonmyeloablative Conditioning and Allogeneic Hematopoietic Cell Transplantation for Advanced Haematologic Malignancies}

Si Ying Tang ${ }^{1,2}$, Yeh Ching Linn ${ }^{3}$, Bee Choo Tait, Michelle LM Poon $^{1}$, Lip Kun Tan ${ }^{1}$, Belinda Tan ${ }^{1}$, Yelly Lim ${ }^{1}$, Teck Guan Soh ${ }^{1}$, Davanaliz Gonzales Ramos ${ }^{1}$, William YK Hwang ${ }^{3}$, Liang Piu Koh ${ }^{1,2}$

${ }^{1}$ National University Cancer Institute, Department of HaematologyOncology, Singapore, Singapore; ${ }^{2}$ National University Hospital, Department of Medicine, Singapore, Singapore; ${ }^{3}$ Singapore General Hospital, Department of Haematology, Singapore, Singapore;

${ }^{4}$ National University of Singapore, Saw Swee Hock School of Public Health, Singapore, Singapore

Background: Nonmyeloablative (NM) hematopoietic cell transplantation (HCT) has extended the potential curative treatment option of allografting to patients in whom it was previously contraindicated due to advanced age or comorbidity.

Methods: From 2001 to 2011, 62 patients aged 16 to 66 years (median age 45 years) with haematologic malignancies, who were poor candidates for conventional HCT, received NM conditioning with fludarabine $90 \mathrm{mg} / \mathrm{m}^{2}$ and total body irradiation (TBI) 200c-Gy, followed by filgrastim-mobilized peripheral blood stem cell transplant from HLA identical $(n=57)$, matched unrelated $(n=2), 5 /$ 6 mismatched sibling $(\mathrm{n}=3)$ donors. A calcineurin inhibitor and mycophenolate mofetil with/without methotrexate were used for postgrafting immunosuppression. Diagnoses include acute leukemia $(\mathrm{n}=18)$, myelodysplastic syndrome and myelofibrosis $(\mathrm{n}=19)$, lymphoma $(\mathrm{n}=$ 7), CML $(\mathrm{n}=8)$ and myeloma $(\mathrm{n}=10)$.

Results: With a median follow up of 10 years (range 0.9 to 13.4 years) in surviving patients, the 10-year overall survival (OS) was 43\%, Based on disease risk index (DRI), the OS was $61 \%, 47 \%$ and $11 \%$ for patients with low risk, intermediate risk and high/very high risk group respectively. The day 120 cumulative incidence of acute graft versus host disease (GVHD) grade 2-4, and grade 3-4 were $40 \%$ and $19 \%$, respectively. The day 60 cumulative incidence of chronic GVHD was $41 \%$. At 5 years, non relapse mortality (NRM) rate was $19 \%$, and relapse mortality rate (RRM) was $37 \%$. Most NRM was due to GVHD. Patients with high or very high risk DRI have the highest risk of RRM (HR 6.96; 95\%CI 1.13-43.1; $\mathrm{p}=$
0.037). In multivariate analysis, improved OS was seen in patients with low risk group according to the DRI (HR 0.2, 95\%CI 0.12-0.68; $\mathrm{p}=0.005$ ).

Conclusions: Allogeneic NM conditioning using fludarabine and low dose TBI, relying almost exclusively on graft-vs-tumour effect, can result in long term remission but it is still limited by high incidence of relapse, especially in high-risk diseases. Novel approaches should be explored to reduce early disease progression after HCT without increasing regimen-related toxicity.

Conflict of interest: [S. Ying Tang]: nothing to disclose

\section{P183}

Long Term Survival Outcomes of AML Patients Undergoing Hematopoietic Cell Transplantation Using FTBI-based Myeloablative Conditioning: A Single Institution Experience

Amandeep Salhotra ${ }^{1}$, Susanta Hui ${ }^{2}$, Dongyun Yang ${ }^{3}$, Joycelynne Palmer $^{3}$, Sally Mokhtari ${ }^{3}$, Haris Ali ${ }^{1}$, Ibrahim Aldoss ${ }^{1}$, Ahmed Aribi $^{1}$, Matt Mei ${ }^{1}$, Sandhu Karamjeet ${ }^{1}$, Monzr Al Malki ${ }^{1}$, Sameer Khaled $^{1}$, David Snyder ${ }^{1}$, Margaret O'Donnell ${ }^{1}$, Joseph Rosenthal ${ }^{1}$, Vinod Pullarkat ${ }^{1}$, Jeffrey Wong $^{2}$, Ryotaro Nakamura ${ }^{1}$, Guido Marcucci $^{1}$, Stephen Forman ${ }^{1}$, Anthony Stein ${ }^{1}$

${ }^{1}$ City of Hope National Medical Center, Hematology/HCT, Duarte, CA, United States; ${ }^{2}$ City of Hope National Medical Center, Radiation Oncology, Duarte, CA, United States; ${ }^{3}$ City of Hope National Medical Center, Biostatistics and Population Sciences, Duarte, CA, United States

Background: Fractionated total body irradiation (FTBI) combined with Cyclophosphamide/etoposide or intravenous (IV) busulfan combined with Fludarabine/ Cyclophosphamide, are currently used as myeloablative conditioning regimens for allogeneic hematopoietic cell transplantation (alloHCT) for patients with acute myeloid leukemia (AML). Recent data has suggested that IV busulfan may be a preferred myeloablative regimen in patients with myeloid malignancies given improved 2-year overall survival of $56 \%(95 \% \text { CI, 53-60\% })^{1,2}$. However, IV busulfan based regimens are associated with higher rates of sinusoidal obstruction syndrome. Here, we are reporting long-term survival outcomes of AML patients undergoing alloHCT who received FTBI combined with cyclophosphamide or etoposide at City of Hope Medical Center.

Methods: We analyzed the clinical outcome of 167 consecutive AML patients in CR1 (77.2\%) or CR2 (22.8\%), who underwent alloHCT from 2005-2015 at our center and were entered in a prospectively maintained institutional registry. Patients received myeloablative conditioning using FTBI (1320cGy) and cyclophosphamide (CY: $120 \mathrm{mg} / \mathrm{kg}$ ) for unrelated donor (MUD; $\mathrm{n}=48.5 \%$ ) transplant or etoposide (VP: $60 \mathrm{mg} / \mathrm{kg}$ ) for related donors $(\mathrm{n}=51.5 \%$ ). 
transplant. Patients received tacrolimus and sirolimus for graft-versus-host disease (GVHD) prophylaxis.

Results: Patient clinical characteristics are summarized in Table 1. The 6- year overall survival (OS) and disease-free survival (DFS) for the whole cohort were $60.2 \%$ (95\% CI: 51.8-67.6) and 54.8\% (95\% CI: 46.4-62.5\%), respectively (Figure 1a). The six year NRM and relapse rate were 14.9\% and $30.3 \%$, respectively. The incidence of acute GVHD grade III-IV at day +100 was $16.8 \%$, and extensive chronic GVHD incidence at 6 years was $61.8 \%$. When patients were divided into two cohorts based on transplant eras (20102015 vs. 2005-2009), we showed no difference in outcome between the two groups with a 6-year OS of $63.4 \%(95 \%$ CI: $53.3-71.9)$ vs. $52.3 \%$ (95\% CI: $36.7-65.7)(\mathrm{P}=0.28)$ and 6-year DFS of $58.2 \%$ (95\% CI: $48.1-66.9)$ vs. $47.7 \%$ (95\% CI: $32.5-61.5 \%)(\mathrm{P}=0.33)$, respectively. No statistical significant differences were observed in outcome between patient undergoing HCT in CR-1 and CR-2 (6 year OS: 62.0 and $54.4 \% ; \mathrm{p}=0.31$ ) and (6 year DFS: $54.7 \%$ and $54.7 \% ; \mathrm{p}=0.55)$, respectively. No statistically significant differences in outcome were detected between patients receiving graft from unrelated (MUD) or sibling donors [6year OS $56.1 \%$ (95\% CI: 43.8-66.6\%) vs. $64.5 \%$ (95\% CI: 52.6-74.0\%)] and 6-year DFS [54.5\% (95\% CI: 42.5-65.1) vs. $55.4 \%$ (95\% CI: 43.3-66.0\%)], respectively. Incidence of chronic GVHD did not reach statistical significance between MUD 75.5\% (95\% CI: 63.9-83.8\%) and sibling donors $65.5 \%$ (95\% CI: 54.1-74.7\%), $(\mathrm{p}=0.13)$.

Conclusions: Our data suggests using FTBI-based conditioning for AML patients results in excellent longterm OS and DFS, compares favorably to IV busulfan-based myeloablative regimens and remains clinically important as a myeloablative conditioning regimen. The majority of events occurred in the first 2 years post- alloHCT thereby resulting in achievement of OS plateau indicating a potential cure in the majority of patients. Relapse remains a major cause of treatment failure as seen in $30 \%$ of patients . Refinements in radiation techniques using targeted dose delivery methods may improve outcomes by allowing further radiation dose-escalation in high risk AML patients. 3,4

\section{Clinical Trial Registry: References:}

1. Bredeson $\mathrm{C}$, LeRademacher $\mathrm{J}$, Kato $\mathrm{K}$, et al. Prospective cohort study comparing intravenous busulfan to total body irradiation in hematopoietic cell transplantation. Blood. 2013;122(24):3871-3878.

2. Copelan EA, Hamilton BK, Avalos B, et al. Better leukemia-free and overall survival in AML in first remission following cyclophosphamide in combination with busulfan compared with TBI. Blood. 2013;122(24):3863-3870.

3. Rosenthal J, Wong J, Stein A, et al. Phase 1/2 trial of total marrow and lymph node irradiation to augment

\section{Kaplan Meier Survival Curves}

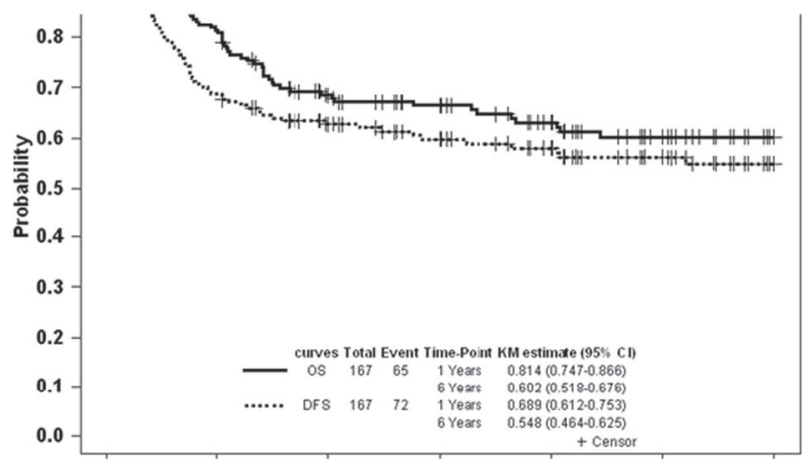

[P183 Figure] [Kaplan Meier Survival Curves]

reduced-intensity transplantation for advanced hematologic malignancies. Blood. 2011;117(1):309-315.

4. Stein A, Palmer J, Tsai NC, et al. Phase I Trial of Total Marrow and Lymphoid Irradiation Transplantation Conditioning in Patients with Relapsed/Refractory Acute Leukemia. Biol Blood Marrow Transplant. 2017;23 (4):618-624.

Conflict of interest: None

\begin{tabular}{llll}
\hline variable & $\begin{array}{l}2005-2009 \\
(\mathrm{~N}=44)\end{array}$ & $\begin{array}{l}2010-2015 \\
(\mathrm{~N}=123)\end{array}$ & $\begin{array}{l}\text { total } \\
(\mathrm{N}=167)\end{array}$ \\
\hline Age & $38.5(18.0-57.0)$ & $42.0(18.0-55.0)$ & $\begin{array}{l}41.0(18.0- \\
57.0)\end{array}$ \\
Complete Remission -1 & $31(70.5 \%)$ & $98(79.7 \%)$ & $129(77.2 \%)$ \\
CR-1 & & & \\
CR-2 and beyond & $13(29.5 \%)$ & $25(20.3 \%)$ & $38(22.8 \%)$ \\
Graft source : PBMC & $33(75 \%)$ & $115(93.5 \%)$ & $148(88.6 \%)$ \\
Bone marrow & $11(25 \%)$ & $8(6.5 \%)$ & $19(11.4 \%)$ \\
Donor Type MSD & $28(63.3 \%)$ & $58(47.2 \%)$ & $86(51.5 \%)$ \\
MUD & $16(36.4 \%)$ & $65(52.8 \%)$ & $81(48.5 \%)$ \\
Conditioning Regimen & $12(27.3 \%)$ & $66(53.7 \%)$ & $78(46.7 \%)$ \\
FTBI/Cy & & & \\
FTBI/VP-16 & $32(72.7 \%$ & $57(46.3 \%)$ & $89(53.3 \%)$ \\
\hline
\end{tabular}

\section{[[P183 Table] Table 1]}

\section{P184}

Long-term follow-up of patients undergoing allogeneic hematopoietic stem cell transplantation for relapsed or refractory follicular lymphoma

Risa Hashida, Jun Kato, Shinichiro Okamoto, Takehiko Mori

Keio University School of Medicine, Division of Hematology, Tokyo, Japan

Background: Although the options for the treatment of follicular lymphoma are increasing, allogeneic hematopoietic stem cell transplantation (allo-HSCT) is considered the 
only curative treatment. We analyzed the long-term outcome of patients with relapsed/refractory follicular lymphoma who underwent allo-HSCT by using reducedintensity conditioning.

Methods: In this single-institute study using the database and medical records, patients who underwent allo-HSCT for relapsed or refractory follicular lymphoma by using fludarabine plus melphalan-based reduced-intensity conditioning at Keio University Hospital (Tokyo, Japan) were selected and analyzed. Twenty-five patients were analyzed, and the median age at transplant was 51 years (range, 3462). Conditioning regimen consisted of fludarabine (125 $\left.\mathrm{mg} / \mathrm{m}^{2}\right)$ and melphalan $\left(140 \mathrm{mg} / \mathrm{m}^{2}\right)$. Types of donor and graft were bone marrow or peripheral blood stem cell from HLA-identical sibling $(\mathrm{N}=7)$, bone marrow from unrelated donor $(\mathrm{N}=16)$, and cord blood from unrelated donor $(\mathrm{N}=2)$. All statistical analyses were performed with EZR software.

Results: There was no case of early death before engraftment and all patients achieved neutrophil engraftment at a median of 17 days (range, 11-25) after transplantation. With a median follow-up period of 104 months (range, 29 to 166), 9-year overall survival and progression-free survival rates were $88.0 \%(95 \% \mathrm{CI}$, 67.3-96.0) and 76.0\% (95\%CI, 54.2-88.4), respectively.

There were three cases of TRM whose causes were GVHD in 2 patients (5.6 and 13.4 months post-transplant) and bacterial infection in 1 (1.2 months post-transplant). Two-year cumulative incidence of TRM was $12.0 \%$ (95\% CI, 2.9-28.1\%). Disease progression/relapse was observed in three patients at 4.0, 5.0, and 9.4 months after transplantation. However, all patients re-achieved complete response solely by the discontinuation of immunosuppressant and were alive without disease at 104, 122, and 125 months after transplantation.

Conclusions: These findings suggest that allo-HSCT using a reduced-intensity conditioning for relapsed or refractory follicular lymphoma could provide a favorable long-term survival rate with low incidences of TRM and disease progression.

In addition, allo-HSCT has a potential to induce clinically significant graft-versus-lymphoma effect leading to a durable disease-free survival even in patients with disease progression after transplantation.

Conflict of interest: None of the authors has anything to disclose.

\section{P185}

Low incidence of interstitial pneumonitis and chronic kidney disease post myeloablative stem cell transplant using novel forward planned intensity modulated IMRT total body irradiation technique
Emily Durie $^{1}$, Emma Nicholson ${ }^{2}$, Chloe Anthias ${ }^{2}$, Emma Dunne $^{1}$, MIke Potter ${ }^{2}$, Mark Ethell' ${ }^{2}$, Christina Messiou ${ }^{3}$, Joy Brennan ${ }^{2}$, Sally Eagle $^{1}$, Westley Ingram ${ }^{4}$, Frank Saran ${ }^{1}$, Henry Mandeville ${ }^{1}$

${ }^{1}$ Royal Marsden Hospital, Radiotherapy, London, United Kingdom; ${ }^{2}$ Royal Marsden Hospital, Haematology, London, United Kingdom;

${ }^{3}$ Royal Marsden Hospital, Raidology, London, United Kingdom;

${ }^{4}$ Institute of Cancer Research, Royal Marsden Hospital, Physics, London, United Kingdom

Background: Total body irradiation (TBI) is an integral component of a variety of myeloablative conditioning regimens prior to allogeneic haematopoetic stem cell transplant (HSCT). TBI can be associated with significant potentially life threatening toxicities post transplant. Reported rates of radiation induced interstitial pneumonitis (IP) post TBI are between 11-31\%, with an associated mortality rate of nearly $50 \%$ and chronic kidney disease is reported in approximately $30-44 \%$ of long term survivors. We undertook a retrospective service evaluation of patients following the introduction of a forward planned, organ sparing, intensity modulated radiotherapy technique (FP IMRT), assessing the incidence of IP and CKD post full intensity HSCT.

Methods: We retrospectively analysed 74 adult patients who received TBI between July 2009 and February 2016. Indication for HSCT were: $\operatorname{ALL} / \mathrm{LBL}(\mathrm{n}=37)$; AML/MDS $(\mathrm{n}=33), \mathrm{CML}-\mathrm{BC}(\mathrm{n}=2)$ and $\mathrm{NHL}(\mathrm{n}=2)$. Patients were conditioned with Cyclophosphamide/TBI, etoposide/TBI or Fludarabine/Cyclophosphamide/TBI and received stem cells from matched sibling donor $(\mathrm{n}=18)$, matched unrelated donor $(\mathrm{n}=50)$, mismatched unrelated donor ( $\mathrm{n}$ $=1,9 / 10 \mathrm{C}$ mismatch) or mismatched cord donor $(\mathrm{n}=6)$. 58 patients received $\mathrm{T}$ cell depletion with Alemtuzumab. GVHD prophylaxis was Ciclosporin $(\mathrm{n}=58)$ or Ciclosporin/Methotrexate $(\mathrm{n}=16)$. TBI was dosed at $14.4 \mathrm{~Gy}$ in 8 fractions over 4 days, prescribed at $100 \%$; lungs and kidneys both receiving a reduced mean dose of maximally 12-12.5Gy. IP was defined as multilobar infiltrates on CT with symptoms of dyspnoea. Renal dysfunction was defined as an eGFR $<60$ for $>3$ months.

Results: Median age at transplant was 27.6 years(range 17.0-46.1 yrs). The estimated 4 year overall survival and progression free survival rate was $71 \%(95 \%$ CI $58-80)$ and $65 \%$ (95\% CI 52-76) respectively with median follow up time of survivors of 3.9 years(range 1.2-7.7 yrs). Cumulative incidence (CI) of relapse at 4 years was $14 \%(95 \%$ CI 7 24). CI of grade III/IV aGVHD was $11(95 \%$ CI 5-19) and of chronic GVHD was 32\%(95\% CI 21-44). Non Relapse Mortality at 100 days was 3\%(95\% CI 1-8) and at 4 years $20 \%(95 \%$ CI 11-32). Rates of IP due to any cause were $30 \%$ with 16 of 22 patients $(73 \%)$ having positive proven evidence of bacterial or viral infection at that time. Seven 
patients $(9.5 \%)$ died from chest sepsis at a median of 246 days after TBI(range 89- 2413 days). Rate of idiopathic IP was $8 \%$ (CTCAE Grade $\geq 1$ ); with only 2 patients suffering IP of CTCAE grade $\geq 3$. IP developed at a median of 146 days (range 10-406 days) post TBI. Of the 53 long term survivors only two developed CKD, one of whom had biopsy confirming thrombotic microangiopathy. No patients died of CKD.

Conclusions: This novel FP IMRT based TBI technique, with reduced lung and kidney dose, resulted in significantly lower rates of radiation-induced IP and CKD compared to the published literature. Idiopathic IP of CTCAE Grade $\geq 3$ was observed in just $2.7 \%$ of patients. The risk of long term renal dysfunction is very low, with 1 secondary to thrombotic microangiopathy, which can be associated with either GVHD or was TBI related. This series has confirmed FP IMRT-based TBI a safer conditioning for myeloablative HSCT than other published radiation techniques.

Clinical Trial Registry: N/A

Conflict of interest: N/A

\section{P186}

\section{Low regimen-related toxicity and high engraftment including in unrelated donor transplants using fludarabine/ treosulfan/thiotepa/alemtuzumab-based conditioning in Diamond Blackfan Anaemia patients with severe iron overload}

Samar Elbahy ${ }^{1,2}$, Denise Bonney ${ }^{1}$, Prashant Hiwarkar ${ }^{1}$, Helen Campbell, Robert Wynn ${ }^{1}$

${ }^{1}$ Royal Manchester Children's Hospital, Department of Bone Marrow Transplant, Manchester, United Kingdom; ${ }^{2}$ Faculty of Medicine, Benha University, Department of Paediatrics, Benha, Egypt

Background: Diamond Blackfan Anaemia (DBA) is a rare, constitutional red cell aplasia in which many patients require regular red cell transfusion, and where the natural history includes bone marrow failure (BMF) and myeloid malignancy. BMF and iron overload constitute indications for Bone marrow transplantation (BMT) but regimenrelated toxicity is increased in patients with significant iron overload, as in haemoglobinopathy. Reduction of intensity of conditioning is associated with an increased risk of graft failure. Fear of transplant-related toxicities in DBA has largely restricted transplant to those with a sibling donor. In this retrospective survey we report the success of transplant in heavily iron-loaded DBA patients, including with unrelated donors, following fludarabine/treosulfan/thiotepa and alemtuzumab conditioning therapy. We achieve the twin goals of high engraftment and low procedure-related morbidities.
Methods: Five DBA patients (one boy and four girls) received allogeneic BMT at Royal Manchester Children's Hospital, between 2014 and 2017. Standard pre-transplant investigations were carried out including ferriscan in all and liver biopsy in most. Fludarabine (150mgs/m2), Treosulfan (42gms $/ \mathrm{m} 2)$, Thiotepa $(10 \mathrm{mgs} / \mathrm{kg})$ and Alemtuzumab $(0.3 \mathrm{mgs} / \mathrm{kg}$ in family donors, and $1 \mathrm{mg} / \mathrm{kg}$ in unrelated donors) conditioning regimen was given. Three patients had identical related donor, and the other two had matched unrelated donor, all donors were 10/10 HLA allele-matched, in the graft versus host $(\mathrm{GvH})$ direction at least, and bonemarrow harvested stem cells were collected. Transplantation was done according to standard protocols. Outcomes and complications following transplantation were recorded.

Results: Median age at transplant was six years and one month old (Range, three years and seven months old to seven years and six months old). Ferriscan showed severe hepatic iron overload in all patients with a median of 14.85 $\mathrm{mg} / \mathrm{gram} / \mathrm{dry}$ weight (Range, 11.3 to $20.5 \mathrm{mg} / \mathrm{g} / \mathrm{dw}$ ). Liver biopsy showed fibrosis and iron overload in all and fibrosis in some, the Ishak stage was recorded (3/6). Prophylactic defibrotide was given where there was hepatic fibrosis. All patients were engrafted successfully after transplant at a median of 12 days (Range, 10 to 14 days). All remain donor-cell engrafted with red cell transfusion independence, and donor chimerism of $95 \%$ or more was established and sustained in all of them. All patients survived with a median follow up of one year and two months (Range, six months to one year and ten months). There was no veno-occlusive disease, or any acute graft versus host disease (GVHD) > grade 2, or any chronic GVHD.

Conclusions: These conditioning regimens were found to be safe and effective in patients with severe iron overload, including in unrelated donor HSCT. We commend such a protocol in those with a red cell disorder and severe transfusion-related iron-overload. We did not find liver biopsy and its results to influence outcomes and have abandoned.

Conflict of interest: The authors have nothing to disclose.

\section{P187}

Abstract previously published

\section{P188}

Modified BEAM schedule with Lomustine instead of Carmustine in the conditioning regimen of autologous stem cell transplantation in Lymphoma patients: analysis of efficacy and toxicity

Elisabetta Terruzzi, Matteo Parma, Marilena Fedele, Elisa Diral, 
Cecilia Brambilla, Andrea Aroldi, Carlo Gambacorti-Passerini, Pietro Enrico Pioltelli

University of Milano-Bicocca, Dept. of Hematology and Bone Marrow Transplantation, Monza, Italy

Background: The treatment of choice for relapsed/ refractory lymphoma consists of high dose chemotherapy (HDC) followed by autologous stem cell transplantation (ASCT). In our Institution the classical BEAM conditioning regimen was modified by using Lomustine $(\mathrm{CCNU})$ in place of Carmustine (BCNU).

Methods: Between 2009 and 2016, 90 lymphomas (79 Non-Hodgkin Lymphoma and 11 Hodgkin Lymphoma) patients (pts) were treated with CEAM regimen followed by ASCT.

The CEAM regimen consisted of: CCNU $200 \mathrm{mg} / \mathrm{m} 2$ on day -6; Etoposide $200 \mathrm{mg} / \mathrm{m} 2$ (total dose $800 \mathrm{mg} / \mathrm{m} 2$ ) on days -5 to -2 ; Cytarabine $400 \mathrm{mg} / \mathrm{m} 2$ (total dose $1600 \mathrm{mg}$ / $\mathrm{m} 2$ ) on days -5 to -2 ; Melphalan $140 \mathrm{mg} / \mathrm{m} 2$ on day -1 . Peripheral Blood Stem Cells (PBSC) were infused on day 0.

Results: The median duration of hospitalization was 20 days (range 16-57). No pts died during hospitalization. The median number of $\mathrm{CD} 34+$ cells infused was $5.22 \times 10^{\wedge} 6 / \mathrm{kg}$ (range $1.72-15.35$ ). Median time to hematopoietic engraftment (neutrophilic one when more than 500/ mmc) occurred on day 16 (range 7-56). Fever of unknown origin during neutropenia occurred in 82 patients $(91 \%)$, whereas etiology was defined in 8 pts $(9 \%)$ and only 2 had a bacterial pneumonia. Gastrointestinal mucositis was observed in 81 pts (90\%), whose 47 had grade I-II (52.3\%), 30 grade III (33.3\%) and 4 pts grade IV (4.4\%).

Median follow-up was 3.56 years (yrs) (range 1.02-7.39); median overall Survival (OS) was not reached at 5 yrs; besides, median event-free survival (EFS) was reached at $4.06 \mathrm{yrs}$ (Figure 1). Transplant-related mortality at +100 was $0 \%$. Finally, 25 pts $(25.5 \%)$ died due to disease progression.

Conclusions: In this series we found that CEAM schedule is well tolerated without significant regimenrelated complications in pts with sensitive disease. No pts died of TRM at +100 .

When compared to the most widely used BEAM regimen, the results of CEAM seem neither to add significant toxicity nor to be inferior in terms of clinical outcomes.

Therefore, we can conclude that Lomustine can be a valid alternative to Carmustine as conditioning regimen for ASCT in lymphoma pts without losing clinical effectiveness.

Anyway, further strategies should be investigated for pts with refractory lymphomas.

\section{Conflict of interest:}

C. Gambacorti-Passerini: consultancy, honoraria and research funding from Pfizer; consultancy from BMS.

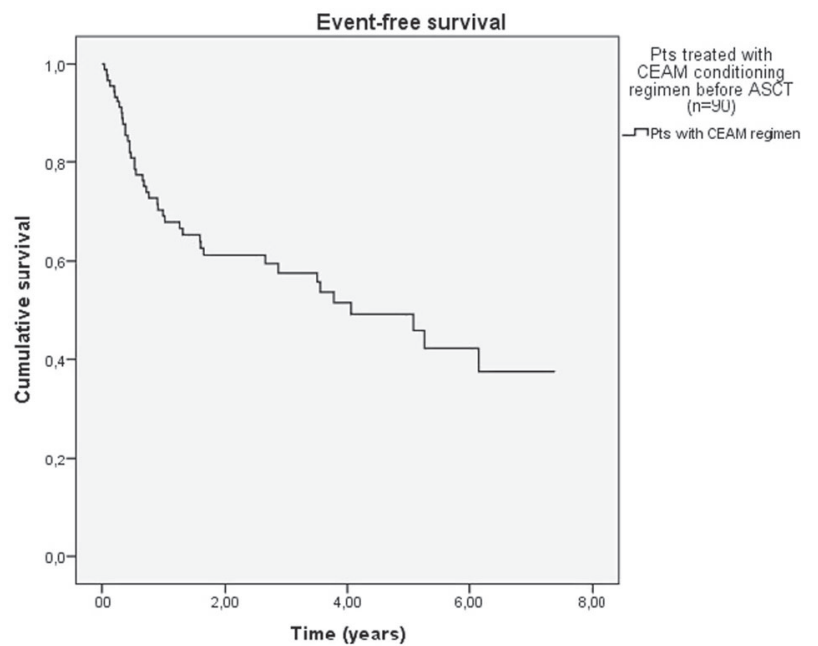

[P188 Figure] [EFS in pts affected by NHL and HL who underwent modified BEAM regimen (CEAM)]

Other authors: nothing to declare.

\section{P189}

Myeloablative versus Reduced-Intensity Hematopoietic Cell
Transplantation for Acute Myeloid Leukemia and
Myelodysplastic Syndrome: single center experience

Khalid Halahleh ${ }^{1}$, Abdallah Al Tell ${ }^{2}$, Sarah Odaily', Tala AlAwabdeh ${ }^{3}$, Shanta Sharma ${ }^{4}$, Rula Najjar, Rawad Rihani ${ }^{6}$, Eman Khatab $^{7}$, Mayada Abu Shanab ${ }^{8}$, Nilly Hussien ${ }^{9}$, Hashem Hasan ${ }^{10}$, Mohammad Ma'kosa ${ }^{11}$, Husam Abu-Jazar ${ }^{1}$, Abdelghani Tbachi' ${ }^{12}$, Mahmoud Sarhan ${ }^{13}$

${ }^{1}$ King Hussein Cancer Center, Adult Medical Oncology Hematology, Bone Marrow Transplantation Program, Amman, Jordan; ${ }^{2}$ King Hussein Cancer Center, Adult Medical Oncology Hematology, Amman, Jordan; ${ }^{3}$ King Hussein Cancer Center, Adult Medical Oncology, Amman, Jordan; ${ }^{4}$ King Hussein Cancer Center, Department of Cell Therapy and Applied Genomics, Amman, Jordan; ${ }^{5}$ King Hussein Cancer Center, Clinical Pharmacy Department, Bone Marrow Transplantation, Amman, Jordan; ${ }^{6}$ King Hussein Cancer Center, Pediatric Medical Oncology Hematology, Bone Marrow Transplantation Program, Amman, Jordan; ${ }^{7}$ King Hussein Cancer Center, Bone Marrow Transplnatation, Amman, Jordan; ${ }^{8}$ King Hussain Cancer Center, Pediatric Oncology Hematology, Bone Marrow Transplnatation Program, Amman, Jordan; ${ }^{9}$ King Hussein Cancer Center, Amman, Jordan; ${ }^{10}$ King Hussein Cancer Center, Pediatric, Medical Oncology Hematology Department, Bone Marrow Transplantation, Amman, Jordan; ${ }^{11}$ King Hussain Cancer Center, Adult Medical Oncology Hematology, Bone Marrow Transplantation Program, Amman, Jordan; ${ }^{12}$ King Hussein Cancer Center, Department of Cell THerapy and Appliedallel Genomics, Amman, Jordan; ${ }^{13}$ King Hussein Cancer Center, Bone Marrow

Transplantation, Amman, Jordan

Background: Allogeneic hematopoietic cell transplantation (HCT) is an effective, potentially curative treatment for advanced or high-risk acute myeloid leukemia (AML) and myelodysplastic syndrome (MDS) using different intensity 
conditioning regimens(1).The optimal regimen intensity before HCT is unknown.Myeloablative conditioning (MAC) is associated with a reduced risk of relapse, but does not translate to improved survival due to increased transplant-related mortality(TRM).Reduced-intensity conditioning (RIC) allowed extension of HCT to a much wider patient population by reducing the toxicity without compromising HCT efficacy. In this retrospective study, we compared the results of myeloablative and reduced intensity conditioning in 65 patients with AML and high risk MDS at King Hussein Cancer center.

Methods: We performed a single center retrospective analysis on 65 adult patients with high risk myelodysplastic syndrome (MDS) or acute myeloid leukemia (AML), who underwent HCT at King Hussein Cancer Center from January-2008 till December-2016. We compared the outcome of patients who underwent MAC versus RIC in regard to overall and progression free survival, cumulative incidence of relapse and TRM using Kaplan-Meier method. Forty-four cases received MAC and 21 received RIC .Fortysix patients had HCT in CR1, 16 in CR2 and 3 in CR3 . MAC was given to 29 patients in CR1 and 15 in CR2 and CR3. RIC was given to 17 patients in CR1 and 4 patients in CR2.

Results: Median age at time of HCT was 42 years (range: 17-69).All patients received PBSC as graft source. After a median follow up of 27.9 months, the probability of 5-year progression free survival (PFS) was $41.5 \% \pm 10.2 \%$ and 5 year overall survival (OS) was $34 \% \pm 8.3 \%$ for the whole group. PFS and OS at 5 years for those who received HCT in CR1/CR2 was $53.4 \pm 10.7 \%$ and $32.3 \% \pm 11.1 \%$ after $\operatorname{MAC}(\mathrm{P}-0.1478)$ and $27.4 \pm 14.1 \%$ and $38.85 \pm 10.8 \%$ after RIC (P-0.5256) respectively. Patients who received MAC experienced more acute GvHD compared to those received RIC (40.0\% versus $34.8 \% \%, \mathrm{P}-0.675)$ and the 5-year TRM was not different $(50 \%$ versus $46.2 \%, \mathrm{P} \quad 0.823)$.Relapse incidence at 5 years in CR1 patients was $38.77 \%$ in MAC,

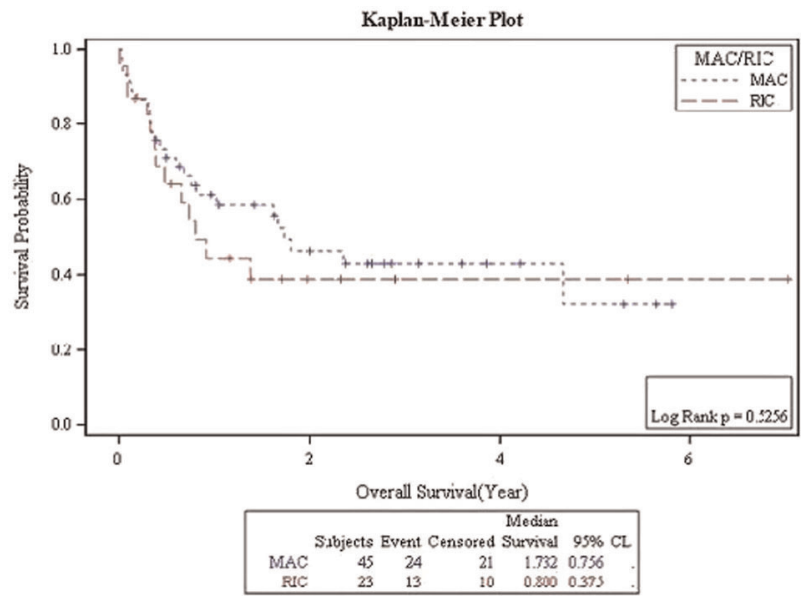

[P189 Figure] [Overall Survival MAC Vs RIC] which is not statistically different from $62.00 \%$ (P-0.1354) in RIC patients.

Conclusions: There was no statistically significant difference in Overall and progression free survival between patients who received MAC vs. RIC. RIC resulted in higher relapse rate compared with MAC and lower transplantrelated mortality but not statistically significant. These data support the use of MAC as the standard of care for fit patients with AML and high risk MDS.

Conflict of interest: No conflict of interest

\section{P190}

Outcomes of Allogeneic Hematopoietic Stem Cell Transplantation with Different Doses of Antithymocyte Globulin Containing Conditioning in Hematological Malignancies

Zafer Gokgoz ${ }^{1}$,Ender Soydan', Ayla Gokmen ${ }^{1}$, Onder Arslan ${ }^{2}$, Osman Ilhan', Muhit Ozcan ${ }^{2}$

${ }^{1}$ Medicana Ankara International Hospital, Hemaology and Bone Marrow Transplantation Unit, Ankara, Turkey; ${ }^{2}$ Ankara University Faculty of Medicine, Hematology, Ankara, Turkey

Background: Allogeneic hematopoietic stem cell transplantation is a potentially curative strategy in many of the hematologic malignancies. Greft versus host disease (GVHD) remains one of the major causes of morbidity and mortality. Antithymocyte globulin (ATG) reduces the incidence of GVHD in the matched unrelated, mismatched unrelated and haploidentical-donor transplantation. The optimal dose of ATG is currently unclear.

The aim of this study was to evaluate the different ATG doses on outcomes of patients with hematological malignancies under HCT.

Methods: Allogeneic hematopoietic stem cell transplantation is a potentially curative strategy in many of the hematologic malignancies. Greft versus host disease (GVHD) remains one of the major causes of morbidity and mortality. Antithymocyte globulin (ATG) reduces the incidence of GVHD in the matched unrelated, mismatched unrelated and haploidentical-donor transplantation.The optimal dose of ATG is currently unclear.

The aim of this study was to evaluate the different ATG doses on outcomes of patients with hematological malignancies under HCT.

Results: Neutrophil and thrombocyte engraftment periods are 15 days and 16.5 in ATLG 30 group vs. 18 days and 17 days in ATG45 group respectively. Acute GVHD (grade II-IV) seen $18 \%(\mathrm{n}=4)$ in ATLG30 group vs $16 \%(\mathrm{n}=4)$ in ATLG45 group. Chronic GVHD (grade II-IV) in ATLG30 group is $38 \%(n=7)$ and $16 \%(n=3)$ in ATLG45 group.Controversly to classical knowledge CMV 
reactivation was more common in ATLG30 group (41.7\% vs $36 \%$ ). Hospitalization was 28.5 days in ATLG30 group and 28.5 days in ATLG45 group. Relaps rates are 25\% (n $=6)$ in ATLG30 group vs $15 \%(\mathrm{n}=4)$ in ATLG45 group. Overall, in ATLG30 group 75\% $(\mathrm{n}=18)$ of patients and in ATLG 45 group $88 \%(\mathrm{n}=23)$ patients are alive. DFS 220 days median (20-799) in ATLG30 group vs 100 (10761) days in ATLG 45 group. OS was 230 days median (20-799) in ATLG30 group and 110 days (20-799) in ATLG 45 group.Table1

Conclusions: ATG as part of allogeneic conditioning regimens is associated with lower acute and chronic GVHD incidence but increases viral infections especially CMV, higher relaps rates and ATG toxicities with dose dependence. Here we compared ATLG $30 \mathrm{mg} / \mathrm{kg}$ and ATLG45 $\mathrm{mg} / \mathrm{kg}$ doses. In ATLG45 group DFS and OS are lower than ATLG30 group. Other parameters were not statistically different. ATLG30 can be safely given in mismatched and mathced unrelated allogeneic transplantations.

Conflict of interest: Nothing to disclose

\section{P191}

\section{Outcomes of Allogeneic Stem Cell Transplant for Elderly Patients with Hematologic Malignancies}

\section{Jingmei Hsu ${ }^{1}$, Usama Gergis ${ }^{1}$, Sebastian Mayer ${ }^{1}$, Adrienne Phillips $^{1}$, Tsipora Shore ${ }^{1}$, Danielle Guarner ${ }^{1}$, Ronit Reich-Slotky ${ }^{2}$, Melissa Cushing ${ }^{2}$, Koen Van Besien ${ }^{1}$ \\ ${ }^{I}$ Weill Cornell Medical Center/New York Presbyterian Hospital, Department of Medicine, Division of Hematology/Oncology, New York, NY, United States; ${ }^{2}$ Weill Cornell Medical Center/New York Presbyterian Hospital, Department of Pathology, New York, NY, United States}

Background: Reduced intensity conditioning regimens, improved HLA matching, and better supportive care allow allogeneic stem cell transplant (alloSCT) to be offered to elderly patients population. CIBMTR data showed that only small percentage of eligible AML patients between 65 to 74 years actually underwent allogeneic $\mathrm{SCT}^{1}$ and comprehensive alloSCT outcome data from aging population is still largely lacking. In this study, we examine the practice and outcome of older patients who underwent allogeneic stem cell transplant for hematologic malignancies at Weill Cornell Medical Center.

Methods: Patients > 65 years old who underwent alloSCT from Jan 2012 to Nov 2017 for hematologic malignancies with HLA-identical related and unrelated donors, or with combined haplo-cord donors were included. Probability of overall survival (OS) and progression-free survival (PFS) were calculated using the Kaplan-Meier estimator. Probabilities of non-relapse related mortality
(NRM), relapse related mortality, acute and chronic GVHD were calculated using $\mathrm{R}$ statistics using cumulative incidence estimates to accommodate competing risks.

Results: We identified 127 patients older than 65 years who underwent allogeneic stem cell transplant for hematologic malignancies. The median age for the cohort was 69 years (range, 66-77). Thirty (24\%) underwent MRD SCT, 46 (44\%) MUD SCT and 39 (31\%) combined halpoidentical/umbilical cord alloSCT. Two patients, age 70 and 72 underwent double cord alloSCT. AML (68\%) was the most common indication for transplant, followed by MDS/MPN (32\%) and NHL (22\%). Forty-seven (37\%), 27 (21\%) and $53(42 \%)$ has low, intermediate and high ASBMT disease risk index prior to transplant. One hundred and eight $(85 \%)$ patients received fludarabine/melphalan-based conditioning regimen with either ATG or Alemtuzumab. Twelve patients $(10 \%)$ received fludarabine/busulfan-based and 7 patient (5\%) with BEAM and other regimens.

The median time to neutrophil engraftment was 13 days (8-37) and platelet engraftment was 17 days (9-169). Cumulative Incidence of NRM was $13 \%$ at 100 days, and $33 \%$ and $38 \%$ at 1 and 2 years respectively. Cumulative incidence of relapse was $25 \%$ and $31 \%$ at 1 and 2 years. Cumulative incidence of grade II/IV aGVHD at 3 and 6 month was $11 \%$ and $25 \%$, and cGVHD at 6,12 and 24 month was only $1.7 \%, 3.5 \%$ and $3.5 \%$ respectively. With a median follow up for survivors of 18 months, 1 and 2 year PFS were $34 \%(95 \% \mathrm{CI}+0.085-0.084)$ and $22 \%$ (95\%CI $+0.087-0.077)$. One and 2 year OS were $43 \%$ (95\% $\mathrm{CI}+0.087-0.09)$ and $31 \%(95 \% \mathrm{CI}+0.09-0.086)$ respectively (Figure 1).

Either age (>70 vs $<70$ ), ASBMT risk score, nor donor type (MUD vs MRD vs Haplo-cord) predicted for the outcome in this patient cohort.

Conclusions: A significant proportion of our elderly patients had high-risk disease risk index. Older patients can

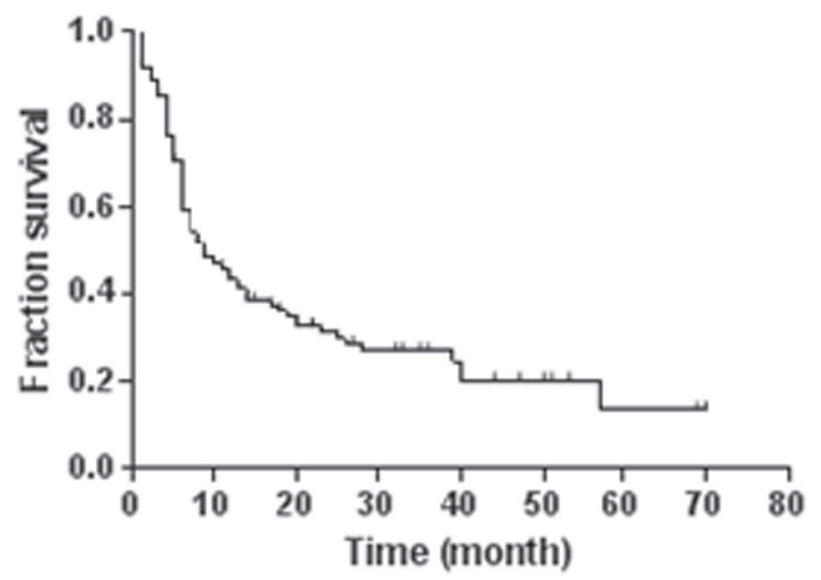

[P191 Figure] [Kaplan-Meier Survival Curve of Elderly Patients Underwent Allogeneic Stem Cell Transplant] 
benefit from RIC alloSCT with HLA matched and alternative donor sources without discernible impact of donor source on outcome. Better predictors for outcome in this patient population need to be identified. Using Alemtuzumab based in vivo - $\mathrm{T}$ cell depletion for MRD, MUD or haplo-cord transplantation, the incidence of chronic GVHD is extremely low. This should contribute to quality of life among survivors.

Conflict of interest: None

\section{P192}

\section{Pharmacokinetics and Safety of Intravenous Busulfan in Children with High Risk Neuroblastoma and Ewing Sarcoma Undergoing Autologous Stem Cell Transplantation using Busulfan based Conditioning Regimen}

\author{
Hila Rosenfeld Keidar ${ }^{1}$, Edna Efrati ${ }^{2}$, Daniel Kurnik ${ }^{2,3}$, Sabina \\ Edelman , Alina Tal ${ }^{1}$, Marcela Broitman ${ }^{1}$, Jawad Abu Rizk ${ }^{1}$, Rina \\ Dvir $^{1}$, Dror Levin ${ }^{1}$, Michal Manisterski ${ }^{1}$, Sivan Achituv ${ }^{1}$, Ronit \\ Elhasid $^{1,4}$ \\ ${ }^{1}$ Sourasky Tel Aviv Medical Center, Department of Pediatric Hemato- \\ Oncology, Tel Aviv, Israel; ${ }^{2}$ Rambam Health Care Campus, \\ Laboratory of Toxicology, Pharmacology and Pharmacogenetics, \\ Haifa, Israel; ${ }^{3}$ Technion-Israel Institute of Technology, Rappaport \\ Faculty of Medicine, Haifa, Israel; ${ }^{4}$ Tel Aviv University, Sackler \\ Faculty of Medicine, Tel Aviv, Israel
}

Background: : Busulfan (BU)-based conditioning regimens is widely used in the pediatric population in the setting of hematopoietic stem cell transplantation. Oral BU has a narrow therapeutic window and previous studies have shown wide intrapatient and interpatient variations in the pharmacokinetic (PK) behavior of the drug, especially in children. Intravenous (IV) BU has a more consistent PK profile and dosing reliability. There are few studies in children evaluating the PK of IV BU in patients undergoing allogeneic bone marrow transplantation. Nevertheless, there are no studies analyzing the safety, efficacy and PK of IV BU in children with solid tumors undergoing autologous stem cell transplantation using $\mathrm{BU}$ and melphalan as conditioning regimen.

Methods: Between November 2010 and November 2017, 26 consecutive pediatric patients undergoing autologous stem cell transplantation using conditioning regimen of IV BU and melphalan were retrospectively analyzed. There were 19 males and 7 females with median age of 10.08 years, Range: 1.08-23.66 years. Fourteen patients had high risk neuroblastoma and 12 patients had metastatic or relapsed Ewing sarcoma. IV BU was administrated every 6 hours for a total of 16 doses. The initial BU dose was determined according to patients' weight: 9 to $<16 \mathrm{~kg}$ $(1.2 \mathrm{mg} / \mathrm{kg}), 16-23 \mathrm{~kg}(1.1 \mathrm{mg} / \mathrm{kg}),>23-34 \mathrm{~kg}(0.95 \mathrm{mg} / \mathrm{kg})$ and $>34 \mathrm{~kg}(0.8 \mathrm{mg} / \mathrm{kg})$. Each dose was given over 2 hours, and sample blood was drawn at nine separate points over 6 hours following the second BU dose. The desired area under the curve (AUC) was 1150 $\mu$ mol-min/L. Dose modification was done if required.

Results: In 15/26 patients (58.8\%) the targeted AUC was achieved using weight-adjusted dosing approach without a need for dose modification. In 11/26 (42.2\%) patients BU dose was modified; increased in $6 / 11$ patients $(54.5 \%)$ and decreased in $5 / 11(45.5 \%)$ patients. BU was well tolerated without associated neurotoxicity. Hepatic veno-occlusive disease developed in one patient only $(3.8 \%)$ who had high AUC of $1700 \mu \mathrm{mol}-\mathrm{min} / \mathrm{L}$ following the second BU dose, although dose reduction of $32 \%$ was applied. All patients engrafted and were alive at 100 days post transplantation.

Conclusions: Although IV BU is considered to have a more consistent $\mathrm{PK}$ profile, our results demonstrated the need for IV BU routine AUC monitoring. In almost half of our patients, weight-adjusted dosing did not result in the desired AUC and dose adjustment was needed. This resulted in optimization of systemic drug exposure and reduced toxicity. Further prospective studies analyzing larger cohorts of patients are needed to consolidate our results.

Conflict of interest: nothing to disclose

\section{P193}

Phase 1 study of myeloablative conditioning combined with plerixafor in high-risk leukemia patients undergoing allogeneic hematopoietic stem cell transplantation

Takehiko Mori, Jun Kato, Masatoshi Sakurai, Yuya Koda, Keiichi Tozawa, Shinichiro Okamoto

Keio University School of Medicine, Division of Hematology, Tokyo, Japan

Background: In high-risk patients, leukemia relapse is the most important factor interfering with the success of allogeneic hematopoietic stem cell transplantation (HSCT). Plerixafor is a CXCR4 inhibitor, which has been approved for stem cell mobilization in autologous HSCT setting. By interacting with $\mathrm{CXCR} 4$, plerixafor also has a potential to mobilize leukemic cells, which could contribute to the chemosensitization. In this Phase 1 study, we evaluated the safety of myeloablative conditioning combined with plerixafor in allogeneic HSCT for high-risk leukemia or myelodysplastic syndrome (MDS) patients.

Methods: Patients were eligible with high-risk leukemia defined as follows: acute leukemia (AML or ALL)

1) not in complete remission,

2) in complete remission (CR) with risk factors (e.g. cytogenetics);

chronic myeloid leukemia (CML) 
1) in accelerated phase or blast crisis,

2) in second chronic phase; advanced MDS (RAEB-1 or -2). Patients were prospectively enrolled and the study was performed by using a $3+3$ design escalating the total dose of plerixafor from 0.36 to 0.48 and $0.72 \mathrm{mg} / \mathrm{kg}$ at maximum. Plerixafor was given subcutaneously 8 hours before delivering total-body irradiation (TBI) or chemotherapeutic agents, which was divided in 2 or 3 days. Conditionings were TBI (12 Gy)-based myeloablative in combination with chemotherapeutic agents such as cyclophosphamide, cytarabine, and etoposide. All sources of stem cells except for that from a haploidentical donor were eligible. Tacrolimus or cyclosporine and short-term methotrexate were given for GVHD prophylaxis. This study was approved by ethics committee of Keio University School of Medicine.

Results: The dose of plerixafor was successfully escalated to the maximum dose without dose-limiting toxicities. In total, 9 patients, 3 each for 3 doses of plerixafor, were enrolled and underwent allogeneic HSCT. Median age was 32 years (range, 22-54). Underlying diseases were AML $(\mathrm{N}=4), \operatorname{ALL}(\mathrm{N}=3), \mathrm{CML}(\mathrm{N}=1)$, and MDS (RAEB-2, $\mathrm{N}=1$ ). In 5 of the 7 evaluated patients, leukemic cells were positive for CXCR4 expression. Stem cell sources were related peripheral blood stem cells (PBSC, $\mathrm{N}=3$ ), unrelated bone marrow or PBSC $(\mathrm{N}=4)$, and cord blood $(\mathrm{N}=2)$. As adverse events, plerixafor administration was associated with Grades 2-3 diarrhea $(\mathrm{N}=7)$ and abdominal pain $(\mathrm{N}=4)$, which was observed shortly after the administration. Other toxicities within 28 days after transplantation were generally consistent with myeloablative conditioning and allogeneic HSCT, and all patients achieved neutrophil engraftment within 22 days without early deaths. In 4 patients, leukemic cell mobilization into the peripheral blood after plerixafor administration was confirmed by morphological or molecular method. At the time of analysis, 5 patients were alive in remission with a median follow-up period of 31 months (range, 23-33 months).

Conclusions: Plerixafor-combined myeloablative conditioning was well tolerated in adult patients undergoing allogeneic HSCT. Leukemic cell mobilization into the peripheral was observed in half of the patients. A future study is required to further evaluate the efficacy and safety of this regimen.

Clinical Trial Registry: Thi study was registered at http://www.umin.ac.jp/ctr/index.htm (UMIN000009485).

Conflict of interest: S. Okamoto: research funding from Sanofi K.K. All other authors: nothing to disclose
P194

Reduced Intensity Conditioning and Dual T-Cell Suppression with Anti-Thymocyte Globulin and PostTransplant Cyclophosphamide for the Prophylaxis of GraftVersus-Host Disease in Haploidentical Stem Cell Transplants

Maria Queralt Salas, Arjun Datt Law, Wilson Lam, Fotios V Michelis, Santhosh Thyagu, Dennis (Dong Hwan) Kim, Jeffrey Howard Lipton, Hans Messner, Auro Viswabandya

Princess Margaret Cancer Centre, Allogeneic Blood and Marrow Transplant Program, Department of Medical Oncology and Hematology, Toronto, Canada

Background: Haploidentical hematopoietic cell transplantation (haploHCT) is potentially curative for patients with high-risk hematologic malignancies. Existing conditioning regimens using post-transplant cyclophosphamide (PTCy) for peripheral blood stem cell (PBSC) grafts have been limited by higher rates of chronic graft-versus-host disease (GVHD). This may be ameliorated by the addition of anti-thymocyte globulin (ATG). This study evaluates the results of haploHCT after reduced intensity conditioning (RIC) with ATG, PTCy \& cyclosporine for prevention of graft rejection and GVHD.

From August 2016-September 2017, 34 adults underwent haploHCT at our center. RIC consisted of fludarabine $\left(30 \mathrm{mg} / \mathrm{m}^{2} /\right.$ day) on days -5 to -2 , busulfan $\left(3.2 \mathrm{mg} / \mathrm{m}^{2} /\right.$ day) on days -3 to -2 and Total Body Irradiation (200 Gy) on day -1. T-cell replete PBSCs were infused on day 0. GVHD prophylaxis included escalating dose ATG (total $4.5 \mathrm{mg} / \mathrm{kg}$ ) from days -3 to -1 , PTCy $(50 \mathrm{mg} / \mathrm{kg} /$ day) days +3 and +4 , and cyclosporine from day +5 until day 30-50. Clinical features, post-transplant outcomes and adverse events were collected through retrospective chart review. Survival rates and time-to-event were calculated from the date of transplant to the date of event or last follow-up. Information was updated on December 2017. Survival rates were calculated at 6 months and 1 year.

Results: Median age was 56 years (22-70 years); 25 $(73.5 \%)$ patients were in first remission (CR1), 5 (14.7\%) in second remission (CR2) and $8(23.5 \%)$ had active disease. Median time to neutrophil engraftment was 16 days (range 843 days). Fifteen patients $(44.1 \%)$ developed acute GVHD (any grade). However, incidence of grades III-IV acute GVHD was only $2.9 \%$. Mild chronic GVHD was seen in $11.4 \%$ cases. No patients developed moderate/severe chronic GVHD. CMV reactivation occurred in 26 (76.5\%) cases and CMV disease in $4(11.5 \%)$. EBV reactivation occurred in 21 $(61.8 \%)$ patients but biopsy proven PTLD in only $5.8 \%$. Four patients received rituximab for symptomatic EBV reactivation or PTLD. There were no CMV, EBV or PTLD-related deaths. Six months overall survival (OS), relapse-free survival (RFS) 


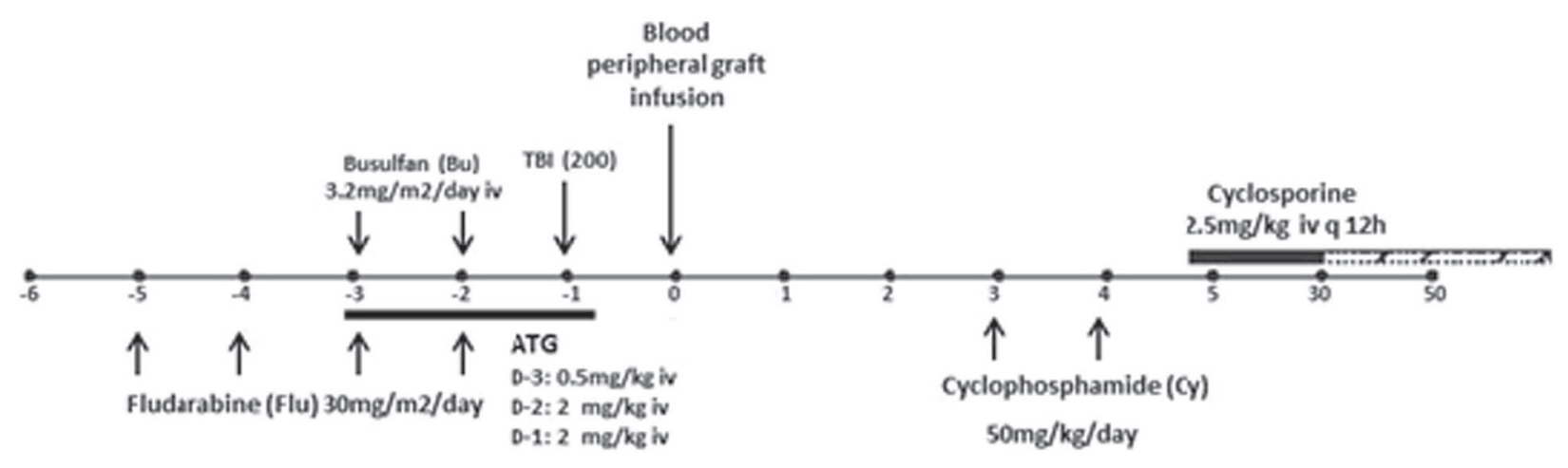

[P194 Figure] [HaploHCT conditioning and post-transplant immunosuppressive regimen]

and non-relapse mortality (NRM) were respectively $78.9 \% \%$, $64.2 \%$ and $18.4 \%$. One year OS, RFS and NRM were respectively $57.4 \%, 46 \%, 34.7 \%$. Infection was the most common cause of death $(23.5 \%)$.

Conclusions: Preliminary data suggest that unmanipulated haploidentical PBSC transplantation following RIC with ATG, PT-CY and CSA as a GVHD prevention strategy results in low rates of acute and chronic-GVHD.

Conflict of interest: Nothing to disclose Methods:

\begin{tabular}{ll}
\hline Recipient Age & Median $56(22-70)$ \\
\hline Sex Female Male & $13(38.2) 21(61.8)$ \\
Diagnosis AML MDS ALL Lymphoma Others & $22(64) 5(14.7) 1(2.9) 2$ \\
& $(5.9) 4(11.7)$ \\
Donor relation Sibling Parent Child & $10(29.4) 6(17.6) 18(59)$ \\
HLA mismatch 8/10 7/10 6/10 5/10 & $2(5.9) 3(8.8)$ None 29 \\
& $(85.3)$ \\
CMV Donor - Recipient Pos / Pos Neg / Neg Pos & $15(44.2) 3(8.8) 10$ (29.4) 6 \\
/Neg Neg / Pos & $(17.6)$ \\
CD34 cell dose/kg & $9.42(3.73-15.3)$ \\
Days to Neutrophil Engraftment & $16(8-43)$ \\
Acute GVHD Grade 3-4 & $1(2.9)$ \\
Chronic GVHD & $4(11.8)$ \\
\hline
\end{tabular}

[[P194 Table] Table 1. Clinical characteristics of donors and re]

\section{P195}

Reduced intensity conditioning with 300 cGY total body irradiation in hematologic malignancy

\footnotetext{
Jeong A Kim ${ }^{1}$, Young Hoon Park ${ }^{1}$, Eunjung Yim ${ }^{1}$, Jae-Yong Kwak ${ }^{2}$

${ }^{1}$ St. Vincent Hospital, College of Medicine, The Catholic University of Korea, Suwon, Korea, Republic of; ${ }^{2}$ Chonbuk National University Medical School \& Hospital, Jeonju, Korea, Republic of
}

Background: Low-dose total body irradiation (TBI) in reduced intensity conditioning (RIC) may reduce host- immune response and disease relapse. However, it is uncertain whether the addition of low-dose TBI to RIC has benefits in the treatment. Therefore, we conducted a prospective study to evaluate the clinical impact of lowdose TBI on outcomes after allo-peripheral blood stem cell transplantation (PBSCT) in the setting of fludarabine-based RIC.

Methods: 300cGy TBI was added to a regimen incorporating busulfan (Bu; $3.2 \mathrm{mg} / \mathrm{kg}$ for 2 days), fludarabine ( $\mathrm{Fu} ; 30 \mathrm{mg} / \mathrm{m}^{2}$ for 5 days) and antithymocyte globulin (ATG; 2.5 to $5 \mathrm{mg} / \mathrm{kg}$ ). GVHD prophylaxis was $\mathrm{CsA}+$ mycophenolate mofetil (MMF).

Results: Ten patients with hematologic malignancies (7 acute leukemia, one MDS, one lymphoma and one multiple myeloma) who underwent allo-PBSCT from HLA-matched related $(n=5)$ and unrelated donors $(n=5)$. Two patients had history of one prior auto-PBSCT. The median number of $\mathrm{CD} 4^{+}$cells was $6.0 \times 10^{6} / \mathrm{kg}$ (range, 2.4 to 23.6 ). Neutrophil engraftment was achieved in $100 \%$ of the patients with the median of 11 days. Overall survival (OS) and relapse free survival (RFS) were $68 \%$ and $90 \%$, respectively at 1 year. The cumulative incidence of acute graft-versus-host disease (GVHD), III to IV acute GVHD, and severe chronic GVHD at 1 year were 53\%, 33\%, and $22 \%$, respectively. Non-relapse mortality rate were $22 \%$ at 1 year.

Conclusions: Combination of 300 cGy TBI and fludarabine-based RIC in allo-PBSCT may stabilize donor engraftment with anti-cancer activities. However, high incidence of acute and chronic GVHD still remains problematic with the current transplantation setting. Optimal GVHD prophylaxis could be identified in this setting, and, hopefully, the outcome of allo-PBSCT would improve.

Clinical Trial Registry: NCT02885623

Conflict of interest: There are no relevant conflicts of interest to disclose 


\section{P196}

\section{REDUCED RELAPSE INCIDENCE WITH FLAMSA-TBI IN COMPARISON TO BU/FLU IN AML PATIENTS TRANSPLANTED IN CR1 OR CR2: ON BEHALF OF THE ALWP OF THE EBMT}

\begin{abstract}
Thomas Heinicke ${ }^{1}$, Myriam Labopiin ${ }^{2}$, Christoph Schmid ${ }^{3}$, Didier Blaise $^{4}$, Arne Brecht ${ }^{5}$, Jean Yves Cahn ${ }^{6}$, Yosr Hicheri ${ }^{7}$, Anne Huynh $^{8}$, Bruno Lioure, ${ }^{9}$ Noel Milpied ${ }^{10}$, Ghulam J Mufti ${ }^{11}$, Christof Scheid $^{12}$, Gérard Socie ${ }^{13}$, Bipin Savani ${ }^{14}$, Arnon Nagler ${ }^{15,16,17}$

${ }^{1}$ Otto-von-Guericke University, Hematology and Oncology, Magdeburg, Germany; ${ }^{2}$ Hospital Saint Antoine Paris France, Department of Hematology and Cell Therapy and ALWP EBMT Office, Paris, France; ${ }^{3}$ Klinikum Augsburg, Augsburg, Germany; ${ }^{4}$ Centre de Recherche en Cancérologie de Marseille (CRCM), Institut Paoli Calmettes, Department of Hematology, Marseille, France; ${ }^{5}$ DKD Helios-Klinikum, KMT- Abteilung, Wiesbaden, Germany; ${ }^{6}$ Grenoble University Hospital, Clinical Hematology, Grenoble, France; ${ }^{7}$ University Hospital, Montpellier, France; ${ }^{8}$ University of Toulouse, Toulouse, France; ${ }^{9}$ Nouvel Hopital Civil, Strasbourg, France; ${ }^{10} \mathrm{CHU}$ Bordeaux, Hôpital Haut-leveque, Bordeaux, France;

${ }^{11}$ King's College London, Haematology Department, London, United Kingdom; ${ }^{12}$ University of Cologne, Department I of Internal Medicine and Center of Integrated Oncology Cologne Bonn, Cologne, Germany; ${ }^{13}$ Hopital Saint-Louis, Paris, France; ${ }^{14}$ Vanderbilt University Medical Center, Department of Medicine, Division of Hematology-Oncology, Brentwood, TN, United States; ${ }^{15}$ Chaim Sheba Medical Center, Tel-Hashomer, Tel-Aviv, Hematology Division and Bone Marrow Transplantation, Tel Aviv, Israel; ${ }^{16}$ Tel Aviv University, Tel Aviv, Israel; ${ }^{17}$ Acute Leukemia Working Party of the EBMT, Paris, France
\end{abstract}

Background: BuFlu is a widely used non-myeloablative (NMA) conditioning regimen for patients (pts) with myeloid malignancies. The sequential reduced intensity regimen FLAMSA (Fludarabine+Ara-C+Amsacrine; Schmid et al. J Clin Oncol 2005; 23: 5675-5687) chemotherapy followed by reduced intensity conditioning (RIC-Cyclophosphamide 80-120mg/kg with TBI 400 cGy) has shown remarkable activity in relapsed/refractory $(\mathrm{r} / \mathrm{r})$ AML and in high risk AML in first or second complete remission (CR). A modification of FLAMSA has been developed in which TBI is replaced by busulfan $(\mathrm{Bu} 6.4$ $\mathrm{mg} / \mathrm{kg}$, FLAMSA-Bu). Here, we compare the outcomes of AML pts transplanted in CR1 or CR2 after conditioning with either BuFlu, FLAMSA-TBI or FLAMSA-Bu.

Methods: In this retrospective registry study pts were eligible if they had a first alloSCT for AML (de novo or secondary) in CR1 or CR2 between 1/2005 and 6/2016. Donors were matched related or unrelated with up to one mismatch at antigen or allele level. Conditioning consisted of either BuFlu (busulfan (8x0.8 $\mathrm{mg} / \mathrm{kg}$ b.w. i.v.), fludarabine $5 \times 30 \mathrm{mg} / \mathrm{m} 2$ ), or FLAMSA-TBI (fludarabine $4 \times 30 \mathrm{mg} / \mathrm{m} 2$, cytarabine 4 x $2000 \mathrm{mg} / \mathrm{m} 2$, amsacrine 4x100 $\mathrm{mg} / \mathrm{m} 2)$-TBI(4Gy), cyclophosphamide (2x40-60 mg/kg b. w.), or FLAMSA-Bu, in which TBI is replaced by busulfan $(8 \times 0.8 \mathrm{mg} / \mathrm{kg}$ b.w.). Pts had to have available information on cytogenetic risks. The three groups were compared using Kruskal-Wallis test for quantitative variables, chi-square test, or Fisher's exact test for categorical variables. Propensity score matching was applied with factors included in the model: age per deciles, status at transplant, donor type, cytogenetics risk group, sex matching, pt and donor CMV serology and use of in vivo $\mathrm{T}$ cell depletion. Comparisons were performed using weighted Cox regression.

Results: BuFlu conditioning was used in 1197 pts., whereas FLAMSA-TBI and FLAMSA-Bu were used in 258 and 141 pts, respectively. Median follow-up of survivors was 24.72 months (0.66-136.59). In univariate analysis, NRM was higher in the FLAMSA-Bu group (26.7\%) compared to FLAMSA-TBI (16.4\%) and Flu/Bu (16.1\%), respectively, $\mathrm{p}<0.01$. RI was lower in both FLAMSA groups (FLAMSA-TBI 21.9\%, FLAMSA-Bu 23.1\%) compared to $\mathrm{Flu} / \mathrm{Bu}(30.3 \%), \mathrm{p}<0.01)$. LFS at two years was significantly higher in the FLAMSA-TBI group (61.6\%) compared to FLAMSA-Bu (50.1\%) and BuFlu $(53.6 \%)$ groups, respectively, $\mathrm{p}=0.03$. A trend for better OS was seen for the FLAMSA-TBI group (68.3\%) compared to the FLAMSA-Bu group (56.4\%) and the BuFlu group (60\%), respectively, $\mathrm{p}=0.07)$. Weighted Cox regression revealed that at two years after transplantation FLAMSA-TBI compared to Bu/Flu was associated with lower RI (HR 0.64 (0.42-0.98), $\mathrm{p}=0.04)$. Also a trend for better LFS for FLAMSA-TBI compared to BuFlu was detected (HR $0.72(0.49-1.06, \mathrm{p}=0.09)$. OS, GRFS, aGVHD II-IV, aGVHD III-IV, cGVHD and ext cGVHD were not different. Comparing FLAMSA-Bu with BuFlu no statistically significant differences in any of the endpoints was detected.

Conclusions: The results of this retrospective study show that compared to BuFlu conditioning with FLAMSA-TBI leads to reduced relapse incidence at two years and to a trend for a better leukemia-free survival in AML patients transplanted in CR1 or CR2. No differences were seen when comparing BuFlu with FLAMSA-Bu. These results need confirmation in a prospective trial.

Conflict of interest: T. Heinicke: Jazz Pharmaceuticals: Travel grants

C. Schmid: MoilMed: Membership on an entity's Board of Directors or advisory committees; Incyte: Research Funding, Speakers Bureau; Novartis: Research Funding, Speakers Bureau; Celgene: Research Funding, Speakers Bureau; Jazz: Membership on an entity's Board of Directors or advisory committees

C. Scheid: Amgen: Consultancy, Honoraria; Takeda: Consultancy, Honoraria; BMS: Consultancy, Honoraria; Novartis: Consultancy, Honoraria; Sanofi: Consultancy, Honoraria; Janssen: Consultancy, Honoraria; Celgene: Consultancy, Honoraria 
G. Socié: Alexion Pharmaceuticals, Inc.: Consultancy

B. Savani: Jazz Pharmaceuticals: Speakers Bureau

All other authors: Nothing to disclose

\section{P197}

\section{Sequential chemotherapy with reduced intensity conditioning is feasible in high risk lymphomas}

Urpu Salmenniemi, Mervi Putkonen, Soile Salomäki, Marjut Kauppila, Tommi Salmi, Juha Ranti, Maija Valta, Pia Ettala, Kari Remes, Maija Itälä-Remes

Turku University Hospital, Turku, Finland

Background: Sequential conditioning regimen combining short course of intensive chemotherapy with reduced intensity conditioning (RIC) allogeneic stem cell transplant (alloSCT) has shown promising results in high risk relapsed and refractory myeloid malignancies. We report the outcome of 18 patients with advanced lymphoma who received alloSCT with sequential conditioning approach.

Methods: This retrospective analysis includes all lymphoma patients treated at Turku University Hospital that have received sequential conditioning regimen. Transplants were performed between Dec 2013 and Aug 2017. Induction chemotherapy contained fludarabine $(4 \times 30 \mathrm{mg} /$ $\left.\mathrm{m}^{2}\right)$ and cytarabine $\left(4 \times 2 \mathrm{~g} / \mathrm{m}^{2}\right)$ with or without idarubicin (2 $\mathrm{x} 10 \mathrm{mg} \mathrm{m}^{2}$ ), and after 3 days rest, was followed by RIC with cyclophosphamide $(2 \times 40-60 \mathrm{mg} / \mathrm{kg})$ and total body irradiation (TBI) 4-6 Gy. In two patients TBI was substituted with busulfan or treosulfan. Thirteen patients received transplant from unrelated donor, 2 from matched sibling donor and 3 from haploidentical family donor. Stem cell source was bone marrow in one case and GCSFmobilized peripheral blood in remaining patients.

Results: Eighteen patients with median age of 51 years (range 21-63) are included. Lymphoma subtypes were HL $(\mathrm{n}=5)$, FL $(\mathrm{n}=4)$, DLBCL $(\mathrm{n}=3)$, PMBL $(\mathrm{n}=2)$, AITL $(\mathrm{n}=2), \operatorname{MF}(\mathrm{n}=1)$ and ALCL $(\mathrm{n}=1)$. All patients had received several lines of chemotherapy and 11 patients (61\%) had received previous autologous SCT. Disease status at transplantation was CR $(\mathrm{n}=4,22 \%), \mathrm{PR}(\mathrm{n}=7$, $39 \%)$, progressive/refractory disease $(\mathrm{n}=6,31 \%)$ and untreated relapse ( $\mathrm{n}=1,6 \%$, FL and tr-MDS). The EBMT risk score was $4(\mathrm{n}=3), 5(\mathrm{n}=5)$ and $6(\mathrm{n}=10)$, predicting TRM between $29 \%$ and $37 \%$ with RIC approach.

Median follow-up time for surviving patients was 19.6 mo (3-56 mo). The incidence of grade $\geq$ II acute GVHD was $56 \%$ ( gr II $\mathrm{n}=9$, gr IV $\mathrm{n}=1$ ). Among the 13 evaluable patients $3(23 \%)$ had moderate chronic GVHD. There were no GVHD related deaths.

Thirteen $(72 \%)$ patients were alive at the time of data analysis, among them $11(61 \%)$ were in CR. TRM was $22 \%$
(4/18) and all deaths were infection-related. Three patients (17\%) have relapsed of whom one patient has died of relapse. The estimated median survival had not been reached.

Conclusions: Our results suggest that sequential chemotherapy with RIC alloSCT provides a good disease control with acceptable toxicity in lymphoma patients with high risk of both TRM and relapse.

Conflict of interest: Authors declare no conflict of interest.

\section{P198}

Sequential chemotherapy/reduced-intensity (FLAMSA-TBI) versus Treosulfan-Fludarabine (Treo-Flu) conditioning preallogeneic transplantation for patients (40-65 years) with acute myeloid leukaemia in remission. On behalf of ALWP of EBMT

Vipul Sheth ${ }^{1}$, Myriam Labopin ${ }^{2}$, Liisa Volin ${ }^{3}$, Arne Brecht ${ }^{4}$, Arnold Ganser $^{5}$, Jiri Mayer ${ }^{6}$, Hélène Labussière- Wallet ${ }^{7}$, Jörg Bittenbring ${ }^{8}$, Bipin Savani, Mohammad Mohty ${ }^{10}$, Arnon Nagler ${ }^{2,11}$

${ }^{1}$ Kings College Hospital, Department of Hematology and Stem Cell Transplant, London, United Kingdom; ${ }^{2}$ EBMT Paris Office, CERESTTC, Saint Antoine Hospital, Department of Haematology, Paris, France; ${ }^{3}$ HUCH Comprehensive Cancer Center, Stem Cell Transplantation Unit, Helsinki, Finland; ${ }^{4}$ Deutsche Klinik fuer Diagnostik, KMT Zentrum., Weisbaden, Germany; ${ }^{5}$ Hannover Medical School, Department of Haematology, Hemostasis, Oncology and Stem Cell Transplantation, Hannover, Germany; ${ }^{6}$ University Hospital Brno, Department of Internal Med. - Hematooncology, Brno, Czech Republic; ${ }^{7}$ Centre Hospitalier Lyon Sud, Pavillon Marcel Bérard - Bat $1 G$, Service Hematologie, Lyon, France; ${ }^{8}$ University of Saarland, University Hospital, Department of Internal Med., BMT Unit, Homburg, Germany; ${ }^{9}$ Vanderbilt University Nashville, Department of Hematology and Stem Cell Transplant, Vanderbilt, TN, United States;

${ }^{10}$ Saint Antoine Hospital, Department of Haematology, Paris, France;

${ }^{11}$ Institute of Hematology and Stem Cell Transplant, Chaim Sheba Medical Centre Tel Hashomer, Tel Aviv, Israel

Background: Allogeneic stem-cell transplantation (HSCT) is the most effective way to control and treat intermediate and high risk acute myeloid leukaemia (AML). FLAMSA (Schmid et al. J Clin Oncol 2005) chemotherapy followed by reduced intensity conditioning (RIC-Cyclophosphamide $120 \mathrm{mg} / \mathrm{kg}$ with $\mathrm{Bu}-4-6 \mathrm{mg} / \mathrm{kg}$, or TBI $4-6 \mathrm{cGy}$ ) is effective regimen in high-risk AML, but has not yet been extensively studied amongst patients in remission. Treosulfan in combination with Fludarabine has been shown (Treo-Flu) to be effective conditioning regimen for AML including in the higher risk setting, especially in patients above 40 years of age. Thus, we wanted to compare these two regimens (FLAMSA/RIC vs Treo-Flu), in patients aged 40-65 years.

Methods: Inclusion criteria included: age 40 to $65 \mathrm{y}$, denovo/secondary AML in CR1 or CR2, transplantation 
between 2007 and 2016, conditioning with Treo-Flu (30$42 \mathrm{~g} / \mathrm{m}^{2}$ ) or FLAMSA/RIC (FLAMSA-TBI, FLAMSA-Bu, $\mathrm{Bu} 6 \mathrm{mg} / \mathrm{kg}$, 4cGY TBI), either with a HLA-matched sibling donor (MSD) or a 9-10/10 HLA-matched unrelated donor (MUD).

Results: Total of 629 patients were included (203 with FLAMSA-TBI, 145 with FLAMSA-Bu and 281 with TreoFlu). Median follow-up was 36,18 and 18 months in the FLAMSA-TBI FLAMSA-Bu and Treo-Flu groups respectively. As compared to other groups, FLAMSA-TBI group included younger patients, who were more likely to have received MSD transplant for de novo FLT3-ITD positive AML in an earlier period. The Treo-Flu group included more patients transplanted in CR1, CMV seropositive, who were more likely to have received $\mathrm{BM}$ as stem cell source without in vivo TCD as compared to the 2 FLAMSA groups. Cytogenetics and performance status were comparable among the 3 groups. All three regimen had similar rates of engraftment (97\% and $95 \%$ and $98 \%$, respectively, $\mathrm{p}=$ 0.3). In the FLAMSA-TBI, FLAMSA-Bu and Treo-Flu groups, two years non- relapse mortality (NRM) were $15 \%$, $25 \%$ and $13 \%$; incidence of grade II-IV acute GVHD were $26 \%, 30 \%$ and $19 \%$ and 2 year incidence of extensive chronic GVHD were $14 \%, 9 \%$ and $21 \%$ respectively. As compared to Treo-Flu, relapse incidence at 2 years was lower in FLAMSA-TBI and FLAMSA-Bu,39\% for the Treo Flu vs $20 \%$ and $28 \%$ for the FLAMSA-TBI and FLAMSA$\mathrm{Bu}$ respectively, the median time being 17, 18 and 14 months respectively. Two year LFS and OS were $48 \%$, 64\% and $69 \%, 47 \%$ and $53 \%$ in the Treo-Flu, FLAMSA-TBI and FLAMSA-Bu groups. In multivariate

\section{LFS}

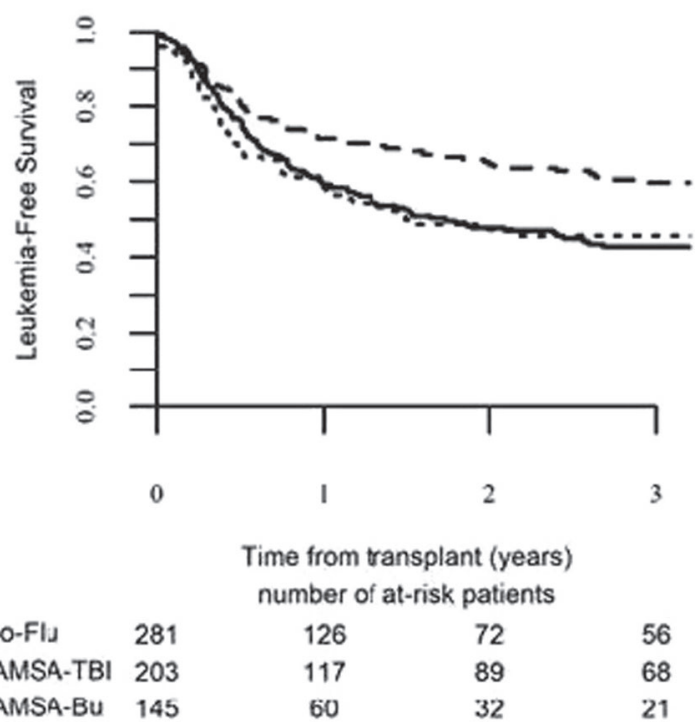

$\begin{array}{lllll}\text { - Treo-FIJ } & 281 & 126 & 72 & 56 \\ \text { - FLAMSA-TBI } & 203 & 117 & 89 & 68 \\ \ldots \text { FLAMSA-BU } & 145 & 60 & 32 & 21\end{array}$

[P198 Figure] [Leukemia free survival FLAMSA-TBI, FLAMSA-Bu and Treo-Flu] analysis, after adjusting for baseline co-variates, there were no significant differences between FLAMSA-Bu and TreoFlu groups for all outcome parameters. FLAMSA-TBI was associated with a higher incidence of acute GVHD (HR: $2.0, \mathrm{p}=0.02)$, a lower relapse rate $(\mathrm{HR} 0.44, \mathrm{p}=0.003)$ and a better LFS (HR 0.67; p = 0.04) compared to Treo-Flu.

Conclusions: In patients age 40-65 years with AML in $\mathrm{CR}$ undergoing allogeneic transplantation from sibling or unrelated donors, FLAMSA-TBI is associated with significantly better LFS and a trend towards better OS as compared to Treo-Flu, mainly due to reduction in relapse rates. While, FLAMSA-Bu is with similar overall efficacy as compared to Treo-Flu. These data may serve as the scientific background for a well design randomized study comparing the FLAMSA/RIC and the Treo Flu conditioning regimens for AML.

Conflict of interest: No conflicts of interest to declare

P199

Abstract previously published

P200

TBF conditioning regiment for allogeneic transplant in high risk acute lymphoblastic leukemia: results of Rome Transplant Network

Alessandra Picardi ${ }^{1}$, Laura Cudillo ${ }^{2}$, Raffaella Cerretti ${ }^{2}$, Gottardo De Angelis ${ }^{2}$, Adriano Venditti ${ }^{3}$, Paolo De Fabritiis ${ }^{4}$, Luca Cupelli, Andrea Mengarelli ${ }^{5}$, Francesco Marchesi ${ }^{5}$, Anna Chierichini, Agostino Tafuri ${ }^{7}$, Antonella Ferrari ${ }^{7}$, Maria Cristina Tirindelli, Ombretta Annibali ${ }^{8}$, Ernesto Pavoni ${ }^{2}$, Fabrizio Bonanni ${ }^{9}$, Antoine Bruno $^{2}$, Giulia Ciangola ${ }^{2}$, Mariarosa Battara ${ }^{10}$, Marco Andreani ${ }^{10}$, Cristina Rapanotti ${ }^{3}$, Giovangiacinto Paterno ${ }^{2}$, William Arcese ${ }^{2}$

${ }^{1}$ Tor Vergata University of Rome, Stem Cell Transplant Unit, Policlinico Universitario Tor Vergata, Biomedicina e Prevenzione, Rome, Italy; ${ }^{2}$ Tor Vergata University of Rome, Stem Cell Transplant Unit, Policlinico Universitario Tor Vergata, Rome, Italy; ${ }^{3}$ Tor Vergata University Hospital, Rome, Italy; ${ }^{4}$ Sant'Eugenio Hospital, Rome, Italy; ${ }^{5}$ IFO Regina Elena National Cancer Institute, Rome, Italy; ${ }^{6}$ Azienda Ospedaliera San Giovanni Addolorata, Rome, Italy; ${ }^{7}$ Azienda Ospedaliera Sant'Andrea, Rome, Italy; ${ }^{8}$ Campus Biomedico University Hospital, Rome, Italy; ${ }^{9}$ Tor Vergata University, Rome, Italy;

${ }^{10}$ Laboratorio di Immunologia e Biologia dei Trapianti Fondazione IME, Rome, Italy

Background: Allogeneic stem cell transplantation (ASCT) represents an important curative therapy for adult patients with high risk acute lymphoblastic leukaemia (ALL). Nowadays, data on ASCT outcomes from alternative donors, such as matched unrelated, cord blood and haploidentical sources, are available whereas few analysis are reported concerning the use of non-TBI conditioning regimen in this subset of patients. 
Methods: Between October 2006 and October 2017, 130 patients with high-risk ALL, all considered eligible for allogeneic transplant (ASCT), were referred to the Rome Transplant Network (RTN), a JACIE accredited metropolitan transplant program. Based on a pre-defined RTN policy, search for a donor followed a hierarchical selection: 1) HLA identical sibling (Id-sib); 2) matched unrelated donor (MUD); 3) Umbilical cord blood (UCB); 4) Haploidentical donor (haplo). All patients received the same myeloablative $(n=68)$ or reduced intensity $(n=13)$ conditioning regimen consisting of thiotepa, single daily dose of i.v. busulphan and fludarabine (TBF), including ATG in MUD, haplo and UCB transplant. As GVHD prophylaxis, CSA/MTX combination was given in all patients but UCB patients who received CSA/PDN, whereas in haplo recipients MMF and an anti- CD-25 MoAb was added. Of 130 patients, 40 were not transplanted because of disease progression and death $(\mathrm{n}=25,63 \%)$, donor unavailability $(\mathrm{n}=4,10 \%)$, loss of eligibility $(\mathrm{n}=6$, $15 \%)$ and 5 patients are ongoing. Finally, 90 patients (72\%) underwent ASCT: 39 MUD, 25 Id-sib, 17 haplo and 9 UCB. Excluding UCB due to the small patient number, 81 patients with a median age of 36 years (18-66) were included in this preliminary analysis. According to the disease status at time of ASCT, 40 patients were in first CR and 41 in $\geq 2$ CR or active disease.

Results: All patients engrafted with full donor chimerism either for PMN or PLTS at a median of 17 (range, 10-35) and 20 days (range, 10-67), respectively. The median number of infused CD34+ was $4.85 \times 10$ (E6)/kg (range, 0.613.5) with a significant correlation between cell dose and median time to PMN ( $\mathrm{p}=0.02)$ and PLTS $(\mathrm{p}=0.0004)$ recovery. Overall, TRM at 100 days and 2-yrs was $12.6 \%$ and $32.5 \%$, respectively; the relapse rate was $27 \%$ and the 5 -yrs OS and LFS was $34 \%$ and $42.8 \%$ respectively. The 5years OS was 55\% for MUD, 20.5\% for Id-sib and 20.6\% for haplo with a significant advantage of MUD compared to haplo-ASCT $(\mathrm{p}=0.05)$. A aGvHD and cGvHD occurred in $59 \%$ and $38 \%$ respectively with $12.5 \%$ of patients who experienced $>2$ grade aGvHD, regardless of donor's type (MUD $\mathrm{n}=2$, Id-sib $\mathrm{n}=2$ and haplo $\mathrm{n}=2$ ). Finally, patients transplanted in first $\mathrm{CR}$ experienced a significantly longer 5-yrs OS compared to the others $(40 \%$ vs $25 \%, \mathrm{p}=$ $0.04)$.

Conclusions: Although disease progression occurring during donor search is the main obstacle (63\%) to get access to ASCT, RTN policy allows a high proportion of eligible patients with high-risk ALL to be transplanted with some specific categories significantly benefiting of a favourable outcome.

Conflict of interest: none
P201

T-cell replete allografting using sequential conditioning regimen in HLA-matched versus HLA-haploidentical transplantation in older high-risk MDS and AML patients: Results of a matched pair analysis

Alessia Fraccaroli, Dusan Prevalsek, Sarah Haebe, Christoph Schul, Heidrun Drolle, Susanne Fritsch, Georg Ledderose, Johanna Tischer

University Hospital of Munich-Grosshadern, Ludwig-Maximilians University, Internal Medicine III, Hematopoietic Stem Cell Transplantation, Munich, Germany

Background: Sequential conditioning regimens are successfully used for high-risk (HR) AML/MDS in matched related (MRD) and unrelated donor (URD) hematopoietic stem cell transplantation (HSCT). However, few data are available for sequential conditioning in the context of HLAhaploidentical transplantation (haplo-HSCT), and even fewer is known regarding sequential therapy in the elderlies. To investigate the relative merits of sequential MRD-, URD- and HAPLO-HSCT, a matched-pair analysis comparing the outcome of recipients aged $\geq 50$ years was performed.

Methods: Thirty-six patients with high-risk (HR) AML/ MDS who underwent haplo-HSCT were considered for potential matching with recipients of MRD $(n=25)$ and URD $(\mathrm{n}=47) \mathrm{HSCT}$. All patients were $\geq 50$ years old, received sequential conditioning with either FLAMSA or clofarabine shortly prior to reduced-intensity conditioning (RIC) and were transplanted between January 2009 and June 2017 at our institution. In the HLA-matched setting in vivo T-cell depletion with anti-thymocyteglobuline (ATG) was performed while in haplo-HSCT PTCY was used. Matching criteria comprised

(1) disease activity (blast yes or no),

(2) disease status (relapse, refractory, high-risk) and (3) age $(+/-5 y)$.

Seventeen pts undergoing haplo-HSCT were successfully pair-matched with seventeen recipients of MSD and USD HSCT, respectively ((1) $p=1.0$; (2) $p=1.0$; (3) $p=0.95$ ). MRD and URD HSCT was uniformly performed using FLAMSA-RIC and peripheral blood stem cells (PBSCs) as stem cell source, whereas in haplo HSCT 4/17 patients received clofarabine-RIC and 9/17 patients a bone marrow graft.

Results: Median age of the entire cohort was 51 years (50-70). Each group consisted of 3 MDS patients with HR profile and 14 AML patients (5 relapse, 3 refractory, 5 unfavorable cytogenetics). Median follow-up was 4.6, 6.2 and 4 years for MRD, URD and HAPLO recipients, respectively $(p=0.32)$. No graft rejection occurred. 
Neutrophil engraftment was achieved after a median of 16 , 16 and 18 days in MSD, URD and HAPLO HSCT, respectively $(\mathrm{p}=0.26)$. Cumulative incidence $(\mathrm{CI})$ of acute GvHD $>{ }^{\circ} \mathrm{I}$ at day +100 was $35 \%$ for each group. Yet, if considering only higher grade aGvHD (III-IV ${ }^{\circ}$ ) CIs were $12 \%$ for MRD, $18 \%$ for URD and $12 \%$ for HAPLO-HSCT. CI of NRM at 1 -year was $0 \%, 6 \%$ and $18 \%$ for MSD, URD and HAPLO recipients, respectively $(\mathrm{p}=0.74)$, whereas CI of relapse was $43 \%, 18 \%$ and $12 \%$ within the first year $(\mathrm{p}=0.18)$. If comparing MRD to HAPLO HSCT a trend towards better disease control was seen in HAPLO $(\mathrm{p}=0.09)$.

No significant difference was observed in 1/3-year OS, which was $82 \% / 52 \%, 77 \% / 70 \%$ and $77 \% / 65 \%(\mathrm{p}=0.53)$ for MSD, URD and haplo recipients, respectively. 1/3-year LFS was $64 \% / 50 \%$ for MRD, $77 \% / 70 \%$ for URD and $71 \%$ / $65 \%$ for HAPLO-recipients, and as such not significantly different between the groups $(\mathrm{p}=0.37)$.

Conclusions: T-cell-replete sequential HAPLO allografting using a PTCY based GvHD prophylaxis in older aged HR MDS/AML patients is feasible and results in an outcome comparable to that of MRD and URD HSCT. Hence, it represents a valuable alternative in HR AML/ MDS of the elderly.

Conflict of interest: nothing to disclose

\section{P202}

\section{The effect of melphalan administration time on engraftment in multiple myeloma patients undergoing autologous stem cell transplantation}

Ali Unal, Esra Yildizhan, Leylagul Kaynar, Fatma Varal, Sibel Ciftci, Elmas Coskun, Gulseren Yuldı, Ayse Yanar, Bulent Eser, Mustafa Cetin

Erciyes University Faculty of Medicine, Hematology Department, Kayseri, Turkey

Background: High dose chemotherapy following by autologous stem cell transplantation is the most important step of multiple myeloma treatment. Melphalan, an alkylating agent, is the most preferable drug for conditioning regimens and dosage and timing is important with regards to side effects or engraftment timing. Engraftment time is determinative on enfections and duration of hospitalisation

Methods: We compared the neutrophil and thrombocyte engraftments retrospectively in patients with multiple myeloma who recived melphalan $200 \mathrm{mg} / \mathrm{m} 2$ single dose on day -3 and day -1 as conditioning regimen

Results: There were 29 patients reciving melphalan on day -1 and and 41 patients on day -3 . The mean neutrophil engraftmet times for day -1 group and day -3 group were
$12,8 \pm 2.4$ days and $10,4 \pm 1.3$ days, respectively $(\mathrm{p}<0,001)$. The mean thrombocyte engraftmet times for day -1 group and day -3 group were $13,48 \pm 3,7$ days and $12,7 \pm 3,3$ days, respectively ( $\mathrm{p}: 0,36)$.

Conclusions: In conclusion administiring melphalan on day -3 is beter than on day -1 in terms of neutrophil engraftment and hence in terms of duration of hospitalisation.

Conflict of interest: all authors declare that there is no conflict of interest

\section{P203}

The effectiveness of the conditioning regimen high dose melphalan with bortezomib with autologous transplantation of hematopoietic stem cells in patients with multiple myeloma

Marina Khorobrykh ${ }^{1}$, Natalia Zorina ${ }^{1}$, Natalia Minaeva ${ }^{1}$, Philipp Sherstnev ${ }^{1}$, Igor Paramonov ${ }^{2}$

${ }^{1}$ Federal State Institute of Science 'Kirov Research Institute of Hematology and Blood Transfusion of the Federal Medical and Biological Agency of Russia', Bone Marrow Transplantation, Kirov, Russian Federation; ${ }^{2}$ Federal State Institute of Science 'Kirov Research Institute of Hematology and Blood Transfusion of the Federal Medical and Biological Agency of Russia', Head, Kirov, Russian Federation

Background: Current tendencies in primary multiple myeloma (MM) patients younger than 65 years include induction with subsequent high dose chemotherapy and autologous hematopoietic stem cell transplantation (autoHSCT).

"The gold standard" of conditioning regimen is considered to be melphalan in high dose $200 \mathrm{mg} / \mathrm{m}^{2}$ (HDmel). Efforts to optimize conditioning protocols by combination of melphalan with bortezomib are made to manage drug resistance and enhance plasmatic cells sensitivity to melphalan.

Methods: During the $2002-2017$ period 131 autoHSCT to $91 \mathrm{MM}$ patient were performed in our clinic. Traditional conditioning regimen was used in 56 autoHSCT, of them 20 single and 18 - double (HDMel group). Combination of high dose melphalan with bortezomib was used in 75 autoHSCT, of them - 31 single and 22 double (Bor-HDMel group).

Before autoHSCT complete remission (CR) or very good partial remission (VGPR) was achieved in 64 patients (70\%): 25 patients (65.8\%) in HDMel group and 39 patients (73.6\%) in Bor-HDMel group. Partial remission (PR) was achieved in 13 patients (34.2\%) in HDMel group and in 14 (26.4\%) patients in Bor-HDMel group.

Toxicity profile of HDMel and Bor-HDMel conditioning regimens was assessed. 
Results: Number of patients with optimal response to treatment after autoHSCT increased to $80(88 \%), \mathrm{p}<0.05$. During monitoring period 8-174 months (median 28 months) median OS was not achieved. 5 - year and 10 -year OS was $77 \%$ and $61 \%$ respectively. 5 - year RFS was $69 \%$ with median OS not achieved. VGPR and CR were achieved in $84 \%$ and $90 \%$ in HDMel and Bor-HDMel groups respectively, $\mathrm{p}>0.05$. Use of bortezomib in conditioning regimen did not influence OS in MM patients $(\mathrm{HR}=0.351,95 \%$ CI $0.1-1.12, \mathrm{p}=0.078)$. Relapse rate in patients, who received Bor-HDMel, was 3.8 times lower, than in those, who received HDMel $(\mathrm{HR}=0.26,95 \% \mathrm{CI}$ $0.08-0.84, \mathrm{p}=0.025$ ).

In the period of myelotoxic hematopoiesis aplasia the most frequent complications was mucositis, grade I-II. The proportion of patients with peripheral polyneuropathy did not increase in Bor-HDMel group.

We didn't observe mortality, associated with autoHSCT. Hematopoietic recovery rates were similar. Addition of bortezomib to standard conditioning regimen did not increase hematologic and non-hematologic toxicity.

Conclusions: Use of conditioning regimen high dose melphalan with bortezomib increased RFS without toxicity increasing. Combination of melphalan with bortezomib could be perspective measure of autoHSCT efficiency enhancement in MM patients.

Conflict of interest: authors declare no conflict of interest.

\section{P204}

\section{Thiotepa $10 \mathrm{mg} / \mathrm{kg}$ seems superior to Thiotepa $5 \mathrm{mg} / \mathrm{kg}$ in the TBF conditioning regimen in patients undergoing allogeneic stem cell transplantation}

Jean El-Cheikh ${ }^{1}$, Radwan Massoud ${ }^{1}$, Nour Moukalled ${ }^{1}$, Basel Haffar $^{1}$, Ammar Zahreddine ${ }^{1}$, Rami Mahfouz ${ }^{2}$, Ali Bazarbachi ${ }^{1}$

${ }^{1}$ American University of Beirut Medical Center, Internal Medicine, Division of Hematology-oncology, Bone Marrow Transplant Unit, Beirut, Lebanon; ${ }^{2}$ American University of Beirut Medical Center, Department of Pathology and Laboratory Medicine, Beirut, Lebanon

Background: Allogeneic hematopoietic stem cell transplantation (allo-SCT) offers a chance of long-term remission and possibly cure for many hematologic malignancies. The conditioning regimen plays an important role and its choice is influenced by various factors, such as age of the patient, performance status, disease risk, and remission status at the time of transplantation. In this study, we compared outcomes between $5 \mathrm{mg} / \mathrm{kg}$ (T5) and $10 \mathrm{mg} / \mathrm{kg}$ (T10) Thiotepa-based conditioning regimen for allogeneic stem cell transplantation in adults with hematological malignancies.
Methods: In this retrospective study, we identified 29 consecutive patients with different hematological malignancies. Thirteen patients $(45 \%)$ received T5; the remaining 16 patients $(55 \%)$ received T10. Thirty eight percent of patients in both groups received stem cells from matched related sibling, and $62 \%$ from haplo-identical donor (haplo). Disease status at transplant was first complete remission (CR1) in 38\% and 56\% in the $\mathrm{T} 5$ and $\mathrm{T} 10$ groups respectively; CR2 was $8 \%$ and $25 \%$ respectively. None of the patients in the T10 group were transplanted in $\mathrm{CR} \geq 3$, while $23 \%$ in the $\mathrm{T} 5$ group were in $\mathrm{CR} \geq 3$. Three patients (10\%) had partial response (PR) (all received T5), while 4 patients (14\%) had progressive disease ( 3 received T10, and one T5) at time of transplant.

Results: The incidence of acute graft-versus-host disease (aGVHD) (grade > II) was 24\%, while chronic (cGVHD) occurred in $6 \%$ of the patients at 1 year. With a median follow-up of 11 months, the transplant related mortality (TRM) was $0 \%$ and $14 \%$ at 100 days and 1 year, respectively, with TRM observed only in the T5 group $(\mathrm{p}=0.016)$. The relapse incidence at 1 year was $20 \%$, none of them was in $\mathrm{CR} 1$ at the time of transplant. The one-year progression-free survival (PFS) and overall survival (OS) were 60\% and 65\%, respectively. At one year the PFS and OS were $30 \%$ vs $87 \%$ $(\mathrm{p}=0.012)$ and $46 \%$ vs $87 \%(\mathrm{p}=0.008)$ in the $\mathrm{T} 5$ and $\mathrm{T} 10$ groups, respectively. In univariate and multivariate analysis, only age at transplant and the total dose of Thiotepa had a significant impact on TRM, PFS and OS.

Conclusions: Acknowledging the retrospective nature, and the small heterogeneous population of our study; our results suggest that a Thiotepa-based conditioning for allogeneic transplantation in high risk hematological malignancies is feasible and effective, including in the haplo setting. The effect of the total dose of Thiotepa on transplant outcomes needs to be confirmed in a larger study.

Conflict of interest: All authors have nothing to disclose.

\begin{tabular}{|c|c|c|c|c|}
\hline & Total N (\%) & T5 N (\%) & $\mathrm{T} 10 \mathrm{~N}(\%)$ & $P$ value \\
\hline Number of patients & $29(100)$ & $13(45)$ & $16(55)$ & NS \\
\hline $\begin{array}{l}\text { Age at diagnosis / at } \\
\text { transplant }\end{array}$ & $\begin{array}{l}36(21-64) / \\
37(21-65)\end{array}$ & $\begin{array}{l}43(24-64) / \\
43(25-65)\end{array}$ & $\begin{array}{l}36(21-54) / \\
37(21-55)\end{array}$ & NS \\
\hline Female / Male & $\begin{array}{l}9(31) / 20 \\
(69)\end{array}$ & $\begin{array}{l}1(8) / 12 \\
(92)\end{array}$ & $\begin{array}{l}8(50) / 8 \\
(50)\end{array}$ & 0.017 \\
\hline AML/ALL & $\begin{array}{l}12(42) / 9 \\
(31)\end{array}$ & $5(38) / 2(15)$ & $\begin{array}{l}7(44) / 7 \\
(44)\end{array}$ & $\begin{array}{l}\text { NS/ } \\
\text { NS }\end{array}$ \\
\hline HL / NHL & $3(10) / 4(14)$ & $1(8) / 4(31)$ & $2(13) / 0(0)$ & $\begin{array}{l}\text { NS / } \\
0.0184\end{array}$ \\
\hline MDS & $1(3)$ & $1(8)$ & $0(0)$ & NS \\
\hline $\begin{array}{l}\text { Karyotype Normal/ } \\
\text { Abnormal }\end{array}$ & $\begin{array}{l}20(69) / 9 \\
(31)\end{array}$ & $\begin{array}{l}11(85) / 2 \\
(15)\end{array}$ & $9(56) / 7(44)$ & NS \\
\hline $\begin{array}{l}\text { Disease risk index } \\
\text { Intermediate / high }\end{array}$ & $\begin{array}{l}15(52) / 14 \\
(48)\end{array}$ & $\begin{array}{l}5(38) / 8 \\
(62)\end{array}$ & $\begin{array}{l}10(63) / 6 \\
(38)\end{array}$ & NS \\
\hline HCT-CI & $0(0-2)$ & $0(0-1)$ & $0(0-2)$ & NS \\
\hline
\end{tabular}

[[P204 Table] patients and transplant characteristics] 
Overall survival according to Thiotepa dose intensity (T5 vs T10)

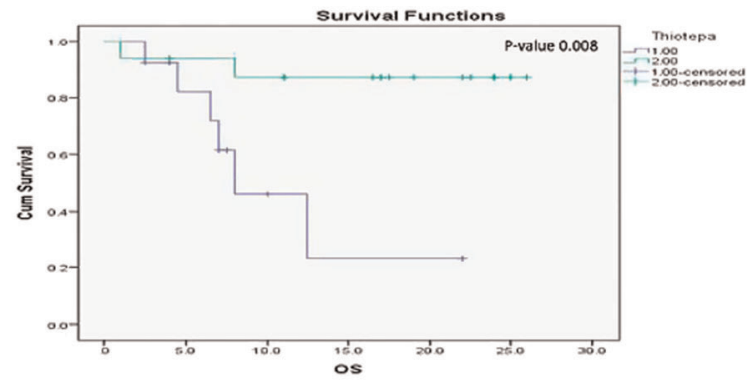

Progression Free survival according to Thiotepa dose intensity (T5 vs T10)

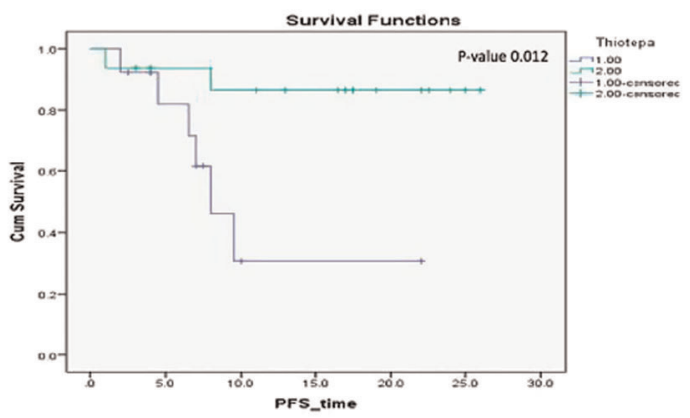

[P204 Figure] [Overall survival and Progression free survival T5vsT10]

\section{P205}

Three-month mixed T-cell chimerism followed by donor lymphocyte infusions is associated with superior outcomes in reduced-intensity alemtuzumab based allogeneic stem cell transplants for haematological malignancies

\section{Alexandros Kanellopoulos ${ }^{1}$, Graham McIlroy ${ }^{1}$, Shankara Paneesha $^{1}$, Maria Kaparou ${ }^{1}$, Bhuvan Kishore ${ }^{1}$, Richard Lovell, Shereef Elmoamly ${ }^{1}$, David Davies ${ }^{1}$, Evgenia Xenou ${ }^{1}$, Claire Horgan $^{1}$, Francesca Kinsella ${ }^{2}$, Carolina Arbuthnot ${ }^{3}$, Anton Borg ${ }^{3}$, Katie Randall, Lynn Ryann ${ }^{1}$, Katie Collins ${ }^{1}$, Kathleen Holder ${ }^{1}$, Rebecca Pryor ${ }^{1}$, Amy Gudger ${ }^{1}$, Hannah Giles ${ }^{1}$, Donald Milligan ${ }^{1}$, Emmanouil Nikolousis ${ }^{1}$}

${ }^{1}$ Heart of England NHS Foundation Trust, Birmingham, United Kingdom; ${ }^{2}$ University of Birmingham, Birmingham, United Kingdom; ${ }^{3}$ South Warwickshire NHS Foundation Trust, Warwick, United Kingdom

Background: Chimerism kinetics and donor lymphocyte infusions (DLI) are important tools for the success of reduced intensity conditioning (RIC) alemtuzumab-based allogeneic stem cell transplants (SCT), although their impact on patient outcomes has been scarcely studied.

Methods: Retrospective analysis of 132 consecutive patients with myeloid $(n=67)$ and lymphoid $(n=65)$ malignancies receiving alemtuzumab-based RIC allogeneic SCT from matched sibling (32/132, alemtuzumab dose
$30 \mathrm{mg}$ ), or matched unrelated (97/132, alemtuzumab dose $50 \mathrm{mg}$ ) donors. Median recipient age was 59 years (range 22 to 72 ) and $48(36 \%)$ were female. For myeloid disease conditioning consisted of fludarabine and melphalan (34/ $67)$, fludarabine and busulphan (32/67) or fludarabine and cyclophosphamide (1/47); whilst lymphomas were treated with fludarabine and melphalan (32/65), BEAM (10/65), fludarabine and BEAM (22/65), or fludarabine, melphalan and gemcitabine (1/65).

Results: With a median follow-up of 13 months, the median overall survival (OS) and relapse free survival (RFS) were 24 months and 15 months, respectively. There were no statistical differences in outcomes between two disease categories $(p=0.86)$. Time to transplant was significantly longer for lymphoid than myeloid transplants (median 25.5 vs 7 months, p < 0.001) but it was not associated with the OS. OS was worse in patients with highrisk lymphoid disease (high-grade, T-cell, or lymphomas in partial remission at transplant) (HR $3.1 \mathrm{p}=0.039)$. Adjusting for age, sex and disease lineage, full chimerism ( $>95 \%$ donor) in whole marrow at 3 months was correlated with superior OS (HR 0.48, p = 0.039) and RFS (HR 0.45, p = 0.009 ). Being $>95 \%$ donor in the CD3+ T-cell compartment at 3-months conferred inferior 2-year transplantrelated mortality (TRM) 8/26 (31\%), whereas no 2-year TRM was seen in 3-month mixed T-cell chimeras $(0 / 34)$ $(\mathrm{p}=0.001)$. Acute GvHD was more common in patients with lymphoid malignancies $(\mathrm{p}=0.017)$ and correlated with 3-month full donor T-cell chimerism (OR 5.7, $\mathrm{p}=0.001$ ) but not with whole blood chimerism. At 3 months, only mixed T-cell chimerism predicted which patients would go on to receive DLI $(\mathrm{p}<0.001)$. At 6 months, both mixed whole blood and T-cell chimerism predicted use of DLI ( $\mathrm{p}=0.042$ and $\mathrm{p}=0.003$, respectively). Of patients with mixed T-cell chimerism at 3 months, $23(70 \%)$ went on to have DLI. The first DLI dose was given a median of 186 days post-transplant, with patients receiving a median of 2 infusions. DLI was associated with improved survival outcomes: 20/23 (87\%) of 3-month mixed T-cell patients treated with DLI were alive at 2 years, compared with $3 / 10$ (33\%) of the mixed T-cell cohort who did not receive DLI $(\mathrm{p}=0.002)$.

Conclusions: In our patient series of RIC alemtuzumabbased allogeneic transplants, three-month full donor status in whole blood and three-month mixed T-cell chimerism followed by DLI are associated with favourable RFS and OS.

Alexandros Kanellopoulos and Graham McIlroy contributed equally to this work.

Conflict of interest: All authors have nothing to disclose. 


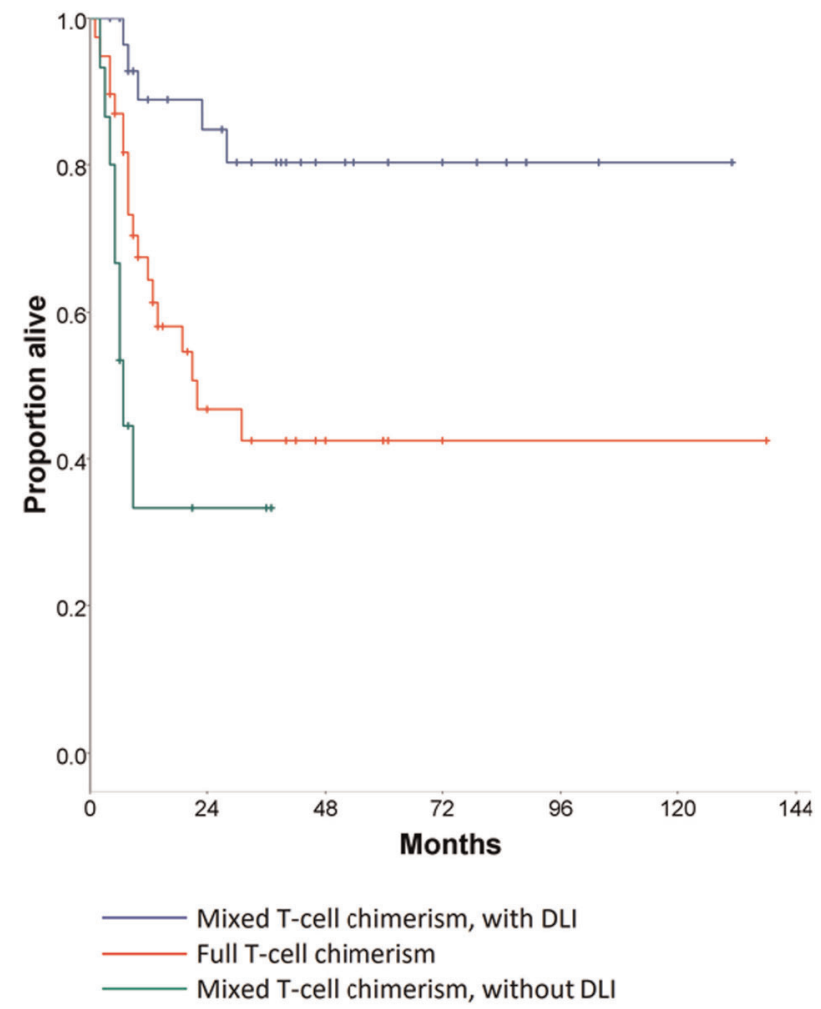

[P205 Figure] [Mixed T-cell chimerism followed by DLI confers favourable overall survival.]

\section{P206}

Treosulfan based conditioning regimen in patients undergoing allogenic stem cell transplant for hematological malignancies - a single centre experience from a tertiary cancer centre in India

Lingaraj Nayak, Rushabh Kothari Kothari, Anant Gokarn, Sachin Punatar, Avinash Bonda, Aniket B Mohite, S Kartik, Libin J Mathew, Sadhana Kannan, Navin Khattry

Tata Memorial Centre, Bone Marrow Transplant Unit, ACTREC, Mumbai, India

Background: Myeloablative conditioning regimens for allogenic transplant have significant toxicities which warrant exploration of reduced intensity regimens. Treosulfan, an alkylating agent, has been shown to have low organ toxicity. We retrospectively analyzed the toxicities and outcomes of treosulfan based conditioning regimen in our patients of hematolymphoid malignancies.

Methods: Twenty-five patients (AML-9, ALL-3, MDS/ MPN-11, lymphoma-2) who underwent allogenic transplant between January 2011 and July 2016 were included in this study. Hematopoietic Cell Transplant-Comorbidity Index (HCT-CI) score, EBMT score and Disease Risk Index (DRI) at transplant were calculated for all patients. All patients received fludarabine $30 \mathrm{mg} / \mathrm{m} 2$ (5 days) and treosulfan $12-14 \mathrm{gm} / \mathrm{m} 2$ (3 days). Ten patients also received 2 Gy TBI and 4 patients received Ara-C $2 \mathrm{gm} / \mathrm{m}^{2}$ (5 days) $+/$ - idarubicin $10 \mathrm{mg} / \mathrm{m}^{2}$ (3 days). GVHD prophylaxis was cyclosporine (CSA) with methotrexate $(\mathrm{N}=7)$ or mycophenolate (MMF, $\mathrm{N}=5$ ) for matched sibling; post transplant cyclophosphamide with CSA + MMF was used for haploidentical $(\mathrm{N}=10)$ and rabbit ATG was used for unrelated donor transplants $(\mathrm{N}=3)$. Chimerism was done on $\mathrm{d}+15, \mathrm{~d}+30$ and then monthly for 1 year. Neutrophil Engraftment (NE) was defined as absolute neutrophil count (ANC) $>0.5 \times 10^{9} / \mathrm{L}$ for 3 consecutive days without GCSF support and platelet engraftment(PE) was defined as platelet count $>20 \times 10^{9} / \mathrm{L}$ for 7 consecutive days unsupported. Acute toxicities were recorded according to CTCAE version 4.03. Overall survival(OS) was calculated from date of transplant to date of death or last follow-up (LFU) and relapse free survival (RFS) was calculated from date of transplant to date of progression, relapse, death or LFU if in remission.

Results: Median age was 29 years (range 3-54) with 17 (68\%) being male. Median EBMT and HCT CI scores were 3 (range 1-6) and $2(0-5)$ respectively. Fourteen (56\%) patients had high, $8(32 \%)$ had intermediate and $3(12 \%)$ had low DRI. Grade III/IV mucositis and diarrhea were seen in $1(4 \%)$ and $4(16 \%)$ patients respectively. Complete donor chimerism at $\mathrm{d}+15$ and $\mathrm{d}+28$ was seen in $19(76 \%)$ and 23 patients $(92 \%)$ respectively. Median time to NE and PE were 16 and 13 days respectively. Five (20\%) patients relapsed and $5(20 \%)$ died due to transplant complications (GVHD-3, sepsis-2). Incidence of grade II-IV acute GVHD and chronic GVHD was $36 \%$ and $30 \%$ respectively. OS and RFS at 2 years were $55 \%$ and $54 \%$ respectively for the entire cohort. According to high, intermediate and low DRI, the OS were $46 \%, 62.5 \%$ and $100 \%(\mathrm{p}=0.55)$ and RFS were $46 \%, 62.5 \%$ and $66.7 \%$ respectively $(\mathrm{p}=0.97)$.

Conclusions: Treosulfan based conditioning regimen has excellent toxicity profile with reasonable relapse free survival even in patients with high DRI. Prospective comparisons with other conditioning regimens are warranted.

Conflict of interest: Conflict of Interest: The authors declare that they have no conflict of interest.

Funding:There was no funding for the above study.

\section{P207}

Unrelated (non-HLA identical) haematopoietic stem cell transplantation with posttransplantation cyclophosphamide in malignant hematologic diseases: A single centre experience 
Eva Torbagyi ${ }^{1}$, Laszlo Gopcsa ${ }^{1}$, Aniko Barta ${ }^{1}$, Arpad Batai ${ }^{1}$, Zoltan Csukly $^{I}$, Lilla Lengyel ${ }^{1}$, Gergely Lakatos ${ }^{1}$, Melinda Paksi ${ }^{1}$, Marienn Reti $^{1}$, Zita Farkas ${ }^{1}$, Janos Sinko ${ }^{1}$, Janos Fabian ${ }^{1}$, Tamás Masszi ${ }^{2}$, Peter Remenyi ${ }^{I}$

${ }^{I}$ St Istvan and St Laszlo Hospital, Hematology and Stem Cell Transplantation, Budapest, Hungary; ${ }^{2}$ Semmelweis University, Hematology, Budapest, Hungary

Background: The severe (grade III-IV) acute and extensive chronic graft versus host disease (GVHD) are the main causes of the non-relapse transplant related mortality (TRM) after allogeneic haematopoietic stem cell transplantation (HSCT) with non-HLA identical donors. Use of posttransplant cyclophosphamid (CY) can improve the results of HSCT in these cases.

In this report we analysed the outcome of 22 patients(pts) with malignant haematological diseases receiving postgraft CY after allo-HSCT with 7/8 HLA identical unrelated donors.

Methods: Between 2015 and 2017 (3 years) 22 patients were transplanted with unrelated donor and posttransplantation CY in our unit. The median follow up is 20 months (435) Median age: 40 years (21-63) Sex: 6 female/16 male.

Diagnosis: AML: 3, ALL: 12, MM: 3, MF: 2, MDS: 2. Conditioning regimen: Myeloablative (20/22): total body irradiation (TBI)/Fludarabine (FLU): 7, TBI/etoposide (VEP):3, TBI/melphalan: 3, BU(16)/FLU/anti-thymocyta globulion (ATG): 2, BU(12)/FLU/ATG: 1, Tiotepa(TT)/ BU/FLU: 4, Non-myeloablative (2/22):TBI(low dose)/FLU/ ATG:1,: BU(8)/FLU: 1, GVHD prophylaxis: postgrafting CY a +3 . és +4 . on days $50 \mathrm{mg} / \mathrm{kg} / \mathrm{d}+$ tacrolimus and mycophenolat mofetil: 21 pts, +tacrolimus and sirolimus: 1 pt.

Stem cell source: G-CSF stimulated, unmanipulated, periferial stem cell.

Results: Acute GVHD observed 13/22 (59\%): grade 1-2: 11 pts, grade 3: 1 pts, grade 4:0, chronic extensive GVHD: 7 pts. Relapse: 4 pts (2 live, 2 died), Non-infections complications: OP mucositis (grade 2-4): 8 pts. Severe haemorrhagic cystitis: 5 pts, MAHA: 5 pts. Infectious complications: Bacterial septicaemia: 6 pts. Fungal infection: 3 pts. Viral infection: 9 pts (CMV, EBV, adeno, RSV, parainfluenza). Live: 13 pts (2 in relapse), died: 9 pts . Cause of death: 2 pts relapse+bacterial+viral infections (CMV, adeno, parainfluenza), 2 pts GVHD+bacterial sepsis, 2 pts GVHD+viral infection (RSV, EBV), 1 pt GVHD+bacterial sepsis+viral infection (CMV), 1 pt CMV pneumonitis without GVHD, 1 pt candida sepsis without GVHD.

Overall survival (OS): 13/22 (59\%), Progression-free survival (PFS): 11/22 (50\%), TRM: 9/22 (40\%), Relapse: 4/12 (18\%), acute GVHD incidence: 13/22 (59\%), grade 3-4 acute GVHD: 1/22 (4\%)
Conclusions: Use of the postgraft $\mathrm{CY}$ in allogeneic HSCT with HLA mismatch (7/8) unrelated donors resulted better outcome than previously used protocols.

Grade 3-4 acute GVHD is almost completely disappearing. Viral infections must to closely monitored. The results show twenty percentage OS benefit compared to the historical patient cohort.

Conflict of interest: E. Torbagyi: nothing to disclose Early complications / late effects \& quality of life

\section{P208}

'Missing in action or is the action missing', Are the exercises for HSCT patients fit for purpose? A systematic review

Jaleel Mohammed ${ }^{1,2}$, Helene Schoemans ${ }^{3}$, Zinaida Peric ${ }^{4}$, Shahrukh Hashmi ${ }^{5}$

${ }^{1}$ King Faisal Specialist Hospital and Research Centre, Riyadh, Saudi Arabia; ${ }^{2}$ Physical Therapy Association for Graft Versus Host Disease, Swindon, United Kingdom; ${ }^{3}$ University Hospitals of Leuven, Leuven, Belgium; ${ }^{4}$ University of Zagreb School of Medicine, Salata, Croatia; ${ }^{5}$ Myo Clinic, Rochester, MN, United States

Background: Quality of life (QoL), return to normal social and work activities is one of the major concern for both patients and healthcare professionals following hematopoietic stem cell transplant.

The evidence behind exercises as an effective intervention to improve the quality of life in HSCT patients is not well established, with contradicting reports, and many studies leaning towards little or no statistically significant benefit of rehabilitation exercises. The exercise prescription is a science which demands individualisation to achieve any meaningful outcome. In this systematic review, we attempt to analyse if the exercises in HSCT patients are prescribed to reflect the patient's needs.

Methods: We have set out to perform a systematic review of the literature to evaluate the current HSCT exercises prescription and its impact on the patient $\hat{A}$ 's function and quality of life. We included both auto and allo HSCT patients undergoing rehabilitation for HSCT with or without GVHD. A search was undertaken using Sage Journals, Wiley-Blackwell, Clinical Key, Ovid, ScienceDirect, PubMed Central, ProQuest Medical Library, SpringerLink, ProQuest Nursing \& Allied Health Source, Directory of Op, from 2007 to 2017 using Boolean logic with the following terms: HSCT, rehabilitation, exercises, physiotherapy, physical therapy, bone marrow transplant, quality of life. Identified articles were searched for additional references.

Results: 2450 articles were identified and after duplicates, we were left with, 2423 articles. Based on inclusion/ exclusion criteria 13 full-text articles were included for this review. 
Out of the 13 articles, only 2 reported using some scientific base for exercise prescription and was individualised to the patients. Most of the studies involving exercise intervention in HSCT scenario were found to be poorly designed, lack details on the type of exercises (eccentric vs concentric loading) and have failed to acknowledge the science of exercise prescription. HSCT patients have individual strengths and failures which demands custom designed exercise prescription to include the appropriate frequency, duration, intensity and type of exercises to match patient's diverse variables like age, cancer type, drugs, radiation, transplant, the presence of specific symptoms cardiopulmonary function, individual lifestyle, obesity, age, prior treatment, associated comorbidities and the impact of drugs on the musculoskeletal system.

Conclusions: Although the science behind exercise prescription has evolved and perfected its application in both patient and athlete population over the last few decades, its application in the HSCT rehabilitation arena appears to be underutilised. In this current review, we found only two studies out of thirteen, that utilized some elements of the scientific basis for exercise prescription, customizing and designing the exercise regime as per the individual patient physical capacity and finally progressing the patient as per the physiological parameters. The other 11 articles did not provide any justification for their chosen exercise prescription and did not provide any details on the scientific rationale for the baseline exercise design calculation and progression. Future studies should include these elements to design reliable and reproducible trials.

\section{Conflict of interest: none}

\section{P209}

\section{A SINGLE CENTER EXPERIENCE IN SINUSOIDAL OBSTRUCTION SYNDROME/ VENO-OCCLUSIVE DISEASE USING, RETROSPECTIVELY, THE NEW EBMT CLASSIFICATION}

\author{
Sara Martín Herrero ${ }^{1}$, Jose Luis López Lorenzo ${ }^{1}$, Teresa Villaescusa \\ de la Rosa ${ }^{1}, M^{o}$ Soledad Sánchez Fernández ${ }^{1}$, Teresa Arquero \\ Portero $^{1}$, María Yuste Platero ${ }^{2}$, Alberto Velasco Valdazo ${ }^{3}$, Pilar \\ Beltrán Alvárez ${ }^{4}$, Pilar Llamas Sillero ${ }^{1}$ \\ ${ }^{1}$ Fundación Jiménez Díaz Hospital, Hematology and Hemotherapy, \\ Madrid, Spain; ${ }^{2}$ Hospital General de Villalba, Hematology and \\ Hemotherapy, Madrid, Spain; ${ }^{3}$ Hospital Rey Juan Carlos, Madrid, \\ Spain; ${ }^{4}$ Hospital Infanta Elena, Hamatology and Hemotherapy, \\ Madrid, Spain
}

Background: Sinusoidal Obstruction Syndrome (SOS), also known as Hepatic Veno-Occlusive Disease (VOD), is a potential devastating complication after hematopoietic stem cell transplantation (HSCT). SOS/VOD incidence varies with risk factors between $5-15 \%$. Mortality rate is higher than $80 \%$ in cases evolving to multiorgan failure. The diagnosis has been based on achievement of Baltimore and Modified Seattle Criteria established in 1987 and 1992 respectively. Grading the severity was also based in clinical criteria but it could only be assigned retrospectively. The advances in HSCT and the development of Defibrotide as an effective treatment, compel to the definition of new clinical criteria as well as a new severity grading system. This new classification was proposed by the European Society of Bone Marrow Transplantation (ESBMT) in 2016. Aim:To describe the experience in our center and to compare the new\&classical classification criteria of SOS/ VOD

Methods: We retrospectively analyzed all the cases of SOS/VOD secondary to allogenic HSCT in our center from 2011 until 2017. We classified them retrospectively following the old and new classification criteria proposed by the EBMT.

Results: 46 related allogenic HSCT have been performed in our center from 2011 to 2017, 4 of them have developed SOS/VOD: 3 (75\%) male and 1 (25\%) female. Mean age 36.8 years (19-45). Diagnosis were: Prolymphocytic T cell leukemia with complex karyotype in $4^{\text {th }}$ line treatment; Acute Lymphoblastic $\mathrm{T}$ cell leukemia in $4^{\text {th }}$ line treatment, Peripheral T cell Lymphoma NOS in 4th line treatment and Pro-T lymphoblastic Leukemia in $5^{\text {th }}$ line treatment. Three (75\%) were haploidentical with cyclophosphamide days $+3 \&+4$ as well as CyA/MMF and one $(25 \%)$ HLA identical sibling with standard CyA/MTX; 3 received myeloablative conditioning with high dose TBI and 1 RIC with intravenous busulfan. Regarding risk factors of SOS/ VOD: $100 \%$ had Karnofsky $<90 \%$, received hepatotoxic drugs before HSCT, and had an advanced disease before HSCT. $100 \%$ had $\geq 4$ risk factors. $100 \%$ had a weight gain between $5-10 \%$ at the moment of diagnosis. $100 \%$ painful hepatomegaly. $75 \%$ (3) had a bilirubin level $\geq 2 \mathrm{~g} / \mathrm{dL}$; 1 had transaminase level 8 times UNL at the moment of diagnosis. $2(50 \%)$ doubled bilirubin level in less than 48 hours and 2 developed multiorgan failure requiring intensive care. Ultrasound doppler was performed to all of them: 3 (75\%) had hepatomegaly and $1(25 \%)$ decreased speed of venous flow. Baltimore criteria were fulfilled by $3(75 \%)$; modified Seattle criteria were fulfilled by $4(100 \%)$. New SOS/VOD EBMT criteria were met by $3(75 \%)$ of them. $100 \%$ were treated with defibrotide, diuretics and fluid restriction; also acetylcysteine and high dose methylprednisolone were used. VOD/SOS was resolved in all and none died of VOD/SOS.

\section{Conclusions:}

- All patients that developed SOS/VOD had $\geq 4$ risk factors, implying a high risk of developing SOS/VOD before HSCT. 


\begin{tabular}{|c|c|c|c|c|c|c|c|c|c|}
\hline & $\begin{array}{l}\text { DIAGNOSIS/DISEASE } \\
\text { SITUATION }\end{array}$ & HSCT TYPE & $\begin{array}{l}\text { CONDITIONING } \\
\text { REGIMEN }\end{array}$ & $\begin{array}{l}\text { WEGHT } \\
\text { GAIN } \\
\text { FROM } \\
\text { BASELINE } \\
\%\end{array}$ & $\begin{array}{l}\text { BILIRUBIN } \\
\text { LEVEL AT } \\
\text { DIAGNOSIS } \\
(\mathrm{g} / \mathrm{dL})\end{array}$ & $\begin{array}{l}\text { MAXIMUM } \\
\text { BILIRUBIN } \\
\text { LEVEL (g/ } \\
\text { dL) }\end{array}$ & MOF & $\begin{array}{l}\text { DIAGNOSIS } \\
\text { DATE FROM } \\
\text { INFUSION }\end{array}$ & $\begin{array}{l}\text { SEVERITY } \\
\text { GRADE }\end{array}$ \\
\hline $\begin{array}{l}\text { CASE } \\
1\end{array}$ & $\begin{array}{l}\text { Prolymphocytic T cell } \\
\text { leukemia with complex } \\
\text { karyotype/ MRD } 0.013\end{array}$ & HAPLODENTICAL & RIC (Busulfan) & 8 & 7 & 7 & NO & +9 & SEVERE \\
\hline $\begin{array}{l}\text { CASE } \\
2\end{array}$ & $\begin{array}{l}\text { Acute Lymphoblastic T } \\
\text { cell leukemia./ Relapse }\end{array}$ & HLA IDENTICAL & MAC (TBI) & 5 & 1.5 & 1.5 & YES & +18 & $\begin{array}{l}\text { VERY } \\
\text { SEVERE }\end{array}$ \\
\hline $\begin{array}{l}\text { CASE } \\
3\end{array}$ & $\begin{array}{l}\text { Peripheral T cell } \\
\text { Lymphoma NOS. } \\
\text { /Relapse }\end{array}$ & HAPLOIDENTICAL & MAC (TBI) & 9 & 2 & 4 & YES & +11 & $\begin{array}{l}\text { VERY } \\
\text { SEVERE }\end{array}$ \\
\hline $\begin{array}{l}\text { CASE } \\
4\end{array}$ & $\begin{array}{l}\text { Pro-T lymphoblastic } \\
\text { Leukemia. / Relapse }\end{array}$ & HAPLOIDENTICAL & MAC (TBI) & 8 & 2.1 & 7 & NO & +10 & SEVERE \\
\hline
\end{tabular}

[[P209 Table] Table 1]

- One patient did not achieve New EBMT criteria for SOS/VOD since it didn't reach de $2 \mathrm{~g} / \mathrm{dL}$ bilirubin level required; modified Seattle criteria are still necessary for some cases.

- Haploidentical transplantation and myeloablative TBI in our review seems to be more associated to SOS/VOD.

Conflict of interest: None of the authors has anything to disclose.

\section{P210}

Abstract previously published

\section{P211}

\section{Aetiology and ADAMTS13 parameters in patients with HSCT Thrombotic Microangiopathy}

Jorge M. Nieto ${ }^{1}$, Clara Cuellar Pa ${ }^{2}$, Fernando Ataulfo Gonzalez ${ }^{1}$, Ana Villegas ${ }^{1}$, Rafael Martinez ${ }^{1}$, Estefanía Bolaños ${ }^{1}$, Alejandro Bobes ${ }^{1}$, Adhemar Velasco ${ }^{1}$, Carmen Vicente ${ }^{1}$, Nahir Moreno ${ }^{1}$, Roberto Trelles ${ }^{1}$, Mayumi Ibarra ${ }^{1}$, Ropero Paloma ${ }^{1}$

${ }^{1}$ Hospital Clinico San Carlos, Madrid, Spain; ${ }^{2}$ Hospital La Princsa, Madrid, Spain

Background: Thrombotic Microangiopathy (TMA) is a relatively common and often misdiagnosed complication of the HSCT. Here we show the main features related to ADAMTS13 and the possible aetiologies of all cases with post HSCT-TMA received in our hospital for ADAMTS13 testing, from 2012 to 2017.

Methods: Clinicians in nearly $60 \%$ of Spanish territory can send us samples for ADAMTS13 activity testing (normal range 40-124\%) due to TMA findings (996 patients). Search for inhibitors and/or IgG auto-antibodies against ADAMTS13 were performed if activity was $<40 \%$. Data at TMA debut (platelets, haemoglobin, creatinine, schistocytes, relevant clinical conditions, etc.) was recorded for further analysis. Next Generation Sequencing (NGS) of ADAMTS13 (1 case) and alternative pathway complement regulators ( 2 cases) were performed when requested.

Results: 89 out of 996 TMA cases were HSCT-TMAs (8.9\%). The percentage of HSCT-TMAs was growing each year (from $1.3 \%$ of total TMAs in 2012 to $16.2 \%$ in 2017).

Only 2 cases with HSCT-TMA have shown a drastic reduction in ADAMTS13 activity $(<10 \%)$, which is characteristic of Thrombotic Thrombocitopenic Purpura (TTP). However, the mechanism responsible for the ADAMTS13 deficiency seems to be different between those 2 cases (Table 1). A mild activity reduction (10-40\%) was found in 20 patients, all of them without inhibitor and/ or IgG auto-antibodies. The remaining cases (67) had normal activities. No statistically significant differences in haematological/biochemical parameters were found between groups (mild reduction versus normal activity).

No pathogenic mutations in complement regulators were found in the 2 patients studied by NGS, but both of them were carriers of risk haplotypes (H3 and CD46GGAAC) for atypical Haemolytic Uremic Syndrome (aHUS).

In many cases, TMA was caused by drug toxicity (withdrawal of the drug resolve the TMA) associated to cyclosporine A, tacrolimus or sirolimus. Infections causing disseminated intravascular coagulation (DIC) could be responsible of some cases of HSCT-TMA.

Finally, severe GVHD was a common finding in this kind of patients.

Conclusions: Incremental percentage of HSCT-TMA respect to all TMAs every year suggests a global crescent awareness of this condition as a serious complication of HSCT.

Different causes can produce the TMA (endothelial drug toxicity, DIC, TTP and probably others). It is unclear if severe GVHD can act as cause of the TMA or alternatively as trigger of the process in predisposed patients. Presence of risk haplotypes for aHUS in the two patients analyzed suggests that conditions related to HCST can act as a trigger for aHUS. 


\begin{tabular}{lllllll}
\hline $\begin{array}{l}\text { Patient } \\
\text { Adtivity }\end{array}$ & $\begin{array}{l}\text { ADAMTS13 } \\
\text { Inhibitor }\end{array}$ & $\begin{array}{l}\text { antiADAMTS13 } \\
\text { IgG }\end{array}$ & $\begin{array}{l}\text { ADAMTS13 } \\
\text { Mutations }\end{array}$ & Commentary & $\begin{array}{l}\text { Possible mechanism for ADAMTS13 } \\
\text { activity deficiency }\end{array}$ \\
\hline$\# 1$ & $0 \%$ & Negative & Positive & -- & $\begin{array}{l}\text { Several TMA episodes after } \\
\text { HSCT. Complete remission with } \\
\text { normal ADAMTS13 activity. }\end{array}$ & $\begin{array}{l}\text { Graft production of Anti- } \\
\text { ADAM13 IgG antibodies. }\end{array}$ \\
$\# 2$ & $3 \%$ & Negative & Negative & Negative & $\begin{array}{l}\text { Severe hepatic damage. } \\
\text { Multiorganic affectation. Death. }\end{array}$ & $\begin{array}{l}\text { Deficiency of ADAMTS13 molecule } \\
\text { due to severe impairment of } \\
\text { production in stellate hepatic cells. }\end{array}$ \\
\hline
\end{tabular}

[[P211 Table] HSCT-TMA patients with ADAMTS13 activity (<10\%).]

TTP is extremely uncommon, thus, plasma exchange therapy is controversial as first line treatment. It is unknown how frequent is any of the other potential aetiologies due to incomplete availability of clinical data in our cohort.

Treatment depends of the aetiology of the TMA and the presence of other complications, so trying to distinguish between the possible causes of the TMA is mandatory in every patient with HSCT-TMA.

Conflict of interest: None of the authors has anything to disclose.

\section{P212}

Antiemetics: Is a new single capsule treatment better than a multi-dosing treatment?

\section{Kristiina Kuusto, Erica Lauraeus-Käkelä, Hanna Pekkanen, Kari Remes, Urpu Salmenniemi}

Turku University Hospital, Dept of Clinical Haematology and Stem Cell Transplantation Unit, Turku, Finland

Background: We compared our old antiemetic prophylaxis to a simple new one. The old protocol included one aprepitant capsule (Emend), a substance P/neurokinin 1 (NK1) receptor antagonist, during days 1 to 3 of chemotherapy and one i.v.-injection of palonosetron (Aloxi), a serotonin-3 (5-HT3) receptor antagonist, on day 1. A new oral combination product (Akynzeo) contains netupitant and palonosetron is given on day 1. I.v. dexamethasone was given similarly in both protocols.

Methods: We collected information on the patients who received cytostatics with a high risk of nausea and vomiting as a part of autologous or allogeneic hematopoietic stem cell transplantation (HSCT). The patients who received the new oral product filled out a questionnaire during the first 5 or 6 days after taking the capsule (Group 1). The data concerning nausea of patients receiving the old antiemetic protocol was analysed from their medical records (Group 2).

Results: In Group 1, there were 21 patients (10 autoHSCT, 11 alloHSCT) and group 2, 20 (10 autoHSCT, 10 alloHSCT), respectively. Only very few in the Group

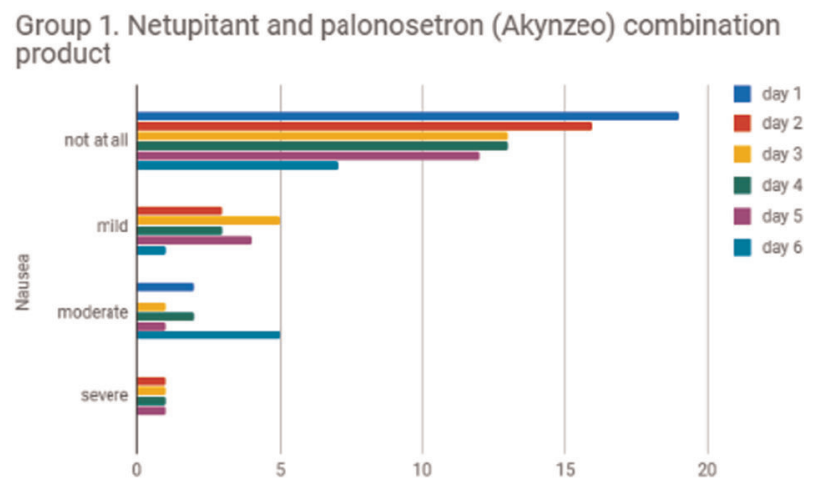

Group 2. Aprepitant (Emend), palonosetron (Aloxi)

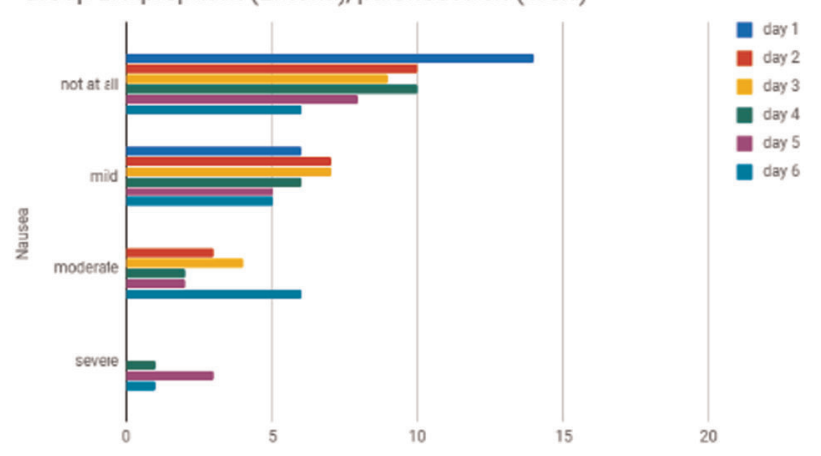

[P212 Figure] [Table 1.]

1 suffered from nausea on the first day of chemotherapy (2/ $21,9,5 \%)$ in comparison to Group $2(6 / 20,30 \%)$. More than half of the patients did not suffer from nausea, not even on the $5^{\text {th }}$ day after starting the antiemetic treatment $(12 / 21$, $57 \%)$ compared to the results of the old treatment $(8 / 20$, 40\%). The main results are shown in Table 1.

Conclusions: Our data support the assumption that the new oral combination product may have a slightly longerlasting effect on preventing nausea. Why then to change the treatment protocol that has given good results for a long time? First, the price for Akynzeo is almost half of the old treatment. Second, a single dose of netupitant has a longer effect (up to 120 hours). The new protocol seems to be more efficient and it is more convenient for the patient too. The easier the nausea prophylaxis is, the more pleasant it is both to the patient and to the nursing staff.

Conflict of interest: None 


\section{P213}

\section{Assessment of Serum Vitamin D in Pediatric Hematopoietic Stem Cell Transplantation Patients And Its Relation With Complications}

\author{
Zeynep Karakas ${ }^{1}$, Selime Aydoğdu ${ }^{1}$, Yasin Yilmaz ${ }^{1}$, Ceyhun \\ Bozkurt', Alper Aykanat ${ }^{3}$, Tunç Fişgın ${ }^{3}$ \\ ${ }^{1}$ Istanbul University, Istanbul Medical Faculty, Pediatric Hematology/ \\ Oncology, Istanbul, Turkey; ${ }^{2}$ Istinye University, Bahcelievler Medical \\ Park Bone Marrow Transplantation Unit, Istanbul, Turkey; \\ ${ }^{3}$ Bahcesehir University, Bahcelievler Medical Park Bone Marrow \\ Transplantation Unit, Istanbul, Turkey
}

Background: Recently, evaluation of serum vitamin D level in pediatric hematopoietic stem cell transplantation (HSCT) patients gained popularity, as there is close relation with development of GVHD and complications. Our aim was to assess the serum vitamin D level and its relation with GVHD and complications such as graft failure in HSCT patients.

Methods: The serum vitamin D level of 66 children (mean age 6.6 years; 36 male, 30 female) with HSCT at baseline, on first and on third month of transplantation was evaluated in Medical Park Hospital, Istanbul, Turkey between 2015 and 2016 years. Patients were divided in groups according to diagnosis as Leukemia $(n=25)$, Anemia $(n=29)$, Immunodeficiency $(\mathrm{n}=4)$ and Solid Tumor $(\mathrm{n}=8)$. The serum vitamin D level was regarded as deficiency $(<12 \mathrm{ng} / \mathrm{ml})$, insufficiency $(12-20 \mathrm{ng} / \mathrm{ml})$ and sufficiency $(>20 \mathrm{ng} / \mathrm{ml})$. Children with vitamin D deficiency were given $50.000 \mathrm{U}$ stoss therapy and then 15 drop/day maintenance therapy. Patients with insufficiency were given only maintenance therapy.

Results: The baseline serum vitamin D level in 61 children was $17.2 \pm 9.5 \mathrm{ng} / \mathrm{ml}$, after one month of transplantation it was $28.0 \pm 17.3 \mathrm{ng} / \mathrm{ml}$ in 47 children and after three month it was measured as $31.0 \pm 15.4 \mathrm{ng} / \mathrm{ml}$ in 29 children $(\mathrm{p}=0.014)$. The forty percent of all patients had vitamin D deficiency and 30\% of patients had insufficiency before transplantation. The baseline level was $18.7 \pm 11.8 \mathrm{ng} / \mathrm{ml}$ in Leukemia group while it was $14.9 \pm 7.5 \mathrm{ng} / \mathrm{ml}$ in anemia group $(\mathrm{p}=0.085)$. Six patients $(10 \%)$ were died during study. Patients with GVHD (\%20, $\mathrm{n}=13)$ and graft failure $(8 \%, \mathrm{n}=5)$ had low level of vitamin $\mathrm{D}$ compared to non-complicated patients $(55 \%, \mathrm{n}=$ 36) $(14.2 \pm 9.5 \mathrm{ng} / \mathrm{ml}, 15.0 \pm 9.1 \mathrm{ng} / \mathrm{ml}, 17.7 \pm 9.3 ; \mathrm{p}=$ 0.53 , respectively). There was a significant difference between first and third month vitamin $\mathrm{D}$ level of noncomplicated patients $(26.9 \pm 15.3 \mathrm{ng} / \mathrm{ml}, 32.3 \pm 14.1 \mathrm{ng} / \mathrm{ml}$; $\mathrm{p}=0.03$, respectively). Although serum level of vitamin $\mathrm{D}$ lowered in patients given maintenance therapy only, it was significantly increased in stoss therapy group on first month (11.8 vs $42.2 ; \mathrm{p}=0.014)$.

Conclusions: The effect of serum vitamin D deficiency on pediatric hematopoietic stem cell transplantation gained importance in terms of GVHD development, graft failure and cure. In this study, the high prevalence of vitamin D deficiency before transplantation was found. Moreover, patients with GVHD and graft failure had lower vitamin D level at baseline. There was no response to maintenance oral therapy, but weekly stoss therapy was successful. The screening of vitamin D level before transplantation and supplying the required support seems to be important for cure and complication.

Conflict of interest: None of the authors has anything to disclose.

\section{P214}

\section{AUTOLOGOUS STEM CELL TRANSPLANTATION FOR LIGHT CHAIN AMYLOIDOSIS}

Xabier Martin, Amaia Balerdi, Laura Posada, Clara Gomez, Elena Amutio, Juan Carlos Garcia Ruiz

Cruces Hospital, Hematology, Barakaldo, Spain

Background: Light chain amyloidosis (AL) is a plasma cell disorder characterized by a small plasma cell clone producing a misfolded light chain, kappa or lambda, that deposits in tissues. In selected patients, high-dose melphalan treatment following autologous blood stem cell transplantation (ASCT) is an effective therapy that can reverse disease. However, this procedure is associated with higher treatment related morbidity and mortality, especially in patients with cardiac involment.

Methods: From 2004 to 2017, we retrospectively analyzed the records of 10 patients with a median age of 55 years (range 43-66) with AL who underwent ASCT. The patients' characteristics are listed in Table I. We analyzed overall survival (OS) as well as transplant related morbidity and mortality. The post-ASCT grade of response was measured 3 months after ASCT in 9 of the 10 patients. The OS was estimated by the Kaplan-Meier method, measured from diagnosis to death by any cause. Any death unrelated to relapse or disease progression during the first 120 days was considered as TRM (procedure-related death).

Results: Median of major viscera organ involment at the time of ASCT was 1 (range 1-3). Nine patients receive induction therapy before ASCT and one patient did not receive any kind of induction and underwent upfront ASCT. Nine patients were mobilizated using granulocyte-colony stimulating factor and one received plerixafor. The 


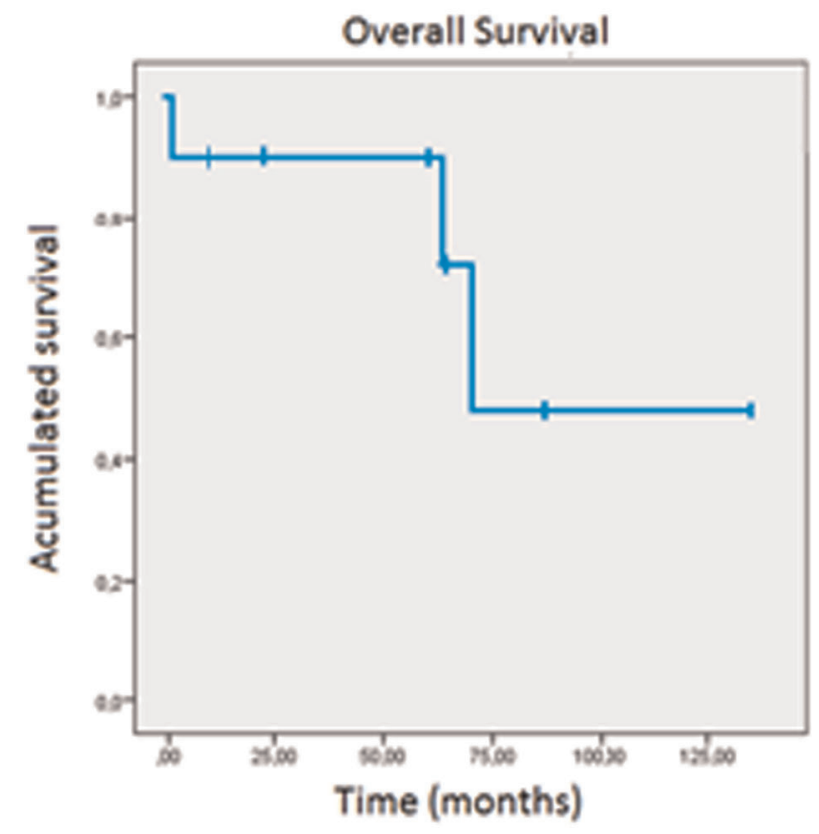

[P214 Figure] [Overall survival]

conditioning regimens consisted of high-dose melphalan, either $140 \mathrm{mg} / \mathrm{m} 2(50 \%)$ or $200 \mathrm{mg} / \mathrm{m} 2(50 \%)$.

Toxicity was frequent and severe. Unusual morbidity was found during stem cell mobilization (40\%) and stem cell component infusion (20\%), including cardiac and hemodynamic complications. The most common complications during post-transplant were neutropenic fever $(90 \%)$ that required broad-spectrum antibiotics and mucositis (90\%: 4 patients moderate and 6 patients severe). Two patients developed renal failure, two developed hemodynamic complications and two cardiac complications. One patient died from consequences of transplant-related complications.

Median OS for the whole group was 70.3 months. Hematological overall response rate, complete remission, very good partial response and partial response were $100 \%$, $77.8 \%, 11.1 \%$ and $11.1 \%$ respectively, whereas organ response was $44.4 \%$. With a median follow up of 61.8 months, 2 patients had died (both for progression at 41 and 59 months after ASCT, respectively).

Conclusions: In our experience, ASCT is an effective therapy for patients with $\mathrm{AL}$, with durable response rates. Despite improvement on patient selection, approach to stem cell mobilization and peri-SCT management, morbidity and mortality are higher than in other hematological malignancies undergoing ASCT. We suggest a strict patient selection based on age and organ involment and risk adapted approach for the treatment of patients with AL.

Conflict of interest: None

\begin{tabular}{ll}
\hline Age median (range) & $55(43-66)$ \\
\hline Gender (male/female) & $3 / 7$ \\
Light chain (Kappa/Lambda) & $3 / 7$ \\
Visceral organ involment $\bullet$ Cardiac $・$ Renal $\bullet$ Hepatic & $\bullet 30 \% \bullet 60 \% \bullet 40 \%$ \\
Proteinuria mg/24h (range) & $1662,5(225-8170)$ \\
Cardiac troponin T ng/L (range) & $19.5(7-77)$ \\
NT-pro-BNP pg/mL (range) & $305(88-742)$ \\
Alkaline phosphatase U/L (range) & $79(41-488)$ \\
\hline
\end{tabular}

\section{[[P214 Table] Table I: Patients characteristics]}

\section{P215}

Autologous transplantation for patients with lymphoproliferative diseases in the terms of outcome: 16 years of experience

Aleksandra Pivkova Veljanovska, Zlate Stojanoski, Irina Panovska Stavridis, Lazar Chadievski, Marija Popova - Labachevska, Sanja Trajkova, Lidija Cevreska, Borce Georgievski

University Clinic for Hematology, Skopje, Skopje, Macedonia, the Former Yugoslav Republic of

Background: Autologous stem cell transplantation (ASCT) improves survival in patients with myeloma and lymphoma but is associated with morbidity and non relapse mortality (NRM). Hematopoietic cell transplant comorbidity index (HCT-CI) was shown to predict risk of NRM and survival after allogeneic transplantation. We tested the utility of HCT-CI as a predictor of NRM and overall survival (OS) in patients undergoing ASCT.

Methods: We analyzed outcomes of 240 patients after high-dose melphalan and high -dose anti lymphoma chemotherapy during year 2000 to 2016. Individual comorbidities were prospectively collected at the time of ASCT. The impact of HCT-CI and other potential prognostic factors, including Karnofsky performance score (KPS), on NRM and survival were studied in multivariate regression models.

Results: HCT-CI score was $0,1,2,3$, and $>3$ in $42 \%$, $18 \%, 13 \%, 13 \%$, and $14 \%$ of the study cohort, respectively. Subjects were stratified into 3 risk groups: HCT-CI score of $0(42 \%)$ versus HCT-CI score of 1 to $2(32 \%)$ versus HCTCI score $>2(26 \%)$. Higher HCT-CI was associated with lower KPS $<90$ (33\% of subject's score of 0 versus $50 \%$ in HCT-CI score $>2$ ). HCT-CI score $>2$ was associated with melphalan dose reduction (22\% versus $10 \%$ in score 0 cohorts). One-year NRM was low at $2 \%$ (95\% confidence 
interval, $1 \%$ to $4 \%$ ). On multivariate analysis, overall survival was inferior in groups with HCT-CI score of 1 to 2 (relative risk, 1.37, [95\% confidence interval, 1.01 to 1.87 ], $\mathrm{P}=.04$ ) and HCT-CI score $>2$ (relative risk, $1.5[95 \%$ confidence interval, 1.09 to 2.08$], \mathrm{P}=.01$ ). Factors that affect $\mathrm{OS}$ in the autologous recipients among lymphoma and myeloma patients were: HCT-CI, Karnofsky score, number of CD34+ cells/kg and time from diagnosis until transplant $(\mathrm{p}<0.05)$. Factors that affect TRM/NRM were HCT-CI, ECOG, Karnofsky score and number of hospital days and body weight. $(\mathrm{p}<0.05)$.

Conclusions: ASCT for MM and lymphoma is associated with low NRM, and death is predominantly related to disease progression. Comorbidity evaluation during autologous transplantation for lymphoproliferative diseases can be a useful tool in predicting transplant outcome

Conflict of interest: No conflict of interest

\section{P216}

Benefits of a coordinated approach between Hematologist and Gynecologist in the management of female health issues after allogeneic hematopoietic stem cell transplantation

María Suárez-Lledó ${ }^{1}$, Marta del Pino ${ }^{2}$, Camilo Castelo-Branco ${ }^{2}$, Teresa Solano ${ }^{1}$, Gonzalo Gutierrez Garcia ${ }^{1}$, Sofia Almeida-Jorge ${ }^{1}$, Luis Gerardo Rodriguez-Lobato ${ }^{1}$, Francesc Fernandez-Aviles ${ }^{1}$, Laura Rosiñol ${ }^{1}$, Montserrat Rovira ${ }^{1}$, Alvaro Urbano-Ispizua ${ }^{1}$, Aureli Torne $^{2}$, Carmen Martinez Muñoz ${ }^{1}$

${ }^{1}$ Hospital Clinic of Barcelona, Stem Cell Trasplantation Unit, Hematology Department, Barcelona, Spain; ${ }^{2}$ Hospital Clinic of Barcelona, Gynecology Oncology Unit, Institut Clinic of Gynecology, Obstetrics and Neonatology, Barcelona, Spain

Background: Female long-term survivors of allogeneic hematopoietic stem cell transplantation (allo-HSCT) incur a significant burden of late effects. Genital chronic graftversus-host disease (cGVHD) is a common and frequently underestimated complication that can dramatically affect quality of life. Ovarian failure, sexual dysfunction, osteoporosis and human papillomavirus (HPV) persistence are other important concerns. In order to improve diagnosis and management of these complications, we started a program of systematic surveillance by Hematologist and Gynecologist for early and late complications in female allo-HSCT recipients. We report here our one year experience.

Methods: Thirty patients (52 years, 19-69) were included in this retrospective analysis. Seventeen patients started surveillance by Hematologist, focusing on specific female health issues, every 3 months immediately after allo-HSCT. Patients who presented genital symptoms and/or who developed cGVHD were referred to a specialized gynecologic consultation. Another 13 patients who had been previously diagnosed of cGVHD were also referred to Gynecologist (Table 1). We collected data on cGVHD, vulvovaginal symptoms, gynecologic examination, cervical cytology, HPV testing, menopausal status, gonadal function, vitamin D levels, and bone densitometry scans.

Results: Genital symptoms (dyspareunia, vaginal dryness, discharge or tightness, and vulvar pain) were presented in $87 \%$ of the patients. Eight out of 17 patients developed cGVHD (mild, $\mathrm{n}=2$; moderate, $\mathrm{n}=5$; severe $\mathrm{n}$ $=1$ ) at median of 10 months (range 5-12) after transplant. Of them, 3 (38\%) had genital involvement (organ score 1, n $=2$; score $2, \mathrm{n}=1)$ that was diagnosed at 1 month $(0.5-2)$ after diagnosis of cGVHD. In the group of 13 patients previously diagnosed of cGVHD (mild, $\mathrm{n}=2$; moderate, $\mathrm{n}$ $=4$; severe, $\mathrm{n}=7$ ), $77 \%$ showed genital GVHD (score $1, \mathrm{n}$ $=8$; score $2, \mathrm{n}=2$ ) diagnosed at a median of 14 months ( 3 120) after cGVHD diagnosis. No patient with mild cGVHD had genital GVHD. A significant correlation between oral mucosa cGVHD and vaginal cGVHD was observed $(63 \%$ of patients with oral had vaginal cGVHD, $x^{2}=0.004$ ). All patients received topical corticosteroids with clinical improvement. Thirty-percent of all included patients showed a positive HPV test (serotype 16 in 33\%) and vaccination was recommended. In 5/30 (16\%) of the patients pre-cancerous lesions were identified leading to cervical conization in 2 patients. Nineteen patients had menopause prior to allo-HSCT; and all women who had normal ovarian function developed ovarian failure after the procedure. Menopausal hormone replacement therapy was recommended and accepted by $50 \%$ of patients leading to resume of menstruations and improvement of menopausal symptoms. Almost all patients (97\%) had low levels of vitamin D, $40 \%$ had osteopenia, and $23 \%$ osteoporosis.

Conclusions: In our series, almost all female allo-HSCT recipients referred genital symptoms, up to $77 \%$ showed genital cGVHD, 30\% HPV positivity, 16\% gynecologic pre-cancerous lesions, $100 \%$ ovarian failure, and almost all had abnormalities in mineral metabolism and bone disease. The recognition of oral mucosa cGVHD should prompt a gynecologic examination owing to their high co-occurrence of genital cGVHD. Systematic gynecologic evaluation within the first year allows an early diagnosis of genital cGVHD. Early diagnosis of these complications is essential to optimize treatment outcomes, avoid severe sequelae, and improve quality of life.

\section{Conflict of interest:}

M. Suárez-Lledó: I received a scholarship from DKMS foundation to dedicate clinical assistance for the LateEffects Follow-up Unit, a new program in the Hospital Clínic of Barcelona, that started one year ago.

None of the other authors has anything to disclose. 


\begin{tabular}{|c|c|c|}
\hline & $\begin{array}{l}\text { Group that initiated } \\
\text { follow-up every } 3 \text { months } \\
\text { after allo-HSCT }(\mathrm{N}=17)\end{array}$ & $\begin{array}{l}\text { Group of patients with } \\
\text { history of } \mathrm{GVHD}(\mathrm{N}=13)\end{array}$ \\
\hline Genital symptoms & $13(76 \%)$ & $13(100 \%)$ \\
\hline CGVHD & $8(47 \%)$ & $13(100 \%)$ \\
\hline Genital cG'VHD & $3(38 \%)$ & $10(77 \%)$ \\
\hline $\begin{array}{l}\text { Time from cGVHD diagnosis } \\
\text { to genital cGVHD diagnosis } \\
\text { (months; median, range) }\end{array}$ & $1(0.5-2)$ & $14(3-120)$ \\
\hline Oral mucosa cGVHD & $7(41 \%)$ & $12(92 \%)$ \\
\hline Oral + genital & $3(18 \%)$ & $9(69 \%)$ \\
\hline HPV & $5(29 \%)$ & $4(31 \%)$ \\
\hline Serotype 16 & 2 & 1 \\
\hline $\begin{array}{l}\text { Pre-cancerous cervical } \\
\text { lesions }\end{array}$ & $2(12 \%)$ & $3(23 \%)$ \\
\hline Ovarian failure & $17(100 \%)$ & $13(100 \%)$ \\
\hline Developed after allo-HSCT & $6(35 \%)$ & $5(38 \%)$ \\
\hline Low vitamin D levels & $16(94 \%)$ & $13(100 \%)$ \\
\hline Osteopenia & $4(24 \%)$ & $8(62 \%)$ \\
\hline Osteoporosis & $3(18 \%)$ & $4(31 \%)$ \\
\hline
\end{tabular}

[P216 Figure] [Fig 1: vaginal cGHVD]

\section{P217}

Bloodless stem cell hematopoietic transplantation
Jose Luis Lopez Lorenzo ${ }^{1}$, Sara Martin Herrero ${ }^{1}$, Teresa Villaescusa de la Rosa ${ }^{2}$, Elham Askari ${ }^{1}$ Elena Prieto Pareja ${ }^{1}$ M $^{a}$ Soledad Sanchez Fernandez ${ }^{1}, M^{a}$ Angeles Perez Saez ${ }^{1}$, Agustin Penedo Coello $^{2}$, Gala Vega Achabal, Tamara Castaño Bonilla ${ }^{2}$, Teresa Arquero Portero $^{2}$, Ines Magdalena Martinez Alfonzo ${ }^{1}$, Juan Manuel Alonso Dominguez ${ }^{2}$, Raquel Mata Serna ${ }^{2}$, Sandra Lopez Gonzalez ${ }^{2}$, Ana Yeguas Bermejo ${ }^{1}$, Diego Velasco Rodriguez ${ }^{1}$, Rosa Vidal Laso ${ }^{1}$, Raul Cordoba Mascuñano ${ }^{2}$, Pilar Llamas Sillero ${ }^{2}$ 
${ }^{1}$ Fundacion Jimenez Diaz, Hematology, Madrid, Spain; ${ }^{2}$ Fundacion Jimenez Diaz, Madrid, Spain

Background: Autologous transplantation is seen as the best treatment option for some patients. Most patients will need red cell or platelet transfusion as part of the supportive care during the aplasia phase after conditioning. Some patients refuse blood transfusión. Interestingly, some of these people agree to a stem cell transplantation, even if progenitors cells are infuse with some red cells\&platelets.

Methods: Our centre has been often choose by Jehova`s witness adherent to religious beliefs that doesn't allow blood transfusion(red cells, platelets or plasma).

We report 2 cases treated with autologous hematopietic transplantation without blood support. We perform it making some changes over the regular procedure: blood draws weekly or if clinically needed, pediatric tubes, tranexamic acid, erythropoietin after conditioning, at least hemoglobin of $11 \mathrm{~g} / \mathrm{dl}$ to proceed.

\section{Results:}

Patient 1. LCG was a 64-year-old man with Mantle cell lymphoma stage IV-B diagnosed October/2013. Past history was relevant for ischemic cardiomiopathy needing revascularization, hypercholesterolemia and acute divetiiculitis. $\mathrm{He}$ was treated with alternating R-CHOP/R-DHAP. Evaluation after fourth cycle showed partial remisión. After failing a first attempt to collect stem cells, a second try was done with plerixafor that was succesful. After 6 cycles, patient was evaluated again and showed to be in complete remission. He was willing to have a hematopoietic transplant without blood support. Conditioning was done with full dose BEAM. He got $2.34 \times 10^{6} \mathrm{CD} 34$ cells $/ \mathrm{kg}$. He engrafted neutrophils at day +10 and platelets at day +21 . Clinical course was complicated with emesis, mucositis, angor, neutropenic fever and need of parenteral nutrition. He was discharged after length of stay of 28 days.

Patient 2. FMG was a 60-year-old woman with IgG multiple mieloma diagnosed may/13 having anemia and extensive bone disease at a different center, Past history was relevant just for high blood pressure. She was treated first line with VCD that was stopped because of hepatotoxicity and zoledronic acid. Then, she was treated lenalidomide plus dexametasone reaching a VGPR. She came to us asking for an autologous transplantation. She sign a informed consent with rejection of blood support. Stem cell movilization and collection was schedulled succesfully. Conditioning with high dose melphalan $200 \mathrm{mg} / \mathrm{m}^{2}$ was done. She got $2.88 \times 10^{6} \mathrm{CD} 34$ cells $/ \mathrm{kg}$. She engrafted neutrophils and platelets at day +11 . No mayor complications were seen. She was discharged after length of stay of 19 days and followed at the outpatient clinic.
Conclusions: In summary, it is possible to perform stem cell transplantation without blood support. Patients need to be aware of the risks, but it is a feasible procedure. Teams doing the procedure to these patients need to implement some changes to address the problem and to avoid transfusión.

Conflict of interest: Nothing to disclose

\section{P218}

\section{Burden of Post-Transplant Lymphoproliferative Disorder- Mortality After Allogeneic Hematopoietic Cell Transplantation}

Aaron Katz ${ }^{1}$, Arie Barlev ${ }^{2}$, AJ Joshi ${ }^{2}$

${ }^{1}$ University of North Carolina at Chapel Hill, Chapel Hill, NC, United States; ${ }^{2}$ Atara Biotherapeutics, Inc, South San Francisco, CA, United States

Background: Post-transplant lymphoproliferative disorder (PTLD) is an uncommon, but serious, life-threatening, and often deadly disease that may occur post transplantation.

Methods: We assessed the burden of PTLD-related mortality after allogeneic hematopoietic stem cell transplantation (HCT) by reviewing and summarizing the published literature. A comprehensive literature search identified studies, published in 2005 and later, reporting patient-level data on patients who developed PTLD following HCT. Patient-level data, including age at HCT, time from HCT to PTLD, and survival following PTLD, were abstracted from identified publications and then aggregated to provide summary statistics.

Results: We identified 9 articles published in 2005 and later with sufficient patient-level data on age at HCT and time until death or end of follow-up. Aggregated data consisted of 120 patients who developed PTLD, including 31 children (age < 18 at HCT) and 89 adults. Mean age at HCT was 9.8 years among children and 35.6 years among adults. Mean time from HCT to PTLD was 138 days (95\% CI, 30 - 247) in children $(n=16)$ and 132 days $(95 \%$ CI, 72 - 191) in adults $(\mathrm{n}=59)$ for the 75 patients with available data. In the overall population, $51(42.5 \%)$ patients died with PTLD, including 11 children and 40 adults. Mean time from PTLD to death was 71.2 days $(95 \%$ CI, 30.3 - 112.2) among children, with mean age of 9.4 years (95\% CI, 5.4 13.4), and 52.8 days (95\% CI, 36.5 - 69.2) among adults, with mean age of 39.4 years (95\% CI, 35.4 - 43.4). Median time from PTLD to death was 55 days (IQR: 15, 111) in children and 48 days (IQR: 10, 76) among adults.

Conclusions: PTLD following HCT is an aggressive disease that impacts a young population. The disease carries 
a significant mortality rate with a relatively short time from diagnosis to death.

Conflict of interest: Employee of Atara Biotherapeutics, Inc.

Source of funding: Atara Biotherapeutics, Inc.

\section{P219}

\section{Cancer risk in survivors of hematopoietic stem cell transplantation for the patients with Fanconi anemia in Japan}

\section{Miharu Yabe ${ }^{1}$, Eri Imai ${ }^{2}$, Keisuke Ohtsubo ${ }^{2}$, Hiromitsu Takakura ${ }^{2}$, Tsuyoshi Morimoto ${ }^{2}$, Takashi Shimizu ${ }^{2}$, Takashi Koike ${ }^{2}$, Shunichi Kato ${ }^{1}$, Hiromasa Yabe ${ }^{1}$ \\ ${ }^{1}$ Tokai University School of Medicine, Department of Cell Transplantation and Regenerative Medicine, Isehara, Japan; ${ }^{2}$ Tokai University School of Medicine, Department of Pediatrics, Isehara, Japan}

Background: Fanconi anemia (FA) patients have high rates of bone marrow failure, acute leukemia, and solid cancers. Hematopoietic stem cell transplantation (HSCT) is effective in curing bone marrow failure, but long-term survivors of HSCT have risks of cancers. We analyzed the incidence of cancer in FA patients undergoing HSCT in Japan, and evaluated the risk factors of secondary cancers.

Methods: We performed a retrospective analysis of 62 patients with FA who underwent a HSCT between 19872016 and survived at least 1 year post HSCT (30 males and 32 females aged 1 to 28 years at HSCT; median 8.6 years). The hematological diagnosis was aplastic anemia $(\mathrm{N}=43)$, 16 with myelodysplastic syndrome (MDS), and 3 with acute myelogenous leukemia (AML). Fifteen patients received HLA-matched related bone marrow (BM), 1 received HLAmatched related cord blood (CB), 5 HLA-mismatched related BM or peripheral blood, 23 HLA-matched unrelated BM, 18 HLA-mismatched unrelated BM. The conditioning regimen consisted of cyclophosphamide (CY) (20-150 mg/ $\mathrm{kg})+$ irradiation $(6-8 \mathrm{~Gy}) \pm$ others $(\mathrm{N}=9)$, other $\mathrm{CY}$ regimen $(\mathrm{N}=1)$ and fludarabine $(\mathrm{Flu})+\mathrm{CY}(40 \mathrm{mg} / \mathrm{kg}) \pm$ irradiation $(2-4.5$ Gy $) \pm$ others $(\mathrm{N}=52)$.

Results: With a maximum follow up of 29 years, including 50 alive $>5$ years after HSCT, two had hematological malignancies; one T-cell lymphoma and one donor-type AML, and thirteen had 17 solid cancers; 4 of those had 2 cancers. Esophagus $(\mathrm{N}=5)$, head and neck $(\mathrm{N}=10)$ squamous cell carcinomas (E/HNSCC) were the common solid cancers occurring in 11 patients. The median time to onset of E/HNSCC was 11 years (range, 5 to 29 years) after HSCT, and median age at diagnosis of E/ HNSCC was 23 years (range, 14 to 41 years). Four of 11 patients with E/HNSCC received from HLA-mismatched donors and, 7 of those received Flu regimen with lower dose irradiation. Moreover, 6 patients had a history of chronic GVHD ( 1 had extensive type and 5 had limited type). Although the small number of patients in each group does not allow for multivariate analyses, univariate analyses showed there was no significant difference in survival free of E/HNSCC between patients received HLA-matched donor and those received HLA-mismatched donor or between patients with chronic GVHD and those without chronic GVHD or between patients received $\geqq 6 \mathrm{~Gy}$ irradiation and those received $<6$ Gy irradiation. The other solid cancers were one skin cancer 15 years after HSCT and one liver cancer with hepatitis B and C 10 years after HSCT. Most solid cancers (excluding the liver cancer) were treated with surgery. The patients with T-cell lymphoma and donor-type AML died of progressive disease. Four patients with E/HNSCC died of cancer progression, and the patient with liver cancer died of bleeding from esophageal varices.

Conclusions: There was a high risk of solid cancers in FA patients after HSCT, and prognosis was very poor. It is important to establish a follow-up system for checking highrisk organs in long-term FA survivors of HSCT, however it is very difficult to predict them.

Conflict of interest: M. Yabe: nothing to disclose

\section{P220}

\section{Donor Cell-derived Leukemia after Allogeneic HCT for Clear-cell Renal Cell Carcinoma}

Federico Monaco ${ }^{1,2}$, Brenda M. Sandmaier ${ }^{2}$

${ }^{1}$ A.O. SS. Antonio e Biagio, Alessandria, Italy; ${ }^{2}$ Fred Hutchinson Cancer Research Center, Seattle, WA, United States

Background: Donor cell-derived leukemia (DCL) is a rare complication of allogeneic hematopoietic cell transplantation (allo-HCT).

It is characterized by emergence of a leukemic clone in donor cells and development of overt acute leukemia. Different methods are used to confirm the origin of the neoplastic cells including cytogenetic, FISH and molecular DNA markers.

The majority of reported cases regards patients that underwent allo-HCT to treat an hematological disease involving bone marrow. We present a case of DCL developed in a patient who underwent allo-HCT to treat metastatic clear-cell renal cell carcinoma (RCC) without prior exposure to chemo-radiotherapy.

Methods: Case report: In September 2000, metastatic RCC was diagnosed in a 40-year old male. No other treatment was given and the patient was enrolled at Fred 
Hutchinson Cancer Research Center in a protocol for an HLA-matched related nonmyeloablative allo-HCT.

He received allo-HCT on April 2001 after conditioning with fludarabine and total body irradiation (TBI) and reinfusion of unmodified peripheral blood stem cell graft. GvHD prophylaxis included mycophenolate mofetil (MMF) and cyclosporine. Unfortunately he had graft rejection on day +94 .

He underwent G-CSF mobilization with apheresis of autologous stem cells and then, on August 2001, he received second allo-HCT from the same donor after conditioning with cyclophosphamide and ATG.

During follow up, chimerism analysis was $100 \%$ donor and there wasn't evidence of GvHD.

Results: On November 2002 (440 days after second alloHCT) the patient developed leukopenia and a bone marrow biopsy was done with diagnosis of acute myelomonocytic leukemia without molecular abnormalities. Chimerism analysis of peripheral blood and marrow cells remained $100 \%$ donor and analysis of flow-sorted blasts was also $100 \%$ donor, confirming diagnosis of donor-cell derived AML.

Induction therapy following $7+3$ scheme was started and complete remission with full-donor chimerism was achieved. Patient received auto-HCT on February 2003 after conditioning with cyclophosphamide and TBI 12Gy.

In February 2007 patient relapsed for RCC and underwent surgery. He started systemic therapy on May 2013 with pazopanib and successively on August 2017 with nivolumab. Currently, he has multiple RCC tumors in right kidney, but no evidence of metastatic disease. There aren't signs of leukemia relapse (14 years after auto-SCT). The patient's donor is alive and healthy.

Conclusions: Until now, mechanisms of leukemogenesis in DCL are still unknown.

In this report, we describe a patient that developed this complication after allo-HCT for solid tumor malignancy. He was subjected to relatively small amounts of chemotherapy compared to analogous patients with a hematological malignancy.

It could be hypothesized that irradiation damage to the marrow microenvironment could have contributed to the development of leukemia, as the donor cells were not directly exposed to the TBI administered as part of the conditioning for his first allo-HCT; otherwise, it can also be hypothesized that donor microchimerism was still present during the second conditioning regimen and that the remaining donor cells were exposed directly to cyclophosphamide with a resulting mutational event.

It is remarkable our patient continues to survive his metastatic RCC 17 years after his diagnosis; it is possible that $\mathrm{T}$ cell alloimmunity has contributed to his survival.

Conflict of interest: The authors have nothing to disclose

\section{P221}

\section{Changes in Bone Mineral Density and Bone Quality One Year Following Allogeneic Stem Cell Transplantation: Effects of Zoledronic Acid Infusion}

\author{
Wasithep Limvorapitak ${ }^{1,2}$, Benjamin Chernoff ${ }^{3}$, Deepesh Lad $^{4}$, \\ David Kendler ${ }^{3,5}$, Raewyn Broady ${ }^{1,3}$ \\ ${ }^{1}$ Leukemia BMT Program of BC, Vancouver, Canada; ${ }^{2}$ Thammasat \\ University, Pathumthani, Thailand; ${ }^{3}$ University of British Columbia, \\ Medicine, Vancouver, Canada; ${ }^{4}$ Post Graduate Institute of Medical \\ Education and Research, Department of Internal Medicine, \\ Chandigarh, India, ${ }^{5}$ Prohealth Clinical Research, Vancouver, Canada
}

Background: Declines in bone mineral density (BMD) is common following allogeneic stem cell transplantation (alloSCT). Multiple factors contribute to accelerated bone loss leading to osteoporosis and fractures. Zoledronic acid (ZA) infusion has been shown to attenuate bone loss in these patients. This study aimed to characterize bone loss 1 year after alloSCT and to identify patient and transplant specific factors associated with declines in BMD and bone quality, including the effect of ZA.

Methods: AlloSCT patients at our Centre have BMD and lumbar spine Trabecular Bone Score (TBS, an indicator of bone architecture) by dual x-ray absorptiometry (DXA) pretransplant, 100 days and 1 year post-alloSCT. ZA was administered to patients at high fracture risk either prealloSCT or at day 100 post-alloSCT. Patients were categorized according to World Health Organization criteria: T-score $\leqslant-2.5$ osteoporosis, $\geqslant-1.0$ normal, and between -1 and -2.5 osteopenia.

Results: We evaluated BMD change at one-year in 147 of 338 adult patients who had alloSCT between 2011 and 2015 and had paired DXA scans. Paired scans were not available for 96 patients (43 missing baseline BMD and 53 did not return for one year), 93 patients died before one year and 2 myeloma patients were excluded. Median age at alloSCT was 52.2 years. There were 74 female patients $(50.3 \%)$. At one year, BMD was normal in 43 patients (28.6\%), osteopenia in 70 patients $(47.6 \%)$, and osteoporosis in 35 patients (23.8\%), compared to baseline of $63(42.9 \%)$ normal, 61 (41.5\%) osteopenia, and 23 (15.6\%) osteoporosis $(\mathrm{p}<0.001)$. Median age for normals was 46.3 years, osteopenia 51.4 years, and osteoporosis 57.4 years $(\mathrm{p}=0.029)$. Factors correlated with BMD included: taking calcium pre-alloSCT $[10(23.8 \%)$ vs $16(22.9 \%)$ vs 17 $(48.6 \%)$, respectively $(\mathrm{p}=0.005)]$; pre-transplant menopause [6 (37.5\%) vs $25(64.1 \%)$ vs $17(89.5 \%), \mathrm{p}=$ 0.005]; family history of osteoporosis/hip fracture [3 $(7.1 \%)$ vs $6(8.6 \%)$ vs $9(25.7 \%), \mathrm{p}=0.031]$; baseline body mass index [median 27.7 vs 24.9 vs $23.2 \mathrm{~kg} / \mathrm{m}^{2}, \mathrm{p}=0.02$ ]; baseline osteopenia/osteoporosis [3 (7.1\%) vs 47 (67.1\%) 
vs $34(97.1 \%), p<0.001]$. In the first year after alloSCT fractures occurred in: normal 2 patients $(4.8 \%)$, osteopenia 11 patients $(15.7 \%)$, osteoporosis 9 patients $(25.7 \%), \mathrm{p}=$
0.031. Median follow-up time was 3.5 years. Three-year fracture-free survivals were: normal $97.2 \%$, osteopenia $87.2 \%$, and osteoporosis $78.8 \%(\mathrm{p}=0.025$, Panel A).
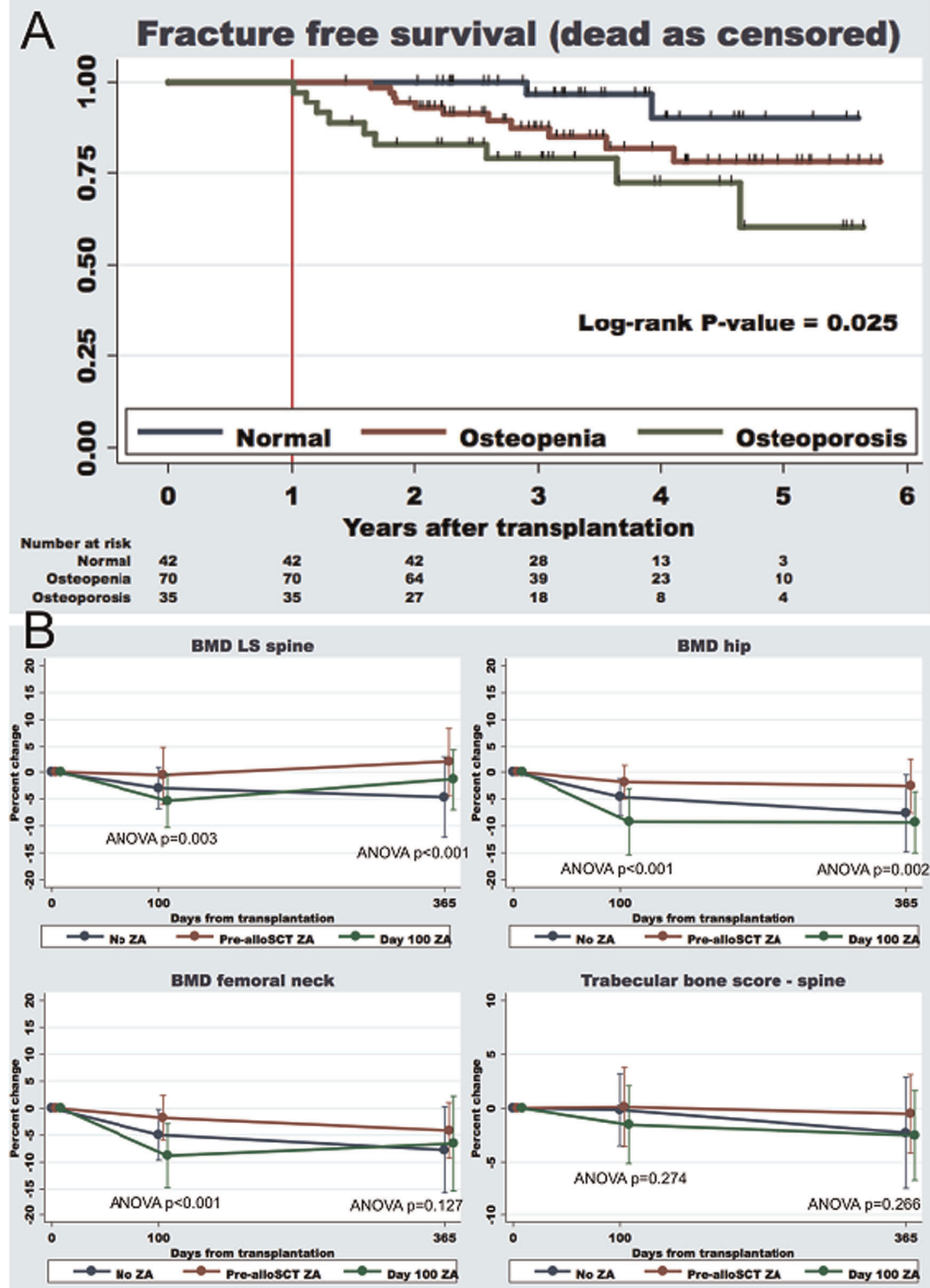

[P221 Figure] [A.Fracture Free Survival. B. Effect of ZA on BMD] 
Patients were classified as: no ZA $\mathrm{n}=108(73.5 \%)$, prealloSCT ZA $n=23(15.6 \%)$, day100 ZA $n=16$ (10.9\%). $\mathrm{BMD}$ and TBS were best maintained in patients with prealloSCT ZA, with lesser protection in patients given ZA at day 100. (Panel B).

Conclusions: Declines in BMD and bone architecture (TBS) are common after alloSCT. Increases in fractures can be seen one-year post alloSCT, especially in patients with osteoporosis by BMD. Clinical risk factors predictive of osteoporosis one-year post alloSCT include older age, menopausal status, positive family history, taking calcium supplements, and lower BMI. ZA is most effective at attenuating bone loss if given pre-alloSCT, with lesser efficacy if given at day 100. Recognizing risks to bone health in this patient population should lead to more aggressive preventive therapy for those at risk.

\section{Conflict of interest:}

R. Broady receives advisory board and/or speaker's honorarium from Janssen, Sanofi, Astellas and Jazz Pharmaceuticals.

D. Kendler receives advisory board and/or speaker's honorarium from Amgen, Pfizer, Alexion, and Eli Lilly.

The other authors have nothing to declare.

\section{P222}

\section{Characterization of Bone Marrow Infiltrate of Immune- mediated Graft Failure after Allogeneic Hematopoietic Stem Cell Transplantation}

Ignazio Caruana ${ }^{1}$, Rita De Vito ${ }^{2}$, Gerrit Weber ${ }^{1}$, Luisa Strocchio ${ }^{1}$, Francesca Del Bufalo ${ }^{1}$, Federica Galaverna ${ }^{1}$, Mattia Algeri ${ }^{1}$, Tamascia Belardinilli ${ }^{1}$, Angela Pitisci ${ }^{1}$, Valentina Cirillo $^{1}$, Franco Locatelli $^{1}$, Pietro Merli ${ }^{1}$

${ }^{1}$ IRCCS Ospedale Pediatrico Bambino Gesù, Pediatric Hematology and Oncology, Rome, Italy; ${ }^{2}$ IRCCS Ospedale Pediatrico Bambino Gesù, Pathology, Rome, Italy

Background: Graft failure (GF) is an important cause of morbidity and mortality after allogeneic hematopoietic stem cell transplantation (HSCT). It has been proven that immune-mediated GF is caused by residual host $\mathrm{T}$ cells surviving the conditioning regimen; however, this population is still poorly characterized.

Methods: .We studied the bone marrow infiltrate of patients experiencing GF after HSCT. We obtained BM aspirates in 7 patients when GF was suspected. We also studied this cell infiltrate through flow-cytometry, immunohistochemistry and immunofluorescence. Expression of cell surface markers was assessed by flow cytometry using the following monoclonal Abs (mAbs): CD3, CD4, CD8, CD25, CD27, CD28, CD45RA, CD45RO, CD56, CD57,
CD62L, CD95, CD127, CD137, CD197, CD223 (Lag3), CD279 (PD1), and CD366 (TIM3)

Results: Between December 2015 and August 2017, 7 patients developed GF and underwent bone marrow sampling (median 14 days (range 7-22). Median age at transplant was 4.0 years (range 2.4-16). They underwent HSCT because of: severe aplastic anemia (3 patients), acute lymphoblastic leukemia (3 patients), acute myeloid leukemia (1 patient). The donor was a partially matched family donor in 6 cases and a matched related donor in the remaining one. Two patients presented anti-HLA antibodies. 7 patients who underwent HSCT in the same period were used as controls.

BM lymphocyte infiltrate was characterized by an increased number and enlargement (number of vacuoles) of CD68+ and CD163+ macrophages indicating activation and phagocytic activity and by a significant increase of CD8 + cells $(\mathrm{p}=0.0018)$, which displayed a predominant effector memory phenotype (CD45RO+/CCR7-) $(\mathrm{p}=0.034)$. Moreover, they significantly expressed markers of prolonged activation (e.g. over-expression of CD95 and downregulation of CD127) and exhaustion (CD57, CD279 (PD1), CD223 and CD366). Finally, when the diverse $\mathrm{T}$ cells repertoire was analyzed by the evaluation of the $\mathrm{V} \beta$ chain on the $\mathrm{CD}^{+}$population, a polyclonal distribution of spectratypes across the TCR V $\beta$ gene families was found. When compared to controls, a higher expansion of the V $\beta$ families 5.2, 5.3, 11, 13.6 and 23 was observed. We further demonstrated that this population besides being activated, was proliferating $(\mathrm{Ki} 67+)$ and cytotoxic expressing specific molecules such as Granzyme B, Perforin and TIA-1, which was conformed by the presence of apoptotic cells in their vicinity. Notably, also other cell populations analyzed showed a markedly increased activation profile, underlining an over-stimulated environment (NK T and $\gamma \delta$ T lymphocytes, $\mathrm{p}<0.05)$.

Conclusions: We demonstrated that the $\mathrm{CD}^{+}$population that infiltrate the $\mathrm{BM}$ in patients experiencing $\mathrm{GF}$ is activated, proliferates and expresses cytotoxic specific molecules such as Granzyme B, Perforin and TIA-1. Interestingly, we observed that the $\mathrm{CD}^{+}$expansion is predominantly polyclonal indicating that the immune response is directed towards several antigens and not against few immunodominant ones. Finally, the expression of several exhaustion and senescence markers confirmed the status of prolonged activation of these $\mathrm{T}$ lymphocytes.

Conflict of interest: None of the authors has anything to disclose. 


\section{P223}

\section{Clinical characteristics and treatment of isolated extramedullary relapses after allogeneic hematopoietic stem cell transplantation for acute myelogenous leukemia: an updated single-center analysis of 401 patients}

Dariusz, Kata, Matgorzata Kopera, Mirostaw Markiewicz, Anna Waclawik, Krzysztof Bialas, Grzegorz, Helbig

Silesian Medical University, Hematology and Bone Marrow Transplantation, Katowice, Poland

Background: Isolated extramedullary relapses (IEMR) are increasingly observed late complications after allogeneic hematopoietic stem cell transplantation (alloHSCT) for acute myeloid leukemia (AML). However, an optimal therapeutic approach to such mode of leukemia relapse remains uncertain.

Methods: We retrospectively analyzed incidence, clinical presentations, treatment options and long-term outcome of IEMR in a cohort of 401 consecutive patients (pts) with AML who underwent allo-HSCT in our center between June 1993 and December 2013. 58 pts relapsed (any site).

Results: 11 (19\%) out of all pts who relapsed (F/M 3/8, median age 40 years, range 25 - 60 years) experienced histologically proven isolated IEMR after a median time of 10 months (mts) (range, 6 - $80 \mathrm{mts}$ ) following allo-HSCT. 3 pts developed skin and/or subcutaneous tissue infiltrates. Other sites of relapse included (No. of cases): central nervous system (CNS) (3), paraspinal soft tissues (1), small intestine (1), lymph nodes (1), paranasal sinuses (1), breast (1).

Treatment plans for those isolated EM relapses included (No. of cases):

$1 /$ systemic chemotherapy (5),

2/ systemic chemotherapy and secondary alloHSCT (4),

$3 /$ imatinib, then dasatinib (1),

4/ surgery (1).

Pts with CNS involvement received intrathecal therapy with cytarabine and in one case additional CNS irradiation was applied.

10/11 pts died after a median time of $9 \mathrm{mts}$ (range, 1 - 25 $\mathrm{mts}$ ) due to resistant systemic relapse, infectious complications or extensive graft versus host disease following alloHSCT. 1 patient remains alive and disease-free at 100+ mts following secondary alloHSCT.

Conclusions: Our data indicate that IEMR following alloHSCT occur in a significant proportion of pts with AML. Anatomic sites of relapses vary widely among the pts with skin and CNS being frequently involved. According to our experience intensive systemic therapy including secondary alloHSCT may produce favorable response in a proportion of cases, however, long-term prognosis for pts with IEMR remains poor. An effective treatment strategy for IEMR still remains a challenge.

Conflict of interest: None of the authors has anything to disclose.

\section{P224}

Clinical factors to predict engraftment in low CD34 count autologous hematopoietic stem cell transplantation in patients with lymphoma and myeloma

Sang-A Kim, Dong-Yeop Shin, Youngil Koh, Junsik Hong, Inho

Kim, Sung-Soo Yoon

Seoul National University Hospital, Internal Medicine, Seoul, Korea, Republic of

Background: Autologous stem cell transplantation (ASCT) is the essential treatment in high-risk lymphoma and multiple myeloma patients. CD34 is one of the most widely used indicators to measure the amount of collected hematopoietic stem/progenitor cell (HSPC), and at least $3.0 \times 10^{6} / \mathrm{kg}$ CD34 positive cells are known to be acceptable for optimal transplantation outcome. But considerable patients have been exposed to chemotherapeutic agents and radiation therapy, which in turn reduces bone marrow function and causes difficulty in collecting sufficient amount of stem cells for ASCT. In this study, we aimed to analyze the factors associated with bone marrow engraftment and clinical outcome among the patients who underwent low CD34 HSPC ASCT.

Methods: We retrospectively reviewed the patients who received ASCT in Seoul National University Hospital from January 2008 to January 2017, and a total of 615 patients were collected. Among these patients, 103 patients underwent HSCT with less than $3.0 \times 10^{6} / \mathrm{kg}$ of CD34 HSPC. Clinical information was obtained by clinical record review. Survival and risk factor analysis was conducted using Kaplan-Meier curve and Cox proportional hazard model.

Results: Total 60 lymphoma patients and 43 myeloma patients were analyzed. With median follow-up duration of 42 months (range, 7.17-119.90), median overall survival (OS) was 105.70 months (95\% Confidence interval (CI), 46.47-164.94) and median relapse-free survival (RFS) was 39.97 months (95\% CI, 27.24-52.70).

In univariate analysis for neutrophil engraftment, lymphoma patients showed delayed engraftment compared to myeloma patients $(\mathrm{p}=.002)$. Age was also a significant factor, indicating patients older than 50 years needs more time to engraftment $(\mathrm{p}=.003)$. And the patients whose duration from stem cell mobilization (SCM) to ASCT was more than one year showed significantly delayed engraftment ( $\mathrm{p}=.006$ ). CD34 count less than $2.0 \times 10^{6} / \mathrm{kg}$ was also associated with poor neutrophil engraftment $(\mathrm{p}=.028)$. 

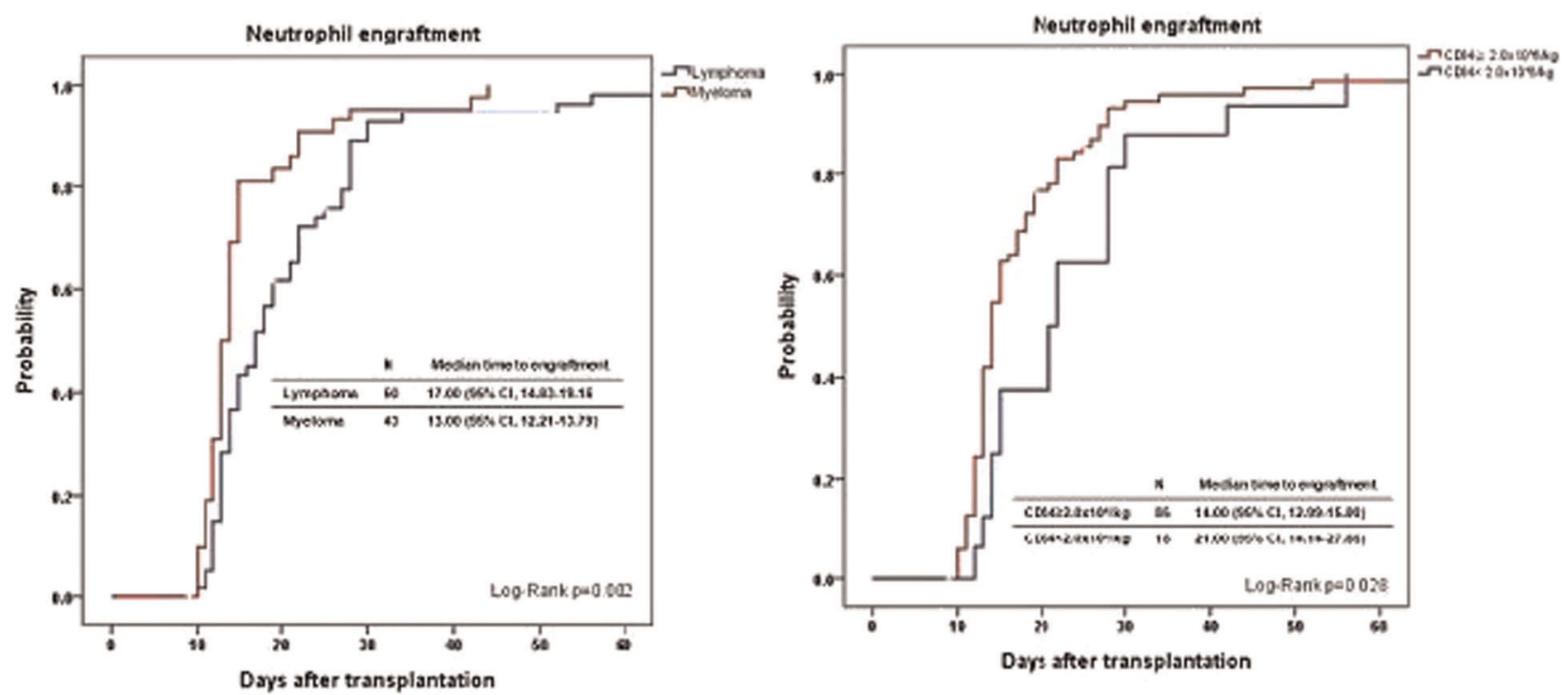

[P224 Figure] [Figure 1. Comparison of time to neutrophil engraftment by CD34 count and underlying disease.]

Among the mobilization methods, bone marrow harvest was the only factor that was meaningfully associated with poor neutrophil engraftment $(\mathrm{p}=.038)$. But sex, initial stage and treatment response before ASCT showed no significant difference in neutrophil engraftment.

By multivariate analysis, lymphoma $(\mathrm{p}=.011, \mathrm{HR} 1.79$, 95\% CI 1.14-2.82), more than 2 lines of previous treatment history ( $\mathrm{p}=.017$, HR $1.87,95 \%$ CI 1.12-3.12), more than one year elapse from SCM to ASCT $(\mathrm{p}=.021$, HR 2.71, 95\% CI 1.17-6.30) and less than $2.0 \times 10^{6} / \mathrm{kgof}$ CD34 count ( $\mathrm{p}=.072$, HR $1.65,95 \%$ CI 0.95 to 2.87 ) were the factors independently associated with neutrophil engraftment.

For the platelet engraftment, multivariate analysis revealed that lymphoma ( $\mathrm{p}=.001$, HR $2.33,95 \%$ CI 1.44 3.77), age more than $50(\mathrm{p}=.045$, HR $1.60,95 \%$ CI 1.01 2.53) and less than $2.0 \times 10^{6} / \mathrm{kg}$ of CD34 count ( $\mathrm{p}=.005$, HR $2.32,95 \%$ HR 1.28 to 4.20 ) was clinically meaningful in expectation of successful platelet engraftment.

Conclusions: By this study focusing on low CD34 count ASCT patient population, we concluded that we could predict the neutrophil and platelet engraftment and clinical outcome using clinical information, suggesting that we can plan patient-tailored strategy in ASCT.

Conflict of interest: None of the authors has anything to disclose.

\section{P225}

\section{CLINICAL IMPACT AND PROGNOSIS ABOUT MULTIPLE TRANSFUSIONS PRIOR TO ALLOGENIC HEMATOPOIETIC STEM CELL TRANSPLANTATION}

Eva Alonso ${ }^{1}$, Maria José Jimenez ${ }^{2}$, Mireia Morgades ${ }^{2}$, Christelle Ferrà $^{2}$, Blanca Xicoy ${ }^{2}$, Montserrat Batlle ${ }^{2}$, Josep Maria Ribera ${ }^{2}$, Joan Ramon Grifols ${ }^{1}$

${ }^{1}$ BST-Hospital Universitari Germans Trias i Pujol, Transfusion Service, Badalona, Spain; ${ }^{2}$ ICO-Hospital Universitari Germans Trias i Pujol, Josep Carreras Leukemia Research Institute, Universitat Autonoma de Barcelona, Hematology Service, Badalona, Spain

Background: Hyperferritinemia in patients that undergo allogeneic hematopoietic stem cell transplantation (alloHSCT) has been related to lower overall survival (OS) and higher non-relapsed mortality (NRM), probably due to iron overload (IO) toxicity in hepatic and cardiac cells, and to an increased risk of fungal and bacterial infections. The aim of this study was to analyse if the number of red blood cells (RBCs) units transfused [with more than $20 \mathrm{RBCs}$ units, chelation therapy (CT) is recommended] prior to alloHSCT could impact on OS or on the probability to develop infections (bacterial or fungal), hepatic sinusoidal obstruction syndrome (SOS) or graft-versus-host disease (GVHD).

Methods: Retrospective analysis of 179 patients undergoes first alloHSCT from January 2008 to July 2016. We compared the patients who had been transfused with less (n $=98)$ or more $(\mathrm{n}=81)$ than 20 RBCs units before allograft. Demographic (image 1) and clinical data such as serum ferritin, C-reactive protein (CRP), erythrocyte sedimentation rate (ESR) and liver function data previous allograft were collected. Previous RBCs transfusion history (and 12 months later) of allograft and liver function data at 3,6 and 12 months later allograft were collected. Previous and post-alloHSCT CT history, bacterial sepsis (two blood positives cultures), invasive fungal infections (IFI) history 
and SOS, grade III-IV mucositis, GVHD (acute and chronic) data as well as cardiac and respiratory events during allograft procedure were collected too. Incidences of OS, NRM and relapse (RI) were analyzed.

Results: The two differentiated groups were divided according RBCs units transfused pre-alloHSCT in [mean (SD)]: < 20RBCs [8,85 (6,35)] and >20RBCs [35,94 $(26,28)]$. There were statistically significant differences between both groups (in the order previously related) on biochemical parameters pre-alloHSCT [mean (SD)]: ferritin [882,34 $(773,80)$ vs. $2236,47(1138,33)$ p < 0.001], IST $[38,41(22,77)$ vs. $53,69(26,49) \mathrm{p}=0.010]$, ESR $[40,21$ $(26,89)$ vs. $57,607(35,41) \mathrm{p}=0.005]$ and glutamic-pyruvic transaminase $[27,49(19,73)$ vs. $40,85(29,86) \mathrm{p}<0.001]$. There were no differences on OS at 5 years, RI or NRM compared with RBCs transfused, IO toxicity and elevated ESR and CRP. There were statistically significant differences between both groups on SOS events [5 (5\%) vs. 11 $(14 \%) \mathrm{p}=0.048]$, without significant differences in III-IV mucositis grade, graft, GVHD type, respiratory or cardiac events and bacterial (multi-resistant bacteria or not) or IFI incidence. RBCs units transfused in both groups at 12 months post-alloHSCT was statistically significant [mean (SD)]: [11,59 $(15,25)$ vs. $14,68(14,68) \mathrm{p}=0,027]$ and although there are no differences in terms of the CT history prior to alloHSCT, this practice increases in the post-alloHSCT [7 (9\%) vs. $1(1 \%) \mathrm{p}=0.017]$ even though it is a scarce practice.

Conclusions: In this study the patients who received more than 20 RBCs units before alloHSCT presented higher serum ferritin values and higher SOS incidence than those who received less than $20 \mathrm{RBC}$ units. It is important to insist on an adequate $\mathrm{CT}$ before submit a patient to alloHSCT whenever possible and to have the patient's transfusion history in order to optimize clinical haematology response assessment.

Conflict of interest: The authors declare that they have no conflict of interest.

\section{P226}

\section{Comparative analysis of allogeneic transplant complications of alternative donors: haploidentical vs unrelated donor}

Nerea Arratibel Zalacain, Victor Higuero, Estefanía Pérez-López, Oscar Rodrigo-Gómez, Melani Irais Otañez, Lucía López-Corral, Eduardo Sobejano, Daniel Presa, Miriam López-Parra, Alba Redondo, Fermín Sánchez-Guijo, Mónica Cabrero, Ana A. Martín, Verónica Gonzalez, Almudena Navarro, Joud Zanabili, Lourdes Vázquez, Dolores Caballero
Hospital Universitario de Salamanca, Salamanca, Spain

Background: In recent years has been extended the use of haploindentical donors in patients lacking a HLA matched donor. Published studies compare the efficacy of these type of transplantation in terms of survival or graft versus host disease (GVHD, but few of them analyzed the early complications of the transplantation. Our aim is to carry out a comparative study of the main complications between haploidentical transplantation (HAPLO) and unrelated donor (URD) transplantation and secondly, the analysis of OS, MRT and EFS in the global serie.

Methods: In this retrospective analysis all the patients receiving an allotransplant from an URD or an HAPLO allogeneic stem cell transplantation in our centre between 2012-2016 have been included. cord blood SCT were excluded.. Statistic analysis is done by SPSS x 22.0.

Results: 169 patients were included, 118 (70\%) from an URD, 18 of them (15\%) with in vivo T depletion (ATG) and $51(30 \%)$ from an Haplo donor with post-transplant cyclophosphamide. General characteristics and differences between the two groups are shown in table 1.

Patients receiving an haplo transplant had a statistically significant $(p<0.05)$ higher incidence of a late graft which lead to a prolonged median admission time, higher number of CMV reactivation and higher hemorrhagic cystitis incidence. In the URD group, a higher incidence of aGVHD grade III-IV and Trombotyc microangiopathy (TMA) was observed, with a statistically significant association between patients with aGVHD and TMA $(p=0.001)$

There were no differences in number of readmissions and the main reason to readmission in both groups were the infections. In URD group, there was a higher percentage of readmissions because of aGVHD (27\%). There were no differences in terms of MRT ( $p 0.36)$, EFS $(p 0.15)$ and OS (p 0.38).

Conclusions: In our experience the use of Haplo and URD is associated with similar days of readmission in hospital however the pattern of complications is different. These analysis are important ir order to organize resources in the era in which most of the patients will have a donor. However, due to the retrospective nature of the study, these results should be confirmed in further studies.

Conflict of interest: Nothing to disclose

\section{P227}

Cost-Effectiveness of Defibrotide for Treatment of VenoOcclusive Disease with Multi-Organ Dysfunction (VOD/ MOD) Post-Hematopoietic Stem Cell Transplantation (HSCT) in Two Countries - Wales and Italy 


\begin{tabular}{|c|c|c|c|}
\hline & $\begin{array}{c}\text { Haplo (n=51) } \\
n(\%)\end{array}$ & $\begin{array}{c}\text { NRD }(n=118) \\
n(\%)\end{array}$ & $\mathbf{P}$ \\
\hline Age (median, range) & $58(17-70)$ & $49(17-68)$ & \\
\hline Diagnosis & & & 0.001 \\
\hline AML & $24(47)$ & $43(36)$ & \\
\hline MDS & $7(14)$ & $20(17)$ & \\
\hline AlL & $4(8)$ & $19(16)$ & \\
\hline HL & $8(16)$ & $1(1)$ & \\
\hline NHL & $5(10)$ & $20(17)$ & \\
\hline SMP & $3(5)$ & $7(6)$ & \\
\hline Others & & $8(7)$ & \\
\hline Status at HSCT & & & 0.093 \\
\hline CR & $27(53)$ & $76(64)$ & \\
\hline PR & $6(12)$ & $8(7)$ & \\
\hline Stable Disease & $6(12)$ & $21(18)$ & \\
\hline Relapse/progression & $12(24)$ & $13(11)$ & \\
\hline $\begin{array}{l}\text { Tiempo desde el diagnostic } \\
\text { (meses) }\end{array}$ & $\begin{array}{c}8 \\
(1-182)\end{array}$ & $\begin{array}{c}8 \\
(2-444)\end{array}$ & 0.6 \\
\hline Missmatch & $\cdots$ & $39(33)$ & \\
\hline Regimen (n,\%) & & & 0.047 \\
\hline RIC & $38(75)$ & $69(59)$ & \\
\hline MAC & $13(25)$ & $49(41)$ & \\
\hline Profilaxis EICH & & & \\
\hline CSA+MMF & 0 & $3(2)$ & \\
\hline Inh.Calcineurina+MMF+CyPT & $51(100)$ & 0 & \\
\hline Tacro+MTX & 0 & $50(42)$ & \\
\hline Tacro+Siro & 0 & $65(55)$ & \\
\hline Admission days (median, range) & $36(23-130)$ & $29(19-221)$ & $<0.001$ \\
\hline Readmission $(n, \%)$ & $41(80)$ & $98(83)$ & 0.72 \\
\hline 1 & $13(32)$ & $25(25)$ & \\
\hline 2 & $7(17)$ & $17(17)$ & \\
\hline$\geq 3$ & $21(51)$ & $57(58)$ & \\
\hline Readmission reason & & & 0.001 \\
\hline GVHD & $3(6)$ & $32(27)$ & \\
\hline Infecciones & $32(63)$ & $47(41)$ & \\
\hline Others & $5(10)$ & $20(17)$ & \\
\hline
\end{tabular}

[P226 Figure] [Table1] 
Jonathan Belsey ${ }^{1}$, Eric Ngonga Kemadjou ${ }^{1}$, Umberto Restelli ${ }^{2,3}$, Davide Croce $^{2,3}$, Fabio Ciceri ${ }^{4,5}$, Kathleen F. Villa ${ }^{6}$

${ }^{1} J B$ Medical Ltd., Sudbury, United Kingdom; ${ }^{2}$ LIUC - Università Cattaneo, Castellanza (VA), Center for Health Economics, Social and Health Care Management, Castellanza, Italy; ${ }^{3}$ University of the Witwatersrand, School of Public Health, Faculty of Health Sciences, Johannesburg, South Africa; ${ }^{4}$ IRCCS San Raffaele Scientific Institute, Hematology and BMT Unit, Milan, Italy; ${ }^{5}$ University Vita-Salute San Raffaele, Milan, Italy; ${ }^{6} \mathrm{Jazz}$ Pharmaceuticals, Health Economics and Outcomes Research, Palo Alto, CA, United States

Background: The current analysis compares costeffectiveness of defibrotide (DF) vs. best supportive care (BSC) in Wales and Italy, based on Phase $3(\mathrm{Ph} 3)$ and country-specific data in the two countries, in patients with veno-occlusive disease with multi-organ dysfunction (VOD with MOD) post-hematopoietic stem cell transplantation (HSCT).

Methods: Country-specific Markov cost-utility models comparing DF treatment with BSC included an acute phase and a long-term phase, with a lifetime horizon. Transition probabilities in the acute phase were based on $\mathrm{Ph} 3$ endpoints of Day +100 survival and complete response (CR). The model included four health states: severe VOD, $\mathrm{CR}$, survival, and death. Survival in the long-term phase was extrapolated using Kaplan-Meier curves and an exponential function, published literature and Welsh and Italian life tables. Sensitivity analyses used propensityadjusted efficacy from the Ph3 study (Richardson, 2016), which used a Cox regression adjustment to account for baseline differences between treatment arms.

Hospital costs were calculated by taking the difference in time to CR in each arm and average HSCT length of stay in Wales and in Italy, assuming $85 \%$ intensive care unit time in Wales and $14.5 \%$ in Italy, and 15\% high dependency unit time in Wales and $85.5 \%$ in Italy. The severe VOD utility value was assumed to be the same as acute liver failure and end-stage liver disease scores (0.208) and the utility for CR was set to the age-matched general population. Costs and outcomes were discounted at $3.5 \%$ per year in Wales and $3.0 \%$ in Italy.

Results: Overall costs were higher in the Wales model compared to the Italian model, while the difference in costs between DF and BSC were higher in Italy with estimates of $£ 19,038$ in Wales and $€ 36,926$ in Italy (Table 1 ). The DF group in the Italian model showed a higher increase in life years (LYs) and quality-adjusted life years (QALYs) compared to the Wales model, and consequently Wales model shows a better incremental cost-effectiveness ratio (ICER) compared to Italy (£18,943/QALY vs $€ 31,117 /$ QALY). The sensitivity analysis showed lower ICERs in both countries (Wales, £7,223/QALY; Italy, €14,671/ QALY).

Conclusions: DF treatment for VOD with MOD represents cost-effective use of health resources in both the Welch and Italian contexts, with ICER estimates compared to BSC that were below accepted willingness to

\begin{tabular}{|c|c|c|c|c|c|c|}
\hline & \multicolumn{3}{|c|}{ Wales - costs in GBP } & \multicolumn{3}{|c|}{ Italy - costs in $€$} \\
\hline & DF & $\mathrm{BSC}$ & Difference & DF & $\mathrm{BSC}$ & Difference \\
\hline \multicolumn{7}{|c|}{ Base case results - unadjusted efficacy estimates from clinical trial (Both countries) } \\
\hline Cost & $£ 74,777$ & $£ 55,739$ & $£ 19,038$ & $€ 53,097$ & $€ 16,171$ & $€ 36,926$ \\
\hline QALYS & 3.829 & 2.824 & 1.005 & 4.544 & 3,357 & 1.187 \\
\hline $\begin{array}{l}\text { ICER } \\
\text { (COST/QALY) }\end{array}$ & & & $£ 18,943$ & & & $€ 31,117$ \\
\hline \multicolumn{7}{|c|}{$\begin{array}{l}\text { Sensitivity analysis results -cfficacy cstimatcs from clinical trial adjustcd for basclinc } \\
\text { differences in patient populations (both countries) }\end{array}$} \\
\hline Cost & $£ 75,725$ & $£ 60,622$ & $£ 15,103$ & $€ 53,770$ & $€ 17,370$ & $€ 36,400$ \\
\hline QALYS & 5.837 & 3.746 & 2.091 & 6.933 & 4.452 & 2.481 \\
\hline $\begin{array}{l}\text { ICER } \\
(\text { COSUQALY) }\end{array}$ & & & $£ 7,223$ & & & $€ 14,671$ \\
\hline
\end{tabular}

[P227 Figure] [Table 1. Cost-utility results by country] 
pay thresholds. Limitations of the analysis include longerterm extrapolation of clinical trial data and assumptions about resource utilization patterns in each country. Results were driven by estimates of more rapid recovery, reduced length of stay and improved Day+100 survival in DFtreated patients. Sensitivity analysis confirmed robust and comparable results for both countries.

Conflict of interest: This analysis was funded by Jazz Pharmaceuticals, Inc. J. Belsey and E. Kemadjou received consultancy payments from Jazz Pharmaceuticals for health economic services in respect of Health Technology Appraisal submissions for defibrotide. U. Restelli and D. Croce have no conflicts of interest. F. Ciceri has served as a consultant to Molmed and Incyte. K. Villa is a full-time employee of Jazz Pharmaceuticals, Inc., who in the course of this employment have received stock options exercisable for, and other stock awards of ordinary shares of Jazz Pharmaceuticals plc.

\section{P228}

CRYOSTEM: a French national Biobank to move research forward in the field of Hematopoietic Stem Cell Transplantation complications

Emilie Robert ${ }^{1}$, Claire Fontenille ${ }^{1}$, Boris Calmels ${ }^{2}$, Jean-Hugues Dalle $^{3}$, Régis Peffault de Latour ${ }^{4}$

${ }^{1}$ CRYOSTEM, Marseille, France; ${ }^{2}$ Institut Paoli-Calmettes, Cell Therapy, Marseille, France; ${ }^{3}$ Hôpital Robert Debré, Pediatric Hematology, Paris, France; ${ }^{4}$ Hôpital Saint-Louis, Transplant \& Hematology, Paris, France

Background: Hematopoietic Stem Cell Transplantation (HSCT) is the only curative treatment for numerous blood diseases. In patients cured of their hematological diseases, complications including notably Graft versus Host Disease (GvHD), infectious or conditioning-related, could be highly injurious. Lack of knowledge in GvHD and other long-term complications physiopathology, along with limited relevant studies explain the absence of curative treatments.

In this context, the CRYOSTEM project was promoted by the Francophone Society for Stem Cell Transplantation and Cell Therapy (SFGM-TC) to set up a unique biobank in Europe firstly dedicated to GvHD, then, since 2016, enlarged to the all HSCT complications. The goal is speed up biomarkers discovery, development of adequate treatments, and in fine improvement of the healthcare provided to transplanted patients.

Methods: CRYOSTEM network brings together $\mathbf{3 3}$ transplant units and 23 biological resource centers (BRCs) throughout France. Transplant units are responsible for the blood sampling, treated by the BRCs and derived in three types of samples (cells in DMSO, plasma and dried pellets) according to harmonized procedures and protocols.

Any patient suffering from blood disorders, such as leukemia, lymphoma or aplastic anemia, justifying an HSCT indication are eligible to be included in the protocol. Similarly, willing related donors for geno- and haploidentical HSCT are also eligible for inclusion. Blood samples are taken pre- and post-HSCT in line with a simple sampling schedule, taking into account acute and/or chronic appearance of GvHD.

CRYOSTEM collection of biological samples associated with clinical data from donors and patients is centralized in the MBioLIMS BioBanking software, enabling homogenization of labelling, traceability, real-time monitoring of operations.

Results: Since 2012, CRYOSTEM operates a standardized collection of high-quality biological samples with well-annotated clinical data from almost 2,500 donors and 6,000 patients, leading to nearly $\mathbf{2 0 0 , 0 0 0}$ aliquots available for research, processed from systematic blood samplings pre- and post-HSCT and at GvHD occurrence.

Moreover, ISO 9001 certification successfully obtained in February 2015 ensures collection quality and focus on user expectations and satisfaction, along with a greater international profile.

CRYOSTEM goal is to provide the national and international scientific community with these samples to improve medical knowledge in HSCT complications field and patient care. To date, five French and one German research projects have been granted with almost 5,000 CRYOSTEM samples to explore GvHD and other complications by identifying markers of risk following transplantation, monitoring and predicting responses to therapies and mapping immune systems of donor and/or recipient to better understand GvHD and complications occurrence. Part of these results and publications are expected for the end of this year. Moreover around ten new projects are to be submitted to CRYOSTEM Scientific Committee and international experts for reviewing in the early 2018, demonstrating the fundamental requirement of a dedicated biobanking to improve research on GvHD.

Conclusions: In conclusion, CRYOSTEM is a unique network in Europe dedicated to HSCT complications, recognized internationally for its high-quality samples. Currently, CRYOSTEM provides the national and international scientific community to improve medical knowledge in HSCT-related fields, such as oncology, hematology and immunology, with the ultimate concern: patients quality of life.

Conflict of interest: None of the authors has anything to disclose. 


\section{P229}

Cryotherapy reduces mucositis incidence in patients submitted to autologous stem cell transplantation after high-dose melphalan conditioning - a prospective cohort study

Adriana Roque ${ }^{1,2}$, Carolina Afonso ${ }^{1}$, Ana Luísa Pinto ${ }^{1}$, Lenka Ruzickova ${ }^{1}$, Diana Mota ${ }^{1,2}$, Rita Tenreiro ${ }^{1}$, Luis Francisco Araújo ${ }^{1}$, Emília Cortesã ${ }^{1,2}$, Ana Isabel Espadana ${ }^{1}$, Ana Bela SarmentoRibeiro $^{1,2}$, Catarina Geraldes ${ }^{1,2}$, Letícia Ribeiro ${ }^{1}$

${ }^{1}$ Centro Hospitalar e Universitário de Coimbra, Clinical Haematology Department, Coimbra, Portugal; ${ }^{2}$ Faculty of Medicine, University of Coimbra, Coimbra, Portugal

Background: Autologous hematopoietic stem cell transplantation (aHSCT) with high-dose melphalan conditioning $\left(200 \mathrm{mg} / \mathrm{m}^{2}\right.$ for multiple myeloma or $140 \mathrm{mg} / \mathrm{m}^{2}$ for lymphoma, both over 30 minutes at day -1) is associated with high morbidity, specially oral $(\mathrm{OM})$ and gastrointestinal (GIM) mucositis.

The concept of cryotherapy came to highlight in 1990's, based on the premise that local vasoconstriction reduces exposure of the oral mucosa to short infusion agents. However, the guidelines do not recommend its usage, mainly due to the low evidence to support them up, as the majority of studies included a reduced number of patients.

Our aim was to evaluate the effectiveness of cryotherapy implementation in our standard practice, in the outcomes of patients submitted to aHSCT.

Methods: All patients submitted to aHSCT in our Center after April/2016 received cryotherapy (group CRY). Patients were instructed to keep on oral cryotherapy shortly before, during, and for $30 \mathrm{~min}$ after melphalan administration.

We prospectively analyzed the group CRY (April/2016September/2017) and compared it with a group of patients submitted to aHSCT between April/2014-March/2016, which did not use cryotherapy (NONCRY).

Patients from both groups received chlorhexidine for mouth washing during hospital stay, and those who developed OM, also received lidocaine/nystatin and calcium phosphate solutions. Toxicities were classified according to CTCAEv.4.03.

Results: During the study period, 151 patients underwent aHSCT - 75 (49.7\%) received cryotherapy and 76 (50.3\%) did not. Both groups were identical for the main clinic and biologic features.

The OM incidence was found to be significantly lower in the CRY group compared with the NONCRY group (89.3\% vs $\quad 98.7 \%)$ (RR $0.91 \quad$ [95\%CI $0.83-0.98] ; \mathrm{p}=0.015)$.
However, the median of OM severity was $1(0-3)$ in both groups $(\mathrm{p}=\mathrm{NS})$ and grade $3-4$ was also similar $(6.7 \%$ and $7.9 \%$ for CRY and NONCRY, respectively)(RR 0.84 [95\% CI $0.27-2.65] ; \mathrm{p}=\mathrm{NS}$ ).

Cryotherapy also seemed to reduce GIM severity, as the incidence of GIM grade $3-4$ is $2.7 \%$ in CRY vs $11.8 \%$ in NONCRY (RR 0.23 [95\%CI 0.05-0.98]; $\mathrm{p}=0.03$ ), although without significant impact in GIM incidence $(\mathrm{p}=\mathrm{NS})$.

The number of patients who needed opioid therapy during hospital stay and its duration were similar in both groups $(\mathrm{p}=\mathrm{NS})$.

No differences were observed for the need of total parenteral nutrition, infections and engraftment parameters $(p=N S)$. The mean length of hospital stay was five days shorter in CRY group, although not statistically significant $(23.4 \pm 1.1$ vs $29.3 \pm 3.3$ days; $\mathrm{p}=\mathrm{NS})$.

Cryotherapy seems to be a protective measure for OM development, even after adjusting for age and diabetes mellitus comorbidity (OR $0.10 ; \mathrm{p}=0.036$ ), for previous radiotherapy and number of treatment lines (OR 0.11 ; $\mathrm{p}=0.038$ ), and for conditioning regimen and volume of DMSO administered (OR 0.09; $\mathrm{p}=0.034$ ).

Cryotherapy procedure was well-tolerated and was not discontinued in any patient.

Conclusions: OM is one of the most prominent toxicities of high-dosage melphalan conditioning regimen. The use of cryotherapy reduces $\mathrm{OM}$ incidence, even after adjusting for patient, disease and aHSCT characteristics.

Cryotherapy is a readily available, inexpensive and welltolerated adjuvant therapy and should be offered to all aHSCT patients. Large randomized trials are necessary to obtain more significant results and to identify the best cryotherapy protocol.

Conflict of interest: None declared.

\section{P230}

Defibrotide for Prophylaxis of Hepatic Veno-Occlusive Disease Hematopoietic Stem Cell Transplantation: Results from the Japanese Prophylactic Use, Phase II, Randomized Trial

Atsushi Kikuta ${ }^{1}$, Takahiro Fukuda ${ }^{2}$, Kazuteru Ohashi ${ }^{3}$, Shuichi Taniguchi', Yuki Asano-Morit, Keizo Horibe ${ }^{5}$, Hiroaki Goto ${ }^{6}$, Hirohisa Nakamae ${ }^{7}$, Hiroyasu Ogawa ${ }^{8}$, Hiroya Tamaki ${ }^{8}$, Yoshiyuki Takahashi ${ }^{9}$, Toshimi Kimura ${ }^{10}$, Kenichi Yoshimura ${ }^{11}$, Kazuo Umemura $^{12}$, Chitose Ogawa ${ }^{13}$

${ }^{1}$ Fukushima Medical University, Department of Pediatric Oncology, Fukushima, Japan; ${ }^{2}$ National Cancer Center Hospital, Department of Hematopoietic Stem Cell Transplantation, Tokyo, Japan; ${ }^{3}$ Tokyo Metropolitan Cancer and Infectious Disease Center, Komagome Hospital, Hematology Division, Tokyo, Japan; ${ }^{4}$ Toranomon Hospital, 
Division of Hematology, Tokyo, Japan; ${ }^{5}$ National Hospital Organization Nagoya Medical Center, Clinical Research Center, Nagoya, Japan; ${ }^{6}$ Kanagawa Children's Medical Center, Division of Hemato-Oncology and Regenerative Medicine, Yokohama, Japan; ${ }^{7}$ Osaka City University, Hematology, Graduate School of Medicine, Osaka, Japan; ${ }^{8}$ Hyogo College of Medicine, Division of Hematology, Hyogo, Japan; ${ }^{9}$ Nagoya University Graduate School of Medicine, Department of Pediatrics, Nagoya, Japan; ${ }^{10}$ Tokyo Women's Medical University Hospital, Department of Pharmacy, Tokyo, Japan;

${ }^{11}$ Kanazawa University, Innovative Clinical Research Center (iCREK), Kanazawa, Japan; ${ }^{12}$ Hamamatsu University School of Medicine, Department of Pharmacology, Hamamatsu, Japan; ${ }^{13}$ National Cancer Center Hospital, Department of Pediatric Oncology, Tokyo, Japan

Background: Hepatic veno-occlusive disease, also called sinusoidal obstruction syndrome (VOD/SOS), is a potentially life-threatening complication of conditioning for hematopoietic stem cell transplantation (HSCT) and is associated with patient and transplant-related risk factors. Overall estimated prevalence is $11 \%$ post-HSCT in Japan. Defibrotide is not approved for treatment and prophylaxis of VOD/SOS post-HSCT in Japan. We report the results of the Japanese prophylactic use program, defibrotide prevention protocol (FMU-DF-003).

Methods: This was a phase 2, multicenter, open-label, randomized, controlled trial in pediatric and adult patients, undergoing myeloablative conditioning before allogeneic HSCT, with $\geq 1$ risk factor for VOD/SOS from 2014-2016. VOD/SOS was diagnosed according to modified Seattle criteria plus $>5 \%$ weight gain. Patients were randomized to standard care with or without defibrotide prophylaxis dosed at $25 \mathrm{mg} / \mathrm{kg} /$ day in 4 divided 2-hour IV infusions q6h. Osteopetrosis was a stratification variable. Defibrotide began on the one day before HSCT conditioning and continued for 30 days post-HSCT. Control patients who developed VOD/SOS received defibrotide treatment. The primary endpoint was incidence of VOD/SOS at day +30 post-HSCT.

Results: The full analysis set population included 50 patients: 33 randomized to defibrotide prophylaxis and 17 in the control group. Mean age was 30.9 years (range 1-49; 12 [24\%] aged $<19$ years). Demographic and clinical characteristics, including VOD/SOS risk factors, were wellmatched in the defibrotide and control groups. The most common risk factors among all patients were active disease (58\%), second myeloablative transplantation $(50 \%)$ and conditioning with busulfan plus melphalan/cyclophosphamide $(44 \%)$.

VOD/SOS occurred by day +30 post-HSCT in $1(3.1 \%)$ patient in the defibrotide prophylaxis group vs no $(0 \%)$ patients in the control group. The estimated day +100 and day +180 survival for defibrotide prophylaxis group and control group were $87.4 \%$ (95\% CI, $69.7-95.1 \%$ ) vs $81.3 \%$ $(52.5-93.5 \%)$ and $84.1 \%(66.0-93.1 \%)$ vs $68.8 \%$
(40.5-85.6\%), respectively. The estimated day +100 grade III-IV acute GVHD for defibrotide prophylaxis group was $17.6 \%$ (7.6-37.8\%) and for control group was $42.9 \%$ (16.1$83.1 \%)$.

At least 1 adverse event was reported for all patients $(100 \%)$ of both group. Overall AE profiles were generally similar between prophylaxis and control group.

Serious adverse events were reported in 8 (24.2\%) patients in the defibrotide group and

$2(11.8 \%)$ were reported in control. Although the most common treatment related $\mathrm{AE}$ was hemorrhage, cumulative hemorrhage incidence was not significant between groups (13 [39.4\%] of 33 in defibrotide group versus 9 [52.9\%] of 17 controls).

Conclusions: The incidence of VOD/SOS was low in both groups. Because the total numbers of patients were small, and this study did not reach planned registration number of cases (defibrotide group; 50 patients, control group; 25 patients) during a registration period, final statistical analysis was not able to judge it. These data are going to be used as safety information in case of the insurance adaptation of the therapeutic use.

Conflict of interest: Support: Jazz Pharmaceuticals

Disclosures: This study was supported by Health and Labour Sciences Research Grants for Clinical Trial on Development of New Drugs and Medical Devices from the Ministry of Health, Labour and Welfare of Japan. Off Label Use: Defibrotide is an investigational prophylaxis for hepatic veno-occlusive disease/sinusoidal obstruction syndrome in Japan.

\section{P231}

Defibrotide for sinusoidal obstruction syndrome/venoocclusive disease prophylaxis in high-risk adult patients

Adrien Picod ${ }^{1}$, Agnès Bonnin ${ }^{1}$, Giorgia Battipaglia ${ }^{1,2}$, Federica Giannotti $^{1}$, Annalisa Ruggeri ${ }^{1}$, Eolia Brissot ${ }^{1,2,3}$, Florent Malard $^{1,2,3}$, Clémence Médiavilla ${ }^{1,2}$, Ramdane Belhocine ${ }^{1}$, Anne Vekhoff ${ }^{1}$, Mor Sény Gueye ${ }^{1}$, Simona Lapusan ${ }^{1}$, Rosa Adaeva ${ }^{1}$, Françoise Isnard ${ }^{1}$, Ollivier Legrand ${ }^{1,2}$, Minh-Tam Baylatry ${ }^{4}$, Anne-Christine Joly ${ }^{4}$, Myriam Labopin $^{1,2}$, Rémy Duléry ${ }^{1,2,3}$, Mohamad Mohty ${ }^{1,2,3}$

${ }^{1}$ Saint Antoine Hospital, Hematology and Cellular Therapy Service, Paris, France; ${ }^{2}$ Université Pierre et Marie Curie (UPMC), Sorbonne Universités, Paris, France; ${ }^{3}$ INSERM, UMRs 938, Paris, France;

${ }^{4}$ Saint Antoine Hospital, UPAC \& C (Unité de Préparation des Anticancéreux et Contrôle), Paris, France

Background: Sinusoidal obstruction syndrome (SOS), formerly known as hepatic veno-occlusive disease (VOD) is a serious complication after hematopoietic stem cell transplantation (HSCT). SOS/VOD usually occurs within 3 weeks of HSCT but the 2016 EBMT diagnosis criteria have been revised to include late forms. Prophylactic use of 
defibrotide is recommended in the paediatric setting. However, data in the general adult population are scarce.

Methods: We retrospectively reviewed the files of 63 consecutive patients who received defibrotide prophylaxis of SOS/VOD while undergoing allogeneic HSCT between May 2012 and August 2016. Donors were matched related $(\mathrm{n}=10 ; 16 \%)$, haploidentical $(\mathrm{n}=26 ; 41 \%)$, or unrelated $(\mathrm{n}=27 ; 43 \%)$. Stem cell source was peripheral blood stem cells $(\mathrm{n}=50 ; 79 \%)$, bone marrow $(\mathrm{n}=8 ; 13 \%)$ and cord blood $(\mathrm{n}=5 ; 8 \%)$. Conditioning regimen consisted in fludarabine - busulfan $(\mathrm{n}=19)$, thiotepa - busulfan fludarabine $(\mathrm{n}=19)$, sequential conditioning (thiotepabased, $\mathrm{n}=15$; clofarabine-based, $\mathrm{n}=3$; amasacrine-based, $\mathrm{n}=1$ ), or total body irradiation-based reduced intensity conditioning $(n=6)$. Incidence of SOS according to 2016 EBMT diagnosis criteria as well as other HSCT-related complications were determined. Tolerance was evaluated by hemorrhagic events incidence.

Results: All 63 patients were at high risk of SOS/VOD according to the EBMT criteria. The median duration of defibrotide therapy was 23 days. All patients also received ursodeoxycholic acid for SOS/VOD prophylaxis until day + 90 after transplant. Bleeding occurred in 14 (21.5\%) patients. Defibrotide prophylaxis was discontinued in 7 (11\%) patients: 4 cases $(6 \%)$ due to bleeding and 3 cases $(5 \%)$ because of the need for antithrombotic therapy. Overall, SOS/VOD occurred in 4 cases (6\%), being in the first 21 days following HSCT (day 13 and 14) in two cases, and late-onset SOS/VOD (day 57 and 58) in the other two cases. SOS/VOD was moderate in one case, very severe in 3 cases with 2 deaths related to SOS/VOD.

The median follow up was 31 months (range, 11-60). The 2-year overall survival, progression free survival, incidence of relapse, and NRM were $56.5 \%, 49 \%, 29 \%$, and $22 \%$, respectively. The cumulative incidence of grade II-IV and grade III-IV acute graft-versus-host disease (GVHD) were $22 \%$ and $8 \%$, respectively. The cumulative incidence of moderate to severe chronic GVHD was $10 \%$. Finally, the cumulative incidence of transplant-associated microangiopathy was 3\% (95\% confidence interval: 0.6-10).

Conclusions: In this cohort of high-risk patients undergoing allogeneic HSCT, defibrotide used in combination with ursodeoxycholic acid proved to be an effective prophylactic strategy, and was associated with a low incidence of SOS/VOD, according to the new EBMT diagnosis criteria, with an acceptable safety profile. Also, defibrotide prophylaxis reduced the incidence of other HSCT complications, such as acute GVHD or endothelium cell activation syndromes. Overall, the prophylactic role of defibrotide against SOS/VOD in adult patients and its potential protective effect against endothelial cells-related complications warrant further investigations in controlled prospective studies.
Conflict of interest: M. Mohty received research support and lectures honoraria from Jazz Pharmaceuticals.

\section{P232}

\section{Defibrotide for Treatment of Hepatic Veno-Occlusive Disease Following Hematopoietic Stem Cell Transplantation: Results from the Japanese Therapeutic Use Protocol}

Atsushi Kikuta ${ }^{1}$, Takahiro Fukuda ${ }^{2}$, Kazuteru Ohashi ${ }^{3}$, Shuichi Taniguchi ${ }^{4}$, Yuki Asano-Mori ${ }^{4}$, Keizo Horibe ${ }^{5}$, Hiroaki Goto ${ }^{6}$, Hirohisa Nakamae ${ }^{7}$, Hiroyasu Ogawa ${ }^{8}$, Hiroya Tamaki ${ }^{8}$, Yoshiyuki Takahashi $^{9}$, Toshimi Kimura ${ }^{10}$, Kenichi Yoshimura ${ }^{11}$, Kazuo Umemura $^{12}$, Chitose Ogawa ${ }^{13}$

${ }^{1}$ Fukushima Medical University, Department of Pediatric Oncology, Fukushima, Japan; ${ }^{2}$ National Cancer Center Hospital, Department of Hematopoietic Stem Cell Transplantation, Tokyo, Japan; ${ }^{3}$ Tokyo Metropolitan Cancer and Infectious Disease Center, Komagome Hospital, Hematology Division, Tokyo, Japan; ${ }^{4}$ Toranomon Hospital, Division of Hematology, Tokyo, Japan; ${ }^{5}$ National Hospital Organization Nagoya Medical Center, Clinical Research Center, Nagoya, Japan; 'Kanagawa Children's Medical Center, Division of Hemato-Oncology and Regenerative Medicine, Yokohama, Japan; ${ }^{7}$ Osaka City University, Hematology, Graduate School of Medicine, Osaka, Japan; ${ }^{8}$ Hyogo College of Medicine, Division of Hematology, Hyogo, Japan; ${ }^{9}$ Nagoya University Graduate School of Medicine, Department of Pediatrics, Nagoya, Japan; ${ }^{10}$ Tokyo Women's Medical University Hospital, Department of Pharmacy, Tokyo, Japan;

${ }^{11}$ Kanazawa University, Innovative Clinical Research Center (iCREK), Kanazawa, Japan; ${ }^{12}$ Hamamatsu University School of Medicine, Department of Pharmacology, Hamamatsu, Japan; ${ }^{13}$ National Cancer Center Hospital, Department of Pediatric Oncology, Tokyo, Japan

Background: Hepatic veno-occlusive disease, also called sinusoidal obstruction syndrome (VOD/SOS), is a potentially life-threatening complication of conditioning for hematopoietic stem cell transplantation (HSCT) and is associated with patient and transplant-related risk factors. Severe hepatic VOD/SOS (with multi-organ failure $[\mathrm{MOF}])$, may be associated with $>80 \%$ mortality. Overall estimated prevalence is $11 \%$ post-HSCT in Japan. Defibrotide is not approved for treatment and prophylaxis of VOD/ SOS post-HSCT in Japan. We report the results of the Japanese therapeutic use program, defibrotide treatment protocol (FMU-DF-002).

Methods: This was a phase 2, multicenter, open-label, non-randomized single-arm trial in pediatric and adult patients with hepatic VOD/SOS, with or without MOF, from 2014-2016.

Eligible patients met modified Baltimore VOD/SOS criteria (total bilirubin $\geq 2.0 \mathrm{mg} / \mathrm{dL}$ with $\geq 2$ of: hepatomegaly, ascites, or $5 \%$ weight gain) by day +35 post-HSCT, plus MOF (renal [trebling of creatinine levels, reduced creatinine clearance, or dialysis] and/or pulmonary [oxygen saturation $\leq 90 \%$, need for oxygen supplementation/ 
ventilator dependence]). Defibrotide dose was $25 \mathrm{mg} / \mathrm{kg} /$ day in 4 divided 2-hour IV infusions q6h; recommended treatment duration was $\geq 21$ days. Primary endpoint was day +100 survival in all patients. Secondary endpoint was day +100 survival in patients with VOD plus MOF, day +100 complete response in all patients and patients with VOD plus MOF and all VOD, safety profile and pharmacokinetic analysis.

Results: A total of 19 patients had hepatic VOD/SOS (100\% had VOD/SOS post-allogeneic HSCT); of these, 13 patients had MOF and 6 patients did not. Median age was 47.0 years ( 2 aged $<19$ years and $17 \geq 19$ years), $63 \%$ were male. The most common primary diseases were acute myelogenous leukemia (32\%), acute lymphoblastic leukemia $(16 \%)$ and lymphoma $(16 \%)$. The most common prophylaxis regimens for graft-vs-host disease (GVHD) were reported as tacrolimus + others regimens $(90 \%)$.

Kaplan-Meier estimated survival rate at Day +100 survival for all patients was $47.4 \%$ (95\% confidence interval [CI], 24.4\%-67.3\%). In the MOF patients group, KaplanMeier estimated Day +100 survival was $38.5 \%$ (95\% CI, $14.1 \%-62.8 \%$ ). Estimated CR rate by day +100 was $36.2 \%$ (95\% CI, 16.3\%-68.0\%) in all patients, $28.6 \%$ (95\% CI, 8.0\%-74.2\%) in the MOF patients group. The survival rate and $\mathrm{CR}$ rate at day +100 for MOF patients were approximately equivalent to the final phase 3 analysis in the US trial.

Adverse events (AEs) were reported in 19/19 (100\%) patients, with treatment-related AEs (TRAEs) in 10 patients $(52.6 \%)$. TRAEs in $>1 \%$ of patients were pulmonary hemorrhage $(\mathrm{n}=5,26.3 \%)$, respiratory failure $(\mathrm{n}=3$, $15.8 \%)$, epistaxis $(\mathrm{n}=3,15.8 \%)$, hematuria $(\mathrm{n}=2$, $10.5 \%)$, post procedural hemorrhage $(\mathrm{n}=1,5.3 \%)$, hemothorax $(\mathrm{n}=1,5.3 \%)$ and mediastinal hemorrhage $(\mathrm{n}$ $=1,5.3 \%)$.

Pharmacokinetics of defibrotide was similar to the report of Japanese healthy volunteers.

Conclusions: In this Japanese treatment study, Day +100 survival rates in patients with VOD/SOS, and plus MOD, were consistent with prior defibrotide studies. No new safety signals were observed.

Conflict of interest: Support: Jazz Pharmaceuticals

Disclosures: This study was supported by Health and Labour Sciences Research Grants for Clinical Trial on Development of New Drugs and Medical Devices from the Ministry of Health, Labour and Welfare of Japan.

Off Label Use: Defibrotide is an investigational treatment for hepatic veno-occlusive disease/sinusoidal obstruction syndrome in Japan.

\section{P233}

Developing a dedicated Dermatology service for allogeneic stem cell transplant recipients

Rubeta N Matin ${ }^{1}$, Robert Danby ${ }^{2}$,Lara Rowley ${ }^{2}$, Daja Barton ${ }^{2}$, Rachel Pawson ${ }^{2}$, Andrew Peniket ${ }^{2}$

${ }^{1}$ Oxford University Hospitals NHS Foundation Trust, Dermatology, Oxford, United Kingdom; ${ }^{2}$ Oxford University Hospitals NHS

Foundation Trust, Haematology, Oxford, United Kingdom

Background: Cutaneous graft-versus-host disease (GVHD) following allogeneic stem cell transplantation is a major cause of morbidity with significant impact on quality of life. The British Committee for Standards in Haematology recognises the value of early input from Dermatologists, but to date, there are no reported models for optimal delivery of a dermatology service for stem cell transplant recipients. We sought to address this potential unmet need by developing a dedicated dermatology service in the bone marrow transplant (BMT) clinic in the haematology outpatient department. The aims of this service development project were to a) quantify clinical need and b) evaluate patient reported outcomes \& experience of this service.

Methods: Demographic and clinical details of cases referred for specialist opinion were recorded (March December 2014) to establish case-mix and assess clinical need. Based on these data, a model involving a dedicated Consultant Dermatologist working in collaboration with the multidisciplinary BMT team was approved and formally operational from July 2015. Patient evaluation of the service was measured using the Outcomes and Experience Questionnaire (OEQ); a validated tool bringing together patients' reports of outcomes (OEQ-O score) and their experience (OEQ-E score) of care. Higher OEQ scores represent better outcomes and enhanced experience. A modified selfadministered OEQ (11 questions) was used. Anonymised data were collected over two months.

Results: Establishing clinical need: 77 patients (48M:29F, median age $51 \mathrm{yrs,} \mathrm{range} \mathrm{17-} 72 \mathrm{yrs}$ ) were referred to and reviewed by the Dermatologist in the service development. Mean number of patients reviewed per clinic was 8 (range $5-12$, accounting for $15-40 \%$ of total attenders). Further review was required in 44\% (34/77), resulting in 86 additional consultations. A broad range of skin conditions were diagnosed; chronic cutaneous and oral GVHD were the commonest presentations (74\% and $61 \%$ respectively).

Service evaluation: response rate 42\%; $27 / 42$ (64\%) responders had used the Dermatology service, $40 \%$ (10/25) reported seeing Dermatology $>3$ times. Median OEQ-O score was 18/20 (range 7-20); 93\% reported seeing 
Dermatology to be 'extremely' or 'very helpful'. Median OEQ-E score was 17/18 (range 13-18); 100\% reported being involved 'as much as they wanted to be' in management decisions. Only 51\% reported information provision to be 'extremely helpful'. Overall, $85 \%$ (22/26) rated the outcome of their visit to the Dermatologist in the BMT clinic as 'excellent'.

Conclusions: We have demonstrated the clinical need for a dedicated Dermatology service in the BMT clinic for managing cutaneous disease. A dedicated parallel Dermatology service enables accurate and early diagnosis of cutaneous and oral GVHD, potentially improving morbidity through appropriate and timely use of local/topical treatments.

Patient-derived data, such as the OEQ, are increasingly being used to evaluate new services. Overall, OEQ scores were high, supporting the clinician's view that a collaborative working environment in the BMT clinic can optimise management of skin disease following allograft transplantation. Improving patient-focused information resources for skin disease could enhance patient experience. Data collection of longterm outcomes in post-transplant patients with skin conditions can provide an objective demonstration of the impact of a dedicated Dermatology service.

Conflict of interest: nothing to declare

\section{P234}

\section{Early toxicities following haploidentical hematopoietic cell transplantation with post-transplant cyclophosphamide}

\author{
Martina Pennisi $^{1}$, Alberto Mussetti ${ }^{1}$, Veronica Sciumbata ${ }^{1}$, Giulia \\ Perrone $^{1}$, Paolo Corradini, ${ }^{1,2}$ \\ ${ }^{1}$ Fondazione IRCCS Istituto Nazionale Tumori, Milano, Italy; \\ ${ }^{2}$ University of Milano, Dept. of Oncology and Hematology,, Milano, \\ Italy
}

Background: Post-transplant cyclophosphamide (PT-Cy) has improved the outcome of haploidentical hematopoietic cell transplantation (haploHCT). Nevertheless, other toxicities are emerging in the early post-transplantation period. Here we report an extensive evaluation of early complications in patients undergoing PT-Cy haploHCT.

Methods: We retrospectively evaluated 30 consecutive patients undergoing PT-Cy haploSCT for lymphoid and myeloid malignancies from April 2012 to December 2016 at our institution.

Results: Median age at transplantation was 44 years (range 19-67). Median lines of previous treatment were 4 (range 1-14), including 18 autologous transplantations. Of the 30 patients, 6 were affected by myeloproliferative disease, 24/30 patients had lymphoma or multiple myeloma. Disease risk index was $\geq$ high in $15 / 30$ patients.
Conditioning regimens were all myeloablative but one. Besides PT-Cy, GVHD prophylaxis was cyclosporine A $(18 / 30)$ or tacrolimus $(12 / 30)$ with mycophenolate mofetil. Graft source was bone marrow in $28 / 30$ patients and peripheral blood stem cells in 2/30.

Infectious and non-infectious toxicities were evaluated until day +180 as cumulative incidences. We recorded $57 \%$ [95\% confidence interval (CI), 40\%-71\%] bacterial infections $\geq$ grade 3 and $10 \%$ (95\% CI, $0-20 \%$ ) invasive fungal infections. CMV reactivation incidence was $70 \%$ (95\%CI, $54 \%-86 \%$ ), with only one CMV disease (colitis). The majority of events occurred during the first month (median day +27 , range $8-45$ ), $33 \%$ requiring more than one line of therapy. EBV reactivation rate was $13 \%(95 \% \mathrm{CI}, 3 \%-23 \%)$, with no post-transplant lymphoproliferative disorder evidence. All patients were successfully treated with preemptive Rituximab. We recorded 33\% (95\%CI, 17\%-49\%) incidence of hemorrhagic cystitis: in 9 of 10 patients symptoms were associated with $\mathrm{BK}$ virus reactivation and one patient had detectable urinary adenovirus DNA. HHV6 and adenovirus related disease incidence was $7 \%$ (95\%CI, $0-14 \%)$.

All grade cytokine release syndrome incidence within day +5 was $40 \%$ (95\%CI, 23\%-57\%) and was mainly related to cyclophosphamide administration. Regarding endothelial toxicities, we encountered 20\% (95\%CI, 7-33) incidence of thrombotic microangiopathy. No veno-occlusive disease was reported. Pulmonary toxicities (idiopathic pneumonia syndrome, diffuse alveolar hemorrhage) were absent. Cardiac events incidence was $13 \%$ (95\%CI, 3-23), specifically 2 arrhythmias and 2 pericarditis.

With a median follow-up in survivors of 33 months (range 1-66), 2-year overall and progression free survival were $79 \%$ (95\% CI, 59\%-90\%] and 45\% (95\% CI, 27\%$62 \%)$. Relapse incidence and non-relapse-mortality (NRM) at 2 years were $39 \%$ (95\% CI, 23\%-57\%) and $13 \%$ (95\%CI, $1 \%-25 \%$ ). GVHD-free relapse-free survival (GFRS) at 2 years was $42 \%$ (95\%CI, 24-59): 13/18 relapses, 3/18 GVHD and 2/18 NRM . In line with previous reports, incidence of acute GVHD grade $\geq 2$ and $\geq 3$ at day +100 were $20 \%(95 \% \mathrm{CI}, 7-33)$ and $5 \%(95 \% \mathrm{CI}, 0-12)$, respectively. Incidence of chronic GVHD $\geq$ moderate at 1 year was $7 \%$ (95\% CI, 0\%-15\%).

Conclusions: Our data show a high incidence of infectious complications in PT-Cy haploHCT. In particular, CMV reactivation is very frequent and often challenging to treat. Moreover, hemorrhagic cystitis associated to BK virus reactivation is the second most common viral complication, and carries severe clinical consequences for patients and high readmission rates. Candidates to PT-Cy haploHCT should be considered at high risk for CMV and BK reactivation and treated accordingly.

Conflict of interest: Nothing to disclose 


\section{P235}

EASIX for Predicting SOS/VOD after Allogeneic Stem Cell Transplantation (alloSCT)

Olaf Penack $^{1}$, Sihe Jiang ${ }^{1}$, Axel Benner ${ }^{2}$, Aleksandar Radujkovic ${ }^{3}$, Peter Dreger ${ }^{3}$, Thomas Luft ${ }^{3}$

${ }^{1}$ Charité Universitätsmedizin Berlin, Berlin, Germany; ${ }^{2}$ Biostatistics
DKFZ, Heidelberg, Germany; ${ }^{3}$ University Hospital, Medicine V, Heidelberg, Germany

Background: No standard laboratory parameters are currently in use to predict sinusoidal obstruction syndrome/ venoocclusive disease (SOS/VOD). The Endothelial Activation and Stress Index (EASIX), based on the simple formula ' $\mathrm{LDH}(\mathrm{U} / \mathrm{l}) \times$ Creatinine $(\mathrm{mg} / \mathrm{dl}) /$ Thrombocytes $\left(10^{9} /\right.$ $l)^{\prime}$, has been proven to predict outcome and overall mortality after of GVHD (Lancet Haematol 2017). The aim of the current study was to assess if the EASIX might be valuable for predicting SOS/VOD after alloSCT.

Figure 1. Cumulative incidence of VOD and OM after alloSCT according to the EASIX-zero Quartiles of the training cohort.

A) VOD incidence and B) 3-year OM in the training cohort, adults $n=524$

C) VOD incidence and D) 3-year OM in the validation cohort, adults $n=447$

A

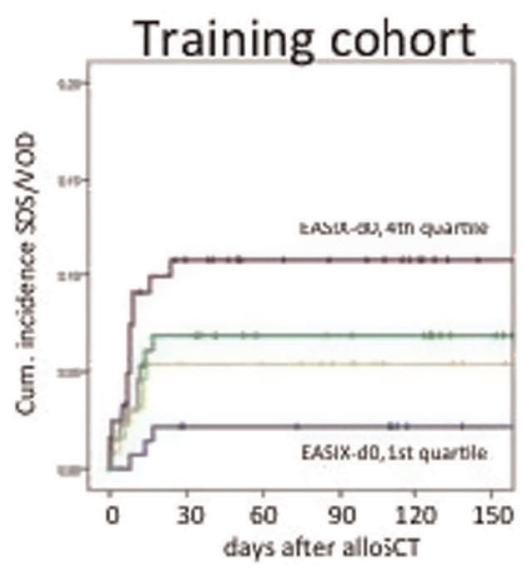

B

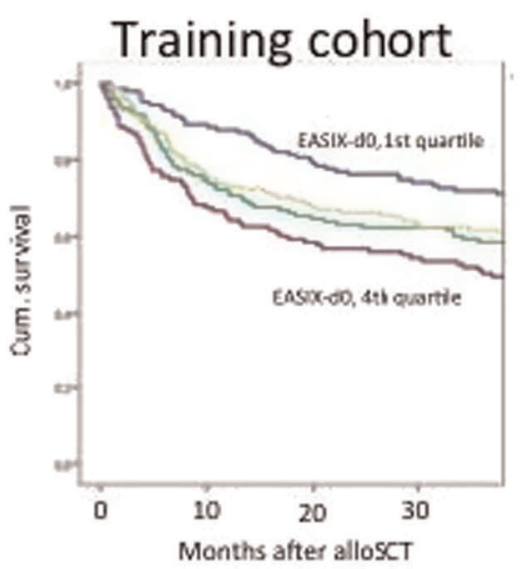

C

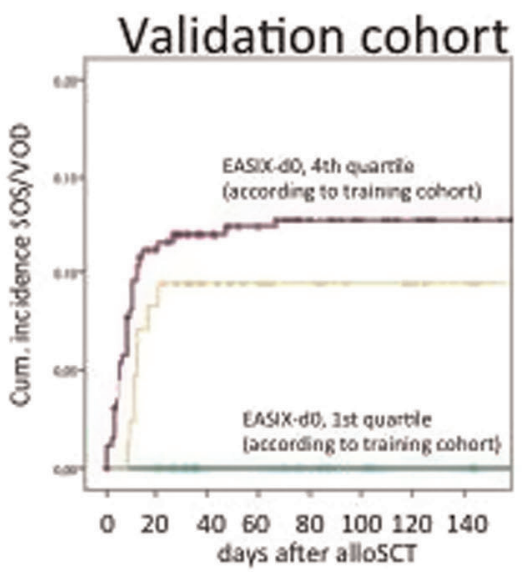

D

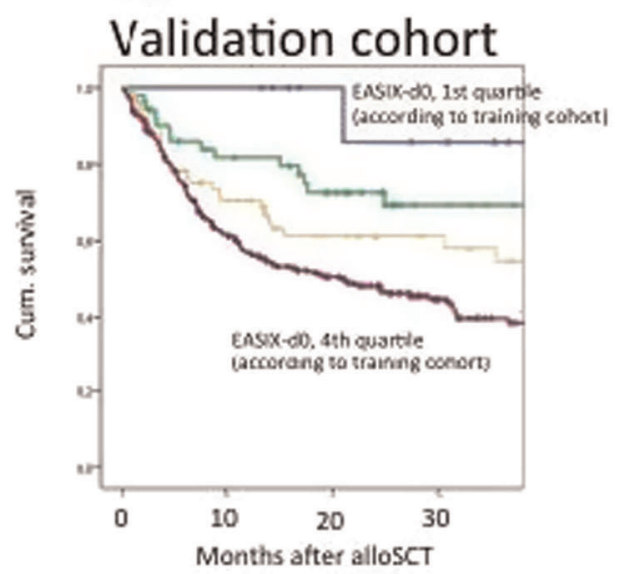

[P235 Figure] [Figure 1] 
Methods: SOS/VOD was defined according to the revised 2016 EBMT criteria. The capacity of EASIX for predicting SOS/VOD was tested retrospectively in 556 consecutive adult patients undergoing alloSCT at a single institution between 2001 and 2013 (training cohort). Primary endpoint was prediction of SOS/VOD when measured at the day of alloSCT (EASIX-zero). Secondary endpoints were prediction of overall mortality $(\mathrm{OM})$ and hyperbilirubinaemia by EASIX-zero and by EASIX taken before start of the conditioning therapy (EASIX-pre). Results were validated in an independent cohort of 447 adult alloSCT recipients from another single institution transplanted between 2013 and 2015.

Results: SOS/VOD was diagnosed in 35 patients $(6.3 \%$, median onset day +8$)$ in the training cohort and in 45 patients $(10.1 \%$, median onset $d+9)$ in the validation cohort, respectively. EASIX was tested in uni- and multivariable Cox regression analyses using age, donor type, mismatch, disease score and strength of conditioning as confounders. In the training cohort, increasing EASIX-zero was significantly associated with SOS/VOD incidence (uni: HR 1.28, 95\%CI 1.06-1.55, $\mathrm{p}=0.010$, multi: HR 1.26, 95\% CI 1.01-1.57, $\mathrm{p}=0.038$ ). Similarly, EASIX-zero predicted the incidence of SOS/VOD in the validation cohort (uni: HR 1.39, 95\%CI 1.20-1.62, p < 0.001, multi: HR 1.38, 95\% CI 1.18-1.62, p < 0.001). In contrast, SOS/ VOD incidence was not predicted by EASIX-pre (uni: $\mathrm{p}=$ 0.707, multi: $p=0.707$ ). However, EASIX-pre was strongly associated with hyperbilirubinaemia $>3 \mathrm{mg} / \mathrm{dl}$ until $\mathrm{d}+28$ after alloSCT (Kruskal-Wallis-Test: $\mathrm{p}<0.001$ in the training cohort, $\mathrm{p}=0.019$ in the validation cohort). Moreover, hyperbilirubinaemia $>3 \mathrm{mg} / \mathrm{dl}$ between until $\mathrm{d}+28$ after alloSCT in patients not diagnosed for SOS/VOD predicted increased overall mortality in both cohorts (training, uni: HR 1.25, 95\% CI 1.08-1.45, $\mathrm{p}=0.003$, multi: HR 1.26, 95\% CI 1.08-1.48, $\mathrm{p}=0.003$; validation, uni: HR $1.80,95 \%$ CI $1.29-2.50, \mathrm{p}=0.001$, multi: HR $1.73,95 \%$ CI 1.24-2.43, $\mathrm{p}=0.001)$. This effect on OM of hyperbilirubinaemia in patients without SOS/VOD was also independent of a later development of acute GVHD. Figure 1 shows Kaplan-Meier estimates of SOS/VOD incidence and OM after alloSCT according to EASIX-zero quartiles in the training set for each cohort separately.

Conclusions: EASIX-zero, reflecting the patient's immediate reaction to conditioning toxicity, is a powerful predictor of SOS/VOD and overall mortality in adult alloSCT recipients. Patients with high EASIX-zero scores might be candidates for clinical evaluation of intensified strategies to prevent SOS/ VOD after alloSCT. The strong association of early hyperbilirubinaemia in the absence of SOS/VOD and increased overall mortality needs to be further explored.

Conflict of interest: All authors have nothing to disclose
P236 EBV associated lymphoproliferations after allogeneic
hematopoietic stem cell transplantation

Anna Pániková, Ivana Bod’ová, Júlia Horáková, Peter Švec, Dominika Tanušková, Tomáš Sýkora, Veronika Urdová, Alexandra Kolenová

Comenius University Children's Hospital, Department of Paediatric Haematology and Oncology, Bratislava, Slovakia

Background: Post-transplant lymphoproliferative disorders (PTLD) are lymphoid and/or plasmacytic proliferations that occur as a result of immunosuppression after allogeneic hematopoietic stem cell transplantation (HSCT) or solid organ transplantation. We are presenting case reports of 3 patients with EBV associated lymphoproliferations after allogeneic HSCT in period of 5 years. EBV infection reactivated in these patients after allogeneic HSCT and they presented with EBV associated lymphoproliferative disorder, as an early post transplant complication.

Methods: In 5 year period (from 1.1.2013 to 30.11.2017) the incidence of this posttransplant complication in our Transplantation unit was 3,8\% (3/79 patients after allogeneic transplantation). We retrospectively compared clinical records and we tried to find features of this potentially fatal post transplant complication the patients had in common. We explored the level of immunosuppression, development of clinical signs and co-existence of other viruses.

Results: All patients were after allogeneic HSCT with conditioning regimen containing anti-thymocyte globulin (ATG), which means T-cell immunosuppression. They received cyclosporin A (CS-A) after HSCT. Before EBV reactivation occured, we had found higher levels of CS-A than we usually try to achieve in all three patients (the highest level was $257 \mathrm{ng} / \mathrm{ml}$ ). None of the patients presented with acute graft-versus-host disease (GvHD). All of them had had cystitis with positive BK viremia and viruria before EBV reactivation (on day 10 to 30 after HSCT). Only one patient was before HSCT EBV seronegative and had EBV seropositive donor, what is considered as a principal risk factor of PTLD along with the degree of T-cell immunosuppression. Median day after HSCT, when first clinical symptoms or first higher EBV load appeared, was day 49 after HSCT. After receiving results of significantly elevated EBV copies in blood, first dose of rituximab was started immediately in all patients. We started preemptive therapy with rituximab only in one patient, who did not have clinical signs of lymphoproliferation when high EBV load (280 000 copies $/ \mathrm{ml}$ ) was detected. In other two of them, which were in ambulatory monitoring, we received results of high EBV load (62000 and 336000 
copies/ml) when they already had clinical symptoms (fever, fatigue) and massive lymphadenopathy with hepatopathy appeared as main clinical signs. Besides 3 or 4 doses of rituximab every 7 days, we reduced the immunosuppression and patients received hepatoprotective and other supportive therapy. We successfully treated all patients with rituximab and no other chemotherapy or cell therapy was needed.

Conclusions: PTLD is a serious complication after HSCT and can be fatal in some cases. Monitoring of EBV copies on a regular basis help us to start preemptive treatment of PTLD with rituximab. What is the optimal schedule for EBV PCR monitoring in ambulatory setting remains unclear, because we did not detect increasing EBV load before clinical signs of disease appeared in ambulatory setting. Relationship between BK virus reactivation and consecutive EBV reactivation was not closely described, but higher rate of immunosuppression could be the reason of both viruses reactivation.

Conflict of interest: none declared

\section{P237}

Efficacy of olanzapine-containing prophylactic antiemetic regimen in autologous hematopoietic stem cell transplantation patients who undergo conditioning with thiotepa, busulfan and cyclophosphamide

\author{
Youree Jung ${ }^{1}$, Bobae Kim ${ }^{1}$, Eunjeong Shin ${ }^{1}$, Sungyun Suh ${ }^{1}$, \\ Yoonsook Cho ${ }^{1}$, Juyeun Lee ${ }^{2}$, Youngil Koh ${ }^{1}$

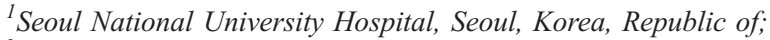 \\ ${ }^{2}$ College of Pharmacy, Hanyang University, Ansan, Korea, Republic of
}

Background: The purpose of this study was to show the efficacy of prophylactic olanzapine-containing antiemetic regimen, which consists of olanzapine (OLN), palonosetron (PAL) and dexamethasone (DEX), for the prevention of nausea and vomiting in patients receiving high dose anticancer agents as conditioning for autologous hematopoietic stem cell transplantation (ASCT).

Methods: A retrospective study was performed in patients receiving thiotepa, busulfan and cyclophosphamide (TBC) as a conditioning regimen for ASCT. The OLN, PAL, DEX (OPD) regimen consisted of $10 \mathrm{mg}$ of oral OLN, $0.25 \mathrm{mg}$ of intravenous PAL, and $8 \mathrm{mg}$ of intravenous DEX on day 1 , and $10 \mathrm{mg}$ of oral OLN alone on days 2-3. Patients who received OPD regimen for emesis prevention during TBC conditioning in Seoul National University Hospital from November 2016 to November 2017, all of whom received OPD in this center, were included.

Results: We analyzed 15 patients (Male:Female $=10: 5$, median age 54 years). For underlying disease, 6 patients had multiple myeloma and 9 patients had non-Hodgkin lymphoma (7 B-cell and 2 T-cell lymphoma). In terms of emesis control, complete response (CR, no vomiting) was achieved in $80 \%$ during the early period (the first 72 hours of conditioning) and in $40 \%$ for the overall period (from day 1 to 13). Patients with response (PR, no vomiting or grade 1 vomiting by NCI-CTCAE) were $93 \%$ and $60 \%$ for the early and overall period respectively. No serious toxicities have occurred. However, there were mild dizziness, hypotension and paresthesia in $13 \%$, which resolved without management in 24 hours after the onset of symptoms.

We compared our results with the data of 27 primary central nervous system lymphoma patients who as well received TBC based ASCT with different antiemetic regimen (Asan Medical Center in Korea, between December 2012 and July 2015): Antiemetic regimen in Asan Medical Center does not contain OLN but includes lorazepam (Table 1). Antiemetic regimen without OPD resulted in $11 \% \mathrm{CR}$ and $63 \%$ PR while $40 \%$ and $60 \%$ of our patients achieved $\mathrm{CR}$ and $\mathrm{PR}$ respectively during the overall period. CR was achieved more frequently in patients with OPD containing regimen $(\mathrm{p}=0.049)$.

Conclusions: OPD regimen has remarkable antiemetic potential during TBC conditioning. We assume that $\mathrm{OPD}$ could also be considered as an effective antiemetic therapy in various highly-emetogenic chemotherapies including conditioning for ASCT.

Conflict of interest: All authors have nothing to disclose

\begin{tabular}{llll}
\hline & Anticancer agents & $\begin{array}{l}\text { Antiemetic regimen of } \\
\text { SNUH }\end{array}$ & $\begin{array}{l}\text { Antiemetic regimen of } \\
\text { AMC }\end{array}$ \\
\hline $\begin{array}{l}\text { Day } \\
1\end{array}$ & Thiotepa & OLN, PAL, DEX & LRZ \\
$\begin{array}{l}\text { Day } \\
2\end{array}$ & Thiotepa & OLN & LRZ \\
$\begin{array}{l}\text { Day } \\
3\end{array}$ & Thiotepa & OLN & LRZ \\
$\begin{array}{l}\text { Day } \\
4\end{array}$ & Busulfan & SRA & \\
$\begin{array}{l}\text { Day } \\
5\end{array}$ & Busulfan & SRA & SRA, LRZ \\
$\begin{array}{l}\text { Day } \\
6\end{array}$ & Busulfan & SRA & SRA, LRZ \\
$\begin{array}{l}\text { Day } \\
7\end{array}$ & Cyclophosphamide & NK1RA, SRA, DEX & NK1RA, SRA, DEX, \\
$\begin{array}{l}\text { Day } \\
8\end{array}$ & Cyclophosphamide & NK1RA, DEX & LRZ \\
$\begin{array}{l}\text { Day } \\
9\end{array}$ & None & & NK1RA, SRA, DEX, \\
\hline
\end{tabular}

[[P237 Table] Antiemetic regimen in each center]

SNUH: Seoul National University Hospital, AMC: Asan Medical Center, OLN: olanzapine, PAL: palonosetron, DEX: dexamethasone, LRZ: lorazepam, SRA: serotonin receptor antagonist, NK1RA: neurokinin-1 (NK-1) receptor antagonist 


\section{P238}

\section{Elevated Cardiovascular Risk Factors at Time of Allotransplant Do Not Impact Survivals in Adult Patients: a Retrospective Study of 194 Cases}

Sophie Vantyghem ${ }^{1}$, Thierry Guillaume ${ }^{1}$, Pierre Peterlin ${ }^{1}$, Alice Garnier $^{1}$, Amandine Le Bourgeois ${ }^{1}$, Béatrice Mahe ${ }^{1}$, Viviane Dubruille $^{1}$, Nicolas Blin ${ }^{1}$, Pierre Gallas ${ }^{1}$, Cyrille Touzeau ${ }^{1,2}$, Thomas Gastinne $^{1}$, Anne Lok ${ }^{1,2}$, Steven Le Gouill, ${ }^{1,2}$, Philippe Moreau ${ }^{1}$, Marie-Christine Béné $e^{3}$, Patrice Chevallier ${ }^{1}$

${ }^{1}$ CHU Nantes, Nantes, France; ${ }^{2}$ CRCINA INSERM U 1232, Nantes, France; ${ }^{3} \mathrm{CHU}$ Nantes, Biology, Nantes, France

Background: Cardiovascular complications are involved in morbi-mortality after allogeneic stem cell transplantation (allo-SCT). However, little is known regarding the impact on outcomes of cardiovascular risk factors at the time of allo-SCT. This retrospective study aimed at examining the impact of pre-transplant cardiovascular risk factors after allo-SCT on overall (OS) and disease-free (DFS) survival.

Methods: All adults ( $\geq 18$ years old) allotransplanted in a single university center between January 2011 and December 2013 ( $n=194)$ were eligible for the study. Ten cardiovascular risk factors out of those previously defined were considered (Mahmood, Lancet 2014). A point of 1 was attributed to each of them if present, allowing to build various scores for statistical analysis.

Results: The cohort comprised 120 males and 74 females (median age: 56 years (range: 21-70). The initial disease was of myeloid lineage for $58 \%$ and lymphoid for $42 \%$. At transplant, 116 patients were in complete remission (CR) $(\mathrm{CR} 1=78$; CR2 or more $=38), 34$ in partial remission $(\mathrm{PR})$ $(\mathrm{PR} 1=4$, PR2 or more=30), and 44 had active disease. Ninety-one patients had received a myeloablative conditioning regimen while 79 had received a reduced-intensity and 24 a sequential regimen. Donors were: sibling: $n=68$, haplo-identical: $\mathrm{n}=4$, matched unrelated donor: $\mathrm{n}=76$, mismatched (9/10) unrelated donor: $n=19$, unrelated cord blood: $\mathrm{n}=27$. Peripheral blood stem cells were the main source of graft $(n=151)$, while 16 cases received bone marrow. All patients had a left ventricular ejection fraction $\geq 50 \%$ at the time of transplant.

With a median OS of 66 months (95\% CI 34-66), 2-year OS and DFS were $59.3 \pm 3.6 \%$ and $51 \pm 3.6 \%$, respectively, for the whole group. Overall incidences of grade 2-4 acute and chronic GVHD were $55 \%$ and $31 \%$. Overall incidences of relapse and non-relapsed mortality(NRM) were $36 \%$ and $16 \%$, respectively.

The number of patients involved for each considered cardiovascular risk factor at transplant was: male $\geq 50$ years: $\mathrm{n}=75$, female $\geq 60$ years: $\mathrm{n}=24$, smokers: $\mathrm{n}=49$, $\mathrm{BMI} \geq 25: \mathrm{n}=85$, hypertension: $\mathrm{n}=26$, diabetes: $\mathrm{n}=9$, first-degree familial history of coronary disease: $\mathrm{n}=2$, total-cholesterol $\geq 2 \mathrm{~g} / \mathrm{L}: \mathrm{n}=96$, triglyceride $\geq 1.5 \mathrm{~g} / \mathrm{L}: \mathrm{n}=$ 100, HDL-cholesterol $\leq 0.4 \mathrm{~g} / \mathrm{L}: \mathrm{n}=60$, and LDLcholesterol $\geq 1.6 \mathrm{~g} / \mathrm{L}: \mathrm{n}=28$.

In univariate analysis, HDL-cholesterol and active disease at transplant were the only factors associated with lower 2year OS $(50.6 \pm 6.1 \%$ vs. $65.6 \pm 4.5 \%$ vs. $\mathrm{p}=0.02$; and 38.3 $\pm 7.6 \%$ vs. CR: $68.3 \pm 4.4 \%$ vs PR: $61 \pm 8.5 \%, \mathrm{p}=0.001$, respectively) and DFS $(55.6 \pm 4.7 \%$ vs. $41.5 \pm 6 \%, \mathrm{p}=0.04$; and $28.7 \pm 7.1 \%$ vs. CR $59.1 \pm 4.6 \%$ vs. PR $51 \pm 7.1 \%, \mathrm{p}=$ 0.0002 , respectively). However, in multivariate analysis, only the disease status at the time of transplant retained significance (OS: HR 1.7. 95\%CI 1.3-2.24, $\mathrm{p}=0.0001$; and DFS: HR 1.7, 95\%CI 1.3-2.17, $\mathrm{p}=0.0001)$.

When considering various cardiovascular risk scores for statistical analysis, none were predictive of survivals in patients.

After transplant, the incidence of cardiovascular events was $16 \%(n=29 / 194)$ within the first year and $13,4 \%(n=$ 17/127) for patients alive after one year. None of the patients died of cardiovascular disease.

Conclusions: These results suggest that elevated cardiovascular risk factors at the time of allo-SCT do not impact survivals in adult patients. Therefore, their presence should not lead to reconsider the procedure and may not make the patients at higher risk for allo-SCT.

Conflict of interest: nothing to disclose

\section{P239}

\section{Endocrinopathies following bone marrow transplantation -} Analysis of 200 cases

\section{Sara Donato, Joana Simões-Pereira, Daniela Dias, Maria Conceição Pereira}

Instituto Português de Oncologia de Lisboa, Francisco Gentil, Endocrinology, Lisbon, Portugal

Background: Bone marrow transplantation (BMT) is a therapeutic option in several hematological diseases either benign or malignant and in a few non-hematological conditions. Due to the reduced mortality after BMT, the survivors of this therapy are increasing over time. These are at risk of developing therapy-related late effects, from which endocrine dysfunctions are the most frequent. The objective of our study was to characterize the endocrine disorders found on BMT survivors followed in our Late Effects Clinic (LEC).

Methods: Retrospective study with analysis of the clinical records from patients submitted to BMT. Inclusion criteria were: being followed in our LEC and having a follow-up of at least three years. Statistics analysis of data 
was made with Microsoft Excel $2010^{\circledR}$ and IBM SPSS Statistics $22^{\circledR}$.

Results: Our study collected 200 patients, 49\% female and $51 \%$ male. The mean age of BMT treatment was 21.84 years, having $52.5 \%$ of the patients done the BMT before the twenties. The mean follow-up time was 140.6 months and during follow-up $80 \%$ of the patients $(89.8 \%$ in women and $70.6 \%$ in men, $p=0.01$ ) developed at least one endocrinopathy. Endocrine complications were significantly more frequent in those patients submitted to BMT after 20 years of age $(71.4 \%$ in $\leq 20$, compared to $89.5 \%$ in those older than twenty, $p=0.01$ ), despite the time of follow-up being shorter in the older group (130.1 vs 166.9). Generally, the occurrence of endocrinopathies were: hypogonadism in $51.5 \%-66 \%$ of women and $36.3 \%$ of men, the only complication with significant difference between genders, $p<0.001$-, dyslipidemia in $29 \%$, osteopenia/osteoporosis in $24 \%$, hypothyroidism in $18 \%$, somatropine deficiency in $13 \%$, nodular thyroid disease in $12.5 \%$, diabetes or prediabetes in $11.5 \%$ and thyroid cancer in $3 \%$. Dividing patients by age of BMT ( $\leq$ and $>20$ years), we found statistical difference on the occurrence of hypogonadism (41.0\% on the lower aged group compared with $67.4 \%$ in the older, $p<0.001)$ and of dyslipidemia $(17.1 \%$ on the first group $v s 42.1 \%$ on the second, $p<0.001$ ).

Conclusions: A higher risk of hypogonadism was seen in older women, possibly due to higher ovarian toxicity plus the natural ovarian reserve decline occurring along the years. Dyslipidemia observed in older group is probably related with life-style/cardiovascular risk factors.

The survivors of BMT have frequent endocrinopathies. The early identification of these complications can represent an improvement in their quality of life and avoid associated morbi-mortality. This is the first Portuguese study analysing this theme, presenting one of the largest samples found in the international literature.

Conflict of interest: The authors deny any conflict of interest on this matter.

\section{P240}

\section{Engraftment syndrome following autologous hematopoietic stem cell transplantation and antimicrobial stewardship opportunities}

Julien Depaus ${ }^{1}$, Marc Andre ${ }^{1}$, Anne Sonet ${ }^{l}$, Carlos Graux ${ }^{1}$, Chantal Doyen $^{1}$, Elodie Collinge ${ }^{I}$, Bérangère Devalet ${ }^{l}$, Florence

Desquesnes $^{1}$, Véronique Deneys ${ }^{2}$, Marc Bourgeois ${ }^{I}$

${ }^{I}$ CHU UCL Namur Mont-Godinne, Hematology, Yvoir, Belgium;
${ }^{2}$ CHU UCL Namur Mont-Godinne, HPC Collection Center, Yvoir, Belgium

Background: Engraftment syndrome (ES) is a clinical condition increasingly reported in the peri-engraftment period following autologous hematopoietic stem cell transplantation (ASCT). Diagnosis criteria and drug management remain widely heterogeneous. We aimed to assess the burden of that condition and the antimicrobial management in adult patients hospitalized for ASCT in a five-year period in a tertiary Belgian referral center.

Methods: Medical data of patients that underwent ASCT between January 2013 and December 2017 were retrospectively reviewed. Hospital discharge reports were screened for following items: engraftment syndrome, unexplained fever and/or diarrhea and/or cutaneous rash shortly ( 2 to 4 days) preceding or following neutrophil recovery. Included patients were subsequently evaluated according to Spitzer (2001) and Maiolino (2003) diagnosis criteria. Antimicrobial data were reviewed in the electronic medical records.

Results: In the five-year period, 199 ASCT were performed at CHU UCL Namur. Main indications were multiple myeloma $(\mathrm{n}=102)$, lymphoid malignancies $(\mathrm{n}=$ $83)$, acute myeloid leukemia $(\mathrm{n}=9)$ and testicular carcinoma $(\mathrm{n}=5)$.

A diagnosis of ES was retained for 37 (19\%) patients. Spitzer and/or Maiolino diagnosis criteria were met for 22 patients (Spitzer $\mathrm{n}=1$; Maiolino $\mathrm{n}=17$; Spitzer/Maiolino $\mathrm{n}=4$ ), while 15 patients didn't meet these criteria mainly because of delayed ES symptoms onset or isolated fever resurgence post-engraftment. Those patients were however considered as probable ES cases after review of the medical records. Fever was present in all patients $(\mathrm{n}=37 ; 100 \%)$, while main secondary symptoms were skin rash and/or diarrhea in $23(62 \%)$ patients. One clinical presentation involved a pulmonary infiltrate, hypoxemia and weight gain.

Thirty $(81 \%)$ patients were treated with Cefepime and 7 (19\%) with Piperacillin-Tazobactam. Empirical antimicrobial therapy was escalated to Meropenem in 22 (59\%) patients. Corticosteroids were administrated in 19 (51\%) patients. Evolution of ES was favorable for all patients.

Conclusions: ES is a clinical syndrome frequently described in the context of ASCT. Current ES diagnosis criteria are too heterogeneous to allow early recognition and appropriate clinical management. Empirical first-line and escalated antimicrobial therapies remain in that context widely prescribed. Refined diagnosis criteria and appropriate initiation of corticosteroid therapy could decrease 
broad spectrum antimicrobial consumption and reduce inhospital lengh of stay.

Conflict of interest: All authors : Nothing to disclose

\section{P241}

Engraftment Syndrome Following High Dose Therapy and Autologous Hematopoietic Cell Transplantation (AHCT) in patients with Light Chain (AL) Amyloidosis

Valkal Bhatt ${ }^{1}$, Evan Diamond ${ }^{2}$, Patrick Hilden ${ }^{3}$, Molly Malloy ${ }^{4}$, Gunjun Shah ${ }^{4}$, Heather Landau ${ }^{4}$

${ }^{1}$ Memorial Sloan Kettering Cancer Center, Bone Marrow Transplant, Department of Pharmacy, New York, NY, United States; ${ }^{2}$ Cornell University, Department of Medicine, New York, NY, United States; ${ }^{3}$ Memorial Sloan Kettering, Department of Biostatistics, New York, NY, United States; ${ }^{4}$ Memorial Sloan Kettering Cancer Center,

Department of Bone Marrow Transplant, New York, NY, United States

Background: Engraftment syndrome (ES) is an increasingly reported complication of AHCT with a incidence of 7$59 \%$. Its characterized by symptoms occurring periengraftment including fever, skin rash, diarrhea, pulmonary infiltrates and neurologic complications. Criteria for diagnosis has been proposed but limited data exists on risk factors and management. We examined patients who underwent AHCT for light chain (AL) amyloidosis to define characteristics associated with ES development.

Methods: We retrospectively examined the incidence, risk factors, characteristics, and treatment of ES in patients with AL amyloidosis that received first AHCT with melphalan $(100-200 \mathrm{mg} / \mathrm{m} 2)$ conditioning between 2005 -2015. Risk factors of ES development was assessed using a logistic regression model. OS and PFS were estimated using Kaplan-Meier method with differences assessed with a logrank test. The cumulative incidence of NRM was estimated using a method for competing risks, with relapse considered a competing risk.

Results: 115 patients (pts) with AL amyloidosis with median age 57 (range 35-76) and 60 (52\%) male, received AHCT with melphalan $100-200 \mathrm{mg} / \mathrm{m} 2$. 69(60\%) received AHCT between 2005-2010, and 46(40\%) between 20102015.75 pts $(65 \%)$ received melphalan $\leq 140 \mathrm{mg} / \mathrm{m}^{2}$ and 40 pts $(35 \%)$ received melphalan $>140 \mathrm{mg} / \mathrm{m} 2$ over $1-2$ days. 67 pts $(58 \%)$ were untreated prior to AHCT and 48 pts (42\%) received a median of one line of therapy (range 1-4). Most common induction therapies were bortezomib/dexamethasone $(\mathrm{n}=28 ; 58 \%)$, melphalan/dexamethasone $(\mathrm{n}=$ $7,14 \%$ ), and cyclophosphamide/bortezomib/dexamethasone $(\mathrm{n}=6 ; 13 \%) .81 \mathrm{pts}(70 \%)$ of patients were mobilized with filgrastim alone, 30 pts (27\%) received plerixafor; 4 pts received chemo-mobilization $(\mathrm{n}=3$ cyclophosphamide, $\mathrm{n}$ $=1$ VDT-PACE). The median infused CD34 dose was

\begin{tabular}{|c|c|c|c|c|c|}
\hline variable & group & $\begin{array}{c}\text { N- } \\
\text { patients }\end{array}$ & $N=25$ & $\begin{array}{l}\text { relative riak } \\
(95 \% \mathrm{CI})\end{array}$ & pivalue \\
\hline $\begin{array}{l}\text { Pre tranglant } \\
\text { eyelopbosphamide expovare }\end{array}$ & $\begin{array}{l}\text { No } \\
\text { Yes }\end{array}$ & $\begin{array}{c}103 \\
12\end{array}$ & $\begin{array}{c}38 \\
1\end{array}$ & $\begin{array}{c}\text { reference } \\
\text { a.16 } \\
(0.01 \cdot 0.85)\end{array}$ & 0,029 \\
\hline Plenciase basedmosilization & $\begin{array}{l}\text { No } \\
\text { Yes }\end{array}$ & $\begin{array}{l}85 \\
90\end{array}$ & $\begin{array}{l}25 \\
14\end{array}$ & $\begin{array}{c}\text { referesce } \\
2.10 \\
(0.89,4.97)\end{array}$ & 0.091 \\
\hline $\begin{array}{c}\text { Median infused } \\
\text { CD34 } \times 10^{\circ} \text { cells } k \text { dose dose }\end{array}$ & $\begin{array}{l}5 \times 10^{\circ} \text { collshkg } \\
\geq 5 \times 10^{\circ} \text { cells } / \mathrm{kg} g\end{array}$ & $\begin{array}{l}57 \\
58\end{array}$ & $\begin{array}{l}15 \\
24\end{array}$ & $\begin{array}{c}\text { reference } \\
1.98 \\
(0.91 .4 .41)\end{array}$ & 0.087 \\
\hline Indaction herapy & $\begin{array}{l}\text { Untreesos } \\
\text { Treated }\end{array}$ & $\begin{array}{l}67 \\
48\end{array}$ & $\begin{array}{l}23 \\
16\end{array}$ & $\begin{array}{c}\text { referesce } \\
0.96 \\
(0.43,209)\end{array}$ & Q911 \\
\hline
\end{tabular}

[P241 Figure] [Table 1 Univariate analysis of risk factors associated with ES]

$5 \times 10^{6}$ cells $/ \mathrm{kg}$ (range $2.2-9.2 \times 10^{6}$ cells $/ \mathrm{kg}$ ). Incidence of ES was $34 \%(\mathrm{n}=39)[\mathrm{n}=32(31 \%) \quad 2005-2010$ and 17 (37\%) 2010-2015; $\mathrm{p}=0.57]$. Clinical features of ES were fever $(>38 \circ \mathrm{C})(\mathrm{n}=38,97 \%)$, infiltrates on imaging $(\mathrm{n}=$ $12,31 \%)$, rash $>25 \%$ BSA $(\mathrm{n}=17,44 \%)$ and noninfectious diarrhea ( $>2$ liquid $\mathrm{BM} / 24$ hours) requiring antimotility agents $(\mathrm{n}=35,90 \%)$. Univariate analysis (Table 1 ) identified a lack of exposure to cyclophosphamide preAHCT as the only statistically significant risk factor for developing ES. 25 pts $(64 \%)$ were treated for ES with corticosteroids (CS) and 14 pts (36\%) were treated supportively. The median CS (prednisone or methylprednisolone) dose was $1 \mathrm{mg} / \mathrm{kg}$ (range $0.5-2 \mathrm{mg} / \mathrm{kg}$ ) for a median of 3 days (1-3 days). 10 pts (40\%) received a CS over median 7 days (range 5-14 days). 6 pts $(24 \%)$ required $2^{\text {nd }}$ CS pulse followed by taper [14 days (7-16 days)]. Median length of hospital stay was 19 days (13-56 days), median 21 days (14-47) and 18 days (13-56 days) in patients with and without ES. The cumulative incidence of NRM at 1 year was $6.1 \%$ (2.7-11.6). PFS and OS at 1 year were 91.2\% (84.3-95.2) and 93\% (86.5-96.4) respectively. There were no differences in PFS or OS among pts who did or did not have ES.

Conclusions: ES occurs in 1/3 of AL patients undergoing AHCT and potentially prolongs hospital stays. Prompt treatment with CS and steroid taper is our favored approach since recrudescence is common. A detailed comparison to pts with multiple myeloma will be presented.

Conflict of interest: Nothing to disclose

\section{P242}

Evaluation of cardiopulmonary exercise testing, heart function and quality of life in children after allogenic hematopoetic stem cell transplantation

Kristof Vandekerckhove ${ }^{1}$, Kathleen De Waele ${ }^{2}$, Aurelie Minne ${ }^{1}$, Ilse Coomans $^{1}$, Katya De Groote ${ }^{1}$, Joseph Panzer ${ }^{1}$, Catherine Dhooge ${ }^{3}$, 
Victoria Bordon ${ }^{3}$, Patricia De Vos ${ }^{3}$, Daniel De Wolf ${ }^{1}$, Jan Boone ${ }^{4}$

${ }^{1}$ Ghent University Hospital, Pediatric Cardiology, Ghent, Belgium; ${ }^{2}$ Ghent University Hospital, Pediatric Endocrinology, Ghent, Belgium; ${ }^{3}$ Ghent University Hospital, Pediatric Hematology, Oncology and Stem Cell Transplantation, Ghent, Belgium; ${ }^{4}$ Ghent University, Movement and Sport Sciences, Ghent, Belgium

Background: Physical fitness is an important determinant of quality of life after hematopoietic stem cell transplantation and since cardiac function can influence exercise performance, the aim of the present study was to assess these factors and their interrelationship.

Methods: Children in stable follow-up for more than 1 year post HSCT were invited to participate in this study. Patients underwent cardiopulmonary exercise testing (CPET) and echocardiography to assess left and right ventricular (LV and RV) systolic and LV diastolic function, and LV wall dimensions. Health related quality of life (HRQOL) was evaluated using child- and adolescent-reported PedsQL ${ }^{\text {TM }}$ questionnaires. Exercise and HR-QOL data were matched to healthy control patients, echocardiographic measurements were compared to their normal values.

Results: Forty-three HSCT-patients performed a maximal exercise test ( 26 boys, $13.6 \pm 3.4 \mathrm{yr}$, weight $45.5 \pm 13.3 \mathrm{~kg}$, length $152.9 \pm 17.5 \mathrm{~cm}$, BSA $1.35 \pm 0.28$ ). Time between transplantation and exercise test was $6.6 \pm 4.5$ years. AML, ALL and Myelodysplasia represent more than 50\% of the indications for HSCT. The patient group was equal to the healthy controls regarding age, height and weight. HSCT patients had lower maximal oxygen consumption (VO2peak $/ \mathrm{kg}, 34.7 \pm 8.4$ vs. $46.3 \pm 7.1 \mathrm{ml} / \mathrm{kg} / \mathrm{min}, \mathrm{P}<0.001$ ), shorter exercise duration $(9.1 \pm 2.5$ vs. $12.9 \pm 2.6 \mathrm{~min}, \mathrm{P}<$ $0.001)$ and lower maximal load (\%Ppeak $70.8 \pm 19.7$ vs. $102.4 \pm 15.9 \%, \mathrm{P}<0.001)$. They reached the ventilatory anaerobic threshold (VAT) earlier ( $48.5 \pm 4.6$ vs. $62.5 \pm 5.5 \%$, $\mathrm{P}=0.050$ ).

Echocardiography demonstrated decreased interventricular septal wall thickness (IVSd Z-value $-0.64 \pm 0.69, \mathrm{P}<$ 0.001). The systolic LV function was diminished in $5 / 43$ children (11.6\%). Measurements of tissue doppler over the mitral valve were higher compared to the normal values for age, gender and weight, pointing towards a degree of diastolic dysfunction (high E/E' Z-value $1.06 \pm 1.13, \mathrm{P}<$ 0.001). Children who had more LV dilatation did have worse oxygen consumption at maximal exercise (VO2max/ $\mathrm{kg})(\mathrm{r}=-0.364, \mathrm{P}=0.017)$. Quality of life was generally good, only the overall HR-QOL-score and the emotional functioning score were slightly lower compared to healthy controls (78.1 vs. $83.6, \mathrm{P}=0.016$ and 74.3 vs. $\mathrm{P}=0.001$ ). Patients after anthracycline therapy had the lowest VO2peak $/ \mathrm{kg}$ (31.1 vs. $37.8 \mathrm{ml} / \mathrm{kg}, \mathrm{P}=0.007$ ). The TAPSE, a parameter for right ventricular function, was lower compared to the reference values in our population. ( $9 / 43$ had TAPSE $z$-value $<-2$ ).

Conclusions: LV and RV dysfunction justify the need for thorough cardiac follow-up in children after HSCT. Lower physical fitness levels and more deconditioning emphasize the importance of CPET and fitness programs. Patients after anthracyclines seem to be the most vulnerable group. Quality of life is only mildly affected long time after HSCT.

\section{Conflict of interest:}

K. Vandekerckhove received a grant as clinical $\mathrm{PhD}$ fellow by the Flemish Scientific Research Board (FWO Vlaanderen) (Grant number 1701418N). The remaining authors have nothing to disclose.

\section{P243} Evaluation of late effects after Hematopoietic stem cell
transplantation in a pediatric cohort

Fernández, Martínez Cristina, López, Duarte Mónica, Pérez, Vázquez, German, Mora Barrios Joan, De Miguel Carlos, Cabero Martínez Almudena, Celis Álvarez Mara, García Ávila Sara, Insunza Andres, Bermúdez, Arancha, Richard Carlos

Hospital Universitario Marqués de Valdecilla, Hematology, Santander, Spain

Background: Hematopoietic stem cell transplantation (HSCT) is a curative strategy for malignant and nonmalignant diseases. The cumulative effect of exposure to chemo and radiotherapy injures tissues leading to long term complications. Up to $70-90 \%$ of the survivors develop at least one late side effect that impacts negatively on their quality of life. The best follow up protocol is not well defined.

Methods: We performed a retrospective study on a pediatric cohort of 94 patients, with at least 6 months survival after HSCT, performed between 1980 and 2017. We compiled Late Effects (LE) following a previously uniformed definition by organ system (cardiovascular, respiratory, hepatic, ocular, endocrine, osteomuscular, central nervous system), second neoplasms and psychosocial issues. Cumulative incidence for each event and overall survival were estimated by Fine-Gray regression and Kaplan-Meier, respectively.

Results: We performed 65 allogenic and 29 autologous stem cell transplants. Seven patients received a second AloHSCT with a median of 3 years between both procedures. There were 55 males and 39 females. Median age at HSCT was 13.1 years [1-18.7]. Twenty-two patients $(23.4 \%)$ had a non-neoplastic disorder. In $63 \%$ of cases, the donor was an HLA matched sibling (2 syngeneic) and $30.7 \%$ were 


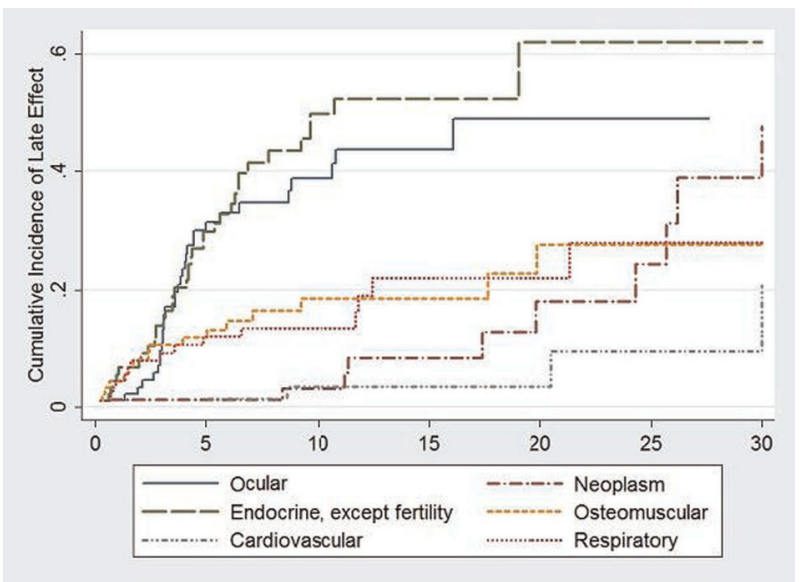

[P243 Figure] [Figure1: Cumulative Incidence of LE.]

unrelated donor. Peripheral blood was used as stem cell source in 4 patients and umbilical cord blood progenitors in 3 patients. T cell depletion was used in 13 patients (20.6\%). ICT was included in the conditioning regimen in $58.5 \%$ of patients. The incidence of severe chronic graft versus host disease was $7.7 \%$.

With a median follow up of 10 years [0.74-33.3] after transplantation, $77.7 \%$ patients developed at least one secondary LE. The median of systems affected per patient was 2 IQR [1-4]. The most frequently observed LE were ocular (mainly cataracts) and endocrine (mainly hypogonadism and hypothyroidism) and secondary neoplasm. The cumulative incidence of endocrinological and ocular at 5, 10 and 20 years were $29.8 \%, 50.0 \%, 62.0 \%$ and $31.5 \%$, $38.8 \%$ and $48.8 \%$ respectively. Cardiovascular, Respiratory and osteomuscular cumulative incidences at 20 years were $9.5 \%, 21.9 \%, 27.6 \%$ respectively. The cumulative incidence of second neoplasms at 10,20 and 30 years was $3.2 \%, 18.1 \%$ and $47.6 \%$ (CI95\% 21.7-69.7\%) (Figure 1)

The overall survival at 10 and 20 years was $94.2 \%$ y $85.4 \%$ respectively.

Conclusions: The incidence of long term secondary LE after stem cell transplantation in our pediatric cohort is high as previously reported. The most frequently observed LE were ocular (mainly cataracts) endocrine (mainly hypogonadism and hypothyroidism) and secondary neoplasm. Although less frequent, other LE such as cardiovascular, respiratory and osteomuscular must be keep in mind. The cumulative incidence of second neoplasm and certain side effects implies a prolonged follow up, so that adherence and education of patients and relatives must be reinforced.

Conflict of interest: Nothing to disclose.

\section{P244}

Evaluation of the Impact of High Dose Chemotherapy and Autologous Hematopoietic Stem Cell Transplantation on the Quality of Life of Elderly Lymphoma Patients

Christopher Lemieux, Imran Ahmad, Nadia M. Bambace, Léa Bernard, Sandra Cohen, Jean-Sébastien Delisle, Thomas Kiss, Jean Roy, Silvy Lachance

Hôpital Maisonneuve-Rosemont, Université de Montréal, Division of Hematology and Medical Oncology, Stem Cell Transplant Program, Department of Medicine, Montreal, Canada

Background: High-dose chemotherapy (HDT) followed by autologous hematopoietic stem cell transplantation (AHSCT) is safe in fit elderly patients with chemosensitive non-Hodgkin lymphoma (NHL). The goal of this study was to assess the quality of life (QoL) of elderly patients following AHSCT.

Methods: This single-center, research and ethic committee approved study, investigated QoL in survivors following AHSCT for the treatment of NHL in the elderly. Inclusion criteria were defined as patient aged $\geq 60$ who received an AHSCT for NHL at our center between January 1st, 2008 and January 1st, 2015 and survived. Fifty-nine patients from the original cohort of 90 met the inclusion criteria. $79.7 \%$ (47) participated in the survey. Patients were required to sign an informed consent. We used the EQ-5D instrument to assess mobility, self-care, usual activities, pain/discomfort and anxiety/depression. We also used the Functional Assessment of Cancer Therapy-Bone Marrow Transplant (FACT-BMT) questionnaire to assess: physical well-being (PWB), social/family well-being (SWB), emotional wellbeing (EWB), functional well-being (FWB) and BMT specific concerns. A higher score indicated better QoL. The different scores were correlated to conditioning regimen, gender, age, Hematopoietic cell transplant comorbidity index score (HCT-CI), Karnofsky index, disease status at transplant and relapse.

Results: The median time from AHSCT was 50 months. $15 \%$ of patients were in relapse at the time of the QoL assessment. Results from the EQ-5D questionnaire are shown in table 1. Few patients (9\%) reported impairments in four or five domain. Lower Karnofsky index at diagnostic was associated with impaired mobility $(\mathrm{p}=0,006)$ and selfcare $(p=0,003)$. Anxiety was significant for patients in relapsed after transplant $(\mathrm{p}=0,002)$. Other comparators did not impact on any of these outcomes.

FACT-BMT questionnaire results demonstrate that physical, social and emotional well-being are good while functional well-being is variable in our cohort. Relapse is associated with impaired EWB $(p=0,05)$ as well as BMT specific concerns $(\mathrm{p}=0,18)$ and lower total FACT-BMT 
results ( $\mathrm{p}=0,035)$. Other comparators did not impact on any of these outcomes.

Conclusions: This study demonstrates that physical, social and functional well-being are preserved for elderly patients following AHSCT. Low Karnosfky score prior transplant is a predictor of disability following AHSCT. Relapse following AHSCT remains the most significant impediment to maintain a good QoL.

Conflict of interest: The authors have nothing to disclose.

\begin{tabular}{llllll}
\hline & \multicolumn{2}{l}{ Score level } & & \\
\cline { 2 - 6 } & 1 & 2 & 3 & 4 & 5 \\
\hline Mobility, \% & 77 & 21 & 0 & 0 & 2 \\
Self-Care, \% & 98 & 0 & 0 & 2 & 0 \\
Usual activities, \% & 72 & 22 & 2 & 0 & 4 \\
Pain/Discomfort, \% & 53 & 30 & 15 & 2 & 0 \\
Anxiety/Depression, \% & 70 & 26 & 4 & 0 & 0 \\
\hline
\end{tabular}

1-no problem, $2=$ slight problems, $3=$ moderate problems, $4=$ severe problems, $5=$ unable to perform

\section{[[P244 Table] EQ-5D overall results]}

\section{P245}

Everyday life after HSCT: fluctuation of patients and their partners mood, support exchange and relationship satisfaction

Malgorzata Sobczyk-Kruszelnicka ${ }^{1}$, Aleksandra Kroemeke ${ }^{2}$, Zuzanna Kwissa-Gajewska ${ }^{2}$, Sebastian Giebel ${ }^{1}$

${ }^{1}$ Maria Sklodowska-Curie Institute - Cancer Center, Gliwice Branch, Department of Bone Marrow Transplantation and Oncohematology, Gliwice, Poland; ${ }^{2}$ SWPS University of Social Sciences and Humanities, Department of Psychology, Warsaw, Poland

Background: Hematopoietic stem cell transplantation (HSCT) is a potentially highly stressful event that can affect the well-being of the patient at any stage of the procedure: pre-, around- and after-HSCT. Until now, research has focused mainly on the functioning of patients after the procedure. The research presented below focuses on the essential element of the patient $\hat{A}$ 's well-being, which is the partnerÂ's support and the relationship between them.

Methods: Two hundred patients (after first autologous or allogeneic HSCT) and their healthy partners (spouse or another relative) independently completed measures of daily positive and negative affect, daily social support (need, received, provided, satisfaction), and daily relationship satisfaction for 28 consecutive evenings after patients $\hat{A}$ ' hospital discharge. Multilevel modeling was used to investigate fixed and random effects for variable changes over time.

Results: The results indicated that negative affect systematically decreased in both, patients and healthy partners over 28 days. In patients, positive affect increased, while relationship satisfaction decreased in time. In partners, the need for social support increased over 28 days, whereas provided support decreased. While patients reported a decrease in received support and support satisfaction, their healthy partners reported opposite direction of change of this support components in time.

Conclusions: The findings suggest a deterioration in support exchange, to the detriment of the patient. Over time, the patient receives less and less support from his partner despite the still high demand for them, which affects the worse assessment of the relationship between them. It may be related to the fact that the patient is in a better shape and his partner gradually withdraws from support, feels exhausted and paradoxically expects himself to be supported by the patient at this stage. The deterioration ought to be taken into consideration in the practical approach.

Conflict of interest: nothing to disclose

\section{P246}

Favourable Outcome of Lung Transplantation for Severe Pulmonary Graft-Versus-Host Disease: An Australian Multicentre Case Series

David Kliman', Sakhee Kotecha ${ }^{2}$, David Abelson ${ }^{3}$, Greg Snell', Allan Glanville ${ }^{3}$, David $\mathbf{M a}^{1}$

${ }^{1}$ St Vincent's Hospital, Department of Haematology, Darlinghurst, Australia; ${ }^{2}$ The Alfred Hospital, Lung Transplant Service, Melbourne, Australia; ${ }^{3}$ St Vincent's Hospital, Lung Transplant Unit, Darlinghurst, Australia

Background: Severe pulmonary chronic graft-versushost disease (cGVHD) is a potentially life-threatening complication affecting up to $5 \%$ of allogeneic stem cell transplant (alloSCT) recipients. Among the treatment options for cGVHD, few influence the outcome of pulmonary involvement, with 5-year overall survival (OS) as low as $13 \%$. For patients who are otherwise fit and free of their primary disease, lung transplantation (LTx) has been reported in small numbers worldwide

$(<100$ transplants). We aimed to investigate the outcomes of LTx performed for this indication in Australia.

Methods: Retrospective review of transplant indications and outcomes from two major Australian lung transplant units. Matched control patients transplanted within the same time period and who developed extensive cGVHD were identified for the purpose of OS comparison. 


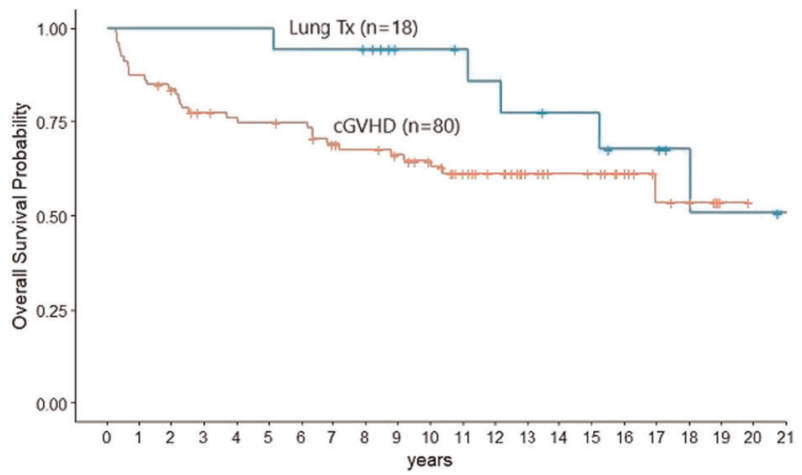

[P246 Figure] [Figure 1. OS from time of alloSCT for LTx and control cGVHD groups. LTx performed at median 8.6 yrs.]

Results: Between 2002-2017, 18 patients (ages 9-64, median 31 years) received bilateral cadaveric lung transplants for pulmonary cGVHD. All but two alloSCT were for malignant diseases, with acute leukaemia the most common indication (12/18). LTx were performed at a median of 8.6 years post alloSCT (range 1.5-22.8) with a median interval of 16 months from time of transplant unit review to LTx. Two early deaths ( $<3$ months) occurred from infectious causes. Three further patients have died, from pulmonary infection (one) and lung allograft rejection (two). There have been three cases of second malignancy but no primary disease relapses. One patient underwent a second LTx at 41 months for lung allograft rejection. At a median follow up of five years (range 0.3-16) the 5-year OS post LTX is $80 \%$. This compares favourably to the ISHLT registry 5-year OS of 55\% for LTx performed for all indications as well as to the published natural history of severe pulmonary cGVHD. The OS curves from time of alloSCT for the LTx and control cGVHD cohort (80 patients, ages 19-45, 10 years median follow up) are shown in figure 1. Outcomes appear similar among patients who developed extensive cGVHD, irrespective of whether LTx was performed.

Conclusions: LTx is a feasible option for selected patients with severe pulmonary GVHD. In this setting, outcomes appear superior to that of non-LTx based therapies for pulmonary cGVHD and likely similar to that of the general LTx population.

Conflict of interest: The authors have nothing to disclose.

\section{P247}

Five-year follow-up of outcomes of older patients with high-risk AML and MDS undergoing two-step peripheral blood haploidentical stem cell transplantation

Ruben Rhoades ${ }^{1}$, Benjamin Leiby ${ }^{2}$, Onder Alpdogan ${ }^{1}$, Matthew Carabasi $^{1}$, Joanne Filicko-O'Hara ${ }^{1}$, Margaret Kasner ${ }^{1}$, Thomas
Klumpp $^{1}$, Ubaldo Martinez-Outschoorn ${ }^{1}$, Neil Palmisiano ${ }^{1}$, Pierluigi Porcu $^{1}$, John Wagner ${ }^{1}$, Neal Flomenberg ${ }^{1}$, Dolores Grosso ${ }^{1}$, Sameh Gaballa $^{1}$

${ }^{1}$ Thomas Jefferson University, Medical Oncology, Philadelphia, PA, United States; ${ }^{2}$ Thomas Jefferson University, Biostastistics, Philadelphia, PA, United States

Background: Haploidentical stem cell transplantation (haploSCT) is a curative therapy for patients with myeloid disorders who lack an HLA-matched donor. These are primarily diseases of the elderly, and recent advances in haploSCT suggest it can be performed safely in older patients. We utilize a peripheral blood (PB) two-step approach that is associated with low graft failure and rapid engraftment, which is crucial for older patients. Here we report their long-term outcomes.

Methods: We retrospectively analyzed all patients over age 55 years with AML $(\mathrm{n}=48)$, biphenotypic leukemia $(\mathrm{n}=2), \operatorname{MDS}(\mathrm{n}=16), \operatorname{MPN}(\mathrm{n}=3)$, and CMML $(\mathrm{n}=2)$ who received a two-step haploSCT between December 2006 and March 2017. Conditioning was myeloablative (MA) (12-Gy TBI) or reduced-intensity (RIC) with fludarabine/busulfan/2-Gy TBI. Afterwards, a large fixed dose of allogeneic T cells was infused $\left(2 \times 10^{8} / \mathrm{kg}\right)$ (step 1), followed by high-dose cyclophosphamide (CY; $60 \mathrm{mg} / \mathrm{kg}$ / day $\mathrm{x} 2$ days) to induce bidirectional tolerance. A CD34+ selected stem cell product was infused after CY (step 2). GVHD prophylaxis comprised tacrolimus and MMF.

Results: Seventy-one patients (50 males, 21 females) received a two-step haploSCT $(\mathrm{MA}=41$; RIC $=30)$ with a median age of 64 years (range 55-78). The median duration of follow-up was 5 years. The MA group had relatively younger patients (median age $=61$ years; range 55-68) compared to the RIC group (median age $=69$ years; range 57-78). Active disease at transplant was: $\mathrm{MA}=63 \%$; RIC $=56 \%$. Two patients had a previous autologous SCT and two patients received prior allogeneic SCT. KPS was $>80 \%$ in $75 \%$ of the MA group and $70 \%$ of the RIC group. HCTCI score was $>3$ in $56 \%$ of the MA group and $63 \%$ of the RIC group. No patients experienced graft failure. The median time to ANC and platelet engraftment were 11 days and 18 days, respectively. The 2-year PFS was $41 \%$ (MA = $46 \%$; RIC $=33 \%$ ), while the 2-year OS was $43 \%$ (MA $=$ $51 \%$; RIC $=33 \%)$. The 1-year cumulative incidence $(\mathrm{CI})$ of non-relapse mortality was $30 \%(\mathrm{MA}=29 \%$; RIC $=34 \%$ ). Complete remission at the time of haploSCT was associated with a trend towards improved 2-year OS (53\% vs 37\%, p $=0.07)$. At 5 years, CI of relapse was $23 \%$ for the whole group $(\mathrm{MA}=22 \%$; RIC $=24 \%)$. The 1 -year $\mathrm{CI}$ of aGVHD was $34 \%$ for grade II-IV and $11 \%$ for grade III-IV. The 2year CI of cGVHD was $17 \%$. On multivariate analysis, age was an independent predictor of NRM (HR 1.16; p = 0.0099 ), while KPS of $70-80 \%$ (HR 2.16; $p=0.069$ ) and 
MA conditioning (HR 2.95; $\mathrm{p}=0.081$ ) showed a strong trend toward greater NRM.

Conclusions: This mature, single-institution data suggests that haploSCT is safe and effective for treating AML and MDS. The two-step PB haploSCT was associated with rapid and uniform engraftment without graft rejection. GVHD rates were comparable to bone marrow graft approaches. These results suggest that age alone should not be a barrier to haploSCT for older fit patients.

Conflict of interest: The authors have nothing to disclose.

\section{P248}

Glutathione S-Transferase Polymorphism Does not Increase the Risk of Hepatic Veno- Occlusive Disease in Patients Undergoing Hematopoietic Stem Cell Transplantation

Intisar Al-Riyami ${ }^{1}$, Khaled Al-Belushi ${ }^{2}$,Murtadha Al-Khabori, Shoaib Al-Zadjali, Mohammed Al-Rawahi ${ }^{3}$, David Dennison ${ }^{3}$

${ }^{1}$ Sultan Qaboos University Hospital, Pharmacy, Muscat, Oman; ${ }^{2}$ Sultan Qaboos University, Pharmacology, Muscat, Oman; ${ }^{3}$ Sultan Qaboos University Hospital, Hematology, Muscat, Oman

Background: Hepatic Veno-Occlusive Disease (VOD) is one of the serious complication post Allogeneic Hematopoietic Stem Cell Transplant (HSCT). Busulfan (Bu) metabolism occurs in the liver and high exposure (defined as Area Under Concentration Time Curve [AUC] > $1500 \mu \mathrm{mol} / \mathrm{min}$ or Steady State Concentration [Css] $>900$ $\mathrm{ng} / \mathrm{ml}$ ) has been shown to correlate with the risk of VOD. The objective of this study is to assess the role of GST polymorphism on the incidence of hepatic VOD in patients undergoing HSCT.

Methods: This is a retrospective single center cohort study. We included 135 patients who underwent HSCT for any indication during the period of January 2003 to October 2016 at our center. Modified Seattle criteria was used to define hepatic VOD. Patients were genotyped for GSTM1 and GSTT1 polymorphism using Capillary Electrophoresis. The GSTA1 (C-69G, A-513G and G-1142C) and GSTP1 (A313G) were genotyped using DNA sequencing (Thermal Cycler Sequencing).The impact of GST polymorphisms on hepatic VOD was examined using $\mathrm{Chi}^{2}$ test. Standard descriptive and analytical statistics and graph were created using STATA version 13.

Results: All patients had a target Css of $\geq 850 \mathrm{ng} / \mathrm{ml}$. The most common diagnosis prior to transplantation was acute leukemias in 48 patients (36\%). Inherited hemoglobinopathy (45 patients; $33 \%$ ) is the second common diagnosis. Overall, 17 patients developed VOD (5\%). The incidence of VOD in patients with GSTM1 deletion was $16 \%$ compared to $15 \%$ in GSTM1 insertion ( $p=0.851)$. The incidence of
VOD in patients with deleted GSTT1 was $16 \%$ compared to $13 \%$ in inserted GSTT1 ( $p=0.514)$. Ten percent, $16 \%$ and $33 \%$ of patients with AA, AG and GG GSTP1 A313G genotypes developed VOD and the differences were not statistically significant $(p=0.158)$. The incidence of VOD in GSTA1 C-69G genotypes (CC, CC and CG) was $14 \%$, $117 \%$ and $15 \%$ respectively with no significant difference $(p=0.999)$. The incidence of VOD in GSTA1 A-513G genotypes (AA and AG was $15 \%$ and $40 \%$ with no significant difference $(p=0.182)$. Similarly, the incidence of VOD in GSTA1 G-1142C genotypes (GG, GC and CC) was $17 \%, 18 \%$ and $\mathrm{CC})$ was $11 \%(p=0.649)$.

Conclusions: Glutathione S-transferase polymorphism (GSTA1\{C-69G, A-513G and G-1142C\}, GSTP1 \{A313G\}, GSTM1 and GSTT1) is not associated with hepatic VOD. This is likely due to the narrow exposure variability in patients receiving pharmacokinetic based $\mathrm{Bu}$ dosing. We recommend that other factors known to be associated with hepatic VOD to be modelled in future studies asking this question.

Conflict of interest: No conflict of interest.

The study was supported by an internal grant from College of Medicine and Health Sciences, Sultan Qaboos University.

\section{P249}

Glutathione S-Transferase Polymorphism is Associated with Overall Survival in Patients Undergoing Hematopoietic Stem Cell Transplantation

Murtadha Al-Khabori ${ }^{1}$, Intisar Al-Riyami ${ }^{2}$, Khaled Al-Belushi ${ }^{3}$, Mohammed Al-Rawahi ${ }^{1}$, Shoaib Al-Zadjali ${ }^{1}$, Salam Al-Kindi ${ }^{1}$

${ }^{1}$ Sultan Qaboos University Hospital, Hematology, Muscat, Oman,

${ }^{2}$ Sultan Qaboos University Hospital, Pharmacy, Muscat, Oman;

${ }^{3}$ Sultan Qaboos University, Pharmacology, Muscat, Oman

Background: Overall Survival (OS) post BMT is associated with the primary disease and the intensity of the preparative regimen used among others. Glutathione Stransferase (GST) polymorphism may change the drug exposure in Busulfan $(\mathrm{Bu})$ based preparative regimens and therefore, it may also be associated with mortality. We aim, herein, to assess the impact of GST polymorphism on the mortality rate of patients undergoing HSCT.

Methods: This is a single center retrospective cohort study of 135 adult and pediatric patients who received intravenous Bu prior to HSCT at Sultan Qaboos University Hospital from January 2003 to October 2016 for any indication. Genotyping for GST polymorphism was done on pre-transplant patients' samples. We used Capillary Electrophoresis for GSTM1 and GSTT1 (insertion or deletion), DNA sequencing for GSTA1 (C-69G, A-513G, and G- 
1142C) and GSTP1 (A313G). The impact of GST polymorphisms on OS was compared using Log-Rank test. Kaplan-Meier curves were used to estimate the OS. Standard descriptive and analytical statistics and graphs were created using the statistical software STATA version 13.

Results: The total number of patients that were included is 135, of which 63 males and 72 females. During the follow up, 5 patients (4\%) died. The median time to death was not reached. The OS at day 100 , one and two years was $97 \%, 92 \%$ and $85 \%$ respectively. The causes of death were mostly due to a combination of graft loss/relapse with or without sepsis $(50 \%)$ followed by severe GvHD with or without sepsis (36\%). Double insertion of GSTM1 and GSTT1 significantly increased the risk of death and was predictive of OS $(p=0.034)$. Additionally, the GSTM1 insertion increased risk of death and was also associated with OS ( $p=0.021)$. All variants of GSTA1 and GSTP1 had no significant effect on the rate of OS (p-values were all $>0.05)$.

Conclusions: Double insertion of GSTM1 and GSTT1 increases the risk of death in patients undergoing HSCT. These results will need to be confirmed in a larger prospective study.

Conflict of interest: No conflict of interest.

The study was supported by an internal grant from College of Medicine and Health Sciences, Sultan Qaboos University.

\section{P250}

\section{Haematocolpos a rare occurrence in adolescents affected with graft versus host disease (GVHD) post allogenic bone marrow transplantation (aBMT)}

\section{Lina Michala ${ }^{1}$, Elpis Vlachopapadopoulou ${ }^{2}$, Pantelis Tsimaris ${ }^{3}$, Stella Roidi ${ }^{1}$, Anna Paisiou ${ }^{4}$, Ioulia Peristeri, ${ }^{5}$, Stefanos Michalacos ${ }^{6}$, Vassiliki Kitra \\ ${ }^{1}$ University of Athens Alexandra Hospital, 1st Department of Obstetrics and Gynecology, Athens, Greece; ${ }^{2}$ Children's Hospital, Endocrine Dept, Athens, Greece; ${ }^{3}$ University of Athens Aretaeion Hospital, 2nd Dept of Obstetrics and Gynecology, Athens, Greece; ${ }^{4}$ Agia Sophia Children's Hospital Athens, Stem Cell Transplantation Unit, Athens, Greece; ${ }^{5}$ Agia Sophia Children's Hospital, Stem Cell Transplantation Unit, Athens, Greece; ${ }^{6}$ Children's Hospital P \& A. Kyriakou, Endocrinology, Athens, Greece; ${ }^{7}$ Agia Sophia Children's Hospital, Athens, Greece}

Background: Chronic graft Versus Host Disease (cGVHD) is a serious complication of allogeneic BMT. Genital cGVHD remains an underrecognised variant. Aim: To present two cases of haematocolpos due to vaginal synechiae formed as a result of GVHD following BMT during late childhood or adolescence.
Methods: 221 allo-transplants have been performed in female patients (1mo-24 years of age). Only in two patients hematocolpos was recognized.

Results: The first patient was diagnosed with Fanconi syndrome and had an allogenic BMT. She developed mild chronic GVHD limited to the skin and mucosas, treated symptomatically without corticosteroids. Eighteen months post-transplant she had no thelarche and the hormonal investigations were consistent with hypergonadotropic hypogonadism (FSH $42.3 \mathrm{mIU} / \mathrm{ml}$ ). At the age of 13 years she was started on estradiol valerate at a dose of $1 \mathrm{mg}$ daily with good clinical response. At 14 years of age, she had a pelvic sonogram which revealed a collection within the upper vagina, assumed to be a hematocolpos. Pelvic MRI confirmed the obstruction and showed a collection of blood. As she was asymptomatic, she remained under surveillance with regular scans, which showed a gradual increase in the size of the haematocolpos. Six months after initial identification of the obstruction, she had a spontaneous discharge of the haematocolpos. On examination, the vagina appeared obliterated still, however a pinhole opening draining brown discharge was identified. This was stented with a small Haegar 3 dilator and gradually dilated with ease until the vagina was fully patent. She reported normal periods, however at a two month follow up, the vagina appeared obliterated at approximately $3 \mathrm{~cm}$ from the introitus and no obvious passage was seen. On examination under anaesthesia, while she had a withdrawal bleed, a pinpoint opening was seen which was bluntly opened with dilators initially and then digitally. The planes were easily identified, and the vagina was made fully patent with minimal bleeding. The second girl age 13 years,underwent allogenic BMT for myelodysplastic syndrome. Her menarche was at 11 years. She developed GVHD limited to the skin for which she was treated with high dose steroid therapy. One year post transplant, she had amenorrhea and the hormonal investigations were consistent with hypergonadotropic hypogonadism (FSH 72.7mIU/ml). She was started on sex steroid replacement therapy with estradiol valerate starting with $1 \mathrm{mg}$ daily which was subsequently increased to $2 \mathrm{mg}$. Three months after increasing the dose to $2 \mathrm{mg}$, a haematocolpos measuring 38x59x65 mm was seen on a pelvic sonogram. The patient was asymptomatic and was kept under surveillance. An increase of the haematocolpos was noticed and eventual spontaneous drainage of the collection. Nevertheless within two months, the vagina restenosed and obliterated. Her oestradiol dose was increased, which led to spontaneous lysis of the vaginal stenosis. Three years later she remains on a cyclical combined preparation of HRT and has regular cyclical bleeding. 
Conclusions: Genital symptoms may not be readily reported by patients post BMT. Physicians should be aware of possible late effects of GVHD on genitalia, inquire about symptoms and be acquainted with addressing complications.

Conflict of interest: There is no conflict of interest

\section{P251}

Hematopoietic stem cell transplant - associated thrombotic microangiopathy: Single centre experience

Anna Torrent Catarineu, Christelle Ferra Coll, Laura Abril Sabater, Maria Jose Jimenez Lorenzo, Mireia Morgades de la Fe, Blanca Xicoy Cirici, Susana Vives Polo, Miriam Moreno Velazquez, Montserrat Batlle Massana, Juan Manuel Sancho Cia, Gladys Ibarra Fernandez, Albert Oriol Rocafiguera, Josep Maria Ribera Santasusana

Hospital Germans Trias i Pujol, Institut Català d'Oncologia, Institut de Recerca contra la Leucèmia Josep Carreras, Hematology,

Badalona, Spain

Background: Hematopoietic stem cell transplantationassociated thrombotic microangiopathy (TA-TMA) is a potentially lethal complication with limited therapeutic options. The incidence of TA-TMA after transplant is variable $(0-74 \%)$ due to the lack of standard diagnostic criteria. Predisposing factors include some conditioning regimens, immunosuppressive agents, GVHD, HLA missmatch, and opportunistic infections. TA-TMA usually occurs within 100 days after HSCT with a mortality rate up to $100 \%$. Treatment strategies for TA-TMA are limited, but therapeutic plasma exchange (TPE) has been shown to be effective when initiated early, suggesting that delayed TPE therapy might be related to the poor outcome rates.

Methods: We performed a retrospective study of 12 episodes of TA-TMA registered from 2004 until november 2017 in a single centre. In a sub-analysis we report the 7 most recent patients with additional information.

Results: The characteristics of the 12 pacients receiving allogeneic HSCT are shown in Table 1. 7 (58\%) were women, with median age of 38 years (range 23-62 years). 3 patients received total body irradiation. GVHD prophylaxis included calcineurin inhibitor (11 cyclosporine, 1 tacrolimus). 6 patients had neurological manifestations and 8 renal involvement, 8 patients had a concomitant infection, and 8 patients had active GVHD ( 3 acute GVHD). Treatment was: withdrawal of calcineurin inhibitor $(\mathrm{n}=12)$, plasma exchange $(\mathrm{n}=8)$, rituximab $(\mathrm{n}=2)$ and defibrotide $(\mathrm{n}=$ 3). 7 patients achieved TA-TMA complete response. Only 2 out of 12 patients (17\%) are long-term survivors. Causes of death were: 4 directly related to TA-TMA, 3 with GVHD and 3 with infections. Data from the 7 most recent patients: refractory hipertension (3/7) requiring more than 2 treatments to control. The first signal of TA-TMA was LDH elevation (5/7), renal failure (1/7) and thrombocytopenia (1/7). The interval between the first signal of TATMA and the onset of treatment was 11.5 days (range 5-16 days). The median time from HSCT to diagnosis of TATMA was 2.75 months (range 0.33-9.97 months) and the probability of OS from TA-TMA was 19\% at 6 months (95\%CI, 0\%-43\%).

Conclusions: TA-TMA is a severe complication after HSCT and is associated to high mortality. The lack of standard diagnostic criteria delays the onset of treatment. The first sign of TA-TMA in our serie was increased LDH level.

Conflict of interest: The autors declare no conflict of interest.

Funding: PI10/01417 (Fondo de Inversiones Sanitarias). RD12/0036/0029 (RTICC, Instituto Carlos III, 2014 SGR225GRE), CERCA Program, Generalitat de Catalunya, España, "La Caixa" Foundation, RD12/0036/0029 RTICCInstituto Carlos III. Proyecto de ayuda a pacientes de la fundación DKMS.

\begin{tabular}{lll}
\hline Haematological disease & AML/ALL/CML & $4 / 4 / 1$ \\
\hline & NHL/HL & $2 / 1$ \\
Donor & RD/URD & $7 / 5$ \\
Stem cell product & PB/BM/CB & $7 / 1 / 4$ \\
HLA missmatch & O/ 1 & $8 / 4$ \\
Conditioning regimen & MA/NMA & $8 / 4$ \\
Disease status at HSCT & CR/PR & $8 / 3$ \\
& Stable disease & 1 \\
\hline
\end{tabular}

[[P251 Table] Pacients characteristics $(N=12)]$

P252

Hemorrhagic cystitis after allogeneic hematopoietic stem cell transplantation in children

Aleksandr Shcherbakov ${ }^{1}$, Maksim Kucher ${ }^{2}$, Aleksandr Shvetcov ${ }^{2}$, Olesya Paina ${ }^{2}$, Olga Slesarchuk2, Ruslana Klementeva ${ }^{2}$, Oleg Goloshchapov', Andrei Kozlov ${ }^{2}$, Ludmila Zubarovskaya', Boris Afanasyev $^{2}$

${ }^{1}$ Pavlov First Saint Petersburg State Medical University, Intensive Care Unit, Saint Petersburg, Russian Federation; ${ }^{2}$ Pavlov First Saint Petersburg State Medical University, Saint Petersburg, Russian Federation

Background: Hemorragic cystitis (HC) after allogeneic hematopoetic stem cell transplantation (allo-HSCT) is a common complication with the incidence of $9-31 \%$. Development of $\mathrm{HC}$ leads to increase in blood transfusions, risk of urinary tract obstruction and renal failure. Etiology of $\mathrm{HC}$ is not well defined but the most probable reason of 
$\mathrm{HC}$ is toxicity of chemotherapy and persisting viral infection (polyomavirus family); in addition impact of aGVHD needs to be investigated. The aim of study - to analyse the epidemiology and risk factors of $\mathrm{HC}$ in children after allo-HSCT with different conditiong regimens.

Methods: From 2011 to 2014172 patients (0-17 y.o., median - 8, g/b - 69/103) with ALL - 73 (42.4\%), AML - 53 (30.8\%), other malignant disiases - 21(12,2\%), inherited diseases - 18(10.5\%), AA - 5(2,9\%), other - 2(1.2\%). Myeloablative conditioning regimen $\mathrm{Bu} 12-16 \mathrm{mg} / \mathrm{kg}+\mathrm{Flu}$ $150-200 \mathrm{mg} / \mathrm{m}^{2}$ or $\mathrm{Cy} 120-200 \mathrm{mg} / \mathrm{kg}$ was used in 79 $(45,9 \%)$ pts; reduced intensity conditioning regimen Flu $150-180 \mathrm{mg} / \mathrm{m}^{2}+\mathrm{Bu} 8 \mathrm{mg} / \mathrm{kg}$ or Mel $140 \mathrm{mg} / \mathrm{m}^{2} \pm$ Thio 8$10 \mathrm{mg} / \mathrm{kg}-93(54,1 \%)$ pts. The source of allo-HSCT: matched related donor $13.9 \%$, haploidentical $-30.2 \%$ and matched unrelated $-55 \%$. Bone marrow (BM) was used in 103 (59.9\%), peripheral blood stem cells (PBSC) - 67 (38,9\%), combination of BM and PBSC - 2(1.2\%). Prophylaxis of aGVHD was on the basis of CsA or Tacro $+\mathrm{MtX} \pm \mathrm{MMF}$ and ATG, CsA or Tacro $+\mathrm{MMF} \pm$ Sirolimus + Pt-Cy on D+4, D+5. Mesna was administered 60-120 $\mathrm{mg} / \mathrm{kg}$ i.v. during 24-hour infusion $30 \mathrm{~min}$. prior to $\mathrm{Cy}$. We study epidemiology of $\mathrm{HC}$ in children, risk factors for the $\mathrm{HC}$, detection of virus (BKPyV or JCPyV) by PCR, transfusion dependance. OS by means of Kaplan-Meier method were assessed.

Results: HC developed in 33 patients (19,2\%). Severe HC (Grade III-IV) developed in 32(97\%) of them. Interval between allo-HSCT and HC onset was 7 - 56 days, the duration of HC was 3-165 days. Factors that increased the risk of HC development were age over 10 years $(p=0,0018)$; cyclophosphamide as part of the conditioning regimen $(\mathrm{p}=0,0046) ; \quad$ graft-versus-host disease grade II-IV $(\mathrm{p}=0,023)$. BKPyV was detected in urine of $24(82,5 \%)$ patients with HC. JCPyV was detected in urine of 10 $(34,5 \%)$ patients with HC. In 28 patients with $\mathrm{HC}(88.4 \%)$ the frequency of blood transfusions was 1 unit per 9 days, the median number of doses was 2 (range 0-6) and didn $\hat{A}^{\prime} t$ differ from patients without HC. In 5 patients with severe $\mathrm{HC}$ and intense bleeding the need for blood transfusions was 1 unit per 3 days, the total number of doses - 8-18, median - 12. Transplant related mortality in children with HC was $21,2 \%$ but in complex with other complications (GVHD, infection). HC was a direct cause of death only in 1 pt. Overall 1-year survival was $60,4 \%$ in patients without $\mathrm{HC}$ and $39,4 \%$ in patients with $\mathrm{HC}$ (95\% CI 155.2-402.7, $\chi 2-3.2, \mathrm{p}=0.072)$.

Conclusions: HC in children after allo-HSCT is a common complication that along with other influence on overall survival. The risk factors of $\mathrm{HC}$ such as $\mathrm{Cy}$ in conditioning regimen, GVHD will allow to develop approaches for prophylaxis and effective treatment.
Conflict of interest: The authors have nothing to disclose.

\section{P253}

\section{Hemostatic complications in hematopoietic stem cell transplantation in a Romanian center}

Cristian Jinca ${ }^{1}$, Andrada Oprisoni ${ }^{2}$, Anca Isac ${ }^{3}$, Andreea Pascalau ${ }^{4}$, Ada Balan ${ }^{1}$, Loredana Balint-Gib ${ }^{4}$, Margit Serban ${ }^{3}$, Smaranda Arghirescu $^{1}$

${ }^{1}$ University of Medicine and Pharmacy 'V. Babes', Pediatrics, Bone Marrow Transplantation, Timisoara, Romania; ${ }^{2}$ University of Medicine and Pharmacy 'V. Babes', Pediatrics, Timisoara, Romania; ${ }^{3}$ Emergency Children's Hospital 'L. Turcanu', Bone Marrow Transplantation, Timisoara, Romania; ${ }^{4}$ Emergency Children's Hospital 'L. Turcanu', Onco-hematology, Timisoara, Romania

Background: Allogeneic hematopoietic stem cell transplantation (alloHSCT) remains a therapeutic approach with high transplant-related morbidity. Among these complications, hemorrhagic and thrombotic disturbances are common, especially in the early post-transplant phase.The aim of the study was to asses transplant-related early and late haemostatic impairments in patients undergoing alloHSCT.

Methods: This is a retrospective study on a number of 65 patients receiving an alloHSCT in the Center for Bone Marrow Transplantation Timisoara Romania between January 2003 and September 2017. Fourty-four of them received grafts from related donors (one cord blood) whereas 21 from unrelated matched donors. Among the studied group, the indications for alloHSCT were: acute lymphoblastic leukemia (ALL) in 18 patients, acute myeloblastic leukemia (AML) in 26 patients, chronic myelogenous leukemia (CML) in 2 patients, 3 cases of myelodisplastic syndrome (MDS), juvenile myelomonocytic leukemia (JMML) in 1 child, severe and very severe aplastic anemia (SAA) in 7 cases, chronic granulomatous disease (CGD) in 3 children, severe combined immunodeficiency in 3 children, hemophagocytic lymphohistiocytosis in 1 child and thalassemia major in one child. All patients received heparine for prophylaxis of veno-occlusive disease. Antithrombin III (ATIII) levels were measured twice weekly and pre-emptive was given if levels decreased below $70 \%$.

Results: Among bleeding complications, $63.63 \%$ were of $2^{\text {nd }}$ and $3^{\text {rd }}$ degree (WHO bleeding score). Upper gastrointestinal (GI) tract bleedings were twice as frequent than lower GI tract bleeds. Intracranial hemorrhage occurred in $6.1 \%$ of the cases. Regarding thrombotic and thrombohemorrhagic disorders, DIC associated with sepsis was encountered in $9.5 \%$ of all cases. Transplantation-related thrombotic microangiopathy was diagnosed in 4 patients and 8 patients (12\%) presented hepatic VOD, among which 
four had a severe form. ATIII administration was necessary at least once in $25.3 \%$.

Conclusions: Hemostatic complications still cause high morbidity among transplant recipients in some cases associated with mortality. Severe forms as VOD may require the use of expensive drugs, contributing to the high costs of the often life-saving bone marrow transplantation procedure. ATIII seemed to be effective in preventing progression of VOD to severe forms.

Conflict of interest: nothing to disclose

\section{P254}

Hepatitis C Virus Infection-Associated Macrofhage Activation Syndrome in a pediatric patient underwent hematopoietic stem cell transplantation for Acute Lymphoblastic Leukemia

Mariacristina Menconi ${ }^{1}$, Gian Luigi Laccetta ${ }^{2}$,Sayla Bernasconi ${ }^{1}$, Gabriella Casazza ${ }^{1}$

${ }^{1}$ Azienda Ospedaliera Universitaria Pisana, Pisa, Italy; ${ }^{2}$ Università degli Studi di Pisa, Pisa, Italy

Background: Hepatitis C virus (HCV) infection causing Macrofhage Activation Syndrome (MAS) in pediatric population has never been reported previously.

Methods: We describe a case of MAS associated with $\mathrm{HCV}$ infection in a girl with acute lymphoblastic leukemia (ALL) which underwent hematopoietic stem cell transplant (HSCT) and attained viral suppression with ledipasvir/ sofosbuvir.

Results: A 13-year-old Ukranian girl with pro-B cell ALL $\mathrm{t}(4 ; 11)(\mathrm{q} 21-\mathrm{q} 23)$, and HCV-genotype1 infection (HCV-RNA $629.500 \mathrm{IU} / \mathrm{mL}$ ) received chemotherapy according to the AIEOP LAL 2000 protocol and a myeloablative allogeneic HSCT from MUD; the engraftment was at day+19 after HSCT (neutrophils 580/ $\mu \mathrm{L}$ ).

At day +22 the patient presented epigastric pain, icteric skin, fever $\left(39^{\circ} \mathrm{C}\right)$, vomiting and diarrhea. Blood exams showed: progressive cytopenia, WBC $670 / \mu \mathrm{L}, \mathrm{N} 120 / \mu \mathrm{L}, \mathrm{Hb}$ $9.0 \mathrm{~g} / \mathrm{dL}$, PLT22.000/ $\mu \mathrm{L}$, hypofibrinogenemia $100 \mathrm{mg} / \mathrm{dL}$, ALT $283 \mathrm{U} / \mathrm{L}$, AST $323 \mathrm{U} / \mathrm{L}$, hyperbilirubinemia tot $11.0 \mathrm{mg} /$ $\mathrm{dL}$, direct $10.4 \mathrm{mg} / \mathrm{dL}$, hypertrigliceridemia $460 \mathrm{mg} / \mathrm{dL}$, hyperferritinemia $67.769 \mathrm{ng} / \mathrm{mL}$. Blood culture was negative, CMV, EBV Adenovirus-PCR negatives; serology for hepatitis $\mathrm{A}$ and HBV-DNA negatives but HCVRNA $>100.000 .000 \mathrm{IU} / \mathrm{mL}$. Clinical examination and abdominal ultrasound revealed hepatosplenomegaly and BOM showed CR for ALL but increased histiocytes and macrophages with hemophagocytosis. No signs of GvHD. MAS secondary to HCV infection was suspected and treatment with high-dose corticosteroids, cyclosporine-A and ledipasvir/sofusbivir was initiated. At day +38 after
HSCT fever disappeared, laboratory exams improved and quantitative PCR for HCV RNA decreased(19.114.275 IU/ $\mathrm{mL}$ ); at 4 weeks of antiviral treatment the HCV-RNA was negative.

At day +82 the patient presented fever $\left(39.1^{\circ} \mathrm{C}\right)$, headache, left facial droop, right convergent strabismus, aphasia, disequilibrium, gait unsteadiness, absence of deep tendon reflexes, clumsiness of both hands and drowsiness. BrainMRI showed multiple supra and infratentorial areas of mass-like appearance with central necrosis surrounded by a hyperintense ring; a broad laboratory workup confirmed cerebral reactivation of MAS. Thus, the dose of intravenous corticosteroids was increased and intravenous mannitol was administered to reduce intracranial pressure; phenobarbital and carbamazepine were initiated. The antiviral therapy with ledipasvir/sofusbivir was discontinued at day +200 (for a total of 6 months)

At follow up at 9 months after HSCT the patient is alert, fully oriented, with fluent speech and normal language; she has a permanent left facial droop, a diffuse left lower extremity weakness, reduced deep tendon reflexes and steppage gait on her left. Brain-MRI shows neither new lesions nor an enlargement of the previously described areas; the last quantitative PCR for HCV-RNA is negative and BM aspirate is in CR for ALL and MAS with full donor chimerism.

Conclusions: The term MAS designates a clinicopathologic entity that occurs in different hemophagocytic syndromes(HSs). Primary hemophagocytic lymphohistiocytosis(HLH) is recognized to have an immunogenetic basis, but the secondary HSs are due to an autoimmune disease or malignancy or an underlying infection. Secondary HS due to viral infections are commonly due to the herpes group. In our case MAS probably triggered by $\mathrm{HCV}$ and the early treatment with Ledipasvir/Sofosbuvir revealed to be highly effective for HCV infection and well-tolerated by the patient; despite viral clearance, HLH persisted over time with permanent neurologic impairment.

Conflict of interest: Nothing to disclose

\section{P255}

High-Dose Cyclophosphamide after allogeneic stem cell transplantation influences on reconstitution of memory CD8 $+\mathrm{T}$ cell subsets

Natalia Popova ${ }^{1}$, Mikhail Drokov ${ }^{1}$, Larisa Kuzmina ${ }^{1}$, Julia Davydova ${ }^{2}$, Nikolay Kapranov ${ }^{2}$, Ekaterina Mikhaltsova $^{1}$, Olga Koroleva $^{1}$, Vera Vasilyeva ${ }^{1}$, Darya Dubnyak ${ }^{1}$, Anna Sidorova ${ }^{1}$, Ekterina Usikova ${ }^{1}$, Zoya Konova ${ }^{1}$, Irina Galtseva ${ }^{2}$, Elena Parovichnikova $^{1}$, Valery Savchenko ${ }^{1}$

${ }^{1}$ National Research Centre for Hematology, Bone Marrow Department, Moscow, Russian Federation; ${ }^{2}$ National Research Centre 

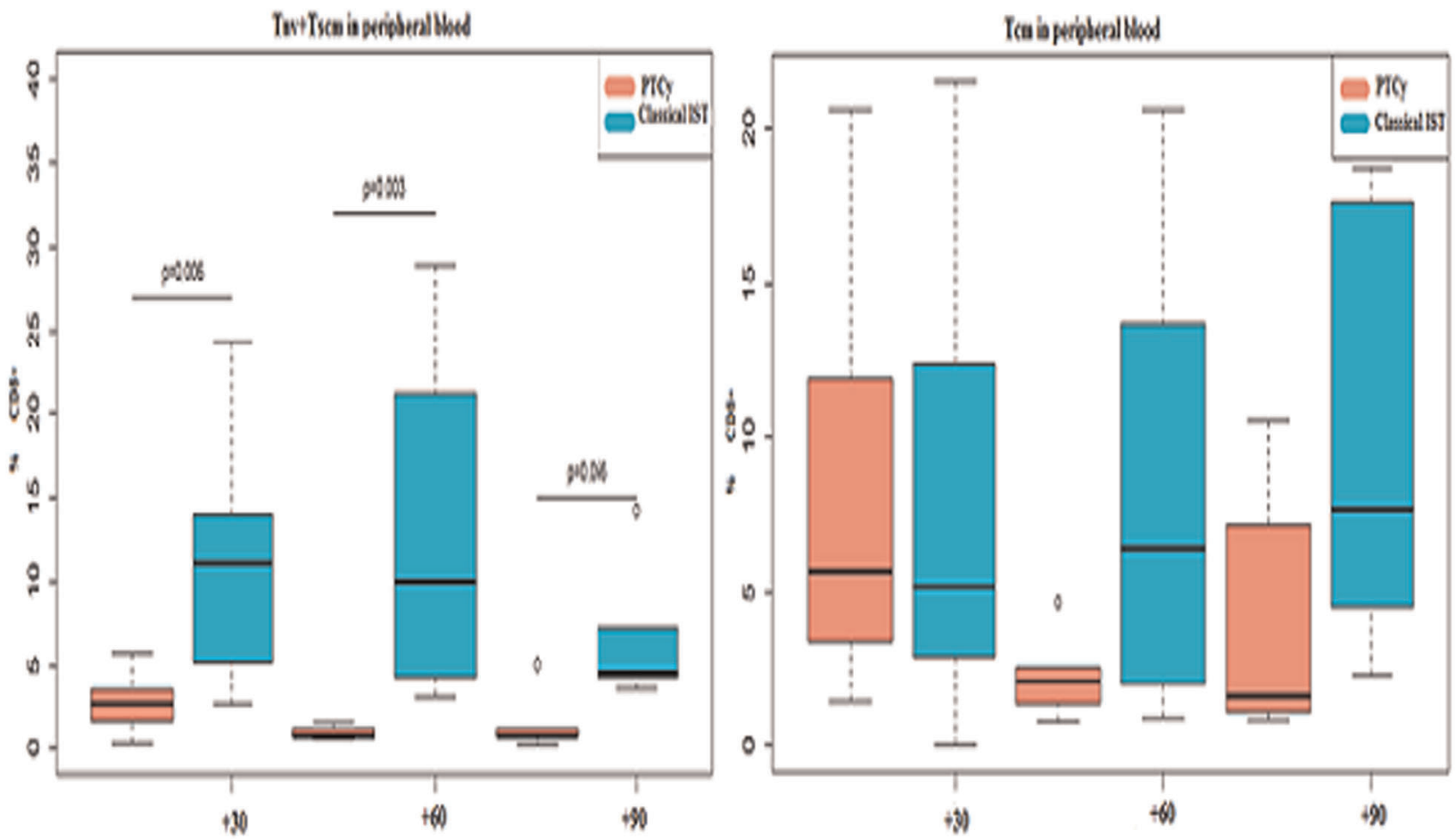

Dụ âtabsact

Itmia peripberal Hood

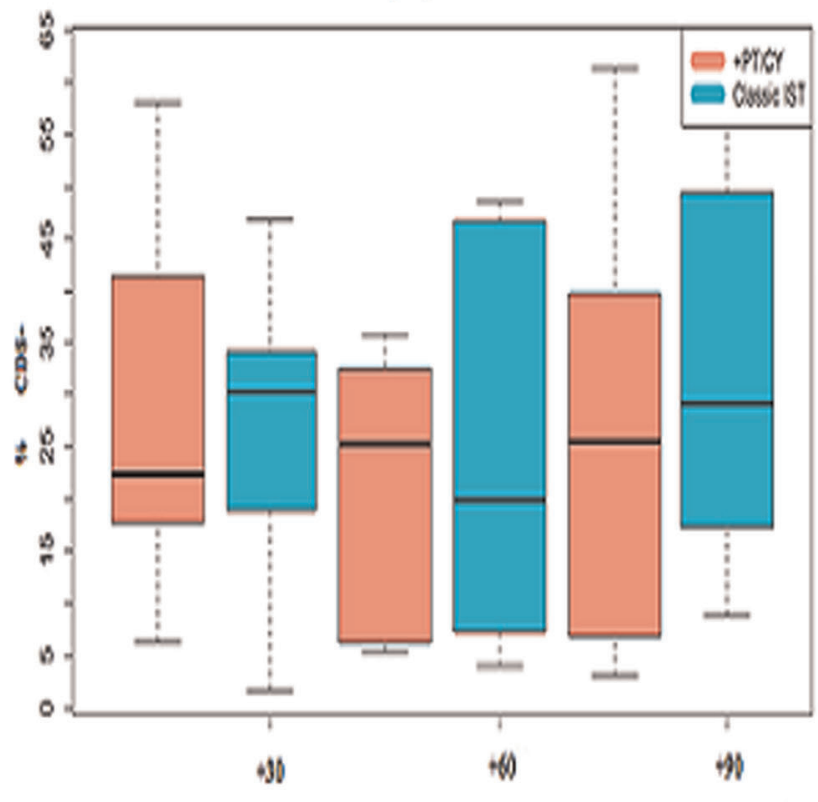

The ia prenpteral Hood

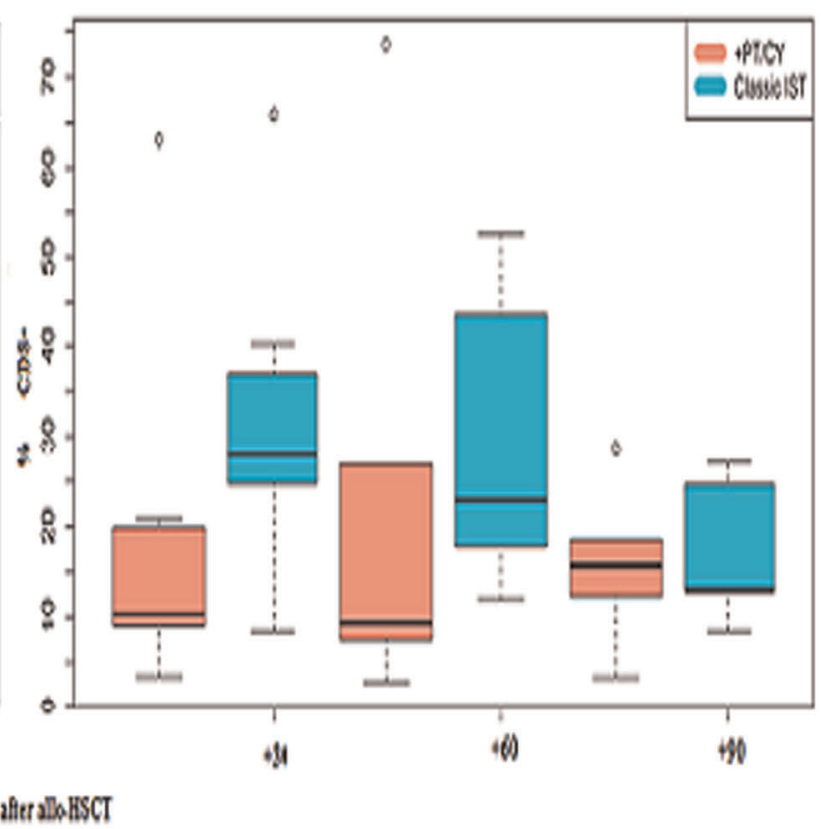

[P255 Figure] [Memory CD8+ subsets recovery after allo-HSCT in terms of different immunosuppressive therapy.] 
for Hematology, Flow Cytometry Laboratory, Moscow, Russian Federation

Background: Allogeneic hematopoietic stem cell transplantation (allo-HSCT) is the effective therapy for leukemia patients. Different memory CD8 $+\mathrm{T}$ cell subsets form the main pool of adaptive immunity against leukemia cells. However it has been shown previously $\mathrm{T}$ naïve cells is going to be the subset responsible for acute graft versus host disease (aGVHD) onset. High dose post-transplant cyclophosphamide (PTCy) is presented as a safe and effective approach to prevent GVHD. In our research we oversee an impact of PTCy on different memory $\mathrm{CD} 8+\mathrm{T}$ cells recovery after allo-HSCT.

Methods: Peripheral blood (PB) samples were collected from patients with an acute leukemia on day $+30,+60$ and +90 after allo-HSCT in EDTA-tubes. The group consisted of 19 patients ( 9 males and 10 females) with a median age of 36 years (20 - 60 years old) who underwent allo-HSCT. All patients received ATG within immunosuppressive therapy. Classical immunosuppressive therapy included on CsA + MMF + MTX $(n=12)$. PTCy on day $+3,+4$ was used in 7 patients: mono-PTCy ( 2 relapsed patients before allo-HSCT) or CsA+MMF+PTCy (5 patients who were transplanted from unrelated mismatched donor). Flow cytometry analysis was performed on BD FACS Canto II (Becton Dickinson, USA). 7-AAD was used to exclude dead cells from the analysis. The anti-CD8-APC-Cy7, antiCCR7-PE-Cy7, anti-CD28-PE, anti-CD45R0-FITC antibodies (Becton Dickinson, USA) were used to define $\mathrm{T}$ memory subsets: $\mathrm{T}$ naive and $\mathrm{T}$ stem cell memory (Tnv $+\mathrm{Tscm})-\mathrm{CD} 8^{+} \mathrm{CD} 45 \mathrm{R} 0{ }^{-} \mathrm{CCR} 7^{+} \mathrm{CD} 28^{+}$; $\mathrm{T}$ central memory (Tcm) - $\mathrm{CD} 8{ }^{+} \mathrm{CD} 45 \mathrm{R} 0{ }^{+} \mathrm{CCR} 7{ }^{+} \mathrm{CD} 28^{+} ; \mathrm{T}$ transitional memory $(\mathrm{Ttm})-\mathrm{CD} 8^{+} \mathrm{CD} 45 \mathrm{R} 0^{+} \mathrm{CCR} 7^{-} \mathrm{CD} 28^{+}$; $\mathrm{T}$ effector memory (Tem) - CD8 ${ }^{+} \mathrm{CD} 45 \mathrm{R} 0^{+} \mathrm{CCR} 7^{-} \mathrm{CD} 28^{-}$; $\mathrm{T}$ terminal effector (Tte) - $\mathrm{CD}^{+} \mathrm{CD} 45 \mathrm{R}^{-} \mathrm{CCR} 7^{-} \mathrm{CD} 28^{-}$. MannWhitney $\mathrm{U}$ test was used for nonparametric data analysis. A p-value less than 0,05 was considered as significant. All data analysis was conducted utilizing SPSS ver. 23. (IBM, Chicago, Ill., USA).

Results: According to our data Tnv+Tscm CD8+ cells are the only subset susceptible to PTCy due to the lowest number of these cells in PB within follow up period after allo-HSCT. On day +30 the number of Tnv+Tscm is $2,36 \%(1,34-2,79 \%)$ after PTCy versus $8,34 \%$ (5,88$12,05 \%)$ after classical immunosuppressive regimen $(\mathrm{p}=0,00)$; on day $+60-0,27 \%(0,22-1,18 \%)$ versus $6,87 \%(3,95-15,18 \%)$ respectively $(\mathrm{p}=0,003)$; on day +90 - $1,85 \%(0,62-2,71 \%)$ versus $7,83 \%(5,13-9,33 \%)$ respectively $(\mathrm{p}=0,015)$. These findings demonstrate PTCy's influence on naïve cell compartment that is going to be associated with its feasible immunosuppressive mechanism.
The other subsets (Tcm, Ttm, Tem, Tte) are not changeable regardless an immunosuppressive regimen.

Conclusions: The successful reconstitution of T memory cells provide the effective immunity and could help to supply anti-leukemia response after allo-HSCT. We found reduced Tnv+Tscm CD8+ cells in PB after PTCy that hypothetically could play a key role in preventing GVHD. Possible immunosuppressive mechanism of PTCy is associated with its influence on naïve cell compartment. At the same time PTCy is seems not to affect considerably the memory and effector pool of CD8+ cells that is going to be a platform for «graft-versus-leukemia» effect after alloHSCT.

Conflict of interest: No relevant conflicts of interest to declare.

\section{P256}

Impact of anti-HLA antibodies on early complications and outcome after unrelated donor haematopoietic stem cell transplantation (HSCT)

Rita Cekauskiene $^{1,2}$, Arturas Jakubauskas ${ }^{1,2}$, Beatrice Valatkaite ${ }^{1,2}$, Igoris Trociukas ${ }^{1}$, Laimonas Griskevicius ${ }^{1}$

${ }^{1}$ Vilnius University Hospital Santaros Klinikos, Hematology, Oncology and Transfusion Medicine Center, Vilnius, Lithuania; ${ }^{2}$ State Research Institute Centre for Innovative Medicine, Vilnius, Lithuania

Background: Prevalence of Anti-HLA antibodies (AHA) among HSCT recipients, reported by different studies is 19.6-39.4\% with higher percentage in parous female. Significance of AHA (especially donor-specific AHA) has been investigated in HSCT field. However, most of findings applied to haploidentical and cord blood settings with the results mostly addressed to impact of donor-specific AHA on graft failure. Donor-specific AHA were found to be associated with primary graft failure, higher transplantrelated mortality (TRM) rate and inferior overall survival (OS) after haploidentical bone marrow and cord blood transplantations. Some studies with unrelated donor (UD) HSCT confirmed that donor-specific AHA were associated with failed engraftment. However, there are quite few studies investigating significance of AHA (non-donorspecific) on HSCT outcomes. The main objective of this prospective study is to evaluate the clinical relevance of anti-HLA Abs in UD alloHSCT.

Methods: From June 2014 to September 2016, 90 adult patients, who reached 6-month follow-up after their first unrelated donor HSCT or died within this period, were recruited into the prospective study. All donor-patient pairs were $10 / 10$ or 9/10 matched at HLA-A, -B, -C, -DRB1, and -DQB1 loci. Additionally, HLA-DRB3,4,5 and -DPB1 loci were typed. Patients' sera sample collection was scheduled 
before the start of conditioning regimen and at 1,2,3, and 6 months after HSCT. Anti-HLA antibodies were screened and specificities were identified by solid-phase immunoassays (Immucor) on the Luminex platform. HLAMatchmaker programs were used to perform structural HLA class I and class II matching at the eplet level.

Results: Patient's median age was 56 years (range 19 74). Of 90 patients, $47(52.2 \%)$ were men. Seventy-four donor - patient pairs (82.2\%) were 10/10 matched. All patients achieved neutrophil engraftment after HSCT and 4 patients $(4.4 \%)$ had secondary graft failure. The overall prevalence of preformed anti-HLA antibodies was $41.1 \%$ (no donor-specific antibodies were found) and 32 of 37 patients retained anti-HLA antibodies after HSCT. The presence of preformed anti-HLA antibodies was found to be associated with more frequest the development of early grade III and IV acute graft-versus-host disease (aGVHD) and post-transplant complications $(\mathrm{p}=0.0446)$, higher transplant-related mortality $(\mathrm{p}=0.011)$, and shorter overall survival $(\mathrm{p}=0.021)$. The preformed anti-HLA antibodies did not affect engraftment, incidence of relapse, graft failure, event free survival. Anti-HLA antibodies detected after HSCT had no impact on development of early complications and outcome of HSCT. Nine patients developed de novo anti-HLA antibodies after HSCT and in the only case they were donor-specific and directed against mismatched DQA1/DQB1.

Conclusions: Preformed anti-HLA antibodies have impact on development of severe aGVHD, higher transplant related mortality and reduced overall survival after unrelated donor HSCT. Therefore, routine testing for antiHLA antibodies may be considered in the pre-transplant work-up of recipients.

Conflict of interest: No conflict of interest. Grant: Research Council of Lithuania (MIP-014/2014).

\section{P257}

Impact of Hepatitis B Core Antibody Seopositivity on the Liver Function Tests after Autologous Hematopoietic Stem Cell Transplantation For Multiple Myeloma

Güldane Cengiz Seval, Elif Sertesen ${ }^{2}$, Sinem Civriz Bozdag ${ }^{1}$, Meltem Kurt Yuksel ${ }^{1}$, Pervin Topcuoglu ${ }^{1}$, Onder Aslan ${ }^{1}$, Muhit Ozcan ${ }^{1}$, Taner Demirer ${ }^{1}$, Gunhan Gurman ${ }^{1}$, Osman Ilhan ${ }^{1}$, Hamdi Akan $^{1}$, Meral Beksac', Selami Koçak Toprak ${ }^{1}$

${ }^{1}$ Ankara University School of Medicine, Department of Hematology and Stem Cell Transplant Unit, Ankara, Turkey; ${ }^{2}$ Ankara University School of Medicine, Department of Internal Medicine, Ankara, Turkey

Background: Hepatitis B virus (HBV) infection is a global disease; an estimated 3.3 million people in Turkiye have chronic hepatitis B infection. Reactivation of $\mathrm{HBV}$ is a well-recognized complication in patients undergoing highdose chemotherapy and hematopoetic stem cell transplant (HCT). The prevalence of HBV infection in multiple myeloma (MM) patients ranges from $6 \%$ to $19 \%$, but the frequency of reverse seroconversion after auto-HSCT is unclear. We performed this retrospective study to assess the incidence of HBV reactivation and liver toxicity.

Methods: We conducted a retrospective study in 79 patients (male/female: 46/33) with MM who had auto HSCT at Ankara University School of Medicine Bone Marrow Transplant Unit in between January 2015 and October 2017. The clinical database and electronic medical records were searched to collect liver function tests, HBV serology and viral load test results. All patients had liver function tests before starting the conditioning regimen. After auto-HSCT day +30 and day +90 liver function tests were performed at three-months intervals during the followup period. HBV serology was not repeated after auto-HSCT except in those who had an abnormal transaminase level.

Results: The median age for our cohort was 58 years (range, 35-75 years). The median follow-up interval was 25.3 months. Bortezomib based regimens were given as induction therapy to majority of the patients. Five patients out of 79 had an active HBV infection at the time of autoHSCT and these patients had been using antiviral treatment. During the follow up after auto-HSCT serology was obtained negative for these patients. Fifty-two patients ad resolved HBV infection (positive hepatitis B core antibody $(\mathrm{HBcAb})$ and negative hepatitis $\mathrm{B}$ surface antigen $(\mathrm{HBsAg}))$. All of the patients with resolved HBV infection received preemptive antiviral therapy $(245 \mathrm{mg} /$ day tenofovir) before auto-HSCT. None of these patients had HBV reactivation after the transplant during the follow-up period. No veno-occlusive disease or fulminant liver failure was observed. Baseline serum transaminases levels were normal in all patients. Five patients had higher ALT and AST (< $1.5 \mathrm{xULN}$ ) levels at day +30 , only 1 patient had higher ALT and normal AST levels at day+90. Mean ALT levels at day +30 and +90 were; $25.9 \pm 15.8$ and 21.2 \pm 9.9 .

Conclusions: Periodic monitoring of liver function and prolonged follow-up may be needed after auto-HSCT to detect HBV reactivation and liver toxicity. This findings needs to be confirmed with prospective studies of larger number of patients.

Conflict of interest: The authors have nothing to disclose.

\section{P258}

Impact of intensity of conditioning regiment on lung function in Lymphoma and myeloma patients undergoing autologous peripheral stem cell transplantation 
Radwan Massoud ${ }^{1}$, Maroun Matar ${ }^{1}$, Basel Haffar ${ }^{2}$, Ammar Zahreddine $^{2}$, Samer Abou Arbid ${ }^{2}$, Ali Bazarbachi', Imad Abou $\mathrm{Akl}^{3}$, Jean El Cheikh ${ }^{2}$

${ }^{1}$ American University of Beirut Medical Center, Internal Medicine, Bone Marrow Transplant Unit, Beirut, Lebanon; ${ }^{2}$ American University of Beirut Medical Center, Internal Medicine, Division of HematologyOncology, Bone Marrow Transplant Unit, Beirut, Lebanon; ${ }^{3}$ American University of Beirut Medical Center, Internal Medicine, Beirut. Lebanon

Background: High dose chemotherapy and autologous peripheral stem cell transplantation (APSCT) has become a standard of care in multiple hematological malignancies. There is limited data investigating the effect of conditioning regiments on lung function post APSCT. In this study we aimed to identify pulmonary function changes occurring post-APSCT for Myeloma and Lymphoma patients receiving Melphalan, and BEAM conditioning respectively.

Methods: This retrospective study was approved by the Institutional Review Board at the American University of Beirut medical center. We included all patients that underwent APSCT with available pre-transplant (1-4 weeks pre-transplant) and D100 Pulmonary function tests results. Measured spirometry values included forced expiratory volume in 1 second (FEV1), forced vital capacity (FVC), the FEV1/FVC ratio, and the forced midexpiratory flow rate (FEF25-75\%) and were obtained from a forced maximal expiratory vital capacity maneuver. Total Lung Capacity (TLC), and Residual volumes (RV) were measured using the helium dilution technique. In addition, the diffusion capacity was measured using the single-breath carbon monoxide method (DLCO).

Results: We included total of 142 patients with available pulmonary function tests results. Forty-five (32\%) patients were transplanted for multiple myeloma and received Melphalan as conditioning regiment, 97 (68\%) patients were transplanted for Lymphoma and received BEAM as conditioning. The median age at transplant was 46 years (range, 39-67) for the Melphalan group, and 32 years (range 14-70) for the BEAM group. Twenty five patients (56\%) in the melphalan group were smokers, compared to 41 patients $(42 \%)$ in the BEAM group. all patients. We observed a greater decrease in lung volumes patients receiving BEAM when compared to the Melphalan group. with FEV1 decreasing $-4.06( \pm 12.51)$ percent in the patients who received BEAM and $-3.43( \pm 13.47)$ in those who received Melphalan $(\mathrm{P}=0.001)$; and FVC decreasing $-4.25( \pm 12.79)$ and $-3.64( \pm 13.70)$ in the BEAM and Melphalan respectively $(\mathrm{P}=0.05)$. However there was no statistically significant decrease in DLCO, and TLC. Smoking, age, disease status at transplant and gender had no significant impact on lung volume changes post-transplant.

Conclusions: Acknowledging the retrospective nature of our study, and the limited sample size, we conclude that
BEAM conditioning regiment is associated with significant decrease in FEV1 and FVC post-transplant when compared to Melphalan conditioning.

Conflict of interest: The authors have nothing to disclose.

\begin{tabular}{llll}
\hline Lung volumes & BEAM & Melphalan & $\begin{array}{l}\text { P } \\
\text { value }\end{array}$ \\
\hline FVC Pre transplant / Post & $96.05( \pm 16.72) /$ & $95.88( \pm 16.69)$ & \\
transplant & $91.77( \pm 19.06)$ & $/ 91.82( \pm 19.01)$ & \\
FVC change & $-4.25( \pm 12.79)$ & $-3.64( \pm 13.70)$ & 0.05 \\
FEV1 Pre transplant / & $99.99( \pm 17.83)$ & $99.76( \pm 18.00) /$ & \\
Post transplant & $195.91( \pm 18.77)$ & $96.30( \pm 18.77)$ & \\
FEV1 change & $-4.06( \pm 12.51)$ & $-3.43( \pm 13.47)$ & 0.001 \\
DLCO Pre transplant / & $84.41( \pm 18.10) /$ & $84.52( \pm 17.93) /$ & \\
Post transplant & $84.94( \pm 20.28)$ & $84.88( \pm 20.23)$ & \\
DLCO change & $-1.32( \pm 20.62)$ & $-1.44( \pm 20.46)$ & 0.078 \\
TLC Pre transplant / Post & $94.70( \pm 15.87) /$ & $94.82( \pm 15.79) /$ & \\
transplant & $92.67( \pm 17.93)$ & $92.83( \pm 17.88)$ & \\
TLC change & $2.68( \pm 13.99)$ & $2.64( \pm 13.88)$ & 0.114 \\
\hline
\end{tabular}

[[P258 Table] PFT changes post APSCT]

\section{P259}

incidence and prognosis of the hepatic sinusoidal obstruction syndrome following haematopoietic stem cell transplantation (SCT). single centre experience

Maria-Jose Jimenez, Mireia Morgades, Anna Torrent, Blanca Xicoy, Montserrat Batlle, Susana Vives, Miriam Moreno, Juan-Manuel Sancho, Gladys Ibarra, Laura Abril, Albert Oriol, Edurne Sarrate, Anna Triguero, Josep-Maria Ribera, Christelle Ferra

Hospital Germans Trias i Pujol, Institut Català d'Oncologia, Institut de Recerca contra la Leucèmia Josep Carreras, Clinical Hematology Department, Badalona, Spain

Background: The hepatic sinusoidal obstruction syndrome (HSOS) generally shows up during the first 20 days after SCT and remains associated with high morbidity and mortality. Clinical symptoms include weight gain, hyperbilirubinemia, painful hepatomegaly and ascites/fluid retention. Main risk factors are: previous SCT, iron overload and treatment with gemtuzumab or inotuzumab ozogamizin. The objective of this study was to evaluate the incidence, treatment and outcome of patients (pts) with HSOS after SCT in a single centre.

Methods: From January 2000 to September 2017, 877 SCT were performed in 810 pts. HSOS severity was graded following the new EBMT criteria. (Mohty M, et al. BMT 2016).

Results: Thirty-three pts (3.8\%) developed HSOS. Characteristics of SCT pts are described on Table 1. Twenty-two out of $31(71 \%)$ pts had at least one risk factor and $15 / 31(48 \%)$ pts with $\geq 2$ risk factors. Prophylactic 
strategy was used in $7 / 30$ pts (23\%) (low weight heparin in 4 and ursodeoxycholic acid in 3). The global cumulated incidence of SOSH on day +30 was $3.4 \%$ (95CI\%, $2.4 \%$ $4.8 \%$ ), been significantly higher for cord SCT that peripheral blood SCT $14.3 \%$ (95CI\%,5.7\%-26.6\%) vs $3 \%$ (95CI\%,2\%-4.3\%), $\mathrm{p}<0.001$. Median time from SCT for HSOS was 9.5 days $[0 ; 38]$ and $26 / 30$ pts $(87 \%)$ showed early onset (before day +21 ). Twenty-one out of 31 pts met the criteria for severe/very severe SOSH. Liver biopsy confirmed the diagnosis in 6/12 pts (50\%), and hepatic venous pressure gradient was $>10 \mathrm{~mm} \mathrm{Hg}$ in $5 / 11$ pts (45\%). Nine out of 31 evaluable pts developed a multiorgan failure. Twenty pts were treated with defibrotide (DFT) $(69 \%)$ and $9(31 \%)$ with supportive treatment (ST). Patients with severe/very severe HSOS received more frequently DFT (16 [80\%]) than ST (4 pts [20\%]). Ten out of 16 pts treated with DFT (62\%) and 6 out of $9(67 \%)$ receiving ST recovered. Sixteen among the 25 allo-SCT pts (64\%) developed acute GVHD (grade IV liver GVHD in 4 pts [25\%]). Only $3 / 33$ pts (9\%) are long-term survivors. Causes of death were: HSOS (13/30; 43\%), GVHD (7/30; $23 \%)$, relapse $(4 / 30 ; 13 \%)$, infection $(4 / 30 ; 13 \%)$, graftfailure $(1 / 30 ; 4 \%)$ and second neoplasm $(1 / 30 ; 4 \%)$. Median OS has been 1.81 months $(95 \% \mathrm{CI}: 0.04 ; 3.59)$. In this study, neither the number of risk factors nor the severity of HSOS significantly influenced OS.

Conclusions: The HSOS incidence in this study was similar to that referred. Even if HSOS recovered in 50\% of pts, their survival remained very low.

Clinical Trial Registry: Not apply

Conflict of interest: Founding: PI10/01417 (Fondo de Investigaciones Sanitarias). RD12/0036/0029 (RTICC, Instituto Carlos III, 2014 SGR225GRE]), CERCA Program, Generalitat de Catalunya, España, "La Caixa" Foundation, RD12/0036/0029 RTICC-Instituto Carlos IIIThe authors declare no conflict of interest.

\begin{tabular}{lll}
\hline Age at SCT (yrs) (Median [range]) & $47[15 ; 65]$ \\
\hline Gender:Male & & $23(70 \%)$ \\
Hematological disease & - Acute leukaemia / MDS & $18(55 \%)$ \\
& - Lymphoproliferative disease & $8(24 \%) / 3$ \\
& / Multiple myeloma & $(9 \%)$ \\
& - Other & $4(12 \%)$ \\
Disease status at SCT & CR / PR & $15(46 \%) /$ \\
& & $6(18 \%)$ \\
Autologous /Allogeneic & Active disease & $12(36 \%)$ \\
Conditioning: Myeloablative & & $8(24 \%) /$ \\
/Non-myeloablative & & $25(76 \%)$ \\
Stem cell source: Peripheral & & $18(72 \%) /$ \\
blood/Umbilical cord blood & & $7(28 \%)$ \\
\hline
\end{tabular}

[[P259 Table] Characteristics of patients $(N=33)]$
P260

Incidence, pre-transplant risk factors and outcomes of severe poor graft function after allogeneic HSCT in adult patients

Tatiana Rudakova, Alexander Kulagin, Olesya Klimova, Tatiana Bykova, Ivan Moiseev, Sergey Bondarenko, Elena Morozova, Elena Darskaya, Anna Smirnova, Mariya Estrina, Ildar Barkhatov, Elena Babenko, Alexander Alyanskiy, Boris Afanasyev

R.M. Gorbacheva Memorial Institute of Hematology, Oncology and Transplantation, Pavlov First Saint Petersburg State Medical University, St. Petersburg, Russian Federation

Background: Severe poor graft function (sPGF) remains a serious multifactorial complication after allo-HSCT. Although incidence and various pre- and posttransplant risk factors for SPGF have been reported, they are still controversial and insufficiently defined. The aim of this study was to investigate incidence and baseline pretransplant risk factors of non-GVHD associated sPGF.

Methods: We performed retrospective and prospective analysis of graft function in a large cohort of adult patients after allo-HSCT between 2008 and 2016. The criteria of SPGF were: 1) $2 / 3$ peripheral blood cytopenia: ANC < $0,5 \times 10^{9} / \mathrm{L}, \mathrm{PLT}<20 \times 10^{9} / \mathrm{L}$, or $\mathrm{Hb}<70 \mathrm{~g} / \mathrm{L}$ any time after sustained engraftment; 2) full or stable mixed donor chimerism $>90 \%$; 3) absence of underlying disease relapse, graft rejection and grade III-IV aGVHD. Factors analyzed included age, gender, diagnosis, ferritin level, donor type (MRD, MUD, haplo), sex- and blood group matching, donor's pregnancy, source of graft (BM, PB), numbers of infused $\mathrm{MNC}$ and $\mathrm{CD} 34^{+}$cells, conditioning regimen (MAC, RIC) and GVHD prophylaxis (conventional, PTCybased). Cumulative incidence of sPGF was calculated considering death, relapse, rejection and severe aGVHD as competing events. Independent risk factors were analyzed in multivariate Fine-Gray proportional hazard

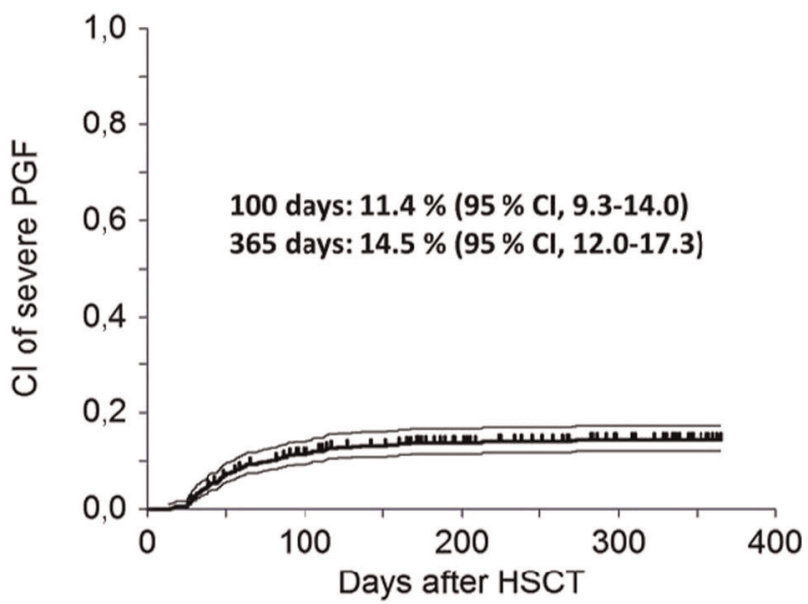

[P260 Figure] [Cumulative incidence of SPGF after allogeneic HSCT] 
regression model for competing events.

Results: The study included 698 adult patients (median age 31 years, range 18-70, M/F 388 / 310) who had successfully engrafted after allo-HSCT for different indications (AML 314, ALL 179, CML 57, HL 38, NHL 18, CLL 13, MDS 35, PMF 13, MDS/MPN 7, SAA 24). Median follow-up after HSCT was 410 days (16-2926). A total of $101(14.5 \%)$ patients developed sPGF with a cumulative incidence of $11.4 \%$ (95\% CI, 9-14) and $14.5 \%$ (95\% CI, 1217) on 100 and 365 days respectively (Fig.1).

Median time between HSCT and onset of sPGF was 50 (13-641) days. Univariate analysis of diagnosis and status at HSCT identified 3 distinct risk groups of sPGF: low (SAA, acute leukemia in CR, CML, lymphomas), intermediate (acute leukemia in non-CR status) and high (MDS, MDS/ MPN, PMF) with the 1-year cumulative incidence of $11 \%$, $20 \%$ and $35 \%$ respectively $(\mathrm{p}=0.00001)$. Another potential risk factors in univariate analysis were donor type (13\% for MRD and MUD vs $42 \%$ for haplo, $\mathrm{p}=0.00009)$ and pretransplant ferritin level $>75$ th percentile $(1250 \mathrm{ng} / \mathrm{mL})$ ( $12 \%$ vs $26 \%, p=0.009)$. Multivariate Fine-Gray proportional hazard regression model confirmed the significance of diagnosis and CR status risk group (HR 1.73, 95\% CI 1.25$2.41, \mathrm{p}=0.0011)$ and pre-transplant ferritin level $>1250$ $\mathrm{ng} / \mathrm{mL}$ (HR 2.22, 95\% CI 1.30-3.79, $\mathrm{p}=0.0037$ ). Fifty (50\%) patients restored good graft function, including those after HSC boost with median SPGF duration of 39 days (7147). The remaining 51 patients had unfavorable outcomes of sPGF, including death in 27 (27\%), relapse in $19(19 \%)$ at a median of 75 days and graft rejection in $5(5 \%)$ at a median of 52 days after sPGF onset.

Conclusions: These data substantially supplemented the current understanding of the incidence, risk factors and outcomes of sPGF after allo-HSCT. Early detection and stratification may contribute to the improvement of sPGF outcomes.

Conflict of interest: Nothing to disclose.

\section{P261}

\section{Intensive care in allogeneic hematopoietic stem cell recipients - Reasons for admission, short- and long-term outcome in a transplant cohort of 942 patients}

Catherina Lueck ${ }^{1,2}$, Michael Stadler ${ }^{1}$, Christian Koenecke ${ }^{1}$, Marius M. Hoeper ${ }^{3}$, Elke Dammann ${ }^{1}$, Andrea S. Schneider ${ }^{4}$, Jan T. Kielstein $^{5,6}$, Arnold Ganser ${ }^{1}$, Matthias Eder ${ }^{1}$, Gernot Beutel ${ }^{1,2}$

${ }^{1}$ Hannover Medical School, Department for Hematology, Hemostasis, Oncology and Stem Cell Transplantation, Hannover, Germany;

${ }^{2}$ Intensive Care in Hematologic and Oncologic Patients, (iCHOP), Germany; ${ }^{3}$ Hannover Medical School, Department for Respiratory Diseases and German Centre of Lung Research (DZL), Hannover, Germany; ${ }^{4}$ Hannover Medical School, Department for Gastroenterology, Hepatology, and Endocrinology, Hannover,
Germany; ${ }^{5}$ Hannover Medical School, Department for Nephrology and Hypertension, Hannover, Germany; ${ }^{6}$ Academic Teaching Hospital Braunschweig, Medical Clinic V; Nephrology, Rheumatology and Blood Purification, Braunschweig, Germany

Background: Intensive care unit (ICU) admission of allogeneic hematopoietic stem cell transplant (HSCT) recipients is associated with relative poor outcome. We analyzed reasons for ICU admission as well as short- and long-term outcome of critically ill HSCT recipients.

Methods: From 942 consecutive adult patients transplanted at the Hannover Medical School from 2000 to 2013, 330 patients were at least once admitted to the ICU and included in this retrospective study.

Results: Main reasons for ICU admission were acute respiratory failure $(\mathrm{ARF})$ in $32 \%(\mathrm{n}=105)$, severe sepsis/ septic shock in $21 \%(\mathrm{n}=70)$, and cardiac problems in $16 \%$ $(\mathrm{n}=53)$. Other reasons like acute renal failure, bleeding, and impaired consciousness occurred in $13 \%(\mathrm{n}=42)$. Admission after emergency surgery encountered in $6 \%$ (n $=20$ ) and Graft-vs-Host-Disease (GvHD) let to ICU admission in $3 \%(\mathrm{n}=10)$. Patients admitted to ICU for monitoring only were excluded from further analysis $(\mathrm{n}=$ 30). Median time between HSCT and ICU transfer was 77 days and depended on the reason of admission. Patients with cardiac events, respiratory failure, severe sepsis or GvHD required intensive care in median 21, 69, 73, and 93 days after HSCT (all n.s.), respectively. In contrast, patients with emergency surgical procedures were admitted after 341 days $(\mathrm{p}<0.01)$. During ICU stay, ARF occurred in $77 \%$ of the patients $(\mathrm{n}=232)$. In half of these patients $(50 \%, \mathrm{n}=116)$ ARF was due to extra-pulmonary reasons (e.g. cardiogenic pulmonary edema, extrapulmonary sepsis resulting in ARDS). Non-invasive ventilation (NIV) was used as primary approach in $39 \%$ of the patients $(n=91)$, with NIV failure requiring secondary intubation in $60 \%$ (n $=55$ ) of those. Invasive mechanical ventilation was used in $65 \%$ of the patients with ARF $(\mathrm{n}=154)$ with a median ventilation time of 4 (2-12) days. Cardiac events occurred in $47 \%$ of the patients $(n=139)$. Treatment with vasopressors

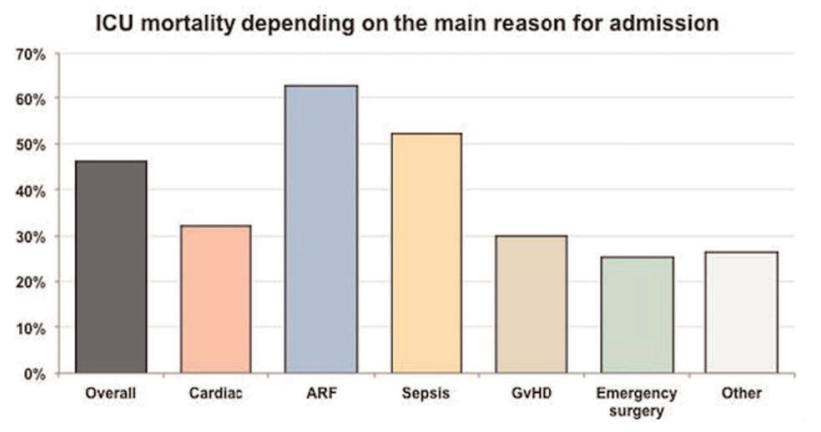

[P261 Figure] [ICU mortality depending on the main reason for admission] 
was necessary in $63 \%$ of the patients $(n=190)$, mainly due to septic shock, occurring in $47 \%(n=142) .75 \%(n=223)$ of the patients suffered from acute kidney injury and half of those required renal replacement therapy.

ICU-, hospital- and 1-year-survival after ICU admission were $54 \%, 36 \%$, and $25 \%$ respectively. ICU mortality rates depending on the main reason for admission are shown in Figure 1. A cox proportional hazard ratio model was used to predict changes and likelihood of ICU mortality in a timedependent manner. While ICU mortality was 13.5-fold higher in the early period after transplantation, a significant decrease was found in patients later than day 100 with a likelihood of mortality only 2.2-fold higher compared to non-ICU patients $(\mathrm{p}<0.05)$. Remarkably, the postdischarge survival of ICU patients was comparable with survival of non-ICU patients at 10 years (44\% vs. 51\%, ns).

Conclusions: Besides ARF and septic shock, cardiac events were the main reason for ICU admission. Short-term survival of critically ill HSCT patients requiring organ support systems still remains poor. The likelihood of mortality decreases continuously from day of transplantation until a plateau is reached after half a year. Long-term survival of HSCT recipients discharged from hospital is not significantly affected by a former ICU stay.

\section{Conflict of interest: None}

\section{P262}

\section{Investigating tolerance and clinical outcomes of early enteral nutrition versus parenteral nutrition support during allogeneic transplantation}

\author{
Sarah Andersen ${ }^{1,2}$, Nicholas Weber ${ }^{1}$, Merrilyn Banks ${ }^{1}$, Teresa \\ Brown $^{1}$, Glen Kennedy ${ }^{1}$, Judy Bauer ${ }^{2}$ \\ ${ }^{1}$ Royal Brisbane and Women's Hospital, Brisbane, Australia; \\ ${ }^{2}$ University of Queensland, Brisbane, Australia
}

Background: Nutrition support during allogeneic haematopoietic stem cell transplantation is an important component of care to prevent malnutrition however there is no consensus on the optimal method of supplementary feeding. Non-randomised studies have indicated that enteral nutrition (EN) may improve patient outcomes when compared to parenteral nutrition (PN) but the level of tolerance of nasogastric feeding is unclear. This randomised study aimed to determine the tolerability of proactive early $\mathrm{EN}$ in comparison to PN (standard care).

Methods: Patients undergoing allogeneic transplantation with reduced intensity (fludarabine/ melphalan) or myeloablative (cyclophosphamide/ TBI) conditioning were eligible to participate. Patients randomized to $\mathrm{EN}$ had a nasogastric tube inserted on day +1 after stem cell infusion and feeding commenced at $30 \mathrm{ml} / \mathrm{hr}$. As oral intake declined the rate of feeding was increased to goal. If patients were intolerant of EN they were converted to PN. The standard care group commenced PN as per unit protocol. The primary endpoint was tolerance in the EN group: the percentage of patients randomized to EN who must discontinue this method and change to PN, or who require 'top-up' PN. Data was also collected on clinical outcomes and assessed using CTCAE version 4 criteria.

Results: Forty four patients (mean age 48 \pm 14.4; 25 male, 19 female) consented to participate and were randomised to $\mathrm{EN}(\mathrm{n}=22)$ or standard care $(\mathrm{n}=22)$. In the EN group eleven tolerated EN (55\%), nine changed to PN and two withdrew from study. The reasons for changing to PN were: gastrointestinal intolerance $(n=3)$, tube dislodgement and patient declining reinsertion $(\mathrm{n}=3)$, tube dislodgement and severe mucositis preventing reinsertion $(n=2)$ and development of an ileus $(n=1)$. No patients required top up PN. Data on complication rates was available for all 15 patients that required $\mathrm{PN}$ in the standard care group and 19 patients in the EN group. There were no statistically significant differences between groups for any clinical outcomes or grade 3-4 complications including elevated liver enzymes $(\mathrm{EN} \mathrm{n}=9, \mathrm{PN} \mathrm{n}=7, \mathrm{p}=1.00)$, hyperglycaemia $(\mathrm{EN} \mathrm{n}=4$, $\mathrm{PN} \mathrm{n}=3, \mathrm{p}=1.00)$, hypertriglyceridaemia $(\mathrm{EN} \mathrm{n}=4, \mathrm{PN}$ $\mathrm{n}=3, \mathrm{p}=1.00)$, mucositis $(\mathrm{EN} \mathrm{n}=9, \mathrm{PN} \mathrm{n}=9, \mathrm{p}=0.46)$, catheter related infections $(\mathrm{EN} \mathrm{n}=2, \mathrm{PN} \mathrm{n}=1, \mathrm{p}=1.00)$, platelet engraftment by Day $100(\mathrm{EN} \mathrm{n}=17, \mathrm{PN} \mathrm{n}=18, \mathrm{p}$ $=1.00)$, time to neutrophil engraftment ( $\mathrm{EN}$ mean of $13.5 \pm$ 4.0 days, $\mathrm{PN} 13.1 \pm 3.6$ days, $\mathrm{p}=0.73$ ) or length of hospital stay (EN median of 28 days, PN 20 days, $\mathrm{p}=0.19$ ). GVHD incidence at Day +100 for the EN group was $50 \%(n=10)$, and $\mathrm{PN} 59 \%(\mathrm{n}=13 ; \mathrm{p}=0.55)$ and incidence of grade $3-5$ GVHD was $15 \%$ in the EN group $(n=3)$ and $22 \%$ in the PN group $(n=5 ; p=0.70)$. Day +100 survival was EN $n$ $=18(90 \%)$, and PN $\mathrm{n}=19(86 \%)(\mathrm{p}=1.00)$.

Conclusions: Half of patients receiving allogeneic transplantation tolerate EN when commenced early postconditioning. Based on relatively small numbers, there is no apparent difference in clinical outcomes between EN and $\mathrm{PN}$. As the use of early EN will reduce the use of PN (and associated costs and risks), it should be considered as first line nutritional support

Clinical Trial Registry: This trial was registered on ANZCTR http://www.anzctr.org.au. Reference number ACTRN12615000284561

\section{Conflict of interest:}

Sarah Andersen: No conflict of interest, funding received from the Royal Brisbane and Women's Hospital Post Graduate Research Scholarship. The remaining authors have nothing to disclose. 


\section{P263}

Is it feasible to conduct a randomised controlled trial of pre-habilitation in multiple myeloma (MM) patients awaiting autologous haematopoietic stem-cell transplantation (AHSCT)? The PREeMPT study

John Snowden ${ }^{1,2}$, Carol Keen ${ }^{3}$, Lauren Smith ${ }^{3}$,Julie Skilbeck ${ }^{4}$, Helen Ross ${ }^{3}$, Karen Collins ${ }^{5}$, Susan Simpson ${ }^{1}$, Joanne Dixey ${ }^{3}$, Stephen Walters ${ }^{6}$, Diana Greenfield ${ }^{1,2}$, Susan Mawson ${ }^{6,7}$

${ }^{1}$ Sheffield Teaching Hospitals NHS Foundation Trust, Haematology, Sheffield, United Kingdom; ${ }^{2}$ University of Sheffield, Oncology \& Metabolism, Sheffield, United Kingdom; ${ }^{3}$ Sheffield Teaching Hospitals NHS Foundation Trust, Physiotherapy, Sheffield, United Kingdom;

${ }^{4}$ Sheffield Hallam University, Nursing and Midwifery, Sheffield, United Kingdom; ${ }^{5}$ Sheffield Hallam University, Health and Social Care Research, Sheffield, United Kingdom; ${ }^{6}$ University of Sheffield, School of Health and Related Research (ScHARR), Sheffield, United Kingdom; ${ }^{7}$ Sheffield Teaching Hospitals NHS Foundation Trust, Collaborations for Leadership in Applied Health Research \& Care (CLAHRC), Sheffield, United Kingdom

Background: AHSCT is an intensive procedure used routinely as consolidation after induction/re-induction chemotherapy at first and second lines of treatment in most MM patients under 70 years and selected older patients. Recovery of quality of life (QoL) post-AHSCT takes an average of 6 months. Only 20\% of MM patients meet national physical activity guidelines post-treatment, with a decline in activity through treatment due to perceived barriers to exercise including pain, fear of injury and fatigue. Exercise rehabilitation programmes have been safely delivered to patients recovering from AHSCT with improvements in physical performance, muscle strength, aerobic capacity, psychological outcomes immunological function and fatigue.

MM patients have a reduced exercise capacity pretransplant and may benefit from 'pre-habilitation', defined as "a process on the continuum of care that occurs between the time of cancer diagnosis and the beginning of acute treatment ... provides targeted interventions that improve a patient's health to reduce the incidence and the severity of current and future impairments". Pre-habilitation has been shown to be effective in other diseases, but to date there has been no research into pre-habilitation in MM patients in the run-up to AHSCT.

Methods: The objectives of this mixed methods study were to determine whether it is feasible to conduct a randomised controlled trial (RCT) of pre-transplant exercise for MM patients awaiting AHSCT. The study (summarised in figure 1) was funded by UK NIHR, with ethics committee approval and fully informed consent.

From referrals to our central HSCT service covering approximately 2 million, we identified patients with MM who had commenced induction/re-induction chemotherapy

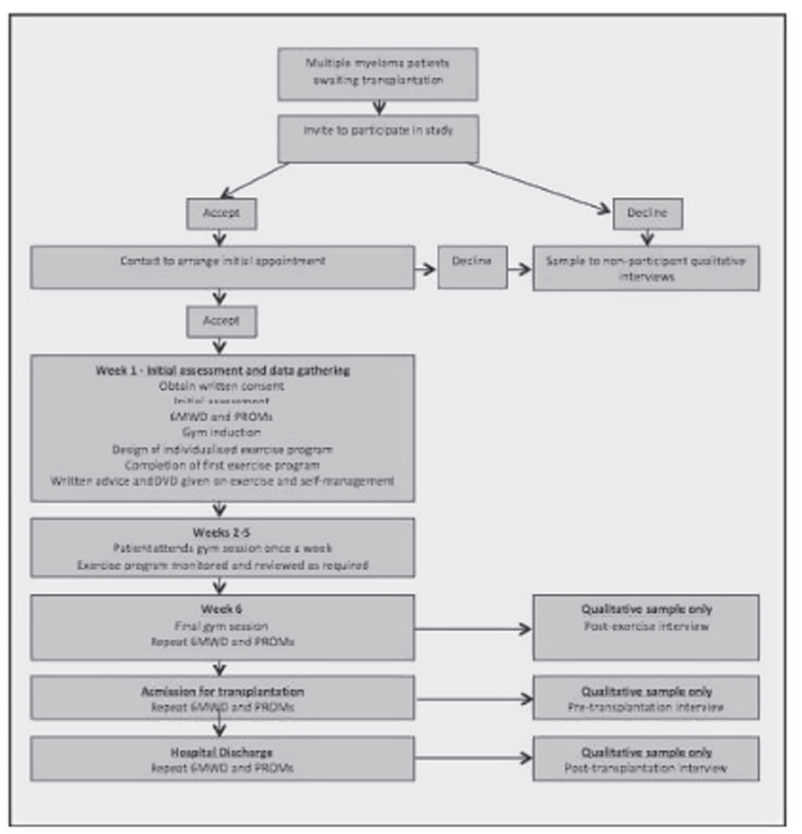

[P263 Figure] [Recruitment and Intervention Flow Chart]

and were appropriate candidates for AHSCT. Patients were invited to participate in 6 weekly sessions of individualised, supervised exercise whilst awaiting AHSCT. The location of the exercise programme was the physiotherapy department in our hospital, co-located with HSCT services.

Quantitative data were obtained to determine feasibility targets include rates of recruitment, adherence and adverse events, and outcome measures including 6-minute walking distance (6MWD) and QoL.

Qualitative interviews were undertaken with a purposive sample of patient to capture their experiences of the study and the intervention.

Results: Over the 12-month study recruitment period, 96 patients were screened with a recruitment target of 24 . Twenty-three $(16 \mathrm{M} / 7 \mathrm{~F}$, average age 64.7 , range 53-78) were recruited to the exercise programme. Of these 14 (61\%) completed the programme. Main reasons for nonparticipation were distance and travel time, managing other commitments and ill-health. The main reason for noncompletion of the programme was commencement of AHSCT.

Conclusions: Pre-habilitation delivered by 6 weekly sessions of individualised, supervised exercise is a potential intervention for MM patients whilst awaiting AHSCT. However, there are significant hurdles combining prehabilitation with ongoing induction/re-induction treatment and only a minority of patients completed the programme. The main themes for non-participation were travel and time commitments during a phase when patients have other hospital appointments and ongoing chemotherapy. Timing 
is important as completion may be compromised by starting AHSCT. Before an RCT can be designed, further evaluation of delivery of pre-habilitation in MM patients pre-AHSCT is warranted, including de-centralised or App based prehabilitation strategies.

Conflict of interest: No authors have conflicts of interest to declare.

\section{P264}

\section{Is post-transplant albumin a predictor of early transplant complications?}

Pervin Topcuoglu ${ }^{1}$, Guldane Cengiz Seval ${ }^{1}$, Erman Akkus ${ }^{2}$, Sinem Civriz Bozdag ${ }^{1}$, Selami Kocak Toprak ${ }^{1}$, Meltem Kurt Yuksel ${ }^{1}$, Onder Arslan $^{1}$, Muhit Ozcan ${ }^{1}$, Taner Demirer ${ }^{1}$, Hamdi Akan ${ }^{1}$, Meral Beksac $^{1}$, Gunhan Gurman ${ }^{1}$

${ }^{I}$ Ankara University School of Medicine, Department of Hematology and Stem Cell Transplant Unit, Ankara, Turkey; ${ }^{2}$ Ankara University School of Medicine, Department of Internal Medicine, Ankara, Turkey

Background: Allogeneic hematopoietic stem cell transplantation is increasing being used for treatment of a wide range of benign and malign hematological disorders. However, prognostic biomarkers in allo-HSCT are needed to improve risk assessment and help guide therapeutic and surveillance strategies to alleviate the risk of death from the procedure. The prognostic value of hypoalbuminemia and high levels of $\mathrm{C}$ reactive protein (CRP) as a determinant for post-transplantation outcomes remains unclear. In this study we aimed to investigate the association between earlier time point serum albumin, CRP levels and acute GVHD, day +100 non-relapse mortality among patients with hematological malignancies who underwent allo-HSCT.

Methods: This analysis included 343 consecutive patients who underwent allo-HCT for various hematological malignancies and bone marrow failure syndrome at Ankara University School of Medicine, Bone Marrow Transplantation Unit between January 2010 and June 2017. We used

\begin{tabular}{|c|c|c|c|c|}
\hline sartables & $\begin{array}{l}\text { Day+100 NRM } \\
\text { HR }(95 \% \mathrm{CI})\end{array}$ & D-value & $\begin{array}{l}\text { Acute GVHD } \\
\text { HR }(95 \% \mathrm{Cl})\end{array}$ & sivalue \\
\hline $\begin{array}{l}\text { Day 0 senum albumin (g/d1) } \\
<3 \\
3 \cdot 3.5 \\
>3.5\end{array}$ & $\begin{array}{l}0.43(0.05-3.04) \\
0.46(0.10-2.81)\end{array}$ & $\begin{array}{l}0.39 \\
0.40\end{array}$ & $\begin{array}{l}i .70(0.67-4.3) \\
1.67(0.76-3.67)\end{array}$ & $\begin{array}{l}0.26 \\
0.2\end{array}$ \\
\hline $\begin{array}{l}\text { Day +30 senum albumin (g/dl) } \\
<3 \\
3-3.5 \\
>3.5\end{array}$ & $\begin{array}{l}7.16(1.57 \cdot 32.75) \\
1.82(0.39-8.51)\end{array}$ & $\begin{array}{l}0.01 \\
0.44\end{array}$ & $\begin{array}{l}2.35(1.09-5.01) \\
1.08(0.53-2.21)\end{array}$ & $\begin{array}{l}0.03 \\
0.83\end{array}$ \\
\hline $\begin{array}{l}\text { Baseline serum CRP }(\mathrm{mg} / \mathrm{L}) \\
<5 \\
5 \cdot 20 \\
>20\end{array}$ & $\begin{array}{l}1.05(0.32-3.50) \\
0.65(0.13 \cdot 3.20)\end{array}$ & $\begin{array}{l}0.94 \\
0.60\end{array}$ & $\begin{array}{l}0.89(0.44-1.78) \\
103(0.46-2.29)\end{array}$ & $\begin{array}{l}0.76 \\
0.94\end{array}$ \\
\hline $\begin{array}{l}\text { Day }+30 \text { sevum CRP }(\mathrm{mg} / \mathrm{L}) \\
<5 \\
\mathrm{~s} \cdot 20 \\
>20\end{array}$ & $\begin{array}{l}0.77(0.23-2.61) \\
0.77(0.12-4.84)\end{array}$ & $\begin{array}{l}0.68 \\
0.78\end{array}$ & $\begin{array}{l}1.23(0.62-2.44) \\
078(0.35-1.74)\end{array}$ & $\begin{array}{l}0.56 \\
0.55\end{array}$ \\
\hline
\end{tabular}

[P264 Figure] [Table 2: Multivariate Logistic Regression Analyses of early time point NRM and occurrence of aGVHD (A] our institutional database to evaluate details and characteristics of patients and treatment outcomes. Serum albumin and CRP levels at the day of infusion and posttransplant day +30 were obtained from patients' files. Demographic features of the patients and donors have been summarized in Table1.

Results: A total of 343 consecutive patients (female/ male: $132 / 211$ ) received an allo-HSCT at our institution for the covering January 2010 and June 2017. The median age of the patients at time of transplantation was 39 years (range, 17-71 years). The five most common diagnosis were acute myeloid leukemia (AML), acute lymphoblastic leukemia (ALL), non-Hodgkin lymphoma (NHL), myelodisplastic syndrome (MDS) and aplastic anemia (AA) (Table 1). Donor sources were generally matched related donor $(58 \%)$. Most patients received a myeloablative regimen $(\mathrm{n}=262,76 \%)$ and cyclosporine-A based acute GVHD prophylaxis $(n=293,85 \%)$. The median serum albumin and CRP levels were $3.2 \mathrm{~g} / \mathrm{dL}$ (range, 1.7-4.6 g/ $\mathrm{dL}$ ), $3.5 \mathrm{~g} / \mathrm{dL}$ (range, 1.8-4.1 g/dL) and $7.5 \mathrm{mg} / \mathrm{L}$ (range, 0.2-50.0 mg/L), $8.8 \mathrm{mg} / \mathrm{L}$ (range, $0.1-316.3 \mathrm{mg} / \mathrm{L}$ ) at stem cell infusion day and day +30 . We observed over a two-fold higher proportion of the occurrence of acute GVHD and day +100 non-relapsed deaths in the subgroups of patients with hypoalbuminemia $(<3 \mathrm{~g} / \mathrm{dL}$ and $3.0-3.5 \mathrm{~g} / \mathrm{dL})$ at day +30 . There is no relationship between CRP subgroups and the presence of acute GVHD and day +100 non-relapsed mortality.

Conclusions: Measurement of serum albumin is routinely performed, cheap, standardized and with readily available results, making it an attractive biomarker. Our data are limited by the retrospective nature of our study and therefore need to be interpreted with caution. Larger and prospective studies are warranted.

Conflict of interest: All authors: nothing to disclose

\begin{tabular}{ll}
\hline Variable & Frequency (n, \%) \\
\hline Median age (range), years & $39(17-71)$ \\
Gender (male/female) & $211(62 \%) / 132(38 \%)$ \\
Donor $\Rightarrow$ patient gender matching F $\Rightarrow \mathrm{M}$ & $73(21.3 \%) 61(17.8 \%) 133(38.8 \%)$ \\
$\mathrm{F} \Rightarrow \mathrm{F} \mathrm{M} \Rightarrow \mathrm{M} \mathrm{M} \Rightarrow \mathrm{F}$ & $76(22.2 \%)$ \\
Donor source MRD MUD UCB mMRD & $185(53.9 \%) 47(13.7 \%) 6(1.7 \%) 22$ \\
mMUD & $(6.4 \%) 83(24.2 \%)$ \\
Cell source PBSC BM UCB PBSC and & $280(81.6 \%) 45(13.1 \%) 6(1.7 \%) 12$ \\
BM & $(3.5 \%)$ \\
Conditioning regimen MAC RIC/NMA & $263(77 \%) 80(23 \%)$ \\
Diagnosis AML ALL MDS NHL AA & 138632819256519535 \\
Idiopathic MF CLL CML MM Others & \\
Median albumin level (g/dL) Day 0 Day & $3.2 \mathrm{~g} / \mathrm{dL}($ range, $1.7-4.6 \mathrm{~g} / \mathrm{dL}) 3.5 \mathrm{~g} /$ \\
30 & $\mathrm{dL}(\mathrm{range}, 1.8-4.1 \mathrm{~g} / \mathrm{dL})$ \\
Serum C-RP level Day 0 Day 30 & $7.5 \mathrm{mg} / \mathrm{L}(\mathrm{range}, 0.2-50.0 \mathrm{mg} / \mathrm{L}) 8.8$ \\
& mg/L (range, 0.1-316.3 mg/L) \\
\hline
\end{tabular}


[[P264 Table] Demographic features of the patients and donors]

\section{P265}

\section{Karnofsky Performance Score and Pre-Transplant Serum Ferritin Level Predict Readmission after Autologous Hematopoietic Stem Cell Transplantation (HCT)}

Vida Jahangiri, Hira Shaikh, Salman Fazal, Santhosh Sadashiv, Cyrus Khan, Anna Koget, Prerna Mewawalla, John Lister

Division of Hematology and Cellular Therapy, Allegheny Health Network Cancer Institute, Pittsburgh, PA, United States

Background: The Hematopoietic Stem Cell Transplantation Comorbidity Index (HCTCI) is predictive of outcome in allogeneic hematopoietic stem cell transplantation. The index correlates with non-relapse mortality (NRM) and 100day readmission rate. The correlation of HCTCI or other variables with complications after autologous transplantation is uncertain.

Methods: We retrospectively analyzed 231 autologous transplant episodes (217 individual recipients) performed at our institution between January 2010 and December 2016. All recipients were discharged to intensive outpatient daily follow-up before re-admission. HCTCI, Karnofsky Performance Score (KPS), Ferritin and Pre-albumin levels, disease and transplant data elements according to CIBMTR criteria were analyzed by univariate methods. We tested these variables for correlation with the rate of readmission within the first 100 days after transplant.

Results: The median age of the recipients $(\mathrm{n}=217)$ was 60 years (range $24-77$ ). The indication for transplant was plasma cell disorder $(n=151)$, lymphoma $(n=65)$, leukemia $(\mathrm{n}=8)$ and testicular cancer $(\mathrm{n}=7)$. Conditioning regimens included Melphalan $(\mathrm{n}=148)$, FluBuTBI $(\mathrm{n}=59)$, BEAM $(\mathrm{n}=10)$, Carboplatin+Etoposide $(\mathrm{n}=7)$ and others $(\mathrm{n}=7)$. The 100-day readmission rate was $73 \%(\mathrm{n}=168)$. The most common indication for readmission was severe mucositis followed by infection and neutropenic fever $(31 \%, 21 \%$ and $19 \%$, respectively). HCTCI and pre-albumin were not predictive of readmission $(\mathrm{p}=0.97, \mathrm{p}=0.81$, respectively). A KPS of 90 or greater predicted a lower readmission rate $(\mathrm{p}=0.019)$. Pre-transplant ferritin level $>500 \mathrm{ng} / \mathrm{mL}$ increased the likelihood of readmission $(\mathrm{p}=0.046)$.

Conclusions: There was no correlation of HCTCI and pre-albumin serum level with the re-admission rate. A KPS of 80 or less and a pre-transplant ferritin level greater than $500 \mathrm{ng} / \mathrm{mL}$ statistically significantly predicted a higher rate of readmission after autologous HCT. A statistically robust model predictive of re-admission would have clinical utility. We intend to construct a multivariate model to predict readmission and will include ferritin and KPS as co-variates.
Conflict of interest: None of the authors has anything to disclose.

\section{P266}

\section{Late effects after treosulfan-based conditioning for allogeneic HSCT in children with hematologic malignancies: a single-center experience}

Yulia Skvortsova ${ }^{1}$, Irina Shipitsina ${ }^{1}$, Dmitrij Balashov ${ }^{1}$, Pavel Trakhtman $^{1}$, Oksana Blagonravova ${ }^{2}$, Marina Persiantseva ${ }^{1}$, Elena Skorobogatova ${ }^{2}$, Lyudmila Papusha ${ }^{1}$,Elena Gutovskaya ${ }^{1}$, Larisa Shelikhova $^{1}$, Elena Kurnikova ${ }^{1}$, Kirill Voronin ${ }^{1}$, Michail Maschan ${ }^{1}$, Alexej Maschan ${ }^{1}$

${ }^{I}$ Dmitry Rogachev National Center of Pediatric Hematology, Oncology and Immunology, Moscow, Russian Federation; ${ }^{2}$ Russian Children's Hospital, Moscow, Russian Federation

Background: Treosulfan-based regimens used in conditioning for allogeneic HSCT (allo-HSCT) in children allow to reduce incidence of transplant-related mortality and early toxicity, but there are no data about long-term effects.

Methods: We propose a retrospective study of late complications after allo-HSCT in children with hematologic malignancies, who got allo-HSCT at BMT Department of Russian Children's Hospital/Dmitry Rogachev National Research Center of Pediatric Hematology, Oncology and Immunology (Moscow, Russia) between 2004 and 2011 and survived at least 1 year after HSCT.

Results: Thirty patients with malignant diseases (AML 21 [high risk and refractory - 10], JMML - 2, lymphomas 3, CML - 1, MDS - 2, X-LPS -1) at median age 8 years (range, 1-17), 24 boys and 6 girls, received treosulfan-based conditioning regimens: treosulfan $36-42 \mathrm{~g} / \mathrm{m}^{2}$, fludarabine $150 \mathrm{mg} / \mathrm{m}^{2}$, thiotepa $300 \mathrm{mg} / \mathrm{m}^{2}$ or melphalan $100 \mathrm{mg} / \mathrm{m}^{2}$, \pm serotherapy. Stem cell sources were: bone marrow in 21 , peripheral stem cells in 6 , cord blood in 2 and combination in 1 patient. Twelve children received a transplant from 10/ 10 HLA-matched related donor (MRD), 15 - from 10/10 HLA-matched unrelated donor (MUD), 3 patients received a $\mathrm{CD} 3+/ \mathrm{CD} 19+$ depleted haploidentical graft. GVHD prophylaxis consisted of cyclosporine $\mathrm{A} \pm$ mycophenolate mofetil in MRD and haploidentical transplants and tacrolimus+mycophenolate mofetil in MUD transplants. Median number of transplanted nucleated cells was $4.1 \times 10^{8} / \mathrm{kg}$ (range, 2.5-42). All patients engrafted at median time to neutrophil recovery 16 days (range, 11-34). At median follow-up of 5.4 years overall survival was $65 \%$, event-free survival - $65 \%$, relapse-free survival - 74\% (risk of relapse incidence $-23 \%$ ). No severe early toxicity was registered. Sixteen patients had acute GVHD grade II-IV (7 children - grade III-IV). Seven patients developed relapses and died. Seventeen children had chronic graft-versus-host 
disease (GVHD): cumulative incidence (CI) of chronic GVHD was 57\% and chronic extensive GVHD - $43 \%$. Eight patients developed endocrine complications: none had hypergonadotropic hypogonadism, CI of thyroid gland pathology was $3.3 \%$ (only 1 patient had subclinical hypothyroidism), CI of physical development delay was 24.8\%. Six patients demonstrated bone mineral density pathology (CI 13.5\%). Nobody except one patient with infectious meningoencephalitis had neurotoxicity with central nervous system impairment, but 8 children demonstrated neurocognitive defects. Cardiovascular abnormalities were revealed in 3 patients. Gastrointestinal, urinary, peripheral neurological and ophthalmological complications were associated predominantly with chronic GVHD. Three patients died due to chronic GVHD and/or infections. One patient developed secondary solid tumor (glioblastoma) and also died. Despite fertility preservation no cases of childbearing are reported till now.

Conclusions: Treosulfan-based conditioning regimen is an effective approach in reducing of serious late complications including hypergonadotropic hypogonadism, thyroid gland pathology, and central nervous system impairment after allogeneic HSCT when compared with historical data of busulfan-based conditioning long-term effects.

Conflict of interest: nothing to disclose

\section{P267}

\section{Lipopolysaccharide-induced endothelial cell gene modulation: effect of defibrotide}

Luciana Teofili, Nicoletta Orlando, Maria Grazia Iachininoto, Caterina Giovanna Valentini, Maria Bianchi, Simona Sica, Valerio De Stefano

Fondazione Policlinico Universitario A Gemelli Università Cattolica, Roma, Italy

Background: Endothelial cell (EC) damage plays a relevant role in the pathogenesis of several lifethreating complications of hematopoietic stem cell transplantation (HSCT), such as sinusoidal obstruction syndrome/venoocclusive disease (SOS/VOD), thrombotic microangiopathy, or graft versus host disease. Chemo- and radio-therapy toxicity, increased circulation of microbial products, allogeneic lymphocyte reaction or exposure to immunosuppressive drugs can activate several EC pathways, including inflammation, adhesion, coagulation and apoptosis. Defibrotide (DF) exhibits several endothelium-protective activities and has been approved for the treatment of SOS/VOD in HSCT patients. Nevertheless, the exact mechanism of action is not precisely recognized. We therefore used a gene
A

\begin{tabular}{|c|c|c|c|c|}
\hline & LPS & p & LPS DF & p \\
\hline \multicolumn{5}{|c|}{ Upनreg.llated gene: } \\
\hline CCL2 & 21,9 & 0,005 & 25,8 & 0,009 \\
\hline CFLAR & 20 & 0,049 & 1,9 & Is \\
\hline $\mathrm{C} \times 3 \mathrm{CLI}$ & 25,0 & 0,041 & 18,1 & 18 \\
\hline $\begin{array}{l}\text { Tisste } \\
\text { Faztor }\end{array}$ & 12,1 & 0,000 & 18,1 & 18 \\
\hline EARI & 29,7 & 0,010 & 29,3 & 0,001 \\
\hline IL6 & 19,6 & 0,010 & 25,0 & 0,001 \\
\hline IL7 & 31,3 & 0,049 & 38,4 & 0,043 \\
\hline रा & 2,9 & 0,032 & 4,9 & 18 \\
\hline PDGFFA & 2,4 & 0,044 & 1,5 & Is \\
\hline $\begin{array}{l}\text { E- } \\
\text { SELECTI } \\
N\end{array}$ & 49,4 & 0,033 & $\$ 5,9$ & 0,000 \\
\hline PAR 1 & 2,0 & 0,018 & 1,5 & 15 \\
\hline VCARI & 31,8 & 0,004 & 12,8 & 0,009 \\
\hline VEGFA & 2,4 & 0,014 & 3,3 & 0,044 \\
\hline \multicolumn{5}{|c|}{ Down-egulated gene: } \\
\hline Nos3 & $-1,9$ & 0,010 & $-2,8$ & 0,013 \\
\hline $\begin{array}{l}\text { Protell C } \\
\text { Reopptir }\end{array}$ & $-1,3$ & $0,0,8$ & $-1,5$ & 18 \\
\hline $\begin{array}{l}\text { Thronlyo } \\
\text { in calilis }\end{array}$ & $-2,2$ & $<0,001$ & $-3,9$ & $<0, \infty, 01$ \\
\hline
\end{tabular}

B

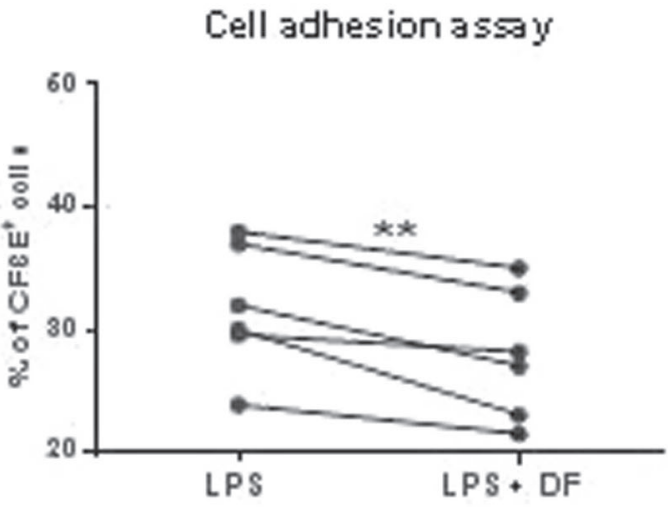

[P267 Figure] [Figure 1] 
profile approach to evaluate which pathways are modulated by DF in EC exposed to lipopolysaccharide (LPS).

Methods: Endothelial colony forming cells (ECFCs) were obtained from umbilical cord blood according to the method of Ingram et al.(Blood 2004;104:2752-2760). Confluent ECFCs at passages II to III were subjected to 6-h exposure to LPS (100 ng/ml, 4 experiments), with or without DF (100 ng/ml). In selected experiments, cells were pre-treated for $2 \mathrm{~h}$ with DF before the addition of LPS. Total RNA was extracted and reverted with a RT2 First Strand cDNA Kit (SABiociences, Qiagen). Genotype profile of control and treated cells was evaluated using the Human Endothelial Cell Biology" (PAHS-015Z, SABiociences, Qiagen). Data were analyzed using the RT2 Profiler PCR array data analysis template v3.5 (SABiociences, Qiagen). Relative changes in gene expression were calculated using the $\Delta \Delta \mathrm{Ct}$ (cycle threshold) method. Adhesion assay was assessed by evaluating the percentage of carboxyfluorescein succinimidyl ester (CFSE)-labeled mononuclear cells remaining adherent to the layer of ECFC after 2-hour incubation.

Results: The microarray included 84 genes involved angiogenesis, cell adhesion, inflammatory response, coagulation and platelet activation, apoptosis, vasoconstriction /vasodilatation. Figure A shows that LPS-treated cells displayed significant up-regulation of several genes involved in the above mentioned pathways. When cells were contemporary exposed to LPS and DF, DF was able to reduce up-regulated gene expression, and, in some cases (CXCL3, tissue factor, PAI-1) to revert it at levels comparable with unstimulated cells. This resulted in an overall decrease of adhesive capacity of LPS + DF treated cells in comparison with cell exposed only to LPS (Figure 1B). Interestingly, DF exposure before LPS treatment, prevented LPS modulation of the majority of involved genes.

Conclusions: These findings suggest that DF effects might be highly dependent on the exposure setting. Moreover, our observations might explain the favorable outcome of SOS/VOD when DF therapy is early started.

Conflict of interest: The authors have nothing to disclose.

\section{P268}

Long-term health outcomes and fertility in survivors of childhood AML treated with allogeneic HSCT: A NOPHO -AML Study

Mari Wilhelmsson ${ }^{1,2}$, Heidi Glosli ${ }^{3}$, Marianne Ifversen ${ }^{4}$, Jonas Abrahamsson $^{5}$, Jacek Winiarski, Kirsi Jahnukainen ${ }^{1,7}$, Henrik Hasle $^{8}$

${ }^{1}$ Karolinska Institutet, Department of Women's and Children's Health,
Stockholm, Sweden; ${ }^{2}$ Astrid Lindgren Children's Hospital, Karolinska University Hospital, Stockholm, Sweden; ${ }^{3}$ Rikshospitalet University Hospital, Department of Pediatrics, Oslo, Norway; ${ }^{4}$ The Juliane Marie Centre, Copenhagen University Hospital, Pediatric Clinic, Copenhagen, Denmark; ${ }^{5}$ Children's Cancer Centre, Queen Silvias Children's and Adolescent's Hospital Gothenburg, Gothenburg, Sweden; ${ }^{6}$ Karolinska Institutet, Department of Clinical Science, Intervention, and Technology, Stockholm, Sweden; ${ }^{7}$ University of Helsinki, Helsinki University Hospital, Division of HaematologyOncology and Stem Cell Transplantation, Children's Hospital, Helsinki, Finland; ${ }^{8}$ Aarhus University Hospital Skejby, Department of Pediatrics, Aarhus, Denmark

Background: Allogeneic hematopoietic stem cell transplantation (allo-HSCT) improves event-free-survival in acute myeloid leukemia (AML) but the indications should be weighed against the burden of late effects.

Methods: In order to assess the additive toxicity associated with allo-HSCT in the treatment of childhood AML we compared self-reported health-related outcomes in childhood AML survivors treated either with or without allo-HSCT. All survivors of childhood AML treated according to the NOPHO-AML protocols $-84,-88,-93$ and 2004 and with allo-HSCT when younger than 21 years alive by August 30, 2012 were sent a questionnaire. Altogether $95(65 \%)$ of the allo-HSCT survivors and 53 (75\%) of their eligible siblings completed the questionnaire. Corresponding data derived previously from AML survivors cured with chemotherapy only $(n=101)$ was used as comparison.

Results: The median follow-up was 12 (range 2-28) years after allo-HSCT. Allo-HSCT survivors reported more frequently physical health limitations $(39 \%$ vs $7 \%, \mathrm{p}<0.005)$, medication for cardiovascular disease $(9.5 \%$ vs $1 \%, \mathrm{p}<0.05)$ and use of analgesics $(32 \%$ vs $11 \%$, p < 0.01$)$ than survivors treated with chemotherapy only. An impairment or health problem prevented $16 \%$ of the allo-HSCT survivors from attending school or managing a job versus $3 \%$ among survivors treated with chemotherapy only $(\mathrm{p}<0.05)$. Among the 73 allo-HSCT survivors 15 years or older (median age 24, range 15-35 years), seven (19\%) of the 37 women reported twelve naturally conceived pregnancies and three $(8 \%)$ of the 36 men reported six naturally conceived pregnancies in partners. From these pregnancies eight children had been born and three female survivors and one partner to a male survivor were pregnant at the time of the study.

Conclusions: Childhood AML survivors treated with allo-HSCT experience more physical health limitations and use more medications than the conventionally treated AML survivors. Several naturally conceived pregnancies were reported after allo-HSCT for childhood AML.

Conflict of interest: The authors declare no conflicts of interest. Financial support was received from the Swedish Childhood Cancer foundation and Mary Béves Childhood 
Cancer foundation. Mari Wilhelmsson was supported by the Stockholm County Council (combined clinical residency and $\mathrm{PhD}$ training program).

\section{P269}

\section{Long-term thyroid abnormalities after total body irradiation preceding paediatric hematopoietic stem cell transplantation}

Natalia Zubarovskaya ${ }^{1}$, Carina Lender ${ }^{2}$, Ece Dila Güclï ${ }^{1}$, Ulrike Poetschger $^{2}$, Christina Peters ${ }^{2}$, Karin Dieckmann ${ }^{3}$, Anita Lawitschka

${ }^{1}$ St Anna Children's Hospital, SCT Outpatient Clinic, Vienna, Austria; ${ }^{2}$ St Anna Children's Hospital, Vienna, Austria; ${ }^{3}$ Medical University of Vienna, Department of Radiotherapy, Vienna, Austria; ${ }^{4}$ St Anna Children's Hospital, SCT Outpatient Clinic, Vienna, Austria

Background: Thyroid dysfunction after total body irradiation (TBI) preceding hematopoietic stem cell transplantation (HSCT) has been well described. Less paediatric data are available regarding the influence of graft versus host disease (GVHD), especially when including nonthyroid illness (NTI) syndrome into the spectrum of dysfunction. Therefore we studied retrospectively thyroid abnormalities (TA) during long-term follow-up (FU) in correlation with various patient and transplant characteristics with a particular focus on National Institutes of Health (NIH)-GVHD-consensus-group criteria.

Methods: We investigated 108 patients after allogeneic and 8 patients after autologous HSCT for malignant diseases having received a TBI over 9 Gy between 19842010 with a median FU of 5,6 years (range 3 months-20 years). Exclusion criteria were thyroid dysfunction before HSCT and death or relapse before day+100 after HSCT. Thyroid function was evaluated by serial measurements of basal TSH, free $\mathrm{T} 3+4$ and thyroid ultrasonography. TA was correlated with various well-known patient-, TBI- and transplant characteristics. GvHD was scored according NIH-GVHD-consensus criteria (reclassified for HSCT between 1984-2006, and prospectively thereafter).

Results: The 5-year cumulative incidence of TA was $53 \%$ with subclinical hypothyroidism in $44 \%$, NTI in $34 \%$, overt hypothyroidism in $13 \%$, pituitary hypothyroidism in $6 \%$ and autoimmune thyroiditis in $3 \%$ of dysfunctions. Significant risk factors for TA were allogeneic HSCT from a matched unrelated donor, HSCT in first complete remission, TBI in 6 fractions and mono-clonal antibodies for conditioning. Regarding GVHD both "acute and/or chronic GVHD" in general and acute GVHD grade IV in detail were significantly associated with the occurrence of TA. There was no further correlation with GVHD subgroups according NIH-definition. Interestingly, additionally applied radiotherapy to the head and upper chest wall did not result in a higher incidence of TA. Ultrasonography was performed in 47 patients and with regard to reduced volume of the thyroid gland the incidence of TA was comparable. In contrast we could observe a correlation between nodular changes and the occurrence of TA. No thyroid carcinoma had to be diagnosed.

Conclusions: Our findings fit well into published data and emphasize the importance of long-term aftercare. GVHD sub-analysis suffers from the problem of small patient numbers in NIH-defined subgroups due to the lower incidence of chronic GVHD in the paediatric population. These results may provide the basis for further multicentre studies to evaluate the impact of TBI, NIH-defined GVHD and immunosuppressive treatment in a larger data set.

Conflict of interest: nothing to disclose

\section{P270}

Abstract previously published

\section{P271}

NEPA (Netupitant plus palonosetron) for the prevention of nausea and vomiting in patients receiving high emetogenic conditioning regimen for Stem Cell Transplantation

Mariana Corrochano ${ }^{1}$, M Estela Moreno Martinez ${ }^{2}$, Irene Garcia Cadenas $^{1}$, Anna Barata ${ }^{1}$, Anna Feliu ${ }^{2}$, Albert Esquirol, Mar Gomis $^{2}$, Rodrigo Martino ${ }^{1}$, Jordi Sierra ${ }^{1}$

${ }^{1}$ Hospital de la Santa Creu i Sant Pau, Hematology, Barcelona, Spain; ${ }^{2}$ Hospital de la Santa Creu i Sant Pau, Pharmacy, Barcelona, Spain

Background: Chemotherapy-induced nausea and vomiting (CINV) is a significant side effect during stem cell transplantation (SCT) despite prophylactic therapy. NEPA (Netupitant plus palonosetron) has proved to be highly effective for high emetogenic chemotherapy; however, data are scarce in SCT recipients.

Methods: The objetive is to evaluate the efficacy and safety of NEPA in a cohort of patients receiving high emetogenic conditioning regimens before SCT.

Since September 2017, NEPA administered orally at fixed-dose combination ( $300 \mathrm{mg}$ netupitant, $0.5 \mathrm{mg}$ palonosetron) the first day of chemotherapy became the standard in our center for all SCT recipients treated with high emetogenic conditioning regimen. Dexamethasone (DXM) was given $8 \mathrm{mg}$ daily during chemotherapy and $4 \mathrm{mg}$ daily in the subsequent 48 hours.

Results: Outcomes of the first 8 patients treated with NEPA and DXM in our institution are described. Baseline characteristics are detailed in Figure 1. 


\begin{tabular}{|l|r|}
\hline Characteristic & N (\%) \\
\hline Gender & \\
\hline Male & $4(50 \%)$ \\
\hline Female & $4(50 \%)$ \\
\hline Age, median (range) & $45(17-59)$ \\
\hline ECOG performance status & \\
\hline 0 & $3(37,5 \%)$ \\
\hline 1 & $5(62,5 \%)$ \\
\hline Baseline disease & \\
\hline AML & $1(12,5 \%)$ \\
\hline ALL & $1(12,5 \%)$ \\
\hline NHL & $5(62,5 \%)$ \\
\hline HL & $1(12,5 \%)$ \\
\hline Disease phase at SCT & \\
\hline Early & $3(37,5 \%)$ \\
\hline Advanced & $5(62,5 \%)$ \\
\hline Conditioning chemoterapy & \\
\hline CyTBI & $2(25 \%)$ \\
\hline BEAM & $5(62,5 \%)$ \\
\hline CBV & $1(12,5 \%)$ \\
\hline Type of SCT & $2(25 \%)$ \\
\hline Allogenic & $6(75 \%)$ \\
\hline Autologous & \\
\hline & \\
\hline
\end{tabular}

"CyTBI: cycloshosphamide and total body iradiation ×BEAM: carmustine, etoposide, cytarabine and melphalan *CBV:cyclophosphamide, carmustine and etoposid:.

[P271 Figure] [Baseline characteristics]

During acute phase (days of chemotherapy) 3 patients (38\%) achieved complete response (CR, defined as no emesis and no rescue antiemetic) and $2(25 \%)$ patients had no emesis but did present mild or moderate nausea during this period. The maximum emetic episodes were 2 in a single patient.

Responses improved in the delayed phase (up to 72 hours after the last dose of chemotherapy) where 5 patients (63\%) achieved CR and only 2 (25\%) patients had mild to moderate nausea.

Five patients had history of significant nausea and emesis with prior chemotherapies, four of them did not develop emesis with NEPA regimen.

No patients required palonosetron for breakthrough emesis, since all of them were successfully rescued with single doses of olanzapine, metoclopramide or lorazepam. Non-hematologic adverse effects attributed to the study medications were minor (hiccup $\mathrm{n}=2$, headache, $\mathrm{n}=3$, somnolence $\mathrm{n}=2$ ); all of them with no further complications.

Conclusions: NEPA-based antiemetic regimen seems to be encouraging in terms of prophylaxis of CINV in the SCT setting. Our results warrant further evaluation in a larger cohort. Based on these promising results, enrolment of new patients is ongoing.

Conflict of interest: M. Moreno Martinez: presentation for Vifor on CINV. The other authors have nothing to disclose.

\section{P272}

\section{Neurological complications in adult allogeneic hematopoietic stem cell transplant patients: results from a retrospective multicentre study}

Cristina Tecchio ${ }^{1}$, Angelo Andreini ${ }^{1}$, Beatrice De Marco ${ }^{1}$, Carlo Borghero $^{2}$, Michele Malagola ${ }^{3}$, Valeria Cancelli ${ }^{3}$, Alessia Orsini ${ }^{1}$, Francesca Elice ${ }^{2}$, Federica Cattina $^{3}$, Cecilia Spina ${ }^{1}$, Enrico Morello $^{3}$, Marco Sorio ${ }^{1}$, Marco Ruggeri ${ }^{2}$, Fabio Benedetti ${ }^{1}$

${ }^{1}$ University of Verona, Hematology and Bone Marrow Transplant Unit, Verona, Italy; ${ }^{2}$ Ospedale San Bortolo, Hematology and Bone Marrow Transplant Unit, Vicenza, Italy; ${ }^{3}$ University of Brescia, Hematology and Bone Marrow Transplant Unit, Brescia, Italy

Background: Patients undergoing allogeneic hematopoietic stem cell transplant (HSCT) are exposed to a number of neurological complications that may be related to drugs, infections, metabolic alterations, cerebrovascular events and immune-mediated disorders including myositis, myasthenia gravis, Guillain-Barrè-like demyelinating polyneuropathy and central nervous system (CNS) manifestations of graft versus host disease (GVHD). The multifactorial etiology of neurological complications in HSCT patients makes diagnosis difficult. However a timely and rigorous characterization of such complications should be obtained in the attempt to avoid fatal outcomes or long-term effects. Data regarding neurological complications in HSCT patients derives from small series and varies largely in respect to incidence and severity. Aim of this study is to describe incidence, characteristics and outcome of neurological complications in a large series of consecutive HSCT patients.

Methods: Data were retrieved from medical records of 777 patients transplanted from January 2007 to January 2017 in 3 Italian centres. Complications affecting either central or peripheral nervous system were classified based on their etiology (Maffini et al. Biol Blood Marrow Transplant. 2017;23:388-397) and time of onset (from days 0 to 30,30 to 120,120 to 180,180 to 365 , and post day $365)$. 


\begin{tabular}{|c|c|c|c|c|c|}
\hline COMPLICATIONS & $\begin{array}{l}0-30 \\
\text { (days) }\end{array}$ & $\begin{array}{l}30-120 \\
\text { (days) }\end{array}$ & $\begin{array}{l}120-180 \\
\text { (days) }\end{array}$ & $\begin{array}{c}180-365 \\
\text { (days) }\end{array}$ & $\begin{array}{l}\text { post-365 } \\
\text { (days) }\end{array}$ \\
\hline Immune-mediated & & 6 [GVHD] $^{*}$ & 2 [GVHD] $*$ & $1[\mathrm{GVHD}]^{* \cdot \cdot}$ & $2[\mathrm{GVHD}]^{\cdots \cdot .}$ \\
\hline Infection-related & 1 [Fusarium] & 1 [virus unspecified] & $\begin{array}{l}1 \text { [Toxoplasma] } \\
2 \text { [JC virus] }\end{array}$ & 1 [Toxoplasma] & $\begin{array}{l}1 \text { [P. meningiditis] } \\
2 \text { [mycotic unspecified] } \\
1 \text { [Varicella Zoster virus] }\end{array}$ \\
\hline Drug-related & $\begin{array}{l}3 \text { [Cyclosporin] } \\
1 \text { [Busulfan] }\end{array}$ & 1 [Cyclosporin] & & & \\
\hline Cerebrovascular & & 1 [unspecified] & 1 [unspecified] & & 1 [hemorragic] \\
\hline Disease relapse & & & 1 [ANL] & $\begin{array}{l}2 \text { [AML] } \\
2 \text { [ALL] }\end{array}$ & \\
\hline CNS malignancy & & & & & 1 [gliobastoma] \\
\hline
\end{tabular}

[P272 Figure] [Classification of neurological complications according to etiology and time of onset.]

Results: Overall 34 [median age: 51.5 (19-66) years; F/ M: 11/23; Caucasian 31] of the 777 patients analysed (4.4\%) presented neurological complications. Among these patients, $19(55.8 \%)$ received a reduced-intensity HSCT, 15 (44.2\%) a myeloablative HSCT. Donors were matched related in $10(29.4 \%)$ cases, haploidentical in $2(5.9 \%)$, and matched or mismatched unrelated in $22(64.7 \%)$. Neurological complications were immune-mediated in $11 / 34$ cases $(32.3 \%)$, infection-related in $9(26.5 \%)$, drug-related in 5 $(14.7 \%)$, relapse-related in $5(14.7 \%)$, cerebrovascular in 3 (8.8\%), and due to a CNS neoplasia in $1(2.9 \%)$. The median time from HSCT to neurological symptoms appearance was $4.7 \quad(0-43.7)$ months (approximately 141 days). Fourteen patients $(41.2 \%)$ presented CMV reactivation and $13(38.2 \%)$ were receiving treatment for GVHD at the onset of neurological symptoms. Three $(75 \%)$ of the 4 drug-related neurological toxicities that occurred within day 30 were observed in patients of Asian (2) and African (1) ethnicity. Details on neurological complications according to etiology and time of onset are depicted in Figure 1.

Thirteen of 34 patients (38.2\%) died due to the neurological complication (relapse of disease 4, immunemediated 3, infection-related 2, drug-related 2, cerebrovascular 1, CNS malignancy 1) with a median time from symptoms onset to death of $2.3(0.2-22.4)$ months. Among the 21 patients who survived, $2(9.5 \%)$ are currently presenting long-term effects (GVHD-related tetraplegia and sensitive neuropathy, respectively).

Conclusions: Although affecting a limited number of patients, in our series neurological complications were associated to a significant mortality and severe long-term disabilities. Multicentre prospective studies using a common classification system are awaited in order to identify the real incidence and risk factors of neurological complications in HSCT patients.

Conflict of interest: The authors of this abstract have nothing to disclose

\section{P273}

Neurological complications post allogeneic hematopoietic cell transplantation: a major determinant of morbidity and mortality

Ioanna Sakellari ${ }^{1}$,Eleni Gavriilaki ${ }^{1}$, Sotirios Papayannopoulos ${ }^{1}$, Despina Mallouri ${ }^{1}$, Ioannis Batsis ${ }^{1}$, Maria Gavriilaki ${ }^{2}$, Anna Vardi ${ }^{1}$, Varnavas Constantinou $^{1}$, Dimitrios Kazis ${ }^{1}$, Marianna Masmanidou ${ }^{1}$, Evangelia Yannaki ${ }^{1}$, Christos Smias ${ }^{1}$, Triantafyllos Geroukis ${ }^{1}$, Vasilis Kimiskidis $^{2}$, Achilles Anagnostopoulos ${ }^{I}$

${ }^{I}$ G. Papanicolaou Hospital, Thessaloniki, Greece; ${ }^{2}$ Aristotle University of Thessaloniki, Thessaloniki, Greece

Background: Neurological manifestations are underrecognized, potentially life-threatening complications post allogeneic hematopoietic cell transplantation (alloHCT). Despite advances in the field, diagnosis and management remain challenging. Therefore, we recorded neurological complications and long-term outcomes in alloHCT recipients.

Methods: We retrospectively reviewed charts of consecutive allogeneic HCT recipients transplanted in our center (7/1990-9/2017). Neurological complications were recorded based on clinical data, imaging, electroencephalography, electromyography, pathology, cerebrospinal fluid testing and neurology consultations of hospitalized patients. Anti-thymocyte globulin (ATG, $2.5-10 \mathrm{mg} / \mathrm{kg}$ ) was administered as part of the conditioning in unrelated and alternative transplantations. 
Results: Among 758 alloHCT recipients (451 sibling, 259 unrelated, 40 haploidentical, 2 twin donors and 6 cord blood), $127 \quad(16.8 \%)$ presented with neurological complications.

Incidence of neurological complications was significantly lower in sibling transplantations $(\mathrm{p}=0.011)$.

The majority developed central nervous system/CNS complications $(89.7 \%)$, late post-transplant (median +140day, interquartile range/IR 232). 11 patients presented with $>1$ episodes (median 10.4 months, IR 25.1). Timing of neurological complications was associated only with acute and chronic graft-versus-host-disease/GVHD ( $\mathrm{p}=0.001$ and $p<0.001$, respectively). Neurological complications included: CNS relapse (24), thrombotic microangiopathy (12), CNS hemorrhage (7), posterior reversible encephalopathy (6), drug-associated polyneuropathy (7) and seizure (6), other leukoencephalopathy (6), thromboembolic events (5), neuralgia (4), myopathy (3), sinusoidal obstruction syndrome (1), Guillain-Barre syndrome (1), Wernicke encephalopathy (1), myelitis (1) and multiple sclerosis (1). CNS infections were attributed to aspergillosis (12), mucormycosis (3), Cytomegalovirus (9), Epstein-Barr encephalitis (3) or lymphoproliferative disease (6), Human herpesvirus 6 (5), Human herpesvirus 7 (2), toxoplasmosis (3); while others were not otherwise specified (10).

With a median follow-up of 11.4 months (IR 30.3), incidence of chronic GVHD was $52.8 \%$, relapse mortality $48.6 \%$, treatment-related mortality $39.1 \%$ and 5-year overall survival (OS) $25.8 \%$ in patients with neurological complications. ATG administration $(\mathrm{p}=0.006)$, chronic GVHD $(\mathrm{p}=0.016)$, sibling transplantation $(\mathrm{p}=0.001)$ and resolution of neurological complications $(\mathrm{p}<0.001)$ were significant predictors of OS. In the multivariate analysis, favorable OS was independently associated with resolution of neurological syndromes $(\mathrm{B}=1.117, \mathrm{p}<0.001)$, absence of chronic GVHD $(\mathrm{B}=0.693$, $\mathrm{p}=0.003)$ and sibling transplantation $(\mathrm{B}=0.775, \mathrm{p}=0.049)$.

Conclusions: Our study highlights the wide spectrum of manifestations and etiologies of neurological complications in alloHCT recipients. Sibling transplantation is associated with lower incidence and better OS of affected patients, irrespectively of other pre-transplant characteristics. Prompt diagnosis is required for adequate management, a major of determinant of survival. Complications occur during the late post-transplant period, particularly in immunocompromised patients. Thus, long-term increased awareness and collaboration between expert physicians is warranted to improve patient outcomes.

Conflict of interest: There are no conflicts of interest to report

\begin{tabular}{ll}
\hline Median age, years (interquartile range) & $36(21)$ \\
\hline Acute lymphoblastic leukemia, n & $49 / 127$ \\
Acute myeloid leukemia, n & $41 / 127$ \\
1st complete remission at transplant, n & $52 / 127$ \\
Other remission at transplant, n & $34 / 127$ \\
Relapsed/Refractory disease at transplant, n & $41 / 127$ \\
Bone marrow graft, n & $31 / 127$ \\
Peripheral blood stem cell graft -Cord blood, n & $93-2 / 127$ \\
Myeloablative conditioning, n & $76 / 127$ \\
Reduced intensity conditioning, n & $51 / 157$ \\
\hline
\end{tabular}

[[P273 Table] Patients with neurological complications]

\section{P274}

\section{Non-Infectious Neurological Complications of Hematopoietic Cell Transplantation}

\author{
Mohammad Sheikh ${ }^{1}$, Michel Toledano', Shahrukh Hashmi ${ }^{3,4}$ \\ ${ }^{1}$ Allama Iqbal Medical College, Lahore, Pakistan; ${ }^{2}$ Mayo Clinic, \\ Neurology, Rochester, MN, United States; ${ }^{3}$ King Faisal Specialist \\ Hospital \& Research Center, Oncology, RIyadh, Saudi Arabia; ${ }^{4}$ Mayo \\ Clinic, Medicine, Rochester, MN, United States
}

Background: Although neurologic complications after hematopoietic cell transplantation (HCT) are common, a literature gap is identified in estimation of the exact burden and outcomes of these conditions in allogeneic and autologous HCT setting.

Methods: Herein, we conducted a systematic review regarding the non-infectious neurological outcomes in HCT recipients to quantify the frequency of each complication occurring across the collected data, and to gauge the importance of relevant risk factors and outcomes. As abundant data already exists on infectious neurological complications, this manuscript primarily focuses on the non-infectious complications.

A search of the medical literature was done as per the PICO criteria. Briefly, case reports, case series, clinical trials, and retrospective studies were included; systematic reviews, meta-analyses, review articles, and pre-clinical studies were excluded. Recipients of all modalities of HCT (i.e. autologous, allogeneic [bone marrow, peripheral blood, umbilical cord]) were eligible.

Complications evaluated included: cerebrovascular accident (CVA); Peripheral Neuropathy (PN); Myasthenia Gravis MG); Seizures; Autoimmune neurological complications other than MG, Guillain-Barre Syndrome (GBS); and Posterior Reversible Encephalopathy Syndrome (PRES).

The databases searched included Amedo Free Medical Journals; Clinical Key; Directory of Open Access Journals (DOAJ); Ovid - MEDLINE; ProQuest Medical Library; PubMed Central; Sage Journals; Science Direct; 
SpringerLink; Wiley-Blackwell. Additionally, manual searching was done of several published articles. Articles from 1983 up to May 2017 were retrieved. Data from a total of 175 eligible articles were extracted according to predefined variables and parameters for quantitative analysis.

Results: Results revealed the following frequencies (compared to US general population): 264 cases of CVA from a sample size $(\mathrm{N})$ of 10,117 (2.6\%), compared to a frequency of $0.246 \%$ per year of the population; 321 cases of PN from an $\mathrm{N}$ of 10,031 (3.2\%), compared to a frequency of $2.4 \%$ in the population; 21 cases of MG from an $\mathrm{N}$ of 1049 (2\%), compared to a frequency of $0.014-0.02 \%$ in the population; of 406 cases of seizures from an $\mathrm{N}$ of 9803 (4.14\%), compared to a frequency of $0.62 \%$ of the population; and of 256 cases of PRES from an $\mathrm{N}$ of 6016 (4.25\%), [unknown incidence in the general population]. See Table 1.

Conclusions: As can be seen from the results above, it may be concluded that HCT is associated with an increased risk of neurological complications. The recognition of this increased risk, in conjunction with the risk factors given and the quantitative extent to which these can be expected in the HCT recipient population, will allow more accurate and vigilant monitoring of patients for the early signs of development of these complications and thus facilitate earlier therapeutic intervention to provide protection from the substantial morbidity these complications may cause in the affected population.

Conflict of interest: The authors have nothing to disclose.

\begin{tabular}{lllll}
\hline $\begin{array}{l}\text { Evaluated } \\
\text { Complication }\end{array}$ & $\begin{array}{l}\mathrm{N} \\
\text { (Sample } \\
\text { Size) }\end{array}$ & $\begin{array}{l}\mathrm{N} \\
\text { Complication }\end{array}$ & $\begin{array}{l}\text { Frequency in } \\
\text { Studied } \\
\text { Population }\end{array}$ & $\begin{array}{l}\text { Frequency in } \\
\text { General } \\
\text { Population }\end{array}$ \\
\hline CVA & 10,117 & 264 & $2.6 \%$ & $0.246 \%$ \\
PN & 10,031 & 321 & $3.2 \%$ & $2.4 \%$ \\
MG & 1049 & 21 & $2 \%$ & $0.014-0.02 \%$ \\
Seizures & 9803 & 406 & $4.14 \%$ & $0.62 \%$ \\
PRES & 6016 & 256 & $4.25 \%$ & Unknown \\
\hline
\end{tabular}

[[P274 Table] Neurological Complications of HCT]

\section{P275}

Non-relapse / non-treatment related mortality after HDC auto-SCT in patients with non-Hodgkin and Hodgkin lymphoma. An important but forgotten outcome not so commonly reported

Saad Akhtar, M.S. Rauf, Tusneem Ahmed M. Elhassan, Irfan Maghfoor
King Faisal Specialist Hospital and Research Center, Oncology Center, Riyadh, Saudi Arabia

Background: HDC auto-SCT is a gold standard treatment for patients with relapsed or refractory diffuse large B cell lymphoma (DLBCL) and Hodgkin lymphoma (HL). Despite improved care and treatment, disease related cause continues to be the main cause of death in long run. Post transplant 100-day treatment related mortality (TRM) account for $2-4 \%$ of all the deaths. Most large studies reports disease and TRM related deaths. We aimed to analyze the causes of death in patients who had HDC autoSCT and died of causes unrelated to disease.

Methods: HDC auto-SCT data was reviewed for all the death. Non-relapse mortality (NRM) is defined as time to deaths without relapse/recurrence. A condition that is not incorporated in NRM is "deaths unrelated to disease but in a patient with disease" (D-un-to-disease-WD). NRM and Dun-to-disease-WD was captured. Any patient who died in a local hospital due to unknown cause or infection before day-100 was counted in TRM.

Results: From 1996 to December 2016, 523 consecutive patients with biopsy proven DLBCL and HL underwent HDC auto-SCT. Median age at HDC auto-SCT was 28 years (14-70 years with only $4 \%>60$ years). NHL:HL 9:13. In total, 203 $(39 \%) / 523$ have died. $155(30 \%) / 523$ died of disease. $26(5 \%) /$ 523 due to TRM and 22 (4.2)/523 due to NRM (19) or D-unto-disease-WD (3). Table 1 has the details of main causes of death. There were 12 (55\%) male, 10 (45\%) female. Median age at HDC auto-SCT was 22 years (16-59 years). Median time to death was 24 months (4-159 months). Causes of deaths are categorized into four groups; infections (27\%), noninfectious organ failure (27\%), second malignancy (23\%) and others/ miscellaneous groups (23\%). Infections (6) site/organ were lung (3), CNS, maxillary/paranasal sinus area and hemophagocytosis/sepsis in 1 each. 6 patients died of noninfectious organ failure; lung complications (2), 1 each adrenal insufficiency, liver failure, multi-organ failure, ruptured-carotid aneurysm. Second malignancy was observed in 5; t-MDS/ leukemia in 3, breast and nasopharyngeal carcinoma in 1 each. Three patients had accidents ( 2 car accidents and 1 drowning). Precise details are not available in 2 patients (unknown cause).

Conclusions: In this large cohort of 523 relatively young patients, NRM and D-un-to-disease-WD was almost equal to TRM. Magnitude of this issue is larger than expected. This important information should be carefully discussed with the patients for informed decision-making. In our set up where people in rural areas generally have less access to healthcare than their urban counterparts which often mean longer response times in emergencies

Conflict of interest: Nothing to disclose for any author 


\begin{tabular}{lll}
\hline Cause of death & Frequency & Percentage \\
\hline Accident (2 car, 1 drowning) & 3 & 14 \\
Infections & 6 & 27 \\
Other medical conditions & 6 & 27 \\
Solid cancers & 2 & 9 \\
t-MDS/leukemia & 3 & 14 \\
Unknown & 2 & 9 \\
Total & 22 & 100 \\
\hline
\end{tabular}

[[P275 Table] Table 1. Main causes of death]

\section{P276}

\section{Nutrition Counseling Improves Malnutrition Scores among Hematopoietic Stem Cell Recipients: Results of a Randomized Controlled Trial}

Jana Jabbour ${ }^{1,2}$, Batoul Manana ${ }^{3}$, May Sakr $^{1}$, Ammar Zahreddine ${ }^{4}$, Hani Tamim ${ }^{5}$, Ali Bazarbachi ${ }^{4}$, Didier Blaise ${ }^{6}$, Jean El-Cheikh ${ }^{4}$

${ }^{1}$ American University of Beirut Medical Center, Department of Clinical Nutrition, Beirut, Lebanon; ${ }^{2}$ Aix Marseille University, Doctoral School of Life Science and Health, Marseille, France; ${ }^{3}$ American University of Beirut Medical Center, Beirut, Lebanon, Division of Hematology/Oncology, Department of Internal Medicine, Beirut, Lebanon; ${ }^{4}$ American University of Beirut Medical Center, Bone Marrow Transplantation Program, Department of Internal Medicine, Beirut, Lebanon; ${ }^{5}$ American University of Beirut Medical Center, Clinical Research Unit, Beirut, Lebanon; ${ }^{6}$ Aix Marseille University, Institut Paoli-calmettes, Marseille, France

Background: Conditioning preceding Hematopoietic Stem Cell Transplantation (HSCT) is associated with elevated rates of malnutrition at hospital discharge and up until 100 days post HSCT. This study aimed to assess the impact of a nutrition intervention provided at hospital discharge on nutritional status 100 days post HSCT.

Methods: This study was a single center randomized controlled trial. Adult patients receiving HSCT were eligible to participate. Data collection was initiated at admission and randomization occurred at hospital discharge. Around discharge from the hospital, recruited patients were randomized to a Control Group (CG) receiving usual care and to an Intervention Group (IG) receiving nutritional counseling on a monthly basis to optimize their nutritional and functional status. The primary outcome was the Patient Generated Subjective Global Assessment (PGSGA) scores at day 100 post HSCT. Malnutrition was also assessed though the American Society for Parenteral and Enteral Nutrition/ Academy of Nutrition and Dietetics malnutrition score. Handgrip Strength (HGS) was compared to normative data to assess diminishment. Fat Mass Index (FMI) was assessed via bioimpedance analysis. Post hospital discharge, assessment was done at days 30, 60 and 100 post HSCT for both groups.

Percent Energy ...

Results: 52 participants were randomized (August 2016 until August 2017) and 46 were analyzed [65\% males, 63\% autologous HSCT, IG $(n=22), C G(n=24)]$. Groups were comparable with no statistically significant changes in characteristics. At hospital admission, percent of patients who were well nourished was low (45\% IG vs $50 \%$ CG with PGSGA category $\mathrm{A}, \mathrm{p}=0.79$ ) and percentage of patients with diminished HGS was high (82\% IG vs $67 \%$ $\mathrm{CG}, \mathrm{p}=0.24$ ) in both groups. At 100 days post HSCT, IG had more well-nourished patients $(45 \%$ vs. $72 \%$ with PGSGA category $\mathrm{A}, \mathrm{p}=0.058)$ and CG had less wellnourished patients (50\% vs $43 \%$ with PGSGA category A, $\mathrm{p}=0.74$ ) compared to admission values in respective groups. Oral caloric intake was better in IG at all assessment points post discharge compared to CG (all $\mathrm{p}<0.05$ ). Compared to admission, percentage of patients with high FMI decreased in the IG $(71 \%$ vs $56 \%, p=0.046)$ and increased in $\mathrm{CG}(65 \%$ vs $62 \%, \mathrm{p}=0.26)$ at 100 days post HSCT. Post discharge, percentage of patients with diminished HGS improved more pronouncedly in IG, yet similar values were reached 100 days post $\mathrm{SCT}(\mathrm{p}=0.96)$.

Graph legend: Left panel: Energy intake refers to percent intake compared to patients' individual needs, $* \mathrm{p}<0.05$ for difference between groups; Right Panel: Malnutrition assessed based on ASPEN/AND malnutrition tool. ASPEN/AND: American Society for Parenteral and Enteral Nutrition/ Academy of Nutrition and Dietetics. $p=0.02$ for
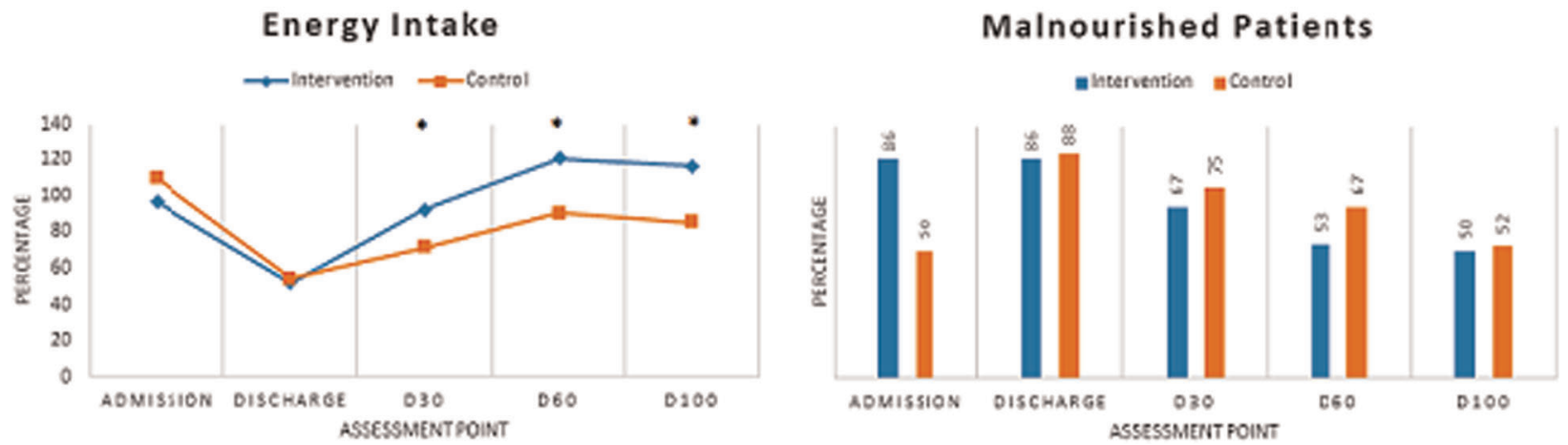

[P276 Figure] [Percent Energy Intake (left panel) and malnourished patients as per ASPEN/AND criteria (right panel)] 
difference between admission and day 100 post HSCT in intervention group; $\mathrm{p}=1$ for difference between admission and day 100 post HSCT in control group.

Conclusions: This RCT showed that nutritional counseling post HSCT can improve nutritional status and reduce obesity post HSCT patients but has no effect on sarcopenia.

Clinical Trial Registry: Clinicaltrial.gov registry: NCT02791347

Conflict of interest: None

\section{P277}

\section{OUTCOME OF PATIENTS WITH DONOR-SPECIFIC ANTI-HLA ANTIBODIES UNDERGOING DESENSITIZATION THERAPY IN MISMATCHED HEMATOPOIETIC STEM CELL TRANSPLANT}

Francesca Patriarca ${ }^{1}$, Davide Lazzarotto ${ }^{1}$, Ursula Larocca ${ }^{2}$, Giulia Perali $^{1}$, Valeria Miotti ${ }^{3}$, Donatella Londero ${ }^{3}$, Alessandra Sperotto ${ }^{4}$, Michela Cerno ${ }^{4}$, Martalisa Battista ${ }^{4}$, Antonella Geromin ${ }^{4}$, Roberta Demarchi $^{1}$, Chiara Cigana ${ }^{1}$, Chiara Savignano ${ }^{3}$, Cristina Rinaldi ${ }^{3}$, Maria Paola Perrone ${ }^{5}$, Paola Cinti ${ }^{5}$, Robin Foa ${ }^{2}$, Renato Fanin ${ }^{1}$, Anna Paola Iori ${ }^{2}$

${ }^{1}$ Hematology, Azienda Sanitaria Universitaria Integrata di Udine, Università di Udine, DAME, Udine, Italy; ${ }^{2}$ Hematology, Policlinico Umberto 1, Sapienza University of Rome, Rome, Italy; ${ }^{3}$ Transfusion Medicine, Azienda Universitaria Integrata di Udine, Udine, Italy;

${ }^{4}$ Hematology,Azienda Sanitaria Universitaria Integrata, Udine, Italy;

${ }^{5}$ Immunoematology and Transfusion Medicine, Sapienza University of Rome, Rome, Italy

Background: Detection of donor-specific anti-HLA antibodies (DSAs) has been reported to be associated with higher rates of graft failure (GF), worse overall survival (OS) and higher transplant-related mortality (TRM) in mismatched hematopoietic stem cell transplant (HSCT), but the efficacy of desensitization treatment remains unclear.

Methods: We prospectively evaluated the presence of DSAs, using Solid Phase system, Luminex (Labscreen Mixed and Single Antigen class I and II, One Lambda), in patients undergoing unmanipulated unrelated mismatched SCT (USCT) and unmanipulated haploidentical SCT (HaploSCT). DSA binding levels were expressed as mean fluorescence intensity (MFI). Subsequently, we compared hematopoietic engrafment, TRM and OS between patients with DSAs who underwent desensitization treatment and patients without DSAs.

Results: Eighty-three consecutive patients- 70 with acute leukemia or myelodysplastic syndrome (84\%), and 13 with lymphoma or myeloma (16\%) - with a median age of 53 years (3 - 71) underwent a HSCT between January 2015 and October 2017. Forty-two/83 (51\%) underwent a HLA minor or major mismatch USCT and 41/83 (49\%) a HaploSCT. GvHD prophylaxis was based on Cyclosporine, ATG and Methotrexate in USCTs, and on Cyclosporine,

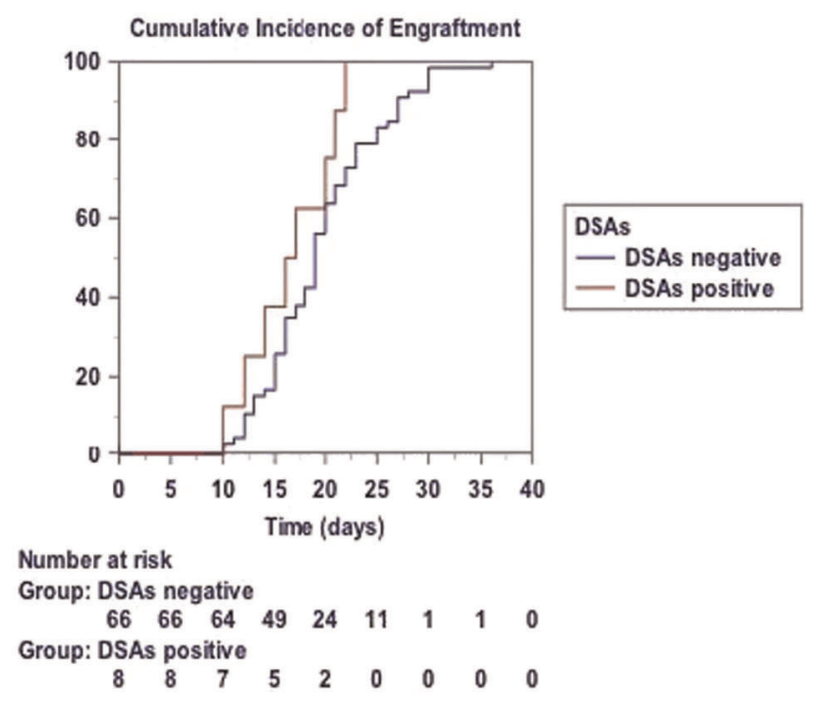

Figure 1

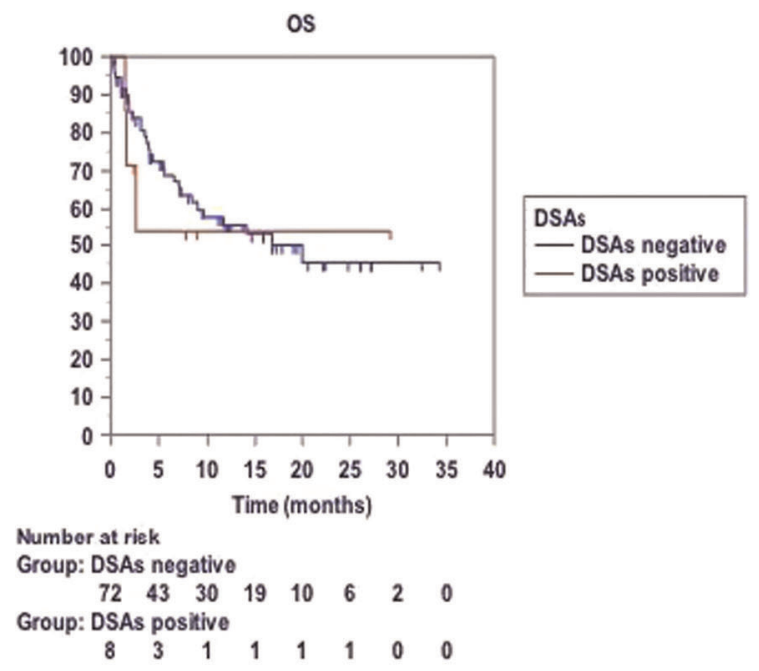

Figure 2

[P277 Figure] [Figures 1 and 2]

Mycophenolate and post-transplant Cyclophosphamide in HaploSCT.

DSAs were detected in 11 patients (14\%) with a median DSA level of 3700 (range 738 - 22400): 7 patients underwent a HaploSCT $(7 / 41,17 \%)$ and 4 an USCT (4/ $42,10 \%)$. Three of them (2 HaploSCT and 1 USCT), who did not receive desensitization treatment and were excluded from the outcome analysis, had primary GF. Desensitization treatment consisted in a median number of 2 plasma exchange (PEX) procedures (range 1 - 4) for all patients, with the addiction of Rituximab and intravenous immunoglobulines (IVIg) in 6/8 patients. We observed no significant differences in the cumulative incidence of neutrophil and platelets engraftment $(p=0.09)$ (Fig. 1) between patients that had DSAs and received 
desensitization treatment and patients that had no DSAs. The overall TRM and OS did not differ between the two groups $(\mathrm{p}=0.32$ and $\mathrm{p}=0.67)$.

Conclusions: DSAs were detected in 4/42 of USCT candidates $(10 \%)$ and in $7 / 41$ of HaploSCT candidates $(17 \%)$, and they were associated with a failure to obtain an allogeneic engrafment in the 3 patients who did not receive desensitization treatment. On the contrary, patients who underwent desensitization treatment showed engraftment, OS and TRM rates similar to those of patients who were DSA negative before transplant. Our study shows that the search of DSAs is mandatory in mismatched transplants. Moreover, it underlines the potential benefit of the desensitization treatment in the setting of HSCT with DSAs.

Conflict of interest: no conflict of interest to disclose

\section{P278}

\section{Outcome of pediatric patients admitted to Pediatric Intensive Care Unit after allogeneic stem cells transplantation}

Zofia Szmit ${ }^{1,2}$, Magdalena Kośmider-Żurawska ${ }^{2}$, Monika Lobos ${ }^{1}$, Justyna Miśkiewicz ${ }^{1}$, Marzena Zielińska ${ }^{2}$, Krzysztof Kalwak ${ }^{1}$, Ewa Gorczyńska

${ }^{1}$ Medical University, Department of Pediatric Hematology, Oncology and Bone Marrow Transplantation, Wroctaw, Poland; ${ }^{2}$ Medical

University, Department of Anesthesiology and Intensive Care, Wroctaw, Poland

Background: Pediatric allogeneic hematopoietic stem cells transplantation (allo-HSCT) is a life-saving therapy for a wide range of diseases. Nevertheless it remains a high risk procedure, associated with many life-threatening complications. Therefore a considerable number of patients require transfer to pediatric intensive care unit (PICU) after transplantation. The aim of this study was to assess the incidence and outcome of PICU admissions after alloHSCT, as well as report a detailed patient characteristic and potential prognostic factors for survival.

Methods: The study was based on retrospective data analysis of 668 children and adolescents who underwent allo-HSCT in Department of Pediatric Hematology, Oncology and Bone Marrow Transplantation in Wrocław in years 2005-2017. Particularly we analyzed those patients who required transfer to PICU within 1 year after transplantation. Clinical and epidemiological features, transplantation procedures, laboratory findings and intensity of applied therapy were assessed. Primary endpoints were death or discharge from PICU. 30-days and 6-months survival for those who were discharged were also evaluated. Statistical analysis was performed using Statistica software.
Results: In analyzed population 58 of $668(8.7 \%)$ patients required 68 admissions to PICU. Five patients were admitted twice to PICU. Forty-six patients $(67.7 \%)$ received stem cells due to a malignant disease and $22(32.3 \%)$ due to non-malignant disorder. Main indications for admission were: respiratory failure $(25 ; 36.8 \%)$, septic shock (16; $23.5 \%)$, neurological disorders $(8 ; 11.8 \%)$, acute kidney injury $(5 ; 7.3 \%)$, other $(14 ; 21.5 \%)$. Median day post HSCT at the moment of transfer was 59 (1-297), length of PICU hospitalization were 7 days (1-104).Twenty-five $(36.8 \%)$ cases had severe granulocytopenia $(<500 / \mathrm{mcl})$ at the day of PICU admission. Fifty-four $(79,4 \%)$ cases required mechanical ventilation, $47(69 \%)$ received cardiac support, 9 $(13,23 \%)$ needed renal support. Thirty-four (50\%) were discharged, $34(50 \%)$ cases died. Among discharged patients 30 days survival was $82 \%$ and 6-months survival was $58 \%$. Compared with survivors, death cases more likely received haploidentical allo-HSCT $(7 ; 20.6 \%$ vs 1 ; $2.9 \% \mathrm{p}=0.04)$, required mechanical ventilation $(31 ; 91.2 \%$ vs $23 ; 67.7 \% \mathrm{p}=0.05)$, received more aggressive $(>=3$ catecholamines) cardiac support $(17 ; 50 \%$ vs $3 ; 8.8 \% \mathrm{p}=$ 0.002 ), and had lower granulocyte level at the day of death comparing to day of discharge $(\mathrm{p}=0.02)$. Differences in age, sex, underlying disease, stem cells source, day and reason of admission, granulocyte count at the moment of admission, acute graft versus host disease were statistically irrelevant. Main causes of death at PICU were: cardiopulmonary failure $(10 ; 29 \%)$, Septic shock $(9 ; 26.5 \%)$, MOF $(4,11.8 \%)$ acute graft versus host disease $(3,8.82 \%)$, relapse $(3,8.82 \%)$ other $(5 ; 14.7 \%)$.

Conclusions: Pediatric recipients of allo-HSCT who had PICU care had a high rate of mortality. Mainly those patients who required more aggressive approach, including mechanical ventilation had greater chance of death. The most curious prognostic factor was granulocyte count at the end of treatment as opposed to day of admission. This may suggest that, beside supportive care, hematological recovery requires more careful consideration in patients hospitalized in PICU after allo-HSCT.

Conflict of interest: None of the authors has anything to declare.

\section{P279}

Outpatient-based autologous stem cell transplantation: feasible, safe and cost effective approach

Hani Al Hashmi, Panayotis Kaloyannidis, Eshrak Al Shibani, Nihad Mokhtar, Solaf Kafnar, Khalid Bakhit, Ann Estanislao, Fuad Abdulrahma, Reem Khalili, John Apostolidis, Khalid Al Anezi 
King Fahad Specialist Hospital, Adult Hematology and Stem Cell Transplantation Department, Dammam, Saudi Arabia

Background: Given the current improvements in the supportive care (broad-spectrum antibiotics, growth factors, sufficient control of nausea/vomiting and mucosal toxicity), autologous hematopoietic stem cell transplantation (ASCT) could be performed in an outpatient basis, for selected patients, offering the benefit of shorter hospitalization, less exposure to hospital pathogens and nosocomial infection, demonstrating thus not only a safe but also a cost effective profile.

Methods: To evaluate the feasibility and safety of the aforementioned approach, we performed 31 ASCTs on an outpatient basis, in a total of 24 pts, diagnosed either as relapsed/refractory Hodgkin's lymphoma $(\mathrm{n}=6)$ or as Multiple Myeloma $(\mathrm{n}=18) ; 7$ pts with MM underwent tandem ASCT. Eight were females and 16 males with a median age of 51 (25-68) ys. The eligibility criteria for the outpatient ASCT, were the standard clinical and laboratory tests, plus psychosocial evaluation, patient's compliance assessment, 24 hours caregiver availability, timely access to the hospital and signed informed consent. The conditioning regimen consisted from single agent Melphalan of 200mg/ $\mathrm{m}^{2}(\mathrm{n}=23)$ or $140 \mathrm{mg} / \mathrm{m}^{2}(\mathrm{n}=8)$, graft infusion and supportive care were given in an allocated room. The antimicrobial, antifungal and antiviral prophylaxis was administered from day -2 and filgrastim $5 \mathrm{mcg} / \mathrm{kg}$ from day +5 till neutrophils recovery. If no infection was documented the antimicrobial and antifungal prophylaxis were discontinued upon stable neutrophils recovery while antiviral prophylaxis was continued for 10-12 months. Patients were evaluated daily or every other day in the outpatient clinic. The criteria for admission were fever $>38^{\circ} \mathrm{C}$, intractable nausea/vomiting or diarrhea, mucositis needing total parenteral nutrition and any other toxicity $\mathrm{WHO}>$ grade 3 .

Results: The median day for neutrophils recovery $\left(>1000 / \mathrm{mm}^{3}\right)$ was $+11(11-18)$ and for platelets recovery $\left(>25000 / \mathrm{mm}^{3}\right)$ was $+11(0-21)$; in 2 pts platelets never dropped lower than $25000 / \mathrm{mm}^{3}$. Fourteen admissions were required in a total, 9 for inability for food/fluid uptake due to severe mucositis, 4 for febrile neutropenia and 1 for engraftment syndrome. The infections were successfully treated with broad spectrum antibiotics and no patient was admitted to intensive care unit. For the whole 31 ASCTs, the total hospitalization days were 65 (median:1, range 0-9), while for the 14 admissions the median hospitalization days were 5 (range 1-9), which favorably compares with the average of 14 hospitalization days for a single "conventional" ASCT. No other toxicities (WHO $>3$ ) were observed. All pts are alive for 9 (1-36) months post AHSCT.
Conclusions: Our data indicate that the outpatient-ASCT is a feasible and safe approach provided a caregiver availability, close patient monitoring and adequate supportive care. Keeping in mind the nosocomial complications and the potential high cost of the prolonged hospitalization, it seems that the outpatient ASCT offers lower risk of infections and significant cost saving compared to the "conventional" inpatient ASCT approach.

Conflict of interest: None of the authors has anything to declare.

\section{P280}

Pain management in children with acute mucositis after haematopoietic stem cell transplantation (HSCT)

Ekaterina Goncharova ${ }^{1,2}$, Marina Ermolova ${ }^{3}$, Olga Ivanova ${ }^{3}$, Julia Oparina $^{3}$, Ilya Kazantsev ${ }^{3}$, Maxim Bogomolny ${ }^{3}$, Alexey Sokolov ${ }^{2}$, Gleb Ulrikh ${ }^{4}$, Liudmila Zubarovskaya ${ }^{3}$, Boris Afanasyev ${ }^{3}$

${ }^{1}$ R.M. Gorbacheva Institute of Pediatric Oncology Hematology and Transplantation, I.P. Pavlov First St. Petersburg State Medical University, Anesthesiology Department, St. Petersburg, Russian Federation; ${ }^{2}$ Valdman Institute of Pharmacology Pavlov First St. Petersburg Medical University, St. Petersburg, Russian Federation;

${ }^{3}$ R.M. Gorbacheva Institute of Pediatric Oncology Hematology and Transplantation, I.P. Pavlov First St. Petersburg State Medical University, St. Petersburg, Russian Federation; ${ }^{4}$ Pediatric Medical University, Department of Anesthesiology and Pediatric Intensive Care, St. Petersburg, Russian Federation

Background: Gastrointestinal tract mucositis (GITM) is one of the most common complications of early posttransplant period developing in $20-40 \%$ of patients with grade ( 0 to 4 ) mostly depending on conditioning regimen used, oral cavity involvement, patient's somatic state and post-transplant cytopenia duration. GITM is usually associated with nausea, vomiting, pain, food and liquids intake impairment, and higher risk of infections and bleeding, thus being a factor with significant influence on patientÂ's quality of life. GITM patients require continuous pain management, most often narcotic analgesics are used. The opiates infusion rate depends on clinical situation and inherited opiate receptors polymorphisms, which determine their sensitivity. Patient-controlled analgesia (PCA) is often an optimal way to achieve the most rational dosing regimen in adults. However, in pediatric post-HSCT patients its utility is often disputed.

Methods: A group of 73 consecutive pediatric HSCT recipients (median age 8.5, range 1.1 to 17 years) with grade 2-4 GITM was evaluated. The GITM severity was evaluated according to WHO toxicity scale, the pain intensity assessment tool depended on patient's age and capability. All patients were divided into two groups based on age: 1 to 6 years $(n=32)$ and 7 to 17 years $(n=41)$. 
According to pain intensity all patients received iv tramadol, morphine or fentanyl via PCA. All patients unable to control the equipment for any reason were assisted by caregiver. The target pain score was 4 points (by any scale used) for younger patients, while in older patients group it was determined individually. In each case the process was controlled by dedicated pain management team members.

Results: The median of initial pain intensity was 5 (range 0 to 8 ) and 5 (range 2 to 8 ) points with median breakthrough pain intensity of 6 (range 2 to 8 ) and 7 (range 2 to 10) points in the first (younger) and second (older) groups, accordingly. After therapy initiation the average baseline / breakthrough pain intensity decreased to $2 / 4$ and $3 / 5$ in the first and second groups, respectively. The ability for fluid intake was preserved in 16 (50\%) first group and 18 (44\%) of second group patients. Eight (25\%) first group and $13(32 \%)$ second group patients were also able to eat solid foods. The bolus button was operated by seven (22\%) of younger group and all but one (98\%) older group patients. The normal daily activities were preserved in 28 (88\%) patients from first group and 36 (88\%) from second. In 4 (12\%) younger patients the PCA had to be ceased due to drowsiness $(n=3)$ and photophobia $(n=1)$. For older group patients the main therapy cessation reasons were nausea and vomiting exacerbation in upright position in 1 $(2 \%)$ and dizziness in $4(10 \%)$ patients.

Conclusions: PCA allows individual selection of analgesic dose providing a good balance of pain relief and side effects regardless mucositis severity. It is manageable in pediatric population and significantly improves quality of life and comfort level of pediatric patients.

\section{Clinical Trial Registry: no}

Conflict of interest: no

\section{P281}

\section{Pegfilgrastim versus Filgrastim in autologous peripheral stem cell transplantation}

José G. Ríos-Contreras, Manuel A. Granja-Morán, Brenda L. Acosta-Maldonado, Liliana Rivera-Fong, Luis M. Valero-Saldaña

Instituto Nacional de Cancerología, Hematology, Ciudad de México, Mexico

Background: In the scenario of high doses of chemotherapy (HDT) and autologous stem cell transplantation (ASCT) in hemato-oncological diseases, there is still controversy about the efficacy and safety of granulocytestimulating cytokines, pegylated versus non-pegylated forms, in hematological recovery, infections risk, hospital stay, overall survival and event-free survival.
Methods: Retrospective, comparative, from January 2009 to November 2017, receiving filgrastim $10 \mathrm{mg} / \mathrm{kg} /$ day until neutrophil recovery or pegfilgrastim $6 \mathrm{mg}$ single dose at day +5 . Febrile neutropenia $\left(\mathrm{Neu}<500 / \mathrm{mm}^{3}\right)$ defined if the patient's temperature was $\geq 38.3^{\circ} \mathrm{C}$ in one measurement or $\geq 38.0^{\circ} \mathrm{C}$ for more than one hour. Haematological recovery defined as the first of 3 consecutive days with neutrophils $\geq 500 / \mathrm{mm}^{3}$ and platelets $\geq 20,000 / \mathrm{mm}^{3}$ for 7 consecutive days without transfusion requirement. Statistical analysis: numerical variables with Student's t test, nominal variables with $\chi^{2}$ and the ordinal ones with Mann Whitney`s U.

Results: A total of 185 patients, the two cohorts were similar in baseline characteristics analyzed in gender, median age, chemotherapy lines prior to ASCT, disease status at ASCT, mobilization scheme, myeloablative conditioning regimen according to disease, and median CD34+ infused cells. Median age of 47 years (18-75), male sex $61 \%$ (112); diagnosed in $37 \%$ (68) with multiple myeloma, $17 \%$ (31) with Hodgkin's lymphoma, 44\% (82) with non-Hodgkin's lymphoma and 2\% (4) with germ-cell tumors; the CD34+ infused cells with a media of $2.77 \times 10^{6} \%$ $\mathrm{kg}$ of body weight (1.38-6.26), 2.21 (1.38-6.26) and 2.87 (1.97-5.80) in filgrastim and pegfilgrastim group, respectively. Forty-one patients (22\%) received filgrastim and 144 (78\%) pegfilgrastim. With media duration of neutropenia < $500 / \mathrm{mm}^{3}$ for 4 days (1-9) and 3 days (1-9) $(\mathrm{p}=0.021)$; febrile neutropenia in 58\% (24) and 55.6\% (80) $(\mathrm{p}=0.438)$, with media duration of 5 days (2-10) and 4 days (1-10) $(\mathrm{p}=0.04)$; clinically or microbiologically documented infections occurred in 32\% (13) and 33\% (48) ( $\mathrm{p}=0.502)$ in the filgrastim and pegfilgrastim groups, respectively. A media of 8 doses (6-18) was required in the filgrastim group and only 1 dose in the pegfilgrastim group ( $<0.0001)$; platelet transfusion requirement in 78\% (32) and $81 \%$ (117) with a media platelet apheresis/pool of 2 (1-5) and 1 (1-9) $(\mathrm{p}=0.117)$ and $\mathrm{RBC}$ concentrates in $19.5 \%$ (8) and 5.6\% (8) $(\mathrm{p}=0.01)$ with a media of $1 \mathrm{RBC}$ concentrate in both groups. A median hospital stay on the day of ASCT at the discharge of 17 days (12-24) and 15 days (11-37) $(\mathrm{p}=0.103)$ in the filgrastim and pegfilgrastim groups, respectively. With a 3 -year overall survival of $83 \%$ and $82 \%(\mathrm{p}=0.87)$, therapy-related mortality to transplantation was not significant either. No serious adverse events (grade 23) were reported.

Conclusions: Equivalence between filgrastim and pegfilgrastim can be considered in terms of efficacy and safety in hematological recovery and febrile neutropenia prophylaxis in ASCT. In this cohort, with shorter duration of neutropenia and transfusion requirement of RBC concentrate, both clinically significant parameters; however, it should be taken with reservation due to the greater number of patients with pegfilgrastim. With regard to cost, $6 \mathrm{mg}$ pegfilgrastim vial $\left(\right.$ Neulasta $\left.^{\circledR}\right)$ cost $\$ 1,700$ USD and 300 
mcg filgrastim vial $\left(\right.$ Neupogen ${ }^{\circledR}$ ) $\$ 275$ USD in Mexico, with superiority in cost-benefit issue.

Conflict of interest: Nothing to disclose.

\section{P282}

Post-transplant hypomethylating agent maintenance is associated with a shortened relapse free survival in myelodysplastic syndrome and acute myeloid leukemia

Michael Byrne ${ }^{1}$, Katie Culos ${ }^{2}$, Nathalie Danielson ${ }^{3}$, Brian Engelhardt ${ }^{1}$, Stacey Goodman ${ }^{1}$, Sanjay Mohan ${ }^{1}$, Caleb Prow ${ }^{2}$, Stephen Strickland ${ }^{1}$, Heidi Chen ${ }^{4}$, Wichai Chinratanalab ${ }^{1}$, Katie Gatwood $^{2}$, Bipin Savani ${ }^{1}$, Salyka Sengsayadeth ${ }^{1}$, Adetola Kassim ${ }^{1}$, Madan Jagasia ${ }^{1}$

${ }^{1}$ Vanderbilt University Medical Center, Medicine, Nashville, TN, United States; ${ }^{2}$ Vanderbilt University Medical Center, Pharmacy, Nashville, TN, United States; ${ }^{3}$ Vanderbilt University Medical Center, Nursing, Nashville, TN, United States; ${ }^{4}$ Vanderbilt University Medical Center, Biostatistics, Nashville, TN, United States

Background: A significant barrier to long-term survival after allogenic hematopoietic cell transplantation (HCT) in myeloid neoplasms is disease relapse. Post-HCT maintenance therapy with hypomethylating agents (HMA) is commonly used to prevent relapse after HCT at many transplant centers, however, data supporting this practice is limited. We hypothesized that post-HCT HMA would have

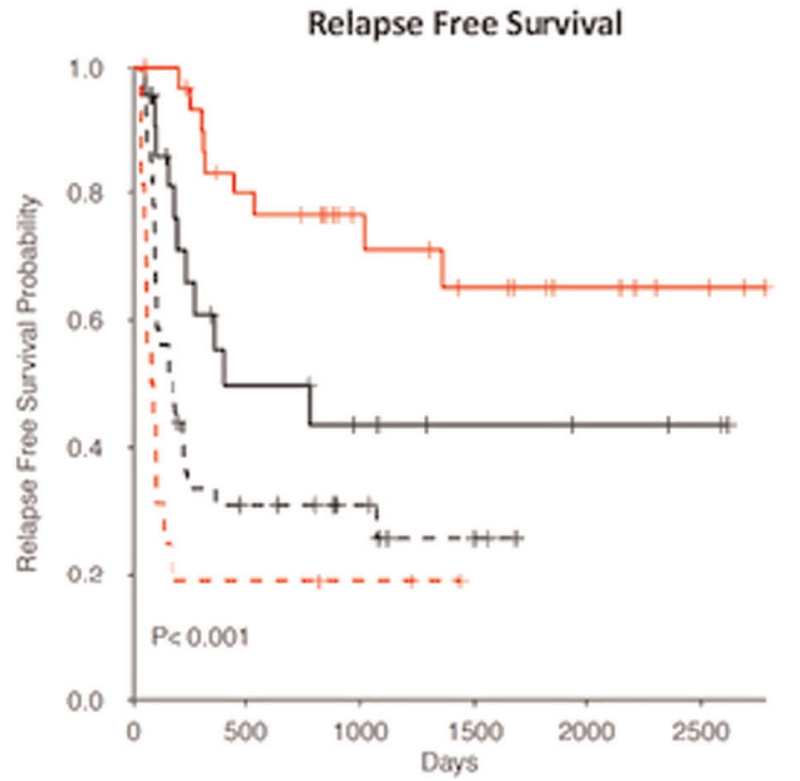

limited impact on disease outcome and practice patterns between physicians would be heterogeneous.

Methods: This is a single institution, retrospective study of patients with myelodysplastic syndrome (MDS) and acute myeloid leukemia (AML) who underwent HCT from 2005-2015. Patients were most frequently treated with azacitidine $100 \mathrm{mg} /$ day x 3 days for 2-4 cycles. Disease risk index (DRI) was utilized in order to draw comparisons between treated (at least one cycle of a HMA post-HCT) and untreated (no post-HCT HMA) patients with comparable disease biology (Armand, Blood 2012). Relapse free survival (RFS) and overall survival (OS) were calculated using the Kaplan-Meier method and compared using logrank test. Cox proportional hazard models were used for multivariate analyses. In order to assess practice variation in post-HCT HMA use, we conducted a blinded review of HCT physicians and non-HCT leukemia physicians using pertinent patient data. Fleiss Kappa agreement assessed for agreement in prescribing practices

Results: A total of 113 patients (57 treated and 56 untreated) were studied. Treated patients were more likely to be older (59.7 vs. 50.5 years, $\mathrm{p}=0.001$ ), have a very high/high $(\mathrm{VH} / \mathrm{H})$ DRI $(72 \%$ vs. $41 \%, \mathrm{p}=0.001)$, and receive reduced-intensity conditioning $(70 \%$ vs. $42 \%, \mathrm{p}=$ 0.03). In addition, fewer treated patients developed acute graft versus host disease (GVHD) (44\% vs. 67\%, p = 0.013). There was a trend toward earlier relapse amongst treated patients with $\mathrm{VH} / \mathrm{H}$ risk disease compared to those

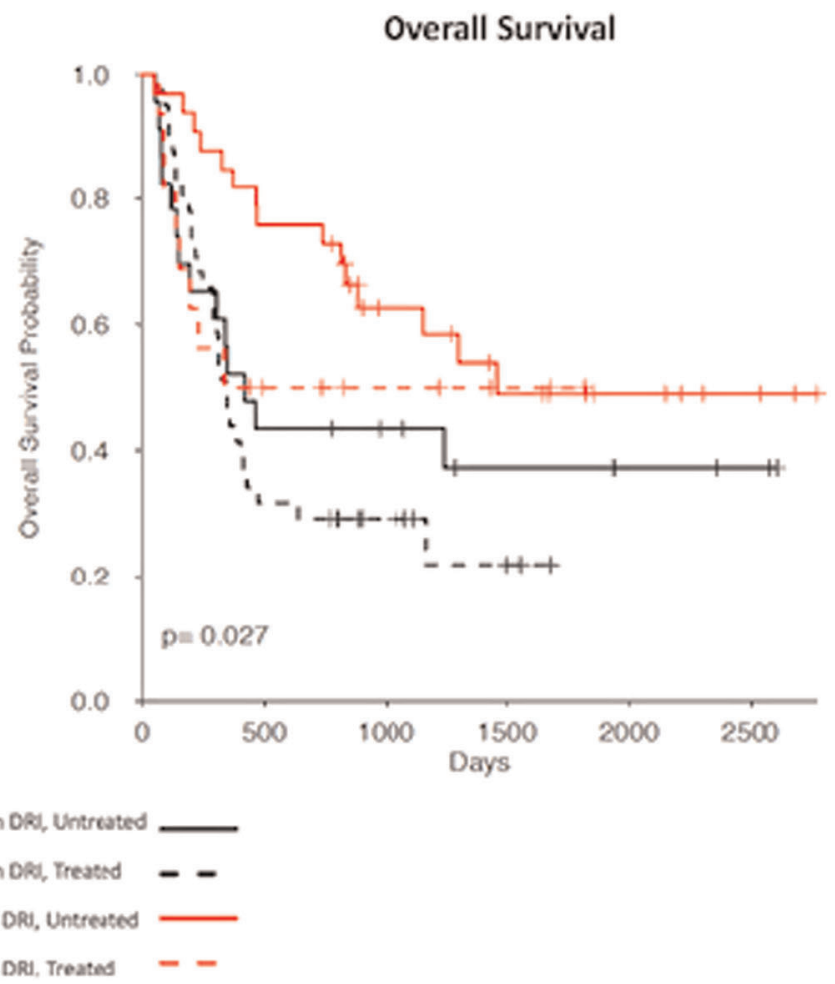

[P282 Figure] [Relapse free and overall survival of patients categorized by DRI and HMA use.] 
untreated (median of 175 vs. 407 days, $\mathrm{p}=0.066$ ). Similarly, HMA use in the intermediate and low-risk (I/L) group was associated with substantially shorter RFS compared to the untreated group (median of 81.5 days vs. not reached, $\mathrm{p}:<0.001)$. An important interaction between HMA use and DRI was identified for RFS ( $p=0.0318)$. In the OS analysis, patients treated with HMAs had a shorter median OS compared to untreated patients (VH/H: 344 days vs. 419 days, $\mathrm{p}=0.353$ and I/L: 336 days vs. 1461 days, $\mathrm{p}$ $=0.234$ ). Peripheral blood chimerism data from the patients that received treatment with post-HCT HMAs demonstrated a higher incidence of mixed myeloid and lymphoid chimerisms. Finally, a blinded review of HCT and leukemia physicians revealed moderate agreement amongst HCT physicians (0.54) and fair agreement between leukemia physicians (0.28) in their prescribing patterns.

Conclusions: Our data does not support the routine use of post-HCT HMA maintenance. Treated patients, particularly those with I/L-risk disease, experienced inferior RFS compared to treated patients. In our cohort, post-HCT HMA maintenance was associated with lower incidence of acute GVHD, possibly by enhancing the effect of Treg lymphocytes, which may have reduced the post-HCT graft versus leukemia effect. These findings, and underlying mechanisms, need further study. The results of a prospective study of post-HCT HMAs (NCT00887068) will better define the role of this intervention.

Conflict of interest: None of the authors has anything to disclose.

\section{P283}

\section{Predicting Survival after Autologous Stem Cell Transplantation. The GATMO score}

Mariano Berro ${ }^{1}$, Jorge Arbelbide ${ }^{2}$, Maria M Rivas ${ }^{1}$, Ana L Basquiera $^{2}$, Gonzalo Ferini ${ }^{2}$, Adriana Vitriu ${ }^{3}$, Cecilia Foncuberta ${ }^{3}$, Nicolas Fernandez Escobar ${ }^{4}$, Alejandro Requejo ${ }^{4}$, Vera Milovic ${ }^{5}$, Sebastian Yantorno ${ }^{6}$, Milagros Szelagowsky, Juliana Martinez Rolon $^{7}$, Gonzalo Bentolila ${ }^{7}$, Juan J Garcia ${ }^{8}$, Pablo Garcia ${ }^{8}$, Gaston Caiero $^{8}$, Martin Castro ${ }^{9}$, Gregorio Jaimovich ${ }^{9}$, Silvina Palmer ${ }^{10}$, Eduardo Bullorsky ${ }^{10}$, Martin Saslavsky ${ }^{11}$, Patricio Duarte ${ }^{12}$, Amalia Cerutti $^{13}$, Gustavo Jarchum ${ }^{14}$, Maria L Rizzi ${ }^{14}$, Jose I Trucco ${ }^{1}$, Lucia A Bet ${ }^{1}$, Matias Tisi Baña ${ }^{1}$, Bronwen E Shaw ${ }^{15}$, Gustavo D Kusminsky ${ }^{1}$

${ }^{1}$ Hospital Universitario Austral, Derqui, Argentina; ${ }^{2}$ Hospital Italiano, Buenos Aires, Argentina; ${ }^{3}$ Instituto Alexander Fleming, Buenos Aires, Argentina; ${ }^{4}$ Fundacion Favaloro, Buenos Aires, Argentina; ${ }^{5}$ Hospital Aleman, Buenos Aires, Argentina; ${ }^{6}$ Hospital Italiano, La Plata, Argentina; ${ }^{7}$ Fundaleu, Buenos Aires, Argentina; ${ }^{8}$ Hospital Privado, Cordoba, Argentina; ${ }^{9}$ Sanatorio Anchorena, Buenos Aires, Argentina; ${ }^{10}$ Hospital Britanico, Buenos Aires, Argentina; ${ }^{11}$ CETRAMOR, Rosario, Argentina; ${ }^{12}$ Cemic, Buenos Aires, Argentina; ${ }^{13}$ Sanatorio Britanico, Rosario, Argentina;
${ }^{14}$ Sanatorio Allende, Cordoba, Argentina; ${ }^{15}$ CIBMTR, Medical College of Wisconsin, Medicine, Milwaukee, WI, United States

Background: There have been several efforts to predict mortality after autologous stem cell transplantation (ASCT), like the HCT-CI. This index has demonstrated its value in ASCT by several groups including us. There is no valid score in the setting of ASCT combining comorbidities with other patient characteristics. Our objective is to describe a comprehensive score including comorbidities with other clinical factors and analyze the impact of this score in early morbidity end-points (mechanical ventilation, shock or dialysis), NRM and OS after ASCT.

Methods: We retrospectively reviewed data of 2068 adult patients who received an ASCT in Argentina (01/2000-04/ 2017) for Multiple Myeloma (MM) or Lymphoma. We compared NRM and Relapse with Grey test, OS with
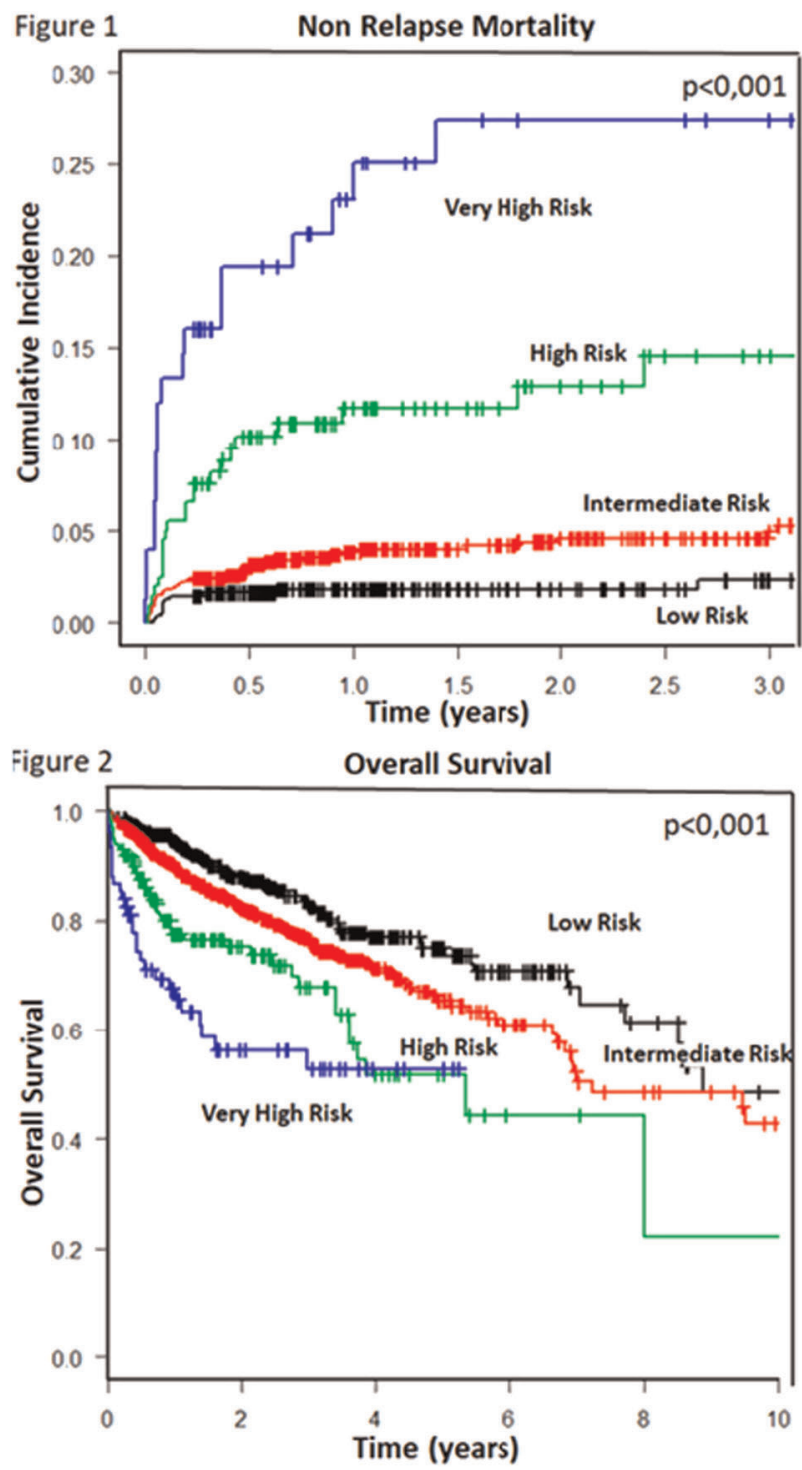

[P283 Figure] [Figures 1 and 2] 
Kaplan Meyer, morbidity end-points with $\mathrm{chi}^{2}$ and long term multivariate analysis (MVA) for NRM with fine-Gray regression. All the factors remaining significant after MVA for NRM were included in the score, and were assigned a value of 1 if the hazard ratio was $<3.5$, or 2 if the HR was $\geq 3.5$.

Results: Mean age was 51 years; 59\% were male, 52\% had MM, 30\% Non-Hodgkin Lymphoma (NHL) and 18\% Hodgkin Lymphoma (HL). Fifty-three percent were in CR, $44 \%$ in $\mathrm{PR}$ and $3 \% \mathrm{SD} / \mathrm{PD} ; 13 \%$ received three or more chemotherapy lines before transplant (heavily pre-treated). Regarding comorbidities, 58\% had HCT-CI 0, 29\% 1-2 and $13 \% \geq 3$. Median follow up was 1.1 years (range 100 days14 years). Early NRM (day 100) was 3.1\%, 1-3 years was 4.7-5.8\% and OS (1-5 years) was $89-66 \%$. On MVA risk factors showing an independent significant impact with NRM were: male patients ( 1 point), age 55-64 (1 point), $\geq 65$ years ( 2 points), HCT-CI $\geq 3$ ( 1 point), HL ( 1 point) and NHL ( 2 points). The hazard ratio for NRM increased proportionally with the score. Patients were grouped as low risk (LR) with a score 0-1 (686, 33\%), intermediate risk (IR) score 2-3 (1109, 53\%), high risk (HR) score $4(198,10 \%)$ and very high risk (VHR) score $\geq 5(4 \%)$. The score was associated with a progressive increase in all the early morbidity end-points (table). Moreover, the score was significantly associated with early NRM (day $100: 1.5 \%$ vs. $2.4 \%$ vs. $7.6 \%$ vs. $17.6 \%)$ as well as long term (1-3 years $1.8-2.3 \%$ vs. $3.8-4.9 \%$ vs. $11.7-14.5 \%$ vs. $25.0-27.4 \%$ respectively, $\mathrm{p}<0.0001$; hazard ratio, $95 \% \mathrm{CI}$, ref LR: 2.2 , $1.2-3.9 ; 6.4,3.3-12.4 ; 14.0,6.9-28.2)$ and OS (1-5 years 94$73 \%$ vs. $8-75 \%$ vs. $76-47 \%$ vs. $65-52 \%$ respectively, p < 0.0001 ; hazard ratio, $95 \% \mathrm{CI}$, ref LR: $1.4,1.1-1.8 ; 2.5,1.8-$ 3.6; 3.9, 2.6-6.1).

Conclusions: [We describe a novel score predicting NRM and OS in a large cohort of more than 2000 autologous transplants performed in Argentina. This tool can serve for tailoring conditioning regimens or defining risk for outpatient transplant programs.

Conflict of interest: There is no relevant conflict of interest to disclose.

\begin{tabular}{llllll}
\hline \multicolumn{6}{l}{ GATMOS score impact in early morbidity end-points } \\
\hline & Low Risk & Interm. Risk & High Risk & Very High Risk & p value \\
\hline OTI & $2.9 \%$ & $4.9 \%$ & $10.6 \%$ & $22.7 \%$ & $<0.0001$ \\
Shock & $1.9 \%$ & $5.1 \%$ & $9.1 \%$ & $18.7 \%$ & $<0.0001$ \\
Dialysis & $1.0 \%$ & $2.1 \%$ & $4.0 \%$ & $5.3 \%$ & $<0.01$ \\
\hline
\end{tabular}

[[P283 Table] Early end-points]

\section{P284}

Abstract previously published

\section{P285}

Prophylactic prostaglandin E1 prevent sinusoidal obstruction syndrome/veno-occlusive disease of the liver after allogeneic hematopoietic stem cell transplantation

Sung-Nam Lim ${ }^{1}$, Won-Sik Lee ${ }^{2}$, Young-Don Joo ${ }^{3}$, Beoung-Suk Sohn ${ }^{4}$, Seon-Yang Park

${ }^{1}$ Haeunda Paik Hospital, Inje University College of Medicine, Internal Medicine, Busan, Korea, Republic of; ${ }^{2}$ Inje University College of Medicine/Busan Paik Hospital, Internal Medicine, Busan, Korea, Republic of: ${ }^{3}$ On Hospital, Internal Medicine, Busan, Korea, Republic of; ${ }^{4}$ Inje University College of Medicine/Sangye Paik Hospital, Internal Medicine, Seoul, Korea, Republic of; ${ }^{5}$ Haeundae Paik Hospital, Internal Medicine, Busan, Korea, Republic of

Background: Prostglandin E1 (PGE1) was used to prevent sinusoidal obstruction/veno-occlusive disease (SOS/VOD) of the liver after allogeneic hematopoietic stem cell transplantation (HSCT) for hematologic malignancies.

Methods: It was given in continuous intravenous infusion from day -8 to day 14 after allogeneic HSCT at a dose of 0.3 micrograms $/ \mathrm{kg} /$ hour. The patients were studied according to the risk factors for SOS/VOD: diagnosis, intensification of the conditioning and previous liver abnormalities. The diagnosis of SOS/VOD was made on revised diagnosis and severity criteria for SOS/VOD in new classification from the European society for blood and marrow transplantation. Seventy eight patients underwent allogenic HSCT between September 2010 and November 2017 and 71 were treated by PEG1 and seven did not received it.

Results: The median age of including patients was 45 year-old (range, 18-64 year-old) and male was 36 patients (46\%). The actual incidence of SOS/VOD was $1.3 \%$ (one patient) in the PGE1 group and $14.3 \%$ (one patient) in the non PGE1 group $(\mathrm{P}<0.001)$. SOS/VOD developed in two of 78 patients (2.6\%) with diagnosis day 7 and day 11 after hematopoietic stem cell infusion. of two patients, one had modrate SOS/VOD and one had severe SOS/VOD as new EBMT criteria for severity grading. Two patients who was diagnosed in SOS/VOD were in acute leukemia.

Conclusions: The prophylactic PGE1 treatment may decrease the incidence of SOS/VOD in patients by allogenic hematopoietic stem cell transplantation.

Conflict of interest: Nothing to disclose

\section{P286}

PROTECTIVE DIET VERSUS NON-RESTRICTIVE DIET IN PATIENTS WITH PROLONGED NEUTROPENIA: PRELIMINARY DATA FROM A MULTICENTER, RANDOMIZED TRIAL 
Alberto Mussetti ${ }^{1}$, Giulia Perrone ${ }^{1}$, Martina Pennisi ${ }^{1}$, Anna Guidetti $^{1,2}$, Paola Matteucci ${ }^{1}$, Daniela Tomaiuolo ${ }^{1}$, Giorgia Gobbi ${ }^{1}$, Lucia Saracino ${ }^{1}$, Rita Russo ${ }^{1}$, Nicola Mordini ${ }^{3}$, Paolo Corradini ${ }^{1,2}$

${ }^{1}$ Fondazione IRCCS Istituto Nazionale Tumori, Milano, Italy;

${ }^{2}$ University of Milano, Dept. of Oncology and Hematology, Milano, Italy; ${ }^{3}$ Ospedale Santa Croce e Carle, Cuneo, Italy

Background: The effect of a protective diet (PD) in patients with prolonged neutropenia has never been tested prospectively in adult hematologic patients versus a non restrictive diet (NRD).

Methods: We started since July 2016 a multicenter randomized interventional study comparing the use of a PD vs NRD in hematological patients with $\geq 18$ years for whom a prolonged neutropenia ( $>7$ days) was expected. Random allocation was 1:1 between the two diet types. Adherence to the study was represented by the ingestion of at least one of the diet-specific foods each day. If the patient was unable to ingest food, this consideration was not applicable. For the $\mathrm{PD}$, foods cooked above $80^{\circ} \mathrm{C}$ and/or thick peel fruit were considered diet-specific. For the NRD, raw fruit and vegetables (washed as per standard hygiene hospital standards) were considered diet-specific. Moreover, patients receiving an allogeneic hematopoietic cell transplant (alloHCT) were allocated 1:1 between the two study cohorts. An interim analysis for safety was planned after the enrollment of the first 60 patients. Results were evaluated by an independent Data Monitoring and Safety Committee.

Results: Of the enrolled 60 patients, there were 4 dropouts due to medical reason or patientÂ's will. For the analysis, each cohort was represented by 28 patients. Seventeen patients received an alloHCT, 8 patients in the PD and 9 patients in the NRD group respectively. Cyclosporine plus methotrexate was used as standard GVHD prophylaxis in all patients. Anti-thymocyte globulin was added in case a matched-unrelated donor according to institutional guidelines.

The number of patients who developed at least 1 event was $15(54 \%)$ for both groups [relative risk $R R=1.00$, confidence interval $(\mathrm{CI}) 95 \%=0.62,1.61$, table 1]. Other secondary endpoints were analyzed. The number of patients developing gastrointestinal infections and fever of unknown origin during hospitalization was $7(25 \%)$ versus $2(7 \%)$ $(\mathrm{RR}=0.29, \mathrm{CI} 95 \%=0.07,1.10)$ and $9(32 \%)$ versus 7 $(25 \%)(\mathrm{RR}=0.78, \mathrm{CI} 95 \%=0.34,1.77)$ between $\mathrm{PD}$ and NRD respectively. Weight variation (\%) from the start of the study to the discharge was $4.50 \%$ vs $5.35 \%$ (difference $\mathrm{D}=0.85$, CI95\% $=-1.33,3.03$ ). Hospitalization length was 26 days vs 21 days $(\mathrm{D}=-5$ days, $\mathrm{CI} 95 \%=-11.9,0.54)$. No deaths were reported at day +30 . For alloHCT patients, patients who developed acute GVHD grade $\geq 2$ (Seattle criteria) at day +100 was $25 \%$ vs $22 \%$ respectively (1 patients died before day +100 ).

Conclusions: The preliminary analysis shows no significant differences between the use of a PD versus a NRD for hematological patients during prolonged neutropenia in terms of infections and deaths. These results support the continuation of the study.

Conflict of interest: Nothing to disclose

\begin{tabular}{|c|c|c|c|}
\hline & $\begin{array}{l}\text { Protective } \\
\text { diet, } \mathrm{n}(\%)\end{array}$ & $\begin{array}{l}\text { Non- } \\
\text { restrictive } \\
\text { diet, } \mathrm{n}(\%)\end{array}$ & $\Delta^{*}$ \\
\hline $\begin{array}{l}\text { PRIMARY ENDPOINT Infections } \\
\text { grade }>2 \text { or deaths during } \\
\text { neutropenia }-1 \text { event }-2 \text { events }\end{array}$ & $\begin{array}{l}15(54 \%) 12 \\
(43 \%) 3(11 \%)\end{array}$ & $\begin{array}{l}15(54 \%) \\
13(46 \%) 2 \\
(7 \%)\end{array}$ & $\begin{array}{l}1.00 \\
(0.62,1.61)\end{array}$ \\
\hline $\begin{array}{l}\text { SECONDARY ENDPOINT GI } \\
\text { tract infections during } \\
\text { hospitalization }-1 \text { event }-2 \text { events }\end{array}$ & $\begin{array}{l}7(25 \%) 6 \\
(21 \%) 1(4 \%)\end{array}$ & $\begin{array}{l}2(7 \%) 2 \\
(7 \%)-\end{array}$ & $\begin{array}{l}0.29 \\
(0.07,1.10)\end{array}$ \\
\hline $\begin{array}{l}\text { SECONDARY ENDPOINT FUO } \\
\text { during hospitalization - } 1 \text { event - } 2 \\
\text { events }\end{array}$ & $\begin{array}{l}9(32 \%) 8 \\
(29 \%) 1(4 \%)\end{array}$ & $\begin{array}{l}7(25 \%) 6 \\
(21 \%) 1 \\
(4 \%)\end{array}$ & $\begin{array}{l}0.78 \\
(0.34,1.77)\end{array}$ \\
\hline $\begin{array}{l}\text { SECONDARY ENDPOINT Weight } \\
\text { variation }(\%) \text { from the start to the } \\
\text { end of hospitalization, mean (SD) }\end{array}$ & $4.50 \%(3.55)$ & $\begin{array}{l}5.35 \% \\
(2.18)\end{array}$ & $\begin{array}{l}0.85 \\
(-1.33,3.03)\end{array}$ \\
\hline $\begin{array}{l}\text { SECONDARY ENDPOINT } \\
\text { Hospitalization length in days, mean } \\
\text { (SD) }\end{array}$ & 26 days (9.04) & $\begin{array}{l}21 \text { days } \\
(7.76)\end{array}$ & $\begin{array}{l}-6 \text { days } \\
(-12,1)\end{array}$ \\
\hline $\begin{array}{l}\text { SECONDARY ENDPOINT Overall } \\
\text { survival at day }+30 \text { from study } \\
\text { initiation }\end{array}$ & NO EVENTS & $\begin{array}{l}\text { NO } \\
\text { EVENTS }\end{array}$ & - \\
\hline $\begin{array}{l}\text { SECONDARY ENDPOINT Acute } \\
\text { GVHD at day }+100 \text { from study } \\
\text { initiation Death before day }+100\end{array}$ & $\begin{array}{l}8 \text { patients } \\
\text { overall } 2(25 \%) \\
1(12 \%)\end{array}$ & $\begin{array}{l}9 \text { patients } \\
\text { overall } 2 \\
(22 \%)\end{array}$ & $\begin{array}{l}0.59 \\
(0.13,2.49)\end{array}$ \\
\hline
\end{tabular}

[[P286 Table] Table 1: Study outcomes accordingly to diet type]

\section{P287}

Pulmonary complications in patients with hematopoietic stem cell transplantation. A Cohort study

Claudia Lucia Sossa Melo ${ }^{1}$, Carlos Machado ${ }^{2}$, Sara Ines Jimenez ${ }^{1}$, Manuel Rosales ${ }^{3}$, Angela Maria Peña Castelllanos ${ }^{1}$, Luis Salazar', Miguel Enrique Ochoa Vera ${ }^{1}$, Manuel Ardila ${ }^{4}$, Jenifer Sandoval ${ }^{4}$, Jenifer Garay ${ }^{4}$

${ }^{1}$ FOSCAL, Bucaramanga, Colombia; ${ }^{2}$ Universidad Autonoma de Bucaramanga/FOSCAL, Internal Medicine - Division Neumology, Bucaramanga, Colombia; ${ }^{3}$ Clinica Carlos Ardila Lulle (FOSCAL), Hematology and Transplantation, Bucaramanga, Colombia;

${ }^{4}$ Universidad Autonoma de Bucaramanga/FOSCAL, Internal Medicine, Bucaramanga, Colombia

Background: Literature described that patients who undergo hematopoietic stem cell transplantation may develop approximately $30 \%$ to $60 \%$ of early or late pulmonary complications, the importance is that it increases the morbidity and mortality, and has been relate to $10 \%$ to $40 \%$ of deaths. 
Table 1

Characteristics of the 6 pasients, with autologous transplantation that had preumonia between follow up 101-365 days. Including Mucositis a known factor for lung infectious complication

\begin{tabular}{|c|c|c|c|}
\hline Patient age and gender & Diagnose & Mucositis & Immunoglobulin A.G. M \\
\hline male, $62 \mathrm{y}$ & B-cell lymphoma & negative & Unknown \\
\hline male, $58 \mathrm{y}$ & Multiple myeloma & negatve & Normal-Low-Low \\
\hline Female $60 y$ & Lymphoma non hodgkin & negative & Normal-low-low \\
\hline Fomale $56 \mathrm{y}$ & Multiple myeloma & negative & Low-normal-normal \\
\hline Male $62 y$ & Lymphoms non hodgkin & negaive & Low-normal.low \\
\hline Male 24 y & Lymphoma non hodgkin & positve & $10 w-10 w-10 w$ \\
\hline
\end{tabular}

Figure 1. Pulmonary infection and non-infection complication in allogeneic stem cell transplantation patients.

\section{ALLOGENEIC STEM CELL TRANSPLANTATION}

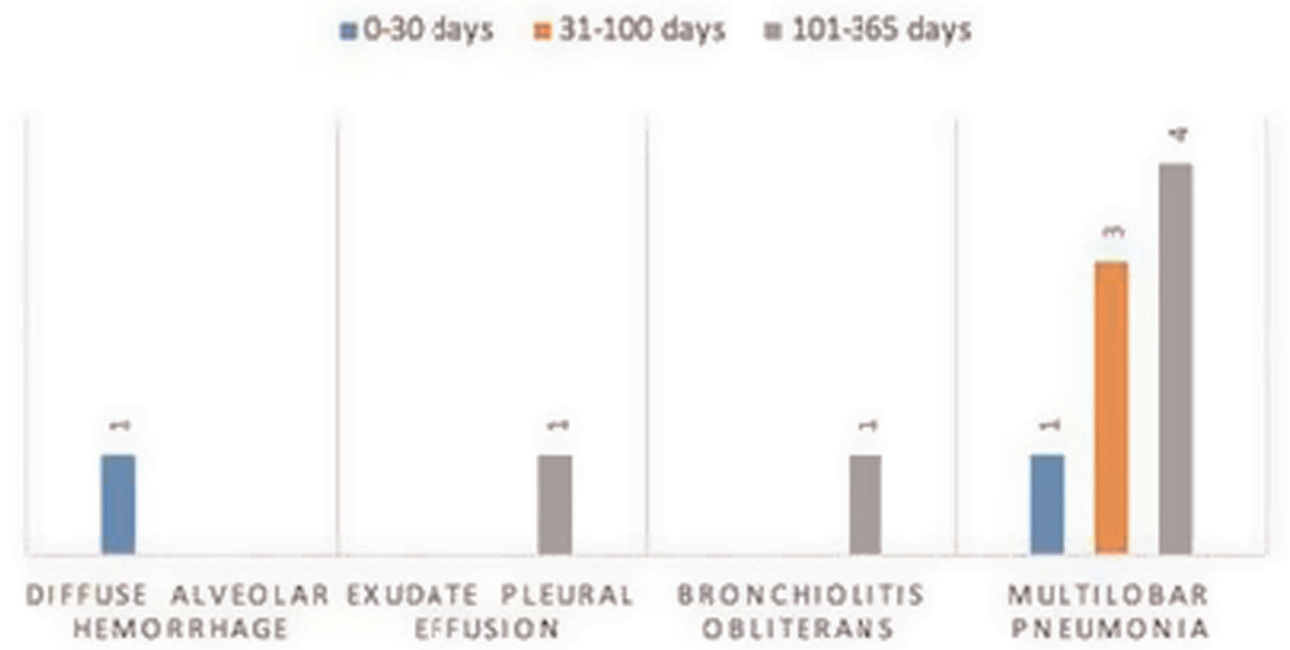

[P287 Figure] [Pulmonary infection and non-infection complication in allogeneic stem cell transplantation patients.]

Methods: We conducted an ambispective study, reviewing the records of all the patients who underwent allogeneic transplant between at our institution between 2010 and 2017. And the ones that underwent autologous stem cell transplantation between 2015 and 2017. The follow up was at 30 days, 31 to 100 days and 100 to 365 days.

Results: A total of 105 patients were studied, 54 autologous transplantation with a mean age 50 years old, $46.30 \%$ were female and $53.70 \%$ were male, BEAM (BCNU, etoposide[Office1], cytarabine, melphalan) conditioning regimen was the most common used, we found 9.3\% $(\mathrm{n}=5)$ incidence of early infection complication at 0 30 days, at $31-100$ days $4 \%(\mathrm{n}=2)$, and late infection complication (101-365 days) was $11.11 \%(\mathrm{n}=6$, see table 1 ), non-infection complication was $2 \%, 0 \%, 2 \%$ respectively. Also 51 allogeneic transplant was reviewed with a mean age of 31 years old, $60.8 \%$ male and $39.22 \%$ female, $66.67 \%$ had myeloablative conditioning regimen (thiopet busulfan fludarabin) meanwhile $31.37 \%$ had reduced intensity. Cyclosporine and methotrexate was most usual used for GVHD prophylaxis. Early infection complication was $2 \%(\mathrm{n}=1,0-30$ days $), 4 \%$ (31-100 days) $0 \%$, late infection complication $(101-365$ days) was $8 \%(\mathrm{n}=4)$ and $4 \%(\mathrm{n}=2)$ for non-infection was $2 \%, 0 \%$ and $2 \%$ complications respectively. $23.5 \%$ had changes in the spirometry after the transplantation, mild restrictive ventilatory defect was the predominantly defect found $(n=9)$ and carbon monoxide diffusing capacity of the lung the usual defect identify was mild decrease of the DLCO with a normal alveolar volume $(21.57 \mathrm{n}=11)$.

Conclusions: In comparison with reports in the literature of other Hematopoietic Stem Cell Transplantation centers 
around the world, the incidence of complications found in our center was much lower, but keeping the tendency to be predominantly infectious causes.

Conflict of interest: The authors declare no conflicts of interest

\section{P288}

\section{Quality of Life Following Haematopoietic Stem Cell Transplantation}

\section{Claudia Lucia Sossa Melo ${ }^{1,2}$, Sara Ines Jimenez ${ }^{1,2}$, Angela Maria Peña Castellanos ${ }^{1,2}$, Manuel Rosales ${ }^{1}$, Oscar Mariano Pinto Saavedra ${ }^{3}$, Gustavo Adolfo Serrano Baez, Miguel Enrique Ochoa Vera $^{4}$, Maria Lucrecia Luna ${ }^{5}$, Claudia Marcela Chalela ${ }^{1}$, David Leonardo Reyes ${ }^{1}$ \\ ${ }^{1}$ Universidad Autonoma de Bucaramanga/FOSCAL, Internal Medicine - Division of Hematology and Hematopoietic Stem Cell Transplantation, Bucaramanga, Colombia; ${ }^{2}$ Universidad Autonoma de Bucaramanga, School of Medicine, Bucaramanga, Colombia; ${ }^{3}$ Universidad Autonoma de Bucaramanga/FOSCAL, Internal Medicine, Bucaramanga, Colombia; ${ }^{4}$ Universidad Autonoma de Bucaramanga/FOSCAL, Epidemiology, Bucaramanga, Colombia; \\ ${ }^{5}$ Universidad Autonoma de Bucaramanga, School of Medicine - Data Manager, Bucaramanga, Colombia}

Background: There has been an important increase in the survival rate in patients with hematopoietic stem cell transplantation (HSCT). Subsequently, the assessment of health-related quality of life (HRQoL) in STC recipients has gained great interest in the medical community.

Methods: A retrospective cohort study was conducted. HSCT adult patients were recruited from a single institution in Colombia, South America between November 2014 and March 2016. HRQL was assessed at 0, 30, 90, and 180 days after HSCT, using the European Organization for Research and Treatment of Cancer Quality of Life Questionnaire Core 30(EORTC QLQ-C30). No exclusion criteria.

Results: Forty-nine patients were included (25 men and 24 women). Average age 47,90 \pm 15 (Ages 18-77). The present study showed a decline in global quality of life (QL) regardless of the type of SCT, especially in the functional scales during the first month, as demonstrated in similar studies (Wettergren et al). There were statistically significant differences in the physical functioning scale in the first and sixth-month post-transplantation $(\mathrm{P}=0.06, \mathrm{P}=$ $0.04)$, role functioning scale in the first month $(\mathrm{P}=0.001)$, and social functioning scale in the first and third month (P $=0.016, \mathrm{P}=0.044$ ) when compared to the mean baseline. Comparing autologous with allogenic SCT, the autologous SCT group had a greater decline in quality of life than the allogenic SCT group. There was a statistically significant decrease in the role functioning scale in autologous SCT patients during the first month post-transplantation, showing that the probability of decrease in the role function is 2.57 times more likely in autologous than in allogenic transplantation (IC95=1.51-4.36). After three months of transplantation, the social functioning scale in the autologous group showed a higher decline rate than the allogenic (RR 4.1, IC95=1.06-15.82).

Analyzing symptom scales between autologous and allogenic SCT, pain was 1.85 more likely in autologous SCT patients than in allogenic at three months posttransplantation (IC: 0.52-6.62). There were no statistically significant differences between the two groups at six months post-transplantation. After excluding nonmyeloablative SCT patients, the study showed statistically significant differences when comparing autologous vs. allogenic SCT. At 30 days post-transplantation, there was a significant decline in global QL (RR 3.08, IC95=1.138.45), physical functioning (RR 1.98, IC95 $=1.12-3.52$ ), role functioning (RR 2.7, IC95=1.58-4.6), fatigue (RR 3.85 , IC95=1.56-9.56), and nausea/vomiting (RR 2.89, IC95 $=1.28-6.53)$ in autologous vs. allogenic transplantation. There were differences in emotional functioning (RR 4.63, IC95=1.13-18.87) and social functioning (RR 6.17, I95 $=1.7-22.34)$ at three months and global QF (RR 2.31, IC 95\%, 1.09-4.92) at six months. There were no differences when comparing myeloablative vs. nonmyeloablative SCT. Mortality at six months post-transplantation was $4.08 \%$.

Conclusions: There is a significant decline in quality of life following hematopoietic stem cell transplantation. HSCT Patients show an improvement in QL after three months post-tranplantation, reaching baseline levels after six months. Autologous SCT patients have a greater decline in QL than allogenic SCT patients.

Conflict of interest: The authors declared no potential conflict of interest.

\section{P289}

Recovery from hypogonadism and male health in adult allogeneic stem cell transplantation

Laila Schneidewind ${ }^{1}$, Thomas Neumann ${ }^{1}$, Kai Probst ${ }^{2}$, Andrzej Plis ${ }^{1}$, Christian Andreas Schmidt ${ }^{1}$, William Krüger ${ }^{1}$

${ }^{1}$ University Medicine Greifswald, Greifswald, Germany; ${ }^{2}$ Westpfalz Hospital, Kaiserslautern, Germany

Background: There is a substantial lack of data about men`s health and hypogonadism in adult allogeneic stem cell transplantation.

Methods: Therefore, we conducted prospective unicentric non-interventional clinical study on men's health with a focus on male hypogonadism in adult allogeneic stem cell transplantation with a follow up time of one year.

Results: Between 11/2013 and 12/2015, we were able to include 27 patients. Acute myeloid leukaemia (AML) was 
the most frequent underlying disease (25.9\%), we mainly used intermediate intense conditioning protocols (77.8\%). In terms of Graft versus host disease (GvHD) prophylaxis this is a very homogenous population since all patients had to receive low dose alemtuzumab (10 $\mathrm{mg}$ at day -2 ; respectively $20 \mathrm{mg}$ in case of mismatch). Erectile dysfunction, loss of libido and loss of efficiency were the most frequent symptoms of hypogonadism. At inclusion of the study hypogonadism was already frequent. Primary hypogonadism was found in eight cases (29.6\%) and secondary hypogonadism in one case $(3.7 \%)$. We did not observe hypogonadism 6 months after inpatient treatment anymore, but there might still be the impairment of fertility because of still rising FSH levels at the end of the observation period. There were no significant associations of hypogonadism with myeloablative conditioning or kind of donor. Interestingly, there is a significant association with nicotine abuse $(\mathrm{p}=0.049)$.

Conclusions: On the whole, we report first data about male hypogonadism in allogeneic stem cell transplantation. Interestingly, no case of hypogonadism was observed 6 months after inpatient treatment. Hypogonadism can have high impact on organ function and quality of live, so further studies, especially with longer follow up periods, are necessary.

Clinical Trial Registry: Ethical approval BB116/2013 from 8 October 2013

Conflict of interest: All authors state that there is no conflict of interest regarding this study.

\section{P290}

\section{Reduced plasma amino acid levels during allogeneic HSCT are associated with systemic inflammation and treatment- related complications}

Sarah Weischendorff, ${ }^{1,2}$, Katrine Kielsen ${ }^{1,2}$, Maria Nederby
${ }^{3}$, Lotte
Svendsen ${ }^{3}$, Douglas Burrin ${ }^{4}$, Carsten Heilmann ${ }^{1}$, Marianne
Ifversen ${ }^{1}$, Henrik Sengelov ${ }^{5}$, Christian Molgaard ${ }^{3}$, Klaus Müller ${ }^{1,2}$
${ }^{1}$ Rigshospitalet, Department of Paediatrics and Adolescent Medicine,
Copenhagen, Denmark; ${ }^{2}$ Rigshospitalet, Institute for Inflammation
Research, Center for Rheumatology and Spine Disease, Copenhagen,
Denmark; ${ }^{3}$ University of Copenhagen, Department of Nutrition,
Exercise and Sports, Copenhagen, Denmark; ${ }^{4}$ Baylor College of
Medicine, USDA/ARS, Children's Nutrition Research Center,
Department of Pediatrics, Houston, TX, United States;
${ }^{5}$ Rigshospitalet, Department of Haematology, Copenhagen, Denmark

Background: Patients undergoing allogeneic haematopoietic stem cell transplantation (HSCT) are challenged by a high degree of systemic inflammation due to cytotoxic effects of the conditioning regimen, which elevates the metabolic demand for amino acids involved in tissue repair, regeneration of the hematopoietic cells, and antioxidant defenses. Few studies have addressed amino acid levels during transplantation, and it remains unknown if amino acid deficiency may aggravate treatment-related morbidity. The aim of the present study was to measure plasma levels of free amino acids (PAA) before and after transplantation, and to investigate whether PAA-levels are related to the level of inflammation and treatment-related complications.

Methods: We included 80 patients (age 1.1 -55.4) treated with allogeneic HSCT between 2010 and 2013 in Copenhagen, Denmark. All patients received pre-treatment with myeloablative conditioning based on total body irradiation combined with chemotherapy $(n=45)$ or chemotherapy alone $(n=35)$. Free plasma concentrations of the 23 human amino acids were measured using ultraperformance liquid chromatography at baseline and on day 7 post-transplant along with C-reactive protein (CRP) and interleukin 6 (IL-6) levels on day 7 post-transplant.

Results: A significant decrease in PAA concentrations from baseline to day 7 was found for tyrosine, aspartate, glutamine, asparagine and citrulline, while an increase was observed for leucine, valine, tryptophan and phenylalanine (all $\mathrm{p}<0.05$ ). On day 7 post-transplant, several amino acids were inversely correlated with both CRP and IL-6, in particular serine $(\mathrm{r}=-0.50, \mathrm{p}<0.0001$ and $\mathrm{r}=-0.41, \mathrm{p}=$ 0.0004), glutamine $(\mathrm{r}=-0.44, \mathrm{p}<0.0001$ and $\mathrm{r}=-0.53, \mathrm{p}<$ $0.0001)$, glycine $(\mathrm{r}=-0.53, \mathrm{p}<0.0001$ and $\mathrm{r}=0.61, \mathrm{p}<$ $0.0001)$ and lysine $(\mathrm{r}=-0.51, \mathrm{p}<0.0001$ and $\mathrm{r}=-0.66, \mathrm{p}<$ 0.0001 ), but also glutamic acid, alanine, arginine, cysteine, histidine, tryptophan, threonine, taurine, proline, and methionine (all $\mathrm{p}<0.05$ ). High levels of CRP and IL-6 were associated with a larger decrease in plasma levels of these amino acids between baseline and day 7, suggesting a dynamic relationship with the inflammatory response; these associations were most pronounced for serine, glutamine, glycine and threonine ( $\mathrm{r}$ between -0.47 and -0.53 , all $\mathrm{p}<$ $0.01)$.

Eleven patients developed severe aGvHD (defined as grade 3-4) and 18 patients developed sinusoidal obstruction syndrome (SOS). Patients with SOS had significantly lower levels of some amino acids compared to patients without SOS on day 7 including glutamic acid $(\mathrm{p}=0.028)$, serine $(\mathrm{p}=0.013)$, arginine $(\mathrm{p}=0.015)$, glycine $(\mathrm{p}=0.004)$, lysine $(\mathrm{p}=0.0008)$, valine $(\mathrm{p}=0.048)$, tryptophan $(\mathrm{p}=0.0007)$, threonine $(\mathrm{p}=0.003)$ and proline $(\mathrm{p}=0.031)$. Furthermore, patients with severe aGvHD had lower levels of serine $(\mathrm{p}=0.004)$, glutamine $(\mathrm{p}=0.029)$, cysteine $(\mathrm{p}=0.021)$, glycine $(\mathrm{p}=0.034)$, lysine $(\mathrm{p}=0.047)$ and threonine $(\mathrm{p}=0.010)$.

Conclusions: Significant changes occur in the plasma concentrations of several human amino acids during HSCT. Associations were found between low plasma concentrations of amino acids involved in anti-inflammatory mechanisms (including tissue renewal) and increased 
systemic inflammation, SOS and severe aGvHD. Further studies should address if reduced PAA is caused by an insufficient intake and/or increased consumption of these amino acids due to stress metabolism, and whether substitution of amino acids during the course of transplantation may reduce treatment-related morbidity.

Conflict of interest: The authors declare no financial conflict of interest.

\section{P291}

\section{REFRACTORY HAEMORRHAGIC CYSTITIS SUCCESSFULLY TREATED WITH SPRAY FIBRIN GLUE ON BLADDER MUCOSA}

\begin{abstract}
Maria Cristina Tirindelli ${ }^{1}$, Alessandra Picardi ${ }^{2}$, Pierluigi Bove ${ }^{3}$, Raffaella Cerretti ${ }^{2}$, Laura Cudillo ${ }^{2}$, Gottardo De Angelis ${ }^{2}$, Ernesto Pavoni $^{2}$, Sonia Soave ${ }^{2}$, Alessandro Lanti ${ }^{4}$, Ombretta Annibali ${ }^{1}$, Carolina Nobile ${ }^{1}$, Anna Paola Iori ${ }^{5}$, Lidia De Felice ${ }^{6}$, Ursula La Rocca $^{6}$, Teresa Dentamaro ${ }^{7}$, Simona Jacobelli ${ }^{8}$, Stella Santarone ${ }^{9}$, Fabrizio Bonanni $^{10}$, Corrado Terella ${ }^{11}$, Giuseppe Avvisati ${ }^{1}$, William Arcese $^{12}$

${ }^{1}$ Campus Biomedico University Hospital, Hematology Stem Cell Transplant Transfusion Medicine and Cellular Therapy, Rome, Italy; ${ }^{2}$ Tor Vergata University of Rome, Stem Cell Transplant Unit, Policlinico Universitario Tor Vergata, Rome, Italy; ${ }^{3}$ Tor Vergata University Hospital, Division of Urology, Rome, Italy; ${ }^{4}$ Tor Vergata University, Division of Blood Bank, Department of

Immunohematology, Rome, Italy; ${ }^{5}$ Sapienza University,, Stem Cell Transplant Unit, Department of Hematology, Rome, Italy; ${ }^{6}$ Sapienza University, Stem Cell Transplant Unit, Department of Hematology, Rome, Italy; ${ }^{7}$ Sant'Eugenio Hospital, Division of Hematology and Transplant, Rome, Italy; ${ }^{8}$ tor Vergata University, Medical Statistics, Department of Biology, Rome, Italy; ${ }^{9}$ Civil Hospital, Stem Cell Transplant Unit, Pescara, Italy; ${ }^{10}$ Tor Vergata University Hospital, Rome, Italy; ${ }^{11}$ European Institute of Oncology, Milan University, Hemato-Oncology Division, Milano, Italy; ${ }^{12}$ Tor Vergata University of Rome, Stem Cell Transplant Unit, Policlinico Universitario Tor Vergata, Biomedicina e Prevenzione, Rome, Italy
\end{abstract}

Background: Haemorrhagic cystitis (HC) occurring after allogeneic transplant significantly affects quality of life and in some cases becomes intractable increasing the risk of death. To date, its therapy is not established. In 2014, we reported the first experience of Rome Transplant Network (RTN) in treating 35 patients with refractory post-transplant $\mathrm{HC}$ using the haemostatic agent fibrin-glue (FG) diffusely sprayed on bleeding mucosa by an endoscopic applicator. The cumulative incidence of pain discontinuation and complete remission, defined as regression of all symptoms and absence of haematuria, was $100 \%$ at 7 days and $87 \pm 7 \%$ at 50 days from FG application, respectively. Herein, we report an update of that experience on 63 patients including 28 new patients entered the multicentre study on FG treatment for $\geq 2^{\text {nd }}$ grade post-transplant HC.

Methods: With respect to the first 35 patients, the 28 new patients were slightly older, received more heterogeneous conditioning regimens, presented more frequently grade 4
$\mathrm{HC}$, received less cidofovir and underwent later FG treatment; however, except for cidofovir therapy, these differences were not statistically significant. A descriptive analysis for the demographic and clinical characteristics of the patients was performed including median, standard deviations and range for continuous variables, absolute and relative frequencies for categorical variables. Taking into account the corresponding competing risks, pain discontinuation, catheter removal and complete response were estimated with the cumulative incidence method. Overall survival was estimated according to the Kaplan-Meier product-limit method.

Results: All 63 patients received an allogeneic transplant for high-risk hematological malignancy: $6(9.5 \%) \mathrm{CB}, 9$ (14.3\%) HLA identical Sib, 19 (30,2\%) MUD and 29 (46\%) Haplo. Of these patients, $19(30 \%)$ developed $\geq 2$ grade acute GVHD occurring at a median of 25 days (range 1069) after transplant. BK viremia and viruria detected at any time before the $\mathrm{HC}$ onset were positive in $65 \%$ and $85 \%$ of patients. HC occurred at a median of 34 days (range 5-146) after transplant and was of grade 2, 3 and 4 in 15 (24\%), 40 $(63 \%)$ and $8(13 \%)$ patients, respectively, at time of FG treatment, which was administered at a median of 14 days (range 1-146) from the $\mathrm{HC}$ onset. The numbers of $\mathrm{FG}$ application were 1 for 33 (53\%), 2 for 21 (33\%) and 3 for 9 patients (14\%). Overall, a response was obtained in 59 (94\%) patients being partial in $10(16 \%)$ and complete in 49 (78\%). Only 4 patients were non responders. For responder patients, the pain discontinuation occurred at a median of 1 day (range 1-7) and catheter removal at a median of 5 days (range 1-33) from FG. In univariate analysis, the complete response was significantly correlated with type of graft donor, grade of $\mathrm{HC}$ and interval time from $\mathrm{HC}$ onset and FG application. The 90-day and 2-year probability of survival for all patients was $78 \%$ and $35 \%$, respectively.

Conclusions: From this last analysis, we can confirm that FG therapy is a feasible, effective, repeatable and inexpensive procedure for treating $\geq 2$ grade $\mathrm{HC}$ after allogeneic transplant. Furthermore, FG should be applied early after the onset of $\mathrm{HC}$.

Conflict of interest: none

\section{P292}

Relationship between employment status and quality of life after allogeneic hematopoietic cell transplantation according to patient age and GVHD status

Saiko Kurosawa ${ }^{1}$, Takuhiro Yamaguchi ${ }^{2}$, Kumi Oshima ${ }^{3}$, Atsumi Yanagisawa $^{4}$, Takahiro Fukuda ${ }^{1}$, Heiwa Kanamori ${ }^{5}$, Takehiko Mori $^{6}$, Satoshi Takahashi , Tadakazu Kondo ${ }^{8}$, Shin Fujisawa ${ }^{9}$, Yasushi Onishi ${ }^{10}$, Shingo Yano ${ }^{11}$, Makoto Onizuka ${ }^{12}$, Yoshinobu Kanda $^{13}$, Ishikazu Mizuno ${ }^{14}$, Shuichi Taniguchi ${ }^{15}$, Takuya Yamashita $^{16}$, Yoshihiro Inamoto ${ }^{1}$, Shinichiro Okamoto ${ }^{6}$, Yoshiko 


\section{Adjusted mean scores of QOL: Employment status \\ \%Change: changes in jobs, employers, or positions af ter allo-HCT \\ SF-36 physical component summary SF-36 mental component summary}

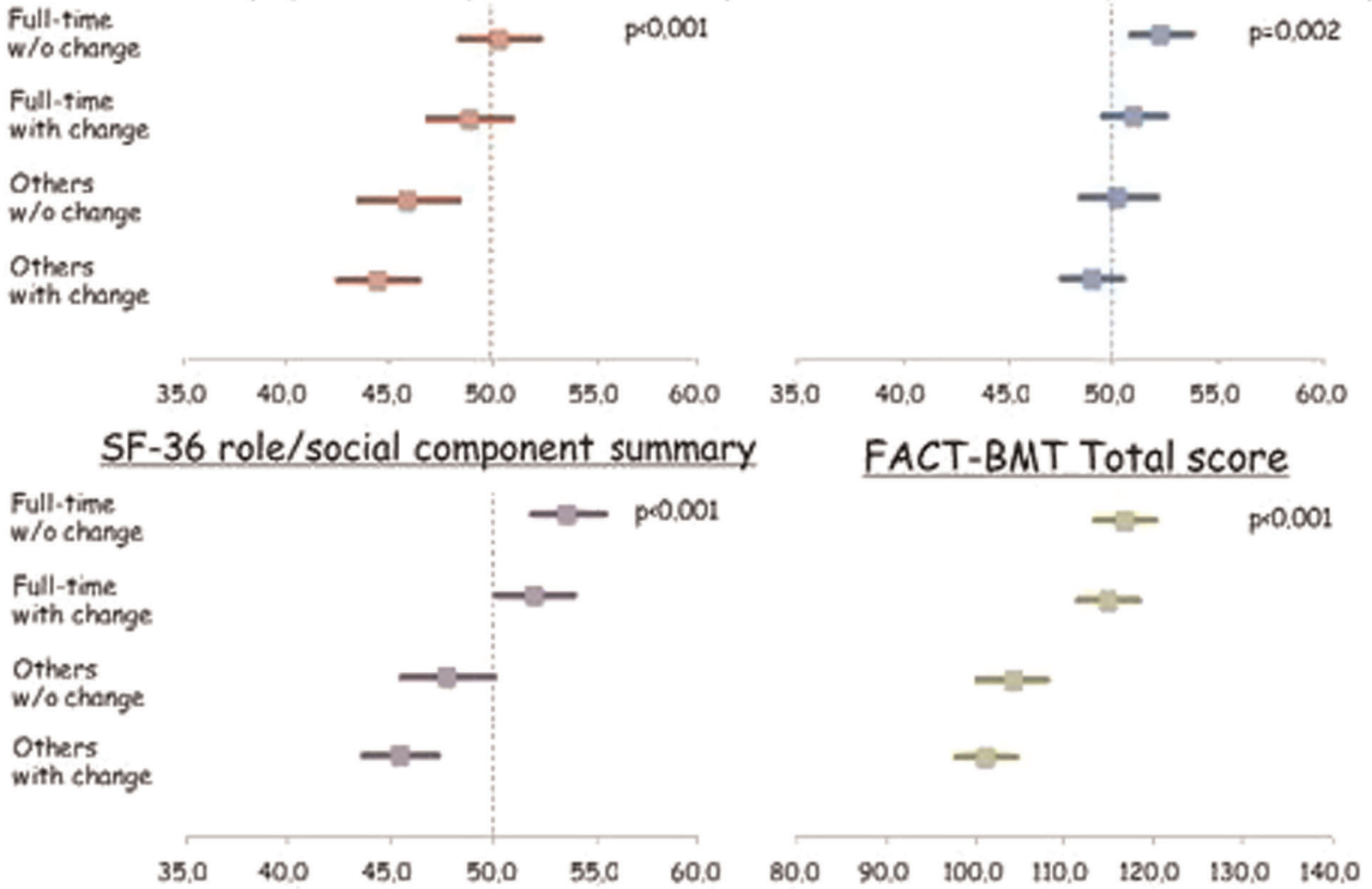

[P292 Figure] [Physical, mental, role/social QOL and FACT-BMT total in 4 groups by employment status]

Atsuta $^{4,17}$

${ }^{1}$ National Cancer Center Hospital, Department of Hematopoietic Stem Cell Transplantation, Tokyo, Japan; ${ }^{2}$ Tohoku University Graduate School of Medicine, Sendai, Japan; ${ }^{3}$ Jyoban Hospital, Tokiwa Foundation, Fukushima, Japan; ${ }^{4}$ Japanese Data Center for Hematopoietic Cell Transplantation, Nagoya, Japan; ${ }^{5}$ Kanagawa Cancer Center, Yokohama, Japan; ${ }^{6}$ Keio University School of Medicine, Tokyo, Japan; ${ }^{7}$ Institute of Medical Science, University of Tokyo, Tokyo, Japan; ${ }^{8}$ Kyoto University, Kyoto, Japan; ${ }^{9}$ Yokohama City University Medical Center, Yokohama, Japan; ${ }^{10}$ Tohoku University Hospital, Sendai, Japan; ${ }^{11}$ Jikei University School of Medicine, Tokyo, Japan; ${ }^{12}$ Tokai University School of Medicine, Isehara, Japan; ${ }^{13}$ Jichi Medical University, Tochigi, Japan; ${ }^{14}$ Hyogo Cancer Center, Akashi, Japan; ${ }^{15}$ Toranomon Hospital, Tokyo, Japan; ${ }^{16}$ St. Luke's International Hospital, Tokyo, Japan; ${ }^{17}$ Nagoya University Graduate School of Medicine, Nagoya, Japan

Background: The relationship of employment status and QOL after allo-HCT remains to be elucidated. To address this critical issue, we conducted a nationwide crosssectional questionnaire study.

Methods: Allo-HCT recipients with hematological diseases, who were aged $\geq 16$ years at transplant, and $\geq 20$ years without relapse at survey were included. QOL was assessed by SF-36 and FACT-BMT, and participants were asked to provide lifestyle information. Multivariable models were constructed to examine the relationship between QOL and employment status after adjusting for background covariates.

Results: In 1250 patients who were informed of the study, consent was obtained from 1216, and 1149 responded. 750 patients who were 30-59 years of age at survey were included in the analysis. The cohort consisted of $52 \%$ male, their median age at survey was 47 , and their median time after allo-HCT was 7.2 years (3.4-18.9). At the time of the survey, $52 \%$ of patients were employed fulltime, $18 \%$ employed part-time, and $29 \%$ were unemployed/ housewife/student. 50\% experienced a career change in jobs/employers/positions after allo-HCT. The cohort was divided into 4 groups by employment status ('full-time/not' and 'with/without change'). Patients employed full-time without a career change (full/same) had the highest scores for the physical (PCS), mental (MCS), role/social (RCS) component summary, and FACT-BMT total. Patients not employed full-time had significantly lower scores $(\mathrm{p}<0.01)$ for most domains compared to the full/same group (Figure). 
Patients in their 30s at survey had PCS comparable to the general population regardless of the employment status with no significant difference among groups. On the other hand, patients in their $40 \mathrm{~s} / 50 \mathrm{~s}$, who were not employed full-time, had a significantly lower PCS compared to the full/same group, while MCS was comparable to the general population with no significant difference among groups. In patients without chronic GVHD at survey, PCS, MCS, and RCS in the full/same group were higher than those of the general population. In patients with GVHD, PCS and RCS in those not employed full-time were significantly lower compared to the full-time groups or the general population.

Conclusions: Employment status was highly correlated with QOL after allo-HCT, and the impact may differ according to patient age and GVHD status.

Clinical Trial Registry: UMIN000009545

Conflict of interest: The authors have no conflict of interest to disclose.

\section{P293}

Risk factors for early transplantation related mortality in patients undergoing allogeneic stem cell transplantation

Da Jung Kim ${ }^{1}$, Sung Mi Jung ${ }^{2}$, Jee-Yeong Jeong ${ }^{3}$, Ji Hyun Lee ${ }^{4}, \mathrm{Su}$ Hee Cho ${ }^{5}$, Ki Sun Jung, Jae-Cheol Jo ${ }^{6}$, Silvia Park ${ }^{7}$, Ho Sup Lee

${ }^{1}$ Kosin University College of Medicine, Department of Internal Medicine, Busan, Korea, Republic of; ${ }^{2}$ Kosin, University Gospel Hospital, Registered Nurse, Stem Cell Transplantation Clinical Nurse Specialist, Busan, Korea, Republic of; ${ }^{3}$ Kosin University College of Medicine, Department of Biochemistry Cancer Research Institute, Busan, Korea, Republic of; ${ }^{4}$ Dong-A University College of Medicine, Department of Internal Medicine, Busan, Korea, Republic of; ${ }^{5}$ Pusan National University Yangsan Hospital, Yangsan, Korea, Republic of; ${ }^{6}$ Ulsan University Hospital, University of Ulsan College of Medicine, Ulsan, Korea, Republic of; ${ }^{7}$ Samsung Medical Center, Sungkyunkwan University School of Medicine, Seoul, Korea, Republic of

Background: Transplantation-related mortality (TRM) is an important factor in patients undergoing allogenic stem cell transplantation (AlloSCT). TRM can be divided into early and late TRM based on 100 days after transplantation. However, causes of TRM have been reported the age of the patient, tumor burden prior to transplantation, comorbid conditions, intensity of conditioning, infection, and acute GVHD etc. However, there have been reported that with the development of transplantation techniques, the incidence of TRM is also decreasing, but there is a lack of reports on the incidence rates of TRM and there are few reports about causes of TRM. Therefore, this study was conducted to analyze the TRM rate in Korea and analyze the cause of TRM.

Methods: This study included patients with acute leukemia, MDS (myelodysplastic syndrome), and CML (chronic myeloid leukemia) who received alloSCT. They underwent conditioning regimen based on fludarabine (30 $\mathrm{mg} / \mathrm{m}^{2}$ daily, for 5days) or cyclophosphamide $\left(50 \mathrm{mg} / \mathrm{m}^{2}\right.$ daily for 2days), intravenous busulfan $(3.2 \mathrm{mg} / \mathrm{kg}$ daily for 2-4days) and r-ATG (total dose of $2.5-9 \mathrm{mg} / \mathrm{kg}$, for 13days) prior to alloSCT. All patients received from peripheral blood stem cell as graft source and they received calcinurin inhibitor as GVHD prophylaxis started at day-1.

Results: Data from patients at five university hospitals in South Korea between Sep 1998 and Jun 2016 were collected retrospectively. We analyzed 978 consecutive patients with a median age of 44 years (range, 10-75) and the male was $58.6 \%$. The $616(63.0 \%)$ patients were acute leukemia and others were MDS or CML. The 364 (37.2\%) patients were received myeloablative conditioning regimen and $523(53.5 \%)$ received reduced intensity of conditioning. The 457 (46.7\%) patients were received alloSCT until 2009, and others were from 2010. The incidence rates of early TRM were $80(8.2 \%)$ in all patients. The predictive factors for early TRM were age (younger less than 50 years vs more than ; $6.9 \%$ vs $10.7 \%, \mathrm{p}=0.042)$, disease status before alloSCT (CR1 vs CR2 or non-CR ; $5.1 \%$ vs $20.2 \%$, p $<0.001$ ), and type of alloSCT (sibling vs unrelated vs haploidentical ; $5.6 \%$ vs $13.1 \%$ vs $8.4 \%, \mathrm{p}=0.005$ ). However, intensity of conditioning, and year of alloSCT were not shown different results comparing previous other reports. Reduced intensity of conditioning (RIC) were shown higher TRM than those of myeloablative conditioning (MAC) $(10.1 \%$ vs $7.0 \%, p=0.050)$ because RIC was performed more frequently from 2010 than until 2009. Acute GVHD more than grade II, CD34 counts, and comorbid conditions were not shown significant difference. In multivariate analysis, CR2 or non-CR and unrelated donor were independent significant risk factors for early TRM (RR; 2.820, and 2.394, p $<0.001$ and 0.004, respectively).

Conclusions: In this study, the incidence of early TRM, defined as death within 100 days after stem cell transplantation, was $8.2 \%$. In addition, patients who were CR2 or non$\mathrm{CR}$ in the pre-transplant disease status and received unrelated donor source of transplanted cells were identified significant high incidence rates of early TRM..

Conflict of interest: All authors have no conflict of interest to report.

\section{P294}

\section{Second Malignant Tumors after Hematopoietic Cell Transplantation: Retrospective Cohort Study}

Sara Alves ${ }^{1}$, Rosa Branca ${ }^{2}$, João Dias ${ }^{1}$, Luís Leite ${ }^{2}$, Carlos Pinho Vaz $^{2}$, Fernando Campilho ${ }^{2}$, Susana Roncon ${ }^{3}$, António Campos Junior ${ }^{2}$ 
${ }^{1}$ Instituto Português de Oncologia do Porto Francisco Gentil, Medical Oncology, Porto, Portugal; ${ }^{2}$ Instituto Português de Oncologia do Porto Francisco Gentil, Bone Marrow Transplantation Department, Porto, Portugal; ${ }^{3}$ Instituto Português de Oncologia do Porto

Francisco Gentil, Cellular Therapy Department, Porto, Portugal

Background: The success of hematopoietic cell transplantation (HCT) has led to development of secondary malignancies in patients who would otherwise be long-term survivors. Among the malignancies are solid tumors, myelodysplastic syndromes, and post-transplant lymphoproliferative disease (PTLD). We aim to describe what were the most common second malignant tumors after HCT at Instituto Português de Oncologia do Porto (IPOP) as well as determinate if there was difference between time to diagnosis of the second neoplasia in allogenic and autologous transplant.

Methods: Retrospective cohort of patients submitted to hematopoietic cell transplantation at IPOP, since june 1989 until june 2017. Data of second malignant tumors, main diagnosis that led to hematopoietic transplantation, and the date of the diagnosis were collected.

Results: Of 2159 patients treated on our center since 1989,89 were eligible for this study.

$29(32,6 \%)$ were women and $60(67,4 \%)$ were men. The median age of 47,9 (7-74). 44 (49,4\%) were submitted to an autologous transplant. The most common second malignant tumor diagnosed were oral cavity (12 patients - 14,1\%), post-transplant lymphoproliferative disease ( 9 patients $10,6 \%$ ), myelodysplastic syndrome (9 patients - 10,6\%) and nonmelanoma skin cancer ( 9 patients - 10,6\%). Median time to diagnosis of the second tumor, since the transplant was 50 months (1-296).

43 patients $(47,8 \%)$ have died. Median overall survival was 9,7 years (1-29), since the diagnosis of first malignant neoplasia. 26 patients $(29,2 \%)$ had the diagnosis of a third neoplasia.

The median time to diagnosis of the second tumor between patients submitted to an autologous transplant was 4,4 years and to an allogenic transplant was 6,6 years, and it was statistically significant $(\mathrm{p}=0,047)$.

Conclusions: The success of HCT has led to the complication of secondary malignancies in patients who would be long-term survivors. The most common second malignant tumor diagnosed at our Institution seems to be concordant with retrospective studies. HCT survivors should be advised of their increased risk of developing a malignancy following HCT and encouraged to report any concerning symptoms to their physician.

Conflict of interest: The authors report no conflict of interest

\section{P295}

\section{Secondary primary malignancies after autologuous hematopoietic stem cell transplantation: Single center experience}

Dilara Tekin ${ }^{1}$, Gizem Dagci ${ }^{1}$, Ipek Yonal Hindilerden ${ }^{2}$, Simge Erdem $^{2}$, Melih Aktan ${ }^{2}$, Meliha Nalcaci ${ }^{2}$, Deniz Sargin ${ }^{3}$, Sevgi Kalayoglu Besisik ${ }^{2}$

${ }^{1}$ Istanbul University Istanbul Faculty of Medicine, Internal Medicine Department, Istanbul, Turkey; ${ }^{2}$ Istanbul University Istanbul Faculty of Medicine, Internal Medicine Department, Division of Hematology, Istanbul, Turkey; ${ }^{3}$ Medipol University, Internal Medicine Department, Division of Hematology, Istanbul, Turkey

Background: The secondary primary malignancies (SPM) including acute leukemia, myelodysplastic syndrome, Hodgkin's lymphoma, non-Hodgkin's lymphoma, and solid tumors arise in hematopoietic stem cell transplant (HSCT) patients, are relatively rare complications of transplantation. We investigated the incidence of posttransplant SPMs in a cohort of 247 patients who had undergone autologous peripheral HSCT.

Methods: The medical records of patients who received autologous HSCT between 1996 - 2016 at our institution and survived at least one year were reviewed.

Results: The median age was 48 (range, 14 - 70) years with a sex ratio 152:95 M/F. Median follow-up since transplantation was 10.7years (range, 1-20 years) and 30 patients died after transplantation mostly due to disease progression. Previous diseases included multiple myeloma $(\mathrm{n}=132)$, and other plasma cell disorders $(\mathrm{n}=2)$, nonHodgkinÂ's lymphoma $(\mathrm{n}=59)$, Hodgkin's lymphoma ( $=39)$, acute leukemia $(\mathrm{n}=11)$, chronic myeloid leukemia $(\mathrm{n}=4)$.

Only 4 patients $(1.84 \%)$ developed SPM, each of them having a different origin. The median latency between transplantation and secondary neoplasms was 3 years (range: 2-11 years).

Case1: 33y/F. CML patient diagnosed before of tyrosine kinase inhibitors (TKI). She was refractory to standard treatment with interferon and cytarabine and had no donor. She achieved hematological response after etoposide based chemotherapy and underwent autologous SCT. During TKI era imatinib followed by nilotinib provided molecular response. At 11th year of transplantation, she was diagnosed as having ovarian cancer.

Case2: $48 \mathrm{y} / \mathrm{M}$. MM patient was in nearly complete response after transplantation. Lenalidomid has been giving as maintenance. At 3 years of HSCT, routine screening with PET-CT revealed a mass lesion which was histologically documented as renal cell carcinoma. 
Case 3: 47/F. MM patient was in complete response and receiving lenalidomide as maintenance. At 2 years after HSCT she developed epithelial tumor localized at her tongue.

Case 4: 58/M. MM patient in nearly complete response developed at 3 years of HSCT concurrent bladder carcinoma and pheocromocytoma. All of the patients are alive and have been cured for their SPM.

Conclusions: The factors effecting secondary cancer occurrence after HSCT are declared as age (>35), late period after HSCT, use of prior alkylating agent, use of topoisomerase II inhibitor, high dose pre transplantation radiotherapy. Our study encompasses all consecutive cases transplanted at our institute. SPM ratio would change with the time, among risk factors; our cases have all of those listed above. Moreover, smoking was an additional risk factor in the last patient. Thus benefit and risks including SPM should be weighed before the decision for autologous HSCT, taken into account that SPMs are curable malignancies in general.

Conflict of interest: All of the authors: Nothing to disclose

\section{P296}

Secondary solid tumor following allogeneic haematopoietic stem cell transplantation for patients with hematological malignancy: a single center analysis

Yuta Katayama $^{1}$, Koji Iwato ${ }^{2}$, Ryouta Imanaka ${ }^{1}$, Tatsuji Mino ${ }^{1}$, Takeshi Okatani ${ }^{1}$, Kouhei Kyo ${ }^{1}$, Mitsuhiro Itagaki ${ }^{1}$, Shinya Katsutani, Hideki Asaoku ${ }^{1,3}$

${ }^{1}$ Hiroshima Red Cross Hospital and Atomic Bomb Survivors Hospital, Division of Haematology, Hiroshima, Japan; ${ }^{2}$ Hiroshima Red Cross Hospital and Atomic Bomb Survivors Hospital, Division of

Transfusion, Hiroshima, Japan; ${ }^{3}$ Hiroshima Red Cross Hospital and Atomic Bomb Survivors Hospital, Division of Laboratory, Hiroshima, Japan

Background: Allogeneic haematopoietic stem cell transplantation (allo-HSCT) is an effective treatment for hematological malignancy. Secondary solid tumor (SST) after allo-HSCT has been reported as an important problem. We performed a retrospective survey of SST development following allo-HSCT.

Methods: We have retrospectively analyzed the records of 422 hematological malignancy adult patients who underwent allo-HSCT at our hospital between January 2007 and November 2017. SST was confirmed by tissue biopsy or surgical specimen.

Results: Seventeen patients (4.0\%) developed 20 SST after allo-HSCT(Table 1.): 3 patients with acute myeloid leukemia (AML), 2 with acute lymphoblastic leukemia, 9 with myelodysplastic syndrome (MDS) or MDS overt AML,
2 with Malignant Lymphoma, and 1 with chronic myeloid leukemia. The median time to SST diagnosis following alloHSCT was day 2021 (range 455 - 3581). Ages ranged from 19 to 62 years at allo-HSCT (median 47). Eight patients were treated with full-intensity conditioning regimens: high dose total body irradiation (TBI) $12 \mathrm{~Gy}$ containing regimens. Nine patients were treated with reduced-intensity conditioning regimens. The donor source was sibling bone marrow (BM) in 3 patients, sibling peripheral blood stem cell in 2 patients, unrelated BM in 10 patients and unrelated cord blood in 2 patients. Fourteen patients had developed chronic graft versus host disease (GvHD); all of those patients had extensive before developing SST. Five patients had taken methylprednisolone (mPSL), and 7 patients taken mPSL and tacrolimus at the time of SST diagnosis. Six patients developed secondary solid tumor in the same organ as chronic GvHD. Three patients developed two secondary solid tumor lesions and two out of those 3 patients developed two solid tumor legions simultaneously. Six patients had received local surgery only, and 3 patients had surgery and radiation, 3 patients had chemotherapy and radiation, 3 patients had surgery, chemotherapy and radiation, and 1 patient had cryoablation. One patient died before the therapy. Currently, 12 of 17 patients are alive and 8 patients are disease-free after therapy for SST. The median time of survival following therapy for SST was day 412 (range 19 - 1525). During therapy for SST, only one patient had relapsed primary hematological malignancy, MDS overt AML. The causes of death are progression of SST (4 patients) and progression of primary hematological malignancy (1 patient).

Conclusions: We suggest that SST development among hematological malignancy patients after allo-HSCT is not rare. There is a need for novel follow up strategies to manage SST of hematological malignancy patients after allo-HSCT.

Conflict of interest: The authors have nothing to disclose.

\begin{tabular}{llll}
\hline Site & Observed & Site & Observed \\
\hline All solid tumors & 20 & & \\
Tongue & 1 & Liver & 1 \\
Lip & 1 & Pancreas & 1 \\
Larynx & 1 & Kidney & 1 \\
Esophagus & 2 & Breast & 2 \\
Stomach & 1 & Cervix & 1 \\
Colon & 3 & Uterine corpus & 1 \\
Anal & 1 & Ovary & 1 \\
Lung & 2 & two sites of SST & 3 \\
\hline
\end{tabular}

[[P296 Table] Site of secondary solid tumor after alloHSCT] 


\section{P297}

\section{Secondary solid tumors of oral cavity and first airways following hematopoietic cell transplantation}

Stella Santarone ${ }^{1}$, Annalisa Natale ${ }^{1}$, Paola Olioso ${ }^{1}$, Mauro Di Ianni $^{1}$, Gabriele Papalinetti ${ }^{1}$, Andrea Di Bartolomeo ${ }^{2}$, Davide Bartolomeo Gissi ${ }^{2}$, Lucio Montebugnoli ${ }^{2}$, Stefano Angelini ${ }^{1}$, Paolo Di Bartolomeo

${ }^{1}$ UTIE, Ospedale Civile, Pescara, Dipartimento di Ematologia, Medicina Trasfusionale e Biotecnologie, Pescara, Italy; ${ }^{2}$ Clinica Odontoiatrica, Alma Mater Studiorum, Università di Bologna, Dipartimento di Scienze Biomediche e Neuromotorie, Bologna, Italy

Background: There is limited information about the occurrence of SSTs in the oral cavity and first airways in patients undergoing allogeneic HCT. The aim of this retrospective study was to determine the incidence and the clinical outcome of SSTs of oral cavity and first airways and to assess potential risk factors in 836 patients allotransplanted in our Center between 1977 and 2013.

Methods: Table 1 shows some characteristics of patient population at time of transplantation.

Results: Full engraftment of donor cells was evident in 794 patients (95\%). Acute and chronic GvHD affected 337 (40\%) and $176(21 \%)$ patients, respectively. The mortality rate due to either transplant-related causes or recurrence of original disease was 22\% (183 patients) and 20\% (165 patients), respectively. Moreover, 71 patients $(8 \%)$ died for late non-transplant related causes. At time of this report, 417 patients (50\%) are living with a median follow-up of 19 years (4-35). During follow-up, 12 patients developed a malignancy of oral cavity $(n=10$, of which 6 of tongue and 4 of oral mucosa) or larynx $(n=2) 3.8$ to 32.6 years (median 13.4 years) after HCT. In all cases, the histologic examination showed a squamous cell carcinoma. The 30-yr cumulative incidence (CI) of developing SST was 2.2 $\pm 0.005 \%$. All patients underwent surgical resection of the tumor and in addition 7 of them received radiotherapy and 6 chemotherapy. Of relevance, 9 out of 12 patients were affected by chronic GvHD with buccal cavity involvement in 8. Currently, 6 patients are living and SST-free, while 6 patients died of tumor progression. In univariate analysis, factors associated with an increased CI of SST were nonmalignant hematologic disease $(5 \%, \mathrm{P}=0.05)$, reduced intensity conditioning $(9.5 \%, \mathrm{P}=0.002)$, drugs used in the conditioning regimen different from irradiation or Busulfan (7\%, P $=0.009)$, chronic GvHD (7.7\%, P < 0.001), and oral chronic GvHD $(11.6 \%, \mathrm{P}<0.001)$. In the Fine and Gray's model of multivariate analysis, 2 factors maintained the significance: non-malignant hematologic disease (HR 3.53; $\mathrm{P}=0.0228$ ), and oral chronic GvHD (HR 13.452; $\mathrm{P}=$
0.0001). The 30-yr overall Kaplan Meyer survival of the 12 patients with STT was $52 \%$.

Conclusions: This study demonstrates that oral chronic GvHD and a diagnosis of non-malignant hematologic disease are strong risk factors in the development of new SSTs of oral cavity and first airways. Patients with cGvHD, especially those with involvement of the oral cavity, must receive a very long careful monitoring and surveillance in order to prevent the development of secondary cancers.

Conflict of interest: No conflict of interest

\begin{tabular}{ll}
\hline N. of patients & 836 \\
\hline Gender, male / female & $477 / 359$ \\
Median age, years (range) & $30(1-71)$ \\
Diagnosis Malignant disease & $\begin{array}{l}654 \text { (AML 236, ALL 145, CML 115, } \\
\text { Lymphoma 42, MM 44, MDS 51, other 21) }\end{array}$ \\
$\begin{array}{l}\text { Diagnosis Non-malignant } \\
\text { disease }\end{array}$ & $\begin{array}{l}\text { anemia 11, other 10) } \\
\text { Donors HLA identical sibling / }\end{array}$ \\
6nrelated / haploidentical & \\
$\begin{array}{l}\text { Stem cell source BM / PBSC / } \\
\text { CB }\end{array}$ & $612 / 216 / 8$ \\
Conditioning MAC / RIC & 695/141 \\
GvHD prophylaxis & $\begin{array}{l}\text { CSA 190 / CSA + MTX 613 / Tcell } \\
\text { depletion 30 / other 3 }\end{array}$ \\
\hline
\end{tabular}

[[P297 Table] Characteristics of patient population at time of $t]$

\section{P298}

Secondary Tumors following Hematopoietic Stem Cell

Tahani Sarrawi, Nilly Hussein, Mayada Abu-Shanap, Eman Khattab, Hasan Hashem, Ghadeer Al-Najjar, Rula Najjar, Mohammad Ma'koseh, Husam Abu Jazar, Khalid Halahleh, Abdelghani Tbakhi, Iyad Sultan, Rawad Rihani

King Hussein Cancer Center, Amman, Jordan

Background: Survival post hematopoietic stem cell transplantation (HSCT) is improving, and the number of patients living free of their original disease is increasing. As a result, the long term effects after HSCTs are increasingly identified. Second malignancies(SM) following HSCTs and their sequelae on survival and quality of life are of a particular concern.

Methods: A retrospective chart review was conducted of all patients who underwent HSCT between Jan, 2003 and Nov, 2017, after obtaining an IRB approval. Analysis was performed using Kaplan Meier curves and log-rank tests for survival and chi-square and Mann-Whitney test for categorical and numeric items, respectively. 


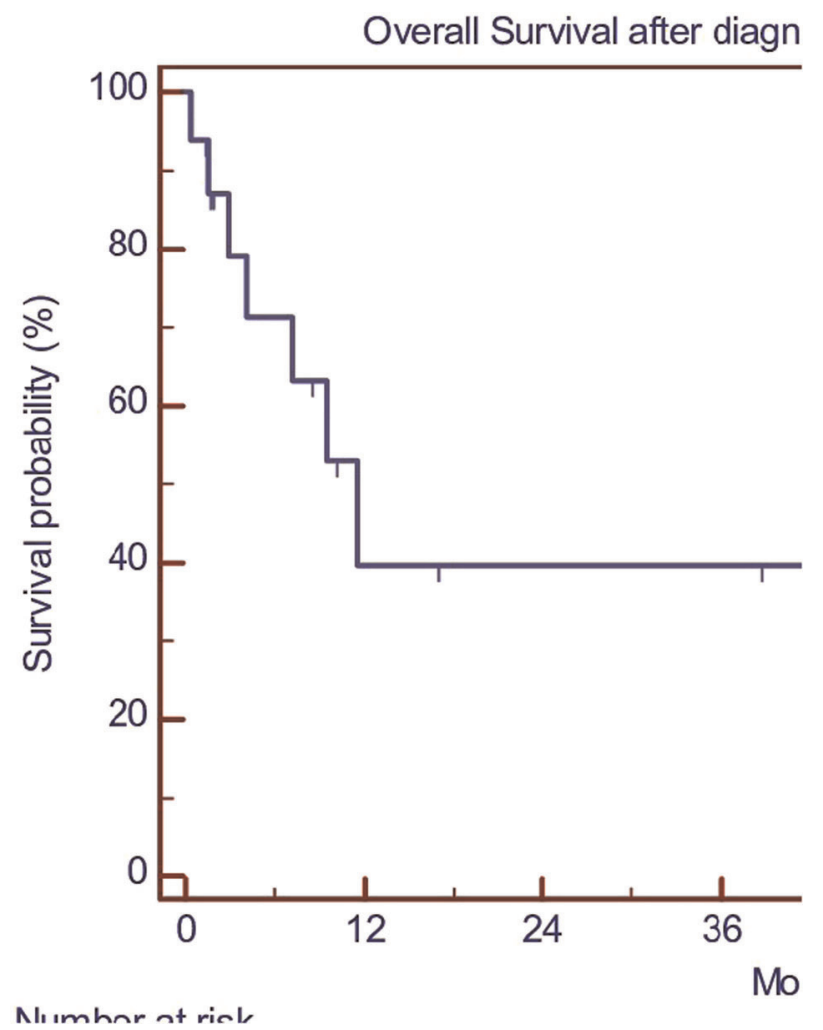

[P298 Figure] [Overall survival after the diagnosis of the secondary tumors]

Results: During the studied period, 1400 transplants were performed with a median follow-up post HSCTs of 63.3 months (range, 2.3-158). Sixteen patients developed SM (1.1\%) after a median of 63 months .The median age at time of HSCT of patients with SM was 20 years (range, 2.9$65)$ and was similar to the whole group. Among patients who developed SM, the primary diagnoses were ALL $(\mathrm{n}=$ 4, 25\%), AML $(\mathrm{n}=3,19 \%)$, Hodgkin lymphoma $(\mathrm{n}=3$, $19), \mathrm{MM}(\mathrm{n}=2,13 \%)$, Fanconi anemia $(\mathrm{n}=2,13 \%), \mathrm{CML}$ in chronic phase $(\mathrm{n}=1,6.3 \%)$ and neuroblastoma $(\mathrm{n}=1$, $6.3 \%$ ). Second hematologic malignancies were diagnosed in $44 \%(\mathrm{n}=7)$ of those who developed second cancers; with myeloid leukemia being the commonest $(n=6,86 \%)$. Second solid tumors were observed in $56 \%(\mathrm{n}=9)$; most commonly squamous cell carcinoma $(33 \% ; n=3)$. The 2 year-overall-survival (OS) after developing second malignancy was $40 \% \pm 16 \%$, with a median survival of 11.6 months. There was no significant difference in OS between second hematological vs. solid tumors $(p=0.33)$. Using Fisher's exact test, there was no significant association for developing SM with age at transplant $(<18$-years, $p=0.80)$, allogeneic transplant $(p=0.80)$, myeloablative conditioning $(p=0.73)$ and use of TBI $(p>0.99)$.

Conclusions: Indefinite follow-up and early detection of SM may improve the quality of life and survival post
HSCTS. Surveillance and reporting of SM is essential for new transplant programs like ours. In our population the rate of secondary tumors is lower than that reported in the literature; possibly, due to short follow-up.

Conflict of interest: all authors declare no conflict of interest.

\section{P299}

Serial lineage chimerism analysis improves early diagnosis of primary and secondary graft failure after allogeneic HSCT

Ignacio Gómez-Centurión ${ }^{1}$, Mi Kwon ${ }^{1,2}$, Pascual Balsalobre ${ }^{1,2}$, María Chicano Lavilla ${ }^{1,2}$, Diego Carbonell ${ }^{1,2}$, David Serrano ${ }^{1,2}$, Nieves Dorado ${ }^{1,2}$, Laura Solán ${ }^{1,2}$, Javier Anguita ${ }^{1,2}$, José Luis DíezMartin $^{1,2}$, Ismael Buño ${ }^{1,2,3}$, Carolina Martínez-Laperche ${ }^{1,2}$

${ }^{1}$ Hospital General Universitario Gregorio Marañón, Hematology, Madrid, Spain; ${ }^{2}$ Instituto de Investigación Sanitaria Gregorio Marañón, Madrid, Spain; ${ }^{3}$ Hospital General Universitario Gregorio Marañón, Genomics Unit, Madrid, Spain

Background: Graft failure (GF) is an unusual but threatening complication after allogeneic HSCT (alloHSCT). Chimerism monitoring has shown to contribute to the early detection of GF. The objective of this study was to describe chimerism dynamics in patients who developed GF after allo-HSCT.

Methods: Characteristics and outcome of 14 patients diagnosed with GF after allo-HSCT since 2003 in a single centre were retrospectively analyzed. Chimerism studies were performed in peripheral blood (PB) and $\mathrm{T}$ cells (TC) weekly since day +14 by STR-PCR. Patients were classified into two groups: primary GF (absence of neutrophil engraftment by day +28 ), and secondary GF (development of severe cytopenias and progressive mixed chimerism (MC) after initial achievement of neutrophil engraftment). Relapse was ruled out in all cases.

Results: Seven patients were diagnosed with primary GF and 7 patients with secondary GF (Table 1). No evident causes were identified in all primary GF cases. Median percentage of recipient cells in chimerism analysis at days $+14,+21,+28$ and +40 were $38,23,18$ and $19 \%$ in PB, and 98, 96, 66 and 65\% in TC, respectively (Figure 1). As salvage, two patients were treated with donor lymphocytes infusion (DLI) and five with second allo-HSCT. Median time to salvage therapy was 45 days (range $35-77$ ). Five patients achieved engraftment, and six complete chimerism (CC). Four-month OS after salvage was $43 \%$. The seven patients diagnosed with secondary GF had achieved initial neutrophil engraftment in a median of 17 days (range 1328). Median time to GF after allo-HSCT was 75 days (range 39-108). Six of them developed CMV reactivation before 


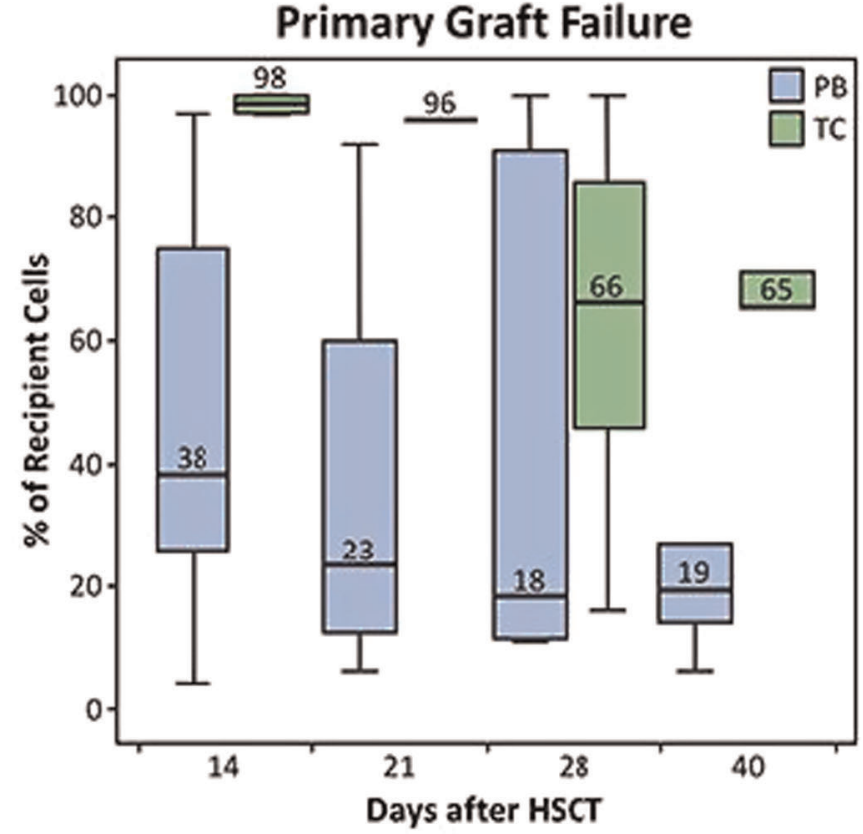

Secondary Graft Failure

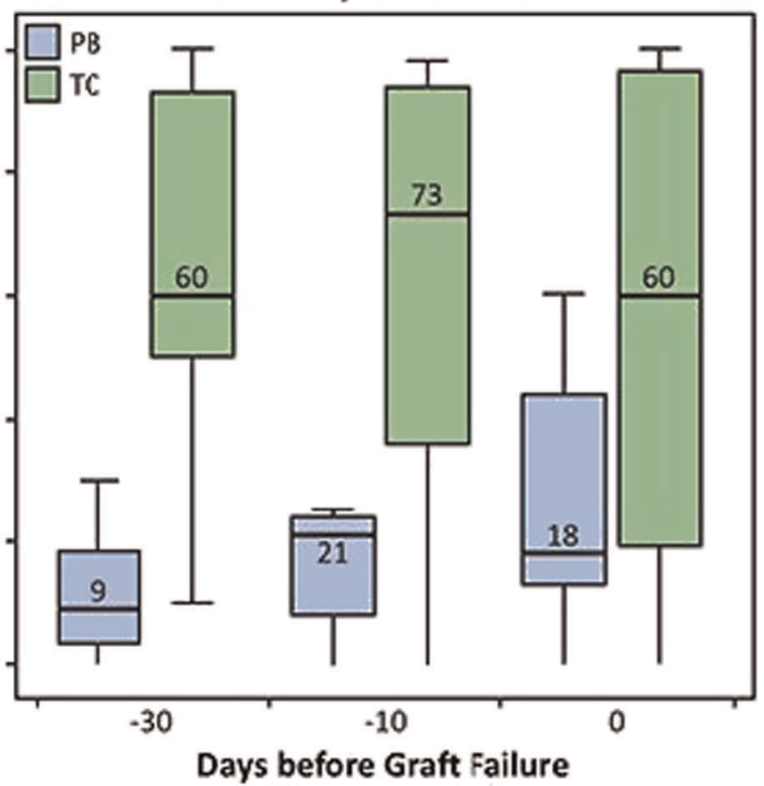

[P299 Figure] [Chimerism Dynamics in Primary and Secondary Graft Failure]

GF. Median percentage of recipient cells in chimerism analysis 30 and 10 days before diagnosis, and at the moment of GF confirmation were 9, 21 and $18 \%$ in PB, and 60, 73 and 60\% in TC, respectively (Figure 1). As salvage, one patient was treated with DLI and six with second alloHSCT. Median time to salvage therapy was 35 days (range 8-817) after GF confirmation. Five patients achieved engraftment and CC. Four-month OS after salvage was $57 \%$.

Conclusions: Patients with primary GF presented persistent MC in early serial chimerism analysis after alloHSCT, with particullary high percentages of recipient cells (65-98\%) in TC. Patients with secondary GF showed increasing percentages of recipients cells, before GF was established. Hence, the use of serial multilineage chimerism, especially in TC, may predict and identify primary and secondary GF, allowing early interventions. In our experience, timely and individualized second allo-HSCT after GF succesfully salvage a significant proportion of patients.

Conflict of interest: The authors have nothing to disclose.

\begin{tabular}{|c|c|c|}
\hline & $\begin{array}{l}\text { PRIMARY } \\
\text { GRAFT } \\
\text { FAILURE }\end{array}$ & $\begin{array}{l}\text { SECONDARY } \\
\text { GRAFT } \\
\text { FAILURE }\end{array}$ \\
\hline Patients & 7 & 7 \\
\hline Median age, y (range) & $29(1-60)$ & $49(18-62)$ \\
\hline $\begin{array}{l}\text { Diagnosis: AML, ALL, SMD/Aplastic } \\
\text { Anemia/Other }\end{array}$ & $6 / 0 / 1$ & $4 / 2 / 1$ \\
\hline
\end{tabular}

SPRINGER NATURE
Table (continued)

\begin{tabular}{lll}
\hline & $\begin{array}{l}\text { PRIMARY } \\
\text { GRAFT } \\
\text { FAILURE }\end{array}$ & $\begin{array}{l}\text { SECONDARY } \\
\text { GRAFT } \\
\text { FAILURE }\end{array}$ \\
\hline $\begin{array}{l}\text { Graft: HLA-id/HLA-mismatched/ } \\
\text { Haplo-id }\end{array}$ & $4 / 1 / 2$ & $4 / 2 / 1$ \\
$\begin{array}{l}\text { Stem Cells Source: PB/BM/CB } \\
\begin{array}{l}\text { Conditioning Regimen Intensity: MAC/ } \\
\text { RIC }\end{array}\end{array}$ & $5 / 1$ & $5 / 2 / 0$ \\
$\begin{array}{l}\text { Use of ATG: Yes/No } \\
\begin{array}{l}\text { Salvage Treatment: HLA-id allo/HLA- } \\
\text { mismatched allo/Haplo-id allo/DLI }\end{array}\end{array}$ & $4 / 3$ & $3 / 4$ \\
\hline
\end{tabular}

[[P299 Table] Characteristics of patients and transplants]

P300

Severe periostitis and myositis as a manifestation of transplant-associated thrombotic microangiopathy (TATMA) after peripheral blood stem cell transplantation from unrelated donor for homozygous $ß$ - thalassemia

Claudia Bettoni da Cunha-Riehm, Britta Maecker-Kolhoff, Kinan Kafa, Britta Lamottke, Martin Sauer, Karl-Walter Sykora

Medical School Hannover, Paediatric Haematology and Oncology, Hannover, Germany

Background: A 16-year-old girl with $\beta$ thalassemia major, Pesaro risk class 2, was transplanted from a 9/10 HLA- 
matched unrelated female donor in May, 2017. Graft: frozen unmanipulated PBSC: 3,5x $10^{6} \mathrm{CD} 34+/ \mathrm{kg}, 6 \times 10^{8} \mathrm{MNC} / \mathrm{kg}$. CMV serostatus donor/recipient positive. Conditioning: fludarabine/treosulfan / thiothepa/ATG. GvHD prophylaxis: CSA/MTX. Post-transplant complications: engraftment syndrome on day +13 treated with prednisone $(10 \mathrm{mg} / \mathrm{kg} /$ day for 3 days) overlapping with steroid-resistant acute skin-GvHD Grade 3, no gut or liver GvHD. Therapy CSA and prednisone $2 \mathrm{mg} /$ day and Ruxolitinib - up day +20 . Engraftment: day +18 leucocytes, platelets $>50.000 / \mu$ was not achieved until discharge on day 32, with $100 \%$ chimerism. Polyomavirusassociated hemorrhagic cystitis on day+ 53. Several CMVreactivations since day +32 , treated with ganciclovir, cidofovir, foscarnet. The patient developed diabetes mellitus treated with insulin and had very poor graft function, requiring innumerous blood transfusions (erythrocytes and thrombocytes). After day +60 post-HSCT she presented with severe diffuse skeletal and muscular pain in the extremities, requiring high dose intravenous hydromorphone and non-opioid analgesics. At that time, she had no signs of GvHD under ruxolitinib and decreasing doses of prednisone. CSAneurotoxicity was suspected, and GvHD treatment was changed to sirolimus. Fluconazole was given as antifungal prophylaxis. No antinuclear or ENA antibodies as well as no antibodies for scleroderma or polymyositis/dermatomyositis were present. The patient progressed with swelling of both knees and distal thighs. MRI examination showed typical signs of extensive myositis and periostitis of the knee, distal femur and tibia. Concomitantly, the patient had 3-4 fold increase in serum LDH. The diagnosis of TA-TMA was confirmed by measuring the soluble membrane attack complex (sC5b-9) levels, which was significantly increased ( $4 \mathrm{x}$ basal level). Additionally, she had proteinuria of $400 \mathrm{mg} /$ $\mathrm{dl}$, increased urine protein-to-creatinine ratio but normal serum creatinine and S-Cystatin. She never had schistocytosis in peripheral blood, possibly due to high transfusion requirements. At the time of diagnosis TA-TMA, she required two antihypertensive drugs with a third one added later on.

Methods: Eculizumab was started on day +65 with 1200 $\mathrm{mg}$, loading dose, followed by $900 \mathrm{mg} \mathrm{2x}$ /week (weeks 1 5), then $900 \mathrm{mg}$ weekly (weeks 6-21). She received one cycle of rituximab on day +80 . Sirolimus was stopped on day +85 . Because of a very slow decrease of the sC5b-9 and not significant reduction of $\mathrm{CH}-50$, etanercept was initiated. The patient received 4 doses of etanercept s.c. (25 mg 1x/week, day +85 to +105 ).

Results: One week later she was discharged with myositis, periostitis and kidney abnormalities completely resolved. On day +128 , after the 11th dose of Eculizumab, she became transfusion-free and for the first time achieved more than $50.000 / \mu$ l platelets. Currently she has no signs TA-TMA, acute or chronic GvHD.
Discussion: So far, periostitis and myositis have never been described in TA-TMA, but in other systemic vasculopathies. Etanercept improves endothelial function and inhibits TNF- $\alpha$, a major trigger for the development of TA-TMA.

Conclusions: The treatment of severe TA-TMA remains challenging, TNF- $\alpha$ inhibitor etanercept combined with eculizumab could potentially contribute to resolve this often treatment-refractory condition.

Conflict of interest: no conflict of interest

\section{P301}

\section{Severe reduction in skeletal muscle mass in long-term survivors of pediatric allogeneic HSCT}

\begin{abstract}
Malene Mejdahl ${ }^{1}$, Sidsel Mathiesen ${ }^{1}$, Anu Suominen ${ }^{2}$, Kaspar $^{2}$ Sorensen $^{1}$, Marianne Ifversen ${ }^{1}$, Christian Molgaard ${ }^{3,4}$, Anders Juul $^{5}$, Kirsi Jahnukainen ${ }^{2,6}$, Klaus Müller ${ }^{1}$

${ }^{1}$ Rigshospitalet, University of Copenhagen, Department of Pediatrics and Adolescent Medicine, Copenhagen, Denmark; ${ }^{2}$ University of Helsinki and Helsinki University Central Hospital, Division of Haematology-Oncology and Stem Cell Transplantation, Children's Hospital, Helsinki, Finland; ${ }^{3}$ University of Copenhagen, Department of Nutrition, Exercise and Sports, Copenhagen, Denmark; ${ }^{4}$ Rigshospitalet, University of Copenhagen, Pediatric Nutrition Unit, Copenhagen, Denmark; ${ }^{5}$ Rigshospitalet, University of Copenhagen, Department of Growth and Reproduction, Copenhagen, Denmark; ${ }^{6}$ Karolinska Institutet and University Hospital, Pediatric Endocrinology Unit, Department of Women's and Children's Health, Stockholm, Sweden
\end{abstract}

Background: Changes in body composition are associated with increased risk of metabolic syndrome, cardiovascular disease and functional impairment. We therefore investigated body composition in male long-term survivors of pediatric allo-HSCT.

Methods: We conducted a cross-sectional follow-up study of 99 males (49 Danish, 50 Finnish) aged 18-47 years with a median (range) follow-up of 18.0 (7.7-34.6) years. By dual-energy X-ray absorptiometry scan we measured body fat and lean mass. We calculated lean body mass index, skeletal muscle mass index and fat mass index that relate lean body mass and fat mass to height $(\mathrm{m})^{2}$. Z-scores were calculated from an American healthy reference population.

Results: The mean (SD) body mass index (BMI) was normal $23.9(4.32) \mathrm{kg} / \mathrm{m}^{2} .9 .1 \%$ of the patients were obese with a BMI $\geq 30.0 \mathrm{~kg} / \mathrm{m}^{2}$, whereas $7.1 \%$ were underweight with a BMI $<18.5 \mathrm{~kg} / \mathrm{m}^{2}$ according to WHO definition. Soft lean mass percentage, lean body mass index and skeletal muscle mass index were all significantly reduced indicating a general loss of skeletal muscle tissue. Fat mass index was similar to the reference population, but the android/gynoid fat mass (AG) ratio was increased reflecting 


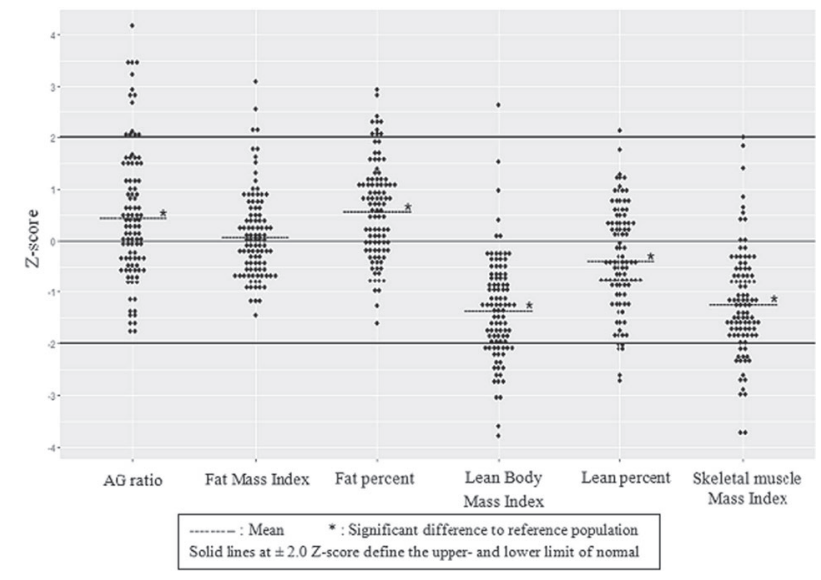

[P301 Figure] [Body composition Z-scores]

an altered fat mass distribution towards abdominal obesity (table, figure).

Lean body mass was significantly reduced and AG ratio was significantly increased in patients with a TBI based conditioning compared to non-TBI (table). Importantly, however, patients in the non-TBI group still had significantly reduced lean body mass index $(p=0.0011)$ and skeletal muscle mass index $(\mathrm{p}=0.0068)$ compared to the reference population (table). In accordance, patients with malignant disease $(n=75)$ had more pronounced reduction in lean body mass index and skeletal muscle mass index than those with benign disease $(n=24)$ with a mean $(S D)$ Z-score of $-1.52(0.90)$ vs. $-0.72(1.07), \mathrm{p}=0.0014$, and $-1.41(0.95)$ vs. 0.55 (1.00), $\mathrm{p}=0.0003$, respectively. Changes in body fat percentage, lean body mass index and skeletal muscle mass index all tended to improve with increased follow-up time from HSCT, while changes in AG ratio became more deteriorated by longer follow-up (table).

Conclusions: The majority of male long-term survivors have markedly decreased skeletal muscle mass. BMI and fat mass appear normal, but AG ratio is increased. These changes in body composition indicate an increased risk of functional impairment as well as metabolic and cardiovascular disease among survivors of pediatric allo-HSCT.

Conflict of interest: The authors have nothing to disclose.

\begin{tabular}{|c|c|c|c|c|c|c|c|c|}
\hline & \multirow{2}{*}{\multicolumn{2}{|c|}{$\frac{\text { All patients }}{\text { allo-HSCT }}$}} & \multicolumn{3}{|c|}{ Total body irradiation } & \multicolumn{3}{|c|}{ Time from HSCT } \\
\hline & & & \multirow{3}{*}{$\begin{array}{l}\text { TBI } \\
\mathrm{n}=73 \\
\begin{array}{l}\text { Mean } \\
\text { (SD) }\end{array}\end{array}$} & \multirow{2}{*}{$\begin{array}{l}\text { nonTBI } \\
\mathrm{n}=26\end{array}$} & \multirow[b]{3}{*}{$\mathrm{p}$ value } & \multirow[b]{3}{*}{ slope } & \multirow[b]{3}{*}{$r^{2}$} & \multirow[b]{3}{*}{$\mathrm{p}$ value } \\
\hline & $\mathrm{n}=9$ & & & & & & & \\
\hline & $\begin{array}{l}\text { Mean } \\
\text { (SD) }\end{array}$ & $\mathrm{p}$ value & & $\begin{array}{l}\text { Mean } \\
\text { (SD) }\end{array}$ & & & & \\
\hline \multirow{2}{*}{$\begin{array}{l}\text { AG } \\
\text { ratio Z- } \\
\text { score }\end{array}$} & $\begin{array}{l}0.48 \\
(1.29)\end{array}$ & $0.0004^{*, 1}$ & $\begin{array}{l}0.702 \\
(1.23)\end{array}$ & $\begin{array}{l}-0.146 \\
(1.25)\end{array}$ & $0.0034^{*, 2}$ & 0.040 & 0.047 & $0.032 *, 4$ \\
\hline & & $<0.0001^{*}, 1$ & & & $0.026^{*, 2}$ & -0.028 & 0.041 & $0.045^{*, 4}$ \\
\hline
\end{tabular}

Table (continued)

\begin{tabular}{|c|c|c|c|c|c|c|c|c|}
\hline & \multirow{2}{*}{\multicolumn{2}{|c|}{$\begin{array}{l}\text { All patients } \\
\text { allo-HSCT }\end{array}$}} & \multicolumn{3}{|c|}{ Total body irradiation } & \multicolumn{3}{|c|}{ Time from HSCT } \\
\hline & & & \multirow{3}{*}{$\begin{array}{l}\text { TBI } \\
\mathrm{n}=73 \\
\text { Mean } \\
\text { (SD) }\end{array}$} & \multirow{2}{*}{$\begin{array}{l}\text { nonTBI } \\
\mathrm{n}=26\end{array}$} & \multirow[b]{3}{*}{$\mathrm{p}$ value } & \multirow[b]{3}{*}{ slope } & \multirow[b]{3}{*}{$r^{2}$} & \multirow[b]{3}{*}{$\mathrm{p}$ value } \\
\hline & \multicolumn{2}{|l|}{$\mathrm{n}=99$} & & & & & & \\
\hline & $\begin{array}{l}\text { Mean } \\
\text { (SD) }\end{array}$ & $\mathrm{p}$ value & & $\begin{array}{l}\text { Mean } \\
\text { (SD) }\end{array}$ & & & & \\
\hline $\begin{array}{l}\text { Fat \% } \\
\text { Z-score }\end{array}$ & $\begin{array}{l}0.60 \\
(0.95)\end{array}$ & & $\begin{array}{l}0.732 \\
(0.88)\end{array}$ & $\begin{array}{l}0.248 \\
(1.08)\end{array}$ & & & & \\
\hline $\begin{array}{l}\text { FMI Z- } \\
\text { score }\end{array}$ & $\begin{array}{l}0.12 \\
(0.85)\end{array}$ & $0.18^{1}$ & $\begin{array}{l}0.153 \\
(0.76)\end{array}$ & $\begin{array}{l}0.009 \\
(1.06)\end{array}$ & $0.165^{3}$ & -0.011 & 0.008 & $0.392^{4}$ \\
\hline $\begin{array}{l}\text { LBMI } \\
\text { Z-score }\end{array}$ & $\begin{array}{l}-1.33 \\
(1.00)\end{array}$ & $<0.0001^{*, 1}$ & $\begin{array}{l}-1.502 \\
(0.89)\end{array}$ & $\begin{array}{l}-0.831 \\
(1.15)\end{array}$ & $0.009^{*}, 3$ & 0.039 & 0.074 & $0.006^{*, 4}$ \\
\hline $\begin{array}{l}\text { Lean\% } \\
\text { Z-score }\end{array}$ & $\begin{array}{l}-0.35 \\
(0.99)\end{array}$ & $0.0007^{*, 1}$ & $\begin{array}{l}-0.503 \\
(0.89)\end{array}$ & $\begin{array}{l}0.078 \\
(1.15)\end{array}$ & $0.0097 *, 2$ & 0.020 & 0.020 & $0.164^{4}$ \\
\hline $\begin{array}{l}\text { SMI Z- } \\
\text { score }\end{array}$ & $\begin{array}{l}-1.20 \\
(1.03)\end{array}$ & $<0.0001^{*, 1}$ & $\begin{array}{l}-1.413 \\
(0.94)\end{array}$ & $\begin{array}{l}-0.616 \\
(1.06)\end{array}$ & $0.0005^{*, 2}$ & 0.038 & 0.066 & $0.010^{*, 4}$ \\
\hline
\end{tabular}

[[P301 Table] Z-score analyzes]

$*=$ Significance, ${ }^{1}=$ One sample t-test, ${ }^{2}=$ Two sample t-test, ${ }^{3}=$ Mann-Whitney $\mathrm{U}$ test, ${ }^{4}=$ Univariate linear regression.

\section{P302}

Simple spirometry monitoring is useful in detecting late post-stem cell transplantation lung function deterioration

Emilia Hardak $^{1,2}$, Eyal Fuchs ${ }^{3}$, Orlee Rak Cohen ${ }^{2}$, Tsila Zuckerman $^{2,4}$

${ }^{1}$ Rambam Health Care Campus, Division of Pulmonology, Haifa, Israel; ${ }^{2}$ Technion - Israel Institute of Technology, The Ruth and Bruce Rappaport Faculty of Medicine, Haifa, Israel; ${ }^{3}$ Rambam Health Care Campus, Internal Medicine C, Haifa, Israel; ${ }^{4}$ Rambam Health Care Campus, Hematology and BMT, Haifa, Israel

Background: Post-allogeneic stem cell transplantation (ASCT) lung injury occurs in about $50 \%$ of patients, which may be secondary to multiple factors, including: high-dose therapies incorporated in conditioning regimens or those administered to treat the primary disease, infections, immunological reactions, immune suppression and graftversus-host disease (GVHD). Signs and symptoms of pulmonary complications are often nonspecific and insidious. Prompt diagnosis is crucial, as treatment delays are associated with rapid deterioration and adverse outcome. The aim of the present study was to identify risk factors and predictors of post-ASCT pulmonary function test (PFT) abnormalities, using regular lung function monitoring.

Methods: Adult patients who underwent ASCT and signed informed consent were followed at 6,12 and 24 months post-ASCT by designated pulmonologists and underwent a detailed respiratory evaluation and PFTs according to the American Thoracic Society (ATS) / European Respiratory Society (ERS) 2005 guidelines, including: diffusing capacity of carbon monoxide (DLCO), 
vital capacity (VC), total lung capacity (TLC), forced volume vital capacity (FVC), forced expiratory volume in 1 second (FEV1) and FEV1/FVC. Findings were compared to those of pre-SCT PFTs.

Results: Between 2012-2016, 114 consecutive adult patients were enrolled in the study. A median age was 53 (range 19-69) years, 59\% had myelodysplastic syndrome (MDS) / acute myeloid leukemia (AML), 32\% were past smokers. HLA-matched related or unrelated donors were used in $96 \%$ of patients, $16 \%$ (14 patients) received a TBIbased conditioning regimen. Up to $49 \%$ of transplant recipients developed acute GVHD, which was grade 2-4 in $62 \%$ of them. Sixty six percent of patients $(n=75)$ had chronic GVHD (cGVHD), mostly mild-moderate.

Following ASCT, 42 patients $(36.8 \%)$ died. The most common cause of death was related to relapse of the primary hematological condition, which occurred in 17 patients. During the first 24 months post-transplant, $71.9 \%$ of patients developed pulmonary complications, which were infectious, non-infectious and both infectious and non-infectious in $64 \%, 1.8 \%$ and $6.1 \%$ of patients, respectively. Six patients were diagnosed with bronchiolitis obliterans. The mean FEV1 declined from 91.9 pre-SCT to 85 at 24 months $(\mathrm{p}=.003)$, while the mean $\mathrm{RV} / \mathrm{TLC}$ ratio increased from 118.5 to 134.5 at 24 months $(p=0.02)$. Although DLCO declined at 6 and 12 months post-SCT, this was not statistically significant. A correlation was observed between the decline in FEV1 and the rise in the RV/TLC ratio $(\mathrm{p}<$ 0.0001, $\mathrm{r}=-0.674$ ) and between the decline in FEV1 and the decline in DLCO ( $p<0.007, r=0.45)$. The FEV1 decline was associated with female gender $(p=0.003)$, cGVHD $(\mathrm{p}=.001)$ and viral infections $(\mathrm{p}=.002)$.

Conclusions: Both FEV1 and RV/TLC ratio have shown a statistically significant change from baseline at 2 years post-ASCT. As there is a correlation between an FEV1 drop and an alteration in DLCO and RV/TLC, simple spirometry testing can be adequately suggestive of pulmonary impairment following SCT. This is particularly important for lowresource centers where spirometry is more accessible than complete PFTs. Moreover, FEV1 is reproducible and easier to perform for patients.

Clinical Trial Registry: NA

Conflict of interest: Nothing to disclose

\section{P303}

Successful pregnancies following stem cell transplantation in childhood - a single centre experience

Petra Keslova, Renata Formankova, Petr Riha, Petr Sedlacek 2nd Faculty of Medicine and University Hospital Motol, Prague, Czech Republic

Background: The prevalence of allogeneic hematopoietic stem cell transplantation (HSCT) survivors continues to increase. Gonadal insufficiency and infertility are the most frequent late complications.

Methods: We analysed the data of successful pregnancies in patients (pts) following HSCT in childhood at our centre. A total 180 patients older 18 years have checked at the outpatient department.

Results: 25 patients $(13.8 \%$, male $=14$, female $=11)$ became parents. Forty children were born to the overall group, 34 out of $40(85 \%)$ in term, all were healthy. First pregnancy was documented 10.4 (2.5 - 24) years after HSCT in patient's age 25.7 (18.3 - 41.2) years. Median age of patients at time of HSCT was $15.5(8.0$ - 19.9) years. 12 patients were treated due to SAA (48\%), 13 patients underwent HSCT due to malignant diagnosis ( $\mathrm{ALL}=6$ $\mathrm{AML}=1 \mathrm{CML}=3 \mathrm{MDS}=3$ ). We detected spontaneous gravidity in 14 pts $(\mathrm{SAA}=86 \%$ with cyclophosphamide regimen only, 2 males after full myeloablative Busulphan based conditioning). 4 pts (all males, 3 following TBI 1214.4 Gy, 1 after full Busulphan use) are biological fathers, own cryopreserved sperms were used in 2 of them, testicular sperms were retrieved in 2 pts (TESE - testicular sperm extraction). Other 7 patients have utilized assisted reproduction techniques - IVF (donor sperm=1, donor oocytes=4), 2 females underwent repeating unsuccessful IVF and hyperstimulation and both became later pregnant spontaneously (even one after TBI 14.4 Gy based regimen).

Conclusions: Gonadotoxicity after HSCT is well known posttransplant complication affecting the quality of life. Fertility issues must be routinely discussed at peritransplant care. In the field of reproductive medicine impressive advances have been achieved to offer the best option for fertility preservation and pregnancy outcome. Better knowledge of pregnancy rate especially in paediatric population following HSCT will require more data for analysis in international cooperation.

Supported by MHCZ for conceptual development 00064203 University Hospital Motol

Conflict of interest: all authors: nothing to disclose

\section{P304}

Systematic follow-up within the first year after allogeneic hematopoietic stem cell transplantation (allo-HSCT) allows early detection of so called late effects after transplant

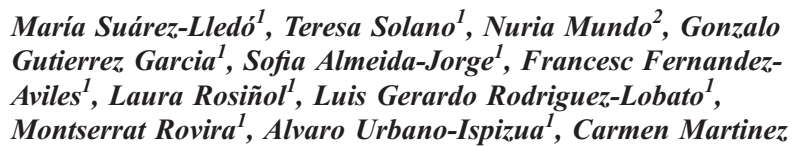




\section{Muñoz ${ }^{1}$}

${ }^{1}$ Hospital Clinic of Barcelona, Stem Cell Trasplantation Unit, Hematology Department, Barcelona, Spain; ${ }^{2}$ Hospital Clinic of Barcelona, Endocrinology and Nutrition Department, Barcelona, Spain

Background: Advances in the allo-HSCT procedure such as supportive care and the use of reduced intensity conditioning regimens for older patients have led to significant improvements in long-term survival after transplant. The survivors have an elevated probability of late morbidity and mortality. As hematologic disease relapse is the predominant cause of mortality in the first 2 years, most studies have analyzed long-term complications in patients who are alive and disease-free at 2-5 years after allo-HSCT. In the present study, we prospectively investigate within the first year after transplant the prevalence of risk factors and the development of so called late effects.

Methods: According to the recommended screening and preventive practices for long-term survivors (Majhail $\mathrm{N}$. et al, 2012), we prospectively evaluate allo-HSCT recipients at 3, 6, 9 and 12 months post-SCT. Patients were also evaluated for chronic GVHD according to NIH Consensus Criteria for GVHD Diagnosis. We analyzed data from physical examination: blood pressure, heart rate, respiratory rate, and body mass index (BMI); laboratory test: blood cells counts, liver and renal function tests, fasting lipids profile, glucose, thyroid function, cortisol test, calcium, PTH and vitamin D, CD4+ T-cells counts, and immunoglobulin levels; iron overload, and respiratory functionalism. Bone density scans were performed in those patients who present risk factors for osteoporosis. Patients were also evaluated by Ophthalmologist, Pneumologist, Dermatologist and Gynecologist.

Results: Thirty-nine patients were evaluated (20 male/19 female, median age of 51 years, 18-69). Chronic GVHD was diagnosed in 12 patients. Almost all patients $(80 \%)$ presented cardiovascular risk factors (in $52 \%$ of them, de novo after transplant): $48 \%$ had dyslipidemia, $29 \%$ hypertension, and $23 \%$ diabetes. Four patients had cardiovascular disease (organ damage), 2 of them after alloHSCT. Twelve patients (30\%) had cortisol levels lower than $7 \mu \mathrm{l} / \mathrm{dl}$ ( $40 \%$ of them had received glucocorticoids). Twelve patients $(30 \%)$ required nutritional intervention due to low or high BMI $\left(<19 \mathrm{~kg} / \mathrm{m}^{2}, \mathrm{n}=6 ; 19-20 \mathrm{~kg} / \mathrm{m}^{2}, \mathrm{n}=5 ;>25\right.$ $\left.\mathrm{kg} / \mathrm{m}^{2}, \mathrm{n}=1\right)$. After dietary supplements, $80 \%$ of them reached normal BMI. Eighty-seven percent of patients had vitamin D level lower than 30ng/ml, 13\% had osteopenia (2 male, 3 female), and $13 \%$ had osteoporosis (2 male, 3 female). Menopause (all women) and glucocorticoids treatment ( $28 \%$ women, $50 \%$ men) were identified as risk factors for bone loss. All of them received treatment with calcifediol and, after that, 6 achieved normal levels of vitamin $\mathrm{D}$ and bone mass increased in one patient.

Conclusions: In this adult group of allo-HSCT recipients, we identify a high prevalence of cardiovascular risk factors, and a significant number of patients with abnormalities in mineral metabolism and bone disease, hypocortisolism and nutritional deficiencies. Early identification and treatment of these conditions could be useful to prevent the severity or frequency of the most common late effects after allo-HSCT.

\section{Conflict of interest:}

M. Suárez-Lledó: I received a scolarship from DKMS foundation to dedicate clinical assitance for the Late-Effects Follow-up Unit, a new program in Hospital Clínic Barcelona, that has started one year ago. The other authors have nothing to disclose.

\section{P305}

Taken by storm - Cytokine release syndrome in children undergoing haploidentical haematopoietic stem cell transplantation for benign haematological disorders

Revathi Raj, Ramya Uppuluri, Dhaarani Jayaraman, Meena Sankaran, Venkateswaran V S, Shivani Patel, Sathishkumar Kandadai

Apollo Speciality Cancer Hospitals, Paediatric Blood and Marrow Transplantation, Chennai, India

Background: Cytokine release syndrome is a well documented phenomenon in patients undergoing novel monoclonal antibody therapy and haematopoietic stem cell transplantation (HSCT). With the increasing use of haploidentical HSCT in benign haematological conditions, there is an urgent need for early recognition and treatment of this entity to reduce morbidity and mortality.

Methods: A retrospective analysis of data from our centre over the last 3 years of children who underwent $\mathrm{T}$ replete haploidentical haematopoietic stem cell transplantation with post transplant cyclophosphamide (PTCy) was performed. $\mathrm{T}$ replete grafts in haploidentical HSCT are known to release cytokines and result in an "allo effect". Serial serum ferritin was performed as a surrogate marker of cytokine release twice a week in all children undergoing haploidentical HSCT from the day of infusion until engraftment. The normal range of serum ferritin at our centre was 7 to $140 \mathrm{ng}$ / $\mathrm{ml}$. Over a 35 to 50 fold increase in serum ferritin level to over 5000 heralded the symptoms of cytokine release storm (CRS). The grading of CRS was performed as per the revised CRS grading system ranging from mild fever, fluid retention and myalgia to hypoxaemia, hypotension needing vasopressors and death.

Results: A total of 52 haploidentical HSCT patient data with $\mathrm{T}$ replete grafts and post transplant cyclophosphamide 
performed between 2014 and 2017 were analysed retrospectively. The children were aged between 3 months to 16 years of age. Forty children were transplanted for benign haematological conditions like primary immune deficiency, aplastic anaemia, adrenoleucodystrophy and haemoglobinopathies. Twelve patients had relapsed or refractory leukaemia. Amongst the children transplanted for benign haematological conditions, Grade 2 CRS was seen in 7 of the 40 children, Grade 3 to 4 in 2 children and Grade 5 resulting in death was documented in 3 children. The ferritin levels increased exponentially from the normal baseline level in all these patients. A ferritin level over 100,000 was seen in the children that died before intervention. Etarnacept was used in 3 children and two children were salvaged using early IL6 inhibitor Tociluzimab at $4 \mathrm{mg} / \mathrm{kg}$. None of the 12 children with malignancy demonstrated the signs of CRS which was seen in $30 \%$ of children with benign conditions ( $\mathrm{p}$ value less than 0.001).

Conclusions: Haploidentical HSCT is increasingly being offered to infants and children with benign haematological disorders as it represents a cost effective option for cure from life threatening disorders. Estimation of serum IL6 levels as a marker of cytokine release is not available in all centres and $\mathrm{C}$ reactive protein and serum ferritin are useful surrogate markers. Early recognition of this clinical entity, the use of easily accessible low cost investigations like serial serum ferritin or CRP will result in appropriate intervention and reduce mortality.

Clinical Trial Registry: N/A

Conflict of interest: None

\section{P306}

\section{The effects of nutritional support on the systemic metabolic} profile early after allogeneic stem cell transplantation

Tor Henrik Anderson Tvedt ${ }^{1,2}$, Kristin Aneta Joan Skaarud ${ }^{3,4}$, Geir Erland Tjonnfjord ${ }^{3,5}$, Tobias Gedde-Dahl ${ }^{3}$, Per Ole Iversen ${ }^{3,4}$, Oystein Bruserud ${ }^{1,2}$

${ }^{I}$ Haukeland University Hospital, Department of Medicine, Bergen, Norway; ${ }^{2}$ University of Bergen, Institute of Clinical Science, Section for Hematology, Bergen, Norway; ${ }^{3}$ Oslo University Hospital

Rikshospitalet, Department of Hematology, Oslo, Norway; ${ }^{4}$ University of Oslo, Institute of Basic Medical Sciences, Department of Nutrition, Oslo, Norway; ${ }^{5}$ University of Oslo, Institute of Clinical Medicine, Oslo, Norway

Background: We have recently performed a randomized controlled trial evaluating the effects of individual nutritional support on quality of life, Graft-versus-host disease (GVDH), infectious complications and nutritional status after allogeneic stem cell transplantation (NASQ trial/ NCT01181076). In this study participants were randomized either to the intervention group receiving early posttransplant nutritional support mainly based on enteral nutrition (oral intake or through tube feeding) or to the control group that received parenteral nutrition initiated and dosed by the attending physician. We wanted to evaluate the effects of enteral and parenteral nutrition on the metabolomic plasma profile of allotransplant recipients three weeks posttransplant.

Methods: We selected 10 patients from the intervention group and 10 from the control group. To minimize differences between the two groups we ensured that all patients were treated with busulphan plus cyclophosphamide or total-body irradiation plus cyclophosphamide; they all received GVHD prophylaxis with ciclosporin A and methotrexate; and only patients being alive without relapse at day +100 posttransplant were included in the analysis. Plasma samples were collected one week prior to and three weeks after graft infusion, and the metabolite profile $(>800$ metabolites) was assessed using mass spectrometry.

Results: There were no significant differences between the two patient groups with respect to GVHD or reported severity of mucositis posttransplant. The goal to secure an adequate total energy intake was met in the intervention group. The control group received a substantially higher dose of parenteral nutrition (average total dose 25,275 kcal, range $13,750-35,400)$ than the intervention group $(13,000$ $\mathrm{kcal}$, range 424-21,920 kcal, p-value < 0.01).

The most prominent posttransplant metabolic changes common for both groups were a significant increase in the levels of secondary bile acids and endocannabinoids together with reduced levels of food preservatives, plasmalogens and retinol metabolites. The main posttransplant differences between the two groups were higher acylcarnitines in the intervention (enteral) group and significantly higher levels of the mitochondrial activation marker $\mathrm{N}$-formylmethionine in the control (parenteral) group. The two last observations suggest an insufficient calorie intake in the control group with a higher degree of fat mobilization and catabolic metabolism. Posttransplant amino acid or carbohydrate metabolites were not significantly altered.

We observed a significant increase in the systemic levels of several primary and secondary bile acids, this was not sees for the potentially liverprotective secondary bile acids isoursodeoxycholate and ursodeoxycholate. Corticosterone showed a significantly higher concentration in the control group. Finally, we were also able to detected significant higher levels of bilirubin metabolites in the control group posttransplant.

Conclusions: Allotransplant recipients showed altered metabolic profiles early after transplantation; this was mainly due to the conditioning/transplantation/reconstitution whereas the type of nutritional support had minor effects. Our observations also suggest that enteral 
nutritional support reduces the need for parenteral nutrition and secures adequate energy intake in this cohort of patients

Clinical Trial Registry: www.clinicaltrials.gov: NCT01181076.

Conflict of interest: The study received financial support from the The Blix Family Foundation, Eivind Møllbach Pedersens Foundation, Helse-Vest, Norwegian Cancer Society, Oslo University Hospital, University of Bergen and Throne Holst Foundation. The enteral feeding was supported by Nutricia.

\section{P307}

\section{Thyroid function post Allogenic Bone Marrow Transplantation in childhood and adolescence}

Elpis Vlachopapadopoulou ${ }^{1}$, Anna Paisiou ${ }^{2}$, Eleni Dikaia Ioannidou $^{2}$, Stefanos Stergiotis ${ }^{3}$, Eugenios Goussetis ${ }^{2}$, Maria Kafetzi ${ }^{4}$, Stefanos Michalacos ${ }^{3}$, Vassiliki Kitra ${ }^{2}$

${ }^{1}$ Children's Hospital, Endocrine Dept, Athens, Greece; ${ }^{2}$ Agia Sophia Children's Hospital, Stem Cell Transplantation Unit, Athens, Greece; ${ }^{3}$ Children's Hospital P \& A. Kyriakou, Endocrinology, Athens, Greece; ${ }^{4}$ Children's Hospital P \& A. Kyriakou, Dept of Biochemistry, Athens, Greece

Background: Allogenic Bone Marrow transplantation (BMT) is the treatment of choice for a number of childhood malignancies, as well as thalassemia major aplastic anemia and immunodeficiency. A number of endocrine long term sequelae are recognized.

Aim: To report the incidence of thyroid dysfunction of patients who underwent allogenic BMT during childhood or adolescence.

Methods: 82 patients (56 boys) who were transplanted from an HLA matched donor at a mean age of $7.5 \pm 4.8$ years (range 0.18-17.5 years) were followed prospectively having measurements of fT4, TSH twice yearly, using chemiluminescence. Patients with elevated TSH higher than $8 \mu \mathrm{IU} / \mathrm{ml}$ had a repeat evaluation in a month time. Thyroid sonogram was performed yearly in patients who have received radiation therapy (RT) and at least once in patients with no history of RT. The initial diagnosis included acute lymphocytic leukemia (ALL), acute myelocytic leukemia, thalassemia, Fanconi anemia, aplastic anemia and severe combined immunodeficiency. The conditioning regimen consisted of Busulfan $(16 \mathrm{mg} / \mathrm{Kg})+$ Cyclophosphamide $(200 \mathrm{mg} / \mathrm{Kg})+/$-Fludarabine $\left(100 \mathrm{mg} / \mathrm{m}^{2}\right)$ and antithymocyte globulin. Four patients received total body irradiation (TBI). Seven patients received CNS prophylaxis with 12 Gys for ALL.

Results: The age of the last evaluation was $11.97 \pm 5.17$ yrs and the years post BMT were $4.47 \pm 3.24$. Fifty patients $(60.9 \%)$ patients had normal thyroid function. Twenty five patients $(30.4 \%)$ had primary hypothyroidism as evidenced by TSH levels higher than $8 \mu \mathrm{IU} / \mathrm{ml}$ and low or low normal fT4 levels verified with a second measurement and they were started on replacement therapy with L-thyroxine. One patient had TSH higher than $10 \mu \mathrm{IU} / \mathrm{ml}$ which returned to normal and she has normal thyroid function up to now. Among the patients with hypothyroidism 2 had received TBI and 3 had received 12Gys prophylaxis RT. Four patients $(4.8 \%)$ had compensated hypothyroidism as evidenced by TSH level range greater than 5 and less than $8 \mu \mathrm{IU} / \mathrm{ml}$. None of them had progressed so far to overt hypothyroidism. One patient with a history of TBI, borderline elevation of TSH and low fT4 was considered to have central hypothyroidism and he was started on replacement therapy. One of the hypothyroid patients who had received TBI had a thyroid nodule for which he had an FNA consistent with a dysplastic nodule BETHESDA 1. One of the normothyroid patients who had also received TBI had papillary Ca and underwent thyroidectomy.

Conclusions: Thyroid dysfunction is quite common complication of BMT in this pediatric population. The exact mechanism is not clear. Patients who have received radiation prior to BMT are at increased risk. Patients who underwent BMT should have evaluation of thyroid function routinely in order to prevent overt hypothyroidism.

Conflict of interest: There is no conflict of interest.

\section{P308}

Abstract previously published

\section{P309}

Treatment of Poor Graft Function after Allogeneic SCT with Eltrombopag

Vera Milovic, Alejandro Requejo, Claudia Nonaka, Gabriela Martinez, Guillermo Drelichman, Leonardo Feldman, Juan Real

Hospital Aleman, Stem Cell Transplant, Buenos Aires, Argentina

Background: Poor graft function (PGF)is a complication of allogeneic stem cell transplantation(SCT) that increases morbility and mortality due to infection and bleeding. Stasia et al,defined it by the presence of cytopenias in at least 2 hemopoietic cell lines: neutroplhil counts $\leq 1.5 \times 10 \pi / \mathrm{L}$, platelets counts $\leq 30 \times 10 \pi / \mathrm{L}$ and hemoglobin $\leq 8.5 \mathrm{gr} / \mathrm{dl}$ for at least 2 consecutive weeks beyond day +14 after achieving engraftment, in the presence of full donor chimerism, and absence of severe GVHD, CMV reactivation, relapse or drug related myelosuppression and hypocellular bone marrow. The mechanisms reported are related to the presence of high proportion of IFN $\mathrm{Y}$ produced by 
alloreactive $\mathrm{T}$ cells or inflammatory stimulation as gvhd, or viral infection, leading to a decreased proliferation of HSC. Appropriated treatment is not well established but second transplants, CD34+ selected stem cell boost infusions, growth factors, have been used with different rates of success. Eltrombopag a trombopoietin mimetic oral molecule, binds to the c-mpl receptor on HSC stimulating proliferation and differentiation, and is approved as a second line treatment for aplastic anemia.

Objective: To report the improvement of hematopoiesis in 3 patients(pts) presenting with PGF, as defined by Stasia, and treated with eltrombopag.

Methods: We report 3 AML pts and 1 ALL pt who received an haploidentical SCT between july 2015 and april 2017. Conditioning regimens used:TBI/Flu in $1 \mathrm{pt} \mathrm{Cy/Flu/}$ TBI200 in 2 pts and Bu/Flu in 1.Age range:28-64y. Hematopoietic recovery : neutrophils $\geq 1 \times 10 \pi / \mathrm{L}:+14$, $+16,+17$ and +18 . Plt recovery $\geq 20 \times 10 \pi / \mathrm{L}:+15,+19$, +30 and +40 , respectively. $2 / 4$ pts presented CMV reactivation on days +36 and +53 . All 4 pts presented GII skin GVHD., responsive to steroid tratment..Etrombopag was initiated on days $+90,+97,+76$ and +62 , due to fall in platelet and neutrophil counts associated to PGF as defined by Stasia, at $50 \mathrm{mg} /$ day and increased to $150 \mathrm{mg}$ after 2 weeks.

Results: 3 pts became platelet transfusion independent and stopped GCS/F administration after 21, 30 and 30 days $.2 / 3$ pts stopped eltrombopag at days +152 and +200 with plt counts over $100 x 10 \pi / \mathrm{L}$. One of them became eltrombopag dependent since her plt dropped every time she stopped the medication. She died on day +367 due to a CNS haemorraghe. The third pt improved his plt counts to 50 $\mathrm{x} 10 \pi / \mathrm{L}$, without evidence of further increase, after 3 months of treatment. The fourth pt stopped eltrombopag administration after 30 days due to severe skin toxicity, and an incipient response. $3 / 4$ pts are in $\mathrm{CR}$ at $+753,+596$ and + 251 days postransplant.

Conclusions: Although responses were heterogenous, eltrombopag was well tolerated improving cell counts and allowing transfusion independence in a short time period. Eltrombopag can be considered as part of the treatment strategy of PGF . In cases a second transplant or a CD34+ cell boost is required, may improve clinical status while waiting for the procedure.

Conflict of interest: V. Milovic has received honoraria from Novartis.

\section{P310}

Abstract previously published
P311

Uneventful pregnancies after allogeneic hematopoietic stem cell transplantation for homozygous beta-thalassemia

Elpis Vlachopapadopoulou ${ }^{1}$, Anna Paisiou ${ }^{2}$, Ioulia Peristeri ${ }^{2}$, Eleni Dikaia Ioannidou ${ }^{2}$, Eugenios Goussetis ${ }^{2}$, Stefanos Michalacos ${ }^{1}$, Vassiliki Kitra $^{2}$

${ }^{1}$ Children's Hospital, Endocrine Dept, Athens, Greece; ${ }^{2}$ Agia Sophia Children's Hospital, Stem Cell Transplantation Unit, Athens, Greece

Background: Homozygous b-thalassemia (b-THAL) is a disease encountered with increased frequency in Mediterranean countries and, traditionally, clinical management involves regular blood transfusions and regular chelation therapy. However, the only curative treatment modality remains allogeneic hematopoietic stem cell transplantation (allo-HSCT). As there is an increasing number of long term survivors after transplantation for homozygous beta thalassemia the post-transplantation infertility is a major concern.

Methods: We performed a retrospective analysis of data regarding pregnancy and childbirth of patients who underwent allo-HSCT for b-THAL between 1994-2016. A total of 126 children with b-THAL received allo-HSCT between 1994-2016 from matched sibling donor (MSD) or voluntary unrelated donor (VUD). Median age at transplantation was 10.1 years (range 1,2-24 year) while 50 patients (25 male and 25 female) were older than 12 years at the time of transplantation. All patients were conditioned with Busulfan $(16 \mathrm{mg} / \mathrm{Kg})+$ Cyclophosphamide(200mg/Kg) +/-Fludarabine $\left(100 \mathrm{mg} / \mathrm{m}^{2}\right)$ and antithymocyte globulin without total body irradiation. Graft versus host disease prophylaxis consisted of cyclosporine and methotrexate. Sperm cryopreservation was

Results: All patients developed hypergonadotropic hypogonadism. Female patients received substitution therapy with estrogen and progesterone. Two male and 7 female long term survivors (two of whom had documented recovery of ovarian function with normalization of gonadotropins and menses resumption) reported childbirth. Two out of 7 female long term survivors achieved childbirth after in vitro fertilization whereas the remaining 5 reported spontaneous conceptions. One out of 2 male long term survivors needed assisted reproduction techniques in order to achieve childbirth.

Conclusions: Secondary amenorrhea and hypergonadotropic hypogonadism occur almost universally in postpubertal females undergoing none marrow transplantation for homozygous beta-thalassemia. Hormonal substitution is mandatory in order to maintain secondary sex characteristics and bone density. Spontaneous recovery can occur; prognostic factors are not fully elucidated and include age, AMH levels, hemosiderosis. Long-term follow-up by 
specialized team is necessary. The documentation of uneventful pregnancies of our patients is promising. The patients should be well informed for all the possibilities before transplantation and during the follow-up. Whenever possible all preventive measures to preserve fertility should be undertaken.

Conflict of interest: There is no comflict of interest.

\section{P312}

\section{Use of basiliximab in allogeneic transplant recipients to replace calcineurin-inhibitors for transplant-associated thrombotic microangiopathy and acute renal failure}

\section{Ana Lerma-Verdejo ${ }^{1}$, Blanca Basagoiti-Carreño ${ }^{2}$, Carlos DE} Miguel $^{I}$, María Esther Martinez-Muñoz ${ }^{1}$, Guiomar Bautista ${ }^{I}$, Ana Morales $^{1}$, Rafael Fores ${ }^{1}$, Amelia Sanchez-Guerrero ${ }^{2}$, José Rafael Cabrera $^{I}$, Rafael Francisco Duarte ${ }^{I}$

${ }^{1}$ Hospital Universitario Puerta de Hierro Majadahonda, Hematology, Majadahonda, Spain; ${ }^{2}$ Hospital Universitario Puerta de Hierro Majadahonda, Pharmacy, Majadahonda, Spain

Background: Calcineurin-inhibitors (CNI) are the backbone of graft-versus-host disease (GVHD) prophylaxis and management in allogeneic hematopoietic cell transplantation (alloHCT), but can associate with early complications including transplant-associated thrombotic microangiopathy (TA-TMA) and acute renal failure (ARF). While removal of CNI is widely recommended in this setting, the question remains what to replace CNI with, in particular early after alloHCT.

Methods: We reviewed all cases of alloHCT in which the anti-CD25 monoclonal antibody basiliximab was used to replace $\mathrm{CNI}$ as a result of CNI-associated TA-TMA or ARF between 2009 and 2017. Cases of TA-TMA had nonimmune hemolytic anemia with high LDH, low haptoglobin and negative Coombs test, $>2$ schistocytes per high-power field and thrombocytopenia $(<50 \times 10 \mathrm{E} 9 / \mathrm{L}$ or $<50 \%$ baseline) [Uderzo C, 2014].

Results: We identified 25 alloHCT recipients in whom basiliximab was used to replace cyclosporine A $(15,60 \%)$ or tacrolimus $(10,40 \%)$ as a result of CNI-associated TATMA $(19,76 \%)$ or $\operatorname{ARF}(6,24 \%): 10$ men, 15 women; median age 34 years (16-65). AlloHCT included cord-blood plus third-party donor cells $(13,52 \%)$, matched siblings and matched unrelated donors $(6,24 \%$ each), following myeloablative $(16,64 \%)$ and non-myeloablative $(9,36 \%)$ conditioning regimens. Basiliximab was used to replace CNI being used for GVHD prophylaxis $(17,68 \%)$ and GVHD treatment $(8,32 \%)$, and started earlier in patients receiving CNI for GVHD prophylaxis than for treatment (median day after alloHCT +23 , IQR 10-44, versus +112 , IQR 43-293; $p=0.028$ ). Basiliximab (20mg IV) was administered twice weekly on days +1 and +4 and repeated over several weeks, as required. The median treatment dose was $40 \mathrm{mg}$ (40-120) for the prophylaxis group and 120mg (40-400) for the treatment group $(p=0.005)$. Of note, only 2 out of 17 cases at risk in the prophylaxis group developed GVHD following CNIreplacement with basiliximab $(11.8 \%$, 95\%CI 3.3-34.3). Out of 13 patients at risk of CMV infection who had not reactivated CMV prior to the start of basiliximab, only 5 developed CMV infection thereafter $(38.5 \%$, 95\% CI 17.7 64.5). Overall, 7 out of 25 patients died in the context of CNI-toxicity and basiliximab replacement: 3 severe infections (2 invasive fungal infections, $1 \mathrm{CMV}$ pneumonitis), 2 multi-organ failures of unknown origin, 1 TA-TMA and 1 veno-occlusive disease. The probability of overall survival in relation to the episode at 60 days after the start of basiliximab was 68\% (95\%CI 46.1-82.5). Beyond CNIreplacement with basiliximab, 14 patients received additional treatments for TA-TMA and for GVHD, including corticosteroids, eculizumab, etanercept, defibrotide, plasmapheresis, rituximab and combinations of these drugs.

Conclusions: The need to withdraw CNI as a result of TA-TMA and ARF after alloHCT is a therapeutic challenge, as there is no current guidance on the choice of alternative drugs in this setting. Our data suggest that basiliximab may be an option for these patients, with good tolerance, low rates of subsequent GVHD and viral reactivations and acceptable survival rates for such a challenging clinical situation. Prospective studies to better explore the role of basiliximab in this context should be warranted.

Conflict of interest: The authors have nothing to disclose.

\section{P313}

Use of defibrotide to treat adult patients with transplantassociated thrombotic microangiopathy

María Esther Martínez-Muñoz ${ }^{1,2}$, Ana Lario $^{1}$, Guiomar Bautista $^{1,2}$, Jose Luis Bueno ${ }^{1,2}$, Belén Navarro ${ }^{1,2}$, Almudena De Laiglesia ${ }^{1,2}$, Jose Rafael Cabrera ${ }^{1,2}$, Rafael Duarte ${ }^{1,2}$

${ }^{1}$ Hospital Universitario Puerta de Hierro Majadahonda, Madrid, Spain; ${ }^{2}$ Instituto de Investigación Sanitaria Puerta de Hierro - Segovia Arana, Madrid, Spain

Background: Transplant-associated thrombotic microangiopathy (TA-TMA) is a severe early transplant complication which results from endothelial injury. Beyond removal or treatment of precipitating factors and, more recently, treatment with eculizumab, TA-TMA remains a therapeutic challenge. Defibrotide may be considered a therapeutic option for this condition. 


\begin{tabular}{|c|c|c|c|}
\hline & $\begin{array}{c}\text { All } \\
\text { TMA episodes }\end{array}$ & $\begin{array}{c}\text { Resolved } \\
\text { TMA episodes }\end{array}$ & $\begin{array}{l}\text { Unresolved } \\
\text { TMA episodes }\end{array}$ \\
\hline Number of episodes & 17 & 11 & 6 \\
\hline Age at HCT (years) & $38(16-57)$ & $39(16-56)$ & $35(21-57)$ \\
\hline \multicolumn{4}{|l|}{ Sex } \\
\hline -Male & 9 & 6 & 3 \\
\hline -Female & 8 & 5 & 3 \\
\hline \multicolumn{4}{|l|}{ Indication for HCT } \\
\hline$-A L L$ & 8 & 5 & 3 \\
\hline -AML/MOS & 4 & 2 & 2 \\
\hline -Lymphora & 3 & 2 & 1 \\
\hline - Multiple Iryeloma & 1 & 1 & 0 \\
\hline - Aplastic Anemia & 1 & 1 & 0 \\
\hline \multicolumn{4}{|l|}{ Potential Risb Factors } \\
\hline - GVHD & 8 & 5 & 3 \\
\hline - CMV reactivation & 3 & 2 & 1 \\
\hline - Infection & 3 & 0 & 3 \\
\hline - CSA / Tacrolimus & $13 / 4$ & $8 / 3$ & $5 / 1$ \\
\hline$-\mathrm{CB} / \mathrm{SD} / \mathrm{UD}$ & $11 / 3 / 3$ & $7 / 1 / 3$ & $4 / 2 / 0$ \\
\hline$-M A C / R I C$ & $14 / 3$ & $8 / 3$ & $6 / 0$ \\
\hline MACTBI & 11 & 7 & 4 \\
\hline - MAC NOTBI & 3 & 1 & 2 \\
\hline \multicolumn{4}{|l|}{ TMA by organ dysfunction } \\
\hline - Probable TMA & 9 & 8 & 1 \\
\hline - With organ dysfunction & 8 & 3 & 5 \\
\hline Day of TMA onset & $+43(2-556)$ & $+40(2-308)$ & $+94(3-556)$ \\
\hline - Carly onset $(s+C$ whks $)$ & 9 & 7 & 2 \\
\hline - Late onset [>+6wks] & 8 & 4 & 4 \\
\hline \multicolumn{4}{|l|}{ TMA treatment } \\
\hline -DF monotherapy & 5 & 3 & 2 \\
\hline - DF in combination & 12 & 8 & 4 \\
\hline - Delay in DF treatment (days) & $0(0-25)$ & $0(0-3)$ & $1(0-25)$ \\
\hline - Length of DF treatment (days) & $14(1-54)$ & $14(1-54)$ & $13(5-25)$ \\
\hline All-cause mortality & 7 & 2 & 5 \\
\hline
\end{tabular}

[P313 Figure] [TA-TMA episodes overall and per response to defibrotide treatment]

Methods: We reviewed all cases of TA-TMA treated with defibrotide in our allogeneic transplant recipients between 2009 and 2016. All cases had non-inmune hemolytic anemia with high LDH, low haptoglobin and negative Coombs test, $>2$ schistocytes per high-power field and thrombocytopenia. Cases without signs of renal or neurological dysfunction were classified as probable TATMA [Cho B et al, 2010].

Results: We identified 17 TA-TMA episodes treated with defibrotide in 16 allogeneic transplant recipients: 9 men; median age 38 years old (16-57); 10 single-cord blood plus third-party donor cells, 3 HLA-identical siblings and 3 unrelated donors; 13 myeloablative conditioning regimen, 10 with TBI (Table 1). Concomitant risk factors at the time of TA- TMA onset were: calcineurin inhibitor (CNI) treatment in all cases (13 cyclosporin, 4 tacrolimus), acute GVHD grade $\geq \mathrm{II} / \mathrm{IV}$ in 8 cases, $3 \mathrm{CMV}$ reactivations and 3 severe fungal (1 pulmonary aspergillosis, 1 Scedosporium Prolificans septicemia) or bacterial (1 E Coli sepsis) infections. Median onset of TA-TAM was on day +43 after transplant (2-556); 9 cases of early onset ( $\leq 6$ weeks) and 8 of late onset. Nine episodes were probable TA-TMA without organ dysfunction, 8 had renal failure, 3 had resistant hypertension and 1 presented with concomitant diffuse alveolar hemorrhage. First line replacement of CNIs for basiliximab or other drugs for GVHD was performed in all cases. Defibrotide was subsequently administered as monotherapy in 5 cases, in combination with rituximab and/ or plasma exchange in 7 , and with other agents in 5 others ( 2 vincristine; 2 mesenchymal stromal cells; 1 eculizumab; 1 bevacizumab;). Median time to start defibrotide treatment was day 0 (0-25) after TA-TMA diagnosis $(6.25 \mathrm{mg} / \mathrm{kg}$ qds in 12 episodes; $10 \mathrm{mg} / \mathrm{kg}$ qds in 5), with a median duration of 14 days (7-54). Complete resolution of TA-TMA (CR) was achieved in 11 episodes $(65 \%)$ at a median time of 15 days (1-58) after defibrotide initiation. CR was associated with a reduced all-cause mortality: $18 \%$ of CR cases (2/11: 1 multilobar pneumonia and 1 toxic encephalopathy) versus $83 \%$ of cases without CR (5/6: 1 TA-TAMrelated diffuse alveolar hemorrhage, 1 lymphocytic encephalitis and 3 severe infections), $P=0.035$. TA-TMA cases triggered by severe infections had early (days 4,11 and 15 after onset) and higher mortality rates $(3 / 3,100 \%$ vs $4 / 14$, $29 \%$ ). Rates of CR were higher in cases of probable TATMA without renal failure $(8 / 9,89 \%$ vs $3 / 8,38 \%)$.

Conclusions: We provide encouraging evidence suggesting that defibrotide, proven to be safe and effective in sinusoidal obstruction syndrome, may also have a role in this other endothelial dysfunction syndrome as monotherapy or in combination with other agents. Our data show complete resolution of TA-TMA in two thirds of cases and even higher in those with early treatment. Validation of single-center experience in prospective controlled studies should be warranted.

Conflict of interest: Authors have nothing to disclose.

\section{P314}

\section{Vaccine preventable diseases: a clinical evaluation of antibody reconstitution after autologous HCT}

Diana Greenfield ${ }^{1,2}$, John Snowden ${ }^{2,3}$, Nick Morley ${ }^{3}$, Josh Wright ${ }^{3}$, Cariad Evans ${ }^{4}$, Thushan DeSilva ${ }^{5}$

${ }^{1}$ Sheffield Teaching Hospitals NHS Foundation Trust, Specialised Cancer Services, Sheffield, United Kingdom; ${ }^{2}$ University of Sheffield, Department of Oncology and Metabolism, Sheffield, United Kingdom; ${ }^{3}$ Sheffield Teaching Hospitals NHS Foundation Trust, Department of Haematology, Sheffield, United Kingdom; ${ }^{4}$ Sheffield Teaching Hospitals NHS Foundation Trust, Department of Microbiology, Sheffield, United Kingdom; ${ }^{5}$ Sheffield Teaching Hospitals NHS Foundation Trust, Department of Infectious Diseases, Sheffield, United Kingdom

Background: Revaccination programmes following HCT is important yet is often not completed. Antibody titres to vaccine preventable diseases (VPD) may reconstitute more readily post-auto than post-allo HCT. We used the platform 
of a Late Effects Clinic to check whether long-term survivors, who did not undergo a revaccination programme post-auto SCT, had adequate antibody titres to a range of VPD.

Methods: 25 consecutive patients $\geq 2$ years post-auto SCT and who attended the late effects clinic were evaluated over a 6 month period between May and November 2017. 1 patient was missed. 24 patients were included in the interim analysis. Blood was drawn on the day of the clinical to measure antibody titres to the following: diphtheria, tetanus, Haemophilus influenzae type b (Hib), Danish pneumococcal subtypes, measles, mumps, rubella and varicella (VZV). Cut-offs were pre-defined by the laboratory's reference intervals.

Patients: Median age of patients was 46.5 years (range 22-72). Median age at auto SCT was 35 years (range 1869). Primary diagnostic categories were haemato-oncology $(\mathrm{n}=16)$, solid tumours $(\mathrm{n}=7)$, and autoimmune disease $(\mathrm{n}$ $=1$ ). $21 / 24$ had previously received documents chemotherapy prior to auto SCT. 11 patients had received previous radiotherapy (TBI (2), mantle (2), whole brain (2), neck (2), mediastinal (1), neck and chest (1), pelvic (1)). 14 patients had received BEAM conditioning and the remainder received a variety of other conditioning regimens (CEM, ATG/Cyclo, carbo/etoposide, TBI/melph, TBI/cyclo, TICE).

Results: Patients were a mean of 11 years from auto SCT (range 2-26). Interim analysis of available results to date revealed low levels of anti-diptheria antibodies were found in most patients (1/20 immune), along with inadequate antipneumococcal immunity (no patients with robust titres to all 12 serotypes tested $(0 / 22)$ ). Protective anti-measles antibody titres were also absent in 13/21 patients. In contrast, 19/19 had good anti-HIB titres and 18/24 had VZV IgG above a threshold considered protective.

Conclusions: Significant deficiencies in protection from VPD were observed. The deficiencies of anti-pneumococcal immunity are of particular concern. Whilst recommending adherence to a routine vaccination programme early after HCT, a strategy involving screening and subsequent selected vaccination should be considered in long term survivors post-auto SCT who did not undergo a routine revaccination programme. Late Effects Clinics provide a good opportunity to facilitate this pragmatic approach.

Conflict of interest: We have no conflict of interest. This study is a clinical evaluation.

\section{P315}

Validation of conventional and EBMT diagnosis and severity criteria for SOS/VOD: A multicenter retrospective study in Korea
Keon Hee Yoo ${ }^{1}$, Ki Woong Sung ${ }^{1}$, Hyoung Jin Kang ${ }^{2}$, Seung Min $\mathrm{Hahn}^{3}$, Jin Seok Kim ${ }^{3}$, Jae-Ho Yoon ${ }^{4}$, Bin Cho ${ }^{4}$, Je-Hwan Lee, Chul Won Jung ${ }^{1}$, Jae-Sook Ahn ${ }^{6}$, Ho Joon Im $^{5}$, Hyery Kim ${ }^{5}$, Hoon Kook $^{6}$, Jong Wook Lee

${ }^{1}$ Samsung Medical Center, Sungkyunkwan University School of Medicine, Seoul, Korea, Republic of: ${ }^{2}$ Seoul National University Hospital, Seoul National University College of Medicine, Seoul, Korea, Republic of; ${ }^{3}$ Severance Hospital, Yonsei University College of Medicine, Seoul, Korea, Republic of; ${ }^{4}$ Seoul St. Mary's Hospital, College of Medicine, the Catholic University of Korea, Seoul, Korea, Republic of; ${ }^{5}$ Asan Medical Center, University of Ulsan College of Medicine, Seoul, Korea, Republic of; ${ }^{6}$ Chonnam National University Hwasun Hopspital, Chonnam National University Medical School, Hwasun, Korea, Republic of

Background: Sinusoidal obstruction syndrome or venoocclusive disease (SOS/VOD) is a potentially lethal complication following hematopoietic stem cell transplantation (HSCT). Conventional severity criteria for SOS/VOD is retrospectively classified and is not appropriate for determining a therapeutic option. In this study, we aimed to validate the revised EBMT severity criteria for SOS/VOD in Korean patients who were diagnosed with SOS/VOD according to the conventional criteria, in regard to their 100 day survival and mortality. Although recently revised EBMT criteria was proposed for adult populations, we tried to see if it could also be applicable to pediatric transplant recipients.

Methods: Six institutions participated in this study. Among patients undergone HSCT between 2011 and 2015 , data of 235 patients who met clinical definition of SOS/VOD were obtained and were assessed to verify if the patients met either conventional (modified Seattle or Baltimore) or EBMT diagnostic criteria.

Results: A total of 208 patients (127 children, 81 adults) were identified to have definitive SOS/VOD. All patients (study subjects) met the modified Seattle criteria, while 134 (B group) and 137 patients (E group) satisfied the Baltimore and EBMT criteria, respectively. Only 78 of 127 children $(61.4 \%)$ and 59 of 81 adults $(72.8 \%)$ among the study subjects fulfilled the EBMT diagnostic criteria of SOS/ VOD. Using the conventional severity criteria, the moderate form predominated $(63.9 \%)$ over the severe one without any mild one. In contrast, the majority of the study subjects belonged to the very severe $(66.8 \%)$ or severe $(15.4 \%)$ form when the EBMT severity criteria were applied. The 100 day survival rates of mild, moderate, severe, and very severe SOS/VOD were $91.7,83.6,90.6$, and $61.9 \%$, respectively $(P=0.001)$. The 100 day survival of patients with very severe SOS/VOD was significantly lower than the others (61.9\% vs $88.3 \%, P<0.0001)$. This result was mainly influenced by pediatric recipients $(70.0 \%$ vs $97.9 \%$, $P=0.0002)$ rather than adults $(50.8 \%$ vs $68.2 \%, P=0.09)$. This trend was also true when we compared the 100 day survival of very severe SOS/VOD with mild/moderate/ 
severe one in $\mathrm{B}$ group $(P=0.04$ in children, $P=0.7$ in adults, $P=0.04$ in total $)$ and in E group $(P=0.005$ in children, $P=0.4$ in adults, $P=0.007$ in total). The 100 day mortalities caused by SOS/VOD were $13.5 \%$ in whole study subjects, $17.2 \%$ in B group, and $16.8 \%$ in E group, respectively. All of the SOS/VOD-related mortality occurred exclusively in patients with very severe SOS/VOD.

Conclusions: Very severe form accounted for the majority of SOS/VOD according to the revised EBMT severity criteria, the presence of which was highly predictive of early post-transplant mortality. Although this new severity criteria could also be applicable to pediatric patients, a significant proportion of children with SOS/VOD might have been missed, which obviates the need for validating a new EBMT diagnosis and severity criteria for SOS/VOD in pediatric patients.

Conflict of interest: This study was financially supported by Handok.

\section{P316}

\section{Why Patients Fail to Proceed To Transplant - The South Wales Blood and Marrow Transplant Programme Experience}

David Davies ${ }^{1}$, Andrew Simmons ${ }^{1}$, Saad Al-Ismail ${ }^{2}$, Hamdi Sati $^{2}$, Sheri Thompson ${ }^{1}$, Emma Kempshall ${ }^{1}$, Wendy Ingram $^{1}$, Keith Wilson ${ }^{1}$

${ }^{1}$ Cardiff and Vale University Health Board, South Wales Blood and Marrow Transplant Programme, Cardiff, United Kingdom; ${ }^{2}$ Abertawe Bro Morgannwg University Health Board, South Wales Blood and Marrow Transplant Programme, Swansea, United Kingdom

Background: Although HSCT is potentially curative many transplant-eligible patients fail to have the procedure, even when identified early. We therefore reviewed the reasons for patients being removed from the transplant waiting list of the South Wales Blood and Marrow Transplant (SWBMT) Programme which serves $77 \%$ of the Welsh population.

\section{Methods:}

1. All new cancer patients in Wales are discussed at multidisciplinary team (MDT) meetings and their cancer type coded and entered on an electronic Welsh cancer database (CANISC). Potential HSCT recipients are identified at that meeting and entered on the SWBMT waiting list. Patient progress is monitored by the BMT team to ensure timely assessment for BMT and to minimise the wait from end of treatment to transplant.

2. Data is presented for the 900 patients with plasma cell disorders (PCD), lymphoma or acute leukaemia (the

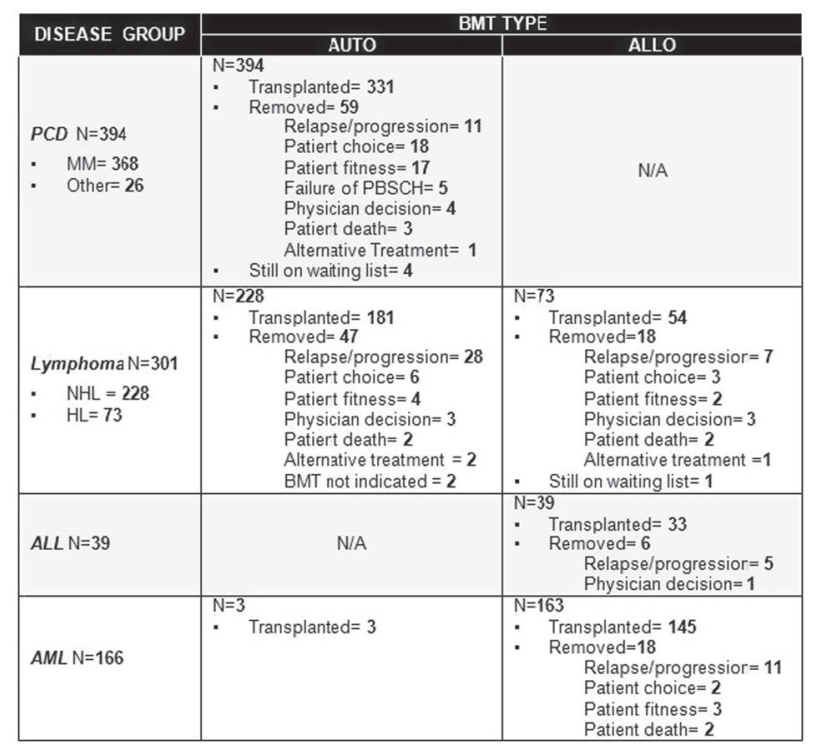

[P316 Figure] [Fate of patients on BMT waiting list]

main indications for BMT) who were assessed in the BMT clinic between 01.04.2010 and 30.09.2017 to determine the rate and reasons for deferral from BMT.

Results: Results are summarised in Figure 1.

Autologous transplant rate for PCD and lymphoma was 84\% (331/394) and 79.4\% (181/228), respectively. For PCD patients the main reasons for not progressing to BMT were patient choice $(18 / 394,4.6 \%)$, patient fitness $(17 / 394$, $4.3 \%$ ), or disease progression $(11 / 394,2.8 \%)$. For lymphoma the main reasons were disease progression $(28 / 228$, $12.3 \%)$, patient choice $(6 / 228,2.6 \%)$ or patient fitness (4/ 228, 1.8\%). All 3 patients with relapsed APL who subsequently attained a molecular CR went to transplant.

For allogeneic transplant 89\% (145/163) of AML patients achieved BMT with relapse $(11 / 163,6.7 \%)$ being the main reason for not being transplanted. Results for ALL were similar with $84.6 \%(33 / 39)$ of patients being transplanted and relapse accounting for $12.8 \%$ (5/39) not being transplanted. For lymphoma 74\% (54/73) were transplanted with relapse being the main reason for not attaining BMT (7/73, 9.6\%).

For the entire cohort $83 \%(747 / 900)$ had a transplant with $6.9 \%$ (62/900) not achieving BMT due to relapse/ progression.

Conclusions: The data, representing real-world experience of a national transplant programme, shows that despite close monitoring, $7 \%$ of patients will fail to achieve transplantation due to disease progression/relapse, representing the precarious position of patients with malignancy.

Conflict of interest: No conflict of interest.

Experimental stem cell transplantation 


\section{P317}

Acute graft-versus-host disease rescued by ex vivo TGF-b1induced CD4+FoxP3 + regulatory $T$ cells in mouse model

Tzeon-Jye Chiou ${ }^{1,2}$, Jhih-Yun Jian ${ }^{1}$, Yi-Chun Ke ${ }^{1}$, Chun-Tse Kuo ${ }^{1}$, Sin-Tak Chu ${ }^{3}$, Woan-Fang Tzeng ${ }^{4}$

${ }^{1}$ Taipei Veterans General Hospital, Division of Transfusion Medicine, Department of Medicine, Taipei, Taiwan, Republic of China; ${ }^{2}$ National Yang-Ming University School of Medicine, Department of Medicine, Taipei, Taiwan, Republic of China; ${ }^{3}$ National Taiwan University, Institute of Biochemical Science, Taipei, Taiwan, Republic of China; ${ }^{4}$ Fu-Jen University, Department of Life Science, New Taipei City, Taiwan, Republic of China

Background: To investigate whether using induced regulatory $\mathrm{T}$ cells (iTregs) instead of natural regulatory $\mathrm{T}$ cells (nTregs) can reduce the incidence of the acute graftversus-host disease (aGVHD) within a short time.

Methods: Splenocytes from C57BL/6 mice were used as a source of naïve $\mathrm{T}$ cells by a $\mathrm{CD} 4+$ naïve $\mathrm{T}$ cell isolation Kit. To induce regulatory $\mathrm{T}$ cells (iTregs), the $\mathrm{CD} 4+$ naïve $\mathrm{T}$ cells were incubated with anti-CD3/CD28 coated 24-well plate in the presence of IL-2 (20U/ml) and TGF-b1 (50ng/ $\mathrm{ml})$ for 3 days. iTregs were harvested and identified as the expressions of CD4+/CD25+/FoxP3+/CD127- via flow

\section{Control}

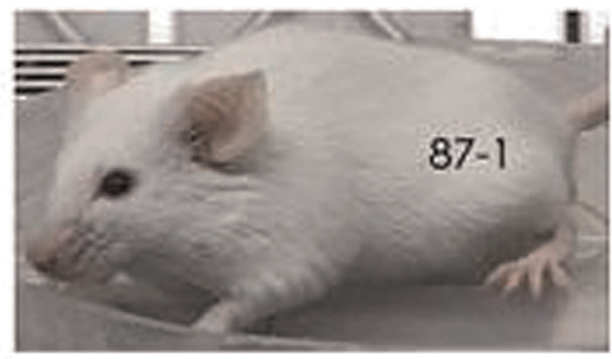

\section{Transplantation Day 5: TCD-BM+T}

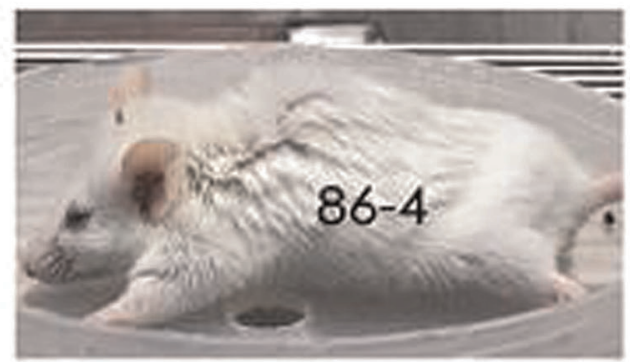

cytometry. The functional iTregs were confirmed via qPCR of the marker genes expression, such as CTLA4, IL-10 and LAG3. In this experiment, recipients $(B A L B / c)$ were irradiated with $800 \mathrm{cGy}$ and then infused with donor (C57BL/6) bone marrow cells with or without donor $\mathrm{T}$ cells by intravenous injection. The expression of CD8 +/CD4+ ratio and CD25, CD197 expressions on the peripheral blood $\mathrm{T}$ cells were as GVHD identification markers. $1 \times 10^{6}$ iTregs were injected into the aGVHD mouse for immunosuppression assay.

Results: The functional iTreg cells were harvested after 3-day induction from naïve T cells. As we known, nTregs collection needs 18-21 days. The iTregs collection time was less than that of nTreg cells. For the CD8+ memory T cells have been reported that are able to induce aGVHD and these effector T cells should be CD197 negative on cell surface. Therefore, aGVHD mice were identified by increase of $\mathrm{CD} 8+/ \mathrm{CD} 4+\mathrm{T}$ cell ratio with the positive expression of CD25 and negative expression of CD197 of these cells. After $\mathrm{T}$ cell depleted-bone marrow cell transfusion to $\mathrm{BALB} / \mathrm{c}$ mice (TCD-BM), the $\mathrm{CD} 8+/ \mathrm{CD} 4$ + ratio was $0.28 \pm 0.08$. However, the $\mathrm{CD} 8+/ \mathrm{CD} 4+$ ratio in aGVHD mice was increase to $1.21 \pm 0.61$. Meanwhile, the CD197+ T cells was decrease from $45.0 \pm 5.6 \%$ to 28.0 $\pm 3.1 \%$, comparing the TCD-BM mice to aGVHD mice. On

\section{Transplantation Day 5 : TCD-BM}

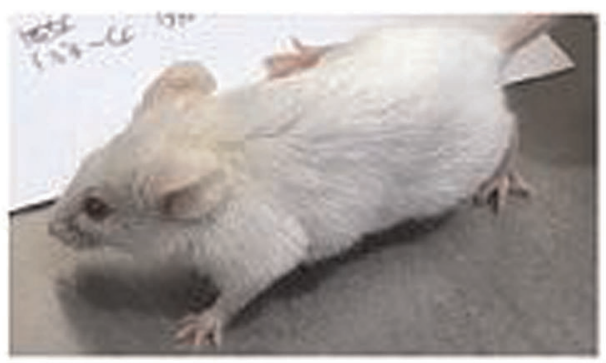

Transplantation Day 5 : TCD-BM+T+iTreg

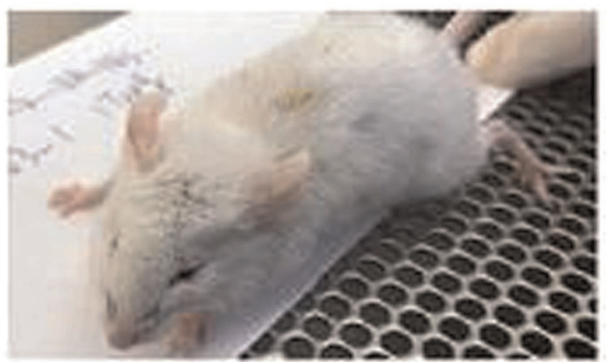

Fig. I The aCVHD mouse model before and after iTregs treatments. TCD-BM: T cell depletel-bone marrow cells infission; TCDBM+T; T cel depleted bone marrow cells with $C D V+T$ for aGVHD induction; BM-TCD+F +iTregs; aGVHD mice with iTregs treatment.

[P317 Figure] [aGVHD mouse model before and after iTreg treatment] 
iTregs treated-aGVHD mice, CD8+/CD4+ ratio decreased and CD197+ T cells increased, indicating the aGVHD mice were recovered after iTregs treatment. The mice phenotypes were similar to bone marrow infusion mice (Fig.1). It indicated that the supplement of iTregs could suppress the incidence of aGVHD.

Conclusions: To collect the enough iTreg cells for aGVHD therapy, the collection time of iTreg cells was shortened significantly as comparing the harvest time of nTreg cells. We concluded that the increase of peripheral blood CD8+ $\mathrm{T}$ cells could be a sensitive marker of aGVHD, iTregs treatment reduces CD8 $+\mathrm{T}$ cells infiltration to the peripheral blood and decreases $\mathrm{CD} 8+/ \mathrm{CD} 4+\mathrm{T}$ cells ratio. The use of iTregs therapy in the prevention of aGVHD is possible.

Conflict of interest: T. Chiou: Nothing to disclose

\section{P318}

\section{Allo-CD19-CAR-T Therapy followed by Allo-HSCT with the same donor to treat refractory or relapsed B-ALL}

Zhang Jian-Ping ${ }^{1}$, Yang Jun-Fang ${ }^{1}$, Li Nan-Nan ${ }^{1}$, Fan Li-Jun ${ }^{1}$, Gao Xin-Xia ${ }^{1}$, Chang Lung-Ji' ${ }^{2}$ Lu Xin-An ${ }^{3}, L u$ Pei-Hua ${ }^{1}$

${ }^{1}$ Hebei Yanda Lu Daopei Hospital, Langfang, China; ${ }^{2}$ Shenzhen Geno-Immune Medical Institute, Shenzhen, China; ${ }^{3}$ ImmunoChina Pharmaceutical Co. Ltd, Beijing, China

Background: Although CD19 chimeric antigen receptormodified T (CD19-CAR-T) treatment has been approved for treating refractory/relapsed B cell acute lymphocytic leukemia (B-ALL), it is still mainly use autologous CD19CAR-T therapy before allogeneic hematopoietic stem cell transplantation (allo-HSCT). We report two patients with relapsed B-ALL treated with allo-CD19-CAR-T cells then bridged into allo-HSCT from the same donor of CAR-T with great success.

Methods: The first case was a 7-year-old girl with relapsed B-ALL, who could not achieve CR after4 courses of re-induction chemotherapy. We could not generate CAR$\mathrm{T}$ cells from her own lymphocytes because of persistent low peripheral blood WBC $<1 \times 10^{9} / \mathrm{L}$. She had $22 \%$ blasts in bone marrow. She received allo-CD19-CAR-T cells (total $5 \times 10^{5} / \mathrm{kg}$ ) from her mother (5/10 matched). She achieved morphologic complete remission (CR) but still had minimal residual disease (MRD), therefore, given autologous CAR$\mathrm{T}$ therapy. The second case was a 6-year-old boy with relapsed B-ALL, who received the allo-CD19-CAR-T cells $\left(3 \times 10^{5} / \mathrm{kg}\right)$ from his HLA $10 / 10$ matched brother. Once CR achieved from CAR-T treatment, both patients were bridged into allo-HSCT from the same donor of their allo-CD19CAR-T
Results: After allo-CD19-CAR-T, the first case achieved morphological CR but the MRD was still positive. This patient had no donor chimerism, therefore, she had autoCD19-CAR-T therapy. Subsequently she achieved MRD negative CR per flow cytometry study. She received alloHSCT (100 days after her allo-CAR-T, 46 days after her auto-CAR-T) from the same donor of her allo-CAR-T. Post allo-HSCT, her chimerism was $100 \%$ donor type. She only had grade I skin acute graft versus host disease (aGVHD). She is currently disease free 28 months post allo-HSCT. The second case achieved MRD negative CR after receiving the allo-CD19-CAR-T cells from his sibling brother. The chimerism of $\mathrm{CD} 3+$ in the peripheral blood went up to $39.58 \%$ on 10 day post CAR-T cells infusion but rapidly dropped to $4.82 \%$ on day 48 . The patient was bridged into allo-HSCT (50 days after her CAR-T therapy) from the same donor of his allo-CD19-CAR-T. His leukocytes were successfully engrafted and the chimerism was $100 \%$ the donor type. He only had grade II skin aGVHD. He is currently disease free 3 months post allo-HSCT.

Conclusions: Allo-CAR-T treatment is feasible for patients prior to allo-HSCT. For those patients if CAR-T cells are not able to be manufactured from their own lymphocytes, allo-CD19-CAR-T therapy can be considered as an option. Once CR achieved with CAR-T therapy, bridging into allo-HSCT using the same donor of alloCD19-CART may result in long-term disease-free survival.

Conflict of interest: None to declare

\section{P319}

An update on haploidentical hematopoietic stem cell transplantation with in vivo T-cell depletion after reduced intensity conditioning regimen in pediatric patients affected by malignancies

Francesco Saglio, Massimo Berger, Francesca Carraro, Rosanna Pessolano, Paola Quarello, Elena Vassallo, Franca Fagioli

AOU Citta della Salute e della Scienza di Torino, Oncoematologia Pediatrica, Torino, Italy

Background: Haploidentical hematopoietic stem cell transplantation with in vivo T-cell depletion with high dose Cyclophosphamide (HaploHSCT-CTX) is currently one of the most largely adopted strategies for patients needing a transplantation and lacking an HLA-matched donor, but long-term results of this procedure are still limited. Main aim of this study is to evaluate the long-term overall survival (OS) of a pediatric population undergoing HaploHSCT-CTX for advanced stage hematological malignancies. Secondary endpoint of this study is the assessment of potential pitfalls of this strategy regarding transplant 
related mortality (TRM), relapse incidence (RI), and acute and chronic GvHD incidence.

Methods: We retrospectively evaluated 19 pediatric patients (median age 9 years, range:1-21) undergoing HaploHSCT-CTX at our Center for hematological malignancies between 2010 and 2015. 13 out of 19 patients (63\%) were beyond the second disease remission or refractory to at least one previous line of chemo or radiation therapy at the transplantation.

Conditioning regimen included Cyclophosphamide (total dose $29 \mathrm{mg} / \mathrm{kg}$ ), Fludarabine (total dose $150 \mathrm{mg} / \mathrm{mq}$ ) and TBI (2 Gy in 1 single fraction). GvHD prophylaxis was based on high-dose Cyclophosphamide $(50 \mathrm{mg} / \mathrm{kg} /$ day on days +3 and +4 ), Tacrolimus and Mofetil Micofenolate. Patients underwent clinical and hematological posttransplantation assessments according to our Center's policy. OS was calculated according to the Kaplan-Meier method. TRM and RI were calculated as a cumulative incidence (CI) to adjust the analysis for competing risks: relapse and transplant-related death were considered competing risks, respectively. aGvHD and cGvHD incidence was calculated as CI as well: in these cases, the death for any reason was considered a competing risk. All the analyses were performed using NCSS software (Hintze, 2001; NCSS PASS, Number Crunched Statistical System, Kaysville, UT, USA).

Results: Median follow up of the study population is of 1096 days (range: 78-2168). At 5 years after HSCT OS is of $84 \%$ (95\%CI: 67-100), TRM of 5\% (95\%CI: 0-35), RI of $32 \%$ (95\% CI: 16-62) and incidence of grade II-IV acute GvHD 10\% (95\%CI: 3-39). All the cases of GvHD just involved the skin and were easily manageable with systemic steroid treatment. No case of chronic GvHD was observed in the study population. Causes of death were disease progression (2 patients), Adenovirus infection (1 patient) and cerebral hemorrhage (1 patient).

Conclusions: Our retrospective data confirm the safety of HaploHSCT-CTX also in a setting of patients previously heavily treated and so at high risk of developing transplantrelated toxicities. Especially the occurrence of immunological complications as acute and chronic GvHD is low.

Conflict of interest: The authors have no commercial, proprietary or financial interests in the products or companies described in this article

\section{P320}

Identification and evaluation of CD34+Microparticles in grafts and patients post allogeneic hematopoietic cell transplantation

\author{
Angeliki Xagorari ${ }^{1,2}$, Michail Iskas ${ }^{1}$, Dimitris Bougiouklis ${ }^{1}$, Eleni \\ Gavriilaki $^{1}$, Giorgos Karavalakis ${ }^{1}$, Despoina Papadopoulou ${ }^{1,2}$,
}

Dionisia Papaioannou ${ }^{1,2}$, Ioannis Batsis ${ }^{1}$, Despoina Mallouri ${ }^{l}$, Ioanna Sakellari ${ }^{1}$, Achilles Anagnostopoulos ${ }^{1,2}$, Damianos Sotiropoulos $^{1,2}$

${ }^{1}$ G. Papanicolaou Hospital, Bone Marrow Transplantation Unit, Dept. of Hematology, Thessaloniki, Greece; ${ }^{2}$ G. Papanicolaou Hospital, Public Cord Blood Bank, Dept. of Hematology, Thessaloniki, Greece

Background: Allogeneic hematopoietic cell transplantation (HCT) main sources are CD34+ from peripheral blood mobilized and umbilical cord blood (UCB). Hematopoietic stem cell homing are connected with different factors that affect hematopoietic reconstitution after HCT. Cells communicate with cytokines, growth factors, adhesion molecules and microparticles (MPs). In this study we analyse the CD34+ MPs in peripheral graft and patient post HCT and in UCB.

Methods: Samples were collected from the graft and from the peripheral blood of 7 patients who underwent HCT in our department. Four patients were diagnosed with acute myeloid leukemia and 3 with acute lymphoblastic leukemia. Informed consent was obtained from all patients. Patients received peripheral blood stem cell (PBSC) transplant from sibling donors. All patients were at complete remission before HCT. Regarding the conditioning 5 patients received myeloablative regimen and 2 patients received reduced intensity conditioning (RIC). The number of stem cell derived-MPs (AnnV+/CD34+MPs) has been estimated in the plasma of the graft as well as the peripheral blood of the patients at days 0,4 and 14 (day0, $+4 \mathrm{~d},+14 \mathrm{~d}$ ) from the transplantation. Cord blood units $(\mathrm{CBUs})(\mathrm{n}=34)$ were processed using the Sepax automated method (Biosafe). Adjusted variables of the CBUs included number of CD34 + per graft and their viability and number of $\mathrm{AnnV}+/ \mathrm{CD} 34$ + MPs was determined by flow cytometry. Mononuclear cells were seeded in semisolid cultures in the presence of a cocktail of growth factors for colony forming unit granulocyte/macrophage (CFU-GM), burst forming unit erythroid (BFU-E) and Colony-Forming Unit-Granulocyte, Erythrocyte, Monocyte/macrophage, Megakaryocyte (CFUGEMM) colony growth. Electron microscope photographs were obtained, after the proceedings for TEM. Statistical analysis was performed using t-test, PearsonÂ's and Spearman $\hat{A}^{\prime}$ s depending on the normality of the distribution of variables.

Results: AnnV+/CD34+MPs has been detected in the graft and in peripheral blood of the patients post HCT. CD34+ per unit were significantly associated with AnnV $+/ \mathrm{CD} 34+\mathrm{MPs}$ at $+4 \mathrm{~d}\left(\mathrm{p}<0,016\right.$,Spearman $\hat{A}^{\prime} \mathrm{s}$ rho $=0,848$ ) whereas at $+0 \mathrm{~d}$ and $+14 \mathrm{~d}$ were not statistical significant correlated.

In UCB AnnV+/CD34+MPs were detected pre- and post-processing with SEPAX. On univariate analysis found that the number of AnnV+/CD34+MPs (pre-processing vs. 
post-processing) has statistically significant mean positive correlation ( $\mathrm{p}<0,000$, PearsonẤs rho $=0,878$ ). Additionally, AnnV+/CD34+MPs (pre-processing vs.post-processing) has statistical significant negative correlation to the CD34+ viability $(\mathrm{p}<0,002$,PearsonÂ's rho $=0,541 ; \mathrm{p}<$ 0,050,Pearson $\hat{A}$ 's rho $=0,339$ ).

Clonal assays for hemopoietic progenitors showed that the number of AnnV+/CD34+MPs has statistically significant mean positive correlation to the number of CFU$\mathrm{GM}(\mathrm{p}<0,031$,Spearman $\hat{A}$ 's rho $=0,370)$ and to BFU-E ( $\mathrm{p}$ $<0,001$, SpearmanÂ's rho $=0,541)$.

Conclusions: The result of the present study indicate the presence of hematopoietic stem cell derived-MPs in the PBSC transplants, the UCB and the peripheral blood of the patients undergoing HCT. Furthermore, in the peripheral blood of HCT patients, the number of AnnV+/CD34+MPs at day 4 was associated with the absolute number of CD34. In UCBs, the number of pre-and post-processing AnnV +/CD34+MPs was statistically correlated with the CFUGM and BFU-E. The role of the CD34+MPs in the engraftment of the transplant has to be determined.

Conflict of interest: All authors declares that they have no conflict of interest. The source of funding is Greek Ministry of Health.

\section{P321}

Iron overload results in poor graft function after allogeneic hematopoietic stem cell transplantation by impairing hematopoiesis

\section{Xiao Min, Ting Yang, Xueqiong Wu, Yuxin Zhang, Zhizhe Chen, Jianda $\mathrm{Hu}$}

Fujian Institute of Hematology, Fujian Provincial Key Laboratory on Hematology, Fujian Medical University Union Hospital, l, Department of Hematology, Fuzhou, China

Background: Clinical data suggest that pre-transplant iron overload due to high transfusion requirements may affect graft function after allogeneic hematopoietic stem cell transplantation (allo-HSCT) .

Methods: In the setting of allo-HSCT, a BALB/C mice and a C57/BL6 (H-2b) were used as recipient and donor, respectively. Recipient mice were injected intraperitoneally with iron sucrose with the cumulative doses of $50 \mathrm{mg}, 100$ $\mathrm{mg}$, and $200 \mathrm{mg}$ in three experimental groups, while the control with phosphate-buffered saline. All mice received total body irradiation for HSCT. Plasma iron levels and Iron stores of liver, spleen and bone marrow (BM) were measured. Hematological parameter, CD34+ cells and the hematopoietic colony-forming capacity were monitored to evaluate the hematopoietic recovery. Bone marrow derived mesenchymal stem cells (BM-MSCs) were isolated and assessed for proliferation. Immunohistochemical analysis assessed the BM microenvironment, expression of haematopoietic chemokines and immunological parameters.

Results: Iron overload aggravated PGF as observed by the results that peripheral blood cells were significantly lower in number and recovered less rapidly with a dosedependend manner, compared with that of controls $(\mathrm{P}<$ 0.05). In the experimental groups, BM was hypocellular, CD34+ cell counts and colony-forming units were significantly decreased $(\mathrm{P}<0.05)$. BM-MSCs showed a longer double time than control $(\mathrm{P}<0.05)$. Furthermore, the immunohistochemical analysis demonstrated the immunological impairment and the low expression of stem cell factor -1 and vascular endothelial growth factor-1.

Conclusions: Current evidence shows that iron overload is an important risk factor for PGF by damaging hematopoietic stem cells and the microenvironment, which highlights the need for optimizing iron management to improve the transplantation outcomes.

Conflict of interest: Without any conflict of interest

\section{P322}

Abstract previously published

P323

Viral-specific $T$ cell infusions for refractory viral infections after allogeneic stem cell transplantation

Sara Lozano, Oscar Pello, Sandra Loaiza, Philippa Woolley, Shab Uddin, Emma Bray, Kemal Ozbey, Shaida Nazir, Graham Smith, Sinju Thomas, Tsembayena Dlamini, Rosalinda Elio, Renuka Palanicawandar, Dragana Milojkovic, Jiri Pavlu, Edward Kanfer, Ian Gabriel, Donald McDonald, Christian Jakob, Aristeidis Chaidos, Anastasios Karadimitris, Jane Apperley, Eduardo Olavarria

Imperial College London, Haematology, London, United Kingdom

Background: Patients undergoing allogeneic stem cell transplantation (allo-SCT) are susceptible to viral infections due to prolonged periods of immunosuppression and a significant number of patients do not respond optimally to pharmacological therapies.

Methods: We have selected viral-specific T-lymphocytes from 14 healthy donors. Using the closed system CliniMACS Prodigy in a grade A GMP room, $1 \times 10^{9}$ cells collected by leukapheresis were stimulated for 6 hours with a pool of viral PepTivators to induce the expression of IFN$\gamma$ in viral-specific memory $\mathrm{T}$ cells. Cells were labelled with an anti-IFN- $\gamma$ antibody coupled to an iron bead and passed through a magnetic column to enrich the CD4+ IFN- $\gamma+$ 


\begin{tabular}{|c|c|c|c|c|c|c|c|c|}
\hline Dotar & Vrus & Yixilose & $\cos +\cos \operatorname{lig}^{2}$ & $\mathrm{COA}+\mathrm{IPS}_{-3}$ & $\mathrm{Cos}+\mathrm{inN}_{\mathrm{a}}$ & cosicol b/a itax & Outrom in vi bas & 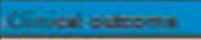 \\
\hline Masie & ADV & 7320 & $2.7 \times 28$ & $34 \%$ & $79 \%$ & $2.7 \times 20$ & 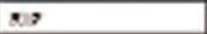 & ovin'estoe \\
\hline Hegle & $\mathrm{BK}$ & $3.2 \times 20^{\circ}$ & $0.24 \times 20$ & $20 \%$ & 476 & $0.24 \times 28$ & es & cx \\
\hline$\therefore 8$ & BK & $200 \times 20$ & $2.00 \times 20$ & 4.46 & $28 \%$ & $2.00 \times 20$ & ca & 0 gina \\
\hline Megle & BK & $20 \times 20$ & $0.22 \times 20$ & $8 / 4$ & 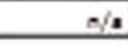 & $0.22 \times 20$ & se & 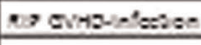 \\
\hline 28 & BK & $92 \times 28$ & $=/ 2$ & 725 & $23 \%$ & $-/ /$ & c* & ce \\
\hline 58 & $\mathrm{CMV}$ & 2232 & $2.22 \times 20$ & 476 & 326 & $2.25 \times 28$ & ex & ex \\
\hline Tesle & $\mathrm{CMV}$ & 20592 & $0.72 \times 20$ & 296 & $e: \%$ & $0.79 \times 20$ & ce & og ovinb-elenter \\
\hline Tesle & $\mathrm{CMN}$ & 2298 & $2.2 \times 22^{4}$ & $27 \%$ & $2 e \%$ & $2 \times: 0$ & ex & ex \\
\hline tosle & CMV & 445 & $0.38 \times 20$ & 746 & 876 & $0.58 \times 22^{4}$ & ce & ce \\
\hline Hesle & $\mathrm{CMV}$ & 8402 & $0.07 \times 20$ & est & 726 & $0.07 \times 22^{4}$ & $c x$ & $\because$ ando \\
\hline mos & CMV & 250 & $2.7 \times 20^{4}$ & $30 \%$ & $32 \%$ & $2 \times 28$ & $c x$ & ca \\
\hline Mus & EDV & 79000 & $22.2 \times 20$ & 736 & 246 & $2 \times 28$ & ex & Oo Nolasue CN3 \\
\hline 28 & EBV & 70000 & $2.08 \times 20$ & $23 \%$ & $20 \%$ & $2 \times 20^{4}$ & c* & $\Rightarrow 8$ \\
\hline mos & EBV & 290000 & $3.2 \times 22^{4}$ & $22 \%$ & 2026 & $2 \times 20^{4}$ & ca & 88 \\
\hline
\end{tabular}

[P323 Figure] [Table 1]

and CD8 + IFN- $\gamma+$ lymphocyte populations in the eluted fraction. The process is fully automated and takes 17 hours.

Results: The median age of the patients was 46 years (range 6-65) and 8 (57\%) patients were male. The diagnosis were Acute Myeloid Leukaemia (4 patients), Acute Lymphoblastic Leukaemia (2 patients) and one patient each: Myelodysplastic Syndrome, Chronic Myeloid Leukaemia, Follicular Lymphoma, Cutaneous T-cell Lymphoma, Severe Aplastic Anaemia, Beta-Thalassemia Major, Sickle Cell Disease and Diamond-Blackfan Anaemia. The transplant donors were 7 haplo-identical, 4 HLAIdentical siblings and 3 matched unrelated donors. Conditioning was Myeloablative in 11 and reduced intensity in 3 transplants.

Viral-specific T-lymphocytes were selected against Cytomegalovirus (CMV) from 6 donors, BK-virus (BKV) from 4, Ebstein-Barr-Virus (EBV) from 3 and Adenovirus (ADV) from one donor respectively. The median viral load at the time of infusion was 1,772 copies/ul (150-20,339) for CMV, 52 x $10^{6}$ copies/ul (3.3-203) for BKV, 76,000 copies/ ul (75,000-250,000) for EBV and 7,326 copies/ul for ADV. Patients had failed to respond to anti-CMV (median 3, Ganciclovir, Foscarnet and Cidofovir), anti-EBV (Rituximab), anti-BKV (Cidofovir, Brincidofovir) and anti-ADV (Cidofovir) therapies. A total of 3 patients had developed acute GVHD and 2 were receiving systemic corticosteroids at the time of the T-cell infusion.

The median viral-specific CD3 + cell dose obtained was $0.73 \times 10^{4} / \mathrm{kg}$ for $\mathrm{CMV}, 0.34 \times 10^{4} / \mathrm{kg}$ for $\mathrm{BKV}, 6.5 \times 10^{4} /$ $\mathrm{kg}$ for $\mathrm{EBV}$ and $4.5 \times 10^{2} / \mathrm{kg}$ for ADV. The characteristics of each selection process are described in table 1. Patients received viral-specific T-cells at a median of 86 days (26130) for BKV, 82 days (56-177) for CMV, 121 days (115288) for EBV and 162 days for ADV. All patients received a single infusion. No infusional reactions were recorded. No evidence of new onset acute GVHD was detected. All 6 patients with CMV infection achieved a CR (negative PCR) and none developed CMV disease. All patients with BKV haemorrhagic cystitis responded (3 CR and one PR), although all remained persistently positive by urine PCR. All 3 patients with EBV viraemia became PCR negative, although only 2 achieved a CR and one a PR by PET-CT scanning. The patient with ADV infection died 3 weeks after ADV-specific T-cell infusion of multiorgan failure without responding.

Conclusions: Viral-specific T-lymphocytes can be manufactured from healthy stem cell donors and infused safely to allogeneic SCT patients refractory to conventional antivirals. Viral load responses could be seen in this patient population without significant side-effects.

Conflict of interest: No conflict of interest

\section{P324}

Will early allogeneic hematopoietic stem cell transplantation yield better outcomes in STAT3 deficient patients?

Claudia Berlin $^{1}$, Anna Eichinger ${ }^{2}$, Mathias Kurzay ${ }^{1}$, Christoph Klein $^{3}$, Heiko-Manuel Teltschik ${ }^{1}$, Tobias Feuchtinger ${ }^{1}$, Fabian Hauck $^{2}$, Michael Albert ${ }^{1}$

${ }^{1}$ Dr.v.Hauner Children's Hospital, Ludwigs-Maximilians-University, Department of Pediatric Hematology, Oncology and Stem Cell Transplantation, Munich, Germany; ${ }^{2}$ Dr.v.Hauner Children's Hospital, Ludwigs-Maximilians-University, Immunodeficiency Unit and Immunological Diagnostics Laboratory, Munich, Germany; ${ }^{3}$ Dr.v. Hauner Children's Hospital, Ludwigs-Maximilians-University, Munich, Germany

Background: The autosomal dominant Hyper-IgESyndrome (AD-HIES) is a rare primary immunodeficiency and multisystem disorder resulting from heterozygous mutations in the STAT3 gene. AD-HIES is characterized by skeletal dysplasia, recurrent pulmonary and skin 
infections (e.g. staphylococcal abscesses, eczematoid dermatitis) due to an increased susceptibility to bacteria and fungi. The immunological work-up for these patients usually reveals an impaired Th17 cell differentiation and markedly elevated IgE-levels. Since many patients do rather well on anti-infective prophylaxis and supportive care and early case reports suggested no benefit of allogeneic hematopoetic stem cell transplantation (HSCT), AD-HIES patients are rarely referred for HSCT.

The literature still contains only a handful of patients who underwent $\mathrm{HSCT}$ after variable conditioning regimens and from matched and mismatched donors. All these patients had experienced severe disease related complications before HSCT and consequently the benefit of HSCT was reported to be variable. Currently, the general consensus is to only consider patients with severe pulmonary disease for HSCT. It could however be postulated that transplanting patients earlier in their disease cause and correcting their immunodeficiency before permanent organ damage due to infectious complications has occurred may extend life-expectancy and improve the quality of life of AD-HIES patients.

Methods: We report on a 15 year old female AD-HIESpatient who presented with one pneumatocele, bronchiectases and modestly impaired pulmonary function following recurrent pneumonias. Her past medical history also included recurrent skin infections, serious haemoptysis, pathological fractures and atopic eczema.

Considering that lung infections are the major lifelimiting complication in AD-HIES and a potentially positively influenced by HSCT, our patient had requested a transplant. After extensive discussion of the pros and cons and obtaining full informed consent from her and her family, she underwent an elective HSCT. She received bone marrow from an HLA-matched sibling donor after a reduced-intensity conditioning consisting of alemtuzumab $(0,6 \mathrm{mg} / \mathrm{kg})$, treosulfan $\left(14 \mathrm{~g} / \mathrm{m}^{2}\right)$, fludarabine $\left(150 \mathrm{mg} / \mathrm{m}^{2}\right)$ and thiotepa $(10 \mathrm{mg} / \mathrm{kg})$, and graft-versus-host disease (GVHD) prophylaxis with cyclosporine A (CSA) and mycophenolate mofetil (MMF).

Results: The peri-transplant course was complicated by acute gastrointestinal bleeding and renal failure of unknown origin. Continued kidney function impairment led to early tapering and discontinuation of cyclosporine on day +132 after HSCT in the absence of any acute GvHD. Neutrophil and platelets engrafted on days +15 and +26 respectively. She is currently on day +136 free of GvHD or infection, exhibiting full donor chimerism and recovered kidney function. In view of the pre-existing pulmonary disease she remains on antibiotic prophylaxis.

Conclusions: This AD-HIES patient who underwent HSCT with few pre-HSCT disease complications and relatively little permanent organ damage may add to our understanding of whether early HSCT will lead to improvement of quality of life and increased lifeexpectancy in AD-HIES patients.

Conflict of interest: Nothing to disclose

Gene therapy

\section{P325}

\section{Footprint-less Gene Correction of Wiskott Aldrich Syndrome Patient-Specific iPSCs Normalizes the Formation of Podosomes in Macrophages}

\author{
Yan Long ${ }^{1}$, Ying Jia Wang ${ }^{2}$, He Huang ${ }^{1}$, Jiing-Kuan Yee ${ }^{3}$
}

${ }^{1}$ Bone Marrow Transplantation Center, The First Affiliated Hospital, Zhejiang University School of Medicine, Hangzhou, China; ${ }^{2}$ School of Medicine, Hangzhou Normal University, Department of Pathology, Hangzhou, China; ${ }^{3}$ Beckman Research Institute, City of Hope National Medical Center, Department of Diabetes and Metabolic Diseases Research, Duarte, CA, United States

Background: Wiskott-Aldrich syndrome (WAS) is an Xlinked immunodeficiency disease caused by mutations in the gene encoding a scaffold protein called WAS protein (WASp). WASp function as an important actin regulator in hematopoietic cells. The combination of RNA- guided clustered regularly interspaced short palindromic repeats (CRISPR)-Cas9 together with the piggyBac technology has been used to direct seamless gene correction. Here, we used this strategy corrected the integration-free induced pluripotent stem cells (iPSCs) generated from a male patient. In the first gene manipulation, we created corrected WAS-iPSCs with selectable markers. After piggyBac excision, we achieved seamless correction with no detectable exogenous sequences can be found in genome DNA of corrected WASiPSCs (cWAS-iPSCs). Monocytes/macrophages can be successfully generated. The failure in podosome formation was restored in cWAS-iPSCs-derived macrophages.

Methods: The non-integrated WAS-iPSCs were created by reprogramming from a male patient's fibroblasts (GM01598) with 3 episomal plasmids expressing OCT3/4, SOX2, KLF4, L-MYC, LIN28 and a TP53 shRNA. The resulting WAS-iPSCs were characterized by the expression of pluripotency markers. The residual vector DNA was detected by PCR and the WAS gene mutation was confirmed by sequencing. To correct the mutation and meanwhile introduce a selectable marker (GFP-puro) for positive selection of homologous recombination, we first examined the sequences at exon10 of the WAS gene in the region of mutation and found a TTAA site serve as an insertion site for the piggyBac cassette. To target the mutation site of WAS locus, two CRISPR/Cas9 sgRNA expressing vectors were cotransfected with a targeting vector containing the piggyBac cassette into one WASiPSC cell line. The correction of WAS gene was detected by 

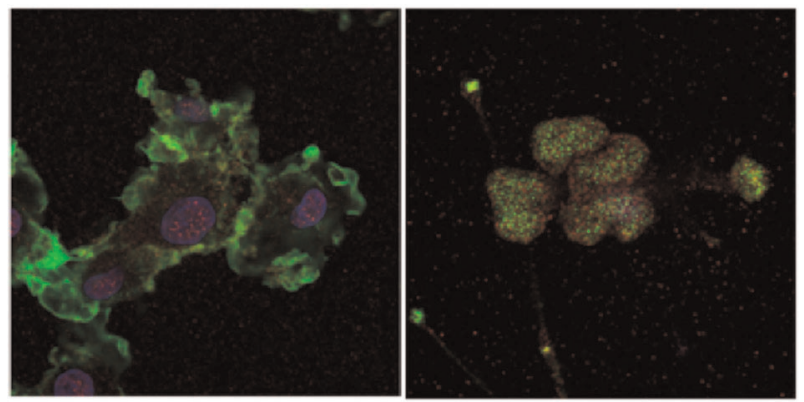

[P325 Figure] [Podosome restored in cWAS-iPSC-derived macrophages [right]]

PCR. Finally, to achieve footprint-less correction, an excision-competent/integration-defective transposase expressing vector was cotransfected with a GFP vector into the cWAS-iPSCs. A three-step differentiation method containing OP9 co-culture was used to direct differentiation toward monocytes/macrophages.

\section{Results:}

1. The WAS-iPS cells we generated by episomal plasmids carried the same mutation in WAS gene as parental patient fibroblasts, and contained no exogenous vector integration in their genome.

2. The combination of CRISPR/Cas9 and piggyBac can achieve gene correction without footprint. The cWAS-iPS cells we created restored the mutation in WAS gene and all the cWAS-iPS clones carried no residual piggyBac segments after the transposon excision.

3. The WAS-iPSCs and cWAS-iPSCs can be differentiated into monocytes/macrophages and macrophages derived from WAS-iPSCs restored the formation of podosome which proved to be impaired in WAS-iPSCderived macrophages.

Conclusions: The combination of CRISPR/Cas9 and the piggyBac system achieved the footprint-less site-specific correction in the WAS-iPSCs. The podosome formation failure caused by the mutation can be restored after gene correction. Integration-free reprogramming and efficient gene correction without leaving any exogenous segments in genome DNA of WAS patient-specific induced pluripotent stem cells (WAS-iPSCs) could offer a safe approach to cure this disease.

Conflict of interest: Yan Long: nothing to disclose

\section{P326}

Abstract previously published

\section{P327}

A mechanistic biomarker may predict clinical responses to MSC in Graft-versus-Host Disease

Antonio Galleu ${ }^{1}$, Malte von Bonin ${ }^{2}$, Dragana Milojkovic ${ }^{3}$, Hannah Hunter $^{4}$, Nick Morley ${ }^{5}$, Eleni Tholouli ${ }^{6}$, Kim Orchard ${ }^{7}$, David I. Marks $^{8}$, Martin Bornhauser ${ }^{1,2}$, Dazzi Francesco ${ }^{1}$

${ }^{1}$ King's College London, London, United Kingdom; ${ }^{2}$ University Hospital Carl Gustav Carus, Dresden, Germany; ${ }^{3}$ Imperial College Healthcare NHS Trust, London, United Kingdom; ${ }^{4}$ Plymouth Hospitals NHS Trust, Plymouth, United Kingdom; ${ }^{5}$ Royal Hallamshire Hospital, Sheffield, United Kingdom; ${ }^{6}$ Central Manchester University Hospital, Manchester, United Kingdom; ${ }^{7}$ University Hospital Southampton, Southampton, United Kingdom; ${ }^{8}$ University Hospitals Bristol NHS Trust, Bristol, United Kingdom

Background: Mesenchymal Stromal Cell (MSC) therapy can dramatically improve overall survival in patients with steroid-resistant acute Graft versus Host Disease (aGvHD). However, only a proportion of patients responds and no criteria for patient stratification are available. We have recently discovered that MSC undergo apoptosis soon after infusion and that this is crucial for their immunosuppressive effect. MSC apoptosis is effected by cytotoxic cells harboured in GvHD recipients. In this study, we tested the feasibility of using the in vitro cytotoxic activity of patient peripheral blood mononuclear cells (PBMC) against MSC to predict clinical responses to MSC in a cohort of 33 patients.

Methods: PBMC from 33 steroid-resistant aGvHD patients were collected within one week before MSC treatment. One patient received two doses of MSC and the cytotoxic assay was performed before each dose independently. Third party MSC were co-cultured with patients' PBMC for 4 hours. The level of MSC apoptosis was assessed by flow-cytometry using Annexin $\mathrm{V}$ and 7-AAD. Cytotoxicity was expressed as the proportion of Annexin V ${ }^{+} / 7-\mathrm{AAD}^{-} \mathrm{MSC}$. When performing the assay, the operator was blind to patients' clinical details. Informed consent was obtained from all patients in accordance with the local ethics committee requirements. Response to treatment was assessed after one week from the MSC infusion. Patients were defined as: a) Responders when an improvement of at least $50 \%$ in at least one organ affected by aGvHD was observed, or b) Non-Responders if they had stable or progressive disease.

Results: Median age of patients was 46 years (range 369). Transplant was performed as part of treatment for the following malignancies: AML (10), ALL (4), CML (3), MDS/MPNs (6) NHL/HL/MM (10). Median time from transplant to MSC treatment was 92 days (range 33-564), while median time from GvHD diagnosis to MSC was 23 (4-279). Before MSC infusion, patients had received 


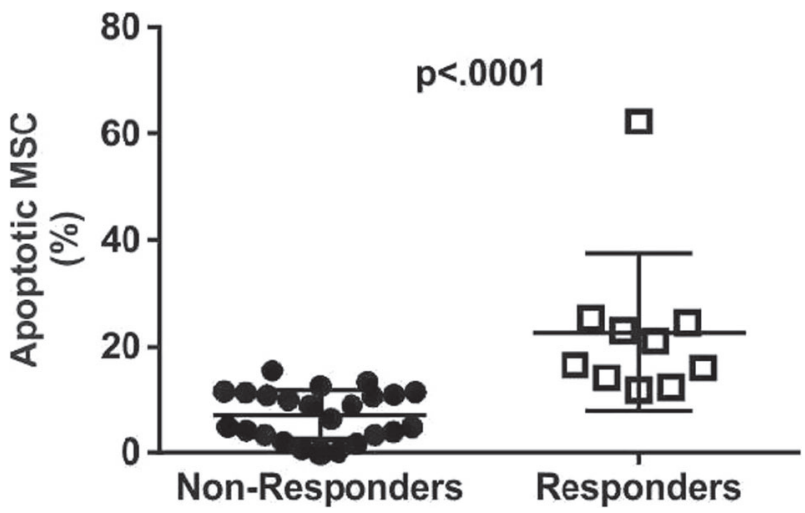

[P327 Figure] [Figure 1. Cytotoxic activity against MSC predicts clinical responses to MSC in GvHD patients]

Methylprednisolone alone (3) or in combination with two or more drugs (28). In 2 cases, patients had received Mycophenolate Mofetil or Cyclosporin only. Response to MSC treatment was achieved after 10 out of 34 infusions (29\%). PBMC from Responders exhibited a significantly higher cytotoxicity against MSC in comparison to nonresponders (mean: $22.6 \% \pm 14.8 \% 95 \%$ CI: $12.0-33.2$ versus $7.0 \% \pm 4.6 \% 95 \%$ CI: 5.0-9.0, respectively. $\mathrm{p}<.0001$ ) (Fig. 1). Importantly, the study of the receiver-operating characteristic curve revealed that a $11.5 \%$ cut-off was predictive of clinical response with $100 \%$ sensitivity and $87.5 \%$ specificity.

Conclusions: These data show that in vitro cytotoxic assay can be used as predictor of clinical response to MSC in GvHD patients.

Conflict of interest: Nothing to disclose

\section{P328}

\section{A National Audit of Paediatric Extracorporeal Photopheresis in the United Kingdom}

Aisling Flinn ${ }^{1}$, Sheba Macheka ${ }^{1}$, Arun Alfred ${ }^{2}$, Gail Smith $^{3}$, Brenda Gibson ${ }^{3}$, Sarah Lawson ${ }^{4}$, Anna Talby, Giovanna Luichinni, Helen New $^{6}$, Beki James ${ }^{7}$, Julia Scarisbrick ${ }^{8}$, Andrew Gennery ${ }^{1}$

${ }^{1}$ Newcastle University, ICM, Newcastle upon Tyne, United Kingdom; ${ }^{2}$ The Rotherham NHS Foundation Trust, Rotherham, United Kingdom; ${ }^{3}$ Royal Hospital for Sick Children, Glasgow, United Kingdom; ${ }^{4}$ Birmingham Children's Hospital, Birmingham, United Kingdom; ${ }^{5}$ Great Ormond Street Hospital, London, United Kingdom; ${ }^{6} \mathrm{NHS}$ Blood and Transplant, London, United Kingdom; ${ }^{7}$ Leeds Children's Hospital, Leeds, United Kingdom; ${ }^{8}$ Queen Elizabeth Hospital, Birmingham, United Kingdom

Background: Extracorporeal Photopheresis (ECP) is an immunomodulatory apheresis therapy used to treat a variety of disorders in children, particularly acute (a) and chronic (c) graft-versus-host disease (GVHD) and solid organ transplant rejection. Advantages include disease control with reduction of systemic immunosuppressive dose and lack of toxic side effects seen with conventional immunosuppression. Potential disadvantages relate to line infection and bleeding, as well as lack of access for low body weight patients. We performed a review of ECP for UK paediatric patients.

Methods: Retrospective data collection of paediatric patients treated with ECP across the UK was performed. Data collected included demographic details, underlying diagnosis, reason for ECP, previous therapies used prior to ECP and response/outcome.

Results: Data were received from six centres. 105 patients received ECP, 72 males. 77 patients had aGVHD, 20 cGVHD and 8 had solid organ transplant rejection. The median age at commencing ECP was 7 years $(0.33-18.41$ years). The median weight at commencing treament was 23 kilograms (6.8-93kg): 10 children weighed $<10 \mathrm{~kg}$. 67 patients were blood-primed with heparin and 25 patients were blood primed with ACDA. A central apheresis line was inserted in 4 patients, 2 patients had a central split-cath line and 74 had a central hickman line for ECP treatment. Venous access type information was not available for 25 patients. 56 children started ECP because of corticosteroiddependent disease, 25 had corticosteroid-refractory disease, 3 had a concurrent systemic infection and in 21 the reason was unavailable. 88 patients were treated with $>1$ immunosuppressive therapy prior to commencing ECP. 6 patients had complications associated with central venous access:- line infection in 5 patients. Thrombocytopaenia in 1 , otherwise ECP was safe and well tolerated by all patients.

The median time to commence ECP from onset of disease was 231 days ( 6 - 3678 days). The median number of ECP cycles was 16 (1 - 89). Overall, 45 children had a complete response to treatment, 22 had a partial response, 19 had no response and in 4 patients the response to ECP treatment was unknown. Treatment is on-going for 15 patients. For patients who completed treatment, 53\% of acute GVHD had a complete response, $42 \%$ of chronic GVHD and $50 \%$ of children with solid organ transplants had a complete response to ECP treatment. 29 patients died from infection or disease progression - $31 \%$ had a partial response to ECP treatment and $13 \%$ had a complete response.

Conclusions: ECP was safe and well-tolerated in paediatric patients including those $<10 \mathrm{~kg}$. The median time to commencing ECP was long, which suggests that ECP was used late in the management of disease complications - likely due to historic issues with access to treatment. Patients with acGVHD were more likely to have a complete response to treatment, in comparison to those with chronic GVHD or solid organ transplant rejection. Easier access to ECP may now result in less delay to starting treatment, which may lead to better outcomes.

Clinical Trial Registry: N/A 
Conflict of interest: None of the authors has anything to disclose.

\section{P329}

\section{A Novel Mesenchymal Stem Cell Therapy in Steroid Refractory Graft-Versus-Host Disease and its Promising Transfusional Effects}

Emese Molnar ${ }^{1}$, Arpad Batai ${ }^{2}$, Aniko Barta ${ }^{2}$, Zoltan Csukly ${ }^{2}$, Zita Farkas $^{2}$, Laszlo Gopcsa ${ }^{2}$, Gabor Tatai ${ }^{2}$, Lilla Lengyel ${ }^{2}$, Gabor Kovacs $^{2}$, Tamas Masszi ${ }^{3}$, Gabor Mikala ${ }^{2}$, Melinda Paksi ${ }^{2}$, Marienn Reti $^{2}$, Eva Torbagyi ${ }^{2}$, Halvard Bönig ${ }^{4}$, Peter Bader ${ }^{4}$, Peter Remenyi ${ }^{2}$

${ }^{1}$ Hungarian National Blood Transfusion Service, Budapest, Hungary; ${ }^{2}$ St. Istvan \& St. Laszlo Hospital, Budapest, Department of Hematology and HSCT, Budapest, Hungary; ${ }^{3}$ Semmelweis University, Budapest, 3rd Department Of Internal Medicine, Budapest, Hungary;

${ }^{4}$ University Hospital Frankfurt/Main, Division for Stem Cell

Transplantation and Immunology, Frankfurt, Germany

Background: Steroid refractory graft-versus-host disease (GvHD) is a serious complication of allogeneic hematopoietic stem cell transplantation (HSCT). More experience accumulates in the immunomodulatory effect of mesenchymal stem cell (MSC) infusion in numerous immunopathological disorders - such as GvHD - and signals.

Methods: We have evaluated the efficacy of a novel MSC product, we exclusively used the licensed MSC-FFM (derived from bone marrow, "MSC-FFM" /Kuci et al. Haematologica 2016/ submitted on a named-patient basis) in cases of GvHD refractory to conventional immunosuppressive treatment. Patients with steroid-resistant GvHD were treated with the MSC-FFM, 4 times per case weekly at a dose of 1 million cells $/ \mathrm{kg}$. Clinical response was assessed 28 days after administering the first dose. Complete remission was defined as the complete disappearance of symptoms. Partial remission was assessed by the significant relief of symptoms and by the general improvement of the patient $\hat{A}$ 's condition. We have evaluated the red blood cell and platelet concentrate needs of the patients before and after the treatment.

Results: Our 12 patients had received a total of 13 cycles of MSC-FFM-treatment ( 4 doses per cycle). The median age was 47 (19-56) years with a male/female ratio of 1:2. Distribution of the underlying malignancies (n): acute myeloid leukemia: 6; acute lymphoblastic leukemia: 2; myelofibrosis: 1; myelodysplastic syndrome: 1 ; multiple myeloma: 1; T-cell lymphoma: 1 . Nine patients had undergone allogeneic HSCT with matched unrelated donors, the other three had stem cells derived from HLAidentic relatives. The first episode of GvHD after HSCT was observed on the median 63rd (7-455) day. The involved organs were skin (2), gut (4), skin and gut combined (7) and lung in 3 cases. We applied an average of 3 lines of treatment (it varies from 1-5 lines) before we started MSCFFM treatment. The median time of MSC-FFMs first infusion was 274 (114-1981) days after stem cell transplantation (HSCT) and 165 (19-1974) days after the first episode of GvHD. Four of the 13 cycles of MSC-FFMtreatment led to complete remission $(30.8 \%)$ and 7 resulted in partial remission (53.8\%). All of the patients GvHD NIH stage score was 3 before MSC-FFM infusions, and it decreased to a median of 1 after treatment. The overall survival on the $180^{\text {th }}$ day after the first GvHD was $75 \%$. After the MSC-FFM treatment the need of red blood cell concentrate were reduced from an average of 12.83 to 8.92 (30.5\% reduction) and the platelet concentrate need from an average of 107.17 to 50.75 (52.6\% reduction).

Conclusions: According to our observation MSC-FFMtherapy is an effective treatment for GvHD in the majority of the observed cases with $83 \%$ overall cumulative response rate. The reduction of the transfusional needs after the MSC-FFM treatment also shows promising results. The application of third-party MSC-FFMs offers a promising alternative in the therapy of GvHD and other GvHDassociated complications after HSCT. Further research is required to warrant the optimal start and dosage of the MSC-FFM treatment, along with the issue of long-term safety.

Clinical Trial Registry: NA

Conflict of interest: nothing to disclose

\section{P330}

Abstract previously published

\section{P331}

Adverse Effects of Therapeutic Treatments for Acute Graft versus Host Disease (aGvHD): A Systematic Review

Fiona Dignan ${ }^{1}$, Vladica Velickovic ${ }^{2}$, Emily McIlwaine ${ }^{2}$, Rongrong Zhang $^{2}$, Tim Spelman ${ }^{2}$

${ }^{1}$ Manchester University Hospitals NHS Foundation Trust, Manchester, United Kingdom; ${ }^{2}$ Synergus AB, Health Economics and Evidence Synthesis, Stockholm, Sweden

Background: Allogeneic hematopoietic stem cell transplantation (allo-HSCT) is associated with an increased risk of GvHD, a strong prognostic predictor of early mortality within the first two years following allo-HSCT. The objective of this study was to review the harm outcomes reported among patients receiving treatment for acute GvHD.

Methods: A systematic literature search was performed in Medline, Embase, and the Cochrane CENTRAL databases. Studies were included if they are clinical trials 


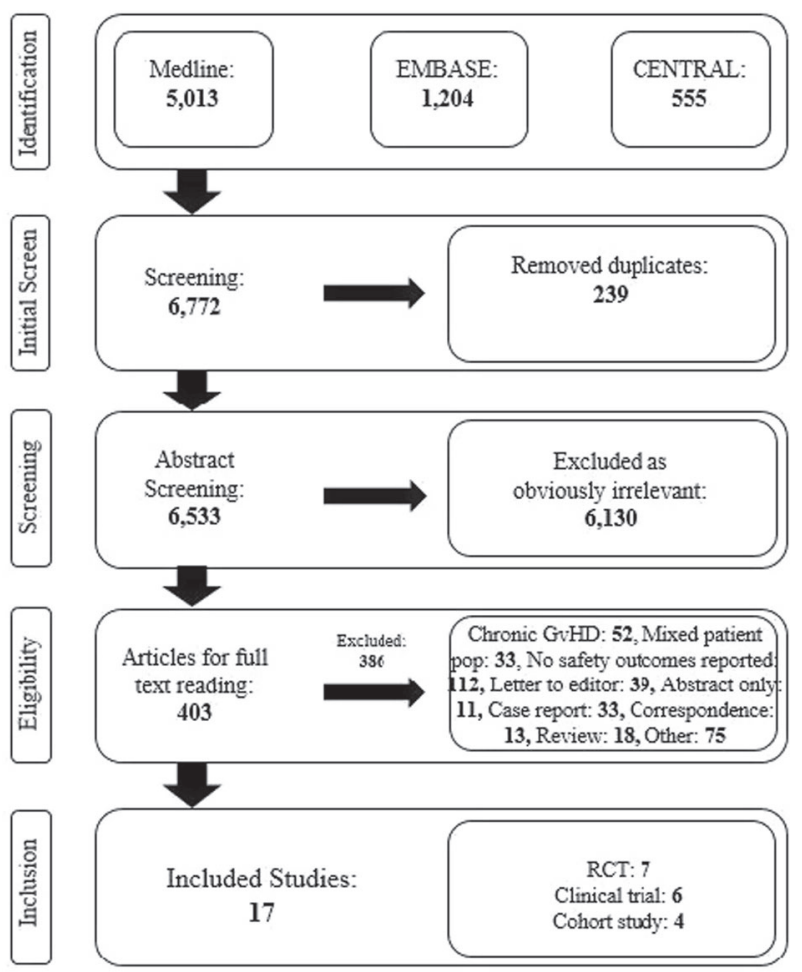

[P331 Figure] [Figure 1: PRISMA flow chart of articles included in this systematic review]

or cohort studies reporting AEs of patients diagnosed with aGvHD after allo-HSCT when received any of the following treatments, in combination with corticosteroids: extracorporeal photopheresis (ECP), basiliximab, inolimomab, etanercept, infliximab, sirolimus, MMF, everolimus, imatinib, pentostatin, rituximab, methotrexate, alemtuzumab, ruxolitinib, or mesenchymal stromal cells (MSCs). Papers reporting prophylactic use of the above-mentioned treatments were excluded from this review. The review was conducted in-line with Preferred Reporting Items for Systematic reviews and Meta-Analyses (PRISMA) guidelines, and the PRISMA harms checklist (Figure 1).

Results: Seventeen studies reporting adverse effects (AEs) of ten different therapies, used in the treatment of aGvHD, were identified. During 3 months of follow-up, 1.63 infectious AEs per patient was reported for etanercept compared to 0.37 per patient for MMF. In the case of MSCs grade 3 / 4 AEs per patient were reported across three months of follow up. Across 6 months of follow-up, infectious AEs per patient were reported as: 0.27 for ECP, 0.85 for etanercept, and 1.35 for infliximab. In a comparison of grade 3-5 infections, the cumulative incidences of AEs for etanercept, MMF, and pentostatin were $47 \%, 80 \%$ and $67 \%$ respectively (during 9 months of follow-up). One study reported a cumulative incidence of grade 3-5 infections as $44.5 \%$ annually for patients treated with
MMF. Infectious AEs per patient during 1 year of follow-up were reported as 0.74 for basiliximab and 1.94 for inolimomab. Laboratory abnormalities with severity grade 3-5 were reported in the cases of etanercept, MMF, and pentostatin with a 2-month cumulative incidence of $76 \%$, $44 \%$, and $57 \%$ respectively; these events were reported as absent for ECP, infliximab, and basiliximab. Serious AEs leading to death were reported as 0.03 per patient during 6 months of follow-up for etanercept, and as 0.17 and 0.53 per patient after 1 year of follow-up for basiliximab and inolimomab respectively. When MSCs infusion were used 1.56 serious AEs per patient annually were reported. During 6 months of follow-up, 0.13 serious AEs per patient were reported for ECP. One retrospective cohort study reported 0.06 central line infections per patient at 6 months of follow-up, and $64 \%$ cumulative incidence of mild hypotensive events during ECP treatment.

Conclusions: These results suggest that ECP may lead to a lower incidence of infectious AEs compared with pharmaceutical treatments. However, due to a lack of standardized reporting of severity grades and inconsistencies in the definition of serious AEs, definitive conclusions can only be drawn after conducting clinical trials with headto-head comparisons.

Conflict of interest: This study was sponsored by Mallinckrodt Pharmaceuticals. All authors received a research grant to conduct the analysis. F.L.D. has received speaker fees, research funding, and travel expenses for conference attendance from Mallinckrodt Pharmaceuticals.

\section{P332}

\section{Adverse Effects of Therapeutic Treatments for Chronic Graft versus Host Disease (cGvHD): Systematic Review}

Fiona L. Dignan ${ }^{1}$, Vladica Velickovic ${ }^{2}$, Emily McIlwaine ${ }^{2}$, Rongrong Zhang', Tim Spelman ${ }^{2}$

${ }^{1}$ Manchester University Hospitals NHS Foundation Trust, Manchester, UK, Department of Haematology, Manchester, United Kingdom;

${ }^{2}$ Synergus AB, Health Economics and Evidence Synthesis, Stockholm, Sweden

Background: cGvHD, which occurs in $~ 30-50 \%$ of allogeneic hematopoietic stem cell transplantation (alloHSCT) recipients, can affect nearly every organ or tissue in the body. Recommended treatment options for cGvHD, after initial immunosuppression with corticosteroids, include extracorporeal photopheresis (ECP), tyrosine kinase inhibitors, mTOR inhibitors, nucleoside analogues, antiCD20 monoclonal antibodies, and immunosuppressants.

Methods: A systematic literature review was conducted using Medline, Embase, and the Cochrane CENTRAL databases. The following inclusion criteria were used: patients 


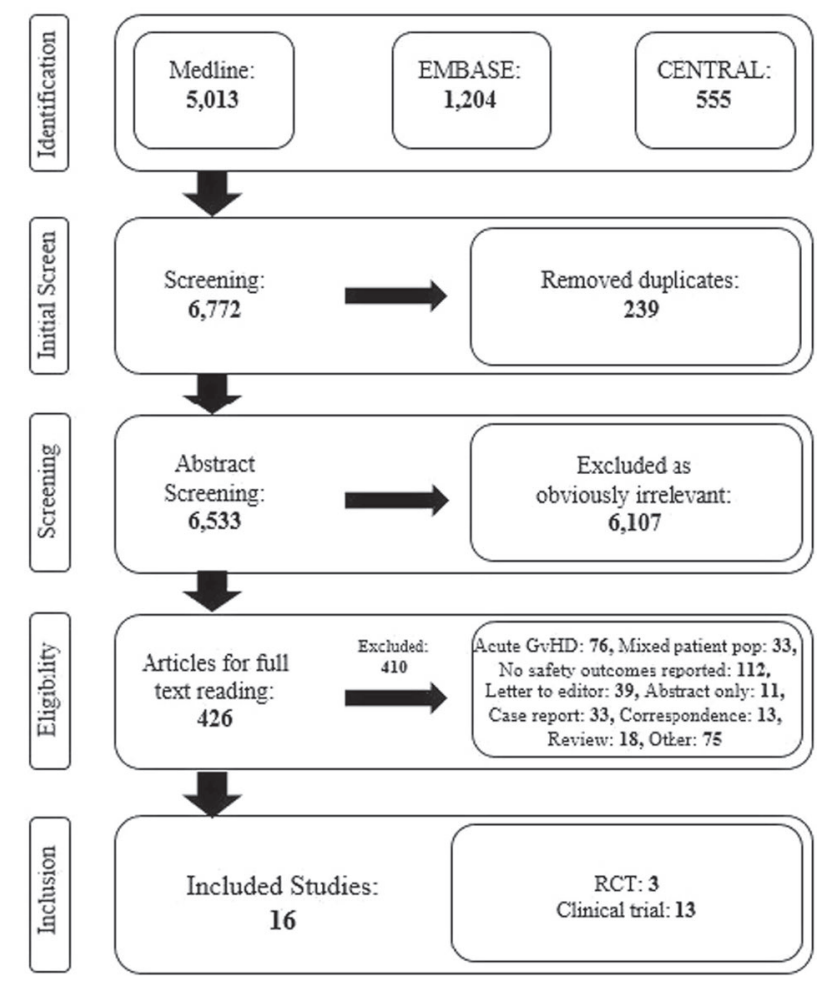

[P332 Figure] [Figure 1: PRISMA flow chart of articles included in this systematic review]

with cGvHD after allo-HSCT treated with ECP, basiliximab, inolimomab, etanercept, infliximab, sirolimus, mycophenolate mofetil (MMF), everolimus, imatinib, pentostatin, rituximab, methotrexate, alemtuzumab, ruxolitinib, mesenchymal stromal cells (MSCs) and reported treatment related adverse effects. Articles were excluded from the review if they were case report or series, literature reviews/meta-analysis and if reported the prophylactic use of the treatments. All identified articles meeting the inclusion criteria were assessed according to the recommendations of the Preferred Reporting Items for Systematic reviews and Meta-Analyses (PRISMA) guidelines, and the PRISMA harms checklist.

Results: Of the 6772 abstracts identified through a database search (Figure 1), 16 studies met the inclusion criteria and reported the adverse effects (AEs) of seven different treatments. Analysis showed that patients receiving ECP treatment had an average of 0.12 grade 3-5 AEs per patient over a follow-up period of 3 months, compared with imatinib at a low dosage $(200 \mathrm{mg}$ ) which had an average of 0.59 grade 3-5 AEs per patient. Reduction to a 100 -mg dose of imatinib reduced the grade 3-5 AEs to 0.26 per patient within 6 months of follow-up. Imatinib showed a dose related toxicity as higher doses (up to $300 \mathrm{mg}$ ) resulted in an average of 0.9 grade 3-5 AEs per patient (6 months follow-up), increasing to $1.17 \mathrm{AE}$ per patient when the dosage increased to $400 \mathrm{mg}$ (14 months of follow-up). In 2 studies in pediatric cGvHD populations reporting with pentostatin, there was a higher $\mathrm{AE}$ rate per patient at 6 months compared with 19 months of follow-up (0.29 vs. 0.69 , respectively). In one study of cGvHD patients treated with sirolimus, the number of AEs per patient was 0.53 over a 9-month follow-up period. Two studies of rituximab treatment in an Asian population with cGvHD reported a very small rate of AEs per patient, from 0.14 and 0.19 annually. However, in the US population AEs per patient were 0.43 at 12 months and 0.46 at 19.5 months of followup as reported by two independent studies. Severe AEs related to MSCs were reported in the phase I/II trial with an average of 0.72 per patient per annum.

Conclusions: Results from this systematic review suggest fewer AEs per patient when ECP is used in the treatment of patients with cGvHD compared with immunosupressive agents; however, the current level of evidence does not permit determination whether a specific drug or class of drug has a significantly lower incidence of AE.

Conflict of interest: This study was sponsored by Mallinckrodt Pharmaceuticals. All authors received a research grant to conduct the analysis. F.L.D. has received speaker fees, research funding, and travel expenses for conference attendance from Mallinckrodt Pharmaceuticals.

\section{P333}

Antihuman T-lymphocyte immune globulin for graft versus host disease prevention in sibling allogeneic stem cell transplant - single center experience at UMC Ljubljana, Slovenia

Matjaz Sever, Nika Zima, Mihela Sajn, Matevz, Skerget, Barbara Skopec, Polona Novak, Samo Zver

\section{UMC Ljubljana, Hematology Department, Ljubljana, Slovenia}

Background: Graft versus host disease (GvHD) is a major complication after allogeneic stem cell transplant (SCT) contributing to reduced quality of life and increased mortality. Antihuman T-lymphocyte immune globulin (ATG) for GvHD prophylaxis was shown to reduce the incidence of chronic GvHD in matched unrelated and recently in matched sibling allogeneic SCT without affecting relapse free survival and overall survival. We adopted ATG (Grafalon, Neovii) in sibling allogeneic SCT after initial publication in January 2016 and analysed results from patients treated at our center.

Methods: We retrospectively collected data from institutional database. Patients with sibling donor and who received ATG were included. We used cumulative incidence analysis to assess GvHD free survival and Kaplan-Meier analysis for overall survival. 

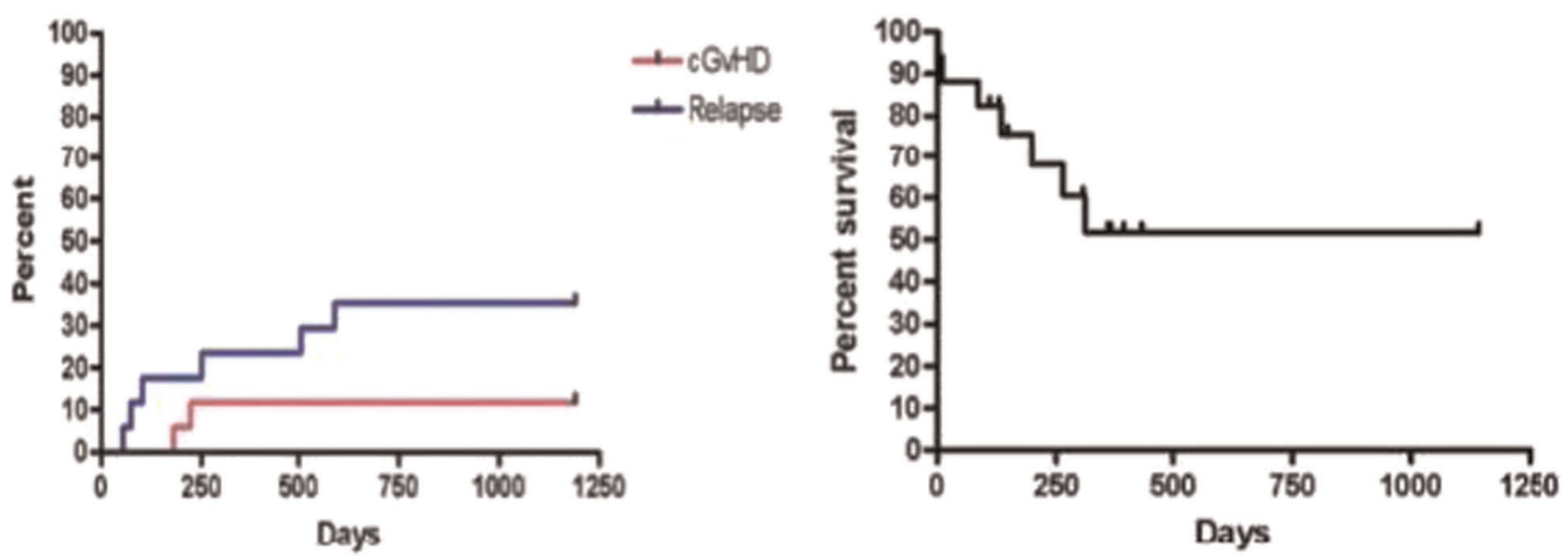

[P333 Figure] [Cumulative incidence of $c G v H D$ and relapse rate (left) and overall survival (right)]

Results: We transplanted 17 patients, one in 2014 and the rest from 2016 on, $10(59 \%)$ men, with median age 59 years. The most common indication was AML in ten $(59 \%)$ patients, $2(12 \%)$ had MDS, $2(12 \%)$ CML, and one $(6 \%)$ each ALL, PMF and CLL. Nine (53\%) patiens had a high risk disease at presentation. Eleven $(65 \%)$ were in first CR and the rest in second CR at the time of SCT. The most common conditioning protocol used was $\mathrm{Cy}-\mathrm{Bu}$ in 9 patients $(53 \%)$, followed by TBF $3(18 \%)$, Flu-Mel 3 (18\%), Cy-TBI 1 (6\%) and TT-Flu-Bu 1 (6\%). All sibling donors were fully HLA matched, all stem cells were collected from peripheral blood. GvHD prophylaxis was CSA-MTX in myeloablative and CSA-MMF in nonmyeloablative setting. All patients received ATG in cumulative dose $30 \mathrm{mg} / \mathrm{kg}$ with no serious side effects. Two (12\%) patients presented with acute GvHD involving skin and gut (grade 3/4) and two (12\%) patients presented with chronic GvHD involving gut and lung (grade 3/4). Six (35\%) patients had relapse at median 177 days (range, 54590). During follow-up seven $(41 \%)$ patients died. Cause of death was relapse in $3(18 \%)$, GvHD in $2(12 \%)$ and infection in 2 (12\%) (Graph 1).

Conclusions: ATG use in sibling allogeneic SCT for GvHD prophylaxis is safe. Both acute and chronic GvHD rates were surprisingly low in our patient group. We observed relatively high relapse rate. Compared to initial report our patient group was older, heterogenous regarding the diagnosis and conditioning, with larger proportion of high risk disease cases at diagnosis. According to our results intensive post SCT relapse detection and/or prevention should be employed with ATG use. Further research is warranted to elucidate the role of ATG in sibling allogeneic SCT.

Conflict of interest: Neovii: honoraria for a lecture.

\section{P334}

Anti-thymocyte globulin (ATG) in patients with haematological malignancies undergoing haematopoietic cell transplantation (HCT) from unrelated donors: Follow up of a CBMTG randomized trial

Irwin Walker ${ }^{1}$, Tony Panzarella ${ }^{2}$, Stephen Couban ${ }^{3}$, Felix Couture $^{4}$, Gerald Devins $^{5}$, Mohamed Elemary ${ }^{6}$, Genevieve Gallagher ${ }^{7}$, John Kuruvilla $^{5}$, Stephanie J Lee ${ }^{8}$, John Moore ${ }^{9}$, Cynthia Toze ${ }^{10}$, Thomas Nevill $^{10}$, Gizelle Popradi ${ }^{11}$, Jean Roy ${ }^{12}$, Kirk R Schultz ${ }^{13}$, David Szwajcer ${ }^{14}$, Ronan Foley ${ }^{15}$

${ }^{1}$ McMaster University, Medicine, Hamilton, Canada; ${ }^{2}$ University of Toronto, Dalla Lana School of Public Health, Toronto, Canada; ${ }^{3}$ Dalhousie University, Halifax, Canada; ${ }^{4}$ Hospitalier Universitaire de Québec, Pavillon Hôtel-dieu de Québec, Quebec City, Canada; ${ }^{5}$ University of Toronto, Princess Margaret Cancer Centre, Toronto, Canada; ${ }^{6}$ University of Saskatchewan, Saskatoon Cancer Centre, Saskatoon, Canada; ${ }^{7}$ Université Laval, CHA Hôpital l'Enfant-Jésus, Quebec City, Canada; ${ }^{8}$ University of Washington, Fred Hutchinson Cancer Research Center, Seattle, WA, United States; ${ }^{9}$ University of New South Wales, St Vincent's Hospital, Sydney, Australia;

${ }^{10}$ Vancouver General Hospital, University of British Columbia, Vancouver, Canada; ${ }^{11}$ University of McGill, McGill University Health Centre, Montreal, Canada; ${ }^{12}$ University of Montreal, Hopital Maisonneuve-Rosemont, Montreal, Canada; ${ }^{13}$ British Columbia Children's Hospital and Child \& Family Research Institute, Michael Cuccione Childhood Cancer Research Program, Vancouver, Canada; ${ }^{14}$ CancerCare Manitoba, Winnipeg, Canada; ${ }^{15}$ Juravinski Hospital and Cancer Centre, Hamilton, Canada

Background: We previously reported a decrease in incidence of steroid use (primary endpoint), chronic graft versus host disease (CGVHD), and symptom burden (by Lee scale) at exactly 12-months following pre-treatment with ATG in patients receiving unrelated donor transplants (Lancet Oncol 2016; 17: 164-73). We now report the results at 24-months follow up of each patient, 23.90 (range 11.726.41) months in the ATG group and 23.95 (range 11.425.95) months in the No ATG group. 
Methods: Eligible patients were aged 16 to 70 years with any haematological malignancy receiving either myeloablative (MA) or non-myeloablative/reduced intensity (NMA/RIC) conditioning before HCT from an unrelated donor. ATG administration consisted of Thymoglobulin ${ }^{\circledR}$ $4.5 \mathrm{mg} / \mathrm{kg}(0.5 \mathrm{mg} / \mathrm{kg}$ on day $-2,2.0$ on day -1 and $2.0 \mathrm{on}$ day +1$)$.

Results: We recruited and assigned 203 eligible patients to treatment, analyzing 196 by modified intention to treat (99 to ATG and 97 to No ATG). At 24-month follow-up of each patient, 50/99 (52\%) patients in the ATG group and 26/97 (26.8\%) in the No ATG group were free from steroid use (adjusted odds ratio 3.47 [95\% CI 1.71-7.03]; $\mathrm{p}=$ $0.0006)$ - the primary endpoint. The cumulative incidence of CGVHD was significantly lower in the ATG group, 26/ $99(26.3 \%)$ compared with $40 / 97(41.2 \%)$ in the No ATG group (adjusted odds ratio 0.58 [95\% CI 0.35-0.96]; $\mathrm{p}=$ 0.035). There was no difference in the incidence of relapse, $16 / 99(16.2 \%)$ vs $17 / 97(17.5 \%)$ in the ATG and No ATG groups respectively $(\mathrm{p}=0.21)$, nor in non-relapse mortality, 21/99 (21.2\%) vs 30/97 (30.9\%) in the ATG and No ATG groups respectively $(\mathrm{p}=0.12)$. Overall survival was greater in those in the ATG group, 70/99 (70.7\%) vs 52/97 (53.6\%) in the ATG and No ATG respectively (adjusted HR 0.57 [95\% CI 0.36-0.92]; $\mathrm{p}=.021$ ). CRFS (chronic graft versus host disease- and relapse- free survival) was higher in the ATG group versus No ATG group $(\mathrm{p}=0.0022)$ - both CRFS and GRFS will be presented. CGVHD symptom burden by Lee scale was both clinically and significantly lower in the ATG group compared with the No ATG group, 13.56 (1.50), adjusted mean (SE), $\mathrm{n}=49$, vs $19.76(2.11), \mathrm{n}=44$ $(\mathrm{p}=.017)$.

Conclusions: This follow up study demonstrates that the benefits of ATG previously reported at 12-months have been sustained for 24 months. Patients receiving ATG have had a lower incidence of CGVHD, lower use of steroids and fewer symptoms of CGVHD. ATG is now standard of care in Canada when HCT is performed with myeloablative conditioning and unrelated donor blood stem cell grafts (independent survey results available).

Clinical Trial Registry: ISRCTN 29899028

Conflict of interest: The author received grant support from Sanofi corporation for this combination-funded (CIHR/Industry) investigator-initiated study, and for travel and honorarium support for Sanofi sponsored corporate symposiums at EBMT in 2016 and 2017.

\section{P335}

Better disease control and lower GVHD: Short-term chidamide, an HDAC inhibitor in matched myeloablative allogeneic peripheral-blood stem-cell transplantation
Jie Ji, Ting Liu, Pu Kuang, Zhigang Liu, Tian Dong, Jian Li, Jiazhuo Liu, Li Zhang

West China Hospital of Sichuan University, Chengdu, China

Background: Relapse of disease and graft-versus-host disease (GVHD) are two major events after allogeneic stemcell transplantation leading to significant mobility and mortality. In preclinical research and clinical trials, histone deacetylase (HDAC) inhibitors increase cytotoxicity of alkylator containing conditioning therapy, and reduced acute GVHD. Therefore, we conducted a phase II clinical trial of a selective HDAC inhibitor chidamide, also named HBI-8000, combine with post-transplantation cyclophosphamide based GVHD prophylaxis in patients undergoing myeloablative conditioning matched allogeneic PBSCT.

Methods: Eligible patients were aged 16 to 65 years, diagnosed with hematologic malignancy, and had a Karnofsky performance score of $\geq 70 \%$ and were candidates for myeloablative HCT. A 10/10 HLA allelic match between the donor and the recipient at HLA-A, HLA-B, HLA-C, and HLA-DRB1 by high-resolution typing was required. The graft source was PBSC.

Patients received a myeloablative conditioning regimen consisting of oral chidamide given twice weekly at a dose of $20 \mathrm{mg}$ from day -7 to 2 weeks post transplantation, intravenous busulfan $3.2 \mathrm{mg} / \mathrm{kg}$ from day -6 to -3 , intravenous fludarabine $30 \mathrm{mg} / \mathrm{m}^{2}$ and cytarabine $1 \mathrm{~g} / \mathrm{m}^{2}$ respectively from day -6 to -2 . PBSCs were infused on day 0 . GVHD prophylaxis was post-transplantation cyclophosphamide $(50 \mathrm{mg} / \mathrm{kg}$ on day $+3,+4)$ and cyclosporine (started from day +5 ). In the absence of GVHD, cyclosporine tapering started on day +100 and discontinued on day +180 . Minimal residual disease (MRD) was determined by multi-parameter flow cytometry.

Results: Between 1 July 2015 and 31 Sep 2017, 49 patients were enrolled into this single-center prospective phase 2 clinical trial. The median age of study participants was 41 years (16-62 years). Thirty patients $(61 \%)$ had a diagnosis of acute myeloid leukemia, 13 (27\%) had acute lymphocytic leukemia, and 6 (12\%) had myelodysplastic syndrome. Twelve (24\%) patients had MRD and 12 (24\%) had active diseases. A median of $3.3 \times 10^{6}$ cells $/ \mathrm{kg}$ of CD34 + cells from sibling donor $(\mathrm{n}=42,86 \%)$ or unrelated donor $(\mathrm{n}=7,16 \%)$ were infused into recipients.

Neutrophils and platelets engrafted a median of 16 days (9-36 days) and 18 days (11-44 days) after transplant, respectively. There were no cases of primary or secondary graft failures. At day 100, 5 of 49 (11\%) had grade 2-4 aGVHD, with median time to onset of 93 days. Only 2 patients developed grade 4 aGVHD (Figure 1A). The median follow-up time of the whole cohort was 11.5 months (2.3-27). The cumulative incidence of relapse at 1 year was 
A

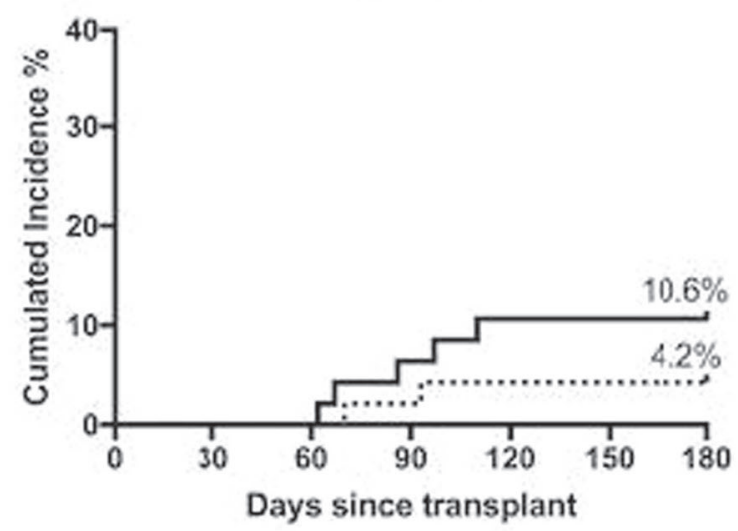

C

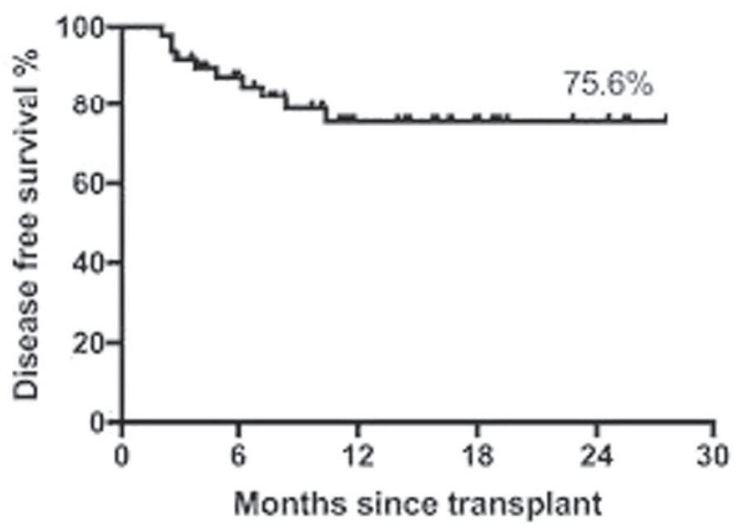

E

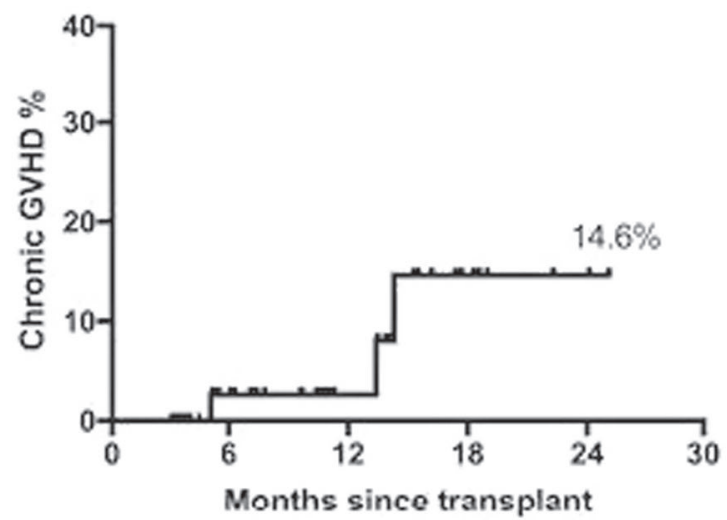

[P335 Figure] [aGVHD, relapse, DFS, OS, cGVHD, and GRFS.]

17\% (Figure 1B). The median time to relapse was 166 days (59-212 days) post-transplant. NRM incidence at 1 year was 7.9\%. Median follow-up time of the whole cohort is 11.5 month (3-24.7). Estimated progression free survival
B

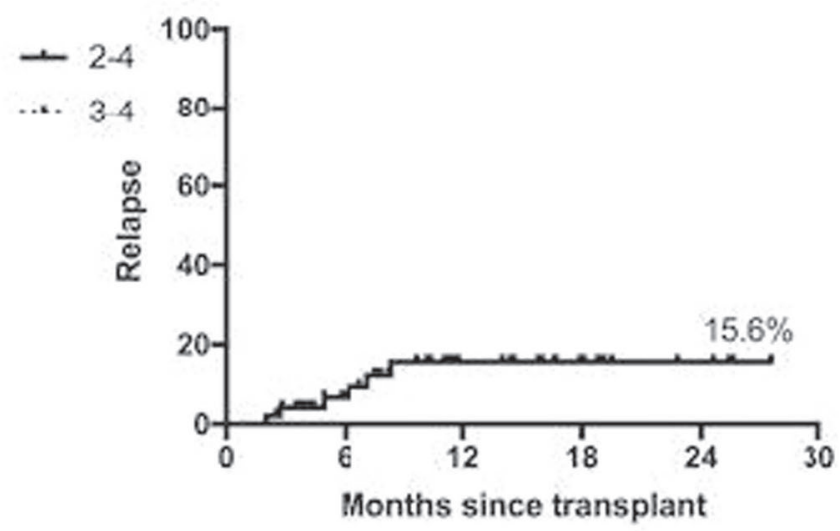

D

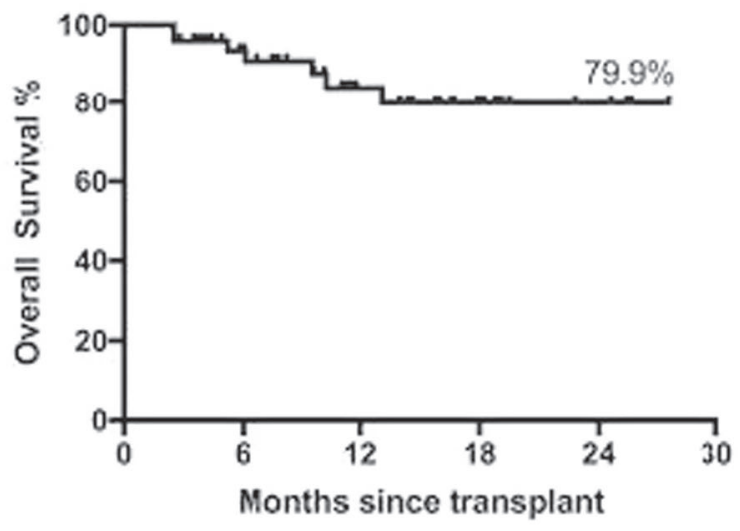

$F$

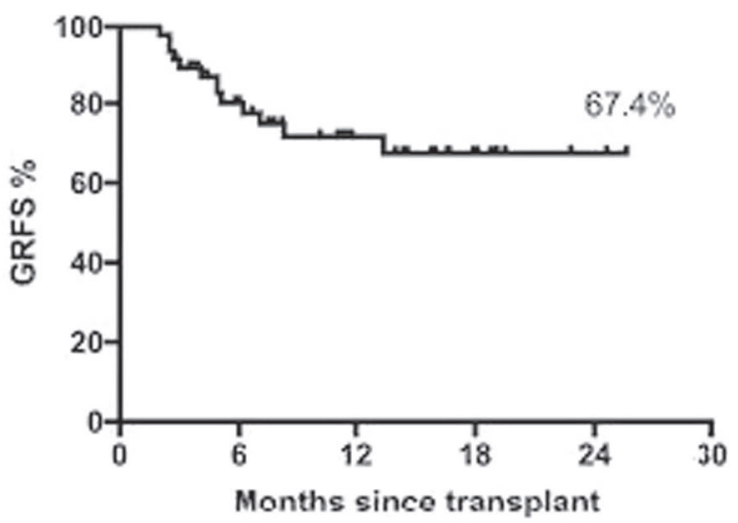

and overall survival at 1 year are $76 \%$ and $80 \%$ respectively (Figure1-C\&D). Chronic extensive GVHD at 1 year is $15.8 \%$ (Figure1-E), which resulted in a GVHD-free relapsefree survival of $67 \%$ (Figure1-F). 
Conclusions: ntroducing chidamide into conditioning and GVHD prophylaxis with post-transplantation cyclophosphamide and cyclosporine in patients undergoing matched myeloablative conditioning HCT is safe and feasible. Chidamide decreases GVHD while preserves sufficient graft-versus-leukemia response.

Conflict of interest: Jie Ji: nothing to disclose

\section{P336}

\section{Brentixumab vedotin for the treatment of steroid-refractory chronic graft-versus-host disease}

Zachariah DeFilipp ${ }^{1}$, Shuli Lit ${ }^{2}$, Maria Kempner ${ }^{1}$, Jami Brown ${ }^{1}$, Candice Del Rio ${ }^{I}$,Betsy Valles ${ }^{1}$, Karen Ballen ${ }^{3}$, Bimalangshu Dey ${ }^{1}$, Areej El-Jawahri, Steven McAfee ${ }^{1}$, Thomas Spitzer ${ }^{1}$, Yi-Bin Chen ${ }^{1}$

${ }^{1}$ Massachusetts General Hospital, Blood and Marrow Transplant Program, Boston, MA, United States; ${ }^{2}$ Dana-Farber Cancer Institute, Department of Biostatistics and Computational Biology, Boston, MA, United States; ${ }^{3}$ University of Virginia School of Medicine, Division of Hematology/Oncology, Charlottesville, VA, United States

Background: Chronic graft-versus-host disease (cGVHD) is an important cause of morbidity and mortality in recipients of allogeneic hematopoietic cell transplantation. Systemic corticosteroids remain the mainstay of cGVHD therapy. Recently, CD30, a cell surface marker of activated lymphocytes, has been identified as a potential novel target of GVHD therapy. Brentuximab vedotin (BV), an antibody-drug conjugate targeting $\mathrm{CD} 30$, was shown to have activity in steroid-refractory acute GVHD in a phase I study (Chen et al. Blood. 2017). Given the overlap in pathophysiology between acute and chronic GVHD, we conducted a phase I study of $\mathrm{BV}$ for the treatment of steroid-refractory cGVHD.

Methods: Patients were at least 18 years of age and had a diagnosis of steroid-refractory chronic GVHD, defined as 1) persistent disease despite $\geq 0.25 \mathrm{mg} / \mathrm{kg} /$ day of prednisone or its equivalent for at least 2 weeks, 2) disease not responding to therapy beyond steroids, or 3 ) clinical scenarios where steroids were contraindicated. A modified $3+3$ study design was used with the primary endpoint to determine the maximum tolerated dose (MTD) of BV in this population. Six escalating doses of BV were planned: $0.3 \mathrm{mg} / \mathrm{kg}$ q3weeks (dose level -1), $0.6 \mathrm{mg} / \mathrm{kg}$ q3weeks (dose level 0), $0.9 \mathrm{mg} / \mathrm{kg}$ q3weeks (dose level +1$), 1.2 \mathrm{mg} / \mathrm{kg} \mathrm{q} 3$ weeks (dose level +2 ), $1.5 \mathrm{mg} / \mathrm{kg}$ q3weeks (dose level +3 ), and $1.8 \mathrm{mg} / \mathrm{kg}$ q3weeks (dose level +4 ). BV was administered IV on Day 1 of 21-day cycles for up to 16 cycles of therapy. The period of dose-limiting toxicity (DLT) evaluation was the first 2 cycles. Grade 2 peripheral neuropathy was included in the definition of DLT.
Results: Nineteen patients were enrolled on the study, with 2 withdrawing consent prior to dosing. Amongst the 17 patients receiving $\mathrm{BV}$, NIH cGVHD severity included mild $(\mathrm{n}=2)$, moderate $(\mathrm{n}=10)$ and severe $(\mathrm{n}=5)$. The median number of cycles of therapy was 4 (range, 1-16). Reasons for stopping therapy included toxicities $(n=9)$, patient decision $(n=3)$, completion of planned therapy $(n=2)$, lack of response $(n=2)$, and death $(n=1)$. There were 3 DLTs observed: peripheral neuropathy (cohort 3, grade 2), PRES (cohort 4, grade 3), and sepsis (cohort 4, grade 4). The MTD was not reached as the trial was prematurely closed due to toxicity. Seven patients $(41 \%)$ developed grade 3 or 4 adverse events that were attributed to therapy. Four patients developed moderate or severe peripheral neuropathy, leading to cessation of treatment in each case. Overall, 13 patients $(76 \%)$ had a partial response as assessed by treating physicians. There were no complete responses observed. One patient had progression of cGVHD, 2 had no response, and 1 patient was unevaluable. Eleven patients (65\%) were able to decrease their systemic corticosteroid dose by $\geq 50 \%$ by 6 months after initiation of $\mathrm{BV}$, including 3 patients who were able to stop corticosteroids completely.

Conclusions: BV has activity in treatment of steroidrefractory cGVHD, yet its use is limited by treatmentrelated toxicities, including peripheral neuropathy. Continued efforts to investigate targeted approaches to cGVHD, which do not cause broad immunosuppression, are needed.

\begin{tabular}{|c|c|}
\hline Characteristic & Value \\
\hline Total patients, $\mathrm{n}$ & 17 \\
\hline Median age, years (range) & $59(23-74)$ \\
\hline $\begin{array}{l}\text { NIH cGVHD severity, n (\%): Mild, Moderate, } \\
\text { Severe }\end{array}$ & $2(12) ; 10(59) ; 5$ (29) \\
\hline $\begin{array}{l}\text { End-organ involvement, n (\%): Skin, Oral, } \\
\text { Liver, Ocular, Musculoskeletal, Genital, Lung }\end{array}$ & $\begin{array}{l}13(76) ; 11(65) ; 8(47) ; 7(41) ; \\
4(24) ; 1(6) ; 1(6)\end{array}$ \\
\hline $\begin{array}{l}\text { Median number of previous or concurrent } \\
\text { systemic cGVHD therapies at study } \\
\text { enrollment, } n \text { (range) }\end{array}$ & $3(2-7)$ \\
\hline $\begin{array}{l}\text { Median time from cGVHD diagnosis to } \\
\text { enrollment, months (range) }\end{array}$ & $6(0-35)$ \\
\hline
\end{tabular}

[[P336 Table] Table 1]

Clinical Trial Registry: ClinicalTrials.gov Identifier: NCT01940796

Conflict of interest: Seattle Genetics, Inc., provided brentuximab vendotin and clinical trial funding. Dr. Chen has received research funding from Adienne and Otsuka for work performed outside of the current study. The other authors declare no conflict of interest. 


\section{P337}

\section{Cardiological involvement during acute GVHD after sibling donor transplantation for AML: a case report}

Ilaria Cutini ${ }^{1}$, Chiara Nozzoli ${ }^{l}$,Antonella Gozzini ${ }^{1}$, Stefano Guidi ${ }^{1}$, Iacopo Olivotto $^{2}$, Anna Arretini ${ }^{2}$, Riccardo Saccardi ${ }^{I}$

${ }^{1} A O U$ Careggi, Cellular Therapies and Transfusional Medicine Unit, Florence, Italy; ${ }^{2} \mathrm{AOU}$ Careggi, Cardiomyopathy Unit, Florence, Italy

Background: Despite prophylaxis with immunosuppressive therapy agents, nearly $50 \%$ of patients experience acute graft-versus-host disease (aGVHD) after allogeneic hematopoietic cell transplantation (HSCT). Cardiac GVHD is rare event and the diagnosis usually carried out by exclusion.

Methods: A 44-year-old female with AML FAB M1 (46 XX), FLT3 ITD and NPM1 mutated (ELN int), diagnosed on November 2016 was admitted to our hospital. Induction treatment had included two cycles, (ICE and high dosage of Ara-C) without achieving complete hematological remission (CHR). She received 4 courses of FLT3 inhibitors achieving CHR with positive flow cytometry minimal residual disease (FC-MRD), 3\% and molecular residual disease (2,3\%). On May 2017, a T cell-repleted PBSCs HSCT from an HLA-identical sibling donor was performed. Echocardiography pre-transplant showed an ejection fraction $(\mathrm{EF})$ of $58 \%$ and normal cardiac parameters. The conditioning consisted of TBF regimen. Ciclosporine and Methotrexate was given as GVHD prophylaxis. On day +30 skin aGVHD grade 1 occurred, treated with topical steroid. +30 bone marrow evaluation showed: CHR, negative FC-MRD, molecular analysis of NPM1 and FLT3 were both negative, full donor bone marrow chimerism. On day +42 aGVHD grade 3 global (skin 0 , liver 0, intestinal 2, ECOG 2) occurred. Stool studies were negative for infection, endoscopy was not performed due to patient condition. Treatment with $2 \mathrm{mg} / \mathrm{kg}$ daily i.v. metilprednisone was started with no response after 7 days. On day +49 treatment with infliximab was started, without improvement. Another GVHD therapy line was added: extracorporeal photopheresis (ECP), a small improvement was detected, aGVHD global grade 2 . +60 bone marrow evaluation revealed: CHR, negative FC-MRD, molecular analysis of NPM1 $0.0023 \%$ and FLT3 was negative, 99.5\% donor bone marrow chimerism. On day +90 a chronic overlap GVHD diagnosis was performed with moderate global score. A pulmonary CT scan revealed plueral, pericardial effusion, and hepatic and splenic serositis. Treatment with $1 \mathrm{mg} / \mathrm{kg}$ daily i.v. metilprednisone was started. Patient condition got worse (ECOG 3): progressive fatigue, dyspnea and hypotension were detected. Therefore, on day +103 a complete cardiological evaluation shown revealing global dysfunction with an $\mathrm{EF}$ of $35 \%$, mild pericardial effusion not compromising heart function. ECG demonstrated a sinus tachycardia no signs of cardiac ischemia, cardio enzymes were negative, NT proBNP was $8800 \mathrm{pg} / \mathrm{ml}$. The CMV, EBV, HHV6, HSV1 and HSV 2, adenovirus, toxoplasma and BK viral load were negative. The cardiac failure was therefore interpreted as autoimmune myocarditis. The level of bilirubin quickly increased to 5.70 $\mathrm{mg} / \mathrm{dl}$. Due to myocarditis, ECP was stopped and Rituximab was started. A Cardio evaluation was repeated on day +105 revealing mild improvement of $\mathrm{EF}(42 \%)$, stable pericardial effusion level, worsening of NT proBNP $(21000 \mathrm{pg} / \mathrm{ml})$. Patient condition got worse, hemorrhagic shock on day +105 occurred. Transfusion therapy was ineffective and patient died. Permission for autopsy was denied.

Results: no results.

Conclusions: Possible cardiac GVHD was the latest event of a severe cGVHD scenario. Serositis, and cardiac involvement are rare manifestations of cGVHD, their attribution is a diagnosis of exclusion. Cardio MRI and myocardial biopsy should be performed whenever if cardiac gvhd is suspected.

Conflict of interest: nothing to disclose

\section{P338}

Abstract previously published

\section{P339}

$\mathrm{CD} 8+\mathrm{CD} 161^{\text {hi }} \mathrm{T}$ cells are associated with acute graft-versushost disease after haploidentical hematopoietic stem cell transplantation

Yan Hong, Long Liu, Yingjun Chang, Yu Wang, Xiaohui Zhang, Lanping Xu, Xiaojun Huang, Xiaosu Zhao

Peking University People's Hospital, Beijing, China

Background: Acute graft-versus-host disease (aGVHD) is a major complication after allogeneic stem cell transplantation (allo-HSCT). The effect of immune cells on the risk of aGVHD has been studied extensively. T lymphocytes expressing the C-type lectin CD161 had been proved to share a transcriptional profile and innate function across these previously defined subsets. Among three main CD161 $+\mathrm{T}$ cell subsets $(\mathrm{CD} 4+, \mathrm{CD} 8+, \mathrm{CD} 4-\mathrm{CD} 8-), \mathrm{CD} 8+\mathrm{CD} 161$ + can be divided into two populations expressing either intermediate $\left(\mathrm{CD} 161^{\mathrm{int}}\right)$ or high levels of CD161 (CD8 $\left.+\mathrm{CD} 161^{\mathrm{hi}}\right)$. Previous study showed CD161+ T Cells in peripheral blood at the day around neutrophil engraftment could be predictive markers for aGVHD under the setting of identical allo-HSCT. However, the impact of CD161+ $\mathrm{T}$ cells in G-CSF-primed grafts (including G-CSF-primed 


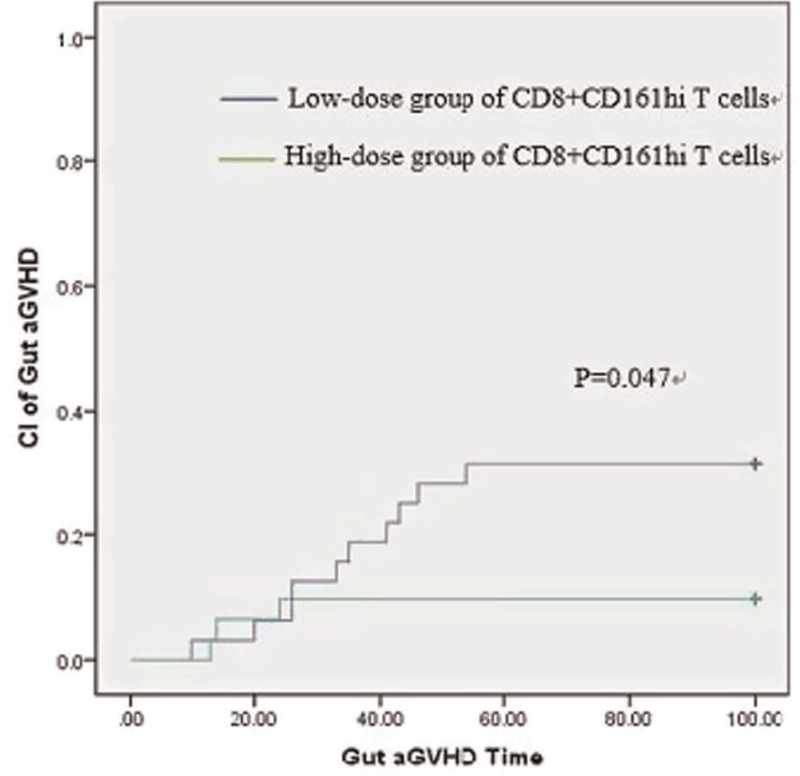

[P339 Figure] [Cumulative incidence of gastrointestinal aGVHD]

bone marrow (G-BM) and G-CSF-prime peripheral blood (G-PB)) and CD161+ T cells reconstitution on aGVHD in haplo-HSCT setting remains unknown. This study aims to investigate whether different $\mathrm{CD} 161+\mathrm{T}$ cell subsets affect aGVHD after haplo-HSCT.

Methods: All patients enrolled were adult (above 14) with hematological malignancies and underwent allo-HSCT from March 2016 to July 2016 in our center. G-BM and GPB from 63 haploidentical donors were examined. PostHSCT peripheral immune reconstitution was analyzed from 43 recipients (31 underwent haplo-HSCT, 12 underwent identical-HSCT) on 30d, 60d, 90d after transplantation. Briefly, fresh cells were stained with the following antibodies: CD3, CD4, CD8, CD161 and detected via multi-color flow cytometery.

Results: In the cohort of 63 patients whose grafts were analyzed, 37 (58.7\%) suffered from aGVHD at a median of 21d (10-72d) and 15 (23.8\%) had grade II-IV aGVHD at a median of 21d (10-46d). Among patients with grade II-IV aGVHD, $13(86.7 \%)$ experienced acute gastrointestinal GVHD at a median of 26d (10-54d). Patients were divided into low- and high-dose groups based on the median dose of $\mathrm{CD} 161+\mathrm{T}$ cell subsets. Our result showed the low-dose group of $\mathrm{CD} 8+\mathrm{CD} 161^{\mathrm{hi}} \mathrm{T}$ cells in G-PB had a higher cumulative incidence of gastrointestinal aGVHD compared with the high-dose group $(\mathrm{P}=0.047)$. Therefore, we further studied the relationship between CD161 $+\mathrm{T}$ cell subsets reconstitution and aGVHD. In the cohort of 43 patients whose peripheral reconstitution was analyzed, 14 of 31 patients undergoing haplo-HSCT suffered from aGVHD and only 2 of 12 patients undergoing identical-HSCT had aGVHD. The median numbers of CD8+CD161 ${ }^{\text {hi }} \mathrm{T}$ cells in haplo-group on 30d, 60d, 90d were 865.4(0-18366.9)cells/ ul, 2339.4(32.9-25419.8)cells/ul, 2372.0(7.3-34801.4)cells/ ul, respectively. The median numbers of $\mathrm{CD} 8+\mathrm{CD} 161^{\text {hi }}$ $\mathrm{T}$ cells in identical-group on $30 \mathrm{~d}, 60 \mathrm{~d}$ and $90 \mathrm{~d}$ were 6444.6 (106.2-57043.4) cells/ul, 13569.8(1973.7-45657.3) cells/ul, 8589.6.0(1790.4-69053.2) cells/ul, respectively. CD8 $+\mathrm{CD} 161^{\mathrm{hi}} \mathrm{T}$ cells reconstitution in haplo-group was slower than identical-group $(\mathrm{P}<0.01)$. Moreover, patients in haplogroup with gastrointestinal aGVHD $(\mathrm{n}=5)$ had a lower frequency of $\mathrm{CD} 8+\mathrm{CD} 161^{\text {hi }} \mathrm{T}$ cells $(\mathrm{P}=0.01)$ and also lower in absolute number $(\mathrm{P}=0.009)$ on $30 \mathrm{~d}$ post-HSCT compared with those without gastrointestinal aGVHD $(\mathrm{n}=$ 26).

Conclusions: $\mathrm{CD} 8+\mathrm{CD} 161^{\text {hi }} \mathrm{T}$ cells in G-PB might predict the occurrence of gastrointestinal aGVHD after haplo-HSCT. CD8+CD161 ${ }^{\text {hi }} \mathrm{T}$ cells reconstitution are associated with gastrointestinal aGVHD.

Conflict of interest: nothing to disclose

\section{P340}

\section{Chronic GVHD Clinical Phenotypes Revealed by Machine Learning Stratify Treatment Response and Survival}

Jocelyn S. Gandelman ${ }^{1,2,3}$, Michael T. Byrne ${ }^{1}$, Akshitkumar M. Mistry $^{3,4}$, Hannah Polikowsky ${ }^{5}$, Kirsten E. Diggins ${ }^{2,3}$, Heidi Chen ${ }^{6}$, Stephanie J. Lee ${ }^{7}$, Mukta Arora ${ }^{8}$, Corey Cutler ${ }^{9}$, Mary E. Flowers ${ }^{7}$, Joseph Pidala ${ }^{10}$, D. Joanne Song ${ }^{3}$, Jonathan M. Irish ${ }^{2,3}$, Madan H. Jagasia $^{1,3}$

${ }^{I}$ Vanderbilt University Medical Center, Medicine, Nashville, TN, United States; ${ }^{2}$ Vanderbilt University, Cell and Developmental Biology, Nashville, TN, United States; ${ }^{3}$ Vanderbilt-Ingram Cancer Center, Nashville, TN, United States; ${ }^{4}$ Vanderbilt University Medical Center, Neurological Surgery, Nashville, TN, United States;

${ }^{5}$ Cytobank, Inc., Mountain View, CA, United States; ${ }^{6}$ Vanderbilt University Medical Center, Biostatistics, Nashville, TN, United States; ${ }^{7}$ Fred Hutchinson Cancer Research Center, Seattle, WA, United States;

${ }^{8}$ University of Minnesota, Medicine, Minneapolis, MN, United States; ${ }^{9}$ Dana Farber Cancer Institute, Boston, MA, United States; ${ }^{10} \mathrm{H}$. Lee Moffitt Cancer Center and Research Institute, Tampa, FL, United States

Background: Classification of chronic graft-versus-host disease (cGVHD) changed with the NIH Consensus Criteria. A score of mild, moderate or severe is calculated from multiple organ domains, but important information from organ scores may be lost when using an overall severity score. We harnessed machine learning to identify clinical phenotypes and determined if these clusters stratified outcomes and could be translated into a clinical prognostic tool.

Methods: Adult patients with cGVHD enrolled in prospective multicenter studies were assessed. Clinical phenotypes were identified using incident patients (cGVHD Consortium 2011, $\mathrm{N}=339,56 \%$ male, median age 51, 


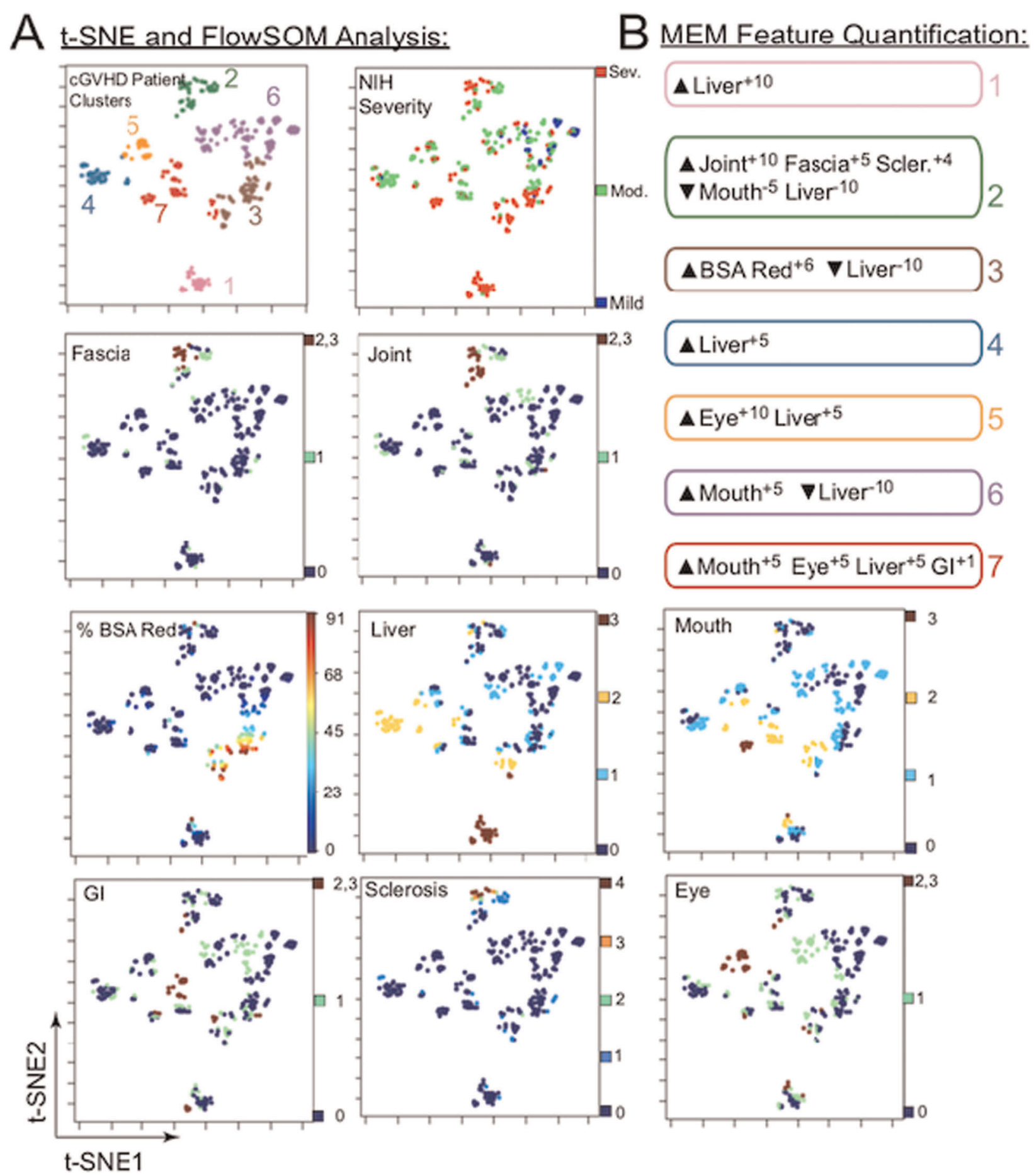

[P340 Figure] [Computational Analysis of NIH Criteria in cGVHD Patients $(n=339)$ Reveals 7 Revised Clusters]

range: 19-79) and tested in 85 additional cases from two studies of extracorporeal photopheresis (ECP). Research was conducted with informed consent, IRB approval and in accordance with the Declaration of Helsinki. For training, enrollment scores from 0 (no involvement) to 3 (most involved) were calculated for eye, liver, joint, mouth, GI, and $\%$ body surface area erythema. Sclerosis and fascia were measured by Hopkins scores. Analysis included viSNE for dimensionality reduction (Amir et al., Nature Biotechnology 2013), flowSOM for patient clustering (Van Gassen et al., Cytometry 2015), marker enrichment modeling (MEM) for feature enrichment scoring (Diggins et al., Nature Methods 2017), Cox proportional hazards for overall survival, and Chi-squared for cluster comparison. Response was 
measured using NIH 2005 criteria. To test clinical applicability, a decision tree based on a series of questions was developed to classify patients into the 7 clusters. MEM was used to compare phenotypes defined by initial machine learning with decision tree analysis. MEM Labels are reported as $\boldsymbol{\Delta}$ enriched organs, $\boldsymbol{\nabla}$ de-enriched organs, and $\operatorname{Organ}^{\mathrm{X}}$ with the superscript on a scale from -10 (most deenriched) to 10 (most enriched).

Results: Machine learning analysis revealed 7 patient clusters (Figure 1), including a sclerotic phenotype (Cluster 2): $\Delta$ Joint $^{+10}$ Fascia $^{+5}$ Scler. $^{+4} \boldsymbol{\nabla}$ Liver $^{-10}$ Mouth $^{-5}$, enriched for joint, fascia and sclerosis while lacking liver and mouth involvement, a liver phenotype (Cluster 1): $\Delta$ Liver $^{+10}$, and mixed phenotype (Cluster 7): $\Delta$ Mouth $^{+5}$ Eye $^{+5}$ Liver $^{+5} \mathrm{GI}^{+1}$, enriched for mouth, eye, liver and GI involvement. This decision tree classified patients into phenotypically comparable clusters and identified clusters with different survival and ECP response. Significantly worse overall survival was observed for severe liver phenotype patients (Cluster 1, HR 1.72 [95\% CI 1.012.93], $\mathrm{p}=0.04$ ) and mixed phenotype (Cluster 7, HR 1.75 [1.02-2.98], $\mathrm{p}=0.04)$, compared with other clusters. Patients classified as sclerotic phenotype $(\mathrm{n}=27)$ had lower response to ECP compared to mixed phenotype patients $(\mathrm{n}=12)(37 \%$ in Cluster 2 vs. $75 \%$ in Cluster $7, \mathrm{p}$ $=0.03$ ). Clusters weren't explained by NIH Severity alone and NIH Severity didn't significantly stratify survival.

Conclusions: Machine learning tools revealed patterns in cGVHD organ scores and characterized phenotypic groups with differing survival and treatment response. A clinically applicable tool for identifying patient groups was created. Identified clusters can now be evaluated for associated biomarkers, immune cell types, and additional clinical responses.

Figure 1. (A) t-SNE analysis shows patient cluster identified by flowSOM (left) and organ scores (heat) for each patient. (B) Patient clusters are labeled by MEM.

Clinical Trial Registry: NCT00637689; NCT01324908; NCT01380535

\section{Conflict of interest:}

J. Gandelman, M. Byrne, A. Mistry, K. Diggins, H. Chen, S. Lee, J. Pidala, J. Song: nothing to disclose.

H. Polikowsky is an employee at Cytobank Inc., Mountain View, CA.

M. Arora is a consultant for Takeda and receives research funding from Pharmacyclics LLC.

C. Cutler is a consultant for Pfizer, Pharmacyclics, Kite, Bristol-Myers Squibb, Incyte and Astellas.

M. Flowers is a consultant for Pharmacyclics LLC.

J. Irish is a co-founder and board member of Cytobank Inc. and received research support from Incyte Corp, Janssen, and Pharmacyclics.
M. Jagasia is a consultant and has received research funding from Mallinckrodt (formerly Therakos, Inc.) and Janssen.

\section{P341}

Clinical outcome and incidence of leukemia relapses with loss of mismatched HLA after T cell rich haploidentical hematopoietic stem cell transplantation with low-dose anti-thymocyte globulin

Shogo Kobayashi, Hideki Sano, Kazuhiro Mochizuki, Yoshihiro Ohara, Nobuhisa Takahashi, Satoshi Ono, Kazuhiko Ikeda, Hitoshi Ohto, Atsushi Kikuta

Fukushima Medical University, Fukushima, Japan

Background: Genomic loss of the mismatched HLA is a recently described mechanism of leukemia immune escape and relapse after allogeneic HSCT. L Crucitti et al. reported the incidence, risk factors and clinical outcome of myeloid malignancy relapses with loss of mismatched HLA after incompatible HSCT. However, features of lymphoid malignancy relapses with loss of mismatched HLA are poorly understood. The aim of this study was to investigate the risk factors and outcome of patients who underwent T-cell-replete haploidentical transplantation using a GvHD prophylactic regimen consisting of low-dose ATG $(2.5 \mathrm{mg} / \mathrm{kg}$ body weight), tacrolimus, methotrexate, and prednisolone.

Methods: Inclusion criteria for the study were the patients who achieved neutrophil engraftment (thus excluding early deaths or cases of immune rejection), and whose genomic HLA loss at relapse were able to be assessed. 'nonHLA loss' relapses were defined as the reappearance of leukemic cells in the relapse site with concomitant detection of patient-specific HLA by molecular typing.

Results: A total of 57 pediatric patients who underwent consecutive haploidentical transplantation were included in the analyses. Indication to HSCT was in all cases the diagnosis of high-risk malignancies. The underlying diagnoses of the 57 transplantation were as follows: ALL $(n=33)$, AML $(n=16)$, myeloid/NK acute precursor leukemia (MNKAL) $(n=5)$, mixed phenotype acute leukemia (MPAL) $(n=2)$, and juvenile myelomonocytic leukemia $(n=1)$. The age distribution was $0-20$ years, (median, 10 years) and the median follow-up for survivors was 428 days (range 23-2947). We documented 25 relapses, 17 of which can be assessed genomic HLA. In eight out of 17 cases of relapse $(47 \%)$ molecular typing revealed the selective genomic loss of the patient-specific HLA haplotype in leukemic cells. Out of the eight cases of HLA loss, four occurred in patients who underwent transplant for ALL, two in AML patients, one in an MNKAL patient, and one in an MPAL patient. Timing since HSCT was not 
significantly different between the HLA loss relapses and non-HLA loss relapses (median: 195.5 vs 309 days, $P=$ 0.426). Seven of the eight HLA loss relapse patients and six of nine non-HLA loss relapse patients underwent transplantation in the presence of active disease. Before the occurrence of relapse, all HLA loss relapse patients and eight non-HLA loss relapse patients experienced acute GvHD. Of the 12 evaluable patients, four HLA loss relapse patients and five non-HLA loss relapse patients experienced chronic GvHD. In the univariate analysis, T-cell dose infused $\left(<4.0 \times 10^{8} / \mathrm{kg}\right)$ with the transplantation was weakly associated with an increased risk of HLA loss $(P=0.06)$. The outcomes after HLA loss and non-HLA loss relapses were similarly poor, and of the 17 patients whose genomic HLA was assessed, only two non-HLA loss relapse patients had undergone transplantation when in complete remission survived in sustained remission.

Conclusions: In conclusion, HLA loss is a frequent mechanism of evasion from T-cell alloreactivity and relapse in patients with lymphoid and myeloid malignancies transplanted from a haploidentical donor, warranting routine screening in this transplantation setting.

Conflict of interest: The authors declare no conflicts of interest

\section{P342}

\section{Combined treatment for severe steroid-refractory acute graft-versus-host disease in acute promyelocytic leukemia case on its third allogeneic hematopoietic stem cell transplantation}

Fatos Dilan Atilla, Nur Soyer, Ayhan Donmez, Murat Tombuloglu

Ege University Faculty of Medicine, Hematology, Izmir, Turkey

Background: Allogeneic hematopoietic stem cell transplantation (HSCT) is potentially curative in a variety of hematological malignancies. Despite the high response rates in acute promyelocytic leukemia a subset of patients still relapse. While the graft-vs.-leukemia $(\mathrm{GvL})$ effect of allogeneic transplant offers the best anti-leukemia activity in patients with advanced disease, its success is notoriously hampered by graftvs.-host disease (GvHD) which remains a life-threatening complication. Standard treatment of GvHD is high-dose corticosteroids and steroid-refractory GvHD is associated with poor prognosis. Second-line treatment includes a number of agents (mesenchymal stromal cells, alemtuzumab, azacitidine, sirolimus, cyclophosphamide, anti-TNF agents and ruxolitinib). However, clinical experience with these agents remains limited. Here we have reported an interesting case which underwent allogeneic HSCT on its third time following acute steroidrefractory GvHD treated with multiple agents.
Methods: A 31-year-old male with refractory promyelocytic leukemia (PML) underwent allogeneic HSCT from full HLA-matched sibling donor for two times but both ended up with relapsed PML. The patient relapsed after second allogeneic HSCT with central nervous system (CNS) involvement and underwent a myeloablative allogeneic HSCT from a 9/10 HLA-matched related donor after conditioning with busulfan and cyclophosphamide. GvHD prophylaxis consisted of cyclosporine and methotrexate. On day 45, the patient developed overall grade IV acute GvHD with biopsy proven stage IV gastrointestinal GvHD refractory to high dose corticosteroids. Patient conditions rapidly deteriorated and became critical despite the addition of mycophenolate mofetil and cyclophosphamide. On day 48 etanercept $25 \mathrm{mg}$ was administered twice a week but due to insufficient response to previous therapies on day 50 , mesenchymal stromal cells of $1 \times 10^{6}$ cells $/ \mathrm{kg}$ bodyweight were given intravenously. Ruxolitinib were started concomitantly with mesenchymal stromal cells with a dose of $20 \mathrm{mg}$ twice a day. On day 54 the patient clinical conditions gradually improved. Complete resolution of aGvHD was also confirmed by histology on day 62. Sufficient response was achieved with bone marrow aspiration on remission and without any evidence of CNS involvement on examinations. After recovery of progressive-acute GvHD, extracorporeal photopheresis of every week and mycophenolate mofetil were given since GvHD was completely controlled. The patient is still on clinic follow up with remission.

Results: We reported a male patient complicated with severe acute GvHD after his third allo-HSCT due to relapsed PML. Combined therapies overcame severe acute GvHD and remission of PML was achieved. Severe GvHD may accompanied an intense GvL effect which presumably provided remission of leukemia.

Conclusions: GvHD remains serious barriers to successful allo-HCT and it is not clear that a major improvement has occurred to prevent or treat these patients. Multiple drugs and treatments have demonstrated clinical improvement in steroid refractory GvHD but clinical evidence is still needs to be highlighted. Nevertheless, there is an increasing understanding of the biology, clinical manifestations, and transplantation-related factors, and GvHD is getting better organized.

Conflict of interest: None declared.

\section{P343}

Comparison of two different anti-thymocytes globulins preparations in pediatric allogeneic hematopoietic stem cell transplantation from unrelated donors for hematological malignancies: results of a retrospective study 
Francesco Saglio, Rosanna Pessolano, Francesca Carraro, Massimo Berger, Paola Quarello, Elena Vassallo, Franca Fagioli

AOU Citta della Salute e della Scienza di Torino, Oncoematologia Pediatrica, Torino, Italy

Background: The addition of rabbit anti-thymocytes globulins to the GvHD prophylaxis has been shown to be highly effective in reducing the transplant related mortality and morbidity but available data about the best antithymocytes globulins preparation to be used in the pediatric population are limited so far. Primary aim of this study is to investigate if there are differences in terms of overall survival (OS) between pediatric patients treated with ATGThymoglobuline $\odot$ (Genzyme-Sanofi, Cambridge, MA) (ATG-Thymo) and patients treated with ATG-Grafalon $\odot$ (Neovii Biotech, Lexington, MA) (ATG-Grafalon). Secondary endpoints of this study are the evaluation of differences regarding transplant related mortality (TRM), relapse incidence (RI), and acute and chronic GvHD incidence.

Methods: We retrospectively evaluated 71 pediatric patients (median age 10 years) undergoing HSCT at our Center for hematological malignancies between 2010 and 2015 and having received as part of the GvHD prophylaxis schedule either ATG-Thymo $(\mathrm{n}=56$, total dose $10,5 \mathrm{mg} /$ $\mathrm{kg}$ ) or ATG-Grafalon $(\mathrm{n}=15$, total dose 15 to $30 \mathrm{mg} / \mathrm{kg}$ ). Patients underwent clinical and hematological posttransplantation assessments according to our Center's policy. OS was calculated according to the Kaplan-Meier method and the significance between the observed differences was established by the log-rank test. TRM and RI were calculated as a cumulative incidence (CI) to adjust the analysis for competing risks: relapse and transplant-related death were considered competing risks, respectively. aGvHD and cGvHD incidence was calculated as $\mathrm{CI}$ as well: in these cases, the death for any reason was considered a competing risk. The differences in terms of CI were compared using Grey's test. All the statistical analyses were performed using NCSS software (Hintze, 2001; NCSS PASS, Number Crunched Statistical System, Kaysville, UT, USA).

Results: Median follow up was of 977 days (range: 222485) for ATG-Thymo group and 1882 days (range: 662727) for ATG-Grafalon group. At 5 years after HSCT patients treated with ATG-Thymo had OS of $67 \%$ (95\%CI: 54-80) and patients treated with ATG-Grafalon OS of $73 \%$ (95\%CI: 50-95) but this difference was not statistically significant $(\mathrm{p}=0,62)$. Patients having received ATG-Thymo showed TRM of $17 \%$ (95\%CI: 9-32) while patients having received ATG-Grafalon $7 \% \quad(95 \% \mathrm{CI}: \quad 1-44)$ but this difference was not statistically significant $(p=0,32)$. RI was similar in both groups: $16 \%$ (95\% CI: 9-30) for ATGThymo and 20\% (95\% CI: 7-55) for ATG-Grafalon.
Similarly, patients showed same occurrence rate of both grade II-IV aGvHD and cGvHD: respectively $24 \%$ (95\%CI: 15-39) and 13\% (95\%CI: 6-31) for the ATG-Thymo group and $13 \%$ (95\%CI: 4-48) and 16\% (95\%CI: 4-57) for the ATG-Grafalon group $(\mathrm{p}=0,38$ and $\mathrm{p}=0,86)$.

Conclusions: Our retrospective data suggest that patients treated with ATG-Grafalon have an improved survival compared to patients treated with ATG-Thymoglobuline and this advantage seems to be related to a reduced TRM. Obviously, these results are preliminary and need to be further confirmed by larger prospective randomized controlled trials aimed also to establish if the use of this drug can be extended to other situation too (i.e. HSCT from sibling donors) as it has been described for adults

Conflict of interest: The authors have no commercial, proprietary or financial interests in the products or companies described in this article.

\section{P344}

Cutaneous graft-versus-host disease after hematopoietic stem cell transplantation; a single institution experience

Fatos Dilan Atilla ${ }^{1}$, Hale Bulbul ${ }^{1}$, Nur Soyer ${ }^{1}$, Bengu Gerceker Turk $^{2}$, Ayda Acar ${ }^{2}$, Murat Tombuloglu, Guray Saydam ${ }^{1}$, Mahmut Tobu ${ }^{1}$, Fahri Sahin ${ }^{1}$, Filiz Vural ${ }^{1}$

${ }^{1}$ Ege University Faculty of Medicine, Hematology, Izmir, Turkey; ${ }^{2}$ Ege University Faculty of Medicine, Dermatology, Izmir, Turkey

Background: Graft-versus-host disease (GvHD) is a major complication of allogeneic hematopoietic stem cell transplants (allo-HSCT) associated with significant morbidity and mortality. GvHD is a major cause of morbidity and mortality, affecting 40-60\% of allo-HSCT recipients, and accounting for $15 \%$ of deaths following this procedure. One of the earliest and most common manifestations of GvHD is cutaneous GvHD, which consists essentially of a maculopapular rash that can begin anywhere in the body but often starts with palm and sole involvement.

Methods: We conducted a retrospective analysis of adult patients after allogeneic hematopoietic stem cell transplantation (allo-HSCT) developed cutaneous GvHD between November 1st, 2014 and November 1st, 2017. Clinical details recorded included demographics, underlying diagnosis, details of the HSCT and conditioning regime, immunosuppressive agents used, grade of cutaneous GvHD and overall outcome. Grade I cutaneous GvHD which responded to immunosuppressive treatment did not included to the study.

Results: Among 95 patients, cutaneous GvHD was found in 38 patients (40\%), and was accompanied by visceral (hepatic/gastrointestinal) cGvHD $(\mathrm{n}=9 / 23)$, mucous involvement $(\mathrm{n}=10)$, lung involvement $(\mathrm{n}=3)$ following 
allogeneic HCT (26 matched related, 10 unrelated, 2 haploidentical) for various indications (22 AML/MDS, 3 Hodgkin lymphomas, 3 non-Hodgkin lymphomas, 2 myelomas, 2 CML, 2 CLL, 4 aplastic anemias). Among 38 patients with cutaneous GvHD median age was 39 years (19-64). Gender Male/Female was 23/15. Peripheral blood was the source in all patients. Reduced-intensity conditioning was used in 55\%. Acute cutaneous GvHD grade II occurred in $40 \%$, grade III occurred in $35 \%$ and grade IV occurred in $25 \%$. Among 38 patients with cutaneous GvHD $76 \%$ of them had failed conventional immunosuppressive therapy or could not be adequately controlled only by corticosteroid treatment. In those patients alternative second line treatments including cyclophosphamide, ruxolitinib, mesenchymal stromal cells, extracorporeal photopheresis (ECP) and UVA-1 phototherapy were used. Thirty-five patients $(92 \%)$ were on ECP which administered twice a week starting at a median of 30 days from the diagnosis of acute GvHD and 15 (40\%) were on UVA-1 phototherapy. The median duration of ECP was 24 months (5-65) and the median number of ECP procedures in each patient was 9 (548). Overall response rate was $73 \%(n=28)$. The complete response rate was $13 \%(\mathrm{n}=5)$ and the partial response rate was $60 \%(n=23)$. Ten patients $(27 \%)$ had no response.

Conclusions: Due to increasing incidence of hematological diseases, HSCT is a common therapy worldwide in parallel with GvHD is highly prevalent in patients receiving allo-HSCT. Cutaneous GvHD is the most common manifestation following HSCT. It can appear as an acute or chronic comorbidity, and it affects greatly patients $\hat{A}^{\prime}$ quality of life. ECP is an effective treatment of steroidrefractory GvHD, especially with cutaneous and mucous involvement of cutaneous GvHD.

Conflict of interest: None declared.

\section{P345}

\section{Cutaneous severe acute graft-versus-host disease mimicking toxic epidermal necrolysis: diagnostic and therapeutic challenge}

\section{Jon Badiola ${ }^{1}$, Andrea Rodriguez-Tejero ${ }^{2}$, Elisa López-Fernández ${ }^{1}$, Pedro González-Sierra ${ }^{1}$, Alejandro Molina-Leyva ${ }^{2}$, Lucia Moratalla $^{1}$, Salvador Arias-Santiago ${ }^{2}$, Manuel Jurado-Chacón ${ }^{1}$ \\ ${ }^{1}$ Hospital Virgen de las Nieves, Department of Hematology, Granada, Spain; ${ }^{2}$ Hospital Virgen de las Nieves, Department of Dermatology, Granada, Spain}

Background: Toxic epidermal necrolysis (TEN) is a lifethreatening blistering skin disease typically induced by drugs with poorly understood pathogenesis. The incidence and main features of TEN in hematopoietic stem cell transplantation (HSCT) patients has not been evaluated as there are only a few documented cases in the literature. The extensive blistering and desquamation presentation of the cutaneous acute graft-versus-host disease (GvHD) is the most severe variant of this condition, mimicking TEN. Both skin diseases have exhibit clinical and histological similarities which makes it virtually impossible to differentiate between them in the context of HSCT.

Methods: We present two recent cases diagnosed with cutaneous severe GvHD/NET after HSCT in our centre (Hospital Virgen de las Nieves, Granada, Spain).

Results: Patient 1: 47-year-old man, with myelodysplastic syndrome, subjected to allogeneic HSCT from a matched related donor with non-myeloablative conditioning regimen. On day +35 , we observed a maculopapular rash that improved after steroid administration. On day +84 , the patient developed a rapidly progressive generalized erythema with mucosal involvement and fever for which sirolimus and extracorporeal photopheresis were added to the current treatment. In the following days, clinical deterioration was observed with blisters, presenting positive Nikolsky sign, in more than $50 \%$ of the body surface area. High dose steroids and ruxolitinib were initiated at this point. After numerous complications, on day +229 , the patient was released from the hospital with cutaneous, hepatic and ocular chronic GvHD treated under immunosuppressive therapy. On day +322 , the patient died from respiratory infection.

Patient 2: An allogeneic matched-related donor HSCT was performed with non-myeloablative conditioning regimen in 61-year-old woman with chronic lymphatic leukemia. On day +16 , she developed gastrointestinal symptoms that were treated with steroids after biopsy confirmation of acute grade $2 \mathrm{GvHD}$. On the day +38 , we observed a worsening of her gastrointestinal condition that progressed with mucosal involvement, disseminated rash and fever. Sirolimus was added to the current treatment. In the following days, multiple blister formation and large areas of epidermal denudation appeared in $>30 \%$ of the body surface area (Figure 1). Intensive plasmapheresis, photopheresis and ruxolitinib were initiated. On day +80 , the patient was released from the hospital and she is still alive on day +187 .

In both cases, the biopsies show morphological findings compatible with grade $4 \mathrm{GvHD}$ and NET, making it impossible to differentiate between them. In the second case, the immunohistochemical study showed a CD8+/CD4 $+\mathrm{T}$ lymphocyte ratio of 2,5 .

Conclusions: - In HSCT patients that exhibit progressive severe skin disease with mucosal involvement and fever, severe cutaneous GvHD/NET should be suspected.

- While the discrimination between both entities is challenging, gastrointestinal or liver involvement and/or a 


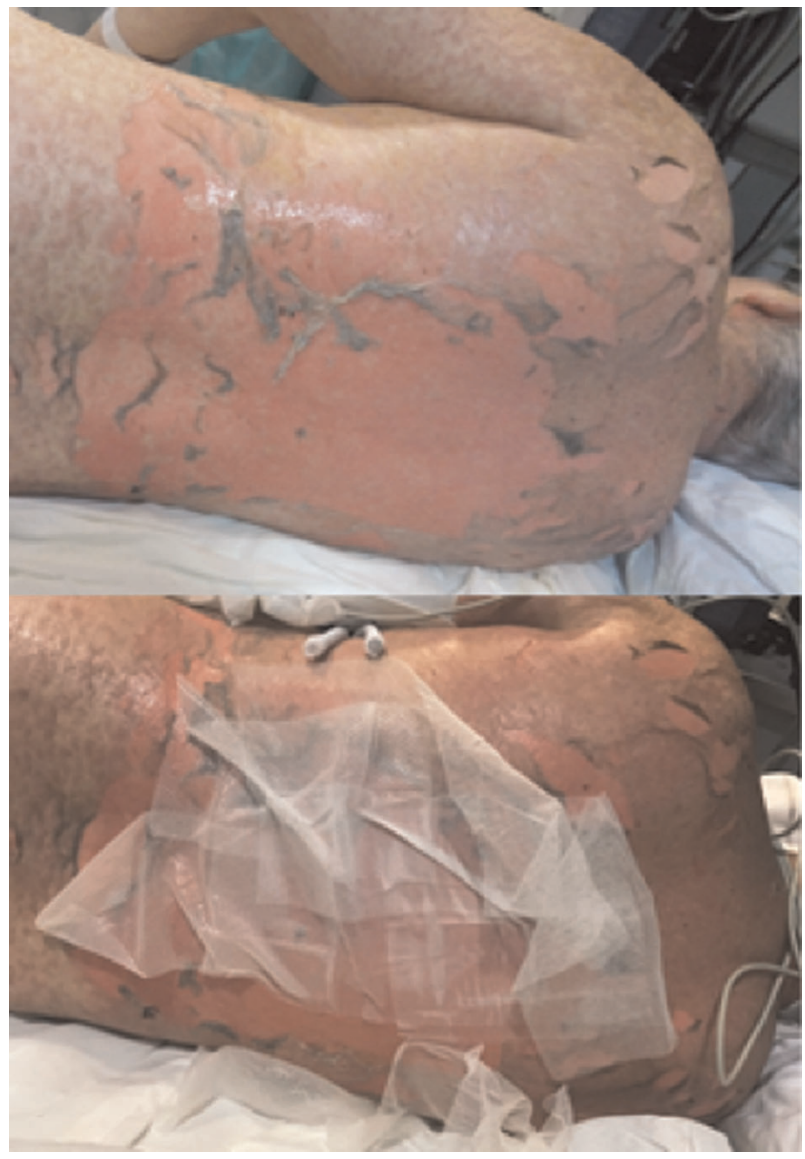

[P345 Figure] [Image 1: patient 2 on day +48.$]$

CD8+/CD4+ T lymphocyte ratio $<4$, should tilt the diagnostic balance towards the GvHD side.

- Probably the best approach to the treatment of these patients is dual, involving an intensive treatment of GvHD and the usual management of NET avoiding those drugs that may trigger the disease.

Conflict of interest: None of the authors has anything to disclose.

\section{P346}

Cyclosporin A (CSA) and Mycophenolate Mofetil (MMF) vs Cyclosporine $A$ and Steroids for graft-versus-host disease (GVHD) prophylaxis after umbilical cord blood (CB) transplantation

Ibrahim Ghemlas, Khawar Siddiqui, Rafat Jafri, Mary Barria, Antonette Amao, Hawazen Alsaedi, Suleimman Alsweedan, Ali Alahmari, Abdullah Aljerfi, Amal Alseraihy, Mouhab Ayas
King Faisal Specialist Hospital \& Research Centre, Pediatric

Hematology / Oncology, Riyadh, Saudi Arabia

Background: The optimal GVHD prophylaxis after CB transplantation has not been established. MMF has been widely used for GVHD prophylaxis after SCT but experience following $\mathrm{CB}$ transplantation is still limited. We retrospectively compared CSA/Steroids and CSA/MMF as GVHD prophylaxis after CB transplantation.

Methods: 252 pediatric transplant naïve recipients underwent CB transplantation from Jan 1993 to December 2016 at King Faisal Specialist Hospital \& Research Centre, Riyadh, Saudi Arabia. Data on diagnosis, transplant-related parameters, and incidence of GVHD, VOD, viral, bacterial and fungal infections during first D100 post-transplant were abstracted. $42.9 \%(108)$ were female. Median age at transplant was 1.9 years (range,0.21-13.5 years). For $30.6 \%(77)$ patients, the indication for SCT was malignancy,8.7\%(22) were in Group A (CSA/MMF) while the remaining 91.3\%(230) were in Group B (CSA/Steroids). $7.1 \%$ (18) patients had fully HLA-matched units while the remaining were 1-2 antigen mismatched.

Results: Acute GVHD occurred in $31.8 \%$ (7) in Group A compared to $41.7 \%$ (96) in Group B (P-Value: 0.497). Severe aGVHD (Grade $>=3$ ) was seen in $9.1 \%$ (2) in group A and in $15.7 \%$ (36) in Group B (P-Value: 0.545). Post infusion chimeric studies were 76.5\% in Group A and 83\% in Group B while median time to neutrophils engraftment was 24.5 days in Group A and 18 days in Group B (PValue:0.077 and 0.033 respectively). Incidence of VOD was higher in Group A $(13.6 \%, 3)$ compared to Group B $(5.7 \%, 13$; P-Value:0.153). Bacterial, viral, fungal of any of the three infection(s) rate was also higher in Group A $(40.9 \%, 27.3 \%, 18.2 \%$ and $68.2 \%)$ vs. Group B (37.8\%, 20.4\%, 10\%, 50\%; P-Values: 0.820, 0.422,0.270, and 0.121 respectively). Cumulative incidence of chronic GVHD (cGVHD) in Group A was $28.6 \%$ in comparison to $18.7 \%$ in Group B.

Conclusions: Despite a trend of lower GVHD incidence in the CSA+MMF group, the difference was not statistically significantly different when compared to CSA/ Steroids group. Rate of VOD, bacterial, viral, fungal or any of these infections during D100 post SCT and cGVHD were higher in CSA/MMF group, while engraftment was Faster with higher chimeric studies in CSA/Steroids group. Further studies are needed with higher number of patients to evaluate the effectiveness of CSA/MMF.

Conflict of interest: None to declare.

\section{P347}

Discriminatory potential of lgG N-glycosylation in chronic graft-versus-disease 
Ema Prenc ${ }^{1}$, Drazen Pulanic ${ }^{1,2}$, Ivo Ugrina ${ }^{3,4}$, Lana Desnica ${ }^{2}$, Maja Pucic Bakovic $^{3}$,Jerko Stambuk ${ }^{3}$, Milan Milosevic ${ }^{1,5}$, Ranka Serventi
Seiwerth $^{2}$, Nadira Durakovic ${ }^{1,2}$, Zinaida Peric ${ }^{1,2}$, Radovan Vrhovac $^{1,2}$, Damir Nemet ${ }^{1,2}$, Gordan Lauc ${ }^{3}$, Steven Z. Pavletic ${ }^{6}$

${ }^{1}$ University of Zagreb School of Medicine, Zagreb, Croatia;

A)

\begin{tabular}{|c|c|}
\hline Glyeopepsist. & p-vates \\
\hline $\mathrm{I}_{5} \mathrm{Cr}_{2} \mathrm{C}-1 \mathrm{NN}$ & 0.0007705 \\
\hline IgG4_G2S & 0.0260003 \\
\hline IgGl_GIFN & 0.0319112 \\
\hline $\mathrm{I}_{5} \mathrm{O}_{2} \mathrm{a}_{1173}$ & 0.0368368 \\
\hline IgG2_G2FS & 0.0422437 \\
\hline IgG2_G-2RN & 0.0596104 \\
\hline$\ldots$ & $\ldots$ \\
\hline
\end{tabular}

F)

B)

\begin{tabular}{|c|c|}
\hline $\begin{array}{l}\text { Glycan } \\
\text { garamotors }\end{array}$ & p-rates \\
\hline Glyczon:PC1 & 0.1736151 \\
\hline GlyesnaPC2: & 0.3510235 \\
\hline GhyesonaPC3 & 0.8573189 \\
\hline Glyoun:P4 & 0.1720769 \\
\hline GlyezonaPs & 0.0314943 \\
\hline ChyerendC6 & 0.0523513 \\
\hline
\end{tabular}

C)

\begin{tabular}{|c|c|}
\hline $\begin{array}{l}\text { Clinical labortsory } \\
\text { paremosors }\end{array}$ & p-rates \\
\hline LabPCl & 0.0426091 \\
\hline $\mathrm{L}_{2} 6 \mathrm{PC} 2$ & 0.0769052 \\
\hline L=6PC3 & 0.0520165 \\
\hline Plasobets & 0.0999667 \\
\hline
\end{tabular}

D)

\begin{tabular}{|c|c|}
\hline $\begin{array}{l}\text { Combinod } \\
\text { pursmoters }\end{array}$ & prevates \\
\hline Plasolats & 0.0177396 \\
\hline LabPCl & 0.0334138 \\
\hline GlycanPCs & 0.0206250 \\
\hline
\end{tabular}

G)

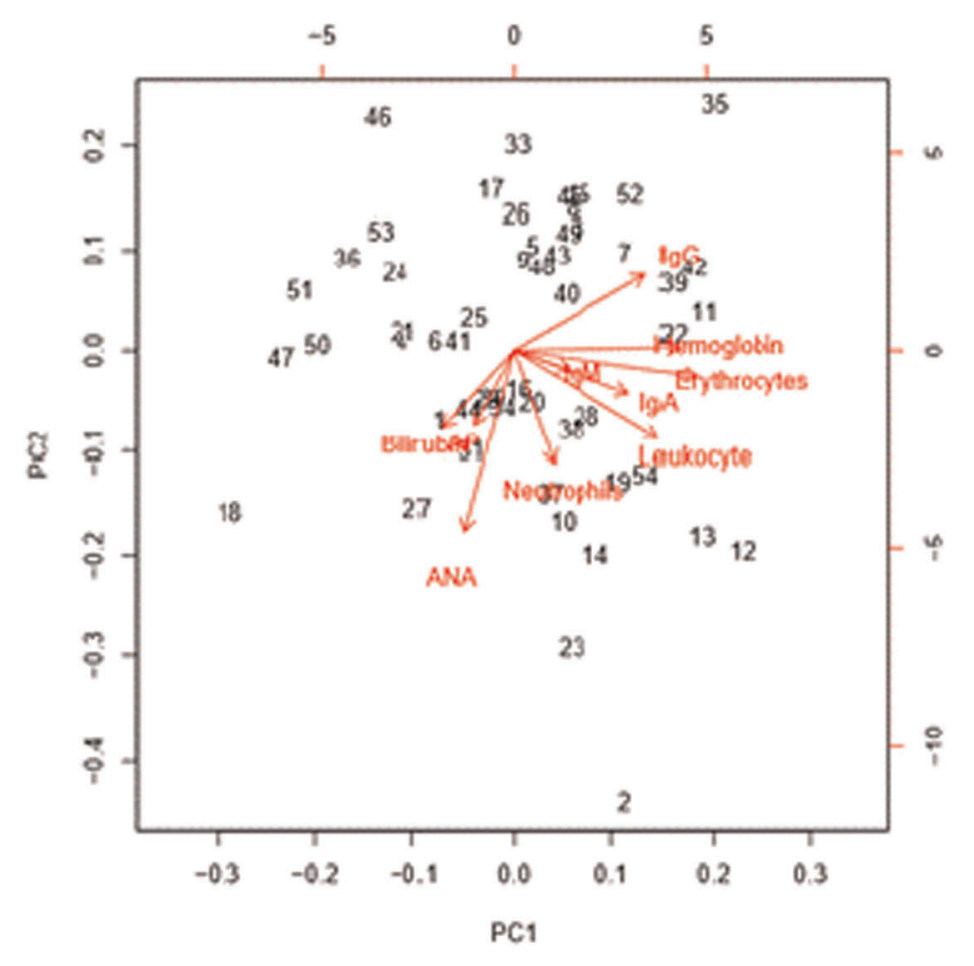

E)

\begin{tabular}{|c|c|}
\hline $\begin{array}{c}\text { Glyeopopits } \\
\text { (erroetion) }\end{array}$ & p-vahes \\
\hline IgG1_G1Fs & 0.0305158 \\
\hline IgG2_G1FS & 0.0497006 \\
\hline
\end{tabular}

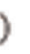
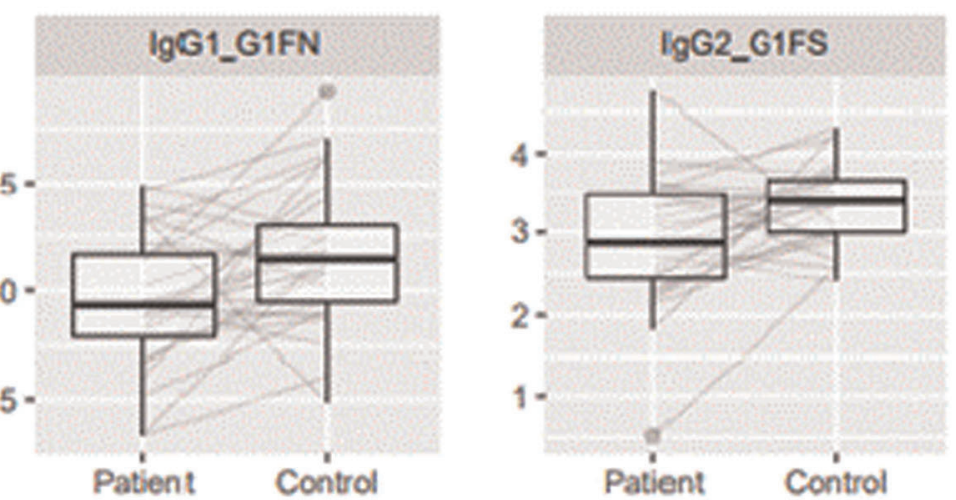

$$
\text { (n) }
$$

[P347 Figure] [Figure 1. Logistic regression of glycopeptide measurements and clinical laboratory results.] 
${ }^{2}$ University Hospital Centre Zagreb, Zagreb, Croatia; ${ }^{3}$ Genos Ltd., Zagreb, Croatia; ${ }^{4}$ Faculty of Science, University of Split, Split, Croatia; ${ }^{5}$ Andrija Stampar School of Public Health, Zagreb, Croatia; ${ }^{6}$ National Institutes of Health, National Cancer Institute, Bethesda, WA, United States

Background: Chronic graft-versus-host disease (cGvHD) is an immune disorder following allogeneic hematopoietic stem cell transplantation (alloHSCT). It is characterized by an altered homeostasis of the humoral immune response with the production of antibodies. Changes in glycosylation of immunoglobulin $\mathrm{G}(\mathrm{IgG})$, the most abundant class of antibody, are associated with autoimmune diseases resembling cGvHD (BBA.2016;1860:1615-22.). In this study we evaluated the potential of IgG N-glycosylation in discriminatory analysis of patients after alloHSCT with and without cGvHD.

Methods: Plasma samples were collected from cGvHD patients $(\mathrm{n}=30)$ and control participants after alloHSCT but without $\mathrm{cGvHD}(\mathrm{n}=30)$ matched by age and gender included into a natural history cGvHD study at the University Hospital Center Zagreb, Croatia (2013-2016). Chronic GvHD was diagnosed according to the established NIH criteria. Laboratory tests were done (complete blood count, coagulation, biochemistry, immunology). IgG was isolated from blood plasma and analyzed by liquid chromatography-mass spectrometry. Discriminatory models were obtained with conditional logistic regression of $\operatorname{IgG}$ glycopeptide measurements, laboratory results and literature based clinically significant laboratory markers associated with cGvHD (Leukemia. 2012;26(4):633-43.). P values below 0.05 were considered significant.

Results: cGvHD patients and controls were similar regarding gender (53.3\% male), age (median 41.5 vs 40.5 years), main disease $(76.7 \%$ leukemias), myeloablative conditioning (53.3\% vs $56.7 \%)$, related donors $(63.3 \%)$ and HSCT source (peripheral blood 53.3\% vs $50.0 \%$ ). cGvHD patients had more frequent previous acute GvHD than control group $(73.3 \%$ vs $26.7 \%, \mathrm{p}=0.001)$. Median time from cGvHD diagnosis to study enrollment was 164 days, majority of patients had severe $(50 \%)$ or moderate $(40 \%)$ global NIH cGvHD score, $50 \%$ had active disease by clinical impression, and $53.3 \%$ patients were receiving systemic immunosuppression. Patients had lower B cells $(\mathrm{p}=0.027)$, albumin $(\mathrm{p}=0.005)$ and vitamin $\mathrm{D}(\mathrm{p}=0.018)$, and higher GGT $(\mathrm{p}=0.004)$, CRP $(\mathrm{p}=0.040)$ and beta 2 microglobuline $(\mathrm{p}=0.049)$ than controls. Logistic regression of individual glycopeptides identified several potentially significant candidates (Figure 1A). Next model was built using glycopeptide measurements principal components (glycanPC; Figure 1B) to reduce the effect of multicolinearity. The third model used literature based clinically significant laboratory markers associated with $\mathrm{cGvHD}$ and other laboratory parameters reduced to principal components (labPC; Figure 1C). Final model combined statistically significant parameters from previous models identified the following parameters as significant to discriminate cGvHD patients from controls (Figure 1D): higher platelet count $(\mathrm{p}=0.0177)$, labPC1 $(\mathrm{p}=0.0334)$ with hemoglobin and erythrocytes as main contributors (Figure 1G), and glycanPC5 $(\mathrm{p}=0.0206)$. Original glycopeptide candidate selection was further corrected by using platelets and labPC1 and identified that cGvHD patients had lower IgG1_G1FN $\quad(p=0.0308)$ and lower IgG2_G1FS $(\mathrm{p}=0.0497)$ (Figure 1E, 1F).

Conclusions: Our study indicates an increased discriminatory power of the model that combines laboratory parameters with IgG-glycopeptide measurements to distinguish cGvHD patients and controls. This method also identified glycan structures that are significantly lower in cGvHD patients indicating a disturbance in antibodydependent cell-mediated cytotoxicity and a reduced antiinflammatory $\operatorname{IgG}$ feature, suggesting that glycans have a great research potential in clarifying events in cGvHD.

Conflict of interest: None of the authors has anything to disclose.

\section{P348}

Donor and recipient hypercholesterolemia before allogeneic stem cell transplantation associates with increased incidence of acute graft-versus-host disease

Monica Magdalena Rivera Franco, Eucario Leon Rodriguez, Isabel Lastra German, Andrea Mendoza Farias

Instituto Nacional de Ciencias Medicas y Nutricion Salvador Zubiran, Hematology and Oncology, Tlalpan, Mexico

Background: Hypercholesterolemia leads to cholesterol accumulation in macrophages and other immune cells, which promotes inflammatory responses. Although the links between cholesterol and inflammation are best exemplified by atherosclerosis, similar mechanisms may also contribute to other metabolic disorders such as obesity or to autoimmune diseases. In this context, acute graft-versushost disease (aGVHD) is an immunological syndrome that involves tissue damage mediated by donor lymphocytes, resulting from an imbalance between the effector and regulatory arms of the immune system that can affect the outcomes of allogeneic hematopoietic stem cell transplantation (allo-HSCT). The aim of this study was to investigate the association of hypercholesterolemia in both, donors and recipients, and incidence of aGVHD.

Methods: A retrospective analysis was performed identifying 121 patients receiving allogeneic HSCT at the National Institute of Medical Sciences and Nutrition 

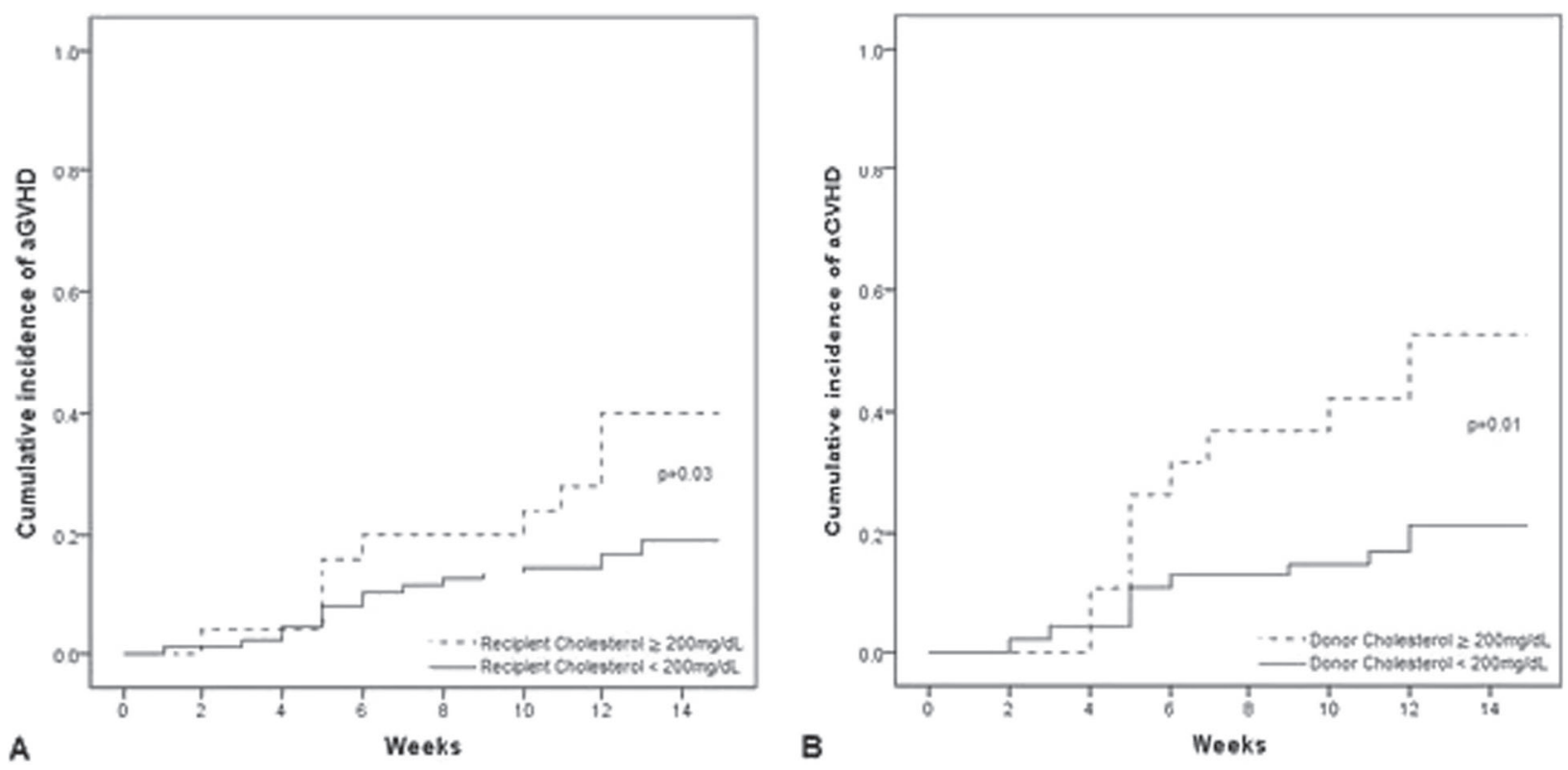

[P348 Figure] [Figure 1. Cumulative incidence of aGVHD according to cholesterol levels in A)recipients and B)donors]

between May 1999 and August 2017. Exclusion criteria included incomplete laboratory information, engraftment failure or 30 day mortality. The final cohort included 114 patients undergoing allo-HSCT. Data was obtained from electronic and paper medical records. Documented donor and recipient cholesterol included levels before performing HSCT. High cholesterol was considered as total cholesterol $\geq 200 \mathrm{mg} / \mathrm{dL}$, according to international guidelines. For the analysis of donor cholesterol levels only 66 donors, who had complete data, were included. For aGVHD, death and relapse without aGVHD were considered competing risks. A p-value of $<0.05$ was considered significant. SPSS v. 21 was used.

Results: Most patients were male $(n=64,56 \%)$. The median age was 31 years (range, 16-62). The underlying diseases were the following: acute lymphoblastic leukemia $(\mathrm{n}=28,25 \%)$, aplastic anemia $(\mathrm{n}=23,20 \%)$, myelodysplastic syndrome $(\mathrm{n}=17,6 \%)$, acute myeloblastic leukemia $(\mathrm{n}=15,13 \%)$, lymphomas $(\mathrm{n}=6,5 \%)$, chronic lymphocytic leukemia $(\mathrm{n}=15,13 \%)$, and others $(\mathrm{n}=10,9 \%)$. Acute graft-versus-host disease was presented in $24 \%(\mathrm{n}=$ 27) patients. Figure $1 \mathrm{~A}$ shows a statistically significant increase of aGVHD associated to hypercholesterolemia of the recipients ( $40 \%$ vs $18 \%, \mathrm{p}=0.03$ ); also, as shown in Figure 1B, hypercholesterolemia of the donors associated with increased aGVHD (53\% vs $21 \%, \mathrm{p}=0.018)$. Hypercholesterolemia in both, donor and receptor, was also associated with increased aGVHD (63\% vs $17 \%, \mathrm{p}=$ 0.016). Other variables such as type of donor (related, unrelated, haploidentical), gender disparity, HSC source, age, CMV infection, and other lab results (LDL, HDL,
$\mathrm{CBC}$, liver function, glucose, iron) were tested in a multivariate analysis and no statistical significance was observed (results not shown).

Conclusions: To date, this is the first study associating hypercholesterolemia with aGHVD. Few studies have reported that some medications, statins in particular, may have a role in modulating graft-versus-host disease. On the other hand, some studies have exclusively reported hypercholesterolemia as a consequence of hepatic GVHD. According to our results testing other variables, we conclude that hypercholesterolemia in the recipient, donor, or in both, is an independent factor for the development of aGVHD, nonetheless, further, prospective studies with a larger cohort, are necessary.

Conflict of interest: None of the authors have any conflict of interest to disclose.

\section{P349}

Abstract previously published

\section{P350}

Durability of Response and Need for Second-Line Topical Therapy in the Management of Oral Chronic Graft-VersusHost Disease

Muhammad Ali Shazib ${ }^{1}$, Jillian Muhlbauer ${ }^{2}$, Shuli $\mathrm{Li}^{3}$, Corey Cutler $^{3,4}$, Nathaniel Simon Treister ${ }^{1}$

${ }^{1}$ Brigham and Women's Hospital and Dana-Farber Cancer Institute, Oral Medicine and Dentistry, Boston, MA, United States; ${ }^{2}$ Harvard 
School of Dental Medicine, Boson, MA, United States; ${ }^{3}$ Dana Farber Cancer Institute, Boston, MA, United States; ${ }^{4}$ Harvard Medical School, Boston, MA, United States

Background: An open label phase 2 study in oral chronic graft-versus-host disease (cGVHD) of topical dexamethasone versus tacrolimus solutions revealed superior efficacy of dexamethasone. The objective of this study was to report long-term management and outcomes in this study cohort after completing the 30-day trial.

Methods: A retrospective record review was performed from the date of study completion to January 2017. Topical therapies, systemic immunosuppressive therapies, NIH oral cGVHD scores, and adverse effects were recorded for all cGVHD-related outpatient visits. Patient follow up (FU) was characterized by the following time intervals: FU1 $=$ 1 month (m), FU2 = 6-12 m, FU3 = 18-24 m, FU4 = >24 $\mathrm{m}$.

Results: Forty patients $(50 \%$ male, median age $=58.8$ y) completed the clinical trial, had persistent oral cGVHD. At FU1 all patients remained on topical therapy (dexamethasone [DEX, 90\% of patients], clobetasol [CLO, 10\%]) and/ or tacrolimus (TAC, 40\%). This reduced to $55 \%, 52 \%$ and $49 \%$ at FU2 $(\mathrm{n}=35), \mathrm{FU} 3(\mathrm{n}=21)$ and FU4 $(\mathrm{n}=28)$, respectively. Mean NIH (0-3) and sensitivity (0-10) scores fluctuated from FU1 $(\mathrm{NIH}=1.4$; severity $=3.5)$, to FU2 $(1.3 ; 3.7)$, to FU3 $(0.7 ; 2.3)$, to FU4 $(0.8,3.2)$. Mean and maximum ulceration scores $(0,3$, or 6$)$ decreased from FU1 $(1.1,6)$, to FU2 months $(0.7,4)$, to FU3 months $(0.05,1)$, and to FU4 $(0.6,3)$. Nine patients $(23 \%)$ developed oral candidiasis and were treated with antifungal therapy.

Conclusions: Patients with de novo symptomatic oral cGVHD are likely to require long-term care with topical immunomodulatory therapy, possibly for years. Topical steroid and tacrolimus therapies are safe and effective in managing the condition. Second-line topical therapy for refractory oral cGVHD requires further investigation.

Clinical Trial Registry: N/A

Conflict of interest: There are no conflict of interests declared by the authors.

\section{P351}

\section{Dynamic of peripheral blood schistocytes in GvHD after allogeneic stem cell transplants assessed by digital microscopy}

Meropi Karakioulaki, Michael Medinger, Jakob Passweg, Dimitrios A. Tsakiris
University Hospital Basel, Diagnostic Hematology, Basel, Switzerland

Background: One of the diagnostic criteria for the transplant-associated thrombotic microangiopathy (TATMA) is the quantification of schistocytes in peripheral blood (detected as schistocytes and helmet cells). The value of this method has been debated, since evaluation of schistocytes can be technically very variable. Semiautomated digital microscopy systems have been developed in order to differentiate peripheral blood cells and erythrocyte morphology in a variable way. We tested the diagnostic accuracy and precision of digital microscopy compared to conventional microscopy with respect to the recognition of schistocytes in the setting of acute graftversus-host-disease (aGvHD).

Methods: Peripheral blood smears from 127 allogeneic and 63 autologous HSCT patients (January 2016-June 2017) were retrospectively examined before, one month and at three months after transplantation. Quantification of schistocytes by digital microscopy (Vision Hema, WestMedica, Austria) was done out of 5'000 erythrocytes and was correlated to clinical signs of aGvHD/TA-TMA. Peripheral blood smears from 31 healthy blood donors were also analyzed as controls.

Results: In table 1 schistocyte counts in percent of erythrocytes (mean \pm standard deviation) are given for the allogeneic HSCT patients with or without aGvHD, the autologous HSCT group, the allogeneic HSCT with or without HLA mismatch (HLAmm) and the control group. Values before and after 3 months in the same group were compared by paired Wilcoxon test $(*)$ and values at 3 months between groups by Mann-Whitney test (**). Patients at 3 months with established aGvHD were already under respective treatment. Schistocytes were significantly increased after 3 months in the allogeneic $(p<0.001)$ or the autologous HSCT $(\mathrm{p}<0.001)$ when compared to controls. Statistically significantly higher schistocyte counts were found at 3 months in patients with aGvHD $(p=0.027)$ and in patients with an HLA mismatch $(\mathrm{p}=0.003)$ compared to the baseline before start of conditioning. Furthermore, allogeneic HSCT patients with high schistocyte counts (upper quartile, $>0.64 \%$ ) had significantly more frequently aGvHD (chi-squared test $p=0.024$ ), high lactate dehydrogenase LDH levels $>225 \mathrm{U} / \mathrm{L}$ (chi-squared test $\mathrm{p}=0.006$ ) and HLA mismatch (chi-squared test $\mathrm{p}=0.035$ ).

Conclusions: Schistocytes can be reliably and precisely counted by means of a digital microscopy system and they are increased in the acute phase after allogeneic HSCT within the context of aGvHD, probably as an indirect sign of microangiopathy. This might be used as an additional indicator of endothelial GvHD, long before a possible TATMA syndrome evolves, as patients with aGvHD and/or 
HLA mismatch have more frequently endothelial damage. Appropriate cut-offs for schistocytes have to be defined and validated prospectively.

Conflict of interest: The authors declare no conflicts of interest.

\begin{tabular}{llllll}
\hline & Baseline & $\begin{array}{l}1 \text { month } \\
\text { after } \\
\text { HSCT }\end{array}$ & $\begin{array}{l}\text { 3 months } \\
\text { after HSCT }\end{array}$ & $\begin{array}{l}\text { Wilcoxon } \\
\text { test }\end{array}$ & $\begin{array}{l}\text { Mann- } \\
\text { Whitney } \\
\text { test }\end{array}$ \\
\hline Allogeneic w/o & 0.526 & 0.474 & 0.433 & N.S.* & $\mathrm{p}=0.05^{* *}$ \\
aGvHD (n=70) & \pm 0.6 & \pm 0.483 & \pm 0.329 & & \\
Allogeneic with & 0.430 & 0.471 & 0.563 & $\mathrm{p}=0.027^{*}$ & \\
aGvHD (n=57) & \pm 0.313 & \pm 0.343 & \pm 0.424 & & \\
Allogeneic w/o & 0.490 & 0.471 & 0.458 & N.S.* & $\mathrm{p}=0.005^{* *}$ \\
HLAmm (n=76) & \pm 0.397 & \pm 0.466 & \pm 0.327 & & \\
Allogeneic with & 0.472 & 0.475 & 0.600 & $\mathrm{p}=0.003^{*}$ & \\
HLAmm (n=51) & \pm 0.613 & \pm 0.358 & \pm 0.441 & & \\
Autologous (n= & 0.463 & 0.511 & 0.519 & N.S.* & \\
63) & \pm 0.560 & \pm 0.469 & \pm 0.535 & & \\
Control group (n & 0.095 & 0.095 & 0.095 & & \\
$=31)$ & \pm 0.060 & \pm 0.060 & \pm 0.060 & & \\
\hline
\end{tabular}

\section{[[P351 Table] Table 1]}

\section{P352}

\section{Effect of extracorporeal photopheresis on production of serum soluble CD163 in chronic graft versus host disease}

\section{Charlotte S Burton, Nick C Matthews, Arun Alfred}

The Rotherham NHS Foundation Trust, Department of Haematology, Rotherham, United Kingdom

Background: Extracorporeal photopheresis (ECP) is used as a second line therapy for steroid refractory, dependent or intolerant chronic GVHD (cGVHD). In cGVHD, activated tissue macrophages produce factors that contribute to the formation of fibrotic lesions. CD163 is a scavenger receptor expressed by monocytes and macrophages, which is proteolytically cleaved into soluble CD163 (sCD163) following activation and is increased in the serum of patients with systemic scleroderma and pulmonary fibrosis. Recent data shows that plasma CD163 is raised in active cGVHD and de novo cGVHD patients compared to HSCT patients without cGVHD and healthy controls. Since ECP involves the periodic infusion of pre-apoptotic leukocytes and engulfment of apoptotic cells by macrophages inducing an anti-inflammatory phenotype, we conducted a pilot study to investigate whether ECP affects the production of SCD163, a putative biomarker of in vivo macrophage activation.

Methods: Serum samples were collected from 18 moderate to severe cGVHD patients (14 male $/ 4$ female; age range: $25-74)$ and 8 age-matched healthy controls (4 male / 4 female) before ECP and at 3 month intervals up to 1 year.
Patients had cGVHD affecting skin (17/18), mucosal membranes (6/18), liver (4/18), joints (2/18), gut (2/18), eye (1/18), genital (1/18), but none with respiratory involvement. Serum sCD163 levels were assessed by ELISA. Data were analysed using GraphPad Prism 6. Statistical tests performed include 2-tailed Mann-Whitney and 2-way ANOVA with repeat measures, as appropriate.

Results: cGVHD patients had significantly elevated serum levels of sCD163 ( $\mathrm{P}=0.0343$; median of 799ng/ $\mathrm{ml}$, IQR 504-1159) compared to healthy controls (median of 386ng/ml, IQR 301-1011). Sub-analysis was performed by grouping patients according to whether levels were above $\left(\mathrm{sCD} 163^{\mathrm{hi}}\right)$ or below $\left(\mathrm{sCD} 163^{\text {lo }}\right)$ the median level of sCD163 at pre-ECP baseline. Retrospective analysis revealed that $\mathrm{sCD} 163^{\mathrm{hi}} \mathrm{cGVHD}$ patients had a significant reduction in serum sCD163 levels after 3 months $(\mathrm{p}<0.01$; mean +/- SD: $1218 \mathrm{ng} / \mathrm{ml}+/-318 \mathrm{ng} / \mathrm{ml}$ vs $857 \mathrm{ng} / \mathrm{ml}+/$ $299 \mathrm{ng} / \mathrm{ml}$, respectively), which was sustained up to 6 months of ECP $(\mathrm{P}<0.05$; mean $+/$ - SD: $924 \mathrm{ng} / \mathrm{ml}+/$ $355 \mathrm{ng} / \mathrm{ml}$ ). In contrast, $\mathrm{sCD} 163^{\mathrm{lo}}$ patients (mean $+/-\mathrm{SD}$ : $534 \mathrm{ng} / \mathrm{ml}+/-121 \mathrm{ng} / \mathrm{ml}$ ) showed no significant change in serum sCD163 levels over the same period. Although glucocorticoids strongly regulate CD163 expression, there was no significant difference in prednisolone dosages between the $\mathrm{sCD} 163^{\mathrm{lo}}$ and $\mathrm{sCD} 163^{\text {hi }}$ patient groups at pre-ECP baseline (mean +/-SD: $31 \mathrm{mg} / \mathrm{d}+/-15 \mathrm{mg} / \mathrm{d}$ vs $20 \mathrm{mg} / \mathrm{d}+/-16 \mathrm{mg} / \mathrm{d}$, respectively). Further, the reduction in sCD $163^{\text {hi }}$ patients at month 3 was independent of significant reduction in immunosuppression by prednisolone (mean +/- SD: $14 \mathrm{mg} / \mathrm{d}+/-9 \mathrm{mg} / \mathrm{d}$ ).

Conclusions: Consistent with recent data, we found that sCD163 is significantly elevated in cGVHD patients compared to healthy controls. Further, the data suggests that ECP therapy reduces in vivo macrophage activation as measured by sCD163 levels in a subset of sCD163 ${ }^{\text {hi }}$ cGVHD patients independent of a reduction in glucocorticoid immunosuppression. These preliminary results may provide insight into a possible macrophage-mediated mechanism of ECP that requires further investigation.

Clinical Trial Registry: N/A

Conflict of interest:

C. Burton and N. Matthews: Nothing to disclose

A. Alfred: speaker fees from Mallinckrodt

\section{P353}

Effect of new drugs in the treatment of bone marrow transplantion: severe complications in a child affected by myelodiplastic syndrome

Fulvio Porta ${ }^{1}$, Elena Soncini ${ }^{2}$, Giulia Carracchia ${ }^{2}$, Fiammetta Zunica $^{3}$, Marianna Maffeis ${ }^{4}$, Ilaria Fracassetti ${ }^{5}$, Elisa Cavalleri ${ }^{5}$, Arnalda Lanfranchi ${ }^{6}$ 
${ }^{1}$ Pediatric Oncohaematological and BMT Unit, Childrens Hospital Chair of Paediatrics University of Brescia, Brescia, Italy; ${ }^{2}$ Pediatric Oncohaematological and BMT Unit, Childrens Hospital University of Brescia, Brescia, Italy; ${ }^{3}$ Pediatric Childrens Hospital, University of Brescia, Brescia, Italy; ${ }^{4}$ Childrens Hospital Brescia, Brescia, Italy; ${ }^{5}$ Childrens Hospital University of Brescia, Brescia, Italy; ${ }^{6}$ Stem Cell Laboratory, Section of Hematology and Blood Coagulation, Children's Hospital, Spedali Civili of Brescia, Brescia, Italy

Background: Heterozygous familiar o sporadic GATA 2 mutations consist of a wide spectrum of phenotypes including cytopenias, myelodysplasia, myeloid leukemia, viral and bacterial infections, pulmonary alveolar proteinosis and lymphedema. We describe the case of a 9 years old child with Kosovar origins, which has been diagnosed a GATA 2 mutation (c 1081C >T; p. R361C) manifested with symptoms like persistent anemia, leukopenia and fever.

Methods: He urgently underwent haploidentical peripheral blood stem cells from father using BusulfanMelphalan-Cyclophosfamide conditioning regimen. The patient subsequently had a graft failure with persistent aplasia. So, after conditioning with Fludarabin-ThiotepaATG-Cyclophosfamide and TBI 200 Rads, he underwent a second haploidentical transplant with maternal bone marrow stem cells. Engraftment was reached at day +18 . From day +20 the patient started to have diffuse bleeding with petechiae and hematomas, mane and hematic vomits, clinical manifestations of thrombotic microangiopathy (TMA). He started octreotide and Eculizumab (anti-C5 monoclonal antibody) once/week and daily transfusions of platelet, erythrocytes and plasma with good response. Since day +40 he had increase levels of bilirubina and liver enzymes; investigations demonstrated an hepatic GVH grade III. For this reason he has been subjected to sessions of extracorporeal photopheresis and high dose of corticosteroids without no significant results.During hematological reconstitution the patient also had a CMV reactivation treated with Gancyclovir and CMV Immunoglobulins and a hemorragic cystitis from Polyomavirus. The patient in July 2017 also developed related steroid side effects such as osteopenia with pathological fracture of the tibia and osteonecrosis. He stopped treatment with Eculizumab 5 months later and Ruxolitinib 8 months later.

Results: Actually the patient is in good health one year after the second blood marrow transplant.

Conclusions: This case illustrated the benefit of Eculizumab therapy in a severe TMA after blood marrow transplantation resistant to classical therapy, furthermore the use of Ruxolitinib is a salvage therapeutic agent in patient with steroid refractory acute GVHD.

Conflict of interest: The authors have no conflicts of interest to declare.
P354

\section{Efficacy of Etanercept in patients with steroid refractory acute graft versus host disease}

Chun Kei Kris $\mathrm{Ma}^{1}$, Irene García-Cadenas ${ }^{2}$, Laura Fox ${ }^{3}$, Sylvia Ai $^{1}$, Ian Nivison-Smith ${ }^{1}$, Samuel Thomas Milliken ${ }^{1}$, Anthony Dodds ${ }^{1}$, Keith Fay ${ }^{1}$, David D F Ma ${ }^{1}$, Rodrigo Martino ${ }^{2}$, Jorge Sierra ${ }^{2}$, John Moore $^{1}$

${ }^{1}$ St Vincent's Hospital Sydney, Haematology, Sydney, Australia; ${ }^{2}$ Hospital de la Santa Creu i Sant Pau, IIB Sant Pau and Jose Carreras Leukemia Research Institutes, Universidad Autónoma de Barcelona, Haematology, Barcelona, Spain; ${ }^{3}$ Hospital Universitario Vall d'Hebrón, Haematology, Barcelona, Spain

Background: Steroid refractory acute graft versus host disease (aGVHD) has a poor prognosis. Etanercept is a potentially effective treatment strategy for this condition, but to date, only small studies are available. Here, we present the results of a large cohort of patients affected with grade II-IV steroid refractory aGVHD who received this monoclonal antibody in 3 different centres in Sydney and Barcelona.

Methods: Allogeneic stem cell transplant recipients diagnosed with moderate or severe steroid refractory aGVHD between April 2006 to June 2016 were included in the analysis. Outcome measures include overall response rate on day +28 and +60 , median survival, infectious complications and cause of death. Statistical analyses include Kaplan-Meier survival curves and Cox-regression analysis, in a univariate and multivariate model to identify independent predictors of response to Etanercept and survival.

Results: Fifty-eight patients, with a median age of 51 years (range: 18-70) were included in the study. Median follow-up for survivors was 26.6 months. Overall response rate at days +28 and +60 was $36 \%$ (CR 22\%, PR 14\%) and $47 \%$, (CR 29\%, PR 17\%) respectively. Median survival was 3.9 months (52 months for day-60 responders and 2.4 months for non-responders respectively, $\mathrm{p}<0.0001$ ), with a 1-year overall survival of $34 \%$ (65\% for responders at day-60 and $6.7 \%$ for non-responders, $\mathrm{p}=<0.0001$ ). Factors associated with response to etanercept were: grade IV acute GVHD (HR 0.27, p=0.003) and low serum albumin level at etanercept start (HR 0.30, p =0.004). Factors adversely associated with overall survival were: grade IV acute GVHD (HR 2.07, $\mathrm{p}=0.03$ ) and nonresponse to etanercept (HR 5.66, $\mathrm{p}<0.001$ ).

Conclusions: Etanercept has a modest effect on moderate to severe steroid refractory aGVHD leading to around 50\% short term responses, however long term outcomes remain poor with only one third of long term survivors. These results highlight the need to identify effective therapies in this setting. 
Conflict of interest: J. Sierra: AGAUR 2014 SGR 1281 and PERIS SLT002/16/00433 from the Catalan Government; RD12/0036/0071 and PI17/01246 from the Instituto de Salud Carlos III, Ministerio de Economía y Competitividad, Spain. The other authors have nothing to disclose.

\section{P355}

Extracorporeal Photopheresis (ECP) Therapy with UVADEX ${ }^{\circ}$ (methoxsalen) Improved Quality of Life (QoL) for National Institutes of Health (NIH)-graded Moderate to Severe Chronic Graft-vs-Host Disease (cGvHD)

Madan Jagasia ${ }^{1}$, Hildegard Greinix ${ }^{2}$, Christoph Scheid ${ }^{3}$, Gérard Socie $^{4}$, Francis Ayuk ${ }^{5}$, Johanna Tischer, Michele Donato ${ }^{7}$, Arpád Bátai $^{8}$, Heidi Chen', Sheau-Chiann Chen ${ }^{9}$, Thomas Chin ${ }^{10}$, Henri Boodée $^{10}$, Ghaith Mitri ${ }^{11}$

${ }^{I}$ Vanderbilt-Ingram Cancer Center, Nashville, TN, United States; ${ }^{2}$ Medical University of Graz, Graz, Austria; ${ }^{3}$ University of Cologne, Köln, Germany; ${ }^{4}$ Hospital St. Louis and University Paris 7, Paris, France; ${ }^{5}$ UKE - Universitätsklinikum Hamburg-Eppendorf, Hamburg, Germany; ${ }^{6}$ University Hospital Munich, LMU Munich, München, Germany; ${ }^{7}$ Hackensack University Medical Center, John Theurer Cancer Center, Hackensack, NJ, United States; ${ }^{8}$ Unified St. Istvan and St. Laszlo Hospital, Budapest, Hungary; ${ }^{9}$ Vanderbilt University Medical Center, Nashville, TN, United States; ${ }^{10}$ Mallinckrodt Pharmaceuticals, Bedminster, NJ, United States; ${ }^{11}$ Formerly of Mallinckrodt Pharmaceuticals, Bedminster, NJ, United States

Background: Moderate to severe cGvHD as defined by $\mathrm{NIH}$ criteria, requiring systemic therapy is associated with impaired QoL. The phase I/pilot prospective randomized, multicenter international study (NCT01380535) confirmed that addition of ECP to standard of care $(\mathrm{SoC})$ (calcineurin inhibitor + corticosteroid) is safe and well tolerated in patients (pts) with cGvHD. ${ }^{1}$ We hypothesized that $\mathrm{SoC}$ would be associated with worsening QoL compared with $\mathrm{ECP}+\mathrm{SoC}$.

Methods: Sixty adult pts with new-onset moderate $(52.8 \%, \mathrm{n}=28)$ or severe $(47.2 \%, \mathrm{n}=25) \mathrm{cGvHD}$ requiring systemic therapy received 26 weeks of SoC vs SoC + ECP. Baseline severity of GvHD (investigatorÂ's assessment of overall status of cGvHD) was similar in the two groups [ $\mathrm{SoC}$ and $\mathrm{SoC}+\mathrm{ECP}$, moderate $45.8 \%(\mathrm{n}=11)$ and 58.6\% $(\mathrm{n}=17)$; severe: $54.2 \%(\mathrm{n}=13)$ and $41.4 \%(\mathrm{n}=$ 12)]. Assessment of QoL using the Functional Assessment of Cancer Therapy-Bone Marrow Transplantation (summation of FACT-G \{well-being [WB] domains including physical $[\mathrm{PWB}\}$, social $[\mathrm{SWB}]$, emotional [EWB] and functional [FWB] , plus BMT specific [BMTS]), and SF-36 tools was a secondary endpoint. BMT Trial Outcome Index (TOI) was calculated as the sum of PWB, EWB and BMTS. Pts completed QoL surveys at baseline, 12, and 28 Weeks, respectively. QoL at various time points, was measured using time trend analyses. Ordinal logistic regression was used to study the association of organ specific score with QoL.

Results: Of the 60 pts, 53 (29, SoC + ECP; 24, SoC) were eligible for QoL assessment. A time trend analysis revealed significant worsening of QoL in PWB (-0.7326; $P=0.0315)$, EWB $(-0.7151 ; P=0.0057)$, and FACT-G $(-1.6618 ; P=0.0183)$ scores in the SoC arm, while no significant changes in any QoL domains were observed in SoC + ECP pts. The FACT-BMT Total score approached significance in favor of SoC + ECP $(-1.7842, P=0.057)$. QoL in SF-36 scores for the bodily pain domain significantly worsened by $-2.3728(P=0.0093)$ in SoC pts, while no significant changes were observed in $\mathrm{SoC}+$ ECP pts. For both the primary assessor and a blinded assessor, increased NIH cGvHD scores were associated with worsened scores in several QoL domains, indicating cross-validity of the FACT-BMT QoL tool in cGvHD studies. Organ domains associated with QoL worsening included skin (EWB, BMTS, BMT total, BMT TOI), gastrointestinal (PWB, BMTS, FACT BMT total, BMT TOI), eyes (SWB, EWB, FWB, FACTG, BMTS, FACT BMT total, BMT TOI), joints/fascia (PWB, SWB, EWB, FWB, FACTG, FACT BMT total, BMT TOI), and lung (PWB, SWB, EWB, FWB, FACTG, FACT BMT total, BMT TOI). In this limited cohort, changes in steroid dose were not associated with change in QoL as measured by the instruments used.

Conclusions: This is the first prospective, randomized therapeutic clinical trial to assess the impact of ECP + SoC on various QoL domains using established tools like FACTBMT and SF-36. Worsening in organ specific NIH scores across multiple organ domains is significantly associated with worsening QoL scores. For pts with moderate to severe cGvHD, QoL is maintained by ECP, while the current SoC is associated with worsening in QoL, notably in the domains mentioned above.

Clinical Trial Registry: Clinical Trial Registry: NCT01380535

https://clinicaltrials.gov/ct2/show/NCT01380535?term= NCT01380535\&rank $=1$

Conflict of interest:

M. Jagasia: speaker bureau honoraria and research support from Mallinckrodt Pharmaceuticals, speaker bureau honoraria from Pharmacyclics, Inc., research support from Janssen ;

H. Greinix: speaker bureau honoraria from Mallinckrodt Pharmaceuticals, Novartis, Amgen, Celgene, Sanofi, Bristol-Myers Squibb;

C. Scheid: honoraria from Amgen, Bristol-Myers Squibb, Celgene, Janssen, Novartis, Sanofi;

G. Socié: speaker bureau honoraria from Therakos, Inc., a Mallinckrodt Pharmaceuticals Company; 
F. Ayuk: speaker bureau honoraria from Therakos Inc., a Mallinckrodt Pharmaceuticals Company;

J. Tischer, M. Donato, Á. Bátai, H. Chen and S. Chen: nothing to disclose;

T. Chin and H. Boodée: employees of Mallinckrodt Pharmaceuticals; G. Mitri: former employee of Mallinckrodt Pharmaceuticals.

\section{P356}

\section{Extracorporeal Photopheresis for Graft versus Host Disease in Pediatric Patients: A Pilot Single Institution Report}

Fatma Visal Okur, Baris Kuskonmaz, Nevin Cetin, Aysegul Demirsu, Ozan Ali Tasdemir, Duygu Uckan Cetinkaya

Hacettepe University, Faculty of Medicine, Pediatrics, Ankara, Turkey

Background: Graft versus host disease (GvHD) remains as a clinical challenge despite aggressive first-line treatments and limits the success of allogeneic hemotopoietic stem cell transplantation. Since children are more vulnerable to long-term effects of GVHD and of prolonged treatment with steroids and other immunosuppressive agents, extracorporeal photopheresis (ECP) could be an attractive steroid-sparing second-line therapy. ECP is a cellbased therapy inducing tolerance of donor T lymphocytes to host antigens. However apheresis in children is associated with unique challenges, which restricts its use.

Methods: We retrospectively analyzed nine patients treated with ECP for steroid-dependent or refractory acute and chronic GVHD from 2006 to 2017. An "off-line" approach was performed using a continuous flow cell separator (Spectra Optia Terumo BCT) and an Ultraviolet A irradiator (UVA-PIT system). This system offers advantages in children, having smaller extracorporeal volume and reduction of final collection volume. Citrate anticoagulation at a blood to anticoagulation ratio of 10:1 was used. Hematocrit $>20 \%$ and platelet count $>50,000 / \mu \mathrm{L}$ were desirable to start leukapheresis. Red blood cell priming of circuit was performed for low-body weight children $(<20$ $\mathrm{kg})$. Double-lumen central catheters were placed prior to procedure. Schedule and duration of ECP treatment and patients' characteristics were given in Table 1 .

Results: Among three acute GVHD patients, complete response was observed in two patients and one having partial response was died due to infection during treatment. One patient with overlap syndrome showed minimal response with stable disease when he died from infection. In the chronic GVHD group, three patients achieved partial response, and one was in complete response. One patient could not be evaluated since he died at the early phase of treatment. In all living patients concomitant

\begin{tabular}{|c|c|c|c|c|c|c|c|c|}
\hline $\begin{array}{l}\text { Patient } \\
\text { ID }\end{array}$ & $\begin{array}{l}\text { Primary } \\
\text { disease }\end{array}$ & $\begin{array}{l}\text { Age / } \\
\text { body } \\
\text { weight }\end{array}$ & $\begin{array}{l}\text { Donor } \\
\text { type }\end{array}$ & $\begin{array}{l}\text { Stem } \\
\text { cell } \\
\text { source }\end{array}$ & GVHD & Treatment of GVHD & $\begin{array}{l}\text { Schedule of ECP ( } 1 \\
\text { cycle }=2 \text { consecutive days/ } \\
\text { week })\end{array}$ & Number of cycles \\
\hline P1 & Thalassemia & $\begin{array}{l}3 \mathrm{yr} / \\
13.5 \mathrm{~kg}\end{array}$ & MRD & $\mathrm{BM}$ & chronic & $\begin{array}{l}\text { FK-506 steroid, MMF } \\
\text { ECP imatinib }\end{array}$ & $\begin{array}{l}\text { one cycle } / 1-2 \text { wk for } 8-12 \\
\text { wk, tapered as one cycle/2- } \\
4 \text { wk }\end{array}$ & $\begin{array}{l}\text { Number of cycles } 19 \text { cycles in } \\
\text { first attack } 29 \text { cycles in } 2 \text { nd, } \\
\text { ongoing }\end{array}$ \\
\hline $\mathrm{P} 2$ & CGD & $\begin{array}{l}4 \mathrm{yr} / \\
15.4 \mathrm{~kg}\end{array}$ & MRD & PBSC & chronic & $\begin{array}{l}\text { FK-506 steroid MMF, } \\
\text { ECP }\end{array}$ & $\begin{array}{l}\text { one cycle } / 1-2 \text { wk for } 8-12 \\
\text { wk, tapered as one cycle/2- } \\
4 \text { wk }\end{array}$ & 18 cycles \\
\hline P3 & JMML & $\begin{array}{l}7 \mathrm{yr} / 17 \\
\mathrm{~kg}\end{array}$ & MRD & PBSC & chronic & $\begin{array}{l}\text { FK-506 steroid MMF } \\
\text { imatinib ECP }\end{array}$ & $\begin{array}{l}\text { one cycle } / 1-2 \text { wk for } 8-12 \\
\text { wk, tapered as one cycle/2- } \\
4 \text { wk }\end{array}$ & 67 cycles \\
\hline P4 & ALL & $\begin{array}{l}16 \mathrm{yr} / \\
45 \mathrm{~kg}\end{array}$ & MRD & $\mathrm{BM}$ & $\begin{array}{l}\text { overlap } \\
\text { syndr. }\end{array}$ & $\begin{array}{l}\text { FK-506 steroid MMF } \\
\text { MSC etanercept, ECP } \\
\text { rituximab }\end{array}$ & $\begin{array}{l}\text { one cycle } / 1-2 \text { wk for } 8-12 \\
\text { wk, tapered as one cycle/2- } \\
4 \text { wk }\end{array}$ & 67 cycles \\
\hline P5 & FAA-AML & $\begin{array}{l}15 \mathrm{yr} / \\
46 \mathrm{~kg}\end{array}$ & Haplo & PBSC & acute & $\begin{array}{l}\text { CSA steroid,MMF MSC } \\
\text { etanercept }\end{array}$ & $\begin{array}{l}\text { one cycle/ wk for } 8 \mathrm{wk} \text {, } \\
\text { tapered acc. to response }\end{array}$ & 8 cycles (exitus) \\
\hline P6 & Thalassemia & $\begin{array}{l}21 \mathrm{yr} / \\
60 \mathrm{~kg}\end{array}$ & MRD & $\mathrm{BM}$ & acute & $\begin{array}{l}\text { CSA steroid MMF } \\
\text { MSC,ECP etanercept }\end{array}$ & $\begin{array}{l}\text { one cycle/ wk for } 8 \text { wk, } \\
\text { tapered acc. to response }\end{array}$ & 12 cycles, ongoing \\
\hline P7 & ALL & $\begin{array}{l}16 \mathrm{yr} / \\
49 \mathrm{~kg}\end{array}$ & MRD & $\mathrm{BM}$ & acute & $\begin{array}{l}\text { CSA steroid MMF } \\
\text { MSC,ECP etanercept }\end{array}$ & $\begin{array}{l}\text { one cycle/ wk for } 8 \mathrm{wk} \text {, } \\
\text { tapered acc. to response }\end{array}$ & 11 cycles, ongoing \\
\hline P8 & AML & $\begin{array}{l}21 \mathrm{yr} / \\
38 \mathrm{~kg}\end{array}$ & MUD & $\mathrm{BM}$ & chronic & $\begin{array}{l}\text { CSA steroid MMF } \\
\text { MSC,ECP etanercept }\end{array}$ & $\begin{array}{l}\text { one cycle } / 1-2 \text { wk for } 8-12 \\
w k \text {, tapered as one cycle/2- } \\
4 \text { wk }\end{array}$ & 17 cycles \\
\hline P9 & AML & $\begin{array}{l}14 \mathrm{yr} / \\
46 \mathrm{~kg}\end{array}$ & MRD & $\mathrm{BM}$ & chronic & $\begin{array}{l}\text { FK-506, imatinib } \\
\text { steroid, MMF }\end{array}$ & $\begin{array}{l}\text { one cycle } / 1-2 \text { wk for } 8-12 \\
w k \text {, tapered as one cycle/2- } \\
4 \text { wk }\end{array}$ & 6 cycles (exitus) \\
\hline
\end{tabular}

[[P356 Table] Characteristics of children treated with ECP] 
immunosuppressive therapies were considerably reduced. Most common adverse effect was catheter-related infections leading treatment interruptions.

Conclusions: ECP might be a safe, effective and welltolerated treatment in children with refractory acute and chronic GVHD. Large-scale prospective studies are needed to establish optimal schedule and duration of ECP. Standardized treatment protocols for pediatric patients with GVHD will help to reduce therapy-related adverse effects.

Clinical Trial Registry: NA

Conflict of interest: nothing to disclose

\section{P357}

\section{Extracorporeal photopheresis for steroid-refractory acute and chronic GVHD: a single center experience}

Javier Carrillo Checa, Miriam López Parra, Olga López, Villar, Alejandro Avendaño Pita, Daniel Esteban Rivera Delgado, Ana África Martín López, Estefania Pérez López, Mónica Cabrero Calvo, Lourdes Vázquez López, María Dolores Caballero Barrigon, Lucía López Corral

Complejo Asistencial Universitario de Salamanca-IBSAL, Hematology, Salamanca, Spain

Background: GVHD is the most common complication after alloSCT. Extracorporeal photopheresis (ECP) is a based inmune-modulatory therapy and is emerging as one of the most used salvage treatment due to a high rate of response with an excellent safety profile.

Methods: We reported a single center retrospective study of 82 patients with steroid-refractory or dependent acute or chronic GVHD treated with ECP (Cellex system) between january-2010 to november-2017.

Patients with aGVHD, received 2 consecutives sessions/ week during weeks 1 to 8 , and then 2 sessions/fortnight, while the protocol for cGHVD was 2 sessions/fortnight during 12 weeks and then 2 sessions/month. ECP was finalized when steroids were stopped or progression of GVHD.

Results: Baseline, transplant characteristics and GVHD data are shown in table 1.

aGHVD ( $\mathrm{n}=33)$ : median time from aGVHD to ECP was 19 days (5-197). 18 had grade IV aGVHD. Skin was the most frequent organ involved $(90.9 \%)$, and $75.8 \%$ had multiorgan disease. Median prior lines was 1 (0-6). ORR was $72,7 \%$ (CR:51.5\%). All but one patient with grade II-III aGVHD responded $(92.8 \%$ ), while 8 out of 18 patients (44\%) with grade IV did not respond. Best responses were achived in skin damage (organ response was skin (ORR: 96.2\%, CR: $80.8 \%$ ), followed by gut (ORR:60\% CR:40\%) and liver (ORR:53.8\% CR:30.7\%). After a median followup after ECP of 31 months (5-197), OS after ECP at 1 and 2 years was $78.4 \%$ and $47.5 \%$, respectively. CR after ECP was favourable predictive factor of OS (median OS not reached vs 5 months, $\mathrm{p}=0.03$ ) while grade IV aGVHD patients had an adverse outcome (median OS not reached vs 7 months, $\mathrm{p}=0.04$ ).

cGVHD ( $\mathrm{n}=49)$ : median time from cGVHD to ECP was 212 (9-1679). 25 patients had moderate and 24 severe cGVHD. Principal organs involved were skin and mucosas. Median prior lines was $2(1-4)$. ORR was $69 \%$ (CR:24.4\%). Responses of moderate cGVHD was superior $(80 \%)$. Best organ responses were observed in oral mucosa

\begin{tabular}{|c|c|c|}
\hline \multirow[t]{2}{*}{ Characteristics } & \multicolumn{2}{|c|}{ All patients $(\mathrm{n}=82)$ Median (range) $/ \mathrm{n}(\%)$} \\
\hline & aGVHD $(n=33)$ & cGVHD $(n=49)$ \\
\hline Age at trasplant /male patients & $48(11-71) / 22(66.7 \%)$ & $55(9-69) / 26(54 \%)$ \\
\hline $\begin{array}{l}\text { Diagnosis: AML or MDS / ALL / Lymphoma / } \\
\text { Others }\end{array}$ & $\begin{array}{l}11(33.3 \%) / 10(30.3 \%) / 4 \\
(12.1 \%) / 8(24,2 \%)\end{array}$ & $24(49 \%) / 3(6 \%) / 7(14 \%) / 15(30 \%)$ \\
\hline $\begin{array}{l}\text { HLA identical related / HLA identical unrelated / } \\
\text { Haploidentical / mismatched unrelated donor }\end{array}$ & $\begin{array}{l}5(15.1 \%) / 13(39.5 \%) / 5 \\
(15.1 \%) / 10(30.3 \%)\end{array}$ & $25(51 \%) / 11(48 \%) / 1(2 \%) / 12(25 \%)$ \\
\hline $\begin{array}{l}\text { Stem cell source: } \mathrm{PB} / \mathrm{BM} / \mathrm{SCU} \text { and } \\
\text { Conditioning regimen: myeloablative/reduced- } \\
\text { intensity }\end{array}$ & $\begin{array}{l}30(90.9 \%) / 2(6.1 \%) / 1(3 \%) \\
\text { and } 20(60.6 \%) / 13(39.4 \%)\end{array}$ & $47(96 \%) / 2(4 \%) / 0$ and $36(73.5 \%) / 13(26.5 \%)$ \\
\hline $\begin{array}{l}\text { GVHD prophylaxis: Tacrolimus + MTX / } \\
\text { Tacrolimus + Sirolimus / Others }\end{array}$ & $\begin{array}{l}16(48.5 \%) / 11(33 \%) / 6 \\
(18.5 \%)\end{array}$ & $26(53 \%) / 17(34.6 \%) / 6(11 \%)$ \\
\hline $\begin{array}{l}\text { Median time (months) from GVHD to ECP / } \\
\text { Grade of GVHD }\end{array}$ & $\begin{array}{l}19 \text { (5-197) / II: } 12(36 \%), \text { III: } 3 \\
(9.1 \%), \text { IV: } 18(54.5 \%)\end{array}$ & 212 (9-1679) / Moderate: 25 (51\%), Severe: 24 (49\%) \\
\hline $\begin{array}{l}\text { Number of organs involved / Organs involved at } \\
\text { ECP treatment }\end{array}$ & $\begin{array}{l}2(1-3) \text { / skin: } 30 \text { (90.9\%), gut: } \\
25(75.8 \%) \text {, liver: } 13(39.4 \%)\end{array}$ & $\begin{array}{l}3 \text { (1-4) / Skin: } 38 \text { (77.5\%), sclerodermiform: } 25(51 \%) \text {, } \\
\text { Mouth: } 32 \text { (65.3\%), eyes: } 25 \text { (51\%), liver: } 11(22.4 \%)\end{array}$ \\
\hline $\begin{array}{l}\text { Overlap Syndrome /Progresive GVHD / Steroids } \\
\text { refractory / dependent }\end{array}$ & $0 / 0 / 28(84.8 \%) / 5(15.2 \%)$ & $4(8 \%) / 5(10 \%) / 43(87.7 \%) / 6(10 \%)$ \\
\hline
\end{tabular}

[[P357 Table] Table 1] 
(ORR:84\%) and skin (ORR:80\%). After a median followup of 29 months (3-96), OS after ECP at 1 and 2 years was $81.2 \%$ and $78.3 \%$. The majority of patients could stop steroids. 3 and 4 patients reactivated aGVHD or cGVHD during ECP, the majority of them responded again with intensification.

Conclusions: ECP is an effective and safe salvage therapy for GVHD, especially in grade IV aGVHD and moderate cGVHD, and allows to stop steroids. Best organ responses are skin in aGVHD and skin and mucosa in cGVHD.

Conflict of interest: Nothing to disclose

\section{P358}

First report of long lasting expansion of $\mathrm{CD} 3{ }^{+} \mathrm{CD} 56^{+}$ NKT cells after anti-cytokine treatments in a patient with steroid-refractory graft-versus-host disease

Lixia Sheng ${ }^{1,2}$, Huarui $\mathrm{Fu}^{1}$, Yanmin $\mathrm{Tan}^{1}$, Yongxian $\mathrm{Hu}^{1}$, Yi Luo ${ }^{1}$, Jianmin Shi ${ }^{1}$, Zhen Cai ${ }^{1}$, Guifang Ouyang ${ }^{2}$, He Huang ${ }^{1}$

${ }^{I}$ The First Affiliated Hospital, Zhejiang University School of Medicine, Hangzhou, China; ${ }^{2}$ Ningbo No.1 Hospital, Ningbo, China

Background: In our previous study the combined anticytokine therapy with basiliximab and etanercept achieved a promising response in patients with steroid-refractory acute graft-versus-host disease(SR-aGVHD). However, the in vivo immune changes following the treatment have not been elucidated.

Methods: A 14-year-old boy with relapsed acute lymphocytic leukemia underwent HLA-haploidentical peripheral blood stem cell transplantation from his mother in the phase of CR2. On +20 day he developed grade 3 aGVHD with skin and gastrointestinal involvement and refractory to steroid treatment. Thereafter, second line treatment with basiliximab and etanercept was prescribed and achieved a complete response. We performed the immunophenotyping, interferon gamma(IFN-- $\gamma$ ) production and cytotoxicity assay of peripheral blood lymphocytes by flow cytometry before and after the combined anti-cytokine treatment. Serum cytokines IL-2, IFN-- $\gamma$, IL-15 were detected by ELISA.

Results: We detected a gradual rise of the cd3 + cd56+ NKT cells after the control of GVHD and STR test showed the expanded NKT cells were of donor origin. The cytokines IFN- $\gamma$ increased at time of aGVHD did not decreased to baseline, but increased again at 4 weeks and reached it's second peak at 8 weeks after treatment. Simultaneously, we found NK, NKT-like and T cells from the patients all produced high level of IFN-gamma. Furthermore, CD3+CD56+ NKT-like cells showed preferred proliferation in response to IFN- $\gamma$. Further analysis showed the expanded NKT like cells were predominately positive for CD8, expressing KIRs, NKG2D, DNAM-1, NKP46,CD27, CD69 and PD-1, while negative for NKG2A, CTLA-4 and TIM-3, and showed potent cytotoxicity against leukemia cells. The expansion of $\mathrm{cd} 3+\mathrm{cd} 56$ + nkt cells lasted 2 years and the patient had leukemia free survival of 61 monthes after HSCT at the time reported.

Conclusions: In this case the increased serum IFN- $\gamma$ caused by the cytokines storm in GVHD and the priority of NKT cells proliferating in response to IFN- $\gamma$ may led to the unusal expansion of NKT cells after the immunecontraction caused by basiliximab and etanercept. The clinical course suggests that the combined anti-cytokine treatments may reset the immune system and the innate immunity cells got the superiority of rapid expansion, which may contribute to the control of GVHD and leukemia as well.

Conflict of interest: The authors have no conflicting financial interests.

\section{P359}

Galectin-3 Levels Are Associated with the Incidence and Severity of Chronic GVHD One Year after Allogeneic Hematopoietic Cell Transplantation

Nalu Navarro-Alvarez ${ }^{1,2}$, Alec R Andrews ${ }^{1}$, Zachariah DeFilipp ${ }^{1}$, YiBin A Chen ${ }^{3}$, Vincent T Ho ${ }^{4}$, Jerome Ritz ${ }^{2,4}$, Thomas R Spitzer ${ }^{2,5}$, Christene A Huang ${ }^{1,2}$

${ }^{I}$ Massachusetts General Hospital, Center for Transplantation Sciences, Boston, MA, United States; ${ }^{2}$ Harvard Medical School, Boston, MA, United States; ${ }^{3}$ Massachusetts General Hospital, Blood and Marrow Transplant Program, Boston, MA, United States; ${ }^{4}$ DanaFarber Cancer Institute, Hematologic Malignancies, Boston, MA, United States; ${ }^{5}$ Massachusetts General Hospital, Hematology/ Oncology, Boston, MA, United States

Background: Chronic graft-versus-host disease (cGVHD) is a frequent complication of allogeneic hematopoietic cell transplantation (HCT) and a leading cause of late morbidity and mortality. What was previously believed to be a $\mathrm{T}$ cell mediated disease has more recently been shown to involve activation of both $\mathrm{T}$ and $\mathrm{B}$ cells, as well as several inflammatory mediators and cytokines. Galectin-3 (Gal-3) is known to modulate T-cell proliferation and B cell fate and has been shown to play a role in systemic inflammation and fibrosis. We measured plasma Gal-3 concentrations at 1 year post-HCT and correlated these results with the presence of cGVHD.

Methods: We retrospectively studied 69 patients who underwent HCT between 2005 and 2014. In an initial analysis, we determined the levels of Gal-3 in 7 healthy controls vs. patients who underwent HCT. Subsequently, we analyzed Gal-3 levels according to development of 


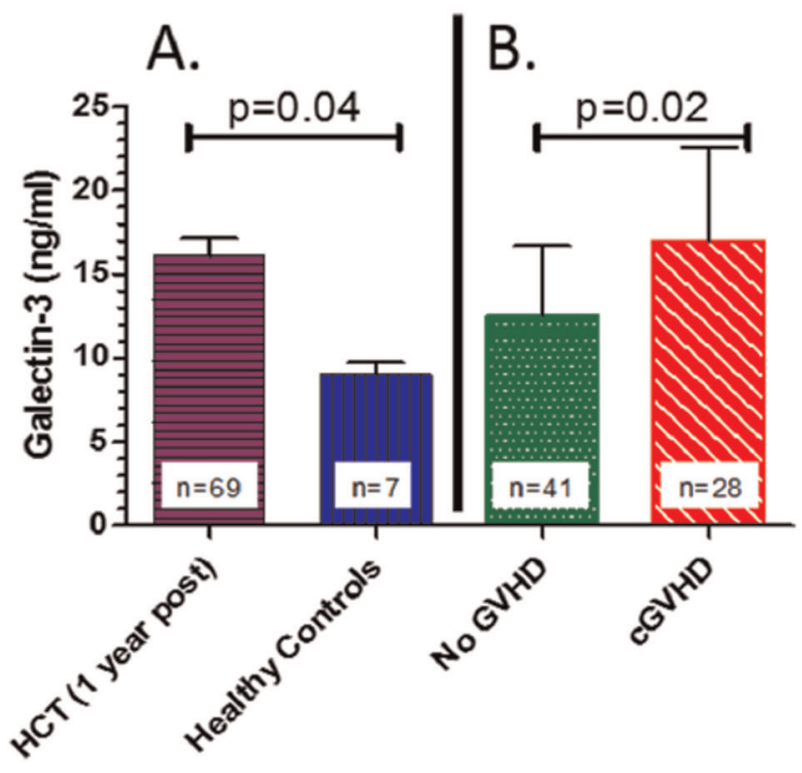

[P359 Figure] [Galectin-3 levels 1 year post- HCT]

cGVHD, and correlated plasma levels with the severity and organ involvement of cGVHD.

Results: Gal-3 levels were significantly higher in HCT patients compared to healthy controls (median $=14.6,8.21$ $\mathrm{ng} / \mathrm{ml}$ respectively, $\mathrm{p}=0.04$ ) (Figure $1 \mathrm{~A}$ ). Among HCT patients, Gal-3 levels were also significantly higher in patients who developed cGVHD $(n=28)$ compared with those with no cGVHD $(\mathrm{n}=41) 1$ year post-HCT (median $=$ $17,12.55 \mathrm{ng} / \mathrm{ml}, \mathrm{p}=0.02$ ) (Figure $1 \mathrm{~B}$ ). Using a cut-off value of $17.8 \mathrm{ng} / \mathrm{ml}$ which is used clinically to predict adverse events in chronic heart failure patients, 48 HCT patients had Gal-3 levels below (range: 5.5-17.6ng/mL; Gal-3-Low) and 21 patients above this cut-off (range: 17.9$61.6 \mathrm{ng} / \mathrm{mL}$; Gal-3-High). Of the 48 Gal-3-Low patients, 15 (31\%) had cGVHD at 1 year post-HCT with $33 \%$ experiencing moderate-to-severe cGVHD over time. Of the 21 Gal-3-High patients, $12(57 \%)$ had cGVHD at 1 year with $47 \%$ experiencing moderate-to severe cGVHD. When analyzed by organ involvement, no clear differences were observed. Among the Gal-3-High patients diagnosed with cGVHD, $80 \%$ presented with multiple organ involvement; individual organ breakdown included skin $(80 \%)$, oral $(60 \%)$, ocular (27\%), liver (60\%) and GI (20\%). Among the Gal-3-Low patients with cGVHD, $71 \%$ experienced multiple organ involvement; individual organ involvement included skin $(70 \%)$, oral $(54 \%)$, ocular $(38 \%)$, liver (63\%), and GI (13\%).

Conclusions: These results demonstrate a significant difference in incidence and severity of cGVHD based on Gal-3 levels at 1 year post-HCT, suggesting a potential role of Gal-3 in the pathobiology of cGVHD. Further investigation will be required to determine whether it can serve as a predictive biomarker for cGVHD in patients undergoing allogeneic HCT.

\section{Conflict of interest:}

C. Huang: nothing to disclose directly relevant to the current study. Related studies involving some company support - EcoNugenics, Inc is supplying a dietary supplement and matched placebo for a clinical trial examining galectin-3 blockade in patients with osteoarthritis. Eliaz Therapeutics, Inc has provided a research gift to examine approaches of removing galectin-3 from circulation in animal models. The other authors have nothing to disclose.

\section{P360}

Gastrointestinal (GI) acute graft versus host disease (aGVHD) and opportunistic viral infections (single center experience)

Ludek Raida ${ }^{1}$, Adam Kuba ${ }^{1}$, Margita Bartkova ${ }^{2}$, Svetlana Brychtova $^{3}$, Ivana Skoumalova ${ }^{1}$

${ }^{1}$ University Hospital and Faculty of Medicine and Dentistry, Palacky University, Dpt. of Hemato-Oncology, Olomouc, Czech Republic;

${ }^{2}$ University Hospital and Faculty of Medicine and Dentistry, Palacky University, Dpt. of Biochemistry, Olomouc, Czech Republic;

${ }^{3}$ University Hospital and Faculty of Medicine and Dentistry, Palacky University, Dpt. of Molecular Pathology, Olomouc, Czech Republic

Background: AGVHD still represents serious early complication of allogeneic stem cell transplantation (alloSCT) associated with significant mortality. Nevertheless, symptoms of aGVHD, particularly its GI form, are not specific. Sometimes they cannot be clinically distinguished from infection and resistance to corticosteroids (CS) has been observed in more than $50 \%$ of cases. Therefore, searching for infections, particularly opportunistic viral ones, has been included into our investigational algorithm used in biopsied involved gut mucosa.

Methods: 37 adult patients allografted for various hematological malignancies developed possible GI symptoms of aGVHD at a median of 40 (range, 12-140) posttransplant days. They underwent a biopsy of rectal/colic mucosa followed by histological and virologic evaluation. Morphology and histopathological grade of gut aGVHD were evaluated by two independent pathologists and PCR analysis of CMV, EBV, HHV6, PVB19 and ADV DNA was included into the complex assessment. Overall clinical grade of aGVHD and resistance to immunosuppressants in the patients treated for aGVHD, including association with the number of viruses detected in gut, were analyzed. Nonrelapse (NRM) and post-transplant overall survival (OS) regarding to immunosuppressants resistance and viral detection in gut were assessed as well. 
Results: No histopathological signs, grade I, II, III and IV of gut aGVHD were found in 9 (24.3\%), 8 (21.6\%), 10 (27\%), $7(18.9 \%)$ and $3(8.1 \%)$ cases, respectively. Skin, liver and the both these systems aGVHD symptoms were concomitantly observed in 10 (27\%), $3(8.1 \%)$ and 18 $(48.6 \%)$ of recipients with GI involvement, respectively. 28 $(75.7 \%)$ patients developed overall clinical manifestation of aGVHD grade III/IV. 24 recipients (64.9\%) also suffered from fever at the onset of suspected aGVHD. There was found no viral DNA in gut mucosa in two cases $(5.4 \%)$ only. On the other hand, CMV, EBV, HHV6 and PVB19 DNA were found in $4(10.8 \%), 12(32.4 \%), 32(86.5 \%)$ and $21(56.8 \%)$ hosts, respectively. Seven (18.9\%) patients (1 with GI stage III, 2 with overall grade III/IV) did not receive any treatment of aGVHD and their symptoms completely resolved on anti-viral therapy and supportive care. All these recipients survive even without chronic GVHD (cGVHD) at the median of 1051 (range, 74-1463) post-transplant days. 18 of treated $30(60 \%)$ patients were refractory to immunosuppressants administered for aGVHD. There was observed some trend to higher number of viruses in gut among these recipients $(\mathrm{p}=0.06)$. Not surprisingly, higher $\mathrm{NRM}$ in refractory patients $(72.2 \%$ vs. $8.3 \% ; \mathrm{p}=0.0007)$ resulted in their worse OS $(11.1 \%$ vs. $91.7 \% ; \mathrm{p}=0.0002)$. However, detection of $\geq 2$ different viral DNA in mucosa was associated with increase risk of NRM $(55.6 \%$ vs. $34.4 \% ; \mathrm{p}=0.001)$ as well as lower OS (38.1\% vs. $65.6 \% ; \mathrm{p}$ $=0.03$ ).

Conclusions: Virologic evaluation (viral DNA detection using PCR technique) of biopsied GI mucosa may be useful in the diagnostics and management of aGVHD, particularly refractory one.

Conflict of interest: Authors declare no conflict of interest.

\section{P361}

\section{G-CSF Mobilized Macrophages Polarization May Prevent Acute Graft-versus-Host Disease after Allogeneic Hematopoietic Stem Cell Transplantation}

Qi Wen ${ }^{1,2}$, Yuan Kong ${ }^{1}$, Hong Yan Zhao ${ }^{1}$, Yuan Yuan Zhang ${ }^{1}$, Ting Ting Han ${ }^{1}$, Yu Wang ${ }^{1}$, Lan Ping $\mathrm{Xu}^{1}$, Xiao Hui Zhang ${ }^{1}$, Xiao Jun Huang ${ }^{1,2}$

${ }^{1}$ Peking University People's Hospital, Peking University Institute of Hematology, Beijing Key Laboratory of Hematopoietic Stem Cell Transplantation, Collaborative Innovation Center of Hematology, Beijing, China; ${ }^{2}$ Nanfang Hospital, Southern Medical University, Guangzhou, China

Background: Macrophage (MФ) is an important population of immune cells. MФs are now classified as M1 (classic) MФs, which produce pro-inflammatory cytokines, and M2 (alternative) MФs, which produce antiinflammatory cytokines. The unbalanced polarization of MФs has been shown to play a critical role in the occurrence and development of disease, such as cancer and diabetes. Therefore repolarization of MФs may be a novel therapeutic option to treat diseases. Allogeneic hematopoietic stem cell transplantation (allo-HSCT) is an effective treatment for malignant hematopoietic diseases. Granulocyte colony-stimulating factor (G-CSF) induced immune tolerance has been shown to play a critical role in allo-HSCT, as G-CSF application in a healthy donor can modulate the balance between Th1/Th2/Th17 cells, promote regulatory $\mathrm{T}$ cell differentiation, as well as affect myeloidderived suppressor cells induction. These cells mentioned above are important factors associated with the occurrence of acute graft-versus-host disease (aGvHD). (Haematologica 2004; Haematologica 2005; Eur J Immunol 2011; Eur J Immunol 2013; Am J Hematol 2015). As the impact of endogenous G-CSF on MФs induction has been described in mouse models. But not in human. However, little is known about the association of $M \Phi$ s subgroups in allografts with the occurrence of aGvHD in patients who underwent allo-HSCT.

Methods: We investigated the effects of G-CSF on the percentages of M1(CD68+CCR2+), M2(CX3CR1 $+\mathrm{CD} 163+)$ МФs both in bone marrow(BM) and peripheral blood(PB) of healthy donors by flow cytometry. Moreover, the effects of G-CSF on MФs function, including DiI-AcLDL uptake, DAPI binding assay and migration assay were investigated in vitro. Subsequently, we evaluated the association of M1/M2 MФs ratio with the occurrence of aGvHD in patients who underwent allo-HSCT.

Results: We found that G-CSF mobilized МФs polarization in both PB and BM. We found a decrease in M1 MФs in the G-CSF mobilized-BM (G-BM, 4 days after G-CSF) and G-CSF mobilized-PB (G-PB, 5 days after G-CSF) compared to the stable-BM (S-BM) and stable-PB (S-PB) pre-G-CSF administration. Moreover, we found that M2 $\mathrm{M} \Phi$ s decreased in the G-PB, whereas a reciprocal increase in M2 MФs in the G-BM. As a result, the M1/M2 MФs ratio was markedly decreased in G-PB and G-BM. After GCSF mobilization, BM MФs showed reduced migration and increased phagocytic activities. Patients who received a higher ratio of M1/M2 MФs infusion exhibited higher incidence of grade II-IV aGvHD.

Conclusions: These findings suggested that a unbalanced polarization of MФs after G-CSF mobilization, characterized by decreased ratio of pro-inflammatory $M 1 M \Phi$ s and anti-inflammatory M2 MФs both in G-PB and G-BM. Moreover,our study demonstrated dysfunctional BM MФs after G-CSF mobilization, characterized by decreased capacities of migration, and increased phagocytic activities. Meanwhile, the cumulative incidence analyses suggested 
that patients who received a lower ratio of M1/M2 MФs infusion exhibited lower incidence of grade II-IV aGvHD. Thus, we speculate that the ratio of M1/M2 MФs may predict the occurrence of grade II-IV aGvHD. Therefore, our data indicate that G-CSF-induced immune tolerance may be mediated by MФs polarization in allo-HSCT. Thereby G-CSF-mobilized MФs are a potential novel immunotherapeutic strategy against aGvHD in the future.

Conflict of interest: nothing to disclose

\section{P362}

\section{Glutathione S-Transferase Polymorphism Predicts the Development of Acute Graft Versus Host Disease in Patients Undergoing Hematopoietic Stem Cell Transplantation}

Intisar Al-Riyami ${ }^{1}$, Murtadha Al-Khabori ${ }^{2}$, Khaled Al-Belushi ${ }^{3}$, Mohammed Al-Rawahi ${ }^{2}$, Shoaib Al-Zadjali', Melanie Tauro ${ }^{2}$, Mohammed Al-Huneini ${ }^{2}$, Abdulhakeem Al-Rawas ${ }^{4}$

${ }^{1}$ Sultan Qaboos University Hospital, Pharmacy, Muscat, Oman; ${ }^{2}$ Sultan Qaboos University Hospital, Hematology, Muscat, Oman; ${ }^{3}$ Sultan Qaboos University, Pharmacology, Muscat, Oman; ${ }^{4}$ Sultan Qaboos University Hospital, Child Health, Muscat, Oman

Background: Acute Graft Versus Host Disease (aGvHD) is an important complication post Hematopoietic Stem Cell Transplantation (HSCT) leading to morbidity and mortality. The intensity of the preparative regimen has been associated with the risk of aGvHD, albeit, with marked variability. We hypothesized that glutathione S-Transferase (GST) polymorphism affects the metabolism of Busulfan $(\mathrm{Bu})$ and hence may impact the risk of aGvHD. Therefore, we planned to assess the role of GST polymorphism on the risk of aGvHD.

Methods: This is a single center retrospective cohort study of 135 adult and pediatric patients who received $\mathrm{Bu}$ prior to HSCT at Sultan Qaboos University Hospital from January 2003 to October 2016. All indications were included. Genotyping for GST polymorphism was done on pre-transplant patients' samples. We used Capillary Electrophoresis for GSTM1 and GSTT1 (insertion or deletion), and DNA sequencing (Thermal Cycle Sequencing) for GSTA1 (C-69G, A-513G, and G-1142C) and GSTP1 (A313G). The impact of GST polymorphisms with aGvHD was assessed using $\mathrm{Chi}^{2}$ test. Multivariable regression was performed on aGvHD and included all predictors variables with a $\mathrm{p}$ value of $<0.05$ in univariable testing. Standard descriptive and analytical statistics and graphs were created using the statistical software STATA version 13.

Results: The incidence rate of aGvHD among the study population was $22 \%$, of which, $14 \%$ had skin, $3.7 \%$ had liver and $9.6 \%$ had gut aGvHD. Grades I, II, III, IV aGvHD were seen in $6.7 \%, 11.1 \%, 0.74 \%$ and $3.7 \%$ of patients respectively. Combined GSTM1 and GSTT1 double deletion has an increased risk of aGvHD with a statistically significant difference in the incidence compared to single or double insertion $(p=0.04)$. In a multivariable regression model which included conditioning medication used and the combination of GSTM1 and GSTT1, the impact remained statistically significant $(p=0.042)$. In the same model, the preparative regimen was also predictor of aGvHD, Patients who received CYCLO/BU/FLU had more risk of aGvHD (55\%) compared to patients receiving other combinations $\mathrm{BU} / \mathrm{CY} \pm \mathrm{ATG}, \mathrm{BU} / \mathrm{MEL} \pm \mathrm{ATG}$, and $\mathrm{FLU} / \mathrm{BU} \pm \mathrm{ATG}$ $(33 \%, 33 \%$, and $17 \%, p=0.024)$. The incidence of aGvHD in different alleles of GSTP1 A313G was not significantly different (AA 19\%, AG 16\% and GG 42\%; $p=0.156$ ). The incidence was not significantly different in all tested polymorphism in all the loci of GSTA1 (-69, -513 and $-1142 ; p=0.832,0.727$ and 0.934 , respectively). The difference in the incidence of aGvHD in different polymorphism groups of GSTM1 and GSTT1 were also not statistically significant $(p=0.766$ and 0.289 respectively).

Conclusions: Combined GSTM1 and GSTT1 double deletion increases the risk of aGvHD in patients undergoing HSCT using Bu-based preparative regimen. To the best of our knowledge, this is the first report of such association. These results should be confirmed and, if true, may be used for predicting aGvHD and stratifying patients for aGvHD prophylaxis.

Conflict of interest: No conflict of interest.

The study was supported by an internal grant from College of Medicine and Health Sciences, Sultan Qaboos University.

\section{P363}

Abstract previously published

\section{P364}

High risk of relapse with high mutation burden at day 21 post-transplant can be overcome by chronic GVHD in patients with AML

Joon-Ho Moon ${ }^{1}$, Taehyung Kim ${ }^{2}$, Jae-Sook Ahn ${ }^{3}$, Hyeoung-Joon $\mathrm{Kim}^{3}$, Seung-Shin Lee ${ }^{3}$, Seo-Yeon $\mathrm{Ahn}^{3}$, Sung-Hoon Jung, DeokHwan Yang ${ }^{3}$, Je-Jung Lee ${ }^{3}$,Yoo-Jin Lee ${ }^{1}$, Sang-Kyun Sohn ${ }^{1}$, JaYeon Lee ${ }^{4}$, Seung-Hyun Choi ${ }^{4}$, Sung-Kyu Park, Zhaolei Zhang ${ }^{6}$, Dennis Dong Hwan Kim ${ }^{7}$

${ }^{1}$ Kyungpook National University Hospital, Hematology/Oncology, Daegu, Korea, Republic of; ${ }^{2}$ University of Toronto, Computer Science, Toronto, Canada; ${ }^{3}$ Chonnam National University Hwasun Hospital, Hematology-Oncology, Hwasun, Korea, Republic of; ${ }^{4}$ Chonnam National University Hwasun Hospital, Genomic Research Center for 
Hematopoietic Diseases, Hwasun, Korea, Republic of; ${ }^{5}$ Soon Chun Hyang University Bucheon Hospital, Hematology-Oncology, Bucheon, Korea, Republic of; ${ }^{6}$ University of Toronto, Molecular Genetics, Toronto, Canada, ${ }^{7}$ Princess Margaret Cancer Centre, Medical Oncology and Hematology, Toronto, Canada

Background: Integration of next generation sequencing (NGS) in the treatment decision algorithm in patients receiving allogeneic hematopoietic cell transplantation (HCT) is demanding. Our previous work demonstrated that higher mutation allelic burden (variant allele frequency $[\mathrm{VAF}] \geq 0.2 \%$ ) at day 21 after HCT correlates with an increased risk of relapse $(\mathrm{HR} 4.8, \mathrm{P}<0.001)$ and poor overall survival $(\mathrm{OS}$; $\mathrm{HR} 3.1, \mathrm{P}=0.003)$ after $\mathrm{HCT}$ in patients with acute myeloid leukemia (AML). The present study evaluated whether the development of chronic GVHD (cGVHD) could overcome the adverse prognostic impact of higher mutation burden (VAF $\geq 0.2 \%$ ) post-HCT by reducing the risk of relapse and improving OS. In addition, we also have incorporated the 2017 ELN risk stratification of AML and analyzed whether VAF post-HCT is also independently prognostic from the ELN risk groups.

Methods: The current study included 104 AML patients who received HCT. We performed NGS targeting 84 myeloid gene panel (Agilent custom probe set) on 529 samples using Illumina HiSeq 2000. Sequenced samples include bone marrow/peripheral blood samples taken at diagnosis, pre-HCT, and post-HCT day 21 as well as samples from longer follow-up, T-cell fraction, and donor. The OS and cumulative incidence of relapse (CIR) were compared according to multiple variables including age, acute GVHD, cGVHD as well as the 2017 ELN genetic risk group. All statistical analyses were performed using $\mathrm{R}$ statistical software.

Results: A total of 256 mutations were detected in the samples taken at diagnosis in 90/104 patients $(86.5 \%$, median 3 mutations per patient) with on target coverage of $1725.6 \mathrm{X}$. In the group with post-HCT $\mathrm{VAF} \geq 0.2 \%$ (VAFHigh), the development of cGVHD lead to improved outcomes with respect to OS: 3-year OS rate of $66.7 \%$ and $15.0 \%$ in the groups with and without cGVHD, respectively $(\mathrm{p}=0.042)$; as well as to relapse risk: 3 -year CIR of $16.7 \%$ and $80.0 \%$ in the groups with and without cGVHD, respectively $(\mathrm{p}=0.015)$. Similarly, in the group with $\mathrm{VAF}<0.2 \%$ post-HCT $\left(\mathrm{VAF}^{\mathrm{Low}}\right)$, the CIR was lower in the group with cGVHD than the group without cGVHD (3-year CIR of $4.0 \%$ vs $34.3 \%, p<0.001$ ) although there was no statistical difference of OS between the groups. In the multivariate analysis, the presence of cGVHD was an independent prognostic factor for CIR both in the $\mathrm{VAF}^{\mathrm{High}}$ (HR 0.11, p = 0.021) and $\mathrm{VAF}^{\text {Low }}$ group (HR 0.10, $\mathrm{p}=$ 0.001). The finding implies that a therapeutic intervention to increase the chance of cGVHD development would bring some therapeutic benefit in the $\mathrm{VAF}^{\mathrm{High}}$ group. In addition, somatic mutation VAF post-HCT was independent of the ELN 2017 risk groups. Excellent stratification of VAF postHCT with regards to OS and relapse was well replicated in the intermediate and adverse subgroups by the ELN 2017, respectively.

Conclusions: Day 21 monitoring of mutation burden using NGS should be considered to be incorporate into the future practice for post-transplant management of AML patients. It could identify the high risk of relapse in patients requiring pre-emptive intervention including rapid tapering of immunosuppressive GVHD prophylaxis as well as preemptive donor lymphocyte infusion attempt in case of the high VAF group.

Conflict of interest: No conflict of interest to disclose.

\section{P365}

High-dose posttransplantation cyclophosphamide (PTCy) plus tacrolimus for HLA-identical 10/10 unrelated donor allogeneic transplantation is associated to a low risk of GVHD: experience of a single-center

Sofia Jorge ${ }^{1}$, María Suárez-Lledón ${ }^{1}$, Gonzalo Gutiérrez-García ${ }^{1,2,3}$, Francesc Fernández-Avilés ${ }^{1,2,3}$, Laura Rosiñol ${ }^{1,2,3}$, Luis Gerardo Rodríguez-Lobato $^{1}$, Teresa Solano ${ }^{1}$, Álvaro Urbano-Ispízua ${ }^{1,2,3}$, Montserrat Rovira ${ }^{1,2,3}$, Carmen Martíne $z^{1,2,3}$

${ }^{1}$ Hospital Clínic, Hematopoietic Stem Cell Transplantation Unit, Hematology Department, Institute of Oncology and Hematology, Barcelona, Spain; ${ }^{2}$ Institut Josep Carreras, Barcelona, Spain; ${ }^{3}$ Institut d'Investigació Biomèdica Agustí Pi I Sunyer (IDIBAPS), Barcelona, Spain

Background: The safety and efficacy of high-dose PTCy when combined with tacrolimus and mycophenolate mofetil were previously demonstrated after nonmyeloablative conditioning and transplantation of $\mathrm{T}$ cell-replete bone marrow from haploidentical related donors. It has been also found to be effective as sole prophylaxis of GVHD after HLA-matched bone marrow transplant. There is, however, little information regarding the efficacy and safety of PTCy when used in the context of matched unrelated donor (MUD) and/or with peripheral blood as stem cell source. We report our experience with the use of PTCy plus tacrolimus as GVHD prophylaxis for MUD (HLA-identical 10/10) allogeneic hematopoietic stem cell transplantation (MUD allo-HSCT).

Methods: Nineteen consecutive MUD allo-HSCT recipients were included (median age 61y, range 36-69). Unrelated donor selection was performed according to standard criteria, including high resolution typing for alleles at HLA-A, -B, -Cw, DRB1 and DQB1.

Results: Most patients $(84 \%)$ had a HCT-CI $\geq 2(\geq 3$ in 11 ), $58 \%$ had acute leukemia, $68 \%$ were in complete 
remission at the time of transplant, and the Disease Risk Index (DRI) was intermediate in 68\%, high in $16 \%$ and very-high in $5 \%$. Fifteen $(79 \%)$ patients received reduced intensity conditioning regimens (fludarabine-busulfan in 12 patients), and $17(89 \%)$ peripheral blood stem cells. One primary graft-failure was observed. Median time to neutrophil $(>500 / \mathrm{mL})$ and platelet $(>20,000 / \mathrm{mL})$ recovery were 21 days (range 13-30) and 26 days (10-185), respectively. Sixty-five percent of patients had full donor chimerism at 1 month after allo-HSCT. Only 2 cases of hemorrhagic cystitis were observed, both were mild. Three patients developed proven or probable invasive pulmonary Aspergillosis, and 5 had severe bacterial infection. Three patients out of 17 at risk (18\%) developed acute grade II-IV GVHD (all of them grade II, and all achieved complete remission after systemic corticosteroid treatment). Only one patient developed chronic GVHD. Three patients died without disease relapse/progression: 2 infections and 1 pulmonary complication. Two patients relapsed (with very high and high DRI, one of each). After a median follow-up for surviving patients of 7 months (range 3-16), overall survival and progression-free survival at 1 -year were $71 \%$ and $70 \%$, respectively.

Conclusions: Our results suggest that MUD allo-HSCT in a high-risk population of patients (advanced age, high HCT-CI) using PTCy plus tacrolimus as GVHD prophylaxis and peripheral blood graft is safe, associated with a low incidence of GVHD and relapse, and encouraging survival outcomes. Prospective studies including a higher number of patients and longer follow-up are needed to validate these findings.

Conflict of interest: nothing to disclose.

\section{P366}

\section{Host-derived tissue-resident memory $\mathrm{T}$ cells with proliferative potential reside in nonlesional skin of allo- HSCT recipients}

\author{
Johanna Strobl ${ }^{1}$, Nadine Bayer ${ }^{1}$, Manuel Mayrdorfer ${ }^{1}$, Bärbel \\ Reininger ${ }^{1}$, Philipp Wohlfarth ${ }^{2}$, Peter Kalhs ${ }^{2}$, Werner Rabitsch', \\ Georg Hopfinger', Georg Stary ${ }^{1}$ \\ ${ }^{1}$ Medical University of Vienna, Department of Dermatology, Vienna, \\ Austria; ${ }^{2}$ Medical University of Vienna, Department of Internal \\ Medicine I, Bone Marrow Transplantation Unit, Vienna, Austria
}

Background: Skin reactions remain one of the most common manifestations of acute and chronic graft versus host disease (GVHD) in allogeneic HSCT recipients. While donor $\mathrm{T}$ cells were shown to be responsible for the graftversus-leukemia effect and are thus regarded as major players in graft-versus-host reaction, little is known about the potential role of host immune cell subsets in GVHD.
Tissue-resident memory $\mathrm{T}$ cells (Trm) were recently described in mice as unique subset of non-recirculating radio-resistant cells in various organs.

Methods: To determine the presence and resilience of Trm in human tissue, we tracked peripheral blood- and skinderived T cells of 32 patients in the process of HSCT until one year after transfer of donor stem cells. Serial samples were taken from nonlesional skin at up to 5 time points before and after HSCT (days $-7,0,+14,+100,+365$ ) and analyzed for the presence of $\mathrm{T}$ cell subtypes using lymphocyte homing molecules and residency markers. Isolated $\mathrm{T}$ cells were evaluated for their proliferative capacity upon $\mathrm{T}$ cell receptor (TCR) stimulation. Skin sections of patients after transplantation of sex- $(n=9)$ and HLA-A $(\mathrm{n}=1)$ mismatched donor cells were assessed for chimerism by fluorescence in situ hybridization of $\mathrm{X} / \mathrm{Y}$ chromosomes and HLA phenotyping, respectively. Sections were processed using an automated imaging acquisition and analysis software (TissueGnostics ${ }^{\circledR}$ ).

Results: Upon myeloablative treatment, recirculating and naïve $\mathrm{T}$ cells $\left(\mathrm{CCR} 7^{+} \mathrm{CD} 62 \mathrm{~L}^{ \pm}\right)$declined in skin and peripheral blood, while a subset of dermal $\alpha \beta T$ cells expressing tissue residency markers $\left(\mathrm{CD} 69^{+} \mathrm{CD} 103^{ \pm}\right)$ remained stable even after HSCT. Moreover, skin-resident $\mathrm{T}$ cells surviving conditioning treatments displayed high proliferative potential after TCR stimulation, comparable to $\mathrm{T}$ cells isolated from skin of non-transplanted donors. Strikingly, chimerism analysis revealed that, unlike in peripheral blood, $\mathrm{CD} 69^{+} \mathrm{CD} 103^{ \pm}$dermal $\mathrm{T}$ cells of the recipient coexisted with donor $\mathrm{T}$ cells and constituted $>30 \%$ of dermal T cells 3 - 4 months after engraftment. When we subdivided patients according to their development of acute GVHD at later time points, Trm were enriched in the skin at early time points after HSCT of patients, who later developed acute GVHD compared to those that had no signs for GVHD.

Conclusions: We have identified a long-lived and remarkably large population of host-derived dermal $\mathrm{T}$ cells, which may play a so far unappreciated role in GVHD. To the best of our knowledge this is the first definitive proof of Trm in human tissue with implications to infectious and chronic-inflammatory diseases.

\section{Conflict of interest:}

J. Strobl: recipient of a DOC fellowship from the Austrian Academy of Science

G. Stary: supported by the Austrian Federal Reserve Bank, Arbeitsgemeinschaft Dermatologische Forschung

The other authors have nothing to disclose. 
P367

Impact of $4^{\text {th }}$ dose of methotrexate associated with calcineurin inhibitors as GVHD prophylaxis in allogeneic stem cell transplant

María José Jiménez ${ }^{1}$, Mireia Morgades ${ }^{1}$, Anna Torrent ${ }^{1}$, Blanca Xicoy $^{1}$, Susana Vives ${ }^{1}$, Montserrat Batlle ${ }^{1}$, Miriam Moreno ${ }^{1}$, Juan Manuel Sancho ${ }^{1}$, Gladys Ibarra ${ }^{1}$, Laura Abril ${ }^{1}$, Albert Oriol ${ }^{1}$,

Edurne Serrate ${ }^{1}$, Ana Triguero ${ }^{1}$, David Cruz ${ }^{1}$, Josep-Maria Ribera ${ }^{1}$, Christelle Ferra ${ }^{2}$

${ }^{1}$ Hospital Germans Trias i Pujol, Institut Català d'Oncologia, Institut de Recerca contra la Leucèmia Josep Carreras, Clinical Hematology, Badalona, Spain; ${ }^{2}$ Clinical Hematology, Badalona, Spain

Background: The combination of a calcineurin inhibitor (CaI) with a short course of methotrexate (MTX) is the most common regimen of GVHD prophylaxis. There are no studies directly comparing the administration of 3 versus 4 doses of MTX. The objective of this study was to evaluate the impact of the $4^{\text {th }}$ dose of MTX omission on outcome.

Methods: Between January 2008 and December 2016, 215 first allogeneic SCT were performed at our institution. 144 patients (pts) (67\%) were planned to receive $\mathrm{CaI}$ and a short course of MTX as GVHD prophylaxis. Only 140 pts were available for final analysis.

Results: Patients characteristics are on Table 1. Seventyseven (55\%) pts received all planned MTX doses, 41 (29\%) received 3 doses per protocol and $22(16 \%)$ did not completed the 4 planned doses due to: severe mucositis (14, $63 \%)$, mucositis plus hyperbilirubinemia $(3,14 \%)$, HSOS $(3,14 \%)$ and hyperbilirubinemia (2, 9\%). ATG was administered for GVHD prophylaxis in 30 pts that had completed 4 MTX doses (39\%) and in 16 who had received 3 MTX doses $(25 \%)(p=0.089)$. Four MTX doses were more frequently administered from 2012-2016 than in the previous period $(\mathrm{P}<0.001)$. Two pts (all had received 3 MTX doses) showed primary graft failure. The 5 ys OS probability for pts receiving 4 or $<4$ MTX doses were $38 \%$ (95\% CI: $25 \%-51 \%)$ and $31 \%$ (95\% CI: $19 \%-43 \%)$ with median follow-up 2,12 ys $(0,30-7,88)$ and 5,41 ys $(0,08$ $9,18)$ respectively. NRM was similar for those patients who completed the 4 planned doses, and for those who received only 3 . There were no differences in engraftment, relapse incidence, NRM and acute GVHD. The 5y-probability of developing severe chronic GVHD was $20 \%$ (95\% CI: $10 \%$ $32 \%$ ) and $11 \%$ (95\% CI: 5\%-21\%) for pts receiving $\leq 4$ and 4 MTX doses $(p=0,098)$. In the subgroup of pts that did not receive ATG (46 pts; $33 \%$ ), the $4^{\text {th }}$ dose of MTX was also associated with a lower probability of developing severe chronic GVHD (8\% [95\% CI: $2 \%-19 \%$ ] vs. $24 \%$ [95\% CI: $11 \%-40 \%] ; p=0,021)$. Moreover, the probability for the composite end-point GVHD-free/relapse-free survival was
$28 \%$ (95\% CI: $16 \%-40 \%)$ and $8 \%$ (95\% CI: $1 \%-15 \%)$ for pts who received 3 versus 4 doses of MTX $(p=0,012)$.

Conclusions: The administration of the $4^{\text {th }}$ dose of MTX associated with a CaI for GVHD prophylaxis in conventional allogeneic SCT was not associated with a worse OS or NRM but was related with a better GRFS survival.

Clinical Trial Registry: No apply.

Conflict of interest: The authors declare no conflict of interest

Funding: PI10/01417 (Fondo de Investigaciones Sanitarias). RD12/0036/0029 (RTICC, Instituto Carlos III, 2014 SGR225GRE]), CERCA Program, Generalitat de Catalunya, España, "La Caixa" Foundation, RD12/0036/0029 RTICC-Instituto Carlos III

\begin{tabular}{lll}
\hline $\begin{array}{l}\text { Age at HSCT (yrs) (Median } \\
\text { [range]) }\end{array}$ & $51.5[15 ; 69]$ \\
\hline Disease & - AML/ MDS / ALL & $\begin{array}{l}59(42) / 19(14) / \\
19(14) \\
\end{array}$ \\
& $\begin{array}{l}\text { - Lymphoma / Multiple } \\
\text { myeloma }\end{array}$ & $20(24) / 5(3)$ \\
& $\begin{array}{l}\text { - Chronic } \\
\text { myeloproliferative disease }\end{array}$ & $7(5)$ \\
& - Other & $11(8)$ \\
& & $14(10)$ \\
Previous SCT & $87(62) / 53(38)$ \\
Sibling / Unrelated donor & & $71(51)$ \\
Myeloablative conditioning & & $129(92) / 10(7) / 1$ \\
Stem cell source: PB /BM & & $11)$ \\
/PB +BM & $17(12)$ \\
HLA mismatch &
\end{tabular}

[[P367 Table] Demographic, disease and SCT characteristics. $N=140]$

\section{P368}

Abstract previously published

\section{P369}

Impact of methotrexate on acute GVHD rate in allo-HSCT from matched unrelated donor due to different graft source

Mikhail Drokov, Larisa Kuzmina, Natalia Popova, Ekaterina Mikhalcova, Daria Dubnyak, Vera Vasilyeva, Olga Koroleva, Anna Sidorova, Ekaterina Usikova, Zoya Konova, Elena Parovichnikova, Valery Savchenko

National Research Center for Hematology, BMT Department, Moscow, Russian Federation

Background: Here we report an impact of methotrexate on acute GVHD rate in allo-HSCT from matched unrelated donor due to different graft source. 
Methods: This study analysis included 106 engrafted patients (47 males and 59 females) with a median age of 35 years (range 18-60), who was underwent allo-HSCT from matched unrelated donor. All patients had hematological malignancy: ALL $(\mathrm{n}=27), \operatorname{AML}(\mathrm{n}=59), \operatorname{LPD}(\mathrm{n}=7)$, $\operatorname{MDS}(n=5), \operatorname{CLL}(n=1), \operatorname{CML}(n=3), \operatorname{CMML}(n=4)$. 25 patients were underwent MAC conditioning regimen and 81 patients - RIC. All patients received standard GVHD prophylaxis with horse ATG (from day -4 to -1 at total dose $40 \mathrm{mg} / \mathrm{kg}$ ), cyclosporine A (CSA, from day -1 at daily dose $3 \mathrm{mg} / \mathrm{kg}$ ), and mycophenolate mofetil (MMF, from day +1 at total daily dose $3000 \mathrm{mg}$ ) with or without methotrexate (MTX) on day $+1,+3,+6,+11$. Study groups were formed based on the type of graft source: bone marrow ( $\mathrm{n}$ $=40)$, PBSC $(\mathrm{n}=66)$. The groups with and without MTX were matched for age, disease phase, number of infused cells. Fisher's exact test was used for $2 \times 2$ tables. KaplanMeier analysis with Wilcoxon test was used for survival analysis and the groups comparison. A p-value less than 0.05 were considered significant.

Results: According to the data cumulative incidence of aGVHD (grade II-IV) in bone marrow recipients was $16.7 \%$ vs $4.5 \%$ without and with MTX respectively. There was no significant variance $(p=0.199)$. In case of PBSC as a graft source using MTX reduced aGVHD cumulative incidence from $40.9 \%$ to $18.2 \%$ ( $\mathrm{p}=0.037$ ).

Conclusions: Here we show that using of methotrexate in allo-HSCT from matched unrelated donor in combination with ATG, CSA and MMF is relevant only in PBSC recipients and leads to significant decrease of aGVHD incidence.

Conflict of interest: nothing to disclose

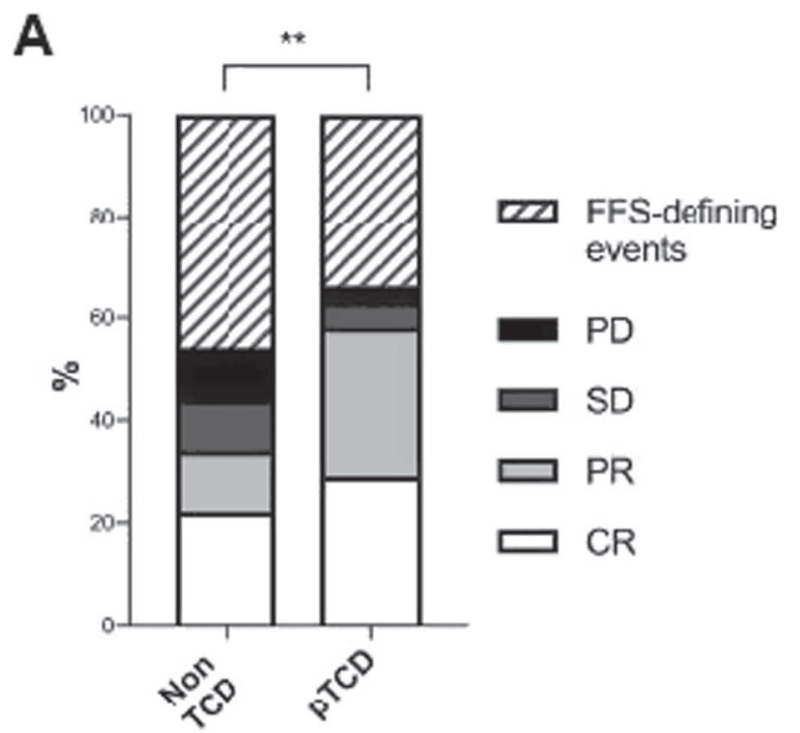

P370

Impact of partial T-cell depletion on a composite endpoint associated with clinical benefit after initial treatment of chronic graft-versus- host disease

Stavroula Masouridi-Levrat, Federico Simonetta, Yordanka Tirefort, Sarah Morin, Carole Dantin, Caroline Stephan, Olga Tsopra, Yan Beauverd, Anne-Claire Mamez, Yves Chalandon

Geneva University Hospitals, Oncology Department, Division of Hematology, Geneva, Switzerland

Background: Chronic graft-versus-host disease (cGvHD) is a serious complication of allogeneic hematopoietic stem cell transplantation (HSCT) associated with significant morbidity and mortality. Survival with a complete or partial response and no previous secondary systemic treatment or recurrent malignancy at 1 year after initial systemic therapy for $\mathrm{cGvHD}$ is a recently reported composite endpoint associated with clinical benefit (Martin PJ et al, Blood 2017). In the present study we investigated the effect of partial T-cell depletion (pTCD), a strategy for GvHD prophylaxis, on this endpoint in order to evaluate its impact on the clinical evolution of cGVHD.

Methods: We performed a retrospective analysis on 79 consecutive patients diagnosed with systemic therapyrequiring cGVHD from 2003 to 2016 at our center after undergoing allogeneic HSCT for hematologic diseases. 38 patients received pTCD grafts, consisting of in vitro alemtuzumab incubation before infusion followed on day +1 by an add-back of donor T CD3+ cells. 41 patients received non-TCD grafts. Donor lymphocyte infusions (DLI) were provided, in the absence of GvHD, at three months to all patients receiving pTCD grafts with reduced

B

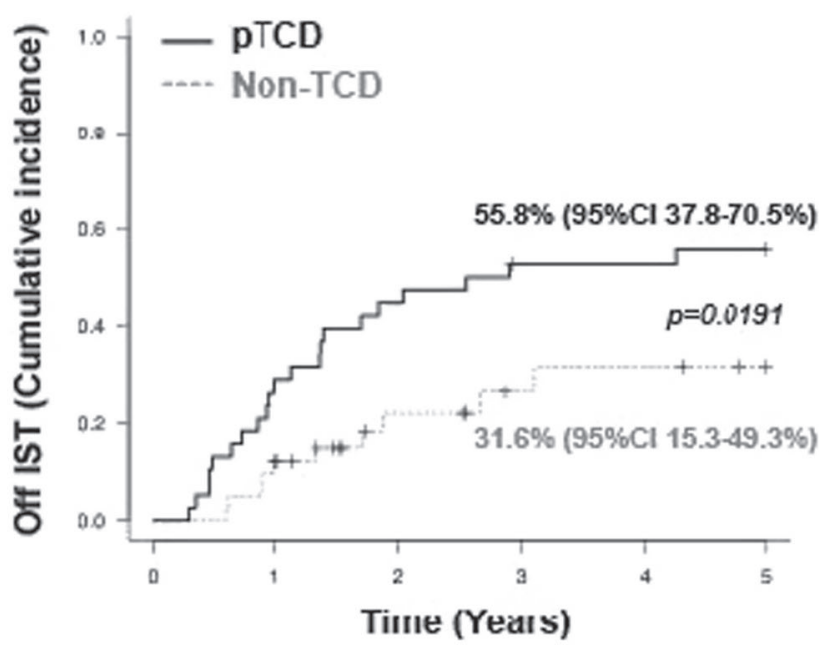

[P370 Figure] [Impact of pTD on endpoint success (A) and on treatment interruption (B)] 
intensity conditioning and as needed to patients, transplanted with either pTCD or non-TCD grafts. Failure free survival (FFS)-defining events were death, disease relapse or secondary systemic treatment. Response at 1 year was assigned as complete response (CR), partial response (PR), stable disease (SD) or progressive disease (PD). Success was defined as FFS with CR or PR at 1 year after instauration of initial treatment. Both cGVHD diagnosis and response to treatment were retrospectively determined according to the $2014 \mathrm{NIH}$ consensus criteria.

Response rates were compared using the chi-square test. Kaplan-Meier estimates were employed to determine the probability of overall survival (OS) and FFS since cGvHD diagnosis. Cumulative incidence of systemic treatment cessation was calculated with relapse or death defined as competitive events and compared using the Gray test.

Results: Median age was 50 years (range, 17-70 years). Of the 79 patients, $70(89 \%)$ had HLA-matched related or unrelated donors and 27 (34\%) were males having female donors. The median time from HSCT to systemic therapyrequiring cGvHD was 10 months (range, 2-627). 22/79 (29\%) patients presented post-DLI cGvHD and 24/79 (30\%) had severe cGVHD.

Endpoint success was observed in 36/79 (45\%) patients while 48/79 (61\%) patients achieved 1-year FFS. pTCD was associated with a significantly higher 1 -year endpoint success achievement [22/38 (58\%)] compared to non-TCD transplantations [14/41 (34\%); $\mathrm{p}=0.0072$, Figure 1A]. Accordingly, patients receiving pTCD had higher 5-year cumulative incidence of systemic treatment interruption [55.8\% (95\%CI 37.8-70.5\%)] compared to patients receiving non-TCD grafts $[31.6 \%(95 \% \mathrm{CI} 15.3-49.3 \%) ; \mathrm{p}=$ 0.0191 , Figure 1B]. Conversely, no effect of pTCD was observed on 5-year OS [pTCD: 72.8\% (95\%CI 55.284.4\%); Non-TCD 68.8\% (95\%CI 51.3-81.1\%); $\mathrm{p}=0.69$ ], and FFS [pTCD: $59.9 \%$ (95\%CI 42.5-73.6\%); Non-TCD: $47.7 \%$ (95\%CI 31.5-62.2\%); $\mathrm{p}=0.27]$.

Conclusions: pTCD appears to improve the endpoint success achievement in systemic therapy-requiring cGvHD, resulting in an earlier cessation of treatment without affecting OS. These results suggest that pTCD could ameliorate the clinical evolution of cGvHD improving patients' quality of life.

Conflict of interest: All authors: nothing to disclose

\section{P371}

Impact of True Naïve and Stem Cell Memory recovery on chronic graft-versus-host disease after allogeneic hematopoietic stem cell transplantation

Ana C. Alho ${ }^{1,2,3}$, Marie J. Chammas ${ }^{1}$, Carol G. Reynolds ${ }^{1}$, Sarah Nikiforow ${ }^{1,4}$, Corey S. Cutler ${ }^{1,4}$, John Koreth ${ }^{1,4}$, Vincent T Ho ${ }^{1,4}$, Joseph H. Antin ${ }^{1,4}$, Edwin P. Alyea ${ }^{1,4}$, Joao F. Lacerda ${ }^{2,3,5}$, Robert J. Soiffer ${ }^{1,4}$, Jerome Ritz,

${ }^{1}$ Dana Farber Institute, Division of Hematologic Malignancies, Boston, MA, United States; ${ }^{2}$ Instituto de Medicina Molecular, Jlacerda Lab, Lisboa, Portugal; ${ }^{3}$ Hospital de Santa Maria, Serviço de Hematologia e Transplantação de Medula, Lisboa, Portugal;

${ }^{4}$ Harvard Medical School, Boston, MA, United States; ${ }^{5}$ Faculdade de Medicina da Universidade de Lisboa, Lisboa, Portugal

Background: The role of $\mathrm{CD}^{2} 5 \mathrm{RA}^{+} \mathrm{CD} 6 \mathrm{~L}^{+}$naive $\mathrm{T}$ cells in the setting of chronic graft-versus-host disease (cGvHD) after allogeneic hematopoietic stem cell transplantation is controversial. Within naïve $\mathrm{CD} 8+$ and $\mathrm{CD} 4+$ T-cells, recent studies have identified a small subset of

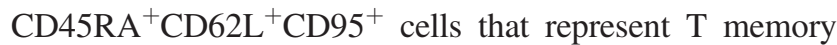
stem cells (SCM). Despite the expression of naïve markers, SCM are less differentiated memory cells, with antigenspecificity, distinguished by their self-renewing capacity and superior T-cell engraftment in allogeneic transplant models. In this study we examined the post-transplant reconstitution of T-cells expressing naïve markers, and the lineage relationship with other $\mathrm{T}$-cell subsets, likely involved in cGvHD.

Methods: For a 2-year period, we prospectively monitored 111 patients who underwent allogeneic hematopoietic stem cell transplantation after myeloablative conditioning
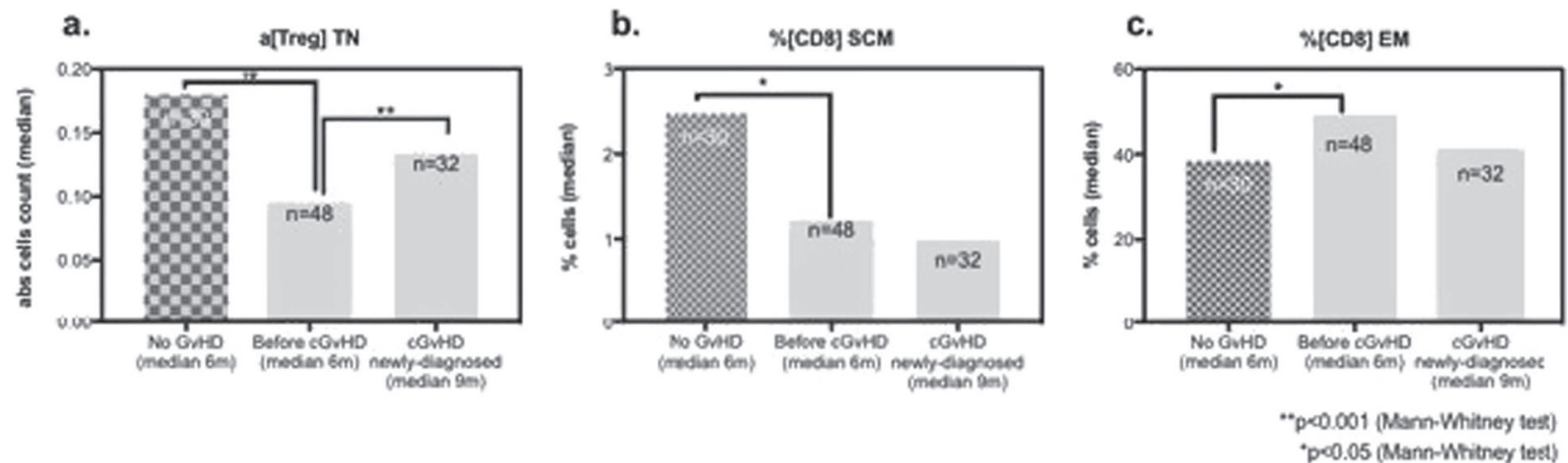

[P371 Figure] [Imbalance between CD4 regulatory and CD8 effector subsets before cGvHD development.] 
without T-cell depletion. During the post-transplant period recovery of $\mathrm{SCM}\left(\mathrm{CD} 45 \mathrm{RA}^{+} \mathrm{CD} 2 \mathrm{~L}^{+} \mathrm{CD} 95^{+}\right)$and true naïve (TN, CD45RA ${ }^{+} \mathrm{CD}_{2} \mathrm{~L}^{+} \mathrm{CD} 95^{-}$) cells was studied within $\mathrm{CD} 4+\mathrm{Foxp}^{+}$regulatory $\mathrm{T}$ cells (Treg), conventional $\mathrm{CD}^{+}$T-cells (Tcon) and $\mathrm{CD}^{+}$T-cells, and correlated with development of cGvHD. To understand the immune-changes that occur before cGvHD, we compared three groups of samples: samples collected at cGvHD diagnosed, samples collected before cGvHD, and samples from noGvHD/control group collected at the same median time as the previous group (Figure 1).

Results: With a median follow-up of 398 days (375-777), 52 patients $(46,85 \%)$ developed cGvHD; the median time to onset of cGvHD was 231 days $\left(\mathrm{Q}_{1}-\mathrm{Q}_{3}\right.$ 168.5-303.5). The absolute count (n) and percentage (\%) of TN and SCM regulatory T-cells (Tregs) in the cGvHD cohort is significantly lower at early time points $(\mathrm{p} \leq 0.05$ at $2 \mathrm{~m}$ for $\mathrm{TN} \%, \mathrm{p} \leq 0.001$ at $6 \mathrm{~m}$ for TN $\mathrm{n} ; \mathrm{p} \leq 0.05$ at $1 \mathrm{~m}$ for $\mathrm{SCM} \%$ and $\mathrm{n})$. No significant differences are observed in the naïve Tcon compartment. Within CD8 T-cells, the $\mathrm{n}$ and\% of $\mathrm{SCM}$ is significantly lower at 1 and $6 \mathrm{~m}$ post-transplant $(\mathrm{p} \leq 0.05)$ in $\mathrm{cGvHD}$ patients. In contrast, the $\mathrm{n}$ and $\%$ of Effector memory (EM) CD8 is significantly higher ( $\mathrm{p} \leq 0.05$ at 3 and $6 \mathrm{~m}$ for $\mathrm{n} ; \mathrm{p} \leq 0.001$ at $1-3 \mathrm{~m}$ for $\%$ ).

Conclusions: In our cohort, the immune compartment of patients who develop cGvHD can be distinguished at early time-points before the onset of disease by reduced recovery of regulatory TN and SCM subsets, and predominance of EM CD8 over SCM CD8 T-cells. Although the lineage relationship within T-cell subsets is difficult to assign, recent studies suggest a differentiation pathway from SCM to EM cells, which may explain the observed increase of EM over SCM CD8 T-cells in our cohort. Increased differentiation of SCM into EM cells likely reflects an uncontrolled effector response to allo-antigens. Overall, our data suggests that an early imbalance between CD4 regulatory and CD8 effector subsets influences the subsequent development of cGvHD.

Conflict of interest: The authors declare no competing financial interests

\section{P372}

Abstract previously published

\section{P373}

Importance of peripheral blood absolute lymphocyte counts (ALC) in predicting transplant outcomes in preparative regimens using anti-thymocyte globulin or alemtuzumab: an international 2 centre experience
Vipul Sheth ${ }^{1}$, Vanessa Kennedy ${ }^{2}$, Hugues de Lavallade ${ }^{1}$, Donal Mclornan ${ }^{1}$, Victoria Potter ${ }^{1}$, Bipin Savani, Wichai Chinratanalab ${ }^{3}$, Brian Engelhardt', Stacey Goodman ${ }^{3}$, John Greer ${ }^{3}$, Adetola Kassim ${ }^{3}$, Sally York ${ }^{3}$, Austin Kulasekararaj ${ }^{1}$, Judith Marsh ${ }^{1}$, Ghulam Mufti ${ }^{1}$, Antonio Pagliuca ${ }^{1}$, Madan Jagasia ${ }^{3}$, Kavita Raj ${ }^{1}$

${ }^{1}$ Department of hematology and stem cell transplant, Kings College hospital., London, United Kingdom; ${ }^{2}$ Department of medicine, Stanford University hospital, Stanford, CA, United States;

${ }^{3}$ Department of hematology and stem cell transplant, Vanderbilt university., Nashville, TN, United States

Background: Antithymocyte globulin (ATG) and antiCD52 antibody (alemtuzumab) are used as prophylaxis for graft-versus-host disease (GVHD) in unrelated donor allografts (URD) for leukaemia. Dosing regimens for these are empiric or weight-based. Furthermore, the target of these agents, recipient $\mathrm{T}$ cells post-cytotoxic chemotherapy, is not a function of recipient weight. Recently, it is shown that recipient peripheral blood absolute lymphocyte count (ALC) on day of ATG administration interacts with the dose of ATG administered to predict transplantation outcomes (Kennedy et al, Soiffer et al). We hypothesized that a similar phenomenon may be occurring when using alemtuzumab, and compared the outcomes of ATG vs alemtuzumab based regimens.

Methods: We retrospectively analyzed 287 patients, 199 patients (alemtuzumab for GVHD prophylaxis) undergoing unrelated allogeneic hematopoietic cell transplantation in leukaemia and myelodysplastic syndrome (MDS) and compared it with 88 similar patients receiving ATG from another centre from 2006-2014.

Results: 68 patients received alemtuzumab $60 \mathrm{mg}$ and 131 patients received $100 \mathrm{mg}$, whereas in ATG group 52 patients received $5 \mathrm{mg} / \mathrm{kg}, 21$ patients received $7.5 \mathrm{mg} / \mathrm{kg}$ and 13 patients received $10 \mathrm{mg} / \mathrm{kg}$ doses respectively. Median follow-up was 35 months in alemtuzumab and 30 months in ATG group. As compared to ATG group, alemtuzumab group had significantly more number of patients receiving $9 / 10$ mismatched donor grafts (3 vs $22 \%, p=0.0001$ ), patients with intermediate disease risk index, $(57 \mathrm{vs} 76 \%$, p $=0.004)$ and higher exposure to myeloablative regimen, (28 vs $40 \%, \mathrm{p}=0.04)$. The 2 -year overall survival $(\mathrm{OS})$,

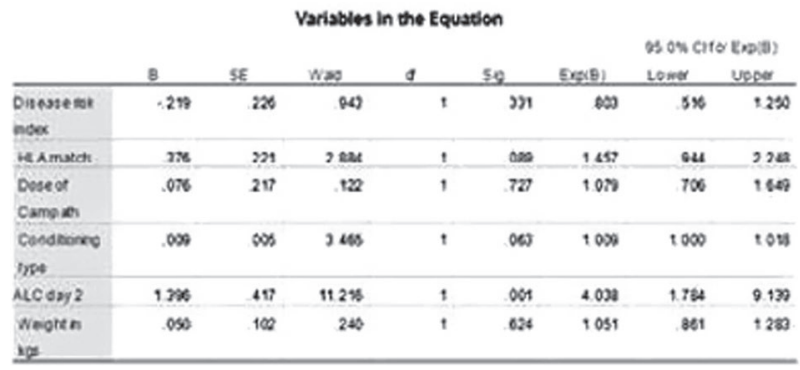

[P373 Figure] [Cox model in multivariate analysis for PFS. ALC day 2 , $p=0.001$ was significant for PFS] 
progression free survival (PFS), relapses and 1 year NRM (non-relapse mortality) for alemtuzumab group was $48 \%$, $42 \% 38 \%$ and $14 \%$, respectively and was comparable to ATG group, being $45 \%, 40 \%$ and $44 \%, 15 \%$ respectively, except for early relapses before 2 year (44 vs $32 \%$, for ATG and alemtuzumab, $\mathrm{p}=0.05$ ) being more significant for ATG group, after adjusting for baseline co-variate disparities. The incidence of severe (grade 3 and 4)acute and chronic graft vs host disease (aGVHD/cGVHD) was $12 \%$ / $11 \%$ and $16 \% / 25 \%$ for alemtuzumab and ATG and mild/ moderate aGVHD and CGVHD was 50\%/40\% and $48 \%$ / $44 \%$ for alemtuzumab and ATG respectively, with only severe chronic GVHD being lower in alemtuzumab arm $(\mathrm{p}=0.06)$. Within alemtuzumab group, only chronic mild/ moderatGVHD was significantly lower with lower doses of 60mg $(\mathrm{p}=0.05)$. In multivariate analysis, the peripheral blood ALC on day of ATG administration and the total amount of ATG interacted to predict OS (hazard ratio, 0 $.09 ; \mathrm{P}=0.03$ ). However, for alemtuzumab group, the absolute lymphocyte count after first dose, irrespective of the dose (day 2 of alemtuzumab) interacted significantly with OS and PFS (HR-4.7, 2.1-10.6, $\mathrm{p}=0.002$ and 4.8, 2.2$10.4, \mathrm{p}=0.001$, respectively), in univariate as well as multivariate analysis.

Conclusions: We concluded, that similar to ATG and peripheral blood ALC interaction, there is an interaction between alemtuzumab and peripheral blood ALC. These findings if prospectively validated will allow for optimization of alemtuzumab and ATG dosing based on patient specific ALC. Studies to explain mechanistic details of these clinical findings need to be undertaken to refine the strategies for acute GVHD prophylaxis using ATG and alemtuzumab. Overall transplant outcomes are similar in ATG or alemtuzumab based URD transplants.

\section{Clinical Trial Registry: No}

Conflict of interest: Authors 1 and 2 have contributed equally. M. Jagasia and K. Raj are corresponding authors.

\section{P374}

Incidence, risk factors and treatment response in patients with chronic graft versus-host disease after haploidentical transplantation with post-transplantation cyclophosphamide

Daniel Rivera ${ }^{1}$, Lucia López-Corral ${ }^{1}$, Mi Kwon ${ }^{2}$, Arancha Bermudez $^{3}$, Lucrecia Yañez ${ }^{3}$, Anabel Gallardo ${ }^{4}$, Monica Cabrero ${ }^{1}$, Diana Champ ${ }^{2}$, Sara Garcia-Avila ${ }^{3}$, Abel García ${ }^{4}$, Estefania Pérez ${ }^{1}$, Pascual Balsalobre $^{2}$, Mercedes Colorado ${ }^{3}$, María Jesús Pascual ${ }^{4}$, Ana Martín ${ }^{1}$, José Luís Diez ${ }^{2}$, Jorge Gayoso ${ }^{2}$, Maria Dolores Caballero $^{I}$

${ }^{1}$ Hospital Universitario de Salamanca/IBSAL, Hematology, Salamanca, Spain; ${ }^{2}$ Hospital 12 de Octubre, Hematology, Madrid,
Spain; ${ }^{3}$ Hospital Marques de Valdecilla, Hematology, Santander, Spain; ${ }^{4}$ Hospital Carlos Haya, Hematology, Malaga, Spain

Background: The use of HLA-haploidentical transplantation (Haplo-SCT) with post-transplantation cyclophosphamide (PT-Cy), has become a more frequent therapeutic approach, for patients without an available HLA-matched related or unrelated donor. According to several reports, the incidence of acute GVHD is similar or even inferior to what has been observed after HLA-matched allogeneic hematopoietic stem cell transplantation. Currently there is limited data regarding chronic GVHD in this setting. Additionally, the impact of an haploidentical donor as graft source along with the conditioning regimen remains unknown.

Objectives: The purpose of this study was to analyze the incidence, risk factors and treatment response of chronic GVHD in patients undergoing Haplo-SCT with PT-Cy.

Methods: We conducted a multi-center retrospective study in 219 patients from four Spanish centers, who received Haplo-SCT between December 2007 and September 2017.

Results: Patient and transplant characteristics are detailed in table 1. The median follow-up time was 14(3-85) months. The median time to neutrophil engraftment was $17(12-45)$ days, and of platelet engraftment was 27(6-150) days. There were 4 primary graft failures. Complete bone marrow chimerism was achieved after a median of 28(13-180) days. Transplant related mortality (TRM) at day 100 and one year was $12 \%$ and $21 \%$ respectively. Cumulative incidence (CI) of grade II-IV acute GVHD was $49 \%$ and $10 \%$ for grade IIIIV cases.

The incidence of chronic GVHD at two years was $28.7 \%$ (mild 14\%; moderate. $7.6 \%$; severe $3.1 \%$ ). Through univariate analysis we observed that, mieloablative conditioning was associated to chronic GVHD ( $p=0.047$ ). However, in multivariate analysis there was not statistical significance. Characteristics related to chronic GVHD are shown in table 2. The median time to chronic GVHD onset was $218(68-1017)$ days. In $17.5 \%(\mathrm{n}=10)$ was progressive and overlapping in $22.8 \%(\mathrm{n}=13)$ of patients. At the onset of chronic GVHD, 49.1\% $(n=28)$ of patients did not had immunosuppressive therapy (IT). Hence, the development of chronic GVHD maybe related either to reducing of stopping IT in $40.3 \%(n=23)$ of patients. The median of treatment lines for chronic GVHD was 1(1-4). Thus, the overall response rate (ORR) was $80 \%$. The $\mathrm{CI}$ of relapse at one year was $14 \%$ (overall 20\%). The composite end point at one and two years was $51.8 \%$ and $46.6 \%$ respectively. The overall survival (OS) at one and two years was $69.3 \%$ and $61.3 \%$ respectively. 
Conclusions: Haplo-SCT is a promising therapeutic approach with low rates of GVHD. Chronic GVHD was predominantly mild, showing good responses to first line treatments. Our data did not show risk factors that may predispose the development of chronic GVHD.

Conflict of interest: The authors do not have relevant conflict of interest to disclose

\begin{tabular}{|c|c|}
\hline Age at traplantation, years & $46(5-74)$ \\
\hline Gender: Male/Female & $143(65,3 \%) / 76(34,4 \%)$ \\
\hline $\begin{array}{l}\text { Diagnosis } \bullet \text { AML/MDS/ALL } \cdot \text { HL/NHL } \\
\cdot \text { Others (MPN,MM,AA) }\end{array}$ & $\begin{array}{l}76(34,7 \%) / 19(8,7 \%) / 17(7.8 \%) \\
62(28,3 \%) / 23(10,5 \%) 19(9.1 \%)\end{array}$ \\
\hline HCT-CI Sorror & $2(0-7)$ \\
\hline $\begin{array}{l}\text { Pre-Haplo disease status } \bullet \text { CR MRD- } \bullet \text { CR } \\
\text { MRD }+\bullet \text { Active disease/Progression }\end{array}$ & $101(46,5 \%) 47(21,7 \%) 71(31,8 \%)$ \\
\hline $\begin{array}{l}\text { Graft source } \bullet \text { Peripheral blood } \bullet \text { Bone } \\
\text { marrow }\end{array}$ & $167(76,3 \%) 52(23,7 \%)$ \\
\hline $\begin{array}{l}\text { Number of CD34 x106 / kg } \bullet \text { CD } 34 \text { PB } \\
\cdot \text { CD34 BM }\end{array}$ & $\begin{array}{l}5,01(0,98-13,68) 5,32(2,24-13,68) \\
2,88(0,98-11,42)\end{array}$ \\
\hline $\begin{array}{l}\text { Conditioning intensity } \bullet \text { Reduced } \\
\text { intensity } \bullet \text { Mieloablative } \bullet \text { Secuntial }\end{array}$ & $68(66 \%) 30(29,1 \%) 5(3 \%)$ \\
\hline $\begin{array}{l}\text { Conditioning type } \bullet \text { Reduced intensity } \bullet \\
\text { Flu }(150 \mathrm{mg} / \mathrm{m} 2)+\mathrm{Bu}(3,2 \text { ó } 6,4 \mathrm{mg} / \mathrm{Kg}) \\
+\mathrm{Cy}(29 \mathrm{mg} / \mathrm{Kg}) \bullet \text { Flu }(150 \mathrm{mg} / \mathrm{m} 2)+\mathrm{TBI} \\
(2 \mathrm{~Gy})+\mathrm{Cy}(29 \mathrm{mg} / \mathrm{kg}) \cdot \text { Mieloablative } \bullet \\
\text { Flu }(160 \mathrm{mg} / \mathrm{m} 2)+\mathrm{Bu}(9,6 \text { ó } 12,8 \mathrm{mg} / \mathrm{Kg}) \\
+\mathrm{Cy}(29 \mathrm{mg} / \mathrm{kg}) \bullet \mathrm{Flu}(150 \mathrm{mg} / \mathrm{m} 2)+\mathrm{Bu} \\
(9,6 \mathrm{mg} / \mathrm{Kg})+\mathrm{Thio}(10 \mathrm{mg} / \mathrm{Kg})\end{array}$ & $\begin{array}{l}128(58.4 \%) 23(10.5 \%) 38(17.3 \%) \\
22(10 \%)\end{array}$ \\
\hline $\begin{array}{l}\text { GVHD prophylaxis } \bullet \mathrm{CSA}+\mathrm{MMF}+\mathrm{Cy} \\
50 \mathrm{mg} / \mathrm{kg} \times 2 \cdot \mathrm{Mtx}+\mathrm{MMF}+\mathrm{Cy} 50 \mathrm{mg} / \\
\mathrm{kg} \times 2 \cdot \text { Others }\end{array}$ & $87(39,7 \%) 131(59,8 \%) 1(0,5 \%)$ \\
\hline
\end{tabular}

[[P374 Table] Table 1: Patient and transplant characteristics]

\section{P375}

Influence of gut decontamination prior to allogeneic hematopoietic stem cell transplantation on the risk of acute graft-versus-host disease: a retrospective single-center cohort study

\section{Lars Klingen Gjaerde, Mette Schmidt, Henrik Sengelov}

Rigshospitalet, Department of Hematology, Copenhagen, Denmark

Background: Recent research have discovered that the gut microbiota plays a major role on the development of acute graft-versus-host disease (aGvHD), yet studies on the impact of gut decontamination prior to allogeneic hematopoietic stem cell transplantation (allo-HSCT) on the risk of aGvHD are limited and conflicting.

Methods: In September 2002, Rigshospitalet changed its prophylactic measures against infections in patients undergoing allo-HSCT, including a discontinuation of the use of oral gut decontamination with cefuroxime, tobramycin and nystatin prior to transplant. We included in a retrospective cohort 283 consecutive patients who underwent their first

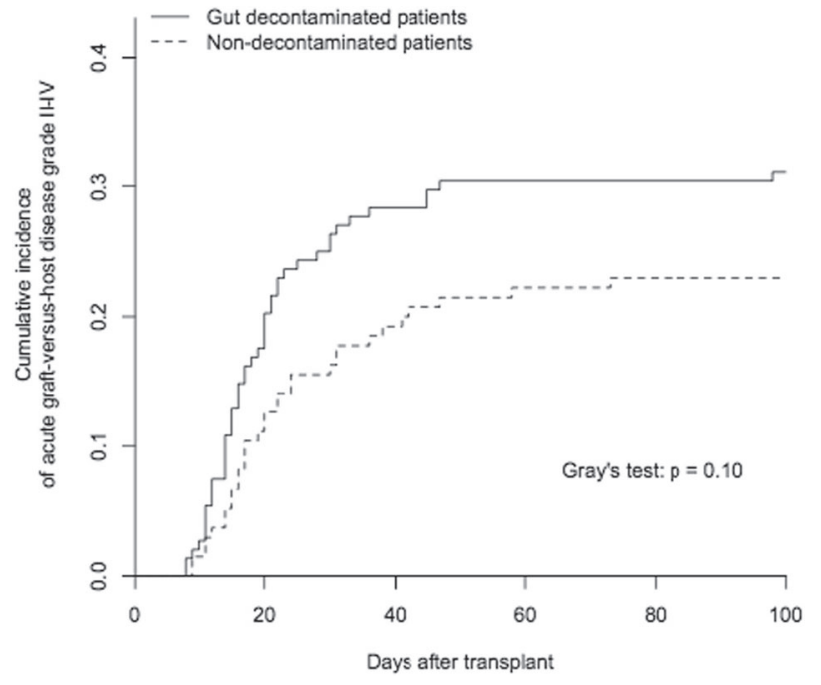

[P375 Figure] [Cumulative incidence of acute graft-versus-host-disease grade II-IV]

allo-HSCT at Rigshospitalet, of which 148 were transplanted before the change (from Jan 1998 to Aug 2002) and 135 were transplanted after the change (from Sep 2002 to Apr 2007), and studied - within the first 100 days after transplant - the development of aGvHD grade II-IV, gastrointestinal aGvHD stage II-IV and non-relapse mortality (NRM), respectively. All patients received myeloablative conditioning and GvHD prophylaxis consisting of ciclosporin and methotrexate. Multivariate Cox proportional hazards models, censoring subjects at death and relapse, were employed to estimate hazard ratios (HR). A priori defined covariates were age, stem cell source, femaledonor-to-male-recipient, donor relation plus matching, use of total body irradiation and use of anti-thymocyte globulin.

Results: Gut decontaminated- and non-decontaminated patients were comparable when regarding age (median of 33 and 36 years, respectively) and sex (34\% and $41 \%$ female, respectively), but differed when regarding diagnosis, stem cell source ( $28 \%$ and $49 \%$ peripheral blood, respectively) and donor relation $(60 \%$ and $43 \%$ matched related donor, respectively). aGvHD grade II-IV occurred in 31\% $(95 \%$ confidence interval (CI): $24 \%, 39 \%$ ) of gut decontaminated patients and in 23\% (95\% CI: $16 \%, 30 \%)$ of nondecontaminated patients (Figure 1).

Gastrointestinal aGvHD stage II-IV occurred in 9\% (95\% CI: $4 \%, 13 \%$ ) of gut decontaminated patients and in $4 \%$ (95\% CI: $1 \%, 7 \%$ ) of non-decontaminated patients, and non-relapse mortality at day 100 after transplant was $10 \%$ (95\% CI: $5 \%, 15 \%$ ) in gut decontaminated patients and $8 \%$ $(95 \%$ CI: $4 \%, 13 \%)$ in non-decontaminated patients. In adjusted multivariate analysis, the receipt of gut decontamination prior to transplant was significantly associated with an increased hazard of aGvHD grade II-IV (HR 1.81, 
95\% CI: 1.07, 3.06), and - however not with statistical significance - with an increased hazard of gastrointestinal aGvHD stage II-IV (HR 3.29, 95\% CI: 0.96, 11.26) and non-relapse mortality (HR 1.99, 95\% CI: 0.76, 5.24).

Conclusions: Patients who received oral gut decontamination prior to allo-HSCT had, after adjustment for potential confounders, an increased risk of developing aGvHD within the first 100 days after transplant. While there also were trends in adjusted analysis towards these patients also having an increased risk of gastrointestinal aGvHD and non-relapse mortality, the associations did not reach statistical significance. Our results support the idea that disruption of the gut microbiota prior to transplant worsens the risk of developing aGvHD.

Conflict of interest: None of the authors has anything to disclose.

\section{P376}

Interferon-Gamma as an Early Biomarker to Predicts GraftVersus-Host Disease in Allogeneic Hematopoietic Stem Cell Transplanted Patients

\author{
Ahmad Abu-Khader ${ }^{1}$, Abdelghani Tbakhi ${ }^{1}$, Ernst Holler ${ }^{2}$ \\ ${ }^{1}$ King Hussein Cancer Center, Cell Therapy and Applied Genomics, \\ Amman, Jordan; ${ }^{2}$ University of Regensburg, Department of \\ Hematology and Oncology, Regensburg, Germany
}

Background: No laboratory test can predict the risk of graft-versus-host disease (GvHD) or monitor its activity after allogeneic hematopoietic cellular transplantation (HSCT). Here, we describe an immunological assay that may help to detect GvHD using interferon-gamma (IFN-g) production using real-time PCR (RT-PCR) assay.

Methods: After obtaining peripheral blood mononuclear cells (PBMNCs) from blood samples of 27 patients' undergoing allogeneic HCST, 70 samples were assessed for IFN-g production using a RT-PCR assay at different time points after HSCT. We measured IFN-g production as a GvHD biomarker using a mixed lymphocyte reaction (MLR) protocol. PBMNCs from patients were challenged in vitro with recipient cells obtained before HSCT (BT) or with an autologous control, which represents the donor (D) cells, in one-sided MLR. After 3 hours of MLR, IFN-g production was measured using RT-PCR, and "IFN-g folds change" was used as a unit to express the production. More than two folds of IFN-g production were considered a significant increase. Patients were grouped according to time points depending on presence of GvHD and immunosuppression status at the time of blood collection (1: never GvHD, on cyclosporine A (CsA) only; 2: never GvHD but on immunosuppression with CsA and corticosteroids, 3: onset of GvHD, CsA only, no corticosteroids, 4: GvHD, on treatment with corticosteroids below $1 \mathrm{mg} / \mathrm{Kg}$, and 5: GvHD on high dose corticosteroids).

Results: Patients with neither GvHD nor immunosuppression showed basal IFN-g production of $1.05 \pm 0.49$ and $1.26 \pm$ 0.47 when patient's PBMNCs were challenged against BT or $\mathrm{D}$, respectively. IFN-g production increased to $2.53 \pm 1.69$ and $0.80 \pm 0.17$ in BT and D settings with no GvHD but with low immunosuppression, respectively. With the onset of GvHD but without any additional immunosuppressive intervention, IFN-g production was significantly higher $(\mathrm{BT}=6.55 \pm 2.05$ vs. $\mathrm{D}=1.47 \pm 0.275$ ) compared to no GvHD and no immunosuppression $(\mathrm{P}=0.04)$ as well to GvHD on treatment with corticosteroids below $1 \mathrm{mg} / \mathrm{Kg}(\mathrm{P}=0.009)$, and even to GvHD on high dose corticosteroids $(\mathrm{P}=0.04)$. The overall IFN-g production in recipient (BT) samples $(3.30 \pm 0.782)$ was found to be higher than control (D) samples (1.52 \pm 0.261 ), which confirm the specificity of our detection assay.

Conclusions: Our findings suggest that recipient PBMNCs can produce significant amounts of INF-g as an indicator of active GvHD, after challenging with recipient HLA-matched cells mimicking an in vivo sensitizing stimulus. Immunosuppression with corticosteroids suppresses the IFN-g response and must be considered in surrogate assays of GvHD responses.

Clinical Trial Registry: not applicable

Conflict of interest: None of the authors has anything to disclose.

\section{P377}

Intestinal microbial diversity might serve as a biomarker for the outcome of acute graft-versus-host disease

Maleen Beck ${ }^{1}$, Eric Galvez ${ }^{2}$, Jan Beneke ${ }^{3}$, Harald Schrem ${ }^{3}$, Melanie Drenker ${ }^{1}$, Elke Dammann ${ }^{1}$, Matthias Eder ${ }^{1}$, Arnold Ganser ${ }^{1}$, Till Strowig $^{2}$, Christian Koenecke ${ }^{1}$

${ }^{1}$ Hannover Medical School, Hematology, Hemostasis, Oncology and Stem Cell Transplantation, Hannover, Germany; ${ }^{2}$ Helmholtz Centre for Infection Research, Braunschweig, Germany; ${ }^{3}$ Hannover Medical School, Core Facility Quality Management and Health Technology Assessment in Transplantation, Hannover, Germany

Background: The gastrointestinal tract is populated by a highly diverse microbial ecosystem, the microbiota, that is a potent modulator of local inflammation and systemic immune responses. However, medical treatments such as broad-spectrum antibiotic treatment, which is often given in the course of allogeneic hematopoietic stem cell transplantation (allo-HSCT), influence the diversity of the microbiota thereby impairing host immunity. The relationship between changes in the diversity of the gut microbiota and the occurrence of major inflammatory as well as immune 


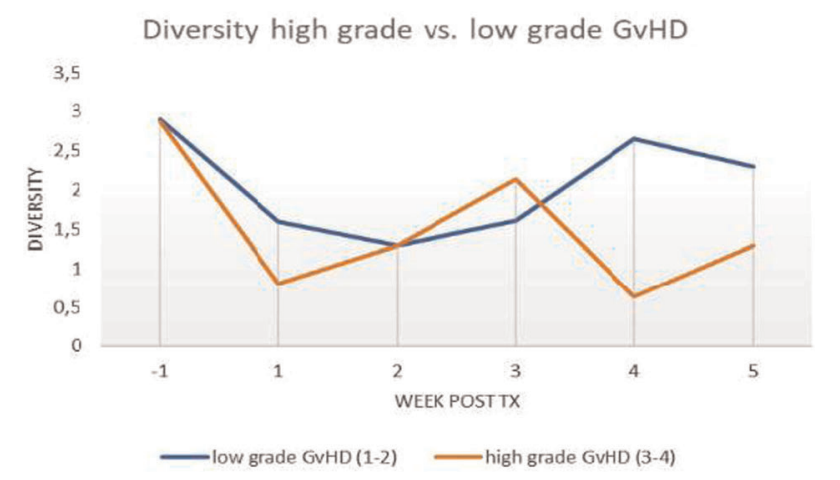

[P377 Figure] [Diversity high grade vs. low grade GvHD]

related complications, especially acute GVHD, during alloHSCT is largely unknown.

Methods: We examined longitudinal changes in intestinal microbial diversity in patients undergoing allo-HSCT and their relationship to subsequent complications, especially acute GvHD. In a prospective clinical trial, we collected fecal specimens from 53 patients admitted to alloHSCT before and weekly after HSCT. 16S rRNA gene sequencing was used to characterize the microbiota and microbial diversity was quantified using the Shannon index. Moreover, we collected approximately 35 different clinical variables, including conditioning regimen, antibiotic use and nutrition to investigate their impact on the gut microbiota. Finally, for comparison of different groups medians (ranges) and means (standard deviation) were calculated and statistical analysis was performed by using Wilcoxon- and t-test.

Results: Allo-HSCT and its accompanying treatment, such as antibiotic treatment and conditioning therapy leads to a marked decrease of the intestinal microbial diversity which is most pronounced two weeks after HSCT (4-fold reduction from samples before HSCT to samples two weeks after HSCT). Contrary to our expectations, we found no difference in the diversity of the microbiota between acute GVHD patients and non-acute GvHD patients before onset of clinical symptoms $(0.7(0.7-1.9)$ vs. $0.7(0.4-1.2))$ at two weeks after HSCT, $p=0.49$ ). However, patients who developed acute GVHD showed a delayed recovery of their microbiota in comparison to patients without acute GvHD. Especially at 8 weeks after HSCT there was a significant difference in the diversity between these groups (2.8 (1.6-2.9 vs. 3 (2.9-3.3), $\mathrm{p}=0.03)$. Among the patients with acute GvHD there was no difference between patients who received a treatment with corticosteroids and those who did not (mean 2.14 (SD 1.31) vs. 2.88 (0.58), p = $0.41)$. Furthermore, more detailed analyses showed around the time of clinical manifestation of GvHD (median 4-6 weeks after HSCT) a lower diversity in patients who suffer from high grade GvHD (3-4) compared to those who suffer from low grade GvHD (1-2) (mean 0.63 (SD 0.26) vs. 2.65 (SD 0.41), 4 weeks after HSCT, $\mathrm{p}=0.001$ ).

Conclusions: During allo-HSCT we observed a drastic decrease of the intestinal microbial diversity in all patients. We were not able to distinguish between patients who subsequently developed an acute GvHD and those who did not. However, a high grade GvHD is reflected by a lower diversity, and therefore microbiome diversity in the course of GvHD might serve as a potential biomarker for GvHD outcome and/ or treatment response. Further analyses with larger number of cases are needed to confirm these results and additional studies of specific bacterial species might deliver more information.

Conflict of interest: The authors have no conflict of interest to disclose.

\section{P378}

Intraosseous injections of mesenchymal stem cells is promising therapeutic option for treatment of children with graft hypofunction associated with severe GvHD

Elena Skorobogatova ${ }^{1}$, Kirill Kirgizov ${ }^{1,2}$, Oksana Blagonravova ${ }^{1}$, Bazarma Purbueva ${ }^{1}$, Ekaterina Pristanskova ${ }^{1}$, Elena Osipova ${ }^{2}$

${ }^{1}$ Russian Children's Research Hospital, Moscow, Russian Federation; ${ }^{2}$ Dmitry Rogachev National Research Center of Pediatric Hematology, Oncology and Immunology, Moscow, Russian Federation

Background: Severe GvHD treatment is always faced with a series of clinical challenges such as severe infections, organ toxicity and graft hypofunction. Hypofunction lead to poor prognosis. Thus, the graft function recovery is crucial for successful outcome of treatment. Mesenchymal stem cells (MSCs) intravenous application together with combined immunosuppressive therapy is widely used method for treatment of severe GvHD. Experience of Intraosseous injections of MSCs is limited. We aimed to investigate the effectiveness and safety of intraosseous injections of MSCs.

Methods: Seven patients with a refractory cytopenia as a result of severe GvHD II-IV gr. were included. Age median - 11 y.o. $(6-16)$. M:F rate - 4:3. Two patients were with acute lymphoblastic leukemia and 5 - with aplastic anemia. HSCT types: MUD - 2, mismatched MUD - 3, MRD - 2. Graft type: BM - 3, PBSC - 4. MSCs were injected with the help of intraosseous needle directly to the iliac crest. MSC dose median - 0.8 millions $/ \mathrm{kg}(0.5-1.5)$. Median of day of first injection was 190 (54-410).

Results: All patients included to the study restored the graft function in 1,3 months (1-2). Graft function improvement was correlated with GvHD symptoms reduction. Median number of injections to recover graft function was 6 (2-9). No complications were found. Median of follow-up is 34 months (5-72). All patients are alive and disease free. 
Conclusions: Thus, intraosseous injections of MSCs safe and well-tolerated method to restore the graft function and reduce GvHD. This method could be a feasible option for patients with complicated course of GvHD due to absence of toxicity and complications. Future studies could be organized.

Conflict of interest: No conflicts of interest.

\section{P379}

\section{Leflunomide- the new trump card for therapy of musculoskeletal GVHD?}

Aniket Mohite, Avinash Bonda, Kalasekhar Vijayshekaran, Sachin Punatar, Kartthik S, Lingaraj Nayak, Anant Gokarn, Navin Khattry

Tata Memorial Centre and ACTREC, Bone Marrow Transplant Unit, Navi Mumbai, India

Background: Musculoskeletal GvHD is a chronic disease with varied manifestations- fasciitis, scleroderma, myositis, joint stiffness and arthritis. Similar to other autoimmune disorders, there is crosstalk between T-cells, B cells, macrophages, fibroblasts and release of various cytokines. Systemic steroids, cyclosporine, tacrolimus, sirolimus, mycophenolatemofetil, etanarcept, imatinib, all have been used with far and few responses but with significant treatment related toxicity and costs. Leflunomide has anti-T cell proliferative action, is inexpensive with minimal adverse effects and is a steroid sparing agent used as disease modifying drug in rheumatoid arthritis. While leflunomide has been shown to be effective in GVHD in animal models, its use in clinic is unknown.

Methods: Leflunomide was used as single agent in patients with chronic musculoskeletal GvHD from March 2016 to October 2017. The dose used was $100 \mathrm{mg}$ daily for 3 days followed by $20 \mathrm{mg}$ daily. The severity was assessed by National Institute Health (NIH) joint score and Photographic Range of Motion (PROM) score at baseline, 1 month and 3 monthly thereafter. Response was defined as $>50 \%$ improvement of joint symptoms and range of motion. The PROM score ranges from 4-25(best score 25) and NIH score ranges from 0-3(best score 0). Baseline MRI of specific site involved was done for all patients at the time of diagnosis of GVHD.

Results: Five patients in above setting received leflunomide. The median age was 33 years (range 14-65) with 4 males and 1 female. One patient had mixed phenotypic acute leukemia (MPAL), 1- primary myelofibrosis (PMF), 1- Ph+ ALL, 1- CML in blast crisis and 1- MDS-AML. Three patients had fully matched sibling, 1- unrelated donor and 1- haploidentical donor grafts respectively. All were GCSF mobilised PBSC grafts. The conditioning regimens used were fludarabine with melphalan, treosulphan or 4 Gy TBI $(\mathrm{n}=4)$ and Cy-TBI with rabbit ATG $(\mathrm{n}=1)$. GVHD prophylaxes included cyclosporine or tacrolimus with methotrexate $(\mathrm{n}=3)$, mycophenolate mofetil $(\mathrm{n}=1)$ and post-transplant cyclophosphamide $(\mathrm{n}=1)$.

The musculoskeletal GVHD course of the 5 patients is shown in table below:

The median duration of onset of musculoskeletal GvHD was D+733 (range:83-1600). The median baseline PROM score and NIH score were 20 (range:9-22) and 2 (range:2-3) respectively. The median time to achieve best response was 30 days (range:4-180). The median PROM and NIH scores at best response were 24 (range:17-25) and 0 . None of the patients received any other adjuvant therapy. There were no toxicities due to leflunomide.

Conclusions: This is the first case series showing efficacy of leflunomide in the setting of musculoskeletal GVHD to our knowledge. A large prospective study in this setting is warranted.

Conflict of interest: None of the authors has anything to disclose.

\begin{tabular}{|c|c|c|c|c|c|c|}
\hline $\begin{array}{l}\text { Serial } \\
\text { number }\end{array}$ & $\begin{array}{l}\text { Symptoms at } \\
\text { presentation }\end{array}$ & $\begin{array}{l}\text { Day of } \\
\text { presentation }\end{array}$ & $\begin{array}{l}\text { Baseline } \\
\text { PROM } \\
\text { score/ } \\
\text { NIH } \\
\text { score }\end{array}$ & $\begin{array}{l}\text { Day of } \\
\text { starting } \\
\text { leflunomide }\end{array}$ & $\begin{array}{l}\text { Time to } \\
\text { achieve } \\
\text { best } \\
\text { response } \\
\text { (days) }\end{array}$ & $\begin{array}{l}\text { PROM } \\
\text { score/ } \\
\text { NIH } \\
\text { score at } \\
\text { best } \\
\text { response }\end{array}$ \\
\hline $\begin{array}{l}\text { Patient } \\
1\end{array}$ & $\begin{array}{l}\text { Painful restriction } \\
\text { of shoulders, knees } \\
\text { and palmar small } \\
\text { joint }\end{array}$ & $D+133$ & $21 / 2$ & $\mathrm{D}+144$ & 30 & $24 / 1$ \\
\hline $\begin{array}{l}\text { Patient } \\
2\end{array}$ & $\begin{array}{l}\text { Bilateral palmar } \\
\text { contractures, diffuse } \\
\text { skin sclerosis, } \\
\text { trismus, restriction } \\
\text { of bilateral elbows }\end{array}$ & $\mathrm{D}+1600$ & $9 / 3$ & $D+1649$ & 180 & $17 / 1$ \\
\hline $\begin{array}{l}\text { Patient } \\
3\end{array}$ & $\begin{array}{l}\text { Restriction of right } \\
\text { shoulder } \\
\text { movements }\end{array}$ & $\mathrm{D}+733$ & $20 / 2$ & $\mathrm{D}+733$ & 30 & $24 / 0$ \\
\hline $\begin{array}{l}\text { Patient } \\
4\end{array}$ & $\begin{array}{l}\text { Right shoulder } \\
\text { movement } \\
\text { restriction }\end{array}$ & $\mathrm{D}+1378$ & $20 / 2$ & $\mathrm{D}+1393$ & 8 & $25 / 0$ \\
\hline $\begin{array}{l}\text { Patient } \\
5\end{array}$ & $\begin{array}{l}\text { Restriction of } \\
\text { bilateral shoulder } \\
\text { movements }\end{array}$ & $\mathrm{D}+83$ & $22 / 2$ & $\mathrm{D}+95$ & 4 & $25 / 0$ \\
\hline
\end{tabular}

[[P379 Table] Course of musculoskeletal GvHD and leflunomide]

P380

Less GvHD but more early deaths after in-vivo T-cell depletion in allogeneic stem cell transplantation from unrelated donors. Six years experience from a single centre

Malin Nicklasson ${ }^{1}$, Mikael Lisak ${ }^{2}$, Yvonne Björk ${ }^{3}$, Harald Anderson $^{4}$, Mats Brune ${ }^{3}$

${ }^{1}$ University of Gothenburg, Section of Hematology, Gothenburg, Sweden; ${ }^{2}$ Sahlgrenska University Hospital, Section of Hematology, Gothenburg, Sweden; ${ }^{3}$ University of Gothenburg, Department of 
Hematology, Gothenburg, Sweden; ${ }^{4}$ Lund University, Department of Cancer Epidemiology, Lund, Sweden

Background: T-cell depletion (TCD) with antithymocyte globuline (ATG) or alemtuzumab decreases risk of chronic GvHD (cGvHD) in alloSCT from unrelated donors (URD). This retrospective study compare outcome between URD/TCD and matched sibling donors (MSD/ noTCD).

Methods: Patients were allografted at Sahlgrenska University Hospital 2010-2015. Patients with aplastic anemia, or with unconventional transplant procedures were excluded $(\mathrm{n}=30)$. Remaining 167 patients, median age 50 (17-71), 54\% males, 40 MSD and 127 URD (HLA 96\% 9$10 / 10)$. Conditioning was RIC $(n=63)$, MAC $(n=104)$, BM $(n=26)$ or PBSC $(n=141)$. Diagnoses were AML/ ALL $(\mathrm{n}=97), \mathrm{MDS} / \mathrm{MPN} / \mathrm{CML}(\mathrm{n}=32), \mathrm{CLL} / \mathrm{HD} / \mathrm{NHL} /$ MM $(\mathrm{n}=35)$ and other $(\mathrm{n}=3)$. Immunosuppression included ciclosporin-A (CSA) and methotrexate. TCD in URDs were thymoglobuline $4-6 \mathrm{mg} / \mathrm{kg}(\mathrm{n}=84)$, antilymphocyte globuline $30-40 \mathrm{mg} / \mathrm{kg}(\mathrm{n}=26)$, or alemtuzumab $(\mathrm{n}=17)$. Chronic GvHD was graded (NIH 2005) at 12 , 24 and 36 months after alloSCT.

Results: At analysis, follow-up for living patients was median 49 (20-93) months. URD patients were older, $51 \mathrm{vs}$ 43 years $(\mathrm{p}=0.052)$, with younger donors ( $27 v s 44$ years; $\mathrm{p}$ $<0.001$ ), whereas risk category, BM/PBSC and RIC/MAC were similar.

Standard methotrexate dose $(45 \mathrm{mg} / \mathrm{sqm})$ was used in $48 \%$ of URD patients, whereas $78 \%$ of MSD patients received $\leq 35 \mathrm{mg} / \mathrm{kg}(\mathrm{p}=0.006)$. Median CSA trough level at Day +30 was higher in URD patients: 170 (53-380) vs 130 $(59-260) \mu \mathrm{g} / \mathrm{L}(\mathrm{p}<0.001)$.

OS at 4 yrs was $75 \%$ and $56 \%$ (K-M estimate) in MSD and URD groups, respectively. At follow-up, cumulative incidence, and causes, of death in MSD and URD groups were relapse (18vs 25\%), GvHD (2.5 vs 6.3\%), infection (0 vs 7.9\%), other (5 vs 5\%). Both OS and LFS were superior in the MSD group (log rank test: $\mathrm{p}=0.020$ and $\mathrm{p}=$ 0.0065). NRM and relapse incidences were higher in MUDs (19 vs 7.5\%, $\mathrm{p}=0.038$, and 31 vs 20\%, $\mathrm{p}=0.066$ ), respectively. URD patients had more EBV infections (30 vs $5 \% ; \mathrm{p}=0.001)$. Incidence of fungal and CMV infections were similar.

Grade II-IV acute GvHD was $43 \%$ in MSD vs $23 \%$ $(\mathrm{p}=0.024)$. Moderate/severe cGvHD at 12 months was $50 \%$ and $11 \%(\mathrm{p}<0.001)$ in MSD and URD patients, respectively. Glucocorticoid treatment at 12 months was ongoing in $69 \%$ of MSD vs $33 \%$ ( $p<0.001)$ with similar (significant) pattern at 24 and 36 months.

A large difference in early ( $\leq 12$ months) mortality was found between MSD $(n=1)$ and URD $(n=32)(p=0.001)$.
Causes of death in URDs: relapse $(\mathrm{n}=18)$, infection $(\mathrm{n}=$ 7), GvHD-related infections $(n=4)$, other $(n=3)$. In a multivariate Cox analysis adjusted for risk, RIC/MAC, diagnosis (acute leukemia vs other), CSA, the higher mortality rate in URD patients during the first year remained $(\mathrm{HR}=8.6(95 \%$ CI 1.1-66.1).

Conclusions: In this study, the prevalence of cGvHD was higher in MSD patients. However, URD patients did significantly worse in terms of OS, LFS and NRM seemingly due to $25 \%$ mortality the first year after transplant. Speculatively, the combination of TCD, more methotrexate and higher CSA levels in URD patients may have increased the risk of relapse and lethal infections, albeit the influence of confounding factors; co-morbidities, previous treatments, is not clear. Our results merit studies on optimal TCD dose.

Conflict of interest: No conflict of interest

\section{P381}

Abstract previously published

\section{P382}

Low dose rabbit anti-thymocyte globulin for graft versus host disease prophylaxis after unrelated hematopoietic stem cell transplantation in pediatric patients

Didem Atay, Fatih Erbey, Arzu Akcay, Aygozel Akmuradova, Florenc Seferkolli, Gulyuz Ozturk

Aclbadem University, Pediatric Hematology/Oncology \& Pediatric BMT Unit, Istanbul, Turkey

Background: Graft versus host disease (GVHD) is a major cause of morbidity and mortality after allogeneic hematopoietic stem cell transplantation (HSCT) from unrelated donors. Anti-thymocyte globulin (ATG) is commonly used as prophylaxis for GVHD. The studies according to optimum dose of ATG especially in pediatric patients are limited.

Methods: We therefore analyzed a retrospective study of patients who received ATG-Fresenius (ATG-F) as GVHD prophylaxis for matched unrelated donor (MUD) HSCT. A total of 91 patients were identified (median age 7.8 years, range 0.7-18.8). The median duration of follow-up was 19.3 months (range, 1.5-47 months). Seventy seven patients received a myeloablative regimen, 14 patients received reduced intensity conditioning regimen. ATG-Fresenius was given to 62 patients ( $68.1 \%$, group 1$)$ at a total dose of $10 \mathrm{mg} / \mathrm{kg}$ (3-3-4 mg/kg on days -3 to -1$)$ and to 29 patients (31.8\%, group 2) at a total dose of $15 \mathrm{mg} / \mathrm{kg}(5 \mathrm{mg} / \mathrm{kg}$ on days -3 to -1). GVHD prophylaxis consisted of cyclosporine (CSA) in 23 patients, CSA+methotrexate (MTX) in 32 
patients, CSA + mycophenolate mofetil (MMF) in 19 patients and CSA + MMF + MTX in 17 patients.

Results: Acute grade I-II and grade III-IV GVHD were observed $22 \%$ and $35.2 \%$ of all patients. $35.5 \%$ of group 1 patients (22/62) and $34.5 \%$ of group 2 patients (10/29) had severe acute GVHD ( $\geq$ grade III). The incidences of mildmoderate chronic GVHD and severe chronic GVHD were $5.5 \%$ (6.5\% in group 1 and $3.4 \%$ in group 2) and 5.5\% (4.8\% in group 1 and $6.9 \%$ in group 2), respectively. The incidences of acute and chronic GVHD were not statistically different between two groups $(\mathrm{p}=0.96$ and $\mathrm{p}=0.78$ ). Sixty four patients $(70.3 \%)$ had one viral infection, 30 patients $(33 \%)$ had more than one viral infection. The cumulative incidence of transplant related mortality (TRM) for the whole patients were $7.7 \%$ at day 100 and $26.2 \%$ at 4year. The probability of overall survival (OS) at day 100 and at 4-year was $91.2 \%$ and $68 \%$. TRM and OS did not differ between two groups $(\mathrm{p}=0.7, \mathrm{p}=0.22)$.

Conclusions: The addition of low-dose ATG $(10 \mathrm{mg} / \mathrm{kg}$ or $15 \mathrm{mg} / \mathrm{kg}$ ) to GVHD prophylaxis in pediatric patients who received MUD HSCT resulted in increased incidences of acute grade I-II and grade III-IV GVHD in comparison with other pediatric studies with higher doses of ATG $(30 \mathrm{mg} / \mathrm{kg})$, but the incidence of chronic GVHD was similar. Studies about ATG pharmacokinetics and pharmacodynamics are ongoing. The effects of low-dose and patient-specific dose ATG should be assessed in a prospective clinical trial.

Conflict of interest: None

\section{P383}

Low incidence of severe acute GvHD and NRM with tacrolimus and methotrexate for GvHD prevention after umbilical cord blood transplantation

Yasushi Onishi, Masahiro Kobayashi, Sayaka Sano, Tetsuro Ohchi, Kentaro Nasu, Satoshi Ichikawa, Yoko Okitsu, Noriko Fukuhara, Tohru Fujiwara, Minami Yamada Fujiwara, Hideo Harigae

Tohoku University Hospital, Department of Hematology and Rheumatology, Sendai, Japan

Background: Although combination of tacrolimus (Tac) and short-term methotrexate (MTX) is one of the standard GvHD prophylaxis, it is unclear whether its use after cord blood transplantation (CBT) is appropriate.

Methods: We retrospectively analyzed the outcomes of patients with hematological malignancies, aged $\geq 16$ years, who underwent single-unit CBT with Tac/MTX for prevention of GvHD. Tac was administered from day -1 at $0.015 \mathrm{mg} / \mathrm{kg}$ continuous intravenously (target concentration of $8-12 \mathrm{ng} / \mathrm{ml}$ ) and MTX at $10 \mathrm{mg} / \mathrm{m}^{2}$ intravenously on day 1 , followed by $7 \mathrm{mg} / \mathrm{m}^{2}$ on days 3 and 6 .
Results: A total of 101 first CBT for 38 AML, 23 ALL, 3 CML-BC, 11 MDS, 1 MPD, 25 lymphoid malignancies were included. Forty-eight patients received CBT in first or second CR(standard risk, SR) and 53 patients did in third or further CR, or non-remission (high-risk, HR). The median age of patients was 36 years (range, 16 to 66). Myeloablative conditioning (MAC, $\mathrm{n}=74$ ); cyclophosphamide (CY) plus 12Gy of TBI with cytarabine, or reducedintensity conditioning (RIC, $n=27$ ); fludarabine plus $C Y$ or melphalan with $4 \mathrm{~Gy}$ of TBI, were given before CBT. The median nucleated and CD34+ cell doses were $2.5 \times 10^{7} / \mathrm{kg}$ (range, 1.6 to 5.9 ) and $1.1 \times 10^{5} / \mathrm{kg}$ (range, 0.34 to 6.2 ), respectively. The cumulative incidence of neutrophil engraftment at day 60 was $80.2 \%$ (95\% confidential interval [CI], 70.9 to 86.8 ), and platelet recovery at day 180 was $74.3 \%$ [95\% CI, 64.3 to 81.8]. The median times to neutrophil engraftment and platelet engraftment were 20 days (range, 12 to 38 ) and 40 days (range, 24 to 127), respectively. The rate of acute GvHD of grade 2-4 was $23.9 \%$ [95\%CI, 16.1 to 32.7], and the rate of acute GvHD of grade 3-4 was $6.1 \%$ [95\% CI, 2.5 to 12 ]. With a median follow-up of 60 months, the cumulative incidence of chronic GvHD was 34.3\% [95\%CI, 24.9 to 43.9]. The use of systemic steroid for GvHD treatment was needed in $25 \%$ of the patients with acute GvHD of grade 2-4, and in $21 \%$ of those with cGvHD. There were no significant differences in the rates of engraftment or GvHD between MAC and RIC groups. The 5-year rate of overall survival was $81.9 \%$ [95\% CI, 68.1 to 90.2] in the SR group and $37.8 \%$ [95\%CI, 22.8 to 52.8] in the HR group. The cumulative incidence of relapse at 2 years was $16.4 \%$ [95\% CI, 7.6 to 28.3 ] in the SD group and $52.2 \%$ [95\%CI, 35.9 to 66.2 ] in the HD group. The cumulative incidence of non-relapse mortality (NRM) at 2 years were $12.5 \%$ [95\% CI, 6.8 to 20.1 ].

Conclusions: GvHD prophylaxis with FK/MTX resulted in a low rate of severe acute GvHD and contributed to low incidence of NRM after CBT. However, the results of engraftment rate and relapse rate were not satisfactory. A prospective study is needed to find the optimal dosage of MTX for GvHD prevention after CBT.

Conflict of interest: The authors have no conflict of interest to disclose.

\section{P384}

Low rates of acute/chronic GVHD using combination of ATG and PTCy as GVHD prophylaxis in MUD transplants for hematological malignancies - a single centre experience

Shruti Prem Sudha ${ }^{1}$, Arjun Law ${ }^{1}$, Wilson Lam ${ }^{1}$, Santhosh Thyagu ${ }^{1}$, Fotios Michelis ${ }^{2}$, Dennis Kim ${ }^{1}$, Jeffrey Lipton ${ }^{1}$, Hans Messner ${ }^{1}$, Auro Viswabandya ${ }^{1}$ 


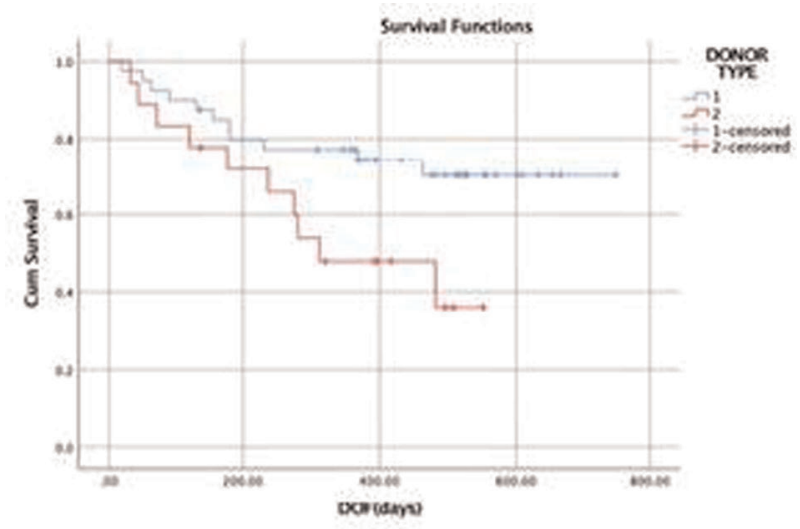

[P384 Figure] [Cumulative OS for donor type 1 (10/10 matched) and donor type 2 (9/10 matched)]

${ }^{1}$ Princess Margaret Hospital, DMOH, Toronto, Canada; ${ }^{2}$ Princess Margaret Hospital, Toronto, Canada

Background: The non-myeloablative conditioning regimen using intra-venous Fludarabine and Busulphan with ATG and post- transplant cyclophosphamide (PTCy) for GVHD prophylaxis has been demonstrated to have good efficacy/safety in several studies in HLA matched and mismatched transplants. PTCy and ATG have been shown to decrease the risk of acute and chronic GVHD respectively. We adopted this combination regimen for GVHD prophylaxis for matched unrelated donor (MUD) PBSCTs in Princess Margaret Hospital, Toronto in order to decrease the rates of acute and chronic GVHD in this patient population.

Methods: 58 adult patients (median age 58y, range 29$73 y$ ) with hematological malignancies (AML-29,MDS-14, MPD-8, ALL-3,CML-2,lymphoma-2) were treated with this protocol in Princess Margaret Hospital from October, 2015 to December 2016. 18 patients received stem cell transplants from 9/10 HLA matched unrelated donors while the remainder received stem cells from fully matched unrelated donors.

Pre-transplant conditioning consisted of iv Fludarabine $(30 \mathrm{mg} / \mathrm{m} 2 /$ day) for 4 days, i.v. Busulphan $(3.2 \mathrm{mg} / \mathrm{kg} /$ day for 2 days), and low dose TBI (200cGy) on day-1. GVHD prophylaxis was with rabbit ATG $4.5 \mathrm{mg} / \mathrm{kg}$ over 3 days (day-3 to -1) and PTCy at $50 \mathrm{mg} / \mathrm{kg} /$ day on days +3 and +4 and cyclosporine $(5 \mathrm{mg} / \mathrm{kg} /$ day $)$ from day +5 .

Results: At a median follow up of 380 days (range 18750 days), the cumulative OS and PFS in our patients was $60 \%$ and $55 \%$ respectively. The 100 day mortality was $12.1 \%$ while the overall mortality was $36.2 \%$ and the nonrelapse mortality (NRM) was $24 \%$. There were 2 primary graft failures and 2 secondary graft failures.

In sub-group analysis of 10/10 versus 9/10 HLA matched transplants, the latter group had significantly inferior OS and DFS (70\% OS for $10 / 10$ vs $36 \%$ OS for $9 / 10, p=$
0.034 ; and $66 \%$ PFS for $10 / 10$ vs $30 \%$ PFS for $9 / 10, p=$ 0.02 ).

The overall incidence of acute GVHD was $34.5 \%$, and of grade 3-4 acute GVHD was $8.5 \%$. Steroid refractory acute GVHD was seen in 2 patients. The overall incidence of chronic GVHD was $21.5 \%$ (extensive chronic GVHD was seen in 3 patients). The overall relapse rate was $15.5 \%$, all relapses occurring within the first one year.

Conclusions: Our experience confirms that PTCy and ATG can be combined safely and effectively for GVHD prophylaxis in matched unrelated donor peripheral blood stem cell transplants with acceptable non relapse mortality and relapse rates and low rates of Gr3-4 acute GVHD and of chronic GVHD.

Conflict of interest: The authors have no conflicts of interest to disclose.

\section{P385}

Abstract previously published

\section{P386}

Multi-state modelling to evaluate strategy of scheduled prophylactic donor lymphocyte infusions after T-cell depleted transplantation following low-toxicity conditioning for elderly patients with high risk leukemia

E.A.S. Koster, L.C. de Wreede, P.A. von dem Borne, E.W.A. Marijt, P. van Balen, S.M. Wallet-Malicka, E.J.M. Bogers, J.H. Veelken, C.J. M. Halkes, J.H.F. Falkenburg

Leiden University Medical Center, Leiden, Netherlands

Background: T-cell depletion reduces the risk of acute graft-versus-host-disease (GVHD) after allogeneic stem cell transplantation (alloSCT), but increases relapse risk. Therefore, prophylactic donor lymphocyte infusions (DLIs) may be necessary to provoke a graft-versus-leukemia-effect. However, after postponed DLI GVHD can still occur, depending on timing and DLI dose. To evaluate the outcomes of the combination of alloSCT and prophylactic DLIs, standard statistical models are not sufficient and novel methods like multi-state models are needed. We report the outcome of a low-toxicity conditioning regimen combining in-vivo and in-vitro T-cell depletion followed by scheduled prophylactic DLIs for frail or elderly (up to 75 years) patients with high risk acute leukemia.

Methods: Between 06/2010 and 07/2016 92 patients (83 AML, 9 ALL) received fludarabin $\left(50 \mathrm{mg} / \mathrm{m}^{2}, 6\right.$ days), busulphan $(4 \times 0.8 \mathrm{mg} / \mathrm{kg}, 2$ days) and $15 \mathrm{mg}$ alemtuzumab (2 days) before infusion of a peripheral blood or bone marrow $(\mathrm{n}=1)$ stem cell graft $(10 / 10$ HLA-matched) to which $20 \mathrm{mg}$ alemtuzumab was added. Patients with an 


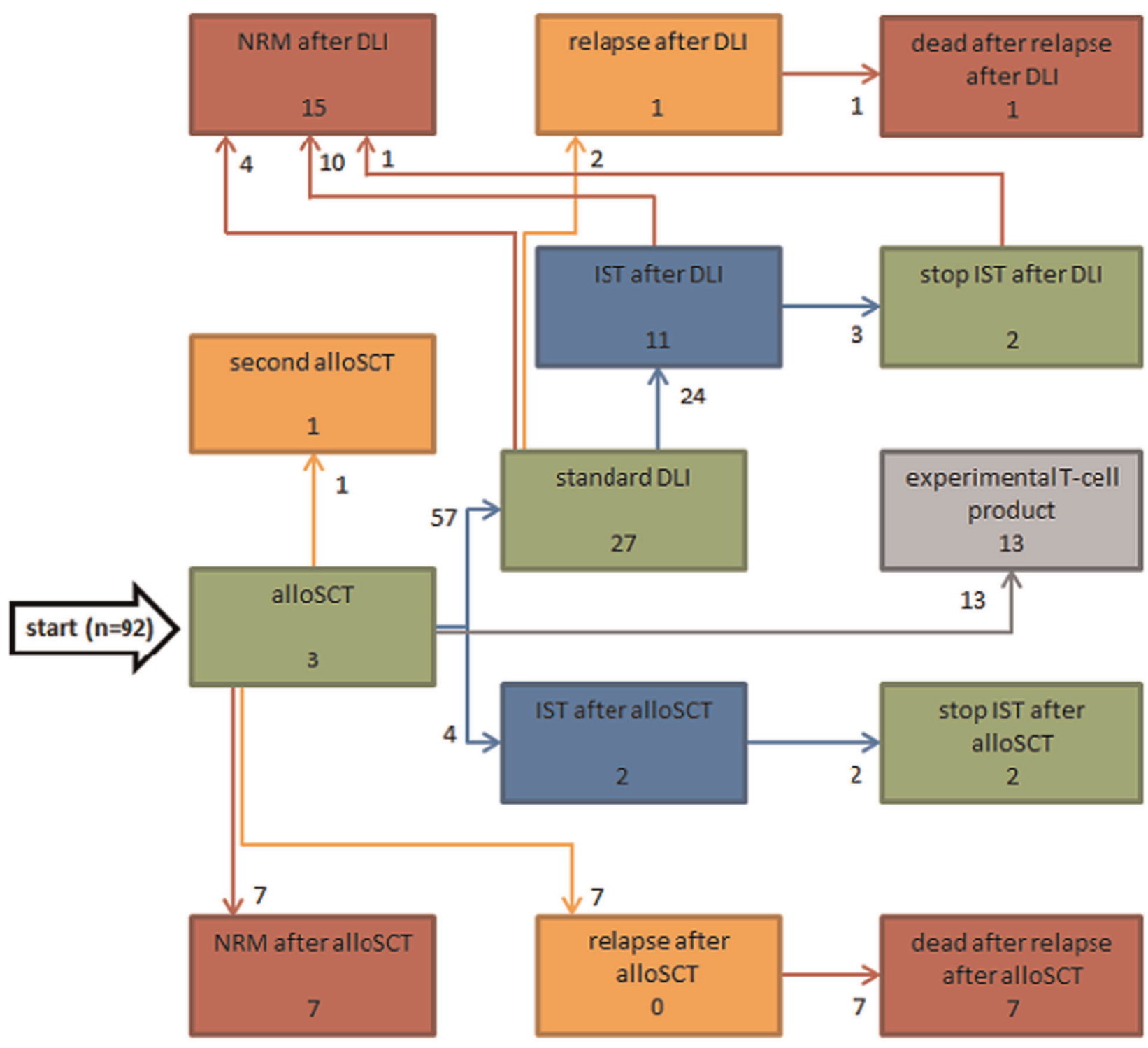

Boxes represent states, arrows transitions. Numbers next to arrows represent the number of patients who made this transition, numbers inside boxes represent the distribution of patients between states at 1 year after alloscT.

[P386 Figure] [Multi-state model]

unrelated donor $(\mathrm{n}=59)$ received $1 \mathrm{mg} / \mathrm{kg}$ Thymoglobulin on day-2. No standard post transplantation immune suppressive therapy (IST) for GVHD prophylaxis was given. If no GVHD requiring systemic IST developed after transplantation, freshly harvested DLI was given at 3 months after alloSCT in patients with very-high risk disease $(\mathrm{n}=$ 19, dose $0.15-0.3 \mathrm{M} \mathrm{T}$-cells $/ \mathrm{kg}$ ), and subsequently at 6 months (dose 1.5-3M T-cells $/ \mathrm{kg}$ ) in all patients. A multi-state model was designed to evaluate the events in relation to alloSCT and DLIs during the first year after transplantation.

Results: Median age was 63 years (range 42-76). All patients engrafted with a median time of 15 days for neutrophils. After 1 and 3 years, overall survival (OS) was 65\%(95\%-confidence interval:56-75) and 52\%(95\%-CI:4063), relapse rate was $12 \%(95 \%$-CI:5-19) and $22 \%(95 \%$ CI:13-32), and non-relapse mortality (NRM) was 25\% (95\%-CI:16-34) and 34\%(95\%-CI:24-45), respectively. One patient received a second alloSCT from a second 
donor as the original unrelated donor refused collection for DLI. Thirteen patients received experimental T-cell products as part of a study protocol. Figure 1 shows the outcomes of the multi-state model at 1 year. Between alloSCT and DLI, 4\%(95\%-CI:0-9) needed therapeutic IST for GVHD, 8\%(95\%-CI:2-13) had a relapse and died and $8 \%(95 \%$-CI:2-13) died due to NRM. 62\%(95\%-CI:52-72) received (1 or 2) standard DLIs. After DLI, 42\%(95\%CI:29-55) needed therapeutic IST, 26\%(95\%-CI:15-38) died due to NRM and 4\%(95\%-CI:0-8) had a relapse. At 1 year, 58 patients were alive without relapse of whom 43 (74\%) were not treated with IST at that time.

Conclusions: T-cell depleted alloSCT after reducedintensity conditioning regimen followed by scheduled prophylactic DLIs is feasible for elderly patients with high risk acute leukemia. At three years, OS was 52\%, with a low relapse rate of $22 \%$ and NRM of $34 \%$. At one year, $74 \%$ of the surviving patients were IST-free. The multi-state model shows that prior to DLI, NRM was low (8\%), but increased following DLI illustrating the necessity to further optimize dosing, timing and manipulation of T-cell therapy.

Conflict of interest: None.

\section{P387}

\section{Oral complications of allogeneic haematopoietic stem cell transplantation: the importance of diagnoses other than graft-versus-host disease}

\author{
Martina K. Shephard ${ }^{1}$, Andy Peniket, Rubeta N. Matin ${ }^{2}$ \\ ${ }^{1}$ University College London Hospitals NHS Foundation Trust, \\ London, United Kingdom; ${ }^{2}$ Oxford University Hospitals NHS \\ Foundation Trust, Oxford, United Kingdom
}

Background: Oral mucosal involvement with graft versus host disease (GVHD) is common and can significantly impact quality of life. However, not all oral mucosal signs and symptoms are due to GVHD and correct diagnosis is essential in order to manage these correctly and avoid unnecessary immunosuppression. The aim of this study was to review all oral mucosal diagnoses presenting in patients reviewed by a dedicated oral medicine specialist service in a Bone Marrow Transplant outpatient clinic.

Methods: Data were collected between May 2015 and October 2017 for all patients reviewed by an oral medicine specialist in a Bone Marrow Transplant (BMT) outpatient clinic. Demographic and clinical data were collected including haematological diagnosis, transplant type, time since transplant, oral medicine diagnosis and treatment, GVHD manifestations and treatment. Oral medicine diagnoses were categorised as active/symptomatic manifestations of GVHD; inactive manifestations of GVHD; late

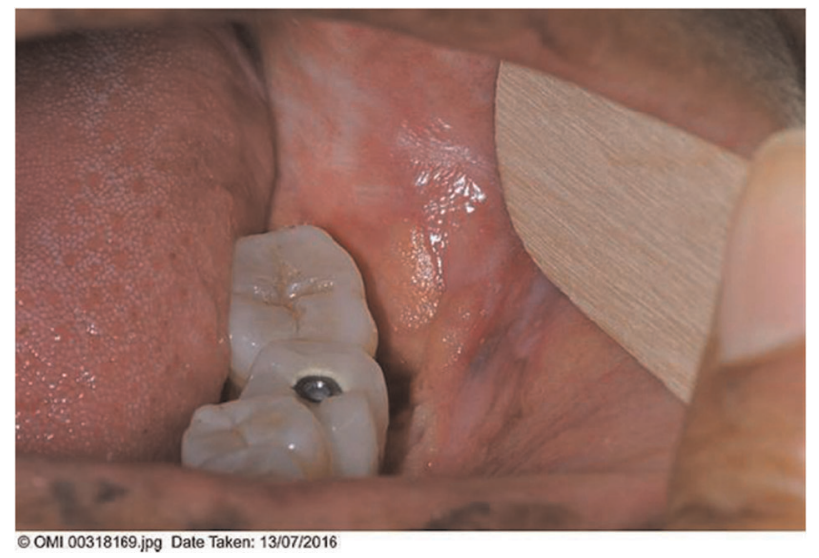

[P387 Figure] [Figure 1: Verruciform xanthoma on the buccal mucosa]

effects related to transplant; or an unrelated diagnosis. Data were analysed descriptively using Microsoft Excel.

Results: Over a 29 month period 67 patient encounters were recorded, consisting of 37 individual patients; mean age 51.9 years (range 25-73 years) and mean time posttransplant 49.4 months (range 1-206 months). Mean number of clinical reviews by the oral medicine specialist was 1.8 (range 1-6).

The commonest haematological diagnoses were acute myeloid leukaemia (35.1\%), follicular lymphoma (16.2\%), acute lymphoblastic leukaemia (10.8\%) and myelodysplasia $(10.8 \%)$. The most common transplant type in this sample was reduced intensity conditioning (RIC) sibling allograft (45.9\%), followed by RIC matched unrelated donor allograft $(29.7 \%)$.

Of the 67 patient encounters, $56.7 \%$ were related to active/symptomatic oral GVHD (mean time post-transplant $66 \mathrm{mths}$ ), $37.3 \%$ were related to inactive oral GVHD manifestations, $7.5 \%$ were late effects of BMT, and $29.9 \%$ were due to an unrelated diagnosis.

In $55.2 \%$ of patient encounters, patients were taking systemic immunosuppressants. 3 patient encounters $(7.9 \%)$ led to an increase in systemic immunosuppression for active/symptomatic oral GVHD. 35 patient encounters (92\%) with active/symptomatic oral GVHD resulted in prescription of topical therapy only.

Unrelated oral mucosal diagnoses included viral papillomata, plaque-induced gingivitis, recurrent aphthous stomatitis, chronic hyperplastic candidiasis, benign hyperkeratosis, pseudomembranous candidiasis, drugrelated pigmentation, small-fibre neuropathies (oral dysaesthesia, dysgeusia, subjective xerostomia and postinflammatory hyperalgesia), dental infection, and white hairy tongue.

Late effects diagnoses included squamous cell carcinoma and verruciform xanthoma (See Figure 1). 
Overall $79.1 \%$ of patients had more than two sites affected with GVHD during at least one encounter. Of these, $60.4 \%$ had active/symptomatic oral GVHD.

Conclusions: Our cohort demonstrates that the oral mucosa is commonly involved in patients with multi-site GVHD. The majority of patients with active/symptomatic oral GVHD can be managed using topical therapy rather than increasing immunosuppression. Moreover, up to a third of patients with oral mucosal symptoms or signs attending BMT clinics have non-GVHD diagnoses. The presence of a dedicated oral medicine specialist in the BMT clinic can ensure correct diagnosis and optimal management.

Conflict of interest: None of the authors has anything to disclose.

\section{P388}

Peripheral blood stem cell transplantation from match related donors do not increases GvHD, but less traumatic for donors

Ekaterina Pristanskova ${ }^{1}$, Kirill Kirgizov ${ }^{1,2}$, Veronika Konstantinova ${ }^{1}$, Natalia Sidorova ${ }^{1}$, Alexandra Burya Burya ${ }^{1}$, Elena Machneva ${ }^{1}$, Elena Skorobogatova ${ }^{1}$

${ }^{1}$ Russian Children's Research Hospital, Moscow, Russian Federation; ${ }^{2}$ Dmitry Rogachev Federal Research Center of Pediatric Hematology, Oncology and Immunology, Moscow, Russian Federation

Background: Usage of peripheral blood stem cells (PBSC) for hematopoietic stem cell transplantation (HSCT) is limited due to increased risk of GvHD. On the other hand it is less traumatic for donors. We aimed to compare GvHD incidence in HSCT with PBSC vs. bone marrow (BM).

Methods: Sixty two patients were transplanted with PBSC from match related donors during the period November 2011 - May 2017 and 30 - with BM. Diagnosis were following - AML: PBSC - 27\% $(\mathrm{n}=17)$ and BM 20\% $(\mathrm{n}=6)$, ALL: $16 \% \quad(\mathrm{n}=10)$ and $7 \% \quad(\mathrm{n}=2 \%)$ accordingly, SAA: $29 \%(\mathrm{n}=18)$ and $13 \%(\mathrm{n}=4)$ accordingly, JMML: $6,5 \%(\mathrm{n}=4)$ and $13 \%(\mathrm{n}=4)$ accordingly, other: $12(19 \%)$ and $50 \%(\mathrm{n}=15)$ accordingly. Gender males: PBSC - 66\% $(\mathrm{n}=41)$ and BM - 57\% ( $\mathrm{n}=17)$, females - 34\% ( $\mathrm{n}=21)$ and $34 \%(\mathrm{n}=13)$ accordingly. Age median - PBSC: 8,5 y.o. ( 7 months - 17 years) and BM - 3,5 y.o. (6 months - 14 years). Conditioning regimens - MAC: PBSC - 69\% ( $\mathrm{n}=43)$ and BM - 73\% $(\mathrm{n}=22)$, RIC $-31 \%$ $(\mathrm{n}=19)$ and $27 \%(\mathrm{n}=8)$ accordingly. Rituximab - PBSC $35 \%(\mathrm{n}=22)$ and BM 20\% $(\mathrm{n}=6)$. ATG - PBSC 45\% (n $=28)$ and BM 57\% $(\mathrm{n}=17)$. Graft in PBSC group: siblings (children in 90\%) - HLA 10/10 - 98\% ( $\mathrm{n}=61), 2 \%$ - 1MM $(\mathrm{n}=1), 2 \%$ - father HLA 10/10 ( $=1)$. CD34+ cell dose $7,3 \times 10^{6} / \mathrm{kg}\left(3,4-15 \times 10^{6} / \mathrm{kg}\right)$. Graft in BM group: siblings -
$83 \%(\mathrm{n}=25)$, parents $-17 \%(\mathrm{n}=5)$, HLA $10 / 10-90 \%(\mathrm{n}=$ 27), HLA $1 \mathrm{MM}-10 \%(\mathrm{n}=3)$. CD $34+8,5 \times 10^{6} / \mathrm{kg}(1,5-$ $29 \times 10^{6} / \mathrm{kg}$ ).

Results were compared with historical data on equal group of patients received BM from match related donors.

Results: All of the donors were without complications. All patients received adequate immunosuppressive therapy. PBSC median time of engraftment - day +13 (9-22 days). Rejection/relapse registered in $14,5 \%(n=9)$ of cases. One patient in PBSC group experienced primary rejection of graft, 9 patients $(14,5 \%)$ - severe aGvHD III-IV st. and seven of these patients transformed to cGvHD (3 patients limited and 4 patients - extensive form of GVHD), one patient had extensive cGvHD and three patients - limited cGVHD without previous history of aGvHD. Chronic GvHD led to severe disability only in four patients. Five patients fully recovered from chronic GvHD. Two patients with extensive chronic GVHD died from infectious complications. At the median follow-up 41,5 months of the OS was $85,5 \%$.

BM median time of engraftment - day +20 (3-30 days). Rejection/relapse - 27\% $(\mathrm{n}=8)$. Two patients in BM group experienced primary rejection, aGvHD III-IV st. diagnosed in $13 \%(n=4)$, extensive cGvHD - 3\% $(n=1)$, limited cGVHD - 3\% $(\mathrm{n}=1)$. Cause of deaths: relapse - 13\% $(\mathrm{n}=$ $4)$, infectious complications $-10 \%(n=3)$. At the median follow-up 14,6 months of the OS was 75,5\%.

Conclusions: Thus, PBSC match related donors transplants not increased risk of GvHD in comparison with BM. PBSC transplants could be feasible option for HSCT and less traumatic for donors. Adequate prevention of GvHD required for successful outcome.

Conflict of interest: None declared

\section{P389}

Pharmacokinetics for once-daily modified release formulation of tacrolimus (Advagraf ${ }^{\oplus}$ ) in allogeneic hematopoietic stem cell transplantation (alloHSCT)

Patrícia Seabra $^{1}$, Mercè Brunet ${ }^{2}$, Cristina Espinosa ${ }^{3}$, Montserrat Rodriguez $^{4}$, María Suárez-Lledó ${ }^{1}$, Gonzalo Gutiérrez ${ }^{5}$, Sofia Almeida $^{1}$, Francesc Fernández-Avilés ${ }^{5}$, Laura Rosiñol ${ }^{5}$, Montserrat Rovira $^{5}$, Carmen Martinez ${ }^{5}$

${ }^{1}$ Institute of Oncology and Hematology, Hospital Clínic, Hematopoietic Stem Cell Transplantation Unit, Hematology Department, Barcelona, Spain; ${ }^{2}$ Biomedical Diagnostic Center, Pharmacology and Toxicology Department, CIBERehd, Barcelona, Spain; ${ }^{3}$ Biomedical Diagnostic Center, Pharmacology and Toxicology Department, Barcelona, Spain; ${ }^{4}$ Hospital Clínic, Pharmacology Department, Barcelona, Spain; ${ }^{5}$ Institute of Hematology and Oncology, Hospital Clínic, IDIBAPS, Institute Josep Carreras, 


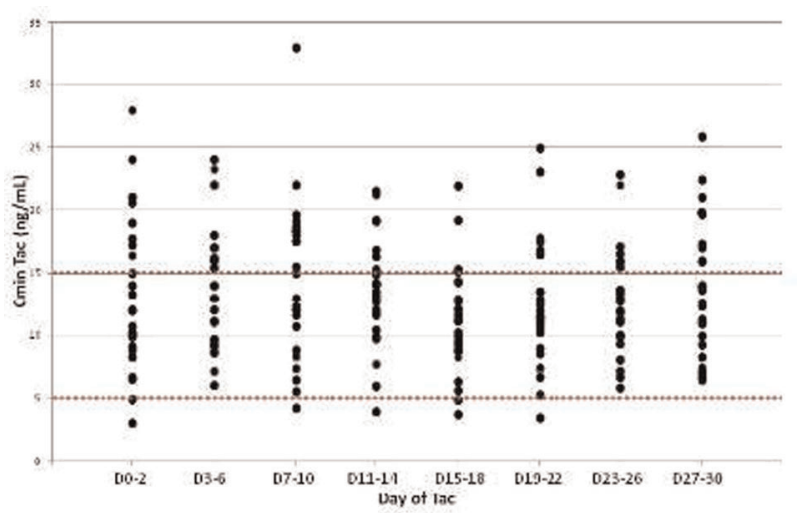

[P389 Figure] [Figure 1. Tac exposure over the therapeutic range during the first month after transplant.]

Hematopoietic Stem Cell Transplantation Unit, Hematology Department, Barcelona, Spain

Background: Tacrolimus (Tac) is a pivotal immunosuppressant agent used in alloHSCT. Besides intravenous (i.v.) and twice-daily oral Tac (Prograf ${ }^{\circledR}$ ), once-daily prolongedrelease formulations (Advagraf ${ }^{\circledR}$, Envarsus ${ }^{\circledR} \mathrm{XR}$ ) are becoming available. Tac has a narrow therapeutic index and small variations in drug exposure due to formulation differences can have a significant impact on patient outcomes. There are scarce data about the use of once-daily Tac in the setting of alloHSCT. We analyzed the pharmacokinetics of Advagraf in the first month after alloHSCT.

Methods: A total of 26 patients with hematological malignancies received alloHSCT with a Tac-based regimen prophylaxis of GVHD. Seventeen patients received Pro$\mathrm{graf}^{\oplus} 0.03 \mathrm{mg} / \mathrm{kg}$ i.v. by continuous infusion followed by conversion to Advagraf at a 1:6 ratio when the patients had recovered from regimen-related gastrointestinal toxicity (group IV/PO). Nine additional patients started immunosuppression directly with Advagraf ${ }^{\circledast}$ at a dose of $0.18 \mathrm{mg} / \mathrm{kg}$ (group PO). All patients received prophylaxis with fluconazol and acyclovir. Through level of Tac (Cmin) was measured three times a week, and in 10 patients we also measured the area under the concentration-time curve from 0 to $24 \mathrm{~h}\left(\mathrm{AUC}_{0-24}\right) 48-72 \mathrm{~h}$ after Advagraf initiation. Tac dose was adjusted to maintain a Cmin of $5-15 \mathrm{ng} / \mathrm{ml}$. A dose reduction was recommended if serum creatinine increased more than the upper limit of normal or if other serious toxicities appeared. Acute kidney injury (AKI) was defined according to Common Terminology Criteria for Adverse Events (CTCAE v4).

Results: In the IV/PO group, $71 \%$ patients with i.v. Tac had median Cmin (10.2ng/ml, 3-13.7) within the therapeutic range; 48-72h after oral conversion, Cmin was within the therapeutic range in $76 \%(\mathrm{n}=13)$ patients, and $24 \%(\mathrm{n}=4)$ had levels $<5 \mathrm{ng} / \mathrm{ml}$. In the PO group $78 \%$ of patients had median Cmin (12ng/ml, 9.2-15.6) within therapeutic range.
No differences were found between groups in the mean peak of Tac concentration (Cmax) (63.5ng/dl vs. 56.6ng/dl) that was attained at median time of $2 \mathrm{~h}$. However, patients in group PO had a significant higher exposition to Tac (mean $\mathrm{AUC}_{0-24} 392 \mathrm{ng} \cdot \mathrm{h} / \mathrm{ml}$ vs. $\left.591.2 \mathrm{ng} \cdot \mathrm{h} / \mathrm{ml}, \mathrm{p}=0.005\right)$. No significant correlation was observed between Tac Cmin and $\mathrm{AUC}_{0-24}$. In the whole population, while $3 \%$ of patients had lower levels of Cmin, $30 \%$ presented a Tac exposure over the therapeutic range during the first month after transplant (Figure 1). No graft failures were observed. Eight patients developed acute GVHD (6 grade I, 1 grade II, 1 grade III), of them, only one had infra-therapeutic levels of Tac. $85 \%$ of patients developed AKI (grade $1 \mathrm{n}=12$, grade $2 \mathrm{n}=9$, grade $3, \mathrm{n}=1$ ), $73 \%$ of them without the addition of other nephrotoxic drugs. Seventeen patients required dose adjustments due to renal toxicity $(\mathrm{n}=13)$, initiation of posaconazol $(\mathrm{n}=3)$, and/or thrombotic microangiopathy ( $\mathrm{n}$ $=1$ ).

Conclusions: Once-daily oral Tac (Advagraf) is a suitable alternative to twice-daily Tac in alloHSCT. Conversion from i.v. Tac to Advagraf on a 1:6 basis in patients who receive fluconazol results in adequate exposure to Tac in most patients. However, a significant proportion of them (30\%) have a high Tac exposure and risk of AKI, and a lower conversion ratio should be considered.

Conflict of interest: No conflict of interest.

\section{P390}

\section{POST-TRANSPLANT CYCLOPHOSPHAMIDE AS GRAFT VERSUS HOST DISEASE PROPHYLAXIS IN HLA-IDENTICAL SIBLING HEMATOPOIETIC STEM CELL TRANSPLANTATION}

Rebeca Bailen ${ }^{1}$, Mi Kwon ${ }^{1,2}$, María Jesús Pascual Cascón ${ }^{3}$, Pascual Balsalobre $^{1,2}$, Carlota Díaz Aizpun ${ }^{3}$, Olga Benítez Hidalgo ${ }^{3}$, David Serrano $^{1,2}$, Javier Anguita ${ }^{1,2}$, Ismael Buño ${ }^{1,2}$, Nieves Dorado ${ }^{1,2}$, Laura Solán ${ }^{1,2}$, José Luis Díez-Martín ${ }^{1,2,4}$

${ }^{1}$ Hospital General Universitario Gregorio Maranon, Hemathology and Hemotherapy, Madrid, Spain; ${ }^{2}$ Instituto de Investigación Sanitaria Gregorio Maranon, Madrid, Spain; ${ }^{3}$ Hospital Regional Universitario de Málaga, Hemathology and Hemotherapy, Málaga, Spain; ${ }^{4}$ Universidad Complutense de Madrid, Madrid, Spain

Background: Post-transplant high dose cyclophosphamide (PT-CY) effectively prevents graft-versus-host disease (GVHD) after HLA-haploidentical hematopoietic stem cell transplantation (HSCT). The use of PT-CY in HLAidentical HSCT is less explored. In this study, we analyzed the results of PT-CY as GVHD prophylaxis in HLAidentical sibling HSCT and compared them with those obtained after prophylaxis with methotrexate (MTX) and cyclosporine (CsA).

Methods: 68 HLA-identical sibling (10/10) HSCT from 2 spanish hospitals have been analyzed: 33 performed 
Figure 1. Acute GVHD grades II-IV cumulative incidence.

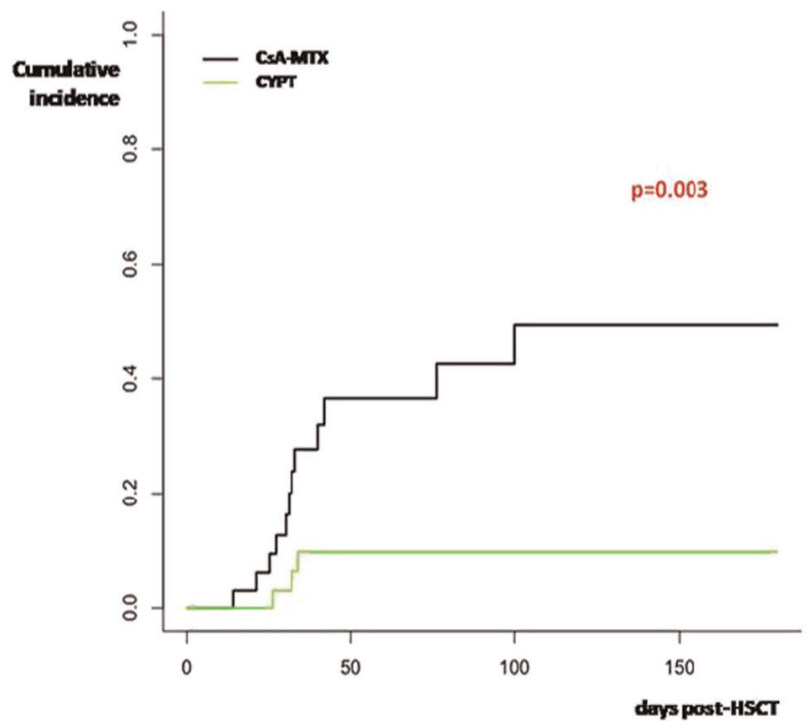

[P390 Figure] [Acute GVHD grades II-IV cumulative incidence.]

between 2012 and 2015 using MTX-CsA as GVHD prophylaxis, and 35 performed between 2014 and 2017 using PT-CY.

Results: Baseline characteristics and post-transplant complications are shown in Table 1. GVHD prophylaxis consisted in MTX days $+1,+3,+6$ and +11 , and CsA from day -1 in the MTX-CsA group. The PT-CY group recieved cyclophosphamide $50 \mathrm{mg} / \mathrm{kg} / \mathrm{d}$ on days +3 and +5 in 25 patients (71\%), combined with CsA from day +5 , and cyclophosphamide on days +3 and +4 in 10 patients (29\%), followed by CsA and mycophenolate mofetil from day +5 . After a median follow-up of 42 months for the MTX-CsA group and 11 months for the PT-CY group, 1year overall survival $(\mathrm{OS})$ was $75 \%$ and $79 \%(\mathrm{p}=0.16)$, and event-free survival (EFS) was $77 \%$ and $55 \%(\mathrm{p}=0.18)$, respectively. No differences were observed in the 1-year cumulative incidence of relapse $(27 \%$ vs $20 \%, \mathrm{p}=0.55)$. Non-relapse mortality (NRM) at 1-year showed a higher trend in the CsA-MTX group (18\% vs $8.7 \%, \mathrm{p}=0.55$ ). Cumulative incidence at 100 days of grade II-IV (49\% vs $10 \%)$ and III-IV (25\% vs 6\%) acute GVHD, were significantily higher in the MTX-CsA group (Figure 1). Cumulative incidence of chronic moderate to severe GVHD showed a higher trend in the MTX cohort (34\% vs $16 \%$, p $=0.05$ ).

Conclusions: In our experience, in spite of the limited number of patients, GVHD prophylaxis using PT-CY combined with additional immunosupresion after HLAidentical sibling HSCT, using mostly peripheral blood as graft source, reduced the cumulative incidence of acute GVHD compared to standard prophylaxis with MTX-CsA. Further studies with longer follow-up are needed to confirm these observations.

Conflict of interest: The authors have nothing to disclose.

\begin{tabular}{|c|c|c|c|}
\hline & $\begin{array}{l}\text { CsA-MTX } \\
\mathrm{N}=33\end{array}$ & $\begin{array}{l}\text { PT-CY } \\
\mathrm{N}=35\end{array}$ & $\mathrm{p}$ \\
\hline Age, years, median (RIC) & $51(41-59)$ & $51(41-57)$ & NS \\
\hline Sex, male $(\%)$ & $20(54)$ & $29(69)$ & NS \\
\hline $\begin{array}{l}\text { Diagnosis, N (\%): - AML and MDS - } \\
\text { ALL - NHL and CLL - Others }\end{array}$ & $\begin{array}{l}17(52) 7 \\
(21) 7(21) \\
2(6)\end{array}$ & $\begin{array}{l}16(46) 7 \\
(20) 8(23) 4 \\
(11)\end{array}$ & NS \\
\hline $\begin{array}{l}\text { Disease Risk Index, N (\%): - Low - } \\
\text { Intermediate - High/Very High }\end{array}$ & $\begin{array}{l}4(12) 8(24) \\
21(64)\end{array}$ & $\begin{array}{l}2(6) 25(71) \\
8(23)\end{array}$ & NS \\
\hline Donor/recipient sex, female/male (\%) & $6(18)$ & $13(37)$ & NS \\
\hline Stem cell source BM/PB, N (\%) & $\begin{array}{l}1(3) / 33 \\
(97)\end{array}$ & $\begin{array}{l}7(20) / 28 \\
(80)\end{array}$ & NS \\
\hline $\begin{array}{l}\text { Conditioning regimen RIC/MAC, N } \\
(\%)\end{array}$ & $\begin{array}{l}12(36) / 21 \\
(64)\end{array}$ & $\begin{array}{l}23(66) / 12 \\
(34)\end{array}$ & 0.03 \\
\hline $\begin{array}{l}\text { Engraftment: - Neutrophil count } \\
>0.5 \times 109 / \mathrm{L} \text { median days }(\%) \text { - Platelet } \\
\text { count }>20 \times 109 / \mathrm{L} \text {, median days }(\%)\end{array}$ & $\begin{array}{l}15(100) 12 \\
(91)\end{array}$ & $\begin{array}{l}18(100) 23 \\
(91)\end{array}$ & $0.02<0.01$ \\
\hline $\begin{array}{l}\text { Post-transplant complications: - } \\
\text { Hepatotoxicty, grade II-IV, N (\%) - } \\
\text { Hemorrhagic cystitis, N (\%) - CMV } \\
\text { reactivation, N }(\%)\end{array}$ & $\begin{array}{l}5(15) 3(9) \\
10(30)\end{array}$ & $\begin{array}{l}3(9) 4(11) \\
16(46)\end{array}$ & NS NS NS \\
\hline
\end{tabular}

[[P390 Table] Baseline characteristics and HSCT complications]

P391

Post-Transplant Cyclophosphamide prevents Graft-versusHost Disease in Acute Myeloid Leukemia treated with Allogeneic Peripheral Blood Hematopoietic Cell Transplantation from HLA-Matched Donors

Paolo Becco $^{1,2}$, Daniela Caravelli ${ }^{1,2}$, Susanna Gallo ${ }^{1,2}$, Elena Vassallo $^{2,3}$, Valentina Coha ${ }^{1,2}$, Monica Mangioni ${ }^{2,4}$, Massimo Berger $^{2,3}$, Alessandra Polo ${ }^{2,4}$, Milena Salierno ${ }^{2,4}$, Pio Manlio Mirko Frascione $^{1,2}$, Francesco Saglio ${ }^{2,3}$, Luca Crotto ${ }^{1,2}$, Massimo Aglietta $^{1,2,5}$, Franca Fagioli ${ }^{2,3}$, Fabrizio Carnevale-Schianca ${ }^{1,2}$

${ }^{1}$ Candiolo Cancer Institute-FPO, IRCCS, Medical Oncology, Hematopoietic Stem Cells Unit, Candiolo, Italy; ${ }^{2}$ Turin Metropolitan Transplant Center, Torino, Italy; ${ }^{3}$ A.O.U. Citta' della Salute e della Scienza di Torino, Ospedale Infantile Regina Margherita, Pediatric Onco-Hematology, Stem Cell Transplantation, and Cellular Therapy Division, Torino, Italy; ${ }^{4}$ Candiolo Cancer Institute-FPO, IRCCS, Collection and Processing Laboratory, Candiolo, Italy; ${ }^{5}$ University of Torino, Department of Oncology, Torino, Italy

Background: Hematopoietic cell transplantation (HCT) is the only curative approach for Acute Myeloid Leukemia (AML) but still life-threatening toxicities, such as graftversus-host disease (GVHD) and infections, limit its fullpotential impact on the disease. Post-transplant Cyclophosphamide (ptCy) has been proven to be effective in reducing 
acute GVHD (aGVHD) and chronic GVHD (cGVHD) incidence after bone-marrow HCT from identical and haploidentical donor, and now it is increasingly adopted in this setting. The use of ptCy after peripheral blood stem cell (alloPBSCT) transplantation from HLA-matched unrelated/related donors is still under investigation. We report the outcomes of a cohort of patients with high/intermediate risk AML treated with this approach (NCT02300571).

Methods: We analyzed 21 patients with high/intermediate risk LAM who had PBSC transplantation from HLAmatched unrelated/related donors between August 2011 and July 2017. All of them were in complete response (CR) at transplant after induction or salvage regimens. GVHD prophylaxis was ptCy $50 \mathrm{mg} / \mathrm{kg}$ (days $+3+4$ ), Tacrolimus from day +5 and Mycophenolate Mofetil (MMF) from day +5 to day +28 . Conditioning regimens were reduced in 2 (9\%) patients and myeloablative in 19(91\%). Primary objectives were cumulative incidence of aGVHD and cGVHD. Secondary objectives were event free survival (EFS), cGVHD-EFS, overall survival (OS) and transplant related mortality (TRM).

Results: Patients median age was 47 (range 20-66) years. 12 patients were in first $\mathrm{CR}, 9$ patients were in second $(\mathrm{n}=$ $7)$ or third $(n=2)$ CR. A median dose of 6.5 (range 3-15) x $10^{\wedge} 6 \mathrm{CD} 34 / \mathrm{kg}$ was infused. All but one patient engrafted. MMF was stopped on day +28 in all patients. The median day of Tacrolimus discontinuation was 120 (range 40-331). CMV reactivation occurred in 11(52\%) patients. In regard to GVHD, $3 / 21$ (14\%) patients developed aGVHD grade IIIII ( 2 GI, 2 skin), median day of onset was day 35 (range 22-119). No grade IV was observed. They were treated with steroids or steroids plus calcineurin inhibitor. Two patients (9.5\%) developed cGVHD, on day +177 and +268 respectively. Systemic treatments were required (steroid at $1 \mathrm{mg} / \mathrm{kg} / \mathrm{day}$ and tacrolimus) but both of them were able to discontinue immunosuppression (IS) on day +385 and on day +330 respectively. With a median follow-up of 27 (range 1-64) months, EFS was 57\%, cGVHD-EFS was $48 \%$ and OS was $67 \%$. TRM incidence was 4\%. Only 1 patient, who developed multiresistant Pseudomonas aeruginosa septicemia, died after transplant.

Nowadays 12 patients are alive with no evidence of disease, completely off IS and completely reintegrated in their normal daily life activities.

Conclusions: In high/intermediate risk AML setting the reported results appear comparable to literature-data. Moreover thee results suggest that ptCy also after PBSC-HCT maintains a favorable profile with limited acute and cGVHD incidence and sharp reduction in TRM. The relevant decrease of transplant toxicities and mortality, if confirmed, might expand the indication of HCT in AML. Leukemia relapses still represent a limit. The low GVHD incidence and the early IS discontinuation might contribute, in the future, to transform HCT into a safe immunologic platform for cellular therapies with potential increase of graft versus leukemia effect.

Clinical Trial Registry: NCT02300571

www.clinicaltrials.gov

Conflict of interest: None of the authors has anything to disclose.

\section{P392}

Potential role of photobiostimulation in the management of hyposalivation and xerostomia in patients with oral cGVHD

Judith Evelyn Raber-Durlacher, ${ }^{1,2}$,Joel. B. Epstein ${ }^{3,4}$, Andrei Barasch $^{5}$, Dan M.J. Milstein ${ }^{1,2}$, Fred R. Rozema ${ }^{2}$, D Tzachanis ${ }^{6}$, Praveen Arany, Rene-Jean Bensadoun ${ }^{8}$

${ }^{1}$ Academic Medical Center, Oral and Maxillofacial Surgery, Amsterdam, Netherlands, ${ }^{2}$ Academic Centre for Dentistry Amsterdam, Oral Medicine, Amsterdam, Netherlands; ${ }^{3}$ Cedars-Sinai Health Center, Los Angeles, CA, United States; ${ }^{4}$ City of Hope, Duarte, CA, United States; ${ }^{5}$ Weill Cornell Medical College, New York, NY, United States; ${ }^{6}$ University of California, Blood and Marrow Transplant, San Diego, CA, United States; ${ }^{7}$ University of Buffalo, Oral Biology, Buffalo, NY, United States; ${ }^{8}$ Centre de Haute Energie, Nice, France

Background: Cancer patients treated with allogeneic hematopoietic stem cell transplantation (HSCT) may develop oral complications associated with chronic Graft versus Host Disease (cGVHD). The salivary glands may be affected resulting in hyposalivation and xerostomia (feeling of dry mouth), which may present a significant burden to patients. Patients may have trouble sleeping, speaking and swallowing and decreased salivary defense mechanisms predispose to mucosal and dental hypersensitivity as well as to opportunistic oral infections including rampant dental caries. Current treatment options for cGVHD-associated salivary gland dysfunction are limited. An additional intervention would thus be a welcome addition to currently available management strategies. Studies suggest that photobiomodulation (PBM) therapy using low dose light therapy (previously called low level laser therapy) reduces inflammation and fibrosis, and promotes tissue repair. We hypothesize that PBM therapy may ameliorate salivary gland dysfunction and aim to explore whether PBM may play a role in the management of hyposalivation and xerostomia associated with oral cGVHD.

Methods: Eight patients with cGVHD-associated hyposalivation/xerostomia were treated with PBM, using a 660 $\mathrm{nm}$ intraoral laser probe $(75 \mathrm{~mW}$, pulsed $2.5 \mathrm{~Hz}$, illuminated area $2.5 \mathrm{~cm} 2,30 \mathrm{~mW} / \mathrm{cm} 2,60 \mathrm{~s}$ per point, $4.5 \mathrm{~J} /$ point, $1.8 \mathrm{~J} /$ $\mathrm{cm} 2$ ) at the orifices of the major salivary glands. Extraorally, a cluster of 69 LEDs $(660 \mathrm{~nm} / 850 \mathrm{~nm}, 1400 \mathrm{~mW}$ total, pulsed $2.5 \mathrm{~Hz}$, illuminated area $28 \mathrm{~cm} 2,50 \mathrm{~mW} / \mathrm{cm} 2$, 
$60 \mathrm{~s}, 84 \mathrm{~J}, 3 \mathrm{~J} / \mathrm{cm} 2$ ) was applied to the major salivary glands and cervical lymph node chain bilaterally. Patients underwent at least one PBM treatment per week. Follow up data are presented for 4 weeks of treatment. Unstimulated and stimulated whole salivary flow was measured during 5 minutes before and after PBM therapy and patients completed a 10-point Visual Analogue Scale (VAS) score in order to assess xerostomia.

Results: PBM therapy significantly attenuated hyposalivation and xerostomia in 5 out of 8 patients, whereas one patient experienced less xerostomia while his whole salivary production improved only marginally. In 2 patients with longstanding GVHD-associated dry mouth, PBM therapy following the protocol described above had no effect.

Conclusions: Our results suggest that controlled studies should be conducted to confirm the efficacy and safety of PBM therapy in cGVHD related salivary gland dysfunction and to determine optimal PBM therapy protocols.

Conflict of interest: PBM devices were provided by THOR Photomedicine Ltd, Chesham, Buckinghamshire, UK

\section{P393}

Preliminary validation of a new software for standardized response evaluation, according to the NIH criteria

Giorgia Mancini ${ }^{1}$, Raul Frulla ${ }^{2}$, Stefano Angelini ${ }^{3}$, Jacopo Olivieri ${ }^{4}$, Federica Monaco ${ }^{1}$, Elena Busilacchi ${ }^{5}$, Ilaria Scortechini ${ }^{1}$, Martina Chiarucci $^{1}$, Chiara Montagna ${ }^{1}$, Monica Poiani ${ }^{1}$, Giuseppina Urbano $^{1}$, Andrea Badaloni ${ }^{6}$, Attilio Olivieri ${ }^{1}$

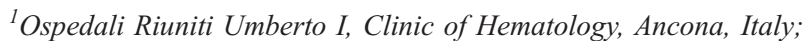
${ }^{2}$ Bimind Software House, Jesi, Italy; ${ }^{3}$ Ospedale Mazzoni, UOC Ematologia e Terapia Cellulare, Ascoli Piceno, Italy; ${ }^{4}$ Ospedale della Misericordia, Dept Hematol, Udine, Italy; ${ }^{5}$ Univerisità Politecnica delle Marche, Dipartimento di Scienze Cliniche e Molecolari - Clinic of Hematology, Ancona, Italy; ${ }^{6}$ Ospedali Riuniti Umberto I, Sistema Informatico Aziendale, Ancona, Italy

Background: Chronic GVHD (cGVHD) represents the first cause of transplant-related mortality and reduced quality of life after transplant. The epidemiology of cGVHD is largely unknown; moreover, diagnosis of cGVHD can be easily missed because its onset is often late in the posttransplant period, requires specific follow up, and general

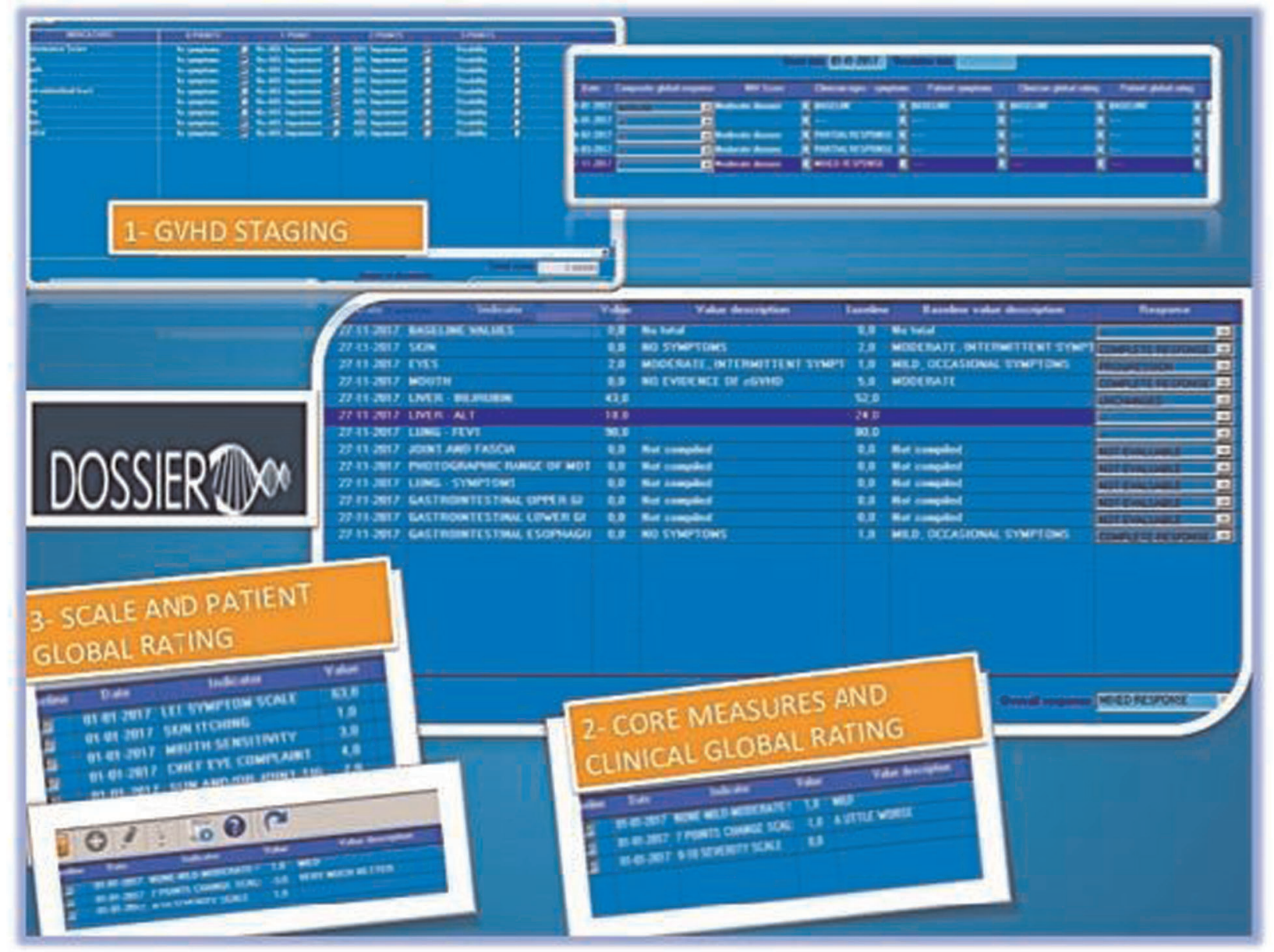

[P393 Figure] [Fig1: Data flow and response calculation]

SPRINGER NATURE 
practitioners are usually not familiar with this entity. To standardize the cGVHD management, the NIH Consortium proposed a consensus definitions for diagnosis, scoring and response criteria in 2006; however these criteria have been used only in a minority of trials. The updated consensus of the 2015 NIH criteria now offers a shared framework to better study this rare disease. These criteria should be validated prospectively in clinical trials; moreover, a learning period is needed and some critical points could emerge from the application of new criteria, including the possible discrepancies between organ specific measures and the global rating scales according to the physician and patient-reported symptoms.

Methods: We have developed an electronic medical record, based on an integrated system called Dossier, which allows complete traceability of the transplanted patientÂ's clinical and laboratory data. Starting from this background we developed an algorithm calculating both the cGVHD score and the overall response, combining the Core specific Measures and the global rating scales, including the Lee scale. The system also keeps track of the criteria that led to the diagnosis, also through photographic support, including the change of immunosuppressive therapies performed and the adverse events, including the infectious complications.

Results: We retrospectively tested the algorithm performance in 21 patients enrolled in a clinical interventional study with NIlotinib in cGVHD, by means of a double check "software-paper support" which showed the perfect overlap of the results and similar outcomes, according to the response evaluated by the conventional and the softwarebased assessment.

Starting from these preliminary data, this tool was extended to all the Italian centers which participate to a prospective multicenter observational study, in newly diagnosed cGVHD (CROSY GITMO STUDY); the aim is to prospectively evaluate the overall incidence of cGVHD and the long-term efficacy of whichever treatment in a large population of adult and pediatric patients, according to the 2015 NIH criteria, and to validate the response assessment with the hard outcomes (Event Free Survival (EFS), Overall Survival (OS) and non-relapsed mortality (NRM).

Figure 1 show the data flow, and response calculation, according to the algorithm. All the data are centrally stored by all the 54 Italian centers which participate to this study; up to now 8 Italian centers have successfully used this tool in a real time fashion and the remaining 46 are now starting.

Conclusions: This software represents an ideal tool for evaluating in a standardized fashion the cGVHD patients and allows both to increase the physician expertise, and to improve the quality of data that can be captured during the patient evaluation. Finally this tool could substantially increase the quality of the future interventional studies in this field, thus helping to select the most effective compounds for this difficult to treat disease.

Conflict of interest: All authors: Nothing to disclose

\section{P394}

Pre-transplant use of glycopeptide tend to increase the incidence of extensive chronic GVHD in adult patients with hematologic malignancies

Ik Chan Song, So Hyun Lee, Deog Young Kim, Hyewon Ryu, Yoon Seok Choi, Hyo Jin Lee, Hwan Jung Yun, Deog Yeon Jo

Chungnam National University Hospital, Department of Internal Medicine, Daejeon, Korea, Republic of

Background: The alteration of fecal microbiota affects the incidence and extent of graft-versus host disease (GVHD) after allogeneic hematopoietic stem cell transplantation (Allo-HSCT). Most patients with hematologic malignancies receive antibiotics for the treatment of febrile neutropenia prior to Allo-HSCT and pre-transplant use of antibiotics may influence the fecal microbiota. Thus, we investigated whether pre-transplant use of antibiotics influence on incidence and extent of GVHD.

Methods: We retrospectively analyzed the consecutive adult patients with hematologic malignancies who received allo-HSCT at Chungnam National University Hospital between 2007 and 2016. Patients receiving haplo-identical donor and cord blood transplantation were excluded. Pretransplant use of antibiotics was defined as usage of antibiotics before conditioning chemotherapy.

Results: This study included 131 patients with median age of 46 (range, 18-71) years: 76 (58\%) patients were AML, 28 (21.4\%) ALL, 23 (17.6\%) MDS, and 4 (3.1\%) CML. Seventy-two (55\%) patients received transplant from sibling. All the patients received calcineurin inhibitor with short-course methotrexate for GVHD prophylaxis. Thirtyone (23.7\%) patients received anti-thymocyte globulin. All the patients received antibiotics prior to HSCT: 70 (53.4\%) patients received glycopeptide, $114(87.0 \%)$ cefepime, 87 (66.4\%) piperacillin/tazobactam, and 51 (38.9\%) carbapenem. Patients who received glycopeptide had more frequent extensive chronic GVHD (cGVHD) than those who had not $(51.1 \%$ vs. $28.1 \%$ at 5 years, $\mathrm{p}=0.026)$ and they had more frequent cGVHD of lung ( $34.8 \%$ vs. $15.8 \%$ at 5 years, $p=$ 0.028). Multivariate analysis, however, revealed that only non-use of ATG [HR=1.97 (1.18-3.30), $\mathrm{p}=0.009]$ and HLA-mismatched donor transplantation $[\mathrm{HR}=1.44$ (1.032.03), $p=0.033)]$ were significant risk factors for extensive cGVHD. Pretransplant use of glycopeptide did not influence on the overall survival (OS) and GVHD-related mortality (median OS; 49 months in glycopeptide vs. not reached in non-glycopeptide, $\mathrm{p}=0.330 ;$ GVHD-related mortality at 5 
year; $15.7 \%$ in glycopeptide vs. $9.8 \%$ in non-glycopeptide, $\mathrm{p}=0.627)$.

Conclusions: Pre-transplant use of glycopeptide tends to increase the incidence of extensive cGVHD, especially in the lung.

Conflict of interest: No conflict of interest.

\section{P395}

Promising role of extracellular vesicles as biomarker of acute graft-versus-host-disease

Giuseppe Lia $^{1,2}$, Lucia Brunello ${ }^{1,2}$, Stefania Bruno ${ }^{3}$, Marta Tapparo $^{3}$, Paola Maria Omedè ${ }^{4}$, Moreno Festuccia ${ }^{1}$, Enrico Maffini $^{1}$, Giovannino Ciccone ${ }^{5}$, Lorenzo Comba ${ }^{2}$, Laura Tosti ${ }^{2}$, Luisa Giaccone ${ }^{1,2}$, Mario Boccadoro ${ }^{2,4}$, Andrea Evangelista ${ }^{5}$, Giovanni Camussi, Benedetto Bruno ${ }^{1,2}$

${ }^{I}$ A.O.U. Città della Salute e della Scienza di Torino, Dipartimento di Oncologia e Ematologia, SSD Trapianto Allogenico di Cellule Staminali, Torino, Italy; ${ }^{2}$ Università degli Studi di Torino,
Dipartimento di Biotecnologie Molecolari e Scienze per la Salute, Torino, Italy; ${ }^{3}$ Università degli Studi di Torino, Centro di Biotecnologie Molecolari, Dipartimento di Scienze Mediche, Torino, Italy; ${ }^{4}$ A.O.U. Città della Salute e della Scienza di Torino,

Dipartimento di Oncologia e Ematologia, Torino, Italy; ${ }^{5}$ A.O.U. Città della Salute e della Scienza di Torino, Clinical Epidemiology, Torino, Italy

Background: Reliable biomarkers could be crucial to define patients at high-risk of developing acute Graft-vs.Host Disease (aGVHD). Extracellular Vesicles (EVs), membrane-enclosed structures secreted by cells in body fluids, may represent an attractive biomarker. We recently carried out a preliminary study to investigate their potential role as biomarkers of aGVHD (Lia G. et al. Leukemia 2017). Three EVs membrane antigens (CD146, CD31, CD140a) were associated with the onset of aGVHD. To confirm our preliminary findings, we designed a prospective study which enrolls patients with various hematological malignancies undergoing an allograft. Moreover, EVs are

\section{A CD146+fluorescencelevel}
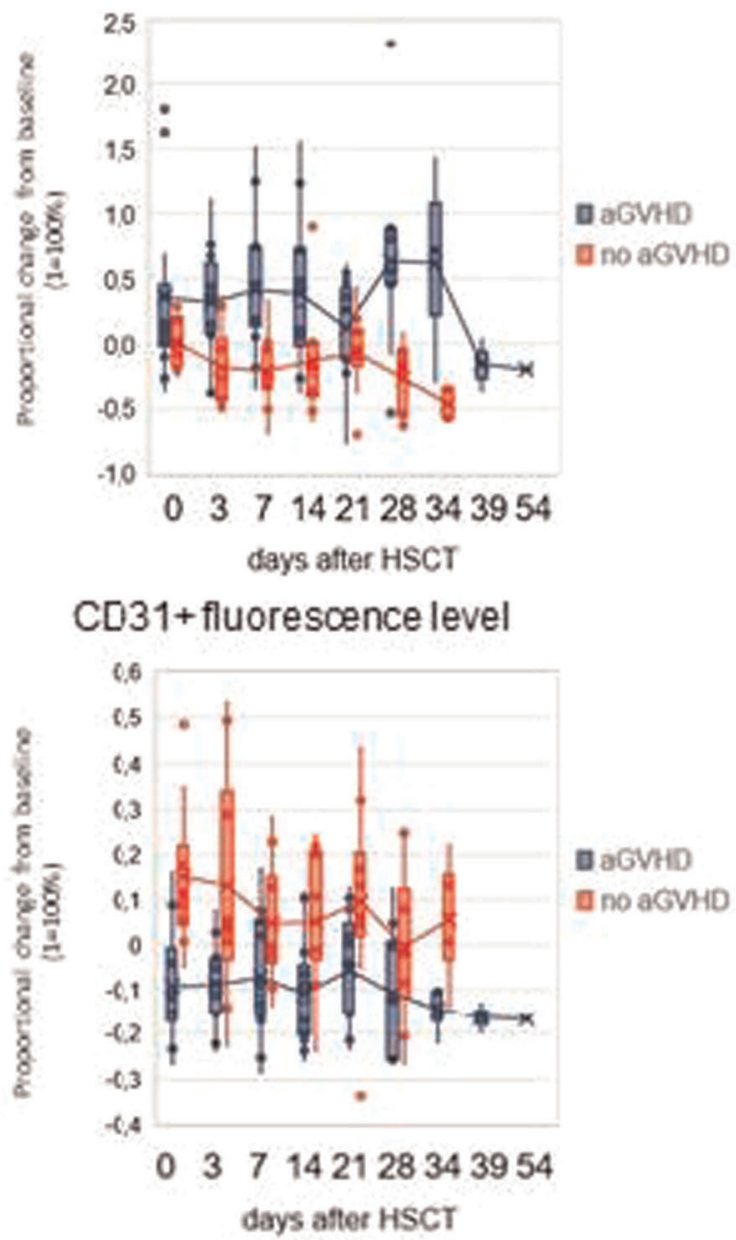

B

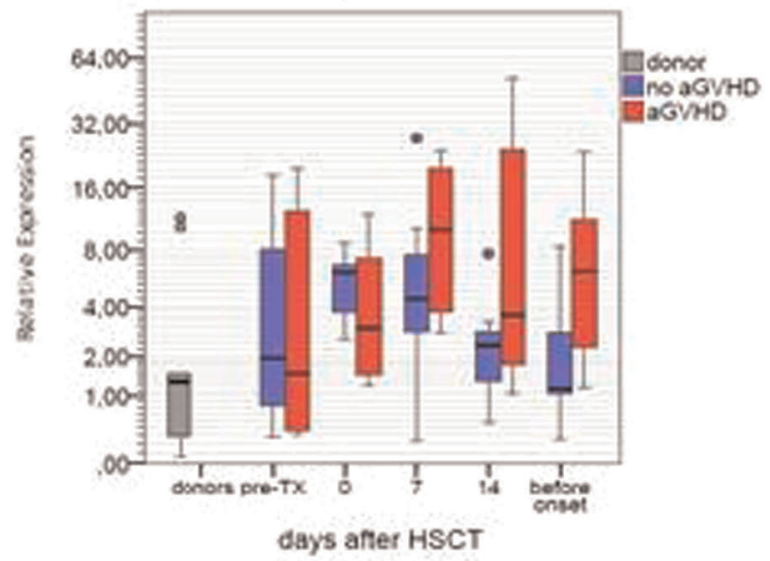

miR100 expression level

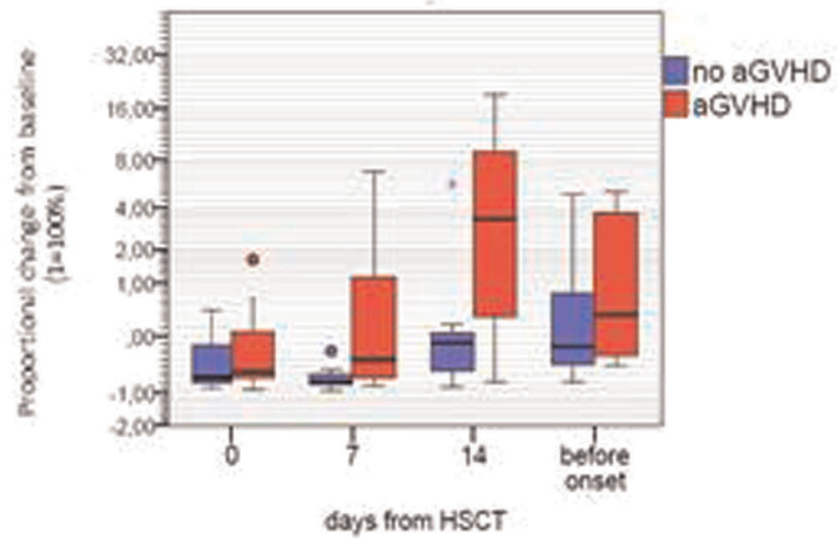

[P395 Figure] [Preliminary results on cytofluorimetry (A) and miRNAs quantification (B) at different days after HSC] 
characterized by their content in miR100, miR92b, miR155, miR194.

Methods: Thirty-five patients have so far been enrolled. Serum samples are collected at the following time-points: pre-transplant, on day $0,+3,+7,+14,+21,+28,+35$, +45 and then monthly up to 1 year. EVs are extracted using a protamine-based precipitation method and analyzed for the expression of membrane proteins (CD44, CD138, CD146, KRT18, CD120a, CD8, CD30, CD106, CD25, CD31, CD144, CD86, and CD140a) by flow-cytometry (Guava EasyCyte Flow Cytometer). Total EVs concentration, fluorescence distribution and percentage of positive EVs are evaluated for all biomarkers. MiRNAs are extracted from EVs using a combination of Trizol and commercial kits (miRNeasy mini kit Qiagen). MiRNAs are then quantified by real time PCR and defined as relative expression level compared to healthy donors. By logistic regression analysis, odds ratio (OR) is calculated as proportional change from pre-transplant levels for each marker.

Results: Overall, 17 patients (48.6\%) developed grade IIIV aGVHD. Preliminary results (20/35 patients studied) showed that the expression of CD146 and CD31 was correlated with the development of aGVHD (Fig. 1). CD146 level (Cell Adhesion Molecule family, MCAM-1) was associated with an increased risk of aGVHD (OR 4.3, $\mathrm{p}=0.008$ ), while CD31 (platelet endothelial cell adhesion molecule, PECAM-1) was associated with a decreased risk (OR 0.36, $\mathrm{p}=0.004)$. These biomarkers showed a proportional signal change from baseline to GVHD onset (gradual increase for CD146 and decrease for CD31). Preliminary results (15/35 patients studied) on miRNAs expression levels showed that an upregulation of miR100 and miR194 (Fig. 1) was associated with increased risk of aGVHD (miR194 absolute level OR 2.3, p =0.048, and miR100 proportional signal change OR 2.7, $\mathrm{p}=0.048$ ).

Conclusions: We confirm a significant association of MCAM-1 and PECAM-1 with the onset of GVHD, underlying a role of the endothelium and immune cells interactions. MiR194 and miR100 are involved in several pathways (including the NOTCH signaling system) and further investigation of their roles may provide insights into the pathogenesis of acute GVHD.

\section{Conflict of interest:}

M. Boccadoro: Honoraria and research funding from: Sanofi, Celgene, Bristol-Myers Squibb, Novartis, Amgen, Janssen; Honoraria from AbbVie; Research funding from Mundipharma.

B. Bruno: Honoraria from Gilead, Pfizer, Hospira; Honoraria and research funding from Celgene; Research funding from Pierre Fabre, Adienne, Hospira italia, MSD Italia.
P396

Abstract previously published

P397

ProTmune $^{\mathrm{TM}}$, the Next Generation Graft for GVHD

Prophylaxis of Allogeneic Hematopoietic Cell Transplantation: Phase 1 Safety and Efficacy Data

Richard Maziarz ${ }^{1}$, John Edwards ${ }^{2}$, James Essell ${ }^{3}$, Cesar Freytes ${ }^{4}$, Chatchada Karanes $^{5}$, Ayman Saad ${ }^{6}$, Dimitrios Tzachanis ${ }^{7}$, Monica Diaz $^{8}$, Amanda Medcalf ${ }^{8}$, Daniel Fremgen ${ }^{8}$, Chris Storgard ${ }^{8}$, Abhinav Deol ${ }^{9}$

${ }^{\text {I}}$ Oregon Health \& Science University, Portland, OR, United States, ${ }^{2}$ Indiana Blood and Marrow Transplantation, Indianapolis, IN, United States; ${ }^{3}$ Jewish Hospital Mercy Health, Cincinnati, OH, United States;

${ }^{4}$ Texas Transplant Physician Group, San Antonio, TX, United States; ${ }^{5}$ City of Hope National Medical Center, Department of Hematology and Hematopoietic Cell Transplantation, Duarte, CA, United States;

${ }^{6}$ University of Alabama at Birmingham, Birmingham, AL, United States; ${ }^{7}$ University of California, San Diego, San Diego, CA, United States; ${ }^{8}$ Fate Therapeutics, Inc, San Diego, CA, United States;

${ }^{9}$ Karmanos Cancer Institute/Wayne State University, Department of Oncology, Blood and Marrow Stem Cell Transplant Program, Detroit, MI, United States

Background: ProTmune, is a next generation graft with FDA fast track designation for the reduction of incidence and severity of acute graft-versus-host disease (GvHD) in patients undergoing allogeneic hematopoietic cell transplantation (HCT).

Methods: PROTECT (NCT02743351) is an ongoing Phase 1 open-label / Phase 2 double-blinded and randomized controlled trial of ProTmune in adult subjects with hematologic malignancies undergoing a mobilized peripheral blood HCT following myeloablative conditioning (MAC) using an HLA A, B, C, DR matched unrelated donor (M-URD) and receiving standard GvHD prophylaxis of methotrexate and tacrolimus. ProTmune is manufactured on-site the day of HCT by pharmacologically modulating the donor peripheral blood stem cell (PBSC) allograft $e x$ vivo with two small molecules (FT1050 and FT4145) to enhance the biologic properties and therapeutic function of the graft.

Results: The Phase 1 stage of PROTECT included seven adult subjects (median age 56, range 34-69) with hematologic malignancies (AML, $\mathrm{n}=3$; ALL, $\mathrm{n}=3$; and MDS) undergoing M-URD HCT following MAC. ProTmune manufacturing was successful in all cases. Pharmacologic modulation was confirmed by the marked increase of the potency marker CXCR4 on $\mathrm{CD} 34^{+}$cells. The mean dose of cells administered was $6.6 \times 10^{6} / \mathrm{kg}$ cells (range 3.0-10.9) for $\mathrm{CD} 4^{+}$cells and $2.3 \times 10^{8} / \mathrm{kg}$ (range $1.2-3.1$ ) for T cells. Overall median time on study is 154 days (range 106- 254). 


\begin{tabular}{|c|c|c|c|c|c|c|c|}
\hline \multicolumn{8}{|c|}{ PROTECT Day 100 Clinical Data } \\
\hline Subject & 1 & 2 & 3 & 4 & 5 & 6 & 7 \\
\hline Hematologic Malignancy & MDS & AML & AML & ALL & ALL & ALL & AML \\
\hline CD34+ cell dose $\left(\times 10^{8} / \mathrm{kg}\right)$ & 10.3 & 4.6 & 10.9 & 4.8 & 3.2 & 3.0 & 9.4 \\
\hline CD3+ cell dose $\left(\times 10^{8} / \mathrm{kg}\right)$ & 3.1 & 1.8 & 2.6 & 2.8 & 2.0 & 1.2 & 2.8 \\
\hline ProTmune-related SAEs & None & None & None & None & None & None & None \\
\hline $\begin{array}{l}\text { Day of Neutrophil } \\
\text { Engraftment }{ }^{1}\end{array}$ & Day 14 & Day 18 & Day 22 & Day 15 & Day 16 & Day 18 & Day 19 \\
\hline $\begin{array}{l}\text { Acute GvHD / Grade } \\
\text { (CIBMTR) }\end{array}$ & None & None & Grade 2 & None & Grade 2 & Grade 3 & None \\
\hline Treatment Responsive & - & - & Yes & - & Yes & Yes & - \\
\hline $\begin{array}{l}\text { Time to Resolution of } \\
\text { Maximum Grade }\end{array}$ & - & - & 7 days & - & 8 days & 5 days & - \\
\hline Cancer Relapse-free & Yes & Yes & Yes & Yes & Yes & Yes & Yes \\
\hline Survival & Yes & Yes & Yes & Yes & Yes & Yes & Yes \\
\hline
\end{tabular}

${ }^{1}$ As measured from the day following $\mathrm{HCT}$

[P397 Figure] [PROTECT Day 100 Clinical Data].

During the first 100 days following HCT, all seven subjects remained alive and relapse-free. There were no events of graft failure with a median time to neutrophil engraftment of 18 days (range 14-22). There were no ProTmune-related serious adverse events reported by investigators. Three of the seven subjects experienced acute GvHD during the first 100 days following HCT, all of whom responded to standard-of-care steroid treatment; the median time to resolution of the maximum GvHD grade was 7 days [range: 5-8 days].

Conclusions: ProTmune can be reproducibly manufactured and delivered as part of standard-of-care HCT. ProTmune was well tolerated with no cases of graftfailure or disease-relapse. The randomized, double-blind, Phase 2 portion has commenced enrollment.

Clinical Trial Registry: NCT02743351

Conflict of interest:
R. Maziarz has received honoraria from Novartis Pharmaceuticals Corporation, Incyte, Juno Therapeutics, Kite Therapeutics, and Athersys.

A. Saad has consulted for Actinium and received honoraria from Spectrum.

M. Diaz, A. Medcalf, D. Fremgen, and C. Storgard are employees of Fate Therapeutics.

J. Edwards, J. Essel, C. Freytes, C. Karanes, D. Tzachanis, and A. Deol have nothing to disclose.

\section{P398}

REACH-3 trial design: A Phase 3, Randomized Study to Assess Ruxolitinib over BAT in Patients with CorticosteroidRefractory Chronic Graft-versus-Host Disease Post AlloHSCT

Robert Zeiser ${ }^{1}$, Ron Ram ${ }^{2,3}$, Stephen Ronan Foley ${ }^{4}$, Moshe Yeshurun $^{3,5}$, Franco Locatelli ${ }^{6,7}$, Brian Gadbaw ${ }^{8}$, Edric Atienza $^{8}$, 
Norbert Hollaender ${ }^{9}$, Takanori Teshima ${ }^{10}$

${ }^{1}$ Department of Hematology, Oncology and Stem Cell Transplantation, University Hospital Freiburg, Freiburg, Germany; ${ }^{2}$ BMT Unit, Tel Aviv Medical Center, Tel Aviv, Israel; ${ }^{3}$ Sackler Faculty of Medicine, Tel Aviv University, Tel Aviv, Israel; ${ }^{4}$ Department of Pathology and Molecular Medicine, McMaster University, Hamilton, Canada; ${ }^{5}$ Institute of Hematology, Davidoff Cancer Center, Rabin Medical Center, Petah Tikva, Israel; ${ }^{6}$ Department of Pediatric Hematology/Oncology, Bambino Gesù Children's Hospital, Rome, Italy; ${ }^{7}$ Department of Pediatric Sciences, University of Pavia, Pavia, Italy; ${ }^{8}$ Novartis Pharmaceuticals Corporation, East Hanover, NJ, United States; ${ }^{9}$ Novartis Pharma AG, Basel, Switzerland;

${ }^{10}$ Department of Hematology, Hokkaido University Faculty of Medicine, Graduate School of Medicine, Sapporo, Japan

Background: Steroid-refractory chronic graft-versus-host disease (SR-cGvHD) is associated with high morbidity and long-term mortality, as well as reduced quality of life, and represents an unmet medical need. In a retrospective study (Zeiser et al. 2015), ruxolitinib has demonstrated promising outcomes, and was well tolerated in patients with SRcGvHD. CMV reactivation, rates of thrombocytopenia, and rate of underlying malignancy relapse are favorable when compared to published literature on the use of best available therapy (BAT) in patients with SR-cGvHD. The present phase 3 study (REACH-3) will investigate the efficacy of ruxolitinib as add-on therapy to corticosteroid therapy in patients with SR-cGvHD.

Methods: REACH-3 is a randomized, open-label, multicenter study. Patients aged $\geq 12$ years who underwent allogeneic-hematopoietic stem cell transplantation (alloHSCT), who have evidence of myeloid and platelet engraftment (absolute neutrophil count $>1000 / \mathrm{mm}^{3}$ and platelets $\geq 25,000 / \mathrm{mm}^{3}$ ) and have been diagnosed with moderate or severe cGvHD according to National Institutes of Health (NIH) Consensus Criteria (Jagasia et al, 2015) are eligible for inclusion. Exclusion criteria comprise patients who have received prior JAK inhibitors for acute GvHD (except patients who achieved complete response [CR] or partial response $[\mathrm{PR}]$ and were off JAK inhibitor for $\geq 8$ weeks prior to cycle 1 day 1 ), have failed prior SCT $\leq 6$ months, or have overlap syndrome.

Eligible patients will be randomized (1:1) to the ruxolitinib (10 mg orally twice daily) arm or investigatordetermined BAT arm. Patients randomized to the BAT arm are allowed to cross over to the ruxolitinib arm (Figure 1). Duration of treatment and/or follow-up is 3 years (39 cycles/156 weeks).

The primary objective is to compare the efficacy of ruxolitinib vs BAT by overall response rate (ORR) at month 6. ORR is defined as the proportion of patients with $\mathrm{CR}$ or

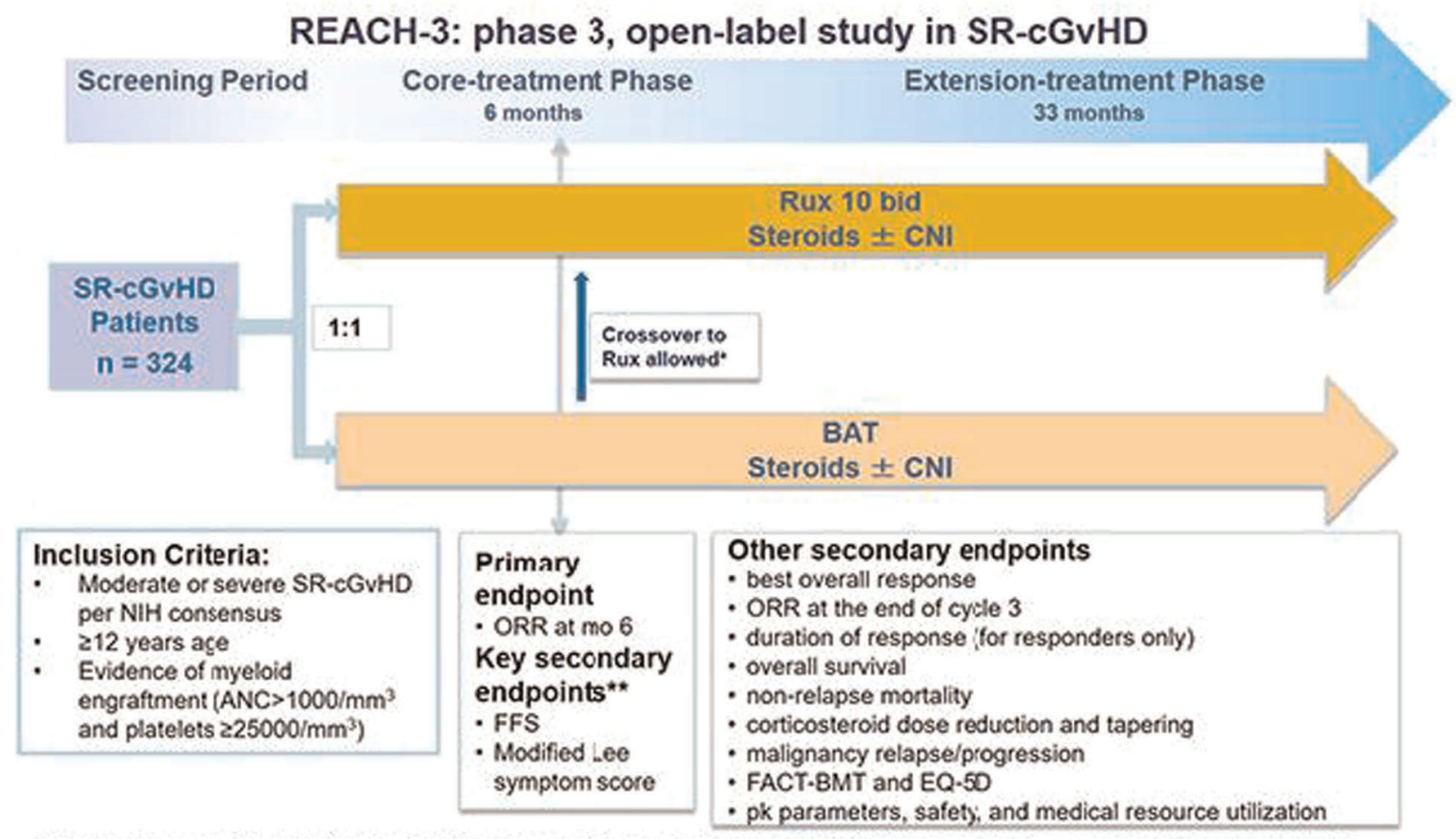

ANC, absolute neutrophil count; BAT, best available therapy; CN1, calcineurin inhibitors; FACT-BMT, Functonal Assossment of Concer Therapy-Bone Marrow Transplant; FFS, falluze-free survival; MO(s), month(s); NIH, National institutes of Health; ORR, overall respcense rate; PK, pharmacokinetic; SR. CGWHD; Steroid-refractory chronic graft-worsus-host disoase: Rux, ruxclitinib.

-Patients randomizes to the BAT arm are allowed to cross over to the ruxolitinib arm after the cycle 7 day 1 visit.

"FFS will bo usad as first koy secondary ondpoint in all rogions axcopt US and tho modified Loo symptom score will bo used as first key sesondary

endpoint for the US.

[P398 Figure] [Study Design] 
PR according to 2014 NIH response guideline (Lee et al, 2015). Overall 324 patients are planned to be enrolled to achieve $90 \%$ power for the ORR comparison (assuming odds ratio $=2.35$ ). Secondary endpoints include failure-free survival and the modified Lee symptom score.

Results: First patient was enrolled in June 2017.

Conclusions: Enrollment is ongoing. This trial was registered at ClinicalTrials.gov: NCT03112603.

Clinical Trial Registry: ClinicalTrials.gov: NCT03112603.

Conflict of interest: Robert Zeiser: Honoraria as speaker in symposium (Jazz Pharmaceuticals); and honoraria as speaker (Novartis)

Stephen Ronan Foley: Consultancy (Novartis) and advisory board (Celgene)

B. Gadbaw and E. Atienza: employees (Novartis)

N. Hollaender: employee (Novartis Pharma AG)

T. Teshima: Consultancy, research funding and advisory board (Novartis)

R. Ram, M. Yeshurun, and F. Locatelli: No conflict of interest to disclose

\section{P399}

Abstract previously published

\section{P400}

Abstract previously published

\section{P401}

Rituximab in treatment of chronic graft-versus-host-disease

Sebastian Klobuch, Daniela Weber, Barbara Holler, Wolfgang Herr, Ernst Holler, Daniel Wolff

Universitätsklinikum Regensburg, Innere Medizin III, Regensburg, Germany

Background: Rituximab was recently described also as first line therapy of chronic graft-versus-host-disease (cGVHD). We retrospectively analyzed the efficacy and safety of all patients receiving Rituximab for treatment of cGVHD between 2005 and 2016 at Regensburg University.

Methods: Responses of 29 allogeneic stem cell transplanted patients (median age 49, range 21-65 years) were evaluated within one year after Rituximab treatment. cGVHD was diagnosed and graded applying the NIH criteria. Median time after stem cell transplantation and after cGVHD onset before Rituximab application was 784 days (range 181-2885) and 349 days (range 45-2129), respectively. Overall severity of cGVHD at start of treatment of Rituximab was moderate $(\mathrm{n}=11)$ and severe $(\mathrm{n}=18)$.
Main organ manifestations were skin (24 patients, 83\%), oral (4 patients, 14\%), and lung (1 patient, 3\%). 5 patients $(17 \%)$ had platelets lower than $100 / \mathrm{nl}$ at onset of cGVHD. All patients failed sustained response to prior steroid treatment with 7 patients $(24 \%)$ had one prior line of therapy, 15 patients (52\%) two lines, and $7(24 \%)$ three or more lines. 25 patients $(86 \%)$ received 4 doses a $375 \mathrm{mg} / \mathrm{m}^{2}$ Rituximab applied weekly, 4 patients $(14 \%)$ received 8 or more doses ( 2 cycles). All patients received additional immunosuppressive agents, in 7 (24\%) patients a new immunosuppressive agent was started in parallel to Rituximab.

Results: Best responses were observed as follows: 3 patients $(10 \%)$ achieved complete response, 7 patients (24\%) partial response, 4 patients (14\%) mixed response, 3 patients $(10 \%)$ had a nondurable response, while 12 patients (42\%) did not respond.

7 patients (24\%) (including 4 lethal infectious complications) developed serious infections requiring hospitalization within 1-8 months after Rituximab therapy. Infectious causes were: fungal $(\mathrm{n}=1)$, viral $(\mathrm{n}=1)$, and bacterial $(\mathrm{n}$ $=5$ ).

Conclusions: Rituximab shows moderate activity in treatment of cGVHD patients failing primary treatment. The significant incidence of severe infectious complications warrants further Evaluation.

Conflict of interest: None of the authors has anything to disclose.

\section{P402}

Role of 2677G $>T>A$ Genetic Polymorphism in the $A B C B 1$ Drug Transporter in the Interaction between Voriconazole and Cyclosporine and Cyclosporine Related Nephrotoxicity After Allogeneic Stem-Cell Transplantation

\author{
Nathalie Khoueiry-Zgheib ${ }^{1}$, Raafat Alameddine ${ }^{2}$, Radwan \\ Massoud ${ }^{3}$, Rihab Nasr' ${ }^{1}$, Jean El Cheikh ${ }^{3}$, Rami Mahfouz ${ }^{4}$, Ali \\ Bazarbachi $^{2}$ \\ ${ }^{I}$ American University of Beirut, Faculty of Medicine, Beirut, Lebanon, \\ ${ }^{2}$ American University of Beirut Medical Center, Internal Medicine, \\ Division of Hematology-Oncology, Bone Marrow Transplant Unit, \\ Beirut, Lebanon; ${ }^{3}$ American University of Beirut Medical Center, \\ Internal Medicine, Bone Marrow Transplant Unit, Beirut, Lebanon; \\ ${ }^{4}$ American University of Beirut Medical Center, Department of \\ Pathology and Laboratory Medicine, Beirut, Lebanon
}

Background: The aims of this pilot non-interventional observational study were to examine genetic polymorphisms of drug metabolizing enzymes (DMEs) and transporters $(\mathrm{T})$ involved in voriconazole and/or cyclosporine disposition among patients undergoing allogeneic stem cell transplantation (SCT), and to assess the relationship between these polymorphisms and clinical and laboratory 


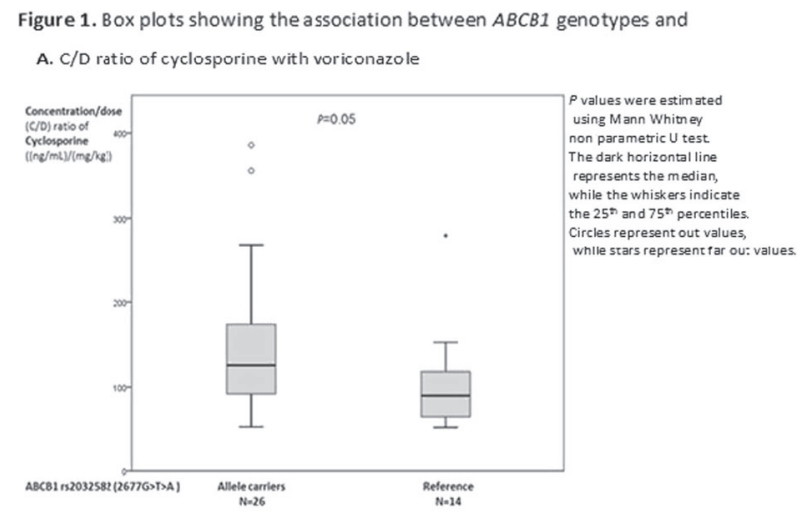

B. Highest serum creatinine values dur ing immunother apy

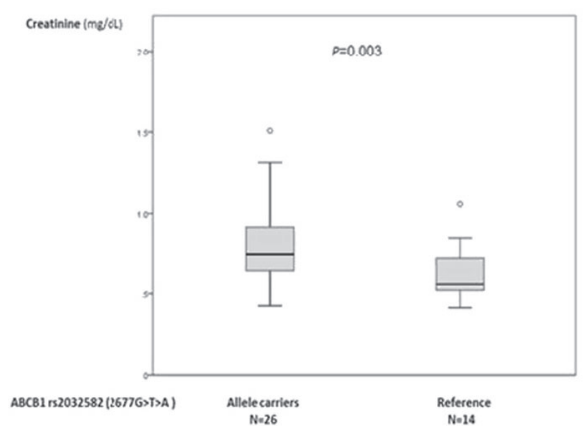

[P402 Figure] [Figure 1. Box plots showing the association between $A B C B 1$ genotypes and]

parameters related to cyclosporine kinetics and toxicity within the same patient population.

Methods: Clinical data and DNA were collected from 40 subjects who underwent SCT and received cyclosporine based immunosuppression and voriconazole prophylaxis for fungal infections. Samples were genotyped using DMET Plus arrays from Affymetrix. Only known candidate genes involved in the disposition (metabolism and transport) of cyclosporine and or voriconazole were analyzed. The average concentration/dose (C/D) ratio of cyclosporine $((\mathrm{ng} / \mathrm{mL}) /(\mathrm{mg} / \mathrm{kg}))$ was calculated using available through levels and daily doses per participant's weight.

Results: As expected, there was a definite interaction between voriconazole and cyclosporine whereby the C/D ratio of cyclosporine was lower when it was administered alone as compared to when it was administered with voriconazole, and this was statistically significant: median (range) $\mathrm{C} / \mathrm{D}$ of cyclosporine $((\mathrm{ng} / \mathrm{mL}) /(\mathrm{mg} / \mathrm{kg}))$ of 27.33 (90.12) vs. 116.75 (333.23) without and with voriconazole respectively; $P<0.001$. There was a statistically significant association between the $2677 G>T>A(r s 2032582) A B C B 1$ genetic polymorphism and the $\mathrm{C} / \mathrm{D}$ ratio of cyclosporine combined with voriconazole $(P=0.05)$ (Figure 1A). In parallel, $A B C B 1$ allele carriers had statistically significantly higher creatinine values during therapy [median (range) creatinine $(\mathrm{mg} / \mathrm{dL})$ were of $0.74(1.08)$ vs. $0.56(0.64)$ for allele carriers vs. reference; $P=0.003$ ]

(Figure 1B). Interestingly, there were trends of associations (though not statistically significant) between phenotypes of the other candidate genes and in the expected direction with $\mathrm{C} / \mathrm{D}$ ratio of cyclosporine combined with voriconazole.

Conclusions: This is one of the few pharmacogenetic studies in patients undergoing SCT, and the first to show that $2677 G>T>A$ genetic polymorphism in the $A B C B 1$ drug transporter plays a role in the interaction between voriconazole and cyclosporine and cyclosporine related nephrotoxicity in patients undergoing SCT. Moving forward is to increase sample size to potentially show significant associations with the additional candidate genes such as CYP2C9, CYP2C19 and CYP3A4/5.

Clinical Trial Registry: all authors have no conflict of interest to declare

Conflict of interest: all authors declare no conflict of interest

\section{P403}

\section{Ruxolitinib and extracorporeal photopheresis (ECP) for steroid refractory acute Graft versus Host Disease after allogeneic stem cell transplantation}

Franziska Modemann ${ }^{1,2}$, Francis Ayuk ${ }^{1}$, Christine Wolschke ${ }^{1}$, Maximilian Christopeit ${ }^{1}$, Ute von Pein ${ }^{1}$, Dietlinde Janson ${ }^{1}$, Nicolaus Kröger ${ }^{1}$

${ }^{1}$ Medical Center - University of Hamburg, Dept of Stem Cell Transplantation, Hamburg, Germany; ${ }^{2}$ Medical Center - University of Hamburg, Dept of Hematology and Oncology, Hamburg, Germany

Background: For steroidrefractory GvHD (SR-GvHD) after allogeneic stem cell transplantation (alloHSCT) the treatment option are limited. Ruxolitinib is a JAK $1 / 2$ inhibitor causing a blockade of the JAK-STAT pathway reducing inflammatory effects and has shown activity in SR-GvHD Extracorporeal photopheresis (ECP) is an immunomodulatory,leukapheresis-based procedure. Here we show a single center experience of the tolerability and efficacy of combining both therapeutic strategies in SRacute GvHD.

Methods: From 2015 to 2017, 28 patients (60\% male, $40 \%$ female) with SR-aGvHD of liver (7), skin (11) and mainly upper (2) and lower GI-tract (26) or a combination of them after alloHSCT were treated with Ruxolitinib. Median age was 56,5 years (r: 19-74), donor sources were: MMUD 25\%, MUD 65\%, MRD 10\%. Steroid refractoriness was defined as no improvement in 7 days or aggravation after 5 days of steroid treatment. At start of Ruxolitinib, $14 \%$ had an overall grade II, $43 \%$ a grade III 
and $43 \%$ a grade IV acute GvHD. Median day of GvHD onset after transplantation was day 28 (r: 9-198), median start of Ruxolitinib day 62 (r: 33-3025). Median duration of Ruxolitinib therapy was 45,5 days (r: 7-192) with a median dosage of $20 \mathrm{mg}$ per day $(2 \times 10 \mathrm{mg})$.

Additional treatment of Ruxolitinib was as follows: All patients received Ruxolitinib and a calcineurin inhibitor, 25 patients with a calcineurin inhibitor alone and 3 patients with additional mycophenolate mofetil (MMF). In 19 cases of patients $(67,9 \%)$, ECP (extracorporeal photopheresis) was added. Steroids were rapidly tapered after starting with Ruxolitinib with a median time of stopping steroids after onset of Ruxolitinib at day 26,5 (r: 9-89).

Results: Overall response to the treatment with Ruxolitinib and the combined immunosuppressive therapies $(67,9 \%$ received ECP) in all patients was $61 \%$, including complete remission of $50 \%(\mathrm{n}=14)$ and partial remission of $11 \%$, while 11 patients (39\%) had no benefit of the therapy. The main reason for stopping the therapy was receiving complete remission status $(\mathrm{n}=10,36 \%)$, followed by severe cytopenia $(n=9,32 \%)$, severe infections $(n=2$, $7 \%)$, no benefit of Ruxolitinib therapy $(\mathrm{n}=2,7 \%)$ and other reasons $(\mathrm{n}=5,18 \%) .25$ patients $(89 \%)$ had Ruxolitinib associated side effects. Main side effect was cytopenia. 9 patients developed worsening of anemia about one to two CTC grades, 11 patients worsening of the platelet count and leukocyte count about one to three CTC grades. Infectious complication were observed in 12 patients (42\%), CMV reactivation during Ruxolitinib therapy has been observed in $46 \%$ of cases $(n=13)$.

One-year estimated survival of all patients after starting with Ruxolitinib was $60,7 \%(n=17), 11$ patients died. 2 of them because of severe refractory aGvHD, 7 because of severe infections in combination with refractory GvHD and associated therapy, one patient died due to sepsis (GvHD was in CR) and two due to relapse.

Conclusions: Treatment of SR-aGvHD with Ruxolitinib in combination with ECP and rapid tapering of steroids could show a high rate of complete remission from overall $50 \%$ and an encouraging one year overall survival of $60,7 \%$. Cytopenia is the most common side effect.

Clinical Trial Registry: N/A

Conflict of interest: N. Kröger received a research grant and honorarium from Novartis.

All other authors have no conflict of interest.

\section{P404}

\section{Ruxolitinib in steroid refractory GVHD treatment: single center experience}

Guldane Cengiz, Seval, Sinem Civriz, Bozdag, Meltem Kurt Yuksel,
Selami Kocak Toprak, Pervin Topcuoglu, Meral Beksac

Ankara University School of Medicine, Department of Hematology and Stem Cell Transplant Unit, Ankara, Turkey

Background: Despite major improvements in allogeneic hematopoetic stem cell transplantation (AHSCT) over last decades, corticosteroid-refractory acute and chronic graftversus-host disease (GVHD) still remains a life-threatening complication. Novel insights into the pathophysiology of GVHD highlighted the relevant role of the host inflamatory response governed by janus kinase family. Ruxolitinib is a potent inhibitor of a janus kinase $1 / 2(\mathrm{JAK} 1 / 2)$ with proven efficacy in myelofibrosis, was recently employed in corticosteroid-refractory GVHD with encouraging overall response rates.

Methods: Cases presentation: Here in, we present our single center experience in 5 steroid refractory GVHD patients who were treated with ruxolitinib. Patient demographics are summarized in Table 1. Patients were treated initially with $10 \mathrm{mg}$ B.I.D and reduced to $5 \mathrm{mg}$ B.I.D in patients with grade $3 / 4$ adverse events. Median follow up of the patients were 15(1-16 months). Three out of four (75\%) acute GVHD patients achieved response ( 2 complete +1 partial response). One of these patients died in the $3^{\text {rd }}$ week of ruxolitinib therapy due to sepsis. One chronic GVHD patient was treated with ruxolitinib and improvement was observed in FEV1 measurements of the patient despite the short treatment duration. Grade $2 / 3$ thrombocytopenia and grade $3 / 4$ neutropenia was observed in all acute GVHD patients. Grade 2 thrombocytopenia was observed in chronic GVHD patient. Cytomegalovirus reactivation was not observed as all of the acute GVHD patients were treated with prophylactic gancyclovir.

Results: See table below

Conclusions: In our series, ruxolitinib was very effective as a salvage therapy for patients with GVHD refractory to standard treatments.

Conflict of interest: All authors: nothing to disclose

\begin{tabular}{|c|c|c|c|c|c|c|}
\hline Patients & $\begin{array}{l}\text { Gender/ } \\
\text { Age }\end{array}$ & Diagnosis & Donor source & $\begin{array}{l}\text { NIH } \\
\text { GVHD } \\
\text { grade }\end{array}$ & $\begin{array}{l}\text { Previous GVHD } \\
\text { therapy }\end{array}$ & $\begin{array}{l}\text { Sites } \\
\text { involved }\end{array}$ \\
\hline 1 & $\begin{array}{l}\text { Male/ } \\
45\end{array}$ & MDS & MUD & 3 acute & $\begin{array}{l}\text { Steroid, } \\
\text { Mesanchymal } \\
\text { SC }\end{array}$ & GI \\
\hline 2 & $\begin{array}{l}\text { Male/ } \\
39\end{array}$ & ALL & MRD & $\begin{array}{l}\text { Chronic } \\
\text { (extensive) }\end{array}$ & $\begin{array}{l}\text { Steroid,ECP, } \\
\text { MMF }\end{array}$ & $\begin{array}{l}\text { Skin, } \\
\text { lung,eye }\end{array}$ \\
\hline 3 & $\begin{array}{l}\text { Male/ } \\
56\end{array}$ & AML & $\begin{array}{l}\text { MMRD } \\
\text { (haploidentic) }\end{array}$ & 4 acute & $\begin{array}{l}\text { Steroid, } \\
\text { Mesanchymal } \\
\text { SC }\end{array}$ & GI \\
\hline 4 & $\begin{array}{l}\text { Male/ } \\
23\end{array}$ & ALL & MRD & 4 acute & $\begin{array}{l}\text { Steroid, } \\
\text { Mesanchymal } \\
\text { SC,MMF }\end{array}$ & skin \\
\hline 5 & $\begin{array}{l}\text { Female/ } \\
55\end{array}$ & Myelofibrosis & MRD & 3 acute & $\begin{array}{l}\text { Steroid, } \\
\text { Mesanchymal } \\
\text { SC,MMF }\end{array}$ & GI,skin \\
\hline
\end{tabular}


[[P404 Table] Patient demographics]

\section{P405}

\section{Ruxolitinib in steroid-refractory chronic GVHD: safety and efficacy}

Zoi Bousiou, Ioannis Batsis, Maria Kaliou, Anastasia Marvaki, Michail Iskas, Apostolia Papalexandri, Despoina Mallouri, Ioanna Sakellari, Achilles Anagnostopoulos

George Papanicolaou Hospital, Hematology and BMT Unit, Thessaloniki, Greece

Background: Steroid-refractory (SR) cGVHD remains a major complication of alloHSCT, associated with high rates of morbidity and mortality. Treatment alternatives are urgently needed and novel agents such as ruxolitinib are currently being investigated. However, limited clinical data exist in the literature, especially regarding the toxicity profile in this challenging group of severely immunocompromised patients.

Methods: We retrospectively studied patients who received ruxolitinib as salvage therapy for SR-GVHD and analyzed response rates and major toxicities.

Results: Fifteen patients received ruxolitinib for SR cGVHD in our institution during 2016-2017. All patients (median age 30 years, range: 18-66) had undergone alloHSCT between 2002-2017 for:

(i) acute leukemia $(n=8)$,

(ii) $\operatorname{MDS}(\mathrm{n}=3)$,

(iii) Hodgkin lymphoma( $\mathrm{n}=2)$,

(iv) CML ( $\mathrm{n}=1)$, and

myelofibrosis $(\mathrm{n}=1)$.

The conditioning regimen was:

(i) myeloablative $(n=11)$, or reduced intensity $(n=4)$. Ten patients were transplanted from well-matched (10/10) unrelated donors and 5 from sibling donors. GVHD prophylaxis consisted of cyclosporine in combination with short-term methotrexate or mycophenolate mofetil (MMF) plus rabbit ATG in the case of MUD alloHSCT. Twelve patients had developed acute GVHD (grade II, $\mathrm{n}=6$ and grade III-IV, $\mathrm{n}$ $=6)$ that was steroid sensitive in patients with grade II but those with grade III-IV refractory aGVHD progressed to overlap syndrome after multiple treatment lines. All presented SR extensive cGVHD involving skin, lungs, liver or intestinal tract. Most were heavily pretreated (median 3, range1-4 prior treatment lines), including corticosteroids, MMF, thalidomide and extracorporeal photopheresis. Ruxolitinib was initiated as an add-on immunosuppressant at a median of 9 months post cGVHD diagnosis (range 1-169 months) starting with a total daily dose of $20 \mathrm{mg}$. Four patients showed partial response enabling reduction of immunosuppression (response rate 26.6\%). Notably, they had received less treatment lines (median 2, range 1-3). Two progressed (12.5\%) and 9 remained stable. Particularly important is that no patient with overlap syndrome responded to ruxolitinib. Regarding toxicity, 60\% $(n=9)$ developed severe cytopenias (grade III-IV), requiring dose modifications and eventually discontinuation in three cases. Infections were another important complication during ruxolitinib administration. In particular, CMV reactivation occurred in 8 patients $(53 \%)$, and one presented with severe recurrence of CMV retinitis with partial visual loss. Except for 2 cases, CMV reactivations responded to antiviral therapy. In addition, almost all cases (13/15, $86.6 \%$ ) were complicated with infections from resistant bacteria, especially of the respiratory system. In one case, possible aspergillosis was diagnosed. Six patients eventually died of these infections without responding to ruxolitinib therapy, 2 of respiratory infection and four of septic shock while on grade IV neutropenia. During follow-up (median 9 months, range: 2-15), there was no recurrence of the original disease.

Conclusions: Our study shows that ruxolitinib may benefit patients with SR-cGVHD, possibly even more in early administration. The drug is associated with major toxicities and high rate of discontinuation which make proper administration challenging and necessitates close monitoring, especially regarding viral and bacterial infections. Prospective studies in large cohorts are needed to confirm these results.

Conflict of interest: nothing to disclose

\section{P406}

Safety and Efficacy of ATG-based GvHD prophylaxis in allogeneic stem cell transplantation for acute leukemia and myelodysplastic syndrome: a single center experience

Benedetta Rambaldi ${ }^{1}$, Michele Malagola ${ }^{1}$, Alessandro Turra ${ }^{1}$, Enrico Morello ${ }^{1}$, Federica Cattina ${ }^{1}$, Valeria Cancelli ${ }^{1}$, Simona Bernardi $^{2}$, Camilla Zanaglio ${ }^{2}$, Simone Perucca ${ }^{2}$, Domenico Russo ${ }^{1}$, Nicola Polverelli ${ }^{1}$

${ }^{1}$ Università degli Studi di Brescia, Unit of Blood Diseases and Stem Cells Transplantation, Department of Clinical and Experimental Sciences, ASST Spedali Civili of Brescia, Brescia, Italy; ${ }^{2}$ Università degli Studi di Brescia, Laboratorio CREA (Centro di Ricerca Ematooncologica AIL), ASST Spedali Civili of Brescia, Brescia, Italy

Background: Relapse occurrence after allogeneic stem cell transplantation (allo-SCT) represents one of the main concerns about ATG use, as GvHD-prophylaxis.

Methods: Clinical and laboratory data of 74 (53\%) ATGexposed and 66 unexposed patients submitted to allo-SCT for acute leukemia and myelodysplastic syndrome from January 2006 to December 2016 were retrospectively collected. Standard GvHD-prophylaxis was mostly 
represented by cyclosporine and methotrexate. The median follow-up was 52 months (range 9-132).

Results: Clinical characteristics of the cohort are detailed in table 1.

Patient characteristics. MAC $=$ myeloablative conditioning regimen.

Acute GvHD (aGvHD) occurred in $38(51 \%)$ and 31 (47\%) patients $(\mathrm{p}=0.5)$, with a cumulative incidence of $50 \%$ vs $36 \%$ at 3 months $(\mathrm{p}=0.39)$, in ATG and control group respectively. Overall, $122(87 \%)$ of patients survived more than 100 days from transplantation and were evaluable for cGvHD occurrence. ATG-exposed patients showed a significant lower rate of global and moderate-severe chronic GvHD (cGvHD) (16\% vs $41 \%, \mathrm{p}=0.002$ and $8 \%$ vs $24 \%$, $\mathrm{p}=0.02$ ), with a cumulative incidence at 2 years of $15 \%$ vs $38 \%,(\mathrm{p}=0.003)$ and $8 \%$ vs $24 \%(\mathrm{p}=0.018)$, in ATG versus standard prophylaxis, respectively. A trend for an increased incidence of CMV reactivation was recorded in the ATG group (54\% vs $39 \%, \mathrm{p}=0.09$ ). No graft failure was recorded. No differences were observed between the two groups in terms of transplant-related mortality (19\% vs $15 \%, \mathrm{p}=0.53)$, relapse-free survival $(62 \%$ vs $62 \%, \mathrm{p}=$ $0.38)$, relapse-incidence $(30 \%$ vs $33 \%, \mathrm{p}=0.34)$ and overall survival $(50 \%$ vs $50 \%, \mathrm{p}=0.71)$ at 2 years for ATG and control group, respectively.

In univariate analysis, previous $\mathrm{aGvHD}(\mathrm{p}=0.02)$, GvHD-prophylaxis without ATG $(\mathrm{p}=0.0048)$, donor age $(\mathrm{p}=0.0007)$, sibling $(\mathrm{p}=0.001)$ and female donor $(\mathrm{p}=0.001)$ did correlate with higher risk of cGvHD occurrence. In multivariate analysis, previous aGvHD (HR 2.43, CI95\% $1,14-5.18, \mathrm{p}=0.02$ ) and female donor (HR 2.91, CI95\% 1,42-5.97, $\mathrm{p}=0.003$ ) confirmed their detrimental role, on the contrary ATG utilization was associated with a significant reduction of cGvHD (HR 0.38, CI95\% 0.17$0.82, \mathrm{p}=0.01$ ).

Conclusions: ATG seems to prevent cGvHD, without affecting relapse and transplant-related mortality.

Conflict of interest: None of the authors has anything to disclose.

\begin{tabular}{llll}
\hline & $\begin{array}{l}\text { No ATG }(\mathrm{n}= \\
66)\end{array}$ & $\begin{array}{l}\text { ATG }(\mathrm{n}= \\
74)\end{array}$ & $\mathrm{p}$ \\
\hline $\begin{array}{l}\text { Age (years) at allo-SCT, median } \\
\text { (range) }\end{array}$ & $54(18-67)$ & $48(19-68)$ & 0.07 \\
Sex, female, N (\%) & $25(37.9 \%)$ & $36(48.6 \%)$ & 0.23 \\
Acute Leukemia, N (\%) & $48(72.7 \%)$ & $53(71.5 \%)$ & 0.29 \\
First complete remission at transplant, & $32(48.5 \%)$ & $35(47.3 \%)$ & 0.12 \\
N (\%) & $27(40.9 \%)$ & $52(70.3 \%)$ & 0.001 \\
MAC, N (\%) & $56(86.2 \%)$ & $62(83.8 \%)$ & 0.81 \\
Peripheral blood as Stem cells source, & & & \\
N (\%) & $34(53.3 \%)$ & $30(40.5 \%)$ & 0.23 \\
Female donor, N (\%) & $15(22.7 \%)$ & $66(89.2 \%)$ & $<0.001$ \\
Matched unrelated donor, N (\%) & $52(9-124)$ & $53(10-132)$ & 0.88 \\
\hline \begin{tabular}{l} 
Follow-up, median (range) \\
\hline
\end{tabular}
\end{tabular}

[[P406 Table] Table 1]

\section{P407}

\section{Severe Chronic Pulmonary Graft Versus Host Disease}

Sandra Marini, Sobrinho Carvalho João, André Pinto, Fernando Campilho, Campos Júnior

IPOFG Porto, Serviço de Transplantação de Medula Óssea, Porto, Portugal

Background: Graft-versus-host disease (GvHD) is an immunological disorder that affects many organ systems including the gastrointestinal tract, liver, skin and lungs. Chronic GVHD (cGVHD) is still a major complication of long-term survivors after allo-HSCT, it occurs in $20 \%-45 \%$ of patients who survive after 6 months of transplantation and pulmonary involvement is common. Pulmonary complications significantly contribute to late mortality after allo-HSCT, furthermore, late-onset non-infectious pulmonary complications can be present with restrictive lung function impairment (restrictive pulmonary function test (PFT) pattern, late interstitial pneumonitis (IP), cryptogenic organizing pneumonia (COP),or airway obstruction (obstructive PFT pattern, bronchiolitis obliteratans (BO). However, $\mathrm{BO}$ is the only pulmonary complication, which is currently considered diagnostic of cGVHD. Late IP as well as COP may occur in association with cGVHD and are regarded as 'associated' manifestations of cGVHD, being rare the cases that occur in isolation.

Methods: Description of a clinical case.

Results: A 49-year-old female diagnosed with Acute Myeloid Leukemia (NMP1 + and FLT3 +) in 1 RC after HOVON 132 arm A protocol, followed by consolidation with high dose cytarabine, is described. Allograft of bone marrow, from related donor (sister, HLA 10/10, Isogrupal, CMV IgG -) was performed in January 2017. Prophylaxis of GVHD was made with Methotrexate and Cyclosporine. In April 2017, the patient had an irritative cough and dyspnea with moderate efforts which deteriorated to dyspnea with minimal efforts motivating the hospitalization in July 2017. Patient denied dry eyes, dry skin or weight lost. A diagnostic study was carried out, excluding infections, cardiac, thromboembolic and other possible manifestations of GVHD. CT scan with evidence of depolyzed glass densification in the upper lobe of the right lung and several others with peri-bronchial distribution, suggestive of COP. Spirometry with mixed ventilatory alteration (FEV1- 29.5\%; CPT- 60.4\%), with no positive response with bronchodilation and decreased $\mathrm{CO}$ diffusion capacity. Pulmonary biopsy showed aspects suggestive of 
organizational pneumonia that may correspond to the manifestation of graft disease. Treatment with $1 \mathrm{mg} / \mathrm{kg} /$ day of Prednisolone, nebulizations with Fluticazone, Aclidinium bromide and Formoterol was initiated with clinical and imaging improvement.

Conclusions: COP is a rare presentation of cGVHD, which sometimes leads to a complex diagnosis leading to a delayed initiation of therapy. The majority of patients with COP have persistent, bothersome, and progressive symptoms, the initial therapy is with oral glucocorticoids (dose of prednisone of 0.75 to $1 \mathrm{mg} / \mathrm{kg}$ per day). Recovery, usually with complete resolution of symptoms and normalization of the chest film, occurs in two-thirds of patients. Symptomatic improvement is occasionally quite dramatic, occurring in one to two weeks, although most patients improve more gradually over several weeks to a few months.

Conflict of interest: Nothing to disclose

\section{P408}

\section{Significance of drug monitoring for mycophenolic acid in GVHD prophylaxis in cord blood transplantation}

Hiroyuki Muranushi ${ }^{1}$, Junya Kanda ${ }^{1}$, Takero Shindo ${ }^{1}$, Masakatsu Hishizawa ${ }^{1}$, Tadakazu Kondo ${ }^{1}$, Kohei Yamashita ${ }^{1}$, Takashi Yamamoto $^{2}$, Kazuo Matsubara ${ }^{2}$, Akifumi Takaori-Kondo ${ }^{1}$

${ }^{1}$ Kyoto University, Department of Hematology and Oncology, Kyoto, Japan; ${ }^{2}$ Kyoto University Hospital, Department of Clinical

Pharmacology and Therapeutics, Kyoto, Japan

Background: Significance of drug monitoring for mycophenolic acid (MPA), active constituent of mycophenolic mofetil (MMF), is unclear in GVHD prophylaxis in cord blood transplantation (CBT).

Methods: We retrospectively analyzed the data of 46 patients who received first CBT with GVHD prophylaxis of calcineurin inhibitor plus MMF in our institute from 2011 to 2016. They received $10 \mathrm{mg} / \mathrm{kg}$ of oral MMF every 8 hours from day -1 to 30 . We measured blood concentration of MPA immediately before and 1,2 and 4 hours after taking $\mathrm{MMF}$, estimated 24-hour area under the curve $\left(\mathrm{AUC}_{0-24}\right)$, and analyzed the impact of $\mathrm{AUC}_{0-24}$ of MPA in day 7 and 21 on transplant outcomes.

Results: Median observation times of survivors were 475 (range: 101 - 1339) days. Median age was 50 (range: 20-66) years. The percentage of patients diagnosed with AML, ALL, MDS and ML was 43\%, 13\%, $15 \%$ and $17 \%$, respectively. The percentage of patients with complete remission and partial remission was $52 \%$ and $7 \%$, respectively. Fifty two percent of patients received myeloablative conditioning and $93 \%$ received TBI. The cumulative incidence of neutrophil engraftment was $94 \%$. Median days of neutrophil engraftment were 25 (range: 11-
43) days. The cumulative incidence of pre-engraftment syndrome (PES), grade II-IV acute GVHD (aGVHD), grade III-IV aGVHD, limited chronic GVHD (cGVHD) and extensive cGVHD was $30 \%, 63 \%, 11 \%, 15 \%$ and $13 \%$, respectively. Two-year overall survival, cumulative incidence of relapse and non-relapse mortality was $80 \%, 14 \%$ and $10 \%$, respectively. $\mathrm{AUC}_{0-24}$ of MPA in day 7 (MPA7) was $49.4 \pm 24.7 \mu \mathrm{g} \mathrm{h} / \mathrm{ml}$ (mean \pm standard deviation). There was no association between MPA7 and the incidence of PES. The rate of sepsis before engraftment in patients with MPA7 of $>60 \mu \mathrm{g} \mathrm{h} / \mathrm{ml}$ was higher than in the other patients (33\% vs $6 \%, p=0.03$ ). $\mathrm{AUC}_{0-24}$ of MPA in day 21 (MPA21) was $47.7 \pm 20.3 \mu \mathrm{g} \mathrm{h} / \mathrm{ml}$. After excluding 3 cases of engraftment failure, we divided patients into two groups by MPA21. The incidence of grade II-IV aGVHD tended to be higher in group A (MPA21: $\leq 50 \mu \mathrm{g} \mathrm{h} / \mathrm{ml}$ ) than in group B (MPA21: $>50 \mu \mathrm{g} \mathrm{h} / \mathrm{ml})(70 \%$ vs $50 \%, p=0.11)$. The rate of HHV6 reactivation in group A was higher than in group B ( $70 \%$ vs $35 \%, p=0.03)$. The reason of this result might be more frequent use of corticosteroids for aGVHD in group A. The level of serum albumin was lower $(p=0.03)$, and the incidence of diarrhea tended to be higher $(57 \%$ vs $25 \%, p=0.06)$ in group A. There was no significant difference between two groups in overall survival, cumulative incidence of relapse and cGVHD.

Conclusions: In CBT, blood concentration of MPA may have an impact on incidence of aGVHD and infection. In case of poor nutrition or diarrhea, frequent monitoring and dose adjustment of MPA may be necessary since blood concentration of MPA tends to be low.

Clinical Trial Registry: No registry.

Conflict of interest: All authors have nothing to disclose.

\section{P409}

Site Specific Diagnostic Yield of Endoscopic biopsies: Case series of Gastrointestinal Graft-versus-Host Disease in a Tertiary Center

Fady Daniel', Mohamad Husni ${ }^{1}$, Lara Hassoun ${ }^{1}$, Ala' Sharara ${ }^{1}$, Assad Soweid ${ }^{1}$,Kassem Barada ${ }^{1}$, Yasser Shaib ${ }^{1}$, Jana Al-Hashash ${ }^{1}$, Ali Bazarbachi', Jean El-Cheikh ${ }^{2}$

${ }^{1}$ American University of Beirut Medical Center, Department of Internal Medicine, Division of Gastroenterology, Beirut, Lebanon; ${ }^{2}$ American University of Beirut Medical Center, Internal Medicine, Division of Hematology-oncology, Bone Marrow Transplant Unit, Beirut, Lebanon

Background: Gastrointestinal (GI) graft versus host disease (GVHD) occurs in up to $40 \%$ of patients undergoing allogenic hematopoietic stem cell transplation (HSCT). The diagnosis relies on the combination of clinical symptoms, exclusion of other causes of diarrhea and histologic 
evaluation of GI biopsies. However, the optimal endoscopic approach is still unclear and the area of the GI tract with the highest diagnostic yield is still a topic of debate.

Methods: We aim to compare the diagnostic yield of different anatomic site biopsies in the diagnosis of GI GVHD and assess the correlation of endosocopic findings with the histolopathology in a series of patients in American University of Beirut Medical Center (AUBMC), a tertiary care center. : All cases of biopsy proven GI GVHD were obtained from pathology database AUBMC between 1/1/ 2005 and 31/8/2017. We retrospectively analyzed the demographical, clinical and endoscopic data.

Results: A total of 19 patients were diagnosed with GI GVHD over 17.6 years. The average age at diagnosis was $41( \pm 12)$ years and $78.9 \%$ (15 patients) were males. The most common indication for HSCT was acute myeloid leukemia (6 patients, 31.6\%), and the most common presenting symptom was severe diarrhea (18 patients, 94.7\%). The diagnosis of GI GVHD occurred between 19 and 840 days, with a median interval of 113 days. Out of 19 patients, 3 patients had a history of skin GVHD (15.8\%). Combining upper endoscopy and sigmoidoscopy with biopsies had the highest diagnostic yield of $90 \%$ in diagnosing GI GVHD compared to $63.6 \%, 78.6 \%$ and $77.8 \%$ for upper endoscopy, sigmoidoscopy and colonoscopy respectively. In macroscopically normal mucosa, the recto-sigmoid and duodenal biopsies had the highest diagnostic yield $(75 \%)$. As for the macroscopically abnormal mucosa, the highest yield was for the rectosigmoid biopsies (100\%) in lower endoscopy and duodenal biopsies in the upper endoscopy $(60 \%)$.

Conclusions: In a patient suspected to have GI GVHD, the best endoscopic approach is the combination of upper endoscopy and flexible sigmoidoscopy with biopsies of normal as well as abnormal mucosa. It should be emphasized that normal mucosa be biopsied especially in the duodenum and recto-sigmoid for a better diagnostic yield.

Conflict of interest: All authors have nothing to disclose.

\begin{tabular}{lllll}
\hline $\begin{array}{l}\text { Biopsies on normal } \\
\text { mucosa by: }\end{array}$ & location & $\begin{array}{l}\text { Positive for } \\
\text { GVHD }\end{array}$ & $\begin{array}{l}\text { Negative for } \\
\text { GVHD }\end{array}$ & sensitivity \\
\hline EGD & Esophagus & $0(0.0 \%)$ & $2(100.0 \%)$ & $0.0 \%$ \\
EGD & Stomach & $2(66.7 \%)$ & $1(33.3 \%)$ & $66.7 \%$ \\
EGD & Duodenum & $3(75.0 \%)$ & $1(25.0 \%)$ & $75.0 \%$ \\
Sigmoidoscopy & Rectosigmoid & $3(75.0 \%)$ & $1(25.0 \%)$ & $75.0 \%$ \\
colonoscopy & Colon and & $1(50.0 \%)$ & $1(50.0 \%)$ & $50.0 \%$ \\
& ileum & & & \\
\hline
\end{tabular}

[[P409 Table] table1]
P410

Specific Anti-Nuclear Antibodies, Clinical Features and Outcome Following Treatment with Nilotinib In Patients with Chronic Graft-Versus-Host Disease

\author{
Elena Marinelli Busilacchi ${ }^{1}$, Andrea Costantini ${ }^{1,2}$, Giorgia \\ Mancini $^{3}$, Federica Monaco ${ }^{1,3}$, Veronica Falciani ${ }^{2}$, Nadia Viola ${ }^{2}$, \\ Ilaria Scortechini ${ }^{3}$, Luca Butini ${ }^{2}$, Attilio Olivieri ${ }^{1,3}$ \\ ${ }^{1}$ Università Politecnica delle Marche, Dipartimento di Scienze \\ Cliniche e Molecolari, Ancona, Italy; ${ }^{2}$ Azienda Ospedaliero \\ Universitaria Ospedali Riuniti, Servizio di Immunologia Clinica, \\ Ancona, Italy; ${ }^{3}$ Azienda Ospedaliero Universitaria Ospedali Riuniti, \\ Clinica di Ematologia, Ancona, Italy
}

Background: Clinical manifestations of chronic graftversus-host diseases (cGVHD) often resemble systemic autoimmune diseases. The association with autoantibodies, including anti-nuclear antibodies (ANA), has been reported, but the clinical and prognostic relevance of this association is still unclear. Tyrosine kinase inhibitor nilotinib is currently under investigation for the treatment of cGVHD; we sought to investigate the relationship between presence of ANA and outcome of cGVHD following administration of nilotinib.

Methods: Fifteen patients with severe cGVHD following hematopoietic stem cell transplantation (HSCT) and nine transplanted patients without cGVHD (controls) were evaluated. Clinical data were collected by patientsẤ files. A panel of 19 specific ANA was assessed by quantitative immunoblot assay (Alifax, Padova, Italy). Proportions of T cell subpopulations (including $\mathrm{T}$ helper, $\mathrm{T}$ cytotoxic and $\mathrm{T}$ regulatory), B lymphocytes, $\mathrm{NK}$ and $\mathrm{NK} / \mathrm{T}$ cells were assessed by flow cytometry (Bekton Dickinson, San Josè, CA). Data were compared by StudentÂ's t test; differences were considered statistically significant when $\mathrm{p}<0.05$.

Results: In patients who developed cGVHD, organs more commonly involved were skin $(80 \%)$, eyes/mouth (Sicca Syndrome, 67\%) and lungs (33\%); ANA were detected in seven of these patients (47\%, figure panel A). Most frequent specificity was anti-SSA/Ro (3 cases); other specificities were anti-SSB, anti-CENP A/B, anti-Nucleosome and anti$\mathrm{Pm}-\mathrm{Scl}$ (1 case each). Sicca Syndrome was the clinical manifestation more associated with the presence of ANA (7/ 10 cases, $70 \%$ ), followed by skin disease (5/12 cases, $42 \%$ ). Only one of the controls (11\%) was tested ANA-positive (anti-Histones/PCNA/DFS-70, figure panel A); of note, this patient suffered from isolated xerostomia. No difference in timing of cGVHD onset following HSCT was observed between ANA-positive and ANA-negative patients (267 vs. 280 days, $\mathrm{p}=0.88$ ). Compared to controls (figure panels BE), cGVHD patients had significantly higher proportions of $\mathrm{T}$ and NK/T cells $(\mathrm{p}<0.05)$ and lower proportions of $\mathrm{B}$ 
A

\begin{tabular}{|l|c|c|}
\cline { 2 - 3 } \multicolumn{1}{|c|}{} & ANA-pos & ANA-neg \\
\hline CGVHD & $7 / 15$ & $8 / 15$ \\
\hline controls & $1 / 10$ & $9 / 10$ \\
\hline
\end{tabular}

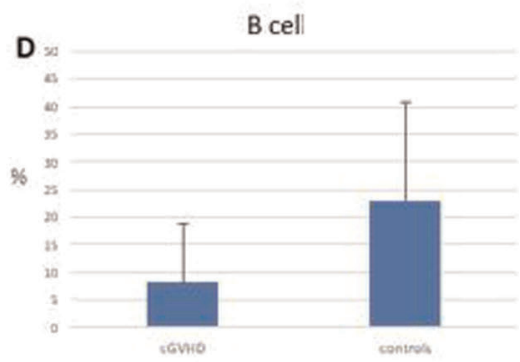

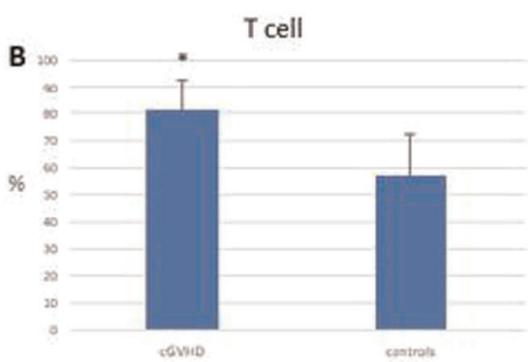

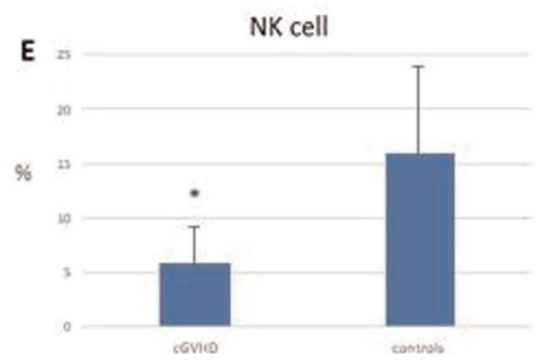

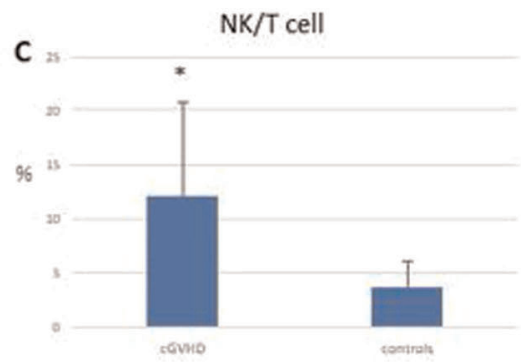

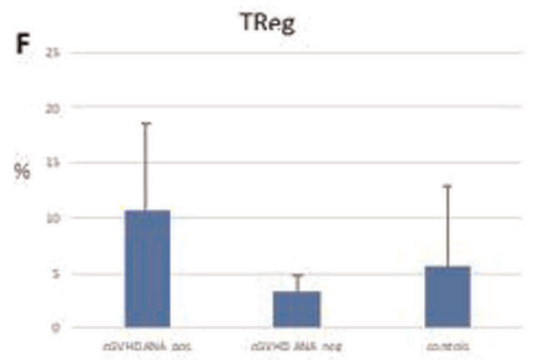

Panel A: ANA-screening results among chronic graft-versus-host (CGVHD) patients and transplanted controls. Panels B-F: Proportions of lymphocyte subpopulation gated by forward scatter (FSC) and side scatter (SSC) profile. T lymphocytes were identified as CD3 positive cells (panel B); NK/T as $C D 3, C D 16$ and $C D 56$ triple-positive cells (panel C); $B$ ymphocytes as CD3-negative CD19-positive cells (panel D); NK cells as CD3-negative, CD16 and CDS6 positive cells (panel E); $T$ regulatory cells as $\mathrm{CD} 3, \mathrm{CD} 4$ and $\mathrm{CD} 25$ triple-positive cells (panel F). Data obtained from cGVHD patients and transplanted controls are compared; means and standard deviations are showed. ${ }^{\circ} p<0.05$

[P410 Figure] [Figure]

$(\mathrm{p}=0.06)$ and NK cells $(\mathrm{p}<0.05)$. CD4/CD8 ratio (not shown) as well as $\mathrm{T}$ regulatory cells (figure panel $\mathrm{F}$ ) were comparable between the two groups; however, this latter subset showed a trend toward higher values among ANApositive compared to ANA-negative cGVHD patients (10,7 $\pm 7.9 \%$ vs. $3.4 \pm 1.4 \%, p=0.12$ ). No difference in cGVHD progression nor in response to nilotinib was observed between ANA-positive and ANA-negative patients; after six months of treatment, eight (4 ANA-positive) remained stable and the other seven (3 ANA-positive) improved.

Conclusions: In agreement with published data, presence of ANA was frequent among patients who developed cGVHD and uncommon among transplanted patients without this condition. Most common cGVHD clinical features were skin disease and Sicca Syndrome; the latter was also the most frequent manifestation among ANA-positive cGVHD individuals. Although the frequency of B cells was not increased among patients developing cGVHD, these data suggest that B cells may play a pathogenic role in the onset of this condition and that presence of ANA may predict the development of particular clinical manifestations, such as Sicca Syndrome. Six-months course of nilotinib stabilized or improved cGVHD in all the study patients, independently of the presence of ANA. The evaluation of autoantibodies and immune population after nilotinib treatment is ongoing.

Conflict of interest: All authors have nothing to disclose.
P411

Successful treatment of steroid refractory chronic Graft vs. Host disease with combination of Bortezomib plus Ruxolitinib

Panayotis Kaloyannidis, Eshrak Al Shibani, Hani Al Hashmi, Solaf Kafnar, Asraf Suhebah, John Apostolidis, Khalid Al Anezi

King Fahd Specialist Hospital, Adult Hematology and Stem Cell Transplantation Department, Dammam, Saudi Arabia

Background: Despite the improvements on chronic Graft vs Host disease (cGvHD) management, it still remains the leading cause of late non-relapse mortality and also contributes in high morbidity rates and poor quality of life post allogeneic stem cell transplantation (alloSCT). Even in the modern-era of GvHD treatment $(\mathrm{NFk} \beta$ or kinases inhibitors), in a considerable number of patients, cGvHD cannot be controlled, so new approaches are highly demanded to overcome its refractoriness

Methods: We herein present the clinical course and the outcome in a patient with a refractory cGvHD after multiple lines of treatment, including Jak-2 inhibitor (Ruxolitinib) and NFk $\beta$ inhibitor (Bortezomib), given as single agents. However, the combination of Bortezomib and Ruxolitinib, proven to be extremely efficacious, suggesting an additive effect of these two agents 
Results: A 22 years old male allografted for hepatosplenic T-cell lymphoma, after a myeloablative regimen consisted of Total Body Irradiation and Cyclophosphamide. The graft source was blood stem cells from his full-matched sister. As GvHD prophylaxis, cyclosporine plus short-term methotrexate were administered. The early course post transplant was uneventful; however the second month post alloSCT the immunosuppression was tapered rigorously because of molecularly detectable disease. Two months later he achieved complete molecular remission with $100 \%$ donor-origin hematopoietic cells, but at the presence of induced-GvHD with extensive skin involvement [scleroderma and lichenoid lesions, [highest modified-Rodnan skin score (mRSS): 25], severe buccal mucosal and liver involvement (highest levels of: SGOT: $300 \mathrm{IU} / \mathrm{L}$ SGPT: $624 \mathrm{UI} / \mathrm{L}$, ALP: $131 \mathrm{UI} / \mathrm{L}$, total billirubin: 14,5 umol/L). He first treated with calcineurin inhibitors plus steroids but no improvement was noticed, so, Mycophenolate Mofetil (MMF) was added, however, no significant response was achieved. Since extracorporeal photopheresis was not available, patient put on Ruxolitinib (10mg bid). After a month the treatment was discontinued because of GvHD progression (skin-liver). He next put on Bortezomib $1.3 \mathrm{mg} /$ $\mathrm{m}^{2}$ (days 1-8-15-22) every 35 days plus MMF and steroids but after 2 cycles, the patient had stable findings being still steroid depended. Because of its different mechanism of action, we decided to add again on his treatment Ruxolitinib (5mg bid). After 2 months on this combination the patient experienced markedly skin improvement, sclerodermatosis was improved (mRSS: 12), the lichenoid lesions disappeared and liver tests became normal. Noticeably, after 3 months of treatment patient was out of MMF while steroids dose was feasible to be reduced by $75 \%$. He is still on Bortezomib and Ruxolitinib on the aforementioned doses. During the treatment period he did not experienced any major toxicity including neuropathy, or myelotoxicity. At his last evaluation the complete metabolic remission status along with $100 \%$ donor chimera were confirmed

Conclusions: In this heavily retreated patient for a refractory cGVHD, the combination of Bortezobib \& Ruxolitinib proved to be highly effective demonstrating also no toxicity. The inhibitory effects on lymphocytes in addition to the anti-inflammatory properties of both bortezomib and ruxolitinib, seemed to resulted in a possible synergistic and complementary effect. Undoubtedly, future clinical trials will clarify the role of this combination in the treatment of $\mathrm{cGvHD}$

Conflict of interest: All authors have nothing to disclose.
P412

Tacrolimus conversion from intravenous continuous infusion to oral once-daily formulation in allogeneic hematopoietic stem cell transplantation

Takuya Yamashita, Reiko Ito, Shin-Ichiro Oshima, Misa Nishitani, Koki Shimizu, Masaya Morimoto, Ryosuke Koyamada, Shin-Ichiro Mori

St. Luke's International Hospital, Department of Hematology, Tokyo, Japan

Background: Tacrolimus (TAC) is commonly adopted in allogeneic hematopoietic stem cell transplantation (allo$\mathrm{SCT}$ ) to prevent graft-versus-host disease (GVHD). TAC plus short-term methotrexate has been used as one of the standard regimens for GVHD prophylaxis. Usually, continuous infusion of intravenous TAC (TAC CIV) is converted to an oral twice-daily formulation (TAC BID) after patients has recovered from regimen-related gastrointestinal toxicity. A once-daily modified release formulation of oral TAC (TAC QD), which has recently been developed, has been reported to be almost equivalent to TAC BID in kidney and liver transplantation. So TAC QD is expected to contribute to improve the convenience and the adherence of all-SCT recipients.

Methods: We retrospectively reviewed the clinical outcomes of the patients who underwent allo-SCT between December 2011 and April 2017 using TAC QD converted from TAC CIV as GVHD prophylaxis.

Results: Twenty-four patients were included in this study. Median age of these patients at the time of SCT was $53(23-70)$ years. One patient received related bone marrow, 3 related peripheral blood stem cells, and 20 unrelated bone marrow. Thirteen patients received myeloablative conditioning. The median time to the conversion from CIV to QD was 33 (13-63) days after SCT. Major conversion rate from CIV to QD was 1:4 in this study. The cumulative incidence of grade II-IV acute GVHD at day 100 was $16.7 \%$. The probability of overall survival and the cumulative incidence of non-relapse mortality at 2 years were $70.8 \%$ and $12.2 \%$, respectively. Ten patients experienced CTCAE grade $\geq 2$ increase of creatinine after conversion to TAC QD, which required transient suspension of this drug in 2 patients. Grade $\geq 2$ increase of asparate aminotransferase (AST) and/or alanine aminotransferase (ALT) occurred in 8 patients. The cumulative incidences of the termination of immunosuppressive agents including TAC for GVHD prophylaxis and/or treatment at 1 and 2 years were $23.9 \%$ and $56.9 \%$, respectively.

Conclusions: This study shows that TAC QD was well tolerated with similar efficacy and safety profiles to TAC BID in allo-SCT. The once-daily medication regimen of 
TAC QD improves the convenience and the drug adherence in GVHD prophylaxis after allo-SCT.

Conflict of interest: All authors have nothing to disclose

\section{P413}

Th1 to Th2 cytokine shift: a possible mechanism behind ECP in the treatment of acute GvHD

\author{
Catherine Roberts ${ }^{1}$, Aisling Flinn ${ }^{2}$, Xiao Nong Wang ${ }^{2}$, Andrew \\ Gennery ${ }^{2}$ \\ ${ }^{1}$ Newcastle University, Newcastle upon Tyne, United Kingdom; \\ ${ }^{2}$ Newcastle University, ICM, Newcastle upon Tyne, United Kingdom
}

Background: Evidence suggests there is an imbalance in Th1 and Th2 cytokines in the setting of acute graft-versushost disease (aGvHD). Restoring the Th1/Th2 balance may be beneficial in the management of aGvHD and has been reported to be one of the mechanisms behind extracorporeal photopheresis (ECP). However, changes in Th1/Th2 cytokines in the setting of aGvHD have not previously been studied. Use of the ratio of interferon gamma (IFN $\gamma$ ) to interleukin 4 (IL-4) has been used in previous studies to represent the balance of Th1/Th2 cytokines.

Methods: We measured Th1/Th2 cytokines in 5 paediatric patients who were receiving ECP treatment for aGvHD following allogeneic haematopoietic cell transplant for malignancy or primary immune deficiency. Serum samples were taken before each ECP treatment and IFN- $\gamma$ and IL-4 cytokines were measured using the Enhanced Cytokine Bead Assay (BD Biosciences) (CBA Human Enhanced Sensitivity Master Buffer Kit). IFN $\gamma$ : IL-4 was used as a marker to represent $\mathrm{Th} 1 / \mathrm{Th} 2$ balance. The ratio was calculated using the mean of the absolute IFN $\gamma$ and IL4 values at each time point for each patient.

Results: Analysis of data showed a reduction of the IFNy:IL-4 ratio during therapy $(\mathrm{r}=-0.278, \mathrm{P}=0.222)$ (Figure 1), calculated using the median ratio of all patients

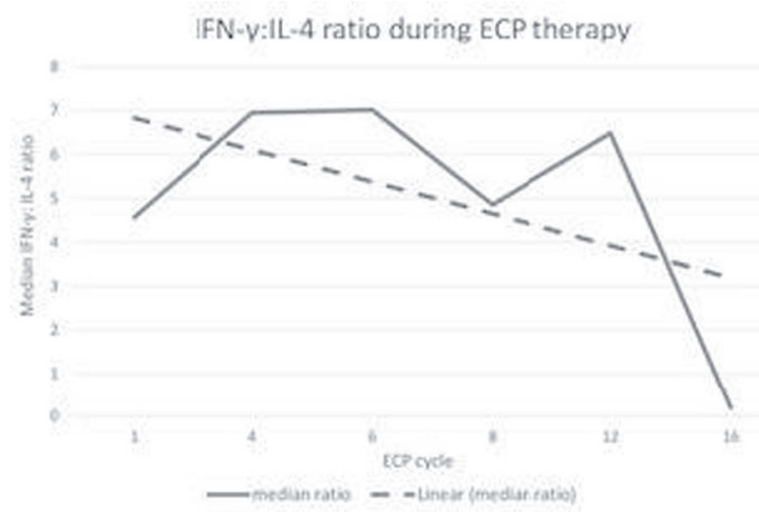

[P413 Figure] [Figure 1] at each time-point, and consistent with resolution of symptoms of aGvHD.

Conclusions: Although further prospective data are needed, this is preliminary evidence suggesting that ECP may modulate the cytokine profile in aGvHD, possibly mediated through an effect on DC polarisation of T-helper cells ${ }^{1}$.

1. Holtick U, et al. Transplantation 2008;85: 757-766

Clinical Trial Registry: N/A

Conflict of interest: The authors have nothing to disclose

\section{P414}

The Addition of Extracorporeal Photopheresis (ECP) to Tacrolimus and Methotrexate to Prevent Acute and Chronic Graft-Versus Host Disease in Myeloablative Hematopoietic Cell Transplant (HCT)

Anthony Accurso ${ }^{1}$, Dean Merkel $^{2}$, Shaun DeJarnette ${ }^{3}$, Jianghua $\mathrm{He}^{4}$, Junqiang Dai ${ }^{4}$, Siddhartha Ganguly ${ }^{3}$, Joseph McGuirk ${ }^{3}$, Sunil Abhyankar ${ }^{3}$

${ }^{1}$ University of Kansas Medical Center, Department of Hematological Malignancy and Cellular Therapy, Westwood, KS, United States;

${ }^{2}$ University of Kansas Medical Center, Pathology and Laboratory Medicine, Westwood, KS, United States; ${ }^{3}$ University of Kansas Medical Center, Hematological Malignancy and Cellular Therapy, Westwood, KS, United States; ${ }^{4}$ University of Kansas Medical Center, Department of Biostatistics, School of Medicine, Westwood, KS, United States

Background: Graft-versus-host disease is a major contributor of morbidity and mortality in allogeneic HCT patients. Incidence of acute GVHD is high, 30-50\% after transplant from HLA identical sibling, and 50-80\% in MUD (Greinix). Chronic GVHD occurs in 40-50\% of allogeneic HCT patients and is fatal in $20-40 \%$ of cases.

Corticosteroids are the standard treatment for GVHD. If steroid-refractory, survival is poor. There is no standard second-line therapy, although options include mycophenylate mofetil, sirolimus, rituximab, other monoclonal antibodies, and recently FDA-approved ibrutinib.

ECP involves removing cells from a patient, treating with psoralens, and exposing to ultraviolet light. This induces apoptosis in lymphocytes. Reinfused apoptotic lymphocytes have a tolerogenic effect on antigen-presenting cells. Response rates in chronic GVHD are 50-80\% (Bredeson). However, response may take $8-12$ weeks to manifest (Flowers, Beaton). Effectiveness of ECP in treating chronic GVHD, along with limited data suggesting efficacy in GVHD prevention (Shaughnessy, Miller) has created interest in studying ECP as prophylaxis.

Methods: This is a single-center, seventeen-patient prospective cohort conducted at Kansas University Cancer Center. Included patients underwent myeloablative 


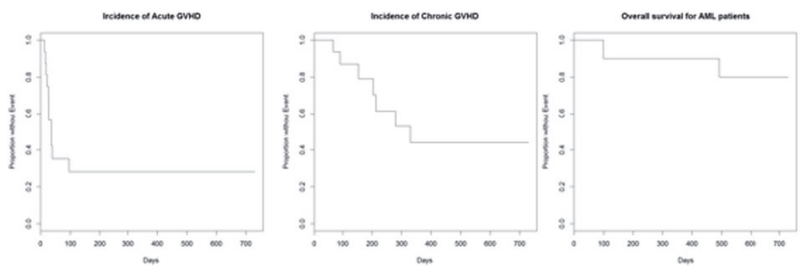

[P414 Figure] [ECP Graphs]

allogeneic HCT for hematologic malignancy in 2011-2012. Patients were excluded if felt unable to tolerate ECP, or if they had received rituximab within 3 months. Most patients were being treated for AML, but CML and lymphomas were also included. Participants provided informed consent, which was approved by the Institutional Review Board at KUMC.

All patients received myeloablative conditioning with either busulphan and cyclophosphamide or cyclophosphamide and total body irradiation (TBI). All patients received GVHD prophylaxis with tacrolimus or cyclosporine, plus methotrexate. ATG was allowed for unrelated donors. Participants received two ECP treatments prior to conditioning regimen, as well as 8 additional treatments between day 0-100. Patients were assessed weekly until day 100 and at least once every three months after day 100 , per NIH protocol.

Results: 17 patients were consented. One patient withdrew consent prior to receiving any ECP treatment. Of the 16 patients who received any ECP treatment, 10 completed the entire protocol. Six patients received partial protocol but were subsequently removed from the protocol or withdrew consent due to medical complications of their malignancy. All 16 patients who received any ECP were included in analysis.

Ten total aGVHD events occurred ( 7 grade two, one grade 3, two grade 4). Actuarial rate of aGVHD was $62.5 \%$. One death was attributable to aGVHD. Six total cGVHD events occurred (5 mild-moderate, 1 severe). Actuarial rate of cGVHD was $37.5 \%$.

Overall survival of AML patients at two years was $88 \%$.

No adverse events were attributable to ECP. Additional transfusion needs, to safely undergo ECP were minimal. Four of sixteen patients required additional blood products (6 units PRBC and 2 units platelets).

Conclusions: Our study is too small to draw conclusions about efficacy. However, in our high-risk cohort, (majority MUD, 1/4 mismatched donors), acute and chronic GVHD rates were within acceptable range and occurred with mild/ moderate severity. AML patient outcomes were excellent, with two-year overall survival of $88 \%$. Randomized controlled trials using prophylactic ECP are warranted.

Conflict of interest: Funded by Therakos.

\section{P415}

The correlation between amount of Th17 lymphocytes and occurrence of early complications after stem cell transplantation - a prognostic factor for acute GvHD development?

\author{
Alicja Sadowska-Klasa ${ }^{1}$, Krzysztof Lewandowski ${ }^{2}$, Agnieszka \\ Piekarska ${ }^{1}$, Andrzej Hellmann ${ }^{1}$
}

${ }^{1}$ Medical University of Gdansk, Department of Hematology and Transplantology, Gdansk, Poland; ${ }^{2}$ Medical University of Gdansk, Department of Monitored Therapy and Pharmacogenetics, Gdansk, Poland

Background: Allogeneic hematopoietic cell transplantation (HCT) is a curative option for patients with haematological malignancies, however non-relapse mortality related to infectious complications and acute graft versus host disease (GvHD) is still important. A subpopulation of pro-inflammatory Th17 lymphocytes may have impact on HCT outcome. The correlation between the amount of Th17 lymphocytes and occurrence of early post-HCT complications was the aim of this study.

Methods: We prospectively analysed data of 19 patients undergoing HCT during last 6 months due to haematological malignancies. The study included 9 female and 10 male patients with a median age of 41 years. Ten patients were transplanted from unrelated donors (MUD/MMUD), 8 from sibling and 1 from haploidentical donor. We analysed the count of Th17 and T-regulatory lymphocytes (Tregs) in peripheral blood and concentrations of IL6 and IL17 in serum. Samples were collected prior to the conditioning and after HCT (day $+14,+21,+30$ and +60 ). With the use of flow cytometry Th17 lymphocytes were defined as cells CD3+, CD4+, CD161+, CD196+ and Tregs as sCD3+, $\mathrm{CD} 4+, \mathrm{CD} 25+, \mathrm{CD} 28+, \mathrm{CD} 45 \mathrm{RO}+, \mathrm{CD} 69+$.

Results: In 7 patients (36\%) acute GvHD grade I-II was diagnosed: in 5 cases with skin involvement, 2 patients with skin and intestinal manifestation. Median time from HCT to aGvHD development was 28 days (range 23-47). The median time to engraftment was 28 days (range 15-25). One patient did not achieve neutrophil engraftment. Serious infectious complications (defined as bacteraemia, septic shock, typhlitis) were observed in 8 cases $(42 \%)$ and fever of unknown origin in 17 (89\%) patients.

There was no correlation between the Th17 lymphocytes count before procedure and infectious events after HCT (median value $0,027 \times 10^{9} / 1$ ). We did not find connection between the amount of Th17 before SCT, day $+14,+30$ after HCT and GvHD development. However, there was a strong correlation between the lower absolute count of Th17 at day +21 and aGvHD development (median value $0,001 \mathrm{x}$ $10^{9} / 1$ in GvHD group vs $0,01 \times 10^{9} / 1, \mathrm{p}<0.05$ ) - one week 
before median time of GvHD occurrence. In addition, we observed a change in median fluorescence intensity (MFI) of Th17: a shift to bright fluorescence of CD161 at the time of aGvHD development (before GvHD onset - MFI $<3.5$ vs. MFI $>6.5$ at the onset). Patients with GvHD also presented lower Th17/Tregs ratio at day $+21(\mathrm{p}<0.05)$. We did not observe differences in Tregs amount between both groups.

The serum levels of IL6 did not correlate with the amount of Th17 cells, except day 21 after SCT $(\mathrm{R}=-0.81, \mathrm{p}<$ $0.001)$. IL17 was detected only in $6 \%$ of examined samples (median value $918 \mathrm{fg} / \mathrm{ml}$ )

\section{Conclusions:}

1. A higher Th17 lymphocytes count before HCT did not have protective value against infectious complications.

2. A lower amount of Th17 during time of regeneration may be a prognostic factor for GvHD development. Changes in MFI values need a further investigation.

Conflict of interest: A. Sadowska-Klasa: Nothing to disclose

\section{P416}

The frequency of ocular GVHD in patients with allogeneic stem cell transplantation: a single center experience

\author{
Nur Soyer ${ }^{1}$, Suzan Guven Yilmaz, Melis Palamar Onay ${ }^{2}$, Sait \\ Egrilmez $^{2}$, Filiz, Vural \\ ${ }^{1}$ Ege University Medical Faculty, Department of Hematology, Izmir, \\ Turkey; ${ }^{2}$ Ege University Medical Faculty, Department of \\ Ophthalmology, Izmir, Turkey
}

Background: Ocular complications develop in a substantial percentage of patients after allogeneic stem cell transplantation (ASCT). One of the most important complications is ocular graft versus host disease (GVHD) that develops in $40-60 \%$ of patients after ASCT. It typically presents with dry eyes [keratoconjunctivitis sicca (KCS)]. Ocular GVHD has the potential to lead blindness and impairment of quality of life.

Methods: One hundred and seventy three patients who underwent ASCT from January 2012 to June 2017 were included in the study. Ocular complications and treatments were retrospectively recorded. Ocular GVHD was evaluated with short tear break-up time, decreased Schirmer 1 test, and ocular surface staining score (according Oxford Scheme; ranging from 0 to 5).

Results: Of the 173 patients (65 female, 108 male) who underwent ASCT, 24 (13.8\%) (19 male, 5 female) developed ocular GVHD. Underlying diseases were acute myeloid leukemia $(\mathrm{n}=8)$, acute lymphoblastic leukemia ( $\mathrm{n}$ $=1$ ), myelodysplastic syndrome $(\mathrm{n}=4)$, myelofibrosis ( $\mathrm{n}$ $=2)$, lymphoma $(\mathrm{n}=6)$ and aplastic anemia $(\mathrm{n}=3)$. All patients received peripheral blood as the stem cell source. There were 18 matched sibling donor (MSD) transplants, 5 matched unrelated donor (MUD) transplants and 1 haploidentical transplant. The median age at first symptoms was 38 (19-67) years and the median time to first symptoms was 11 (2- 44) months. The most common ocular manifestation of ocular GVHD was KCS (95.8\%). Severity scores in ocular GVHD are shown in Table 1. Oxford Staining score was grade 0 in $3(12.5 \%)$, grade 1 in $6(25 \%)$, grade 2 in $7(29.2 \%)$, grade 3 in $2(8.3 \%)$, grade 4 in 5 (20.8\%), and grade 5 in $1(4.2 \%)$ patients. Of the other manifestations, 2 of $24(8.3 \%)$ had cataract requiring surgery and 1 of 24 (4.1) had corneal perforation due to grade 4 KCS. This patient treated with the bandage contact lens. All patients diagnosed as KCS were treated with artificial tears. Twenty required additional topical cyclosporine $(83.3 \%)$ and 5 required additional topical steroids (fluorometholone, or loteprednol; 20.8\%).

Conclusions: Ocular GVHD presenting with KCS is the most common ocular complication in ASCT. In severe KCS, even corneal perforation that might end up with the loss of eye may develop. In order to prevent undesired complications, patients who underwent ASCT should carefully be monitored for possible ocular GVHD, and appropriate treatment should promptly be started when needed.

Conflict of interest: The authors have nothing to disclose.

\begin{tabular}{lllll}
\hline $\begin{array}{l}\text { Severity } \\
\text { Score }\end{array}$ & $\begin{array}{l}\text { Schirmer 1 } \\
\text { test }(\mathrm{mm})\end{array}$ & $\begin{array}{l}\text { Patients }(\mathrm{n}= \\
24)(\%)\end{array}$ & $\begin{array}{l}\text { Break-up time test } \\
(\text { second })\end{array}$ & $\begin{array}{l}\text { Patients }(\mathrm{n}= \\
24)(\%)\end{array}$ \\
\hline 0 & $>15$ & $1(4.2)$ & $>15$ & $2(8.3)$ \\
1 & $11-15$ & 0 & $11-15$ & 0 \\
2 & $6-10$ & $6(25)$ & $6-10$ & $3(12.5)$ \\
3 & $\leq 5$ & $17(70.8)$ & $\leq 5$ & $19(79.2)$ \\
\hline
\end{tabular}

[[P416 Table] Severity score in ocular GVHD]

\section{P417}

The prognostic impact of serum ferritin level at time of diagnosis of chronic graft-versus-host disease on survival and mortality following allogeneic hematopoietic stem cell transplantation

Warren Fingrut, Arjun Law, Wilson Lam, Fotios V. Michelis, Santhosh Thyagu, Auro Viswabandya, Jeffrey H. Lipton, Hans A. Messner, Dennis (Dong Hwan) Kim

University of Toronto, Allogeneic Blood and Marrow Transplant Program, Princess Margaret Cancer Centre, Toronto, Canada

Background: Chronic graft versus host disease (cGVHD) is a major complication following allogeneic hematopoietic stem cell transplantation (HCT). Many 


\section{A.Distribution of ferritin level at diagnosis of cGVHD}
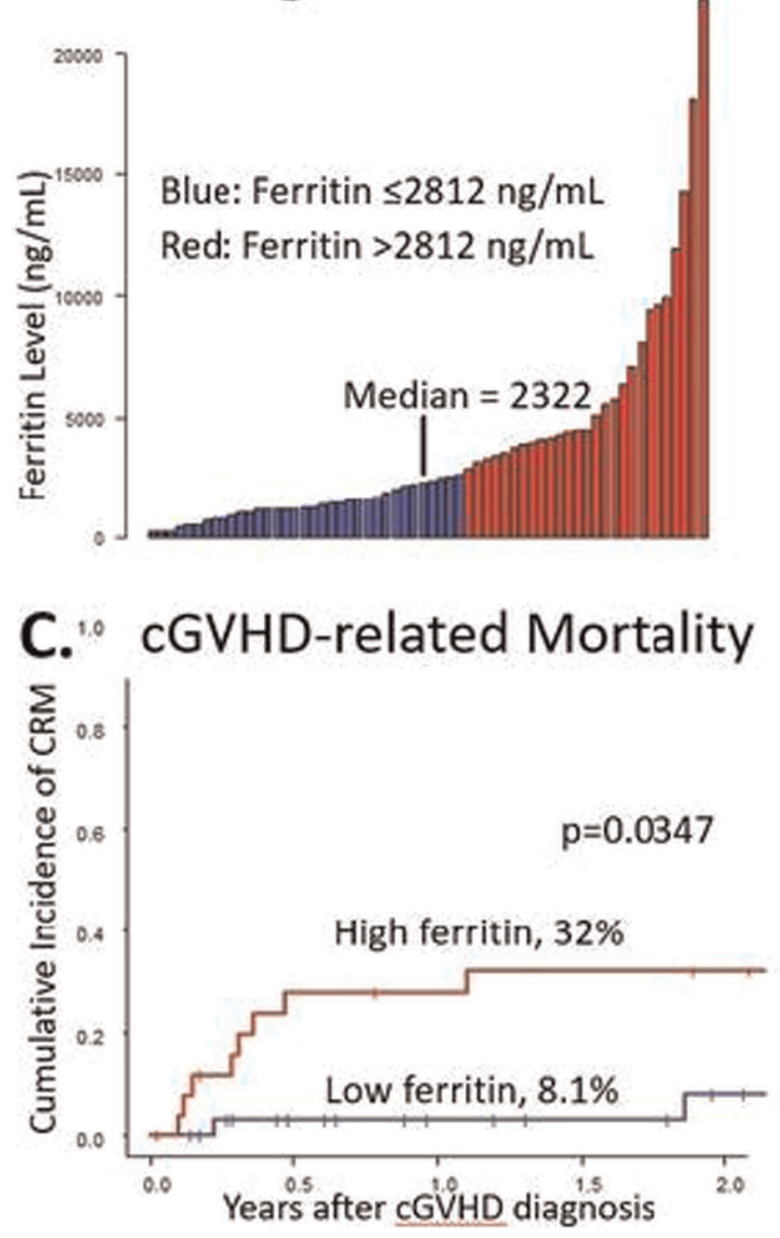

[P417 Figure] [A. Distribution of ferritin level at diagnosis of $c G V H D$;

studies have shown that elevated pre-transplant ferritin levels are associated with increased risk of morbidity and mortality following HCT. Given ferritin's association with systemic inflammation, it is a plausible potential prognostic marker after onset of cGVHD. The present study attempts to determine the prognostic impact of serum ferritin level at time of cGVHD diagnosis.

Methods: Sixty-two patients who had ferritin level data available at cGVHD diagnosis were enrolled into this study. These patients underwent HCT and were diagnosed with cGVHD at Princess Margaret Cancer Centre, Toronto, Canada. Patients' characteristics were: median age: 51 (range:19-70); refined disease risk index: low $(\mathrm{n}=11)$, intermediate $(\mathrm{n}=33)$, high $(\mathrm{n}=18)$; donor type: HLAmatched related donors $(n=41)$, HLA-matched or partiallymatched unrelated donors $(\mathrm{n}=21)$; conditioning regimen: myeloablative $(n=24)$, reduced intensity $(n=38)$.
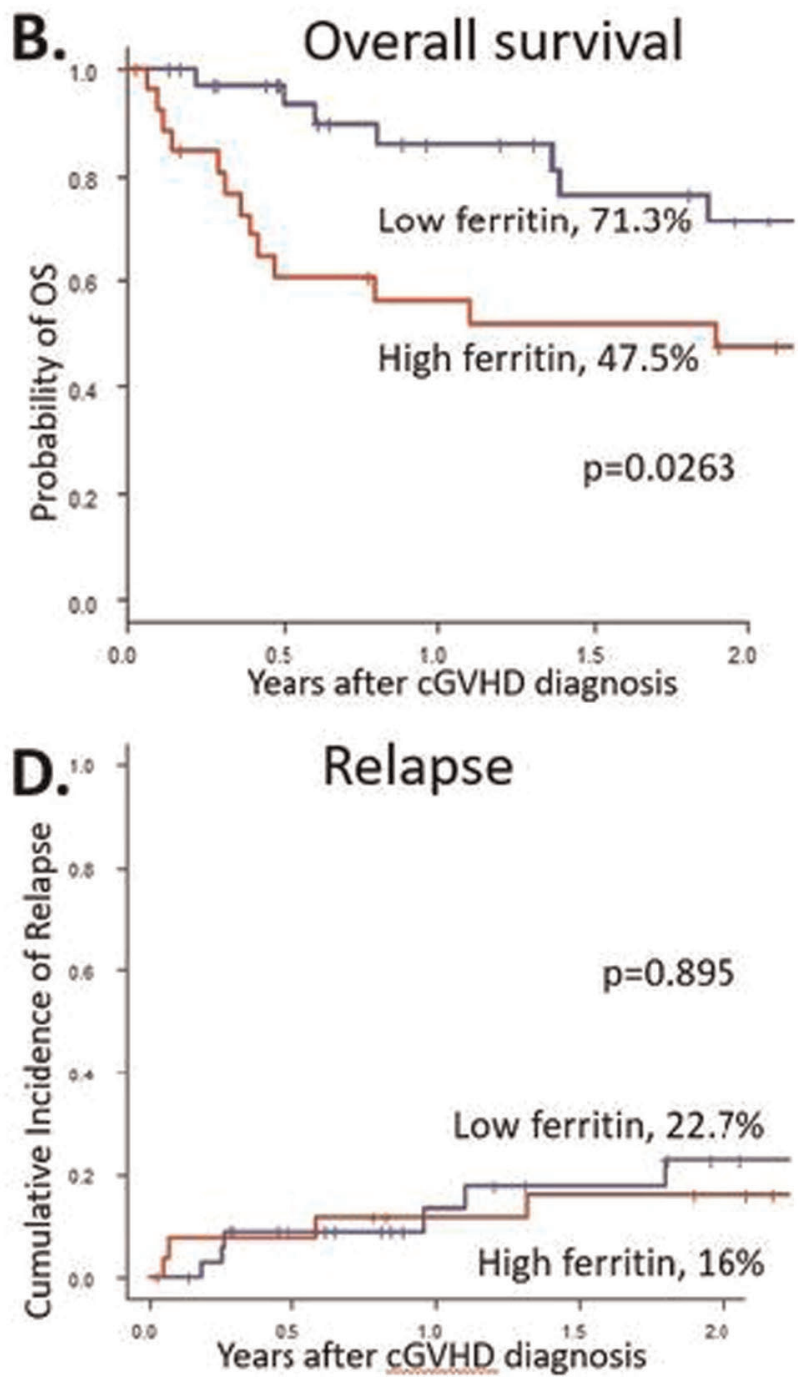
(2005). Primary outcomes were overall survival (OS), cGVHD-related mortality (CRM), and relapse. KaplanMeier method was used for OS; cumulative incidences were used for CRM and relapse, considering competing risks. Multivariate analysis employed Cox proportional hazard regression model for OS and Fine-Gray method for incidences of CRM and relapse. Factors evaluated included recipient age, donor-recipient sex and CMV matching, comorbidity score, performance index, GVHD prophylaxis, ferritin level at cGVHD diagnosis, and ferritin level prior to HCT. Recursive partitioning was used to stratify patients into low or high risk groups based on serum ferritin level at cGVHD diagnosis $(<=2812 \mathrm{vs}>2812 \mathrm{ng} / \mathrm{mL}$ ) and prior to HCT ( $<=942$ vs $>942 \mathrm{ng} / \mathrm{mL}$ ). Only factors with $\mathrm{P}<0.10$ in univariate analysis were included in multivariate analysis. 
Results: There were no significant differences in patient characteristics between the low $(\mathrm{n}=35)$ and high $(\mathrm{n}=27)$ ferritin level at cGVHD groups. Median follow-up duration among survivors was 25.1 months after diagnosis of cGVHD. Median onset of cGVHD was 143 days. Median ferritin level at cGVHD was $2322 \mathrm{ng} / \mathrm{mL}$ (range:28222304; Fig A). There was no correlation between ferritin levels at cGVHD diagnosis and prior to HCT $\left(\mathrm{R}^{2}=0.0187\right.$, $\mathrm{p}=0.400)$.

Univariate analysis revealed, compared to the low-ferritin at cGVHD group, the high-ferritin group had decreased 2year OS rate $(47.5 \%$ vs $71.3 \%, \mathrm{p}<0.0263$; Fig B) and increased CRM rate (32\% vs $8.1 \%, \mathrm{p}<0.0347$; Fig C), but no difference in relapse incidence $(16 \%$ vs $22.7 \%$, $\mathrm{p}=$ 0.895; Fig D).

In multivariate analysis, ferritin level at cGVHD diagnosis was found to be an independent prognostic factor for OS ( $p<0.02148$, HR 2.696, 95\% CI [1.158-6.278]) and for CRM ( $<<0.038$, HR 3.877, 95\% CI [1.075-13.98]). High ferritin level at cGVHD diagnosis also showed good correlation with increased cGVHD grade $(\mathrm{p}=0.049)$.

Conclusions: In our cohort, elevated ferritin levels at time of cGVHD diagnosis were significantly associated with decreased OS and increased CRM, independent of other prognostic factors including pre-HCT ferritin level. Our findings suggest that ferritin levels at cGVHD could be used to stratify patients according to their prognosis at the time of diagnosis of cGVHD. Additional studies including larger sample sizes and prospective investigation are warranted.

Conflict of interest: The authors report no conflicts of interest.

\section{P418}

Tissue damage in the skin caused by myeloablative conditioning before allogeneic stem cell transplantation results in dermal recruitment of macrophages without inflammation

Peter Van Balen ${ }^{1}$, Boris Van der Zouwen ${ }^{1}$, Alwine B. Kruisselbrink $^{1,2}$, Matthijs Eefting ${ }^{1}$, Karoly Szuhai ${ }^{3}$, Ekaterina $S$. Jordanova $^{2,4}$, J.H.Frederik Falkenburg ${ }^{1}$, Inge Jedema ${ }^{1}$

${ }^{1}$ Leiden University Medical Center, Hematology, Leiden, Netherlands; ${ }^{2}$ Leiden University Medical Center, Pathology, Leiden, Netherlands;

${ }^{3}$ Leiden University Medical Center, Molecular Cell Biology, Leiden, Netherlands; ${ }^{4} V U$ University Medical Center, Center for Gynaecologic Oncology, Amsterdam, Netherlands

Background: The conditioning regimen preceding allogeneic stem cell transplantation (alloSCT) can cause tissue damage and acceleration of the development of graftversus-host disease (GVHD). T-cell depleted alloSCT with postponed application of donor lymphocyte infusion (DLI) may reduce GVHD because tissue injury can be restored at the time of DLI. In this study, we investigated the presence of tissue injury and inflammatory conditions in skin during the period of hematologic recovery and immune reconstitution after alloSCT.

Methods: Skin biopsies were immunohistochemically stained for HLA class II, CD1a, CD11c, CD40, CD54, CD68, CD86, CD206, CD3 and CD8. Using these markers, HLA class II expressing cells were further characterized as activated T-cells, activated professional antigen presenting cells (APC) or tissue repairing macrophages. In sex mismatched patient and donor couples, the origin of cells in the skin biopsies was determined by multiplex analysis combining XY-FISH and fluorescent immunohistochemistry.

Results: No inflammatory environment due to pretransplant conditioning was detected at the time of infusion of the stem cell graft, irrespective of the type of conditioning regimen that was applied. A significant increase in HLA class II positive macrophages and an increase in CD3 Tcells was observed in the immune reconstitution period 1224 weeks after total body irradiation containing myeloablative (MA) alloSCT, but these macrophages did not show signs of interaction with the co-localized patient or donor Tcells. In contrast, in skin biopsies taken during GVHD, an increase in HLA class II expressing cells coinciding with Tcell interaction was observed, resulting in an overt inflammatory reaction with presence of activated APC, activated donor T-cells and localized upregulation of HLA class II expression on epidermal cells. In the absence of GVHD, HLA class II positive macrophages of patient origin were gradually replaced over time by donor derived macrophages, although patient derived macrophages were detectable even 24 weeks after alloSCT.

Conclusions: Conditioning regimens applied prior to alloSCT cause tissue damage in the skin, but this tissue damage does not result in a local increase of activated APC. In contrast to the inflamed situation in GVHD, when interaction takes place between activated APC and activated donor T-cells, the tissue damage caused by MA alloSCT results in dermal recruitment of HLA class II positive tissue repairing macrophages co-existing with increased numbers of patient and donor derived T-cells, but without signs of specific interaction and initiation of an immune response. Thus, the local skin damage caused by the conditioning regimen appears to be insufficient as single factor to provoke GVHD induction.

Conflict of interest: All authors have no conflicts of interest to disclose. 


\section{P419}

Total Nucleated Cell Dose and Allele-level HLA Matching Have Associations with Graft-versus-Host Disease after Umbilical Cord Blood Transplantation for Children with Hematologic Malignancies

Peifang Xiao, Sh H

Children's Hospital of Soochow University, Suzhou, China

Background: Umbilical Cord Blood Transplantation (UCBT) is widely used as an effective treatment for Children with hematologic malignancies. For good immune reconstitution and fewer viral reactivations, thymoglobulin was omitted in UCBT for Children with hematologic malignancies. However, Graft-versus-host disease (GVHD) is a common complication after transplantation and affects the outcome of UCBT. Both total nucleated cell (TNC) dose and human leukocyte antigen (HLA) match affect the probability of GVHD and the outcome of UCBT. However, how to prioritize these characteristics in unit selection is not well established.

Methods: We reviewed outcomes of 30 UCBT children transplanted for hematologic malignancies with myeloablative conditioning and calcineurin-inhibitor/mycophenolate mofetil immunosuppression. The median age at UCBT was 62 months (range, 8-166 months), and the median weight was $18.5 \mathrm{~kg}$ (range, $8.5-43.5 \mathrm{~kg}$ ). All patients survived more than 30 days and had myeloid engraftment.

Results: The median dose of TNC was $7.78 \times 10^{7} / \mathrm{kg}$ (range, $3.27-31.85 \times 10^{7} / \mathrm{kg}$ ), $12(40 \%)$ patients infused TNC were $\geq 8.00 \times 10^{7} / \mathrm{kg}$ and $18(60 \%)$ patients infused TNC were $<8.00 \times 10^{7} / \mathrm{kg}$. Notably, a higher probability of III-IV acute GVHD (aGVHD) was found in the patients infused higher dose of TNC: $75 \%$ versus $22 \%(\mathrm{P}=0.008)$. However, there were no difference in the rates of chronic GVHD (cGVHD) and 1-year proportions of survival: $29 \%$ versus $18 \%(\mathrm{P}=$ $0.61), 75 \%$ versus $83 \%(\mathrm{P}=0.66)$, respectively. HLA-A,B,-C,-DRB1, and -DQB1 allele match $\geq 6 / 10,22$ (73\%) were mismatched $(\mathrm{MM})$ at $<3$ alleles and $8(27 \%)$ were $\mathrm{MM}$ at $\geq 3$ alleles. Compared with recipients of $<3 \mathrm{MM}$ units, recipients of $\geq 3 \mathrm{MM}$ units had the higher rate of cGVHD, $9.5 \%$ versus $50 \%(\mathrm{P}=0.05)$; lower rate of survival, $91 \%$ versus $50 \%(\mathrm{P}=0.03)$; but there was no difference in the rate of aGVHD, 36\% versus $63 \%(\mathrm{P}=$ $0.24)$.

Conclusions: Our findings support the consideration of HLA- A,-B,-DRB1, -DQB1 allele donor-recipient HLAmatch in unit selection and to control the infusion dose of TNC in a practice change in the field.

Conflict of interest: The authors declared that they have no conflicts of interest to this work.We declare that we do not have any commercial or associative interest that represents a conflict of interest in connection with the work submitted.

\section{P420}

Treatment of refractory chronic graft-versus-host disease with Imatinib: a single center experience

Javier Carrillo Checa, Daniel Esteban Rivera Delgado, Alejandro Avendaño Pita, Daniel Presa Morales, Eduardo Sobejano Fuertes, Victor Higuero Saavedra, Nerea Arratibel Zalacain, Mónica Baile González, Alvaro Veiga Vaz, Félix López Cadenas, Ana A. Martín López, Estefania Pérez López, Mónica Cabrero Calvo, Lourdes Vázquez López, Fermin Sanchez Guijo, María Dolores Caballero Barrigon, Lucia López Corral

Complejo Asistencial Universitario de Salamanca-IBSAL, Hematology, Salamanca, Spain

Background: Chronic GVHD remains a therapeutic challenge. Steroids are the standard treatment but the outcomes are not satisfactory. Imatinib has been testing for steroids-refractory GVHD (SR-GVHD) because it inhibits autoantibodies againts the PDGFR, which are identified in patients with sclerotic chronic GVHD. Retrospective studies have been shown variable responses, but the results of the only randomized phase II (Sally Arai et al, 2016) at 6 months was lower. Probably, long-term follow-up beyond 6 months is neccesary due to the sclerotic process of GVHD reverse slowly.

Methods: We reported a retrospective analysis of 24 patients with SR-cGVHD treated with Imatinib between jan-2000 and dec-2016. cGVHD diagnosis and response evaluation were stablished by NIH criteria. Responses were evaluated at 6 and 12 months. Imatinib was started at 100 $\mathrm{mg} / \mathrm{day}$, with increase up to $400 \mathrm{mg}$ according to response and tolerance.

Results: Baseline, transplant characteristics and chronic GVHD data before starting Imatinib are shown in Table 1. Median time from cGVHD to Imatinib treatment was 14 months (0-82). 16 patients $(66.7 \%)$ received $\geq 3$ lines of treatment prior to Imatinib. $62.5 \%(\mathrm{n}=15)$ had severe and $37.5 \%(\mathrm{n}=9)$ moderate cGVHD.

Median duration of treatment was 8.5 months (0.5-102) and median dosis was $100 \mathrm{mg} /$ day (0-400). 8 patients couldn't be evaluated at 6 and 12 months because 2 were exitus and 6 discontinued treatment before 6 months for intolerance $(\mathrm{n}=3)$, potencial toxicity $(\mathrm{n}=1)$ and non response $(\mathrm{n}=2)$. 6-months evaluation showed an ORR of $25 \%$ (partial response, $\mathrm{n}=4$ ) while 12 patients $(75 \%)$ didn't response (stable disease, $\mathrm{n}=11$ and progression disease, $\mathrm{n}$ $=1$ ). At 12-months evaluation the ORR was $56.25 \%$, (complete response, $\mathrm{n}=2$ and partial response, $\mathrm{n}=7$ ), while 7 patients $(43.75 \%)$ were stable disease. We could 
stop or lower steroids in all 7 patients which were being treated with steroids at the time of start Imatinib.

Regarding safety data, no grade $3 / 4$ side effects were observed but 3 patients discontinued Imatinib due to gastrointestinal intolerance or edema.

With a median follow-up from start of treatment of 42.5 months (0.5-102), 7 patients are still under treatment and 19 are still alive (5 exitus, 4 due to cGVHD).

Conclusions: Imatinib success rates were low at 6-month, but assessments after a longer duration of treatment showed higher ORR. Prospective trials with long-term follow-up are necessary to verify its efficacy.

Conflict of interest: Nothing to disclose.

\begin{tabular}{|c|c|}
\hline Characteristic & $\begin{array}{l}\text { All patients }(\mathrm{n}=24) \text { Median (range) / } \\
\mathrm{n}(\%)\end{array}$ \\
\hline Age / Male patient & $45(24-68) / 12(50 \%)$ \\
\hline $\begin{array}{l}\text { Diagnosis AML / MDS / ALL / NHL / } \\
\text { Other }\end{array}$ & $\begin{array}{l}9(37.5 \%) / 7(29.2 \%) / 1(4.2 \%) / 4 \\
(16.7 \%) / 3(12.5 \%)\end{array}$ \\
\hline $\begin{array}{l}\text { Donor: Related / Unrelated 9/10 / } \\
\text { Unrelated 10/10 }\end{array}$ & $12(50 \%) / 3(12.5 \%) / 9(37.5 \%)$ \\
\hline $\begin{array}{l}\text { Conditioning regimen: Myeloablative / } \\
\text { RIC }\end{array}$ & $9(37.5 \%) / 15(62.5 \%)$ \\
\hline $\begin{array}{l}\text { Time (months) from SCT to cGVHD } \\
\text { and from cGVHD to Imatinib }\end{array}$ & $8(2-54)$ and $14(0-82)$ \\
\hline $\begin{array}{l}\text { GVHD sites involved at Imatinib } \\
\text { treatment: Skin / Sclerodermal features } \\
\text { / Eyes / Mouth / Liver / Lung Joint or } \\
\text { fascia }\end{array}$ & $\begin{array}{l}17(70.8 \%) / 16(66.7 \%) / 6(25 \%) / 5 \\
(20.8 \%) / 1(4.2 \%) / 12(50 \%) / 11 \\
(45.8 \%)\end{array}$ \\
\hline Prednisone at Imatinib treatment & $7(29.1 \%)$ \\
\hline $\begin{array}{l}\text { NIH global score at Imatinib treatment: } \\
\text { moderate/severe }\end{array}$ & $9(37.5 \%) / 15(62.5 \%)$ \\
\hline $\begin{array}{l}\text { Other treatment at Imatinib therapy: } \\
\text { Topical treatment / ECP / Tacrolimus / } \\
\text { Cyclosporin / Sirolimus / Oral steroids } \\
\text { / Methotrexate }\end{array}$ & $\begin{array}{l}2(8.3 \%) / 2(8.3 \%) / 13(54.2 \%) / 3 \\
(12.5 \%) / 10(41.7 \%) / 7(29.1 \%) / 2 \\
(8.3 \%)\end{array}$ \\
\hline
\end{tabular}

\section{[[P420 Table] Table 1]}

\section{P421}

Treatment of steroid-refractory acute and chronic graftversus-host disease with ruxolitinib in adults and children

Ivan Moiseev, Elena Morozova, Maria Barabanshikova, Andrey Kozlov, Tatyana Rudakova, Olesya Klimova, Nikolai Tcvetkov, Olga Pirogova, Evgeniya Borzenkova, Polina Kozhokar, Kirill Ekushev, Olesya Paina, Tatyana Bykova, Elena Darskaya, Sergey Bondarenko, Ludmila Zubarovskaya, Boris Afanasyev

Pavlov First Saint Petersburg State Medical University, R.M. Gorbacheva Memorial Institute of Hematology, Oncology and Transplantation, Saint-Petersburg, Russian Federation

Background: Steroid-refractory graft-versus-host disease (srGVHD) is a life-threatening complication of allogeneic stem cell transplantation. Currently there is no standard of care for this complication and with existing modalities the survival remains relatively low. A pilot study was

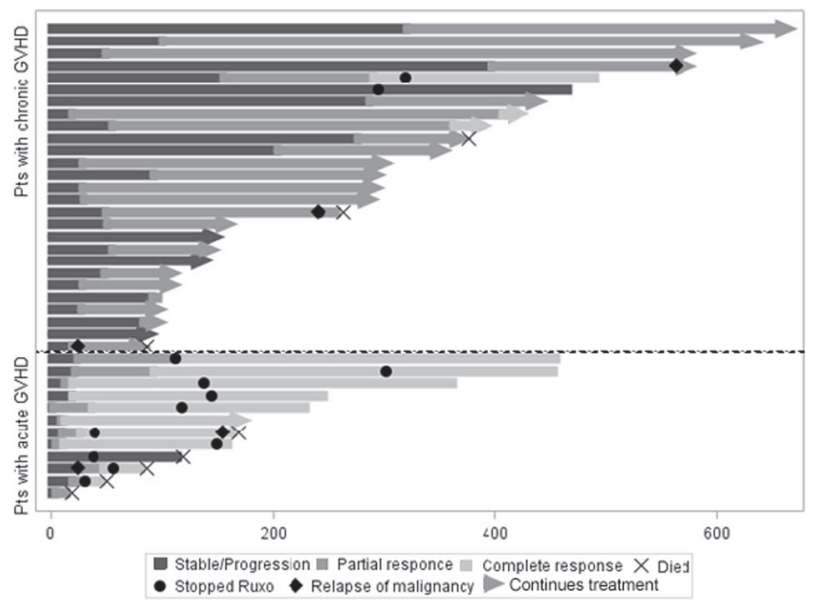

[P421 Figure] [Figure 1. Response to treatment and survival of patients in the study]

conducted in children and adults who developed steroidrefractory or steroid-dependent acute and chronic GVHD.

Methods: 39 patients (age 1-62 years, $46 \%$ children and $54 \%$ adults) were included in the prospective study (NCT02997280). EBMT/ELN criteria were used for steroid refractory disease (T. Ruutu et al., 2014). 12 had acute srGVHD and 27 had moderate or severe chronic srGVHD. $28 \%$ had acute myeloid leukemia, $28 \%$ acute lymphoblastic leukemia, 23\% - non-malignant diseases, $21 \%$ - other malignant diseases and. $10 \%$ were grafted from related donor, 64\% from unrelated and 26\% from haploidentical. Patients with acute GVHD had a median of 2 prior lines of therapy (range 1-2), patients with chronic GVHD had a median of 3 prior lines (range 1-6). 11/12 acute GVHD patients had grade III-IV disease, and 23/27 chronic GVHD patients had severe (NIH) disease. Ruxolinib was administered at the starting dose of $10 \mathrm{mg}$ bid in adults and $0.3 \mathrm{mg} /$ $\mathrm{kg} / \mathrm{day}$ (bid or tid) in children. Dose modifications were performed in patients with grade 4 hematologic toxicities. Ruxolinib was continued until complete response or absence of response in 28 days for acute GVHD and six months for chronic GVHD.

Results: Median follow-up for alive patients was 10 months (range 3-22). Overall response for acute GVHD was $92 \%$. Complete response (CR) was observed in 10/12 patients with median time to CR 28 days (range 10-112). One patient is in continued partial response (PR) and one had progressive disease. Overall response for chronic GVHD was $81 \%$. 3/27 patients achieved CR after median 12 months of treatment (range 10-14 months). 19/27 achieved PR after median 2 months of treatment (range 113). Five patients relapsed while on therapy, and four died due to progression of underlying malignancy. Non-relapse mortality occurred in 4/39 patients with multidrug-resistant sepsis as the cause of death in all cases. 
Overall survival was higher in chronic than in acute srGVHD patients $(89 \%$ vs $58 \%, \mathrm{p}=0.017)$. There was no difference in response rate and mortality between adults and children $(p>0.05)$ with stratification by the type of GVHD. Three severe adverse events were documented in chronic GVHD group (sepsis, myasthenia gravis and RS-virus pneumonia), and eight in acute GVHD (6/12 patients had sepsis, one BK-virus encephalitis, one viral gastritis with recurrent severe bleeding and one mixed-pathogen pneumonia requiring mechanical ventilation). Dose reduction/ drug interruption due to cytopenia was required in $3 / 12$ acute GVHD and 1/27 chronic GVHD patients.

Conclusions: Despite the small group size, our preliminary results indicate that ruxolitinib is a promising agent for srGVHD. Randomized prospective studies are required to confirm that ruxolitinib is superior to the other approaches.

Clinical Trial Registry: Clinicaltrials.gov, NCT02997280

Conflict of interest: Moiseev: Novartis - honoraria, consulting fees, travel grants. Other authors have nothing to disclose.

\section{P422}

Trial Design of a Phase 3, Randomized, Open-Label, Multicenter Study (REACH-2) to evaluate Ruxolitinib over BAT in Patients with Corticosteroid-Refractory Acute GraftVersus-Host Disease Post Allo-HSCT

Gérard Socie $^{1}$, Dietger Niederwieser ${ }^{2}$, Jeff Szer ${ }^{3}$, Mohamad Mohty ${ }^{4}$, Robert Zeiser, Brian Gadbaw ${ }^{6}$, Betty Molloy, Leslie O'SullivanDjentuh $^{7}$, Nikolas von Bubnoff

${ }^{1}$ APHP, Hématologie-Transplantation Hôpital St Louis, Université Denis Diderot and INSERM UMR 1160, Paris, France; ${ }^{2}$ University of Leipzig, Department of Hematology and Oncology, Leipzig, Germany; ${ }^{3}$ Royal Melbourne Hospital, Melbourne, Australia; ${ }^{4}$ Hopital SaintAntoine, Paris, France; ${ }^{5}$ University Hospital Freiburg, Department of Hematology, Oncology and Stem Cell Transplantation, Freiburg, Germany; ${ }^{6}$ Novartis Pharmaceuticals Corporation, East Hanover, NJ, United States; ${ }^{7}$ Novartis Pharma AG, Basel, Switzerland

Background: Success of allogeneic hematopoietic stem cell transplantation (allo-HSCT) is hampered by graftversus-host disease (GvHD). There is an increased mortality risk in patients with steroid refractory (SR) acute GvHD $(\mathrm{aGvHD})$ and treatment for this population remains as an unmet need. Use of ruxolitinib for treatment of patients with SR-aGvHD was supported in a retrospective analysis (Zeiser et al, 2015). The present global study was developed to further evaluate ruxolitinib in a phase 3 trial.

Methods: REACH-2 is a randomized (1:1), open-label, multicenter study of ruxolitinib (10 mg twice daily) vs investigator-determined best available therapy (BAT) in patients with SR-aGvHD post allo-HSCT (Figure 1). Patients aged $\geq 12$ years, clinically diagnosed with grade 2-4 SR-aGvHD, with evidence of myeloid and platelet engraftment (absolute neutrophil count $>1000 / \mathrm{mm}^{3}$ and platelets $\geq 20000 / \mathrm{mm}^{3}$ ) were eligible for inclusion. Exclusion criteria comprise of patients who have received $>1$ systemic treatment for SR-aGvHD, have clinical presentation resembling de novo chronic GvHD or GvHD overlap syndrome with both acute and chronic GvHD features, have failed prior SCT $<6$ months, or have an active uncontrolled infection.

The primary objective is to compare the efficacy of ruxolitinib vs BAT by overall response rate (ORR) at day 28 of treatment. ORR is defined as the proportion of patients in each arm exhibiting a complete response (CR) or partial response (PR), without receiving additional systemic therapies for an earlier progression, mixed response, or nonresponse. The key secondary objective is to compare the rate of durable ORR at day 56 between ruxolitinib and BAT, defined as proportion of all patients in each arm who achieve a CR/PR at day 28 and maintain a CR/PR at day 56 . Other secondary objectives include ORR at day 14 , duration of response, cumulative steroid dose until day 56, overall survival, event-free survival, failure-free survival, nonrelapse mortality, malignancy relapse/progression, incidence of cGvHD, pharmacokinetic parameters, exposure-efficacy relationship, patient-reported outcomes, and safety.

Target enrollment is 308 randomized patients.

Results: First patient first visit occurred in March 2017.

Conclusions: Enrollment is ongoing. As of December 14, 2017, 95 patients have been randomized.

Clinical Trial Registry: ClinicalTrials.gov: NCT0291326

Conflict of interest:

G. Socié: honoraria as speaker in symposium (Novartis)

D. Niederwieser: consultancy (Novartis)

J. Szer: advisory board, consultancy and honoraria (Alexion); advisory board, consultancy and honoraria as consultant and speaker (Shire Pharmaceutical); advisory board and honoraria as consultant and speaker (Sanofi/ Genzyme); advisory board, honoraria and consultancy as consultant and speaker (Pfizer); advisory board (Amgen); and advisory board (Novartis)

M. Mohty: consultancy (Novartis); and consultancy, honoraria and research funding for consultancy and speaking (Sanofi)

R. Zeiser: honoraria as invited speaker for symposium (Jazz Pharmaceuticals); and honoraria as invited speaker (Novartis)

B. Gadbaw and B. Molloy: employees (Novartis)

L. O'Sullivan-Djentuh: no conflict of interest to disclose 


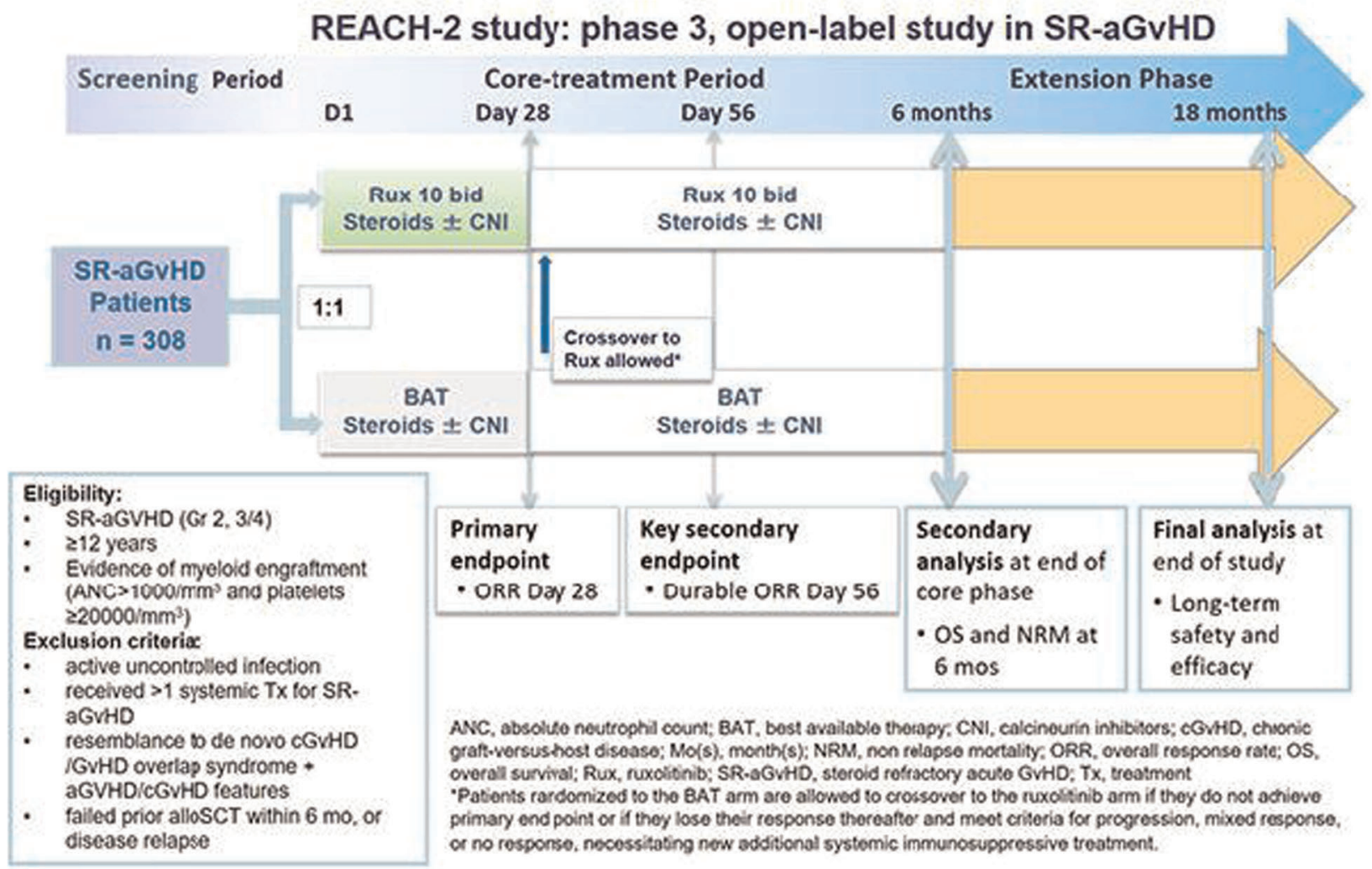

[P422 Figure] [Study Design]

N. von Bubnoff: consultancy for role in REACH-2 trial steering committee (Novartis); research funding (Novartis); and honoraria (BMS)

REACH-2 trial is registered at ClinicalTrials.gov: NCT0291326.

\section{P423}

Trial in Progress: GRAVITAS-119, a Single-Arm, Open-Label, Phase 1 Study of Itacitinib With Calcineurin Inhibitor-Based Interventions for Prophylaxis of Graft-Versus-Host Disease [GVHD]

\section{Miguel-Angel Perales ${ }^{1}$, Mark Schroeder ${ }^{2}$, Rafael F. Duarte ${ }^{3}$, Michael Arbushites ${ }^{4}$, Peter Langmuir ${ }^{4}$, Ying Yan ${ }^{4}$, Alessandro Rambaldi $^{5}$}

${ }^{1}$ Memorial Sloan Kettering Cancer Center, New York, NY, United States; ${ }^{2}$ Washington University School of Medicine, St. Louis, MO, United States; ${ }^{3}$ Hospital Universitario Puerta de Hierro Majadahonda, Madrid, Spain; ${ }^{4}$ Incyte Corporation, Wilmington, DE, United States; ${ }^{5}$ University of Milan and Azienda Socio Sanitaria Territoriale Papa Giovanni XXIII, Bergamo, Italy

Background: GVHD is a significant source of patient morbidity and mortality after allogeneic hematopoietic stem cell transplantation [allo-HSCT]. While there is currently no standard of care for GVHD prophylaxis, calcineurin inhibitors [CNIs] combined with methotrexate [MTX], mycophenolate mofetil [MMF], or anti-thymocyte globulin [ATG] are the most commonly used regimens. Itacitinib, a potent, selective Janus kinase [JAK] 1 inhibitor, demonstrated an acceptable safety profile in combination with steroids in a phase 1 study of patients with acute GVHD. This report describes the study design for an ongoing trial assessing the efficacy and safety of itacitinib added to CNIbased GVHD prophylaxis regimens.

Methods: GRAVITAS-119 is a single-arm, open-label, phase 1 study evaluating itacitinib in combination with CNI-based regimens for GVHD prophylaxis. The study will enroll patients age $\geq 18$ years who are undergoing alloHSCT using peripheral blood stem cells [PBSCs] from $8 / 8$ or 7/8 matched related or unrelated donors for a hematologic malignancy, are candidates for reducedintensity conditioning regimens, and have a Karnofsky Performance Status [PS] score $\geq 70 \%$ or ECOG PS 0-2. Patients are excluded if they received prior allo-HSCT or JAK inhibitor therapy, or have an active uncontrolled infection or severe organ dysfunction. Patients will initiate oral itacitinib $200 \mathrm{mg}$ QD 3 days before allo-HSCT; dose reduction/interruption will be permitted for toxicity management. Treatment will be tapered by Day 90 and 


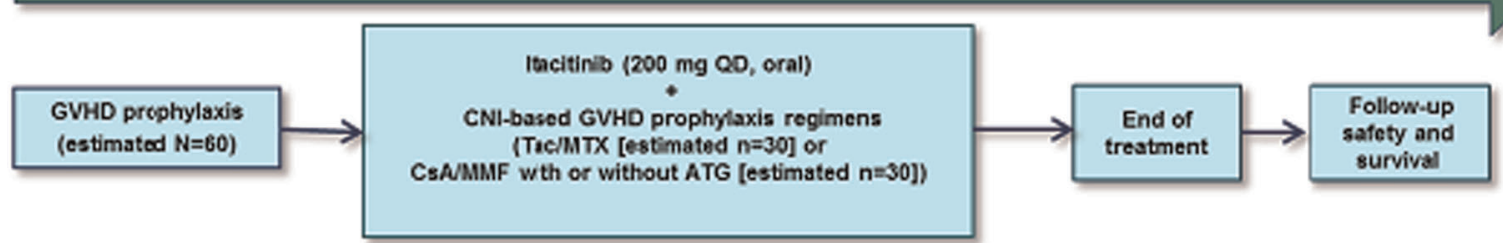

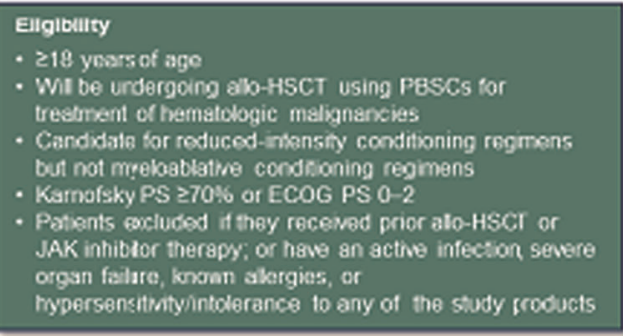

¿18 yosrs of ago

ing regmens

organ failure, knoan akargies, or

mypersensitintyintolerance to any of the study croducts
Primary endpoint

- Hamatologic recovery.

proportion of petients

with nautrophil resovery

(absoute neutroph!

count $2500 \mathrm{~mm}^{3}$ for 3

consocutiva

messurements) and

platelet reconery

(Platalat count

$\left.220.000 \mathrm{~mm}^{3}\right)$ at $28 \mathrm{~d}$
Secondsry endpolnts

- GRFS rates at 180 d and $3860 \mathrm{~d}$

- RFS, TRM, onerall sumal

- Modisn time to neutroptil and platekat engraflmert end proportion of petents who achieve enoraftiment \&t $7,14,21$, and 280

- Incidonso of actio GVHD (by 190 d) and chronic

GVHD (by $180 \mathrm{~d}$ and $385 \mathrm{~d})$

- Infection rate

- Safoty grades 3-5 adverse events by incidence

end senverity

[P423 Figure] [GRAVITAS-119 Study Design]

discontinued by Day 180, unless patients are diagnosed with GVHD, experience malignancy relapse or unacceptable toxicity, or withdraw consent. CNI-based GVHD therapy with tacrolimus/MTX or cyclosporine A/MMF with or without ATG will be administered per institutional practices.

Results: Approximately 60 patients will be enrolled. The primary endpoint is hematologic recovery, defined as the proportion of patients with both neutrophil and platelet recovery at Day 28. Secondary endpoints include GVHD relapse-free survival [GRFS], relapse-free survival [RFS], transplant-related mortality [TRM], and safety assessments; additional secondary endpoints are described in Figure 1.

Conclusions: GRAVITAS-119 will be important for evaluating the efficacy and safety of itacitinib in combination with CNI-based regimens for GVHD prophylaxis and is expected to provide key insights for informing future GVHD prophylaxis studies.

\section{Clinical Trial Registry: NCT03320642}

\section{Conflict of interest:}

M. Perales and M. Schroeder have received honoraria and research funding from Incyte Corporation.

R. Duarte has received honoraria from and participated in advisory boards for Incyte Corporation.

M. Arbushites, P. Langmuir, and Y. Yan are employed by and own stock in Incyte Corporation.

A. Rambaldi declares no potential conflicts of interest.

This study was funded by Incyte Corporation.

\section{P424}

Trial in Progress: GRAVITAS-301, a Randomized, DoubleBlind Phase 3 Study of Itacitinib or Placebo With Corticosteroids for the First-Line Treatment of Patients With Acute GVHD Robert Zeiser ${ }^{1}$, Michael Arbushites ${ }^{2}$, Peter Langmuir $^{2}$, Ying Yan $^{2}$,
Yi-Bin Chen

${ }^{1}$ University Hospital Freiburg, Freiburg im Breisgau, Germany; ${ }^{2}$ Incyte Corporation, Wilmington, DE, United States; ${ }^{3}$ Massachusetts General Hospital, Boston, MA, United States

Background: Approximately 50\% of patients who receive allogeneic hematopoietic stem cell transplantation [aHSCT] for hematologic disorders develop acute graftversus-host disease [aGVHD], a leading cause of nonrelapse mortality [NRM]. There are no FDA-approved treatments for aGVHD and only a minority of patients experience sustained responses to corticosteroids [CS], the standard-of-care treatment. Janus activated kinase [JAK] has been implicated in GVHD pathogenesis. JAK inhibitors have demonstrated improvements in aGVHD outcomes while preserving the graft-versus-leukemia effect in preclinical models, and have shown preliminary clinical efficacy in patients with CS-refractory aGVHD. Itacitinib is a potent, selective JAK1 inhibitor that demonstrated an acceptable safety profile in a phase 1 study of patients with CS-naive and CS-refractory aGVHD. This report 


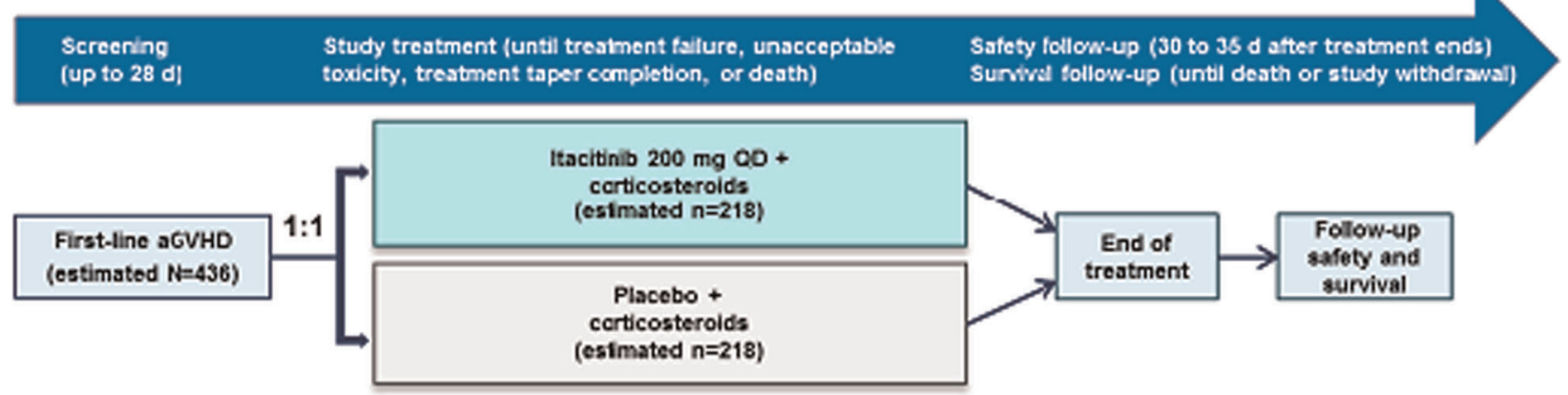

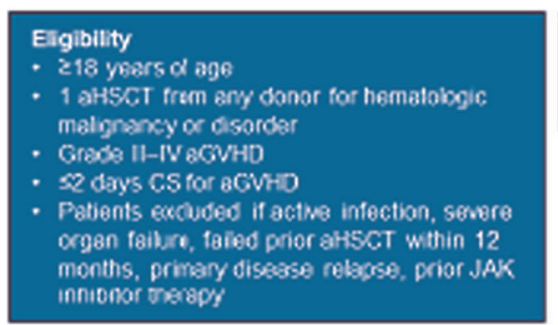

Primary Endpoint

- orr at 0ay 28
Secondsry Endpoints

- Kay secondary: URU at Month 6

CAR at Days $14,5 t$, and 100

NRM at Uerths 9, 12, and 24

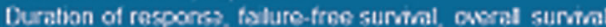

Incidence of gratt talure, aGVHD thares, and chronic GVHD

Satoty

Pharmsocionatics

Discontinumion of CSimmunosuppresstre madicetion

[P424 Figure] [GRAVITAS-301 Study Design]

summarizes the study design for an ongoing phase 3 trial of first-line treatment with itacitinib in patients with aGVHD.

Methods: GRAVITAS-301 is a randomized, doubleblind, placebo-controlled, multicenter, phase 3 study comparing the efficacy of itacitinib or placebo in combination with CS as a first-line treatment for aGVHD. Eligible patients are $\geq 18$ years of age who received an aHSCT for a hematologic malignancy/disorder and have grade II-IV aGVHD (MAGIC criteria). Patients are excluded if they received $>1$ aHSCT, $>2$ days of $\mathrm{CS}$ for aGVHD, CS for indications other than GVHD within 7 days of randomization, or prior JAK inhibitor therapy since aHSCT; or have GVHD overlap syndrome, an active infection, evidence of relapsed primary disease, or severe organ dysfunction. An estimated 436 patients will be randomized 1:1 to itacitinib (200 mg orally, QD) or placebo with methylprednisolone (or prednisone equivalent) at $2 \mathrm{mg} / \mathrm{kg} / \mathrm{d}$ or a dose appropriate for disease severity per local treatment guidelines. Patients will receive study treatment until treatment failure, unacceptable toxicity, completion of treatment taper, or death.

Results: The primary endpoint is overall response rate [ORR] at Day 28. The key secondary endpoint is NRM at Month 6. Additional secondary endpoints are described in Figure 1.

Conclusions: The GRAVITAS-301 trial is expected to provide comprehensive efficacy and safety data for itacitinib as initial therapy in combination with CS in patients with aGVHD and may inform future treatment strategies.

Clinical Trial Registry: NCT03139604
https://clinicaltrials.gov/ct2/show/NCT03139604

Conflict of interest:

R. Zeiser has served as a steering committee member for Novartis and Incyte Corporation.

M. Arbushites, P. Langmuir, and Y. Yan are employed by and own stock in Incyte Corporation.

Y. Bin Chen has served as a consultant for Takeda Pharmaceuticals, Magenta Therapeutics, Jazz Pharmaceuticals, Engene, and Incyte Corporation. This study was funded by Incyte Corporation. This abstract is an encore of an abstract that was submitted for presentation at BMT Tandem 2018.

\section{P425}

Updated safety results of the phase 3 randomized trial comparing inolimomab versus antithymocyte globulin (ATG) in adults with steroid-resistant acute GVHD

Gerard Socie $^{1}$, Stephane Vigouroux ${ }^{2}$, Ibrahim Yakoub-Agha ${ }^{3}$, Jacques-Olivier Bay ${ }^{4}$, Sabine Furst ${ }^{5}$, Karin Bilger ${ }^{6}$, Felipe Suarez, $^{7}$, Mauricette Michallet ${ }^{8}$, Dominique Bron ${ }^{9}$, Philippe Lehert ${ }^{10}$, David Liens $^{11}$, Catherine Mathis ${ }^{11}$, Eric Guemas ${ }^{12}$, Jean-Paul Vernant ${ }^{13}$

${ }^{1}$ Hopital St-Louis Lariboisiere, Hematology, Paris, France; ${ }^{2}$ Hopital Haut-Leveque, Hematology, Pessac, France; ${ }^{3} \mathrm{CHU}$ de Lille,

Hematology, Lille, France; ${ }^{4} \mathrm{CHU}$ Clermont Ferrand, Hematology, Clermont Ferrand, France; ${ }^{5}$ Institut Paoli Calmettes, Hematology, Marseille, France; ${ }^{6} \mathrm{CHU}$ Hautepierre, Hematology, Strasbourg, France; ${ }^{7}$ Hopital Necker, Hematology, Paris, France; ${ }^{8}$ Centre Leon Berard, Hematology, Lyon, France; ${ }^{9}$ Institut Jules Bordet,

Hematology, Bruxelles, Belgium; ${ }^{10}$ University of Melbourne, Faculty of Medicine, Melbourne, Australia; ${ }^{11}$ Elsalysbiotech, Lyon, France; 


\section{${ }^{12}$ Biossec, Paris, France; ${ }^{13}$ Hopital Pitie Salpetriere, Hematology, Paris, France}

Background: Despite improvements in allogeneic hematopoietic stem cell transplantation, acute graft-versus-host disease (aGvHD) remains a significant issue after transplantation and a major cause of non-relapse mortality, especially in case of steroid-resistance (SR). We recently published results of a phase 3 randomized study comparing inolimomab, a monoclonal antibody to $\mathrm{CD} 25$, versus antithymocyte globulin (ATG) in SR-a GvHD (Socié et al. Blood 2017). The composite primary objective was to evaluate overall survival (OS) at 1 year without changing baseline allocated therapy. The study concluded that there was no statistically significant difference between the two arms regarding the primary endpoint $(\mathrm{HR}=0,722$ in favor of inolimomab, $\mathrm{p}=0.0941$ one sided). Extensive safety analysis could not be completed at that time, it showed although no difference between the two groups with regard to number of patients experiencing at least one adverse event (AE) grade $\geq 3$ (100\% vs $98 \%$ in the inolimomab and ATG group respectively). We report here the complete safety results of the study.

Methods: Forty-nine and 51 patients were randomized in the inolimomab and ATG groups respectively. Median time to onset of aGvHD was day 23 (range, 15-52). The secondline treatment was started 6 days after initial steroid treatment (range, 3-9) without significant difference between the two arms. Inolimomab-treated patients received $0.3 \mathrm{mg} / \mathrm{kg} /$ day during induction that lasted up to 2 weeks and $0.4 \mathrm{mg} / \mathrm{kg} 3$ times per week during maintenance. The total treatment duration was $28 \pm 2$ days. Patients in the control arm received rabbit ATG (Genzyme) at the dose of $2.5 \mathrm{mg} / \mathrm{kg}$ for 4 consecutive days.

Results: The absolute number of AEs and grade $\geq 3$ AEs was significantly increased in the ATG group compared to the inolimomab group (1401 vs 1087 and 328 vs 269 respectively, $\mathrm{p}<0.001)$. The number of patients with related AEs of any grade or related AEs grade $\geq 3$ was significantly increased in the ATG group compared to the inolimomab group (41\% vs $14 \%, \mathrm{p}=0.004$ and $29 \%$ vs $12 \%, \mathrm{p}=0.04$ respectively). Furthermore, a statistically significant difference in overall incidence of infectious episodes was apparent in favor of inolimomab $(\mathrm{p}<0.001)$ with reduction in potentially life-threatening AEs like sepsis and septic shock (14\% vs $24 \%$ and $4 \%$ vs $16 \%$ for inolimomab and ATG groups respectively). In addition, exploratory analysis of each parameter of the primary composite endpoint showed that the trend in favor of inolimomab was mostly driven by a difference in OS at 1 year $(\mathrm{HR}=0.628, \mathrm{p}=0.055$ one sided $)$ and not a difference in change of initial allocated treatment $(\mathrm{HR}=0.767, \mathrm{p}=$ 0199 one sided).

Conclusions: These results point out the improved tolerability profile of inolimomab, especially with regard to life-threatening infections when compared to ATG which was at that period the only available agent in this indication of aGvHD. Given that efficacy on survival appears to be at least equivalent to that of ATG but with a significantly better safety profile, inolimomab may represent a suitable alternative in patients with SR-aGvHD, especially those not candidate for clinical trials evaluating new drugs.

Clinical Trial Registry: https://www.clinicaltria 1sregister.eu/ctr-search/search as EUDRACT 2007005009-24.

Conflict of interest: Nothing to disclose

Graft-versus-host disease - preclinical and animal models

\section{P426}

\section{A new Data Integration Platform for the Development of} Predictive Models in Stem Cell Transplantation (XplOit)

Amin T. Turki ${ }^{1}$, Jochen Rauch ${ }^{2}$, Kerstin Rohm ${ }^{2}$, Ulf Schwarz ${ }^{3}$, Thorsten Lehr ${ }^{4}$, Stefan Theobald ${ }^{5}$, Stephan Kiefer ${ }^{2}$, Katharina Och ${ }^{4}$, Katharina Götz, Christian Brossette ${ }^{5}$, Nico Pfeifer ${ }^{6}$, Lisa Handl, Sigrun Smola ${ }^{7}$, Matthias Ihle ${ }^{8}$, Jürgen Rissland ${ }^{7}$, Jörg Bittenbring $^{9}$, Norbert Graf ${ }^{5}$, Dietrich W. Beelen ${ }^{1}$, Gabriele Weiler ${ }^{2}$

${ }^{1}$ University Duisburg-Essen/ University Hospital Essen, Department of Bone Marrow Transplantation, West-German Cancer Center, Essen, Germany; ${ }^{2}$ Fraunhofer Institute for Biomedical Engineering, St. Ingbert, Germany; ${ }^{3}$ Saarland University, Institute for Formal Ontologies and Medical Information Science, Saarbrücken, Germany; ${ }^{4}$ Saarland University, Department of Clinical Pharmacy, Saarbrücken, Germany; ${ }^{5}$ Saarland University, Department of Pediatric Oncology and Hematology, Homburg (Saar), Germany; ${ }^{6}$ University of Tübingen, Department of Computer Science, Tübingen, Germany; ${ }^{7}$ Saarland University, Institute of Virology, Homburg (Saar), Germany; ${ }^{8}$ Averbis GmbH, Freiburg, Germany; ${ }^{9}$ Saarland University, Department. of Internal Medicine 1, Homburg (Saar), Germany

Background: In allogeneic stem cell transplantation, models and scores for risk assessment such as the SororScore are frequently-used in order to evaluate patients' fitness for transplantation. Ideally, these would translate into individual risk assessment. The incidence of viral infections and transplantation-associated disorders such as graftversus-host disease (GvHD) could be reduced through a new generation of models. For their development, high amounts of heterogeneous medical data need to be harmonized, integrated, stored and analyzed, while providing the highest level of data protection and IT security. The European Union General Data Protection Regulation (EUGDPR) 2018 further impacts the development of such models. We present an ontology-based platform that 
supports data owners and model developers to share and harmonize data for both machine-learning and systems biology driven models in stem cell transplantation.

\section{Methods:}

Different hospitals upload pseudonymized data. Deidentification and pseudonymisation-services run locally to semi-automatically process structured (e.g. laboratory data) and unstructured data (e.g. medical reports). Unlike most current medical data integration approaches which use merely concepts from the ontology as annotations, we have chosen an ontology-based data integration approach that allows complex descriptions to realize deep expressiveness. Hospital researchers perform the data integration tasks, i.e. extend ontology and create data annotations, since they are familiar with the kind of data. Instead of creating annotations manually, semi-automatic tools such as the Viral Disease Ontology Trunk (VDOT) aggregator tool provide formal, semantic descriptions of concepts, using a framework of established ontologies for the description of biomedical data and predictive models. VDOT extensions can be semi-automatically generated by searching a semantic repository with standardized ontologies and relating needed concepts with others in VDOT. Semantic data annotation service supports data owners to easily annotate similar data from the ontology, using a stringmatching algorithm. For the matched concepts, potential ontology paths are created by iteratively searching VDOT utilizing semantic constraints. Path selection is performed through likelihood ranking.

After annotation, an information extraction framework stores harmonized data as triples into the data warehouse, the platform's central database, which can be searched and analyzed by model developers. Security services ensure data protection and data integrity via data access and audit trail. The modeling workbench enables model developers to search and analyze the integrated data to check data quality, possible correlations and generate hypotheses.

Results: We developed a web-based platform (XplOit) to integrate processes of pseudonymization, semantic annotation, data aggregation, information extraction and model development, in order to develop machine-learning and systems biology driven models in stem cell transplantation. The platform facilitated interaction between modeldevelopers and clinicians and accelerated data-integration according to EU-GDPR 2018. The integration of both structured and unstructured data sources may impact model development.

Conclusions: We developed a semantic data integration platform for enhancing the development of predictive models. Data integration tools allow clinicians and model developers to work together efficiently. Machine learning model development may reveal new insights and enhance predictive models in stem cell transplantation. The platform will be opened to further partner hospitals to enlarge the data basis for model development.

Conflict of interest: None of the authors has anything to disclose.

Funding: The XplOit project is funded by the German Federal Ministry of Education and Research (BMBF, grant id: 031L0027A).

\section{P427}

Autologous hematopoietic transplantation with clonal deletion of alloreactive clones prevents acute rejection and prolongs immunologic tolerance of skin allograft in rat

Krzysztof Bogusz ${ }^{1}$, Kaja Kasarello ${ }^{2}$, Robert Wrzesien ${ }^{3}$, Renata Podkowińska - Polak ${ }^{4}$, Barbara Górnicka ${ }^{4}$, Agnieszka Cudnoch Jędrzejewska ${ }^{2}$, Wiestaw Wiktor-Jędrzejczak ${ }^{1}$, Emilian Snarski ${ }^{l}$

${ }^{1}$ Medical University of Warsaw, Hematology, Oncology and Internal Diseases, Warszawa, Poland; ${ }^{2}$ Medical University of Warsaw, Department of Experimental and Clinical Physiology, Laboratory of Centre for Preclinical Research, Warszawa, Poland; ${ }^{3}$ Medical University of Warsaw, Central Laboratory of Animal Experiments, Warszawa, Poland; ${ }^{4}$ Medical University of Warsaw, Department of Pathology, Warszawa, Poland

Background: The major obstacles in organ transplantation are rejection of transplanted organ and need for life long immunosuppression. We hypothesized that immunoablation connected with autologous hematopoietic transplantation with simple cyclophosphamide-induced posttransplant clonal deletion of lymphocyte clones crossreactive to transplanted allograft would lead to reset of immune system of recipient and block acute rejection with immune tolerance of allograft without need for lifelong immunosuppression. The clinical rationale for this hypothesis is tolerance of the recipient by haploidentical hematopoietic cell transplants in humans, where posttransplant cyclophosphamide deletes autoreactive lymphocyte clones and allows long term tolerance of the recipient despite the differences in HLA antigens. We hypothesized that autograft of bone marrow could be modulated in similar way by post-transplant cyclophosphamide to tolerate transplanted allogenic tissues (which are haploidentical or fully incompatible).

Methods: Two different rat strains were used in the experiment. Sprague Dawley (SPRD) rats were used as recipients of skin graft from (Spontaneously Hypertensive Rat) SHR rats as this model shows very high immunogenicity and very rapid rejection of graft. To further expand the model we also used SPDR/SHR rats as skin donors as a model of haploidentical organ transplantation (model of "family grafts").

Results: The skin grafts from SHR rats were transplanted to SPRD rats. All grafts were acutely rejected by the day 3 
in all control rats (including rats that underwent autologous bone marrow transplantation without post-transplant cyclophosphamide and rats with post-transplant cyclophosphamide without previous autologous hematopoietic stem cell transplantation). In the SPRD rats who received autologous HSCT and SHR skin graft with post-transplant cyclophosphamide the acute rejection was blocked and rats were able to tolerate graft for the 14 days without other immunosuppression. Also the haploidentical SPDR/SHR skin grafts were not rejected after the treatment and were well tolerated up to 14 days after the autologous HSCT without additional immunosuppression.

Conclusions: The reset of the immune system of the donor with autologous hematopoietic transplantation and post-transplantation cyclophosphamide leads to deletion of highly active lymphocyte clones and can prevent the acute rejection of allograft in rat. The rejection of graft was slowed down in similar fashion in fully incompatible and haploidentical transplants. The results give intriguing question if this type of simple and straightforward treatment supported by short term immunosuppressive therapy could: a) stop acute organ rejection and b) lead to lifelong immunotlerance of less immunogenic tissues such as liver and kidney and or c) could be used as novel conditioning approach in autoimmune diseases - especially in patients who require organ transplantation due to the terminal organ failure caused by autoimmunity.

Clinical Trial Registry: The protocol of a study has been approved and registered by local Bioethics Committee for Animal Experiments (Medical University of Warsaw)

Conflict of interest: Nothing to disclose

\section{P428}

\section{Control of graft-versus-host responses by mesenchymal stem cells expressing tryptophan metabolic enzymes}

Won Sik Lee ${ }^{1}$,Soung-Min Lee ${ }^{2}$,Young-Don Joo ${ }^{3}$, Su-Kil Seo ${ }^{2}$

${ }^{1}$ Inje University Busan Paik Hospital, Internal Medicine, HematoOncology, Busan, Korea, Republic of: ${ }^{2}$ Inje University, Microbiology and Immunology, Busan, Korea, Republic of; ${ }^{3}$ Busan ON General Hospital, Hemato-Oncology, Busan, Korea, Republic of

Background: Although mesenchymal stem cells (MSCs) are applied as a cellular therapeutic to control graft-versushost disease (GVHD) in clinic, they are still needed to improve the immunomodulatory activity. Recently, indoleamine 2,3 dioxygenas (IDO)-mediated tryptophan metabolism has been recognized as one of the critical mechanisms in the immunomodulatory activity of MSCs. Here, we investigated the full tryptophan metabolism in mouse MSCs and its effect on GVH responses.
Methods: MSCs were isolated from bone marrow of WT. B6 or IDO KO.B6 mice and expanded in vitro with MesenCult medium. mRNA expression of tryptophan metabolic enzymes included in IDO as well as downstream enzymes kynurenine 3-hydroxylase (KMO) and kynureninase (KYNU) in the cells were determined using RT-PCR in the presence or absence of IFN-gamma. KMO and KYNU expressing MSCs were generated by transfection of pBI-cmv1-KMO-KYNU plasmid vector. The suppressive activity of the MSCs was measured on allogeneic mixed lymphocyte reactions.

Results: The meek suppressive activity of IDO on allogeneic $\mathrm{T}$ cell responses was observed through comparison between WT-MSCs and IDO KO-MSCs. We found that IDO was induced by IFN-gamma but there was no downstream enzymes KMO and KYNU in the cells regardless of IFN-gamma stimulation. We generated MSCs expressing $\mathrm{KMO}$ and $\mathrm{KYNU}$ by gene transfection and observed that their inhibitory activity against alloreactive $\mathrm{T}$ cell responses was significantly increased.

Conclusions: Our results demonstrate that mouse MSCs are induced IDO expression by IFN-gamma but not downstream enzymes, indicating that the cells are partially induced on tryptophan metabolism when they are stimulated with IFN-gamma. Our study suggests that MSCs may be able to improve their immunoregulatory activity with producing active regulatory metabolites by transduction of the downstream tryptophan enzymes.

Clinical Trial Registry: N/A

Conflict of interest: None of the authors has anything to disclose.

\section{P429}

\section{Discovery of a new minor histocompatibility antigen presented in HLA-B*13:02 allele}

Nadia Bykova, Dmitrii Romaniuk, Grigory Efimov, Zoya Konova

National Research Center for Hematology, Laboratory of Transplantation Immunology, Moscow, Russian Federation

Background: Allogenic hematopoetic stem cells transplantation (allo-HSCT) is commonly used to treat hematopoetic malignancies. It was shown that after allo-HSCT donor $\mathrm{T}$ cells facilitate eradication of residual disease targeting allo-antigens to a large extent. Hence, such alloantigens, also called minor histocompatibility antigens (MiHAs) in the context of HLA-matched allo-HSCT, are recognized as promising targets for $\mathrm{T}$ cell immunotherapy of relapsed leukemia. Up to date, around 50 MiHAs are known, most of them are restricted by the most common 
and well studied HLA*02:01 allele, while only a few MiHAs are described for other HLA alleles.

Here we report a second MiHA restricted by allele HLAB*13:02.

Methods: HLA-B*13:02 donors was genotyped for GEMIN4-1Q coding polymorphism (rs2740348) using real time allele specific PCR to select GEMIN4-1Q -/individuals. Dendritic cells were differentiated from peripheral blood monocytes and co-cultured with autologous PBMC in the presence of GEMIN4-1Q peptide. Culture was restimulated with fresh peptide-pulsed DC weekly, and IL-2,7,15 were added every 2-3 days after first restimulation. After second restimulation cells were stained with CD137 antibodies, or IFN-gamma intracellular staining was performed to detect functional response.Lymphoblastoid cell lines of different HLA and GEMIN4-1Q genotype were used as target cells for cytotoxic assay. Cytotoxic effect was measured in caspase 3/7 assay.

Results: Here we report, that peptide GEMIN4-1Q (RQPDLVLRL) that was previously suggested as a potential MiHA presented in HLA-A*02:01 by Granados et al., is indeed an immunogenic MiHA but restricted by allele HLA-B*13:02.

Originally suggested HLA-A*02:01 restriction was predicted by NetMHC algorithm. However, other MHCbinding algorithms make contradicting predictions about affinity of GEMIN4-1Q peptide to HLA-A*02:01. The prediction about wrong HLA restriction was made on the basis of analysis of immunopeptidome datasets, that showed strong correlation of peptide occurrence with HLA-B*13:02 genotype. To test this hypothesis we attempted GEMIN41Q-specific T cell expansion in GEMIN4-1Q -/- HLA-A2HLA-B*13:02+ donor, and succeeded. The obtained T cell clone specifically recognized GEMIN4-1Q on HLAB*13:02+ cells, showing strong cytotoxic effect.

Conclusions: Recent studies suggest that tutor cells can efficiently escape immune response by dowregulating expression of a particular HLA allele on the cells surface. This suggests that targeting MiHAs restricted by different alleles simultaneously can be sufficiently more effective. Accordingly, the discovery of new MiHAs restricted by less frequent HLA alleles is important as well. Another interesting side of the work is that HLA restriction can sometimes be refined using data analysis. Funding was provided by RFBR grant number 17-04-02186.

Conflict of interest: None of the authors has anything to disclose.

\section{P430}

Enhanced immunosuppressive properties of human mesenchymal stem cells primed by inflammatory stimuli
Keon Hee Yoo ${ }^{1}$, Young Rok Do ${ }^{2}$, Yoojin Park ${ }^{1}$, Dae Seong Kim ${ }^{1}$, Myoung Woo Lee ${ }^{1}$, Hyun Jin Park', Jung Jun Chae ${ }^{1}$, Ji Won Lee ${ }^{1}$, Ki Woong Sung ${ }^{1}$, Hong Hoe Koo ${ }^{1}$

${ }^{1}$ Samsung Medical Center, Sungkyunkwan University School of Medicine, Seoul, Korea, Republic of; ${ }^{2}$ Dongsan Medical Center, Keimyung University School of Medicine, Daegu, Korea, Republic of

Background: Although human mesenchymal stem cells (MSCs) have been of particular interest owing to their immunosuppressive effect, their clinical benefit is often limited. Therefore, strategies to promote MSCs' immunosuppressive properties need to be sought. In this study, we investigated the effects of ex vivo culture conditions under the presence of inflammatory stimuli including IFN- $\gamma$, TNF$\alpha$, and poly(I:C) on the immunosuppressive properties of MSCs.

Methods: The gene expression profiles under IFN- $\gamma$, TNF- $\alpha$, or poly(I:C) treatments were compared using RTPCR analysis and microarray. In addition, the impact of IFN- $\gamma$ treatment, regulation of IDO, and PGES expression on MSCs' in vitro and in vivo function was analyzed.

Results: The expression of chemokine genes (CXCL9, CXCL10, CXCL11), immunomodulation-associated genes (IDO1, TLR3, B7H1, SOCS1, SOCS3), anticancer effectassociated genes (TNFSF10, BAFF), and an adhesionassociated gene (VCAM1) were significantly upregulated in IFN- $\gamma$-treated MSCs, whereas the expressions of these genes in TNF- $\alpha$ - or Poly(I:C)-treated MSCs were slightly increased or not changed. Furthermore, IFN- $\gamma$-treated MSCs significantly increased the survival rates of graft-versus-host disease (GVHD) mice compared with MSCs without IFN- $\gamma$ stimulation. Downregulation of IDO in MSCs by shRNA resulted in restoration of T-cell proliferation in vitro and decreased the survival rates of GVHD mice, while overexpression of IDO significantly increased MSCs' Tcell inhibitory function and the survival rates of GVHD mice. We also investigated the impact of inflammatory stimuli on the expression of PGE2 pathway members and PGE2 production by bone marrow (BM)-MSCs. Both IFN$\gamma$ and TNF- $\alpha$ increased the expression of cyclooxygenase (COX)-2, but had no impact on the expression of prostaglandin E synthase (PGES) or PGE2 production. On the other hand, the toll like receptor 3 (TLR3) stimulant poly(I:C) increased expression of both COX-2 and PGES, resulting in a significant increase in PGE2 levels. This effect was reversed upon COX-2 inhibition with indomethacin or PGES downregulation by siRNA. Reduced PGE2 levels decreased MSCs' capacity to inhibit hPBMC proliferation. Administration of MSCs with inhibited PGES expression did not reduce the mortality of GVHD mice. These results indicate that upregulation of PGES via TLR3 is critical for BM-MSCs-mediated immunosuppression by PGE2 secretion via the COX-2/PGE2 pathway. 
Conclusions: Given that ex vivo culture expansion is essential for the clinical application of MSCs, these results suggest that IFN- $\gamma$ or Poly(I:C) priming during culture expansion could be an effective strategy to enhance MSCs' immunosuppressive properties.

Conflict of interest: Nothing to disclose.

\section{P431}

Human mesenchymal stem cells primed by interferon- $\gamma$ ameliorate GVHD in NOD/SICD mice through indoleamine 2,3-dioxygenase activity

Hong Hoe Koo ${ }^{1}$, Young Tak Lim ${ }^{2}$, Yoo Jin Park ${ }^{1}$, Hyun Jin Park', Myoung Woo Lee ${ }^{1}$, Dae Sung Kim ${ }^{1}$, Ki Woong Sung ${ }^{1}$, Keon Hee Yoo $^{1}$

${ }^{1}$ Samsung Medical Center, Sungkyunkwan University School of Medicine, Pediatrics, Seoul, Korea, Republic of; ${ }^{2}$ Pusan National University Chidren's Hospital, Pediatrics, Pusan, Korea, Republic of

Background: Mesenchymal stem cells (MSCs) have the capability of acting as modulators of the immune response, and as such may have applicability for the treatment of allogeneic diseases such as graft-versus host disease (GVHD). The clinical use of MSCs has been limited because of heterogeneity that arises due to differences in MSC source and in the methodology used for isolation. In this study, we aimed to characterize the effect and mechanism of interferon (IFN) $\gamma$ priming on MSCs, and their subsequent immunomodulatory activity.

Methods: MSCs were isolated from four different human tissues, namely bone marrow, adipose tissue, cord blood, and Wharton's jelly from the umbilical cord and were stimulated with IFN- $\gamma$ to induce their immunomodulatory capacities. T-cell proliferation was measured in phytohemagglutinin stimulated co-cultures of human peripheral blood-derived mononuclear cells (hPBMCs) and mitomycin C-treated MSCs using a BrdU assay. An animal model of GVHD was established in irradiated NOD/SCID mice injected with hPBMCs, and the effect of immunomodulatory MSCs was assessed. Gene expression profiling was conducted in MSCs treated, or not, with IFN- $\gamma$.

Results: Five hundred twelve genes were up-regulated in IFN- $\gamma$-primed MSCs. One of these genes, indoleamine 2,3dioxygenase (IDO), was found to be highly expressed when MSCs were stimulated with IFN- $\gamma$. Up-regulation of IDO occurred through the JAK/STAT1 pathway, whereas it was only marginally upregulated with either poly I:C, a stimulator of Toll-like receptor 3, or TNF- $\alpha$. Moreover, proliferation of T-cells was suppressed after co-culture with MSCs, and proliferation was further suppressed after coculture with IFN- $\gamma$-primed MSCs. In addition, infusion of MSCs primed with IFN- $\gamma$ significantly reduced the symptoms of GVHD in NOD/SCID mice, which resulted in an increase in their survival rate compared with that in naïve MSC-infused mice. Furthermore, infusion of IDOoverexpressing MSCs increased the survival rate of GVHD mice to a similar extent to what was observed with IFN- $\gamma$ primed MSCs. Moreover, infusion of IFN- $\gamma$-primed MSCs, in which IDO was down-regulated, did not elicit this effect.

Conclusions: Our data indicate that IFN- $\gamma$ stimulation induces IDO expression in MSCs through the IFN- $\gamma / \mathrm{JAK} /$ STAT1 pathway, which is a key modulator in their immunosuppressive properties. These data further suggest that cell therapy using MSCs primed with IFN- $\gamma$ could be highly effective in treating allogeneic diseases, including GVHD.

Conflict of interest: This research was supported by a grant of the Korea Health Technology R\&D Project through the Korea Health Industry Development Institute (KHIDI) funded by the Ministry of Health \& Welfare, Republic of Korea (Grant number: HI14C3484 and HI15C2963).

The authors have nothing to disclose.

\section{P432}

\section{IL-22 as treatment for GVHD in a preclinical model}

Amy Publicover ${ }^{1}$, Xiao Nong Wang $^{1}$, Venetia Bigley ${ }^{1}$, Erin Hurst ${ }^{2}$, Arian Laurence $^{1}$, Graham Jackson ${ }^{2}$, Matthew Collin ${ }^{1}$

${ }^{1}$ Newcastle University, Institute of Cellular Medicine, Newcastle upon Tyne, United Kingdom; ${ }^{2}$ Northern Centre for Bone Marrow Transplantation, Newcastle upon Tyne Hospitals, Newcastle upon Tyne, United Kingdom

Background: Although there are many agents available for prophylaxis, treatment options for established graft versus host disease (GVHD), especially when steroid resistant, remain limited. IL-22 offers an exciting therapeutic opportunity, as the absence of IL-22 receptors on leucocytes gives the potential for separation of GVHD from the graft versus tumour effect. The role of IL-22 in GVHD, however, remains controversial.

Methods: Using an adaptation of a previously described human skin explant model we have explored the potential for use of recombinant IL-22 (rIL-22) to treat GVHD in an in vitro model. Briefly, PBMCs from two healthy donors were isolated, combined, suspended in culture medium and cultured at $37^{\circ} \mathrm{C}$. After five or seven days the mixed lymphocyte reaction (MLR) supernatant was removed and stored at $-80^{\circ} \mathrm{C}$. $6 \mathrm{~mm}$ skin punch biopsies (normal donor excess skin from plastic surgery) were cut into four pieces of equal size, and trimmed of excess dermis. Fragments were then incubated for 72 hours at $37^{\circ} \mathrm{C}$ with culture medium alone, MLR supernatant alone, MLR supernatant plus $30 \mathrm{pg} / \mathrm{ml}$ rIL-22 or MLR supernatant plus $150 \mathrm{pg} / \mathrm{ml}$ 


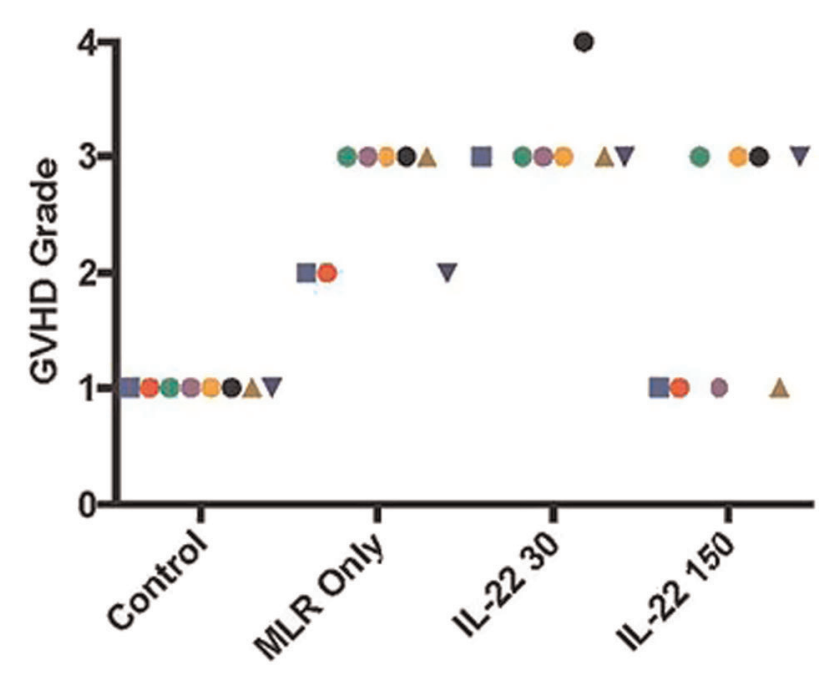

[P432 Figure] [Impact of IL-22 concentration on GVHD Grade]

rIL-22. After 72 hours the samples were formalin fixed, paraffin embedded and Haemotoxylin and Eosin stained according to local GVHD protocols. GVHD grading was assessed using the Lerner grading system and performed blinded by an independent assessor.

Results: The samples in culture medium alone showed consistent Grade 1 damage. Those in the MLR supernatant showed Grade 2-3 damage. The addition of 30pg/ml rIL-22 did not always have an effect, but where it did, this was always to increase the grade of GVHD. The addition of $150 \mathrm{pg} / \mathrm{ml} \mathrm{rIL}-22$, however, resulted in resolution to Grade 1 in $50 \%$ of experiments performed (Figure 1), an almost identical response rate to that which we had previously observed with methylprednisolone (XN Wang, unpublished). The IL-22 produced by the MLR reaction itself was measured by ELISA, so the total IL-22 concentration (baseline and recombinant) could be calculated. IL-22 concentration in the MLR/MLR plus 30pg/ml IL-22 ranged from $10-155 \mathrm{pg} / \mathrm{ml}$. Total IL-22 in the MLR plus $150 \mathrm{pg} / \mathrm{ml}$ ranged from $160-275 \mathrm{pg} / \mathrm{ml}$.

Conclusions: The total IL-22 concentration in the MLR supernatant with or without $30 \mathrm{pg} / \mathrm{ml} \mathrm{rIL-22}$ is in the same range as that measured in the serum of patients with GVHD, while the addition of $150 \mathrm{pg} / \mathrm{ml}$ rIL-22 results in a supraphysiological concentration. The response seen at the higher dose raises the possibility of a biphasic response to IL-22, with a therapeutic effect seen above the physiological range. The presence of the IL-22 receptor on the target organs of GVHD, but not on leucocytes gives the potential for treatment without loss of the graft versus tumour effect. RNA sequencing is being used to establish a signature that separates responders from non-responders.

\section{Conflict of interest:}

Amy Publicover's Clinical Research Training Fellowship is funded by Bloodwise. None of the authors have any conflict of interest to disclose in relation to this work.

\section{P433}

Imidazoline Scaffold Proteasome Modulator TCH-013 Ameliorates Experimental Graft-versus-Host Disease (GvHD)

\author{
A. Samer Al-Homsi ${ }^{1}$, Austin Goodyke ${ }^{2}$, Ulrich Duffner ${ }^{2}$, Kelli Cole ${ }^{1}$, \\ Jetze Tepe ${ }^{3}$ \\ ${ }^{I}$ New York University Langone Health, Blood and Marrow \\ Transplantation, New York, NY, United States; ${ }^{2}$ Spectrum Health, \\ Pediatric Blood and Marrow Transplantation, Grand Rapids, MI, \\ United States; ${ }^{3}$ Michigan State University, Department of Chemistry, \\ East Lansing, MI, United States
}

Background: Substrate competitive proteasome inhibitor, bortezomib, has been shown to prevent GvHD in murine models and in early phase clinical trials. However, sustained administration of bortezomib aggravates GvHDrelated intestinal injury, in part due to paradoxical increase in interleukin (IL) $1 \beta$. Imidazoline scaffold TCH-013 compound prevents proteasome cap binding to $\alpha$ rings and thus modulates proteasome activity. We aimed to study the effects of TCH-013 on dendritic cell (DC) maturation and cytokine production and on $\mathrm{T}$ cell proliferation and differentiation in vitro. We also examined the ability of TCH-013 to protect mice in an MHC-mismatched GvHD model.

Methods: DCs were isolated from the peripheral blood of healthy volunteers and treated with TCH-013 at different concentrations (12 and $24 \mu \mathrm{M}$ ) for $4 \mathrm{hrs}$ and were then stimulated with lipopolysaccharide (LPS) for $16 \mathrm{hrs}$. DCs were then stained with antibodies against maturation markers and analyzed by flow cytometry. DC viability was assessed after 7AAD staining. Pro-inflammatory cytokines were measured in the supernatant of DC cultures treated before or after stimulation with LPS. Similarly, the effects of TCH-013 on $\mathrm{T}$ cell differentiation was studied in healthy volunteer mononuclear cells stimulated with $\mathrm{T}$ cell activating antibodies without and with the addition of TCH-013. $\mathrm{T}_{\mathrm{h}} 1, \mathrm{~T}_{\mathrm{h}} 17$, and regulatory $\mathrm{T}$ cells $\left(\mathrm{T}_{\text {regs }}\right)$ were enumerated after staining with antibodies to IFNy, IL-17, and FOXP3. At a minimum, all experiments were done in triplicate. ANOVA with Tukey post hoc tests was used for statistical analysis. A $p$ value $<0.05$ was considered significant. A B6 $\rightarrow$ BALB/c pre-clinical GvHD model was adopted to evaluate the effect of TCH-013 on GvHD development. Mice were transplanted in 3 groups with intraperitoneal injection of either a vehicle or TCH-013 $250 \mathrm{mg} / \mathrm{kg}$ on days 0 to +3 or TCH-013 $250 \mathrm{mg} / \mathrm{kg}$ on days 0 to +6 . 
Results: TCH-013 inhibited the expression of DC maturation markers including CD40, CD54, CD80, and CD86. DCs viability remained unchanged compared to the control. TCH-013 significantly decreased DC production of IL- $1 \beta$ and IL- 6 . TCH-013 decreased TNF- $\alpha$ at the higher concentration. Importantly, TCH-013 did not cause paradoxical IL- $1 \beta$ by pre-stimulated DCs. In mononuclear cell cultures, TCH-013 decreased the percentage of $\mathrm{Th}_{1}$ and $\mathrm{Th}_{17}$ cells and increased the $\mathrm{T}_{\text {regs }}: \mathrm{T}_{\mathrm{h}} 1$ and $\mathrm{T}_{\text {regs }}: \mathrm{T}_{\mathrm{h}} 17$ ratio. In vivo, TCH-013 improved GvHD scores and survival compared to the control. Noticeably, TCH-013 did not cause GvHD exacerbation in mice treated from days 0 to +6 .

Conclusions: $\mathrm{T}$ cell differentiation, by reducing $\mathrm{T}_{\mathrm{h}} 1$ and $T_{h} 17$ and increasing $T_{\text {regs }}$ ratio to $T_{h} 1$ and $T_{h} 17 ; 3$ ) TCH013 ameliorates GvHD in an aggressive murine model; 4) Contrary to the effects of bortezomib, TCH-013 does not cause a paradoxical surge in IL- $1 \beta$ in pre-stimulated DCs and does not exacerbate GvHD in mice treated in a sustained fashion.

Conflict of interest: A. Samer Al-Homsi: nothing to disclose

\section{P434}

Itacitinib, a JAK1 Selective Inhibitor is highly efficacious in a MHC-mismatched mouse model of acute GvHD

Ashish Juvekar ${ }^{1}$, Bruce Ruggeri ${ }^{1}$, Sindy Condon ${ }^{1}$, Paul Smith ${ }^{1}$, Peggy Scherle $^{1}$, Michael Montgomery ${ }^{1}$, Patricia Delaite ${ }^{1}$, Dominic Beal $^{2}$, Andrew Borkowski ${ }^{2}$

${ }^{1}$ Incyte Corporation, Wilmington, DE, United States; ${ }^{2}$ Biomodels LLC, Watertown, MA, United States

Background: Graft-versus-host-disease (GvHD) is a severe complication arising in patients undergoing allogeneic hematopoietic stem cell transplantation (allo-HSCT). Potent and selective modulation of JAK1/STAT-mediated signaling is an attractive therapeutic strategy for the management of acute GvHD.

Methods: Acute GvHD was induced in BALB/c mice using the established MHC-mismatched mouse model. $\mathrm{BALB} / \mathrm{c}(\mathrm{H}-2 \mathrm{Kd})$ recipients were given an intravenous injection of a combination of splenocytes and T-cell depleted bone marrow cells received from allogeneic cell transfer from donor C57BL/6 $(\mathrm{H}-2 \mathrm{~Kb})$ mice. Animals were dosed orally with vehicle or the potent and selective JAK1 inhibitor, itacitinib $(60 \mathrm{mg} / \mathrm{kg}$ or $120 \mathrm{mg} / \mathrm{kg}$ BID). GvHD clinical scores were assessed by standard methods and TH1 and TH2 cytokine profiles quantified by Luminex analyses. The efficacy of itacitinib was evaluated against targeted therapeutic agents (filgotinib and ibrutinib) that could be useful in the management of GvHD.
Results: Itacitinib administration was highly effective in both prophylactic (day-3) and therapeutic (day14) dosing regimens in ameliorating body weight loss and improving GvHD scores and had no detrimental effects on engraftment. Similar efficacy was observed with $60 \mathrm{mg} / \mathrm{kg}$ versus $120 \mathrm{mg} / \mathrm{kg}$ BID dosing regimens. Filgotinib, a JAK1 selective inhibitor (50mg/kg BID) was not efficacious in this model, and ibrutinib, a potent BTK and ITK inhibitor $(15 \mathrm{mg} / \mathrm{kg}$ BID) showed modest effects on recovery of body weight loss but no effect on GvHD score. Combinations of ibrutinib with itacitinib had no additive therapeutic benefit relative to single agents. Oral itacitinib administration in BALB/c mice achieved JAK1 $\mathrm{IC}_{50}$ coverage for $4 \mathrm{~h}$ and $12 \mathrm{~h}$ at $60 \mathrm{mg} / \mathrm{kg}$ BID and $120 \mathrm{mg} / \mathrm{kg}$ BID, respectively. Maximal upregulation of $\mathrm{TH} 1$ and $\mathrm{TH} 2$ relevant cytokines was observed in peripheral blood on Day 17 and in colon on Day28 and significant differences in cytokine profiles at these disease stages were observed with treatment of $120 \mathrm{mg} / \mathrm{kg}$ itacitinib. No differences were observed in absolute number of $\mathrm{CD} 4^{+} \mathrm{T}$-cells and $\mathrm{CD} 8^{+} \mathrm{T}$-cells in blood and spleen.

Conclusions: Itacitinib a JAK1 selective inhibitor administered prophylactically or therapeutically had significant effects on ameliorating disease severity in a MHCmismatched model of acute GvHD and modulating levels of TH1 and TH2 relevant cytokines important in the pathophysiology of acute GVHD.

Conflict of interest: A. Juvekar, B. Ruggeri, S. Condon, P. Smith, P. Scherle, M. Montgomery, and P. Delaite are Incyte Corporation employees.

\section{P435}

MicroRNA-155 (miR-155) Inhibit T Lymphocyte Prenylation to Protect Against Acute Graft Versus Host Disease (aGVHD)

\section{Xiaoxiao Wang, Linghui Xia, Qiuling Wu}

Union Hospital, Tongji Medical College, Huazhong University of Science and Technology, Wuhan, China

Background: It was reported that farnesyl-transferase and geranylgeranyl-transferase inhibitors could help to reduce graft-versus-host (GVHD) disease significantly without having a negative impact on immune reconstitution. Previously, we have reported that miR-155 levels in $\mathrm{T}$ lymphocyte from peripheral blood of acute GVHD (aGVHD) patients and mice were significantly elevated and the inhibition of miR-155 can relieve the severe murine aGVHD. This work presents a regulatory mechanism of miR-155 in aGVHD.

Methods: TNF- $\alpha$, which participates in the initiating events that culminate in aGVHD as well as amplifies the 

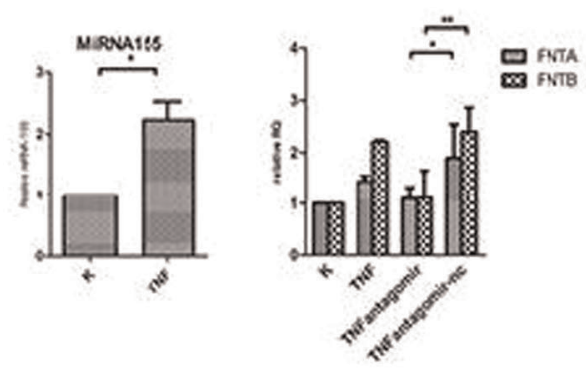

b

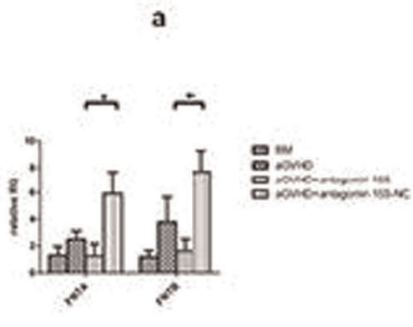

Figurel

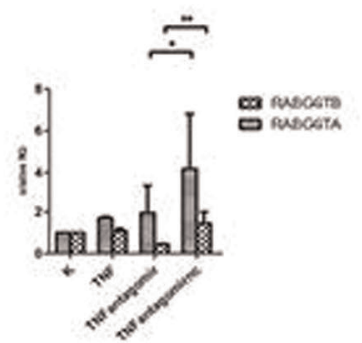

c
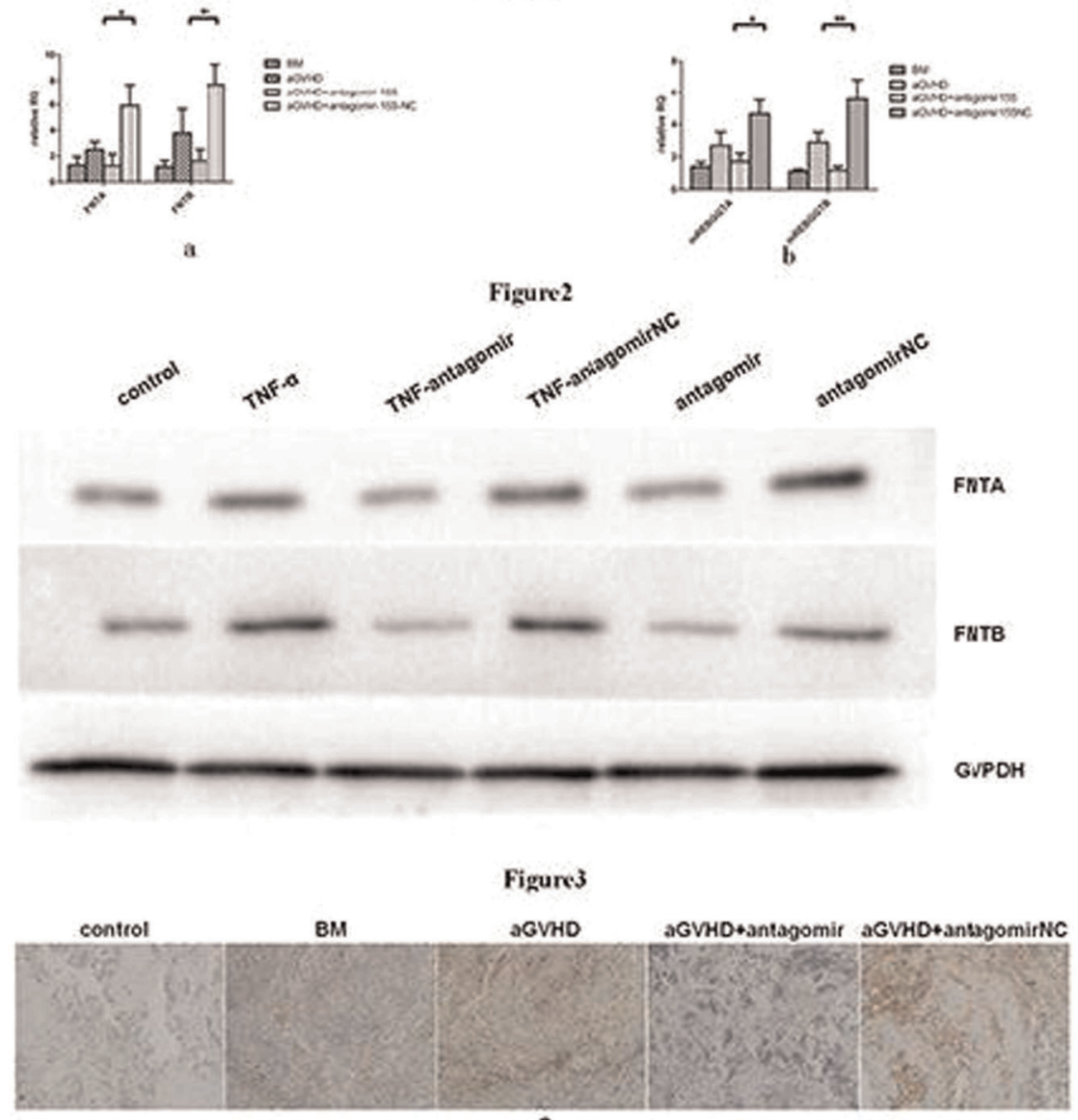

a
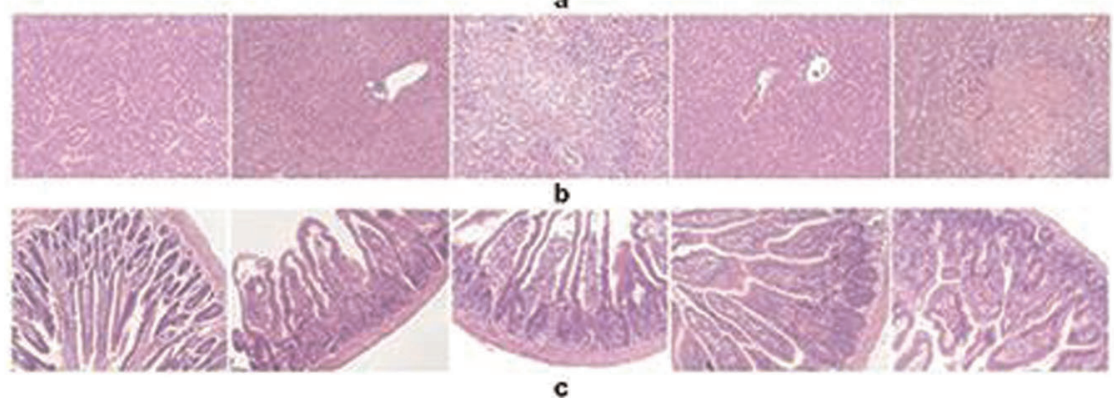

Figuret

[P435 Figure] [miR-155 inhibit T lymphocyte prenylation to protect against aGVHD in vitro and vivo.]

disease process once established. Therefore, TNF- $\alpha$ induced

$\mathrm{T}$ cell injury was used to simulate the course of aGVHD in vitro. To assess the contribution of miR-155 to prenylation of human $\mathrm{T}$ lymphocyte, $\mathrm{T}$ lymphocyte from 
human peripheral blood mononuclear cells (PBMCs) was selected through negative selection using magnetic bead, which was effected for $48 \mathrm{~h}$ with antagomir-155 and antagomir-NC at a concentration of $150 \mathrm{nM}$ before stimulated with TNF- $\alpha(100 \mathrm{ng} / \mathrm{ml})$ for $24 \mathrm{~h}$. To assess the contribution of miR-155 to prenylation in vivo, a wellestablished MHC mismatched bone marrow transplantation (BMT) model (C57BL/6 to $\mathrm{BALB} / \mathrm{c})$ was used to investigate the effect of deficiency of miR-155 on prenylation in vivo. $800 \mathrm{cGy}$ X-rays irradiated BALB/c mice transplanted with $1 \times 10^{7} \mathrm{BM}$ cells and $2 \times 10^{7}$ spleen cells from C57BL/6 mice, were treated with antagomir- 155 and antagomir-NC at a loading dose of $25 \mathrm{mg} / \mathrm{kg}$ on $+7 \mathrm{~d}, 5$ $\mathrm{mg} / \mathrm{kg}$ intravenously twice weekly up to $+21 \mathrm{~d}$ posttransplant. Mice T lymphocyte magnetically isolated from PBMCs. Then, we examined expression of prenyltransferase of both human $\mathrm{T}$ lymphocyte and mice $\mathrm{T}$ lymphocyte by Realtime quantitative-PCR and western blotting. We evaluated the severity of aGVHD using a histopathology scoring system and the farnesyltransferase activity by immunohistochemistry.

Results: The results indicated TNF- $\alpha$ stimulated T cells to imitate aGVHD in vitro induced a higher expression of miR-155 (Figure1a, $\mathrm{P}<0.05$ ), coincidentally, TNF- $\alpha$ stimulated $\mathrm{T}$ cells resulted a higher expression of farnesyltransferase(FT) subunits farnesyltransferase- $\alpha$ (FNTA) which is common to geranylgeranyltransferaseI- $\alpha$ and farnesyltransferase- $\beta$ (FNTB) mRNA (Figure1b) and protein levels (Figure3), similarly increased geranylgeranyltransferaseII (GGTII) subunits RABgeranylgeranyltransferase- $\alpha$ (RABGGTA) and RABgeranylgeranyltransferase- $\beta$ (RABGGTB) mRNA expression(Figure1c). Intriguingly, the suppression of miR-155 could reverse this phenomenon, which not only restrained FNTA, FNTB mRNA and protein expression (Figure1b, Figure3, $\mathrm{P}<0.01$ ), but also decreased RABGGTA and RABGGTB (Figure1c, P< 0.05) mRNA levels. Simultaneously, in aGVHD mice models, compared with control groups, aGVHD group caused higher expression of FNTA, FNTB, REBGGTA and RABGGTB, however, which exacerbation can be reversed by inhibition of miR-155 (Figure2a, b, P< 0.05), all of this suggested that during the initiation and development of aGVHD, prenyltransferase and miR-155 levels were both increased, the suppression of miR-155 decreased the expression of prenyltransferase in vivo and vitro. Next, we consistently discovered that the miR-155 deficient aGVHD mice which had a lower histopathology score of live(Figure4b) and small intestine(Figure4c) possessed of decreased farnesylated proteins and farnesyltransferase activity in spleen (Figure4a), this results confirmed that miR-155 simulate the role of farnesyl-transferase and geranylgeranyl-transferase inhibitors to relieve the effect of aGVHD.
Conclusions: Our work suggests an original notion that miR-155 inhibit $\mathrm{T}$ lymphocyte prenylation to protect against aGVHD.

Conflict of interest: The authors declare no conflict of interest

\section{P436}

Minor antigen-specific T cell clones detected after in vitro expansion demonstrate substantial sequence homology

Nadia Bykova, Zoya Konova, Savely Sheetikov, Dmitrii Romaniuk, Anna Kuchmiy, Grigory Efimov

National Research Center for Hematology, Laboratory of Transplantation Immunology, Moscow, Russian Federation

Background: Minor histocompatibility antigens (MiHAs) are polymorphic peptides encoded by nonsynonymous single nucleotide polymorphisms (SNPs) which could be immunogenic after alloHSCT. In the HLA-matched setting MiHAs solely mediate both antitumor $(\mathrm{GvL})$ effect, and detrimental graft-versus host effect (GvHD). Up to date, more than 50 MiHAs presented in different HLA alleles are described, but only a few MiHAspecific $\mathrm{T}$ cell receptor (TCR) sequences are known. Accordingly, the diversity of MiHA-specific repertoire remains largely undescribed.

Here we present a study of MiHA specific immune response after alloHSC and analysis of TCR sequences specific to several MiHAs. Our approach is based on in vitro MiHA-specific expansion of $\mathrm{T}$ cells and NGS sequencing of TCR repertoire.

Methods: HLA-matched donors and patients undergoing alloHST were genotyped for the panel of $>20$ known MiHAs. Peripheral blood samples were taken from patients mismatched for MiHAs at various time points after alloHSCT. Cells were further cultivated in the presence of MiHA peptides and feeder cells. Percentage of MiHAspecific cells in expansions and peripheral blood samples were measured by MHC-tetramer staining. After restimulation expanded cells were magnetically separated to CD137and CD137+ fractions, both were further subjected to TCR repertoire sequencing on Illumina platform.

Separately a panel of healthy HLA-genotyped volunteers was genotyped for SNPs coding for MiHAs. MiHA -/dendritic cells were differentiated from peripheral blood monocytes and co-cultured with autologous naïve CD8+ cells in the presence of MiHA peptides. After restimulation cells were separated to CD137- and CD137+ fractions and their TCR repertoire were analyzed.

Results: We have demonstrated that MiHA specific $\mathrm{T}$ cells in MiHA-mismatched HLA-matched alloHSCT could rarely be detected by direct MHC-tetramer staining of 
peripheral blood. Nevertheless, in some patients with GvHD and known MiHA mismatches, MiHA-specific cells could be detected after in vitro cultivation.

We have sequenced TCR repertoire of T cells specific for HA-1,HA-2 and ACC1-Y MiHAs both from patients and from healthy MiHA negative volunteers. Analysis of the TCR sequences demonstrated that TCRs specific to a particular MiHAs can have a substantial level of similarity.

Conclusions: It has been recently demonstrated that sequences of TCRs specific to a common antigen are similar to a large extent, suggesting that TCR specificity could be deduced from its sequence given the substantial number of previously described sequences specific to the same antigen. Thus, if MiHA-specific TCR clusters are sufficiently well described, MiHA specific $\mathrm{T}$ cells might be detected by analysis of the bulk repertoire sequencing. If succeeded, this will be an alternative for MHC-tetramer approach which allows detection only of MiHA-specific T cells expanded at the moment. Funding was provided by Russian Science Foundation grant 17-15-01512.

Conflict of interest: The authors have nothing to disclose.

\section{P437}

\section{Prevention of Acute Graft-Versus-Host Disease After Allogeneic Bone Marrow Transplantation by Molecular Targeting of Anti-Apoptotic Proteins in Activated Donor T Cells}

Ivan Odak ${ }^{1}$, Maleen Beck ${ }^{2}$, Hanna Kirchhoff ${ }^{2}$, Matthias Eder $^{2}$, Christian Könecke ${ }^{1,2}$

${ }^{1}$ Hannover Medical University, Institute of Immunology, Hannover, Germany; ${ }^{2}$ Hannover Medical University, Department of Hematology, Hemostasis, Oncology and Stem Cell Transplantation, Hannover, Germany

Background: Graft-versus-Host-Disease (GvHD) accounts for the most frequent complication following Bone Marrow Transplantation (BMT). Current therapies with corticosteroids are often inefficient, and usually accompanied by a variety of adverse effects. The BCL-2 family of proteins is crucial for the regulation of cell survival in normal and malignant cells by controlling the mitochondrial apoptotic pathway. The competitive binding of $\alpha$-helical BH3-domains of pro-apoptotic proteins to the BH3-binding grooves of either pro- or anti-apoptotic proteins controls the induction of apoptosis. By targeting the BH3-binding domains of the pro-survival molecules in T cells using the BH3-mimetics ABT-737 and ABT-199, we aim to evaluate the possibility of efficiently preventing GvHD.

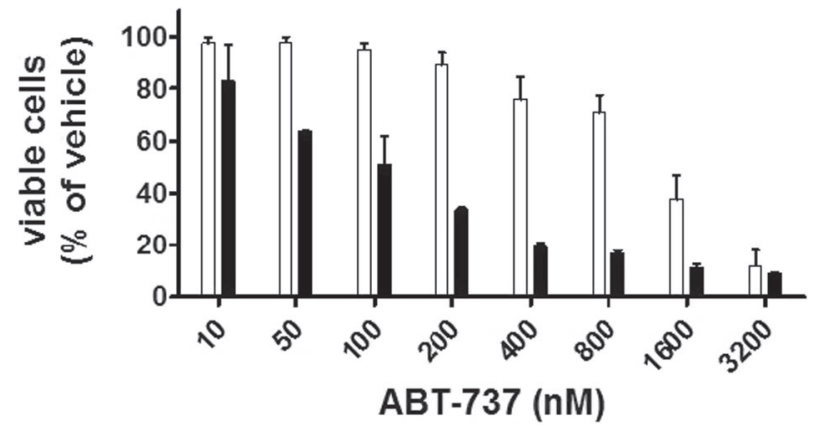

[P437 Figure] [ABT-737 has differential effect on T cell subsets]

Methods: Primary T cells (B6.Cg-Foxp3tm1Mal/J) were segregated on $\mathrm{CD} 4+$ and $\mathrm{CD} 8+$ fractions by magnetic separation, and activated in vitro either with $\alpha-\mathrm{CD} 3$ and $\alpha$ CD28 antibodies or by co-culture with allogeneic (BALB/c) BM-derived dendritic cells (BMDCs). Apoptosis and Mitochondrial Outer Membrane Permeabilization (MOMP) rates were assessed by flow cytometry. In survival experiments, mice were treated either with ABT-199 intraperitoneal or control-vehicle for 10 consecutive days, starting from $24 \mathrm{~h}$ after BMT. Mice were scored daily for signs of clinical GvHD.

\section{Results:}

Comparing the levels of the proteins involved in the MOMP induction, depending on T cell activation stage, we show a distinct kinetics of expression of BCL-2 proteins. Following the incubation of resting and activated CD8+ $\mathrm{T}$ cells with the ABT-199, we report a greater induction of apoptosis in activated as compared to resting CD8+ cells (Figure 1). Such a differential effect is abrogated in the presence of high IL-2 levels. Using immunoblotting and flow cytometry, we demonstrate an upregulation of the prosurvival proteins BCL-2, BCL-xL and MCL-1, and in parallel, a downregulation of the pro-apoptotic protein PUMA in direct proportion to the concentration of added IL-2. This hints towards an antagonistic effect of IL-2 on the induction of apoptosis via BH-3 mimetics. Furthermore we could show a greater resistance of regulatory $\mathrm{T}$ cells (Tregs) to ABT-737 compared to conventional CD4+ cells, suggesting different regulatory elements regulating the mitochondrial apoptosis pathway in Tregs.

Using the murine $\mathrm{BL} / 6 \rightarrow \mathrm{BALB} / \mathrm{c}$ acute $\mathrm{GvHD}$ model, we demonstrate that treatment with ABT-199 led to a significantly delayed onset of GvHD and a prolonged survival after BMT.

Conclusions: Here we report a differential effect of the BH3-mimetics on CD8 + T cells, and a different expression profile of BCL-2 proteins depending on their activation state. Furthermore, we could show that BH3-mimetics prolong survival after BMT by delaying the onset of acute GvHD. However, we observed a functional antagonism of 
IL-2 signaling towards the induction of mitochondrial apoptosis in $\mathrm{T}$ cells and this might be the reason for incomplete GvHD-prevention.

Conflict of interest: None of the authors has anything to disclose.

\section{P438}

\section{Regulatory T cell function in patients with GvHD after allogeneic stem cell transplantation}

\author{
Pascal Woelfinger ${ }^{1}$, Eva-Maria Wagner-Drouet ${ }^{2}$, Matthias \\ Theobald $^{2}$, Bopp Tobias ${ }^{3}$ \\ ${ }^{1} J o h a n n e s$ Gutenberg-University Medical Centre, Mainz, Germany; \\ ${ }^{2}$ Johannes Gutenberg-University Medical Centre, Haematology, \\ Oncology, Pneumology, UCT, Mainz, Germany; ${ }^{3}$ Institute of \\ Immunology, University Medical Center of the Johannes Gutenberg- \\ University Mainz, Germany, Mainz, Germany
}

Background: Different types of $\mathrm{T}$ cell depletion (TCD) are used to prevent Graft versus Host disease (GVHD) after allogeneic stem cell transplantation like Anti-Thymocyte Globulin (ATG) or the monoclonal CD52 antibody Alemtuzumab. Despite TCD nearly half of the patients develop acute or chronic GvHD. Regulatory T cells (Treg) are required to control GvHD. Therefore we focus on Treg function after different TCD regimens to investigate their role in the development of GvHD.

Methods: We analysed peripheral blood samples of patients with acute and chronic GVHD as well as without GVHD (Altemtuzumab $\mathrm{n}=70$, ATG $\mathrm{n}=10$ ) and 10 healthy individuals. Treg (CD3, CD4, CD25, CD127, FoxP3) were stained for CD52, activation markers GARP, TGF-beta, HLA-DR, CD45RA as well as functional markers CD39, CD44, CD62L, Granzyme A, CCR5, ILT3, CTLA4, CXCR3 and GITR. Supressive Treg function was evaluated in CSFE suppression assays. To rule out any influence of immunosuppression (prednisolone, tacrolimus) used in patients with GVHD we analysed Treg function and expression of activation markers in vitro using healthy Treg. In addition we analysed the effect of patient serum on Treg function.

Results: Treg numbers showed no significant differences in patients with acute, chronic or no GVHD. Treg markers did not differ in patients with Alemtuzumab or ATG, despite CD52 expression: after Alemtuzumab treatment patients with aGvHD showed significant elevated levels of CD52neg Treg (mean 78\%) in comparison to $11 \%$ in patients without GvHD and 27\% in patients with chronic GVHD.

Treg dervied from patients with GVHD were showed reduced supressive capacity ex vivo compared to healthy Treg. Especially in patients with acute GVHD after
Alemtuzumab treatment CD52neg Treg were less functional compared to CD52pos Treg of the same patient. GARP/ TGF-beta as well as ILT3 were upregulated in CD52pos Treg (compraed to CD52neg Treg), in Treg derived from patients without GVHD (compared to acute GVHD), and in healthy Treg (compared to GVHD patients). There were no significant differences between other Treg markers in both GVHD-groups in comparison with healthy individuals.

Cyclosporin A or corticosteroids ex vivo (and in vitro) did not influence Treg marker expression or supressive capacity.

Serum of patients with acute and chronic GVHD without immunosuppressive drugs had showed an antiproliferative effect on effector CD4 / CD8 T cells and enhanced the supressive capacity of healthy Treg.

Conclusions: Treg of patients with GVHD showed a reduced supressive function.Higher GARP/ TGF-beta expression seems to be associated with less development of acute GVHD.

Especially Alemtuzumab leads to an increase of CD52neg Treg with reduced supressive capacity and different activation markers in patients with aGVHD. Patients with $>50 \%$ CD52pos Treg before $\mathrm{d}+50$ post allogeneic stem cell transplantation never developed any acute GVHD.

Serum of patients with GVHD enhances supressive Treg function.

Clinical Trial Registry: No

Conflict of interest: None

Granulocyte and osteoclast disorders

\section{P439}

\section{Successful Hematopoietic Stem Cell Transplantation of Infantile Malignant Osteopetrosis Using Reduced Intensity Conditioning}

Bella Shadur ${ }^{1}$, Irina Zaidman ${ }^{1}$, Adeeb Naser Eddin ${ }^{1}$, Batia Avni ${ }^{1}$, Elena Lokshin ${ }^{1}$, Fatma Hussein ${ }^{1}$, Hodaya Cohen Oron ${ }^{1}$, Sigal Grisariu $^{1}$, Polina Stepensky ${ }^{2}$

${ }^{I}$ Department of Bone Marrow Transplantation Hadassah University Medical Center, Jerusalem, Israel; ${ }^{2}$ Hadassah Hebrew University Medical Center Ein Kerem, Department of Bone Marrow

Transplantation and Cancer Immunotherapy, Jerusalem, Israel

Background: Infantile malignant osteopetrosis (IMO) is an autosomal recessive condition characterized by defective osteoclast activity, with hematopoietic bone marrow transplant the only available cure. Over the past several years, new conditioning regimes and donor options have emerged thus extending the possibility of cure to a greater number of patients and improving the outcomes of bone marrow transplant. Here we detail the outcomes of bone marrow transplant in a cohort of 31 patients treated with a 
combination of fludarabine, treosulphan, thiotepa and antithymocyte globulin.

Methods: 31 patients with IMO who underwent HSCT with fludarabine, treosulphan, thiotepa and anti-thymocyte globulin at our center between 2012 to 2017 are retrospectively reviewed in this study.

Results: Overall survival was $100 \%$ with 12 cases of GvHD, no cases of VOD, and eight cases of hypercalcemia. Almost $80 \%$ of patients suffered viral reactivations which were successfully treated in all cases.

Conclusions: We conclude that transplantation of children with IMO using fludarabine, treosulphan, thiotepa and anti-thymocyte globulin is safe and effective and should be performed as early as possible following diagnosis, prior to the development of severe disease sequelae.

Clinical Trial Registry: Not applicable

Conflict of interest: No conflict of interest for all authors Haemoglobinopathy and inborn errors of metabolism

\section{P440}

\section{Allogeneic hematopoietic stem cell transplantation for children with Hurler syndrome}

\section{Tatiana Bykova, Anastasya Borovkova, Anna Osipova, Varvara Ovetchkina, Andrew Kozlow, Alexander Alyanskiy, Elena Semenova, Ludmilla Zubarovskaya, Boris Afanasyev}

R. Gorbacheva Memorial Research Institute for Pediatric Oncology, Hematology and Transplantation, the First State Pavlov Medical University of St. Petersburg, St. Petersburg, Russian Federation

Background: Hurler syndrome (MPS IH) is the most severe form of mucopolysaccharidosis, rare genetic disorder, caused by deficiency of alpha-L-iduronidase enzyme. Intravenous enzyme replacement therapy (ERT) reverses some aspects of MPS IH (hepatomegaly, splenomegaly and glycosaminoglycanuria) and ameliorates others (pulmonary function, cardiac disease, arthropathy and exercise tolerance), but neurologic benefits are thought to be inconsiderable because the blood brain barrier blocks enzyme from reaching the CNS. Hematopoietic stem cell transplantation (HSCT) is the only curative treatment option in Hurler syndrome. Myeloablative conditioning regimens (MAC) allow to achieve good engraftment, but have a higher probability of toxic complications. Reduced intensity conditioning regimens (RIC) are associated with less transplant related mortality but higher risks of graft failure. 96 800x600 Normal 0 false false false RU X-NONE XNONE /* Style Definitions */ table.MsoNormalTable \{msostyle-name:"Обычная таблица"; mso-tstyle-rowbandsize:0; mso-tstyle-colband-size:0; mso-style-noshow:yes; mso-style-priority:99; mso-style-parent:"'; mso-paddingalt:0cm 5.4pt $0 \mathrm{~cm} 5.4 \mathrm{pt}$; mso-para-margin:0cm; mso-para- margin-bottom:.0001pt; mso-pagination:widow-orphan; font-size: $10.0 \mathrm{pt}$; font-family:Cambria;

Aim: To assess effectiveness of allo-HSCT in children with Hurler syndrome, define prognostic factors and compare RIC and MAC in this clinical setting and evaluate efficacy and safety of PTCY as prophylaxis of GVHD.

Methods: Study included 18 pts with MPS IH after allogeneic unrelated HSCT. Median age at diagnosis was 13 months (range 3 - 24), median time to the start of ERT 17 months (range 4 - 30), median time to allo-HSCT 25 months (range 16 - 41). Conditioning regimens were MAC (Bu16+Flu150/Treo42+Flu160+Thio8) - 5 pts, RIC (Flu150+Mel140) - 13 pts. GVHD prophylaxis: 12 pts received classical regimens $(\mathrm{CsA} / \mathrm{Tx} \pm \mathrm{MTX} \pm \mathrm{MMF}), 6$ pts received combinations with posttransplant cyclophosphamide (PTCY). All pts received antithymocyte globulin (ATG). In 12 cases rabbit ATG was administered $20 \mathrm{mg} / \mathrm{m}^{2}$ on Days $-4,-3,-2$ and in 6 cases combination of PtCy and horse ATG (ATG was used at a dose of $0.5 \mathrm{mg} / \mathrm{m}^{2}$ on Day -10 and $2 \mathrm{mg} / \mathrm{m} 2$ on Days-9,-8). Graft source: bone marrow (BM) - 11 pts with median number of CD34+cells $/ \mathrm{kg}$ $4,2 \times 10^{6}(2,7-10)$, peripheral blood stem cells (PBSC) - 7 pts

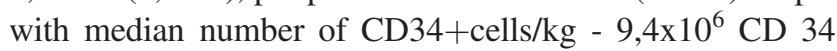
$+/ \mathrm{kg}(7,1-13,1)$.

Results: Engraftment was achieved in all pts. Five-year OS was $83,3 \%$. Age of diagnosis, time to start of ERT and time to allo-HSCT were important prognostic factors. If MPS IH was established prior to 10 months OS resulted in $100 \%$ and if Hurler syndrome was diagnosed later OS was $77 \%$. In case of ERT start prior to 14 months OS was $100 \%$ if later $-75 \%$. In children with allo-HSCT prior to 24 months OS was $100 \%$ if later - $70 \%$. There was no statistically significant difference in OS of children with MAC and RIC ( $80 \%$ vs $85 \%$ ). Cumulative incidence (CI) of acute GVHD was $61 \%$, chronic GVHD - 43\%. PTCY decreased CI of acute GVHD significantly ( $33 \%$ vs $75 \%, \mathrm{p}=0,05$ ).

Conclusions: Allo-HSCT is a standard of care in MPS IH. RIC and MAC regimens demonstrate equal efficiency in children with Hurler syndrome. PTCY decreases CI of acute GVHD and is a promising option that needs further investigation in this clinical setting.

Conflict of interest: nothing to disclose

\section{P441}

Characterization of Candidates for Hematopoietic Stem Cell Transplantation in a Sickle Cell Disease Cohort and Estimation of Number of Potential Donors for Candidates

Miriam Verônica Flor-Park ${ }^{1}$, Shannon Kelly ${ }^{2,3}$, Liliana Preiss ${ }^{4}$, Brian Custer ${ }^{2}$, Anna Barbara Carneiro-Proietti, Aderson Araujo ${ }^{6}$, Paula Loureiro $^{7}$, Claudia Maximo ${ }^{8}$, Daniela Werneck Rodrigues ${ }^{9}$, Rosimere Afonso ${ }^{10}$, Ester Sabino ${ }^{11,12}$, Vanderson Rocha ${ }^{13,14}$ 
${ }^{1}$ Instituto da Criança Hospital das Clínicas da Universidade de São Paulo, Sao Paulo, Brazil; ${ }^{2}$ Blood Systems Research Institute, San Francisco, CA, United States; ${ }^{3}$ University of California San Francisco Benioff Children's Hospital Oakland, Oakland, CA, United States; ${ }^{4}$ Research Triangle Institute, Rockville, MD, United States; ${ }^{5}$ Fundação Hemominas, Belo Horizonte, Brazil; ${ }^{6}$ Fundação Hemope, Recife, Brazil; ${ }^{7}$ Fundação HEMOPE, Recife, Brazil; ${ }^{8}$ Fundação HEMORIO, Rio de Janeiro, Brazil; ' ${ }^{9}$ Fundação Hemominas, Juiz de Fora, Brazil; ${ }^{10}$ Fundação Hemominas, Montes Claros, Brazil; ${ }^{11}$ Universidade de São Paulo, Instituto de Medicina Tropical, São Paulo, Brazil;

${ }^{12}$ Hospital das Clínicas da Faculdade de Medicina da Universidade de São Paulo, São Paulo, Brazil, ${ }^{13}$ Hospital das Clínicas da Faculdade de Medicina da Universidade de São Paulo, Serviço de Hematologia, Hemoterapia e Terapia Celular, São Paulo, Brazil; ${ }^{14}$ University of Oxford, Churchill Hospital Department of Haematology NHS-BT,

Oxford, United Kingdom

Background: Sickle cell disease (SCD) is a worldwide public health problem. In Brazil there are an estimated 3050,000 affected patients. Despite advances in quality of life and reduced mortality, the only curative treatment is hematopoietic stem cell transplantation (HSCT). Recently, the Brazilian Ministry of Health $(\mathrm{MoH})$ established a regulation, based on international recommendations, to require the public health system pay for HSCT for SCD patients with defined indications. However, few Brazilian centers have performed HSCT for SCD, and the number of eligible HSCT candidates according to the regulation is unknown.

Methods: The REDS-III SCD Cohort was established to investigate outcomes in a large sample of SCD patients in Brazil. Patients from six Brazilian cities (Belo Horizonte, Juiz de Fora, Montes Claros, Rio de Janeiro, Recife and Sao Paulo) were selected and recruited at routine visits. A cross sectional analysis of enrollment data was conducted to identify the number of HSCT candidates in the cohort according to Brazilian $\mathrm{MoH}$ criteria: patients with sickle cell anemia $(\mathrm{SCA}=\mathrm{SS} / \mathrm{Sb} 0 / \mathrm{SD} / \mathrm{SQuebec})<16$ years old with stroke, cerebrovascular disease, $\geq 2$ antibodies while under chronic transfusion therapy (CTT) or $\geq 1$ of the following despite treatment with hydroxyurea: $\geq 2$ vasocclusive crises (VOC) or acute chest syndrome (ACS) episodes in the last year, priapism, avascular necrosis (AVN).

Results: There were 2795 patients enrolled in the REDSIII cohort from 2013-2015. Of the 2064 SCA patients, 172 $(17.7 \%)$ of 974 children $<16$ years had at least 1 HSCT indication (121 with 1,45 with 2 and 6 with $\geq 3$ indications). The most common indication for transplant was stroke ( $\mathrm{n}=$ 97) followed by $\geq 2$ VOC $(n=39)$, cerebrovascular disease $(\mathrm{n}=33)$, priapism $(\mathrm{n}=20), \geq 2$ ACS $(\mathrm{n}=14)$, CTT with alloantibodies and AVN. Comparisons of patients with and without a HSCT indication are summarized in table 1. Of the 172 children who would be eligible for HSCT, 87 $(50.6 \%)$ had at least 1 non-SCD full sibling. If the eligibility criteria were expanded to include patients $\geq 16$ years, there would be additional 296 (27.2\% of 1090) HSCT candidates.
Conclusions: This study provides useful, real world data regarding how the recently enacted HSCT regulations would impact Brazilian SCD patients. We have estimated that $18 \%$ to $27 \%$ of SCA patients (depending on age) could benefit from HSCT. This estimation could be applied in other countries with a high prevalence of SCD.

This study provides critical data to estimate financial cost and infrastructure that would be required to implement the new regulation.

Clinical Trial Registry: it is not applicable.

Conflict of interest: All authors declare they do not have conflicts of interest related to this manuscript

\begin{tabular}{|c|c|c|c|c|c|}
\hline & \multicolumn{2}{|c|}{$\begin{array}{l}0 \text { HSCT } \\
\text { Indication }(\mathrm{n}= \\
802)\end{array}$} & \multicolumn{2}{|c|}{$\begin{array}{l}\geq 1 \text { HSCT } \\
\text { Indication }(\mathrm{n}= \\
172)\end{array}$} & \multirow[t]{2}{*}{ P-value } \\
\hline & $\begin{array}{l}\mathrm{N} \text { or } \\
\text { Mean }\end{array}$ & $\begin{array}{l}\% \text { or } \\
\text { Range }\end{array}$ & $\begin{array}{l}\mathrm{N} \text { or } \\
\text { Mean }\end{array}$ & $\begin{array}{l}\% \text { or } \\
\text { Range }\end{array}$ & \\
\hline $\begin{array}{l}\text { Abnormal Transcranial } \\
\text { Doppler }\end{array}$ & 193 & $24.1 \%$ & 70 & $40.7 \%$ & 0.0002 \\
\hline $\begin{array}{l}\text { Number VOC Hospitalizations } \\
\text { previous year }\end{array}$ & 0.3 & $0-5$ & 0.8 & $0-6$ & $<0.0001$ \\
\hline $\begin{array}{l}\text { Number ACS Hospitalizations } \\
\text { previous year }\end{array}$ & 0.2 & $0-4$ & 0.4 & $0-4$ & 0.08 \\
\hline $\begin{array}{l}\text { Ever received red blood cell } \\
\text { transfusion }\end{array}$ & 672 & $83.8 \%$ & 172 & $100 \%$ & $<0.0001$ \\
\hline Chronic transfusion therapy & 46 & $5.7 \%$ & 90 & $52.3 \%$ & $<0.001$ \\
\hline Hydroxyurea & 260 & $32.4 \%$ & 110 & $63.9 \%$ & $<0.0001$ \\
\hline Hemoglobin (g/dL) & 8.2 & $\begin{array}{l}5.2- \\
13.8\end{array}$ & 8.6 & $\begin{array}{l}5.9- \\
11.2\end{array}$ & $<0.0001$ \\
\hline Hemoglobin F & 12.7 & $0.7-47$ & 7.3 & $0.01-42$ & $<0.0001$ \\
\hline
\end{tabular}

\section{[[P441 Table] Comparison between patients]}

\section{P442}

Cord blood transplantation or matched unrelated donor transplantation for children with Hurler syndrome who lack of a HLA-matched healthy family donor?

Su Han Lum ${ }^{1}$, Giorgio Ottaviano ${ }^{2}$, Heather Church ${ }^{3}$, Karen Tylee ${ }^{3}$, Jean Mercer ${ }^{3}$, Paul Veys ${ }^{2}$, Persis Amrolia ${ }^{2}$, Simon Jones ${ }^{3}$, Robert Chiesa $^{2}$, Robert Wynn ${ }^{1}$

${ }^{1}$ Royal Manchester Children's Hospital, Manchester, United Kingdom; ${ }^{2}$ Great Ormond Street Hospital for Children NHS Foundation Trust, London, United Kingdom; ${ }^{3}$ Manchester Center for Genomic Medicine, Manchester, United Kingdom

Background: Cord blood (CB) is the preferred cell source for haematopoietic cell transplantation (HCT) children with Hurler syndrome (HS) who lack a noncarrier, HLA-matched family donor. CB was previously shown to be associated with greater donor chimerism and better $\alpha$-L-iduranidase (IDUA) enzyme level. Our recent report demonstrated that graft failure occurred exclusively in $\mathrm{CB}$ transplantation (CBT) after implementation of 
pharmacokinetic guided myeloablative-busulfan (Bu-pK). Here we compared the outcome of these children undergoing CBT or matched unrelated donor (MUD) transplantation in the modern era of HCT.

Methods: We examined the outcome of 60 children with HS received first unrelated HCT at Royal Manchester Children's hospital and Great Ormond Street Hospital from 2004 to 2016. Busulfan with pK monitoring and cyclophosphamide were used from 2004 to 2009. From 2010, the conditioning regimen was switched to busulfan and fludarabine. ATG was used with $\mathrm{CB}$ while alemtuzumab was used in MUD. Outcomes of interest included overall survival (OS), transplant-related mortality (TRM), engrafted survival (ES), donor chimerism and leucocyte IDUA. Comparison of survival outcomes between CBT and MUD was analysed using log-rank analysis.

Results: The median age at transplant was 14.8 months in the MUD $(n=22)$ and 13.3 months in the CBT $(n=38)$ $(p=0.44)$. The interval from diagnosis to HCT was significantly shorter in the CBT (median 4.1 months) compared to the MUD (5.7 months) $(p=0.04)$. The 5-year
OS was $95 \%$ in the MUD and $92 \%$ in the CBT $(p=0.60)$. The one-year TRM was $2.6 \%$ in the CBT while no TRM in the MUD. ES was $95 \%$ in the MUD and $83 \%$ in the CBT $(p=0.14)$. All the six graft failure occurred in CBT and were aplastic type. There were no differences between incidences of aGvHD, cGvHD, viral reactivations (CMV, EBV and adenovirus) between CB and MUD recipients.

Donor chimerism and IDUA level were available for 44 patients (CBT, $\mathrm{n}=22$; MUD $=18$ ) engrafted more than one year after first HCT. There was no significant difference in full donor chimerism $(p=0.47)$ between MUD $(n=15 /$ 18) and CBT $(n=20 / 22)$. Of 35 patients with donor chimerism $\geq 90 \%$, a greater proportion of CB recipients (n $=11 / 20$ ) had supraphysiological IDUA enzyme (above institutional reference range) compared to MUD recipients $(\mathrm{n}=1 / 14)(p=0.01)$.

Conclusions: Our findings reiterated that the survival rates after unrelated donor transplant are good for HS children. Once engrafted, CB provides superior IDUA level and remains an important cell source for affected children. The reasons that underpin the graft loss should be addressed
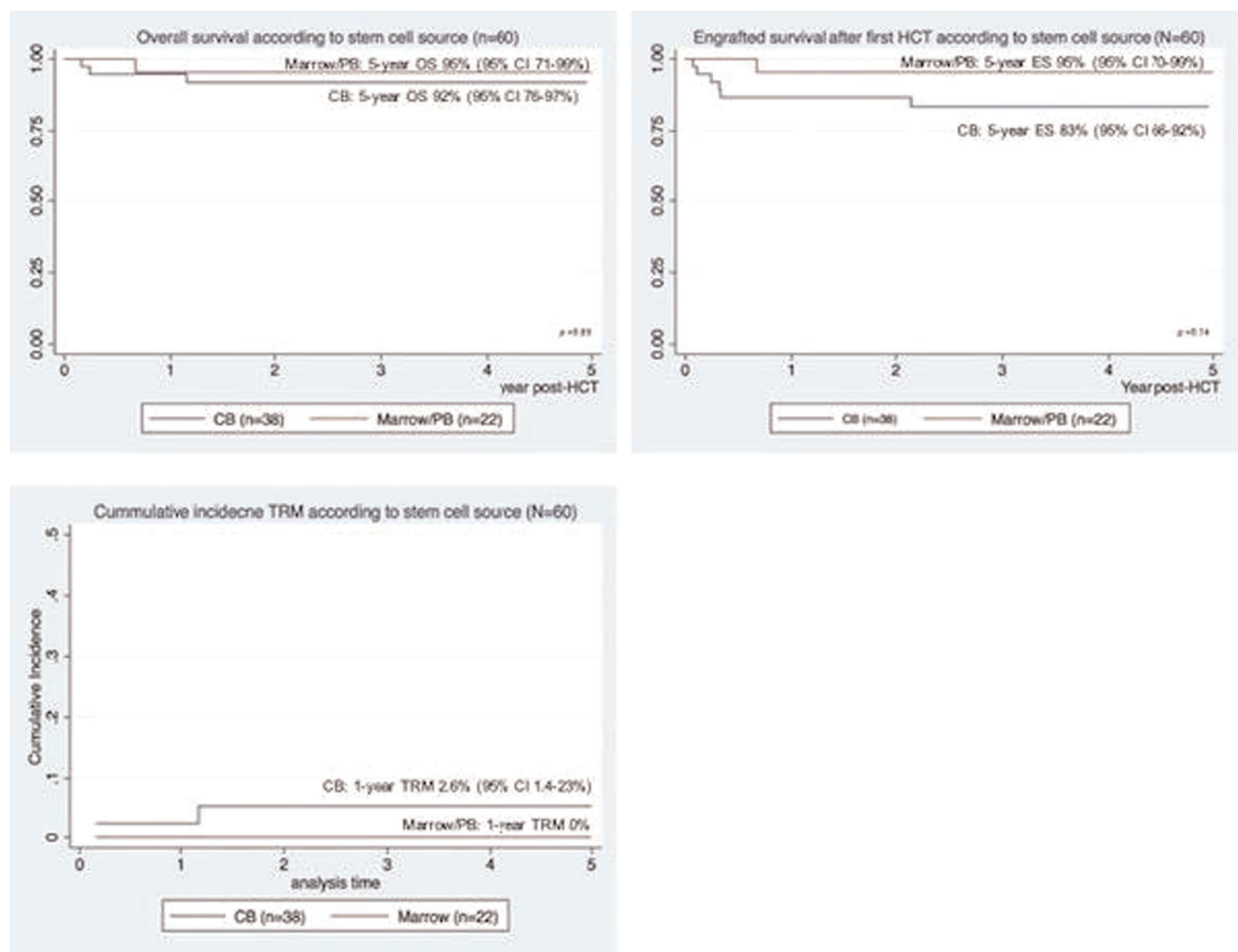

[P442 Figure] [Figure 1: Transplant outcomes according to stem cell source $(N=60)]$ 
imminently to prevent the untoward consequences of delayed engraftment in $\mathrm{CB}$ recipients.

Clinical Trial Registry: None

Conflict of interest: None

\begin{tabular}{llll}
\hline & $\begin{array}{l}\text { Marrow/PB } \\
(\mathrm{n}=18)\end{array}$ & $\begin{array}{l}\mathrm{CB}(\mathrm{n}= \\
22)\end{array}$ & $\begin{array}{l}\mathrm{p}- \\
\text { value }\end{array}$ \\
\hline $\begin{array}{l}\text { Interval between transplant and last assessment, } \\
\text { years, median (range) }\end{array}$ & $2.9(1.0-11.8)$ & $\begin{array}{l}7.8(1.07- \\
11.5)\end{array}$ & 0.007 \\
$\begin{array}{l}\text { Donor chimerism } \\
\text { Full chimerism (>=90\%), n (\%) }\end{array}$ & $20(91)$ & 0.47 \\
Mixed chimerism (20-89\%), n (\%) & $15(83)$ & $2(8)$ & \\
$\begin{array}{l}\text { IDUA level in patients with donor chimerims }>=90 \% \\
\text { Supraphysiological level (according to }\end{array}$ & $3(17)$ & $11(55)$ & 0.013 \\
institutional reference range), n $(\%)$ & $14(93)$ & $9(45)$ & \\
$\quad$ Normal level, n $(\%)$ & & & \\
\hline
\end{tabular}

[[P442 Table] Latest graft function (chimerism and enzyme level)]

\section{P443}

Early graft rejection in a child with heavily transfused Bthalassemia major resolved after T-cell depleted peripheral blood stem cell (PBSC)-boost

Claudia Bettoni da Cunha-Riehm ${ }^{1}$, Kinan Kafa ${ }^{1}$, Karl-Walter Sykora $^{1}$, Britta Maecker-Kolhoff ${ }^{\prime}$, Lubomir Arseniev ${ }^{2}$, Lilia Goudeva ${ }^{3}$, Martin Sauer ${ }^{1}$

${ }^{1}$ Medical School Hannover, Paediatric Haematology and Oncology, Hannover, Germany; ${ }^{2}$ Medical School Hannover, Cellular Therapy Centre, Hannover, Germany; ${ }^{3}$ Medical School Hannover, Institute of Transfusion Medicine, Hannover, Germany

Background: A boy with $\beta$ thalassemia major, from consanguineous parents received his first transfusion at the age of four in Turkey. He received blood transfusions almost every second day and he had innumerous episodes of hemoglobinuria. He was treated with corticosteroids and immunoglobulin but continued to have hemolysis. Six months after the first transfusion, he presented at our hospital with severe anemia $(3 \mathrm{~g} / \mathrm{dl} \mathrm{Hb})$ and congestive heart failure. We identified $\mathrm{Jk}(\mathrm{b})$ and $\mathrm{Kp}(\mathrm{a})$ antibodies, so that phenotype-matched, compatible RBC units were used further on, but hemolysis persisted. A diagnosis of hyperhemolysis syndrome was made. After two methylprednisolone-pulses $\left(20 \mathrm{mg} / \mathrm{m}^{2} /\right.$ day-4 days, every 3 weeks) he stabilized and with transfusions every 3 weeks. Six months later he again presented with hemolysis; however, no irregular antibodies were detected. Hemolysis resolved with two pulses of methylprednisolone, immunoglobulin and mycophenolate mofetil (MMF). MMF was given until conditioning for HSCT. Deferasirox was used as chelation therapy. In September 2016, at the age of 6 years he received a transplant from his 10/10 HLA identical mother. Blood type: Donor 0, CcDee, Jk(b) and $\mathrm{Kp}(\mathrm{a})$ negative. Recipient: A, CcDee. The patient had no HLAantibodies. Graft: bone marrow: 4,7x $10^{8} \mathrm{MNC} / \mathrm{kg}$ and $2,8 \mathrm{x}$ $10^{6} \mathrm{CD} 34+/ \mathrm{kg}$. Conditioning: fludarabine/treosulfan/ thiothepa/ATG; GvHD prophylaxis: CSA/MTX. Early posttransplantat course was uneventful. Engraftment of platelets and leucocytes on day +26 . Initially, mixed chimerism (99\% neutrophils, $80 \%$ lymphocytes from donor) was observed. CSA was rapidly reduced and withdrawn on day +90 . No GvHD occurred. He had full donor erythropoiesis (Blood type 0 ) on day +121 , but on day +142 his $\mathrm{Hb}$ fell to $5 \mathrm{~g} / \mathrm{dl}$, with leucocytes and platelets still in the normal range. Blood type showed a mixed donor and recipient population. No new antibodies against red blood cells were identified .Molecular genetic analysis showed B-thalassemia major status, confirming complete erythropoietic loss of graft.

Methods: Three weeks later he received a PBSC boost with $11 \times 10^{6} / \mathrm{kg}$ purified CD34+ and $1 \times 10^{6} / \mathrm{kg} \mathrm{CD} 3+$ cells. No further GvHD prophylaxis was used.

Results: The patient's hematopoiesis reverted to a complete donor 's blood type, and molecular genetics showed B-thalassemia minor status again. No GVHD occurred. Nine months later, he is transfusion-free, with full donor erythropoiesis, stable mixed myeloid chimerism (60\% donor) and $100 \%$ Lansky score.

Conclusions: The use of of CD34+ stem cell boost with a low-dose T-cell addback was effective in the treatment of early complete graft rejection without GVHD. This approach shows promising potential for treatment of early transplant rejection in hemoglobinopathies avoiding the controversial use of donor lymphocyte infusions in nonmalignant diseases.

Conflict of interest: None declared

\section{P444}

Abstract previously published

\section{P445}

Full-dose busulfan-based conditioning regimen for pediatric non-malignant disease

Hiromasa Yabe ${ }^{1}$, Eri Imai ${ }^{2}$, Akiko Sugimoto ${ }^{2}$, Keisuke Otsubo ${ }^{2}$, Tsuyoshi Morimoto ${ }^{2}$, Hiroyuki Mochiduki', Takashi Koike ${ }^{2}$, Shunichi Kato ${ }^{1}$, Miharu Yabe ${ }^{1}$

${ }^{1}$ Tokai University School of Medicine, Department of Cell Transplantation and Regenerative Medicine, Isehara, Japan; ${ }^{2}$ Tokai 
University School of Medicine, Department of Pediatrics, Isehara, Japan

Background: Graft rejection is a major obstacle after hematopoietic stem cell transplantation (HSCT) for the treatment of inherited metabolic disorders or primary immunodeficiencies such as Wiskott-Aldrich syndrome. We retrospectively investigated the feasibility of a full-dose intravenous busulfan (ivBu)-based conditioning regimen for pediatric non-malignant diseases.

Methods: Sixteen patients including 14 males and 2 females with inherited metabolic disorders or primary immunodeficiencies who underwent HSCT with a fulldose ivBu-based conditioning regimen between 2006 and 2017 were enrolled. The median (range) age at HSCT was 2 years (5 months to 7 years), and the underlying diseases were mucopolysaccharidosis $(n=11)$, Wiskott-Aldrich syndrome $(n=2)$, I-cell disease $(n=1)$, metachromatic leukodystrophy $(n=1)$, and reticular dysgenesis $(n=1)$. The conditioning regimen ranged from $0.8-1.2 \mathrm{mg} / \mathrm{kg} / \mathrm{dose}$ (according to body weight) of ivBu 4 times per day for 4 consecutive days followed by both cyclophosphamide (CY) $50 \mathrm{mg} / \mathrm{kg} /$ day and anti-thymocyte globulin $1.25 \mathrm{mg} / \mathrm{kg}$ for 4 consecutive days. Most patients received ivBu and CY/ ATG in that order, but 4 patients received them in reverse order (i.e., CY and ivBu/ATG). Graft-versus-host disease prophylaxis was selected according to donor status.

Results: Two infantile patients developed hepatic sinusoidal obstruction syndrome, which responded well to treatment with defibrotide or recombinant thrombomodulin. All patients achieved both neutrophil count $>0.5 \times 10^{9} / \mathrm{L}$ and erythrocyte $>20 \%$ at a median of 16 and 19 days, respectively. Donor elements 4 weeks after HSCT measured by the short-tandem repeat method using whole bone marrow specimens reached $100 \%$ in 8 patients, $95-99 \%$ in 5, 90-94\% in 1, and 85-89\% in 2. Eight weeks after HSCT, 4 other patients achieved $100 \%$ donor type. Chimeric analyses using peripheral blood specimens were also performed by the short-tandem repeat method as whole blood or after separating granulocytes, T-cells, and others. At 8 weeks, among 11 patients who showed $100 \%$ donor type in whole peripheral blood, 3 patients exhibited T-cell mixed chimerism with $12-27 \%$ recipient type, which eventually decreased to $0-4 \%$. Three patients developed recipient-derived chimerism $>20 \%$ in the granulocyte fraction at 8 weeks; two of them improved after administration of granulocyte colony-stimulating factor or donor lymphocyte infusion, while 1 patient was lost in follow-up. Late graft rejection was observed in 2 patients with I-cell disease or mucopolysaccharidosis. The patient with I-cell disease deteriorated rapidly after graft rejection and died of disease 20 months after HSCT. The patient with mucopolysaccharidosis lost bone marrow graft owing to post-transplant-emerging, donor-specific anti-HLA antibodies and underwent successful re-HSCT with unrelated cord blood. Fifteen patients were alive and well 9 months to 11 years after HSCT.

Conclusions: A full-dose ivBu-based conditioning regimen provides excellent short-term toxicity and myeloid engraftment in inherited metabolic disorders or primary immunodeficiencies. However, serial chimerism analysis to confirm lineage-specific engraftment is important to detect mixed chimerism and administer early intervention such as donor lymphocyte infusion.

Conflict of interest: The authors declare no conflicts of interest.

\section{P446}

\section{Haemopoietic cell transplantation is a safe, long-term disease-modifying therapy for transplant-permissive inherited metabolic disease in the modern era}

Su Han Lum ${ }^{1}$, Andrew Will, Denise Bonney ${ }^{1}$, Prashant Hiwarkar ${ }^{1}$, Kay Poulton ${ }^{2}$, Heather Church ${ }^{3}$, James Edmond Wraith ${ }^{3}$, Simon A Jones $^{3}$, Robert Wynn ${ }^{1}$

${ }^{1}$ Royal Manchester Children's Hospital, Manchester, United Kingdom; ${ }^{2}$ Manchester Royal Infirmary, Manchester, United Kingdom;

${ }^{3}$ Manchester Center for Genomic Medicine, Manchester, United Kingdom

Background: Haemopoietic cell transplantation (HCT) confers a long-term disease-modifying therapy for transplant-permissive inherited metabolic diseases (IMD). Donor-derived leucocyte engraftment serves as a stable source of the deficient enzyme for in-situ cross-correction of neighbouring tissues, including neurons as well as an immunomodulatory effect where there is a significant inflammatory component to the disease.

Methods: We examined the outcome of children with IMD received a first HCT at Royal Manchester Children's hospital from 1985 to 2016. The outcomes of interest included overall survival (OS), transplant-related mortality (TRM), and engrafted survival (ES). The predictors of OS, ES and TRM were analysed using Cox proportional-hazard models and Fine-gray competing risk models.

Results: 137 children with IMD were included in this analysis (historical cohort, 1985 and 2006, $\mathrm{n}=65$; current cohort, 2007 and 2016, $\mathrm{n}=72$ ). Primary diagnoses were mucopolysaccharidoses (MPS, $\mathrm{n}=112,81 \%$ ), X-linked adrenoleukodystrophy (XALD, $\mathrm{n}=8,6 \%$ ), metachromatic leukodystrophy ( $\mathrm{n}=5,4 \%)$, alpha-mannosidosis $(\mathrm{n}=4$, $3 \%)$, Wolman disease $(\mathrm{n}=3,2 \%)$ and others $(\mathrm{n}=5,4 \%$; Aspartylglucosaminuria, $\mathrm{n}=1$; Fucosidosis, $\mathrm{n}=1$; Glycogen storage disease $I B, n=1$; Niemann-Pick $C 2, n=1$; 
Tay-Sachs disease, $\mathrm{n}=1$ ). The 5-year OS improved from $65 \%(95 \%$ CI, 53-76\%) in the historical cohort to $91 \%$ (95\%CI, 81-96\%) in the current cohort $(p<0.001)$. The oneyear TRM reduced from $23 \%$ (95\% CI, $13-40 \%)$ in the historical cohort to $7 \%$ (95\% CI, 3-18\%) in the current cohort $(p=0.005)$. On Cox model, year of transplant $(\mathrm{p}=0.01)$, age at transplant $(\mathrm{p}=0.02)$, male $(\mathrm{p}=0.001)$ and grade III-IV GVHD $(\mathrm{p}=0.003)$ were predictors for overall survival. In current cohort, none of the pre-transplant factors, transplant factors and transplant related complications were associated with OS. The 5-year OS in children transplanted in the current cohort was $100 \%$ for XALD (n $=5), 98 \%(95 \%$ CI, $83-98 \%)$ for MPS $(\mathrm{n}=55)$ and $75 \%$ (95\% CI, 42-92\%) for other IMD. The engrafted survival (ES) has doubled from $41 \%$ (95\% CI, 29-53\%) in the historical cohort to $86 \%$ (95\% CI, 75-92\%) in the current cohort $(p<0.001)$. The proportion of patients with graft failure has decreased from $37 \%$ in the historical cohort to $8 \%$ in the current cohort $(p<0.001)$. Thirteen out of 18 patients $(71 \%)$ in the historical cohort and all four patients (100\%) in the current cohort receiving a second transplant are alive-and-engrafted. In the historical cohort, the incidence of GvHD was $4 \%$ for grade III-IV aGvHD and $4 \%$ for cGvHD. In current cohort, the incidence of GvHD was $4 \%$ for grade III-IV aGvHD and $1 \%$ for cGvHD. Of the 82 survivors followed-up in Manchester, $80 \%$ have full chimerism and $20 \%$ have mixed chimerism.

Conclusions: Our findings confirm that HCT performed in an experienced metabolic transplant centre is increasingly safe and provides life-long therapy for children with IMD. These results will lead to a reappraisal of the role of transplant in such centres, including in diseases where it is currently not practised because of risk, where that perception of risk is based on survival data from a previous era. This is likely to include those diseases where ERT is currently standard treatment, such as in attenuated MPSI and MPSII.

Conflict of interest: None of the authors has anything to declare.

\section{P447}

\section{Hematopoietic Stem Cell Transplant for Sickle Cell Disease with End Stage Renal Disease: A Case Report}

\section{Gaurav Kharya, Divya Bansal, Silky Jain}

Artemis Hospital, Pediatric Hematology Oncology Immunology and Bone Marrow Transplant, Gurugram, India

Background: Sickle cell disease (SCD) remains associated with high risks of morbidity and early death. Even best of supportive care fails to improve quality of life. End stage renal disease (ESRD) is one rare complication of SCD warranting hematopoietic stem cell transplant (HSCT) and kidney transplant one after other. HSCT in a patient with ESRD is a challenging task. We report a case of sickle cell nephropathy (SCN) with ESRD transplanted using reduced intensity conditioning (RIC) along with $\mathrm{T}$ cell depletion (TCR alpha/beta, CD19 depletion).

Methods: 18 year old boy R/O Nigeria known case of SCD diagnosed at 1 year of age. He had multiple episodes of veno-occlusive crisis which was managed symptomatically. He was started on hydroxyurea at 2 yrs of age but did not benefit him much. He presented to us with worsening renal function, oligourea and odema. On evaluation he was diagnosed as ESRD. Renal biopsy was suggestive of SCN. $\mathrm{He}$ was put on renal replacement therapy (RRT). His siblings were screened for a potential donor and younger sibling was found to be a 10/10 HLA match. As conventional BuCyATG was not the right choice considering his ESRD, he was conditioned with Fludarabine@20mg/m2 (day-6 till day-2; dose adjusted based on $\mathrm{CrCl}$ ); Busulfan@3.2mg/kg/day (day-8 till day-5) and ATG (PfizerATGAM)@30mg/kg/day (day-3 till day-1). He subsequently received GCSF primed, peripherally harvested $\mathrm{T}$ cell depleted graft from his brother (absolute CD34 cells 9.7 million cells $/ \mathrm{kg}, 4 \mathrm{log}$ reduction of alpha beta cells and $3.3 \mathrm{log}$ reduction of CD19 cells). No additional immune suppression was given. He continued to be on RRT during the course of HSCT.

Results: Neutrophil and platelet engrafted on day +8 . He was successfully discharged on day +14 . Post BMT course was complicated with febrile neutropenia and mild CMV reactivation which was managed with IV antibiotics and ganciclovir. His day +14 donor chimerism was $92 \%$. He continues to be on RRT. Plan is to consider him for renal transplant in due course preferably from the same donor.

Conclusions: HSCT followed by renal transplant is the only possible long term curative option for SCN with ESRD. RIC with FluBuATG and T cell depletion is the safest way to do HSCT in such cases.

Clinical Trial Registry: None.

Conflict of interest: None.

\section{P448}

Abstract previously published

\section{P449}

Abstract previously published 


\section{P450}

\section{Myeloablative versus Reduced Intensity Conditioning For Matched Related Donor Transplant For Sickle Cell Disease}

Gaurav Kharya ${ }^{1}$, Edward Kasirye ${ }^{2}$, Francis Ssali ${ }^{3}$, Musa Waiswa ${ }^{4}$, Henry Ddungu ${ }^{5}$, Deogratius Munube ${ }^{6}$, Yemi Onijala ${ }^{7}$, Sola Festus ${ }^{8}$

${ }^{1}$ Artemis Hospital, Pediatric Hematology Oncology Immunology and Bone Marrow Transplant, Gurugram, India; ${ }^{2}$ Childrens Clinic,

Kampala, Uganda; ${ }^{3}$ Kampala Medical Chambers, Kampala, Uganda; ${ }^{4}$ Case Clinic, Kampala, Uganda; ${ }^{5}$ Mulago Teaching Hospital, Department of Haematology, Kampala, Uganda; ${ }^{6}$ Mulago Teaching Hospital, Department of Pediatrics, Kampala, Uganda; ${ }^{7}$ Sickle Cell Foundation, Lagos, Nigeria; ${ }^{8}$ University Teaching Hospital, Lagos, Nigeria

Background: Sickle cell disease (SCD) remains associated with high risks of morbidity and early death. Hematopoietic stem cell transplant (HSCT) is the only curative option. HLA matched related donors (MRD) are considered to be better donors. Traditionally myeloablative (MAB) conditioning regimen had been used for MRD HSCT and reduced intensity conditioning (RIC) is used less often. We present comparative outcomes of MAB vs RIC for MRD HSCT for SCD.

Methods: 25 consecutive patients underwent HSCT for SCD from MRD between Jan 2015 till date. Median age of HSCT was 7 yrs (range 1 to 31). Five patients more than 14 yrs were considered as adults and received RIC with Bu@3.2 mg/kg/day x 4 days, Cy@60mg/kg/day x 2 days and hATG (ATGAM@30mg/kg/day x 3 days). All received GCSF primed peripherally harvested stem cells with a median CD34 dose of $5.18 \times 10^{6} / \mathrm{kg}$ (range 2.1 to 8.5 ). Twenty patients < 14 yrs received MAB Bu@3.2 mg/kg/ day x 4 days, Cy@50mg/kg/day x 4 days and hATG (ATGAM@30mg/kg/day x 3 days). Two received GCSF primed peripherally harvested stem cells whereas seventeen received bone marrow and one patient received cord blood product with a median CD34 dose of $5 \times 10^{6} / \mathrm{kg}$ (range 0.92 to 10.09 ) for $\mathrm{PB} / \mathrm{BM}$ and $\mathrm{MNC}$ of $0.5 \times 10^{5} / \mathrm{kg}$ for cord blood unit. Graft versus host disease $(\mathrm{GVH})$ prophylaxis for BM/PBSC patients was cyclosporine@3mg/ $\mathrm{kg} /$ day in 2 divided doses starting D-4 and methotrexate@10/m2 on D +1 followed by $7 \mathrm{mg} / \mathrm{m} 2$ on day 3,6 and 11 post BMT and cyclosporine@3mg/kg/day + methylprednisolone for the CBT.

Results: 25 patients are alive with a median follow up of 531 days (range 22 till 1338) with overall survival and disease free survival of $100 \%$. 22/25 (88\%) have donor chimerism $>95 \%$ whereas $3 / 25(12 \%)$ have mixed stable chimerism at last follow up. 2/5 (40\%) experienced Grade I/ II gvh (skin/gut) in RIC group and 2/19 (10\%) in MAB group which was successfully managed with either topical steroids or short course of parenteral steroids. 1/25 (4\%) experienced chronic musculoskeletal GVH. CMV reactivation was seen in 3/5(60\%) patients in RIC and 3/20 (15\%) patients in MAB group.

Conclusions: RIC with reduced dose of cyclophosphamide can be considered for all the SCD patients undergoing MRD HSCT. This helps in preventing exposure to higher doses of cyclophosphamide in turn decreasing immediate and long term complications. Prospective studies with larger cohort is required to prove this.

Clinical Trial Registry: None.

Conflict of interest: None.

\section{P451}

Non-myeloablative Transplant in Severe Sickle Cell Disease is Safe \& Effective. Single Center Experience from Saudi Arabia

Mohsen Alzahrani, Moussab Damlaj, Samer Ghazi, Hind Salama, Giamal Gmati, Osama Ali, Khadega Ahmed, Ayman Hejazi, Ahmed Alaskar

King Abdulaziz Medical City, Oncology, Riyadh, Saudi Arabia

Background: Patients with severe Sickle cell disease (SCD) phenotype manifested with stroke or other complications may benefit from allogeneic hematopoietic stem cell transplant (SCT). Recent reports in pediatric patients using myeloablative regimen showed excellent outcomes but similar progress in adults has been hampered by higher rates of transplant related mortality (TRM) and infertility.

Methods: We conducted a retrospective study of adult patients at our institution with SCD whom underwent a matched related SCT during the period of 2014-2017. All patients received hydroxyurea (HU) at maximally tolerated doses with hyper-transfusion 2-3 months prior to SCT. The preperative regimen was NMA consisting of alemtuzumab 1 $\mathrm{mg} / \mathrm{kg}$ divided over 5 days (day -7 to -3 ) followed by TBI 300 cGy on day -1 . Stem cells were collected from PBSC apheresis after 5 days of GCSF with target CD34 cells of $10 \times 10^{6} / \mathrm{kg}$. GVHD prophylaxis with sirolimus from day -2 and continued for one year post SCT with dose modification based on chimerism. EFS was defined from time to SCT to graft failure, development of GVHD or death from any cause. EFS and OS via time to end point analysis was computed using the Kaplan-Meir test.

Results: A total of 24 adults patients enrolled. Median age was $26(14$ - 39) years. Indications for transplant were recurrent/refractory VOC $61 \%$, stroke $38 \%$, recurrent AVN $38 \%$, recurrent ACS $22 \%$. All donors were related siblings and fully matched with median age of 25 years. A total of $13(54 \%)$ of donors were SC carriers with average $\mathrm{Hgb} \mathrm{S}$ $33.9 \%$. Stem cell mobilization was with $10 \mathrm{ug} / \mathrm{kg}$ GCSF in all donors and the average stem cell collected $10.7 \times 10^{6} / \mathrm{kg}$. 


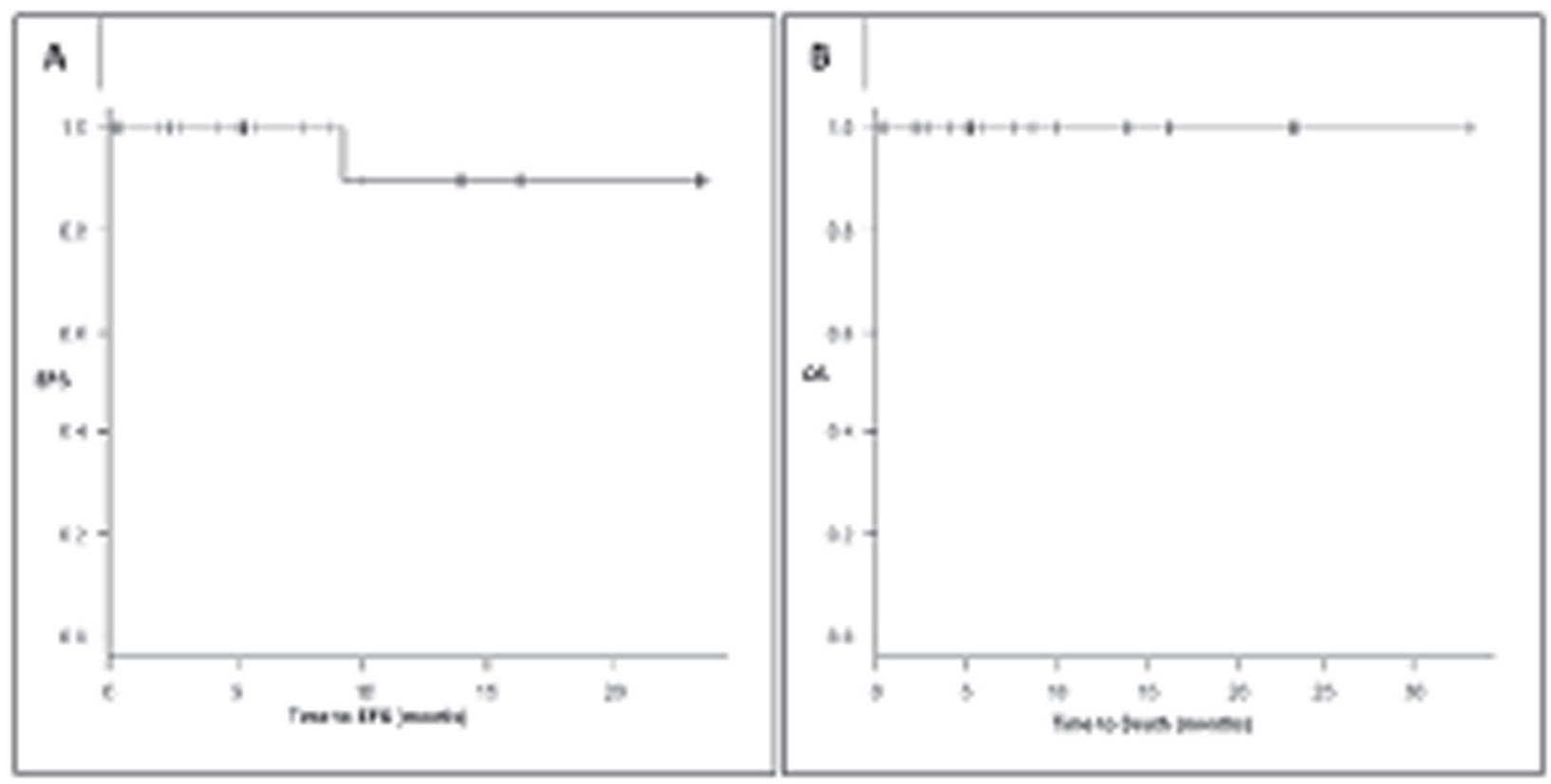

[P451 Figure] [Figure 1. Event free survival (A) and overall survival (B) following HSCT in sickle cell disease]

No significant complications resulted from GCSF use in donors with SC trait. 23 patients were conditioned with alemtuzumab / TBI regimen and GVHD prophylaxis with sirolmus. One patient who failed prior transplant received Flu-Bu and ATG with GVHD prophylaxis as CSA + MTX. Median duration of ANC and platelet engraftment was 20 and 13 days respectively. Average $\mathrm{Hb}$ pre- and post-SCT was $81.5 \mathrm{~g} / \mathrm{L}$ and $138 \mathrm{~g} / \mathrm{L}$ respectively. Average $\mathrm{HbS}$ preand post-SCT was $83 \%$ and $10.7 \%$ respectively. None of the patients developed acute or chronic GVHD. No SCD crises developed post SCT. One patient developed aplastic anemia while retaining $71 \%$ donor cell chimerism and underwent a second successful SCT from the same donor with Flu-Cyclo + ATG. All patients have stable mixed chimerism resulting in full donor myeloid engraftment and normalization of blood count. With a median follow up of 190 days (5-970), all patients are alive and free of SC crises. At last follow up, OS and EFS were $100 \%$ and $88.9 \%$, respectively as shown in Figure 1.

Conclusions: Herein, we report that SCT in adults with severe SCD using NMA regimen is safe, feasible and results in amelioration of disease severity. These excellent results are encouraging given the high prevalence and debilitating nature of SCD in our region.

Clinical Trial Registry: Not applicable

Conflict of interest: No conflict of interest

\section{P452}

Setting-up a transplant service for thalassemia in a middleincome country
Khaled M. Ghanem ${ }^{1}$, Nariman Hariss ${ }^{1}$, Suzanne Koussa ${ }^{2}$, Samar Muwakkit $^{1}$, Raya Saab ${ }^{1}$, Nidale Tarek ${ }^{1}$, Dima Hamideh ${ }^{1}$, Hassan ElSolh $^{1}$, Adlette Inati ${ }^{3}$, Jean El Cheikh ${ }^{1}$, Ali Taher ${ }^{1}$, Ali Bazarbachi', Miguel R. Abboud ${ }^{1}$

${ }^{1}$ American University of Beirut, Beirut, Lebanon; ${ }^{2}$ Chronic Care Center, Beirut, Lebanon; ${ }^{3}$ Lebanese American University, Beirut, Lebanon

Background: Allogeneic hematopoietic stem cell transplantation (allo-HSCT) is a curative therapy for transfusiondependent thalassemia. Multiple barriers prevent the implementation of allo- HSCT for thalassemia patients in low- and middle-income countries, including status of patients pre-transplant, financial barriers, and need for extended follow up post-transplant.

Methods: To overcome the major barriers in this setting in Lebanon, a collaboration was developed between an academic transplant service and a philanthropic center. Thalassemia care, including chelation and monitoring for complications, has been provided for many years by the Chronic Care Center (CCC). Financial support was provided by government insurance, the CCC, the Al Walid Ben Talal foundation and the GNK foundation. Transplant and post-transplant care is provided by the pediatric BMT program at the American University of Beirut. In this abstract, we retrospectively review the outcome of alloHSCT of all consecutive transfusion-dependent thalassemia patients who underwent transplant between 2012 and 2017 with the support of this partnership.

Results: Twelve patients ( 8 males and 4 females) with a median age of 10 (range: 2 - 24) years underwent allo- 
HSCT in the previously-mentioned period. All donors were matched-related. The median serum ferrtin at the time of transplant was 1842 (range: 718-3700) mg/dl. The median liver and cardiac iron contents by MRI were $4.7 \mathrm{mg} / \mathrm{g}$ (range: 1-9.5) and 0.8 (range: $0.3-1.9) \mathrm{mg} / \mathrm{g}$, respectively. Conditioning regimen consisted of busulfan (fixed weightdependent dose), cyclophosphamide (50 mg/ $\mathrm{kg} /$ day $\mathrm{X}$ 4 days) and ATG (Genzyme, rabbit, $2.5 \mathrm{mg} / \mathrm{kg} /$ day $\mathrm{X}$ 3 days). GvHD prophylaxis consisted of cyclosporin and methotrexate. Stem cell source was BM for 10 patients and G- CSF mobilized PBSC for two patients. The median CD34 cell dose was 6.3 (range: 5.1 - 7.8) X 106 per kg. All patients achieved sustained neutrophil and platelet engraftment at a median of 20 (range: 16-29) and 20.2 (range: 1528 ) days. Eight patients had complete chimerism ( $>95 \%)$ while four patients had partial chimerism (range: 52-89\%), however, all patients became transfusion-independent after complete recovery. Acute grade II skin GvHD occurred in one patient. None of the patients developed chronic GvHD. One patient developed severe VOD that required intensivecare admission but recovered fully. No other major toxicities were observed. After a median follow-up of 29 (range: 1-64) months, the 2-year GvHD-free survival rate was $100 \%$.

Conclusions: Barriers to allo-BMT for thalassemia could be overcome by a partnership between an academic transplant service, government insurance and communitybased philanthropic institutions in middle-income countries. We plan to introduce busalfan pharmacokinetics in the near future.

Conflict of interest: No relevant conflict of interest

\section{P453}

\section{Twenty-six Years OF Experience on Allogeneic HSCT in Thalassemia Major Patients: A Long- term Survey and Co- transplantation of Mesenchymal Stem Cells (MSCs)}

\section{Ardeshir Ghavamzadeh, Tahereh Rostami, Mohsen Nikbakht, Amir Kasaeian, Azadeh Kiumarsi \\ Tehran University of Medical Sciences,Hematology, Oncology and Stem Cell Transplant Research Center, Tehran, Iran, Islamic Republic of}

Background: Allogeneic hematopoietic stem cell transplantation (HSCT) as the only available curative option in thalassemia major (TM) patients is currently being widely used worldwide with a high probability of event-free survival.

Recently, it has been shown that MSCs can transdifferentiate into hepatocyte-like cells in vitro with the ability to perform normal metabolic functions of liver cells. However, the in vivo ability of hepatocytes differentiated from donor's MSCs to replace the damaged hepatocytes in TM patients has not yet been thoroughly demonstrated.

Methods: We extracted data from Hematology-Oncology and Stem Cell Transplantation Research Center (HORCSCT) registry database to illustrate the characteristics of TM patients at their long-term follow-ups. Between February 1991 and December 2015, a total of 717 TM patients underwent HSCT following myeloablative conditioning regimen consecutively. Co-transplantation of MSCs was conducted in 127 patients in a prospective randomized intervention study to assay liver fibrosis improvement.

Results: Peripheral blood (PB) was used as the most common source of allogeneic hematopoietic stem cells in patients (63.6\%). Although rejection rate and chronic GvHD were significantly higher in patients whose graft source was bone marrow (BM), no significant difference in 5- year OS was found $(74.9 \% \pm 2.1$ vs $78.5 \% \pm 2.7)$ [p:0.22] compared to PB grafts [P: 0.00].

There was no significant difference in OS among the following age groups: $2-5$ years old $(82.5 \pm 2.9 \%)$, 6-10 years old $(78.9 \pm 2.7 \%)$ and $11-15$ years old $(74.3 \pm 3.8 \%)$ [p>0.05], but OS was significantly lower in patients older than 15 years $(64.6 \pm 4.3 \%)$ [p $<0.001]$.

In a randomized trial, 85 patients under the age of 17 classified as LRC III who received co-transplantation of MSCs were compared with 101 non-MSCs patients. There was no difference in rejection rate between the two groups (18.8\% vs 21\%) [p: 0.74]. Interestingly, the incidence of acute GvHD was higher in MSCs compared to non-MSCs patients (47\% vs 30\%) [p:0.02]. The incidence of chronic GvHD was not significantly different between the two groups (33\% in MSCs vs 28\% in non-MSCs) [p: 0.53].

Fibroscan and T2* MRI were performed in 13 MSCs and 11 non-MSCs patients before transplantation and one year after HSCT.T2* MRI mean score increased significantly within both groups [p: 0.00] without significant difference [p: 0.7]. The Fibroscan score also increased one year after HSCT, but these changes were not significantly different between MSCs and non-MSCs patients [P: 0.95].

Conclusions: HSCT offers promising outcomes in TM patients all over the world, and the results

are superior if perform at an earlier age, so we strongly suggest that the age would be incorporated into risk classifications as an important influencing factor. Moreover, we observed no considerable improvement of liver fibrosis evaluated by Fibroscan following the co-transplantation of MSCs. However, larger studies are required for clearly defining the effectiveness of MSCs in the improvement of hepatic fibrosis.

Conflict of interest: There are no conflicts of interest to report.

Hematopoietic stem cells 
P454

A comparison of clinical outcomes in umbilial cord blood stem cell transplantation between humam leucocyte antigen matched and mismatched in the treatment of hematological diseases

Zong Zhai, Sh H

Children's Hospital of Soochow University, Suzhou, China

Background: To compare the clinical outcomes between human leucocyte antigen matched(HLA-m) and HLA mismatched(HLA-mis) umbilial cord blood stem cell transplantation(UCBT) in the treatment of children with hematological diseases.

Methods: 6 patients in low resolution received HLA-mis of 4/6, 35 patients received HLA-mis of 5/6 and 26 patients received HLA-m for UCBT. 22 patients in high resolution received HLA-mis less than 8/10, 25 patients received HLA-mis of $8-9 / 10$ and 9 patients received HLA-m for UCBT in Children's Hospital of Soochow University from April 2011 to September 2017.

Results: In low resolution,the engraftment of neutrophil were $6(100 \%) 31(88.6 \%) 25(96.2 \%)(\mathrm{P}=0.619)$, as platelet were $6(100 \%) \quad 29(82.9 \%) \quad 22(84.6 \%)$, respectively $(\mathrm{P}=$ $0.780)$. In high resolution, the implantion of neutrophil were $19(86.4 \%) 24(96 \%) 9(100 \%)(\mathrm{P}=0.37)$, as platelet were $16(72.3 \%) \quad 23(92.0 \%) \quad 9(100 \%)$,respectively $(\mathrm{P}=$ $0.082)$. Acute graft versus host disease(aGVHD) rate in low resolution were $3(50 \%) 19(54.3 \%) 15(57.7 \%)(\mathrm{P}=$ 0.93 ) and those in high resolution were 14(63.6\%) 16(64\%) $4(44.4 \%)(\mathrm{P}=0.62)$ (Table 1 and 2$) .5$-year overall survival (OS) rate were $83.3 \% 77.1 \% 76.9 \%$ in low resolution respectively $(\mathrm{P}=0.94)$ while $54.5 \% \quad 88.0 \% \quad 100 \%$ in high resolution $(\mathrm{P}=0.007)$ (Figure 1).

Conclusions: For children with hematological diseases in UCBT, there's no different of HLA-m and HLA-mis ((4-5)/ 6) in engraftment and aGVHD. By the decreased mismatched loci in high resolution, transplant-related mortality (TRM) increased. For children lacking of matched donor, HLA-mis((4-5)/6) cord blood stem cell was an alternative stem cell source and the high resolution would help us to choose a better donor.

Conflict of interest: The authors declared that they have no conflicts of interest to this work.We declare that we do not have any commercial or associative interest that represents a conflict of interest in connection with the work submitted.
P455

Allogeneic Heamatopoietic Stem Cell Transplantation in Elderly Patients; Single Centre Experience

Hale Bülbül, Fatoş Dilan Atilla, Nur Akad Soyer, Murat Tombuloğlu, Mahmut Töbü, Fahri Şahin, Güray Saydam, Filiz Vural

Ege University Hospital, Department of Internal Medicine Division of Hematology, Izmir, Turkey

Background: Allogeneic hematopoietic stem cell transplant (HSCT) survivors are at risk of developing therapyrelated complications that may be mortal. Data of outcomes with elderly patients are very limited. In recent years, the development of reduced-intensity conditioning (RIC) and nonmyeloablative (NMA) regimens have extended the performing of HSCT in elderly population. The goal of this retrospective study was to identify factors impacting the safety and efficacy of allogeneic HSCT in the elderly patients.

Methods: This single-center, retrospective study examine outcomes of allogeneic HSCT in elderly patients ( $\geq 60$ years old). Between January 1st, 2011 and December 1st, 2016, 30 patients met the inclusion criteria and were included in the study. Diagnosis, age, regimens, toxicities, overall survival (OS) and progression free survival (PFS) were analyzed according to age at the time of transplantation.

Results: Among 30 patients $66 \%$ were male and $34 \%$ were female. Median age at transplantation time was 62 years (range 60 to 67). Approximately one-third (36\%) of our cohort was $\geq 65$ years old. According to diagnosis; $50 \%$ had AML, 20\% had MDS, 14\% had MM, 10\% had KLL, $3 \%$ had KML and 3\% had PMF. The incidence of febrile neutropenia was $92 \%$ with $2 \%$ admission to the intensive care unit. Among patients; $\% 80$ had HLA matched sibling, $10 \%$ had HLA matched unrelated and 10\% had haploidentical donors. RIC/NMA regimens were administered to $80 \%$ $(\mathrm{n}: 24)$ for conditioning regimen before transplantation. The median follow-up was 27 months (range, 1 to 87). Disease relapse observed $10 \%$ of patients (OS; 7,58 \pm 1.61 months) and grade 3-4 acute and chronic GvHD was observed at $23.3 \%$ of patients (OS;3,25 \pm 1.33 months) at 1 year post HSCT. At 1 year post HSCT,OS,PFS and NRM were $56.7 \%, 60 \%, 28 \%$ respectively .At 2 year post HSCT OS was $40 \%$. HLA matched siblings donor associated with best outcomes (median OS; 20,9 $\pm 6,7$ months and at 1 year post HSCT OS; 66.7\%) whereas median OS for HLA matched unrelated and haploidentical were 12,68 $\pm 9,3$ months and 2,23 $\pm 1,6$ months respectively. Haploidentical donors and grade 3-4 GvHD were associated with higher NRM $(P<0.05, \mathrm{p}=0,02$ and $P<0.05, \mathrm{p}=0,04$ respectively).Also ablative conditioning regimens were 
associated with inferior outcomes.(RIC/NMA regimens OS;

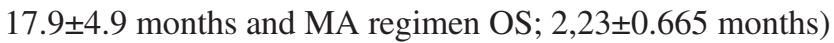

Conclusions: Consequently, elderly age had no adverse effect on NRM, relapse and OS. Allogeneic HSCT was well tolerated. Allogeneic hematopoietic stem cell transplantation with RIC/NMA regimens may be a feasible treatment option for elderly patients who have low comorbidity index. For this reason, advanced age alone should not be considered a contraindication to allogeneic HSCT.

Conflict of interest: None declared

\section{P456}

\section{Allogeneic Hematopoietic Stem Cell Transplant in Elderly Patients using Reduce Intensity Conditioning Regimen: A Single Centre Experience}

Riwa Sakr ${ }^{1}$, Sylvain Pilorge ${ }^{1}$, Stephane De Botton ${ }^{1,2,3}$, Claude Chahine $^{1}$, Tereza Coman ${ }^{1}$, Christophe Willekens ${ }^{1,2}$, Jean-Baptiste Micol $^{1}$, Florence Pasquier ${ }^{1}$, Alina Danu ${ }^{1}$, David Ghez ${ }^{1}$, Julien Lazarovici $^{1}$, Valerie Lapierre ${ }^{1}$, Eric Solary ${ }^{1,2,3}$, Vincent Ribrag ${ }^{1,2}$, Jean-Henri Bourhis ${ }^{1,2}$, Cristina Castilla-Llorente ${ }^{1}$

${ }^{I}$ Gustave Roussy Cancer Campus, Hematology, Villejuif, France; ${ }^{2}$ Gustave Roussy Cancer Campus, INSERM U1170, Villejuif, France; ${ }^{3}$ Faculty of Medicine, Université Paris- Sud, Le Kremlin- Bicêtre, France

Background: Allogeneic stem cell transplantation (SCT) remains a cornerstone in the treatment strategy of most hematologic malignancies. Previously, age and comorbidities were considered limiting factors. With the introduction of reduced intensity conditioning regimens (RIC) and the understanding of donor immune function, allogeneic SCT in adults aged more than 60 years gained its place.

Objectives: To determine overall survival (OS; defined as time to death irrespective of the cause), Relapse-free survival (RFS; defined as survival without death or relapse), non-relapse mortality (NRM; defined as time to death without evidence of disease relapse) and cumulative incidence of relapse (RI; defined as time to relapse).

Methods: It is a retrospective study of a total of 102 patients aged 60 years or older undergoing RIC allogeneic hematopoietic SCT for hematologic malignancies, all diseases combined from 2010 to 2016 in our institution.

Results: With a median follow-up of 3.1 years, OS and RFS at 3 years were $49 \%$ (95\% CI: $38-60 \%$ ) and 39\% (95\% CI: $29-50 \%)$ respectively. The cumulative incidence of NRM and relapse at 3-year were $24 \%$ (95\% CI: $17-36 \%$ ) and $44 \%$ (95\% CI: $34-57 \%$ ) respectively. The differences in OS by term of HCT-CI comorbidity risk score, donor types and RIC conditioning regimen types were not statistically significant ( $P=0.147 ; 0.51$ and 0.102 respectively). Grade II-IV acute GVHD occurred in $36(35 \%)$ patients. The cumulative incidence of grades II to IV acute GVHD was $27 \%$ at 100 days. Eighteen $(17 \%)$ patients developed chronic GVHD.

\section{P457}

Allogeneic hematopoietic stem cell transplantation for Blastic Plazmositoid Dentritic Cell Neoplazm: Is The Best Treatment Option?

\section{Hale Bülbüll${ }^{1}$, Mine Hekimgil ${ }^{2}$, Nazan Özsan ${ }^{2}$,Mahmut Töbü̈}

${ }^{1}$ Ege University Faculty of Medicine, Department of Hematology, Izmir, Turkey; ${ }^{2}$ Ege University Faculty of Medicine, Department of Pathology, Izmir, Turkey

Background: Blastic plasmacytoid dendritic cell neoplasm (BPDCN) is a clinically aggressive tumor derived from the precursors of plasmacytoid dendritic cells. It is a rare aggressive hematologic neoplasm, included among acute myeloid leukemia and related precursor disorders in

\section{Overall Survival}

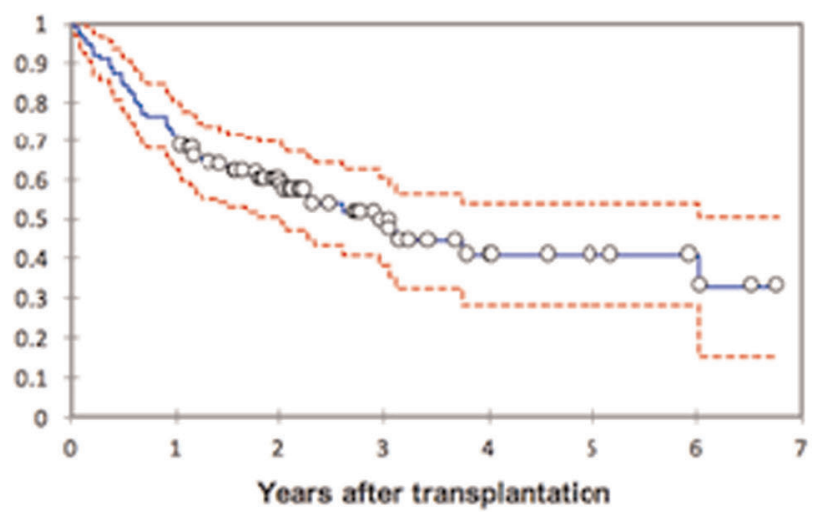

Relapse Free Survival

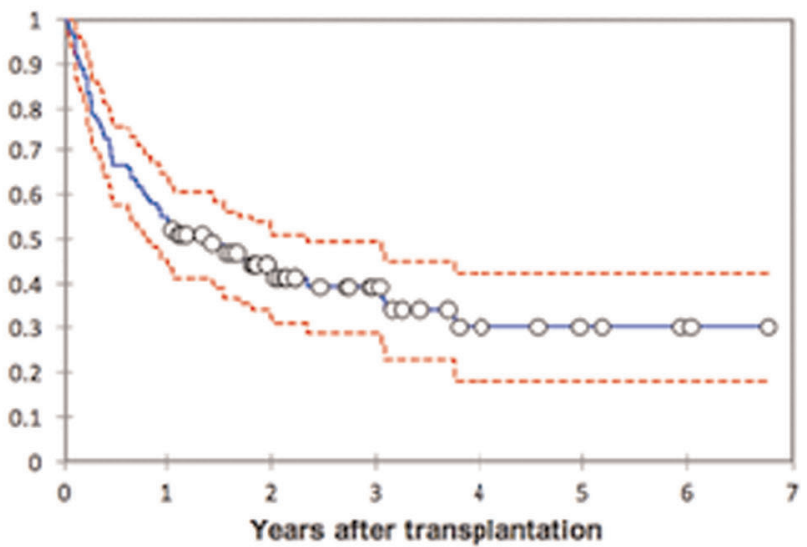

[P456 Figure] [overall and relapse-free survival] 
the 2008 WHO classification of hematological diseases and then classified as a distinct entity among myeloid neoplasms in the 2016 revision. BPDCN, typically occurs in elderly patients, with a mean age between 60 and 70 years and is more prevalent in males, (male/female:3:1). Most patients present with cutaneous lesions with or without bone marrow involvement and leukemic dissemination. A minority of cases present with leukemia without skin involvement. Cytopenias, lymphadenopathy, and/or splenomegaly are present in a significant majority of patients. We report our experience with allogeneic transplantation for BPCDN

Methods: Three patients with blastic plazmositoid dentritic cell neoplazm were treated with HyperCVAD regimene, in the period from September 2016 to June 2017. Clinical datas of patients were collected retrospectively

Results: All patients were male, aged 20 to 65 and were admitted to the hospital because of fever, weight loss, weakness and lymphadenopathy. General examination revealed that all of them had lymphadenopathy (two of them had multiple lymphadenopathy), one of them had hepatosplenomegaly and two of them had skin lesions. Skin lesions were bruise-like" brown to violaceous infiltrated plaques on the back, body, extremities and one patient had brown-purple tumoral mass and also brown-purple nodular lesions on the head region. On laboratory tests revealed that all patients had pancytopenia (two of them had severe thrombocytopenia $<20.000 \times 10^{9} / \mathrm{L}$ ). All patients' bone marrow and lymph node biopsies showed diffuse infiltration by medium sized blasts with irregular nuclear contour, slightly large cytoplasm, high mitotic index and IHC expression CD4(+), CD56(+), CD123(+), TCL1(+). Skin biopsies of two patients revealed diffuse infiltration by analogous cells. They were diagnosed with blastic plasmacytoid dentritic cell neoplasm. One of them developed confusion and a lumbar puncture was performed because of his neurological symptoms. Cranial MR imaging was found to have clivus involvement and also CNS involvement pathologically proven by cerebrospinal fluid cytology. All patients were started on HyperCVAD chemotherapy. After one course of chemotherapy, two of them achieved complete remission .One of two patients who achieved $\mathrm{CR}$ and the other patient who couldn't achieve CR presented with persistent fever, hypotension, tachycardia,organ dysfunction developed and died from sepsis. One of them who achieved complete remission after one course of chemoterapy was treated three courses of chemoterapy as maintenance and afterwards he underwent transplantation with peripheral blood progenitor cells from a related one missmatched donor.BuCy administered for conditioning regimen before transplantation. At present,one year after diagnosis, he is still in $\mathrm{CR}$

Conclusions: Two of three patients achieved CR with HyperCVAD chemotherapy. Optimal treatment is unknown but CNS prophylaxis must be routinely incorporated into all treatment protocols. BPDCN is highly malignant and prognosis is poor, with overall survival reported between 9-20 months. Most adult patients will relapse within two years. For this reason,hematopoietic stem cell transplantation, either autologous or allogeneic, is further required for attaining sustained remissions

Conflict of interest: Not declared.

\section{P458}

Allogeneic hematopoietic stem cells transplant in a patient with contemporary diagnosis of acquired immune deficiency syndrome, disseminated tuberculosis and classical Hodgkin lymphoma

Carlotta Cerva ${ }^{1}$, Gaetano Maffongelli ${ }^{1}$, Ida Provenzano ${ }^{2}$, Daniela Nasso $^{2}$, Federico Meconi ${ }^{2}$, Loredana Sarmati ${ }^{1}$, Maria Cantonetti ${ }^{2}$, Andreoni Massimo ${ }^{1}$, William Arcese ${ }^{2}$

${ }^{1}$ Tor Vergata University, Clinical Infectious Disease, Rome, Italy; ${ }^{2}$ Tor Vergata University, Department of Hematology, Rome, Italy

Background: The survival of HIV-positive patients with hematological diseases, in the active antiretroviral therapy (HAART) era, continue to improve. Hodgkin (HL) and nonHodgkin lymphoma (NHL) remain the most common malignancies in this population. Allogeneic hematopoietic cell transplantation (alloHSCT) is recommended therapy for relapsed/refractory HL disease after autologous stem cells mobilization but only few cases have been reported for patient with HIV.

We report the case of refractory classical HL in a patient with disseminated tuberculosis at the time of HIV diagnosis

Methods: Our patient is a 40-years old man admitted to the hospital with fever, lymphopenia and asthenia. On physical examination, generalized lymphadenopathy was detected. Laboratory tests showed anemia, lymphopenia, QuantiferonTB Gold test and Mantoux skin test resulted negative.

Total body PET CT scan showed paraaortic, aortocaval and iliac lymphadenopathy and splenomegaly with bone involvement.

HIV test resulted positive, the lymph node biopsy revealed positivity for Mycobacterium tuberculosis PCR and classical Hodgkin's lymphoma EBV-related. Diagnosis was IV B stage disease according to Ann Arbor staging system.

CD4 count was 96 cells (12\%), HIV viremia was 54865 $\mathrm{cp} / \mathrm{mL}$, EBV viremia was $3102 \mathrm{cp} / \mathrm{mL}$. Prophylaxis for opportunistic infections and standard anti tubercular drugs were started and, considered clinical conditions and the need to start chemotherapy, cART with TDF/FTC and raltegravir was started. 
Two weeks later the patient started ABVD regimen (doxorubicin, bleomycin, vinblastine and dacarbazine).

The PET-CT scan after 2 cycles resulted negative.

PET-CT scan after 4 cycles however revealed disease progression and resistance to first-line chemotherapy.

Second-line treatment was started with IGEV (ifosfamide, gemcitabine, vinorelbine and prednisolone) combined regimen. After 2 cycles of therapy the patient was declared resistant and first stem cells mobilization with G-CSF failed.

In consideration of clinical conditions and comorbidities, third-line therapy with Brentuximab Vedotin $(1.8 \mathrm{mg} / \mathrm{kg})$ was administered.

Four cycles were concluded but a PET-CT scan revaluation documented resistant disease.

The case was discussed and an off label treatment with Brentuximab-Bendamustine was decided.

Revaluation PET-CT scan after 4 cycles showed a complete remission.

The patient failed a second attempt in PBSC mobilization with plerixafor and G-CSF and he was admitted in Blood Marrow Transplant Unit 20 months after the diagnosis with a complete remission of hematological disease. At the time CD4 cells count was $48(8 \%)$ and HIV viremia resulted undetectable.

Results: The patient underwent alloHSCT (HLA compatibility 9/10), after myeloablative conditioning. Engraftment was obtained at days 18, no acute GvHD was detected. Anti-tubercular therapy was prolonged until 100 days after stem cells transplantation and PET-CT scan resulted negative.

We are now 9 months post-transplant, immunological parameters are improving, CD4 cells count is 167 (7\%), HIV and EBV viremia are undetectable.

Conclusions: Despite the severe immunosuppression induced by HIV infection, alloHSCT is a valid option in patients with refractory/relapsed Hodgkin lymphoma who failed autoHSCT. Clinical follow up and close monitoring are essential.

Conflict of interest: None of the authors has anything to disclose.

\section{P459}

\section{Allogeneic Stem Cell Transplantation in Children: Single Centre Experience from North India}

Chintan Vyas, Neha Singh, Faizuddin Ansari, Aniruddha Dayama, Santosh Kurmi, Rahul Bhargava, Vikas Dua

Fortis Memorial Research Institute, Gurugram, India

Background: Allogeneic stem cell transplant (Allo-SCT) services in children are still far behind in India as compared to the developed countries because of the resource constraints. There is a paucity of literature for the same from developing world. We present our experience of AlloSCT for various benign and malignant paediatric diseases.

Methods: A retrospective analysis of total 101 children (61 male and 40 female) who underwent allo-SCT between January 2014 and May 2017 at a tertiary care hospital in North India was done. The mean age of the patients was 9.2 years (range: 14 months-17 years). The indications for benign diseases were hemoglobinopathy [thalassemia major (TM)-22, sickle cell disease(SCD)-6], aplastic anemia-16, Fanconi anaemia-10, Diamond-Blackfan anemia-1, immunodeficiency (mucopolysaacharidoisis-2, one each for Wiskott-Aldrich syndrome and Chediak-Higashi syndrome), while for malignancies were acute myeloid leukaemia (AML)-14, acute lymphoblastic leukaemia (ALL)-20, juvenile myelomocytic leukaemia (JMML)-1, myelodysplastic syndrome-1, relapse neuroblastoma-1 and chronic myeloid leukaemia (CML)-5. Donors were HLAmatched sibling-58, haploidentical-33, single antigen mismatch-5 and matched unrelated- 5. The source of stem cells was peripheral blood in 98 patients and bone marrow in 3 patients. We chose a post-transplant cyclophosphamide based approach in majority of haploidentical transplants except in 4 patients, where T cell receptor alpha beta CD 19 depletion was done.

Results: Out of total 101 patients, 70 patients are alive and disease free at a median follow-up of 223 days (range: 55-1213 days). The median time for neutrophil engraftment was at day 13 (range: 11- 18 days) in 96/101 patients, including 5 patients who underwent second allo-SCT. Five patients had rejection (one each with TM, SCD, AML, CML and JMML), out of which 2 patients are alive and disease free. Acute graft versus host disease (GVHD) was reported in $31 \%$ patients (grade III/IV-12\%) while chronic GVHD in $14 \%$ patients. Day 100 mortality was 31/101 (30\%). Causes of non relapse mortality were infection-18, GVHD-5, veno-occlusive disease-1. Seven patients died due to relapse and/or progressive disease. None of the patients succumbed to cytomegalovirus, BK virus, EpsteinBarr virus or adenovirus disease.

Conclusions: Our results are comparable to many national and international published reports in terms of complications and outcomes of the treatment. This gives hope to many children who need allogeneic stem cell transplant in the developing world.

Clinical Trial Registry: Not applicable

Conflict of interest: None

\section{P460}

Cardiovascular risk factors and subclinical organ damage in children after hematopoietic stem cell transplantation 
Bianca Borchert-Moerlins ${ }^{1}$, Nima Memaran Dadgar ${ }^{1}$, Martin Sauer $^{2}$, Britta Maecker-Kolhoff ${ }^{2}$, Karl-Walter Sykora ${ }^{2}$, Ricarda Blöte $^{1}$, Elena Bauer ${ }^{1}$, Bernahrd M.W. Schmidt ${ }^{3}$, Anette Melk ${ }^{1}$, Rita Beier $^{4}$

${ }^{1}$ Hanover Medical School, Ped. Liver-, Kidney- and Metabolic Diseases, Hanover, Germany; ${ }^{2}$ Hanover Medical School, Ped. Haematology, Hanover, Germany; ${ }^{3}$ Hanover Medical School, Nephrology, Hanover, Germany; ${ }^{4}$ Essen University Hospital, Ped. Haematology, Essen, Germany

Background: Advances in allogeneic hematopoietic stem cell transplantation (HSCT) in malignant and non-malignant diseases have resulted in more long-term survivors, in whom cardiovascular (CV) disease is one leading noncancer cause of mortality. This study aimed to evaluate risk factors (RF) and subclinical $\mathrm{CV}$ organ damage in survivors after HSCT in paediatric age.

Methods: We enrolled 64 children $3.3 \pm 3.1$ years after HSCT in a cross-sectional approach. Anthropometric data, laboratory values, office- and 24-hour ambulatory blood pressure monitoring (ABPM) were evaluated.CV organ damage was determined by non-invasive measurements of aortic pulse wave velocity (PWV), carotid intima media thickness (IMT), and left ventricular mass index (LVMI). SDS scores for every measurement was calculated to age if appropriate.

Results: The mean age of the children at enrolment was $13.9 \pm 4.5$ years, $60 \%$ males. There was high prevalence of obesity and dyslipidemia in 22\% (14/64) and 23\% (15/64), respectively. Hypertension was present in $17 \%$ (11/64) of children, of those, 9 had masked hypertension that was only deteced by ABPM. Increased IMT and elevated PWV were detected in 48\% (IMT) and 6\% (PWV). For IMT, physical activity had a positive impact and was worsened by time after HSCT. Elevated PWV SDS was associated in multivariate analysis with higher waist circumference and if HSCT was carried out before the age of 12 years. Anthracycline therapy and history of veno-occlusive disease were associated with lower LVMI.

Conclusions: Our results show a surprisingly high rate of subclinical CV organ damage and classical RFs in children after HSCT. Therefore, diagnosis and management of wellknown CV risk factors belong to clinical care after HSCT.

Conflict of interest: The authors declare that no conflicts of interest exist.

\section{P461}

Changing trends in haemopoietic stem cell transplantation in the UK: $\mathbf{4 0}$ years of data from the British Society for Blood and Marrow Transplantation (BSBMT)

Jennifer Byrne ${ }^{1,2}$, Julia Perry ${ }^{1}$, Charles Crawley ${ }^{1,3}$, Kim Orchard $^{1,4}$,
Rachel Pearce ${ }^{1}$

${ }^{1}$ British Society of Blood and Marrow Transplantation, London, United Kingdom; ${ }^{2}$ Nottingham University Hospitals Trust, Clinical Haematology, Nottingham, United Kingdom, ${ }^{3}$ Addenbrookes Hospital, Haematology, Cambridge, United Kingdom; ${ }^{4}$ University Hospital Southampton, Haematology, Southampton, United Kingdom

Background: 2017 marked the $60^{\text {th }}$ anniversary of HSCT since the pioneering work by Donall Thomas, and 20 years since the BSBMT was founded by John Goldman and Tony Goldstone. Since that time transplantation has evolved with changes in the source of stem cells; conditioning regimens; diseases treated; patient demographics; GVHD strategies and supportive care methods.

Methods: Activity and overall survival data from over 66,500 UK HSC transplants performed between 1974-2016 were analysed to determine how transplantation has evolved in the UK over this time period.

Results: During this time period 59,561 patients underwent 66,585 transplant procedures, of which 27,443 were allografts and 39,142 were autologous. The total number of transplant procedures per year continues to rise, although this increased activity is mainly in the autologous setting, whilst allografts numbers appear to have plateaued. In 2016, $100 \%$ of autologous procedures and in children were done using PBSC whereas in adults this has been the case for 10 years. In the allograft setting $30 \%$ of paediatric and $95 \%$ of adult transplants are done with PBSC. The number of cord transplants appears also to have plateaued. The use of TBI has declined in both adult and paediatric settings and reduced intensity conditioning accounts for $75 \%$ of adult and approx $20 \%$ of paediatric procedures. In both adult autograft and allograft setting there has been a marked increase in the number of patients aged $>60$ years undergoing HSCT, and the number of patients aged $>70$ years being transplanted is rapidly rising.

Not surprisingly the number of allografts for CML has steeply declined since 2002 and there has been a similar, more recent, reduction in the number of allografts for CLL, both due to the introduction of effective new drugs for these diseases. In contrast allografts for AML has continued to rise due to the lack of new drugs for this disorder. In the autograft setting there has been an increase in the number of transplants performed for autoimmune diseases and the number of myeloma autografts is steadily rising.

Overall survival post autograft has increased steadily across the 4 decades for NHL, HD and myeloma, possibly due partly to reduced TRM but also improved salvage therapies after relapse. Following allografts, a continuous improvement in OS is also seen in SAA, MDS and ALL, whilst for CML there was marked improvement in OS in the 1998-2007 era (likely due to the introduction of TKIs) but 


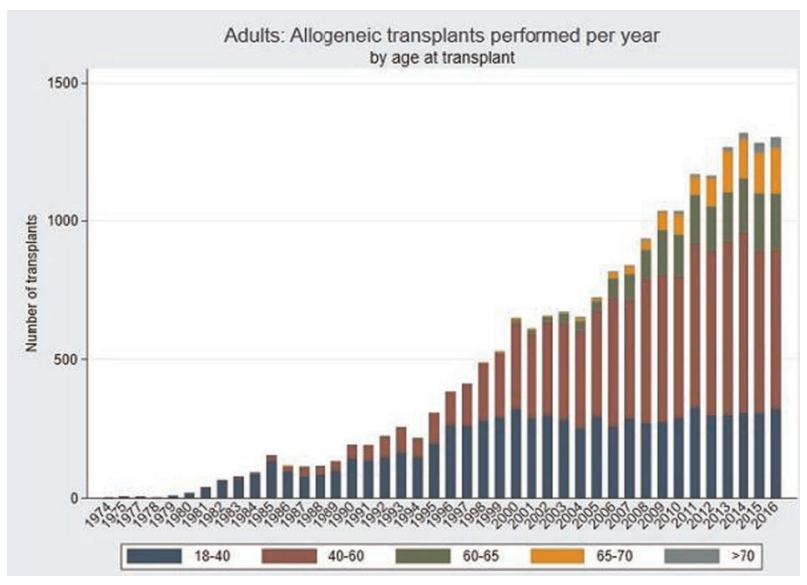

[P461 Figure] [BSBMT: adult allografts by age group 1974-2016]

no further improvement since then. In contrast AML outcomes have not improved across the last 3 decades.

Conclusions: This BSBMT registry data demonstrates how transplantation has evolved over the past 4 decades with new techniques allowing more patients to benefit and transplants to be done more safely. New drug treatments have influenced survival and reduced transplant activity for some diseases but new indications have replaced them resulting in overall continued expansion

\section{Conflict of interest:}

J. Byrne, C. Crawley: honoraria Jazz pharmaceuticals

J. Perry, K. Orchard, R. Pearce: nothing to declare

\section{P462}

CMV reactivation after haploidentical hematopoietic stem cell transplantation with postransplant cyclophosphamide promotes NK cell maturation

Nieves Dorado Herrero', ${ }^{1}$ Virginia Pradillo ${ }^{2}$, Ana Perez-Corral', Laura Solan ${ }^{2}$, Pascual Balsalobre ${ }^{2}$, Carolina Martinez-Laperche ${ }^{2}$, Ismael Buño ${ }^{2}$, Mi Kwon ${ }^{2}$, Jose Luis Diez-Martin ${ }^{2}$

${ }^{1}$ Hospital General Universitario Gregorio Marañón, HematologyBMT Unit, Madrid, Spain; ${ }^{2}$ Hospital General Universitario Gregorio Marañón, Madrid, Spain

Background: CMV reactivation is a frequent complication after allogeneic hematopoietic stem cell transplantation (HSCT). Some groups have reported its association with the improvement in the relapse free survival and overall survival after HSCT from HLA-identical donors. Natural killer cells (NK) are important effectors of innate immune responses against virally infected cells and therefore critical in limiting CMV replication.

The aim of this study is was to evaluate the impact of CMV reactivation after HSCT, and its association with NK maturation profile and transplantation outcome, in patients

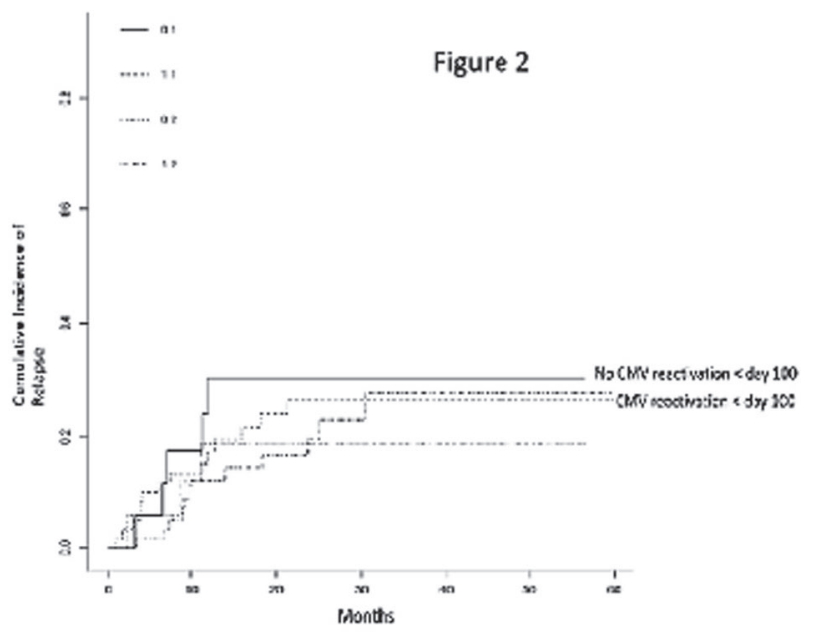

[P462 Figure] [Figure 2]

who underwent peripheral blood unmanipulated haploidentical HSCT (Haplo-HSCT) with postransplant cyclophosphamide (PTCy) at our institution.

Methods: From July 2011 to June 2016, 84 consecutive patients underwent Haplo-HSCT. Three died before day 30 after Haplo-SCT, so 81 patients were retrospectively analyzed (Table 1).CMV reactivation monitoring was performed twice weekly during admission for HSCT and later on weekly, using a plasma-real time PCR assay.

NK cells were assessed at days $+30,+90$ and +180 of NK CD56 ${ }^{\mathrm{dim}}\left(\mathrm{NK}^{\mathrm{dim}} 30, \mathrm{NK}^{\mathrm{dim}} 90, \mathrm{NK}^{\mathrm{dim}} 180\right)$ absolute count and percentage over all NK cells.

Results: Median follow-up was 18 months (8-31). Cumulative incidence of CMV reactivation was observed in $23 \%$ before day $+30,72 \%$ at day +100 , and $28 \%$ after day +100 . None of them developed CMV infection. Analysis of NK cells show median of $\mathrm{NK}^{\mathrm{dim}} 3026 \%$ (12.5-42.0) and 13 (3-39) absolute count; $\mathrm{NK}^{\mathrm{dim}} 9066 \%$ (56-77.25) and 77 (34-123) absolute count; $\mathrm{NK}^{\mathrm{dim}} 18073 \%$ (61-82) and 82 (50-125) absolute count.

Patients who developed CMV reactivation before day +100 showed higher $\mathrm{NK}^{\mathrm{dim}} 90$ percentage $(\mathrm{p}=0.024)$. No relationship was detected among patients with CMV reactivation before day +30 .

Overall cumulative incidence of DFS was $24 \%$ at 2 years. Patients with CMV reactivation before day +100 , showed a trend towards better DFS rate compared to those with no CMV reactivation $(p=0.295$, Figure 1). Cumulative incidence of relapse in patients with CMV reactivation before day +100 was $30 \%$ and $23 \%$ in patients without reactivation $(\mathrm{p}=0.689$, figure 2$)$.

Conclusions: Our study showed a significant correlation between NK maturation and CMV reactivation after haploHSCT with PTCy, with a trend towards a better DFS in those with CMV reactivation before day +100 . Further studies are needed to confirm these observations. 
Conflict of interest: Nothing to disclose

\begin{tabular}{ll}
\hline Age (y.o) & $46(33-59)$ \\
\hline Sex of patients & Men 72\% (58) Women 28\% (23) \\
Diagnosis & AML 36\% (29) ALL 9\% (7) HL/NHL 35\% (28) MDS \\
& 7 (6) Other (MF, AA) 13\% (11) \\
$\begin{array}{l}\text { Disease status } \\
\text { pretransplantation }\end{array}$ & CR 54\% (44) PR 19\% (15) Overt disease 23\% (19) \\
$\begin{array}{l}\text { Donors } \\
\text { Conditioning Regimen }\end{array}$ & Men 53\% (43) Women 47\% (38) \\
CD34+ x106/kg & 5.4 (4.6-6.4) \\
infused & \\
$\begin{array}{l}\text { Neutrophil } \\
\text { engraftment (day) }\end{array}$ & 17 (16-19) \\
\hline
\end{tabular}

\section{[[P462 Table] Table 1]}

\section{P463}

\section{Collection of peripheral hematopoietic cells in family donors after mobilization using biosimilar G-CSF-our experience}

Zdenka Bhuiyan Ludvikova ${ }^{1}$, Zdenka Gasova ${ }^{1}$, Martina Böhmova ${ }^{1}$, Marketa Sloukova ${ }^{1}$, Antonin Vitek ${ }^{2}$

${ }^{1}$ Institute of Hematology and Blood Transfusion, Department of Apheresis, Prague, Czech Republic; ${ }^{2}$ Institute of Hematology and Blood Transfusion, Clinical Department, Prague, Czech Republic

Background: Peripheral hematopoietic stem cells (PBPCs) are usually obtained from donors after using the growth factor mobilization (G-CSF). There had been using the original product Neupogen in the past. Today, biosimilar preparations are being introduced from economical aspect. We've started using the biosimilar G-CSF (Zarzio) since January 2017 in our department. Our goal was to show whether the mobilization is same effective as of the original and whether there are any differences in donor-based mobilization from the age point of view.

Methods: There were family donors mobilized by original G-CSF in period from $1 / 2015$ to $1 / 2017$ and 21 donors, mobilized by biosimilar G-CSF in period from 1/ 2017 to $9 / 2017$. Groups of the donors were indicated as N (Neupogen) and Z (Zarzio). G-CSF s.c. in one dose was administered in the evening.Group $\mathrm{N}$ received 10.8 (7.213.4) $\mu \mathrm{g} / \mathrm{kg}$ per day and group $\mathrm{Z} 10.1$ (8.8-12) $\mu \mathrm{g} / \mathrm{kg}$ per day. Separation was started after the $4^{\text {th }}$ dose of G-CSF. All separations were performed in Spectra Optia (Terumo) in CMNC mode. Only first separations were evaluated. We considered the mobilization as failure when the value of $\mathrm{CD}_{3}{ }^{+}$in peripheral blood was less than $20 \mu \mathrm{l}$. The optimum transplant dose was $4-5 \times 10^{6} \mathrm{CD} 34^{+} / \mathrm{kg}$ of the recipient and minimum dose more than $2 \times 10^{6} \mathrm{CD} 34^{+} / \mathrm{kg}$.
Results: There were no significant differences in both groups by age and gender. Side effects of the stimulation $29 \%$ in group $\mathrm{N}$ and $35 \%$ in $\mathrm{Z}$. The rate of the failure of mobilization: $3,8 \%$ in group $\mathrm{N}$ and $4,7 \%$ in $\mathrm{Z}$. Collection of PBPC was performed as most similar in both groups (N 3.3 $\mathrm{TBV}, \mathrm{Z} 3.03 \mathrm{TBV})$. Median of $\mathrm{CD}^{+} 4^{+}$cells from one collection was $5.9(1.3-18.6) \mathrm{CD} 34^{+}\left(10^{6}\right) / \mathrm{kg}$ of the recipient in $\mathrm{N}$ and $6.4(1.8-14.4) \mathrm{CD} 34^{+}\left(10^{6}\right) / \mathrm{kg}$ in group Z. Minimum transplant dose was collected and in the most of cases reached optimum transplant dose $(88 \%$ for N, $90 \%$ for $\mathrm{Z}$ group). Median number of collections was one. However, the donors had to complete in some cases two separations (27\% in $\mathrm{N}$ and $33 \%$ in $\mathrm{Z}$ ). We also divided the both groups into two subgroups by the age (less and more 40 yrs).Better mobilization was detected in group $\mathrm{N}$ age more 40 yrs. However this fact didn $\hat{A}^{\prime} t$ have any impact in the resulting collection.

Conclusions: We did not find any significant differences in the mobilization of family donors by the use of different types of G-CSF. Utility of different types of G-CSF from age seems to be not important issue. We routinely follow up the donors after mobilization for a period longer than 10 years after using the original product. WeÂ're currently in the beginning in using biosimilar G-CSF. We believe that the outcome of both types of G-CSF will be the same. This is our expectation.

Conflict of interest: Nothing to disclose

\section{P464}

\section{Comparison of Different Concentrations of the Extracellular Cryoprotectant in Hematopoietic Stem Cell Freezing}

Ivan Tonev, Chavdar Botev, Svetlana Hristova, Zhana MinchevaGove, Milcho Mincheff

National Specialized Hospital for Active Treatment of Haematological Diseases, Sofia, Bulgaria

Background: Hematopoietic stem cell (HSC) transplantation is an established method for treatment of malignant and benign hematological diseases. HSC have intensive metabolism, therefore the duration of their storage at $2-8^{\circ} \mathrm{C}$ is short - up to 5 days, and their long term storage is possible only if they are frozen. The main reasons for the cellular damage during slow freezing is cell dehydration due to extracellular ice formation, leading to irreversible deformation and loss of cell membrane. The presence in the cellular suspension of cryoprotective agents reduces these damages. We use dimethyl sulfoxide as an intracellular cryoprotectant and hydroxyethyl starch, plasma, albumin as extracellular ones. To establish the most appropriate content of the cryoprotective solution, we 
investigated combinations of DMSO with different extracellular cryoprotectants.

Methods: Apheresis-derived hematopoietic stem cells were mixed with cryoprotective solution and frozen at $-80^{\circ} \mathrm{C}$. The cryoprotective solution contained DMSO with $5 \%$ final concentration, human albumin with or without isotonic solutions of hydroxyethyl starch (HES) with different relative molecular weight $(130,000,200,000$ and $450,000)$ and autologous plasma. The cells vitality was tested before mixing with the cryoprotective solution, after the first 3 days, the third, the sixth and the twelfth month of storage by trypan blue exclusion.

Results: Viability of the cells following storage in different cryoprotective solutions exceeded $87 \%$. The presence or absence of HES in the cryoprotective solution did not affect viability, however, samples with no HES had better viability when the human albumin concentration was higher.

Conclusions: DMSO is toxic for the cells and might lead to serious adverse reactions (heart rhythm disorders, hemodynamic disturbance, bronchial spasm, etc.) after infusion. The addition of extracellular cryoprotectants gives opportunity to use smaller concentration of DMSO in the final suspension, thus reducing the incidence and severity of the adverse events after the infusion of the thawed cells. Human albumin may be used as a substitute for high molecular weight hydroxyethyl starch as an extracellular cryoprotectant.

Conflict of interest: none

\section{P465}

\section{CONDITIONING REGIMEN FOR HEMATOPOIETIC STEM CELL TRANSPLANTATION IN NK/T-CELL LYMPHOMA AND LEUKEMIA}

\author{
Min Xiong ${ }^{1}$, Xing-Yu Cao ${ }^{2}$, De-Yan Liu ${ }^{2}$
}

${ }^{1}$ Hebei Yanda Lu Daopei Hospital, Division of Bone Marrow Transplantation, Beijing, China; ${ }^{2}$ Hebei Yanda Lu Daopei Hospital, Beijing, China

Background: The aim of this study is to determine the safety and efficacy of conditioning regimen consisting of total body irradiation (TBI)/cyclophosphamide (Cy) /Vp-16 prior to allogeneic hematopoietic stem cell transplantation (allo-HSCT) forpatients with NK/T-cell lymphoma and leukemia.

Methods: We retrospectively analyzed 9 patients with NK/T-cell lymphoma and leukemia who received TBI/Cy/ $\mathrm{Vp}-16$ as the conditioning regimen for myeloablativealloHSCT in our hospital between July 2013 and April 2017. Five of the nine patients had aggressive natural killer cell leukemia (ANKL), three had refractory extranodal NK/T- cell lymphoma, and one had EBV-associated T/NK-cell lymphoproliferative disease (T/NK-LPD). The median age was 11 (4-46) years. Male to female ratio was 5:4. The median disease course from diagnosis to transplant was 14 (4-41) months. Eight patients were persistently EBVpositive before allo-HSCT. Of the eight patients, five had hemophagocytic lymphohistiocytosis (HLH). For six patients, the disease was not under control prior to alloHSCT. One patient underwent matched but unrelated donor HSCT; four received haploid HSCT (1 CRD) and the other four received HLA-matched sibling transplantation. The conditioning regimen used consists of TBI (4Gy) for 3 days; cyclophosphamide $\left(1.8 \mathrm{~g} / \mathrm{m}^{2}\right)$ for 2 days; $\mathrm{Vp}-16(15 \mathrm{mg} / \mathrm{kg})$ for 2 days; and a total dose of $5 \mathrm{mg} / \mathrm{kg}$ of anti-thymocyte globulin (ATG) was given in 3 cases; and $20 \mathrm{mg} / \mathrm{kg}$ of ATG-Fresennius in 1 case. No ATG was given in HLAmatched sibling transplantation. For GVHD prophylaxis, cyclosporine, tacrolimus, mycophenolate mofetil and methotrexate were given.

Results: All 9 patients underwent treatment successfully. The median time to neutrophil engraftment was 12 (9-20) days; the median time to platelet engraftment was 19.7 (962) days. Grade II-IVaGVHD occurred in 5 cases. Cumulative incidence of grade II-IV aGVHD was 55.5\%. Five cases had limited cGVHD. EBV in infected patients was undetectable after transplant. With a median follow-up 30 (7-53) months, seven patients remained disease-free. Two patients died of pulmonary infection and thrombotic microangiopathy respectively. The overall survival rate was $78 \%$.

Conclusions: Our results indicate that conditioning regimen of $\mathrm{TBI} / \mathrm{Cy} / \mathrm{Vp}-16$ for allo-HSCT can improve disease-free survival of patients with NK/T-cell lymphoma and leukemia.

Conflict of interest: nothing to disclose

\section{P466}

Delayed non-infectious rabbit anti-thymocyte globulin related fever and pseudoflare of neurologic disability in Multiple Sclerosis patients undergoing autologous hematopoietic stem cell transplant

\footnotetext{
Regan M Buckley ${ }^{1}$, Bailie Giesel ${ }^{1}$, Rachel Schmitt ${ }^{1}$, Allison M Clendenan ${ }^{1}$, Jacquelyn L Henry', Amy L Morgan', Michelle A Calvario $^{1}$, Roumen D Balabanov ${ }^{2}$, Richard K Burt ${ }^{1}$

${ }^{1}$ Northwestern University Feinberg School of Medicine, Division of Immunotherapy for Autoimmune Diseases, Chicago, IL, United States; ${ }^{2}$ Northwestern University Feinberg School of Medicine, Department of Neurology, Chicago, IL, United States
}

Background: Patients with Multiple Sclerosis (MS) undergoing autologous hematopoietic stem cell transplant 
(HSCT) experience delayed fevers associated with rabbit anti-thymocyte globulin (rATG) that induce temporary neurologic pseudoflares.

Methods: Inpatient records were reviewed of 104 MS patients who underwent HSCT at Northwestern Medicine in Chicago, Illinois over a 12 month period from July 2016 through July 2017. Data collected included: recorded temperatures $>38^{\circ} \mathrm{C}$, neurological symptoms, day of transplant that the fever occurred, blood culture results, steroid protocol given, recurrent fevers, and neurological symptoms reported by the patient or provider related to the fever.

Results: Out of the 104 patients reviewed, there were 49 patients with fevers and 55 patients with no fevers. The mean temperature was $38.3^{\circ} \mathrm{C}$ (ranging $37.3^{\circ} \mathrm{C}$ to $39.2^{\circ} \mathrm{C}$ ). Sixty-seven percent of patients (33 of 49) developed fever on day +1 post HSCT. Fevers were also reported on days $-1,0,+2,+4,+5,+6$, and +8 . Sixty-five percent of patients (30 of 49) developed neurologic symptoms, predominately weakness and fatigue, during the febrile episodes. After the first temperature spike, blood cultures were drawn, a chest x-ray was obtained, methylprednisolone $250 \mathrm{mg}$ IV was given, vancomycin was added in addition to prophylactic cefepime to broaden antibiotic coverage, and methylprednisolone $125 \mathrm{mg}$ IV was ordered for two consecutive days after the spike. Twenty-two patients had recurrent fevers and then received a second course of methylprednisolone $125 \mathrm{mg}$ IV for three consecutive days. Two patients had positive Escherichia coli urine cultures that were not associated with febrile episodes. One patient was positive for Clostridium difficile on the day of fever and was treated with oral vancomycin for 14 days. All blood cultures were negative for growth. Fevers resolved within three hours of the steroid administration along with resolution of neurologic symptoms.

Conclusions: Fevers during neutropenia after rATG and cyclophosphamide conditioning for MS are usually secondary to rATG and fever associated pseudoflares resolve rapidly after the administration of methylprednisolone, but expanded antibiotic coverage and vigilance is important to rule out infection.

Conflict of interest: All authors have nothing to disclose

\section{P467}

Donor specific anti-HLA antibodies(DSA) in High Risk patients of beta- thalassemia major predicts poor outcome with T-replete Haploidentical Hematopoietic Stem Cell Transplantation using Post-transplant Cyclophosphamide
Satyendra Katewa ${ }^{1}$, Mohammed Ramzan ${ }^{1}$, Ajay Narayan Sharma ${ }^{1}$, Ramesh Bairwa ${ }^{1}$, Rahul Sharma ${ }^{1}$, Kamal Goyal ${ }^{1}$, Shiv Kumawat, Rakesh Repaswal ${ }^{1}$, Monica Singh ${ }^{2}$, Sunil Dutt Sharma ${ }^{3}$, Ravikant Porwal $^{4}$, Ravi Dara ${ }^{5}$

${ }^{1}$ Manipal Hospital, Pediatric Hemato-Oncology \& BMT, Jaipur, India; ${ }^{2}$ Manipal Hospital, Hemato-pathology and Molecular Diagnostics, Jaipur, India; ${ }^{3}$ Manipal Hospital, Pediatric Critical Care, Jaipur, India; ${ }^{4}$ Manipal Hospital, Infcetious Disease, Jaipur, India; ${ }^{5}$ Manipal Hospital, Immuno-Hematology, Jaipur, India

Background: Hematopoietic stem cell transplant (HSCT) is the only cure for patients with thalassemia major but probability of finding a suitable match is just $25-50 \%$ (including matched unrelated donor) in India. Moved by the published results of T-replete haploidentical HSCT(HaploSCT) with post-transplant cyclophosphamide (pt-CY), we investigated the Haplo-SCT in Indian pts.

Methods: From August 2015 to September 2017, 28 pts of thalassemia major underwent haplo-SCT with pt-Cy strategy at our centre. Sixteen subjects were males and 12 were females. The median age was 14 yrs (range 2-18). In pre-transplant Pesaro risk groups, 2 were class-I, 12 were class-II and 14 were Class-III. Donor source was peripheral stem cells (PBSC) in 26 and PBSC+Cord cells in 2 pts. Donor was mother in 17 pts, father in 6 and brother in 5 pts. All pts were given two cycles of Dexamethasone+Fludarabine, every 28 days. Two conditioning regimens were used. Regimen-A was ATG(Rabbit@ $1.5 \mathrm{mg} / \mathrm{kg} /$ day from Day-12 to-10) + Flu(35mg/m² from D-7to-2) \& Bu(130mg/ $\mathrm{m}^{2} /$ day from D-6to-3) in 18 pts. Regimen-B was regimen A +Thiotepa $(8-10 \mathrm{mg} / \mathrm{kg} /$ day on D-4) and was used in $10 \mathrm{pts}$. Donor specific anti- HLA antibodies (DSA) were present in 10 pts. None of class-I while 2 from class-II and 8 of classIII pts. Treatment for DSA was given if patient had MFI $>2500$. GvHD prophylaxis was pt-Cy $50 \mathrm{mg} / \mathrm{kg} / \mathrm{d}(\mathrm{D}+3 \& 4)$ and tacrolimus and mycophenolate mofetil (MMF) started on $\mathrm{D}+5$. Tacrolimus given till 9-12 months and MMF tapered off by $\mathrm{D}+100$. T replete $\mathrm{PBSC}$ or $\mathrm{PBSC}+\mathrm{Cord}$ cells were given with a targeted $\mathrm{CD}_{3} 4^{+}$dose between 5 $15 \times 10^{6}$ cells $/ \mathrm{kg}$.

Results: Both the regimens were tolerated and VOD of liver was seen in two cases with regimen-B. None rejected the graft. The median time to neutrophil engraftment was 18 (range;14-22) while for platelet 40 days(range;18-60). Acute GvHD was seen in 6pts and 4/6 had Liver and skin GvHD(grade I-II). Gut GvHD (grade III\&IV) was observed in 2 pts and one died at D+150. Chronic GvHD was observed as limited skin in 2 pts. Viral re-activation was observed in 11 pts, BKV in 3 pts, CMV in 7 while Adenovirus in $1 \mathrm{pt}$. Transplant related mortality(TRM) was $6 / 28(21 \%)$ and total deaths were 8/28 (28.5\%). In TRM, two pts were of class-II and four of class-III. All six pts had multidrug resistance sepsis. Four out of these six patients (1 class-II and 3 class-III) had refractory macrophage 
activation syndrome along with sepsis. The two late deaths were at $\mathrm{D}+150$ and $\mathrm{D}+210$ and were attributed to macrophage activation syndrome(MAS) with gut GvHD and MAS respectively. Both pts were class-III. All patients who died had a pre-transplant DSA titres with MFI $>10,000$ (against HLA class-I and II both). Study report thalassemia free and overall survival of $71.5 \%$ while for class-I, II \& III, It's $100 \%, 84 \%$ and $58 \%$ respectively. The median followup is 16 months (range 4-27 months).

Conclusions: T replete haplo-SCT with pt-Cy produced excellent results in class I and II pts but a combination of DSA with Class-III pts predicted very poor survival (probably MAS) and warrants more novel interventions to overcome this hurdle

\section{Conflict of interest: None}

\section{P468}

\section{Early detection of lack of chimerism allows prompt regraftment}

Arnalda Lanfranchi ${ }^{1}$, Marta Comini ${ }^{1}$, Alessandra Beghin ${ }^{1}$, Samanta Villanova $^{1}$, Elena Soncini ${ }^{2}$, Giulia Carracchia ${ }^{2}$, Fulvio Porta ${ }^{3}$

${ }^{1}$ Stem Cell Laboratory, Section of Hematology and Blood Coagulation, Children's Hospital, Spedali Civili of Brescia, Brescia, Italy; ${ }^{2}$ Pediatric Onco-Hematology and BMT Unit, Children's Hospital, Spedali Civili of Brescia, Brescia, Italy; ${ }^{3}$ Pediatric OncoHematology and BMT Unit, Childrens Hospital Chair of Paediatrics University of Brescia, Brescia, Italy

Background: Analysis of short tandem repeats (STR) is the predominant method for post Hematopoietic Stem Cell Transplantation (HSCT) monitoring of donor engraftment. It can enable early detection of disease relapse, level of engraftment and provide useful information on the graftversus-host disease (GvHD) / graft-versus-tumor (GvT) effect, facilitating therapeutic intervention.

The good standardization of this method in our center allowed the analysis of donor chimerism also in patients with a very low white cell count due to the high sensibility of the test $(2.5 \%)$.

Methods: We analyzed the early donor chimerism in 12 paediatric patients (3 LLA, 2 Talassemia, 1 SCID, 1 CID, 1 Hyper IgE Syndrome, 1 Aplastic Anemia, 1 MDS, 1 LAD, 1 WAS) after HSCT. The STR analysis was performed on peripheral blood (PB) in presence of low number of white blood cell (WBC) count. The blood populations analyzed were PBL (Peripheral Blood Lymphocytes) and PMN (Polimorphonucleated cells).

Results: A total of 12 paediatric patients were evaluated for donor chimerism after HSCT. The average WBC count at the moment of analysis, was $0.315 \times 10^{\wedge} 3 / \mathrm{ml}$ (range: $0.04-0.83 \times 10^{\wedge} 3 / \mathrm{ml}$ ) at the first time-point.
5 patients had total donor chimerism on PBL and PMN (100\% of donor cells), 3 mixed chimerism on PBL ( $>82 \%$ of donor cells, range $82-89.4 \%$ ) and total on PMN, 3 mixed chimerism on PBL $(>70.8 \%$ of donor cells, range 70.8 $78.1 \%)$ and PMN (>81.4\% of donor cells, range 81.4$98.9 \%$ ), and 1 patient was autologous ( $0 \%$ of donor cells).

This method is helpful as predictive tool in monitoring HSCT outcome. In particular in the patient with autologous reconstitution, analysis was performed after 19 days post HSCT with WBC of $0.04 \times 10^{\wedge} 3 / \mathrm{ml}$ and this allowed to go for a second transplant after 42 days. In a patient affected by Severe Aplastic Anemia that had a very slow hematological reconstitution, STR analysis was performed with WBC of $0.2 \times 10^{\wedge} 3 / \mathrm{ml}$ after 24 days post HSCT. The result, in condition that would not allow the standard analysis of chimerism, confirmed donor engraftment $(89.4 \%$ of donor cells on PBL and $100 \%$ of donor cells on PMN).

Conclusions: STR method is the best and appropriate tool in our hand for predicting events, such as disease relapse and graft rejection. In our experience this assay allows prompt salvage therapeutic approaches in case of unsuccess of the procedure since if chimerism analysis is performed as soon as possible it gives the opportunity to rapidly find another donor or modify the conditioning regimen

Conflict of interest: The authors have no conflicts of interest to declare.

\section{P469}

Effectiveness of allogeneic hematopoietic stem cell transplantation (allo-HCT) in patients transplanted with active myeloid disease

Maria Liga, Nikolaos Spyridis, Evi Triantafyllou, Markos Marangos, Alexandros Spyridonidis

University Hospital of Patras, Bone Marrow Transplantation Unit, Patras, Greece

Background: The clinical outcome of patients transplanted with active myeloid leukemia (AML) is extremely poor, with recent retrospective studies reporting an overall survival $<10 \%$. Encouraging results have been reported with the sequential administration of pre-conditioning along with the conditioning regimen during the neutropenia (FLAMSA-like protocol, Schmidt et al). Aim of this study is the effectiveness of allogeneic transplantation with a FLAMSA-like sequential conditioning regimen in patients with active myeloid disease (sequential chemo-allo).

Methods: There have been analysed patients that underwent allo-HCT with active myeloid leukemia. All patients have received chemotherapy pre-conditioning one week before the beginning of the conditioning regimen, according 
an approved protocol by the Ethic Comitee (344/04.08.06). Overall survival (OS) and relapse free survival (RFS) were obtained by the method of Kaplan-Meier where patients were censored at last follow up if still alive. Relapse (REL) and non relapse mortality (NRM) were considered to be competing risk.

Results: Overall, 15 patients (10 male, 5 female) with median age of 52 years (range 30-70) were transplanted with active myeloid disease (de novo AML 8, secAML 6, OMF 1). Thirteen patients had refractory disease, while 2 patients with MDS/AML hadn't received any chemotherapy. The median cycles of chemotherapy were 2 (range 0 5 ). The median number of blasts at the beginning of preconditioning were $10 \%$ (range 6\%-45\%). Preconditioning was given on day -14 and included administration of Aracytin at a variable dosage $1-4 \mathrm{gr} / \mathrm{mq}$, one patient received Etoposide $100 \mathrm{mg} / \mathrm{mq}$ and one patient received GCLAC. The conditioning regimen was given from d-7 to d-3 (BCNU/Flu/Mel or TT $n=8$, Busilvex/Flu/TT $n=7)$. The donors were related siblings $\mathrm{n}=5,10 / 10$ - matched unrelated donors $\mathrm{n}=5,9 / 10$-matched unrelated donors $\mathrm{n}=$ 4 and 1 haploidentical donor. All patients engrafted. The median time to recover leukocyte counts $(>1000 / \mu \mathrm{l})$ and to achieve untransfused platelets were 13 days and 14 days, respectively. Cyclosporine was interrupted in all patients before day +120 . The median follow up was 322 days (range 14-3535). From the 15 patients, 6 are alive, 4 of them being in complete remission. Seven patients died due to NRM (median day +184 , range 123-546) and 2 due to relapse. The estimated OS and DFS at 1 year was $49 \%$ and $40 \%$, respectively and at 2 years $31 \%$ and $24 \%$, respectively. The cumulative incidence of NRM at +1 and +2 years were $38.5 \%$ and $63 \%$, respectively and of REL $35 \%$ and $35 \%$, respectively. Cause of NRM were predominantly viral infections (JC, H1N1).

Conclusions: Carrying out allogeneic transplantation with a combination of intensive chemotherapy and administering a conditioning regimen during the neutropenia is a therapeutic option for patients with active myeloid disease. Compared to published results, the present "sequential chemo-allo" strategy shows survival superiority over classical allo-HCT (Ferguson et al), but such a comparison is necessary in further prospective studies with a larger number of patients.

Conflict of interest: Nothing to disclose

\section{P470}

Eltrombopag for the treatment of late cytopenia following allogeneic stem cell transplants
Sabrina Giammarco ${ }^{1}$, Simona Sica ${ }^{2}$, Patrizia Chiusolo ${ }^{1}$, Luca Laurenti $^{2}$, Federica Sorá ${ }^{2}$, Massimo Martino ${ }^{3}$, Alessandro Busca ${ }^{4}$, Andrea Bacigalupo $^{2}$

${ }^{1}$ Universitá Cattolica del Sacro Cuore, Haematology, Rome, Italy; ${ }^{2}$ Università Cattolica del Sacro Cuore, Haematology, Rome, Italy; ${ }^{3}$ Azienda Ospedaliera BMM, Centro Trapianti di Midollo Osseo, Reggio Calabria, Italy; ${ }^{4}$.A.O.U. Cittá della Salute e Della Scienza di Torino. Presidio Molinette, Haematology, Torino, Italy

Background: The occurrence of cytopenia following an allogeneic haemopoietic stem cell transplant (HSCT), has been reported to occur in $25 \%$ of patients within day +100 (Brit J Haematol, 2001, 112, 219 \pm 227 ). Infections and/or graft-vs.-host disease, have been reported as predictive factors (GvHD) (NEJM,1991, 324, 667 \pm 674 ; Annals of Hematology,1992， 64，A128 \pm 131$)$. Treatment with CD34 selected cells from the same donor, has been reported as an effective form of therapy. (Biol Blood Marrow Transplant. 2014 Sep;20(9):1440-3) However this implies the availability for a second donation from the same donor; in addition the median interval between CD34 boost infusion and trilineage recovery is over 3 months. Eltrombopag (EPAG) has been recently shown to be effective in the management of patients with acquired aplastic anemia (N Engl J Med. 2017 Apr 20;376(16).

Methods: Ten patients were retrospectively collected among 3 Centers (Rome, Reggio Calabria and Torino), and had received EPAG for uni, bi or trilineage cytopenia. The diagnoses were AML $(n=4) T$, ALL $(n=3), \operatorname{MDS}(n=3)$; there were 7 male $/ 3$ female with a median age of 48 years (rang: 37-65). Median CD34 cell dose infused was 3.7 $\mathrm{x} 10^{\wedge} 6 / \mathrm{kg}$ (range 1.1-7.6). Engraftment was achieved in all patients, with a median interval from HSCT to reach $0.5 \times 10^{\wedge} 9 / \mathrm{L}$ neutrophils of 21 days (14-28) and to reach 20x10^9/L platelets of 19 days (13-57). EPAG was started at a median interval from HSCT, on day +134 (range 71877 ) and was given for a median number of 164 days (range 45-242), at a dose of 25-100 mg/day . Two patients also received EPO and GCSF together with EPAG, for trilinear cytopenia. The hypothesis is that EPAG would be effective to treat late cytopenia developing after engraftment of an allogeneic HSCT.

Results: Nine of 10 patients had a complete response, and 1 patient had a partial response, as shown In Table 1. Three patients with thrombocytopenia increased teir median Plt counts from 12 to $128 \times 10^{\wedge} 9 / \mathrm{L}$. Two patients with PRCA associated with major $\mathrm{ABO}$ mismatch increased their median $\mathrm{Hb}$ levels from 7.5 to 14.2. One patient with neutropenia recovered completely. Four patients with pancytopenia, showed a response, which was complete in 3 patients; one patient remains anemic.

Conclusions: EPAG is effective in patients with unilinear, bi-linear, tri-linear cytopenia, followjg an allogeneic 
HSCT. We believe these data may warrant a prospective trial.

Conflict of interest: No conflicts of interest to declare

\begin{tabular}{llllll}
\hline EPAG & $\begin{array}{l}\text { n. } \\
\text { pts }\end{array}$ & $\begin{array}{l}\text { EPAG } \\
\text { start } \\
\text { post } \\
\text { HSCT } \\
\text { (median) }\end{array}$ & $\begin{array}{l}\text { EPAG } \\
\text { Duration } \\
\text { (median) }\end{array}$ & $\begin{array}{l}\text { Peripheral } \\
\text { Blood count } \\
\text { Before EPAG } \\
\text { Hb WBC Plts } \\
\text { g/dl 10^9/L }\end{array}$ & $\begin{array}{l}\text { Peripheral } \\
\text { Blood count } \\
\text { After EPAG } \\
\text { Hb WBC Plt g/ } \\
\text { dl 10^9/L }\end{array}$ \\
\hline $\begin{array}{l}\text { Thrombocytopenia } \\
\text { Neutropenia }\end{array}$ & 3 & 108 & 203 & 11.83 .512 & 10.95 .9128 \\
$\begin{array}{l}\text { Pure Red cell } \\
\text { Anemia }\end{array}$ & 1 & 542 & 101 & 13.50 .6117 & 13.53 .9175 \\
Pancytopenia & 652 & 140 & 7.53 .75203 & 14.28 .4279 \\
Total & 4 & 134 & 185 & 81.19 & 11.74 .691 \\
\hline
\end{tabular}

\section{[[P470 Table] Table 1]}

\section{P471}

\section{Evaluation of Cardiac Parameters in Bone Marrow Transplant Patients: Effect of pulmonary artery pressure on survival}

Ali Caner Ozdover ${ }^{1}$, Ilknur Gundes ${ }^{1}$, Melya Pelin Kirik ${ }^{1}$, Handan Haydaroglu Sahin', Murat Sucu ${ }^{3}$, Mustafa Pehlivan ${ }^{4}$

${ }^{1}$ Gaziantep University Faculty of Medicine, Internal Medicine, Gaziantep, Turkey; ${ }^{2}$ Gaziantep University Faculty of Medicine, Hematology, Gaziantep, Turkey; ${ }^{3}$ Gaziantep University Faculty of Medicine, Cardiology,, Gaziantep, Turkey; ${ }^{4}$ Gaziantep University Faculty of Medicine, Hematology, Bone Marrow Tranplantation Unit, Gaziantep, Turkey

Background: Hematopoietic stem cell transplantation (HSCT) is the process of delivering progenitor stem cells to the patient. It is the choice of treatment for various malignant and non-malignant diseases. Patients are treated with mobilization regimens before HSCT. These mobilization regimens include myeloablative, non-myeloablative or reduced-dose regimens. Complications can develop after HSCT. Among the most common complications are those of cardiac origin, which can be seen at all 3 stages of the treatment (acute, subacute or chronic), and can include heart failure (HF). The cardiovascular side effects of the mobilization regimens and cytoreductive therapies can be cumulative. The anthracycline chemotherapeutics, in particular, may have severe cardiac side effects. Cardiac side effects are among the very important determinants of survival. PAB has not been investigated in this group of diseases.

Methods: In this study, 428 patients who underwent HSCT in the Bone Marrow Transplantation Unit of Gaziantep University, Medical Faculty, Hematology Department were investigated. In the retrospective analysis, the patients who underwent transplantation once between
09.09.2009 and 09.09.2016 were evaluated by the official "medulla" system and file scanning from the archive. Normal 021 false false false TR X-NONE X-NONE /* Style Definitions */ table.MsoNormalTable \{mso-stylename:"Normal Tablo"; mso-tstyle-rowband-size:0; msotstyle-colband-size:0; mso-style-noshow:yes; mso-stylepriority:99; mso-style-parent:"'; mso-padding-alt:0cm $5.4 \mathrm{pt} 0 \mathrm{~cm} 5.4 \mathrm{pt}$; mso-para-margin:0cm; mso-para-marginbottom:.0001pt; mso-pagination:widow-orphan; fontsize:10.0pt; font-family:Calibri; \} EF and PAP values were analyzed during symptom-oriented ECHO examinations performed in pretransplant and postransplant periods. The cut off EF value was 50\% according to ESC (European Society of Cardiology) 2016 guide $^{1}$ and that of PAP was 25 mmHg according to ESC 2015 guide $^{2}$.

Results: In our study, 428 HSCT patients (allogeneic or autologous) were analyzed. EF and PAP values were investigated in these patients using symptom-oriented echocardiography $(\mathrm{ECHO})$ examinations during pretransplant and postransplant period. In both groups, pretransplant EF values were similar (median: 60\%). An increase of more than $10 \%$ in post-transplant $\mathrm{EF}$ was observed in 2 allo-HSCT (4.4\%) and 2 autologous HSCT $(3.3 \%)$ patients.

In patients who underwent AHSCT, the five-year mortality rate was $48.6 \%$ in patients with a PAP level above $25 \mathrm{mmHg}$ and $25.5 \%$ in patients with a normal PAP value $(\mathrm{p}<0,046)$. Overall five-year survival rate was found to be $38 \%$ (median 33.6 months) in 35 patients with pretransplant PAP> $25 \mathrm{mmHg}$ and $61 \%$ in 239 patients with PAP $<25$ mmHg $(\mathrm{p}<0,001)$.

Conclusions: In conclusion, pre-transplant PAP> 25 $\mathrm{mmHg}$ is an important risk factor affecting mortality and overall survival in patients with AHSCT.

Conflict of interest: We are submitting a paper entitled;

Evaluation of Cardiac Parameters in Bone Marrow Transplant Patients: Effect of pulmonary artery pressure on survival in your congress. This paper has not been published before. All authors have read and approved the manuscript. None of the authors have any potential conflicts of interest regarding this paper.

\section{P472}

Gender and time to relapse post hematopoietic cell transplant for AML predict the risk of death irrespective of the relapse type

Mona Hassanein, Mahmoud Aljurf, Naeem Chaudhri, Shahrukh Hashmi, Hazzaa Alzahrani, Walid Rasheed, Fahad Alsharif, Amr Hanbali, Syed Osman Ahmed, Marwan Shaheen, Feras Alfraih, Saud Alhayli, Maamoun Alsermani, Husam Alsaadi, Muhned Alhumaid, Ahmad Alshomar, Mohammed Assiri, Ghada Elgohary, Fahad Almohareb, Riad El Fakih 
King Faisal Specialist Hospital and Research Center, Adult Hematology/BMT, Riyadh, Saudi Arabia

Background: Acute myeloid leukemia (AML) relapse after allogeneic hematopoietic cell transplant (allo-HCT) is associated with a high risk of death and reported survival rates of 4-7 months. Bone marrow relapse (BMR) is the most frequent presentation. Extramedullary relapse (EMR), in isolation, or along with BMR is less common.

Methods: We studied 215 patients with AML who underwent allo-HCT in our institution between January 2005 and December 2015. We limited this retrospective analysis to patients who received a myeloablative conditioning, were in complete remission (CR) at the time of transplant and who received a matched related transplant, all other patients were excluded to avoid heterogeneity.

Results: Seventy seven of the studied patients (35.8\%) experienced disease relapse, 45 had BMR and 32 had EMR. Univariate analysis revealed that female patients, and relapse $<12$ months post allo-HCT have a significant association with the post-relapse death (Table 1). Both gender and Time to relapse remained significance in multivariate analysis after forcing the relapse type variable into the final model ( $\mathrm{p}$-value $=0.04$, and 0.002 respectively). However, relapse type either BMR or EMR did not show significant association with the risk of death $(\mathrm{HR}=0.8, \mathrm{p}$-value $=0.4)$. By logistic regression, chronic GVHD as a time-dependent covariate did not prove to be significantly associated with $\mathrm{EMR}(\mathrm{HR}=0.9, \mathrm{p}$-value $=0.1)$.

Conclusions: Our study concluded that regardless of the relapse type (BMR or EMR) patient gender and time from transplant to relapse are the most significant factors predicting the risk of death. While the late relapse is a known risk factor influencing survival, research into this gender disparity affecting prognosis must be sought.

Conflict of interest: No conflict of interest

\begin{tabular}{llll}
\hline Risk factors & Hazard Ratio (HR) & $95 \%$ CI & $\begin{array}{l}\text { p- } \\
\text { value }\end{array}$ \\
\hline Cytogenetic at diagnosis & $\begin{array}{l}\text { High risk(1), Low/ } \\
\text { intermediat risk(0.6) }\end{array}$ & $(0.3,1.09)$ & 0.1 \\
Disease status at HCT & CR1(1), Beyond CR1 (0.5) & $(0.4 .1 .5)$ & 0.5 \\
Sex & Female (1), Male (0.5) & $(0.3,0.9)$ & 0.04 \\
Graft source & BM (1), PB (0.9) & $(0.5,1.6)$ & 0.7 \\
Conditiong & TBI (1), Non-TBI(1.02) & $(0.51,2.04)$ & 0.9 \\
Time from allo-HCT to & $\leq 12$ months (1), >12 months & $(0.1,0.38)$ & $<0.001$ \\
relapse & $(0.2)$ & & \\
$\begin{array}{l}\text { Time from diagnosis to } \\
\text { allo-HCT }\end{array}$ & $\leq 6$ months (1), >6 months & $(0.5,2.15)$ & 0.8 \\
\hline
\end{tabular}

[[P472 Table] Univariate analysis using Cox regression]

\section{P473}

\section{Hematopoietic Stem Cell Transplantation in Children with Hemoglobinopathy: a Single-Centre Experience}

\author{
Muhammad Matloob Alam ${ }^{1}$, Ibraheem Abosoudah ${ }^{1}$, Hassan Al- \\ trabolsi ${ }^{1}$, Reem Zabani ${ }^{2}$, Mohamed Bayoumy ${ }^{1}$
}

${ }^{1}$ King Faisal Specialist Hospital \& Research Center, Jeddah, Saudi Arabia; ${ }^{2}$ Ibn Sina National College, Jeddah, Saudi Arabia

Background: Hematopoietic Stem Cell Transplantation (HSCT) has become an accepted therapeutic modality for a wide variety of diseases and is increasingly utilized for the treatment of nonmalignant disorders and frequently considered as a curative treatment for children with serious hematological disorders including thalassemia major (TM) and sickle cell disease (SCD).

Methods: We retrospectively analyzed the clinical, laboratory and outcome data of all pediatric patients with hemoglobinopathy consecutively underwent BMT between 2005 and 2016.

Results: Total number of transplantation in hemoglobinopathy patients during this study period were 32 , out of them 19 (59.4\%) were TM and remaining 13 (40.6\%) were $\mathrm{SCD}$. The mean age at the time of transplant was $8.14+/$ 4.6 years. Males were $16(50 \%)$ and females were $16(50 \%)$. All patients received allogeneic full matched related donor transplantation. (14 brother, 14 sister, 3 mother and 1 father). Conditioning regimen was Bu/Cy/ATG ( $\mathrm{n}=17$, $53.1 \%$, Flu/Bu/Cy/ATG $(\mathrm{n}=14,43.8 \%)$ Italian protocol $(\mathrm{n}$ $=1,3.1 \%$ ) Source of stem cell in all patients were bone marrow. Mean stem cell dose CD 34+ was $6.25(+/$ - 2.9) x $10^{6} / \mathrm{kg}$. All patient were received CSA/MTX for GVHD prophylaxis.

Acute GVHD was observed in 10 (31.25\%); out of them skin was involved in $(\mathrm{n}=5,15.6 \%)$ gut $(\mathrm{n}=2,6.3 \%)$, liver $(\mathrm{n}=2,6.3 \%)$ and both skin and gut $(\mathrm{n}=1,3.1 \%)$. Grade I, II, III and IV GVHD were observed in $1(3.1 \%), 4$ (12.5\%) and $4(12.5 \%)$ and $1(3.1 \%)$ patients respectively. Chronic GVHD were noticed in $2(6.3 \%)$ patients and both had skin involvement.

In most patients median duration of ANC engraftment was is 22 (Range: 12-27). Only one patients have delayed ANC engraftment at day 57 and another patients were expired before day 28 post transplantation. Median platelet engraftment was 32 (Range: 18- 45) days in 28 patients and remaining 4 patients had delayed platelet engraftment (6290 days).

CMV reactivation was observed in $18(56.3 \%)$ cases within 100 day of post BMT. Out of them majority were asymptomatic $(\mathrm{n}=17 ; 53.1 \%)$ and remaining $(\mathrm{n}=1 ; 3.2 \%)$ had clinical manifestation/organ involvement (pneumonia and skin manifestation). Most of CMV cases were resolved 
( $\mathrm{n}=17 ; 53.1 \%)$. In most case $(16 / 18 ; 88.8 \%)$, the level of antigenemia declined within 2 weeks as a result of the continuation of GCV therapy.

Overall survival (OS) rate were $93.7 \%$ and Event free survival (EFS) rate were $90.6 \%$ with median duration of follow up $33.72(+/-25.3)$ months. Two beta thalassemia major patient lost engraftment at 6 month and 9 month of transplantation. Both patients who expired was TM, one on day +16 post BMT with pulmonary hemorrhage and massive ICH and another at Day +75 with ARDS.

Conclusions: The results of the pediatric BMT program at our institution have been nearly comparable to those reported in the literature as far as transplant-related morbidity and mortality is concerned. However, the duration of follow-up is short and the long-term outcome is yet to be determined.

\section{Conflict of interest: None}

\section{P474}

\section{High volume and erythrocyte content of umbilical cord blood units predict risk of poor engraftment}

\author{
Jacqueline Overdevest ${ }^{1}$, Arjan Lankester ${ }^{2}$, Peter von dem Borne ${ }^{3}$, \\ Jaap Jan Zwaginga ${ }^{1}$, Tanja Netelenbos ${ }^{1}$ \\ ${ }^{1}$ Leiden University Medical Center, Department of \\ Immunohematology and Blood Transfusion, Leiden, Netherlands; \\ ${ }^{2}$ Leiden University Medical Center, Pediatrics, Division of Stem Cell \\ Transplantation, Leiden, Netherlands; ${ }^{3}$ Leiden University Medical \\ Center, Department of Hematology, Leiden, Netherlands
}

Background: Umbilical cord blood (UCB) units from unrelated donors used for stem cell transplantation (SCT) differ in type of bag, volume size and erythrocyte content. Although, modern UCB units are small in volume and contain little red blood cells (RBC's), older units are still used for SCT. In our center we use criteria for washing the product based on the NMDP BB-IND\#7555 safety report 2009. When the number of RBC's is $>190 \times 10 \mathrm{e} 9$ or in case of major blood group incompatibility $>150 \times 10 \mathrm{e} 9$, the product is washed to remove erythrocytes and Dimethylsulfoxide (DMSO). We analyzed a large cohort of patients that were transplanted with UCB and studied the impact of UCB volume, erythrocyte content and washing on quality controls and engraftment.

Methods: Retrospectively, data were collected on all UCB transplants performed in our center between 2003 and 2017 in adults and pediatric patients for various indications. The following data were collected provided by the UCB bank where the product was released: the volume, the number of RBC's and whether RBC depletion or volume reduction was performed prior to freezing the product. Data were collected on CD34 viability (ISHAGE) after thawing and after washing if performed. Furthermore, patient engraftment was studied.

Results: Data from 122 UCB units were analyzed. Most UCB units had a volume $<30 \mathrm{ml}(66 \%)$. Sixteen UCB units were washed after thawing (13\%). Eleven UCB contained RBC's $>150 * 10 \mathrm{e} 9$ and all of these units had a large volume (median: $53.2 \mathrm{ml}$, range $40--200 \mathrm{ml}$ )). From 61 units it was unknown whether $\mathrm{RBC}$ reduction was performed in the UCB bank. From 74 units we could retrieve the collection date. In the group of older products (collected $<2009$ ) there were relatively more units with a volume $>30 \mathrm{ml}(\mathrm{n}=24)$ and a high ( $>150)$ or unknown number of RBC's $(n=20)$. From the washed UCB units, six (38\%) showed a CD34 viability $<25 \%$ after washing. However, viability after thawing and washing was reasonably comparable with viability before washing. An inverse correlation was found between the volume of the unit and the viability after thawing. From 90 patients that were transplanted with single CB (57) or double CB (33), 15 patients failed to show engraftment $(9 \%)$. Eight of these patients received washed CB units.

Conclusions: Our data show a clear correlation between washed UCB units and risk of non-engraftment (50\% versus $9 \%$ ). This is not necessarily due to the washing procedure itself, but more likely due to the pre-existing high amount of RBC's in the units. High volume UCB units $(>30 \mathrm{ml})$ therefore need to be avoided due to a higher risk for nonengraftment. The causal mechanism is not clear, but RBC's might influence the post thawing quality of the UCB units by hemolysis.

Conflict of interest: J. Overdevest: nothing to disclose

\section{P475}

HLA phased haplotypes in Switzerland, predictive value for matched unrelated donor searches and clinical outcome after HSCT

Jean Villard ${ }^{1}$, Stephane Buhler ${ }^{1}$, Helen Baldomero ${ }^{2}$, Sylvie FerrariLacraz ${ }^{1}$, Stravoula Massouridi-Levrat ${ }^{3}$, Dominik Heim ${ }^{2}$, Joerg Halter $^{2}$, Gayathri Nair ${ }^{4}$, Yves Chalandon ${ }^{3}$, Urs Schanz ${ }^{4}$, Tayfun Guengoer $^{5}$, Grazia Nicoloso ${ }^{6}$, Jean-Marie Tiercy ${ }^{1}$, Jakob Passweg ${ }^{2}$

${ }^{1}$ Geneva University Hospitals, Dpt of Nephrology / UIT-LNRH, Genève, Switzerland; ' ${ }^{2}$ Basel University Hospital, Dpt of Haematology, Basel, Switzerland; ${ }^{3}$ Geneva University Hospitals, Dpt of OncoHaematology, Genève, Switzerland; ${ }^{4}$ University Hospital of Zurich, Dpt of Haematology, Zurich, Switzerland; ${ }^{5}$ Children's Hospital, Zurich, Switzerland; ' ${ }^{6}$ wiss Blood Stem Cells Registry, Bern, Switzerland

Background: A crucial criterion for successful allogeneic hematopoietic stem cell transplantation (HSCT) is HLA matching between the recipient and donor. In practice HLA matching is performed at the phenotypic level but recent 


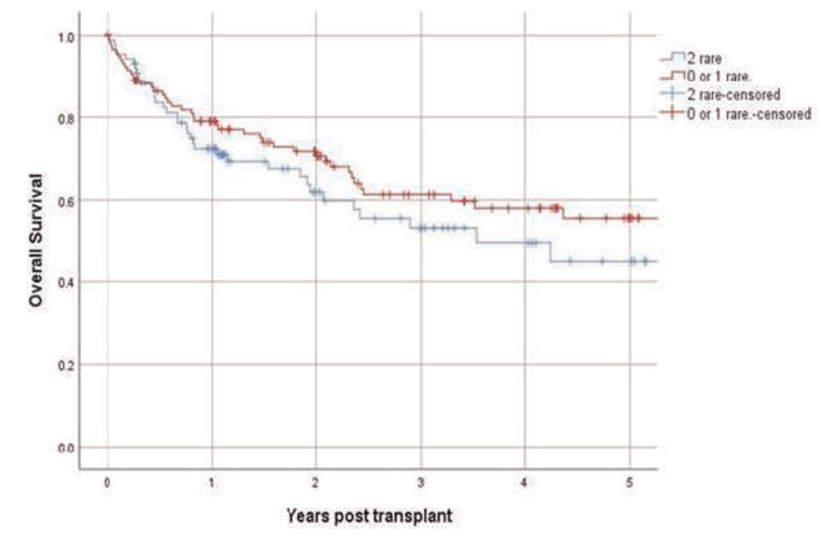

[P475 Figure] [Survival curves for patient's carrying 2 rare vs 0 or 1 rare HLA haplotypes ( $\log$ rank test $p=0.27)$ ]

studies have proposed that further matching at the haplotypic level could be beneficial (e.g. reduced graft versus host disease) if several 10/10 MUD (matched unrelated donors) are available.

Methods: The aims of this study were twofold. First, we used the high resolution typings of 291 patients living in Switzerland and potential candidates for an unrelated donor search to determine their HLA haplotypes by descent using family members and segregation analysis. The sum of ranks of the two haplotypes for each patient (as compared to the frequencies estimated for 6114 volunteer donors of the Swiss registry) was used as a surrogate predictor of a successful search (i.e. finding a 10/10 MUD) in the BMDW database. Second, the putative impact of phased HLA haplotypes (i.e. considered as 'frequent' or 'rare' as an indirect parameter of haplotype matching) on the clinical outcome of HSCT was analysed in a cohort of 212 patients transplanted with 10/10 MUD. These 212 patients were mainly affected by malignancy diseases, a large majority being represented by acute leukaemia (100), myelodysplastic and myeloproliferative syndromes (42). About $40 \%$ of the patients were aged between 40-60, a quarter of them below 20 and the rest evenly distributed between 20-40 and greater than 60 years old.

Results: Logistic regression analysis showed a highly significant effect of the haplotype ranks in the outcome of a search in BMDW $(p=2.25 e-14)$. Furthermore, a ROC curve analysis showed that the best trade-off between true and false positive rates was to choose a cut off corresponding to a sum of ranks of 1000 (i.e. equivalent to a patient carrying at least one very rare haplotype).

However, we could not find any significant effect on overall survival (Figure 1), acute or chronic graft versus host disease and relapse following HSCT despite considering different frequency cut offs and genotype subgroups for categorizing HLA haplotypes.
Conclusions: This study provides useful data for the optimization of unrelated bone marrow donor searches in Switzerland and beyond, but could not confirm previous findings that matching at the haplotypic level has a clinical impact following HSCT. Due to the extreme polymorphism of HLA genes further studies are warranted to better comprehend the many factors at play.

Clinical Trial Registry: NA

Conflict of interest: None

\section{P476}

HPC count with Sysmex XN-2000 is a good predictor of the $\mathrm{CD} 34+$ cell in patients' blood

Iwona Mitrus ${ }^{1}$, Magdalena Prokop ${ }^{2}$,Malwina Czech ${ }^{2}$, Wojciech Fidyk $^{1}$, Agata Chwieduk ${ }^{1}$, Magdalena Glowala-Kosinska ${ }^{1}$, Sebastian Giebel $^{1}$

${ }^{1}$ Maria Sklodowska-Curie Institute - Oncology Center, Gliwice Branch, Department of Bone Marrow Transplantation and Oncohematology, Gliwice, Poland; ${ }^{2}$ Maria Sklodowska-Curie Institute - Oncology Center, Gliwice Branch, Analytics and Clinical Biochemistry Department, Gliwice, Poland

Background: The hematopoietic stem and/or progenitor cells (named HSCs or HPCs) are traditionally identified by flow cytometry, as CD34 positive cells. Flow cytometry is a gold standard, unfortunately this methodology is timeconsuming and operator-dependent. Currently, many producers of diagnostic equipment offer the automated hematology analyzers that have the possibility of HPC enumeration. This technique is fast, easy to perform and does not require the use of expensive antibodies.

The first aim of this study was to examine a correlation between enumeration of HPCs (performed by Sysmex XN2000) and CD34+ cells in peripheral blood of mobilized patients. The second aim was to determine the optimal cutoff point of HPCs that predicts CD34+ cells count above $20 / \mu \mathrm{L}$. This is sufficient number to perform an affective apheresis.

Methods: HPCs and CD34+ cells were counted in the same fresh samples of peripheral blood. 131 samples were collected from 80 patients ( 44 men and 36 women, median age: 59 years, range 24-74 years) mobilized with intermediate-dose cytosine arabinoside followed by GCSF. In case of 3 patients, a single injection of Plerixafor was used to increase the number of circulating HSCs. The patients carried a diagnosis of MM $(\mathrm{n}=42)$, NHL $(\mathrm{n}=27)$, HL $(n=7)$ and others $(n=4)$.

Flow cytometry analysis was performed according to the ISHAGE guidelines. Cells were double-labeled with phycoerythrin (PE) conjugated anti-CD34 and fluorescein isothiocyanate (FITC) conjugated anti-CD45 monoclonal 
antibodies (BD Biosciences) and analyzed using fluorocytometer FACS Canto II. HPC enumeration was performed using the Sysmex XN-2000 (Sysmex, Kobe, Japan).

Results: The median number of $\mathrm{CD} 34+$ cells/ $\mu \mathrm{L}$ in blood was 50 (range: 0-813), the median number of HPCs/ $\mu$ was 75 (range: 0-1269). There was a strong correlation between the numbers of HPCs and CD34+ cells $\left(\mathrm{R}^{2}=0.8298, \mathrm{p}<\right.$ $0.001)$.

Next, ROC curve was created as a marker of the test quality. The HPC cut-off values to distinguish CD34+ cell counts below or above $20 / \mu \mathrm{L}$ were determined to be 34 (AUC $=0.951$, Youden Index 0.76).

Conclusions: There is a strong correlation between the numbers of HPCs counted by Sysmex XN-2000 and CD34 + cells in samples of peripheral blood. This predictor may be useful for making a decision to start apheresis.

Conflict of interest: Nothing to disclose

\section{P477}

Immune-hematologic monitoring after allogeneic stem cell transplantation: unicentric prospective study on 104 patients

Ursula La Rocca ${ }^{1}$, Serelina Coluzzi ${ }^{2}$, Walter Barberi ${ }^{1}$, Gianluca Giovannetti $^{2}$ Isabella Santilio ${ }^{2}$, Alessia Neri ${ }^{2}$, Giovanni Fernando Torelli $^{1}$, Luisa Quattrocchi ${ }^{1}$, Sara Mohamed ${ }^{1}$, Serena Rosati ${ }^{1}$, Giulia De Luca ${ }^{1}$, Michela Ansuinelli ${ }^{1}$, Sara Pepe ${ }^{1}$, Robin Fò̀ ${ }^{1}$, Gabriella Girelli ${ }^{2}$, Anna Paola Iori ${ }^{1}$

${ }^{1}$ Policlinico Umberto 1, Sapienza University of Rome, Hematology, Rome, Italy; ${ }^{2}$ Sapienza University of Rome, Immunoematology and Transfusion Medicine, Rome, Italy

Background: The impact of $\mathrm{ABO}$ incompatibility between recipient and donor on the outcome of hematopoietic stem cell transplantation (HSCT), on the immunehematologic (IH) complications and on the role of $\mathrm{IH}$ monitoring before and after HSCT are still debated. The aim of this study was to evaluate the impact of $\mathrm{ABO}$ mismatch on the development of immediate and late IH complications, DFS, OS, GVHD and TRM. Moreover, we analyzed the efficacy of the protocol used at the "Sapienza"University of Rome to manage $\mathrm{ABO}$ incompatibility in patients undergoing a HSCT.

Methods: From January 2013 to December 2016, we prospectively analyzed 104 consecutive patients (64 males/ 40 females) receiving a HSCT:29.8\% from a matched related donor, $53.8 \%$ from a matched unrelated donors, $1.9 \%$ from a cord blood, $14.4 \%$ from a haploidentical donor. Within 30 days prior to the HSCT and every week up to 30 days post-HSCT, then every 15 days until RBC engraftment, patients underwent a complete IH assessment, including $\mathrm{ABO}$ and $\mathrm{Rh}$ blood phenotype, indirect antiglobulin test, direct antiglobulin test, anti-A and anti-B IgM, and IgG titration in ABO-incompatible cases. Graft manipulation or desensitization strategies were defined according to ABO incompatibility, donor sex (all female donor grafts were plasma depleted), donor's transfusion history. RBC and PLT transfusion support was established according to $\mathrm{IH}$ features. Data were prospectively collected in a database, including age, sex, diagnosis, donor hematopoietic stem cell source, conditioning regimen and post-HSCT complications such as CMV/EBV infections, venous-occlusive disease, hemorrhagic cystitis, hemolyticuremic syndrome and GVHD.

Results: Fourty-nine of 104 patients (47\%) were ABOidentical and 55 (53\%) ABO-incompatible (23 major, 25 minor, 7 bidirectional). Seven patients $(6.7 \%)$ received a non-myeloablative conditioning regimen, $33(31.7 \%)$ a reduced intensity and 64 patients $(61.5 \%)$ a myeloablative regimen. Stem cell source was bone marrow for $52 \%$ of patients, peripheral blood for $46 \%$ of patients, cord blood for $2 \%$. In 61 cases $(58.7 \%$ ), a plasma depletion from the graft was carried out, in 2 cases the graft was subjected to erythro-sedimentation, in 2 other cases both plasma and RBC depletion were performed. Two patients were treated with plasmapheresis (1 ABO bidirectional incompatibility and 1 ABO major + high titer anti-K alloantibody). Hemolysis during infusion was observed in $17 \%$ of patients with $\mathrm{ABO}$ major and bidirectional incompatibility. Donor engraftment in PMN, PLTs and RBCs, graft failure or other complications did not differ between patients with or without $\mathrm{ABO}$ incompatibility; only 1 patient with a major ABO incompatibility suffered from a pure red cell aplasia. Two patients experienced autoimmune hemolytic anemia. Factors associated with a prolonged blood support (RBC) were ABO incompatibility $(p=0.01)$, hemorrhagic cystitis $(\mathrm{p}=0.01)$ and haploidentical transplant $(\mathrm{p}=0,004)$. ABO incompatibility did not show a significant impact on GVHD, OS and DFS.

Conclusions: In this prospective study, we confirm that ABO incompatibility does not represent a barrier to alloHSCT. It is, however, associated with a prolonged transfusional requirement. In our experience, ABO incompatibility does not influence the risk of GVHD. A strict IH monitoring, as a shared standard procedure, allows to perform an appropriate transfusion support and to treat patients efficiently, limiting post-HSCT IH complications.

Conflict of interest: All authors declare no conflict of interest

\section{P478}

Impact of geographic distance from transplant center for patients undergoing Allogeneic Stem Cell transplantation: A retrospective, single study 
Basel Haffar ${ }^{1}$, Radwan Massoud ${ }^{1}$, Nour Moukalled ${ }^{1}$, Elie Akl', Ammar Zahreddine ${ }^{1}$, Rita Nehme ${ }^{1}$, Ali Bazarbachi ${ }^{1}$, Jean El Cheikh ${ }^{1}$

${ }^{1}$ American University of Beirut Medical Center, Internal Medicine, Division of Hematology-oncology, Bone Marrow Transplant Unit, Beirut, Lebanon; ${ }^{2}$ American University of Beirut Medical Center, Faculty of Medicine, Beirut, Lebanon

Background: It has been reported that proximity to transplant center affects outcomes of allogeneic stem cell transplantation (Allo-SCT). We conducted this study at the American University of Beirut Medical-Center (AUBMC), a referral center for the Middle-East located in Lebanon to determine the differences in the survival between patients residing in Lebanon and those referred from abroad.

Methods: We identified 161 consecutive adult patients who underwent allo-SCT for hematologic malignancies between January-2007 and December-2016. one hundred and three $(64 \%)$ patients were residing in Lebanon $(68$ males, 35 females), and 58 patients $(36 \%)$ were referred from abroad (41 males, 17 females). Follow up on patients residing in Lebanon was done via clinic visits, while for those residing abroad was either by clinic appointment, and/ or phone-calls in case they could not travel to Lebanon. All patients and transplant characteristics are listed in table-1.

Results: Median age at transplant was 40 (range 18-70) in the Local-group, versus 39 (range, 23-69) in the foreigngroup. All patients engrafted, the median time to neutrophil engraftment was 15 days in both groups but with ranges (10-21) and (9-35) days in the local and foreign groups respectively. In the local-group $73(71 \%)$ patients received ATG, and $45(77 \%)$ patients in Foreign group.

The incidence of acute-graft-versus-host-disease was higher in local-group with 30 cases (29\%) and 11 cases $(19 \%)$ in foreign group. Similarly, the incidence of chronicgraft-versus-host disease in local-group was superior to foreign-group, with 23 (22\%) patients vs. 11(19\%) patients respectively. After a median follow- up of 16 months (range, 1-99), 59 (57\%) and 34 (59\%) patients were alive in the local and foreign groups respectively. The median OS was 55 months in local groups and has not been reached in foreign group. The median progression-free-survival (PFS) has not been reached in both groups. The cause of death was classified into two categories, disease related and TRM. In the local group, $44(43 \%)$ patients died, from those, 27 (26\%) were disease related and $17(14 \%)$ due to TRM. Whereas $24(41 \%)$ patients died in foreign group, from those $12(21 \%)$ deaths were disease related, while $7(12 \%)$ deaths were TRM.

Conclusions: Our results imply that the geographical distance has no impact on OS, however, it might have impact on PFS, but we should take into consideration that more
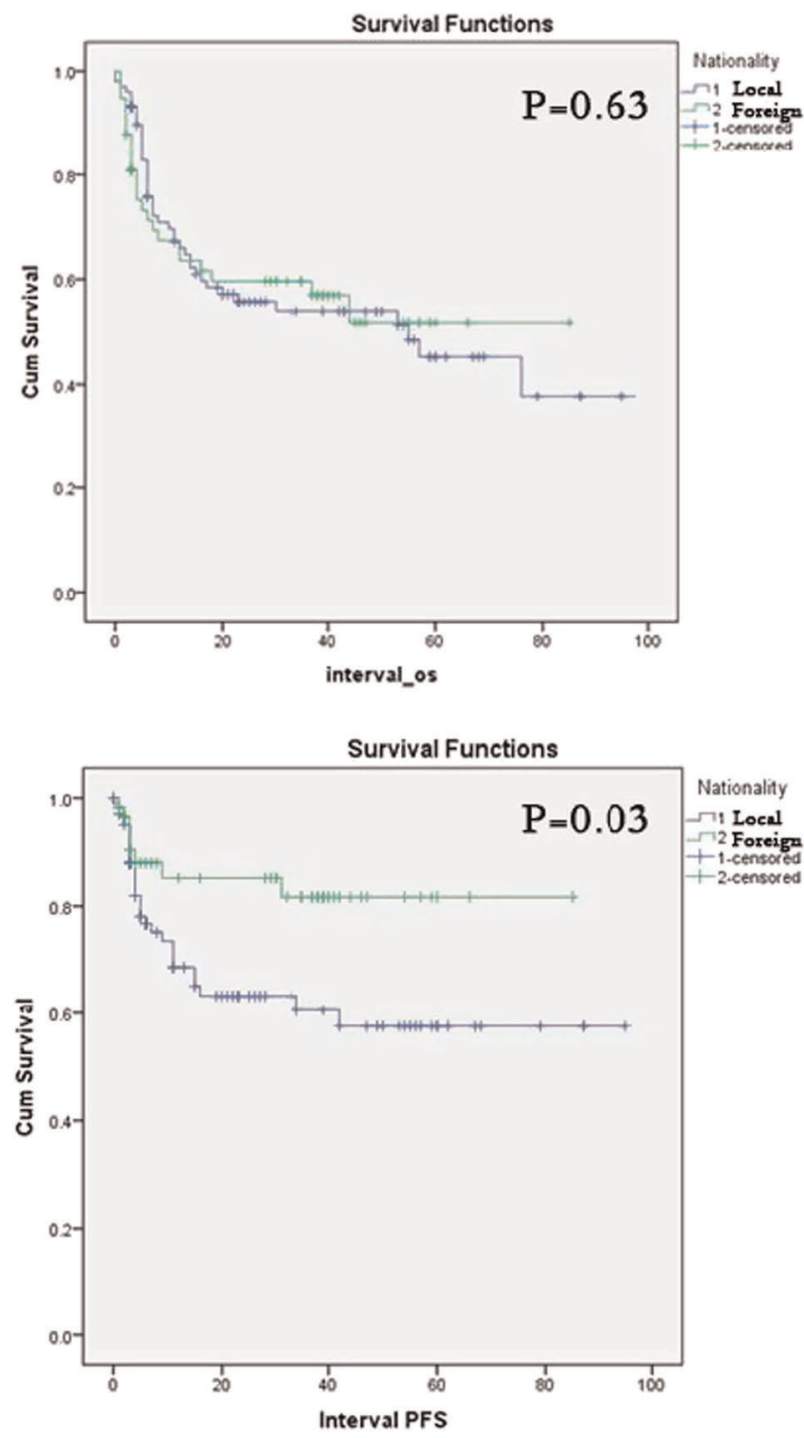

[P478 Figure] [Overall survival and Progression free survival local vs foreign groups]

patients in local group underwent Haploidentical transplant. These findings need to be verified in larger scale studies.

Conflict of interest: All authors have nothing to declare.

\begin{tabular}{llll}
\hline & $\begin{array}{l}\text { Local group N } \\
(\%)\end{array}$ & $\begin{array}{l}\text { Foreign group } \\
\mathrm{N}(\%)\end{array}$ & P value \\
\hline $\begin{array}{l}\text { induction at AUBMC/ } \\
\text { referred for transplant }\end{array}$ & $44(43) / 59$ & $19(33) / 39$ & $\mathrm{NS} / \mathrm{NS}$ \\
$\begin{array}{l}\text { Referred from Iraqi/ Syrian/ } / \\
\text { UA }\end{array}$ & $\begin{array}{l}67) \\
46(79) / 8(14) /\end{array}$ & $\mathrm{NS} / \mathrm{NS} / \mathrm{NS}$ \\
UAE/jordanian & & $3(5) / 1(2)$ & \\
$\begin{array}{l}\text { Disease: AML / ALL/ NHL/ } \\
\text { HL/aplastic anemia/others }\end{array}$ & $48(47) / 16(16) /$ & $31(53) / 13$ & $\mathrm{NS} / \mathrm{NS} / \mathrm{NS} /$ \\
& $5(5) / 8(8)$ & $(22) / 3(5) / 1(2) /$ & $0.006 / \mathrm{NS} /$ \\
Type of donor MRD/Haplo/ $/ 76(7) / 4(7)$ & $\mathrm{NS}$ \\
MUD & $5(5)$ & $(2)$ & $0.0007 /$ \\
DRI Low / intermediate/ & $6(6) / 59(57) /$ & $5(8) / 27(46) /$ & $\mathrm{NS} / \mathrm{NS} / \mathrm{NS}$ \\
high & $31(30)$ & $20(34)$ & \\
Status At transplant: CR/ & $68(66) 39(57)$ & $34(59) / 20(59) /$ & $\mathrm{NS} / \mathrm{NS} /$ \\
CR1/ CR2/ CR3/ & $18(26) 11(16)$ & $8(23) / 6(18) /$ & $\mathrm{NS} / \mathrm{NS} /$
\end{tabular}


Table (continued)

\begin{tabular}{|c|c|c|c|}
\hline & $\begin{array}{l}\text { Local group N } \\
(\%)\end{array}$ & $\begin{array}{l}\text { Foreign group } \\
\mathrm{N}(\%)\end{array}$ & $P$ value \\
\hline $\begin{array}{l}\text { Stable disease-Partial } \\
\text { Response/progression } \\
\text { relapse }\end{array}$ & $18(17) / 17(17)$ & $10(17) / 14(24)$ & NS \\
\hline $\begin{array}{l}\text { Conditioning Regimen TBF- } \\
\text { ATG / FB-ATG / FB-ATG- } \\
\text { TBI / Sequential / Others }\end{array}$ & $\begin{array}{l}21(20) / 39 \\
(38) / 5(5) / 8 \\
(8) / 30(29)\end{array}$ & $\begin{array}{l}4(7) / 30(52) / \\
2(3) / 3(5) / 19 \\
(33) /\end{array}$ & $\begin{array}{l}0.038 / \mathrm{NS} \\
\mathrm{NS} / \mathrm{NS} / \\
\mathrm{NS} / \mathrm{NS}\end{array}$ \\
\hline $\begin{array}{l}\text { Last Follow up Complete } \\
\text { remission/ Relapse- } \\
\text { Progression/ lost to follow } \\
\text { up }\end{array}$ & $\begin{array}{l}71(69) / 32 \\
(31) / 0(0)\end{array}$ & $\begin{array}{l}34(58) / 12(21) \\
/ 12(21)\end{array}$ & NS/NS \\
\hline
\end{tabular}

[[P478 Table] patients and transplant characteristics]

\section{P479}

Investigating the impact of patient's non-shared HLA-C
expression levels in a single HLA-C mismatched HSCT
setting using two different HLA-C expression proxy models

Chrysanthi Tsamadou ${ }^{1,2}$, Daniel Fürst ${ }^{1,2}$, Markus Rojewski ${ }^{2}$, Dietger Niederwieser $^{3}$, Donald Bunjes ${ }^{4}$, Christine Neuchel ${ }^{1,2}$, Martin Gramatzki $^{5}$, Renate Arnold ${ }^{6}$, Eva Wagner ${ }^{7}$, Hermann Einsele ${ }^{8}$, Sixten Körper ${ }^{9}$, Hubert Schrezenmeier ${ }^{2,9}$, Joannis Mytilineos ${ }^{1,2,10}$

${ }^{1}$ Institute of Clinical Transfusion Medicine and Immunogenetics Ulm, German Red Cross Blood Transfusion Service, Baden Wuerttemberg Hessen, and University Hospital Ulm, Transplantation Immunology, Ulm, Germany; ${ }^{2}$ Institute of Transfusion Medicine, University of Ulm, Ulm, Germany; ${ }^{3}$ University of Leipzig, Hematology/Oncology, Leipzig, Germany; ${ }^{4}$ University of Ulm, Department of Internal Medicine III, Ulm, Germany; ${ }^{5}$ University of Kiel, Division of Stem Cell Transplantion and Immunotherapy, 2nd Department of Internal Medicine, Kiel, Germany; ${ }^{6}$ Charité Campus Virchow Berlin, Hematology/Oncology, Berlin, Germany; ${ }^{7}$ Johannes GutenbergUniversity Mainz, Department of Internal Medicine III, Mainz, Germany; ${ }^{8}$ University Hospital Würzburg, Department of Internal Medicine II, Würzburg, Germany; ${ }^{9}$ Institute of Clinical Transfusion Medicine and Immunogenetics Ulm, German Red Cross Blood Transfusion Service, Baden Wuerttemberg - Hessen, and University Hospital Ulm, Ulm, Germany; ${ }^{10}$ DRST - German Registry for Stem Cell Transplantation, Ulm, Germany

Background: Petersdorf et al. were the first to report an association between patient's high expressed HLA-C (C) allotypes and worse HSCT outcome by using data on $\mathrm{C}$ expression mainly derived from African-Americans (Apps et al. 2013). Main objectives of this study were:

1) To examine the applicability of Apps et al. model in Caucasians by using the same quantification methodology in a much larger sample of 400 healthy German blood donors and to subsequently generate a second proxy expression model .

2) To specifically investigate the effect of patient's nonshared (PNS) C expression levels on outcome by applying the previously mentioned expression models in a 9/10 HLA C-mismatched HSCT setting.
Methods: Buffy coats from 400 healthy German blood donors were tested by flow cytometry as previously described (Apps et al. 2013) in order to determine C expression on lymphocytes. With reference to the mean fluorescence intensity (MFI) values measured, C antigens were subsequently categorized as high- or low- expressed.

In a cohort of 324 single $\mathrm{C}$-mismatched transplant pairs both $\mathrm{C}$ expression models were used in order to investigate the impact of high- and low-expressed PNS-C allotypes on HSCT outcome. Overall survival (OS), disease free survival (DFS), relapse incidence (RI) and non-relapse mortality (NRM) were set as outcome endpoints, while statistical significance was set at $\mathrm{p}<0.05$.

Results: With the exception of HLA-C*15, the two expression models were found fully concordant as to the definition of low- and high-expressed $\mathrm{C}$ antigens. Analysis of PNS-C expression's effect on HSCT outcome revealed an unexpected correlation between high expression and better OS due to lower NRM. Although similar trends were observed, statistical significance was only met with the Apps et al. model.

Death cause analysis revealed a tendency for lower infection-related mortality in the high expressed PNS-C (10\% vs $14 \%$ ), while no differences were observed as to GvHD-associated death. Interestingly, overall patient's C expression had no influence on any outcome endpoint.

Conclusions: Basic conclusions of this study are:

1) HLA-C antigens exhibit similar expression patterns regardless of race.

2) High expressed PNS-C may play a role in better infection control in a single HLA-C mismatched HSCT setting. Albeit noteworthy, these findings must be confirmed by independent and significantly larger cohorts, before definitive conclusions can be drawn.

Conflict of interest: None of the authors has anything to declare.

\begin{tabular}{|c|c|c|c|c|c|}
\hline $\begin{array}{l}\text { Univariate } \\
\text { Analysis }\end{array}$ & $\begin{array}{l}\text { Apps } \\
\text { model } \\
\text { high vs } \\
\text { low }\end{array}$ & $\begin{array}{l}\text { German } \\
\text { model } \\
\text { high vs } \\
\text { low }\end{array}$ & $\begin{array}{l}\text { Multivariate } \\
\text { Analysis }\end{array}$ & $\begin{array}{l}\text { Apps model } \\
\text { high vs low }\end{array}$ & $\begin{array}{l}\text { German } \\
\text { model high vs } \\
\text { low }\end{array}$ \\
\hline $5 y O S$ & $\begin{array}{l}(49 \% \text { vs } \\
33 \%) \mathrm{p} \\
=0.04\end{array}$ & $\begin{array}{l}(45 \% \text { vs } \\
35 \%) \mathrm{p} \\
=0.13\end{array}$ & OS & $\begin{array}{l}\mathrm{HR}=0.43, \mathrm{CI} \\
95 \%=0.25- \\
0.74, \mathrm{p}= \\
0.002\end{array}$ & $\begin{array}{l}\mathrm{HR}=0.65, \mathrm{CI} \\
95 \%=0.35- \\
1.20), \mathrm{p}= \\
0.17\end{array}$ \\
\hline $5 y$ DFS & $\begin{array}{l}(37 \% \mathrm{vs} \\
25 \%) \mathrm{p} \\
=0.07\end{array}$ & $\begin{array}{l}(35 \% \text { vs } \\
27 \%) \mathrm{P} \\
=0.16\end{array}$ & DFS & $\begin{array}{l}\mathrm{HR}=0.50, \mathrm{CI} \\
95 \%=0.30- \\
0.83, \mathrm{p}= \\
0.008\end{array}$ & $\begin{array}{l}\mathrm{HR}=0.64, \mathrm{CI} \\
95 \%=0.36- \\
1.14, \mathrm{p}=0.13\end{array}$ \\
\hline 5yNRM & $\begin{array}{l}(30 \% \text { vs } \\
47 \%) \mathrm{p} \\
=0.02\end{array}$ & $\begin{array}{l}(35 \% \text { vs } \\
44 \%) \mathrm{p} \\
=0.17\end{array}$ & NRM & $\begin{array}{l}\mathrm{HR}=0.29, \mathrm{CI} \\
95 \%=0.15- \\
0.59, \mathrm{p}= \\
0.0006\end{array}$ & $\begin{array}{l}\mathrm{HR}=0.69, \mathrm{CI} \\
95 \%=0.31- \\
1.53, \mathrm{p}=0.36\end{array}$ \\
\hline $5 y R I$ & $\begin{array}{l}(43 \% \text { vs } \\
40 \%) \mathrm{p} \\
=0.74\end{array}$ & $\begin{array}{l}(41 \% \text { vs } \\
41 \%) \mathrm{p} \\
=0.90\end{array}$ & RI & $\begin{array}{l}\mathrm{HR}=1.11, \mathrm{CI} \\
95 \%=0.59- \\
2.07, \mathrm{p}=0.75\end{array}$ & $\begin{array}{l}\mathrm{HR}=0.69, \mathrm{CI} \\
95 \%=0.35- \\
1.37, \mathrm{p}=0.29\end{array}$ \\
\hline
\end{tabular}

[[P479 Table] Univariate and multivariate analyses] 
P480

\section{LONG TERM OUTCOME OF 138 ALLOGENEIC HEMATOPOIETIC STEM CELL TRANSPLANTATION (HSCT) IN ADULT ACUTE LYMPHOBLASTIC LEUKEMIA: RESULTS OF A TRANSPLANT CENTER}

Irati Ormazabal Velez, Javier Cornago Navascues, Angela Figuera, Adrian Alegre Amor

Hospital Universitario La Princesa, Hematology and Hemotherapy, Madrid, Spain

Background: High rates of relapse remains the main barrier to long term survival though the great improvement in first complete remission (CR1) rates achieved in adult Acute lymphoblastic leukemia (ALL). HSCT could be the only curative option for high-risk patients in CR1 and all patients who experience relapse. Because some controversy on indication and timing exists, large patient cohorts should be evaluated.

Methods: Between 1990 and 2016, 138 HSCT were performed in 128 patients with ALL in our hospital (10 double alloHSCT; 7,2\%). Median age was 28 years (12-65) and $76 \%$ of the patients were $<40$ years. There were $27,5 \%$ $(\mathrm{n}=38)$ Philadelphia positive $(\mathrm{Ph}+)$ ALL. Disease status before transplant was divided in $42 \%$ CR 1 and, $60,5 \%$ ( $\mathrm{n}=$ 23) in $\mathrm{Ph}+$ and $35 \%(\mathrm{n}=35)$ in $\mathrm{Ph}-$, and $58 \%$ advanced disease $(A D=\geq R C 2, M R D+$, active disease), 39,5\% ( $\mathrm{n}=$ $15)$ in $\mathrm{Ph}+$ and $65 \%(\mathrm{n}=65)$ in $\mathrm{Ph}-$.

The primary endpoint was disease-free survival (DFS) and overall survival (OS). Secondary endpoints refined acute graft vs. host disease (aGvHD) and chronic GvHD (cGvHD) incidence, relapse incidence (RI), and non-relapse mortality (NRM).

Results: With a median follow up of 11 years (21 days26 years), the whole cohort DFS an OS rates were 37,7\% and $42,8 \%$, respectively. OS \& DFS in $\mathrm{Ph}+$ and $\mathrm{Ph}-\mathrm{ALL}$ patients who underwent HSCT in CR1 were 66,7\% \& $66,6 \%$ and $57,6 \% \& 51,4 \%$, respectively . OS and PFS in AD were similar in $\mathrm{Ph}+$ and Ph- ALL: 28,6 and 13,3\% and $30,8 \& 24,6 \%$, respectively.

The incidence of aGvHD was $32,6 \%(\mathrm{n}=45)$ and cGvHD 29\% $(n=40)$. Twenty six cases $(18,8 \%)$ of extensive cGvHD occurred.

RI was $36,2 \%(n=50)$ with a median time of 6 months. Late relapse seemed unlikely 3 years after HSCT $(n=3)$. The incidence of early NRM ( $<100$ days) was $18,8 \%(\mathrm{n}=$ 26 ), being the most frequent death causes pneumonia or respiratory failure $(\mathrm{n}=11 ; 36,7 \%)$, sinusoidal obstructive syndrome $(n=4 ; 13,3 \%)$, septic shock $(n=3 ; 10 \%)$ and acute GvHD $(\mathrm{n}=2 ; 6,7 \%)$.

Conclusions: In our cohort was highlighted a high OS for both $\mathrm{Ph}+$ and $\mathrm{Ph}-$ ALL underwent HSCT in CR1, indicating the importance of achieving the best response before HSCT. However quite an acceptable percentage of Ph- ALL with AD benefit from HSCT, taking into account it was the most common group.

It seems the principal objective for the future should be the improvement of strategies to decrease RI in ALL in order to achieve a higher OS and DFS.

Conflict of interest: Nothing to disclosure

\begin{tabular}{ll}
\hline CHARACTERISTIC & RESULTS \\
\hline Median age (years) & $28(12-65)$ \\
$<40 />40$ years & $105(76 \%) / 36(34 \%)$ \\
Gender (male/female) & $77(55,8 \%) / 61(44,2 \%)$ \\
$\mathrm{Ph}+/$ Ph- ALL & $38(27,5 \%) / 100(72,5 \%)$ \\
$\mathrm{B}$ ALL/ T ALL/ biphenotypic AL & $113(81,9 \%) / 18(13 \%) / 7(5,1 \%)$ \\
Years of HSCT: 1990-2000/2001-2008 & $61(44,2 \%) / 57(41,3 \%) / 20(14,5 \%)$ \\
HSC source: BM/PB/CB & $90(65,2 \%) / 46(33,3 \%) / 2(14,5 \%)$ \\
Conditioning regimen: CyTBI/CyBu/ & $108(78,1 \%) / 17(11,7 \%) / 14$ \\
other & $(10,2 \%)$ \\
\hline
\end{tabular}

[[P480 Table] Table 1]

\section{P481}

Long-term Storage At $-80^{\circ} \mathrm{C}$ And 5\% DMSO Does Not Affect Stem Cell Viability And Transplantation Outcome In Patients Receiving Second Autologous Transplantation

Ivan Tonev, Chavdar Botev, Stanislav Simeonov, Zhana MinchevaGove, Svetlana Hristova, Georgi Arnaudov, Penka Ganeva, Yavor Petrov, Georgi Mihaylov, Branimir Spassov, Milcho Mincheff

National Specialized Hospital For Active Treatment Of Haematological Diseases, Sofia, Bulgaria

Background: : Dimethylsulfoxide (DMSO) is the main intracellular cryoprotective agent and, for a long-term storage of hematopoietic stem cells (HSC), it is usually used at a final $10 \%$ concentration. Lower concentrations of DMSO, that are sparing side effects (cell toxicity, heart rhythm disorders, hemodynamic disturbance, bronchial spasm, etc.) to patients after transfusion, have been used for a shorter period of storage (up to eighteen months). We report here of stem cell viability and transplant results with samples, frozen with $5 \% \mathrm{DMSO}$ and stored at $-80^{\circ} \mathrm{C}$ for periods between 35 and 82 months.

Methods: Peripheral hemopoetic stem cells were processed and frozen in a cryoprotectant solution containing a final concentration of 5\% DMSO, 6\% HES and 3\% HSA. The cells were stored at $-80^{\circ} \mathrm{C}$ in mechanical freezers. Eight patients (one with NHL and seven with MM), were cytapheresed and their cells cryopreserved. Since some of the patients required a second transplant several years later, 
autologous cells that were collected after the first mobilization and stored for up to 82 months were used.

Results: There was no difference in the cell viability, tested with trypan blue exclusion test. All patients engrafted successfully with similar engraftment time for WBC and platelets after the first and second transplantation.

Conclusions: DMSO is toxic for the cells and leads to serious adverse reactions (heart rhythm disorders, hemodynamic disturbance, bronchial spasm, etc.) after infusion. The addition of extracellular cryoprotectants gives the opportunity to use smaller concentration of DMSO in the final suspension, thus reducing the incidence and severity of the posttransfusion adverse events. This small group of patients shows that long-term storage at $-80^{\circ} \mathrm{C}$ degrees and $5 \%$ DMSO is safe and reduces the adverse events resulting from DMSO toxicity. Storage at $-80^{\circ} \mathrm{C}$ is easier, less time and space consuming.

Conflict of interest: none

\section{P482}

\section{LYMPHOCYTE SUBSET RECONSTITUTION AND OUTCOMES AFTER AUTO STEM CELL TRANSPLANTATION IN CHILDREN- SINGLE CENTRE EXPERIENCE}

Ana Maria Fita ${ }^{1}$, Maria Victoria Martínez Sánchez ${ }^{2}$, María Esther Llinares Riestra ${ }^{1}$, María Mar Bermúdez Cortés ${ }^{1}$, Ana María Galera Miñarro ${ }^{1}$, Miguel Blanquer Blanquer ${ }^{3}$, Andrés Sánchez Salinas ${ }^{3}$, José Luis Fuster Soler ${ }^{1}$, José María Moraleda ${ }^{3}$

${ }^{1}$ Hospital Clínico Universitario Virgen de la Arrixaca, Paediatrics Oncology and Haematology Unit, El Palmar, Murcia, Spain;

${ }^{2}$ Hospital Clínico Universitario Virgen de la Arrixaca, Immunology Service, El Palmar, Murcia, Spain; ${ }^{3}$ Hospital Clínico Universitario Virgen de la Arrixaca, IMIB, University of Murcia, Haematology Service and Stem Cell Transplant Unit, El Palmar, Murcia, Spain

Background: Long-term follow-up data of immune reconstitution and engraftment stability in pediatric patients who undergo autologous stem cell transplatation (ASCT) remains poorly reported.

Methods: Retrospective, observational study of patients aged between 0 and 15 years undergoing ASCT at a tertiary hospital between January 2007 and December 2015. Time to engraftment was defined as the first of 3 consecutive days on which neutrophils exceeded $0.5 \times 10^{9} / \mathrm{L}$, platelets $20 \mathrm{X}$ $10^{9} / \mathrm{L}$ without transfusion, Immunologic reconstitution was assessed by total lymphocytes; CD3+, CD4+ and CD8+ T lymphocytes; CD19+ B lymphocytes, CD16+/CD56+ natural killer $(\mathrm{NK})$ and $\mathrm{CD} 4+/ \mathrm{CD} 8+$ ratio at day +30 , $+60,+90,+180$ and +360 after transplantation. Normal lymphocyte population values were defined as those included in The Pediatric AIDS Clinical Trials Group P1009 study.
Results: 38 ASCT procedures were performed in 35 patients with a median follow-up of $18(1-86)$ months. The median age at HSCT was $5(0-13)$ years, and $66 \%$ were male. The indication for HSCT was a solid tumor in $89 \%$ of the patients and malignant hemopathies in the remaining $11 \%$. Bu-Mel accounted for $50 \%$ of conditioning protocols used. Thiotepa-based (24\%), Mel-VP16 (13\%) and other regimens $(13 \%)$ were also used. The median number of CD34+ (x 106/kg) infused was 4,45 (0,96 - 15,77). We observed rapid neutrophil and platelet engraftment kinetics (median time to engraft, 11 days and 13 days, respectively). The immune reconstitution was assessed in 17 (48\%) patients. Full recovery of $\mathrm{CD} 3+$ lymphocytes along with $\mathrm{CD} 4+\mathrm{T}$ lymphocytes count, was achieved at day +360 , while CD8+ T lymphocytes showed prompt recovery one month post ASCT (Figure 1A). Similarly, NK cells showed normal and sustained counts from day +30 . By contrast, CD19+ B lymphocytes showed a slower recovery with normal counts from day +180 . This evolution resulted in a reversion of the CD4/CD8 ratio (Figure 1B).

During the first post SCT year we documented bacterial, viral and fungal infections, in $65 \%, 24 \%$ and $8 \%$ of the patients respectively. Only one patient died of infection (bronchopulmonary aspergillosis) at day +57 post SCT. The event-free survival (EFS) at day +100 and one year post SCT, was $82 \pm 6 \%$ and $64 \pm 8 \%$ respectively. The overall survival (OS) at day +100 and one year post SCT was $94 \pm 4 \%$ and $79 \pm 7 \%$ respectively.

Conclusions: Our data show a rapid immune reconstitution and low transplant mortality rate in patients undergoing ASCT. The observed deficiencies are transient, followed by a relatively reliable pattern of recovery.

Conflict of interest: None

\section{P483}

\section{Mapping the Cellular Heterogeneity of CD34-Selected Umbilical Cord Blood Products}

Sruthi Mantri ${ }^{1}$, Andreas Reinisch ${ }^{2}$, Kenneth Weinberg ${ }^{1}$, Deirdre Lyell $^{3}$, David DiGiusto ${ }^{1}$, Rajni Agarwal-Hashmi ${ }^{1}$, Matthew Porteus ${ }^{I}$

${ }^{1}$ Stanford University, Pediatrics, Stanford, CA, United States;

${ }^{2}$ Stanford University, Medicine, Stanford, CA, United States; ${ }^{3}$ Stanford University, Obstetrics and Gynecology, Stanford, CA, United States

Background: Using umbilical cord blood (UCB) as a source of hematopoietic stem cells (HSCs) for allogeneic hematopoietic stem cell transplantation (HSCT) often results in delayed engraftment, immune reconstitution and platelet recovery when compared with marrow. Standard characterization of cord blood products includes CD34+ and total nucleated cell counts, as colony forming unit assays have not been widely adopted as a measure of 

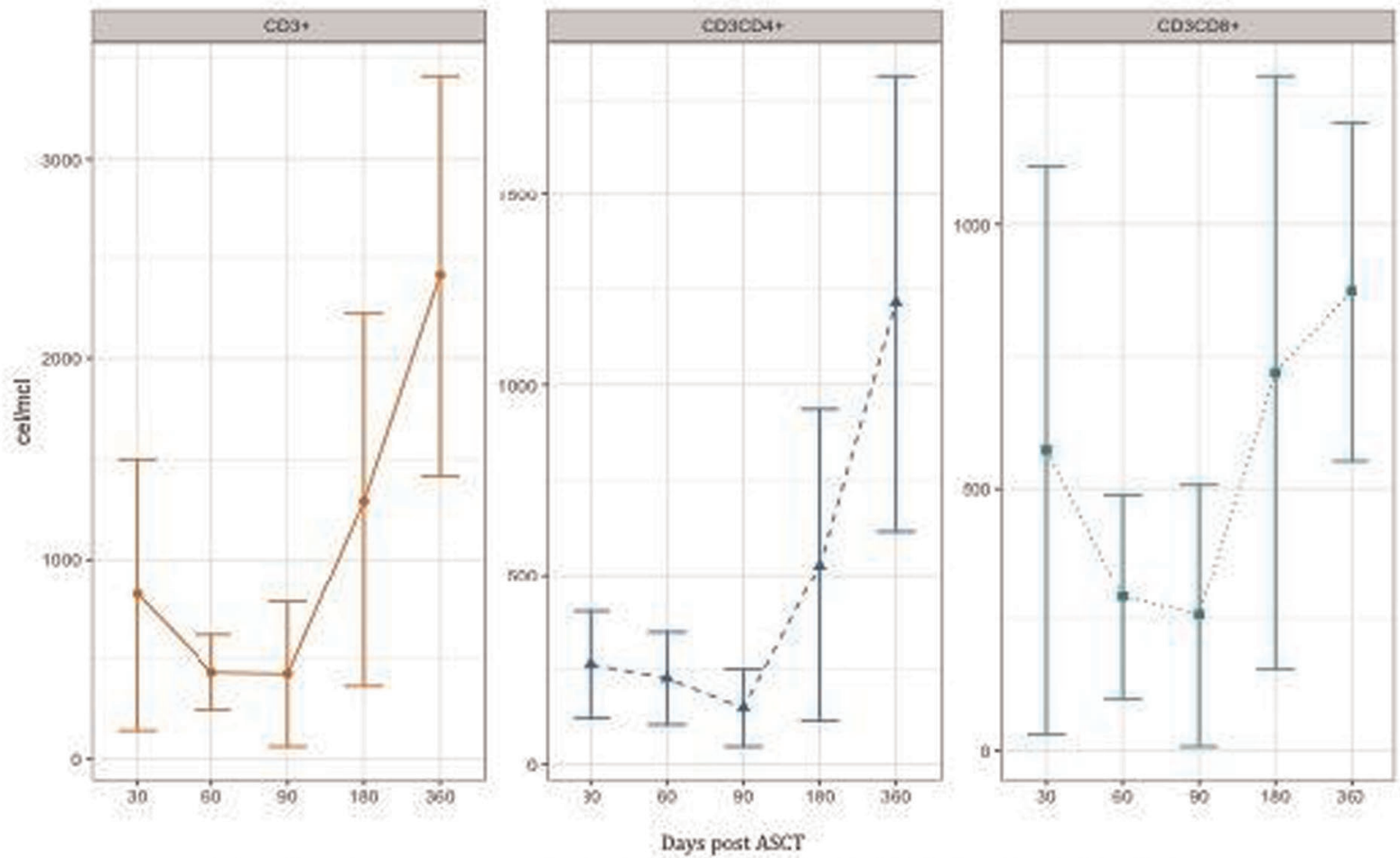

Figure 1A. Immune reconstitution in ASCT: CD3+, CD3CD4+, CD3CD8+.
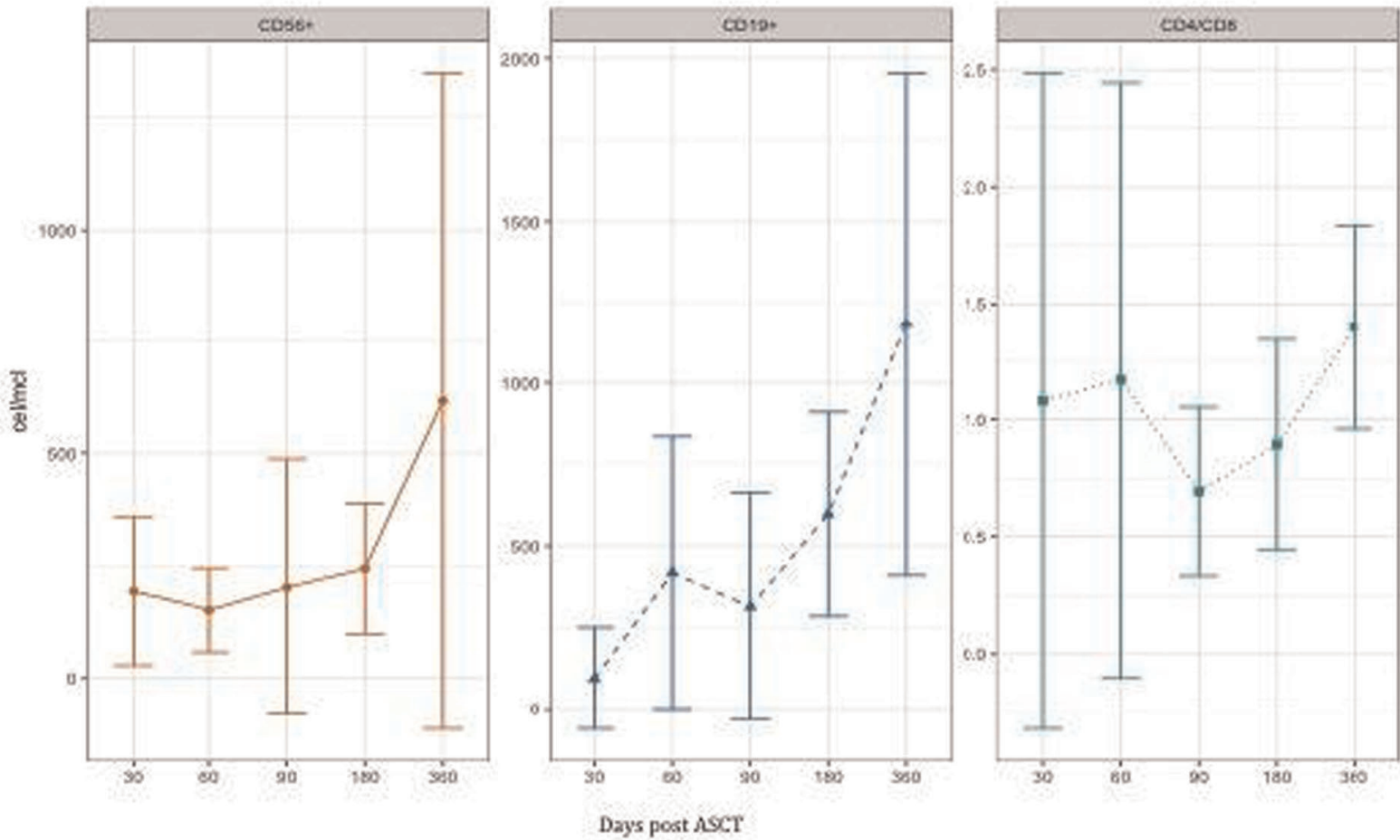

Figure 1B. Immune reconstitution in ASCT: CD56+, CD19+, CD4/CD8+.

[P482 Figure] [Figure 1. Immune reconstitution in ASCT. Data expressed as the means and standard deviations.]

potency. While we now recognize the diversity of hematopoietic stem and progenitor cell (HSPC) populations within the CD34-positive pool, the proportion of these populations is not well understood. To this end, we present 
a novel data set revealing the frequencies of different HSPC populations in human UCB.

Methods: Between November 2015 and November 2017, 553 UCB samples were collected at the Labor and Delivery unit under IRB-approved protocols. Mononuclear cells were isolated by density gradient centrifugation using Ficoll Paque Plus and labeled using the CD34+ Microbead Kit Ultrapure (Miltenyi Biotec) for positive selection of HSPCs. Enriched cells were stained with human anti-CD34, -CD38, -CD45RA, -CD90, -CD10, -CD123, -CD127, and a cocktail targeting the following lineage markers: CD2, CD3, CD4, CD8, CD14, CD16, CD19, CD20, CD56, and CD235a prior to analysis on a BD FACS Aria II flow cytometer. All antibodies were obtained through BD, Biolegend, and eBioscience.

Results: Cord blood collected from 50 individuals was immunophenotyped for primitive, early and committed progenitor cell surface markers (Table 1). High degrees of heterogeneity were observed between individuals, with a frequency range spanning one log in most cell types. Although the median HSC immunophenotype was $6.7 \%$ of all CD34+ cells, the range was large (0.1-17.3\%). Phenotypic common lymphoid progenitors were consistently found in less than $0.1 \%$ of all CD34+ cells (median: $0.007 \%$ ). In striking contrast, the common myeloid progenitor phenotype was expressed by the largest proportion of all early progenitors (median: $32.8 \%$ ). Preliminary analysis showed that the median frequency of phenotypic megakaryocte progenitors is $<1 \%$, with a range of 0.1 $17 \%$.

Conclusions: Significant variation in the cellular content of human UCB suggests that immunophenotyping samples prior to HSCT may provide important information regarding individual graft potency. Low CLP and MKP immunophenotype frequencies provide a potential new explanation for the prolonged period for immune reconstitution and platelet recovery, a key feature of UCB HSCT. Taken together, these results provide a rapid method of mapping the cellular content of UCB and may pave the way for functional studies and improved graft selection for UCB HSCT.

Conflict of interest: None of the authors has anything to disclose.

\begin{tabular}{|c|c|c|c|}
\hline Cell Type & Immunophenotype & Median & Range \\
\hline Hematopoietic stem cell (HSC) & $\begin{array}{l}\text { Lin-34+38-45RA-90 } \\
+\end{array}$ & 6.7 & $0.1-17.3$ \\
\hline Multipotent progenitor (MPP) & Lin-34+38-45RA-90- & 10.6 & $1.1-37.5$ \\
\hline Common myeloid progenitor (CMP) & $\begin{array}{l}\text { Lin-34+38+45RA- } \\
123+\end{array}$ & 32.8 & $3.1-45.3$ \\
\hline Granulocyte / monocyte progenitor (GMP) & $\begin{array}{l}\text { Lin-34+38+45RA } \\
+123+\end{array}$ & 20.1 & $6.3-50.4$ \\
\hline $\begin{array}{l}\text { Megakaryocyte / erythrocyte progenitor } \\
\text { (MEP) }\end{array}$ & $\begin{array}{l}\text { Lin-34+38+45RA- } \\
123-\end{array}$ & 11.2 & $3.7-22.7$ \\
\hline Megakaryocyte progenitor (MKP) & $34+41+$ & 0.4 & $0.1-17$ \\
\hline
\end{tabular}

Table (continued)

\begin{tabular}{llll}
\hline Cell Type & Immunophenotype & Median & Range \\
\hline Common lymphoid progenitor (CLP) & Lin-34+38+127+ & 0.0071 & $0.000-$ \\
& Lin-34+38-45RA-90- & 1.1 & $0.064-4.6$ \\
$\begin{array}{l}\text { Multipotent lymphoid progenitor (MLP) } \\
\begin{array}{l}\text { Lymphoid-primed multipotent progenitor } \\
\text { (LMPP) }\end{array}\end{array}$ & $\begin{array}{l}\text { Lin-34+38-45RA } \\
+90 \mathrm{v}\end{array}$ & 3.3 & $0.9-16.5$ \\
\hline
\end{tabular}

[[P483 Table] Population frequencies in UCB-derived CD34+ pool]

\section{P484}

\section{Micronutrients Intake among Hematopoietic Stem Cell Recipients: Result of a Randomized Controlled Trial Post Hospital Discharge}

\author{
Batoul Manana ${ }^{1}$, Jana Jabbour ${ }^{2,3}$, Ali Bazarbachi ${ }^{4}$, Didier Blaise ${ }^{5}$, \\ Jean El-Cheikh ${ }^{4}$ \\ ${ }^{1}$ American University of Beirut Medical Center, Division of \\ Hematology/Oncology, Department of Internal Medicine, Beirut, \\ Lebanon; ${ }^{2}$ American University of Beirut Medical Center, Department \\ of Clinical Nutrition, Beirut, Lebanon; ${ }^{3}$ Aix Marseille Université, \\ Doctoral School of Life Science and Health, Marseille, France; \\ ${ }^{4}$ American University of Beirut Medical Center, Bone Marrow \\ Transplantation Program, Department of Internal Medicine, Beirut, \\ Lebanon; ${ }^{5}$ Aix Marseille Université, CNRS, INSERM, CRCM, Institut \\ Paoli-calmettes, Marseille, France
}

Background: Among hematopoietic stem cell transplant (HSCT) patients, micronutrient profile is altered due to increased excretion, decreased intake, and increased need of some nutrients. The nutrition care in HSCT is focused on meeting energy and protein needs with minimal attention to micronutrient needs. We aimed to assess adequacy of vitamins and minerals intake among HSCT patients in the peritransplant phase.

Methods: This is a randomized control trial approved by the Institutional Review Board. Patients admitted to undergo HSCT at the American University of Beirut Medical Center were invited to join the study. Assessment points were at admission for transplant, at hospital discharge, and at days 30, 60, and 100 post HSCT. Study participants $(n=46)$ were randomized at hospital discharge into intervention group ( $\mathrm{IG}, \mathrm{n}=22$ ) and control group (CG, $\mathrm{n}=24)$. IG received standardized meal plan and nutritional counselling at the assessment points post hospital discharge aiming at optimizing energy and protein intake while CG received the standard of hospital care. At assessment points, quality of life (QoL) was assessed using Functional Assessment of Cancer Therapy (FACT) - Bone Marrow Transplant (BMT) questionnaire, the intake of patients was monitored, and 24-hour recalls were collected and analyzed via Nutritionist ProÒsoftware. Data was analyzed by age 

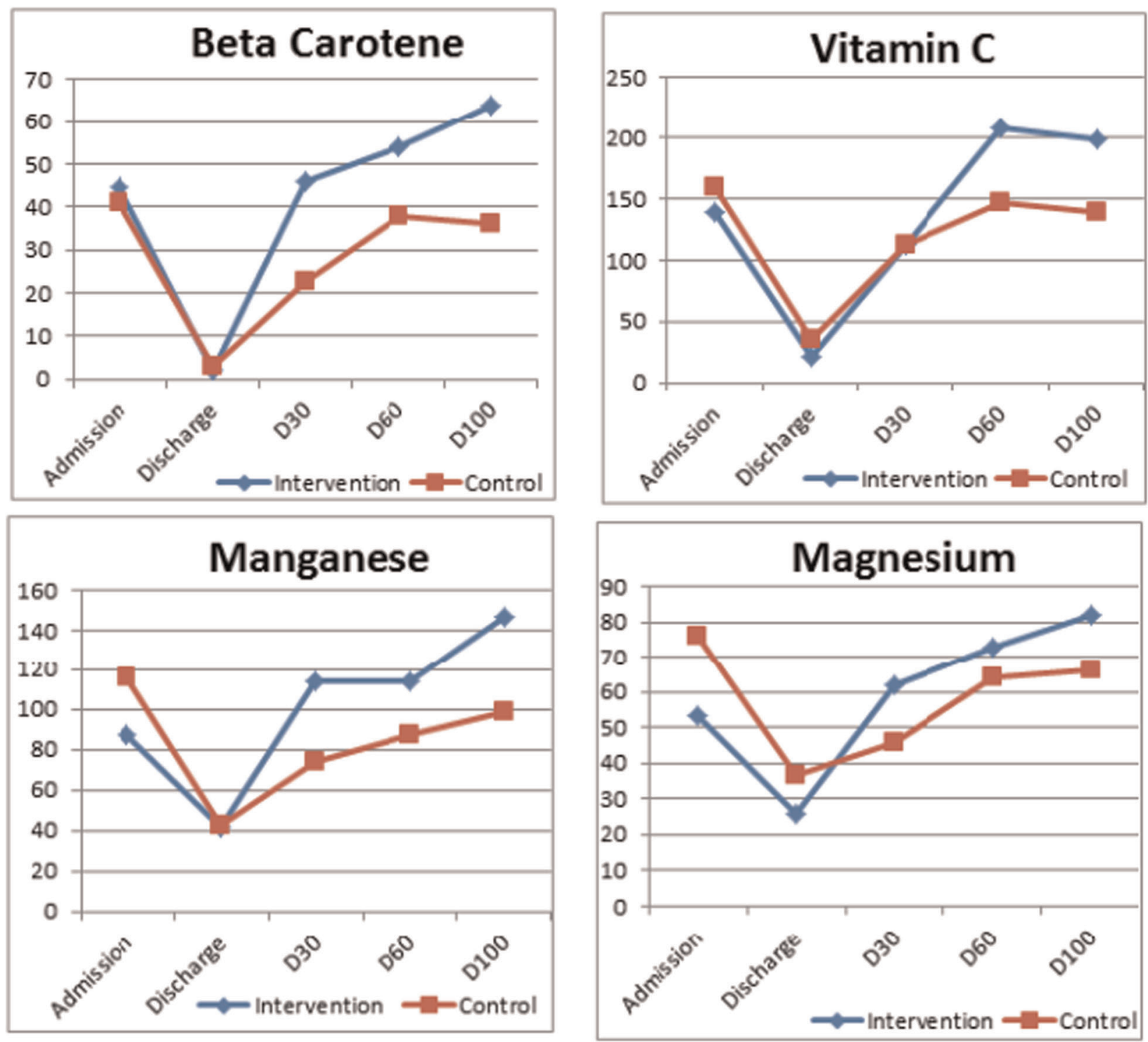

[P484 Figure] [Graphical Representation of selected micronutrients' intake in the peri-transplantation phase, pres]

and gender based on the dietary reference intake (DRI) values and results were reported as percentages of DRI.

Results: The IG participants were of median age (years) of 52 vs. 39 in $\mathrm{CG}(\mathrm{p}=0.15)$, both groups had more males, 15 (68\%) in IG and 15 (63\%) in CG. At hospital Discharge, QoL was clinically better in IG (median $=109$ for IG vs. 100 for $\mathrm{CG}, \mathrm{p}=0.24)$. Results post hospital discharge showed alarmingly low intake levels of vitamin D in both groups at all assessment points, median intake was below 18\% DRI in IG and 23\% DRI in CG. At all assessment points, median vitamin $\mathrm{E}$ intake remained below $70 \%$ DRI in IG and $40 \%$ DRI in CG. The intake of many micronutrients showed a significant improvement from discharge until day 100 in the IG. Intake was statistically better for beta carotene, vitamins K, B2, B12 and C and for zinc, potassium, phosphorous, magnesium, manganese and iron in IG Changes in CG were of a lower magnitude. Fiber intake was better in IG but did not reach $100 \%$ of needs at all assessment points.

Conclusions: This study fills a research gap by providing an overview of nutrients intake among HSCT patients in the peri-transplant phase. Results showed that nutrition counseling improves intake of some micronutrients, while some remains at risk in both control and intervention groups.

Clinical Trial Registry: Clinicaltrials.gov NCT02791347, June 2016.

Conflict of interest: The authors report no conflict of interest. 
P485

Peripheral blood versus bone marrow as a source of hematopoietic stem cells for allogeneic transplantation in children. A comparative study

Juan Carlos Jaime Fagundo ${ }^{1}$, Sandra Sarduy Sáez ${ }^{1}$, Dunahisy Llerena Moreno ${ }^{1}$, Carsten Heilmann ${ }^{2}$, Wilfredo Roque ${ }^{1}$

${ }^{1}$ Institute of Hematology and Immunology, La Habana, Cuba, La Habana, Cuba; ${ }^{2}$ University Children's HospitalHospital

Rigshospitalet, Paediatric and Adolescent Medicine, Copenhagen, Denmark

Background: Hematopoietic progenitors obtained from peripheral blood (PB) are used increasingly in autologous and allogeneic transplantations. Both advantages and disadvantages have been reported when $\mathrm{PB}$ is used in instead of bone marrow (BM) as a graft source in children.

Methods: We evaluated 37 patients aged between 3 and 18 years, with hematological diseases (14 with acute lymphoblastic leukemia, 10 with acute myeloid leukemia, 6 with chronic myeloid leukemia, 5 with aplastic anemia, one with Hodgkin lymphoma and one with sickle cell disease) that received allogeneic hematopoietic transplantation from a sibling donors at The Institute of Hematology and Immunology, Cuba from June 1996 to April 2014. In 23 patients the BM was used as a source of cells, and in 14 progenitors were obtained from the $\mathrm{PB}$ and various variables were compared between the two sources.

Results: The number of mononuclear cells in the graft was $2,87 \times 10^{8} / \mathrm{kg}$ in the BM group and $9,20 \times 10^{8} / \mathrm{kg}$ in the PB group. Neutrophil and platelet recovery were faster and transfusion requirements were lower when PB was used. All the patients presented febrile episodes before neutrophil engraftment. In the PB group, the majority of episodes corresponded to fever of unknown origin while in the BM group the microorganism were identified more often. In the two groups, acute graft-versus-host disease was the same; both with regard to frequency and severity, but chronic graft-versus-host disease had a greater frequency and severity in patients who received $\mathrm{PB}$. The probability of overall survival at day 100 with BM was 0.82 , and with $\mathrm{PB}$ 0.92 ; whereas the probability of overall survival at 6 months was 0.68 and 0.92 respectively.

Conclusions: : It was observed that the transplantation of hematopoietic progenitor cells mobilized to the PB offers advantages when compared with BM transplantation. However, the small number of patients studied and the fact that it is not a randomized study, do not allow for definitive conclusions.

Conflict of interest: Juan Carlos Jaime Fagundo: nothing to disclose.
P486

\section{Planning the evaluation of stability of cryopreserved cellular therapy products-preliminary results}

Fátima Amado, Sérgio Machado Lopes, Filipa Bordalo, Sara Ferreira, Ana Catarina Pinho, Margarida Dias, Ana Salselas, Susana Roncon

Instituto Português de Oncologia do Porto, Porto, Portugal

Background: Cryopreservation is the method used for the storage of hematopoietic progenitor cells (HPC) in the transplantation setting; this study aims to evaluate the impact of several techniques during the freezing, cryopreservation and thawing processes, as well as to determine the quality criteria to include in the stability program.

Methods: Between April and October 2017, 22 units composed of remaining HPC from four allogeneic donors were frozen and cryopreserved, according to standardized procedures of our facility and after the approval of the ethics committee. A cryoprotector solution was added to the cellular suspension composed by dimethyl sulfoxide $10 \%$ of final volume and 5\% human serum albumin (ratio $>2$ ). The products were assessed regarding: sterility (blood cultures in aerobic/anaerobic conditions and mycology); functionality (quantification of granulocyte-monocyte colony-forming units, CFU-GM); cellular viability (total nucleated cells, TNC, by the Trypan blue exclusion method and CD34+ and CD45+ cells by 7-aminoactinomycin D staining); TNC recovery was evaluated with a hematologic counter and CD34+ cells by flow cytometry. Finally the cells were cryofreezed in EVA bags with a sterile secondary container, at controlled temperature, and stored in liquid nitrogen tanks at $-196^{\circ} \mathrm{C}$.

Six units were thawed in a water bath at $37^{\circ} \mathrm{C}$ (with continuous agitation) until the crystals disappeared. At the end, the pre-freezing assays were repeated and additionally a macroscopic assessment of the product and of the bags' integrity was made. The HPC traceability was assured by electronic and paper registries of the physical localization within the tank and by data compliance of the cryogenic bag labels concerning identification and legibility. In the liquid nitrogen tanks it was evaluated the nitrogen quantity, temperature, corrosion level and integrity of the boxes.

Results: The median time of cryopreservation was 103 (50-228) days; all unities complied with the established criteria. From the 6 samples tested, none presented haemolysis neither aggregates; $100 \%$ were sterile both in freezing and thawing, proving that there was no contamination during storage. Regarding the functionality, there was CFU-GM growth in all tested samples. The results obtained for the viability and recovery are shown in table 1 . 


\begin{tabular}{|c|c|c|}
\hline \multirow{2}{*}{ Cell viability } & TNC & $81.5 \%(78.0-84.0)$ \\
\cline { 2 - 3 } & CD45+ & $76.0 \%(73.0-81.0)$ \\
\cline { 2 - 3 } & CD34+ & $99.0 \%(99.0-100.0)$ \\
\hline \multirow{2}{*}{ Hematologic recovery } & TNC & $98.2 \%(95.6-102.8)$ \\
\cline { 2 - 3 } & CD34+ & $86.1 \%(62.9-93.2)$ \\
\hline
\end{tabular}

[P486 Figure] [Table 1- Results of cell viability and recovery evaluation (Median, Minimum-Maximum)]

All the cryogenic and secondary bags were undamaged, as well as the nitrogen tanks and metal boxes. The tracking of the cellular product from the donor to the patient and the tracing from the patient to the donor were ensured in all the cases.

Conclusions: All pre-established quality criteria (sterility, functionality, cellular viability and haematological recovery) were met and physical parameters were unaffected. This shows that cryopreservation for a short period of time does not affect the quality of the product, if the operational procedures in place are followed.

We intend to prolong the study in order to evaluate the stability of long term cryopreservation of HPC, and eventually define a maximum storage period, with predefined time frames by each donor.

Conflict of interest: All authors: Nothing to disclose.

\section{P487}

\section{Preclinical and Clinical Applications of 17-color Whole Blood Dissection Protocol for Studying the Human Hematopoietic System}

\section{Luca Basso-Ricci ${ }^{1}$, Serena Scala ${ }^{1}$, Raisa Hernandez ${ }^{1}$, Raffaella Milani $^{2}$, Maddalena Migliavacca ${ }^{3}$, Attilio Rovelli ${ }^{4}$, Maria Ester Bernardo $^{1,3}$, Fabio Ciceri ${ }^{5}$, Luca Biasco ${ }^{1}$, Alessandro Aiuti ${ }^{1,3,6}$ \\ ${ }^{1}$ SR-TIGET, Milan, Italy; ${ }^{2}$ San Raffaele Scientific Institute, Cytometry Laboratory, Milan, Italy; ${ }^{3}$ San Raffaele Scientific Institute, Pediatric Immunohematology and Bone Marrow Transplantation Unit, Milan, Italy; ${ }^{4}$ MBBM Foundation/ASST San Gerardo, BMT Unit, Pediatric Department, Monza, Italy; ${ }^{5}$ San Raffaele Scientific Institute, Hematology and Bone Marrow Transplantation Unit, Milan, Italy; ${ }^{6}$ Università Vita-Salute San Raffaele, Milan, Italy}

Background: Human hematopoiesis is a complex and dynamic system where morphologically and functionally diverse mature cell types are generated and maintained throughout life by bone marrow (BM) Hematopoietic Stem/ Progenitor Cells (HSPC). Bone marrow transplantation (BMT) or gene therapy (GT) based on autologous infusion of gene-corrected HSPC can be definitive treatments for several human blood disorders such as immunodeficiencies and hematological tumors. The generation of tools for the analysis of the hematopoietic system composition remains one crucial goal to understand the causes of hematological diseases, to monitor hematopoietic reconstitution in patients after transplantation, to design novel therapeutic approaches and to test their efficacy.

Methods: We designed a novel multi-parametric flowcytometry protocol called "Whole Blood Dissection" (WBD) (object of international patent application, waiting for publication) capable of unambiguously identifying and quantifying the absolute numbers of 23 different blood cell types, including HSPC subtypes and all the major myeloid and lymphoid lineage compartments at different stages of maturation, in a single test-tube on either bone-marrow (BM) or peripheral blood (PB) samples. Through a combination of 17 surface markers, 1 viability cell marker and count beads, WBD covers, on average, $99,7 \%$ of each sample in a reproducible, standardized, cost/time-efficient manner.

Results: We validated our technology performing morphological evaluation and analyzing $\mathrm{BM}$ and $\mathrm{PB}$ samples from adult $(n=19)$ and pediatric $(n=10)$ healthy donors highlighting age-related shifts in blood cells composition. We tested the efficacy of our protocol on clinical BM samples unveiling known and novel hematopoietic unbalances in 14 immune-deficient patients including adult and pediatric Wiskott-Aldrich Syndrome (WAS) individuals, pediatric Adenosine-Deaminase Deficient (ADA-SCID) patients and a subject with Chronic Granulomatous Disease (CGD). Through the application of WBD on 8 leukemic samples at diagnosis and after treatment we assessed both type and maturation state of leukemic blasts. We are currently analyzing the effect of G-CSF or G-CSF +Plerixafor administration on the mobilization of HSPC from the $\mathrm{BM}$ niche to the $\mathrm{PB}$, with the aim of quantifying and characterizing the mobilized HSPC (mHSPC) population and the dynamics of cell mobilization. Our preliminary analyses show that the relative distribution of HSPC subpopulations composing BM HSPC or mHSPC with the two types of mobilization is different, suggesting a diverse capacity of different HSPC subsets to respond to distinct mobilizing agents.

Finally we applied WBD protocol to comprehensively analyze the human-in-mouse graft in immunodeficient mice transplanted with human cord blood HSPC. WBD was able to detect, characterize and quantify the hematopoietic output and the composition of engrafted human HSPC in humanin-mouse transplantation settings, fundamental for assessing the safety and efficacy of treatments and for studying the biology of human hematopoietic system.

Conclusions: Overall, our WBD protocol provides a oneshot comprehensive evaluation of human hematopoietic system in a wide range of pre-clinical and clinical 
applications going from the characterization of hematopoietic disorders to the monitoring of hematopoietic reconstitution and HSPC maintenance in patients after transplant or gene therapy.

Conflict of interest: WBD is object of international patent application (waiting for publication). Basso-Ricci L., Aiuti A. and Biasco L. are co-inventors of the WBD.

\section{P488}

Predictive factors for engraftment after allogeneic hematopoietic stem cell transplantation in pediatric patients with myelodysplastic syndrome

Anna Osipova ${ }^{1}$, Tatyana Bykova ${ }^{1}$, Varvara Ovechkina ${ }^{1}$, Anastasya Borovkova ${ }^{1}$, Olesya Paina ${ }^{2}$, Polina Kozhokar ${ }^{2}$, Anastasya Frolova ${ }^{2}$, Kirill Ekushov', Svetlana Razumova ${ }^{2}$, Alexander Alyanskiy ${ }^{2}$, Elena Morozova $^{2}$, Elena Babenko ${ }^{2}$, Tatiana Gindina ${ }^{2}$, Alena Semenova ${ }^{2}$, Boris Smirnov ${ }^{3}$, Ludmilla Zubarovskay ${ }^{2}$, Boris Afanasyev ${ }^{2}$
${ }^{1}$ First State Pavlov Medical University of St. Petersburg, Raisa Gorbacheva Memorial Institute for Children Oncology, Hematology and Transplantation, St. Petersburg, Russian Federation; ${ }^{2}$ First Pavlov State Medical University of St. Petersburg, Raisa Gorbacheva Memorial Research Institute for Pediatric Oncology, Hematology and Transplantation, St. Petersburg, Russian Federation; ${ }^{3}$ Fist

Electrotechnical University, St. Petersburg, Russian Federation

Background: Allogeneic hematopoietic stem cell transplantation (allo-HSCT) is a curative therapy for pediatric myelodysplastic syndrome (MDS). Successful engraftment is crucial for survival of patients after allo-HSCT. To analyze the factors influencing the hematopoietic engraftment and outcome in children with myelodysplastic syndrome (MDS) after allo-HSCT.

Methods: The study included 59 MDS patients (pts) with median age of 7 (range 1-18) years. Distribution of MDS subtypes was as follows: refractory cytopenia of childhood (RCC) $(\mathrm{n}=12,20 \%)$, refractory anemia with excess of

\begin{tabular}{|c|c|c|c|c|}
\hline Characteristics & $\begin{array}{l}\text { All } \\
\text { patients (n) }\end{array}$ & $\begin{array}{l}\text { Engraftment } \\
(\mathrm{n}, \%)\end{array}$ & graft failure (n,\%) & $\mathrm{p}$-value \\
\hline Number of patients & 59 & $46(78 \%)$ & $13(22 \%)$ & \\
\hline \multicolumn{5}{|l|}{ Disease } \\
\hline $\begin{array}{l}\text { MDS (RCC,RAEB, RAEB- } \\
\text { T/AML) }\end{array}$ & 47 & $39(83 \%)$ & $8(17 \%)$ & \multirow[t]{3}{*}{$\mathrm{P}=0.017$} \\
\hline MML & 12 & $7(58 \%)$ & $5(42 \%)$ & \\
\hline \multicolumn{4}{|l|}{ Cytogenetic findings } & \\
\hline Low risk & 21 & $17(81 \%)$ & $4(19 \%)$ & \multirow[t]{2}{*}{$\mathrm{P}=0.033$} \\
\hline High risk & 38 & $29(76 \%)$ & $9(24 \%)$ & \\
\hline \multicolumn{5}{|l|}{ Cellular bone marrow } \\
\hline Normacellalur & 34 & $24(71 \%)$ & $10(29 \%)$ & \multirow[t]{2}{*}{$P=0.045$} \\
\hline Hypo/hypercellar & 25 & $22(88 \%)$ & $3(12 \%)$ & \\
\hline \multicolumn{5}{|c|}{ Time interval from diagnosis to $\mathrm{HSCT}$} \\
\hline$<451$ days & 35 & $30(86 \%)$ & $5(14 \%)$ & \multirow[t]{2}{*}{$\mathrm{P}=0.047$} \\
\hline$>451$ days & 24 & $16(67 \%)$ & $8(33 \%)$ & \\
\hline \multicolumn{5}{|l|}{ HLA-match } \\
\hline $\begin{array}{l}\text { Related (HLA- identical siblings } \\
\text { thap lordentical) }\end{array}$ & 16 & $11(69 \%)$ & $5(31 \%)$ & \multirow[t]{2}{*}{$\mathrm{P}=0.051$} \\
\hline HLA -matched unrelated donor & 43 & $35(81 \%)$ & $8(19 \%)$ & \\
\hline \multicolumn{5}{|l|}{ Donor age } \\
\hline Under 31 y.o & 27 & $24(89 \%)$ & $3(11 \%)$ & \multirow[t]{3}{*}{$P=0.025$} \\
\hline Over 31 y.o & 32 & $22(69 \%)$ & $10(31 \%)$ & \\
\hline \multicolumn{4}{|l|}{ CD34+Cell dose $\left(x 10^{6} / \mathrm{kg}\right)$} & \\
\hline$>5,0$ & 32 & $29(91 \%)$ & $3(9 \%)$ & \multirow[t]{2}{*}{$\mathrm{P}=0.011$} \\
\hline$<5,0$ & 27 & $17\langle 63 \%)$ & $10\langle 37 \%)$ & \\
\hline
\end{tabular}

[P488 Figure] [Univariate analysis of risk factors for engraftment] 
blasts and refractory anemia with excess blasts in transformation (RAEB and RAEB/T-AML) $(\mathrm{n}=36$, $61 \%)$, juvenile myelomonocytic leukemia (JMML) $(\mathrm{n}=$ 11, 19\%). Thirty-nine male and 20 female children were enrolled. At the moment of allo-HSCT the hypocellular bone marrow was in 20 pts (34\%); normocellular - 33 pts (56\%); hypercellular - 6 pts (10\%). Pts were divided into two groups according to the cytogenetic findings: high risk (monosomy 7 and/or complex karyotype) in 38 children (64\%) and low risk (others cytogenetic abnormalities and normal karyotype) - 21 (36\%). The time from diagnosis to allo-HSCT was 1-139 months (median - 13). The source of allo-HSCT: matched related donor in 9 pts (15\%), haploidentical - $7(12 \%)$ and matched unrelated - 43 (73\%). Myeloablative conditioning (MAC) regimen was used in 30 (51\%) pts; reduced intensity conditioning (RIC) $-29(49 \%)$ pts. Bone marrow (BM) was used in 43 pts (73\%), peripheral blood stem cells (PBSC) - 13 pts (22\%), combination of BM and PBSC - 3 pts (5\%).

Statistical analysis was performed using SPSS 19.0. The logistic regression model was used in univariate and multivariate analyses.

Results: Five-year overall survival (OS) in children with MDS after allo-HSCT was $44 \%$ with median follow up of 21 months (range 2-308). OS after MAC - 55\% and after RIC $-61 \%(\mathrm{p}=0.495)$.

Engraftment was achieved in 46 pts (78\%), after MAC 22 pts $(73 \%)$, RIC - 24 pts $(83 \%)$.

The results of univariate analysis of risk factors for engraftment are presented in Table1.

All significant factors from univariate analysis included in multivariate.

In multivariate analysis favorable predictive factors for the engraftment were type of MDS (other types of MDS vs JMML, $\mathrm{p}=0.004$ ), cytogenetic findings (low risk vs high risk $(\mathrm{p}=0.010)$, hematopoietic stem cell dose (CD34 $+>5 \times 10^{6} / \mathrm{kg}$ vs $\left.\mathrm{CD} 34+<5 \times 10^{6} / \mathrm{kg} \quad(\mathrm{p}=0.008)\right)$. At the same time graft failure was observed more frequently in pts with hypocellular bone marrow $(\mathrm{p}=0.07)$ and in cases, when donors were elder than 31 y.o $(\mathrm{p}=0.007)$. Univariate anal...

Conclusions: In the present study we defined five predictive factors for engraftment in children with MDS after allo-HSCT. However, only two of them (donor age and number of $\mathrm{CD} 34+$ cells in the graft) are of practical use.

Conflict of interest: No conflict of interest.

\section{P489}

Predictors Of Early Engraftment In Autologous Peripheral Stem Cell Transplantation. A single center experience
Radwan Massoud ${ }^{1}$, Zaher Otrock ${ }^{2}$, Basel Haffar ${ }^{1}$, Rami Mahfouz $z^{3}$, Ammar Zahreddine ${ }^{1}$, Nabila Kreidieh ${ }^{3}$, Rita Nehme ${ }^{1}$, Ali Bazarbachi', Jean El Cheikh ${ }^{1}$

${ }^{1}$ American University of Beirut Medical Center, Internal Medicine, Division of Hematology-Oncology, Bone Marrow Transplant Unit, Beirut, Lebanon; ${ }^{2}$ Henry Ford Health System, Department of Pathology and Laboratory Medicine, Detroit, MI, United States;

${ }^{3}$ American University of Beirut Medical Center, Department of Pathology and Laboratory Medicine, Beirut, Lebanon

Background: Autologous peripheral blood stem cells transplantation (APSCT) is a treatment option used in various hematological malignancies. APSCT can prolong disease free survival, improve survival, and may be curative. We investigated the variables affecting engraftment among patients treated with high dose-chemotherapy and APSCT.

Methods: 364 adult patients underwent 419 APSCT from 2001 to 2016, at the American University of Beirut Medical Center. There were 207 males and 157 females, with a median age of 45 years (17-74). The cohort included 126 multiple myeloma (MM), 120 non-Hodgkin lymphoma, and 108 Hodgkin lymphoma, while 10 patients had other malignancies. All patients received high-dose chemotherapy regimens without total body irradiation. The median time from diagnosis to first transplant is 11.9 months (range 2288). The median number of apheresis was 1 (range 1-5).

Results: The median number of infused CD34+ cells was $5.8 \times 10^{6} / \mathrm{kg}$ (range $0.71-24.9$ ) which contained DMSO at a median dose of $0.2 \mathrm{~g} / \mathrm{kg} \mathrm{BW}$ (range 0.0375-0.91). Status of the disease at transplant of the 419 APSCT was as follows: 130 patients were in complete remission (CR), 242 in partial remission (PR), and 47 patients were in stable or relapse disease status. The absolute neutrophil count (ANC) recovery $\left(\geq 0.5 \times 10^{9} / \mathrm{L}\right)$ was reached after a median of 10 days (range 1-97) and Platelet recovery $\left(\geq 20 \times 10^{9} / \mathrm{L}\right)$ occurred after a median of 15 days (range 7-46).

The findings of univariate and multivariate analyses are summarized in Table 1. In multivariate analysis only the diagnosis other MM, low DMSO dose $(<0.2 \mathrm{~g} / \mathrm{kg} \mathrm{BW})$, and high infused $\mathrm{CD} 34+\left(>6 \times 10^{6} / \mathrm{kg}\right)$ were associated with rapid engraftment of neutrophils within 10 days from APST. Low DMSO dose and high infused CD34+ x10 $/ \mathrm{kg}$ were associated with engraftment of platelets within 15 days.

Conclusions: In conclusion, our results imply that low DMSO is associated with earlier engraftment; it indicates a high yield collection which requires a smaller volume for freezing. Hence DMSO dose is predictive of early engraftment and can be used as a biomarker of richer stem cell collection. In addition the number of CD34+ x10 $/ \mathrm{kg}$ infused is an important predictor of neutrophil and platelet engraftment. However these findings need to be confirmed in larger studies. 
Conflict of interest: None of the authors has anything to declare.

\begin{tabular}{|c|c|c|c|c|c|}
\hline $\begin{array}{l}\text { Parameters } \\
\text { associated with } \\
\text { early neutrophil } \\
\text { engraftment ( } \leq \\
10 \text { days) }\end{array}$ & $\begin{array}{l}\text { OR }(95 \% \\
\text { CI) P } \\
\text { value } \\
\text { univariate }\end{array}$ & $\begin{array}{l}\text { OR }(95 \% \\
\text { CI) P value } \\
\text { multivariate }\end{array}$ & $\begin{array}{l}\text { Parameters } \\
\text { associated with } \\
\text { early platelet } \\
\text { engraftment } \\
\text { ( } \leq 15 \text { days) }\end{array}$ & $\begin{array}{l}\text { OR }(95 \% \\
\text { CI) P } \\
\text { value } \\
\text { univariate }\end{array}$ & $\begin{array}{l}\text { OR }(95 \% \\
\text { CI)/ P value } \\
\text { multivariate }\end{array}$ \\
\hline Age $\leq 45$ years & $\begin{array}{l}1.28 \\
(1.08- \\
1.51) \\
0.003\end{array}$ & NS & $\begin{array}{l}\text { Disease status } \\
\text { at transplant } \\
\text { (in CR) }\end{array}$ & $\begin{array}{l}1.32 \\
(1.11- \\
1.59) \\
0.004\end{array}$ & NS \\
\hline $\begin{array}{l}\text { Diagnosis } \\
\text { (other than } \\
\text { MM) }\end{array}$ & $\begin{array}{l}1.56 \\
(1.26- \\
1.95) \\
<0.0001\end{array}$ & $\begin{array}{l}2.07(1.33- \\
3.23) 0.001\end{array}$ & $\begin{array}{l}\text { DMSO dose } \\
(<0.2 \mathrm{~g} / \mathrm{kg} \\
\mathrm{BW})\end{array}$ & $\begin{array}{l}1.48 \\
(1.19- \\
1.83) \\
<0.0001\end{array}$ & $\begin{array}{l}1.84(1.22- \\
2.78) 0.004\end{array}$ \\
\hline $\begin{array}{l}\text { Number of } \\
\text { apheresis (one } \\
\text { apheresis) }\end{array}$ & $\begin{array}{l}1.38 \\
(1.07- \\
1.77) \\
0.006\end{array}$ & NS & $\begin{array}{l}\text { CD34+ } \\
(>6 \times 106 / k g)\end{array}$ & $\begin{array}{l}1.52 \\
(1.22- \\
1.89) \\
<0.0001\end{array}$ & $\begin{array}{l}1.96(1.29- \\
2.96) 0.001\end{array}$ \\
\hline $\begin{array}{l}\text { Disease status } \\
\text { at transplant } \\
\text { (in CR) }\end{array}$ & $\begin{array}{l}1.23 \\
(1.05- \\
1.45) \\
0.016\end{array}$ & NS & & & \\
\hline $\begin{array}{l}\text { DMSO dose } \\
(<0.2 \mathrm{~g} / \mathrm{kg} \mathrm{BW})\end{array}$ & $\begin{array}{l}1.29 \\
(1.03- \\
1.62) \\
0.025\end{array}$ & $\begin{array}{l}1.61(1.03- \\
2.51) 0.035\end{array}$ & & & \\
\hline $\begin{array}{l}\text { CD34 }+ \\
(>6 \times 106 / \mathrm{kg})\end{array}$ & $\begin{array}{l}1.56 \\
(1.23- \\
1.97) \\
<0.0001\end{array}$ & $\begin{array}{l}1.66(1.06- \\
2.58) 0.025\end{array}$ & & & \\
\hline
\end{tabular}

[[P489 Table] Factors affecting engraftment in APSCT]

\section{P490}

\section{Pre-transplant mutational burden predicts for post- transplant outcomes in myelodysplastic syndrome and acute myeloid leukemia}

\section{Katherine Prendergast ${ }^{1}$, Adam Seegmiller ${ }^{2}$, Heidi Chen ${ }^{3}$, Wichai Chinratanalab $^{4}$, Brian Engelhardt ${ }^{4}$, Stacey Goodman ${ }^{4}$, Carole Hunt, Adetola Kassim ${ }^{4}$, Bipin Savani, Michael Savona ${ }^{4}$, Salyka Sengsayadeth $^{4}$, Madan Jagasia ${ }^{4}$, Michael Byrne $^{4}$}

\footnotetext{
${ }^{1}$ Vanderbilt University School of Medicine, Nashville, TN, United States; ${ }^{2}$ Vanderbilt University Medical Center, Department of Pathology, Microbiology, and Immunology, Nashville, TN, United States; ${ }^{3}$ Vanderbilt University Medical Center, Department of Biostatistics, Nashville, TN, United States; ${ }^{4}$ Vanderbilt University Medical Center, Department of Hematology/Oncology, Nashville, TN, United States; ${ }^{5}$ Vanderbilt University Medical Center, Nashville, TN, United States
}

Background: In addition to patient history and cytogenetics, clinicians have begun using next generation sequencing (NGS) to guide treatment decisions in patients with acute myeloid leukemia (AML) and myelodysplastic syndromes (MDS); the impact of molecular aberrations on hematopoietic cell transplant (HCT) outcomes is still being studied.

Methods: We evaluated patients with AML and MDS undergoing $\mathrm{HCT}$ at a single center with available NGS data

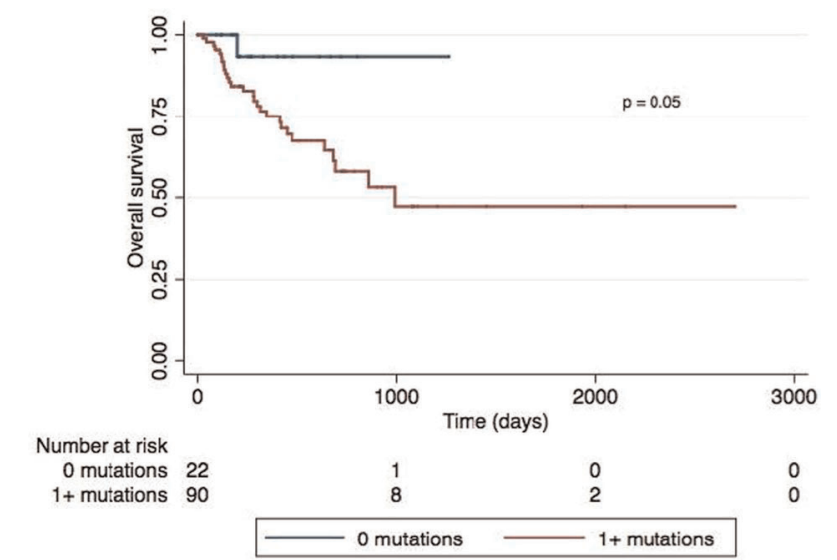

[P490 Figure] [Overall Survival by Mutation Category]

from a time of active disease prior to transplant. NGS was done on a panel of commonly mutated loci in 37 diseaserelated genes. Patients were grouped by number of mutations ( 0 vs. $\geq 1)$ and differences between groups, including disease risk index (DRI)(Armand, Blood 2012), were summarized using descriptive statistics. Progression free survival (PFS) and overall survival (OS) estimates were performed using the log rank test to assess for differences between groups. Multivariate analysis was done using a Cox proportional hazard regression.

Results: The cohort consisted of 112 patients. The majority of patients $(68 \%)$ were transplanted for AML (including secondary AML) and $32 \%$ were transplanted for MDS. Eighty percent of patients had at least 1 mutation. These mutations occurred in a total of 25 genes; the most commonly mutated were NPM1 (21\%), DNMT3A (19\%), and FLT3-ITD/TKD (19\%). There were no significant differences in age, gender, or very high DRI between patients with 0 mutations versus $\geq 1$ mutation. In a univariate analysis, patients with at least one mutation had decreased OS (992 days vs. not reached, $\mathrm{p}=0.05$ ); there was no significant difference in PFS.

Mutations in TP53 were associated with decreased PFS and OS (112 days vs. not reached and 228 days vs. not reached, respectively; $\mathrm{p}<0.01)$. Mutations in WT1 were associated with decreased PFS (194 days vs. not reached, $p$ $<0.01$ ) but not OS. As expected, TP53 was correlated with very high DRI (Spearman's rho 0.80, p $<0.001$ ). In multivariate analysis, very high DRI (HR 4.67, 95\% CI 1.81-12.01, $\mathrm{p}=0.001$ ) was an independent risk factor associated with inferior survival, while $\geq 1$ mutation trended towards significance (HR 6.325, $\mathrm{p}=0.071$ ).

Conclusions: In this analysis, presence of $\geq 1$ mutation in a myeloid-specific NGS panel was associated with decreased OS but not PFS. Very high DRI continued to be an independent risk factor associated with poor outcome. 
Patients with TP53 mutation continue to do poorly after HCT.

Conflict of interest: None of the authors has anything to disclose.

\section{P491}

Prevention of transfusion-transmitted CMV viremia using leukocyte-reduced blood products in seronegative UCBT patients

\author{
Tomonari Shigemura ${ }^{1}$, Takashi Kurata ${ }^{2}$, Kazutoshi Komori ${ }^{1}$, \\ Daisuke Morita ${ }^{1}$, Miyuki Tanaka ${ }^{1}$, Kazuo Sakashita ${ }^{2}$, Yozo \\ Nakazawa ${ }^{l}$ \\ ${ }^{1}$ Shinshu University, Pediatrics, Matsumoto, Japan; ${ }^{2}$ Nagano \\ Children's Hospital, Pediatrics, Azumino, Japan
}

Background: Cytomegalovirus (CMV) infection causes significant morbidity following allogenic stem cell transplantation (SCT). Umbilical cord blood transplantation (UCBT) in particular is at increased risk for CMV complications because of the lack in CMV-specific immunity and the delay in immune reconstitution. Morbidity can arise due to either primary infection or CMV reactivation. CMV transmission through blood components (transfusion-transmitted CMV; TT-CMV) could be a source of primary infection in seronegative SCT patients. Historically, blood components from CMV-negative donors have been providing to high-risk patients to minimize risk of TTCMV, but its supply is limited with increasing demand. Leukocyte-reduced blood components have been widely implemented as an alternative method. However, it is unclear whether leukocyte-reduced cellular components are as safe as seronegative blood products in high-risk patients, such as UCBT patients.

Methods: We retrospectively analyzed the incidence of CMV viremia in UCBT patients who had received leukocyte-reduced, CMV-unselected blood components between 2007 and 2017 in single Japanese pediatric center. Since January 2007, pre-storage leukocyte-reduction was implemented for all blood components in Japan (less than $1.0 \times 10^{6}$ residual white blood cells per unit). Pre-transplant CMV serostatus was determined in onset on all patients except patients who had received granulocyte transfusions and patients who could not be determined only with $\operatorname{IgG}(9$ infants including 5 SCID patients) because of maternal transmitted immunoglobulin (Ig)G or absent ability to produce IgG. In patients who could not be determined serostatus, evidence for CMV infection was decided by frequent CMV PCR or CMV IgM before UCBT. Monitoring for CMV viremia was performed at least once weakly, until day 100. CMV viremia was defined as a positive pp65 CMV antigenemia, which was a $1 \geq$ antigen-positive cell in single slide or detection of CMV DNA in the whole blood by PCR; CMV disease was defined as end-organ disease.

Results: Among 71 patients treated with UCBT, 41 CMV-seronegative patients were identified. These 41 patients received 922 leukocyte-reduced blood products (178 red cells and 744 platelets). All patients including 9 patients receiving antithymocyte globulin, remained negative for CMV viremia and none developed CMV disease. On the other hand, 25 patients $(35 \%)$ developed CMV viremia. Of 25 patients, CMV viremia was detected in all three patietns who had received granulocyte transfusions (100\%), all two SCID patients who had infected CMV before UCBT (100\%) and nineteen of the 23 CMVseropositive patients $(82.6 \%$ ). (In one patient who developed CMV viremia, CMV serostatus was unknown).

Conclusions: Using pre-storage leukocyte-reduction, zero cases of CMV viremia were detected in seronegative UCBT patients. Our finding showed the safety of leukocytereduction as an alternative strategy in preventing TT-CMV in this patient group.

Conflict of interest: The authors declare no conflicts of interest associated with this work.

\section{P492}

Primary graft failure in allogeneic hematopoietic stem cell transplantation - single center experience 1989 - 2017

Diana Mota ${ }^{1}$, Edgar Pratas ${ }^{1}$, Carlos Pinho Vaz ${ }^{1}$, Luis Leite ${ }^{1}$, Rosa Branca $^{1}$, Susana Roncon ${ }^{2}$, Fernando Campilho ${ }^{1}$, António Campos ${ }^{1}$

${ }^{1}$ IPOFG Porto, Serviço de Transplantação de Medula Óssea, Porto, Portugal; ${ }^{2}$ IPOFG Porto, Serviço de Terapia Celular, Porto, Portugal

Background: Allogeneic hematopoietic stem cell transplantation (allo-HSCT) is standard of care for many highrisk malignant and non-malignant diseases, being engraftment the primary goal of this treatment strategy. Graft failure, in spite of its variable low incidence, still remains an important complication that increases morbidity and mortality in allo-HSCT. In this report we describe the outcome of all 25 patients which presented primary graft failure (PGF).

Methods: In this retrospective study, we analysed 1093 patients who were submitted to an allo-HSCT between 1989 and 2017 in our institution. Incidence of graft failure was $4.3 \%, 2.29 \%$ in 25 patients that had failure to reach an absolute neutrophil count of $\geq 500$ cells $/ \mu \mathrm{L}$ and $2.01 \%$ in 22 patients that had graft failure after achieve engraftment.

Results: From 25 patients described, 54.5\% had PGF between 1997-2003, 48\% $(\mathrm{n}=12)$ were male and the median age was 25 years (8-64). Patients were transplanted for malignant (acute leukaemia, $\mathrm{n}=8$; chronic myeloid leukaemia, $\mathrm{n}=4$; myelodysplastic and myeloproliferative 
neoplasms, $\mathrm{n}=5$; non-Hodgkin lymphoma, $\mathrm{n}=1$ ) and nonmalignant diseases (fanconi's anaemia, $\mathrm{n}=4$; others, $\mathrm{n}=3$ ). In the $1^{\text {st }}$ allo-HSCT, cell source was cord blood $(\mathrm{n}=8)$, peripheral hematopoietic blood stem cell $(n=13$; CD34+ selection, $n=6)$ and bone marrow $(n=4)$. Twenty-two patients performed a $2^{\text {nd }}$ transplant, 2 died of infection and 1 presented late autologous recovery. In $2^{\text {nd }}$ transplant, conditioning regimen was myeloblative (MA) in $13.6 \%$ $(\mathrm{n}=3)$, non-myeloablative/reduced intensity (NMA/RIC) \pm ATG/Alemtuzumab in $50 \%(\mathrm{n}=11)$ and without chemotherapy $9.1 \%(n=2), 27.3 \%(n=6)$ had no conditioning. All patients received unmanipulated grafts. Fifteen patients had the same donnor in $2^{\text {nd }}$ allo-HSCT. Median time between $1^{\text {st }}$ and $2^{\text {nd }}$ transplant was 39 days (19-111). Median overall survival (OS) after $1^{\text {st }}$ and $2^{\text {nd }}$ allo-HSCT was 11.1 and 6.1 months respectively. Median OS after $2^{\text {nd }}$ allo-HSCT in NMA/RIC vs myeloablative regimens was 76.9 vs 0.4 months, with ATG 6.1 month and Alemtuzumab NR. In patients that performed NMA/RIC regimens the hazard ratio for death $(\mathrm{HR})$ was $0.4(95 \% \mathrm{CI}, 0.14$ to 1.17 ; $\mathrm{p}=0.095)$. In the group that received ATG the $\mathrm{HR}=2.03$ (95\% CI, 0.61-6.74; $\mathrm{p}=0.250$ ) and for alemtuzumab $\mathrm{HR}=0.31 \quad(95 \% \quad C I, 0.07-1.43 ; \mathrm{p}=0.133) . \quad$ After $2^{\text {nd }}$ transplant, median OS was 129.9 vs 1.4 months for engraftment and non-engraftment patients respectively. In the group that had engraftment criteria, $\mathrm{HR}=0.16(95 \% \mathrm{CI}$, $0.05-0.53 ; \mathrm{p}=0.003)$. OS 1 year after PGF was $45.5(95 \%$ CI, 25.1-67.5). After $2^{\text {nd }}$ allo-HSCT in patients with malignant diseases OS $1 \mathrm{y}=43.8 \%$ (IC 95\%, 20.4-70.2\%). Seventeen patients died $(68 \% ; 17 / 25), 32 \%(n=8)$ of graft failure, $12 \%(n=3)$ of infection, $8 \%(n=2)$ from GVHD and $16 \%(n=4)$ of other causes.

Conclusions: PGF is infrequent, with low incidence rate. This complication is declining over time since many risk factors have been discovered, still the increasing use of RIC and wider application of HLA-mismatched donors might reverse this trend. There is a heterogeneity in the therapeutic strategy when PGF occur, we verified a tendency of superiority in NMA/RIC regimens and in regimens which contain alemtuzumab in our population. Larger and randomized studies in parallel with new strategies to determine an ideal conditioning are needed.

Conflict of interest: No author has any conflict of interest.

\section{P493}

Prophylactic donor lymphocyte infusions after hematopoietic stem cell transplantation in high risk acute leukemia and myelodysplastic syndromes: a single-center retrospective study

Elodie Collinge ${ }^{I}$, Hélène Labussière-Wallet ${ }^{2}$, Carlos Graux ${ }^{I}$, Marc
André $^{1}$, Mohamad Sobh ${ }^{3}$, Gilles Salles ${ }^{2}$, Mauricette Michallet $^{3}$

${ }^{1}$ CHU UCL Namur Mont-Godinne, Hematology, Mont-Godinne (Yvoir), Belgium; ${ }^{2}$ Hospices Civils de Lyon, Centre Hospitalier LyonSud, Hematology, Lyon, France; ${ }^{3}$ Centre Léon Bérard Lyon, Hematology, Lyon, France

Background: As demonstrated by Schmid et al. in 2005, the sequential use of intensive chemotherapy, followed by reduced intensity conditioning (RIC) before allo-SCT and prophylactic DLI (pDLI) after allo-SCT represents an effective treatment in HR AML and MDS. Despite encouraging data, the efficacy/toxicity of pDLI remains poorly assessed. We report a single-center retrospective study about pDLI after allo-SCT, in HR AML and MDS.

Methods: From 2006 to 2016 in the Centre Hospitalier Lyon-Sud (France), 34 patients (median age, 53.73 years) with HR AML and MDS received pDLI after allo-SCT, within a sequential treatment based on: (1) cytoreduction with FLAMSA-regimen, (2) RIC/MAC-allo-SCT, (3) pDLI after allo-SCT (started around day +120 or 30 days after discontinuation of immunosuppression, in CR patients without GVHD and any infection, at initial dose of $1 \mathrm{x}$ $10^{6} \mathrm{CD} 3$ cells $/ \mathrm{kg}$, followed in the absence of GVHD by escalating cell doses at 4- to 6-week intervals, up to three times). HR disease included : refractory leukemia, delayed response to induction, relapse, second remission, unfavorable genetic features, secondary AML, progressive MDS and/or RAEB (HR-IPSS).

Endpoints of this study included: pDLI feasibility, achievement of durable $\mathrm{CR}$ and full donor chimerism, treatment related toxicities, impact on survival.

Results: Repeated and gradual administrations of pDLI were feasible: $65 \%$ of patients received 3 DLI and $23.5 \%$ received 2 DLI.

Before pDLI, aGVHD had concerned $53 \%$ of patients (almost only low grade (1-2)) and was resolved for all. GVHD (acute and chronic) was the major complication (38\%) observed during DLI procedure, requiring systemic treatment for more than half, but resolutive in $69 \%$ of cases. In total, chronic GVHD concerned $44 \%$ of patients (limited for 10/15, extensive for 5), persisted for a third (15\%) and remained extensive for the majority. The 2-year cumulative incidence of TRM was low (7\%).

Although $44 \%$ of patients were not in CR at time of alloSCT (3 PR, 1 SD, 9 relapses, 2 primary refractories), all were in $\mathrm{CR}$ at 1 month after transplant with $94 \%$ of hematopoietic recovery and $88 \%$ of full donor chimerism. Twenty-seven of 33 patients in CR before pDLI (79\% of studied population) were in persistent $\mathrm{CR}$ after $\mathrm{pDLI}$ and 23/34 patients $(68 \%)$ remained in CR at last follow-up.

After pDLI, chimerism was improved for 13 patients (38\%), remained stable for $14(41 \%)$ and was deteriorated for $3(9 \%)$. Seven of 34 patients not totally in full chimerism 
before DLI, obtained full donor chimerism in BM and PB after DLI.

Relapse concerned $32 \%$ of patients after a median of 416 days from allo-SCT, with 2-year cumulative incidence of relapse at $35 \%$.

With a median follow-up of 32 months, the median OS was 86.6 months (95\% CI: 41-NR) with 2-year OS at $88.2 \%$ (95\% CI: 71.6-95.4) and 5-year OS at $61.1 \%$ (95\% CI: 34.6-79.6).

Conclusions: This study confirms the feasibility and the positive effect on chimerism and long-term disease control of pDLI, administrated after FLAMSA regimen-allo-SCT in HR AML and MDS, at the price of GVHD mostly resolutive, with a low rate of chronic GVHD and TRM.

Conflict of interest: No conflict of interest.

\section{P494}

Prospective unicentric analysis on the systematic use of peripherically inserted central catheters (picc lines) in allogenic hematopoietic stem cell transplantation

Elena Mariggiò, Anna Paola Iori, Roberto Latagliata, Paola Berneschi, Massimo Giampaoletti, Agostino Iammarrone, Cristina Morgia, Daniele Ciocchetti, Arianna Sammarco, Federico Vozella, Walter Barberi, Ursula La Rocca, Luisa Quattrocchi, Giulia De Luca, Sara Mohamed, Serena Rosati, Antonella Bruzzese, Robin Foà, Salvatore Giacomo Morano

Policlinico Umberto 1, Sapienza University of Rome, Hematology, Rome, Italy

Background: In patients undergoing chemotherapy peripherally inserted central catheters (PICCs) seem to be associated with a lower rate of complications compared to conventional percutaneously inserted devices (CVADs). Moreover, the insertion and removal of PICCs are easier and the costs lower compared to CVADs. So far, there is limited experience on the use of PICCs in the setting of allogeneic hematopoietic stem cell transplantation (HSCT). In view of the advantages of PICCs vs CVADs, we decided to use PICCs in patients candidates to receive a HSCT. Here, we report the experience on the systematic use of PICCs in HSCT patients at the 'Sapienza' University of Rome.

Methods: A total of 100 silicone or polyurethane PICCs have been inserted between October 2012 and August 2017. Catheter-related bloodstream infections (CRBSI), mechanical (malfunction, obstruction, dislocation, rupture) and catheter-related thrombotic complications (CRTCs) have been prospectively analyzed.

Results: One hundred PICCs were insert into 100 patients (male/female $62 \% / 38 \%$; median age 47.5 years, range 18.7 64.8). Fifty-one patients received a HSCT from an unrelated donor, 33 from a sibling donor and 16 were haploidentical transplants. Fifty-six patients had an acute leukemia and 44 a chronic lymphoproliferative disease. Myeloablative (MAC) and reduced (RIC) intensity condition regimens were employed in 62 and 38 patients, respectively. Polyurethane and silicone PICCs were used in 52 and 48 patients, respectively. The devices were single lumen in 52 patients and double lumen in 48. Eighty-five and 15 PICCS were placed in the basilica and brachial vein, respectively. All devices were inserted without complications. CRBSIs occurred in $32 \%$ of cases, with a rate of 2.5 CRBIs per 1000 PICC days. A coagulase-negative Staphylococcus was the most common agent, not associated with clinically significant complications. The PICC was removed in all cases. In univariate analysis, PICC material was the only factor affecting CRBSIs: polyurethane $42 \%$ vs $20 \%$ silicone; $p=0.02$. CRTCs were observed in $17 \%$ cases, with a rate of 1.3 CRTS per 1000 PICC days. Patients received anti-coagulant therapy with low-weight heparin followed by resolution. In univariate analysis, no variable showed a correlation with CRTC. Mechanical complications occurred in $15 \%$ of cases, corresponding to 1.2 mechanical complications per 1000 PICC days. The type of conditioning (MAC 33\% vs RIC 56\%; $\mathrm{p}=0.014$ ) and place of insertion (basilic 12\% vs brachial vein 33\%; $\mathrm{p}=0.03$ ) influenced mechanical complications. Both variables remained significant in multivariate analysis. Globally, the median duration of in situ PICC placement was 117 days (range16-561).

Conclusions: In our experience, no complications have been observed with the use of PICCs in patients undergoing a HSCTs. Adverse events related to PICCs were manageable and did not affect transplant outcome. Silicone PICCs and a basilic vein access seem to correlate with a lower incidence of infectious and mechanical complications. We conclude that PICCs are a safe and reliable long-term venous access for patients undergoing an allogenic HSCT.

Conflict of interest: All authors declare no conflict of interest.

\section{P495}

Results of Hematopoietic Stem Cell Transplantation in Fanconi Anemia; Single Regimen, Single Center Experience

Gulen Tuysuz ${ }^{1}$, Funda Tayfun Kupesiz ${ }^{2}$, Alphan Kupesiz ${ }^{1}$, Elif Guler $^{1}$

${ }^{1}$ Akdeniz University Pediatric Hematology and Oncology Department, Antalya, Turkey; ${ }^{2}$ Health of Ministry Antalya Education and Research Hospital, Antalya, Turkey

Background: Hematopoietic stem cell transplantation (HSCT) is the only curative treatment of bone marrow 
failure in Fanconi anemia (FA). Because of the underlying DNA repair defect Reduced intensity conditioning (RIC) regimens are preferred in this group of patients.

Methods: In our study, we planned to present the results of Fludarabine-based, radiotherapy free preparative regimen in patients with FA who underwent HSCT at Pediatric Stem Cell Transplantation Unit of Akdeniz University Hospital. The data of FA patients who have been transplanted between 2005-2017 with a RIC preparative regimen including Fludarabine (30/mg/m2, for 5 days), Cyclophosphamide $(10 \mathrm{mg} / \mathrm{kg}$ for 4 days $)$ and anti-thymocyte globulin (Fresenius) were retrospectively analyzed from the files.

Results: A total of 41 patients underwent HSCT during this period. There were 22 girls and 19 boys with a median age of 10 years (range: 4.4-16) at transplantation. The constitutional characteristics associated with FA, such as short stature, microcephaly and skin pigmentation, were absent in 2 of these patients. Diagnosed was confirmed with DEB test in all the group. All patients had moderate or severe bone marrow failure at transplantation and 2 patients had chromosomal abnormalities. The source of HSCT was bone marrow (BM) in 20, peripheral stem cell (PSC) in 17, and cord blood $(\mathrm{CB})$ in the rest 4 patients. Twelve patients received HSCT from a matched sibling donor (29\%), 7 patients from a matched family donor (17\%) and 22 from a matched unrelated donor (54\%). The median infused CD34 + cell dose was $4.8 \times 106 / \mathrm{kg}$ (range:0.8-9.6×106/kg) in BM or PBSC receivers, and 3.5x 105/kg (range: $1.1-4.3$ ) in CB receivers. Graft versus host disease (GVHD) prophylaxis included cyclosporine (in all group) and mycophenolate mofetil (only in MUD). All but 2 patients engrafted (95\%). Within a median follow up of 5 years (range 1-12), overall survival was $73 \%$ in the total group. Severe acute graft versus host disease (grade 3-4) occurred in 3 patients (7\%). Three patients were complicated with veno-occlusive disease (VOD) and 12 of the 41 patients (29\%) suffered from hemorrhagic cystitis. Eleven patients were lost with a median of 5 months (range: 1-12) after HSCT. Most of these patients have received stem source from an unrelated donor (n:9). In both groups with relative transplantation 1 patient was lost (1 out of 12 MSD and 1 out of 7 MFD transplants). Severe infection caused by GVHD related immunosuppressive treatments were the major cause of death (n:5). Four patients were lost due severe hemorrhagic cystitis leading to renal failure, 1 patient because of VOD related complications, and 1 due to regimen related toxicity. All patients that are alive have full chimerism and are in good clinical status.

Conclusions: Our results showed excellent outcome of HSCT with Fludarabine-based, radiotherapy free preparative regimen in MSD and MFD recipients. Even though we had good engraftment rates, interventing complications increased mortality in MUD recipients. We conclude that more effort is warranted to improve the outcome in MUD group.

Conflict of interest: None of the authors has anything to disclose.

\section{P496}

Screening, Monitoring, and Treatment of Adenovirus Infections in Pediatric and Adult Recipients of Allogeneic Hematopoietic Cell Transplants: Multicenter Survey of European Transplant Centers

Kanchan Rao ${ }^{1}$, Marco Zecca ${ }^{2}$, Antonio Pérez-Martínez ${ }^{3}$, Sebastian Voigt $^{4}$, Cécile Pochon ${ }^{5}$, Enrikas Vainorius ${ }^{6}$, Tom Brundage ${ }^{6}$, Artak Khachatryan $^{7}$, Essy Mozaffari ${ }^{6}$, Garrett Nichols ${ }^{6}$

${ }^{1}$ Great Ormond Street Hospital for Children NHS Foundation Trust, London, United Kingdom; ${ }^{2}$ Fondazione IRCCS Policlinico San Matteo, Pediatric Hematology / Oncology, Pavia, Italy; ${ }^{3}$ Hospital Universitario La Paz, Pediatric Hemato-Oncology, Madrid, Spain; ${ }^{4}$ Charité-Universitätsmedizin Berlin, Department of Pediatric Oncology/Hematology/Stem Cell Transplantation, Berlin, Germany; ${ }^{5}$ University Hospital of Nancy, Allogeneic Hematopoietic Stem Cell Transplantation Unit, Department of Hematology, Vandoeuvre-lèsNancy, France; ${ }^{6}$ Chimerix, Durham, NC, United States; ${ }^{7}$ AnalyticaLaser, London, United Kingdom

Background: Allogeneic hematopoietic cell transplant (allo-HCT) recipients, particularly pediatric patients, are at risk of life-threatening adenovirus (AdV) viremia. While this risk is well recognized, there is a lack of multicenter data on the most common practices regarding AdV screening, monitoring, and treatment.

Methods: AdVance is a retrospective, multicenter, multinational study of the incidence, management, and clinical outcomes of AdV infections in European adult and pediatric allo-HCT recipients. Physicians at participating institutions were asked to complete a survey outlining their current practice in the management of $\mathrm{AdV}$ infections in allo-HCT recipients. Questions specifically addressed screening, monitoring, and treatment.

Results: Survey data were available from 33 physicians, 20 of whom treat pediatric patients and 13 of whom treat adult patients (see table). Almost all (95\%) pediatric physicians responded that their center employed a screening protocol while a minority of adult physicians (39\%) reported that their center had a screening protocol. All pediatric physicians indicated that they screened for $\mathrm{AdV}$ infection weekly or bi-weekly using blood $(45 \%)$, stool $(10 \%)$ or both blood and stool $(45 \%)$. In contrast, routine screening was used by only $31 \%$ of adult physicians who responded; though adult patients at high risk of $\mathrm{AdV}$ infection, such as those with graft-versus-host disease (GVHD) or recipients of T-cell depleted, haploidentical, 


\begin{tabular}{lcc}
\hline & Pediatric (N=20) & Adult (N=13) \\
\hline Screening & $19(95 \%)$ & $5(39 \%)$ \\
Protocol available & $20 / 20(100 \%)$ & $4 / 13(31 \%)$ \\
\hline Monitoring & $19 / 20(95 \%)$ & $3 / 4(75 \%)$ \\
Weekly or more frequently & $1 / 20(5 \%)$ & $1 / 4(25 \%)$ \\
Every 2 weeks & & \\
Matrix monitored & $2 / 20(10 \%)$ & $0(0 \%)$ \\
Stool only & $9 / 20(45 \%)$ & $3 / 4(75 \%)$ \\
Blood only & $9 / 20(45 \%)$ & $1 / 4(25 \%)$ \\
Blood and stool & $3 / 20(15 \%)$ & $2 / 13(15 \%)$ \\
\hline Treatment trigger & 0 & $1 / 2(50 \%)$ \\
Stool positive & $3 / 3(100 \%)$ & $1 / 2(50 \%)$ \\
$\geq 1000$ c/mL & $15 / 20(75 \%)$ & $4 / 13(31 \%)$ \\
No threshold defined & $8 / 15(53 \%)$ & $1 / 4(25 \%)$ \\
Blood positive & $7 / 15(47 \%)$ & $3 / 4(75 \%)$ \\
z1000 c/mL & $6 / 20(30 \%)$ & $1 / 13(8 \%)$ \\
No threshold defined & $14 / 20(70 \%)$ & 0 \\
\hline IV CDV starting dose & & $11 / 13(85 \%)$ \\
1 mg/kg 3 times per week & $16 / 20(80 \%)$ & \\
5 mg/kg per week & & \\
IV CDV maintenance dose* & & \\
5 mg/kg 3 times per week & & \\
\hline
\end{tabular}

*For typical patient.

[P496 Figure] [Table]

cord blood, or mismatched transplants were screened more frequently. A positive blood sample was noted as a treatment trigger by $75 \%$ of pediatric physicians, over half of whom $(53 \%)$ defined a threshold of $\geq 1000$ copies $/ \mathrm{mL}$; and $31 \%$ of adult physicians treated pre-emptively for AdV viremia, the majority of whom $(75 \%)$ did not define a threshold. The most frequently reported first-line treatments for both pediatric and adult patients were off-label IV cidofovir and the investigational medication brincidofovir, followed by cell-based therapies.

Conclusions: The AdVance practice patterns survey confirms that pediatric allo-HCT recipients, who are at higher risk of $\mathrm{AdV}$ infections, are monitored closely; whereas adult patients are generally monitored less frequently. Adults with characteristics that could place them at higher risk of AdV infections, such as GVHD, are monitored more closely. For both pediatric and adult patients, viremia is the most common treatment trigger, with off-label IV cidofovir commonly considered for treatment in spite of toxicity concerns. Treatment practices in the centers surveyed are generally consistent with the guidelines of the European Conference on Infections in Leukaemia (ECIL). ${ }^{1}$

\section{References:}

Matthes-Martin, S. Feuchtinger, T., Shaw, P., Engelhard, D., Ljungman, P. Management of adenovirus (ADV) infections. $4^{\text {th }}$ Conference on Infections in Leukaemia. 2012. Available at: https://www.ebmt.org/Contents/ Resources/Library/ECIL/Documents/ECIL4\%20\% 202011\%20Adenovirus.pdf

\section{Conflict of interest:}


K. Rao, M. Zecca, A. Pérez-Martínez, S. Voigt, and C. Pochon are investigators in the AdVance study sponsored by Chimerix.

E. Vainorius, T. Brundage, E. Mozaffari, and G. Nichols are employees of the study sponsor, Chimerix.

A. Khachatryan is an employee of Analytica-Laser, a research consultancy who conducted the study on behalf of the sponsor, Chimerix.

\section{P497}

\section{SIMPLY DECEPTIVE: CAN A SINGLE SPREADSHEET PERFORM ALL CALCULATIONS RELATED TO CELL COLLECTION AND PROCESSING, MONITOR QUALITY CONTROL AND BECOME A RUNNING PRODUCT INVENTORY DATABASE?}

\author{
Shanta Sharma, Heba Alya, Doha Daoud, Mai Rousan, Dania \\ Sarhan, Dania Abuhatab, Abdelghani Tbakhi
}

King Hussein Cancer Center, Cell Therapy and Applied Genomics, Amman, Jordan

Background: Cellular product manufacturing is a complex operation, constituted of cell collection, cell processing, product storage and release. Additionally, many calculations, a robust documentation and quality control systems are required. A database is maintain for the inventory of stored products. If performed manually, the calculations are time consuming and prone to manual error.

Methods: We have captured, listed key steps, in a single, password protected Excel spreadsheet that is a worksheet as well as a documentation of quality and quantity of stored products. A carefully orchestrated design of this spreadsheet allows the user, a subtle advantage of tracking and trending the ongoing operation during simple scrolling.

Results: Password protected formulas ensure error-proof calculations and save time. The user can identify both positive, and negative traits by simple scrolling. With a simple change in its design, this spreadsheet can be used for conventional proliferative potential assay of colony forming cells in methyl cellulose and for various cell types.

Conclusions: This creative spreadsheet is a real asset for our cord blood bank operation and can be used in any cellular product bank to save time, to prevent calculation errors, and to track and trend ongoing operation and institute timely corrective action.

Clinical Trial Registry: Not applicable

Conflict of interest: None of the authors has anything to disclose.

\section{P498}

Survival of hematopoietic stem cell transplantation
Claudia Lucia Sossa Melo ${ }^{1,2}$, Angela Maria Peña Castelllanos ${ }^{1,2}$, Sara Ines Jimenez, ${ }^{1,2}$, Manuel Rosales ${ }^{1}$, Sonia Osma ${ }^{2,3}$, Claudia Marcela Chalela ${ }^{1}$, David Leonardo Reyes ${ }^{1}$, Maria Lucrecia Luna ${ }^{2}$, Miguel Enrique Ochoa Vera ${ }^{4}$, Luís Salazar ${ }^{1}$, Edgar David Gomez Laiton $^{5}$

${ }^{1}$ Universidad Autonoma de Bucaramanga/FOSCAL, Internal Medicine - Division of Hematology and Hematopoietic Stem Cell Transplantation, Bucaramanga, Colombia; ${ }^{2}$ Universidad Autonoma de Bucaramanga, School of Medicine, Bucaramanga, Colombia; ${ }^{3}$ Universidad Autonoma de Bucaramanga/FOSCAL, Obstetrics and Gynecology, Bucaramanga, Colombia; ${ }^{4}$ Universidad Autonoma de Bucaramanga/FOSCAL, Epidemiology, Bucaramanga, Colombia;

${ }^{5}$ Universidad Autonoma de Bucaramanga/FOSCAL, Internal Medicine, Bucaramanga, Colombia

Background: During the past decades, hematopoietic stem cell transplantation (HSCT) has evolved from being an experimental procedure to become the standard treatment for several hematopoietic disorders. Short-term morbidity and mortality from disease relapse and HSCT-related complications are well documented. The aim of the present study is to assess survival rate for patients who underwent HSCT.

Methods: A retrospective cohort study was conducted at a tertiary referral center in Colombia, South America, on 138 patients who were transplanted either using autologous or allogeneic HSCT November 2009 and December 2015. The Kaplan-Meier method was used to assess overall survival and relapse-free survival rates at 100 days, one year, and five years following HSCT.

Results: All patients were older than 18 years with a mean age of 49.5 years (range 14-71). Seventy-two were men and 66 were women. Patients had different types of hematologic diseases. The most common hematologic disease was Multiple Myeloma (32.6\%), followed by Non-Hodgkin Lymphoma (26.08\%), and Acute Leukemia (17.39\%). One hundred and six patients received autologous HSCT and 32 allogenic HSCT. Multiple Myeloma (32.6\%) was the most common indication for autologous transplantation, and Acute Leukemia (65.62\%) for allogenic transplantation.

Overall survival for autologous HSCT was $85.84 \%$ (IC 77.71- 91.34) at one year and $66.6 \%$ (IC 37.68-86.86) at five years, and for allogenic HSCT $90.62 \%$ (IC 74.08 -97.03) at one year and 33\% (2.06 - 92.21) at five years. Relapse-free survival for allogenic transplantation was $84 \%$ (IC 64-94) at one year and $66.67 \%$ at five years, and $74 \%$ (63-83) at one year and $53.33 \%$ at five years for autologous transplantation.

There were 44 (31.88\%) deaths, due to relapse or disease progression for both types of transplants. Mortality rate for autologous HSCT was 3.8 per 10,000 population and 4.9 per 10,000 population for allogenic HSCT.

Conclusions: Overall and relapse-free survival of autologous and allogenic HSCT were very similar to those 
reported in the existing literature. The most common cause of death in HSCT patients was primary disease, and transplant-related mortality was low.

Conflict of interest: The authors declare no potential conflict of interest.

\section{P499}

The clinical features of cytomegalovirus infection and its effect on hematopoietic stem cell transplantation and effectiveness of antiviral treatment protocols

Elif Melis Baloglu Akyol, Handan Haydaroglu Sahin ${ }^{2}$, Fahriye Eksi $^{3}$, Sadettin Ozturk ${ }^{3}$, Ilknur Gundes ${ }^{1}$, Melya Pelin Kirik ${ }^{1}$, Selin Budeyri $^{3}$, Mustafa Pehlivan ${ }^{4}$

${ }^{1}$ Gaziantep University Faculty of Medicine, Internal Medicine, Gaziantep, Turkey; ${ }^{2}$ Gaziantep University, Department of Hematology, Gaziantep, Turkey; ${ }^{3}$ Gaziantep University Faculty of Medicine, Gaziantep, Turkey; ${ }^{4}$ Gaziantep University Faculty of Medicine, Bone Marrow Transplant Unit, Gaziantep, Turkey

Background: The aim of this study was to investigate the clinical features of cytomegalovirus (CMV) infection and its effect on hematopoietic stem cell transplantation (HSCT) and effectiveness of antiviral treatment protocols.

Methods: This study was performed in 2009-2016 at Gaziantep University Faculty of Medicine, Bone Marrow Transplantation Center, retrospectively. A total of 447 patients with 166 allogeneic, 281 autologous stem cell transplants were included in the study. CMV viremia was performed by polymerase-chain-reaction (PCR) routinely twice a week for the first 24 months, and in case of clinical suspicion.

Results: CMV-PCR positivity was detected in 90 allogeneic HSCT patients (54\%) and 41 autologous HSCT patients $(14 \%)$. There was a statistically significant increase in the number of CMV-PCR values of 150-1000 copies $/ \mathrm{mL}$ in the non-myeloablative (NMA) group compared to the myeloablative (MA) group $(\mathrm{p}=0.002)$. Acute/chronic GVHD were observed in 30 of allogeneic HSCT patients. CMV viremia was detected in 29 of these patients $(56 \%)$ and not detected in 1 patient $(1.3 \%)$. There was a statistically significant relationship between the development of acute / chronic GVHD and CMV viremia ( $\mathrm{p}=$ 0.001 ). The first 100 -day and 365-day mortality were higher in the group without CMV viremia in allogeneic HSCT patients $(\mathrm{p}=0.022$ and $\mathrm{p}=0.024)$. The 5 -year survival rate was $61 \%$ in the viremia group; $62 \%$ in the non-viremia group. There was no statistically significant difference between the two groups in terms of 5-year survival ( $p>0.05$ ). In patients receiving valganciclovir treatment because of viremia, CMV disease did not develop and no adverse effect that required the drug to be discontinued was observed.

Conclusions: GVHD was associated with CMV viremia. Early and late mortality rate were smiliar between the patients with and without CMV-antigenemia. The 5-year survival was similar in HSCT patients with or without CMV viremia. Pre-emptive therapy in the CMV viremia with valganciclovir is safe an effective in HSCT.

Conflict of interest: All authors have read and approved the manuscript. None of the authors have any potential conflicts of interest regarding this paper.

\section{P500}

The effect of GLUT-1 levels on leucocyte and thrombocyte engraftment in myeloma patients at autologous stem cell transplantation

Irfan Yavasoglu ${ }^{1}$, Eylem Akkaya ${ }^{2}$, Oktay Asici ${ }^{3}$, Furat Okan ${ }^{4}$, Filiz $^{2}$ Ergin $^{3}$, Mustafa Yilmaz ${ }^{3}$, Ali Zahit Bolaman ${ }^{1}$

${ }^{1}$ Adnan Menderes University, Hematology, Aydin, Turkey;

${ }^{2}$ Addenbrookes Hospital, Hematology, Aydin, Turkmenistan; ${ }^{3}$ Adnan Menderes University, Aydin, Turkey; ${ }^{4}$ Kent Hospital, Izmir, Turkey

Background: Glucose transport protein 1 (GLUT-1) is expressed on malign cells and has a role of cell proliferation. We aimed the effect of GLUT-1 levels on leucocyte and thrombocyte engraftment in myeloma patients during autologous stem cell transplantation in this prospective trial.

Methods: The investigation were done at stem cells harvest of 51 patients from two transplantational center. Exclusion critera were diabetes mellitus and body mass index (BMI) above 30. GLUT 1 levels were determined by ELISA. Mean value accepted if two harvest were collected. Adnan Menderes University Noninterventional Ethical Comission approved our study. Human GLUT-1 elisa kit (catalog: E-EL-H1822) were used fore GLUT-1 test by sandwich-ELISA procedure. The results evaluated by spectrophotometric analysis at absorbance microplate reader DAR 800 with optic density $450 \pm 2 \mathrm{~nm}$ wavelength. The results analysed with SPSS for Windows version 19.0 module.

Results: Median age was $58 \pm 9$, female/male rate is $26 /$ 25. Complet remission rate was 26 of 51 while partial remission rate was $25 / 51$. There was positive correlation between GLUT-1 level and neutrophil engraftment (p:0,004) and no correlation with thrombocyte engraftment. The positive correlation was more marked in patients with partial remisssion (p:0.0000). Engraftment time was independent from CD 34 positive cell count.

Conclusions: In this study showed that pretransplant GLUT-1 levels are high in myeloma patient with partially 
remission and neutrophil engraftment is delayed at these patients whereas GLUT-1 level is low in myeloma patients with complete remission and posttransplatation neutrophil engraftment became earlier.

Conflict of interest: None of the authors has anything to declare.

\section{P501}

The effect of Mesenchymal stem cells derived from adipose tissue (ADMSC) on ovarian cancer

Charbel Khalil ${ }^{1}$, Mayssam Moussam ${ }^{2}$, Albert Azar ${ }^{3}$, Georges Hilal ${ }^{4}$, Rawad Salameh $^{5}$, Ahmad Ibrahim ${ }^{6}$, Nada Alaaddine

${ }^{I}$ Middle East Institute of Health University Hospital-Saint Joseph University-Lebanese University, Cellular Therapy Unit-Regenerative Medicine and Inflammation Laboratory, Bsalim, Lebanon; ${ }^{2}$ Saint Antoine Joseph University, Regenerative Medicine and Inflammation Laboratory, Beirut, Lebanon; ${ }^{3}$ Middle East Institute of Health University Hospital, Bsalim, Lebanon; ${ }^{4}$ Saint Antoine Joseph University, Cancer and Metabolism Lab, Beirut, Lebanon; ${ }^{5}$ Middle East Institute of Health University Hospital, Cellular Therapy, Bsalim, Lebanon; ${ }^{6}$ Makassed and Middle East Institute of Health University Hospitals-Lebanese University, Hematology Oncology-BMT, Beirut,

Lebanon; ${ }^{7}$ Saint Joseph University, Regenerative Medicine and Inflammation Laboratory, Beirut, Lebanon

Background: The adipose tissue is an abundant and accessible source of autologous stem cells. It is also capable of multilineage cell differentiation. Over the past few years, clinical trials have been looking into developing new therapies for different kinds of diseases such as neurodegenerative diseases, cutaneous diseases, heart problems, as well as autoimmune diseases. However, only hematopoietic stem cells were used in cancer treatments. Adipose derived stem cells (ADMSC) have therapeutic properties due to the trophic effect of the cytokines and chemokines they produce. The objective of the study is the evaluation of the effect of stem cells derived from adipose tissue ADMSC on the ovarian cancer, especially in terms of proliferation, apoptosis, Immunophenotyping and tumor markers

Methods: The effect will be measured in vitro on both cell and molecular levels, and that by incubating cancerous ovarian cells (primary cells) along with the supernatant stem cells. Another approach would be cultivating these cells together with the ADMSC. The amount of tumor markers protein (CA-125, CA19-9, HCG-Beta...) that marks ovarian cancer will also be measured by ELISA. Proliferation and immunomodulation effect of the ADMSC along with the apoptosis of cancer cells will be measured by culture and counting of the colony formation. Evaluation of variation of the immunophenotyping in co-culture will be performed using the flow cytometry technique.

Results: During the third subculture, the cells noticeably proliferated without any modification to their immunophenotyping. The CFU-F test has showed a proliferation of the ADMSC along with cell lines in multiplication and healthy cell duplication, all with no changes to their phenotyping. The supernatant of ADMSC increased the cell death of the OVCAR3 $(43.45 \%)$ and the apoptosis of the SKOV3 (54.8\%). Decrease of the level of CA125 (75-90\%), LDH (10-20\%) and HCG-Beta (10-20\%) in co-culture in both cell lines.

The tumorigenicity and aggressiveness of the cancer are reduced by decreasing the CD24 (14.74\%) of the OVCAR3 in co-culture with the supernatant of the ADMSC; however, the rate of the CD24 and CD44 of the SKOV3 asserts their stem state.

Conclusions: The reduction of the CA125 (75-90\%), LDH (10-20\%) and HCG-Beta (10-20\%) in co-culture show the therapeutic characteristics of the ADMSC in the ovarian cancer.

Conflict of interest: Nothing to disclose

\section{P502}

The efficacy of the second allogeneic hematopoietic stem cell transplantation in children with refractory hematological malignancies

Polina Kozhokar, Olesya Paina, Anastasia Borovkova, Anastasia Frolova, Elena Semenova, Anna Osipova, Tatyana Bykova, Kirill Ekushov, Olga Slesarchuk, Elena Babenko, Ludmila Zubarovskaya, Boris Afanasyev

First Pavlov State Medical University of St. Petersburg, Russian Federation,Raisa Gorbacheva Memorial Research Institute for Pediatric Oncology, Hematology and Transplantation, St. Petersburg, Russian Federation

Background: Allo-HSCT is the standard treatment in high risk hematological malignancies.Relapse after alloHSCT is the most common reason of treatment failure and rates from $30 \%$ to $60 \%$.Possible therapeutic options include chemotherapy,immuadoptive therapy(DLI), target therapy, second allo-HSCT.The presented study is retrospective single-institute experience of second allo-HSCT in pts with oncohematological diseases.

Aims: Evaluate the efficiency and factors affecting the outcome after second allo-HSCT in children that relapsed after first allo-HSCT.

Methods: We analyzed the outcome of 36 children after second allo-HSCT with hematological malignancies: ALL18 pts,AML-11pts,JMML-4pts,CML-1pt,MPAL-1 pt, sMDS -1 pt.First allo-HSCT was performed from MUD in 15 pts,MRD in 11 pts,haplo-donors in 9pts, syngeneic donor -1 pt.Condition regimen was MAC in 19 pts,RTC-5 pts and RIC in 12 pts.Indications for $2^{\text {nd }}$ allo-HSCT were relapse in 28 pts,graft failure (including pts with 


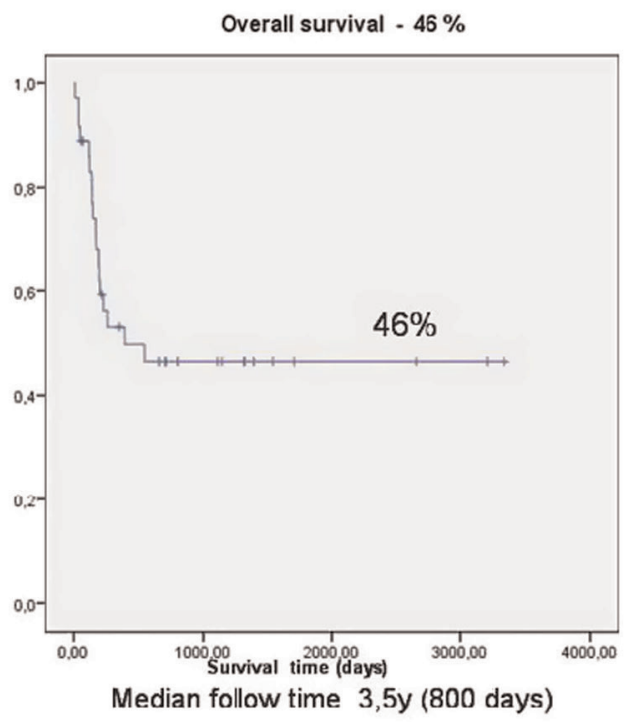

[P502 Figure] [Overall survival]

progression of the disease)- 8 pts.Median age at the time of the first allo-HSCT was 5 y.o.(1-17),median age at second HSCT was 6 y.o.(1,5-20).Median time between $1^{\text {st }}$ and $2^{\text {nd }}$ allo-HSCT was 8,5 month(1-39).The CR for the second allo-HSCT was MAC in 10 pts (28\%), RIC in 26 pts (72\%). GVHD prophylaxis included post-transplant Cy on Days $+3,+4$ in 24 pts(66\%),Tx/+Sir/+MMF-22 pts (61\%), ATG/+Csa-7pts (19\%).At the $2^{\text {nd }}$ allo-HSCT haplo-donors were chosen in $31 \mathrm{pts}$, including 5 pts with the same haplo donor as at first allo-HSCT. In other pts: MUD(donor substitution) - 2 pts,MUD(without donor substitution)-2 pts, MRD(without donor substitution)-1 pt.Median number of CD34+/kg was 4,8 $(0,9$ - 14,8).Cytoreductive therapy prior to $2^{\text {nd }}$ HSCT was performed in $31 \mathrm{pts}(86 \%)$ from $36 \mathrm{pts}, 5$ pts $(14 \%)$ were transplanted in aplasia.No response in $17 \mathrm{pts}$ (54\%),1pts (3\%) achieved remission and blasts cytoreduction achieved in 13pts (42\%).The transplant source was GCSF primed unmanipulated BM in 26 pts (72\%), unmanipulated BM -3 pts (8\%), unmanipulated PBSC-6pts (17\%), combination of PBSC and BM-1 pt (3\%).16pts underwent PT-therapy:DLI-7 pts, (HMA) - 6 pts,maintenance therapy3 pts.

Results: Thirty-three of 36 patients achieved engraftment, median time of neutrophil engraftment 21 days(12-41). Clinical remission was achieved in 33 pts (92\%).OS according to Kaplan-Meier method is $46 \%$.Median follow-up of 3.5 years. There was no statistically significant impact of conditional regimen on OS (MAC vs RIC, $40 \%$ vs.47\%).Donor type didn't affect OS, with or without donor substitution $(50 \%$ vs.37\%), $(\mathrm{p}=0.9)$. OS in pts with cytoreduction(13 pts) or remission (1pt) was better than in pts who did not achieve remission or cytoreduction $(68 \%$ vs. $25 \%, \mathrm{p}=0.03)$.Frequent transplant complications were
Grade I and II aGVHD in 26 pts(72\%),mild and moderate cGVHD in 24 pts (67\%).Causes of death:relapse/progression-13 pts (72\%),TRM in 5 pts (28\%).In pts with aGVHD OS was better than without aGVHD $(59 \%$ vs. $11 \%, \mathrm{p}=0.04)$ and in pts with cGVHD compared without cGVHD $(65 \%$ vs. $15 \%, p=0.01)$.OS was significantly better in patients with post transplant therapy (DLI,HMA,target therapy,maintenance therapy) compared to patients without therapy (62\% vs $31 \%, \mathrm{p}=0.05$ ).

Conclusions: $2^{\text {nd }}$ allo-HSCT is an effective treatment option of relapse after $1^{\text {st }}$ allo-HSCT.Patients that achieved blast cytoreduction or remission prior to $2^{\text {nd }}$ allo-HSCT had improved outcome.RIC in $2^{\text {nd }}$ allo-HSCT was comparable to MAC.Post transplant therapy is required to improve results after $2^{\text {nd }}$ HSCT.

Conflict of interest: nothing to disclose

\section{P503}

\section{The impact of donor selection on HSCT outcomes in children with malignancies: a 13 years single center experience}

Alessandro Di Gangi ${ }^{1}$, Sayla Bernasconi ${ }^{2}$, Gabriella Casazza ${ }^{2}$, Claudio Favre ${ }^{3}$, Mariacristina Menconi ${ }^{2}$

${ }^{1}$ Sant'Anna School of Advanced Studies, Pisa, Italy; ${ }^{2}$ Azienda Ospedaliera Universitaria Pisana, Pisa, Italy; ${ }^{3}$ Azienda Ospedaliera Universitaria Anna Meyer, Firenze, Italy

Background: Over the last 15 years priorities in allogeneic donor selection have been changing, but the need for a stringent HLA-matching remains elusive especially in pediatric HSCT.

Methods: Children (aged $<18$ years) who underwent first allogeneic HSCT for malignancies at our institution between 01/2003 and 12/2016 were included. Patients were classified according to donor source: umbilical cord blood unit (UCBU), matched unrelated (MUD), mismatched unrelated (MMUD), matched sibling (MSD) or haploidentical (HAPLO) donor. 5-years probabilities of OS, DFS and GVHD-free relapse-free survival (GRFS) were calculated according to Kaplan-Meier method and log-rank test was used for statistical analysis. The cumulative incidence procedure and Gray's test were used to assess differences in relapse, NRM, aGVHD grades 2-4, cGVHD, neutrophils and platelets engraftment.

Results: 95 patients were categorized as MSD $(\mathrm{N}=14)$, MUD $(\mathrm{N}=32)$, MMUD $(\mathrm{N}=22)$, HAPLO $(\mathrm{N}=12)$ and UCBU $(\mathrm{N}=15)$ grafts. Groups were uniform for age, sex and graft composition with the exception of lower median cellularity in UCBU. Eleven HAPLO were lymphocytedepleted $(6 \alpha / \beta+$ and 5 CD3+/CD19+) while one received post-transplantation cyclophosphamide. HAPLO received 


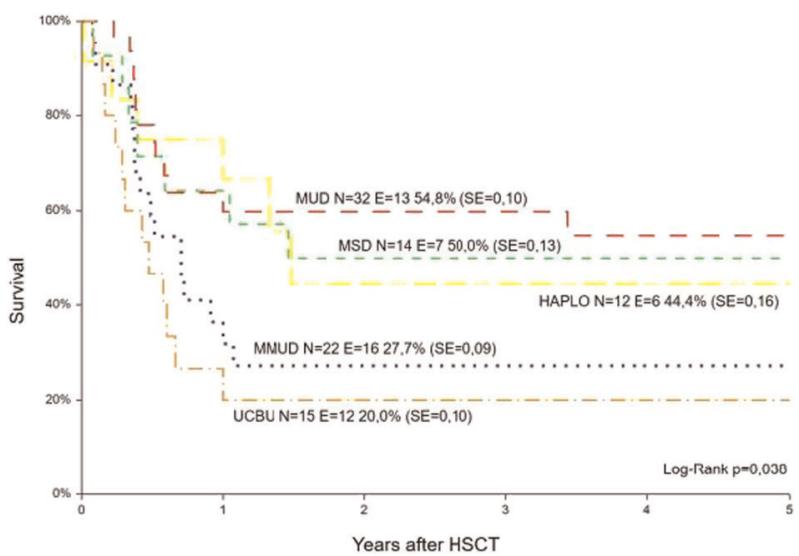

[P503 Figure] [GVHD-free relapse-free survival (GRFS)]

mainly chemotherapy-based RIC regimens and PBSC grafts while others received predominantly TBI-based MA regimens and BM grafts. Comparable post-HSCT CMV disease rates were observed. Major endpoints are reported in Table 1 together with standard errors (SE).

OS $(\mathrm{p}<0.05)$ and DFS $(\mathrm{p}<0.02)$ were significantly higher in MUD, MSD and HAPLO compared to MMUD and UCBU. Although statistically non-significant, relapse was more frequent among MMUD, UCBU and HAPLO. Probably, relapse rate in HAPLO was negatively affected by small sample size. Primary graft failure occurred in UCBU $(\mathrm{N}=1)$, MUD $(\mathrm{N}=1)$ and HAPLO $(\mathrm{N}=1)$, while one secondary graft failure was recorded in the MUD group. The cumulative incidence of aGVHD, cGVHD, neutrophils and platelets engraftment were comparable among groups except for absence of aGVHD and delayed platelets engraftment in the UCBU group. Non-relapse deaths were observed only in MMUD $(\mathrm{N}=2)$ and UCBU $(\mathrm{N}=2)$. MSD, MUD and HAPLO showed significantly higher GRFS ( $p<$ 0.05) compared to others. Figure 1shows GRFS with SE and the number of events $(\mathrm{E})$.

Conclusions: Despite variations in HSCT procedures and the inclusion of small dissimilar cohorts, MSD, MUD and HAPLO have shown similar outcomes, yet favorable when compared to MMUD and UCBU. Although HLA-matching plays a paramount role in donor selection, the use of different haploidentical HSCT platforms may allow for expansion of the donor pool while yielding comparable outcomes.

Conflict of interest: Nothing to disclose.

\begin{tabular}{lllllll}
\hline Endpoints (SE) & MSD & MUD & HAPLO & MMUD & UCBU & p \\
\hline OS & $78.6 \%$ & $79.2 \%$ & $69.5 \%$ & $50.0 \%$ & $46.7 \%$ & $<0.05$ \\
& $(0.11)$ & $(0.08)$ & $(0.16)$ & $(0.11)$ & $(0.13)$ & \\
DFS & $71.4 \%$ & $75.0 \%$ & $72.9 \%$ & $45.5 \%$ & $33.3 \%$ & $<0.02$ \\
& $(0.12)$ & $(0.08)$ & $(0.14)$ & $(0.11)$ & $(0.12)$ & \\
Relapse & $14.3 \%$ & $16.7 \%$ & $27.0 \%$ & $36.4 \%$ & $33.3 \%$ & ns \\
& $(0.09)$ & $(0.07)$ & $(0.14)$ & $(0.10)$ & $(0.12)$ &
\end{tabular}

\begin{tabular}{llllllll} 
Table (continued) & & & & & & \\
\hline Endpoints (SE) & MSD & MUD & HAPLO & MMUD & UCBU & $\mathrm{p}$ \\
\hline aGVHD & $14.3 \%$ & $12.5 \%$ & $25.0 \%$ & $18.2 \%$ & $0 \%$ & $\mathrm{~ns}$ \\
& $(0.09)$ & $(0.06)$ & $(0.13)$ & $(0.08)$ & & \\
cGVHD & $23.1 \%$ & $27.8 \%$ & $31.9 \%$ & $31.7 \%$ & $28.9 \%$ & $\mathrm{~ns}$ \\
& $(0.12)$ & $(0.10)$ & $(0.16)$ & $(0.11)$ & $(0.18)$ & \\
\%Neutrophils & $92.9 \%$ & $93.8 \%$ & $83.3 \%$ & $100 \%$ & $86.7 \%$ & $\mathrm{~ns}$ \\
engraftment at d+100 & $(0.07)$ & $(0.04)$ & $(0.11)$ & & $(0.09)$ & \\
\%Platelets & $85.7 \%$ & $87.5 \%$ & $77.3 \%$ & $75.0 \%$ & $66.7 \%$ & $\mathrm{~ns}$ \\
engraftment at d+100 & $(0.09)$ & $(0.06)$ & $(0.13)$ & $(0.09)$ & $(0.10)$ & \\
\hline
\end{tabular}

\section{[[P503 Table] Major endpoints]}

\section{P504}

\section{The Impact of Foundation of the National Turkish Donor Registry, Turkok on Donor Matching Kinetics}

\author{
Mutlu Arat ${ }^{1}$, Nurcan Ozcelik ${ }^{1}$, Deniz Goren Sahin ${ }^{1,2}$, Medine \\ Hascuhadar ${ }^{3}$, Burcu Kurt', Emine Tulay Ozcelik ${ }^{1,2}$ \\ ${ }^{1}$ Sisli Florence Nightingale Hospital, Hematopoietic Stem Cell \\ Transplantation Unit, Istanbul, Turkey; ${ }^{2}$ Istanbul Bilim University, \\ Hematology Department, Istanbul, Turkey; ${ }^{3}$ Turkish Stem Cell \\ Coordination Center (TURKOK), Turkish Ministry of Health, Istanbul, \\ Turkey
}

Background: Turkish Stem Cell Coordination Center (TURKOK) was established by the Turkish Ministry of Health in April 2015 in order to facilitate unrelated hematopoietic stem cell (HSC) transplantation in Turkey. As of July 2017 they have facilitated more than 300 HSCTs. Transplant Registry Istanbul (TRIS) and Transplant Registry Ankara (TRAN) are two University affiliated donor search - co-ordination centers, which are officially responsible for the search, matching and organization of the delivery for unrelated donor HSC in Turkey. The foundation of TURKOK should have theoretically decreased our time spent to find a suitable matched donor. The aim of this study is to compare the time frames between admission, confirmation, work-up, and infusion of HSC between TURKOK and other WMDA or NMDP facilitated International Volunteer Donor Registries.

Methods: In 20151000 allogeneic HSCTs were peformed in Turkey and only $18 \%$ were from MUDs. This is a single center analysis of a dedicated active HSCT center, which is performing $>70$ allogeneic HSCTs per year and utilizing alternative donor sources $>50 \%$. Patient charts were reviewed retrospectively and 51 patients who admitted to our center within 2015-2017 for various high risk hematological malignancies (mostly acute leukemia) were identified for this study. Patients were divided into two groups: (1) Patients who had received HSC from matched unrelated donors (MUD-T) (10/10-9/10) facilitated by TURKOK $(\mathrm{N}=36)$; (2) 15 patients who had received 
HSC from International Registries facilitated by TRIS or TRAN (MUD-I) . The duration between application and finding a suitable match (tAM), duration between matching and confirmation (tMC) and time from application to HSC transplantation (tATX) were calculated for each of these two groups. The time duration between admission and donor identification and admission to HSCT were not distributed normally. The mean values were compared between two groups by Mann-Whitney U test. $\mathrm{p}<0.05$ was considered statistically significant.

Results: In 51 HSC recipients the overall median durations for tAM, tMC and tATX were 71 (range, 1172), 33 (range, 1-181) and 97 (range, 13-172), respectively. In MUD-I overall median durations for tAM, tMC and tATX were 90 (range, 1-170), 40.5 (range, 1-181) and 98 (range, 13-172), whereas in MUD-T the timelines for tAM, tMC and tATX were 37 (range, 4-172), 17 (range, 6151) and 96 (range, 73-128), respectively. The $p$ values for comparative analysis betwen MUD-I and MUD-T for tAM, tMC and tATX were $\mathrm{p}=0.005, \mathrm{p}=0.007$ and $\mathrm{p}>0.05$, respectively.

Conclusions: In this relative limited size of HSC recipients from MUDs we observed accelerated kinetics for tAM and tMC, which means having a National Registry have a positive impact on finding an available match and confirmation process. On the other hand this improvement did not translate into rapid HSCT. The tATX between MUD-I and MUD-T are not significant. TURKOK has to establish and improve the organization of confirmed donor for collection of HSCs. The decreased timeline from application to confirmation is encouraging, but the time spent from confirmation to work/up and collection are still high. As TURKOK is a recently established donor bank, there is room for improvement.

Conflict of interest: Nothing to disclose

\section{P505}

\section{The relationship between CD133+CD34- cells and CD34+ haematopoietic progenitor cells in cord blood}

Kap-Hyoun Ko ${ }^{1}$, Guy Klamer ${ }^{1}$, Annette Trickett ${ }^{2}$, Joanna Youngson ${ }^{1}$

${ }^{1}$ Sydney Children's Hospital, Sydney Cord Blood Bank, Randwick, Australia; ${ }^{2}$ NSW Health Pathology, BMT Laboratory, Randwick, Australia

Background: Cord blood (CB) banks perform haematopoietic progenitor cell (HPC) enumeration assays to provide qualitative data that correlates with engraftment outcome. The standardised HPC assays that enumerate CD34+ cell content do not incorporate the most primitive HPC compartment, which is CD34-CD133+. This project aims to develop an assay that calculates the content of CD34+ and CD34- HPCs to address the hypothesis that both of these compartments contribute to engraftment and clinical outcome.

Methods: All samples met national (Therapeutic Goods Administration) and international (NetCord-Foundation for the Accreditation of Cellular Therapy) regulatory requirements for cord blood banking. The mean collection volume of $\mathrm{CB}$ was $114 \pm 34 \mathrm{~mL}$ and the mean total nucleated cell (TNC) count before processing was $16 \pm 6 \times 10^{8}$. The range of CD34+ cell percentage in the white blood cell (WBC) compartment was $0.05 \%$ to $1.8 \%$. The CD133 enumeration kit used for this study contains anti-CD45 and anti-CD34 antibodies, and 7AAD viability dye. Annexin- $\mathrm{V}$ was combined with antibodies mentioned above to detect cells undergoing apoptosis in different HPC stages.

Results: A non-linear regression and inverse correlation between $\mathrm{CD} 133+\mathrm{CD} 34-$ cells and $\mathrm{CD} 34+$ cells was observed for 40 tested samples $\left(r^{2}=0.76\right)$. For example, when $\mathrm{CD} 34+$ cell percentage in WBC was $0.08 \%$, CD34$\mathrm{CD} 133+$ in $\mathrm{WBC}$ was $46 \%$ (TNC $16 \times 10^{8}$ ). Conversely, when CD34+ cell percentage in WBC was $1.72 \%$, CD34CD133+ in WBC was $1.71 \%$ (TNC $12 \times 10^{8}$ ).

CD133+CD34- cells were significantly smaller than CD34+ cells $(p<0.0001)$ as determined by forward scatter analysis. CD45 expression in the CD133+ cell population could be divided into $\mathrm{CD} 45^{\text {Bright }}$ and $\mathrm{CD} 45^{\text {Dim }}$ according to the proportion of the different HPC compartments. The percentage of $\mathrm{CD} 133+\mathrm{CD} 34+\mathrm{CD} 45+{ }^{\mathrm{Dim}}$ cells increased as the percentage of $\mathrm{CD} 34+$ cells in WBC increased $\left(\mathrm{r}^{2}=0.81\right)$. Our results demonstrate that the level of CD45 expression shifts with HPC development from primitive $\mathrm{CD} 45^{\text {bright }}$, to intermediate $\mathrm{CD} 45^{\mathrm{dim}}$, to later stage CD45 $5^{\text {bright }}$.

Using One way ANOVA, we demonstrate that HPC viability differs among subsets characterised by the CD34 and CD133 biomarkers $(\mathrm{p}<0.0001, \mathrm{n}=10)$. The viability of CD133+CD34- cells decreased when the percentage of CD $34+$ cells in WBC increased $\left(r^{2}=0.6\right)$. However, the viability of $\mathrm{CD} 34+$ cells did not change with increasing CD34+ cell percentage in WBC. In addition to HPC viability, the relationship between $\mathrm{CD} 133+\mathrm{CD} 34$ - cells and CD34+ cells was verified with the incorporation of Annexin-V $\left(\mathrm{r}^{2}=0.98, \mathrm{n}=10\right)$.

Conclusions: With the advancements in flow cytometric technology and clinical grade reagents, and with access to high quality $\mathrm{CBU}$ final product, we were able to demonstrate that primitive CD133+CD34- cells can be enumerated. Our results show that HPC subsets can be classified into the three compartments: CD133+CD34CD45 Bright (primitive), CD133+CD34+CD45 ${ }^{\text {Dim }}$ (intermediate) and $\mathrm{CD} 133+\mathrm{CD} 34+\mathrm{CD} 45{ }^{\text {Bright }}$ (later stage). In conclusion, incorporation of CD133 into standard HPC enumeration methodology may provide additional 
qualitative data that improves the correlation between HPC content and clinical engraftment.

Conflict of interest: The authors have nothing to disclose.

\section{P506}

The Successful Treatment of Severe Aplastic Anemia With Autologous Cord Blood Transplantation: Will We Need to Change Our Idea About Private Cord Blood Bank?

Gholamreza Bahoush Mehdiabadi

Iran University of Medical Sciences, Tehran, Iran, Islamic Republic of

Background: Umbilical cord blood stem cells transplantation has been used extensively in the allogeneic setting for acquired and genetic disorders of hematopoiesis. There is less experience in the utility of autologous cord blood transplantation, and there is great controversy about the role of autologous cord blood collection and storage. Over 400 cases of pediatric severe aplastic anemia occur annually in the United States. A growing number of children with severe aplastic anemia may have had their stem cells harvested through cord blood collection.

Methods: We report on the successful use of autologous cord blood transplantation for the treatment of 5 years-old boy with idiopathic severe aplastic anemia from Ali-Asghar Children Hospital. He received ATGAM $20 \mathrm{mg} / \mathrm{kg}$ for eight days just before infusing the stem cells and Sandimmune 5 $\mathrm{mg} / \mathrm{kg}$ orally per 12 hours. Then, he received $2.5 \times 10^{7} / \mathrm{kg}$ total nucleated cells.

Results: Platelet engraftment (more than 50000 per micro-liter for three consecutive days)was occurred four weeks after infusing the cells. Sandimmune was discontinued after one month from platelet engraftment. Complete blood count was normal after 3 months from infusing the cells. He is well completely 30 months after doing transplant.

Conclusions: With the increasing number of people cryopreserving autologous cord blood the use of autologous cord blood in the treatment of severe aplstic anemia might be considered as initial therapy. It appears that private cord blood banks will have more applications to the past. However, we need to more extensive studies for confirming of this idea.

Clinical Trial Registry: No clinical trial registration

Conflict of interest: This study was supported by Iran University of Medical Sciences.

\section{P507}

Abstract previously published
P508

Use of Eltrombopag in Patients with Platelet Engraftment Failure Following Second Allogeneic Peripheral Stem Cell Transplantation

Aliye Serpil Sarifakiogullari ${ }^{1}$, Melya Pelin Kirik ${ }^{2}$, Salih Sertac Durusoy ${ }^{1}$, Handan Haydaroglu Sahin ${ }^{1}$, Ilknur Gundes ${ }^{2}$, Mustafa Pehlivan $^{1,3}$

${ }^{1}$ Gaziantep University Faculty of Medicine, Department of Hematology, Gaziantep, Turkey; ${ }^{2}$ Gaziantep University Faculty of Medicine, Department of Internal Medicine, Gaziantep, Turkey;

${ }^{3}$ Gaziantep University Faculty of Medicine, Bone Marrow

Transplantation Center, Gaziantep, Turkey

Background: Thrombocytopenia after peripheral hematopoietic stem cell transplantation (PHSCT) is associated with morbidity and mortality. Eltrombopag, a thrombopoietin receptor agonist (TPA), is successfully used primarily in the treatment of chronic ITP and other thrombocytopenias associated with Aplastic Anemia and Myelodysplastic Syndrome. Recently, the use of Eltrombopag in the treatment of thrombocytopenia after allogeneic PHSCT is promising.

Methods: The use of eltrombopag in 3 patients with hematologic malignancy who experienced graft failure after the first PHSCT and developed platelet engraftment failure following the second bone marrow transplantation at the University of Gaziantep Bone Marrow Unit was presented retrospectively.

Results: Of the 3 patients; 2 were males and one was female, with the mean age of 52 (45-57) years. The diagnoses were AML, NHL and ALL. All 3 patients underwent allogenic PHSCT with myeloablative regimen. Platelet engraftment failure was detected during the followup of the patients. Acute grade 3 skin GVHD developed in NHL patient. Mycophenolate mofetil, CsA and steroid immunosuppression were given complete response at 1 week. However, thrombocytopenia persisted. None of the patients had any viral infection or relapse. Bone marrow biopsies of patients were hypocellular and the number of megakaryocytes decreased. Eltrombopag was initiated in 3 patients 110 days (60-144) after transplantation on average. Response was obtained in 3 patients; $\geq 30 \times 109$ / L (plt: 30.000-247.000). The mean duration of response was 27 days (20-35). No side effects associated with eltrombopag was observed.

Conclusions: Eltrombopag can be used safely and effectively in patients with platelet engraftment failure after PHSCT.

Conflict of interest: The authors have nothing to disclose.

Immunodeficiency diseases and macrophage 


\section{P509}

ADA-SCID: qualitative assessment of caregiver perceptions of treatment options

Rito Bergemann ${ }^{1}$, Angela Gaffuri ${ }^{2}$, Jane Barrett ${ }^{2}$, Ellie Bouchard ${ }^{2}$, Esther Mustchin ${ }^{2}$

${ }^{1}$ GSK, Value Evidence Outcomes, Brentford, United Kingdom; ${ }^{2}$ CelloHealth Insight, London, United Kingdom

Background: Current treatment options for adenosine deaminase-severe combined immunodeficiency (ADASCID) include haematopoietic stem cell transplantation (HSCT), enzyme replacement therapy (ERT) and gene therapy (GT). Little is known regarding caregivers' perceptions of these treatments or the emotional impact of making treatment decisions.

Methods: Research was conducted through telephone interviews of caregivers of patients with ADA-SCID. Participants were recruited in the USA, France, the UK and Italy through patient associations and direct referral from healthcare professionals. The study inclusion criteria were: caregiver's child must have a diagnosis of ADA-SCID with onset within the first year of life and must not be involved in GSK GT studies (including siblings). The research was compliant with the relevant country's market research regulations.

Results: Nine caregivers (all parents; 3 USA, 1 France, 3 UK, 2 Italy) were recruited. They are closely involved in treatment decisions, though there is a great reliance on expert advice in the absence of public information. Once under the care of a centre of excellence, caregivers have confidence in the specialist team; however, the journey to this point involving primary care and local hospitals is frustrating for the majority of caregivers. Caregivers report that the time spent considering treatment options is a highly emotional and challenging time, which is hardest when contemplating HSCT or GT. HSCT is considered better established than GT; however, the decision to proceed is influenced by donor availability. All respondents were aware of GT; related concerns include a perceived risk of leukaemia and lack of long-term data. For both treatments, the greatest concern for caregivers is the need for chemotherapy and the consequential impact this can have on their child's health. The experience of treatments to date is mixed, with varying success reported for both GT and HSCT. Most patients have received ERT and their caregivers are satisfied with this treatment.

Conclusions: Caregivers would benefit from a detailed and comprehensive information package explaining the different treatments for ADA-SCID. This should cover the side effects of transplants, chemotherapy and steroids as well as the benefit-risk profile and possibilities for sequencing treatments. Improved emotional support mechanisms would also be welcomed.

Conflict of interest: R. Bergemann is an employee and shareholder of GlaxoSmithKline (GSK); E. Mustchin, J. Barrett, A. Gaffuri, and E. Bouchard were commissioned by GSK to conduct the research.

Acknowledgements: The study was funded by GSK. Editorial support was provided by Clem Hindley of Fishawack Indicia Ltd. and was funded by GSK.

\section{P510}

\section{Hematopoietic Stem Cell Transplantation for 21 Patients with MHC Class II Deficiency - The Newcastle Experience}

Claire Anderson, Mohamed Najib Mohamed Unni, Terence Flood, Sophie Hambleton, Roderick Skinner, Geoff Shenton, Stephen Owens, Andrew J Cant, Andrew R Gennery, Mary Slatter

Great North Children's Hospital, Newcastle upon Tyne Hospital NHS Foundation Trust, Paediatric Bone Marrow Transplant, Newcastle upon Tyne, United Kingdom

Background: MHC Class II (MHC II) deficiency (bare lymphocyte syndrome) is a rare primary immunodeficiency which is amenable to hematopoietic stem cell transplant (HSCT). However reported outcomes are still limited. The objective of our study was to analyze pre-transplant comorbidities and outcome of HSCT in patients with MHC II deficiency.

Methods: We performed a retrospective study of 21 patients who underwent 23 HSCT procedures for MHC II deficiency at our centre between 1995 and 2017.

Results: The average (median) age at HSCT was 1.35 years (range 26 days to 7.7 years). Eleven patients were male and 10 female. Two patients required a second transplant due to graft failure. Sixteen of 21 patients had viral infections pre-transplant. Donors were as follows: 13 matched (MUD 6, MRD 7); 4 MMUD; and 6 haploidentical donors. Stem cell source was: 14 PBSC; 8 BM; and 1 cord blood. Conditioning was: 12 myeloablative (Busulfan/ Cyclophosphamide +/-Alemtuzumab or Anti-LFA and Anti-CD2, 5; Treosulfan/Cyclophosphamide, 2; Treosulfan/Fludarabine/Thiotepa/Anti-thymocyte Globulin/Rituximab, 5); 10 reduced toxicity (Treosulfan/Fludarabine/ Alemtuzumab); and 1 reduced intensity conditioning (Fludarabine/Melphalan/Alemtuzumab). Overall survival was $76 \%$ (5 patients died). Causes of death were severe interstitial pneumonia $(\mathrm{n}=4)$ with two patients also having multi-organ failure and 1 late death 4 years post-transplant from sepsis. Skin GVHD, maximum grade II, was seen in 13 patients. Only 2 patients required systemic steroids; the rest responded to topical treatment. One patient had chronic GVHD; no autoimmunity was observed. Length of follow 
up was 2.3 years (median; range 87 days to 14.3 years). Viral reactivation was common.

Conclusions: HSCT is a successful treatment for MHC II deficiency. Of note, all 15 patients transplanted since 2008 are alive and well, including 5 post $\mathrm{CD} 3+\mathrm{TCR} \alpha \beta / \mathrm{CD} 19+$ depleted haploidentical grafts.

Conflict of interest: The authors have no conflicts of interest to disclose.

\section{P511}

Abstract previously published

\section{P512}

Immunity and Tolerance after Bilateral Orthotopic Lung Transplant(BOLT) in Tandem with CD3+/CD19+Depleted Vertebral Bone Marrow Transplant(BOLT+BMT) from 1 of 8 HLA-Matched Cadaveric Donors in Immunodeficiency Patients
Paul Szabolcs ${ }^{1,2}$, Xiaohua Chen ${ }^{1}$, Albert Donnenberg ${ }^{3,4}$, Memphis Hill $^{1}$, Jason Rowan ${ }^{1}$, Shawna Mcintyre ${ }^{1}$, Heather Stanczak ${ }^{1,4}$, Nicole Nastasi ${ }^{1,4}$, Zarreen Amin ${ }^{1}$, Jessie Barnum ${ }^{1}$, Geoffrey Kurland $^{5}$, John McDyer ${ }^{6}$

${ }^{1}$ Children's Hospital of Pittsburgh, UPMC, BMT \& CT, Pittsburgh, PA, United States; ${ }^{2}$ University of Pittsburgh, Department of Immunology, Pittsburgh, PA, United States; ${ }^{3}$ University of Pittsburgh, School of Medicine and Department of Infectious Diseases and Microbiology, Pittsburgh, PA, United States; ${ }^{4}$ UPMC, Hematopoietic Stem Cell Laboratories, Pittsburgh, PA, United States; ${ }^{5}$ Children's Hospital of Pittsburgh, UPMC, Division of Pulmonary Medicine, Allergy \& Immunology, Pittsburgh, PA, United States; ${ }^{6} U P M C$, Division of Pulmonary, Allergy and Critical Care Medicine, Pittsburgh, PA, United States

Background: Primary immunodeficiency patients may develop pulmonary complications and most are ineligible for either lung transplant or BMT due to futility. We report on our first 2 subjects enrolled on a first in human prospective clinical trial, ClinicalTrials.gov: NCT01852370 where they receive tandem BOLT and subsequent BMT from the same deceased UNOS donor. We hypothesized:
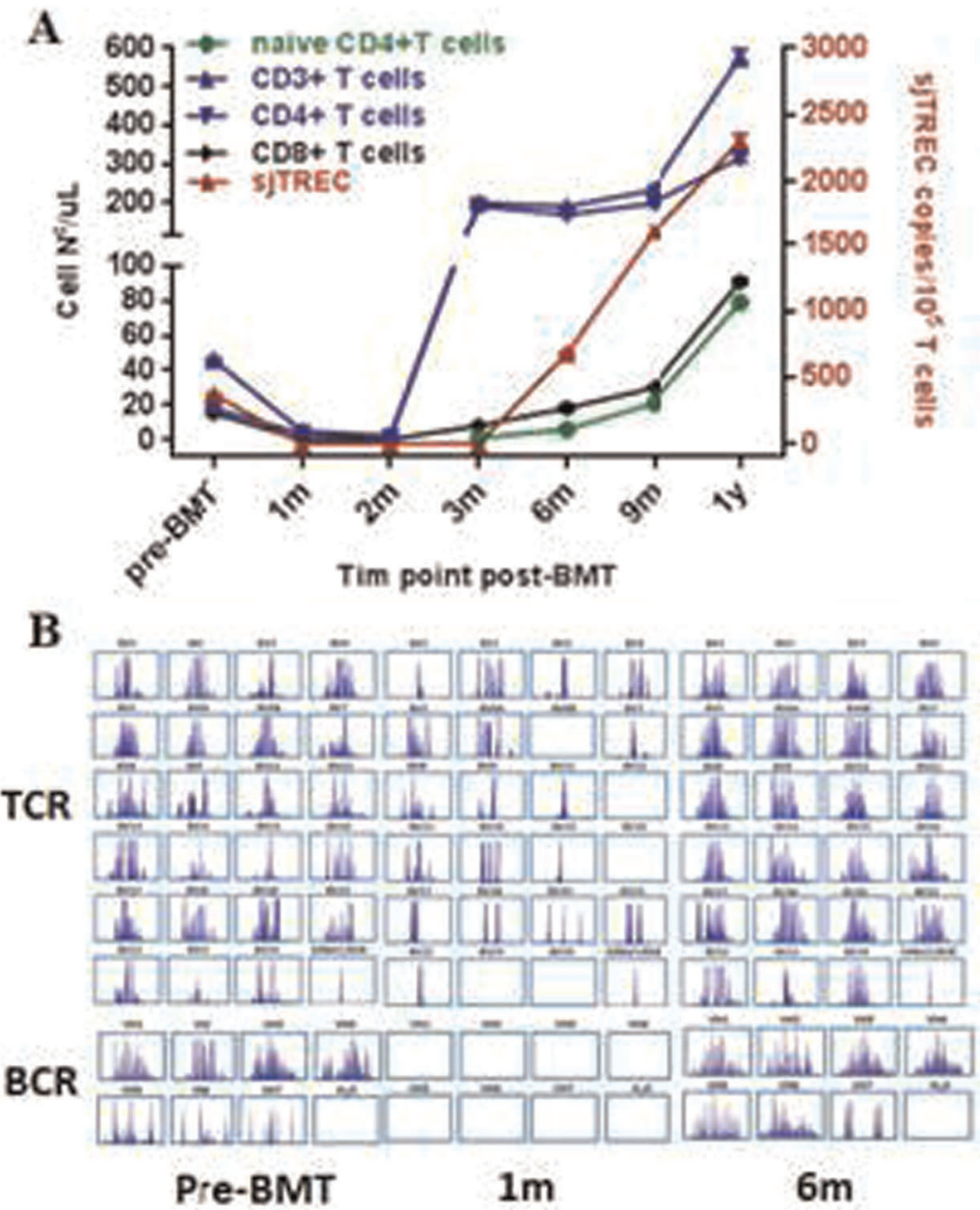

\section{T cell response to BKV (ELISPOT assay)}

\section{BKV-stimulated}
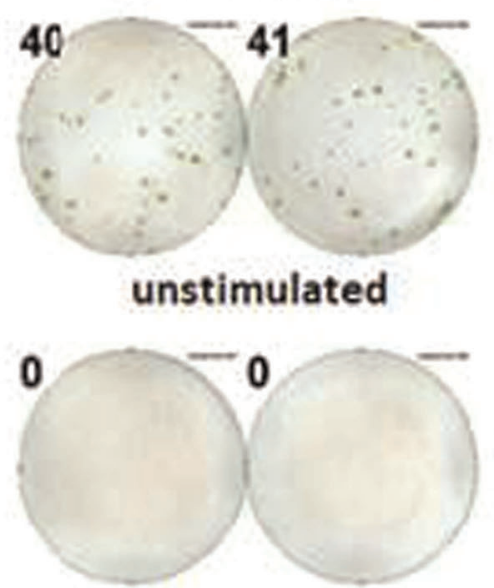

CMV-stimulated

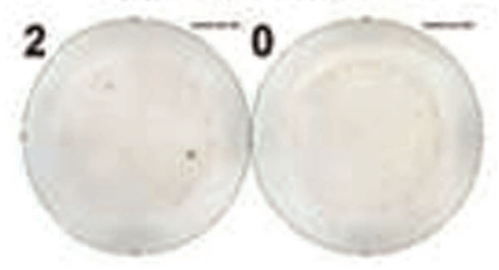

[P512 Figure] [Figure 1] 
1) hematopoietic stem cells prepared from organ donor vertebral bodies (VB) would yield greater CD34+ cell dose compared to iliac crest aspirates-with limited by harvest time due to parallel organ recovery teams;

2) cryopreserved CD3/CD19 depleted bone marrow could generate durable hematopoiesis and donor derived immunity;

3) not only full donor but persistent mixed chimerism could establish tolerance (bidirectional) following withdrawal of immunosuppressive drugs (ISD).

Methods: Case 1: 14 year old female with IL-7R null SCID and recurrent bacterial, fungal pneumonia underwent BOLT from a 2/6 HLA antigen (1/8 at allele level) matched donor. Marrow suspension was prepared from T11-L4 VB logs followed by $\mathrm{CD} 3+/ \mathrm{CD} 19+$ depletion $\left(\right.$ CliniMACS $^{\circ}$ ) and cryopreservation along with $\sim 20$ fold fewer TNC and CD34+ cells from the iliac crest. At 3 months post-BOLT, she started BMT conditioning with Hydroxyurea, Thiotepa. TBI (200cGy) and combined with serotherapy to deplete host B, T and NK lymphocytes. A month later, marrow was thawed and infused $(5 \mathrm{E}+06 \mathrm{CD} 34+$ cells $/ \mathrm{kg}$ and $8 \mathrm{E}+04$ $\mathrm{CD} 3+\mathrm{T}$ cells $/ \mathrm{kg}$ ). She engrafted with $100 \%$ donor cells. Noting persistent $\mathrm{T}$ cell lymphopenia (mostly of host origin) she received DLI $\sim 10$ weeks later $(5 \mathrm{E}+04 \mathrm{~T}$ cells $/ \mathrm{kg})$.

Results: DLI resulted in dominant donor T (>97\%) and B cell (91-95\%) chimerism. Donor myeloid cells (granulocyte, monocytes and myeloid DC) along with NK lymphocytes had declined steadily in the first 3-5 months but have stabilized at $\sim 12 \% \pm 3 \%$. BAL values exhibited different kinetics.

$\mathrm{T}$ and $\mathrm{B}$ cells, TCR/BCR repertoires, and TREC exceeded pre-BMT values by 3-6 months (Fig1A, B). BK virus specific $\mathrm{T}$ cells cleared viremia (Fig1C), suggesting effective reconstitution of pathogen specific immunity. (Donor/recipient each were CMV negative).

Mild skin GVHD post-DLI cleared in $~ 2$ weeks with $<1$ $\mathrm{mg} / \mathrm{kg}$ prednisone. Serial lung biopsies have been negative for rejection at last follow-up report (Nov17; 5m off ISD). At 18 months post-BMT ( 2 months post-ISD withdrawal), circulating donor $\mathrm{T}$ cells were unresponsive to host $\mathrm{DC}$ while responded to third party APC, fulfilling a critical tenet of tolerance. Tolerance towards host APC was Treg and Tr1 independent.

Case 2: 38 years old woman with CVID on ECMO underwent BOLT. She had 3 episodes of lung rejection before becoming eligible for BMT (1/8 match) 14 months later (Nov'17). Total of 3E+06 CD34+ VB cells/kg and 2E $+03 \mathrm{CD} 3+\mathrm{VB}$ cells $/ \mathrm{kg}$ were infused. She engrafted by day 12 with $95 \%$ whole blood chimerism but $\mathrm{T}$ cells of host origin that is converting following DLI.

Conclusions: Case 1 is the first in human to demonstrate

1) durable engraftment,

2) immune competence, and
3) acquisition of tolerance from deceased donor VB marrow

4) matched only at a single class I MHC allele, providing proof of principle, that is currently also tested in adults.

Clinical Trial Registry: NCT01852370

https://clinicaltrials.gov/ct2/show/NCT01852370

Conflict of interest: None of the authors has anything to disclose.

\section{P513}

Less is better - Low cost haploidentical haematopoietic stem cell transplantation with post transplant cyclophosphamide in children with primary immune deficiency disorders

Revathi Raj, Ramya Uppuluri, Dhaarani Jayaraman, Meena Sankaran, Venkateswaran V S, Shivani Patel, Lakshman Vaidhyanathan

Apollo Speciality Cancer Hospitals, Paediatric Blood and Marrow Transplantation, Chennai, India

Background: Haploidentical haematopoietic stem cell transplantation (HSCT) offers a possibility of cure in children with primary immune deficiency disorders with no matched donors or access to gene therapy. We describe encouraging results with the use of post transplant cyclophosphamide in this cohort of children.

Methods: Data presented here is a retrospective analysis of children who underwent $\mathrm{T}$ replete haploidentical haematopoietic stem cell transplantation with post transplant cyclophosphamide (PTCy) for primary immunedeficiency disorder at our centre over a three year period. All children had reduced intensity conditioning using fludarabine with melphalan or treosufan with additional 2 Gy total body radiotherapy in a few children. Infants less than 6 months had a cyclophosphamide dose of $25 \mathrm{mg} / \mathrm{kg} / \mathrm{day}$ for 2 days. The incidence of engraftment, graft rejection, infections, graft versus host disease, event free and overall survival and unusual complications were documented.

Results: A total of 15 children underwent haploidentical HSCT with PTCy with a diagnosis of severe combined immune deficiency in 2, Wiskott Aldrich syndrome in 4, haemophagocytic lymphohistiocytosis in 5, Hyper Ig M syndrome in 1 and Mendelian susceptibility to mycobacterial disease in 2 children. Graft failure was seen in 3 children with 2 surviving after autologous reconstitution. All 3 infants transplanted had cytokine release syndrome and one baby could be salvaged with the use of tociluzimab. Viral infections resulting in death was seen in 1 child and HLH progression resulted in the death of 1 child. None of the children had grade 3 to 4 graft versus host disease. The 
follow up period has been from 109 days to 600 days. The overall survival was $74 \%$ and disease free survival $60 \%$.

Conclusions: This is a unique study of children with primary immune deficiency disorders of different aetiologies to undergo haploidentical HSCT with PTCy with a $74 \%$ survival. The average cost of HSCT was 40,000 USD. Universal access to care is still an issue in developing countries and PTCy offers a cost effective modality of therapy where gene therapy, unrelated HSCT and ex vivo T cell depletion techniques are still beyond the reach of many families with no insurance cover. Unusual complication like cytokine release storm has been documented especially in infants and in children undergoing HSCT for HLH and this needs prospective evaluation.

Clinical Trial Registry: N/A

Conflict of interest: None

\section{P514}

\section{Lights and shadows of haematopoietic stem cell transplantation for DOCK8 deficiency: report of five cases}

Giulia Carracchia $^{1}$, Elena Soncini ${ }^{1}$, Marianna Maffeis ${ }^{2}$, Ilaria Fracassetti $^{3}$, Raffaele Badolato ${ }^{4}$, Arnalda Lanfranchi ${ }^{5}$, Fulvio Porta $^{6}$

${ }^{1}$ Pediatric Oncohaematological and BMT Unit, Children Hospital Univerisity of Brescia, Brescia, Italy; ${ }^{2}$ Childrens Hospital University of Brescia, Brescia, Italy; ${ }^{3}$ Childrens Hospital Paediatrics University of Brescia, Brescia, Italy; ${ }^{4}$ Pediatric Immunology Childrens Hospital University of Brescia, Brescia, Italy; ${ }^{5}$ Stem Cell Laboratory, Section of haematology and Blood Coagulation, Spedali Civili Brescia, Brescia, Italy; ${ }^{6}$ Pediatric Oncohaematological and BMT Unit, Childrens Hospital Chair of Paediatrics University of Brescia, Brescia, Italy

Background: Autosomal recessive Hyper IgE syndrome (HIES), caused by mutations in DOCK8, is associated with severe cellular immunodeficiency, viral and bacterial infections, eczema, food allergies and malignancies. Since 2010 hematopoietic stem cell transplantation has been proposed as curative treatment .

Methods: We report five cases of DOCK8-deficient patients treated with bone marrow transplant (median age at transplant: 11 years and 3 months). A fifth 15-year-old patient cames from a low economic conditions country, so she didn't receive an early bone marrow transplant.

Before transplantation all patients presented with eczema, sinopulmonary infections and food allergies. Other common features were mucocutaneous candidiasis, skin abscesses, bronchiectasis, viral and other serious infections (50\%). Skeletal anomalies (retained primary teeth, fractures and hyperextensibility of joints), vascular abnormalities with lacunar brain infarcts and malignancies (neuroblastoma) were less frequent $(1 / 4)$.
A myeloablative conditioning regimen with busulfan or treosulfan, fludarabine, thiotepa and ATG was used in three patients while one patient was treated with a reducedintensity regimen (fludarabine, alemtuzumab and melphalan). Three patients received allograft from matched unrelated donors and one from haploidentical related donor. The source of stem cells was peripheral blood $(50 \%)$ or bone marrow (50\%). GVHD prophylaxis was based on cyclosporine, mycophenolate mofetil and steroids.

Results: Complete engraftment of neutrophils and platelets was achieved in three patients (at a median day of 18 and 13, respectively). One patient, who had an active infection at the time of transplant, didn't achieve donor engraftment and a boost was necessary. The patient died due to severe pneumonia. A stable $100 \%$ donor chimerism was observed in the other patients and the only adverse event after transplant was an acute GVHD (1/4).

At a follow-up of 12 months the complications were progressive multifocal leukoencephalopathy, papillary thyroid carcinoma, papilledema and pneumonia with sepsis. The other features of HIES, except for food allergen sensitization, weren't observed. All patients showed a reduction of serum $\mathrm{IgE}$ (median range from $4640 \mathrm{IU} / \mathrm{mL}$ to $360 \mathrm{IU} / \mathrm{mL}$ ) and of eosinophil count (median range from 1810 cells/ $\mu \mathrm{L}$ to 290 cells/ $\mu \mathrm{L}$ ). Lymphocyte count increased (median range from 1075 cells $/ \mu \mathrm{L}$ to 1290 cells $/ \mu \mathrm{L}$ ) and lymphocyte proliferation became normal.

The fifth patient is a 15-year-old girl with a congenital immune deficiency type DOCK8 associated with systemic lupus erythematosus, optic atrophy, maculopathy, neurolupus and an antiphospholipid syndrome. She didn't receive an early bone marrow transplant so she developed warts, herpes virus type 1 cutaneous infection, superinfection by Staphilococcus aureus, Pseudomonas aeruginosa and Candida albicans and a cutaneous non-HodgkinÂ's lymphoma $\mathrm{T}$.

Conclusions: Allogenic bone marrow transplant in DOCK8 deficiency results in immunological reconstitution and reversal of HIES features, but it isn't event-free.

Our experience shows the importance of correct timing for bone marrow transplant in these patients.

Conflict of interest: The authors have no conflicts of interest to declare.

\section{P515}

Outcome of Patients with Severe Combined Immunodeficiency/ Combined Immunodeficiency due to ADA Deficiency

Deniz Cagdas Ayvaz ${ }^{1}$, Pınar Gür Çetinkaya ${ }^{2}$, Betül Karaatmaca ${ }^{2}$, Saliha Esenboğa ${ }^{2}$, Çağman Tan ${ }^{1}$, Togay Yılmaz, , Safa Barış ${ }^{4}$, Kaan Boztuğ ${ }^{5}$, Tuba Tuba Turul Ozgur ${ }^{1}$, Michael Hershfield ${ }^{6}$, Ozden 
Sanal, İlhan Tezcan ${ }^{1}$

${ }^{1}$ Hacettepe University Faculty of Medicine, Pediatric Immunology, Ankara, Turkey; ${ }^{2}$ Hacettepe University Faculty of Medicine, Pediatric Allergy and Immunology, Ankara, Turkey; ${ }^{3}$ Hacettepe University Faculty of Medicine, Ankara, Turkey; ${ }^{4}$ Marmara University Pendik Training and Research Hospital, Pediatric Allergy and Immunology, Istanbul, Turkey; ${ }^{5}$ Medical University of Vienna, Pediatric Hematology, Oncology and Immunology, Vienna, Austria; ${ }^{6}$ Duke University School of Medicine, Duke Medical Center, Rheumatology and Immunology, Durham, NC, United States

Background: Adenosine deaminase (ADA) deficiency is an autosomal recessive primary immunodeficiency, at the same time a metabolic disorder which leads to accumulation of toxic metabolites of purine pathway. ADA deficiency accounts about 10-20\% of all severe combined immunodeficiency (SCID). Generally, more than $85 \%$ of the patients present in the first 6 months of life with the manifestations of recurrent and opportunistic fungal, viral and bacterial infections, lymphopenia, and failure to thrive. Late onset forms of disease are also known, but the manifestations appear to be milder.

The aim of this study is to evaluate the outcome of ADA deficient patients, their clinical and laboratory findings before and after ERT, Hematopoietic stem cell transplantation (HSCT) and gene therapy.

Methods: Twelve patients with ADA deficiency was followed up in Hacettepe University Pediatric Immunology from 2000-2017. Nine out of 12 were diagnosed as SCID, while 3 out of 12 were dignosed as late onset ADA deficiency having cliinical and laboratory findings of CID. Immunophenotyping was done with flow cytometry. After ADA enzyme activity and metabolites were measured and found to be defective, Sanger sequencing of the ADA gene was performed.

Results: The ages of onset varies from 3 months to 20 years. Nine out of 12 patients were put on pegylated ADA enzyme replacement therapy (ERT), other two, born before ERT and one, died quickly could not be given. In 6 , it is used for a definite period before HSCT or gene therapy. In two patients, who have late-onset ADA deficiency, ERT is still being used, and sufficient to control the symptoms in addition to IVIG and prophylactic atibacterial therapy. In one of the patients with ADA deficiency, ERT was insufficient to control the immunodeficiency symptoms.

Four out of 12, who were transplanted from matched family donors without conditioning are alive, while one was transplanted from matched unrelated donor with conditioning therapy died soon after HSCT. Gene therapy was performed to one. Four of them, two diagnosed before ERT and having no donors, one after HSCT died. One out of 4 tranplanted/ alive patients had poliposis coli and needed IVIG and albumin therapy also after HSCT, in other $3 \mathrm{f}$ IVIG therapy was stopped.
One whom gene therapy was performed is well, alive and out of IVIG therapy.

Conclusions: The genetic diagnosis of SCID and CID is utmost important, as there is chance to give ERT before planning the definitive therapy if the patient has ADA deficiency. Enzyme replacement therapy is most succesful in patients with partial ADA deficiency who presented with the features of combined immunodeficiency. Although ERT is insufficient to control the symptoms in severe ADA deficiency, it is useful for a short period to gain time before definite therapy, such as HSCT or gene therapy. If there is no associating disease with protein loss, it is possible to stop the IVIG therapy after HSCT performed without conditioning therapy.

Clinical Trial Registry: -

Conflict of interest: Nothing to disclose.

\section{P516}

Outcomes of children with Primary Hemophagocytic Lymphohistiocytosis (HLH) treated at king Hussein Cancer Center

Mayada Abu Shanap, Hasan Hashem, Iyad Sultan, Rawad Rihani, Eman Khattab, Osama Alsmadi, Farid Barakat, Roubi Abu Obeid, Abdelghani Tbakhi, Faris Madanat

King Hussein Cancer Center, Amman, Jordan

Background: Hemophagocytic Lymphohistiocytosis (HLH) is a hyperinflammatory syndrome with high mortality even with appropriate treatment. Allogeneic hematopoietic cell transplantation (HCT) is considered the only curative treatment for patients with primary HLH, with reported survival ranging from $50 \%$ to $70 \%$.

Methods: We retrospectively analyzed outcomes of a cohort of patients diagnosed with primary HLH at King Hussein Cancer Center (KHCC) between 2003 and 2017.

Results: We identified 19 patients (13 males, 6 females); median age at diagnosis was 13.9 months (range, 2.87-238). There were 3 patients with Chediak-Higashi and 2 with Griscelli syndrome. Six other patients had family history of HLH with identical twin boys developing their disease simultaneously. CNS involvement at diagnosis was present in one patient. All patients received standard immunochemotherapy per HLH-1994/2004 protocol. Eleven of 19 patients underwent HCT, median age at HCT was 32 months (range, 6.9-251); median time from diagnosis to HCT was 8.2 months (range, 0.87-13.2). At time of HCT, 2 patients achieved PR and 9 patients were in CR. Seven patients received myeloablative conditioning with busulfan /cyclophosphamide / rabbit antithymocyte globulin (ATG)) +/etoposide. Four patients received reduced intensity conditioning with fludarabine/melphalan/ATG \pm thiotepa $(\mathrm{n}=$ 
3), fludarabine/ cyclophosphamide /ATG $(\mathrm{n}=1)$. Donors were HLA-matched siblings in 8 patients and unrelated cord blood in 3 patients. Median time to neutrophil and platelet engraftment was 16 days (range: 11-29), and 26 days (range: 17-68), respectively. Acute GvHD developed in $63 \%$ (7/11) of patients (grade III in 4 patients) while limited chronic GvHD developed in 18\% (2/11). Median follow up of surviving patients with primary HLH post HCT is 75.23 months (range: 1.27-134.3). Nine of 11 patients are alive at last follow up with 5-year overall survival of $80 \%$ and 5-year event free survival of $70 \%$. Two patients died, one at day +38 post HCT due to CMV infection, and the other patient relapsed at day +290 post HCT and died of the disease. Of surviving patients, 5 patients had mixed donor chimerism while full donor chimerism was noted in 4 patients. Nine patients are off immune suppression. Immune reconstitution studies were available for seven patients; six patients had normal lymphocyte subsets, while one patient had low B cell counts.

Conclusions: Primary HLH is fatal disease; making definitive diagnosis is essential as allogeneic hematopoietic stem cell transplantation (HCT) is the only curative treatment. Prospective study is warranted to validate the previous findings; genetic study is helpful to guide definitive therapy, early referral to transplant and family counseling.

Conflict of interest: Mayada Abu Shanap: Nothing to disclose

\section{P517}

Preliminary results of novel Treosulfan-based conditioning regimen for allogenic HSCT with TCRaß/CD19 graft depletion in Nijmegen breakage syndrome after five years of Busulfan experience

Alexandra Laberko, Elena Gutovskaya, Svetlana Radigina, Svetlana Kozlovskaya, Irina Shipitsina, Elena Deripapa, Anna Shcherbina, Michael Maschan, Alexei Maschan, Dmitry Balashov

Dmitry Rogachev National Research Centre for Pediatric Hematology, Oncology and Immunology, Moscow, Russian Federation

Background: NBS is a DNA repair disorder. Low dose Busulfan based conditioning is usually used for hematopoietic stem transplantation in NBS patients, as its safety and efficacy was demonstrated in patients with another DNA repair defect - Fanconi anemia. Treosulfan is a new alkylating agent thus far there is no reports of use of Treosulfan containing conditioning in HSCT for DNA repair disorders.

Methods: From October 2013 to November 201718 HSCT with TCR $\alpha \beta / C D 19$ depletion of graft were performed in our center in 16 NBS patients (all have "slavic" homozygous mutation 657del5 in NBS1). Median age at HSCT - 8,7 years. Male/female ratio - 12/4. In 8 patients - indication for HSCT was malignancy, in 8 immunodeficiency (autoimmune and infectious complications). 16 patients were transplanted from matched unrelated (including 2 second HSCT), 2 from related sibling donors.

From 2012 to 2016 in 12 patients Busulfan (Bu) based conditioning was used: $\mathrm{Bu} 4 \mathrm{mg} / \mathrm{kg}+$ Fludarabin (Flu) $150 \mathrm{mg} / \mathrm{m}^{2}+$ Cyclophosphamide $(\mathrm{Cy}) 20-40 \mathrm{mg} / \mathrm{kg}$. From 2017 new approach with Treosulfan (Treo) $30 \mathrm{~g} / \mathrm{m}^{2}$ is used, 2 patients received it in combination with Flu $150 \mathrm{mg} / \mathrm{m}^{2}, 4$ ( 2 had second HSCT) with Flu $150 \mathrm{mg} / \mathrm{m}^{2}$ and Cy $40 \mathrm{mg} / \mathrm{kg}$. All patients received Thymoglobulin $5 \mathrm{mg} / \mathrm{kg}, 15$ - Rituximab $100 \mathrm{mg} / \mathrm{m}^{2}$. GVHD prophylaxis included Tacrolimus and in 6 patients short course of Methotrexate.

Results: All patients after Bu containing conditioning had primary engraftment. Bu containing conditioning was well tolerated: only 1 of 12 patients had grade 2 acute GVHD of GUT, 3 patients died - 1 of adenoviral hepatitis, 2 of lymphoma relapse. Unfortunately, 4 patients rejected grafts, median time of rejection - 6,6 months after HSCT (2 of them had second HSCT, 1 died of high grade lymphoma, 1 is going to receive second transplantation).

Since January 20176 patients received Treo containing conditioning, all of them engrafted. To date the median FU is only 6 months, but we observed no early complications (drug induced organ damage, GVHD, severe infections) after HSCT. One of 2 patients with Treo+Flu conditioning has mixed predominantly donor chimerism, 4 patients with addition of $\mathrm{Cy}$ have full donor chimerism at last FU.

Conclusions: Herein we report first experience of safety and efficacy of low dose Treosulfan containing conditioning for HSCT with TCR $\alpha \beta / C D 19$ graft depletion in patients with DNA repair disorder. We also report good tolerance of second transplantations with reduced intensity conditioning in NBS patients.

Conflict of interest: Nothing to disclose

\section{P518}

Successful bone marrow transplantation after orthotopic liver transplantation in a child with familial hemophagocytic lymphohistiocytosis
Tekin Aksu ${ }^{1}$, Lale Olcay ${ }^{2}$, Figen Özçay ${ }^{3}$, Namık Y. Özbek ${ }^{1}$
${ }^{1}$ University of Health Sciences, Ankara Child Health and Diseases Hematology Oncology Training and Research Hospital, Pediatric Hematology and Oncology, Ankara, Turkey; ${ }^{2}$ Baskent University Medical Faculty, Pediatric Hematology and Oncology, Ankara, 
Turkey; ${ }^{3}$ Baskent University Medical Faculty, Pediatric

Gastroenterology Hepatology and Nutrition, Ankara, Turkey

Background: Familial hemophagocytic lymphohistiocytosis (HLH) is a fatal disorder which may progress to acute fulminant liver failure. There are reports concerning hematopoietic stem cell transplantation (HSCT) after liver transplantation in patients with acquired aplastic anemia, but experience in patients with HLH is limited. Here we present a child with HLH who underwent successful HSCT after orthotopic liver transplantation (OLT).

Methods: Characteristics of patient, transplantation and treatment were recorded.

Results: A 3.5-year-old girl was presented with acute liver failure. She underwent OLT from his haploidentical father. Afterwards, her liver pathology disclosed findings of HLH and molecular analysis revealed homozygous syntaxin (STX11) mutation. After OLT, she treated with a HLH directed therapy. She experienced posterior reversible encephalopathy syndrome (PRES) and managed with antiepileptics during treatment. She underwent HSCT 21 months after OLT from 10/10 HLA-matched sibling, who was heterozygous for STX11 mutation. She received reduced intensity conditioning with fludarabine $\left(40 \mathrm{mg} / \mathrm{m}^{2} /\right.$ day; -5 to -2 ), busulfan (qid, target area under curve (AUC): $60 \pm 5 \mathrm{mg} / \mathrm{L} \mathrm{x} \mathrm{h;-5} \mathrm{to} \mathrm{-2),} \mathrm{and} \mathrm{anti-thmocyte} \mathrm{globulin}$ (ATG-Fresenius ${ }^{\circledast}$ ) $20 \mathrm{mg} / \mathrm{kg} / \mathrm{total}(-5$ to -2$)$. N-acetylcystein (NAC) was applied during conditioning in order to prevent liver toxicity. Defibrotide $(25 \mathrm{mg} / \mathrm{kg} /$ day $)$ and ursodeoxycholic acid were prophylactically applied in order to prevent sinus obstruction syndrome (SOS) until day +29 . Bone marrow harvested and yielded $2.14 \times 10^{8} / \mathrm{kg}$ nucleated cells and $4.2 \times 10^{6} / \mathrm{kg} \mathrm{CD} 34+$ cells that were given to the patient. Cyclosporin A (target $175-250 \mathrm{ng} / \mathrm{ml}$ ) and methotrexate $\left(10 \mathrm{mg} / \mathrm{m}^{2},+1,+3\right)$ were used for graft versus host disease (GVHD) prophylaxis. The early posttransplant period was uneventful except for mild increase in liver transaminases at day +6 (ALT $297 \mathrm{U} / \mathrm{L}$, AST $171 \mathrm{U} / \mathrm{L}$ ). A short course of methylprednisolone $(1 \mathrm{mg} / \mathrm{kg} /$ day $)$ was given instead of +6 methotrexate. Myeloid and thrombocyte recoveries occured at day +14 and +15 , respectively. She has not experienced GVHD, organ toxicity, febrile neutropenia, fungal or viral infection. She experienced epileptic seizures 3.5 months after HSCT without clinical or laboratory findings of HLH. We changed her immunosuppressive treatment from cyclosporine to everolimus (target $3-8 \mathrm{ng} / \mathrm{mL}$ ). The seizures are under control for the last 6 months. Analysis revealed 80.5 and $87.3 \%$ donor chimerism at day +30 , and at month +10 , respectively. She is stil well +11 months after HSCT.

Conclusions: There is very limited experience in literature concerning subsequent OLT and HSCT from different donors. Although our patient has three different
HLA groups (somatic, liver and bone marrow), she has not experienced GVHD or liver rejection. We believe reduced intensity conditioning containing low busulfan doses, and prophylaxis containing NAC and defibrotide prevented SOS in the orthotopic liver. Reduced intensity conditioning was sufficient to provide donor chimerism without GVHD or organ toxicity.

Conflict of interest: None of the authors has anything to disclose.

\section{P519}

T-cell replete haploidentical bone marrow transplantation, myeloablative conditioning and post-transplant cyclophosphamide for pediatric patients with nonmalignant disorders

Mathias Kurzay, Fabian Hauck, Tobias Feuchtinger, Christoph Klein, Michael Albert

Dr. von Hauner University Children's Hospital, Munich, Germany

Background: Allogeneic hematopoietic stem cell transplantation (HSCT) from matched related and unrelated donors results in excellent cure rates of around $90 \%$ in children with most inborn, non-malignant disorders. In patients lacking a matched donor, the use of mismatched (haploidentical) family donors was historically associated with inferior outcomes due to rejection, GVHD and infectious complications. In-vivo T-cell depletion with post-transplant cyclophosphamide (pTCy) has been shown to result in outcomes comparable to those with matched family or unrelated donors in adults with malignant diseases. We explored its use in combination with upfront serotherapy and an established reduced toxicity myeloablative conditioning regimen in children with nonmalignant diseases.

Methods: We describe our institutional experience with eleven patients who received T-cell replete haploidentical parental bone marrow grafts at a median age of 9.4 years (range 0.2-21.6). Four patients suffered from primary immunodeficiencies [IPEX syndrome $(\mathrm{n}=1)$, IL-10R deficiency $(\mathrm{n}=1)$, XIAP $(\mathrm{n}=1)$, WAS $(\mathrm{n}=1)$, PNP deficiency $(n=1)$, five patients from sickle cell disease (SCD) and two from thalassaemia. One patient had undergone a previous unsuccessful HSCT. The grafts contained a median of $5.0 \times 10^{\wedge} 8 / \mathrm{kg}$ (2.6-9.1) total nucleated, $4.0 \times 10^{\wedge} 6 / \mathrm{kg}(1.5-8.7) \mathrm{CD} 34^{+}$and $4.0 \times 10^{\wedge} 7 / \mathrm{kg}$ (1.1-7.4) $\mathrm{CD}^{+}$cells. Conditioning consisted of alemtuzumab $2 \times 0.2 \mathrm{mg} / \mathrm{kg} / \mathrm{d}$ (day- 9 to -8 ), fludarabine $5 \times 30 \mathrm{mg} / \mathrm{m}^{2} / \mathrm{d}$ (day-7 to -3 ), treosulfan $3 \times 14 \mathrm{~g} / \mathrm{m}^{2} / \mathrm{d}$ (day-7 to -5 ), thiotepa $10 \mathrm{mg} / \mathrm{kg} / \mathrm{d}$ (day-4) and cyclophosphamide $2 \times 14.5 \mathrm{mg} / \mathrm{kg} / \mathrm{d}$ (day-3 to -2) in all patients except one (PNP) who received 
submyeloablative busulfan (AUC 55.000ngxh/ml) instead of treosulfan/thiotepa. Five patients received rituximab $375 \mathrm{mg} / \mathrm{m}^{2} / \mathrm{d}$, three of whom had pre-transplant plasmapheresis for the presence of anti-donor HLA antibodies. GVHD prophylaxis consisted of cyclophosphamide $50 \mathrm{mg} / \mathrm{kg} / \mathrm{d}$ on days +3 and +4 , followed by MMF and tacrolimus.

Results: After a median follow-up of 23.3 months (2.757.3) all patients are alive and 9 of 11 patients have 90$100 \%$ donor chimerism. Two patients experienced secondary graft loss with autologous reconstitution. One was retransplanted successfully from the same donor (PNP), one is alive with disease (SCD). Neutrophil and platelet engraftment occurred on day +17 (median; 6-30) and +25 (14-180) respectively. T-cell immune reconstitution was timely with a median of $384 / \mu \mathrm{l} \mathrm{CD} 3^{+}$cells (17-2013),

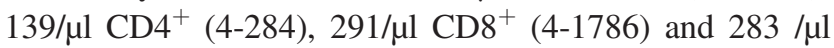

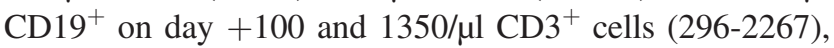

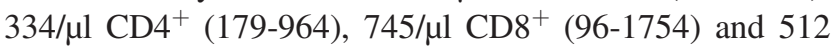
$/ \mu \mathrm{l} \mathrm{CD} 19^{+}$on day +180 . Seven CMV reactivations, one VZV reactivation but no CMV disease were observed. One patient experienced mild VOD. Acute GVHD was limited to overall grade I (skin only) in two patients. Chronic GVHD was not observed.

Conclusions: In our experience a pTCy haplo approach containing upfront serotherapy resulted in excellent engraftment and absence of severe GVHD or relevant transplantation related morbidity and mortality in children with nonmalignant diseases. The pTCy haplo strategy may compare favourably with respect to technically and financially more demanding in vitro T-cell depletion strategies, yet larger prospective and preferably comparative studies are warranted to evaluate this approach.

Clinical Trial Registry: $\mathrm{n} / \mathrm{a}$

Conflict of interest: The authors declare no conflict of interest.

\section{P520}

The Outcome of Matched Unrelated Allogeneic Hematopoietic Stem Cell Transplantation for Isolated CD4 (+) T-cell Deficiency with Interleukin-2 Inducible T-cell Kinase (ITK) Gene Defect

Meltem Kurt Yuksel ${ }^{1}$, Zehra Narli Ozdemir ${ }^{1}$, Ugur Sahin ${ }^{1}$, Deniz Cagdas Ayvaz ${ }^{2}$, Deniz Turkum Atikcan ${ }^{3}$, Elif Sertesen ${ }^{3}$, Sinem Civriz Bozdag ${ }^{1}$, Selami Kocak Toprak ${ }^{1}$, Pervin Topcuoglu ${ }^{1}$, Onder Aslan ${ }^{1}$, Muhit Ozcan ${ }^{1}$, Taner Demirer ${ }^{1}$, Hamdi Akan ${ }^{1}$, Osman Ilhan ${ }^{1}$, Meral Beksac $^{1}$, Gunhan Gurman ${ }^{1}$, Aydan Ikinciogullart ${ }^{4}$

${ }^{1}$ Ankara University Faculty of Medicine, Hematology, Ankara, Turkey; ${ }^{2}$ Hacettepe University Faculty of Medicine, Pediatric Immunology, Ankara, Turkey; ${ }^{3}$ Ankara University Faculty of Medicine, Internal
Medicine, Ankara, Turkey; ${ }^{4}$ Ankara University Faculty of Medicine, Pediatric Immunology, Ankara, Turkey

Background: Primary immunodeficiencies (PIDs) are associated with recurrent infections with multipl microbial agents. They are thought to be rare conditions and have been neglected for many years. However, awareness among pediatricians help us to notice the imporance of PIDs. There is huge heterogeneity underlying conventional phenotypes. The majority of the PIDs are inherited as autosomal recessive (AR) forms. The prevelance of PID diseases are high in Turkey. The high consanguineous marriages and high fertility are the leading factors. Hematopoietic stem cell transplantations (HSCTs) are mostly performed during chilhood.

The aim of this presentation is, for the first time, to report an allogeneic HSCT from a matched unrelated donor (MUD) in an adult patient who was diagnosed with isolated CD4 deficieny due to interleukin-2 inducible $\mathrm{T}$ cell kinase (ITK) gene mutation.

Methods: The patient is a 21 year old male born from a mother married to her maternal cousin. His one brother and two sisters have no known immune deficiency status. Despite the patient had a significant medical history of recurrent infections, the confirmation of immune deficiency was delayed until 2016. Thus, he had to be transplanted at a relatively older age. He was conditioned with a nonmyeloablative regimen contatining fludarabine $30 \mathrm{mg} / \mathrm{m}^{2} /$ day on days -8 to -4 and busulfan $1,8 \mathrm{mg} / \mathrm{kg} /$ day days -5 to -2 . Busulfan dose was calculated considering pharmacokinetic data including area under curve (AUC) assessment. The donor was HLA 10/10 matched unrelated donor and the graft versus host disease (GvHD) prophylaxis involved cyclosporin $3 \mathrm{mg} / \mathrm{kg} / \mathrm{day}$, mycophenolate mofetil $15 \mathrm{mg} / \mathrm{kg} /$ day and anti-thymocyte globulin at a dose of $10 \mathrm{mg} / \mathrm{kg} / \mathrm{day}$ on days -7 to -5 .

Results: Following day- 0 he had lower respiratory symptoms and perianal infection, for which imipenem and teicoplanin were given. The sputum culture revealed Pseudomonas aeruginosa. He was also ordered prophylactic intravenous immunoglobulin (IVIg) $0.4 \mathrm{~g} / \mathrm{kg}$ weekly. Viral loads for EBV and CMV were weekly screened by PCR. Due to an increase in CMV viral load (1256 copies/ $\mathrm{mL}$ ), the patient was ordered parenteral ganciclovir at a dose of $10 \mathrm{mg} / \mathrm{kg} / \mathrm{day}$. The EBV viral load was also high (peaked at 1978 copies $/ \mathrm{mL}$ ) and spontaneous regression was observed after engraftment. Neutrophil and platelet engraftment took place on days 17 and 16, respectively. He required no more than three units of packed red blood cells post-transplantation period. The donor chimerism on day 28 was found to be $95 \%$. The distribution of lymphocyte 
subtypes by flow cytometric analysis demonstrated $16 \%$ CD4(+), 80\% CD8(+) T-cells and 0\% B-cells.

Conclusions: The timing of HSCT for PID diseases in adulthood is considered to be rather late. However, HSCT yet offers a curative potential without causing a serious toxicity due to conditioning regimens and an increased rate of infections during neutropenic period. These patients should be strictly followed-up for the risk of acute/chronic GvHD, although we did not observe GvHD in our case. The long term effects of immunosupressive treatment among these patients is also a serious concern.

Clinical Trial Registry: Not applicaple

Conflict of interest: None of the authors has anything to disclose.

\section{P521}

The Use of Post-transplantation Cyclophosphamide (PT-CY) after Alternative Donor Transplantation for the Treatment of Primary Immunodeficiencies: The Brazilian Experience

Carmem Bonfim ${ }^{1,2,3}$, Samantha Nichele ${ }^{1}$, Juliana Folloni

Fernandes ${ }^{4}$, Adriana Koliski ${ }^{1}$, Adriana Seber ${ }^{5}$, Liane Daudt ${ }^{6}$, Lisandro Ribeiro ${ }^{1,3}$, Cilmara Kuwahara ${ }^{2}$, Luiz Guilherme Darrigo $\mathrm{Jr}^{7}$, Ana Luiza Fabro ${ }^{2}$, Mary Evelyn Flowers ${ }^{8}$, Nelson Hamerschlak $^{4}$, Ricardo Pasquini, ${ }^{1,3}$

${ }^{1}$ BMT Unit Federal University of Parana, Curitiba, Brazil; ${ }^{2}$ Hospital Infantil Pequeno Principe, Curitiba, Brazil; ${ }^{3}$ Hospital Nossa Senhora das Graças, Curitiba, Brazil; ${ }^{4}$ Hospital Israelita Albert Einstein, Sao Paulo, Brazil; ${ }^{5}$ Hospital Samaritano de Sao Paulo, Sao Paulo, Brazil; ${ }^{6}$ Hospital de Clínicas da Universidade Federal do Rio Grande do Sul, Porto Alegre, Brazil; ${ }^{7}$ Ribeirão Preto Medical School, University of São Paulo., Ribeirao Preto, Brazil; ${ }^{8}$ Fred Hutchinson Cancer Research Center, Seattle, WA, United States

Background: In the absence of an unaffected matched sibling, alternative donor transplantation may be curative for a variety of primary immunodeficiencies (PID). The use of PT-Cy has significantly decreased the upfront cost of haploidentical hematopoietic stem cell transplants (HSCT) and allowed its use worldwide. The results of this strategy to treat PID is, however, unknown. The aim of this study is to analyze the outcome of children with PID who had HSCT from an alternative donor using PT-Cy for GVHD prophylaxis.

Methods: This is a multicenter, retrospective study of 47 transplants in 44 patients (pts). These pts were transplanted between 02.2012 and 09.2017 in 8 Brazilian BMT centers. The median age was 20 months (range: 2 months to 19 years), $80 \%$ male. Diagnosis were SCID $(\mathrm{n}=23)$; Wiskott-Aldrich Syndrome $(n=9)$, CGD $(n=5), \operatorname{HLH}(n=2)$; and one each: Kostmann Syndrome, LRBA,LAD,IPEX, and ChediakHigashi. Alternative donors included haploidentical related donors $(\mathrm{n}=40)$, 9/10 related donor $(\mathrm{n}=1)$ and matched unrelated donors $(\mathrm{n}=3)$.. Bone marrow was used in $91 \%$ of the HSCT and GvHD prophylaxis included PT-Cy associated with a calcineurin inhibitor and mycophenolate mofetil. Most pts had active infections, failure to thrive, or severe autoimmune complications before transplant. Four SCID were transplanted while on mechanical ventilation. 32 were upfront transplants and 15 transplants were performed to rescue pts from primary or secondary graft failure, 3 of them already after a haploidentical HSCT with PT-Cy.

Results: With a median follow-up of 2 years (range: 1082124 days), 26 pts are alive with an overall survival (OS) of $60 \%$. OS for SCID was $56 \%$ and for other PID it was $62 \%$ (p:NS). Eighteen pts received a RIC regimen as upfront HSCT and 8 are alive and well, one after a second haploidentical HSCT. Mixed chimerism was very frequent (12/17 evaluable pts) and graft failure was an important complication in the RIC group, 1 primary graft failure (PGF) and 6 progressive losses of donor chimerism, with only 1 long-term survivor. Fourteen pts received a busulfanbased regimen (upfront transplants), 11 of 13 achieved full donor chimerism and 9 are alive and well. One pt with CGD developed PGF and died due to disseminate fungal infection after a second unrelated cord blood HSCT. All 12 pts who were rescued after primary or secondary graft failures from other HSCT received a RIC regimen and 9/12 are alive with full donor chimerism. Altogether, 18 pts died at a median of 150 days after transplant (range: 4-1075). Progressive loss of donor chimerism associated with severe infections were the major causes of death in this group.

Conclusions: Allo-HCT using alternative donors and PT$\mathrm{Cy}$ is promising to cure PID but the best conditioning regimen is yet to be defined since pts are referred in our country with disseminated BCGits and/or severe bacterial, viral or fungal infections. Early diagnosis and delaying BCG vaccination could improve the outcome for these patients.

Conflict of interest: all authors declare no conflict of interest

\section{P522}

Abstract previously published

\section{P523}

Abstract previously published

Infectious complications

\section{P524}

A comparison of two strategies for gut decontamination in allogeneic stem cell transplantation in a single center study 
Kira Beckmann ${ }^{1}$, Natalie Schub ${ }^{1}$, Thomas Spanberger ${ }^{1}$, Martin Gramatzki $^{1}$, Andreas Günther ${ }^{1,2}$

${ }^{1}$ Division of Stem Cell Transplantation and Immunotherapy, Medical Department 2, Kiel, Germany; ${ }^{2}$ Helios Kliniken Schwerin,

Hematology, Oncology and Stem Cell Transplantation, Schwerin, Germany

Background: Recently new insights in the mechanism of immune reconstitution after allogeneic transplantation were made. The microbiome of the gut plays an important role and may be responsible not only for many infectious complications during and after transplantation, but might also be relevant for the development of GvHD. New strategies of gut decontamination, such as the use of rifaximin, an antibiotic with more selective activity than conventional drugs, try to prevent bacterial infections without disrupting putative helpful probiotics. In our center we changed from conventional gut decontamination (GD) with ciprofloxacin and colistin to unconventional GD with rifaximin in all patients receiving allogeneic stem cell transplantation. Here, we present a comparison of both strategies.

Methods: In this study 133 consecutive patients were included, which underwent allogeneic stem cell transplantation from February 2013 to June 2016 in our center. The conventional GD decontamination was used in 60 patients, while 73 were treated with rifaximin. The patient characteristics of both groups was comparable regarding median age, disease, conditioning regimen, stem cell source and donor characteristics. The following endpoints were analyzed to compare both strategies of GD decontamination: fever, bacteremia, infection of interest (clostridium difficile, multi-resistant germs), septic death, acute and chronic GvHD, rate of relapse and survival.

Results: The overall survival rate in both cohorts was nearly similar (conventional GD decontamination: 57\%, rifaximin treatment: $58 \%$ ). No significant difference was found in the endpoints addressing infectious complications including the rate of septic death, although the rate of bacteremia was slightly higher in the rifaximin group compared to the conventional GD decontamination cohort ( $22 \%$ versus $13 \%)$. Interestingly, only one infection with clostridium difficile was found in the rifaximin group, whereas 8 cases were observed after conventional GD decontamination, what was statistically significant $(\mathrm{p}=0.01)$. The rate of acute GvHD tended to be lower in the group treated with rifaximin (40\% compared to $53 \%$ in the conventional GD decontamination cohort) not achieving statistical significance, whereas the rate of chronic GvHD was comparable. No difference in the rate of relapse was noted.

Conclusions: Unconventional gut decontamination with rifaximin is feasible and seems to prevent infections with clostridium difficile more effectively than conventional strategies. However, to test the potential benefit of this approach prospective studies with randomization between the treatment groups are warranted.

Conflict of interest: None

\section{P525}

\section{Adenovirus viremia in pediatric hematopoietic stem cell transplantation}

Victoria Bordon ${ }^{1}$, Elizaveta Padalko ${ }^{2}$, Tiene Bauters ${ }^{3}$, Sofie Colman $^{2}$, Catharina Dhooge ${ }^{1}$

${ }^{1}$ Ghent University Hospital, Pediatric Hematology, Oncology and Stem Cell Transplantation, Ghent, Belgium; ${ }^{2}$ Ghent University Hospital, Centrum Molecular Diagnose, Ghent, Belgium; ${ }^{3}$ Ghent University Hospital, Pharmacy, Pediatric Hemato-Oncology and Stem Cell Transplantation, Ghent, Belgium

Background: Human adenovirus (HAdV) has a DNA genome. Portions of this viral DNA persist in host cells after viral replication has stopped, either as a circular extra chromosome or by integration into the host DNA. HAdV can persist in epithelial cells and lymphoid tissue causing reactivation during immunosuppression.

HAdV viremia is frequently observed after hematopoietic stem cell transplantation (HSCT) and is associated with morbidity and mortality in children.

Methods: Data from 66 consecutive transplants by pediatric patients. The patients underwent allogeneic (allo) and autologous (auto) HSCT at the Ghent University Hospital (period January 2014 to August 2017) are presented. Patients were followed with quantitative PCR from start of the conditioning to at least up to day +100 and longer in case of complications. PCR method used (following instructions of the manufacturer): ADV Virus R-gene TM (bioMérieux) on CFX96 Real-Time PCR Detection System (Bio-Rad) after generic extraction protocol on NucliSens easyMagTM (bioMérieux).

Results: Eleven patients underwent 13 auto- HSCTs for high risk solid tumors, mostly brain tumors and neuroblastoma. Conditioning regimens were according to standard protocols. None of the autologous transplant pts experienced an adenovirus reactivation.

In the allogeneic setting, 51 pts. underwent 53 HSCTs. Thirty-eight received a graft from a Matched Unrelated Donor (MUD), 10 from a sibling and 5 from a haploidentical parent after extensive T-cell depletion. Conditioning regimens were myeloablative in 46 alloHSCT.

In the allogeneic group, HAdV viremia was frequently found. HAdV viremia of any level (33\%) was found in 18 allo-HSCT, in 12 allo-HSCT was found a viremia of $>1000$ 
copies $/ \mathrm{mL}(22 \%)$ while in $8(15 \%)$ the viral load of $>10000$ copies/mL. No patient had symptoms of HAdV disease. No patient died of HAdV. The immunosuppressive therapy was tapered whenever possible with an acceptable response and decrease of the viremia in most of the cases. Three patients needed cidofovir in order to control HAdV. Two patients with active HAdV viremia died, because of invasive Aspergillus fumigatus infection in one case and severe GvHD in the other.

HAdV viremia was only observed in the MUD transplants with in vivo T-cell depletion with ATG. Remarkebly, the 5 pts who underwent a $\mathrm{T}$ cell depleted haploidentical transplant did not experience HAdV. However, this might be due to the low number of patients.

HAdV viremia is an early event after HSCT. In the whole cohort the mean time of detection is at day +26 (5-122), but those patients in whom HAdV was detected before the day +15 had much longer and higher levels viremias $(>1000)$ than those who had a reactivation after day +15 (t-test, $\mathrm{p}<$ 0.005 ).

Conclusions: HAdV infection is still a problem in pediatric HSCT. We recommend weekly monitoring with PCR for HAdV in MUD pts. In pts with a high viral load tapering immunosuppression is usually sufficient. In pts with an early onset and rapid evolving HAdV viremia preemptive therapy with cidofovir must be considered.

Clinical Trial Registry: N/A

Conflict of interest: None

\section{P526}

\section{Adenovirus Viremia is Associated with Substantially Prolonged Hospitalization in Pediatric Allogeneic Hematopoietic Cell Transplant Recipients}

Antonio Pérez-Martínez ${ }^{1}$, Cécile Pochon ${ }^{2}$, Marco Zecca ${ }^{3}$, Kanchan Rao $^{4}$, Sebastian Voigt ${ }^{5}$, Enrikas Vainorius ${ }^{6}$, Tom Brundage ${ }^{6}$, Jie Meng $^{7}$, Essy Mozaffari ${ }^{6}$, Garrett Nichols ${ }^{6}$

${ }^{1}$ Hospital Universitario La Paz, Pediatric Hemato-Oncology, Madrid, Spain; ${ }^{2}$ University Hospital of Nancy, Allogeneic Hematopoietic Stem Cell Transplantation Unit, Department of Hematology, Vandoeuvrelès-Nancy, France; ${ }^{3}$ Fondazione IRCCS Policlinico San Matteo, Pediatric Hematology / Oncology, Pavia, Italy; ${ }^{4}$ Great Ormond Street Hospital for Children NHS Foundation Trust, London, United Kingdom; ${ }^{5}$ Charité-Universitätsmedizin Berlin, Department of Pediatric Oncology/Hematology/Stem Cell Transplantation, Berlin, Germany; ${ }^{6}$ Chimerix, Durham, NC, United States; ${ }^{7}$ Analytica-Laser, New York, NY, United States

Background: Allogeneic hematopoietic cell transplant (allo-HCT) is a resource-intensive procedure necessitating a prolonged stay in hospital post-transplant. An adenovirus $(\mathrm{AdV})$ infection during the post-transplant period has the potential to prolong the transplant hospitalization, or may

\begin{tabular}{|c|c|c|c|c|}
\hline Length of stay & $\begin{array}{l}\text { No positive } \\
\text { AdV test }^{\star}\end{array}$ & $\begin{array}{l}\text { Positive AdV } \\
\text { test* }^{*}\end{array}$ & $\begin{array}{l}\text { Any AdV } \\
\text { viremia* }\end{array}$ & $\begin{array}{c}\text { AdV viremia } \\
\geq 1000 \\
\text { copies/mL* }\end{array}$ \\
\hline Transplant hospitalization & $\mathrm{N}=94$ & $\mathrm{~N}=179$ & $\mathrm{~N}=109$ & $N=79$ \\
\hline Mean days (SD) & $48(20)$ & $82(55)$ & $89(62)$ & $93(57)$ \\
\hline Median (range) & $46(14-121)$ & $65(18-388)$ & $69(27-388)$ & $76(27-305)$ \\
\hline$p$-value & reference & $p<0.01 \#$ & $p<0.01^{\#}$ & $p<0.01^{\#}$ \\
\hline Readmissions & $N=188$ & $\mathrm{~N}=114$ & $\mathrm{~N}=46$ & $\mathrm{~N}=27$ \\
\hline Mean days (SD) & $12(22)$ & $26(33)$ & $32(32)$ & $37(38)$ \\
\hline Median (range) & $6(1-195)$ & $13(2-169)$ & $21(2-131)$ & $22(2-131)$ \\
\hline
\end{tabular}

than transplant hospitalization with no positive AdV test (Mann-Whitney-Wilcoxon test).

[P526 Figure] [Table]

lead to readmission. In order to assess the potential impact of AdV infection on healthcare utilization, we analyzed the duration of hospitalizations among pediatric allo-HCT recipients with and without $\mathrm{AdV}$ infection from a large multicenter study.

Methods: AdVance is a multicenter, multinational study of the incidence, management, and clinical outcomes of AdV infections in adult and pediatric allo-HCT recipients across Europe. This study was conducted retrospectively and included recipients of allo-HCT between January 2013 and September 2015. In this analysis, pediatric allo-HCT recipients $(<18$ years) who had AdV infection diagnosed in the first 6 months after transplantation were identified. Hospitalizations were categorized as transplant hospitalization or readmission (any hospitalization after discharge from the transplant hospitalization). Hospitalizations were further categorized according to whether $\mathrm{AdV}$ was noted in the discharge summary or if any positive $\mathrm{AdV}$ test was noted during the hospitalization. Hospitalizations during which the patient died were analyzed separately. Two subgroups of hospitalizations during which a positive AdV test was noted were also analyzed: those with any viremia and those with clinically relevant viremia $\geq 1000$ copies $/ \mathrm{mL}$ in blood.

Results: Overall, there were 1103 pediatric allo-HCT patients, including 296 who had an AdV infection within 6 months following allo-HCT, 211 of whom developed viremia. The median length of stay during the transplant hospitalization was 69 days for patients who had any AdV viremia and 76 days for those with clinically relevant viremia $\geq 1000$ copies $/ \mathrm{mL}$ (see table). In contrast, for patients without any AdV infection during transplant hospitalization, the median length of stay was 46 days $(p<$ 0.01 ). For readmissions, median length of stay was also longer for those during which the patient had AdV viremia or viremia $\geq 1000$ copies $/ \mathrm{mL}$, compared with those in which the patient did not have AdV. In this analysis, patients who reached clinically relevant viremia $\geq 1000$ copies $/ \mathrm{mL}$ during their hospitalization had the longest duration of stay during both the transplant hospitalization and subsequent readmissions. 
Conclusions: AdV infection in pediatric allo-HCT recipients is associated with significant medical resource utilization, as measured by duration of hospital stay. Pediatric allo-HCT recipients with clinically relevant AdV viremia stayed in the hospital 30 days longer than those without AdV infection during the transplant hospitalization. Mandated screening, identification, monitoring, and treatment of AdV infections is likely to have significant clinical and economic impacts in this patient population.

\section{Conflict of interest:}

A. Pérez-Martínez, C. Pochon, M. Zecca, K. Rao, and S. Voigt are investigators in the AdVance study sponsored by Chimerix.

E. Vainorius, T. Brundage, E. Mozaffari, and G. Nichols are employees of the study sponsor, Chimerix.

J. Meng is an employee of Analytica-Laser, a research consultancy that conducted the study on behalf of the sponsor, Chimerix.

\section{P527}

\section{ALTERNARIA INFECTION IN A CHILD AFFECTED BY SEVERE APLASTIC ANAEMIA AFTER SECOND BONE MARROW TRANSPLANT}

\author{
Elena Soncini ${ }^{1}$, Ilaria Fracassetti ${ }^{1}$, Tiziana Lorenzini ${ }^{1}$, Giulia \\ Carracchia $^{1}$, Marco Berlucchi ${ }^{2}$, Arnalda Lanfranchi ${ }^{3}$, Fulvio Porta $^{1}$ \\ ${ }^{1}$ Pediatric Oncohaematology and BMT Unit, Pediatric Chidren's \\ Hospital Brescia, Brescia, Italy; ${ }^{2}$ Otorhinolaryngology Pediatric \\ Children's Hospital Brescia, Brescia, Italy; ${ }^{3}$ Stem Cell Laboratory, \\ Section of Hematology and Blood Coagulation, Children's Hospital, \\ Spedali Civili of Brescia, Brescia, Italy
}

Background: Acute invasive fungal is a potentially fatal infection in immunocompromised patients as bone marrow transplant recipients. Although Candida and Aspergillus are the most common fungal isolates, other less common pathogens such as Alternaria species are emerging as opportunistic agents associated with clinical syndromes.

Methods: We describe the case of a 9 years old child affected by severe aplastic anaemia characterized by severe leukopenia, thrombocytopenia and anaemia, who developed alternaria infection of parasanal sinuses during the aplasia phase of a second match unrelated bone marrow transplant.

The patient was underwent to a first urgent haploidentical PBSC transplant because of worsening respiratory distress requiring PICU admission and non-invasive ventilation support. Conditioning was performed with TBI 200 rds, fludarabine, cyclophosphamide, ATG and cyclophosphamide on day +3 and +5 .

Fluconazole was used as antifungal prophylaxis. Unfortunately secondary graft failure occurred with recurrence of severe leukopenia. A second bone marrow transplant was planned with a 8/10 match unrelated donor. Conditioning with fludarabine, TBI 200 rds, cyclophosphamide and ATG was well tolerated and peripheral CD34 positive selected cells were infused.

During the conditioning on day - 7 , the patient complained of pain at the upper dental arch and at right cheek with no evidence of swelling, mild gingivitis was noted in the site of pain. On day +9 after transplant, due to the appearance of fever and the worsening of symptoms, a CT scan of paranasal sinuses and brain was performed. Secretions in sphenoidal, frontal and maxillary sinuses with ethmoidal erosion were detected, findings compatible with mycotic osteonecrosis of paranasal sinuses.

Prompt endoscopic surgery was performed the day after. Procedures included intranasal antrotomy with right sphenoidotomy, frontal senotomy and partial ethmoidectomy. Pathologic analysis demonstrated necrotic tissue and fungal hyphae.

The ongoing antifungal therapy with micafungin was enhanced with oral posaconazole and daily liposomal amphotericin B. The culture examination of secretions leaded to the diagnosis of Alternaria alternata infection. Micafungin was stopped according to the sensitivity profile.

Results: The pain and fever quickly disappeared after the surgical intervention but further surgical debridement were necessary in order to achieve a complete resolution of the complication. Neutrophils and platelets engrafted on Day +16 and 10 respectively; the antifungal combination therapy was carried on for six months. The patient healed from aplastic anaemia and had no sequelae related to this infection.

Conclusions: Rapid recovery of neutrophil count, extensive and aggressive treatment including surgical debridement and antifungal combination therapy have been essential in overcoming this potential fatal complication.

\section{References:}

1. Alternaria alternate Invasive Fungal infection in a patient with Fanconi's anemia after Unrelated Bone marrow Transplant. Clin Drug Investigation Feb 2103, Vol 33, Suppl 33-36

2. Pediatric invasive fungal rhinosinusitis in immunocompromised children with cancer. Otolaryngol Head Neck Srg 2005 Sep; 133 (3): 411-6.

Conflict of interest: The authors have no conflicts of interest to declare.

\section{P528}

Analysis of invasive fungal infection incidence in children with hematological malignancies, undergoing hematopoietic stem cell transplantation. Multicenter retrospective polish study 
Jowita Fraczkiewicz ${ }^{1}$, Malgorzata Salamonowicz ${ }^{1}$, Ewa Gorczynska ${ }^{1}$, Anna Krol ${ }^{1}$, Krzysztof Kalwak ${ }^{1}$, Alicja Chybicka ${ }^{1}$, Krzysztof Czyzewski ${ }^{2}$, Mariusz, Wysocki ${ }^{2}$,Patrycja Zalas-Wiecek ${ }^{3}$, Jolanta Gozdzik, Olga Zajac-Spychala ${ }^{5}$, Jacek Wachowiak, Agnieszka Zaucha-Prazmo ${ }^{6}$, Jerzy Kowalczyk ${ }^{6}$, Jan Styczynski ${ }^{2}$

${ }^{1}$ Department and Clinic of Pediatric Oncology, Haematology and Bone Marrow Transplantation, Medical University Wroclaw, Wroclaw, Poland; ${ }^{2}$ Department and Clinic of Pediatric Oncology and Haematology, Collegium Medicum, Bydgoszcz, Bydgoszcz, Poland; ${ }^{3}$ Department of Microbiology, Medical University Bydgoszcz, Bydgoszcz, Poland; ${ }^{4}$ Department of Immunology, and Clinical Transplantology, Collegium Medicum, Krakow, Poland; ${ }^{5}$ Department of Pediatric Oncology, Haematology, and Bone Marrow Transplantation Poznan Medical University, Poznan, Poland;

${ }^{6}$ Department of Pediatric Oncology, Haematology, and Bone Marrow Transplantation Medical University, Lublin, Lublin, Poland

Background: Fungal infections become one of the main cause of morbidity and mortality in children after hematopoietic stem cell transplantation (HSCT). The main risk factors are prolonged neutropenia and broad-spectrum antibiotics. The aim of this study was to analyze an incidence and outcome of invasive fungal infections in 5 Polish Pediatric Hematopoietic Stem Cell Transplantations Centers over a period of 48 months (years 2012-2015).

Methods: Between 2012-2015 a total number of 650 patients received HSCT. The group consisted of children diagnosed with acute lymphoblastic leukemia (ALL), acute myeloblastic leukemia (AML), Hodgkin lymphoma (HL), non-Hodgkin lymphoma (NHL), severe aplastic anemia (SAA) and myelodysplastic syndromes (MDS), solid tumors (ST), and other (non-hematological diseases).

Results: Over 4-year period 164 IFI were diagnosed. 84 IFI $(51,3 \%)$ were diagnosed between years 2012-2013, and 81 cases $(48,7 \%)$ between years 2014-2015. Median age was 9,7 years. Severe neutropenia was present in 108 (55\%) patients. Proven IFI was found in 24 cases $(14,6 \%)$. Probable IFI was diagnosed in 52 patients $(31,7 \%)$. Culture proven agents were Candida spp 29, Aspergillus spp. 18, Fusarium spp. 2 and Mucor 2, whereas 13 proven IFI were identified by histopathology. Among proven IFI the lung was the most frequently affected organ, being involved in 95 of 164 IFI, followed by paranasal sinuses in 17 cases, gastrointestinal tract (liver and spleen) in 12 cases, genitourinary tract in two. Antifungal prophylaxis consisted of: 78 posakonazole $(68,4 \%)$, fluconazole $15(13,2 \%)$, caspofungin $4(3,5 \%)$, voriconazole $8(7 \%)$, liposomal amphotericin B $3(2,6 \%)$ and micafungin $6(5,1 \%)$. In 50 cases there was no antifungal prophylaxis used. There were 38 deaths, but only 9 due to IFI. The main reason of death were progression of malignancy and multi-organ failure.

Conclusions: The incidence IFI is decreasing at the turn of years 2012 and 2015s, probably due to effective antifungal prophylaxis. The most common site of IFI remains respiratory tract. The majority (78\%) of pediatric patients after HSCT with PP-IFI survive. Further prospective studies are required to assess the impact of antifungal prophylaxis on survival.

\section{Conflict of interest: None}

\section{P529}

\section{ANALYSIS OF PATIENTS WITH HEMATOLOGIC MALIGNANCIES UNDERGONE STEM CELL TRANSPLANTATION UNDER VORICONAZOLE PROPHYLAXIS}

Eren Arslan Davulcu, Nur Soyer, Murat Tombuloğlu, Mahmut Töbü, Güray Saydam, Fahri Sahin, Filiz, Vural

Ege University Medical Faculty, Hematology Department, Izmir, Turkey

Background: Invasive fungal infection (IFI) is one of the most prevalent reason for morbidity and mortality in patients with hematologic malignancies either receiving chemotherapy or hematopoietic stem cell transplantation (HSCT). Fluconazole is one of the drugs for antifungal prophylaxis in HSCT. In order to reduce the recurrence of prior IFI, secondary prophylaxis is required. Voriconazole is effective in also secondary prophylaxis in HSCT patients.

Methods: We retrospectively evaluated 602 patients with hematological diseases undergoing autologous (autoHSCT) and allogeneic HSCT (aHSCT) in Ege University Hematology Department Bone Marrow Transplantation Unit between 2012-2017. IFIs were classified according to EORTC criteria as possible, probable, and proven. Probable IFI needed certain microbiological criteria (e.g., serum galactomannan) with host factors and clinical features, whereas proven IFI requires histopathological findings or positive culture from a primary sterile site rather than microbiological criteria. Possible IFI defines cases that are highly likely to be caused by a fungal etiology, although mycological evidence is lacking.Galactomannan testing was performed biweekly during hospitalization. Complete response was defined as the resolution of all signs of IFI, and partial response as clinical and radiological improvement or resolution of all attributable symptoms and signs of fungal disease. Radiological stabilization can be equated with a partial response. Stable response was defined as minor or no improvement in signs of disease and radiological stabilization, whereas progressive disease was defined as worsening clinical symptoms or signs of disease and new sites of disease or radiological worsening of preexisting lesions.

Results: There were 185 autoHSCT and 417 aHSCT patients evaluated. Three patients in autoHSCT and 12 in aHSCT group received voriconazole prophylaxis. Patient characteristics were summarized in table 1 . In all patients, timing of the fungal infection was at induction 
Table 1: Patient characteristics

\begin{tabular}{|c|c|c|c|c|c|c|c|c|c|}
\hline Agevgender & Dlsgnosis & $\begin{array}{l}\text { Type o } \\
\text { traneplat }\end{array}$ & Antifungal therapy & $\begin{array}{l}\text { Duration of } \\
\text { worlconseol } \\
\text { prophylaxls } \\
\text { (asys) }\end{array}$ & $\begin{array}{l}\text { EORTC } \\
\text { criterla }\end{array}$ & $\begin{array}{l}\text { Site of } \\
\text { fungal } \\
\text { Infectlon }\end{array}$ & Galact. & $\begin{array}{l}\text { Response } \\
\text { to } \\
\text { antifungal } \\
\text { treatment }\end{array}$ & Outcome \\
\hline $67, \mathrm{M}$ & AML & GHSCT & LAmpB & 120 & Posside & Lung & - & $\begin{array}{l}\text { Complete } \\
\text { response }\end{array}$ & CR \\
\hline $31, F$ & AML & aHSCT & LAmpB & 60 & Possible & Lung & - & $\begin{array}{l}\text { Complete } \\
\text { response }\end{array}$ & Death,PD \\
\hline $52, \mathrm{M}$ & HL & aUIOHSCT & Voricon36ole, LAmps & 30 & Possible & Lung & - & $\begin{array}{l}\text { Complete } \\
\text { response }\end{array}$ & CR \\
\hline $26, F$ & MES & aHSCT & LAmpB, C36po & 120 & Probable & Lung & + & $\begin{array}{l}\text { Progressive } \\
\text { dsesse }\end{array}$ & Death,PD \\
\hline $58, M$ & $\mathrm{NHL}$ & aUloHSCT & LampB & 30 & Possible & Lung & - & $\begin{array}{l}\text { Partlal } \\
\text { response }\end{array}$ & $\mathrm{CR}$ \\
\hline $53, M$ & ALL & aHSCT & $\begin{array}{l}\text { LampB, c3spo, } \\
\text { worlcon3sole }\end{array}$ & 60 & Possible & Lung & - & $\begin{array}{l}\text { Progressive } \\
\text { dsease }\end{array}$ & Death,seps/5 \\
\hline $32, \mathrm{M}$ & ALL & aHSCT & Lamps & 70 & Posside & Lung & - & $\begin{array}{l}\text { Progre6sive } \\
\text { dsease }\end{array}$ & CR \\
\hline $32, \mathrm{M}$ & ALL & aHSCT & Lamp5 & 170 & Posside & Lung & - & $\begin{array}{l}\text { Complata } \\
\text { remission }\end{array}$ & $\mathrm{CR}$ \\
\hline $36, F$ & ALL & aHSCT & $\begin{array}{l}\text { LAmpB, worlcon36ol, } \\
\text { posaconzzole }\end{array}$ & 180 & Possible & Lung & - & $\begin{array}{l}\text { Progressive } \\
\text { dsesse }\end{array}$ & Death,pneumonl3e \\
\hline$\Delta 2, M$ & AML & autoHSCT & Voricon3sole & 60 & Possible & Lung & - & $\begin{array}{l}\text { Complete } \\
\text { response }\end{array}$ & $\mathrm{CR}$ \\
\hline $22, \mathrm{M}$ & ALL & aHSCT & LampB & 50 & Possible & Lung & - & $\begin{array}{l}\text { Complete } \\
\text { response }\end{array}$ & CR \\
\hline $65, \mathrm{~F}$ & AML & aHSCT & Lamp5 & 60 & Possilde & Lung & - & $\begin{array}{l}\text { Partial } \\
\text { response }\end{array}$ & Death,PD \\
\hline $24, \mathrm{M}$ & $\mathrm{NHL}$ & BHSCT & Voricon36ole & 160 & Possidie & Lung & - & $\begin{array}{l}\text { Comlete } \\
\text { response }\end{array}$ & Partal remission \\
\hline $47, \mathrm{M}$ & AML & aHSCT & $\begin{array}{l}\text { LampB, } \\
\text { woricon36ole, pos } 3 \text { ccn3sole }\end{array}$ & 120 & Defintwe & Sinusits & + & $\begin{array}{l}\text { Complate } \\
\text { remission }\end{array}$ & $C R$ \\
\hline $52, M$ & NHL & aHSCT & LampB & 40 & Posside & Lung & - & $\begin{array}{l}\text { Progressive } \\
\text { disezse }\end{array}$ & Death, sepsis, PD \\
\hline
\end{tabular}

[P529 Figure] [Table 1: Patient characteristics]

chemotherapy before transplantation. There were no patients with active fungal infection during HSCT. Only one patient had pathologically and microbiologically proven fungal infection (mucormicosis sinusitis) which was successfully treated. Eight patients (66\%) responded well to secondary voriconazole prophylaxis and did not have breakthrough infections. One patient had mildly elevated liver enzymes after voriconazole administration.

Conclusions: In our study group, voriconazole was $66 \%$ successful in preventing breakthrough infections that shows voriconazole is effective and safe in secondary prophylaxis for HSCT. We need more comprehensive studies with more patients.

Conflict of interest: The authors whose names are listed certify that they have no affiliations with or involvement in any organization or entity with any financial interest or nonfinancial interest in this abstract.

\section{P530}

Analysis of the Burden of Cytomegalovirus Infection and
Disease in Hematopoietic Stem Cell Transplant Recipients

Silvia Monsalvo, Sara Lozano, Daniele Avenoso, Belen Sevillano,
Philippa Woolley, Nadine Farah, Christos Paliompeis, Dragana Milojkovic, Eduardo Olavarria

Imperial College London, Haematology, London, United Kingdom

Background: CMV infection and disease can be managed with pre-emptive antiviral therapy but this strategy is associated with significant morbidities and subsequent economic burden.

Methods: We have analysed 303 consecutive patients undergoing allogeneic stem cell transplantation (AlloSCT) that were at risk of CMV infection (patient and/or donor CMV seropositive) focusing on the incidence of CMV infection and the healthcare resource utilization by estimating length of inpatient stay and recurrent hospitalization rates.

Results: Median age was 45 years (18-66). There were 185 (61\%) males. CMV serology (Recipient/Donor) distinguished 3 groups: Pos/Pos 182 (60\%); Pos/Neg 85 (28\%); Neg/Pos 36 (12\%). Transplant conditioning was myeloablative in 147 (49\%) and reduced intensity in 156 (51\%). Donor types were 148 (49\%) HLA-identical sibling, 89 (29\%) matched unrelated, 37 (12\%) mismatched unrelated and 29 (10\%) haploidentical donors. T-cell depletion with Alemtuzumab was performed in 164 (54\%). 
All patients were managed with pre-emptive therapy after CMV reactivation was confirmed in 2 consecutive PCR samples. CMV reactivation occurred in $129(43 \%)$ patients at a median of 33 (6-331) days post SCT. In $39(30 \%)$ cases, CMV reactivated a median of $22(2-314)$ days before the diagnosis of GVHD. Acute GVHD developed in 27 patients before CMV reactivation after a median of 22 (1-103) days post AlloSCT. Systemic steroids were started in $35(27 \%)$ patients a median of 9 (1-75) days before CMV reactivation.

A total of $126(98 \%)$ patients received antiviral therapy as per pre-emptive protocol. Intravenous Ganciclovir was given as first line antiviral therapy to 75 (58\%) patients while Foscarnet was given to 29, oral Valganciclovir to 20 and Cidofovir to 2 patients. Three patients did not receive anti-CMV therapy. The median time between CMV reactivation and starting antiviral therapy was 1.5 days (026) with $>90 \%$ of patients starting antivirals within 72 hours of reactivation. The median duration of antiviral therapy was 28 (3-130) days.

The overall response to antiviral therapies was $81 \%$ complete remission (negative CMVPCR) and 9\% partial response (reduction in CMV PCR copies). 13 (10\%) patients died before their response could be evaluated (9 on Ganciclovir and 4 on Foscarnet). The respective response rates according with the different initial treatments were: Ganciclovir 76\% CR and 12\% PR, Foscarnet $76 \%$ CR and $10 \%$ PR and Valganciclovir $90 \%$ CR and $10 \%$ PR. A total of $64(51 \%)$ patients required second-line antiviral therapy: 11 patients after a partial response and 53 patients (52\% of CR patients) because of a second CMV reactivation (CMV relapse). In addition, 56 (44\%) patients changed their initial antiviral for different reasons: renal toxicity $(15 \%)$, intolerance $(16 \%)$ and de-escalation to oral Valganciclovir (13\%). A total of 47 patients required rehospitalisation for their treatment (36\% of CMV reactivations, $15 \%$ of all patients at risk of CMV reactivation). The median number of days in hospital was 20 (3-125).

Conclusions: CMV infection occurs in over $40 \%$ of patients at risk. Responses to first line antivirals are satisfactory, although there is a significant risk of relapse. Anti-CMV therapies impact on healthcare resources with a high rate of re-hospitalisation.

Conflict of interest: Nothing to disclose

\section{P531}

\section{ANTI-CMV IMMUNOGLOBULINS IN ASSOCIATION WITH ANTI-CMV DRUGS IN PATIENTS WITH HEMATOLOGICAL MALIGNANCIES SUBMITTED TO ALLOGENEIC STEM CELL TRANSPLANTATION: A MULTI-CENTRE RETROSPECTIVE EXPERIENCE}

Michele Malagola ${ }^{1}$, Benedetta Rambaldi ${ }^{1}$,Jacopo Peccatori ${ }^{2}$, Anna Paola Iori $^{3}$, Raffaella Greco ${ }^{2}$, Elisabetta Xue ${ }^{2}$, Giuseppe Gentile ${ }^{3}$, Luisa Quatrocchi ${ }^{3}$, Giulia De Luca ${ }^{3}$, Enrico Morello ${ }^{1}$, Nicola Polverelli $^{1}$, Alessandro Turra ${ }^{1}$, Federica Cattina $^{1}$, Valeria Cancelli ${ }^{1}$, Robin Foa ${ }^{3}$, Fabio Ciceri ${ }^{2}$, Domenico Russo ${ }^{1}$

${ }^{1}$ Bone Marrow Transplant Unit, Department of Clinical and Experimental Sciences, University of Brescia, Brescia, Italy; ${ }^{2}$ Hematology and Bone Marrow Transplantation Unit, IRCCS San Raffaele Scientific Institute, Vita-Salute San Raffaele University, Milano, Italy; ' 'Sapienza' University, Department of Cellular Biotechologies and Hematology, Policlinico Umberto 1, Rome, Italy

Background: CMV represents one of the most serious life-threatening complications of allogeneic stem cell transplantaion (allo-SCT). Pre-emptive treatment with anti-CMV drugs is highly effective, but toxicity and repetitive reactivation of $\mathrm{CMV}$ represent a major challenge in the clinical practice. The use of anti-CMV specific immunoglobulins (Megalotect) is controversial.

Methods: We retrospectively collected data on 294 patients with hematological malignancies submitted to allo-SCT in 3 Italian Bone Marrow Transplant Units between 2016 and 2017. One-hundred and fifteen of the 294 patients (39\%) developed at least one CMV reactivation with CMV copies $>1000 / \mathrm{ml}$ at a median of 35 days (range: 2-120) from transplant. In 19/115 cases (17\%), Megalotect was used with the following schedule: 9 patients received $50 \mathrm{UI} / \mathrm{Kg}$ weekly, 4 patients $50 \mathrm{U} / \mathrm{Kg}$ every 2 weeks, 6 patients $50 \mathrm{UI} / \mathrm{Kg}$ every 3 weeks up to CMV viremia negativity. Megalotect was added in high-risk patients, based on: haploidentical allo-SCT $(9 / 19 ; 47 \%)$, steroid therapy for aGVHD $(11 / 19 ; 58 \%)$ and unfavorable CMV serology combination $(12 / 19 ; 63 \%$; $\mathrm{D}-/ \mathrm{R}+$ in 11 cases and D-/R- in 1 case). In 12 cases, Megalotect was used as pre-emptive therapy and in 7 cases as prophylaxis. Moreover, a specific anti-CMV drug was used first-line, together with Megalotect, in 12/19 cases: foscarnet (4 cases), valgancyclovir (6 cases), gancyclovir ( 2 cases).

Results: Overall, the treatment was well tolerated, with no reported infusion reactions nor other adverse events. Megalotect was administered for a median of 12 doses (range 5-33) and 2 doses (range 2-9) in patients treated preemptively or prophylactically. Focusing on the 12 cases treated with Megalotect pre-emptively, all patients except 1 achieved at least one CMV viremia negativity during tratment. Median time to first negativity was 24 days (range $6-120)$. In $7 / 12$ cases (58\%), a second-line treatment was used. The median interval from subsequent anti-CMV treatment after first negativity was 30 days (range 10-80). In $5 / 12$ patients $(42 \%)$, maintenance treatment with Megalotect alone was able to control a persistent low-level CMV viremia $(<1000$ copies/ml) without any other anti-CMV specific treatment. Focusing on the 7 cases treated with Megalotect as prophylaxis, 1 patient only developed CMV 
reactivation, treated with gancyclovir with no response. Interestingly, in these 19 high-risk patients CMV disease was observed in 2 cases only (10\%): a pneumonitis in 1 case and a colitis in 1 the other.

Conclusions: Our preliminary experience with Megalotect suggests that it is safe and well tolerated both in the pre-emptive and prophylaxis setting. We have no conclusive data regarding the efficacy of this treatment in reducing the cumulative dose of anti-CMV specific drugs. Interestingly, when used as pre-emptive therapy, Megalotect was able to maintain low levels of CMV viremia allowing to spare drug toxicity in 5/12 cases (42\%). Further prospective randomized trials are warrented to address this specific issue, particulary in patients at high-risk of multiple CMV reactivation and CMV disease.

Conflict of interest: M. Malagola, J. Peccatori, and A. Iori: advisory board, Biotest

\section{P532}

BKV infection in pediatric allogeneic transplantation: incidence, risk factors, outcome - single center experience

Anca Colita ${ }^{1}$, Cristina Georgiana Jercan ${ }^{2}$, Ana Moise ${ }^{3}$, Ileana Constantinescu ${ }^{3}$, Alina Daniela Tanase ${ }^{4}$, Andrei Colita ${ }^{3}$

1'Carol Davila' University of Medicine, Pediatric Hematology \& BMT, Bucharest, Romania; ${ }^{2}$ Carol Davila University of Medicine, Pediatrics, Bucharest, Romania; ${ }^{3}$ Carol Davila University of Medicine, Bucharest, Romania; ${ }^{4}$ Fundeni Clinical Institute, Bucharest, Romania

Background: BK virus of Polyomaviridae family usually affects all individuals, mainly in childhood, and remains latent in urothelial cells. BKV reactivation in HSCT allotransplant recipients may have different presentations, ranging from completely asymptomatic to severe hemorrhagic cystitis (HC). Rarely patients develop nephritis, pneumonia or CNS disease. The aim of our study is to evaluate patients with $\mathrm{BKV}$ reactivation after allogeneic HSCT in order to analyze the incidence, severity, risk factors and outcome of viral reactivation in a single center.

Methods: We retrospectively analyzed pediatric patients with BKV reactivation admitted at Fundeni Clinical Institute between 2012 and 2017. Diagnosis of viral reactivation was established based on BKV PCR from urine and plasma samples, clinical signs and imagistic investigations. The conditioning regimens used were according to the type of the disease. GvHD prophylaxis consisted of standard methotrexate + ciclosporin/tacrolimus $+/$ - ATG and posttransplant ciclophosphamide and MMF + Tacrolimus in haplotransplant recipients. All parents signed informed consent forms.
Results: In our center, between 2012 and 2017, we performed 41 procedures of allogeneic transplantation: 18 MUD (44\%), 16 MSD (39\%), 7 Haplo (17\%). The patients diagnosis were: ALL 10 patients (25.6\%), AML 10 patients (25.6\%), SAA - 10 patients (25.6\%), MDS - 4 patients (10.2\%), Diamond Blackfan Anemia - 1 patient (2.8\%), Fanconi Anemia - 4 patients (10.2\%). 13/41 (31.7\%) patients presented BKV reactivation following HSCT procedure. The incidence of BKV reactivation was $42.8 \%$ in haplotransplants, $38.88 \%$ in MUD and $18.7 \%$ in MSD. The symptoms consisted of hematuria 6/13 (2/13 cases presented severe hematuria), dysuria (11/13), polakyuria (6/ 13), CNS symptoms (2/13). Symptoms appeared from day 10 to day 65 (median 27 days), lasted from 4 to 33 days (median 14 days). In two haplotransplant patients, BKV infection induced a severe cytokine release syndrome (CRS), with IL-6 level $>1000 \mathrm{pg} / \mathrm{ml}$. The PCR levels of $\mathrm{BKV}$ in urine sample at onset ranged from 100 to $1.69 \times 10^{7}$ copies/ml (median $366 \times 10^{6}$ ), plasma sample levels ranged from 0 to 6546 copies $/ \mathrm{ml}$ (median 1206 copies $/ \mathrm{ml}$ ). 4/13 (30.7\%) patients presented co-infection with CMV. 1/13 patients had concomitant severe intestinal GvHD, 1/13 developed mild skin GvHD afterwards and 2/13 developed severe skin and intestinal GvHD after BKV reactivation. Imagistic investigations (abdominal ultrasound) were performed to $7 / 13$ patients, and all presented thickening of bladder mucosa, 2/13 patients had clots in the bladder. Treatment included extensive hydration (13/13 patients), Levofloxacin (12/13 patients), Cidofovir (1/13 patients), rFVII (2/13 patients), supportive treatment (3/13 patients), 2/ 13 patients required continuous bladder irrigation, 1/13 patient required emergency bladder electro-cauterization, 2/ 13 patients developed acute kidney injury. 7/13 patients are alive, fully recovered, and with $100 \%$ donor chimerism.

Conclusions: BKV reactivation is a common complication of HSCT, and associates significant morbidity. We could not detect any correlation between $\mathrm{BKV}$ reactivation and grade III/IV acute GVHD, cyclophosphamide use and donor type. The incidence of BKV reactivation in our study was $31.7 \%$, $\mathrm{HC}$ appeared only in $14.63 \%$ of patients. CRS associated in patients with BKV PCR levels in urine over 1 billion copies/ml. Further studies are needed in order to completely understand the pathophysiology and mechanisms of BKV disease.

Conflict of interest: Colita Anca: nothing to disclose

\section{P533}

BKV related haemorrhagic cystitis - an insight into risk factors and later complications. A peliminary analysis on behalf of Polish Adult Leukemia Group

Jaroslaw Dybko ${ }^{1}$, Agnieszka Piekarska ${ }^{2}$, Donata Urbaniak-Kujda ${ }^{3}$, Monika Biernat ${ }^{3}$,Magdalena Dutka ${ }^{2}$, Alicja Sadowska-Klasa ${ }^{2}$, 
Sebastian Giebel ${ }^{4}$, Tomasz Wróbel ${ }^{3}$, Kazimierz Kuliczkowski ${ }^{3}$, Lidia $G_{i l}^{5}$

${ }^{1}$ Wroclaw Medical University, Department of Internal and Occupational Diseases, Hypertension and Clinical Oncology, Wroclaw, Poland; ${ }^{2}$ Medical University of Gdansk, Department of Hematology and Transplantology, Gdańsk, Poland; ${ }^{3}$ Wroclaw Medical University, Hematology and Bone Marrow Transplantation, Wroclaw, Poland; ${ }^{4}$ Cancer Center and Institute of Oncology, Department of Bone Marrow Transplantation and Hematology-Oncology, Gliwice, Poland; ${ }^{5}$ Poznan University of Medical Sciences, Department of Hematology and Bone Marrow Transplantation, Poznań, Poland

Background: BKV related haemorrhagic cystitis (BKVHC) after allogeneic stem cells transplantation (HSCT) has still remained an issue for many years. There have been some risk factors recognized although any specific prophylaxis does not exist. Some promising results were achieved with Cidofovir and hyperbaric oxygen therapy but there are no ranomized trials results. In this study we are trying to establish the role of preemptive monitoring of BKV-DNA copies in selecting the highest risk patients.

Methods: We analyzed a cohort of 90 patients (F/M - 37/ 53, median age 42 (21-67)) after allogeneic stem cell transplantation mainly due to acute leukemias diagnosis (acute leukemias vs other indications 67/23). A majority of patients were transplanted from unrelated donor (MUD/ MRD - 68/18 and 4 haploidentical cases) based on myeloablative conditioning (MAC/RIC - 63/27). All patients were monitored for BKV-DNA by PCR at -10 , $+7,+14,+21$ and +28 day (the observation on subsequent days is still ongoing). We identified two groups of patients with BKV reactivation: developing haemorrhagic cystitis (HC) and not developing this complication (nHC).

Results: In the whole cohort of patients we identified 48 cases $(53,34 \%)$ of BKV reactivation in the period of monitoring consisting of $21 \mathrm{HC}$ and $27 \mathrm{nHC}$ cases. We did not find any significant differences between the groups regarding to patients' age, sex and diagnosis, conditioning (MAC vs RIC), stem cells source, CD34 cells number and the donor origin (MUD vs MRD). The only significant difference concerned the number of mismatched HSCT (including 4 haploidentical transplantations) with the higher rate in $\mathrm{HC}$ group $(5 / 21$ vs $3 / 27, \mathrm{p}=0.037)$. All haploidentical HSCT were included in HC group as well. The median time of $\mathrm{HC}$ onset was 20 days (11-50). Analyzing the number of BKV-DNA urine copies before the conditioning (day -10) we did not any difference between the groups.We did find the difference between the groups in urine BKVDNA at days $+14,+21$ and +28 but surprisingly the median number of copies was higher in $\mathrm{nHC}$ groups in all measure points $(1.13 \mathrm{E}+6$ vs $1.45 \mathrm{E}+10, \mathrm{p}=0.0002 ; 1.13 \mathrm{E}$ +10 vs $1.95 \mathrm{E}=10, \mathrm{p}=0.02 ; 5.2 \mathrm{E}+9$ vs $1.22 \mathrm{E}+10, \mathrm{p}=$ 0.008 respectively) although in serum the results were reversed (higher number of copies in HC group) but only at
+ the day +21 the difference was significant (mean 20529 vs $677, \mathrm{p}=0.005$ ). The difference in acute GvHD onset was not significant although in chronic GvHD the higher rate was observed in $\mathrm{HC}$ group $(7 / 21$ vs $3 / 27, \mathrm{p}=0.03)$. There were no differences in OS between the groups. All but two patients were successfully treated with Cidofovir 5 mg per kg b. once a week (2-4 doses, median 3).

Conclusions: Our preliminary results showed the essential role of HLA mismatching and serum viremia in developing HC. The preemptive monitoring seems to be useful in selecting BKV-DNA serum positive patients to indroduce the proper treatment in the future (i.e low dose Cidofovir after randmized trials results will be delivered). The infulence of BKV reactivation on cGVHD onset needs some further investigation.

Clinical Trial Registry: Not applicable

Conflict of interest: Nothing to declare

\section{P534}

Brincidofovir for severe adenovirus infection in adult patients after allo-HSCT: report on 7 cases under compassionate use treatment

Elisabetta Xue, Raffaella Greco, Fabio Giglio, Daniela Clerici, Francesca Farina, Maria Teresa Lupo Stanghellini, Chiara Oltolini, Massimo Bernardi, Andrea Assanelli, Consuelo Corti, Jacopo Peccatori, Fabio Ciceri

IRCCS San Raffaele Scientific Institute, Milan, Italy

Background: Adenovirus (AdV) infections, though uncommon, show a high mortality in immunocompromised patients, especially after allogeneic hematopoietic transplantation (allo-HSCT). Brincidofovir (BCV, CMX001) is an orally bioavailable lipid conjugate of cidofovir that has in vitro activity against $\mathrm{AdV}$ and other double-strand DNA viruses.

Methods: From 2015 to 2017 we treated 7 patients with brincidofovir (tablet formulation) for disseminated and local AdV infection. All patients were adults $(\mathrm{AML}=4 ; \mathrm{NHL}=1$; $\mathrm{HL}=1 ; \mathrm{ALL}=1$ ), median age at transplant was 41 year old. Stem cell donors were unrelated $(n=1)$ and family haploidentical $(n=6)$. All patients received PBSC, and all but one received myeloablative conditioning. GvHD prophylaxis was based on sirolimus plus MMF, and all but one (who received anti-thymoglobulin - ATG) received post-transplant cyclophosphamide. All patients received acyclovir as antiviral prophylaxis.

Results: All pts engrafted after transplantation with a median time of 19 days. Median follow up was 254 days. Median time to AdV reactivation was 43 days from transplant. Six out of 7 pts had a documented GvHD (n $=2$ grade $3, \mathrm{n}=4$ grade $4 ; 7 / 7$ gut involvement), requiring 
high dose steroids in all cases and second line treatments in 5 cases. Six pts have high AdV load at diagnosis (range 8470 - > 10^6 copies/ml) on peripheral blood (PB), and 6 pts has also AdV isolation on organ specimen, which was gastrointestinal tract in all cases plus urine in one case. Concomitantly, in four out of 7 cases we documented a BK virus infection, with severe hemorrhagic cystitis. All pts had also other DNA viruses reactivation: CMV in 5 and Herpes virus six in 3 cases. Five out of 7 pts were treated with endovenous cidofovir, with a median of 2.5 administration, but in all cases a progressive renal dysfunction prevented us to proceed with this drug; two patients were never treated with cidofovir due to concomitant renal dysfunction. Brincidofovir treatment was given for a median time of 36 days, twice weekly. AdV load decreased on PB in 5 out of 6 pts, reaching negativity in 4 cases with a median time to negativization of 70 days from treatment start; clinical improvement in case of organ involvement was seen in 4 out of 6 pts. In two cases AdV disease was hyperacute, without any possibility to see treatment benefit since the patients died early after diagnosis. Decrease of CMV and HSV6 load on PB was documented in 4 out of 7 pts, suggesting a potential activity also on viruses other than AdV. Two out of 7 pts, despite clinical and laboratory improvement, developed worsening diarrhea (grade 3 according to CTCAE scoring) probably related to a concomitant intestinal GvHD. No certain adverse events were reported after BCV therapy.

Conclusions: Although performed on a small cohort, this analysis suggests that BCV is manageable and efficacious for AdV disease in post-transplant frail pts, and should be considered as a treatment option due to its low toxicity profile.

Conflict of interest: Nothing to disclose.

\section{P535}

\section{CATHETER-RELATED INFECTIONS IN PATIENTS SUBMITTED TO HEMATOPOIETIC STEM CELL TRANSPLANTATION. SINGLE CENTER STUDY}

\author{
Montserrat Batlle, Anna Triguero, Mireia Morgades, Montserrat \\ Giménez, Ma José Jiménez, Edurne Serrate, Marta Sitges, Marta \\ Peña, Christelle Ferrà, Juan Manuel Sancho, Miriam Moreno, \\ Blanca Xicoy, Susana Vives, Anna Torrent, Josep M Ribera \\ ICO-Hospital Universitari Germans Trias i Pujol, Josep Carreras \\ Leukemia Research Institute, Universitat Autonoma de Barcelona, \\ Clinical Hematology, Badalona, Spain
}

Background: Bacterial infections are a frequent complication in HSCT, with elevated morbidity and mortality. Catheter-related bacteremias are one of the most frequent.
Methods: We studied catheter-related infections during HSCT in a single institution between 2011 and 2016, to identify the type of catheter infected and the responsible microorganisms.

Results: We observed 39 catheter-related infections out of 318 HSCT (12\%) (autologous 20/159, 12.5\%; allogeneic $19 / 159,12 \%)$. The main clinical characteristics of the patients are summarized in Table 1.

The median of days between catheter emplacement and infection was 17 [2;102]. The most frequently isolated bacteria were: Staphylococus epidermidis $(\mathrm{n}=16)$, Staphylococcus aureus $(\mathrm{n}=6)$ and coagulase-negative Staphylococcus $(\mathrm{n}=3)$ and Staphylococcus hominis, Staphylococus haemolyticus, Escherichia coli, Pseudomonas aeruginosa, Rotia mucilaginosa, Serratia marcesens and Klebsiella pneumonia (one patient each). We also noted polymicrobian infections: Acinetobacter baumanii/Stenotrophomona maltophilia, Staphylococcus epidermidis/Enterobacter cloacae, Stenotrophomonas maltophilia/Enterobacter cloacae and coagulase-negative Staphylococcus/Corynebacterium (one patient each). No bacteria were identified in 3 cases with cellulitis in the area of emplacement.

In autologous HSCT, the most frequently infected catheter was the Niagara $^{\mathrm{TM}}$ (17/20 infections) followed by the conventional subclavian (2/20 infections) and the conventional jugular (1/20 infections). We did not observe infections in the Port-a-Cath ${ }^{\circledast}$ or in the PICC, but their use in this type of transplantation is unusual. In allogeneic HSCT the most frequently infected catheter was the Hickman $^{\circledR}$ (11/19 infections) followed by conventional subclavian (4/19 infections), conventional jugular (3/19 infections) and PICC (1/19 infections). We did not observe Port-a-Cath ${ }^{\circledR}$ infections. The PICC catheter has been recently included in our clinical practice. No relation between catheter infection and the site of emplacement was observed.

Conclusions: The most frequently type of infected catheter in the autologous HSCT was the Niagara ${ }^{\mathrm{TM}}$ (thicker and not tunnelled) being the Hickman ${ }^{\circledR}$ in the allogeneic HSCT the most frequently involved. Staphylococcus spp was the most frequent bacteria but polymicrobian infections with concomitant cellulitis were frequently observed. Catheter-related infections were not related with the site of emplacement. Home care is the most important factor for catheter-related infections.

Supported in part by grants: Funding PI10/01417 (Fondo de Investigaciones Sanitarias). RD12/0036/0029 (RTICC, Instituto Carlos III, 2014 SGR225GRE), CERCA Program, Generalitat de Catalunya, España, "La Caixa" Foundation, RD12/0036/0029 RTICC-Instituto Carlos III.

Conflict of interest: No potential conflict of interest was reported by the authors. 


\begin{tabular}{|c|c|c|}
\hline Variables & Variables & Results \\
\hline Age & Median [range] & $56[20 ; 71]$ \\
\hline Gender, $\mathrm{n}(\%)$ & Male, Female & $29(74), 10(26)$ \\
\hline Disease, $\mathrm{n}(\%)$ & $\begin{array}{l}\text { MM, AML, NHL, MDS, } \\
\text { ALL, HL, CLL, BMF, MF }\end{array}$ & $\begin{array}{l}11(28), 8(21), 7(18), 5(13), 4 \\
(10), 1(2.5), 1(2.5), 1(2.5), 1 \\
(2.5)\end{array}$ \\
\hline $\begin{array}{l}\text { Type of } \\
\text { transplant, } n(\%)\end{array}$ & Autologous, Allogeneic & $20(51), 19(49)$ \\
\hline $\begin{array}{l}\text { Stem cell } \\
\text { source, } \mathrm{n}(\%)\end{array}$ & PB, BM, UCB & $33(84,5), 1(2,5), 5(13)$ \\
\hline $\begin{array}{l}\text { Catheter type, } \mathrm{n} \\
(\%)\end{array}$ & $\begin{array}{l}\text { Jugular, Subclavian } \\
\text { conventional, Hickman }^{\circledast}, \\
\text { Niagara }^{\mathrm{TM}}, \text { PICC }\end{array}$ & $\begin{array}{l}4(10), 6(15) 11(28), 17 \text { (44), } \\
1 \text { (3) }\end{array}$ \\
\hline $\begin{array}{l}\text { Department } \\
\text { catheter } \\
\text { insertion, } \mathrm{n}(\%)\end{array}$ & $\begin{array}{l}\text { Vascular radiology, } \\
\text { Anaesthesiology }\end{array}$ & 29 (74), $10(26)$ \\
\hline
\end{tabular}

[[P535 Table] Clinical characteristics of the series.]

\section{P536}

\section{Central nervous system (CNS) tuberculosis following allogeneic stem cell transplantation}

\section{Mehrdad Payandeh}

KUMS Imam Reza Hospital, Kermanshah, Iran, Islamic Republic of

Background: Tuberculosis is an uncommon infectious complication after stem cell transplantation. We report a patient who presented with a FUO, 14 days after allograft transplantation in 5 days after engraftment phase.

His performance statue deteriorated during this time, he evaluated for all possible diagnosis for fever after transplantation especially most common like HSV, CMV, Aspergillosis, bacterial infection.

$\mathrm{He}$ had acute Gastrointestinal GVHD grade 2 that controlled by mehylprednisolone $2 \mathrm{mg}$ per $\mathrm{kg}$.

Despite wide spectrum of empiric antibacterial and antifungal fever continue and not decrease.

Methods: The diagnosis was made after blood with TB PCR positive. The cerebral mass was detected by brain CT scan, antituberculous treatment was initiated.

Results: He treated with four drug combination therapy but due to ICH he death.

Conclusions: Mycobacterial infections should be considered in allograft recipients (till without pulmonary TB) with GVHD therapy and FUO specially in endemic world area and with sensitive assay like PCR method we can detected sooner this poor prognosis disease in immunocompromised patients.with wide spread evaluation in this patient a rare presention of common disease aould diagnosed.

\section{Conflict of interest: None}

\section{P537}

\section{Chagas Disease Reactivation after Autologous Hematopoietic Stem Cell Transplantation: A Case Report}

Claudia Lucia Sossa Melo ${ }^{1,2}$, Sara Ines Jimenez ${ }^{1,2}$, Angela Maria Peña Castellanos ${ }^{1,2}$, Luis Salazar ${ }^{1,2}$, Manuel Rosales ${ }^{1}$, Maria Fernanda Ortiz Tarazona ${ }^{3}$, Edgar Gomez ${ }^{4}$, Edgar Bernal ${ }^{5}$, David Leonardo Reyes ${ }^{1}$, Claudia Marcela Chalela ${ }^{1}$

${ }^{1}$ Universidad Autonoma de Bucaramanga/FOSCAL, Internal Medicine, Division of Hematology and Hematopoietic Stem Cell Transplantation, Bucaramanga, Colombia; ${ }^{2}$ Universidad Autonoma de Bucaramanga, School of Medicine, Bucaramanga, Colombia; ${ }^{3}$ Clinica Carlos Ardila Lulle (FOSCAL), Division of Hematology and Hematopoietic Stem Cell Transplantation, Bucaramanga, Colombia;

${ }^{4}$ Universidad Autonoma de Bucaramanga/FOSCAL, Internal Medicine, Bucaramanga, Colombia; ${ }^{5}$ Universidad Autonoma de Bucaramanga/FOSCAL, Internal Medicine, Division of Infectious Diseases, Bucaramanga, Colombia

Background: Chagas disease (CD) is a protozoan zoonosis caused by Trypanosoma cruzi (T. cruzi). Patients previously infected with the parasite are at higher risk of disease reactivation by drug-induced immunosuppression before and during transplant. T. cruzi reactivations have been reported to occur between $10 \%$ and $75 \%$ of the cases, and mostly after hematopoietic stem cell transplantation (HSTC) [1].

Methods: Case report of a 62-year old Colombian man diagnosed with classic Hodgkin Lymphoma (HL) in October 2014, treated with six cycles of ABVD (doxorubicin, bleomycin, vinblastine, and dacarbazine) with complete remission. After relapsed disease in January 2016 the next management strategy was hematopoietic transplantation. Pre-transplant evaluation reported positive serology studies for $\mathrm{CD}$, with no evidence of electrocardiography and echocardiography abnormalities.

Results: The patient received salvage chemotherapy with 3 cycles of ESHAP (etoposide, methylprednisolone, cytarabine, and cisplatin) followed by conditioning regimen with BEAM (carmustine, etoposide, cytarabine, and melphalan). In June 2016, on day -3 of autologous HSCT, the patient was found to have fever with elevated C-reactive protein, without clinical evidence of infection and normal blood cultures. Treatment with meropenem was initiated and three days later, $11.14 \times 10^{6} / \mathrm{Kg}$ of hematopoietic cells were infused. On day +2 , after another episode of fever, vancomycin was added to the treatment therapy. Blood and urine cultures were normal, and imaging studies showed no evidence of foci of infection. Despite empiric antibiotic treatment for 10 days, patient persisted with fever and elevated C-reactive protein levels. Management was escalated to meropenem + tigecycline + amikacin + caspofungin. Myeloid engraftment occurred on day +12 . On day +17 , fever persists and antibiotic treatment is 


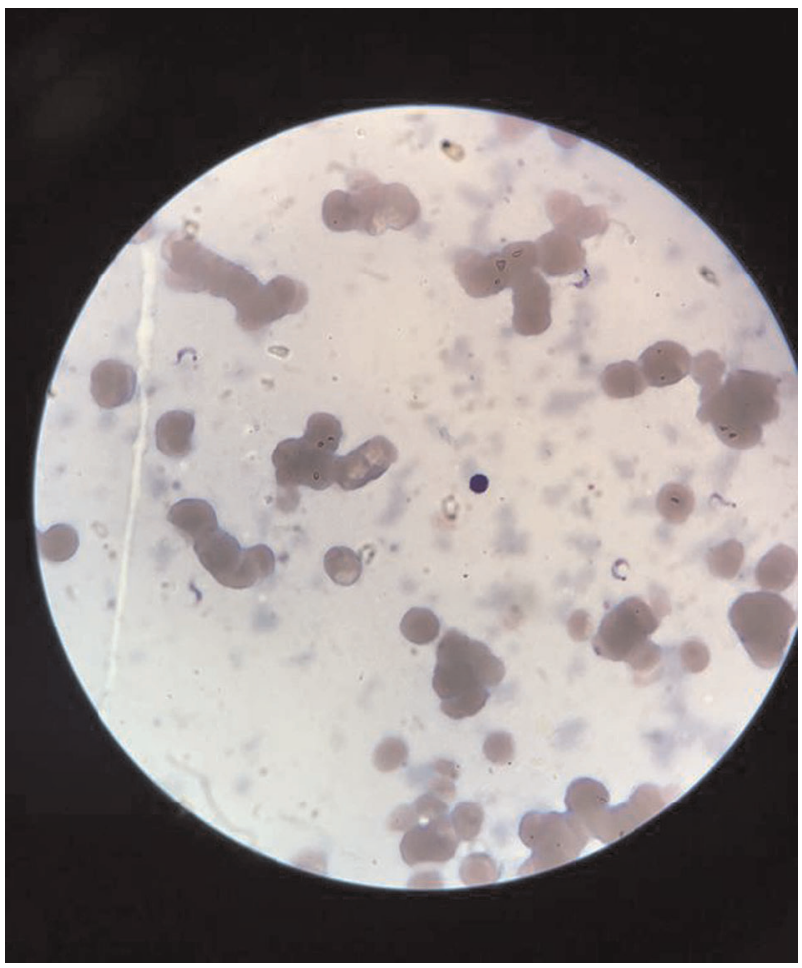

[P537 Figure] [Image 1. Micro-Strout test from patient showing trypomastigotes in peripheral blood sample.]

switched to polymyxin B. Micro-Strout test was performed and showed evidence of Trypomastigotes (see image 1). Once reactivation of the disease was confirmed, therapy with nifurtimox $120 \mathrm{mg} /$ day for 60 days was initiated. Parasitological after treatment follow-up was performed with micro-Strout weekly tests, which became negative three weeks later. Additionally, patient presented graft failure requiring several transfusions. In October 2016, the patient was admitted for generalized weakness, fatigue, intermittent fever, facial edema, and positive micro-Strout test. Treatment was initiated with benznidazole $300 \mathrm{mg} / \mathrm{day}$ for 60 days and follow-up micro-Strout test was negative three weeks after beginning treatment. Currently, the patient has not presented any other symptoms or positive microStrout tests suggestive of disease reactivation. The patient relapsed six months after transplantation and is on treatment with brentuximab.

Conclusions: Immunosuppression by salvage and conditioning chemotherapy previous to transplantation was the most probable cause for $\mathrm{CD}$ reactivation in this patient. HSCT candidates with previous infection by T. cruzi should initiate monitoring of possible reactivation with frequent parasitological studies in the conditioning phase prior to transplantation. In case of febrile syndrome of unknown etiology, urgent parasitological screening is required [1].

Conflict of interest: The authors declared no potential conflict of interest.
P538

\section{CHARACTERISTICS AND OUTCOME OF POLYMICROBIAL BLOODSTREAM INFECTIONS (p-BSI) IN HEMATOLOGIC PATIENTS UNDERGOING STEM CELL TRANSPLANTATION}

\author{
Maria Elena Zannier ${ }^{1}$, Maria Vittoria Dubbini ${ }^{1}$, Anna Candoni, ${ }^{1}$, \\ Carla Fili' ${ }^{1}$, Davide Lazzarotto ${ }^{1}$, Nicholas Rabassi ${ }^{1}$, Claudio \\ Scarparo $^{2}$, Assunta Sartor ${ }^{3}$, Renato Fanin ${ }^{1}$, Antonella Geromin ${ }^{4}$ \\ ${ }^{1}$ Asuiud Santa Maria Della Misericordia, Hematology and Bone \\ Marrow Transplantation, Udine, Italy; ${ }^{2}$ Asuiud Santa Maria Della \\ Misericordia, Microbiology, Udine, Italy; ${ }^{3}$ ASUI Trieste, UO \\ Ematologia, Microbiology, Udine, Italy; ${ }^{4}$ Azienda Sanitaria \\ Universitaria Integrata, Hematology, Udine, Italy
}

Background: Polymicrobial bloodstream infections (pBSI) may complicate the course of hematological diseases although they represent a rare event. The clinical characteristics and outcome of p-BSI occurring after stem cell transplantation (SCT) is still poorly understood.

Methods: We analyze clinical characteristics and outcome of 109 p-BSI occurring in 103 patients with hematologic diseases over a 9-year period (2008-2017) comparing SCT cases $(50 / 109-46 \%)$ with not-transplanted patients $(59 / 109-54 \%)$.

Results: In SCT cases p-BSI occurred both after AutoSCT (13/50-26\%) and Allo-SCT (37/50-74\%; MUD 22, HLA-id 7, Haplo 8); p-BSI occurred in the first 30 days after SCT in $34(68 \%)$ cases, between the $30^{\text {th }}$ and $60^{\text {th }}$ day after SCT in 6(12\%) cases and after $90^{\text {th }}$ day in $10(20 \%)$ cases. The most common underling hematological disease was Acute Myeloid Leukemia (AML) and the median age at onset of p-BSI of the entire population was $60 \mathrm{yrs}$. An acute GvHD grade $>2$ was present in $9(18 \%)$ patients and a chronic GvHD only in 2(4\%).

As reported in table 1 the majority of cases had severe neutropenia (87\%) and the most common bacterial association was Gram-negative plus Gram-positive bacteria (56\%) without differences between two groups. The most common isolates were coagulase-neg Staphylococcus spp, Enterococcus spp, E. Coli and P. aeruginosa. Susceptibility and antibiotic treatment have been recorded. A concomitant pneumonia and enteritis were reported in $15 \%$ of cases and the resolution rate of p-BSI was $83 \%$, without differences between two groups. The mortality p-BSI related was $16 \%$ (14\% in SCT cases). Septic shock occurred in $17 \%$ cases and the septic shock related mortality was $63 \%$ (78\% in SCT and 50\% in not-SCT cases). Multidrug Resistant (MDR) bacteria were involved in $21 \%$ of p-BSI (22\% in SCT-cases) and the mortality MDR p-BSI related was significantly higher in SCT patients (64\% vs $25 \%$ ).

Conclusions: This observational study confirms that $\mathrm{p}$ BSI is a rare infectious complication in SCT-patients; it is 
more frequent in the first 30 days after SCT during a severe neutropenia. A concomitant GVHD is uncommon. The mortality p-BSI related is lower than $20 \%$ but if septic shock occurs the mortality shock related was higher in SCT cases (78\% vs 50\%). MDR bacteria were involved in $21 \%$ of p-BSI and the mortality MDR p-BSI related was significantly higher in SCT cases (64\% vs $25 \%$ ).

Conflict of interest: Maria Elens Zannier: nothing to disclose

\begin{tabular}{|c|c|c|c|}
\hline & $\begin{array}{l}\left.\text { ALL CASES N }{ }^{\circ} \%\right) \\
109(100 \%)\end{array}$ & $\begin{array}{l}\text { SCT cases } \\
50(46 \%)\end{array}$ & $\begin{array}{l}\text { not-SCT cases } 59 \\
(54 \%)\end{array}$ \\
\hline Severe neutropenia & $95(87 \%)$ & $43(86 \%)$ & $52(88 \%)$ \\
\hline Pneumonia + p-BSI & $16(15 \%)$ & $7(14 \%)$ & $9(15 \%)$ \\
\hline Enteritis + p-BSI & $10(9 \%)$ & $4(8 \%)$ & $6(10 \%)$ \\
\hline $\begin{array}{l}\text { Gram pos + Gram } \\
\text { neg bacteria }\end{array}$ & $61(56 \%)$ & $30(60 \%)$ & $31(53 \%)$ \\
\hline MDR bacteria & $23(21 \%)$ & $11(22 \%)$ & $12(20 \%)$ \\
\hline $\begin{array}{l}\text { Mortality p-BSI } \\
\text { related }\end{array}$ & $18(16 \%)$ & $7(14 \%)$ & $11(18 \%)$ \\
\hline Septic shock & $19(17 \%)$ & $9(18 \%)$ & $10(17 \%)$ \\
\hline $\begin{array}{l}\text { Septic shock } \\
\text { Mortality }\end{array}$ & $12 / 19(63 \%)$ & $7 / 9(78 \%)^{\circ \circ}$ & $\begin{array}{l}5 / 10(50 \%)^{\circ}\left[\mathrm{p}^{\circ \circ} \mathrm{vs}\right. \\
\left.{ }^{\circ}=0.34\right]\end{array}$ \\
\hline $\begin{array}{l}\text { Mortality rate in } \\
\text { MDR p-BSI }\end{array}$ & $10 / 23(43 \%)$ & $\begin{array}{l}7 / 11(64 \%) \\
* *\end{array}$ & $\begin{array}{l}3 / 12(25 \%) *[\mathrm{P} * * \\
\mathrm{vs} *=0,09]\end{array}$ \\
\hline
\end{tabular}

[[P538 Table] TABLE-1. Characteristics and Outcome of $p-B S I]$

\section{P539}

Clinical impact of pre-transplant multidrug-resistant Gramnegative colonization in autologous and allogeneic hematopoietic stem cell transplantation

Alessandra Forcina ${ }^{1}$, Francesca Lorentino ${ }^{1}$, Vincenzo Marasco ${ }^{1}$, Chiara Oltolini ${ }^{2}$, Magda Marcatti ${ }^{1}$, Raffaella Greco ${ }^{1}$, Maria Teresa Lupo Stanghellini ${ }^{I}$, Matteo Carrabba ${ }^{I}$, Massimo Bernardi ${ }^{1}$, Jacopo Peccatori $^{I}$, Consuelo Corti ${ }^{l}$, Fabio Ciceri ${ }^{I}$

${ }^{1}$ IRCCS San Raffaele Hospital, Hematology and Bone Marrow Transplant Unit, Milano, Italy; ${ }^{2}$ IRCCS San Raffaele Hospital, Infectious Disease Unit, Milan, Italy

Background: Multidrug-resistant Gram-negative bacteria (MDRO-GN) are an emerging cause of morbidity and mortality after hematopoietic stem cell transplantation (HSCT). Therefore, a pre-transplant MDRO-GN colonization is often considered as a relative contra-indication to transplant. The aim of this study was to assess the impact of a pre-transplant colonization on clinical outcomes in HSCT.

Methods: Three-hundred-forty-eight consecutive patients transplanted at our Hospital from July 2012 to January 2016 (254 allogeneic and 94 autologous HSCT) were screened for a pre-transplant MDRO-GN colonization. Patients undergoing a second transplant were excluded. Univariate comparisons of survival curves were made using the Logrank test, while the Gray's test was used for univariate comparisons of CI functions. To analyze the association between transplantation endpoints and pre-transplant colonization by MDRO-GN pathogens, multivariate Cox proportional hazard models were built with the use of a backward selection procedure, to adjust for other clinical factors known to influence outcomes. Pre-transplant colonization was the main effect term and was held in all steps of model building.

Results: A pre-transplant MDRO-GN colonization was found in $16.9 \%$ of allo-HSCT and in $9.6 \%$ of auto-HSCT recipients. Both in auto- and in allo-HSCT, carriers of a pretransplant MDRO-GN showed no significant differences in overall survival (OS), transplant-related mortality (TRM) nor infection-related mortality (IRM), compared to noncarriers. OS at 2-yrs for carriers compared to non-carriers was $85 \%$ vs $81 \%(\mathrm{P}=0.262)$ in auto-HSCT and $50 \%$ vs $43 \%(\mathrm{P}=0.091)$ in allo-HSCT; TRM at 2 -yrs was $14 \%$ vs $5 \%$; $(\mathrm{P}=0.405)$ in auto-HSCT and $31 \%$ vs $25 \%(\mathrm{P}=$ $0.301)$ in allo-HSCT; IRM at $2 \mathrm{yrs}$ was $14 \%$ vs $2 \%(\mathrm{P}=$ $0.142)$ in auto-HSCT and $23 \%$ vs $14 \%(\mathrm{P}=0.304)$ in alloHSCT. In multivariate analysis, only grade III-IV acute graft-versus-host disease (aGVHD) was an independent factor for a detrimental OS $(\mathrm{P}<0.001)$, TRM $(\mathrm{P}<0.001)$ and IRM $(\mathrm{P}<0.001)$. In the same cohort of patients, 167 posttransplant Gram-negative bloodstream infections were reported, 41/167 (24\%) of which caused by a MDRO-GN (6/41 in auto-HSCT, 35/41 in allo-HSCT), with a 30-day related mortality of $14.7 \%$ overall. Noticeably, survival at 4 months for patients developing a Carbapenem-resistant Klebsiella Pneumonia infection was up to $70 \%$.

Conclusions: We conclude that in this extended singlecenter experience, a pre-transplant MDRO-GN colonization does not significantly impact on transplant outcomes, while the onset of aGVHD is the major risk factor for a detrimental outcome. We think that in this cohort the adoption of institutional protocols of active surveillance and early initiation of targeted antimicrobial therapies in carriers at the onset of neutropenic fever have successfully impacted on clinical outcomes, thus making OS, TRM and IRM not significantly different between pre-transplant MDRO-GN carriers and non-carriers.

Clinical Trial Registry: not applicable.

Conflict of interest: A. Forcina has Biotest Consultancy.

\section{P540}

Clinical patterns of nocardiosis in hematopoietic stem cell transplantation

Joao Dias ${ }^{1}$, Rosa Branca ${ }^{2}$, Sara Alves ${ }^{1}$, Luís Leite ${ }^{2}$, Carlos Pinho Vaz $^{2}$, Fernando Campilho ${ }^{2}$, Susana Roncon ${ }^{3}$, António Campos 


\section{Junior $^{2}$}

${ }^{1} I P O$ - Porto, Medical Oncology, Porto, Portugal; ${ }^{2} I P O$ - Porto, Bone Marrow Transplantation Department, Porto, Portugal; ${ }^{3} I P O$ - Porto, Cellular Therapy Department, Porto, Portugal

Background: Nocardia $s p p$ is a genus of gram-positive saprophyte bacteria that may cause disease in human. The most common disease sites are the lung, skin and central nervous systems, and disseminated disease may occur. Immunocompromised hosts, including patients who have undergone hematopoietic stem cell transplant, are at increased risk for infection.

Methods: We performed a single-centre retrospective analysis of cases from 2009 to july 1st 2017 with laboratory confirmed nocardiosis, defined as a compatible clinical presentation and either isolation of Nocardia spp or its identification by PCR.

Results: We identified 6 patients who met inclusion criteria, of whom 5 had isolation of Nocardia spp and 1 with a PCR-based diagnosis. Median age at diagnosis was $44,5$ years (min-max: $34-58)$, and $5(83,3 \%)$ were male. The most common type of transplant was allogeneic matchedunrelated donor $(\mathrm{n}=3 ; 50 \%)$, allogeneic matched-related donor $(\mathrm{n}=2 ; 33,3 \%)$ and autologous stem cell transplant ( $\mathrm{n}$ $=1 ; 16,7 \%)$. Transplant was due in to acute myeloid leukemia in 4 patients, non-Hodgkin lymphoma in 1 patient and myelodysplastic syndrome in 1 patient. The median time from transplant to the diagnosis of nocardiosis was 251 days (min-max: 3-629). The majority of patients was under prophylactic cotrimoxazol $(n=4 ; 66,7 \%)$. A greater than $20 \mathrm{mg}$ of prednisone was being administered in 5 $(83,3 \%)$ patients and all were taking at least one other immunosuppressant drug. All patients had fever (median temperature $38,5^{\circ} \mathrm{C}$; min-max: $38,1-40^{\circ} \mathrm{C}$ ) and all had lung involvement. In three cases a SNC involvement was suspected (two cases of brain abscess and one case of aseptic refractory meningitis, in whom Nocardia spp was identified by PCR). No agent was isolated in LCR cultures. Cultural identification of Nocardia spp was done in blood cultures in 2 patients (out of 6 patients in whom a sample was taken), in lung biopsy in 2(out of 3 ), in respiratory samples in 2 (out of 6), on the skin in 1 (out of 6) and on the pleura in 1(out of 6). In 3 cases a lumbar puncture was performed, in one on whom a PCR identification of Nocardia spp was done. The mortality rate as $83,3 \%$ (n $=5$ ). Median survival was 108 days after diagnosis, largely due to concomitant invasive opportunistic infections.

Conclusions: Nocardiosis is a rare and in our sample late complication of hematopoietic stem cell transplant, with a common involvement of the lung and a poor prognosis that was mostly due to opportunistic infections. One patient had a diagnosis of nocardiosis 6 days after the stem cell transplant and died of neutropenic sepsis in the engraftment period, meaning that inoculation had likely occurred before the procedure due to previous immunosuppression. A high index of suspicion is required and immunosuppression and antibiotic therapy must be carefully managed in these patients in order to improve this dismal outcome.

Conflict of interest: The authors report no conflicts of interest

\section{P541}

\section{CMV prophylactic regimen with high titer CMV immunoglobulin (Cytotect/Megalotect) for high risk ASCT patients}

Monica Tozzi, Alessandro Bucalossi, Mariapia Lenoci, Francesca Toraldo, Giuseppe Marotta

Azienda Ospedaliera Universitaria Senese, UOC Terapie Cellulari e Officina Trasfusionale, Siena, Italy

Background: Cytomegalovirus (CMV) infection is very common after allogeneic stem cell transplantation (ASCT) and may cause serious, even fatal disease. Specific prophylaxis of CMV infection is possible with antiviral drugs and CMV hyperimmune globulin. The prophylaxis may be particularly important for high risk patients who receive high levels of immunosuppression.

Methods: Several randomized trials on the effectiveness of CMV hyperimmune globulin in the prevention of CMV infection and disease in ASCT have given conflicting results. We have carried out a retrospective single center experience to evaluate the safety and the effectiveness of a prophylactic regimen with high titer CMV immunoglobulin (Cytotect/Megalotect) for high risk ASCT patients. Thirty adult patients who underwent myeloablative ASCT from an unrelated or haploidentical donor for Acute Myeloid or Lymphoblastic Leukemia at our Institute between January 2014 to June 2017 were evaluated. Seventeen patients received peripheral blood stem cell transplant from unrelated donor after a Busulphan based conditioning regimen with a graft versus host disease (GVHD) prophylaxis with short course Methotrexate, Cyclosporine and Antilymphocyte Globulin. Thirteen patients were treated with a Thiotepa Fludarabine Busulphan based regimen followed by bone marrow haploidentical transplant and GVHD prophylaxis with high dose post-transplantation Cyclophosphamide, Mycophenolic acid and Cyclosporine. All patients received a prophylactic regimen with administration of CMV hyperimmune globulin (Cytotect/Megalotect $^{\circledR}$, Biotest, Germany) $0,5 \mathrm{ml} / \mathrm{kg}$ every three weeks, from day 30 until 6 months after transplantation. This preparation has been reported to contain a titer of neutralizing antibodies of 1:1,000. 
Results: No CMV reactivation occurred in 13 of 30 patients $(43 \%)$ while 17 patients $(57 \%)$ showed a whole blood CMV DNA copies number $>1.000 / \mathrm{ml}$ between fifth and twentieth week after transplant. Nine of the 17 patients with arising in CMV DNA copies seroconverted spontaneously without any other therapy despite the high levels of CMV DNA copies/ml (median value 22.760, range 355565.293). Eight of the remaining patient (26\%) showed clinical signs of CMV infection and received an antiviral therapy (Ganciclovir) to control the viral load.

Conclusions: In our experience CMV hyperimmune globulin prophylaxis appears to be well-tolerated, no adverse reaction are recorded, and seems to be effective to control and reduce CMV viral load in high risk ASCT patients.

The CMV hyperimmune globulin schedule seems to be effective to reduce the number of patients who need to be treated with myelotoxic antiviral drugs particulary in the first weeks after ASCT, when hematological recovery often is not achieved.

Finally, our data indicate that CMV hyperimmune globulin prophylaxis is safe for use in the ASCT population. These findings support further trials of the efficacy of a prophylaxis for the CMV infection in the ASCT population.

Conflict of interest: Monica Tozzi have consulted for Biotest Germany; all the other authors declare no competing interests.

\section{P542}

Combined C-reactive protein, presepsin and procalcitonin levels evaluation for sepsis identification in patients after hematopoietic stem cell transplantation

Oleg Goloshchapov, Evgenii Goncharov, Alexandr Kiselev, Andrey Chekalov, Maxim Kucher, Ruslana Klementeva, Lyudmila Zubarovskaya, Boris Afanasyev

Pavlov First Saint Petersburg State Medical University, Gorbacheva Memorial Research Institute for Pediatric Oncology, Hematology and Transplantation, Saint Petersburg, Russian Federation

Background: Infectious complications are the main mortality cause after hematopoietic stem cell transplantation (HSCT). Diagnostic value of inflammation markers in septic immunocompromised patients remains controversial.

Methods: In Raisa Gorbacheva Memorial Research Institute for Pediatric Oncology, Hematology and Transplantation 26 patients with acute myeloid leukemia - 29\% $(\mathrm{n}=8)$, acute lymphoblastic leukemia - 19\% $(\mathrm{n}=5)$, inherited diseases - $12 \%(\mathrm{n}=3)$, non-Hodgkin's lymphoma - $12 \%(n=3)$, myelodysplastic syndrome - $8 \%(n=2)$, chronic myelogenous leukemia - $8 \%(\mathrm{n}=2)$, chronic lymphoblastic leukemia - $4 \% \quad(\mathrm{n}=1)$, chronic myeloproliferative disease - $4 \%(\mathrm{n}=1)$, medulloblastoma $-4 \%(\mathrm{n}=1)$ were enrolled to the prospective study. Median age was 20 years (1-57 years). All patients underwent HSCT: allogeneic unrelated - 61\% $(\mathrm{n}=16)$, allogeneic related $-12 \%(n=3)$, haploidentical - 23\% $(n=6)$ and autologous - $4 \%(n=1)$. The main inclusion criterion was $>10$ times exceed of C-reactive protein (CRP) level (normal rate $5 \mathrm{mg} / \mathrm{l})$. Levels of CRP, PSP, PCT, cystatin C (CysC), interleukins (IL6, IL8, IL10), interferon $\gamma$ (IFN $\gamma$ ), CD163, $\mathrm{CD} 25$ and routine laboratory tests were estimated in three check-points: admission to the ICU department, 48 hours after admission and in the case of 3 times CRP level decrease as compared with initial value or in patient terminal state.

Results: Sepsis was established in $70 \%$ of patients $(\mathrm{n}=$ 18 ), based on clinical and laboratory criteria. In $30 \%$ of patients $(\mathrm{n}=8)$ with elevated CRP, the diagnosis of sepsis was not confirmed. Median values of inflammation markers in the group of patients with and without sepsis were CRP 165 and $99 \mathrm{mg} / \mathrm{dl}$, PCT 2,94 and 0,3 ng/dl, PSP 1085 and $1403 \mathrm{ng} / \mathrm{ml}$, IL6 20,5 and 5 pg/ml, IL8 267 and $110 \mathrm{pg} / \mathrm{ml}$, IL10 1,4 and $0,8 \mathrm{pg} / \mathrm{ml}$, respectively. Initial decrease in glomerular filtration rate (GFR) of different etiology was observed in 20 patients (77\%). In patients with or without sepsis renal failure was observed in $72,2 \%$ of cases $(n=13)$ and in $87,5 \%(n=7)$ respectively. We revealed correlation between PSP, PCT, IL6, IL8, IL10 and increased CysC level and decreased GFR $(p<0,01)$. Whereas in group of patients without sepsis there was a significant correlation only between increased PSP level and decreased GFR ( $p<$ $0.05)$. PSP level reliably indicated the presence of sepsis only in patients with normal kidney function (AUC 854, CI $0,674-1, \mathrm{p}<0,05$, sensitivity (Se) - 53\%, specificity (Sp) $100 \%)$. Among other biomarkers the most valuable were PCT (AUC 844,CI 0,743-0,945, p < 0,001, Se - 53\%, Sp 93\%), CRP (AUC 801,CI 0,684-0,918, p < 0,001, Se - 65\%, Sp - 89\%).

Conclusions: PCT and CRP are highly specific and sensitive sepsis markers in patients after HSCT. PSP level in patients with sepsis suspicion with normal renal function may also be a highly specific diagnostic marker but is not valuable while renal dysfunction.

Conflict of interest: No conflict of interests.

\section{P543}

Comparative Analysis of Posaconazole Suspension vs. Delayed-release Tablet as Antifungal Prophylaxis in Allogeneic Hematopoietic Cell Transplant Patients

Chin Menn Woon ${ }^{1}$, Yi Ching Yuen ${ }^{1}$, Lip Kun Tan ${ }^{2}$, Michelle Li Mei Poon $^{2}$, Liang Piu Koh ${ }^{2}$ 
${ }^{1}$ National University Hospital, Pharmacy, Singapore, Singapore;

${ }^{2}$ National University Cancer Institute, Department of Haematology-

Oncology, Singapore, Singapore

Background: Posaconazole oral suspension has been the choice of antifungal prophylaxis agent in our institution for allogeneic hematopoietic cell transplantation (alloHCT) recipients. The delayed-release tablet formulation which was designed to overcome absorption limitations associated with the suspension was recently approved in Singapore. This study aims to compare the efficacy and safety of posaconazole suspension vs. tablet as antifungal prophylaxis within the first 100 days of alloHCT.

Methods: Twenty-four patients receiving alloHCT between November 2016 and August 2017 were included in this retrospective study. Patients were given either posaconazole suspension $200 \mathrm{mg}$ three times daily $(\mathrm{n}=$ 12 ) or tablet $300 \mathrm{mg}$ once daily $(\mathrm{n}=12)$ beginning the day of alloHCT onwards until 75 days after alloHCT, suspected drug-associated toxicity, or occurrence of invasive fungal disease (IFD). Additionally, posaconazole was switched to fluconazole $200 \mathrm{mg}$ once daily upon neutrophil engraftment for cost saving reason. Therapeutic antifungal therapy was started for fungal infections when indicated according to the standardized EORTC/MSG criteria.

Results: The two study groups were comparable in demographics except that patients in the posaconazole tablet group were older (60-year-old vs. 48-year-old) and comprised of less Chinese (41.5\% vs $83 \%$ ) as compared to the suspension group. There was also a larger proportion of patients who received $>0.5 \mathrm{mg} / \mathrm{kg}$ of prednisolone in the tablet group (58\% vs 17\%). Only one patient who received posaconazole tablet developed IFD in this study. This patient developed Candida Krusei fungemia on Day 31 of alloHCT. However, this was unlikely due to posaconazole prophylaxis failure as posaconazole was switched to IV anidulafungin on Day 17 because of hyperbilirubinemia from veno-occlusive disease (VOD). Treatment or drug related toxicity resulting in posaconazole interruption occurred more in patients who received tablet vs. suspension ( 8 vs. $5, \mathrm{p}=0.319)$. The most common reason leading to temporary switch to intravenous antifungal prophylaxis agent was mucositis in both groups. Grade 2 and above liver toxicity were observed in two tablet recipients. Both are thought to be unrelated to posaconazole as one was due to VOD and the other patient's liver function improved with continuation of posaconazole tablet.

Conclusions: This retrospective study suggests that posaconazole delayed-release tablet and suspension are comparably effective. The sample size is too small to conclude if the better absorption of posaconazole tablet will confer better protection against IFD during the early phase of alloHCT. Mucositis leading to temporary discontinuation of posaconazole prophylaxis remains a major concern in both the new tablet formulation and suspension. Liver function test should be monitored closely for treatment and drug toxicity in alloHCT patients receiving posaconazole.

Conflict of interest: Nothing to disclose

\section{P544}

Cost effectiveness of letermovir as prophylaxis of clinically significant cytomegalovirus reactivation and disease in adult CMV-seropositive allogeneic haematopoietic stem cell transplant: a Scottish payer perspective

Chloe Brown ${ }^{1}$, Jonathan Schelfhout ${ }^{2}$, Emilie Taymor ${ }^{1}$, Yiling

Jiang ${ }^{3}$, Matthew Glover ${ }^{4}$

${ }^{1}$ MSD UK, Hoddesdon, United Kingdom; ${ }^{2}$ Merck \& Co., Inc., North Wales, PA, United States; ${ }^{3}$ Merck \& Co., Inc., Kenilworth, NJ, United States; ${ }^{4}$ PHMR, Berkley Grove, United Kingdom

Background: Data from a recent phase III clinical trial has demonstrated the efficacy of letermovir as prophylaxis against CMV reactivation in adult CMV-seropositive recipients of an allogeneic haematopoietic stem-cell transplant (HSCT). The objective of this cost-effectiveness analysis is to evaluate the clinical and economic value of letermovir when compared to pre-emptive treatment from the payer perspective in Scotland.

Methods: A decision-analytic model evaluated total costs and lifetime quality-adjusted life-years (QALY's) from the perspective of the National Health Service (NHS) and social work in Scotland. Efficacy data, including rates of preemptive therapy for CMV infection, CMV disease, rehospitalization, mortality, and quality of life were incorporated from clinical trial data at 24 weeks posttransplant. The primary endpoint of the trial was clinically significant CMV-infection at week 24 post-transplant. Costs of preemptive treatment, CMV disease, and other trial outcomes were obtained from published sources. Life-years during the first 24 weeks were estimated from the mortality observed in the clinical trial. Life-years post 24 weeks were estimated for the week 24 survivors by applying a relative risk of death for HSCT to the general mortality risk from the life tables for Scotland (2014-16). Utility values were applied based on EQ-5D data collected in the clinical trial and longer-term data from published sources. The annual cost to treat 1-year survivors was based on a proportion of patients receiving 2 years of immunosuppressive agent treatment for chronic graft-versus-host disease (onset 100 days after transplant). Sensitivity analysis explored the impact of including data from the extended follow-up period, where various endpoints were collected through 48 weeks post-transplant. The model used an annual discount rate of $3.5 \%$. 
Results: In the base-case analysis, letermovir reduced the number CMV infections requiring pre-emptive treatment vs standard of care (SoC) $(20.0 \%$ vs $49.3 \%)$ at 24 weeks posttransplant. Prophylaxis with letermovir resulted in lower allcause mortality at week 24 in comparison to SoC $(10.2 \%$ vs. $15.9 \%)$, and a mean increase in life-years $(+0.46)$, QALY's $(+0.41)$ and total costs up to week 24 post-HSCT per patient. This resulted in an incremental costeffectiveness ratio (ICER) of $£ 12,659$ per QALY gained. The sensitivity analysis using data from 48 weeks posttransplant did not have a significant effect on the results. Results of a probabilistic sensitivity analysis indicated that letermovir was cost effective in $77.4 \%$ of iterations at $£ 20,000$ per QALY gained and $87.6 \%$ of iterations at $£ 30,000$ per QALY gained.

Conclusions: The results of this model would suggest that the prophylaxis use of letermovir is a cost-effective treatment strategy at commonly accepted ICER thresholds. The model inputs with a greatest impact on the results were a reduction in the rate of mortality, increasing the age of patients treated with letermovir, and an increase in the cost of prophylaxis for letermovir.

\section{Conflict of interest:}

C. Brown and E. Taymor: employees of Merck Sharp \& Dohme, Limited, UK [receive salary and other benefits]

J. Schelfhout and Y. Jiang: employees of Merck \& Co., Inc. [receive salary and other benefits]

M. Glover: Employee of PHMR [Grant funding from MSD to conduct model]
P545

Cytomegalovirus (CMV) impact on clinical outcomes and resource use after allogeneic hematopoietic cell transplantation: The influence of recurrent episodes of CMV infection

Carlos de Miguel ${ }^{1}$, Ana Royuela ${ }^{2}$, María Francisca Portero ${ }^{3}$, Juan Luis Cruz ${ }^{4,5}$, María Esther Martínez-Muñoz ${ }^{1}$, María Isabel Sánchez Romero $^{3}$, Guiomar Bautista ${ }^{1}$, Rafael Cabrera ${ }^{1}$, Rafael F. Duarte ${ }^{1}$

${ }^{1}$ Hospital Universitario Puerta de Hierro Majadahonda, Hematology, Majadahonda, Spain; ${ }^{2}$ Biomedical Sciences Research Institute Puerta de Hierro-Segovia de Arana, Clinical Biostatistics Unit, Majadahonda, Spain; ${ }^{3}$ Hospital Universitario Puerta de Hierro Majadahonda, Microbiology, Majadahonda, Spain; ${ }^{4}$ Hospital Universitario Puerta de Hierro Majadahonda, IT Department, Majadahonda, Spain; ${ }^{5}$ Universidad Politécnica de Madrid, Madrid, Spain

Background: Despite pre-emptive therapy (PET), Cytomegalovirus infection (CMVi) poses a negative impact on the outcome of allogeneic HCT recipients, and potentially on the use of resources for patient management.

Methods: This retrospective study analyses the impact of $\mathrm{CMVi}$ on clinical outcomes and resource use including hospital length of stay (LOS) in consecutive allogeneic HCT recipients (2009-2016), with a particular focus on recurrent CMVi episodes.

Results: 183 allogeneic HCT in 172 recipients were included: 101 men (58.7\%); median age 44 years (16-68); 51\% AML/MDS, 16\% ALL, 16\% chronic lymphoproliferative disorders, $8 \%$ myeloma and $9 \%$ other; $45 \%$

Table 1. Impact of CMV infection and recurrent infections on hospital admission length of stay.

\begin{tabular}{|c|c|c|c|c|c|c|}
\hline \multirow[t]{2}{*}{ Hospital LOS } & \multirow{2}{*}{$\begin{array}{l}\text { No CMV } \\
\text { infection }\end{array}$} & \multirow{2}{*}{$p$} & \multirow{2}{*}{$\begin{array}{c}\text { CMV } \\
\text { infection } \\
\text { (Any) }\end{array}$} & \multicolumn{2}{|c|}{$\begin{array}{c}\text { Number of CMV } \\
\text { infection episodes }\end{array}$} & \multirow[t]{2}{*}{$p$} \\
\hline & & & & Only 1 & $>=2$ & \\
\hline $1^{\text {st }} \mathrm{HCT}$ Hospital Admission & $\begin{array}{c}44.59 \\
+/-21.7\end{array}$ & 0.029 & $\begin{array}{c}53.98 \\
+/-33.9\end{array}$ & & & \\
\hline $1^{\text {st }}$ Discharge to $d+100$ & $\begin{array}{c}11.90 \\
+/-28.4\end{array}$ & 0.864 & $\begin{array}{c}12.59 \\
+/-26.5\end{array}$ & $\begin{array}{r}12.32 \\
+/-26.8\end{array}$ & $\begin{array}{c}12.89 \\
+/-26.8\end{array}$ & 0.976 \\
\hline $1^{\text {st }}$ Discharge to $d+365$ & $\begin{array}{c}24.75 \\
+/-39.1\end{array}$ & 0.002 & $\begin{array}{c}47.30 \\
+/-56.5\end{array}$ & $\begin{array}{c}35.93 \\
+/-46.2\end{array}$ & $\begin{array}{c}54.59 \\
+/-62.1\end{array}$ & 0.004 \\
\hline$d+100$ to $d+365$ & $\begin{array}{c}12.86 \\
+/-24.1\end{array}$ & $<0.001$ & $\begin{array}{c}34.65 \\
+/-52.3\end{array}$ & $\begin{array}{c}23.59 \\
+/-37.9\end{array}$ & $\begin{array}{c}41.69 \\
+/-59.7\end{array}$ & 0.001 \\
\hline HCT Admission to d +365 & $\begin{array}{c}69.34 \\
+!-43.9\end{array}$ & $<0.001$ & $\begin{array}{c}101.28 \\
+/-63.2\end{array}$ & $\begin{array}{r}88.49 \\
+/-52.5\end{array}$ & $\begin{array}{c}109.7 \\
+/-69.2\end{array}$ & $<0.001$ \\
\hline
\end{tabular}

Abbreviations: CMV: Cytomegalovirus; HCT: Hematopoietic Cell Transplantation; LOS: Length of stay (expressed in days, $d$ ). 
matched related, $30 \%$ cord-blood, $20 \%$ unrelated and 5\% haploidentical donors; $52 \%$ myeloablative conditioning; 161 (88\%) at risk of CMVi (donor and/or recipient positive serology); 61 (33\%) had acute GvHD grades II-IV. CMV disease occurred in only 4 cases $(2 \%)$. CMVi occurred in $56 \%$ of patients ( $60 \%$ of patients at risk), at a median day +35 post-HCT (15-58), and had an impact on overall survival ( $44 \%$ vs $60 \%$ at 2 years; $p=0.027)$. Patients above median age had higher CMV serology at risk ( $97 \%$ vs $80 \%$; $\mathrm{p}<0.001)$ and CMVi rates (65\% vs $48 \% ; \mathrm{p}=0.005)$. Compared to matched-related HCT, cord-blood and haploidentical HCT-recipients had higher CMVi rates $(68 \%$ vs $49 \% ; \mathrm{p}=0.009$, respectively) and earlier onset (70\% vs $32 \%$ within day +35 post-HCT; $p<0.001$ ). Acute GVHD also increased CMVi rates (87\% vs 43\%; $p<0.001$ ). Among patients with a first CMVi, 57\% had $\geq 2$ and $20 \%$ had $\geq 4$ recurrent CMVi episodes. CMVi recurrence $\geq 2$ was also higher in cord-blood and haploidentical versus matched related HCT (HR 2.38; 95\%CI 1.61-3.52; $\mathrm{p}<0.001$ ), age at the time of HCT $(+1.6 \%$ per year OR HR 1.02 (1.016); 95\% CI 1-1.03; $\mathrm{p}=0.024)$ and acute GVHD (HR 2.3; 95\% CI 1.62-3.26; $\mathrm{p}<0.001)$. In terms of clinical burden, hospital LOS throughout the first year post-HCT was overall $>30$ days longer in patients with CMVi (vs without, $\mathrm{p}<0.001$; Table 1). This increase in LOS was $>40$ additional days in patients with $\geq 2$ recurrent CMVi, significantly higher than in those with only one CMVi episode (19 days; $p<0.001$ ). In terms of treatment, $73.7 \%$ of CMV reactivations responded to $1^{\text {st }}$ line of treatment, $60 \%$ to $2^{\text {nd }}$ line and only $30 \%$ to $3^{\text {rd }}$ line. The rate of adverse effects with PET was $31 \%$ after 1 st line of treatment, and increased with usual drugs to $60 \%$ after 2 nd line.

Conclusions: Despite preemptive therapy, CMVi remains a hurdle to the success of allogeneic HCT, with a significant impact on patients' outcome and use of resources. Recurrent CMVi episodes are very frequent, in particular in high-risk HCT-groups such as cord-blood, haploidentical and acute GVHD, pose a significant impact on outcomes and are a strong driver of hospital LOS and resource burden for the HCT program.

Conflict of interest: None of the authors has anything to declare.

\section{P546}

\section{CYTOMEGALOVIRUS INFECTION IN ALLOGENEIC HEMATOPOIETIC CTEM CELL TRANSPLANT PATIENTS WITH HEMATOLOGICAL DISEASES: A SINGLE CENTER EXPERIENCE}

\author{
Eren Arslan Davulcu, Nur Soyer, Fahri Sahin, Güray Saydam,
} Murat Tombuloğlu, Mahmut Töbü, Filiz, Vural
Ege University Medical Faculty, Hematology Department, Izmir, Turkey

Background: Cytomegalovirus (CMV) infection is one of the most serious cause of morbidity and mortality in allogeneic hematopoietic stem cell transplantation (aHSCT). Risk factors for CMV infection are diverse but monitoring CMV status is crucial in all aHSCT recipients.

Methods: We retrospectively evaluated 185 patients with hematological diseases undergoing aHSCT in Ege University Hematology Department Bone Marrow Transplantation Unit between 2012-2017. Patient characteristics, type of the donor, transplantation regimen, donor and patient CMV status, CMV monitoring and CMV antiviral treatment results, clinical status of patients during CMV infection were recorded. Patients and donors were screened for CMV serologic status (CMV IgM and IgG). Post-transplant CMV DNA monitoring was performed starting at the day of transplantation, biweekly for 100 days or longer in the case of any complications, weekly up to 180 days, and monthly until 1 year after aHSCT.

Results: Fifty onepatients developed CMV infection $(25,7 \%), 40$ patients had viral reactivation and 11 patients had symptomatic infection. Symptomatic CMV infections were 4 colitis, 4 pneumonia, 2 cystitis, and 1 retinitis. Stem cell source was peripheral blood in all patients. Underlying disease and number of patients were as follows: acute myeloid leukemia (17), acute lymphocytic leukemia (10), myelodisplastic syndrome (5), multiple miyeloma (5), HodgkinÂ's lymphoma (4), non-Hodgkin Â's lymphoma (3), aplastic anemia (3), myelofibrosis (3), and chronic myeloid leukemia (1). Conditioning regimen was reducedintensity conditioning in 15 and myeloablative in 36 patients. Baseline characteristics of the study population are summarized in table 1.

CMV infection occurred between 7-240 days after aHSCT. Two patients have pathologically proven CMV infections ( 1 colon and 1 lung biopsies). Eight patients have two or more CMV infection attacks. Number of patients treated with gancyclovir only 27 (52\%), valgancyclovir only $1(0,1 \%)$, gancyclovir+valgancyclovir $22(43 \%)$, and gancyclovir+foscarnet $1 \quad(0,1 \%)$. Thirty eight $(78 \%)$ patients have negative CMV DNA levels after antiviral treatment. Nineteen patients (31\%) have concomitant graftversus host disease. Twenty patients are alive. Reasons of death were 11 (35\%) infecitons/sepsis, $11(35 \%)$ transplant complications, $5(16 \%)$ other reasons, 3 (10\%) progressive disease, 1 (4\%) CMV pneumonitis.

Conclusions: In our study population most of the recipients were seropositive (94\%) therefore, CMV infections occur because of viral reactivation rather than transmission from the donor. CMV monitoring should be made regularly in order to reduce CMV-related mortality. 
Table 1: Baseline characteristics of the study population

\begin{tabular}{|l|l|}
\hline Gender (Male/female) & $38 / 13$ \\
\hline Mean age (years) & 43,9 \\
\hline $\begin{array}{l}\text { Number of patients } \\
18-40 \text { years/ }>40 \text { years }\end{array}$ & $22 / 29$ \\
\hline $\begin{array}{l}\text { Donor (Related/Mismatched- } \\
\text { unrelated) }\end{array}$ & $42 / 7$ \\
\hline $\begin{array}{l}\text { Recipient CMV status } \\
\text { (seropositive/seronegative/unknown) }\end{array}$ & $48 / 1 / 2$ \\
\hline $\begin{array}{l}\text { Donor CMV status } \\
\text { (seropositive/seronegative/unknown) }\end{array}$ & $37 / 4 / 10$ \\
\hline CMV recipient donor status & 1 \\
\hline R-/D+ & 36 \\
\hline R+/D+ & 4 \\
\hline R+/D- & \\
\hline
\end{tabular}

[P546 Figure] [Table 1: Baseline characteristics of the study population]

Conflict of interest: The authors whose names are listedcertify that they have no affiliations with or involvement in any organization or entity with any financial interest or non-financial interest in this abstract.

\section{P547}

\section{CYTOMEGALOVIRUS INFECTION IN AUTOLOGOUS HEMATOPOIETIC STEM CELL TRANSPLANT PATIENTS WITH HEMATOLOGICAL DISEASES: A SINGLE CENTER EXPERIENCE}

Eren Arslan Davulcu, Nur Soyer, Fahri Sahin, Mahmut Töbü, Murat Tombuloğlu, Güray Saydam, Filiz, Vural

Ege Uviversity Medical Faculty, Hematology Department, Izmir, Turkey

Background: Cytomegalovirus (CMV) infection after autologous stem cell transplantation (auto-HSCT) is less frequent than allogeneic hematopoietic stem cell transplantation. However, CMV infection may be prevalent and severe in auto-HSCT cases.

Methods: We retrospectively evaluated 417 patients with hematological diseases undergoing auto-HSCT in Ege University Hematology Department Bone Marrow Transplantation Unit between 2012-2017. Patient characteristics, transplantation regimen, CMV monitoring and CMV antiviral treatment results, clinical status of patients during CMV infection were recorded. Post-transplant CMV DNA monitoring was performed starting at the day of transplantation, biweekly until engraftment. If CMV DNA was found positive, screening continued monthly for three months after discharge.

Results: Twenty two patients developed CMV viral reactivation $(5 \%)$, none of them had symptomatic infection. Underlying disease and number of patients were as follows: multiple miyeloma (10), non-HodgkinÂ's lymphoma (6), Hodgkin $\hat{A}$ 's lymphoma (4), acute myeloid leukemia (1), andamiloidosis (1).Conditioning regimen was melphalan 140-200 mg/m² (on day -1) in multiple miyeloma, BuCy (busulfan $3,2 \mathrm{mg} / \mathrm{kg}$ on days $-7,-6,-5,-4$, cyclophasphamide $60 \mathrm{mg} / \mathrm{kg}$ on days $-3,-2)$ in acute myeloid leukemia, BEAM (BCNU $300 \mathrm{mg} / \mathrm{m}^{2}$ on day, -6 etoposide $2 \times 200 \mathrm{mg} / \mathrm{m}^{2}$ ondays $-5,-4,-3,-2$, ARA-C $2 \times 200 \mathrm{mg} / \mathrm{m}^{2}$ on days $-5,-4,-3$,2, melphalan $140 \mathrm{mg} / \mathrm{m}^{2}$ on day -1 ) or TECAM (etoposide $250 \mathrm{mg} / \mathrm{m}^{2}$ on days $-6,-5,-4,-3$, thiotepa $40 \mathrm{mg} / \mathrm{m}^{2}$ on days $-5,-4,-3,-2$, cytarabine $200 \mathrm{mg} / \mathrm{m}^{2}$ on days $-4,-3,-2,-1$, cyclophosphamide $60 \mathrm{mg} / \mathrm{kg}$ day -3 , melphalan $60 \mathrm{mg} / \mathrm{m}^{2}$ on days -2 and -1$)$. Mean age of the study population was 54.8 years. There were 14 male and 8 female patients. CMV infection occurred between 8-50 days after auto-HSCT. All of our patients had one CMV infection attack. Number of patients treated with gancyclovir only 12 (55\%), valgancyclovir only 1 (4\%), and gancyclovir+valgancyclovir 9 (41\%). Twenty (90\%) patients have negative CMV DNA levels after antiviral treatment. Two non-Hodgkin's lymphoma patients died because of progressive hematologic diseases while they were also having gancyclovir treatment. Ten patients $(45 \%)$ had concomitant pneumonia (4 of them were fungal), and $3(13 \%)$ patients had systemic bacterial infection.Thirteen (59\%) patients are alive. Reasons of death were $3(14 \%)$ infection/sepsis, 5 (23\%) progressive disease, and 1 (4\%) other reasons.

Conclusions: $\mathrm{CMV}$ is one of the major infectious problems in auto-HSCT, associated with drugs that make severe cell-mediated immunodeficiency. Furthermore, in highly CMV seropositive populations like ours, viral reactivation may be more prevalent.

Conflict of interest: The authors whose names are listed certify that they have no affiliations with or involvement in any organization or entity with any financial interest or nonfinancial interest in this abstract.

\section{P548}

Abstract previously published

\section{P549}

Delayed Epstein-Barr virus (EBV) infection associated hemophagocytic lymphohistiocytosis (HLH) in 2 patients after allogeneic hematopoietic stem cell transplantation (allo-HSCT)

János Fábián ${ }^{1}$, Anikó Barta ${ }^{1}$, Arpád Bátai ${ }^{1}$, Zoltán Csukly ${ }^{1}$, Zita Farkas $^{1}$, László Gopcsa ${ }^{1}$, Lilla Lengyel ${ }^{1}$, Melinda Paksi', Marienn Réti $^{1}$, Éva Torbágyi ${ }^{1}$, Tamás Masszi ${ }^{2}$, Péter Reményi ${ }^{1}$ 
${ }^{1}$ St. Istvan and St. Laszlo Hospital, Department of Hematology and Stem Cell Transplantation, Budapest, Hungary; ${ }^{2}$ Semmelweis University, 3rd Department of Internal Medicine, Budapest, Hungary

Background: Hemophagocytic lymphohistiocytosis syndrome is a potentially life-threatening condition often preceded by EBV infection. EBV-induced HLH after an allo-HSCT in adults is a rarity about which there are only case reports in the literature.

Methods: We report 2 cases of posttransplant, EBVinduced HLH-syndrome which were observed at the same time in our department. The 2 cases share some features: both patients underwent a matched unrelated donor (MUD) allo-HSCT because of myelodysplastic syndrome (MDS) and the HLH-initiating event was a delayed EBV infection; they presented with rapid-onset severe cytopenias and fever.

\section{Results:}

Patient 1 is a 22-year-old woman who underwent a 11/12 MUD allo-HSCT in 2014.

Due to extensive chronic graft-versus-host disease (GVHD) she had received combined immunosuppressive therapy (tacrolimus + ruxolitinib) continuously.

More than 3 years after HSCT, rapidly progressing severe anemia and thrombocytopenia developed. Immunosuppression was stopped. Bone marrow histology showed some dysplastic features but the patient remained full-donor chimera. She became febrile and required hospitalization. Blood tests showed significantly elevated LDH/CRP/Ddimer levels $(830 \mathrm{U} / \mathrm{l}, 170 \mathrm{mg} / \mathrm{l}$ and $>40 \mathrm{ug} / \mathrm{ml}$, respectively) and a high serum EBV viral load (16286/ml). Suspecting posttransplant lymphoproliferative disease (PTLD), R$\mathrm{CHOP}$ therapy was initiated. After the treatment the patient became transfusion-independent, fever ceased and the aforementioned enzyme levels dropped rapidly; EBV copy number fell to zero. 2 weeks later all symptoms recurred. A very high serum ferritin level $(15419 \mathrm{ng} / \mathrm{ml})$ could be detected. The patient fulfilled the criteria of HLHsyndrome. Etoposide $(140 \mathrm{mg} / \mathrm{m} 2$ iv.once weekly) + dexamethasone $(10 \mathrm{mg} / \mathrm{m} 2 / \mathrm{d}$ p.o.) was started immediately. Fever stopped promptly, LDH/CRP/D-dimer levels dropped rapidly again. 4 weeks later etoposide therapy had to be suspended due to myelotoxicity. 6 weeks after starting the treatment the patient is still pancytopenic, but LDH/CRP/Ddimer levels are only markedly elevated $(583 \mathrm{U} / 1,28 \mathrm{mg} / \mathrm{l}$, and $2,97 \mathrm{ug} / \mathrm{ml}$, respectively) and ferritin level decreased significantly $(6636 \mathrm{ng} / \mathrm{ml})$.

Patient 2 is a 66-year-old man who underwent a 11/12 MUD allo-HSCT in 2016.

No GVHD has developed. 10 months after HSCT mixed chimerism, severe anemia and thrombocytopenia could be observed. Bone marrow histology revealed suppressed granulopoiesis, monocytosis, lymphocytosis. A high serum EBV copy number $(6605 / \mathrm{ml})$ could be detected.

With the suspicion of PTLD, R-CHOP therapy was administered. EBV copy number fell to zero, but blood counts had not improved, the patient had persistent fever. A second bone marrow histology showed signs of hemophagocytosis, serum ferritin level was high $(8103 \mathrm{ng} / \mathrm{ml})$. The patient fulfilled the criteria of HLH-syndrome; tacrolimus + methylprednisolone therapy was started. 4 weeks later the patient is afebrile, anemia, thrombocytopenia and even chimerism have improved.

Conclusions: EBV-associated HLH-syndrome can occur even years after an allogeneic ASCT.

Rapid recognition of this rare condition and immediate therapy is essential.

We observed rapid clinical and laboratory improvement in both patients after

initiating the treatment. Unfortunately, the required immunosuppressive therapy is not easily applicable in this highly vulnerable population.

Conflict of interest: nothing to disclose

\section{P550}

Differences between children and adults in the incidence and outcomes of infectious complications after stem cell transplantation: a nationwide cross-sectional study

Jan Styczynski ${ }^{1}$, Krzysztof Czyzewski ${ }^{1}$, Sebastian Giebel ${ }^{2}$, Jowita Fraczkiewicz ${ }^{3}$, Malgorzata Salamonowicz, Krzysztof Kalwak ${ }^{3}$, Mariusz, Wysocki ${ }^{1}$, Agnieszka Piekarska ${ }^{4}$, Alicja Sadowska-Klasa ${ }^{4}$, Slawomira Kyrcz-Krzemien ${ }^{5}$, Olga Zajac-Spychala ${ }^{6}$, Jacek

Wachowiak $^{6}$, Agnieszka Zaucha-Prazmo ${ }^{7}$,Jerzy Kowalczyk, Monika Adamska ${ }^{6}$, Mieczyslaw Komarnicki ${ }^{6}$, Anna WaszczukGajda $^{8}$, Joanna Drozd-Sokolowska ${ }^{8}$, Grzegorz Basak $^{8}$, Joanna Manko , Marek Hus ${ }^{7}$, Agnieszka Wierzbowska ${ }^{9}$, Patrycja MensachGlanowska $^{10}$, Jolanta Gozdzik ${ }^{11}$, Jaroslaw Dybko ${ }^{3}$, Monika Biernat ${ }^{3}$, Piotr Rzepecki ${ }^{12}$, Agnieszka Tomaszewska ${ }^{13}$, Kazimierz Halaburda ${ }^{13}$, Wieslaw Jedrzejczak ${ }^{8}$, Lidia Gil $^{6}$

${ }^{1}$ Collegium Medicum UMK, Bydgoszcz, Poland; ${ }^{2}$ Cancer Center and Institute of Oncology, Gliwice, Poland; ${ }^{3}$ Medical University, Wroclaw, Poland; ${ }^{4}$ Medical University, Gdansk, Poland; ${ }^{5}$ Medical University, Katowice, Poland; ${ }^{6}$ Medical University, Poznan, Poland; ${ }^{7}$ Medical University, Lublin, Poland; ${ }^{8}$ Medical University, Warszawa, Poland; ${ }^{9}$ Medical University, Lodz, Poland; ${ }^{10}$ Jagiellonian University Medical Collage, Krakow, Poland; "11Jagiellonian University Medical College, Krakow, Poland; ${ }^{12}$ Military Institute of Medicine, Warszawa, Poland;

${ }^{13}$ Institute of Hematology and Transfusion Medicine, Warszawa, Poland

Background: Infections are a significant cause of morbidity, mortality, and resource utilization after HSCT in children and adults.

Objective: Comparison of the incidence, type (fungal, viral, bacterial) and outcome of infections in pediatric and 
adult transplant centers (TC) in Poland in cross-sectional study over a period 2012-2015.

Methods: All consecutive patients transplanted in 5/5 pediatric and 11/13 adult TC were included in the retrospective study. Only reports with known etiology of infections were analyzed in the study. Differences between pediatric and adult TC were shown with odds ratio (OR).

Results: Data on follow-up of 650 pediatric and 3200 adult HSCT recipients were analyzed.

Bacterial infections: The incidence was $37.2 \%$ for children and $22.5 \%$ for adult patients $(\mathrm{OR}=2.0, \mathrm{p}<$ $0.0001)$. It was similar for allo-HSCTs $(38.7 \%$ vs $37.5 \%$, ns), while higher in pediatric auto-HSCTs $(33.1 \%$ vs $15.0 \%$; $\mathrm{OR}=2.8, \mathrm{p}<0.0001)$ in comparison to adults. The incidence was comparable in subgroups of patients with ALL, AML/MDS, NHL/HD, and SAA, however only $12.9 \%$ adult myeloma patients after auto-HSCT had bacterial infections. Infection-related mortality (IRM) from bacterial infections was lower in pediatric patients (3.8\% vs $11.1 \%$; $\mathrm{OR}=3.2, \mathrm{p}=0.0011$ ).

Invasive fungal disease (IFD): The incidence was $25.1 \%$ for children and $5.7 \%$ for adult patients $(\mathrm{OR}=5.5, \mathrm{p}<$ $0.001)$. It was higher both for allo-HSCT ( $28.5 \%$ vs $12.3 \%$; $\mathrm{OR}=2.8, \mathrm{p}<0.0001)$ and auto-HSCT $(13.9 \%$ vs $2.3 \%$; $\mathrm{OR}=6.6, \mathrm{p}<0.0001)$ in children, regardless of the level of diagnosis: proven $(4.8 \%$ vs $1.3 \%$; $\mathrm{OR}=3.7, \mathrm{p}<0.0001)$, probable $(6.5 \%$ vs $1.5 \%$; $\mathrm{OR}=4.4, \mathrm{p}<0.0001)$ or possible IFD $(15.1 \%$ vs $2.8 \%$; OR $=6.1, \mathrm{p}<0.0001)$. The incidence was higher both in pediatric patients with ALL (29.4\% vs $11.3 \% ; \mathrm{OR}=3.2, \mathrm{p}<0.0001)$ and AML/MDS $(41.2 \%$ vs $13.2 \%$; $\mathrm{OR}=4.6, \mathrm{p}<0.0001$ ), when compared to adults. IRM from IFD was slightly decreased in pediatric patients ( $14.9 \%$ vs $17.5 \%$, ns), however no significant differences were found with respect to the level of IFD diagnosis: proven $(15.2 \%$ vs $16.3 \%)$, probable ( $25 \%$ vs $32.7 \%$ ) or possible (10.3\% vs $9.9 \%)$.

Viral infections: The incidence was $56.3 \%$ for children and $29.3 \%$ for adults $(\mathrm{OR}=3.1, \mathrm{p}<0.0001)$ after alloHSCT, and $6.6 \%$ vs $0.8 \%(\mathrm{OR}=8.8, \mathrm{p}<0.0001)$ after autoHSCT. The respective incidences were higher in children with following infections: CMV (28.7\% vs $19.3 \%$; $\mathrm{OR}=1.7, \mathrm{p}<0.0001)$, BKV (20.8\% vs $4.7 \%$; OR $=5.2, \mathrm{p}$ $<0.0001)$, $\operatorname{EBV}(20.2 \%$ vs $1.6 \%$; OR=15.7, $p<0.0001)$, ADV (7.2\% vs $2.1 \%$; OR $=3.5, \mathrm{p}<0.0001)$, and influenza ( $2.2 \%$ vs $0.5 \%$; $\mathrm{OR}=4.8, \mathrm{p}=0.0038)$. Viral infections contributed to mortality usually when coexisted with other infections. IRM from viral infections was similar for children and adults, both for all viral infections $(5.7 \%$ vs $5.4 \%, \mathrm{~ns})$, and for each specific infection: CMV (4.9\% vs $5.8 \%, \mathrm{~ns})$, BKV (2.9\% vs $0 \%$, ns), EBV (4.0\% vs $11.7 \%$, ns), $\operatorname{ADV}(5.6 \%$ vs $8.7 \%$, ns), and influenza ( $0 \%$ vs $30 \%$, ns).
Conclusions: In comparison to adults, pediatric patients have a higher incidence of infections. Pediatric patients have a better outcome in bacterial infections, while in fungal and viral infections the IRM was comparable between children and adults.

Conflict of interest: None of the authors has anything to disclose.

\section{P551}

Abstract previously published

\section{P552}

EBV infection, reactivation and post-transplant lymphoproliferative disease in children undergoing allogeneic hematopoietic stem cell transplant

Melanie Finkbeiner ${ }^{1}$, Victor Lewis ${ }^{1}$, Kedra Peterson ${ }^{2}$, Greg Guilcher ${ }^{1}$, Tony Truong ${ }^{1}$

${ }^{1}$ University of Calgary, Alberta Children's Hospital, Calgary, Canada; ${ }^{2}$ University of Calgary, Calgary, Canada

Background: Epstein Barr Virus (EBV) infection and reactivation are common occurrences after allogeneic hematopoietic stem cell transplantation (HSCT). We sought to determine the overall incidence of EBV reactivation and EBV-related Post-Transplant Lymphoproliferative Disease (PTLD) in children.

Methods: Retrospective chart review of children aged 024 years who underwent allogeneic hematopoietic stem cell transplant at Alberta Children's Hospital in Calgary, Alberta, Canada between January 2007 and January 2017.

Results: Over a 10-year period, 162 allogeneic stem cell transplants in 153 patients were performed for malignant and non-malignant conditions. The population was 55\% male with an average age of 8 years. The overall incidence of any non-zero EBV level was $44 \%$. The overall incidence of EBV greater than $20,000 \mathrm{IU} / \mathrm{ml}$ was $24 \%$ with an incidence of PTLD of $7 \%$. As has been found in other studies, the use of anti-thymocyte globulin (ATG) in conditioning was significantly associated with the development of increased EBV titres. EBV reactivation was associated with the presence of an EBV positive donor but the EBV status of the recipient was not a statistically significant factor, nor when there was a mismatch in EBV status between the donor and the recipient. There was a higher risk of EBV reactivation in our population who received peripheral blood stem cells compared to cord blood or bone marrow but the use of cord blood was protective. On multivariate analysis, the use of ATG and the presence of an EBV positive donor remained significant factors. Of the 10 patients who received treatment for EBV, all had 
levels greater than 100,000 IU/ml and all received rituximab as part of their therapy. There was no association between EBV reactivation and the development of graft versus host disease (GVHD), graft failure or death in our population.

Conclusions: EBV reactivation is common in children who have undergone allogeneic HSCT with an overall incidence of greater than $20,000 \mathrm{IU} / \mathrm{ml}$ of $24 \%$. It was statistically associated with the use of ATG, an EBV positive donor and the use of peripheral blood stem cells. Despite a high frequency of any non-zero level of EBV of $44 \%$, the development of clinical symptoms and features of PTLD was uncommon and treatment was initiated at the onset of symptoms in the majority of patients.

Conflict of interest: None of the authors has anything to disclose.

\section{P553}

EBV-related post-transplant lymphoproliferative disease: single center experience and considerations regarding CNS involvement

Anna Vardi, Ioanna Sakellari, ${ }^{1}$, Andriana Lazaridou ${ }^{1}$, Apostolia Papalexandri ${ }^{1}$, Ioannis Batsis ${ }^{1}$, Despina Mallouri ${ }^{1}$, Anastasia Marvaki ${ }^{1}$, Tasoula Touloumenidou ${ }^{1}$, Evangelia Yannaki ${ }^{1}$, Michail Iskas $^{1}$, Varnavas Konstantinou ${ }^{1}$, Christos Smias ${ }^{1}$, Styliani Papaemmanouil $^{2}$, Achilles Anagnostopoulos ${ }^{1}$

${ }^{\text {I}}$ G. Papanicolaou Hospital, Hematology Department - BMT Unit, Thessaloniki, Greece; ${ }^{2}$ G. Papanicolaou Hospital, Pathology Department, Thessaloniki, Greece

Background: EBV-related post-transplant lymphoproliferative disease (EBV-PTLD) is a serious complication of allo-HCT, and antithymocyte globulin (ATG) administration for aGVHD prophylaxis has been identified as the most important predisposing factor.

Methods: We determined the incidence of EBV-PTLD through chart review of 758 consecutive allo-HCT recipients transplanted in our center (7/1990-9/2017) and evaluated factors potentially influencing EBV-PTLD development and outcome.

Results: Among 758 allo-HCT recipients $(\mathrm{n}=451$ sibling, $\mathrm{n}=259$ MUD, $\mathrm{n}=40$ haploidentical, $\mathrm{n}=2$ twin and $\mathrm{n}=6$ cord blood), $13(1.7 \%)$ patients developed EBVPTLD. The diagnosis was confirmed by biopsy in $8 / 12$ cases (6 monomorphic, 1 polymorphic and 1 HL-like PTLD), one case developed sole EBV encephalitis, and the remaining three cases were clinically diagnosed on the basis of generalized lymphadenopathy coupled with high peripheral blood (PB) EBV title. The patients suffered from ALL $(n=6)$, AML $(n=2)$, CML $(n=2)$, severe aplastic anemia $(\mathrm{n}=2)$, and plasmacytic leukemia $(\mathrm{n}=1)$. Notably, $5 / 10$ leukemia patients had relapsed/refractory disease or, in the case of CML, blastic crisis at the time of transplantation. EBV-PTLD incidence was significantly higher in haploidentical versus conventional $(7.5 \%$ versus $1.4 \%, \mathrm{p}=0.004)$ and in MUD versus sibling allo-HCTs $(3.1 \%$ versus $0.4 \%$, $\mathrm{p}=0.004$ ). Of note, $2 / 3$ haploidentical allo-HCTs complicated with EBV-PTLD were T-cell depleted with add-back infusions of transduced donor lymphocytes, and one was Tcell replete. The majority of EBV-PTLD cases (11/13, $84.6 \%$ ) had received rabbit ATG as part of the conditioning regimen for aGVHD prophylaxis, at doses ranging from 5$10 \mathrm{mg} / \mathrm{kg}$ (median $5 \mathrm{mg} / \mathrm{kg}$ ). An additional case had received ATG $20 \mathrm{mg} / \mathrm{kg}$ as second-line treatment for hyperacute GVHD. The conditioning intensity was not found significantly associated with EBV-PTLD occurrence (classic myeloablative in $7 / 13$, reduced-toxicity in $3 / 13$ and reduced-intensity in 3/13 cases). EBV-PTLD occurred early in the post-transplant course (median: 72, range 41-603 days). In 6/13 cases, it was preceded by the onset of aGVHD (median interval from aGVHD diagnosis: 82.5 days), in one case it coincided with aGVHD diagnosis +45 days from allo-HCT, and in one case it developed soon (+55 days) after induction of GVHD through DLIs. Most EBV-PTLD cases presented with generalized lymphadenopathy and fever in 11/13 cases. Clear evidence of concurrent infection existed in $7 / 11$ cases, therefore at least for the remaining 4 cases fever was considered a $\mathrm{B}$ symptom. At EBV-PTLD diagnosis, the median EBV title in PB was 499,000 copies/ml (range 20,000-2,860,000). Nine patients were treated with intravenous rituximab: in six patients the disease resolved, but all cases with CNS involvement $(\mathrm{n}=$ 3) succumbed. The remaining four patients were unsuccessfully treated with combinations of bleomycin/vindesine/ IFNa/immunoglobulins/DLIs.

Conclusions: EBV-PTLD is an early complication of alloHCT, particularly following ATG administration for aGVHD prophylaxis or treatment. The highest incidence of EBV-PTLD in MUD versus sibling alloHCT may be explained by selective ATG administration to the former, however we identified a trend for higher incidence among haploidentical alloHCTs. Prompt treatment with rituximab is very effective, except for CNS involvement. Taking into account the particularly dismal outcome, CNS involvement should be sought for and, if confirmed, intrathecal rituximab should be considered.

Conflict of interest: No conflict of interest

\section{P554}

Efficacy and toxicity of ganciclovir and foscarnet combination therapy for refractory Cytomegalovirus reactivation in adult allogeneic stem cell transplant recipients 
Melanie Kohlschmidt ${ }^{1}$, Maximilian Christopeit ${ }^{1}$, Christine Wolschke $^{1}$, Francis Ayuk ${ }^{1}$, Nicolaus Kröger ${ }^{1}$, Claudia Langebrake ${ }^{1,2}$

${ }^{1}$ University Medical Center Hamburg-Eppendorf, Stem Cell Transplantation, Hamburg, Germany; ${ }^{2}$ University Medical Center Hamburg-Eppendorf, Hospital Pharmacy, Hamburg, Germany

Background: Cytomegalovirus (CMV) reactivation and disease are important complications after allogeneic hematopoietic stem cell transplant (allo-SCT) leading to increased transplant related mortality. In case of treatment failure of monotherapy with either ganciclovir or foscarnet, no standard salvage therapy exists. The combination of ganciclovir and foscarnet seems a promising strategy, but there is few evidence regarding efficacy and toxicity of this regimen.

Methods: We conducted a retrospective chart review including all adult patients with CMV reactivation after allo-SCT who received a combination therapy of ganciclovir and foscarnet both at half of the initial dosage after failure of monotherapy for at least 7 days from October 2010 to September 2017 at the University Medical Center Hamburg-Eppendorf, Germany. Only patients with at least 7 consecutive days of combination were included. Microbiological response was assessed by quantitative Polymerase Chain Reaction (PCR) monitoring of viremia twice weekly. Toxicity was evaluated regarding leukocyte count and glomerular filtration rate (GFR) daily during combination treatment.

Results: 30 patients with 32 episodes of combination treatment were eligible for evaluation. The 32 episodes showed a median onset of viremia at day 37 after transplant (range: day (-3)-146) and the median duration of combination therapy was 12 days (7-36). Combination therapy was started after failure of monotherapy of either ganciclovir (n $=11)$ or foscarnet $(\mathrm{n}=16)$ or both $(\mathrm{n}=5)$. Median follow up after complete PCR response was 31 days (0-288).

27 episodes provided evaluable PCR data with $63.0 \%$ (17/27) showing complete PCR response after a median of 7 days of combination treatment (1-25). Another 29.6\% (8/ 27) showed at least partial response after a median of 10 days (4-23). Patients received different maintenance regimens after combination treatment. Five episodes (29.4\%) of the initial 17 with complete response developed a relapse of viremia after a median of 28 days (11-38). All 32 episodes could be evaluated for toxicity. Evaluation of toxicity was performed until day 22 of treatment as afterwards the sample size became too small $(\mathrm{n}<5)$. Median baseline leukocyte count was $3.0 \times 10^{9} / \mathrm{L}$ (range: $0.4-14.2 \times 10^{9} / \mathrm{L}$ ). Median leukocyte count was stable up to day 11 of treatment $(\mathrm{n}=20)$ (deviation from baseline less than $+/-10 \%)$ and then varied between a deviation of $-34.8 \%$ (day $14 ; \mathrm{n}=13$ ) and $+31.6 \%$ (day $21 ; \mathrm{n}=6$ ). Median baseline GFR was $62.8 \mathrm{ml} / \mathrm{min}(26.8-190.8 \mathrm{ml} /$ min). Median GFR stayed stable until day 9 of treatment (n =24) (median deviation from baseline ranging between $-0.4 \%$ and $+5.7 \%)$, but decreased from day $10 \quad(n=24$; median deviation $-9.8 \%)$ to day 20 of treatment $(\mathrm{n}=7$; median deviation $-40.2 \%$ ).

Conclusions: Combination of ganciclovir and foscarnet at half their initial dosage seems to be an effective strategy to treat CMV reactivation after failure of monotherapy in allogeneic stem cell transplant recipients leading to complete response in almost two thirds of patients in our sample. While there were no major differences in median leukocyte count during combination treatment, there seems to be a relation between the duration of combination therapy and nephrotoxicity, which should be monitored carefully.

Conflict of interest: None of the authors has anything to disclose.

\section{P555}

Efficacy of primary prophylaxis with piperacillintazobactam in lymphoma patients managed at-home after autologous stem cell transplantation: a propensity score analysis

Luis Gerardo Rodríguez-Lobato, David Moreno-Fajardo, Gonzalo Gutiérrez-García, Montserrat Rovira, Carmen Martínez, María Suárez-Lledo, Laura Rosiñol, Sofia Jorge, Anna Gaya, Cristina Gallego, Adelina Hernando, Susana Segura, Alvaro UrbanoIspizua, Francesc Fernández-Avilés

Hospital Clínic de Barcelona, Barcelona, Spain

Background: Infections remain a major cause of morbidity in patients undergoing autologous stem cell transplantation (ASCT). About $80 \%$ of patients experience fever during aplasia and early engraftment despite conventional oral antibacterial prophylaxis. We implement a strategy to reduce the incidence of neutropenic fever (NF) in lymphoma patients managed at-home after ASCT.

Methods: Between January 2002 and June 2017, 143 lymphoma patients were managed at-home since day +1 of ASCT. All were conditioned with BEAM. All patients received prophylaxis with oral quinolone and fluconazole, aerolized pentamidine and low-dose oral acyclovir if seropositive for herpes simplex virus. Sixty-one patients received prophylactic ceftriaxone $(\mathrm{Ct}) 1 \mathrm{~g} /$ day i.v. $(\mathrm{Ly}-\mathrm{Ct})$ and 82 piperacillin-tazobactam (PT) 4.5 g/8h i.v. (Ly-PT) using a portable intermittent infusion pump. First-line therapy at-home of NF was PT $4.5 \mathrm{~g} / 6 \mathrm{~h}$ i.v. (if prophylactic $\mathrm{Ct}$ ) or refrigerated meropenem $1 \mathrm{~g} / 8 \mathrm{~h}$ i.v. (if prophylactic PT), using a portable intermittent infusion pump for both drugs. We used propensity score (PS) analysis with 1:1 matching with the nearest neighbor matching method using calipers of width equal to 0.2 of the standard deviation of 
Table 1. Main patient characteristics and clinical outcones before and after propensity score matching.

\begin{tabular}{|c|c|c|c|c|c|c|}
\hline \multirow[t]{2}{*}{ Onaracteristics } & \multicolumn{3}{|c|}{ Before Matching } & \multicolumn{3}{|c|}{ After Matching } \\
\hline & $\alpha(n-61)$ & PT $(n-32)$ & $\mathbf{P}$ & $\alpha(n=41)$ & PT $(n=41)$ & $P$ \\
\hline Ago brangel & $44(1767)$ & $45\{1971)$ & 0.13 & $47\{2067\}$ & $20(1968)$ & 051 \\
\hline Gonder, malo (x) & 42 (588.9) & $30(51.0)$ & 0.33 & 28 (58.3) & $26,\{6.3,4\}$ & 0,4 \\
\hline $\begin{array}{l}\text { Types of Irmohores (PS] } \\
\text { Hodghin } \\
\text { Follicular } \\
\text { DuRC } \\
\text { Others }\end{array}$ & $\begin{array}{l}31(30.8) \\
8(23.1) \\
9\langle 14.3) \\
13(2.13)\end{array}$ & $\begin{array}{l}28(34.27 \\
4(4.8) \\
21(25.69) \\
29(35.4)\end{array}$ & 0.02 & $\begin{array}{l}17(41.5) \\
4(9.7) \\
7(17.2) \\
13(31.7)\end{array}$ & $\begin{array}{l}17(4.1 .5) \\
3(7.3) \\
14(34.1) \\
7(17.1)\end{array}$ & DSA \\
\hline $\begin{array}{l}\text { Diacaso stage fic } \\
\text { Complete responese } \\
\text { Partial respones } \\
\text { Resiatance }\end{array}$ & $\begin{array}{l}37(30.6) \\
17(27.9) \\
7(113)\end{array}$ & $\begin{array}{l}62(75.5) \\
15(28.3) \\
3(5.1)\end{array}$ & 0.15 & $\begin{array}{l}31\langle 75.6) \\
8(19.3) \\
2(4.9)\end{array}$ & $\begin{array}{c}29(\pi 0.7) \\
10(24.4) \\
2(49)\end{array}$ & 0.92 \\
\hline $\mathrm{HCT} C \mathrm{Cl}>2 \mathrm{AM}$ & $10\langle 15.4\}$ & $37 \mid 45.2\}$ & 0.0003 & $9(220)$ & $8\{193\}$ & 079 \\
\hline CD34+\{range) & $3.4\{1.321 .6\}$ & $3.5\langle 1.6 .17 .6\rangle$ & 0.001 & $3.6(2.221 .6)$ & $25\langle 2.622 .03)$ & 038 \\
\hline Days of ncutrophls $\leq 0.5 \times 10^{6} /$ Mrango) & $9(5.19)$ & 9 (4) 17) & 0.20 & $8(5.19)$ & $8(6.14)$ & 050 \\
\hline WhiO grado 2 ill nucositis irangel & $23\langle 37.7\rangle$ & $13\langle 159|$ & 0.003 & $14\langle 34.1\}$ & $6\langle 14.5\rangle$ & 004 \\
\hline Nesutropenic fever (2382C) (8.) & $51 / 83.6 \mid$ & $35(22.7)$ & $<0.00001$ & $36(87.8)$ & $15\{39\}$ & $<0.0001$ \\
\hline Durstion of tever (rangel & $2(1.11)$ & $2(16)$ & 0.04 & $25(1 \cdot 11)$ & $2\langle 16\rangle$ & 007 \\
\hline Positive blood cutures [s] & $10\langle 16.4\rangle$ & $4(29)$ & 0.02 & $8\langle 29.3\rangle$ & $2(4.9)$ & 0.10 \\
\hline Ricsdmiasions & $9(14.8)$ & $1\{1.2\}$ & 0.002 & $5(14.6)$ & $1(2.4)$ & 0.11 \\
\hline
\end{tabular}

ASCT: autalogsus stem cell transplan tation; Ct: ceftriaxone; DLBC diffuse large B-cell lymphoma; HCT-Qt: hernatopoietic cell trans plantation-comarbid by indec; PT: piperacillin / tazoboctam.

[P555 Figure] [Main patient characteristics and clinical outcomes before and after propensity score matching.]

the logit of the PS. The following factors were included in the PS model: age, gender, type of lymphoma, number of pre-ASCT lines, disease stage, HCT-CI and CD34+ cell dose. After matching the final study population was 41 for each group.

Results: The main characteristics of the patients and outcomes after PS matching are shown in table 1 .

There were no differences between the variables included in the PS model. Regarding outcomes, there were no differences between groups with respect duration of neutropenia, and the first day with fever. The incidence of grades $\geq 2$ mucositis (NCI-CTC-score) was significantly higher in group Ly-Ct (34.1\% vs. $14.6 \%, \mathrm{p}=0.04)$, as well as fever (87.8\% vs. 39\%; p < 0.0001; relative risk (RR): 0.44; CI95\%: 0.3-0.7; Number Needed to Treat (NNT): 2.05). Bacterial infection (Ly-Ct vs. Ly-PT) was documented in $19.5 \%$ vs. $4.9 \%(\mathrm{p}=0.1)$, and coagulase-negative Staphylococci was the more frequent bacteria isolated. Readmission was required in $6(14.6 \%)$ patients in group Ly-Ct and $1(2.4 \%)$ in Ly-PT $(p=0.1)$. In the multivariate binary logistic regression model, PT retained its independent protective factor (OR: $0.13 ; \mathrm{P}<0.002$ ) and mucositis $\geq$ 2 as risk factor $(\mathrm{OR}: 1.93 ; \mathrm{P}=0.035)$ for $\mathrm{NF}$.

Conclusions: The use of piperacillin-tazobactam prophylaxis in an at-home ASCT program for lymphoma patients allows us to significantly reduce the incidence of neutropenic fever and minimizes the readmission rate.

Conflict of interest: The authors declare no disclosures.

\section{P556}

\section{Emerging pathogen: Candida kefyr (Kluvyeromyces marxianus) in pediatric hematopoietic stem cell transplant recipients}

Ebru Yilmaz ${ }^{1}$, Arda Erdogmus ${ }^{1,2}$, Gulsah Ucan ${ }^{1}$, Altay Atalay ${ }^{2}$, Alper Ozcan ${ }^{1}$, Turkan Patıroglu ${ }^{1}$, Musa Karakukcu ${ }^{1}$, Ekrem Unal ${ }^{1}$

${ }^{1}$ Erciyes University Faculty of Medicine, Department of Pediatrics, Division of Pediatric Hematology Oncology \& Hematopoietic Stem Cell Transplant Unit, Kayseri, Turkey; ${ }^{2}$ Erciyes University Faculty of Medicine, Department of Microbiology, Division of Mycology, Kayseri, Turkey

Background: Candida kefyr, (formerly Candida pseudotropicalis), currently recognized as Kluyveromyces marxianus is topic of interest with an increasing incidence, especially among immune compromised patients with hematological disorders. Higher incidence of colonization up to $8.7 \%$ in patients with acute myelogenous leukemia receiving induction chemotherapy were reported. It was also underlined that the 30-day mortality rate caused by $C$. kefyr is higher than that of $C$. albicans. In addition, $C$. kefyr was also reported as a remarkable agent in catheter-related infections with a four-fold higher adhering affinity to polystyrene surfaces compared to C. albicans.

Methods: We aimed to report our experience of C. kefyr infection in two children who underwent haploidentical and autologous hematopoietic stem cell transplantation (HSCT).

Results: 
Patient 1: 2.5-year old girl with primitive neuroectodermal tumor of lung underwent autologous HSCT with a regimen of busulfan/melphalan according to the EUROEwing 99 protocol. +30 days after HSCT she was hospitalized with fever. The physical examination was unremarkable. C. kefyr grew from catheter culture. She was treated with GCSF, 3 doses of granulocyte suspension for neutropenia, liposomal amphotericin B for 10 days with wide spectrum antibiotics.

Patient 2: 11-months old boy with infant leukemia underwent $\mathrm{T}$ cell receptor alfa beta depleted haploidentical HSCT from his mother. +62 days after HSCT, he was hospitalized for bloody diarrhea. C. kefyr grew from the stool culture. He was treated with fluconazole.

Conclusions: The transplant physicians should be vigilant about the increasing incidence of Candida keyfr as an emerging yeast. In addition, the bloody diarrhea related to C. kefyr after HSCT may mimic gastrointestinal graft versus host disease, which may cause a diagnostic problem who are not familiar to this yeast.

Conflict of interest: All of the co-authors declare nothing to disclose.

\section{P557}

Escalation and de-escalation regimens mandatory for individualized treatment against bacterial infections in stem cell recipients - single center experience

Zlate Stojanoski, Borce Georgievski, Aleksandra PivkovaVeljanovska, Irina Panovska-Stavridis, Lidija Cevreska, Martin Ivanovski, Oliver Karanfilski, Lazar Cadievski, Marija Popova, Bozidar Kocovski, Svetlana Stankovik

University Clinic for Hematology, Skopje, Macedonia, the Former Yugoslav Republic of

Background: Multidrug resistant (MDR) bacteria are increasingly frequent in HSCT recipients, but significant differences in etiology of bacterial infections and prevalence of resistant strains exist between different transplant centers Extended-spectrum beta-lactamase producing Escherichia coli, Vancomicyn resistant enterococci, Penicillin-resistant Pneumococci Methicillin-resistant Staphylococcus aureus and are major problem to manage.

Aim: To evaluate the frequency of multidrug resistant bacteria in our center. Our clinic is a part of CAESAR network for multidrug resistant bacterial isolates in Europa.

Methods: During a 17 years period we have transplanted 440 patients with different hematological malignant and nonmalignant diseases. All patients were treated in sterile room conditioned with HEPA filters and low bacterial diet. Antibacterial prophylaxis consisted Ciprofloxacine 1,0gr/ day. As empirical antibiotics regimen we administered combination of third-generation cephalosporin + amynoglicoside, while as second line therapy Imipenem + Vancomycin.

Results: In every patient 3 times a week we monitor: blood culture, central venous catheter culture, sputum, urine culture. From all 288 bacterial isolates the distribution of MDR bacteria were: ESBL positive Escherichia coli 3 $(0,10 \%)$ VR Enterococcus $2(0,06 \%)$ MDR Pseudomonas aer. $1(0,03 \%)$ Klebsiella aerogenes $2(0,06 \%)$ MRSA 17 $(0,06 \%)$ Acinetobacter baumanii $2(0,06 \%)$ Stenotrophomonas Maltophilia 2(0,06\%). There were fatal outcome due to sepsis in 3 patients (VRE 1, MDR Pseudomonas 1, Stenotrophomonas maltophilia 1).

Conclusions: The monitoring of local microflora is crucial for every transplant center. Empirical first-line therapy must be individualized and according to ECIL 4 escalation and de-escalation strategy is necessery for better outcome in this group of immunocompromised patients.

Conflict of interest: Nothing to declare

\section{P558}

Hepatitis B reactivation after hematopoietic stem cell tranplantation: incidence, risk factors and prophylaxis in $\mathrm{HBCAb}+$ and/orHBsAb+ recipients in a single transplant centre

Lucia Amico ${ }^{1}$, Franca Falzetti ${ }^{1}$, Adelmo Terenzi ${ }^{1}$, Rita Felicini ${ }^{1}$, Loredana Ruggeri ${ }^{1}$, Antonio Pierini, ${ }^{1}$, Sara Tricarico ${ }^{1}$, Genni Casarola $^{1}$, Francesco Di Candilo ${ }^{2}$, Franco Baldelli ${ }^{2}$, Andrea Velardi $^{1}$, Alessandra Carotti ${ }^{1}$

${ }^{1}$ Division of Hematology and HSCT Programme, University of Perugia, Perugia, Italy; ${ }^{2}$ Division of Infectious Disease, University of Perugia, Perugia, Italy

Background: After hematopoietic stem cell transplantation (HSCT) Hepatitis B virus (HBV) reactivation is of particular interest as the infection is very common worldwide. In fact over 2 billion people are infected, with HBV causing chronic infection in over 350 million. HBV reactivation after HSCT was reported in recipients with evidence of resolved HBV infection with negative HBV surface antigen, positive hepatitis B core antibody, $(\mathrm{HBcAb})+,/$-positive hepatitis surface antibody (HBsAb) or isolatedHBsAg, in the absence of vaccination before transplantation. This single-centre retrospective study investigated the incidence, risk factors and timing of HBV reactivation in patients with a history of resolved infection or isolated HBsAb who underwent allogeneic HSCT

Methods: From January 2012 to May 2017, 171 allogeneic HSCTrecipients were enrolled.Neoplastic disease was present in 169/171 and Fanconi anemiain 2/171.62 patients received matched sibling and unrelated donor 
transplants, $10 \mathrm{~T}$ cell depleted haplo-HSCT, 84haplo-HSCT with regulatory and conventional $\mathrm{T}$ cell adoptive immunotherapy (T-reghaplo-HSCT) and 15 unmanipulatedhaploHSCT with post-transplant cyclophosphamide. Conditioning regimens included high-dose chemotherapy with or without TBI. No post-transplant pharmacological immunosoppressionwas given to most recipients. Before transplantation, all donorsand HSCT candidates were routinely tested for $\mathrm{HbsAg}, \mathrm{HBcAb}$ and $\mathrm{HBsAb}$. All recipients were also tested for serum HBV DNA, asdetermined by polymerase chain reaction (Versant 1.0 K PCR).Evidence of HBV reactivation was the development of positive $\mathrm{HBsAg}$ after transplant and changes in serum levels of HBV DNA.

Results: At risk of HBV reactivation were 20/171 transplant recipients as $11 / 20$ presented evidence of resolved HBV infection ( $\mathrm{HBcAb}$ positive $+/$-HbsAb) and 9/20 had isolated HBsAb. None was HBsAgpositive before transplant.7/171 donors wereHBcAb positive $+/$ - HbsAb. HBV reactivation occurred in 6/20 (30\%)transplant recipients at risk with a median onset of 2.3 years after HSCT. Antiviral prophylaxix with lamivudine (LMV) was administered to 5/20 patients at risk and none reactivated (T test $\mathrm{P}<0,001)$. All 6 patients who reactivated had received haplo-HSCT(5T-reg haplo-HSCT and 1 unmanipulated haplo-HSCT). None developed acute or chronic graft-vshost disease or received immunosoppressive therapy. No HBV-related death occurred in patients who reactivated. None developed acute hepatitis; all were treated with entecavir.

Conclusions: HBV infection is a major clinical issue in immunocompromised patients including allo-HSCT recipients, considering the number of infected patients is increasing. The present study demonstrated that HBVreactivation is a late but frequent complication after HSCT among patients with serological evidence of resolved or occult HBV infection and confirmed LMV prophylaxis protected against $\mathrm{HBV}$ reactivation.

Conflict of interest: None

\section{P559}

\section{Herpes zoster after autologous hematopoietic stem cell transplantation without antiviral prophylaxis}

\section{Akihito Shinohara, Satoko Osanai, Yuki Izuka, Kentaro Yoshinaga, Masayuki Shiseki, Junji Tanaka}

Tokyo Women's Medical University, Hematology, Tokyo, Japan

Background: Herpes simplex virus (HSV) and varicella zoster virus (VZV) diseases are common in autologous hematopoietic stem cell transplantation (auto-HSCT). While some reports showed the efficacy of prophylactic antiviral drugs during and after it, the routine use of the prophylaxis remains controversial.

Methods: In this single center retrospective study, we surveyed patients who received auto-HSCT at Tokyo Women's Medical University Hospital between 2005 and 2014. The patients with lymphomas received modified BEAM regimens (ranimustine, etoposide, cytarabine, melphaln), and those with plasma cell disorder (PCD) received high-dose melphalan as conditioning regimens. They received no antiviral prophylaxis during and after HSCT. The diagnosis of herpes zoster was made based on the presence of characteristic vesicular skin lesions, and was confirmed by antibody-to-membrane antigen testing in most cases. This study was performed in accordance with the Helsinki Declaration and approved by the Ethics Committee of Tokyo Women's Medical University.

Results: A total of 72 patients were analyzed. The median age of them was 56 years old, and the median follow up for survivors was 75 months. They consisted of 41 lymphoma patients, 31 patients with PCD. Ten-year overall survival rate in lymphoma patients was $75.6 \%$, and that in $\mathrm{PCD}$ patients was $45.5 \%$. Total 19 of 72 patients experienced herpes zoster during a year after auto-HSCT, and one-year cumulative incidence $(1 \mathrm{yCI})$ of herpes zoster was $26.4 \%$. Five of 19 patients (26.3\%) developed disseminated disease. Among them, a patient experienced it 7 days after auto-HSCT, before neutrophil engraftment. In contrast, early intervention with antiviral drugs ameliorated the disease, and there were neither fatal case nor visceral infection. Additionally, four of 19 patients experienced both HSV disease and herpes zoster. Number of chemotherapy cycles (above 7) before auto-HSCT had an increased risk of $1 \mathrm{yCI}$ of herpes zoster (hazard ratio 3.05, 95\% confidential interval 1.18 to $7.90, \mathrm{P}=0.02$ ). Furthermore, all four patients with past history of herpes zoster experienced it again, and two of them developed disseminated disease.

Conclusions: Cumulative incidence of herpes zoster after auto-HSCT without antiviral prophylaxis was higher than that in previously reported cases with long-term antiviral prophylaxis. Prophylactic antiviral drugs early after autoHSCT is recommended, because there were some disseminated herpes zoster cases, or the case before engraftment, which excess the risk for outbreak and fatal infection. 1yCI in patients with high number of chemotherapy cycles and with past history of it were $40.6 \%$ and $100 \%$, respectively, which were significant risk factor for herpes zoster after auto-HSCT. Long-term antiviral prophylaxis may be effective for the patients with those risk factors.

Conflict of interest: This work was supported by JPSS KAKENHI Grant Number 26463337. 


\section{P560}

IgM-enriched immunoglobulins significantly improve severe sepsis outcome in neutropenic hematopoietic stem cell transplantation recipients

\section{Alessandra Forcina ${ }^{1}$, Anna Maria Mazzone ${ }^{2}$, Giulia Palazzo ${ }^{2}$, Fabio} Ciceri $^{1}$, Giovanni Pisapia ${ }^{2}$

${ }^{1}$ IRCCS San Raffaele Hospital, Hematology and Bone Marrow Transplant Unit, Milano, Italy; ${ }^{2}$ San Giuseppe Moscati Hospital, Hematology and Bone Marrow Transplant Unit, Taranto, Italy

Background: Mortality due to severe bacterial sepsis (SS) occurring during the post-transplant aplasia remains unacceptably high, ranging from $40-75 \%$. This is especially true in case of multi-drug resistant bacteria or when firstline antimicrobial therapy is inadequate, suggesting the opportunity of an immune-intervention approach to synergize with standard treatments for reducing infection-related mortality (IRM). The aim of the study was to explore whether the addition of IgM-enriched immunoglobulins (IgM-IGIV) to standard antimicrobial therapy has the potential to reduce IRM and improve overall survival (OS) at 120 days after the infection onset.

Methods: A total of 28 adult patients developing SS according to standard criteria were retrospectively evaluated (April 2014-November 2017). All patients received IgMIGIV $5 \mathrm{ml} / \mathrm{kg} / \mathrm{d}$ for 3 consecutive days as adjunctive firstline therapy to antimicrobial agents. Kaplan-Meier curves were used to estimate OS. Disease recurrence, GvHD and death from any other cause were competing risks for IRM. Univariate comparisons of survival curves were made using the Log-rank test, while the Gray's test was used for univariate comparisons of $\mathrm{CI}$ functions.

Results: Sixteen out of $28(57.1 \%)$ patients received autologous HSCT (autoHSCT), 6/28 (21.4\%) allogeneic HSCT (alloHSCT) and 6/28 (21.4\%) chemotherapy alone (CT). Median follow-up from infection onset was 327 days (range 2-953). The majority of patients was diagnosed with acute leukemia $(14 / 28,50 \%)$, had active disease $(18 / 28$, $64.2 \%$ ) at SS onset and was beyond the first-line therapy. Nine out of $23(39.1 \%)$ carried Carbapenem-resistant Klebsiella Pneumoniae (KPC). All patients had grade IV neutropenia. Clinically-documented SS were mostly due to KPC $(\mathrm{n}=11,44 \%)$ followed by Escherichia Coli $(\mathrm{n}=3$, 12\%), Pseudomonas Aeruginosa (PA) ( $\mathrm{n}=2,8 \%)$, Acinetobacter baumanii $(\mathrm{n}=1,4 \%)$, Gram-positive bacteria ( $\mathrm{n}$ $=8,32 \%)$ or no isolate $(\mathrm{n}=3,12 \%)$. First-line antimicrobial therapy was considered inadequate in $89.3 \%$ of patients and despite this only $17.8 \%$ (5/28) progressed to septic shock when IgM-IGIV were used. Survival at 120 days from SS onset was $74.4 \%$ overall $(93.3 \%$ for autoHSCT, $66.6 \%$ for alloHSCT and $22.2 \%$ for CT). In the entire cohort, 120-day survival from SS onset according to pathogen was: $100 \%$ in case of no isolate, $85.7 \%$ for Gram-positive, $80.8 \%$ for KPC and $33.3 \%$ for other Gramnegative bacteria. Overall survival at 120 day after transplant was similar comparing autoHSCT to alloHSCT (93.3\% vs $83.3 \% \mathrm{P}=0.13$ ), regardless the prevalence of SS caused from KPC and PA in the alloHSCT group (66.6\% vs $37.5 \%$ ). Interestingly, no deaths were registered after KPCrelated SS in HSCT patients $(\mathrm{n}=8)$ when IgM-IGIV were used as adjunctive therapy. IRM was $0 \%$ both for auto- and allo-HSCT at 120-day and of $0 \%$ and $20 \%$ at 1-yr, respectively.

Conclusions: The early addition of IgM-IGIV to antimicrobial therapy in HSCT neutropenic patients developing SS could ameliorate OS and reduce infection-related mortality thus improving the overall transplant outcome. This is to our knowledge the largest study investigating the role of IgM-IGIV in HSCT patients, which certainly warrant further confirmation in a prospective, wider cohort of patients.

Clinical Trial Registry: not applicable

Conflict of interest: A. Forcina has Biotest Consultancy.

\section{P561}

Impact of Human Herpes Virus (HHV)-6 infection in manipulated haploidentical stem cell transplantation in children

Katia Perruccio ${ }^{1}$, Luisa Sisinni ${ }^{2}$, Antonio Perez-Martinez ${ }^{3}$, Ilaria Capolsini $^{1}$, Maria Speranza Massei ${ }^{1}$, Maurizio Caniglia ${ }^{1}$, Katherine N. Ward $^{4}$, Simone Cesaro 5

${ }^{1}$ Santa Maria della Misericordia Hospital, Pediatric Oncology Hematology, Perugia, Italy; ${ }^{2}$ Santa Creu Hospital, Pediatric Hematology, Sant Pau, Spain; ${ }^{3}$ La Paz Hospital, Pediatric Hematology, Madrid, Spain; ${ }^{4}$ University College Medical School, Division of Infection and Immunity, London, United Kingdom;

${ }^{5}$ University of Verona, Pediatric Oncology Hematology, Verona, Italy

Background: Human Herpes Virus (HHV)-6 infection is increasingly recognized among allogeneic hematopietic stem cell transplantation (HSCT) recipients, 30\% being the risk of reactivation in high-risk patients. Although HHV-6 infection is transient and self-limiting, it has been sometimes associated with encephalitis, acute Graft-versusHost Disease (aGVHD) and graft failure. Here we report two cohorts of pediatric manipulated HSCT in which HHV6 reactivation and disease represented the main early posttransplant infectious complication.

Methods: The two cohorts were recruited in the pediatric (median age 13 years) Transplant Centers of Perugia $(\mathrm{n}=$ 12), Barcelona $(n=9)$ and Madrid $(n=15)$. Patients with acute leukemia received myeloablative conditioning regimens with TBI in 19 and TLI in 16. Ex-vivo graft 
manipulation was CD34+ selection and Treg/Tcon infusion in 12 patients (Martelli MF et al.Blood 2014;124:638-44) and CD45RA T-cell depletion in 24 patients. Median CD34 + cell content was $11.5 \times 10 \mathrm{e} 6 / \mathrm{Kg}$ and $6.75 \times 10 \mathrm{e} 6 / \mathrm{Kg}$, respectively. Antiviral prophylaxis was Aciclovir in 32 or Foscarnet in 4 patients. DNAemia for CMV, HSV-1, HHV6 and Adenovirus were checked by quantitative or qualitative PCR twice a week from conditioning regimen start until CD4+T-cell recovery (>200/ml). HHV-6 reactivation and encephalitis were defined as ECIL 7 guidelines (www.ecil-leukemia.com).

Results: All patients engrafted at a median of 15 and 11 days, respectively, and reactivated HHV-6 at median of 9 days from infusion (range: 5-16); median viral load in blood was 60,000 copies/ml (range: 10,000-120,000). Eight patients $(25 \%)$ developed limbic encephalitis with HHV-6 in CNS fluid. They received antiviral treatment with Foscarnet $90 \mathrm{mg} / \mathrm{Kg}$ twice a day $(\mathrm{n}=3)$, Ganciclovir 5 $\mathrm{mg} / \mathrm{Kg}$ twice a day $(\mathrm{n}=1)$, or Foscarnet plus Ganciclovir $(n=4)$ for 14 days (range: 14-21). All patients responded to treatment with symptoms improvement, and negativization of DNAemia in blood and in the CNS. None died, even if three patients experienced long-term neurological sequelae (37.5\%). Moreover, we suspected symptomatic HHV-6 organ disease in 5 patients with different localizations: liver, lung, stomach, and multi-organ in 2 (encephalitis, pneumonia, gastritis; pneumonia and enteritis). Infection was documented in organ samples (broncoalveolar lavage, and liver, gastric or bowel biopsies), in which DNAemia was higher than in blood. Patients responded to antiviral treatment with Foscarnet $90 \mathrm{mg}(\mathrm{n}=2)$ or $60 \mathrm{mg} / \mathrm{Kg}(\mathrm{n}$ $=3$ ) twice a day. HHV-6 infection was associated to grade $>2$ aGvHD in 2 patients ( 1 in the gut, 1 in the liver) and 1 graft failure. No differences in term of efficacy were observed between 2 Foscarnet dosages, but the lowest dose was associated to less toxicity. No differences in terms of immunological reconstitution were observed between patients who developed HHV-6 disease and patients who did not.

Conclusions: HHV-6 infection is the most frequent viral complication in pediatric patients undergoing haploidentical HSCT. Despite the absence of lethal cases and good response to current antiviral drugs, it determined prolonged hospitalization for significant morbidity, organ disease, aGvHD and graft failure occurrence, and treatment could be associated with toxicity. Moreover, the central nervous system involvement may cause neurological long-term sequelae. We suggest that HHV-6 DNAemia monitoring is useful in these transplant settings in order to diagnose and treat early or pre-emptively HHV-6 infection.

Conflict of interest: K. Perruccio: nothing to disclose
P562

Impact of Procalcitonin and C-Reactive Protein in the diagnosis of infectious complications after allogeneic hematopoietic stem cell transplantation

Sara Butera ${ }^{1,2}$, Andrea Evangelista ${ }^{3}$, Luisa Giaccone $^{1,2}$, Chiara Maria Dellacasa ${ }^{2}$, Sara Vassallo ${ }^{1,2}$, Lucia Brunello ${ }^{1,2}$, Semra Aydin ${ }^{2}$, Giovannino Ciccone ${ }^{3}$, Giulio Mengozzi ${ }^{4}$, Alessandro Busca ${ }^{2}$, Benedetto Bruno ${ }^{1,2}$

${ }^{1}$ University of Torino, Department of Molecular Biotechnology and Health Sciences, Torino, Italy; ${ }^{2}$ SSD Trapianto Allogenico di Cellule Staminali, A.O.U. Città della Salute e della Scienza di Torino,

Department of Oncology, Torino, Italy; ${ }^{3}$ A.O.U. Città della Salute e della Scienza di Torino, Clinical Epidemiology, Torino, Italy; ${ }^{4}$ S.C. Baldi e Riberi, A.O.U. Città della Salute e della Scienza di Torino, Department of Clinical Chemistry, Torino, Italy

Background: C-reactive protein (CRP) and procalcitonin (PCT) have been proposed as potential biomarkers for differential diagnosis of inflammatory complications, bacterial and non-bacterial infections in patients with hematological malignancies. The purpose of this study was to determine the potential role of PCT and CRP testing to differentiate febrile complications in recipients of an allograft during the first 100 days after transplant.

Methods: Four-hundred-forty-five patients underwent an allograft between January 2007 and December 2014 at our Center. Simultaneous determination of PCT and CRP was performed on 250 patients who were included in the current analysis. Donors were HLA-identical siblings (no. 77); haploidentical (no. 36); or unrelated (no. 137). A total of 1900 simultaneous PCT and CRP determinations from day 0 to day +100 were performed during febrile episodes. PCT and CRP serum values were defined as positive for values of $\geq 2.0 \mathrm{ng} / \mathrm{mL}$ and $\geq 5.0 \mathrm{mg} / \mathrm{L}$ respectively. All data were retrospectively retrieved from the computerised database of the Department of Clinical Chemistry. Standard fever workup also included blood cultures, chest $\mathrm{X}$ ray, blood chemistry and blood counts. Simultaneous CRP and PCT levels were used for defining 3 study groups: both levels of PCT and CRP positive; negative PCT and positive CRP; both PCT and CRP negative. Correlation between PCT and CRP levels, and clinical complications were evaluated by marginal effects (predicted probability) according to multivariate multinomial logistic models.

Results: Overall, only $10.26 \%$ of PCT values were positive as compared with $92.42 \%$ positive CRP values. Most PCT/CRP determinations were done in the first 35 days after transplant (median day: +15 ). There was a constant increase of the simultaneous determination of PCT/ CRP from 2007 throughout the study period. Determination groups were categorized as follows: both negative PCT/ CRP: 144 (7.58\%); negative PCT/positive CRP: 1561 
(82.16\%); both positive PCT/CRP 195 (10.26\%). No determination showed positive PCT and negative CRP. At onset of documented gram-neg infections, positive PCT/ CRP values were seen in $43.1 \%$, highly significant when compared with patients with no documented infections ( $\mathrm{p}<$ 0.0001) In patients with documented invasive fungal infections the probability of having discordant values (PCT-negative/CRP positive) was $83.5 \%$ while only $8.7 \%$ showed both positive values. In patients developing cytokine release syndrome (CRS) on day 0 through 4 after haplo-identical allografts, the probability of positive PCT levels was only $10.1 \%$ whereas CRP was almost invariably high $(86 \%)$. No statistically significant difference between patients with and without CRS was reported $(p=0.85)$. The probability of positive PCT/CRP during anti-thymocyte globulin (ATG) treatment as graft-vs.-host disease prophylaxis was high $(55.1 \%)$ and significantly higher than those who did not receive ATG $(<0.0001)$.

Conclusions: Despite the limitations of the retrospective design of our study, simultaneous PCT/CRP determination was helpful in the differential diagnosis of different complications after allografting. Our findings form the basis for the investigation of a specific diagnostic algorithm that includes PCT/CRP determination to monitor inflammatory and infectious complications after allografting.

Conflict of interest: Nothing to disclose.

\section{P563}

Incidence and epidemiology of invasive fungal diseases in patients after allogeneic hematopoietic stem cell transplantion receiving antifungal prophylaxis: a five years single-center experience

\section{Julia Moehrke, Eva Maria Wagner-Drouet, Matthias Theobald, Markus Philipp Radsak, Daniel Teschner}

University Medical Center of the Johannes Gutenberg University, Department of Hematology, Medical Oncology, \& Pneumology, Mainz, Germany

Background: Invasive fungal diseases (IFD) still represent a major threat to immunocompromised patients especially after allogeneic hematopoietic stem cell transplantion (HSCT). Despite that fact, there are only limited data available regarding incidence and epidemiology of IFD after HSCT in the era of antifungal prophylaxis. Therefore, the aim of our study is to analyze IFD in our local HSCT patients to ensure the adequacy of our antifungal management.

Methods: Patients treated by allogeneic HSCT between January 2011 and December 2015 were retrospectively analyzed regarding their patient characteristics like age, gender underlying disease, disease status, type of transplant, and overall survival (OS). Additionally, patients suspected to have IFD were investigated regarding diagnostic procedures, type of IFD, IFD-related mortality, and others.

Results: Between January 2011 and December 2015410 patients underwent allogeneic HSCT in our center. Patients were mainly male with a median age of 51 years (range 1775). Most patients suffer from an acute myeloid leukemia (AML; 208/410) or a myelodysplastic syndrome (MDS; 47/ 410) prior to HSCT. Patients were treated mainly using a reduced intensity conditioning (RIC) regiment (270/410) with an unrelated matched donor (MUD) serving as stem cell source. All patients received prophylactic or prolonged therapeutic antifungal broad-spectrum medication (mainly posaconazole). 70 patients were found to have a possible IFD according to the revised European Organization for the Research and Treatment of Cancer/Mycoses Study Group (EORTC/MSG) criteria. 27 patients presented with probable IFD, whereas in 8 patients a fungal pathogen could be isolated representing a proven IFD. Detected isolates were Candida spp. (6/8), and Aspergillus spp. (2/8). Patients with probable/proven IFD (35/410; incidence of $8,5 \%)$ had a median age of 51 years (range 20-69), mainly suffered from AML (16/35), and mainly underwent RIC allogeneic HSCT with a MUD. Invasive pulmonary disease was the most common site of probable/proven fungal infection. Transplant recipients with proven/probable IFD had to be treated more often on intensive care unit (ICU) indicating a higher morbidity in this population by trend (17\% [IFD] vs. $13 \%$ [non-IFD], $\mathrm{p}=0.5)$. In univariate analysis progressive underlying disease prior to transplant $(8.6 \%$ [IFD] vs. $1.9 \%$ [non-IFD], $\mathrm{p}=0.59)$ was associated with higher incidence of probable/proven IFD by trend. However, patients receiving posaconazole (POS) as antifungal agent were less frequently diagnosed for IFD compared to nonPOS treated transplant recipients (POS: 54\% [IFD] vs. $80 \%$ [non-IFD], $\mathrm{p}=0.39$ ) One-year OS in IFD-patients was $63 \%$ which was lower by trend than in the non-IFD population $(74 \%, \mathrm{p}=0.4)$.

Conclusions: Even in presence of broad-spectrum antifungal medication a significant percentage of our patients after allogeneic HSCT were suspected to have an IFD resulting in higher morbitiy and mortality. Presence of refractory malignant disease was associated with IFD as shown before. However, posaconazole medication seems to be more protective than other antifungal agents as posaconazole treated transplant recipients showed less frequent probable/proven IFD. Although, the relatively high incidence of possible IFD indicates a lack of sufficient diagnostic workup, which has to be addressed in the future to optimize antifungal management in our patients cohort.

Conflict of interest:

J. Moehrke, E. Wagner-Drouet, M. Theobald: nothing to disclose 
M. Radsak: honoraria, consultant and travel grant from Celgene and Novartis. Travel grant from Daiichi Sankyo, Astellas and Gilead

D. Teschner: honoraria and travel grant from Gilead and MSD. Honoraria from Pfizer. Travel grant from Astellas and Jazz. Consultant of advisory board for MSD, Gilead and Pfizer

\section{P564}

Increased Incidence of Hypogammaglobulinemia in Patients with Acute Graft-versus-Host Disease after Allogeneic stem cell transplantation - A single center retrospective analysis

Wasithep Limvorapitak ${ }^{1,2}$, Yasser Abou Mourad ${ }^{1}$, Michael Barnett, Raewyn Broady, Donna Forrest ${ }^{1}$, Alina Gerrie ${ }^{1}$, Donna Hogge ${ }^{1}$, Stephen Nantel ${ }^{1}$, Sujaatha Narayanan ${ }^{1}$, Thomas Nevill ${ }^{1}$, David Sanford $^{1}$, Kevin Song ${ }^{1}$, Heather Sutherland ${ }^{1}$, Cynthia Toze ${ }^{1}$, Jennifer White ${ }^{1}$, Maryse Power ${ }^{1}$

${ }^{1}$ BC Cancer, Leukemia/ Bone Marrow Transplant Program of British Columbia, Vancouver, Canada; ${ }^{2}$ Thammasat University, Div. of Hematology, Dept. of Internal Medicine, Pathumthani, Thailand

Background: Hypogammaglobulinemia (HGG) is a common complication after allogeneic stem cell transplantation (alloSCT). Practices vary across centers with respect to immunoglobulin (Ig) level monitoring and replacement strategies. This study aims to identify risk factors for HGG and the effect of intravenous immunoglobulin (IVIg) replacement on alloSCT outcomes in a cohort of adult acute leukemias and myelodysplastic syndrome (MDS) patients.

Methods: We analyzed risk factors for HGG on day +100 in patients who underwent alloSCT for acute myeloid leukemia (AML), MDS or acute lymphoblastic leukemia (ALL) during 2013-2016. Patients were categorized into: normal Ig level $(\operatorname{IgG}>6.0 \mathrm{~g} / \mathrm{L})$, mild HGG $(\operatorname{IgG} 4.1-6.0 \mathrm{~g} /$ L) and severe HGG $(\operatorname{IgG}<4.0 \mathrm{~g} / \mathrm{L})$. Risk factors for developing HGG were analyzed using multivariable ordinal logistic regression. Overall survival (OS), event-free survival (EFS), and incidences of infection were compared.

Results: We identified 202 patients who underwent alloSCT for MDS (36), AML (111) and ALL (55) over a three-year period, in whom $\operatorname{IgG}$ levels at day +100 were available. A myeloablative regimen was used in 172 cases $(85.2 \%)$ and reduced-intensity 30 cases (14.9\%). Donors were: siblings 80 cases (39.6\%), unrelated-donor 101 cases $(50.0 \%)$, cord blood 15 cases $(7.4 \%)$ and haploidentical 6 cases $(3.0 \%)$. Ig levels on D+100 were: normal 76 cases (37.6\%), mild HGG 82 cases (40.6\%) and severe HGG 44 cases $(21.8 \%)$. After adjusting for disease status prealloSCT, alloSCT type, receipt of $>80 \%$ methotrexate,

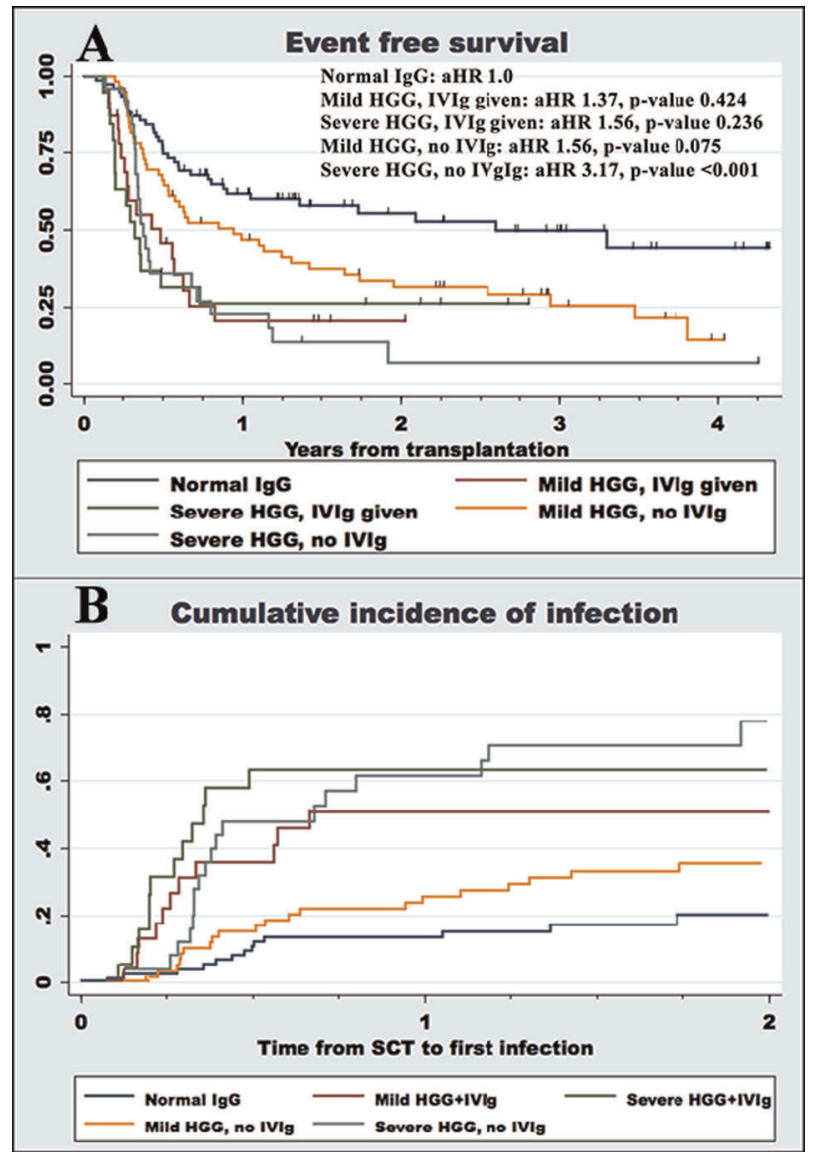

[P564 Figure] [Event free survival between HGG groups (A) and cumulative incidences of infection $(B)]$

donor type, and prednisone dose on day +100 , acute graftversus-host disease (aGVHD) [grade 1-2 Odds ratio (OR) $3.25, \mathrm{p}=0.001$, grade 3-4 OR 8.24, $<<0.001$ ] predicted for HGG, while Cytomegalovirus (CMV) positive donor (D + ) mitigated the risk of $\mathrm{HGG}$ compared to CMV negative pair (D-/R-) as followed: D+/R- OR 0.22, p =0.013, D+/R + OR $0.31, p=0.006$. For the purpose of survival analysis, patients were further sub-classified as: normal Ig 76 cases (37.6\%), mild HGG with IVIg 23 cases (11.4\%), severe HGG with IVIg 19 cases (9.4\%), mild HGG without IVIg 59 cases $(29.2 \%)$ and severe HGG without IVIg 25 cases $(12.4 \%)$. Severe HGG without IVIg was significantly associated with worse EFS [aHR 3.17, p $<0.001$ (Figure)], after adjusting for disease status pre-alloSCT, co-morbidity index, GVHD prophylaxis regimen, receipt of $>80 \%$ methotrexate dose, donor type, histocompatibility mismatch, graft source, aGVHD, CMV reactivation, and prednisone dose on day +100 . However, IVIg replacement and HGG status did not associated with OS in multivariable analysis. Cumulative incidences of infection for each group are shown in Figure, with a significantly increased 
incidence $(77.5 \%$ at 2 years) in severe HGG without $\mathrm{Ig}$ replacement.

Conclusions: Our results show that severe HGG occurs in one fifth of patients. Acute GVHD increases risk of HGG. Patients receiving transplant from CMV reactive donors have a reduced risk of HGG.

HGG and IVIg replacement does not affect OS, but severe HGG without IVIg replacement is associated with an inferior EFS. Our data suggests that replacement therapy should be given in patients with severe HGG. Infection rates were reduced with Ig replacement in both the severe and non-severe HGG groups.

Conflict of interest:

A. Gerrie: honoraria, Seattle Genetics and Lundbeck; advisory committee, Janssen

D. Hogge: consultancy, Novartis, Roche and Sanofi

H. Sutherland: honoraria, Janssen

C. Toze: honoraria, CSL Bering

All other authors have no conflict of interest to declare.

\section{P565}

Infections profile after allogeneic peripheral blood stem cell transplantation (allo-PBSCT) from matched or partially mismatched donor and post-transplant Cyclophosphamide (PTCY): a single Institution experience

Susanna Gallo ${ }^{1,2}$, Daniela Caravelli ${ }^{1,2}$, Paolo Becco ${ }^{1,2}$, Elena Vassallo $^{2,3}$, Valentina Coha ${ }^{1,2}$, Monica Mangioni ${ }^{2,4}$, Massimo Berger $^{2,3}$, Alessandra Polo ${ }^{2,4}$, Pio Manlio Mirko Frascione ${ }^{1,2}$, Milena Salierno ${ }^{2,4}$, Francesca Carraro ${ }^{2,3}$, Luca Crotto ${ }^{1,2}$, Massimo Aglietta $^{1,2,5}$, Franca Fagioli ${ }^{2,3}$, Fabrizio Carnevale-Schianca ${ }^{1,2}$

${ }^{1}$ Candiolo Cancer Institute-FPO, IRCCS, Medical Oncology, Hematopoietic Stem Cells Unit, Candiolo, Italy; ${ }^{2}$ Turin Metropolitan Transplant Center, Torino, Italy; ${ }^{3}$ A.O.U. Citta' della Salute e della Scienza di Torino, Ospedale Infantile Regina Margherita, Pediatric Onco-Hematology, Stem Cell Transplantation, and Cellular Therapy Division, Torino, Italy; ${ }^{4}$ Candiolo Cancer Institute-FPO, IRCCS, Collection and Processing Laboratory, Candiolo, Italy; ${ }^{5}$ University of Torino, Department of Oncology, Torino, Italy

Background: Post-transplant Cyclophosphamide (PTCY) after HLA-matched bone marrow and haploidentical hematopoietic cell transplantation has been increasingly used due to its effective graft-versus-host disease (GVHD) control. Recently we reported as PTCY after allogeneic peripheral blood stem cell transplantation (alloPBSCT) exerts control on GVHD, but its impact on infections complications is still under investigation. We performed a retrospective study about infectious complications in adult patients receiving PTCY allo-PBSCT.

Methods: 58 consecutive patients, who underwent alloPBSCT with PTCY between March 2011 and July 2017 in FPO-IRCCs (Candiolo, Italy) were included. They received
PBSCT and PTCY 50mg/Kg (days +3 and +4 ), Tacrolimus and Mycophenolate Mofetil as GVHD prophylaxis. Reduced intensity conditioning regimens have been used in $18(31 \%)$ patients and myeloablative regimens in 40 (69\%). 54 recipients $(93 \%)$ and 37 donors $(64 \%)$ were CMV-positive. Main transplant outcomes and incidence of bacterial, fungal and viral infections have been evaluated.

Results: Patients median age at transplantation was 49 (range 19-69) years. 30 patients (52\%) had myeloid malignancies while 28 (48\%) lymphoid malignancies. A median dose of 6.2 (range 2-15) x $10^{\wedge} 6 \mathrm{CD} 34 / \mathrm{kg}$ was infused. Median follow-up was 14 (range 2-80) months. 1year overall survival, progression-free survival and nonrelapse mortality were respectively $83 \%, 67 \%$ and $2 \%$. Grade II-IV acute-GVHD was observed in $14 \%$ of patients, 1 -year chronic-GVHD cumulative incidence(CI) was $7 \%$.

One-year infection CI was $45 \%$ (33 episodes occurred in 26 patients) and 13 (39\%) events were identified during neutrophil aplasia before day +14 . Median day of occurrence was +28 (range 2-294). 3 patients developed pneumonia due to Gram-negative (GN) rods, 2 due to Gram-positive (GP) bacteria. 4 patients experienced GN urosepsis. Twenty-four bloodstream infections were due to GP bacteria (33\%) and to GN rods (67\%). One patient died because of Pseudomonas Aeruginosa sepsis (day+46). Infection related mortality (IRM) was $2 \%$. Hospitalization was required for 5 patients $(9 \%)$ because of fever $(n=2)$, pneumonia $(\mathrm{n}=2)$ and Clostridium Difficile infection $(\mathrm{n}=$ 1), all successfully treated and discharged. One patient had Giardia Lamblia gastrointestinal infestation, cured with Metronidazole.

$35 \%$ of patients had Cytomegalovirus (CMV) reactivation, median time was day +36 (range 21-54). They were treated with Valganciclovir as preemptive treatment, none developed CMV associated diseases. Five (8\%) patients had BK hemorrhagic cystitis, 2 had Herpes Zoster Virus (VZV) reactivations resolved with Valacyclovir. 3 of the 4 patients, who were hepatitis B virus (HBV)-positive before HCT, had viral reactivation while on Lamivudine prophylaxis requiring Entecavir. One hepatitis $\mathrm{C}$ virus (HCV) positive patient was started on Sofosbuvir/Velpatasvir after HCT because increasing viral load. 1 patient had a probable fungal pneumonia (evidence at chest CT scan + Galactomannan positive), cured with Voriconazole.

Conclusions: In conclusion, these data show that alloPBSCT followed by PTCY is associated with a low incidence of infections and, these complications, occur mainly within the first 100 days after allo-HCT. Possible explanations could be in the effective control of acute and chronic GVHD and, consequently, in the early discontinuation of immunosuppression in the majority of the patients.

Conflict of interest: All authors have nothing to disclose 


\section{P566}

Infectious complications after allo-stem cell transplantation in pediatric patients; differences according to stem cell source

Ana Maria Fita ${ }^{1}$, Miguel Blanquer Blanquer ${ }^{2}$, Andrés Sánchez Salinas $^{2}$, María Esther Llinares Riestra ${ }^{1}$, María Mar Bermúdez Cortés $^{1}$, Ana Maria Galera Miñarro ${ }^{1}$, José Luis Fuster Soler ${ }^{1}$, José María Moraleda

${ }^{1}$ Hospital Clínico Universitario Virgen de la Arrixaca, Paediatrics Oncology and Haematology Unit, El Palmar, Murcia, Spain; ${ }^{2}$ Hospital Clínico Universitario Virgen de la Arrixaca, IMIB,

University of Murcia, Haematology Service and Stem Cell Transplant Unit, El Palmar, Murcia, Spain

Background: Infections are the most common and significant cause of mortality and morbidity after allogeneic hematopoietic stem cell transplantation (allo-HSCT).

Methods: Retrospective, observational study of patients aged between 0 and 15 years undergoing allo-HSCT at a tertiary hospital, from January 2007 to December 2015. We studied a total of 56 pediatric patients who underwent 69 allo- HSCT with a median follow-up of $19(1-87)$ months. Of these, 6 received cord blood (CB), 36 peripheral blood (PB), 11 haploidentical PB, and 16 bone marrow (BM). We assessed infection incidence during early (day 0 to +30 ), intermediate $(+31$ to +100$)$ and late (since day +101$)$ periods post allo-HSCT. Infection was defined as isolation of a bacterial, viral or fungal pathogen associated with symptoms or disease. Bacteremia, sepsis and septic shock were defined as by the consensus criteria from Pediatric Intensive Care Spanish Society - Pediatric Emergencies Spanish Society (SECIP-SEUP; Alonso Salas MT et al, 2017).

Results: Regarding viral infections: $100 \%$ of $\mathrm{CB}$ recipients developed viral infection, with a $67 \%$ rate during the three post allo-HSCT periods. The most frequent viral pathogen detected in blood stream was CMV in all groups, followed by BK virus in the intermediate period and Epstein Barr virus (EBV) in the 3 post allo-HSCT periods. The most frequent pathogen isolated in urine was BK virus. In stools it was adenovirus during the 3 periods. In the upper respiratory tract the most frequent pathogen isolated was parainfluenza virus, followed by rhinovirus.

Regarding bacterial infections: during early post alloHSCT, $50 \%$ of patients suffered febrile syndrome, being $33 \%$ sepsis with bacterial pathogen isolation. Along the whole follow-up the most frequent pathogen isolated from blood stream with and without symptoms of sepsis was Staphylococcus epidermidis, followed by Staphylococcus hominis in early and intermediate post allo-HSCT. Urinary tract infection was mainly due to Eschecherichia Coli, followed by Klebsiella Pneumoniae. In stools the most frequent pathogen isolated was Clostridium Difficile (along all follow-up), followed by Camylobacter jejunii (intermediate and late period). In the upper respiratory tract, we found Haemophilus influenzae in the late period.

Regarding fungal infections: we detected cutaneous and oral candidiasis in the 3 periods post allo-HSCT. There was a case of isolation of Aspergillus Niger, with possible invasive fungal infection in the lower respiratory tract and another case with isolation of Candida glabrata in stools that associated pneumonia and spleen abscesses.

The mortality rate was $34 \%$ and only one patient died of septic shock, while 14 died of relapse/progression and 4 of GVHD.

Conclusions: The data describes the incidence of infections in our pediatric Allo-HSCT program. We did not find any statistical difference in the incidence of viral, bacterial or fungal infections during early, intermediate and late period between patients that received $\mathrm{CB}$, haploidentical, PB or BM stem cells. Stem cell source seems not to affect the incidence of infectous complications after alloHSCT, but the number of patients were small in some subgroups.

\section{Conflict of interest: None}

\section{P567}

Abstract previously published

\section{P568}

Infectious Diseases Working Party Retrospective Study on HHV-6 encephalitis: clinical characteristics and outcome after allogeneic HSCT

Katherine Nora Ward ${ }^{1}$, Per Ljungman', Jennifer Hoek ${ }^{3}$, Jürgen Finke $^{4}$, Dietger, Niederwieser, Herman Einsele ${ }^{6}$, Nathalie Fegueux ${ }^{7}$, Miguel Angel Diaz, , José Maria Fernandez, Anne-Lise Ménard $^{10}$, Matthew Collin ${ }^{11}$, Rodrigo Martino ${ }^{12}$, Christine Robin, Robin $^{13}$, Malgorzata Mikulska, ${ }^{14}$, Henrik Sengeloev ${ }^{15}$, Jakob Passweg $^{16}$, John A Snowden ${ }^{17}$, Arnon Nagler ${ }^{18}$, Boris Afanasyev ${ }^{19}$, Peter Shaw ${ }^{20}$, Nicolaus Kröger ${ }^{21}$, María Jesús Pascual ${ }^{22}$, Moshe Yeshurun $^{23}$, Tayfun Güngör ${ }^{24}$, Grant McQuaker ${ }^{25}$, Lucrecia Yañez ${ }^{26}$, Rafael de la Camara ${ }^{27}$, Urpu Salmenniemi ${ }^{28}$, Simone Cesaro ${ }^{29}$, Jan Styczynski ${ }^{30}$

${ }^{1}$ University College London, Infection, London, United Kingdom; ${ }^{2}$ Karolinska University Hospital, Stockholm, Sweden; ${ }^{3}$ EBMT Data Office, Leiden, Netherlands; ${ }^{4}$ University of Freiburg, Freiburg, Germany; ${ }^{5}$ University Hospital Leipzig, Leipzig, Germany; ${ }^{6}$ Universitätsklinikum Würzburg, Würzburg, Germany; ${ }^{7} \mathrm{CHU}$ Lapeyronie, Montpellier, France; ${ }^{8}$ Niño Jesus Children's Hospital, Madrid, Spain; ${ }^{9}$ Hospital Universitari I Politècnic la Fe, Valencia, Spain; ${ }^{10}$ Centre Henri Becquerel, Rouen, France; ${ }^{11}$ Freeman Hospital,, Newcastle-Upon-Tyne, United Kingdom; ${ }^{12}$ Hospital Santa Creu I Sant Pau, Barcelona, Spain; ${ }^{13}$ Hôpital Henri Mondor, Creteil, France; ${ }^{14}$ Ospedale San Martino, Genova, Italy; ${ }^{15}$ Rigshospitalet, 
Copenhagen, Denmark; ${ }^{16}$ University Hospital, Basel, Switzerland; ${ }^{17}$ Sheffield Teaching Hospitals NHS Foundation Trust, Sheffield, United Kingdom; ${ }^{18}$ Chaim Sheba Medical Center, Tel Hashomer, Israel; ${ }^{19}$ First State Pavlov Medical University of St Petersburg;, St Petersburg, Russian Federation; ${ }^{20}$ The Children's Hospital at Westmead, Sydney, Australia; ${ }^{21}$ University Hospital Eppendorf, Hamburg, Germany; ${ }^{22}$ Hospital Regional de Málaga, Malaga, Spain;

${ }^{23}$ Beilinson Hospital, Petach-Tivka, Israel; ${ }^{24}$ University Children's Hospital, Zürich, Switzerland; ${ }^{25}$ Gartnaval General Hospital, Glasgow, United Kingdom; ${ }^{26}$ Hospital U. Marqués de Valdecilla, Santander, Spain; ${ }^{27}$ Hospital de la Princesa, Madrid, Spain, Madrid, Spain; ${ }^{28}$ Turku University Hospital, Turku, Finland; ${ }^{29}$ Ospedale Donna Bambino, Verona, Italy; ${ }^{30}$ University Hospital, Collegium Medicum UMK, Bydgoszcz, Poland

Background: HHV-6 is an important cause of encephalitis post-HSCT. Epidemiology and risk factors are not well characterised. Only a few cases have been previously reported in children. Optimal antiviral therapy is unknown.

Methods: Cases transplanted between January 2005 and December 2014 were collected. HHV-6 encephalitis was defined as HHV-6 DNA detected in CSF coinciding with acute-onset altered mental status (encephalopathy), or short term memory loss or seizures. Data were collected on CT or MRI, clinical outcome (including neurological sequelae) and antiviral therapy (timing, start, duration).

Results: Of 128 cases, 101 from 42 centres fulfilled the definition of HHV-6 encephalitis. Fifty two had a myeloid neoplasm, 44 lymphoid and 5 other disease; 21 cases were children ( $\leq 18 \mathrm{yrs})$ and 80 adults. 69 patients were male and 32 female.

Mortality after HHV-6 encephalitis was high (48/101; $48 \%)$, lower in children $(6 / 21 ; 29 \%)$ versus older adults $(16 /$ $35 ; 46 \%$ and $26 / 45 ; 58 \%$, respectively). After CB HSCT it was lower $(6 / 23 ; 26 \%)$ than after BM/PB HSCT $(42 / 78$; $54 \%$ ) Mortality was associated with GVHD in about half of the cases except after CB HSCT.

Abbreviations for Table: $\mathrm{BM}$ bone marrow; $\mathrm{CB}$ cord blood; MA myeloablative; NMA non-myeloablative; NK Not known; PB peripheral blood.

Of the $78 \mathrm{BM} / \mathrm{PB} \mathrm{HSCT}, 3 / 4$ identical sibling, 3/5 mismatched related and 36/69 unrelated died within 3 months of HHV-6 encephalitis. 16/101 cases had received $\geq 2$ transplants and 7 were dead within 3 months. Ninety one cases received antiviral therapy: 32 foscarnet; 32 ganciclovir; 25 ganciclovir and foscarnet; 2 cidofovir. Mortality within 3 months of encephalitis was 20 (63\%) for foscarnet, $13(41 \%)$ for ganciclovir, $10 \quad(40 \%)$ for ganciclovir and foscarnet, and none for cidofovir.

Of the 101 cases the median time after HSCT to detection of HHV-6 DNA in CSF was 27 days (19-1289) but in 37\% the interval was $>42$ days. The median time in the children to detection of HHV-6 DNA was 27 days (19-225), 33 (19-317) in adults aged $19-50$ and 35 (16-1289) in those $>50$ years. The median time to HHV-6 DNA detection in CSF after CB transplant was 38 days (19-1289) versus 33 (16-317) after $\mathrm{BM}$ or PB HSCT.

Conclusions: Mortality after HHV-6 encephalitis was high in both children and adults occurring after both myeloablative and non-myeloablative HSCT. Antiviral therapy had a similarly dismal outcome. HHV-6 encephalitis is usually expected to occur within 6 weeks after transplant but in this study one third of cases occurred later.

Conflict of interest: K. Ward: Nothing to disclose.

\begin{tabular}{lllll}
\hline & \multirow{2}{*}{$\begin{array}{l}\text { No. } \\
\text { case }\end{array}$} & \multicolumn{3}{l}{ No. dead within 3 months of HHV-6 encephalitis } \\
\cline { 3 - 5 } & & $\begin{array}{l}\text { All } \\
\text { cases }\end{array}$ & $\begin{array}{l}\text { GVHD } \\
\text { contributory cause }\end{array}$ & $\begin{array}{l}\text { Relapse } \\
\text { contributory cause }\end{array}$ \\
\hline Pt age yrs - Up to 18 & 21 & 6 & 3 & 1 \\
Pt age yrs 19-50 & 35 & 16 & 8 & 3 \\
Pt age yrs $>50$ & 45 & 26 & 9 & 2 \\
Cells transplanted - & 78 & 42 & 19 & 4 \\
BM/PB & & & & 2 \\
$\begin{array}{l}\text { Cells transplanted - } \\
\text { CB }\end{array}$ & 23 & 6 & 1 & 2 \\
Conditioning - MA & 58 & 24 & 10 & 3 \\
Conditioning - & 41 & 22 & 9 & 1 \\
NMA & & & 1 & \\
Conditioning - NK & 2 & 2 & 1 & \\
\hline
\end{tabular}

\section{[[P568 Table] Patient characteristics and outcome]}

\section{P569}

Infectious epidemiology in Multiple Myeloma patients after Autologous Stem Cell Transplantation: A single-center analysis from Turkey

Shirkhan Amikishiyev ${ }^{1}$, Ipek Yonal Hindilerden ${ }^{2}$, Simge Erdem ${ }^{2}$, Melih Aktan' ${ }^{2}$,Meliha Nalcaci ${ }^{2}$, Deniz Sargin ${ }^{3}$, Sevgi Kalayoglu Besisik $^{2}$

${ }^{1}$ Istanbul University Istanbul Faculty of Medicine, Internal Medicine Department, Istanbul, Turkey; ${ }^{2}$ Istanbul University Istanbul Faculty of Medicine, Internal Medicine Department, Division of Hematology, Istanbul, Turkey; ${ }^{3}$ Medipol University, Department of Internal Medicine, Division of Hematology, Istanbul, Turkey

Background: Multiple myeloma (MM) is a B cell lymphoproliferative disorder. Immune paralysis related to the biology of the disease and the treatment itself cause infections along the course of the disease. We aimed to characterize the epidemiology of infections in MM patients after autologous HSCT in the era new drug induction.

Methods: The medical records of MM patients who received autologous HSCT between May 2007 and November 2016 at our institution, were reviewed to evaluate infection epidemiology. Primary analysis included data of infectious outcome and mortality for the first 30 days post-HSCT. Secondary analysis compared outcomes among 
different prior therapy when VAD regimen was obligatory as first line induction regimen for transplant eligible patients and bortezomib based therapy could be used only in patients with renal failure or cardiac co-morbidity in our country. All patients received antimicrobial prophylaxis as fluconazole, ciprofloxacin and acyclovir. High dose melphalan was the sole conditioning regimen.

Results: Nearly all patients received two lines of induction regimen as VAD followed by bortezomib based induction regimen. Median duration of neutropenia (granulocyte count $<0.5 \times 10(9) / 1$ was 8 days, the overall incidence of fever requiring antimicrobial treatment was 90.6\%. 53\% of patients had fever of unknown origin (FUO), whereas primary bacteremia occurred in $12.6 \%$, pneumonia in $12.6 \%$ and severe skin infection in $5.3 \%$ of patients. $10.6 \%$ of patients experienced candidial and/or herpetic stomatitis despite prophylaxis. Invasive fungal infections occurred in 11 (8\%), and enterocolitis in one patient. There was no infection related death during the study period. Only one patient $(0.6 \%)$ died on disease progression. The most frequently isolated pathogens were Gram-positive cocci. 6 patients experienced CMV infection (4\%). First-line antimicrobial therapy was successful in $75 \%$ of patients with FUO. Survival of patients with a positive blood culture was found to be significantly shorter $(\mathrm{p}=0.063)$. Positive urine culture has also an unfavorable effect on survival which was statistically not significant. There was no significant association between CD34 cell dose and febrile neutropenia. The disease age did not have any influence on antimicrobial treatment lines.

Conclusions: This retrospective study is a single center experience and shows the historical motion in $\mathrm{MM}$ treatment in Turkey. The epidemiology of infection reflects local flora and build up a base for empiric antimicrobial treatment but indicates also infections are not major morbidity reasons and are not major cause of death after autologous HSCT.

Conflict of interest: All authors: Nothing to disclose

\begin{tabular}{llll}
\hline & & $\mathrm{n}$ & $\%$ \\
\hline Blood & Not & 122 & 81,3 \\
Culture & Methicillin sensitive coagulase negative & 9 & 6,0 \\
& Staphylococcus & & \\
& Methicillin resistant coagulase negative & 8 & 5,3 \\
& Staphylococcus & & \\
& E. Coli & 4 & 2,7 \\
& Methicillin sensitive Staphylococcus aureus/ & $3 / 3 /$ & $2,0 / 2,0 /$ \\
& Klebsiella Pneumonia/Enterococcus & 1 & 0,7 \\
Urine & Not & 120 & 79,3 \\
Culture & E. Coli & 10 & 6,7 \\
& Enterococcus & 6 & 4,0 \\
& Klebsiella Pneumonia/Candida/Others & $5 / 3 /$ & $3,3 / 2,0 /$ \\
& & 6 & 4,0 \\
\hline
\end{tabular}

[[P569 Table] Table-1 Blood and Urine Cultures]

\section{P570}

\section{Influenza A and B infection are clinically similar in HSCT recipients}

Carlos S. Sabaini ${ }^{1}$, Anderson J. Siminone ${ }^{1}$, Paula M. da Silva ${ }^{1}$, Lilian P. Zanetti ${ }^{1}$, Leila C.S. de Oliveira ${ }^{1}$, Marina de Oliveira e Souza ${ }^{1}$, Juliana P. Moreno ${ }^{1}$, Fernanda S. Franceschi ${ }^{1}$, Iago Colturato $^{1}$, Mair P. Souza ${ }^{1}$, Vergilio R. Colturato ${ }^{1}$, Clarisse M. Machado $^{1,2}$

${ }^{1}$ Amaral Carvalho Foundation, HSCT Program, Jahu, Brazil;

${ }^{2}$ University of São Paulo, Institute of Tropical Medicine, Sao Paulo, Brazil

Background: Influenza (INF) is a viral infection that can be serious even in immunocompetent hosts. In HSCT recipients, early diagnosis and treatment are necessary to avoid complications of INF, especially the progression to pneumonia. At the Amaral Carvalho Hospital (ACH), INF control policies in HSCT units include annual vaccination, daily monitoring of symptoms, respiratory sampling in the presence of symptoms, and treatment with oseltamivir if INF A or B is detected. We retrospectively compared the frequency of complications and mortality of the proven cases of INF A and B, diagnosed from 2010 to 2016 at the HSCT Program of ACH.

Methods: TCTH candidates performed respiratory virus screening before admission at the HSCT unit. After transplantation, nasal wash samples were taken in the presence of respiratory symptoms. INF A and B diagnosis was made by DFA or RT-PCR. Oseltamivir was introduced at physician discretion. Patient charts and images were retrospectively reviewed by the investigators.

Results: : From 2010 to 2016, we identified 55 cases of INF. Of these, 14 cases occurred in transplant candidates or during conditioning (median day -7 , varying from day -2 to -62). In the remaining 41 cases, the median age was 34 years, ranging from 2 to 64 years. Thirty patients had INF A (73.2\%) and 11 had INF B (26.8\%). The median duration of excretion, evaluated in 38 patients, was 7 (3 to 36) days. At diagnosis, 39 patients (95\%) had upper respiratory symptoms and $2(5 \%)$ had radiological alterations suggestive of pneumonia. The most frequent symptoms were cough (75.6\%), fever $(65.9 \%)$, coryza $(61 \%)$, nasal congestion $(36.6 \%)$ and sore throat $(34.1 \%)$. Oseltamivir was given to 33 patients $(80.5 \%)$. Treatment duration ranged from 5 to 10 days (median 5 days). Quinolone was used in 16 of the 35 patients who received antibiotic therapy $(45.7 \%)$. Ten patients developed pneumonia $(24.4 \%)$, despite the use of Oseltamivir in 8 of them. Two patients died (4.9\%) despite treatment with Oseltamivir; 3 (7.3\%) presented resolution of 
the process with complications and $36(87.8 \%)$ without complications. The two deaths were by INF A. Regarding morbidity, there was no difference between INF A and INF B.

Conclusions: Clinical manifestation and complications were similar in patients with INF A or INF B infection. INF A mortality was $6.6 \%$.

Conflict of interest: Clarisse M. Machado: Nothing to disclose.

\section{P571}

Intensive pre-emptive therapy of CMV reactivation is related to inferior outcome: a single center experience

Apostolia Papalexandri, Despina Mallouri, Panagiota Zerva, Stella Bouziana, Ioannis Batsis, Marianna Masmanidou, Konstantinos Leontopoulos, Evangelia-Evdoxia Koravou, Eirini Baldoumi, Anna Vardi, Tasoula Touloumenidou, Achilles Anagnostopoulos, Ioanna Sakellari

G. Papanikolaou Hospital, Hematology Department and HCT Unit, Thessaloniki, Greece

Background: CMV infection post allogeneic hematopoetic cell transplantation (allo-HCT) remains a significant factor of morbidity in transplantation, despite the close monitoring of CMV viral load and the pre-emptive use of specific anti-viral treatment. A positive impact, however, with a Graft versus Leukemia $(\mathrm{GvL})$ effect of the cytotoxic lymphocytic expansions has been suggested.

Methods: We have retrospectively studied the incidence of CMV reactivation in 193 patients that underwent alloHCT between 2008-2015, when intensive pre-emptive antiviral therapy was a standard of care in our clinic and possible clinico-biological correlations were sought. Patients suffered from hematologic malignancy (188) or aplastic anemia (5) and received a graft from a matched sibling (59), volunteer (122) or haplo-identical relative donor (12) after a myeloablative (M: 171) or reduced intensity conditioning (RIC: 22). Ninety-two out of 193 patients were transplanted in $1^{\text {st }} \mathrm{CR}$. CMV reactivation was considered when viral load $>1000$ viral genomic copies (VGC)/ml in plasma was documented during the regular weekly molecular monitoring of the viral load with RQPCR. In this case, patients received pre-emptive therapy with valacyclovir, gancyclovir or foscarnet. Two consecutive samples with viral load $<100 \mathrm{VGC} / \mathrm{ml}$ were considered as successful clearance of viremia.

Results: : Overall 71/193 (37\%) patients presented with CMV reactivation in a median of 49 (12-1213) days. Resolution was achieved in 52/71 patients (73\%), in a median of 38 days. Among them, 16 patients presented with a new reactivation. Nineteen out of 71 (26\%) patients succumbed, 22 (9-69) days after the reactivation. Reactivation was significantly correlated to a myeloablative conditioning ( $35 \% \mathrm{M}$ vs $22 \% \mathrm{RIC})$, an haploidentical donor (69\% haplo vs $36 \%$ VUD vs $28 \%$ sibling) and EBV viremia $(>10.000 \mathrm{VGC} / \mathrm{ml})(47 \%$ in patients with $\mathrm{EBV}$ vs $32 \%$ without), $\mathrm{p}<0.05$ for all comparisons. Relapse free survival (RFS) was similar regardless the presence of CMV, but overall survival (OS) was significantly worse in patients with CMV (46.3 vs 69.4 months, 95\%CI: $34.9-57$ vs 60.7 $78.2, \mathrm{p}=0.002)$ and $\mathrm{EBV}$ reactivation (34.9 vs 67.5 months, 95\%CI: $25.3-44,4$ vs $59.3-75.7, p=0.002$ ). OS did not differ among patients with different type of transplantation, donor, pre-transplant status or chronic GvHD. When only patients who presented early $(<100$ days) reactivation of CMV were considered, RFS and OS did not significantly change.

Conclusions: In the current study, despite the regular monitoring and the early use of preemptive therapy, reactivation of CMV is related to a worse overall survival and similar RFS, without a benefit from the possible GvL action. Use of specific antiviral cytotoxic cell lines promises a better outcome in immunocompromised allografted patients.

Conflict of interest: None of the authors has anything to disclose.

\section{P572}

Invasive aspergillosis is becoming a late complication of allogeneic hematopoietic stem cell transplantation

Christine Robin ${ }^{1}$, Catherine Cordonnier ${ }^{1}$, Nicole Raus ${ }^{2}$, Karine Sitbon $^{3}$, Olivier Lortholary, Sébastien Maury ${ }^{1}$, Regis Peffault de Latour $^{4}$, Stéphane Bretagne ${ }^{3}$, Sylvie Bastuji-Garin ${ }^{5}$

${ }^{1}$ Henri Mondor Hospital, Hematology, Creteil, France; ${ }^{2}$ Lyon Sud Hospital, Hematology, Lyon, France; ${ }^{3}$ Pasteur Institute, Mycology, Paris, France; ${ }^{4}$ Saint Louis Hospital, Paris, France; ${ }^{5}$ Henri Mondor Hospital, Public Health, Creteil, France

Background: Invasive aspergillosis (IA) remains a lifethreatening complication of allogeneic hematopoietic stem cell transplantation (HSCT), mainly driven by GVHD. However, HSCT procedures and indications change over time and could modify this risk when compared to historical series. We conducted this study on 836 HSCT recipients in order to identify the pre- and post-transplant factors associated with occurrence of IA after allogeneic HSCT.

Methods: This retrospective, multicenter, case-control study was carried out from two prospective registries: SAIF (Surveillance des Aspergilloses Invasives en France, Institut Pasteur Paris) a French 2005-2010 registry of IA, and the HSCT ProMISe registry of the EBMT. Nineteen centers participated, representing 3881 allogeneic HSCT performed 
during that period of time. We identified all consecutive cases of proven and probable IA occurring in allogeneic HSCT recipients from SAIF. Four controls individually matched on center, age and year of transplant were recruited among patients who did not develop IA after HSCT from the ProMISe registry. We analyzed pre- and post-transplant risk factors according to the timing of IA.

Results: We identified 185 cases (166 probable, 19 proven IAs according to the EORTC-MSG criteria) and 651 controls. Median date of IA was 133 days (64-233), with 35 $(19 \%)$ early (< day 40) IA, 33 (18\%) late (d40-d100) IA and $117(63 \%)$ very late (>d100) IA. When considering only pre-transplant factors, early IA tended to be associated with unrelated donor and the absence of complete remission ; late IA with ATG use as conditioning. Very late IA was associated with D-R+ CMV serology and tended to be associated with the underlying disease. When considering pre and post-transplant factors, early IA was significantly associated with lack of engraftment and tended to be associated with the absence of complete remission, late IA with grade $>2$ acute GvHD and tended to be associated with relapse after transplant and very late IA with grade $\geq 2$ acute GvHD, relapse after transplant and secondary neutropenia.

Conclusions: IA observed after allogeneic HSCT nowadays is a late event, with two thirds of the cases occurring after d100 while at least two thirds of the cases were observed before d100 ten years ago. This late cases have different risk factors than earlier cases and raise different issues. Prophylactic strategies should take in account the specific risk factors for IA occurring after d100, especially GVHD, relapse and secondary neutropenia.

Clinical Trial Registry: not applicable

Conflict of interest: nothing to disclose

\begin{tabular}{|c|c|c|c|c|c|c|c|}
\hline & & Early IA & & $\begin{array}{l}\text { Late } \\
\text { IA }\end{array}$ & & $\begin{array}{l}\text { Very } \\
\text { late } \\
\text { IA }\end{array}$ & \\
\hline & & $\mathrm{aOR}$ & $\mathrm{p}$ & $\mathrm{aOR}$ & $\mathrm{p}$ & aOR & $\mathrm{p}$ \\
\hline $\begin{array}{l}\text { Disease } \\
\text { status }\end{array}$ & $\begin{array}{l}\text { Absence } \\
\text { of } \\
\text { complete } \\
\text { remission }\end{array}$ & $2.6[0.9-7.2]$ & 0.06 & & & & \\
\hline $\begin{array}{l}\text { Absence of } \\
\text { engraftment }\end{array}$ & & $\begin{array}{l}\text { Absence of } \\
\text { engraftment } \\
16.4[2.9-92.6] \\
0.002\end{array}$ & 0.002 & & & & \\
\hline \multirow[t]{2}{*}{$\begin{array}{l}\text { Acute } \\
\text { GvHD }\end{array}$} & grade 2 & & & $\begin{array}{l}1.2 \\
{[0.3-} \\
4.4]\end{array}$ & 0.76 & $\begin{array}{l}2.6 \\
{[1.3-} \\
5.3]\end{array}$ & 0.006 \\
\hline & grade 3-4 & & & $\begin{array}{l}4.7 \\
{[1.8-} \\
12.0]\end{array}$ & 0.001 & $\begin{array}{l}6.9 \\
{[3.1-} \\
15.3]\end{array}$ & $<0.001$ \\
\hline $\begin{array}{l}\text { Relapse } \\
\text { after } \\
\text { transplant }\end{array}$ & & & & $\begin{array}{l}11.4 \\
{[0.9-} \\
142.5]\end{array}$ & 0.06 & $\begin{array}{l}24.1 \\
{[7.3-} \\
79.6]\end{array}$ & $<0.001$ \\
\hline $\begin{array}{l}\text { Secondary } \\
\text { neutropenia }\end{array}$ & & & & & & $\begin{array}{l}2.2 \\
{[1.1-} \\
4.1]\end{array}$ & 0.02 \\
\hline
\end{tabular}

[[P572 Table] Multivariate analysis of pre- and posttransplant]

\section{P573}

\section{Legionellosis after haematopoietic stem cell transplantation}

Malgorzata Mikulska ${ }^{1}$, Gloria Tridello ${ }^{2}$, Jennifer Hoek ${ }^{3}$, Lidia Gil $^{4}$, Mercedes Colorado ${ }^{5}$, Hélène Labussière-Wallet ${ }^{6}$, Jakob Passweg ${ }^{7}$, Gerard Socie ${ }^{8}$, Pietro Pioltelli, ${ }^{9}$, Denis Caillot ${ }^{10}$, Gerard Michel ${ }^{11}$, Joan Veelken ${ }^{12}$, Didier Blaise ${ }^{13}$, Benedetto Bruno ${ }^{14}$, Noel Milpied ${ }^{15}$, Maija Itälä-Remes ${ }^{16}$, Charles Crawley ${ }^{17}$, Jean Henri Bourhis ${ }^{18}$, Ain Kaare $^{19}$, William Arcese ${ }^{20}$, Rocio Parody ${ }^{21}$, Jan Styczynski ${ }^{22}$

${ }^{1}$ University of Genoa (DISSAL) and Ospedale Policlinico San Martino, Division of Infectious Diseases, Genoa, Italy; ${ }^{2}$ Policlinico $G$. B. Rossi, Verona, Italy; ${ }^{3}$ EBMT Data Office, Leiden, Netherlands; ${ }^{4}$ Poznan University of Medical Sciences, Poznan, Poland; ${ }^{5}$ Hospital U. Marqués de Valdecilla, Santander, Spain; ${ }^{6}$ Centre Hospitalier Lyon-Sud, Lyon, France; ${ }^{7}$ University Hospital, Basel, Switzerland; ${ }^{8}$ Hôpital St. Louis, Paris, France; ${ }^{9}$ Ospedale San Gerardo, Monza, Italy; ${ }^{10} \mathrm{CHU}$ de Dijon, Dijon, France; ${ }^{11}$ Hôpital Timone, Marseille, France; ${ }^{12}$ Leiden University Hospital, Leiden, Netherlands; ${ }^{13}$ Institut Paoli Calmettes, Marseille, France; ${ }^{14}$ A.O.U. Citta della Salute e della Scienza di Torino, Torino, Italy; ${ }^{15} \mathrm{CHU}$ Bordeaux, Bordeaux, France; ${ }^{16}$ HUCH Comprehensive Cancer Center, Helsinki, Finland;

${ }^{17}$ Addenbrookes Hospital, Cambridge, United Kingdom; ${ }^{18}$ Gustave Roussy, Villejuif, France; ${ }^{19}$ Tartu University Hospital, Tartu, Estonia; ${ }^{20}$ Tor Vergata University, Rome, Italy; ${ }^{21}$ ICO- Hospital Duran I Reynals, Barcelona, Spain; ${ }^{22}$ University Hospital, Collegium Medicum UMK, Bydgoszcz, Poland

Background: Immunocompromised patients, particularly transplant recipients, are at increased risk of Legionella infections. However, data on legionellosis after HSCT come mainly from cases and single-centre case series and the predictors and factors influencing outcome remain unknown.

The aim of this study was to report the cases of legionellosis after HSCT present in EBMT registry and to identify predictors of legionellosis, of legionella-associated death and of non-relapse mortality (NRM) in patients with this infection.

Methods: Registry-based study was performed identifying all the cases of post-HSCT legionellosis. For the analyses of risk factors, controls were identified in 3:1 ratio, with the following matching criteria: the same centre, year of transplant and the length of follow-up at least until the day of diagnosis of legionellosis by the case.

Results: In years 1995-2016, 80 cases from 52 centres in 14 countries were identified (main countries: France, Italy and Spain). In median 4 cases per year were reported (range, 1-8). The median time from HSCT to the diagnosis of legionellosis was 203 days (range, 0-4099); 47.5\% of patients developed legionellosis within 6 months post- 
HSCT, $17.5 \%$ between 7-12 months from HSCT, 21.3\% between 13 and 24 months, and $13.8 \%>24$ months after HSCT.

Patients were mostly male (70\%), the median age was 46.6 years (range, 7.2-68.2). They were diagnosed mainly with acute leukaemia (27.5\%), lymphoma (23.8\%), multiple myeloma (21.3\%) and chronic myeloid leukaemia (10\%). In only $20 \%$ of patients, the underlying disease was in relapse during 2 years before developing legionellosis. Most of patients $(66.3 \%)$ developed legionellosis after first alloHSCT and 30\% after first autoHSCT.

63 patients were included in the analysis of predictors of legionellosis. Compared to controls, cases were more likely to have undergone first and not second HSCT $(p<0.0001)$, to be in complete remission $(p=0.001)$ and to be free of other infections in the 6 months before developing legionellosis $(\mathrm{p}=0.003)$. They were also slightly more likely to receive alloHSCT than auto HSCT $(\mathrm{p}=0.09)$.

Overall, 23 (28.8\%) of patients died due to legionellosis. Among patients with legionellosis, no predictors of death due to Legionella were identified, but fewer patients died in decade 2006-2016 compared to the previous one: $21.7 \%$ vs $38.2 \%, \mathrm{p}=0.09$. Predictors of NRM in this cohort were time from HSCT to legionellosis (HR 0.97, 95\%CI 0.93$1.00, \mathrm{p}=0.035)$ and female sex (HR 2.05, 95\% CI 1.06$3.92, \mathrm{p}=0.033$ ). In particular, patients developing legionellosis within 1 month HSCT had twice as high NRM compared to cases developing later (HR 2.26, 95\% CI 1.14$4.49, \mathrm{p}=0.02$ ).

Conclusions: Legionellosis is a rare complication after HSCT which occurs mainly in the first year post HSCT. It has the associated mortality rate of almost $30 \%$, which decreased in the last decade probably due to improvements in rapid diagnosis and treatment. Since legionellosis frequently affects patients in remission and without other infectious complications, its correct management is important in order to preserve benefits conferred by transplantation.

Conflict of interest: All authors: Nothing to disclose

\section{P574}

\section{Lung ultrasound for evaluation of invasive fungal disease after allogeneic transplant}

Raffaella Greco, Elisabetta Xue, Andrea Assanelli, Sarah Marktel, Fabio Giglio, Daniela Clerici, Maria Teresa Lupo Stanghellini, Consuelo Corti, Massimo Bernardi, Fabio Ciceri, Jacopo Peccatori

IRCCS San Raffaele Scientific Institute, Hematology and BMT Unit, Milan, Italy

Background: Invasive fungal diseases (IFD) are a leading cause of morbidity and infection-related mortality among allogeneic hematopoietic stem cell transplantation (HSCT) recipients. Considering it is a bedside, reliable, real-time and noninvasive technique, lung ultrasound (US) could have a significant role in the diagnostic workup and follow-up of lung lesions also in the oncohematological area.

Methods: In this prospective non-interventional study, bedside lung US examination was performed at diagnosis and 10 days after the start of antifungal treatment. A convex 3.5- to 5-MHz probe (GE Healthcare) was used. In agreement with literature (Lichtenstein et al., 2004), each hemithorax was divided into 5 areas: 2 anterior, 2 lateral, and 1 posterior. The criterion to determine the echographic diagnosis of pneumonia was the finding of subpleural lung consolidation with evidence of static or dynamic air bronchograms.

Results: Ten consecutive patients were included in the study within 18 months recruitment period. All patients had an established diagnosis of pulmonary invasive fungal disease (IFD) according to EORTC/MSG definitions. All patients were considered at high risk for developing posttransplant IFD according to GITMO criteria. Patients (median age $44.5 \mathrm{y}$ ) received allogeneic HSCT for highrisk hematological malignancies (6 acute leukemia, $1 \mathrm{CML}$, $1 \mathrm{HL}$ and $1 \mathrm{NHL}$ ); $50 \%$ of patients were not in complete remission. Stem cell donors were family haploidentical (n $=6$ ), HLA identical sibling $(\mathrm{n}=2)$, unrelated volunteer ( $\mathrm{n}$ $=2$ ). Stem cell source was T-cell replete PBSCs. All patients received a treosulfan-based conditioning regimen and GvHD prophylaxis with PT-Cy and sirolimus. Early after HSCT procedure (before neutrophil engraftment for the majority of patients), we documented 8 possible and 2 probable pulmonary IFD. Posaconazole was used as antifungal therapy in 2 cases, voriconazole in 2, isavuconazole in 2 patients, and a combination of ambisome plus azole was necessary in 4 patients.

Lung US was performed in all cases, concomitantly to the gold standard high-resolution CT scan.

The diagnosis of pneumonia was confirmed by baseline US in all patients. Patients showed consolidations most frequently on both sides of the lungs $(n=7)$, rather than on the right $(n=1)$ or the left $(n=2)$ side of the lungs; one presented an air bronchogram. Contiguous focal B-lines and a pleural line attenuation corresponding to the affected area were evident in 8 cases. Pleural basal effusion was detected in 4 patients. During follow-up, we documented a clinical improvement, demonstrated also by CT scan and lung US, in all patients except one. Pneumonic lesions appeared sonographically smaller and the volume of pleural effusion decreased in patients with a documented IFD response. Importantly, all US findings resulted comparable to CT scan results. 
Conclusions: Lung US has been shown to be a very promising technique due to its high sensitivity in the detection of lung lesions, but also for follow-up examinations, with the advantage of saving time and money. In particular, the contribution of US techniques could be potentially relevant for a multidisciplinary IFD management in this high-risk population, especially if compared to the time and radiation exposure required to obtain the results of radiologic surveys.

Clinical Trial Registry: NA

Conflict of interest: Nothing to disclose

\section{P575}

Lymphocyte-AUC (area under the lymphocyte count-time curve) as a predictive value for immune-reconstitution after allogenic hematopoietic stem cell transplantation and its impact on viral infection

Mizuki Watanabe, Junya Kanda, Tadakazu Kondo, Kohei Yamashita, Masakatsu Hishizawa, Akifumi Takaori-Kondo

Kyoto University, Department of Hematology, Graduate School of Medicine, Kyoto, Japan

Background: Viral infections is a serious complication that affects survival after allogenic hematopoietic stem cell transplantation (allo-HSCT). Therefore, it is important to identify high-risk patients for viral infection in advance. In this present study, we developed lymphocyte-AUC as a new predictive value for immune reconstitution and evaluated its impact on viral infection after allo-HSCT.

Methods: A total of 286 patients who underwent their first allo-HSCT in Kyoto University Hospital from 2005 to 2017 were included. In the analysis of human herpesvirus-6 (HHV-6), whose reactivation is mostly detected before neutrophil engraftment, we included patients who survived 15 days or more after transplantation and estimated lymphocyte-AUC from day 0 to day 15 . In the analysis of cytomegalovirus (CMV) and other viruses including adenovirus (ADV), BK virus (BKV), JC virus (JCV), varicella zoster virus (VZV) and Epstein-Barr virus (EBV), we included patients who survived over 30 days after transplantation and estimated lymphocyte-AUC from day 0 to day 30 . We analyzed an association between lymphocyte-AUC and the risk of virus activation after the landmark of day 15 for HHV reactivation or day 30 for CMV reactivation and other virus infections.

Results: A total 181, 26, and 79 patients underwent bone marrow transplantation, peripheral blood stem cell transplantation, and single cord blood transplantation (CBT), respectively. Median age was 51 (range, 17-68) and median period for first lymphocyte-count was 15 days after transplantation. Forty-eight patients were diagnosed with
HHV-6 reactivation, in whom 7 patients developed HHV-6 encephalitis. CMV antigenemia was detected in 146 patients, in whom 13 patients developed CMV infection. Twenty patients were diagnosed with other viral infections (ADV in 7, BKV in 13, JCV in 5, VZV in 1, EBV in 1). Increase in lymphocyte-AUC was marginally associated with high frequency for HHV6-reactivation $(\mathrm{P}=0.072)$ and significantly associated with low frequency for CMV antigenemia $(\mathrm{P}=0.014)$. There was no apparent association between lymphocyte-AUC and the other viruses. Malignant lymphoma as primary disease $(\mathrm{aHR} 3.97, \mathrm{P}<0.001)$ and CBT as donor source (aHR 6.11 $\mathrm{P}<0.001$ ) were suggested as other risk factors for HHV-6 reactivation and the occurrence of acute GVHD (aHR 2.01, P< 0.001) and recipient age (aHR 1.02, $\mathrm{P}=0.003$ ) for $\mathrm{CMV}$ antigenemia.

Conclusions: Lymphocyte-AUC could be a good predictive value for immune reconstitution against CMV reactivation. Regarding HHV-6 reactivation, lymphocyteAUC were associated with the growth of viral load, which might suggest rapid growth of HHV-6 in donor lymphocyte, especially in immune-naïve cord blood cells. Our data suggests that lymphocyte-AUC could be an informative value to be used in clinical strategies against viral infection after allo-HSCT, along with new insights into HHV-6 reactivation in recipients after HSCT.

Conflict of interest: no conflict of interest

\section{P576}

Management of HCV Hepatitis after HLA-haploidentical Stem Cell Transplantation in two pediatric patients affected by High-Risk Acute Lymphoblastic Leukemia (HR-ALL) treated with Off-Label direct-acting antivirals

Maria Speranza Massei ${ }^{1}$, Olivia Morelli ${ }^{2}$, Ilaria Capolsini ${ }^{1}$, Francesco Arcioni ${ }^{1}$, Katia Perruccio ${ }^{1}$, Elena Mastrodicasa ${ }^{1}$, Grazia Gurdo $^{1}$, Carla Cerri ${ }^{1}$, Maurizio Caniglia ${ }^{1}$

${ }^{I}$ Santa Maria della Misericordia Hospital, Pediatric OncologyHematology, Perugia, Italy; ${ }^{2}$ University of Perugia, Gastroenterology and Hepatology Unit, Department of Medicine, Perugia, Italy

Background: Children affected by HR-ALL with HCV infection in absence of cytolysis before HLA-haploidentical Stem Cell Transplantation (HSCT) were not treated with Off-Label direct-acting antivirals (DAA) because they are considered an off label therapy in pediatric population. Of course they could present after transplant liver dysfunctions due to toxicity, viral infections and GVHD. The main problem is differential diagnosis in order to discriminate between viral hepatitis and GVHD; liver biopsy is needed before a specific therapy.

Methods: 8, 16 and 10 years old Hispanic patients, affected by HR-ALL were transplanted from familiar 
haploidentical donors. They had a $2 \mathrm{a} / 2 \mathrm{c}$ genotype $\mathrm{HCV}$ chronic infection before transplant (HCV RNA was respectively $1042000 \mathrm{IU} / \mathrm{mL}, 43670 \mathrm{IU} / \mathrm{mL}, 244300 \mathrm{IU} /$ $\mathrm{mL})$. Conditioning regimen consisted in: fractioned TBI (total dose: $13.5 \mathrm{~Gy})$, Thiotepa $(10 \mathrm{mg} / \mathrm{kg})$, Fludarabine $\left(200 \mathrm{mg} / \mathrm{m}^{2}\right)$, Ciclophosphamide $(30 \mathrm{mg} / \mathrm{kg})$. The graft was $\mathrm{T}$ cell depleted with $11,5 \times 10^{\wedge} 6 / \mathrm{Kg}$ (range $7,2-13,9 \times 10^{\wedge} 6 /$ $\mathrm{Kg}) \mathrm{CD} 34+$ associated with adoptive immune therapy of $2 \times 10^{\wedge} 6 / \mathrm{kg}$ regulatory $\mathrm{T}$ cells and $0.5 \times 10^{\wedge} 6 / \mathrm{kg}$ conventional $\mathrm{T}$ cells, without post transplant GVHD prophylaxis.

Results: All patients engrafted; the 8 year-old and 16 year-old patients developed severe cholestasis and cytolysis (bilirubin up to $6.9 \mathrm{mg} / \mathrm{dl}$ and $11.52 \mathrm{mg} / \mathrm{dl}, \gamma \mathrm{GT} 1048 \mathrm{IU} / \mathrm{L}$ and $1317 \mathrm{IU} / \mathrm{L}$, ALT $445 \mathrm{IU} / \mathrm{L}$ and $277 \mathrm{IU} / \mathrm{L}$, AST $464 \mathrm{IU} /$ $\mathrm{L}$ and $527 \mathrm{IU} / \mathrm{L}$ ) at respectively 68 days and 25 days after transplant; a liver biopsy was made with evidence of histological signs of aGVHD associated with hepatitis. A significant increase of HCV viremia (respectively 35190000 $\mathrm{IU} / \mathrm{mL}$ and $6545000 \mathrm{IU} / \mathrm{mL}$ ) was revealed. The third patient didn't present post transplant liver dysfunction. GVHD was treated with short course of steroids, associated with photopheresis and mofetil micofenolate. HCV therapy was administered: ribavirin $(15 \mathrm{mg} / \mathrm{kg})$ and sofosbuvir $(200 \mathrm{mg})$ to the 8 year-old patient, $(400 \mathrm{mg})$ to the 16 year-old patient, for 12 weeks. Viremia was monitored at weeks 1, 2, 4, 8, 12 from the beginning of the therapy. After the start of the DAA, significant improvement was observed in terms of liver function and negativity of the viremia at $8^{\text {th }}$ week. The $\mathrm{HCV}$ viremia in the 8 year-old patient was still negative at the $12^{\text {th }}$ week from the end of therapy (Sustained Virological Response: highly predictive of definitive viral eradication); while in the 16 year-old patient an increase of bilirubin up to $6.5 \mathrm{mg} / \mathrm{dl}$, AST $80 \mathrm{IU} / \mathrm{L}$, ALT $88 \mathrm{IU} / \mathrm{L}, \gamma \mathrm{GT}$ $502 \mathrm{IU} / \mathrm{L}$ was observed after two weeks from the end of the anti-viral treatment with recurrence of HCV RNA viremia $(6371000 \mathrm{IU} / \mathrm{mL})$. Analysis of viral resistance was performed and a new pediatric off-label anti-viral therapy based on sofosbuvir (400mg)/valpatasvir (100mg) and ribavirine $(15 \mathrm{mg} / \mathrm{kg})$ was administered with rapid normalization of liver function and negativisation of viremia after two weeks.

Conclusions: GVHD and viral hepatitis in HCV infected patients are frequently associated in the post transplant period. The off-label DAA therapy in pediatric patients is safe and effective treatment of HCV hepatitis after allogeneic HSCT, even if associated at the immunosuppressive treatement for the GVHD without side-effects. In the future the approval of DAA in pediatric patients could provide the early cure of HCV hepatitis in HR-leukemic patients before/after transplant.

Conflict of interest: All authors have nothing to disclose.
P577

Novel Echinocandin Rezafungin (CD101) In vitro Potency Translates to Efficacious Treatment/Prophylaxis in Mouse Models of Disseminated/Pulmonary Aspergillosis

\author{
Voon Ong ${ }^{1}$, Grayson Hough ${ }^{1}$, Shawn Flanagan ${ }^{1}$, Ken Bartizal, \\ Abdul Sattar ${ }^{2}$, Andrew Sharp ${ }^{2}$, Pia Thommes ${ }^{2}$ \\ ${ }^{1}$ Cidara Therapeutics, San Diego, CA, United States; ${ }^{2}$ Evotec, \\ Manchester, United Kingdom
}

Background: Rezafungin (CD101) is a novel echinocandin which exhibits potent in vitro activity against $A$. fumigatus and is in development for treatment and prophylaxis of invasive fungal infections. Its efficacy in multiple mouse aspergillosis infection models is presented to support potential use in treatment or prevention of aspergillosis.

Methods: The in vitro activity of rezafungin was evaluated against 153 A. fumigatus clinical isolates collected during the 2014 and 2015 JMI international SENTRY surveillance program. Susceptibility was determined as the minimum effective concentration (MEC) values in accordance with CLSI broth microdilution guidelines (M38-A2).

Disseminated aspergillosis: Neutropenic ICR mice (6/grp) were infected with A. fumigatus ATCC 13073 (IV, 10 CFU/mouse) on day 0 . Treatment ( $2 \mathrm{~h}$ after infection) with rezafungin was given as either a single $(2 \mathrm{mg} / \mathrm{kg}$ IV and IP) or daily $(0.5 \mathrm{mg} / \mathrm{kg}$ BID) dose. Survival was monitored for $\geq 10$ days. The same model was used for prophylaxis except rezafungin (SC; $5,10,20 \mathrm{mg} / \mathrm{kg}$ ) was dosed on days $-1,-3$ or -5 prior to infection.

Pulmonary aspergillosis: ICR mice (10/grp) were made neutropenic by cyclophosphamide on day $-4(150 \mathrm{mg} / \mathrm{kg})$, and cyclophosphamide/cortisone was given on day -1 (150/ $175 \mathrm{mg} / \mathrm{kg}$ ). Intranasal challenge with A. fumigatus AF293 $\left(10^{5} \mathrm{CFU} / \mathrm{mouse}\right)$ was initiated on day 0 . Prophylaxis with rezafungin as a single dose (IP; 5, 10, $20 \mathrm{mg} / \mathrm{kg}$ ) or posaconazole (PO; 2 and $10 \mathrm{mg} / \mathrm{kg}$ ) or micafungin (IP; 2 $\mathrm{mg} / \mathrm{kg}$ ) was started 1 day prior to infection. Survival was monitored for 10 days.

Results: Rezafungin showed potent in vitro activity against clinical A. fumigatus isolates with $\mathrm{MEC}_{50}, \mathrm{MEC}_{90}$ and MEC range values of $0.015,0.015$, and $\leq 0.0078$ $0.03 \mu \mathrm{g} / \mathrm{mL}$, respectively. Lung epithelial lining fluid concentrations from a single human AUC equivalent dose of rezafungin in the mouse were found to be considerably higher $(>10 \mu \mathrm{g} / \mathrm{mL}$ at 24 hours post-dose or after one halflife interval) than A. fumigatus $\mathrm{MEC}_{90}$ of $0.015 \mu \mathrm{g} / \mathrm{mL}$.

For treatment of disseminated aspergillosis, rezafungin by IV $/ \mathrm{IP}$ at $0.2,1$, or $5 \mathrm{mg} / \mathrm{kg}$ BID $\times 5 \mathrm{~d}$ showed a significant increase in survival compared to vehicle. Survival was 
Figure 1. Survival rate of mice given prophylaxis rezafungin (CD101) or micafungin (Study 1 , top graph) and rezafungin or posaconazole (Study 2, bottom graph) as a single dose one day prior to infection in pulmonary aspergillosis.

\section{Study 1}

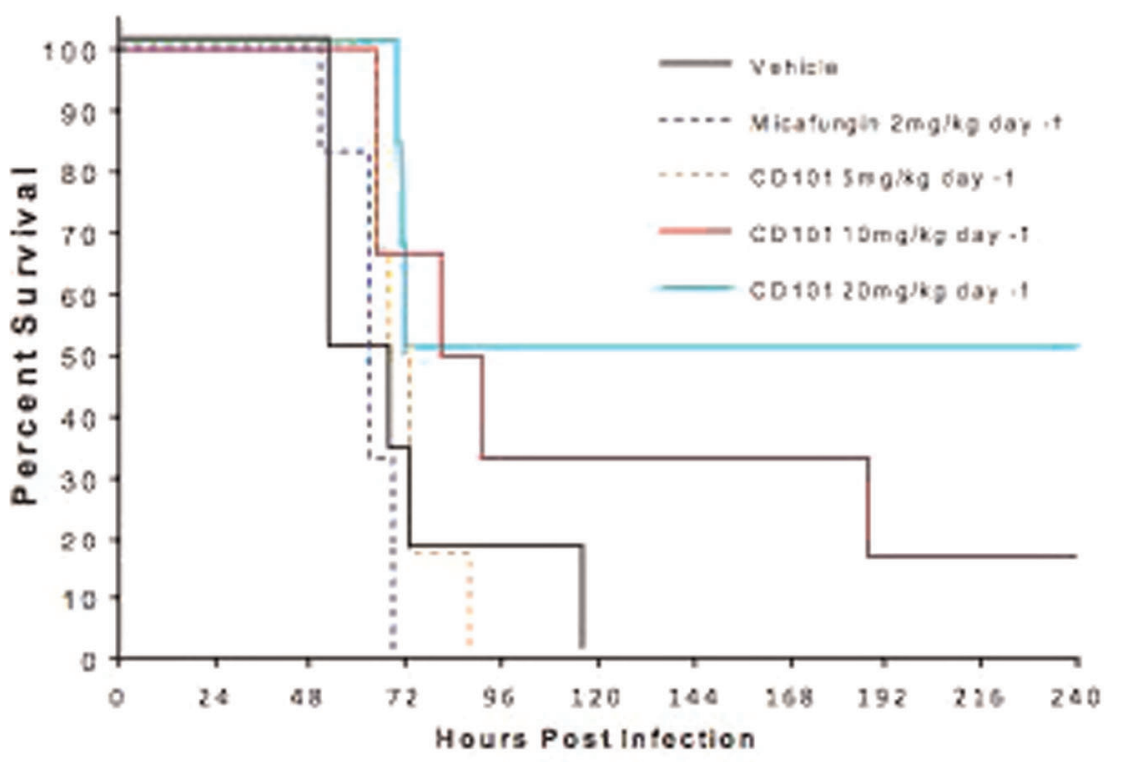

\section{Study 2}

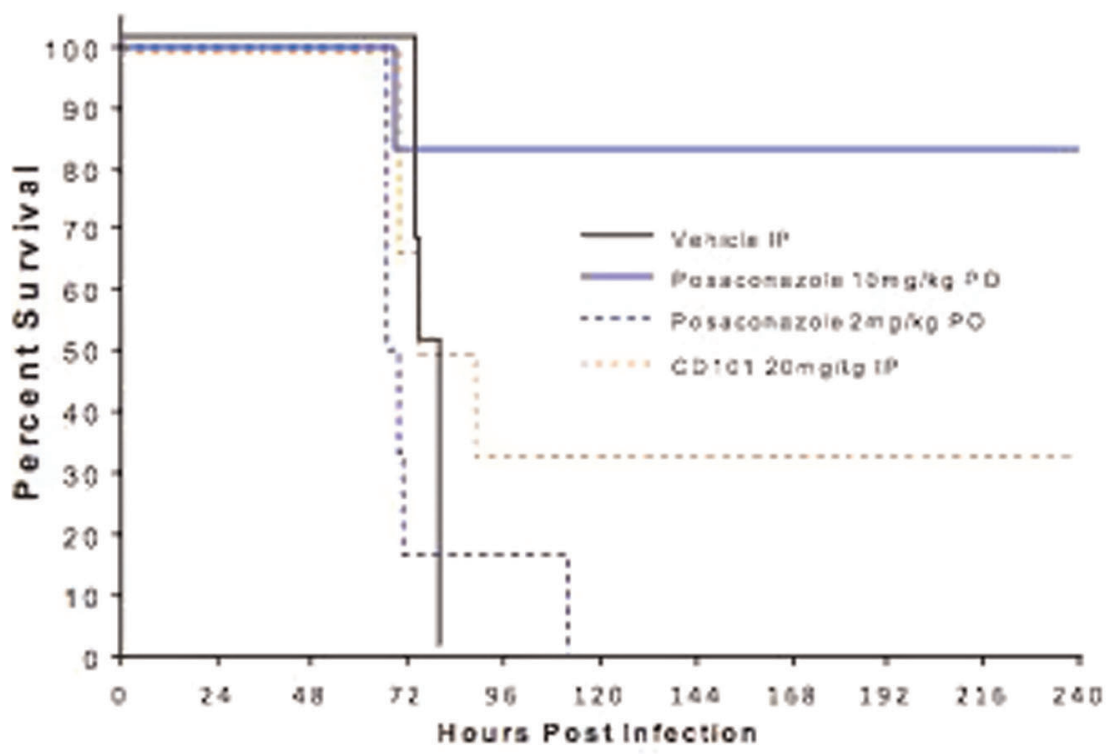

[P577 Figure] [Figure]

comparable with either a single $2 \mathrm{mg} / \mathrm{kg}$ dose or $0.2 \mathrm{mg} / \mathrm{kg}$ BID x $5 \mathrm{~d}$. For prophylaxis, a single $5 \mathrm{mg} / \mathrm{kg}$ dose given up to 5 days prior to infection showed improved survival. Doses $\geq 10 \mathrm{mg} / \mathrm{kg}$ showed $100 \%$ survival.
In the more challenging pulmonary aspergillosis model, dose-dependent increase in survival was observed from a single prophylaxis rezafungin dose. The human (400 mg) AUC equivalent dose of $20 \mathrm{mg} / \mathrm{kg}$ in mice of rezafungin 
showed an increase in survival relative to control. Further comparison with micafungin or posaconazole suggests an advantage for rezafungin with higher survival rate compared to micafungin or posaconazole at their respective human AUC equivalent doses (Figure 1). Only posaconazole at $10 \mathrm{mg} / \mathrm{kg}$ (5x higher than human AUC) showed a statistically-significant increase in survival rate relative to control.

Conclusions: Rezafungin was effective as treatment and as prophylaxis in neutropenic mouse disseminated and pulmonary aspergillosis infection models. Given its long $\mathrm{t}_{1 / 2}$ in human $(133 \mathrm{~h})$, rezafungin could prove useful for intermittent outpatient and inpatient echinocandin treatment and prophylaxis.

\section{Conflict of interest:}

V. Ong, S. Flanagan, K. Bartizal: employees and ownership interests, Cidara Therapeutics

G. Hough: employee, Cidara Therapeutics

A. Sattar, A. Sharp, P. Thommes: employees, Evotec

\section{P578}

\section{Novel Once-Weekly Echinocandin Rezafungin (CD101) Prevention and Treatment of Pneumocystis Biofilms}

\author{
Melanie T. Cushion ${ }^{1}$, Margaret S. Collins ${ }^{1}$, Jeffrey B. Locke ${ }^{2}$, Voon \\ Ong $^{2}$, Ken Bartizal ${ }^{2}$ \\ ${ }^{1}$ University of Cincinnati College of Medicine, Cincinnati, OH, United \\ States; ${ }^{2}$ Cidara Therapeutics, San Diego, CA, United States
}

Background: Rezafungin (formerly, CD101) is a novel long-acting echinocandin with pharmacokinetics that achieve high, front-loaded plasma exposure and allow once-weekly dosing. Rezafungin, currently in development for prophylaxis against Candida, Aspergillus, and Pneumocystis, has minimal potential for myelosuppression and drug-drug interactions that often complicate today's antifungal prophylaxis in patients undergoing bone marrow transplant (BMT).

Biofilm formation by fungi is considered a survival mechanism as biofilms are notoriously difficult to treat. It has been hypothesized that the morphology of Pneumocystis in mammalian lungs is akin to biofilms. In the present study, in vitro biofilm formation by $P$. carinii was used to explore the ability of rezafungin to prevent biofilm formation and to treat mature biofilms.

Methods: Biofilms using $P$. carinii and $P$. murina isolated from rat and mouse lung tissue were grown in Millicell culture inserts in multi-well plates with supplemented RPMI 1640 medium. Mature biofilms were grown for 7 days prior to treatment; nascent biofilm inserts received test compounds at the time of inoculation. Media alone was used as a negative control. The entire contents of
3 inserts per time point were harvested for assessment of ATP content using a luciferin-luciferase based assay at 1, 3, and 7 days of treatment.

Results: Seven days of treatment of mature biofilms with $100 \mu \mathrm{g} / \mathrm{ml}$ rezafungin was most efficacious, with a $97 \%$ decrease in ATP compared to untreated controls. The same dose was effective in preventing biofilm formation by $98 \%$. Previous studies using the same biofilm assays reported a decrease of $95 \%$ of ATP for mature biofilms with $100 \mu \mathrm{g} / \mathrm{ml}$ anidulafungin; $46 \%$ for caspofungin; and $0 \%$ for micafungin. Rezafungin efficacy in preventing biofilm formation was similar to that of anidulafungin at the same concentrations, which had shown a $98 \%$ decrease in ATP vs untreated biofilms. In contrast, caspofungin had little (14\%) and micafungin, no effect $(0 \%)$ on biofilm formation.

Conclusions: The long-acting echinocandin, rezafungin (formerly, CD101) was efficacious in preventing biofilm formation and reducing the viability of mature biofilms of $P$. jirovecii. These findings, in conjunction with previously published data demonstrating effective Pneumocystis, Candida, and Aspergillus prophylaxis in immunosuppressed preclinical models, further suggest utility of rezafungin in the setting of BMT.

Conflict of interest: Cushion: research funding, Cidara Therapeutics; NIH HHSN2722011000181. Task order HHSN27200008. Subcontractor. Patterson T, PI. In vitro screening for antifungal activity; US Veterans Affairs Merit Review

M. Collins: none to disclose

J. Locke, V. Ong, K. Bartizal: employees and ownership interests, Cidara Therapeutics

\section{P579}

Phase 2b, Randomized, Double-Blind, Placebo-Controlled Trial of Presatovir for treatment of Hematopoietic-Cell Transplantation (HCT) Patients with Respiratory Syncytial Virus (RSV) Lower Respiratory Tract Infection (LRTI)

Sanjeet Dadwal', Roy Chemaly ${ }^{2}$, Anne Bergeron ${ }^{3}$, Per Ljungman ${ }^{4}$, Yae-Jean Kim ${ }^{5}$, Michael Boeckh, Alpana Waghmare ${ }^{6}$, Kathleen Mullane $^{7}$, Dong-Gun Lee ${ }^{8}$, Catherine Small ${ }^{9}$, Hans Hirsch ${ }^{10}$, Elodie Blanchard $^{11}$, Roberto Patron ${ }^{12}$, Matt McKevitt ${ }^{13}$, Danielle Porter ${ }^{13}$, Robert Jordan $^{13}$, Ying Guo ${ }^{13}$, Polina German ${ }^{13}$, Timothy Watkins ${ }^{13}$, Jason Chien $^{13}$, Francisco Marty ${ }^{14,15}$

${ }^{1}$ City of Hope National Medical Center, Duarte, CA, United States; ${ }^{2}$ MD Anderson Cancer Center, Houston, TX, United States; ${ }^{3}$ Hopital St. Louis, Paris, France; ${ }^{4}$ Karolinska University Hospital, Stockholm, Sweden; ${ }^{5}$ Samsung Medical Center, Sungkyunkwan University, Seoul, Korea, Republic of: ${ }^{6}$ Fred Hutchinson Cancer Research Center, Seattle, WA, United States; ${ }^{7}$ University of Chicago Medical Center, Chicago, IL, United States; ${ }^{8}$ Seoul St. Mary's Hospital, Catholic University of Korea, Seoul, Korea, Republic of; ${ }^{9}$ Weil Cornell Medical Center, New York, NY, United States; ${ }^{10}$ University Hospital Basel, Basel, Switzerland; ${ }^{11} \mathrm{CHU}$ de Bordeaux, Bordeaux, France; ${ }^{12}$ Mayo 
Clinic, Phoenix, AZ, United States, ${ }^{13}$ Gilead Sciences Inc., Foster City, CA, United States; ${ }^{14}$ Dana Farber Cancer Institute, Boston, MA, United States; ${ }^{15}$ Brigham and Women's Hospital, Boston, MA, United States

Background: Presatovir (GS-5806) significantly reduced nasal viral load, signs and symptoms of RSV infection in a healthy human challenge study. We evaluated whether presatovir is safe and effective in the treatment of HCT patients with RSV LRTI.

Methods: HCT patients with confirmed RSV in upper and lower respiratory tract and evidence of new chest X-ray abnormalities consistent with LRTI were randomized $(1: 1)$ to receive oral presatovir 200mg every 4 days for 5 doses or placebo, in addition to each center's standard of care. Enrollment was stratified by supplemental oxygen requirement $(\leq 2 \mathrm{~L} / \mathrm{m}$ or $>2 \mathrm{~L} / \mathrm{min})$ and treatment with ribavirin. Primary endpoint was time weighted average change in RSV viral load through Day 9, measured by nasal sampling. Secondary endpoints included number of days without supplemental oxygen use, development of respiratory failure requiring mechanical ventilation, and all-cause mortality.

Results: From January 2015 to April 2017, 60 subjects from 17 centers were enrolled (Table). The median age was 57 years [interquartile range (IQR), 40-63]) in the presatovir and 55 years (IQR, 50-63]) in the placebo arm. Most subjects were males (presatovir 21 [70\%], placebo 23 [79\%]). The median duration of symptoms prior to first dose was similar between presatovir (6 days [IQR, 4-8]) and placebo (5 days [IQR, 3-7]) arms, as was median baseline RSV viral load (presatovir $6.2 \log _{10}$ copies/mL [IQR 5.6, 7.3], placebo $6.0 \log _{10}$ copies/mL [IQR 5.4, 7.3]). 10 (32\%; 5 inhaled, 5 oral) presatovir and 11 (38\%; 7 inhaled, 3 oral, 1 intravenous) placebo treated subjects were given Ribavirin.

Despite maintaining mean plasma troughs above 4-fold $\operatorname{paEC}_{95}$ for at least 17 days, presatovir treatment did not reduce the mean time weighted average change in viral load (presatovir $-1.00 \quad \log _{10}$ copies $/ \mathrm{mL}$, placebo $-0.97 \quad \log _{10}$ copies/mL; mean treatment difference $-0.02 \log _{10}$ copies/ $\mathrm{mL}, 95 \%$ confidence interval $-0.62,0.57, \mathrm{p}=0.94)$, the median number of supplemental oxygen-free days (presatovir 26 [IQR 10, 28] days, placebo 28 [IQR 9, 28] days, $\mathrm{p}=0.84$ ), the proportion of subjects developing respiratory failure (presatovir 3 , placebo $3, \mathrm{p}=0.98$ ), or all-cause mortality (presatovir 0 , placebo $2, \mathrm{p}=0.19$ ). Results were similar in exploratory analyses evaluating whether treatment effects were modified by duration of symptoms, baseline supplemental oxygen use, ribavirin use, lymphopenia, time since HCT, or graft-versus-host disease. Treatmentemergent adverse events (TEAE) were similar in both arms (presatovir 80\%, placebo 79\%); there were fewer $\geq$ Grade 3
TEAEs (23\% vs. 31\%), including fewer infection- and respiratory-related Grade $\geq 3$ TEAEs, among presatovirtreated patients.

Conclusions: Presatovir treatment was well tolerated in HCT patients with RSV LRTI but did not result in improved viral or overall clinical outcomes.

Clinical Trial Registry: ClinicalTrials.gov Identifier: NCT02254421

\section{Conflict of interest:}

S. Dadwal: Research funding/site investigator: Gilead, Ansun biopharma, Oxford Immunotec, GlaxoSmithKline, AiCuris, Shire, Merck. Consultant/advisory board, Merck

R. Chemaly: Research funding/site investigator: Gilead, Merck, Chimerix, Ansun Biopharma, Pulmotec, Novartis, Shire, Oxford Immunotec. Advisory board: Ablynx, Janssen, Adama biologics, Merck, Chimerix, Astellas, Medicines company, Workhard. Consultant: Ansun biopharma, Xenex, Oxford immunotec

P. Ljungman: Site investigator/research funding: Astellas, Gilead, Oxford immunotec, Merck. Advisory board: Merck, Aicuris, Ablynx. Honoraria: Shire

Y. Kim: Site investigator/research funding/ teaching/ speaking: Gilead, Janssen, Celltrion

M. Boeckh: Investigator/consulting/research funding: Merck, Astellas, Chimerix, Shire, Gilead. Consulting: Abbvie, Helocyte

A. Waghmare: Site Investigator/research funding: Gilead, Aviragen therapeutics

K. Mullane:Site investigator/research funding: Gilead, Astellas, Chimerix, Contrafect, Crestovo, GlaxosmithKline, Leonard Merlin Biopharm, Medimmune, Merck, Sage, Shire, Synexis. Advisory board: Chimerix, Merck, Synexis. Speaker/honoraria: Astellas, Merck, Nohla for DSMB member

Catherine Small: Site investigator/research funding: Gilead, Merck, Viiv, Glaxo

Hans H. Hirsch: Site investigator/research funding: Gilead, Advisory board: Chimerix, Speaker/honoraria: Novartis

A. Bergeron, D. Lee, E. Blanchard and R. Patron: Site investigators/research funding: Gilead

M. McKevitt, D. Porte, R. Jordan, Y. Guo, P. German, J. Chien: Employee/salary: Gilead

Francisco Marty: Site investigator/research funding: Gilead, Chimerix, GlaxoSmithKline, Merck, Shire, Honoraria: Visterra (DSMB member), United Medical (speaking), Roche molecular diagnostics (consultant), Alexion (DSMB member), Basilea (speaking), Fate therapeutics (Endpoint committee), GlaxoSmithKline (consultant), Merck (consultant) 


\begin{tabular}{lll}
\hline Baseline characteristics & $\begin{array}{l}\text { Presatovir } \mathrm{n}= \\
31\end{array}$ & $\begin{array}{l}\text { Placebo } \mathrm{n}= \\
29\end{array}$ \\
\hline Donor type, $\mathrm{n}(\%)$ & & \\
Autologous & $4(13)$ & $5(17)$ \\
Matched related & $5(17)$ & $9(31)$ \\
Mismatched related & $3(10)$ & 0 \\
Unrelated & $18(60)$ & $15(52)$ \\
Acute or cGVHD present, n(\%) & $17(57)$ & $17(59)$ \\
Days post HCT at RSV diagnosis, median & $451(169,863)$ & $517(182,703)$ \\
(IQR) & & \\
\hline
\end{tabular}

[[P579 Table] LRTI HCT STUDY]

\section{P580}

\section{Phlegmon of the face after allogeneic stem cell transplantation in T-LGL-Leukemia (a case report)}

Ulrich Ritter ${ }^{1}$, Stephan Kaun ${ }^{1}$, Jürgen Spehn ${ }^{2}$, Andreas Naumann ${ }^{3}$, Erik Chankiewitz ${ }^{4}$, Bernd Hertenstein ${ }^{1}$

${ }^{1}$ Klinikum Bremen Mitte, Hematology/Oncology, Bremen, Germany; ${ }^{2}$ Klinikum Bremen Links der Weser, Hematology/Oncology, Bremen, Germany; ${ }^{3}$ Klinikum Bremen Mitte, Ear, Nose and Throat Surgery, Bremen, Germany; ${ }^{4}$ Klinikum Bremen Mitte, Opthalmology, Bremen, Germany

Background: T-cell large granular lymphocytic leukemia (T-LGL-Leukemia) is a rare lymphoproliferative disorder that exhibits an chronic (> 6 months) elevation in large granular lymphocytes (LGLs) in the peripheral blood. It is characterized by a clonal expansion of $\mathrm{CD} 3+$ cytotoxic $\mathrm{T}$ cells and has been recognized by the WHO classification among mature T cell and NK cell neoplasms (WHO 2016). Prominent clinical features of T-LGL leukemia include neutropenia, anemia and rheumatoid arthritis (RA). Prognosis is normally good with 10 -year OS of $70 \%$. Main course of death is severe infection due to neutropenia.

Indications for treatment include severe neutropenia (ANC $<0.5 \times 10.9 / \mathrm{L})$, moderate

neutropenia (ANC $>0.5 \times 10.9 / \mathrm{L}$ ) associated with recurrent infections, symptomatic or

transfusion dependent anemia and associated autoimmune conditions requiring

therapy. Standard treatment of LGL leukemia is immunosuppressive therapy.

The most clinical experience has been reported using lowdose methotrexate,

cyclophosphamide, and cyclosporine as single agents. Only few cases of allogeneic stem cell transplantation have been described.

Methods: Case report

Results: The 60-year-old patient was diagnosed with TLGL-Leukemia in 2012. Treatment indication was mild neutropenia and recurring infection. There was no lasting

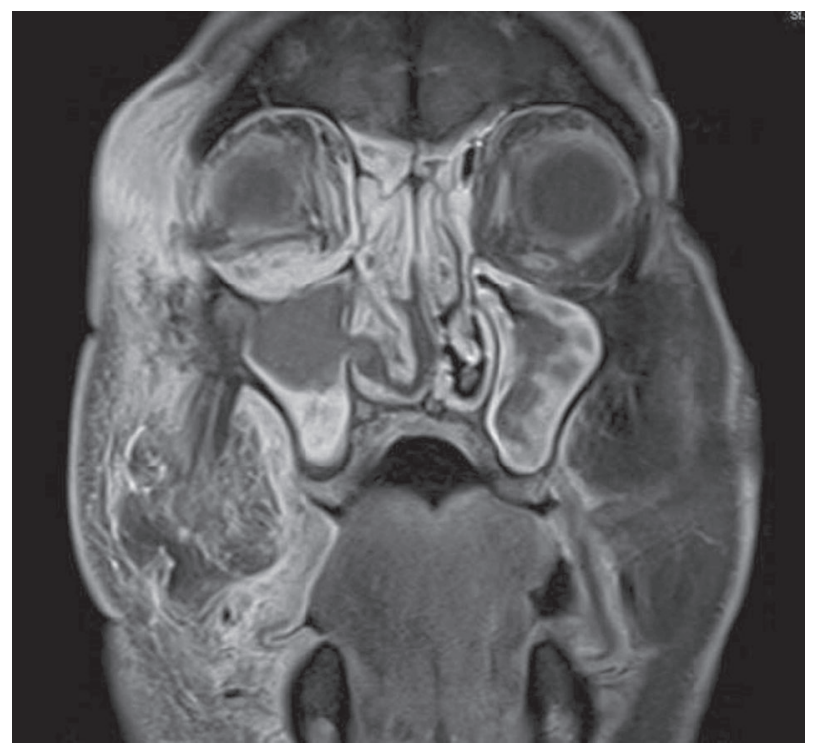

[P580 Figure] [MRI (coronar view) showing the vast plegmonous infection of right maxillary sinus and orbita]

response to G-CSF, MTX, Cyclophosphamide, Fludarabine and CHOP. In november 2016 bone marrow biopsy showed $85 \%$ infiltration with T-LGL, resulting in severe pancytopenia with regular transfusions and daily application of GCSF. From january to may 2017 we administered MabCampath (named patient program). BM-Infitration went down to $40 \%$, severe pancytopenia persisted, several episodes of life threatening neutropenic fever occurred. In search of other treatment options a HLA-ident sibling (sister) was identified and in july 2017 we performed allogeneic transplantation following a RIC conditioning protocol with Flu/Cy.

During aplasia the patient developed a vast phegmonous infection of the right face, origin being the maxillary sinus, spreading to the right orbita. Bacterial infection was assumed but could not be proven in multiple biopsies and in the bloodstream. Aspergillus fumigatus was identified by PAS-staining.

Treatment of the infection consisted of broad antibacterial and antifungal agents. Hematologic regeneration was timely, on day 30 G-CSF could be discontinued permanently (first time since diagnosis). Due to good operational management of our ENT and ophthalmology teams no persisting damage occurred.

BM-Biopsy on day 100 showed 98\% donor chimerism but $25 \%$ residual infiltration of T-LGL-Leukemia. Right now the patient is off immunosuppression and we gave a first DLI to booster Graft versus Lymphoma effect.

Conclusions: T-LGL-Leukemia is a rare lymphoproliferative disorder. Most patients are eventually refractory to conventional immunosuppression and chemotherapy and 
are in urgent need of alternative treatments. Allogeneic transplantation with a suitable donor (MRD, MUD) after RIC may provide cure for selected patients. Our patient benefits from a partial response with freedom from transfusions and G-CSF. Infectious complications during transplantation occur regular due to long lasting immunosuppression and neutropenia.

Conflict of interest: Main authors (U. Ritter and B. Hertenstein): Nothing to disclose

\section{P581}

Pneumocystis jirovecii prophylaxis: From guidelines to real life

Rabah Redjoul ${ }^{1}$, Christine Robin ${ }^{2,3}$, Francoise Foulet ${ }^{4}$, Mathieu Leclerc $^{3,5}$, Florence Beckerich ${ }^{5}$, Ludovic Cabanne ${ }^{5}$, Roberta di Blasi $^{5}$, Cecile Pautas, Andrea Toma ${ }^{5}$, Sebastien Maury ${ }^{3,5}$, Francoise Botterel $^{3,4}$, Catherine Cordonnier ${ }^{3,6}$

${ }^{1}$ Henri Mondor Hospital-Assistance Publique Hopitaux de Paris (APHP), Hematology, Créteil, France; ${ }^{2}$ Henri Mondor Hospital, Créteil, France; ${ }^{3}$ University Paris Est Créteil, Créteil, France; ${ }^{4}$ Henri Mondor Hospital, Parasitology, Créteil, France; ${ }^{5}$ Henri Mondor Hospital, Hematology, Créteil, France; ${ }^{6}$ Henri Mondor Hospital University Hospital, Hematology, Créteil, France

Background: Pneumocystis jirovecii pneumonia (PcP) has a very high mortality rate after allogeneic HSCT. Trimethoprim-sulfamethoxazole (TMP-SMX) is the preferred primary prophylaxis, any alternative being of lower efficacy ${ }^{1}$. According to our local procedures based on the ECIL guidelines, we looked at all patients transplanted in our department between Jan 2013-Nov 2016 in order to evaluate whether patients were correctly prophylaxed for $\mathrm{PcP}$ and in how many cases they receive TMP-SMX.

Methods: We assessed 139 consecutive patients transplanted in our center, all with a follow-up $\geq 12$ months. Our local JACIE procedure mentions that TMP-SMX is the first option for PcP prophylaxis (1 double-strength tablet 3 times a week) associated with folinic acid, starting from engraftment. The alternative in case of TMP-SMX intolerance is atovaquone (ATO $(750 \mathrm{mg} \times 2 / \mathrm{d})$. The period at risk is defined as $\geq 6$ months after myeloablative conditioning regimens, $\geq 12$ months after other regimens, and in any case as long as immunosuppressive drugs (IS) are given. PcP prophylaxis is prolonged 3-4 weeks after stopping the last IS. The period at risk was censored at time of death or PcP onset. We collected the number of PcP cases, the number of days at risk and of days not covered by prophylaxis during the at-risk period, and the reasons to withdraw TMP-SMX.

Results: Eigthteen cases of PcP occurred: 3 before engraftment, 1 under TMP-SMX, 7 under ATO, 4 while prophylaxis was transiently withdrawn for possible side effect, and 4 between 3-7 months after stopping IS. The median period at risk of PcP was 313 (9-1475) days. The median number of days not covered by prophylaxis was 17 (9-281) days. The ratio no. days without prophylaxis/no. days at risk was $20 \%$ in patients who developed PcP vs. $8 \%$ in those who did not $(\mathrm{p}=0.34)$ Thirteen $(8.6 \%)$ patients never received prophylaxis because they died before engraftment $(n=7$, including 3 who developed $\mathrm{PcP}$ ) or because of severe complications at engraftment. Among the 126 patients who received $\mathrm{PcP}$ prophylaxis, the first drug given after transplant was TMP-SMX in $122(97 \%)$ and ATO in $4(3 \%)$ patients (pretransplant intolerance to TMPSMX or poor engraftment). However, only 51 (37\%) patients received TMP-SMX as the sole prophylactic drug during the at-risk period. Indeed, TMP-SMX was stopped for suspected intolerance in 61 patients: cytopenia $(n=15)$, liver test abnormalities $(n=41)$, rash $(n=4)$ or unknown cause $(\mathrm{n}=1)$. As a result, 60 patients consecutively received TMP-SMX then ATO and 4 received pentamidine aerosolized as a $3^{\text {rd }}$ option.

Conclusions: Despite good compliance to guidelines, many concerns still remain about PcP prophylaxis after HSCT: (1) Patients may develop PcP before engraftment, especially in case of graft rejection. (2)TMP-SMX is often replaced by ATO: only 37\% keep TMP-SMX during the atrisk period. (3) Some cases occur several months after stopping IS, raising the issue of finding parameters to decide how long prophylaxis should be continued. TMP-SMX is too often replaced by ATO and probably not given enough time after stopping IS.

${ }^{1}$ Maertens J et al. 2016

Clinical Trial Registry: na

Conflict of interest: Nothing to disclose

\section{P582}

\section{Potential candidates for CMV prophylaxis considering serological status in allogeneic hematopoietic stem cell transplantation (allo-HSCT)}

Sara Aida Jiménez Julià ${ }^{1}$, Leyre Bento ${ }^{1}$, Antonio Gutiérrez ${ }^{1}$, Rubén Barrón ${ }^{2}$, Jordi Ginés ${ }^{3}$, Andrés Novo ${ }^{1}$, Jordi Reina ${ }^{2}$, José Luis Pérez $^{2}$, Antonia Sampol ${ }^{1}$

${ }^{1}$ Son Espases University Hospital, Hematology, Palma de Mallorca, Spain; ${ }^{2}$ Son Espases University Hospital, Microbiology, Palma de Mallorca, Spain; ${ }^{3}$ Son Espases University Hospital, Pharmacology, Palma de Mallorca, Spain

Background: The CMV infection is one of the most important complications leading morbimortality during the first year post-allo-HSCT. The use of high-dose steroids, graft versus host disease (GVHD), T-cell depletion and mismatched or unrelated donors constitute the main predisposing factors. The highest risk of CMV infection is 
associated with seronegative donors(D-)/seropositive recipients $(\mathrm{R}+)$ as the graft does not contain $\mathrm{CMV}$-specific $\mathrm{T}$ cells inside an immunocompromised recipient with latent CMV infection. Although effective, the current anti-CMV drugs are associated with myelosuppression and renal toxicity. Thus, novel agents with a better safety profile in prophylaxis have been developed (Maribavir and Letermovir). As these new drugs increase significantly health costs, we aim to determine in our series, which patients could benefit from this prophylaxis.

Methods: Patients undergoing allo-HSCT in our center were retrospectively reviewed from 2012 to November 2017, analyzing the CMV reactivation by PCR. CMV serostatus before allo-HSCT was checked, as well as the pre-emptive treatment and the rate of response, considering the incidence of CMV reactivation or CMV-related disease. Additionally, relation with GVHD and co-infection with BK virus was examined.

Results: 83 patients were analyzed with a median follow up for alive patients of 19 months. The characteristics of the patients are summarized in Table 1.

43 out of $55 \mathrm{R}+$ patients $(78 \%)$ presented a reactivation while only 3 out of $15 \mathrm{R}$ - patients $(17 \%)$ did it $(\mathrm{p}<0.001)$. Considering the donor serostatus in $\mathrm{R}+$ patients, the incidence or CMV reactivation was similar, 31 of $39 \mathrm{D}$ $+/ \mathrm{R}+(79.5 \%)$ and 3 of $14 \mathrm{D}-/ \mathrm{R}+(79 \%)$ but the risk of CMV disease was higher in $\mathrm{D}-/ \mathrm{R}+$ patients $(14.3 \%$ versus $5.1 \%) .57$ patients $(68.7 \%)$ had GVHD and $40(70.2 \%)$ of them presented a CMV-reactivation. 19 patients had BK virus hemorrhagic cystitis of whom $16(84.2 \%)$ presented CMV reactivation concomitantly. In our series patients with haploidentical donors had the higher rates of CMV reactivation (79\% versus around $60 \%$ in the others), being haploidentical and non-related donors those who presented the highest rates of CMV disease (14\% versus $6 \%$ in HLAidentical sibling donors). The 2 year-overall survival for the whole series was $56 \%$. 40 patients died, 18 of them due to an infection (45\%), 14/18 of these patients also had an active GVHD. We reported 7 CMV-related diseases, 5 of them resulting in death.

Conclusions: In our series those patients $\mathrm{CMV} \mathrm{D}+/ \mathrm{R}+$ together with $\mathrm{D}-/ \mathrm{R}+$ had the highest rate of $\mathrm{CMV}$ reactivations. This group of patients could benefit from prophylaxis with the new anti-CMV drugs in the future. Also, haploidentical or unrelated-donor allo-HSCT could be considered for this new approach.

Conflict of interest: All authors: Nothing to disclose.

\begin{tabular}{ll}
\hline Age(median,R) & $45(0-69)$ \\
\hline Gender(M/F) & $50(60 \%) / 33(40 \%)$ \\
Diagnose: AML or MDS/ ALL/ HL or NHL/ & $44(53 \%) / 11(13 \%) / 12$ \\
Others & $(14 \%) / 16(19 \%)$ \\
& \\
\hline
\end{tabular}

Table (continued)

\begin{tabular}{ll}
\hline Age(median,R) & $45(0-69)$ \\
\hline $\begin{array}{l}\text { Donor type: HLA-matched sibling/ Non-related// } \\
\text { Haploidentical/ UCB }\end{array}$ & $\begin{array}{l}44(53 \%) / 16(19 \%) / 21 \\
(25 \%) / 2(2 \%)\end{array}$ \\
$\begin{array}{l}\text { Disease status pre-HSCT: 1CR/ } \geq 2 \mathrm{CR} / \mathrm{PR} / \text { Stable } \\
\text { disease/progression }\end{array}$ & $30(37 \%) / 12(15 \%) / 6(7 \%) /$ \\
Conditioning: Myeloablative/ Non-myeloablative/ & $44(41 \%)$ \\
Sequential & $44(53 \%) / 32(39 \%) / 7(8 \%)$ \\
GVHD Prophylaxis: MMF+Cyclosporine or & $29(38 \%) / 7(9 \%) / 7(9 \%) / 6$ \\
Tacrolimus/ MTX+Cyclosporine/ Tacrolimus & $(8 \%) / 28(36 \%)$ \\
+Sirolimus/ ATG+Others/ Post- & \\
Cyclophosphamide+MMF+Cyclosporine & \\
\hline
\end{tabular}

[[P582 Table] Table 1: Patients' characteristics]

\section{P583}

Abstract previously published

\section{P584}

Prevalence and Risk factors of Resistant Gram-Negative Bacilli colonization in hospitalized haematological patients

Ana Pilar Gonzalez-Rodriguez ${ }^{1}$, Antonio Sole ${ }^{2}$, Ana Julia GonzalezHuerta ${ }^{3}$, Alejando Alonso Cabrero ${ }^{4}$, Pilar Palomo ${ }^{3}$, Covadonga Valdes-Arias $^{3}$, Ana Maria Fernandez-Verdugo ${ }^{5}$, Teresa Bernal ${ }^{6}$, Soledad Gonzalez-Muñiz ${ }^{3}$

${ }^{1}$ Hospital Universitario Central de Asturias, Hematology. Stem Cell Trasplantation, Oviedo, Spain; ${ }^{2}$ Hospital Central de Asturias, Oviedo, Spain; ${ }^{3}$ Hospital Central de Asturias, Hematology. Stem Cell Trasplantation, Oviedo, Spain; ${ }^{4}$ Universidad de Oviedo, Oviedo, Spain; ${ }^{5}$ Hospital Central de Asturias, Microbiology, Oviedo, Spain;

${ }^{6}$ Hospital Central de Asturias, Hematology, Oviedo, Spain

Background: Antimicrobial resistance is rapidly increasing worldwide and constitute a universal threat for health care. There is limited data of multiple drug resistant bacteria (MDR) in haematological hospitalized patients, without information about resistant Gram-negative bacteria (MRGN).

Methods: A screening programme for MDR strains associated by guidelines of contact isolation was implemented in 2016 in our hospital to know prevalence of MDR colonization among haematological patients -extended spectrum beta-lactamase (ESBL) producing Enterobacteriaceae, multiresistant Acinetobacter Baumannii (MRAB) and mulitresistant Pseudomonas aeruginosa (MRPA)- to indentificate risk factors for colonization and infection among those colonized. We collected samples on 130 hospitalized patients in Hematology Unit of Hospital Universitario Central de Asturias between $1^{\text {st }}$ January 2016 and $30^{\text {st }}$ October 2017. The main reason for admission was acute leukemia or myelodysplastic syndrome $(n=74)$ followed by stem cell transplantation (12 allogeneic and 10 


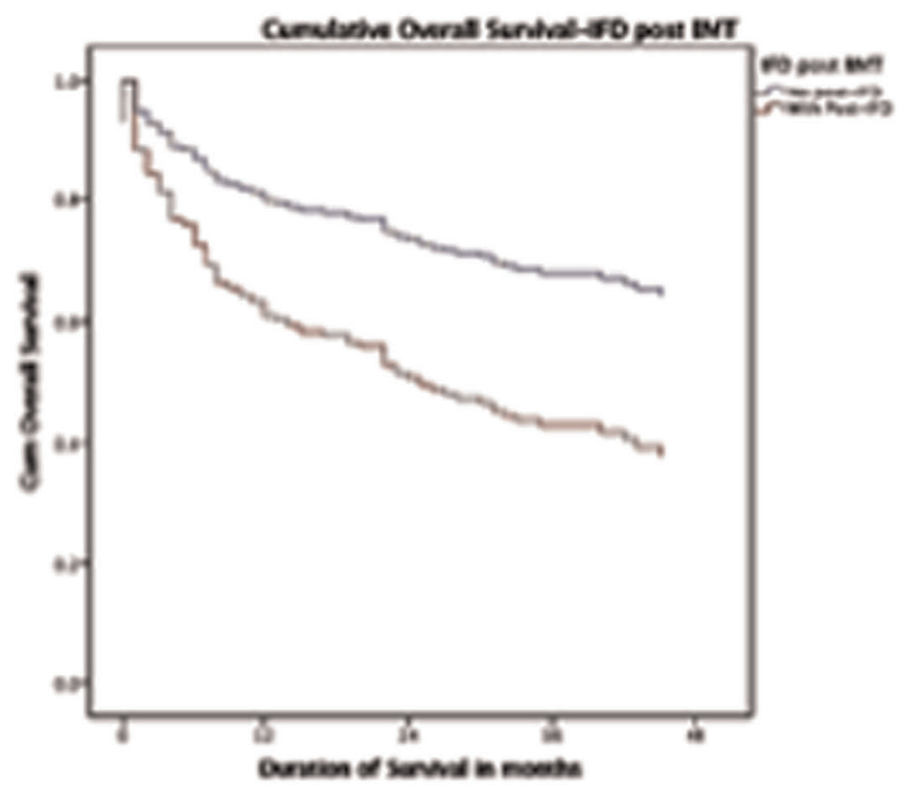

Fig 1: Univariate Cox regression with OS in post BMT IFD independent of pre-IFD status

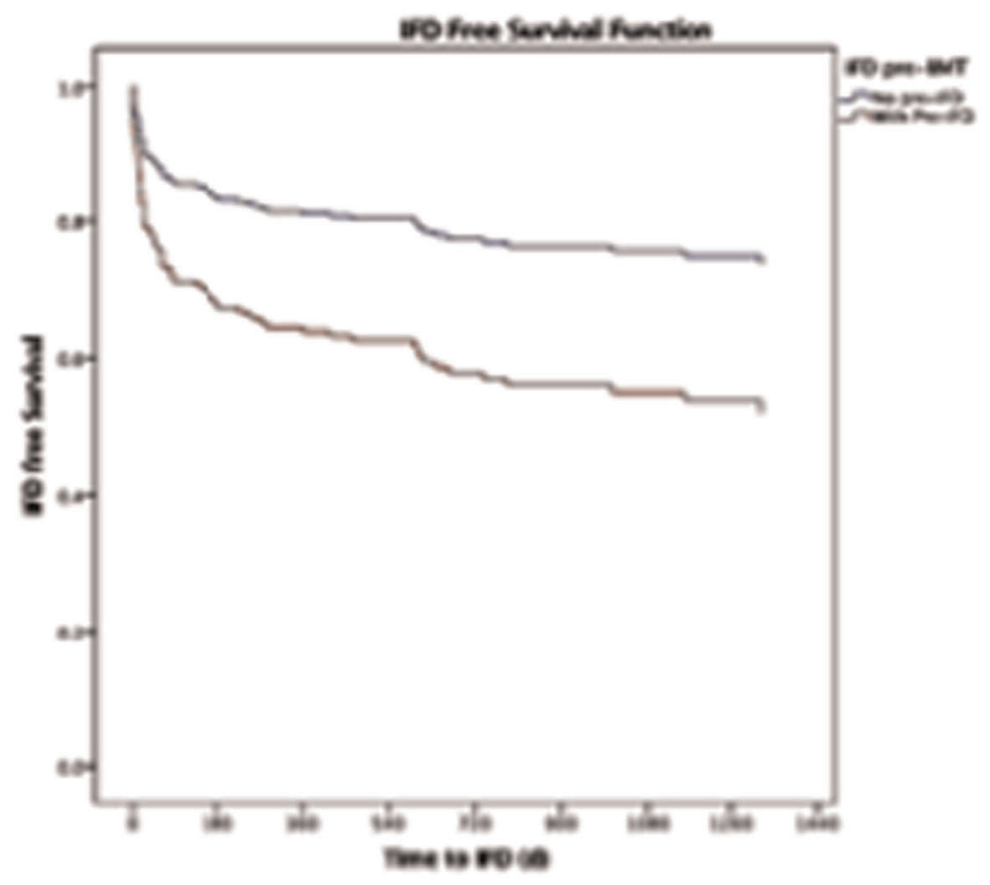

Fig 3- IFD free survival and pre-IFD status- Multivariate model [P583 Figure] [IFD abstract Fig 1 and 3] 
autologous). Routine surveillance cultures and active screening (throat and anus in parallel) were obtained weekly until obtain two consecutive negative results.

Results: We screened 1843 samples: 306 rectal and 70 throats were positive for MRD. 65 patients $(50 \%)$ were colonized with a MRGN with a median duration of 84 days (0-414). K pneumoniae was the most common isolates $(\mathrm{n}=$ 49; 37.7\%) (4 carbapenem-resistant), ESBL Enterobacter cloacae $(\mathrm{n}=4,3,1 \%)$ (2 carbapenem resistant), ESBL Escherichia coli $(\mathrm{n}=2,1,5 \%)$; MRBA $(\mathrm{n}=44(3,1 \%)$ MRPA $(n=6 ; 4,6 \%)$. All patients with throat positive were positive in rectal samples. Risk factors for colonization are showed in table 1.34 patients developed MRGN infection and in 12 patients' carriage of ESBL producers seems to be the consequence of infection. Colonized patients had increased risk for infection and admission in intensive care unit $(\mathrm{p}<0,001)$.

Conclusions: Rate of MRGN colonization is very high in our hospitalized patients and was associated with significantly higher frequency of subsequent infection. In high risk hospitalized patiens is mandatory the development of a screening programme for MDR strains associated by guidelines of contact isolation. Rectal samples to identificate colonizated patients have higher performance than pharyngeal. There is a growing problem of antimicrobial resistance among pathogens isolated in hematological patients and hematopoietic stem cell transplantation and the paucity of new antibacterial drugs should limit use of drugs where there is no other alternative and to develop strategies to decrease these infections.

\section{Conflict of interest: None}

\begin{tabular}{llll}
\hline Colonization & Yes & No & $\mathrm{p}$ \\
\hline Days of hospitalization & 35 & 26 & 0.02 \\
Prior quinolones prophylaxis & $22(64,7 \%)$ & $12(35,3 \%)$ & 0.024 \\
Prior exposure to beta lactam antibiotics & $36(73,5 \%)$ & $13(26,5 \%)$ & $<0,001$ \\
Recent hospitalization & $16(80 \%)$ & $4(20 \%)$ & 0,004 \\
Prior mucositis & $32(80 \%)$ & $8(20 \%)$ & $<0,001$ \\
Catheter venous central & $55(62,7 \%)$ & $33(37,5 \%)$ & $<0,001$ \\
Catheter urinary & $17(77,3 \%)$ & $5(22,7 \%)$ & 0,04 \\
\hline
\end{tabular}

[[P584 Table] Table 1. Risk factors for colonization]

\section{P585}

\section{Primary Antifungal Prophylaxis with caspofungin after haploidentical transplantation with post-transplant cyclophosphamide: a retrospective analysis}

Jacopo Mariotti ${ }^{1}$, Chiara De Philippis ${ }^{1}$, Stefania Bramanti, ${ }^{1}$, Barbara Sarina ${ }^{1}$, Federica Tordato ${ }^{2}$, Daria Pocaterra ${ }^{2}$, Erminia Casari $^{2}$, Carmelo Carlo-Stella ${ }^{3}$, Armando Santoro $^{3}$, Luca Castagna $^{1}$
${ }^{1}$ Humanitas Cancer Center, Bone Marrow Transplant Unit, Rozzano (Milan), Italy; ${ }^{2}$ Humanitas Cancer Center, Infectious Disease Unit, Rozzano (Milan), Italy; ${ }^{3}$ Humanitas Cancer Center, Hematology, Rozzano (Milan), Italy

Background: Invasive fungal infections (IFI) represent one of the most common side effect of allogeneic hematopoietic stem cell transplant (allo-SCT). Donor type, graft-versus-host-disease, pre-transplant disease status, duration of neutropenic phase are the most relevant variables associated with the risk of developing IFI in the early, late and very late phase post-allo SCT. In particular, the incidence of IFI is higher after a mismatched related donor (Haplo-SCT) relative to a matched-related donor (MRD) transplant: $8 \%$ vs $4 \%$. Recent guidelines recommended anti-mold prophylaxis for patients at high risk of IFI. In the attempt to reduce the risk of IFI after Haplo-SCT with post-transplant cyclophosphamide (PT-Cy), we retrospectively analyzed the efficacy of caspofungin as antifungal prophylaxis (AP) at our institution

Methods: Between November 2013 and June 2017, 84 consecutive patients undergoing a Haplo-SCT with PT-Cy for a hemotological malignancy received anti-mold prophylaxis with caspofungin from day -6 to the day of neutrophil engraftment. Patients' characteristics were as follows: median age 52, 57\% were male, $53 \%$ had lymphoproliferative disease and $37 \%$ acute myeloid leukemia, 59\% were in complete remission (CR). Conditioning regimens were non-myeloablative (NMA) in most cases $(86 \%)$ and graft source was either represented by bone marrow (44\%) or peripheral blood stem cells (PBSC). Median day of neutrophil engraftment was 20 (15-73). According to GITMO stratification of IFI risk, in the early and late phase, $58 \%$ and $55 \%$ of the patients, respectively, were at high risk of IFI.

Results: Median follow-up was 1 year. Three-year OS, 3year progression-free survival (PFS) and 1-year NRM were $57 \%$ (42-68), 51\% (36-64) and 19\% (11-28), respectively. Cumulative incidence (CI) of 6-months grade 2-4 acute graft-versus-host-disease (GVHD) and 2-years moderatesevere chronic GVHD was 30\% (20-40) and 6.5\% (2-15), respectively. Five patients in the early phase and 2 in the late phase were diagnosed with a proven-probable (PP) IFI due to CT scan imaging and detection of galactomannan in bronchoalveolar lavage. Median day of PP-IFI was 19 days (15-49) and 100-day CI of IFI was 8.4\% (4-15). No patients died of IFI. By univariate analysis (Table I), having an active disease at the time of transplant was the only variable associated with a trend for increased risk of IFI compared with patients in CR: $15 \%$ (5-29) vs $4 \%(0-12), p=0.08$. Of note, no increased risk of IFI was observed for patients within the high-risk cohort according to GITMO stratification. By multivariate analysis adverse disease status was the 
Table I Univariase analyss of the variables associaned with EORTC PP-IFI within the firs: 100 days after haplo-SCT

\begin{tabular}{|c|c|c|}
\hline Characteristics & CIPP-II & $\mathbf{P}$ \\
\hline $\begin{array}{l}\text { Sex } \\
\text { Male } \\
\text { Eamale }\end{array}$ & $\begin{array}{c}6 \%(2-15) \\
11 \%(2-24)\end{array}$ & 0.4 \\
\hline $\begin{array}{l}\text { Disease Type } \\
\text { Myeloid } \\
\text { HI } \\
\text { NFII } \\
\text { ALL }\end{array}$ & $\begin{array}{l}6 \%(1-19) \\
12 \%(3-28) \\
9 \%(1-27) \\
0 \%\end{array}$ & 0.8 \\
\hline $\begin{array}{l}\text { Disease Status before Transplant } \\
C R \\
\text { PR/SD/PD }\end{array}$ & $\begin{array}{l}4 \%(0-12) \\
15 \%(5-29)\end{array}$ & 0.08 \\
\hline $\begin{array}{l}\text { Armand Risik (DRT) } \\
\text { LR/RR } \\
\text { HR VHR }\end{array}$ & $\begin{array}{c}7 \%(2-15) \\
13 \%(3-30)\end{array}$ & 0.3 \\
\hline $\begin{array}{l}\text { Sex mismatch } \\
F \rightarrow M \\
\text { Others }\end{array}$ & $\begin{array}{l}6 \%(0-25) \\
9 \%(4-17)\end{array}$ & 0.7 \\
\hline $\begin{array}{l}\text { HCT-CI } \\
a-2 \\
23\end{array}$ & $\begin{array}{l}7 \%(2-17) \\
10 \%(3-22)\end{array}$ & 0.5 \\
\hline $\begin{array}{l}\text { Condationing } \\
\text { NMA RIC } \\
\text { MAC }\end{array}$ & $\begin{array}{l}10 \%(4-18) \\
0 \%\end{array}$ & 0.2 \\
\hline $\begin{array}{l}\text { Graft Type } \\
\text { BM } \\
\text { PBSC }\end{array}$ & $\begin{array}{l}11 \%(3-23) \\
0.5 \%(2-10\end{array}$ & 0.4 \\
\hline $\begin{array}{l}\text { CMrV Serostatus } \\
\text { Others } \\
\text { Neg/Pos }\end{array}$ & $\begin{array}{l}9 \%(4-17) \\
6 \%(0-26)\end{array}$ & 0.7 \\
\hline $\begin{array}{l}\text { Domor Type } \\
\text { Child } \\
\text { Sibling } \\
\text { Parent }\end{array}$ & $\begin{array}{l}9 \%(2-22) \\
9 \%(2-22) \\
9 \%(0-34)\end{array}$ & 0.8 \\
\hline $\begin{array}{l}\text { Crrico earty phase } \\
\text { Standard Risi } \\
\text { High Risk }\end{array}$ & $\begin{array}{l}7 \%(1-20) \\
10 \%(4-20)\end{array}$ & 0.7 \\
\hline $\begin{array}{l}\text { GIMO bate phase } \\
\text { Low riah } \\
\text { High Risk }\end{array}$ & $\begin{array}{l}11 \%(4-23) \\
8 \%(1-23)\end{array}$ & 0.4 \\
\hline
\end{tabular}

[P585 Figure] [TableI]

only variable associated with higher risk of IFI: HR 8,5 $(0,9-77), \mathrm{p}=0.05$. When we compared AP with caspofungin to a historical cohort of patients $(n=64)$ treated with ev itraconazole at our institution, we found that 100-day CI of IFI was lower with caspofungin (8.4\%, range 4-16) relative to itraconazole $(16 \%, 8-26)$, but this difference did not reach statistical significance $(\mathrm{p}=0.1)$.

Conclusions: In summary, AP with caspofungin is an effective strategy to prevent PP-IFI after Haplo-SCT with PT-Cy in high-risk patients. Further studies are warranted to reduce the incidence of IFI for patients at higher risk, especially those with active disease at the time of transplantation.

Conflict of interest: The authors have no conflict of interest to disclose

\section{P586}

Primary antifungal prophylaxis with Micafungin in alternative donor transplant patients with active acute leukemia at transplant (GANDALF): a prospective Study from GITMO

Corrado Girmenia ${ }^{1}$, Fabio Ciceri ${ }^{2}$, Paolo Bernasconi $^{3}$, Alessandra Picardi, Maria Teresa Van Lint ${ }^{5}$, Cristina De Pau ${ }^{6}$, Stefano Guidi ${ }^{7}$, Alessandro Busca ${ }^{8}$, Angelo Michele Carella ${ }^{9}$, Irene Cavattoni ${ }^{10}$, Jacopo Peccatori', Daniela Cilloni ${ }^{11}$, Giuseppe Console ${ }^{12}$, Paolo Corradini ${ }^{13}$, Francesco Onida ${ }^{14}$, Anna Paola Iori ${ }^{1}$, Giovanni Grillo $^{15}$, Anna Proia ${ }^{16}$, Giuseppe Milone ${ }^{17}$, Roberto Sorasio ${ }^{18}$, Alessandra Crescimanno ${ }^{19}$, Franco Narni ${ }^{20}$, Francesca Patriarca ${ }^{21}$, Francesco Zallio ${ }^{22}$, Matteo Parma ${ }^{23}$, Giovanni Pisapia ${ }^{24}$, Stella Santarone $^{25}$, Rosanna Scimè ${ }^{26}$, Simona Sica ${ }^{27}$, Paola Carluccio ${ }^{28}$, Adriana Vacca ${ }^{29}$, Daniele Vallisa ${ }^{30}$, Arianna Masciulli ${ }^{31}$, Chiara 
Pavoni $^{31}$, Sonia Mammoliti ${ }^{32}$, Francesca Bonifazi ${ }^{33}$, Alessandro Rambaldi $^{34}$

${ }^{1}$ Policlinico Umberto I, Roma, Italy; ${ }^{2}$ IRCCS San Raffaele Scientific Institute, Milano, Italy; ${ }^{3}$ IRCCS Policlinico S Matteo, Pavia, Italy; ${ }^{4}$ Policlinico Tor Vergata, Roma, Italy; ${ }^{5}$ IRCCS H San Martino, Genova, Italy; ${ }^{6}$ Ospedale Oncologico Businco, Cagliari, Italy; ${ }^{7}$ Ospedale Careggi, Firenze, Italy; ${ }^{8}$ AO Citta della Salute e della Scienza, Torino, Italy; ${ }^{9}$ Casa Sollievo della Sofferenza, San Giovanni Rotondo, Italy; ${ }^{10}$ Comprensorio Sanitario di Bolzano, Bolzano, Italy; ${ }^{11}$ Centro Trapianti Metropolitano, Torinio, Italy; ${ }^{12}$ AZ. Osp. Bianchi Melacrino Morelli, Reggio Calabria, Italy; ${ }^{13}$ Fondazione IRCCS Istituto Nazionale dei Tumori, Milano, Italy; ${ }^{14}$ IRCCS Ca' Granda Ospedale Maggiore Policlinico, Milano, Italy; ${ }^{15}$ Ospedale Niguarda, Milano, Italy; ${ }^{16}$ AO San Camillo Forlanini, Roma, Italy; ${ }^{17}$ Ospedale Ferrarotto - Università degli Studi di Catania, Catania, Italy; ${ }^{18} \mathrm{AOS}$ Croce e Carlo, Cuneo, Italy; ${ }^{19}$ Ospedale La Maddalena, Palermo, Italy; ${ }^{20}$ Università Modena, Modena, Italy; ${ }^{21}$ Azienda OspedalieroUniversitaria "Santa Maria della Misericordia", Udine, Italy; ${ }^{22} \mathrm{AO}$ SS Antonio e Biagio, Alessandria, Italy; ${ }^{23}$ Ospedale San Gerardo, Clinica Ematologica dell Universita Milano-Bicocca, MONZA, Italy; ${ }^{24}$ Ospedale San Giuseppe Moscato, Taranto, Italy; ${ }^{25}$ Ospedale Civile, Pescara, Italy; ${ }^{26}$ AO Ospedali Riuniti Villa Sofia - Cervello, Palermo, Italy; ${ }^{27}$ Policlinico Gemelli, Univ. Cattolica Sacro Cuore, Roma, Italy; ${ }^{28}$ Policlinico Consorziale di Bari, Bari, Italy; ${ }^{29}$ PO R. Binaghi, Cagliari, Italy; ${ }^{30}$ Ospedale G. Da Saliceto di Piacenza, Piacenza, Italy; ${ }^{31}$ Ospedale Papa Giovanni XXIII, Bergamo, Italy; ${ }^{32}$ IRCCS AUO IST- San Martino, Genova, Italy; ${ }^{33}$ Istituto Seragnoli, Bologna, Italy; ${ }^{34}$ AO Papa Giovanni XXIII, Bergamo, Italy

Background: Patients with acute leukemia (AL) refractory to initial or salvage chemotherapy undergoing allogeneic hematopoietic stem-cell transplantation (allo-HSCT) are at high risk of invasive fungal diseases (IFD), in particular invasive aspergillosis, during the early (40 days) post transplant phase. The risk is particularly high in patients receiving transplant from an unrelated or mismatched related donor (Girmenia et al 2014). A mould active primary antifungal prophylaxis is recommended in this setting.

Methods: Across GITMO centres, a treatment algorithm was promoted including an alternative donor search early in the treatment plan of adults patients with Primary Induction Failure or relapsed AL. Patients without an available unrelated donor received a transplant from CBT or family haploidentical donor (GANDALF-01 trial, Gitmo Against Non-responding anD Acute Leukemia Failures; Eudract 2012-004008-37). Patients without a history of pretransplant IFD received primary antifungal prophylaxis (PAP) with micafungin at the dose of $50 \mathrm{mg} /$ day until engraftment. The rate of proven/probable and possible IFDs documented during micafungin prophylaxis, and within the first 40 and 100 days after transplantation was evaluated. An historical comparison with patients with the same characteristics among a previous GITMO survey (20082010) was performed.

Results: From July 2013 to October 2014, 87 patients were submitted to allo-HSCT and 59 of them received micafungin PAP. Median age was 53 (19-69) years. Donor

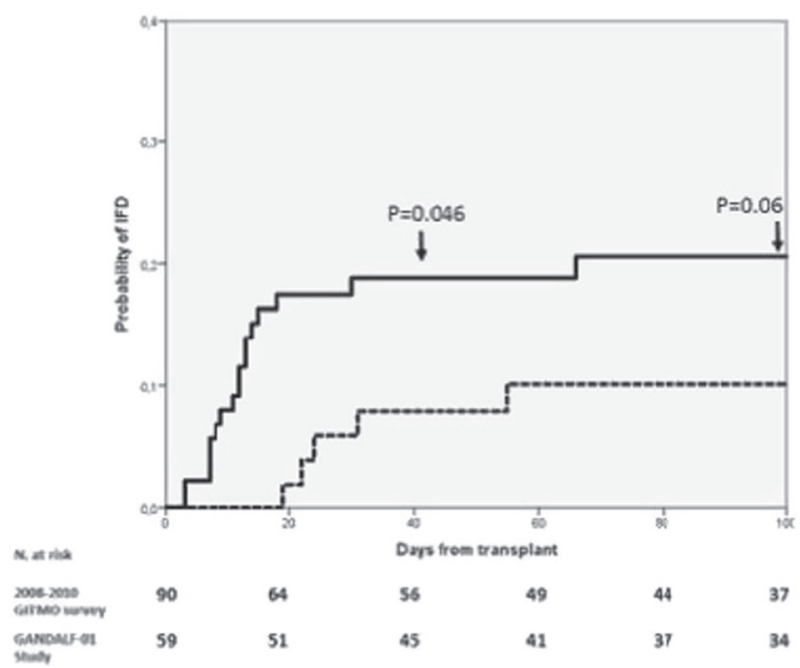

[P586 Figure] [Figure 1. Historical comparison of probability of IFD in Gandalf-01 and 2008-1010 GITMO]

was unrelated in 34, CB in 1 and haplo in 24. Overall, 4 $(6.8 \%)$ and $5(8.5 \%)$ of 59 patients developed a proven/ probale IFD (invasive aspergillosis in 3 cases) within 40 days and 100 days from transplant, respectively. Only 2 (3.4\%) IFDs were documented while the patient was receiving antifungal prophylaxis (breakthrough infection). A possible breakthrough pulmonary IFD was documented in a further patient. The cumulative incidence of proven/ probable IFDs in 59 patients of the GANDALF-01 study and in 90 patients of the 2008-2010 GITMO survey (considering only patients with active AL at transplant, who received transplant from unrelated or mismatched related donors and not received any mould-active antifungal prophylaxis) showed a significantly lower probability of IFD at 40 days from transplant in the GANDALF-01 study population ( $9 \%$ vs $19 \%, \mathrm{p}=0.046$ ) (figure 1). Only two patients discontinued micafungin PAP due to a presumed drug-related side effect.

Conclusions: This prospective multicenter study seems to show that micafungin is a safe and effective option in the early PAP of patients with active AL undergoing alloHSCT from unrelated or mismatched related donors.

Clinical Trial Registry: Eudract 2012-004008-37

Conflict of interest: nothing to disclose

\section{P587}

\section{Probiotics and allogeneic Hematopoietic Stem Cell} Transplant: a feasibility study

Nicola Mordini ${ }^{1}$, Assunta Melaccio ${ }^{2}$, Margherita Bonferroni ${ }^{1}$, Laura Bertolotti $^{1}$, Claudia Castellino ${ }^{1}$, Sandra Cavallero ${ }^{1}$, Mariella Grasso $^{1}$, Daniele Mattei ${ }^{1}$, Luca Prette ${ }^{1}$, Davide Rapezzi ${ }^{1}$, Massimo Massaia $^{1}$ 
${ }^{1}$ AO S Croce e Carle, Hematology, Cuneo, Italy; ${ }^{2}$ Universita' di Bari, AO Policlinico di Bari, Internal Medicine and Oncology, Bari, Italy

Background: Gram-negative Bacteremia (GNB) is a major cause of morbidity and mortality in allogeneic Hematopoietic Stem Cell Transplant (aHSCT). In particular Carbapenem-Resistant Klebsiella pneumoniae(CRKp) infections or colonization before HSCT represent a predictive condition of a subsequent infection with high morbidity and mortality rates. Perturbed microbiota composition results in aberrant systemic immune responses as well as pathogen colonization and mucosal invasion. Probiotics are nutritional supplements that contain a defined amount of viable microorganism preserving the microbiota and upon administration confer a benefit to the host. The proposed mechanism of probiotics are of great interest in aHSCT setting: block the adhesion and suppress the growth of pathogenic bacteria to the intestinal epithelium by the production of inhibitory agents and by directly binding to gram-negative bacteria, enhance and modulate the intestinal immune response and maintain normal levels of short-chain fatty acids, repair intestinal permeability, upregulate intestinal electrolyte absorption. Howewer, safety of administering viable bacteria to severely immunecompromised and neutropenic aHSCT adult patients has not been evaluated, yet.

Methods: CRKp is endemic in our Hospital . In order to minimize the impact of CRKp infection or colonization in our aHSCT recipients, we evaluated the safety and feasibility of 4 multistrain probiotic preparations (commercially available from Bromatech, Italy). They were somministered in a fixed weekly schedule beginning on day -18 and continued during neutropenic phase until discharge. The study cohort comprised 25 consecutive adults recipients of aHSCT treated between January 2016 and June 2017. Sixteen patients suffered from acute leukemia or myelodisplastic syndrome and only 10 were in complete remission at transplant.The conditioning regimen was busulfan -fludarabin based in 18/25 patients and was intended to be myeloablative in 11. Donors were HLA identical (10/10) in 14/25 transplants and stem cells source was peripheral blood in 18 procedures. Sorror was $\geq 2$ in 12/25 cases. Of note, 2 patients experienced a CRKp infection before transplant and 4 were CRKp carrier; they were at high risk to develop a pre-engrafment infection with high mortality rate.

Results: Median time to wbc engrafment was 15 days (11-22) .Severe mucositis (grade III) was seen in 7 patients. Thirtheen patient developed grade II aGVHD and one grade III. All the patients were discharged from the hospital and no infections or adverse events due to probiotics were observed. At day +100 all except one were alive. Only 2 patients were not compliant to the protocol and suspended intake of probiotics before admission for aHSCT. Four patients stopped intake (2-5 days) for mucositis. Among the 4 pre-engrafment GNB documented, we observed 2 CRKp and 2 E.Coli cases in patients who were colonized from the the same pathogen before transplant.

Conclusions: In our small experience multistrain probiotics appear safe and effective in preventig CRK p morbidity and mortality in the aHSCT setting. In particular, their somministration during the neutropenic phase seems possible.

Conflict of interest: None of the authors has anything to disclose.

\section{P588}

Prospective evaluation of the impact of a previous fungal infection on the of patients $\hat{A}^{\prime}$ eligibility to allogeneic hematopoietic stem cell transplantation

Gaetano Maffongelli, Laura Cudillo ${ }^{2}$, Filippo Barreca ${ }^{1}$, Carlotta Cerva $^{1}$, Benedetta Mariotti ${ }^{2}$, Eleonora Ceresoli ${ }^{2}$, Raffaella Cerretti ${ }^{2}$, Alessandra Picardi ${ }^{2}$, Massimo Andreoni ${ }^{1}$, William Arcese ${ }^{2}$, Loredana Sarmati ${ }^{1}$

${ }^{1}$ Tor Vergata University, Clinical Infectious Disease, Department of System Medicine, Rome, Italy; ${ }^{2}$ Tor Vergata University, Department of Hematology, Stem Cell Transplant Unit, Rome, Italy

Background: Fungal infections are a possible complications in patients with haematological neoplasia. Although it is known that these infections may complicate the phases following chemotherapy or allogeneic hematopoietic stem cells transplantion (HSCT), there is no data concerning if fungal infections can affect the eligibility of patients who are candidates for HSCT.

Methods: We collected the clinical data of patients considered to be candidates for allogeneic HSCT between July 2015 and December 2016 at the Rome Transplant Network (RTN), a JACIE accredited metropolitan transplant program established in Rome since 2006. The observation of patients was continued until November 2017. The diagnosis of IFI were defined as possible, probable and proven as established by European Organization for Research and treatment of Cancer.

Results: 120 patients with a haematological malignancy were discussed: $21(18 \%)$ of these were not considered eligible for HSCT at. A further 17 (14\%) patients lost their eligibility during the observation period (6 patients died, 5 had a progression of the disease not compatible with HSCT, 6 patients changed centre). At november 2017, 45 out of 82 evaluated patients received HSCT. The median time from the date of eligibility to transplant was of 160 days (range 35-524). Eleven (23\%) had a previous fungal infection: 1 proven, 9 probable and 1 possible; [8 pneumonia (73\%), 
1 sinusitis (9\%), 1 candida sepsis(9\%), 1 hepato splenic candidias (9\%) were registered]. These patients were considered eligible, but 3 lost their eligibility for a worsening of health conditions during observations, 6 out of 8 remaining patients were transplanted. The absence of fungal infection (79 patients out of 82 eligible patients) appears to be a factor favoring the eligibility of patients for HSCT [OR 7,02, CI 95\% 1,74-28,25, $p$ value 0,004 Yates Test]. Among the eligible patients, those with a previous fungal infection, wait for HSCT for longer period of time than those without a previous history of fungal infection [mean days: 264 days vs 164 days, $T$ student $p$ value 0,014].

Conclusions: A fungal infection is a complication that may preclude access to HSCT, causing a slowdown in the treatment that leads to the transplant itself. Our data showed that a the diagnosis of a fungal infection before transplantation may be a factor that could extend the time before receiving transplantation.

Clinical Trial Registry: Not applicable

Conflict of interest: The project was awarded a grant from Gilead.

\section{P589}

\section{Reconstitution of Natural Killer cells in the context of complications after Hematopoietic Stem Cell Transplantation}

\section{Débora Basílio-Queirós, Susanne Luther-Wolf, Eva Mischak- Weissinger}

Hannover Medical School, Hematology, Hemostasis, Oncology and Stem Cell Transplantation, Hannover, Germany

Background: Allogeneic hematopoietic stem cell transplantation (HSCT) is a curative treatment for malignant hematological diseases in adults. Due to the delayed immune reconstitution after HSCT, human Cytomegalovirus (CMV) often reactivates leading to increased morbidity, prolonged hospitalization and also contributing to a higher mortality rate. Natural Killer (NK) cells have recently been described to undergo persistent reconfiguration in response to CMV reactivation.

Methods: A multicolor flow cytometry panel for monitoring the CMV-specific NK cell reconstitution was established. Peripheral blood from 57 patients was analyzed between day 0 and 100 post-HSCT in an interval of 7-10 days. The median age of the patients was 54 years (range: 24-73), 38 (66.7\%) were transplanted for acute leukaemias, $47(82.5 \%)$ received reduced intensity conditioning (RIC) and $54(94.7 \%)$ received anti T-lymphocyte immune globulin (ATG). GvHD-prophylaxis was cyclosporine A (CsA) in combination with mycophenolate motefil (MMF) for $78.9 \%$ of the patients and $80.7 \%$ were transplanted from matched donors. Twenty-four patients reactivated CMV (median age: 59.5 years, range 28-73; median day of reactivation: 38 days post-HSCT, range: 19-54).

PBMCs from CMV-seropositive healthy individuals were isolated by density gradient from Leukoreduction system chambers. NK cells were negatively selected by magnetic separation. Further purification of $\mathrm{CD}^{2} 6^{+} \mathrm{CD} 57^{+} \mathrm{NKG} 2 \mathrm{C}^{+}$ NK cells was achieved by cell sorting. Selected NK cells were expanded by co-culture with irradiated allogeneic PBMCs as feeder cells and the medium was supplemented with $1 \mu \mathrm{g} / \mathrm{ml}$ PHA and $500 \mathrm{U} / \mathrm{ml} \mathrm{IL}-2$.

Results: A significant increase in the absolute cell counts of $\mathrm{NKG}_{2} \mathrm{C}^{+} \mathrm{CD} 57^{+} \mathrm{NK}$ cells was observed post-CMV reactivation, when compared to patients who do not reactivate CMV $(p=0.0003)$. Interestingly, a slight decreased on the expression of CD8 by NK cells, linked to a more cytotoxic phenotype of these cells, was observed during CMV-reactivation, possibly a consequence of cell death after lytic activity. NKG2D, in turn, shows a significant increase post-CMV reactivation, demonstrating not only a regulation of the activation capacity of NK cells, but also, the co-stimulatory ability for $\mathrm{T}$ cells proliferation and cytokine production. Remarkably, when comparing CMV-specific $\mathrm{T}$ cells, routinely monitored and quantified by tetramer staining, with $\mathrm{NKG} 2 \mathrm{C}^{+} \mathrm{CD} 57^{+} \mathrm{NK}$ cells, both cell populations show similar kinetics, with an increase in the absolute cell counts during CMV-reactivation, and more impressively, the high cell counts of $\mathrm{NKG}_{2} \mathrm{C}^{+} \mathrm{CD} 57^{+} \mathrm{NK}$ cells are maintained post-reactivation.

Preliminary studies aiming to expand $\mathrm{CD} 56^{+} \mathrm{CD} 57$ ${ }^{+} \mathrm{NKG} 2 \mathrm{C}^{+}$population were performed using CMVseropositive healthy individuals. After fourteen days in culture, an expansion of up to 3100-fold was achieved. Further studies aiming to assess the proliferative capacity of this particular NK cell subpopulation and its functional properties, post-HSCT, are ongoing and will be presented. Considering the crosstalk between innate and adaptive immune cells is crucial for an effective response to CMVinfection, further studies investigating the production of key cytokines and chemokines are warranted.

Conclusions: Taken together our results indicate that NK cells undergo a dynamic modulation in response to CMVreactivation and that expansion of this population occurs in response to CMV. In addition, our results also suggest that $\mathrm{CD} 57^{+} \mathrm{NKG} 2 \mathrm{C}^{+} \mathrm{NK}$ cells may be able to substitute for missing CMV-specific T cells, shortly after HSCT.

Conflict of interest: The authors declare no conflicts of interest 


\section{P590}

Relationship of immune response genes polymorphism with the spectrum of infectious complications in patients with multiple myeloma in the early post-transplant period

Elena Nazarova, Natalija Minaeva, Marina Horobryh, Nataljia Zorina, Jemilija Suhorukova, Igor Paramonov, Victor Shardakov

Federal State Institute of Science 'Kirov Research Institute of Hematology and Blood Transfusion of the Federal Medical and Biological Agency of Russia', Kirov, Russian Federation

Background: Transplantation of autologous hematopoietic stem cells (auto-HSCT) is a common therapy for patients with newly diagnosed multiple myeloma (MM), who have no contraindications to the use of this method. The period of myelotoxic hematopoiesis is often accompanied by the infectious complications. The spectrum of causative agents of infectious complications and the severity of their clinical manifestations are very diverse. The purpose of this study was to evaluate the relationship between the polymorphic status of the immune response genes and the etiology of infectious complications in the early post-transplant period in patients with MM.

Methods: Were evaluated 11 patients with the MM aged 47 to 59 years (median - 55 years) who underwent autoHSCT and in the neutropenia period, clinically and/or microbiologically confirmed infectious complications were observed. Among the examined: four men $(36.4 \%)$ and seven women $(63.6 \%)$. Genotyping of 20 polymorphic locus of 13 immune response genes was performed by polymerase chain reaction with allele-specific primers in the pre-transplant period.

Results: Group 1 included five patients who experienced mucositis in the post-transplant period as a form of stomatitis, esophagitis, enteropathy with predominant discharge from the mucous membranes of the upper respiratory tract and urine of bacterial pathogens (Enterococcus faecium, Enterococcus faecalis, Enterococcus aeruginosa, Streptococcus viridans), and two patients with invasive pulmonary aspergillosis. The 2 group consisted of four patients with clinical manifestations of pneumonia (DNA$\mathrm{CMV+}$ ), hepatitis (DNA-HBV+, RNA-HCV+), vesicular rashes on mucous membranes and skin (DNA-HSV 1/2+ and Herpes zoster), respectively. When comparing the detected haplotypes of the immune response genes with the presence of bacterial/fungal and viral complications, some differences were found that affected the genes of interleukin (IL)- $1 \beta$ and FCGR2A (Table 1).

In patients with MM after auto-HSCT, the complications of bacterial and fungal etiology (compared to patients with viral diseases) correlated with the presence of homozygotes and heterozygotes of the wild-type allele of the $I L 1 \beta$ gene at the locus $-1473(\mathrm{OR}=25.00, p=0.02)$ and the presence of a mutant allele in the haplotypes of the FCGR2A gene at the mutation point $-166(\mathrm{OR}=18.00, p=0.04)$.

Conclusions: Taking into account that the revealed mutations affect the promoter regions of the genes $I L 1 \beta$ and FCGR2A and, consequently, influence the level of the final proteins - IL-1 and the receptor for the immunoglobulin $G$ involved in the implementation of anti-microbial immunity, it can be concluded that the study of these polymorphisms at the pre-transplant period will allow individualizing the prognosis of the development of infectious complications different etiology after autoHSCT in patients with MM and develop a personalized tactic to prevent their occurrence. Considering the pilot nature of the studies, further research is needed in this direction.

Clinical Trial Registry: None.

Conflict of interest: Authors declare no conflict of interest.

\begin{tabular}{lllllll}
\hline Gene, haplotype & $\begin{array}{c}1 \text { group } \\
(\mathrm{n}=7)\end{array}$ & $\begin{array}{l}2 \text { group } \\
(\mathrm{n}=4)\end{array}$ & $\chi 2$ & $\mathrm{p}$ & & OR \\
\cline { 6 - 7 } & & & & value & $95 \% \mathrm{CI}$ \\
\hline $\mathrm{IL1} \beta$ (G-1473C) GG & $1.000 /$ & $0.333 /$ & 5.83 & 0.02 & $25.00 /$ & $0.75-833.00$ \\
+GC/ CC & 0.000 & 0.667 & & & 0.04 & $0.00-1.33$ \\
FCGR2A & $0.143 /$ & $0.750 /$ & 4.05 & 0.04 & $0.06 /$ & $0.00-1.23$ \\
(His166Arg) GG/ GA & 0.857 & 0.250 & & & 18.00 & $0.81-399.18$ \\
+AA & & & & & & \\
\hline
\end{tabular}

[[P590 Table] Haplotypes of IL1 $\beta$ and FCGR2A genes in $M M]$

\section{P591}

Abstract previously published

\section{P592}

Revisiting viral surveillance in haploidentical stem cell transplantation (haploSCT). A case-control study

Iago Colturato ${ }^{1}$, Juliana P. Moreno ${ }^{1}$, Lilian P. Zanetti ${ }^{1}$, Leila C.S. de Oliveira ${ }^{1}$, Fernanda S. Franceschi ${ }^{1}$, Carlos S. Sabaini ${ }^{1}$, Ana Cláudia F. dos Santos ${ }^{1}$, Paula M. da Silva ${ }^{2}$, Mair P. de Souza ${ }^{1}$, Vergilio R. Colturato $^{1}$, Clarisse M. Machado ${ }^{1,3}$

${ }^{1}$ Amaral Carvalho Foundation, HSCT Program, Jahu, Brazil; ${ }^{2}$ Amaral Carvalho Foundation, HSCT, Jahu, Brazil; ${ }^{3}$ University of São Paulo, Institute of Tropical Medicine, Sao Paulo, Brazil

Background: Haploidentical stem cell transplantation (haploSCT) is an attractive option for patients requiring a hematopoietic stem cell transplant (HSCT) who do not have 
an HLA-matched donor, because it may extend transplantation to virtually all patients. The use of post-transplant cyclophosphamide for GVHD prevention has dramatically improved the outcomes, and has become the new standard in how this transplant is performed. Similar posttransplantation outcomes have been observed, including post-transplantation survival, but different engraftment kinetics and patterns of DNA virus reactivation. In the present study, we aimed to compare the episodes of viral reactivations of 30 HaploSCT recipients, 30 matchedrelated donor (MRD), and 30 matched unrelated donor (MUD) HSCT recipients.

Methods: To better characterize the frequency of viral reactivations, we selected 30 MRD and 30 MUD consecutive recipients with follow-up longer than 120 days, submitted to HSCT in 2015 and 2016 at the HSCT Program of Amaral Carvalho Foundation. For the haploSCT recipients, no inclusion criteria was applied and all were included. Control policies for viral infection include RSV screening at admission, and weekly viral surveillance by antigenemia pp65 (CMV) and quantitative PCR (EBV and HHV-6) from conditioning up to $d+120$. HHV-6/EBV surveillance is not performed in MRD HSCT. Reconstitution of CMV-immunity was evaluated with QuantiFeronCMV (QTF-CMV, Qiagen, USA). After HSCT, patients with respiratory symptoms or hemorrhagic cystitis were taken nasal washes and urine/blood samples to detect respiratory viruses (RV) and BK virus/adenovirus, respectively.

Results: CMV was the virus with the highest incidence of reactivations (over 70\%). Before transplant, QTF-CMV was reactive in $67.4 \%, 78.6 \%$ and $50 \%$ of the MRD, MUD and haploSCT, respectively. By day30, all haploSCT were nonreactive, while $33.3 \%$ and $36 \%$ of the MRD and MUD were reactive. At day $90,65.4 \%, 80 \%$ and $100 \%$ of the MRD, MUD and haploSCT reconstituted CMV-immunity, respectively. Two patients died of HHV-6 encephalitis. Viral infections and reactivations according to type of HSCT are shown in the table below:

Conclusions: We observed similar frequencies of hemorrhagic cystitis, $\mathrm{RV}$ infections and $\mathrm{CMV}$ reactivation among haploSCT, MRD and MUD HSCT recipients. EBV reactivation was significantly higher in MUD HSCT in comparison to haploSCT. Oppositely, HHV-6 reactivations were more frequently observed in haploSCT. HHV-6 surveillance is necessary not only in MUD HSCT as well as in haploHSCT.

Conflict of interest: Clarisse Machado: nothing to disclose

\begin{tabular}{lllll}
\hline Viral infection & MRD $(\mathrm{N}=30)$ & Haplo $(\mathrm{N}=30)$ & MUD $(\mathrm{N}=30$ & $\mathrm{p}$ value \\
\hline HHV-6 & Not done & $13(43.3 \%)$ & $4(13.3 \%)$ & 0.02
\end{tabular}

Table (continued)

\begin{tabular}{lllll}
\hline Viral infection & MRD $(\mathrm{N}=30)$ & Haplo $(\mathrm{N}=30)$ & MUD $(\mathrm{N}=30$ & p value \\
\hline EBV & Not done & $7(23.3 \%)$ & $25(83.3 \%)$ & $0.0001<$ \\
CMV & $22(73.3 \%)$ & $21(70 \%)$ & $25(83.3 \%)$ & 0.45 \\
Hemorrhagic cystitis & $1(3.3 \%)$ & $1(3.3 \%)$ & $1(3.3 \%)$ & 0.79 \\
Respiratory Viruses & $10(33.3 \%)$ & $10(33.3 \%)$ & $11(36.7 \%)$ & NS \\
\hline
\end{tabular}

[[P592 Table] Viral infections according to HSCT type]

P593

Risk factors and mortality of parainfluenza virus lower respiratory tract disease without prior manifestation of upper respiratory tract infection

Sachiko Seo ${ }^{1,2}, \mathrm{Hu} \mathrm{Xie}^{1}$, Wendy Leisenring ${ }^{1}$, Jane Kuypers ${ }^{1}$, Farah Sahoo $^{1,3}$, Sonia Goyal ${ }^{1}$, Louise Kimball ${ }^{1}$, Angela Campbell ${ }^{1,4}$, Keith Jerome $^{1,3}$, Janet Englund ${ }^{3,5}$, Michael Boeck ${ }^{1,3}$

${ }^{1}$ Fred Hutchinson Cancer Research Center, Seattle, WA, United States; ${ }^{2}$ National Cancer Research Center East, Chiba, Japan;

${ }^{3}$ University of Washington, Seattle, WA, United States; ${ }^{4}$ Center for Disease Control and Prevention, Atlanta, GA, United States; ${ }^{5}$ Seattle Children's Hospital, Seattle, WA, United States

Background: Respiratory viruses are frequently detected after hematopoietic cell transplantation (HCT). Most infections start with an upper respiratory tract infection (URI) and can progress to lower respiratory tract disease (LRD). The literature indicates that $7-41 \%$ of patients with LRD present without prior URI. Parainfluenza virus (PIV) is one of common respiratory viruses and the mortality after PIV LRD is approximately 50\%. The aim of this study was to evaluate factors associated with PIV LRD without URI as first manifestation in HCT recipients with PIV infection.

Methods: HCT recipients with PIV LRD were retrospectively analyzed. LRD was defined as PIV detection in the lower respiratory tract using conventional culture, direct fluorescent antibody testing, and/or polymerase chain reaction (PCR) (CID 2014: 58: 1357-68). URI was defined as PIV detection in a nasopharyngeal sample. LRD diagnosed on the same day or one day after URI diagnosis was also considered as LRD without prior URI. Logistic regression models were used to evaluate cross-sectional associations between each risk factor and occurrence of LRD without prior URI.

Results: There were 121 LRD cases; 64 (53\%) had LRD without prior URI symptoms. The median time to PIV LRD after HCT was 76 days (range, 3-2343) and 81 days (range, 5-2862) in LRD cases with and without prior URI, respectively $(\mathrm{p}=0.07)$. The median time to progression was 7 days (range, 2-40) among 57 patients with prior URI. Of the 64 patients with LRD without prior URI, 13 (20\%) 
had a nasopharyngeal test performed and the result was negative; 6 of the 13 (46\%) samples were examined using PCR. In multivariable logistic regression models, factors associated with the development of LRD without prior URI versus LRD that progressed from URI were low total lung capacity (TLC) before PIV infection, oxygen requirement at diagnosis, and co-pathogen detection in lower respiratory tract (Table). Peripheral blood stem cell as the transplant cell source (OR 2.6, $\mathrm{p}=0.012$ ) and PIV-1, 2, or 4 serotype (OR 3.8, $\mathrm{p}=0.049$ ) were significantly associated with LRD without prior URI in the univariable analysis, but they were not significant in the multivariable analysis. Among 48 cases with calculated viral loads in bronchoalveolar lavage (BAL) samples, the viral loads were not significantly different between LRD cases with and without prior URI. The mortality rates after LRD were comparable between the two groups (HR 1.2, $\mathrm{p}=0.48$ ).

Conclusions: Approximately half of HCT recipients with PIV LRD presented without URI. This scenario was more likely in patients with restrictive lung function, a coinfection in the lungs, or oxygen requirement at diagnosis. These results suggest that intensive virologic testing in BAL fluid is required even in asymptomatic patients.

\section{Conflict of interest:}

S. Seo and M. Boeckh received research support from Ansun Biopharma, Inc.

M. Boeckh also serves as a consultant to Ansun Biopharma.

J. Englund has received research support from Chimerix and GlaxoSmithKline.

All other authors declare no competing financial interests.

\begin{tabular}{lllll}
\hline Variable & & OR & $95 \%$ CI & p-value \\
\hline \% TLC pre PIV LRD & $<80$ vs $\geq 80$ & 5.56 & $1.1-25.0$ & 0.036 \\
URI symptoms at Dx. & Yes vs No & 0.17 & $0.0-0.7$ & 0.017 \\
Co-pathogen & Lower respiratory tract vs No & 43.70 & $6.0-321$ & 0.006 \\
Oxygen requirement at Dx. & Yes vs No & 9.51 & $2.2-41.2$ & 0.003 \\
\hline
\end{tabular}

[[P593 Table] Risk factors for LRD without prior URI]

\section{P594}

\section{Risk Factors Associated with Response to Rituximab Therapy in Allogeneic Stem Cell Transplant Patients with EBV viremia or Post-Transplant Lymphoproliferative Disease}

\author{
Beth Carella ${ }^{1,2}$, Rachel Lehrman ${ }^{1}$, Glenn Heller ${ }^{3}$, Susan Prockop ${ }^{1}$ \\ ${ }^{1}$ Memorial Sloan Kettering Cancer Center, Pediatrics, Blood and \\ Marrow Transplant, New York, NY, United States; ${ }^{2}$ Children's \\ Hospital of Pittsburgh of University of Pittsburgh Medical Center, \\ Pediatrics, Division of Blood and Marrow Transplant and Cellular
}

Therapies, Pittsburgh, PA, United States; ${ }^{3}$ Memorial Sloan Kettering Cancer Center, Biostatistics, New York, NY, United States

Background: EBV-driven lymphoproliferative disease continues to be a significant contributor to transplant related morbidity and mortality amongst patients receiving allogeneic HSCT, especially those receiving $\mathrm{T}$ cell depleted grafts. While treatment with monoclonal anti-CD20 antibody Rituximab is considered to be standard upfront therapy, there remains considerable variation in thresholds used to begin therapy, as well as timing and number of doses administered to reach a complete remission. Because Rituximab therapy is associated with infusion reactions, cytopenias, and prolonged hypogammaglobulinemia, we investigated which clinical features might predict response leading to a risk-adapted approach to therapy.

Methods: A retrospective review of both adult and pediatric patients who underwent allogeneic HSCT at our institution from 2005 through 2015, and received rituximab therapy for EBV viremia with or without PTLD was completed. Patients were stratified by response to therapy as early complete responders, complete responders or nonresponders based on number of doses needed for resolution of viremia or clinically evident PTLD. Details of Rituximab therapy as well as transplant type, incidence of Graft versus Host Disease, and status of immune reconstitution were evaluated in both univariate and multivariate analyses to investigate statistical significance of these factors amongst the 3 response groups.

Results: 156 patients met eligibility criteria for data analysis. $64 \%, 24 \%$ and $12 \%$ of patients were early complete responders, late complete responders and nonresponders, respectively. None of the early complete responders or complete responders relapsed. After univariate and multivariate analyses, the presence of PTLD (radiographically and/or clinically), use of an accelerated dosing schedule, and CD4 count, remained significant between the 3 groups. The number of days between diagnosis and the onset of therapy neared, but did not reach statistical significance.

Conclusions: Our data suggests that patients who achieve an early complete response after 2 doses of rituximab do not relapse. It further suggests that clinicians can use the presence of PTLD and CD4 count as risk factors that indicate that an accelerated dosing schedule and a complete course of Rituximab are likely necessary to achieve a complete response. Overall, our data provides information for treating clinicians who are trying to balance treating EBV with the untoward side effects of Rituximab therapy.

Conflict of interest: Nothing to disclose 


\section{P595}

\section{Risk Factors for Viral Infections in Pediatric Patients Undergoing Allogeneic Hematopoietic Stem Cell Transplantation}

Atsushi Narita, Yoshinori Ito, Yuka Torii, Jun-Ichi Kawada, Shunsuke Miwata, Kotaro Narita, Hironobu Kitazawa, Motoharu Hamada, Shinsuke Kataoka, Daisuke Ichikawa, Rieko Ito, Norihiro Murakami, Daiei Kojima, Kyogo Suzuki, Eri Nishikawa, Nozomu Kawashima, Yusuke Okuno, Hideki Muramatsu, Asahito Hama, Seiji Kojima, Yoshiyuki Takahashi

Nagoya University Graduate School of Medicine, Department of Pediatrics, Nagoya, Japan

Background: Viral infections constitute a significant cause of morbidity and mortality after allogeneic hematopoietic stem cell transplantation (HSCT). The establishment of polymerase chain reaction (PCR)-based qualitative and quantitative monitoring of virus reactivation has allowed the optimal management of antiviral treatment. However, only a few studies in pediatric patients have analyzed the risks for viral reactivation following allogeneic HSCT.

Methods: We retrospectively analyzed the outcomes of 116 Japanese pediatric patients who underwent allogeneic HSCT between 2011 and 2015 at our institute. Peripheral blood specimens were obtained weekly during the patients' hospitalization for up to 180 days post HSCT. Real-time quantitative PCR monitoring for cytomegalovirus (CMV), Epstein-Barr virus (EBV), and human herpesvirus 6 (HHV6) was performed and the results were expressed as viral copies per $1 \mathrm{~mL}$ of whole blood sample. The patients were diagnosed to have viral reactivation if $\mathrm{CMV}$, EBV, or
HHV-6 loads were $>600,>1000$, and $>1000$ copies $/ \mathrm{mL}$, respectively, on 2 consecutive PCRs.

Results: The median age of the patients was 8.7 years (range, 0.4-18.0 years). Graft sources included 5-6/6 HLA matched bone marrow $(\mathrm{n}=60), 3-4 / 6$ HLA matched haploidentical bone marrow $(\mathrm{n}=21)$, and 4-6/6 HLA matched cord blood $(n=35)$. Fifty-seven patients were administered with anti-thymocyte globulin (ATG) in conditioning regimen. Sixty-two patients received steroid treatment for engraftment syndrome and/or graft versus host disease. The overall incidence of viral reactivation in all patients 180 days following allogeneic HSCT was 65\% (n $=75$ ). PCR-based blood analysis revealed significant CMV reactivation in $20 \%(\mathrm{n}=23), \mathrm{EBV}$ reactivation in $41 \%(\mathrm{n}=$ $48)$, and HHV-6 reactivation in $25 \%(\mathrm{n}=29)$ of the patients. The median time to onset of CMV, EBV, or HHV6 reactivation was 26 days (range, 5-54 days), 47 days (range, 19-270 days), and 26 days (range, 12-145 days), respectively. Three of 116 patients developed viral disease (1 CMV pneumonitis, 1 CMV encephalitis, and 1 EBV post-transplantation lymphoproliferative disorder). In multivariate analysis, haploidentical bone marrow graft was significantly associated with CMV reactivation (OR, 3.61; 95\% CI, 1.32-9.85; $\mathrm{p}=0.012)$ and EBV reactivation (OR, $2.08 ; 95 \% \mathrm{CI}, 1.01-4.30 ; \mathrm{p}=0.048$ ) while cord blood graft was the only independent factor associated with HHV-6 reactivation (OR, 3.98; 95\% $\mathrm{CI}, 1.29-12.25 ; \mathrm{p}=0.016$ ). EBV reactivation-associated risk factors included ATG treatment (OR, 2.93; 95\% CI, 1.27-6.78; $\mathrm{p}=0.012$ ). Difference in 3-year OS between patients with and without viral reactivation was not significant $[76 \%$ (95\% CI, 61\%$86 \%$ ) vs. $76 \%$ (95\% CI, 48\%-91\%); $p=0.660)$ ], possibly because of effective preemptive treatment. However, a high

\section{Cumulative incidence of virus reactivation}

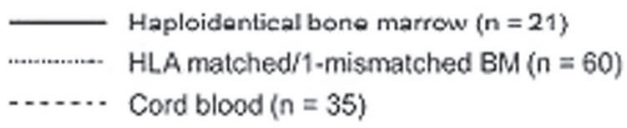

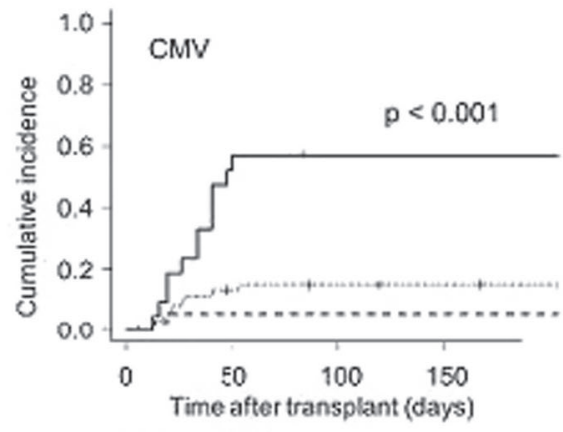

Number at risk

$\begin{array}{llll}60 & 51 & 49 & 48 \\ 35 & 33 & 33 & 33\end{array}$

$21 \quad 10 \quad 3 \quad 8$

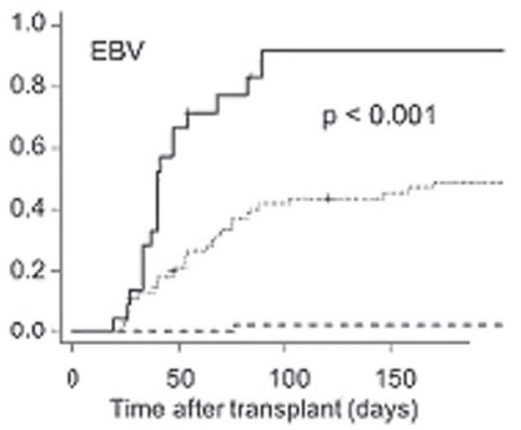

Number at risk

$\begin{array}{cccc}60 & 46 & 34 & 3 \\ 35 & 35 & 34 & 34 \\ 21 & 7 & 1 & 1\end{array}$

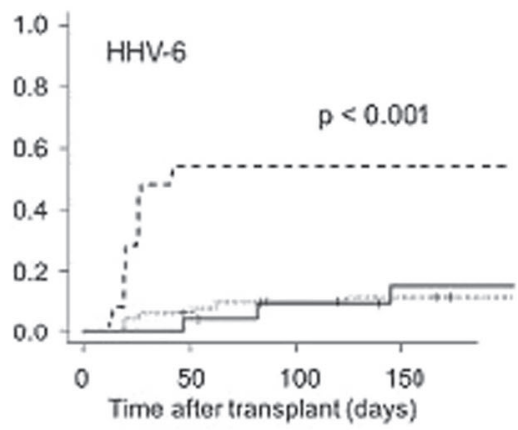

Number at risk

$60 \quad 55 \quad 52 \quad 50$

$35 \quad 16 \quad 16 \quad 16$

$\begin{array}{llll}21 & 20 & 17 & 15\end{array}$

[P595 Figure] [Cumulative incidence of virus reactivation] 
EBV load ( $>10,000$ copies/mL) in 2 consecutive PCRs was associated with poor prognosis [58\% (95\% CI, 30\%-79\%) vs. $80 \%(95 \%$ CI, $65 \%-89 \%) ; \mathrm{p}=0.039)]$.

Conclusions: We demonstrated a significant correlation of cord blood transplantation and HHV-6 reactivation, although there was no HHV-6 disease including encephalitis in our children's cohort. On the other hand, the early detection of CMV and EBV reactivations followed by preemptive antiviral treatment are effective for controlling infections without tissue or organ damage.

Clinical Trial Registry: None

Conflict of interest: None

\section{P596}

Safety of levofloxacin as antibacterial prophylaxis for patients receiving autologous haematopoietic stem cell transplantation: a single-center experience

Rocío Zapata Bautista, María Solé Rodríguez, Encarnación Gil Espárraga, María Antonia Ruiz Cobo, Antonio José Palma Vallellano

Hospital Juan Ramón Jiménez, Huelva, Spain

Background: The benefit of using antibacterial prophylaxis in patients undergoing autologous hematopoietic stem cell transplantation (ASCT) remains unclear. Although the scientific data supporting this practice is limited and wich antibiotic should be choose is not defined, some guides encourage the use of fluorquinolones in this seting. On the other hand, antibiotic resistence is one of the most important challenges in our days and Clostridium difficile infection is also a matter of concern. In our center, levofloxacin prophylaxis is administered orally (500mg OD) to all patients undergoing ASCT. The aim of this study is to review the impact of levofloxacin prophylaxis in terms of bacterian resistence and Clostridiumdifficile infection rates.

Methods: We retrospectively evaluted the febrile episodes, the pathogens isolated and the outcome of the patients with onco-hematological diseases undergoing ASCT in one single center from January 2011 to May 2017.

Results: Eighty-three ASCT were performed in our centre in this period. The average age of the patients was 49 years (range 21-69) and the 68\% were male. The most frequent underlying disease was lymphoproliferative syndrom (SLPC) (52\%), followed by monoclonal gammapathy (GM) (47\%) and there was only one case of acute promyelocytic leukemia in complete response. The disease status before ASCT was partial response or above in the $100 \%$ of SLPC and partial response or above in the $87 \%$ of patients with GM.

Sixty-seven patients $(80,7 \%)$ had fever episodes in wich infection was suspected during the conditioning regimen or before 100 days after ASCT, happening the $97 \%$ of them in the neutropenic period (neutrophil count $<500 \times 10 \mathrm{e} 9 / \mathrm{L}$ ). The patients who had any infection episode had mucositis grade 3-4 in the $73,8 \%$ while the patients without suspected infection only in the $31,3 \%$.

Infection of the central venous catheter was the suspected focus in the $18,4 \%$ of the patients followed by respiratory focus in $7,7 \%$. Patients were given empiric antibiotic with antipseudomonal activity in the $100 \%$ of the cases, adding teicoplanin in the $35 \%$ and empirical antifungal therapy in only the $3 \%$ of the cases due to persistent fever. Seven patients developed septic shock.

Blood cultures were positive in the $35,4 \%$ of the cases: $56,5 \%$ were Gram-positive pathogens and 39\% Gramnegative and there was only one case of Candida. No extended-spectrum beta-lactamase-producing organisms were isolated. There was only one case of A. Baumanii infection in one of the patients who developed septic shock during his hospitalization in the intensive care unit. None of the patients presented with Clostridium difficile diarrhoea.

There was no deaths related to the procedure for infection or other causes before the first 100 days after ASCT.

Conclusions: In our serie the majority of isolated pathogens are Gram-positive as it is also published in other studies in wich fluorquinolones prophylaxis is given.

Levofloxacin prophylaxis in patients underwent ASCT in our environment can be consider a safe procedure but more studies are needed to determine its efficacy and costeffectiveness.

Conflict of interest: None

\section{P597}

Shorter-term prophylaxis with mycophenolate mofetil favorites $V \delta 2^{+} T$-cells recovery and decreases EBV reactivation after hematopoietic transplantation

Jiangying Liu, Lan-Ping Xu, Haitao Gao, Xiaoyu Wang, Xiang-Yu Zhao, Xiao-Jun Huang

Peking University People's Hospital, Beijing, China

Background: Following an increasing use of haploidentical stem cell transplantation (haploHSCT) in the treatment of hematopoietic diseases, complications attributed to viral infections remain the challenging problems for further improving the transplant outcome. Epstein-Barr virus (EBV) reactivation and its related diseases are the common threats with high mortality in the context of haploHSCT. Our previous study demonstrated that the impaired recovery of $\mathrm{V}^{2} 2^{+} \mathrm{T}$ cells at 30 days after haploHSCT significantly correlated to the occurrence of EBV reactivation. The immunosuppressive drug mycophenolate mofetil (MMF) is 
commonly used for prevention and treatment of acute graft versus host disease (aGVHD) following HSCT, which effectively inhibits $\mathrm{T}$ cell proliferation and reasonably can lead to a delay in the recoveries of T-cell subsets. However, the influences of MMF on the $\mathrm{V} \delta 2^{+} \mathrm{T}$ cells and whether early withdrawal of prophylactic MMF administration could favorite ${\mathrm{V} \delta 2^{+}}^{+} \mathrm{T}$ cells recovery and decrease EBV reactivation in the context of haploHSCT are unknown.

Methods: This study prospectively included 173 adult patients receiving haploHSCT from 2016 to 2017 at Peking University People's Hospital. The inclusion criteria is as follows:

1) diagnosed as hematopoietic malignancy;

2) 18 to 60 years old;

3) achieved complete remission before transplantation;

4) $10 \mathrm{mg} / \mathrm{kg}$ of ATG in conditioning regimen.

Patients were divided into 2 groups based on the treatment time of MMF for GVHD prophylaxis [defined as longer-term treatment $(\mathrm{n}=87)$, usually withdrew at 45 60 days after transplant; and shorter-term treatment $(\mathrm{n}=$ 86), withdrew by engraftment after transplant]. The incidences of EBV and CMV reactivation and aGVHD were statistically compared between the groups. Recovery characteristics of T-cell subsets were monitored by flow cytometry at 30,60 , and 90 days following transplant. The effects of MMF on the survival, activation, and EBVassociated cytotoxic function of ${\mathrm{V} \delta 2^{+}}^{+} \mathrm{T}$ cells were investigated by in-vitro experiments.

Results: The median administration times of MMF prophylaxis were 27 days $(15-58 \mathrm{~d})$ in the shorter-term group and 43 days $(25-69 \mathrm{~d})$ in the longer-term group $(P<$ $0.001)$. There is no statistical differences in patients $\hat{A}^{\prime}$ age, gender ratio, types of primary disease, and status of EBV serology between the two cohorts. The incidences of aGVHD were comparable regardless of the different time courses of MMF prophylaxis $(P=0.755)$. Strikingly, the incidence of EBV reactivation in patients with shorter-term MMF was markedly decreased $(9 / 86,10.5 \%)$ compared with that in the longer-term group $(22 / 87,25.3 \%), P=$ 0.016 . Meanwhile, the median counts of peripheral $\mathrm{V} \delta 2^{+}$ $\mathrm{T}$ cells in the shorter MMF group was continuously higher than those in the longer-term group from 30 to 90 days posthaploHSCT. Furthermore, in-vitro treatment with active metabolite mycophenolic acid (MPA) significantly induced the apoptosis of peripheral ${\mathrm{V} \delta 2^{+}}^{\mathrm{T}}$ cells. The IFN- $\gamma$ secretion capacity and cytotoxic effect of ${\mathrm{V} \delta 2^{+}}^{\mathrm{T}}$ on the EBV-targeted cells were also dramatically inhibited upon MPA treatment.

Conclusions: Shorter-term prophylaxis with MMF significantly improved the recovery of $\mathrm{V} \delta 2^{+} \mathrm{T}$ cells and decreased the incidence of EBV reactivation, which was not accompanied with an increase in aGVHD after haploHSCT.
These findings may help further improve the outcome of hematopoietic transplantation.

Clinical Trial Registry: Clinical trial nos. NCT02978274 and ChiCTR-COC-16008052

Conflict of interest: The authors declare no conflicts of interest.

This study is supported by the National Natural Science Foundation of China (Nos. 81770191 and 81670167), and Foundation for Innovative Research Groups of the National Natural Science Foundation of China (No. 81621001).

\section{P598}

The management of adenovirus infection among EBMT centres: a survey from Infectious Diseases Working Party of EBMT

Simone Cesaro ${ }^{1}$, Malgorzata Mikulska ${ }^{2}$, Massimo Berger ${ }^{3}$, Katherine $N$ Ward $^{4}$, Per Ljungman ${ }^{5}$, Gloria Tridello ${ }^{1}$, Steffie van der Werf $^{6}$, Jan Styczynski

${ }^{1}$ Azienda Ospedaliera Universitaria Integrata, Verona, Italy;

${ }^{2}$ University of Genova, Ospedale Policlinico San Martino, Genua, Italy; ${ }^{3}$ Pediatric Onco-Hematology, Stem Cell Transplantation and Cellular Therapy Division, AOU Città della Salute e della Scienza, Regina Margherita Childrens Hospital, Turin, Italy; ${ }^{4}$ Royal Free and University College Medical School, London, United Kingdom; ${ }^{5}$ Karolinska University Hospital, Stockholm, Sweden; ${ }^{6}$ EBMT Data Office, Leiden, Netherlands; ${ }^{7}$ University Hospital, Collegium Medicum UMK, Bydgoszcz, Poland

Background: The diagnosis, monitoring, pre-emptive treatment and treatment of Adenovirus (ADV) infection may vary among centres. The IDWP undertook a survey to assess the management of ADV infection

Methods: A questionnaire was mailed to EBMT centers in July with the deadline for the response in November 2017. The replay was returned by 81 centres (response rate: $18 \%)$.

Results: Center profile: $41 \%$ had adult, $38 \%$ pediatric, and $21 \%$ had a mixuture. The median number of ADV infections diagnosed in the years 2013-2017 amongst 81 centres was 2 , range $0-44$.

ADV diagnosis: $93 \%$ of centres (75) test regularly the patients for ADV infection by ADV-PCR (74) or other method (immunofluorescence 1). 63\% of centers (47) test for ADV all allogeneic hematopoietic stem cell transplants (aHSCT) recipients whereas in the other centers ADV testing is performed only in specific subgroups (haploidentical, mismatched MUD, unrelated cord blood).

Sample source: $52 \%$ of centres (39) use the whole blood, $36 \%$ use plasma (27), and 1 centre use serum for ADV$\mathrm{PCR}$; in 7 centers the ADV-PCR is not tested (response missing in 1 centre). 95\% of centres (71) test for ADV in 
other sites/organs, mainly in stool (65), upper (49) and lower (37) respiratory tract specimen, and urine (9).

$A D V$ surveillance (up to day +100 ): ADV-PCR is performed in the blood once weekly in 37\%, twice weekly in $9 \%$, every 2 weeks in $4 \%$, and according to patient's symptoms or immune recovery in $35 \%$ of centres. ADVPCR is performPCR-ed in stool sample once weekly in $17 \%$, every 2 weeks in $4 \%$, according to patient's symptoms or immune recovery in $67 \%$. ADV-PCR is performed in respiratory sample once weekly in $8 \%$, and according to patient's symptoms or immune recovery in $71 \%$.

Treatment of $A D V$ infection: the routine therapeutic strategy is administration of cidofovir (CDV) $85 \%$, reduction of immunosuppression $84 \%$, adoptive immunotherapy $27 \%$, other $20 \%$. CDV is available in most of centres (63/81) and the most used dosage is $5 \mathrm{mg} / \mathrm{kg} / \mathrm{week}$ with probenecid.The threshold to start CDV is two consecutive PCR positives in $33 \%$ of centres or $\geq 1000$ copies $/ \mathrm{ml}$ in high and intermediate risk patients, and $\geq$ 10.000 copies $/ \mathrm{ml}$ in the low risk patients. The duration of CDV treatment is until the level decreases below 1000 copies $/ \mathrm{ml}$ in $63 \%$ (51) and for a median time of 3 weeks in $16 \% 1(3 / 81)$. In the last 5 years, brincidofovir was used in $26 \%$ of centres whereas ADV-CTL treatment was used in $21 \%$ of centres mainly after failure of CDV.

Conclusions: Although ADV infection is usually not frequent, but almost responding centres consider it a serious enough to deserve a regular testing usually with ADV-PCR in blood. CDV represents the main therapeutic option although one fourth of responding centers uses the novel drug and/or immunotherapy.

Conflict of interest: No conflict of interest

\section{P599}

\section{The significance of pre-transplant cytomegalovirus (CMV) serostatus for viral reactivation after allogeneic stem cell transplantation}

Yavor Petrov ${ }^{1}$, Penka Ganeva ${ }^{1}$, Georgi Arnaudov ${ }^{1}$, Ivan Tonev ${ }^{2}$, Stanislav Simeonov ${ }^{2}$, Milcho Mincheff ${ }^{2}$, Georgui Balatzenko ${ }^{3}$, Dora Alexandrova ${ }^{4}$, Milan Jagurinoski ${ }^{5}$, Margarita Guenova ${ }^{5}$, Branimir Spassov $^{6}$

${ }^{1}$ University National Specialised Hospital for Active Treatment of Haematological Diseases, BMT Unit, Sofia, Bulgaria; ${ }^{2}$ University National Specialised Hospital for Active Treatment of Haematological Diseases, BM and PBSC Apheresis Department, Sofia, Bulgaria;

${ }^{3}$ University National Specialised Hospital for Active Treatment of

Haematological Diseases, Laboratory of Cytogenetics and Molecular Biology, Sofia, Bulgaria; ${ }^{4}$ University National Specialised Hospital for Active Treatment of Haematological Diseases, Virology and Microbiology, Sofia, Bulgaria; ${ }^{5}$ University National Specialised Hospital for Active Treatment of Haematological Diseases,

Laboratory of Hemopathology and Immunology, Sofia, Bulgaria;
${ }^{6}$ University National Specialised Hospital for Active Treatment of Haematological Diseases, Sofia, Bulgaria

Background: Human cytomegalovirus (CMV) infection remains one of the major complications following hematopoietic stem cell transplantation (HSCT) and is associated with considerable morbidity and mortality. The incidence of CMV infection approaches $70 \%$ in allogeneic bone marrow transplant recipients who are CMV seropositive $(\mathrm{R}+)$ and/ or receive a transplant from a CMV-seropositive donor (D + ). The present approach to the management of CMV consists of serial monitoring of peripheral blood using either CMV pp65 antigen (antigenemia assay) or polymerase chain reaction (PCR) and then initiating preemptive therapy with either ganciclovir or foscarnet in patients who become positive. Factors identified for CMV reactivation include CMV seropositivity of the donor, type of conditioning regimen, use of T-cell depletion, and graft-versus-host disease (GVHD).

Methods: Our study includes 110 adult patients 70 men and 40 women, undergoing allogeneic stem cell transplantation (HSCT) between 2010 and 2017, divided into three risk groups : low risk (D-/R-), intermediate risk (D+/R-), and high risk ( $\mathrm{D}-/ \mathrm{R}+$ or $\mathrm{D}+/ \mathrm{R}+)$. Serial weekly monitoring for CMV viremia was performed using a quantitative polymerase chain reaction (PCR). The majority patients (74\%) had conventional myeloablative transplant and $26 \%$ had reduced intensity conditioning (RIC), using either matched sibling/family (48\%) or unrelated donors $(52 \%)$. Graft source was PBSC (85\%), bone marrow in (15\%). In vivo T-cell depletion with ATG was performed in $13 \%$ of the recipients.

Results: CMV reactivation was seen in 37 patients at a median of 56 days post HSCT (range 8 - 95). All patients were treated with antiviral therapy with responses seen in 95\%. Ten patients developed CMV disease at a median of 55 days post HSCT, 3 of whom died. At a median follow up of 36 months patients without CMV reactivation (57.2\%) had significantly higher survival compared with patients with CMV reactivation $(42.8 \%)(\mathrm{p}=0.049)$.

CMV serostatus was the most important risk factor with incidence of $62 \%$ viral reactivation in the high-risk group compared with $23 \%$ in the intermediate risk and $0 \%$ in the low-risk group $(\mathrm{p}<0.0001)$. In addition, the presence of acute graft-versus-host disease (GVHD) $(\mathrm{p}=0.0024)$, and the HLA incompatibility $(\mathrm{p}=0.047)$ were associated with increased risk in RIC transplants $(p=0.051)$. On a multivariate analysis, CMV serostatus, RIC transplants, acute GVHD and HLA incompatibility remained independent predictors of CMV reactivation.

Conclusions: Recipient CMV serostatus continues to be an important variable for overall survival after 
transplantation in the era of antiviral preemptive therapy and prophylaxis. This survival disadvantage appears largely restricted to highly immunosuppressed patients, such as recipients of T-cell-depleted grafts or grafts from HLAmismatched or unrelated donors. Our analysis demonstrates the importance of defining the pre-transplant CMV serostatus for the outcome after the transplant. CMV reactivation and disease remains a major problem in highrisk patients undergoing allogeneic HSCT.

Conflict of interest: There is no conflict of interest

\section{P600}

Towards monitoring adenovirus using serial faecal PCR after allogeneic stem cell transplantation in paediatric recipients

Ryan Malcolm Hum ${ }^{1}$, Andrew Turner ${ }^{2}$, Su Han Lum ${ }^{3}$, Emma Davies $^{2}$, Denise Bonney ${ }^{3}$, David Deambrosis ${ }^{3}$, Malcolm Guiver ${ }^{4}$, Robert F Wynn ${ }^{3}$, Prashant Hiwarkar ${ }^{3}$

${ }^{1}$ University of Manchester, Faculty of Biology, Medicine and Health, Manchester, United Kingdom; ${ }^{2}$ Royal Manchester Children's Hospital, Department of Virology, Manchester, United Kingdom; ${ }^{3}$ Royal Manchester Children's Hospital, Department of Blood and Marrow Transplant, Manchester, United Kingdom; ${ }^{4}$ Royal Manchester Children's Hospital, Department of Molecular Diagnostics, Manchester, United Kingdom

Background: Since faecal shedding of adenovirus (ADV) is an early sign of loss of immune control over $\mathrm{ADV}$, we sought to determine the role of faecal adenoviral kinetics in predicting adenoviraemia and survival outcomes following allogeneic stem cell transplantation in children.

Methods: A total of 341 recipients of allogeneic haematopoietic stem cell transplant from February 2003 to September 2016 were screened weekly for adenoviraemia till immune-recovery and/or cessation of immunosuppression. Pre-transplant blood and faecal polymerase chain reaction (PCR) was performed in all patients. Patients with diarrhoea were also tested for faecal ADV and, weekly faecal PCRs were performed in those with symptomatic diarrhoea. ADV PCR technique with a sensitivity of 500 copies/ml of blood or 500 copies/gm of faeces was used.

Results: ADV was detected in a total of 178 patients. Faecal ADV was positive in $173(51 \%)$ and adenoviraemia was detected in $63(18.5 \%)$ transplant recipients. Fifty-eight of 63 patients with adenoviraemia were tested for faecal PCR and all 58 patients had positive faecal PCR. Median time to detection of faecal ADV was 12 days (IQR: -2.5 to 40.5 ) and median time to detection of adenoviraemia was 26 days (IQR: 12 to 73 ).

Interestingly, we found a linear relationship between peak blood PCR value and faecal PCR value just prior to adenoviraemia (Figure 1 (a)). This relationship was more linear in patients with malignancy than those transplanted for non-malignant disorders. A threshold ADV level of 5 $\log _{10}$ copies/gm of feces predicted adenoviraemia with sensitivity and specificity of $86.4 \%$ and $87.5 \%$ respectively in malignant patients (Figure 1 (b)). Twenty-six of 30 patients with high faecal virus load

( $>$ threshold value of $5 \log _{10}$ copies/gm) had transplant for non-malignant conditions. Thus, the proliferative kinetics of virus in the gastro-intestinal (GI) tract determines the severity of adenoviraemia and pre-transplant immune status

(not compromised by previous chemotherapy) is likely to determine the ability of host to localize ADV infection to the GI tract

$(\mathrm{p}<0.01)$.

In 28 patients with symptomatic diarrhoea, faecal PCR was serially monitored prior to dissemination. In these patients, median time of 8 days (IQR: 3.5 to 22.5 ) was observed between threshold faecal PCR level of $5 \log _{10}$ copies/gm and onset of adenoviraemia.

Our most interesting finding was similar non-relapse mortality (NRM) in patients without any ADV and those with faecal ADV only (10\% and 8\%). However, significantly higher NRM was observed in patients with adenoviraemia (30\%) (Figure 1 (c)). Thus, adenoviraemia increases the likelihood of NRM. Hence, preventing adenoviraemia by controlling the proliferation of ADV in patients reaching threshold faecal PCR level may reduce the likelihood of NRM.

Conclusions: GI tract is the primary site of adenoviral reactivation and, proliferative kinetics of virus in the GI tract determines the risk and severity of adenoviraemia. Since, NRM is significantly higher in patients with adenoviraemia, pre-emptive interventions such as reduction of immune-supression and effective antiviral therapy should aim at preventing adenoviraemia in patients reaching threshold level of faecal adenovirus.

Clinical Trial Registry: $n / a$

Conflict of interest: None

\section{P601}

Toxoplasma PCR screening should start from transplant

Christine Robin ${ }^{1}$, Cécile Angebault ${ }^{2}$, Rabah Redjoul ${ }^{1}$, Mathieu Leclerc $^{1}$, Florence Beckerich ${ }^{1}$, Roberta Di Blasi ${ }^{1}$, Ludovic Cabanne $^{1}$, Françoise Foulet ${ }^{2}$, Cécile Pautas ${ }^{1}$, Andrea Toma ${ }^{1}$, Sébastien Maury', Françoise Botterel ${ }^{2}$, Catherine Cordonnier ${ }^{1}$

${ }^{1}$ Henri Mondor Hospital, Hematology, Creteil, France; ${ }^{2}$ Henri Mondor Hospital, Mycology, Creteil, France

Background: Toxoplasmosis is a rare but well known complication after allogeneic HSCT. Most cases are due to reactivation in seropositive recipients $(\mathrm{R}+)$ for 


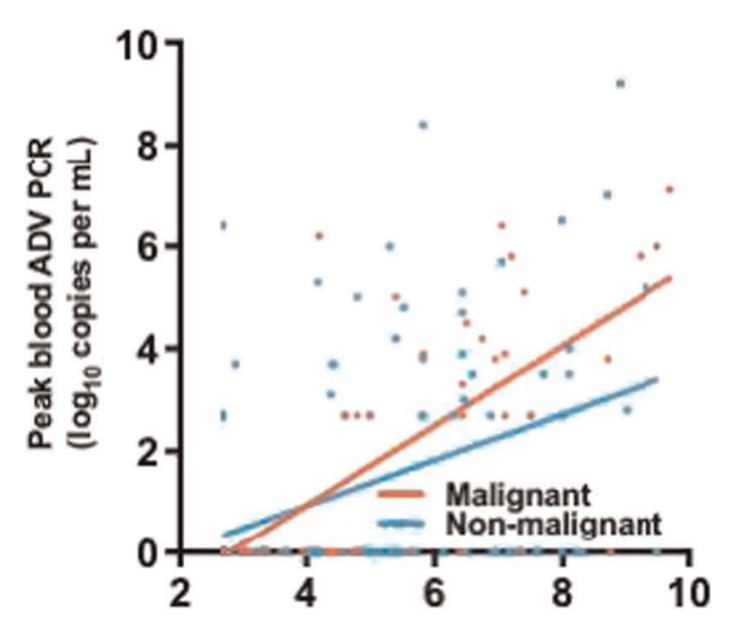

Faecal ADV PCR prior to dissemination Figure 1a

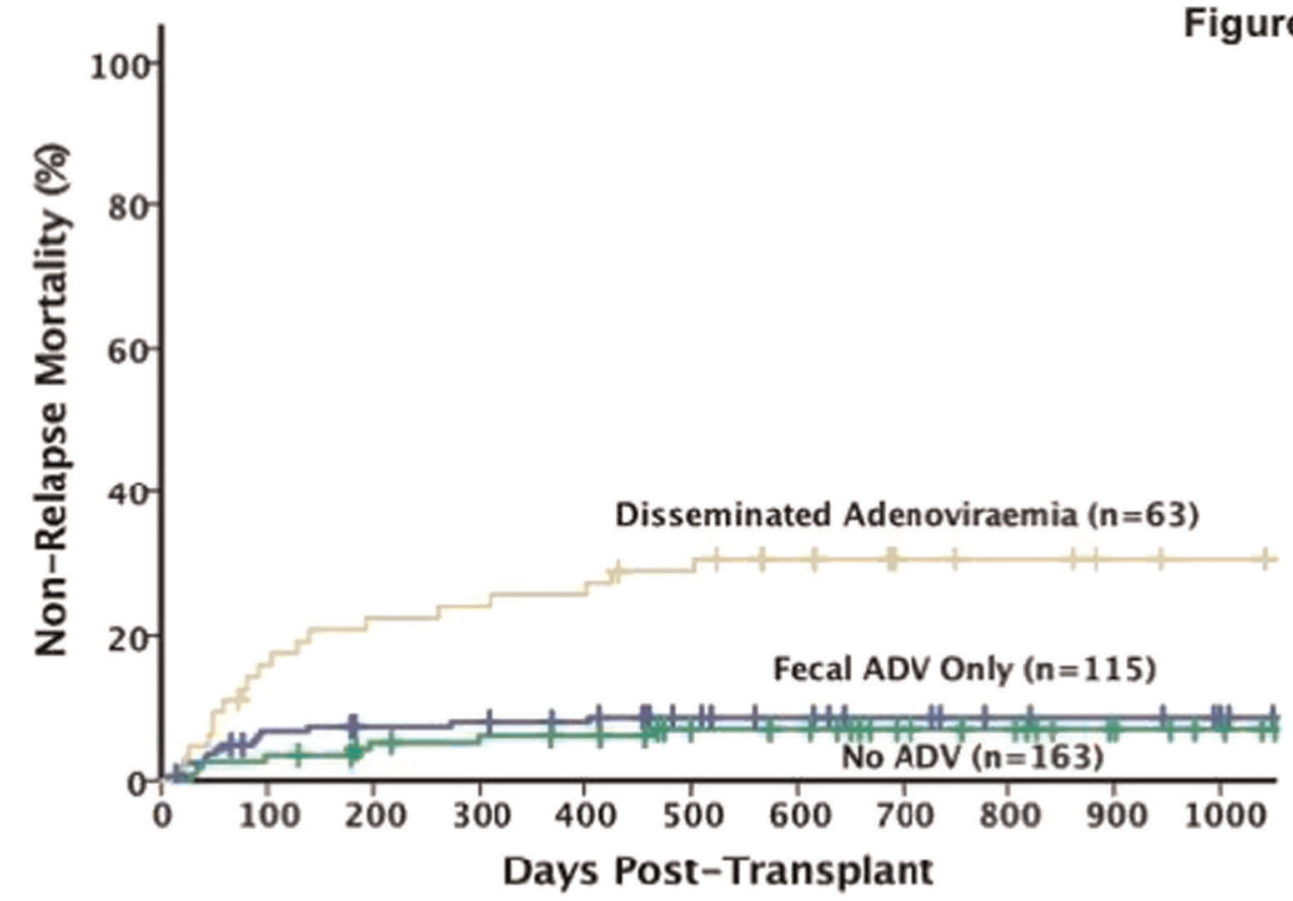

Figure 1a - Linear relationship between faecal ADV prior to dissemination and peak level in blood. This shows proliferative kinetics of virus in the GI tract determines severity of adenoviraemia.

Figure 1b - Patients with disseminated adenoviraemia have ADV faecal PCR above a threshold of $5 \log _{10}$ copies /gm, especially in those with malignant disorders

Figure 1c - Survival curve showing higher NRM in patients with adenoviraemia than those with faecal ADV only or no ADV disease ( $p<0.01)$

[P600 Figure] [Figure 1]

toxoplasmosis, and occur between months 2 to 6 after transplant. Pneumocystis jirovecii prophylaxis with

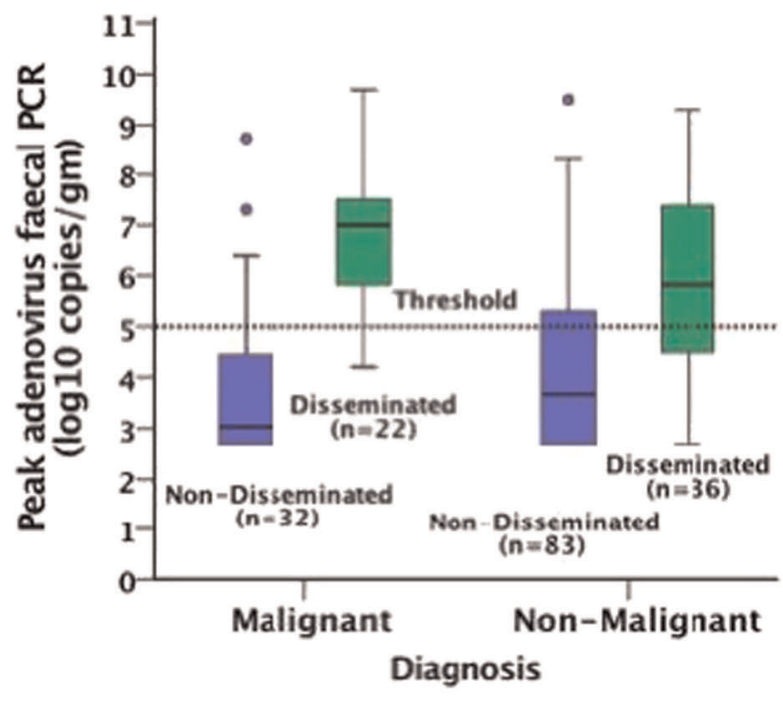

Figure 1b

Figure 1c 
weekly screening with qPCR of $\mathrm{R}+$ patients is recommended by the international guidelines for early identification of reactivation, especially when TMP-SMX is not given, in order to intervene before development of toxoplasmosis disease. In our center until now, we systematically screened $\mathrm{R}+$ patients using 529bp-repeat-DNA fragment qPCR in blood from engraftment to 6 months, even for patients under TMP-SMX, as the absorption and compliance to TMP-SMX may be variable. Due to recent early toxoplasmosis, we reviewed our cases to reconsider the pertinence of this procedure, especially focusing on cases observed before d30 after HSCT.

Methods: We assessed all patients who developed $\geq 1$ blood or BAL positive qPCR and those who had any positive cytology exam showing characteristic trophozoïtes among patients transplanted between 01/01/2008 and 01/10/ 2017. Patients were screened from engraftment, initially weekly, and every 1-2 weeks from 3 months according to their visits in the daycare center. Positive qPCR patients had a diagnostic workup including fundus oculi examination, CNS CT scan, lung CT scan, and additional investigations according to clinical presentation. Toxoplasmosis infection and disease were defined according to Martino et al. 2005.

Results: Among 362 consecutive patients, 16 (4.4\%) patients developed toxoplasmosis: 7 (44\%) before d30 (d6$\mathrm{d} 28$ ) and 9 after $\mathrm{d} 30$ (d43-d322). Among the 7 early cases $(<\mathrm{d} 30)$, there were 4 infections documented with positive blood qPCR and 3 toxoplasmosis diseases. All patients were seropositive for toxoplasmosis and all donors except one were seronegative. These patients were especially immunosuppressed because transplanted with a cordblood $(\mathrm{n}=2)$ or an unrelated donor $(\mathrm{n}=5)$, one had received 2 previous autologous transplants, two were conditioned with a sequential regimen, and all received ATG in the conditioning. None of them was receiving TMP-SMX at time of first positive sample. Five of these 7 patients (71\%) died 1 to 27 days after the first positive sample from disseminated toxoplasmosis with multivisceral failure (MVF) without other documented cause $(\mathrm{n}=3)$ or from MVF associated with veno-occlusive disease $(\mathrm{n}=2)$. According to our procedures, the qPCR blood screening was not started at time of diagnosis.

Conclusions: Early toxoplasmosis after allogeneic HSCT is a rare event but is associated with a high mortality rate. It is probably favored by the fact that TMP-SMX is usually started from engraftment. However, these cases should be anticipated by a weekly blood qPCR screening from transplant and not from engraftment. In patients seropositive for toxoplasmosis before transplant, we recommend to start the screening from transplant and no later. As the seroprevalence for toxoplasmosis increases with age but has decreased over the last decades, we should face, as for $\mathrm{CMV}$, the situation of more seronegative donors giving for older seropositive patients, increasing the risk of reactivation.

Clinical Trial Registry: not applicable

Conflict of interest: nothing to disclose

\section{P602}

Treatment of respiratory syncytial virus (RSV) infection with combined oral Ribavirin and IVIG following allogeneic HSCT is effective and safe

Edmund Watson ${ }^{1}$, Marcin Lubowiecki ${ }^{1}$, Katalin Balassa ${ }^{1,2}$, Rebecca Cash $^{1}$, Nadjoua Maouche ${ }^{1}$, Daja Barton ${ }^{1}$, Mandy Ellis ${ }^{1}$, Lara Rowley $^{I}$, Denise Wareham ${ }^{1}$, Claudia Costa ${ }^{1}$, Rachel Miller ${ }^{1}$, Katie Jeffery $^{3}$, Siraj Misbah ${ }^{4}$, Rachel Pawson ${ }^{1,2}$, Robert Danby ${ }^{1}$, Vanderson Rocha ${ }^{1,2}$, Andy Peniket ${ }^{1}$

${ }^{1}$ Oxford University Hospitals NHS Foundation Trust, Department of Haematology, Oxford, United Kingdom; ${ }^{2}$ NHS Blood and Transplant, Oxford, United Kingdom; ${ }^{3}$ Oxford University Hospitals NHS Foundation Trust, Department of Microbiology, Oxford, United Kingdom; ${ }^{4}$ Oxford University Hospitals NHS Foundation Trust, Department of Clinical Immunology, Oxford, United Kingdom

Background: Respiratory syncytial virus (RSV) infection accounts for significant morbidity and mortality among allogeneic HSCT patients. Retrospective studies suggest that Ribavirin treatment can prevent the progression of upper respiratory tract infection (URTI) to lower respiratory tract infection (LRTI) and can reduce mortality. Aerosolized Ribavirin administration is clinically challenging and raises safety concerns due to its teratogenicity and mutagenicity. Oral Ribavirin offers an alternative treatment modality but there remains a relative lack of data as to the outcomes of patients treated in this way.

Methods: Data of 22 consecutive adult patients, who received oral Ribavirin treatment at our centre in a two-year period between November 2015 and November 2017 were analysed. The patients underwent first allogeneic HSCT for a malignant haematological condition between 2010 and 2017. RSV was tested by PCR from throat swab. According to our institutional protocol patients with confirmed RSV and evidence of LRTI clinically and/or radiologically are treated with oral Ribavirin $(15-20 \mathrm{mg} / \mathrm{kg} /$ day divided into three daily doses) and intravenous immunoglobulin (IVIG, $2 \mathrm{~g} / \mathrm{kg}$ over 2-4 days).

Results: The median age at RSV infection was 53 years (range 19-73). Concomitant acute or chronic GVHD affected eight patients (36\%) and seven $(32 \%)$ of them were treated with Prednisolone at the time of RSV diagnosis.

The median time from transplant to presentation with RSV infection was 10 months (range -0 - 69) posttransplant. All but one patient were treated as inpatients, and the median length of stay and duration of treatment were 8 
and 7 days, respectively. More than half of the patients $(\mathrm{n}=$ $13,59 \%)$ suffered from co-infections. All patients underwent chest XR investigation, which was compatible with infection in 13 cases (59\%). CT chest was performed in 15 cases, which confirmed features in keeping with infection in all cases. The most common radiological findings were ground glass opacification and nodularity. Three patients (14\%) required admission to intensive care unit, of whom two were diagnosed with RSV pre-engraftment and the third patient had been on long term immunosuppression for chronic GVHD. Ribavirin was generally well tolerated. The major side effect was transfusion-dependent haemolytic anaemia $(\mathrm{n}=4,18 \%)$. Six patients died after a median follow-up of 11 months (range 1-23) following RSV diagnosis. One death was attributable to RSV. This was the only death that occurred within 100 days of the diagnosis of RSV infection giving a mortality rate of $5 \%$ following RSV infection.

Conclusions: This study has been one of the largest to date reporting experience with oral Ribavirin in the allogeneic HSCT setting for the treatment of RSV infection. Our data suggest that oral Ribavirin with IVIG is a safe approach for the management of RSV LRTI post-transplant. Although RSV-attributable mortality was low (5\%), a significant proportion of patients were clinically unwell at the time of diagnosis requiring aggressive management in the inpatient setting.

Conflict of interest: Authors declare no conflict of interest.

\section{Lymphoma}

\section{P603}

\section{A SUBGROUP ANALYSIS OF BEAM AND FEAM CONDITIONING FOR MANTLE CELL LYMPHOMA: A MULTICENTER STUDY FROM FONDAZIONE ITALIANA LINFOMI (FIL)}

\author{
Matteo Pelosini, ${ }^{1}$, Jacopo Olivieri ${ }^{2}$, Federico Mosna ${ }^{3}$, Teresa \\ Calimeri $^{4}$, Giuseppe Carli ${ }^{5}$, Angelo Fama ${ }^{6}$, Simone Ferrero ${ }^{7}$, \\ Margherita Giannoccaro $^{8}$, Giacomo Loseto ${ }^{9}$, Dario Marino ${ }^{10}$, Anna \\ Maria Mazzone ${ }^{11}$, Sara Rattotti ${ }^{12}$, Nicola Sgherza ${ }^{13}$, Maria Chiara \\ Tisi $^{5}$, Gabriella Tomei ${ }^{14}$, A. $_{\text {Billio }}{ }^{3}$, Cascavilla $^{13}$, R Centurioni $^{15}, P$ \\ Chiusolo $^{16}$, K Codeluppi ${ }^{17}$, I. Federici ${ }^{18}$, F Finotto $^{19}$, F.

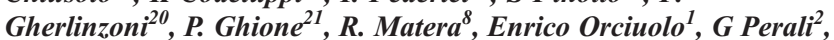 \\ P.M. Stefani ${ }^{20}$, Renato Fanin ${ }^{2}$, Francesco Zaja ${ }^{2}$ \\ ${ }^{1}$ Azienda Ospedaliera Universitaria Pisana, U.O. Ematologia \\ Universitaria, Pisa, Italy; ${ }^{2}$ A.O.U. Santa Maria della Misericordia di \\ Udine, Ematologia, Udine, Italy; ${ }^{3}$ Ospedale Regionale 'S. Maurizio', \\ Ematologia e Centro Trapianto di Midollo Osseo, Bolzano, Italy; \\ ${ }^{4}$ IRCCS Ospedale San Raffaele, Divisione di Ematologia, Milano, \\ Italy; ${ }^{5}$ Ospedale San Bortolo, Ematologia, Vicenza, Italy; ${ }^{6}$ IRCCS, \\ Ematologia ASMN, Reggio Emilia, Italy; ${ }^{7}$ AOU Città della Salute e \\ della Scienza di Torino, • Ematologia 1, Dipartimento di Biotecnologie \\ Molecolari e Scienze per la Salute, Università di Torino, Torino, Italy;
}

${ }^{8}$ ASL Lel1 P.O. Vito Fazzi, Ematologia, Lecce, Italy; ${ }^{9}$ IRCCS Ospedale Oncologico, Ematologia, Bari, Italy; ${ }^{10} \mathrm{IOV}$, IRCCS, UOC di Oncologia Medica 1, Padova, Italy; ${ }^{11}$ Ospedale 'S. Giuseppe Moscati, Ematologia, Taranto, Italy; ${ }^{12}$ Fondazione IRCCS Policlinico San Matteo, Ematologia, Pavia, Italy; ${ }^{13}$ IRCCS Ospedale Casa Sollievo Sofferenza, Ematologia, San Giovanni Rotondo, Italy; ${ }^{14}$ Ospedale di Ivrea-ASL TO4,, Ematologia, Ivrea, Italy; ${ }^{15}$ ASUR - AV3, UOC Medicina Interna ed Ematologia, Civitanova Marche, Italy;

${ }^{16}$ Università Cattolica del Sacro Cuore, Istituto di Ematologia, Roma, Italy; ${ }^{17}$ ASMN - IRCCS, Ematologia, Reggio Emilia, Italy; ${ }^{18}$ Azienda Ospedali Riuniti di Ancona, Ematologia, Ancona, Italy; ${ }^{19}$ IOV, IRCCS, UOC di Oncologia Medica 1, I, Padova, Italy; ${ }^{20}$ Azienda ULSS 9 , Ematologia, Treviso, Italy; ${ }^{21}$ AOU Città della Salute e della Scienza di Torino, Ematologia 1, Dipartimento di Biotecnologie Molecolari e Scienze per la Salute, Università di Torino, Torino, Italy

Background: Due to BCNU unavailability, several transplant centers in Europe switched from BEAM to Fotemustine-based conditioning (FEAM), although no studies support equivalence of the two regimens.

Methods: We retrospectively compared safety and efficacy outcomes of FEAM with respect to BEAM in 1038 patients. Here we report subgroup analysis in mantle cell lymphoma patients. We analyzed 145 ASCTs performed for lymphomas in 16 Italian centers from 2008 to 2015. Selection for BEAM or FEAM conditioning linked on BCNU availability and not on clinical characteristics.

Results: Patients characteristic were similar between FEAM $(n=76)$ and BEAM $(n=69)$ patients. The majority of patients had stage IV at diagnosis (94,7\%BEAM, 94,2\% FEAM), B symptoms (23,7\% BEAM, FEAM 27,3\%). Patients were female in 23,7\% (BEAM) and 26\% (FEAM). As for clinical practice patients had transplant as first line consolidation $(77,6 \%$ BEAM, $72 \%$ FEAM). Induction treatment was comparable with HyperCVAD in $20 \%$ (BEAM) and 15\% (FEAM), CHOP like regimen in 25\% (BEAM) and $17 \%$ (FEAM) and CHOP/HD ARA-C in 54\% (BEAM) and 65\% (FEAM). Response status before ASCT was: CR in $85 \%$, PR in $11,6 \%$, in the BEAM group while FEAM patient were in CR in $73 \%$, PR in $23,1 \%$. More patient had a PET negative scan in BEAM group $(65 \%$ vs $53 \%$ ), even in PET data were missing in the FEAM group. Neutrophil engraftment was similar in both groups (10 days). Oral mucositis was a sligher more severe in the FEAM group (grade 3-4: 37\% FEAM vs 26\% BEAM). Incidence of other severe toxicities (CTCAE grade $\geq 3$ ) did not differ between the two groups. Grade 3 and 4 Febrile neutropenias (FN) occurred at a similar rate (grade $3+4$ FN: $44,9 \%$ FEAM vs $41,73 \%$ BEAM). Response status did not differ at day 100 post ASCT (CR 89\% BEAM vs 82,\% FEAM) and neither at last follow-up (continuous CR 61,8\% vs 66\%). Non-relapse mortality (BEAM 3,9\% vs FEAM $5,8 \%$ ) was comparable as well. Death occurred respectively in $18 \%$ and $21 \%$ for BEAM and FEAM due to disease progression in the majority of patients. Overall survival 
(OS) at 1 years was not different (BEAM 96\% vs FEAM $94 \%, \mathrm{p}=0.1342$ ) and neither was relapse rate (BEAM $36,8 \%$ vs FEAM $28 \%$ ) and progression-free survival at 1 year (BEAM 82\% vs FEAM 77\%, p=0.28).

Conclusions: Substitution with Fotemustine did not increase general toxicities or infectious episodes; however we observed a sligher gastrointestinal toxicities in FEAM patients that did not lead to higher NRM neither significantly worsened OS. PFS and cumulative relapse incidence were comparable as well. Induction therapy do not have any impact of patients outcome even if disease assessment at transplant is biased by PET data missing in the FEAM group. In conclusion no statisticant significant difference emerged between BEAM and FEAM in MCL patients

Conflict of interest: None

\section{P604}

\section{Allogeneic Hematopoietic Cell Transplantation in Cutaneous T Cell Lymphomas - a Portuguese Experience}

Filipe Gonçalves $^{1}$, Joana Desterro ${ }^{1}$, Susana Esteves ${ }^{2}$, Teresa Mendonça ${ }^{1}$, Mariana Cravo ${ }^{3}$, Fernanda Sachse ${ }^{3}$, Gilda Teixeira ${ }^{1}$, Isabelina Ferreira ${ }^{1}$, Filipa Moita ${ }^{1}$, Maria João Gutierrez ${ }^{1}$, Nuno Miranda $^{1}$, Manuel Abecasis ${ }^{1}$

${ }^{1}$ Portuguese Institute of Oncology, Hematology, Lisbon, Portugal; ${ }^{2}$ Portuguese Institute of Oncology, Clinical Research Unit, Lisbon, Portugal; ${ }^{3}$ Portuguese Institute of Oncology, Dermatology, Lisbon, Portugal

Background: Primary Cutaneous T-cell lymphomas (PCTCL) are a heterogeneous and rare group of lymphoproliferative disorders. Treatment is generally not curative and allogeneic cell transplantation (alloHCT) could potentially cure some of these patients, because of potential graftversus-lymphoma effect. Its role has been explored mainly in Mycosis Fungoides/Sézary Syndrome (MF/SS). The largest study published included 129 patients from CIBMTR (2000-2009) with only 39\% patients with stage IV MF (Lechowicz MJ et al. BMT 2014). Few studies suggest that alloHCT provides disease control in heavily treated advanced MF/SS.

Methods: Retrospective review of PCTCL patients submitted to alloHCT in our Centre (2013-2017). Statistics was performed with SPSS and survival analysis by KaplanMeier methodology.

Results: Seven patients with PCTCL performed alloHCT from 2013-2017. Five had a diagnosis of MF/SS and two patients had primary cutaneous cytotoxic and peripheral $\mathrm{T}$ cell lymphoma NOS. All patients had advanced stage disease with a median age of 40 years before transplant (3549) and a median Hematopoietic cell transplant comorbidity index (HCT-I) of 2 (0-6). Our series had a median of 3 previous lines of therapy (2-4). At the time of transplant, 3 patients had stable disease, 2 partial remission, 1 complete remission and 1 progressive disease. A reduced intensity conditioning with Fludarabine/Melphalan was chosen. Acute graft versus host disease (aGvHD) occurred in five of seven patients. One had liver, three had skin and one had skin, gastrointestinal and liver involvement. One patient had mixed MF/GvHD skin manifestations and responded well to extracorporeal photopheresis. Four patients required escalation of immunosuppression and two are still on calcineurin inhibitors. Four of five patients evolved to chronic cutaneous GvHD. Two patients died (one hepatic aGvHD and one pneumonia). One patient progressed and is currently stable with skin-directed therapy. The most frequent complications observed were infections and hemorrhagic cystitis, followed by vascular (veno-occlusive disease requiring defibrotide - 1) and haematological complications (thrombotic thrombocytopenic purpura - 1). Transplant-related toxicities were acute and chronic renal failure (3), metabolic syndrome (5), endocrinopathy (2) and depression (4), requiring psychiatric support.

With a median follow-up of 41 months, we estimated an overall survival (OS) of $71.4 \%$ at 6, 12 and 24 months (95CI: 0.447-1). Progression free survival (PFS) was $71.5 \%$ at 12 months (95CI: 0.447-1). As one patient had progressive disease, the PFS dropped to $53.6 \%$ at 24 months (95CI: 0.257-1). The robustness of our estimations were likely affected by the small sample size.

Conclusions: Our single center experience confirms that alloHCT is feasible in multi-treated advanced stage CTCL patients. Toxicity and tolerability were acceptable. In our small series, we noticed that disease stability before transplant seems to be important for a better response. Prospective studies would be required to confirm this impression. Still, disease control was achievable in one patient with progressive disease. Nonetheless, two patients died in the first 100 days, illustrating that serious complications are expected. In conclusion, our small series adds to the few published data regarding alloHCT in CTCL and confirms the feasibility of the procedure, highlighting its potential regarding disease control and possibly a cure in patients with aggressive CTCL.

Conflict of interest: No conflicts to declare

\section{P605}

Allogeneic Hematopoietic Stem Cell Transplantation in Advanced Hodgkin Lymphoma: A Curative Procedure and a Platform for Immunotherapy

Chara Giatra $^{1}$, Ioannis Tsonis ${ }^{1}$, Dimitra Oikonomopoulou ${ }^{1}$, Ioannis Konstantellos $^{1}$, Konstantinos Girkas ${ }^{2}$, Maria Stamouli ${ }^{2}$, Tatiana 
Tzenou $^{1}$, Anastasios Loidoris ${ }^{3}$, Maria Garofalaki ${ }^{1}$, Eirini Tziotziou ${ }^{1}$, Eirini Grispou ${ }^{1}$, Panagiotis Repousis ${ }^{3}$, Themistoklis Karmiris ${ }^{1}$, Stavros Gigantes ${ }^{1}$, Panagiotis Tsirigotis $^{1}$, Ioannis Baltadakis ${ }^{1}$, Dimitrios Karakasis $^{1}$, Nicholas Harhalakis ${ }^{1}$

${ }^{I}$ Evaggelismos General Hospital, Department of Haematology and Bone Marrow Transplantation Unit, Athens, Greece; ${ }^{2}$ Attikon University Hospital Athens Greece, 2nd Department of Internal Medicine, Athens, Greece; ${ }^{3}$ Metaxa Hospital, Department of Haematology, Piraeus, Greece

Background: Allogeneic hematopoietic stem cell transplantation (allo-HSCT) is a therapeutic approach with curative potential for Hodgkin Lymphoma (HL) after failure of autologous stem cell transplantation (ASCT). Currently, allo-HSCT is feasible for an increasing number of patients, mainly due to novel therapeutic agents serving as a "bridge" to allo-SCT and the availability of an appropriate donor for the majority of patients. The purpose of this study is to analyze the efficacy, prognostic factors and outcomes of allo-HSCT in patients with advanced HL.

Methods: Between 5/2004 and 6/2017, 34 patients, aged 20-54 (median, 28.5) years, underwent allo-HSCT for HL at a median time of 52 (range, 22-143) months from initial diagnosis. Patients had previously been exposed to a median of 4 (range, 3-8) lines of treatment. All patients had undergone ASCT, 11 had received brentuximab vedotin, and 1 nivolumab prior to allo-HSCT. At the time of allo-HSCT, $9(26.5 \%)$ patients were in complete remission (CR), $16(47.05 \%)$ in partial remission (PR), and $9(26.47 \%)$ in refractory relapse. The conditioning regimen was reduced-intensity (Fludarabine/Melphalan, n $=33$ ) or non-myeloablative (Fludarabine/low-dose total body irradiation, $\mathrm{n}=1)$, and included alemtuzumab $(\mathrm{n}=$ 22 ) or antithymocyte globulin $(n=7)$. Donors were HLA identical siblings $(\mathrm{n}=11)$ or volunteer unrelated with $8 / 8$ $(\mathrm{n}=15), 7 / 8(\mathrm{n}=7)$ and $6 / 8(\mathrm{n}=1)$ match at HLA-A, $-\mathrm{B}$, $-\mathrm{C}$, and -DRB1 loci. Graft source was peripheral blood in all but one patient, who received bone marrow due to previous salvage treatment with nivolumab.

Results: At a median follow-up of 53 (range: 3-160) months, 16/34 patients were alive and in CR. Estimated overall survival (OS) was 64\% (95\% CI, 49.6-82.6\%) at 2 years and $50.8 \%(95 \%$ CI, 36-71.5\%) at 4 years. Cumulative incidence (CI) of non-relapse mortality (NRM) was $24.09 \%$ (95\% CI, 11.13-39.74\%) at 24 months. Progression-free survival (PFS) was 37\% (95\% CI, 23.7$57.8 \%$ ) at 2 years. Relapse occurred in 12 patients at a median time of 6.5 (range, 2-21) months after allo-HSCT. Nine patients had overt clinical relapse and 3 metabolic relapse (by PET/CT criteria). CI of relapse or progression was $33.25 \%(95 \% \mathrm{CI}, 17.77-49.56 \%)$ at 24 months. In multivariate analysis, chemosensitive disease was the only factor that was significantly associated with better PFS (46.4\% vs $11.1 \%$ at 2 years, HR $0.24, \mathrm{p}=0.004)$. Twelve

\section{Progression-Free Survival}

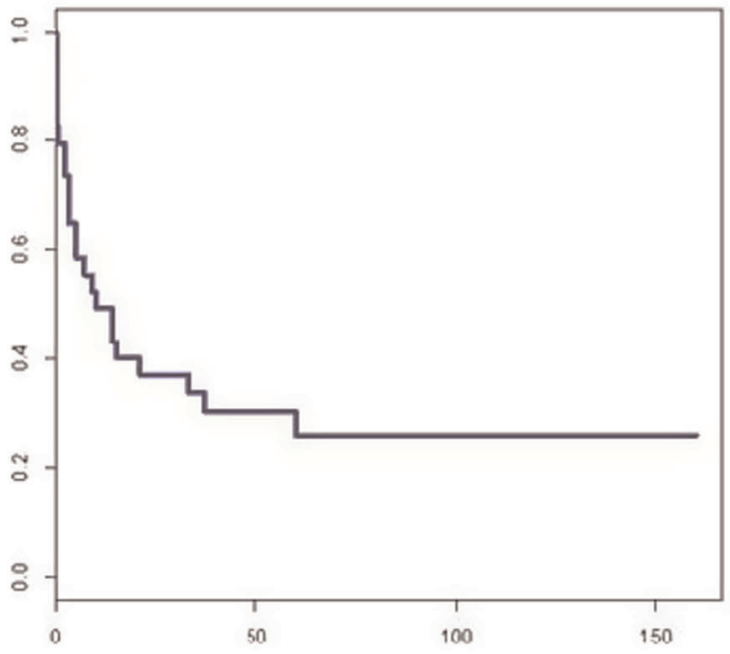

\section{Current Progression-Free Survival}

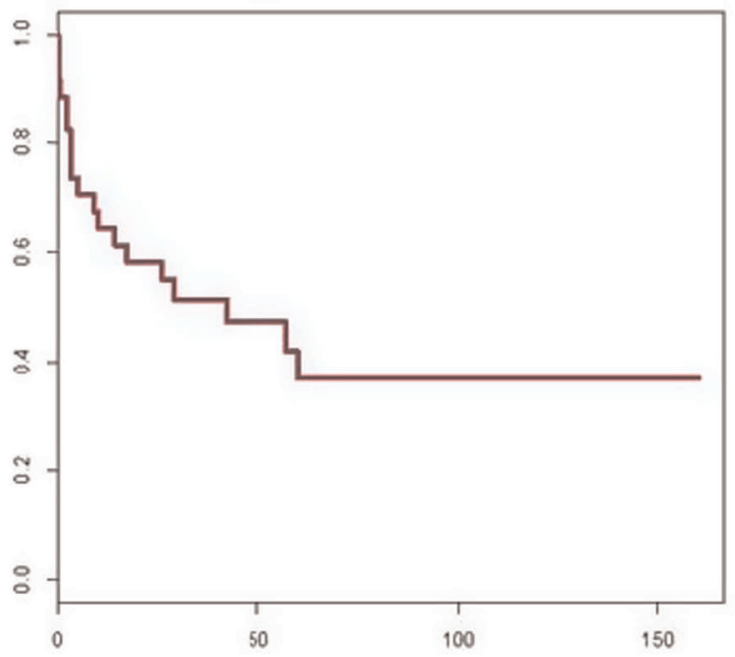

[P605 Figure] [Figure 1. Improvement of PFS by Post-Transplant Immune Interventions]

patients received donor lymphocyte infusions (DLI), due to overt clinical relapse $(n=7)$, metabolic relapse $(n=3)$ or persistent mixed chimerism $(n=2)$. Three patients received DLI in combination with brentuximab vedotin. 10/12 (83\%) responded to DLI (partial response: 4, metabolic remission: 4, full donor chimerism: 2). Immunotherapy with low-dose nivolumab (0.25-1 mg/kg every 2 weeks) was administered in 2 patients, who exhibited prompt responses. Taking into account the response to immune manipulations, current PFS (cPFS) reached $58.2 \%$ at 2 years.

Conclusions: In conclusion, allo-HSCT can be a curative modality for approximately half of the patients with relapsed/refractory HL. Relapse remains the major concern following allotransplant. However, disease control may 
become feasible by means of preemptive DLI or checkpoint inhibitors.

Conflict of interest: All authors have nothing to disclose.

\section{P606}

\section{Allogeneic stem cell transplantation for patients with natural killer/T-cell lymphoid malignancy: a multicentre analysis comparing upfront and salvage transplantation}

Seok Jin Kim ${ }^{1}$, Haa-Na Song ${ }^{2}$, Youngil Koh ${ }^{3}$, Hye Won Lee ${ }^{4}$, Seong Hyun Jeong ${ }^{5}$, Joon Seong Park, Deok-Hwan Yang ${ }^{6}$, Hyeon Seok Eom $^{4}$, Sung-Soo Yoon ${ }^{3}$, Jong-Ho $\mathrm{Won}^{7}$, Won Seog Kim ${ }^{8}$

${ }^{1}$ Samsung Medical Center, Sungkyunkwan University, Medicine, Seoul, Korea, Republic of; ${ }^{2}$ Gyoung Sang National University, Changwon, Korea, Republic of; ${ }^{3}$ Seoul National University, Seoul, Korea, Republic of; ${ }^{4}$ National Cancer Center Hospital, Goyang-city, Korea, Republic of; ${ }^{5}$ Ajou University, Suwon, Korea, Republic of; ${ }^{6}$ Chonnam National Univeristy, Hwasoon, Korea, Republic of; ${ }^{7}$ Soon Chun Hyang University Hospital, Seoul, Korea, Republic of:

${ }^{8}$ Samsung Medical Center, Sungkyunkwan University, Seoul, Korea, Republic of

Background: Natural killer (NK)/T-cell lymphoid malignancies comprise extra-nodal NK/T-cell lymphoma (ENKTL) and aggressive NK-cell leukaemia (ANKL). The cells of these tumours are infected with Epstein-Barr virus (EBV). The prognoses for ENKTL and ANKL remain poor; in particular, the survival outcome for patients with relapsed or refractory disease is extremely poor. Allogeneic stem cell transplantation (SCT) can be used as a front-line consolidation treatment to prevent relapse of advanced disease or as salvage treatment after chemotherapy for relapsed-sensitive disease. However, there are limited data concerning the outcome of front-line or salvage SCT in patients with ENKTL and ANKL.

Methods: We retrospectively analysed 36 patients who underwent allogeneic SCT between February 2004 and February 2017. Newly diagnosed patients with high risk disease $(\mathrm{n}=19)$ underwent upfront allogeneic SCT, whereas patients with relapsed or refractory disease $(\mathrm{n}=$ 17) received allogeneic SCT after salvage chemotherapy. Patients received myelo-ablative $(\mathrm{n}=25)$ or reducedintensity $(n=11)$ conditioning regimens depending on the institute's policy. The most recent update of survival and disease status was in November 2017.

Results: ENKTL $(n=26)$ was more common than ANKL ( $\mathrm{n}=10)$, and more than $80 \%$ of the patients had stage IV disease. The median age at the time of allogeneic SCT was 37 years (range, 17-62 years). The disease status of the patients prior to SCT was complete response $(\mathrm{n}=$ 13), partial response $(n=16)$ and persistent disease $(n=7)$. A higher proportion of ANKL patients (8/10) than ENKTL patients (11/26) received upfront allogeneic SCT. However,
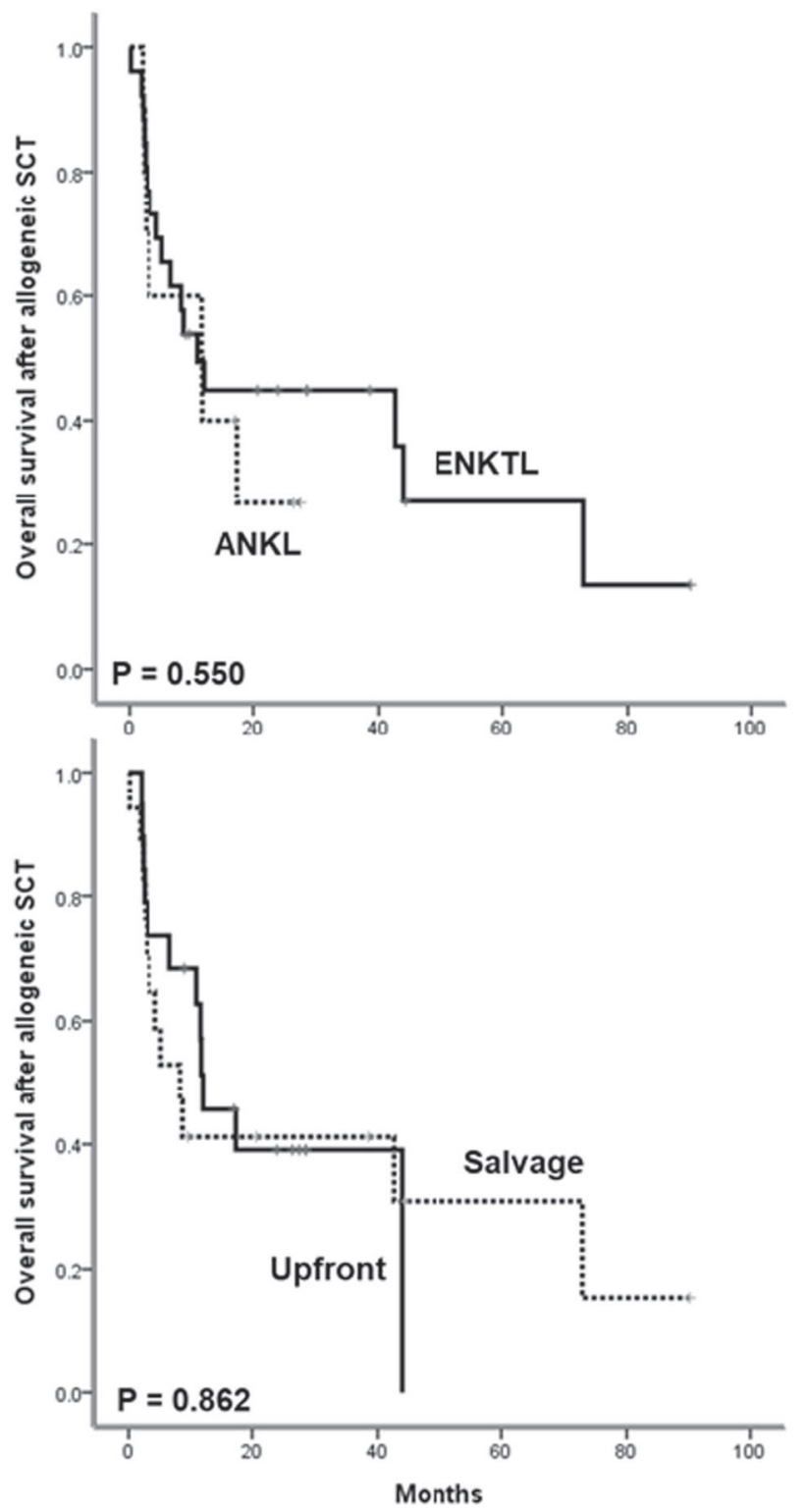

[P606 Figure] [Figure 1. Overall survival after allogeneic SCT in ENKTL/ANKL (Top); Upfront/Salvage (Bottom)]

disease status prior to allogeneic SCT, conditioning regimens and donor sources did not differ between upfront and salvage allogeneic SCT groups. Febrile neutropenia occurred in more than half the patients $(\mathrm{n}=20)$, and 16 patients experienced acute graft-versus-host disease as follows: skin $(\mathrm{n}=9)$, liver $(\mathrm{n}=6)$ and gut $(\mathrm{n}=1)$. With a median follow-up of 28.5 months after allogeneic SCT, the median overall survival (OS) and progression-free survival (PFS) after allogeneic SCT were 11.8 months (95\% confidence interval $[\mathrm{CI}], 7.4-16.2)$ and 10.0 months $(95 \%$ CI, 4.2-15.8), respectively. Twelve patients died because of disease relapse and 12 from non-disease-related causes. Of these 24 deaths, 10 occurred within 100 days after allogeneic SCT (10/24); these were mostly related to 
disease relapse $(n=8)$. The OS after allogeneic SCT did not differ between ENKTL and ANKL $(\mathrm{P}=0.550)$, or between upfront and salvage SCT $(\mathrm{P}=0.862)$. Preallogeneic SCT disease status and elevated serum lactate dehydrogenase were significantly associated with post-SCT PFS. Complete chimerism was significantly associated with better PFS $(P<0.001)$. No significant differences were observed between PFS according to conditioning regimen or source of stem cells $(\mathrm{P}>0.05)$.

Conclusions: Allogeneic SCT may be beneficial for patients with ENKTL and ANKL given that some patients could maintain their remission after allogeneic SCT. However, upfront allogeneic SCT should only be performed in highly selected patients because the risks of disease relapse and non-disease-related mortality remain high.

Clinical Trial Registry: Not applicable

Conflict of interest: All the authors have nothing to disclose.

\section{P607}

\section{Analysis of Data Collected in the European Group for Blood and Marrow Transplantation (EBMT) Registry on a Cohort of Patients with Lymphoma Receiving Plerixafor}

\section{Anna Sureda ${ }^{1}$, Christian Chabannon ${ }^{2}$, Tamás Masszi ${ }^{3}$, David Pohlreich $^{4}$, Christof Scheid ${ }^{5}$, Catherine Thieblemont ${ }^{6}$, Björn E Wahlin $^{7}$, Ioanna Sakellari ${ }^{8}$, Nigel Russell ${ }^{9}$, Andrea Janikova ${ }^{10}$, Anna Dabrowska-Iwanicka ${ }^{11}$, Cyrille Touzeau ${ }^{12}$, Albert Esquirol ${ }^{13}$, Esa Jantunen ${ }^{14}$, Steffie van der Werf ${ }^{15}$, Paul Bosman ${ }^{15}$, Ariane Boumendil $^{16}$, Qianying Liu ${ }^{17}$, Marina Celanovic ${ }^{17}$, Silvia Montoto ${ }^{18}$, Peter Dreger ${ }^{19}$}

${ }^{1}$ Institut Català d'Oncologia, Hospital Duran i Reynals, Barcelona, Spain; ${ }^{2}$ Institut Paoli-Calmettes, Marseille, France; ${ }^{3}$ Semmelweiss University, Budapest, Hungary; ${ }^{4}$ Charles University Hospital, Prague, Czech Republic; ${ }^{5}$ University of Cologne, Cologne, Germany;

${ }^{6}$ HAPHP, Hôpital Saint-Louis, Service d'hémato-oncologie, Université Diderot -Sorbonne Paris Cité, Paris, France; ${ }^{7}$ Karolinska University Hospital, Stockholm, Sweden; ${ }^{8}$ George Papanicolaou General Hospital, Thessaloniki, Greece; ${ }^{9}$ Nottingham University Hospital, Nottingham, United Kingdom; ${ }^{10}$ University Hospital Brno, Brno, Czech Republic; ${ }^{11}$ Maria Sklodowska-Curie Institute-Oncology Center, Warsaw, Poland; ${ }^{12}$ CHU Nantes, Nantes, France; ${ }^{13}$ Hospital de la Santa Creu Sant Pau, Barcelona, Spain; ${ }^{14}$ Kuopio University Hospital, Kuopio, Finland; ${ }^{15}$ EBMT Data Office, Leiden, Netherlands; ${ }^{16}$ EBMT Statistical Unit, Paris, France; ${ }^{17}$ Sanofi Genzyme, Cambridge, MA, United States; ${ }^{18}$ St Bartholomew's hospital, Barts Health NHS Trust, London, United Kingdom; ${ }^{19}$ University of Heidelberg, Heidelberg, Germany

Background: Plerixafor plus granulocyte colony stimulating factor (G-CSF) is administered to patients with lymphoma who are poor mobilisers of haematopoietic stem cells (HSCs) to enhance mobilisation of HSCs for collection and subsequent autologous haematopoietic stem cell transplantation (HSCT).
Methods: This international, multicentre, noninterventional registry study (NCT01362972) evaluated long-term follow up of patients with lymphoma (3.5-7.5 years) who received plerixafor for stem cell mobilisation followed by HSCT compared with patients who received other mobilisation methods and completed HSCT. All patients completed their first autologous HSCT during 2008-2012. To balance the main baseline characteristics between comparison groups propensity score matching was conducted. The following mobilisation regimens were compared: G-CSF + plerixafor $(\mathrm{G}+\mathrm{P})$ vs. G-CSF alone; $\mathrm{G}+\mathrm{P}$ vs. G-CSF + chemotherapy $(\mathrm{G}+\mathrm{C})$; G-CSF + plerixafor + chemotherapy $(\mathrm{G}+\mathrm{P}+\mathrm{C})$ vs. $\mathrm{G}+\mathrm{C}$. The primary outcomes measured were progression free survival (PFS), overall survival (OS) and relapse rate (RR). Proven poor mobilisers were defined as patients who had failed previous attempts at mobilisation.

Results: In total, 3749 patients with lymphoma in the EBMT registry met the eligible study criteria and 313/3749 (8.3\%) patients were mobilised with plerixafor-based regimens. After propensity scoring, 70 vs. 36 patients were matched in the $\mathrm{G}+\mathrm{P}$ vs G-CSF alone cohort, 124 vs 124 in the $\mathrm{G}+\mathrm{P}$ vs $\mathrm{G}+\mathrm{C}$ cohort and 130 vs. 130 in the $\mathrm{G}+\mathrm{P}+\mathrm{C}$ vs $\mathrm{G}+\mathrm{C}$ cohort. There was a loss of statistical power due to propensity scoring which did not overcome all imbalances in the comparison arms. In all comparison groups treated with plerixafor there were a greater proportion of patients who were proven poor mobilisers than in the comparison groups not treated with plerixafor $(97.1 \%$ vs $75.0 \%$ for $\mathrm{G}$ $+\mathrm{P}$ vs G-CSF; $98.4 \%$ vs $68.3 \%$ for $\mathrm{G}+\mathrm{P}$ vs $\mathrm{G}+\mathrm{C} ; 97.7 \%$ vs $69.0 \%$ for $\mathrm{G}+\mathrm{P}$ vs $\mathrm{G}+\mathrm{P}+\mathrm{C}$ ). Estimated median $\mathrm{PFS}$ [months] was 56.6 (95\% CI: 27.17, not calculated [NC]) vs 34.4 (95\% CI: $20.93, \mathrm{NC})$ for G+P vs G-CSF, 57.3 (95\% CI: $27.17, \mathrm{NC})$ vs NC $(95 \%$ CI: 31.31, NC) for $\mathrm{G}+\mathrm{P}$ vs G $+\mathrm{C}$, and 32.4 (95\% CI: $18.86, \mathrm{NC}$ ) vs 57.6 (95\% CI: 20.50, $\mathrm{NC}$ ) for $\mathrm{G}+\mathrm{P}+\mathrm{C}$ vs $\mathrm{G}+\mathrm{C}$. OS and PFS were lower in $\mathrm{G}+\mathrm{P}$ versus $\mathrm{G}+\mathrm{P}+\mathrm{C}$, as well as in $\mathrm{G}+\mathrm{P}+\mathrm{C}$ versus $\mathrm{G}+\mathrm{C}$. The $\mathrm{OS}$ and PFS were higher in the $\mathrm{G}+\mathrm{P}$ versus $\mathrm{G}-\mathrm{CSF}$. However, differences between the groups in OS and PFS were generally small. RR rates were similar between groups. Infection and infestations was the most common standard organ class adverse event in all plerixafor and comparator groups.

Conclusions: This registry study indicates that plerixafor treatment can overcome the mobilisation issues in patients who are difficult to mobilise. The rate of adverse events were generally similar for plerixafor and comparator cohorts. Without plerixafor treatment some patients may not have proceeded to transplantation. However, no firm conclusions can be made as the statistical analyses were underpowered within study groups identified with propensity score matching. The groups were also imbalanced, as a higher proportion of patients treated with plerixafor were 
proven poor mobilisers and therefore represented a patient group that were predisposed to worse outcomes.

Clinical Trial Registry: NCT01362972

Conflict of interest: This study was funded by Sanofi Genzyme

Anna Sureda speaker fees and travel grants from Sanofi.

Silvia Montoto grants for travel from Gilead, and speaker fees from Roche, outside the scope of submitted work

Peter Dreger has received speaker's honoraria from Gilead, Kite; Consultancy: AbbVie, Gilead, Janssen, Roche

Christian Chabannon reports receiving compensation for data transfer from EBMT during the conduct of the study and grants, personal fees and non-financial support from Sanofi S.A outside the scope of the submitted work

Tamás Masszi received financial supports for advisory board membership for AbbVie, BMS, Janssen-Cilag, Novartis, Pfizer and Takeda outside the scope of the submitted work

Christof Scheid reports honoraria and travel support from Sanofi.

Catherine Thieblemont received financial supports for advisory board membership for Roche, Takeda, JanssenCilag, Bayer, Celgene outside the scope of the submitted work

Anna Dabrowska-Iwanicka received personal fees from the EBMT foundation for data transfer during the conduct of the study

Esa Jantunen received speaker's fee and research grant from Sanofi

Qianying Liu and Marina Celanovic are employees of Sanofi

Steffie van der Werf, Paul Bosman, Ariane Boumendi, David Pohlreich, Björn E Wahlin, Ioanna Sakellari, Nigel Russell, Andrea Janikova, Cyrille Touzeau and Albert Esquirol: nothing to disclose

\section{P608}

\section{AUTOLOGOUS PERIPHERAL STEM CELL TRANSPLANTATION TREATMENT IN RELAPSED / REFRACTORY HODGKIN LYMPHOMA PATIENT: ONE CENTRE EXPERIENCE}

\author{
Aliye Serpil Sarifakiogullari ${ }^{1}$, Salih Sertac Durusoy ${ }^{1}$, Handan \\ Haydaroglu Sahin ${ }^{1}$, Mustafa Pehlivan ${ }^{1,2}$ \\ ${ }^{1}$ Gaziantep University Faculty of Medicine, Department of \\ Hematology, Gaziantep, Turkey; ${ }^{2}$ Gaziantep University Faculty of \\ Medicine, Bone Marrow Transplantation Center, Gaziantep, Turkey
}

Background: Hodgkin lymphoma (HL) is a potentially curative malignant disease of the lymphoid tissue. Although it is possible to treat successfully more than $80 \%$ of patients with current treatment methods, some cases maybe refractory or early/late relapse to the treatment. Autologous peripheral stem cell transplantation (APSCT) following high-dose rescue chemotherapy in relapsed / refractory HL has become the standard treatment option in guidelines. In this study, we present the results of 34 refractory/relapsed HL patients treated with APSCT.

Methods: The files of patients who underwent APSCT between July 2010 and July 2017 were reviewed retrospectively and the results of 34 patients with HodgkinÂ's lymphoma were presented at the Gaziantep University Bone Marrow Transplant Unit.

Results: The mean age at diagnosis was 40 (18-72) years old, $18(53 \%)$ male and $16(47 \%)$ female of 34 patients who were diagnosed as Relaps / Refractor HL with APSCT. The median time between diagnosis and administration is 13 (3.3-49.2) months. Distribution of histologic subtypes in diagnosis; 19 nodular sclerosis (56\%), 7 mixed cellularity (20\%), 5 lymphocytes poor (15\%), 2 nodular lymphocyte predominant (6\%) and 1 lymphocyte rich (3\%). According to international prognostic scoring (IPS); 12 patients were low (35\%), 14 patients were middle (41\%) and 8 patients were high (24\%). Nine of the patients were stage I-II (26\%) at diagnosis, 25 patients were stage III-IV (74\%). Bone marrow involvement in 6 patients and Bulky mass in 3 patients. Cisplatin-based (71\%) relapses / refractures were obtained in 24 patients and remission was obtained in 10 patients with rescue treatment based on gemcitabine (29\%). In the mobilization regimen, cisplatin-based G-CSF was used in 24 patients and gemcitabine-based G-CSF regimen was used in 10 patients. Median 1 (1-2) was collected by apheresis procedure. Products containing the median 8 (3.211) $x 106 \mathrm{CD} 34 / \mathrm{kg} \mathrm{CD} 34$ (+) cells were infused after the preparation regimen (24 BEAM, 5 Mito / Mel, 5 BuOtoCyc). During the application, 24 patients were in the second full remission and 10 patients were in the third full remission. A neutrophil scar on the 9th day of median (9-20), platelet engraftment on the 11th day (9-21). A febrile neutropenic attack was detected in 23 (68\%) of the patients. During the follow-up period of 34.2 (3-95) months, relapse developed in 10 patients. Six patients died of progressive disease. There are currently 28 patients, 24 of whom are in complete remission. The expected overall survival rate at 7 years is $78 \%$ and the disease-free survival rate is $65 \%$. There was no clinical risk factor affecting survival after APSCT.

Conclusions: Our results; autologous stem cell transplant suggests an important treatment option with relapse / refractor HL patients with low transplant-related mortality risk and long-term survival advantage.

Conflict of interest: All the authors have nothing to disclose. 


\section{P609}

\section{Autologous Stem Cell Transplantation may potentially abrogate the negative prognostic effect of early relapse after chemo or inmunochemotherapy in Follicular Lymphoma}

Ana Jiménez Ubieto ${ }^{1}$, Carlos Grande $^{2}$, Dolores Caballero ${ }^{3}$, Lucrecia Yañez ${ }^{4}$, Silvana Novelli ${ }^{5}$, Miguel-Teodoro Hernández Garcia ${ }^{6}$, María Manzanares $^{7}$, Reyes Arranz, José Javier Ferreiro ${ }^{8}$, Sabella Bobillo $^{10}$, Santiago Mercadel ${ }^{11}$, Andrea Galego ${ }^{12}$, Javier López Jiménez $^{13}$, José Maria Moraleda ${ }^{14}$, Carlos Vallejo ${ }^{15}$, Carmen Albo ${ }^{16}$, Elena Pérez ${ }^{17}$, Carmen Marrero ${ }^{18}$, Laura Magnano ${ }^{19}$, Luis Palomera $^{20}$, Isidro Jarque ${ }^{21}$, Pilar Martinez-Sánchez ${ }^{2}$, Alejandro Marin $^{22}$, Armando López-Guillermo ${ }^{23}$, Antonio Salar ${ }^{24}$, Juan José Lahuerta $^{2}$

${ }^{1}$ Hospital 12 de Octubre, Hematologia, Madrid, Spain; ${ }^{2}$ Hospital 12 de Octubre, Madrid, Spain; ${ }^{3}$ Hospital de Salamanca, Salamanca, Spain; ${ }^{4}$ Hospital Marques de Valdecilla, Santander, Spain; ${ }^{5}$ Hospital Santa Creu I Sant Pau, Barcelona, Spain; ${ }^{6}$ Hospital Universitario de Canarias, Tenerife, Spain; ${ }^{7}$ Hospital Universitario de Jerez, Jerez, Spain; ${ }^{8}$ Hospital la Princesa, Madrid, Spain; ${ }^{9}$ Hospital Universitario de Aranzazú, Madrid, Spain; ${ }^{10}$ Hospital Vall dHebron, Barcelona, Spain; ${ }^{11}$ Hospital Universitario de Bellvitge, Bellvitge, Spain;

${ }^{12}$ Hospital Universitario A Coruña, A Coruña, Spain; ${ }^{13}$ Hospital Ramón y Cajal, Madrid, Spain; ${ }^{14}$ Hospital Universitario Virgen de Arraxiaca, Murcia, Spain; ${ }^{15}$ Hospital Central de Asturias, Oviedo, Spain; ${ }^{16}$ Hospital Universitario de Vigo, Vigo, Spain; ${ }^{17}$ Hospital Universitario Morales de Meseguer, Murcia, Spain; ${ }^{18}$ Hospital La Candelaria, Tenerife, Spain; ${ }^{19}$ Hospital Clinic de Barcelona,

Barcelona, Spain; ${ }^{20}$ Hospital Clinico Lozano Blesa, Zaragoza, Spain;

${ }^{21}$ Hospital La Fe, Valencia, Spain; ${ }^{22}$ Hospital Universitario

Salamanca, Salamanca, Spain; ${ }^{23}$ Hospital Clínic Barcelona,

Barcelona, Spain; ${ }^{24}$ Hospital del Mar \& IMIM (Hospital del Mar

Medical Research Institute), Barcelona, Spain

Background: Patients with follicular lymphoma (FL) experiencing early therapy failure (ETF) within two years of frontline chemotherapy/immunochemotherapy have poor overall survival (OS). Autologous Stem Cell Transplantation (ASCT) have shown an OS benefit in relapsed FL after a rituximab-free induction regimen, nevertheless its effect in early relapse FL is uncertain.

Methods: : In the Spanish GELTAMO registry we identified 217 patients who underwent ASCT with primary refractory disease $(n=15)$, second complete response (CR2) $(n=45)$ or second partial response (PR2) $(n=57)$ between 1989-2007. ETF was defined as failure to achieve at least PR after frontline frontline chemotherapy/immunochemotherapy or lymphoma progression within two years of frontline chemotherapy/immunochemotherapy. We identified two groups: the EFT cohort $(\mathrm{n}=155$ : 15 primary refractory patients, 95 transplanted in CR2 and 45 in PR2] and the non-EFT cohort [FL patients who received ASCT in either CR2 or PR2 but who did not experience ETF following first-line therapy $(n=62: 50$ transplanted in CR2 and 12 in PR2].

Results: In the ETF cohort an non-ETF cohort, a total 54 $(35 \%)$ and $16(31 \%)$ patients were exposed to rituximab before ASCT.Time interval from first relapse after primary treatment to ASCT was 10 months in both cohorts. In the ETF group a total of $74 \%$ of the patients received ASCT within the first year after treatment failure. Median followup from ASCT was 12 years. Median time to progression within first-line therapy was 9 months and 47 months for patients in the ETF and non-ETF cohorts, respectively $(\mathrm{P}<$ .00001). Patient/disease characteristics were well balanced between the cohorts, except for age, Ann Arbor stage at diagnosis and status of disease at the moment of ASCT. There was a significant difference in PFS $(P=0 \cdot 016)$ between the ETF and non-ETF cohorts, with 5-year PFS rates from the time of ASCT being $44 \%$ and $58 \%$, respectively. Nevertheless, in patients experimenting EFT with an interval from first relapse after primary treatment to ASCT $<1$ year, 5-year PFS was 55\%; similar to that of the non-EFT cohort. There was no significant difference in OS $(P=.27)$ between the ETF and non-ETF cohorts, with 5-
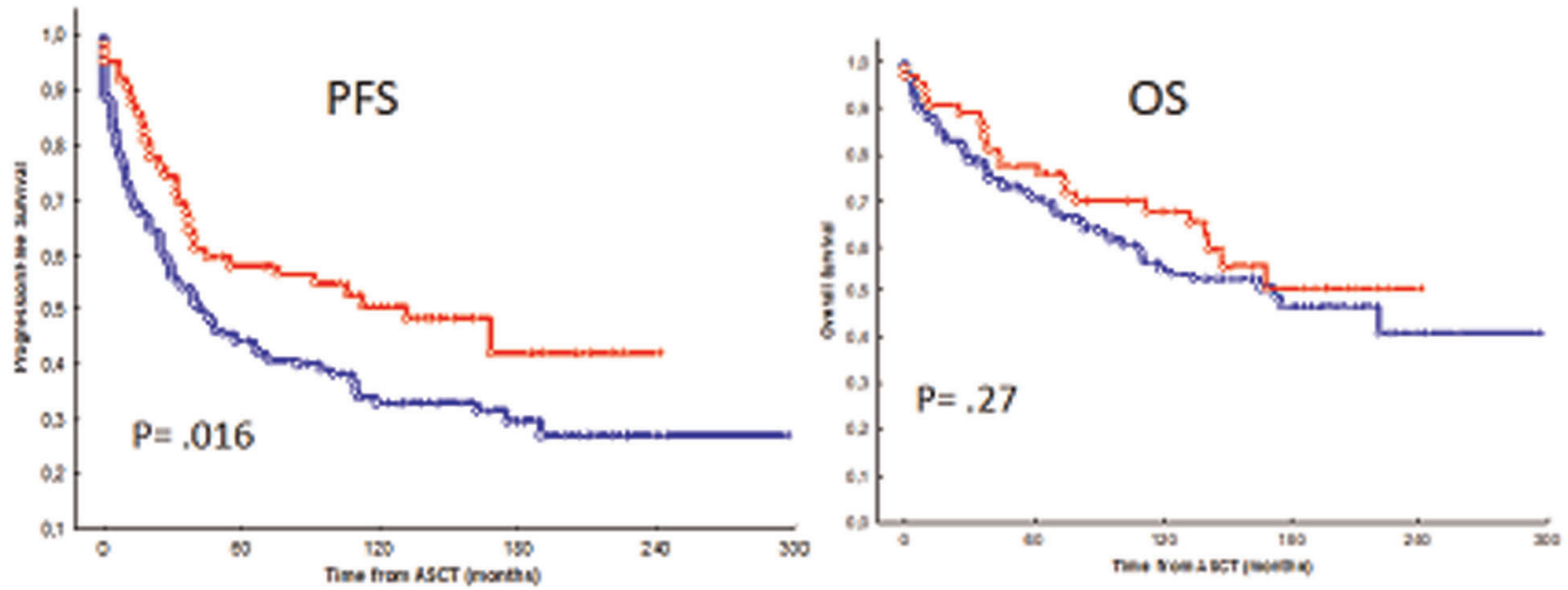

[P609 Figure] [Kaplan-Meier curves ofPFS and OS from the time of ASCT, according to whether patients had E] 
year OS rates from the time of ASCT being 66\% and 79\%, respectively (figure 1). In the rituximab exposed patients, there were no differences in PFS $(P=0 \cdot 8)$, nor in OS $(P=0 \cdot 8)$, between the EFT and non-EFT cohorts. In the EFT-rituximab exposed patients 5-year PFS and OS were $49 \%$ and $76 \%$, respectively; with a plateau in the PFS curves beyond 7.1 years of follow-up, at $45 \%$.

Conclusions: : ASCT may potentially abrogate the negative prognostic effect of early relapse after chemo or R-chemotherapy. Patients experimenting EFT with an interval from first relapse after primary treatment to ASCT of less than 1 year have similar outcome than those who undergo ASCT without EFT. Very long-term FL disease free survival is $45 \%$ in patients who relapse after a rituximab-containing regimen and who are sensitive to a new regimen of R-chemotherapy; however, the value of ASCT in refractory patients must be elucidated.

Conflict of interest: There is no conflict of interest.

\section{P610}

\section{Brentuximab vedotin in children and adolescents with CD30-positive lymphoproliferative disorders}

Andrey Kozlov, Asmik Gevorgian, Ilya Kazantsev, Polina Tolkunova, Elena Vigovskaya, Anton Eltsov, Ilya Nikolaev, Elena Morozova, Natalya Mihailova, Ludmila Zubarovskaya, Boris Afanasyev

R. Gorbacheva Memorial Research Institute for Pediatric Oncology, Hematology and Transplantation, the First State Pavlov Medical University of St. Petersburg, St. Petersburg, Russian Federation

Background: Up to $80-90 \%$ of children with classical Hodgkin lymphoma (cHL) and non-Hodgkin lymphoma (NHL) are cured with first line therapy but the rest demonstrate relapsed or refractory $(\mathrm{R} / \mathrm{R})$ course of the disease. Approximately half of the patients in this unfavorable risk group can be salvaged with second line chemotherapy and autologous hematopoietic stem cell transplantation (HSCT). Immunotherapy is very promising approach in this clinical situation. Endpoints of the study included response to Brentuximab vedotin (BV) or combination of $\mathrm{BV}$ and Bendamustine (B), progression free survival (PFS) and safety in children and adolescents with $\mathrm{R} / \mathrm{R}$ cHL and NHL.

Methods: The analysis included 31 children and adolescents with R/R cHL and NHL that expressed CD30. In patients with NHL the distribution of subtypes was as follows: anaplastic large cell lymphoma $(\mathrm{n}=2)$, primary mediastinal B-cell lymphoma $(\mathrm{n}=2)$ and diffuse large Bcell lymphoma $(\mathrm{n}=1)$. Ann Arbor staging system was used in cHL and Murphy staging system - in NHL.

Treatment regimen for R/R cHL consisted of BV $1.8 \mathrm{mg} /$ $\mathrm{kg}$ triweekly $(\mathrm{n}=19)$ or combination of BV $1.8 \mathrm{mg} / \mathrm{kg}$ on

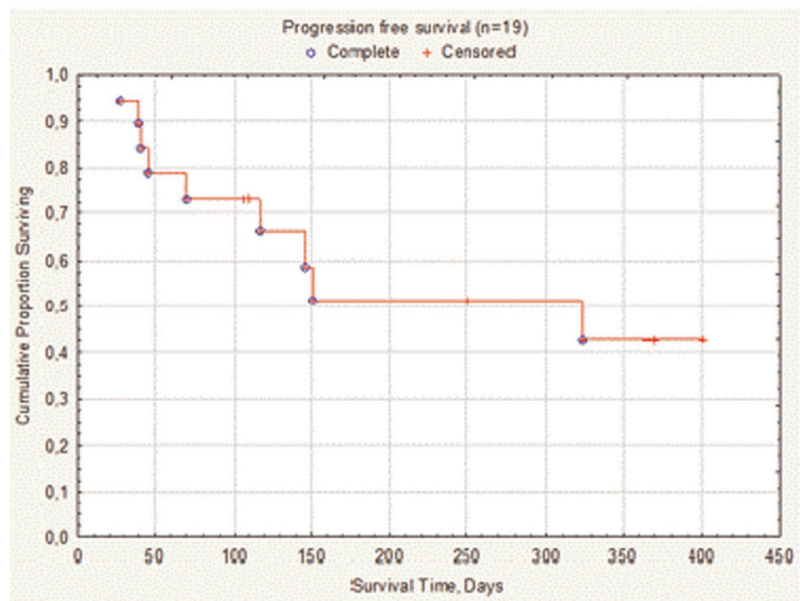

[P610 Figure] [Progression free survival]

Day 1 with B $90 \mathrm{mg} / \mathrm{m}^{2}$ on Days 1 and 2 of 3-week cycles $(\mathrm{n}=7)$. In $\mathrm{R} / \mathrm{R} \quad \mathrm{NHL}$ all children received $\mathrm{BV}$ as monotherapy $1.8 \mathrm{mg} / \mathrm{kg}$ triweekly. Median number of BV cycles in cHL was 4.5 (range 2-12) and in NHL - 8 (3-13).

Results: In children with $\mathrm{R} / \mathrm{R}$ cHL overall response rate (ORR) to BV was achieved in 10 patients (53\%), complete response (CR) - 3 (16\%), partial response (PR) - 7 (37\%), stable disease - $4(21 \%)$ and progression - $5(26 \%)$. Oneyear PFS calculated using the Kaplan-Meier method in this group was $43 \%$.

Median time to progression was 148 days (range 27-324). Combination of BV and B in R/R cHL resulted in ORR in 7 patients (100\%), CR - 2 (29\%), PR - 5 (71\%). All 5 patients with R/R NHL achieved CR after BV. After BV treatment $18(58 \%)$ children proceeded to HSCT: 11 autologous, 7 allogeneic. Several serious side effects were registered in patients: polyneuropathy $(\mathrm{n}=1,3 \%)$, acute pancreatitis ( $\mathrm{n}$ $=1,3 \%)$, anaphylactic shock $(\mathrm{n}=1,3 \%)$. All these side effects were successfully cured.

Conclusions: More than half of children and adolescents with R/R cHL responded to monotherapy with BV but PFS remained low. $\mathrm{BV}$ proved to be an effective and safe option to achieve a remission prior to HSCT. BV in R/R NHL and combination of $\mathrm{BV}$ and $\mathrm{B}$ in $\mathrm{R} / \mathrm{R}$ cHL demonstrated high response rates but groups were too small to draw final conclusions.

Conflict of interest: Nothing to disclose

\begin{tabular}{|c|c|c|c|}
\hline & $\begin{array}{l}\text { Hodgkin } \\
\text { lymphoma (BV } \\
\text { monotherapy) }\end{array}$ & $\begin{array}{l}\text { Hodgkin lymphoma } \\
\text { (Combination of BV and } \\
\text { Bendamustine) }\end{array}$ & $\begin{array}{l}\text { Non- } \\
\text { Hodgkin } \\
\text { lymphoma }\end{array}$ \\
\hline $\begin{array}{l}\text { Patient number } \mathrm{n} \text {, } \\
(\%)\end{array}$ & $19(61 \%)$ & $7(23 \%)$ & $5(16 \%)$ \\
\hline Median age, range & $16(9-18)$ & $17(15-18)$ & $17(4-18)$ \\
\hline Stage III/IV n, (\%) & $13(68 \%)$ & $7(100 \%)$ & $4(80 \%)$ \\
\hline $\begin{array}{l}\text { Therapy lines prior } \\
\text { to BV (median, } \\
\text { range) }\end{array}$ & $4(1-8)$ & $4(1-5)$ & $2(1-3)$ \\
\hline
\end{tabular}




\begin{tabular}{llll}
$\begin{array}{l}\text { Refractory/ } \\
\text { Relapsed disease }\end{array}$ & $10 / 9$ & $4 / 3$ & $3 / 2$ \\
$\begin{array}{l}\text { Median follow-up, } \\
\text { days }\end{array}$ & & & \\
$\begin{array}{l}\text { Remission/ } \\
\begin{array}{l}\text { Progression after } \\
\text { BV }\end{array}\end{array}$ & $11 / 869)$ & $195(100-1068)$ & $373(259-$ \\
Alive/Dead & $15 / 4$ & $3 / 4$ & $4 / 1$ \\
\hline
\end{tabular}

\section{[[P610 Table] Patient's characteristics.]}

\section{P611}

\section{Brentuximab vedotin in relapsed/refractory Hodgkin's lymphoma after an ASCT, Bulgarian experience}

Penka Ganeva ${ }^{1}$, Yavor Petrov ${ }^{1}$, Georgi Arnaudov ${ }^{1}$, Veselina Goranova-Marinova ${ }^{2}$, Janet Grudeva-Popova ${ }^{2}$, Ilina Micheva ${ }^{3}$, Liana Gercheva ${ }^{4}$, Gergana Tsvetkova $^{5}$, Ewgeni Hadjiev ${ }^{5}$, Atanas Radinov ${ }^{6}$, Branimir Spassov ${ }^{7}$, Raya Petrova ${ }^{8}$

${ }^{1}$ University National Specialised Hospital for Active Treatment of Haematological Diseases, BMT Unit, Sofia, Bulgaria; ${ }^{2}$ University Hospital 'St. George', Hematology Department, Plovdiv, Bulgaria; ${ }^{3}$ University Hospital 'St. Marina', BMT Unit, Varna, Bulgaria;

${ }^{4}$ University Hospital 'St. Marina', Hematology Department, Varna, Bulgaria; ${ }^{5}$ University Hospital 'Alexandrovska', Hematology Department, Sofia, Bulgaria; ' Universtity Hospital 'St. Ivan Rilski', Hematology Department, Sofia, Bulgaria; ${ }^{7}$ University National Specialised Hospital for Active Treatment of Haematological Diseases, Sofia, Bulgaria; ${ }^{8}$ University Hospital 'St. Ivan Rilski', Hematology Department, Sofia, Bulgaria

Background: Patients with Hodgkin' lymphoma (HL) who relapsed after an autologous stem cell transplantation (ASCT) have a very poor long- term outcome. Therapeutic options in this subgroup of patients are heterogeneous and include salvage chemotherapy or radiotherapy, palliative care, new drugs and biological agents. In August 2011, the FDA approved Brentuximab vedotin (BV) for two indications in patients with HL: for patients who failed after ASCT or patients in whom ASCT is not an option after failure of at least two previous lines therapies. The introduction of $\mathrm{BV}$ onto clinical practice has improved the prognosis and survival of patients with HL, relapsed after ASCT. The primary endpoint was the overall response rate (ORR), type of response, toxicity profile, event free survival (EFS) and overall survival (OS) in patients with HL relapsed after ASCT, treated with BV.

Methods: Thirty three (33) patients with HL treated with BV relapsed after ASCT in 5 hematology clinics in Bulgaria were studied. The male / female ratio is $14 / 19$, the mean age is $33.1(17-63 \mathrm{y})$. The most common histological type is nodular sclerosis in $26(78.7 \%)$. B - symptoms had 28 patients $(84.8 \%)$. ASCT was performed in all patients, 2 of which were two auto and in one auto/ allo SCT.

The median number of prior anti cancer therapy was 3 (range 2- 5) including ASCT. 21 patients (63.6\%) had received prior radiotherapy. BV was introduced for 21 (64.6\%) patients with relapse/progression after ASCT and for $12(35.3 \%)$ as consolidation therapy after ASCT. The median number of cycles was 10 (range 3-16).

Results: ORR $(\mathrm{CR}+\mathrm{PR})$ was confirmed in 18 patients (54.5\%): CR in $13(39.3 \%)$, $\mathrm{PR}$ in $5(15.1 \%)$, $\mathrm{SD}$ in $2(6 \%)$, progression in $13(39.3 \%)$. The status at the start of BV therapy may be have relation with $\mathrm{PFS}(\mathrm{P}=0.281)$. Patients who were treated with $\leq 10 \mathrm{BV}$ cycles had a lower OS than those received $\geq 10$ courses $(80.8 \%$ vs. $87.5 \% \mathrm{p}=$ $0.060)$. Patients who were treated with $\leq 10$ cycles of BV had a lower PFS than those with $\geq 10$ cycles. $(42.2 \%$ vs. $59.3 \% \mathrm{p}=0.023)$. Patients who responded with progression after treatment with BV had less OS than those who responded with $\mathrm{CR}, \mathrm{PR}, \mathrm{SD}(40.1 \%$ vs. $100 \% \mathrm{p}=0.010)$. The number of courses conducted with $\mathrm{BV}$ is an independent factor that affects OS and PFS. In general the treatment was well tolerated and the toxicity profile was similar to that previously reported. Neurological toxicity was seen in 6 , one patients stop the therapy because of allergic reaction; aspergilosis of lung $(n=1)$, hepatitis $B$ reactivation in one.

Conclusions: The report indicates that $\mathrm{BV}$ as a single agent is effective, safe and good tolerated in standard clinical practice. Our results confirm the international experience.

Conflict of interest: there is no conflict of interest

\section{P612}

Case of successful treatment of Hodgkin lymphoma progression after allogeneic haploidentical stem cell transplantation with combination of brentuximab vedotin therapy and donor lymphocyte infusions

Dmitry Motorin, Darina Zammoeva, Renat Badaev, Diana Babenetskaya, Natalia Ilyina, Yulia Alexeeva, Andrey Zaritskey

Almazov National Medical Research Centre, St. Petersburg, Russian Federation

Background: Relapse of HL after alloSCT still present major clinical problem. Treatment options are limited, and results of relapse treatment are unsatisfactory. We present case of treatment of Hodgkin lymphoma relapse after alloSCT with combination of DLI and brentuximab vedotin.

Methods: 23 year old female with primary refractory Hodgkin lymphoma IVB, nodular sclerosis type, with involvement of intrathoracic lymphnodes and lungs, diagnosed on February 2014.

First line therapy : 6 cycles of BEACOPP with only partial response achieved.

Second line therapy : 2 cycles of DHAP and maintained partial response. 
After LACE conditioning regimen autologous stem cell transplantation was performed in August 2015, but early progression on November 2016 occurred - CT scanning detected increase of target lymph nodes size.

She preceded to allogeneic stem cell transplantation. The donor was her mother, haploidentical by HLA.

Conditioning regimen was nonmyeloablative $\mathrm{Cy} / \mathrm{Flu} / \mathrm{Mel}$, acute GVHD prophylaxis with PTCY +CsA+MMF. Sourse of transplant - PBSC, dose

Engraftment by leukocytes on Day +15 and platelets on Day +18 . On day 30 - complete donor chimerism.

CT scanning on Day +30 showed tumor mass reduction on $70 \%$, so partial remission was achieved. In follow-ups she maintained partial response and no signs of GVHD.

On 6 months after stem cell transplantation PET CT detected progression of the disease with increase in FDG uptake up to Deauville 4.

Treatment of relapse:

Withdrawal of immunosupressive therapy. No GVHD observed. On 8 months after stem cell transplantation the patient was still in progression, with B-symptoms occurred and new metabolic active lymphnodes by PET-CT.

Treatment with brentuximab vedotin since Month $+8+$ DLI. BV $1,8 \mathrm{mg} / \mathrm{kg}$ once in 21 days. Donor leucocyte infusions after $2^{\text {nd }}$ and $6^{\text {th }}$ cycle of treatment she received donor lymphocyte infusions in escalating schedule : $1 \times 10 * 6$ and $1 \times 10 * 7 \mathrm{CD} 3+$ per kilo respectively.

Results: After $5^{\text {th }}$ cycle of brentuximab vedotin and DLI №1 partial metabolic response was archived. By 12 cycles of brentuximab vedotin and DLI№2 patient achieved complete metabolic response. Total 15 cycles of BV were performed. No activation of GVHD was detected.

Now she has no adverse events and feels well.

Conclusions: Brentuximab vedotin in combination with donor lymphocyte infusions seems to be effective treatment option for Hodgkin lymphoma relapse after haploidentical stem cell transplantation.

\section{Conflict of interest: none}

\section{P613}

\section{Checkpoint Inhibitor Therapy for Hodgkin Lymphoma after Allogeneic Bone Marrow Transplantation: A retrospective analysis of the EBMT-Lymphoma Working Party}

\author{
Sascha Dietrich ${ }^{1}$, Anna Sureda ${ }^{2}$, Luca Castagna ${ }^{3}$, Noel Milpied ${ }^{4}$, \\ Herve Ghesquieres, Ali Bazarbachi ${ }^{6}$, Roch Houot ${ }^{7}$, Georg \\ Maschmeyer ${ }^{8}$, Domenico Russo ${ }^{9}$, Jakob Passweg ${ }^{10}$, Alexandros \\ Spyridonidis $^{11}$, Michael Stadler ${ }^{12}$, Stephen Robinson ${ }^{13}$, Peter \\ Dreger $^{14}$, Silvia Montoto ${ }^{15}$ \\ ${ }^{1}$ University and University Hospital of Heidelberg, Hematology, \\ Heidelberg, Germany; ${ }^{2}$ Hospital Universitari Dexeus, Barcelona, \\ Spain; ${ }^{3}$ Istituto Clinico Humanitas, Milano, Italy; ${ }^{4}$ Hôpital Haut- \\ Leveque, Bordeaux, France; ${ }^{5}$ Centre Hospitalier Lyon Sud, Lyon,
}

France; ${ }^{6}$ American University of Beirut Medical Center, Beirut, Lebanon; ${ }^{7}$ Centre Hospitalier Universitaire de Rennes, Rennes, France; ${ }^{8}$ Klinikum Ernst von Bergmann, Potsdam, Germany; ${ }^{9}$ Domenico Russo, Azienda Ospedaliera Spedali Civili Di Brescia, Brescia, Italy; ${ }^{10}$ University Hospital Basel, Basel, Switzerland; ${ }^{11}$ Alexandros Spyridonidis, University Hospital of Patras, Patras, Greece; ${ }^{12}$ Hannover Medical School, Hannover, Germany; ${ }^{13} \mathrm{UH}$ Bristol, Bristol, United Kingdom; ${ }^{14}$ University Hospital Heidelberg, Heidelberg, Germany; ${ }^{15}$ Queen Mary, University of London, London, United Kingdom

Background: Patients with Hodgkin lymphoma (HL) who have relapsed after allogeneic stem cell transplantation (alloSCT) have a very poor prognosis and limited treatment options. Immunotherapy with check point inhibitors (CI) targeting the PD-1 receptor have demonstrated striking clinical activity with durable responses in HL, thus CI have been tested for patients with HL relapsed after alloSCT. However, concerns about reactivation of graft-versus-host disease (GVHD) in this setting have been raised.

Methods: We conducted a retrospective survey on patients who received treatment with CI for relapse of $\mathrm{HL}$ after alloSCT. We collected information on 20 patients who fulfilled these criteria. The patients had received a median of 4 prior lines of treatment before alloSCT (range: 2-7). Eight patients were transplanted from an unrelated donor, three patients from a related donor and nine from a mismatched relative donor.

Results: The median time from alloSCT to relapse was 12 (95\% confidence interval 6.8-20.3) months and the median time from alloSCT to start of CI treatment was 21 (95\% confidence interval 19-41) months. For all patients a median of 11 (range: 1 to 37) CI treatment cycles were administered. Upon CI treatment 10 patients achieved a complete remission (CR: 53\%), 8 patients a partial remission (PR: 42\%) and one patient was not evaluable. The median time to response was 4.3 months $(95 \%$ confidence interval: 2.9-11). The median follow-up after initiation of CI treatment was 12.2 months $(95 \%$ confidence interval: 11.1-20.5). Two of 20 patients (10\%) died due to a fungal pneumonia and a steroid refractory acute GVHD, respectively (Figure 1). Upon CI treatment an episode of acute GVHD occurred in four of 17 (24\%) patients. One patient experienced a steroid refractory grade 3-4 GVHD, whereas acute grade 3-4 GVHD was treatment sensitive in the remaining 3 patients and GVHD severity decreased with subsequent doses of CI treatment. An episode of chronic GVHD during CI treatment occurred in three of 12 patients $(33 \%)$.

Conclusions: In line with other reports, this study confirms that treatment with CI that target the PD-1 receptor can be safely administered to patients with HL relapse after an alloSCT and this results in good tumor responses.

Conflict of interest: no conflict of interest 


\section{P614}

Chemo-immunotherapy with Brentuximab Vedotin combined with Ifosfamide, Gemcitabine and Vinorelbine Results in Impressive Activity in Relapsed Refractory Hodgkin Lymphoma

Khadega A Abuelgasim ${ }^{1,2}$, Mohsen Alzahrani ${ }^{1}$, Yousef Alsharhan ${ }^{3}$, Moataz Khairi ${ }^{3}$, Mohammed Hommady ${ }^{3}$, Giamal Gmati ${ }^{1}$, Hind Salama $^{1}$, Osama Ali ${ }^{1}$, Emad M Masuadi, Ahmed Alaskar ${ }^{2}$, Ayman Alhejazi ${ }^{1}$, Moussab Damlaj ${ }^{1,2,3}$

${ }^{1}$ King Abdulaziz Medical City, Oncology, Riyadh, Saudi Arabia; ${ }^{2}$ King Abdullah International Medical Research Center, Riyadh, Saudi Arabia; ${ }^{3}$ King Saud bin Abdulaziz University for Health Sciences, Riyadh, Saudi Arabia

Background: Attainment of complete metabolic response (CMR) prior to autologous transplantation (HSCT) in relapsed / refractory classical Hodgkin Lymphoma (R/R cHL) is highly predictive of prolonged remissions. Addition of Brentuximab Vedotin ( $\mathrm{Bv}$ ) to Bendamustine was shown to produce higher complete responses than previously achieved with chemotherapy alone (LaCasce et al, Blood 2015). Ifosfamide, gemcitabine, and vinorelbine (IGEV) is an active salvage regimen with excellent stem cells mobilizing potential. Our aim was to examine the efficacy of IGEV combined with $\mathrm{Bv}$ (IGEV-Bv) as first or subsequent salvage in R/R cHL prior to HSCT.

Methods: Patients with R/R cHL whom were candidates for curative HSCT were identified and all records were retrieved retrospectively after IRB approval. $\mathrm{Bv}$ was given as an intravenous infusion of $1.8 \mathrm{mg} / \mathrm{kg}$ on day 1 with IGEV administered as previously reported (Santoro et al., Haematologica 2007). Deauville score of $\leq 3$ was regarded as a negative Positron Emission Tomography (PET) preHSCT. Patients proceeded to HSCT once partial remission (PR) or better was achieved. Categorical and continuous variables were compared using Pearson's chi-squared and Wilcoxon / Kruskal-Wallis, respectively. Overall survival (OS) and progression free survival (PFS) were computed using Kaplan-Meir method with log ranks test.

Results: A total of 24 patients received IGEV-Bv; 10 (42\%) as first salvage and $10(42 \%)$ as second and $4(16 \%)$ as third salvage. The median age was 24 years with 13 (54\%) being male. Ten (42\%) patients had refractory disease and median time to progression was 7.6 months (1.9-132.9). Pre-HSCT PET was negative in 16/23 (70\%)

Table L. Patiznt Cohort Struified by Sequewe of IGEV-Ev Salvage pre-HSCT

\begin{tabular}{|c|c|c|c|}
\hline Characteristic & IGEV-By First Salrage $(n=10)$ & IGEV-Br Subsequent Salvage $(n=14)$ & P value \\
\hline Mals, n $(\%)$ & $4(40)$ & $9|64\rangle$ & 0.23 \\
\hline Age at HSCT, metian (rangc) & $3](15.46)$ & $22(15-49)$ & 0.33 \\
\hline Refractory ( $\leq 3$ wonths remission) & $3(30)$ & $7(50)$ & 0.32 \\
\hline Time to Relapse, dars (range) & $229\{58-2162\}$ & $212(61-3988)$ & 1 \\
\hline Rdapse $\leq 6$ montls, $n(\%)$ & $5(50)$ & $8(57)$ & 0.73 \\
\hline Relapse $\leq 12$ monihs, n $(\%)$ & $7(70)$ & lọili & 0.94 \\
\hline No. of Salvage Reģimens & & & $<0.0001$ \\
\hline One & $9(90)$ & 0 & \\
\hline Two & $1,10)$ & $9(64)$ & \\
\hline$\geq$ Three & 0 & $5(36)$ & \\
\hline Order of IGEV-BSalvage & & & $<0.0001$ \\
\hline $1^{*}$ & J01001 & $\mathrm{N} / \mathrm{A}$ & \\
\hline $2^{\mathrm{ul}}$ & $\mathrm{NA}$ & lộili & \\
\hline $3^{\text {nd }}$ & $\mathrm{NAS}$ & $4(29)$ & \\
\hline No. of Salvage (yales, median (range) & $2(2-2)$ & $4(2-8)$ & $<0.0001$ \\
\hline PETiCT Status pre-HSCT, u $(\%)$ & & & 0.01 \\
\hline Negative (Deaurille $\leq 3$ ) & $8(89)$ & $8(5 i)$ & \\
\hline Pusilive & (111) & $6\{43\}$ & \\
\hline Consolidation pos HSCT, $\mathrm{n}(\%)$ & $6(60)$ & $11179 !$ & 0.049 \\
\hline Median follow up post HSCT, dars (range) & $171\langle 17.1189\rangle$ & $681(39 \cdot 1568)$ & 0,03 \\
\hline
\end{tabular}

[P614 Figure] [Table I] 
evaluable patients. A total of nine (37\%) patients required a single salvage line, $10(42 \%)$ required two while five $(21 \%)$ required three lines with a median number of three salvage cycles (2-8). Twenty two (91.7\%) patients achieved PR or better and were able to proceed to HSCT following IGEV$\mathrm{Bv}$ while the remaining $2(8.3 \%)$ required a subsequent line before HSCT. With a median follow up post HSCT of 13.9 months (0.6-52.3), the estimated 2-year PFS and OS were $63.7 \% \quad(47.9-75.8 \%)$ and $84.5 \% \quad(70-92.4)$, respectively.

The cohort was subsequently stratified whether IGEV-Bv was given as first $(n=10)$ or subsequent $(n=14)$ salvage after failure of at least one other regimen with corresponding baseline characteristics as shown in table 1. Patients receiving IGEV-Bv as first salvage received significantly fewer salvage cycles (median 2 vs. $4 ; p<0.0001$ ) as well as $\mathrm{Bv}$ consolidation post $\mathrm{HSCT}(60 \%$ vs. $79 \% ; p=0.049)$ with a trend towards higher incidence of pre-HSCT CMR (89\% vs. $57 \% ; p=0.09$ ). The observed 2 -year PFS and OS for IGEV-Bv as first vs. subsequent salvage was $100 \%$ vs. $44 \%(16.8-68.4)(p=0.037)$ and $100 \%$ vs. $75.2 \%(40.7-$ 91.4) $(p=0.22)$, respectively.

Conclusions: IGEV-Bv especially when given as first salvage, was associated with a high CMR rate and fewer salvage cycles. This has resulted in impressive remissions post HSCT. Given the limitation of this analysis, longer follow up and prospective validation is warranted.

Conflict of interest: All authors declare no conflicts of interest.

\section{P615}

\section{DA-EPOCH-R followed by consolidation with autologous} stem cell transplantation in the first-line treatment of poorrisk, diffuse large B-cell lymphoma - a single center experience

Zeljko Prka ${ }^{1}$, Marko Lucijanic ${ }^{1}$, Tamara Vasilj ${ }^{1}$, Zdravko Mitrovic ${ }^{1}$, Ozren Jaksic ${ }^{1,2}$, Vlatko Pejsa ${ }^{1,2}$

${ }^{1}$ University Hospital Dubrava, Zagreb, Croatia; ${ }^{2}$ University of Zagreb, School of Medicine, Zagreb, Croatia

Background: Dose adjusted EPOCH + rituximab (DAEPOCH-R) (etoposide, prednisone, vincristine, cyclophosphamide, doxorubicin) is very efficient and feasible regimen for treatment of diffuse large B-cell lymphoma (DLBCL). However, there is a continous need for improvement in therapy of younger, poor risk subgroup. The role of autologous stem cell transplantation (ASCT) as a part od first line therapy is not clear.

Methods: From May 2007 to October 2017 a total of 31 patients with poor risk DLBCL, defined as aaIPI $\geq 2$ and/or Ki67+ $\geq 90 \%$, were planned to receive total of six to eight

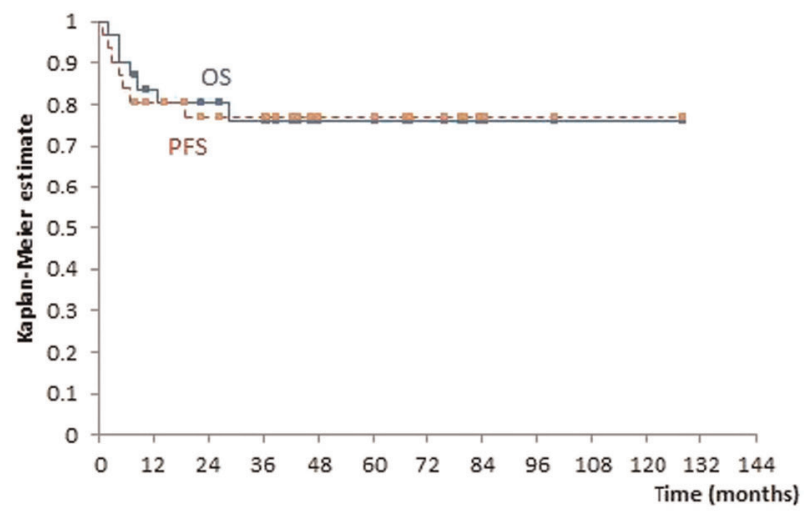

[P615 Figure] [Figure 1. OS and PFS curves for intention to ASCT population]

cycles of DA-EPOCH-R followed by consolidation with ASCT as a part of first- line therapy. Criteria needed for transplant eligibility were: age $<65$, Ki- $67+\geq 90 \%$ or aaIPI $\geq 2$, and absence of transplant-limiting comorbidities. Mean age was $46 \pm 14$ years, 17 (55\%) patients were males. A total of 27 patients (87\%) had increased LDH, $25(81 \%)$ were Ann Arbor III/IV, and 25 (81\%) patients had aaIPI $\geq 2$. Out of 31 patients, 23 proceeded to ASCT. Four patients died during DA-EPOCH-R therapy, one patient withdrew the consent for the transplantation procedure, and three patients failed to collect adequate graft. Myeloablation was performed with BEAM in 16 (70\%) and BeEAM regimen in $7(30 \%)$ patients.

Results: Median follow up of whole cohort was 37 months. OS and PFS rates at 5 years were $76 \%$ and $77 \%$, respectively (Figure 1.)

Out of 27 patients who completed DA-EPOCH-R chemotherapy, in 24 (87\%) patients, last cycle of DAEPOCH was successfully used for stem cell mobilization. In transplanted patients, ORR, CR and PR prior to ASCT were $100 \%, 71 \%$ and $29 \%$, respectively. Median follow up of transplanted patients was 39 months. OS nad PFS at 5 years were $85 \%$ and $86 \%$, respectively. During treatment and follow-up period there was no unexpected toxicities, cardiovascular complications or, although too early, secondary malignancies. One patient died due to transplant related complications. Median time to neutrophil recovery was 10 (9 - 11), and platelet recovery was $11(10-13)$ days. One patient received additonal radiotherapy. Two patients relapsed after ASCT, both with PR after chemotherapy.

Conclusions: DA-EPOCH-R and ASCT as a first line therapy in poor risk aggressive DLBCL is highly efficient and feasible therapeutic option. Our results show that, although generally not recommended, ASCT as consolidation therapy may be beneficial in subgroup of poor risk patients without significant additional toxicities.

Conflict of interest: All the authors have nothing to disclose. 


\section{P616}

Destiny of elderly patients ( $\geq 60$ years) indicated for ASCT for relapsed/refractory (R/R) lymphomas

Robert Pytlik ${ }^{1,2}$, Blanka Vackova ${ }^{2,3}$, Marie Trnkova ${ }^{3}$, Petra Blahovcova $^{3}$, David Pohlreich ${ }^{3}$, Kamila Polgarova ${ }^{2,3}$, Eva Konirova $^{2,3}$, Pavel Klener ${ }^{3,4}$, Katerina Benesova ${ }^{2,3}$, Marek Trneny ${ }^{2,3}$

${ }^{1}$ Institute of Hematology and Blood Transfusion, Department of Cellular Therapy, Praha, Czech Republic; ${ }^{2}$ First Faculty of Medicine, Charles University, Prague, 1st Department of Medicine, Praha, Czech Republic; ${ }^{3}$ General University Hospital Prague, 1 st Department of Medicine, Praha, Czech Republic; ${ }^{4}$ First Faculty of Medicine, Charles University, Prague, Department of Pathological Physiology, Praha, Czech Republic

Background: In comparison to younger patients, there are only limited data for patients $\geq 60$ years old transplanted for $\mathrm{R} / \mathrm{R}$ lymphoma. Furthermore, most of the studies describe only patients actually transplanted and do not take in account the whole cohort of elderly patients, who may be availlable for ASCT.

Methods: This is a single-centre study of consecutive 119 patients $\geq 60$ years with R/R lymphoma indicated for ASCT between 2006-2016. Patients indicated for transplant including those not transplanted for various reasons, were identified from comprehensive records of weekly meetings of the transplant group. Categorical data were compared by Pearson's chi-square tests, continuous data by MannWhitney U test. Survival curves were constructed according to Kaplan-Meier and compared by log-rank tests.

Results: Median age of 119 identified patients was 64 years (range, 60-72), 52\% of them were men. Most frequent diagnoses were diffuse large B-cell lymphoma (DLBCL) 49 patients (41\%), and follicular lymphoma (FL) - 36 patients (30\%). Only 5 patients (4\%) had Hodgkin $\hat{A}$ 's lymphoma (HL). Of 99 mobilized patients, 85 (86\%) collected $\geq 2 \times 10^{6} \mathrm{CD} 34+$ cells $/ \mathrm{kg}$. Of 19 patients indicated for treatment with plerixafor, $14(74 \%)$ had successful harvest. Actually transplanted were 66 patients (55\% of the whole cohort). Reasons for drop-out were: resistant disease (20 patients, 17\%), poor mobilization

(14 patients, 12\%), infections (10 patients, $8 \%$ ), other toxicity (7 patients, $6 \%$ ), and refusal of transplant

(2 patients, $2 \%$ ).

Median PFS and OS were 20.7 months and 55.9 months for the whole group, with median follow-up 46.5 months for living patients. Transplanted patients had PFS and OS 43.5, resp. 103.7 months, while those not transplanted for other causes than progressive disease had PFS and OS 15.7, resp. 61.3 months (differences not significant). For DLBCL, median PFS and OS were 7.9 and 20 months respectively, again, with no significance between transplanted and not transplanted patients. For follicular lymphoma, PFS was
66.6 months for transplanted and 20.5 months for nontransplanted patients and OS were 108.1 v. 61.3 months (p $=0.06$ and 0.07 respectively). Of five patients with HodgkinÂ's lymphoma, three are alive at 15, 25 and 30 months after indication for ASCT.

Conclusions: Of patients $\geq 60$ years with $\mathrm{R} / \mathrm{R}$ lymphoma indicated for transplantation, more than one half were actually transplanted, which compares favorably with prospective studies that recruited younger patients. Overall and progression-free survivals of the whole cohort and especially of transplanted patients are encouraging. Autologous transplantation should be offered to patients $\geq 60$ years in good overall health status. As one of major obstacles for proceeding to transplant was poor mobilization, new mobilization strategies are urgently needed.

Financial support: grants AZV16-310092A and AZV 1728980A from Czech Ministry of Public Health.

Clinical Trial Registry: Not applicable.

Conflict of interest: Robert Pytlik received honoraria from Sanofi in previous 12 months.

No conflict of interest for other authors.

\section{P617}

Evaluation of PET/CT as a tool for the timely detection of relapse / progression of lymphomas after autologous hematopoietic progenitor cell transplantation (ASCT)

Manuel Antonio Granja Morán, Brenda Acosta Maldonado, Liliana Rivera Fong, José Guadalupe Ríos Contreras, Silvia Rivas Vera, Luis Manuel Valero Saldaña

Instituto Nacional de Cancerologia Mexico, Ciudad de Mexico, Mexico

Background: Numerous studies have shown that a negative PET-CT prior to ASCT has prognostic value and predicts overall and progression-free survival in patients with aggressive non-HodgkinÂ's lymphoma (NHL) and HodgkinÂ's lymphoma (HL). The role of PET-CT in posttransplant follow-up has not yet been established. The majority of relapses occurs in the first 18 months for diffuse B large cell lymphoma and 12 months for LH therefore it is necessary to have a study that identifies these patients early. According to the 2014 Lugano recommendations, long-term surveillance with PET-CT in lymphomas is discouraged. However, this practice may be valuable in high-risk lymphoma populations such as those patients undergoing ASCT

Objective: To evaluate the prognostic value of PET-CT for the timely detection of relapse / progression in lymphomas after ASCT.

Methods: Retrospective, cross-sectional analytical study. HodgkinẤs lymphoma (HL) and non-HodgkinÂ's 
lymphoma (NHL) patients undergoing ASCT between January 2007 and December 2016 were included. First PET - CT was performed 6 to 8 weeks after ASCT. Followup PET CT scans were performed at intervals of 3 to 6 months.

Results: 101 patients were eligible for the study (34 NHL and $67 \mathrm{HL}$ ). NHL group, the median age was 44 years. $76.1 \%$ of patients had stages III-IV. $64 \%$ presented extranodal involvement. $64.2 \%$ had a negative PET CT prior to the procedure, $35.8 \%$ had partial response with a positive PET-CT.

In the LH group, the median age was 27 years. $73.5 \%$ of patients had stages III-IV. $41.2 \%$ with extranodal involvement. 53\% had a negative PET-CT prior to the procedure and $47 \%$ had partial response with a positive PET/CT .

In first post-transplant control, 12(50\%) of NHL and 6 patients $(60 \%)$ LH who had PET-CT positive prior to transplantation, the study became negative after the procedure. Forty-two percent (26 NHL and 6 LH patients) had follow-up PET-CT compatible with relapse so patients starting rescue treatment. Majority of patients had no clinical manifestations suspicious of relapse at time of detections.54\% of patients with relapse underwent a biopsy and only the same type of lymphoma was corroborated in $39 \%$.

In our study a positive PET-CT after ASCT had 72\% probability of having a relapse in HL. None of the patients who obtained negative post-transplant PET CT relapsed (NPV 100\%).

Conclusions: The positivity of the first post-ASCT PETCT was associated with a high risk of relapse in our population, these patients should be kept under close surveillance. Contrary to what was reported by some groups, the study allowed us to detect early relapse in the absence of clinical data (28-38\%). In patients with negative early PET-CT, it was not justified to perform negative studies for follow-up, unlike those with a positive study. It is necessary to define the periodicity to detect relapse and adopt the Deauville score.

Clinical Trial Registry: Rev 54/17

Conflict of interest: None

\begin{tabular}{|c|c|c|c|c|}
\hline & $\begin{array}{l}\text { NHL } \\
\text { First post- } \\
\text { transplant }\end{array}$ & $\begin{array}{l}\text { Post-trasplant } \\
\text { (follow-up) }\end{array}$ & $\begin{array}{l}\mathrm{HL} \\
\text { First post- } \\
\text { transplant }\end{array}$ & $\begin{array}{l}\text { Post-trasplant } \\
\text { (follow-up) }\end{array}$ \\
\hline $\begin{array}{l}\text { Positive } \\
\text { Predictive value }\end{array}$ & $72 \%$ & $64 \%$ & $50 \%$ & $58 \%$ \\
\hline $\begin{array}{l}\text { Negative } \\
\text { Predictive } \\
\text { Value }\end{array}$ & $63 \%$ & $100 \%$ & $70 \%$ & $100 \%$ \\
\hline
\end{tabular}

[[P617 Table] values of PET CT according to the time of the ASCT]
P618

First experience of high-dose chemotherapy with autologous hematopoietic stem cell transplantation in patients with HIV-related lymphoma

Marina Popova, Yulia Rogacheva, Anastasia Nekrasova, Ivan Tsygankov, Ali Basahel, Kirill Lepik, Olga Pirogova, Elena Darskaya, Yurii Zalylov, Lilia Stelmah, Ivan Moiseev, Sergey Bondarenko, Natalia Mikhaylova, Boris Afanasyev

First Pavlov State Medical University of Saint Petersburg, Raisa Gorbacheva Memorial Institute of Children Oncology, Hematology and Transplantation, Saint Petersburg, Russian Federation

Background: Human immunodeficiency virus (HIV) infection is associated with an increased incidence of nonHodgkin lymphoma (NHL) and Hodgkin lymphoma (HL). Throughout the HAART era, autologous stem cell transplantation (ASCT) has been reported as a feasible, safe, and useful approach to either rescue or consolidate HIV-related lymphoma patients. However, the number of published comparative studies according to the HIV status is limited.

Methods: Since the Jan 2016 seven patients with HIVrelated lymphoma who have undergone ASCT were included in prospective singe centre study (study group HIV group, $\mathrm{n}=7$ ). The data of the non-HIV-infected patients with lymphoma who have undergone ASCT at the same period of time (control group, $\mathrm{n}=35$ ) were collected to compare the efficacy and safety of the procedure (1:5). Median follow up time was 12 (3-18) months. The primary end points were overall survival (OS) and relapse rate at 12 months after ASCT. Secondary end points were time to hematopoietic recovery and organ toxicity. Common Terminology Criteria for Adverse Events (CTCAE 4.0) for the toxicity analyse have been used. The underlying diseases in HIV-group were HL $\mathrm{n}=4(57 \%)$ and NHL $\mathrm{n}=$ $3(43 \%)$. Conditioning regimen was BEAM with BCNU replacement by Bendamustine $160 \mathrm{mg} / \mathrm{m} 2 /$ day at D-7, D-6. HIV viral load was undetectable (100\%); the median number of CD4+ cells was 265 cells $/ \mathrm{mcl}$; all patients were on HAART. An assessment of potential differences in patients, disease and treatment characteristics between the 2 groups showed no significant differences.

Results: Overall survival (OS) at 12 months for all patients $(\mathrm{n}=42)$ was $93 \%$. OS at 12 months was $100 \%$ in HIV-group vs $91,4 \%(\mathrm{p}=0,3)$ in control group. Relapse rate of the underlying disease at 12 months was $14 \%$ in HIVgroup, in control group - $11 \%(\mathrm{p}=0,8)$. The median time of leukocytes, neutrophils, and platelets recovery was $\mathrm{D}+13$, $\mathrm{D}+15, \mathrm{D}+15$ respectively in HIV-group and $\mathrm{D}+13, \mathrm{D}+16$, $\mathrm{D}+14$ in control group. The rate of organ toxicity according to CTCAE are outlined in table 1 . 
Conclusions: One-year overall survival in patients with HIV-related lymphoma was $100 \%$, relapse rate $-14 \%$ and did not differ from the control group. There were no found significant differences between two groups in hematopoietic recovery and toxicity rate. Preliminary data provide further evidence that HIV status does not affect the outcome of ASCT for lymphoma. Patients with HIV-related lymphoma should be considered candidates for ASCT if they meet standard transplant criteria.

Conflict of interest: All the authors have nothing to disclose.

\begin{tabular}{llll}
\hline Toxicities CTCAE & HIV-group & Control group & P value \\
\hline Enteropathy // Maximum grade & $3(42 \%) / / 3$ & $8(22 \%) / / 3$ & 1,2 \\
Nephrotoxicity // Maximum grade & $1(14 \%) / / 3$ & $3(8 \%) / / 1$ & 0,2 \\
Hepatotoxicity // Maximum grade & $4(57 \%) / / 3$ & $13(35 \%) / / 2$ & 0,9 \\
Mucositis // Maximum grade & $3(42 \%) / / 2$ & $13(35 \%) / / 3$ & 0,08 \\
\hline
\end{tabular}

\section{[[P618 Table] Table 1]}

\section{P619}

\section{First-Line High-Dose Therapy and Autologous Blood Stem Cell Transplantation in Patients with Primary CNS Non- Hodgkin Lymphomas}

\author{
Tomas Brezina ${ }^{1}$, Helena von Dewitz ${ }^{1}$, Mustafa Kondakci, Ariane \\ Dienst $^{1}$, Thomas Schroeder ${ }^{1}$, Sebastian Ullrich ${ }^{2}$, Christina \\ Rautenberg ${ }^{1}$, Kathrin Nachtkamp ${ }^{1}$, Guido Reifenberger ${ }^{3}$, Bastian \\ Malzkorn $^{3}$, Michael Sabel ${ }^{4}$, Rainer Haas ${ }^{1}$, Guido Kobbe ${ }^{1}$ \\ ${ }^{1}$ University Hospital Duesseldorf, Clinic for Haematology, \\ Duesseldorf, Germany; ${ }^{2}$ Life Science Center, .05 Statistic, \\ Duesseldorf, Germany; ${ }^{3}$ University Hospital Duesseldorf, Clinic for \\ Neuropathology, Duesseldorf, Germany; ${ }^{4}$ University Hospital \\ Duesseldorf, Clinic for Neurosurgery, Duesseldorf, Germany
}

Background: Primary central nervous system NonHodgkin-Lymphomas (PCNSL-NHL) are extranodal Bcell lymphomas with inferior prognosis after conventional treatment when compared to their peripheral nodal counterpart. High-dose therapy (HDT) followed by autologous blood stem cell transplantation (ASCT) has shown to induce high rates of long term disease free survival in patients with PCNSL, but its definite role in comparison to intensive conventional chemo- immunotherapy (cCIT) is still not clear.

Methods: We retrospectively collected data of 61 patients (41\% female, median age 63.9 years, range 29-80) with PCNS-NHL who were diagnosed and treated at the University Hospital Düsseldorf between January 2004 and December 2016.

In the conventional chemotherapy (CT) group 36 patients were treated with cCIT, including HD-MTX and
Rituximab. Two patients received consolidating radiotherapy. In the high-dose therapy (HDT) group, 17 patients received an induction with cCIT followed by HDT with ASCT. Three patients were treated solely with radiotherapy and 5 patients received best supportive care only.

Results: In the CT-group treated with cCIT (median 3 cycles of MTX +/- Rituximab, R) but without HDT and ASCT, the overall response rate was $61 \%$ and there were $8 \%$ treatment-related deaths. PFS was 32,2 months and OS 49,2 months with a median follow-up of 22,9 months (range 1-127). In the HDT-group, following a cCIT induction (median 3 cycles of R-MTX and 2 cycles of R-HDCytarabine + Thiotepa) $29 \%$ achieved a CR and $71 \%$ a PR prior to HDT. Conditioning regimens were $\mathrm{BCNU}+$ Thiotepa +/- R (88\%) or BEAM +/- R (12\%). With a median follow-up of 23,3 months (range 6-79), the overall response rate was $82 \%$ and there were $6 \%$ treatment-related deaths. PFS and OS were 66,3 and 57,7 months, respectively. After HDT+ASCT, 11 patients $(64,7 \%)$ are alive in complete or partial remission and 6 died (35,3\%, 5 PD, 1 TRM). All patients achieving CR prior to HDT achieved a durable CR. In the CT-group 12 patients $(33,3 \%)$ are alive in complete or partial remission. Of these, 6 patients are in continuous CR/PR, 2 after salvage HDT+ASCT, 2 after salvage radiotherapy and 2 after salvage chemotherapy. Twenty-one patients $(58,3 \%)$ died of progressive disease.

When compared to the CT-group, patients in the HDTgroup had significantly better PFS (66,3 vs 32,2 months, $p$ $=0,015)$, whereas OS was not significantly different $(57,7$ vs 49,2 months, $p=0,101)$. However, patients in the HDTgroup were also significantly younger when compared to the CT-group $(54,6$ years vs 66,5 years, $\mathrm{p}<0.05)$. There was no significant difference in PFS and OS between the groups treated with or without Rituximab.

Conclusions: Intensive cCIT induction followed by HDT with ASCT is feasible with low treatment related mortality in selected patients with PCNS-NHL. In this single center retrospective analysis response and PFS were better than with conventional cCIT alone. However, patients who received HDT + ASCT were younger and several patients in the CT-group achieved a second response with salvage therapy including ASCT. Randomized studies are needed to define the role of HDT and ASCT as primary treatment for PCNS-NHL.

Conflict of interest: The authors have no conflict of interest.

\section{P620}

HIGH -DOSE CHEMOTHERAPY WITH BEEAM PROTOCOL (BENDAMUSTINE, ETOPOSIDE, ARACYTIN, MELPHALAN) FOLLOWED BY AUTOLOGOUS STEM CELL TRANSPLANTATION IN 30 PATIENTS WITH HODGKIN LYMPHOMA 
Sabrina Akhrouf, Imene Abderahim, Nacera Ait Amer, Farida Tensaout, Fatiha Belhadri, Hanane Moussaoui, Rachida Belhadj, Farih Mehdid, Malek Benakli, Rose-Marie Hamladji, Redhouane Ahmed Nacer

Hematology and Bone Marrow Transplantation Department, Algiers, Algeria

Background: The efficacy of high dose therapy (HDT) followed by autologous stem cell transplantation (ASCT) in refractory or relapsed Hodgkin lymphoma (HL) has been reported, but an optimal HDT regim has not been determined. One of the standards is BEAM which includes Carmustine (BCNU), Etoposide, Aracytine and Melphalan. In view of the unavailability of the BCNU, we have substitute this drug with Bendamustine in the BEAM protocol (BeEAM).

Methods: This is a retrospective study from August 2015 to December 2016 where 30 patients (pts) with HL were treated with ASCT after HDT protocol BeEAM with Bendamustine, it is mainly HL Ann Arbor stage IV (15 pts) and refractory HL (Ann Arbor stage III in 9 pts and stage II in 6 pts), the median age is 27 years (16-47) and a sex ratio of $0.7(13 \mathrm{M} / 17 \mathrm{~F})$. The status of the pts at the graft is $17 \mathrm{pts}$ in partiel response (PR), 08pts in complete response (CR) after 1 to 4 therapeutic lines and 5 pts in unconfirmed $\mathrm{CR}(\mathrm{CR} \mu)$ after 2 to 3 therapeutic lines. The HDT used is BeEAM (Bendamustine: 300mg / $\mathrm{m}^{2}$, Aracytine: $800 \mathrm{mg} /$ $\mathrm{m}^{2}$, Etoposide: $800 \mathrm{mg} / \mathrm{m}^{2}$ and Melphalan: $140 \mathrm{mg} / \mathrm{m}^{2}$ ). Peripheral HSC was collected by cytapheresis after mobilization with G-CSF alone. The median rate CD34 + cells is $3.52 \times 10^{6} / \mathrm{kg}(1.21-10.48)$. The median follow-up at $31 / 10 / 2017$ is 17 months (9-25).

Results: The average duration of hospitalization is 22 days $(20$ - 32) and all the pts had an aplasia of an average duration of 9 days (7-13) during which a transfusion support was assured in red blood cells in 18 pts $(60 \%)$ and in platelet transfusion in 28 pts $(93 \%)$. The median time to graft is 12 days (9-17). Twenty-nine patients had an hyperthermia $(96.6 \%)$ that was clinically documented in $11 \mathrm{pts}$ (catheter infection: 3 , pneumonitis: 2, diarrhea: 6) and microbiologically documented by blood cultures in 17 pts (Staphylococcus Coagulase Negative: 11, Staphylococcus Aureus: 3, E. Coli: 02, Klebsiella pneomonae: 02, Acinetobacter: 01). Renal toxicity was noted in 4 pts (grade 1: 2 pts, grade 3: $1 \mathrm{pt}$ ), hepatic toxicity in $7 \mathrm{pts}$ (grade 1: $5 \mathrm{pts}$, grade 2: 1pt, grade 3: $1 \mathrm{pt}$ ). A death is observed in $1 \mathrm{pt}$ (TRM: $3 \%$ ) following a renal toxicity in a setting of severe sepsis.Of the 29 pts that can be evaluated in posttransplantation, 13 pts $(45 \%)$ are in CR, 9pts (31\%) in $\mathrm{CR} \mu$ and 7 pts (24\%) in PR. A relapse was observed in 8 pts $(27.5 \%)$ after a median duration of 3.5 months $(2-10)$. At 31 of October 2017, 25 pts (83\%) are alive.
Conclusions: The good tolerance of the Bendamustinebased HDT is observed. The BeEAM protocol seems an interesting therapeutic alternative because $\mathrm{BCNU}$ is unavailable.

Conflict of interest: nothing to disclose

\section{P621}

Abstract previously published

\section{P622}

Improving the outcome in patients with relapsed/refractory classical Hodgkin lymphoma undergoing allogeneic stem cells transplantation

Boris Afanasyev, Eugenia Borzenkova, Kirill Lepik, Elena Darskaya, Olga Pirogova, Ivan Moiseev, Natalia Mikhailova, Julia Rogacheva, Andrew Kozlov, Asmik Gevorgian, Yuri Zalyalov, Alexander Alyansky, Marina Popova, Sergey Bondarenko

R.M. Gorbacheva Memorial Institute of Children Oncology, Hematology and Transplantation, I.P. Pavlov First St Petersburg State Medical University, St Petersburg, Russian Federation

Background: AlloHSCT has the curative potential in patients with $\mathrm{r} / \mathrm{r} \mathrm{cHL}$. In recent years, novel RIC regimen Fludarabine-Bendamustine (FluBe) (Khouri et al., Blood2014), the posttransplant cyclophosphamide-based GVHD prophylaxis (PT/Cy) (Luznik., BBMT2008) and new compounds for immunotherapy before the alloSCT (anti-CD30 monoclonal antibody Brentuximab vedotine (BV) (Chen, Blood2016) and PD-1 inhibitor Nivolumab (Nivo) (Younes., Lancet2016)) have the potential for the improvement of alloHSCT outcome.

Purpose of this analysis is to evaluate the outcome of patients with $\mathrm{r} / \mathrm{r} \mathrm{cHL}$ undergoing alloHSCT with FluBe, PT/ Cy-based GVHD prophylaxis, treated with BV or Nivo before or after alloHSCT.

Methods: We retrospectively evaluated alloHSCT in 76 patients with median age 27 (14-49), 57\% male, with $\mathrm{r} / \mathrm{r}$ cHL who had undergone alloHSCT in 2002-2017. The analysis included two groups of patients: group A $(\mathrm{n}=34)$ received the FluBe conditioning, PTCy GVHD prophylaxis and BV/Nivo bridge therapy, and group B patients $(\mathrm{n}=42)$ received melphalan or busulfan-based RIC conditioning, conventional GVHD prophylaxis or chemotherapy-based bridges. In group A, BV was used as bridge in 22 patients, and Nivo in 11 patients. At the time of analysis, median follow-up was 5 (1-43) months for group A, and 15 (1-64) months for group B. There were no differences between patients in the two groups according to median age (26vs 28 years, $\mathrm{p}=0.3$ ), gender (53\%vs60\% male $\mathrm{p}=0.6)$, previous history of autoHSCT (59\%vs54\% $\mathrm{p}=0.8)$, median 


\begin{tabular}{|c|c|c|c|c|c|c|}
\hline \multicolumn{7}{|c|}{ Figure 1A Safety and efficacy of Nivolumab treatment after alloHSCT } \\
\hline Patient & $\begin{array}{c}\text { Best } \\
\text { response }\end{array}$ & $\begin{array}{c}\text { Relapse } \\
\text { /progression } \\
?\end{array}$ & $\begin{array}{c}\text { GvHD } \\
\text { exacerbation }\end{array}$ & AE (gr 3-4) & $\begin{array}{c}\text { Current } \\
\text { treatment }\end{array}$ & $\begin{array}{c}\text { Follow up, } \\
\text { mo }\end{array}$ \\
\hline TSV & PR & $\begin{array}{c}\text { Yes } \\
3 \text { mo }\end{array}$ & No & $\begin{array}{c}\text { Autoimmun } \\
\text { e hepatitis }\end{array}$ & Nivo & $9+$ PD \\
\hline VVK & PR & $\begin{array}{c}\text { Yes } \\
6 \text { mo }\end{array}$ & No & No & Nivo-Benda & $15+$ CR \\
\hline YVM & CR & Yes & No & Meningitis & Nivo & $18+$ CR \\
\hline VIN & PR & No & No & No & Nivo & $8+$ PD \\
\hline DAS & PR & $\begin{array}{c}\text { Yes } \\
\text { PNo }\end{array}$ & No & Meningitis & - & $15+$ PD \\
\hline VNN & CR & No & No & No & Nivo & $6+$ CR \\
\hline Overall 6 & $6 / 6(100 \%)$ & $3 / 5$ & & $3 / 6(50 \%)$ & & $6 / 6$ (100\%) \\
\hline
\end{tabular}

Figure 1B Impact of individual factors on overall survival

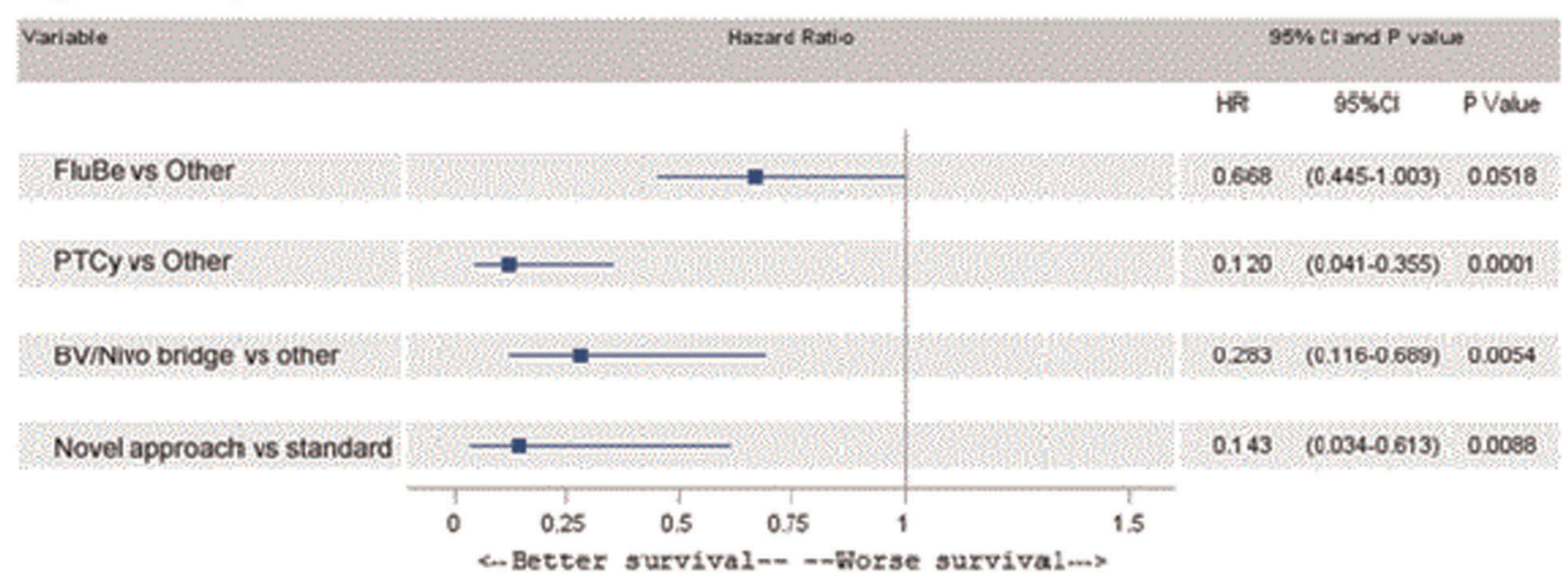

[P622 Figure] [Figure 1]

treatment lines (5vs5 p $=0.8)$, radiation therapy $(58 \%$ vs $56 \%, \mathrm{p}=1.0)$ and type of donors $(24 \%-56 \%-21 \%$ vs $29 \%$ $63 \%-8 \%$ of related, unrelated and haplo, respectively, $\mathrm{p}=$ $0.35)$. Patients in group A achieved better remission status before alloHSCT (CR 65\%; PR 18\%; SD/PD; 17\%) when compared with group B (CR 24\%; PR 30\%; SD/PD 46\%), $\mathrm{p}=0.002$.

Results: Two-year OS and EFS were $70 \%$ and $43 \%$ for the whole population. Cumulative incidence of non-relapse mortality at 2 years was $6 \%$ and $40 \%$ in group A, and group $\mathrm{B}$, respectively $(\mathrm{p}=0,003)$, while relapse incidence was $9 \%$ vs $45 \%$, respectively $(p=0,002)$. There was significant improvement in OS $(\mathrm{p}=0,03)$ and $\operatorname{EFS}(\mathrm{p}=0,001)$ for group A versus group B: $94 \%$ and $85 \%$ vs $51 \%$ and $19 \%$, respectively. Six patients relapsed after alloHSCT were successfully treated with different doses of Nivo $(0,5-3 \mathrm{mg} /$ $\mathrm{kg}$ ) (Figure 1A). There was no difference in grade II-IV $(18 \%$ vs $25 \%, \mathrm{p}=0.6)$ and grade III-IV (3\% vs $10 \%, \mathrm{p}=$ $0.4)$ aGVHD, but reduced incidence of moderate and severe cGVHD in the Group A (3\% vs $27 \%, \mathrm{p}=0.008$ ). The rate of the composite endpoint of extensive cGVHD and relapse-free survival was significantly better for group A compared with group B $(78 \%$ vs $12 \%, \mathrm{p}<0,0001)$. The 
impact of components in the group A strategy is demonstrated in Figure 1B.

Conclusions: We have observed the improvement in alloHSCT outcome with the introduction of FluBe conditioning, PT/Cy GVHD prophylaxis and bridge therapy with BV or Nivo. We observed corresponding improvement of disease status prior to alloHSCT. It should be considered, that in group A, median survival was short, and further observation is needed. The Nivo demonstrate promising efficacy in treatment of relapses after alloHSCT. The impact of individual factors cannot be interpreted in this analysis due to overlap of strategies.

Conflict of interest: All of the co-authors have no conflicts of interest and nothing to disclose.

\section{P623}

Low toxicity and efficacy of high-dose chemotherapy TEAM (Thiotepa, Etoposide, Aracytine, Melphalan) followed by autologous peripheral blood stem cell transplantation in lymphoma

Ahmad Ibrahim ${ }^{1}$, Ali Youssef ${ }^{2}$, Kamal Zahran ${ }^{3}$, Dany Abi Gerges ${ }^{4}$, Mohammad Haidar, Ghada Awad ${ }^{2}$, Berthe Hachem ${ }^{6}$, Anas Mogharbel $^{2}$, Charbel Khalil ${ }^{7}$, Tamima Jisr ${ }^{8}$

${ }^{1}$ Makassed and Middle East Institute of Health University HospitalsLebanese University, Hematology Oncology-BMT, Beirut, Lebanon; ${ }^{2}$ Makassed University Hospitals, Hematology Oncology-BMT, Beirut, Lebanon; ${ }^{3}$ Middle East Institute of Health University Hospital, BMT, Beirut, Lebanon; ${ }^{4}$ Middle East Institute of Health University Hospital, Hematology Oncology, Beirut, Lebanon; ${ }^{5}$ Bahman Hospital, Hematology Oncology, Beirut, Lebanon; ${ }^{6}$ Middle East Institute of Health University Hospital, Blood Bank, Beirut, Lebanon; ${ }^{7}$ Middle East Institute of Health, University Hospital-Saint Joseph, UniversityLebanese University, Cellular Therapy, Beirut, Lebanon; ${ }^{8}$ Makassed University Hospitals, Laboratory Medicine, Beirut, Lebanon

Background: High-dose chemotherapy (HDCT) followed by autologous peripheral stem cell transplantation (APBSCT) improves survival and reduces the risk of relapse in patients (pts) with lymphoma (L) in relapse after first line treatment. BEAM is the most frequently HDCT used in L. BCNU is no longer available and replaced by Thiotepa which is approved by French national agency for the security of drugs for use as part of HDCT followed by APBSCT . A retrospective study from the EBMT showed no differences between TEAM and BEAM in terms of toxicities and survival in pts auto transplanted for $\mathrm{L}$ (Sellner L et al. Bone Marrow Transplant-2016). A French prospective non interventional multi-center study is ongoing in pts with L evaluating the efficacy and toxicity of TEAM: Thiotepa: $8 \mathrm{mg} / \mathrm{kg}$, day(d)-6; Etoposide: $100 \mathrm{mg} / \mathrm{m}^{2} / 12 \mathrm{~h}, \mathrm{~d}-5$ to d-2; Aracytine:200mg/ $\mathrm{m}^{2} / 12 \mathrm{~h}, \mathrm{~d}-5$ to $\mathrm{d}-2$; Melphalan $140 \mathrm{mg} / \mathrm{m}^{2}, \mathrm{~d}-1$ of ASCT. We present the preliminary results of our experience in 15 pts with Hodgkin's lymphoma (HL) and diffuse large B cell non-Hodgkin's lymphoma (DLBCL) who underwent TEAM followed by APBSCT for relapse after first-line treatment between 3/ 2017 and 10/2017

Methods: TEAM was similar to the French study by Thiotepa was given as a dose of $4 \mathrm{mg} / \mathrm{kg} / \mathrm{day}, \mathrm{d}-7$, d- 6 . APBSCT was performed on d0. The minimal number of CD34 + cells infused was $\geq 2.10^{6} / \mathrm{kg}$. Management of febrile neutropenia was performed according to ECIL6 guidelines. Evaluation of toxicities was done according to WHO grading scale.

Results: Median age was 31 y o (18-58). There were 7 females and 8 males. The diagnosis was HL in 10 pts and DLBCL in 5 pts. Four of the 10 pts with HL underwent APBSCT in second complete remission (CR), and 6 were refractory or in relapse after ABVD and obtained CR after $\geq$ 4 lines of salvage therapy including anti-CD30 in one patient(pt), anti-CD30 and anti-PD1 in 5 pts. The 5 pts with DLBCL underwent APBSCT in second CR. The median number of CD34+ cells transfused was $5.10^{6} / \mathrm{kg}(2-18)$. The median times for neutrophil and platelet recovery were $10 \mathrm{~d}(8-23)$ and $9 \mathrm{~d}$ (5-22), respectively. All the $15 \mathrm{pts}$ developed vomiting which was of grade 3 in 1 pt. Oral mucositis occurred in 13 pts and was grade 3 in one pt. Diarrhea occurred in all the pts and was of grade 3 in 4 pts. All the pts developed febrile neutropenia. Two pts developed pneumonia, one bacteremia due to E.Coli ESBL+, and 2 pts colitis due to clostridium difficile. All the 15 pts are alive in CR at a median of $5 \mathrm{~m}(2-9)$.

Conclusions: HDCT TEAM in HL and DLBCL induced low toxicities and high efficacy with a short follow up. TEAM could be a valuable alternative to BEAM but must be investigated in larger prospective clinical trials.

Conflict of interest: Nothing to disclose

\section{P624}

Outcome analysis of high-dose chemotherapy follow by autologous stem cell transplantation for Hodgkin lymphoma in a resource limited center in Asia

Sen Mui Tan, Kim Wah Ho, Sharifah Shahnaz, Syed Abd Kadir, Tee Chuan Ong

Hospital Ampang, Haematology, Ampang, Malaysia

Background: In this new era, the role of autologous stem transplantation (ASCT) in Hodgkin Lymphoma (HL) is evolving with the advent of targeted drugs such as brentuximab or checkpoint inhibiotrs following better understanding of pathogenesis and molecular biology of disease. However, such novel agents are expensive and not widely feasible for all including our center. Therefore, for 
us, ASCT remains the standard approach for relapsed/ refractory HL that failed frontline multiagent chemotherapy with/without radiotherapy after salvage treatment. This study analyzes the results of ASCT performed among HL patients in our center, Hospital Ampang.

Methods: All medical records of HL patients who underwent ASCT will be retrieved from Hospital Ampang database and evaluated retrospectively. Patient's progress post ASCT can be updated through the electronic clinic follow up notes. National Registration Department will be contacted for patients who lost follow up to confirmed their survival status.

Results: A total of $281 \mathrm{HL}$ patients underwent ASCT were studied from June 2000 to June 2017. There was male preponderance $(53 \%)$ with the median age of 26 years (range 13-64 years) and Malays remained majority ethnic group (69\%). Most of them were of nodular sclerosing subtype (55\%) and at advance stage (stage IV, 44\%). Majority of them received chemotherapy mobilization with an average CD34+ cell dose collected at $5.4 \times 10^{\wedge} 6 / \mathrm{kg}$. The conditioning was performed with BEAM (75\%), FLAM $(8 \%)$ or LACE $(7 \%)$ and the grafting with an average of 10.6 days. Transplant outcome evaluated on 15 Dec 2017 showed an overall survival (OS) of $70 \%$ at 5 years and 55\% at 10 years respectively. Detail assessment at Day +100 and further multivariate analysis to ascertain predictors of transplant outcome among this cohort of patients included disease status, chemosensitivity and ethnic difference will be presented. Among this cohort of patients, 14 of them who relapsed post ASCT proceeded with RIC with allografting from matched related sibling (13) and one from haploidentical donor.

Conclusions: In conclusion, despite emergent of effective and potent novel targeted therapy, high-dose chemotherapy follow by ASCT still holds its important role as an excellent rescue therapy regarding tolerance either physically or financially, as well as the overall survival.

Conflict of interest: nothing to disclose

\section{P625}

Outcome of autologous hematopoietic cell transplantation in high-risk lymphoma patients in first complete remission

\footnotetext{
Joanna Romejko-Jarosinska ${ }^{l}$, Katarzyna Domanska-Czyz ${ }^{1}$, Grzegorz Rymkiewicz $^{2}$, Beata Ostrowska ${ }^{1}$, Anna Borawska ${ }^{1}$, Ewa Paszkiewicz-Kozik ${ }^{1}$, Michal Osowiecki ${ }^{1}$, Lidia Poplawska ${ }^{1}$, Lukasz Targonski ${ }^{l}$, Monika Swierkowska-Czeneszew ${ }^{1}$, Anna DabrowskaIwanicka ${ }^{I}$, Beata Grygalewicz ${ }^{2}$, AgnieszKa Druzd-Sitek ${ }^{I}$, Martyna Kotarska $^{I}$, Marcin Szymanski ${ }^{1}$, Robert Konecki ${ }^{I}$, Jan Walewski ${ }^{I}$

${ }^{I}$ Maria Sklodowska-Curie Cancer Centre and Institute, Department of Lymphoproliferative Diseases, Warszawa, Poland; ${ }^{2}$ Maria
}

Sklodowska-Curie Cancer Centre and Institute, Department of Pathology and Laboratoty Diagnostics, Warszawa, Poland

Background: High grade B-cell lymphoma with MYC and BCL2 and/or BCL6 rearrangement (HGBCL-R), HGBCL, NOS, MYC and BCL2 double expresser (DE), CD5+ diffuse large B cell lymphoma (DLBCL) or other high-risk DLBCL by IPI usually progress after standard immunochemotherapy. The value of myeloablative chemotherapy followed by autologous hematopoietic cell transplantation (auto-HCT) in first remission for high-risk lymphoma patients remains to be confirmed. Here we report on the outcome of auto-HCT consolidation of the first remission in patients with aggressive B-cell lymphoma and risk factors.

Methods: Between 2011- 2017, a total of 31 consecutive patients with high-risk aggressive B-cell lymphoma (HGBCL-R - 4, HGBL,NOS - 7, DE - 12, CD5+ or other high-risk DLBCL - 8) were planned to receive R-CHOP (n $=14)$ or DA-EPOCH-R $(\mathrm{n}=17)$ followed by BEAM and auto-HCT as the first-line treatment. Median age was 50 (range 24-66). Three or more IPI risk factors were present in $80 \%$ of patients.

Results: Only 18 patients (58\%) achieved complete remission after R-CHOP $(n=4) / D A-E P O C H-R(n=12)$ induction therapy and proceeded to auto-HCT, and remaining $13(42 \%)$ received 2 or 3 lines of therapy before autoHCT. One patient died early after transplantation. At a median follow-up of 12 months from auto-HCT, the 1-year progression free survival (PFS) and overall survival (OS) were $61 \%$ (95\%CI: $43,79 \%)$ and $81 \%$ (95\% CI: 65, 96\%). The HR (Hazard Ratio) for death and progression in patients who received one line vs two or more lines of therapy before auto-HCT was 6.05 (95\%CI: 0.7, 51.9), $\mathrm{p}=$ 0.05 and 2.05 (95\%CI: $0.57,7.27$ ), $\mathrm{p}=0.26$, respectively. The HR for death was increased in DE-DLBCL vs other high-risk DLBCL and DE-DLBCL vs HGBCL subgroups: 3.39 (95\% CI: 0.37, 30), $\mathrm{p}=\mathrm{NS}$ and 3.25 (95\% CI: 0.36, $29), p=N S$, respectively. The HR for progression in DEDLBCL vs other high-risk DLBCL was increased: 2.27 (95\%CI: $0.43,11.7)$ but the difference was not statistically significant. There was no significant difference in HR for progression between DE-DLBCL vs HGBCL: 1.2 (95\%CI: $0.34,4.05), \mathrm{p}=0.88$ and between HGBCL vs other highrisk DLBCL: 1.36 (95\%CI: 0.58, 3.19). The HR for death was increased in patients, who received R-CHOP vs DAEPOCH-R: 4.28 (0.49-37,1), not statistically significant, and there was no significant difference in HR for progression between R-CHOP and DA-EPOCH-R groups: 1.34(0.4-4.4), $\mathrm{p}=0.64$.

Conclusions: Less than $60 \%$ of patients with aggressive high-risk lymphoma achieved a complete remission after 
the first line treatment. The failure to achieve complete remission after induction therapy was associated with increased risk of death. The diagnosis of DE-DLBCL increased the risk of death and the risk of progression compared to diagnosis of other high-risk DLBCL and nonsignificantly increased the risk of death compared to HGBCL. These single-institution data suggest that RCHOP followed by consolidation auto-HCT is a suboptimal treatment for high-risk aggressive lymphoma patients, in particular in patients with double expresser DLBCL.

Conflict of interest: Nothing to disclose

\section{P626}

Poor outcome of double-protein expressor diffuse large Bcell lymphoma can be overcome by early-treatment intensification (single-center analysis of 223 patients in Russia)

Evgeniya Kharchenko $^{1}$, Sergey Alexeev ${ }^{1}$, Anna Artemeva ${ }^{2}$, Tatiana Semiglazova $^{3}$, Polina Shilo ${ }^{1}$, Vladimir Kushnarev ${ }^{2}$

${ }^{l}$ National Medical Reserch Institute of Oncology n.a. Petrov, Oncology/Hematology and Bone Marrow Transplantation, Saint Petersburg, Russian Federation; ${ }^{2}$ National Medical Reserch Institute of Oncology n.a. Petrov, Pathology, Saint Petersburg, Russian Federation; ${ }^{3}$ National Medical Reserch Institute of Oncology n.a. Petrov, Saint Petersburg, Russian Federation

Background: Double expression of both c-myc and bcl-2 is independent risk factor of aggressive course in Diffuse Large B-cell lymphoma patients (DLBCL) and can be overcome by early treatment intensification.

Methods: To analyze data we used chi-square test for independence, odds ratio (OR), full analisys test. Progression free survival (PFS) were estimated by Kaplan-Meier method, comparison of PFS in experimental groups (longrank test), multifactor analysis of PFS (multinomial logistic regression). Statistically significant difference if $\mathrm{p}<0,05$.

Results: We analyzed 223 pts with DLBCL, from 18 to 85 years old (median age 54 y.o), who underwent treatment in National Medical Research Center of Oncology n.a. Petrov from 2008 to 2017. Sixty four pts (29\%) were performed with early stage of disease (I,II) and 159 pts (64\%) with advance stage (III,IV). Extranodal involvements were observed in 58 pts (26\%) and B-symptoms in 103 pts $(46 \%)$. We determined GCB-subtype in $47 \%$ and non-GCB in 53\% using Hans algorithm (estimated by immunohistochemicals markers CD 10, mum 1 and bcl-6). DA-EPOCH$\mathrm{R}$, Hyper-CVAD-R as first line received $24 \mathrm{pts}(11 \%)$ and up-front high-dose chemotherapy with AutoSCT received 13 pts $(6 \%)$.

2-years PFS was $83 \%$ for all pts [95\% CI 76-92]. PFS was significantly lower in pts with B-symptoms (36\% vs $87 \%$;
RR 3,3 [95\% CI 0,85 - 1,4], p < 0,05); advance stage ( $40 \%$ vs $91 \%$; RR 3,0 [95\% CI 1,7 - 2,0], p < 0,05); c-myc expression (47\% vs 88\%); RR 1,8 [95\% CI 0,25 - 0,9], p < 0,05); bcl-2-expression (57\% vs 80\%) RR 1,9 [95\% CI 1,3 $2,0], \mathrm{p}<0,05)$; Ki-67 higher than $70 \%$ (57\% vs $91 \%$; RR $2,8$ [95\% CI $0,85-1,4], \mathrm{p}<0,05)$.

Double-expressor lymphoma (DEL) (double expression of both c-myc and bcl-2) were observed in 34\% (76 patients). Among patients with DEL, PFS were lower but not significantly different comparing with non-DEL $(p=0,053)$. These data were related to early treatment intensification in this subgroup (DA-EPOCH-R, HyperCVAD-R, up-front high-dose chemotherapy with AutoSCT). DEL subgroup was also associated with advance stage of the disease (III-IV $71 \%$ vs I-II $42 \%$, p < $0,05)$, B-symptoms $(67 \%$ vs $44 \%, \mathrm{p}<0,05)$ and $\mathrm{Ki}-67$ higher than $70 \%$ ( $82 \%$ vs $69 \%$, p $>0,05$ ).

Conclusions: These data confirm more aggressive course of the disease in patients with DEL comparing with standard group. This may suggest benefit for pts to undergo early-treatment intensification, such as R-DA-EPOCH, RHyper-CVAD or up-front high dose chemotherapy with autologous stem cell transplantation.

Clinical Trial Registry: N/A

Conflict of interest: No conflict of interests

\section{P627}

Predictive role of soluble interleukin-2 receptor levels in autologous stem cell transplantation for non-Hodgkin lymphoma

Atsushi Tanaka, Tomohiro Yabushita, Satoshi Yoshioka, Takayuki Ishikawa

Kobe City Medical Center General Hospital, Haematology, Kobe, Japan

Background: In patients with relapsed non-Hodgkin lymphoma (NHL), who have undergone autologous stem cell transplantation (ASCT), there are no well-established serum biomarkers that correlate with post-ASCT survival. The present study investigated the prognostic significance of serum soluble interleukin-2 receptor (sIL-2R) levels prior to ASCT.

Methods: This study retrospectively analysed patients who underwent ASCT for relapsed NHL between January 2011 and September 2016. The primary endpoint was 2year overall survival (OS). The secondary endpoint was 2year progression free survival (PFS). OS and PFS curves were constructed using the Kaplan-Meier method, and compared with the log-rank test. This study complied with the standards of the Declaration of Helsinki and the current 
ethical guidelines, and was approved by the institutional ethics board.

Results: The study included 42 patients. At the time of ASCT, the median patient age was 59.5 years (range, 32-71 years). The histological subtypes of NHL in these patients were as follows: aggressive B-cell lymphoma (32 patients), indolent B-cell lymphoma (4 patients), peripheral T-cell lymphoma (5 patients), and extranodal NK/T-cell lymphoma, nasal type (1 patient). The disease status at ASCT was complete response (CR) in 29 patients and partial response in 13 patients. Ranimustine, etoposide, cytarabine and methotrexate were administered to all patients in the conditioning regimen. Rituximab was co-administered in 12 patients. The median sIL-2R level at ASCT was $465 \mathrm{U} / \mathrm{ml}$ (range, 244$3370 \mathrm{U} / \mathrm{ml}$ ). Receiver operating characteristic curve analysis identified $505 \mathrm{U} / \mathrm{ml}$ as a satisfactory cut-off value to predict survival. The 2-year OS rate was significantly worse in patients with higher sIL-2R levels $(\mathrm{n}=17)$ than in those with lower sIL-2R levels $(\mathrm{n}=25)(64.7 \%$ vs. $91.4 \%, \mathrm{p}=0.009)$. Additionally, the 2-year progression free survival rate was significantly worse in patients with higher sIL-2R levels than in those with lower sIL-2R levels $(41.2 \%$ vs. $88.0 \%, \mathrm{p}=$ 0.002). Among patients with CR at the time of ASCT, the 2year OS rate was significantly worse in those with higher sIL$2 \mathrm{R}$ levels $(\mathrm{n}=9)$ than in those with lower sIL-2R levels $(\mathrm{n}=$ 20) $(66.7 \%$ vs. $94.1 \%, \mathrm{p}=0.04)$.

Conclusions: In patients with relapsed NHL who show response to salvage chemotherapy and have undergone ASCT, the sIL-2R level at the time of ASCT might be prognostic.

Conflict of interest: A. Tanaka: nothing to disclose

\section{P628}

\section{Prognostic value of ${ }^{18} \mathrm{~F}$-FDG PET-CT in patients with lymphoma who underwent allogeneic stem cell transplant}

Luis Gonzaga Diaz', Monica Baile Gonzalez ${ }^{2}$, Lucia Lopez-Corral ${ }^{3}$, Berta Perez ${ }^{1}$, Jose Cañadas ${ }^{1}$, Alejandro Martin ${ }^{3}$, Norma Gutierrez ${ }^{3}$, Ramon Garcia ${ }^{3}$, Andres Achury, Estefania Perez ${ }^{2}$, Dolores Caballero ${ }^{3}$, Pilar Tamayo

${ }^{1}$ University Hospital of Salamanca, Nuclear Medicine Department, Salamanca, Spain; ${ }^{2}$ University Hospital of Salamanca, Hematology Department, Salamanca, Spain; ${ }^{3}$ University Hospital of Salamancal IBSAL, Hematology Department, Salamanca, Spain; ${ }^{4}$ University Hospital of Salamanca/IBSAL, Nuclear Medicine Department, Salamanca, Spain

Background: Stem Cell Transplantation (SCT) has become a potentially curative treatment in patients diagnosed with Hodgkin (HL) and Non Hodgkin Lymphoma (NHL). In autologous SCT, a positive ${ }^{18} \mathrm{~F}$-FDG PET is associated with high rates of relapse and/or progression. In allogeneic SCT (alloSCT), PET-CT has lower Positive
Predictive Value (PPV) due to the graft vs. lymphoma effect, although a positive pretransplant PET-CT may be associated with a poorer outcome. However, the role of ${ }^{18} \mathrm{~F}$ FDG PET-CT after alloSCT is still less well known. The aim of our study has been to assess the predictive and prognostic value of pre-SCT, 100 days and 12 months after SCT ${ }^{18}$ F-FDG PET-CT in patients with HL and NHL who underwent alloSCT.

Methods: Prospective study of 27 patients, 4 diagnosed with HL and 23 with NHL, who underwent alloSCT in our center between 2014 and 2016. Three ${ }^{18}$ F-FDG PET-CT studies were scheduled in each patient: before (PET0), 100 days (PET1) and a year (PET2) after SCT. PET0 was performed within 30 days before SCT. All PET results were analyzed and correlated with clinical status and parameters of each patient. Metabolic response by ${ }^{18}$ F-FDG PET-CT was assessed following the Lugano Criteria.

Results: The mean age of the patients was 47,1 years old and $56,5 \%$ were male. The average number of previous treatment lines received was 3 (12 Allo-SCT patient had received previous Auto-SCT).

Most patients are from a donor-related (DR)-SCT (36\%) or Haplo-SCT (33\%) with source peripheral blood progenitors in $(97.5 \%)$ and with reduced intensity conditioning (RIC) (90\%). Patients status at SCT were: $57 \%$ in Complete metabolic response (CR), $9 \%$ in metabolic progresion and $34 \%$ in stable/refractary disease.

- 15 patients were PET0 negative. Of these, 94\% (14) showed no abnormalities in PET 1 and PET2, with no clinical evidence of relapse, nor progression 1 year after allo-SCT. The remaining patient showed relapse in PET1, with a later negative PET2 (this patient is alive and disease free after 2 years of follow up).

- In 12 patients PET0 was positive and 92\% (11) were PET1 and PET2 negative. The other patient was PET1 negative, but PET2 showed relapse and also later progression was confirmed by PET surveillance. After a mean follow up of 1,5 years, $100 \%$ of them are alive and $92 \%$ disease free.

Conclusions: Our study demonstrated that a positive prealloSCT ${ }^{18}$ F-FDG PET-CT is not associated with a poorer outcome. Additionally, it suggests that a ${ }^{18}$ F-FDG PET-CT study performed 1 year after alloSCT can be a better outcome predictor than a 100 days after SCT PET-CT.

Conflict of interest: The authors state no conflict of interests.

\begin{tabular}{lll}
\hline Patients $(\mathrm{n}=27)$ & PET1 $(\%)$ & PET2 $(\%)$ \\
\hline Positive result in positive PET0 patients & 0 & 8 \\
Negative result in negative PET0 patients & 93 & 100 \\
\hline
\end{tabular}

[[P628 Table] Prognostic value of PET1 and PET2] 


\section{P629}

Promising Remissions in Relapsed Refractory Hodgkin Lymphoma Patients Requiring More than One Line of Salvage Chemotherapy Prior to Autologous Transplantation in the Brentuximab Vedotin Era

Moussab Damlaj ${ }^{1,2,3}$, Ayman Alhejazi ${ }^{1}$, Samer Ghazi, Bader Alahmari $^{1}$, Ahmed Alaskar ${ }^{1}$, Mohsen Alzahrani ${ }^{1}$

${ }^{1}$ King Abdulaziz Medical City, Riyadh, Saudi Arabia; ${ }^{2}$ King Abdullah International Medical Research Center, Riyadh, Saudi Arabia; ${ }^{3}$ King Saud bin Abdulaziz University for Health Sciences, Riyadh, Saudi Arabia

Background: Patients with relapsed / refractory classical Hodgkin lymphoma (R/R cHL) who achieve less than partial response (PR) following first line salvage chemotherapy have poor outcomes (Villa et al., Haematologica 2011). The antibody drug conjugate (ADC) brentuximab vedotin $(\mathrm{Bv})$ has been used as part of salvage regimens or as post HCT consolidation with improved remission rates. Our aim was to examine the outcome of $\mathrm{R} / \mathrm{R}$ cHL patients requiring $\geq$ two lines of salvage compared to patients requiring a single line of salvage in the contemporary era of chemo-immunotherapy.

Methods: After due IRB approval, pts with R/R cHL whom were candidates for curative HCT were identified and all records were retrieved retrospectively. $\mathrm{Bv}$ was given as an intravenous infusion of $1.8 \mathrm{mg} / \mathrm{kg}$ on day 1 with salvage chemotherapy and / or every 3 weeks starting day 30-45 post HCT. Deauville score of $\leq 3$ was regarded as complete

Table I. Patient Cohort Stratified by Number of Salvage Lines pre-HCT

\begin{tabular}{|c|c|c|c|}
\hline Characteristic & Single Salvage $(\mathrm{n}=33)$ & $\geq$ Two Salvage $(n=22)$ & P value \\
\hline Male, n (\%) & $17(52)$ & $13(59)$ & 0.58 \\
\hline$\Delta$ ge at HCT, median (range) & $31(15-57)$ & $22(14-49)$ & 0.041 \\
\hline Refractory ( $\leq 3$ months remission) & $25(76)$ & $12(55)$ & 0.1 \\
\hline Time to Relapse. days (range) & $223(28-3870)$ & $260(26-3988)$ & 0.87 \\
\hline Relapse $\leq 6$ months, $\mathrm{n}(\%)$ & $15(45)$ & $11(5)$ & 0.74 \\
\hline Relapse $\leq 12$ months, $\mathrm{n}(\%)$ & $20(61)$ & $17(77)$ & 0.19 \\
\hline No. of Salvage Regimens & & & $<0.0001$ \\
\hline One & $33(100)$ & $\mathrm{N} / \mathrm{A}$ & \\
\hline Two & N/A & $15(68)$ & \\
\hline$\geq$ Three & N/A & $7(3 z)$ & \\
\hline $\begin{array}{l}\text { No. of Salvage Cycles, median } \\
\text { (range) }\end{array}$ & $2(2-3)$ & $4(2-8)$ & $<0.0001$ \\
\hline PET/CT Status pre-HCT, n (\%) & & & 0,14 \\
\hline Negative (Deauville $\leq 3$ ) & $21 / 29(72)$ & $11 / 21(52)$ & \\
\hline Positive & $8 / 29(28)$ & $10 / 21(48)$ & \\
\hline Bv Part of Salvage, n (\%) & $14(42)$ & $16(73)$ & 0.025 \\
\hline Bv Consolidation, n (\%) & $10(30)$ & $13(5)$ & 0.1 \\
\hline Mediar follow up, months (range) & $21(1-89)$ & $30(0.5-76)$ & 0.68 \\
\hline
\end{tabular}

Ahbreviations: PET/CT, positron emission tomography / computed tomography: HCT. hematopoietic stem cell transplant: Bv, brentuximab vedxtin

Table II. Univariable and Multivariable Risk Factors Influencing PFS

\begin{tabular}{|c|c|c|}
\hline PFS & Univariable HR (95\% CI; P value) & Multivariable HR ( $95 \%$ CI; P value) \\
\hline Age at $\mathrm{HCT}$ & $1.0003(0.96-1.04: p=0.99$ & \\
\hline Age $>$ Median & $0.65(0.23-1.67: p=0.38)$ & \\
\hline Male Geader & $3.27(1.17 .11 .57: p=0.023 i$ & $4.5(1.41-19.92: p=0.0095)$ \\
\hline Refractory Disease & $0.9(0.29-2.38: p=0.83)$ & \\
\hline Remission $<6$ months & $0.55(0.19-1.41: p=0.22)$ & \\
\hline Remission $<12$ months & $0.94(0.37-2.7: p=0.91)$ & \\
\hline$\geq 2$ Salvage Lines & $1.97(0.75-5.25: p=0.16)$ & $2.19(0.78-6.65 ; p=0.14)$ \\
\hline Bv Part of Salvage & $1.75(0.67-4.84: p=0.25)$ & \\
\hline Bv Consolidation & $0.77(0.27-2.04: p=0.61)$ & \\
\hline Deauville $\leq 3$ & $0.24\left(0.074-0.67 ; p=0.006^{2}\right)$ & $0.27(0.08 \cdot 0.76: p=0.013)$ \\
\hline
\end{tabular}

Abbreviations: HR, hazard ratio: $\mathrm{Cl}$, confidence interval: $\mathrm{HCT}$. hematopoietic stem cell transplant: $\mathrm{Bv}$.

brentuximab vedotin

[P629 Figure] [Table] 
metabolic response (CMR) pre-HCT. Patients proceeded to HCT once partial remission (PR) or better were achieved irrespective of PET/CT status. Categorical and continuous variables were compared using Pearson's chi-squared and Wilcoxon / Kruskal-Wallis, respectively. Overall survival (OS) and progression free survival (PFS) were computed using Kaplan-Meir method with log ranks test. Multivariate analyses were performed using Cox regression modeling.

Results: A total of 55 pts with R/R cHL were identified and included for further analysis. Among the cohort, 33 $(60 \%)$ required one line of salvage, $15(27 \%)$ required two lines while the remaining 7 (13\%) required three lines. All patients were able to achieve PR or better and proceed to HCT. The cohort was subsequently stratified to those requiring one or $\geq$ two salvage lines prior to HCT. Baseline characteristics of the strata are shown in table I. No significant difference was found with regards to gender, proportion of refractory disease or time to relapse. In cases requiring $\geq$ two salvage lines prior to $\mathrm{HCT}$, patients were significantly younger $(\mathrm{p}=0.041)$, received $\mathrm{Bv}$ more often as part of salvage chemotherapy (0.025) or post HCT consolidation $(\mathrm{p}=0.1)$. There was a trend of higher CMR status pre-HCT for those requiring single vs. $\geq$ two salvages lines $(72 \%$ vs. $52 \% ; \mathrm{p}=0.14)$. On multivariate analysis for PFS, male gender was an adverse prognostic factor whereas a negative PET/CT was protective as shown in table II. At 2 years, the estimated PFS and OS for patients requiring one vs. $\geq$ two lines of salvage was $71.2 \%(50.1-84.7)$ vs. $51.9 \%$ (27.6-71.6), $\mathrm{p}=0.16$ and $84.6 \%$ (63-94) vs. $84 \%$ (58-95), $\mathrm{p}=0.88$, respectively.

Conclusions: We observed that patients requiring $\geq$ two lines of salvage prior to HCT can achieve comparable outcomes to those requiring a single line of salvage. This is likely in part due to incorporation of $\mathrm{Bv}$ with resultant higher CMR rates than expected pre-HCT. These results have important implications as such patients may not be referred for HCT given the historically reported poor outcomes. Given the limitations of such analysis, these findings should be further examined.

Conflict of interest: No relevant conflicts of interest

\section{P630}

\section{Remission status as a predictor of survival in autologous stem cell transplantation in patients with non-Hodgkin lymphoma - a single center experience}

Ana Luísa Pinto ${ }^{1}$, Adriana Roque ${ }^{1,2}$, Marília Gomes ${ }^{1}$, Emília Cortesão $^{1,2}$, Ana Isabel Espadana ${ }^{1}$, Ana Bela Sarmento ${ }^{1,2}$, Catarina Geraldes $^{1,2}$, M. Letícia Ribeiro ${ }^{1}$
${ }^{1}$ Centro Hospitalar e Universitário de Coimbra, Hematology Department, Coimbra, Portugal; ${ }^{2}$ University of Coimbra, Faculty of Medicine and CIMAGO, Coimbra, Portugal

Background: High dose chemotherapy (HDC) followed by autologous stem cell transplantation (ASCT) is used for treatment of relapsed/refractory non-Hodgkin lymphoma (NHL) and first-remission consolidation in mantle-cell lymphoma. It is unclear whether complete response before or after ASCT correlates with better survival. We evaluated the prognostic value of disease status - complete remission (CR) vs partial remission (PR) - in a cohort of patients with NHL.

Methods: Unicentric, retrospective analysis of patients with NHL treated with HDT and ASCT between 2007 and 2016. All patients received peripheral blood stem cell support after conditioning with BEAM regimen (carmustine $300 \mathrm{mg} / \mathrm{m}^{2}$, etoposide $800 \mathrm{mg} / \mathrm{m}^{2}$, Ara-c $1600 \mathrm{mg} / \mathrm{m}^{2}$ and melphalan $140 \mathrm{mg} / \mathrm{m}^{2}$ ). Response was assessed according to The Lugano Classification. We used Kaplan-Meier method to estimate progression free survival (PFS) and overall survival (OS) and log-rank test to compare risk groups. Univariate analysis was performed and significant predictors were used to adjust a multivariate Cox regression model.

Results: We included 83 patients, mainly males (72.3\%) with a median age of 51 years (18-65). The most prevalent histological subtypes were diffuse large B-cell lymphoma (53.0\%), mantle-cell lymphoma (36.1\%) and follicular lymphoma $(15.7 \%)$. The median number of therapeutic lines was 2 (1-5). Patients with diffuse large B-cell lymphoma and follicular lymphoma were mainly treated with R-CHOP/R-CVP (82.5\%) at first-line. For those who did not achieve a CR or relapsed, (R)-ESHAP/DHAP/ICE (78.8\%) was performed as second-line followed by ASCT as salvage therapy in order to achieve and consolidate CR. The majority of patients with mantle-cell lymphoma received R-CHOP/R-DHAP (55.0\%) followed by consolidation with ASCT in first remission. With a median followup time from ASCT of 39.7 months (0.3-117.6), OS at 2 and 5 years was $84.8 \%$ and $74.5 \%$ and PFS was $76.8 \%$ and $58.2 \%$, respectively. Before ASCT, 60 patients $(72.3 \%)$ were in CR and 23 (27.7\%) were in PR. After ACST, 4 patients were not assessed for response due to early death by toxicity. Of the remaining, $70(88.6 \%)$ achieved a CR, 4 $(5.1 \%)$ a PR and $5(6.3 \%)$ failed to respond. Patients in CR before ASCT presented significantly longer PFS compared with those in PR (107.9 vs 44.0 months, $\mathrm{p}=0.01)$. Additionally, patients with CR after ASCT presented longer OS and PFS compared with those in PR (107.9 vs 8.0 and 107.9 vs 7.3 months, $\mathrm{p}<0.001)$. However, these patients had significantly lower PFS compared to patients that 
continued in CR after ASCT (45.3 vs 107.9 months, $\mathrm{p}=$ 0.041). Univariate analysis indicated that remission status pre-ASCT is a significant predictor of PFS after ASCT (HR $0.39 ; 95 \%$ CI $0.19-0.82, p=0.013)$. Multivariate analysis showed this factor retains prognostic value after adjustment for age, histology, Ann Arbor stage and number of previous treatments.

Conclusions: This data provides evidence for CR previous to ASCT as an independent predictor of PFS in patients with NHL. A CR after ASCT also correlates with higher PFS and OS, with the highest PFS in patients on continued CR. The use of more effective induction regimens in order to improve initial response may be advantageous in terms of clinical benefits post-ASCT.

Conflict of interest: None of the authors has anything to disclose.

\section{P631}

TEAM conditioning (Thiotepa, Etoposide, Cytarabine, Melphalan) prior to autologous hematopoietic stem cell transplantation for Hodgkin and non-Hodgkin lymphoma: first results from a prospective multicenter study

Rémy Duléry ${ }^{1,2,3}$, Laure Lebras ${ }^{4}$, Sylvain Choquet ${ }^{2,5}$, Sandy Amorim $^{6}$, Ahmad Al Jijakli ${ }^{7}$, Laurence Heuberger ${ }^{8}$, Eolia Brissot $^{1,2,3}$, Giorgia Battipaglia ${ }^{1,2}$, Federica Giannotti, ${ }^{1,2}$, Florent Malard $^{1,2,3}$, Annalisa Ruggeri ${ }^{1}$, Clémence Mediavilla ${ }^{1,2}$, Anne Vekhoff $^{1}$, Paul Coppo ${ }^{1,2}$, Nabaz Jaff ${ }^{1}$, Zora Marjanovic ${ }^{1}$, Elise Corre $^{I}$, Marie-Paule Lemonnier ${ }^{1}$, Ramdane Belhocine ${ }^{1}$, Mohamad Mohty $^{1,2,3}$

${ }^{1}$ Saint Antoine Hospital, AP-HP, Hematology and Cellular Therapy, Paris, France; ${ }^{2}$ Sorbonne University, Université Pierre et Marie Curie (UPMC), Paris, France; ${ }^{3}$ INSERM, UMRs 938, Paris, France; ${ }^{4}$ Léon Bérard Center, Hematology, Lyon, France; ${ }^{5}$ Pitié Salpêtrière Hospital, Hematology, Paris, France; ${ }^{6}$ Saint Louis Hospital, Paris, France;

${ }^{7}$ Victor Dupouy Hospital, Hematology, Argenteuil, France; ${ }^{8}$ French Polynesia Hospital, Hematology, Papeete, French Polynesia

Background: Autologous hematopoietic stem cell transplantation (autoSCT) is considered the standard approach for high risk or relapsed/refractory non-Hodgkin and Hodgkin lymphoma. Although a large variety of conditioning regimens are available, including the widely used BEAM (carmustine, etoposide, cytarabine, melphalan), there is no consensus regarding a standard approach. In the context of carmustine shortage, we have chosen to replace it by thiotepa. However, clinical data about TEAM (thiotepa, etoposide, cytarabine, melphalan) conditioning regimen are still sparse and only retrospective. Thus, we designed a multicenter prospective study (NCT02504190) to assess the efficacy and toxicity of a TEAM conditioning regimen.

Methods: TEAM regimen consisted in total dose thiotepa of $8 \mathrm{mg} / \mathrm{kg}$ on day-6; etoposide $100 \mathrm{mg} / \mathrm{m}^{2} / 12 \mathrm{~h}$ and cytarabine $200 \mathrm{mg} / \mathrm{m}^{2} / 12 \mathrm{~h}$ (day-5 to -2); melphalan $200 \mathrm{mg} /$ $\mathrm{m}^{2}$ on day-1. Patients underwent autoSCT with TEAM conditioning, and were included in this analysis if they have fullfilled the following criteria: age between 18 and 65 years, biopsy-proven Hodgkin or non-Hodgkin lymphoma, HIV seronegative, and first autoSCT.

Results: Fifty-eight male and fourteen female with a median age of 53 years (range, 19-64) were analyzed thus far. Karnofsky score was $<90 \%$ in 15 patients $(21 \%)$. Lymphoma were diffuse large B cell $(\mathrm{n}=39)$, mantle cell $(n=7)$, follicular $(n=7)$, marginal zone $(n=1)$, grey zone $(\mathrm{n}=1)$, Hodgkin $(\mathrm{n}=9)$, peripheral T-cell $(\mathrm{n}=7)$ and grade 3 lymphoid granulomatosis $(\mathrm{n}=1)$. Advanced stage disease (Ann Arbor stage III-IV) was noted in 69 patients (94\%). Disease status at time of autoSCT was complete response (CR) in 52 patients (72\%), partial response in 19 (26.5\%), including 11 patients who never reached CR, and progressive disease in 1 (1.5\%). Median CD34+ cells infused in the graft were $4.6 \times 10 \mathrm{e} 6 / \mathrm{kg}$ (range, 1.39-12.7).

Median time to neutrophil recovery was 11 days (range, 9-48) and median time to platelet recovery $>20 \mathrm{G} / \mathrm{L}$ was 13 days (range, 7-48). The most significant regimen-related toxicities were mucositis in $100 \%$ of patients (median grade $=3$, range, 2-4) and diarrhea in 98\% of patients (median grade $=1$, range, $0-3$ ). Other non-hematologic grade 3 adverse events occurred in 7 patients (10\%). Central line-associated bloodstream infection occurred in 16 patients $(22 \%)$. Four patients required intensive care unit transfer.

At day $+100,69$ patients were evaluable for response and all were in CR. Deaths directly attributed to disease progression or relapse occurred in 2 patients. After a median follow-up of 16 months (range, 3.5-29), the nonrelapse mortality (NRM) was $4.2 \%$. Two patients died during aplasia of infection and one patient died 67 days after autoSCT of necrotizing fasciitis. At last follow-up, 7 patients relapsed, 5 died and 67 are still alive. The estimated 2-year overall survival and progression-free survival were $93.1 \%$ and $86.1 \%$, respectively.

Conclusions: The TEAM conditioning regimen seems to be a safe and valid platform in autoSCT for patients with high-risk or relapsed/refractory lymphoma. Although mucositis and diarrhea were frequent, the NRM was similar to the reported NRM of BEAM conditioning regimen.

Clinical Trial Registry: The study is registered on clinicaltrials.gov (NCT02504190)

\section{Conflict of interest:}

Mohamad Mohty, Remy Dulery and Florent Malard received honoraria for lectures from Keocyt whose drug was included in this study.

The other authors declare no competing financial interests. 


\section{P632}

The EBMT/ERIC 2014 high-risk CLL transplant algorithm is feasible and safe: a single-center intent-to-treat analysis

\author{
Almuth Hoffmann ${ }^{1}$, Sascha Dietrich ${ }^{1}$, Susanne Hain' ${ }^{2}$, Michael \\ Rieger $^{3}$, Ute Hegenbart ${ }^{1}$, Leopold Sellner ${ }^{1}$, Andrea Bondong ${ }^{1}$, \\ Anthony $\mathrm{D} \mathrm{Ho}^{4}$, Carsten Müller-Tidow ${ }^{4}$, Peter Dreger ${ }^{4}$ \\ ${ }^{1}$ Heidelberg University Hospital, Medicine V, Heidelberg, Germany; \\ ${ }^{2}$ St. Marienkrankenhaus Siegen, Siegen, Germany; ${ }^{3}$ Oncology \\ Practice Darmstadt, Darmstadt, Germany; ${ }^{4}$ Heidelberg University \\ Hospital, Heidelberg, Germany
}

Background: In 2014, EBMT and ERIC proposed a treatment algorithm for patients with high-risk chronic lymphocytic leukemia (CLL) in the pathway inhibitor (PI) era. This algorithm suggested allogeneic hematopoietic cell transplantation (alloHCT) as an option for patients with relapsed/refractory (R/R) CLL responding to a $1^{\text {st }} \mathrm{PI}$ if they had a high disease-specific risk (TP53 abnormalities and/or del 11q) along with a low transplant risk (well-matched donor and lack of comorbidities; algorithm level 1, L1). AlloHCT should be also considered in patients with $\mathrm{R} / \mathrm{R}$ CLL who have progressed under a $1^{\text {st }}$ PI even with a higher transplant risk (algorithm level 2, L2).

Methods: Objectives of this single centre retrospective analysis were to investigate if this algorithm is feasible and how it compares to non-transplant care in terms of safety and efficacy by intent-to-treat. Eligible were all patients with CLL or B-prolymphocytic leukemia (B-PLL) meeting the entry criteria for the algorithm at L1 or L2 who were referred to our institution for alloHCT evaluation between Jan 2014 and Oct 2017. Primary endpoint was progressionfree survival (PFS) from entering the algorithm.

Results: In total 23 patients entered the algorithm (19 at L1, 4 at L2). Diagnoses were CLL in 20 patients and B-PLL in 3 patients. Median age at entry was 59y (38-63), with $70 \%$ of the patients harbouring TP53 lesions and $70 \%$ being resistant to chemoimmunotherapy after a median of 2 (1-7) pretreatment regimens. First PI was ibrutinib (70\%), idelalisib (22\%), or venetoclax (9\%). With a median follow-up of 31 (2-46) months, 2-year PFS and overall survival (OS) from algorithm entry of all 23 patients was $59 \%(32-82 \%)$ and $100 \%$, respectively. Of the 19 patients entering at L1, $8(42 \%)$ did not meet the requirements for proceeding to alloHCT at this level (no 10/10 donor 6, comorbidity 1 , refusal 1 ). Five of these 8 patients progressed under their $1^{\text {st }} \mathrm{PI}$ and thus proceeded to L2, as did 2 patients of the 11 patients where alloHCT was intended, translating into a 2-year PFS of $44 \%$ (7-83\%) vs $80 \%(55-100 \%)$ in the no-HCT vs intent-to-HCT groups (p 0.11 ). Together with the 4 patients entering directly L2, a total of 11 patients progressed to L2 where the higher disease-specific risk justifies mismatch transplants. Rescue treatments in these 11 patients were venetoclax (6), ibrutinib (2), idelalisib (1), rituximab (1), and direct alloHCT (1). 2year PFS of the 11 patients reaching L2 was $54 \%$ (20-88\%) compared $100 \%$ of the 12 patients who remained at $\mathrm{L} 1$ ( 0.011). Altogether 17 patients proceeded to alloHCT (9 at L1, 8 at L2) and showed an MRD-negative PFS and OS of $87 \%(69-100 \%)$ and $93 \%(79-100 \%)$ at 2 years after transplant.

Conclusions: The EBMT/ERIC algorithm seems to be not inferior to PI-only strategies in patients with high-risk R/R CLL/B-PLL, with promising outlook for patients undergoing alloHCT while responding to their $1^{\text {st }}$ PI. Patients progressing under a $1^{\text {st }}$ PI can be effectively rescued with subsequent alternate PI and alloHCT consolidation.

Conflict of interest: nothing to disclose

\section{P633}

The hematopoietic cell transplantation comorbidity index predicts clinical outcomes after autologous stem cell transplantation

Alexandra Martínez-Roca ${ }^{1}$, Carme Talarn Forcadell ${ }^{1}$, Luis Gerardo Rodríguez-Lobato $^{2}$, Lourdes Escoda Teigell ${ }^{1}$, Rolando Vallansot ${ }^{1}$, Marta Cervera Calvo ${ }^{1}$, Janilson Do Nascimento ${ }^{1}$, Reyes Aguinaco Culebras $^{1}$, Teresa Giménez Pérez ${ }^{1}$, Carmen Araguás Arasanz ${ }^{1}$, Ana Vicent $^{1}$, Maria Sola ${ }^{1}$, Josep Sarrà Escarre ${ }^{1}$

${ }^{1}$ ICO-Hospital Universitari Joan XXIII, Hematology, Tarragona, Spain; ${ }^{2}$ Hospital Clínic, Hematology, Barcelona, Spain

Background: Autologous stem cell transplantation (ASCT) is a standard of care in patients with multiple myeloma and lymphoma, nevertheless is associated with morbidity and mortality. The Hematopoietic cell transplant comorbidity index (HCT-CI) is a score used to evaluate the comorbidities for outcomes after allogenic hematopoietic cell transplant (HCT), proving to be a predictor of non-relapse mortality (NRM) after allogenic HCT. There are few publications that evaluate the use of HCT-CI in the setting of ASCT with heterogeneous results on the score ability to predict NRM and overall survivor (OS) in this group of patients.

Methods: We analyzed 130 ASCT performed in our center between November 2008 and April 2017. Individual comorbidities were prospectively collected at the time of ASCT. All patients were assigned an HCT-CI score. We tested the utility of HCT-CI as a predictor of NRM and survival in patients undergoing ASCT.

Survival curves and probabilities were estimated using the Kaplan-Meier method and log-rank analysis. Multivariate analysis was performed using Cox proportional hazard regression model. Variables analyzed included 


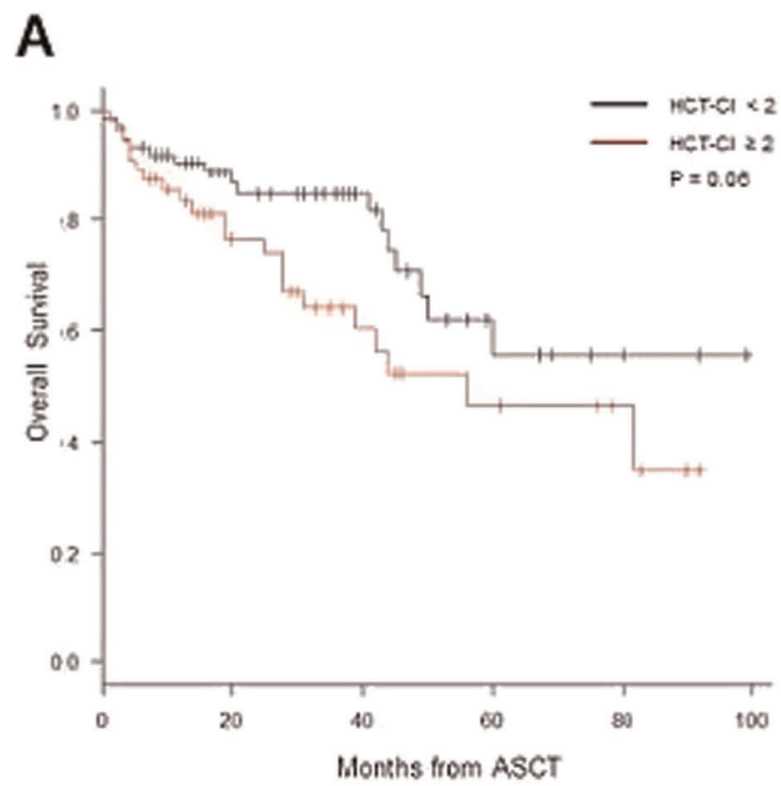

B

[P633 Figure] [OS and NRM of the 2 risk groups (HCT-CI Score <2 and $\geq 2$ ).]

HCT-CI, age, gender, type of disease (myeloma and lymphoma), disease stage and Karnofsky performance score (KPS) (Table 1).

NRM was defined as mortality after transplant in the absence of disease relapse/progression Thus, the cumulative incidence probabilities for NRM were calculated accounting for relapse as a competing risk.

Results: Median follow-up was 29,5 months (CI, 1144,25 ). Patients with a HCT-CI score $\geq 2$ presented a median OS of 56 months $(\mathrm{P}=0.06)$ and trend towards to an inferior OS. In the multivariate analysis HCT-CI score $\geq 2$ retained its independence adverse prognostic factor $(\mathrm{HR}=2.34 ; 95 \%$ CI, 1.166-4.71; $\mathrm{P}=0.016)$. NRM was 43 months in HCTCI score $<2$, and 26 months in the HCT-CI score $\geq 2$ group $(\mathrm{P}=0.083)$ (Figure 1).

Conclusions: A higher HCT-CI score did not predict NRM but was associated with inferior survival in patients undergoing ASCT in our cohort.

Conflict of interest: Nothing to disclose.

\begin{tabular}{|c|c|c|c|c|}
\hline Characteristics & Total & HCT-CI $<2$ & HCT-CI $\geq 2$ & $\mathrm{P}$ \\
\hline Transplants & 130 & 96 & 34 & \\
\hline $\begin{array}{l}\text { Age at transplant (range) } \\
\geq 50\end{array}$ & $94(72.3)$ & $47(62.7)$ & $47(85.5)$ & 0.004 \\
\hline Gender (\%) Male & $71(54.6)$ & $39(52)$ & $32(58.8)$ & 0.48 \\
\hline KPS $(\%)<90$ & $74(56.9)$ & $40(53.3)$ & $34(61.8)$ & 0.33 \\
\hline $\begin{array}{l}\text { Diagnose (\%) Myeloma - } \\
\text { Lymphoma }\end{array}$ & $\begin{array}{l}70(53.8)-60 \\
(46.2)\end{array}$ & $\begin{array}{l}36(48)-39 \\
(52)\end{array}$ & $\begin{array}{l}34(61.8)-21 \\
(38.2)\end{array}$ & 0.12 \\
\hline Disease stage (\%) CR & $54(41.5)$ & $31(41.3)$ & $23(41.8)$ & 0.95 \\
\hline
\end{tabular}

[[P633 Table] Patient Characteristics (HCT-CI Score $<2$ and $\geq 2)]$

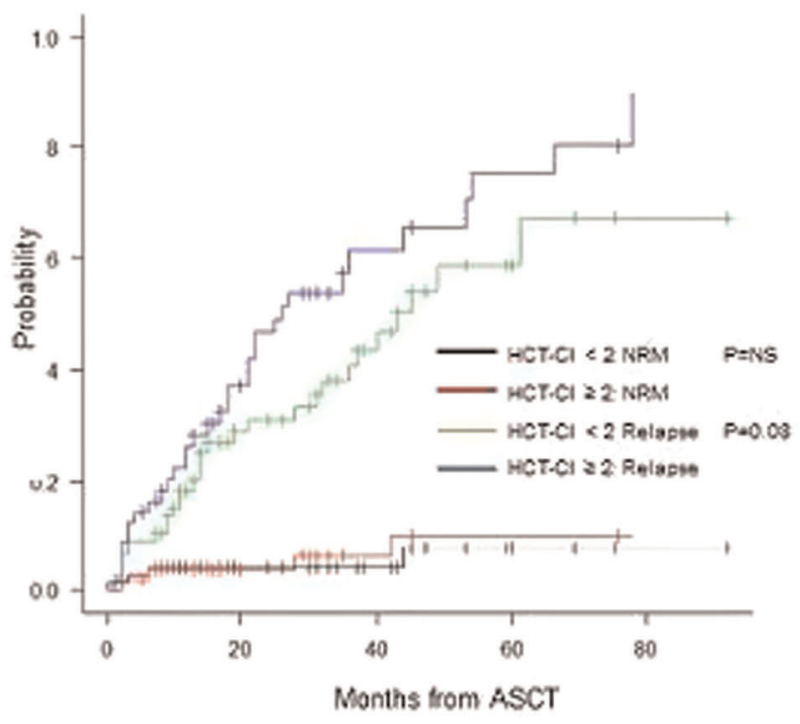

P634

The predictable role of FDG PET/CT on determining upfront autologous stem cell transplantation for the treatment of peripheral $\mathrm{T}$ cell lymphomas

Deok-Hwan Yang ${ }^{1}$, Seo-Yeon Ahn ${ }^{1}$, Sung-Hoon Jung ${ }^{1}$, Jae-Sook Ahn', Je-Jung Lee ${ }^{1}$, Hyeoung-Joon Kim ${ }^{1}$, Jae-Yong Kwak ${ }^{2}$, HoYoung Yhim ${ }^{2}$

${ }^{1}$ Chonnam National University Hwasun Hospital, HematologyOncology, Hwasun-gun, Korea, Republic of; ${ }^{2}$ Chonbuk National University Hospital, Hematology-Oncology, Jeonju, Korea, Republic of

Background: Peripheral T-cell lymphomas (PTCLs) respond poorly to conventional chemotherapy. A few studies demonstrated that upfront high-dose chemotherapy (HDT) and autologous stem cell transplantation (ASCT) as a consolidation could lead to long term progression-free survival (PFS) in PTCLs. However, there is no predictable factor to determine to undergo HDT/ASCT during primary treatment. This study investigated that response assessment using FDG PET/CT could be a predictable indicator of determining the upfront HDT/ASCT in the treatment of PTCLs.

Methods: To evaluate the role of PET/CT on proceeding upfront HDT/ASCT, we analyzed newly diagnosed patients with PTCLs who aged under 65 years between Jan. 2005 and Dec. 2016. We compared the clinical outcome according to interim and final response of PET/CT in two groups, HDT/ASCT group (test cohort) and non-HDT/ ASCT validated group (validation cohort), respectively. A total of 38 patients were enrolled to test cohort, and 54 


\section{Figure 1. PFS according to interim $\mathrm{PET} / \mathrm{C} T$ response $(\mathrm{A}$ : Test cohort, $\mathrm{C}$ : Validation cohort), PFS according to final PET/CT response (B : Test cohort, D : Validation cohort)}

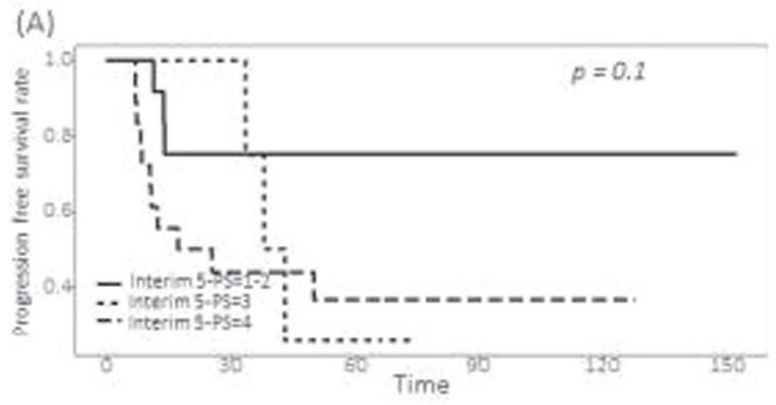

(C)

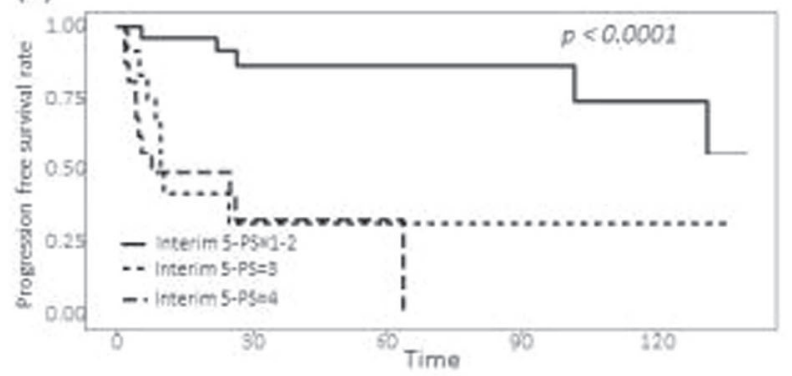

[P634 Figure] [Figure 1. PFS according to PET/CT response]

patients were to validation cohort. All patients underwent interim and final PET/CT during or after primary treatment. The response of interim and final PET/CT was assessed by visual analysis using Deauville five-point scale (5-PS).

Results: The pathologic frequency of HDT/ASCT group included nodal type $(\mathrm{n}=20,52.6 \%)$ and extranodal type ( $\mathrm{n}$ $=18,47.4 \%$ ). Treatment regimen mainly consisted of anthracycline-based $(\mathrm{n}=28,73.7 \%)$ CHOP-like and nonanthracyline-based $(\mathrm{n}=10,26.3 \%)$ chemotherapy. The IPI score at diagnosis was 0 factor $(\mathrm{n}=10,26.3 \%) ; 1(\mathrm{n}=10$, $26.3 \%) ; 2(\mathrm{n}=11,28.9 \%) ; 3(\mathrm{n}=5,13.2 \%) ; 4(\mathrm{n}=2$, $5.3 \%$ ), respectively. After a median follow-up of 55.8 months, 5-year PFS rates according to interim PET/ CT response were $75 \%$ of Deauville score $1-2,25 \%$ of Deauville score 3 and $36.5 \%$ of Deauville score 4 , respectively (Figure $1(\mathrm{~A}), P=0.1$ ). On the other hand, when analyzing PFS rates based on final PET/CT response, 5-year PFS rates were well stratified into $70 \%$ of Deauville score $1-2,21.8 \%$ of Deauville score 3 and $0 \%$ of Deauville score 4, respectively (Figure $1(\mathrm{~B}), P<0.01$ ). In the validated cohort, 3-year PFS rates according to interim PET/CT response were $86.7 \%$ of Deauville score $1-2,31.3 \%$ of Deauville score 3 and $32.8 \%$ of Deauville score 4, respectively (Figure $1(\mathrm{C}), P<0.01$ ). However, the PFS rates based on final PET/CT response were well discriminated as well as test cohort (Figure 1(D), $P<0.01$ ).
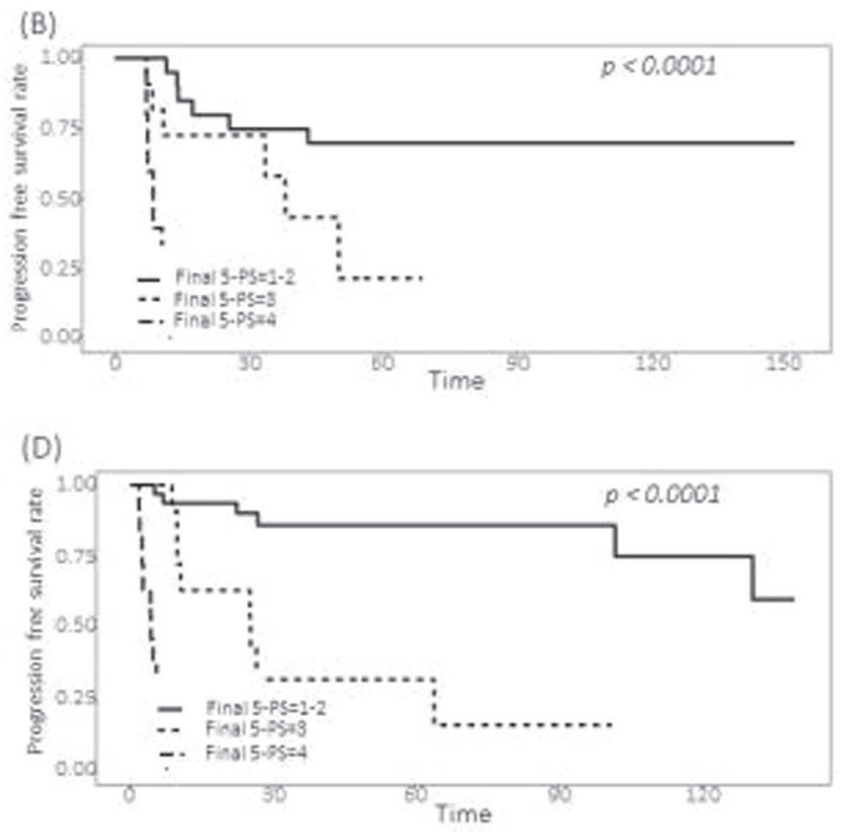

Conclusions: Final PET/CT assessment based on Deauville 5-PS showed the prognostic significance in the outcome of upfront HDT/ASCT, however, interim PET/ $\mathrm{CT}$ response could not predict the outcome in patients with PTCLs.

Conflict of interest: nothing to disclose

\section{P635}

Variation in the Use of Stem Cell Transplantation in the Management of Follicular Lymphoma (FL) and Indolent Lymphomas: a Study of the Lymphoma Working PartyEBMT

Stephen Robinson ${ }^{1}$, Karan Wadhera ${ }^{1}$, Ariane Boumendil ${ }^{2}$, Herve Finel $^{2}$, Irma Khvedelidze ${ }^{3}$, Sascha Dietrich ${ }^{4}$, Peter Dreger, ${ }^{5}$, Olivier Hermine $^{6}$, Chara Kyriakou ${ }^{7}$, Norbert Schmitz ${ }^{8}$, Harry Schouten ${ }^{9}$, Anna Sureda ${ }^{10}$, Alina Tanase ${ }^{11}$, Silvia Montoto ${ }^{12}$

${ }^{1}$ University Hospital Bristol, Bristol, United Kingdom; ${ }^{2}$ Lymphoma Working Party, EBMT, Paris, France; ${ }^{3}$ EBMT Lymphoma Working Party, Paris, France; ${ }^{4}$ University of Heidelberg, Heidelberg, Germany; ${ }^{5}$ University of Heidelberg, Haematology, Heidelberg, Germany; ${ }^{6}$ Hopital Necker, Paris, France; ${ }^{7}$ UCLH, London, United Kingdom; ${ }^{8}$ Asklepios Clinic St. Georg, Hamburg, Germany;

${ }^{9}$ University of Maastrich, Maastrich, Netherlands; ${ }^{10}$ Hospital Duran $i$ Reynals, Haematology, Barcelona, Spain; ${ }^{11}$ Fundeni Clinical Institute, 
Bucharest, Romania; ${ }^{12}$ St Bartholemews Hospital, London, United Kingdom

Background: Autologous (autoSCT) and allogeneic stem cell transplantation (alloSCT) are employed in the management of patients with indolent lymphoma (indNHL) but the optimal sequencing of these therapies is not well established. The role of SCT in the management of indNHL has been challenged recently by the advent of new therapeutic options. We analysed the transplant activity for indNHL as reported to the EBMT registry over the last 4 decades to determine geographic and temporal changes in the utilisation of SCT.

Methods: Retrospective analysis of the EBMT database. Inclusion criteria were autoSCT or allo SCT between 1982 and 2015 inclusive for indNHL (pre 1999) or FL (post 1999).

Results: 14631 autoSCT and 3469 alloSCT fullfilled the inclusion criteria. The percentage of EBMT registered centres reporting a SCT for indNHL has risen from $13 \%$ in 1990 to $60 \%$ in 2006 . The maximum number of autoSCT occurred in 2005 (917) and of alloSCT in 2011 (252). The ratio of autoSCT:alloSCT per country showed marked variation (range 0.4-10.8) with differences persistent over time. The median age at autoSCT and alloSCT has risen from 43 yrs (range 20-61) and 37 yrs (25-47) in 1982-90 to 57 yrs (22-80) and 53 yrs (19-73) in 2010-2015 respectively.

Regarding autoSCT, the median time from diagnosis to SCT has increased from 26 to 37 months and the percentage of patients transplanted with primary refractory/progressive disease has fallen from $30 \%$ to $14 \%$. The use of autoSCT in first response peaked at $57 \%$ of all autoSCT in the period 1996-2000. There has been a reduction in the use of TBI based conditioning from $52 \%$ to $2 \%$ whilst the use of peripheral blood stem cells has increased to $99 \%$.

With respect to alloSCT, the median time from diagnosis to alloSCT has increased from 29 to 54 months, with the percentage performed after a prior autoSCT increasing to $48 \%$. The percentage of patients with primary refractory/ progressive disease at the time of alloSCT has fallen from $40 \%$ to $24 \%$. Unrelated donors now account for $55 \%$ of all alloSCT, cord blood $2.5 \%$ and haploidentical family donors $0.8 \%$. The use of myeloablative conditioning has fallen from $100 \%$ of transplants prior to 1993 to $23 \%$ in 2010 2015 and the use of TBI based conditioning has fallen from $81 \%$ to $21 \%$.

Conclusions: There is marked national and individual transplant centre variation in the use of autoSCT and alloSCT in indNHL probably reflecting the difficulties in defining the right timing for SCT in indNHL. The increasing time from diagnosis to SCT may relate to improvements in non-transplant therapies. There has been an increase in the use of alternative donors and reduced intensity conditioning for alloSCT and more alloSCTs performed after a prior autoSCT. In addition, there is a better selection of patients with less patients receiving either an autoSCT or an alloSCT with refractory/progressive disease.

Conflict of interest: The authors have nothing to disclose Minimal residual disease, tolerance, chimerism and immune reconstitution

\section{P636}

Assessment of immune reconstitution after allogeneic hematopoietic stem cell transplantation by extensive flow cytometry analysis and possible correlations with clinical outcome

Giorgia Saporiti $^{1}$, Giulia Soverini ${ }^{1}$, Antonella Montemurro ${ }^{2}$, Alessandra Cattaneo ${ }^{2}$, Gianluca Cavallaro ${ }^{1}$, Elena Tagliaferri, Federica Grifoni ${ }^{1}$, Marco Fornili ${ }^{3}$, Laura Porretti ${ }^{2}$, Agostino Cortelezzi $^{1}$, Francesco Onida ${ }^{1}$

${ }^{1}$ Fondazione IRCCS Ca' Granda Ospedale Maggiore Policlinico, University of Milan, BMT-Hematology Unit, Milano, Italy;

${ }^{2}$ Fondazione IRCCS Ca' Granda Ospedale Maggiore Policlinico, Flow Cytometry Facility, Service Department, Milano, Italy; ${ }^{3}$ Unit of Medical Statistics, Biometry and Bioinformatics 'Giulio A.

Maccacaro", University of Milan Campus Cascina Rosa, Fondazione IRCCS Istituto Nazionale Tumori, Department of Clinical Sciences and Community Health, Milano, Italy

Background: Following allogeneic hematopoietic stem cell transplantation (allo-HSCT), prompt immune reconstitution appears to be essential for most clinical outcomes; however, complete immunological recovery may require up to years, with prolonged immunodeficiency both being affected and leading to potentially severe complications, including post-transplant infections, relapse or secondary malignancies.

Methods: Aiming to prospectively evaluate immunological reconstitution and possible association with clinical outcomes, blood samples from patients undergoing alloHSCT in our Center from January 2015 to June 2017 were analyzed at baseline (before the start of conditioning) and at different time points following transplantation $(+1,+2,+3$, $+6,+9,+12,+24$ months) by extensive flow cytometry assays including: double positive thymocytes $\left(\mathrm{CD} 4^{+} \mathrm{CD} 8^{+}\right)$; naïve $\left(\mathrm{CD}^{2} 5 \mathrm{RA}^{+} \mathrm{CCR} 7^{+}\right)$, effector $\left(\mathrm{CD}^{2} 5 \mathrm{RA}^{+} \mathrm{CCR} 7^{-}\right)$, central memory - $\mathrm{T}_{\mathrm{CM}}\left(\mathrm{CD} 45 \mathrm{RA}^{-} \mathrm{CCR} 7^{+}\right)$, effector memory - $\mathrm{T}_{\mathrm{EM}}\left(\mathrm{CD} 45 \mathrm{RA}^{-} \mathrm{CCR} 7^{-}\right) \mathrm{CD}^{+}$and $\mathrm{CD}^{+} \mathrm{T}$ cells; regulatory $\left(\mathrm{CD} 25^{\text {bright }} \mathrm{CD} 127^{\text {low }}\right) \mathrm{CD}^{+} \mathrm{T}$ cells; nä̈ve $\left(\mathrm{CD} 27^{-} \operatorname{IgD}{ }^{+}\right)$, IgM-memory $\left(\mathrm{CD} 27^{+} \mathrm{IgD}^{+}\right)$and switchedmemory - $\mathrm{SM}\left(\mathrm{CD} 27^{+} \mathrm{IgD}-\right) \mathrm{CD}^{-} 9^{+} \mathrm{B}$ cells; regulatory 
$\left(\mathrm{CD} 3-\mathrm{CD} 56^{\text {bright }} \mathrm{CD} 16^{-}\right)$and cytotoxic $\left(\mathrm{CD} 3{ }^{-\mathrm{CD}} 56^{\mathrm{dim}} \mathrm{CD} 16\right.$ ${ }^{+}$) Natural Killer (NK) cells.

Results: 38 patients (median age 51 yrs, range 19-68 yrs) were included in the study. Indications for allo-HSCT were acute leukemia in 27 (17 myeloid, 10 lymphoblastic), myelodysplastic syndrome in 4, non-Hodgkin's lymphoma in 5, Hodgkin's disease in 1 and primary myelofibrosis in 1 . As expected, NK were the first cell subset to return to a normal level after transplantation, with an early expansion of regulatory cells during the $1^{\text {st }}$ month and a subsequent expansion of cytotoxic cells from the $2^{\text {nd }}$ month on. With regard to $\mathrm{T}$ cells, we observed a progressive peripheral expansion of the mature subsets (especially $\mathrm{T}_{\mathrm{EM}}$ ) from the $2^{\text {nd }}$ month on, most likely originating from donor mature $\mathrm{T}$ cells, with a subsequent maintained prevalence of the $\mathrm{CD}^{+}$over the $\mathrm{CD} 4+$ cells. The reconstitution of $\mathrm{CD} 4^{+}$ and $\mathrm{CD} 8^{+}$naïve $\mathrm{T}$ cells from donor hematopoietic cells through the thymic pathway was delayed, starting to increase only from the $9^{\text {th }}$ month and leading to a longlasting limited T-cell repertoire. B cell tended to return to normal counts within 9 months, mainly thanks to naïve cells, while memory and SM cells remained low up to 2 years after allo-HSCT.

In patients who were treated for acute-GvHD, we observed a marked reduction of NK cells, mainly belonging to the cytotoxic subset, and a subsequent decrease of both CD4+ and CD8+ effector and memory $\mathrm{T}$ cells, most evident at the +6 time point evaluation. On the other hand, occurrence of treatment-requiring chronic GvHD primarily affected B-cell reconstitution, both quantitatively and functionally. In patients experiencing early CMV reactivation, at the +6 and +12 time points we observed a significant reduction of almost all $\mathrm{CD} 4+$ and $\mathrm{CD} 8+\mathrm{T}$ lymphocytes, with the only exception of $\mathrm{T}_{\mathrm{CM}}$ and $\mathrm{T}_{\mathrm{EM}} \mathrm{CD} 8$ + subsets, and of B-lymphocytes. Worth mentioning, patients who showed EBV reactivation appeared to have lower counts of $\mathrm{T}_{\mathrm{CM}} \mathrm{CD} 4+$ and $\mathrm{CD} 8+$ cells at the baseline evaluation in comparison to patients who did not experience EBV reactivation.

Conclusions: Extensive flow cytometric analysis allows to study specific immune reconstitution after allo-HSCT, suggesting possible correlations with clinical outcome.

Conflict of interest: None of the authors has anything to disclose.

\section{P637}

BEAM-Campath allogeneic stem-cell transplant for patients with relapsed/refractory lymphoma - high incidence of long term mixed donor-recipient chimerism and the response to donor lymphocyte infusions
Claire N Burney ${ }^{1}$, Karan Wadehra ${ }^{2}$, Patricia Breslin ${ }^{1}$, Rajesh Alajangi $^{1}$, James Griffin ${ }^{1}$, David Marks ${ }^{1}$, Rachel Protheroe ${ }^{1}$, Stephen Robinson ${ }^{1}$

${ }^{1}$ University Hospitals Bristol NHS Trust, Haematology, Bristol, United Kingdom; ${ }^{2}$ Epsom and St. Helier University Hospitals NHS Trust, Haematology, London, United Kingdom

Background: BEAM-Campath conditioned allogeneic stem cell transplants (alloSCT) were developed to reduce the high transplant-related mortality in patients with lymphoma whilst simultaneously delivering intensive antineoplastic therapy and a platform for allogeneic stem-cell engraftment. Here we describe outcomes of BEAMCampath alloSCT for lymphoma treated at a single centre and report a high incidence of long term mixed donorrecipient chimerism (MDRC).

Methods: Retrospective analysis of all patients with lymphoma undergoing a BEAM-Campath alloSCT at our centre between 2003-2017. Chimerism analysis was performed with peripheral blood utilising short tandem repeat PCR. MDRC was defined as $>5 \%$ recipient and $>5 \%$ donor. Statistical analysis was performed with SPSS.

Results: 52 patients ( 22 female, 30 male), with a median age at transplant of 45 years (range 19-65), were identified. Histological diagnoses were Hodgkin's lymphoma $(\mathrm{n}=13)$, DLBCL $(\mathrm{n}=7)$, low-grade NHL $(\mathrm{n}=16)$, mantle cell $(\mathrm{n}=$ 10) and T-cell lymphoma $(n=6)$. Patients received a median of 3 prior lines of therapy (range 1-7), including 3 autoSCT and $96 \%$ were in CR or PR pre-transplant. Donors were matched sibling $(n=21)$, matched unrelated $(n=24)$, mismatched unrelated ( $\mathrm{n}=6$, all 9/10 HLA-matched) and syngeneic $(\mathrm{n}=1)$. Median CD34+ and CD3+ cell dose was $5.47 \times 10^{6} / \mathrm{kg}$ and $198.35 \times 10^{6} / \mathrm{kg}$, respectively.

Median time to platelet and neutrophil engraftment was 12 days. 2 patients developed graft failure, 4 received a stem-cell top up (3 graft failure/poor function, 1 myeloid MDRC). $49 \%$ developed acute GvHD (84\% grade $1 / 2)$ and $11 \%$ developed chronic GvHD (83\% extensive). 10 patients died from non-relapse mortality (NRM) (6 infection, 3 GvHD, 1 PTLD) with a cumulative incidence of NRM of $5.8 \%$ at $\mathrm{D} 100$, and $15.4 \%$ at 1 year.

The prevalence of T-cell lineage MDRC peaked at D100 and subsequently declined over time whilst myeloid lineage MDRC remained relatively stable over 10 years (16.7$27.8 \%$ ) (see Figure 1). At 5 years 33\%, 33\% and $23.8 \%$ of patients had MDRC in whole blood, T-cell and myeloid lineages, respectively. Full recipient myelopoiesis developed in one patient (developing recipient-derived AML) and full recipient T-cell lymphopoiesis in one patient (who subsequently relapsed).

23 patients received a median of 2 (range 1-5) donor lymphocyte infusions (DLI) for MDRC (74\%) or relapse $(26 \%)$ at a median starting dose of $1 \times 10^{6} / \mathrm{kg}$. 9 patients 


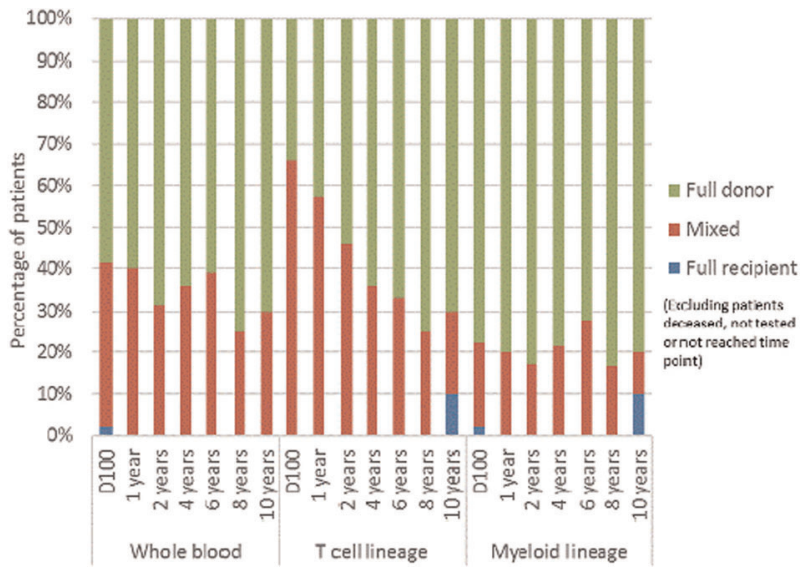

[P637 Figure] [Lineage-specific chimerism over time post transplant (day 100 to 10 years)]

developed acute post-DLI GvHD (5 grade 1/2) and 2 chronic GVHD (both limited). Of those with T-cell $(\mathrm{n}=22)$ or myeloid MDRC $(\mathrm{n}=10), 52.2 \%$ and $50 \%$, respectively, converted to full donor chimerism after DLI(s).

With a median follow up of 5.8 years, 13 patients have relapsed of whom 4 achieved a CR or PR with further therapy or DLI and 7 died from lymphoma (cumulative incidence of relapse was $5.8 \%$ at 1 year and $13.5 \%$ at 5 years). The 5 year PFS and OS were 62\% and 63\% respectively.

Conclusions: BEAM-Campath conditioned alloSCT is curative for many patients with high risk lymphoma. MDRC is common in both T-cell and myeloid lineages and persists. Of those with MDRC, half convert to full donor in both myeloid and T-cell lineages following DLI. Further analysis is indicated to identify pre-transplant factors associated with MDRC and its impact on relapse rates.

Conflict of interest: All authors: Nothing to disclose

\section{P638}

\section{Clinical usefulness of pre-transplant and early post- transplant MRD quantification in patients with FLT3 + AML}

Gillen Oarbeascoa ${ }^{1}$,Carolina Martinez-Laperche ${ }^{1,2}$, Ana PérezCorral $^{1,2}$, Cristina Pascual ${ }^{1,2}$, Nieves Dorado ${ }^{1,2}$, Laura Solan ${ }^{1,2}$, David Serrano ${ }^{1,2}$, Pascual Balsalobre ${ }^{1,2}$, Javier Anguita ${ }^{1,2}$, Jose Luis Díez-Martín $^{1,2}$, Ismael Buño ${ }^{1,2,3}$, Mi Kwon ${ }^{1,2}$

${ }^{1}$ Hospital General Universitario Gregorio Marañon, Hematology Department, Madrid, Spain; ${ }^{2}$ Instituto de Investigación Sanitaria Gregorio Marañon (IiSGM), Madrid, Spain; ${ }^{3}$ Hospital General Universitario Gregorio Marañon, Genomics Unit, Madrid, Spain

Background: Relapse remains the main cause of treatment failure in this patients after HSCT. Identification of high-risk patients is crucial for post-transplant management, including maintenance targeted therapy. FMS-like tyrosine kinase 3 (FLT3) mutations are associated with unfavorable prognosis in acute myeloid leukemia (AML). The objective of this study was to assess the significance of pre-HSCT and early post-HSCT minimal residual disease (MRD) studied by multiparametric flow cytometry (MFC) and molecular analysis in patients with FLT3 mutated AML.

Methods: 29 consecutive adult patients transplanted for FLT3-ITD+ AML in complete remission (CR) between 2008 and 2017 were retrospectively analyzed (Table 1). Patients with persistent or refractory disease were excluded. MRD was studied by 4-color MFC on BM aspirates, and quantitative RT-PCR (NPM1, RUNX1-RUNX1T1, MLL, WT1) was assessed on BM and/or peripheral blood (PB) samples obtained within 30 days before HSCT and on day +30 after HSCT.

Results: Conditioning regimen was myeloablative and stem cell source used was PBSC in most cases (Table 1). 16 patients showed negative pre-HSCT MRD whereas 13 showed positive values: 2 by RT-PCR, 4 by MFC, and 7 by both methods. Characteristics of patients in both groups were not significantly different. With a median follow-up of 30 months, 2-year overall survival (OS) was $92 \%$ vs $63 \%(\mathrm{HR}=3.84, \mathrm{p}=0.10)$ for pre-HSCT MRDnegative and MRD-positive patients, respectively. Event free survival (EFS) was 80 vs $65 \%$ (HR 2.16, p =0.3). Cumulative incidence of grades II-IV acute GvHD was $44 \%$ vs $46 \%(\mathrm{p}=0.87)$, and for moderate-severe chronic GvHD $14 \%$ vs $36 \%(\mathrm{p}=0.23)$, respectively. Relapse incidence was $13 \%$ vs $19 \%(\mathrm{p}=0.73)$, and TRM was $8 \%$ vs $15 \%(\mathrm{p}=0.36)$. At day +180 post-HSCT, $31 \%$ pre-HSCT MRD negative patients and $72 \%$ pre-HSCT MRD patients had ceased immunosuppressive therapy $(\mathrm{p}=0.3)$. From the group with pre-HSCT positive MRD, 23\% presented positive MRD at day +30 , whereas in $6 \%$ MRD was detectable in the group with pre-HSCT negative MRD. Patients who showed positive MRD at day +30 presented a 2-year OS of $75 \%$ vs $84 \%(\mathrm{p}=0.17)$ compared to patients with negative day $+30 \mathrm{MRD}$, and a 2 -year EFS of $50 \%$ vs $81 \%(\mathrm{p}=0.95)$, respectively.

Conclusions: In this unicentric experience with posttransplant homogeneous management, patients with FLT3+ AML transplanted with detectable MRD showed reduced OS and EFS. Differences were not significant due to the limited number of cases. Differences in EFS occur in spite that most patients with detectable pre-HSCT MRD show negativization early after HSCT, and immunosuppression is stopped earlier. Hence, patients with positive MRD before transplantation represent a high-risk group. Further studies are needed to confirm these higher-risk patients who may benefit from programmed post-transplant interventions 


\begin{tabular}{l|c|c|}
\cline { 2 - 3 } \multicolumn{1}{l|}{$\begin{array}{c}\text { Table 1. Characteristics of } \\
\text { patients }\end{array}$} & $\begin{array}{c}\text { MRD } \\
\text { negative }\end{array}$ & $\begin{array}{c}\text { MRD } \\
\text { positive }\end{array}$ \\
\hline N=29 & $\mathrm{N}=16$ & $\mathrm{~N}=13$ \\
\hline Age (medan, R) & $42(37-47)$ & $43(35-56)$ \\
\hline Gender (male/female) & $10 / 6$ & $8 / 5$ \\
\hline Pre-SCT status & 15 & 12 \\
CR1 & 1 & 1 \\
CR) & & \\
\hline Donor & 2 & 2 \\
HLA-identical sibling & 4 & 6 \\
HLA-matched unrelated & 1 & 1 \\
HLA-mismatched & 2 & 1 \\
UCB-dual & 7 & 6 \\
HLA-haploidentical related & & \\
\hline Stem cell source & 13 & 11 \\
PB & 1 & 1 \\
BM & 2 & 1 \\
UCB & 14 & 12 \\
\hline Conditioning intensity & 2 & 1 \\
MAC & & \\
RIC & & \\
\hline
\end{tabular}

\section{Overall Survival}

MRD Status + Negative MRD + Positive MRD

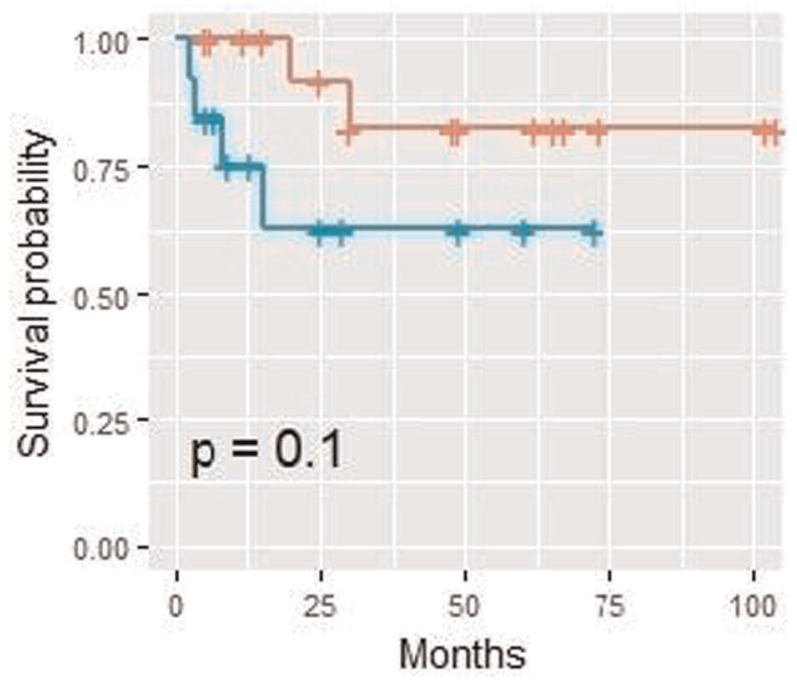

MRD: minimal residual disease.

UCB-dual: single cord blood with $c 0$-infusion of selected CD 34 + cells from a third party HLAmismatched donor

Haploidentical Unmanipulated graft, with posttransplant high dose cidophosphamide

\section{Event Free Survival}

MRD Gtatus + Negative MRD + Positive MRD

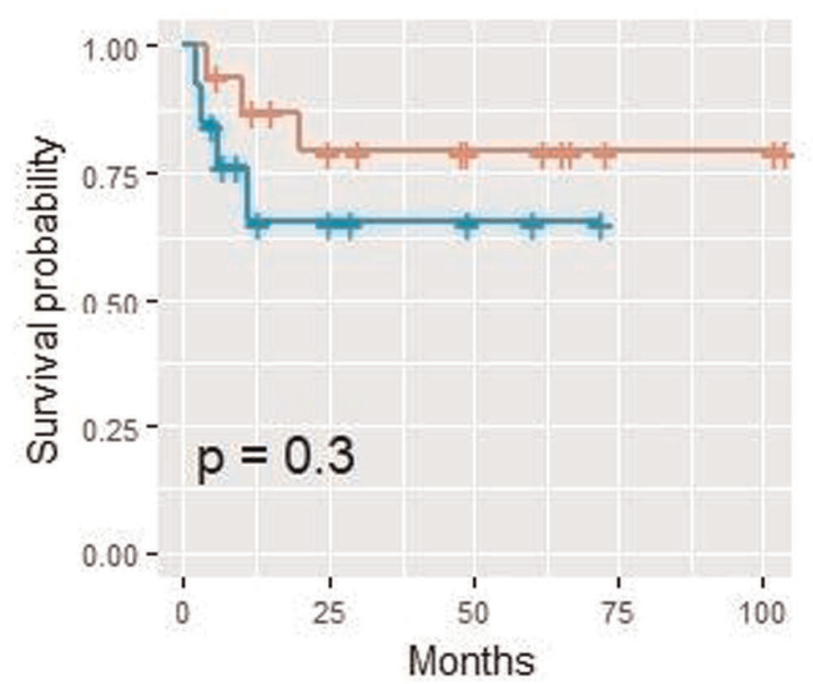

[P638 Figure] [Patient characteristics, overall survival and event free survival]

including early immunosuppression withdrawal, DLI or new target-directed drugs.

Conflict of interest: All authors: Nothing to disclose.
P639

Comparable outcomes after transplantation for FCM-MRD highly-positive $A M L$ and non-hematological remission $A M L$ 
Tomomi Oka, Junya Kanda, Katsuyuki Ohmori, Masakatsu Hishizawa, Toshiyuki Kitano, Tadakazu Kondo, Kouhei Yamashita, Akifumi Takaori-Kondo

Kyoto University, Kyoto, Japan

Background: We have previously reported that flowcytometry (FCM)-based detection $(\geq 0.6 \%)$ of minimal residual disease (MRD) before hematopoietic stem cell transplantation (HSCT) was associated with poor overall survival in patients with acute myelogenous leukemia (AML) in hematological complete remission (CR). In this study, we compared MRD-positive patients with non-CR patients.

Methods: We included 105 adult patients with AML (myeloablative conditioning (MAC):62, reduced-intensity conditioning:43), who underwent their first allogeneic HSCT between April 2005 and February 2016 at Kyoto University Hospital. Median age was 48 (range: 17-66). MRD of bone marrow before HSCT was measured using FCM. To search for target antigens to detect MRD, threecolor FCM analyses were performed using a differential panel for every disease and patient, which allowed us to detect $\geq 0.1 \%$ of MRD.

Results: Sixty-two and forty-three patients had hematological CR and non-CR disease status before HSCT, respectively. Of hematological CR patients, 15 patients were included in the MRD-positive group (MRD $\geq 0.1 \%$ ), whereas 47 were included in the MRD-negative group (MRD $<0.1 \%$ ). In the MRD-positive group, 9 patients were included in the MRD-high group (MRD $\geq 0.6 \%$ ), and 6 were included in the MRD-low group (MRD< $0.6 \%)$. MAC was used in $67 \%, 56 \%, 50 \%$, and $53 \%$ of the non-CR, MRDhigh, MRD-low, and MRD-negative groups, respectively (P $=0.538)$. The 3 -year overall survival rates for the non-CR, MRD-high, MRD-low, and MRD-negative groups were $35 \%, 30 \%, 67 \%$, and $79 \%$, respectively $(\mathrm{p}<0.001$, figure 1). In a multiple regression analysis, the MRD-high group showed comparative overall mortality with the non-CR group (MRD-high vs. non-CR, hazard ration [HR] 0.93, p $=0.874$; MRD-low vs. non-CR, HR 0.27, $\mathrm{p}=0.074$; MRD-negative vs. non-CR, HR 0.23, p < 0.001). The 3year relapse rates for the non-CR, MRD-high, MRD-low, and MRD-negative groups were 50\%, 67\%, 0\%, and $15 \%$, respectively $(\mathrm{p}<0.001)$. There were no significant differences in non-relapse mortality among the four groups $(\mathrm{p}=0.328)$.

Conclusions: The analysis of FCM-based detection of MRD revealed that an MRD positivity of $\geq 0.6 \%$ was significantly associated with high risk of relapse and death even in patients with AML in hematological CR, and transplant outcomes were comparable between MRD-high and non-CR groups. The stronger conditioning therapy
Overall su'vivals ascording to disease status

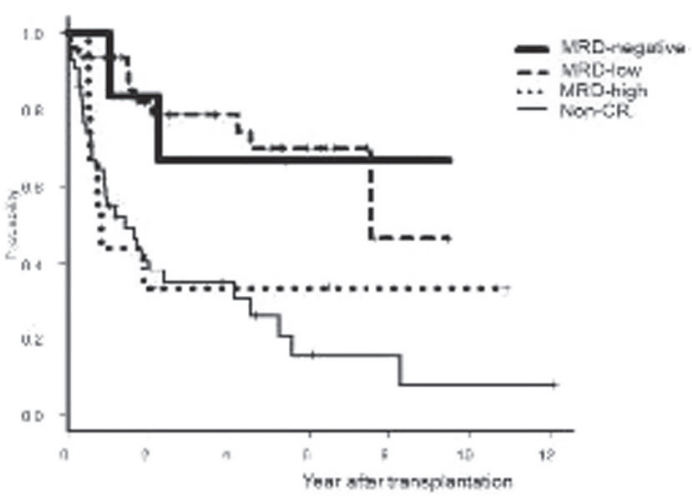

[P639 Figure] [figure 1]

before HSCT would be a treatment option for patients with highly-positive MRD.

Conflict of interest: no conflict of interest

\section{P640}

Comparative analysis of flow cytometry and RQ-PCR for the detection of minimal residual disease in Philadelphia chromosome-positive acute lymphoblastic leukemia after hematopoietic stem cell transplantation

Xiangyu Zhao, Xiaosu Zhao, Huan Chen, Yazhen Qin, Lanping Xu, Xiaohui Zhang, Kaiyan Liu, Xiaojun Huang, Yingjun Chang

Peking University People's Hospital, Peking University Institute of Hematology, Beijing, China

Background: The effect on relapse of achieving a complete molecular response (CMR, BCR-ABL/ABL < $0.01 \%$ ) or minimal residual disease (MRD) negativity by multiparameter flow cytometry (MFC) in Philadelphia chromosome-positive ALL (Ph+-ALL) at the early stage after hematopoietic stem cell transplantation (HSCT) remains unknown.

Methods: The aim of this study was to examine the value of MRD detection by MFC and RQ-PCR at the early stage after transplantation for predicting relapse and leukemiafree survival (LFS).

Results: Patients who maintained CMR status at 1 and 3 months were associated with a lower relapse rate $(\mathrm{P}=$ 0.02 and $<0.001)$ and better LFS $(P=0.014$ and 0.013$)$ than those without a CMR; however, the CMR status at 2 months could not predict relapse $(\mathrm{P}=0.445)$ and LFS ( $\mathrm{P}$ $=0.935)$. Negative MFC at 1,2 and 3 months was associated with a lower relapse rate $(\mathrm{P}=0.01,0.004$, and 0.04 respectively) and better LFS $(\mathrm{P}=0.044,<0.0001$ and 0.013 , respectively). Multivariate analysis showed that MRD status identified by MFC at 1 month and a CMR at 
3 months exhibited better discrimination for relapse (HR 6.969 (95\% CI 1.843-26.352), $\mathrm{P}=0.004$; HR 10.285 (95\% CI 3.787-27.937), $\mathrm{P}<0.0001)$ and DFS (HR 4.418 (95\% CI 1.442-13.535), $\mathrm{P}=0.009$; HR 3.205 (95\% CI 1.238-8.297), $\mathrm{P}=0.016$ ). Among the 155 patients, 21 patients relapsed, and 17 of 21 were MRD-positive according to MFC or RQPCR detection of BCR-ABL1 post-transplantation.

Conclusions: In summary, MRD detection by MFC and RQ-PCR detection of BCR-ABL1 at the early stage were important predictors of outcome in patients with $\mathrm{Ph}+$-ALL, and these tests played complementary roles in predicting prognosis.

Conflict of interest: Xiangyu Zhao: Nothing to disclose.

\section{P641}

\section{Comparative analysis of immune reconstitution shows better recovery of $B, N K$ and plasmacytoid DC after allogeneic cord blood as compared to PBSC and BM transplantation}

Mario Arpinati, Francesca Ulbar, Gabriella Chirumbolo, Valentina Pensato, Giulia Tolomelli, Francesca Bonifazi

University Hospital Bologna, Hematology, Bologna, Italy

Background: The reconstitution of immune cell subsets after allogeneic hematopoietic stem cell transplantation (HSCT) is relevant to patient outcome. We have studied the impact of several factors (including stem cell source, type of disease, conditioning regimen, Graft Versus Host Disease (GVHD) on immune reconstitution in a large (>500) monocentric cohort of patients

Methods: We have studied 503 adult patients receiving PBSC (262), BM (191) or UCB (48) transplant at the Bologna University Hospital. Data were collected between 2000 and 2014. We have analysed the number of immune cell subsets in the peripheral blood at specific time points from 1 month up to 1 year after transplant. A total of 1368 samples were processed. Multicolor flow cytometry was employed to measure the numbers of circulating $\mathrm{T}$ and B lymphocytes, NK cells and APC subtypes (monocytes, plasmacytoid (pDC) and myeloid (mDC) dendritic cells).

Results: The recovery of pDC at 3 through 12 months after transplant was significantly increased in patients receiving UCB as compared to both PBSC and BM transplants ( $\mathrm{p}$ 0,0001 at all time points). Moreover we have confirmed the increase of $\mathrm{B}$ and NK cell numbers and the delay in the recovery of T cell subsets in UCB transplants as compared to both PBSC and BM transplants. The only other factor affecting immune recovery was chronic GVHD. The number of $\mathrm{pDC}$ was reduced in patients with extensive cGVHD compared to patients with limited GVHD or without cGVHD at 3 months $<0,001$ ) and 6 and 12 months ( $p<0,01)$. The recovery of $\mathrm{T}$ cells and NK cells was unaffected by cGVHD, whereas the B cell recovery was also reduced by cGVHD ( $\mathrm{p}<0,01$ only at 9 months).

Conclusions: Our study shows that patients undergoing UCB transplantation have better reconstitution of plasmacytoid DC, as well as NK cells and B cells, as compared to patients receiving PBSC and $\mathrm{BM}$ grafts. Also, pDC recovery was significantly delayed in patients with cGVHD.

Conflict of interest: Mario Arpinati: nothing to disclose

\section{P642}

Dendritic cells content is associated with the recovery of ${\mathrm{V} \delta 2^{+}}^{+} \mathrm{T}$ cells after haploidentical hematopoietic stem cell transplantation

\author{
Xiaoyu Wang ${ }^{1,2}$, Jiangying Liu ${ }^{1}$, Haitao Gao ${ }^{1}$, Xiaojun Huang ${ }^{1}$ \\ ${ }^{1}$ Peking University People's Hospital, Beijing, China; ${ }^{2}$ Beijing \\ Hightrust Diagnostics, Co., Ltd, Beijing, China
}

Background: Immune reconstruction is critical to the therapeutic efficacy and outcomes of patients undergone haploidentical hematopoietic stem cell transplantation (haploHSCT). Our previous study has demonstrated that recovery of ${\mathrm{V} \delta 2^{+}}^{+} \mathrm{T}$ cells inversely correlated with $\mathrm{EBV}$ reactivation after haploHSCT. Moreover, the proportions of $\mathrm{V} \delta 2^{+} \mathrm{T}$ cells were continuously decreased in recipients regardless of the status of EBV reactivation, from 30 days to 90 days after haploHSCT. The reasons that caused the delay of $\mathrm{V} \delta 2^{+} \mathrm{T}$ cells recovery remain elusive. $\mathrm{V} \delta 2^{+} \mathrm{T}$ cells can be specifically activated and expanded by small nonpeptidic phosphoantigens in an HLA-unrestricted manner. Aminobisphosphonates, such as pamidronate and zoledronic acid

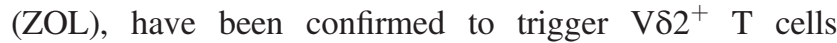
expansion in vitro and in vivo. Many immunotherapeutic strategies targeting $\gamma \delta \mathrm{T}$ cells with $\mathrm{ZOL}$ have been conducted in patients with various hematologic malignancies and solid tumors. Dendritic cells (DCs), the most potent antigen presenting cells, were reported to promote $\mathrm{V} \delta 2^{+}$ $\mathrm{T}$ cells proliferation by presenting phosphoantigens through CD277/butyrophilin-3A1 (BTN3A1) glycoprotein. However, whether bisphosphonates could stimulate the proliferation of $\mathrm{V} \delta 2^{+} \mathrm{T}$ cells after unmanipulated haploHSCT and DCs play a role in this process are still unknown.

Methods: The recovery characteristics of ${\mathrm{V} \delta 2^{+}}^{+} \mathrm{T}$ cells and DCs were monitored on 35 patients around 180 days after unmanipulated haploHSCT, which were compared with those of 20 healthy donors. Immunophenotyping was performed using flow cytometry. Furthermore, in-vitro effects of pamidronate on the recovered $\mathrm{V} \delta 2^{+} \mathrm{T}$ cells from haploHSCT recipients were investigated. The expression of 
BTN3A1 was measured using both flow cytometry and realtime quantitative PCR. Statistical analyses were performed using SPSS 22.0.

Results: The concentrations of recovered $\mathrm{V} \delta 2^{+} \mathrm{T}$ cells were significant lower in recipients until 180 days after haploHSCT, compared with the healthy donors $(P<$ 0.0001). In-vitro functional experiments showed that pamidronate stimulated a strong proliferation of $\mathrm{V} \delta 2^{+}$ $\mathrm{T}$ cells from healthy donors, whereas failed to trigger V $\delta 2$ ${ }^{+} \mathrm{T}$ cells from haploHSCT recipients. Quantitative RT-PCR analysis showed that the expression levels of BTN3A1 and B30.2 were not statistically different between donors and haploHSCT recipients $(P=0.944$ and $P=0.802$, respectively). No difference was found when comparing the proportions of $\mathrm{CD} 277^{+} \mathrm{V} \delta 2^{+} \mathrm{T}$ cells between the groups $(P=0.747)$. The percentages of DCs, including pDCs and $\mathrm{mDCs}$, were strikingly decreased in recipients after haploHSCT $(P=0.029, P=0.022$ and $P=0.022$, respectively). Correlation analysis found that the proportion of $\mathrm{mDCs}$, but not DCs or pDCs, significantly associated with the ratio of recovered ${\mathrm{V} \delta 2^{+}}^{+} \mathrm{T}$ cells after haploHSCT $(P<0.001)$.

Conclusions: The current study indicated that reduced

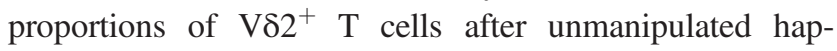
loHSCT inhibited their proliferation in response to pamidronate stimulation in vitro. Correlation analysis found that decreased DCs content was associated with the delay in

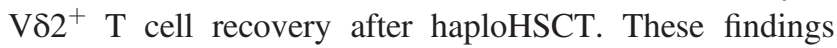
provide evidences for exploring new strategies to rescue the impaired recovery of ${\mathrm{V} \delta 2^{+}}^{\mathrm{T}}$ cells after haploHSCT recipients, by using in-vitro induced and bisphosphonatepretreated autologous or allologous DCs.

Conflict of interest: The authors declare no conflicts of interest.

This study is supported by the Beijing Postdoctoral Research Foundation (2017-ZZ-058), National Natural Science Foundation of China (No. 81770191), and Foundation for Innovative Research Groups of the National Natural Science Foundation of China (No. 81621001).

\section{P643}

\section{Engraftment Follow Up with New CBC Parameters of The Children in Hematopoietic Stem Cell Transplantation}

\section{Musa Karakukcu, Ekrem Unal, Gulsah Ucan, Alper Ozcan, Turkan Patiroglu}

Erciyes University Faculty of Medicine, Pediatric Hematology Oncology, Kayseri, Turkey

Background: Hematopoietic stem cell transplantation (HSCT) has been used successfully in the treatment of many hematologic malignancies, genetic and metabolic disorders. Stem cell engraftment as well as stem cell recovery is a complex medical process. During the first few days after transplantation, the reinfused allogeneic or autologous stem cells migrate to the bone marrow and begin the process of producing replacement blood cells, a process called engraftment. The stem cells start to produce new blood cells within 2 to 3 weeks following infusion. After HSCT, early detection of engraftment would be of critical value for clinicians. The aim of the study is to help identify engraftment earlier with new automatic CBC analyzer parameters.

Methods: Sixteen patients who underwent HSCT and achieved myeloid and platelet engraftment between March 2017 and August 2017 were included in the study. The diagnoses of these patients were AML (3), ALL (3), Primer Immunodeficiency (5), Thalassemia Major (2), Ewing Sarcoma, Neuroblastoma and Hodgkin Lymphoma. The patients were treated with 13 allogeneic (4 haploidentical (HAPLO), 2 unrelated (MUD), 2 relative (MFD), 5 siblings (MSD)) and 3 autologous HSCT. The myeloid and platelet engraftments at the time of transplantation of these patients were followed by the new automated CBC parameters. CBC and differentials were administered by XN-9000Hematology-Analyzer (Sysmex, Kobe, Japan). The day of myeloid engraftment was defined as the first of 3 consecutive days on which the absolute neutrophil count (ANC) exceeded $>0.5 \times 10^{9} / \mathrm{L}$ at any time after transplantation. Platelet engraftment was considered to have occurred when the platelet count was $>50 \times 10^{9} / \mathrm{L}$, without transfusion, for 7 days.

Results: All patients achieved an absolute neutrophil count (ANC) of $0.5 \times 10^{9} / \mathrm{L}$ in a median of 14 th days (range, 10 to 23th days) (Figure 1). The median absolute neutrophil and immature granulocyte (IG) count were $0.690 \times 10^{9} / \mathrm{L}$ $(0.510-2.500)$ and $0.040 \times 10^{9} / \mathrm{L}(0.010-0.350)$ in the same myeloid engraftment day, respectively. The IG/ANC ratio was $5.8 \%$. The percentages of neutrophils (NE\%) and immature granulocytes (IG\%) were found $42.1 \%(7,1-76,6)$ and 2,5\% (0.5-9,6), respectively. IG count and IG\% values began to increase median 3 th $(0-8)$ and 2 nd $(0-8)$ days before myeloid engraftment days, respectively (Figure 2). At the same time, absolute monocyte counts (MO) and monocyte percentage $(\mathrm{MO} \%)$ were $0.420 \times 10^{9} / \mathrm{L}$ (290$1680)$ and $26.2 \%(3,6-71,3)$, respectively. The percentage of the monocytes values were above the normal value (10\%) median 5 (0-14) days before the engraftment.

In 14 of 16 patients, median 18th day (10-25) platelet count without transfusion reached over $50 \times 10^{9} / \mathrm{L}$. The percentage of immature platelet fraction (IPF) was $5.1 \%$ $(2,7-9,5)$ on the same platelet engraftment day.

Conclusions: According to the results of this study, new CBC parameters such as IG and IPF may be helpful in engraftment follow up of patients with HSCT. Although the 
number of patients in this study is few, the increase in IG count and IG\% suggests the myeloid engraftment earlier. For new platelet parameters such as IPF, the determination of cut-off values in large group studies will be helpful in following the platelet engraftment of HSCT patients.

Conflict of interest: nothing to disclose

\section{P644}

\section{Extramedullary Relapse of Myeloid Malignancies after Allogeneic Blood Stem Cell Transplantation}

Esther Schuler ${ }^{1}$, Sarah Boughoufala ${ }^{1}$, Christina Rautenberg ${ }^{1}$, Kathrin Nachtkamp ${ }^{1}$, Ariane Dienst ${ }^{1}$, Roland Fenk ${ }^{1}$, Mustafa Kondakci ${ }^{1}$, Ulrich Germing ${ }^{1}$, Martina Rudelius ${ }^{2}$, Thomas Schroeder $^{1}$, Rainer Haas ${ }^{1}$, Guido Kobbe ${ }^{1}$

${ }^{1}$ University Hospital Duesseldorf, Department of Hematology, Oncology and Clinical Immunology, Duesseldorf, Germany; ${ }^{2}$ Heinrich Heine University, Institute of Pathology, Duesseldorf, Germany

Background: In order elucidate pathomechanisms, risk factors and treatment options of extramedullary relapse (XR) after allogeneic blood stem cell transplantation (aSCT) we retrospectively collected patients with myeloid malignancies (MyM) and XR from our transplant database.

Methods: Of 446 adult patients, who received their first aSCT for MyM at our center between 02-2002 and 04-2015, we identified 167 (37\%) with relapse.

Results: Of these $1^{\text {st }}$ relapses $38(23 \%)$ were molecular (MR), 119 (71\%) isolated bone marrow (BR), 5 (3\%) isolated XR and $5(3 \%)$ combined BR and XR. When also considering later relapses a total of 26 patients $(6 \%)$ experienced XR in median 15.7 months (4.2-90.1) after aSCT. Sixteen patients (62\%) had already been treated with DLI before XR and 35\% had evidence of cGvHD before or at diagnosis of XR. Primary diagnoses were 17 AML (65\%), 3 MDS (12\%), 4 MPN (15\%), 2 sAML (8\%) and $31 \%$ had high-risk cytogenectis according to ELN or IPSSR.

Initially affected sites were: 7 CNS ( 3 isolated meningiosis leukaemica), 5 skin, 1 skin and muscle, 1 skin and lymph nodes, 1 lymph nodes and gastric mucosa, 4 muscle, 2 bone, 2 bone and soft tissue, 1 pleural/pericardial effusion, 1 testis and 1 disseminated at multiple sites[W1]. Seven patients had multiple chloromas at follow up[W2] . In 9 patients chloromas were tested for PD-L1 expression resulting in a Cologne score of 0 in $100 \%$.

The most frequent therapy was radiation (14), either alone (5) or in combination with systemic therapy (ST,6), DLI (1) or ST and intrathecal therapy (2). Five patients received ST without radiation. STs were HMA in 3 patients. Of these 2 were escalated to intensive chemotherapy (IC). In total IC was given to 11 patients. Six patients received a second
aSCT[W3] . One patient with testicular relapse had orchiectomy and 5 patients received best supportive care only. Four patients with CNS XR received intrathecal chemotherapy[W4] .

Nine of 26 patients (35\%) died early and 17 were available for response assessment. Complete remission was achieved in 9 (35\%, 5 IC and second aSCT, 1 sorafenib, 1 radiation, 1 orchiectomy). Partial remission was induced in 3 patients (12\%, ORR $46 \%)$. Local response with radiation was seen in another 3 patients, but with progression at other locations. Median remission duration was 8.4 months (range 0.4-54.6). Two patients are alive in CR with median follow up of 22 and 55 months, both after subsequent aSCT. One- and two- year survival were $23 \%$ and $12 \%$. Median survival of all patients was 5.3 months. Patients with isolated XR had better overall survival (11.7 vs 2.7 months, $p=0.054$ ), as had patients with isolated CNS disease (14.2 vs. 5.3 months, $\mathrm{p}=$ n.s.).

Conclusions: $\mathrm{XR}$ is a relevant, prognostically dismal event after aSCT, occurring as a late and often subsequent relapse in heavily treated (including DLI) patients despite cGvHD arguing for an evolutionary immune escape mechanism. The pathological background is still not understood and we found no evidence for PD-L1 expression. As a consequence, conventional chemo/ immunotherapy fails and new treatment strategies are needed.

Conflict of interest: The authors have nothing to disclose.

\section{P645}

Glutathione S-Transferase Polymorphism Impacts the Risk of Graft Loss Post Hematopoietic Stem Cell Transplantation

Khaled Al-Belushi ${ }^{1}$, Intisar Al-Riyami ${ }^{2}$,Murtadha Al-Khabori, Shoaib Al-Zadjali ${ }^{3}$, Mohammed Al-Rawahi ${ }^{3}$, Khalil Al-Farsi ${ }^{3}$, Yaser Wali $^{4}$

${ }^{1}$ Sultan Qaboos University, Pharmacology, Muscat, Oman; ${ }^{2}$ Sultan Qaboos University Hospital, Pharmacy, Muscat, Oman; ${ }^{3}$ Sultan Qaboos University Hospital, Hematology, Muscat, Oman; ${ }^{4}$ Sultan Qaboos University Hospital, Child Health, Muscat, Oman

Background: In Busulfan (Bu) based preparative regimen, $\mathrm{Bu}$ exposure is associated with the risk of graft loss. Glutathione S-Transferase (GST) Polymorphism affects Bu drug exposure and therefore may affect the risk of graft loss. $\mathrm{Bu}$ metabolism occurs in the liver, where it initially gets conjugated to glutathione catalyzed by mainly GST isoenzyme A1-1 (GSTA1). There are other isoenzymes (GSTM1, GSTT1 and GSTP1) involved but to a lower extent. As soon as $\mathrm{Bu}$ is conjugated, it loses its alkylating property. Herein, we plan to study the impact of GST polymorphism on the risk of graft loss in patients 
undergoing Hematopoietic Stem Cell Transplantation (HSCT) with $\mathrm{Bu}$ based preparative regimen.

Methods: This is a single center retrospective cohort study of 135 adult and pediatric patients who received intravenous Bu prior to HSCT at Sultan Qaboos University Hospital from January 2003 to October 2016 for any indication. Genotyping for GST polymorphism was done on pre-transplant patients' samples. We used Capillary Electrophoresis for GSTM1 and GSTT1 (insertion or deletion), DNA sequencing for GSTA1 (C-69G, A-513G, and G1142C) and GSTP1 (A313G). The impact of GST polymorphisms on graft loss was assessed using LogRank test. Standard descriptive and analytical statistics and graphs were created using the statistical software STATA version 13.

Results: Four patients out of 135 had graft loss during the follow up period. The median time to graft loss was not reached. Graft loss free survival was $97 \%$ at day $90,94 \%$ at day 180 , and $89 \%$ at day 360 . The incidence of graft loss in the heterozygous genotype (AG) of GSTA1 A-513G was $40 \%$ compared to $8 \%$ in the wild type genotype (AA) and the difference was statistically significant $(p=0.006)$. The difference in the incidence was not statistically significant in the other GSTA1 loci (p-values were all higher than 0.05). There were no significant difference in the incidence of graft loss in GSTM1 deleted and inserted groups ( $p=$ 0.263). Similarly, GSTP1 insertion and deletion had no significant impact on graft loss ( $p$-value $=0.898)$.

Conclusions: Patients with heterozygous GSTA1 A-513G have a higher risk of graft loss likely related to the lower $\mathrm{Bu}$ clearance. These results need to be confirmed in a larger different population. Patients with heterozygous GSTA1 A$513 \mathrm{G}$ should undergo therapeutic drug monitoring during the preparative regimen to guide the appropriate dose of $\mathrm{Bu}$ to lower the risk of relapse.

Conflict of interest: No conflict of interest.

The study was supported by an internal grant from College of Medicine and Health Sciences, Sultan Qaboos University.
P646

GvL without GvHD ? Role of different NK-cell subpopulations after allogeneic stem cell transplantation

Jonas Wißkirchen ${ }^{1}$, Diana Wolff ${ }^{1}$, Pascal Wölfinger ${ }^{1}$, Evelyn Ullich $^{2}$, Matthias Theobald ${ }^{1}$, Eva M. Wagner-Drouet ${ }^{1}$

${ }^{1}$ University Medical Center Mainz, Haematology, Oncology and Pneumology, Mainz, Germany; ${ }^{2}$ Goethe University Frankfurt am Main, Hospital of Children and Adolescents, Cellular Immunology, Frankfurt, Germany

Background: Allogeneic haematopoietic stem cell transplantion (HSCT) is the only curative treatment option for many haematological malignancies. The long-term effect of the engrafted immune system is crucial for treatment success; on one hand there is the desired effect on leucemia cells $(\mathrm{GvL})$, whereas on the other hand the new immune system can also act against healthy tissue and cause graftversus-host disease (GvHD). In this context NK-cells are of particular interest as they can mediate GVL-enhancing and GvHD-limiting effects.

We examined patient blood samples after HSCT to determine the relevance of different NK-cell subpopulations in the development of GvHD and relapse (GvL).

Methods: We analysed blood samples from 30 patients (aGvHD, cGvHD and relapse) and 5 healthy controls. NKcells were divided into different groups depending on their expression of CD16 and CD56 using flow cytometry (FACS): $\quad$ CD $56^{\text {bright }} / \mathrm{CD} 16^{\text {neg }}, \quad \mathrm{CD} 56^{\text {bright }} / \mathrm{CD} 16^{\text {pos }}$, CD56 ${ }^{\text {dim }} / \mathrm{CD} 16^{\text {pos. }}$ CD56 $6^{\text {dim }} /$ CD $16^{\text {neg }}$. Additionally we examined expression of Nkp44, Nkp46, NKG2A, NKG2C, NKG2D, CD69, CD62L, Tigit and Nkp30.

As immunosuppressives are routinely administered in the setting of HSCT we performed functional tests to evaluate their effect on the expression of those markers: PBMCs were incubated for 24 hours with either prednisolone, Tacrolimus or both drugs at different concentrations. Subsequent flow cytometry was performed.

\begin{tabular}{|c|c|c|c|}
\hline Marker & aGvHD & cGvHD & Relapse \\
\hline Nkp44 & + & + & + \\
\hline Nkp46 & $+^{-}$ & + & + \\
\hline Nkp30 & + & + & + \\
\hline NKG2A & $+^{-}$ & $+^{+}$ & $+^{*}$ \\
\hline NKG2D & $+^{-}$ & + & + \\
\hline CD69 & + & + & + \\
\hline CD52L & + & + & + \\
\hline Tigit & + & & + \\
\hline
\end{tabular}

Key: +increase; -decrease; "significant

[P646 Figure] [Results] 
Results: In all patients after HSCT the whole NK-cell population exhibits an increased expression of the activation marker Nkp44. In the CD56 $6^{\text {bright } / C D 16-~ s u b p o p u l a t i o n ~ o f ~}$ patients with cGVHD this increase is significant (p:0.0052).

Patients with cGvHD and relapse showed the highest expression of the activation marker CD69 (p:0.1557 and 0.2247 compared to buffycoat).

The expression of the activating receptor NKG2D was increased in NK-cells of all patients compared to healthy controls ( $\mathrm{p}$ values: aGvHD:0.0001;cGvHD:0.0074; relapse:0.0365) whereas expression of the inhibitory receptor NKG2A is only significantly increased in patients with aGvHD (p:0.0131) (cGvHD p:0.2735, relapse $\mathrm{p}: 0.3202)$.

In all patients expression of Nkp46 is increased compared to healthy controls but only reaches statistical significance for patients with aGvHD (p: 0.0324).

CD62L is increased in all patient-derived NK-cells compared to healthy controls (p:aGvHD:0.1782, cGvHD:0.4991, relapse: 0.1712).

For the whole NK-cell population there was a reduced expression of Nkp30 in patients with cGvHD and relapse (p:0.6820 and 0.9226)

In contrast patients with aGvHD showed an increased expression of Nkp30 in the CD56 $6^{\text {bright }} / \mathrm{CD} 16$ - subpopulation (p:0.0110).

In all patients the expression of the inhibitory receptor Tigit is increased but this is not significant.

Immunosuppressive therapy (in vitro) did not influence the expression of activating or inhibitory receptors on NKcells with exception of high dose steroid resulting in a not significant increase of CD69.

Conclusions: NK-cells from patients after HSCT show distinctive changes in the expression of certain markers. Especially the increased expression of inhibitory markers (NKG2A and Tigit) may account for an impaired function of these cells. Albeit the increase of activating receptors the decreased expression of Nkp30 in patients with cGvHD and relapse may play a decisive role in the pathophysiology of these complications. A better understanding of the individual composition of NK-cells in patients could lead to future individualized NK-cell-based donor lymphocyte infusions.

Conflict of interest: None of the authors has anything to disclose.

\section{P647}

\section{IgG Fc Glycosylation After Hematopoietic Stem Cell Transplantation is Dissimilar to Donor Profiles}

Noortje de Haan ${ }^{1}$, Maarten van Tol ${ }^{1}$, Gertjan Driessen ${ }^{2,3}$, Manfred Wuhrer $^{1}$, Arjan Lankester ${ }^{1}$
${ }^{1}$ Leiden University Medical Center, Leiden, Netherlands; ${ }^{2} J u l i a n a$ Children's Hospital, Haga Teaching Hospital, Den Haag,

Netherlands; ${ }^{3}$ Erasmus Medical Center, Sophia Children's Hospital, Rotterdam, Netherlands

Background: The $\mathrm{N}$-glycan attached to the conserved glycosylation site of the fragment crystallizable $(\mathrm{Fc})$ region of immunoglobulin $\mathrm{G}(\mathrm{IgG})$ has a large influence on the structure and function of the antibody. The presence or absence of specific monosaccharides have proven to be crucial both in modulating the affinity of $\operatorname{IgG}$ binding to $\mathrm{Fc} \gamma$-receptors (FcyRs) and in the activation of the complement system. Following allogeneic hematopoietic stem cell transplantation (HSCT), and in the presence of (almost) complete donor chimerism, $\operatorname{IgG}$ is expected to be produced by, and glycosylated in, B cells of donor origin. We investigated to what extent $\operatorname{IgG}$ glycosylation in patients after transplantation is determined by factors of the donor (genetics) or the recipient (environment).

Methods: In the current study we characterized in a selected group of patients, at a steady state post-HSCT, their IgG Fc glycosylation profiles. Using an IgG subclassspecific liquid chromatography-mass spectrometry method, we analyzed the plasma/serum IgG Fc glycosylation profiles of 34 pediatric patients pre-HSCT and at six and twelve months post-HSCT and compared these to the profiles of their donors and age-matched healthy controls. Data analysis was performed separately for patients treated for malignant and non-malignant hematological diseases.

Results: For patients treated for hematological malignancies, the most prominent IgG Fc glycosylation differences between donors and recipients pre-HSCT were observed in the galactosylation, which was lower in the patients as compared to the donors independently of the $\mathrm{IgG}$ subclass. Furthermore, a significant difference was present for the galactosylation when comparing recipients preHSCT with healthy age-matched controls. Therefore, a low galactosylation state most likely reflects glycosylation changes caused by the disease and/or the treatment thereof. Twelve months after HSCT, the patients treated for hematological malignancies still showed a lower $\operatorname{IgG} \mathrm{Fc}$ galactosylation as compared to their donors, which indicates that the recipients produce $\operatorname{IgG}$ with a Fc glycosylation pattern that partly mimics the profile of the recipient preHSCT and not the donor profile. Of note, although for the patient-subgroup treated for non-malignant hematological diseases the pre-HSCT galactosylation level did not differ from the donor, twelve months post-HSCT their galactosylation was also lower than that of the donors. The low level of galactosylation at this stage after HSCT, when all study patients were in a stable and uncomplicated clinical condition, was comparable between the two subgroups. Furthermore, pre-HSCT, the patient group treated for 
hematological malignancies showed as distinctive feature a decrease in sialylation and in hybrid-type glycans as compared to healthy controls, which both normalized after transplantation.

Conclusions: While the B cells in children after HSCT are mainly of donor origin and patients were investigated after reaching independency of $\mathrm{IgG}$ supplementation, we found that $\mathrm{IgG}$ Fc glycosylation in our pediatric cohort did not reflect the glycosylation pattern of the donors. These data suggest that external local influences on the $\operatorname{IgG~Fc}$ glycosylation are operative before HSCT as well long term after HSCT, independently of the original disease.

Conflict of interest: The authors declare that the research was conducted in the absence of any commercial or financial relationships that could be construed as a potential conflict of interest.

\section{P648}

Immune Reconstitution and Lineage-specific Chimerism in a Busulfan/Fludarabin/Serotherapy-based Reduced Intensity Conditioning Regimen for Pediatric Patients with Non-Malignant Diseases

Tiziana Burkart, Veronika Haunerdinger, Ulrike Zeilhofer, Tayfun Güngör, Mathias Hauri-Hohl

University Children's Hospital, Department of Stem Cell Transplantation, Zürich, Switzerland

Background: Reduced Intensity Conditioning (RIC) has significantly contributed to the improved outcome of HSCT for non-malignant diseases in pediatric patients. Yet, early graft rejection or delayed development of waning donor chimerism with subsequent graft loss remain significant challenges. Timely diagnosis and immunotherapeutic interventions may prevent graft loss but may entail substantial side effects such as GvHD.

Methods: We retrospectively assessed immune reconstitution and lineage-specific donor chimerism in 45 pediatric patients (median age 48 months, range 4 216 months) consecutively transplanted in our center for non-malignant diseases (immunodeficiency, HLH, hemoglobinopathy) with a uniform conditioning regimen. Reduced intensity conditioning consisted of Busulfan (PK-adjusted, targeted to a cumulative exposure of 45$75 \mathrm{mg} / \mathrm{lxh}$ ), high-dose Fludarabine and serotherapy (Alemtuzumab in 24 patients or ATG in 21 patients). Grafts were derived from matched unrelated (MUD, 20 patients), matched related (MRD, 19 patients), mismatched unrelated (MMUD, 4 patients) and haploidentical (haplo-donor, 2 patients) donors. Assessment of T-cell reconstitution included naïve and memory T-cell subtypes (including regulatory $\mathrm{T}$ cells) as well as $\mathrm{CD} 31+$ recent thymic emigrants (RTE) in the naïve CD4 T-cell compartment. Lineage-specific chimerism was performed by means of immunomagnetic bead or FACS separation followed by subsequent analysis of VNTR or FISH for XY (gendermismatched transplants) if required.

Results: Acute GvHD $\geq$ Grade II occurred in 7 patients (15\%), 2 patients (4\%) experienced limited chronic GvHD. Viral reactivation was detected in 22 patients (CMV 11 patients, EBV 9 patients, HHV6 2 patients; without manifestation of disease). Myeloid chimerism at primary engraftment was donor-specific in all cases. Three patients (2 MUD, 1 haplo-donor) developed mixed myeloid chimerism beyond 3 months post-transplant. The two recipients of MUD grafts achieved a stable long-term mixed chimerism without immunotherapeutic intervention. The third patient (haplo-donor) became aplastic 8 months post-transplant in the course of an EBV reactivation and partially reconstituted with autologous myeloid cells despite increasing donor chimerism in the $\mathrm{T}$ - and NK-cell compartment. Following Rituximab-treatment and stem cell boost the patient reverted to full DC.

Early T-cell chimerism ( $\leq$ day 100 post-transplant) was assessed in 20 patients, of which $61 \%$ demonstrated predominantly recipient $\mathrm{T}$-cell chimerism, with a high content in regulatory $\mathrm{T}$ cells. In none of the patients an immunotherapeutic intervention to promote DC was performed.

Acute GvHD rapidly and permanently converted the Tcell compartment towards donor-type, whereas in the context of viral reactivation the origin of the antiviral $\mathrm{T}$ cell response transiently influenced the chimerism of the $\mathrm{T}$ cell compartment. Thymic output - as measured by an increase in naïve T-cells and RTE lead to a slow but steady conversion of the T-cell pool to donor-type.

Conclusions: The RIC regimen applied in this study allows the persistence of recipient $\mathrm{T}$ cells in a significant proportion of patients. Nevertheless, only few patients experienced graft loss, indicating promotion of tolerance between the donor and host immune system in the majority of patients.

\section{Clinical Trial Registry: -}

Conflict of interest: None

\section{P649}

Immune reconstitution of TV $\delta 1, T V \delta 2$ and TV $\gamma 9$ cells after autologous hematopoietic stem cell transplantation in multiple myeloma patients

Adriana Roque ${ }^{1,2}$, Ana Luísa Pinto ${ }^{1}$, Isabel Silva ${ }^{3}$, Lenka Ruzickova $^{1}$, Diana Mota ${ }^{1,2}$, Carolina Afonso ${ }^{1}$, Emília Cortesão ${ }^{1,2}$, Luís Francisco Araújo ${ }^{1}$, Ana Isabel Espadana , Ana Bela SarmentoRibeiro $^{1,2}$, Catarina Geraldes ${ }^{1,2}$, Artur Paiva ${ }^{3}$, Letícia Ribeiro ${ }^{1}$ 
${ }^{1}$ Centro Hospitalar e Universitário de Coimbra, Clinical Haematology Department, Coimbra, Portugal; ${ }^{2}$ Faculty of Medicine, University of Coimbra, Coimbra, Portugal; ${ }^{3}$ Centro Hospitalar e Universitário de Coimbra, Flow Cytometry Unit, Clinical Pathology Department, Coimbra, Portugal

Background: $T \gamma \delta$ cells are effector/cytotoxic cells involved in immune surveillance, and there is pre-clinical evidence supporting an anticancer activity for TV $\delta 1$.

The successful of autologous hematopoietic stem cell transplantation (aHSCT) depends on previous eradication of neoplastic clones and the reconstitution of hematopoietic and immune system.

Currently, in vitro modulation of immune cells has growing importance and, although the lymphocytes recover after transplantation has been extensively studied, there are limited data about $\mathrm{T} \gamma \delta$ cells.

Our aim was to evaluate the number of TV $\delta 1$ cells and its main functional subpopulations (CD27+ and CD27-), as well as other $\mathrm{T} \gamma \delta$ populations (V反2 and V89) in multiple myeloma (MM) patients submitted to aHSCT, in order to understand how TV $\delta$ reconstitution occurs after aHSCT and what is its impact in post-aHSCT recovery.

Methods: We analyzed $31 \mathrm{MM}$ patients who underwent aHSCT, previously to conditioning with melphalan $200 \mathrm{mg} /$ $\mathrm{m} 2$ (Dc) and at 30 (D30), 60 (D60), and 100 (D100) days after aHSCT.

The quantification and characterization of $T \gamma \delta$ cells in peripheral blood samples were performed by flow cytometry based in the expression of $\mathrm{CD} 3 / \mathrm{CD} 45 / \mathrm{V} \delta 1 / \mathrm{V} \delta 2 / \mathrm{V} \gamma 9$ / CD27.

Results: The median age of the cohort was 64 years (4170), with $61.3 \%$ of males.

Our results show that TV $\delta 1+, \mathrm{TV} \delta 1+\mathrm{V} \gamma 9-, \mathrm{TV} \delta 1+\mathrm{V} \gamma 9$ $\mathrm{CD} 27+, \mathrm{TV} \gamma 9+$ and $\mathrm{TV} \gamma 9+\mathrm{CD} 27+$ subpopulations decrease immediately after aHSCT, with a subsequent significant increase at D60 $(\mathrm{p}=0.041, \mathrm{p}=0.042, \mathrm{p}=0.033$, $\mathrm{p}=0.019$ and $\mathrm{p}=0.018$, respectively), and return to preaHSCT levels at D100. Otherwise, TV $82+\mathrm{V} \gamma 9+$ and TV $\delta 2$ $+\mathrm{V} \gamma 9+\mathrm{CD} 27+$ subpopulations decrease significantly at D100 compared with Dc $(\mathrm{p}=0.041$ and $\mathrm{p}=0.029)$.

A higher number of CD34+ cells infusion correlates with higher proportion of $\mathrm{TV} \delta 1+\mathrm{V} \gamma 9+$ at D60 (coef. $=7.3$; $\mathrm{p}=0.021$ ) and at D100 (coef. $=1.6 ; \mathrm{p}=0.016$ ), while a higher volume of dimethylsulfoxide infused correlates with a lower proportion of $\mathrm{TV} \gamma 9+\mathrm{CD} 27+($ coef. $=-0.001 ; \mathrm{p}=0.046)$.

Increasing numbers of TV $\delta 1+\mathrm{V} \gamma 9+$ (coef.0.26; $\mathrm{p}=0.001$ ), TV $81+\mathrm{V} \gamma 9+\mathrm{CD} 27+$ (coef.0.57; $<0.001)$, TV $\delta 1+\mathrm{V} \gamma 9$ $\mathrm{CD} 27+($ coef. $0.26 ; \mathrm{p}=0.002), \quad \mathrm{TV} \gamma 9+($ coef. $0.25 ; \mathrm{p}=0.001)$ and $\mathrm{TV} \gamma 9+\mathrm{CD} 27+($ coef.0.48; $=0.022)$ at $\mathrm{D} 60$ as well as TV $\delta 1+$ (coef. $0.15 ; \mathrm{p}=0.023$ ) and TV $\delta 1+\mathrm{V} \gamma 9$-(coef.0.16; $\mathrm{p}=0.019)$ at D100 are associated with an extended time to engraftment. Furthermore, an increased proportion of TV $\delta 1$ $+\mathrm{V} \gamma 9+\mathrm{CD} 27+($ coef.0.13; $=0.010), \quad \mathrm{TV} \gamma 9+($ coef.0.06; $\mathrm{p}=0.036)$ and $\mathrm{TV} \gamma 9+\mathrm{CD} 27+($ coef. $0.03 ; \mathrm{p}=0.039)$ at $\mathrm{D} 60$ is associated with higher need for packed RBC units, while the increase of $\mathrm{TV} \delta 1+\mathrm{V} \gamma 9+($ coef. $0.10 ; \mathrm{p}=0.001), \mathrm{TV} \delta 1$ $+\mathrm{V} \gamma 9+\mathrm{CD} 27+($ coef. $0.22 ; \mathrm{p}=0.018)$ and $\mathrm{TV} \gamma 9+$ (coef. 0.08 ; $\mathrm{p}=0.018$ ) cells at D60 are associated with greater necessity for platelet transfusion.

The length of hospital stay is lower in the patients that have higher number of TV $\delta 1+\mathrm{V} \gamma 9+$ (coef.- $0.21 ; \mathrm{p}=0.013$ ) and $\mathrm{TV} \delta 1+\mathrm{V} \gamma 9+\mathrm{CD} 27+($ coef. $-0.84 ; \mathrm{p}=0.041)$ at $\mathrm{D} 30$ as well as TV $\delta 1+$ (coef. $-0.24 ; \mathrm{p}=0.023$ ) and $\mathrm{TV} \delta 1+\mathrm{V} \gamma 9+$ (coef.-9.85; $\mathrm{p}=0.039$ ) at D100.

There was no association between $\mathrm{T} \gamma \delta$ subpopulations and oral and gastrointestinal mucositis or the incidence of febrile neutropenia.

There was no correlation between pre-aHSCT disease response and $\mathrm{T} \gamma \delta$ subpopulations (Dc) as well as between D100 response and $\mathrm{T} \gamma \delta$ subpopulations (D100).

Conclusions: Our results, although preliminary, show that $\mathrm{T} \gamma \delta$ cells subpopulations have a role in post-aHSCT recovery, being the increase in $\mathrm{TV} \delta 1+\mathrm{V} \gamma 9+, \mathrm{TV} \delta 1+\mathrm{V} \gamma 9$ and $\mathrm{TV} \gamma 9+$ cells associated with the delay in hematological recovery. On the other hand, a lower dimethylsulfoxide volume and a higher number of CD34+ cells infused are associated with a higher number of $\mathrm{TV} \gamma 9+$ and $\mathrm{TV} \delta 1+\mathrm{V} \gamma 9$ + , respectively.

There are necessary more studies to evaluate the relationship between these $\mathrm{T}$ cells subpopulations and the response to therapeutics/aHSCT, as well as the utility of their modulation.

Conflict of interest: None declared.

\section{P650}

Immune status in long term survivors after allogeneic stem cell transplant is influenced by GvHD occurrence, donor type and timing: a cross-sectional evaluation

Maria Teresa Lupo-Stanghellini ${ }^{1}$, Francesca Lorentino ${ }^{1}$, Fabio Serpenti $^{1,2}$, Miriana Belloni ${ }^{3}$, Raffaella Milani ${ }^{4}$, Raffaella Greco $^{1}$, Magda Marcatti ${ }^{1}$, Andrea Assanelli, Matteo Giovanni Carrabba ${ }^{1}$, Fabio Giglio $^{1}$, Elisabetta Xue ${ }^{1}$, Gabriele Casirati ${ }^{1}$, Francesca Farina $^{1}$, Sarah Marktel ${ }^{1}$, Francesca Lunghi ${ }^{1}$, Elena Guggiari ${ }^{1}$, Luca Vago ${ }^{1,5}$, Massimo Bernardi ${ }^{1}$, Jacopo Peccatori ${ }^{1}$, Consuelo Corti $^{1}$, Chiara Bonini ${ }^{2,6}$, Fabio Ciceri $^{1,2}$

${ }^{1}$ San Raffaele Scientific Institute, Hematology and Bone Marrow Transplantation Unit, Milano, Italy; ${ }^{2}$ Vita-Salute San Raffaele University, San Raffaele Scientific Institute, Milano, Italy; ${ }^{3}$ Istituto d'Istruzione Superiore 'Racchetti-Da Vinci', Liceo Scientifico, Crema, Italy; ${ }^{4}$ San Raffaele Scientific Institute, Immunohematology and Transfusion Medicine Unit, Milano, Italy; ${ }^{5}$ San Raffaele Scientific Institute, Unit of Immunogenetics, Leukemia Genomics and Immunobiology, Division of Regenerative Medicine, Stem Cells and Gene Therapy, Milano, Italy; ${ }^{6}$ San Raffaele Scientific Institute, Experimental Hematology Unit, Division of Immunology, 
Transplantation and Infectious Diseases, Program in Immunology and Bio-Immunotherapy of Cancer (PIBIC), Milano, Italy

Background: Allogeneic stem cell transplantation (alloHCT) survivors are at a defined relevant risk of developing long-term complications, consequently, life expectancy remains lower compared to the age and sex matched population. Recent registry studies from the CIBMTR have outlined how late fatal infections (LFI) in allo-HCT remain high, contributing to third of all deaths occurring $>2$ years after HCT. History of GvHD, age and HLA-match are associated with increased mortality and morbidity from LFI.

So far, few details regarding immune-reconstitutions (IR) in long-term follow-up (LTFU) are available. The aim of our study is to evaluate in a cross-sectional analysis the status of IR in survivors in respect of donor type, distance from HCT and GvHD occurrence.

Methods: A standardized LTFU of allo-HCT survivors is applied at our center. We here analyze data consecutively collected in an Institutional database, starting from 2006, including 376 adult pts who underwent an allo-HCT between 1992 and 2015 at our Institution. A written consent was given by pts allowing the use of medical records for research in accordance with the Declaration of Helsinki.

We considered for the analysis pts with an overall survival $>/=2 \mathrm{y}$. Status of immune recovery is analyzed by $\mathrm{CD} 3+, \mathrm{CD} 3+\mathrm{CD} 4+, \mathrm{CD} 3+\mathrm{CD} 8+, \mathrm{B}$ cell, ratio 4/8, IgG, IgA, IgM collected at 2 years and beyond.

Results: With a median follow-up of 6y (r 2-24y) 263 pts were evaluable; donor was a match unrelated donor (MUD) in 89 cases, HLA identical sibling (MRD) in 76, haploidentical relative (HAPLO) in 96 and cord blood in 2. A follow-up of 2-5y, 5-10y, >10y was available for 106, 98,59 pts respectively.

In respect of follow-up, comparing IR in pts with a follow-up of $2-5 \mathrm{y}$ vs $5-10 \mathrm{y}$ vs $>10 \mathrm{y}$ there was a significant difference for $\operatorname{Ig} A(p<.0001), \operatorname{IgM}(\mathrm{p}<.0001), \mathrm{CD} 3+\mathrm{CD} 4$ + (p.001), CD3+CD8+ (p.02), ratio 4/8 $(\mathrm{p}<.0001)$ with better recovery in the longest follow-up period.

In respect of type of donor, overall pts receiving a HCT from a MRD show better IgA (p.017), CD3+CD4+ (p.002) and ratio $4 / 8$ (p.005) than MUD/HAPLO. The same evaluation at early time point (2-5y) show better CD3+ (p.027), CD3+CD4+ (p.039), ratio 4/8 (p.027) for MRD vs HAPLO/MUD, while there was no difference at late time point $(>5 \mathrm{y})$.

IgA recovery was the only significant variable when comparing pts with both acute and chronic GvHD vs the entire cohort $(\mathrm{p}<.0001)$, while comparing moderate/severe chronic GvHD with the entire cohort both $\operatorname{IgA}(\mathrm{p} .023)$ and CD3+ (p.021) were significant.
Conclusions: Immune status after MRD allo-HCT is better in the early phase $(<5 y)$ vs HAPLO/MUD, while it is comparable in the late phase $(>5 \mathrm{y})$.

Allo-HCT survivors are still affected by IR impairment at very LTFU, providing a biological justification of occurrence of LFI especially in presence of GVHD-history.

This study provide justification for monitoring IR to individualize treatment and follow-up.

Conflict of interest: Nothing to disclose

\section{P651}

Abstract previously published

\section{P652}

\section{Large Granular Lymphocytes Expansion Following Allogeneic Hematopoietic Cell Transplantation: A Retrospective Study}

Lou Soret ${ }^{1}$, Anne Roggy ${ }^{1}$, Estelle Seilles ${ }^{1}$, Francine GarnacheOttou $^{1}$, Francoise Schillinger ${ }^{1}$, Fabrice Larosa ${ }^{2}$, Etienne Daguindau $^{3}$, Yohan Desbrosses ${ }^{2}$,Eric Deconinck ${ }^{3}$, Ana Berceanu ${ }^{4}$

${ }^{1}$ EFS Bourgogne Franche Comte, Besancon, France; ${ }^{2}$ CHU Jean Minjoz, Hematology, Besancon, France; ${ }^{3}$ Université Franche Comte, Besancon, France; ${ }^{4}$ CHU JEAN MINJOZ, HEMATOLOGY, Besancon, France

Background: Large granular lymphocyte (LGL) proliferation is a chronic lymphoproliferative disorders usually associated along with cytopenia and immune abnormalities. However, the elevated numbers of LGL following allogeneic hematopoietic cell transplantation (allo-HCT) is of unknown significance.

Methods: We performed a retrospective analysis on 450 consecutive patients who underwent an allo-HCT at University hospital of Besançon, France, between 2008 and 2016. In our study, LGL lymphocytosis was defined as persistence of LGL in the peripheral blood more than one month. LGL were identified by immunophenotypic criteria (lymphocytes CD8+CD57+ $\geq 30 \%$ ) and/or morphological criteria in blood smear. We performed deeper analyzes of clonality by PCR $(n=6)$ and broader immunophenotyping characterization by flow cytometry $(n=10)$ for a subset of samples from patients with circulating LGL.

Results: Among these 450 patients, 19 (4\%) met the defined criteria for LGL lymphocytosis post allo-SCT. The median onset of LGL lymphocytosis was 15 months [2 at 82 months] and the average duration was 12,15 months (1 at 48 months). The repartition of hematopoietic diseases in patient with LGL was: 58\% of AML/ALL, $16 \%$ of MDS/ MPN, $21 \%$ of Lymphoma/Multiple Myeloma and 5\% of other hematopoietic malignancies The stem cell source was 
$74 \%$ of peripheral blood hematopoietic stem cell, $10 \%$ umbilical cord blood and $16 \%$ bone marrow. Graft compatilibity was phenoidentical $(\mathrm{n}=11)$, genoidentical $(n=7)$ or haploidentical $(n=1)$. Sex ratio was identical in the two groups with or without LGL $(p=0.92)$. There was not significant difference between patients with and without LGL lymphocytosis in conditioning regimen: attenuated conditioning $(\mathrm{n}=5$ in LGL group and $\mathrm{n}=81$ in other group $\mathrm{p}=0.58)$ or standard conditioning $(\mathrm{n}=14$ in LGL group and $\mathrm{n}=350$ in other group $\mathrm{p}=0.11$ ) respectively. The development of acute Graft-versus-Host $(\mathrm{GvH})(\mathrm{p}=$ $0.7)$ and chronic $\mathrm{GvH}(\mathrm{p}=0.13)$ between the two groups was not different. On the other hand, we noticed an increase of CMV infection in patients displaying LGL lymphocytosis compared to control group without LGL (58\% vs $18 \%$, $\mathrm{p}<0.005$ ). All the subset of 6 samples from LGL lymphocytosis analyzed for $\mathrm{T}$-cell receptor gene rearrangement shown a clonal rearrangement. Moreover, 10 patients benefit from an expanded immunophenotyping, loss of CD7 expression was found for 2 patients and we did not detect alteration in T-cell surface markers for the 8 others patients.

Conclusions: : Few studies have reported variable incidence of LGL lymphocytosis after allo-HCT.In our retrospective monocentric cohort, this incidence rises $4 \%$. We identified a statistic correlation between CMV infection and LGL expansion. Although the T-LGL proliferation were clonal and persistent, we did not detect pejorative impact on clinical outcome for the patients with LGL. This suggests that the clonal LGL expansion is a reactive, potentially beneficial, immune response in a context of allogenic graft.

Conflict of interest: None of the authors has anything to disclose.

\section{P653}

Leukemic stem cell (LSC) quantification by Multiparameter Flow Cytometry (MFC): a tool to evaluate pediatric AML patients with greater benefit from allogeneic HSCT?

Adriana Plesa ${ }^{1}$, Christophe Roumier ${ }^{2}$, Nathalie Bleyzac ${ }^{3}$, Cecile Renard $^{3}$, Aline Renneville ${ }^{4}$, Florent Dumezy ${ }^{4}$, Delphine Manzoni, Meyling Cheok ${ }^{6}$, Marie Pierre Pages ${ }^{5}$, Pierre Sujobert ${ }^{5}$, Xavier Thomas $^{7}$, Claude Preudhomme ${ }^{4}$, Yves Bertrand ${ }^{3}$

${ }^{1}$ Lyon Sud University Hospital, Laboratory of Hematology, Pierre Benite, France, ${ }^{2}$ Lille University Hospital, Hematology Laboratory, Lille, France; ${ }^{3}$ Lyon University Hospital, Department of Pediatric Hematology, IHOP, Lyon, France; ${ }^{4}$ Lille University Hospital, Laboratory of Hematology, Lille, France; ${ }^{5}$ Lyon University Hospital, Laboratory of Hematology, Lyon, France; ${ }^{6} U M R-S .1172$, Institute for
Cancer Research in Lille, Lille, France; ${ }^{7}$ Lyon University Hospital, Department of Hematology, Lyon Sud Hospital, Lyon, France

Background: Clonal heterogeneity of the AML blast cell population has been well documented using next generation sequencing (NGS) strategies. This heterogeneity extends to the presence of LSC fraction among the leukemic population: a dynamic compartment evolving to overcome cell selection pressure during disease progression and its treatment including allogeneic Hematopoietic Stem Cell Transplantation (HSCT). Here we propose a strategy of quantification of LSC at the time of diagnosis and during follow-up to identify patients with adverse outcome.

Methods: We designed MFC panel based on 2-tube antibody combinations to identify the pattern of LSC in the CD34+CD38- cell compartment. CD34/CD38/CD45/ CD117 « backbone » was used in both tubes, completed by lineage defined markers CD7/CD56/CD19/CD13/CD33/ and/or LSC identified markers CD123 +/- CD90/CD45RA.

Results: 54 pediatric AML patients were treated in Lyon clinical center (IHOP) between 2008 and 2016 according to the ELAM02 clinical trial. Among them 40 children (aged 0,3-19 years) undergoing HSCT based on the following criteria: high-risk AML in CR1 (29/40) and 11/40 in CR2. The pre-transplant conditioning regimen was based on busulfan combined with cyclophosphamide or fludarabine for all patients. GvHD prophylaxis consisted in CsA alone after in vivo $\mathrm{T}$ cell depletion (using anti-thymocyte globulin) for bone marrow unrelated donors and prednisolone for cord blood cell stem cell sources. Half of the HSCT were from HLA-MSD (matched sibling donor) and the others from MURD (matched unrelated donor) or MMURD (miss-matched unrelated donor). Statistical analysis was performed using SPSS for windows. The Kaplan-Meyer methods was used for survival or probability estimates. The log-Rank test was used for comparison between groups.

Our preliminary data tend to show: 1) the clinical impact in terms of survival (DFS and OS) of LSC CD34+CD38CD123+ levels at diagnosis; 2) the relevance to establish a scoring system including the level of LSC at diagnosis and in MRD after chemotherapy to distinguish patients with good outcome. In our series (see Fig 1), even the high-risk patients had good outcome if the LSC was $<0,9 \%$ at diagnosis and/or MRD LSC undetectable during follow up. At the other hands, some patients belonging to favorable group having LSC $>0,9 \%$ and/or MRD LSC positivity had poor outcome.

Conclusions: In summary, we confirmed that the presence of the LSC CD34+CD38-CD123+ subpopulation representing more than $0,9 \%$ of total "bulk leukemic cells" at diagnosis could help to identify patients with poor outcome. Despite heterogeneity and complexity of the AML 

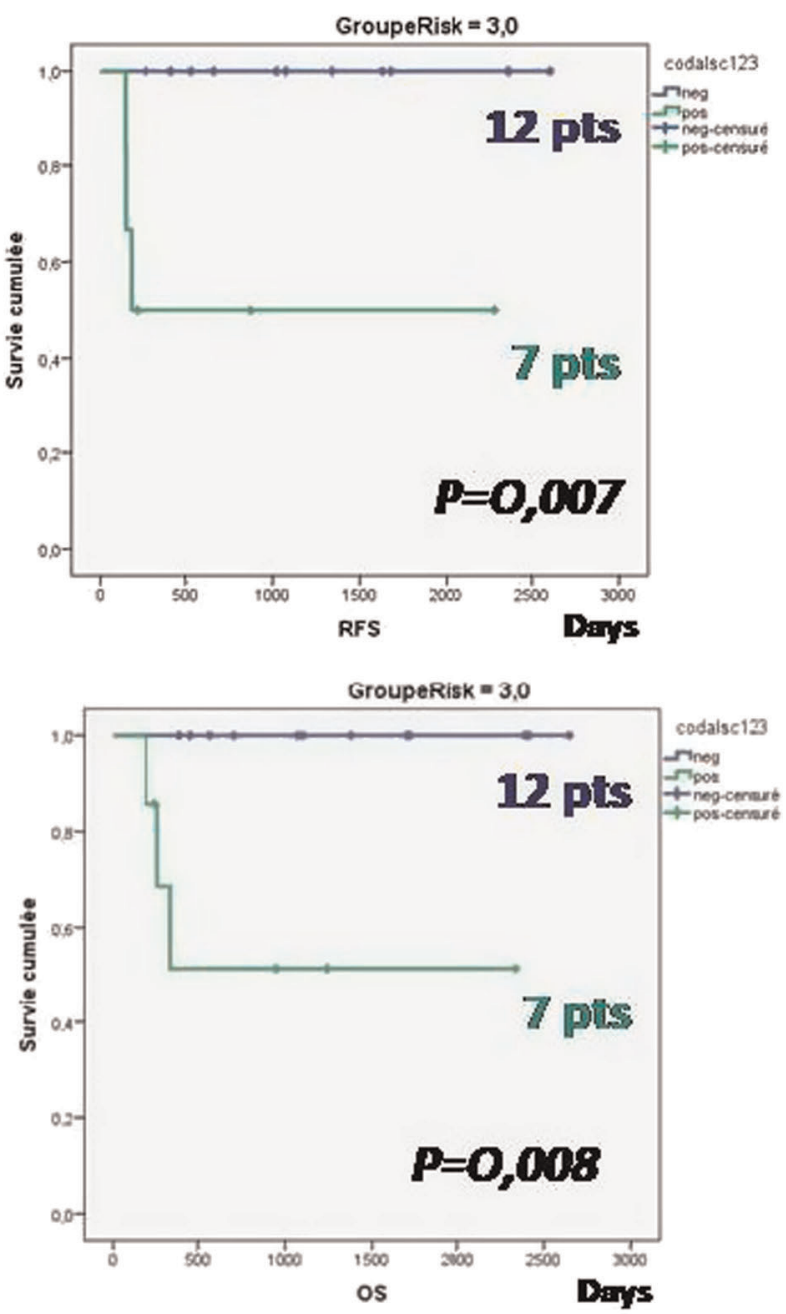

Legend: DFS and OS according to LSC level ( $>$ vs $<0,9 \%$ ) at diagnosis on Pediatric AML Patients belongig to adverse prognosis group

[P653 Figure] [Figure 1]

LSC compartment, we should still use LSC quantification as a biomarker of response to HSCT therapy. In our study, greater benefit of GVL effect seems to be observed in the patients with low-level of LSC. For the patients with highlevel of LSC other therapeutic modalities should be chosen to eradicate LSC using targeting immunotherapy before allograft. Monitoring of the LSC fraction should be useful in most clinical trials to overcome chemoresistance of LSC.

These results should be confirmed in a prospectively larger cohort of patients and could be considered as useful complementary prognostic parameter for risk-stratification AML patients in future clinical trials.

Clinical Trial Registry: ELAM02

Conflict of interest: No disclosure.
P654

Level of MN1 gene expression as a prognostic marker in patients with acute myeloid leukemia in the context of hematopoietic stem cells allogeneic transplantation

Ildar Barkhatov, Ksenya Afanaseva, Daniil Ershov, Ivan Moiseev, Ludmila Zubarovskaya

Academician I.P.Pavlov First St.Petersburg State Medical University, R.M. Gorbacheva Memorial Institute of Children Oncology,

Hematology and Transplantation, St.Petersburg, Russian Federation

Background: The meningioma 1 (MN1) gene is located at chromosome band 22q12 and it's overexpression is associated with a poor prognosis in patients with acute myeloid leukemia (Baldus et al., 2007, Heuser et al., 2006). Therefore we checked the clinical significance of aberrant MN1 expression on patients after HSC. In addition, we investigated the possibility of using the MN1 gene as a marker of minimal residual disease.

Methods: The material was obtained from 68 AML patients both before and after the alloHSCT. After the RNA isolation and reverse transcription reaction, gene expression was analyzed by using the MN1 Oncoprime system (Inogene). The expression results were normalized to the ABL1 gene. As a threshold level, two values were used 500 and 1000 copies of the MN1 gene per 10,000 copies of the ABL1 gene.

Results: To assess the possibility of using the MN1 gene expression in order to estimate the minimal residual disease, we compared the expression data with the number of blast cells and could not detect the presence of correlation dependence. A similar picture was observed when compared the expression data of MN1 and chimeric transcripts as well as with a level of donor chimerism.

In addition, in patients characterized by resistance to therapy prior to transplantation, we detected a significant increase of the MN1 expression level. Higher values were observed in patients with adverse cytogenetic risk $(\mathrm{p}<$ $0.05)$. Moreover, we obtained data that the detection of MN1 overexpression after HSC did not have a significant prognostic value, while in patients with overexpression of this gene more than 1000 copies of MN1 to HSC, a decrease in overall survival was noted. At the same time, as the achievement of an expression level of 500 copies, 1000 copies of the MN1 gene were associated with a greater probability of posttransplant relapses $(\mathrm{p}=0.02$ and $\mathrm{p}=$ 0.07 , respectively). When assessing the effectiveness of the conditioning regime type, we did not reveal they significant differences in prognostic significance in patients with registered hyperexpression before HSCT $(\mathrm{p}=0.9)$.

Conclusions: Our results indicate that MN1 is not a marker of therapy efficiency and can not be recommended 
for estimating the minimum residual disease. At the same time, the obtained results indicate the most aggressive characteristics of the MN1 positive tumor clone, which may cause the development of posttransplantation relapses. The lack of prognostic significance of high MN1 expression in the posttransplant period allow to assume the presence of response from ongoing posttransplant immunoadoptive therapy (infusion of donor lymphocytes).

Conflict of interest: The authors declare the absence of conflicts of interest.

\section{P655}

Pre-emptive Donor Lymphocyte Infusion in T cell-depleted Reduced Intensity Allogeneic Stem Cell Transplant

\author{
Nuria Martinez, Cibrian', Wendy Ogden ${ }^{2}$, Muhammad Saif ${ }^{1}$, Eleni \\ Tholouli', Fiona Dignan ${ }^{1}$ \\ ${ }^{1}$ Manchester Royal Infirmary, Clinical Haematology, Manchester, \\ United Kingdom; ${ }^{2}$ Manchester Royal Infirmary, Therapeutic Stem Cell \\ Laboratory, Manchester, United Kingdom
}

Background: Allogeneic stem cell transplant (allo-SCT) is a curative option for many haematological malignancies. Reduced intensity conditioning (RIC) regimen and T-cell depleted (TCD) strategies have extended the use of alloSCT in elderly patients and in those with comorbidities, as it carries low rates of non-relapse mortality and low incidence of severe graft-versus-host disease (GvHD). However, in TCD allo-SCT there is a slow immune reconstitution and high incidence of mixed donor chimerisms (MDC) which is associated with impending relapse. Donor lymphocytes infusion (DLI) enhances the graft-versus-leukaemia effect and has been given to prevent relapse in patients with MDC.

Methods: Fifty-seven patients who underwent a RIC TCD allo-SCT received pre-emptive DLI due to MDC between January 2010 and July 2017 in our institution. Median age at transplant was 59 years (range 30-74). Diagnoses were acute myeloid leukaemia $(\mathrm{n}=36)$, myelodysplastic syndrome $(\mathrm{n}=11)$, acute lymphoblastic leukaemia $(n=6)$ and atypical chronic myeloid leukaemia $(n=1)$. Eighty-four percent were in complete remission at the time of allo-SCT, $4 \%$ in partial remission and $12 \%$ received an allo-SCT without any previous treatment. Thirty-four patients had a matched sibling donor, 22 a matched unrelated donor and 1 a mismatched unrelated donor. Chimerisms were assessed by polymerase chain reaction (PCR) of short tandem repeats (STR) in peripheral blood or bone marrow samples and fluorescent in situ hybridisation (FISH) for donor-recipient sex mismatched every month during the first six months and then every 2-3 months. MDC was defined as donor CD3 percentage $<95 \%$. DLI was administered using escalated doses until patients achieved full donor chimerisms (FDC), developed GvHD or relapsed.

Results: Median donor chimerism for $\mathrm{CD} 3$ at the time of DLI was $61 \%$ (range 5-94), median days from transplant to first DLI was 152 days (range 74-715) and median DLI administration was 1 (range 1-6). Median follow up was 28 months. A total of 30 patients (53\%) developed GvHD post-DLI; 16 patients (28\%) had acute GvHD and 14 (25\%) chronic GvHD. Acute GvHD grade 1-2 was seen in 10 patients (18\%), grade 3 in $5(9 \%)$ and grade 4 in $2(3 \%)$, limited chronic GvHD in 10 (18\%) and extensive chronic GvHD in $3(5 \%)$. GvHD was treated with 1 systemic agent in $47 \%$ of the cases, with 2 systemic agents in $27 \%$, with 3 systemic agents in $10 \%$ and $26 \%$ received only topical treatment. Two patients (3\%) died due to GvHD. Median time between last DLI and GvHD was 51 days (range 13112). FDC was achieved in 45 patients (79\%). Eleven patients (19\%) relapsed. Overall survival (OS) at 1, 2 and 5 years after the first DLI was $84 \%, 78 \%$ and $52 \%$. The presence of FDC and chronic GvHD after DLI was associated with better survival $(\mathrm{p}=0.01$ and $\mathrm{p}=0.03$, respectively).

Conclusions: Pre-emptive DLI post TCD RIC allo-HSCT in patients with MDC reduced the risk of relapse in patients who achieved FDC. Chronic GvHD post-DLI was associated with better outcomes.

Conflict of interest: None

\section{P656}

Quantitative PCR-Based Chimerism Monitoring in Bone Marrow or Peripheral Blood to Predict Leukemia Relapse in High-Risk Patients: Final Results of the KIM-PB Prospective Study

Valentina Gambacorta ${ }^{1,2}$, Riccardo Parolini ${ }^{1}$, Elisabetta Xue ${ }^{1,3}$, Raffaella Greco $^{3}$, Evelien Bouwmans ${ }^{4}$, Cristina Toffalori ${ }^{1}$, Fabio Giglio $^{3}$, Andrea Assanelli, Maria Teresa Lupo Stanghellini, Alessandro Ambrosi ${ }^{5}$, Wietse Mulder ${ }^{4}$, Consuelo Corti ${ }^{3}$, Jacopo Peccatori $^{3}$, Fabio Ciceri ${ }^{3}$, Luca Vago ${ }^{1,3}$

${ }^{1}$ San Raffaele Scientific Institute, Unit of Immunogenetics, Leukemia Genomics and Immunobiology, Milano, Italy; ${ }^{2}$ San Raffaele Scientific Institute, Unit of Senescence in Stem Cell Aging, Differentiation and Cancer; SR-TIGET, Milano, Italy; ${ }^{3}$ San Raffaele Scientific Institute, Hematology and Bone Marrow Transplantation Unit, Milano, Italy; ${ }^{4}$ GenDx, Utrecht, Netherlands; ${ }^{5}$ University Vita-Salute San Raffaele, Center for Statistics in Biomedical Sciences, Milano, Italy

Background: Despite considerable progress in the understanding of leukemia biology, numerous patients that receive allogeneic HSCT still lack reliable markers to monitor minimal residual disease (MRD): an issue for which hematopoietic chimerism can serve as a valid surrogate. Recently, the development of quantitative-PCR 
based assays increased dramatically the sensitivity of chimerism monitoring, but prospective studies on their clinical utility are still lacking.

Methods: We designed a prospective, non-interventional, single-center study to compare chimerism monitoring in peripheral blood $(\mathrm{PB})$ or bone marrow $(\mathrm{BM})$ in patients undergoing myeloablative allo-HSCT for high-risk leukemia. The study group comprised 30 AML patients with a high or very high disease risk index (DRI), and the control group 15 patients with Hodgkin or non-Hodgkin lymphomas without BM disease involvement transplanted with the same protocol. Chimerism was monitored using a commercial qPCR-based assay (KMRType and KMRTrack, GenDx, Utrecht). BM evaluations were performed at day $30,60,90,180,270$ and 360 after allo-HSCT, according to the practice of our center ( 6 total BM TPs per patient). PB chimerism monitoring was performed at the same timepoints (TPs) and at least an additional TP in-between each BM evaluation (17 total PB TPs per patient).

Results: Twenty patients from the study group were evaluable for donor HSC engraftment, 6 of which subsequently relapsed (median time time to relapse: 79 days; range, 60-91), 12 were alive and in complete remission at the end of the one-year follow-up (FU) and 2 died in remission before the end of the FU (at day 112 and 213). In the control group, 7 patients were evaluable for engraftment and 2 died before the end of FU (at day 26 and 114), with no patient developing $\mathrm{BM}$ involvement throughout the study.

Altogether, we collected and analyzed 484 samples (PB, $\mathrm{n}=374 ; \mathrm{BM}, \mathrm{n}=110$ ). Pairwise comparison of PB and BM samples collected at the same TP demonstrated higher host chimerism in BM compared to PB (mean 1.51 and 0.75 respectively), with a surprising lack of correlation between the two measurements $\left(\mathrm{R}^{2}=0.12\right)$.

By analyzing our entire dataset, we found that the most predictive model was obtained by considering as threshold the maximal reproducible sensitivity of the method $(0.136 \%$ for PB samples and $0.2 \%$ for BM samples), and by scoring as positive the cases with an increasing mixed chimerism (IMC) that exceeded the threshold value. Amongst the 6 study group patients who relapsed, IMC preceded the relapse in PB in 4 cases (66\%) and in BM in 2 cases (33\%). Time from IMC detection to relapse was on average 18 days for PB (range: 7-44) and 33 days for BM (range: 29-63). False positive IMC in non-relapsed patients from the study group were $28.6 \%$ for $\mathrm{PB}$ and $50 \%$ for $\mathrm{BM}$, and similar frequencies were documented also in the control group.

Conclusions: The present study is the first to our knowledge to prospectively address qPCR-based chimerism monitoring in high-risk AML patients undergoing alloHSCT. PB resulted superior to BM for chimerism monitoring with this high-sensitivity technique, allowing also shorter interval monitoring due to its reduced invasiveness.

Conflict of interest: This study was supported by the European Commission (ERA-NET TRANSCAN JTC2012 Cancer12-045-HLALOSS) to LV; by the Italian Ministry of Health RF-2011-02351998 to FC and LV and RF-201102348034 to $\mathrm{LV}$; by the Associazione Italiana per la Ricerca sul Cancro Start-Up Grant \#14162 to LV; and by the DKMS Mechtild Harf Research Grant to LV. Reagents were kindly provided by GenDx, Utrecht. LV received research funding from GenDx, all other Authors declare no relevant conflicts of interest to disclose.

\section{P657}

Study on the Immune Reconstitution in Reduced Intensity Alemtuzumab Based Allogeneic Haematopoeitic Stem Cell Transplant for Lymphoid Malignancies

Shereef Elmoamly, Claire Horgan, Maria Kaparou, Manos Nikolousis, Bhuvan Kishore, Shankara Paneesha, Ricahrd Lovell, Kathy Holder, Lynn Ryan, David Davies, Alex Kanellopoulos

Heart of England NHS Trust, Haematology Department, Birmingham, United Kingdom

Background: Reduced intensity conditioned (RIC) allogeneic stem cell transplant (allo-SCT) incorporating Alemtuzumab has enabled the transplant of heavily pretreated patients with low grade as well as high grade lymphomas but there is a paucity of data regarding the impact of various reconstitution markers on the transplant outcomes.

Methods: We retrospectively analysed data from a 64 patients treated in the haematology department in Heart of England NHS trust, 22 female and 42 male, median age was 55 years, with a diagnosis of lymphoma who underwent RIC Alemtuzumab based allo-SCT with Carmustine, Etoposide, Cytarabine, and Melphalan (BEAM)-Campath (10 patients), Fludarabine-BEAM-Campath (22 patients) and Fludarabine-Melphalan-Campath (FMC) (32 patients) as conditioning regimens. Alemtuzumab dose was $50 \mathrm{mg}$ daily for day-1, -2 for volunteer unrelated donors (n: 42) and $30 \mathrm{mg}$ once for sibling donor transplants (n: 22).

Results: Patients were followed up for a median of 26 months, 30/64 patients (46.9\%) had acute graft versus host disease (GVHD) of which 10/30 grade II, IV, 18/64 patients $(28.1 \%)$ had chronic GVHD. Median CD4 T-cell counts at $3,6,12$ months were $67,119,224 / \mathrm{mm}^{3}$ respectively, median CD8 T-cell counts at 3, 6, 12 months were 31, 111, $304 / \mathrm{mm}^{3}$ respectively, median absolute lymphocyte count (ALC) at 15,30 days were $50,150 / \mathrm{mm}^{3}$ respectively.

CD4 T-count at 12 months was associated with better overall survival (OS) (median OS for CD4 T-cells >200/ 
Overall survival according to CD4 count at 1 year ( $P$ value $\left.0.02^{*}\right)$

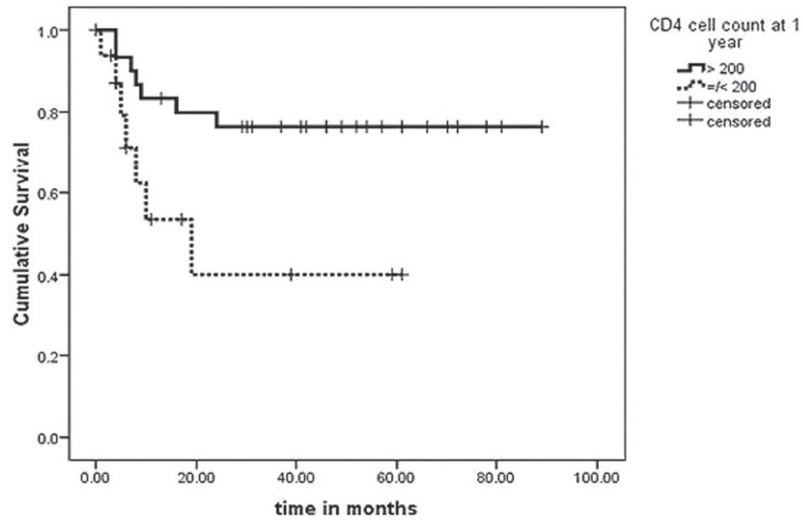

[P657 Figure] [CD4 T-cell count/OS]

$\mathrm{mm}^{3}$ patients at 12 months $=46$ months Vs 15 months for patients with CD4 T-cells $<200 / \mathrm{mm}^{3}$ at 12 months) (P value: 0.02) but not progression free survival (PFS) (P value: 0.9). No other immune reconstitution marker was correlating with OS or PFS.

Cumulative incidence of life threatening bacterial infection, CMV reactivation, EBV reactivation and respiratory viral infection was $51.6 \%, 42.2 \%, 14.1 \%, 43.8 \%$ respectively during the period of follow up. CD4 T-cell count $>$ $75 / \mathrm{mm}^{3}$ at 3 months was associated with significantly less life threatening bacterial infection ( $\mathrm{P}$ value: 0.03 ) but not with $\mathrm{CMV}$ or $\mathrm{EBV}$ reactivation or respiratory viral infection, likewise ALC $>100 / \mathrm{mm}^{3}$ at 1 month was associated with significantly less life threatening bacterial infection (P value: 0.004 ). CD8 T-cell count $>30 / \mathrm{mm}^{3}$ was associated with significantly less $\mathrm{CMV}$ reactivation ( $\mathrm{P}$ value: 0.015 ) but not with $\mathrm{EBV}$ reactivation or respiratory viral infection or life threatening bacterial infection, none of the immune reconstitution markers were associated with occurrence of acute or chronic GVHD. Finally there was a trend with reduced overall survival with CMV reactivation although that was not statistically significant (P value: 0.1 ).

Conclusions: Our data suggests that CD4 T-cell count > $200 \mathrm{~mm}^{3}$ at 1 year, may confer a survival advantage in lymphoma patients treated with RIC-allo SCT, and that immune reconstitution markers at 1,3 months has a significant correlation with bacterial infection and CMV reactivation but not on GVHD in these patients.

Clinical Trial Registry: Not applicable

Conflict of interest: nothing to disclose

\section{P658}

The Impact of Vitamin D Replacement on Survival, Relapse and GvHD after Allogeneic Stem Cell Transplantation: A Matched Cohort Analysis
Sara Mastaglio ${ }^{1}$, Eric Wong ${ }^{1}$, Travis Perera ${ }^{1}$, Andrew Lim ${ }^{2}$, Kate Mason ${ }^{1}$, Jenny Collins ${ }^{1}$, Jeff Szer ${ }^{1}$, Andrew Grigg ${ }^{3}$, Rachel Koldej ${ }^{1}$, David Ritchie ${ }^{I}$

${ }^{1}$ Royal Melbourne Hospital, Melbourne, Australia; ${ }^{2}$ University of Melbourne, Melbourne, Australia; ${ }^{3}$ Austin Hospital, Austin, Australia

Background: Vitamin D (VitD) has normal physiological roles in innate and adaptive immunity in addition to its classical effects on calcium and phosphate homeostasis. In recipients of allogeneic haematopoietic stem cell transplantation (alloHSCT), pre-transplant VitD deficiency is associated with increased cancer relapse and inferior overall survival in retrospective cross-sectional studies. It is unclear, however, whether VitD deficiency is an indicator of pre-transplant comorbidity or a modifiable risk factor for post-transplant outcomes. The aim of this study was to examine the impact of VitD replacement on survival and GvHD post-transplant.

Methods: We performed a retrospective, matched cohort analysis on patients undergoing alloHSCT at The Royal Melbourne Hospital between 2000 and 2013. Seventy-eight patients received VitD supplementation (1000iu cholecalciferol or $0.25 \mathrm{mg}$ calcitriol daily) starting before alloHSCT until 1 year after transplant as part of two sequential prospective clinical trials. These cases were matched in a 1:2 ratio with control patients who did not receive VitD supplementation, using propensity score matching according to 7 variables: patient age, graft type, donor type, conditioning intensity, year of transplant, disease risk index (DRI) and pre-transplant VitD level. Analysis endpoints included overall survival (OS), cumulative incidences of non relapse mortality (NRM), relapse (CIR), relapse free survival (RFS), acute and chronic graft versus host disease (GvHD). Survival analysis was performed using the Kaplan-Meier method. Cumulative incidences were calculated with competing risk analysis and with comparison via Gray's test.

Results: VitD replaced and non-replaced groups were well matched for key baseline characteristics (Table 1).

Median follow-up was 66 months. Pre transplant VitD levels did not influence patient outcome after alloHSCT. Moreover, VitD replacement did not impact on OS (5 year OS replaced $68 . \%$ vs non-replaced $65 . \%, P=0.51$ ), NRM (replaced $12 \%$ vs non-replaced $12 \%, P=0.85$ ), or cumulative incidence of relapse (replaced $26 . \%$ vs non-replaced $31 \%, P=0.38$ ). There were also no significant differences in the rates of acute GVHD or chronic GVHD. However, among patients with a high risk of cancer relapse (high or very high DRI), VitD replacement was associated with improved OS (5 year replaced OS 79\% [n=10/12] vs nonreplaced $36 \%,[\mathrm{n}=12 / 30], P=0.02)$ and $\mathrm{RFS}$ at 5 years ( $72 \%$ vs $34 \%, P=0.05$ ). There was a trend towards reduced 
Table 1. Patient characteristics

\begin{tabular}{|c|c|c|c|}
\hline & Non-Replaced $(n=156)$ & Replaced $(n=78)$ & $p$ \\
\hline $\operatorname{Sex}(M / F)$ & $98 / 58$ & $40 / 38$ & \\
\hline Age, median (range) & $44(17-64)$ & $46(19.65)$ & 0.8 \\
\hline \multicolumn{4}{|l|}{ Disease, $n(\%)$} \\
\hline Aoute leukaemia & $73(46.8)$ & $33(42.3)$ & \\
\hline Lymphoma & $35(22.4)$ & $13(16.7)$ & \\
\hline Chronic lymphocytic leukaemia & $15(9.6)$ & $6(7.7)$ & \\
\hline Myelodysplastic syndrome & $3(1.9)$ & $3(3.8)$ & \\
\hline Chronic myeloid leukzemia & $14(9)$ & $16(20.5)$ & \\
\hline Myeloproliferative neoplasms & $7(4.5)$ & $7(9)$ & \\
\hline Maltiple myeloma & $6(3.8)$ & $0(0)$ & \\
\hline Other & $3(1.9)$ & $0(0)$ & \\
\hline \multicolumn{3}{|l|}{ Graft, n $(\%)$} & 0.12 \\
\hline Bene marrow & $37(23.7)$ & $26(33.3)$ & \\
\hline Peripheral blood & $119(76.3)$ & $52(66.7)$ & \\
\hline \multicolumn{3}{|l|}{ Donor, $\mathrm{n}(\%)$} & 0.9 \\
\hline Matched related & $106(67.9)$ & $52(66.7)$ & \\
\hline Urrelated & $50(32.1)$ & $26(33.3)$ & \\
\hline \multicolumn{3}{|l|}{ Conditioning, $\mathrm{n}(\%)$} & 0.3 \\
\hline Myeloablative & $96(61.5)$ & $54(69.2)$ & \\
\hline Reduced intensity/non myeloablative & $60(38.5)$ & $24(30.8)$ & \\
\hline \multicolumn{3}{|l|}{ Year of allo-HSCT, $n(\%)$} & 0.9 \\
\hline $2000 \cdot 2006$ & $72(46,2)$ & $35(44.9)$ & \\
\hline $2007-2013$ & $84(53.8)$ & $43(55.1)$ & \\
\hline \multicolumn{3}{|l|}{ DR1, n $(\%)$} & 0.9 \\
\hline Low & $42(26.9)$ & $22(28.2)$ & \\
\hline Intermediate & $73(46.8)$ & $37(47.4)$ & \\
\hline Hizh & $20(12.8)$ & $9(11.5)$ & \\
\hline Very high & $10(6.4)$ & $3(3.8)$ & \\
\hline na & $11(7)$ & $7(9)$ & \\
\hline $\begin{array}{l}\text { Pre allo-HSCT VitD, } \\
\text { median (range) }\end{array}$ & $50.5(7 \cdot 160)$ & $54.5(18 \cdot 100)$ & 0.6 \\
\hline
\end{tabular}

[P658 Figure] [Patient characteristics]

cancer relapse in VitD replaced patients (5 year CIR $28 \%$ vs $63 \%, P=0.07)$. No significant differences in the rates of NRM and acute or chronic GVHD between replaced and non-replaced patients were observed in this high-risk subcohort. VitD replacement did not impact posttransplant survival or GVHD incidence in patients with low or intermediate DRI.

Conclusions: Vitamin D replacement may improve survival after alloHSCT for patients with high or very high DRI, perhaps by reducing the incidence of relapse. Further work is required to determine the immunological impact of VitD replacement on the post-transplant graft versus leukaemia effect.

Conflict of interest: None of the authors has anything to disclose.
P659

The occurrence of incompatibilities of rare blood group antigens in donor-recipient pairs and their impact on the course of transplantation

Maria Bieniaszewska, Magdalena Dutka, Michat Taszner, Andrzej Hellmann

Medical University of Gdańsk, Department of Hematology and Transplantology, Gdańsk, Poland

Background: The impact of blood group incompatibility on the transplantation effect remains unclear. The aim of the study was to assess the frequency of occurrence of 
incompatibilities in the main and rare blood group antigens and their impact on the results of transplantation.

Methods: In 128 patients with AML in CR1 and CR2, before allogeneic transplantation of peripheral blood stem cells with myeloablative conditioning and their donors an extended panel of blood group antigens was examined: ABO, Rh, Kell, Duffy, Kidd, Ss, MN and P. Antigens of $\mathrm{ABO}$, Rh system and Kell was determined by the tube method, the remaining using the microplate method.

Results: In the study group, $\mathrm{ABO}$ compatibility was found in $43 \%$ of patients and their donors, $\mathrm{Rh}$ system assessment reduced the compatibility rate to $42 \%$. Full compatibility of antigens $\mathrm{ABO}, \mathrm{Rh}$ and Kell groups was found in $37 \%$ pairs. Analysis of all examined systems showed compatibility of all group antigens in only $17 \%$ of donor-recipient pairs. There was no influence of any of the incompatibilities examined on the incidence of acute and chronic graft versus host disease and transplant related mortality. A significantly higher rate of relapses was found in the period of 2 years after transplantation in the group of patients with full compatibility in all the examined systems regardless of transplant time (CR1, CR2).

Conclusions: Full compatibility in many blood groups could be a risk factor of relapse after alloSCT.

Conflict of interest: None of the authors has anything to disclose.

\section{P660}

The utility of whole- blood and lineage- specific chimerism in predicting graft failure after stem cell transplantation for nonmalignant disease in paediatric patients

\section{Maria Neocleous ${ }^{1}$, Robert Wynn ${ }^{2}$, Su Han Lum ${ }^{2}$, Helena Lee ${ }^{3}$, Heather Church ${ }^{4}$}

${ }^{1}$ University of Manchester, School of Medicine, Manchester, United Kingdom; ${ }^{2}$ Royal Manchester Children's Hospital, Bone Marrow Transplant Unit, Manchester, United Kingdom; ${ }^{3}$ Manchester Royal Infirmary, Transplantation Laboratory, Manchester, United Kingdom; ${ }^{4}$ St Mary's Hospital, Willink Biochemical Genetics Unit, Manchester, United Kingdom

Background: Allogeneic haematopoietic stem cell transplantation (HSCT) is widely used to treat nonmalignant conditions such as inborn errors of metabolism, immunodeficiencies and bone marrow failures. Mixed chimerism (MC) is a commonly observed phenomenon in such cases, with a conflicting body of evidence on whether a consequent, additional intervention is necessary or not. Lineage- specific chimerism (myeloid and T-cell) is increasingly been explored in an attempt to explain chimerism kinetics and immune reconstitution post- transplant as well as inform best management of patients with a
MC status. This study's purpose was to explore predictors of $\mathrm{MC}$ and graft failure, variations in outcome between patients with complete chimerism (CC) and $\mathrm{MC}$, and the utility of whole- blood and lineage- specific chimerism in predicting graft outcomes.

Methods: Our patient sample included all HSCTs performed in children with nonmalignant conditions between July 2000 and March 2017 at our centre. A total of 296 HSCTs were identified, however, 12 of them had to be excluded due to insufficient data present, resulting in 284 cases being included in this study. The following variables were considered in each patient: gender, age at transplant, date of transplant, disease, conditioning regimen, T-cell depletion, donor \& stem cell source, alive/ deceased status, chimerism status. Variables were assessed using univariate and multivariate logistic regression analysis on the statistical programme STATA14. The relationship between myeloid chimerism, T-cell chimerism and transplant outcomes was also investigated in those patients with lineagespecific analysis.

Results: In total, 186 patients exhibited CC while 98 patients exhibited MC. A reduced- intensity conditioning regimen as well as a young age at the time of transplantation were identified as significant predictors of MC. The year of transplant (pre-2006) and a MC status were identified as significant predictors of graft failure. In 29 out of 49 patients with high-level MC, defined as $<70 \%$ donor, graft failure was developed. In contrast, none of the patients with low-level MC, defined as $\geq 70 \%$ donor, developed graft failure. Early full donor myeloid chimerism was identified as a useful predictor of long- term engraftment and was further associated with a rising donor T-cell chimerism. Subsequently, an algorithm was created to guide clinical management of chimerism in nonmalignant disease, taking into consideration the nature of the disease (T-cell or non Tcell disease) as well as the lineage- specific chimerism status.

Conclusions: High-level MC is a better predictor of graft failure than low-level MC. Myeloid chimerism could be used as a reliable indicator of the transplant outcome. Additionally, T-cell chimerism monitoring can be important in patients with T-cell immunodeficiencies. Finally, the algorithm suggested is used to inform and predict graft outcomes as well as the need for specific interventions in patients with a nonmalignant disease, whilst utilising lineage- specific chimerism analysis.

Conflict of interest: All authors declare no conflict of interest.

\section{P661}

Abstract previously published

Multiple myeloma 


\section{P662}

Allogeneic Hematopoietic Cell Transplantation for Multiple Myeloma - a Multicenter Retrospective Study of Polish Myeloma Group

Aleksandra Golos ${ }^{1}$, Lidia Gil ${ }^{2}$, Piotr Boguradzki ${ }^{3}$, Kazimierz Hataburda ${ }^{4}$, Waldemar Sawicki, Matgorzata SobczykKruszelnicka ${ }^{6}$, Grzegorz Helbig ${ }^{7}$, Jarostaw Dybko ${ }^{8}$, Artur Jurczyszyn ${ }^{9}$, Bartosz Pula ${ }^{l}$, Krzysztof Warzocha ${ }^{1}$, Krzysztof Jamroziak $^{1}$

${ }^{1}$ Institute of Hematology and Transfusion Medicine, Department of Hematology, Warsaw, Poland; ${ }^{2}$ Poznan University of Medical Sciences, Department of Hematology and Bone Marrow Transplantation, Poznan, Poland; ${ }^{3}$ Medical University of Warsaw, Department of Hematology, Oncology and Internal Medicine, Warsaw, Poland; ${ }^{4}$ Institute of Hematology and Transfusion Medicine, Department of Hematopoietic Stem Cell Transplantation, Warsaw, Poland; ${ }^{5}$ Military Institute of Medicine, Department of Hematology and Bone Marrow Transplantation Unit, Warsaw, Poland; ${ }^{6}$ Maria Sklodowska-Curie Memorial Cancer Center and Institute of Oncology, Gliwice Branch, Department of Bone Marrow Transplantation and Oncohematology, Gliwice, Poland; ${ }^{7}$ Medical University of Silesia, Department of Hematology and Bone Marrow Transplantation, Katowice, Poland; ${ }^{8}$ Medical University, Department of Hematology, Blood Neoplasms and Bone Marrow Transplantation, Wroclaw, Poland; 9 Jagiellonian University Medical College, Department of Hematology, Kraków, Poland

Background: The role of allogeneic hematopoietic cell transplantation (alloHCT) in therapy of multiple myeloma (MM) despite curative, is not well established, mainly due to high transplant-related mortality (TRM). The objective of the study is to describe the use of alloHCT in Poland outside clinical trials and to evaluate it outcome.

Methods: Data of 60 patients (pts) with median age 46 years (range: 22-59), who received alloHCT at any timepoint in their MM course between 1993 and 2016 were collected and analyzed based on questionnaire sent to all transplant center in Poland. In the study cohort $68 \%$ pts were in stage III according to Salmon-Durie classification at time of alloHCT. The median number of treatment regimens before alloHCT was 3 (range: 1-8). In 45 (75\%) pts autoHSCT preceded allotransplant: in $23 \%$ pts as a part of tandem auto-allo protocol and in $68 \%$ because of relapsed/ refractory disease. $32 \%$ patients received alloHCT as a consolidation of first-line treatment. Before alloHCT 15 pts were at least in partial remission. For conditioning, RIC protocols based on fludarabine were used in $44(70 \%)$ pts and $16 \mathrm{pts}$ were treated with myeloablative conditioning, mainly based on melphalan (8), TBI (5), or treosulphan (2). Stem cells from related $(50 \%)$ or unrelated donors were grafted in median dose $4.8(0.5-8.6) \times 10^{6}$ of CD34+ cells/ $\mathrm{kg}$. Peripheral blood (PB) stem cells were transplanted in 52 (87\%) pts.

Results: Engrafment occurred in $98 \%$ of pts. Infectious complications until day +100 were observed in $38(67 \%)$ pts as FUO (8), bacterial (12), viral (10) or fungal (3). Severe toxic complications (CTCAE grade 3-4) developed in $26(43 \%)$ pts. Acute GvHD occurred in 45\% patients, in $18 \%$ at grades III or IV. Eight patients developed limited chronic GvHD, 5 extensive. The relapse rate was 38\%. 74\% transplanted in complete remission or very good partial remission had not relapsed until the last follow up. In median follow up of 23 (range: 0-183) months 37 (62\%) pts died (early deaths 12). Causes of deaths included relapsed/ refractory disease (43\%), infections (27\%), GvHD (19\%) and others (suicide 1, MOF 1, cardiac arrest 1, HUS 1). The median OS was 23 months (0-183) and PFS 9 months (0183), (Figure $1 \mathrm{~A}$ and $\mathrm{B}$, respectively). The absence of aGvHD correlated with longer OS (61 vs 11 months, $\mathrm{p}=$ $0.009)$ and PFS (14 vs 8 months, $p=0.05$ ). The presence of
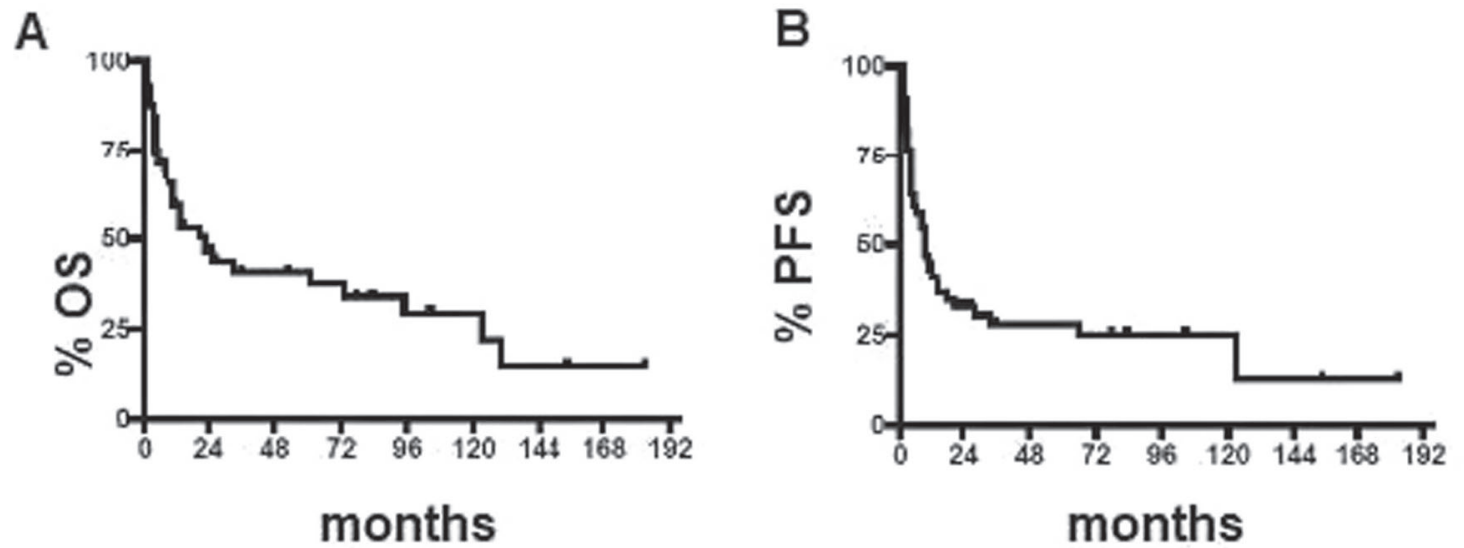

\section{Figure 1. Analysis of Kaplan-Meier estimated (A) overall survival (OS) and (B) progression-free survival (PFS) of the whole cohort.}

[P662 Figure] [Analysis of Kaplan-Meier estimated (A) overall survival (OS) and (B) progression-free survival.] 
chGvHD was associated with longer PFS (28 vs 6 months, $\mathrm{p}=0.05$ ) and OS (73 vs 8 months, $\mathrm{p}=0.09$ ). OS or PFS did not differ significantly a ccording to conditioning regimen, disease stage, the year of alloHCT.

Conclusions: Our results suggest that alloHCT in MM is associated with high risk of complications, including TRM, however, it may give long term survival in some patients. However, the identification of the most beneficial group remains elusive. The positive effect of chGvHD on survival confirms the presence of anti-myeloma effect. Due to the long time scale in our study and many patients treated before novel agents era, the results must be interpreted carefully.

Conflict of interest: None of the authors has anything to disclose.

\section{P663}

\section{Allogeneic hematopoietic stem cell transplantation for multiple myeloma in the novel agent era: Study from the Japanese Society of Myeloma}

Koji Kawamura ${ }^{1}$, Nobuhiro Tsukada ${ }^{2}$, Yoshinobu Kanda ${ }^{1}$, Takashi Ikeda ${ }^{3}$, Akiyo Yoshida ${ }^{4}$, Yasunori Ueda ${ }^{5}$, Tadao Ishida ${ }^{2}$, Kenshi Suzuki $^{2}$, Hirokazu Murakami ${ }^{6}$

${ }^{1}$ Saitama Medical Center, Jichi Medical University, Division of

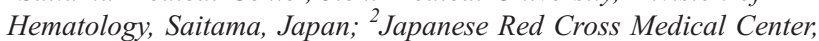
Tokyo, Japan; ${ }^{3}$ Shizuoka Cancer Center, Shizuoka, Japan; ${ }^{4}$ Toyama Prefectural Central Hospital, Toyoma, Japan; ${ }^{5}$ Kurashiki Central Hospital, Kurashiki, Japan; ${ }^{6}$ Gunma University Graduate School of Health Sciences, Maebashi, Japan

Background: Allogeneic hematopoietic stem cell transplantation (allo-HCT) is considered a potentially curative therapy for patients with multiple myeloma because of graft-versus-myeloma effect. However, relatively higher transplant-related mortality and relapse rate have restricted its use, and the role of allo-HCT remains unclear in the era of novel agents.

Methods: We conducted a retrospective study of 65 patients with pretreated multiple myeloma who underwent allo-HCT at 19 institutions from 2009 to 2016 in Japan. The primary endpoint was progression-free survival (PFS) after allo-HCT, and secondary endpoints were overall survival (OS), non-relapse mortality (NRM) and overall response rate.

Results: The median number of previous therapies was 3 (range 1-7), and all patients received at least one novel agent before allo-HCT. The probabilities of PFS at 1 year and 3 years were $46.6 \%$ (95\% confidence interval (CI), 34.0-58.3) and $18.8 \%$ (95\% CI, 9.6-30.3), respectively. The probabilities of OS at 1 year and 3 years were $65.5 \%$ (95\% CI, 52.5-75.8) and 47.2\% (95\% CI, 33.9-59.4), respectively. In the multivariate analysis, 50 years of age or older and less than very good partial response (VGPR) before allo-HCT were independent significant adverse factors for PFS (hazard ratio (HR) 2.30; 95\% CI, 1.27-4.17; $\mathrm{p}=0.0063$ and HR 2.86; 95\% CI, 1.35-6.05; $\mathrm{p}=0.0059)$ and OS (HR 2.37; 95\% CI, 1.20-4.69; $\mathrm{p}=0.013$ and HR 2.74; 95\% CI, 1.04-7.20; $p=0.040$ ). Conversely, the probabilities of PFS and OS at 3 years in patients younger than 50 years who achieved VGPR or better before allo-HCT was $64.3 \%$ (95\% CI, 29.8-85.1) and 80.2\% (95\% CI, 40.3-94.8), respectively. Overall response rate was $86.4 \%$ (95\% CI, 75.0\%-94.0\%). The proportion of VGPR or better was $29 \%$ before alloHCT, and was increased up to $71 \%$ after allo-HCT. The incidences of NRM at 1 year and 3 years were both $23.4 \%$ (95\% CI, 13.8-34.4). Only 50 years of age or older was associated with higher NRM (HR 4.71; 95\% CI, 1.34-16.5; $\mathrm{p}=0.015$ ). The incidence of NRM at 2 years in patients younger than 50 years was $9.3 \%$ (95\% CI 2.3-22.3), although that in patients aged 50 years or older was $37.5 \%$ (95\% CI 20.9-54.1).

Conclusions: We showed that allo-HCT is feasible for heavily pretreated patients with multiple myeloma even in the novel agent era. Especially, allo-HCT can be a promising therapy for patients younger than 50 years with VGPR or better before allo-HCT.

Conflict of interest: None of the authors has anything to disclose.

\section{P664}

Allogeneic HSCT in multiple myeloma: number of therapy lines prior to transplantation is associated with survival outcomes

Christine Eisfeld ${ }^{1}$, Eva Esseling ${ }^{1}$, Martin Kropff ${ }^{2}$, Andrea Kerkhoff ${ }^{l}$, Christian Reicherts ${ }^{1}$, Jörn C. Albring ${ }^{1}$, Jan-Henrik Mikesch ${ }^{1}$, Christoph Groth ${ }^{1}$, Rolf Mesters ${ }^{I}$, Christoph Schliemann ${ }^{I}$, Torsten Kessler $^{1}$, Georg Lenz ${ }^{I}$,Wolfgang E. Berdel ${ }^{\text {, Matthias Stelljes }}{ }^{1}$

${ }^{1}$ University Hospital Münster, Department of Medicine A, Münster, Germany; ${ }^{2}$ Klinikum Osnabrück, Department of Medicine III, Osnabrück, Germany

Background: The role of allogeneic hematopoietic stem cell transplantation (HSCT) in multiple myeloma (MM) therapy is not well defined and often being reserved for selected patients in later stages of their disease. In addition, for patients with high-risk disease there might be some benefit from allogeneic HSCT, although evidence is weak and there is uncertainty about the best timing in the sequence of therapy.

Methods: We retrospectively analyzed the outcomes of 88 MM patients who received allogeneic HSCT in our center between 1999 and 2016 with respect to cytogenetic risk factors, prior therapies and transplant characteristics. 
Statistical analysis was performed using the Kaplan-Meier approach, the cumulative incidence method and Cox proportional hazards model.

Results: Median age of patients at time of transplant was 51 years. Among a total of 88 patients, 38 patients (43\%) received stem cell grafts from a HLA-identical sibling donor, 50 patients $(57 \%)$ either from an unrelated donor of whom $40(80 \%)$ were 10/10 HLA matched unrelated donors (MUD) or from a mismatched related donor. 30 patients (34\%) received a tandem autologous-allogeneic transplantation upfront, while $58(66 \%)$ patients received allogeneic HSCT in relapse setting after two to seven treatment lines including one or two autologous HSCT. Conditioning regimens mainly consisted of busulfan- $(\mathrm{n}=29)$, melphalan- $(\mathrm{n}=34)$, or TBI-based (8-12 Gy, $\mathrm{n}=21)$ regimens. Median follow-up of surviving patients was 5.6 years. Median overall survival (OS) and progression free survival (PFS) from the time of allogeneic HSCT was 30.5 and 12.3 months, respectively. PFS and OS at 5.6 years was $24 \%$ (95\% CI 14-33\%) and 37\% (95\% CI 26-48\%), respectively. The cumulative incidence of non-relapse death was $14 \%$ at three months, $24 \%$ at one year and $29 \%$ at three years. Cumulative incidence of relapse at one, two, and three years was $31 \%, 46 \%$, and $54 \%$, respectively. For 64 (73\%) patients cytogenetic abnormalities were reported, 23 (26\%) were considered as high-risk MM (17/17p deletion, 4;14 translocation, 14;16 translocation and/or 1q amplification). OS was significantly reduced in patients receiving more than two lines of therapy prior to allogeneic HSCT (HR 3.4, 95\% CI 1.3-8.6, $\mathrm{p}=0.01$ ). This association was still present in the subgroup of patients who received allogeneic HSCT in relapse setting (HR 3.4, 95\% CI 1.39.1, $\mathrm{p}=0.01)$. OS was not associated with adverse cytogenetics, age, type of donor, or remission status prior to allogeneic HSCT.

Conclusions: The graft-versus-myeloma effect following allogeneic HSCT offers an additional treatment element that might be valuable even in combination with subsequent novel antibody based strategies. Despite recent improvements in outcome, allogeneic HSCT should still be considered in selected MM patients with poor prognosis. Whether these patients benefit from allogeneic HSCT in earlier phases of the disease, e.g. after re-induction following first or second relapse rather than in late stage disease, deserves further investigation in randomized trials including novel agents.

Conflict of interest: The authors have no conflict of interest to disclose.

\section{P665}

Atraumatic splenic rupture in light chain amyloidosis (AL) after autologous stem cell transplantation (ASCT)

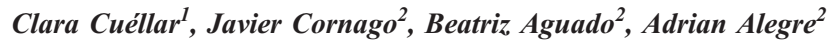

${ }^{1}$ Hospital Universitario La Princesa, Madrid, Spain; ${ }^{2}$ Hospital La Princesa, Madrid, Spain

Background: We present a case of spontaneous rupture of the spleen, an uncommon and life-threatening complication of AL amyloidosis.

Methods: A 62-year-old woman diagnosed with AL amyloidosis, treated with 6 cycles of Bortezomib and Dexamethasone, was referred to our hospital for ASCT.

The patient had renal infiltration of amyloid confirmed by biopsy, wtith nephrotic syndrome and chronic renal failure (creatinine $1.6 \mathrm{mg} / \mathrm{dl}$, clearence $30 \mathrm{~mL} / \mathrm{min} / 1.73 \mathrm{~m}^{2}$ ), peripheral neuropathy and anemia.

No cardiac or gastrointestinal infiltration was found. Abdominal ecography with no evidence of visceromegalies. Coagulation parameters in normal range, including factor X. We assigned her a score of 1 (low risk) according to Mayo Clinic staging system (NT-proBNP $369 \mathrm{pg} / \mathrm{ml}$, Troponina T $0.6 \mathrm{ng} / \mathrm{mL}, d F L C 8.77 \mathrm{mg} / \mathrm{dl}$ ), and proceed to the transplant procedure.

Mobilization of hematopoietic progrenitors was carried out in a hospital admission regimen, with G-CSF at a dose of $5 \mathrm{mcg}$ every 12 hours for 3 days, obtaining a product of $3.2 \times 10^{6} \mathrm{CD} 34+/ \mathrm{kg}$, suitable for ASCT. Conditioning regimen was Melphalan $140 \mathrm{mg} / \mathrm{m}^{2}$ and the transplant took place initially without complications, with leukocyte engraftment $\left(>1000 / \mathrm{mm}^{3} \mathrm{NT}\right)$ on day +11 .

Results: That same day, the patient required admission to the ICU due to hypotension, tachycardia, sudden worsening of anemia and abdominal pain in the left iliac fossa. Abdominal CT showed splenic rupture with acute subcapsular hematoma and secondary hemoperitoneum (Figure 1). The patient had cardiorespiratory arrest secondary to hemorrhagic shock. She was reanimated and stabilized. Once stable, an urgent splenectomy was performed with favorable outcome. The histopathological report of the spleen revealed extensive amyloid infiltration.

In the following days, the patient presented clinical improvement, so she was discharged from the ICU and transferred to Hematology Transplant Unit. At 24 hours, the patient suffered a cardiorespiratory arrest and died.

Conclusions: Splenic involvement in amyloidosis is rather frequent $(5-10 \%)$. Non-traumatic splenic ruptures can be fatal. In our case, the patient recovered favorably from the acute episode but later died from sudden death. It is probable that cardiorespiratory arrest was consequence of sequelae from splenic rupture (hemorrhagic shock and electromechanical dissociation) and subclinical amyloid deposition. 


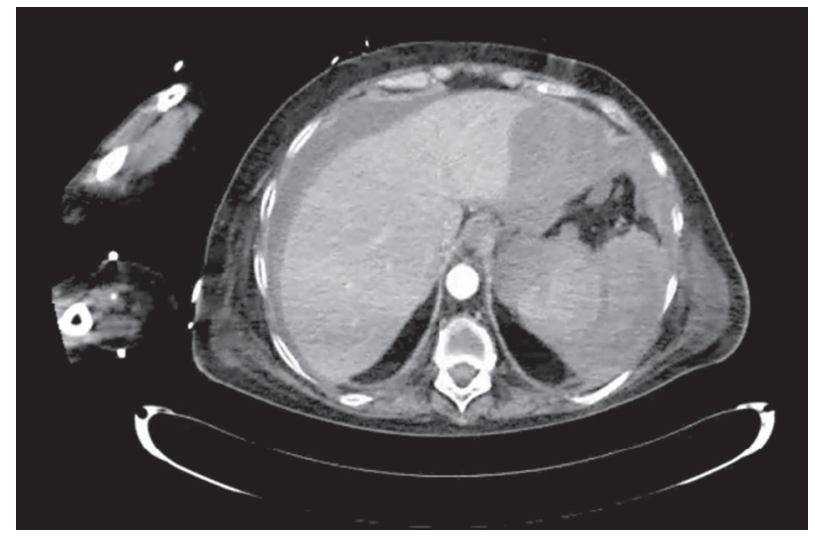

[P665 Figure] [Figure 1. Abdominal CT: splenic rupture with acute subcapsular hematoma and secondary hemoperi]

It has been published that the alteration of coagulation and splenomegaly are factors that predispose to splenic rupture in patients who undergo ASCT. However, our patient did not present any of these conditions. Therefore, this case illustrates that patients with AL amyloidosis, even at low risk, can have severe acute complications that can compromise patient's life.

Conflict of interest: None of the authors has anything to disclose.

\section{P666}

\section{AUTOLOGOUS STEM CELL TRANSPLANTATION IN ELDERLY MULTIPLE MYELOMA PATIENTS}

Michela Staderini ${ }^{1}$, Chiara Nozzoli ${ }^{2}$, Gabriele Buda ${ }^{3}$, Elisabetta Antonioli $^{4}$, Claudia Schifone ${ }^{5}$, Ilaria Cutini ${ }^{2}$, Antonella Gozzini ${ }^{2}$, Stefano Guidi ${ }^{2}$, Agata Suellen Guarrera ${ }^{2}$, Alberto Bosi ${ }^{1}$, Mario Petrini $^{5}$, Riccardo Saccardi ${ }^{2}$

${ }^{1}$ SODc Hematology AOU Careggi, Florence, Italy; ${ }^{2}$ BMT Unit Azienda Ospedaliera Universitaria Careggi, Florence, Italy;

${ }^{3}$ Hematology, Pisa, Italy; ${ }^{4}$ Hematology AOU Careggi, Florence, Italy; ${ }^{5}$ Hematology and Bone Marrow Transplantation Unit, Pisa, Italy

Background: Autologous stem cell transplantation (ASCT) is currently approved as the "gold standard" first line treatment for multiple myeloma (MM) patients (pts) younger than 65 years, furthermore it is considered feasible in fit elderly pts in some retrospective studies.

Methods: To evaluate the tolerability and the efficacy of high dose chemotherapy followed by ASCT we retrospectively analyzed consecutive MM pts aged 65 or older who underwent upfront ASCT at Hematology Unit of Florence and Pisa from January 2009 to November 2017.

Results: We analyzed 80 pts, 48 male and 32 female; median age 67 (range 65-74). Among 61 pts evaluable for FISH 6 pts $(15 \%)$ were high cytogenetic risk. ISS stage was available in 58/80 pts: $29 / 58$ were ISS $1,14 / 58$ were ISS 2, $15 / 58$ were ISS 3 . Induction therapy was bortezomib-based in $67(85 \%)$ pts, (VD in 10/80 pts, VTD in 57/80 pts), while $2 \%$ received thalidomide plus dexamethasone. Eleven pts received other type of induction. Peripheral blood stem cells (PBSC) were collected after high-dose cyclophosphamide in $89 \%$ of pts ( $2 \mathrm{~g} / \mathrm{sqm}$ in $4 / 80 \mathrm{pts}, 3 \mathrm{~g} / \mathrm{sqm}$ in $26 / 80 \mathrm{pts}, 4$ $\mathrm{g} / \mathrm{sqm}$ in 41/80 pts) plus G-CSF; 9 pts received other type of mobilization therapies . Plerixafor was administered in 19/ 80 pts.

Before ASCT, $14 / 80$ pts (18\%) were in complete response/stringent complete response (CR/sCR), 41/80 $(51 \%)$ in very good partial response (VGPR), 25/80 $(31 \%)$ in partial response (PR). The conditioning regimen consisted of melphalan $100 \mathrm{mg} / \mathrm{sqm}$ in 36/80 pts (45\%), 140 $\mathrm{mg} / \mathrm{sqm}$ in $13 / 80 \mathrm{pts}(16 \%)$, or $200 \mathrm{mg} / \mathrm{sqm}$ in $28 / 80 \mathrm{pts}$ (35\%); $3 / 80$ pts $(4 \%)$ underwent other conditioning therapies.

A median of $4.32 \times 10^{\wedge} 6 \mathrm{CD} 34+$ cells $/ \mathrm{Kg}$ was reinfused (range 2.09-10.44). All 80 pts achieved neutrophils recovery after a median of 11 days (range 8-25) and platelets recovery after a median of 12 days (range 7-45) after ASCT. The most frequent non hematologic complication was fever grade $1-3$ in $28 \%$ of patients (23/80 pts). Other adverse events were represented by grade 1-2 gastrointestinal toxicity (nausea, vomiting, diarrhea, mucositis) in $8 \%$ of pts, cardiovascular events in $6 \%$ of patients (4 atrial fibrillation and 1 TVP), 3 cases of pneumonia, 2 cases of acute renal impairment, 2 cases of viral reactivations (CMV and HZV reactivation). No transplant-related mortality was recorded within 100 days post transplantation. For 75/80 pts a 3 months or more follow-up period was available. Three months after ASCT, among 67 evaluable pts, 25/67 pts (37\%) were in CR/sCR, 33/67 pts (49\%) in VGPR and 7/67 pts $(11 \%)$ in PR while $2 / 67$ pts $(3 \%)$ were in disease progression. Three pts underwent tandem ASCT. After a median follow-up after transplant of 29 months (range 1104), 46/80 experienced disease relapse and 10 deaths occurred. Median PFS and OS were 28 and 41 months.

Conclusions: ASCT is an effective and safe first-line treatment approach also in elderly MM. A careful pts selection reduces the toxicity of the procedure.

Conflict of interest: Staderini M nothing to disclosure

\section{P667}

Autologous stem cell transplantation performed in Multiple Myeloma patients older and younger than 65 years old. Results from two Spanish Hospitals

Sally Franco $^{1}$, Marta Canet ${ }^{2}$, Josep Borras ${ }^{1}$, Sandra Jara ${ }^{2}$, Raquel Del Campo ${ }^{1}$, Marta Juliá ${ }^{2}$, Antonia Cladera ${ }^{1}$, Ana Muntañola ${ }^{2}$, Martín Mascaró ${ }^{1}$, Ferran Vall-Llovera ${ }^{2}$, Jose Maria Guerra ${ }^{1}$, 
Patricia Velez ${ }^{2}$, Delia Gómez ${ }^{1}$, Josep Marti ${ }^{2}$, Elena González ${ }^{1}$, Inés Herráez $^{1}$, Julia Domínguez, ${ }^{1}$,Joan Bargay ${ }^{1}$

${ }^{1}$ Son Llatzer Hospital, Palma de Mallorca, Spain; ${ }^{2}$ Mutua Terrassa Hospital, Barcelona, Spain

Background: Autologous stem cell transplantation (ASCT) should be performed in all eligible Multiple Myeloma (MM) patients, even in those older than 65 years. Although most clinical trials exclude them, age must be considered in conjunction with performance status and comorbidities when assessing suitability for ASCT.

Methods: We performed a retrospective observational study involving $189 \mathrm{MM}$ patients from 2 Spanish hospitals (Hospital Mutua Terrassa, Barcelona; Hospital Son Llatzer, Palma de Mallorca), who underwent ASCT from December 2003 to June 2017. Patients were separated into two groups depending on its age at the moment of transplantation (group 1: < 65 years old; group 2: 65 years old or older). Data were collected from electronic medical records. For statistical analyses, IBM SPSS ${ }^{\circledR}$ software was used. KaplanMeir method was used to perform survival analysis; and the Log-Rank test to compare cohorts ( $\mathrm{p}<0.05$ was considered statistically significant).

Results: Out of 189 patients analyzed, 143 (75.7\%) were under 65 years old and $46(24.3 \%)$ were 65 or older. Median age at transplantation was 57.8 years (22-73.1). Median follow-up was 44.6 months (1.09-200.9) and median overall survival (OS) 106.6 months (CI 95\%: 84.39-128.96).

Treatment response was evaluated according to the IMWG criteria. Most patients in both groups achieved at least a very good partial response (VGPR) before ASCT (58.74\% in the group $<65$ years old, and $56.52 \%$ in patients $>65$ years old).

No differences were observed between groups in regard to ECOG and HCT-CI at the time of transplantation, and the hematological recovery was also similar (14 days and 15 days for the group $<65$ and $>65$ years old, respectively). There was no significant difference in the occurrence of infection in the post-ASCT period $(88.8 \%$ vs $91.3 \%$ for the group $<65$ and $>65$ years old, respectively, $p=0.38$ ).

100-day re-evaluation showed a VGPR or better in $64.4 \%$ of patients aged $<65$ years and $71.8 \%$ for patients $>65$ years.

Median OS after transplant was better in the group $<65$ years old, but this difference was not significant (107.27 vs 71.61, $p=0.134$ ).

Conclusions: Upfront ASCT should be considered in all eligible MM patients, even in those $>65$ years with excellent functional status.

This retrospective analysis of 189 myeloma patients treated with ASCT in the period 2003 to 2017, confirms ASCT as an effective strategy even in patients $>65$ years old, with toxicity and outcome comparable to younger patients.

A frailty index should consider measures of activities of daily living plus comorbidities to identify a population with low risk of transplant-related complications; this would be a more realistic approach to eligibility than age alone.

Conflict of interest: The authors declare no conflicts of interest.

\begin{tabular}{lll}
\hline Parameter & $<65$ years old $>65 \mathrm{~N}(\%)$ \\
& $\mathrm{N}(\%)$ & \\
\hline $\mathrm{N}$ & $143(75.7 \%)$ & $46(24.3)$ \\
Median ECOG & $0(0-2)$ & $0(0-2)$ \\
Median HCT-CI & $0(0-6)$ & $1(0-6)$ \\
Hematological recovery median (days)** & $14(4-43)$ & $15(4-71)$ \\
$0.5 \times 109 / 1$ neutrophils and $>20 \times 109 / 1$ platelets & & \\
Median OS after ASCT, CI $(95 \%) \mathrm{p}=0.134$ & $107.27(84.3-$ & $71.61(71.5-$ \\
& $130.23)$ & $71.72)$ \\
Median PFS from ASCT, CI $(95 \%) \mathrm{p}=0.275$ & $32.43(18.79-$ & 43.22 \\
& $46.07)$ & $(30.34-56-$ \\
& & $1)$ \\
\hline
\end{tabular}

[[P667 Table] Basic information]

\section{P668}

Bortezomib Induces Apoptosis with Increasing Oxidative Stress by Mitochondria-specific Reactive Oxygen Species via a Consequence of Peroxiredoxin III Oxidation with Multimer Formation in Multiple Myeloma

Chu Myong Seong ${ }^{1}$, Yeung-Chul Mun ${ }^{1}$, Jee-Young Ahn ${ }^{1}$, Eun-Sun Yoo $^{2}$, Soon Nam Lee ${ }^{1}$, Hyun Ae Woo ${ }^{3}$, Sue Goo Rhee ${ }^{4}$

${ }^{1}$ Ewha Womans University School of Medicine, Department of Internal Medicine, Seoul, Korea, Republic of; ${ }^{2}$ Ewha Womans University School of Medicine, Department of Pediatrics, Seoul, Korea, Republic of; ${ }^{3}$ Ewha Womans University, Graduate School of Parmaceutical Science, Seoul, Korea, Republic of; ${ }^{4}$ Yonsei University College of Medicine, Yonsei Biomedical Research Institute, Seoul, Korea, Republic of

Background: Though the Bortezomib(BTZ) is a effective drug that achieves excellent response in newly diagnosed Multiple Myeloma(MM) via increasing oxidative stress, we need to elucidate not only the mechanisms that how ROS be increased and the roles of antioxidants especially PRX but also the ways that potentiates antimyeloma treatment in proteosome inhibitors, BTZ. Aims of this study are to identify the source of ROS during the treatment of BTZ in MM cell lines and how antioxidant enzyme, especially Trx 2 and Trx-R2, have the roles in ROS generation with BTZ. We also tried to find the way to potentiate the effect of ROS production to have more apoptosis of MM cell lins by down regulation of Trx 2 and Trx-R2. 
Methods: The human MM cell lines were treated with BTZ to induce apoptosis in RPMI-1640 medium supplemented with $10 \%$ FBS in $\mathrm{CO} 2$ humidified atmosphere at $37^{\circ} \mathrm{C}$. Apoptosis was measured by staining with 7 -aminoactinomycin $\mathrm{D}(7-\mathrm{AAD})$ with flow cytometry. 2, 7-dichlrodihydro-fluorescein-diacetate(H2DCF-DA) and MitoSOX Red were used to detect cellular and mitochondrial levels of ROS. SO2 form for PRX I, PRX II, and PRX III were detected by western blot assay. Steady state level of sulfiredoxin(SRX) and caspase 3, 9 were also studied by western blot analysis. To evaluate of the effect of SRX depletion, NB4 cells were transfected with small interfering RNA(siRNA).

Results: Apoptosis of MM cell after BTZ treatment was increased in concordance with mitochondrial ROS increment of MM cells. Monomer, indicated active form of Prx III was decreased and dimer, indicated inactive form, of Prx III was increased in MM cells after treatment of BTZ among Prxs. Down regulation of Trx-R2 by siRNA upregulated ROS generation and apoptosis in MM cells, together with decrease in Trx 2 and increase in multimer of PrxIII. In addition, there was synergistic effect for apoptosis of $\mathrm{MM}$ cells with Trx-R2 inhibition by siRNA and BTZ treatment simultaneously. However, increment of cysteine SO2 PrxIII was not observed. Meanwhile, Srx, reducing enzyme of SO2 Prx, was induced in MM cells after BTZ treatment. Down regulation of Srx by siRNA did not promote ROS generation or apoptosis in BTZ-treated MM cells.

Conclusions: Our results showed inactivation of PrxIII by multimer formation as hyperoxidation is found during BTZ-induced mitochondrial ROS generation and apoptosis in MM cells. This PrxIII oxidation was due to down regulation of reduced Trx2 and Trx-R2 in BTZ-treated MM cells. To design of raising ROS stress and down regulation of anti-oxidants (ie, Trx), as a treatment-strategies may be useful to potentiate BTZ-induced apoptosis in MM cells.

Conflict of interest: All of the authors have nothing to disclose.

\section{P669}

Abstract previously published

\section{P670}

Abstract previously published

\section{P671}

Comparison of two post-transplant predictive indexes: Day 100 is a better time-point for response evaluation after autologous stem cell transplantation in Multiple Myeloma
Mohammad Ma'koseh, Shanta Sharma, Ahmed Al-Shyoukh, Salwa Sa'deh, Khaled Halahleh, Eman Khattab, Mayada Abu Shanap, Hussam Abu-Jazar, Nilly Hussein, Rula Najjar, Laith Al Dahabreh, Maher Sughayer, Hasan Hashem, Rawad Rihani, Abdelghani Tbakhi

King Hussein Cancer Center, Amman, Jordan

Background: High-dose chemotherapy (HDC) followed by autologous stem cell transplantation (ASCT) remains the standard of care for young patients with multiple myeloma (MM). Timing for response evaluation after ASCT is not well studied. We retrospectively evaluated the correlation between response on day 30 and day 100 with in terms of progression free survival (PFS) and overall survival (OS).

Methods: Charts of patients diagnosed with multiple myeloma who received HDC and ASCT at King Hussein Cancer Center (KHCC) from January 2008 till December 2015 were retrospectively reviewed. Results of serum protein electrophoresis and urine protein electrophoresis on day 30 and day 100 post ASCT were collected, and response was determined according to the criteria of International Working Group For Myeloma. PFS and OS were calculated using Kaplan Meier method.

Results: One hundred and nineteen patients were included. Median follow-up was 39.8 months. Complete response (CR) was achieved in $53.8 \%$ and $55.5 \%$ of patients on day 30 and day 100 , respectively. On day 100 , $17 \%$ of patients had better response than day 30 , while $17 \%$ had worse response and $66 \%$ of patients had the same response. On day 30, patients achieved CR didn't have significantly better PFS or OS compared to patients not achieving CR (35.4 vs 22.1 months, $p$ : 0.058$)$ and (92.6 vs not reached $p: 0.96$ ), respectively, while on day 100 , they had significantly better PFS (33.8 vs 18.1 months, $p: 0.0047$ ) but results were not significant for OS (92.6 vs 52.1 months $p: 0.46)$. Moreover, patients who achieved a response on day 30, didn't have better PFS or OS compared to patient whom response was stable disease (SD) or disease progression (DP) (97.8 vs 47.1 months $p$ : 0.08 ) and (30.2 vs 18.9 months, $p$ : 0.09 ) respectively, while on day 100 , PFS was significantly better in responders (30.6 vs 16.9 months, $p: 0.015)$ but was not significant for OS (92.6 months vs not reached $p: .0,88$ ).

Conclusions: After HDC and ASCT for MM, we recommend doing response evaluation on day 100 rather than day 30 as it better correlates with PFS . Further studies are required to confirm this finding in the era of consolidation and maintenance treatment.

Conflict of interest: [M. Ma'koseh]: nothing to disclose. 


\section{P672}

Correlation of IgA and IgG Heavy/Light chain analysis with M-protein measurement by Capillary Zone Electrophoresis

Lidia Gartcheva ${ }^{1}$, Penka Ganeva ${ }^{2}$, Violeta Petkova ${ }^{1}$, Lidia Petrov ${ }^{2}$, Keranka Dimitrova ${ }^{1}$, Branimir Spassov ${ }^{3}$, Margarita Guenova ${ }^{1}$

${ }^{1}$ University National Specialised Hospital for Active Treatment of Haematological Diseases, Laboratory Haematopathology and Immunology, Sofia, Bulgaria; ${ }^{2}$ University National Specialised Hospital for Active Treatment of Haematological Diseases, Transplantation Unit, Sofia, Bulgaria; ${ }^{3}$ University National Specialised Hospital for Active Treatment of Haematological Diseases, Sofia, Bulgaria

Background: Capillary zone electrophoresis (CZE) and total immunoglobulins measurement (tIg) are standard techniques for the quantification of monoclonal protein (M-Ig). Hevylite ${ }^{\mathrm{TM}}$ allows quantitative discrimination between heavy/light chain (HLC) Igא and Ig $\lambda$ (involved/ uninvolved) immunoglobulin pairs. We compared measurement of M-Ig by CZE and tIg to Hevylite ${ }^{\circledR}$.

Methods: 102 serial serum samples from 8 patients with $\operatorname{IgA}$ and 168 serial serum samples from $19 \mathrm{IgG}$ intact multiple myeloma patients were prospectively evaluated. M-Ig was measured using CZE (Sebia Capillarys 2 system) and $\operatorname{tg} A, \operatorname{IgA\kappa } / \operatorname{Ig} A \lambda$ and $\operatorname{IgG} \kappa / \operatorname{IgG} \lambda$ HLC concentrations on a SPAPLUS analyser ${ }^{\circledast}$. Passing Bablok fit analysis was used to determine correlation between assays.

Results: A total of 51/102 samples from IgA MM patients had detectable M-Ig by CZE HLC analysis was performed with 34/51 samples of which 32/34 (94\%) exhibited an abnormal HLC ratio. Measurement of involved HLC (iHLC) (median: 12.45g/L; range:0.27-44.71 g/L) compared well with M-Ig measurement by CZE (median: $11.04 \mathrm{~g} / \mathrm{L} ; \quad$ range: $1.2-37.17 \mathrm{~g} / \mathrm{L}) \quad \mathrm{y}=1.2 \mathrm{x}+2.40 \quad \mathrm{R}^{2}=0.94$. TIgA was measured in 65/102 serial samples. Further, tIgA was above the normal range in 15/65 (23.1\%) samples and in all 15 samples the HLC ratio was also abnormal. iHLC (median:0.88g/L range:0.05-21.55g/L) correlated well with total IgA (median: $1.44 \mathrm{~g} /$;range: $0.227-21.11 \mathrm{~g} / \mathrm{L} \mathrm{1}$ ) $\mathrm{y}=0.85 \mathrm{x}$ $+-0.85, \mathrm{R}^{2}=0.98$. Changes in iHLC (median:32.20\% range:-96.36-347.8\%) correlated with changes in M-Ig (median:8.13\%; range -96.6-210\%) $\mathrm{y}=1.59 \mathrm{x}+0.15 \mathrm{R}^{2}=$ 0.94 and tIgA ((iHLC median:-81.98\% range:-97.74$80.66 \%$ TIgA range $-96.78-57.04 \% \quad(\mathrm{y}=1.06 \mathrm{x} \quad+0.01$ $\left.\mathrm{R}^{2}=0.95\right)$ ). For $\mathrm{IgG} \mathrm{MM}$ patients, $144 / 180$ samples had detectable M-Ig with 132/144 samples analysed for HLC. An abnormal HLC ratio was recorded in 92/132 (70\%). Measurement of iHLC (median:11.32g/L; Range:3.72$60.53 \mathrm{~g} / \mathrm{L}$ ) correlated with M-Ig measured by CZE ((median:7.16g/L; range0.29-66.64g/L) $\left.\mathrm{y}=0.83+5.2 \mathrm{R}^{2}=0.81\right)$. Changes in iHLC (median:-4.53\%; range:-81.62-489.7\%) from baseline also correlated with changes to M-protein ((median:-43.46\%; range: $-97.91-748.7 \%) \quad \mathrm{y}=0.90 \mathrm{x}+0.20$ $\left.\mathrm{R}^{2}=0.87\right)$.

Conclusions: M-Ig measurement is comparable between Hevylite $^{\circledR}$ and CZE for monoclonal protein detection.

Conflict of interest: Nothing to disclose

\section{P673}

CXCL13 chemokine induction is dependent on BTK signaling and is involved in $\mathrm{M} 2 \mathrm{C}$ macrophage polarization and tumor-supportive osteolytic microenvironment: novel targets in multiple myeloma milieu

Katia Beider ${ }^{1}$, Valeria Voevoda ${ }^{1}$, Hanna Bitner ${ }^{1}$, Evgenia Rosenberg $^{1}$, Hila Magen ${ }^{1}$, Olga Ostrovsky ${ }^{1}$, Robert Mikhelashvili, Elena Ribakovsky ${ }^{1}$, Avichai Shimoni ${ }^{1}$, Lola Weiss ${ }^{2}$, Michal Abraham $^{2}$, Amnon Peled ${ }^{2}$, Arnon Nagler ${ }^{1}$

${ }^{I}$ Sheba Medical Center, Ramat Gan, Israel; ${ }^{2}$ Hadassah Hebrew University Hospital, Jerusalem, Israel

Background: Studying the mechanism by which neoplastic plasma cells shape the BM microenvironment may help to develop new therapeutic targets to treat MM. Here we identify B-cell chemo-attractant CXCL13 (BCA-1) being a novel factor expressed in the tumor microenvironment of MM, associated with M2-like macrophage polarization, osteoclastogenesis and extramedullary progression.

Methods: We utilized in vivo xenograft model of BMdisseminated myeloma, as well as analysis of MM cell lines, stromal components and primary samples from patients with MM.

Results: Analysis of the BM of MM-bearing mice inoculated with human CXCR4-expressing RPMI8226 cells revealed a significant increase in $\mathrm{M} 2 \mathrm{M} \Phi$ in comparison to control mice $(\mathrm{p}<0.01)$. Attempts to characterize the MMassociated changes in BM milieu revealed murine chemoattractant CXCL13 being one of the most increased factors upon MM development. Furthermore, elevated amounts of CXCL13 were detected in blood of MM-bearing animals in comparison to healthy controls. Immunohistochemical staining identified myeloid cells as the main source of increased murine CXCL13 in MM-occupied BM. Importantly, murine CXCL13 mRNA expression strongly correlated with human $\beta 2$-microgllobulin mRNA levels $(\mathrm{p}<$ $0.0001, R^{2}=0.64$ ), demonstrating the interrelation between tumor burden and CXCL13 induction. Moreover, reduction in the MM load with anti-myeloma agents including bortezomib and panobinostat, resulted in parallel decrease in murine CXCL13, both in the BM and blood, suggesting the possible use of CXCL13 levels as a surrogate marker for response to anti-MM novel therapies. 
CXCL13 expression by M $\Phi$ is associated with antiinflammatory M2c phenotype. Indeed, we observed a significant up-regulation of additional M2c markers, such as MERTK and MRC1 in the BM infiltrated with MM in strong correlation with CXCL13 expression $(\mathrm{p}<0.001$, $\left.\mathrm{R}^{2}=0.65\right)$. In vitro studies confirmed the ability of MM cell lines $(n=6)$ to induce CXCL13 production and concurrent expression of M2c markers (MERTK, CD206, CD163) in co-cultured MФ. Furthermore, interaction with $\mathrm{M} \Phi$ reciprocally induced CXCL13 expression in MM cell lines and in primary human CD138+ cells. Mechanistically, TLR4 and Bruton tyrosine kinase (BTK) signaling were found to be involved in the MM-mediated induction of CXCL13 in MФs. In addition, TLR4 stimulation with LPS or soluble CD138 increased CXCL13 secretion from MФ, while BTK inhibitor ibrutinib interfered with the CXCL13 induction resulted from the MM cell interaction.

We than used osteoclastogenic assays to elucidate the down-stream effects of the elevated CXCL13. Recombinant CXCL13 as well as medium produced by co-cultured of MM and M $\Phi$ increased RANKL expression and induced TRAP + osteoclast formation in vitro, while CXCL13 neutralization blocked these activities.

Finally, the presence of CXCL13 in primary MM samples was evaluated. Elevated levels of CXCL13 transcript and protein were detected in BM aspirates from MM patients ( $\mathrm{n}$ $=24)$ in comparison to normal BM $(n=5)$.The elevated CXCL13 levels were in correlation with gene expression signature associated with osteoclast activation and M2c MФ phenotype. Furthermore, markedly increased expression of CXCL13 was observed within plasmacytoma samples $(\mathrm{n}=$ 6), suggesting that elevated CXCL13 levels may be associated with extramedullary disease.

Conclusions: Together, our findings suggest that CXCL13 may thus serve as potential novel target for the diagnosis and treatment of MM.

Conflict of interest: No conflict of interest to declare

\section{P674}

High overall survival rate, in the pre-novel agent era, following tandem autologous stem cell transplantation in newly-diagnosed patients with multiple myeloma: Results of long-term follow-up

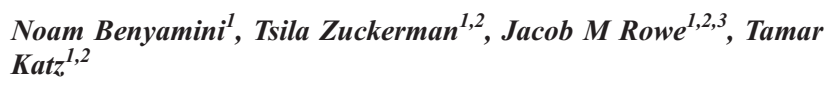

${ }^{I}$ Rambam Health Care Campus, Hematology and BMT, Haifa, Israel; ${ }^{2}$ Technion - Israel Institute of Technology, Ruth and Bruce Rappaport
Faculty of Medicine, Haifa, Israel; ${ }^{3}$ Shaare Zedek Medical Center, Hematology, Jerusalem, Israel

Background: High-dose therapy followed by autologous stem cell transplantation (ASCT) in multiple myeloma (MM) improves overall responses, increasing their depth, progression-free survival (PFS) and overall survival (OS). Studies comparing single to tandem ASCT provided no conclusive results. With introduction of novel MM treatments, tandem ASCT has become rarely used, albeit with a hint of resurgence in recent years. The current retrospective analysis evaluated safety and efficacy of tandem ASCT in MM patients, based on long-term follow-up.

Methods: Data on newly-diagnosed MM patients who underwent tandem ASCT between 1998-2009 at the Rambam Department of Hematology were evaluated (Approval \#0110-13RMB). Patients received 3-4 courses of VAD (vincristine, adriamycin, dexamethasone) induction and cyclophosphamide $3 \mathrm{~g} / \mathrm{m}^{2}$ for stem cell mobilization. High-dose melphalan $\left(140-200 \mathrm{mg} / \mathrm{m}^{2}\right)$ was used for conditioning. Second ASCT was preformed within 3-6 months from the first one. Patients who underwent second ASCT in relapse were excluded from the analysis. Novel agents were administered to progressed patients only. Outcome parameters of both transplants, including engraftment, adverse events, clinical responses, OS and PFS for a median observational period of 16.7 years (range 7.6-21.4) were analyzed.

Results: The final analysis included 69 consecutive patients [median age 54 years (range 33-66); 48 males, 21 females]. MM subtypes were $\operatorname{IgG}, \operatorname{IgA}$ and free light chain in $42(60.8 \%), 14(20.2 \%)$ and 10 (14.5\%) patients, respectively. Three patients $(4.5 \%)$ presented with nonsecretory myeloma. Median treatment duration from diagnosis to the first ASCT was 6 months (range 3.138.2); median time between ASCTs was 4.5 months (range $3.1-9.4)$. A median of $12.8 \times 10^{6}(2.5-46.8)$ and $11.3 \times 10^{6}(3-$ 38) of $\mathrm{CD}_{3}{ }^{+}$cells/kg were administered in first and second transplant, respectively. Engraftment was comparable in both ASCTs: median time to neutrophil engraftment $(>500$ cells/ $\mu \mathrm{l})$ being 10 days and to platelet engraftment $(>25,000$ cells/ $\mu \mathrm{l}) 13$ and 12 days for the first and second transplants, respectively. Hospitalization length was similar in both transplantations (15 versus 14 days). No significant adverse events were observed during ASCTs; 2 therapy-related deaths occurred 6 and 8 weeks after second ASCT. Response rate to induction was $84.8 \% ; 19.6 \%$ of patients achieved very good partial response (VGPR) or better. The overall response rate increased following the first ASCT (95.7\%, with $53.2 \%$ of patients achieving VGPR or better) and was further improved after the second transplant (97.9\%, with $76.6 \%$ of patients demonstrating VGPR or 
better). Median follow-up (from diagnosis until death/latest evaluation) was 7.7 years (range 1.0-19.6), with 5-year, 7year and 10-year OS being 57\%, 46\% and 36\%, respectively. The 10-year PFS was $15 \%$. At a median of 16.9 years from diagnosis, $18(26 \%)$ are still alive.

Conclusions: The current analysis of the long-term follow-up in 69 consecutive MM patients demonstrates that tandem ASCT, given as part of initial induction, is safe and efficacious, providing high survival rates. This outcome is at least comparable to that observed with novel and potentially toxic agents that need to be continuously administered in this clinical setting. Hence, as the most potent chemotherapeutic modality, tandem ASCT needs to be reevaluated in the current era.

Clinical Trial Registry: NA

Conflict of interest: nothing to disclose

\section{P675}

Impact of induction regimen duration before autologous stem cell transplantation in myeloma

Laurent Garderet ${ }^{1}$, Giulia Sbianchi ${ }^{2}$, Steffie van der Werf ${ }^{3}$, Didier Blaise $^{4}$, Nigel Russell ${ }^{5}$, Holger Auner ${ }^{6}$, Patrice Chevallier ${ }^{7}$, Hareth Nahi $^{8}$, Péter Reményi', Ibrahim Yakoub-Agha ${ }^{10}$, Cecilia Isaksson ${ }^{11}$, Marta Krejci ${ }^{12}$, Paul Browne ${ }^{13}$, Stig Lenhoff ${ }^{4}$, Muñiz Soledad González $^{15}$, Rocio Parody Porras ${ }^{16}$, Wieslaw Wiktor-Jedrzejczak ${ }^{17}$,

A

Overall survival by induction - collection group

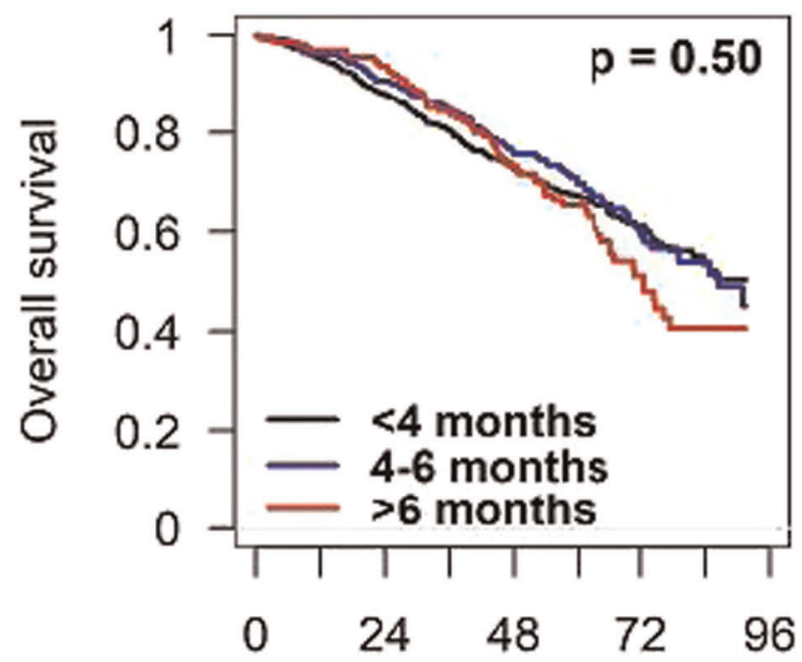

Months since transplant
Keith M. O. Wilson ${ }^{18}$, Marek Trneny ${ }^{19}$, Xavier Poire ${ }^{20}$, Jakob Passweg $^{21}$, Esa Jantunen ${ }^{22}$, Edgar Jost ${ }^{23}$, Stefan Schönland ${ }^{24}$, Nicolaus Kröger ${ }^{25}$

${ }^{1}$ Hopital Saint Antoine, Paris, France; ${ }^{2}$ Tor Vergata University of Rome, Rome, Italy; ${ }^{3}$ EBMT Data Office Leiden, Leiden, Netherlands; ${ }^{4}$ Institut Paoli Calmettes, Marseille, France; ${ }^{5}$ Nottingham University, Nottingham, United Kingdom; ${ }^{6}$ Hammersmith Hospital, London, United Kingdom; ${ }^{7}$ CHU Nantes, Nantes, France; ${ }^{8}$ Karolinska University Hospital, Stockholm, Sweden; ${ }^{9}$ St. István \& St. Laszlo Hospital, Budapest, Hungary; ${ }^{10} \mathrm{CHU}$ de Lille, Lille, France; ${ }^{11}$ Umea University Hospital, Umea, Sweden; ${ }^{12}$ University Hospital Brno, Brno, Czech Republic; ${ }^{13}$ St. James 's Hospital, Dublin, Ireland;

${ }^{14}$ Skanes University Hospital, Lund, Sweden; ${ }^{15}$ Hospital Universitario Central de Asturias, Asturias, Spain; ${ }^{16}$ ICO - Hospital Duran $i$ Reynals, Barcelona, Spain; ${ }^{17}$ Central Clinical Hospital, Warsaw, Poland; ${ }^{18}$ University Hospital of Wales, Cardiff, United Kingdom; ${ }^{19}$ Charles University Hospital, Prague, Czech Republic; ${ }^{20}$ Cliniques Universitaires St. Luc, Brussels, Belgium; ${ }^{21}$ University Hospital, Basel, Switzerland; ${ }^{22}$ Kuopio University Hospital, Kuopio, Finland; ${ }^{23}$ University Hospital Aachen, Aachen, Germany; ${ }^{24}$ University of Heidelberg, Heidelberg, Germany; ${ }^{25}$ University Hospital Eppendorf, Hamburg, Germany

Background: In newly diagnosed myeloma, induction regimen aims to reach the best response before proceeding to ASCT. Numerous regimens have been tested with different length. A longer treatment does not necessarily improve the response.

Methods: Within the Calm database, we selected 1064 patients between 2008 and 2012 who were not progressive

B

Overall survival by induction treatment

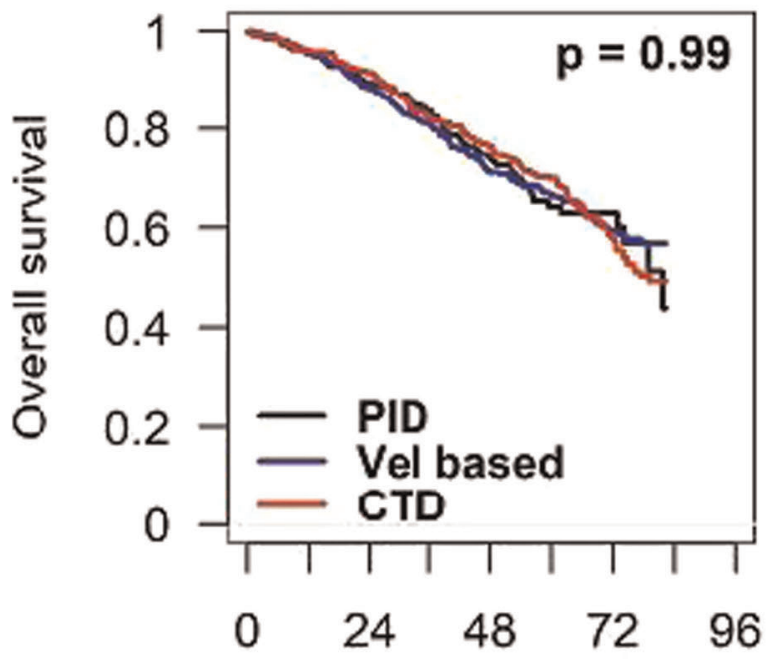

Months since transplant

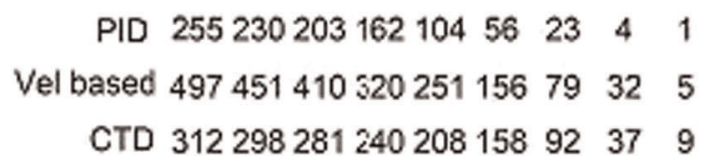

[P675 Figure] [OS according to treatment duration and regimen] 
before proceeding to ASCT after a single line of therapy. We split the patients according to the time from the beginning of induction to the time of stem cell collection as a surrogate marker for the number of treatment cycles: less than 4 months (mo), between 4-6 mo and $>6$ mo. We analyzed three types of regimen: Velcade plus an IMiDs plus steroids ( $\mathrm{PID}=\mathrm{VTD}$ or VRD), Velcade-based regimen (VD or VCD), and cyclophosphamide plus thalidomide plus dexamethasone (CTD).

Results: Patient characteristics (sex, myeloma isotype) were not different between the three time periods but a Karnofsky score $<90$ was higher in the $<4$ mo $(\mathrm{p}<0.01)$. Median age was 60 years (25-77). The median time from initiation to collection was 3.4 months $(0.4-20),<4$ months: 631 pts, $4-6$ months: 265 pts, $>6$ mo: 168 pts. 255 pts (24\%) had PID, 497 (46\%) Vel, 312 pts (30\%) CTD. Vel was mostly given $<4$ mo while CTD in the $4-6$ and $>6$ mo periods: $35 \%$ vs $55 \%$ vs $10 \%$ (<4 mo); $7 \%$ vs $40 \%$ vs $53 \%$ (4-6 mo); $6 \%$ vs $27 \%$ vs $67 \%$ ( $>6 \mathrm{mo}$ ) (PID vs Vel vs CTD, respectively) $(\mathrm{p}<0.01)$. The rate of response $(\mathrm{MR} / \mathrm{stable}$, PR, VGPR) post induction treatment were not different between 4 mo vs $4-6$ mo and $>6 \mathrm{mo}$ ( $\mathrm{p}=0.5$ ): $5 \%$ vs $55 \%$ vs $40 \%$ (< $4 \mathrm{mo}) ; 3 \%$ vs $55 \%$ vs $42 \%$ ( $4-6 \mathrm{mo}) ; 3 \%$ vs $60 \%$ vs $37 \%$ ( $>6 \mathrm{mo}$ ). The median follow-up was 56 months (1-105) and similar in the three time periods. Median OS was 85 mo (76-NR) with no difference between $<4$ mo (93 mo), 4-6 mo $(87 \mathrm{mo})$ and $>6 \mathrm{mo}(73 \mathrm{mo})(\mathrm{p}=0.5)$ (Figure $1 \mathrm{~A})$ and no difference between the three induction regimens: PID (82 mo); Vel (92 mo) and CTD (79 mo) $(\mathrm{p}=0.99)$ (Figure 1B). Median PFS was 29 mo (27-31) similar in the three time periods $(p=0.5)$ and the three regimens $(p=0.3)$. In multivariate analysis, only ISS III and an interval between diagnosis and initiation of treatment less than 1 month had a worse OS. Type of induction regimen and duration of induction regimen had no impact on OS. PFS was influenced by response to induction $(\mathrm{p}=0.01)$, response at transplant $(\mathrm{p}=0.006)$ and interval between diagnosis and initiation less than 1 month $(\mathrm{p}=0.016)$. Similar to OS, PFS was not related to type of induction regimen nor duration of induction. High risk cytogenetic defined by del $17 \mathrm{p}$ and/or $\mathrm{t}$ $(4-14)$ was associated with a worse OS $(\mathrm{p}<0.01)$ and PFS $(\mathrm{p}=0.04)$.

Conclusions: Response to induction regimen and response at transplant influenced PFS but not OS. The three types of induction regimen gave similar results and so, whatever their length of treatment.

Conflict of interest: Supported by a grant from Sanofi

No conflict of interest from any co-authors related to this work.

\section{P676}

Improving Safety of Autologous Hematopoietic Stem Cell Transplant in Patients with Light Chain (AL) Amyloidosis

Gonzalo Gutiérrez-García, María Teresa Cibeira, Laura Rosiñol, Ana B. Moreno, Àlex Bataller, Carlos Fernández de Larrea, Marta Garrote, Luis Gerardo Rodríguez, María Suárez-Lledó, Sofia Jorge, Monserrat Rovira, Carmen Martínez, Alvaro Urbano-Ispizua, Joan Bladé, Francesc Fernández-Avilés

Hospital Clinic de Barcelona, Hematology, Barcelona, Spain

Background: Autologous Hematopoietic Stem Cell Transplant (ASCT) is able to achieve hematological and organic responses and improve survival in patients with amyloidosis. However, ASCT role has been controversial due to high transplant-related mortality (TRM) rate. A tailored patient selection policy, performing ASCT in specialized centers and improving supportive measures are the main strategies to decrease TRM. The purpose of this study was to analyze the impact of post-transplant GCSF administration and primary prophylaxis of the engraftment syndrome with corticosteroids in the outcome of patients with amyloidosis undergoing an ASCT beyond a tailored patient selection policy and improving supportive care measures.

Methods: 66 patients with amyloidosis who underwent an ASCT between 1997 and 2017 in a single institution were included. The median age was 57.7 years (34 to 72). All patients received melphalan as conditioning $\left[200 \mathrm{mg} / \mathrm{m}^{2}\right.$ in $53(80 \%)$ and $140 \mathrm{mg} / \mathrm{m}^{2}$ in $\left.13(20 \%)\right]$. In our amyloidosis transplant program we are making a tailored selection policy since 2007, we have not used GCSF posttransplant $(300 \mu \mathrm{g} / 24 \mathrm{~h}$ from day +7 until neutrophils recovery) since 2009 and we are using corticosteroids prophylaxis $(0.5 \mathrm{mg} / \mathrm{kg} / \mathrm{day}$ from day +7 until neutrophils $\geq 500 / \mathrm{mm}^{3}$ ) from 2014 . We have established 4 patients risk subgroups according to selection policy, use of GCSF and corticosteroids: group A [non-selection and GCSF, 29 (44\%)], group B [selection and GCSF, $13(20 \%)$ ], group C [selection and non-GCSF, 14 (21\%)] and group D [selection, non-GCSF and corticosteroids prophylaxis, 10 $(15 \%)]$.

Results: The median follow-up was 9.46 years (CI 95\%= 8.13-10.8). No significant differences regarding age, gender, comorbidity index and median CD34+ dose among different subgroups (A, B, C and D) were found ( $>0.05$ ). In group $\mathrm{A}$, there was a higher patients proportion who received melphalan $140 \mathrm{mg} / \mathrm{m}^{2} \quad[10 \quad(35 \%)$ than other subgroups, $p=0.04]$. The incidence of mucositis and intestinal toxicity grade $\geq 3$ was $8 \%$ and $2.2 \%$, respectively. The diagnosis of engraftment syndrome established based on Maiolino criteria and infectious fever with 
microbiological isolation were $41(62 \%)$ and 19 (29\%), respectively. The Global TRM was $21 \%$ and the 5-years OS was $68 \%$. The complete analysis among subgroups is detailed in the table. In the multivariate analysis, variables with impact in TRM were: subgroups of risk $(\mathrm{p}=0.01$, HR $2.4)$ and comorbidity index ( $p=0.04$, HR 2.1$)$.

\begin{tabular}{llllll}
\hline N=66 & $\begin{array}{l}\text { Group A, } \\
\mathrm{N}=29 \\
(44 \%)\end{array}$ & $\begin{array}{l}\text { Group B } \\
\mathrm{N}=13 \\
(20 \%)\end{array}$ & $\begin{array}{l}\text { Group C, } \\
\mathrm{N}=14 \\
(21 \%)\end{array}$ & $\begin{array}{l}\text { Group D, } \\
\mathrm{N}=10 \\
(15 \%)\end{array}$ & $\mathrm{p}$ \\
\hline $\begin{array}{l}\text { 2 organs involved } \\
\text { Cardiac involvement }\end{array}$ & $12(41 \%)$ & $3(23 \%)$ & 0 & $2(20 \%)$ & $0.03^{*}$ \\
$\begin{array}{l}\text { Engraftment } \\
\text { syndrome }\end{array}$ & $22(76 \%)$ & $10(77 \%)$ & $7(50 \%)$ & $2(20 \%)$ & $0.008^{*}$ \\
$\begin{array}{l}\text { Infection fever } \\
\text { (microbiolgical }\end{array}$ & $11(38 \%)$ & $5(39 \%)$ & $2(14 \%)$ & $1(10 \%)$ & 0.1 \\
$\begin{array}{l}\text { isolation +) } \\
\text { TRM }\end{array}$ & $11(38 \%)$ & $2(15 \%)$ & $1(7 \%)$ & $0(0)$ & $0.02^{*}$ \\
\begin{tabular}{l} 
5-years OS \\
\hline
\end{tabular} & $35 \%$ & $62 \%$ & $86 \%$ & $100 \%$ & $0.05^{*}$ \\
\hline
\end{tabular}

\section{[[P676 Table] Table 1]}

Conclusions: A tailored selection policy together with the avoidance of GCSF and use of corticosteroids posttransplant in patients with amyloidosis undergoing an ASCT seem to contribute to reduce TRM.

Conflict of interest: All of the authors have nothing to disclose.

\section{P677}

Induction treatment with RVD-lite for transplant-eligible patients with multiple myeloma

Nobuhiro Tsukada, Kota Sato, Kanji Miyazaki, Yumiko Yoshiki, Yu Abe, Kiyoshi Okazuka, Tadao Ishida, Kenshi Suzuki

Japanese Red Cross Medical Center, Division of Hematology, Tokyo, Japan

Background: Lenalidomide, bortezomib, and dexamethasone combination (RVD) is one of the effective induction treatment regimens for transplant-eligible newly diagnosed patients with multiple myeloma (NDMM), but modifications are often required because of adverse events (e.g. skin rash, neutropenia, and others). We have introduced modified RVD (RVD-lite) regimen for transplant-eligible NDMM since March 2016. Here, we report a retrospective analysis of the efficacy and safety of RVD-lite regimen for Japanese patients.

Methods: RVD-lite regimen was introduced for 24 patients between March 2016 and April 2017 at Japanese Red Cross Medical Center. RVD-lite regimen is consisted of lenalidomide $15 \mathrm{mg} /$ day (days 1-21), bortezomib $1.3 \mathrm{mg} /$ $\mathrm{m}^{2}$ (days 1, 8, 15, 22), and dexamethasone $40 \mathrm{mg} /$ body (days $1,8,15,22$ ).
The response, adverse events, progression free survival, and efficacy of autologous stem cell collection were retrospectively analyzed.

Results: Median age was 57 (range 46-67), M/F 10/14, $\operatorname{IgG/IgA/IgD/BJ~13/3/1/7,~and~ISS~I/II/III~5/17/2.~Eight~out~}$ of 24 had $\mathrm{t}(11 ; 14)$ and 4 had $\mathrm{t}(4 ; 14)$. Grade 4 neutropenia (< $500 / \mu \mathrm{L}$ ) was observed in 1 patient and no grade 2 thrombocytopenia $\left(<7.5 \times 10^{4} / \mu \mathrm{L}\right)$ was observed. Grade 3 non-hematologic adverse event was observed in 5 patients with skin rash. Response after RVD-lite was $\geq \mathrm{CR} 13 \%$, $\geq$ VGPR $50 \%$, and $\geq$ PR $96 \%$. Peripheral blood stem cells were successfully collected from all patients and preemptive plerixafor was used in 11 out of 24 patients. Median numbers of CD34-positive cells was $5.3 \times 10^{6} / \mathrm{kg}$ (range 1.2-9.5). Up-front high-dose melphalan and autologous stem-cell transplantation (HDM/ASCT) was performed in 19 patients and best response after HDM/ASCT was $\geq \mathrm{CR}$ $58 \%$ (including sCR 53\%), $\geq$ VGPR $74 \%$, and $\geq \mathrm{PR} 100 \%$.

Conclusions: RVD-lite regimen was well tolerated and the efficacy was comparable to previous reports of the RVD regimen. Weekly administration of bortezomib is convenient for patients and $15 \mathrm{mg} /$ day of lenalidomide may lead to high compliance. Our results suggest that RVD-lite regimen is safe and useful for transplant-eligible NDMM.

Conflict of interest: The authors declare that there are no conflicts of interest.

\section{P678}

\section{Melphalan 140 versus Melphalan 200 in frail patients}

Nuno Miranda ${ }^{1}$, Joana Santos ${ }^{2}$, Filipa Moita ${ }^{1}$, Gilda Teixeira ${ }^{1}$, Isabelina Ferreira ${ }^{1}$, Fernando Leal-da-Costa ${ }^{1}$, Maria-João Gutierrez, ${ }^{1}$, Teresa Mendonça ${ }^{3}$, Manuela Bernardo ${ }^{3}$, Ines Coelho ${ }^{3}$, Manuel Abecasis ${ }^{1}$

${ }^{1}$ Instituto Portugues de Oncologia de Lisboa Francisco Gentil, BMT Unit, Lisboa, Portugal; ${ }^{2}$ Centro Hospitalar de Setúbal, Oncology, Setubal, Portugal; ${ }^{3}$ Instituto Portugues de Oncologia de Lisboa Francisco Gentil, Haematology, Lisboa, Portugal

Background: High dose chemotherapy /HDCT) remains an essential treatment modality in multiple myeloma patients, prolonging survival. Median age of presentation, and several comorbidities, mainly renal failure, present a particular challenge, in the choice of conditioning regimen. In our Unit patients with Creatinine Clearence $<60$, with severe comorbidities or considered frail by assistant physician are conditioned with Melphalan $140 \mathrm{sqm}$.

Methods: We preform a retrospective and comparative analysis, between patients above 60 years old submitted to melphalan $200 \mathrm{mg}$ sqm versus all patients treated with 140 mg sqm, during the period of 2005 to 2014 . 
Results: Seventy two patients were evaluated, 34 with Mel200 and 38 with Mel140.The median age at TMO was 63 yo in both groups. $62 \%$ and $53 \%$ were males, respectively. ISS stage was equivalent in both groups (Mel 200- ISS1, 44\%; ISS2, 24\%, ISS3 24\%, ND 8\%: Mel 140 - ISS1, 26\%, ISS2, 29\%, ISS3, 32\%, ND 13\%). Bortezomib based regimens were used in first line in $85 \%$ vs $66 \%$, thalidomide $6 \%$ vs $18 \%$. Disease status at transplant, and comparing Mel 200 pts with Mel140 was CR in 32 vs $15 \%$, PR/VGPR 56 vs $77 \%$, PD/SD 12 vs $8 \%$ $\%$. In the cohort Mel140 26\% have age below 60 yo, 8 have Creatinine $\mathrm{Cl}<60$, one has heart ischemic disease and one pulmonary disease.. Median time to neutrophil recovery was the same (12,5 versus 13 days) platelet recovery 11 days in both cohorts and median stay in the Unit 19.5 versus 20 days. Best response after BMT was CR (65 vs $42 \%, \mathrm{p}=0.53$ ), PR/VGPR (32 vs $47 \%, \mathrm{p}=0.36$ ), $\mathrm{PD} / \mathrm{SD}$ (3 vs $8 \%, p=0.09$ ). Median follow-up was 60 versus 41 months. Median PFS was 30 (16-49) vs 20 months (1032) $\mathrm{p}=0.2797$, Time to new treatment 45 (32-74) vs 25 months (15-37) $\mathrm{p}=0.0824$, and Overall Survival 68 (4193) vs 40 months (35-83) $\mathrm{p}=0.0056$.

Conclusions: The dosis of melphalan in frail patients remains a matter of discussion. This study is not a comparative trial, but populations in both cohorts were quiet similar, except for creatinine clearence, and there was no significant differences in toxicity. Although PFS was not significantly different, Mel 200 was associated with a significantly better outcome, in terms of overall survival, refleting a better PFS2, and a trend in prolongation time for new treatment was also present. When feasible, Mel 200 should be preferred.

Conflict of interest: No conflicts of interest to declare

\section{P679}

\section{Multiple Myeloma: Allogeneic or Autologous Hematopoietic Stem Cell Transplantation?}

\section{Mohammad Vaezi ${ }^{1}$, Sahar Tavakkoli ${ }^{2}$, Amir Kasaeian ${ }^{1}$, Kamran Alimoghadam $^{1}$, Hosein Kamranzadeh ${ }^{1}$, Seyed Asadillah Mousavi ${ }^{1}$, Ardeshir Ghavamzadeh ${ }^{1}$}

${ }^{1}$ Tehran University of Medical Sciences, Hematology, Oncology and Stem Cell Transplant Research Center, Tehran, Iran, Islamic Republic of; ${ }^{2}$ Tehran University of Medical Sciences, Tehran, Iran, Islamic Republic of

Background: Despite remarkable progress in survival with the availability of novel agents, a majority of patients with multiple myeloma (MM) relapse and the curability of MM remains limited.Autologous stem cells transplantation (auto-HCT) is an accepted method in multiple myeloma (MM) patients, but usually it is not curative. The issue of
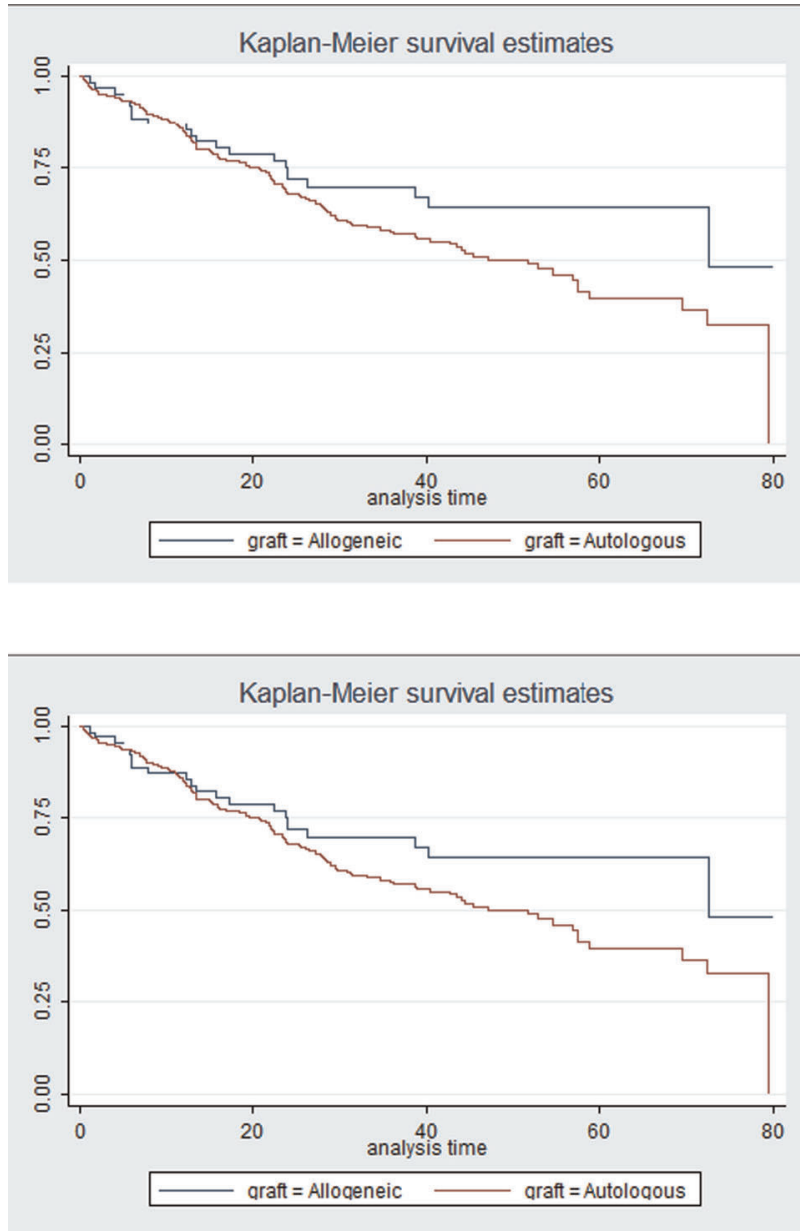

[P679 Figure] [OS]

allogeneic hematopoietic stem cells transplantation (alloHCT) is challenging yet for myeloma. We investigated alloHCT in MM and compared with auto-HCT.

Methods: In this retrospective study, we recruited 437 patients from January 2011 to January 2016285 (82.13\%) patients in autologous group and $62(17.87 \%)$ in allogeneic group.

Both group of our patients, underwent stem cells transplantation after achieving a complete respons or partial respons after first line induction therapy or salvage.

We performed allogeneic HCT by using peripheral blood stem cells in our center for patients who were relatively young (less than 55 years old) with good performance, who had matched sibling donor and accepted to undergo allogeneic HCT. The conditioning regimens in autologous groups was only Melphalan $200 \mathrm{mg} / \mathrm{m} 2$ and for allogeneic group was Fludarabine $30 \mathrm{mg} / \mathrm{m} 2$ plus Melphalan 140mg/ $\mathrm{m} 2$ in 5 days. GVHD prophylaxis consisted of Methotrexate and Cyclosporine. Then the outcomes were compared between two groups using log-rank and Gray tests and cox proportional hazard 
Results: The median follow-up in the autologous and allogeneic group was $\mathbf{4 0 . 5 7 \pm 1 . 7 1}$ months.

Five years disease-free survival of auto-HCT was $\mathbf{3 9 . 8 5 \%}$ (SD: $4.48 \%$ ) and $\mathbf{6 4 . 3 0 \% ( S D ~ : ~} \mathbf{6 . 6 5 \%}$ ) for allo-HCT patients $(\mathrm{P}$-value $=0.05)$.

Five years overall survival of auto-HCT was $\mathbf{7 3 . 9 5 \%}$ (SD: $3.49 \%$ ) and $\mathbf{7 4 . 8 7 \% ( S D : ~ 6 . 3 7 \% ) ~ f o r ~ a l l o - H C T ~ p a t i e n t s ~}$ $(\mathrm{P}$-value $=\mathbf{0 . 9 3})$ showing no significant statistical difference between two groups.

Mortality rate was $\mathbf{1 8 . 6 0 \%}$ for auto-HCT and for alloHCT was $20.97 \%$. The most common cause of death between two groups was relapse and progression of disease of primary disease. Five years relapse incidence was $\mathbf{2 7 . 1 4 \% ( C I : ~} 9.04 \%, 35.30 \%$ ) for allo-HCT and $\mathbf{5 4 . 6 8 \%}$ (CI: $42.02 \%, 65.09 \%)$ for auto-HCT (Gray's test p-value = 0.01). The five years TRM incidence was $\mathbf{8 . 4 7 \%}$ (CI: $2.92 \%, 23.33 \%$ ) and $\mathbf{5 . 4 6 \%}$ (CI: $3.14 \%, 12.98 \%$ ) in allogeneic and autologous patients respectively (Gray's test $\mathrm{p}$-value $=0.36$ ).

Conclusions: Despite there was no statistically significant difference between two groups in terms of OS but DFS and relapse incidence was meaningfully better in allogeneic group. So, perhaps the reason of non-significant OS improvement in allogeneic group is higher early death due to higher TRM. We suggest that this study needs longer follow up to see whether allo-HCT resulted in OS improvement.

Conflict of interest: The authors have no conflict of interest regarding this study.
P680

Overall survival of patients post-transplant: study results from two phase 3 trials ASPIRE and ENDEAVOR

Hartmut Goldschmidt ${ }^{1}$, Maria-Victoria Mateos ${ }^{2}$, David Siegel ${ }^{3}$, Rafat Abonour ${ }^{4}$, Heinz Ludwig, Mihaela Obreja ${ }^{6}$, Karim Saad Iskander ${ }^{7}$, Parameswaran Hari $^{8}$

${ }^{1}$ Universität Heidelberg, Heidelberg, Germany; ${ }^{2}$ University Hospital Salamanca, Salamanca, Spain; ${ }^{3}$ Hackensack University Medical Center, John Theurer Cancer Center, Hackensack, NJ, United States; ${ }^{4}$ Indiana Cancer Pavilion, Indianapolis, IN, United States;

${ }^{5}$ Wilhelminen Cancer Research Institute, Department of Medicine I, Vienna, Australia; ${ }^{6}$ Amgen Inc., South San Francisco, CA, United States; ${ }^{7}$ Amgen Inc., Thousand Oaks, CA, United States; ${ }^{8}$ Medical College of Wisconsin, Milwaukee, WI, United States

Background: In the phase 3 ASPIRE (carfilzomiblenalidomide-dexamethasone [KRd] vs lenalidomidedexamethasone $[\mathrm{Rd}]$ ) and ENDEAVOR trials (carfilzomib-dexamethasone [Kd56] vs bortezomib-dexamethasone $[\mathrm{Vd}]$ ), carfilzomib-based regimens demonstrated superior overall survival (OS) vs standard therapies in relapsed/ refractory multiple myeloma (RRMM).

Methods: In this subgroup ad-hoc analysis of ASPIRE and ENDEAVOR, OS was evaluated based on prior autologous stem cell transplant (ASCT) status.

Results: Of the patients with prior ASCT in ASPIRE (n $=446)$ and ENDEAVOR $(\mathrm{n}=538)$, baseline patient and disease characteristics were generally balanced between treatment groups. In ASPIRE, patients with prior ASCT treated with $\mathrm{KRd}$ had an 11.4 month improvement in median OS and, for those with first relapse after ASCT, an

\begin{tabular}{|c|c|c|c|c|c|c|c|c|}
\hline & $\begin{array}{l}\text { ASPIREa } \\
\text { Prior } \\
\text { ASCT } \\
\text { KRd }\end{array}$ & $\begin{array}{l}\text { ASPIREa } \\
\text { Prior } \\
\text { ASCT Rd }\end{array}$ & $\begin{array}{l}\text { ASPIREa } \\
\text { No Prior } \\
\text { ASCT KRd }\end{array}$ & $\begin{array}{l}\text { ASPIREa } \\
\text { No Prior } \\
\text { ASCT Rd }\end{array}$ & $\begin{array}{l}\text { ENDEAVORb } \\
\text { Prior ASCT } \\
\text { Kd56 }\end{array}$ & $\begin{array}{l}\text { ENDEAVORb } \\
\text { Prior ASCT Vd }\end{array}$ & $\begin{array}{l}\text { ENDEAVORb } \\
\text { No Prior ASCT } \\
\text { Kd56 }\end{array}$ & $\begin{array}{l}\text { ENDEAVORb } \\
\text { No Prior ASCT } \\
\text { Vd }\end{array}$ \\
\hline ITT population, $\mathrm{n}$ & 217 & 229 & 179 & 167 & 266 & 272 & 198 & 193 \\
\hline $\begin{array}{l}\text { Median OS }(95 \% \text { CI }) \text {, } \\
\text { months }\end{array}$ & $52(46,66)$ & $\begin{array}{l}41(31, \\
48)\end{array}$ & $40(32,49)$ & $40(31,46)$ & $48(44, \mathrm{NE})$ & $41(33,42)$ & $51(40, \mathrm{NE})$ & $37(25, \mathrm{NE})$ \\
\hline $\begin{array}{l}\text { Median PFS }(95 \% \\
\text { CI), months }\end{array}$ & $\begin{array}{l}26(23,32) \\
\mathrm{c}\end{array}$ & $\begin{array}{l}18(14, \\
22) \mathrm{c}\end{array}$ & $26(21,31)$ & $17(14,22)$ & $\mathrm{NE}(16, \mathrm{NE}) \mathrm{c}$ & $10(9,12) \mathrm{c}$ & $18(14, \mathrm{NE})$ & $9(6,10)$ \\
\hline $\begin{array}{l}\text { First relapse after } \\
\text { frontline ASCT, } \mathrm{n}\end{array}$ & 88 & 78 & 96 & 79 & 123 & $141 d$ & 109 & 90 \\
\hline $\begin{array}{l}\text { 3-year OS }(95 \% \text { CI), } \\
\%\end{array}$ & $68(57,76)$ & $\begin{array}{l}52(40, \\
63)\end{array}$ & $51(41,61)$ & $48(36,58)$ & $66(56,74)$ & $64(55,71)$ & $64(54,73)$ & $57(45,68)$ \\
\hline $\begin{array}{l}\text { Median PFS }(95 \% \\
\text { CI), months }\end{array}$ & $\begin{array}{l}30(21,39) \\
\mathrm{c}\end{array}$ & $\begin{array}{l}18(13, \\
26) \mathrm{c}\end{array}$ & $28(20, \mathrm{NE})$ & $17(13,26)$ & $\mathrm{NE}(17, \mathrm{NE}) \mathrm{c}$ & $11(9,15) \mathrm{c}$ & $19[15, \mathrm{NE})$ & $10(7,13)$ \\
\hline $\begin{array}{l}\text { Patients with at } \geq 1 \\
\text { treatment-related } \\
\text { adverse event, } \mathrm{n}\end{array}$ & 215 & 224 & 177 & 165 & 266 & 268 & 197 & 188 \\
\hline Grade $\geq 3$ & $73 \%$ & $60 \%$ & $66 \%$ & $64 \%$ & $58 \%$ & $51 \%$ & $62 \%$ & $57 \%$ \\
\hline $\begin{array}{l}\text { Patients discontinuing } \\
\text { due to AEs }\end{array}$ & $8 \%$ & $10 \%$ & $9 \%$ & $12 \%$ & $19 \%$ & $21 \%$ & $18 \%$ & $20 \%$ \\
\hline
\end{tabular}

[[P680 Table] Study Results from Carfilzomib Studies] 
18.6 month improvement in median OS (58 months vs 39 months; $\mathrm{HR}=0.71$ [0.48, 1.0]). In ENDEAVOR, a consistent OS benefit was observed for Kd56 vs Vd across ASCT subgroups; the benefit was more evident in the no prior transplant groups. In the updated safety analysis, adverse events were comparable between patients with and without prior ASCT and consistent with previous reports (Hari et al, Leukemia 2017 Apr 25).

aData cutoff 28Apr2017.

${ }^{\mathrm{b}}$ Data cutoff 3Jan2017.

${ }^{\mathrm{c}}$ Hari et al, Leukemia 2017 Apr 25.

${ }^{\mathrm{d}}$ For PFS, $\mathrm{n}=142$.

ASCT, autologous stem cell transplant; KRd, carfilzomiblenalidomide-dexamethasone; $\mathrm{Rd}$, lenalidomide-dexamethasone; Kd56, carfilzomib-dexamethasone; Vd, bortezomib-dexamethasone; ITT, intent-to-treat; OS, overall survival; NE, not estimable.

Conclusions: These results suggest carfilzomib-based regimens led to clinically meaningful improvements in survival for patients who had received prior ASCT. For patients who were treated at first relapse, more differences were observed for KRd vs Rd after ASCT and Kd56 vs Vd for transplant-ineligible patients.

The ASPIRE and ENDEAVOR studies were supported by Onyx Pharmaceuticals, Inc., an Amgen subsidiary.

Clinical Trial Registry: NCT01568866

Conflict of interest: Hartmut Goldschmidt received research support from Amgen, BMS, Celgene, Chugai, Janssen, Sanofi, Mundipharma, Takeda, Novartis; participated in advisory boards of Adaptive Biotechnology, Amgen, BMS, Celgene, Janssen, Sanofi, Takeda; and speakers bureaus of Celgene, Janssen, Novartis, Chugai, BMS, ArtTempi.

Maria-Victoria Mateos received consulting fees from Celgene, Janssen, Amgen, Takeda.

David Siegel participated in speakers bureaus and ad boards for Amgen, Celgene, Takeda, BMS, Janssen.

Rafat Abonour consulted for Celgene, Takeda and Jensen.

Heinz Ludwig received research grants from Amgen, Takeda; and participated in speakers bureaus of Amgen, Takeda, Janssen, Celgene, BMS.

Mihaela Obreja and Karim Iskander are employees of and own stock in Amgen.

Parameswaran Hari received research grants from Amgen.

\section{P681}

Relapse and progression patterns in multiple myeloma after upfront hematopoietic stem cell transplant
Pedro Chorão ${ }^{1}$, Tânia Maia ${ }^{1}$, Rui Bergantim ${ }^{1}$, Eliana Aguiar ${ }^{1}$, Jorge Cancela Pires ${ }^{1}$, Fernanda Trigo ${ }^{1}$, José E Guimarães ${ }^{1,2}$

${ }^{1}$ Centro Hospitalar de São João, Porto, Portugal; ${ }^{2}$ Faculdade de Medicina da Universidade do Porto, Porto, Portugal

Background: Although autologous hematopoietic stem cell transplantation (ASCT) is widely performed in multiple myeloma (MM) to intensify response and improve overall survival (OS), virtually all patients experience relapse or progression $(\mathrm{R} / \mathrm{P})$. We aim to describe patterns of $\mathrm{R} / \mathrm{P}$ and determinants of OS and progression free survival (PFS) in these patients.

Methods: Raw clinical features of MM patients referred from 2008 to 2015 for melphalan-based ASCT after first line induction chemotherapy were retrospectively analyzed. Available data from 100 patients was obtained for the analysis of diagnosis and R/P characteristics. OS and PFS at 3 years (3y) were calculated and multivariate logistic regression analysis performed.

Results: Median time to $\mathrm{R} / \mathrm{P}$ was 17 months (mo) (interval 2-100) and median follow-up after R/P was $13 \mathrm{mo}$ (interval 0-94). Fifty-five per cent of patients relapsed from complete response.

In $64 \%$ of patients, the first sign of $\mathrm{MM} \mathrm{R/P}$ was biochemical. Overall, biochemical criteria for $\mathrm{R} / \mathrm{P}$ was detected in $80 \%$ of patients, of which $47 \%$ had reappearance and $42 \%$ progression of serum M-protein. Before salvage treatment was required, clinical $\mathrm{R} / \mathrm{P}$ was experienced by $66 \%$ of patients, most frequently due to hemoglobin decrease $>2 \mathrm{gr} / \mathrm{dL}$ attributable to $\mathrm{MM}(42 \%)$ and extramedullary involvement $(31 \%)$.

At R/P, anemia (28\%), bone lytic lesions (55\%) and extramedullary involvement $(25 \%)$ were the most common features and were also more frequent when present at diagnosis $(\mathrm{p}<0.05)$.

The $3 y-O S$ after R/P was $21 \%$ (median 13mo), and the $3 y-P F S$ for the 86 patients who required salvage treatment was $12 \%$ (median 9mo). Table 1 summarizes the analysis for determinants of 3y-OS and 3y-PFS. Survival was shorter for patients relapsing less than $12 \mathrm{mo}$ after upfront ASCT (median 9mo versus 14mo, $\mathrm{p}=0.027$ ). For patients requiring treatment, PFS was better for women (median $10 \mathrm{mo}$ versus $7 \mathrm{mo}, \mathrm{p}=0.136$ ). Although not statistically significant in logistic regression analysis, patients with clinical R/P at least $4 \mathrm{mo}$ after biochemical R/P seem to have better OS (median $22 \mathrm{mo}$ versus $11 \mathrm{mo}, \mathrm{p}=0.011$ ) and PFS (median $23 \mathrm{mo}$ versus $11 \mathrm{mo}, \mathrm{p}=0.005$ ), showing lower LDH and serum M-protein levels $(\mathrm{p}<0.001)$ but similar serum free light chain ratio $(\mathrm{p}=0.111)$. Patients who had a retransplant after $\mathrm{R} / \mathrm{P}$ had superior OS (median $24 \mathrm{mo}$ versus $10 \mathrm{mo}, \mathrm{p}=0.002$ ) and PFS (median $14 \mathrm{mo}$ versus $6 \mathrm{mo}, \mathrm{p}=$ $0.016)$. 


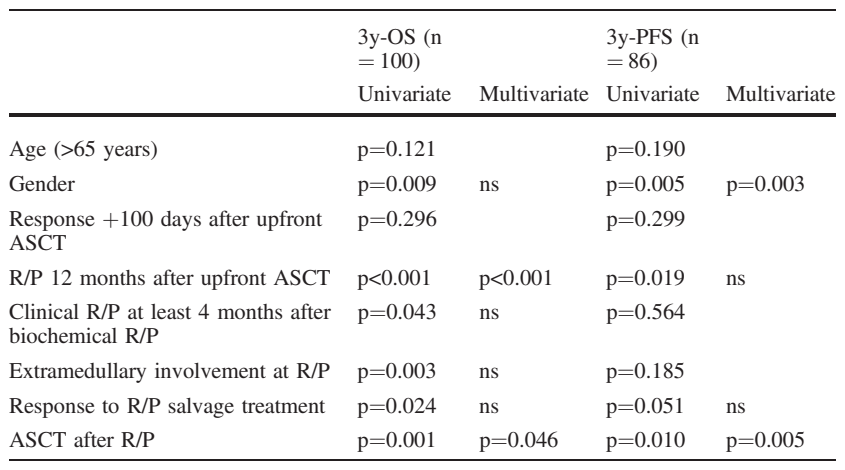

[[P681 Table] Table 1. Variables related to 3y-OS and $3 y-P F S]$

Conclusions: In our patients, the most common pattern of $\mathrm{R} / \mathrm{P}$ was biochemical. Anemia, lytic bone lesions and extramedullary involvement should be closely monitored, especially if present at diagnosis. After R/P, a new ASCT seems the best therapeutic approach to improve OS and PFS.

Conflict of interest: None of the authors has anything to disclose.

\section{P682}

Role of disease status prior autologous stem cell transplantation (ASCT) on survival in patients with multiple myeloma

Nour Ben Abdeljelil, Rym El Fatmi, Dorra Belloumi, Lamia Torjemane, Amel Lakhal, Saloua Ladeb, Tarek Ben Othman

Tunis, Centre National de Greffe de Moelle Osseuse, Tunis, Tunisia

Background: Evaluate the impact of disease status before and after autologous stem cell transplantation (ASCT) on overall survival (OS) and progressive free survival (PFS) in patients with newly diagnosed multiple myeloma under the age of 65 years.

Methods: Retrospective study conducted in 192 patients aged less than 65 years who received ASCT between February 2007 and December 2014. Induction therapy consisted of dexamethasone and thalidomide. Conditioning before autologous is based on melphalan $200 \mathrm{mg} / \mathrm{m}^{2}$. The majority of patients received maintenance therapy with thalidomide started on month 3 post-ASCT and continued until 12 months. Responses were assessed using the IMWG criteria. OS and PFS were compared according disease status using the log-rank test.

Results: The median age was 55 years (25-65yrs). Sexratio was 1.3. Monoclonal component type was complete immunoglobulin in $80.7 \%$ of cases $(n=155)$, light chain in $11.4 \%(\mathrm{n}=22)$, non-secretory myeloma in $6.3 \%(\mathrm{n}=12)$ and multiple plasmacytoma in $1.6 \%(\mathrm{n}=3)$. Before ASCT, 82 patients $(42.7 \%)$ were in CR/VGPR, $58(30.2 \%)$ in partial response (PR) and $52(26.5 \%)$ in progressive disease or induction failure. One hundred and thirty two patients $(67.3 \%)$ received maintenance therapy. Forty-four patients received salvage treatment after the first ASCT: thalidomide $(n=14)$, bortezomib $(n=22)$, lenalidomide $(n=3)$, others $(\mathrm{n}=5)$. A second ASCT was performed in 19 patients. Transplant related mortality was $1 \%$. After a median followup of 31 months (range, 15 days -112 months), the OS and the PFS were not significantly different between patients in CR/VGPR and those achieving less than VGPR before ASCT: 61 vs 58 months $(\mathrm{p}=0.6)$ and 32 vs 26 months $(\mathrm{p}=0.8)$, respectively. However, patients with continued CR/VGPR after ASCT $(n=27)$ had significantly longer PFS than those who obtained CR/VGPR after ASCT ( $\mathrm{n}=$ 58): not reached vs 34 months $(\mathrm{p}=0.01)$. Patients who Achieved CR/VGPR after ASCT $(\mathrm{n}=58)$ had higher OS compared to those with less than VGPR $(\mathrm{n}=53)$ : not reached vs 58 months $(\mathrm{p}=0.02)$, respectively.

Conclusions: Our results provide further evidence that achieving and maintaining of CR/VGPR after ASCT had a positive impact on OS and PFS. Patients with less than VGPR after induction with TD should undergo ASCT.

Conflict of interest: None

\section{P683}

Safety of undertaking ASCT in patients with daratumumabinduced suppression of normal immunoglogulins

Raymond Powles $^{1}$, Linda Little ${ }^{1}$, Joanna Sheldon ${ }^{2}$, Rachel Wheeler ${ }^{2}$

${ }^{1}$ Cancer Centre London, London, United Kingdom; ${ }^{2}$ St George's Hospital, Immunology, London, United Kingdom

Background: In the current treatment pathway for MM patients, autologous stem cell transplantation (ASCT) is central to their management (IMWG 2017), but inevitably if they have previously received, or are receiving Daratumumab (dara)then the cellular inmmunological status in the host will altered(ASH 2017 abstract 3148), This and serum immunoglogulin alterations may impinge on the safety of ASCT.

Methods: Serum immunoglogulins; In this study 21 patients with MM (1 and 13 previous lines of treatment) aged $65 \mathrm{y}(36-83) 14 \operatorname{IgG} ; 4 \operatorname{IgA} ; 3 \mathrm{BJ}$ were treated in our Institution with dara, plus/minus Velcade or Revlimid, with minimum follow-up 6 months. We undertook ASCT in three of these patients. ASCT Dara induced altered immune status may cause post ASCT issues such as delayed engraftment, infections, possibly autologous GVHD and a 
dara infusion related reaction when re-challenged with the next dose of dara post ASCT.

Results: All but one patient responded to dara and there was marked severe suppression of normal unaffected immunoglogulins levels at 6 months from baseline in all but one patient, even those in CR.

\begin{tabular}{llllllll}
\hline $\begin{array}{l}\text { IgA } \\
\text { baseline }\end{array}$ & $\begin{array}{l}\text { IgA at } \\
1 \text { month }\end{array}$ & $\begin{array}{l}\text { IgA at } 6 \\
\text { Months }\end{array}$ & $\begin{array}{l}\text { Max } \\
\text { percentage } \\
\text { change }\end{array}$ & $\begin{array}{l}\text { IgM at } \\
\text { baseline }\end{array}$ & $\begin{array}{l}\text { IgM at } \\
\text { 1 month }\end{array}$ & $\begin{array}{l}\text { IgM at } \\
6 \text { months }\end{array}$ & $\begin{array}{l}\text { Max } \\
\text { Percentage } \\
\text { change }\end{array}$ \\
\hline $\mathbf{9 . 3}$ & 9.3 & 4.8 & $\mathbf{- 4 8 . 4}$ & $\mathbf{2 . 3}$ & 2.3 & 0.3 & $\mathbf{- 8 6 . 9 6}$ \\
$\mathbf{1 . 5}$ & 0.3 & 0.2 & $\mathbf{- 8 6 . 6 7}$ & $\mathbf{0 . 6}$ & 0.3 & 0.2 & $\mathbf{- 6 6 . 7}$ \\
$\mathbf{1 . 3}$ & 0.5 & 0.5 & $\mathbf{- 6 1 . 5}$ & $\mathbf{0 . 6}$ & 0.3 & 0.2 & $\mathbf{- 6 6 . 7}$ \\
$\mathbf{0 . 6}$ & 0.2 & 0.1 & $\mathbf{- 8 3 . 3}$ & $\mathbf{0 . 6}$ & 0.3 & 0.5 & $\mathbf{- 1 6 . 7}$ \\
$\mathbf{0 . 5}$ & 0.1 & 0.1 & $\mathbf{- 8 0}$ & $\mathbf{0 . 4}$ & 0.3 & 0.3 & $\mathbf{- 2 5}$ \\
$\mathbf{0 . 2}$ & 0.1 & 0.2 & $\mathbf{- 5 0}$ & $\mathbf{0 . 5}$ & 0.3 & 0.4 & $\mathbf{- 4 0}$ \\
$\mathbf{0 . 1}$ & $<0.07$ & 0.07 & $\mathbf{- 3 0}$ & $\mathbf{0 . 0 8}$ & $<0.1$ & 0.08 & $\mathbf{0}$ \\
\hline
\end{tabular}

\section{[[P683 Table] dara-induced immune paresis]}

We undertook a pilot ASCT study on three patients in which we had stem cells collected and a commitment to transplant prior to starting dara.

ASCT's Patient 1: F 72, IgA. 13 previous lines treatment/9 years treatment. 6 cycles dara prior to ASCT 6 cycles; 10 cycles post. ANC Engraftment 12 days. Inpatient stay: 14 days. Pre ASCT: MRD+ve (PP35). Post ASCT MRD negative.

Patient 2: F49, IgGK. 7previous lines/9 years treatment. 7 cycles Dara prior to ASCT ; 10 post. ANC Engraftment 13 days. Inpatient stay: 15 days. MDR Status Pre ASCT: BJ P trace. Post ASCT MRD-ve.

Patient 3: M63 IgA /AL. 2 previous lines/2? years of treatment. 7 cycles Dara pre ASCT; 10 post. ANC Engraftment 13 days. Inpatient stay 22 days. MRD status pre ASCT IP pos. Post ASCT MRD-ve. All three patients had Dara circulating at time of transplant.

Conclusions: We describe profound immunoglogulin suppression for patients on dara, and even with dara circulating in their blood, ASCT patients engrafted and were discharged from hospital promptly. They had no evidence of autologous GVHD, unexpected infections, or an IRR with their next infusion of dara.

Clinical Trial Registry: none.

Conflict of interest: Janssen honorarium.

\section{P684}

\section{Study of PD-1 inhibitor (nivolumab) with autologous hematopoietic stem cell transplantation in multiple myeloma patients}

Olga Pirogova, Elena Darskaya, Valentina Porunova, Elena Davidova, Olga Kudyasheva, Sergey Bondarenko, Ivan Moiseev, Boris Afanasyev
Pavlov First St. Petersburg State Medical University, R.M. Gorbacheva Memorial Institute of Hematology, Oncology and Transplantation, St. Petersburg, Russian Federation

Background: Patients (pts) with multiple myeloma (MM) who do not achieve complete response (CR) after primary therapy, including autologous hematopoietic cell transplantation (auto-HSCT), have poor outcome. Preclinical and clinical evidence suggests that the immune checkpoint programmed death-1 (PD-1) receptor/PD-1 ligand axis plays an important role in suppressing immune surveillance against MM, but monotherapy with PD-1 inhibitor was not effective in pts with MM. We hypothesized that the administration of nivolumab (an PD-1 inhibitor) during the lymphodepleted state post-autoHSCT can improve therapeutic efficacy in MM.

Methods: We conducted a phase 1-2, single-arm study nivolumab with auto-HSCT in MM pts who had not achieved pre- auto-HSCT complete response (CR) to induction therapy (NCT03292263). Nivolumab was administered $100 \mathrm{mg}$ IV fixed dose on day -3 before and day +17 after auto-HSCT. The endpoints were safety and overall response rate (ORR). Pts aged 18-70 years with $\mathrm{MM}$, of any molecular risk group and with $<\mathrm{CR}$ status after induction therapy were eligible and received high-dose melphalan (140-200 mg/m2 IV).

Results: Currently twelve pts are enrolled, 8 males and 4 females with the median age 56 years (range, 45-62). Two pts $(16,6 \%)$ had light chain MM, four pts $(33,4 \%)$ had IgA, six pts (50\%) -IgG MM. All pts except 2 received a tripleregimen primary therapy, a median of 6,5 cycles (range, 49). After induction therapy, all pts achieved partial response (PR), but three pts (25\%) lost response and auto-HSCT was in progressive disease. Among these 12 pts, grade 3 toxicities were in 2 pts ( 1 patient had infusion reaction and 1 patient had colitis). None of the other pts did have immunemediated adverse reactions. There were no primary or secondary graft failures, and the median time to neutrophil and platelet engraftment was 12 days (range, 10-17) and 14 days (range, 9-18), respectively. Ten pts were considered evaluable for response assessment. The median follow-up was 188 days (range, 93-244) none had progressive disease or relapse. Of the10 pts who had a complete response evaluation by serology and bone marrow (BM) study (morphology and flow cytometry), the ORR was 50\% (5/ $10)$, the CR rate was $30 \%(3 / 10)$, two pts (20\%) achieved VGPR, four pts (40\%) stayed in PR, one of pts with progressive disease had stabilization.

Conclusions: Preliminary results of the addition of nivolumab to auto-HSCT show the relative safety of the therapy. The efficacy of this combination requires further evaluation. 


\section{Clinical Trial Registry: https://clinicaltrials.gov NCT03292263}

Conflict of interest: Authors have nothing to disclose.

\section{P685}

The evaluation of consolidation and maintenance therapies after HDT/ASCT for symptomatic MM

Aya Nakaya ${ }^{1,2}$, Eiji Nakatani ${ }^{2,3}$, Hitomi Kaneko ${ }^{2,4}$, Hirohiko Shibayama $^{2,5}$, Yuji Shimura ${ }^{2,6}$, Junya Kanda ${ }^{2,7}$, Maki Shindo ${ }^{2,7}$, Kensuke Ohta ${ }^{2,8}$, Satoru Kosugi ${ }^{2,9}$, Shin-Ichi Fuchida ${ }^{2,10}$, Hideo Yagi $^{2,11}$, Hirokazu Tanaka ${ }^{2,12}$, Yuri Kamitsuji ${ }^{2,13}$, Takahiro Karasuno $^{2,14}$, Nobuhiko Uoshima ${ }^{2,15}$, Eri Kawata ${ }^{2,15}$, Yoko Adachi $^{2,16}$, Hitoji Uchiyama ${ }^{2,17}$, Toshimitsu Matsui ${ }^{2,18}$, Mitsuhiro Matsuda $^{2,19}$, Jun Ishikawa ${ }^{2,20}$, Tsuneyoshi Hamada ${ }^{2,21}$, Ryoichi Takahashi2,22, Kazunori Imada ${ }^{2,4}$, Chihiro Shimazaki ${ }^{2,10}$, Masayuki Hino $^{2,23}$, Junya Kuroda ${ }^{2,6}$, Yuzuru Kanakura ${ }^{2,5}$, Akifumi TakaoriKondo $^{2,7}$, Shosaku Nomura ${ }^{1,2}$, Itaru Matsumura ${ }^{2,12}$

${ }^{1}$ Kansai Medical University, First Department of Internal Medicine, Osaka, Japan; ${ }^{2}$ Kansai Myeloma Forum, Osaka, Japan; ${ }^{3}$ Osaka University Graduate School of Medicine, Department of Biostatistics and Data Science, Osaka, Japan; ${ }^{4}$ Japanese Red Cross Osaka Hospital, Department of Hematology, Osaka, Japan; ${ }^{5}$ Osaka University Graduate School of Medicine, Department of Hematology and Oncology, Osaka, Japan; ${ }^{6}$ Kyoto Prefectural University of Medicine, Division of Hematology and Oncology, Department of Medicine, Kyoto, Japan; ${ }^{7}$ Graduate School of Medicine, Kyoto University, Department of Hematology and Oncology, Kyoto, Japan; ${ }^{8}$ Osaka Saiseikai Nakatsu Hospital, Department of Hematology, Osaka, Japan; ${ }^{9}$ Toyonaka Municipal Hospital, Department of Internal Medicine (Hematology), Osaka, Japan; ${ }^{10}$ JCHO Kyoto Kuramaguchi Medical Center, Department of Hematology, Kyoto, Japan; ${ }^{11}$ Nara Hospital, Kinki University School of Medicine, Department of Hematology, Nara, Japan; ${ }^{2}$ Kindai University Faculty of Medicine, Department of Hematology and Rheumatology, Osaka, Japan;

${ }^{13}$ Matsushita Memorial Hospital, Department of Hematology, Osaka, Japan; ${ }^{14}$ Rinku General Medical Center, Department of Hematology, Osaka, Japan; ${ }^{15}$ Japanese Red Cross Kyoto Daini Hospital,

Department of Hematology, Kyoto, Japan; ${ }^{16} \mathrm{JCHO}$ Kobe Central Hospital, Department of Internal Medicine, Hyogo, Japan; ${ }^{17}$ Japanese Red Cross Kyoto Daiichi Hospital, Department of Hematology, Kyoto, Japan; ${ }^{18}$ NIshiwaki Municipal Hospital, Department of Hematology, Hyogo, Japan; ${ }^{19}$ PL General Hospital, Department of Hematology, Osaka, Japan; ${ }^{20}$ Osaka International Cancer Center, Department of Hematology, Osaka, Japan; ${ }^{21}$ Kyoto-Katsura Hospital, Kyoto, Japan; ${ }^{22}$ Omihachiman Community Medical Center, Department of

Hematology, Shiga, Japan; ${ }^{23}$ Osaka City University Graduate School of Medicine, Hematology, Osaka, Japan

Background: The clinical significance of consolidation and maintenance therapies after high dose chemotherapy with autologous stem cell transplantation (HDT/ASCT) remains controversial. Therefore, we evaluated effects for these therapies using the database of Kansai Myeloma Forum (KMF).

Methods: 284 of symptomatic multiple myeloma patients received HDT/ASCT was analyzed. Consolidation therapies were defined second HDT/ASCT and short-term intensive therapies after HDT/ASCT, and maintenance therapies were long-term continuous therapies.

Results: Median age was 56.8 (31-74) y/o. Consolidation therapies included second HDT/ASCT (54\%), Bortezomib (B)-based regimen (23\%), Lenalidomide (L)-based (15\%), Thalidomide (T)-based (4\%). Median overall survival (OS) of patients with consolidation $(\mathrm{n}=77)$ was $7.12 \mathrm{y}$, whereas without consolidation $(\mathrm{n}=207)$ was $6.39 \mathrm{y} \quad(\mathrm{p}=0.363)$. Median time to next treatment (TTNT) with and without consolidation was 1.96 and $1.90 \mathrm{y}(\mathrm{p}=0.524)$. According to regimens, second HDT/ASCT significantly prolonged TTNT (second HDT/ASCT: 2.54y, B-based: $1.74 \mathrm{y}, \mathrm{L}-$ based: $1.39 y, p<0.001)$. However, there was no difference in OS according to regimens. Maintenance therapies included L-based (62\%), T-based (15\%), B-based (14\%). Median OS of patients with maintenance $(n=170)$ was $6.4 \mathrm{y}$, whereas that of patients without maintenance $(\mathrm{n}=$ 114) was 6.6y $(\mathrm{p}=0.721)$. Median TTNT with and without maintenance was 4.2 and $4.1 \mathrm{y}(\mathrm{p}=0.81)$. In subgroup analyses, there was no significant prolongation of TTNT and OS according to regimens.

Conclusions: In our cohort, there was no significant difference between with/without consolidation or maintenance therapies after HDT/ASCT. However, second HDT/ ASCT as a consolidation therapy significantly prolonged TTNT. The value of consolidation and maintenance therapies after HDT/ASCT should be further evaluated in the clinical practice.

Clinical Trial Registry: https://kmu.bvits.com/rinri/ Apply/project.aspx?PROJECT_ID $=788$

\section{\#2017084}

Conflict of interest: JK has been paid honoraria by Celgene, Bristol-Myers Squibb, Ono, Janssen. JK's institution has received research funding from Celgene and Bristol-Myers Squibb. HS has been paid honoraria by Takeda, Celgene, Novartis. CS has been paid honoraria by Fujimoto, Ono, Celgene. AT has been paid by BristolMyers Squibb, Janssen, Celgene. JKa's institution has received research funding from Takeda. AT and IM's institution have received research funding from Celgene. All members have received grants from Celgene, Takeda, Fujimoto, Ono.

\section{P686}

Toxicity and Outcome following High Dose Chemotherapy and Autologous Stem Cell Transplantation in Older Patients with Multiple Myeloma

Christopher Lemieux, Vincent Laroche, Guy Cantin, Julie Côté, Christine Demers, Geneviève Gallagher

Hôpital de l'Enfant-Jésus, CHU de Québec - Université Laval, 
Division of Hematology and Medical Oncology, Department of Medicine, Ville de Québec, Canada

Background: High-dose chemotherapy followed by autologous stem cell transplantation (ASCT) is considered the standard of care as first-line therapy for eligible multiple myeloma patients (Barlogie et al. 1987). With an expected myeloma incidence rising by $57 \%$ from 2010 to 2030 (Smith et al. 2009), the number of older patients considered for ASCT will increase significantly. The goal of this study was to identify factors impacting the safety and efficacy of ASCT in older patients with multiple myeloma in order to better select those who will benefit from such an intervention.

Methods: This single center, retrospective study examined outcomes of ASCT in elderly patients ( $\geq 60$ years old) with multiple myeloma compared to younger patients $(<60$ years old). Between January 1, 2011, and September 1, 2015, 93 patients met the inclusion criteria and were included in the study. Patients signed an informed consent and the ethics committee of our institution approved the study. Toxicity was analyzed according to age and Hematopoietic cell transplant comorbidity index score (HCT-CI). Progression-free-survival (PFS) and overall survival (OS) were analyzed according to age at the time of transplantation, HCT-CI, Revised International Staging System (R-ISS) and disease status at the time of transplant.

Results: From the 93 patients included in the study, 49 were included in the younger group and 44 were included in the older cohort. The median age at transplant was 54 (range 33-59) and 65 (range 60-69) years-old, respectively. Distribution of R-ISS and HCT-CI was comparable in both groups. After transplantation, no parenteral alimentation was required in the older patient group while 4 patients required it in the younger patient group $(\mathrm{p}=0,053)$. The incidence of febrile neutropenia was universal in all patients $(100 \%)$. The incidence of septic shock and admission to intensive care unit was only $1 \%$. Median engraftment for neutrophils was 14 days and 16 days for platelets. Median hospital stay duration was not significantly different between groups at 21 days. With a median follow-up of 45 months(range 2 to 75 ), the older cohort had a better median PFS of 24 months compared to 20 months $(p=0,049)$ in the younger cohort. The median OS was not reached at the time of evaluation for both groups (figure1). The estimated 5 years OS is $56 \%$ for the younger group compared to $72 \%$ for the older group $(p=0,218)$. In the entire cohort, patients who did not obtain a partial response (PR) or better before transplantation had significantly earlier progression (median of 9 compared to 26 months, p < $0,001)$. Our 1 year NRM was $0 \%$. Surprisingly, HCT-CI did not correlate with toxicity or outcomes in both groups.

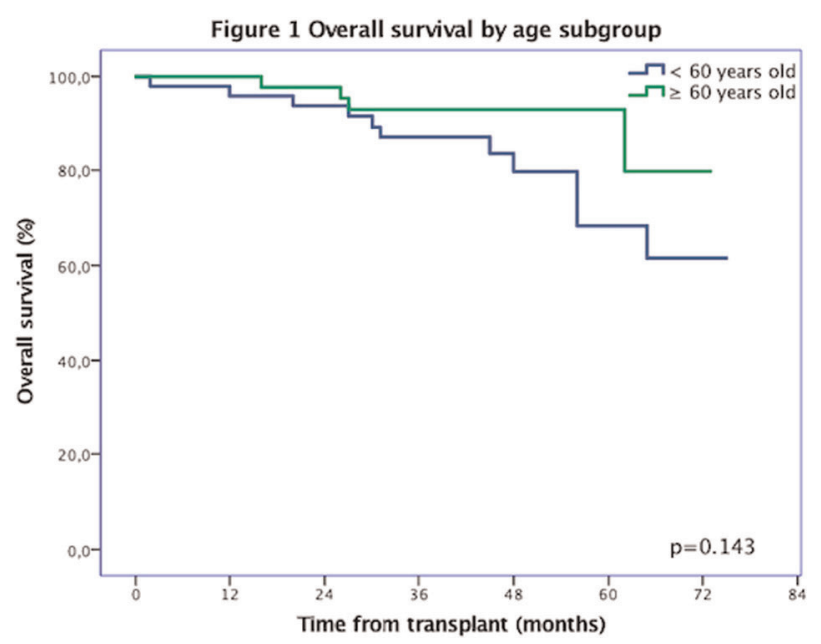

[P686 Figure] [Figure 1]

Conclusions: ASCT is a beneficial treatment option in well-selected older multiple myeloma patients. Comorbidities must be considered and HCT-CI can guide clinicians in the selection of eligible patient. Our data showed that in older patients, toxicity and OS were comparable to younger patients. Obtaining at least a PR was a more powerful predictor of a greater survival. Therefore, age should not be the limiting factor when considering ASCT in older patients with multiple myeloma.

Conflict of interest: None of the authors has anything to disclose.

\section{P687}

Treosulfan conditioning for allogenic hematopoietic stem cell transplantation in multiple myeloma, advantages for non-relapse mortality and survival, but with high incidence of relapse

Charlotte Gran ${ }^{1}$, Junfeng Wang ${ }^{2}$, Hareth $\mathrm{Nahi}^{3}$, Linda Koster ${ }^{4}$, Herman Einsele ${ }^{5}$, Maija Itälä-Remes ${ }^{6}$, Ernst Holler ${ }^{7}$, Dietrich Beelen $^{8}$, Fabio Ciceri ${ }^{9}$, Gerhard Ehninger ${ }^{10}$, Jürgen Finke ${ }^{11}$, Liesbeth de Wreede ${ }^{12}$, Per Ljungman ${ }^{3}$, Johanna Tischer ${ }^{13}$, Mareike Verbeek $^{14}$, Evgeny Klyuchnikov ${ }^{15}$, Inken Hilgendorf ${ }^{16}$, Kerstin Schäfer-Eckart ${ }^{17}$, Christof Scheid ${ }^{18}$, Donald Bunjes ${ }^{19}$, Nadezda Basara $^{20}$, Mathias Haenel ${ }^{21}$,Lutz Peter Müller ${ }^{22}$, Harald Biersack ${ }^{23}$, Gösta Gahrton ${ }^{3}$, Laurent Garderet ${ }^{24}$, Nicolaus Kröger ${ }^{15}$

${ }^{1}$ Karolinska Institutet and University Hospital, Department of Medicine, Huddinge, Stockholm, Sweden; ${ }^{2}$ EBMT Statistical Unit Data Office Leiden, Leiden, Netherlands; ${ }^{3}$ Karolinska Institutet and University Hospital, Stockholm, Sweden; ${ }^{4}$ EBMT Data Office Leiden, Leide, Netherlands; ${ }^{5}$ Universitätsklinikum Würzburg, Wûrxburg, Germany; ${ }^{6} \mathrm{HUCH}$ Comprehensive Cancer Center, Helsinki, Finland; ${ }^{7}$ University Regensburg, Regensburg, Germany; ${ }^{8}$ University Hospital, Essen, Germany; ${ }^{9}$ Ospedale San Raffaele s.r.l., Milano, Italy;

${ }^{10}$ Universitaetsklinikum Dresden, Dresden, Germany; ${ }^{11}$ University of Freiburg, Freiburg, Germany; ${ }^{12}$ Leiden University Medical Centre, Leiden, Netherlands; ${ }^{13}$ Klinikum Grosshadern, Munich, Germany; 
${ }^{14}$ Klinkum Rechts der Isar, Munich, Germany; ${ }^{15}$ University Hospital Eppendorf, Hamburg, Germany; ${ }^{16}$ Universitätsklinikum Jena, Jena, Germany; ${ }^{17}$ Klinikum Nuernberg, Nuernberg, Germany; ${ }^{18}$ University of Cologne, Colonge, Germany; ${ }^{19}$ Universitätsklinikum Ulm, Ulm, Germany; ${ }^{20}$ St. Franziskus Hospital, Flensburg, Germany; ${ }^{21}$ Klinikum Chemnitz gGmbH, Chemnitz, Germany; ${ }^{22}$ Martin-Luther-Universität Halle-Wittenberg, Hallo, Germany; ${ }^{23}$ University Medical Center Schleswig-Holstein, Lûbeck, Germany; ${ }^{24}$ Hospital Saint Antoine, Paris, France

Background: Allogeneic hematopoietic stem cell transplantation (AlloSCT) for treatment of multiple myeloma $(\mathrm{MM})$ is controversial mainly due to high non-relapse mortality (NRM) with myeloablative conditioning. However, AlloSCT is probably the only treatment that may result in cure of a small fraction of patients. Treosulfan is a prodrug of a bifunctional alkylating agent which has both myelotoxic and immunosuppressive properties. Conditioning regimes with treosulfan have been tried in various hematologic neoplasia such as AML and MDS with suggested results of low NRM and increased progression free survival. A previous published single centre study on use of treosulfan condition in AlloSCT for MM indicated improved overall survival (OS) and low NRM ${ }^{1}$.

Methods: We conducted a retrospective analysis of all patients with MM undergoing Allo-SCT 2008-2016 reported to the EBMT data registry. Patients were analysed in three cohorts; those receiving treosulfan in their conditioning regimen, regardless of upfront or subsequent line of allo, $\mathrm{n}=508$, non-treosulfan reduced intensity conditioning (RIC), $\mathrm{n}=2830$, and those undergoing nontreosulfan myeloablative conditioning (MAC), $\mathrm{n}=1177$. Patient characteristic presented in table 1.

Results: The 5-year overall survival showed a slight improvement by the use of treosulfan regimes, $41 \%$, compared to $39 \%$ and $37 \%$ for RIC and MAC respectively $(\mathrm{p}=0.05)$. There was no significant difference in progression free survival between the cohorts, $19 \%$ treosulfan, $19 \%$ RIC and 21\% MAC ( $\mathrm{p}=0.1)$. Long term, 5-year, cumulative incidence $(\mathrm{CI})$ of relapse in treosulfan conditioning cohort was $64 \%$ contrasted to $60 \%$ for RIC and $55 \%$ for MAC $(\mathrm{p}=<0.001)$. Noteworthy is the lower 5 -year CI of NR of $17 \%$ in the Treosulfan cohort compared to $21 \%$ in the RIC and $23 \%$ in MAC $(\mathrm{p}=0.017)$.

Conclusions: In this retrospective study treosulfan reduced the NRM and tended to improve OS. However, the risk for relapse was increased in treosulfan treated patients especially compared to patients receiving MAC. Addition of maintenance treatment might reduce the relapse rate after AlloSCT and improve survival for multiple myeloma patients. Prospective studies on treosulfan conditioning are warranted to further define suitable dosing in MM AlloSCT.
Reference: 1. Nahi H, Afram G, Liwing J, et al. Treosulfan Plus Fludarabine - Encouraging Conditioning for Allogeneic Hematopoietic Stem Cell Transplantation in Multiple Myeloma. Journal of Cancer Science and Clinical Oncology 2016; 3(2).

Conflict of interest: None of the authors has anything to disclose.

\begin{tabular}{|c|c|c|c|}
\hline & $\begin{array}{l}\text { Treosulfan } \\
\text { conditioning }\end{array}$ & $\begin{array}{l}\text { Reduced intensity } \\
\text { conditioning }\end{array}$ & $\begin{array}{l}\text { Myeloablative } \\
\text { conditioning }\end{array}$ \\
\hline $\begin{array}{l}\text { Gender male\% (n } \\
\text { male/female }\end{array}$ & $60 \%(307 / 201)$ & $62 \%(1763 / 1067)$ & $65 \%(765 / 412$ \\
\hline \multicolumn{4}{|c|}{ Type on myeloma, no (\%) } \\
\hline $\operatorname{IgG}$ & $20(41 \%)$ & $1366(48 \%)$ & $556(47 \%)$ \\
\hline $\operatorname{IgA}$ & $78(15 \%)$ & $543(19 \%)$ & $197(17 \%)$ \\
\hline light chain & $191(38 \%)$ & $761(27 \%)$ & $334(28 \%)$ \\
\hline non-secretory & $9(2 \%)$ & $64(2 \%)$ & $37(3 \%)$ \\
\hline \multicolumn{4}{|c|}{ Line of treatment, no $(\%)$} \\
\hline Upfront & $136(27 \%)$ & $587(21 \%)$ & $375(32 \%)$ \\
\hline $\begin{array}{l}\text { Second line or } \\
\text { beyond }\end{array}$ & $372(73 \%)$ & $2243(79 \%)$ & $802(68 \%)$ \\
\hline
\end{tabular}

\section{[[P687 Table] Table 1 Patient characteristics]}

\section{P688}

Abstract previously published

\section{P689}

\section{Web-based symptom tracking of Multiple Myeloma patients}

Mervi Putkonen', Tommi Salmi ${ }^{1}$, Henri Virtanen ${ }^{2}$, Jaakko Laukkanen $^{2}$, Marjut Kauppila ${ }^{3}$, Urpu Salmenniemi ${ }^{3}$, Soile Salomäki ${ }^{3}$, Maija Valta ${ }^{3}$, Pia Ettala ${ }^{3}$, Kari Remes ${ }^{3}$

${ }^{1}$ Turku University Hospital, Dept of Medicine, Turku, Finland; ${ }^{2}$ Kaikuhealth, Helsinki, Finland; ${ }^{3}$ Turku University Hospital, Turku, Finland

Background: The web-based symptom tracking has been shown to improve overall survival and quality of life amongst metastatic cancer patients undergoing chemotherapy. ${ }^{1,2,3}$ However, similar approach has not been piloted amongst Multiple Myeloma patients.

Methods: Together with a patient-reported outcomes web application provider, Kaiku Health, we developed an adaptive symptom questionnaire covering 9 of the most common symptoms during treatments of MM (numbness, pain, fatigue, etc.) at Turku University Hospital. Patients also have possibility to report other symptoms, and contact medical staff electronically through the application. The application reminds patients to complete symptom reports on a weekly basis via e-mail, but other reminders are not 
sent to patients. The application highlights severe symptoms for medical staff. Nurses were following the symptom reports through application, and they had an opportunity to consult doctors if necessary.

Results: 14 out of 15 invited patients registered to the application, and 13 out of 14 registered patients started to follow on symptoms through the application. Experiences were collected from 5 patients, 2 nurses and 2 doctors through in-depth interviews, and the key performance indicators on the usage of the platform were analyzed. Patients reported that application is easy to use and they would rather use it than call to the clinic. The median time to complete the symptom report through the application was 3 minutes and altogether patients have completed $80,1 \%$ of the symptom questionnaires in time (expected to complete the report once a week). Medical staff reported that they caught patients' symptoms that would have been missed in traditional communication. There was also a clear decrease in the number of phone calls to the clinic. By completing symptom reports, patients simultaneously learned to recognize and follow their most relevant symptoms, which reduced patients' symptom-related uncertainty.

Conclusions: Web-based symptom tracking is a suitable way to follow-up on symptoms of Multiple Myeloma patients. Patients are engaged to their follow-up. Web-based tracking improves effectiveness of clinical appointments and reduces the patients' need for phone calls. A larger study to further assess the impact of the follow-up is recommended.

Conflict of interest: Authors declare no conflict of interest.

Myelodysplastic Syndromes

\section{P690}

\section{ALLOGENEIC HEMATOPOIETIC STEM CELL TRANSPLANTATION: RESULTS OF THE LATIN AMERICAN BONE MARROW TRANSPLANT REGISTRY IN MYELODYSPLASTIC SYNDROME (MDS)}

Fernando Barroso Duarte ${ }^{1}$, Talyta Ellen de Jesus Santos ${ }^{2}$, Anna Thawanny Gadelha Moura ${ }^{2}$, Vaneuza Funke ${ }^{3}$, Vergilio Antonio Rensi Colturato ${ }^{4}$, Nelson Hamerschlak ${ }^{5}$, Neysimelia Costa Villela ${ }^{6}$, Luis Fernando Lopes ${ }^{6}$, Maria Cristina Martins de Almeida Macedo ${ }^{7}$, Afonso Celso Vigorito ${ }^{8}$, Rodolfo Daniel de Almeida Soares', Alessandra Paz ${ }^{10}$, Mariana Stevenazzi ${ }^{11}$, Lilián Diaz ${ }^{11}$, Abraão Elias Halack-Neto $^{12}$, Gustavo Bettarello ${ }^{13}$, Breno Moreno Gusmao ${ }^{14}$, Marco Aurélio Salvino ${ }^{15}$, Rodolfo Calixto ${ }^{16}$, Maria Claudia Rodrigues Moreira ${ }^{17}$, Romélia Pinheiro Gonçalves Lemes ${ }^{18}$

${ }^{1}$ Universidade Federal do Ceará - Hospital das Clínicas, Fortaleza, Brazil; ${ }^{2}$ Universidade Federal do Ceará, Fortaleza, Brazil;

${ }^{3}$ Universidade Federal do Paraná, Curitiba, Brazil; ${ }^{4}$ Fundação Amaral Carvalho, Jaú, Brazil; ${ }^{5}$ Hospital Israelita Albert Einstein, São Paulo, Brazil; ${ }^{6}$ Hospital de Câncer Infanto-Juvenil de Barretos, Barretos, Brazil; ${ }^{7}$ Intituto Brasileiro de Controle do Câncer, São Paulo, Brazil; ${ }^{8}$ Universidade Estadual de Campinas, Campinas, Brazil; ${ }^{9}$ Natal Hospital Center, Natal, Brazil; ${ }^{10}$ Hospital das Clinicas de Porto Alegre, Porto Alegre, Brazil; ${ }^{11}$ Hospital de Clínicas do
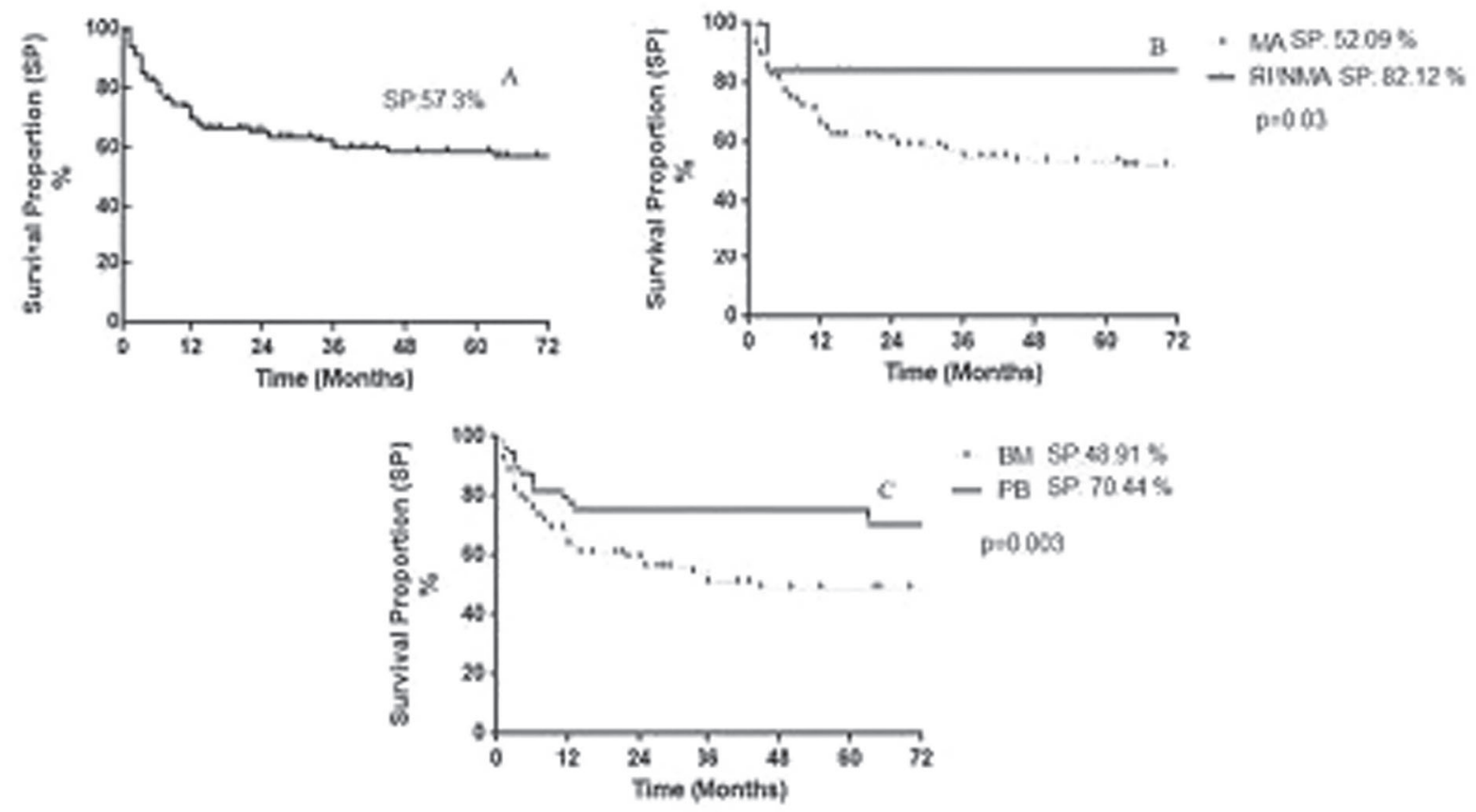

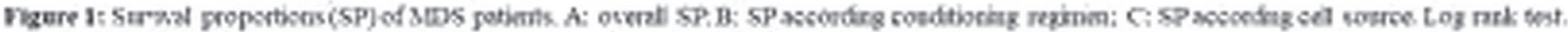

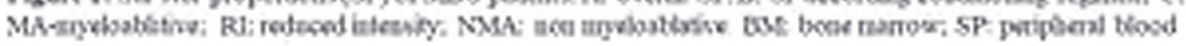

[P690 Figure] [Survival proportions] 
Uruguai, Montevideo, Uruguay; ${ }^{12}$ Hospital Universitário de Juiz de Fora, Juiz de Fora, Brazil;; ${ }^{13}$ Pietro Albuquerque ICDFBMT, São Paulo, Brazil; ${ }^{14}$ Hospital São José, São Paulo, Brazil; ${ }^{15}$ Universidade Federal da Bahia, Salvador, Brazil; ${ }^{16}$ Real Hospital Português de Beneficência em Pernambuco, Recife, Brazil; ${ }^{17}$ CEMO-Instituto Nacional do Câncer, Rio de Janeiro, Brazil; ${ }^{18}$ Universidade Federal do Ceara, Fortaleza, Brazil

Background: Hematopoietic stem cell transplantation (HSCT) is the only curative procedure in Myelodysplastic Syndrome (MDS).

Methods: This is a multicenter study of 150 patients with MDS submitted to HSCT in Brazil and Uruguay from 1988 to 2017. Data were obtained from the Latin American transplantation registry. Statistical analyses were performed utilizingthe SPSS software, version 23.1 and GraphPad Prism version 5.0 with a significance level of $\mathrm{p}<0.05$.

Results: There was a predominance of male $(53 \%)$ and Caucasians $(80 \%)$ patients. The predominant age group was 51 to 60 years $(26 \%)$. According to the IPSS-R classification, the sample was classified as "high risk" (28\%), "very high risk" (3\%), and "low risk" (11\%). Chemotherapy (42\%) and hypomethylating agents (29\%) were used. Azacitidine was the most commonly used drug (24\%). More than 20 red blood cell units $(65 \%)$ and more than 15 platelet units $(69 \%)$ were used.The myeloablative conditioning was performed in $80 \%$ and the reduced intensity in $5 \%$. The cell sources were bone marrow (BM) $(57 \%)$ and peripheral blood (PB) (41\%). The donor was related in $71 \%$ of the cases. Post-HSCT complications were infections (65\%), mucositis (49\%), acute GVHD (43\%) and chronic GVHD (32\%). For acute GVHD, the bivariate analysis showed an association with myeloablative conditioning ( $\mathrm{p}$ $=0.003)$ and with BM cell source $(\mathrm{p}=0.001)$.In the binary logistic regression analysis, conditioning $(\mathrm{OR}=0.286$; $95 \% \mathrm{CI}=0.094-0.875)$ and cell source $(\mathrm{OR}=0.395 ; 95 \%$ $\mathrm{CI}=0.178-0.875)$ were significant predictors of acute GVHD. As for chronic GVHD, an association with myeloablative conditioning was observed in the bivariate analysis $(\mathrm{p}=0.008)$. In the binary logistic regression, this variable was considered a significant predictor $(\mathrm{OR}=$ $0.288 ; 95 \% \mathrm{CI}=0.090-0.923$ ) for chronic GVHD development. Overall patient survival over the 6-year period was $57.3 \%$.It was observed that patients submitted to reduced intensity/non-myeloablative conditioning had higher survival when compared to the myeloablative regimen $(p=0.03)$. Survival was higher in cases where peripheral blood was the cell source $(\mathrm{p}=0.003)$.

In the chi-square test, the myeloablative regimen $(\mathrm{p}<$ $0.0001)$ and BM as cell source $(\mathrm{p}<0.0001)$ were associated with death after HSCT. The variables conditioning, cell source and donor type constitute predictors of acute GVHD, with a positive result being observed for myeloablative conditioning $(\mathrm{p}=0.005)$ and BM cell source $(\mathrm{p}=0.002)$.

Conclusions: The results showed that reduced intensity conditioning and peripheral blood cell source were associated with longer survival. Myeloablative conditioning and BM cell source were associated with acute GVHD. These data open a discussion about the ideal cell conditioning and source in MDS, considering patient age and prognostic factors for post-transplantation therapies aiming to optimize results.

Conflict of interest: None to be declared

\section{P691}

Allogeneic transplantation for patient with myelodisplastic sindromes: a single centre experience

Valeria Cancelli, Michele Malagola, Enrico Morello, Alessandro Turra, Nicola Polverelli, Federica Cattina, Benedetta Rambaldi, Domenico Russo

Chair of Hematology, Unit of Blood Diseases and Bone Marrow Transplantation, ASST Spedali Civili, Brescia, Italy

Background: Myelodysplastic syndromes (MDS) are a heterogeneous group of myeloid malignancies characterized by dysplastic features and cytopenias that have a high variable clinical course leading from rapid transformation to acute leukemia to stable disease for many months or even years. Treatment options for patients with MDS are still limited and allogeneic hematopoietic stem cell transplantation (alloSCT) is the only curative treatment option. The advent of less toxic conditioning regimens and the availability of stem cells donors (sibling, matched unrelated or haploidentical) in the great majority of patients led to an increasingly use of alloSCT, even in older MDS patients, often following hypometilating agents (HMA), with the aim to reduce the disease burden.

Methods: We report here a series of 17 patients submitted to allo-SCT for MDS from January 2010 to June 2017 in our Institution. Six of 17 (35\%) were secondary MDS, mostly related to chemotherapy for other hematological malignancies. Ten of 17 (59\%) were RAEB 2 and 7 of $17(41 \%)$ were RAEB 1. Four of 17 (24\%) MDS patients were high IPSS risk and 13 of $17(76 \%)$ were INT-2 IPSS risk. Fifteen of 17 patients (88\%) were treated with HMA agents as "bridge" to transplant and 2 patients (12\%) were transplanted frontline. In the group of HMA patients, 4 of $15(26 \%)$ achieved a hematologic response and 11 of 15 (74\%) were transplanted with a stable disease. Median course of HMA was 6 cycles (range 1 - 12). Median age at transplant was 50 years (range 46-68). Six of 17 patients $(35 \%)$ received a myeloablative conditioning regimen. The donor was a matched sibling in 3 of 17 cases (18\%), a 
matched unrelated in 13 of 17 cases (76\%) and a haploidentical donor in 1 case $(6 \%)$. Median hematopoietic cell transplantation (HCT)-specific comorbidity index score was 2.2 (range 3-7). Median time from diagnosis to alloSCT was 10.5 months (range 4-65) and median follow up time from alloSCT was 55.6 months (range 1 - 84).

Results: Nine of 17 (53\%) patients submitted to alloSCT for MDS are alive at last follow up. Six of $8(75 \%)$ deaths were considered as transplant-related mortality (4 infections and 2 conditioning toxicity). Only one patient died for a recurrence of the disease and another one for a second neoplasia.

Ten of 17 (59\%) patients developed grade II aGVHD after allo-SCT, successfully treated with standard steroid therapy in all but one case. Two of 17 (12\%) developed limited cGvHD, not requiring any treatment.

Conclusions: Our experience, although limited, suggests that alloSCT is an effective treatment option for MDS, even in older patients. In our experience the recurrence of MDS after alloSCT is low but the toxicity of the conditioning (especially in the secondary MDS group) is high, probably related to a worse HCT score for these patients. Further studies are needed to evaluate less toxic conditioning regimen, specially for secondary MDS patients, frequently transplanted with active (although stable) disease and with higher HCT score.

Conflict of interest: None of the authors has anything to disclose.

\section{P692}

Clonal Evolution in Patients with Relapsed Myelodysplastic Syndrome after Hematopoietic Stem-cell Transplantation

Zuzana Urbanová, Jitka Veselá, Monika Hrubá, Hana Votavová, Hana Čechová, Markéta Št’astná Marková, Jaroslav Čermák, Monika Beličková

Institute of Hematology and Blood Transfusion, Prague, Czech Republic

Background: Disease relapse represents the major cause of treatment failure after allogeneic hematopoietic stem-cell transplantation (HSCT) in myelodysplastic syndrome (MDS). It is well characterized that various combinations of somatic mutations are associated with different clinical outcomes in MDS but there are limited data on changes of mutational profile of relapsed autologous chimerism after HSCT.
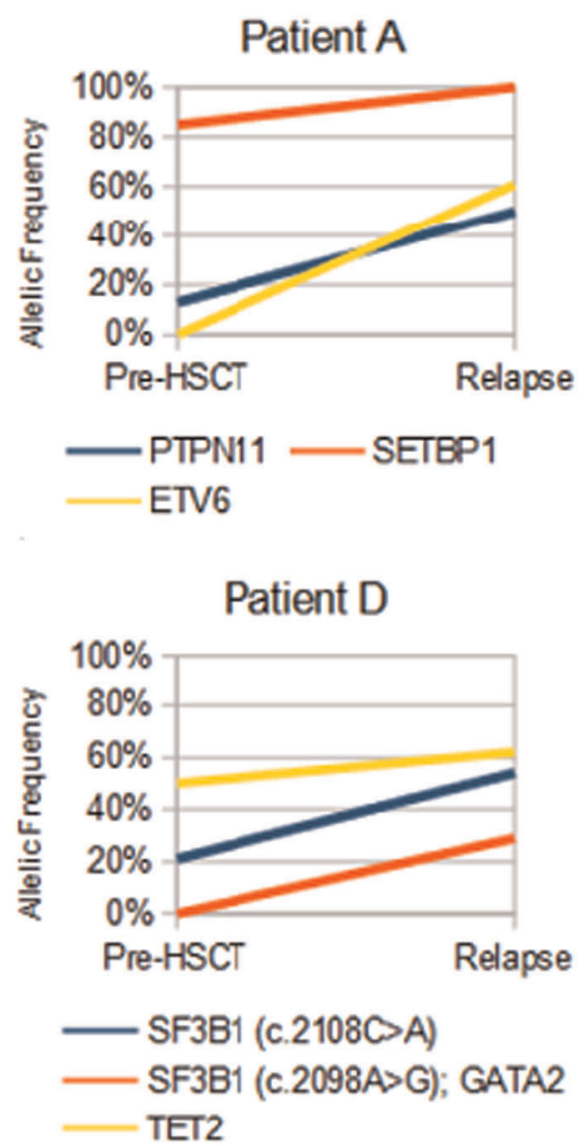
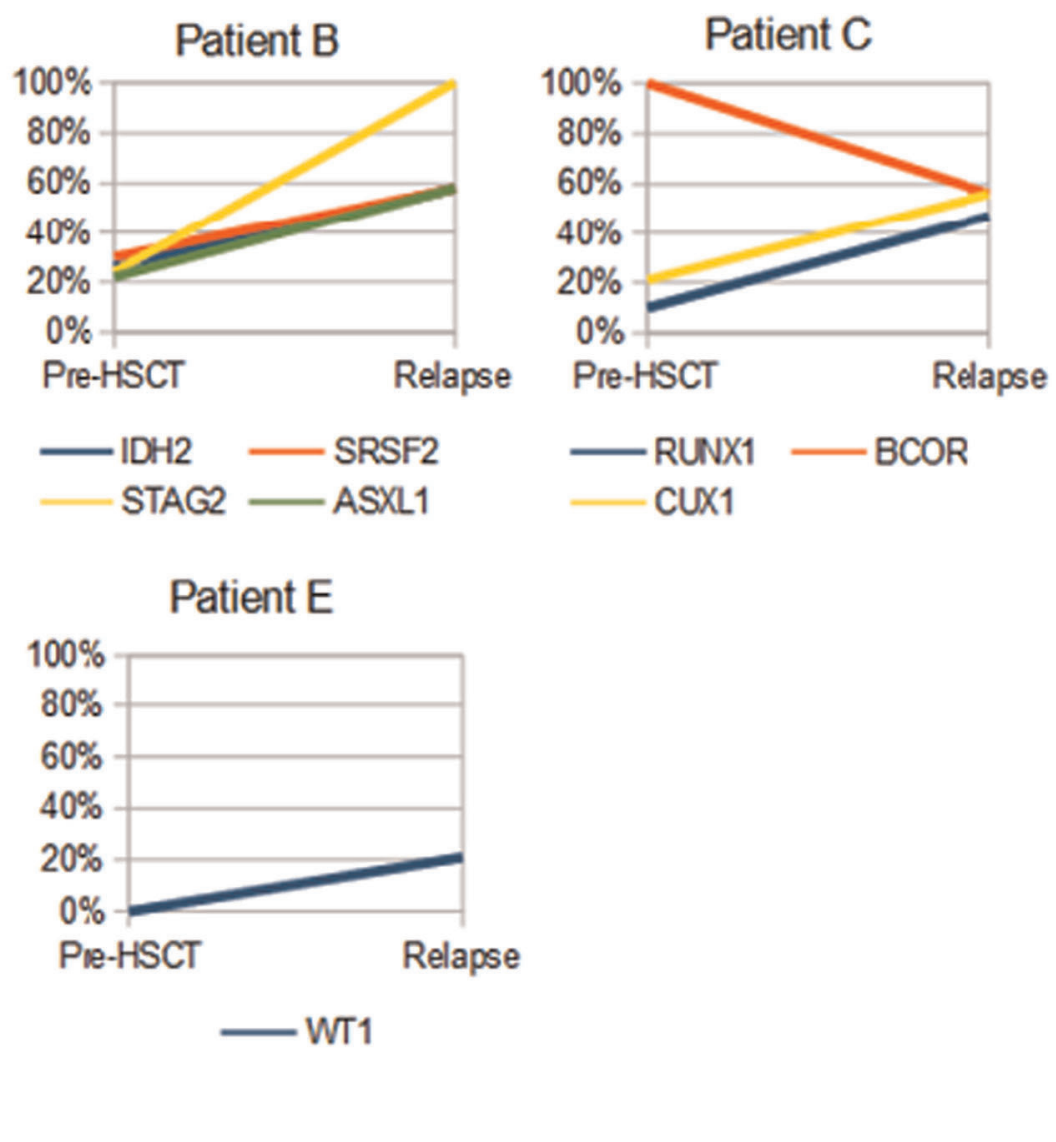

[P692 Figure] [Evolution of the mutational profile in MDS patients at the time of relapse after HSCT.] 
Methods: We analyzed bone marrow or peripheral blood from 6 patients with relapsed MDS after HSCT which was characterized by rising autologous chimerism (4-36\%). We used Illumina next-generation sequencing platform to examine tumor samples collected a day before starting conditioning regimen and at the time of relapse after HSCT for somatic mutations in 54 genes frequently mutated in myeloid malignancies.

Results: Fifteen mutations were identified in 5 of 6 patients, namely in genes: ASXL1, RUNX1, SF3B1, TET2, ETV6, WT1, PTPN11, SETBP1, IDH2, SRSF2, STAG2, $B C O R, C U X 1$ and GATA2. Most of these mutations (11/15) were detected before transplantation but predominantly in a lower percentage than at the time of relapse. Only one mutation $(B C O R)$ showed lower frequency at relapse than in pre-transplant period, which was characterized by loss of homozygosity. Most patients (4/6) relapsed with reexpansion of mutated clones existing prior HSCT. Other 2 patients were treated intensively before transplantation and therefore we did not detect any mutated clones in pretransplant samples in a higher percentage than $1 \%$. Three of 6 patients carried at least one new driver mutation after HSCT. For each patient, allelic frequencies of mutations in the pre-transplant and post-transplant relapse samples are shown in Figure 1.

Conclusions: Our pilot study demonstrates the evolution of clonal structure in the relapsed MDS patients after HSCT who show the considerable heterogeneity in the mutational profiles. The data suggest that post-transplant relapse is mainly driven by reexpansion of pre-HSCT persistent mutated clones that is accompanied by the acquisition of new mutations after HSCT.

Post-transplant frequencies of mutations were calculated according to autologous chimerism.

Supported by the Ministry of Health of the Czech Republic (The project for conceptual development of research organization No. 00023736).

Conflict of interest: There is no conflict of interest to report.

\section{P693}

Comparison of the predictive ability for transplant-specific prognostic scoring systems in the myelodysplastic syndrome patients receiving haploidentical stem cell transplantation

Seung-Hwan Shin, Young-Woo Jeon, Jae-Ho Yoon, Seung-Ah Yahng, Sung-Eun Lee, Byung-Sik Cho, Ki-Sung Eom, Seok Lee, Hee-Je Kim, Chang-Ki Min, Seok-Goo Cho, Dong-Wook Kim, JongWook Lee, Woo-Sung Min, Yoo-Jin Kim
The Catholic University of Korea, Hematology, Seoul, Korea, Republic of

Background: The decision to perform haploidentical stem cell transplantation for patients with myelodysplastic syndrome (MDS) should be made after weighing the potential benefit versus risk. Therefore, it is very important to identify patients who may gain an advantage from this procedure with reliable transplant-specific prognostic scoring tools. The purpose of this study was to validate the predictive ability with respect to survival of the MDS transplantation prognostic scoring system (MTPSS) in MDS patients who received haploidentical SCT, compared with that of the MDS transplantation risk index (TRI) and the scoring system from the Center for International Blood and Marrow Transplant Research (CIBMTR).

Methods: We evaluated the predictive ability of the MTPSS compared with two other transplant-specific prognostic systems: the TRI and the CIBMTR. We retrospectively analyzed 73 MDS patients receiving haploidentical transplantation.

Results: The median pre-transplant age was 50 (range, 19-69) years, and the worst point of disease course was categorized as refractory anemia in one (1.4\%), refractory cytopenia of multilineage dysplasia in 16 (21.9\%), refractory anemia with excess blasts in 38 (52.1\%), secondary acute myeloid leukemia in $16(21.9 \%)$ and other in two $(2.7 \%)$ patients. The IPSS-R cytogenetic risk of very good/good, intermediate, and poor/very poor was observed in 35 (48.0\%), 25 (34.2\%), and $13(17.8 \%)$ patients, respectively, including $4(5.5 \%)$ with a monosomal karyotype. Additionally, $30(41.1 \%)$ and 35 (48.0\%) patients had pre-transplant treatment failure and high hematopoietic cell transplant co-morbidity indices, respectively. The MTPSS $(100 \%, 77.3 \%, 62.5 \%$, and $42.0 \%$ at 4 years; $P=0.02)$ and the TRI $(100 \%, 79.9 \%, 76.0 \%$, and $17.1 \%$ at 4 years; $P<0.01)$ discriminated the overall survival of patients according to their 4 risk groups, whereas the CIBMTR $(93.3 \%, 60.6 \%, 46.9 \%$, and $100 \%$ at 4 years; $P=0.17)$ did not. The $c$-indices for overall survival of the MTPSS (0.675) and the TRI (0.662) were similar, whereas that of the CIBMTR (0.560) was relatively lower.

Conclusions: Our results suggest the potential of the MPTSS and the TRI, but not the CIBMTR, as prognostic tools for haploidentical recipients with MDS.

Conflict of interest: nothing to disclose

\section{P694}

External validation of scores predicting non-relapse mortality in patients with myelodysplastic syndrome: a study from MDS subcommittee of the CMWP EBMT 
Martin Carre ${ }^{1}$, Raphaël Porcher ${ }^{2}$, Jürgen Finke ${ }^{3}$, Gerhard Ehninger $^{4}$, Linda Koster ${ }^{5}$, Dietrich Beelen ${ }^{6}$, Arnold Ganser ${ }^{7}$, Liisa Volin $^{8}$, Sara Lozano ${ }^{9}$, Henrik Sengeloev ${ }^{10}$, Mauricette Michallet ${ }^{11}$, Johanna Tischer ${ }^{12}$, Eduardo Olavarria ${ }^{13}$, Maria Jesús Pascual
Cascon $^{14}$, Simona Iacobelli ${ }^{15}$, Yener Koc ${ }^{16}$, Pavel Jindra ${ }^{17}$, Mutlu Arat $^{18}$, Theo de Witte $^{19}$, Nicolaus Kröger ${ }^{20}$, Marie Robin ${ }^{21}$

${ }^{1}$ CHU Grenoble Alpes, Grenoble, France; ${ }^{2}$ Hôpital de l'Hôtel Dieu,
A

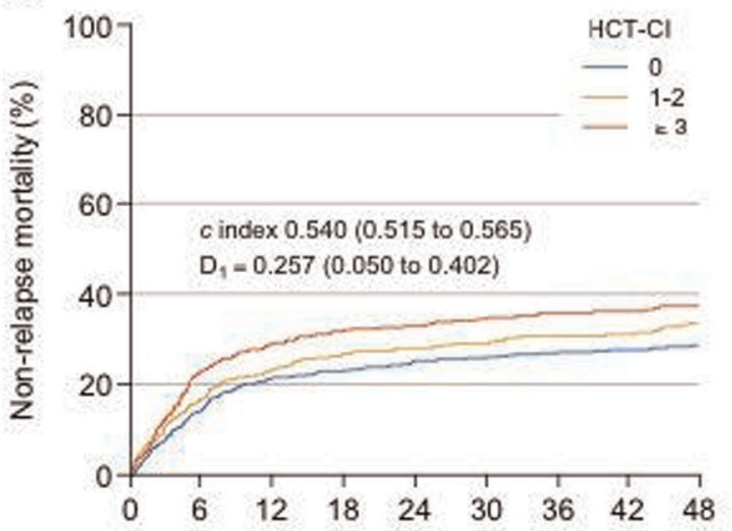

Months since transplantation

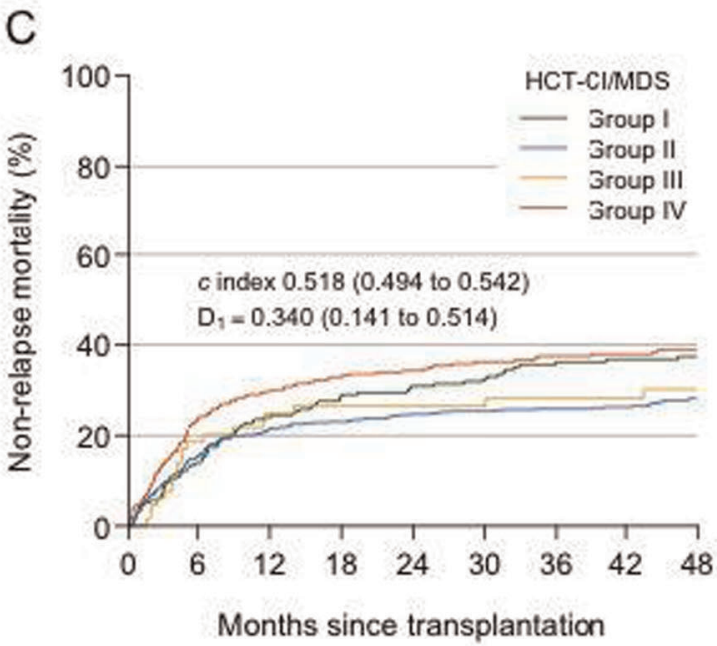

E

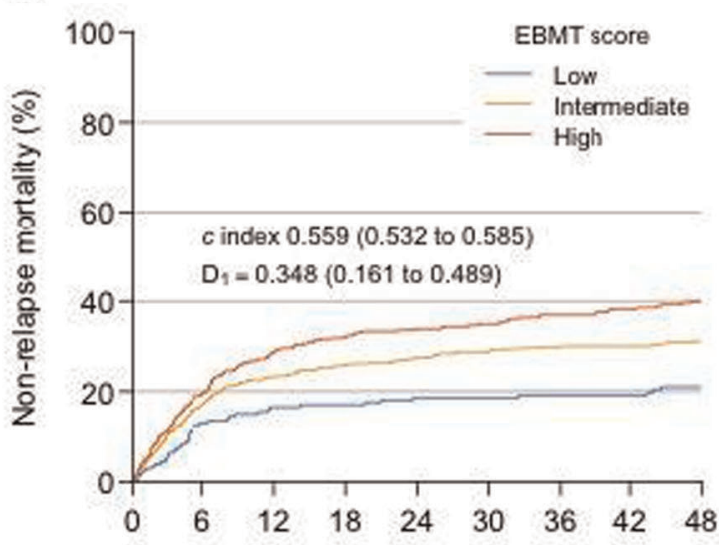

Monthe since transplantation
B

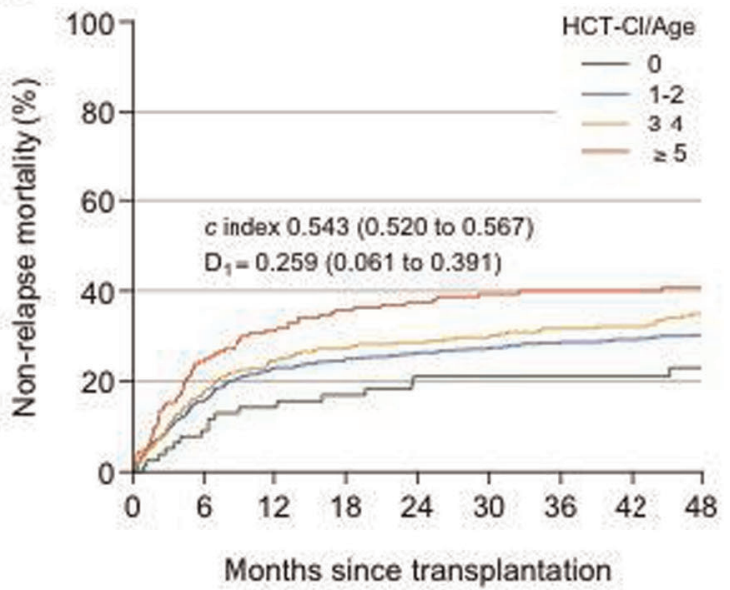

D

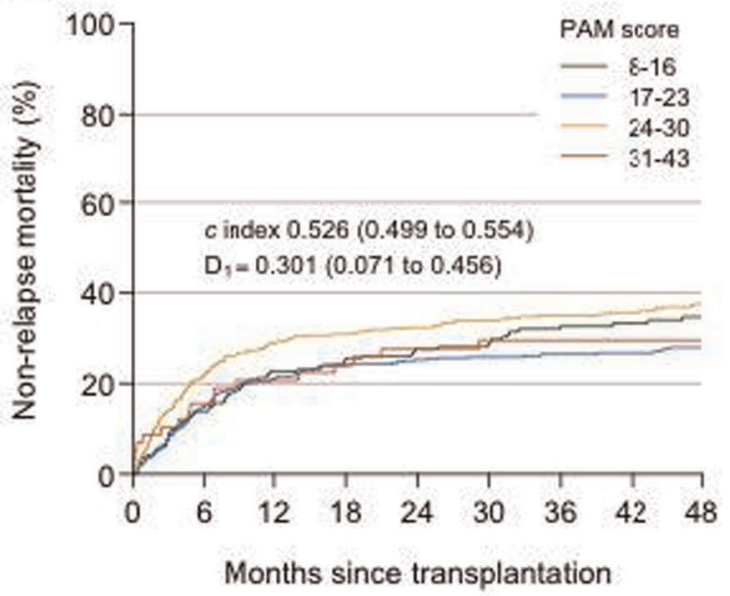

$\mathrm{F}$

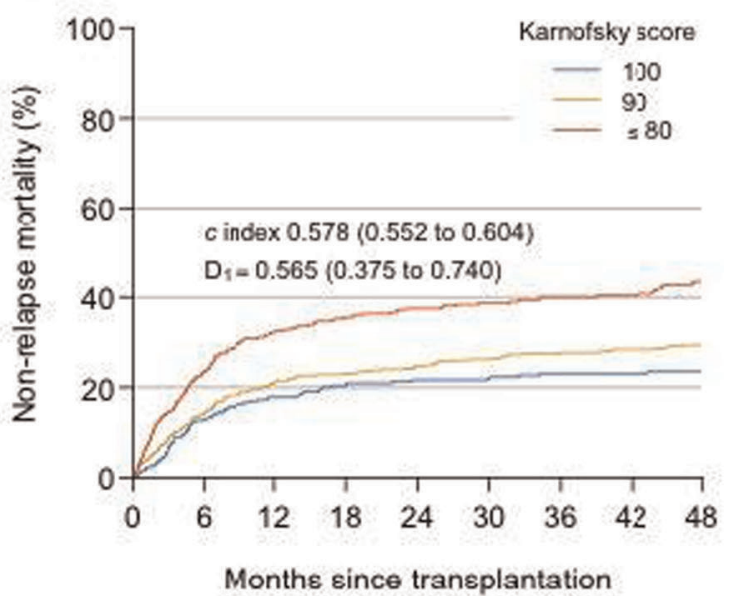

[P694 Figure] [Figure 1. NRM and discrimination indexes according to scores] 
Paris, France; ${ }^{3}$ University of Freiburg, Freiburg, Germany;

${ }^{4}$ Universitaetsklinikum Dresden, Dresden, Germany; ${ }^{5}$ EBMT Data

Office, Leiden, Netherlands; ${ }^{6}$ University Hospital, Essen, Germany;

${ }^{7}$ Hannover Medical School, Hannover, Germany; ${ }^{8} \mathrm{HUCH}$

Comprehensive Cancer Center, Helsinky, Finland; ${ }^{9}$ Hospital

Universitario Ramon y Cajal, Madrid, Spain; ${ }^{10}$ Rigshospitalet,

Copenhagen, Denmark; ${ }^{11}$ Centre Léon Bérard, Lyon, France;

${ }^{12}$ Klinikum Grosshadern, Munchen, Germany; ${ }^{13}$ Imperial College

Healthcare NHS Trust, London, United Kingdom, ${ }^{14}$ Hospital Regional de Málaga, Malaga, Spain; ${ }^{15}$ University of Tor Vergata, Roma, Italy;

${ }^{16}$ Medical Park Hospitals, Antalya, Turkey; ${ }^{17}$ Charles University Hospital, Pilsen, Czech Republic; ${ }^{18}$ Florence Nightingale Sisli

Hospital, Istanbul, Turkey; ${ }^{19}$ Radboud University - Nijmegen Medical Centre, Nijmegen, Netherlands; ${ }^{20}$ University Hospital Eppendorf,

Hamburg, Germany; ${ }^{21}$ Hopital St. Louis, Paris, France, APHP, Paris,

France

Background: Allogeneic stem cell transplantation (HSCT) is the only potential curative treatment for myelodysplastic syndromes (MDS). MDS occurring mainly in an elderly population, non-relapse mortality (NRM) remains a matter of concern despite the introduction of reduced-intensity conditioning. Here, we aim to evaluate the predictive role of different pre-transplant score.

Methods: 1245 MDS patients who were transplanted between 2003 and 2014 in centers who participated to the EBMT Data Quality Initiative were included. The performance of the HCT-CI (Hematopoietic cell transplantationspecific comorbidity index), PAM (Pretransplant Assessment of Mortality) scores and EBMT score (Sorror. Blood 2005 \& JCO 2014; Parimon Ann Intern Med 2006; Gratwohl. BMT 2012) in predicting NRM and overall survival (OS) was investigated according to principles and methods that have been described for external validation of prediction models (Royston BMC Medical Research Methodology 2013; 13:33). Besides, model discrimination was assessed by the concordance (c) index and its adaptation for competing risks analyses (Harrell JAMA 1982; Wolbers. Biostatistics 2014), and by the D1 index. The latter corresponds to the mean of absolute deviations of the sample individual log hazard ratios around the sample mean.

Results: Median age was 59 (18-79) years. Four hundred patients had MDS transformed into AML at time of transplant. In patients still in MDS status at time of transplant, 358 (47\%) had good, 146 (19\%) intermediate and $250(33 \%)$ poor cytogenetics according to IPSS, $91 \mathrm{had}$ no cytogenetics available. Donors were mostly matched related $(34.1 \%)$ or unrelated $(62.1 \%)$, use of peripheral blood stem cells was predominant $(89.7 \%)$ and conditioning regimen was reduced in 744 (59.8\%) of the transplants.

In multivariate analysis adjusted for disease risk, cytogenetics, sex mismatch and conditioning regimen, age (HR: $1.01, p=0.017)$, disease status $(p=0.029$ for intermediate risk, HR:1.46), donor type (unrelated, HR:1.76, $\mathrm{p}<$
$0.0001)$ and karnofsky $(<80 \%$, HR: $2.05, \mathrm{p}<0.0001)$ had significant impact on NRM. When similar variables were tested for OS, age (HR: 1.01, $\mathrm{p}=0.001$ ), disease risk (intermediate, HR 1.32, $\mathrm{p}=0.043$ ), donor type (unrelated, HR 1.32, $\mathrm{p}=0.031$ ), sex mismatch (female donor to male recipient, $1.26, \mathrm{p}=0.029)$, karnofsky $(<80 \%$, HR: $1.72, \mathrm{p}$ $<0.0001$ ) and cytogenetics (poor, HR: 1.59, p < 0.0001) had significant impact.

Figure 1 shows NRM according to scores and the performance of these scores according to the c-index and D1 index. C-index was not significant for HCT-CI/MDS and PAM scores while HCT-CI, HCT-CI/age, EBMT and Karnofsky had a significant c-index meaning that they can be considered as predicting NRM. The best D1 index was given by Karnofsky and EBMT score. HCT-CI/age score has the advantage to predict NRM in a low risk group (21\% NRM, only $6 \%$ of the cohort) not predicted by the original HCT-CI score.

The regression analysis confirms that HCT-CI/MDS and PAM scores had no gradual increasing of their Hazard ratio according to the categories. For OS prediction, all scores except HCT-CI/MDS predicted OS, including PAM score which was one of the best.

Conclusions: We validated in an EBMT cohort that HCT-CI, HCT-CI/age and EBMT scores predict NRM while PAM only predicts OS.

Conflict of interest: There is no conflict of interest

\section{P695}

Abstract previously published

\section{P696}

\section{Role of Hypomethylating Therapy in Patients with Myelodysplastic Syndrome Before Allogeneic Hematopoietic Stem Cell Transplantation}

Varvara Ovechkina, Elena Morozova, Sergey Bondarenko, Tatiana Gindina, Ivan Moiseev, Elena Darskaya, Ludmila Zubarovskaya, Boris Afanasyev

R.M.Gorbacheva Scientific Research Institute of Pediatric Oncology, Hematology and Transplantation, Pavlov First Saint Petersburg State Medical University, Saint Petersburg, Russian Federation

Background: The aim of this study was to assess the impact of hypomethylating therapy (HMT) before allogeneic hematopoietic stem cell transplantation (allo-HSCT) on the outcome in myelodysplastic syndrome (MDS) patients after allo-SCT.

Methods: We analyzed 46 patients (pts) with MDS that received allo-HSCT after HMT. Pts characteristics are in Tabl.1. Cytogenetic risk group at the moment of diagnosis 
was very good/good - 25 pts (54\%), intermediate - 7 pts (15\%), poor/very poor - 14 pts (31\%). Before allo-HSCT 36 pts $(78 \%)$ received 5-azacytidine, 10 pts $(22 \%)$ - decitabine in standard doses. Median number of cycles - 4 (range, 110). Response to the therapy was estimated according to the criteria of international working group (Cheson et al., 2006). At allo-HSCT RIC regimen was used in 35 pts (76\%), MAC in 11 pts (24\%). 12 (26\%) pts were grafted from MRD, 32 (69\%) - from M/MUD, 2 (5\%) - from haplodonors. Prophylaxis of GVHD on the basis CsA or Tacro \pm ATG or \pm PT-Cy.

Results: During HMT 5 pts (11\%) achieved complete response (CR), 14 pts (30\%) - partial response (PR), 26 pts $(56 \%)$ - stabilization $(\mathrm{S}), 1$ pts $(3 \%)$ - progression $(\mathrm{P})$. Before allo-HCST best response to HMT was associated with very good/good vs intermediate/poor/very poor cytogenetic risk group (100\% vs $79 \%, p=0.02)$, instead of poor/very poor vs intermediate/good/very good cytogenetic risk group (74\% vs $97 \%, \mathrm{p}=0.04)$. Worst response to HMT was described in the group of complex cytogenetic abnormalities vs other karyotype (62\% vs $95 \%, \mathrm{p}=0.02)$. Overall survival (OS) after allo-HSCT was $47 \%$, event-free survival (EFS) - 34\%, relapse incidence (RI) was $43 \%$, transplant-related mortality (TRM) - 31\%. Pts, who achieved partial/complete response or stabilization after HMT demonstrated significantly better EFS after allo-HCT than pts, who had progression $(40 \%$ vs $0 \%, \mathrm{p}=0.001)$. Median OS for the survivals was 730 days.

Conclusions: HMT can be the bridging option for pts with MDS before allo-HSCT. Cytogenetic risk group and type of the response to HMT before allo-HSCT influence on post-transplant EFS in patients with MDS.

Conflict of interest: Nothing to disclose.

\begin{tabular}{ll}
\hline Characteristic & $\mathrm{N}$ \\
\hline Number of patients & 46 \\
Gender n,\% Male; Female & $30,65 \% ; 16,35 \%$ \\
Age, years median (range) & $48(25-58)$ \\
Secondary n, $\%$ & $4,9 \%$ \\
Diagnosis (WHO) n,\% RA; RARS; RCMD; & $4,9 \% ; 1,2 \% ; 9,19 \% ; 16,35 \% ;$ \\
RAEB1; RAEB2 & $16,35 \%$ \\
IPSS median, range & $1.5(0-3)$ \\
WPSS median, range & $3(0-8)$ \\
\hline
\end{tabular}

[[P696 Table] Characteristics of patients]

\section{P697}

Stem Cell Transplantation (SCT) in Pediatric (Age $\leq 14$ years) Myelodysplastic Syndrome (MDS) patients at King Faisal Specialist Hospital and Research Centre, Riyadh Saudi Arabia (1993 - 2016)
Suleimman AlSweedan, Khawar Siddiqui, Rafat Jafri, Mary Barria, Ali Al-Ahmari, Ibrahim Ghemlas, Hawazen Al-Saedi, Abdullah AlJefri, Mona Assiri, Mouhab Ayas, Amal Al-Seraihy

King Faisal Specialist Hospital \& Research Center, Pediatric Hematology / Oncology, Riyadh, Saudi Arabia

Background: We analyzed the data on our cohort of MDS patients undergoing Stem Cell Transplantation to review the results of our treatment efforts.

Methods: A total of 37 patients were transplanted from Jan 1993 to December 2016 at our institution. Two (2) were excluded for having previously transplanted at other hospitals. Data on demographics, diagnosis, transplantrelated parameters and outcome was abstracted from clinical databases and through medical charts review for this retrospective cohort study and processed and analyzed using IBM-SPSS. The study was pre-approved by the IRB of the hospital.

Results: $62.9 \%(22)$ were boys. Median age at transplant was 4 years $(0.8-14.8)$ with median time to transplant from diagnosis was 8.1 (2.3-102.5) months. In $88.6 \%(31)$ we used bone marrow as source of stem cells, remaining $11.4 \%$ (4) were transplanted with cord blood. 51.4\%(18) got Bu/ $\mathrm{Cy} / \mathrm{VP}-16$ as conditioning regimen followed by $\mathrm{Bu} / \mathrm{Cy}$ in 28.6\%(10) and Bu/Cy/ATG in 20\%(7). 74.3\%(26) received GCSF. $80 \%$ (28) donors were HLA-Identical siblings. Our transplant related mortality rate was $5.7 \%(2)$ on D100, $88.6 \%(31)$ were engrafted, $2.9 \%(1)$ had primary and secondary Graft Failure each. VOD was seen in $5.7 \%(2)$ and $14.3 \%$ (5) had hemorrhagic cystitis within first 100 days. No Grade III-IV aGVHD was observed. With a median follow-up of 112.4 months and 12 events of mortality, 3year overall and event free survival of our cohort of patients was $0.681 \pm 0.080$. No significant risk factor including age at diagnosis, time to transplant from diagnosis, pre-transplant disease status, donor and recipient gender, conditioning regimen, source of Stem Cells, or occurrence of aGVHD was found through uni- or multi-variable analyses.

Conclusions: In our cohort of patients, results of stem cell transplantation in terms of transplant related toxicity and overall survival are promising.

Conflict of interest: None declared

\section{P698}

TP53 mutation in allogeneic hematopoietic cell transplantation for de novo myelodysplastic syndrome

Yoo-Jin Kim ${ }^{1}$, Seung-Hyun Jung ${ }^{2}$, Eun-Hye Hur ${ }^{3}$, Eun-Ji Choi ${ }^{3}$, Kyoo-Hyung Lee ${ }^{3}$, Seon-Hee Yim $^{2}$, Hye-Jung Kim ${ }^{1}$, Yong-Rim Kwon', Young-Woo Jeon', Sug Hyung Lee ${ }^{4}$, Yeun-Jun Chung ${ }^{2}$, JeHwan Lee

${ }^{1}$ Seoul St. Mary's Hospital, College of Medicine, The Catholic 
University of Korea, Hematology, Seoul, Korea, Republic of; ${ }^{2}$ College of Medicine, The Catholic University of Korea, Microbiology, Seoul, Korea, Republic of; ${ }^{3}$ Asan Medical Center, University of Ulsan College of Medicine, Hematology, Seoul, Korea, Republic of; ${ }^{4}$ College of Medicine, The Catholic University of Korea, Pathology, Seoul, Korea, Republic of

Background: Somatic mutations, which are involved in the pathogenesis of MDS, have become relevant in MDS prognostication. We investigated prognostic role of somatic mutations including TP53 mutation in patients with de novo myelodysplastic syndrome (MDS) who underwent allogeneic hematopoietic cell transplantation (HCT).

Methods: We performed targeted deep sequencing analysis of 26 genes on bone marrow samples obtained within 6 weeks before HCT from 202 patients with de novo MDS at two Korean institutes. We analyzed association of somatic mutations with post-transplant outcomes: overall survival (OS), relapse-free survival (RFS), event-free survival (EFS), and non-relapse mortality (NRM).

Results: The median age of the patients, 74 females and 128 males, was 51 years. Overall, $76 \%$ of patients carried one or more somatic mutations, and TP53 mutation was present in 23 patients (11.4\%). The 5-year probabilities of OS, RFS, EFS, and NRM were $63.6 \%, 78.4 \%, 58.5 \%$, and $25.4 \%$, respectively. TP53 mutation was an independent risk factor for shorter OS (41\% vs. 67\%; hazard ratio [HR], 2.897; $\mathrm{P}=0.001$ ), RFS (47\% vs. 83\%; HR, 4.178; $\mathrm{P}<$ 0.001 ), and EFS (38\% vs. $61 \%$; HR, 2.400; $\mathrm{P}=0.005)$, but it was not for NRM (18\% vs. $26 \%)$. N-RAS mutation was an independent risk factor for shorter RFS (HR, 5.505; $\mathrm{P}=$ 0.009). Among clinical variables, male sex (OS, EFS, NRM), thrombocytopenia (OS), old age (RFS), poor cytogenetics (RFS, EFS), higher HCT-comorbidity index (RFS, EFS), and older donor (RFS) were independent poor prognostic factors. TP53 mutations were more frequently found in patients with poor IPSS cytogenetic risk and those with higher scores of IPSS or R-IPSS. Of 76 patients with IPSS higher-risk scores, 18 patients $(23.7 \%)$ had TP53 mutations and the patients with TP53 mutations showed significantly lower OS (25.0\%. vs. $59.1 \%, P=.001)$, lower RFS $(27.5 \%$ vs. $86.0 \%, P<.001)$, lower EFS $(20.0 \%$ vs. $58.1 \%, P<.001)$, and similar NRM $(27.1 \%$ vs. $32.5 \%, P=$ .832). TP53 mutation did not have significant interactions with conditioning intensity or occurrence of graft-versushost disease regarding post-transplant outcomes.

Conclusions: TP53 mutation was significantly associated with poor outcomes after HCT for patients with de novo MDS, mainly due to higher incidence of disease relapse.

Conflict of interest: None of the authors has anything to disclose.
P699

\section{Treatment and clinical outcomes of patients relapsing after allogeneic hematopoietic cell transplantation for myelodysplastic syndrome}

Eun-Ji Choi, Je-Hwan Lee, Jung-Hee Lee, Han-Seung Park, SunHye Ko, Miee Seol, Young-Shin Lee, Young-Ah Kang, Mijin Jeon, Kyoo-Hyung Lee

Asan Medical Center, University of Ulsan College of Medicine, Department of Hematology, Seoul, Korea, Republic of

Background: Allogeneic hematopoietic cell transplantation (HCT) is considered the only curative treatment option for patients with myelodysplastic syndrome (MDS). However, even after HCT, substantial number of patients experience disease relapse and there is no standard treatment for relapsed patients. We reviewed clinical outcomes after relapse of MDS patients who had received allogeneic HCT.

Methods: Thirty patients, who experienced relapse or progression after allogeneic HCT for MDS between July 2000 and May 2016, were included in this study.

Results: The median age of patients was 54 years (range, 19-70). Of total patients, 8,19 , and 3 were diagnosed with MDS-refractory anemia of excess blasts (RAEB)-1, RAEB2 , and refractory cytopenia with multilineage dysplasia (RCMD) before HCT, respectively. The pre-transplantation international prognostic scoring system was recorded as low (13.3\%), intermediate-1 (36.7\%), intermediate-2 (33.3\%), and high (16.7\%). Patients experienced disease relapse in a median of 6.6 months (range, 0.9-136.3) after HCT including 13 patients who presented with transformation to acute myeloid leukemia (AML). Donor lymphocyte infusion (DLI) was delivered to four patients: one who relapsed with RCMD was alive in complete remission (CR) and three showed mixed chimerism after DLI and eventually died of disease. Of seven patients who received hypomethylating agents (HMA), one continued to receive 5 cycles of decitabine at the time of analysis in stable disease. Other 3 patients died during the HMA treatment with persistent disease and pneumonia, and 3 patients who progressed to AML after 3 to 4 cycles of decitabine underwent salvage HCT, but died of disease and/or bleeding complication. Six Of 13 patients progressing to AML were treated with AML induction-like intensive chemotherapy and 3 achieved CR. Of those 3 patients, 2 underwent second HCT and one received DLI. One who received second HCT after CR was alive without disease and other two eventually relapsed and died. All 3 patients who did not respond to intensive chemotherapy died of disease. Four patients treated with low-dose cytarabine did not respond to treatment and died from refractory disease. 
One patient who experienced cytogenetic relapse at 2 years after HCT received second transplantation and was alive at the time of analysis without evidence of disease. All eight patients treated with best supportive care died from disease. The median overall survival (OS) after relapse of all patients was 4.4 months (95\% confidence interval [CI], 0.78.2). The longer interval between HCT and relapse was associated with improved OS; $\geq 6$ months vs. $<6$ months, hazard ratio, $0.195 ; 95 \% \mathrm{CI}, 0.070-0.547, \mathrm{P}=0.002$.

Conclusions: Though various therapeutic approaches have been attempted, the outcome of MDS patients relapsed or progressed after HCT was disappointing. Early detection of disease relapse and application of therapeutic strategies such as prophylactic DLI or second HCT to patients with minimal disease burden could be an option to induce longterm response and survival.

Conflict of interest: The authors declare no conflict of interest.

New drug- and cell-based immune therapies

\section{P700}

\section{A standardized methodical approach to characterize the key parameters influencing the in vitro extracorporeal photopheresis efficiency}

Marie Laulhé, Sylvie Lefebvre, Delphine Le Broc - Ryckewaert, Maxime Pierre, Bruno Delorme

Macopharma, R\&D Biotherapy, Mouvaux, France

Background: Extracorporeal photopheresis (ECP) is an autologous cellular immunomodulatory treatment that involves the ex vivo collection of mononuclear cells (MNC), their UVA irradiation in the presence of the photosensitizing agent 8-methoxypsoralen (8-MOP) to induce cell apoptosis (mainly of T-cells) and the reinfusion of the treated cells into the patients. Although ECP benefits are well-known in clinic for a number of T-cell mediated diseases, no studies was ever reported to link the variability of the different components entering in the composition of the cell preparation to treat with the in vitro efficacy of ECP treatment.

Methods: A standardized methodical approach mimicking clinical procedures was defined as follow:

1) each component present in the irradiation container (plasma, hematocrit, cells, saline and 8-MOP solutions) was studied separately by changing one component ratio or system setting at a time

2) a human lymphoma T-cell line model was used to analyze in a reproducible way the in vitro efficacy of the ECP treatment.
We systematically analyzed and compared for all conditions studied two different cellular responses as relevant acceptance criteria of the ECP technique: cell apoptosis and inhibition of cell proliferation. 8-MOP consumption during irradiation was also systematically determined before and after each treatment.

Results: This in vitro performance study allowed us to highlight a decrease of cellular response following the increase of hematocrit or plasma ratio in a dose-dependent manner. In addition, only plasma ratio had an influence on 8-MOP consumption. Finally, our study also provided a workaround to counteract extreme variations in blood components using system setting adjustments, notably the irradiation dose, to reach optimal in vitro ECP efficiency.

Conclusions: This methodical approach could be especially useful to evaluate other leukapheresis preparation protocols and better define the specifications of use of ECP technique towards a blood composition-dependent ECP procedure standardization.

Conflict of interest: Maco Pharma provided support in the form of salaries for authors ML, SL, DLBR, AF, MP and BD but did not have any additional role in the study design, data collection and analysis, decision to publish, or preparation of the manuscript. Maco Pharma produces and distributes the MacoGenic G2, irradiation containers, 8MOP solution, $0,9 \% \mathrm{NaCl}$ solution and ACD-A solution that were used in this study.

\section{P701}

\section{Bridges to pediatric stem cell transplant: a new lane through}

Bárbara Torres Guerola, Sandra Carreño Sánchez, José María Fernández Navarro

La Fe, Valencia, Spain

Background: Relapsed and/or refractory acute lymphoblastic leukemia (RRAAL) in children achieve remissions often fragile. In this setting, urgent SCT is mandatory. Several options could be considered to maintain remission until transplant. We describe our experience with inotuzumab ozogamicin (InO) as a bridge to transplant in a pediatric cohort.

Methods: We describe three pediatric cases of $\mathrm{CD} 22$ positive RRALL in second remission treated with $\mathrm{InO}$ as a single agent (after rescue treatment) unable to receive further chemotherapy because of their clinical condition. InO was obtained as per compassionate use local regulations after informed parental consent. Dose for cycle 1 was: $0,4 \mathrm{mg} / \mathrm{m} 2$ day 1 and $0,2 \mathrm{mg} / \mathrm{m} 2$ on days 8 and 15 . Cycle 2 
and further: $0,2 \mathrm{mg} / \mathrm{m} 2$ on days 1,8 and 15 . Dosage was adapted for patients under $10 \mathrm{Kg}$.

Case 1: 9 year old boy. Isolated bone marrow relapsed ALL during maintenance therapy. Started treatment on Spanish National Relapsed ALL protocol. Admitted in PICU in week 2 of induction therapy for E coli sepsis. Ventilated for two weeks. Inotropic support needed. He presented seizures during PICU admission and developed tetraparesis. MRI and electromyography compatible with myopathy due to prolonged use of muscle relaxants. It was decided in multidisciplinary meeting to avoid the use of any neurotoxic drugs. His bone marrow was in complete remission. To favor patient full recovery before transplant and while an appropriate donor was identified, three cycles of $\mathrm{InO}$ were administered. MUD performed.

Case 2: 6 year old girl. Isolated bone marrow relapse during maintenance therapy. Refractory to induction as per Spanish National Relapsed ALL protocol. Combined clofarabine based chemotherapy was then administered. Grade 4 toxicity: hematological and neurological. She also suffered from several septic episodes and systemic adenovirus (ADV) infection. She achieved her second remission but no MRD nor MUD were identified. It was decided in multidisciplinary meeting because of previous toxicity and ADV infection to avoid further chemo. Once the infections were resolved and to support her second remission until haploidentical transplant, one cycle of InO was given. Haploidentical transplant performed.

Case 3 : 5 months old girl. Refractory to infant ALL Spanish Protocol. We administered FLAG- Myocet as salvage chemotherapy. Grade 4 hematological and gastrointestinal toxicity. Several septic episodes. It was decided in multidisciplinary meeting because of previous toxicity to avoid further chemotherapy. No MRD neither MUD were identified. While her haploidentical transplant was arranged, we gave her one dose of InO. Haploidentical transplant performed.

Results: No immediate toxicity was found related to InO administration. Nine out ot eleven doses were administered in our day beds unit. All three patients underwent transplant in complete remission with negative minimal residual disease with excellent pre SCT performance status. Prophylactic defibrotide was given to every patient. Case 3 developed SOS that was resolved with standard treatment.

Conclusions: InO has been safe in our cohort. It could be considered a suitable option as a bridge to transplant in pediatric CD22 RRALL with fragile remission and bad tolerance to chemotherapy.

Clinical Trial Registry: non applicable

Conflict of interest: N/A
P702

CD8+PD-1+ lymphocytes preferentially reside in the marrow as compared to the blood and may witness antileukemic activity of the immune system

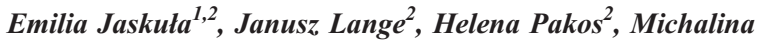
Krydel $^{2}$, Jolanta Bocheńska ${ }^{2}$, Iwona Wodzińska-Maszko ${ }^{2}$, Andrzej Lange $^{1,2}$

${ }^{1}$ L. Hirszfeld Institute of Immunology and Experimental Therapy, Polish Academy of Sciences, Wroclaw, Poland; ${ }^{2}$ Lower Silesian Center for Cellular Transplantation with National Bone Marrow Donor Registry, Wroclaw, Poland

Background: The issue of exhausted tumour infiltrating lymphocytes gained a great deal of interest fuelled by solid scientific data on restoring the function of $\mathrm{T}$ cells by blocking some receptors on their membrane, representative examples being CXCR4 and PD-1. In fighting leukaemia two approaches were employed in our institution: (i) donor lymphocytes injected directly into the bone leukaemic infiltrates or the marrow cavity (lower risk of graft-versushost disease (GvHD), direct contact between mononuclear cell population and the blasts usually at a low level), (ii) a multikinase inhibitor blocking the outcome of FLT3 ITD mutation and boosting independently the immune function of the patients.

Methods: The clinical effect was monitored and highlighted by a thorough observation of the immune environment in the marrow. Altogether, 21 intra-bone donor lymphocyte infusion (IB-DLI) were delivered to 6 patients and in 3 sorafenib was used. Overall, a positive effect of the IB injection was seen; however, temporally it led to a decrease in the blast count and restoration of haematopoiesis.

Results: The body of the observation is as follows:

1. In no situation did GvHD symptoms appeared or were exaggerated if present before in patients after IB-DLI.

2. From the primary observation it was found that proportions of CD8+ cells and CD8+PD-1+ lymphocytes were higher in the marrow as compared to the blood $(7.294 \pm 0.957 \%$ vs $11.18 \pm 1.28 \%, \mathrm{p}<$ $0.001)$. The high proportion of CD8+PD-1 positive lymphocytes was in the blood, but even so prevalence in the marrow ( $32 \%$ vs $21 \%$ ) was seen in a patient lacking any myeloblasts in the marrow but suffering from extra-medullar relapse. The leukemic skin lesions were, however, infiltrated by CD3+ cells.

3. An increase of CD8+PD-1+ lymphocytes in the marrow was associated with a well-documented retreat of blasts from the marrow. In addition, morphology of lymphocytes depicted their activation 
and in the marrow biopsies blast-lymphocyte conjugates could be spotted.

4. Consecutive IB injection there rather decreased than increased of a proportion of exhausted lymphocytes $(\mathrm{CD} 8+\mathrm{PD}-1+)$ in the marrow $(11.327 \pm 4.555$ vs $9.688 \pm 3.191, \mathrm{p}=0.003)$.

5. In sorafenib-receiving patients parallel to the decrease in the proportions of $\mathrm{CD} 4+\mathrm{CD} 25$ hight + lymphocytes $(0.36 \% \pm 0.03 \%$, vs $0.13 \% \pm 0.03 \%, p=0.036$, values registered prior and during the sorafenib treatment) had marrow infiltrated with $\mathrm{CD} 8+$ lymphocytes which co-expressed PD-1 in higher proportions than those in the blood $(163 \pm 32 \times 103$ cells $/ \mu$ l vs $38 \pm 8$ $\mathrm{x} 103$ cells/ $\mu \mathrm{l}, \mathrm{p}<0.001)$. All patients on sorafenib developed skin lesion of alloreactive character.

Conclusions: In spite of the high proportion of CD8 +CDPD-1+ lymphocytes in the marrow and their apparent prevalent contribution to the marrow lymphocytes, an antileukemic effect of both implemented procedures was documented.

Therefore, PD-1 positivity of CD8+ cells are not necessarily associated with $\mathrm{T}$ cells' poor function and may represent the presence of an active immune response in the marrow environment to the leukemic blasts.

Conflict of interest: The authors declare that there is no conflict of interest regarding the publication of this abstract

\section{P703}

\section{Characterization of CD19 CAR T cells from patients with B- cell chronic lymphocytic leukemia}

\section{Isabelle Magalhaes ${ }^{1}$, Anders Öterborg $^{1}$, Michael Uhlin ${ }^{2}$, Jonas Mattsson $^{1}$}

${ }^{1}$ Karolinska Institutet, Oncology and Pathology, Stockholm, Sweden; ${ }^{2}$ Karolinska University Hospital, Transfusion Medicine and Clinical Immunology, Stockholm, Sweden

Background: CD19 CAR T cell immunotherapy has demonstrated dramatic results for the treatment of B-cell malignancies such as chronic lymphocytic leukemia (CLL) and acute lymphoblastic leukemia. Since T cell defects are characteristic of patients with CLL, we compared T cells from patients with CLL and healthy donors (HDs), and subsequently the CD19 CAR T cells produced from either patients or HDs.

Methods: The MSGV-FMC63-28Z PG13 packaging cell clone $\mathrm{H} 3$ producing the retroviral vector encoding the second generation anti-CD19 CAR with a CD28 costimulatory domain used in this study, was kindly provided by Prof. Steven Rosenberg(NCI). The production of retroviral supernatant and CAR T cell manufacturing were performed according to published methods. Immunophenotyping was performed using monoclonal antibodies directed against multiple surface antigens. CD19 CAR $+\mathrm{T}$ cells were detected using Biotin-SP-conjugated goat anti-mouse specific antibody. CD19 CAR transduced $\mathrm{T}$ cells were incubated at an effector target ratio of $1: 1$ with either CD19+ K562, NGFR + K562 or autologous B cells (CLL cells or EBV-transformed B cells). After incubation, monoclonal antibodies directed against $\mathrm{CD} 3, \mathrm{CD} 4$ and CD8 were used for cell surface staining, and after fixation and permeabilisation antibodies against IL-2, TNF, IFN- $\gamma$ and IL-17 or in other experiments granzyme B were used for intracellular staining. One week after transduction, CD19 CAR T cells were stimulated and re-stimulated 7 days later by the addition to the cultures of CD19+ K562 cells. After 14 days CD19 CAR T cells were re-stimulated for 6 hours as with CD19+ K562 cells for determination of CD107a expression and cytokine production. CD19+ K562 and NGFR + K562 cells were labelled using the CellTrace Violet dye. K562 cells were then added at a ratio of 1:1 to CD19 CAR transduced T cells. After 24 hours, the cells were analyzed by flow cytometry.

Results: In patients with CLL, circulating $\mathrm{T}$ cell frequency within lymphocytes was lower (4\%) as compared to HDs (64\%). In patients, the majority of CD8+ and CD4 $+\mathrm{T}$ cells were significantly enriched in the effector memory compartment (CD45RA-CCR7-), and CD8 $+\mathrm{T}$ cells were more frequent in the terminally differentiated phenotype compartment (CD45RA+CCR7-). However, T cells' frequency of expression of the checkpoints inhibitors PD-1, LAG-3 and TIM-3 was similar between patients and HDs. After retroviral transduction, $T$ cells expressing the CD19 CAR construct from either patient's or HD's cells showed a similar phenotype (effector memory or terminally differentiated), were specifically activated by and killed CD19+ target cells, and secreted cytokines (i.e. IL-2, TNF, and IFN- $\gamma$ ). Interestingly, the frequency of CD4+ and CD8+ $\mathrm{CD} 19 \mathrm{CAR}+\mathrm{T}$ cells producing IFN- $\gamma$ was significantly higher in cells produced from patients as compared to those produced from HDs. This was true using either autologous B cells or K562-CD19+ cells as target cells.

Conclusions: In conclusion, patients with CLL expressing CD19 CAR were phenotypically, and functionally (as measured by CD107a expression and cytokine production) comparable to HDs, however, their increased IFN- $\gamma$ production measured in this study in vitro, may if this is also the case in vivo, contribute to a higher risk of a cytokine release syndrome in patients.

Conflict of interest: Nothing to disclose 


\section{P704}

\section{Clinical-scale production of ARI-0001 cells using the ClinicMACS Prodigy system}

Maria Castella ${ }^{1}$, Anna Boronat ${ }^{2}$, Raquel Martin-Ibañez $z^{3}$, Vanina Rodríguez, ${ }^{4}$ Miguel Caballero ${ }^{2}$, Berta Marzal ${ }^{2}$, Julio Castaño 5 , Clara Bueno $^{5}$, Olga Balague ${ }^{6}$, Daniel Benitez ${ }^{2}$, Valentin OrtizMaldonado $^{1}$, Tycho Baumann ${ }^{1}$, Elias Campo ${ }^{6}$, Pablo Menendez, Susana Rives ${ }^{7}$, Julio Delgado ${ }^{1}$, Jordi Yagüe ${ }^{2}$, Patricia Perez-Galan ${ }^{4}$, Alvaro Urbano-Ispizua ${ }^{1,5}$, Josep Maria Canals ${ }^{3}$, Manel Juan ${ }^{2}$

${ }^{1}$ Hospital Clinic, Hematology, Barcelona, Spain; ${ }^{2}$ Hospital Clinic, Immunology, Barcelona, Spain; ${ }^{3}$ University of Barcelona, Biomedical Sciences, Barcelona, Spain; ${ }^{4}$ IDIBAPS, Hemato-Oncology, Barcelona, Spain; ${ }^{5}$ Josep Carreras Leukemia Research Institute, Biomedicine, Barcelona, Spain; ${ }^{6}$ Hospital Clinic, Pathology, Barcelona, Spain;

${ }^{7}$ Hospital Sant Joan de Deu, Hematology, Barcelona, Spain

Background: We have shown that our A3B1 anti-CD19 CAR performs competently in pre-clinical studies, with an effectiveness comparable to other CART19 constructs currently used in the clinic. The next step was to set up the infrastructure and procedures to take our CAR19 to clinical stage. This represents a big challenge for a relatively small publicly funded institution. Here we present the production system that we successfully implemented in Hospital Clinic, Barcelona.

Our CAR19 T cells (ARI-0001) production system includes two separated processes: 1) Large-scale CAR19 lentivirus production; and 2) Ex vivo transduction and expansion of ARI-0001 cells. Here we describe the validation of the production method for both processes ( 3 validation testings each).

\section{Methods:}

1. Large-scale CAR19 lentivirus production:

2. $80 \times 10^{6}$ HEK293T cells from Master Cell Bank were thawed on day 1 . Cells were expanded on T175 flasks until $2900 \times 10^{6}$ cells are obtained on day 8 , then transferred to $4 \times 1 \mathrm{~L}$ cell factories. $24 \mathrm{~h}$ later, cells were transfected using $3.86 \mathrm{mg}$ PEI, $763 \mathrm{mg}$ transfer vector, $377 \mathrm{mg} \mathrm{pMDLg} / \mathrm{pRRE}, 188 \mathrm{mg}$ pRSV-Rev and $221 \mathrm{mg}$ pMD2.G per liter. Viral supernatant was collected on day 11, centrifuged and filtered through a $0.45 \mathrm{~mm}$ PVDF membrane. On day 12, viral supernatants were concentrated to $100 \mathrm{ml}$ using Tangential Flow Filtration (Hollow fibers $500 \mathrm{kDa}$ ), also diafiltered using $\mathrm{PBS}$, and finally preserved at $-80^{\circ} \mathrm{C}$ until use.

3. Ex vivo transduction and expansion of ARI-0001

\begin{tabular}{|c|c|c|c|c|c|}
\hline Parameter & Method & Acceptance criteria & ARI0001/01 & ARI0001/02 & ARI0001/03 \\
\hline Appereance & Visualinspection & Cloudy liquid solution & doudy liquid solution & Cloudy Equid solution & Cloudy Equid solution \\
\hline Number of CAR19+ cells & $\begin{array}{c}\text { Neubaercell } \\
\text { counting and flow } \\
\text { sytometry }\end{array}$ & $\begin{array}{l}35 \times 10^{6} \mathrm{cells} \text { for a } 70 \mathrm{~kg} \\
\text { patient }\left(>0.5 \times 10^{6} \text { cells } / \mathrm{kg}\right)\end{array}$ & $1495 \times \times 10^{\circ}$ & $1745 \times \times 10^{4}$ & $1340 \times \times 10^{5}$ \\
\hline$\%$ CAR $19+$ cells & Elow cytometry & $220 \%$ & 26.0 & 41.9 & 39.7 \\
\hline$\% \mathrm{CD}_{3}+$ cells & Flow cytometry & 2708 & 97.9 & 93.6 & 98.6 \\
\hline \%Call viability & $\begin{array}{l}\text { Neubsuercell } \\
\text { counting withtryp nn } \\
\text { blue exclusion }\end{array}$ & $270 \%$ & 93.9 & 93.1 & 98.0 \\
\hline sterility & Microbial growth & Sterie & Sterile & Sterile & Sterile \\
\hline Mricoplasma & $P C R$ & Absent & Absent & Abient & Absent \\
\hline Endotoxin & Chromegenic assty & $\leq 0.5 E U / \mathrm{ml}$ & $\leq 0.580 / \mathrm{ml}$ & $\leq 0.500 / \mathrm{ml}$ & $\leq 0.5 E U / \mathrm{ml}$ \\
\hline Adventitious viruses & $P C R$ & Absent & Absent & Abient & Absent \\
\hline $\begin{array}{c}\text { Numberof transgene } \\
\text { cepies/cell }\end{array}$ & Real-time $P C R$ & s10 copies/cell & 1.45 & 232 & 2.51 \\
\hline $\begin{array}{c}\text { RCL (Replication- } \\
\text { competent lentivirus) }\end{array}$ & Real-time $P C R$ & absent & Absent & Abient & Absent \\
\hline Cytotoxicpotential & floweytometry & $\begin{array}{l}\text { (a) NXL1A6 coll } \\
\text { surviving fraction } \\
\text { using CART19 } \\
\text { ratio ET } 1: 1< \\
\text { T0\% } \\
\text { or } \\
\text { Difference in } \\
\text { NALI.16 cell } \\
\text { survivingfraction } \\
\text { between CART19 } \\
\text { vsUntransduced } \\
\text { Tusing ratio E:T } \\
\text { A:1 }>50 \%\end{array}$ & $14.9 ;$ (b) 90.9 & 4), 4 ; (b) 64.6 & $43.0 ;(b) \in 7.0$ \\
\hline
\end{tabular}

[P704 Figure] [ARI0001 product specification list and acceptance criteria] 
cells:

4. Leukoaphereses obtained from healthy donors were obtained, after informed consent, using the Amicus device (Fresenius Kabi). A minimum of $1 \times 10^{8} \mathrm{~T}$ cells was required. Cells were introduced into the CliniMACS Prodigy system (Miltenyi Biotec), were the whole process was carried out in a completely closed and sterile system under GMP conditions. CD4/CD8 $\mathrm{T}$ cells underwent magnetic positive selection. $100 \times 10^{6} \mathrm{CD} 4+/ \mathrm{CD} 8+$ were cultured in the CliniMACS Prodigy system using TexMACS media supplemented with 3\% human AB serum, IL-7 and IL-15. Cells were immediately activated using TransACT GMP Grade. 24h after activation, T cells were transduced with our CAR19 lentivirus at $\mathrm{MOI}=10$. T-cell expansion was continued for 8-11 days under recommended perfusion and shaking conditions until the desired number of cells was reached.

\section{Results:}

1. Lentiviral titer and quality control:

2. Viral titer of frozen-concentrated virus ranged between 1.1- $2.2 \times 10^{8} \mathrm{TU} / \mathrm{ml}$. Viral supernatant lots were negative for bacterial-fungal growth, endotoxin, mycoplasma or replication-competent lentivirus (RCL). Virus identity was also confirmed by PCR amplification of principal virus components.

3. ARI-0001 production validation:

4. The expansion time varied between 8-11 days. A mean of $3780 \times 10^{6}$ total cells were obtained, and the percentage of transduction averaged $35.8 \%$ at the time the cell expansion was terminated. Therefore, the acceptance criteria were reached in all three processes.

5. Product characterization and quality control (see table below):

Conclusions: We have developed a novel CART19 cell product (ARI-0001) composed by our anti-CD19 MAb (A3B1) scFv plus the $4-1 \mathrm{BB} / \mathrm{CD} 3 \mathrm{z}$ signaling domains. Although the CliniMACS Prodigy has some disadvantages compared to other technologies available, it has shown a good process robustness and reproducibility in our hands, allowing clinical scale ARI-0001 cell production even for medium-size publicly funded institutions like ours.

Clinical Trial Registry: After positively fulfilling preclinical stage and demonstrating quality and reproducibility of our lentivirus and ARI-0001 cells production systems, this production system is currently being used in the recently launched, phase I, CART19-BE-01 clinical trial (EudraCT: 2016-002972-29, NCT03144583) for relapsed/ refractory CD19+ leukemia and lymphoma at the Hospital Clínic de Barcelona and Hospital Sant Joan de Déu, Barcelona (Spain).

Conflict of interest: Source of funding: Projecte ARI, ISCIII (PI13/00676; PIE13/00033; PICIS14/00122)

\section{P705}

\section{Comparison of in vitro efficacies of a T-cell utilizing bispecific anti-CD3/GD2 construct and $\mathrm{CH} 14.18 \mathrm{mAb}$ for treatment of neuroblastoma patients post autologous / allogeneic SCT}

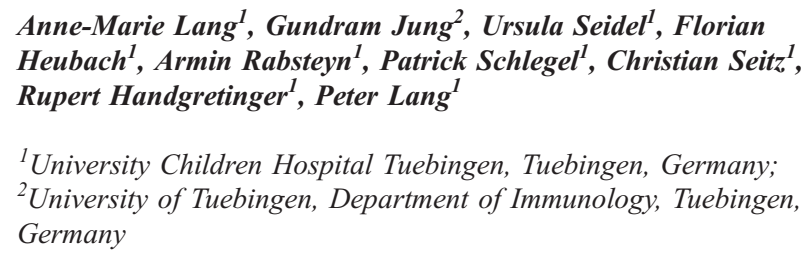

${ }^{1}$ University Children Hospital Tuebingen, Tuebingen, Germany;

${ }^{2}$ University of Tuebingen, Department of Immunology, Tuebingen, Germany

Background: Whereas immunotherapy in high-risk/ relapsed neuroblastoma with standard antibody formats targeting the GD2 antigen is currently used after autologous/allogeneic stem cell transplantation (SCT), T-cells have rarely been addressed as effectors. Bispecific antibodies can bring $\mathrm{T}$-cells into close proximity to targets and activate them through the CD3 region. They lack the Fcdomain and cannot trigger ADCC or CDC. We investigated whether the bispecific antibody NG-CU, which recognizes CD3 and GD2 and utilizes T-cells, might be an alternative to the therapeutic antibody $\mathrm{CH} 14.18$, which mediates CDC and ADCC through NK-cells.

Methods: Different antibody concentrations and effector to target ratios $(\mathrm{E}: \mathrm{T})$ were evaluated using the $\mathrm{xCELLigence}$ RTCA system, PBMCs (healthy donors and patients after allogeneic/autologous SCT) and the LS cell line. CDC was evaluated using autologous serum.

Results: NG-CU showed enhanced cytotoxicity compared to CH14.18. Median specific lysis $(\mathrm{n}=3)$ after 12/24/ 48 hrs (effectors: healthy PBMCs, E:T=5:1) was: 55/73/ $77 \%(\mathrm{CH} 14.18,1 \mu \mathrm{g} / \mathrm{ml})$ vs. $70 / 100 / 100 \%$ (NG-CU, 100 $\mathrm{ng} / \mathrm{ml})$. Taking CDC into account by adding autologous serum, cytotoxicity of $\mathrm{CH} 14.18$ was drastically increased. ADCC and CDC eradicated targets with $64 \%$ after $4 \mathrm{hrs}$ and 93\% after 48 hrs $(\mathrm{E}: \mathrm{T}=5: 1)$. However, using a lower E:T ratio (1:1) lysis of $\mathrm{CH} 14.18$ remained static, but not for NGCU. Median lysis $(\mathrm{n}=3)$ after 12/24/48 hrs: $71 / 74 / 77 \%$ (CH14.18) vs. $25 / 42 / 67 \%$ (NG-CU). After $>48$ hrs targets were completely eradicated by NG-CU, while targets treated with $\mathrm{CH} 14.18$ still proliferated.

Using patient PBMCs, significant lysis with both constructs was detected dependent on percentages and total numbers of T- and NK-cells. In patients early after SCT, 
NK-cells represented the majority of effectors. Here, $\mathrm{CH} 14.18$ produced higher lysis, whereas later on, associated with increasing T-cell counts, NG-CU was more effective.

Conclusions: NG-CU showed enhanced cytotoxicity compared to $\mathrm{CH} 14.18$ even in lower concentrations and E:T ratios. The bispecific design represents an alternative to classical CH14.18 based therapies. Dependent on the differential recovery of effector cells posttransplant either NK-cell based or T-cell based antibody constructs might result in optimal anti tumor activity.

Conflict of interest: None of the authors has anything to disclose.

\section{P706}

\section{Development of A VHH Based Chimeric Antigen Receptor $\mathrm{T}$ cells Targeting $\mathrm{MUCl}$}

\author{
Amir Ali Hamidieh ${ }^{1}$, Alireza Rajabzadeh ${ }^{1}$, Fatemeh Rahbarizadeh ${ }^{2}$ \\ ${ }^{1}$ Tehran University of Medical Sciences, Tehran, Iran, Islamic \\ Republic of; ${ }^{2}$ Tarbiat Modarres University, Biotechnology, Tehran, \\ Iran, Islamic Republic of
}

Background: Most recently chimeric antigen receptor (CAR) $\mathrm{T}$ cell therapy provide very promising approach in cancer therapy. Heavy-chain antibodies (HCAbs) are antibodies that found in camelidae and lack the light chain. The antigen binding domain of camelid HCAbs consist of only one domain, termed 'nanobody' a term inspired by their small size. Here, we have evaluated permanent expression of chimeric antigen receptor containing anti-MUC-1 specific VHH on surface of $\mathrm{T}$ cells and activity of these MUC-1 specific CAR T cells.

Methods: pRSV-Rev, pMDLg/pRRE, pMD2.G and pLJM1-EGFP vectors transformed to Stbl3 E.coli and then were purified using mega prep kit (MN). Anti-MUCI CAR construct contain VHH (anti-MUCI)- $\mathrm{IGg}_{3}$ (hinge)-CD28CD3z inserted to transfer vector (pLJM1-EGFP). For virus packaging transfer vector contain CAR construct along with helper vectors transfected to HEK293T cells.

Results: Anti-MUCI VHH-IGg3-CD28-CD3z construct obtained from the previous study was cloned to pLJM1EGFP vector using digestion-ligation method. Enzyme digestions and sequencing data confirmed that these processes have been done successfully. Vector transfected into HEK293T cells successfully and viruses was produce. Jurkat cells was transduced by that lentiviruses and they had increasing proliferation upon co-culture whit MUCI positive cells.

Conclusions: Our result demonstrated constantly expression of chimeric antigen receptor contain anti-MUCI VHH and also this recombinant receptor is able to targeting specific antigen (MUCI) on cell surface and promote proliferation of engineered Jurkat cells.

Conflict of interest: The authors report no conflicts of interest.

\section{P707}

Efficacy and safety of nivolumab-based therapy in children with relapse of hematologic malignancies after allogenic HSCT

Olga Molostova, Larisa Shelikhova, Maria Alyabieva, Dmitriy Balashov, Svetlana Radygina, Irina Kalinina, Sergei Kovrygin, Michael Maschan, Alexei Maschan

Dmitry Rogachev National Medical Research Center of Pediatric Hematology, Oncology and Immunology, Moscow, Russian Federation

Background: Patients with post-transplant acute leukemia relapse are hardly curable and the outcome is extremely poor. The effectiveness of standard chemotherapy and donor lymphocyte infusions is limited. A possible alternative to standard chemotherapy and DLI is use of PD-1 inhibitors, which may potentiate the allogeneic immune response in order to harness graft-versus-leukemia effect.

Methods: We analyzed retrospectively 8 patients, age 3 to 14 years, who had received therapy with PD-1-inhibitor after HSCT. The cohort included 6 patients with acute myeloid leukemia, $1 \mathrm{pt}$ with acute lymphoblastic leukemia and $1 \mathrm{pt}$ with hepato-splenic gamma/delta $\mathrm{T}$ cell lymphoma. At the moment of HSCT 5 patients had an advanced stage of their disease, 2 were in complete remission and 1 had positive minimal residual disease. All patients received alloHSCT (5 patients from haploidentical donors, 3 patients from HLA-identical siblings). At day +30 after HSCT all patients were in clinical and molecular remission, $50 \%$ of the patients developed grade 1-2 acute GVHD with skin and GI impairment. The average time to relapse was 180 (97420) days. Bone marrow relapse was detected in 6 patients while 2 patients had molecular relapse. All patients received 1 injection of nivolumab at a dose of $3 \mathrm{mg} / \mathrm{kg}$. One patient with AML received the injection of nivolumab already being in the remission after second HSCT because of high risk of relapse.

Results: Adverse events were registered in $6(75 \%)$ patients. Two patients developed immune meningoencephalitis, $1 \mathrm{pt}$ had grade 4 immune-mediated liver toxicity, 2 pt had grade 3 skin GVHD flare and immune fever was registered in 4 pts. Four patients required prolonged systemic immunosuppressive therapy to control immunemediated adverse effects. No mortality related to checkpoint-inhibitor toxicity was registered. One patient developed progressive liver disease. Bone marrow aspiration was done to evaluate efficiency of therapy after the first 
injection of nivolumab. Seven patients were evaluable for response. Six of 7 patients did not respond to therapy. Average time to disease progression was 21 day. Stable remission was achieved in 1 patient with ALL who received injection of nivolumab being in the full-blown relapse after HSCT. One year later this patient remains in clinical and molecular remission, although immune-mediated liver damage progressed to end-stage liver disease. Patient with AML received the injection of nivolumab already being in the remission and 18 months after therapy remains in clinical and molecular remission.

Conclusions: Our limited experience suggests that use of PD-1 inhibitors after allo-HSCT is associated with significant toxicity. Further prospective study is required to establish the safety and efficiency of this approach.

Conflict of interest: The authors declare they have no competing interests.

A

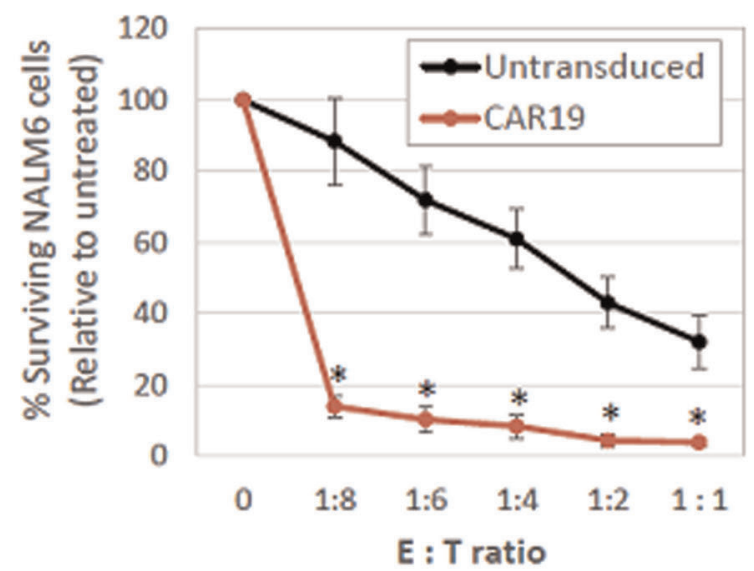

B

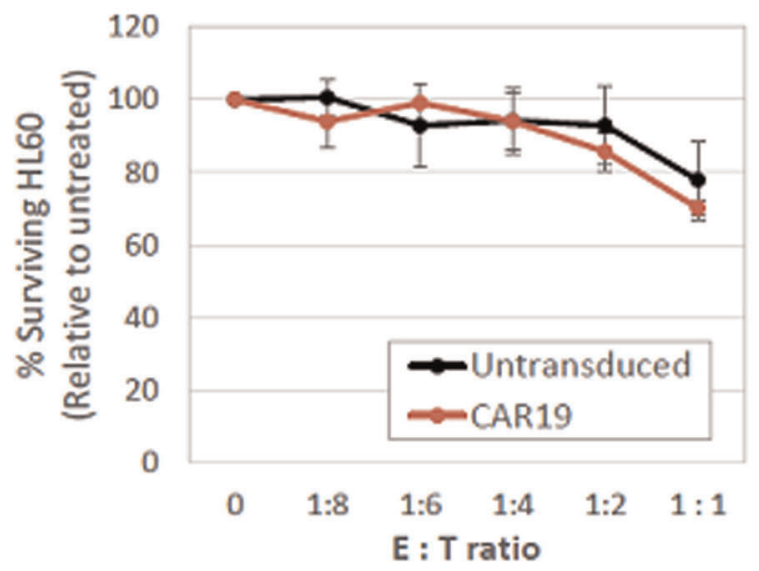

P708

Abstract previously published

\section{P709}

Preclinical development of a new type of anti-CD19 chimeric antigen receptor T cells (ARI-0001 cells)

Maria Castella ${ }^{1}$, Anna Boronat ${ }^{2}$, Raquel Martin-Ibañez $z^{3}$, Vanina Rodriguez $^{4}$, Miguel Caballero ${ }^{2}$, Berta Marzal ${ }^{2}$, Julio Castaño ${ }^{5}$, Clara Bueno ${ }^{5}$, Olga Balague ${ }^{6}$, Daniel Benitez, ${ }^{2}$, Valentín OrtizMaldonado ${ }^{1}$, Tycho Baumann ${ }^{1}$, Elias Campo ${ }^{6}$, Pablo Menendez, Susana Rives ${ }^{7}$, Julio Delgado ${ }^{1}$, Jordi Yagüe ${ }^{2}$, Patricia Perez-Galan ${ }^{4}$, Josep Maria Canals ${ }^{3}$, Alvaro Urbano-Ispizua ${ }^{1,5}$, Manel Juan ${ }^{2}$

${ }^{1}$ Hospital Clinic, Hematology, Barcelona, Spain; ${ }^{2}$ Hospital Clinic, Immunology, Barcelona, Spain; ${ }^{3}$ University of Barcelona, Department of Biomedical Sciences, Barcelona, Spain; ${ }^{4}$ IDIBAPS, Hemato-

C
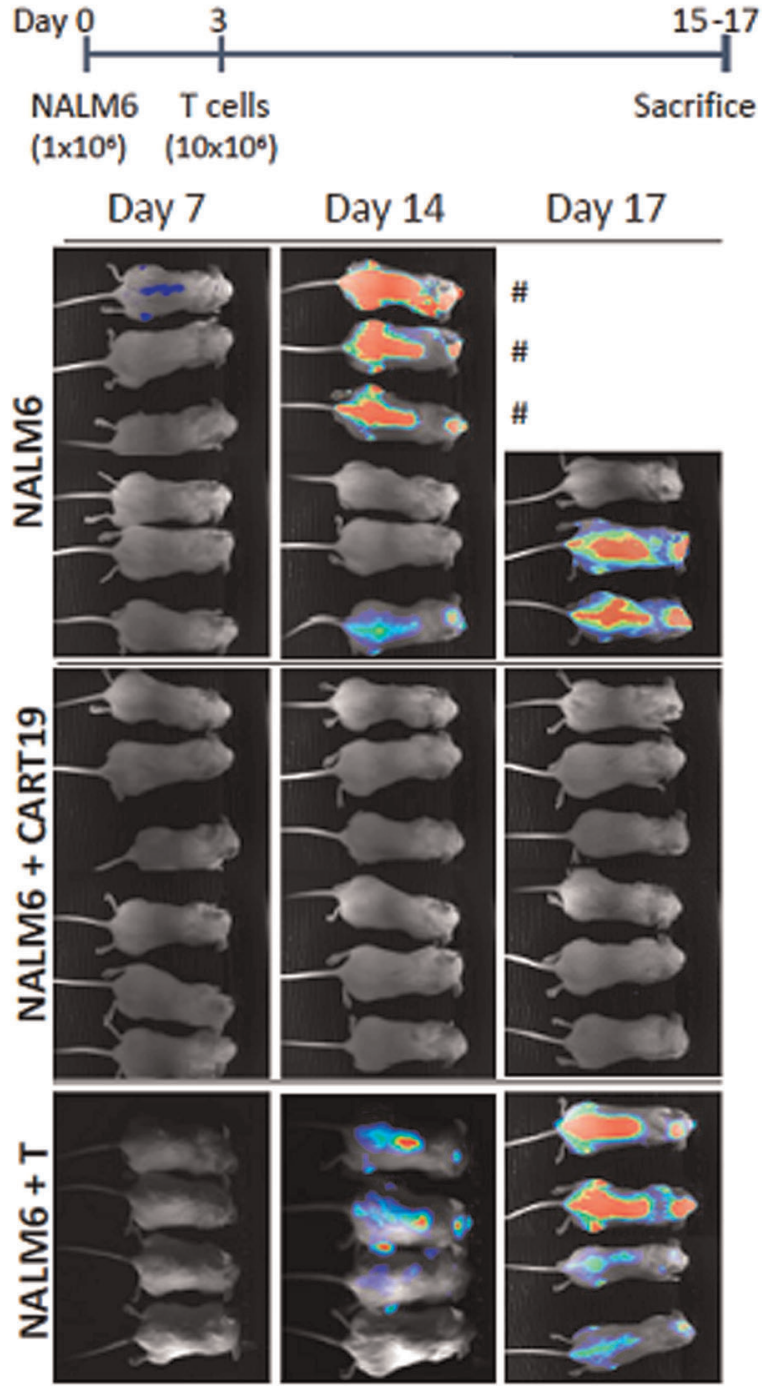

[P709 Figure] [In vitro and in vivo assays] 
Oncology, Barcelona, Spain; ${ }^{5}$ Josep Carreras Leukemia Research Institute, Barcelona, Spain; ${ }^{6}$ Hospital Clinic, Pathology, Barcelona, Spain; ${ }^{7}$ Hospital Sant Joan de Deu, Hematology, Barcelona, Spain

Background: Anti-CD19 chimeric antigen receptor T (CART19) cells have shown remarkable responses in the treatment of relapsed/refractory CD19-positive hematological malignancies across several clinical trials, leading to the consolidation of immunotherapy as one of the building blocks of modern cancer therapy. Here we present the preclinical work conducted to test the efficacy and specificity of our own CART19 cells (named ARI-0001). ARI-0001 cells were prepared through lentiviral transduction of a construct comprising our own anti-CD19 scFv (A3B1) plus CD8 (hinge and transmembrane region), 4$1 \mathrm{BB}$ and $\mathrm{CD} 3 \mathrm{z}$ moieties.

Methods: In vitro cytotoxicity assays were performed using ARI-0001 or untransduced T (UT) cells co-cultured with tumor target cell lines (NALM6 [CD19 positive] or HL60 [CD19 negative]) or primary B-ALL tumor cells, at different effector to target (E:T) ratios. Cytotoxicity was determined by the number of surviving target cells, identified as 7-AAD negative by flow cytometry. Coculture supernatants were analyzed for cytokine production (IFN $\gamma$, TNF $\alpha$ and IL-10) by ELISA. ARI-0001 cell proliferation in response to $\mathrm{CD} 19$ antigen was measured using CFSE assay. In vivo assays were performed using three-month old NSG mice infused intravenously with NALM6 tumor cells $\left(1 \times 10^{6}\right.$ cells/mice $)$ expressing greenfluorescent protein (GFP) and luciferase. Mice were then randomly allocated to the infusion of either ARI-0001 cells $\left(10 \times 10^{6} /\right.$ mice $)$, UT cells $\left(10 \times 10^{6}\right.$ cells/mice $)$ or vehicle. Tumor growth was evaluated weekly by bioluminiscence imaging (Hamamatsu detector) after the intravenous administration of D-luciferin.

Results: We detected the expression of our construct in a significant proportion of T cells (20 to 56\%). As shown in

Figure 1A, NALM6 cells were almost completely eliminated after $16 \mathrm{~h}$ of co-culture even with very low E:T ratios

( 1 effector cell for every 8 target cells). Similar cytotoxic efficacy was demonstrated against primary B-ALL cells. As shown in Figure 1B, ARI-0001 cells specificity was shown by its co-culture with a CD19 negative cell line (HL60), where no CART19-mediated killing was appreciated. Also, no significant efficacy differences were seen on in vitro cytotoxic assays in comparison to FMC63 CART19 cells, which are currently used in the clinic. Cytokine production measured in effector-target cell co-cultures (16h), showed that ARI-0001 cells produced a significant increase in IFN $\gamma$ and TNF $\alpha$ compared to UT cells. As shown in Figure 1C, disease progression was clearly observed during a period of
2 weeks following NALM6 cell infusion in NALM6 cells group (5/6) and NALM6 + UT cells group (4/4). However, no disease was detected in any of the mice belonging to the NALM6 + ARI-0001 group (0/6). Also, overall survival was significantly improved in the ARI-0001 treated mice when compared to vehicle or UT cell groups ( $\mathrm{P}$ values= 0.0066 and 0.0012 , respectively).

\section{Conclusions:}

- ARI-0001 cells showed a robust and specific cytotoxic and proliferative effect against CD19 positive cells in vitro.

- ARI-0001 cells exposure to CD19+ cells induced a robust production of pro-inflammatory cytokines.

- ARI-0001 cells cytotoxicity was comparable to FMC63 CART19 cells (currently approved by the FDA).

- ARI-0001 cells could also eliminate CD19-positive leukemia cells in xenograph mouse models and prolong survival.

Conflict of interest: There are no conflicts of interest related to this study.

Source of funding: Projecte ARI, ISCIII (PI13/00676; PIE13/00033; PICIS14/00122)

\section{P710}

\section{Regulation of HMGB1 release protects chemoradiotherapy- associated mucositis}

Keon-Il Im, Nayoun Kim, Young-Sun Nam, Yunejin Song, YoungWoo Jeon, Seok-Goo Cho

Seoul St. Mary's Hospital, The Catholic University of Korea College of Medicine, Institute for Translational Research and Molecular Imaging, Seoul, Korea, Republic of

Background: Oral mucositis (OM) is a common complication in cancer patients undergoing anticancer treatment. Despite the clinical and economic consequences of $\mathrm{OM}$, there are no drugs available for its fundamental control. Here, we show that high-mobility group box 1 (HMGB1), a "danger signal" that acts as a potent innate immune mediator, plays a critical role in the pathogenesis of OM.

Methods: We investigated treatment of OM through HMGB1 blockade using NecroX-7 (tetrahydropyran-4-yl)[2-phenyl-5-(1,1-dioxo-thiomorpholin-4-yl)methyl-1Hindole-7-yl]amine). Mice were administered 5-FU (50 mg/kg, intraperitoneally [i.p.]) once daily for 5 days (days $0-4$ ) to induce OM. NecroX-7 (0.3, 3, and $30 \mathrm{mg} / \mathrm{kg}$, intravenously [i.v.]) or phosphate-buffered saline (PBS) was administered once daily for 5 days. 
Results: NecroX-7 ameliorated basal-layer epithelial cell death and ulcer size in OM induced by chemotherapy or radiotherapy. This protective effect of NecroX-7 was mediated by inhibition of HMGB1 release and downregulation of mitochondrial oxidative stress. Additionally, NecroX-7 inhibited the HMGB1-induced release of TNF- $\alpha$, IL $1 \beta$ and MIP-1 $\beta$, as well as the expression of $\mathrm{p} 53$ upregulated modulator of apoptosis (PUMA) and the excessive inflammatory microenvironment, including nuclear factor kB (NF-kB) pathways.

Conclusions: Our findings suggest that HMGB1 plays a key role in the pathogenesis of OM; therefore, blockade of HMGB 1 by NecroX-7 may be a novel therapeutic strategy for OM.

Conflict of interest: This work was supported by Grant HI14C3417 from the Korea Healthcare Technology Research and Development Project, Ministry for Health, Welfare, and Family Affairs, Republic of Korea.

\section{P711}

Self-differentiating induced DCs co-expressing GM-CSF, IFNa and tWT1 as first-in-man immune-gene therapy of MRD $^{+}$ AML patients

Angela Cornelius ${ }^{1}$, Laura Gerasch ${ }^{1}$, Ulrike Koehl ${ }^{2}$, Britta EizVesper $^{3}$, Farzin Farzaneh ${ }^{4}$, Michael Schmitt ${ }^{5}$, Arnold Ganser ${ }^{1}$, Renata Stripecke ${ }^{1}$

${ }^{1}$ Hannover Medical School, Hemalotogy Oncology Stem Cell Transplantation, Hannover, Germany; ${ }^{2}$ Hannover Medical School, Institute of Cellular Therapeutics and GMP Core Facility, Hannover, Germany; ${ }^{3}$ Hannover Medical School, Institute for Transfusion Medicine, Hannover, Germany; ${ }^{4}$ King's College London, Cell and Gene Therapy - King's, London, United Kingdom; ${ }^{5}$ University Heidelberg, GMP-Core-Facility and Clinic for Internal Medicine, Heidelberg, Germany

Background: Acute myeloid leukemia (AML) patients in morphologic complete remission (CR) with positive measurable residual disease $\left(\mathrm{MRD}^{+}\right)$have a poor prognosis. MRD is associated with immune suppressive processes and predisposes to high risks of relapse. Therefore, novel immune therapies counteracting immune suppression and leading to long-term control of genetically heterogeneous leukemia clones are warranted. WT1 is a broadly studied antigen over-expressed in leukemia, lymphomas, and other malignancies. Recently, promising results were obtained in a phase II trial (\#NCT00965224) investigating the effect of vaccination with ex vivo cultivated DCs electroporated with WT1 mRNA as post-remission treatment in 30 AML patients at very high risk of relapse (Anguille et al, 2017 Blood).

Methods: We propose a novel DC modality as an advanced therapeutic medicinal product (ATMP) consisting of $\mathrm{CD}_{14}{ }^{+}$monocytes manipulated for 16 hours with an integrase-defective lentiviral vector (IDLV) and cryopreserved. Upon cell thawing, the vector expresses GM-CSF and IFN-a, resulting in in vivo self-differentiation of monocytes into highly viable and activated induced dendritic cells (iDCs). Studies in mice humanized with human $\mathrm{CD}_{3} 4^{+}$stem cells obtained from adult donors or cord blood and immunized with the same donor iDC expressing the HCMV antigen pp65 showed effective biodistribution to adjacent and distal lymph nodes promoting accelerated reconstitution of mature and functional $\mathrm{T}$ and $\mathrm{B}$ cells (Salguero et al, 2014 Journal of Immunology). Upscaled production of the IDLV and iDCpp65 under GMPcompliant conditions was validated (Sundarasetty et al, 2015 Journal of Translational Medicine). A safety longterm ( $>33$ weeks) study of iDCpp65 immunizations in humanized mice showed no development of malignancies (Sundarasetty et al, 2017 American Journal of Pathology).

Results: Here, a tricistronic vector co-expressing GMCSF, IFN-a and truncated WT1 (tWT1, lacking the zinc finger domain) was constructed and the full vector sequence was determined. Expression of the transgenes in transduced 293T cells was confirmed by ELISA (GM-CSF, IFN-a) and flow cytometry analyses (tWT1). After 7 days in culture, monocytes transduced with IDLV self-differentiated into viable iDCtWT co-expressing $\mathrm{HLA}-\mathrm{DR}^{+} / \mathrm{CD} 86^{+}(67 \%$ double positivity) and showing secretion of GM-CSF (5 $\mathrm{ng} / \mathrm{ml})$ and IFN-a $(3.5 \mathrm{ng} / \mathrm{ml})$. After cryopreservation and thawing, high viability of iDCtWT1 in vitro was observed for 3 weeks.

Conclusions: In vitro potency assays with iDCtWT1 are ongoing to determine activation of autologous $\mathrm{CD} 4^{+}$and $\mathrm{CD} 8^{+} \mathrm{T}$ cells and WT1-restricted anti-leukemia responses. Humanized mice will be immunized with iDCtWT1 generated with chromatography-purified IDLV vector. After prime-boost iDCtWT1 immunizations, mice will be maintained for 28 days or 26 weeks to evaluate immune responses against WT1, anti-leukemia responses and toxicity. For clinical trials, we will explore an automated system for GMP validation of iDCtWT1 cell manufacturing.

Conflict of interest: International patent pending "Induced Dendritic Cells and Uses Thereof" (WO2014/ 121840).

\section{P712}

Skin rash after CART19 (ARI-0001) therapy in a prior recipient of allogeneic HCT: cutaneous GvHD vs. ARI-0001 expansion

Valentín Ortiz-Maldonado $^{1}$, David Moreno ${ }^{1}$, Andrea Rivero ${ }^{1}$, Marta Garrote $^{1}$, Ana Moreno ${ }^{1}$, Tycho Baumann ${ }^{1}$, Clara Fernandez ${ }^{2}$, 

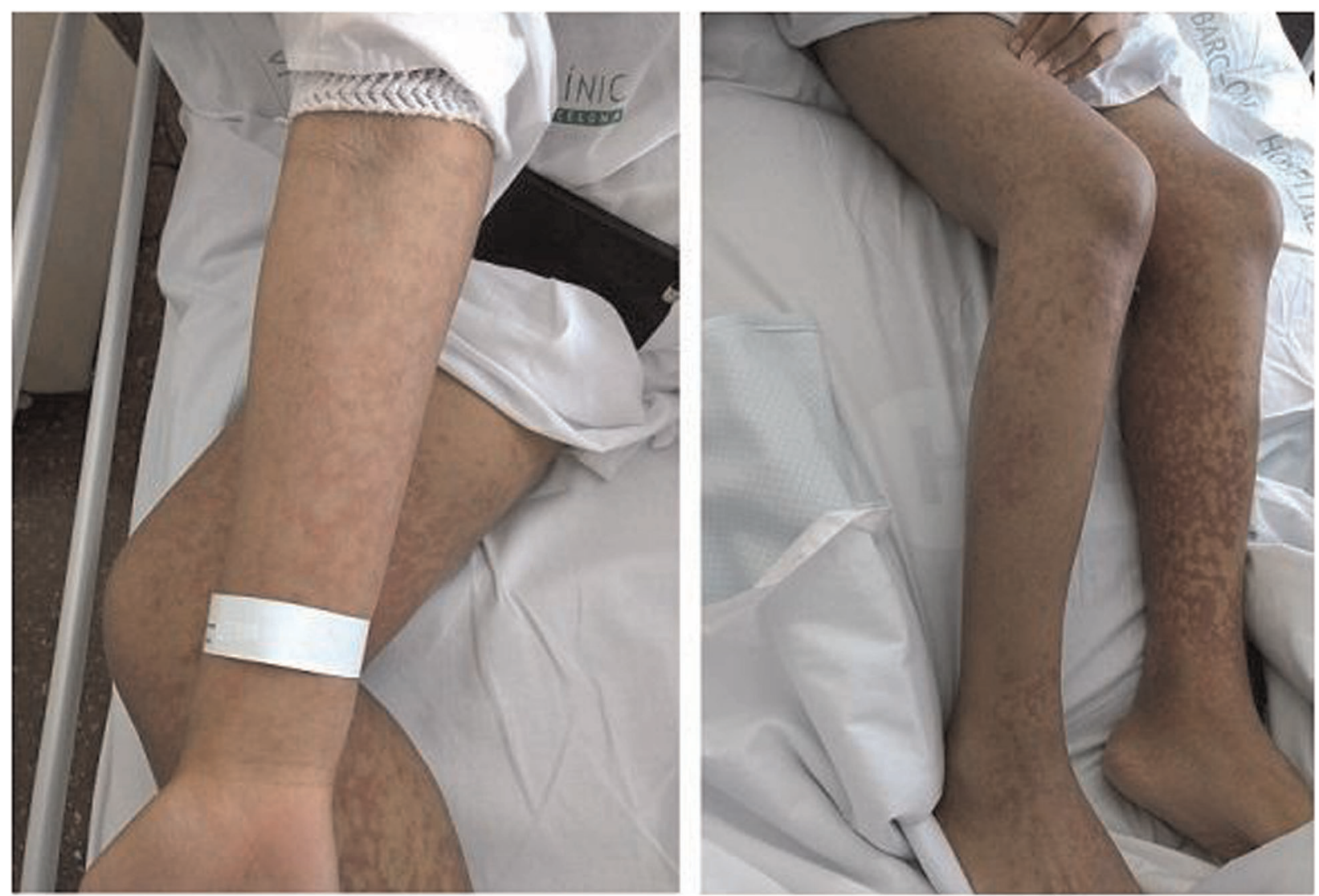

[P712 Figure] [Cutaneous rash]

Adriana García ${ }^{3}$, María Castella ${ }^{1}$, Anna Boronat ${ }^{4}$, Miguel Caballero ${ }^{4}$, Berta Marzal ${ }^{4}$, Alvaro Urbano-Ispizua ${ }^{1,5}$, Manel Juan ${ }^{4}$, Julio Delgado ${ }^{1,6}$

${ }^{1}$ Hospital Clínic de Barcelona, Department of Hematology, Barcelona, Spain; ${ }^{2}$ Hospital Clínic de Barcelona, Department of Dermatology, Barcelona, Spain; ${ }^{3}$ Hospital Clinic de Barcelona, Department of Pathology, Barcelona, Spain; ${ }^{4}$ Hospital Clinic de Barcelona,

Department of Immunology, Barcelona, Spain; ${ }^{5}$ Josep Carreras Leukemia Research Institute, Department of Biomedicine, Barcelona, Spain; ${ }^{6}$ CIBERONC, Barcelona, Spain

Background: Chimeric antigen receptor T (CART) cell therapy has been a major breakthrough in the treatment of relapsed/refractory hematological malignancies. CART cells targeting CD19+ hematologic malignancies have showed promising results across several clinical trials worldwide, remaining the most commonly used target to date. Even so, certain adverse effects are still yet to be observed and remain poorly understood. CART cells are usually prepared from autologous $\mathrm{T}$ cells. Even when generated from allogeneic HCT recipients, no CART cell mediated GvHD has been described so far.

Here we present the case of a 20-year-old male with CD19+ B-ALL whose disease relapsed 5 months after an HLA-identical sibling alloHCT. He received $\mathrm{T}$ cells modified with a CART19 composed of the A3B1 anti-
CD19 linked to the $\mathrm{CD} 3 \mathrm{z}$ and $\mathrm{CD} 137$ (4-1BB) costimulatory domains (ARI-0001) and experienced a generalized skin rash coinciding with the proliferation peak of the ARI-0001.

Methods: The skin rash (Figure 1) started 3 days after the infusion of $1 \times 10^{6}$ ARI-0001 cells / Kg and a skin biopsy was performed. Histological analysis was carried out using the usual staining techniques in addition to an immunohistochemical panel for the detection of $\mathrm{CD} 3+$ and $\mathrm{CD} 8+$ cells. Follow-up was carried out by skin examination on a daily basis. Also, daily analysis was performed for blood counts and biochemical markers of ARI-0001 cell proliferation, such as leukocyte counts, CRP and ferritin levels. Also, ARI-0001 cells were measured using flow cytometry during hospital admission.

Results: The histopathological evaluation of the skin biopsy showed discrete changes of spongiotic dermatitis, with no evidence of interface injury. The presence in the dermis of a slight perivascular lymphocytic infiltration was detected, with some lymphocytes of activated appearance in which in immunohistochemistry stainings were positive for CD3 and CD8 and negative for CD19. On the same day $(+3)$ onwards, clinical and biochemical signs of cytokine release syndrome (CRS) were also evident. The patient developed daily fever of up to $39^{\circ} \mathrm{C}$ with increasing levels 
of CRP and ferritin. ARI-0001 cells also started to rise, peaking at $9 \%$ of all circulating lymphocytes on day +7 . The clinical and biochemical manifestations of CRS ameliorated by day +11 , when ARI-0001 cells could no longer be detected by flow cytometry. The patient had no diarrhea or signs of liver inflammation, and the skin rash improved with topical steroids.

Conclusions: In this particular case, even though the ARI-0001 cells were technically allogeneic, they were extracted from a patient with a complete donor chimerism who had no previous history of GvHD, and, therefore, the possibility of cutaneous GvHD was unlikely. There are few reports of cutaneous reactions of this magnitude after the infusion of CART19 cells. There are yet many uncertainties regarding the mechanisms leading to this phenomenon and there were no conclusive signs of GvHD in the sample examined. It may be linked to ARI-0001 proliferation and not to the same tissue injury mechanisms of classic GvHD.

Clinical Trial Registry: This patient participated in the recently launched CART19-BE-01 clinical trial (EudraCT: 2016-002972-29, NCT03144583) for relapsed/refractory CD19+ leukemia and lymphoma at the Hospital Clinic and Hospital Sant Joan de Deu, both in Barcelona. www. clinicaltrialsregister.eu

Conflict of interest: There are no conflicts of interest related to this study.

Source of funding: Projecte ARI, ISCIII (PI13/00676; PIE13/00033; PICIS14/00122)

\section{P713}

The evaluation of the safety of decitabine as bridging pretreatment regimen before hematopoietic stem cell transplantation in pediatric malignant blood disorders

\section{Liyan Fan, Sh H}

Children's Hospital of Soochow University, Suzhou, China

Background: Objective Refractory acute leukemia (AL) showed resistant to chemotherapy which resulted in high minimal residual disease (MRD). In such a situation, patients easily relapsed after hematopoietic stem cell transplantation(HSCT). It is recommended to reduce MRD by optimizing conditional regimen before HSCT to improve the outcome of HSCT in refractory malignant blood disorders. In this study, we evaluated the safety of bridging pretreatment regimen with decitabine (CHIATAI TIANQING Pharmaceutical Group) before pediatric HSCT in children with refractory hematological malignancies and the efficacy of HSCT in pediatric hematological malignancies.

Methods: Retrospectively analyzing the clinical data of the 11 cases (4cases for myelodysplastic syndrome, 5 cases for acute myeloid leukemia, 2 relapse cases of acute lymphoblastic leulkemia, and 1 case for myeloproliferative disorders) from children's hospital of Soochow University who were given decitabine bridging pretreatment regimen before HSCT from March 2015 to January 2017. The safety of decitabine was evaluated in terms of engraftment, the grade of graft versus host disease (GVHD), side effect of hematologic and non-hematologic systems.

Results: Eleven cases of children enrolled in this study succeeded in implantation. Four cases showed Grade IV hematologic toxicity, 2 cases with Grade III hematologic toxicity, 4 cases with Grade II hematologic toxicity. None of them suffered infection, drug-induced liver damage, and renal impairment during decitabine administration. Most cases showed I II degrees of gastrointestinal impairment, only 1 case showed III degrees of gastrointestinal impairment. Four cases transplanted with haploid donor had IV grade of gastrointestinal acute GVHD. Amont them, one case was diagnosed as super aGVHD and died of intracranial hemorrhage on day 61 after HSCT. Follow up to Nov. 30, 2017, 10 patients survived.

Conclusions: Decitabine pretreatment is safe as a combined conditional regimen in allogeneic HSCT (alloHSCT) which is an effective treatment for pediatric refractory hematologic malignancies.

Conflict of interest: The authors declared that they have no conflicts of interest to this work.

We declare that we do not have any commercial or associative interest that represents a conflict of interest in connection with the work submitted.

\section{P714}

Abstract previously published

Non-hematopoietic stem cells

\section{P715}

Bone marrow derived Mesenchymal Stromal Cells from patients with Immunoglobulin light-chain amyloidosis

Alice Maltese $^{1}$, Melissa Mantelli ${ }^{1}$, Stefania Croce $^{2}$, Elisa Lenta $^{1}$, Laura Caliogna ${ }^{1}$, Maria Antonietta Avanzini, Laura Catenacci, ${ }^{1}$, Margherita Massa $^{1}$, Mario Nuvolone $e^{1,2}$, Margherita Bozzola ${ }^{1,2}$, Paolo Milani $^{1,2}$, Giovanni Palladini, ${ }^{1,2}$, Giampaolo Merlini, ${ }^{1,2}$

${ }^{1}$ Fondazione IRCCS Policlinico San Matteo, Pavia, Italy; ${ }^{2}$ University of Pavia, Pavia, Italy

Background: Immunoglobulin light-chain (AL) amyloidosis is a disorder caused by a misfolded light chain, produced by a clonal population of plasma cells in the bone marrow, that deposits in tissues and causes organ dysfunction. It is recently reported that microenvironment defect 


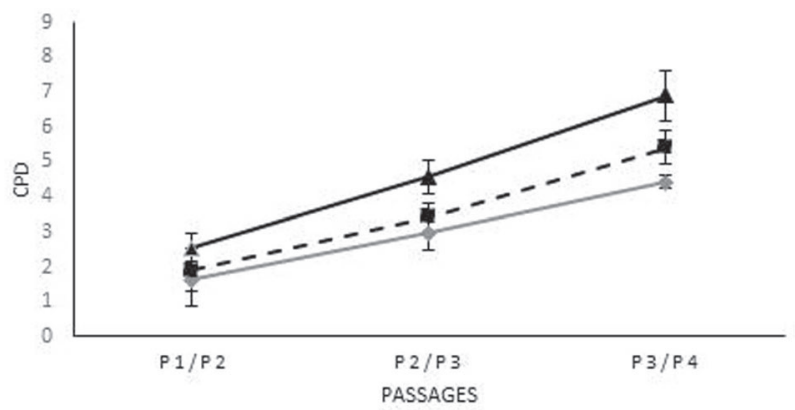

[P715 Figure] [Fig.1. CPD in HD-MSC, AL-MSC, AL-CD138negMSC at different culture P. Data are shown as median \pm SD.]

may either lead to or favor the pathogenesis of different hematologic disorders. Since Mesenchymal Stromal Cells (MSC) represent one of the key components of the BM niche, in the present study we evaluated MSC of $\mathrm{AL}$ patients.

Methods: MSC from Bone Marrow (BM) of $9 \mathrm{AL}$ patients and from BM of 3 Healthy Donors (HD) were expanded following standard in vitro culture procedure (Avanzini M.A. et al Leukemia 2014). MSC were characterized by plastic adhesion, morphology, clonogenic capacity (defined as CFU-F $/ 10^{6}$ plated MNC at P0), immunophenotype by flow-cytometry, adipogenic and osteogenic differentiation and proliferation capacity defined as Population Doubling (PD) $[\log ($ harvested cells/seeded cells)/log2]. BM Mononuclear cells (MNC) from 7 AL patients were cultured both before (AL-MSC) and after CD138 immunomagnetic depletion (AL-CD138 ${ }^{\text {neg }}$-MSC).

Results: MSC were isolated from all patients and HD. Morphology, immunophenotype, osteogenic and adipogenic differentiation, clonogenic capacity (mean: 8.0 vs 9.2 in $\mathrm{AL}$ and $\mathrm{HD}$, respectively), proliferative capacity [Fig.1, CPD in HD-MSC (black line), AL-MSC (broken line), AL-CD138 ${ }^{\text {neg }}-\mathrm{MSC}$ (grey line)] were comparable in MSC from AL patients and HD (mean:1.92 vs 2.56 in AL and $\mathrm{HD}$, respectively). Among patient group, clonogenic capacity was significantly higher $(\mathrm{p}=0.039)$ in AL-MSC than AL-CD138 ${ }^{\text {neg }}$-MSC (mean: 9 and 5.3, respectively). AL-MSC arrested their growth (number of harvested cells $\leq$ number of plated cells) at significantly earlier passages $(\mathrm{p}=0.049)$ compared to HD-MSC [median P2 (range 2-7) vs P11 (range 5-16), respectively].

Conclusions: Our preliminary data suggest some discrepancies between AL-MSC and HD-MSC; whether these features are involved in the pathophysiology of the disease or are caused by the disease itself, deserves further investigation.

Conflict of interest: nothing to disclose

\section{P716}

Characterization of mesenchymal stromal cells generated from patients with non-malignant hematopoietic diseases: an option for the treatment of post-transplant complications?

Zyrafete Kuci ${ }^{1}$, Christiane Jordan ${ }^{1}$, Sibylle Wehner ${ }^{1}$, Jan Soerensen $^{1}$, Emilia Salzmann-Manrique ${ }^{1}$, Ralf Lieberz ${ }^{2}$, Thomas Klingebiel $^{\text {, }}$ Peter Bader ${ }^{1}$, Selim Kuci ${ }^{1}$

${ }^{1}$ University Hospital Frankfurt, Goethe University, Department for Children and Adolescents, Division for Stem Cell Transplantation and Immunology, Frankfurt am Main, Germany; ${ }^{2}$ Institute of Pathology, Goethe University, Frankfurt am Main, Germany

Background: Hematopoietic cell transplantation is the only cure for patients with severe forms of non-malignant hematopoietic diseases (NM-HD) such as thalassemia major, sickle cell disease and congenital neutropenia. In this study, we asked whether mesenchymal stromal cells (MSCs) generated from readily available bone marrow backups of patients with NM-HD are functionally equivalent to MSCs from healthy donors and may be used as an autologous cell-based therapy to treat post-transplant complications such as graft failure, GvHD and osteonecrosis.

Methods: To generate MSCs we used bone marrow (BM) samples from 12 pediatric patients (4-15 years old). Six patients suffered from thalassemia major (TM), four from sickle cell disease (SCD) and two of them from severe congenital neutropenia ( $\mathrm{SCN})$.

As control group served MSCs generated from 12 pediatric donors (4-16 years old). We assessed the frequency of progenitor cells for MSCs (CFU-Fs) per 1x10e6 BM-MNCs as well per ml BM, MSC phenotype, proliferation capacity (doubling time-DT) as well as their differentiation potential in osteoblasts, adipocytes and chondrocytes. Their allosuppressive effect was evaluated by means of mixed lymphocyte reaction (MLR). In MLR we assessed the percentage of T-regs and PGE2 levels.

Results: Bone marrow of TM patients possessed a significantly higher number of nucleated cells (BM$\mathrm{MNCs}) / \mathrm{ml}$ BM compared to control $(18.31 \pm 3.36 \times 10 \mathrm{e} 6, \mathrm{n}$ $=6$ vs. $6.64 \times 10 \mathrm{e} 6, \mathrm{n}=11, \mathrm{p}<0.001)$. This higher number of MNCs has contributed mostly to the higher number of BM-MNCs /ml BM in a whole group of NM-HD patients $(12.66 \times 10 \mathrm{e} 6, \mathrm{n}=12$ vs. control, $\mathrm{p}<0.04)$. But this higher number of BM-MNCs did not result in a higher number of CFU-F/ml BM compared to control and other diseases. Evaluation of the number of CFU-F per 1x10e6 BM-MNCs showed that BM-MNCs of SCD patients generated more CFU-F than TM-BM-MNCs $(301.3 \pm 59, \mathrm{n}=4$ vs. $78 \pm$ $24.38, \mathrm{n}=6, \mathrm{p}<0.004)$. Despite the higher number of 
CFU-Fs in SCD patients, this difference compared to control did not reach the significance $(p<0.0509)$.

Patients' MSCs showed a normal phenotype and equal proliferation capacity (one PD per $46.81 \pm 1.3$ hours) compared to control group $(44.98 \pm 1.9$ hours). They differentiated in three lineages as the control group, except MSCs from TM patients which differentiated weakly in adipocytes. In the MLR, the immunosuppressive potential of NM-HDs MSCs was not significantly different to control group $(52.4 \pm 4.3 \%$ vs. $54.8 \pm 1.3 \%)$. However, this effect was impaired in TM-MSCs $(46 \pm 5.1 \%, \mathrm{p}<0.04)$ vs. control MSCs. Indomethacin as an inhibitor of PGE2 synthesis, was able to partially reverse the effect of MSCs. In supernatants of MLR with patients MSCs, PGE2 amount was not significantly different compared to healthy donors (28.9 $\pm 1.7 \mathrm{ng} / \mathrm{ml}$ vs. $30.8 \pm 2.5 \mathrm{ng} / \mathrm{ml}$ ). In MLR, MSCs of all patients were as efficient as control MSCs in expansion of T-regulatory cells.

Conclusions: Our results suggest that MSCs from patients with NM-HD except MSCs of patients with TM, may be used as an autologous cell-based therapy to treat post-transplant complications such as graft failure, GvHD and osteonecrosis.

Conflict of interest: The authors have nothing to disclose

\section{P717}

\section{Comprehensive Evaluation the Impact of Total Body Irradiation on the Characteristics of Bone Marrow-Derived Mesenchymal Stromal Stem Cell: Implication for Late Effect of Transplantation}

Su-Peng Yeh ${ }^{1}$, Wen-Jyi Lo ${ }^{1}$, Chiao-Lin Lin ${ }^{2}$, Yu-Chien Chang ${ }^{2}, J_{-}$ An Liang ${ }^{3}$, Hsin-Ting Lin ${ }^{1}$

${ }^{1}$ China Medical University Hospital, Hematology and Oncology, Taichung, Taiwan, Republic of China; ${ }^{2}$ China Medical University Hospital, Medical Research, Taichung, Taiwan, Republic of China; ${ }^{3}$ China Medical University Hospital, Radiation Oncology, Taichung, Taiwan, Republic of China

Background: Total body irradiation (TBI) is associated with many late complications due to radiation injury to the normal cells, specifically normal stem cells. Mesenchymal stromal stem cells (MSCs) are cells responsible for adipogenesis and osteogenesis and are important in supporting hematopoiesis. Nevertheless, the whole picture of how TBI affects the characteristics of MSCs and the relevant clinical implication remain unknown.

Methods: Bone marrow-derived MSCs (BM-MSCs) isolated from normal adults were irradiated with 200cGy twice daily for consecutive three days, a regimen identical to that used in the TBI-conditioning HSCT. The morphology, surface markers, proliferation potential, apoptosis,
DNA damage (by Comet assay), abilities of adipocytic and osteocytic differentiation, hematopoiesis-supporting function (by co-culture of mobilized peripheral blood mononuclear cells with MSCs for 10 days and evaluate the CD34+ and long-term culture initiating cell [LTC-IC] counts), and cytogenetics of the irradiated BM-MSCs were examined and were compared to the non-irradiated control. Since clonal cytogenetic abnormalities were found in all irradiated MSCs, carcinogenicity was evaluated in vitro by transformation assay and in vivo by implanting irradiated MSCs onto 4 Balb/c Nude mice to look at tumor formation. In parallel with the in vitro study, paired BM-MSCs harvested from 9 transplant recipients before and after TBI were also examined in the same way to validate the findings of in vitro experiments.

Results: The irradiated and non-irradiated MSCs shared similar morphology and surface markers. However, irradiated MSCs showed much lower proliferative ability (Figure A), higher percentage of apoptosis (Figure B), more cells with comet tails, and much lower differentiative potential (Figure $\mathrm{C} 1 / \mathrm{C} 2$ : Oil red $\mathrm{O}$ stain for adipogenic and Von Kossa stain for osteogenic differentiation respectively). Irradiation also induced clonal cytogenetic abnormalities, which was persisted after prolonged ex vivo culture. Nevertheless, transformation assay showed no colony formation (Figure D, A549 lung cancer cell line as positive control). Pathological examination of the $4 \mathrm{Balb} / \mathrm{c}$ nude mice showed no tumor formation after an observation for 100 days. The hematopoiesis-supporting function was similar between irradiated and non-irradiated MSCs (Figure E, relative fold change of LTC-IC number). Paired BMMSCs harvested from transplant recipients before and after TBI conditioning showed markedly decreased proliferative and differentiative abilities of TBI-exposed MSCs. Cytogenetic test of paired-MSCs were done in 6 recipients of sex-mismatched, TBI-conditioning HSCT. All the posttransplant MSCs were of recipient-origin and clonal cytogenetic abnormalities could be identified in all the 6 cases, including 1 case having clonal evolution.

Conclusions: TBI do not affect the hematopoiesissupporting function of MSCs, which reflects the fact that engraftment is not a problem after TBI-based conditioning. However, TBI drastically impairs the proliferation and differentiation of MSCs, which likely contribute to the acquired lipodystrophy and bone growth retardation specifically in children receiving TBI-based HSCT. Furthermore, although the incidence of second soft tissue malignancy after TBI-based HSCT is low, as supported by the observation of low carcinogenicity of irradiated MSCs in this study, the impaired chromosomal integrity of irradiated MSCs poses a real risk and increased incidence of second soft tissue malignancy in these patients comparing to general population. 


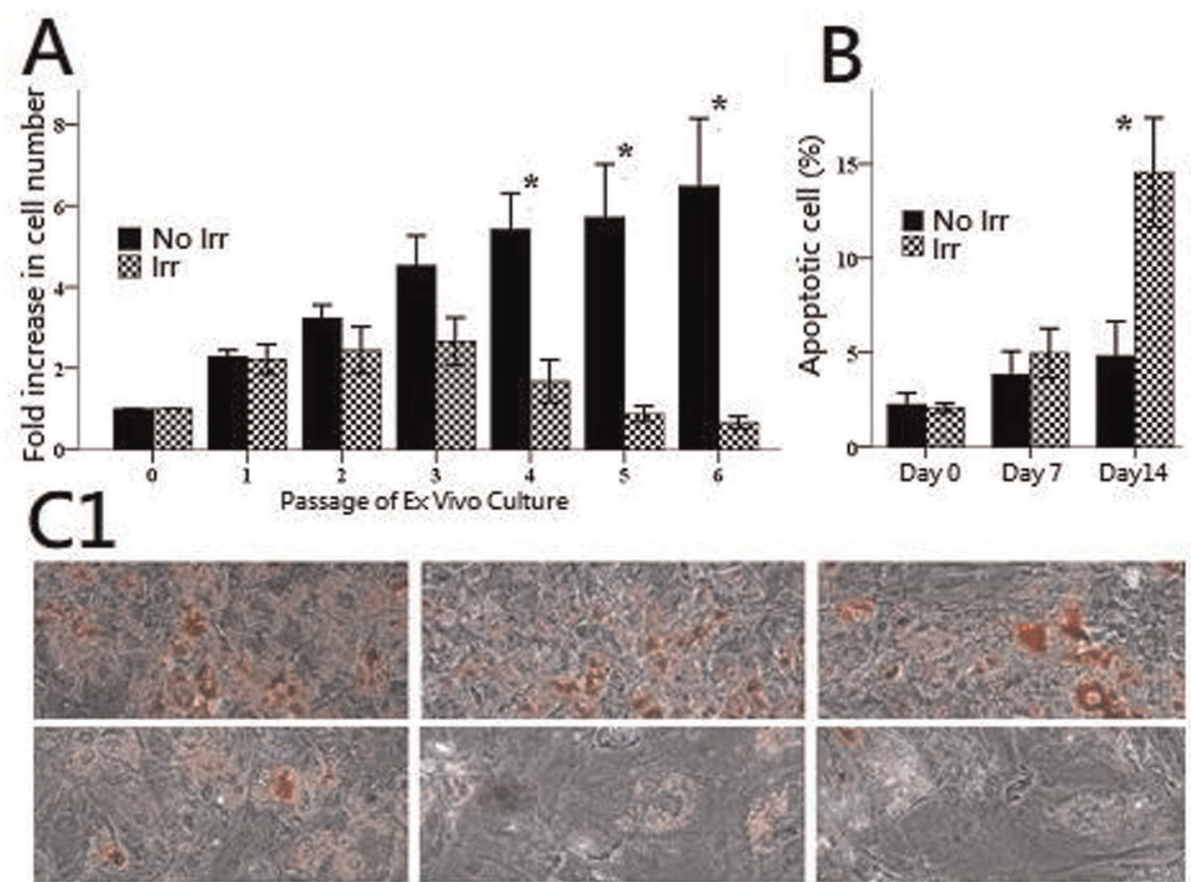

Oil red O stain for adipoqenic differentiation. Upper: 3 cases of non-irradiated MSCs. Lower: corresponding irradiated MSCS

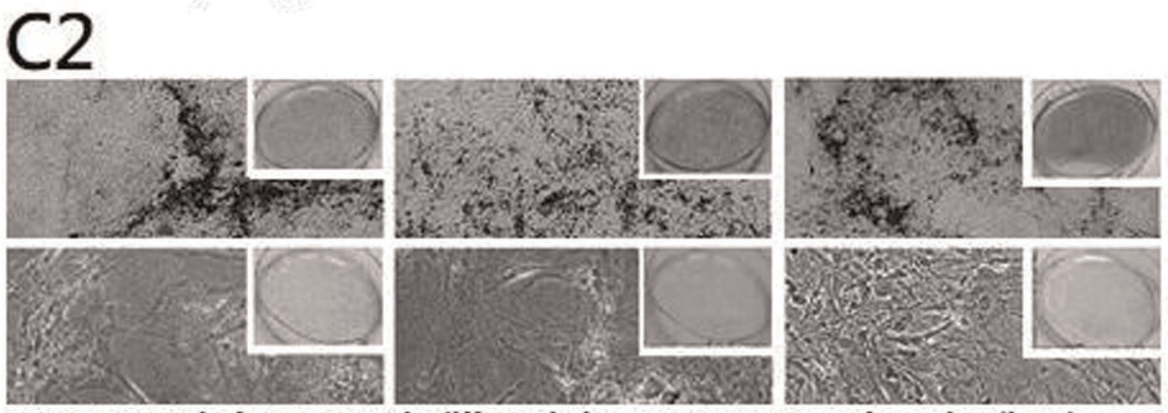

Von Kossa stain for osteogenic differentiation. Upper: 3 cases of non-irradiated MSCs. Lower: corresponding irradiated MSCs (Insert: gross picture of dish)
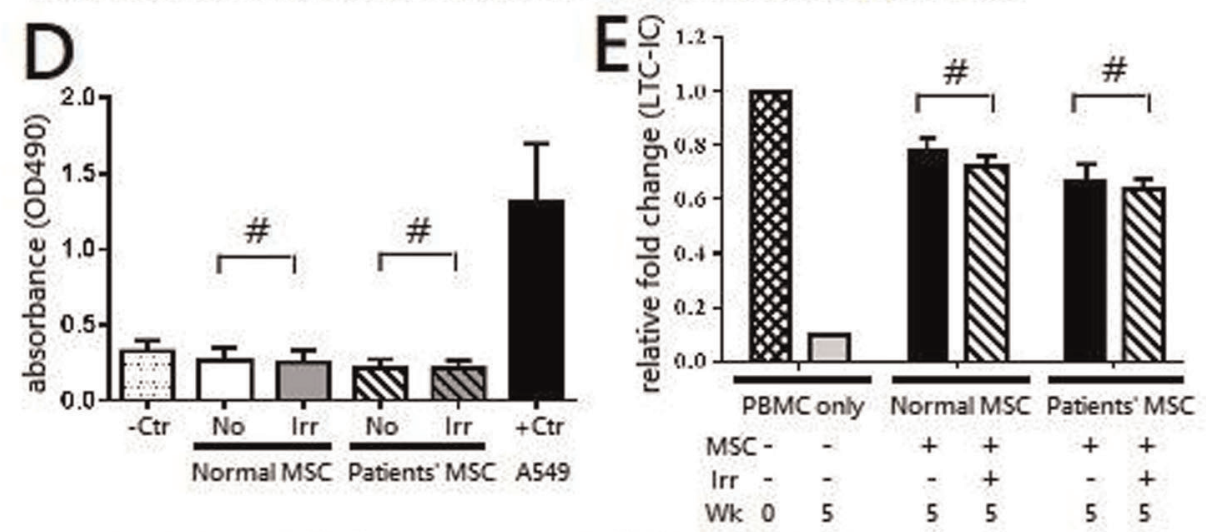

Bar = 1 standard error; Irr = Irradiation; * $p<0.05 ; \# p>0.05$

[P717 Figure] [Impact of irradiation on characteristics, transformation, \& hematopoiesis-supporting function of MSC]

Clinical Trial Registry: NA

Conflict of interest: Nothing to disclose of all authors 


\section{P718}

\section{Molecular fingerprint of subsets of human bone marrow- derived mesenchymal stromal cells}

Selim Kuci ${ }^{1}$, Zyrafete Kuci ${ }^{1}$, Richard Schäfer ${ }^{2}$, Gabriele Spohn ${ }^{2}$, Stefan Winter $^{3}$, Thomas Klingebiel ${ }^{1}$, Peter Bader ${ }^{1}$

${ }^{1}$ University Hospital Frankfurt, Goethe University, Department for Children and Adolescents, Division for Stem Cell Transplantation and Immunology, Frankfurt am Main, Germany; ${ }^{2}$ German Red Cross Blood Center Frankfurt and Institute of Transfusion Medicine and Immunohematology, Frankfurt am Main, Germany; ${ }^{3}$ Dr. Margarete Fischer-Bosch-Institute of Clinical Pharmacology, Stuttgart, Germany

Background: There are thus far only few reports on genetic signature of subsets of mesenchymal stromal cell (MSCs). In the current study we assessed the molecular fingerprint (global gene expression) of the MSC-subset derived from positively selected $\mathrm{CD} 271^{+}$bone marrow mononuclear cells (CD271-MSCs) and compared it with the transcriptomics of MSCs generated from non-selected bone marrow mononuclear cells (PA-MSCs).

Methods: We expanded CD271-MSCs and PA-MSCs from three donors until passage 3. Both MSC types were compared against each other in gene expression microarray experiments. Raw intensity data were extracted from Feature Extraction output files for Agilent Whole Human Genome Oligo Microarrays 8X60K (Agilent Technologies, Inc) using Rosetta Resolver software (Rosetta, Inpharmatics, LLC.). The set of up- or down-regulated genes between CD271-MSCs and PA-MSCs were hierarchically clustered and displayed in heatmap images using Multiple Experiment Viewer software. Reporters identified in the discriminatory genes analysis were annotated with information from Gene Ontology (GO), which provides information on molecular function, as well as various pathway resources for information on involvement in biological signalling pathways. Differences between the sample group means of CD271-MSCs and PA-MSCs were assessed with Student's t-test (two-tailed, equal variance). Reporters were considered as differentially expressed when they passed the filtering criteria of an uncorrected p-value of 0.05 or less, and fold change difference of at least 1.5-fold up- or downregulation of the mean of the CD271-MSC sample group compared to the PA-MSC sample group.

Results: Discriminatory gene analysis (DGA) demonstrated that in CD271-MSCs 124 genes were upregulated and 139 other genes were down-regulated when compared to PA-MSCs. The upregulated genes in CD271-MSCs were primarily cell surface molecules, particularly IL12RB, CD3G and NCAM1, CXCR7 etc. The highest differences in the magnitude for lower expressed genes encode cell surface molecules, or components of the cytoskeleton including AMIGO3, ACTG2, and KRT28, among others. In addition, functional grouping analysis revealed that highly expressed genes in CD271-MSCs are significantly associated with extracellular matrix and cell adhesion processes. Genes lower expressed in CD271-MSCs than in PA-MSCs are mainly associated with differentiation, particularly known for cells involved in immunoregulatory processes. The following categories are significantly enriched: cell proliferation and differentiation, innate immunity and inflammation, T-cell immunity, receptor signaling, including kinase/phosphatase signaling particularly of the SAP-signaling cascade and angiogenesis. Only the set of downregulated genes showed significant members of pathways. Consistent with the findings of the functional processes are signaling pathways of TGF-beta and Wnt affected, which may relate more to alterations in cytoskeleton and proliferation. In addition, cytokine/chemokine signaling pathways are significantly enriched, confirming the aforementioned altered expression of immunoregulatory molecules.

Conclusions: Our results may therefore explain the genetic basis for the functional differences between CD271-MSCs and PA-MSCs concerning their proliferative, differentiation and engraftment-promoting properties.

Conflict of interest: The authors have nothing to disclose.

\section{Paediatric issues}

\section{P719}

Allogeneic Hematopoietic Stem Cell Transplantation for children with Juvenile Myelomonocytic Leukemia: A single Center experience of 22 patients

Ali Al-Ahmari ${ }^{1,2}$, Nada Elshaikh ${ }^{3}$, Amal Alseraihy $^{3}$, Mouhab Ayas $^{3}$, Abdullah Al-Jefri ${ }^{3}$, Suleiman Alsweedan ${ }^{3}$, Ibrahim Al-Ghemlas, Hawazen Alsaedi ${ }^{3}$,Hasan Shaheen ${ }^{3}$, Neamah Farhan ${ }^{3}$, Khawar Siddiqui ${ }^{3}$

${ }^{1}$ King Faisal Hospital and Research Center, Pediatric Hematology/ Oncology, Riyadh, Saudi Arabia; ${ }^{2}$ Alfaisal University, College of Medicine, Riyadh, Saudi Arabia; ${ }^{3}$ King Faisal Specialist Hospital \& Research Center, PHO, Riyadh, Saudi Arabia

Background: Juvenile Myelomonocytic Leukemia (JMML) is a rare type of leukemia affecting infants and early childhood, with an incidence rate of 1.2 per million per year, it is characterized by an aggressive clinical behavior with a median survival of 12 month if left untreated. Currently, Hematopoietic Stem Cell Transplantation (HSCT) is the only curative treatment available; however, success is limited by engraftment failure or early relapse. We are reporting the outcome of patients with 
JMML who underwent HSCT at our institution over the last 22 years.

Methods: The medical records of 22 children who fulfilled the International Juvenile Myelomonocytic Leukaemia Working Group Criteria for a diagnosis of JMML and underwent HSCT at our institution from January 1993 through December 2015 were reviewed.

Results: The outcome of 22 children $(13$ males and 9 females) with JMML given unmanipulated HSCT after preparative regimens including busulfan and cyclophosphamide in 21 patients with the addition of etoposide in fifteen cases. One patient received busulfan and fludarabine. Donors were HLA-identical siblings $(n=11)$, unrelated Cord Blood $(n=9)$ or mismatched family members $(n=2)$. Splenectomy had been performed before HSCT in 9 children. Twenty patients $(90 \%)$ received chemotherapy as frontline treatment. Three patients had active disease at time of transplant despite chemotherapy. Engraftment was documented in eighteen patients, whereas graft failure occurred in four, three of whom had received HLAmismatched UCB.

Six patients relapsed including the three patients with active disease at HSCT. All five patients but one who developed acute GVHD are long term survivors. Five and four patients succumbed to disease recurrence and transplant-related complications respectively. A total of 13 patients survive in CR (median follow-up 10.7 years, range 2.6-18.6 years). Relapse was observed in five patients who subsequently succumbed to disease progression. Transplant related mortality occurred in four patients. Overall survival at 10 years was $59 \%$. The 10-year probability of overall survival for children given HSCT from either a familyrelated donor or a UCB was $69 \%$ and $44 \%$, respectively $(P$ $=0.27$ ). Overall survival was poorly associated with age of $>3$ years and platelet count of $<50 \times 10^{9} / \mathrm{L}$ at diagnosis and active disease at HSCT $(\mathrm{P}=0.04, \mathrm{P}=0.005$ and $\mathrm{P}=0.00 .6$ respectively).

Conclusions: Our results showed that about two thirds of the JMML patients could be potentially cured with allogeneic HSCT. Factors such as age and platelets count at diagnosis and disease status at transplant was statistically associated with poorer outcome. Disease recurrence remains the major cause of treatment failure, and novel strategies to lower the risk of relapse are warranted.

Conflict of interest: Nothing to disclose

\section{P720}

\section{Allogeneic Hematopoietic Stem Cell Transplantation for Myelodysplastic Syndrome in Children and Adolescents: Single-Center Experience}

Jae Won Yoo, Sung Han Kang, Hyery Kim, Kyung-Nam Koh, Eun
Seok Choi, Ho Joon Im, Jong Jin Seo

Asan Medical Center Children's Hospital, Pediatrics, Seoul, Korea, Republic of

Background: Allogeneic hematopoietic stem cell transplantation (HSCT) is the only curable approach for myelodysplastic syndromes (MDS). However, limited data are available focusing on children. In this study, we evaluated the outcome of HSCT in childhood MDS at a single center.

Methods: Thirty-six patients with MDS underwent allogeneic HSCT at Asan Medical Center Children's Hospital (AMCCH) between December 1997 and September 2017. All 36 patients were diagnosed based on the WHO 1999/2008 criteria. At the time of transplantation, 24 were low grade MDS (Refractory anemia, refractory cytopenia with multilineage dysplasia and refractory cytopenia of childhood) and 12 were advanced MDS (refractory anemia with excess blasts-1 and 2). Patients with $\geq 20 \%$ blasts in PB or BM at any time before transplantation were excluded. Cytogenetic risk group was stratified according to revised IPSS (23 Good, 8 Intermediate, 4 Poor, 1 Very poor). Conditioning regimen, GVHD prophylaxis and supportive care were accomplished according to AMCCH's protocol. The final follow-up visits for endpoint analysis were conducted on November 30, 2017.

Results: Median age at transplantation of the 36 patients (22 male and 14 female) was 12 years (range, 2-24). Median time from diagnosis to transplantation was 5.9 months (range, 1-139). Five out of 14 patients with advanced MDS received AML-type chemotherapy prior to transplantation. Ten (28\%) transplants were performed between 1997 and 2007, and 26 (72\%) between 2008 and 2017. Thirty two (89\%) patients achieved engraftment and the median time to engraftment of absolute neutrophil count and platelet were 11 and 18 days, respectively. Grade II to III acute GVHD occurred in 13 patients $(36 \%)$ and no patient developed grade IV acute GVHD. Extensive chronic GVHD occurred in 4 patients $(11 \%)$. With a median follow-up of 53 months (range, 2-239), the probability of EFS and OS at 4 years for all patients were $71 \%$ and $79 \%$, respectively. Four patients died of transplant-related causes, leading to TRM of $8.7 \%$ at 1 year and $12.3 \%$ at 2 years. Patients with low grade MDS represented significantly higher OS compared to advanced MDS (90\% vs. $58 \%, p=0.02$ ) but EFS and TRM were not different between two groups. The univariate analysis identified disease status of MDS and year of transplantation as variables affecting the OS. In the multivariate analysis, patients who transplanted between 1997 and 2007 were associated with lower OS (OR, 8.0; 95\% CI 1.5-42). Among the patients with advanced MDS, the OS was improved according to the time of transplantation (33\% for 
$1997-2007$ vs. $83 \%$ for $2008-2017, \mathrm{p}=0.07)$. Nine $(25 \%)$ out of 36 patients underwent haploidentical HSCT with RIC regimen (8 low grade MDS, 1 advanced MDS), all the patients except one achieved sustained engraftment and were alive in remission at the time of analysis.

Conclusions: Our study demonstrated that allogeneic HSCT is feasible treatment option for childhood MDS especially in patients with low grade MDS. The outcome of patients with advanced MDS was improved over time. Furthermore, haploidentical HSCT with RIC regimen could be a realistic alternative for children with MDS.

Conflict of interest: Jae Won Yoo: nothing to disclose

\section{P721}

Antiemetic prophylaxis in pediatric HSCT setting: a survey of the current practice in Europe. Supportive care meeting of the EBMT-PDWP - Frankfurt, September 2017

Tiago Nava ${ }^{1,2}$, Chakradhara Rao S. Uppugunduri ${ }^{1,2}$, Simona Mlakar $^{1,2}$, Frédérique Rodieux ${ }^{2}$, Dominik Turkiewicz ${ }^{3}$, Riita Niinimäki ${ }^{4}$, Kim Vettenranta ${ }^{5}$, Tamara Diesch ${ }^{6}$, Christina Peters ${ }^{7}$, Jean-Hugues Dalle ${ }^{8}$, Peter Bader ${ }^{9}$, Adriana Balduzzi ${ }^{10}$, Marc Ansari $^{1,2}$

${ }^{1}$ CANSEARCH Research Laboratory, University of Geneva, Faculty of Medicine, Geneva, Switzerland; ${ }^{2}$ University Hospital of Geneval University of Geneva, Geneve, Switzerland; ${ }^{3}$ Wroclaw Medical University, Wroclaw, Poland; ${ }^{4}$ Oulu University Hospital, Oulu, Finland; ${ }^{5}$ University of Helsinki Children's Hospital, Helsinki,

Finland; ${ }^{6}$ University Children's Hospital of Basel, Basel, Switzerland; ${ }^{7}$ St Anna Children's Hospital, Vienna, Austria; ${ }^{8}$ Hôpital Robert Debré, Paris, France; ${ }^{9}$ University Hospital Frankfurt, Frankfurt, Germany;

${ }^{10}$ Ospedale San Gerardo, Monza, Italy

Background: Chemotherapy-induced nausea and vomiting (CINV) is a frequent concern of oncological patients during antineoplastic treatment leading to a reduced quality of life. With the implementation of an adequate prophylaxis, based on evidence-based guidelines, CINV could be improved. Despite these guidelines in pediatric oncology, recommendations are lacking for HSCT in children. The present survey intended to assess the current practice regarding antiemetic prophylaxis across European pediatricHSCT centers as part of a taskforce of EBMT-PDWP aiming to improve the supportive care in children during HSCT.

Methods: A standardized questionnaire was sent to 25 EBMT centers containing a list of well-known conditioning regimens used before allogenic and autologous HSCT. Each center was asked to classify the locally used regimens according to their emetogenic potential and to indicate the antiemetic drugs, if any, employed as prophylaxis in each case, according to the local practice. The emetogenic classification was compared with the current recommendations in oncology (drug- and dose-based). The availability of a standardized operational procedure (SOP) for antiemetic prophylaxis in HSCT setting was also assessed.

Results: Out of 25 center, 17 replied and declared to use 37 different regimens (median of 13 regimens per center, from 8 to 29). Totally 22 regimens, which were used by at least $20 \%$ of the centers, were included in further analysis. $\operatorname{Busulfan}(\mathrm{Bu})+\mathrm{Cyclophosphamide}(\mathrm{Cy})$ and $\mathrm{Bu}+\mathrm{Cy}+\mathrm{Mel}-$ phalan(Mel) were the most frequently used across the centers (15 out of 17). Regarding the emetogenic potential, most of the regimens were classified as moderately emetogenic (52\%). As antimemetic drug, 5- $\mathrm{HT}_{3}$ antagonists were used in all regimens classified as highly or moderatly emetogenic and in $69.7 \%$ of the low emetogenic potential. In regimes with high emetogenic potential, a second drug was added in $37.1 \%$ of cases and in $49.1 \%$ of those with a moderate one. In highly emetogenic regimen, lorazepam (28.6\%) and dexamethasone $(22.4 \%)$ were the preferable applied medications whereas in regimens with moderate potential, dimenhydrinate was preferred (34.8\%). Based on the current recommendation, the overall rate of discordance on the classification of the emetogenic potential was of $55 \%$.

Only 7 centers $(41.2 \%)$ declared to have an SOP and 6 were available for analysis. Across the SOPs, some disagreements were noticed such as disparate criteria in emetogenic potential ( $\mathrm{Bu}, \mathrm{Mel}$ and $\mathrm{Cy}$ doses), drug-drug interactions (DDIs) and dexamethasone contraindications (e.g. AML, HSCT, CNS tumors).

Conclusions: Current guidelines in pediatric oncology recommends $5-\mathrm{HT}_{3}$ antagonists for every patients receiving at least a lowly emetogenic regimen. Patients receiving a moderately emetogenic conditioning would benefit from adding dexamethasone and for those receiving a higly emotogenic regimen a third prophylactic medication should be associated. Otherwise, the compliance of that practice seems to be low in HSCT setting. The high level of disagreement on emetogenic classification might explain some degree of discordance, but some concerns such as the use of dexamethasone or potential DDIs may also contribute to it. Assessing the hurdles of the applicability of the current recommendations may allow adapting them to the HSCT setting, which can improve the quality of life of those patients.

Conflict of interest: The authors declare no conflict of interest

\section{P722}

Bears get sick too: preparing children for admission to the transplant unit

Dana Ashkenazi Lustig ${ }^{1}$, Isaac Yaniv ${ }^{2}$, Jerry Stein ${ }^{2}$ 
${ }^{1}$ Schneider Children's Medical Center of Israel, Pediatric Oncology, Petah Tikva, Israel; ${ }^{2}$ Schneider Children's Medical Center of Israel, Oncology, Petah Tikva, Israel

Background: Stem cell transplant (SCT) is frightening for adult patients, and all the more so for young children. Anticipation of the unknown adds to the stress that many children and their families experience on the cusp of admission to the transplant unit. On-line and interactive tools have been developed in many centers to facilitate age appropriate preparation of patients for the rigors of SCT.

Methods: We have developed a decidedly low-tech tool (a book) which would permit children and their parents to share a reading experience that transmits information about the medical and psycho-social challenges that children aged 4-11 years (or their siblings) experience in the SCT period. The complex process of SCT is told in a culture neutral narrative from the perspective of a teddy-bear who undergoes SCT, and experiences the side effects and isolation that are part and parcel of a childÂ's experience before, during, and after SCT.

Results: Our book, Bears Get Sick, Too, was written by a physician in consultation with psychologists and social workers from a busy pediatric SCT Unit. It tells the story of a bear who must leave his home in the forest, receive treatment for a malignant disease, and then enter the previously unknown world of 'transplant'. The book is illustrated with soft, warm images and discusses common questions that parents and children often raise. The story follows the process of donor search/selection, pre-transplant conditioning and prolonged isolation and separation from family and friends. Side effects and lifestyle changes are addressed in a forthright but gentle and age-appropriate manner. Children and care-givers can use the book as a platform on which to develop dialogues that facilitate coping with a long and often painful ordeal. The procedure of the transplant itself is explained using simple metaphors to which children can relate. Originally written in Hebrew, we now present an English translation of this book. Initial acceptance of this modality in a handful of families has been enthusiastically positive.

Conclusions: Enhancing parent-child communication with the aid of the book we have designed should help lessen anxiety in advance of admission to the stem cell unit. Copies of the book can be viewed at our website.

Conflict of interest: nothing to disclose

\section{P723}

\section{CD45 RA DEPLETION AS AN ALLOGENIC HEMATOPOIETIC TRANSPLANTATION PLATFORM IN CHILDREN FROM HLA- IDENTICAL DONORS}

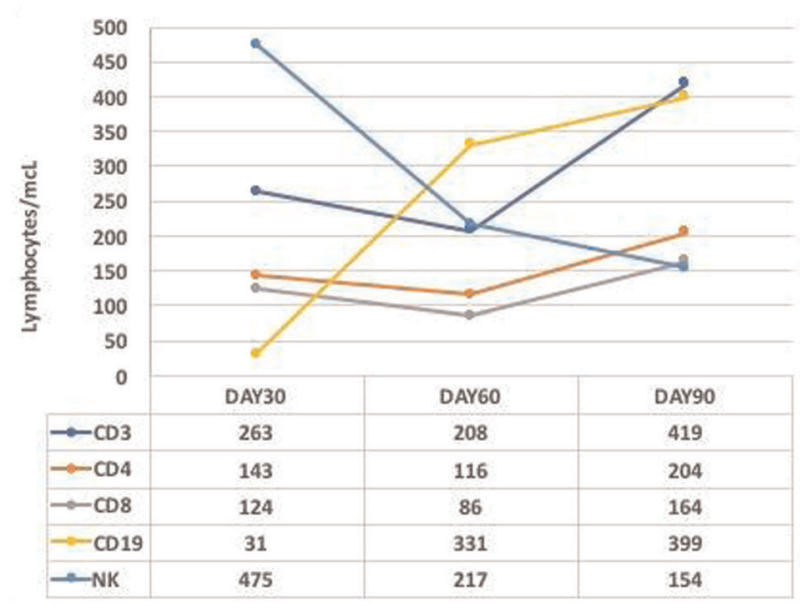

[P723 Figure] [Figure 1. Immune reconstitution.]

Miguel Angel Diaz ${ }^{1}$, Marta Gonzalez-Vicent ${ }^{1}$, Blanca Molina ${ }^{1}$, Elena Sebastian ${ }^{2}$, Josune Zubicaray ${ }^{2}$, Julian Sevilla ${ }^{2}$

${ }^{1}$ Hospital Niño Jesus, Stem Cell Transplant Unit, Madrid, Spain; ${ }^{2}$ Hospital Niño Jesus, Blood Bank, Madrid, Spain

Background: In hematopoietic transplantation, the $\mathrm{T}$ lymphocytes of the inoculum play a determining role in promoting hematopoiesis, transferring immunity to pathogens and acting as mediators of the graft-versus-leukemia effect (GVL). However, they are also responsible for graftversus-host disease (GVHD), the main cause of posttransplant morbidity and mortality. The depletion of CD45 RA lymphocytes, by eliminating naive $\mathrm{T}$ lymphocytes from the inoculum, aims to conserve the GVL without producing GVHD.

Methods: Since April 2016, 14 patients (8 boys and 6 girls), with a median age of 8 years, have undergone an allogeneic hematopoietic transplant from an HLA donor identical with CD45 RA/CD19 depletion. The indication for transplant was: acute lymphoblastic leukemia (4), acute myeloblastic leukemia (4), myelodysplasia (5) and medullary aplasia (1). The donor was familiar in 4 cases and unrelated in 10 . The conditioning regimen was with fludarabine, busulfan and thiotepa. The median of CD34 + cells infused was $6.82 \times 106 / \mathrm{Kg}$. On the day $0,+15$ and +30 a programmed infusion of $1 \times 10^{6} / \mathrm{Kg}$ lymphocytes CD45RA- was performed.

Results: All the patients grafted with a median leukocyte $(>0.5 \times 109 / L)$ and platelet (> 20x109/L) engraftment time of 15 and 10 days, respectively. Only one patient has developed acute GVHD grade I and no patient has developed chronic GVHD. Immune reconstitution was early and rapid in all $\mathrm{T}$ cell subsets (see Figure 1).

No patient has relapsed so far and only 1 patient with myelodysplasia has developed an AML. She has received a 2nd transplant and has died of relapse. There was no case of 
toxic mortality. The event-free survival (SLE) was $90 \pm$ $10 \%$ with a median follow-up of 10 months. At present, 13 patients are alive, out of immunosuppressive treatment and doing well.

Conclusions: Allogeneic transplantation with CD45 lymphocytes RA depletion resulted on very encouraging results, with a very low incidence of acute and chronic GVHD, but preserving the GVL effect by infusing CD45 RA- donor lymphocytes.

Conflict of interest: None of the authors has anything to disclose.

\section{P724}

\section{Change in the Disease-Scape of Pediatric Patients Underwent Hematopoietic Stem Cell Transplantation in Iran}

Amir Ali Hamidieh, Alieh Safari Sharari, Nazanin Khodayari, Leila Hejazipour, Hamideh Keykani, Zeynab Nikfetrat, Fahimeh Jaafari, Maryam Behfar, Poorya Salajegheh, Seyed Mostafa Monzavi

Children's Medical Center, Tehran University of Medical Sciences, Department of Pediatric Stem Cell Transplantation, Tehran, Iran, Islamic Republic of

Background: Hematopoietic stem cell transplantation (HSCT) for pediatric patients started in 2007 in Iran. At first, it was part of a joint program with adult patients, based in an adult general hospital affiliated to Tehran University of Medical Sciences. The pediatric team has moved to an exclusive independent department at the Children's Medical Center (CMC), the largest children hospital of Iran and the country's pediatric center of excellence, since September

Table. Characteristics of the patients

\begin{tabular}{|c|c|c|}
\hline \multirow[t]{3}{*}{ Parameters } & \multicolumn{2}{|c|}{ Time period } \\
\hline & 2007-Aug & Sep 2016-Sep \\
\hline & 2016 & 2017 \\
\hline Number of patients & 1029 & 79 \\
\hline Median age (range), years & $7.5(0.4-15)$ & $7(0.4-15)$ \\
\hline Male sex, \% & 57.4 & 60.8 \\
\hline \multicolumn{3}{|l|}{ HSCT type, \% } \\
\hline Autologous & 11.8 & 11.1 \\
\hline Allogeneic (Related) & 83.9 & 78.5 \\
\hline Allogeneic (Unrelated) & 4.3 & 11.4 \\
\hline \multicolumn{3}{|l|}{ Underlying disease, $\mathrm{n}(\%)$} \\
\hline $\begin{array}{l}\text { Inherited abnormalities } \\
\text { of RBC }\end{array}$ & $454(44.1)$ & $18(22.8)$ \\
\hline Leukemias & $284(27.6)$ & $17(22.8)$ \\
\hline Bone marrow failure & $128(12.4)$ & $9(11.4)$ \\
\hline $\begin{array}{l}\text { Primary } \\
\text { immunodeficiency }\end{array}$ & $48(4.7)$ & $16(20.3)$ \\
\hline $\begin{array}{l}\text { Inborn errors of } \\
\text { metabolism }\end{array}$ & $43(4.2)$ & $11(13.9)$ \\
\hline Solid tumors & $72(7.0)$ & $7(10.1)$ \\
\hline
\end{tabular}

[P724 Figure] [Characteristics of the patients]
2016. The new department serves to most of the country's HSCTs performed for children. Following this transition, the disease-scape of the patients transplanted shows changes compared with the roughly ten years earlier.

Methods: In this study, yearly epidemiologic profile of patients underwent HSCT at CMC compared with the data of 2007 to August 2016.

Results: Comparing the two time periods, a slight decrease in the annual number of patients transplanted can be seen (79 vs. 103), which is largely due to the fact that the new department needed technical preparations to be completed at the first few months. There are no significant differences in patients' sex and age distribution between the two time periods (Table). Although inherited abnormalities of erythrocytes are still the leading indication for HSCT, $50 \%$ reduction in the rate of such patients has occurred after the establishment of the new department. On the other hand, marked increases can be seen in the rate of HSCT-treated patients with primary immunodeficiencies $(15.6 \%)$ and inborn errors of metabolism (9.7\%) after starting the new department. Interestingly, with the commencement of the new department, the rate of unrelated allogeneic HSCTs increased by $7.1 \%$.

Conclusions: After separation of the pediatric program from the joint program with adults, the disease-scape of the patients underwent HSCT has changed, showing a growth in the rate of patients with primary immunodeficiencies and inborn errors of metabolism. This might be explained by three underlying reasons: 1 . Public awareness about the diseases that can be cured by HSCT has been improved during the recent years, and so there has been an increased visits to HSCT clinic by couples who have children with such genetic disorders, 2. The development of Iranian HLA registry program in 2007 has resulted in the increase in the number of unrelated allogeneic HSCTs, 3. Owing to the national thalassemia prevention program, the annual incidence rate of the disease in Iran has reduced from 800 in the beginning of the new century to 200 in recent years, while the rate of other inherited disorders and malignant diseases has remained relatively unchanged.

Conflict of interest: There are no conflicts of interest to report.

\section{P725}

Complement and Interferon Pathway Activation Triggers Thrombotic Microangiopathy after Stem Cell Transplantation

\author{
Sonata Jodele ${ }^{1}$, Nathan Luebbering ${ }^{2}$, Mario Medvedovic ${ }^{3}$, Stella \\ Davies $^{1}$ \\ ${ }^{1}$ Cincinnati Children's Hospital, Bone Marrow Transplant, Cincinnati, \\ OH, United States; ${ }^{2}$ Cincinnati Children's Hospital, Cincinnati, OH,
}


United States: ${ }^{3}$ University of Cincinnati College of Medicine, Cincinnati, $\mathrm{OH}$, United States

Background: Transplant-associated thrombotic microangiopathy (TA-TMA) is an important cause of morbidity and mortality after hematopoietic cell transplant (HCT). The pathogenesis of TA-TMA is poorly understood, but our recent data show clinical response to terminal complement blockade using eculizumab. However, we recognize that there are still patients with refractory disease requiring additional therapeutic options. Moreover, recent basic science studies have identified important local activities of extrahepatic complement components produced by immune cells, suggesting a potential direct role of transplanted stem cells in pathological complement activation. The goal of our study was to examine complement system in TA-TMA and to identify other novel therapeutic targets.

Methods: We prospectively collected serial weekly peripheral blood mononuclear cells (PBMC) from children receiving autologous transplant for neuroblastoma. We identified four children who developed TA-TMA and were treated with eculizumab and three receiving the same preparative regimen who did not develop TA-TMA. We selected PBMC collected prior to start of transplant, at time of diagnosis of TA-TMA (or equivalent time in those without TA-TMA) and at resolution of TA-TMA (or equivalent time in those without TA-TMA) and performed RNAseq to identify activated and deactivated pathways contributing to pathogenesis of TA-TMA.

Results: Transcriptome analysis at onset of TA-TMA showed significant up-regulation of both classical and alternative complement pathways during active TA-TMA, in particular markedly increased expression of the initiator molecule $\mathrm{Clq}$ and the alternative pathway rapid loop activator CFD, which may serve to amplify complement activation. Lectin pathways showed modest up-regulation. There were no significant elevation of expression of components of the coagulation cascade Analysis of the transcriptome after treatment with complement blockade using eculizumab showed the up-regulated genes and pathways returned to baseline expression and correlated with clinical resolution of TA-TMA. Further analysis of the global transcriptional regulatory network showed a notable interferon signature associated with TA-TMA (Figure 1).

Conclusions: Our data demonstrated a broad increase in expression of complement related genes in children in the acute phase of TA-TMA before complement blocking therapy was initiated. Gene expression returned essentially to baseline after use of eculizumab with no residual elevation of complement-related pathways, indicating the efficacy of the therapy. Our data also show a significant elevation in expression of interferon-responsive genes in
Fig 1. Change in gene expression between clinical time points

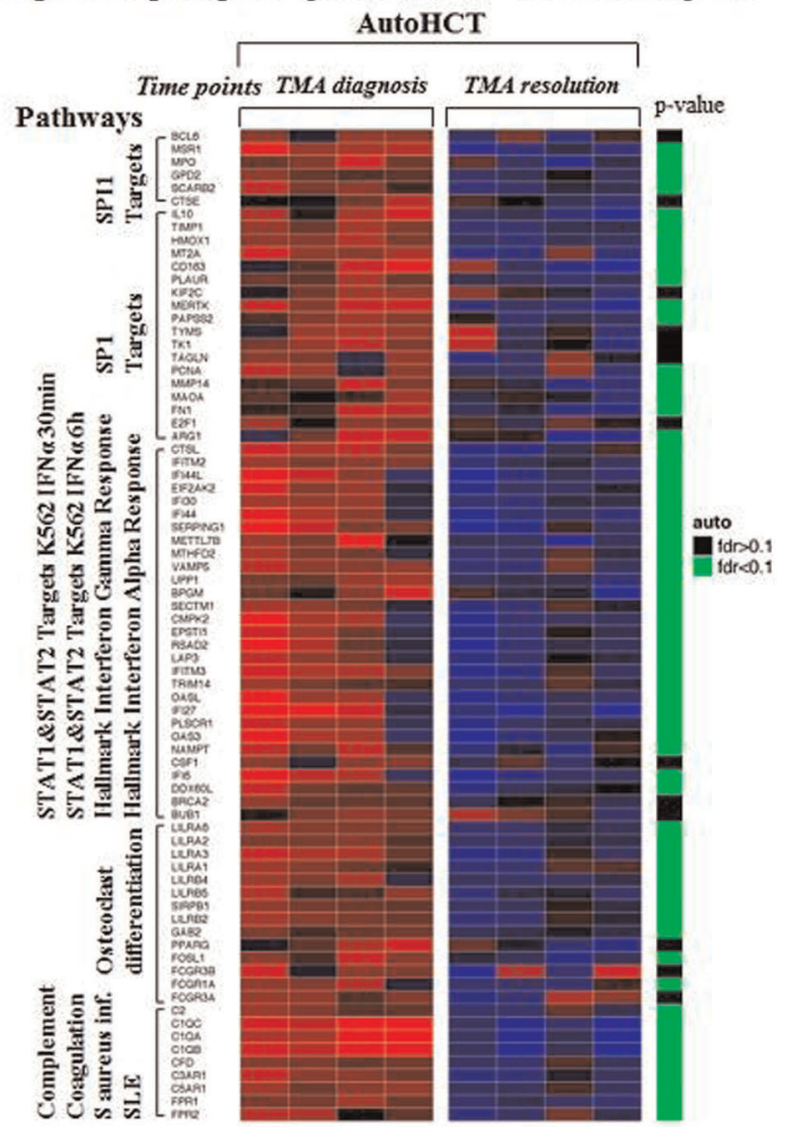

[P725 Figure] [Change in gene expression between clinical time points]

children with TA-TMA that is not seen in children without TA-TMA. This finding is in accordance with the highly inflammatory clinical phenotype of children presenting with TA-TMA and may explain, at least in part, lack of complete response to complement blocking therapy alone in some patients with severe disease. Interferon is a novel druggable target in TA-TMA that could be beneficial in severe or eculizumab refractory TA-TMA cases. Anti-interferon therapeutics, including antibodies and other targeted therapies are in clinical trials and might offer an alternative strategy for immediate treatment of the most critically ill children. In addition, our data offer an explanation for the occurrence of TA-TMA in interferon driven disease such as systemic lupus erythematosus (SLE) hemophagocytic lymphohistiocytosis (HLH), endothelial injury with CART therapies, and for the occurrence of TMA with therapeutic use of interferon.

Conflict of interest: SJ, SMD have a US Provisional patent application pending. Other authors have no disclosures to report. 


\section{P726}

\section{Correlation Analysis of WAS Gene mutations with Clinical Phenotype}

Yunjing Zheng, Peifang Xiao, Jun Lu, Jie Li, Shaoyan Hu

Children's Hospital of Soochow University, Suzhou, China

Background: The WAS-related disorders such as WAS, X-linked thrombocytopenia (XLT), and X-linked congenital neutropenia $(\mathrm{XLN})$ have been recognized. However, with more and more novel mutations identified by next generation sequencing, novel phenotype may be accompanied. In this study, we investigated the phenotypic characteristics of 30 patients with WAS gene defects.

Methods: The hot spots mutations and related clinical phenotypes of 30 patients with WAS gene defects were retrospectively analyzed, including inpatients and outpatients of department of Hematology, ChildrenÂ's Hospital, Soochow University from January 2013 to August 2017. The important role of WASP p.P460S mutation was reevaluated.

\section{Results:}

1. A total of 30 WAS patients were all male. The median onset age was 1 month (range, 0-24 months). The median age of diagnose was 4 months (1-96 months). There were 24 cases of classic WAS, 2 cases of X-linked thrombocytopenia (XLT), 1 case of intermittent X-linked thrombocytopenia (IXLT), 2 cases of X-linked pancytopenia and 1 case of X-linked thrombocytopenia with granulocytopenia among 30 patients.

2. Nine mutants were never reported before among 24 mutants detected in 30 patients, including c.1234_1235dupCC, c.1093-1097delG, c.28-30dupC, c.436G> T, c. $273+10 \_273+$ 11dupCC, c.995_996insG, c.1010T> A, c.332_333delCC and c.683C > T mutations. One patient's mutation was spontaneous among, and the others were inherited from their mothers. Mutations were found in all WASP functional regions, with WH1 region predominantly $(45.5 \%)$, and the most common mutation was missense mutation $(45.5 \%)$.

3. The same WASP c.C1378T mutation that caused WAS protein p.P460S change was identified in 4 patients, in two cases whose complete blood count showed pancytopenia, of which 1 patient developed into $\mathrm{T}$ lymphoblastic leukemia, and the remaining 2 patients, 1 case of onset manifestation showed only hematochezia and thrombocytopenia, and the other patient showed thrombocytopenia with repeated neutropenia.

4. Immune laboratory examination showed that CD3 + was decreased in 14 patients (58.3\%), CD19 + was decreased in 3 patients $(12.5 \%)$, and CD56 $+16+$ in 4 patients was decreased, accounting for $16.7 \%$. 5. Twentyfive patients survived and 5 died. Among 25 survivors, 22 patients were treated with hematopoietic stem cell transplantation (HSCT), 2 are preparing for HSCT, and the last 1 was IXLT patient who was stable after treatment. Five patients died, two died of post-transplant complications, one died of cerebral hemorrhage before transplantation, and two gave up treatment because of economic reasons.

Conclusions: WASP gene defect is an important basis for the diagnosis of WAS and related diseases. As the number of cases increased, some of the gene functions previously thought to be SNP have been redefined. HSCT is an effective treatment for WAS.

Conflict of interest: There is no potential conflict of interest among authors.

\section{P727}

Abstract previously published

\section{P728}

\section{CXCL12 AND CYP1A1 GENE POLYMORPHISMS IN CHILDHOOD SOLID TUMORS}

\section{Evangelia-Dimitra Ampazoglou, Marilena Lourou, Eleni Kampouraki, Emmanouil Athanasopoulos, Nikolaos Katzilakis, Eftichia Stiakaki}

University of Crete, Laboratory of Blood Diseases and Childhood Cancer Biology, University Hospital of Heraklion, Pediatric Hematology-Oncology, Heraklion, Greece

Background: Genetic association studies on cancer risk have focused on the effects of single nucleotide polymorphisms in genes that regulate inflammation and tumor suppression such as chemokines and P450 cytochrome. Chemokines induce the motility of endothelial and tumor cells. CXCL12 binds to chemokine receptor 4 (CXCR4), is selectively expressed on $\mathrm{B}$ and $\mathrm{T}$ lymphocytes and is involved in hematopoiesis, thymocyte trafficking, stem cell motility, neovascularization and tumorigenesis. The polymorphism rs1801157 of this gene has been investigated concerning breast cancer, Wilms' tumor, nasopharyngeal carcinoma, renal cell carcinoma and leukemia. CYP1A1 gene belongs to family 1 , subfamily $1 \mathrm{~A} 1$ of cytochrome P450. CYP1A1 protein is a phase I xenobiotic metabolizing enzyme that activates the conversion of environmental chemicals into carcinogens. The above gene contains two important single nucleotide polymorphisms, CYP1A $1 * 2 \mathrm{~A}$ (rs4646903) and CYP1A1*2C (rs1048943), which have been investigated concerning breast cancer, testicular cancer, lung cancer and leukemia. The aim of this study is to investigate the role of single nucleotide polymorphisms 
rs1801157 of CXCL12 and CYP1A1*2C (rs1048943) in children and adolescents with solid tumors, including central nervous system (CNS) tumors, neuroblastoma, sarcomas, lymphomas, Wilms' tumors and histiocytosis ( $\mathrm{LCH})$.

Methods: Fifty one children with solid tumors and 92 healthy individuals ( 20 children and 72 adults blood donors) were included in the present study. Genomic DNA was isolated from peripheral blood of participants and was analyzed for the existence of polymorphisms rs1801157 of CXCL12, and CYP1A1*2C (rs1048943) with Polymerase Chain Reaction (PCR). The PCR products were digested with the restriction enzyme MsPI for CXCL12 and BsrDI for CYP1A1. Descriptive statistics and logistic regression analysis were used to examine for differences between children with solid tumors and controls.

Results: As shown in the table below, regarding the CXCL12 loci, there was a positive association for the GG genotype and solid tumors [OR: 2.826 (95\% CI: 1.264, 6.318), $\mathrm{p}=0.011]$ and a positive association for the $\mathrm{AG}$ genotype and normal phenotypes [OR: 0.324 (95\% CI: -), p $=0.001]$. No statistical significant differences in CYP1A1 polymorphism were revealed between children with solid tumors and control group using logistic regression analysis.

Conclusions: A higher frequency of CXCL12 homozygote allele $\mathrm{G}$ was observed among children with solid tumors whereas no differences were observed regarding CYP1A1 polymorphism. Future studies in larger populations are needed in order to specify the role of the above polymorphism in childhood solid tumors.

Conflict of interest: No conflict of Interest

\begin{tabular}{lllll}
\hline Genotype & Solid tumors $(\mathrm{n}=49)$ & Control group $(\mathrm{n}=87)$ & OR $(95 \% \mathrm{CI})$ & P value \\
\hline CXCL12 & & & & \\
AA & $4(8.16 \%)$ & $14(16.09 \%)$ & $0.881(0.243,3.194)$ & \\
GG & $33(67.35 \%)$ & $36(41.38 \%)$ & $2.826(1.264,6.318)$ & 0.011 \\
AG & $12(24.49 \%)$ & $37(42.53 \%)$ & 0.324 & 0.001 \\
Genotype & Solid tumors $(\mathrm{n}=51)$ & Control group $(\mathrm{n}=92)$ & OR $(95 \% \mathrm{CI})$ & \\
CYP1A1 & & & & \\
AA & $47(92.16 \%)$ & $80(86.96 \%)$ & $1.616(0.487,5.363)$ & \\
GG & $0(0 \%)$ & $1(1.09 \%)$ & 0 & \\
AG & $4(7.84 \%)$ & $11(11.96 \%)$ & 0.364 & \\
\hline
\end{tabular}

[[P728 Table] Table 1]

\section{P729}

Donor lymphocyte infusion after CD3+TcRaß-depleted haploidentical haematopoietic stem cell transplantation in pediatric patients with hematologic malignancies

Francesca Compagno $^{1}$, Stella Boghen ${ }^{1}$, Tommaso Mina ${ }^{1}$, Sabrina Basso $^{2}$, Antonella Gurrado ${ }^{2}$, Ilaria Pisani ${ }^{2}$, Alessandra Tolva ${ }^{1}$, Santina Recupero ${ }^{1}$, Elena Bergami ${ }^{1}$, Nunzia Decembrino ${ }^{1}$, Federico
Bonetti $^{1}$, Antonia Moretta $^{2}$, Gianluca Viarengo ${ }^{3}$, Claudia Del Fante $^{3}$, Cesare Perotti ${ }^{3}$, Giovanna Giorgiani ${ }^{1}$, Patrizia Comoli ${ }^{1}$, Marco Zecca ${ }^{1}$

${ }^{1}$ Fondazione IRCCS Policlinico San Matteo, Pediatric Hematology/ Oncology, Pavia, Italy; ${ }^{2}$ Fondazione IRCCS Policlinico San Matteo, Pediatric Hematology/Oncology \& Cell Factory, Pavia, Italy;

${ }^{3}$ Fondazione IRCCS Policlinico San Matteo, Immunohematology and Transfusion Service, Pavia, Italy

Background: Haploidentical haematopoietic stem cell transplantation (haplo-HSCT) after negative depletion of Tcell receptor (TcR) $\alpha \beta+\mathrm{T}$ and CD19+ B lymphocytes from mobilized peripheral stem cell grafts, is a therapeutic option for high risk or relapsed leukaemia patients without HLAcompatible sibling or unrelated donors. Relapse and opportunistic infections due to delayed immune reconstitution are the most common post-transplant complications. Post-HSCT administration of donor lymphocyte infusions (DLI) has been proved able to enhance immune reconstitution and prevent these complications, but the advantages are blurred by the risk of uncontrolled graft-versus-host disease (GvHD). The routine use of unmanipulated DLI is therefore still discussed and data on paediatric patients are still limited.

Methods: We report data on 14 pediatric recipients of CD3 $+\alpha \beta$-depleted haplo-HSCT transplanted between 2016 and 2017 for hematologic malignancies, who received a DLI schedule starting with $1 \times 10^{4} \mathrm{CD} 3+$ cells $/ \mathrm{kg}$ dose and proceeding with escalating doses every 2-3 weeks $\left(5 \times 10^{4}\right.$ cells $/ \mathrm{kg}, 1 \times 10^{5}$ cells $/ \mathrm{kg}, 5 \times 10^{5}$ cells $/ \mathrm{kg}, 1 \times 10^{6}$ cells $/ \mathrm{kg}$, $2 \times 10^{6}$ cells $/ \mathrm{kg}, 4 \times 10^{6}$ cells $/ \mathrm{kg}, 8 \times 10^{6}$ cells $/ \mathrm{kg}$ ). We retrospectively compared these patients with 17 patients who underwent $\mathrm{CD} 3+\alpha \beta$-depleted haplo-HSCT during 2014-2015, and did not receive DLI. Patients were aged between 0.5 and 16 years and received HSCT for relapsed or high-risk ALL, AML and MDS.

Results: The first DLI was administered at a median time of 48 days (range 30-109) after HSCT. The patients had a minimum follow up of 6 months after DLI initiation. The patients received a median of 6 DLI infusions (range 1-12). In both study and control groups, 3 patients developed grade I-II acute GVHD early after HSCT and before DLI; after DLI, 2 patients developed aGVHD (1 grade I and 1 grade IV), and 3 experienced chronic GvHD that resolved after steroid treatment and extracorporeal photopheresis. Three patients relapsed in the DLI group at a median time of 238 days from HSCT, while 4 patients relapsed in the control group (median time 113 days). Relapse mortality was not significantly different in the 2 groups. One patient died of aGVHD progression in the DLI group while no GVHD-related mortality was observed in the control group. Regarding infection mortality, no patients died of infection- 
related disease in the DLI group, while 3 patients succumbed to infectious complications in the control group.

Conclusions: The use of DLI appears to be a feasible therapeutic option after pediatric $\mathrm{CD} 3+\alpha \beta$-depleted haploHSCT for high-risk or relapsed malignancy. The main benefit in this cohort was due to infection control rather than prevention of disease relapse.

Conflict of interest: None to declare

\section{P730}

\section{DONOR LYMPHOCYTE INFUSION FOR TREATMENT OF MIXED DONOR CHIMERISM FOR PEDIATRIC HEMATOPOIETIC STEM CELL TRANSPLANTATION IN NONMALIGNANT DISEASES}

\section{Lizbeth Estefania Díaz Polo ${ }^{1}$, Luisa Sisinni ${ }^{1}$, Laura Medina ${ }^{2}$, Montserrat Torrent ${ }^{1}$, Vicente Serna ${ }^{1}$, Alba Bosch ${ }^{3}$, Querol Sergi ${ }^{2}$, Isabel Badell}

${ }^{1}$ Hospital de la Santa Creu i Sant Pau, Pediatric Unit of Hematology, Oncology and Stem Cell Transplantation., Barcelona, Spain; ${ }^{2}$ Banc de Sang $i$ Teixits, Cord Blood Unit, Barcelona, Spain; ${ }^{3}$ Banc de Sang $i$ Teixits, Transfusion Medicine, Barcelona, Spain

Background: Mixed donor chimerism (MC) is increasingly common in the pediatric HSCT setting because of the increased use of reduced-intensity conditioning regimens for nonmalignant diseases. Most of the patients with MC may spontaneously evolve to complete donor chimerism (CC) or maintain stable MC, but up to one-third of the patients may have graft failure. Pre-emptive usage of DLI for managing patients with unstable MC after transplantation is very limited in nonmalignant diseases.

Methods: Between January 2010 and December 2017 we retrospectively studied the chimerism course of 7 children with nonmalignant diseases who received an HSCT and afterwards received pre-emptive DLI due to unstable MC. Five patients received reduced-intensity conditioning regimens and two received myeloablative reduced toxicity regimens.

The underlying diseases were: Thalassemia maior, Hurler syndrome, Mucopolysaccharidosis VII, Hyper IgM Syndrome, Familial Hemophagocytic Lymphohistiocytosis, Pyruvate kinase deficiency and chronic granulomatous disease.

Donor chimerism was monitored by PCR amplification of 15 highly variable short tandem repeats.

Lymphocytes for DLI were either frozen aliquots collected from the donor at the time of the original harvest or were collected peripherally from the donor before DLI. We followed the institutional protocol consisting of progressively increasing doses, starting with $0.01 \times 10^{8}$
$\mathrm{CD} 3 / \mathrm{Kg}$, followed by $0.05 \times 10^{8} \mathrm{CD} 3 / \mathrm{Kg}$ and $0.1 \times 10^{8}$ $\mathrm{CD} 3 / \mathrm{Kg}$.

The first DLI was given at a median of 9 months posttransplant (range, 2 to 21 months). A median of 8 DLIs (range, 4 to 13) was given, with a median interval between infusions of 21 days (14 to 28 days). Median donor chimerism before DLI was $55 \%$ for both lymphocytes (range, $27 \%$ to $89 \%$ ) and granulocytes (range, $10 \%$ to $100 \%)$.

Results: Five out of seven patients showed an improvement in donor chimerism after DLI. Three patients reached full donor chimerism at a median of 8 months after the first DLI. Two patients (Chronic granulomatous disease and Hurler Syndrome) maintained mixed donor chimerism but having $100 \%$ donor chimerism in granulocytes and increased in Lymphocytes chimerism. Two patients continued with mixed donor chimerism for lymphocytes and granulocytes until complete failure of engrafment. One of them received a successful second myeloablative allogeneic HSCT, at 18 months from first HSCT, reaching complete donor chimerism.

Two patients developed GvHD after the last DLI: in one patient hepatic GvHD with chronic characteristics was confirmed after 18 days of last DLI (liver biopsy) and the second one initiated an acute GvHD of skin (grade II) 44 days after last DLI. Both patients showed good response to corticoids. The development of GVHD coincides with the improvement of the mixed chimerism. All patients are alive.

Conclusions: In our experience DLI showed efficacy in treatment of mixed donor chimerism in nonmalignant diseases, in children receiving reduced-intensity conditioning regimens and myeloablative regimens, with no mortality related to the procedure and low incidence of GVHD. It would be a possible strategy to avoid the graft failure and the need of a second transplant.

Conflict of interest: None of the authors has anything to disclose.

\section{P731}

Eculizumab therapy followed by BMT in a pediatric patient with paroxismal nocturnal hemoglobinuria

Maria Trabazo del Castillo ${ }^{1}$, Júlia Marsal Ricoma ${ }^{1}$, Izaskun Elorza Alvarez ${ }^{1}$, Catalina Montoya Tamayo ${ }^{2}$, Albert Catalá Temprano ${ }^{2}$, Anna Ruiz Llobet ${ }^{2}$, Rubén Berrueco Moreno ${ }^{2}$, Susana Rives Sola ${ }^{2}$, Mireia Camós Guijosa ${ }^{3}$, Montserrat Torrebadell Burriel ${ }^{3}$, Susana Gassiot Riu ${ }^{3}$, Isabel Badell Serra ${ }^{1}$

${ }^{1}$ Hospital Sant Joan de Deu, HSCT Unit, Esplugues de Llobregat (Barcelona), Spain; ${ }^{2}$ Hospital Sant Joan de Deu, Pediatric Hematology, Esplugues de Llobregat (Barcelona), Spain; ${ }^{3}$ Hospital 
Sant Joan de Déu, Hematology Laboratory, Esplugues de Llobregat (Barcelona), Spain

Background: Paroxysmal nocturnal hemoglobinuria (PNH) is a rare disease that develops as a result of somatic mutation of hematopoietic stem cells, and is characterized by clonal, complement-mediated intravascular hemolysis. $\mathrm{PNH}$ is characterized by hemoglobinuria, thrombosis, infection and marrow failure. While hemoglobinuria and thrombosis are major presenting symptoms in adult patients, bone marrow failure (BMF) is the initial symptom in most pediatric cases.

Eculizumab is a humanized anti-C5 monoclonal antibody functioning as a terminal complement inhibitor. In patients with PNH, it reduces intravascular hemolysis, the need for transfusions and the risk of thrombosis, and improves quality of life.

Hematopoietic Stem Cell Transplant (HSCT) is the only curative option for patients with $\mathrm{PNH}$, and if a suitable matched donor is available, transplant should be considered.

Methods: We present the case of a 13-year-old girl with PNH, who successfully underwent unrelated bone marrow transplantation (uBMT) after receiving treatment with eculizumab.

Results: A previously healthy 13-year-old girl initially presented with a 1-month history of asthenia, pallor and ecchymosis. Blood tests showed pancytopenia (Hemoglobin 5 g/dl, Leukocytes 1.9x10e9/L, Neutrophils 0.5x10e9/L and platelets $8 \times 10 \mathrm{e} 9 / \mathrm{L})$. Bone marrow aspirate and biopsy (BMA+BMB) were performed; the results were compatible with $\mathrm{BMF}$ and were confirmed 3 weeks later with a new BMA and BMB. The study of BMF showed in the flow cytometry a PNH clone in $67 \%$ of neutrophils, $50 \%$ of monocytes and $6 \%$ of erythrocytes. Remaining study results were negative.

The patient did not present hemoglobinuria, thrombosis or impaired renal function, but presented heavy menorrhagia due to thrombocytopenia, and required multiple transfusions ( $\geq 2$ transfusions / week). She started GnRH analogue.

The patient had no HLA-matched related donor, so an HLA-matched unrelated donor was sought. Meanwhile, due to high transfusion requirements, the presence of large $\mathrm{PNH}$ clone $(>50 \%)$, and to avoid the thrombotic and renal complications, we started with a weekly treatment of eculizumab, then moved to every two weeks. After starting eculizumab, the patient became transfusion independent.

A matched unrelated donor was found and, after 6 doses of eculizumab, the patient underwent a uBMT.

A conditioning regimen was used according to EWOGSAA 2010 guidelines, including Cyclophosphamide (3 g/ $\mathrm{m} 2)$, Fludarabine $(120 \mathrm{mg} / \mathrm{m} 2)$ and ATG (10 mg/Kg).
During the transplant, the patient presented the following complications: Pseudomona aeuriginosa septicemia, a Pneumocystis jirovecii pneumonia and grade II aGvHD of the upper digestive tract. All complications were resolved. Platelet and neutrophil engraftment were in day +18 and +22 respectively, and the $\mathrm{PNH}$ clone disappeared.

Currently, 11 months after uBMT, the patient does not present GvHD and PNH clone has not reappeared. Total donor quimerism persists.

Conclusions: Eculizumab given before the HSCT can be effective in preventing thrombotic events and nephropathy, which can compromise the result of the HSCT.

In cases of $\mathrm{PNH}$, if a matched donor is available and the HSCT procedure can be performed in a short time, HSCT is an alternative to the usual immunosuppressive treatment.

Conflict of interest: We have no conflicts of interest to declare.

\section{P732}

ERN TRANSPLANTCHILD, an European strategy to attend "the secondary rare disease" induced by Paediatric Transplantation

Antonio Pérez-Martínez ${ }^{1,2}$, Jaap Jan Boelens ${ }^{3}$, Caroline Lindemans $^{4}$, Marc Bierings ${ }^{4}$, Jelena Rascon ${ }^{5}$, Jacek Toporski ${ }^{6}$, Dominik Turkiewicz ${ }^{7}$, Esteban Frauca ${ }^{8,9}$, Eduardo López Granados $^{9,10}$, Francisco Hernández Oliveros ${ }^{9,11}$, Juan Torres', Marisa Tejedor', José María Muñoz ${ }^{12}$, Ana Coloma ${ }^{9}$, Javier Cobas $^{13}$, Paloma Jara on behalf of Transplantchild ERN ${ }^{14,15}$

${ }^{1}$ Hospital Universitario La Paz, Pediatric Hemato-Oncology and Stem Cell Transplantation, Madrid, Spain; ${ }^{2}$ Hospital La Paz Institute for Health Research' (IdiPAZ), Madrid, Spain; ${ }^{3}$ Wilhelmina Children's Hospital, Pediatric Blood and Marrow Transplantation Program, Utrecht, Netherlands; ${ }^{4}$ Wilhelmina Children's Hospital, Utrecht, Netherlands; ${ }^{5}$ University Hospital Santariskiu Klinikos, Vilna, Lithuania; ${ }^{6}$ Skåne University Hospital, Lund, Sweden; ${ }^{7}$ Skåne University Hospital, Lund, Sweden, Lund, Sweden; ${ }^{8}$ Hospital Universitario La Paz, Hepatology, Madrid, Spain; ${ }^{9}$ La Paz Biomedical Research Institute-IdiPAZ, Madrid, Spain; ${ }^{10}$ Hospital Universitario La Paz, Clinical Immunology, Madrid, Spain, ${ }^{11}$ Hospital Universitario La Paz, Pediatric Surgery, Madrid, Spain; ${ }^{12}$ Hospital Universitario La Paz, Medical Director, Madrid, Spain; ${ }^{13}$ Hospital Universitario La Paz, Madrid, Spain; ${ }^{14}$ La Paz Biomedical Research Institute-IdiPAZ, Transplantchild Coordinator, Madrid, Spain; ${ }^{15}$ Hospital Universitario La Paz, Pediatric Hepatology, Spain

Background: Paediatric transplantation (PT), both hematopoietic stem cell transplantation (HCST) and solid organ (SOT) has greatly improved survival in children with endstage disease, becoming the main treatment options in this population. PT approach requires a multidisciplinary team that supports the transplantation procedure in common processes as immunosuppression, immune reconstitution, rejection, tolerance, risk of infection and psychosocial wellbeing. These areas are common in different types of 
transplants; therefore a holistic procedure with a crosscutting approach will improve the expectancy and long-term quality of life of children and families. Herewith we aim to describe the strategic approach of TRANSplantCHILD to the improvement of life expectancy and quality of life for EU paediatric patients.

Methods: TRANSplantCHILD is an European reference network (ERN) focuses on a low prevalent and complex clinical condition in children, as trasplantation is and includes SOT and HSCT, with a specific 'cross-cutting approach. TRANSplantCHILD aims are:

(1) Ensuring patient and families access through the network to the best possible care practices and support procedures related to a transversal and multidisciplinary approach.

(2) Developing and gathering efforts within the network for integrative, innovative and better procedures, information, training, knowledge and expertise.

(3) Integrating stakeholders in the transplantation process and making available the knowledge and information. As established in the Strategic approach of TRANSplantCHILD, the strategic areas have been approved in order to ensure the achievement of the mission and vision of the Network:

(1) To improve patient healthcare;

(2) To harmonise clinical best practices;

(3) To harmonise research and innovation;

(4) Spread knowledge;

(5) Education and training;

(6) Network organization, quality and safety.

Results: TRANSplantCHILD integrates 18 Healthcare providers from 11 Members states which have proven experience in PT and addressing the related complications in order to gather the knowledge and experience available throughout Europe. The holistic model ensures permanent efforts for the routinely implementation and standardization of the most recent improvements in the transplantation process as a whole (at pre-transplant, transplant and posttransplant stage). The crosscutting approach of TRANSplantCHILD allow the identification of common topics to all transplants such as clinical, personal and socio-economic issues, and The improvement of the transplanted patient handling, their life expectancy and long-term quality of life of children and their family by:

(1) preventive practices to anticipate and minimise patients' risks;

(2) Treatment standardisation;

(3) Harmonisation of clinical best practices;

(4) Improving chronicity approach and the treatment of secondary diseases related to PT;

(5) The provision of psycho-social support in different phases of patients' life;

(6) The provision of grounds for patients' empowerment.
The first case has been recently upload to the network to address a pre-HSCT question in a patient who previously received a deceased liver transplantation.

Conclusions: PT care constitutes yet a medical challenge due to the lack of robust data In comparison with the adult transplantation. TRANSplantCHILD focuses the PT as process approach instead of a disease/organ approach, developing

an integrative model for share within the network the knowledge or expertise from highly specialised support and advice at national healthcare providers for the transplanted children.

Conflict of interest: Nothing to disclose

\section{P733}

Abstract previously published

\section{P734}

Extracorporeal photopheresis for treatment of acute GVHD in children with Beta-Thalassemia Major

Giorgia Guiducci', Javid Gaziev ${ }^{2}$, Francesca Docimo ${ }^{3}$, Katia Paciaroni $^{2}$, Antonella Isgrò ${ }^{2}$, Ardita Aleti ${ }^{1}$, Gaspare Adorno ${ }^{1}$, Alessandro Lanti ${ }^{3}$

${ }^{1}$ Università Tor Vergata, Rome, Italy; ${ }^{2}$ Mediterranean Istitute of Hematology, Rome, Italy; ${ }^{3}$ Policlinico Tor Vergata, Rome, Italy

Background: Allogenic hematopoietic stem cell transplantation is the only potentially curative treatment for patients with major beta-thalassemia. Graft-versus-host disease (GvHD) is a leading cause of post-allogeneic haematopoietic stem cell transplantation (HSCT) morbidity and mortality. Extracorporeal photopheresis (ECP) is a second-line treatment for steroid refractory or dependent GvHD that facilitates the reduction of immunosuppression.

Methods: A seven-year-old boy with class 3 thalassemia major who underwent Allogenic BMT transplantation from UNRELATED DONOR and MAJOR ABO incompatibility, in June 2016. Prophylaxis therapy of GVHD: Cyclosporine + Methylprednisolone + short MTX.

On the +20 day a Mixed Chimerism with $100 \%$ donor cell was identified.

Early complications after BMT were: at day $+6 \mathrm{CMV}$ reactivation, day +14 Hemorrhagic cystitis BKV-related, day +16 Engraftment syndrome with capillary leak and pulmonary distress.

Severe acute GvHD grade IV involving skin and gut evolved, with progression of skin rushes ( $>80 \%$ of skin involvement) itching and fourth degree skin lesions located mainly in the head, therefore Thymoglobulin and mycophenolate mofetil were added to the ongoing 
immunotherapy, CSA Methyl-PDN 2 mg/kg.In September 2016 he had a reactivation of the Intestinal GVHD of grade IV with severe gastrointestinal bleeding, treated with Sandostatin, replacing Defibrotide and Methyl-PDN, up to the remission of the symptomatology when immunotherapy for GVHD was restored with high dose of Methyl-PDN $4 \mathrm{mg} / \mathrm{kg} / \mathrm{day}$, increased up to $4,5 \mathrm{mg} / \mathrm{kg} / \mathrm{die}$ until the moment we started Extracoerporeal Photopheresis.Photochemotherapy was administered 3 times a week for the first month, after which we switched to twice and than once weekly until present day.During the procedures we processed one blood volume at a rate of $10 \mathrm{ml} / \mathrm{min}$, via a central venous catheter. During the whole cycle there have never been adverse events.

Results: During the period from December 2016 to March 2016, GVHD of skin and gastrointestinal visibly decreased, with a liver abnormality documented by abdominal ultrasound that described in December 2016 mild hepatosplenomegaly, with widespread dishomogenity of the liver parenchyma, improved in the subsequent control in March 2017. The ECP was interrupted in September, during which, he had a worsening of cutaneous GVHD for which he increased Methyl-PDN to $1.5 \mathrm{mg} / \mathrm{kg} / \mathrm{day}$. After this episode the patient restarted the photopheresis, once a week, which allowed the current state to reduce the PDN to $0.5 \mathrm{mg} / \mathrm{kg} / \mathrm{day}$, with remission of cutaneous GVHD, showed by the pallor of the skin, and gastrointestinal evidence seen by EGD that showed no signs of bleeding from the stomach and duodenum, and an important thickness of the duodenal mucosa that quickly normalized.(fig.1)

Conclusions: Patients who suffer from acute and chronic GVHD have limited therapeutic treatment. ECP treatment might improve clinical situation of patients suffering from GvHD.

Our experience has shown that ECP remains an important therapeutic option, since during ECP therapy the concurrent immunosuppression could be reduced, without appearance of an increase in opportunistic infection. Furthermore from the beginning of the ECP a clear improvement of the cutaneous symptomatology was evident, after only one month.

Conflict of interest: None

\section{P735}

\section{First use of hematopoetic stem cell transplantation in a patient with Fanconi anemia in Croatia}

Toni Matic ${ }^{1}$, Zrinko Salek $^{1}$, Radovan Vrhovac ${ }^{2}$, Nadira Durakovic ${ }^{2}$, Ruzica Lasan-Trcic ${ }^{3}$, Sanda Basic-Kinda ${ }^{2}$, Dino Dujmovic ${ }^{2}$,Ernest Bilic $^{1}$

${ }^{1}$ University Hospital Center Zagreb, Dep. of Pediatrics, Zagreb,
Croatia; ${ }^{2}$ University Hospital Center Zagreb, Dep. of Internal Medicine, Zagreb, Croatia; ${ }^{3}$ University Hospital Center Zagreb, Dep. of Laboratory Diagnostics, Zagreb, Croatia

Background: Fanconi anaemia (FA) is a rare inherited disorder associated with congenital abnormalities, progressive bone marrow failure (BMF) and a predisposition to develop malignant diseases. The BMF ususaly occurs in the first decade of life. With the characteristic phenotypic appearance and pancytopenia, the main characteristic is an increased risk for development of malignant hematological (MDS, AML) and nonhematological diseases (squamous cell carcinoma of head and neck, skull, oesophagus, vulva, anus and cervix). The incidence increases with age, and by the age of 40 it is $90 \%$ for BMF and $30 \%$ for neoplasms. Mortality rate of the patients with FA is very high with a median age of death of 30 years. Allogenic hematopoietic stem cell transplantation (HSCT) is the only curative option for the haematological diseases of FA.

Methods: Our patient is a boy who was admitted to our clinic at the age of 5 because of pancytopenia. Bone marrow aspiration (BMA) showed moderately decreased cellularity and deletion $7 \mathrm{q} 22$ in $13 \%$ interphase chromosomes. Prolonged pancytopenia, RBC macrocytosis, microcephaly and skin hyperpigmentation raised suspicion of FA. In the culture without fragility stimulation abnormal chromosome fragility was found, and was enhanced by adding diepoxybutane, which confirmed the diagnosis of FA. HSCT was indicated. Since there was no related donor we initiated androgenic steroid therapy which was not beneficial and patient developed signs of precocious puberty. When matched unrelated donor (HLA 10/10) was found, HSCT was performed. Engraftment was successful without GVHD. At day +100 post HSCT BMA was normal without $7 \mathrm{q} 22$ deletion.

Our team decided to follow Consensus of German Transplant Centres on Hematopoietic Stem Cell Transplantation in Fanconi Anemia, to be more precise GEFA03 protocol. Conditioning regimens nowadays avoid total body irradiation and high dose alkylating agents since they are associated with increased risk for later malignancy. Most recent reduced intensity conditioning regimens include low dose fludarabine, cylophosphamide and busulfan, which were also used in our patient according to GEFA03 protocol. In vivo T-cell depleting agents are used to avoid GVHD. Whether one lymphocyte-depleting antibody should be given priority over the other is difficult to say. However immunosuppression with alemtuzumab showed better 5 -year survival rate, better overall survival and lower risk for GVHD compared to ATG. For GVHD prophylaxis alemtuzumab was used with cyclosporine and mycophenolate mofetil. 
Results: The case we are presenting is the first FA patient treated with HSCT in Croatia, and also the first paediatric patient in Croatia in whom alemtuzumab was given, without any complications or side effects.

Conclusions: Our patient is now 6 months after HSCT, with good engraftment and without complications. Despite improved outcomes for patients with FA post-HSCT in recent years, long term survival is still affected with secondary malignancies (of which $89 \%$ are solid tumours). Risk factors for development of secondary malignancies (older age, peripheral stem cells transplant and chronic GVHD) have so far not been present in our patient, but long term regular monitoring with emphasis on early recognition of possible secondary malignancy is warranted.

Conflict of interest: None of the authors has anything to disclose.

\section{P736}

Abstract previously published

\section{P737}

GST-M1 and -T1 null polymorphisms are associated with lower event-free survival and higher rate of relapse in children with malignancies undergoing allogeneic HSCT

Simona Jurkovic Mlakar ${ }^{1}$, Chakradhara Rao S Uppugunduri ${ }^{1}$, Vid Mlakar $^{1}$, Med Aziz Rezgui ${ }^{2}$, Fannete Bernard ${ }^{3}$, Laurence Lesne ${ }^{1}$, Denis Marino ${ }^{1}$, Michel Duval ${ }^{4}$, Rodolfo Lo Piccolo ${ }^{3}$, Yves Chalandon $^{5}$, Lee Dupuis ${ }^{6}$, Tal Schechter ${ }^{7}$, Imke H. Bartelink ${ }^{8}$, Jaap Jan Boelens ${ }^{8}$, Robbert Bredius ${ }^{9}$, Jean Hugues Dalle ${ }^{10}$, Christina Peters $^{11}$, Peter Bader ${ }^{12}$, Henrique Bittencourt ${ }^{4}$, Maja Krajinovic ${ }^{2}$, Marc Ansari ${ }^{1}$

${ }^{1}$ CANSEARCH Research Laboratory, University of Geneva, Faculty of Medicine, Pediatric Oncology and Hematology Unit, Department of Pediatrics, Geneva, Switzerland; ${ }^{2}$ Charles-Bruneau Cancer Center, CHU Sainte-Justine Research Center, Hematology-Oncology Division, Montreal, Canada; ${ }^{3}$ Onco-Hematology Unit, Geneva University Hospital, Department of Pediatrics, Geneva, Switzerland; ${ }^{4}$ Faculty of Medicine, University of Montreal, Department of Pediatrics, Montreal, Canada; ${ }^{5}$ Division of Hematology, Geneva University Hospital, Department of Medical Specialties, Geneva, Switzerland; ${ }^{6}$ The Hospital for Sick Children, Leslie Dan Faculty of Pharmacy, University of Toronto, Department of Pharmacy, Toronto, Canada; ${ }^{7}$ The Hospital for Sick Children, Blood and Marrow Transplant Unit, Department of Haematology/Oncology, Toronto, Canada; ${ }^{8}$ University Medical Center Utrecht, Pediatric Blood and Marrow Transplantation Program, Utrecht, Netherlands; ${ }^{9}$ Leiden University Medical Center, Department of Pediatrics, Center of Infectious Diseases, Leiden, Netherlands; ${ }^{10}$ Robert Debré Hospital, Pediatric Immuno-Hematology Unit, Paris, France; " 1 St Anna Children's Hospital, Department of Pediatrics, Stem Cell Transplantation Unit, Vienna, Austria;
${ }^{12}$ University Hospital Frankfurt, Division for Stem Cell Transplantation and Immunology, Frankfurt, Germany

Background: On Behalf of the Pediatric Disease Working Party of the European Society for Blood and Marrow Transplantation.

Busulfan and Cyclophosphamide (BU/CY) is the conditioning regimen most widely used in allogeneic hematopoietic stem-cell transplantation (HSCT) in children. BU is conjugated with glutathione by glutathione S-transferase enzymes (GSTs), following oxidation by cytochromes P450 (CYPs), cystathionine gamma-lyase (CTH) and flavincontaining monooxygenases (FMOs). $\mathrm{CY}$ is bioactivated into 4-hydroxy-cyclophosphamide (4-OH-CY) through cytochrome $\mathrm{P} 450$ enzymes and its active metabolites are eliminated by aldehyde dehydrogenase (ALDH3A1) and GSTs. The aim of this study was to evaluate the association of genetic polymorphisms of main BU/CY - related metabolizing enzymes and transporters with event-free survival (EFS) and relapse in children with malignancies undergoing HSCT in order to personalize treatment efficiency.

Methods: In this retrospective study, association of common genetic variants in BU/CY metabolizing enzyme genes with EFS and relapse post HSCT was assessed individually or in combination in 86 pediatric patients with malignancies by using both univariate and multivariate analysis. In this study we also explored the role of GSTMI and $-T 1$ in determining the cellular sensitivity to BU. Halfmaximal inhibitory concentration (IC50) for BU to inhibit cell viability was screened in 56 different lymphoblastoid cell lines (LCLs). BU-induced activation of cellular apoptosis and necrosis was screened in 20 LCLs, grouped on the basis of GSTM1- and GSTT1-null genotypes. Activation of various cellular pathways, leading to cell death, were assessed in relation to the GSTM1/GSTT1 genotypes in LCLs.

Results: We observed the significant reduction in EFS and increased incidence of relapse in GSTM1/GSTT1-null/ null carriers compared to both or either non null carriers ( $88.9 \%$ vs $40.3 \%, \mathrm{p}<0.001$ and $44,4 \%$ vs $15,6 \%$, p < 0.001, respectively). We observed for $38.9 \%$ higher BUIC50 values in LCLs carrying GSTM1-null/null alleles compared to GSTM1-non null carriers ( $\mathrm{p}=0.013, \mathrm{~N}=56$ ). Interestingly, ratios between necrosis and early apoptosis after the induction with BU were significantly lower in carriers with GSTM1-null/null and combined GSTM1/ GSTT1-null/null ( $\mathrm{p}=0.015$ and 0.031 , respectively), suggesting slower progression rate of GSTM1/GSTT1-null carriers through apoptosis. This observation was further confirmed in real time experiments with measurements from $0 \mathrm{~h}$ to $72 \mathrm{~h}$. Apoptosis triggered by $\mathrm{BU}$ was primarily due to 
caspases 3 and 7 activation and involvement of caspase 1 activation was ruled out. Total GST activity, autophagy, ROS production and mitochondrial membrane potential showed no significant difference when stratified to GSTMI/ GSTT1-null genotypes, however GSH levels were significantly higher in GSTM1/GSTT1-non null carriers $(\mathrm{p}<0.01)$ suggesting the potential compensatory mechanism in conjugating process of BU in LCLs.

Conclusions: Our results indicate that the combined GSTM1 and GSTT1 null carrier status was associated with reduced event-free survival and increased incidence of relapse in children undergoing allogeneic HSCT possibly through their influence on cellular survival.

Clinical Trial Registry: No Clinicaltrials.gov identifier: NCT01257854.

Website: https:/clinicaltrials.gov/ct2/show/results/ NCT01257854

Conflict of interest: None of the authors has anything to disclose.

\section{P738}

GvHD prophylaxis with post-transplant cyclophosphamide is associated with earlier immune reconstitution as compared to ATG or Campath

Herbert Pichler ${ }^{1}$, Evgenia Glogova ${ }^{2}$, Volker Witt ${ }^{1}$, Anita Lawitschka $^{1}$, Wolfgang Holter ${ }^{1}$, Rene Geyeregger ${ }^{2}$, Gerhard Fritsch $^{2}$, Susanne Matthes-Martin ${ }^{1}$

${ }^{1}$ Pediatric Hematology and Oncology, St Anna Children's Hospital, Department of Pediatrics, Medical University of Vienna, Vienna, Austria; ${ }^{2}$ Children's Cancer Research Institute (CCRI), St. Anna Kinderkrebsforschung, Vienna, Austria

Background: Serotherapy with anti-thymocyte globulin (ATG) or Campath has a significant impact on immune reconstitution after haematopoietic stem cell transplantation (HSCT).

Methods: We retrospectively evaluated the impact of GvHD prophylaxis (serotherapy versus post-transplant cyclophosphamide) in children and adolescents receiving a uniform reduced intensity (RIC) conditioning regimen. The reconstitution of $\mathrm{CD} 3^{+} \mathrm{CD} 4^{+}$or $\mathrm{CD} 3^{+} \mathrm{CD} 8^{+}$T-cells, naïve $\mathrm{T}$-cell subsets and $\mathrm{CD} 3^{-} \mathrm{CD} 16^{+} \mathrm{CD} 56^{+} \mathrm{NK}$-cells as detected by FACS analysis on day +50 and +100 was evaluated in 40 consecutive children undergoing HSCT for malignant and non-malignant diseases following a RIC regimen consisting of Fludarabine $160 \mathrm{mg} / \mathrm{m} 2$, Thiotepa $10 \mathrm{mg} / \mathrm{kg}$ and Melphalan $140 \mathrm{mg} / \mathrm{m} 2$. The GvHDprophylaxis consisted of ATG in 26 and Campath in one patient (Group 1), and post-transplant Cyclophosphamide in 13 patients (Group 2). Furthermore, both groups were analysed for Cytomegalovirus (CMV), Adenovirus (AdV) viremia and the frequency of acute GvHD.

The median patient age was 5.4 years. Underlying diseases were leukaemias or solid tumors in 11 and nonmalignant diseases in 29 patients. Twenty-nine patients received bone marrow and 11 peripheral-blood stem cells. Twelve patients were grafted from HLA-matched siblings, 20 from matched unrelated donors and nine patients received transplants from haploidentical donors. Clinical characteristics such as the diagnosis, gender, recipient age and donor type or graft source were equally distributed within the two groups.

Results: As shown in Table 1 below, the detected median T- and NK cell counts generally were higher among patients who received a GvHD prophylaxis with post-transplant cyclophosphamide (Group 2), and reached statistical significance for NK cells on day $+50(p=0.034)$. Similarly, we found higher $\mathrm{CD}^{+}{ }^{+} \mathrm{T}$-cell numbers on day +100 in Group $2(\mathrm{p}=0.039)$. The recovery of naïve $\mathrm{CD} 4^{+}$and CD8 ${ }^{+}$T-cell subsets did not differ between both groups.

CMV viremia was detected among 14/27 patients who received serotherapy (Group 1) and in four/13 patients after post-transplant cyclophosphamide (Group 2). AdV viremia (2/27) was only observed in Group 1. However, this difference was not statistically significant.

The GvHD rates were comparable in both groups: $22 \%$ (Group 1) vs. 23\% (Group 2) for any acute GvHD and 11\% vs $8 \%$ for acute GvHD Grade 2 or higher.

Conclusions: Post-transplant cyclophosphamide-based GvHD prophylaxis as compared to serotherapy after Fludarabin-based RIC conditioning in children is associated with enhanced immune reconstitution of the NK- and CD4 ${ }^{+}$ T-cell compartment, which in turn might reduce the risk for virus reactivations.

Conflict of interest: The authors have nothing to disclose.

\begin{tabular}{llll}
\hline Parameter & Group 1 (n or\%) & Group 2 (n or\%) & $\begin{array}{l}\text { p- } \\
\text { value }\end{array}$ \\
\hline Median NK/microL day+50 & 156 & 332,5 & 0,034 \\
Median NK/microL day+100 & 105 & 205 & 0,062 \\
Median CD4+/microL day+50 & 80 & 137,5 & 0,063 \\
Median CD4+/microL day+100 & 125 & 185 & 0,039 \\
Median CD8+/microL day+50 & 195 & 320 & 0,198 \\
Median CD8+/microL day+100 & 250 & 360 & 0,284 \\
CMV Viremia & $52 \%$ & $31 \%$ & 0,148 \\
AdV Viremia & $7 \%$ & $0 \%$ & 0,073 \\
\hline
\end{tabular}

[[P738 Table] Table 1] 


\section{P739}

\section{Haemopoetic stem cell transplantation is a curative treatment for severe Glanzmann's thrombasthenia: Experience in Leeds Paediatric Stem Cell Transplant Unit}

\section{Charlotte Crone, Beki James}

Leeds Children's Hospital, Paediatric Haematology and Oncology Services, Leeds, United Kingdom

Background: Glanzmann's thrombasthenia is a rare autosomal recessive bleeding disorder of platelet function caused by a qualitative or quantitative defect of the platelet membrane glycoprotein IIb/IIIa. For patients with a severe form of the condition haemopoietic stem cell transplantation (HSCT) is the only curative option. Since 1985 there have been 19 allogeneic stem cell transplants reported in 18 patients with Glanzmann's thrombasthenia. We report two cases of patients with severe Glanzmann's thrombasthenia with anti-platelet antibodies who had a successful allogeneic HSCT in our centre.

Methods:

Patient 1 is a 13 year old boy who underwent a HSCT after he suffered an intracerebral bleed.

Patient 2 is a 11 year old girl who had a HSCT following increased freqency of episodes of severe bleeding.

Stem cell source:

Patient 1 received paternal haploidentical alpha/beta $\mathrm{T}$ lymphocyte depleted G-CSF mobilised peripheral blood stem cells.

Patient 2 received 10/10 HLA matched sibling bone marrow.

Conditioning:

Conditioning regimes of a combination of Fludarabine, Thiotepa and Treosulfan and ATG were used in both cases:

Patient 1: ATG $2.5 \mathrm{mg} / \mathrm{kg}$ for 3 days (day -10 to -8 ); Fludarabine $30 \mathrm{mg} / \mathrm{m} 2$ for 5 days (day -7 to -3 ); Treosulfan $14 \mathrm{~g} / \mathrm{m} 2$ for 3 days (day -6 to -4 ) and Thiotepa $5 \mathrm{mg} / \mathrm{m} 2$ bd for 1 day (day -4).

Patient 2: Thiotepa $5 \mathrm{mg} / \mathrm{m} 2$ bd for 1 day (day -7); Treosulfan for 3 days (day -6 to -4 ); Fludarabine $40 \mathrm{mg} / \mathrm{m} 2$ for 4 days (day -6 to -3 ); Treosulfan for 3 days (day -6 to $-4)$; ATG $2.5 \mathrm{mg} / \mathrm{m} 2$ for 3 days (day-4 to day -2 )

\section{GvHD prophylaxis:}

Patient 1: Ciclosporin $1.5 \mathrm{mg} / \mathrm{kg}$ bd from day -1 onwards and Methotrexate $15 \mathrm{mg} / \mathrm{m} 2$ on day $+1,10 \mathrm{mg} / \mathrm{m} 2$ on day +3 , day +6 and day +11 . Ciclosporin was later switched to Tacrolimus.

Patient 2: Ciclosporin $1.5 \mathrm{mg} / \mathrm{kg}$ bd from day -1 onwards and Mycophenolate Mofetil $15 \mathrm{mg} / \mathrm{kg}$ bd from day -3 to day +35 . Ciclosporin was later switched to Tacrolimus.
Immunosuppression was weaned at 6 months post HSCT in both patients.

Results:

Engraftment:

Patient 1 engrafted neutrophils at day +13 and platelets at day +11 .

Patient 2 engrafted neutrophils at day +16 and platelets at day +13 .

Chimerism:

In both patients, $100 \%$ donor chimerism on all cell fractions at day 28 and on all subsequent tests up to 6 months post HSCT.

Transplant related morbidity:

Patient 1: Mild gut GvHD which responded to oral prednisolone and budesonide, and switching of immunosuppression from Ciclosporin to Tacrolimus. He also had CMV reactivation which was treated with intravenous Ganciclovir and oral Valganciclovir.

Patient 2: Ciclosporin induced gum hypertrophy and hirsuitism which resolved following switching of Ciclosporin to Tacrolimus.

At the 2 year and 18 month follow up point respectively, both patients have no symptoms of Glanzmann's thrombasthenia and no residual complications from HSCT.

Conclusions: Both these cases demonstrate that HSCT can be a curative treatment option with minimal transplant related complications, rapid engraftment, and short duration of neutropenia for patients with severe Glanzmann's thrombasthenia.

Conflict of interest: [Dr Charlotte Crone]: nothing to disclose

\section{P740}

Haploidentical hematopoietic stem cell transplantation experience in children in France: a retrospective study from the SFGM-TC

Claire Freycon ${ }^{1}$, Benedicte Neven ${ }^{2}$, Blanche Stephane ${ }^{2}$, Karima Yacouben $^{3}$, Yves Bertrand ${ }^{4}$, Gerard Michel ${ }^{5}$, Claire Galambrun ${ }^{5}$, Patrick Lutz ${ }^{6}$, Catherine Paillard ${ }^{7}$, Justina Kanold ${ }^{8}$, Anne Sirvent ${ }^{9}$, Gerard Socie $^{10}$, Charlotte Jubert ${ }^{11}$, Benedicte Bruno ${ }^{12}$, Mohamad Mohty $^{13}$, Marie Therese Rubio ${ }^{14}$, Dominique Plantaz ${ }^{1}$

${ }^{1}$ Grenoble University Hospital, Department of Pediatric OncoHematology, Grenoble, France; ${ }^{2}$ Necker Enfants Malades University Hospital, Assistance Publique-Hôpitaux de Paris, Pediatric ImmunoHaematology and Rheumatology Unit, Paris, France; ${ }^{3}$ Robert Debré Hospital, APHP, Hematology Department, Paris, France; ${ }^{4}$ University Hospital of Lyon, Department of Pediatric Hematology and Oncology, Lyon, France; ${ }^{5}$ Timone Hospital, Department of Pediatric Hematology and Oncology, Marseille, France, ${ }^{6}$ University Hospital, Strasbourg, Department of Pediatric Hematology-Oncology, Strasbourg, France;

${ }^{7}$ University Hospital Strasbourg, Department of Pediatric

Hematology-Oncology, Strasbourg, France; ${ }^{8}$ Children University Hospital, Department of Hematology-Oncology, Clermont Ferrand, 
France; ${ }^{9}$ University Hospital, Pediatric Hematology and Oncology Department, Montpellier, France; ${ }^{10}$ Paris-St-Louis Hospital, ParisDiderot University, Hematology Department, Paris, France;

${ }^{11}$ Bordeaux-Hospital, Bordeaux University, Pediatric Hematology Department, Bordeaux, France; ${ }^{12}$ Jeanne de Flandre Hospital, Lille Nord de France University, Department of Pediatric Hematology, Lille, France; ${ }^{13}$ Hopital Saint Antoine, Hematology Department, Paris, France; ${ }^{14}$ Saint Antoine Hospital, AP-HP, Hematology and Cellular Therapy Service, Paris, France

Background: The aim of this study was to perform a review of haplo-hematopoietic stem cell transplantation pediatric experience in France between 2005 and 2016

Methods: This epidemiologic observational multicenter descriptive and retrospective study was based on data of the French section of ProMISe register of EBMT

Results: A total of 145 patients were included for having received a haplo-HSCT from January 2005 to June 2016. The median age of patients was 0.3 (0.04-18.6) years old. The main diagnosis were primary immune deficiency (48\%), hemophagocytic lymphohistiocytosis (14\%) and bone marrow failure (12\%). 112 patients (77\%) received an ex-vivo manipulated graft while 9 patients received posttransplant high-dose Cyclophosphamide. A preparatory regimen was given in $99 \%$ of patients including $67 \%$ of myeloablative. The median follow-up was 354 (0-3692) days. Durable hematopoietic reconstitution was seen in $66 \%$ of patients. Neutrophil and platelet engraftment was achieved with median delay of 20 and 23 days. The cumulative incidences of grade II to IV acute graft-versushost disease at day 100 (mainly skin aGVHD) and chronic GvHD at one year were $16 \%$ and $8 \%$, respectively. Infectious complications were diagnosed in 96 patients $(66 \%)$ including $51 \%$ of viral infections. The incidence of relapse or progression was $13 \%$. One-year overall survival (OS) rate was $70 \%$ and one year transplant related mortality was $26 \%$.

Conclusions: Haplo-HSCT is an interesting alternative in patients lacking an HLA matched donor. Prolonged follow up and prospective studies will be necessary to assess the role of haplo-HSCT in children and to establish guidelines for their management

Conflict of interest: No conflict of interest.

\section{P741}

\section{haploidentical peripheral blood stem cell transplants with drug-free GVHD prophylaxis in paediatric patients with acute leukaemia}

Poh Lin Tan ${ }^{1,2,3}$, Mariflor $S$ Villegas ${ }^{2,3}$, Teck Guan Soh ${ }^{4}$, Allen Eng

${ }^{1}$ National University of Singapore, Paediatrics, Singapore, Singapore;

${ }^{2}$ National University Health System, Khoo Teck Puat - National
University Children's Medical Institute, Singapore, Singapore; ${ }^{3}$ National University Health System, National University Cancer Institute, Singapore, Singapore; ${ }^{4}$ National University Health System, Laboratory Medicine, Singapore, Singapore

Background: In paediatric haploidentical peripheral blood stem cell transplantation (PBSCT) using ex-vivo T cell depletion (TCD) with negative selection approaches such as CD3/CD19 or TCRab depletion, reported acute and chronic GVHD rates ranges between 30 to $46 \%$ and 5 to $21 \%$, respectively. In addition to the TCD, majority of these patients receive additional GVHD prophylaxis which could include pre-transplant ATG and/ or post-transplant mycophenolate motfetil (MMF) with a calcineurin inhibitor or FK506. A disadvantage of post-transplant immunosuppression in TCD setting is delayed immune reconstitution and resultant infective and relapse risks. A novel graft engineering technique that depletes naïve $\mathrm{T}$ cells and retains functional pathogen-specific memory $\mathrm{T}$ cells used in adult patients with acute leukaemia receiving HLA matched sibling donor PBSCT resulted in excellent outcome. With FK506 as GVHD prophylaxis, 66\% and 9\% of patients developed acute (grade I to III) and chronic GVHD, respectively (Bleakley 2015). When used in the setting of HLA haploidentical PBSCT in 17 paediatric patients with leukaemia, additional GVHD prophylaxis included Sirolimus or MMF. Grade III/IV acute and chronic GVHD occurred in $17.6 \%$ and $35 \%$ of patients, respectively (Triplett 2015).

Methods: Between 2014 - 2016, we treated 18 consecutive paediatric patients with acute leukaemia (lymphoid, 10; myeloid, 6; mixed, 2) using similar approach with naïve $\mathrm{T}$ cell depleted haploidentical PBSCT without additional drug prophylaxis against GVHD in an attempt to improve quality of life and overall outcome. Stem cell grafts were either $\mathrm{CD} 34+$ enriched $(\mathrm{N}=3)$ or $\mathrm{CD} 3$ + depleted $(\mathrm{N}=15)$.

Results: Median age was 7.7 (range, 0.8 to 15.4) years with 11 in CR1, 4 in CR2 and 2 not in CR at time of PBSCT. Preparative regimens were either TLI/ Thiotepa $(\mathrm{N}=14)$ or Treosulfan based. No GCSF was given. Median TNC, CD34+, CD3+/CD45RO+ were 21.7 (range, 7.7 65) $\times 10^{8} / \mathrm{kg}, 21.4$ (range, $9.8-43$ ) x 10 $/ \mathrm{kg}, 6.72$ (range, 0.48 - 330) x $10^{6} / \mathrm{kg}$, respectively. Myeloid and platelet engraftment occurred at a median of 12 (range, $10-26$ ) and 13 (range, 10 - 29) days, respectively. Secondary graft rejection rescued with an alternate donor and progressive leukaemia was noted in 1 and 2 patients, respectively. Cytomegalovirus and adenovirus DNAemia without disease was noted in $40 \%$ and $12 \%$ of patients, respectively. There was no EBV or fungal infections. Acute GVHD grade II-III occurred in 50\%. No grade IV acute GVHD was seen. De novo, limited chronic GVHD occurred in $28 \%$ of patients. 
Extensive chronic GVHD was seen only in patients (16\%) who received repeated dosing of DLI. GVHD were steroid responsive in all. In 8 evaluable patients, median duration of post-transplant immunosuppression was 121 (range, 79 333) days. At a median follow-up of 346 (range, 55 - 878) days, 16 of 18 patients were alive in remission.

Conclusions: Our haploidentical PBSCT with drug-free GVHD prophylaxis in paediatric patients with acute leukaemia is safe with no grade IV acute GVHD and provided an overall survival of $88 \%$.

Clinical Trial Registry: NA

Conflict of interest: None of the authors has anything to disclose

\section{P742}

\section{Haploidentical stem cell transplant with post- transplantation cyclophosphamide and mini- dose methotrexate in children}

Diego Medina Valencia ${ }^{1}$, Mayra Estacio Benavides ${ }^{2}$, Maria Cecilia Rosales Garcia ${ }^{1}$, Eliana Manzi ${ }^{2}$

${ }^{1}$ Fundacion Valle del Lili, Stem Cell Transplant, Cali, Colombia;

${ }^{2}$ Fundacion Valle del Lili, Clinical Research Center, Cali, Colombia

Background: Haploidentical stem cell transplantation (haplo-SCT) is an option for patients without HLA-matched related or unrelated donor. Post-transplantation cyclophosphamide (PTCy) is an effective method of graft versus host disease (GVHD) prophylaxis and permits the use of T-cell replete grafts in settings were ex-vivo manipulation is not available.

Methods: We retrospectively reviewed all pediatric with hematologic malignancies who underwent haplo-SCT, between years 2012 and 2016. Patients received a preparative regimen of fludarabine, busulfan, and 400cgy total body irradiation or melphalan. GvHD prophylaxis consisted in PTCy $(50 \mathrm{mgr} / \mathrm{Kg}$ on days +3 and +4$)$ and cyclosporine (CSA) plus mycophenolate (MMF) $(15 \mathrm{mg} / \mathrm{kg} /$ dose TID, PO) or mini- dose methotrexate $\left(5 \mathrm{mg} / \mathrm{m}^{2}\right.$ dose $)$ (MTX) from day $+5,+7,+10$ and +15 .

Results: A total of 52 children were enrolled, median age, 9 years(IQR 4.9-14; range, 1.2-17 years), 63\% were males, the Indication for HSCT was acute lymphoblastic leukemia (ALL) 32(61\%), acute myeloid leukemia (AML) 13 (25\%), myelodysplastic síndrome (MDS) 4 (8\%), chronic myeloid leukemia (CML) 1(2\%), Non Hodgkin's lymphoma (NHL) $1(2 \%)$, Hodgkin's lymphoma $1(2 \%)$. The disease status in patients with acute leukemia $(\mathrm{n}=45)$ was 19 patients had SCT in first complete remission, 22 in second or more remission and 4 with active disease. The median donor age was 22 years; IQR (11-30; range, 3-52 years). The donor source was bone marrow for $31(60 \%)$ patients and peripheral blood stem cells (PBSCs) for $21(40 \%)$ patients. The most common donor was the father $19(36 \%)$, followed by a sibling $18(35 \%)$, mother $15(29 \%) .35(67 \%)$ of the donors were $\mathrm{ABO}$ Compatible, minor $\mathrm{ABO}$-mismatched 9 $(17.3 \%)$, major ABO-mismatched 5 (9.6\%). The median CD34+ cell dose was $6.4 \times 10^{6}$ cells $/ \mathrm{kg}$ body weight (IQR 3.5-11.2; range 1.4- $18.5 \times 10^{6}$ ). The median time to engraftment of neutrophils 16 days (range 12-29), the median time to engraftment of platelets 16 days (range 1043). As complications, we reported $36 \%$ of hemorrhagic cystitis, $57 \%$ of cytomegalovirus (CMV) reactivation, $13 \%$ CMV disease, One patient had primary graft failure. For aGVHD prophylaxis $42(81 \%)$ patients received PTCy, CSA, and mini-dose MTX; 10 (19\%) patients received PTCy, CSA, and MMF. Patients who surviving less than 28 days and who with graft failure were excluded from GVHD analysis $(n=47)$. The cumulative incidence of acute GvHD II-IV, acute GvHD III-IV, and chronic GvHD were $42 \%, 8.5 \%, 19 \%$ respectively. Grade I to IV aGvHD occurred in $100 \%$ of the patients who received prophylaxis with CSA and MFM and $62 \%$ who received CSA and minidose MTX. Transplant related mortality (TRM) at 100 days was $18 \%$. 5-year overall and event-free survival are 59\% and $57 \%$, respectively.

Conclusions: The haplo-SCT with PT/Cy can be an available, safe, and feasible option for children with hematologic malignancies, the use of mini-dose of methotrexate was associated with lower rates of acute GVHD. However, our results require further support from prospective randomized studies to improve the efficacy of this prophylaxis strategy.

Conflict of interest: We have no conflict of interest to declare.

\section{P743}

Haploidentical Stem Cell transplantation with PostTransplant Cyclochosphamide in children: prolonged single center experience

Julia Bazaluk, Oleg Ryzhak, Yana Kovgan, Natalia Kavardakova, Sophia Matsibokh, Oxana Leontyeva, Alyona Zinchuk, Igor Kireiev, Olena Kreminska, Tatyana Ponomarenko, Tamara Smolenska, Olexandra Sharamok, Svitlana Donska

BMT-Department and Reference-Laboratory for Diagnostic of Oncohematological Diseases in Children, Center of Pediatric Oncohematology and BMT, National Children`s Specialized Hospital 'Okhmatdyt', Kyiv, Ukraine

Background: Patients with high-risk hematological malignancies have a poor prognosis without hematopoetic stem cell transplant. Haploidentical SCT is a potential cure for patients without HLA-identical donors. The experience 
of haplo-SCT with post-transplant HD-Cyclophosphamide in the pediatric population is still limited.

Methods: From April 2015 to December 2017 totally 14 pts (1-16 y) with different malignancies (Table.) underwent haploidentical HSCT with post-transplant HD-Cy. 4 patients at the moment are alive, but they are in early posttransplant period ( $<100$ days after transplantation) so we report about other 10 patients. The different reduced toxycity conditioning regimens were used (Table.). All patients received high-dose $\mathrm{Cy}(50 \mathrm{mg} / \mathrm{kg})$ on days +3 and +4. Cyclosporine A (CyA; 3mg/kg/d i.v., then $6 \mathrm{mg} / \mathrm{kg} / \mathrm{d} \mathrm{p}$. o. adjusting for blood levels 200-400 ng/ml) and mycophenolate mofetil (MMF; $15 \mathrm{mg} / \mathrm{kg}$ two times daily per os) were started on day +5 . MMF was discontinued on day +35 . All patients received anti-microbial prophylaxis for bacterias, fungals, herpes viruses infection and Pneumocystis jiroveci according to institutional practices. Firstly chimerism in PB and BM was controlled at day +30 , then at days +60 , +100 , +180 . Patient, donor and stem-cell harvest characteristics are described in Table.

5 patients underwent transplant in $1^{\text {st }} \mathrm{CR}$ (high risk AML, AHL, sNHL). All other patients had $>1{ }^{\text {st }}$ CR before SCT.

Table. Patient, donor and harvest characteristics

Variable $\mathrm{N}=10$

Age, median(range in years) 11 (2-15)

Gender, $\mathbf{n}$

Male, 6

Female, 4

Diagnosis, $n$

ALL IICR, 1

ALL III CR, 1

AML I CR,3

AML II CR, 2

$\mathrm{NB}, 1$

NHL, 1

AHL, 1

Conditioning regimen, $\mathbf{n}$

Flu 150mg/m2 + Treo 36g/m2+Mel140 mg/m2, 1

Flu 150mg/m2 + ARA-C 8000 mg/m2+Mel 180 mg/m2, 1

FLAME- Flu 150 mg/m2+CCNU 300mg/m2(p.o.)+ ARA- C 4000 mg/m2+ VP-16 1000 mg/m2+Mel 140 $\mathrm{mg} / \mathrm{m} 2,8$

Donor, $\mathbf{n}$

Father, 5

Mother, 3

Sibling, 2

Stem cells source

Peripheral blood, 7

Bone marrow,3

Results: $1 / 10$ pts died before engraftment at day +20 (fulminant Ps. Aeruginosa sepsis).

$2 / 10$ pts died because relapse after day +100 .
$1 / 10$ pt was LFU at day 250.

$7 / 10$ pts achieved full engraftment. $1 / 10 \mathrm{pt}$ had no neutrophil and platelet engraftment by day +30 , despite of complete donor chimerism. He was transplanted additionally with the same donor at day +49 after the transplant. 1/ $10 \mathrm{pt}$ achieved only 2-lineas engraftment by day +100 , despite of complete donor chimerism.

$6 / 10$ pts at $15^{\text {th }}$ of Dec., 2017 remain alive with median follow-up 346 days (range 239-539 days).

4/10 pts had grade 2 aGVHD; 2 of them have cGVHD.

1 pt with T-ALL relapsed at day +194 now is under salvage-CT.

1 pt had secondary MDS (at day +154) and was transplanted again from the same donor.

Conclusions: HLA-haploidentical HSCT with posttransplant T-cell in vivo depletion using high-dose cyclophosphamide is feasible in children and adolescents.

Conflict of interest: None

\section{P744}

Haploidentical transplantation in high risk pediatric leukaemia: a retrospective analysis of the Spanish Working Party for Blood and Marrow Transplantation in Children (GETMON/GETH)

Antonio Pérez-Martínez ${ }^{1}$, Antonia Pascual ${ }^{2}$, Marta GonzalezVicent ${ }^{3}$, Laura Alonso ${ }^{4}$, Isabel Badell, Jose María Fernández Navarro $^{6}$, Alexandra Regueiro ${ }^{7}$, Mercedes Plaza ${ }^{8}$, Jose María Pérez Hurtado $^{9}$, Ana Benito ${ }^{10}$, Cristina Beléndez ${ }^{11}$, José Miguel Couselo ${ }^{7}$, José Luis Fuster ${ }^{8}$, Ana Sastre ${ }^{1}$, Julia Marsal ${ }^{2}$, Luisa Sisinni, Cristina Díaz de Heredia ${ }^{4}$, Miguel Ángel Díaz,

${ }^{1}$ Hospital La Paz, Madrid, Spain; ${ }^{2}$ Hospital Carlos Haya, Malaga, Spain; ${ }^{3}$ Hospital Niño Jesus, Madrid, Spain; ${ }^{4}$ Hospital Vall d'Hebron, Barcelona, Spain; ${ }^{5}$ Hospital Santa Creu I Sant Pau, Barcelona, Spain; ${ }^{6}$ Hospital La Fe, Valencia, Spain; ${ }^{7}$ Hospital Santiago de Compostela, Santiago de Compostela, Spain; ${ }^{8}$ Hospital Virgen de la Arreixaca, Murcia, Spain; ${ }^{9}$ Hospital Virgen del Rocio, Sevilla, Spain; ${ }^{10}$ Hospital de Salamanca, Salamanca, Spain; ${ }^{11}$ Hospiital Gregorio Marañón, Madrid, Spain; ${ }^{12}$ Hospital Sant Joan de Déu, Barcelona, Spain

Background: Haploidentical hematopoietic stem cell transplantation (haplo-HSCT) has emerged as an alternative transplant strategy for patients without HLA match related donors or without time to wait for a HLA match unrelated donor. However, graft failure, graft versus host disease (GvHD) and delay immune recovery are major concerns.

Methods: We report a retrospective analysis of 193 consecutive children and adolescents with high-risk haematological malignancies, who received a haplo-HSCT using post-transplantation cyclophosphamide $(\mathrm{n}=42)$ or ex vivo T-cell depletion $(\mathrm{n}=151)$ as graft versus host disease prophylaxis between January 1999 to December 2016, in 11 centers in Spain. 
Results: A total of 119 (62\%) children were male. The median age at transplant was 8.6 years (0.4-19.7). The underlying disease was acute lymphoblastic leukaemia $(55 \%)$, acute myeloid leukaemia (32\%), myelodysplastic syndrome $(6 \%)$, myelopoliferative syndrome $(5 \%)$ and biphenotipyc leukaemia (2\%). Donors were: mother 109 , father 69 , brother 5 and sister 10 . Stem cell source was: peripheral blood stem cells, 184 and bone marrow 9. Median CD34 dose was $6 \times 10^{6} / \mathrm{kg}(0.4-41)$. One hundred sixty-five $(87 \%)$ of the 193 patients engrafted. The cumulative incidence rate of severe acute GVHD was $19 \%$, and the 2-year cumulative incidence of total chronic GVHD was $46 \%$. Trasplant related mortality at day 100 was $11 \%$. Two-year estimated relapse probability was $36 \%$. Of the 193 patients, 114 survived throughout the follow-up period of 623 days (range: 23-4739 days) with the 2-year overall survival rates of $54 \%$. No statistical difference was found between post-transplantation cyclophosphamide or ex vivo $\mathrm{T}$ cell depletion. Univariate analysis showed lower overall survival in lymphoid malignancies $(\mathrm{P}=0.01)$, previous HSCT $(\mathrm{P}=0.001)$, positive minimal residual disease $(\mathrm{P}=.001)$, and KIR A haplotype donor $(\mathrm{P}=$ $.002)$. Univariate analysis showed higher probability of relapse in lymphoid malignancies $(\mathrm{P}=0.05)$, patients with positive minimal residual disease $(\mathrm{P}=.000)$, and KIR A haplotype donor $(\mathrm{P}=.001)$. Multivariate analysis showed higher overall survival in myeloid diseases $(\mathrm{HR}=0,439)$ and KIR B haplotype donor $(\mathrm{HR}=0.348)$ and lower overall survival when minimal residual disease was positive $(\mathrm{HR}=2.76)$. Multivariate analysis showed lower relapse probability when KIR B haplotype donor $(\mathrm{HR}=0.465)$ was used.

Conclusions: Haplo-HSCT is a feasible therapeutic option for children and adolescents with high-risk haematological malignancies. We suggest that outcomes of haploHSCT may be improved by selecting KIR B haplotype donors, acute myeloid leukaemia patients and pretransplantation negative minimal residual disease.

Conflict of interest: Nothing to disclose

\section{P745}

\section{Hematopoietic stem cell transplantation from unrelated donor in children with beta thalassemia major}

Gülsün Karasu ${ }^{l}$, Vedat Uygun ${ }^{1}$, Hayriye Daloğlu ${ }^{l}$, Suar Kiliçç ${ }^{2}$, Seda Öztürkmen $^{1}$,Zeynep Dinçer ${ }^{1}$,Volkan Hazar ${ }^{2}$, Akif Yeşilipek ${ }^{I}$

${ }^{1}$ Medical Park Göztepe, Pediatric Bone Marrow Transplantation Unit, Antalya, Turkey; ${ }^{2}$ Medical Park Göztepe, Pediatric Bone Marrow Transplantation Unit, Istanbul, Turkey

Background: Although regular red cell transfusion and improved iron chelation therapy alternatives have prolonged life, allogeneic hematopoietic stem cell transplantation (HSCT) is the only curative treatment option for patients with beta thalassemia major. However, the presence of the HLA-matched related donor remains the main barrier for allogeneic HSCT. A few promising studies have been reported, but there is still insufficient data on unrelated transplantation. We aimed to evaluate the result of 51 children with beta thalassemia major who received allogeneic HSCT from HLA-matched unrelated donors

Methods: We retrospectively evaluated 51 unrelated HSCT in children with beta thalassemia major. All patients received myeloablative regimen with weight adjusted doses of busulfan. In addition, all patients received fludarabine $150 \mathrm{mg} / \mathrm{m} 2$ in five days, cyclophosphamide $120 \mathrm{mg} / \mathrm{kg}$ in three days, thiotepa $10 \mathrm{mg} / \mathrm{kg}$ for one day and ATG $30 \mathrm{mg}$ $/ \mathrm{kg}$ in three days. Cyclosporin-A and MTX were used for prophylaxis of graft-host disease (GVHD). Peripheral blood was assessed for donor chimerism on days $+30,+100$ and +180 .

Results: The median age of the patients was 87 months (range 14-184 months). Two of the patients were class I and the rest were class II. The median serum ferritin level was $1.277 \mathrm{ng} / \mathrm{ml}$ (range, 425-5832). Although 9 of the donors were 9/10 matched with HLA high resolution in graft failure direction, all of the patients were 10/10 matched in GVHD direction. BM was used as the stem cell source of thirty-four transplants (median TNC: $6.6 \times 10^{8}$, range; $1.62-$ 17.60) and PBSC for 17 transplants (median MNC: $7.05 \times 10^{8}$, range; 5.0-13.0) with median $\mathrm{CD} 34+$ cell number $7.23 \times 10^{6}$ (range 1,54-15,70). The median neutrophil and platelet engraftment days were 14 and 18 days in PBSC and 17 and 23 days in BM group, respectively. Graft failure was experienced in one and graft rejection in 2 patients. Moderate VOD was observed in 15 patients (29\%) and all were treated with defibrotide successfully. Grade IIV acute GVHD was observed in 11 patients (22\%), six of which experienced Grade II-IV (12\%). However, no chronic GVHD was observed. All patients are alive with full donor chimerism (between 95.47-100\%) with a median 14 months (range 3-46 months) follow up, except one patient was lost because of sepsis on post-transplant day 124. Probability of thalassemia-free (TFS) and overall survival (OS) were found $92 \%$ and $98 \%$, respectively.

Conclusions: Our data showed that the results of HSCT from unrelated donors in selected low risk thalassemia patients (Class I \& II) may be comparable to HSCT from matched sibling donors. We believe that the most important event that provides this successful outcome in unrelated stem cell transplantation is having a fully matched donor. However, it needs further studies with long term follow up and larger study population.

Conflict of interest: nothing to disclose 


\section{P746}

Hematopoietic stem cell transplantation in children with Wiskott-Aldrich syndrome with myeloablative conditioning

Baris Kuskonmaz ${ }^{1}$, Fatma Visal Okur ${ }^{1}$, Deniz Çă̆daş Ayvaz ${ }^{2}$, Selin Aytac Eyüpoglu, ${ }^{3}$,Ihan Tezcan ${ }^{2}$, Duygu Uckan Cetinkaya ${ }^{1}$

${ }^{1}$ Hacettepe University Faculty of Medicine, Department of Pediatrics, Division of BMT Unit, Ankara, Turkey; ${ }^{2}$ Hacettepe University Faculty of Medicine, Departmet of Pediatrics, Division of Immunology, Ankara, Turkey; ${ }^{3}$ Hacettepe University Faculty of Medicine,

Department of Pediatrics, Division of Hematology, Ankara, Turkey

Background: Wiskott-Aldrich syndrome (WAS) is a severe X-linked disorder characterized by immunodeficiency, microthrombocytopenia and eczema. This syndrome is caused by homozygous mutations in the WAS gene, which encodes the WAS protein (WASp). Despite advances in clinical care, patients with classic WAS have a poor prognosis and the median life expectancy is only 15 years, unless hematologic and immune reconstitution is achieved by hematopoietic stem cell transplantation (HSCT). Here, we report the results of HSCT in 9 children with WAS.

Methods: This study includes nine male patients with WAS underwent HSCT between May 1999- March 2016 at our center. The details of HSCT were shown on Table 1. The median age of the patients was 16 months, ranging between 6 months-58 months. One patient (Case 1) underwent second HSCT due to engraftment failure. All patients received myeloablative conditioning regimen including busulfan (between $12.8 \mathrm{mg} / \mathrm{kg}$ and $20.4 \mathrm{mg} / \mathrm{kg}$ according to the conditioning protocol and body weight of the patients)+cyclophosphamide (200 mg/kg)/fludarabine $(160 \mathrm{mg} / \mathrm{kg}) \pm$ antithymocyte globulin $(12.5 \mathrm{mg} / \mathrm{kg}$ for Thymoglobulin, $20 \mathrm{mg} / \mathrm{kg}$ for ATG-Fresenius).
Cyclosporine $\mathrm{A}+$ methotrexate were used for graft versus host disease (GVHD) prophylaxis.

Results: The results of transplantation were shown on Table 1. Engraftment was achieved in all patients except case 1. Two patients developed acute and chronic GVHD. The other complications were as follows: cytomegalovirus (CMV) reactivation in three patients, CMV retinitis in two patients, engraftment syndrome in one patient, venoocclussive disease in one patient, pulmonary infection in one patient and skin infection in one patient. One patient (case 1) died due to engraftment failure and pulmonary infection six days after second transplantation. The remaining eight patients are alive and all of them are disease free. Seven of these eight patients have full donor chimerism ( $\geq 97 \%$ donor profile). Case 4 had full donor chimerism at first two months, then he developed mixed chimerism (79\% donor profile). One patient still has active chronic GVHD. The median duration of follow-up was 89 months (ranged between 9-161 months) (Table 1).

Conclusions: This single center study evaluated the results of HSCT in WAS after myeloablative conditioning regimen. Three of the patients were above 5 years of age, which is a bad prognostic factor. Eight of nine patients are alive and disease free. The ratio of full donor chimerism is found to be 7/8. CMV reactivation and infection were seen as the major complication of HSCT in this study.

Conflict of interest: There is no conflict of interest.

\section{P747}

\section{HEMATOPOIETIC STEM CELL TRANSPLANTATION IN PEDIATRIC ACUTE LYMPHOBLASTIC LEUKEMIA: A SINGLE CENTER EXPERIENCE}

\begin{tabular}{|c|c|c|c|c|c|c|c|c|c|}
\hline $\begin{array}{l}\text { Patient } \\
\text { no }\end{array}$ & $\begin{array}{l}\text { Age } \\
\text { (years) }\end{array}$ & Donor & Stem cell source & $\begin{array}{l}\text { Conditioning } \\
\text { regimen }\end{array}$ & $\begin{array}{l}\text { Neutrophil } \\
\text { engraftment day }\end{array}$ & $\begin{array}{l}\text { Acute } \\
\text { GVHD }\end{array}$ & $\begin{array}{l}\text { Chronic } \\
\text { GVHD }\end{array}$ & Other complications & Outcome \\
\hline 1 & 1.3 & $\begin{array}{l}\text { HLA identical } \\
\text { father }\end{array}$ & Peripheral blood & $\mathrm{Bu}, \mathrm{Cy}, \mathrm{ATG}$ & - & $\begin{array}{l}\text { Not } \\
\text { applicable }\end{array}$ & $\begin{array}{l}\text { Not } \\
\text { applicable }\end{array}$ & $\begin{array}{l}\text { VOD, pulmonary } \\
\text { infection }\end{array}$ & Dead (day +66) \\
\hline 2 & 3.2 & $\begin{array}{l}\text { HLA identical } \\
\text { sibling }\end{array}$ & $\begin{array}{l}\text { Bone marrow }+ \text { cord } \\
\text { blood }\end{array}$ & $\mathrm{Bu}, \mathrm{Cy}, \mathrm{ATG}$ & Day +16 & No & No & CMV retinitis & $\begin{array}{l}\text { Alive } \\
(+161 \text { months })\end{array}$ \\
\hline 3 & 6.8 & $\begin{array}{l}\text { HLA identical } \\
\text { sibling }\end{array}$ & Bone marrow & $\mathrm{Bu}, \mathrm{Cy}$ & Day +13 & No & No & - & $\begin{array}{l}\text { Alive } \\
(+103 \text { months })\end{array}$ \\
\hline 4 & 1.1 & $\begin{array}{l}\text { HLA identical } \\
\text { sibling }\end{array}$ & Bone marrow & $\mathrm{Bu}, \mathrm{Cy}$ & Day +15 & No & No & CMV reactivation & $\begin{array}{l}\text { Alive } \\
(+92 \text { months })\end{array}$ \\
\hline 5 & 6.8 & $\begin{array}{l}\text { HLA identical } \\
\text { sibling }\end{array}$ & Bone marrow & $\mathrm{Bu}, \mathrm{Cy}$ & Day +16 & No & No & CMV recativation & $\begin{array}{l}\text { Alive } \\
(+89 \text { months })\end{array}$ \\
\hline 6 & 14.1 & $\begin{array}{l}\text { HLA identical } \\
\text { sibling }\end{array}$ & Bone marrow & $\mathrm{Bu}, \mathrm{Cy}$ & Day +11 & Yes & Yes & Skin infection & $\begin{array}{l}\text { Alive } \\
(+68 \text { months })\end{array}$ \\
\hline 7 & 4.3 & $\begin{array}{l}\text { HLA identical } \\
\text { sibling }\end{array}$ & Bone marrow & Bu, Flu, ATG & Day +14 & Yes & Yes & CMV reactivation & $\begin{array}{l}\text { Alive } \\
(+48 \text { months })\end{array}$ \\
\hline 8 & 4.5 & $\begin{array}{l}\text { HLA identical } \\
\text { mother }\end{array}$ & Bone marrow & $\mathrm{Bu}, \mathrm{Flu}$ & Day +17 & No & No & $\begin{array}{l}\text { Engraftment } \\
\text { syndrome }\end{array}$ & $\begin{array}{l}\text { Alive } \\
(+11 \text { months })\end{array}$ \\
\hline 9 & 0.3 & $\begin{array}{l}\text { HLA identical } \\
\text { sibling }\end{array}$ & Bone marrow & Bu, Flu & Day +16 & No & No & CMV retinitis & $\begin{array}{l}\text { Alive } \\
\text { (+9 months) }\end{array}$ \\
\hline
\end{tabular}

[[P746 Table] Table 1. Characteristics of patients and results of] 
Serap Aksoylar ${ }^{1}$, Burcu Deveci ${ }^{1}$, Salih Gözmen ${ }^{1}$, Serra Kamer ${ }^{2}$, Savas Kansoy ${ }^{1}$

${ }^{1}$ Ege University Faculty of Medicine, Pediatric Hematology and Oncology, Izmir, Turkey; ${ }^{2}$ Ege University Faculty of Medicine, Radiation Oncology, Izmir, Turkey

Background: Hematopoietic stem cell transplantation (HSCT) is the major indication for acute lymphoblastic leukemia (ALL) in children. In this study, we retrospectively analyzed the patients who transplanted for ALL for 16 years. We aimed to investigate clinical outcomes of ALL patients underwent HSCT and risk factors (donor type, TBI based regimen, stem cell source) that may have affected the survival of the patients.

Methods: Between January 2001 and 2017, 59 patients with acute lymphoblastic leukemia underwent HSCT at Ege University Hospital. Information of these procedures was collected retrospectively from the patient's medical records. Data analyses were primarily conducted using SPSS software. The mean age at transplant was $9,4 \pm 4,6$ years (2,5-19 years) and the ratio of males to females was 3.2:1. Twentysix patients had achieved first complete remission (CR) at the time of transplant (29 patients were beyond 2nd CR). Four patients underwent transplant with active or refractory leukemia.

Results: All transplants were allogeneic and the donors were matched sibling, matched related, matched unrelated and mismatched donor in 35 (60\%), 6 (10\%), 16 (27\%), and $2(3 \%)$ patients, respectively. Stem cell source was bone marrow in 31 patients, peripheral blood stem cells (PBSC) in 23 patients and unrelated cord blood in 3 patients. Bone marrow with cord blood from the sibling was used in two other patients. Total body irradiation was used as a part of the conditioning regimen in 37 acute lymphoblastic leukemia patients. One patients had graft failure and 6 patients died before the engraftment. Among the others who had neutrophil engrafment 13 (22\%) resulted in relapse. The relapse was isolated extramedullary in $5(38 \%)$ patients. The median time from transplant to relapse was 5 months (range: 1-58 months). The 5 year estimate of overall survival (OS) and event free survival (EFS) for the patients was $63 \%$ and $57 \%$ respectively. EFS was $61 \%$ and $62 \%$ when the donor was MRD or MUD $(\mathrm{p}=0.82)$. Transplant related mortality was $18 \%$.

Conclusions: Unrelated donor is a good option if the matched related donor is not available in pediatric ALL patients.

Conflict of interest: Nothing to disclose
P748

High Rate Of Health Care Utilization Associated With
Chronic Graft-Versus-Host Disease Among Canadian
Childhood Cancer Survivors Treated With Blood And
Marrow Transplantation

Kirk R. Schultz ${ }^{1}$, Yang Zhang ${ }^{2}$, Mary L. McBride ${ }^{2}$

${ }^{1} B C$ Children's Hospital Research Institute/UBC, Michael Cuccione Childhood Cancer Research Program, Vancouver, Canada; ${ }^{2} B C$ Cancer Agency, Cancer Control Research, Vancouver, Canada

Background: Blood and Marrow Transplant (BMT) has been increasingly used as a treatment for children and adolescents with hematologic malignancies and a number of non-malignant indications in children and adolescents. Chronic graft-versus-host disease (cGvHD) remains the primary late complication in about $15-20 \%$ of children receiving a BMT. Moreover, cGvHD has a significant morbidity and increased mortality that is poorly recognized in Canadian healthcare resource allocation. While the effect of cGvHD on children's quality of life is increasingly being studied, there is little known regarding the impact of cGvHD on health service utilization. Due to the centralized, single payer system in British Columbia, we are able to evaluate the impact in all children and adolescents receiving a allogeneic BMT for a cancer diagnosis.

Methods: Our analysis was restricted to all cancer patients identified from the provincial cancer registry and diagnosed 0-19 years of age in British Columbia (BC) between 1970 and 2010. Patient records were linked to clinical data including the BC Children's Hospital BMT Program dataset of those who received an allogeneic BMT in BC from 1981 (start of program) to 2010. These files were linked to provincial healthcare administrative datasets that provided information on all medically-necessary hospitalizations, outpatient physician services, and prescription drugs.

Results: Of 223 BMT oncology patients identified, 28 received their BMT between 1980-1989; 113 from 19901999; and 82 from 2000 to 2010. cGvHD developed in 16 (7.2\%) patients. In the follow-up period, the hospitalization rate was the same for both patients with and without cGvHD (14.4 per case). By contrast, the rate of physician service utilization in the community was significantly higher in cGvHD patients (1561 versus 801 per case) compared to those without cGVHD $(\mathrm{p}<0.05)$. Similarly, the prescription rate per case was higher in those with cGvHD compared to those with no cGvHD (223 versus $151 ; \mathrm{p}<0.05)$.

Conclusions: Since cGvHD is primarily treated as an outpatient disease it was not surprising that there was no increase in hospitalizations. By contrast, ultization of 
physician services and drugs was between 50 - 100\% higher in children with cGvHD. The low rate of cGvHD (7.2\% versus $15 \%$ ) we believe is due to the difficulty of cGvHD diagnosis documentation and probably under estimates the cost of cGvHD with some cGvHD patients included in the control group. These results indicate the impact of cGvHD on later health services demand significant and should be considered in allocation of resources to help this highly vulnerable population.

Conflict of interest: There are no conflicts of Interest to declare by any of the authors.

\section{P749}

HLA-haploidentical transplantation in children: a retrospective analysis of the Spanish Working Party for Blood and Marrow Transplantation in Children (GETMONGETH)

Antonia Pascual ${ }^{1}$, Antonio Perez-Martinez ${ }^{2}$, Marta GonzálezVicent ${ }^{3}$, Mercedes Plaza ${ }^{4}$, Jose Maria Fernández-Navarro ${ }^{5}$, Alexandra Regueiro ${ }^{6}$, Laura Alonso ${ }^{7}$, Jose Maria Pérez-Hurtado ${ }^{8}$, Luisa Sissini ${ }^{9}$, Isabel Badell ${ }^{10}$, Ana Benito ${ }^{11}$, Cristina Belendez ${ }^{12}$, Jose Miguel Couselo ${ }^{13}$, Jose Luis Fuster ${ }^{4}$, Ana Sastre ${ }^{14}$, Cristina Diaz de Heredia ${ }^{15}$, Miguel Angel Diaz ${ }^{16}$

${ }^{1}$ Hospital Carlos Haya. Hospital MaternoInfantil, Pediatric Hematology, Malaga, Spain; ${ }^{2}$ Hospital La Paz, Pediatric Hematology and Oncology, Madrid, Spain; ${ }^{3}$ Hospital Niño Jesus, SCT Unit, Madrid, Spain; ${ }^{4}$ Hospital Virgen de la Arreixaca, Pediatric

Hematology and Oncology, Murcia, Spain; ${ }^{5}$ Hospital La Fe, Pediatric Hematology and Oncology, Valencia, Spain; ${ }^{6}$ Hospital Santiago de Compostela, Pediatric Hematology and Oncology, Santiago de Compostela, Spain; ${ }^{7}$ Hospital Vall d'Hebron, Pediatric Hematology and Onclogy, Barcelona, Spain, ${ }^{8}$ Hospital Virgen del Rocio, Pediatric Hematology, Sevilla, Spain; ${ }^{9}$ Hospital Santa Creu I Sant Pau, Pediatric Hematology and Oncology, Barcerlona, Spain; ${ }^{10}$ Hospital Sant Joan de Deu, Pediatric, Barcelona, Spain; ${ }^{11}$ Hospital de Salamanca, Pediatric Hematology and Oncology, Salamanca, Spain;

${ }^{12}$ Hospital Gregorio Marañón, Pediatrica Hematology and Oncology, Madrid, Spain; ${ }^{13}$ Hospital de Santiago, Pediatric Hematology and Oncology, Santiago de Compostela, Spain; ${ }^{14}$ Hospital La Paz, Pediatric Hematolgy and Oncology, Madrid, Spain; ${ }^{15}$ Hospital Vall d 'Hebrón, Pediatric Hematology and Oncology, Barcelona, Spain;

${ }^{16}$ Hospital Niño Jesus, Pediatric SCT Unit, Madrid, Spain

Background: Allogeneic HSCT is widely used for pediatric patients with malignant and non-malignant diseases. With smaller family sizes, it is difficult to find a matched sibling donor for many children, so the only option of such patients is alternative donor sources. HLAhaploidentical HSCT (haplo-HSCT) have provided an alternative option for those patients who are lacking available HLA-identical sibling donors or unrelated donors or in need of urgent allograft.

Methods: We report a retrospective analysis of 232 consecutive children and adolescents with malignant and non-malignant diseases, who received a haplo-HSCT using post-transplant cyclophosphamide (PT Cy) $(\mathrm{n}=49)$ or ex vivo T-cell depletion $(\mathrm{n}=183)$ as graft versus host disease prophylaxis in 12 hospital centers in Spain.

Results: Median age at transplant was 8 years $(0.26-19)$. The underlying disease was malignant diseases: $86.6 \%$ (acute leukaemia $82.8 \%$ and solid tumours $3.9 \%$ ) and nonmalignant diseases: $13.4 \%$ (Immunodeficiencies 8.6\%, aplastic anaemia $2.2 \%$, Osteopetrosis $0.9 \%$, Thalassemia $0.9 \%$ and congenital neutropenia $0.9 \%$ ). Stem cell source was: peripheral blood stem cells $222(95.7 \%)$ and bone marrow $10(4.3 \%)$. One hundred eighty-five $(79.7 \%)$ of the 232 patients engrafted. The cumulative incidence rate of severe acute GVHD was 18.1, and the 2-year cumulative incidence of total chronic GVHD was $22 \%$. Trasplant related mortality at day 100 was $11.8 \%$. Two-year estimated relapse probability was $33 \%$. Of the 232 patients, 131 survived throughout the follow-up period of 643 days (range: 2- 5079 days) with the 2-year overall survival rates of $48 \%$. No statistical difference was found between PT Cy or ex vivo $\mathrm{T}$ cell depletion. Univariate analysis showed lower overall survival in: PT Cy group compared with CD34+ positive selection group, $\mathrm{CD} 3+$ and $\mathrm{CD} 19+$ negative selection group, TCR $\alpha \beta+$ and $\mathrm{CD} 19+$ negative selection group and CD45+ Ra negative selection group ( $\mathrm{P}$ $=0.02)$, no previous HSCT $(P=0.015)$, positive minimal residual disease $(\mathrm{P}=0.000)$, no chronic GvHD, $(\mathrm{P} 0,000)$ and KIR A haplotype donor (p 0.02). Multivariate analysis showed lower overall survival when minimal residual disease was positive $(\mathrm{HR}=2.4)$.

Conclusions: Haplo-HSCT is an effective therapeutic option for children with malignant and non-malignant diseases who need a HSCT and lack of a HLA match donor.

In our results minimal residual disease pretransplantation affect global and disease free survival.

Prospective studies design are needed to compare both ex vivo and in vitro $\mathrm{T}$ cell depletion.

Conflict of interest: None

P750

Increase in recipient chimerism by real time quantitative PCR in blood after Haematopoietic Cell Transplantation in Childhood Leukaemia is associated with relapse

Anna Karen Haugaard ${ }^{1}$, Hans Ole Madsen ${ }^{2}$, Hanne Vibeke Marquart $^{2}$, Susanne Rosthoj ${ }^{3}$, Tania Nicole Masmas ${ }^{1}$, Carsten Heilmann ${ }^{1}$, Marianne Ifversen ${ }^{1}$

${ }^{1}$ Rigshospitalet, University of Copenhagen, Department for Children And Adolescents, Copenhagen Ø, Denmark; ${ }^{2}$ Rigshospitalet, University of Copenhagen, Department of Clinical Immunology, 
Copenhagen, Denmark; ${ }^{3}$ University of Copenhagen, Department of Public Health, Section of Biostatistics, Copenhagen, Denmark

Background: Relapse remains the primary treatment failure after HCT for acute childhood leukaemia. The development of novel therapies accentuates the need for early detection of relapse. Measurement of chimerism can be performed in peripheral blood, enabling frequent testing in children. STR-PCR chimerism is recommended by the Eurochimerism consortium and has a sensitivity of $1-5 \%$. RQ-PCR based chimerism is 10-100 fold more sensitive, but clinical studies in children are sparse. We aimed to describe the applicability of the method by three different cut-offs using relapse as primary endpoint.

Methods: We retrospectively included children $<18$ years of age, transplanted for ALL or AML at the national paediatric transplant centre in Copenhagen between 2008 and 2014. RQ-PCR chimerism was performed using a commercial kit (KMRtype/track, GenDx, Utrecht, Netherlands). Increasing mixed chimerism (IMC) was defined as an increase of recipient DNA above the cut-offs $0.1 \%$, $0.05 \%$ and $0.01 \%$, respectively. For STR-PCR IMC was defined as an increase of $1 \%$ or above. Results by RQ-PCR and STR-PCR were compared and association between IMC and relapse was analysed using a Cox regression model with IMC as a time-dependent variable.

Results: Fifty-seven children with acute leukaemia received $62 \mathrm{HCT}$ during the study period. In total, 619 samples of whole peripheral blood were analysed, with a median of 10 (range: 2-29) samples per child. In total, 16 children relapsed, a median of 9 (1-52) months from transplantation, with a 5 year relapse incidence of 25.8\% (CI 15.6-37.2\%). Median follow-up time was 47 months (15-91) from transplantation. By STR-PCR, 9 of 619 samples had IMC, and for RQ-PCR this was 26/619 for cut-off $0.1 \%, 44 / 619$ for cut-of $0.05 \%$ and $132 / 619$ for cut-off $0.01 \%$. STR-PCR and RQ-PCR chimerism results were equally distributed $\left(X^{2}=109, \quad P<0.0001\right)$ when applying cut-off $0.1 \%$ for RQ-PCR.

The risk of relapse was significantly higher in children with IMC compared to children without IMC at all cut-offs with hazard ratio (HR) for relapse of 13.2 (95\% CI: 4.538.8); $\mathrm{P}<0.01,9.0$ (95\% CI: 3.0-26.8); $\mathrm{P}<0.01$ and 3.7 (95\% CI: $1.3-10.7$ ); $\mathrm{P}=0.02$, for IMC above $0.1 \%, 0.05 \%$ and $0.01 \%$ recipient DNA, respectively. Among the children who relapsed, an IMC above $0.1 \%, 0.05 \%$ and $0.01 \%$ was observed at median 37 ( 1 - 1554), 90 (0-1554) and 178 (10-1554) days prior to relapse. In comparison, for STR-PCR IMC was detected 2 (0-84) days prior to relapse, and was not associated with relapse (HR 3.6 (95\% CI: 0.528.0); $\mathrm{p}=0.228$ ). The 5-year cumulative incidence of relapse (CIR) for children with IMC was $55.2 \%$ (CI 22.0- respectively (Figure 1).
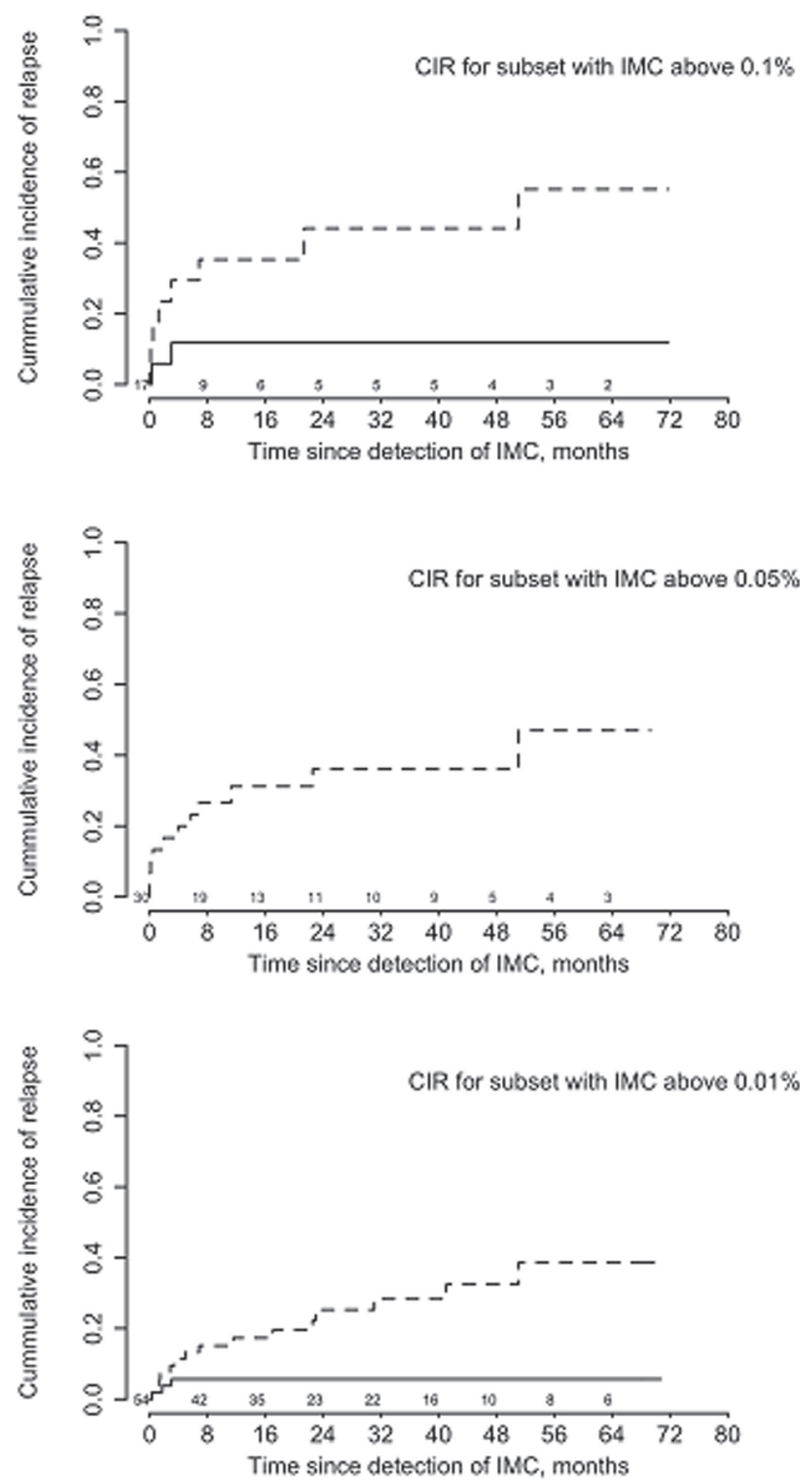

[P750 Figure] [CIR in children with IMC. Continuous line: incidence of death in remission, dashed line: CIR.]

79.1), 46.9\% (CI 20.3-69.7) and 32.4\% (18.1-47.6) for IMC above $0.1 \%, 0.05 \%$ and $0.01 \%$ respectively (Figure 1 ).

Conclusions: Changes in chimerism by RQ-PCR were associated with subsequent relapse at all three cut-off levels 1-6 months prior to relapse. Although limited by a retrospective design, these results indicate that monitoring of chimerism by RQ-PCR is applicable for early detection of relapse.

Conflict of interest:

The study was supported by the Childhood Cancer Foundation, the Danish Cancer Society and Dansk Kræftforskningsfond. None of the authors has anything to disclose. 


\section{P751}

Insulin-like growth factor deficiency in children referred for allogeneic hematopoietic stem cell transplantation: impact on hematologic reconstitution and clinical outcomes

Maria Schou Ebbesen ${ }^{1}$, Anders Juul', Kaspar Sorensen ${ }^{1}$, Carsten Heilmann ${ }^{1}$, Klaus Müller ${ }^{1}$

${ }^{1}$ Rigshospitalet, University of Copenhagen, Department of Paediatrics and Adolescent Medicine, Copenhagen, Denmark; ${ }^{2}$ Rigshospitalet,

University of Copenhagen, Department of Growth and Reproduction, Copenhagen, Denmark

Background: Allo-HSCT is challenged by significant toxicities mainly related to infections and inflammatory complications that are induced due to depressed marrow function and damage of the epithelial barriers. Insulin-like growth factor 1 (IGF-1) plays a key role in growth and repair of most tissues including epithelium, endothelium and hematopoietic cells. Previous studies in animals and in adults undergoing HSCT indicate that reduction in IGF-1 leads to a poorer outcome. We investigated retrospectively levels of IGF-1 at baseline and until more than 2 years postHSCT in a pediatric cohort and explored associations with outcomes and hematopoietic reconstitution.

Methods: We included 213 children (age range: 0.3 17.9) undergoing HSCT at the National Danish HSCT center between January 2004 and December 2016. Diagnoses included malignancies $(\mathrm{n}=135)$ and nonmalignant disorders $(n=78)$. Donors were either matched siblings $(\mathrm{n}=64)$ or matched unrelated donors $(\mathrm{n}=149)$ and grafts included marrow $(\mathrm{n}=201)$ or peripheral stem cells $(\mathrm{n}=12)$. Due to the clinical routine at our institution scheduled measurements of IGF-1 were available pretransplant and at regular intervals until several years postHSCT. Due to age and sex-related differences in IGF-1 levels SD-scores were applied in the analyses based on healthy Danish children.

Results: Median pre-HSCT IGF-1 levels were significantly reduced (median: -1.09 SD-score, $\mathrm{p}<0.001$ ) and remained reduced until two years post-HSCT (figure 1a). Pre-transplant levels were not related to sex, age or malign/ non-malignant diagnosis.

Patients with IGF-1 levels $<-2$ SD-score pre-HSCT remained below $-2 \mathrm{SD}$-score during the first year postHSCT but then tended to normalize (figure $1 \mathrm{~b}$ ). This group of patients had significantly slower hematologic recovery (median time to: ANC $>500 / \mathrm{mm}^{3}: 24$ vs. 21 days, $\mathrm{p}=$ 0.02 ; thrombocytes $>50 \times 10^{9} / \mathrm{L}: 28.5$ vs 25 days, $\mathrm{p}=0.01$; thrombocytes $>100 \times 10^{9} / \mathrm{L}$ : 40 vs 29 days, $\mathrm{p}=0.03$ ).

Patients with a malignant diagnosis and patients undergoing high-intensity myeloablative conditioning (TBI $\geq$ $12 \mathrm{~Gy}$ or a non-TBI regimen without Fludarabine) had

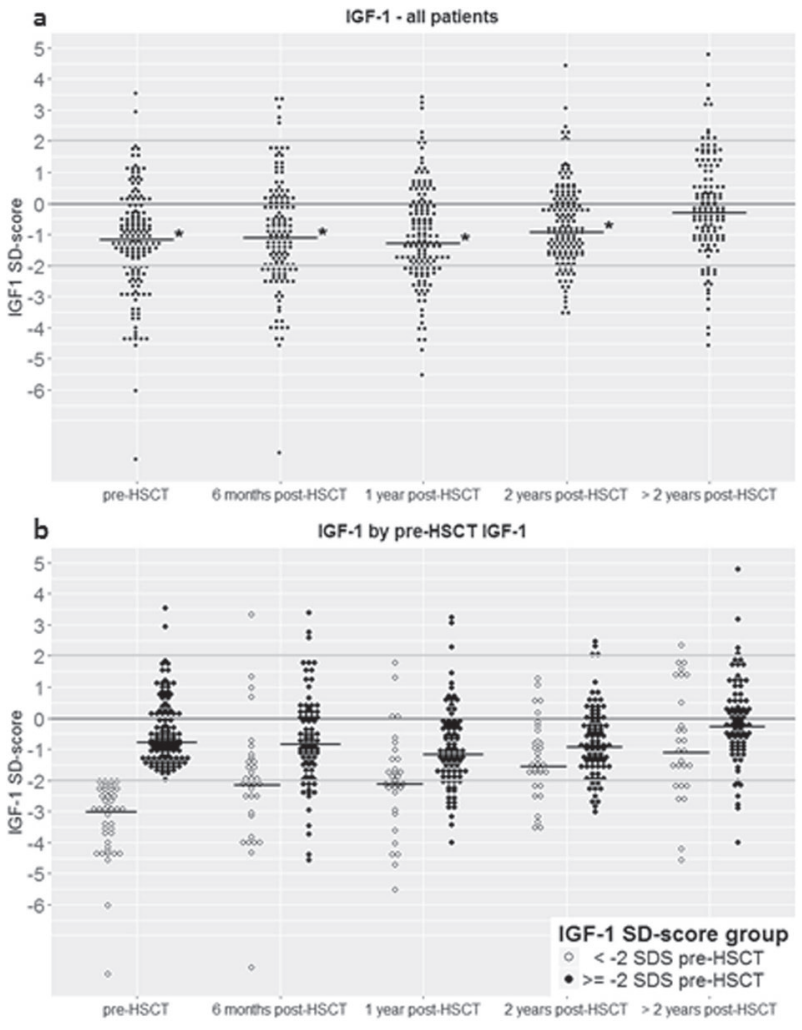

[P751 Figure] [Horizontal line: median IGF-1 at timepoint. *: median IGF-1 SD-score significantly different from 0.]

generally lower median IGF-1 levels post-transplant (at year 1: median: -1.4 vs $-0.67 \mathrm{SD}$-score, $\mathrm{p}=0.03$ and -1.3 vs -1.0 SD-score, $\mathrm{p}=0.04$, respectively). Furthermore, IGF-1 reduction was most pronounced in patients with higher degree of systemic inflammation during the phase of aplasia (CRP day 7- 21 post-transplant; $\mathrm{R}_{\mathrm{S}}=-0.22, \mathrm{p}=0.008$ ). Pre- and post-transplant IGF-1 levels were not related to aGvHD, relapse or OS.

Conclusions: This study has demonstrated that children referred to allogeneic HSCT have a reduced and longstanding reduction in IGF-1 levels with an impact on hematologic reconstitution but does not translate into a poorer survival. These finding should be further explored and validated in prospective studies, including studies on growth and tissue composition.

Conflict of interest: None of the authors has anything to disclose.

\section{P752}

Kinetics of engraftment and incidence of cytokine release syndrome in Pediatric Haploidentical Stem Cell Transplantation

Mariflor Villegas ${ }^{1,2}$, Teck Guan Soh $^{3}$, Vinod Gunasekaran ${ }^{1}$, Thuan 
Chong Quah ${ }^{1,2,4}$, Allen Yeoh ${ }^{1,2,4}$, Poh Lin Tan ${ }^{1,2,4}$

${ }^{1}$ National University Health System, Khoo Teck Puat-National University Children's Medical Institute, Singapore, Singapore;

${ }^{2}$ National University Cancer Institute, Singapore, Singapore;

${ }^{3}$ National University Health System, Laboratory Medicine, Singapore,

Singapore; ${ }^{4}$ National University of Singapore, Singapore, Singapore

Background: Haploidentical Stem Cell Transplant (HaploSCT) with its varying degrees of T-cell manipulation has changed the kinetics of engraftment with higher incidence of cytokine release syndrome.

Methods: Retrospective review of pediatric HaploSCT s done in our center from 2012 to 2017

Results: Thirty-eight pediatric HaploSCTs were performed from September 2012 through 09 December 2017. There were 35 patients who received T-deplete (TCD) HaploSCTs (majority (20) were CD3/CD45RA TCD and 6 TCR ab TCD) and 3 patients with T-replete $\mathrm{pCy}$ HaploSCTs. There were 4 graft failures. The TCD HaploSCTs show a fast engraftment.

We also noted that monocyte engraftment precedes neutrophil engraftment. In the cohort of CD3/CD45RA TCD 90\% had fever at the time of neutrophil engraftment majority had CRS grade 1=16; one patient had Grade 3 CRS. Majority (12) only required supportive care, 4 required short course of hydrocortisone, and only 1 required Tocilizumab. Out of those patients who had CRS, 72\% developed aGVHD (38\% grade $1 ; 38 \%$ grade $2 ; 23 \%$ grade $3)$. By 1 year, only 1 of the patients who required steroids + other immunosuppression has not discontinued them.

Conclusions: TCD Haploidentical SCT shows rapid engraftment accompanied by low grade CRS, that rarely needs systemic steroid treatment. Despite the high incidence of CRS, aGVHD that develops is mostly grade1-2; there is no incidence of grade 4 GVHD

Conflict of interest: None

\begin{tabular}{llll}
\hline & $\begin{array}{l}\text { CD3/CD45RA TCD } \\
\text { (No GCSF) N=20 }\end{array}$ & $\begin{array}{l}\text { TCR } \alpha \\
\beta T C D ~ N=4\end{array}$ & $\begin{array}{l}\text { T-replete } \\
\text { N=3 }\end{array}$ \\
\hline $\begin{array}{l}\text { Median time to } \\
\text { neutrophil engraftment }\end{array}$ & 13.5 (Range 8-26) days & $\begin{array}{l}\text { 10.5(Range } \\
\text { 9-17) days }\end{array}$ & $\begin{array}{l}\text { 16(Range } \\
15-17) \text { days }\end{array}$ \\
$\begin{array}{l}\text { Median time to } \\
\text { monocyte engraftment }\end{array}$ & 10(Range 7-21) days & $\begin{array}{l}\text { 10.5(Range } \\
\text { 8-13) days }\end{array}$ & >30 days \\
$\begin{array}{l}\text { Median time to complete } \\
\text { donor chimerism }\end{array}$ & 9(Range 7-12) days & $\begin{array}{l}\text { 11(Range 8- } \\
\text { 12) days }\end{array}$ & $\begin{array}{l}\text { 16(Range } \\
14-21) \text { days }\end{array}$ \\
\hline
\end{tabular}

[[P752 Table] Kinetics of Engraftment]

\section{P753}

Leucoderma and leucotrichia in association with chronic cutaneous graft-versus-host disease in a child with WiskottAldrich syndrome
Baris Kuskonmaz, ${ }^{1}$ Fatma Visal Okur ${ }^{2}$, Nevin Cetin ${ }^{3}$, Siar Teker ${ }^{3}$, Duygu Uckan Cetinkaya ${ }^{1}$

${ }^{1}$ Hacettepe University, Faculty of Medicine, Department of Pediatrics, Division of BMT Unit, Ankara, Turkey; ${ }^{2}$ Hacettepe University Faculty of Medicine, Department of Pediatrics, Division of BMT Unit, Ankara, Turkey; ${ }^{3}$ Hacettepe University Faculty of Medicine, Ankara, Turkey

Background: The skin is the most commonly affected organ in chronic graft-vs-host disease (GVHD). Cutaneous manifestations of chronic GVHD) vary from non-sclerotic lichen planus-like eruptions or poikiloderma to morphealike or deep sclerotic conditions. Vitiligo is not well characterized in the chronic GvHD setting and extensive leucoderma and leucotrichia are only rarely reported. Here we present leucoderma and leucotrichia associated with GVHD in a 14-year-old boy who had undergone allogeneic bone marrow transplantation for Wiskott-Aldrich syndrome (WAS).

Methods: Clinical and transplantation characteristics of the patient was recorded.

Results: 14.1 years old boy with Wiskott-Aldrich syndrome underwent HSCT from HLA identical sister. The source of the stem cells was peripheral blood and the number of $\mathrm{CD} 34^{+}$cells was $9.2 \times 10^{6} / \mathrm{kg}$. The conditioning regimen included busulfan $(4 \times 0.8 \mathrm{mg} / \mathrm{kg} /$ day for 4 days $/ \mathrm{mg} /$ $\mathrm{m}^{2}$ ), cyclophosphamide $(50 \mathrm{mg} / \mathrm{kg} /$ day for 4 days). Cyclosporine A (CsA) and methotrexate were used as graft versus host disease prophylaxis. Neutrophil and platelet engraftment were achieved at day +11 and +28 respectively. During the post-transplantation period, the patient developed skin infection and neutropenic fever and broad spectrum antibacterial and antifungal treatment were given. The patient developed acute GVHD (grade 2) with skin and intestinal involvement $($ day +20$)$. Methyl prednisolone $(2$ $\mathrm{mg} / \mathrm{kg}$ ) was started. At +6 months the patient developed chronic GVHD involving skin and oral mucosa. The skin lesions included vitiligo on hands. At +15 months, vitiligo lesions progressed to generalized leucoderma and leucotrichia. During the follow period, chronic GVHD also involved other organs including liver, eye and lungs. The patient was given methyl prednisolone, $\mathrm{CsA}$ /tacrolimus and mycophenolate mofetil for chronic GVHD. Although chronic GVHD in the other organ systems responded partially or completely to these agents, leucoderma and leucotrichia were non-responsive. PUVA (psoralen ultraviolet $\mathrm{A}$ ) treatment is planned. At present the patient is at +68 months with full donor chimerism.

Conclusions: Several case reports and small series have reported vitiligo following HSCT, most occurring in the setting of GvHD. GVHD seems to play a part in the destruction of melanocytes by skin-homing autoreactive melanocyte-specific cytotoxic T lymphocytes. Premature graying of the hair and eyelashes and vitiligo-like 
leukoderma have been reported in association with chronic GVHD, and most of these cases are consequences of sclerodermatous lesions with poikilodermal aspects. In our patient, leucoderma and leucotrichia appeared in the absence of sclerodermatous skin lesions.

Conflict of interest: There is no conflict of interest.

\section{P754}

New pediatric EBMT criteria for SOS/VOD may prove useful in identifying patients with increased risk of treatmentrelated complications

\author{
Marte B. Kammersgaard ${ }^{1,2}$, Katrine Kielsen ${ }^{1,2}$, Carsten Heilmann ${ }^{1}$, \\ Marianne Ifversen ${ }^{1}$, Klaus Müller ${ }^{1,2}$ \\ ${ }^{1}$ Copenhagen University Hospital Rigshospitalet, Department of \\ Pediatrics and Adolescent Medicine, Copenhagen, Denmark; \\ ${ }^{2}$ Copenhagen University Hospital Rigshospitalet, Institute for \\ Inflammation Research, Copenhagen, Denmark
}

Background: SOS/VOD is a life-threatening complication of allogeneic HSCT. New pediatric diagnostic criteria have been suggested due to age-related differences in presentation, incidence and outcomes (Corbacioglu et al. 2017). These criteria score bilirubin and weight gain by an individual baseline. Moreover, transfusion refractory thrombocytopenia (RT) is added as an additional criterion, and there is no time restriction.

In this retrospective study we assessed the pediatric EBMT criteria along with the Baltimore and modified Seattle criteria.

Methods: From 2010-2017, 87 children aged 7.8 years (range 1.1-17.3) undergoing allo-HSCT in Denmark were included. Diagnoses were malignant $(\mathrm{n}=51)$ or benign diseases $(n=36) .10$ patients had diagnoses associated with increased risk of SOS/VOD. Donors were MSD $(n=22)$, MUD $(n=51)$, haploidentical $(n=6)$ or $\operatorname{UCB}(n=8)$. Stem cell sources were BM $(n=69)$, PBSC $(n=10)$ or UCB grafts $(n=8)$. Conditioning regimens were TBI-based $(n=21)$, Busulfan-based $(n=42)$ or other $(n=24)$.

Clinical parameters were registered for the first year following HSCT. Bilirubin was considered increased if above normal range or 4 times above the baseline value. RT was defined as unexplained platelet transfusions daily for $\geq$ 3 days.

Results: 39 (44.8\%) patients fulfilled the EBMT criteria, $9(10.3 \%)$ patients fulfilled modified Seattle criteria and none met the Baltimore criteria. Patients fulfilling the EBMT criteria presented with: rising bilirubin $(n=33)$, weight gain $(\mathrm{n}=26)$, consumptive RT $(\mathrm{n}=31)$, hepatomegaly $(n=1)$ and ascites $(n=1)$. This group included all patients fulfilling Seattle criteria, except one.
To evaluate the clinical relevance of applying EBMT criteria, we looked into the clinical course of the transplantation. Patients fulfilling the EBMT SOS/VOD criteria had a longer duration of their primary admission (31 days (23-183) vs. 27 days $(17-61), \mathrm{p}=0.001)$. Furthermore, patients fulfilling the EBMT SOS/VOD criteria received diuretics for more days within 3 months (29 days $(0-90)$ vs. 3.5 days $(0-90), \mathrm{p}<0.0001)$, and had a longer time to platelet engraftment (29 days (16-111) vs. 22 days $(14-68), \mathrm{p}<0.0001)$.

We found no significant difference between patients with and without SOS/VOD regarding development of acute or chronic GVHD, time to neutrophil engraftment or admission to the ICU (all $\mathrm{p}>0.1$ ).

Two patients who selectively fulfilled the EMBT criteria died of treatment-related complications within one year post-HSCT (pulmonary SOS/VOD and macrophage activation syndrome). The clinical details of these patients will be presented.

Conclusions: Application of the pediatric EBMT criteria defined a broader group of patients than the group defined by the Seattle criteria, still containing the patients fulfilling Seattle criteria. Patients meeting EBMT criteria had generally longer duration of the primary admission, but for the majority the course of transplant was without severe complications. Importantly however, application of EBMT criteria identified patients with fatal inflammatory complications that were not identified by the Seattle criteria. These findings suggest that application of the pediatric EBMT criteria may be helpful in identifying patients at increased risk of severe treatment-related complications and mortality, although there is a risk of over-diagnosis. Further assessment of the EBMT criteria based on prospective studies is needed.

Conflict of interest: None of the authors has anything to disclose.

\section{P755}

\section{Non-alcoholic fatty liver disease as a late effect of childhood acute lymphoblastic leukemia and lymphomas}

Ioanna Chranioti ${ }^{1}$, Nikolaos Katzilakis ${ }^{1}$, Dimitrios Samonakis ${ }^{2}$, Gregory Chlouverakis ${ }^{3}$, Ioannis Mouzas ${ }^{2}$, Eftichia Stiakaki ${ }^{1}$

${ }^{1}$ University of Crete, Laboratory of Blood Diseases and Childhood Cancer Biology, University Hospital of Heraklion, Pediatric Hematology-Oncology, Heraklion, Greece; ${ }^{2}$ University of Crete, Medical School, University Hospital of Heraklion, GastrenterologyHepatology, Heraklion, Greece; ${ }^{3}$ University of Crete, Medical School, Clinic of Social and Family Medicine, Heraklion, Greece

Background: Non-alcoholic fatty liver disease (NAFLD) represents a common cause of liver disease in children and 
adolescents in the Western world, affecting 3-10\% of pediatric population, while exceeds $70 \%$ amongst obese patients. Children and adolescents treated for Acute Lymphoblastic Leukemia (ALL) and lymphomas are at risk of developing metabolic disorders as obesity, hyperglycemia, insulin resistance (IR), hypertriglyceridemia and hypertension leading to increased morbidity and mortality in early adulthood. We aimed to study the prevalence of NAFLD in childhood ALL and lymphoma survivors and assess diagnostic and prognostic markers.

Methods: Eighty six (55 male, 31 female) children, adolescents and young adults with median age 17.6 years, diagnosed with hematologic malignancy between 2000 and 2014 (62 ALL, 24 lymphomas) were included in this study. Antineoplastic treatment ceased at least one year (median post-treatment 9.1 years). All patients underwent body measurements, biochemical assay and liver ultrasound study (a small group had Fibroscan /CAP). HOMA-IR and QUICKI were calculated from fasting plasma glucose and insulin values. History of impaired glucose metabolism and other causes of liver toxicity were excluded.

Results: US findings due to NAFLD were observed in $21.1 \%$ of the study population. Overweight and obese patients, based on BMI adjusted for age, accounted for $44.2 \%$ and had 8-times greater risk for NAFLD $(\mathrm{p}<0.01)$. Patients with NAFLD had significantly higher BMI, waist circumference and waist/height ratio (WHtR) than those without NAFLD $(\mathrm{p}<0.01)$. The correlation analysis showed that NAFLD were positively correlated with ALT:AST ratio $(\mathrm{p}<0.01)$, total cholesterol $(\mathrm{p}=0.02)$, LDL and triglycerides $(\mathrm{p}<0.01)$. HOMA-IR was significantly correlated with BMI $(\mathrm{p}<0.01)$, waist circumference $(\mathrm{p}<0.01)$, WHtR $(\mathrm{p}<0.01)$, LDL $(\mathrm{p}=0.014)$, HDL $(\mathrm{p}<0.01)$, triglycerides $(\mathrm{p}<0.01)$ and NAFLD fibrosis score $(\mathrm{p}=0.033) .39 .3 \%$ of patients had HOMA-IR values $>2.31$ indicating early insulin resistance. When we considered all the variables with a stepwise analysis, age, BMI, waist circumference, WHtR and ALT: AST ratio were independently associated with NAFLD ( $p<$ 0.05).

Conclusions: This ongoing study showed that childhood ALL and lymphoma survivors have increased prevalence of NAFLD, IR and visceral adiposity. These data support the need to prevent visceral adiposity, search for metabolic syndrome and ephasize the need of intervention on diet and lifestyle in the follow-up of these patients.

Conflict of interest: No conflict of interest

\section{P756}

Outcome of Second Hematopoietic Stem Cell Transplantations in Children with malignant and nonmalignant diseases. A single Center Retrospective Analysis
Irina Zaidman ${ }^{1}$, Sigal Grisariu ${ }^{2}$, Batia Avni, Yevgeni FrankKamensky, Adeeb Nasereddin ${ }^{3}$, Elena Lokshin ${ }^{3}$, Sharon Pantel Bakst ${ }^{3}$, Aliza Nagler ${ }^{3}$, Polina Stepensky ${ }^{3}$

${ }^{1}$ Hadassah Hebrew University Medical Center, Department of Clinical Immunology and Bone Marrow Transplantation, Jerusalem, Israel;

${ }^{2}$ Hadassah-Hebrew University Medical Center, Department of Bone Marrow Transplantation, Jerusalem, Israel; ${ }^{3}$ Hadassah Hebrew University Medical Center, Jerusalem, Israel

Background: Relapse following Hematopoietic Stem Cell Transplantation (HSCT) is the leading indication for a second transplant in patients with malignant diseases. HSCT has been shown to be superior to chemotherapy alone or palliative measures in these patients. For nonmalignant diseases a second transplantation may be considered for graft failure after first transplant. Data regarding the outcome after second HSCT especially for non malignant diseases is a scare. We retrospectively analyzed all pediatric patients who underwent more than one HSCT in Hadassah Hospital for survival and toxicity

Methods: 75 children (age 0-18) received 158 HSCT at our institution from 1992 to 2017.

50 patients had an underlying malignancy and 25 - were transplanted for non malignant indications, including inborn errors of metabolism, non malignant hematological diseases and immune deficiency. In group of non malignant diseases, 23 patients received 2 HSCT and 2 children - 3 HSCTs. Among the patients with malignancy, 44 - received 2 HSCTs and 6 children underwent 3 HSCTs.

Results: In malignant group, only 6 of 50 patients survived, OS- $12 \%, 5$ of 6 survived patients were transplanted after 2010 and all had symptoms of GVHD. 19 of 44 children died from relapse of malignant disease $(43 \%)$ and 25 of 44 - died from different complications during and after HSCT - TRM - 56\%. In group of non malignant diseases, 12 of 25 patients survived without evidence of disease with EFS od 48\%. The rest 13 died, most of them from Transplant Related toxicity and Graft Versus Host Disease.

Conclusions: Treatment related mortality following second HSCT is higher compared to first transplant. The higher survival rate in the non malignant group suggest that transplant following graft failure should be considered in patients with otherwise incurable underlying disease. Though the outcome for patients with relapse of malignant disease following HSCT is poor, a second transplant may benefit a subset of these patients. Attempts to achieve complete remission prior to transplant should be made to improve outcome. Due to the small number of patients in our cohort, further multi-center trials are needed.

Conflict of interest: Nothing to disclose 


\section{P757}

Outcomes of a pediatric program of allogeneic stem cell transplantation at an university hospital in the south-east of Spain

Ana Maria Fita, Maria Victoria Martínez Sánchez ${ }^{2}$, María Esther Llinares Riestra ${ }^{1}$, Maria Mar Bermúdez Cortés ${ }^{1}$, Ana Maria Galera Miñarro $^{1}$, Miguel Blanquer Blanquer ${ }^{3}$, Andrés Sánchez Salinas ${ }^{3}$, José Luis Fuster Soler ${ }^{1}$, José María Moraleda ${ }^{3}$

${ }^{1}$ Hospital Clínico Universitario Virgen de la Arrixaca, Paediatrics Oncology and Haematology Unit, El Palmar, Murcia, Spain;

${ }^{2}$ Hospital Clínico Universitario Virgen de la Arrixaca, Immunology Service, El Palmar, Murcia, Spain; ${ }^{3}$ Hospital Clínico Universitario Virgen de la Arrixaca, IMIB, University of Murcia, Haematology Service and Stem Cell Transplant Unit, El Palmar, Murcia, Spain

Background: Allogeneic stem cell transplantation (alloSCT) is widely used in the treatment of a variety of hematologic diseases and solid tumors. Main problems are the treatment-related mortality (TRM), mostly due to regimen-related toxicities (RRT) after myeloablative conditionings (MAC) and graft-versus-host disease (GVHD), and disease relapse that is usually higher with reducedintensity or nonmyeloablative regimens.

Methods: This is a retrospective, observational study based on medical records of patients aged between 0 and 15 years, that underwent allogeneic stem cell transplantation at a tertiary hospital, between January 2007 and December 2015.

Results: Overall, 69 allo-SCT were performed in 56 pediatric patients (54\% male), with a median age of $5(0,4$ 15) years, and a follow-up of $19(1-87)$ months. The patients were transplanted for malignant hemopathies (26 ALL, 8 AML, 5 MDS, 4 JMML), solid tumors (3 Ewing sarcoma, 3 neuroblastoma, 1 osteosarcoma) and non malignant disorders (4 inborn errors of metabolism, 5 haemoglobinopathies, 1 SCID, 3 inherited and 1 acquired bone marrow failure syndrome, 3 primary hemophagocytic lymphohistiocytosis, 1 sideroblastic anemia). The source of stem cells was peripheral blood in 47 procedures $(68 \%$, $21 \%$ from unrelated donor, $79 \%$ from related donor), bone marrow in 16 procedures $(23 \%, 63 \%$ unrelated donor, $38 \%$ related donor - $83 \%$ of whom were identical sibling) and umbilical cord in 6 procedures (9\%). The matched related donors (MRD) accounted for 31 cases, mismatch in one case and haploidentical in 11 cases. On the other side, the unrelated donors were matched in 24 cases and mismatched in 2 cases.

Two main types of conditioning regimens were used: high intensive regimens in $71 \%$ of patients (busulfan melphalan based, or Cyclophosphamide - TBI based) and low intensive regimen (fludarabine based) in 27,5\%. GVHD prophylaxis consisted in CSP - MTX (71\%), CSP- MMF
(11\%) and others (CSP; MMF; Cy-CSP-MMF) in 11,5\% of cases. Veno-occlusive disease (VOD) prophylaxis was made with ursodeoxycholic acid and low molecular weight heparin.

Graft failure occurred in $9,7 \%$ of the procedures. The most frequent early toxicities were mucositis in $83 \%$ of the patients, being $65 \%$ grade 3 and grade 4; acute GVHD grade $\geq$ II in $36 \%$ (GRADE III-IV 25\%); acute transient hepatic toxicity $22 \%$; pulmonary complications $19 \%(16 \%$ infectious and 3\% transfusion related acute lung injury); Cyclosporine induced acute renal injury $12,5 \%$; neurologic impairment $11 \%$ (posterior reversible encephalopathy syndrome most frequent); metabolic complications $7 \%$; VOD $4 \%$ and engraftment syndrome $1,5 \%$. Late toxicities were endocrine anomalies (18\%) followed by chronic GVHD (11\%).

The progression-free survival was $83 \pm 5 \%$ at day +100 and $75 \pm 6 \%$ at day +365 ; overall survival was $95 \pm 3 \%$ at day +100 and $72 \pm 6 \%$ at day +365 . The mortality rate was $34 \%$ (19 cases): relapse/progression of the disease in 14 cases, 4 chronic GVHD and 1 multiorganic failure secondary to septic shock.

Conclusions: Allo-SCT in pediatric patients is a safe and effective treatment for hematological and non hematological malignancies. The overall results of our pediatric program that started in 2007 are similar to those obtained in other pediatric series.

\section{Conflict of interest: None}

\section{P758}

\section{Pediatric Haplo-identical and Cord Blood Transplantation: A single Center Experience}

Eman Khattab ${ }^{1}$, Iyad Sultan ${ }^{2}$, Rawad Rihani, Hasan Hashem ${ }^{1}$, Mayada Abu Shanap ${ }^{1}$, Rula Najjar ${ }^{3}$, Shanta Sharma ${ }^{4}$, Abdelghani Tbakhi ${ }^{4}$

${ }^{l}$ King Hussein Cancer Center, Pediatric Stem Cell Transplant, Amman, Jordan; ${ }^{2}$ King Hussein Cancer Center, Department of Pediatrics, Amman, Jordan; ${ }^{3}$ King Hussein Cancer Center, Clinical Pharmacy, Amman, Jordan; ${ }^{4}$ King Hussein Cancer Center, Bone Marrow/Stem Cell Lab, Amman, Jordan

Background: Haplo-identical stem cell, umbilical cord blood (UCB) and matched unrelated donor (MUD) transplantation are common alternative donor strategies used, when a matched related sibling donor (MRD) is not available. In Jordan, $65 \%$ of patients will have MRD and alternative donors are pursued in the remaining $35 \%$. In this study, we compare the outcomes of our Haplo-identical stem cell and umbilical cord blood (UCB) transplantation programs. 
Methods: A retrospective analysis was conducted for pediatric patients who underwent Haplo-identical stem cell and umbilical cord blood (UCB) transplantation at King Hussein Cancer Center (KHCC) between Janu2003 and Dec2016, after obtaining an IRB approval. Data collected included transplant indications, acute and chronic graftversus-host disease (aGVHD and cGVHD) and cytomegalovirus (CMV) reactivation. Of note, Cyclophosphamide post unmanipulated stem cell infusion has been used for Tcell depletion after 2013 for Haplo-identical transplants.

Results: A total of fifty six pediatric patients were transplanted using either Haplo-identical stem cell $(\mathrm{N}=28$, $50 \%$ ) or umbilical cord blood (UCB) transplantation $(\mathrm{N}=28,50 \%)$. Transplant indications for 28 Haploidentical were: primary immune deficiency syndromes $(\mathrm{N}=10)$, acute leukemia $(\mathrm{N}=8)$, severe aplastic anemia $(\mathrm{N}=3)$, Fanconi anemia $(\mathrm{N}=2)$, inborn error of metabolism $(\mathrm{N}=2)$, relapsed Hodgkin disease $(\mathrm{N}=1)$, osteopetrosis $(\mathrm{N}=1)$, and congenital amegakaryocytic thrombocytopenia $(\mathrm{N}=1)$. Transplant indications for UCB were: acute leukemia $(\mathrm{N}=16)$, Chediak-Higashi syndrome $(\mathrm{N}=4)$, thalassemia $(\mathrm{N}=2)$, Fanconi anemia $(\mathrm{N}=3)$, JMML $(\mathrm{N}=1)$, severe aplastic anemia $(\mathrm{N}=1)$, and Leukocyte adhesion deficiency $(\mathrm{N}=1)$. The transplant conditioning intensity and post-transplant complications are depicted in Table 1.

Conclusions: Haplo-identical stem cell and umbilical cord blood (UCB) transplantation are viable and life-saving alternative donor programs. Complications at our center were manageable and mortality was similar to what was reported in the literature. Timely availability of Haploidentical donors make this option plausible in places where umbilical cord transplant is not feasible.

Conflict of interest: no conflict of interest

\begin{tabular}{lll}
\hline & $\begin{array}{l}\text { Haplo-identical } \\
\text { transplant }\end{array}$ & $\begin{array}{l}\text { Umbilical Cord } \\
\text { transplant }\end{array}$ \\
\hline Total number & $\begin{array}{l}\text { 28 (age range 1-18 } \\
\text { years) }\end{array}$ & $\begin{array}{l}\text { 28(age range 0.25-18 } \\
\text { years) }\end{array}$ \\
$\begin{array}{l}\text { Overall survival (median } \\
\text { follow up) }\end{array}$ & $52 \%(29.8$ months) & $58 \%$ (52.2 months) \\
$\begin{array}{l}\text { Conditioning intensity } \\
\text { Myeloablative }\end{array}$ & $6(21.4 \%)$ & $23(82.1 \%)$ \\
Reduced intensity & $14(50 \%)$ & $5(17.9 \%)$ \\
Without conditioning & $8(28.6 \%)$ & 0 \\
CMV reactivation & $10(35.7 \%)$ & $16(57.1 \%)$ \\
aGVHD & $13(46.4 \%)$ & $7(25.0 \%)$ \\
cGVHD & $7(25.0 \%)$ unknown 1 & $3(10.7 \%)$ \\
\hline
\end{tabular}

[[P758 Table] Table 1 transplant conditioning intensity and post]
P759

Pediatric Hematopoietic Stem Cell Transplantation in India: A report by Indian Pediatric Hematology Oncology Group

Sunil Bhat ${ }^{1}$, Ravi Joshi', Satyendra Katewa ${ }^{2}$, Gaurav Kharya ${ }^{3}$, Santanu Sen ${ }^{4}$, Ruchira Misra ${ }^{5}$, Intezar Mehdi, Deenadayalan Munirathnam $^{7}$, Anand Kumar ${ }^{8}$, Shobha Badiger ${ }^{1}$, Pooja Mallya ${ }^{1}$, Neha Rastogi ${ }^{5}$, Mohammed Ramzan ${ }^{2}$, Sachin Suresh Jadhav ${ }^{8}$, Vimal Kumar ${ }^{7}$, Sharat Damodar', Anupam Sachdeva ${ }^{10}$, Satya Prakash Yadav ${ }^{5}$

${ }^{1}$ Mazumdar Shaw Cancer Center, Narayana Health City, Pediatric Hematology Oncology, Bangalore, India; ${ }^{2}$ Manipal Hospital, Jaipur, India; ${ }^{3}$ Artemis Hospital, Gurgaon, India; ${ }^{4}$ Kokilaben Dhirubhai Ambani Hospital, Mumbai, India; ${ }^{5}$ Medanta Medicity Hospital, Gurgaon, India; ${ }^{6}$ HCG Hospital, Bangalore, India; ${ }^{7}$ Gleneagles Global Health City, Chennai, India; ${ }^{8}$ BGS Gleneagles Global Hospital, Bangalore, India; ${ }^{9}$ Mazumdar Shaw Cancer Center, Narayana Health City, Bangalore, India; ${ }^{10}$ Sir Gangaram Hospital, Delhi, India

Background: Hematopoietic stem cell transplantation (HSCT) is a life saving and sometimes only treatment option for management of many children with malignant and non-malignant diseases. India has a population of 1.21 billion of which $39 \%$ are children. There is a need to have a robust HSCT program for children across multiple centers. There is paucity of data on HSCT in pediatric patients from India. Indian Pediatric Hematology Oncology Group (IPHOG) is a collaborative research group and reports here the outcomes of HSCT in children from various centers across India.

Methods: In our retrospective study HSCT data from 8 pediatric transplant centers across India from 2005 till Dec 2017 was collected on a standard format for analysis. Data was collected on demographic profile, indication for transplant, conditioning regimen, source of stem cells, graft versus host disease (GvHD) and outcome.

Results: Total number of HSCTs performed in all these centers during this time frame was 717 , of which complete data was available for 691 transplants. The mean age at transplant was 7.4 years (5 months to 18 years) with $65.1 \%$ male and $34.9 \%$ female patients. Common indications for HSCT were hemoglobinopathies $(33.71 \%)$, with thalassemia contributing to the majority, acute and chronic leukemia constituted (18.2\%), bone marrow failure syndromes (13.8\%), and autologous transplant for solid tumors (20.4\%), primary immunodeficiency disorders $(2.1 \%)$ and various other conditions (11.8\%). Majority of transplants done were first transplants (91.8\%). Allogeneic and autologous transplants comprised $78.3 \%$ and $21.7 \%$ of the patients respectively. Among the allogeneic HSCTs fully matched sibling/family donor comprised $62.1 \%$, while as haplo-identical transplants, matched unrelated donor (MUD) and cord blood comprised 23.2\%, 6.4\% and 1.6\% 


\section{Total number of transplants}

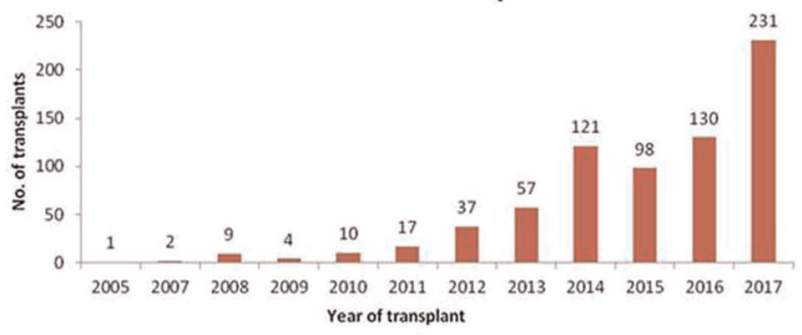

[P759 Figure] [Graph 1]

transplants respectively. Stem cells were collected from peripheral blood in $71.2 \%$ of donors, while in $24.7 \%$ bone marrow was the source. Cord blood was used in $1.6 \%$, while in $2.5 \%$ of the cases combination of stem cell sources were used. Acute GvHD was reported in $22.7 \%$ of patients and chronic GvHD in $6.1 \%$ of patients. Acute GvHD was more commonly seen in patients with haploidentical transplant $(34.1 \%)$ and matched unrelated transplants (28\%). Among all the patients undergoing HSCT the overall mortality comprised $18.4 \%$ with infection $(60.6 \%)$ being the most common cause followed by disease recurrence (15.7\%) and GVHD (6.3\%). The overall survival in autologous, matched sibling/family donor, haploidentical and in MUD transplants is $83.4 \%, 78.7 \%, 69.4 \%, 68 \%$ respectively. Trend over the years is shown in graph 1 . Graph 1

Conclusions: We report the largest Indian data on HSCT in pediatric patients from India. Thalassemia is the commonest indication. The outcomes of allogeneic HSCT are best for matched sibling donor category however outcomes for haplo-identical and MUD transplants are encouraging. Haplo-identical transplants are on a rise.

\section{Conflict of interest: None}

\section{P760}

\section{Peripheral blood stem cell collection in a hemophiliac child} with high-risk neuroblastoma - a case report

Juliana Moutinho ${ }^{1}$, Ana Maia Ferreira ${ }^{2}$, Fernando Campilho ${ }^{3}$, Sara Morais $^{4}$, Armando Correia Pinto ${ }^{2}$, Luisa Lopes dos Santos ${ }^{1}$, António Campos Júnior ${ }^{3}$, Susana Roncon ${ }^{5}$

${ }^{1}$ Instituto Português de Oncologia do Porto, Department of ImmuneHemotherapy, Porto, Portugal; ${ }^{2}$ Instituto Português de Oncologia do Porto, Department of Pediatrics, Porto, Portugal; ${ }^{3}$ Instituto Português de Oncologia do Porto, Bone Marrow Transplant Unit, Porto, Portugal; ${ }^{4}$ Centro Hospitalar do Porto, Department of Clinical Hematology, Thrombosis and Haemostasis Unit, Porto, Portugal; ${ }^{5}$ Instituto Português de Oncologia do Porto, Department of Cellular Therapy, Porto, Portugal

Background: This paper reports the first case of a severe congenital A hemophiliac child with high-risk neuroblastoma, proposed for high-dose chemotherapy with peripheral blood stem cell (PBSC) support.

Methods: This child presented at 20-months of age left adrenal mass accompanied by retroperitoneal lymph nodes, renal and bone marrow metastasis (stage IV INSS neuroblastoma, undifferentiated, large cell variant). FISHmutations were presented at diagnosis (relative loss of $1 \mathrm{p} 36$; relative gain of $17 \mathrm{q} 12$ and $\mathrm{MYCN}$-gene). Standard chemotherapy was started (RapidCOJEC-protocol + 3TVD-cycles), leading to a very good partial response; after tumor surgical excision, 2TVD-cycles were done and he was proposed to the MATIN-protocol (MIBG+Topotecan) followed by double autologous PBSC transplantation. The patient initiated prophylaxis with FVIII concentrate (25IU/Kg, 3x/week), immediately after tumor diagnosis. On-demand treatments were used, adapting the FVIII dose to the hemorrhagic risk associated with different therapies or invasive procedures (insertion/replacement of central venous catheter; abdominal surgery; PBSC collection/ infusion).

Results: At 28-months old (weight $=13 \mathrm{~kg}$ ), patient collected PBSC by apheresis with the separator SPECTRA OPTIA $^{\circledast}$ (version 11.2, MNC program) after inserting in the right subclavian vein a 2-way $9 \mathrm{Fr}$ Hickman catheter. At the $5^{\text {th }}$-day of mobilization (Table1), the number of $\mathrm{CD} 34^{+}$ cells in peripheral blood was $63 / \mu \mathrm{L}$ and a one-day collection was performed: $3850 \mathrm{ml}$ of whole blood processed during 5.5 hours; extracorporeal circuit anticoagulated with citrate, ratio 14:1. The graft presented good cellular content $\left(7.7 \times 10^{6} \mathrm{CD} 4^{+}\right.$cells $\left./ \mathrm{kg}\right)$, was functional $\left(7.2 \times 10^{5} \mathrm{CFU}-\right.$ $\mathrm{GM} / \mathrm{kg}$ ), sterile (negative bacterial/mycological cultures) and FISH-negative; it was subsequently cryopreserved in liquid nitrogen. Ten and twelve months after the diagnosis, $3.5 \times 10^{6} \mathrm{CD} 34^{+}$cells/ $\mathrm{Kg}$ were thawed and infused for each transplant; hematological recovery $\left(>500\right.$ Neutrophils $\left./ \mathrm{mm}^{3}\right)$ occurred at day +7 and day +11 , respectively. Despite his hemorrhagic disease, the patient did not experience severe bleeding events nor needed an increased transfusional use. The bone marrow trephine biopsy and MIBG-scintigraphy showed absence of metastatic involvement; however CTscan revealed 3 metastatic renal lesions and patient is currently proposed to localized radiotherapy.

Conclusions: The PBSC collection, that has demonstrated to be safe and effective in adults, has proved to be successful also in pediatrics, relying on the optimization of techniques in this age group and the timing of the harvest. The product obtained met all the quality requirements necessary to ensure hematopoietic grafting. The multidisciplinary team, integrating clinical and laboratorial experts, allowed the realization of the proposed treatment, namely high-dose chemotherapy, in a low weight child with severe coagulopathy and metastatic tumor. 
Conflict of interest: The authors declare that there is no conflict of interest in the accomplishment of this work.

\begin{tabular}{|c|c|c|c|}
\hline Clinical settings & Graft collection & 1st graft infusion & 2nd graft infusion \\
\hline $\begin{array}{l}\text { Hospitalization } \\
\text { (days) }\end{array}$ & 2 & 15 & 24 \\
\hline $\begin{array}{l}\text { G-CSF } \\
\text { mobilization* } \\
\text { Conditioning } \\
\text { regimen** }\end{array}$ & $* 12 \mu \mathrm{g} / \mathrm{kg} /$ day & $\begin{array}{l}15 \text { days after } 2 \text { nd } \\
\text { MIBG therapy }\end{array}$ & $\begin{array}{l}* * \text { Bussulfan } \\
+ \text { Melphalan }\end{array}$ \\
\hline $\begin{array}{l}\text { Transfusion of RBC } \\
\text { concentrate / Platelet } \\
\text { concentrate }\end{array}$ & $\begin{array}{l}1 \text { (priming) / } 1 \\
\text { (platelets }>100000 / \\
\mathrm{mm} 3 \text { ) }\end{array}$ & $\begin{array}{l}3 \\
\text { (hemoglobin }>7.5 \mathrm{~g} / \\
\text { dl) } / 12 \\
\text { (platelets }>50000 / \\
\text { mm3) }\end{array}$ & $1 / 8$ (same criteria) \\
\hline $\begin{array}{l}\text { FVIII prophylaxis } \\
\text { (IU/Kg) } \\
\text { (Immediately before: } \\
\text { IB; Aplasia period: } \\
\text { AP) }\end{array}$ & $\begin{array}{l}\text { IB: } 50 ; 1 \text { st day: } 50 \\
1 \text { st dose }+408 / \\
8 \text { h; } \geq 2 \text { nd day: } 25 \\
3 x / \text { week }\end{array}$ & $\begin{array}{l}\text { IB: } 25 \text { 3x/week; } 1 \text { st } \\
\text { and } 2 \text { nd day: } 40 \text { 8/ } \\
\text { 8h; } \geq 3 \text { rd day: } 25 / \text { day } \\
\text { during AP }\end{array}$ & $\begin{array}{l}\text { IB: } 25 \text { 3x/week; } \\
\geq 1 \text { st day: } 25 / \text { day } \\
\text { during AP }\end{array}$ \\
\hline Adverse events & No & Febrile neutropenia & $\begin{array}{l}\text { Mucositis (grade } \\
\text { 4), Hepatotoxicity, } \\
\text { Febrile syndrome } \\
\text { + bacteremia }\end{array}$ \\
\hline
\end{tabular}

[[P760 Table] Table1: Clinical settings]

\section{P761}

Abstract previously published

\section{P762}

Post-transplant cyclophosphamide as a graft versus host prophylaxis in pediatric haploidentical hematopoietic stem cell transplantation - case series

Elżbieta Wawrzyniak-Dzierżek, Kornelia Gajek, Ewa Gorczyńska, Krzysztof Kalwak, Marek Ussowicz
Wroclaw Medical University, Dept of Pediatric Hematology and Oncology, BMT Unit CIC 817, Wroctaw, Poland

Background: Haploidentical stem cell transplantation (haploSCT) is an alternative for patients with advanced hematologic malignancies without matched family donors, especially due to increased alloreactivity from HLA and KIR mismatch. The risk of severe GvHD can be prevented by ex vivo T-cell depletion or post-transplant cyclophosphamide (PTCY).

Methods: We present six pediatric patients transplanted from haploidentical donors with PTCY. Three girls and three boys aged 1 to 17 years underwent haploSCT between August 2012 and November 2017.

Results: In 2 patients classical Baltimore protocol was used with 2 Gy total body irradiation, Fludarabine $(150 \mathrm{mg} /$ $\left.\mathrm{m}^{\wedge} 2\right)$, Cyclophosphamide $(2 * 14,5 \mathrm{mg} / \mathrm{kg})$, two patients were conditioned with original protocol FCM- Fludarabine (150 $\left.\mathrm{mg} / \mathrm{m}^{\wedge} 2\right)$, Cyclophosphamide $(2 * 14,5 \mathrm{mg} / \mathrm{kg})$, Melphalan $(4 \mathrm{mg} / \mathrm{kg})$, one patient was treated with Treosulfan $(30 \mathrm{~g} /$ $\left.\mathrm{m}^{\wedge} 2\right)$, Fludarabine $\left(120 \mathrm{mg} / \mathrm{m}^{\wedge} 2\right)$ and one with Treosulfan $\left(20 \mathrm{~g} / \mathrm{m}^{\wedge} 2\right)$, Fludarabine $\left(150 \mathrm{mg} / \mathrm{m}^{\wedge} 2\right)$, Cyclophosphamide $(2 * 14,5 \mathrm{mg} / \mathrm{kg})$. The source of $\mathrm{CD} 34+$ cells was bone marrow in five cases and peripheral blood apheresis in one case. Cyclosporine was used in all recipients and mycophenolate mofetil in four. Engraftment with complete donor chimerism was achieved in four patients, one patient rejected and one died before leukocyte recovery. All 4 engrafted patients developed skin aGvHD (maximal stage III), two of them - gut aGvHD (both stage III), one - liver aGvHD (stage II). In 3 recipients cGvHD was observed (2limited, 1-extensive). 3 of 4 engrafted patients are alive: two patients after FCM conditioning live in complete remission: 17 and 21 months after transplantation, one patient after Baltimore conditioning died due to the relapse diagnosed 6 months after transplantation, one patient after

\begin{tabular}{|c|c|c|c|c|c|c|c|c|c|}
\hline $\begin{array}{l}\text { Patient } \\
\text { initials/ } \\
\text { sex/age) }\end{array}$ & Diagnosis & Previous SCT & $\begin{array}{l}\text { Date of } \\
\text { PTCY } \\
\text { HaploSCT }\end{array}$ & $\begin{array}{l}\text { Graft } \\
\text { source } \\
\text { (donor) }\end{array}$ & $\begin{array}{l}\text { Conditioning } \\
\text { protocol }\end{array}$ & Immunosupression & Engraftment & $\begin{array}{l}\text { GvHD (acute/ } \\
\text { chronic) }\end{array}$ & $\begin{array}{l}\text { Follow } \\
\text { up }\end{array}$ \\
\hline $\begin{array}{l}\text { M.M./F/ } \\
14\end{array}$ & $\begin{array}{l}\text { MDS/ } \\
\text { AML }\end{array}$ & $\begin{array}{l}\text { I MUD (Treo+Cy+Mel+ATG) II } \\
\text { MUD (Bu+Flu+Cy+ATG) III } \\
\text { MUD (TBI+Cy+Thymo) }\end{array}$ & 17.08.2012 & $\begin{array}{l}\text { BM } \\
\text { (father) }\end{array}$ & $\begin{array}{l}2 \text { Gy sTBI+Flu } \\
\left(150 \mathrm{mg} / \mathrm{m}^{\wedge} 2\right)+\mathrm{Cy} \\
(2 * 14,5 \mathrm{mg} / \mathrm{kg})\end{array}$ & $\begin{array}{l}\text { PTCY }(+3,+4) \\
\text { CSA, MMF }\end{array}$ & donor $100 \%$ & $\begin{array}{l}\text { Acute skin I, } \\
\text { gut III/chronic } \\
\text { limited }\end{array}$ & $\begin{array}{l}\text { relapse, } \\
\text { died }\end{array}$ \\
\hline $\begin{array}{l}\text { K.L./M/ } \\
17\end{array}$ & AML & $\begin{array}{l}\text { I MUD }(\mathrm{Bu}+\mathrm{Cy}+\mathrm{Mel}+\mathrm{Thymo}) \mathrm{II} \\
\text { MUD (Treo+Flu+ATG) }\end{array}$ & 19.08.2014 & $\begin{array}{l}\text { BM } \\
\text { (brother) }\end{array}$ & $\begin{array}{l}2 \text { Gy sTBI+Flu } \\
\left(150 \mathrm{mg} / \mathrm{m}^{\wedge} 2\right)+\mathrm{Cy} \\
(2 * 14,5 \mathrm{mg} / \mathrm{kg})\end{array}$ & $\begin{array}{l}\text { PTCY }(+3,+4) \\
\text { CSA, }\end{array}$ & $\begin{array}{l}\text { rejection } \\
(100 \% \\
\text { autologous) }\end{array}$ & $\mathrm{n} / \mathrm{a}$ & $\begin{array}{l}\text { alive in } \\
\text { 3. CR }\end{array}$ \\
\hline L.F./F/2 & ALL & $\begin{array}{l}\text { I MUD }(\mathrm{Bu}+\mathrm{Flu}+\mathrm{TT}+\mathrm{ATG}) \mathrm{II} \\
\text { MUD (Treo+Cy+VP-16+Thymo) }\end{array}$ & 27.08 .2015 & $\begin{array}{l}\text { PBPC } \\
\text { (mother) }\end{array}$ & $\begin{array}{l}\text { Flu }\left(150 \mathrm{mg} / \mathrm{m}^{\wedge} 2\right) \\
+ \text { Cy }(2 * 14,5 \mathrm{mg} / \mathrm{kg}) \\
+\operatorname{Mel}(4 \mathrm{mg} / \mathrm{kg})\end{array}$ & $\begin{array}{l}\text { PTCY }(+3,+4) \\
\text { CSA, MMF }\end{array}$ & donor $100 \%$ & $\begin{array}{l}\text { Acute skin III, } \\
\text { gut III/chronic } \\
\text { extensive }\end{array}$ & $\begin{array}{l}\text { alive in } \\
4 . \mathrm{CR}\end{array}$ \\
\hline E.S./F/7 & ALL & MSD (TBI+VP-16) & 18.12 .2015 & $\begin{array}{l}\text { BM } \\
\text { (father) }\end{array}$ & $\begin{array}{l}\text { Flu }\left(150 \mathrm{mg} / \mathrm{m}^{\wedge} 2\right) \\
+ \text { Cy }\left(2^{*} 14,5 \mathrm{mg} / \mathrm{kg}\right) \\
+\operatorname{Mel}(4 \mathrm{mg} / \mathrm{kg})\end{array}$ & $\begin{array}{l}\text { PTCY }(+3,+4) \\
\text { CSA, MMF }\end{array}$ & donor $100 \%$ & $\begin{array}{l}\text { Acute skin I/ } \\
\text { chronic } \\
\text { limited }\end{array}$ & $\begin{array}{l}\text { alive in } \\
3 . \mathrm{CR}\end{array}$ \\
\hline $\begin{array}{l}\text { J.K./M/ } \\
10\end{array}$ & $\begin{array}{l}\text { ALL/ } \\
\text { MDS/ } \\
\text { AML }\end{array}$ & $\begin{array}{l}\text { Alpha-Beta T-cell depleted } \\
\text { HAPLO (Bu+Cy+Mel+Thymo) }\end{array}$ & 13.04 .2017 & $\begin{array}{l}\text { BM } \\
\text { (father) }\end{array}$ & $\begin{array}{l}\text { Treo }\left(30 \mathrm{~g} / \mathrm{m}^{\wedge} 2\right)+\text { Flu } \\
\left(120 \mathrm{mg} / \mathrm{m}^{\wedge} 2\right)\end{array}$ & $\begin{array}{l}\text { PTCY }(+3,+4) \\
\text { CSA, MMF }\end{array}$ & $\begin{array}{l}\text { not } \\
\text { achieved }\end{array}$ & $\mathrm{n} / \mathrm{a}$ & died \\
\hline $\begin{array}{l}\text { S.C./M/ } \\
1\end{array}$ & HLH & $\begin{array}{l}\text { Alpha-Beta T-cell depleted } \\
\text { HAPLO (Bu+Flu+TT+Mabthera) }\end{array}$ & 10.11 .2017 & $\begin{array}{l}\text { BM } \\
\text { (mother) }\end{array}$ & $\begin{array}{l}\text { Treo }\left(20 \mathrm{~g} / \mathrm{m}^{\wedge} 2\right)+\text { Flu } \\
\left(150 \mathrm{mg} / \mathrm{m}^{\wedge} 2\right)+\text { Cy } \\
(2 * 14,5 \mathrm{mg} / \mathrm{kg})\end{array}$ & $\begin{array}{l}\text { PTCY }(+3,+4) \\
\text { CSA, MMF }\end{array}$ & donor $100 \%$ & $\begin{array}{l}\text { Acute skin II, } \\
\text { liver II }\end{array}$ & alive \\
\hline
\end{tabular}

[[P762 Table] Haploidentical SCT with PTCY in pediatric patients] 
conditioning Treo-Flu-Cy conditioning is alive 1 month after transplantation. T-cell count of 200 cells/uL was reached 90 days after haploSCT in all patients. The patient who rejected graft was salvaged with stem cell transplant ation from an unrelated donor.

Conclusions: HaploSCT can be a viable option for patients with advanced malignancies, who relapse after transplantation and are heavily pretreated. Alternative, TBIfree protocols can be used as conditioning in PTCY haploSCT. The aGVHD rate in these protocols needs more investigation.

Conflict of interest: None of the authors has anything to disclose.

\section{P763}

\section{Predicting outcomes after pediatric stem cell} transplantation for hematological malignancies: a decisiontree-based model

Antonio Pérez-Martínez ${ }^{1}$, Alicia Gómez López ${ }^{2}$, Yasmina Mozo ${ }^{1}$, Blanca Rosich ${ }^{1}$, David Bueno ${ }^{1}$, Berta González ${ }^{1}$, Adela Escudero ${ }^{1}$, Isabel Mirones ${ }^{1}$, Alba Fernández ${ }^{3}$, Ana Sastre ${ }^{3}$, Carlos Óscar Sorzano $^{4}$

${ }^{1}$ Hospital Universitario La Paz, Madrid, Spain; ${ }^{2}$ Universidad Autonoma de Madrid, Medicine School, Madrid, Spain; ${ }^{3}$ Hospital Universitario La Paz, Pediatric Hemato-Oncology and Stem Cell Transplantation, Madrid, Spain; ${ }^{4}$ Universidad Autonoma de Madrid, National Center of Biotechnology (CSIC), Madrid, Spain

Background: Identification of pre-transplantation variables by risk of mortality after paediatric haematopoietic stem cell transplantation (HSCT) might be helpful for accurate patient counselling and clinical decision-making. Some scores, as European Bone Marrow Transplantation (EBMT) risk score, have shown predictable as pretransplant risk assessment in adult HSCT. However, it has not been address yet in paediatric HSCT. In fact some variables, as age, cannot be incorporate from the original one. Survival tree models are an easy-to-interpret alternative statistical approach to identify subgroups, offering cut-off points for different covariates. This study aimed propose a survival tree models that could help to predict mortality after paediatric HSCT in a cohort of high risk leukemia patients.

Methods: We analysed 185 leukaemia patients who received a HSCT at our center between 1996 and 2017. We constructed a random forest classifier on the database of patients with malignant disease transplanted during the last 20 years. The random forest identified four variables that were highly related to the final result (death or survival) of the patient after transplantation: disease state, patient age, time from diagnosis to transplantation and EBMT risk score. With these variables we constructed a classification tree in order to predict outcome after HSCT.
Results: The disease survival (DFS) was $66 \%$ whereas the cumulative non-relapse mortality (NRM) and relapse incidences were $28 \%$ and $11 \%$. The values of EBMT risk score was $0(8.7 \%), 1(32.9 \%), 2(20.5 \%), 3(23.6 \%), 4$ $(11.8 \%), 5(2.5 \%)$. According to univariate analysis there were not associated between the increase in score intervals with a decreasing probability of 2-year OS and LFS. The tree shows that there are three groups significantly different from the general population. On the negative side, patients with 1) an advanced disease state or 2) non-advanced, but an age between 102 and 146 months (between 8.5 and 12 years) with a EBMT risk score larger than 0 and whose transplantation occurs before 3 years after diagnostic have a mortality significantly larger than the general population. On the positive side, small children (age $<=68$ months, about 5.5 years) with a transplantation have a prognostic significantly better than the general population. There are two groups that point out promising positive prognostic (1: Non-advanced disease, Age $>68$ and $\mathrm{T}>35.5 ; 2$ : Nonadvanced disease, Age $>68, \mathrm{~T}<35.5$ and Score=0). However, the observed number of patients does not allow showing a statistically significant difference (1: pvalue $=0.1928 ; 2$ : $p$-value $=0.39$ ).

Conclusions: In conclusion, we demonstrated the tree model to be an accurate and attractive tool to predict pediatric HSCT outcome in our institution. This may aid the evaluation of individual patient prognosis and thereby the design of measures to improve HSCT outcome in the poor prognosis groups

Conflict of interest: Nothing to disclose

\section{P764}

Predictive markers for B-cell function after HSCT in patients with B-positive SCID (IL2RG/ JAK3-deficiency)

Abdallah Khazaleh, Eva-Maria Jacobsen, Ansgar Schulz, KlausMichael Debatin, Manfred Hoenig

University Medical Center Ulm, Pediatrics, Ulm, Germany

Background: Genetic defects in $I L 2 R G$ or $J A K 3$ result in severe combined immunodeficiency (SCID) with absent $\mathrm{T}$ cells and non-functional B cells (T-B + SCID). B-cell function after hematopoietic stem cell transplantation (HSCT) depends on the engraftment of donor B-cells. The objective of the study was to identify a B-cell subpopulation as an early predictor for maturation and function of B-cells after HSCT in addition to the detection of $\operatorname{IgA}$ or IgM.

Methods: We retrospectively analysed 25 long term surviving patients with $\mathrm{B}+\mathrm{SCID}$ due to mutations in JAK3 $(9 / 2536 \%)$ and IL2RG (16/25 64\%) after HSCT in our institution between 1980 and 2017. 13/25 patients were 
independent of, whereas $12 / 25$ patients remained dependent on regular immunoglobulin substitution. Flow cytometry analysis was performed from blood samples (MNCs) which had been frozen after HSCT between day +80 to +250 .

Results: Total numbers of memory B-cells or their proportion in CD19 positive cells did not correlate well with B-cell function after HSCT as they are dependent on B-cell chimerism. The presence of more than $4.7 \%$ of class switched memory B-cells (CD27+IgM-) in relation to total memory B cells $(\mathrm{CD} 27+)$ though proved to be a reliable marker for positive B-cell function with a sensitivity of 1.0 and a specificity of 0.93 .

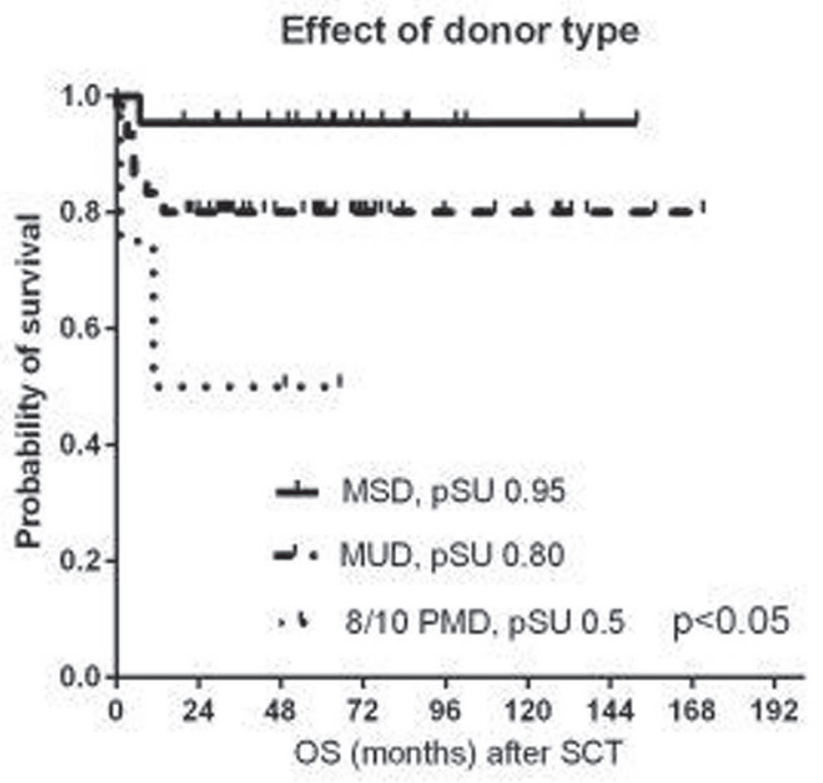

Effect of KIR GVH mismatch

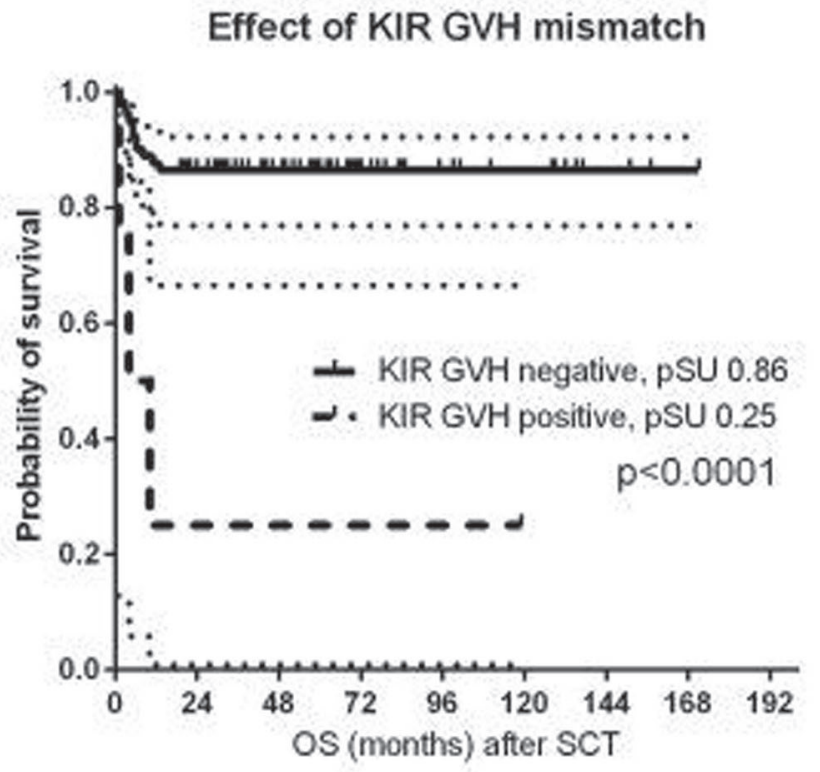

[P765 Figure] [Survival outcome of 86 children after allo-SCT.]
Conclusions: For patients with $\mathrm{B}+\mathrm{SCID}$ the ratio of $\mathrm{CD} 27+/ \mathrm{IgM}$ - to $\mathrm{CD} 27+\mathrm{B}$-cells is a specific and sensitive marker at day +100 to identify those who will need Igsubstitution after HSCT.

Conflict of interest: The authors have nothing to disclose

\section{P765}

Presence of KIR-ligand graft versus host mismatch decreases overall survival after allogeneic matched donor SCT in children

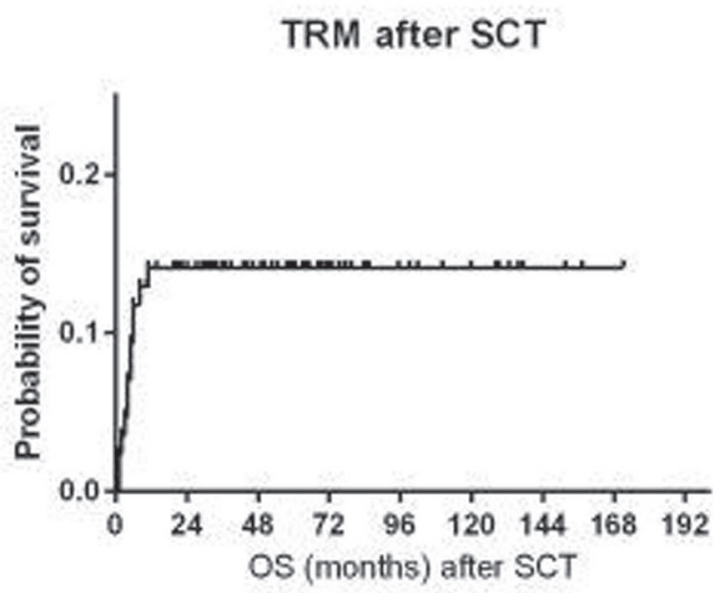


Kornelia Gajek, Elżbieta Wawrzyniak-Dzierżek, Blanka Rybka, Renata Ryczan-Krawczyk, Krzysztof Kahwak, Marek Ussowicz,

Wroclaw Medical University, Dept of Pediatric Hematology and Oncology, BMT Unit CIC 817, Wroctaw, Poland

Background: Killer immunoglobulin-like (KIR) receptors play role in regulation of NK-lymphocyte reactivity and affect immunological response in stem cell transplantation (SCT) recipients. The inclusion of KIR-associated factors can result in better donor selection in allogeneic SCT.

Methods: We analysed the role of donor- and recipientrelated factors in 86 children after allogeneic SCT from matched sibling (22 cases) or matched unrelated donor (64 cases). 62 children were transplanted due to non-malignant indications (SAA-21, primary immunodeficiencies-18, BDA-6, Fanconi anemia - 4, HLH - 4, PNH-2, storage diseases-5, other-2), and 24 for malignant diseases (MDS11, JMML-3, ALL-2, AML-7, JXG-1). The 86 donor samples were tested for KIR genotype using KIR typing kit (Miltenyi, DE). The impact of diagnosis (malignant vs nonmalignant), degree of HLA-match for each locus separately, pre-transplant conditioning (busulfan-, treosulfan, or TBIcontaining), and KIR-genotyping results were analysed using Statistica 13.1 and GraphPad Prism 6.0.

Results: 22 patients had 10/10 HLA matched sibling donor (MSD), 60 pts $-9 / 10$ or 10/10 matched unrelated donor (MUD), 4 pts - 8/10 partially matched donor (PMD). The mismatches in HLA antigens were present in locus A in 8 patients, $\mathrm{B}$ in $\mathrm{B}-3 \mathrm{pts}$, in $\mathrm{C}-10 \mathrm{pts}$, in DRB1 - $1 \mathrm{pt}$, in HLA DQB1 - 4 pts. The calculated B-content was best-10/ better-36/neutral-25/other-12. The graft-versus-host (GVH) KIR-ligand mismatch was observed in 4 patients and hostversus-graft (HVG) in 2 cases. Probability of 5-year OS in the analysed group was $83 \%$. Better donor choice was associated with higher probability of survival (MSD vs MUD vs PMD, $95 \%$ vs $80 \%$ vs $50 \%, \mathrm{p}=0.05)$. The probability of OS was decreased in the presence of GVH KIR mismatch ( $86 \%$ vs $25 \%, \mathrm{p}=0.0001)$, but other analysed factors did not affect OS. The multivariate analysis using the Cox regression model revealed GVH KIR mismatch (HR 7.9, 95\% CI 1.98-31.48) as the only relevant factor for OS. TRM in the whole group was $14 \%$, and no statistically significant factors affecting TRM were identified.

Conclusions: In case of partially matched donors, the optimal donor choice algorithm should eliminate presence of graft-versus-host KIR ligand mismatch. The effect of KIR genotype and presence of single activating or inhibiting KIR alleles on SCT outcome can be elusive and need cautious consideration during donor selection.

Conflict of interest: None of the authors has anything to disclose.
P766

Progressive refractory pediatric Hodgkin lymphoma after salvage treatment is associated with poor prognosis after autologous stem cell transplant - single center experience

Anca Colita ${ }^{1}$, Cristina Georgiana Jercan ${ }^{2}$, Ana Maria Bica ${ }^{3}$, Andrei Colita $^{2}$, Constantin Arion ${ }^{2}$, Carmen Calugaroiu ${ }^{3}$, Alexandra Ionete ${ }^{3}$, Alina Daniela Tanase ${ }^{3}$

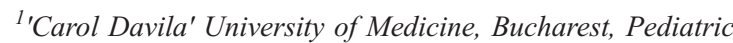
Hematology \& BMT, Bucharest, Romania; ${ }^{2}$ Carol Davila University of Medicine, Bucharest, Romania, ${ }^{3}$ Fundeni Clinical Institute, Bucharest, Romania

Background: Hodgkin Lymphoma (HL) represents 6\% of pediatric cancer, and has a highly curable rate with standard chemotherapy, but despite this good prognosis, approximately $15 \%$ patients relapse. Treatment options for relapsed or refractory HL are high-dose chemotherapy (CHT) with autologous SCT. Our study evaluate single center experience over 15 years in treatment of pediatric Hodgkin lymphoma patients.

Methods: We retrospectively evaluated pediatric patients with refractory or relapsed HL, who received autologous SCT in Pediatric Hematology \& Bone Marrow Transplant Department, Fundeni Clinical Institute between 2001 2017. Patients records were demographic data, clinical findings, diagnosis, treatment course and follow-up. First line treatment included several chemotherapy regimens: ABVD, COPP-ABV, OEPA, BEACOPP, local radiotherapy (RT). Second line treatment for refractory or relapsed HL consisted of salvage therapy with DHAP or IGEV regimens followed by high-dose chemotherapy and autologous HSCT. After salvage therapy, all patients were evaluated with PET-CT. All parents signed informed consent forms.

Results: We analyzed 34 consecutive pediatric patients with HL, sex ratio $25 \mathrm{~F} / 9 \mathrm{M}$, age range $4-18 \mathrm{y}$ referred to our center between 2002-2017. Based on hystopathological results patients were diagnosed with: nodular sclerosis $76 \%$, mixed cellularity $24 \%$, lymphocyte depleted $2 \%$; early stage at diagnosis $32 \%$, advanced stage $67 \%$. Salvage therapy consisted of 2 cycles of DHAP in 30 cases and 2 cycles of IGEV in 4 cases. PET evaluation before transplant showed: complete remission 21 cases (70.5\%), partial remission 10 cases $(29.4 \%)$ and progressive disease 3 cases $(8.8 \%)$. BEAM regimen was used in $88.6 \%$ or LEAM in $11.4 \%$. Median time to transplantation was 10.5 months (range 6 to 75 moths). Disease free survival according to pretransplant status showed $85.7 \%$ in PET negative patients, $60 \%$ in PET positive partial remission and $0 \%$ in PET positive, progressive disease. 
Conclusions: In conclusion, 24/34 patients (70\%) maintained CR after autologous SCT, 10/34 (30\%) relapsed. 8/ 10 relapsed patients died. Autologous HSCT should be recommended in patients with relapsed or refractory disease, but chemosensitive disease, demonstrated by PET-CT. For patients with progressive disease after salvage chemotherapy, other therapeutic options should be considered.

Conflict of interest: Nothing to disclose.

\section{P767}

Results of a clinical phase II trial of treosulfan/fludarabinebased conditioning therapy prior to allogeneic haematopoietic stem cell transplantation in paediatric patients with haematological malignancies

Krzysztof Kalwak ${ }^{1}$, Monika Mielcarek ${ }^{1}$, Katharine Patrick ${ }^{2}$, Jan Styczynski $^{3}$, Peter Bader ${ }^{4}$, Selim Corbacioglu ${ }^{5}$, Birgit Burkhardt ${ }^{6}$, Karl-Walter Sykora ${ }^{7}$, Katarzyna Drabko $^{8}$, Jolanta Gozdzik ${ }^{9}$, Franca Fagioli $^{10}$, Johann Greil ${ }^{11}$, Bernd Gruhn ${ }^{12}$, Bernhard Kremens ${ }^{13}$, Franco Locatelli $^{14}$, Ingo Mueller ${ }^{15}$, Paul-Gerhardt Schlegel ${ }^{16}$, Petr Sedlacek $^{17}$, Klaus Daniel Stachel ${ }^{18}$, Ajay Vora $^{19}$

${ }^{1}$ Wroclaw Medical University, Department of Pediatric Hematology, Oncology and BMT, Wroclaw, Poland; 'Sheffield Children's Hospital, Department of Paediatric Haematology, Sheffield, United Kingdom;

${ }^{3}$ University Hospital Collegium Medicum UMK, Department of

Paediatrics, Haematology and Oncology, Bydgoszcz, Poland;

${ }^{4}$ University Hospital Frankfurt, Goethe University, Department for Children and Adolescents, Division for Stem Cell Transplantation and Immunology, Frankfurt, Germany; ${ }^{5}$ University Hospital Regensburg, Department of Paediatric Oncology and Haematology, Regensburg, Germany; ${ }^{6}$ University Hospital Muenster, Department for Children and Adolescents, Pediatric Hematology and Oncology, Muenster, Germany; ${ }^{7}$ Hannover Medical School, Center for Pediatrics and Adolescent Medicine, Pediatric Hematology and Oncology, Hannover, Germany; ${ }^{8}$ Medical University of Lublin, Department of Pediatric Hematology and Oncology, Lublin, Poland; ${ }^{9}$ Jagiellonian University Medical Collage, University Children's Hospital in Cracow.,

Department of Clinical Immunology and Transplantology, Cracow,

Poland; ${ }^{10}$ Children's Hospital Regina Margherita, Clinical Research Unit, Turin, Italy; ${ }^{11}$ University Hospital Heidelberg, Department of Pediatric Hematology and Oncology, Heidelberg, Germany;

${ }^{12}$ University Hospital Jena, Department of Pediatrics, Unit for Hematology, Oncology and Stem Cell Transplantation, Jena,

Germany; ${ }^{13}$ University Hospital Essen, Department of Pediatrics III, Essen, Germany; ${ }^{14}$ IRCCS Ospedale Pediatrico Bambino Gesù, Department of Pediatric Hematology and Oncology, Rome, Italy;

${ }^{15}$ University Hospital Hamburg Eppendorf, Clinic for Pediatric Hematology and Oncology, Hamburg, Germany; ${ }^{16}$ Children's Hospital Julius Maximilians University, Department of Pediatric Hematology, Oncology and Stem Cell Transplantation, Wuerzburg, Germany; ${ }^{17}$ 2nd Medical School, Charles University Motol,

Department of Pediatric Hematology and Oncology, Prague, Czech Republic; ${ }^{18}$ University Hospital Erlangen, Department of Pediatric Hematology and Oncology, Erlangen, Germany; ${ }^{19}$ Great Ormond
Street Hospital, Department of Haematology, London, United Kingdom

Background: Standard myeloablative conditioning regimens prior to allogeneic haematopoietic stem cell transplantation (alloHSCT) are associated with a considerable risk of severe early and late adverse events (AEs), especially in young children. Clinical studies have indicated that treosulfan-based conditioning has myeloablative, immunosuppressive, and anti-neoplastic effects with a favourable toxicity profile. Here, we present efficacy and safety results from a multicentre, international, open-label, prospective phase II trial evaluating a treosulfan-based preparative regimen in paediatric patients undergoing alloHSCT.

Methods: Patients $(\mathrm{n}=70,62.9 \%$ male, $37.1 \%$ female $)$ less than 18 years old with acute lymphoblastic leukaemia $(38.6 \%)$, acute myeloid leukaemia $(41.4 \%)$ in haematological remission, myelodysplastic syndrome (14.3\%), or juvenile myelomonocytic leukaemia $(5.7 \%)$ received treosulfan intravenously (10 [8.6\%], 12 [37.1\%], or 14 [54.3\%] $\mathrm{g} / \mathrm{m}^{2} /$ day according to their body surface area of $\leq 0.5,>0.5$ to 1.0 , or $>1.0 \mathrm{~m}^{2}$ ) on Days -6 to -4 . Fludarabine $(7.1 \%)$ or fludarabine and thiotepa (92.9\%) were administered additionally at the investigators' discretion. A total of $51.4 \%$ of patients received bone marrow, whereas $48.6 \%$ of patients received peripheral blood stem cells. The primary objective of the study was freedom from transplantation (treatment)related mortality from start of conditioning treatment until 100 days after alloHSCT.

Results: Data presented in this abstract describe the Day +100 trial results, while the final 12-month follow-up will be available at the conference. Median patient age was 9.5 years. A total of $80.0 \%$ of the patients underwent a matched (ie, $\geq 9 / 10$ allele-matched) unrelated donor alloHSCT, the remaining $20.0 \%$ were given matched sibling donor transplant. The freedom from transplantation (treatment)related mortality at 100 days after alloHSCT was $98.6 \%$ (90\% confidence interval [CI; 93.4\%, 99.9\%]). There was only 1 transplantation-related death before Day +100 (sepsis; Stenotrophomonas maltophilia). The incidence of complete donor-type chimerism was $94.2 \%$ (90\% CI $[87.2 \%, 98.0 \%])$ on visit Day +28 and $91.3 \%(90 \% \mathrm{CI}$ $[83.6 \%, 96.1 \%])$ on visit Day +100 . At 100 days, the cumulative incidence of grade I to IV acute graft-versushost disease was $43.5 \%$ (90\% CI [33.7\%, 53.3\%]) and that of grade III to IV was $8.7 \%$ (90\% CI [3.1\%, 14.3\%]). The median duration of CTCAE grade IV neutropenia was 21 days (range: 7 to 44 days). No primary and only 1 secondary graft failure (patient received a stem cell boost) occurred.

The most common AEs of at least CTCAE grade III were mucositis (41.4\%), infections (28.6\%), and nausea (18.6\%). 
Only 1 patient (1.4\%) developed hepatic sinusoidal occlusion syndrome.

Conclusions: Treosulfan-based conditioning resulted in excellent engraftment and low transplantation (treatment)related mortality at 100 days after alloHSCT in paediatric patients with haematological malignancies. Additionally, regimen-related toxicity was limited. Chimerism data on Day +100 highlighted the myeloablative properties of this conditioning regimen.

Clinical Trial Registry: EudraCT number: 2013003604-39; ClinicalTrials.gov identifier: NCT02333058

\section{Conflict of interest:}

A. Vora: advisory board member for Jazz, Pfizer and Novartis

K. Kalwak: travel grants medac, Sanofi; Sykora: speaker fees Jazz, research and travel grants medac, travel grant Neovii

P. Bader: research grants: Neovii, medac

B. Kremens: DSMC Member for Miltenyi Biotec

I. Mueller and K. Patrick: travel grants medac

The remaining authors have nothing to disclose.

\section{P768}

\section{Results of allogeneic hematopoietic stem cell} transplantation in infants with acute leukemias

Olesia Paina, Anastasiai Borovkova, Polina Kozhokar, Anastasia Frolova, Kirill Ekushov, Elena Babenko, Ildar Barkhatov, Tatiana Gindina, Alexander Alianskii, Elena Semenova, Ludmila Zubarovskaya, Boris Afanasyev

First State Pavlov Medical University of Saint Petersburg, Raisa Gorbacheva Memorial Institute for Children Oncology, Hematology and Transplantation, Saint Petersburg, Russian Federation

Background: The eventual outcomes for infants with leukemia ( $<1$ yo) in terms of relapse-free survival (RFS) are poor compared with leukemia in older children despite the use of maximally intensified standard therapies. When comparing the effectiveness of chemotherapy only or with allogeneic hematopoietic stem cell transplantation (alloHSCT) the data is contradictory but several studies suggested that allo-HSCT improved outcome of infant acute leukemia (AL). Primary aim: to evaluate the feasibility and outcome of allo-HSCT in infant with AL. Secondary aim: assess factors that contribute on long-term survival.

Methods: Between May 2003 and July 2016, 21 infants (range from 0-12 month of birth median 6,5 month) with AL (ALL-13pts, AML-7pts, AMLL-1pt; 11q23 abnormality-16pts, $\mathrm{t}(4 ; 11)(\mathrm{q} 21 ; \mathrm{q} 23)$ or MLL-AF4-8pts, $\mathrm{t}(9 ; 11)$ (p22;q23) or MLL-AF9-2pts, t(11;19)(q23;p13) or MLLENL-1pt, other 11q23 abnormalities-5pts, normal karyotype-5pts) were analyzed. All pts received chemotherapy before allo-HSCT. Prednisolone good responder (PGR)-15pts, prednisolone poor responder (PPR) - 6pts. Median time to allo-HSCT-237 days. Status at the moment of allo-HSCT: CR1+/-MRD status-9pts; 12pts received "bridge" chemotherapy before allo-HSCT because of primary resistant and resistant relapse (3pts achieved response). Type of allo-HSCT: MSD-1pt, MUD-8pts, haplo-12pts. Conditioning regimen: MAC (Bu+Cy/ GIAC)-13pts; MAC-reduced toxicity (Treo+Flu)-5pts; RIC (Flu+Mel)-3pts. All pts received prophylaxis of aGVHD based on CsA-10pts, Tacro+/-Sir-11pts. In addition ATG received in 7 pts., PTCy $\mathrm{D}+3 ;+4-13 \mathrm{pts}$, $1 \mathrm{pt}-$ without ATG and PTCy. Median CD34+ cells 7,9x10 $/ \mathrm{kg}$. The median follow-up of surviving patients was 3,5 years. Statistical analyses were performed using SPSS V17.

Results: In all group 3-year OS was 48\%. Pts transplanted in CR1+/-MRD had 3y. OS better than pts after "bridge" chemotherapy before allo-HSCT (77,8\% vs $25 \%$ respectively $\mathrm{p}=0,02)$. There are no statistical significance in OS after allo-HSCT in patient with ALL vs AML (43\% vs 57\% p =0,4), MLL-r +/- (44\% vs $60 \%$ p $=0,7$ ), type of allo-HSCT (haplo - 50\% vs MSD or MUD $44,4 \% \mathrm{p}=0,8)$, age at the moment of allo-HSCT $(<$ $6 \mathrm{~m} .33,3 \%$ vs $>6 \mathrm{~m}$. 53,3\% $\mathrm{p}=0,2)$, conditioning regimen (MAC 53,8\% vs MACr $40 \%$ vs RIC $33,8 \% \quad \mathrm{p}=0,7$ ). Cumulative incidence of grade 2-3 aGVHD was $28,6 \%$ (4pts), grade 4 aGVHD-7,1\%(1pt); moderate cGVHD$14,3 \%$ (2pts). Cumulative incidence of relapse was $33 \%$ (7pts). Mortality: VOD - 14,3\% (3pts), bloodstream - 4,8\% (1pt), relapse - 33,3\% (7pts).

Conclusions: HSCT in 1CR with or without MRD allows achieving the long-term survival in $77.8 \%$ infants with AL. Despite the acceptable frequency of acute and chronic GVHD, the main cause of death in our study was relapse after allo-HSCT. Encouraging overall survival (25\%) was achieved in an unfavorable group of "bridge" therapy. Taking into account the frequency of posttransplant relapses in this group of patients, it is necessary to carry out preventive or prophylaxis therapy in early post-transplant period.

Conflict of interest: The autorths declare no conflict of interest.

\section{P769}

Role of HSCT in children and adolescents with refractory or relapsed $\mathrm{NHL}$

Birgit Burkhardt ${ }^{1}$, Marta Pillon ${ }^{2}$, Mary Taj $^{3}$, Nathalie Garnier ${ }^{4}$, Veronique Minard-Colin ${ }^{5}$, Volkan Hazar ${ }^{6}$, Karin Mellgren ${ }^{7}$, Tomoo Osumi $^{8}$, Alina Fedorova ${ }^{9}$, Natalia Myakova ${ }^{10}$, Jaime Verdú Amorós $^{11}$, Mara Andrés Moreno ${ }^{12}$, Edita Kabíčková ${ }^{13}$, Andishe Attarbaschi $^{14}$, Alan Chiang ${ }^{15}$, Eva Bubanská ${ }^{16}$, Svetlana Donska ${ }^{17}$, 
Lisa Lyngsie Hjalgrim ${ }^{18}$, Jacek Wachowiak ${ }^{19}$, Anna Pieczonka ${ }^{19}$, Anne Uyttebroeck ${ }^{20}$, Jan Loeffen ${ }^{21}$, Jochen Buechner ${ }^{22}$, Felix Niggli $^{23}$, Julia Palma ${ }^{24}$, Amos Burke ${ }^{25}$, Auke Beishuizen ${ }^{21}$, Kristin Koeppen $^{1}$, Heidi Herbrueggen ${ }^{1}$, Willi Woessmann ${ }^{26}$, Martin Zimmermann $^{27}$, Adriana Balduzzi ${ }^{28}$

${ }^{1}$ University Hospital Muenster, Department of Pediatric Hematology, Oncology and BMT, Muenster, Germany; ${ }^{2}$ University-Hospital of Padua, Department of Child and Woman Health, Oncology Hematology Division, Padua, Italy; ${ }^{3}$ Royal Marsden Hospital, Department of Paediatric Oncology, Sutton, United Kingdom; ${ }^{4}$ Hospices Civils de Lyon, Institute of Pediatric Hematology and Oncology, Lyon cedex, France; ${ }^{5}$ Gustave Roussy Cancer Campus, Department of Pediatric Oncology, Villejuif, France; ${ }^{6}$ Medical Park Göztepe Hospital, Departments of Pediatric Hematology and Oncology and BMT Unit, Istanbul, Turkey; ${ }^{7}$ Sahlgrenska University Hospital, Department of Pediatrics, Institution for Clinical Sciences, Gothenburg, Sweden; ${ }^{8}$ Children's Cancer Center, National Center for Child Health and Development, Tokyo, Japan; ${ }^{9}$ Belarusian Research Center for Pediatric Oncology, Hematology and Immunology, Clinical Research Department, Minsk, Belarus; ${ }^{10}$ Federal Center for Pediatric Hematology, Oncology and Immunology, Pediatric Hematology and Oncology, Moscow, Russian Federation; ${ }^{11}$ University Hospital Valencia, Department of Pediatric Hematology and Oncology, Valencia, Spain; ${ }^{12}$ University Hospital La Fé, Department of Pediatric Oncology, Valencia, Spain; ${ }^{13}$ Charles University and University Hospital Motol, Department of Paediatric Haematology and Oncology, Prague, Czech Republic; ${ }^{14}$ St. Anna Children's Hospital, Medical University of Vienna, Department of Pediatric Hematology and Oncology, Vienna, Austria; ${ }^{15}$ University of Hong Kong, Li Ka Shing Faculty of Medicine, Queen Mary Hospital, Department of Pediatrics and Adolescent Medicine, Hong Kong, China; ${ }^{16}$ Slovak Health University and Children's Faculty Hospital, Clinic of Pediatric Oncology and Hematology, Banská Bystrica, Slovakia; ${ }^{17}$ National Ukrainian Children 's Hospital 'Ochmatdyt', Center of Pediatric Oncohematology and BMT, Kiev, Ukraine; ${ }^{18}$ University Hospital of Copenhagen, Department of Paediatrics and Adolescent Medicine, Rigshospitalet, Copenhagen, Denmark; ${ }^{19}$ Poznan University of Medical Sciences, Department of Pediatric Oncology, Hematology and Transplantology, Poznan, Poland; ${ }^{20}$ University Hospital Gasthuisberg, Department of Paediatric Haematology and Oncology, Leuven, Belgium; ${ }^{21}$ Erasmus Medical Center Rotterdam, Sophia Children's Hospital, Department of Pediatric Oncology and Hematology, Rotterdam, Netherlands; ${ }^{22}$ Oslo University Hospital, Department of Pediatric Hematology and Oncology, Oslo, Norway; ${ }^{23}$ University Children's Hospital Zurich, Department of Pediatric Oncology, Zurich, Switzerland; ${ }^{24}$ University of Chile - Faculty of Medicine, Hospital Dr. Luis Calvo Mackenna, Department of Pediatrics - Bone Marrow Transplantation Unit, Santiago, Chile;

${ }^{25}$ Cambridge University Hospitals NHS Foundation Trust, Department of Paediatric Haematology and Oncology and Palliative Care, Addenbrooke's Hospital, Cambridge, United Kingdom; ${ }^{26}$ JustusLiebig-University, Department of Pediatric Hematology and Oncology, Giessen, Germany; ${ }^{27}$ Hannover Medical School, Department of Pediatric Hematology and Oncology, Hannover, Germany; ${ }^{28}$ Clinica Pediatrica Università degli Studi di Milano Bicocca, Ospedale San Gerardo, Monza, Italy

Background: For Non-Hodgkin Lymphoma (NHL) in children and adolescents systematic treatment protocols resulted in improved event-free survival rates without transplant indications in first CR. However, so far no standard treatment approach could be established for relapsed or refractory NHL ( $r / \mathrm{r}$ NHL). The aim of the current project was to gather international experiences on re-induction strategies in children and adolescents with $\mathrm{r} / \mathrm{r}$ NHL and to identify factors predictive of outcome.

Methods: Retrospective analysis of eligible cases collected on a common project specific CRF. Inclusion criteria were: a) NHL diagnosis, any subtype except ALCL, B) age at diagnosis $<18$ years, c) diagnosis in/after Jan 2000, d) refractory disease, disease progression or relapse. The primary endpoint of the study was overall survival (pOS). Secondary endpoints were treatment-related mortality (TRM) and disease-related mortality (DOD).

Results: A total of 695 eligible patients were reported by the following national groups: Germany (146), Italy (118), UK (67), France (66), Turkey (45), Sweden and Finland (35), Japan (33), Belarus (26), Moscow (23), Spain (23), Czech Republic (19), Austria (18), Hong Kong (18), Slovakia (11), Ukraine (9), Denmark (9), Poland (8), Belgium (6), Netherlands (5), Norway (5), Switzerland (4) and Chile (1). Median age at initial diagnosis was 10.8 years (0.3 to 17.9) and $499(72 \%)$ of the patients were male. The distribution of histological subtypes was Burkitt 282, DLBCL 118, PMLBL 46, mature B-NHL not further specified 11, T-LBL 181, pB-LBL 37, PTCL 15 and other NHL subtypes 5 .

Eight-year pOS for the total cohort was $37 \pm 2 \%$ with highly significant differences according to NHL subtypes, with pOS of $30 \pm 3 \%$ in Burkitt, $51 \pm 5 \%$ in DLBCL, $47 \pm 8 \%$ in PMLBL, $35 \pm 2 \%$ in LBL and $41 \pm 10 \%$ in rare subtypes.

Late relapses $\geq 9$ months from diagnosis $(\mathrm{n}=233$, pOS 47 $\pm 4 \%$ ) were associated with superior pOS compared to $\mathrm{r} / \mathrm{r}$ NHL diagnosed 6 to $<9$ months $(\mathrm{n}=107$, pOS $35 \pm 5 \%), 3$ to $<6$ months $(\mathrm{n}=264$, pOS $31 \pm 3 \%)$ and $<3$ months $(\mathrm{n}=$

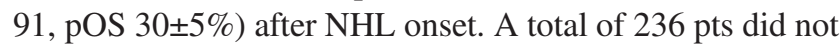
receive $\mathrm{HSCT}$ as it was not planned in one third of those pts and not achieved because of disease progression in the remaining two thirds. Outcome was poor for patients who did not receive high-dose treatment $(\mathrm{n}=236$, pOS $11 \pm 2 \%)$ compared to autologous $(\mathrm{n}=197, \mathrm{pOS} 55 \pm 4 \%)$ or allogeneic $(\mathrm{n}=243$, pOS $49 \pm 3 \%)$ transplant. The 5-year cumulative incidences of TRM and DOD were $2 \pm 1 \%$ and $80 \pm 3 \%$ for patients without HSCT, $6 \pm 2 \%$ and $32 \pm 3 \%$ for autologous HSCT and $14 \pm 2 \%$ and $35 \pm 3 \%$ for allo HSCT. Among those patients who underwent HSCT, the remission status at HSCT was highly significant associated with outcome (pOS of $59 \pm 3 \%$ for CR, $60 \pm 9 \%$ for $\mathrm{CRu}$ and VGPR, $35 \pm 7 \%$ for PR, $20 \pm 10 \%$ for SD and $11 \pm 6 \%$ for progressive disease).

Conclusions: International co-operative efforts provided by far the largest series of $\mathrm{r} / \mathrm{r}$ NHL which allowed the identification of prognostic parameters in $\mathrm{r} / \mathrm{r} \mathrm{NHL}$ and may support transplantation strategies.

Clinical Trial Registry: not applicable

Conflict of interest: 
A. Burke: consultancy for Janssen re Ibrutinib and Daratumomab; payment for lecture at London Lymphoma Forum 2017. The remaining authors have nothing to disclose.

\section{P770}

Role of Lung Clearance Index in early detection of pulmonary complications and prediction of pulmonary outcomes following allo-HSCT in children

Linnea Skov Jensen ${ }^{1,2}$, Hilde Hylland Uhlving ${ }^{2}$, Frederik Buchvald $^{1,2}$, Carsten Heilmann ${ }^{2}$, Marianne Ifversen ${ }^{2}$, Klaus Müller ${ }^{2}$, Kim G. Nielsen ${ }^{1,2}$

${ }^{1}$ Rigshospitalet, Copenhagen University Hospital, Paediatric Pulmonary Service, Copenhagen, Denmark; ${ }^{2}$ Rigshospitalet, Copenhagen University Hospital, National Paediatric HSCT Centre, Department of Paediatrics and Adolescent Medicine, Copenhagen, Denmark

Background: Pulmonary chronic Graft-versus-Host Disease (cGvHD) as complication following allo-HSCT in children is primarily characterized by impairment of the small airways. Assessment of lung clearance index (LCI) gained from Multiple Breath Washout-test (MBW) might be more sensitive than spirometry in detection of small airway impairment and is feasible even in infants and might therefore potentially lead to earlier detection of pulmonary complications. The aim of this study was to evaluate MBW for early diagnosis of cGvHD-related pulmonary complications and as predictor of pulmonary outcome following HSCT in children.

Methods: We performed a prospective, longitudinal study in children treated with allo-HSCT between 2010 and 2013. Spirometry, Sulfur hexafluoride $\left(\mathrm{SF}_{6}\right) \mathrm{MBW}$ (Amis 2000, Innovision, Denmark), total lung capacity (TLC) and diffusing capacity (DLCO) were performed before (baseline) and at day 90, 180, 270 and 365 after alloHSCT. Respiratory symptoms and signs of cGvHD were recorded at each visit according to the Common Terminology Criteria for Adverse Events v.4.0 and the NIH criteria, respectively. LCI was calculated using customized software (TestPoint, Capital Equipment, MA).

Results: Twenty-eight children were included, at a median age (range) of 11.1 (3.6-15.8) years. Median zscore values of LCI increased and FEV 1 , DLCO and TLC decreased during the first 90-180 days, however the values were not significantly different from baseline measurements. A tendency towards normalization of z-scores for these parameters was seen from day 180-270 and onwards.

Eight patients fulfilled criteria of cGvHD at median (range) 150 (70-856) days after HSCT, one verified by lung biopsy. Of the seven remaining patients with cGvHD, five experienced pulmonary symptoms, a decrease in $\mathrm{FEV}_{1}$ to $<$ $75 \%$ of predicted and/or gastrapping on HRCT. At day 90 , 270 and 365 a significant higher LCI was seen in children who developed cGvHD compared to patients without cGvHD after HSCT (median LCI z-score 2.69 vs 1.48 , p $=0.046 ; 4.59$ vs. $1.71, \mathrm{p}=0.044$ and 3.98 vs. $1.26, \mathrm{p}=$ 0.040 respectively), and with a similar non-significant trend at day 180 . However, $\mathrm{FEV}_{1}$ was also significantly lower in children with cGvHD at day 90, 180 and 270 (median FEV $_{1}$ z-score -2.28 vs. $-0.50, p=0.004 ;-2.07$ vs. $-0.82, p=$ 0.004 and -2.12 vs. $-0.70, p=0.003)$ as well as significantly lower TLC at day 90 (median TLC z-score -3.12 vs -1.52 , p $=0.004$, respectively). Patients who still reported respiratory symptoms at day 365 tended to deteriorate whereas children without respiratory symptoms improved in median LCI z-score between baseline and day 90, indicating a possible role of LCI as predictor of persistent late pulmonary complications.

Conclusions: We found a significant association between increased LCI at day 90, 270 and 365 following HSCT and later development of $\mathrm{cGvHD}$ although a signal from $\mathrm{FEV}_{1}$ also was seen. We believe further larger studies are needed, preferably with closer measurements during the first months following HSCT in order to further evaluate the potential role of $\mathrm{MBW}$ in early detection of $\mathrm{cGvHD}$ and as predictor of pulmonary outcome in allo-HSCT.

Conflict of interest: None of the authors has anything to disclose.

\section{P771}

Salvage Haploidentical Stem Cell Transplantation with Post-Transplant Cyclophosphamide for graft failure in children with non-malignant diseases

Juliana Folloni Fernandes ${ }^{1,2}$, Andreza Alice Feitosa Ribeiro ${ }^{1}$, Fabio Rodrigues Kerbauy ${ }^{1}$, Luiz Fernando Mantovani ${ }^{1,2}$, Gabriele Zamperlini Netto ${ }^{1}$, Angela Mandelli Venancio ${ }^{1}$, Cinthya Correa Silva $^{1}$, Andrea Tiemi Kondo ${ }^{1}$, Maria Dulce Silveira Collassanti ${ }^{2}$, Maria Aparecida Zanichelli ${ }^{2}$, Carolina Sgarioni Camargo Vince ${ }^{1}$, Melina Brumatti ${ }^{1}$, Vicente Odone Filho ${ }^{2}$, Nelson Hamerschlak ${ }^{1}$

${ }^{1}$ Hospital Israelita Albert Einstein, Hematology and Hematopoietic Stem Cell Transplantation, Sao Paulo, Brazil; ${ }^{2}$ Instituto da Criança Hospital das Clínicas da Universidade de São Paulo, Sao Paulo, Brazil

Background: Graft failure (GF) is a severe complication after hematopoietic stem cell transplantation (HSCT) and has a high mortality rate. Reported incidences of GF are higher in patients receiving HSCT for non-malignant diseases. Salvage second HSCT can rescue patients after GF, but optimal conditioning regimen and donor source are yet to be determined. 
Methods: In this study, we retrospectively analysed our experience with 12 paediatric patients with non-malignant diseases treated with T-cell replete haploidentical HSCT with post-transplant cyclophosphamide for graft failure after first HSCT.

Results: Between January 2011 and November 2017, 127 patients with non-malignant diseases were submitted to HSCT in two different institutions caring for paediatric patients. From these, twelve patients developed early graft failure and underwent a salvage second transplant. Median age was 3 years, range 1 to 13. Diagnoses were: primary immune deficiencies $(n=8)$, Diamond-Blackfan Anemia $(\mathrm{n}=1)$ and Leukodystrophies $(\mathrm{n}=3)$. Source of stem cells and donors for the first transplants were: haploidentical donor (bone marrow or peripheral blood stem cells), $n=6$; unrelated cord blood units, $\mathrm{n}=4$; and unrelated bone marrow, $n=2$. Four patients had received myeloablative conditioning regimens and 8 had received reduced toxicity regimens. Graft failure was diagnosed as either failure to recover neutrophil counts (in 8 cases) or autologous reconstitution with recipient chimerism (in 4 cases). Median interval between the first and the second transplant was 48 days (range $=32$ to 93 days). For the second transplant, all patients received T-cell replete grafts from a haploidentical relative (first or second degree). Cell source was bone marrow in 11 cases and granulocyte-colony stimulating factor was used in five donors before harvest. All patients received the same reduced toxicity conditioning regimen consisting of: fludarabine $30 \mathrm{mg} / \mathrm{m}^{2}$ on days -6 to -2 , cyclophosphamide $14.5 \mathrm{mg} / \mathrm{kg}$ on days -6 and -5 , and total body irradiation 2 Gy on day -1 . GVHD prophylaxis consisted of cyclophosphamide $50 \mathrm{mg} / \mathrm{kg} / \mathrm{d}$ on days +3 and +4 , tacrolimus or cyclosporine $\mathrm{A}$ and mycophenolate mofetil starting on day +5 . All patients engrafted, 12 to 20 days after the second transplant, with complete donor chimerism. One patient died early after engraftment (Day + 19) from pulmonary haemorrhage. Three patients developed Acute GVHD (grades II-III), and nine patients had viral infections (most part CMV). Five patients developed chronic GVHD, that was severe in 3 cases. One patient died 22 months after transplant from severe pulmonary GVHD. With a median follow-up of 45 months, ten patients are alive with complete donor chimerism from 2 to 60 months after second transplants, and seven are off any immunosuppression.
Conclusions: In conclusion, T-cell replete haploidentical HSCT with post-transplant cyclophosphamide can rescue patients after early graft failure with excellent engraftment. The high incidence of chronic GVHD in our cohort is an issue that has to be better prevented.

Conflict of interest: Nothing to disclose.

\section{P772}

\section{Selective Splenic Artery Embolization in Two Cases of Pediatric Patients post HSCT}

Massimo Berger ${ }^{1}$, Gaetana Ferraro ${ }^{2}$, Elisabetta Teruzzi ${ }^{3}$, Rosanna Pessolano $^{1}$, Francesco Saglio ${ }^{1}$, Francesca Carraro ${ }^{1}$, Elena Vassallo $^{1}$, Pierluigi Muratore ${ }^{4}$, Gabriella Agnoletti ${ }^{2}$, Franca Fagioli ${ }^{1}$

${ }^{1}$ City of Health and Science, Regina Margherita Children Hospital, Pediatric Onco-Hematology, Turin, Italy; ${ }^{2}$ City of Health and Science, Regina Margherita Children Hospital, Pediatric Cardiology, Turin, Italy; ${ }^{3}$ City of Health and Science, Regina Margherita Children Hospital, Pediatric Surgery, Turin, Italy; ${ }^{4}$ City of Health and Science, San Giovanni Battista Hospital, Radiology Department, Turin, Italy

Background: Selective splenic artery embolization (SSAE) followed by splenectomy has been show as a feasible procedure for adult patients with hypersplenism. The purpose of this article is to describe two cases of pediatric patients with SCID and JMML having hypersplenism and severe thrombocytopenia after HSCT.

Methods: Case 1: a 6 months old girl was diagnosed with SCID T-B $+\mathrm{NK}+$ with a IL7R mutation. She underwent haploidentical alfa/beta and CD19+ depleted peripheral blood HSCT from her mother at 9 months of age. The conditioning was based on Treosulan-Fludarabine and Serotherapy, no post transplant immunosuppression was given. She achieved full donor engraftment but at +6 months from HSCT she developed a atypical mycobatterium infection (mycobacterium avium) and despite intensive treatment a progressive hypersplenism occurred.

Cases 2: a three old boy was diagnosed with JMML. Following an unsuccessful search for an unrelated donor, he received an haploidentical bone marrow HSCT from his mother. The conditioning regimen was based on BusulfanThiotepa-Fludarabine, while GvHD prophylaxis was based on post HSCT Cyclophosphamide together with Tacrolimus and Mofetil Mycofenolate. Despite an early partial engraftment, the splenomegaly did not reduce and the hypersplenism dramatically worsened.

\begin{tabular}{|c|c|c|c|c|c|c|c|c|c|}
\hline & Diagnosis & $\begin{array}{l}\text { Age at } \\
\text { Dgn }\end{array}$ & HSCT & Reason of SSAE & $\begin{array}{l}\text { Patient } \\
\text { weight }\end{array}$ & $\begin{array}{l}\text { Spleen } \\
\text { size }\end{array}$ & $\begin{array}{l}\text { Plts at SSAE } \\
(x 109 / \mathrm{L})\end{array}$ & $\begin{array}{l}\text { Plts post }+24 \text { hrs } \\
\left(\times 10^{\wedge} 9 / \mathrm{L}\right)\end{array}$ & Outcome \\
\hline $\begin{array}{l}\text { Pt. } \\
1\end{array}$ & SCID & 6 months & $\begin{array}{l}\text { Haplo alfa/beta CD19+ } \\
\text { depleted }\end{array}$ & $\begin{array}{l}\text { Hypersplenism and } \\
\text { infection tank }\end{array}$ & 9 & 16.5 & 14 & 63 & $\mathrm{~A} \& \mathrm{~W}+29$ months \\
\hline Pt.2 & JMML & 3 years & $\begin{array}{l}\text { Haplo with Cyclo post- } \\
\text { HSCT }\end{array}$ & $\begin{array}{l}\text { Hypersplenism and } \\
\text { disease tank }\end{array}$ & 12 & 15.4 & 9 & 19 & $\begin{array}{l}\text { DOD }+5 \text { months from } \\
1 \text { st HSCT }\end{array}$ \\
\hline
\end{tabular}

[[P772 Table] Clinical data of patients having SSAE] 
Selective Splenic Artery Embolization and Splenectomy. For both patients splenectomy was indicated, but they were at very high risk for severe trombocytopenia, so we decided to perform a preoperative SSAE. The SSAE were performed in the catheterization laboratory by a pediatric interventional cardiologist and by a interventional radiologist. Both patients received platelets and plasma infusion pre- and during the procedure. In both cases splenic artery was incannulated with a 4Fr Judkins Righy coronary catheter. Through a $2.5 \mathrm{Fr}$ microcatheter in the distal branches of splenic arter 7.700 micron microparticles were slowly injected. In the proximal part of the artery several platinum coils were implanted until total occlusion of the splenic artery. Both patients immediately after the procedure were moved to the operation room and a laparoscopic splenectomy was performed, without any further significative complications.

Results: The post-surgery outcome was uneventful. Pt. 1 had a quickly returns of normal counts while Pt. 2 presented only an early improvement in the transfusion needs but three months after first haploidentical HSCT he received a second haploidentical HSCT for primary graft failure. The histological review showed a massive infiltration by atypycal mycobatterium and a diffuse leukemic infiltration for Pt. 1 and 2, respectively.

Conclusions: SSAE is a feasible procedure in pediatric patients also early after HSCT. Both patient presented a post-surgical course without significant complications and returned to the Bone Marrow Ward after one night in ICU only.

Clinical Trial Registry: NA

Conflict of interest: None of the authors has anything to disclose.

\section{P773}

\section{Similar outcome in children undergoing unrelated donor HSCT after the use of ATG Fresenius (Grafalon ${ }^{\circledR}$ ) or Thymoglobulin in the conditioning regimen}

\author{
Anna Krol', Joanna Owoc-Lempach ${ }^{2}$, Monika Mielcarek $^{2}$, \\ Malgorzata Salamonowicz ${ }^{2}$, Jowita Fraczkiewicz ${ }^{2}$, Malgorzata \\ Janeczko-Czarnecka ${ }^{2}$, Mateusz, Stachowiak ${ }^{2}$, Aleksander \\ Krzywulski², Tomasz, Jarmolinski ${ }^{2}$, Marek Ussowicz ${ }^{2}$, Ewa \\ Gorczynska ${ }^{2}$, Krzysztof Kalwak ${ }^{2}$ \\ ${ }^{1}$ Wroclaw University of Economics, Department of Management, \\ Finance and Computer Studies, Wroclaw, Poland; ${ }^{2}$ Wroclaw Medical \\ University, Department of Pediatric Hematology, Oncology and BMT, \\ Wroclaw, Poland
}

Background: Rabbit ATG remains a gold standard for GvHD prophylaxis in pediatric patients undergoing unrelated donor transplants (UD-HSCT). There is no clear data, which ATG formulation would result in better outcome.
The aim of our study was to compare the influence of the type of ATG (Grafalon ${ }^{\circledR}$, formerly ATG Fresenius vs. Thymoglobulin) on the outcome, including overall survival, event-free survival, as well as the incidence of aGvHD and cGvHD.

Methods: This retrospective single-centre study included 207 children (131 males and 76 females) with malignant ( $\mathrm{n}$ $=144)$ and non-malignant $(\mathrm{n}=63)$ diseases who underwent UD-HSCT with pre-transplant rabbit ATG infusion (day -3 to day -1). There were 150 matched and 57 mismatched donor-recipient pairs. 116 patients $(56 \%)$ received ATG Fresenius(or Grafalon ${ }^{\circledR}$ ) with median dose $45 \mathrm{mg} / \mathrm{kg}$ and 91 (44\%) Thymoglobulin with median dose $10 \mathrm{mg} / \mathrm{kg}$. The analyzed outcomes were overall survival, event-free survival, as well as cumulative incidence of aGvHD and cGvHD. Multivariate analysis of the influence of various risk factors (including the type of ATG) on OS and EFS was done using Cox proportional hazard model and log-rank test. The cumulative incidence of aGvHD and cGvHD were assessed using competing risk regression and Gray's test, with death and graft rejection as competing events.

Results: The study included patients who underwent UDHSCT in our centre in the years 2010-2017. The median follow-up for the whole cohort was 589 days (IQR 2401114). The 5-year overall survival probability for the entire study population was $77.6 \%$, whereas $73.2 \%$ of all patients survived 5 years without relapse and graft rejection. Among all 207 patients, 83 (40\%) developed grade II-IV aGvHD, $33(16 \%)$ grade III-IV aGvHD, and 13 (6.3\%) severe cGvHD. While comparing the influence of ATG Fresenius vs. Thymoglobulin, we have found no evidence of statistically significant between-group differences in terms of the analyzed outcomes. Thymoglobulin was used mainly in patients with non-malignant disorders, whereas ATG Fresenius in malignant diseases $(p<0.0001)$. The overall survival probability among the patients receiving ATG Fresenius was $77.6 \%$ vs. $76.4 \%$ in the Thymoglobulin group (hazard ratio HR 0.83, $\mathrm{p}=0.55$ ). The event-free survival was $74.5 \%$ in the ATG Fresenius group and $72.5 \%$ in the Thymoglobulin group (HR 0.94, $\mathrm{p}=0.83$ ). The cumulative incidence of grade II-IV aGvHD was $40 \%$ in both groups (HR 0.96, $\mathrm{p}=0.84$ ), whereas grade III-IV aGvHD was developed in $13.8 \%$ of the ATG Fresenius group and in $18.7 \%$ of the Thymoglobulin group (HR 0.71 , $\mathrm{p}=0.31)$. The cumulative incidence of severe cGvHD was close to $6 \%$ in both groups (HR 0.96, $\mathrm{p}=0.94$ ).

Conclusions: Pre-transplant rabbit ATG remains a gold standard in pediatric UD-HSCT. Both types of rabbit ATG proved to be successful in optimizing the outcome of patients. It is worth mentioning, that despite widespread use of ATG Fresenius in patients with malignant disorders, our results, including OS and incidence of GvHD, do not differ 
from those in patients with non-malignant diseases, where the use of Thymoglobulin predominated. It may be interesting to see in future, whether ATG Fresenius (nowadays Grafalon ${ }^{\circledR}$ ) could further improve the outcome of children with non-malignant disorders undergoing UDHSCT.

Conflict of interest: K. Kalwak: travel grants Sanofi. The remaining authors have nothing to disclose.

\section{P774}

\section{Successful Hematopoetic Stem Cell Transplantation with Non-Myeloablative conditioning in a patient with Primary Pulmonary Alveolar Proteinosis}

Ugur Ozcelik ${ }^{1}$, Selin Aytac ${ }^{2}$, Baris Kuskonmaz ${ }^{3}$, Ebru Yalcin $^{1}$, Deniz Dogru Ersoz ${ }^{1}$, Visal Okur ${ }^{2}$, Nural Kiper ${ }^{1}$, Duygu Uckan Cetinkaya ${ }^{3}$

${ }^{1}$ Hacettepe University Faculty of Medicine, Department of Pediatric Pulmonology, Ankara, Turkey; ${ }^{2}$ Hacettepe University Faculty of Medicine, Department of Pediatric Hematology, Ankara, Turkey; ${ }^{3}$ Hacettepe University, Faculty of Medicine, Department of Pediatric Hematology, Bone Marrow Transplantation Unit, Ankara, Turkey

Background: Pulmonary alveolar proteinosis (PAP) is a rare disorder characterized by accumulation of surfactant like lipo-proteinous material within the alveolar lumen which may lead to progressive respiratory failure. Primary PAP is the hereditary form caused by granulocytemacrophage colony-stimulating factor(GM-CSF) receptor $\alpha$-chain (CSF2RA) deficiency that lead a functional insufficiency of alveolar macrophages which require GMCSF signaling for terminal differentiation and effective degradation of alveolar proteins and phospholipids. Therapy choices for the primary PAPs are extremely limited, whole lung lavage is the treatment of choice. Here, we want to describe a primary PAP patient who was successfully treated with allogeneic hematopoetic stem cell transplantation(HSCT). To the best of our knowledge this is the first case report successfully transplantated in the literature.

Methods: Clinical and transplantation characteristics of the patient was recorded.

Results: An 18 years old boy who was presented with cough,dyspnea when he was 4 years old diagnosed as primary PAP with a homozygous mutation in the gene coding CSF2RA was treated with bilateral partial lung lavage for years however his lung conditions get worse gradually and he was oxygen dependent. As a progressive disorder, he may progress to fatal respiratory insufficiency which led to the emergence of alternative experimental treatments such as HSCT with an idea of having a functional macrophages after transplantation. At the age of 18 years he underwent HSCT from his matched father donor. Mesenchymal stem cell were also given before
3 weeks and on the day of tranplantation to ameoliorate the success of HSCT. Non-myeloablative conditioning regimen including fludarabine $\left(30 \mathrm{mg} / \mathrm{m}^{2} / \mathrm{d}\right.$ for 5 days), melphalan $\left(70 \mathrm{mg} / \mathrm{m}^{2} / \mathrm{d}\right.$ for 2 days) and antithymocyte globuline(ATGFresenius: $7.5 \mathrm{mg} / \mathrm{kg} / \mathrm{d}$ for 4 days) was given to the patient. Cyclosporine A and methotrexate were used as graft versus host disease (GVHD) prophylaxis. Bone marrow was used as the stem cell source and the number of $\mathrm{CD} 34^{+}$cells was $2.3 \times 10^{6} / \mathrm{kg}$. Neutrophil and platelet engraftment were achieved on day +14 and day +38 respectively. Chimerism analysis showed $94 \%$ and $96 \%$ donor profile on day +23 and day +170 respectively. After the transplantation he no longer requires continuous oxygen therapy. However he experienced acute GVHD and CMV reactivation after transplantation. He had a cytopenia on the day +75 which may related to CMV infection and/or acute GVHD. During this period he had a complaint of hoarseness and he was diagnosis as a biopsy proven vocal cord aspergillosis with pulmonary nodules, received antifungal treatment. After 6 months of transplantation now, there was no evidence of clinical worsening and hypoxia.

Conclusions: The only effective treatment for primary PAP is repetitive whole-lung lavage. Novel therapeutic strategies for primary PAP cases are urgently required. Moreover, HSCT which requires myeloablation may further aggravate the respiratory insufficiency and may lead to an increased risk for infections. Therefore in this case we choose non-myeloablative regimen and we think that mixed chiemerism is sufficient to decreased the progression of pulmonary symptomps. This is the first case represent a successful non-myeloablative HSCT for primary PAP which will be a new treatment option for these patients.

\section{Conflict of interest: None}

\section{P775}

\section{Successful second allogeneic hematopoietic stem cell transplantation in a child with XIAP deficiency}

Renata Formankova ${ }^{1}$, Petra Keslova ${ }^{1}$, Petr Riha ${ }^{1}$, Ondrej Hrusak ${ }^{1}$, Ester Mejstrikova ${ }^{1}$, Tomas Freiberger ${ }^{2}$, Hana Cechova ${ }^{3}$, Jan Stary ${ }^{1}$, Petr Sedlacek ${ }^{I}$

${ }^{1}$ 2nd Faculty of Medicine and University Hospital Motol, Prague, Czech Republic; ${ }^{2}$ Center for Cardiovascular Surgery and Transplantation, Brno, Czech Republic; ${ }^{3}$ Institution of Hematology and Blood Transfusion, Prague, Czech Republic

Background: Deficiency of X-linked inhibitor of apoptosis (XIAP) is associated with $\mathrm{X}$-linked lymphoproliferative disease, hemophagocytic lymphohistiocytosis (HLH) phenotype and inflammatory bowel disease. Allogeneic hematopoietic stem cell transplantation (HSCT) is curative for affected patients but the loss of XIAP and its apoptotic 
functions contributes to high risk for treatment-related toxicity. The use of reduced intensity conditioning (RIC) regimens improved survival rates at the cost of more frequent mixed chimerism and potential risk of disease recurrence. To date, little has been published concerning the outcome of second HSCT for XIAP deficiency.

Methods: Our patient was manifested with HLH at the age of 4 months, XIAP deficiency was diagnosed by identifying a hemizygous nonsense mutation in BIRC4 gene (c.712C $>$ T p.Arg238Ter). He was treated with thymoglobulin, etoposide, dexamethasone and intrathecal methotrexate (Euro-HIT-HLH study), disease reactivation was documented in 6th week of therapy with response to increase of dexamethasone and etoposide dose. He was transplanted in remission of HLH at the age of 7 months with peripheral blood stem cells (PBSC) from matched unrelated donor (MUD). Conditioning regimen included alemtuzumab $(1 \mathrm{mg} / \mathrm{kg}$, started on day -14$)$, fludarabine $(150 \mathrm{mg} / \mathrm{m} 2)$ and melfalan $(140 \mathrm{mg} / \mathrm{m} 2)$, GVHD prophylaxis consisted of cyclosporin (CsA) and mycophenolate mofetil (MMF).

Results: Engraftment of granulocytes was documented on day +14 , GVHD gr. II with response to corticosteroids was manifested on day +53 . Chimerism was above $99 \%$ of donor until day +60 , but rapid decrease of donor signal was documented from day +100 , up to $30 \%$ on day +180 . MMF was stopped on day +65 , corticosteroids on day +129 and CsA on day +188 . He suffered from recurrent automimmune hemolytic anemia (AIHA) from day +224 , treated with corticoseroids, rituximab, HD-IVIG, MMF and sirolimus. Recurrent cytopenias, intermitent diarrhea, fever, hepatosplenomegaly and elevation of ferritin, triglycerides and solubile IL2-R were documented from day +300 . Donor chimerism decreased to $10 \%$ in non-separated peripheral blood, $25 \%$ in T-lymphocytes and less than 5\% in NK cells. The indication for second allogeneic HSCT was very low level of donor chimerism in the presence of clinical and laboratory signs of incipient disease recurrence. The boy underwent retransplantation with defrosted PBSC from the same MUD one year after the first HSCT. Second RIC regimen included alemtuzumab $(1 \mathrm{mg} / \mathrm{kg}$ from day $-14)$, fludarabine $(150 \mathrm{mg} / \mathrm{m} 2)$ and treosulfan $(36 \mathrm{mg} / \mathrm{m} 2)$, ATG (total dose $24 \mathrm{mg} / \mathrm{kg}$ ) was added on day $-3,-2$ for disease acceleration. He engrafted granulocytes on day +20 , trombocytes on day +15 . No serious toxicity, GVHD or viral reactivation was documented. The post-transplant course was again complicated by AIHA, manifested on day +189 , with good response to corticosteroids, HD-IVIG and rituximab treatment. The patient remains in remission of HLH, with stable level of donor chimerism (above $80 \%$ in non-separated peripheral blood, $90 \%$ in T-lymfocytes, $65 \%$ in NK cells) 9 months after second HSCT.
Conclusions: We performed second allogeneic HSCT using different RIC regimen in patient with XIAP deficiency without any serious toxicity. We believe that second transplant allowed us to achieve stable level of donor chimerism which should be protective against disease recurrence.

Supported by MHCZ 00064203

Conflict of interest: All authors: nothing to disclose

\section{P776}

Tandem high dose chemotherapy and autologous hematopoietic cell transplantation in newly diagnosed pediatric patients with malignant brain tumors - a Portuguese transplantation center experience

Joana Desterro ${ }^{1}$, Filipe Gonçalves ${ }^{1}$, Sofia Nunes ${ }^{2}$, Duarte Salgado ${ }^{2}$, Filipa Moita $^{1}$, Maria João Gutierrez ${ }^{1}$, Gilda Teixeira ${ }^{1}$, Nuno Miranda $^{1}$, Isabelina Ferreira ${ }^{1}$, Manuel Abecasis ${ }^{1}$

${ }^{1}$ Instituto Português de Oncologia de Lisboa, Hematologia, Lisboa, Portugal; ${ }^{2}$ Instituto Português de Oncologia de Lisboa, Pediatria, Lisboa, Portugal

Background: Central nervous system (CNS) tumors are the most common pediatric solid tumors with dismal prognosis and low cure rates.

There is no universally accepted treatment for these tumors but high-dose chemotherapy followed by tandem autologous hematopoietic stem cell transplantation (HDCT/ autoHSCT) has been incorporated in different protocols as consolidation of either induction RT or chemotherapy, the later most relevant in very young children to decrease toxicity and avoid adverse effects of brain irradiation.

In this study we present our experience using HDCT/ autoHSCT protocols to treat CNS tumors.

Methods: Retrospective review from medical records of newly diagnosed children with CNS tumors treated in our Institution between December 2008 and November 2017.

All patients (pts) had surgical resection followed by treatment using two different protocols according to age: I) children $<4$ yo were treated with induction chemotherapy (cisplatin, VP16, vincristine, cyclophosphamide) followed by 3 HDCT/autoHSCT using a carboplatin and thiotepa conditioning (Thorarinsdottir et al., 2007), if relapse, salvage RT was used; II) children $>4$ yo were treated with risk-adapted RT and subsequent consolidation with 4 HDCT/autoHSCT using cisplatin, vincristine and cyclophosphamide conditioning (Chintagumpala et al., 2009).

Event-free survival (EFS) was calculated from diagnosis date until relapse, progression or death whichever occurred first. Overall survival (OS) was calculated from diagnosis date until death from any cause or last follow-up. KaplanMeier method was used for the survival analysis. 
Results: Our study included a total of 16 pts, 8 males, median age of 5,8 years (range 0,6-16 years) with newly diagnosed malignant CNS tumors. Histological analysis revealed 5 medulloblastomas, 5 supratentorial primitive neuroectodermal tumors (PNET), 4 glioblastomas, 1 pineoblastoma and 1 CNS embryonal tumor SOE.

Nine pts were treated according to protocol I. Five pts received the intended 3 tandem transplants (tts), 1 suspended protocol after 2 tts (hematological toxicity) and 3 after 1 tts due to progressive disease. Relapse occurred in 6 pts, 2 died and 4 were rescued with RT - 2 relapsed 10 and 21 months after tts and are in CR and 2 relapsed after first transplant, currently under salvage.

Seven pts were treated according to protocol II. Five pts completed the total number of 4 tts, 2 pts suspended protocol after 3 tts (neurological toxicity). Relapsed occurred in one patient 20 months after tts and died 8 months later.

Eleven of the 16 pts received RT during treatment, 7 prior to tts as planned in protocol II and 4 after transplant relapse in protocol I.

With a median follow-up of 4.3 years, 9 pts are alive, in CR. The 4-year EFS and OS estimates were 55.0\% [95\% CI: $0.35-0.86$ ] and $76.4 \%$ [95\% CI: 0.55-1] respectively. There was no treatment related mortality.

Conclusions: Although our study presents limitations, mainly imposed by the sample size, the data shows that RT remains an important component for treatment of brain tumors but HDCT/autoHSCT must be considered as an important adjuvant tool to avoid or defer RT.

In this population tandem HDCT/autoHSCT was effective for treating pediatric aggressive brain tumors with low toxicities and encouraging OS, EFS.

Conflict of interest: None

\section{P777}

TCRaß and CD19-depletion in hematopoietic stem cells transplantation from haploidentical donors in pediatric patients with chemorefractory acute myeloid leukemia

Larisa Shelikhova, Maria Ilyushina, Zhanna Shekhovtsova, Dmitriy Balashov, Elena Kurnikova, Yakov Muzalevsky, Irina Shipitsina, Daria Shasheleva, Rimma Khismatullina, Elena Gutovskaya, Sergey Blagov, Svetlana Kozlovskaya, Anna Bogoyavlenskaya, Irina Kalinina, Galina Novichkova, Alexei Maschan, Michael Maschan

'Dmitry Rogachev National Medical Research Centre for Pediatric Hematology, Oncology and Immunology', Moscow, Russian Federation

Background: The outcome hematopoietic stem cell transplantation (HSCT) in a cohort of children with chemorefractory acute myeloid leukemia is poor. Additional attempts at remission induction with various combinations of chemotherapy are unlikely to improve the outcome and will contribute to toxicity. TCR $\alpha \beta+/ \mathrm{CD} 19+$ depletion is a technology of graft manipulation with a potential to increase GvL effect and improve GvHD control and immune reconstitution in this group of patients.

Methods: A total of 36 pts with chemorefractory AML (induction failure $(\mathrm{n}=14)$, refractory relapse $(\mathrm{n}=22), 19$ female/17male, median age 9,4 years (1,1-18), underwent HSCT between May 2012 and September 2017, median FU 3,3 years $(0,2-4,8)$. All pts were transplanted from haploidentical donors, had active disease (AD) at the moment of SCT and received treosulfan-based conditioning regimen. Three regimens of GvHD prophylaxis were used. Regimen $1(\mathrm{n}=5)$ : ATGAM $50 \mathrm{mg} / \mathrm{kg}+$ Tacro/MTX; regimen $2(\mathrm{n}=9)$ : thymoglobulin $5 \mathrm{mg} / \mathrm{kg}$, rituximab 200 $\mathrm{mg} / \mathrm{m}^{2}$ and bortezomib on day $+2,+5(\mathrm{n}=9)$; regimen $3(\mathrm{n}$ $=22$ ): tocilizumab at $8 \mathrm{mg} / \mathrm{kg}$ on day -1 and post-transplant bortezomib $(\mathrm{n}=8), 14 \mathrm{pts}$ receive additional abatacept at 10 $\mathrm{mg} / \mathrm{kg}$ on day $+2,+7,+14,+28$.

TCR $\alpha \beta+/ C D 19+-$ depletion of SCT with CliniMACS technology was implemented in all cases. All engrafted pts. received additional post-transplant therapy courses, including hypomethylating agents AND/OR modified donor lymphocyte infusions (DLI). DLIs included either parts of primary graft ( $\alpha \beta \mathrm{T}$ cell depleted MNC) or memory $\mathrm{T}$ cell enriched fraction (CD45RA-depleted DLI).

Results: Primary engraftment was achieved in 32 of 36 pts ( 2 pts. had disease progression, 1 died at the moment of engraftment, 1 had (immune-mediated? Idiopathic?) graft failure and is alive after 2nd SCT), the median time to ANC and platelets recovery was 12 days (10-25). All engrafted pts achieved complete chimerism by day +30 . The cumulative incidence of aGVHD grade 2-4 was 25 $(+/-7) \%$, chronic GVHD - 17(+/-6)\%. Use of serotherapy (ATG) did not affect the incidence of GVHD . Among all patients transplant-related mortality is $6(+/-4) \%$, cumulative incidence of relapse is $53(+/-9) \%$. The incidence of relapse in a subgroup without ATG was 43(+/- 12)\%, which was less than a subgroup with ATG - $69(+/-12) \%$, p $=0,04$. At a median follow-up of 3,3 years, event-free survival is $41(+/-9) \%$ and overall survival is $40(+/-10) \%$. NK-cell recovery at day +30 was significantly associated with decreased incidence of relapse, CI of relapse of 65 $(+/-8) \%$ in those with peripheral blood NK recovery < median vs $30(+/-11) \%$ in those with NK-cell count $>$ median, $\mathrm{p}=0,02$, with improved event-free survival, EFS in NK"high" - 69(+/-11)\% vs NK"low" $34(+/-13) \%$, $\mathrm{p}=0,02$.

Conclusions: We confirm that the depletion of TCRa/b/ CD19+ lymphocytes from the haploidenticalgraft ensures high engraftment rate and low transplant-related mortality. Combined administration of tocilizumab, abatacept and 
bortezomib post-TCR a/b+/CD19+depleted grafting without serotherapy might improve GvL effects without extensive GvHD-related morbidity and mortality. Attempts at improving early NK cell recovery may open the possibility to effectively enhance the anti-tumor effect and decrease relapse rate in children with chemorefractory AML.

Conflict of interest: The authors declare they have no competing interests.

\section{P778}

\section{The Effect Of Early Warning Scorıng System In Pediatric Intensive Care Unit On Mortality After Pediatric Hematopoietic Stem Cell Transplantation}

\author{
S.Sema Anak ${ }^{1}$, Leyla Telhan ${ }^{2}$, Yöntem Yaman ${ }^{1}$, Şifa Şahin ${ }^{3}$, Murat \\ Elli $^{1}$ \\ ${ }^{1}$ Medipol University, Pediatric Hematology/Oncology/BMT, Istanbul, \\ Turkey; ${ }^{2}$ Medipol University, Pediatric Intensive Care Unit, Istanbul, \\ Turkey; ${ }^{3}$ Medipol University, Depart. of Pediatrics, Istanbul, Turkey
}

Background: Pediatric early warning scoring system (PEUSS) is a nurse-administered clinical identification tool that provides early detection of clinical impairment in hospitalized patients. Early diagnosis of critical patients have an effect on the condensation monitoring and mortality. Pediatric hemato-oncology patients, constitute the highest risk group in terms of sudden clinical deterioration and the need for intensive care.

Methods: In our study, the NHS Institute for Innovation $\&$ Improvement PEWS was used on the scoring chart . This application contains every patient $>$ PEWSS 3 and above was evaluated by the physician; $>5$ was transferred to the pediatric intensive care unit.

Between January 2015 and July 2017, 24 HSCT patients (25 hospitalizations) requiring pediatric intensive care in PICU were evaluated retrospectively. Demographic data such as age, sex ; diagnosis and mortality rates were evaluated and compared rates before and after the usage of pediatric early warning system (PEWS) (March 2016) .

Results: 25 pediatric patients (15 male -60\%) (10 female $-40 \%$ ) with the diagnosis of ALL (4 cases), thalassemia majör( 9 cases), aplastic anemia (3 cases), neuroblastoma (3 cases), Hurler(1 case), Diamond-Black anemia(1 case), AML (1 case), Hodgkin's lymphoma(1 case), non-hodgkin 's lymphoma (1 case), and Omenn's Syndrome (1 case) were transplanted. The etiology of intensive care admission was determined as septic shock (10 patients), venoocclusive disease (7 patients), gastrointestinal GVHD (3 patients), pneumonia (2 patients), hyponatremic convulsions (2 patients), hypovolemic shock (2 patients) and idiopatic generalized tonic clonic seizure (2 patients). The mean number of days in the intensive care unit was 9.2 days. It was 5.7 days before the pediatric early warning system and 11 days after the usage of PEWS.

Between 2014 and 2017, a total of 144 transplants were performed in our transplant unit and 34 patients $(23,6 \%)$ died during the follow-up period. PICU mortality rates were 4 patient out of $9(\% 44.4)$ before the usage of PEWS and 4 patient out of 16 (\%25) after the PEWS .

Conclusions: This study shows the early warning system is a feasible and effective application decreasing the rate of mortality. There was no significant difference in intensive care hospitalization period,because of some patients hospitalized for longer unexpected periods. There is a need for a prospective, multicenter study to assess the value of early warning system.

Conflict of interest: None of the authors has anything to report.

\section{P779}

The experience of BK polyoma virus infection treatment after allogeneic hematopoietic stem cell transplantation in pediatric patients

Bulent Antmen ${ }^{1}$, Ilgen Sasmaz ${ }^{2,3}$, Barbaros Karagun ${ }^{1}$, Murat Serbest ${ }^{2}$

${ }^{1}$ Adana Acibadem Hospital, Adana, Turkey; ${ }^{2}$ Adana Acibadem Hospital, Bone Marrow Transplantation Unit, Adana, Turkey;

${ }^{3}$ Cukurova University, Pediatric Hematololy, Adana, Turkey

Background: BK polyoma virus infection treatment modalities are an evolving challenge following allogeneic pediatric hematopoietic stem cell transplantation (alloHSCT). Several risk factors have been reported including BU-containing myeloablative conditioning, unrelated donors and GVHD. In contrast to early -onset HC, lateonset $\mathrm{HC}$ mainly results from polyomavirus BK and less frequently adenovirus or cytomegalovirus. Reactivation of human polyomavirus BK (BKV) may cause polyomavirusassociated nephropathy or polyoma virus-associated hemorrhagic cystitis in bone marrow-transplant patients. We present 33 patients with $\mathrm{BK}$ polyoma virus (BKV) ascociated hemorrhagic cystitis and 2 patients with BK polyoma virus associated hemorrhagic cystitis and nephritis.

Methods: Between 2013 and 2017, 198 patients received an allogeneic BMT at Acıbadem Adana Hospital Pediatric Bone Marrow Transplantation Unit. Thirty five patients occurred BKV associated hemorrhagic cystitis and nephritis. We checked BKV by PCR in all transplanted patients before pretransplant period. BKV was detected in the urine analysis and blood by PCR (polymerase chain reaction) in patients. 
Results: We presented 35 patients with BKV infection, age ranging from 3 to 20 with a average of 11.8 years. They underwent allogeneic BMT due to thalassemia major (20 patients), aplastic anemia (6 patients), acute leukemia ( 8 patients) and kostmann syndrome (one patient). The patients were treated firstly with hydration, continuous bladder irrigation, ciprofloxacin, and weekly intravesical hyaluronic acid instillation for four weeks, and cidofovir. Thirty three patients showed complete resolution of hematuria. One patient with refractory above these therapy also received hyperbaric oxygen and recombinant factor VIIa (rFVIIa, NovoSeven; Novo Nordisk,Bagsvaerd, Denmark). Hemodialysis was performed in two patients who developed renal failure due to nephritis.

Conclusions: BKV is ubiquitously present in the general population. Reactivation of infection occurs under conditions of severe immunosuppression, particularly HSCT or renal transplantation, and causes late-onset $\mathrm{HC}$ of $\mathrm{BKV}$. The management of BKV cystitis and nephritis sometimes may be very difficult and could be refractory for all treatments modalities. In this retrospective study, we presented our experience of BKV infection and management and treatment modalities in pediatric transplanted patients in our center.

Conflict of interest: Nothing to disclose

\section{P780}

The experience of using defibrotide for the prevention and treatment of hepatic veno-occlusive disease after pediatric hematopoietic stem cell transplantation: A single center experience

\author{
Bulent Antmen', Ilgen Sasmaz ${ }^{1,2}$, Barbaros Karagun', Murat \\ Serbest ${ }^{1}$ \\ ${ }^{1}$ Adana Acibadem Hospital, Bone Marrow Transplantation Unit, \\ Adana, Turkey; ${ }^{2}$ Cukurova University, Pediatric Hematology, Adana, \\ Turkey
}

Background: Hepatic veno-occlusive disease (VOD) is a common and serious complication of hemotopoietic stem cell transplantation (HSCT) in children. We aimed to assess prospectively the use of prophylactic defibrotide in pediatric patients undergoing HSCT and present of treatment results.

Methods: In this study, 157 patients who underwent HSCT were given defibrotide prophylaxis as $25 \mathrm{mg} / \mathrm{kg}$ per day in four divided intravenous infusions over $2 \mathrm{~h}$, starting on the same day as the pre-transplantation conditioning regimen. The mean duration of use of defibrotide is 25 days as a prophylaxis.

Results: In this study, 157 patients were recruited, 95 male patients and 62 female patients, with the average of 8.7 years, range $1-20 ; 9 \%$ infants, $58 \%$ children and $33 \%$ adolescent. There were 69 patients with thalassemia major, 58 patients with leukemia, 12 patients with aplastic anemia, one patient with Diamond Blackfan anemia, two patients with congenitale dyserythropoetic anemia, one patient with osteopetrosis, five patients with famial hemophagocytic lymphohistiocytosis, three patients with severe immune deficiency, threee patients with sickle cell disease and threee patients with Kostman syndrome. All transplants were allogeneic. No serious side effects were seen. In 13 patients developed clinical VOD (Seattle criteria). In these patients, defibrotide dose was increased to a treatment dose of 40-60 $\mathrm{mg} / \mathrm{kg}$ per day. One infant patient with Kostman syndrome died due to hepatic and pulmonary VOD. After 48 months of follow up, 12 patients who developed VOD are being well and no patient have transplant related complications.

Conclusions: Hepatic veno-occlusive disease, which is caused by hepatocyte and sinusoidal vessel endothelium damage, can ocur usually early phase after HSCT, and in its severe form, may lead to liver failure, hepatorenal syndrome, portal hypertension, and eventually death from multiorgan faillure. In this prospective study, we demonstrated that the use of defibrotide is safe and effective in preventing and treating VOD in pediatric patients at high risk group.

Conflict of interest: Nothing to disclose

\section{P781}

The experience using of eltrombopag for treatment of thrombocytopenia after allogeneic hematopoetic stem cell transplantation in pediatric patients

Ilgen Sasmaz, ${ }^{1,2}$, Bulent Antmen ${ }^{1}$, Barbaros Karagün ${ }^{1}$, Murat Serbest ${ }^{1}$

${ }^{1}$ Adana Acibadem Hospital, Bone Marrow Transplantation Unit, Adana, Turkey; ${ }^{2}$ Cukurova University, Pediatric Hematology, Adana, Turkey

Background: Thrombocytopenia after allogeneic hematopoetic stem cell transplantation is a common complication. Eltrombopag is an oral thrombopoietin receptor agonist which interacts with the transmembrane domain of the receptor on bone marrow megakartocytes and upstream progenitor stem cells. There are few reports eltrombopag treatment after allogeneic hematopoetic stem cell transplantation. We report our eltrombopag experiences with 7 pediatric patients treated with eltrombopag for severe thrombocytopenia after allogeneic hematopoetic stem cell transplantation at our center.

Methods: A total of 198 allogeneic hematopoetic stem cell transplantation were performed at Acibadem Adana Hospital Pediatric Bone Marrow Transplantation Unit in Turkey from 2013 to 2017. Seven cases of 198 patients 
were treated eltrombopag for persistan thrombocytopenia after allogeneic hematopoetic stem cell transplantation. Bone marrow aspiration and biyopsy were performed in all patients before starting eltrombopag treatment. Medical records and treatment modalities were evaluated retrospectively.

Results: In this study, 7 patients were treated with eltrombopag, age ranging from 5 to 17 years with a average of 12 years. There were 3 patients with thalassemia major, 2 patients with acute lymphoblastic leukemia, one patients with aplastic anemia and one patient with myelodysplastic syndrome. Four patients were males, three were female. Five patients had hematopoetic stem cell transplantation from an unrelated donor. Two patients had haploidentical transplantation from mother. Five patients had bone marrow transplantation, 2 had peripheral blood stem cell transplantation. Eltrombopag was started in 6 patients with prolonged isolated thrombocytopenia and one patient with secondary failure of platelet recovery. The number of megacaryocytes was decreased in 6 patients and was within the normal range in one patient. Before starting eltrombopag treatment 7 patients were dependent on platelet transfusions weekly. Eltrombopag was started at a median 228 days after HSCT (range, 77 to 582 days) in patients with PIT. Eltrombopag were started at the dose of $25-50 \mathrm{mg}$ once daily. Median duration for treatment was 177 days. No serious side effects were seen. The incidence rate of successful platelet recovery to $>50000 /$ mikrolitre without transfusion support was $71 \%$ ( 5 of 7 patients).

Conclusions: Eltrombopag is currently available oral thrombopoietin receptor agonist. We showed that our experience using of eltrombopag is very effective with minimal side effects for persistent thrombocytopenia after allogeneic hematopoetic stem cell transplantation in pediatric patients who had depended on platelet transfusion.

Conflict of interest: Nothing to disclose

\section{P782}

The impact of percutaneous endoscopic gastrostomy on weight change and survival in children after allogeneic hematopoetic stem cell transplantation

\author{
Mantas Vaisvilas ${ }^{1}$, Giedrius Semys ${ }^{2}$, Audrone Jakaitiene ${ }^{2}$, Jelena \\ Rascon $^{2,3}$, Goda Elizabeta Vaitkeviciene ${ }^{I}$ \\ ${ }^{1}$ Vilnius University Hospital Santaros Klinikos, Hematology, Oncology \\ and Transfusion Medicine Center, Vilnius, Lithuania; ${ }^{2}$ Vilnius \\ University, Vilnius, Lithuania; ${ }^{3}$ Childrens Hospital, affiliate to Vilnius \\ University Hospital Santaros Kliniko, Center for Pediatric Oncology \\ and Hematology, Vilnius, Lithuania
}

Background: A lower body mass and body mass index are associated with an inferior outcome in children undergoing allogeneic hematopoietic stem cell transplantation (alloHSCT). Percutaneous endoscopic gastrostomy (PEG) is a safe and effective way to ensure enteral nutrition. Since 2012 PEG placement prior to alloHSCT has become a standard procedure at our institution. In the current study we aimed to assess the impact of PEG placement on weight kinetics and overall survival.

Methods: A retrospective single-center analysis of 71 children below 18 years old who underwent an alloHSCT from 2004 until 2016 was performed. The entire cohort comprised 34 patients with PEG placed prior to alloHSCT (transplanted in 2012-2016) and 37 non-PEG patients (transplanted in 2004-2016). Data on patient weight and relevant HSCT characteristics (primary disease, donor type, conditioning, graft source, GvHD) were retrieved from medical records. Weight data on days $0,+30,+60$ and +100 after HSCT were included to evaluated weight change after alloHSCT. Primary study end points were defined as overall survival (OS) on day +100 and at 4 years following alloHSCT.

Results: Comparative analysis of weight kinetics revealed an increase of a median weight from $32.8 \mathrm{~kg}$ on day 0 to $34.2 \mathrm{~kg}$ on day $+100(\mathrm{p}=0.01)$ in PEG group whereas in the non-PEG group median weight on the respective time-points declined from $41.5 \mathrm{~kg}$ to $37.3 \mathrm{~kg}$ (p $=0.006)$. OS on +100 day following PEG placement was $94 \%$ as compared to $69 \%$ in the non-PEG group ( $\mathrm{p}=$ $0.014)$ ). OS at 4 years after alloHSCT in the PEG and nonPEG groups was $83 \%$ vs $36 \%(\mathrm{p}=0.0001)$ with a median follow up period of 2.07 (range 0.15-4.54) years and 5.35 (range 1.19-12.68) years respectively. In a univariate analysis PEG placement was associated with better OS (HR 0.183, 95\% CI [0.07-0.478], $\mathrm{p}=0.001$ ) and remained the most significant factor for OS in a multivariate analysis (HR 0.179, 95\%CI [0.055-0.585], p = 0.004) when transplantation period (before or after 2012), disease type (malignancy vs inborn error), preparative regimen (RIC vs MAC), donor type (HLA identical sibling vs MUD), stem cell course (BM vs PBSC), acute GvHD (I-II vs III-IV) and PEG placement (PEG vs non-PEG) were included in the model. No severe PEG-associated complications were observed within the study cohort.

Conclusions: PEG placement in children undergoing alloHSCT appeared to be feasible and effective to prevent weight loss in early post-transplant period. Our retrospective analysis indicated that PEG placement was associated with improved OS however larger prospective trials are warranted to confirm the results.

Conflict of interest: The authors have no financial or any kind of other bias to disclose. 


\section{P783}

The role of insulin growth factors binding proteins (IGFBP$1,-2,-3,-4,-6$ and -7 ) and insulin growth factors (IGF-I and -II) in pediatric $\mathrm{HSCT}$

Danuta Pietrys ${ }^{1}$, Przemyslaw J. Tomasik ${ }^{2}$, Kinga Kwiecinska ${ }^{3}$, Wojciech Strojny, Michal Korostynski ${ }^{4}$, Marcin Piechota ${ }^{5}$, Katarzyna Herbut ${ }^{6}$, Walentyna Balwierz ${ }^{3}$, Szymon Skoczen $^{3}$

${ }^{1}$ University Chldren's Hospital, Department of Oncology and Hematology, Krakow, Poland; ${ }^{2}$ Institute of Pediatrics, Jagiellonian University Medical College, Department of Clinical Biochemistry, Krakow, Poland; ${ }^{3}$ Institute of Pediatrics, Jagiellonian University Medical College, Department of Oncology and Hematology, Krakow, Poland; ${ }^{4}$ University Children's Hospital, Department of Oncology and Hematology, Krakow, Poland; ${ }^{5}$ Institute of Pharmacology of Polish Academy of Sciences, Department of Molecular Neuropharmacology, Krakow, Poland; ' ${ }^{6}$ agiellonian University Medical College, Krakow, Poland

Background: The IGF pathway is important for regulating proliferation, differentiation and apoptosis in normal tissues and tumor cells. As in obesity this pathway is hyperstimulated obese children were recruited as additional control. The goal of the study was determination of chosen IGFs and IGFBPs concentrations and their genes expression profiles in children treated with stem cell transplantation.

Methods: Plasma total IGF-I and -II, IGFBP-1, $-2,-3,-4$, -6 and -7 concentrations were measured using ELISA in fasting state in 19 children 1.5-19 (average 9) years old, 15 boys and 4 girls, referred to allogeneic haematopoietic stem cell transplantation (HSCT) due to neoplastic - 74\% (ALL8, AML-5, MDS-1) and non-neoplastic - 26\% (CGD-2, SAA-1, ALPS-1, HiperIgM-1) diseases. HSCT group was studied twice - before transplantation and with a median 6.4 months after transplantation. HSCT types were as follows: MUD-11, MSD-7, MFD-1.Two groups were recruited as a control: healthy group (HC) consisted of 35 patients 4.3-21 (average 12) years old, 14 boys and 21 girls, and obesity group (OB) including 27 children 4-18 (average 14) years old, 12 boys and 15 girls. Additionally the studied proteins genes expression in peripheral blood leukocytes using GeneChip Human Gene 1.0 ST Array (Affymetrix, Santa Clara, USA) were assessed in both HSCT and OB groups.

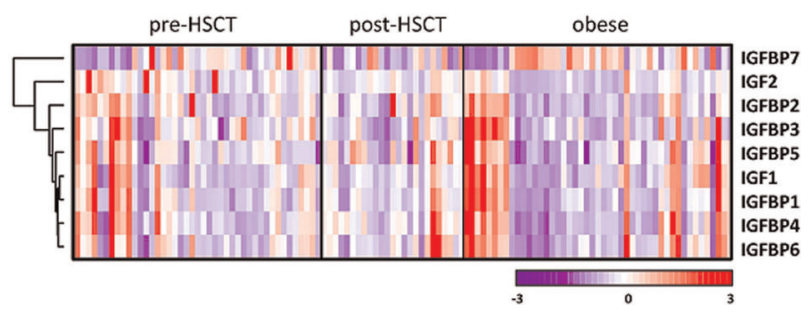

[P783 Figure] [Clustering of signals of the studied genes]
Results: Significant differences of peptides concentrations and genes expression values are presented in the Table 1 and Figure 1.

\begin{tabular}{|c|c|c|c|c|c|}
\hline $\begin{array}{l}\text { Parameter } \\
(\mathrm{ng} / \mathrm{ml})\end{array}$ & $\begin{array}{l}\text { pre- } \\
\text { HSCT }\end{array}$ & $\begin{array}{l}\text { post- } \\
\text { HSCT }\end{array}$ & $\mathrm{HC}$ & OB & Significant differences \\
\hline IGF-I & 151 & 106 & 203 & 265 & $\begin{array}{l}\text { pre-HSCT vs. post-HCST, pre-HSCT vs. OB, } \\
\text { post-HSCT vs. HC, Post-HSCT vs. OB }\end{array}$ \\
\hline IGF-II & 110 & 265 & 113 & 21,7 & $\begin{array}{l}\text { pre-HSCT vs. OB, post-HSCT vs. OB, HC vs. } \\
\text { OB }\end{array}$ \\
\hline IGFBP-1 & 7,56 & 15,6 & 4,39 & 1,74 & $\begin{array}{l}\text { pre-HSCT vs. post-HCST, pre-HSCT vs. OB, } \\
\text { post-HSCT vs. HC, Post-HSCT vs. OB, HC vs. } \\
\text { OB }\end{array}$ \\
\hline IGFBP-2 & 159 & 300 & 102 & 67,1 & $\begin{array}{l}\text { pre-HSCT vs. HC, post-HSCT vs. HC, Post- } \\
\text { HSCT vs. OB, HC vs. OB }\end{array}$ \\
\hline IGFBP-3 & 1818 & 1908 & 1811 & 1903 & $\begin{array}{l}\text { pre-HSCT vs. HC, pre-HSCT vs. OB, post- } \\
\text { HSCT vs. HC, HC vs. OB }\end{array}$ \\
\hline IGFBP-4 & 50,9 & 47,8 & 21,4 & 32,8 & $\begin{array}{l}\text { pre-HSCT vs. HC, pre-HSCT vs. OB, post- } \\
\text { HSCT vs. HC, post-HSCT vs. OB, HC vs. OB }\end{array}$ \\
\hline IGFBP-6 & 84,1 & 108 & 139 & 73,6 & $\begin{array}{l}\text { pre-HSCT vs. post-HSCT, pre-HSCT vs. HC, } \\
\text { post-HSCT vs. HC, HC vs. OB }\end{array}$ \\
\hline IGFBP-7 & 28,1 & 33,1 & 59,5 & 33,8 & $\begin{array}{l}\text { pre-HSCT vs. post-HSCT, pre-HSCT vs. OB, } \\
\text { post-HSCT vs. OB }\end{array}$ \\
\hline
\end{tabular}

\section{[[P783 Table] Concentrations of studied peptides]}

Conclusions: Concentrations of all peptides except IGF-I and IGFBP-4 increased after HSCT (differences significant for IGFBP-1, -6, -7). Concentrations of IGF-II, IGFBP-1, -2 , and -4 in Post-HSCT were significantly higher than in OB. Concentrations of IGFBP-1, -2, -3 , and -4 in PostHSCT were significantly higher than in HC. Interestingly gene expression analysis did not show comparable differences as in biochemical analysis. The biochemical results suggest contribution of IGF pathways in reconstitution and regeneration processes in children after HSCT.

Conflict of interest: The study was sponsored by national grant number NN 407198737.

None of the authors has anything to disclose.

\section{P784}

Toll-like receptor expression pattern on T- and NKlymphocytes in children after allogenic stem cell transplantation is associated with viral infections and aGVHD incidence

Kornelia Gajek ${ }^{1}$, Benita Wiatrak ${ }^{2}$, Dorota Noworolska-Sauren ${ }^{1}$, Blanka Rybka ${ }^{1}$, Renata Ryczan-Krawczyk ${ }^{1}$, Aleksandra Ślęzak ${ }^{1}$, Marek Ussowicz ${ }^{1}$

${ }^{1}$ Medical University of Wroclaw, Department and Clinic of Paediatric Oncology, Haematology and Bone Marrow Transplantation, Wroclaw, Poland; ${ }^{2}$ Medical University of Wroclaw, Department of Basic Medical Sciences, Wroclaw, Poland

Background: Viral opportunistic infections and acute graft versus host disease (aGvHD) are leading causes of morbidity and mortality after allogenic hematopoietic stem 
cell transplantation (allo-HSCT). The sophisticated balance between infections and aGvHD in the early period after HSCT depends on the innate immune system and reconstitution of adaptive immune system. Toll-like receptors (TLRs) expressed on the T-lymphocytes and NK cells form specific link between innate and adaptive immunity. We analyzed possible connection between frequency of viral infections or aGvHD incidence and expression of TLRs on the reconstituted lymphocytes.

Methods: 55 pediatric patients who underwent alloHSCT between February 2016 and February 2017 were monitored for the expression level of TLR2, TLR3, TLR4, TLR7, TLR8 and TLR9 on T- and NK-lymphocytes. The expression of listed receptors was measured by flow cytometry at five time points: $+15,+30,+45,+60$ and +90 days post-HSCT. TLR expression was further analysed for connection with viral infections (EBV, ADV, BKV, and CMV) and aGvHD incidence.

Results: The risk of aGvHD was associated with high expression level of TLR2 on $\mathrm{T}$ lymphocytes $(\mathrm{P}=0.005)$ and on NK cells $(\mathrm{P}=0.04)$, TLR4 $(\mathrm{P}>0.001)$ on $\mathrm{T}$ lymphocytes and TLR9 $(\mathrm{P}=0.03)$ on $\mathrm{T}$ lymphocytes and on NK cells $(\mathrm{P}=0.03)$. Levels of TLR7 $(\mathrm{P}<0.05)$ and TLR8 $(\mathrm{P}<0.05)$ on NK lymphocytes and TLR8 $(\mathrm{P}<0.05)$ on $\mathrm{T}$ lymphocytes were positively correlated with occurrence of cytomegalovirus (CMV) infection. The expression level of TLR7 $(\mathrm{P}<0.05)$ on the natural killer cells was also positively correlated with adenoviral (ADV) infection. Otherwise, values of TLR8 on $\mathrm{T}$ lymphocytes were correlated negatively with BK polyomavirus (BKV) infection in urine $(\mathrm{P}<0.05)$.

Conclusions: Obtained results suggest a connection between expression level of some TLRs on T lymphocytes or NK cells and viral infection/aGvHD events in patients after allo-HSCT. TLR2 can contribute to induction of aGVHD through activation of NF- $\mathrm{KB}$ signalling pathway. Precise determination of these correlations and their influence for the condition of patients can help identify the patients population prone to viral infections and aGvHD providing with opportunity to early intervention.

Conflict of interest: None.

\section{P785}

Treatment dilemmas in asymptomatic children with primary haemophagocytic lymphohistiocytosis

Giovanna Lucchini ${ }^{1}$, Rebecca Marsh ${ }^{2}$, Kimberly Gilmour ${ }^{1}$, Austen Worth $^{1}$, Zohreh Nademi ${ }^{1}$, Anupama Rao ${ }^{1}$, Claire Booth ${ }^{1}$, Persis Amrolia $^{1}$, Juliana Silva ${ }^{1}$, Robert Chiesa ${ }^{1}$, Robert Wynn ${ }^{3}$, Kai Lehmberg $^{4}$, Itziar Astigarraga ${ }^{5}$, Tayfun Guengoer ${ }^{6}$, Jan Stary ${ }^{7}$, Despina Moshous ${ }^{8}$, Marianne Ifversen ${ }^{9}$, Daniel Zinn ${ }^{10}$, Hirofumi Shibata $^{11}$, Paul Veys ${ }^{1}$, Kanchan Rao ${ }^{1}$
${ }^{1}$ Great Ormond Street Hospital for Children NHS Foundation Trust, London, United Kingdom; ${ }^{2}$ Cincinnati Children's Hospital,

Cincinnati, OH, United States; ${ }^{3}$ Royal Manchester Children Hospital, Manchester, United Kingdom; ${ }^{4}$ University Medical Center Hamburg Eppendorf, Hamburg, Germany; ${ }^{5}$ Hospital Universitario Cruces., Barakaldo, Spain; ${ }^{6}$ University Children Hospital, Zurich, Switzerland; ${ }^{7}$ Motol University Hospital, Prague, Czech Republic; ${ }^{8}$ Hôpital NeckerEnfants Malades, Paris, France; ${ }^{9}$ Rigshospitalet, Copenhagen,

Denmark; ${ }^{10}$ Baylor College of Medicine, Houston, TX, United States;

${ }^{11}$ Kyoto University Hospital, Kyoto, Japan

Background: Asymptomatic carriers of pathogenic mutations in causative genes for primary HLH are at high-risk of developing a life-threatening condition, which requires allogeneic SCT to be cured once activated. There is currently no data to inform the treatment pathway for asymptomatic pts. We analysed the outcomes of pairs of index cases (ICs)/asymptomatic family members (ACs) with a genetic defect predisposing to primary HLH .

Methods: We collected data from 22 SCT Centres worldwide. 30 analysable pairs/triads of ICs/ACs were reported (11 perforin, 6 MUNC18-2, 4 MUNC13-4, 2 syntaxin deficiency, 6 Griscelli II, 1 Chediak Higashi syndrome).

Results: ICs presented at a median age of 1.5 years. 21/ 31 underwent SCT. 14/21 are alive in CR. 6/21 transplanted pts died from toxicity, 1 from progression. Of the 10 nontransplanted pts, 2 are alive in CR, 7 died (6 progression, 1 toxicity), 1 is lost to follow-up.

Median age of ACs at diagnosis was 0.6 years. Of these, 10/30 pts activated HLH whilst on observation. 9/10 patients underwent SCT; 5 are alive in CR, 4 died (3 progression, 1 toxicity). 20/30 ACs remained asymptomatic. 6/20 have received no treatment and are in CR. 14/19 underwent pre-emptive SCT, 13/14 are alive in CR at a median follow-up of 29 months.

OS in non-active ACs was higher than in ICs $(95.0 \%$ vs $51.6 \%, \mathrm{p}<0.003)$, but OS in ACs who activated HLH while on observation was not $(50.0 \%$ vs $51.6 \%, \mathrm{p}=1.0)$.

Conclusions: Pre-emptive SCT in non-active ACs proved safe (OS 92.9\%) and should be considered as treatment option.

Conflict of interest: No conflict of interest to declare

\section{P786}

Treatment outcome of hematopoietic stem cell transplantation in children with acute lymphoblastic leukemia in the Yeungnam region of Korea: A multicenter retrospective study

Young Tak Lim ${ }^{1}$, Kyung Mi Park', Eu Jeen Yang ${ }^{1}$, Jae Min Lee', Jeong Ok $\mathrm{Ha}^{3}$, Sang Kyu Park, Jae Young Lim ${ }^{4}$, Eun Sil Park, Heewon Chueh ${ }^{6}$, Seom Gim Kong ${ }^{7}$

${ }^{1}$ Pusan Nationak University Children's Hospital, Department of 
Pediatrics, Yangsan, Korea, Republic of; ${ }^{2}$ Yeungnam University College of Medicine, Department of Pediatrics, Daegu, Korea, Republic of; ${ }^{3}$ Daegu Fatima Hospital, Pediatrics, Daegu, Korea, Republic of; ${ }^{4}$ Ulsan University Hospital, Pediatrics, Ulsan, Korea, Republic of; ${ }^{5}$ Gyeongsang National University School of Medicine, Pediatrics, Jinju, Korea, Republic of; ${ }^{6}$ Dong-A University College of Medicine, Pediatrics, Busan, Korea, Republic of; ${ }^{7}$ Kosin University College of Medicine, Pediatrics, Busan, Korea, Republic of

Background: The prognosis of high-risk or advanced childhood acute lymphoblastic leukemia (ALL) is still unsatisfactory and allogeneic hematopoietic stem cell transplantation (HSCT) is still the only curative option. We report the transplantation outcomes of 36 children with relapsed ALL and very high risk ALL at initial presentation who received HSCT in Yeungnam region of Korea.

Methods: From January 2004 to December 2013, children (up to age 18 years) with ALL who received HSCT $(n=36)$ at Yeungnam region of Korea were reviewed retrospectively. Patients transplanted in the first complete remission $(\mathrm{CR} 1, \mathrm{n}=19)$ had very high-risk features at presentation, including high-risk chromosome translocation, white blood cell count exceeding 200,000/ $\mathrm{l}$, induction failure and others. Of the patients who transplanted after relapse $(n=17)$, most were in the second remission at HSCT.

Results: Median age at transplantation was 6.75 years (range 0.92 - 14.25 years). 7 patients received human leukocyte antigen matched related donor transplants, 13 received matched unrelated donor transplants, 10 received partially matched unrelated donor (URD) transplants. Stem cell sources included 19 peripheral blood stem cells (PBSC), 4 bone marrows (BM) and 13 umbilical cord bloods (CB). 7 patients received conditioning regimens including fractionated total body irradiation (TBI) with cyclophosphamide or melphalan and 26 patients received busulfan based conditioning regimens. The median infused CD34+ cells were $4.3 \times 10^{6} / \mathrm{kg}$ of $\mathrm{BM}$ and PBSC transplants $(\mathrm{n}=23)$ and $2.6 \times 10^{5} / \mathrm{kg}$ of CB transplants (n $=13$ ). All but 3 patients who received unrelated cord blood transplants showed successful engraftment. The median time to ANC recovery was 14 days (range, 9-23 days). The incidence of acute graft versus host disease (GVHD) was $50 \%$ including $15.6 \%$ of grade III-IV acute GVHD. Chronic GVHD developed in 8 of $29(27.6 \%)$ evaluable patients. Patients who transplanted on CR2 suffered from significantly higher incidence of grade III-IV acute GVHD (26.7\%) compared to those transplanted on CR1 (5.9\%) $(p=0.001)$. The estimated 5-year event-free survival (EFS) and overall survival $(\mathrm{OS})$ were $69.4 \pm 9.2 \%$ and $75.0 \pm$ $8.5 \%$, respectively. The 5-yr EFS and OS were significantly higher in CR1 patients than in CR2 patients $(p=0.045)$. However, there were no significant differences in outcomes between patients who received with TBI and those without TBI. In addition, the 5-year EFS and OS were not significantly different between CBT and URD transplantation ( $p=0.187, p=0.28)$. The cumulative incidence of relapse in CR2 patients was $23.5 \%$, but there was no relapse in CR1 patients.

Conclusions: HSCT is effective treatment for pediatric patients with ALL who had very high-risk features in CR1 or had already relapsed. In addition, the outcome after CB transplantation was comparable to the outcome after URD transplantation.

Conflict of interest: No conflict of interest to disclose.

\section{P787}

\section{Unexpected high incidence of HHV-6 encephalitis after naive $T$ cell depleted graft haploidentical stem cell transplantation in pediatric patients}

Luisa Sisinni ${ }^{1}$, Mercedes Gasior ${ }^{2}$, Rachel de Paz ${ }^{2}$, Sergio Querol ${ }^{3}$, David Bueno ${ }^{4}$, Lucia Fernandez ${ }^{5}$, Julia Marsal, Isabel Badell, Adela Escudero $^{7}$, Laura Medina ${ }^{3}$, Ana Sastre ${ }^{4}$, Montserrat Torrent ${ }^{1}$, Ramon Gimeno ${ }^{8}$, Eduardo Lopez, Juan Torres ${ }^{9}$, Antonio PérezMartínez ${ }^{4}$

${ }^{1}$ Hospital Santa Creu i Sant Pau-Universitat Autònoma, Pediatric Hematology, Oncology and HSCT Unit, Barcelona, Spain; ${ }^{2}$ Hospital La Paz, Hematology, Madrid, Spain; ${ }^{3}$ Banc de Sang i Teixits, Celular Therapy Unit, Barcelona, Spain; ${ }^{4}$ Hospital La Paz, Pediatric Hematology-Oncology, Madrid, Spain; ${ }^{5}$ Hospital 12 de Octubre, Clinical Research Unit Spanish National Cancer Research Centre, Madrid, Spain; ${ }^{6}$ Hospital Sant Joan de Deu, Pediatric HSCT Unit, Barcelona, Spain; ${ }^{7}$ Hospital La Paz-Instituto de Genética Médica y Molecular (INGEMM), Madrid, Spain; ${ }^{8}$ Hospital del Mar \& IMIM (Hospital del Mar Medical Research Institute), Immunology Department, Barcelona, Spain; ${ }^{9}$ Hospital La Paz, Immunology, Madrid, Spain

Background: Haploidentical stem cell transplantation (haplo-HSCT) has presented a major clinical challenge during the past decades, due mainly to the high incidence of GvHD induced by strong donor T-cell alloreaction. The CD45RA $T$ cell depletion method has been performed to deplete naïve $\mathrm{T}$ cells preventing GvHD but preserving memory cells providing immediate functional $\mathrm{T}$ cells with antiinfection, antileukemia and anti-rejection effects. Although reactivation of human herpesvirus-6 (HHV-6) is common after HSCT it has not yet been described after this type of T-cell depletion.

Methods: Twenty-four consecutive high-risk leukemia patients have received haploidentical transplantation following non-myeloablative conditioning regimen. Each patient received two cell products, one created by CD34 positive selection and the other through CD45RA depletion from the CD34 negative fraction by CliniMACS device. The CD34+ enriched product provided a median CD34+ 
and $\mathrm{CD} 3+$ dose of $6.29 \times 10^{6} / \mathrm{kg}$ (range 4.04-18.1) and $5.75 \times 10^{3} / \mathrm{kg}(1-490)$, respectively. The $\mathrm{CD} 45 \mathrm{RO}+$ cell dose was a median of $10.5 \times 10^{7} / \mathrm{kg}$ (range 3.8-102.9) and the $\mathrm{CD} 45 \mathrm{RA}+$ cell dose was a median of $4.9 \times 10^{3} / \mathrm{kg}$ (range 0 14.6). The median $\log 10$ depletion of CD45RA + cells was 4.75 (range 2.2 to 6.37 ).

Results: All patients achieved neutrophil engraftment at a median of 10 days (range 8 to 15) post-transplant. Two patients experienced a secondary graft rejection. Immune recovery was fast with a median absolute lymphocyte count (ALC) of $1,200 \times 10^{\wedge} 9 / \mathrm{L}$ at 30 days post-transplant. Eleven patients $(46 \%)$ have developed acute GvHD grade I-II and four patients (19\%) have developed acute GvHD grade IIIIV. Three patients $(12.5 \%)$ showed chronic GvHD. The overall survival (OS) and disease-free survival (DFS) were $56 \%$ and $53 \%$ respectively at 30 months of follow-up. We observed an unexpected high rate of limbic encephalitis due to HHV6 (8 cases), proved by demonstration of HHV6 viral DNA in CSF samples. The infection appeared early just after the engraftment with a median onset of 14 days (8-15) after transplantation. Characteristically the infection followed a similar pattern in almost all cases: clinical signs compatible with a strong "pre-engraftment syndrome" (fluid retention, erythematous rash, fever) preceded the start of neurological symptoms. On the other hand, none of the patients that didn't develop the infection showed the preengraftment syndrome or at most they showed only mild signs like fever. No patient died due to the encephalitis. One patient experienced secondary graft failure just after the infection. One patient showed neuropsychological sequelae and hyponatremia that are persisting after one and half year post-infection. The median ALC was $665 \times 10^{\wedge} 9 / \mathrm{L}$ when the encephalitis started and was similarly high that in patients withouth the infection.

Conclusions: CD45RA depleted haplo-HSCT is well tolerated with a rapid engraftment and low risk of severe acute GvHD and chronic GvHD. We have seen that the robust and fast immune recovery allowed to a rapid control of viral reactivations such as CMV but, unexpectedly, we observed a high rate of HHV6 encephalitis and we should need further studies to find out the mechanisms related to it.

Conflict of interest: No potential conflicts of interest were disclosed.

Regenerative medicine

\section{P788}

Development of a cell culture model based on biocompatible polymeric scaffolds engineered with human Mesenchymal Stromal Cells (MSCs) for cartilage and bone regenerative therapy
Federica Re $^{1}$, Simone Perucca ${ }^{1}$, Simona Bernardi ${ }^{1}$, Luciana Sartore $^{2}$, Fabio Bignotti ${ }^{2}$, Camillo Almici ${ }^{3}$, Andrea Bianchetti ${ }^{3}$, Luigi Rodella ${ }^{1}$, Manuel Salmeron-Sanchez ${ }^{4}$, Emilio Sardini ${ }^{5}$, Francesco Lopomo ${ }^{5}$, Muhammad Ahmed Khan ${ }^{5}$, Pierangelo Guizzi $^{6}$, Domenico Russo ${ }^{1}$, Michele Malagola ${ }^{1}$

${ }^{1}$ Università degli Studi di Brescia, Dipartimento di Scienze Cliniche e Sperimentali, Brescia, Italy; ${ }^{2}$ Università degli Studi di Brescia, Dipartimento di Ingegneria Meccanica e Industriale, Brescia, Italy; ${ }^{3}$ ASST Spedali Civili, Servizio Immuno-Trasfusionale, Brescia, Italy; ${ }^{4}$ University of Glasgow, Department of Biomedical Engineering, Glasgow, United Kingdom; ${ }^{5}$ Università degli Studi di Brescia, Dipartimento di Ingegneria dell'Informazione, Brescia, Italy; ${ }^{6}$ ASST Spedali Civili, U.O. di Ortopedia e Traumatologia, Brescia, Italy

Background: Regenerative medicine aims to restore normal tissue function by repairing or replacing damaged cells and tissues in patients. Scaffolds acts as a temporary matrix for cell proliferation and extracellular matrix deposition until the tissues are totally restored. Mesenchymal Stromal Cells (MSCs) promotes tissue repair because of their proliferative potential, ability to migrate to injured tissues, as well as immunomodulatory and trophic effect.

Methods: Different natural hydrogel scaffolds were characterized; A first series obtained from gelatin, hydroxyethylcellulose (HEC) and PEG (Polyethylene Glycol) as a cross-linker and a second series based on gelatin, different concentrations of chitosan (Ch) and PEG. Scaffolds were characterized for their physico-chemical, thermomechanical and morphological properties. MSCs were seeded into the scaffolds, in both dry and wet (hydrated) states, at a cellular density of $1 \times 10^{6}$ cells/scaffolds and $4 \times$ $10^{6}$ cells/scaffolds. In this context, the MSCs proliferation in presence of Fetal Bovine Serum (FBS) versus HPL (Human Platelet Lysate) were tested. The expansion of MSC from bone marrow (BM) and adipose tissue (AT) was evaluated aiming to find the best source of MSCs. The viability of MSCs was determined using MTT cell proliferation assay after 2, 7 and 14 days of culture. For inducing osteogenic and chondrogenic differentiation, cells/ scaffolds constructs were cultured in 24-well plates for 3 weeks with osteogenic differentiation media consisting of a high-glucose DMEM supplemented with $10 \%$ FBS or $5 \%$ HPL, $10^{-7} \mathrm{M}$ Dex, $25 \mathrm{mg} / \mathrm{ml}$ l-ascorbic acid, and $3 \mathrm{mM}$ $\mathrm{NaH}_{2} \mathrm{PO}_{4}$ or chondrogenic media composed of highglucose DMEM supplemented with Dex $10^{-7} \mathrm{M}$, sodium pyruvate $1 \mathrm{mM}, 50 \mu \mathrm{g} / \mathrm{ml}$ l-ascorbic acid-2- phosphate, $40 \mu \mathrm{g} / \mathrm{ml}$ proline, $1 \%$ ITS and $10 \mathrm{ng} / \mathrm{ml}$ of human transforming growth factor $\beta 3$. These media were renewed every 3 days. Scaffolds without cells were used as controls.

Results: Hydrogel scaffolds with $\mathrm{Ch}$ seem to be an optimal model to host MSCs compared to scaffolds based on HEC. Moreover $\mathrm{Ch}$ materials show highly porous structure and interconnected pores. Tensile properties, specific pore size, wettability, weight loss and hydrolytic 
degradation were different between the HEC and $\mathrm{Ch}$ as well as in Ch concentrations. MSCs seeded into the Ch scaffolds at a cellular density of $1 \times 10^{6}$ cells/scaffolds were able to occupy the whole volume, showing good viability, proliferation and adhesion. Furthermore, the synergistic effects of HPL in combination with the proper scaffold is useful to support the MSCs proliferation better than FBS. Currently, we are exploring the effects of scaffolds on modulating MSCs osteogenic and chondrogenic differentiation as well as assessing MSC differentation by immunofluorescence to characterise the expression of osteogenic and chondrogenic specific markers.

Conclusions: Hydrogel scaffolds with $\mathrm{Ch}$ were selected to design a potential substrate to support MSCs from different sources. Moreover, the use of HPL for cell expansion offers the possibility to obtain a safer cellular products without xenogenic contaminants and able to be transferred to clinical applications in humans.

Conflict of interest: Federica Re: nothing to disclose

\section{P789}

Dividing the marrow mononuclear cell population into cells improving degenerated joint function and those leading to revascularization if injected locally

Agata Borowik $^{1,2}$, Emilia Jaskuta ${ }^{1,2}$, Janusz Lange ${ }^{2}$, Andrzej Lange $^{1,2}$

${ }^{1}$ L. Hirszfeld Institute of Immunology and Experimental Therapy, Polish Academy of Sciences, Wroclaw, Poland; ${ }^{2}$ Lower Silesian Center for Cellular Transplantation with National Bone Marrow Donor Registry, Wroclaw, Poland

Background: We showed (Blood 2005, 106:4319) that mesenchymal stem cells (MSC) retain the recipient phenotype after HSCT in spite of the presence of full haematological chimera. This and other observations led to the conclusion that in the marrow there are several zones with hematopoietic stem/progenitor cells and mesenchymal stem cells (MSC) at different stages of differentiation kept in a dormant state. MSC are recognized as master cells having a broad potential to differentiate into different tissues. The presence of more differentiated MSC in the quiescent stage in the marrow is not unequivocally confirmed.

Methods: To address this issue we evaluated the efficacy of marrow cells having MSC markers to improve vascularization of limbs with ischaemia in 28 patients (4F/24M, age median: 51 range 33-64 years old) or to improve the function of worn-out hips and knees (14 procedures in 13 patients, $9 \mathrm{~F} / 4 \mathrm{M}$, age median: 58 , range 45 71 years old). These two groups of patients received marrow-derived mononuclear cells injected either into the ischemic leg calf muscles or intra-articular into the hip or knee. Ischemic leg patients received autologous leukophoretic product freshly taken from the marrow cavity. Cells were injected into the calf muscles in about $0.7 \mathrm{~mL}$ small portions. Patients with of worn-out hips received to the joint marrow cells harvested after preparation od $50 \mathrm{ml}$ of the marrow in the Auto Stem Cell Kit (Pharmed) according to manufacture's procedure. The dose of injected cells was standardized with the beginning volume of the marrow processed. The patients who composed these two groups were subsequently subdivided into patients responding favourably to the treatment (healing of foot ulceration and cessation of intermittent claudication or pain release and improvement in the joint function, respectively, in two groups investigated) and those who failed.

Results: It was found that the patients who improved in joint function had a higher contribution of CD90+ and $\mathrm{CD} 73$ + cells to the CD45-CD34- marrow cell population in cells harvested after preparation in the Auto Stem Cell Kit than sufferers from recurrence of symptoms who reported that in 6 months after the procedure $(5.470 \pm 2.045 \%$ vs $1.607 \pm 0.128 \%, \mathrm{p}=0.012$ for $\mathrm{CD} 90+$ in $\mathrm{CD} 45-\mathrm{CD} 34-$ population; $13.154 \pm 12.550 \%$ vs $0.150 \pm 0.015, \mathrm{p}=0.020$ for CD73 in CD45-CD34- cell population).

In contrast, patients with critical limb ischemia who showed remission until 12 months after intervention received less $\mathrm{CD} 73+$ than those who deteriorated $(0.061$ $\pm 0.011 \%$ vs $0.112 \pm 0.015 \%, \mathrm{p}=0.022$ ).

Conclusions: Intra-articular injection of $\mathrm{CD} 73+$ and CD90+ cells of marrow origin improves joint function but does not play a positive role in the revascularization process of ischemic legs. The latter effect is rather due to the endothelial stem cell (ESC) which were found in inoculum employed for revascularisation purposes.

Supported by the National Centre for Research and Development (grant number INNOMED/I/I/NCBR/2014) from Innovative Economy Operational Programme funds, in the framework of the European Regional Development Fund.

Conflict of interest: The authors declare that there is no conflict of interest regarding the publication of this abstract.

\section{P790}

In vitro analysis of spontaneous fusion of two populations of stem/progenitor cells of bone marrow and myogenicorigin for potential application to treat muscular dystrophies

Aleksandra Klimczak ${ }^{1,2}$, Urszula Kozlowska ${ }^{1,2}$, Agnieszka Malcher ${ }^{2}$, Agnieszka Zimna ${ }^{2}$ Maciej Kurpisz, 
${ }^{1}$ Hirszfeld Institute of Immunology and Experimental Therapy PAS, Wroclaw, Poland; ${ }^{2}$ Institute of Human Genetics PAS, Poznan, Poland

Background: Muscular dystrophies represent a group of diseases which may develop in several forms, and severity of disease is usually associated with gene mutation. Therapeutic effect of individually transplanted MSC of bone marrow-origin (BM-MSC) or stem/progenitor cells of myogenic-origin is limited due to complexity of muscular dystrophies and biological activity of stem/progenitor cells. We analyzed biological properties of BM-MSC and myogenic stem/progenitor cells for potential application for treatment of patients suffering on Duchenne muscular dystrophy (DMD).

Methods: Bone marrow samples and skeletal muscle oligobiopsies were collected from three healthy donors with approval by the local Bioethics Committee. MSC were isolated from $30 \mathrm{ml}$ of donor BM. Nucleated cell concentrate (NCC) were obtained by using Marrow Stim Mini instrument. NCC were resuspended in alpha-MEM medium supplemented with $8 \%$ of platelet lysate and antibiotics, and were placed in MC3 cell culture system (Macopharma). The myogenic progenitor cells were isolated from skeletal muscle oligobiopsies. Procedure included combined method of mechanical agitation with enzymatic digestion. Separated cells were placed in tissue culture gelatin-coated flasks in standard DMEM medium supplemented with $10 \%$ FBS, FGF and antibiotics. Both, MSC of BM-origin and myogenic progenitor cells were kept in standard in vitro culture conditions in humidified atmosphere $5 \% \mathrm{CO}_{2}, 37^{\circ} \mathrm{C}$. Culture medium was changed every three days until adherent cells reached confluence $70-80 \%$.

The phenotype of the cultured cells BM-MSC and myogenic progenitor cells were characterized by flow cytometry and immunocytochemistry for: CD45, CD31, CD34, CD56, CD73, CD90, CD105, CD146, HLA-ABC, HLA-DR, and costimulatory molecules CD28, CD40, CD80, CD154. Co-culture of BM-MSC labeled with PKH-26 (red) and myogenic progenitor cells labeled with PKH-67 (green) were performed in DMEM medium supplemented with $10 \%$ FBS and antibiotics.

Results: Adherent cells of BM- and myogenic-origin exhibit similar phenotype $\mathrm{CD} 90+, \mathrm{CD} 73+, \mathrm{CD} 105+$ characteristic for naïve MSC (85-99\% of adherent cells). Myogenic progenitor cells (but not from BM) after culture additionally expressed CD56 (63-68\% of adherent cells), and this observation suggest that those population of MSC is muscle-specific. All cultured cells of BM- and myogenicorigin were negative for: HLA-DR antigens, hematopoietic markers CD45, CD34 and co-stimulatory molecules. Proangiogenic properties of BM-MSC were confirmed by expression of CD146 (68-72\% of adherent cells). Spontaneous fusion of BM-MSC and myogenic progenitors was observed as early as 12 hours after co-culture was started and plateau was seen between 18 to 36 hours.

Conclusions: Stem/progenitor cells generated from BM and from skeletal muscle express phenotype characteristic for naïve MSC, do not express immunogenic phenotype, and are able to generate spontaneous fusion in in vitro conditions.

Thus, the development of cell-based therapies for muscular dystrophies by the delivery of two populations of normal stem/progenitor cells (BM-MSC and myogenic progenitors) would be a promising tool to treat muscular dystrophies. We hypothesize that both populations of cells will fuse with damaged muscle cells in vivo facilitating skeletal muscle recovery.

Conflict of interest: Study supported by National Science Center grant N N407 121940

\section{P791}

\section{Psychological distress in mothers of children with oncological malignancies}

Olga Chernenko, Dmitriy Bakun, Svetlana Oleshko-Gortinskaya, Olga Ushakova, Asmik Gevorgian, Olesya Paina, Julia Tarakanova, Inna Markova, Alisa Volkova, Ludmila Zubarovskaya, Boris Afanasyev

First Pavlov State Medical University of Saint Petersburg, $R$. Gorbacheva Memorial Institute of Children Oncology, Hematology and Transplantation, Saint Petersburg, Russian Federation

Background: Parenting a child with cancer is profoundly distressing. Parents who permanently support and take care of children during their treatment at an oncology clinic are at risk of developing psychological trauma.

Methods: In a prospective single-center study, we collected data from 52 mothers whose children were undergoing treatment in Raisa Gorbacheva Memorial Institute of Children Oncology, Hematology and Transplantation. Several methods were used, including a clinical psychological method aimed at assessing the personal response of a mother to her childÂ's illness, and an experimental psychological method: Impact of Event Scale - Revised (IES-R), assessment of self-actualization (POI) and a questionnaire for diagnostics of a mother's reaction to her child's illness.

Results: The study of the level of traumatic stress (IES-R) allowed to identify three groups of mothers: those with a low $(32.1 \%)$, high (33.9\%) and extremely high (34\%) level of psychological distress. The group of women with an extremely high level of psychological distress predominantly included mothers raising an only child who was undergoing relapse treatment. Most of the women in this group came from outside the city. The analysis of self- 
actualization assessment (POI) showed that mothers with a high level of psychological distress tended to express their feelings spontaneously and directly: the higher the degree of traumatic stress, the higher the expressiveness in the behaviour, which was often manifested in their interactions with other people - primarily with doctors - rather than with their children. Mothers with a high level of psychological distress were more likely to accept their irritability, anger, and aggression $(p \leq 0.01)$. The study of the motherÂ's reaction to the childÂ's illness showed that mothers with a high level of psychological distress felt their impotence and inability to influence the outcome of the disease, which increased proportionally to the duration of the disease, especially in case of a relapse. The general exhaustion of a mother both at the psychological and physical levels reduced her control over the behaviour of her child undergoing treatment, which led to a decrease in her responsibility and could be a sign of her asthenization as a parent. Such mothers tended to seek external support: the more dramatically a mother perceived her child's disease, the more she needed the support of her husband or family.

Conclusions: The correlation analysis of the results showed that the parents' ability to cope with their psychological and emotional state was important for successful and timely treatment of their child, since it largely determined the behaviour of the sick child, his/her attitude to treatment and to the doctor. Oncological malignancies in children are traumatic experiences for their mothers. $68 \%$ of the women included in the study exhibited a high to extremely high level of psychological distress. Their reaction and behaviour are determined by their emotional state, which must be taken into account when communicating; thus, mothers of sick children are in need of psychological help.

Conflict of interest: No conflict of interest

\section{P792}

\section{Regenerative medicine as applied to solid organ transplantation: Mesenchymal stromal cell on extracellular matrix-scaffold for complex organ engineering}

\section{Stefania Croce ${ }^{1}$, Melissa Mantelli ${ }^{2}$, Elisa Lenta ${ }^{2}$, Alice Maltese ${ }^{2}$, Laura Caliogna ${ }^{2}$, M.Antonietta Avanzini ${ }^{2}$, Stefania Mantovani ${ }^{2}$, Szandra Brambilla ${ }^{1}$, Livia Visai ${ }^{1}$, Francesco Calabrese ${ }^{1}$, Tamara Zoro $^{1}$, Marco Zecca ${ }^{2}$, Andrea Peloso ${ }^{2}$, Lorenzo Cobianchi ${ }^{1,2}$ \\ ${ }^{1}$ University of Pavia, Pavia, Italy; ${ }^{2}$ Fondazione IRCCS Policlinico San Matteo, Pavia, Italy}

Background: Liver dysfunction is a major health problem in the world. The restricted availability of donor organs limit liver transplantation, the only established successful treatment. Currently, extracellular matrix
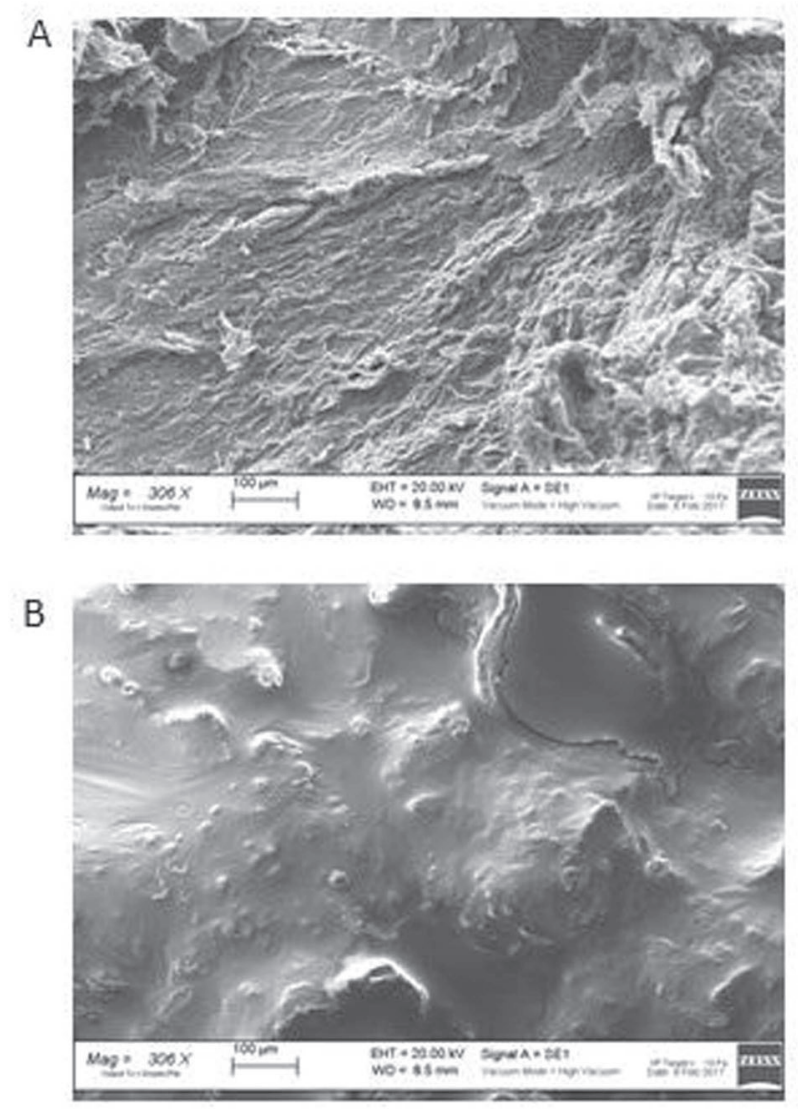

[P792 Figure] [Fig.1: SEM images of decellularized scaffold (A) and $M S C$ recellularized scaffold $(B)]$

(ECM)-scaffolds obtained through decellularization of discarded organs are considered a good three-dimensional structure for subsequent recellularization in order to create a functional organ. Stem cells, such as mesenchymal stromal cells (MSC), are capable of self-renewal and have the capacity to differentiate into specific cell types. It has been observed that MSC fate could be influenced by ECM. The aim of the present study is to investigate the capacity of MSC, to differentiate into functional hepatocyte-like cells, after interaction with liver ECM.

Methods: Porcine livers were decellularized with a SDS solution, following previously published procedure (Peloso A. et al, Annals of Surgery. 2016). The ECM-scaffolds were stained with haematoxylin/eosin to test the absence of nuclei, while the integrity of matrix was evaluated by immunofluorescence. MSC were isolated and expanded from porcine bone marrow and characterized by plastic adhesion, morphology, differentiation capacity and immunophenotype (Comite P. et al, Transplant Proc. 2010). $1 \times 10^{6}$ cells/scaffold were seeded on 6 ECM-scaffolds $(0.5$ $\mathrm{cm}^{3}$ dimension each) into a 48 well plate and cultured at $37^{\circ}$ C, $5 \% \mathrm{CO}_{2}$. After 10 days culture, samples were goldsputtered and high vacuum analyzed by scanning electron microscope SEM (Zeiss EVO MA10, Carl Zeiss). In 
addition, adherent cells, after trypsinization, were analyzed for intracellular albumin (R\&D System) by flow cytometry (Navios, Beckman Coulter).

Results: We observed i) complete and homogeneous coverage of inner scaffold surfaces by MSC (Fig.1), ii) acquisition of polygonal cell morphology, typical of hepatocytes and, iii) weak intracellular albumin production, main function of hepatocyte.

Conclusions: Our preliminary results suggest that the interaction of MSC with liver ECM promote differentiation into hepatocyte-like cell, showed by change in morphology and by the ability to produce albumin. Further studies are needed to confirm this approach suitable for complex organ engineering in regenerative medicine.

Conflict of interest: Nothing to disclose.

\section{Solid tumours}

\section{P793}

\section{Autologous Stem Cell Transplantation in Ewing's Sarcoma: Single-centre Retrospective Cohort Study}

Diogo Martins-Branco ${ }^{1}$, Ana Joao Pissarra ${ }^{2}$, Maria Teresa Alexandre $^{1}$, Filipa Moita ${ }^{3}$, Margarida Ferreira ${ }^{1}$, Nuno Miranda ${ }^{3}$, Gilda Teixeira ${ }^{3}$, Isabelina Ferreira ${ }^{3}$, Fernando Leal-da-Costa ${ }^{3}$, Manuel Abecassis ${ }^{3}$

${ }^{1}$ Instituto Português de Oncologia de Lisboa, Francisco Gentil, Medical Oncology Department, Lisboa, Portugal; ${ }^{2}$ Centro Hospitalar Lisboa Ocidental, Medical Oncology Department, Lisboa, Portugal; ${ }^{3}$ Instituto Português de Oncologia de Lisboa, Francisco Gentil, Blood and Marrow Transplantation Unit, Lisboa, Portugal

Background: High dose therapy (HDT) with autologous stem cell transplantation (ASCT) has been used with high risk or relapsed Ewing's sarcoma (ES). Although HDT had been evaluated in smaller, uncontrolled studies, the EUROE.W.I.N.G 99 trials were the first to evaluate this approach in a randomised fashion showing that may be an effective treatment for selected patients with localised high-risk ES but does not provide a significant efficacy benefit in patients with lung metastases.

Methods: Retrospective cohort study of all the patients with ES diagnosis treated with HDT with ASCT at a single cancer centre, between January 1995 and December 2017. Indications for ASCT were: a) 1st-line treatment in metastatic or high-volume localised disease and b) 2ndline treatment after chemo-sensitive recurrence. Clinical data were obtained by individual medical chart review. Time to relapse and survival after ASCT were calculated by Kaplan-Meier method and survival analysis for indications was performed by Log-Rank test, using the software Stata $12.1^{\circledR}$ and considering a 5\% statistical significance level.

Results: We identified 44 patients: the median age at ASCT was 17 (IQR 14-21) and 72.7\% were male $(\mathrm{n}=32$ ).
Regarding poor prognosis factors: i) age at diagnosis was $>15$ in $50.0 \%(\mathrm{n}=22)$, ii) tumour location was axial in $18.2 \%(\mathrm{n}=8)$ and, when reported, iii) the tumour volume of localised disease $(\mathrm{n}=10)$ was $>200 \mathrm{~mL}$ in $60.0 \%(\mathrm{n}=6)$ and iv) LDH $(\mathrm{n}=37)$ was elevated in $64.9 \%(\mathrm{n}=24)$. $79.5 \%(\mathrm{n}=35)$ of the primary tumours were from bone. The disease status was metastatic at the diagnosis in $68.2 \%$ $(\mathrm{n}=30)$. Secondary disease was localised at lung in $54.5 \%$ $(\mathrm{n}=24)$, bone in $20.5 \%(\mathrm{n}=9)$ and bone marrow in $13.6 \%$ $(n=6)$. Most of the patients were initially treated with EuroEwing $(54.5 \%, \mathrm{n}=24)$ or EICESS-92 $(20.5 \%, \mathrm{n}=9)$ protocols. $40.9 \%(\mathrm{n}=18)$ surgically removed the primary tumour, $27.3 \%(\mathrm{n}=12)$ after chemotherapy. From these, $66.7 \%(\mathrm{n}=8)$ present $<10 \%$ residual viable tumour in the specimen. $43.2 \%(n=19)$ received radiation therapy. The indication for ASCT distribution was as follows: a1) $34.1 \%$ $(\mathrm{n}=15)$ 1st-line with complete response (CR), a2) 43.2\% $(\mathrm{n}=19) 1$ st-line with very good partial response (VGPR) and b) $22.7 \% \quad(n=10)$ 2nd-line after chemo-sensitive recurrence. HDT was performed with busulphan and melphalan in $97.7 \%(n=43)$. Febrile neutropenia was a complication in $88.6 \%$ of patients $(\mathrm{n}=39)$. The median length of stay was 25 (IQR 22-29) days. Median time to progression was 27.8 months and median survival was 33.7 months. 1-year overall survival (OS) was $76.2 \%(\mathrm{n}=$ $32 / 42)$ and 5 -year OS was $35.1 \%(n=13 / 37)$. Median survival by indication group was significantly different $(\mathrm{p}=0.025)$ : a1) not reached for 1 st-line with $\mathrm{CR}$, a2) 38.1 months for 1st-line with VGPR and b) 22.1 months for 2nd-line after chemo-sensitive recurrence.

Conclusions: HDT with ASCT showed to be an effective treatment in our cohort of patients, mainly as a 1st-line treatment either with or without CR. Patients with $\mathrm{CR}$ are those that better benefit from this treatment with longer survival, thus all the efforts should be made to obtain CR.

Conflict of interest: Nothing to disclose

\section{P794}

High dose chemotherapy and autologous stem cell transplantation improves survival of patients with osteosarcoma which respond poorly to neoadjuvant chemotherapy

Che Ry Hong ${ }^{1}$, Kyung Taek Hong ${ }^{1}$, Jung Yoon Choi ${ }^{1}$, Han-Soo Kim $^{2}$, Sung-Hye Park, Hyoung Jin Kang ${ }^{1}$, Hee Young Shin ${ }^{1}$

${ }^{1}$ Seoul National University Children's Hospital, Pediatrics, Seoul, Korea, Republic of; ${ }^{2}$ Seoul National University College of Medicine, Orthopedic Surgery, Seoul, Korea, Republic of; ${ }^{3}$ Seoul National University College of Medicine, Pathology, Seoul, Korea, Republic of

Background: Despite the great advancement in outcome for osteosarcoma, patients who respond poorly to neo- 
adjuvant chemotherapy show poor outcome with 5-year event free survival (EFS) of $35-45 \%$. This retrospective analysis evaluated the role of high dose chemotherapy and autologous stem cell transplantation (HDCT \& ASCT) in osteosarcoma patients with poor response to neoadjuvant chemotherapy as a sole risk factor.

Methods: A retrospective medical chart review was done on all the patients who were diagnosed with osteosarcoma between June 1997 and May 2015 at Seoul National University Children's Hospital and the patients with tumor necrosis below $90 \%$ after neoadjuvant chemotherapy as a sole risk factor were identified. The outcome of the patients treated with conventional chemotherapy only (Group 1) and that of those treated with HDCT \& ASCT (Group 2) were compared. Conditioning regimen for HDCT \& ASCT consisted of melphalan $140 \mathrm{mg} / \mathrm{m}^{2}$ on day -7 and $70 \mathrm{mg} /$ $\mathrm{m}^{2}$ on day -6 , etoposide $200 \mathrm{mg} / \mathrm{m}^{2}$ and carboplatin $400 \mathrm{mg} /$ $\mathrm{m}^{2}$ from day -8 to day -5 .

Results: This review included 21 patients (17 male, 4 female) who were diagnosed at a median age of 11.7 years old and all of them had a single primary mass in the extremities and none of them had metastatic lesions. All of them underwent neoadjuvant chemotherapy and subsequent wide excision and limb salvage operation. The EFS of the patients in Group $1(\mathrm{n}=11)$ was $63.6 \%$ at median 7.8 (range, 0.8-16.2) years from diagnosis whereas that of those in Group $2(\mathrm{n}=10)$ was $100 \%$ at median 5.6 (range, 1.78.9) years from diagnosis and at 5.1 (range, 0.7-8.0) years from ASCT $(p=0.042)$. Of the patients in Group 1, 2 patients had lung metastases at median 0.8 years from diagnosis, and 2 patients had primary site relapses at median 1.4 years from off-therapy. The overall survival of the patients in Group 1 was $72.7 \%$ at median 8.3 (range, 2.216.2) years from diagnosis whereas that of those in Group 2 was $100 \%$ at median $5.6(1.7-8.9)$ years from diagnosis and at $5.1(0.7-8.0)$ years from ASCT $(p=0.113)$. Three patients in Group 1 died of disease. During ASCT, 1 patient experienced moderate-grade veno-occlusive disease which resolved with supportive care.

Conclusions: HDCT \& ASCT with melphalan, etoposide and carboplatin improves the EFS of the osteosarcoma patients with poor response to neoadjuvant chemotherapy as a sole risk factor.

Conflict of interest: The authors have no relevant financial relationships to disclose or conflicts of interest to solve.

\section{P795}

High Dose Chemotherapy followed by Autologous Stem Cell Transplant for Metastatic Germ Cell Tumours; the Scottish Experience
Bahaa Al-Bubseeree ${ }^{1}$, Yun Yi Tan ${ }^{2}$, David Irvine ${ }^{1}$, Graham MacDonald $^{3}$, Grant McQuaker', Anne Parker ${ }^{1}$, Ashita Waterston ${ }^{2}$, Jeff White $^{2}$

${ }^{1}$ NHS Greater Glasgow \& Clyde, BMT, Glasgow, United Kingdom; ${ }^{2}$ Beatson West of Scotland Cancer Centre, NHS Greater Glasgow and Clyde, Oncology, Glasgow, United Kingdom; ${ }^{3}$ Aberdeen Royal Infirmary, Aberdeen, United Kingdom

Background: Patients with germ cell tumours that respond poorly or relapse following first line therapy have a poor prognosis. Optimal salvage treatment in these patients is uncertain; both conventional dose salvage regimens and high dose chemotherapy with autologous stem cell transplantation (HDCT) are utilised.

Methods: All patients with relapsed/refractory metastatic germ cell tumours treated with HDCT between 2001-2016 at the Beatson West of Scotland Cancer Centre were identified and their medical records reviewed for baseline patient and tumour characteristics, prognostic features, toxicity and survival outcomes.

Results: Eighteen patients (15 male and 3 female) received HDCT salvage. Most had gonadal primaries of non-seminomatous or mixed pathology and $11(61 \%)$ had poor prognostic score at diagnosis (IGCCCG-1). All patients had platinum-based chemotherapy and most had surgery. Seven (39\%) had no evidence of disease following 1 st line therapy and $2(11 \%)$ were refractory.

The IPFSG prognostic score was calculated for the 14 male patients, $8(57 \%)$ were high or very high risk. Patients received between 1-4 cycles of induction chemotherapy containing paclitaxel, ifosfamide and cisplatin. Twelve patients $(66 \%)$ received carboplatin, etoposide tandem HDCT according to the Einhorn protocol, 4 patients (22\%) received palitaxel, etoposide, carboplatin, cyclpohosphamide HDCT according to the McNeish protocol and 1 patient had 3 cycles of HDCT according to the TI-CE protocol.

Median CD $34^{+}$dose transfused was $3.43 \times 10^{6} / \mathrm{kg} \mathrm{CD} 4^{+}$ cells (range $2.38 \times 10^{6}-9.7 \times 10^{6} \mathrm{CD} 34^{+} / \mathrm{kg}$ ). All patients engrafted. Median time to neutrophil recovery to $>0.5 \times 10^{9} /$ $\mathrm{L}$ was 11 days (range 9-16) and the median time to platelet recovery above $20 \times 10^{9} / \mathrm{L}$ and $50 \times 10^{9} / \mathrm{L}$ was 11 days (range 8-16) and 14 days (range 11-30) respectively.

Toxicities were common following HDCT. Supportive transfusion was required in all patients; a median 4 units of red cells (range 1-14) and 6 platelet transfusions (range 147) were given. All patients had at least 1 infective episode requiring broad-spectrum intravenous antibiotics. Grade 3-4 mucositis was common ( $46 \%$ transplant episodes). All patients lost weight during HDCT and 10 patients required nutritional support. Two patients required critical care due to sepsis and multi-organ failure. The most common longterm toxicities were neurotoxicity in 12 patients $(67 \%)$ and 
ototoxicity in 7 patients (39\%) due to cumulative platinum exposure. Time between HDCT cycles was significantly longer than in the published literature (mean 8.6 weeks compared with 3-4 weeks) reflecting the toxicity of the procedures.

The median length of follow-up post HDCT is 61 months. By September 2017, 5 patients (28\%) had died, 1 of posttransplant complications (intrapulmonary haemorrhage) and 4 following relapse. The 2-year PFS and OS for the 17 patients who have had at least 2 years of follow-up is $63 \%$ and $69 \%$ respectively.

Conclusions: Delivery of HDCT and ASCT as salvage treatment for metastatic germ cell cancer in a tertiary cancer centre in Scotland resulted in survival outcomes comparable to published literature, though toxicity is significant and maintaining dose intensity is challenging. We hope to recruit to the international TIGER trial (NCT02375204) which will attempt to clarify if HDCT is superior to conventional-dose chemotherapy in the salvage setting.

Conflict of interest: No conflict of interest

\section{P796}

\section{Human endogenous retroviruses (HERV)- $\mathrm{H}$ and $-\mathrm{K}$ sequences are hypomethylated and present in the circulating microvescicles of colon cancer patients}

Serena Del Bue ${ }^{1}$, Maria Dolci, Letizia Tarantini, ${ }^{1}$, Valentina Bollati $^{1}$, Chiara Favero ${ }^{1}$, Sonia Villani, Alberto Della Valle $^{2}$, Rosalia Ticozzi ${ }^{1}$, Pasquale Ferrante ${ }^{1}$, Elena Parrello ${ }^{1}$, Filippo Crivelli ${ }^{3}$, Marco Bregnit

${ }^{1}$ University of Milan, Milano, Italy; ${ }^{2}$ Istituto Clinico Città Studi, Milano, Italy; ${ }^{3}$ ASST Valle Olona, Busto Arsizio, Italy; ${ }^{4}$ ASST Valle Olona, Oncology, Busto Arsizio, Italy

Background: Human Endogenous Retroviruses (HERVs) are remnants of ancient exogenous retroviral infections of the humans, representing about $8 \%$ of the human genome. Their regulation is mainly based on epigenetic mechanisms, such as methylation of the proviral Long Terminal Repeats (LTRs). A few reports has shown the existence of a relationship between the HERVs expression and colon cancer, based on mRNA and/or protein expression profile in normal and cancer tissues, but conclusive evidences are still lacking.

Methods: The expressions of the env genomic region of HERV-H, HERV-K, HERV-P, HERV-R and W were evaluated in the tumor, in the adjacent normal tissues, and in the circulating plasma microvesicles of 63 colorectal cancer patients. RNA was isolated from the clinical specimens, reverse-transcribed, and subjected to relative quantitative Real-Time PCR. The env expressions were related to the expression of the housekeeping GAPDH gene.
The quantification was conducted using comparative $\mathrm{Ct}$ method and the difference between the levels of env gene expression in the different specimens was given by folddifference. Methylation status of the HERV LTRs was evaluated by means of bisulfite-PCR and pyro-sequencing.

Results: Higher levels of expression of HERV-R were found in tumor tissues, as compared to adjacent normal tissues (1.45-fold), while HERV-H, -K, -P, and -W expression levels were comparable or decreased in tumor tissues compared to normal tissues (from 0.9 to 0.5 folds). HERV-H and -K LTRs were significantly $(\mathrm{p}<0.0001)$ hypomethylated in the tumor tissues $(56.1 \%$, and $54.0 \%$ respectively) compared to normal adjacent tissues $(71.6 \%$, and $58.1 \%$ respectively), while HERV-W methylation did not change between tumor (93.4\%), and normal adjacent (94.4\%) tissues. HERV-H,-K,-R, and -P env sequences were present in the plasma circulating microvesicles of $31 \%, 24 \%, 12 \%$ and $4 \%$ of patients respectively, while HERV-W env sequences were absent.

Conclusions: HERVs expression levels did not significantly differ among tumor and normal clinical samples. Demethylation of HERV-H and -K LTRs might support the hypothesis of specific release of HERV-H and -K LTRs from epigenetic controls in colon cancer. If this hypothesis holds true, specific viral sequences might be the targets of antigen-specific $\mathrm{T}$ cell therapy in colon cancer.

As known, cancer-secreted microvesicles influence tumor microenvironment and support cancer growth and metastasis: HERV $-\mathrm{H}$ and $-\mathrm{K}$ sequences present in the circulating plasma microvesicles may be transferred from one cell to another, and favour cellular transforming mechanisms.

Clinical Trial Registry: not applicable

Conflict of interest: None

\section{P797}

\section{Therapeutic effects of high-dose chemotherapy with autologous peripheral blood stem cell transplantation for high-risk advanced neuroblastoma}

Lei Mao ${ }^{1}$, Bing Xia ${ }^{1}$, Hongliang Yang ${ }^{1}$, Yafei Wang ${ }^{1}$, Yong $\mathrm{Yu}^{1}$, Xiaofeng Wang ${ }^{1}$, Zhigang Zhao ${ }^{1}$, Jingfu Wang ${ }^{2}$, Jie L $i^{2}$, Zhanglin Li $^{2}$, Qiang Zhao ${ }^{2}$, Jie Yan', Yizhuo Zhang ${ }^{1}$

${ }^{1}$ Tianjin Medical University Cancer Institute and Hospital, National Clinical Research Center for Cancer, Key Laboratory of Cancer Prevention and Therapy, Department of Hemotology, Tianjin, China; ${ }^{2}$ Tianjin Medical University Cancer Institute and Hospital, National Clinical Research Center for Cancer, Key Laboratory of Cancer Prevention and Therapy, Department of Children Oncology, Tianjin, China

Background: The prognosis for neuroblastoma in highrisk advanced stage is still poor, high-dose chemotherapy followed by single or tandem autologous peripheral blood 
stem cell transplantation(APBSCT) may improve the prognosis of these children .This study aimed to investigate the prognosis of children with highrisk neuroblastoma who received intensive chemotherapy followed by APBSCT, and further analyse the difference of therapeutic effects between single and tandem transplantation.

Methods: The medical records of 33 high-risk advanced neuroblastoma patients treated with high-dose chemotherapy with APBSCT in our hospital between 2010 and 2017 were retrospectively reviewed. Primary sites of the diseases were in the abdominal cavity $(\mathrm{n}=15)$ adrenal gland $(\mathrm{n}=$ 17) and mediastinum $(n=1) .27$ out of 33patients had bone marrow metastasis, 15 had multiple bone metastasis and 11 had distant lymphnode metastasis. All of the patients received very high dose chemotherapy, surgery, local radiation(20-30 Gy), and APBSCT as well as maintenance treatment. The conditioning regimen consisted of busulfan and melphalan(busulfan $1 \mathrm{mg} / \mathrm{kg} \times 4 \mathrm{~d}$, melphalan $140 \mathrm{mg} /$ $\mathrm{m}^{2} \times 1 \mathrm{~d}$ ) or CEM regimen (Carboplatin $600 \mathrm{mg} / \mathrm{m}^{2} \times 3 \mathrm{~d}$, etoposide $500 \mathrm{mg} / \mathrm{m}^{2} \times 3 \mathrm{~d}$, cyclophosphamide $1800 \mathrm{mg} /$ $\mathrm{m}^{2} \times 2 \mathrm{~d}$ ); Relapse patients were treated with salvage chemotherapy and radiation therapy.

Results: The median age at diagnosis was 40.5 months (range 12-96 months).After a median number of 8 (range 517) induction therapy,23 patients $(69.69 \%)$ got complete remission (CR), while 10 patients failed got CR(non-CR:5 got very good partial remission (VGPR) and 5 got partial response (PR) before APBSCT.In our study, a total of 13 patients received tandem transplantation.All the patients successfully underwent mobilization, collection and reinfusion without treatment-associated death occoured.Infectious fever $(n=22)$ and gastrointestinal $\operatorname{symptom}(n=20)$ are the main toxicity after APBSCT.By the end of follow up, a total of 9 patients(27.27\%) got relapsed and 7 (21.21\%) of them died .The mean time from diagnosis to relapse is $(24.77$ \pm 19.36 ) months. The 3-year event-free survival and overall survival of allpatients were $50 \%$ and $75 \%$, respectively. In the CR group,patients who received single transplatation got a better outcome than tandem transplantation:mean EFS:55 \pm 8 months VS $22 \pm 3$ months, whereas the non-CR group showed the opposite outcome : mean EFS:47 \pm 18 months VS $72 \pm 10$ months in the single transplatation and tandem transplantation.

Conclusions: The findings in this small cohort indicate that high-dose chemotherapy followed by APBSCT is feasible and tolerable in pediatric patients with high-risk neuroblastoma with a lower relapse rate and better survival. Tandem transplantation might benefit more for patients who failed getting CR.There is need for long-term following up and more cases to investigate the respective advantages of single and tandem transplantation.

\section{Conflict of interest:}

Corresponding author: Jie Yan, E-mail: bingxianew2016@163.com

Yizhuo Zhang, Email:yizhuozhang111@163.com

* Lei Mao and Bing Xia contributed equally as co-first authors.

Grant and Financial Support: This work was supported by grants from the National Natural Science Foundation of China (81600163, 81570201).

\section{Stem cell donor}

\section{P798}

\section{An Epidemiological Characterization of the Portuguese Bone Marrow Donor Registry}

Eduardo Espada ${ }^{1}$, Dário Ligeiro ${ }^{2}$, Hélder Trindade $^{2}$, João Forjaz de Lacerda $^{1}$

${ }^{1}$ Hospital de Santa Maria - Centro Hospitalar de Lisboa Norte, EPE, Serviço de Hematologia e Transplantação de Medula, Lisboa, Portugal; ${ }^{2}$ Centro de Sangue e Transplantação de Lisboa, IPST, IP, Lisboa, Portugal

Background: The Portuguese Bone Marrow Donor Registry, also known as CEDACE (National Center of Bone Marrow or Stem Cells, or Cord Blood Donors) is a database of hematopoietic stem cell donors that was created in 1995 and is available for finding a matched graft for both national and foreign patients. CEDACE has had a steady growth over the years, constituting the tenth largest bone marrow donor registry in the world, and the third largest per capita.

Methods: As a part of our analysis of the CEDACE Registry, we queried the database regarding the selfdeclared epidemiological characteristics of the population, namely age, gender, ethnicity, country of origin, and residence. We then cross-referenced the distribution with the official INE (National Statistics Institute) data, available on the website of the institution.

Results: As of August 2017, the CEDACE Registry comprised 396545 donors, totaling $3.85 \%$ of the Portuguese population. $99.51 \%$ had HLA-A/-B/-DRB1 typing, $43.91 \%$ also included HLA-Cw, and $1.28 \%$ included typing for 5 HLA loci (including HLA-DQB1). Median donor age was 39 years $\left(Q_{1}-Q_{3} 33-45\right)$. There was a greater representation of women when compared to men $(60.4 \%$ vs. $39.6 \%$, respectively), even larger than the actual gender difference in the country $(52.6 \%$ vs. $47.4 \%)$. Considering that $91.5 \%$ of the resident population in Portugal was born in the country, the representation of Portuguese nationals in the Registry is much larger (98.98\%), although 40 other nationalities are present. Most foreign donors come from Brazil ( $\mathrm{n}=1693$; 0.43\%), with Portuguese-speaking African countries (Cape Verde, Angola, Mozambique, Guinea- 
Bissau, and São Tomé and Príncipe) also comprising a significant proportion of the remaining foreign donor population $(\mathrm{n}=1230 ; 0.31 \%)$; only three other countries are represented with more than 100 donors, namely France $(\mathrm{n}=241 ; 0.06 \%)$, Ukraine $(\mathrm{n}=192 ; 0.05 \%)$, and Spain ( $\mathrm{n}$ $=137 ; 0.03 \%$ ). Of the $76.19 \%$ of donors who voluntarily self-declared their ethnicity/origin, $99.12 \%$ identified as "Western", $0.66 \%$ as "African", $0.13 \%$ as "Mixed", $0.03 \%$ as "South Asian", $0.02 \%$ as "Eastern", and $0.004 \%$ as "Hindu". Of the seven NUTS (Nomenclature of Territorial Units for Statistics) 2 regions of Portugal, the Center was the region that had the most per capita donors (4.10\%), followed by the Metropolitan Area of Lisbon (3.97\%), the North $(3.77 \%)$, the Alentejo (3.35\%), the Algarve (2.29\%), and lastly the autonomous regions of Madeira (1.91\%) and Azores (1.66\%). Despite the fact that donors that reside in the subregions of Lisbon and Oporto represented $40.82 \%$ of the registry (23.44\% and $17.37 \%$, respectively), of the 20 NUTS 3 subregions the one that had the most per capita donors was Coimbra (4.81\%), followed by Portalegre (4.43\%), and Aveiro (4.40\%); the one that had the least was Azores (1.66\%), followed by Madeira (1.91\%), and Faro (2.29\%), with Lisbon and Oporto having $4.13 \%$ and $3.79 \%$ per capita donors, respectively.

Conclusions: Besides the ongoing HLA data analysis, the knowledge of the epidemiological characteristics of the CEDACE Registry enables us to redirect efforts to recruit donors in more underrepresented regions of the country, namely the autonomous regions, while also encouraging us to mobilize donor recruitment campaigns to underrepresented ethnic minorities.

Conflict of interest: The authors have nothing to disclose.

\section{P799}

\section{Analysis of single nucleotide polymorphism in healthy peripheral stem cell donors}

Soo-Zin Kim-Wanner ${ }^{1}$, Elena Schmidt ${ }^{1}$, Katharina Landwehr ${ }^{1}$, Erhard Seifried $^{1}$, Halvard Bönig ${ }^{1,2}$

${ }^{1}$ German Red Cross Blood Service and Johann-Wolfgang-Goethe Medical University, Institute of Transfusione Medicine and Immune Hematology, Frankfurt, Germany; ${ }^{2}$ University of Washington School of Medicine, Division of Hematology, Seattle, WA, United States

Background: Annually over 40.000 allogeneic stem cell transplantations (HSCT) are performed as the often remaining curative treatment option for hematological malignancies. Mobilised peripheral blood stem cells have been established as the main source of stem cells. Among healthy donors the mobilisation efficacy after application of granulocyte colony stimulating factors (G-CSF) vary dramatically. So far, no reliable factors are established that can predict satisfactory stem cell collection yield in healthy donors. Therefore this study intends to analyze genomewide single nucleotide polymorphism to discover predictive markers for stem cell yield in peripheral stem cell donors.

Methods: Genomic DNA of 28 healthy stem cell donors were analyzed for single nucleotide polymorphism using the Illumina HumanOmni 5 v.1.1 Bead Chip. For stem cell mobilisation G-CSF (Lenograstim, Chugai) was administered at a dosage of 7,5-10 $\mu \mathrm{g} / \mathrm{kg} / \mathrm{d}$ s.c. for 5 consecutive days and apheresis was performed on day 5 . The dosage was split into twice injections daily. CD34+cell yield was measured before apheresis. Bioinformatical analysis was performed using PLINK and the R/Bioconductor package GWASTools. The data were compared to public available 1000 genome data set.

Results: 15 donors showed a poor CD34+ yield with < $30 \mathrm{CD} 34+$ cells/ $/ \mathrm{l}, 10$ donors with a good mobilisation efficiency of $>200 \mathrm{CD} 34+$ cells $/ \mu \mathrm{l}$ and 3 donors showed values in a range from $80-120 \mathrm{CD} 34+$ cells/ $\mu$. After quality control (missing call rate $>0.15$, minor allele freqency $(\mathrm{MAF})<0.06) 1.624 .796$ variants passed the filter criterias and were used for quantitative phenotype association analysis. 73542 SNPs with a p-value $<0.02$ in the association tests were used for further downstream analysis. Especially, genomic regions of chr 9:132150001132550001, chr 2:230350001-230750001, chr 7:17400001-17750001 and chr16:8795000188300001 showed enrichment of signifcant SNPs in the association studies.

Conclusions: The knowledge of predictive markers for stem cell yield after G-CSF mobilisation may help to optimize mobilisation regimens and selection for potential donors. In this genome wide association study SNPs that may associate with CD34 cell yield could be detected. Further analysis in larger donor cohorts are needed to validate the identified SNPs.

Conflict of interest: Nothing to disclose.

The study was funded by German Red Cross Blood Service.

\section{P800}

Clinical impact of the immune reconstitution status on 100 day after haploidentical stem cell transplantation Se Hyung Kim ${ }^{1}$, Seong Kyu Park ${ }^{1}$, Dae Sik Hong ${ }^{1}$, Kyoung Ha
Kim $^{2}$, Jong Ho Won

${ }^{1}$ Soonchunhyang University Hospital, Hematology, Bucheon, Korea, 
Republic of; ${ }^{2}$ Soonchunhyang University Hospital, Hematology, Seoul, Korea, Republic of

Background: Immune reconstitution following allogeneic hematopoietic stem cell transplantation (HSCT) can be affected by many variables in the transplant process such as the particular conditioning regimen, donor type, GVHD prophylaxis, and so on. Haploidentical HSCT has been accepted worldwide as an alternative treatment for patients with hematologic malignancies who do not have a completely HLA-matched sibling or who require urgent transplantation. We assessed clinical outcomes according to the immune recovery status at 100 days after haploidentical SCT compared to those of HSCT from related donors or unrelated donors.

Methods: 39 patients received allogeneic HSCT using peripheral blood stem cells after fludarabine/busulfan/antithymocyte globulin conditioning. Immunophenotypic analysis using flowcytometry was performed to evaluate the percentage and the absolute numbers of $\mathrm{T}$ cell subsets (CD3 ${ }^{+}, \mathrm{CD}^{+}{ }^{+}$, and $\mathrm{CD}^{+}$cells), $\mathrm{NK}$ cells $\left(\mathrm{CD} 16^{+} \mathrm{CD}^{+} 6^{+}\right.$cells $)$, and $\mathrm{B}$ cells $\left(\mathrm{CD} 19^{+}\right.$cells) with serum immunoglobulin levels as well as clinical outcomes were investigated.

Results: While 34 patients $(89.5 \%)$ achieved the normal range of NK cells, T-cell subset recovery was achieved in the reference range in 9 patients (23.7\%). And B-cell recovery was achieved in the reference range in 26 patients $(66.7 \%)$. Haploidentical HSCT seemed to be associated with delayed immune reconstitution of $\mathrm{T}$ cell subsets and $\mathrm{B}$ cells. Although the incidence of grade II IV acute GVHD was slightly increased, the incidence of chronic GVHD was lower in haploidentical HSCT group compared to those of HSCT using other donor types. Events of all viral reactivation including CMV reactivation was more frequent in abnormal group of $\mathrm{T}$ cell subsets $(\mathrm{p}=0.045)$. In addition, the incidence of relapse seemed to be more frequent in abnormal group of $\mathrm{T}$ cell subsets.

Conclusions: Immune reconstitution after haploidentical HSCT tends to be delayed, especially of $\mathrm{T}$ cell subsets. Delayed immune reconstitution might cause increased morbidity from viral reactivation. We need strategies to prevent infectious complications and enhance immune reconstitution according the immune recovery status following haploidentical SCT.

Clinical Trial Registry: Nothing

Conflict of interest: Nothing to disclose

\section{P801}

Comparing Alternative Donor Transplant using Unrelated Cord Blood or Unmanipulated Haploidentical Transplant with Post-transplant Cyclophosphamide against Matched
Related, Matched Unrelated Donor Transplant for Haematological Malignancies

Cheryl XQ Lim ${ }^{1}$, Yeh Ching Linn ${ }^{2}$, Bee Choo Tai ${ }^{3}$, William YK Hwang $^{2}$, Lip Kun Tan', Michelle LM Poon ${ }^{1}$, Belinda LE Tan ${ }^{1}, A$. Yelly $^{1}$, Teck Guan Soh ${ }^{1}$, Davanaliz R.Del Rosario ${ }^{1}$, Zi Yi Lim ${ }^{1}$, Liang Piu Koh ${ }^{1}$

${ }^{I}$ National University Hospital, Department of Haematology Oncology, National University Cancer Institute, Singapore, Singapore;

${ }^{2}$ Singapore General Hospital, Department of Haematology, Singapore, Singapore; ${ }^{3}$ National University of Singapore, Saw Swee Hock School of Public Health, Singapore, Singapore

Background: Outcomes after unmanipulated haploidentical haematopoietic cell transplantation (HCT) and after unrelated cord blood transplantation (UCBT) are encouraging and have become alternative options to treat patients with high-risk haematological malignancies without human leukocyte antigen (HLA) matched donor.

Methods: We studied 459 adults patients receiving allogeneic HCT for various haematologic malignancies between Jan 2005 and Dec 2016. The patients received HCT using 7-8/8 HLA-matched related donor (MRD, $\mathrm{n}=$ 223), HLA-matched unrelated donor (MUD, $\mathrm{n}=118$ ), Haploidentical $(\mathrm{n}=28)$ or 4-6/6 HLA matched UCB $(\mathrm{n}=$ 90) graft and after myeloablative (MAC, $\mathrm{n}=288$ ) or reduced intensity conditioning ( $\mathrm{RIC}, \mathrm{N}=171$ ) regimen. Haploidentical recipients received calcineurin inhibitor (CNI), mycophenolate, and post-transplant cyclophosphamide (PT Cy) for graft-versus-host disease (GVHD) prophylaxis.

Results: 5 year overall survival for patients undergoing MRD, MUD, UCB and Haploidentical transplantation were $45 \%, 52 \%, 41 \%$ and $44 \%$ and of disease free survival (DFS) were $42 \%, 50 \%, 35 \%$ and $34 \%$, respectively; these were not significantly different among the 4 donor groups. The 24month cumulative incidences (C.I.) of non-relapse mortality (NRM) were 18\%, 22\%, 37\% and 33\% ( $\mathrm{p}=0.007)$ and of relapse were $40 \%, 28 \%, 37 \%$ and $31 \%$, respectively $(\mathrm{P}=$ $0.226)$. C.I. of grades 3 to 4 acute GVHD at 6 months were $10 \%, 6 \%, 9 \%$ and $8 \%$, respectively $(\mathrm{P}=0.653)$; chronic GVHD occurred in $45 \%, 17 \%, 8 \%$ and $24 \%$ of patients, respectively $(\mathrm{P}<0.001)$. Despite a higher NRM using Haploidentical donor (HR 2.54, 95\% CI 1.19-5.34; $\mathrm{p}=$ 0.015 ) and UCB (HR 2.41, 95\% CI 1.09-2.32; $\mathrm{p}=0.001$ ), the use of these two alternative donors did not result in any statistically significant difference in DFS $(\mathrm{p}=0.075)$ as compared with other donors.

Conclusions: UCBT and haploidentical HCT using unmanipulated graft and PT $\mathrm{Cy}$ result in equivalent outcome to those contemporaneous HCT performed using MRDs and MUDs. They provides additional alternative for patients lacking HLA matched donors.

Conflict of interest: Nothing to disclose 
P802

Dissecting genetic control of HLA-DPB1 expression and its relation to structural mismatch models in hematopoietic stem cell transplantation

Thuja Meurer ${ }^{1}$, Esteban Arrieta-Bolanos ${ }^{1}$, Maximilian Metzing ${ }^{1}$, Mona-May Langer ${ }^{2}$, Peter van Balen , J.H. Frederik Falkenburg, Dietrich W. Beelen ${ }^{4}$, Peter A. Horn ${ }^{2}$, Katharina Fleischhauer', Pietro Crivello

${ }^{1}$ University Hospital Essen, Institute for Experimental Cellular Therapy, Essen, Germany; ${ }^{2}$ University Hospital Essen, Institute for Transfusion Medicine, Essen, Germany; ${ }^{3}$ Leiden University Medical Center, Department of Hematology, Leiden, Netherlands; ${ }^{4}$ University Hospital Essen, Department of Bone Marrow Transplantation, West German Cancer Center, Essen, Germany

Background: Donor-recipient HLA-DPB1 mismatches are frequent in stem cell transplantation (SCT) and associated with the risks of graft-versus-host-disease, relapse and non-relapse mortality. High or low HLADPB1 expression levels have been linked to the singlenucleotide polymorphism (SNP) rs9277534 G/A in the 3'untranslated region (3'UTR), and mismatches involving highly expressed HLA-DPB1 alleles in the patient were associated with higher risk of acute GvHD after unrelated SCT (Expression-model). On the other hand, the SNP rs9277534 G/A is in linkage with structurally defined HLADPB1 T-cell epitope (TCE) groups associated with highrisk (non-permissive) or low-risk (permissive) mismatch combinations after unrelated SCT (TCE-model). A comprehensive analysis of HLA-DP expression in immune cells is lacking, and the relationship between the Expression- and the TCE-model in SCT remains unclear. Here we investigated the potential mechanisms at the basis of HLA-DPB1 differential expression and its relation to invitro alloreactivity predicted by structurally different TCE groups.

Methods: The Expression- and the TCE-model were comparatively applied to 416 donor-recipient pairs who received unrelated SCT at the University Hospital Essen. Transcriptional and protein cell surface expression levels of HLA-DPB1 were investigated in B cell-lines (BCL), primary $\mathrm{B}$ cells and monocytes before or after exposure to interferon-g, as well as in mature and immature dendritic cells (DC), by quantitative PCR and flow cytometry. The HLA-DPB1 3'UTR was mapped into eight overlapping fragments and analysed for transcriptional activity by dual luciferase assays in BCL. HeLa cells transduced to express single HLA-DP molecules under a viral promoter in the absence of the 3'UTR were used as targets for CD4+ Tcells alloreactive to TCE-mismatched or TCE-matched HLA-DP, and the strength of the response was quantified by CD137 upregulation assays.
Results: Low- and high-risk HLA-DPB1 mismatch combinations were concordantly assigned by the Expression and the TCE-model in 69\%. Significant differences in HLA-DPB1 transcript and cell surface expression levels were found between rs9277534 G/A homozygous BCL and unstimulated primary B-cells and monocytes, but not in the same cells after interferon-g exposure or in DC. In the 29 most common HLA-DPB1 alleles, we identified at least 7 HLA-DPB1 3'UTR rs9277534 G/A haplotypes characterized by $36 \mathrm{SNP}$, the number of short tandem repeats (STR) in intron 2 and their TCE classification. 3'UTR mapping of two representative haplotypes revealed no significant differences in luciferase activity for any of the overlapping fragments including those encompassing rs9277534 G/A. There was no evidence for alternative splicing associated with the intron 2 STR. In 189 independent T-cell cultures, we observed significant differences in the percentage of responding T-cells between TCE-matched compared to TCE-mismatched combinations $(36 \pm 12 \%$ vs $19 \pm 15 \%$; $\mathrm{p}<$ 0.001 ), despite similar HLA-DP expression levels on transduced target cells.

Conclusions: While the mechanism underlying genetic control of HLA-DPB1 expression by rs9277534 G/A in $\mathrm{BCL}$ remains unclear, our data challenge its relevance in professional antigen-presenting-cells and suggest that structural HLA-DPB1 TCE variability is associated with differential allo-immunogenicity, independently from expression levels. The clinical risk associations in SCT of HLA-DPB1 mismatches discordantly assigned as high or low risk by the Expression- and the TCE-model remain to be investigated.

Conflict of interest: The authors have no conflict of interest to disclose.

This work was supported by a grant from the Deutsche José Carreras Leukämie Stiftung (DJCLS R 15/02).

\section{P803}

Double haplo-identical and/or cord-blood allogeneic stem cell transplantation in hematologic malignancies: a retrospective study from the SFGM-TC

\footnotetext{
Doriane Cavalieri ${ }^{1}$, Bruno Pereira ${ }^{2}$, Reda Chebel ${ }^{3}$, Hélène Labussière-Wallet ${ }^{4}$, Gerard Socie ${ }^{5}$, Anne Calleja ${ }^{6}$, Edouard Forcade $^{7}$, Patrice Chevallier ${ }^{8}$, Marie-Thérèse Rubio ${ }^{9}$, Nicole Raus ${ }^{3}$, Jacques-Olivier Bay ${ }^{1}$, Aurélie Ravinet ${ }^{1}$

${ }^{1}$ CHU de Clermont Ferrand, Service d'Hématologie Clinique Adulte et de Thérapie Cellulaire, Clermont-Ferrand, France; ${ }^{2} \mathrm{CHU}$ de Clermont Ferrand, Biostatistics Unit (DRCI), Clermont-Ferrand, France; ${ }^{3}$ Centre Hospitalier Lyon Sud, SFGM-TC, Pierre-Bénite, France; ${ }^{4}$ Centre Hospitalier Lyon Sud, Hematology Department, Pierre-Bénite, France; ${ }^{5}$ Hopital Saint Louis, APHP, Department of Hematology, Paris, France; ${ }^{6} \mathrm{CHU}$ de Nice, Department of Hematology, Nice, France, ${ }^{7}$ Hopital Haut Leveque, CHU de
} 
Bordeaux, Service Hématologie Clinique et Thérapie Cellulaire, Pessac, France, ${ }^{8} \mathrm{CHU}$ de Nantes, Department of Hematology, Nantes, France; ${ }^{9} \mathrm{CHU}$ de Nancy, Department of Hematology, Nancy, France

Background: Haplo-identical donors and cord-blood stem cells provide alternative transplant options in patients lacking HLA-matched donor. For patients with relapsed disease or graft failure, a second « alternative » allogeneic hematopoietic stem cell transplant (AHSCT) is usually not considered because of high risk of transplant-related mortality (TRM). We report here the outcome of 70 patients who received two allogeneic transplants from alternative donors.

Methods: We performed a retrospective multicenter analysis of 70 patients who underwent two allogeneic transplants from alternative donors between 2000 and 2016, using the ProMISE database. We analysed the 3-year progression-free survival (PFS), GVH and relapse-free survival (GRFS), TRM, and overall survival (OS).

Results: Forty patients received two cord-blood AHSCT, 10 patients received two haplo-identical AHSCT, 3 patients received first a haplo-identical AHSCT and cord-blood as second AHSCT, and 17 patients had a cord-blood AHSCT and then a haplo-identical AHSCT. The main diagnosis at AHSCT1 was de novo AML $(\mathrm{n}=23)$, and other diagnoses were: ALL $(n=16)$, MDS $(n=10), \operatorname{MPD}(n=9)$, secondary AML $(\mathrm{n}=8)$, and lymphoproliferative disorder $(\mathrm{n}=4)$. Patients had a median age of 42 years at AHSCT1. Thirty-one $(44.3 \%)$ received a myeloablative regimen and 19 (27.1\%) ATG for AHSCT1; they were respectively 6 $(8.5 \%)$ and $15(21.4 \%)$ for AHSCT2. Median delay between the two AHSCT was 3.27 months. Indications for AHSCT2 were graft failure $(n=44,62.9 \%)$, and relapse or disease progression $(n=26,37.1 \%)$. Fourty-two patients $(60.0 \%)$ were in complete remission at time of AHSCT1, and $35(50.0 \%)$ at time of AHSCT2. The 3-year probabilities for OS, PFS, GRFS and TRM after AHSCT2 were $15.8 \%, 14.8 \%, 8.9 \%$ and $68.9 \%$ respectively. Among the 55 evaluable patients who survived more than 28 days, $43(78.2 \%)$ engrafted. Twenty-seven patients (38.6\%) developed acute GVHD, and 17 patients chronic GVHD. Fifty-nine patients $(84.3 \%)$ died, including 40 of TRM (57.1\%), in a median delay of 2,07 months after AHSCT2 (range: 0.13-44.16). Eleven patients are alive, at a median follow-up of 27,9 months (range: 17.3-106.2). In multivariate analysis, aGVHD after second AHSCT, and complete remission status at second HSCT were significantly associated with a better outcome $(\mathrm{p}=0.001$ and $\mathrm{p}<$ 10-4), whereas use of ATG at AHSCT1 was associated with a worst outcome $(p=0.05)$, with higher TRM $(p=0.01)$. Choice of donor (haplo-identical or cord-blood stem cells)

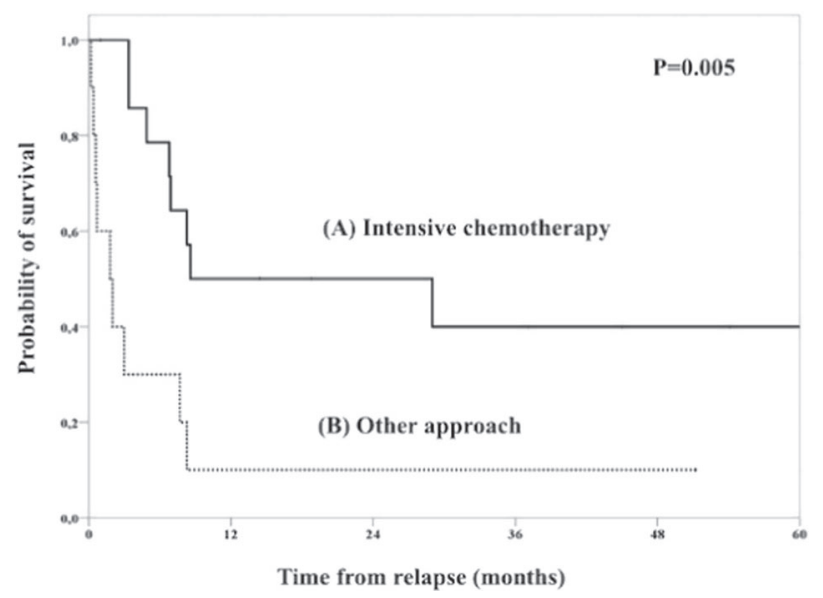

[P803 Figure] [Overall Survival according to disease status at AHSCT2]

for both AHSCT had no impact on OS, TRM, PFS and GRFS.

Conclusions: Our results suggest that a second haploidentical or cord-blood stem cell transplantation is a very high-risk procedure, but could be considered in patients achieving a complete remission before receiving the second AHSCT.

Conflict of interest: Nothing to disclose

\section{P804}

Duration of hospital stay in the first year post-transplant is similar in patients transplanted from matched unrelated and haploidentical donors - a single institution analysis

Antonela Samardžić ${ }^{1}$, Nadira Durakovic ${ }^{1,2}$, Zinaida Perić ${ }^{1,2}$, Ranka Serventi Seiwerth $^{1}$, Alen Ostojić ${ }^{1}$, Lana Desnica ${ }^{1}$, Dražen Pulanic ${ }^{1,2}$, Pavle Rončević ${ }^{1}$, Radovan Vrhovac ${ }^{1,2}$

${ }^{1}$ UHC Zagreb, Hematology, Zagreb, Croatia; ${ }^{2}$ University of Zagreb School of Medicine, Internal Medicine, Zagreb, Croatia

Background: With the introduction of post-transplant cyclophosphamide (PT-Cy), haploidentical transplantation became increasingly used. That coupled with the expanding number of indications results in more transplantations done. We analyzed length of initial hospital stay, number of rehospitalizations, hospital days in the first year posttransplant and GVHD incidence comparing transplantation using haploidentical (haplo), matched related (MRD) and unrelated donors (MUD).

Methods: In the time period between 1/2012 and 07/2017 we performed a total of 311 allogeneic transplantations in adults: 106 using MRD, 176 using MUD and 29 using haploidentical donor. All patients being transplanted from haploidentical donors received PT-Cy. All data were computed using the $\mathrm{R}$ package. The link between variables 
was assessed using Kruskal-Wallis test. The probability of GVHD was calculated using the cumulative incidence method and subgroups were compared using the Gray test.

Results: Median age of entire group was 47.5 (18.6-67) years; 43.1 (18.9-63.1), 45.8 (18.5-65.8) and 49.6 (19.4-67) years for the haplo group. In haplo patients $25(86 \%)$ received bone marrow (BM), 4 (14\%) peripheral blood stem cells (PBSC). Of MRD patients, 31 (29\%) and 75 (71\%) received BM and PBSC, while of MUD patients $32(18 \%)$ and $144(82 \%)$ received BM and PBSC respectively. In haploidentical group 23 (79\%) patients received NMA ("Baltimore") conditioning, and 6 (21\%) standard myeloablative conditioning (MAC). In MRD and MUD group, 63 (59\%) and $115(65 \%)$ received reduced intensity conditioning, while $43(41 \%)$ and $61(35 \%)$ received MAC, respectively.

With a median follow up of 380 days, overall survival was 50\% (95\%CI, 43-57\%); with no significant difference between groups. Median number of days to discharge posttransplant was significantly longer in haplo group, 37 (range 24-243) vs MRD, 28 (18-96) or MUD, 28 (15-198) $(\mathrm{p}=0.0007)$. During the first year post transplantation patients had an average of $0.86,0.82$ and 1.0 rehospitalization in haplo, MRD and MUD group, respectively, $(p=0.22)$. Median number of hospital days during the first year was significantly shorter after MRD with 35.5 (19-209) days, while it was similar after MUD 44 days (15$198)$ and haplo 46 days (25-243) transplantation $(\mathrm{p}=0.04)$. Cumulative incidence of aGVHD II-IV was 33\% (95\%CI, $28-38 \%$ ) for the entire group; and even though it was highest after MUD (38\%, 95\% CI 31-45), particularly in comparison to haplo $(24 \%, 95 \%$ CI 10-41), statistical significance was not reached. Cumulative incidence of cGVHD was $24 \%$ (95\% CI, 17-30\%), with significantly less cGVHD after haplo $(8 \%, 95 \%$ CI 1-23) in comparison to MRD $(16 \%, 95 \%$ CI 9-24) and MUD (27\%, 95\% CI 20-35), $\mathrm{p}=0.03$.

Conclusions: Haploidentical transplantation program in our center has considerably expanded, and even though the number of haplo transplantations in comparison to other two groups is still modest, we believe this analysis reflects its value. Longer time to discharge in haplo group is surely partly influenced by our cautiousness in the early days of program development, but overall days spent in hospital, less re-hospitalizations and particularly incidence of cGVHD in the haplo group speaks to the overall better quality of life (QoL) post-transplant of our haplo patients.

Conflict of interest: Authors declare no conflict of interest

\section{P805}

Abstract previously published
P806 Establishment of Haploidentical Bone Marrow
Transplantation Program at KHCC

Khalid Halahleh ${ }^{1}$, Eman Khatab ${ }^{2}$, Mayada Abu Shanab ${ }^{3}$, Mohammad Ma'kosa ${ }^{1}$, Nilly Hussein ${ }^{4}$, Maha Yousef, Rula Najjar ${ }^{6}$, Shanta Sharma ${ }^{7}$,Husam Abu-Jazar', Rawad Rihani, Abdelghani Tbakhi $^{7}$, Mahmoud Sarhan ${ }^{9}$

${ }^{1}$ King Hussein Cancer Center, Adult Medical Oncology Hematology, Bone Marrow Transplantation Program, Amman, Jordan; ${ }^{2}$ King Hussein Cancer Center, Oncology Hematology, Bone Marrow Transplantation Program, Amman, Jordan; ${ }^{3}$ King Hussein Cancer Center, Pediatric Oncolgy Hematology, Bone Marrow Transplantation Program, Amman, Jordan; ${ }^{4}$ King Hussein Cancer Center, Amman, Jordan; ${ }^{5}$ King Hussein Cancer Center, Nursing Department, Amman, Jordan; ${ }^{6}$ King Hussein Cancer Center, Clinical Pharmacy Department, Bone Marrpw Transplantation Program, Amman, Jordan; ${ }^{7}$ King Hussein Cancer Center, Department of Cell Therapy and Applied Genomics, Amman, Jordan; ${ }^{8}$ King Hussein Cancer Center, Pediatric Medical Oncology Hematology, Bone Marrpw Transplantation Program, Amman, Jordan; ${ }^{9}$ King Hussein Cancer Center, Bone Marrow Transplnatation Program, Amman, Jordan

Background: Hematopoietic cell transplantation (HCT) is a promising method for the treatment of patients with malignant and non-malignant conditions. Only half of patients are able to find an HLA-matched related or unrelated donor.Haploidentical HCT(HIT) is an alternative transplant strategy for patients without an HLA-matched donor. Haploidentical donor is readily available for most patients in need for immediate HCT.At King Hussien Cancer Cente,HIT started since 2004, but with limited number of cases. In 2015, we adapted and validated screening method for Anti-HLA and Anti-DSA at our laboratory and started desensetization protocol adapted from MDACC regimen.

Methods: Medical records of patients who underwent HIT at King Hussein Cancer Center bone marrow transplant between January-2015 till september-2017 were retrospectively reveiwed.Database was created in Microsoft Access. Descriptive analyses of relevant demographic, clinical and laboratory data,including transplant indication, donor source, conditioning regimen and GvHD prophylaxis, engaftment and chimerism dynamics were performed . Summary statistics of demographic and clinical parameters were summerized. Relapse incidence, TRM and aGvHD at 100 days post transplant were calculated using Kaplan-Meir method.

Results: Total of 31 patients were included.Two third with malignant conditions,most were in CR2 or CR3 and in six patients,HIT was the second transplant. Fourteen received myeloablative(MAC) and 17 reduced intensitity conditioning(RIC).RIC conditioning was classic Hopkin's protocol and Fludarabine/TBI for MAC with PICY on day 3 
and 4 used in both regimens.All patients received PBSC as graft source, but 4 recieved GCSF primed bone marrow graft . The age of the donors was variable with different sources. $51.6 \%$ of transplants were sex-mismatch,half female to male, one third with blood group mismatch and only three with CMV IgG D-R mismatch. $86 \%$ engarfted and $90 \%$ had sustained full donor chimerism.At last encounter, $80.6 \%$ of patients were alive, $64.5 \%$ were in complete remission, $19.4 \%$ relapsed and four had graft failure,all of them were with bone marrow failure syndromes.

At 100 days post HCT relapse was $9.7 \%$ and TRM was $9.7 \%$.

Conclusions: The trend of HIT is increasing, feasible nowadays in Jordan especially with the use of post infusional Cyclophosphamide and no need for complex TCD procedures.

Conflict of interest: No conflict of interest

\begin{tabular}{lll}
\hline Age & Adults/Pediatrics & $7(22.6 \%) 24(77.4 \%)$ \\
\hline Gender & Female/Male & $16(51.6 \%) 15(48.4 \%)$ \\
Donor & $\begin{array}{l}\text { Parents/Siblings/Son } \\
\text { daughters }\end{array}$ & $\begin{array}{l}16(51.7 \%) \\
2(6.5)\end{array}$ \\
& Malignant/Non- & $19(61.5 \%) 12(38.5 \%)$ \\
Disease Category & Malignant & \\
aGVHD & No/Yes & $10(32.3 \%) 21(67.7 \%)$ \\
Disease status & Relapse/Remission & $6(19.4 \%) 20(64.5 \%)$ \\
Patient Status & Alive/Dead & $25(80.6 \%) 6(19.4 \%)$ \\
Death $\leq 100$ days & Yes & $4(12.6 \%)$ \\
Transplant related deaths(TRM) & Yes & $4(9.5 \%$ \\
100 days & & $9.7 \%$ \\
Relapse at 100 days & Yes & \\
\hline
\end{tabular}

[[P806 Table] Tab-1]

\section{P807}

\section{Experiences with Haploidentical Allogeneic Blood STEM CELL Transplantation in a Colombian Reference Center}

Claudia Lucia Sossa Melo ${ }^{1}$, Sara Ines Jimenez ${ }^{1}$, Angela Maria Peña Castelllanos $^{1}$, Luis Salazar ${ }^{1}$, Manuel Rosales ${ }^{1}$, Claudia Marcela Chalela $^{1}$, Jenifer Garay ${ }^{1}$, Jenifer Sandoval ${ }^{1}$, Sandra Rios ${ }^{2}$, Sonia Osma ${ }^{1}$, David Leonardo Reyes ${ }^{1}$, Maria Lucrecia Luna ${ }^{1}$, Manuel Ardila $^{1}$

${ }^{1}$ FOSCAL, Santander, Bucaramanga, Colombia; ${ }^{2}$ Clínica Carlos Ardila Lulle, Hematology and Transplantation, Bucaramanga, Colombia

Background: Hematopoietic stem cell transplant (SCT), is an alternative curative treatment for patients diagnosed with different hematological malignancies, metabolic and immunologic diseases. In this context, the ideal donor would be one that shares HLA identity, however the probability of finding a HLA identical brother is about $25 \%$.
Nowadays a new modality of SCT is being used, the haploidentical SCT with post-transplant cyclophosphamide (PT-Cy). Even thought antigenic differences are essential to eradicate minimal residual disease through the graft against tumor effect, haploidentical SCT is associated with greater Graft vs host disease, graft rejection, slow immune reconstitution and less survival. Despite these considerations, the advantages of these transplant modality include: high donor availability, reduced costs, easy application and the results are similar compared to HLA matched SCT with regard to progression free survival and overall survival.

Methods: We reviewed all patients taken to (HHSCT) at our institution between January 2014 and December 2017. Twenty patients received conditioning therapy with tiothepa, fludarabine and busulfan, one patient received busulfan, fludarabine and one received melphalan- thiotepa, fludarabine preparative regimen. All patients received posttransplantation cyclophosphamide cycle. OS was estimated at 100 days and at 1 year of follow up.

Results: The median age was 23 (range 16-57) years, 10 patients $(45,4 \%)$ were male and $12(54,5 \%)$ were female. . The number of patients with a sibling donor was $14(63 \%)$, for parent donor $6(27 \%)$, there was 2 patient with son donor (9\%). Conditioning therapy with tiothepa, fludarabine, busulfan and posttransplantation cyclophosphamide was utilized in $20(90 \%)$ of patients and fludarabine, busulfan without tiothepa and posttransplantation cyclophosphamide was utilized in $1(5 \%)$ patient, and melphalan, fludarabine, tiothepa in 1 patient $(\%)$. The majority of patients had acute lymphocytic leukemia 17 (72\%), followed by myelodysplastic syndrome $2(9 \%)$, two with hodgkin disease $(9 \%)$, one with acute myeloid leukemia associated $(5 \%)$, one patient had acute leukemia mixed phenotype $(5 \%)$. At the time of transplant 20 patients $(90 \%)$ were in complete remission, whereas 2 patients (10\%) was not in remission at transplant.

The majority of patients (77\%) achieved $100 \%$ donor chimerism at day +28 . The median time to neutrophil $\geq 0,5$ x $10^{9} / \mathrm{L}$ was 16 days (range 12-29) and platelets $\geq 20 \times 10^{9} /$ $\mathrm{L}$ was 16,5 (range 10-100). Acute GVHD was seen in eleven patients. Chronic GVHD or GVHD beyond day +100 of mild severity was observed in five patients. The major posttransplant complication was hemorrhagic cystitis (HC). Evidence of CMV reactivation was detected in 13 patients successfully pre-emptive eradicated, while one patient got prophylactic valganciclovir from day +15 . Evidence of EBV viremia/reactivation was found in 6 patients of whom three patients got rituximab. In all patients EBV virus load vanished without illness. The OS within a median follow up of 12 months and three years was $89 \%$ and $51 \%$ respectively. Four patients have relapsed, one with B-ALL, MDS, AML and hodgkin disease.

Allograft and t... 


\begin{tabular}{|c|c|c|}
\hline \multicolumn{3}{|c|}{ TABLE 1. PATIENT CHARACTERISTICS $\mathrm{N}=22$} \\
\hline Gender male, $N(\%)$ & \multicolumn{2}{|c|}{$10(45 \%)$} \\
\hline Age $\cdot$ & \multicolumn{2}{|c|}{$24,5(16-57)$} \\
\hline $\begin{array}{l}\text { Score HCT-CI (Sorter et } \\
\text { al), N (\%) }\end{array}$ & \multicolumn{2}{|c|}{$s 2: 19(86,4 \%)>2: 3(13,6 \%)$} \\
\hline Donor relation, $N(\%)$ & $\begin{array}{l}\text { - Father: } 2(9 \%) \\
\text { - } \text { Mother: } 4(18 \%) \\
\text { - Sister: } 0\end{array}$ & - Son: $2(9 \%)$ \\
\hline $\begin{array}{l}\text { Female donor/male } \\
\text { recipient, } \mathrm{N}(\%)\end{array}$ & \multicolumn{2}{|c|}{$1(4,5 \%)$} \\
\hline Diagnosis, $\mathrm{N}(\%)$ & $\begin{array}{ll}\text { - } & \text { HL: } 2(9 \%) \\
\text { - } & \text { B-ALL: } 15(68 \%) \\
\text { - } & \text { T-ALL: } 1(4,5 \%) \\
\text { - } & \text { AML: } 2(9 \%)\end{array}$ & $\begin{array}{l}\text { MSD: } 1(4,5 \%) \\
\text { Acute leukemia } \\
\text { mixed phenotype: } 1 \\
(4,5 \%)\end{array}$ \\
\hline $\begin{array}{l}\text { Disease status before pre } \\
\text { hapleidentical HSCT, N } \\
(\%)\end{array}$ & $\begin{array}{ll}\text { - } & \text { CR1: } 9(40,5 \%) \\
\text { - } & \text { CR2: } 9(40,5 \%) \\
\text { - } & \text { CR3: } 1(4,5 \%)\end{array}$ & $\begin{array}{l}\text { - CR4: } 1(4,5 \%) \\
\text { - Refractory: } 1(4,5 \%) \\
\text { - PR: } 1(4,5 \%)\end{array}$ \\
\hline Previous HSCT, N (\%) & \multicolumn{2}{|c|}{$3(13,6)$} \\
\hline Previous HSCT type, N (\%) & Haplo-HSCT: $1(4,5 \%)$ & Auto-HSCT: $2(9 \%)$ \\
\hline Conditioning, $\mathrm{N}(\%)$ & \multicolumn{2}{|c|}{$\begin{array}{l}\text { - TT/Bu/ElutCEEM post: } 20(91 \%) \\
\text { - Bu/ElutCEEM post: } 1(4,5 \%) \\
\text { - MTF: } 1(4,5 \%)\end{array}$} \\
\hline GVHD profilaxis, $N(\%)$ & \multicolumn{2}{|c|}{$\begin{array}{l}\text { - Ciclosporine/mycophenolate: } 2(9 \%) \\
\text { - Tacrolimus/ mycophenolate: } 20(91 \%)\end{array}$} \\
\hline $\operatorname{cD} 34+\times 10^{8} / \mathrm{Kg}^{*}$ & \multicolumn{2}{|c|}{$7,55(1,4-22,4)$} \\
\hline
\end{tabular}

Pedian. CR: complete response. PR: Partial response. TT/Bu/Flu+CFM post: Thiotepa-Busulfan-Fludarabine-Cyclophosphamide. MTF: Melfalan-ThiotepaFludarabine

[P807 Figure] [Allograft and transplantation related characteristics]

Conclusions: Our experience regarding HHSCT suggests that this treatment modality could cause a long awaited increase in curative allogeneic transplantations and more patients with a transplant option.

Conflict of interest: The authors declare no conflict of interest

\section{P808}

Haploidentical transplantation compared with matched sibling donor transplantation for adults with Hodgkin lymphoma: a single center experience

Félix López Cadenas, Ana Africa Martín López, Nerea Arratibel Zalacain, Alvaro Veiga Vaz, Monica Baile Gonzalez, Alejandro Avendaño Pita, Daniel Rivera Delgado, Javier Carrillo Checa, Lourdes Vázquez López, Monica Cabrero Calvo, Estefania Perez López, M Dolores Caballero Barrigon, Ramón García Sanz, Lucia López, Corral

Hospital Clinico Univerisario de Salamanca, Hematology, Salamanca, Spain

Background: Allogeneic transplant (HCT) is one of the option for high refractory Hodgkin Lymphoma (HL), especially indicated for chemosensitive relapse after autologous stem cell transplant. In the last years post-transplant cyclophosphamide-based haploidentical HCT (Haplo-PT$\mathrm{Cy}$ ) has greatly developed and many studies have shown its efficacy and safety, especially in $\mathrm{H}$.

Methods: From 2007 to nowadays, $22 \mathrm{HL}$ patients underwent alloHCT from a related donor in our institution [Haplo-PT-Cy:13 and matched sibling donor (iHLASIB):9]. Our aim was to analyze the clinical and biological characteristics of Haplo-PT-Cy HL patients and their outcomes, and to compare these results with a wellmatched group of iHLA-SIB HL patients.

Results: Patients and alloHCT transplant: Patients age, number of treatment lines and percentage of patients with active disease prior alloHCT were similar in both groups. All patients in both groups were treated with reduced intensity conditioning regimens and peripheral blood was the stem cell source used.

\section{Results after Haplo-PT-Cy:}

Hematopoietic Recovery: All patients engrafted. The median time for neutrophil $[>500 / \mu \mathrm{L}]$ and platelet recovery [>20.000/ $\mu \mathrm{L}]$ was 18 days (13-30) and 27 days (13-103), respectively.

Complications: 7 patients (54\%) developed acute GVHD: 6 (46\%) grade I/II and $1(8 \%)$ grade IV, after a median of 26 days (21-48). Chronic GVHD (cGVHD) was evidenced in 3 patients (23\%): 2 (16\%) mild cGHVD and 1 $(8 \%)$ moderate cGVHD. We observed a tendency for more $\operatorname{aGVHD}(p=0,194)$ and cGVHD $(p=0.049[\mathrm{~L} 2])$ in patients who received anti-PD1 drugs prior Haplo-PT-Cy. CMV infection was diagnosed in 6 patients $(46 \%)$ without evidence of CMV disease. Four patients suffered hemorrhagic cystitis and one developed severe sinusoidal obstruction syndrome (resolved with defibrotide). No data of thrombotic microangiopathy were observed.

Outcome: After a median follow-up of 34 months (5 56) two patients died, one due to sepsis and the other one because of aGVHD. Patients were reevaluated by PET-CT at day +100 , reaching complete response all the evaluable patients. Only one patient relapsed at day +221 . Immunosuppressive treatment was discontinued in 7 of the 11 alive patients (64\%), after a median of 297 days (150-270).

Comparative outcomes between Haplo-PT-Cy and iHLA-SIB HL group of patients: Overall survival at 24 months for Haplo-PT-Cy and iHLA-SIB groups was $85 \%$ and $66 \%$, respectively $(p=0.273)$ [median follow-up for iHLA-SIB was 80 months (52-106)]. Relapse free survival (RFS) at 18 months was statistically superior in Haplo-PT-Cy group comparing to iHLA-SIB patients: $92 \%$ and $50 \%$, respectively $(p=0.026)$. The incidence of aGVHD $(p=0.204)$ and cGVHD $(p=0.183)$ was similar in both groups, but there was a trend towards more incidence of severe cGVHD in iHLA-SIB group.

Conclusions: Haplo-PT-Cy is an interesting approach for high-risk HL patients that show good results. These 
outcomes are similar to the standard procedure (iHLA-SIB) and even superior in some parameters like RFS without more incidence of aGVHD or cGVHD.

Randomized studies and a longer follow-up are necessary to confirm this data.

Conflict of interest: Nothing to disclose

\section{P809}

\section{Haploidentical transplantation does not have survival advantage over unrelated donor transplantation in haematological diseases}

Ender Soydan', Ayla Gokmen ${ }^{1}$, Zafer Gokgoz ${ }^{1}$, Osman Ilhan $^{2}$, Onder Arslan ${ }^{2}$, Muhit Ozcan ${ }^{2}$

${ }^{1}$ Medicana International Ankara Hospital, Ankara, Turkey; ${ }^{2}$ Ankara University School of Medicine, Ankara, Turkey

Background: Allogeneic hematopoietic stem cell transplantation is still the only curative treatment option for certain malign and non malign hematologic disease. Unfortunately less than $30 \%$ of patients are lucky enough to have a HLA match sibling donor. Alternative donor options are cord blood, HLA match and mismatch unrelated donors and haploidentical family donors such as parents, children and siblings.

Methods: We retrospectively compared the outcomes of 130 alternative donor transplants performed in our center according to donor source. The donors were 90 unrelated and 40 haploidentical, where as 52 out of 90 unrelated donors were HLA mismatched .

Results: TabPatient characteristics were similar in means of diagnosis, age of the patients and donors, recipient-donor gender match. But the percentage of refractory leukaemia patients were high in the haploidentical donor group (29\% vs $7 \%, p=0,01)$. The conditioning regiments were mostly reduced intensity in the haploidentical donor group while 47 out of 90 patients were treated with ablative conditioning regiments all with antithymocyte globulin for graft versus host disease prophylaxis. The engraftment kinetics were better with advantage of early neutrophil and platelet recovery in the match/mismatch unrelated donor group $(\mathrm{p}=$ 0,00 and 0.03 respectively). Acute and chronic graft versus host disease incidence, cytomegalovirus reactivation, transplant related mortality and relapse incidence were similar in both groups.

When we compare disease free (DFS) and overall survival (OS) the match and mismatch unrelated donor group was better in the whole patient cohort as well as in the leukaemia patients. Estimated 2 year DFS for all patients was $22 \%$ vs $51 \%$ for according to haploidentical and match and mismatched unrelated donor $(\mathrm{p}=0,003)$ and OS was 40 vs $59 \%(\mathrm{p}=0,02)$. For leukaemia patients DFS was 29 vs $56 \%(\mathrm{p}=0,02)$ and OS was 43 vs $59 \%(\mathrm{p}=0,1)$.

Conclusions: In conclusion, haploidentical donor transplantation provides quick donor availability in patients especially with high risk of relapse and mortality but did not show advantage of disease and overall survival both in leukaemia and other haematological disease in our cohort of patients.

Clinical Trial Registry: No

Conflict of interest: None

\section{P810}

Hematopoietic stem cells transplantation from relative donors' perspective: donors' quality of life and correlated psychological variables

Serena Giuliani ${ }^{1}$, Eleonora Franchini ${ }^{1}$, Raffaella Greco $^{2}$, Lucia Malabarba $^{3}$, Salvatore Gattillo ${ }^{3}$, Laura Bellio ${ }^{3}$, Luca Santoleri ${ }^{3}$, Lucio Sarno ${ }^{I}$, Fabio Ciceri ${ }^{4}$

${ }^{1}$ San Raffaele Scientific Institute, Clinical Health Psychology Unit, Milano, Italy; ${ }^{2}$ IRCCS San Raffaele Scientific Institute, Hematology and BMT Unit, Milan, Italy; ${ }^{3}$ San Raffaele Scientific Institute, Immunohematology and Transfusional Medicine Unit, Milano, Italy; ${ }^{4}$ San Raffaele Scientific Institute, Hematology and Bone Marrow Transplantation Unit, Milano, Italy

Background: The care of the donors during allogeneic hematopoietic stem cell transplantation (HSCT) requires to identify factors that predict poorer donation experiences.

Since pre-donation quality of life (QoL) scores predict time to recovery from donation (Billen, 2015), factors potentially associated to the donors' perception of QoL should be investigated.

Particularly interesting could be to assess if donors' psychological condition (psychopathological symptoms) and/or the relationship with the patient (caregiving style; family functioning) could be associated to the donors' perception of QoL, as far as specific psychological intervention (individual or focused on the couple donor-patient) can be timely addressed to help the donor to face the donation.

Methods: From May 2015 to June 2017 in San Raffaele Hospital in Milan, 35 related donors (43\% female, 57\% male; mean age: $41,4 \mathrm{y}$; $82 \%$ donors of acute leukemia patients) were studied during the period before the donation. Self-administered questionnaires were used to evaluate donors' QoL [Medical Outcomes Study SF-36 (Ware e Sherbourne, 1992)], psychopathological symptoms [SCL90-R (Daniels, 1994)], caregiving styles [Caregiving Questionnaire (Kunce \& Shaver, 1994)] and family functioning [Family Apgar (Smilkstein, 1978)]. The association between QoL and all these variables was studied in univariate analysis (statistical test SPSS-20). 
Results: The results show that donors describe a good physical QoL, but not psychological: they describe a good family functioning (Tot Apgar range 7.8), do not report psychopathological symptoms (Global Severity Index $<1$ ) and show a responsive caregiving style $(41,31)$ and a degree of proximity with the patient $(36,70)$ above average. Mental QoL is negatively associated $(\mathrm{p}<0.05)$ with controlling and compulsive caregiving styles and psychopathological symptoms, while physical QoL is positively associated (p $<0.05$ ) with a good degree of proximity with the patient, a responsive caregiving style and the perception of an adequate family functioning.

Conclusions: These data suggest that a multidisciplinary approach for donors should be developed in order to assess and to act on psychological variables in the pre donation phase. The relationship between donor and patient appears to be a key variable to be evaluated since it's correlated not only with both mental and physical QoL, but also because can influence donors' compliance to treatment and could be modified by donation side effects. Considering the great importance of the donor's role in the treatment of HSCT patients, should be important to notice that donors would seem to benefit from being treated in a multidisciplinary setting with a structural implementation of psychological assessment.

Conflict of interest: The authors have nothing to disclose.

\section{P811}

\section{HLA-Haploidentical Hematopoietic Cell Transplantation after TCR- $\alpha \beta$ and CD45RA + Depletion Following Reduced Intensity Conditioning in Adults and Children with Hematological Malignancies}

L M Poon ${ }^{1}$, Yeh Ching Linn ${ }^{2}$, Poh Lin Tan ${ }^{3}$, Rajat Bhattacharyya ${ }^{4}$, Ah Moy Tan', Lip Kun Tan ${ }^{1}$, Teck Guan Soh ${ }^{1}$, Kee Khiang Heng ${ }^{2}$, Gina PL Gan', Wing Leung ${ }^{4}$, Liang Piи Koh ${ }^{1}$

${ }^{l}$ National University Cancer Institute, Dept of Hematology Oncology, Singapore, Singapore; ${ }^{2}$ Singapore General Hospital, Dept of Hematology, Singapore, Singapore; ${ }^{3}$ National University Hospital, Dept of Paediatrics, Singapore, Singapore; ${ }^{4}$ Kandang Kerbau Children and Women Hospital, Singapore, Dept of Paediatrics, Singapore, Singapore

Background: Outcomes after haploidentical (Haplo) hematopoietic cell transplantation (HCT) are encouraging and has become an alternative option for patients without HLA-matched donor. GVHD, engraftment failure, and infectious complications continue to be the main causes of transplant-related morbidity (TRM). We hypothesized that selective depletion of $\mathrm{TCR} \alpha \beta+$ and $\mathrm{CD} 45 \mathrm{RA}+$ naïve $\mathrm{T}$ cells subset will permit hematopoietic engraftment, while effectively reducing GVHD, and provide improved donor

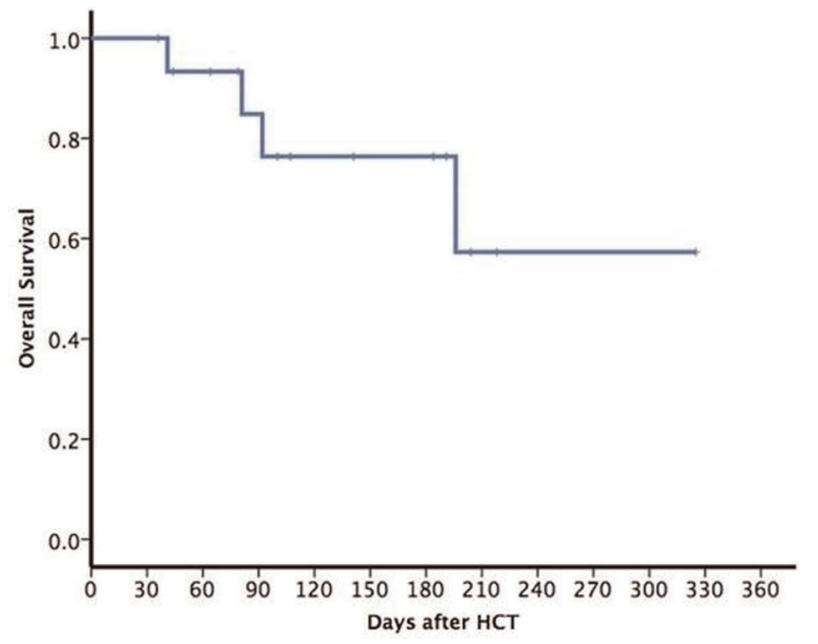

[P811 Figure] [Overall Survival]

immune reconstitution through adoptive transfer of donor's mature NK and $\gamma \delta$ T cells and CD45RA- memory T cells. Herein, we present results from the first 16 patients with poor-prognosis hematologic malignancies, who received haplo-HCT with TCR $\alpha \beta$ and CD45RA+ depleted grafts following a reduced-intensity conditioning (RIC) regimen.

Methods: The conditioning regimen consisted of total nodal irradiation 6 - 7.5 Gy over 3 equal fractions, $160 \mathrm{mg} /$ $\mathrm{m} 2$ fludarabine divided daily over 4 days, thiotepa $10 \mathrm{mg} / \mathrm{kg}$ divided twice daily for 1 day and melphalan $70-140 \mathrm{mg} / \mathrm{m} 2$ for 1 day. Short term GVHD prophylaxis for 30 days was given to 1 patient using MMF, 4 using tacrolimus or 2 using Sirolimus.

Results: We transplanted 13 adults (median age 41 years, range 21 - 68) and 3 children (age range 7-14 years) with high risk AML $(\mathrm{n}=9)$, ALL $(\mathrm{n}=2)$, and MDS $(\mathrm{n}=2)$. The graft contained a median of $9.73 \times 10^{6}$ (range, 6.10 - 20.78) $\mathrm{CD} 34+$ cells $/ \mathrm{kg}$ and $0.0 \times 10^{4}$ (range, $0.0-0.30$ ) CD $45 \mathrm{RA}$ $+\mathrm{CD} 3+$ cells $/ \mathrm{kg}$. In addition, the graft contained a median of $10.05 \times 10^{6}$ (range $\left.1.73-29.74\right) \mathrm{TCR} \gamma \delta+$ cells $/ \mathrm{kg}$, and $4.21 \times 10^{6}$ (range, $0.15-9.86$ ) CD $45 \mathrm{RO}+\mathrm{CD} 3+$ cells $/ \mathrm{kg}$. Fifteen patients had engraftment of ANC $>500$ cells $/ \mu \mathrm{L}$ at a median of 11 days (range, 9 - 18) and PLT $>20,000$ cells/ $\mu \mathrm{L}$ at a median of 13 days (range, 8 - 19). Complete donor chimerism was seen at the time of engraftment in all patients with primary engraftment. One patient experienced primary graft failure, requiring re-transplant using a different mismatched family member. There was no secondary graft failure.

Six patients developed acute GVHD of grade II-IV with the cumulative incidence on day 90 of $58 \%$. One patient experienced chronic GVHD. Cumulative incidence of TRM on Day 100, and relapse on day 180 were $23 \%$ and $0 \%$, respectively.

Viral reactivation included CMV $(n=6)$, HHV6 $(\mathrm{N}=2)$, EBV $(\mathrm{N}=2)$ and adenovirus $(\mathrm{N}=1)$, with no fatal viral 
infection occurred within 100 days. Additionally, there was no fatal blood stream infection within 100 days.

Kaplan-Meier estimated 3 month and 6 months overall survival with a median follow up of 124 days (range 36$325)$ are $76 \%$ and $57 \%$, respectively.

Conclusions: Our preliminary results suggest that haploHCT with TCR $\alpha \beta$ and CD45RA + depleted grafts following a RIC regimen allows successful allograft in high-risk patients lacking a suitable donor. TRM due to GVHD remains the major obstacle for the successful HCT. More effective strategies are needed to eliminate the high risk of severe GVHD, while preserving antitumor and antimicrobial immunocompetence.

Clinical Trial Registry: NA

Conflict of interest: W. Leung: Works with Miltenyi Biotec. None of the authors has anything to disclose.

\section{P812}

\section{How we managed a family donor and a PBSC donation for a related recipient transplanted in a different Country: experience of Verona Collection Center}

\author{
Aurora Vassanelli ${ }^{1}$, Nicoletta Sacchi ${ }^{2}$ \\ ${ }^{1}$ Azienda Ospedaliera Universitaria Integrata, Transfusion Medicine \\ Department, Verona, Italy; ${ }^{2}$ Ente Ospedaliero Ospedai Galliera, \\ Italian Bone Marrow Donor Registry, Genova, Italy
}

Background: Stem Cell Transplantation in family setting (related donor and recipient) is usually performed in Transplant Centre (TC) and Collection Center (CC) that are part of the same Transplantation Program, at the same Hospital, or in the same City; it rarely happens that donor and recipient live in different cities : in these cases the donor normally moves to the Donor Center/ Collection Center closer to the Transplant Center.

We describe our experience in the management of a family donor who lives in Italy and who donated PBSC (Peripheral Blood Stem Cell) to his brother who lives in Germany and transplanted in a german TC

Methods: At the end of October 2017 we received the formal request to PBSC donation from the italian familiy donor for his brother who lives in Germany. The donor had already declared his difficulty in making the donation in Germany and asked to donate in Italy.

The request was sent from the german TC to the Registry ZKRD and from this to the Italian Registry IBMDR in order to ask the nearest Donor Center for availability to contact the donor and to evaluate his eligibility for the donation.

IBMDR forwarded the request to the $\mathrm{CD}$ closest to the donor $\hat{A}^{\prime}$ s home and put the $\mathrm{CT}$ in contact with the $\mathrm{CD}$. The CD (TN01), has contacted both our CC (VR01) and the donor for the pre-donation work-up. As $\mathrm{CC}$, in strict agreement with the $\mathrm{CD}$, we agreed with the $\mathrm{CT}$ the work-up program, the donation date, the donor clearance, the start of mobilization therapy. The CT was responsible for the transport of PBSC.

For the administrative aspects, the fee applied at IBMDR, which the CT has declared to accept, has also been applied.

For all organizational aspects and for management of the donor and the PBSC unit, the operating protocol established by the IBMDR standards was followed, as if it were an unrelated donor in favor of an international recipient.

IBMDR has been updated in real time for all phases of the donation.

Results: The donation took place on 28 November 2017, the unit was transported to the CT, after authorization to export issued by the italian competent authority and the SEC was assigned, according to European legislation. The transplant was successfully performd with full haematological recovery in 14 days. The donor is currently followed by the CD for post-donation follow-up.

Conclusions: Our experience shows that in the case of family donors who donate PBSC or BM to their family members living in different Countries, the same operative protocol followed for unrelated donors can be applied, and that related and unrelated donor management should be the same. It also emphasizes the important role played by the Registry (IBMDR and ZKRD in this case) as facilitator and as important reference for the organizational aspects of Stem Cell donation when different Countries are involved.

Conflict of interest: A. Vassanelli: nothing to disclose

\section{P813}

Immune Reconstitution after Allo-stem Cell Transplantation in Pediatric Patients: Differences According Donor Type

Ana Maria Fita ${ }^{1}$, Maria Victoria Martínez Sánchez ${ }^{2}$, Maria Esther Llinares Riestra ${ }^{1}$, Maria Mar Bermúdez Cortés ${ }^{1}$, Ana Maria Galera Miñarro ${ }^{1}$, Miguel Blanquer Blanquer ${ }^{3}$, Andrés Sánchez Salinas ${ }^{3}$, José Luis Fuster Soler ${ }^{1}$, José María Moraleda ${ }^{3}$

${ }^{1}$ Hospital Clínico Universitario Virgen de la Arrixaca, Paediatrics Oncology and Haematology Unit, El Palmar, Murcia, Spain;

${ }^{2}$ Hospital Clínico Universitario Virgen de la Arrixaca, Immunology Service, El Palmar, Murcia, Spain; ${ }^{3}$ Hospital Clínico Universitario Virgen de la Arrixaca, IMIB, University of Murcia, Haematology Service and Stem Cell Transplant Unit, El Palmar, Murcia, Spain

Background: Recovery of the immune system after allogeneic haematopoietic stem cell transplantation (alloHSCT) is a highly dynamic process, often delayed as a result of the preparative regimen used prior to transplant, predisposing the recipients to infections.

Methods: Retrospective, observational study of patients aged between 0 and 15 years undergoing allo-HSCT at a tertiary hospital, from January 2007 to December 2015. We 

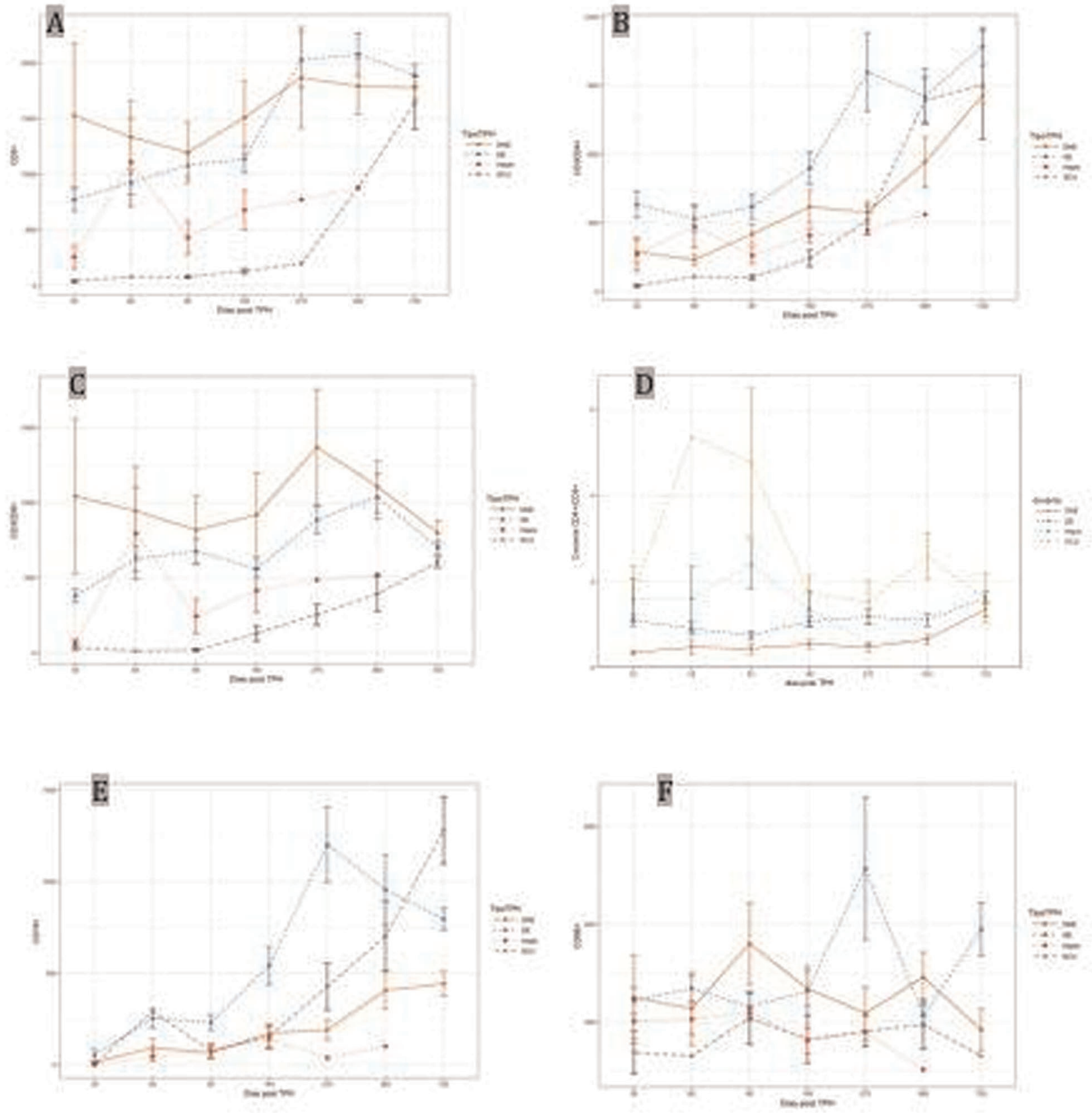

[P813 Figure] [Immune recovery post allo-HSTC according donor type (RD, URD, Haplo, CB)]

studied a total of 56 pediatric patients who underwent 69 allo- HSCT with a median follow-up of 19 (1 - 87) months. Immunologic reconstitution was assessed by total lymphocytes; CD3+, CD4+ and CD8+ T lymphocytes; CD4 $+/ \mathrm{CD} 8+$ ratio; CD19+ B lymphocytes and CD16+/CD56 + natural killer $(\mathrm{NK})$ at day $+30,+60,+90,+180,+360$ and +720 post allo-HSCT. Normal lymphocyte population values were defined as those included in The Pediatric AIDS Clinical Trials Group P1009 study. We performed 4 groups according to donor type: related donor (RD), unrelated donor (URD), haploidentical (Haplo) and cord blood (CB). The immune recovery data was available in 25/ 32 RD-HSCT, 19/20 URD-HSCT, 4/11 haplo-HSCT (depletion CD3 $\alpha \beta$ CD19+) and 4/6 CB-HSCT.

Results: We depict the immune evolution post alloHSCT of each type of lymphocyte according to donor type.

There were no significant differences between groups in the $\mathrm{CD} 3+$ and $\mathrm{CD} 4+$ recovery.

Normal CD3 + values were found in the URD group since day +30 and since day +180 in the RD group. The other 
groups showed slow and steady increase, achieving normal values 2 years after transplant in the $\mathrm{CB}$ group.

The CD4+ recovery was faster in the RD group, showing normal values at day +270 . The $\mathrm{CB}$ group achieved them at day +360 and the URD group at 2-years post-transplant.

The CD8+ lymphocyte recovery was significantly lower $(\mathrm{p}<0.05)$ at day +30 in the haplo group than in the RD group. At day +180 it also was significantly lower $(\mathrm{p}<$ 0.05 ) in the CB group than in the RD group. The RD and URD group showed normal CD8+ values since day +30 , while haplo group recovered normal values since day +60 and $\mathrm{CB}$ group since day +360 .

The $\mathrm{CD} 4+/ \mathrm{CD} 8+$ ratio at day +30 was significantly lower $(\mathrm{p}<0.05)$ in the URD group than in RD and haplo groups. Both haplo and CB groups showed a CD4+/CD8+ ratio greater than 1 since day +30 , while $\mathrm{RD}$ and URD groups showed ratio inversion until day +180 in the RD group and day +720 in the URD group.

RD was the first group in recovering normal CD19+ values, at day +90 (significantly different to the URD group, $\mathrm{p}<0.05$ ) followed by CB at day +270 and URD at day +360 . By contrast, the haplo group did not reach normal level of CD19+ during one year of follow-up.

The CD16+/CD56+ lymphocyte showed an early recovery with normal values since day +30 , without significant difference between groups.

Conclusions: Immune reconstitution is variable according to donor SC source. Overall, innate immunity recovers within the first weeks of HCT, followed by that of the adaptive immune system.

\section{Conflict of interest: None}

\section{P814}

\section{Occurrence and dynamics of HLA-antibodies in the setting of matched related hematopoietic stem cell transplantation}

Michelle Bräutigam ${ }^{1,2}$, Thomas Volken ${ }^{3}$, Alexandra Plattner ${ }^{2}$, Jörg Halter $^{1}$, Jakob Passweg ${ }^{1}$, Beatrice Drexler ${ }^{1,2}$, Dominik Heim ${ }^{1}$, Andreas Buser ${ }^{1,2}$, Laura Infanti, ${ }^{1,2}$, Andreas Holbro ${ }^{1,2}$

${ }^{1}$ University Hospital Basel, Division of Hematology, Basel, Switzerland; ${ }^{2}$ Swiss Red Cross, Blood Transfusion Center, Basel, Switzerland; ${ }^{3}$ Zurich University of Applied Sciences, School of Health Professions, Winterthur, Switzerland

Background: HLA-antibodies are increasingly recognized to play an important role in the setting of HSCT. The aim of the current prospective study was to evaluate occurrence and dynamics of HLA-antibodies after matched related HSCT.

Methods: Patients and their matched related donors were prospectively included in the IRB approved study. HLAantibodies were determined by Luminex technique at predefined time points. For patients, samples were drawn at baseline (before HSCT), at HSCT and weekly thereafter until 4 weeks after HSCT and for donors at eligibility assessment and at donation. We used generalized estimating equation models of the Gaussian and negative binomial family with log links and robust standard errors in order to assess temporal trajectories of patients' average mean fluorescence intensity (MFI), highest MFI, and the number of antibodies with MFI > 500.

Results: Among the 50 patients included in the study, 26 $(51 \%)$ were female and median age at transplantation was 51 years. The majority of patients had AML (37\%) and MM $(15.7 \%)$, received myeloablative conditioning (58.8\%) and GvHD prophylaxis consisted mainly of cyclosporine containing regimens.

At baseline, HLA-antibodies were detected in 49 patients (98\%) (mean number of antibody specifities: 13; range 0-102) and in only 25 donors $(50 \%)$ (mean number of antibody specifities: 6; range 0-51). Overall, both number and MFI of class I antibodies were higher compared to those of class II antibodies. At baseline, the total number was 348 for class I (mean MFI: 2330) and 310 for class II antibodies (mean MFI: 1637). The highest mean MFI for class I and class II antibodies were 4126 and 3735, respectively.

Surprisingly, a considerable increase of the number and intensity of antibodies was observed within a few days, from baseline to the day of transplantation. At HSCT the total number of antibodies was 706 for class I with a mean highest MFI of 6007 and 353 for class II antibodies with a mean highest MFI of 3482, respectively. Thereafter, the number of antibodies as well as MFI-levels - measured weekly - remained stable until the end of observation. This finding was similar after adjusting for gender, age, diagnosis, conditioning, GVHD prophylaxis, CMV status, and $\mathrm{ABO}$ incompatibility (Figure).

Furthermore, 14 of the 50 patients $(28 \%)$ developed new HLA antibodies increasing steadily in number and intensity over the observed time period. New class II antibodies (mean number: 184, mean MFI: 2549) occurred more often and at higher intensities than class I antibodies (mean number: 42, mean MFI: 1415). Some of the molecular specifities of these antibodies emerging in the patients were the same as to those found in their corresponding donors.

Conclusions: Our data show that HLA antibodies are frequently present in patients undergoing HSCT and that they should be measured at the day of transplantation. Additionally, some patients develop new, including presumably donor-derived antibodies. This might have some impact regarding both transfusion strategies (platelet transfusion refractoriness) as well as transplant outcome. Since HLA-mismatched (incl. haploidentical) HSCT are increasingly performed worldwide, further studies on the significance of HLA-antibodies in these settings are warranted. 
Conflict of interest: The authors declare no conflict of interest.

\section{P815}

Pediatric allogeneic transplantation from a matched family donor with seropositive rheumatoid arthritis: Development of rheumatoid arthritis related autoantibodies nine years after transplantation

Sebnem Yilmaz, Ozlem Tufekci, Melek Erdem, Hale Oren

Dokuz Eylul University, Pediatric Hematology, Izmir, Turkey

Background: There are few reports of donor-derived autoimmune diseases by allogeneic stem cell transplantation. In cases of post-transplant adoptive autoimmunity, autoantibodies are produced by a clone of programmed donor lymphocytes. Here we describe a pediatric acute lymphoblastic leukemia (ALL) patient, who was transplanted from his mother with the diagnosis of rheumatoid arthritis (RA). Nine years after transplant, he developed autoantibodies without clinical findings of RA.

Methods: A 5-year-old male with high risk T cell ALL underwent myeloablative allogeneic peripheral blood stem cell transplantation in his first complete remission from his 10/10 HLA matched mother in July 2008. The 33-year-old donor was diagnosed with seropositive RA when she was 22 years old. Her clinical disease was under full control with $8 \mathrm{mg} / \mathrm{day}$ methylprednisolone, but high titers of rheumatoid factor $(113.6 \mathrm{IU} / \mathrm{mL})$ and anti-cyclic citrullinated antibody (anti-CCP antibody) $(198 \mathrm{U} / \mathrm{mL})$ were detected at the time of peripheral stem cell collection. The patient and donor had both HLA-DRB1*0408 alleles, a HLA-DRB1 phenotype that may be involved in susceptibility to RA.

The patient received busulfan, etoposide and cyclophosphamide as conditioning. Graft versus host disease (GVHD) prophylaxis consisted of cylosporine and shortcourse methotrexate. Neutrophil and thrombocyte engraftment was on day +14 and +21 , respectively. The posttransplant period was uncomplicated except culture negative febrile neutropenia and mucositis. There was no occurence of acute or chronic GVHD during follow-up. Bone marrow samples demonstrated complete donor chimerism with fluorescence in situ hybridization.

Results: During the post-transplant nine-year follow-up period, no complaints or physical examination findings that might be associated with rheumatoid RA developed. Rheumatoid factor was negative, but in November 2017, the highly specific anti-CCP antibody for RA was detected positive at a low titer $(21.5 \mathrm{U} / \mathrm{mL})$. Additionally, anti- nuclear antibody was positive (1/1000-1/3200 nucleolar and 1/320-1/1000 granular pattern).

Conclusions: We think that donor derived memory B cell may have been contributed to the production of autoantibodies in this patient. But we cannot exlude the possibility of de novo autoimmune antibody development. First-degree relatives of a person with RA are slightly more likely to develop the condition, and our patient had the HLA-DRB $1 * 0408$ alleles.

The appearance of autoantibodies in autoimmune diaseases often appear before the development of clinical disease. Even low anti-CCP antibody levels are known to be associated with progression to RA. Therefore the patient will be closely followed for development of RA.

Conflict of interest: None of the authors has anything to disclose.

\section{P816}

Peripheral blood stem cell RIC haploidentical transplantation low incidence of GVHD and non relapse mortality: Preliminary results from the prospective phase II UK Haplo study

Kavita Raj ${ }^{1}$, Rachel Protheroe ${ }^{2}$, Matthew Smith ${ }^{3}$, Maria Gilleece, John.A. Snowden ${ }^{5}$, Ben Uttenthal ${ }^{6}$, David Irvine ${ }^{7}$, Matthew Collin ${ }^{8}$, Shankaranarayana Paneesha ${ }^{9}$, Rahuman Salim ${ }^{10}$, Eleni Tholouli' ${ }^{11}$, Ellice Marwood ${ }^{12}$, Pavlina Mesiri ${ }^{12}$, Husne Timur ${ }^{12}$, Charlotte O'Connor $^{12}$, Laura Clifton-hadley ${ }^{12}$, Pip Patrick ${ }^{12}$, David Marks ${ }^{13}$, Stephen Mackinnon ${ }^{14}$, Antonio Pagliuca ${ }^{15}$

${ }^{1}$ Kings College Hospital and Guys and St Thomas' NHS Foundation Trust, Haematology, London, United Kingdom; ${ }^{2}$ University Hospital Bristol, Haematology, Bristol, United Kingdom; ${ }^{3}$ Bart's Health NHS Trust, Haematology, London, United Kingdom; ${ }^{4}$ Leeds Teaching Hospitals NHS Trust, Haematology, Leeds, United Kingdom; ${ }^{5}$ Sheffield Teaching Hospitals NHS Foundation Trust, Haematology, Sheffield, United Kingdom; ${ }^{6}$ Cambridge University Hospitals NHS

Foundation Trust, Haematology, Cambridge, United Kingdom; ${ }^{7}$ NHS Greater Glasgow \& Clyde, Haematology, Glasgow, United Kingdom; ${ }^{8}$ Newcastle upon Tyne Hospitals NHS Foundation Trust, Northern Centre for Cancer Care, Newcastle, United Kingdom; ${ }^{9}$ Heart of England NHS Foundation Trust, Haematology and Stem cell Transplantation, Birmingham, United Kingdom; ${ }^{10}$ The Royal Liverpool University Hospital, Haematology and Stem cell transplantation, Liverpool, United Kingdom; ${ }^{11}$ Manchester Royal Infirmary, Manchester University Foundation Trust, Manchester, United Kingdom; ${ }^{12}$ CR UK and UCL Cancer Trials Centre, London, United Kingdom; ${ }^{13}$ University Hospital Bristol, Bristol, United Kingdom; ${ }^{14}$ University College London Hospitals NHS Foundation Trust, London, United Kingdom; ${ }^{15}$ Kings College Hospital and Guys and Kings College, Haematology, London, United Kingdom

Background: T-cell replete haploidentical (haplo) stem cell transplants with post-transplant cyclophosphamide were developed with bone marrow grafts. Peripheral blood stem cells (PBSC) are the commonest source in use internationally for all donors but have higher rates of 


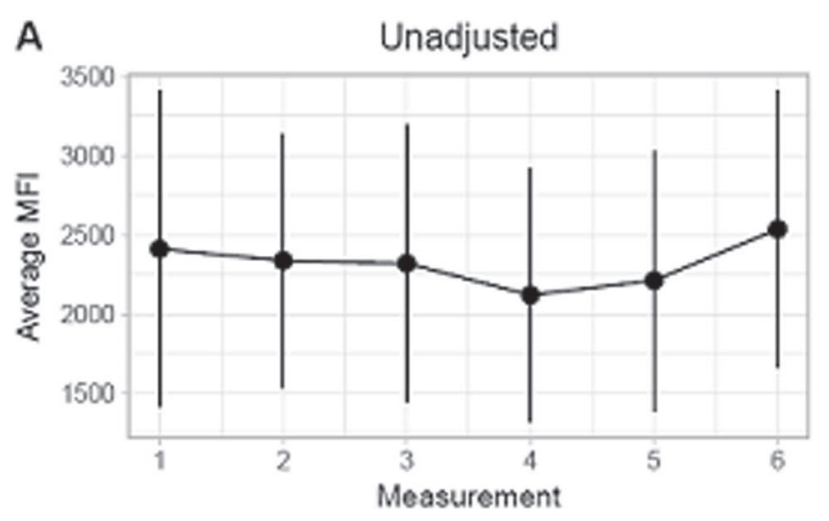

B
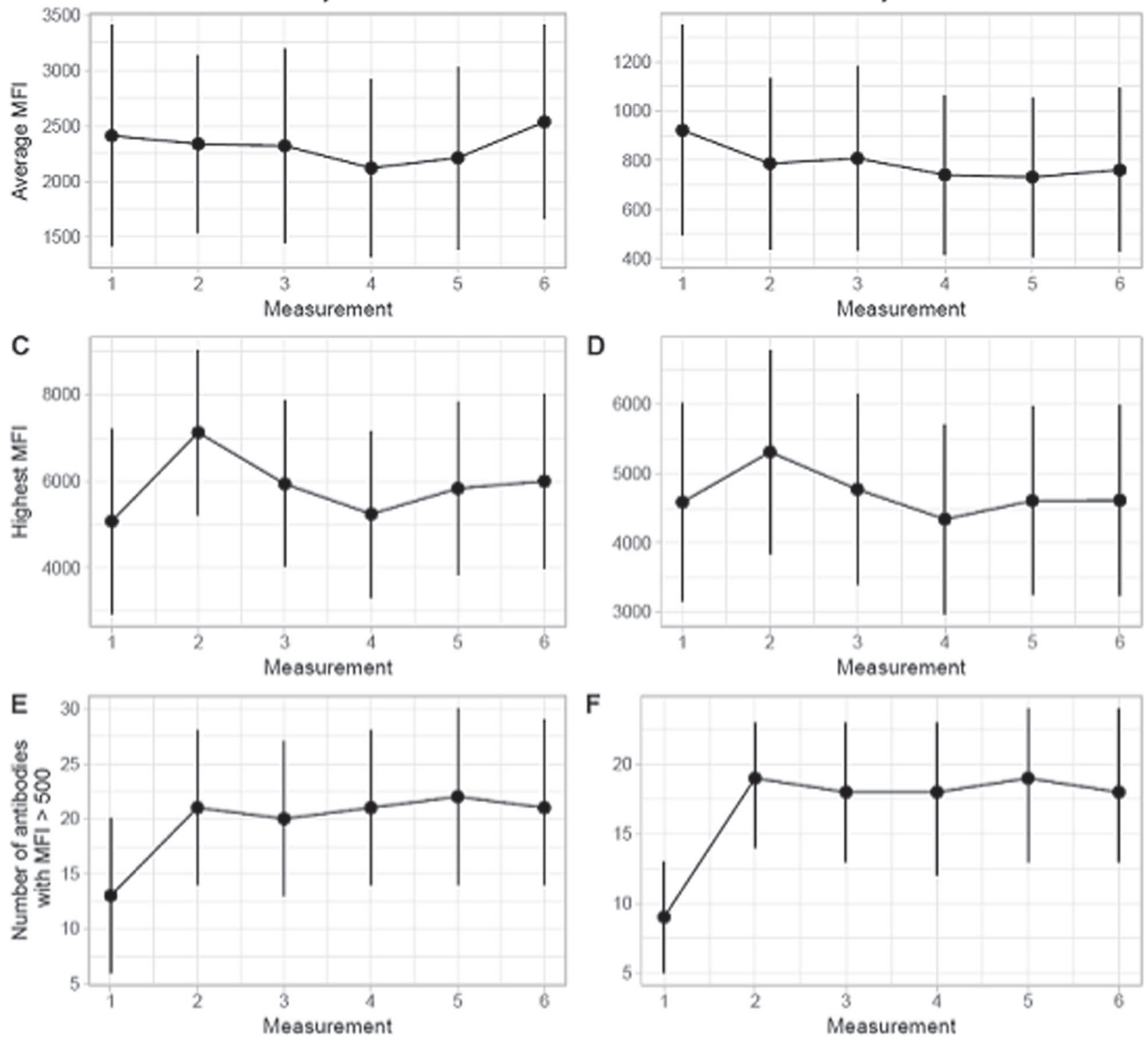

D

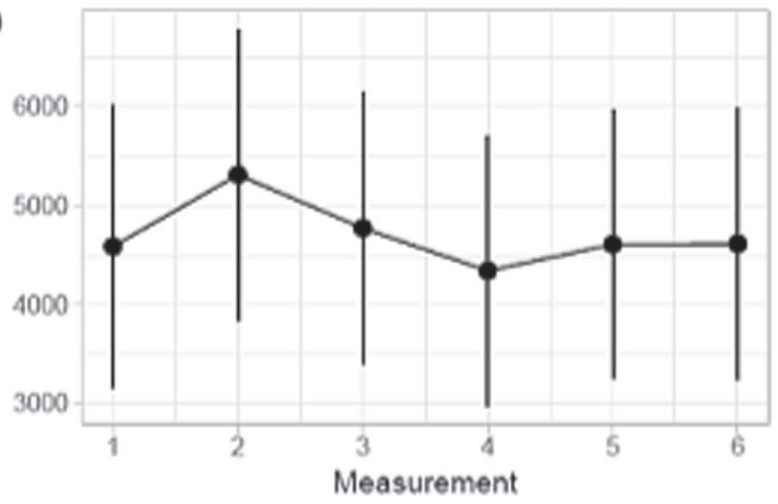

$\mathbf{F}$

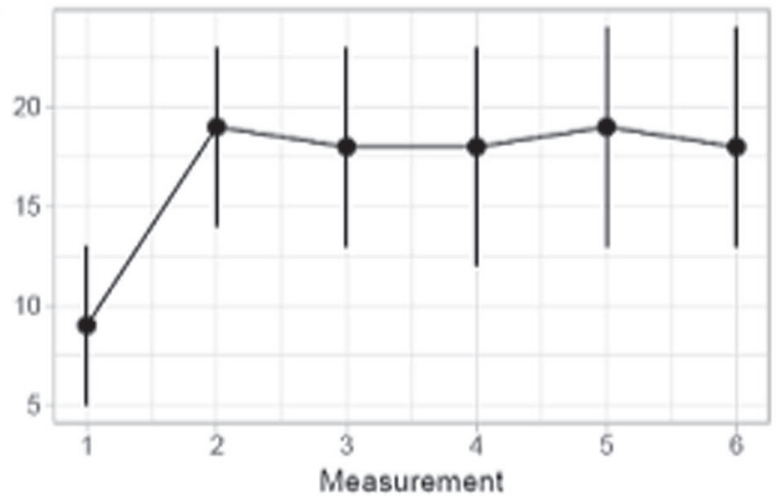

[P814 Figure] [Number of Antibodies and MFIs (Average/highest) unadjusted (A,C,E) and adjusted (B,D,F) (see text)]

graft-versus-host-disease (GVHD). We studied PBSC in RIC haplo transplants.

Methods: A multicentre, single arm prospective phase II study of haplo HSCT in patients without donor directed HLA antibodies. RIC conditioning was Fludarabine 30mg/ $\mathrm{m}^{2} \times 5$ days, Cyclophosphamide $14.5 \mathrm{mg} / \mathrm{kg} \times 2$ days and 2 Gy TBI; post-transplant cyclophosphamide, day +3 and day +4 , at $50 \mathrm{mg} / \mathrm{kg}$ as GVHD prophylaxis. Tacrolimus or cyclosporine and mycophenolate mofetil (MMF) at day +5 .

The primary outcome was overall survival (OS) at 1 year. Secondary outcomes included progression free survival (PFS), non-relapse mortality (NRM), incidence of GVHD and engraftment.
Results: Fifty patients enrolled between 2011 and 2017 at 11 UK centres, the median age was 60 (19-70). Patient characteristics in table 1.

The median CD34+ cells administered was $5 \times 10^{6} / \mathrm{kg}$ $\left(1.3-10.4 \times 10^{6} / \mathrm{kg}\right)$. Acute classic GVHD grade I occurred in 9 (21.4\%), grade II in $8(19.0 \%)$ and grade IV in $1(2.4 \%)$. Acute persistent, recurrent or late onset GVHD grade I occurred in $8(19.0 \%)$, grade II and III in $2(4.8 \%)$ and grade IV in $1(2.4 \%)$. No patient developed chronic classic GvHD but chronic overlap syndrome occurred in 5 (11.9\%). The Cumulative incidence (CI) of grade II-IV aGVHD at day +90 was $22.3 \%(10.9-45.6)$ respectively. The median time to GVHD was 44 days (33-385). No deaths were attributable to GVHD. 
Median follow-up of survivors was 12 (1-26) months. The CI of NRM at 1 year was 7.4\% (2.7-20.7). OS at 1 and 2 years was $81.0 \%$ (63.0-90.0) and $71.0 \%$ (43.0-87.0), respectively. PFS at 1 and 2 years was $78.0 \%(60.0-88.0)$ and $56.0 \%(30.0-76.0)$ and 1 year relapse rate was $14.3 \%$ (0.7-46.0). Graft rejection occurred in 4(8.0\%); two failed despite adequate cell doses, the third harvested an inadequate dose and the final had early withdrawal of IST leading to rejection and salvage cord blood transplant.

Eight patients died, 4 post-relapse (3 disease, 1 unknown), 2 from infection (1 before day 100), 2 from TRM following a second SCT post graft failure.

Conclusions: Preliminary results demonstrate that PBSC Haplo RIC transplantation is safe in a multicentre setting, with low levels of severe GVHD. The OS and PFS at 1 year is comparable to that of $\mathrm{BM}$ haplo, sib and matched unrelated donors, with low NRM and relapse rates but further follow-up is needed.

Clinical Trial Registry: Clinicaltrials.gov: NCT01597219

EUDRACT 2011-005889-38

Conflict of interest: There are no conflicts of interest for any author.

This trial was funded by a research grant from Blood wise (grant no 11034).

\begin{tabular}{lll}
\hline $\begin{array}{l}\text { Male } \\
\text { Non-Caucasian }\end{array}$ & & $29(58.0 \%)$ \\
Co-morbidity score & $0-2$ & $14(28.0 \%)$ \\
& $3+$ & $32(64.0 \%)$ \\
Karnofsky & $70 \%-90 \%$ & $17(34.0 \%)$ \\
Performance Status & & $15(30.0 \%)$ \\
& $>90 \%$ & $35(70.0 \%)$ \\
Diagnoses & Acquired Bone Marrow Failure & $2(4.0 \%)$ \\
& Syndromes & \\
& Acute Leukaemia: ALL, AML, & $25(50 \%): 6$, \\
& Biphenotypic & $42,2 \%$ \\
& Myelodysplastic Syndrome & $15(30.0 \%)$ \\
& Lymphoma: Hodgkins, Non & $5(10.0 \%): 2$, \\
& Hodgkins & $8 \%$ \\
\hline
\end{tabular}

[[P816 Table] Patient Characteristics]

\section{P817}

Peripheral Hematopoeitic Stem Cell Mobilization Utilizing Growth Factors in Donors with Sickle Cell Trait is Safe and Effective

\author{
Moussab Damlaj ${ }^{1,2,3}$, Ahmed Alaskar ${ }^{1}$, Samer Ghazi, Bader \\ Alahmari $^{1}$, Ayman Alhejazi ${ }^{1}$, Mohsen Alzahrani ${ }^{1}$
}

${ }^{1}$ King Abdulaziz Medical City, Riyadh, Saudi Arabia; ${ }^{2}$ King Abdullah International Medical Research Center, Riyadh, Saudi Arabia; ${ }^{3}$ King
Saud bin Abdulaziz University for Health Sciences, Riyadh, Saudi Arabia

Background: Peripheral hematopoietic stem cell mobilization using GCSF is widely used in allogeneic HCT. Patients with sickle cell disease (SCD) are prone to significant risks with GCSF including splenic rupture or death, in part due to precipitation of vaso-occlusive crises (VOC). Such VOC can also be seen in patients with sickle cell trait (SCT) during conditions of severe stress. Although allogeneic HCT is curative in SCD, donor availability is a major limitation as sibling donors are often affected. Furthermore, there is paucity in the literature with regards to the safety of GCSF in SCT carriers. Our aim was to examine the safety and efficacy of utilizing growth factors in donors with SCT.

Methods: After due IRB approval, allogeneic donors for sibling recipients with SCD were identified and medical records were retrospectively extracted. A sickle cell screen was performed and if positive, high performance liquid chromatography (HPLC) was completed. All donors were interviewed post mobilization and a survey was completed to inquire about adverse effects, ranging in severity from none, mild, moderate or severe. GCSF was administered at a dose of $10 \mathrm{mcg} / \mathrm{kg}$ rounded to the nearest vial size for 5 days followed by high flow apheresis. CD34 enumeration was measured on days 4 and 5 . Goal of collection was $\geq$ $10 \times 10^{6} / \mathrm{kg}$ CD34 cells of recipient weight to allow safe engraftment. Categorical and continuous variables were compared using Pearson's chi-squared and Wilcoxon / Kruskal-Wallis, respectively.

Results: A total of 27 donors were identified, 14 (52\%) of whom were SCT carriers. The cohort was stratified based on the presence of SCT and their corresponding baseline characteristics were similar with regards to age, gender, weight, CMV status or GCSF dose/kg used. There was a trend towards multiple day collections in control donors. Complete blood counts (CBC) and peripheral CD34 count on day of collection was also similar. The donor reported symptoms on the post mobilization survey are shown in table below. The incidence and severity of adverse events were similar between the cohorts including the percentage of analgesia utilization and duration of symptoms. The majority of donors reported symptoms and $>80 \%$ required analgesia to control pain. None of the donors developed infections, thrombosis, seizures or required transfusion support. There were no serious adverse events in both cohorts including VOC, acute chest syndrome, hospital admission due to GCSF, splenic rupture or death.

Conclusions: We demonstrate that GCSF can be used safely in donors with SCT as it results in a similar adverse event profile to control subjects. Furthermore; all SCT 


\begin{tabular}{|c|c|c|c|}
\hline Symptom / Severity & SCT Donors $(n=14)$ & Control Donors $(n=13)$ & P value \\
\hline Fever / Chills, n (\%) & $4(29)$ & $2(15)$ & 0.41 \\
\hline Fatigue, n (\%) & & & 0.63 \\
\hline None & $6(43)$ & $4(30.8)$ & \\
\hline Mild & $4(28.5)$ & $4(30.8)$ & \\
\hline Moderate & $4(28.5)$ & $4(30.8)$ & \\
\hline Severe & 0 & $1(7.6)$ & \\
\hline Nausea, n (\%) & & & 0,12 \\
\hline None & $13(93)$ & $11(85)$ & \\
\hline Mild & 0 & $2(15)$ & \\
\hline Moderate & $1(7)$ & 0 & \\
\hline Severe & 0 & 0 & \\
\hline Insomnia, n (\%) & & & 0.55 \\
\hline None & $8(57)$ & $10(77)$ & \\
\hline Mild & $4(29)$ & $2(15)$ & \\
\hline Moderate & $2(14)$ & I (8) & \\
\hline Severe & 0 & 0 & \\
\hline Headache, n (\%) & & & 0.44 \\
\hline None & $8(57)$ & $9(69)$ & \\
\hline Mild & $3(22)$ & $1(8)$ & \\
\hline Moderate & $2(14)$ & $3(23)$ & \\
\hline Severe & $1(7)$ & 0 & \\
\hline Myalgia, n (\%) & & & 0,29 \\
\hline None & $4(28.5)$ & $6(46)$ & \\
\hline Mild & $4(28.5)$ & $1(8)$ & \\
\hline Moderate & $6(43)$ & $5(38)$ & \\
\hline Severe & 0 & $1(8)$ & \\
\hline Arthralgia, n (\%) & & & 0.82 \\
\hline None & $3(22)$ & $2(15)$ & \\
\hline Mild & $2(14)$ & $3(23)$ & \\
\hline Moderate & $7(50)$ & $5(39)$ & \\
\hline Severe & $2(14)$ & $3(23)$ & \\
\hline Abdominal Pain, n (\%) & & & 0.96 \\
\hline None & $13(93)$ & $12(92)$ & \\
\hline Mild & $1(7)$ & $1(8)$ & \\
\hline Moderate & 0 & 0 & \\
\hline Severe & 0 & 0 & \\
\hline Pain Elsewhere, n ( $\%)$ & & & 0.2 \\
\hline None & $14(100)$ & $11(85)$ & \\
\hline Mild & 0 & $1(7.5)$ & \\
\hline Moderate & 0 & $1(7.5)$ & \\
\hline Severe & 0 & 0 & \\
\hline Analgesia Required, n (\%) & $12(86)$ & $11(85)$ & 0,94 \\
\hline $\begin{array}{l}\text { Duration of Pain (days), } \\
\text { median (range) }\end{array}$ & $3(2-10)$ & $4(2-5)$ & 0.77 \\
\hline
\end{tabular}

[P817 Figure] [Table]

donors were able to mobilize target yield of stem cells with one apheresis session. Such results are important to appropriately counsel prospective donors with SCT thus potentially increasing the donor pool for patients afflicted with SCD.

Conflict of interest: No relevant conflicts of interest

\section{P818}

Prep@gref: elaboration of an on-line computerized tool to pilot parallel care pathways of donor and patient for allogenic HSCT (donor search and qualification, patient qualification)

Claude Lemarie $^{1,2}$, Sabine Furst ${ }^{3}$, Didier Blaise ${ }^{3}$, Christian Chabannon ${ }^{1,2}$, Sylvain Fluzin ${ }^{4}$ 
${ }^{1}$ Institut Paoli-Calmettes, Centre de Thérapie Cellulaire, Marseille, France; ${ }^{2}$ Centre d'Investigations Cliniques en Biothérapie, CBT-1409, Marseille, France; ${ }^{3}$ Institut Paoli-Calmettes, Unité de Transplantation et de Thérapie Cellulaire, Marseille, France; ${ }^{4}$ Institut Paoli-

Calmettes, Direction du Système d'Information et de l'Organisation, Marseille, France

Background: Given the constant increase in the number of allogenic transplantations carried out in the establishment and the lack of computer tools available on the market, the creation of a software has become essential, in order to optimize the management of the pre-transplant parallel steps for donors and recipients. This allows the multiple actors involved to reduce the risk of oversights or errors and limit the workload of each.

Methods: The main objectives and tools are:

1) Reduce delays in the preparatory process for allogenic transplantation by creating:

- work lists specific to each function, updated in real time,

- automated alerts for non-compliance with deadlines for accomplishing the tasks of each.

2) Facilitate interactions between professionals (internal and external to the institution) through real-time sharing of all information and associated documents.

3) Avoid the loss of information and secure exchanges.

Results: The main benefits observed are:

- increased probability of identifying a donor in a timely manner $(13 \%$ reduction in the frequency of match related donor searches lasting more than 45 days in the first year of use ; figure 1)

- greatly reduced risk that a patient disappears from the active file.

- real-time access to all information, reducing the workload, telephone calls and the work disruption of the various actors,

- archiving of source documents (HLA typing reports for example) allowing online access by all stakeholders,

- reduction of the frequency / duration of meetings between the actors of the process,

- automatic calculation of indicators.

Since the process was optimized prior to the use of this software (3 months reduction in search time for an unrelated donor between 2007 and 2010), the other efficacy indicators have remained stable since the introduction of the software.

Conclusions: The Prep@gref tool represents a prototype of complex care process modeling that can be adapted to piloting other activities in the field of health (autograft for example), or even outside.

The tools available on the market do not allow to track a flow of patients dynamically. The tool developed makes it possible to go beyond the stage of the usual and traditional organization set up in many hospitals to pilot complex care processes. The preallograft process includes 120 steps, not
Figure 1: Frequency of match related donor searches lasting more than 45 days:

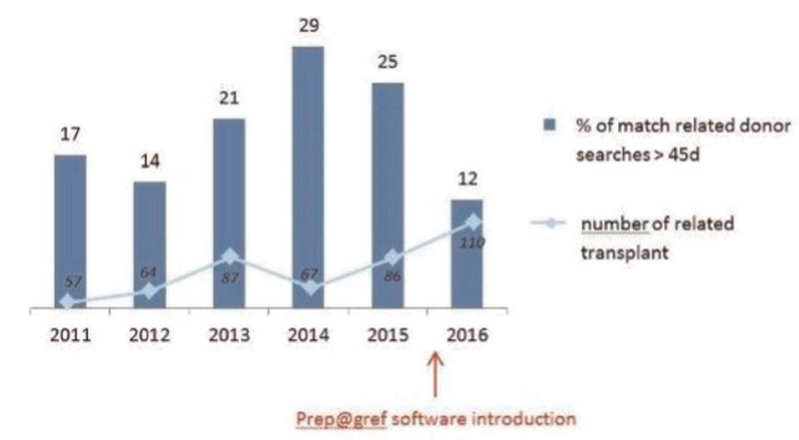

[P818 Figure] [Figure 1: Frequency of match related donor searches lasting more than 45 days:]

systematically performed in the same order. The software adapts in real time, and redefines the patient / donor path according to the response provided at each step (600 possible values). In the first year of use, 10400 actions were identified in the software, carried out by the 20 internal or external actors in the institution.

Conflict of interest: None

\section{P819}

Re-modeling of public cord blood bank inventory in Korea: to enhance cord blood transplantation as well as basic and clinical researches

Young-Ho Lee ${ }^{1}$, Meerim Park ${ }^{2}$,Hani Koh ${ }^{1}$

${ }^{1}$ Hanyang University Medical Center, Department of Pediatrics, Seoul, Korea, Republic of; ${ }^{2}$ Chungbuk National University College of Medicine, Department of Pediatrics, Cheongju, Korea, Republic of

Background: Currently, the utilization rate of cord bloods (CBs) is as low as $2-3 \%$ of stored $\mathrm{CB}$ units in Korea. We tried to remodel the public $\mathrm{CB}$ inventory to enhance the utilization of $\mathrm{CB}$ for clinical cord blood transplantation (CBT) as well as basic and clinical research.

Methods: Data were collected from the 5 public $\mathrm{CB}$ banks and $\mathrm{CB}$ information center as well as the published papers regarding $\mathrm{CBT}$ in Korea. We compared the characteristics of stored and transplanted CB units between the pre-legislation (2000-2010) and post-legislation era (2011. July - 2016. Dec) in Korea. We optimized the public CB inventory based on the current status of CBT and research requirements as well as national budget from Korean government.

Results: Compared with the pre-legislation period, the stored $\mathrm{CB}$ units $>10 \times 10^{8}$ of total nucleated cell (TNC) were increased from $25.5 \%$ to $49.0 \%$ in public CB inventory. The proportion of transplanted $\mathrm{CB}$ units $>10 \times 10^{8}$ of TNC were increased from $52.1 \%$ to $82 \%$. The TNCs of stored/ 
transplanted $\mathrm{CB}$ units were increased from $8.5 \pm 3.0 \times 10^{8}$ \% $10.8 \pm 3.8 \times 10^{8}$ to $10.7 \pm 3.1 \times 10^{8} / 13.7 \pm 4.3 \times 10^{8}$. In the retrospective data analysis from several researches regarding CBT in Korea, median TNC of CBs which were used for CBT was $10.9 \times 10^{8}, 11.9 \times 10^{8}, 12.5 \times 10^{8}, 11.9 \times 10^{8}, 8.2$ $\mathrm{x} 10^{8}$, respectively, according to the patients category. The age of transplanted CBs $(n=479)$ between 2011 and 2016, among stored CBs since 16 yrs ago $(n=39,753)$, was 4.58 $\pm 2.72(0.5-14.2) \mathrm{yrs}$. CBs of $<5 \mathrm{yrs}$ of age were used in $60.3 \%, 5-10 \mathrm{yrs}$ in $34.5 \%$, and $>10 \mathrm{yrs}$ in $5.2 \%$. The requested $\mathrm{CB}$ units for research since post-legislation era were total 5,757 units and about 1,000 units annually. These requirement could be satisfied in the $\mathrm{CB}$ inventory which were $\mathrm{TNC}<10 \times 10^{8}$ and/or $>15$ yrs of age.

Conclusions: Based on these data, we increased the cutoff level of post-processing TNC for upcoming storage of public CBs from $7 \times 10^{8}$ to $10 \times 10^{8}$. Furthermore, we remodeled the public $\mathrm{CB}$ inventory as follows: $\mathrm{CBs}$ of $\mathrm{TNC}>10 \times 10^{8}$ and $<15 \mathrm{yrs}$ of stored age are available for clinical $\mathrm{CBT}$ and $\mathrm{CBs}$ of $\mathrm{TNC}<10 \times 10^{8}$ and/or $>15 \mathrm{yrs}$ of age are for basic or clinical research.

Conflict of interest: Nothing to disclose

\section{P820}

\section{Screening and Management for Clonal Hematopoiesis in Related Allogeneic Donors: An International Survey on behalf of The CIBMTR}

\section{Matthew Seftel ${ }^{1}$, Michelle Formanek ${ }^{2}$, Linda Burns ${ }^{3}$, Pintip Chitphakdithai ${ }^{2}$, Dennis Confer ${ }^{3}$, Deidre Kiefer ${ }^{2}$, Stephanie Lee ${ }^{4}$, Brent Logan $^{5}$, Paul O'Donnell ${ }^{6}$, Michael Pulsipher ${ }^{7}$, Nirali Shah $^{8}$, Galen Switzer, Bronwen Shaw ${ }^{5}$}

${ }^{1}$ University of Manitoba, Internal Medicine, Hematology/Oncology, Winnipeg, Canada; ${ }^{2}$ Center for International Blood and Marrow Transplant Research, Minneapolis, MN, United States; ${ }^{3}$ National Marrow Donor Program, Minneapolis, MN, United States; ${ }^{4}$ Fred Hutchinson Cancer Research Center, Clinical Research, Seattle, WA, United States; ${ }^{5}$ Medical College of Wisconsin, Center for International Blood and Marrow Transplant Research, Milwaukee, WI, United States; ${ }^{6}$ Massachusetts General Hospital, Harvard Medical School, Boston, MA, United States; ${ }^{7}$ Children's Hospital Los Angeles, Los Angeles, CA, United States; ${ }^{8}$ National Cancer Institute - NIH, Bethesda, MD, United States; ${ }^{9}$ University of Pittsburgh, Pittsburgh, $P A$, United States

Background: Hematopoietic Cell Transplantation (HCT) donors must be evaluated for conditions that are transmissible to the intended recipient. There is increasing interest in the risk of transferring potentially serious non-infectious syndromes, in particular clonal hematopoiesis $(\mathrm{CH})$. It is unknown to what extent HCT centres screen for $\mathrm{CH}$ in blood or marrow from related donors (RDs), and if found, how such findings are managed. We conducted a survey to better understand: (i) the process that HCT centers utilize to

\begin{tabular}{|l|r|r|l|}
\hline & $\begin{array}{c}\text { Responders } \\
(n=280)\end{array}$ & $\begin{array}{c}\text { Non- } \\
\text { Responders } \\
(n=329)\end{array}$ & p-value \\
\hline Donor Age & & & $<0.001$ \\
\hline Adult donors only & $98(35)$ & $88(27)$ & \\
\hline Pediatric donors & $51(18)$ & $35(11)$ & \\
\hline Both of the above & $131(47)$ & $206(63)$ & \\
\hline Geographic Region & & & $<0.001$ \\
\hline North America (US/Canada) & $131(47)$ & $107(33)$ & \\
\hline International & $149(53)$ & $222(67)$ & \\
\hline Center Size & $147(53)$ & $227(69)$ & \\
\hline Less than 30 & $20(7)$ & $31(9)$ & \\
\hline $31-50$ & $47(17)$ & $25(8)$ & \\
\hline $51-99$ & $53(19)$ & $41(12)$ & \\
\hline $100-299$ & $13(5)$ & $5(2)$ & \\
\hline 300 and greater & & & \\
\hline
\end{tabular}

tThe Pearson chi-square test was used for comparing discrete variables

[P820 Figure] [Selected Characteristics for Survey Responders and Non-responders]

identify RDs who may have $\mathrm{CH}$; (ii) whether such RDs would be excluded from donation.

Methods: We sent a confidential online survey to medical directors of all CIBMTR-registered HCT centres that evaluate RDs. The survey consisted of questions that prompted respondents about their approach to assessing otherwise healthy RDs with a normal complete blood count (CBC). For HCT centres with adult and pediatric programs, a separate response from both the adult and pediatric program directors was requested.

Results: Of the 609 survey requests that were sent, we received 280 responses (46\%). Figure 1 summarizes differences between responding and non-responding centres. $86.5 \%$ of respondents collected both bone marrow $(\mathrm{BM})$ and peripheral blood (PB) hematopoietic cells. Most centers collected cells from adults (32\%) or from both adults and children (59\%). 63\% of centers were FACT/ JACIE accredited. Lower volume HCT centers $(<30$ HCTs/ year) constituted $51 \%$ of the respondents.

The majority of HCT centers reported that they do not routinely screen RDs for $\mathrm{CH}$. However, routine peripheral blood testing for $\mathrm{CH}$ is most frequently performed for monoclonal gammopathy (20.2\% of respondents), monoclonal B-cell lymphocytosis [MBL] (5.3\%), and gene sequencing for somatic mutations (3.8\%). Routine BM aspirates are performed by $6 \%$ of respondents, with an additional $6.7 \%$ performing this test in specific sub-groups of RDs, especially in older adults. Routine BM aspirates are more frequently performed at lower volume HCT centers vs. larger centers (17.2\% vs. $9.2 \%$ of respondents). Besides standard morphological assessment, the most common routine tests on $\mathrm{BM}$ are cytogenetics $(57.7 \%)$, MBL (23.1\%) and somatic mutation testing (12\%). Respondents recalled detecting at least one case of the following abnormities during routine work-up of RDs: Monoclonal gammopathy (17.8\%), MBL (9.9\%), and clonal cytogenetic abnormalities (7.7\%). Although $>50 \%$ of respondents would defer a donor with these findings, a significant 
minority would not defer or were uncertain about whether to defer the donor: Deferral was definitely recommended in $62 \%$ of respondents for monoclonal gammopathy, $60.4 \%$ for MBL, $74 \%$ for clonal cytogenetics, and $62.8 \%$ for somatic mutations.

Conclusions: In otherwise healthy RDs with a normal $\mathrm{CBC}$, a small but appreciable number of HCT centres actively look for $\mathrm{CH}$ by $\mathrm{PB}$ or BM testing. Routine $\mathrm{BM}$ aspirates are performed in RDs at some HCT centers, especially in older RDs and in those RDs at lower volume HCT centers. However, there is considerable uncertainty about whether the detection of $\mathrm{CH}$ in the $\mathrm{PB}$ and $\mathrm{BM}$ constitutes grounds for donor exclusion. We recommend the development of international guidelines and standardization specific to the evaluation and management of potentially transmissible disorders of clonal hematopoiesis in donors.

Clinical Trial Registry: N/A

Conflict of interest: nothing to disclose

\section{P821}

\section{SECOND TRANSPLANTS FOR PRIMARY GRAFT FAILURE, FOLLOWING HAPLOIDENTICAL GRAFTS WITH POST- TRANSPLANT CYCLOPHOSPHAMIDE}

\section{Andrea Bacigalupo $^{1}$, Anna Maria Raiola ${ }^{2}$, Sabrina Giammarco ${ }^{1}$, Federica Sora' ${ }^{\prime}$, Carmen Di Grazia ${ }^{2}$, SImona Sica ${ }^{1}$, Francesca Gualandi $^{2}$, Riccardo Varaldo ${ }^{2}$, Emanuele Angelucci ${ }^{2}$}

${ }^{1}$ Universita' Cattolica, Policlinico Gemelli, Istituto di Ematologia, Roma, Italy; ${ }^{2}$ IRCCS AUO IST- San Martino, Divisione Ematologia, Genova, Italy

Background: Rejection, following haploidentical transplants (HAPLO) with post-transplant high dose cyclophosphamide (PT-CY) has been described and has been associated with donor specific antibodies (DSA) (BMT 2015;21:1392).

Aim of the study: To assess the incidence of rejection in patients with hematologic malignancies, prepared with a myeloablative conditioning regimen and the outcome of a second transplant.

Methods: We studied 403 patients with hematologic malignancies, receiving an unmanipulated HAPLO marrow graft. Median age was 52 (17-74) and 38\% had advanced disease. All patients received GvHD prophylaxis with Cyclosporin, mycophenolate and PTCY. The conditioning regimen used, was one of the following three:

\# Fludarabine (FLU) $40 \mathrm{mg} / \mathrm{m}^{\wedge} 2 \times 3+$ Total body irradiation (TBI) 9.9-12 Gy $(\mathrm{n}=75)$

\# Thiotepa (THIO) $5 \mathrm{mg} / \mathrm{kgx} 2$, busulfan (BU) $3.2 \mathrm{mg} / \mathrm{kg} /$ dayx3, FLU $50 \mathrm{mg} / \mathrm{m}^{\wedge} 2 \times 3(\mathrm{TBF})(\mathrm{n}=180)$

\# THIO $5 \mathrm{mg} / \mathrm{kgx} 2$, BU $3.2 \mathrm{mg} / \mathrm{kg} /$ dayx2, FLU $50 \mathrm{mg} /$ $\mathrm{m}^{\wedge} 2 \times 3(\mathrm{n}=148)$.
Results: Rejection was seen in $0 / 75$ patients after TBI (0\%), 5/180 after TBF with 3 days of Busulfan $(2,7 \%)$ and in 10/148 in patients receiving TBF with 2 days of Busulfan $(p=0.02)$. We could not find a correlation of rejection with donor age, patients age, diagnosis, phase of the disease, $\mathrm{ABO}$ mismatch, or bone marrow cell dose infused.

Busulfan. We did find a trend for more rejections in Rome $(6,8 \%)$, where BU is given on 2 divided daily doses (q12 h), as compared to Genova (3,2\%), where BU is given in a single daily dose, (q24h): rejection with full dose TBF (BU3 days) was 3/47 for patients receiving BU q12h (6.38\%), vs $2 / 133(1,50 \%)$ for BU given $\mathrm{q} 24 \mathrm{~h}(\mathrm{p}=0.08)$.

Second transplant: 15 patients failed to recover neutrophils by day +28 , and were found to have $100 \%$ recipient chimerism. Of these 15 patients, 1 is not evaluable, since he received a second graft only 10 days ago. A second patient was infused with CD34 selected cells from the same donor, without a conditioning regimen, and died without recovery, after having failed a third transplant.

13 patients received a second graft (at a median interval of 43 days, range 33-66) with the Baltimore protocol and PB from the same donor $(\mathrm{n}=10)$ or another family member $(\mathrm{n}=$ 3). GvHD prophylaxis was again CyA and PTCY. 11/13 had trilineage recovery (85\%) and became $100 \%$ donor: a neutrophil count of $0.5 \times 10^{\wedge} 9 / \mathrm{L}$ was reached on day 18 (1621) and a platelet count of $20 \times 10^{\wedge} 9 / \mathrm{L}$ on day 35 (18-80). Acute GvHD occurred in 6 patients (grade I)(46\%) and in 1 patient grade II (8\%). Moderate-severe chronic GvHD developed in 3/13 patients (23\%). One year actuarial survival of the 13 patients who received a second graft is $85 \%$.

\section{Conclusions:}

(a) the risk of rejection is dependent on the intensity of the conditioning regimen : it does not occur after TBI, and is low with full dose TBF (2.7\%);

(b) there is a trend for more rejection with Busulfan given q12h;

(c) full engraftment can be achieved with a second transplant, in $85 \%$ of patients rejecting. Mortality due to rejection, in this series of 403 patients, was $0.75 \%$.

Conflict of interest: the authors declare no conflict of interest

\section{P822}

The Effect of Sex-Mismatch on Pediatric Allogeneic Hematopoietic Stem Cell Transplantation Outcome: Optimal Donor Selection

Nariman Hariss, Khaled M. Ghanem, Radwan Massoud, Nabila Kreidieh, Rita Nehme, Samar Muwakkit, Raya Saab, Nidale Tarek, Dima Hamideh, Miguel R. Abboud 
Mauricio Sarmiento, Veronica Jara, Carla Lorca, Isabel Pizarro, Pablo Bertin, Mauricio Galleguillos, Isabel Rodriguez, Mauricio Ocqueteau, Jaime Pereira

Background: Donor selection is considered a key element in improving the outcomes of hematopoietic stem cell transplantation (HSCT). Sex-mismatched HSCT has been linked to increased graft-versus-host disease (GVHD), transplant-related mortality (TRM) and overall mortality in adults. No current consensus is present regarding this effect on the outcome of HSCT in children and adolescents.

Methods: Retrospective chart review was conducted on 70 patients aging less than 21 years who underwent allogeneic HSCT from related fully-matched donor at the American University of Beirut between 2005 and 2016. The effect of sex-mismatch on TRM, rejection, relapse, acute GvHD, chronic GvHD was done using cox-regression model.

Results: Donor- recipient sex combination was categorized as follows: $24.3 \%(\mathrm{~N}=17)$ were male donors to male recipients $(\mathrm{M}-\mathrm{M}), 28.6 \%(\mathrm{~N}=20)$ were male donors to female recipients $(\mathrm{M}-\mathrm{F}), 25.7 \%(\mathrm{~N}=18)$ were female donors to female recipients $(\mathrm{F}-\mathrm{F})$, and $21.4 \%(\mathrm{~N}=15)$ were female donors to male recipients (F-M). Transplant was done for the following diseases: leukemia $(\mathrm{N}=35)$, lymphoma $(\mathrm{N}=2)$, hemoglobinopathies $(\mathrm{N}=9)$, inherited immune system disorders $(\mathrm{N}=8)$, inherited metabolic disorders $(\mathrm{N}=1)$, familial hemophagocytic lymphohistiocytosis $(\mathrm{N}=3)$, bone marrow failure diseases $(\mathrm{N}=12)$. Stem cell source was bone marrow for 56 patients (80\%) and mobilized peripheral blood stem cells for 14 patients (20\%). After a median follow-up of 47 months (range: 4 135 months), the 2-year overall survival rate was $70 \%(95 \%$ CI: 57 - 79\%). Using univariate analysis, sex-mismatch had no effect on overall mortality (HR: 0.978, P: 0.905), TRM (HR: 0.837, P: 0.463), relapse (HR: 0.793, P: 0.474), rejection (HR: 0.608, P: 0.403), acute GvHD (HR: 1.563, P: 0.473 ) and chronic GvHD (HR: 0.961, P: 0.956). With subgroup analysis, neither female-to-male nor male-female transplants were predictors for worse survival, rejection, acute or chronic GvHD.

Conclusions: Sex-mismatch has no effect on the major outcomes of allogeneic matched-related HSCT in our pediatrics population. Further larger studies are needed for confirmation.

Conflict of interest: No relevant conflict of interest Stem cell mobilization, collection and engineering

\section{P823}

\section{A PLERIXAFOR JUST IN TIME BASED PROTOCOL RESULTS IN SUCCESFULL CD34 COLLECTIONS AND ALLOWS AUTOLOGOUS STEM CELL TRANSPLANTATION}

PUC, Santiago, Chile

Background: Autologous hematopoietic stem cell transplantation is a proven therapy for several diseases. To perform it, an adequate harvest of stem cells is required. In patients that are poor mobilizers the collection of stem cells can be a challenge, because they do not achieve satisfactory CD34 cell counts with GCSF + - chemotherapy. Plerixafor is a potent drug that promotes the release of stem cells from bone marrow niche to peripheral blood and allows satisfactory harvests but at a high cost.

Methods: We conducted a retrospective analysis of the harvests performed in patients treated with autologous transplantation in our institution.

Results: 84 patients, mainly with myeloma and lymphoma, there were no harvest failures when using plerixafor in $50 \%$ of them. Satisfactory harvests were obtained in patients mobilized with GCSF or plerixafor. In patients who used plerixafor, it was necessary to perform less apheresis ( $\mathrm{p}=0.05$ ). In multivariate analysis, the only factor that predicted the need for plerixafor was the presence of less than 30,000 CD34 / ul at the day of the apheresis (OR 0.3.p $<0.001)$.

Conclusions: We can conclude that the rational and standardized use of plerixafor favors satisfactory harvests in patients who require autologous transplantation.

Conflict of interest: nothing to disclose

\section{P824}

A single-center retrospective multivariate analysis of factors influencing collecting efficiency in a population of adult patients and donors undergoing apheresis over a calendar year

Anne-Line Chateau ${ }^{1,2}$, Claude Lemarie ${ }^{1,2}$, Patrick Sfumato ${ }^{3}$, Boris Calmels $^{1,2}$, Ilhem Rahal, Angela Granata ${ }^{4}$, Catherine Faucher ${ }^{4}$, Christian Chabannon ${ }^{1,2,5}$

${ }^{1}$ Institut Paoli-Calmettes, Centre de Thérapie Cellulaire, Marseille, France; ${ }^{2}$ Inserm CBT-1409, Centre d'Investigations Cliniques en Biothérapies de Marseille, Marseille, France; ${ }^{3}$ Institut PaoliCalmettes, Department of biostatistics, Marseille, France; ${ }^{4}$ Institut Paoli-Calmettes, Department of Hematology, Transplantation Program, Marseille, France; ${ }^{5}$ Aix Marseille University, Marseille, France

Background: Apheresis is a technique with wide usage in a selective collection or depletion of various blood components for therapeutic purposes. In the context of autologous and allogeneic hematopoietic cell transplantation, "peripheral blood stem cells" (PBSC) nowadays 
represent the exclusive and most widely used source of stem cells respectively. The graft quality can be evaluated through flow cytometric enumeration of collected CD34+ cells, and thereafter by their clonogenecity. The "collection efficiency" or CE is a gross estimate of the proportion of circulating CD34 + cells harvested by the separator and can be considered as a measure of the performance of the procedure. The goal of this study was to identify patientrelated or procedural factors that could influence CE.

Methods: A total of 350 first apheresis procedures performed on 139 donors and 211 patients carried out over calendar year 2016 were retrospectively studied. Factors investigated included patient-related criteria (age, sex, body mass index, disease status, collection type, mobilisation, pre-apheresis biological parameters, pre-procedure CD34+ cell concentration) and procedural criteria (operator, continuous or discontinuous mononuclear cell collection, total processed blood volume, number of processed blood masses). Statistical relationships between $\mathrm{CE}$ and various characteristics were determined by multiple linear regression analysis.

Results: The median value for CE was $51,1 \%$ for all individuals, $54,1 \%$ for donors and $48,7 \%$ for patients respectively $(\mathrm{p}=0,001)$. By stepwise multiple regression analysis, disease status had no significant impact on $\mathrm{CE}$ value. Conversely, procedural criteria and patient-related criteria may explain the variability of CE. In our study, we demonstrate that $\mathrm{CE}$ value is stable whatever operator or type of cell collection considered. Total processed blood volume is significantly correlated to CE (beta $=0,016, \mathrm{p}=$ $0,028)$. For allogeneic aphereses, pre-apheresis leucocyte counts (beta $=-0,006, \mathrm{p}=0,005$ ), haemoglobin (beta $=$ $0,0568, p=0,003$ ), and percentage of CD34 cells (beta $=$ $-0,509, \mathrm{p}=0,036)$ are independent predictive factors affecting CE. For autologous aphereses, leucocyte counts (beta $=-0,028, p<0,0001$ ), neutrophils counts (beta $=$ $-0,025, \mathrm{p}=0,0004$ ), hematocrit (beta $=0,014, \mathrm{p}=0,005$ ), and introduction of plerixafor (beta $=0,1998, p=0,009$ ) significantly contribute to variability of $\mathrm{CE}$ value.

Conclusions: $\mathrm{CE}$ can be used as a criteria to monitor the efficiency and performance of apheresis procedures over time. Value of CE is dependent of pre-apheresis biological factors that must be considered independently in allogenic or autologous procedures, due to clinical and biological differences between these two populations. A better inclusion of pre-apheresis biological parameters can be considered in order to limit the variability of PBSC collection.

Conflict of interest: No conflict of interest
P825

ABO-mismatched bone marrow processing for transplantation: results of 17 procedures performed with Spectra Optia ${ }^{\circledR}$ device

Gala Aglaia Méndez ${ }^{1}$, Cristina Amunárriz ${ }^{2}$, José Luis Arroyo ${ }^{2}$

${ }^{1}$ Hospital Universitario Marqués de Valdecilla, Santander, España, Hematology, Santander, Spain; ${ }^{2}$ Banco de Sangre y Tejidos de Cantrabria, Liencres, Spain

Background: In ABO-mismatched bone marrow (BM) transplant, red blood cell (RBC) removal in essential to minimize the risk of inmunohematological complications. The maximum volume of mismatched RBCs that can be safely infused in not clearly defined and practice differs between hospitals, although it is generally accepted that $<$ $0.2-0.4 \mathrm{ml} / \mathrm{kg}$ recipient bodyweight can be tolerated without significant acute haemolysis [1].

Different techniques have been used to reduce RBCs while maximizing $\mathrm{CD} 34+$ stem cells recovery, as apheresis devices. For instance, the Cobe Spectra ${ }^{\circledR}$ separator has been utilized succesfully for years until its withdrawal from the market and replacement by the Spectra Optia ${ }^{\circledR}$. We have evaluated the Spectra Optia ${ }^{\circledR}$ apheresis system to determine its effectiveness in ABO-incompatible BM processing.

Methods: We analized ABO-incompatible processed BM transplants performed in our center since 2015 (date of Optia device implementation in our practice). Samples were processed using the Spectra Optia separator ${ }^{\circledR}$ as directed by the manufacturer, running in the semi-automatic RBC depletion protocol with an initial collection preference of 50. The entire BM volume was processed on average, 4.7 times on 90 minutes, with a typical flow velocity of 100 $120 \mathrm{ml} / \mathrm{min}$.

Volume reduction, RBC depletion and recovery of total nucleated cells (TNCs), mononuclear cells (MNCs), CD34 +cells and CD3+cells were calculated and hematopoietic engraftment was assesed. Data was analyzed with IBM SPSS software ${ }^{\circledR}$, version 24 . Results are expressed as mean \pm standard desviation and as median (range) for engraftments.

Results: 17 samples were included, all of them allogeneic fresh $\mathrm{ABO}$-incompatible $\mathrm{BM}$ transplants. All processes proceeded successfully, without technical incidents or serious post infusion adverse effects.

The mean volume collected was $1363.3 \mathrm{ml} \pm 155.7$ and it was reduced to $130.7 \mathrm{ml} \pm 43.9$ after processing. Inicial and residual mean RBCs volumes were $435 \mathrm{ml} \pm 71$ and $6.3 \mathrm{ml}$ \pm 2.2 , respectively, corresponding to a depletion of $98.5 \pm$ $0.5 \%$. Residual RBC volumes were no larger than $0.14 \mathrm{ml} /$ $\mathrm{kg}$ (mean: $0.09 \mathrm{ml} / \mathrm{kg} \pm 0.03$; maximum 0.13 ); thus, all cases 
were below the maximal recomended volume of $0,2-0.4 \mathrm{ml} /$ $\mathrm{kg}$.

Mean percentage of recovery was $64.6 \% \pm 22.5$ for TNCs, $108.3 \% \pm 38.6$ for MNCs and $106.4 \% \pm 28.3$ for CD34 cells. Cell recoveries were, therefore, optimal and in line with previous reports [2]; full data is shown in Table 1.

Median engraftment for neutrophils, platelets and RBCs was 18 (10-27), 22 (13-109) and 37 (1-64) respectively. All patients but two engrafted (one due to toxic mortality and the other to early relapse).

Conclusions: Spectra Optia ${ }^{\circledR}$ is an efficient device for $\mathrm{RBC}$ and volumen depletion in $\mathrm{ABO}$-incompatible $\mathrm{BM}$ transplants, while providing excellent $\mathrm{CD} 34+$ recovery in clinical routine.

\section{References:}

1. Daniel-Johnson J et al. Transfusion 2011; 51: 11431149.

2. Kim-Wanner SZ et al. Journal of Translational Medicine 2017; 15: 174

Conflict of interest: None of the authors has disclosed any conflicts of interest.

\begin{tabular}{llll}
\hline & Inicial BM & Post-processing BM & Recovery $(\%)$ \\
\hline TNCs $\left(10^{\wedge} 8\right)$ & $413.3 \pm 283.1$ & $237.5 \pm 134.5$ & $64.6 \pm 22.5$ \\
MNCs $\left(10^{\wedge} 8\right)$ & $52.1 \pm 24.8$ & $57.6 \pm 42.1$ & $108.3 \pm 38.6$ \\
CD34 cells $\left(10^{\wedge} 6\right)$ & $262.4 \pm 139$ & $267.7 \pm 138.1$ & $106.4 \pm 28.3$ \\
CD3 cells $\left(10^{\wedge} 7\right)$ & $260.5 \pm 116.7$ & $275.5 \pm 144.9$ & $94.7 \pm 28.5$ \\
\hline
\end{tabular}

\section{[[P825 Table] Table 1]}

\section{P826}

\section{Allogeneic Peripheral blood stem-cell harvest using percutaneous arterial lines in adults}

Dana Ashkenazi Lustig ${ }^{1,2}$, Michal Pearl-Yafe ${ }^{3}$, Limor HorevAzaria $^{3}$, Yakov Sverdlov ${ }^{3}$, Lili Cabas ${ }^{4}$, Anat Yahel ${ }^{1}$, Bracha Zisser ${ }^{4}$, Isaac Yaniv ${ }^{1,2}$, Jerry Stein ${ }^{1,2}$

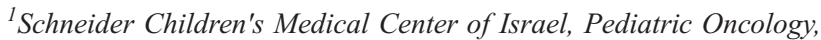
Bone Marrow Transplant Unit, Petah Tikva, Israel; ${ }^{2}$ Tel Aviv University, Sackler Faculty of Medicine, Tel Aviv, Israel; ${ }^{3}$ Schneider Children's Medical Center of Israel, Bone Marrow Transplant Lab, Petah Tikva, Israel; ${ }^{4}$ Schneider Children's Medical Center of Israel, Ezer Mizion, Petah Tikva, Israel

Background: Adequate vascular access is a prerequisite to successful Peripheral Blood Stem Cell Collection (PBSCC). In adults with "difficult" veins and in children, central venous catheters have been used for PBSCC, but placement of these lines is fraught with complications that can sometimes be severe. We have previously reported a large series of children who underwent PBSCC using radial artery cannulation. We now report the use of this procedure for allogeneic donations in adults with "difficult" veins who were donating PBSCC.

Methods: Data from adult peripheral allogeneic stem cell collections performed from January 2013 to December 2017 using radial arterial lines were collected. Lines were inserted percutaneously using local anesthetic by physicians trained in this procedure.

Results: Nineteen APBSCC procedures (15 for unrelated donors and four for family donors) were performed during the study period using an arterial line. The average donor age and weight were 22 years and $57 \mathrm{~kg}$, respectively. The average total volume processed was $3699 \mathrm{ml}$, and the average number of blood volumes processed was 3.26. Average collection time for a single blood volume was 48 minutes. The average number of CD34+ cells collected per donation was $6 \times 10(6) / \mathrm{kg}$. Three donors $(18 \%)$ required more than one collection to meet the requested CD34+ cell target. No serious adverse effects associated with vascular access occurred in this cohort, although a hematoma (not requiring treatment) developed in one donor. Donor satisfaction with the procedure was high, as collection and return lines were inserted in the same arm, and as the collection could proceed in a timely fashion without undue alarms caused by high pressure in the collection line.

Conclusions: Percutaneous radial artery catheters are an easy, rapid, safe alternative to the placement of central venous catheters in adults donating PBSCC for allogeneic transplant.

Conflict of interest: nothing to disclose

\section{P827}

Analysis of Data Collected in the European Group for Blood and Marrow Transplantation (EBMT) Registry on a Cohort of Myeloma Patients Receiving Plerixafor

Curly Morris ${ }^{1}$, Christian Chabannon ${ }^{2}$, Tomas Masszi ${ }^{3}$, Nigel Russell $^{4}$, Hareth Nahi ${ }^{5}$, Guido Kobbe ${ }^{6}$, Marta Krejci ${ }^{7}$, Holger Auner $^{8}$, David Pohlreich ${ }^{9}$, Patrick Hayden ${ }^{10}$, Grzegorz, W Basak ${ }^{11}$, Stig Lenhoff ${ }^{2}$, Nicolaas Schaap ${ }^{13}$, Anja van Biezen ${ }^{14}$, Cora Knol ${ }^{14}$, Simona Lacobelli ${ }^{15}$, Qianying Liu ${ }^{16}$, Marina Celanovic ${ }^{16}$, Laurent Garderet $^{17}$, Nicolaus Kröger ${ }^{18}$

${ }^{1}$ Queens University, Belfast, United Kingdom; ${ }^{2}$ Institute Paoli Calmettes, Marseille, France; ${ }^{3}$ Semmelweiss University, Budapest, Hungary; ${ }^{4}$ Nottingham University, Nottingham, United Kingdom; ${ }^{5}$ Karolinska University Hospital, Stockholm, Sweden; ${ }^{6}$ University Hospital of Dusseldorf, Dusseldorf, Germany; ${ }^{7}$ University Hospital, Brno, Czech Republic; ${ }^{8}$ Hammersmith Hospital, London, United Kingdom; ${ }^{9}$ Charles University Hospital, Prague, Czech Republic; ${ }^{10} \mathrm{St}$ James Hospital, Dublin, Ireland; ${ }^{11}$ Medical University of Warsaw, Warsaw, Poland; ${ }^{12}$ Skanes University Hospital, Lund, Sweden;

${ }^{13}$ Radboud University Medical Centre, Nijmegen, Netherlands;

${ }^{14}$ European Bone Marrow Transplantation Society, Leiden,

Netherlands; ${ }^{15}$ University Tor Vergata, Rome, Italy; ${ }^{16}$ Sanofi Genzyme, 
Cambridge, MA, United States, ${ }^{17}$ Hospital Saint Antoine, Paris, France; ${ }^{18}$ University Hospital, Hamburg, Germany

Background: Plerixafor plus granulocyte colony stimulating factor (G-CSF) enhances the mobilisation of haematopoietic stem cells (HSCs) for collection and subsequent autologous haematopoietic stem cell transplantation (HSCT) in patients with multiple myeloma (MM) who are poor mobilisers of HSCs.

Methods: This international, multicentre, noninterventional registry study (NCT01362972), with 3.5 to 7.5 years follow-up, evaluated the long-term outcomes for MM patients who received plerixafor compared with other HSC mobilisation regimens. Patients achieved their first autologous HSCT between 2008 and 2012. Patients who did not receive a transplant were not included in the analyses. The mobilisation comparisons were: G-CSF plus plerixafor $(\mathrm{G}-\mathrm{CSF}+\mathrm{P})$ versus $\mathrm{G}-\mathrm{CSF}$ alone (comparison 1); $\mathrm{G}-\mathrm{CSF}+\mathrm{P}$ versus $\mathrm{G}-\mathrm{CSF}$ plus chemotherapy $(\mathrm{G}-\mathrm{CSF}+\mathrm{C})$ (comparison 2); and G-CSF $+\mathrm{P}$ plus chemotherapy (G-CSF $+\mathrm{P}+\mathrm{C}$ ) versus $\mathrm{G}-\mathrm{CSF}+\mathrm{C}$ (comparison 3 ). Primary outcomes were progression free survival (PFS), overall survival (OS) and relapse rates (RR) from transplantation. Patients who failed previous mobilisation attempts were defined as proven poor mobilisers.

Results: Overall, 3566 patients met the eligibility criteria for the study, with 260 patients receiving plerixafor. Baseline characteristics between cohorts were balanced using propensity score matching. After propensity analysis, 77 versus 41 patients were matched in the $\mathrm{G}-\mathrm{CSF}+\mathrm{P}$ versus G-CSF alone cohort, 129 versus 129 in the G-CSF+P versus $\mathrm{G}-\mathrm{CSF}+\mathrm{C}$ cohort and 117 versus 117 in the G-CSF $+\mathrm{P}+\mathrm{C}$ versus $\mathrm{G}-\mathrm{CSF}+\mathrm{C}$ cohort. Propensity score matching resulted in a loss of statistical power and all imbalances in the comparison arms were not overcome by propensity scoring. There were more proven poor mobilisers in the plerixafor cohorts versus the comparator cohorts. Proven poor mobilisers were 77/77 (100\%) G-CSF+P versus 35/41 (85.4\%) G-CSF alone, 128/129 (99.2\%) G-CSF+P versus 93/129 (72.1\%) $\mathrm{G}+\mathrm{C}$ and 115/117 (98.3\%) G-CSF+P+C versus 85/117 (72.6\%) G-CSF+C. The estimated median PFS for the G-CSF+P cohort was 19.6 months $(95 \% \mathrm{CI}$ : 15.5, 25.9 months) versus 28.6 months (95\% CI: 12.2 , 42.8 months) for G-CSF alone cohort. PFS for the G-CSF $+\mathrm{P}$ cohort was 19.3 months (95\% CI: 16.7, 24.3 months) versus 25.4 months (95\% CI: 22.5, 28.9 months) for the G$\mathrm{CSF}+\mathrm{C}$ cohort and PFS for the G-CSF+P+C cohort was 25.1 months (95\% CI: 19.7, 29.9 months) versus 26.3 months (95\% CI: 20.5, 31.7 months) for the G-CSF $+\mathrm{C}$ cohort. Kaplan-Meier curves showed a trend towards lower PFS and OS in the plerixafor cohorts versus G-CSF comparators. RR rates were higher in the plerixafor cohorts.
The proportion of patients with any adverse event was similar between the plerixafor and comparator cohorts.

Conclusions: This registry study showed that mobilisation issues in patients who are difficult to mobilise can be overcome by plerixafor treatment. Some patients in the study may not have received a transplant without plerixafor. The cohorts treated with plerixafor had shorter PFS and OS times and higher RR with similar safety than their respective comparators. No firm conclusions can be made from the study due to imbalances between the cohorts, with more proven poor mobilisers in the plerixafor cohort possibly selecting patients with worse outcomes. The absence of information on genetic risk and maintenance treatment, and the observational nature of the study are further limitations of the study.

Clinical Trial Registry: NCT01362972

Conflict of interest: This study was funded by Sanofi Genzyme.

N. Kröger: reports research grants from EBMT and Sanofi and honoraria from Sanofi.

L. Garderet: reports advisory boards for Amgen and Takeda.

C. Chabannon: reports grants from EBMT during the study as well as grants, personal fees and non-financial support from Sanofi outside of the submitted work.

T. Masszi: reports advisory board membership for AbbVie, BMS, Janssen-Cilag, Novartis, Pfizer, and Takeda, outside the submitted work.

G. Kobbe: reports grants from EBMT during the study.

P. Hayden: reports receiving Sanofi support for a meeting in 2012.

G. Basak: reports personal fees from EBMT during the study, as well as personal fees and non-financial support from Sanofi/Genzyme outside the submitted work.

Q. Liu and M. Celanovic are Sanofi employees.

The remaining authors have nothing to disclose.

\section{P828}

\section{Bone Marrow Processing - COBE 2991 vs Spectra Optia}

Ana Lúcia Barbosa, Carla Espadinha, Maria João Gutierrez, Elisabete Pereira, Manuel Abecasis

Instituto Português de Oncologia de Lisboa Francisco Gentil, EPE, BMT Unit, Lisbon, Portugal

Background: Adult bone marrow (BM) harvests are typically in the order of 1-2L in volume (smaller volumes if paediatrics harvests) and contain a high number of red cells. These volumes represent a clinical risk to the patient in terms of fluid and lysed red cell load and are impractical to cryopreserve. For these reasons, bone marrow harvests are processed through a cell separator to yield a reduced volume, 


\begin{tabular}{|c|c|c|c|c|c|c|c|c|}
\hline & \multicolumn{2}{|c|}{ TNC recovery efficiency $(\%)$} & \multicolumn{2}{|c|}{ CD34+ recovery efficiency $(\%)$} & \multicolumn{2}{|c|}{ RBC volume reduction (\%) } & \multicolumn{2}{|c|}{ Total volume reduction (\%) } \\
\hline & $\begin{array}{l}\text { COBE } 2991 \\
(\mathrm{~N}=35)\end{array}$ & $\begin{array}{l}\text { SPECTRA OPTIA } \\
(\mathrm{N}=(8)\end{array}$ & $\begin{array}{l}\text { COBE } 2991 \\
(\mathrm{~N}=35)\end{array}$ & $\begin{array}{l}\text { SPECTRA OPTIA } \\
(\mathrm{N}=(8)\end{array}$ & $\begin{array}{l}\text { COBE } 2991 \\
(\mathrm{~N}=35)\end{array}$ & $\begin{array}{l}\text { SPECTRA OPTIA } \\
(\mathrm{N}=(8)\end{array}$ & $\begin{array}{l}\text { COBE } 2991 \\
(\mathrm{~N}=35)\end{array}$ & $\begin{array}{l}\text { SPECTRA OPTIA } \\
(\mathrm{N}=(8)\end{array}$ \\
\hline Mean & 89.61 & 57.97 & 86.79 & 88.02 & 82.49 & 98.46 & 75.74 & 89.98 \\
\hline $\begin{array}{l}\text { Standard } \\
\text { Deviation }\end{array}$ & 11.82 & 17.46 & 13.40 & 15.67 & 11.99 & 0.23 & 8.79 & 0.88 \\
\hline Minimum & 64.31 & 34.80 & 51.49 & 63.78 & 29.08 & 98.12 & 42.40 & 88.35 \\
\hline Maximum & 109.32 & 86.76 & 108.73 & 107.09 & 94.68 & 98.83 & 88.14 & 91.63 \\
\hline
\end{tabular}

[[P828 Table] Results of BM processing]

stem cell enriched buffy coat depleted in red blood cells and plasma. Buffy coat preparation is also indicated for fresh infusions in the allogeneic setting, if there is a donor>recipient $\mathrm{ABO}$ incompatibility (mandatory if a major ABO incompatibility). In this work we aim to compare the efficiency of two cell separators: COBE 2991 vs Spectra Optia.

Methods: In a retrospective study, all BM processed in our Institute between January 2011 and December 2017 were divided in two groups, according to the equipment used. The efficiency of the process was assessed by total nucleated cells (TNC) recovery, CD34+ cell recovery, red blood cell (RBC) reduction and total volume reduction. All $\mathrm{BM}$ were analysed pre and post processing regarding TNC, $\mathrm{CD}^{+} 4^{+}$cells, $\mathrm{RBC}$ and volumes.

Results: In the last 7 years, $43 \mathrm{BM}$ required processing: $35(81 \%)$ were performed on COBE 2991 and 8 (19\%) on Spectra Optia. From data analysed, Spectra Optia presented a mean of TNC recovery lower than COBE 2991, but that lower TNC recovery was mainly due to the loss of neutrophils and eosinophils, which do not have importance in the transplant context. Regarding CD34+ cell recovery, the results were similar using both cell separators. Spectra Optia presented a higher RBC volume reduction and that value remained with a rate of $98 \%$ among all procedures, while using COBE 2991 that value was variable and depended of the operator decision to collect more or less $\mathrm{RBC}$. For this reason, the mean of total volume reduction was also higher with Spectra Optia utilization. Comparative results are presented in the table below.

Note: Values up to $110 \%$ were accepted considering the cell counting errors. Values above $110 \%$ were excluded.

Conclusions: Compared with COBE 2991, Spectra Optia appears to present some advantages regarding bone marrow processing, especially in the RBC volume reduction, which is very important in an allogeneic transplant with a major donor>recipient ABO incompatibility. Spectra Optia is also less time consuming. Both equipments presented a good and similar $\mathrm{CD} 34^{+}$cell recovery.

Conflict of interest: Nothing to disclose.

\section{P829}

CD34-positive monocytes mobilized by G-CSF in donor peripheral blood and clinical outcomes after allogeneic hematopoietic cell transplantation from related donors

Hideki Nakasone, Misato Kikuchi, Yu Akahoshi, Koji Kawamura, Yuko Ishihara, Miki Sato, Kiriko Terasako-Saito, Kazuki Yoshimura, Yukiko Misaki, Ayumi Gomyo, Jin Hayakawa, Masaharu Tamaki, Machiko Kusuda, Aki Tanihara, Kazuaki Kameda, Hidenori Wada, Shun-Ichi Kimura, Shinichi Kako, Yoshinobu Kanda

Saitama Medical Center, Jichi Medical University, Division of Hematology, Saitama, Japan

Background: CD34-positive monocytes (CD34+mono) have recently been identified following mobilization by granulocyte-colony stimulating factor (G-CSF), and have been suggested to have a potential to modulate immune functions. However, the impact of CD34+mono on clinical outcomes after allogeneic hematopoietic cell transplantation (allo-HCT) still remains unclear.

Methods: Among patients who received allo-HCT from related donors between 2009 and 2016, we identified 74 donors whose peripheral blood cells during G-CSF mobilization were available for flow-cytometry analyses. CD34+mono was defined as CD34+CD14+CD11b+ CD $33+$ cells. When $>0.001 \%$ of CD34+mono was detected, the donor was considered to test positive.

Results: Of 74 donors, CD34+mono was detected in 46 [0.0019\% (range: 0-0.034\%)]. CD34+mmono was significantly more detected in male donors $(77 \%$ vs. $51 \%, \mathrm{P}=$ $0.029)$, while the distributions of donor age and donor body weight were not different. Furthermore, a ratio of total CD34+cells in peripheral blood was significantly higher in donors who tested positive for CD34+mono (0.6\% vs. $0.19 \%, \mathrm{P}<0.001)$.

Patient diseases were acute leukemia in 49, lymphoma in 9 , myelodysplastic syndrome in 7 , and others in 9. Of them, high-risk disease was included in 31. The detection of CD34+mono in donors was not different in patient backgrounds including age, gender, disease, disease risk, HLA-mismatch, conditioning intensity, and graft-versushost disease (GVHD) prophylaxis. In the whole cohort, no 


\section{Postive case}

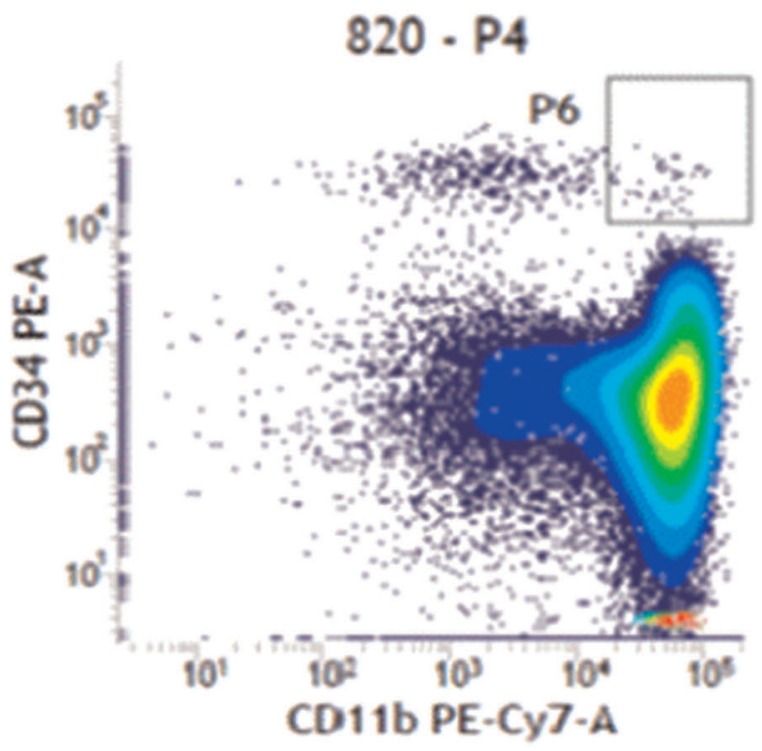

Negatlve case

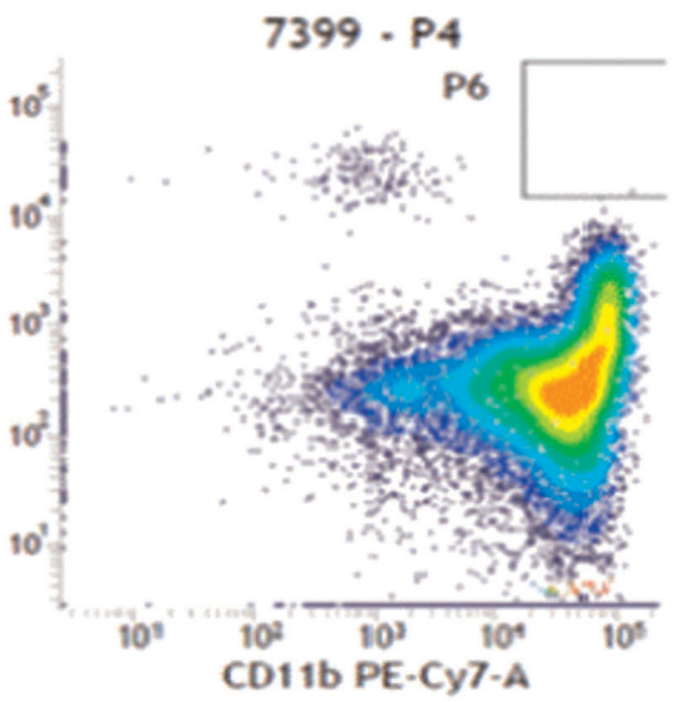

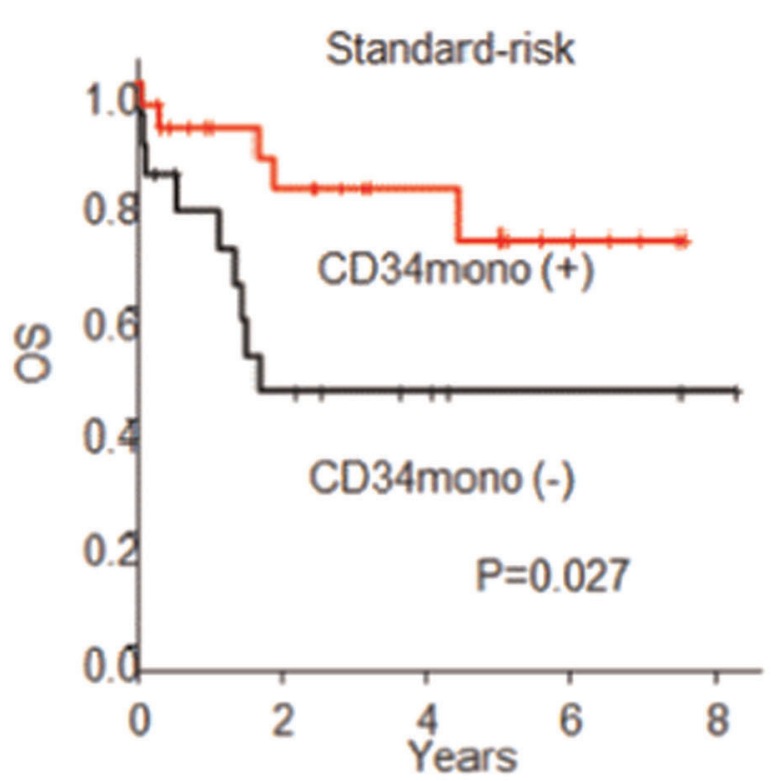

High risk

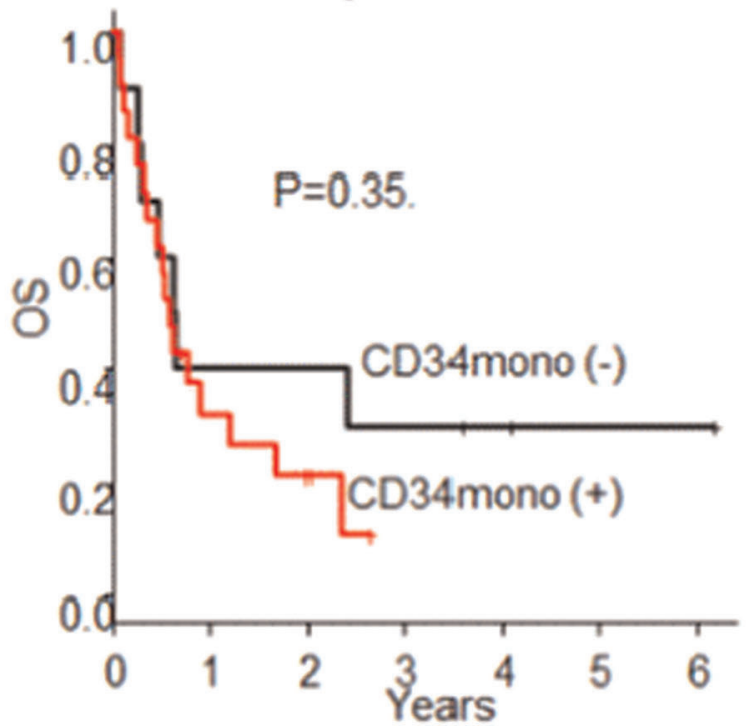

[P829 Figure] [Typical cases of CD34+mono and OS according to the presence of CD34+mono]

significant difference was observed in overall survival (OS), neutrophil engraftment, acute GVHD, non-relapse mortality (NRM), and relapse incidence. However, focusing on the subgroup of standard-risk diseases $(n=41)$, OS of the donor group with $\mathrm{CD} 34+$ mono was significantly superior to that of the donor group without $\mathrm{CD} 34+$ mono (81\% vs. $45 \%$ at $3 \mathrm{y}$ after allo-HCT, $\mathrm{P}=0.027$ ). In addition, NRM was significantly lower in the donor groups with CD34 + mono $(8 \%$ vs. $37 \%, \mathrm{P}=0.046)$, while the relapse incidence was not different (30\% vs. $30 \%$ at $3 y$ after alloHCT, $\mathrm{P}=0.62$ ). The incidence of CMV infections tended to be lower in the donor group with CD34+mono ( $0 \%$ vs. $17 \%, \mathrm{P}=0.07)$. On the other hand, no difference was observed in the incidence of acute GVHD.

Conclusions: $\mathrm{CD} 34+$ mono was detected in $62 \%$ of donors during G-CSF mobilization. No difference was observed in the incidence of acute GVHD as well as other clinical outcomes in the whole cohort. Focusing on the standard-risk disease, the detection of $\mathrm{CD} 34+$ mono in donor may have a favorable effect on survival. However, the study population included various diseases, and the total doses of CD34+mono in actually infused graft were not 
assessed. Therefore, a further investigation is still necessary to conclude the impact of $\mathrm{CD} 34+$ mono in donor or graft on clinical outcomes following allo-HCT.

Conflict of interest: None of the authors has anything to disclose.

\section{P830}

\section{Change of apheresis device decreased the incidence of severe acute graft-versus-host disease among patients after allogeneic stem cell transplantation with sibling donors}

Tengyu Wang ${ }^{1}$, Mats Remberger ${ }^{2}$, Ulla Axdorph-Nygell ${ }^{3}$, Andreas Björklund ${ }^{4}$,Jonas Mattsson ${ }^{2}$, Michael Uhlin ${ }^{3}$,Emma Watz ${ }^{5}$

${ }^{1}$ Karolinska Institute, Department of Clinical Sciences, Intervention and Technology, Huddinge, Sweden; ${ }^{2}$ Karolinska Institute, Department of Oncology and Pathology, Stockholm, Sweden; ${ }^{3}$ Karolinska Institute, Department of Clinical Science, Intervention and Technology (CLINTEC), Stockholm, Sweden; ${ }^{4}$ Karolinska University Hospital, Department of Haematology, Huddinge, Sweden; ${ }^{5}$ Karolinska University Hospital, Department of Clinical Immunology and Transfusion Medicine, Huddinge, Sweden

Background: The composition of the graft used for allogeneic stem cell transplantation (HSCT) is important for the treatment outcome. Different apheresis devices may yield significant differences in the in peripheral blood stem cell grafts cellular composition. We have compared stem cell grafts produced by Cobe Spectra (Cobe) and Spectra Optia (Optia), and evaluated clinical outcome parameters such as graft-versus-host disease (GvHD), transplant related mortality (TRM), relapse and overall survival (OS).

Methods: During three years, 31 Cobe Spectra and 40 Spectra Optia grafts were analyzed for CD34, CD3, CD4, CD8, CD19, and CD56 cell content. Clinical outcome parameters were correlated and compared between the two patient groups using different apheresis devices.

Results: Optia grafts contained less lymphocytes compared to Cobe $(\mathrm{p}<0.001)$. Optia grafts had significantly lower incidence of acute GvHD grades II-IV (Cobe $45 \%$ vs Optia $23 \%, \mathrm{p}=0.039$ ), grades III-IV (Cobe $23 \%$ vs Optia $5 \%, \mathrm{p}=0.03)$, and TRM (16\% vs. $2.5 \%, \mathrm{p}<0.05)$ but higher chronic GvHD (32\% vs $67 \%, \mathrm{p}=0.005)$ compared to Cobe grafts. No difference in relapse incidence and OS was observed. Finally, the multivariate analysis showed a significant correlation between the different apheresis devices and both aGvHD II-IV and severe cGVHD. The multivariate analysis also showed a significant correlation between the $\mathrm{CD} 3+$ cell dose the incidence of severe aGVHD.

Conclusions: Optia-obtained grafts yielded a lower aGvHD grades II-IV and TRM risk, but had no impact on relapse or OS in this study. Understanding and further improvement of PBSC apheresis techniques may be used in the future to personalize HSCT by e.g. fine-tuning the GvHD incidence.

Conflict of interest: No conflict of interest.

\section{P831}

Chemomobilization with reduced-dose etoposide is effective in lymphoma and myeloma patients undergoing autologous stem cell transplantation

\section{Seok Jin Kim ${ }^{1}$, Silvia Park ${ }^{1}$, Kihyun Kim ${ }^{1}$, Won Seog Kim ${ }^{1}$, Jong-Ho Won $^{2}$}

${ }^{1}$ Samsung Medical Center, Sungkyunkwan University, Seoul, Korea, Republic of; ${ }^{2}$ Soon Chun Hyang University Hospital, Seoul, Korea, Republic of

Background: High-dose chemotherapy followed by autologous stem cell transplantation (ASCT) is one of essential treatment options for patients with lymphoma and myeloma. For successful ASCT, collecting a sufficient number of peripheral blood stem cells is mandatory. However, repeated mobilization attempts increase medical costs and the risk of morbidity related with apheresis. In general, mobilization with G-CSF alone is less effective than chemomobilization consisting of chemotherapy and GCSF administration. Thus, the development of more effective and less toxic chemomobilization regimen is extremely important to avoid mobilization failure as well as successful ASCT.

Methods: We analyzed the outcome of chemomobilization with reduced-dose etoposide in patients with lymphoma and myeloma between January-2016 and Novemeber-2017. Etoposide $\left(375 \mathrm{mg} / \mathrm{m}^{2}\right)$ was intravenously administered to lymphoma patients on day 1 and 2 , and myeloma patients on day 1 . G-CSF was administered at a dose of $10 \mu \mathrm{g} / \mathrm{kg}$ daily starting on days 8 and continuing through the last day of stem cell collection. Apheresis was initiated when white blood cell and platelet count was more than $4,500 / \mathrm{mm}^{3}$ and $75,000 / \mathrm{mm}^{3}$, respectively. The target number of CD34+ cell count was $4 \times 10^{6} / \mathrm{kg}$ of recipient body weight.

Results: A total of 82 patients were analyzed: lymphoma $(n=64)$ and myeloma $(n+18)$. Lymphoma patients underwent ASCT in the upfront $(\mathrm{n}=29)$ or salvage $(\mathrm{n}=$ 35) setting, however, all patients with myeloma underwent upfront ASCT. The mean number of CD34+ cells per kg of body weight was 8.9 for lymphoma and 7.8 for myeloma patients. All patients performed on the average two cycles of apheresis to collect peripheral blood stem cells. $100 \%$ of patients with myeloma reached the goal of apheresis $(>4 \times$ $10^{6}$ of CD34+ cells) and $81 \%$ of patients with lymphoma collected more than $4 \times 10^{6}$ of CD34+ cells. The efficacy of stem cell collection was not different in upfront and salvage 
ASCT for lymphoma. The mean time interval between the first date of etoposide administration and the first date of apheresis was 14 , and the $44 \%$ of patients $(n=36)$ collected more than $4 \times 10^{6}$ of CD34+ cells on the first cycle of apheresis. After etoposide administration, no event of grade 3 and 4 febrile neutropenia was observed, and no severe adverse event related with hematologic toxicity occurred. There was no case of engraftment failure in lymphoma and myeloma patients.

Conclusions: Taken together, chemomobilization with reduced-dose of etoposide was effective for stem cell collection in lymphoma and myeloma patients. Its efficacy and toxicity of etoposide administration was comparable to that of high-dose etoposide. Especially, one dose of etoposide $\left(375 \mathrm{mg} / \mathrm{m}^{2}\right)$ could be effective for ASCT in myeloma patients.

Clinical Trial Registry: Not applicable

Conflict of interest: None of the authors has anything to disclose.

\section{P832}

Comparable efficacy of peripheral stem cell mobilization using biosimilar Filgrastim Nivestim ${ }^{\circledast}$ to original Neupogen ${ }^{\circledast}$ in healthy donors

Rula Najjar ${ }^{1}$, Shanta Sharma ${ }^{2}$, Rand Farraj ${ }^{1}$, Duaa Muffarrej ${ }^{1}$, Anas Haroun ${ }^{3}$, Hasan Hashem ${ }^{4}$, Eman Khattab ${ }^{5}$, Rawad Rihani, Husam Abu Jazar, Khalid Halahleh ${ }^{5}$,Mayada Abu Shanab ${ }^{4}$, Mohammad Ma'koseh ${ }^{5}$, Nilly Hussein ${ }^{6}$, Abdelghani Tbakhi ${ }^{2}$

${ }^{1}$ King Hussein Cancer Center, Pharmacy, Amman, Jordan; ${ }^{2}$ King Hussein Cancer Center, Cell Therapy \& Applied Genomics, Amman, Jordan; ${ }^{3}$ King Hussein Cancer Center, Nursing, Amman, Jordan;

${ }^{4}$ King Hussein Cancer Center, Pediatrics, Amman, Jordan; ${ }^{5}$ King Hussein Cancer Center, Bone Marrow Transplant Program, Amman, Jordan; ${ }^{6}$ King Hussein Cancer Center, Cancer Registry, Amman, Jordan

Background: Introduction of Biosimilar products is common practice that can decrease cost of therapy especially in oncology with similar efficacy and safety profile. Filgrastim biosimilar products have been studied for post chemotherapy effects. We aim to review and compare efficacy of biosimilar product Nivestim ${ }^{\circledR}$ (filgrastim), a recombinant human granulocyte colony-stimulating factor, to the reference product, Neupogen ${ }^{\circledR}$ in terms of peripheral CD34+ cell mobilization in healthy donors subsequent to its replacement in the formulary list in a single institution at King Hussein Cancer Center.

Methods: We conducted a retrospective comparative analysis on 160 healthy related donors who received either Neupogen ${ }^{\circledR}$ or Nivestim ${ }^{\circledR}$ for peripheral stem cell mobilization during a 2 year period from September 2014 to September 2016 in a single center institution. Donors received filgrastim at a dose of $5 \mathrm{mcg} / \mathrm{kg} / \mathrm{dose}$ subcutaneously every 12 hours for 4 days. Complete blood count (CBC) and $\mathrm{CD} 34+$ cell enumeration was done on donor's peripheral blood samples during the mobilization period and immediately prior to leukapheresis (between day 4 to day 6 of mobilization). The actual number of circulating $\mathrm{CD} 34+$ cells per microliter of peripheral blood was used to determine the optimum time for stem cell collection.

Results: A total of 160 healthy subjects received either products of filgrastim for stem cell mobilization over a period of 2 years; 2 were excluded because they received both products during the transition period from one product to the other. Neupogen ${ }^{\circledR}$ was used in 89 donors, while 69 donors received Nivestim ${ }^{\circledast}$ product. Mean age of donors at harvest was 24 years (2-57). Fifty-seven percent of donors were males.

The average circulating CD34+ cells per microliter was 97.18 in the Neupogen ${ }^{\circledR}$ group, while it was 83.75 in the Nivestim $^{\circledast}$ group. This was not considered statistically significant $(\mathrm{p}=0.287)$. However, the mean total number of doses needed for stem cell mobilization was 8.79 doses and 7.93 doses, for Neupogen ${ }^{\circledR}$ and Nivestim ${ }^{\circledR}$ respectively. This was considered as statistically different $(\mathrm{p}=.028)$.

Conclusions: Biosimilar Nivestim ${ }^{\circledR}$ showed comparable efficacy profile to the published data for the reference product, Neupogen ${ }^{\circledast}$ in terms of peripheral stem cell mobilization in healthy donors with less number of doses required. The use of Nivestim may lead to significant cost containment. Presented observation should be verified with wider prospective research.

Conflict of interest: No conflict of interest to declare

\section{P833}

Cost-effectivness and budget impact analysis of Lenograstim vs Filgrastim for mobilization of peripheral blood progenitors cells in patients with lymphoma and myeloma treated with autotransplant

Fabio Benedetti ${ }^{1}$, Umberto Restelli ${ }^{2}$, Davide Croce ${ }^{2}$, Mario Marzanatti ${ }^{3}$, Erminio Bonizzoni ${ }^{3}$, Claudio Costantini ${ }^{1}$, Angelo Andreini $^{1}$, Cristina Tecchio ${ }^{1}$, Erika Barison ${ }^{1}$

${ }^{1}$ Bone Marrow Transplant Unit - Hematology, Internal Medicine, Verona, Italy; ${ }^{2}$ Università Cattaneo, Centro Ricerche Economiche, Milan, Italy; ${ }^{3}$ Università di Milano, Dipartimento di Scienze Cliniche e di Comunità, Statistica Medica Biometria ed Epidemiologia, Milan, Italy

Background: The current economic context led the National Health Services to implement cost-effectiveness criteria to sustain decision-making processes of health technology use. 
Lenograstim (Leno), Filgrastim (Fil) and its biosimilars are Granulocyte-Colony Stimulator Factors (G-CSFs) used for clinical transplantation to induce hematopoietic stem cells (HSCs) mobilization.

Methods: At Azienda Ospedaliera Universitaria Integrata in Verona, a budget impact analysis and an incremental cost-effectiveness study of Leno use vs Fil have been performed.

248 patients undergoing autologous HSCs mobilization and HSCT have been evaluated and assigned to 3 groups according to G-CSF used for HSCs mobilization and hematopoietic cells recovery after transplant: 100 were treated with Leno both for mobilization and hematological recovery (Leno-Leno), 93 initially treated with Leno and then with biosimilar Fil (Leno-Fil), 55 were treated with biosimilar Fil both for mobilization and post-transplatantion support (Fil-Fil).

The efficacy of post-transplant recovery was evaluated considering the number of G-CSF vials used, the required days to recover an adequate level of WBCs and PLTs, hospitalization days, percentage of patients who experienced adverse events (AEs).

Results: The analysis of the 3 cohorts of patients showed statistically significant differences: a higher number of harvested $\mathrm{CD}_{3}{ }^{+}$cells in Leno group vs Fil group. No statistically significant differences were found regarding the number of G-CSF vials, apheresis number and CD $34^{+}$cells peak. The post-transplant hematological recovery was faster in Leno group compared with Fil group: median neutrophil $>500 / \mathrm{mm}^{3} 12$ vs 13 days; median platelets $>20.000 / \mathrm{mm}^{3}$ 13 vs 15 days $(\mathrm{p}<0.0001)$.

Regarding AEs, in Leno group was observed a lower pneumonia and fever incidence and a higher sepsis incidence to Fil group.

In terms of budget impact, Leno use would allow a lower funds absorption amounting to -€566/patient. These lower costs are related to a fewer days of hospitalization (-€822), overall lower incidence of AEs (-€182), laboratory tests, transfusions for PLTs recovery following discharge (-€115), that balance the higher cost associated with the G-CSF (Leno) used $(+€ 302$ for apheresis and $+€ 251$ for bone marrow recovery).

Conclusions: In terms of cost-effectiveness, Leno use allows to obtain better results for all measured parameters. Therefore, Leno is dominant to Fil in terms of efficacy and lower global costs of transplantation.

Treatment with biosimilar Fil entails an increase in the mean duration of hospitalization (1.5 days/transplant). Considering 50 transplants/year in our department this increase corresponds to: 75 additional days of hospitalization (corresponding to a total cost amounting to $+€ 61696.5$ ) and 5 transplants lost per year (loss of earnings accounting to $-€ 198793.6)$ (table 1).
The study revealed a clinical superiority of Leno-Leno group vs Fil-Fil group postulating a potential cost-savings favouring Leno over Fil, as well as a significant economic impact in terms of higher costs and lower earnings.

Conflict of interest: nothing to disclose

\begin{tabular}{llcl}
\hline ADDITIONAL COSTS & $\begin{array}{l}\text { Euro/ } \\
\text { days }\end{array}$ & x1,5 days & $\begin{array}{l}\text { Euro/ } \\
\text { Transplant }\end{array}$ \\
\hline Cost of hospitalization & 822.62 & 1233.93 & \\
Total Costs (75 days of hospitalization) & & 61696.5 & \\
DRG Veneto Region (per Transplant) & & & 39758.72 \\
Total Cost (loss of earnings) & & & 198793.6 \\
\hline
\end{tabular}

\section{[[P833 Table] table 1]}

\section{P834}

\section{Determining the optimal day for leukapheresis after chemomobilization and pegylated filgrastim}

Martina Lukić1, Njetočka Gredelj Šimec ${ }^{2}$, Viktor Zatezalo ${ }^{2}$, Antonio Hanžek $^{2}$, Silvana Jurenec ${ }^{3}$, Delfa Radić-Krišto ${ }^{2,4}$, Slobodanka Ostojić Kolonic ${ }^{2,5}$

${ }^{1}$ Croatian Institute of Transfusion Medicine, Zagreb, Croatia; ${ }^{2}$ Clinical Hospital Merkur, Department of Hematology, Zagreb, Croatia; ${ }^{3}$ Clinical Hospital Merkur, Transfusion Medicine, Zagreb, Croatia; ${ }^{4}$ School of Medicine, University of Osijek, Osijek, Croatia: ${ }^{5}$ School of Medicine, University of Zagreb, Zagreb, Croatia

Background: Chemomobilization is generally accepted approach for the collection of peripheral blood stem cells (CD34+) for autoPBSCT. The use of pegylated filgrastim is not yet widely accepted, but it is good alternative due to simplicity of its use and shorter hospital stay. The aim of this study was to determine the optimal time of CD34+ cells collection, after chemomobilization protocols and pegfilgrastim administration 24 hours after chemotherapy, as well as to find out if there is a group of patients in which we could reduce the number of leukapheresis if the leukapheresis day is changed.

Methods: We analyzed data of 199 patients in period from January 2013 to October 2017. The minimum number of CD34+ cells required for one autoPBSCT was $2 \times 10^{6} / \mathrm{L}$ per kilogram of patient body mass. For multiple myeloma patients we aimed to collect $\mathrm{CD} 34+$ cells for two transplantations. Previous therapies with fludarabine or melphalan, low platelet count $\left(<100 \times 10^{9} / \mathrm{L}\right)$ before protocol and fever prior to harvesting were considered risk factors for adequate mobilization. Collection started if the number of CD34 + in peripheral blood was $>10 \times 10^{6} / \mathrm{L}$.

Results: All patients with multiple myeloma $(\mathrm{N}=102)$ received cyclophosphamide $3 \mathrm{mg} / \mathrm{m}^{2}$. Sufficient number of stem cells was collected with one leukapheresis in 73 
patients $(71,6 \%)$, with two leukapheresis in 25 patients $(24,5 \%)$ and in 4 patients $(3,9 \%)$ with three leukapheresis. Harvest days were between days 7-16 (median 10). In 8 patients $(7.8 \%)$, the harvest was started too early and only a single procedure on the following day would be enough for collecting an adequate number of cells.

Lymphoma patients received following protocols: 45 miniBEAM protocol, 24 ICE protocol, 12 DHAP protocol, and the remaining 10 patients some other protocol. Sufficient number of stem cells was collected with one harvest in $84.53 \%$ patients, and in $13,4 \%$ and $2,06 \%$ patients with two and three leukapheresis, respectively. According to each protocol, days for collection were: for miniBEAM day 13-24 (median 17), for ICE day 9-21 (median 13) and for DHAP protocol day 10-14 (median 11.5). Harvesting started too early in $6,18 \%$ patients.

The risk of poor mobilization was present in $19 \%$ of all patients. These patients had mobilized later than others. For the group who received cyclophosphamide harvest was between day 9-14 (median 13) and for those who received miniBEAM was between days 15-24 (median 18). Also, by comparing groups of patients who received cyclophosphamide, number of collected CD34+ cells was significantly higher on the first leukapheresis day in patients without risk factors $(p=0,0316)$. There wasn't difference between the groups of patient who received other chemomobilization protocols.

Conclusions: According to the analyzed data of patients who have received pegfilgrastim $24 \mathrm{~h}$ after chemomobilization protocol, we can conclude that only in small number of patients, $8,04 \%$, an adequate number of CD34+ cells could have been collected with a single procedure on the following day. We can infer that it is not necessary to change the collection strategy of any group, including those with a risk of poor mobilization.

Conflict of interest: None of the authors has anything to disclose.

\section{P835}

\section{Different CD34+ recruitment during large volume leukapheresis after mobilization with filgrastim and pegfilgrastim in multiple myeloma and lymphoma patients}

Ozren Jaksic, Ivana Maricic, Zeljko Prka, Gorana Dzepina, Zdravko Mitrovic, Visnja Haris, Mario Pirsic, Zeljko Jonjic, Vlatko Pejsa

University Hospital Dubrava, Zagreb, Croatia

Background: During last decade along with filgrastim several new drugs become available for stem cell mobilization including peg-filgrastim, biosimilar filgrastims and plerixafor for poor mobilizers. In real life settings there are currently multiple possibilities with different availability and cost. Due to our positive experience with pegfilgrastim in treatment of non-Hodgkin lymphoma (NHL) with DAEPOCH (which we also predominantly use as chemomobilization protocol for NHL) and due to changing availability of filgrastim preparations we have increasingly used peg-filgrastim in stem cell mobilizations. To compare pegfilgrastim and filgrastim based mobilizations with respect to $\mathrm{CD} 34+$ mobilization dynamics, efficacy and cost, we have analyzed retrospectively a cohort of consecutive patients mobilized at our hospital.

Methods: From January 2011 to December 2017 we have performed 117 chemomobilizations and 9 steady/state mobilizations in 73 multiple multiple myeloma (MM) and 53 NHL and Hodgkin lymphoma (HL) patients. In 81 mobilizations we used filgrastim and in 45 mobilzations we have used pegfilgrastim. For chemomobilizations we have used high dose cyclophosphamide (HDC), DA-EPOCH \pm R, DHAP or mini-BEAM protocols. Filgrastim or pegfilgrastim was administered from/at the leukocyte (L) nadir or from/at $\mathrm{L}<1 \times 10^{9} / 1$. Apheresis was started when CD34+ cells reached $>20 \times 10^{6} / 1$ in peripheral blood.

Results: There was no difference between filgrastim and pegfilgrastim groups regarding sex (M/F, 45/36 vs 22/23), age (median age 56 vs 56), diagnosis (MM/NHL/HL, 47/29/ 5 vs $26 / 16 / 3)$ and BMI $\left(28.0\right.$ vs $\left.26.7 \mathrm{~kg} / \mathrm{m}^{2}\right)$. There were significant differences between chemotherapy protocols in median times from chemotherapy start to filgrastim or pegfilgrastim start i.e. 9 days for HDC $(n=66), 12$ days for DA-EPOCH $(\mathrm{n}=31), 11.5$ days for DHAP $(\mathrm{n}=8)$ and 14 days for miniBEAM $(n=12)$, but there were no differences between filgrastim and pegfilgrastim subgroups. There was no difference in time from filgrastim or pegfilgrastim start to apheresis and it was median 4 days for the whole group including steady state mobilizations. However, there was significantly less variability in pegfilgrastim vs filgrastim group (S.D. 1.29 vs 2.07 , p < 0.05). There was no significant difference in CD34+ numbers in peripheral blood prior to apheresis. There was no difference between filgrastim and peg-filgrastim regarding blood volumes processed per apheresis $(4.7 \mathrm{BV})$, nor in mean $\mathrm{CD} 34+\times 10^{6} / \mathrm{kg}$ yield ( 8.0 vs 8.6 ). However, in pegfilgrastim there is significantly higher mean $\mathrm{CD} 34+$ yield (total CD34+ collected/total CD34+ in PB prior to apheresis) per first apheresis (3.2 vs $2.1, \mathrm{p}<0,05)$ indicating more significant CD34+ cell recruitment form bone marrow during LVL. Difference was independent of chemomobilization protocol used and it was less pronounced in subsequent aphereses. That resulted in significantly more completed procedures in single apheresis in pegfilgrastim group $(51 \%$ vs $33 \%, \mathrm{p}<0.05)$. Although drug cost of pegfilgrastim compared to filgrastim is significantly higher there was no significant difference in total cost of 
mobilizations in our series (probably compensated by smaller number of apheresis).

Conclusions: Our results show comparable efficacy and cost of filgrastim and pegfilgrastim based mobilizations. However, pegfilgrastim group showed more predictable dynamics of mobilization and better intra-apheresis recruitment during large volume leukapheresis leading to less apheresis procedures. Pegilgrastim is also more convenient for patient.

Conflict of interest: None

\section{P836}

Early upfront plerixafor administration on day 3 in the "chemo-free" mobilization regimen G-CSF+Plerixafor, for Multiple Myeloma patients: a retrospective single center experience

Valentina Bozzoli $^{1}$, Paola Mazzotta ${ }^{1}$, Rosella Matera ${ }^{1}$, Anna Rita Messa $^{1}$, Michelina Dargenio ${ }^{1}$, Donato Canaris ${ }^{2}$, Nicola Di Renzo ${ }^{1}$

${ }^{1}$ Ospedale 'vito Fazzi', Hematology Department, Lecce, Italy;

${ }^{2}$ Ospedale 'Vito Fazzi', Servizio di Medicina Trasfusionale ed Immunoematologia, Lecce, Italy

Background: Recent studies suggested that additional exposure to cytoreductive agents, as part of the mobilisation regimen, do not significantly impact on progression-free or overall survival after autologus Stem cell Transplantation (ASCT) in Multple Myeloma (MM), but rather increase the morbidity associated with SC mobilisation (y GL et al, BMT. 2015;50(12):1513-1518).

Plerixafor plus granulocyte-colony stimulating factor $(\mathrm{G}-$ $\mathrm{CSF}$ ) is a safe and effective Stem cell (SC) mobilization regimen in Myeloma patients, and has been proven to be superior to G-CSF alone.

Basing on the phase III study by DiPersio and coworkers, Plerixafor has been used in the setting of "chemo-free" mobilization regimens for multiple myeloma (MM) patients, at the following schedule: G-CSF $10 \mathrm{~g} / \mathrm{kg}$ per day subcutaneously in the morning and, on the evening of day 4, plerixafor $0.24 \mathrm{mg} / \mathrm{kg}$ subcutaneously daily (DiPersio et al, Blood 2009).

Methods: We here retrospectively evaluated thirteen consecutive MM patients treated in our center, who underwent "chemo-free" stem cell mobilization from January 2015 to October 2017. The focus of this study was to evaluate the efficacy of an early Plerixafor admnistration on the night of day 3, namely after three days of G-CSF administration.

Results: The mean age of our 4 women and 9 men cohort, was 59 years old (range 51-73). Stem cell mobilisation was started after 4 cycles of induction therapy: twelve patients received VTD (Velcade, Talidomide, Desametasone) and one patient underwent Vel-Dex (Velcade, Desametasone) induction. Nine patients obtained VGPR after induction therapy, while the remaining four were in CR. Just one patient $(7.69 \%)$ received prior radiotherapy.

All thirteen patients except one, required two apheresis days to collect more than or equal to $6 \times 10^{6} \mathrm{CD} 34$ cells $/ \mathrm{kg}$. The median of CD34 cells $/ \mathrm{kg}$ collected was $9,45 \times 10^{6}$ (range 6,18- 12,64).

Day 4 median peripheral blood CD34 cell count was 49,5 cells/ $\mu \mathrm{L}$ (range 19,3-64,6), while on day 5 was 43,1 cells/ $\mu \mathrm{L}$ (range 33,5-144,3).

All patients in the study underwent at least one ASCT, the mean number of CD34 cells transplanted was $3,82 \times 10^{6}$ (median 3,87; range 2,83-4,98). For patients who underwent tandem transplantation

( 8 patients), the mean number of CD34 cells transplanted for the second transplant was 4,3 x $10^{6}$ (median 4,08; range 2,86-5,64).

The transplantation outcomes were in line with data published in literature: $100 \%$ of the patients had successful neutrophil and platelet engrafment, whose median time was respectively 11 days for neutrophil and 14 days for platelet.

Conclusions: Considering the limitations of a retrospective study, and the small number of patients included, we could show that, in comparison with data reported from DiPersio et al, an early administration of Plerixafor on third day did not show any advantage neither in term of collection (number of apheresis and stem cells collected) nor in transplantation outcome.

To confirm these data a multicenter prospective study, promoted by the Rete Ematologica Pugliese (REP), on the early use of plerixafor in patients with Multiple Myeloma is now uderway.

Conflict of interest: The authors declare they have no conflict of interest

\section{P837}

Effectiveness of the original granulocyte-colony stimulating factor (G-CSF) (Neupogen ${ }^{\circ}$ ) and the biosimilar G-CSF $\left(\right.$ Zarzio $\left.^{\circ}\right)$ in autologous peripheral blood stem cell mobilization: a single-center experience

Rocío Zapata Bautista, Antonio José Palma Vallellano, María Solé Rodríguez, María Antonia Ruiz Cobo, Encarnación Gil Espárraga

Hospital Juan Ramón Jiménez, Huelva, Spain

Background: In recent years Zarzio , a biosimilar GCSF, has been approved as a mobilization agent. The vast majority of clinical trials supporting its effectiveness were performed in healthy donors used as a single agent while in patients it is mostly used combined with chemotherapy as 
well as Plerixafor. The aim of this study is to compare the effectiveness between Neupogen ${ }^{\circ}$ and Zarzio as mobilization agents with and without chemotherapy and/or Plerixafor in a real clinical context.

Methods: We retrospectively evaluated the outcome of mobilization attempts and apheresis sessions carried out from October 2013 to May 2017 in one single center, defined by: maximum number of mobilized CD34+ cells/ $\mu \mathrm{L}$ in peripheral blood, number of first apheresis collecting more than $2 \times 10 \mathrm{e} 6 \mathrm{CD} 34+$ cells $/ \mathrm{kg}$, rate of patients in whom Plerixafor was needed to obtain an adequate apheresis product and number of episodes in which apheresis was not performed because insufficient amount of $\mathrm{CD} 34+$ cells $/ \mu \mathrm{L}$ in peripheral blood. The patients were allocated to Neupogen ${ }^{\circ}$ or Zarzio ${ }^{\circ}$ group depending on the date: before May 2016 they all received Neupogen while after that date they all received Zarzio ${ }^{\circ}$.

Results: We performed 82 apheresis sessions in 72 patients in this period. In the Neupogen group 33 patients had lymphoproliferative syndromes (SLPC) and 21 monoclonal gammapathies (GM). In the Zarzio group we performed 9 mobilizations procedures in 8 patients with SLPC and 11 in 10 patients with GM.

In the Neupogen group patients received $22,5 \%$ only Neupogen ${ }^{\circ}$, 64,5\% chemotherapy plus Neupogen and $13 \%$ received also Plerixafor. In the Zarzio group, $25 \%$ only received Zarzio ${ }^{\circ}$ 65\% Zarzio ${ }^{\circ}$ plus chemotherapy and in $10 \%$ Plerixafor was also given.

We reached a maximun concentration of $\mathrm{CD} 34+$ cells preapheresis of 80.06 per microliter $(/ \mu \mathrm{L})$ with Neupogen ${ }^{\circ}$ and $67.01 / \mu \mathrm{L}$ with Zarzio ${ }^{\circ}$. The number of episodes in which more than $2 \times 10^{6} \mathrm{CD} 34+$ cells were collected during the first apheresis procedure was $70.9 \%$ with Neupogen and $80 \%$ with Zarzio ${ }^{\circ}$. The rate of mobilization failure because of very low concentration of $\mathrm{CD} 34+$ cells preapheresis $(<10-15 \mathrm{CD} 34+$ cells $/ \mu \mathrm{L})$ was $10 \%$ in both groups. Plerixafor was necessary to obtain an adequate amount of $\mathrm{CD} 34+$ cells for trasplant in $14.8 \%$ in the Neupogen ${ }^{\circ}$ group and $11.1 \%$ in patients who were given Zarzio ${ }^{\circ}$. Approximately $94 \%$ of patients with a median of $6.84 \mathrm{CD} 34+$ cells/ $\mathrm{Kg}$ (range between 2.81-21.9 cells $/ \mathrm{kg}$ ) have been appropiately mobilized. None of the observed differences between the groups were statistically significant.

Conclusions: In our experience we do not find any differences between Neupogen ${ }^{\circ}$ and Zarzio ${ }^{\circ}$ effectiveness but this could be so because our serie is small and the majority of patients received Neupogen. Therefore, we will continue evaluating our data in the future to obtain more comparable groups.

Conflict of interest: None

\section{P838}

\section{Ex vivo depletion using biotinylated anti-TCRa $\beta$ monoclonal antibody for haploidentical hematopoietic cell transplantation}

Eun Seok Choi ${ }^{1}$, Jae Won Yoo $^{1}$, Sung Han Kang ${ }^{1}$, Hyery Kim ${ }^{1}$, Kyung Nam Koh ${ }^{1}$, Ho Joon Im ${ }^{1}$, Jong Jin Seo ${ }^{1}$, Ho Ran Park ${ }^{2}$

${ }^{1}$ Asan Medical Center, Pediatrics, Seoul, Korea, Republic of; ${ }^{2}$ College of Nursing, The Catholic University of Korea, Seoul, Korea, Republic of

Background: Depletion of T cells to prevent GVHD is an essential component for haploidentical hematopoietic cell transplantation (haplo-HCT). Moreover, chronic GVHD is a critical issue in pediatric patients undergoing allogeneic HCT which is associated with quality of life. Recent depletion method targeting $\alpha \beta^{+} \mathrm{T}$ cells produces grafts containing many $\gamma \delta^{+}$lymphocytes which are not implicated in the development of GVHD. In addition, the establishment of a consistent and reproducible depletion technique is critical for the successful haplo-HCT. Since 2012, $\alpha \beta^{+} \mathrm{T}$ cell-depletion method has been applied for haplo-HCT in pediatric patients at our center. In this study, we evaluated the efficacy of ex vivo $\alpha \beta^{+}$T cell-depletion technique which was performed by one clinical nurse specialist.

Methods: Between May 2012 and December 2017, 119 depletion procedures using biotinylated anti-TCR $\alpha \beta$-monoclonal antibody were performed in 105 children and adolescents using the CliniMACS ${ }^{\oplus}$ system (MiltenyiBioTec, Germany). Initially, depletion procedures were performed from the combined product of 2-day collections on day-1 and 0. Since December 2016, the harvesting strategy was modified with one-day collection on day-1. If sufficient number of $\mathrm{CD}_{3} 4^{+}$cells were not obtained after first collection, then the $2^{\text {nd }}$ apheresis was carried out. If the number of $\mathrm{CD}_{3} 4^{+}$cells were $<3^{\prime} 10^{6} / \mathrm{kg}$ after depletion process, booster infusion of $\mathrm{CD}^{+} 4^{+}$stem cells was performed.

Results: Of 105 patients, 61 had hematologic malignancy, 36 had non-malignant disease, and 8 had solid tumor. Donors were mothers in 47 cases, fathers in 45 cases and siblings in 27 cases. The median log depletion was 4.0 for $\alpha \beta^{+} \mathrm{T}$ cells (range, 2.5-5.0) and 1.5 for $\mathrm{CD}^{+} \mathrm{T}$ cells. The median yields were $90.4 \%$ for $\mathrm{CD}^{3} 4^{+}$cells, $44.7 \%$ for B cells, $74.9 \%$ for NK cells and $75.9 \%$ for $\gamma \delta^{+} \mathrm{T}$ cells, respectively. TNC exceeding $6^{\prime} 10^{10}$ cells in preprocessedproducts was associated with lower depletion efficacy of $\alpha \beta^{+} \mathrm{T}$ cells (4.0 $\log$ versus $3.6 \log , P=0.008$ ). In chronological analysis, the depletion efficacy of $\alpha \beta^{+} \mathrm{T}$ cells was significantly improved over time $\left(\mathrm{R}^{2}=0.187, P<\right.$ $0.001)$. In addition, the total elapse times for manipulation 
was significantly shorter over time (median 280 minutes, $P<$ 0.001). However, there was no significant change in recovery of $\mathrm{CD} 34^{+}$cells, B cells, and NK cells.

Of 30 cases that were intended to receive 1-day collection, 22 cases obtained sufficient number of CD34 ${ }^{+}$ cells with 1-day collection. On the other hand, 8 donors underwent $2^{\text {nd }}$ collection as the $\mathrm{CD} 34^{+}$cells collected on the first day were not enough. The cases that required $2^{\text {nd }}$ collection were associated with a higher weight percentage of recipient over donor, and a less $\mathrm{CD} 34^{+}$cells in raw bag. Sixteen cases intended to performone-day collection. Of them, 4required $2^{\text {nd }}$ apheresis. Of total 119 transplants, 8 cases necessitated booster infusion of $\mathrm{CD} 34+$ stem cells.

Conclusions: This is our 5-year experience with $\alpha \beta^{+} \mathrm{T}$ cell-depletion procedure, which was performed by one technical staff. Our study suggests that this depletion method by skilled personnel is highly efficient and reproducible to remove $\alpha \beta^{+}$T cells for haplo-HCT.

Conflict of interest: nothing to disclose

\section{P839}

Factors affecting colony forming unit assay of cord blood units in the first Egyptian public cord blood bank

\begin{abstract}
Mohamed Saleh ${ }^{1,2}$, Ahmad Darwish ${ }^{2,3}$, Heba Mosaad ${ }^{1}$, Mohamed Darwish $^{1,2}$, Sara Mohamed ${ }^{2,4}$, Farha El-Chennawi ${ }^{1,2}$

${ }^{1}$ Faculty of Medicine, Mansoura University, Clinical Pathology, Mansoura, Egypt; ${ }^{2}$ Faculty of Medicine, Mansoura University, Research Center for Cord Stem Cells, Mansoura, Egypt; ${ }^{3}$ Faculty of Medicine, Mansoura University, Pediatrics - Hematology/Oncology Unit, Mansoura, Egypt; ${ }^{4}$ Faculty of Medicine, Mansoura University, Obstetrics and Gynecology, Mansoura, Egypt
\end{abstract}

Background: Umbilical cord blood (UCB) is a promising alternative to bone marrow as a source of stem cells. Quality of UCB is a leading factor to determine success of transplantation. Therefore, donor selection became a worldwide issue. In this study, we try to identify the effect of many maternal and neonatal factors on the quality of UCB units which affect the bankability of these units. These factors may vary according to the geographical distribution. Egypt is a new comer to the field of public cord blood banking. Therefore, it will be of great benefit to study these factors in our area.

Methods: Informed consent was obtained from 50 eligible mothers for donation of cord blood. The UCB units were collected in utero at Mansoura University Hospital. The cord blood volume was measured. The quality of UCB units were evaluated by measuring the cord blood volume, the total nucleated cell (TNC) count, the $\mathrm{CD} 34+$ cell count and the colony forming units (CFU) assay. Colony forming unit assay is considered the gold standard for assaying potency of UCB units as it assess viability of cells as well as their differentiation ability.

Results: We have found that factors which are associated with high quality UCB units are: volume $\geq 80 \mathrm{ml}$, birth weight $\geq 3500 \mathrm{gm}$, male gender of the baby, younger gestational age and maternal age $\geq 20$ years.

Conclusions: Umbilical cord blood donations associated with high quality are collected from heavier babies at birth, younger gestational age, larger cord blood volumes, older mothers and male babies.

Conflict of interest: This study is funded by Science and Technology Development Fund (STDF), Egypt

\section{P840}

Feasibility and efficiency of mobilized peripheral blood stem cell apheresis via Hickman catheter in pediatric patients

Nora Doberschuetz ${ }^{1}$, Jan Soerensen ${ }^{1}$, Bonig Halvard ${ }^{2}$, Andre Willasch ${ }^{1}$ Eva Rettinger ${ }^{1}$, Verena Pfirrmann ${ }^{1}$,Emilia Salzmann ${ }^{1}$, Richard Schaefer ${ }^{2}$, Thomas Klingebiel ${ }^{1}$, Peter Bader ${ }^{1}$, Andrea Jarisch $^{1}$

${ }^{1}$ Universitätsklinikum Frankfurt am Main, Pediatric Stem Cell Transplantation, Frankfurt/M., Germany; ${ }^{2}$ German Red Cross Blood Center Frankfurt and Institute of Transfusion Medicine and Immunohematology, Frankfurt/M., Germany

Background: Autologous stem cell transplantation remains an integral tool in the treatment of certain childhood malignancies. In order to facilitate preparative apheresis in pediatric patients, typically a central venous catheter is necessary to provide adequate flow rates. Pediatric patients in our center however, have preimplanted Hickman catheters for chemotherapy. We evaluated the feasibility of collecting adequate numbers of CD34+ cells via Hickman catheter instead of placing an additional temporary central venous dialysis catheter.

Methods: Aphereses of pediatric patients undergoing leukapheresis for autologous HSCT between February 2011 and March 2017 were reviewed. Where pre-implanted Hickman catheters were available, these were used for apheresis. The other patients received a temporary Shaldon catheter. Apheresis was performed using Spectra Optia MNC v.3.0 and was considered successful if a dose of $2 \times 10^{6} \mathrm{CD} 34+\mathrm{PBSC} / \mathrm{kg}$ body weight $(\mathrm{kgBW})$ was achieved.

Results: In 43 (89.6\%) of the 48 patients, the Hickman catheter was used for leukapheresis. Only 5 patients $(10.4 \%)$ received a temporary Shaldon catheter. In both groups, apheresis was tolerated well and without apparent adverse reactions. The dose of CD34+ PBSC in the apheresis product amounted to $12.7 \times 10^{6}$ (range 0.6- 
$70.7 \times 10^{6}$ ) cells/kgBW in the Hickman and $16.2 \times 10^{6}$ (range $1.7-48.4 \times 10^{6}$ ) cells $/ \mathrm{kgBW}$ in the Shaldon group, showing no statistically significant difference $(p=0.67)$. In both groups, the primary endpoint of a minimal CD34+ cell concentration of $2 \times 10^{6}$ cells $/ \mathrm{kgBW}$ was achieved in all patients by at maximum two leukapheresis sessions. The efficacy of apheresis is further confirmed by the collection efficiency (CE2) of $45 \%$ in the Hickman and $36 \%$ in the Shaldon Group ( $\mathrm{p}=0.32$ ).

Conclusions: Our data indicate reliable feasibility and efficacy of mobilized apheresis via an indwelling central line. In light of this, routine insertion of a dialysis catheter for the purpose of stem cell apheresis should be reconsidered.

Conflict of interest: None of the authors has anything to disclose.

\section{P841}

\section{Haploidentical Stem Cell Transplantation in Adult Patients Using TCR-alpha/beta and CD19 Depleted Stem Cells: First Results of a Prospective Multicenter Phase I/II Study}

Wolfgang Bethge ${ }^{1}$, Stephan Mielke ${ }^{2}$, Dietger Niederwieser ${ }^{3}$, Roland Meisel $^{4}$, Donald Bunjes ${ }^{5}$, Arne Brecht ${ }^{6}$, Jürgen Kuball, Sandra Karitzky $^{8}$, Silke Holtkamp ${ }^{8}$, Christiane Siewert ${ }^{8}$, Stefanie Pflitsch $^{8}$, Michael Schumm ${ }^{9}$, Matthias Eyrich ${ }^{10}$, Vladan Vucinic ${ }^{3}$, Markus Wiesneth $^{11}$, Kasper Westinga ${ }^{12}$, Rupert Handgretinger ${ }^{9}$

${ }^{1}$ Medical Center University Hospital Tuebingen, Hematology/ Oncology, Tuebingen, Germany; ${ }^{2}$ Medical Center University Hospital Wuerzburg, Hematology/Oncology, Wuerzburg, Germany; ${ }^{3}$ Medical Center University Hospital Leipzig, Hematology/Oncology, Leipzig, Germany; ${ }^{4}$ University Hospital Duesseldorf, Pediatric Oncology, Duesseldorf, Germany; ${ }^{5}$ Medical Center University Hospital Ulm, Hematology/Oncology, Ulm, Germany; ${ }^{6}$ DKD HELIOS Hospital Wiesbaden, Bone Marrow Transplantation, Wiesbaden, Germany; ${ }^{7}$ Medical Center University Hospital Utrecht, Hematology, Utrecht, Netherlands; ${ }^{8}$ Miltenyi Biotec GmbH, Bergisch Gladbach, Germany; ${ }^{9}$ Children's University Hospital Tuebingen, Tuebingen, Germany;

${ }^{10}$ Children's University Hospital Wuerzburg, Wuerzburg, Germany;

${ }^{11}$ Institute for Clinical Transfusion Medicine and Immunogenetics Ulm, Ulm, Germany; ${ }^{12}$ Medical Center University Hospital Utrecht, Pharmacy Celltherapy Facility, Utrecht, Netherlands

Background: We report the first results of a prospective, multi-center, single-arm phase I/II study (EudraCT No. 2011-005562-38) of haploidentical stem cell transplantation (SCT) with TCR-alpha/beta and CD19 depleted peripheral blood stem cell grafts.

Methods: Graft depletion was performed using the CliniMACS plus System (Miltenyi Biotec) in. Patients received a RIC regimen consisting of 15 or $30 \mathrm{mg}$ ATG (Neovii/Grafalon) or $7 \mathrm{~Gy}$ total nodal irradiation, $160 \mathrm{mg} /$ $\mathrm{m}^{2}$ fludarabine, $10 \mathrm{mg} / \mathrm{kg}$ thiotepa, and $140 \mathrm{mg} / \mathrm{m}^{2}$ melphalan. MMF (40 mg/kg/day) was used for GVHD prophylaxis until Day 30.
Results: 30 adult patients (median age 38 years, range 20-63, 13 female, 17 male) were enrolled in 7 centers. Patient's diagnosis included 7 ALL, 17 AML, 3 MDS/MPS, 1 each with multiple myeloma, acute undifferentiated leukemia or sickle cell anemia. Malignant diseases were in CR $(n=19)$, PR $(n=6)$ or non-remission $(n=4)$. Eight had $\geq 1$ previous SCTs. The median follow-up was 379 days (range, 18 - 776).

The median graft content $/ \mathrm{kg}$ recipient weight was for CD34 + cells $8.1 \times 10^{6}$ (range, $4-18$ ), TCRab + cells $13.5 \times$ $10^{3}$ (range, $0.22-64.3$ ), CD20+ cells $28 \times 10^{3}$ (range, 2 727), CD56+ NK cells $39.3 \times 10^{6}$ (range, $9.4-102$ ) and TCRgd + cells $8.9 \times 10^{6}$ (range, $1.1-30.7$ ). 28/30 patients had primary engraftment with ANC $>500$ cells $/ \mu \mathrm{L}$ at a median of 14 days (range, 9 - 41) and PLT $>20,000$ cells $/ \mu \mathrm{L}$ at a median of 15 days (range, 12 - 38). 2 patients experienced primary graft failure. 1 patient had a secondary graft failure. All 3 patients were successfully retransplanted. Peripheral donor T-cell chimerism at the time of engraftment was complete in 27 patients, and mixed in 1 .

No patient developed acute GVHD grade III/IV. 5 patients (16.7\%) grade II, 7 (23.3\%) grade I, and 18 (60\%) had no GVHD.

28 patients were evaluable for immune reconstitution (Figure 1).

The median cell counts d 28 were: NK cells $272 / \mu \mathrm{L}$ (range, 10 - 1092), CD3 + cells 112/ $\mu \mathrm{L}$ (range, 9 - 911), TCRgd + cells $87 / \mu \mathrm{L}$ (range, 7-891). Between day 28 and day 100 , the median TCRab + cells increased from 36 to 116 cells/ $\mu \mathrm{L}$.

Following infectious events occurred: viral reactivation $(\mathrm{CMV} \mathrm{n}=13, \mathrm{HHV} 6 \mathrm{n}=10, \mathrm{BKV}=4, \mathrm{EBV}=2, \mathrm{ADV}$ in stool $n=4)$, viral disease (CMV colitis $=1$, BKV positive hemorrhagic cystitis $=1$ ), bacterial sepsis $=3$.

At a median follow-up of 379 days 21/30 patients are alive; 9 patients died: 4 relapses, 5 organ failures (2 lung, 1 each liver, CNS, cardiac) resulting in a Kaplan-Meier estimated OS and DFS of $93 \%$ and $86 \%$ on day 100 and $75 \%$ and $67 \%$ at 1 year, respectively.

After 100 days and 1 year the cumulative incidences of NRM and relapse were $7 \%$ and $8 \%$ and $14 \%$ and $17 \%$, respectively.

Conclusions: Haploidentical SCT using TCR-alpha/beta/ CD19-depleted grafts and RIC resulted in primary engraftment in $93 \%$, no acute GVHD grade III/IV, NRM of $7 \%$ and OS of $93 \%$ at day 100 and an estimated OS of $75 \%$ after 1 year. The immune reconstitution of innate immunity was rapid and there was no lethal viral and fungal infection.

Clinical Trial Registry: EudraCT No. 2011-005562-38

Conflict of interest: Study sponsored by Miltenyi Biotec $\mathrm{GmbH}$

W. Bethge: Honoraria and research funding, Miltenyi Biotech $\mathrm{GmbH}$ and Neovii $\mathrm{GmbH}$ 

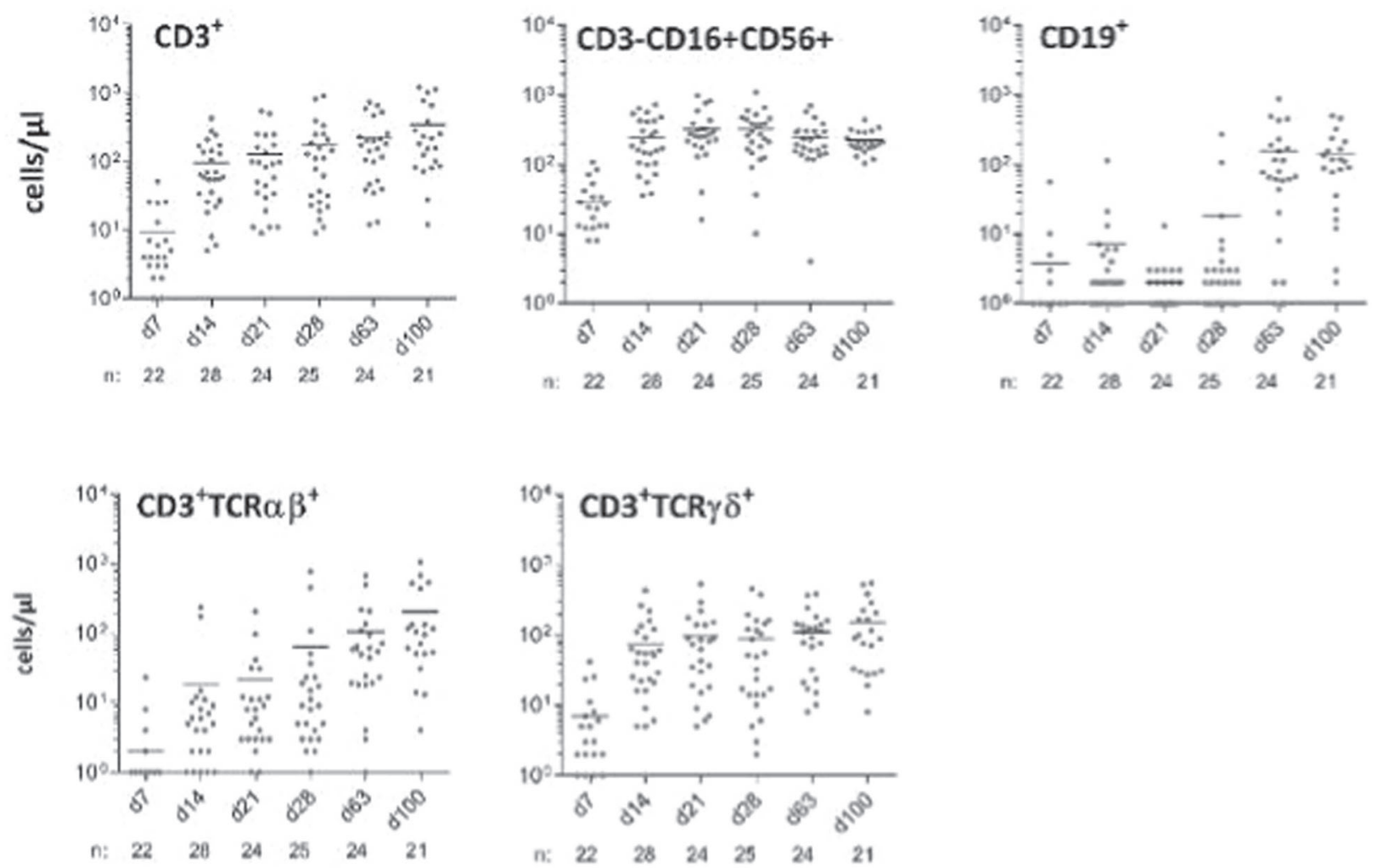

\section{Days Post Transplantation}

[P841 Figure] [immune reconstitution adults TCRab]

S. Mielke: Travel grants, KIADIS Pharma, Celgene, MSD, Gilead and Cellex GmbH; consultancy, Novartis, MSD

D. Niederwieser: research funding, Novartis

R. Meisel: research funding, Miltenyi Biotech $\mathrm{GmbH}$ and Neovii GmbH

J. Kuball: Research funding, Miltenyi $\mathrm{GmbH}$, consultancy and equity ownership, Gadeta

R. Handretinger: Co-patent holder of TcR alpha/beta depletion technologies and research funding, Miltenyi Biotech GmbH

S. Karitzky, S. Holtkamp, C. Siewert, and S. Pflitsch are employees of Miltenyi GmbH

M. Schumm, M. Eyrich, V. Vucinic, M. Wiesneth, K. Westinga, D. Bunjes, and A. Brecht: Nothing to disclose

\section{P842}

Hematopietic Recovery after a second autologous transplant confirms the effectiveness of long term progenitor cell cryopreservation and storage: experience from Rome Transplant Network (RTN)

Antoine Bruno ${ }^{1}$, Alessandra Picardi ${ }^{1}$, Gottardo De Angelis ${ }^{1}$, Pavoni Ernesto $^{1}$, Raffaella Cerretti ${ }^{1}$, Laura Cudillo ${ }^{1}$, Benedetta Mariotti ${ }^{1}$,
Federico Meconi ${ }^{1}$, Andrea Mengarelli ${ }^{2}$, Francesco Marchesi ${ }^{2}$, Maria Cristina Tirindelli, Alessandra Scardocci ${ }^{3}$, Agostino Tafuri ${ }^{4}$, Paolo De Fabritiis ${ }^{5}$, Teresa Dentamaro ${ }^{5}$, Anna Chierichini ${ }^{6}$, Roberto Cotumaccio $^{6}$, Gaspare Adorno ${ }^{7}$, Alessandro Lanti ${ }^{7}$, Maria Cantonetti ${ }^{7}$, Luca Franceschini ${ }^{7}$, Manuela Rizzo ${ }^{7}$, William Arcese ${ }^{1}$

${ }^{1}$ Tor Vergata University of Rome, Stem Cell Transplant Unit, Policlinico Universitario Tor Vergata, Rome, Italy; ${ }^{2}$ IFO Regina Elena National Cancer Institute, Rome, Italy; ${ }^{3}$ Campus Biomedico

University Hospital, Rome, Italy; ${ }^{4}$ Azienda Ospedaliera Sant'Andrea, Rome, Italy; ${ }^{5}$ Sant'Eugenio Hospital, Rome, Italy; ${ }^{6}$ Azienda

Ospedaliera San Giovanni Addolorata, Rome, Italy; ${ }^{7}$ Tor Vergata University Hospital, Rome, Italy

Background: The variability in processing autologous hematopoietic stem cells (AHSC) represents a major quality control challenge. As requested by JACIE standards, method validation assuring adequate potency of the graft is essential. The widely accepted criteria of $\mathrm{CD} 34+$ cells quantification and viability tests do not guarantee the functional activity of AHSC post long-term storage and thawing. Moreover, in vitro colony assays standardization remains a barrier for widespread adoption. To study the effect of long-term cryopreservation in vivo, we compared neutrophil and platelet engraftment timing in patients who received two AHSC transplantations for multiple myeloma (MM). 
Methods: Inclusion criteria consisted of double transplant from single AHSC mobilization with equivalent cell dose of CD34+ cells. The two transplant procedures have been spaced out for a period of at least two years. From January 2006 to June 2017, 26 patients affected by MM (16 M/10 F) with a median age at first transplant of 56 years (16-68) entered the study. The disease status at time of the first and the second transplant were respectively: $13(50 \%)$ and 8 (30\%) CR, 2 (7\%) VGPR for both, 8 (30\%) and $13(50 \%)$ PR, $2(7 \%)$ and 1 (4\%) SD, $1(4 \%)$ and 2 (7\%) PD. After apheresis collection, AHSC products were splitted in two equivalent fractions and mixed 1:1 volume with the cryoprotectant solution (20\% DMSO in 5\% human albumin) and cryopreserved using a controlled rate freezer to minimize freezing damage. According to patient age and comorbidities, the conditioning regimen consisted of melphalan at high dose in $21(81 \%)$ patients at first transplant and in 15 (58\%) patients at second one intensity in . Myeloid engraftment was defined as the first of 3 consecutive days with a neutrophil (ANC) $>0,5 \times 10 \mathrm{e} 9 / \mathrm{L}$, whereas platelet engraftment was defined as the day with platelets (Plts) $>20 \times 10 \mathrm{e} 9 / 1$ in absence of transfusion support over a week.

Results: The median storage timing for the first and second transplant was 2 months (1-20) and 42 months (2472), respectively. The median CD34+ cell dose at the first AHSCT was 4,6x10e6/kg (2,1-6,8) while at the second AHSCT the dose was $4,8(2-6,6)$. The median time to neutrophil and platelet engraftment was 11 and 12 days for both transplants (ANC 8-17 days and Plts 10-31 days for the first; ANC 8-25 and Plts 9-30 for the second one, respectively). No significant correlation was found between time to engraftment, disease status and CD34/ $\mathrm{kg}$ dose in both AHSC transplant.

Conclusions: Quality methods to validate cellular therapy product storage are still matter of debate in AHSC transplant setting. Our data indicate that long-term storage of AHSC does not negatively impact on neutrophil and platelet engraftment. In conclusion this in vivo quality control could be a useful tool for the validation of graft function during long-term storage as required by JACIE Standards.

Conflict of interest: None

\section{P843}

\section{Hematopoietic Stem Cell Mobilization with Biosimilar Filgrastim in Related Donors: Its Safety and Efficacy Assessment}

Riko Tsumanuma ${ }^{1}$, Eijiro Omoto ${ }^{1}$, Hiroaki Kumagai ${ }^{1}$, Yuta Katayama $^{2}$, Koji Iwato ${ }^{2}$, Go Aoki $^{3}$, Yuji Sato ${ }^{4}$, Yutaka Tsutsumi, Kanji Miyazaki ${ }^{6}$, Nobuhiro Tsukada ${ }^{6}$, Masaki Iino ${ }^{7}$, Yoshiko Astuta ${ }^{8}$,
Yoshihisa Kodera ${ }^{9}$, Shinichiro Okamoto ${ }^{10}$, Hiromasa Yabe ${ }^{11}$

${ }^{1}$ Yamagata Prefectural Central Hospital, Department of Hematology, Yamagata, Japan; ${ }^{2}$ Hiroshima Red Cross Hospital \& Automic-bomb Survivors Hospital, Department of Hematology, Hiroshima, Japan;

${ }^{3}$ Kanazawa University Hospital, Department of Haematology, Kanazawa, Japan; ${ }^{4}$ Tsukuba Memorial Hospital, Department of Hematology, Tsukuba, Japan; ${ }^{5}$ Hakodate City Hospital, Department of

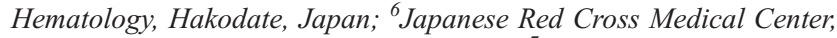
Department of Hematology, Tokyo, Japan; ${ }^{7}$ Yamanashi Prefectural Central Hospital, Department of Hematology and Medical Oncology, Kofu, Japan; ${ }^{8}$ Japanese Date Center for Hematopoietic Cell Transplantation, Nagoya, Japan; ${ }^{9}$ Aichi Medical University School of Medicine, Department of Promotion for blood and Marrow Transplantation, Nagakute, Japan; ${ }^{10}$ Keio University School of Medicine, Division of Hematology Department of Medicine, Tokyo, Japan; ${ }^{11}$ Tokai University School of Medicine, Department of Cell Transplantation and Regenerative Medicine, Isehara, Japan

Background: Biosimilar granulocyte colony-stimulating factor (G-CSF) was approved for mobilizing hematopoietic stem cells (HSCs) in healthy donors; however, its safety and efficacy has not been fully elucidated by clinical trials.

Methods: The Donor Committee of the Japan Society for Hematopoietic Cell Transplantation has set a prospective observational study entitled "A short-term follow-up investigation of relative hematopoietic stem cell donors receiving biosimilar G-CSF to mobilize peripheral blood stem cells." in April 2014 as part of the related donor follow-up initiative. A total of 106 donors received biosimilar G-CSF for mobilization were registered from 25 transplant facilities by the end of March 2017, and their data were analyzed.

Results: There were 48 men and 58 women, and their median age: 38.5 years (range: $15-65$ years). Devices used for apheresis were COBE Spectra ${ }^{\mathrm{TM}}$, Spectra Optia ${ }^{\mathrm{TM}}$, COMTEC $^{\mathrm{TM}}$, and AS.TEC204TM. The collection of HSCs were performed by one-, two-, and three-day apheresis in 57, 47, and 2 donors, respectively. The first apheresis was started on day 4 of bio-similar administration in 93 donors, or on day 5 in 13 donors. Collection of sufficient number of HSCs was completed on day 4 only in 49 donors and on day 5 in 9 donors. Forty six donors required more than one apheresis for collecting sufficient number of HSCs (42 had days 4 and 5, 4 had days 5 and 6, and 2 had days 4, 5, and 6 apheresis). The CBC of donor on day 4 of biosimilar G-CSF showed a mean white blood cell (WBC) count of 40,462 \pm $10,318 / \mu \mathrm{L}$, red blood cell (RBC) count, $451 \pm 39 \times 10^{4} / \mu \mathrm{L}$, and platelet count of $21.0 \pm 4.4 \times 10^{4} / \mu \mathrm{L}$. On day 5 those values showed a WBC count of $38,035 \pm 10,517 / \mu \mathrm{L}$; RBC count, $444 \pm 41 \times 10^{4} / \mu \mathrm{L}$; and a platelet count of $16.8 \pm$ $4.3 \times 10^{4} / \mu \mathrm{L}$. Mean count of collected CD34-positive cells per kilogram recipient weight was $4.28 \pm 6.5 \times 10^{6}$ on day 4 and $3.84 \pm 6.0 \times 10^{6}$ on day 5 . Mean cell count per liter processed blood was $0.46 \pm 0.6 \times 10^{6} / \mathrm{kg} / \mathrm{L}$ on day 4 and $0.43 \pm 0.7 \times 10^{6} / \mathrm{kg} / \mathrm{L}$ on day 5 . In 13 donors started 
apheresis on day 5 mean CD34-positive cell count by donor body weight and processed blood volume were $4.03 \pm 2.0 \times$ $10^{6} / \mathrm{kg}$ and $0.48 \pm 0.3 \times 10^{6} / \mathrm{kg} / \mathrm{L}$, respectively. In this study donor age, gender, WBC count on day 4, G-CSF dose reduction, type of apheresis apparatus, blood collection speed, and treated blood volume had no significant impact on HSCs collection efficacy. Regarding safety of biosimilar G-CSF, there were 126 adverse events in 73 donors were reported, but none of them were serious. The most common adverse event that could attributed to biosimilar G-SCF include low back pain, headache, and bone pain, but none were serious.

Conclusions: When biosimilar G-CSF is administered to healthy related donors, it is comparable with original GCSF with respect to quality and efficacy, and with safety at least over a short term observation.

\section{Conflict of interest:}

S. Okamoto: Honoraria from Chugai, Mochida, Kyowa Kirin, Sanofi

Grant support from Chugai, Kyowa Kirin, Mochida, Sanofi
The other authors state that they have no relationship to disclose.

\section{P844}

Identification of the mechanism of autologous stem cell mobilization using cyclophosphamide or etoposide

Ka-Won Kang ${ }^{1}$, Dae Sik Kim ${ }^{1}$, Eun Sang Yu ${ }^{1}$, Se Ryeon Lee ${ }^{1}$, Hwa Jung Sung ${ }^{1}$, Seok Jin Kim ${ }^{2}$, Chul Won Choi ${ }^{1}$, Yong Park', Byung Soo $\operatorname{Kim}^{1}$

${ }^{1}$ Korea University College of Medicine, Seoul, Korea, Republic of; ${ }^{2}$ Sungkyunkwan University School of Medicine, Seoul, Korea, Republic of

Background: Peripheral blood hematopoietic stem cell collection has been used increasingly, thus, how to mobilize hematopoietic stem cells from bone marrow to peripheral blood becomes an important issue. There are several mobilization methods, and one of these is using chemotherapies (cyclophosphamide or etoposide). In a previous study, hematopoietic stem cell mobilization using etoposide with granulocyte-colony stimulating factor (G-

\section{Drugs 24 hours}
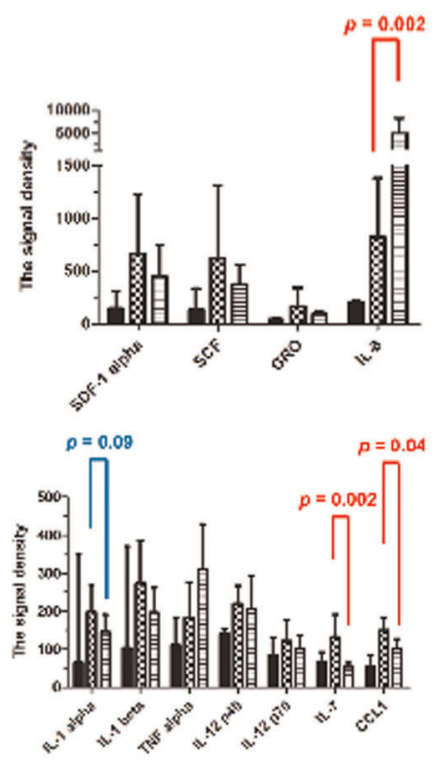

Figure 1. Cytokine antibody arrays
Drugs 72 hours
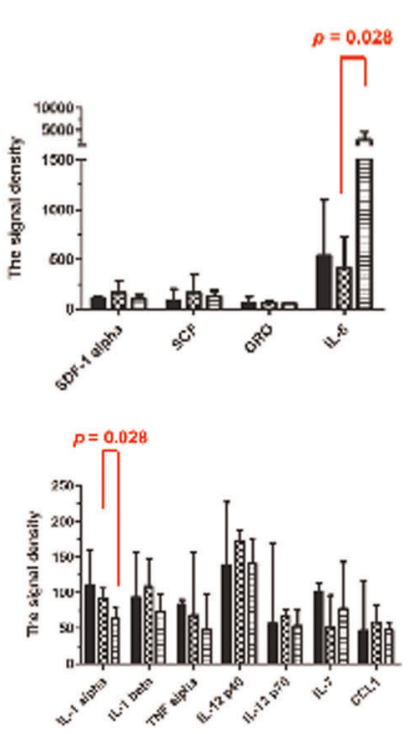

- Coritrul Cycloptosphanide Etoposide

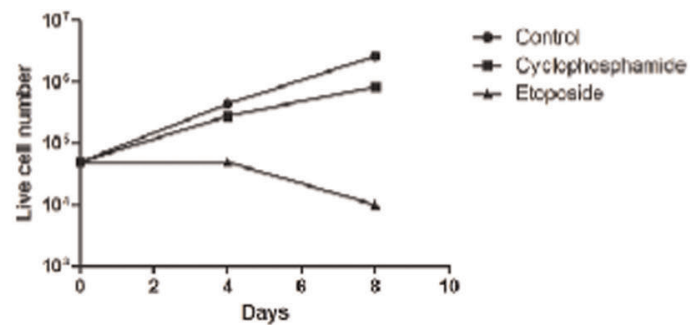

Figure 2. Cell growth curve
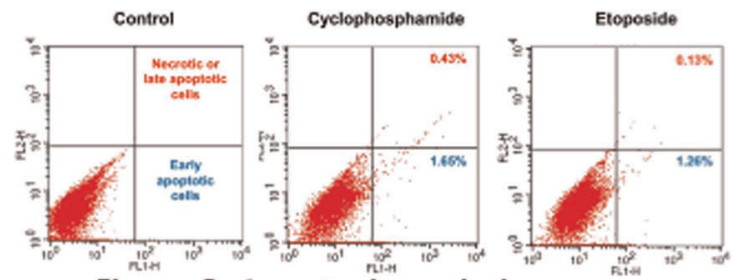

Figure 3. Apoptosis analysis

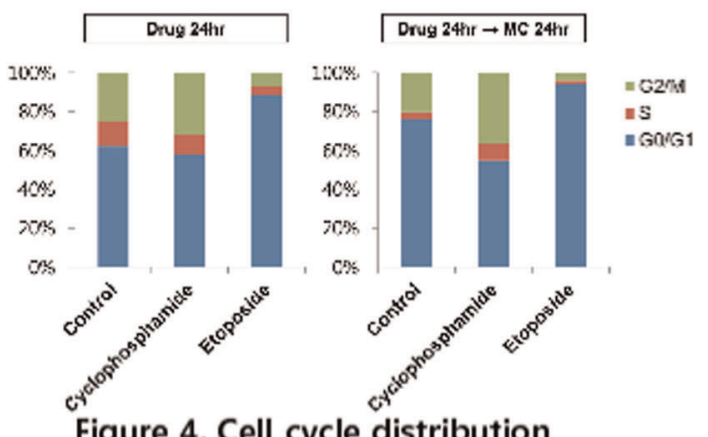

Figure 4. Cell cycle distribution 
CSF) collected more CD34+ cell $/ \mathrm{kg}$ than using cyclophosphamide with G-CSF. However, the mechanism of autologous stem cell mobilization between cyclophosphamide and etoposide is unclear. To identify of this, we analyzed the mechanism of mobilization of hematopoietic stem cells by examining the expression of cytokines.

Methods: Mesenchymal stem cells (MSC) were obtained from the healthy controls who were hospitalized to collect hematopoietic stem cells of the bone marrow. First, the cell viability curves were drawn using the CCK- 8 assay (Dojindo) to determine concentrations that inhibit the growth of mesenchymal stem cells. In subsequent experiments, cyclophosphamide and etoposide were used at a concentration of $10 \%$ inhibition of mesenchymal stem cell growth. Second, $5 \times 10^{\wedge} 4$ mesenchymal stem cells were dispensed per well of a 6-well plate, and treated with cyclophosphamide and etoposide. After one day, and three days after the treatment, the supernatant was collected, and cytokine analysis was carried out using Human Cytokine Array C Series 1000(Ray Biotech). Third, cell growth curve, apoptosis analysis, and cell cycle distribution of MSC treated with cyclophosphamide or etoposide were performed.

Results: In MSC treated with etoposide, SCF, SDF, and TPO, which interfere with the mobilization of hematopoietic stem cells, were shown a trend of decrease, and IL-8, which is a cytokine that promotes hematopoietic stem cell mobilization, were shown a statistically significant increase. In MSC treated with cyclophosphamide, the secretion of cytokines related to inflammation was increased. When MSCs were repeatedly treated with cyclophosphamide or etoposide at intervals of three days, it was identified that the MSCs growth was inhibited by etoposide. In apoptosis analysis, there was no difference between MSCs treated with cyclophosphamide or etoposide. In cell cycle distribution analysis, MSCs treated with etoposide showed cell cycle arrest of G0/G1 phase.

Conclusions: Etoposide could induce the mobilization of HSCs with IL-8 mediated mechanism. Mobilization using etoposide with G-CSF may show synergy effect compared to cyclophosphamide with G-CSF. However, the accumulation of etoposide may interfere with the growth of MSCs, and it is thought to be toxic to MSCs through cell cycle arrest.

Clinical Trial Registry: N/A

Conflict of interest: Nothing to disclose

\section{P845}

Immunophenotypic characterization of autologous, DMSOtreated stem cell transplants

Jan Dirks, Albert Baschong, Dominik Heim, Jacob Passweg,

\section{Dimitrios A. Tsakiris}

University Hospital Basel, Diagnostic Hematology, Basel, Switzerland

Background: Dimethylsulfoxide (DMSO) at final concentrations of $5-10 \%$ is used as cryoprotectant for deepfreezing and storing of autologous stem cell transplants. Recovery of CD34 positive stem cells after thawing can variate considerably, affecting stem cell engraftment. Low CD34 recovery can only be partially attributed to DMSO itself as it does not explain the striking inter-patient differences in products treated with the same DMSO concentrations. We characterized the cell composition of the autologous transplants after collection in order to find out additional reasons that could explain low CD34 recovery post-thawing and test the hypothesis, that increased numbers of leukocyte subsets known to be cytotoxic, such as neutrophils, are responsible for stem cell loss post-thawing.

Methods: Flow cytometry and clinical data of 44 consecutive autologous stem cell transplants (01/2016 $06 / 2016$, single center) were retrospectively analyzed.

Stem cell content before and after thawing of the transplants was registered and correlated to neutrophil and platelet engraftment. Possible DMSO-related complications were recorded immediately after transplantation. Immunophenotyping data of the stem cell products were retrospectively analyzed to elucidate the influence of leukocyte subsets such as neutrophils, monocytes and lymphocytes on CD34 recovery. Furthermore, we correlated time to engraftment with counts of viable CD34 cells pre-freezing and post-thawing.

Results: Viability after stem cell harvest was high for CD34 (99.91\% [99.35-100\%], as well as for all leukocyte subsets. There was no correlation between CD34 or leukocyte subset viability pre-freezing and CD34 recovery. CD34 viability post-thawing was very heterogeneous (88\% [10-98\%]). Relative and absolute counts of monocytes and lymphocytes in the stem cells product had no influence on CD34 recovery. In contrast, a clear negative correlation between relative and absolute neutrophil counts prefreezing and CD34 recovery post-thawing was found $(\mathrm{r}=$ $-0.4 ; \mathrm{p}<0.01$ and $\mathrm{r}=-0.6$ and $\mathrm{p}<0.0001$, respectively).

Stem cell engraftment was categorized as "normal" $(\mathrm{n}=$ $29)$ vs. "late" $(n=13)$ using day 17 as cut-off. Late engrafters had significantly less viable CD34 cells*10\% $\mathrm{kgBW}$ in the transplant post-thawing, compared to normal engrafters (1.6 [0.3-3.2] vs. 2.4 [0.7-4.9]; $p=0.04)$, whereas there was no difference pre-freezing between these groups (2.4 [2.0-5.5] vs. 3.4 [1.1-8.3]; ns).

Conclusions: In the setting of autologous stem cell transplantation high neutrophil counts in the stem cell product correlate with poor CD34 recovery post-thawing 
and delayed engraftment. As viability of neutrophils themselves was very low post-thawing in all products, we postulate that release of cytotoxic substances from apoptotic neutrophils have a detrimental influence on CD34 viability in a dose dependent manner. Although not mandatory by current JACIE standards, CD34 viability measurement postthawing is a valuable tool for quality control of stem cell products. In addition, regular monitoring of neutrophil counts pre-freezing might help explain low CD34 recoveries and if obtained in real-time might help achieve optimal stem cell apheresis conditions.

Conflict of interest: Authors declare no conflicts of interest.

\section{P846}

Abstract previously published

\section{P847}

Lenograstim $5 \mathrm{mcg} / \mathrm{Kg}$ vs Biosimilar Filgrastim $10 \mathrm{mcg} / \mathrm{Kg}$ in lymphoma patients undergoing peripheral blood stem cell mobilization: interim analysis of a randomized study

Francesco Marchesi ${ }^{1}$, Michele Vacca ${ }^{2}$, Svitlana Gumenyuk ${ }^{1}$, Annino Pandolf $^{2}$, Daniela Renzi ${ }^{1}$, Francesca Palombi ${ }^{1}$, Francesco Pisani ${ }^{1}$, Atelda Romano ${ }^{1}$, Antonio Spadea ${ }^{1}$, Francesco Ipsevich ${ }^{2}$, Elena Papa $^{1}$, Marco Canfora ${ }^{3}$, Luca Pierelli ${ }^{2}$, Andrea Mengarelli ${ }^{1}$

${ }^{1}$ Regina Elena National Cancer Institute, Hematology and Stem Cell Transplant Unit, Rome, Italy; ${ }^{2}$ S. Camillo-Forlanini Hospital, Leukapheresis and Cellular Therapy Unit, Rome, Italy; ${ }^{3}$ Regina Elena National Cancer Institute, Scientific Direction, Rome, Italy

Background: So far there are many studies comparing efficacy and safety of biosimilar Filgrastim with the originators but only few data have been published about the comparison with Lenograstim. Our previously published study suggested that biosimilar Filgrastim $\left(\right.$ Zarzio $\left.^{\circledR}\right)$ could be less effective than Lenograstim when used at standard dosage of $5 \mathrm{mcg} / \mathrm{Kg}$ in patients with lymphoma undergoing peripheral blood stem cell (PBSC) mobilization.

Methods: From October 2014 to September 2017, 40 patients with lymphoma (non-Hodgkin $n=32$, Hodgkin $n$ $=8$ ) were randomly assigned to receiving Lenograstim 5 $\mathrm{mcg} / \mathrm{Kg}(\mathrm{n}=20)$ or Zarzio ${ }^{\circledR} 10 \mathrm{mcg} / \mathrm{Kg}(\mathrm{n}=20)$ for PBSC mobilization. The patients were stratified according to treatment line at the time of PBSC mobilization (after a first-line treatment or after a salvage treatment for refractory/relapsed disease). The target of $\mathrm{CD} 34+$ cell dose was $4 \times 10^{\wedge} 6 / \mathrm{Kg}$. All patients underwent the same standardized procedures, including criteria for Plerixafor administration. The primary end-point of the study was to compare the rate of mobilization failures and of patients unable to reach the planned CD34+ cell dose.

Results: In October 2017, we planned the first interimanalysis of the study. The two cohort of patients were well balanced for all baseline clinical features analyzed. As for primary end-point, we observed an identical rate of mobilization failures (5\% in both cohorts; $\mathrm{P}=\mathrm{NS}$ ) and of patient unable to reach the target of CD34+ cell planned dose $(10 \%$ in both cohorts; $\mathrm{P}=\mathrm{NS})$. We also observed a similar rate of patients needing Plerixafor administration due to a suboptimal level of CD34+/mcL at peak under GCSF (20\% in Zarzio ${ }^{\circledR}$ group vs $25 \%$ in Lenograstim group; $\mathrm{P}=\mathrm{NS}$ ). Moreover, median days of G-CSF administration, median $\mathrm{CD} 34+/ \mathrm{mcL}$ at leukepheresis and median number of $\mathrm{CD} 34 \times 10^{\wedge} 6 / \mathrm{Kg}$ collected at first leukapheresis were similar between the two cohorts of patients. No difference in terms of drug-related adverse events was observed in the two groups of patients, with no reported serious adverse events.

Conclusions: At first interim-analysis, our data clearly demonstrate that Lenograstim $5 \mathrm{mcg} / \mathrm{Kg}$ could be considered substantially equivalent in terms of efficacy and safety compared with Zarzio ${ }^{\circledR}$ at dosage of $10 \mathrm{mcg} / \mathrm{Kg}$ to in lymphoma patients undergoing PBSC mobilization and collection.

Conflict of interest: None of the authors has anything to disclose.

\section{P848}

Low dose vs intermediate-high dose cyclophosphamide vs G-CSF stem cell mobilization in multiple myeloma in the era of novel agents: a multicenter study

Francesco Saraceni ${ }^{1}$, Annalisa Pezzi ${ }^{1}$, Martina Chiarucci ${ }^{2}$, Attilio Olivieri $^{2}$, Eliana Zuffa ${ }^{1}$, Massimo Martino ${ }^{3}$, Daniele Lasz lo $^{4}$, Francesco Lanza ${ }^{1}$

${ }^{1}$ Ravenna Hospital, Ravenna, Italy; ${ }^{2}$ Ospedali Riuniti Ancona, Ancona, Italy; ${ }^{3}$ Ospedale Bianchi-Melacrino-Morelli, Reggio Calabria, Italy; ${ }^{4}$ European Institute of Oncology, Milano, Italy

Background: The optimal stem cell mobilization strategy for patients (pts) with multiple myeloma (MM) is still a matter of debate.Possible approaches include low or intermediate-high doses of cyclophosphamide (CY) or granulocyte colony-stimulating factor (G-CSF) alone.

Methods: We retrospectively analyzed MM patients undergoing stem cell mobilization in four Italian centers (Ravenna, Ancona, Milano, Reggio Calabria) between January 2010 and August 2017.Sixty-two (25\%) patients received low dose cyclophosphamide (LD-CY group, defined as $2 \mathrm{~g} / \mathrm{m} 2), 142$ (57\%) intermediate-high dose 
cyclophosphamide (HD-CY group, defined as 3-4 g/m2) and $45(18 \%)$ received G-CSF alone (G-CSF group).

Results: A total of 261 patients fulfilled the inclusion criteria.Median age at the time of mobilization was 62 years (IQR 56-65), without any significant difference among the 3 groups.All patients had received a previous treatment before the mobilization attempt, mainly bortezomib-based (50\%) or lenalidomide-based (13\%) protocols; $10 \%$ of patients had received a karfilzomib-including regimen prior to stem cell mobilization. CY patients tended to had received mainly lenalidomide (23\%) or karfilzomib (37\%)-based regimens $(\mathrm{p}=0.043$ and $\mathrm{p}<0.001$, respectively) while G-CSF patients tended to had received mainly bortezomib-based regimen $(84 \% \mathrm{p}<0.001)$. At day 0 of the planned mobilization schedule, median platelet count was inferior in LD-CY group as compared to HD-CY and G-CSF groups $(\mathrm{p}=0.02)$, while Hemoglobin and WBC count did not differ.

Median peak of circulating CD34+ was 73, 88 and 56/ $\mathrm{mmc}$ in LD-CY, HD-CY and G-CSF group, respectively (p $<0.01)$. The median amount of CD34+ stem cells collected was $8.1,9.7$ and 7 respectively $(\mathrm{p}=0.03)$. Ninety-six percent of G-CSF patients achieved a stem cell collection of at least $2 \times 10^{6} \mathrm{CD} 34+/ \mathrm{kg}$, as compared to $94 \%$ of LD$\mathrm{CY}$ and $96 \%$ of HD-CY group $(\mathrm{p}=0.78)$. No statistical difference was observed in the number of days of apheresis necessary to achieve the mimimum yield.Similarly, no significant difference was observed in probability of achieving a collection of at least $4 \times 10^{6} \mathrm{CD} 34+/ \mathrm{kg}$ (target dose for single transplant, minimum dose for double transplant), which was successfully collected in $89 \%$ of LD-CY, 91\% if HD-CY and $87 \%$ of G-CSF patients $(p=0.66)$. Twenty-four percent of G-CSF patients achieved a collection greater than $8 \times 10^{6} \mathrm{CD} 34+/ \mathrm{kg}$ (target dose for double transplant) as compared to 58\% of LD-CY and 59\% of HD-CY $(\mathrm{p}<0.01)$. Plerixafor was added to the planned mobilization regimen in $18 \%, 12 \%$ and $82 \%$ of $\mathrm{LD}-\mathrm{CY}$, HD-CY and G-CSF groups, respectively $(\mathrm{p}<0.01)$.

In multivariate analysis, the kind of mobilization strategy did not significantly affect stem cell collection. Similarly no impact of age, previous treatment with lenalidomide, bortezomib or karfilzomib, Hb, PLT or WBC counts was observed. Grade 3-4 adverse events during mobilization were neutropenic fever (2 pts in CY group) and diarrhea (2 pts in G-CSF group).Median days of hospitalization were 0 for LD-CTX and G-CSF as compared to 3 for HD-CTX (p $<0.01$ ).

Conclusions: Given the limits of a retrospective analysis, our results suggest that low dose CY, intermediate-high dose CY and G-CSF have comparable efficacy for stem cell mobilization in MM patients in the modern era of novel agents.

Conflict of interest: [all authors]: nothing to declare
P849

Novel protocol for ambulatory mobilization of hematopoietic stem cells in multiple myeloma patients very low doses of Ara-C and tailored G-CSF

\begin{abstract}
Martyna Maciejewska, Elżbieta Urbanowska, Magdalena Feliksbrot-Bratosiewicz, Karol Lis, Anna Waszczuk-Gajda, Kamila Skwierawska, Michal Górka, Hubert Heleniak, Malgorzata Król, Maria Król, Wiesław Wiktor Jędrzejczak, Emilian Snarski
\end{abstract}

Medical University of Warsaw, Hematology, Oncology and Internal Diseases, Warszawa, Poland

Background: High-dose chemotherapy followed by autologous stem cell transplantation (ASCT) remains a standard of care in eligible patients with multiple myeloma. The perfect mobilization regimen should have all the advantages of known protocols: predictable time of CD34+ mobilization to the blood (G-CSF mobilization), lack of serious side effects (G-CSF mobilization), high stem cell collection number with low number of apheresis (High dose Ara-C mobilization) and ability to be held in outpatient setting (G-CSF). It should also be free of the major disadvantages of known protocols: severe cytopenias (High dose Ara-C or Cyclophosphamide mobilization), unpredictability of optimal day of apheresis (Cyclophosphamide), relatively low number of collected cells (G-CSF) or price (plerixafor). In aim to create a protocol which would meet all of those expectations, we reduced the Ara-C dose and adjusted the doses of G-CSF given prior to apheresis.

Methods: The medical records of 37 patients who were mobilized in ambulatory setting in 2016 - 2017 with this new protocol were analysed retrospectively. Mobilization protocols: $400 \mathrm{mg} / \mathrm{m}^{2}$ of $\mathrm{AraC}$ on days +1 and +2 and G$\mathrm{CSF}$ at the dose $5 \mu \mathrm{g} / \mathrm{kg} /$ day from day +5 and escalated to $10 \mu \mathrm{g} / \mathrm{kg} /$ day split into two doses on days +12 and +13 or $+10,+11,+12,+13$, apheresis started on day +14 in all patients, regardless of CD34+ count. The potential side effects were monitored.

Results: 37 patients underwent and completed ambulatory mobilization with very low dose Ara-C in outpatient setting. The patients who received $400 \mathrm{mg}$ Ara-C on days 1 and 2 and tailored G-CSF (5ug/kg from day +5 and 10ug/ $\mathrm{kg}$ from day +10 ) had the best results. The average collection of CD34+ cells was $11.3 \times 10^{6}$ per $\mathrm{kg}$ on the first apheresis. The target CD34+ cell count $>5 \times 10^{6} \mathrm{CD} 34+$ cells $/ \mathrm{kg}$ was reached in all but one patient (who collected $4.2 \times 10^{6} \mathrm{CD} 34+$ cells $/ \mathrm{kg}$ ). The average number of apheresis needed for collection of $>5 \times 10^{6} \mathrm{CD} 34+$ cells/ $\mathrm{kg}$ was 1.125 - and more than 2 aphereses were never performed. All but one collection were performed with peripheral venous access (no PICCs and CVCs were used). 
Moreover $100 \%$ of those patients proceeded to transplantation.

None of the analysed patients experienced severe adverse events including cytopenia, infections, transfusions, that required hospital stay during mobilization. The most commonly reported adverse events were connected with G-CSF - mild bone pain with no need for drugs intake or treatable with OTC drugs including paracetamol. As for the economic analysis at least 800 Euros were saved per patient due to the lower cost of medical care.

Conclusions: Very low dose cytarabine and tailored GCSF protocol became preferred regimen for stem cell mobilization in patients with multiple myeloma at our centre according to its advantages over all known mobilization protocols.

Clinical Trial Registry: NOT APPLICABLE

Conflict of interest: None to declare

\section{P850}

\section{Optimized timing of chemomobilization, G-CSF administration and leukapheresis for a more efficient hematopoietic stem cell collection}

Katharina Kriegsmann ${ }^{1}$, Anita Schmitt ${ }^{1}$, Mark Kriegsmann ${ }^{2}$, Thomas Bruckner ${ }^{3}$, Stefan Klein ${ }^{4}$, Patrick Wuchter ${ }^{5}$

${ }^{1}$ Heidelberg University, Department of Medicine V, Heidelberg, Germany; ${ }^{2}$ Heidelberg University, Institute of Pathology, Heidelberg, Germany; ${ }^{3}$ Heidelberg University, Institute of Medical Biometry und Informatics, Heidelberg, Germany; ${ }^{4}$ Medical Faculty Mannheim, Heidelberg University, Department of Medicine III, University Medical Center Mannheim (UMM), Mannheim, Germany; ${ }^{5}$ Medical Faculty Mannheim, Heidelberg University, Institute of Transfusion Medicine and Immunology, Mannheim, Germany

Background: Efficient collection of autologous peripheral blood stem cells (PBSCs) depends on the optimal timing of mobilization chemotherapy, application of granulocyte-colony-stimulating factor (G-CSF), and assessment of $\mathrm{CD}_{3}{ }^{+}$cell number in the peripheral blood (PB). Therefore, implementing a valid timetable depending on the applied chemomobilization regimen is essential.

Methods: For eight of the most commonly used chemomobilization regimens we developed a comprehensive time schedule for PBSC mobilization. A retrospective analysis of the mobilization process of representatively selected patients with a variety of diagnoses, including multiple myeloma, malignant lymphoma, and sarcoma was performed (6 to 10 patients per regimen). Clinical and PBSC collection parameters were analyzed including duration of G-CSF application, time point of $\mathrm{CD}_{3}{ }^{+}$ assessment, $\mathrm{PB} \mathrm{CD} 34^{+}$cell count, number of leukapheresis sessions, processed blood volume, and $\mathrm{CD}_{3} 4^{+}$collection results.

\begin{tabular}{|c|c|c|c|}
\hline \multicolumn{2}{|c|}{ Mobilization chemotherapy } & \multirow{2}{*}{$\begin{array}{l}\text { G-CSF application } \\
\text { Start }\end{array}$} & \multirow{2}{*}{$\begin{array}{l}\text { PB CD34+ assessmen } \\
\text { Start }\end{array}$} \\
\hline Name & Duration & & \\
\hline CAD & d $1-4$ & d 9 & d 13 \\
\hline Cyclophoshamide & d $1-2$ & d 5 & d 10 \\
\hline (R)CHOP & d $1-5$ & d 6 & d 11 \\
\hline (R)DHAP & d $1-4$ & d 9 & d 14 \\
\hline (R)ICE & d $1-3$ & d 6 & d 12 \\
\hline PEI & d $1-5$ & d 6 & d 12 \\
\hline VIDE & d $1-3$ & d 5 & d 12 \\
\hline GMALL C1 & d $1-5$ & d 7 & d 14 \\
\hline
\end{tabular}

\section{[[P850 Table] Timing of mobilization]}

Results: All patients successfully collected at least one transplant (i.e. $>2 \times 10^{6} \mathrm{CD} 34^{+}$cells $/ \mathrm{kg}$ body weight) and reached their individual collection goal in adherence to the given schedule of chemotherapy, application of G-CSF, measurement of CD34+ cells in the PB and subsequent leukapheresis. The proposed schedule provides the earliest time point one should start monitoring CD34+ cells in PB, while considering that the first LP session may well start one or several days later.

Conclusions: In this study, we analyzed the most commonly used chemomobilization regimens regarding the optimal timing of subsequent G-CSF application and stem cell collection. As a result, we present a validated blueprint of a timing schedule, which improves the efficiency of the mobilization attempt and also contributes to patient satisfaction by increasing the transparency and calculability of the approach.

Conflict of interest: The first author and all co-authors confirm that there are no potential conflicts of interest to disclose except the following: A. Schmitt: travel grants from Hexal AG; P. Wuchter: honoraria and membership on advisory boards for Sanofi-Aventis, membership on advisory boards and travel grants from Hexal AG.

\section{P851}

PAR1 expression level is a positive predictive marker for efficiency of G-CSF induced human CD34+ hematopoietic stem cells mobilization and repopulation

Neta Nevo ${ }^{1,2}$, Shiri Gur-Cohen ${ }^{2}$, Orit Kollet', Tzila Zuckerman ${ }^{3}$, Benjamin Brenner ${ }^{3}$, Myriam Ben-Arush ${ }^{1}$, Tsvee Lapidot ${ }^{2}$

${ }^{1}$ Ruth Rappaport children's Hospital, Pediatric Hematology Oncology and BMT, Haifa, Israel; ${ }^{2}$ The Weizmann Institute of Sciense, Department of Immunology, Rehovot, Israel; ${ }^{3}$ Rambam Medical Center, Hematology and BMT, Haifa, Israel

Background: Hematopoietic stem and progenitor cell (HSPC) mobilization by repeated Granulocyte Colony Stimulating Factor (G-CSF) treatment is the most frequently used protocol for allogenic transplantations. The 
coagulation system regulates murine bone marrow (BM) HSPC retention, egress and rapid mobilization. Herein we aim to assess the function and clinical importance of thrombin receptor, Protease Activated Receptor 1 (PAR1), in human G-CSF induced CD34+ cell mobilization.

Methods: Mononuclear cells (MNCs) of 20 healthy matched sibling stem cells donors were isolated, stained and analyzed by FACS before and after treatment with G-CSF. Next, we used pre-clinical functional mice model. Results were statistical significant with at least 3 repeats.

Results: We revealed that PAR1 expression levels on circulating donor leukocytes before G-CSF treatment were positively correlated with higher yields of mobilized leukocytes after stimulation $(\mathrm{P}<0.0001)$. In addition, the percentage of PAR1-expressing CD $34^{+}$HSPCs in the blood and their absolute numbers before initiation of mobilization positively correlated with peripheral blood leukocyte counts $(\mathrm{P}<0.0001)$ and yield of mobilized $\mathrm{CD} 34^{+}$HSPCs $(\mathrm{P}<$ $0.001)$.

To further gain insight into the role played by PAR1 signaling in the regulation of G-CSF-induced HSPC mobilization, we used a functional preclinical C57BL mouse model. We observed a significant up regulation in PAR1 expression on BM HSPC following G-CSF treatment. This elevation is critical since interfering with PAR1 signaling with a specific PAR1 antagonist, SCH79797, inhibited stem cell mobilization. G-CSF treatment dramatically accelerated PAR1 dependent nitric oxide (NO) production by BM leukocytes including HSPC. Notably F2r -/- mice which are deficient in PAR1, express high levels of NO on BM HSPC at baseline, failed to elevate NO after treatment with G-CSF and as a result mobilized to a much lower extent as compared to their wild type (WT) counterpart. Pharmacological inhibition of NO generation by L-NAME during G-CSF mobilization also abrogated mobilization via inhibition of BM HSPC PAR1 elevation, concomitant with expansion of BM HSPC Endothelial Protein C Receptor (EPCR) + stem cells with high adhesion and repopulation potential. Interestingly, mobilized stem cells lack EPCR expression which is part of the mobilization process, and it reappears following their transplantation and $\mathrm{BM}$ repopulation. Antagonizing PAR1 signaling during G-CSF stimuli expanded BM $\mathrm{EPCR}^{+}$Long Term (LT)-HSPC and supportive stromal progenitor cells (MSPCs), as confirmed by increased repopulation in transplanted mice.

Importantly, we further evaluated the clinical influence of PAR1 on HSPC function. We report that blocking PAR1 signaling significantly blocked human MNCs migration towards a gradient of CXCL-12 in vitro. In line, as PAR1 expression on human CD34+ cells is higher after stimulation with G-CSF among healthy stem cells donors the cells migrate better toward CXCL-12. Finally, tracing the consequences of the follow-up stem cell transplantation, we found accelerated neutrophil and platelet recovery in patients transplanted with mobilized cells expressing higher surface PAR1 levels at baseline $(\mathrm{P}<0.01, \mathrm{P}<0.05)$.

Conclusions: Our study indentifies PAR1 signaling by NO generation as an essential regulator of G-CSF induced HSPC mobilization and highlights PAR1 expression level as a predictor of HSPCs mobilization, migration and repopulation.

Conflict of interest: None of the authors has anything to disclose.

\section{P852}

Plerixafor Combinded with Standard Regimens for Hematopoietic Stem Cell Mobilisation in Paediatric Patients with Solid Tumours Eligible for Autologous Transplants: 2Arm Phase I/II Study (MOZAIC)

Bruce Morland ${ }^{1}$, Tomas Kepak ${ }^{2}$, Sandro Dallorso ${ }^{3}$, Julian Sevilla ${ }^{4}$, Dermot Murphy ${ }^{5}$, Roberto Luksch, Isaac Yaniv ${ }^{7}$, Peter Bader ${ }^{8}$, Jochen Rößler ${ }^{9}$, Gianni Bisogno ${ }^{10}$, Britta Maecker-Kolhoff ${ }^{11}$, Peter Lang $^{12}$, C. Michel Zwaan ${ }^{13}$, David Sumerauer ${ }^{14}$, Gergely Kriván ${ }^{15}$, John Bernard ${ }^{16}$, Qianying Liu ${ }^{16}$, Eileen Doyle ${ }^{16}$, Franco Locatelli ${ }^{17}$

${ }^{1}$ Birmingham Women's and Children's Hospital, Birmingham, United Kingdom; ${ }^{2}$ University Hospital Brno and St. Anna University Hospital, Brno, Czech Republic; ${ }^{3}$ IRCCS Giannina Gaslini, Genova, Italy; ${ }^{4}$ Hospital Infantil Universitario Niño Jesus, FIB HIUNJ, CIBERER, Madrid, Spain; ${ }^{5}$ Royal Hospital for Children, Glasgow, United Kingdom; ${ }^{6}$ Fondazione IRCCS Istituto Nazionale dei Tumori, Milano, Italy; ${ }^{7}$ Schneider Children's Medical Center of Israel, Tel Aviv, Israel; ${ }^{8}$ Universitätsklinikum Frankfurt am Main, Frankfurt, Germany; ${ }^{9}$ Medical Center - University of Freiburg, Freiburg, Germany; ${ }^{10}$ University of Padova, Padova, Italy; ${ }^{11}$ Hannover Medical School, Hannover, Germany; ${ }^{12}$ Universitätsklinikum Tübingen, Tübingen, Germany; ${ }^{13}$ Erasmus MC-Sophia Children's Hospital, Rotterdam, Netherlands; ${ }^{14}$ Faculty Hospital Motol, Praha, Czech Republic; ${ }^{15}$ United St Istvan and St Laszlo Hospital, Budapest, Hungary; ${ }^{16}$ Sanofi Genzyme, Cambridge, MA, United States; ${ }^{17}$ IRCCS Bambino Gesù Children's Hospital, Rome, Italy

Background: Plerixafor promotes release of $\mathrm{CD} 34+$ cells to the peripheral blood for collection and subsequent autologous transplantation. The safety and efficacy of plerixafor has been established in adults. This study (NCT01288573) investigated plerixafor's safety and efficacy in children.

Methods: Stage 1 investigated the dosage, pharmacokinetics (PK), pharmacodynamics (PD) and safety of plerixafor in addition to standard mobilisation (G-CSF \pm chemotherapy) in paediatric cancer patients. The Stage 2 primary endpoint was the difference in a proportion of patients achieving doubling of $\mathrm{CD} 34+$ cell count in the plerixafor+standard mobilisation group versus standard mobilisation alone. Secondary Stage 2 endpoints were number of aphereses required to reach $\geq 2 \times 10^{6} \mathrm{CD} 34+$ cells/ 
$\mathrm{kg}$, total and per-apheresis CD34+ cell yield, percentages of patients proceeding to transplantation, and sustained engraftment at 3, 6, 12 and 24 months post-transplantation.

Results: Stage 1: 27 patients were treated across 3 age groups (ages 2 to $<6,6$ to $<12$, and 12 to $<18$ years). 3 patients in each age group were treated at each dose level $(160,240,320 \mu \mathrm{g} / \mathrm{kg})$. Based on PK and PD data, the dose proposed for Stage 2 was $240 \mu \mathrm{g} / \mathrm{kg}$. 16/27 (59\%) patients experienced a treatment-emergent adverse event (TEAE); 9 (33\%) patients experienced a serious TEAE. 2 deaths occurred after the 30-day period following the last plerixafor dose; both deaths were due to disease progression and considered unrelated to study drug.

Stage 2: 45 patients were randomised $2: 1$ to receive plerixafor + standard mobilisation versus standard mobilisation alone. Demographic characteristics were similar across groups. 26 patients aged 1 to $<6,12$ aged 6 to $<12$, and 7 aged 12 to $<18$ years were treated. Primary cancer histology was similar in each treatment arm. Most (35/45, $77.8 \%$ ) patients completed the study period. 3 patients in each cohort died due to disease progression. 24/30 (80\%) patients in the plerixafor arm versus $4 / 14(28.6 \%)$ patients in the standard arm met the primary endpoint $(\mathrm{p}=0.0019)$. Median percent change in $\mathrm{CD} 34+$ cell counts was overwhelmingly in favour of the plerixafor arm $(220.8 \%$ versus $39.0 \%)$. More patients $(76.7 \%$ plerixafor vs $66.7 \%$ standard) proceeded to transplantation in the plerixafor arm. Sustained engraftment rate was similar between treatment arms at 6, 12, 18 and 24 months. Most patients experienced a TEAE; $23 / 30(76.7 \%)$ versus 10/15 (66.7\%) patients in the plerixafor and standard arms, respectively. TEAEs $\geq$ Grade 3 were reported in $43.3 \%$ of patients in the plerixafor arm versus $40.0 \%$ of patients in the standard arm. TEAEs related to study treatment (all Grade 1) were reported for 4 patients in the plerixafor arm. Serious TEAEs occurred in $30.0 \%$ of patients in the plerixafor arm versus $26.7 \%$ of patients in the standard arm (none related to study treatment). No patient discontinued treatment or experienced death due to a serious TEAE.

Conclusions: Plerixafor is effective in children, with $80.0 \%$ versus $28.6 \%$ of patients meeting the primary endpoint for the plerixafor and standard mobilisation arms, respectively, and increasing the peripheral blood CD34+ cell count in children by $>200 \%$ compared with standard mobilisation treatment. Plerixafor had an acceptable safety profile in children.

Clinical Trial Registry: (NCT01288573)

Conflict of interest: This study was funded by Sanofi Genzyme

J. Sevilla reports that payments from Sanofi Genzyme to the institution were received for conducting this clinical trial; reports financial support outside the submitted work for educational lectures by Novartis, advisory board member for Rocket Pharma, educational lectures for Miltenyi, and advisory board member for Novartis.

J. Bernard, Q. Liu, and E. Doyle are employees of Sanofi Genzyme.

Jochen Rößler reports grants from Pfizer, personal fees from Pierre Fabre, outside the submitted work

Britta Maecker-Kolhoff reports personal fees and nonfinancial support from Jazz Pharmaceuticals, outside the submitted work.

The remaining authors have nothing to disclose.

\section{P853}

\section{PLERIXAFOR ON DEMNAD IN FIVE HEALTHY FAMILY DONORS TO MOBILIZATION FOR STEM CELL TRANSPLANTATION}

\begin{tabular}{|c|c|c|c|c|c|c|c|c|c|}
\hline Case & Weight $D / R$ & Day & Mobilization & $\begin{array}{c}\text { Donor CD } 34+ \\
\text { count } / \mathrm{ml}\end{array}$ & $\begin{array}{c}\text { Graft CD34+ } \\
\text { (x106/kg RBW) }\end{array}$ & $\begin{array}{l}\text { Graft } \mathrm{CD}^{4} 4+ \\
(\times 106)\end{array}$ & Graft $\operatorname{co} 3 \%$ & $\begin{array}{l}\text { Total CD34+ } \\
\text { x106 infused }\end{array}$ & $\begin{array}{c}\text { Total CD34+ } \\
\times 106 / \mathrm{kg} \text { infused }\end{array}$ \\
\hline \multirow[t]{3}{*}{1} & $80 / 0.73$ & 1 & GCSF & 16.000 & 1.06 & 111 & 40 & 530 & 5.12 \\
\hline & & 2 & GCSF & 15.060 & 0.93 & 96 & 20 & & \\
\hline & & 3 & GCSF $+P L$ & 44.570 & 3.13 & 123 & 22 & & \\
\hline \multirow[t]{3}{*}{2} & $72 / 1.18$ & 1 & GCSF & 25.580 & 1.98 & 121 & 24 & 458 & 7.5 \\
\hline & & 2 & GCSF & 12.663 & 1.88 & 115 & 35 & & \\
\hline & & 3 & GCSF+PL & 46.323 & 3.64 & 222 & 30 & & \\
\hline \multirow[t]{2}{*}{3} & $55 / 0.98$ & 1 & GCSF & 21.053 & 1.42 & 81 & 65 & 305 & 5,35 \\
\hline & & 2 & GCSF+PL & $\$ 9.860$ & 3.93 & 224 & 53 & & \\
\hline \multirow[t]{2}{*}{4} & $48 / 0.81$ & 1 & GCSF & 32.500 & 2.10 & 128 & 42 & 568 & 9.63 \\
\hline & & 2 & GCSF+PL & 86.000 & 7.53 & 449 & 32 & & \\
\hline \multirow[t]{2}{*}{5} & $98 / 0.9$ & 1 & GCSF & 16.072 & 1.63 & 180 & 24 & 789 & 7.18 \\
\hline & & 2 & GCSF $+P L$ & 54.390 & 5.54 & 610 & 22 & & \\
\hline
\end{tabular}

[P853 Figure] [Table 2. Cellularity obtained] 
Virginia Pradillo Fernandez, Cristina Pascual, Ana Perez-Corral, David Serrano, Nieves Dorado, Laura Solan, Mi Kwon, Jose Luis Diez, Martin, Javier Anguita

Hospital General Universitario Gregorio Maranon, Madrid, Spain

Background: Plerixafor (PL) is a selective antagonist receptor CXCR4 that blocks the union of CD34+ hematopoietic progenitor cells to marrow stromal. PL mobilizes CD34+ cells to peripheral blood (pb) by its own and potencies G-CSF capacity when they use in combination. PL is licensed for patients with multiple myeloma or non-Hodgkin`s lymphoma achieving poor mobilization with GCS-F only. We present five allogenic healthy donors with a poor mobilization which G-CSF and where using PL "off label" to achieve an adequate graft for stem cell transplantation.

Methods: We report five healthy donors from June 2016 to November 2017 in which we use PL in case of poor mobilization with G-CSF, in our institution. The median follow-up was 15 months. Patient's characteristics are described in Table 1. We started mobilization with GCSF in a dosage of $10 \mathrm{ug} / \mathrm{kg} /$ day subcutaneous for four days. CD34+ cell count in blood was determined by flow cytometry.

PL $(0.24 \mathrm{mg} / \mathrm{kg} /$ day $)$ was added, 12 hours before second or third leukapheresis, due to a poor mobilization (CD34+ $<20 \mathrm{x} 10^{6} / \mathrm{ml}$ in $\mathrm{pb}$ pre-apheresis) and the short time available previous Cyclophosphamide post-transplant (Cypost). In one case, PL was added due to the need of high $\mathrm{CD} 34+$ graft because of previous failure engraftment. Cell blood count (CBC) was performed with Unicell DHX 800 (Beckman Coulter $^{\circledR}$ ) and CD34+ cells were quantificated by flow cytometry with FC500 (BeckmanCoulter ${ }^{\circledR}$ ) cytometer according to protocol ISHAGE.

Results: After PL administration, CD $34+\mathrm{x} 10^{6} / \mathrm{ml}$ count pre-apheresis increased 3 times media and optimal cellularity was reached in the five donors (Table 2). In terms of side effects, emetic syndrome was described with duration of 24 hours in two donors, without other side effects

attributable to PL. No retarded neutrophils and platelet engrafted time was described except third case which developed failure engraftment in presence of another risk factors (severe aplastic anaemia). Graft CD3 + cellularity was similar to the literature reported and there were no acute GVHD grade II-IV.

Conclusions: PL was well tolerated in all cases and ensured procurement of an adequate graft for transplantation resulting in a normal hematopoietic engraftment. In our cases reported, the use of PL, without severe side effects, permitted achieving the enough cellularity in case of poor mobilization and in transplant with need of high CD34+ dose just in time before Cypost.
Although used in a few healthy donors, we consider the use of PL can be useful and safe in combination with GCSF to reach a satisfactory mobilization.

Conflict of interest: There is no conflict of interest.

\begin{tabular}{lllll}
\hline Patient & Gender/Age & Diagnosis & Type transplant & GVHD prophylaxis \\
\hline 1 & M/44 & Myelofibrosis & Haplo & Cypost \\
2 & V/31 & AML & Id-HLA & Cypost \\
3 & V/18 & Aplastic Anemia & Haplo & Cypost \\
4 & V/51 & AML & Haplo & Cypost \\
5 & V/58 & CLL & Haplo & Cypost \\
\hline
\end{tabular}

[[P853 Table] Table 1. Patients' characteristics]

\section{P854}

Predicting the duration of Peripheral Blood Stem Cell (PBSCC) collection: Mid collection CD34 ${ }^{+}$cell count is more reliable than pre-collection counts

Michal Pearl-Yafe ${ }^{1}$, Limor Horev-Azaria ${ }^{1}$, Yakov Sverdlov ${ }^{1}$, Bracha Zisser $^{2}$, Isaac Yaniv ${ }^{1,3}$, Jerry Stein ${ }^{1,3}$

${ }^{1}$ Schneider Children's Medical Center of Israel, Petach Tikva, Israel; ${ }^{2}$ Ezer Mizion, Petach Tikva, Israel; ${ }^{3}$ Tel Aviv University, Sackler Faculty of Medicine, Tel Aviv, Israel

Background: The duration of a Peripheral Blood Stem Cell Collection (PBSCC) is a function of the target CD34 ${ }^{+}$ cell count, of the $\mathrm{CD} 34^{+}$cell count in donor blood, and of the efficiency of the pheresis process. Projection of anticipated PBSCC yield is important to the collection staff, as it permits customization of pheresis duration. Formulae for predicting CD34 ${ }^{+}$yields of PBSCC have been published. We evaluated two models to predict endcollection (End-C) $\mathrm{CD}_{4} 4^{+}$cell yield, each predicated on either a pre-collection (Pre-C) donor blood sample or a midcollection (Mid-C) sample obtained from the collection bag after the processing of one blood volume.

Methods: PBSCC was performed using the Optia ${ }^{\circledR}$ Spectra apheresis system version 11 (Terumo, Denver Colorado, USA) using $\mathrm{CMNC/MNC}$ procedures. The default collection volume at our center is 4 total blood volumes (TBV). CD34 ${ }^{+}$cells were enumerated by flow cytometry using a two-stage ISHAGE protocol. We compared the predictive value of using Pre-C and Mid-C samples from each donor to forecast $\mathrm{CD} 34^{+}$cell $/ \mathrm{kg}$ (recipient) at the end of the collection (End-C), the need to extend the collection (or arrange for a second day of apheresis) or the ability to shorten the collection. 45 consecutive PBSCCÂ's performed during $<3$ months from healthy donors were analyzed. Calculations were based on the efficiency coefficient validated at our center. Collections 
were complete if $>4 \times 10^{\wedge} 6 \mathrm{CD} 34^{+}$cells $/ \mathrm{kg}$ (recipient) were obtained in the End-C sample.

Results: Mean End-C of 45 PBSCC's was 9.9+4.9 x $10^{\wedge} 6 \mathrm{CD} 4^{+}$cells/Kg and mean recipient weight was 73.4 $+20.1 \mathrm{Kg}$. Mid-C CD34 ${ }^{+}$cell counts correctly forecast adequate collections with $100 \%$ accuracy and correctly predicted that $13 \%$ of collections would need to be lengthened. Mid-C counts allowed us to shorten $77 \%$ of PBSCCÂ's to $<4$ blood volumes, with a mean decrease of $32 \%$ of the initially predicted collection duration. Pre-C determinations would have unnecessarily prolonged PBSCC in $20.5 \%$ of cases; in $56 \%$ of these we would have collected $>4 \mathrm{TBV}$ based on Pre-C. In $4.5 \%$ of collections, Pre-C counts falsely predicted that the target $\mathrm{CD} 34^{+}$count would be achieved in one procedure. Correlations $\left(\mathrm{r}^{2}\right)$ of Pre- and Mid-C with End-C were 0.8852 and 0.9762, respectively. Mid-C counts, obtained after processing of one blood volume, reflect adequacy of venous access and the actual progress of the collection in addition to the efficacy of G-CSF-mediated stem cell mobilization.

Conclusions: Mid-collection $\mathrm{CD}_{3} 4^{+}$cell counts are more reliable predictors of PBSCC $\mathrm{CD}_{3} 4^{+}$cell $/ \mathrm{kg}$ yield as compared to samples obtained from donor blood precollections.

\section{Conflict of interest: None}

\section{P855}

\section{Predictive factors for poor peripheral blood stem cell mobilization in patients with malignant lymphoma and multiple myeloma}

Da Jung Kim ${ }^{1}$, Sung Mi Jung ${ }^{2}$, Jee-Yeong Jeong ${ }^{3}$, Ji Hyun Lee ${ }^{4}$, SuHee Cho ${ }^{5}$, Ki Sun Jung, Jae-Cheol Jo ${ }^{6}$, Silvia Park ${ }^{7}, H_{o}$ Sup Lee ${ }^{1}$

${ }^{1}$ Kosin University College of Medicine, Department of Internal Medicine, Busan, Korea, Republic of; ${ }^{2}$ Kosin, University Gospel Hospital, Registered Nurse, Stem Cell Transplantation Clinical Nurse Specialist, Busan, Korea, Republic of; ${ }^{3}$ Kosin University College of Medicine, Department of Biochemistry Cancer Research Institute, Busan, Korea, Republic of; ${ }^{4}$ Dong-A University College of Medicine, Department of Internal Medicine, Busan, Korea, Republic of; ${ }^{5}$ Pusan National University Yangsan Hospital, Yangsan, Korea, Republic of; ${ }^{6}$ Ulsan University Hospital, University of Ulsan College of Medicine, Ulsan, Korea, Republic of; ' 7 Samsung Medical Center, Sungkyunkwan University School of Medicine, Seoul, Korea, Republic of

Background: Autologous stem cell transplantation (ASCT) is an important treatment option in multiple myeloma and malignant lymphoma, but some patients can not receive ASCT due to poor mobilization. Therefore, we assessed the incidence rates and investigated the predictive factors for poor mobilization in patients with lymphoma or multiple myeloma who were performed peripheral blood stem cell collection (PBSCC).
Methods: This study included patients with histological confirmed malignant lymphoma and multiple myeloma confirmed by bone marrow aspiration who received chemotherapy followed by PBSCC. Mobilization failure is defined as the inability to procure $2 \times 10^{6} \mathrm{CD} 34+$ cells $/ \mathrm{kg}$ at least 2 apheresis sessions. This study was conducted as a case-control study, we constructed a sample in the population and compared the successful mobilization group with the mobilization failure group.

Results: Data of total 572 patients at four university hospitals in South Korea between Jan 2006 and Sep 2017 were collected retrospectively. Of the total 572 patients, 61 patients had failed in sufficient mobilization and incidence of PBSCC failure rate was $10.7 \%$. We analyzed 122 patients (success, $\mathrm{n}=61$ and failure, $\mathrm{n}=61$ ) with a median age of 56 years (range, 18-68) and the male was $55.7 \%$. The $73(59.8 \%)$ patients received first line chemotherapy (CTx) and 36 (29.5) patients received second line CTx and 13 $(10.7 \%)$ patients received more than third line treatment prior to mobilization. The $76(62.3 \%)$ patients were complete remission after first line chemotherapy (CR1) and 32 (26.2) patients were non-CR1 before PBSCC. The $72(59.0 \%)$ patients used G-CSF only for mobilization and $49(40.2 \%)$ patients used CTx plus G-CSF. The median interval from diagnosis to PBSCC was 5.03 months (range, 0.03-146.47). The univariate analysis showed that the factors affecting poor mobilization were age (younger less than 56 years vs more than; $32.8 \%$ vs $65.6 \%, \mathrm{p}=0.001$ ), risk of disease (low vs intermediate vs high ; $55.0 \%$ vs $36.2 \%$ vs $71.4 \%, \mathrm{p}=0.006$ ), numbers of CTx line (first line vs more than second line; $38.4 \%$ vs $67.3 \%, p=0.003$ ), disease status before PBSCC (CR1 vs CR2 or non-CR ; $24.5 \%$ vs $65.6 \%, \mathrm{p}<0.001$ ), ALC before PBSCC (less than $1,000$ vs more than 1,$000 ; 59.1 \%$ vs $38.2 \%, \mathrm{p}=0.029)$ and interval from diagnosis to PBSCC (less than 5 months vs more than 5 months ; $33.3 \%$ vs $66.1 \%$, p=0.001). In multivariate analysis, 56 or more years old (RR; $0.275, \mathrm{p}=$ 0.015 ), non-CR1 before mobilization ( $R R$; 0.250, $\mathrm{p}=$ 0.018 ) and interval from diagnosis to PBSCC of more than 5 months (RR; 0.344, $\mathrm{p}=0.047)$ were independent significant poor risk factors for successful PBSCC.

Conclusions: In this study, the incidence rates of poor mobilization, defined as defined as the inability to procure $2 \times 10^{6} \mathrm{CD} 34+$ cells $/ \mathrm{kg}$ at least 2 apheresis sessions, was $10.7 \%$. And the patients who were 56 or more years old, $\mathrm{CR} 2$ or non-CR prior to mobilization and interval from diagnosis to mobilization of more than 5 months showed significant high incidence rates of poor mobilization.

Conflict of interest: All authors have no conflict of interest to report. 
P856

Progenitor cell thresholds and hematopoietic engraftment in 900 autologous transplantations. Implications for quality control

\section{Vanessa Oliveira ${ }^{1}$, Fatima Costa ${ }^{1}$, Gilda Ferreira ${ }^{1}$, Aida Botelho de} Sousa ${ }^{2}$

${ }^{1}$ Hospital dos Capuchos, Lisboa, Portugal; ${ }^{2}$ Hospital dos Capuchos, Hematologia, Lisboa, Portugal

Background: In autologous transplantation the hematopoietic progenitor cells (PC) needed for marrow engraftment are usually harvested from peripheral blood after mobilization with granulocyte colony-stimulating factor + chemotherapy, as this source has many advantages over bone marrow (confort, resource utilisation, speed of engraftment). Due to the highly variable efficacy of the mobilization procedure, it became necessary to devise PC thresholds to guide clinical decisions relating to the number of apheresis needed and to the safey of proceeding to transplant, and the established threshold is $2 \times 10>6 / \mathrm{kg}$ CD34+ cells. However CD34+ assays may fail to reflect quantitative and functional losses during the subsequent handling, freezing and thawing processes, and the ultimate measure of successful cell processing must be prompt and reproducible engraftment. Clinical criteria for transplantation with sub-optimal numbers of PC are not unusual in heavily pretreated "poor mobilisers", and each center should evaluate the influence of these factors on the quality of engraftment. We analised hematological recovery in 900 consecutive transplantations performed in our center.

Methods: From 4/97 to 12/16 900 transplants were given to 735 patients, median age 53 (17-73), for myeloma (48\%), non Hodgkin (25\%) and Hodgkin (20\%) lymphoma, acute leukemia (4\%) and solid tumors (3\%). There were 165 second transplants, 130 of which were tandem and 35 were salvage transplants at relapse. The first mobilisation attempt was successful in $89 \%$ patients. One apheresis was sufficient in $40 \%$ patients, 2 days were necessary in $40 \%$, 3 days in $10 \%$, and in $10 \%$ a pool from multiple mobilisations was used (2-6 days). PH were cryopreserved at $-80^{\circ} \mathrm{C}$. Conditionning was MEL200 (56\%), BEAM/ BEAC $(37 \%)$ or other regimens $(7 \%)$. Eight transplants $(0.9 \%)$ resulted in death before neutrophil recovery, and these patients are censored for the regeneration analysis.

Results: A median of $3.3 \times 10>6$ CD $34+/ \mathrm{kg}$ cells $(0.93-$ 70 ) was reinfused. The dose was $<2 \times 10>6$ in $14 \%$ of the transplants, and $>5.0 \times 10>6$ in $27 \%$. Median time of recovery of 500 neutrophils /ul was $\mathrm{d}+12$ (7-172), and this value was achieved until $d+14$ in $84 \%$ and until $d+28$ in
$99 \%$; for transplants with $<2.0 \times 10>6$, in $92 \%$ and $100 \%$ respectively, and for $>5.0 \times 10>6$ in 83 and $98 \%$. Recovery of 20000 platelets/ul occured at $\mathrm{d}+15$ (median;6-195) and was achieved until $\mathrm{d}+14$ in $64 \%$ e until $\mathrm{d}+28$ in $93 \%$; for transplants with $<2.0 \times 10>6$, until $\mathrm{d}+28$ in $92 \%$, e for $>$ $5.0 \times 10>6$ in $93 \%$ ( $\mathrm{p}=\mathrm{ns}$ for all comparisons). Of 24 cases with a platelet nadir $>20000 /$ ul, none had received $<2 \times 10$ $\mathrm{CD} 34+/ \mathrm{kg}$.

Conclusions: Our data confirm that optimised cell processing allows neutrophil engraftment within 14 days in $84 \%$ of cases and within 28 days in $99 \%$, and platelet engraftment within 28 days in $93 \%$, even with sub-optimal PC doses, with no significant differences for doses $>5$ $\mathrm{x} 10>6$. Delays beyond these limits should be indicators for exaustive case reviews in the context of the quality control programme of each center.

Conflict of interest: No conflict

\section{P857}

Prospective comparison of filgrastim, pegfilgrastim and lipegfilgrastim added to chemotherapy for mobilization of blood grafts in non-Hodgkin lymphoma patients

Anu Partanen ${ }^{1}$, Jaakko Valtola ${ }^{1}$, Antti Ropponen ${ }^{2}$, Kaija Vasala ${ }^{3}$, Hanne Kuitunen ${ }^{4}$ Outi Kuittinen ${ }^{4}$, Lasse Agren ${ }^{5}$, Karri Penttilä̈ ${ }^{6,7}$, Leena Keskinen ${ }^{8}$, Marja Pyörälä ${ }^{1}$, Tapio Nousiainen ${ }^{1}$, Tuomas Selander ${ }^{9}$, Pentti Mäntymaa ${ }^{10}$, Jukka Pelkonen ${ }^{2,10}$, Ville Varmavuo $^{11}$, Esa Jantunen ${ }^{1}$

${ }^{1}$ Kuopio University Hospital, Kuopio, Finland; ${ }^{2}$ University of Eastern Finland, Kuopio, Finland; ${ }^{3}$ Central Hospital of Central Finland, Jyväskylä, Finland; ${ }^{4}$ Oulu University Hospital, Oulu, Finland; ${ }^{5}$ North Karelia Central Hospital, Joensuu, Finland; ${ }^{6}$ Central Hospital of Savonlinna, Savonlinna, Finland; ${ }^{7}$ Finnish Medicines Agency, Kuopio, Finland; ${ }^{8}$ Tampere University Hospital, Tampere, Finland; ${ }^{9}$ Kuopio University Hospital, Science Service Center, Kuopio, Finland;

${ }^{10}$ Eastern Finland Laboratory Centre, Kuopio, Finland;

${ }^{11}$ Kymenlaakso Central Hospital, Kotka, Finland

Background: Autologous stem cell transplantation (autoSCT) is commonly employed in non-Hodgkin lymphoma (NHL) patients after a relapse or as a consolidation after the first line therapy. Granulocyte stimulating factors (G-CSF s) are a prerequisite for the mobilization of blood progenitor cells and historically short-acting filgrastim has been used. Limited data is available on the effects of novel long-acting cytokines lipegfilgrastim as well as pegfilgrastim after chemotherapy for mobilization purposes in regard to the mobilization efficiency, graft cellular composition and posttransplant engraftment.

Methods: This prospective, multicenter study included, as a part of the Graft and Outcome of Autologous transplantation (GOA) study, altogether 119 NHL patients who were mobilized with chemotherapy plus G-CSF by the end of 2016. Thirty-nine patients received filgrastim (FIL 


\begin{tabular}{|c|c|c|c|c|c|c|}
\hline Variable & $\begin{array}{l}\text { FIL } \\
\text { group } \\
(n=39)\end{array}$ & $\begin{array}{l}\text { PEG } \\
\text { group } \\
(n=64)\end{array}$ & $\begin{array}{l}\text { LIPEG } \\
\text { group } \\
(n=16)\end{array}$ & $\begin{array}{l}\text { Significance }(p) \text { between } \\
\text { FIL group and PEG group }\end{array}$ & $\begin{array}{l}\text { Significance }(p) \text { between FIL } \\
\text { group and LIPEG group }\end{array}$ & $\begin{array}{l}\text { Significance }(\mathrm{p}) \text { between } \\
\text { PEG group and LIPEG group }\end{array}$ \\
\hline $\begin{array}{l}\text { WBC count }(\mathrm{x} 109 / \mathrm{L}) \text { at the time of } \\
\text { first apheresis, median (range) }\end{array}$ & $\begin{array}{l}20.3(1.0- \\
116.2)\end{array}$ & $\begin{array}{l}8.6(1.2- \\
37.3)\end{array}$ & $\begin{array}{l}11.7(3.7- \\
45.6)\end{array}$ & 0.003 & 0.161 & 0.320 \\
\hline $\begin{array}{l}\text { B-CD34+ }(\times 106 / L) \text { at the time of } \\
\text { the first apheresis, median (range) }\end{array}$ & $\begin{array}{l}28.4(5- \\
538)\end{array}$ & $25(2-168)$ & $\begin{array}{l}59.5(11- \\
238)\end{array}$ & 0.643 & 0.017 & 0.005 \\
\hline $\begin{array}{l}\text { CD34+ cell yield } \times 106 / \mathrm{kg} \text { of the } \\
\text { first apheresis, median (range) }\end{array}$ & $\begin{array}{l}2.3(0.1- \\
25.5)\end{array}$ & $\begin{array}{l}1.8(0.4- \\
13.6)\end{array}$ & $\begin{array}{l}5.1(0.8- \\
16.5)\end{array}$ & 0.245 & 0.023 & 0.006 \\
\hline $\begin{array}{l}\text { Total yield CD } 34+\text { cells x106/kg } \\
\text { harvested, median (range) }\end{array}$ & $\begin{array}{l}4.7(2- \\
25.5)\end{array}$ & $\begin{array}{l}3(1.1- \\
13.6)\end{array}$ & $\begin{array}{l}5.1(2.1- \\
16.5)\end{array}$ & $<0.001$ & 0.726 & 0.019 \\
\hline $\begin{array}{l}\text { Peak B-CD34+ count }<20 \times 106 / L \text { n } \\
(\%)\end{array}$ & 7 (18) & 17 (27) & $3(19)$ & 0.316 & 1.0 & 0.749 \\
\hline $\begin{array}{l}\text { Peak B-CD34+ cell count x106/L, } \\
\text { median (range) }\end{array}$ & $\begin{array}{l}35(3- \\
538)\end{array}$ & $\begin{array}{l}30.5(1- \\
168)\end{array}$ & $\begin{array}{l}59.5(1- \\
238)\end{array}$ & 0.222 & 0.258 & 0.051 \\
\hline $\begin{array}{l}\text { The number of apheresis, median } \\
\text { (range) }\end{array}$ & $2(1-4)$ & $2(1-4)$ & $1(1-2)$ & 0.128 & 0.002 & 0.022 \\
\hline
\end{tabular}

[[P857 Table] Table 1.]

group), sixty-four pegfilgrastim (PEG group) and sixteen lipegfilgrastim (LIPEG group) as a cytokine in chemomobilization, thus forming the three study groups. The efficacy of $\mathrm{CD}_{3} 4^{+}$cell mobilization as well as the blood graft cellular composition from the thawed cryopreserved samples was analyzed with flow cytometry. The hematological recovery by total blood counts until 12 months posttransplant as well as outcome was analyzed.

Results: Patients in the LIPEG group had significantly less apheresis sessions as well as approximately $60 \%$ higher $\mathrm{CD} 34^{+}$yields of the first apheresis day compared to FIL group or PEG group (Table1). The graft cellular composition was comparable between the groups except for significantly higher $\mathrm{CD} 34^{+}$counts with and without 7AAD addition in the LIPEG group. The engraftment kinetics was significantly slower in FIL group compared to the other groups. The hematological recovery was more rapid in LIPEG group in terms of leukocyte as well as neutrophil counts at day 15 after auto-SCT.

Conclusions: LIPEG added to chemotherapy appeared to be more efficient to mobilize blood grafts for auto-SCT compared to PEG or especially to FIL. LIPEG group achieved adequate grafts to proceed to transplantation with significantly less apheresis sessions. Neither PFS nor OS was affected by the type of cytokine in mobilization procedures.

\section{Conflict of interest:}

A. Partanen reports honoraria from Behringer.

M. Valtola reports honoraria from Sanofi and JansenCilag.

Dr. Siitonen reports honoraria from Amgen and Celgene and participated Advisory Boards organized by Pfizer and Amgen.

E. Jantunen reports honoraria from Genzyme, Amgen and Sanofi and has participated in EU Leadership meeting organized by Genzyme as well as Medical Advisory Board meeting organized by Genzyme, Amgen and Takeda.
V. Varmavuo reports consultancy fees from Abbvie, Roche, Celgene, Amgen and Sanofi. The other authors declare no conflicts of interest.

\section{P858}

Abstract previously published

\section{P859}

\section{Sandoz biosimilar filgrastim in stem cell mobilization - a decade of clinical experience}

Samir Agrawal', Nicola Mathieson ${ }^{2}$, Andriy Krendyukov ${ }^{2}$

${ }^{1}$ St Bartholomew's Hospital, Haemato-Oncology, London, United Kingdom; ${ }^{2}$ Hexal AG, Hematology/Nephrology, Holzkirchen, Germany

Background: The granulocyte colony-stimulating factor (G-CSF) filgrastim and its biosimilars are widely used for prevention and treatment of chemotherapy-induced neutropenia (CIN) and for stem cell mobilization (SCM). Biosimilars of filgrastim have been available in Europe since 2008, including Sandoz biosimilar filgrastim (Zarzio $\%$ Zarxio ${ }^{\circ}$, filgrastim-sndz, Hexal AG). Approval studies for Sandoz biosimilar filgrastim were performed for prevention of CIN in patients with breast cancer, considered the most sensitive indication in which to confirm biosimilarity. Confirmation of the matching efficacy, safety and immunogenicity of Sandoz biosimilar filgrastim to the reference filgrastim in this sensitive patient population allowed extrapolation of the other indications of the reference medicine, including SCM, based on totality of evidence approach. Consequently, the biosimilar regulatory process does not require a comparative study on the safety and efficacy of biosimilar versus reference biologic in SCM. A post-approval long-term safety study is currently underway 
in healthy donors receiving Sandoz filgrastim biosimilar for SCM. ${ }^{1,2}$ With almost 10 years of clinical experience of Sandoz biosimilar filgrastim use in healthy donors, there is a large body of clinical evidence to support its safety profile.

Methods: A Medline literature search was performed to identify published evidence available regarding use of Sandoz biosimilar filgrastim for both autologous and allogeneic SCM, including a 10-year post-approval safety study in healthy donors. Abstracts and posters from relevant congresses were also identified.

Results: More than fifteen prospective and retrospective studies were identified which reported the efficacy and safety of Sandoz biosimilar filgrastim when used for SCM in an autologous setting (a total of 882 patients receiving Zarzio). When comparative data were available, these studies consistently reported similar mobilization yields and adverse events (AEs) between Sandoz biosimilar filgrastim and the reference filgrastim.

A significant body of evidence is emerging in allogeneic SCM. The 10-year post-approval safety study reported on 244 healthy volunteers who received Sandoz biosimilar filgrastim. ${ }^{1}$ At a mean follow-up of 433 days (range: 2-1528), both the SCM efficacy and AEs of Sandoz biosimilar filgrastim were similar to that reported previously with the reference biologic. ${ }^{1}$ All harvests achieved a yield of $>3 \times 10^{6} \mathrm{CD} 34$-positive cells $/ \mathrm{kg}$ recipient weight, with $98.8 \%$ achieving $>4\left(\right.$ range $\left.3-52 \times 10^{6}\right)$. ${ }^{1}$ Other long-term data are becoming available. A retrospective analysis in 51 healthy sibling donors demonstrated effective mobilization without notable AEs after 2 years' follow-up; median time to neutrophil and platelet engraftment was 16 days (range: 10-28) and 13 days (range: 9-54), respectively.,4

Conclusions: A decade of clinical experience is reassuring in terms of the safety and efficacy of Sandoz biosimilar filgrastim in SCM with evidence from long-term studies now emerging in the allogeneic setting, including an ongoing 10-year safety study in healthy donors. Our review suggests that, to date, the safety and efficacy of Sandoz biosimilar filgrastim match that of reference biologic in SCM.

\section{References:}

1. Becker P, et al. Transfusion 2016;56:3055-64.

2. Barosi G, et al. Haematologica 2011;96:937-42.

3. Taylor et al. Bone Marrow Transplant 2017;52(S1): S145.

4. Uddin S, et al. Ther Adv Hematol 2015; 6(2) 53-60.

Clinical Trial Registry: N/A

Conflict of interest:

S. Agrawal has acted as a consultant for Sandoz.

N. Mathieson and A. Krendyukov are employees of Hexal AG.
P860

Abstract previously published

\section{P861}

Standard and Large volume leukapheresis (LVL) using the new CMNC protocol Spectra Optia

Zdenka Gašováa ${ }^{1,2}$, Blanka Vackováa ${ }^{3}$ Zdenka BhuiyanLudvíkovál, Martina Bohmovál, Markéta Sloukovál, Iuri Marinov $^{4}$, Pavla Pecherková ${ }^{5}$

${ }^{1}$ Institute of Haematology and Blood Transfusion, Apheresis Department, Prague, Czech Republic; ${ }^{2}$ Institute of Clinical and Experimental Medicine, Charles University, Prague, Czech Republic; ${ }^{3}$ General Faculty Hospital, 1st. Internal Clinics - Hematology, Prague, Czech Republic; ${ }^{4}$ Institute of Haematology and Blood Transfusion, Laboratory of Flow Cytometry, Prague, Czech Republic; ${ }^{5}$ Institute of Haematology and Transfusion Medicine, Department for Statistical Analysis, Prague, Czech Republic

Background: The aim of the study was to evaluate and optimize the new CMNC protocol Spectra Optia v. 11 (Terumo) for PBPC collections in patients with haematooncological malignant diseases.

Methods: The results of 159 autologous PBPC collections were evaluated in the case of: (a) well mobilized patients with precollection CD $34+$ cells concentration in blood higher than $20 / \mu \mathrm{l}$, (b) from only the first collections, which were performed either by the use CMNC Spectra Optia v. 11 or Cobe Spectra v. 6, v. 7, Terumo (c) collections were performed in the Standard and Large volume Leukapheresis regimen, LVL. Engraftment data in 56 transplanted patients were assessed. Statistical analysis was performed by means of Mann-Whitney $\mathrm{U}$ test and $\mathrm{F}$ test, alfa $=0,05$. The results are expressed as medians and their ranges.

Results: Standard collections were performed in 52 patients. The yield of CD $34+$ cells was high, and no significant differences were found between the numbers of $\mathrm{CD}$ $34+$ cells prepared from Spectra Optia 8,6 $(1,3-41) \times 10^{6}$ and Cobe Spectra $10,9(1,8-45,6) \times 10^{6} / \mathrm{kg}$ b. w. $(\mathrm{a}=0,05$; pval 0,619$)$. The dependence of CD $34+$ cell yield on the precollection concentration of CD 34+ cells in blood can be considered as linear with high correlation coefficients in CMNC Spectra Optia $\mathrm{R}=0,95$, and Cobe Spectra $\mathrm{R}=0,93$.

LVL collections were performed in 107 of patients, and there were no significant differences between the numbers of CD 34+ cells prepared by CMNC Spectra Optia 10,9 (2$61,2) \times 10^{6}$ and Cobe Spectra 9,3 (2,4-86) $\times 10^{6} / \mathrm{kg}$ b.w. $(a=0,05 ;$ pval 0,35$)$. The relations between the precollection CD 34+ cells concentration in blood and the numbers of CD $34+$ cells from collections can also be considered as 
linear with the correlation coefficients in CMNC Spectra Optia $\mathrm{R}=0,93$, and Cobe Spectra $\mathrm{R}=0,78$, respectively. In $L V L$, the median platelet loss was significantly lower in CMNC Spectra Optia (45\%) than in Cobe Spectra (57\%). A group of 12 patients was transplanted by means of PBPC prepared in the standard regimen. Median time in the neutrophil reconstitution was in CMNC Spectra Optia as well as Cobe Spectra 11 days, while in platelets from CMNC 14 days, and from Cobe Spectra 12 days, respectively. The number of 44 patients obtained PBPC from LVL. The median time in neutrophils and platelets reconstitution was in CMNC Spectra Optia as well as Cobe Spectra the same, and corresponded with 11 and 13 days, respectively.

Conclusions: CMNC protocol Spectra Optia is a modern, efficient and the safe system, which can be used for both Standard and LVL procedures. In well mobilized patients the sufficient dose of CD 34+ cells for transplantation could be prepared from one Standard or one LVL procedure. No serious adverse reaction have been observed.

Conflict of interest: None of the authors has anything to disclose.

\section{P862}

\section{Success of Stem Cell Collection and Neutrophil Recovery in Pediatric Patients with Brain Tumours}

George Michaiel $^{1}$, Tony Truong ${ }^{1}$, Nicole Prokopishyn ${ }^{1,2}$, Henry Luu $^{3}$, Lucie Lafay-Cousin ${ }^{1}$, Douglas Strother ${ }^{1}$, Gregory Guilcher ${ }^{1}$, Victor Lewis ${ }^{1}$

${ }^{1}$ University of Calgary, Calgary, Canada; ${ }^{2}$ Calgary Laboratory Services, Calgary, Canada; ${ }^{3}$ Alberta Children's Hospital, Calgary, Canada

Background: The use of high dose chemotherapy (HDC) and autologous hematopoietic stem cell transplant (HSCT) results in improved survival in certain patients with central nervous system (CNS) tumours, and has been used as a radiotherapy delaying or sparing approach. We aimed to study factors related to the success of stem cell collection (SCC) and correlate stem cell dose infused with HSCT outcomes.

Methods: A retrospective chart review was undertaken for pediatric patients with CNS tumours treated at our centre with HDC and HSCT between December 2004 and December 2016.

Results: Fifty-five patients were identified (32 male, 23 female) with a median age of 6.3 years at time of SCC (range 0.4-18.7). Patient diagnoses were medulloblastoma $(62 \%)$, ATRT $(20 \%)$ and PNET $(18 \%)$. The majority of patients $(45 / 55,82 \%)$ underwent a single (1-day) SCC, while the remaining 10 underwent 2 SCC procedures.
Peripheral blood stem cells were the source in most collections (95\%). Successful SCC (defined as CD34 dose collected $\geq 2 \times 10^{6} / \mathrm{kg} /$ transplant) was achieved in $47 / 55$ $(85 \%)$ of patients, whereas an ideal SCC (defined as CD34 dose collected $\geq 5 \times 10^{6} / \mathrm{kg} /$ transplant) was achieved in $25 / 55$ (45\%) of patients. Use of chemotherapy with G-CSF for mobilization was significantly associated with achieving an ideal collection compared to use of G-CSF alone (OR 41.5, CI 4.9 - 350; $\mathrm{P}=0.0006)$. Gender, age, stem cell source, and pre-apheresis peripheral blood CD34 count were not found to be significant predictors of ideal collection. Median time to neutrophil engraftment was 10 days for HSCT courses 1-3 and 12 days for course 4 . Median time to platelet engraftment was 15 days for courses 1 and 3, and 16 days for courses 2 and 4 . Higher doses of CD34 per kg infused was associated with faster neutrophil recovery in the first 3 courses of chemotherapy $(\mathrm{P}=0.0171, \mathrm{P}=0.0442$, $\mathrm{P}$ $=0.0455$ respectively for courses $1-3)$ and faster platelet engraftment on only the first course $(\mathrm{P}=0.0224)$.

Conclusions: Patients with CNS tumours who are mobilized with both chemotherapy and G-CSF have higher CD34 yield compared to patients mobilized with G-CSF alone. The majority of SCC for autologous HSCT can be successfully completed with a single apheresis session. Higher dose of CD34/kg infused was associated with faster neutrophil recovery post-HSCT, and to a more limited scale faster platelet recovery.

Conflict of interest: Nothing to disclose

\section{P863}

The Binns Program for Cord Blood Research: a novel program for cord blood procurement in an academic setting for biomedical research

Sruthi Mantri ${ }^{1}$, Deirdre Lyell ${ }^{2}$, David DiGiusto ${ }^{1}$, Matthew Porteus ${ }^{1}$, Rajni Agarwal-Hashmi ${ }^{1}$

${ }^{1}$ Stanford University, Pediatrics, Stanford, CA, United States;

${ }^{2}$ Stanford University, Obstetrics and Gynecology, Stanford, CA, United States

Background: Umbilical cord blood (UCB) is a vital source of hematopoietic stem and progenitor cells (HSPCs). Since 1991, UCB has been collected and stored in public cord blood banks to be primarily used as a source for allogeneic hematopoietic stem cell transplantation (HSCT). Since the seminal UCB transplant reported in 1988 by Gluckman et al., UCB has contributed to advances in HSCT and clinical trials. However, UCB-derived cells remain costly and difficult to procure for pre-clinical research. Here we present a protocol for a self-sustaining cord blood program dedicated to biomedical research: The Binns 


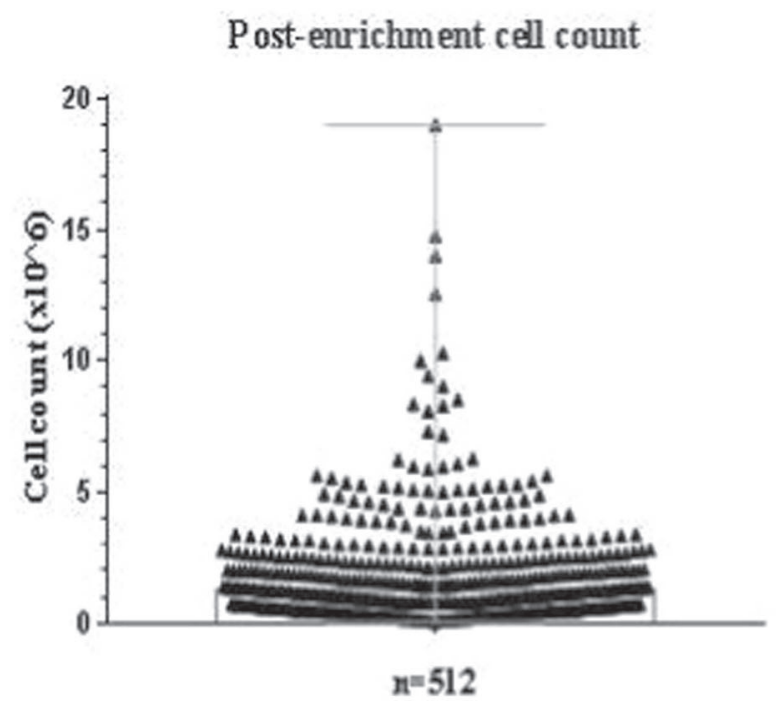

[P863 Figure] [Post-enrichment CD34+ cell count and sample purity]

Program for Cord Blood Research, piloted at the Stanford University School of Medicine.

Methods: Eligible donors are identified using IRBapproved inclusion criteria. UCB samples are then collected in utero, with informed consent, by obstetric providers at the time of delivery. Within 24-48 hours of isolation, samples are processed into three fractions:

(1) plasma,

(2) hematopoietic stem and progenitor cells (HSPCs) by immunomagnetic separation, and

(3) non-HSPC cellular flow-through. UCB-derived fractions are then distributed to researchers upon request.

Results: Between November 2015 and November 2017, 609 expecting mothers were consented to collect cord blood at delivery. $553(91 \%)$ UCB samples were collected, of which 534 (97\%) were processed for distribution. Consent forms and informational materials were developed in both English and Spanish. With our streamlined protocol in place, the median HSPC yield per cord, accounting for obstetric parameters that might affect sample quality (most notably delayed cord clamping), was 1.25 million (range: 40,000 - 19 million, Figure 1, Table 1). Manual processing of UCB samples resulted in a median purity of $90 \%$ following one-step purification (range: 5 - 99, Figure 1, Table 1). By October 2016, a protocol had been developed and optimized for the Binns Program to begin providing UCB-derived products to six laboratories. By June 2017, this number nearly tripled to 16 laboratories as the program responded to increased interest from researchers and opened to 47 laboratories. Research supported by the Binns Program spans a broad array of topics including normal and abnormal hematopoiesis, genetic engineering including genome editing, and novel approaches to HSCT.

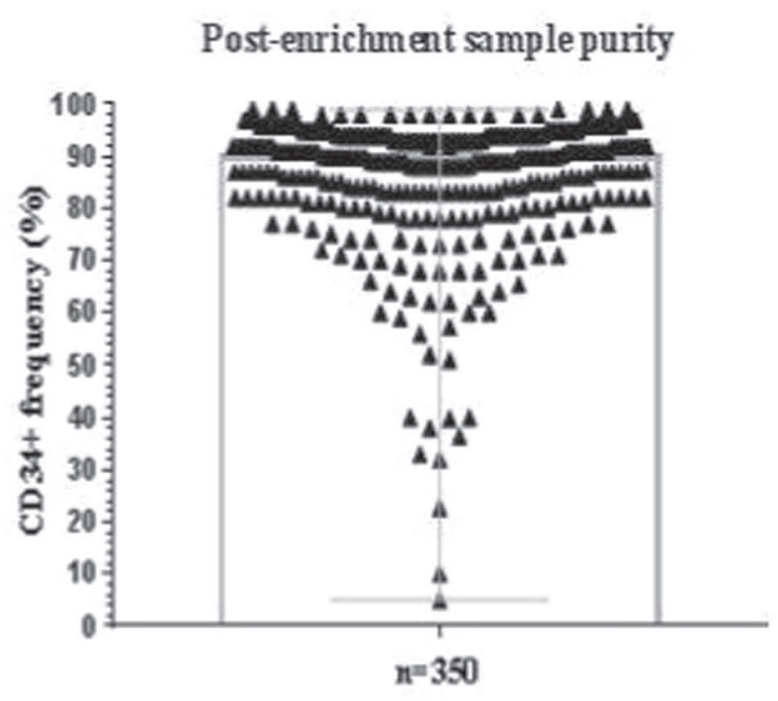

*As reported, collection volume is inclusive of $35 \mathrm{~mL}$ anticoagulant (citrate phosphate dextrose). Therefore, whole blood collection volumes range from $0 \mathrm{~mL}$ to $135 \mathrm{~mL}$.

Conclusions: The Binns Program for Cord Blood Research represents a feasible, financially self-sustaining model for cord blood banking to support the advancement of biomedical research by providing fresh or cryopreserved UCB-derived cells to the research community at a fraction of the commercial cost. We hope that this model may be useful for other institutions and centers where the need to support research is high and the traditional model for public cord blood banking, which requires extensive financial infrastructure, may not be as suitable an option.

Conflict of interest: None of the author has anything to disclose.

\begin{tabular}{ll}
\hline Number of collections (Nov. 2015 - Nov. 2017) & 553 \\
\hline Median collection volume (mL) & $77(35-170)$ \\
Median CD34+ cell yield & $1.25 \times 10^{6}\left(4 \times 10^{4}-1.9 \times 10^{6}\right)$ \\
Median sample purity (\%CD34+) & $90(5-99)$ \\
\hline
\end{tabular}

[[P863 Table] Key parameters of UCB collection and processing]

\section{P864}

The effect of a second mobilization and collection of peripheral blood stem cells on the cell yield: 22-year experience of an apheresis center

Ana Catarina Pinho, Filipa Bordalo, Sérgio Machado Lopes, Sara Ferreira, Fátima Amado, Margarida Dias, Susana Roncon 
Instituto Português de Oncologia do Porto, Porto, Portugal

Background: Graft failure or progressive disease are frequent indications for a second allogeneic hematopoietic stem cell transplantation, bringing the need for a new collection from the same donor. The effects of repeated peripheral blood stem cell (PBSC) mobilization on the graft quality have been poorly studied and still unknown.

Methods: We retrospectively evaluated the results of all two cycles of PBSC mobilization and collection from unrelated or related donors cared for at our Institution between 1995 and 2017. In both cycles, apheresis started at the fifth day of mobilization, using a COBE Spectra in first years and Spectra Optia in the last five. For each donor, age, weight, GCSF dose, circulating and collected CD34+ cells counts were recorded. In order to evaluate the impact of the interval between the two cycles, donor population was divided in two groups: $<180$ days (group A) and $>180$ days (group B).

Results: Among the total of 37 donors, 7 were volunteer donors of the Portuguese Donor Registry and 30 were familiar. Twenty-one donors were re-mobilized because of recipient graft failure and 16 because of disease progression/ relapse. At the time of first collection, median donor age was 36 years (range 18-71) and median weight $71 \mathrm{~kg}(50-100)$. The median delay between both collections was 222 days (211519). Although the mean dose of G-CSF administered was very similar at the first and second mobilization $(13.2 \pm 0.5$ versus $13.5 \pm 0.6 \mu \mathrm{g} / \mathrm{kg}$ ), the mean number of circulating $\mathrm{CD} 34+$ cells $/ \mu \mathrm{L}$ was lower at the second mobilization $(82 \pm 10$ versus $72 \pm 8$ ). Concerning the number of apheresis days per collection, 9 donors (24\%) needed more than one day to obtain the requested number of $\mathrm{CD} 34+$ cells during the first cycle, comparing to $12(32 \%)$ at the second. The mean total number of CD34+ cells collected was higher after the first mobilization than after the second (556.9 versus $491.0 \times 10^{6}$, respectively). Five of the 37 donors had a CD34+ cells yield $<4 \times 10^{\wedge} 6 / \mathrm{kg}$ at the second mobilization, whereas only 1 failed at the first. The analysis of graft cell content according to the time interval between both collections show that $82 \%$ of group A donors yielded fewer CD34+ cells after the second mobilization than after the first, comparing with only $40 \%$ of group B.

Conclusions: Our study shows that a second cycle of PBSC mobilization and collection from donors is associated with a reduction of the circulating and collected CD34+ cells, even using the same dose of hematopoietic growth factor. Moreover, shorter time intervals between both collections seem to be associated with lower cell yield. However, the minimum number of cells required was in general accomplished at both cycles. In that way, is our opinion that a second PBSC collection may be considered when it is first preference, trying to maintain the G-CSF dose and to respect the minimum period of 180 days (as stated by donor registries). Despite this study not addressing the donor safety, we consider that follow-up period must be extended in order to detect possible long-term effects of repeated G-CSF exposure.

Conflict of interest: All authors: nothing to disclose

\section{P865}

Two versus three day upfront use of granulocyte-colony stimulating factor in healthy bone marrow donors for pediatric bone marrow transplantation

Namık Yaşar Ö̈bek ${ }^{1}$, Tekin Aksu ${ }^{1}$, İkbal Ok Bozkaya ${ }^{1}$, Sibel Akptnar Tekgündü̈ $z^{2}$, Mehtap Olcar Kanbur ${ }^{1}$, Yasin Köksal ${ }^{3}$, Meltem $\ddot{O ̈ g u ̈ n e r}^{3}$

${ }^{1}$ University of Health Sciences, Ankara Child Health and Diseases Hematology Oncology Training and Research Hospital, Department of Pediatric Hematology and Oncology, Ankara, Turkey; ${ }^{2}$ University of Health Sciences, Ankara Keçiören Training and Research Hospital, Department of Pediatric Hematology and Oncology, Ankara, Turkey; ${ }^{3}$ University of Health Sciences, Ankara Child Health and Diseases Hematology Oncology Training and Research Hospital, Stem Cell Research Laboratory, Ankara, Turkey

Background: In disproportionately small bone marrow (BM) donors compared to the recipient's weight, mobilization with granulocyte-colony stimulating factor (G-CSF) has been used. Studies concerning upfront use of G-CSF in $\mathrm{BM}$ donors to boost graft yield established the safety as well as the efficacy to obtain secure engrafment. However, optimal dosing and the schedule of G-CSF priming before stem cell harvest were not clearly defined in the literature. In order to decrease exposure to G-CSF, we compared the effect of two versus three days of G-CSF priming on BM CD34+ yield of donors.

Methods: Donors were primed with G-CSF at $10 \mu \mathrm{g} / \mathrm{kg}$ daily for 2 (G-CSF2 group) or 3 (G-CSF3 group) consecutive days. Peripheral blood (PB) samples were analyzed for white blood cells (WBC), mononuclear cells (MNC) and CD34+ cell numbers 24, 48 hours after the first day of G-CSF and at the day of harvest in all donors. Also, $\mathrm{BM}$ samples of the donors were analyzed for WBC, MNC and CD34+ cell numbers.

Results: Seventeen donors included in the study. Eleven donors [median age15 years (1-48), median weight $47 \mathrm{~kg}$ (14.8-92), M/F 6/5] included in the G-CSF2 group and 6 donors [median age 6.5 years (4-13) and $22.7 \mathrm{~kg}$ (12.7-34), $\mathrm{M} / \mathrm{F}$ 4/2] included in the G-CSF3 group. They were all matched sibling donors with HLA compatibility $6 / 6$ or $10 /$ 10 except one first-degree cousin with 10/10 HLA compatibility. No donor experienced major adverse events related to G-CSF administration. There were no difference in myeloid or platelet engrafment days between two groups. 
Peripheral blood analysis revealed median CD34+ cell numbers 24, 48, and 72 hours after G-CSF priming as $2(0$ -

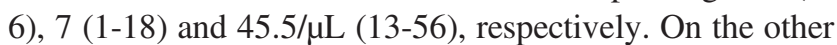
hand, BM graft analysis disclosed median CD34+ numbers as $164 / \mu \mathrm{L}(66$ - 962) in G-CSF2 group and 227/ $\mu \mathrm{L}(112$ 770) in G-CSF3 group. Bone marrow harvest yielded similar total nucleated cells (TNC), MNC and CD34+ cells per kilogram of the recipient's weight in all patients (Table $1)$. When donors were grouped according to the age, the results demonstrated that younger donors ( $\leq 10$ year) had more $\mathrm{PB}$ and $\mathrm{BM} \mathrm{MNC}$, and $\mathrm{BM} \mathrm{CD} 34+$ cell numbers compared to those in donors $>10$ years of age.

Conclusions: Although the number of BM-CD34+ cells was higher in 3 day G-CSF priming, we achieved the same number of $\mathrm{CD} 34+$ cells per recipient's weight in 2 day GCSF priming group, too. In addition, the number of TNC harvested from BM were similar with two or three day regimen. Another interesting finding of this study was obtaining adequate number of peripheral blood stem cells for leukapheresis with three day G-CSF administration. Since engrafment times were also similar in two groups, we concluded that 2-days G-CSF priming was resulted in sufficient mobilization of BM stem cells.

Conflict of interest: All authors: nothing to disclose

\begin{tabular}{llll}
\hline & G-CSF2 $\mathrm{n}=11$ & G-CSF3 $\mathrm{n}=6$ & P value \\
\hline CD34 $(/ \mu \mathrm{L})$ & $164(66-962)$ & $227(112-770)$ & 0.26 \\
TNC $(\mathrm{x} 108 / \mathrm{kg}$ recipient BW $)$ & $6.73(2.1-12.5)$ & $5.76(1.2-10.9)$ & 0.54 \\
CD34+ (x106/kg recipient BW) & $3.4(1.8-5.4)$ & $3.13(1.07-4.17)$ & 0.31 \\
\hline
\end{tabular}

[[P865 Table] Bone marrow cell counts according to GCSF priming]

\section{P866}

\section{Understanding collection efficiency and recovery in stimulated and non-stimulated blood- and bone marrow apheresis}

Jacqueline Overdevest, Patricia Spruitenburg, Jaap Jan Zwaginga, Tanja Netelenbos

Leiden University Medical Center, Department of Immunohematology and Blood Transfusion, Leiden, Netherlands

Background: In peripheral blood stem cell (PBSC) collection using an apheresis device, collection efficiency (CE) of mononuclear cells (MNC) is often calculated as an indicator for a successful procedure. $\mathrm{CE}$ is determined by the MNC concentration in the blood and is under influence of continuous mobilization of white blood cells through chemotherapy and/or G-CSF. This mobilizing effect is lacking in unstimulated donors, however, the apheresis procedure itself could stimulate a cellular shift from extrato intravascular. We aimed to separate effects of PBSC mobilization and the apheresis procedure itself on MNC homeostasis by comparing apheresis procedures of GCSFmobilized donors, unstimulated donors and bone marrow harvests.

Methods: Data of apheresis procedures performed between January 2015 and June 2017 on the Spectra Optia were collected. For donors with leukocytes < 15 x 10e9/1 collection with the elutriation chamber was used; otherwise the continuous MNC collection. For BM, the BMprocessing program was used. Before and after apheresis, donors and BM harvest samples were measured for MNC numbers by flow cytometric analysis using CD45, CD14 and scatter patterns in stimulated donors and BM, and using the sum of monocyte and lymphocytes measured routinely in unstimulated donors. CE2 was calculated according to: MNC in product (x10e9)/((pre-procedural MNC count $(\mathrm{x} 10 \mathrm{e} 9 / 1) *$ Processed volume $(\mathrm{L}))^{*} 100 \%$. Recovery was defined as MNC in product/ MNC in total blood volume (using Nadlers formula) $* 100 \%$. In unstimulated donors, the estimated number of harvested MNC was calculated by subtracting the absolute intravascular MNC count post from the absolute intravascular MNC count before apheresis.

Results: The highest CE2 was found in procedures performed on mobilized allogeneic compared to autologous donors due to higher MNC pre-counts (median 7.0 and 2.6 x10e9/1 respectively). In unstimulated MNC apheresis, CE2 was much lower, while being lowest in BM. Recovery however, was higher in BM compared to unstimulated MNC apheresis while in stimulated donors, recovery was highest. In unstimulated donors, the real number of MNC harvested was higher than the estimated number (median $4 \times 10 \mathrm{e} 9$ ) representing an intravascular replenishment of MNC during apheresis.

Conclusions: Large differences exist in $\mathrm{CE}$ and recovery with respect to donor type and source of MNC. In healthy stimulated- compared to autologous donors, the higher CE2 can be explained by higher amount of MNC pre-apheresis. The high recovery is due to the release of MNCs from the marrow to the intravascular space during the procedure. The lower CE2 in unstimulated MNC apheresis can be explained by lack of ongoing production. This situation is not entirely steady state, as a shift occurs from extra- to intravascular. In BM apheresis, the aim is get a recovery $>75 \%$, therefore many cycles are performed on the apheresis machine, leading to higher processed volumes and therefore low CE2. This knowledge helps interpreting CE outcomes.

Conflict of interest: J. Overdevest: nothing to disclose 


\begin{tabular}{llll}
\hline & $\mathrm{N}$ & CE median (range) & Recovery median (range) \\
\hline PBSC autologous & 10 & $36(21-80)$ & $79(32-237)$ \\
PBSC allogeneic & 40 & $52(41-105)$ & $121(47-320)$ \\
MNC unstimulated & 11 & $29(20-36)$ & $48(29-110)$ \\
BM & 33 & $11(3-20)$ & $80(28-121)$ \\
\hline
\end{tabular}

\section{[[P866 Table] Results]}

\section{P867}

\section{USE OF PLERIXAFOR IN THE MORNING BEFORE THE APHERESIS}

Juan Jose Mateos Mazon, Amaia Uresandi, Miriam Vara Pampliega, Francisco Ignacio Zuazua Verde, Juan Carlos Garcia Ruiz

Department of Haematology, Cruces University Hospital, BioCruces Health Research Institute, Barakaldo, Spain

Background: Plerixafor is molecule use for mobilization of Hematopoietic Stem and Progenitor Cells (HSPC) at patients who will be treated with an autologous transplantation. It is very useful at patients who were poors or bad mobilizers, and reduce mobilization failure rates. Plerixafor is administered beetween 11 and 4 hours before planned apheresis.

At our center we had administered Plerixafor just before the apheresis and we have seen that Plerixafor mobilize very fast the HSPC after his administration.

Methods: From 2008 to 2017 we had made 82 mobilization with Plerixafor to 61 patients (women 26), with differents pathologies. Sometimes we used just in time or a remobilization strategy. Some patients have used more than one dose and have need more than one mobilization with or without Plerixafor. We haved used plerixafor at $8 \mathrm{~h}$ in the morning, 1 hour before to start the apheresis (45 patients), or at $23 \mathrm{~h}$ of the night, 10 hours before start the apheresis (30 patients). Fewer times we used at $3 \mathrm{~h}$ in the morning (7) 6 hours before start the apheresis.

Results: There aren't significative differences beetween the 3 groups, the amount of $\mathrm{CD} 34+/ \mathrm{Kg}$ is a little bit bigger at the group of $23 \mathrm{~h}$, but we observed that the efficiency of the apheresis has good results at patients who received the drug before the apheresis (Efficiency; $C D 34+$ of product $X$ volume collected / CD34+/uL of the day before Plerixafor administration $X$ volume processed), wich could mean that Plerixafor is capable to mobilize HSPC very fast to peripherical blood. Table 1.

Conclusions: The use of Plerixafor avoid to reduce the fail of mobilization, and reduce the number of apheresis sessions. Some problems with his administration are that sometimes you need to register a patients to give the drug the night before. Another situation is when you don't have the results of the collection till some hours later, then maybe you can't give the drug to the patients because he is not there. At this cases the use of Plerixafor before the Apheresis could be a good strategy, because we have seen than Plerixafor enhaces the HSPC at peripheral blood very fast, and avoid a very "just in time" strategy, although the use of Plerixafor some hours before maybe have a little better results.

Conflict of interest: Collaboration with Sanofi at some meetings, and round tables about Plerixafor

\begin{tabular}{llll}
\hline & $\begin{array}{l}\text { Number of } \\
\text { apheresis }\end{array}$ & $\begin{array}{l}\text { CD34/Kg } \\
\text { collected }\end{array}$ & Efficiency \\
\hline $\begin{array}{l}\text { At 23 hours (9 h before } \\
\text { paheresis) }\end{array}$ & 30 & $2,01(1,26-$ & $207(304-$ \\
At 8 hours (1 h before & 45 & $2,76)$ & $110)$ \\
apheresis) & & $1,32(1,67-$ & $282(391-$ \\
At 3h (6 h before apheresis) & 7 & $0,97)$ & $172)$ \\
& & $1,27(2,61-$ & $149,03(36-$ \\
p & $0,67)$ & $261)$ \\
\hline
\end{tabular}

\section{[[P867 Table] Table 1]}

\section{P868}

Viability of CD34-selected cells in vials, a poor quality predictor for cryopreserved CD34-enriched stem cell transplants

Joost van den Muijsenberg, Jacqueline Overdevest, Paulien te Marvelde, Yolanda Rozier, Jaap Jan Zwaginga, Tanja Netelenbos

Leiden University Medical Center, Immuno Hematology and Bloodtransfusion, Leiden, Netherlands

Background: High risk systemic sclerosis patients can be treated by an autologous stem cell transplantation after conditioning with cyclophosphamide. After stem cell mobilization and apheresis a CliniMACS CD34 selection procedure is performed to eradicate autoimmune cells allowing subsequent cryopreservation of purified CD34 cells. Post thawing viability of transplants is an important quality criterion in our center and routinely assessed in reference vials that undergo the same freezing procedure as the product. When viability is above $50 \%$, the product is approved for clinical use. The viability of a sample of the thawed product is always assessed additionally. While vials of non-selected products show median post thawing viability of $95 \%$ (range $63-100 \%, \mathrm{n}=154$ ), it was noticed that viabilities of the vials of the CD34-selected products seemed lower. To investigate this, we studied viability of both vials and products after thawing in relation with engraftment. 
Methods: Between July 2005 and November 2017 we cryopreserved CD34-selected cells from 29 patients using the CliniMACS system (Miltenyi). Data were collected on CD34 viability of thawed reference vials and the transplants before infusion and engraftment of platelets and granulocytes in the recipient. CD34 viability testing was performed using the ISHAGE gating strategy with 7AAD as marker for dead cells. Engraftment data on transplanted patients were collected from patient records. Engraftment was defined as the first day granulocytes were $>0.5 * 10 \mathrm{e} 9 / 1$ and the first day of platelet counts $>20 * 10 \mathrm{e} 9 / 1$.

Results: Vials of $31 \mathrm{CD} 34$-selected cell products, showed a median viability of $49 \%$ (range $7-100 \%$ ). Of 16 vials (52\%) viability was below $50 \%$ with 3 even below $20 \%$. Upon thawing, the products for administration showed a viability below $50 \%$ in only 4 cases (median $65 \%$, range 31 $100 \%)$. A clear relation between viability in vials and stem cell products however was present $(\mathrm{p}=0.0164$, Wilcoxon signed-rank test), although the reference vial showed a lower viability than that of the thawed product in $70 \%$ of cases. In two patients it was decided to do a second stem cell mobilization due to an insufficient amount of viable stem cells. This decision was taken after sacrificing part of the product for $\mathrm{CD} 34$ viability testing revealing also too little viable CD34 cells (37 and 22\%). The median neutrophil and platelet engraftment in the 29 transplanted scleroderma patients took 13 (range 10 -18) and 12 (range 7 -15) days respectively. Illustrative in this respect was that the only engraftment failure occurred with a transplant of $5.7 \times 10 \mathrm{e} 6 / \mathrm{kg}$ CD34 cells and a viability of $87 \%$ (49\% in the reference vial).

Conclusions: For CD34-selected products, viabilities below 50\% occurred in more than half of the thawed reference vials. This might be explained by the selection itself, the cell concentration and/or medium conditions in which the selected cells are frozen and thawed making them more vulnerable than their unselected counterparts. Alternatively, in unselected transplants, other cells might protect CD34 cells. The good overall engraftment of CD34-selected transplants, seems to suggest that the present cut off values for vial viability could be adjusted for these products.

Conflict of interest: None of the authors has anything to disclose.

Stem cell source

\section{P869}

A case of severe aplastic anemia infused with multiple low doses of CD34 cells from poorly mobilized matched sibling donor at allogeneic stem cell transplantation

Chang Dae Kum ${ }^{1}$, Jun Eun Park, Kwang Chul Lee ${ }^{2}$, In Keun $\mathrm{Jang}^{3}$, O Kyu Noh${ }^{1}$, Jaesung Heo ${ }^{1}$
${ }^{1}$ Ajou University School of Medicine, Pediatrics, Suwon, Korea, Republic of; ${ }^{2}$ Korea University College of Medicine, Pediatrics, Seoul, Korea, Republic of; ${ }^{3}$ Biomedical Research Institute, Lifeliver Co Ltd, Yongin, Korea, Republic of

Background: Severe aplastic anemia (SAA) is a rare and potentially fatal disease that is characterized by an immunemediated functional impairment of hematopoietic stem cells (HSCT), with peripheral blood stem cell transplantation (PBSCT) or bone marrow transplantation (BMT), is the treatment of choice for young patients who have a matched related donor (MRD). Especially, CD34 cell dose is known to influence outcome of peripheral stem cell and/or T-celldepleted transplantation. Previous study showed that $2 \times 10^{6}$ $\mathrm{CD} 4^{+}$cells $/ \mathrm{kg}$ may be the ideal minimum cell dose for allogeneic transplantation although lower doses do not preclude successful therapy.

Methods: We reviewed the chart about the patient to report here

Results: A 6-years old patient diagnosed aplastic anemia was treated hematopoietic cell transplantation from matched sibling donor. Because of not sufficient amount of harvested CD34+ cells, so we gave total five times transplantation for five days (Day 0 Day 4), including cord blood (cryopreserved at birth of the same donor) (D4). After 12 days from first transplantation, White blood cell engraftment was achieved. The result of bone marrow biopsy and PCR with STR(short tandem repeat) markers showed completely engraftment at 1 month after Day 0 when the first date of stem cell infused.

Conclusions: Multiple infusions of hematopoietic stem cells, including umbilical cord blood stem cells, could be engrafted even if the amount of total count of hematopoietic stem cells was insufficient.

Conflict of interest: no conflict of interest.

\section{P870}

Clinical risk factors for graft failure following allogeneic stem cell transplantation in acute leukemia patients

Anna A. Sidorova, Mikhail Y. Drokov, Larisa A. Kuzmina, Ekaterina D. Mikhaltsova, Olga M. Koroleva, Natalia N. Popova, Vera A. Vasilyeva, Darya S. Dubnyak, Ekterina V. Usikova, Zoya V. Konova, Elena N. Parovichnikova, Valery G. Savchenko

National Research Center for Hematology, Moscow, Russian Federation

Background: Primary and secondary graft failure (GF) following allogeneic hematopoietic stem cell transplantation (allo-HSCT) leads to prolonged neutropenia, infection complications and as a consequence lowered overall survival. Here we report feasible risk factors which can 


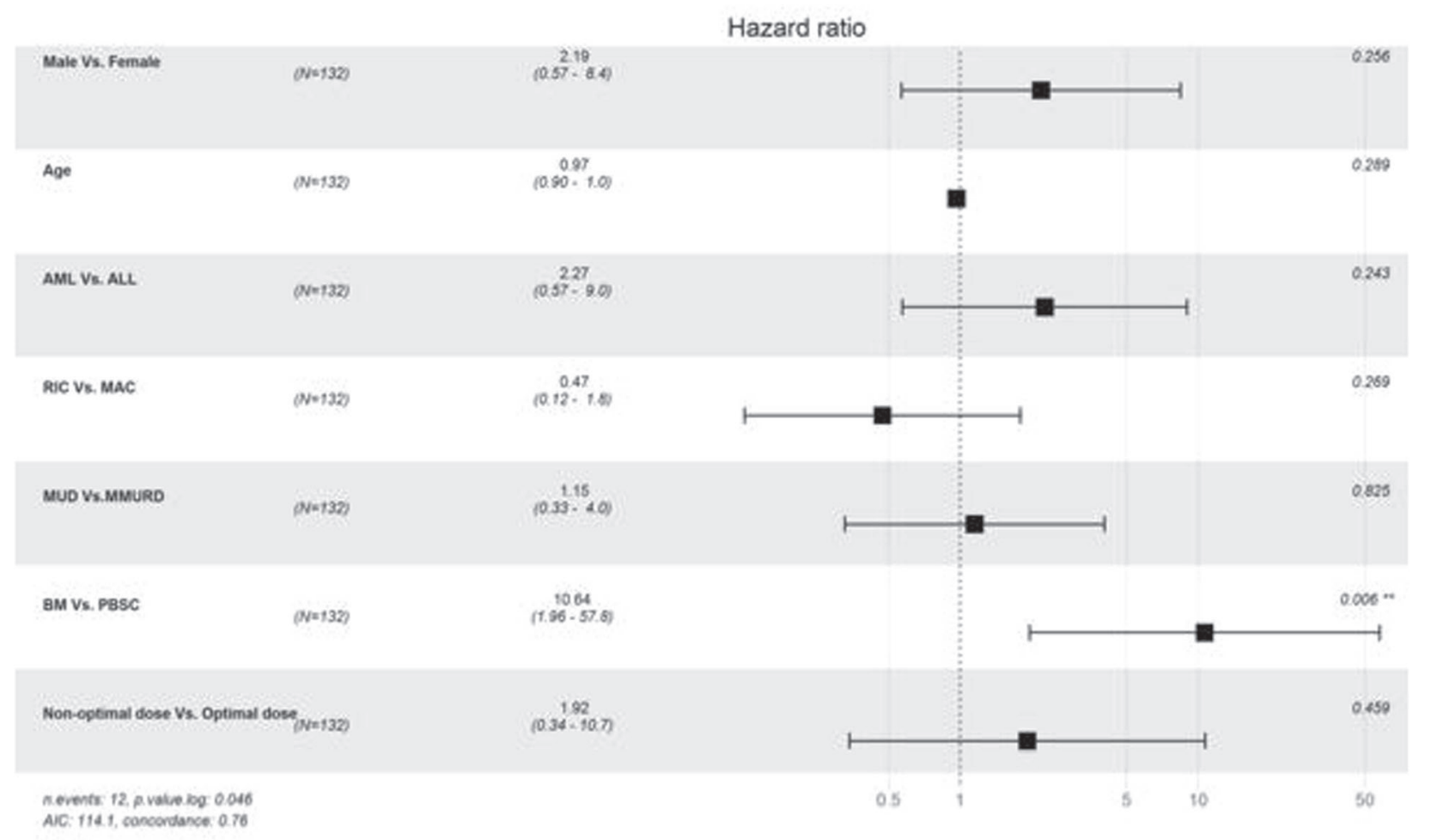

[P870 Figure] [Results of Cox Proportional-Hazards model]

influence GF in acute leukemia patients (pts) during first six months after allo-HSCT from unrelated donor.

Methods: We analyzed medical records of 132 pts with acute leukemia who underwent allo-HSCT from unrelated donors between 2011 and 2017 at National Research Center for Hematology and was alive for more than 28 days after allo-HSCT. A median age of patients was 34.5 (18-64) years, $58(43.9 \%)$ males and $74(56.1 \%)$ females. At the time of transplantation $106(80.3 \%)$ pts were in complete remission and $26(19.7 \%)$ pts had advanced disease. Most pts $(\mathrm{n}=101 ; 76.5 \%)$ received reduced intensity conditioning regimen (RIC) and 31 pts $(23.5 \%)$ underwent myeloablative conditioning (MAC). All donors were unrelated: HLA-matched - $65.9 \%(\mathrm{n}=87)$, mismatched $34.1 \%(\mathrm{n}=45)$. Bone marrow (BM) was used as a graft source in $42.4 \%$ (56 pts) and peripheral blood stem cell (PBSC) - in 57.6\% (76 pts). In our analysis GF was defined as neutropenia and host chimerism in bone marrow more than 50\%. Cox Proportional-Hazards model was used to identify independent prognostic factors. Age, sex, conditioning regimen, donor type, type of leukemia, graft source and graft cell dose were entered as covariates. Kaplan-Meier survival analysis with long-rank test was used to compare two groups.

Results: Results of Cox Proportional-Hazards model are shown on Figure 1. According to our data using of BM as a graft source for allo-HSCT from unrelated donors was associated with high risk of GF within six months of follow up period after allo-HSCT HR-10.64 (95\% Cl, 1.96-57.8, p $=0.006$ ). The probability of GF during six month depending on graft source was $19.1 \%$ for BM recipients compared to $2.7 \%$ for patients who received PBSC $(\mathrm{p}=0.0026)$.

Conclusions: BM as a graft source is characterized by low risk of graft-versus-host disease onset. But apart that fact using of BM increases probability of GF. For this reason it could be considered that $\mathrm{BM}$ as a graft source seems to be controversial for acute leukemia pts who is going to undergo allo-HSCT from unrelated donor.

Conflict of interest: nothing to disclose

\section{P871}

Comparable outcomes of haplo-identical and matched sibling allogeneic stem cell transplants for AML in first remission: experience of single center from northern India

Meet Kumar, Dharma Choudhury, Sanjeev Sharma, Vipin Khandelwal, Divya Doval, Mamata Sister

BLK Hospital, New Delhi, India

Background: Allogeneic stem cell transplant is an accepted post-remission therapy for patients with high and intermediate risk acute myeloid leukemia. For patients without a matched sibling donor (MSD), alternative donor 
transplant is indicated. In this scenario, haplo-identical donor (HID) transplant makes a strong point considering its ready availability, and especially its better applicability in the Indian setting due to the cost implications involved in procuring matched unrelated stem cells, where most of the transplants are self-funded. Data comparing matched unrelated donor vs. haplo-identical donor suggests equivalent outcomes in AML, however, there is paucity of literature comparing matched sibling vs. haplo-identical donor transplant for patients with AML in CR1. This prompted us to compare our single center experience of patients with AML in first remission undergoing allogeneic stem cell transplant with either MSD or HID as the donor our center.

Methods: We retrospectively compared all AML patients in first remission who underwent allogeneic transplants, with either MSD donor $(\mathrm{N}=45)$ or T-cell replete HID donor $(\mathrm{N}=15)$ between January 2011 and July 2017. Conditioning regimes were variable, and all HID transplants used posttransplant cyclophosphamide approach as GVH prophylaxis. Primary end points were overall survival (OS) and disease free survival (DFS). Secondary end points were acute and chronic GVHD and relapse rates.

Results: Patients were comparable with regard to median age, follow-up duration, CMV serostatus and conditioning intensity. Three year overall survival (OS) were comparable between the groups, with a trend for better survival for MSD transplants $(60 \%$ vs $48 \%, \mathrm{p}=0.58, \mathrm{DF}=1)$. Three year disease free survival (DFS) was also comparable, with trend for better survival favoring MSD donor (57\% vs. $38 \%, \mathrm{P}=0.13, \mathrm{DF}=1)$. Acute GVHD of all grades was comparable between the groups. In MSD, acute grade 1-2 GVHD was $19.5 \%$, compared to $17.3 \%$ in HID $(\mathrm{p}=0.12)$. Grade 3-4 acute GVHD was $16.8 \%$ in MSD, compared to $13.1 \%$ in HID $(p=0.11)$. Incidence of chronic GVHD was higher in HID transplants $(20.8 \%$ in MSD, compared to $30.4 \%$ in HID transplants $\mathrm{p}=0.055)$. Detailed data analysis will be presented later.

Conclusions: In the present study, we found similar overall and disease free survival outcomes between patients undergoing MSD vs. HID transplants for patients with AML in first remission. However, there was a trend for more chronic GVHD in patients with HID compared to MSD transplants. Also, there is no much difference between the donor sources in terms of GVHD and relapse mortality. It appears that for AML patients in first remission lacking a MSD, HID is a readily available donor source with comparable efficacy, probably due to recently updated better understanding in the field of haplo-identical transplants.

Clinical Trial Registry: Not applicable

Conflict of interest: None of the authors has anything to disclose.

\section{P872}

Does post-thaw washing of cryopreserved bone marrow reduce the adverse events of infusion in pediatric patients?

Maria Isabel Benitez Carabante ${ }^{1}$, Jesus Fernandez Sojo ${ }^{2}$, Laura Alonso $^{3}$, Laura Medina ${ }^{2}$, Carmen Azqueta ${ }^{2}$, Elena Valdivia ${ }^{2}$, Cristina Díaz de Heredia ${ }^{3}$, Sergi Querol ${ }^{2}$

${ }^{1}$ Vall d'Hebron Hospital, Hematology, Oncology and HSCT Unit, Barcelona, Spain; ${ }^{2}$ Banc de Sang $i$ Teixits, Barcelona, Spain; ${ }^{3}$ Vall d 'Hebron Hospital, Barcelona, Spain

Background: Cryopreservation of bone marrow stem cells (BMSC) grafts is occasionally needed due to problems related to the donor and recipient and logistical issues. Nevertheless, direct infusion of cryopreserved bone marrow has been associated with the development of adverse events (AE). These AE are known to be associated with the use of dimethyl sulfoxide (DMSO), red cells volume and nonmononuclear cells.

Methods: Data on patients who received a cryopreserved bone marrow transplant from 2005 to 2017 were retrospectively collected. All products were red cell depleted prior cryopreservation. Patients were classified in two different groups according to the use or non- use of a washing method post bone marrow thawing prior to the graft infusion. AEs were analyzed and classified following the CTCAE version 4.0. All patients received premedication with dexchlorpheniramine and methylprednisolone before the infusion of the graft.

Results: Sixteen patients were studied: 12 received thawed and non-washed BMSC grafts and 4 received thawed and washed BMSC grafts. Patients and product characteristics and adverse events are described in table 1. Eight patients $(50 \%)$ presented AEs, 3 in the washed

\begin{tabular}{|c|c|c|c|c|c|c|c|}
\hline $\begin{array}{l}\text { Washed } \\
\text { product after } \\
\text { thawing }\end{array}$ & $\begin{array}{l}\text { Age at } \\
\text { transplant }\end{array}$ & $\begin{array}{l}\text { Major ABO } \\
\text { incompatibility } \\
\text { (Yes/No) }\end{array}$ & $\begin{array}{l}\text { Total product } \\
\text { volume }(\mathrm{ml} / \mathrm{kg})\end{array}$ & $\begin{array}{l}\text { Red cells volume } \\
(\mathrm{ml})\end{array}$ & $\begin{array}{l}\text { Red cells volume } \\
(\mathrm{ml} / \mathrm{kg})\end{array}$ & $\begin{array}{l}\text { Non-MNC (x10e8)/ } \\
\mathrm{kg}\end{array}$ & $\begin{array}{l}\text { Adverse event, YES/NO } \\
\text { (GRADE) }\end{array}$ \\
\hline $\begin{array}{l}\text { Yes, patients: } 1 \\
234\end{array}$ & $\begin{array}{l}131311 \\
14\end{array}$ & NO NO NO YES & 1.844 .483 .152 .58 & $\begin{array}{l}22.454 .1215 .48 \\
6.48\end{array}$ & 0.411 .641 .020 .15 & $0.795 .01 \quad 1.852 .04$ & $\begin{array}{l}\text { NO YES (3) YES (2) } \\
\text { YES (2) }\end{array}$ \\
\hline $\begin{array}{l}\text { No, Patients: } 5 \\
6789101112 \\
13141516\end{array}$ & $\begin{array}{llll}1 & 2 & 0.3 & 8 \\
11 & 4 & 12 & 4 \\
8 & 15 & 4 & 15\end{array}$ & $\begin{array}{l}\text { NO NO NO NO } \\
\text { NO YES NO NO } \\
\text { NO NO NO NO }\end{array}$ & $\begin{array}{llll}8.07 & 8.63 & 8.53 & 3.31 \\
1.91 & 4.61 & 1.47 & 5.38 \\
5.68 & 1.93 & 5.29 & 2.92\end{array}$ & $\begin{array}{lllll}11 & 56.9 & 0.99 & 22 & 24 \\
37.17 & 10.72 & 8.25 \\
20.65 & 21.6 & 24 & 4.25\end{array}$ & $\begin{array}{llll}1.57 & 5.17 & 0.22 & 1 \\
0.5 & 1.77 & 0.16 & 0.66 \\
0.59 & 0.6 & 1.20 & 0.08\end{array}$ & $\begin{array}{llll}1.94 & 5.89 & 3.44 & 2.65 \\
0.38 & 3.48 & 0.30 & 1.14 \\
4.15 & 0.93 & 4.10 & 0.95\end{array}$ & $\begin{array}{l}\text { NO YES (1) NO NO YES } \\
\text { (2) NO NO NO YES (3) } \\
\text { YES (3) NO YES (2) }\end{array}$ \\
\hline
\end{tabular}

[[P872 Table] Table 1. Characteristic of the patients and product] 
product group and 5 in the non-washed product group. AEs were hypertension (7), hemoglobinuria (6), headache (2), nausea and vomiting (2), bradycardia (2), allergic reaction (1) and acute renal failure (1), related to the hemolysis and DMSO. Three patients presented Grade 3 AEs, 4 Grade 2, one Grade 1. Six of eight patients who presented AEs received bone marrow grafts with more than $20 \mathrm{ml}$ of red cells.

Conclusions: AEs are frequent after BMSC thawing even in absence of red cell incompatibility. Graft washing not seems to reduce incidence. The actual volume of red cells infused could be an important factor regarding the occurrence of AEs after cyopreserved BM infusion. Further investigation is needed to ascertain the impact of washing cryopreserved BM grafts in the occurrence of AEs during infusion.

Clinical Trial Registry: Not applicable

Conflict of interest: We do not have any conflict of interest

\section{P873}

\section{Evaluation of hematopoietic recovery after autologous stem cell transplant in patients with hematological malignancies}

Ricardo Pinto $^{1}$, Filipa Bordalo ${ }^{2}$, Sara Ferreira ${ }^{2}$, Catarina Pinho ${ }^{2}$, Sérgio Lopes ${ }^{2}$, Fátima Amado , Ana Salselas ${ }^{2}$, Susana Roncon ${ }^{2}$

${ }^{1}$ Instituto Português de Oncologia do Porto Francisco Gentil, Immunohemotherapy, Porto, Portugal; ${ }^{2}$ Instituto Português de Oncologia do Porto Francisco Gentil, Cellular Therapy, Porto, Portugal

Background: Autologous stem cell transplant (ASCT) is essential in consolidation treatment in some hematological malignancies. Cellularity content in apheresis products and evaluating cellular efficacy after cryopreservation seem to influence directly the efficiency and stability of the bone marrow engraftment. This clinical methodology definitely contributes to patients' overall and long time periods of disease-free survival.

Methods: Evaluation of hematological recovery (defined as neutrophil and platelets' recovery) in adult patients who underwent ASCT due to multiple myeloma (MM), Hodgkin's (HL) and non-Hodgkin's lymphoma (NHL) between the years 2015 and 2016.

Descriptive and retrospective study, performed by reviewing patients' electronic clinical records. Cryopreservation was done in liquid nitrogen at $-196^{\circ} \mathrm{C}$ (at $-80^{\circ} \mathrm{C}$ in hepatitis $\mathrm{B}$ positive patients). Statistical analysis was performed with Excel ${ }^{\circledR}$ and SPSS $^{\circledR}$.

Results: We evaluated 133 patients that were submitted to ASCT, $62.4 \%$ were male, with median age of 59 years (min. 20; max. 72) and median weight of $74 \mathrm{~kg}$ (min. 44; max. 141); 84 patients had MM (ISS I 25.6\%, ISS II 26.3\%, ISS III 10.5\%), 14 had HL and 35 had NHL (stage II $6.8 \%$, III $8.3 \%$, IV $22.5 \%$ ). The median pre-ASCT follow-up time was of 16 months (min. 1; max. 224).

Mobilized peripheral blood was the stem cell source in all patients. Regarding cryopreservation times, significant differences $(p=0,003)$ were found between lymphomas (3 months) and MM (4 months); 3 patients were excluded due to shorter preservation time motivated by positive viral serology.

In MM, melphalan was used as conditioning chemotherapy, $140 \mathrm{mg} / \mathrm{m}^{2}$ in 7 patients $(5,3 \%)$ and $200 \mathrm{mg} / \mathrm{m}^{2}$ in 77 $(57,8 \%)$. In lymphomas, fotemustine, etoposide, cytarabine and melphalan (FEAM) was used in 48 patients $(36.1 \%)$.

Three groups were defined by $\mathrm{CD}^{+}\left(\mathrm{x} 14^{6} / \mathrm{Kg}\right)$ cellularity after thawing $(\leq 2.5-60.9 \% ; 2.5-5-30.1 \%$; $\geq 5$ - 9\%), with median CFU-GM of $189.9 \times 10^{5} / \mathrm{Kg}$. Cellular viability, determined by tripane blue method, was $87 \%$ in lymphomas and $79.5 \%$ in MM, with statistical significance $(\mathrm{p}=0.01)$. Bacterial evaluation was negative in all product samples.

Patients were admitted in the hospital for a median period of 21 days (min. 14; max. 87), and cellular viability had statistical impact in shortening this period $(p=0.015$ in lymphomas and $\mathrm{p}=0.035$ in $\mathrm{MM})$. The 71 (53.4\%) MM patients and $23(17.3 \%)$ lymphoma patients had neutrophil and platelets recovery at the appropriate time, while 13 (9.8\%) MM patients and 22 (16.5\%) lymphoma patients had neutrophil recovery maintaining platelet transfusion needs. Almost patients, $91.7 \%$ are alive at the end of this study, with post-ASCT median follow-up time of 26 months.

Conclusions: High $\mathrm{CD} 34^{+}$cellularity and viability after thawing seem to determine better hematological recoveries and shorter inpatient periods. It is important to develop similar studies with larger number of patients to establish more definitive conclusions.

Conflict of interest: Nothing to disclose

\section{P874}

Experience of a developing country using G-CSF-Primed Bone Marrow as the source for Allogeneic Hematopoietic Stem Cell Transplantation

\section{Eucario Leon Rodriguez, Monica Magdalena Rivera Franco}

Instituto Nacional de Ciencias Medicas y Nutricion Salvador Zubiran, Hematology and Oncology, Tlalpan, Mexico

Background: Currently, peripheral blood (PBSC) is the most commonly used source for allogeneic hematopoietic stem cell transplantation (allo-HSCT) due to faster 
engraftment, faster immunological reconstitution, and practicability. However, it is associated with higher incidence of graft-versus-host-disease (GVHD). On the other hand, some cohort studies have described the safety and efficacy of using related G-CSF-primed bone marrow (G-BM) allografts. In addition to accelerating engraftment, the use of G-BM as the source of HSC associates with decreased incidence of GVHD. Nonetheless, to date, G-BM as the main source of hematopoietic stem cells is rarely used worldwide. The aim of this study was to describe the outcomes of patients undergoing an allo-HSCT using G$\mathrm{BM}$ at a tertiary/referral center in a developing country.

Methods: Consecutive patients who underwent alloHSCT from identical HLA donors, using G-BM, at the National Institute of Medical Sciences and Nutrition in Mexico City, from May 1999 to April 2017, were retrospectively analyzed. The dataset used for this study derived from medical records collected at the HSCT Program and electronic Institutional medical records. Informed consent was obtained from all patients and donors before undergoing allo-HSCT. HSCs were collected from donors by multiple aspirations of the iliac crests; G-CSF $(10 \mu \mathrm{g} / \mathrm{kg} / \mathrm{day})$ was administered 3 days (every 8 hours) prior the procedure. The volume of harvest was adapted to the recipients' body weight.

Results: A total of 66 consecutive patients were included. Overall patient characteristics are shown in Table 1.

The median age was 29 years and the most common underlying diseases were: aplastic anemia $(\mathrm{n}=17,26 \%)$, acute lymphoblastic leukemia $(\mathrm{n}=15,23 \%)$, and myelodysplastic syndrome $(\mathrm{n}=15,23 \%)$. All patients had identical HLA-identical sibling donors. Engraftment occurred in all patients. No late post-transplant graft failure was observed. Toxicity was presented in $89 \%$ patients $(54 \%$ grades I-II). Acute GVHD was developed in $15 \%$ of patients (60\% grades I-II); 22 patients $(33 \%)$ had chronic GVHD, which was limited in $82 \%$. Thirty and 100-day NRM was $3 \%$ and $4.5 \%$, respectively. One and 3-year NRM was $7.5 \%$ and $12 \%$, respectively. Only $8 \%$ TRM was accounted to GVHD. At last follow up, relapse free and overall survival was $73 \%$ and $68 \%$, respectively.

Conclusions: Allo-HSCT has become an established therapy for many hematological diseases. However, serious complications, including GVHD, sepsis, and infections, still limit the success of the procedure also increasing the overall financial costs. We reported that using G-BM associates with platelet engraftment similar to reported literature with PBSC, and although few days longer for neutrophils, all patients included in our study engrafted. Further, the frequency of acute and chronic GVHD was lower compared to published literature using SS-BM or PBSC. In conclusion, G-BM generated favorable outcomes including low frequency of aGVHD, which produced measurable clinical

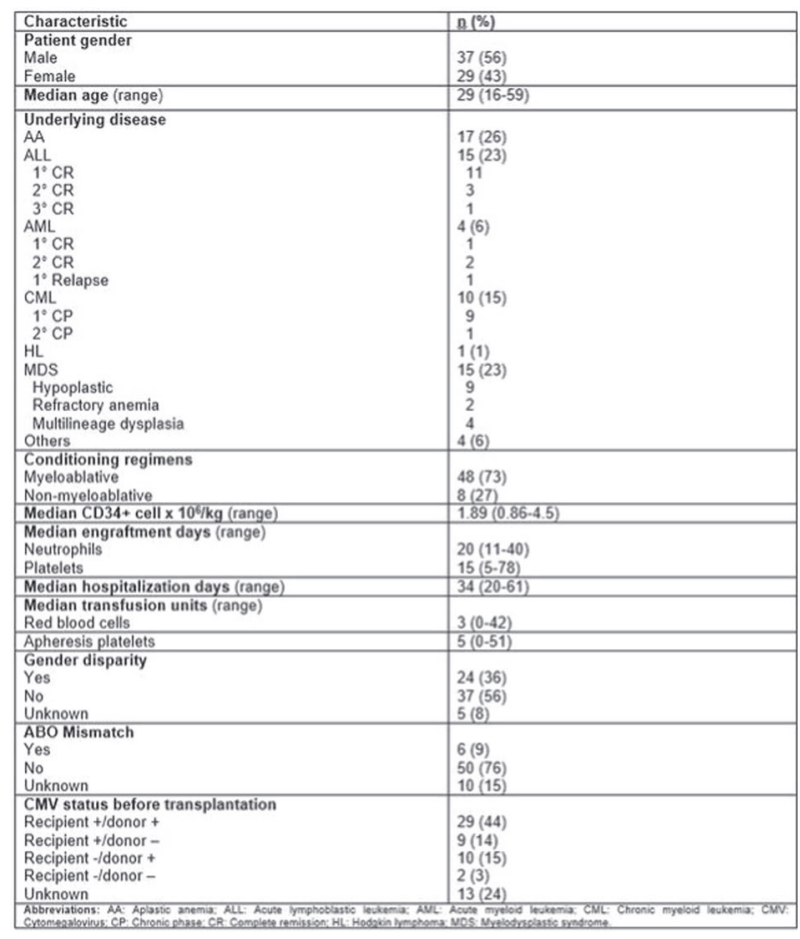

[P874 Figure] [Table 1. Patient and HSCT Demographics.]

benefits such as minimal NRM. Further, this reduction in GVHD can reduce costs in low-middle income countries making G-BM the best source for allo-HSCT in these scenarios.

Conflict of interest: None of the authors have any conflict of interest to disclose.

\section{P875}

Haploidentical Transplantation Of T-Cell Replete, GCSF Mobilized Peripheral Blood Stem Cells With High Dose PostTransplant Cyclophosphamide: An effective Therapeutic Option For Heavily Pre-Treated Lymphoma Patients

Rocco Pastano, Federica Gigli, Simona Sammassimo, Daniele Avenoso, Alberto Agazzi, Anna Vanazzi, Enrico Derenzini, Tommaso Radice, Safaa Ramadan, Elisabetta Todisco, Corrado Tarella

European Institute of Oncology, Haemato-Oncology, Milano, Italy

Background: Hematopoietic stem cell transplant (HSCT) from haploidentical donor using high-dose, post-transplant cyclophosphamide $(\mathrm{Cy})$ is an alternative procedure for patients with advanced haematological malignancies lacking an HLA identical donor. A prospective study based on the use of peripheral blood stem cells (PBSC) as graft source was launched at European Institute of Oncology in 2010. The updated experience in 63 patients is here reported. 
Methods: Haploidentical HSCT was performed with GCSF mobilized PBSC. Conditioning regimen was either non-myeloablative (56 patients) (Cy $14.5 \mathrm{mg} / \mathrm{kg} / \mathrm{die}$ days $-6,-5$, fludarabine $30 \mathrm{mg} / \mathrm{m}^{2} /$ die days -6 to -2 , and $200 \mathrm{cGy}$ Total Body Irradiation day -1), or myeloablative (treosulfane $14 \mathrm{mg} / \mathrm{m}^{2} /$ die days -6 to -3 and fludarabine $30 \mathrm{mg} / \mathrm{m}^{2} /$ die days -6 to -2$)$. GVHD prophylaxis included posttransplant high dose Cy days $+3,+4$, mycophenolate and tacrolimus. Among 63 evaluable patients, 31 (49\%) received haplo-HSCT for refractory/relapsed lymphoma (25 non-Hodgkin and 6 Hodgkin Lymphoma); the remaining patients included 23 (36\%) high-risk Acute Leukemia (AL), 7 (11\%) Multiple Myeloma (MM), and 2 Myelodysplastic Syndrome (MDS). Median age was 54 (19-73), disease status included 26 (41\%) complete remission (10 CR1, 16 CR $\geq 2), 14$ (22\%) partial response (PR), 9 (14\%) stable disease (SD), 14 (22\%) progressive disease (PD); one MDS patient received HSCT upfront. A median of $5.5 \times 10^{6}$ (range 2.45 -13.4) CD34+ cells $/ \mathrm{kg}$ was infused, with a median of $2.8 \times 10^{8}$ (range 0.3-5.4) CD3 + cells $/ \mathrm{kg}$.

Results: There were four toxic deaths due to infections before engraftment. For the remaining patients, median time to absolute neutrophils $\geq 500 / \mu \mathrm{L}$ was 18 days (range 12-34), and 21 days (range 12-65) to platelets $\geq 20.000 / \mu \mathrm{L}$. Incidence of grade I-II acute GVHD was $32 \%$, with grade III of 3\%. Mild chronic GvHD was observed in 11/60 evaluable patients (1-year cumulative incidence: 18\%). Mixed donor chimerism was achieved in all 60 evaluable patients, with $\mathrm{CD} 3+$ chimerism $>50 \%$ at day $+28,>90 \%$ at day +84 . CMV reactivation occurred in $70 \%$ of patients, at a median of 30 days (range 5-82) post-HSCT, infection resolved with pre-emptive therapy in 43 patients, was fatal in 1 heavily pre-treated patient. At a median follow-up of 322 days (6-2,417), 31 patients are alive (49\%), 26 of them (41\% of the whole series) in CR, while the cumulative incidence of relapse is $29 \%$. Overall, 32 patients (51\%) died; causes of death were PD in 20 patients (31\%), infections in 10 (16\%), non-infection complications in 2 (cumulative transplant related mortality: 19\%). Among 31 lymphoma patients, $17(55 \%)$ are alive in CR at a median follow-up of 288 days $(6-2,417)$. Their pre-HSCT disease status included: $\geq \mathrm{CR} 2$ (7 patients), PR (6), and PD (4). The 5 -year PFS and OS for the lymphoma patients is $72 \%$ and $51 \%$, respectively, with $87 \%$ and $62.5 \%$ for patients transplanted in $\mathrm{CR} / \mathrm{PR}$ and $46 \%$ and $33.3 \%$ for those transplanted in SD/PD.

Conclusions: Haploidentical T-cell replete PBSC transplantation with high-dose post-transplant $\mathrm{Cy}$ is a feasible procedure for high-risk haematological malignancies, with an overall toxicity analogous to HSCT with HLA-identical donors. This approach is an effective salvage option for heavily pre-treated lymphoma patients.

Conflict of interest: All authors have nothing to disclose.

\section{P876}

Harvesting from the fountain of youth - superior stem cell
concentrations in BM from young donors

Sofia Frändberg, Berit Waldner, Lisa Lagerlöf, Lena Eriksson, Linn Zimmerman, Marie Elving, Anna Persson

Sahlgrenska University Hospital, Stem Cell Laboratory, Clinical Immunology and Transfusion Medicine, Gothenburg, Sweden

Background: Hematopoietic stem and progenitor cells (HSPC) for use in hematopoietic stem cell transplantation (HSCT) can be harvested from bone marrow (BM). BM is retrieved from the posterior iliac crest under general anesthesia in volumes of 10-20 ml per kilogram donor weight. Donor and collection related factors can affect the concentration of HSPC in the resulting cellular product and the harvesting physician frequently requests evaluation of the quality of the BM harvest during the procedure. The outcome after HSCT weighs heavily on the number of transplanted HSPC. We aimed to calculate reference values for HSPC concentration in BM harvests and investigate how donor age affected HSPC concentration.

Methods: 62 BM harvests performed 2002-2017 (without G-CSF mobilization), were investigated for mononuclear cell (MNC) concentration (manual count under the microscope) and the concentration of viable CD34+ cells (cells/ $\mu l$, BD FACSCalibur, BD Biosciences) Descriptive statistics including median and percentiles were calculated for donors $<12$ years $(\mathrm{n}=29)$, donors $12-60$ years $(\mathrm{n}=27)$ and donors $>60$ years $(n=6)$ for MNC and CD34+ cells concentrations. Correlations (Spearman's Rho) were calculated for MNC and CD34+ cells concentration and age. Group comparisons between results for age groups $(<12$ years, 12-60 years and $>60$ years) were performed using Kruskall-Wallis test.

Results: The median MNC and CD34+ cells concentration were; $124^{\wedge} 10^{8} \mathrm{MNC} / \mathrm{L}$ and $408 \mathrm{CD} 34+$ cells/ $\mu$ l for donors $<12$ years, $108^{\wedge} 10^{8} \mathrm{MNC} / \mathrm{L}$ and $190 \mathrm{CD} 34+$ cells/ $\mu \mathrm{l}$ for donors $12-60$ years and $92^{\wedge} 10^{8} \mathrm{MNC} / \mathrm{L}$ and 106 $\mathrm{CD} 34+$ cells $/ \mu \mathrm{l}$ for donors $>60$ years. There was a significant correlation between the age of the donor and the concentration of CD34+ cells $(r=0.70 \mathrm{p}<0.0001)$ in the $\mathrm{BM}$ harvest. There was no correlation between age and MNC concentration $(\mathrm{p}=0.4)$. The Kruskall-Wallis test revealed a significant difference in $\mathrm{CD} 34+$ cells concentration across the 3 different age groups $(\mathrm{p}<0.0001)$, but not for MNC concentration $(\mathrm{p}=0.5)$

Conclusions: We have established reference values relative the age of the donor for HSPC concentrations in BM harvests, enabling us to give feedback on the quality of the BM harvest during and after the harvesting procedure. Younger donors have significantly higher concentrations of 
$\mathrm{CD} 34+$ cells in BM indicating age related differences in hematopoiesis. This means that smaller volumes of BM per kilogram donor weight might be harvested from younger donors, without compromising the total dose of HSPC available for the HSCT recipient. The lack of correlation between age and MNC concentration is probably due to lesser precision in the manual counting of cells and the greater heterogeneity of this cell population compared to CD34+ cells.

Conflict of interest: None of the authors has anything to disclose.

\section{P877}

HLA-haploidentical hematopoietic stem cell transplantation with in vivo or in vitro T-cell depletion strategies in different hematological diseases. A single centre experience

László Gopcsa ${ }^{1}$, Anikó Barta ${ }^{1}$, Arpád Bátai ${ }^{1}$, Zoltán Csukly ${ }^{1}$, János Dolgos ${ }^{1}$, János Fábián ${ }^{1}$, Zita Farkas ${ }^{1}$, Agnes Király ${ }^{1}$, Gergely Lakatos $^{1}$, Enikö Lehoczky ${ }^{1}$, Lilla Lengyel ${ }^{1}$, Nóra Lovas ${ }^{1}$, Zoltán Mátrai $^{1}$, Gábor Mikala ${ }^{1}$, Melinda Paksi ${ }^{1}$, Mónika Petô ${ }^{1}$, Marienn Réti $^{1}$, János Sinkó ${ }^{1}$, Andrea Sipos ${ }^{1}, E^{\prime} v a$ Torbágyi ${ }^{1}$, Tamás Masszi ${ }^{2}$, Péter Reményi ${ }^{1}$

${ }^{1}$ St Istvan and St László Hospital, Haematology and Stem Cell Transplantation, Budapest, Hungary; ${ }^{2}$ Semmelweiss University, $3 \mathrm{rd}$. Department of Internal Medicine, Budapest, Hungary

Background: Haploidentical hematopoietic stem cell transplantation (HSCT) is increasingly considered as a viable therapeutic option for patients lacking HLA-identical siblings or HLA-matched unrelated donors. Initial approaches with haplo-HSCT lead to unacceptably high complication rates. Nowadays, new in vivo approaches such as posttransplantation Cyclophosphamide (PTCY) and in vitro graft manipulation strategies marked improved clinical outcome.

Methods: We retrospectively analyzed data from 28 consecutive patients who underwent haploidentical-HSCT between June 2014 and November 2017 in our Unit. According to the Baltimore group, we employed PTCY in 19 patients. Nine patients with acute leukemia were treated with $\alpha \beta$ T-cell depleted allograft. In total, 17 patients were male $(60 \%)$ and 11 female (40\%). Median age was 36 years (range:21-64). Baseline disease was:16 AML (57\%), 6 ALL (21\%), 2 MDS (7\%), 2 SAA (7\%), $1 \mathrm{HD}(3,5 \%)$ and $1 \mathrm{CML}$ $(3,5 \%)$. Disease state at time of haplo-HSCT was the following: 12 patients in CR1 (43\%), $6>\mathrm{CR} 1(21 \%)$ and 10 no response/active disease (36\%). Myeloablative conditionining (MAC) was used in 23 patients $(82 \%)$ and reduced intensity regimen (RIC) in 5 patients (18\%). The MAC regimen consisted of thiotepa $(2 \times 5 \mathrm{mg} / \mathrm{kgBW})$, busulfan $(3 \times 3,2 \mathrm{mg} / \mathrm{kgBW})$ and fludarabine $\left(3 \times 50 \mathrm{mg} / \mathrm{m}^{2}\right)$ or TBI
(12Gy) and fludarabine $\left(4 \times 30 \mathrm{mg} / \mathrm{m}^{2}\right)$. The stem cell source was PBSC in $46 \%$, and BM in $54 \%$.

Results: With a median follow-up of 112 days (range:141058), 21 patients are alive and in remission. Seven patient died including 6 due to NRM and one patient had disease relapse. OS and DFS were $75 \%$ and $75 \%$. The OS was higher in CR1 compared with other ( $>$ CR1 and active disease) disease state ( $84 \%$ vs $69 \%$ ). TRM in the first 100 days were $10 \%$. Grade 2 acute GVHD, mainly with skin involvement was observed 32\%. Grade 3-4 acute GVHD occured in two (7\%) patients and chronic extensive GVHD in two (7\%). Median time of $>0,5 \mathrm{G} / 1$ and $>1,0 \mathrm{G} / \mathrm{l}$ absolute neutrophyl leukocyte engraftment were 16 days (range:8-31) and 17 days (range:9-34), respectively. Median time of 20G/1 platelet engraftment was 17 days (range:10112). All the patients who successfully engrafted had $>95 \%$ chimerism shortly after HSCT. With a median follow-up of 250 days (range:14-1058), the OS was $63 \%$ in the PTCY group and $100 \%$ in the TCRab selected group, but the follow-up period was (median 56 days, range: 14-135) too short. Median time for neutrophyl engraftment $(1 \mathrm{G} / \mathrm{l})$ was 16,5 (range:13-34) and 10 days (range:9-11) after PTCY and $\alpha \beta$ TCR selected subgroup. Median platelet engraftment was 25 (range:13-112) and 12 days (range:10-16) after PTCY and $\alpha \beta T C R$ selected subgroup. All patients are engrafted in the TCR $\alpha \beta$ T-cell-depleted goup, which slightly was lower in the PTCY group (88\%). Incidence of severe GVHD was same in both groups. Severe hemorrhagic cystitis was observed only in the PTCY group.

Conclusions: Based on our experiences, haplo-HSCT showed better outcome than our earlier results with $7 / 8$ matched MUD HSCT. Both PTCY and TCR $\alpha \beta$ T-celldepletion after haplo-HSCT are safe and feasible treatment options for patients who waiting for allografting. We emphasize that use of in vitro graft manipulation technics might provide further improvement of the effectivity of haplo-HSCT.

Conflict of interest: Disclosure of conflict of interest: None.

\section{P878}

Immune reconstitution after allo-stem cell transplantation in pediatric patients: differences between bone marrow and peripheral blood stem cell source

Ana Maria Fita ${ }^{1}$, Maria Victoria Martínez Sánchez ${ }^{2}$, Maria Esther Llinares Riestra ${ }^{3}$, María Mar Bermúdez Cortés ${ }^{3}$, Ana Maria Galera Miñarro ${ }^{3}$, Miguel Blanquer Blanquer ${ }^{4}$, Andrés Sánchez Salinas ${ }^{4}$, José Luis Fuster Soler ${ }^{3}$, José María Moraleda ${ }^{4}$

${ }^{1}$ Hospital Clínico Universitario Virgen de la Arrixaca, Paediatrics Oncology and Haematology Unit, El Palmar, Spain; ${ }^{2}$ Hospital Clínico Universitario Virgen de la Arrixaca, Immunology Service, El Palmar, 
Murcia, Spain; ${ }^{3}$ Hospital Clínico Universitario Virgen de la Arrixaca, Paediatrics Oncology and Haematology Unit, El Palmar, Murcia, Spain; ${ }^{4}$ Hospital Clínico Universitario Virgen de la Arrixaca, IMIB, University of Murcia, Haematology Service and Stem Cell Transplant Unit, El Palmar, Murcia, Spain

Background: Recovery of the immune system after allogeneic haematopoietic stem cell transplantation (alloHSCT) is a highly dynamic process, often delayed as a result of the preparative regimen used prior to transplant, predisposing the recipients to infections.

Methods: Retrospective, observational study of patients aged between 0 and 15 years undergoing allo-HSCT at a tertiary hospital, from January 2007 to December 2015. We studied a total of 56 pediatric patients who underwent 69 allo- HSCT with a median follow-up of $19(1-87)$ months. Immunologic reconstitution was assessed by total lymphocytes; CD3+, CD4+ and CD8+ T lymphocytes; CD4
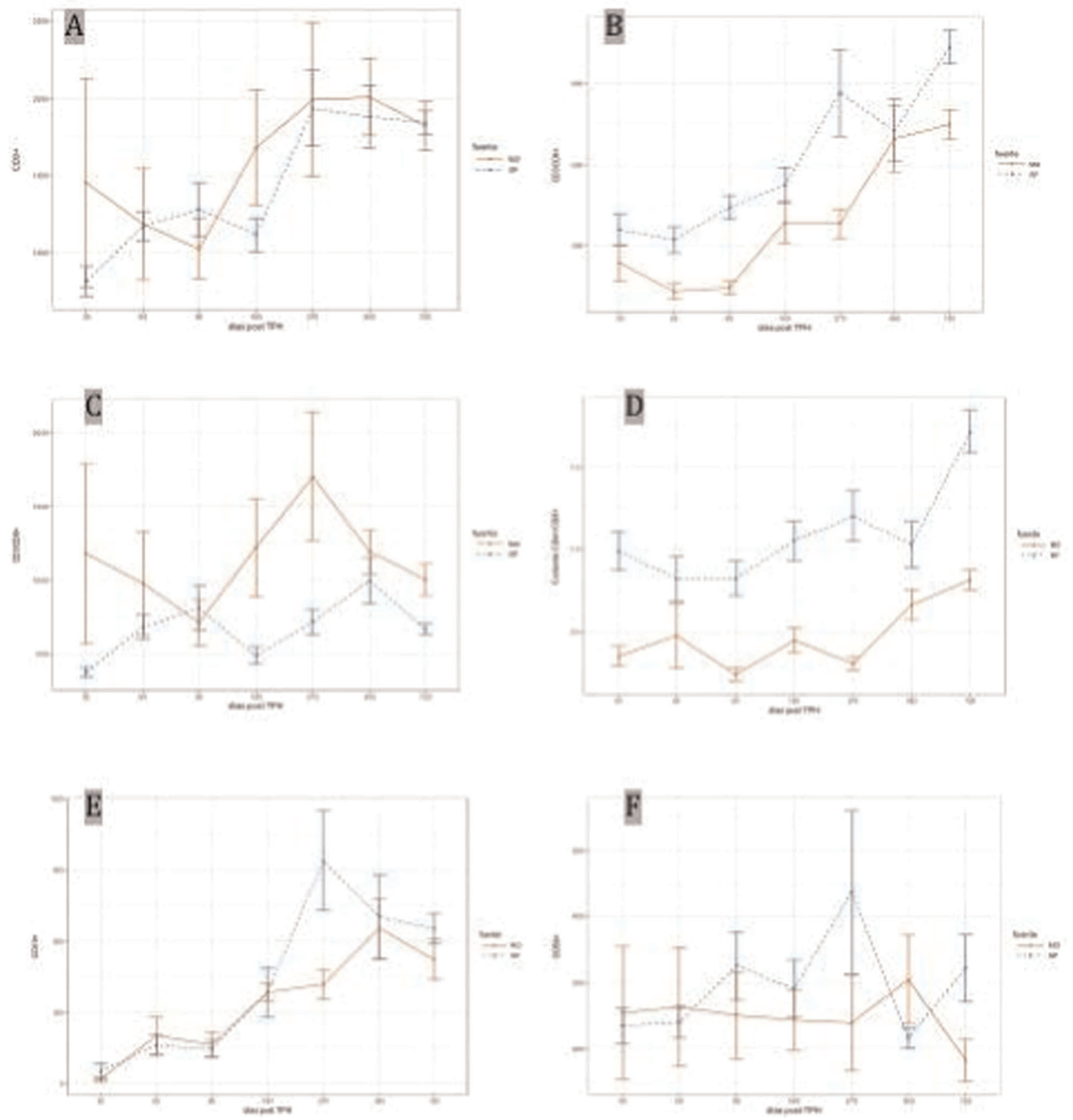

[P878 Figure] [Immune recovery post allo-HSTC according stem cell source (PB vs BM).] 
+/CD8+ ratio; CD19+ B lymphocytes and CD16+/CD56 + natural killer $(\mathrm{NK})$ at day $+30,+60,+90,+180,+360$ and +720 post allo-HSCT. Normal lymphocyte population values were defined as those included in The Pediatric AIDS Clinical Trials Group P1009 study. We divided the data in 2 groups according to stem cell source: peripheral blood (PB) and bone marrow (BM).

Results: We depict the immune evolution post alloHSCT of each type of lymphocyte, according to the stem cell source.

The CD3+ lymphocytes presented normal counts since day +270 in the PB group, while since day +180 in the BM group. Similarly, the CD4+ lymphocytes presented normal counts since day +270 in the PB group, but was delayed up until day +360 in the $\mathrm{BM}$ group. By contrast, the $\mathrm{CD} 8+$ lymphocytes presented normal counts since day +60 in the $\mathrm{PB}$ group and since day +30 in the $\mathrm{BM}$ group. Consequently, the $\mathrm{CD} 4+/ \mathrm{CD} 8+$ ratio showed initial inversion in both groups, with normalization since day +180 in the PB group while in the BM group persisted inverted until day +360 . The $\mathrm{CD} 19+$ lymphocytes presented normal counts in both groups since day +180 . The CD16+/CD56+ lymphocytes exhibited a similar course in both groups, with normal counts since day +30 . We encountered a significant difference $(\mathrm{p}<0.05)$ between $\mathrm{PB}$ and $\mathrm{BM}$ in $\mathrm{CD} 4+$ lymphocytes recovery at days +60 and $+90 ; \mathrm{CD} 8+$ at day +180 ; and $\mathrm{CD} 4+/ \mathrm{CD} 8+$ ratio at days $+30,+90,+180,+270$ and +720 .

Conclusions: Our results confirm that the kinetics of immune reconstitution is different among bone marrow and peripheral blood SC source. Overall, innate immunity recovers within the first weeks of HCT, followed by that of the adaptive immune system. The inversion of CD4 $+/ \mathrm{CD} 8+$ ratio is one of the earliest features of $\mathrm{T}$ cell reconstitution and can persist for up to several years after HSCT.

Conflict of interest: None

\section{P879}

\section{Improvement of overall survival and continuous reduced non-relapse mortality after allogeneic hematopoietic stem cell transplantation over a 30 -year period at a single institution}

Manuel Abecasis ${ }^{1}$, Nuno Miranda ${ }^{2}$, Isabelina Ferreira ${ }^{2}$, Gilda Teixiera $^{2}$, Filipa Moita ${ }^{2}$, Fernando Leal da Costa ${ }^{2}$, Ines Nolasco ${ }^{1}$, Antonio Guimaraes ${ }^{2}$, Jose Luis Passos Coelho ${ }^{2}$, Alexandra Machado $^{2}$, Ernesto Fernandez ${ }^{2}$, Maria Joao Gutierrez ${ }^{2}$, Monica Cunha $^{2}$, Susana Eteves ${ }^{2}$
${ }^{1}$ Instituto Portugues Oncologia, BMT, Lisbon, Portugal; ${ }^{2}$ Instituto Portugues Oncologia, Lisbon, Portugal

Background: Allo-HSCT is a potentially curative treatment for life-threating malignant and nonmalignant disorders. Since the implementation of our transplant program in May 1987 we have introduced numerous modifications following the trends in the field, aiming to reduce transplant associated morbidity and mortality, and improving results. We now evaluate whether the changes implemented over the past 3 decades had a positive reflection in the outcome.

Methods: A retrospective review of 682 consecutive first allo-HSCT was conducted. Data from 28 May 1987 to 31 May 2016 were analyzed from our transplant database. Cutoff date for analyses was 31 May 2017. Survivors data were censored at the date of last follow-up. We compared the cumulative incidence of OS, PFS, NRM and relapse in 10year cohorts over the study 3 decades. OS and PFS were calculated using the Kaplan-Meier method and log-rank test for group comparison. Probability of relapse and NRM were calculated using the cumulative incidence procedure for competing risk models and the Gray test for group comparison. Patients with acute leukemia (AL) in CR1, CML in first chronic phase (CP1), aplastic anemia and nonmalignant conditions were considered standard risk. Patients with AL beyond CR1, CML beyond CP1, lymphomas and all malignancies in partial remission, progression or relapse were included in the high risk group. TBI-cyclophosphamide and busulfan with cyclophosphamide were the most commonly used conditioning regimens for $\mathrm{AL}$ and $\mathrm{CML}$. Reduced intensity regimens were introduced over the last 2 decades. GVHD prophylaxis was with calcineurin inhibitors and methotrexate or mycophenolate mofetil. "In vivo" T cell depletion with ATG/ALG was added when using unrelated donors. Patients with an HLA-C mismatch were given alemtuzumab. Infection prophylaxis included low dose acyclovir, oral quinolone, an azole and trimethoprimsulfamethoxazole.

Results: The median age of patients transplanted increased from 22 years in the first decade to 33 years in the last decade $(\mathrm{P}<0.001)$. The proportion of patients transplanted for AML increased over the past 3 decades $(\mathrm{P}<$ 0.001) whereas the proportion of ALL and CML patients decreased significantly $(\mathrm{P}=0.007$ and $\mathrm{P}<0001)$. Regarding disease status at transplantation there was a significant increase of high risk patients $(\mathrm{P}=0.02)$. The use of peripheral blood $(\mathrm{P}<0.0001)$, and the number of unrelated donors $(\mathrm{P}<0.0001)$ increased dramatically over the study period.

372 patients are alive at the date of last follow-up, the median FU being 23, 13 and 3.5 years respectively for the 3 
consecutive decades. The 3 -year OS between the first 2 decades was $58 \%$ and $55 \%$ increasing to $64 \%$ over the last decade. PFS at 3 -years remained relatively stable at $54 \%$, $49 \%$ and $62 \%$ over the 3 decades. NRM at 3 -years decreased significantly from $29 \%$ in the first decade to $20 \%$ on the last one $(\mathrm{P}=0.045)$. The cumulative incidence of relapse/progression at 3 -years was $25 \%$, being stable over the 3 decades.

Conclusions: Despite increasing age, higher risk patients and the use of unrelated donors our results in the last 30 years show a continuous and significantly reduced NRM together with an improvement in overall survival, with a stable PFS and relapse rate.

Conflict of interest: nothing to disclose

\section{P880}

\section{In PBSC mobilization of Multiple Myeloma, after cyclophosphamide, G-CSF at dose of $10 \mathrm{mcg} / \mathrm{Kg}$ is more effective than at dose of $5 \mathrm{mcg} / \mathrm{Kg}$}

\section{Giuseppe Milone $^{1}$, Massimo Martino ${ }^{2}$, Maria Grazia Michieli,} Giuseppe Sapienza ${ }^{4}$, Luca Scalise ${ }^{4}$, Andrea Spadaro ${ }^{4}$, Giuseppe Avola ${ }^{5}$, Maria Grazia Camuglia ${ }^{5}$, Anna Lia Di Marcos, Giovanni Tripepi $^{6}$

${ }^{1}$ Azienda Ospedaliera Policlinico, Hematology and BMT UNIT, Catania, Italy; ${ }^{2}$ Azienda Ospedaliera Bianchi Melacrino Morelli, Trapianti di Midollo, Reggio Calabria, Italy; ${ }^{3}$ Centro Riferimento Oncologico Aviano, High Dose Chemotherapy, Aviano, Italy; ${ }^{4}$ Azienda Ospedaliera Policlinico, Catania, Italy; ${ }^{5}$ Azienda Ospedaliera Policlinico, Hematology and BMT Unit, Catania, Italy; ${ }^{6} \mathrm{CNR}$, IBIM, Reggio Calabria, Italy

Background: The use of algorithm to administer PLX has increased the success rate of the mobilization treatments. On the other hand, also the increase of the dose of G-CSF from $5 \mathrm{mcg} / \mathrm{Kg}$ to $10 \mathrm{mcg} / \mathrm{Kg}$ could be important to the end of harvesting an optimal amount of CD34+ cells. It is not known if "high dose G-CSF" and "on demand PLX" show a synergistic effect so that their combined used may be of additive usefulness

Methods: 404 patients were retrospectively studied, all were affected by MM and were in first mobilization attempt. All 404 patients received CTX at 2-4 gr/sqm, 180/404 patients were enrolled in on demand PLX (PLX GROUP) and 224/404 were not (NO PLX GROUP). In NO PLX GROUP 112 patients received G-CSF at $10 \mathrm{mcg} / \mathrm{Kg}$ and 68 received it at dose of $5 \mathrm{mcg} / \mathrm{Kg}$. In PLX GROUP 153 received G-CSF at dose of $10 \mathrm{mcg} / \mathrm{Kg}$ and 71 at dose of 5 $\mathrm{mcg} / \mathrm{Kg}$.

Results: In NO PLX GROUP, percentage of patients failing to harvest the minimum CD34+ of $2 \times 10 \mathrm{e} 6 / \mathrm{Kg}$ was $19 \%$ in patients receiving $5 \mathrm{mcg} / \mathrm{Kg}$ versus $8.8 \%$ in patients receiving G-CSF at $10 \mathrm{mcg} / \mathrm{Kg}(\mathrm{p}=0.04)$. Patients receiving
G-CSF at dose of $5 \mathrm{mcg} / \mathrm{Kg}$ reached a harvest $>6 \times 10 \mathrm{e} 6$ CD34+ cells in $42 \%$ of cases while this outcome was reached in $72 \%$ of patients receiving G-CSF at dose of 10 $\mathrm{mcg} / \mathrm{Kg}(\mathrm{p}=0.0001)$. Mean harvested CD34+ cells was 6.3 $\mathrm{x} 10 \mathrm{e} 6 / \mathrm{Kg}$ versus $13.0 \mathrm{x} 10 \mathrm{e} 6 / \mathrm{Kg}(\mathrm{p}=0.0001)$.

In PLX GROUP, patients failing the minimum CD34 harvest $(2 \times 10 \mathrm{e} 6 / \mathrm{Kg})$ was $5.0 \%$ and $1.7 \%$ in the two groups treated, respectively, with $5 \mathrm{mcg} / \mathrm{Kg}$ and in $10 \mathrm{mcg} / \mathrm{Kg}$ ( $\mathrm{p}=0.08$ ) the optimal threshold of $6 \times 10 \mathrm{e} 6 / \mathrm{Kg}$ was reached In $67 \%$ of those receiving G-CSF at $5 \mathrm{mcg} / \mathrm{Kg}$ and in $73 \%$ of patients treated with G-CSF at $10 \mathrm{mcg} / \mathrm{Kg} \quad(\mathrm{p}=$ not significant). The mean number of harvested CD34+ cells was $10.4 \times 10 \mathrm{e} 6 / \mathrm{Kg}$ versus $11.2 \times 10 \mathrm{e} 6 / \mathrm{Kg}$, respectively in 5 $\mathrm{mcg} / \mathrm{Kg}$ and in $10 \mathrm{mcg} / \mathrm{Kg}$ groups $(\mathrm{p}=\mathrm{NS})$.

Thus, high dose G-CSF seems more advantageous when PLX on demand is not used while its efficacy is less evident when PLX on demand is programmed. We therefore wished to study the existence of an interaction between these two factors. In ANOVA test the amount of CD34 cells harvested was used as dependent variable and "dose of G-CSF" and "to be enrolled in a PLX ON DEMAND study" were evaluated. Interaction between the two factors was significant $(\mathrm{F}=14.1 ; \mathrm{p}=0.0002)$.

Conclusions: After CTX based mobilization, a dose of G-CSF of $10 \mathrm{mcg} / \mathrm{Kg}$ allows to reach an optimal amount of $\mathrm{CD} 34+$ cells $(>6 \mathrm{x} 10 \mathrm{e} 6 / \mathrm{Kg})$ more frequently in respect to the dose of $5 \mathrm{mcg} / \mathrm{Kg}$. However, this hold true only when PLX is not used. In fact, in patients enrolled in PLX studies, there is no evidence that a higher dose of G-CSF determines further improvement in respect of that obtained with the use of the on demand PLX.

Conflict of interest: no conflict of interest

\section{P881}

Abstract previously published

Clinical Trial Registry: Not applicable

Conflict of interest: None of the authors has anything to disclose.

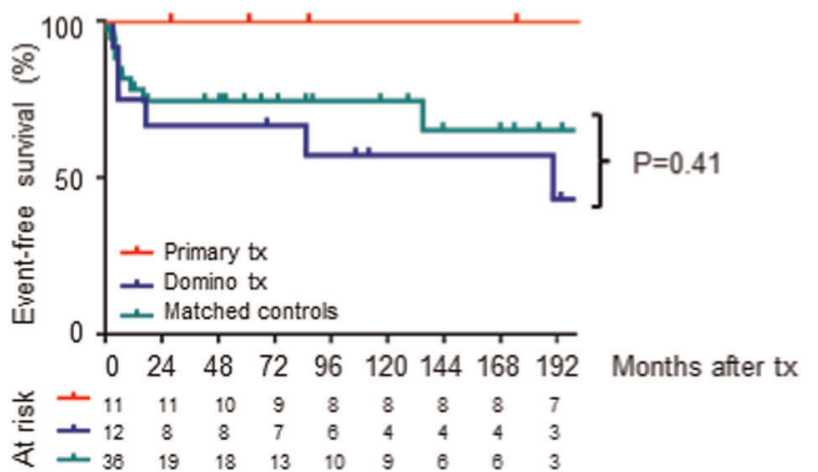

[P881 Figure] [Figure 1: Outcome of domino-HSCT in human recipients.] 
P882

PBSC for paediatric HSCT is associated with increased non relapse mortality without a better GVL effect and an inferior outcome compared to bone marrow

Adam Gassas, Patricia Breslin, Colin Steward, Christina Moris, Jessica Fuidge, Stephen Palmer, Hemalatha Doss, Ponni Sivaprakasam

University of Bristol, Bristol, United Kingdom

Background: Transplantation of peripheral blood hematopoietic stem cells (PBSC) is now the predominant graft source for unrelated adult allogeneic hematopoietic stem cell transplantation (HSCT). However, for paediatrics, bone marrow (BM) remains the first option for allogeneic unrelated HSCT mainly due the increased risk of acute and chronic GVHD that is associated with PBSC grafts.

Methods: Our objective herein was to compare the outcome of children receiving allo unrelated BMT utilizing BM vs PBSC graft source in a large dedicated UK paediatric HSCT programme in Bristol. Study design was a retrospective analysis.

Results: Between 2005 and 2016, 204 children received allogenic unrelated HSCT [ALL $=62 ; \mathrm{AML}=57$; other malignant (CML, HL, NHL, MDS, JMML) $=40$, nonmalignant $=45] . \quad \mathrm{BM}=161, \quad \mathrm{PBSC}=43$. The majority received a myeloablative conditioning regimen and all patients received campath $1 \mathrm{H}$. Survival was superior for $\mathrm{BM}$ recipients compared to PBSC (72\% vs $55.8 \% p=0.03$ ). The inferior outcome for PBSC recipients was due to the higher non relapse mortality (NRM) (21\% for PBSC vs $7.5 \%$ for $\mathrm{BM}$ recipients, $p=0.02$ ). Interestingly, for malignant diagnosis, ALL and AML, relapse rate was similar regardless of stem cell source. For ALL, relapse rate

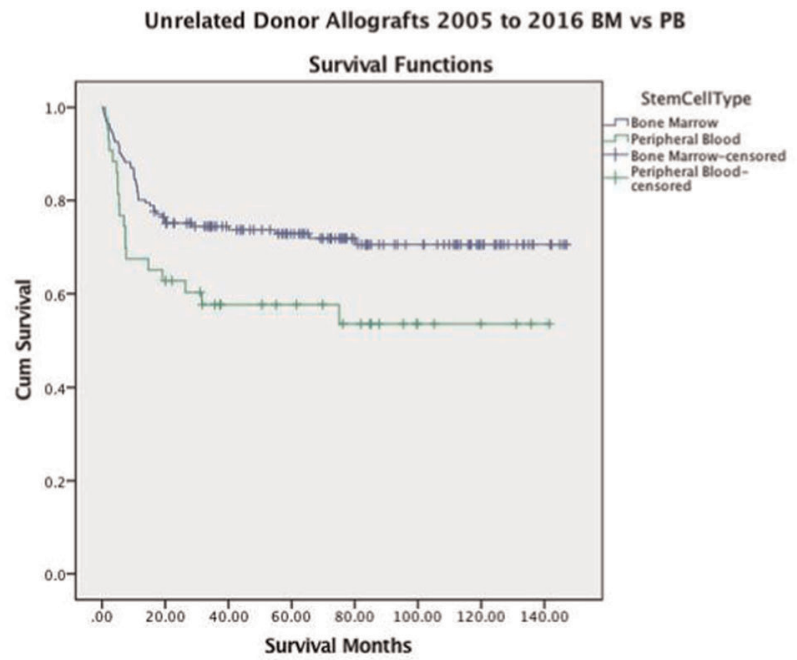

[P882 Figure] [Graph 1: Survival Curve PBSC vs BM] was $33 \%$ and $26 \%$ for PBSC and BM, respectively; $p=0.7$. For AML, relapse rate was $23 \%$ and $29 \%$ for PBSC and $\mathrm{BM}$, respectively; $p=0.7$.

Conclusions: Our study suggests that, for children, PBSC as a source for stem cell is associated with a higher NRM without a better graft versus leukemia (GVL) effect leading to an inferior outcome. Unlike adults, a BM source for stem cell continues to be the preferred option for paediatric HCST's.

Conflict of interest: nothing to declare

\section{P883}

Plerixafor on demand in 12 healthy family donors PBSC mobilization: long term follow-up of the recipients

Maria Teresa Lupo-Stanghellini ${ }^{1}$, Fabio Serpenti ${ }^{1,2}$, Salvatore Gattillo $^{3}$, Sarah Marktel ${ }^{1}$, Magda Marcatti ${ }^{1}$, Andrea Assanelli, Fabio Giglio $^{1}$, Raffaella Greco ${ }^{1}$, Simona Malato ${ }^{3}$, Daniele Mannina $^{1}$, Lucia Malabarba ${ }^{3}$, Raffaella Milani ${ }^{3}$, Consuelo Corti, Massimo Bernardi ${ }^{1}$, Jacopo Peccatori ${ }^{1}$, Luca Santoleri ${ }^{3}$, Fabio Ciceri $^{1,2}$

${ }^{1}$ San Raffaele Scientific Institute, Hematology and Bone Marrow Transplantation Unit, Milano, Italy; ${ }^{2}$ Vita-Salute San Raffaele University, San Raffaele Scientific Institute, Milano, Italy; ${ }^{3}$ San Raffaele Scientific Institute, Immunohematology and Transfusion Medicine Unit, Milano, Italy

Background: Procurement of an adequate amount of hematopoietic stem cells (HSC) is essential for engraftment in recipient of allogeneic stem cell transplantation (alloHCT). It is estimated that $5-10 \%$ of healthy donors undergoing $\mathrm{HSC}$ mobilization do not reach the minimum target dose of $2 \times 10^{\wedge} 6 / \mathrm{Kg} \mathrm{CD} 34+$ cells. Plerixafor (PL) is a chemokine receptor CXC Type-4-stromal-derived factor-1 inhibitor; its HSC-mobilizing properties are synergistic with G-CSF in poor mobilizing patients (pts). We and others previously reported that the use of PL, as adjuvant or alternative to G-CSF, in healthy donors is safe with only transient adverse effects.

The aim of the present study is to evaluate the outcome in long-term-follow-up (LTFU) of pts that received HSC mobilized with PL (+/- G-CSF) from a healthy family donor.

Methods: We report 12 cases of HCT performed with HSC collected with PL. PL was given off label after provision of an informed signed consent and in the absence of alternative options including clinical trials. Data were prospectively collected in an Institutional database. A written consent was given by pts allowing the use of medical records for research in accordance with the Declaration of Helsinki. 


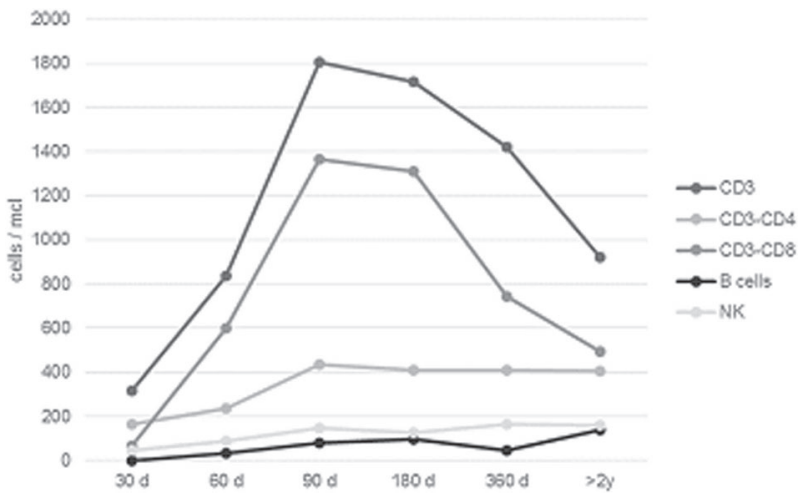

[P883 Figure] [Immune-reconstitution (median values)]

Results: Between 2010 and 2016 in our Institution, 416 healthy family donors underwent HSC harvest, in 12 exceptional cases (3\%) PL was used (2 PL, $10 \mathrm{PL}+\mathrm{G}-\mathrm{CSF}$ ).

Overall 9 female and 3 male pts received HCT for Acute Leukemia (9), Myelodisplasia (1), non-HodgkinLymphoma (1) and Renal Cancer (1). Median age at transplant was 47y (r27-64), median time from diagnosis to transplant was 277 days (r85-1336). Donor was a match sibling in 3 cases, an haploidentical in 9. Conditioning was in 11/12 cases treosulphan based and GvHD prophylaxis was rapamycin based in 8 cases, cyclosporine in 2, in 2 cases we performed an ex-vivo T-cell depletion. In all but 1 case the planned harvest consisted of peripheral HSC. Engraftment was possible in 10/12 cases - median time to neutrophils/platelets take 19/15 days; in 2 pts engraftment was not evaluable due to early death $(+4,+22)$. Overall 2 pts experienced peri-engraftment syndrome. Acute GvHD GII-IV was diagnosed in 5 pts (GIII-IV 3) - median time 48 days (r14-209). Chronic GvHD was diagnosed in 6 pts (3 classic, 3 overlap syndrome) - median time 196 days (r41-363). According to NIH 2004/2015 4 were severe and 2 moderate, $4 / 6$ obtained meaningful response.

By day-60 all the evaluable pts reached the goal of sustained immune-reconstitution, with CD3CD4 >200/mcl and B cells $>20 / \mathrm{mcl}$ (figure 1).

No evidence of second solid-tumor, severe adverse events or unexpected adverse events were registered at last followup.

Overall 3 pts deceased before day 100 and 4 pts between day 100 and 365; cause of death was disease relapse (1), GvHD (2), infection (4). So far 5 pts are alive - median follow-up 925 days (r443-2059).

Conclusions: In our experience PL ensured an adequate graft for HCT in all pts in need, providing a normal hematopoietic engraftment, effective immune reconstitution and a compoarable rate of transplant related complications with GvHD outcome not different from Institutional experience.
Conflict of interest: Nothing to disclose

\section{P884}

\section{Survival Outcomes of Haploidentical Hematopoietic Stem Cell Transplantation: A small retrospective study}

\section{Lan Wang, Mojtaba Akhtari}

University of Southern California Norris Cancer Center, Internal Medicine, Los Angeles, CA, United States

Background: Haploidentical hematopoietic stem cell transplant (HSCT) is an increasingly popular alternative source of stem cells for allogeneic HSCT due to its widespread availability and comparable outcomes to HLAmatched donors.

Methods: We conducted a retrospective study of patients who underwent haploidentical HSCT at Norris Comprehensive Cancer Center (NCCC, private hospital) between 2014 and 2016. We identified patients who had undergone haploidentical HSCT from administrative records. Patient demographics, treatment related factors and outcomes were collected. The primary endpoint was survival at one year post haploidentical HSCT. Descriptive statistics were used to describe the population and survival analysis was done with chi-square and log-rank tests.

Results: A total of 29 patients (median age 45 years) with $55 \%$ Hispanic, 21\% non-Hispanic Whites (NHWs), 14\% African-American, and 10\% Asian, were included. The primary diagnoses included: $27.6 \%$ acute lymphocytic leukemia (62.5\% Philadelphia chromosome $(\mathrm{Ph})$ positive, $37.5 \% \mathrm{Ph}$ negative), $37.9 \%$ acute myeloid leukemia, $6.9 \%$ myelodysplastic syndrome, $6.9 \%$ severe aplastic anemia, $3.4 \%$ mixed acute lymphoid and acute myeloid leukemia, 3.4\% non-Hodgkin's Lymphoma, 3.4\% Hodgkin's Lymphoma, 3.4\% systemic mastocytosis, 3.4\% myelofibrosis, and $3.4 \%$ chronic myeloid leukemia. Of the 23 patients who had staging prior to transplant, $52.2 \%$ was in complete remission (CR) $1,26.1 \%$ was in CR2, $4.3 \%$ was in CR4, and $17.4 \%$ had active disease. $51.7 \%$ of the patients experienced acute graft versus host disease (GvHD) most commonly of the skin and GI, and $51.7 \%$ of patients experienced chronic GvHD, most commonly of the skin and liver. Overall survival at one year was achieved in $76 \%$ of patients with $55 \%$ of those patients alive and in complete remission at one year. Median days after transplant to death was 58 days and cause of death included septic shock (28.6\%), hemorrhagic stroke $(28.6 \%)$, cardiac arrest $(14.3 \%)$, relapse disease $(14.3 \%)$ and diffuse alveolar hemorrhage $(14.3 \%)$. There was a significant difference in overall survival between younger patients $(<60$ years $)$ and older patients $(90 \%(\mathrm{n}=20)$ vs. $33.3 \%(\mathrm{n}=9) ; 95 \%$ CI 
14.0 - 83.96; $\mathrm{p}=0.0019)$. No prognostic impact was seen with primary disease type, stage at transplant, or race on overall survival. However, patients with AML as their primary disease process trended towards lower one year survival than other primary diseases but were not significant.

Conclusions: Haploidentical HSCT is a promising alternative treatment option for patients with hematologic malignancies without HLA-matched donors regardless of primary disease type, stage at transplant or patient's race. However, one year overall survival was significantly lower in patients 60 years or older.

Conflict of interest: The authors have nothing to disclose.

\section{P885}

Transplantation of pediatric patients from matched unrelated donors with Peripheral Blood Stem Cells (PBSCs) containing a defined number of $\mathrm{CD}^{+}$cells

Katerina Kaisari ${ }^{1}$, Anna Paisiou ${ }^{1}$, Christina Oikonomopoulou ${ }^{1}$, Eleni-Dikaia Ioannidou ${ }^{1}$, Eutichia Petrakou ${ }^{1}$, Maria Theodosaki ${ }^{1}$, Marianna Tzanoudaki ${ }^{2}$, George Vessalas ${ }^{1}$, Kalliopi Zisaki ${ }^{1}$, Ioulia Peristeri $^{1}$, Stelios Graphakos ${ }^{1}$, Eugenios Gousetis ${ }^{1}$, Vasiliki Kitra ${ }^{1}$

${ }^{1}$ Agia Sophia Children's Hospital Athens, Stem Cell Transplantation Unit, Athens, Greece; ${ }^{2}$ Agia Sophia Children's Hospital Athens, Immunology and Histocompatibility Department, Athens, Greece

Background: Transplantation of adults and children with PBSCs is associated with increased incidence of chronic Graft versus Host Disease (cGvHD). The number of $\mathrm{CD}^{+}$ lymphocytes in PBSCs is ten-fold higher compared to bone marrow. The role of $\mathrm{CD}^{+}$number on the incidence of cGvHD is not clear. In our study we processed PBSC grafts from unrelated donors in order to decrease $\mathrm{CD} 3+$ lymphocytes at a number comparable with bone marrow grafts $\left(1-5 \times 10^{7} / \mathrm{kgr}\right)$. Our aim was mainly the assessment of the incidence of GvHD, but also the outcome of the transplantation in general.

Methods: Thirty seven patients - 11 girls - with a median age 8,4 years were transplanted with tailored PBSC grafts from HLA-matched unrelated donors. Twenty seven of them had hematological malignancies and ten nonmalignant diseases. The conditioning was myeloablative in all patients (only in six patients TBI-based) and GvHD prophylaxis consisted of cyclosporine, methotrexate and antithymocyte globulin. The procession of the grafts was done with positive selection of CD34+ cells (CLINIMACS) and then the addition of a certain number of CD3+ lymphocytes (1-5 x 107/ kgr). Endpoints were the incidence of acute and chronic GvHD, the Overall Survival (OS), the
Leukemia Free Survival (LFS), the Relapse Rate (RR) and the Treatment Related Mortality (TRM).

Results: The median number of $\mathrm{CD} 34+$ and $\mathrm{CD} 3+$ of the grafts was $8,8 \times 10^{6} / \mathrm{kgr}\left(2.58-23.6 \times 10^{6} / \mathrm{kgr}\right)$ and $4 \mathrm{x}$ $10^{7} / \mathrm{kgr}\left(1-5 \times 10^{7} / \mathrm{kgr}\right)$ respectively. All patients engrafted. The median time of engraftment for neutrophils $>500 / \mu \mathrm{l}$ was $16 \pm 4$ days and for platelets $>20000 / \mu \mathrm{l} 22 \pm 6$ days. With a median follow-up of two years the OS was 52,2\% (95\%CI,) and the EFS 43,8\% (95\% CI). The incidence of aGvHD was $40 \%$ (95\% CI, $17 \%-57 \%$ ) and of cGvHD 5\% (95\% CI, 1$12 \%)$. The RR was 36\% (95\%CI, 24-62\%) and TRM until day +100 was $20 \%(95 \% \mathrm{CI}, 10-34 \%)$. Only two children manifested cGvHD, one limited and the other extensive.

Conclusions: According to the results of our study, transplantation with tailored PBSC grafts containing similar number of CD3 + lymphocytes as bone marrow grafts, leads to a low incidence of cGvHD. Additionally, OS and LFS are comparable with the survival of children who have been transplanted with bone marrow grafts from unrelated donors.

Clinical Trial Registry: Not applicable

Conflict of interest: All authors: Nothing to disclose

\section{P886}

Unmanipulated haploidentical hematopoietic stem cell transplantation in children with hematologic diseases and primary immunodeficiency diseases

Youngeun $\mathrm{Ma}^{1}$, Heewon Cho ${ }^{1}$, Ji Won Lee ${ }^{1}$, Ki Woong Sung ${ }^{1}$, EunSuk Kang ${ }^{2}$, Yae-Jean Kim ${ }^{1}$, Hong Hoe Koo ${ }^{1}$, Geon Hee Yoo ${ }^{1}$

${ }^{1}$ Samsung Medical Center, Sungkyunkwan University School of Medicine, Pediatrics, Seoul, Korea, Republic of; ${ }^{2}$ Samsung Medical Center, Sungkyunkwan University School of Medicine, Laboratory medicine \& Genetics, Seoul, Korea, Republic of

Background: Given the limited availability of HLAmatched donors, haploidentical hematopoietic stem cell transplantation (Haplo-HSCT) is increasingly used. Current strategies to deplete T-cells include ex vivo T-cells depletion, incorporation of antithymocyte globulin during conditioning, and administration of post-transplant cyclophosphamide. The aim of this study was to analyze the outcomes of unmanipulated haplo-HSCT using antithymocyte globulin in children.

Methods: We retrospectively reviewed the data of 18 patients who received an unmanipulated haplo-HSCT for various hematologic diseases or primary immunodeficiency diseases from January 2011 to March 2017. Transplant outcomes and related prognostic factors were analyzed.

Results: Patients' underlying diseases were hematologic malignancies $(n=9)$, severe aplastic anemia $(n=3)$ and primary immunodeficiency diseases $(n=6)$. Median age at 
haplo-HSCT was 14.9 years and their median follow-up was 14.2 months from transplantation. Twenty-one transplants were performed for 18 patients with 3 patients (17\%) re-transplanted for engraftment failure from the same haploidentical donor which resulted in successful engraftment in all. The incidence of acute graft-versus-host disease (GVHD) was $83 \%$ for grade II-IV and $28 \%$ for grade III-IV. Seven patients (39\%) developed chronic GVHD with 4 extensive diseases. No patient developed CMV disease although 13 patients (72\%) had CMV antigenemia. Four $(22 \%)$ patients died from the primary disease, $1(6 \%)$ from GVHD, $1(6 \%)$ from sepsis and $1(6 \%)$ from post-transplant lymphoproliferative disease. The 4-y overall survival rate was $60.6 \%$. We developed a scoring system using 3 parameters such as disease status (nonmalignant disease $=0$, malignancy $=1$, advanced malignancy $=2$ ), the number of previous transplant (none $=0$, once $=1$, more than once $=2$ ), and the donor gender (male $=0$, female $=1$ ). When patients were grouped according to the sum of risk scores, the OS rates were $100 \%$ in the low risk (score $0-1$ ), $75 \%$ in the intermediate risk (score 2), and $14.3 \%$ in the high risk (score $\geq 3$ ) group $(P=0.0018)$.

Conclusions: Unmanipulated haplo-HSCT seems a reasonable treatment option for children without an HLAmatched donor. Our scoring system may serve as a useful reference when an unmanipulated haplo-HSCT is considered.

Conflict of interest: nothing to disclose 